\title{
Web-based Tools for Vaccine Design
}

Lund, Ole; Nielsen, Morten; Kesmir, Can; Christensen, Jens K.; Lundegaard, Claus; Worning, Peder; Brunak, Søren

Published in:

HIV molecular immunology 2002

Publication date:

2002

Document Version

Publisher's PDF, also known as Version of record

Link back to DTU Orbit

Citation (APA):

Lund, O., Nielsen, M., Kesmir, C., Christensen, J. K., Lundegaard, C., Worning, P., \& Brunak, S. (2002). Webbased Tools for Vaccine Design. In HIV molecular immunology 2002 (pp. 45-51). Theoretical Biology \& Biophysics.

\section{General rights}

Copyright and moral rights for the publications made accessible in the public portal are retained by the authors and/or other copyright owners and it is a condition of accessing publications that users recognise and abide by the legal requirements associated with these rights.

- Users may download and print one copy of any publication from the public portal for the purpose of private study or research.

- You may not further distribute the material or use it for any profit-making activity or commercial gain

- You may freely distribute the URL identifying the publication in the public portal

If you believe that this document breaches copyright please contact us providing details, and we will remove access to the work immediately and investigate your claim. 


\title{
HIV Molecular Immunology 2002
}

Bette T. M. Korber

Theoretical Division

Los Alamos National Laboratory

Christian Brander

Infectious Disease Unit

Massachusetts general Hospital

Barton F. Haynes

Duke Center for AIDS Research

Duke University

\section{Editors}

Richard Koup

Vaccine Research Center

National Institutes of Health

Carla Kuiken

Theoretical Division

Los Alamos National Laboratory

John J. Moore

Weill Medical College

Cornell University

\author{
Bruce D. Walker \\ Harvard Medical School \\ Harvard University \\ David I. Watkins \\ Wisconsin Regional Primate \\ Research Center
}

\section{Project Officer \\ James A. Bradac \\ Division of AIDS}

National Institute of Allergy and Infectious Diseases

\author{
Staff \\ Charles Calef, Kristina Kommander, Catherine Miller, James J. Szinger \\ Los Alamos National Laboratory
}

This publication is funded by the Dept. of Health and Human Sevices, USA, and the National Institute of Health (Division of AIDS, National Institute of Allergy and Infectious Diseases), through an interagency agreement with the U.S. Department of Energy.

Published by Theoretical Biology \& Biophysics

Group T-10, Mail Stop K710

Los Alamos National Laboratory, Los Alamos, New Mexico 87545 U.S.A.

http://hiv-web.lanl.gov/immunology

LA-UR 03-5816 



\section{Contents}

\section{Contents}

\section{Preface}

Scope and Purpose of the HIV Molecular Immunology Database . . Citing the Database $\ldots \ldots \ldots \ldots \ldots \ldots \ldots$ About the Cover . . . . . . . . . . . . . . . . . . . . . .

About the PDF

Genome Maps

HIVISIV Proteins . . . . . . . . . . . . . . . . . . . . vii

Abbreviations .

\section{Part I. Review Articles}

Total Assessment of HIV-Specific CTL Responses: Epitope Clustering, Processing Preferences, and the Impact of HIV Sequence Heterogeneity

Nicole Frahm, Philip J.R. Goulder, and Christian Brander

Mutational Analyses and Natural Variability of the gp41 Ectodomain 23 Rogier W. Sanders, Bette Korber, Min Lu, Ben Berkhout, and John P. Moore

\section{Web-based Tools for Vaccine Design}

Ole Lund, Morten Nielsen, Can Kesmir, Jens K. Christensen, Claus Lunde gaard, Peder Worning, and Søren Brunak

\section{Part II. HIV CTL Epitopes}

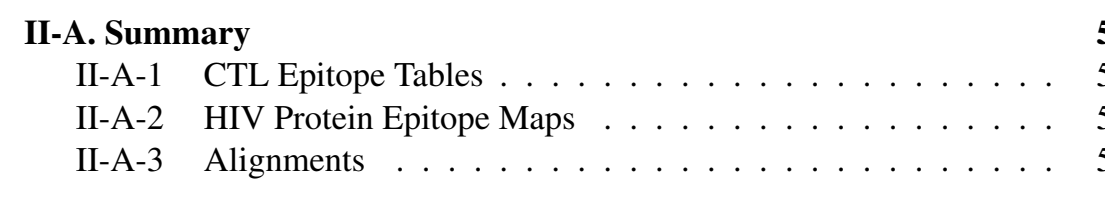

\section{II-B. HIV CTL Epitope Tables} II-B-1 1 p17 CTL Epitopes

\begin{tabular}{|c|c|}
\hline $\mathrm{B}-2$ & p17-p24 CTL Epitopes \\
\hline$-\overline{B-3}$ & p24 CTL Epitopes \\
\hline$\overline{\mathrm{B}-4}$ & p24-p2p7p1p6 CTL Epitopes \\
\hline$\overline{\mathrm{B}-5}$ & p2p7p1p6 CTL Epitopes \\
\hline$-B-6$ & Gag CTL Epitopes \\
\hline -B-7 & Gag/Pol CTL Epitopes \\
\hline$\overline{\mathrm{B}-8}$ & Protease CTL Epitopes \\
\hline -B-9 & RT CTL Epitopes \\
\hline $\mathrm{B}-10$ & RT CTL Epitopes \\
\hline$-\mathrm{B}-11$ & Integrase CTL Epitopes \\
\hline$\overline{-\mathrm{B}-12 \mathrm{I}}$ & Pol CTL Epitopes \\
\hline$\overline{\mid-B-13}$ & Vif CTL Epitopes \\
\hline$\overline{\mathrm{I}-\mathrm{B}-14}$ & Vpr CTL Epitopes \\
\hline$-B-15$ & Tat CTL Epitopes \\
\hline$-\mathrm{B}-16 \mathrm{~F}$ & Rev CTL Epitopes \\
\hline$\overline{-\overline{-B-17}}$ & Vpu CTL Epitopes \\
\hline$\overline{-\overline{-B}-18 \xi}$ & gp160 CTL Epitopes \\
\hline$-\mathrm{B}-19 \quad \mathrm{~F}$ & Env CTL Epitopes. \\
\hline $\begin{array}{cc}\mathrm{B}-20 & \mathrm{~N} \\
\end{array}$ & Nef CTL Epitopes \\
\hline $\begin{array}{cc}-\mathrm{B}-21 \quad \mathrm{H} \\
\end{array}$ & \\
\hline
\end{tabular}

II-C. Maps of CTL Epitope Locations Plotted by Protein

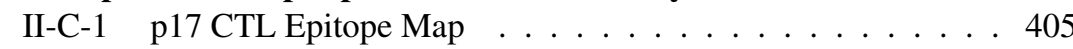
III-C-2 p24 CTL Epitope Map . . . . . . . . . . . . . . . . . . . . 407

III-C-3 p2p7p1p6 CTL Epitope Map . . . . . . . . . . . . . . . 411

II-C-4 Protease CTL Epitope Map . . . . . . . . . . . . . . . . . . 411

III-C-5 $\quad$ RT CTL Epitope Map . . . . . . . . . . . . . . . . . . . . . 412

II-C-6 $\quad$ Integrase CTL Epitope Map. . . . . . . . . . . . . . . . . . . 414

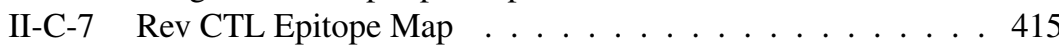

II-C-8 $\quad$ Tat CTL Epitope Map . . . . . . . . . . . . . . . . . . . . . 415

III-C-9 Vif CTL Epitope Map . . . . . . . . . . . . . . . . . . . 416

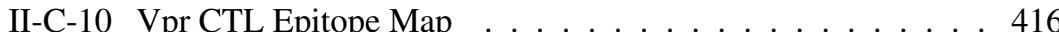

III-C-11 Vpu CTL Epitope Map . . . . . . . . . . . . . . . . . . . 417

II-C-12 gp160 CTL Epitope Map . . . . . . . . . . . . . . . . . . . 417

III-C-13 Nef CTL Epitope Map . . . . . . . . . . . . . . . . . 422 


\section{Part III. HIV Helper T-Cell Epitopes}

\section{III-A. Summary}

III-A-1 Tables

III-A-3 Alignments . . . . . . . . . . . . . . . . . . . . . . . 428

\section{III-B. HIV Helper T-Cell Epitope Tables}

III-B-1 p17 Helper T-Cell Epitopes

IIII-B-2 p24 Helper T-Cell Epitopes

III-B-3 p2p7p1p6 Helper T-Cell Epitopes. . . . . . . . . . . . . . . 440

III-B-4 Gag Helper T-Cell Epitopes . . . . . . . . . . . . . . . . . . 441

III-B-5 $\quad$ RT Helper T-Cell Epitopes

III-B-6 RT-Integrase Helper T-Cell Epitopes . . . . . . . . . . . . . 455

III-B-7 Integrase Helper T-Cell Epitopes . . . . . . . . . . . . . 456

III-B-8 Pol Helper T-Cell Epitopes . . . . . . . . . . . . . . . . . . 457

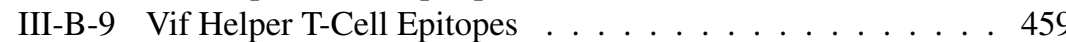

III-B-10 Vpr Helper T-Cell Epitopes . . . . . . . . . . . . . . . . . 460

III-B-11 Tat Helper T-Cell Epitopes . . . . . . . . . . . . . . . . . . 461

III-B-12 Rev Helper T-Cell Epitopes . . . . . . . . . . . . . . . . . 464

III-B-13 Vpu Helper T-Cell Epitopes . . . . . . . . . . . . . . . . . 466

III-B-14 gp160 Helper T-Cell Epitopes. . . . . . . . . . . . . . . . 467

III-B-15 Env Helper T-Cell Epitopes . . . . . . . . . . . . . . . . . 524

III-B-16 Nef Helper T-Cell Epitopes . . . . . . . . . . . . . . . . . . 530

III-B-17 HIV-1 Helper T-Cell Epitopes. . . . . . . . . . . . . . . 535

III-C. Maps of T-Helper Epitope Locations Plotted by Protein 537

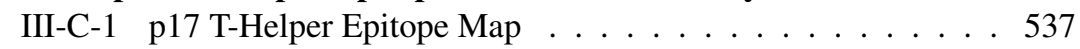

III-C-2 p24 T-Helper Epitope Map . . . . . . . . . . . . . . . . . . 538

III-C-3 p2p7p1p6 T-Helper Epitope Map . . . . . . . . . . . . . . . 539

III-C-4 Protease T-Helper Epitope Map . . . . . . . . . . . . . . . . 539

III-C-5 $\quad$ RT T-Helper Epitope Map. . . . . . . . . . . . . . . . . . . 540

III-C-6 Integrase T-Helper Epitope Map ... . . . . . . . . . . . . 541

III-C-7 $\quad$ Rev T-Helper Epitope Map . . . . . . . . . . . . . . . . . . 541

IIII-C-8 Tat T-Helper Epitope Map. . . . . . . . . . . . . . . . . . . 542

III-C-9 Vif T-Helper Epitope Map. . . . . . . . . . . . . . . . . . . 542

III-C-10 Vpr T-Helper Epitope Map . . . . . . . . . . . . . . . . . . 543

III-C-11 Vpu T-Helper Epitope Map . . . . . . . . . . . . . . . . 543

III-C-12 gp160 T-Helper Epitope Map . . . . . . . . . . . . . . . . 544

III-C-13 Nef T-Helper Epitope Map . . . . . . . . . . . . . . . . 548
549

IV-A. Summary

IV-A-1 Indices . . . . . . . . . . . . . . . . . . . . . . . . . . 551

IV-A-2 Tables . . . . . . . . . . . . . . . . . . . . . . . 551

IV-A-3 HIV Protein Binding Site Maps . . . . . . . . . . . . . . . 552

IV-A-4 Alignments . . . . . . . . . . . . . . . . . . . 552

IV-B. Cross Reference Listing of MAbs 553

IV-B-1 MAbs by binding type. . . . . . . . . . . . . . . . . 553

IV-B-2 Alphabetical listing of MAbs . . . . . . . . . . . . . . . 557

IV-B-3 MAbs by order of appearance in tables . . . . . . . . . 563

IV-C. HIV Antibodies Tables

IV-C-1 p17 Antibodies . . . . . . . . . . . . . . . . . . . . . . . . 569

IV-C-2 p24 Antibodies . . . . . . . . . . . . . . . . . . . . . . 575

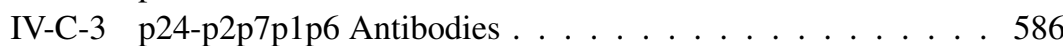

IV-C-4 $\quad$ p2p7p1p6 Antibodies . . . . . . . . . . . . . . . . . . . . . 587

IV-C-5 Gag Antibodies . . . . . . . . . . . . . . . . . . . . . 589

IV-C-6 Protease Antibodies . . . . . . . . . . . . . . . . . . . . 595

IV-C-7 $\quad$ RT Antibodies . . . . . . . . . . . . . . . . . . . . . . . 597

IV-C-8 Integrase Antibodies . . . . . . . . . . . . . . . . . . 600

IV-C-9 $\quad$ Pol Antibodies . . . . . . . . . . . . . . . . . . . . . 605

IV-C-10 Vif Antibodies . . . . . . . . . . . . . . . . . . . . . . . . 609

IV-C-11 Tat Antibodies . . . . . . . . . . . . . . . . . . . . . . . 610

IV-C-12 Rev Antibodies . . . . . . . . . . . . . . . . . . . . 612

IV-C-13 gp160 Antibodies . . . . . . . . . . . . . . . . . . . 615

IV-C-14 Env Antibodies . . . . . . . . . . . . . . . . . . . . . 728

IV-C-15 Nef Antibodies . . . . . . . . . . . . . . . . . . . . . . 835

IV-C-16 HIV-1 Antibodies . . . . . . . . . . . . . . . . . . 842

IV-D. Maps of MAb Locations Plotted by Protein 845

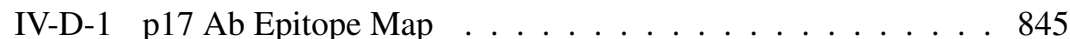

IV-D-2 p24 Ab Epitope Map . . . . . . . . . . . . . . . . . . . . . . 846

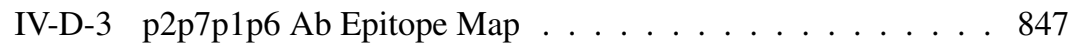

IV-D-4 Protease Ab Epitope Map . . . . . . . . . . . . . . . . . . . 848

IV-D-5 $\quad$ RT Ab Epitope Map . . . . . . . . . . . . . . . . . . . . . . . 848

IV-D-6 $\quad$ Integrase Ab Epitope Map . . . . . . . . . . . . . . . . . 849

IV-D-7 Rev Ab Epitope Map . . . . . . . . . . . . . . . . . . 850 
IV-D-8 Tat Ab Epitope Map . . . . . . . . . . . . . . . . . . . . . . 850

IV-D-9 Vif Ab Epitope Map . . . . . . . . . . . . . . . . . . . . . 851

IV-D-10 Vpr Ab Epitope Map . . . . . . . . . . . . . . . . . . . 851

IV-D-11 Vpu Ab Epitope Map . . . . . . . . . . . . . . . . . . . . . 852

IV-D-12 gp160 Ab Epitope Map
IV-D-13 Nef Ab Epitope Map

Part V. HIV Immunology References
860

861

iii

DEC 2002 



\section{Preface}

\section{Scope and Purpose of the HIV Molecular Immunology Database}

HIV Molecular Immunology is a companion volume to Human Retroviruse and AIDS Genetic Sequence Compendium. This publication, the 2002 issue, is the printed version of the Web-based HIV Immunology Database (http: //hiv-web.lanl.gov/immunology). The web interface for this relational database has many search options, as well as interactive tools to help immunologists design reagents and interpret their results.

The data included in this database is extracted from the HIV immunology literature. HIV-specific B-cell and T-cell responses are summarized and annotated. Immunological responses are divided into three sections, CTL, T helper, and antibody. Within these sections, defined epitopes are organized by protein and binding sites within each protein, moving from left to right through the coding regions spanning the HIV genome. We include human responses to natural HIV infections, as well as vaccine studies in a range of animal models and human trials. Responses that are not specifically defined, such as responses to whole proteins or monoclonal antibody responses to discontinuous epitopes, are summarized at the end of each protein sub-section. Studies describing general HIV responses to the virus, but not to any specific protein, are included at the end of each section.

The annotation includes information such as cross-reactivity, escape mutations, antibody sequence, TCR usage, functional domains that overlap with an epitope, immune response associations with rates of progression and therapy, and how specific epitopes were experimentally defined. Basic information such as HLA specificities for T-cell epitopes, isotypes of monoclonal antibodies, and epitope sequences are included whenever possible. All studies that we can find that incorporate the use of a specific monoclonal antibody are included in the entry for that antibody. A single $\mathrm{T}$ cell epitope can have multiple entries, generally one entry per study.

Finally, maps of all defined linear epitopes relative to the HXB2 reference proteins are provided. Alignments of CTL, helper T-cell, and antibody epitopes are available through the search interface on our web site at http:// hiv-web.lanl.gov/immunology.

Only responses to HIV-1 and HIV-2 are included in the database. CTL responses to SIVs have been periodically summarized in our review section by $\mathrm{Dr}$.
Dave Watkins and colleagues. (For their most recent review, please see: Where Have All The Monkeys Gone?: Evaluating SIV-Specific CTL in the Post-MamuA*01 Era David H. O'Connor, Todd M. Allen, and David I. Watkins, in the 2001 HIV Immunology compendium). Dr. Christian Brander and colleagues annually provide a concise listing of optimal CTL epitopes. Additional reviews that our editorial board deems of general interest to the HIV research immunology community are solicited each year. This year's reviews are printed in the first section of this database; reviews from previous years can be found at: http: / / www . hiv.lanl.gov/content/hiv-db/REVIEWS/reviews.html

Comments on the database or requests for the hard copy can be sent via email to immuno@t10.lanl.gov

\section{Citing the Database}

This publication may be cited as

HIV Molecular Immunology 2002. Bette T. M. Korber, Christian Brander, Barton F. Haynes, Richard Koup, Carla Kuiken, John P. Moore, Bruce D. Walker, and David I. Watkins, editors. Publisher: Los Alamos National Laboratory, Theoretical Biology and Biophysics, Los Alamos, New Mexico. LA-UR 03-5816.

\section{About the Cover}

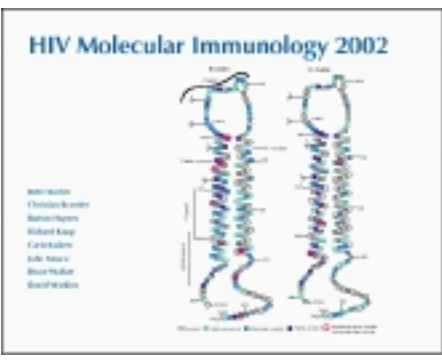

The illustration used for the cover of this year's immunology compendium highlights the location of the most variable amino acids in gp41, and was extracted from the review: Mutational Analyses and Natural Variability of the gp41 Ectodomain, by Rogier W. Sanders, Bette Korber, Min Lu, Ben Berkhout, and John P. Moore (this volume). 


\section{About the PDF}

The complete HIV Molecular Immunology 2002 is available in Adobe Portable Document Format (PDF) from our website, http://hiv-web.lanl.gov/ immunology. The PDF version is hypertext enabled and features 'clickable' table-of-contents, indexes, references and links to external web sites. 


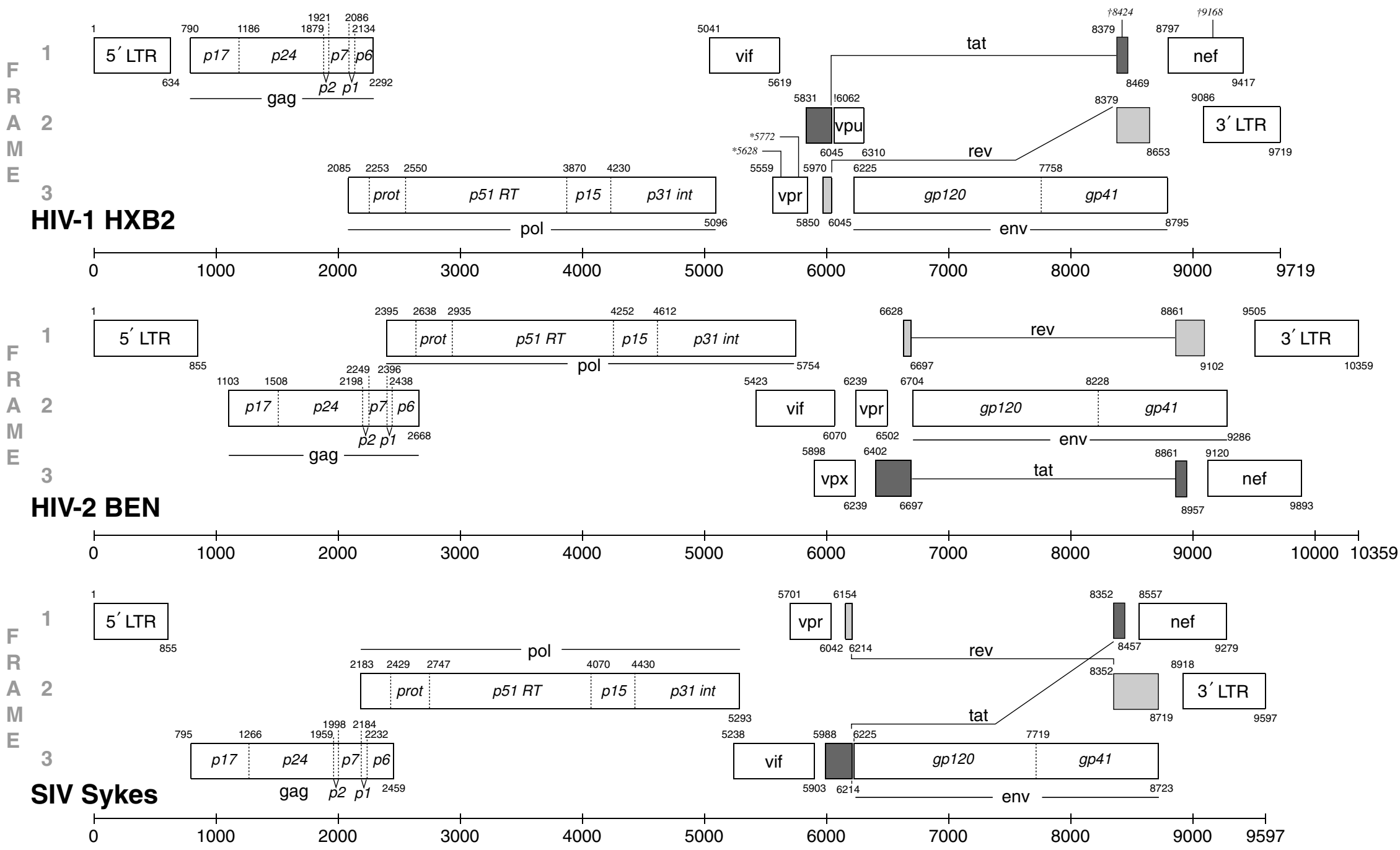

Landmarks of the HIV-1, HIV-2, and SIV genomes. The gene start, indicated by the small number in the upper left corner of each rectangle normally records the position of the a in the atg start codon for that gene while the number in the lower right records the last position of the stop codon. For pol, the start is taken to be the first $t$ in the sequence $t$ tt tt tag which forms part of the stem loop that potentiates ribosomal slippage on the RNA and a resulting -1 frameshift and the translation of the Gag-Pol polyprotein. The tat and rev spliced exons are shown as shaded rectangles. In HXB2, *5628 and *5772 mark positions of frameshifts in the $v p r$ gene; !6062 indicates a defective acg start codon in vpu; †8424 and †9168 mark premature stop codons in tat and nef. See Korber et al., Numbering Positions in HIV Relative to HXB2CG, in Human Retroviruses and AIDS, 1998, p. 102. Available from/http://hiv-web.lanl.gov/HTML/reviews/HXB2.html. 


\section{HIV/SIV Proteins}

\begin{tabular}{|c|c|c|c|}
\hline Name & Size & Function & Localization \\
\hline Gag MA & p17 & $\begin{array}{l}\text { membrane anchoring; env interaction; nuclear transport of } \\
\text { viral core. (myristylated protein) }\end{array}$ & virion \\
\hline $\mathrm{CA}$ & p24 & core capsid & virion \\
\hline \multirow[t]{2}{*}{$\mathrm{NC}$} & p7 & nucleocapsid, binds RNA & virion \\
\hline & p6 & binds $\mathrm{Vpr}$ & virion \\
\hline Protease (PR) & p15 & gag/pol cleavage and maturation & virion \\
\hline Reverse Transcriptase (RT) & p66, p51 & reverse transcription & virion \\
\hline RNase H & (heterodimer) & RNAse $\mathrm{H}$ activity & virion \\
\hline Integrase (IN) & & DNA provirus integration & virion \\
\hline Env & gp120/gp41 & $\begin{array}{l}\text { external viral glycoproteins bind to CD4 and chemokine } \\
\text { co-receptors }\end{array}$ & plasma membrane, virion envelope \\
\hline Tat & $\mathrm{p} 16 / \mathrm{p} 14$ & viral transcriptional transactivator & primarily in nucleolus/nucleus \\
\hline Rev & p19 & RNA transport, stability and utilization factor (phosphoprotein) & $\begin{array}{l}\text { primarily in nuleolus/nucleus shuttling } \\
\text { between nucleolus and cytoplasm }\end{array}$ \\
\hline Vif & $\mathrm{p} 23$ & $\begin{array}{l}\text { viral infectivity factor, inhibits minus-strand viral DNA } \\
\text { hypermutation }\end{array}$ & cytoplasm (cytosol, membranes), virion \\
\hline Vpr & p10-15 & $\begin{array}{l}\text { promotes nuclear localization of preintegration complex, } \\
\text { inhibits cell division, arrests infected cells at G2/M }\end{array}$ & virion nucleus (nuclear membrane?) \\
\hline Vpu & p16 & $\begin{array}{l}\text { promotes extracellular release of viral particles; degrades CD4 } \\
\text { in the ER; (phosphoprotein only in HIV-1 and SIVcpz) }\end{array}$ & integral membrane protein \\
\hline Nef & p27-p25 & CD4 and class I downregulation (myristylated protein) & plasma membrane, cytoplasm, (virion?) \\
\hline $\mathrm{Vpx}$ & p12-16 & Vpr homolog present in HIV-2 and some SIVs absent in HIV-1 & virion (nucleus?) \\
\hline Tev & $\mathrm{p} 28$ & tripartite tat-env-rev protein (also named Tnv) & primarily in nucleolus/nucleus \\
\hline
\end{tabular}




\section{Abbreviations}

Common abbreviations used in this database.

\begin{tabular}{ll}
\hline Abbrev. & Meaning \\
\hline Ab & Antibody \\
ADCC & Antibody-Dependent Cell-medicated Cytotoxicity \\
ADE & Antibody-Dependent Enhancement \\
APC & Antigen Presenting Cell \\
AZT & Azidothymidine \\
CD4BS & CD4 Binding Site \\
CD4i & Antibody that has enhanced binding to gp120 in the \\
& presence of SCD4 (CD4 induced) \\
CSF & Cerebrospinal Fluid \\
CTL & Cytotoxic T Lymphocyte \\
CTLp & CTL precursor \\
DTT & Dithiothrietol \\
EIA & Enzyme Immuno Assay \\
ELISA & Enzyme Linked ImmunoSorbent Assay \\
ER & Endoplasmic reticulum \\
Fabs & Fragment Antigen Binding-univalent antibody \\
FIV & fragment \\
gp & Feline Immunodeficiency Virus \\
HIV & Glycoprotein \\
HLA & Human Immunodeficiency Virus \\
HLA-MHC & Human Leukocyte Antigens \\
IFN & Histocompan Leukocyte Antigens-Major \\
IL & Interferon \\
IN & Interleukin \\
\hline & Integrase \\
\hline
\end{tabular}

\begin{tabular}{ll}
\hline Abbrev. & Meaning \\
\hline Ig & Immunoglobulin \\
MAb & Monoclonal Antibody \\
MHC & Major Histocompatibility Complex \\
MRC & Medical Research Council, UK \\
NAb & Neutralizing Antibody \\
NIBSC & National Institute for Biological Standards and \\
& Control, UK \\
NIH & National Institutes of Health \\
PBLs & Peripheral Blood Lymphocyte \\
PBMC & Peripheral Blood Mononuclear Cell \\
PR & Protease \\
RAC & Ricin A Chain \\
rec/r & recombinant \\
RIP & Recombinant Identification Program \\
RIPA & Radio Immuno Precipitation assay \\
rsgp160 & recombinant soluble gp160 \\
RT & Reverse Transcriptase \\
sCD4 & soluble CD4 \\
SDS & Sodium Duodecyl Sulfate \\
SIV & Simian Immunodeficiency Virus \\
Th & T-helper cell \\
TNF & Tumor Necrosis Factor \\
VLP & Virus like particle, assembled from p55 gag \\
VV & Vaccinia virus \\
WB & Western Blot \\
\hline
\end{tabular}



Part I

Review Articles

DEC 2002 



\section{Total Assessment of HIV-Specific CTL Responses: Epitope Clustering, Processing Preferences, and the Impact of HIV Sequence Heterogeneity}

\author{
Nicole Frahm ${ }^{1}$, Philip J.R. Goulder ${ }^{1,2}$, and Christian Brander ${ }^{1}$ \\ ${ }^{1}$ Partners AIDS Research Center, Massachusetts General Hospital, Boston, USA. \\ ${ }^{2}$ The Peter Medawar Building for Pathogen Research, Oxford, UK.
}

The HIV Immunology database at the Los Alamos National Laboratory has collected data on HIV-specific cellular immune responses for over 8 years now and the list of targeted regions within the HIV protein sequences has been growing steadily. These compiled data and our own studies using comprehensive sets of overlapping peptides indicate that almost all parts of the viral protein sequence can be targeted by virus-specific T cells, especially CTL Addo2003 Frahm2003]. HIV is the pathogen that has been characterized most extensively in terms of T-cell epitope distribution and the well-defined epitope landscape of HIV has allowed for a number of studies beyond assessing CTL activity in relation to HIV disease progression [Brander2002].

\section{Targets of HIV-specific CTL}

Whilst in the early years of HIV CTL epitope mapping, attention was focused on structural proteins, more recent studies have included regulatory and accessory proteins as well [Tomiyama1999a, Altfeld2001a, van Baalen1997, Addo2001 Addo2002b]. High-throughput assays such as intracellular cytokine staining (ICS), and the Elispot assay are now routinely used to assess genome wide immune responses to HIV [Edwards2002, Frahm2003, Betts2001, Addo2003 Novitsky2001, Novitsky2002]. This is especially true for the characterization of CD8+ CTL responses, but newer data also include the identification of Th cell activity. Studies from several labs, including ours, using overlapping peptide sets spanning the entire HIV protein sequence have now shown that at least $90 \%$ of these peptides can be targeted by HIV-specific CTL, indicating

In HIV Molecular Immunology 2002. Bette T. M. Korber, Christian Brander, Barton F. Haynes, Richard Koup, Carla Kuiken, John P. Moore, Bruce D. Walker, and David I. Watkins, editors. Publisher: Los Alamos National Laboratory, Theoretical Biology and Biophysics, Los Alamos, New Mexico. LA-UR 03-5816. pp.3 31 that all viral proteins undergo appropriate antigen processing in vivo and that epitopes from all HIV proteins can be effectively presented to CD8 T cells [Addo2003, Frahm2003]. However, there are specific patterns among these responses which will impact HIV vaccine design and which can potentially help to address more fundamental aspects of antigen processing, antigen presentation and T-cell repertoire development [Yusim2002].

Of special interest for these extended studies, but also for questions of CTL escape and (sub-unit)-vaccine development, is the identification of optimally defined CTL epitopes. Since 1995, largely through the voluntary contributions of unpublished data from many laboratories, regularly updated lists of "optimal CTL epitopes" have been made accessible through the Los Alamos National Laboratory's HIV database [Brander1995]. This year's update again adds a number of new epitopes whilst some others were removed as they were erroneously included before (mainly some HLA-A*0201 restricted epitopes from our own lab which were based on epitope prediction only and which were not defined with the same stringency as the other epitopes in this list). While the earliest reports clearly focused on alleles common in individuals infected early in the US epidemic, more attention is now given to individuals of non-Caucasian descent [Frahm2003, Sabbaj2003]. In addition, epitopes from non-clade B infections are increasingly identified |Novitsky2002, Novitsky2003, Bond2001, Fukada2002 Lynch1998, Sriwanthana2001, Goulder2001]. The identification of these epitopes provides valuable information for vaccine development in non-Caucasians and non-clade B infection.

In addition, these new epitopes, when characterized in full detail, can provide important insights into HLA binding motifs for these less well characterized alleles; again facilitating the design of a potential HIV vaccine. To support this work, the HIV database offers additional tools such as EPILIGN:

http:

//hiv-web.lanl.gov/content/hiv-db/EPILIGN/EPI.html

FeptGen:

http://hiv-web.lanl.gov/content/hiv-db/PEPTGEN/

PeptGenSubmitForm.html 
MotifScan:

http://hiv-web.lanl.gov/content/hiv-db/MOTIFSCAN/ MotifScanner.html

as well as valuable links to other sites, including the SYFPEITHI HLA binding motifs database:

http://www.syfpeithi.de/ and others:

http://hiv.basic.nwu.edu/HLA, http://bimas.dcrt.nih.gov/cgi-bin/molbio/ken_parker comboform,

http://www.jenner.ac.uk/JenPep/

Clearly, these databases and prediction softwares can profit from each other and facilitate the future identification of T-cell targets in HIV and other infections.

\section{Immunodominant regions in HIV protein sequences}

As mentioned above, the described optimal CTL epitopes are not evenly distributed over the entire viral genome. Rather, there are regions where many epitopes overlap. This phenomenon has been described as early as 1993 and various explanations have been put forward [Goulder2000a, Buseyne1993]. Two factors that seem to significantly contribute to this epitope clustering appear to be viral sequence heterogeneity and processing preferences [Yusim2002].

Sequence heterogeneity affects all HIV proteins, albeit to variable degrees. Relatively conserved regions in Gag and Nef have been identified as immunodominant regions in a study of more than 150 individuals of different ethnicities [Frahm2003]. Independently of the HLA background, these clade B infected individuals made strong responses to the peptides spanning these regions. When comparing the sequence heterogeneity in published clade B sequences, these data also show that peptides with low sequence entropy (more conserved) are targeted more frequently than epitopes with higher entropy. It is likely that these differences are due to the fact that the average phylogenetic distance of the test reagent (consensus B sequence) to an individual's autologous viral sequence is larger in higher variable regions than in more conserved ones and thus, responses against the less conserved peptides are not detected due to differences between test reagent and inoculum sequence [Yusim2002, Gaschen2002].

In addition to sequence incompatibility between test reagent and autologous virus, certain regions of the HIV protein sequence may not be processed and presented very effectively. Although $86 \%$ of our overlapping peptide sets used in the study above were targeted by at least one individual in the cohort of 150 people, there are still some relatively conserved peptides that do not seem to induce a detectable CTL response in natural HIV infection [Frahm2003]. These peptides may lie within stretches of viral proteins that are relatively resistant to proteasomal digestions or may lack adequate "Transporter associated with Antigen Processing" (TAP) binding motifs [Brander2002, Yusim2002]. The HIV Immunology database provides valuable web links to software where sequences of choice can be analyzed for proteasomal processing preferences (NetChop by C. Kesmir et al., http://www.cbs.dtu.dk/services/NetChop/). Recent work by Yusim et al., demonstrates the accuracy and predictive potential of this algorithm and its usefulness in identifying CTL epitopes [Yusim2002].

Together, these studies indicate that CTL epitope clustering may reflect the biased detection of these responses in rather conserved regions and that processing preferences may play an important role in providing processed antigen. In addition, sequence variability may not only affect CTL recognition but could also have an effect on processing of viral proteins [Yellen-Shaw1997]. Although we have been unable to show such an effect for the flanking regions of the immunodominant, HLA-A*0201 restricted CTL epitope SL9 (SLYNTVATL) in HIV Gag p17, other studies outside the HIV field suggest that escape from processing may be an effective means of immune evasion [Yellen-Shaw1997. Kuckelkorn2002, Gileadi1999, Brander1999]. These studies also highlight the importance of defining T-cell targets in maximal detail, so that prediction algorithm such as NetChop and binding motif algorithms can be optimized by a precisely characterized training set of defined epitopes. In addition, in order to discriminate between processing escape and escape from T-cell receptor recognition or HLA binding, the boundaries of targeted epitopes need to be optimally determined. The present listing is designed to provide these data specifically for HIV derived epitopes and we therefore still separate CTL epitopes in a list of optimally and suboptimally defined epitopes. We hope that this discrimination continues to provide support for the HIV immunologists and laboratories involved in antigen processing and presentation, who want to take advantage of the exceptionally well defined epitope landscape of HIV.

As every year, we would like to express our gratitude to the large number of researchers in the field who continuously contribute to this database. We very much welcome any criticism, comments and additions to this list since we are sure that some epitopes will unintentionally escape our attention, despite close monitoring of the literature. Also, pertinent information, such as resources for single HLA allele expressing cell lines, HLA subtype information and new technologies for CTL epitope mapping could be listed or referenced in this list, providing additional help to problems encountered by investigators. 


\section{Acknowledgments}

The mostly unpublished data added to this years update stemming from the AIDS Research Center at Massachussetts General Hospital have been largely funded by an NIH contract (\#NO1-A1-15442) supporting HLA typing and HIV CTL epitope definition in non-Caucasian populations and non-clade B HIV infection.

Please write or call us with any comments you may have at:

Nicole Frahm

phone: (617) 726-2648

FAX: (617) 726-5411

nfrahm@partners.org

Philip J. R. Goulder

phone: (617) 726-5787 or 01144-1865-221335

FAX: (617) 726-5411 or 01144-1865-220993

goulder@helix.mgh.harvard.edu

or philip.goulder@ndm.ox.ac.uk

\section{Bette Korber}

phone: (505) 665-4453

FAX: (505) 665-3493

btk@t10.lanl.gov
Christian Brander

phone: (617) 724-5789

FAX: (617) 726-5411

brander@helix.mgh.harvard.edu

\section{Bruce D. Walker}

phone: (617) 724-8332

FAX: (617) 726-4691

bwalker@helix.mgh.harvard.edu 
Table 1: Best Defined HIV CTL Epitopes

\begin{tabular}{|c|c|c|c|c|}
\hline HLA & Protein & AA & Sequence & Reference \\
\hline A*0101 (A1) & gp160 & $787-795$ & RRGWEVLKY & [Cao2002 \\
\hline \multirow[t]{4}{*}{$A * 0201(A 2)$} & & & $6 \mathrm{C}$ & [Falk1991, Barouch1995] \\
\hline & & \multirow[t]{2}{*}{$1^{\circ}$ anchor } & $r \quad L$ & \\
\hline & & & $\mathbf{M}$ & \\
\hline & & \multicolumn{2}{|c|}{$2^{\circ}$ anchor } & \\
\hline A*0201 (A2) & p17 & $77-85$ & \multirow{2}{*}{$\begin{array}{r}\text { SLYNTVATL } \\
\text { FLGKIWPSYK }\end{array}$} & [Johnson1991, Parker1992, Parker1994] \\
\hline A*0201 (A2) & p1 & $1-10$ & & Yu2002b \\
\hline $\mathrm{A}^{*} 0201(\mathrm{~A} 2)$ & RT & $33-41$ & ALVEICTEM & [Haas1998, Haas1999] \\
\hline $\mathrm{A} * 0201(\mathrm{~A} 2)$ & RT & $179-187$ & VIYQYMDDL & Harrer1996a \\
\hline A*0201 (A2) & RT & $309-317$ & ILKEPVHGV & [Walker1989, Tsomides1991] \\
\hline$A * 0201(\mathrm{~A} 2)$ & Vpr & $59-67$ & AIIRILQQL & [Altfeld2001a, Altfeld2001b \\
\hline A*0201 (A2) & gp160 & $311-320$ & RGP GRAFVTI & Alexander-Miller1996 \\
\hline A*0201 (A2) & gp160 & $813-822$ & SLLNATDIAV & [Dupuis 1995] \\
\hline A*0201 (A2) & Nef & $136-145$ & PLTFGWCYKL & [Haas1996, Maier1999] \\
\hline $\mathrm{A} * 0201(\mathrm{~A} 2)$ & Nef & $180-189$ & VLEWRFDSRL & Haas1996, Maier1999 \\
\hline \multirow[t]{3}{*}{ A*0202 (A2) } & & & \multirow{4}{*}{\begin{tabular}{rr}
2 & C \\
$\mathbf{L}$ & $\mathbf{L}$ \\
& $\mathbf{V}$ \\
& \multicolumn{2}{l}{ SLYNTVATL }
\end{tabular}} & Barouch1995] \\
\hline & & & & \\
\hline & & & & \\
\hline $\mathrm{A} * 0202(\mathrm{~A} 2)$ & p17 & $77-85$ & & Goulder1999 \\
\hline $\mathrm{A} * 0205(\mathrm{~A} 2)$ & p17 & $77-85$ & SLYNTVATL & Goulder1999] \\
\hline $\mathrm{A} * 0205$ (A2) & gp41 & $335-343$ & RIRQGLERA & Sabbaj2003 \\
\hline A*0207 (A2) & p24 & $164-172$ & YVDRFYKTL & Currier2002 \\
\hline $\mathrm{A} * 03(\mathrm{~A} 3)$ & RT & $73-82$ & KLVDFRELNK & Yu2002a \\
\hline $\mathrm{A} * 03(\mathrm{~A} 3)$ & RT & $356-366$ & RMRGAHTNDVK & [Yu2002a] \\
\hline A*03 (A3) & Integrase & 179-188 & AVF I HNF KRK & [Yu2002a] \\
\hline $\mathrm{A} * 03(\mathrm{~A} 3)$ & Vif & $28-36$ & HMYISKKAK & [Yu2002a] \\
\hline A*03 (A3) & Vif & $158-168$ & KTKPP LP SVKK & [Yu2002a \\
\hline $\mathrm{A} * 03(\mathrm{~A} 3)$ & Rev & $57-66$ & ERILSTYLGR & Addo2002a, Yu2002a \\
\hline $\mathrm{A} * 03(\mathrm{~A} 3)$ & Nef & $84-92$ & AVDLSHFLK & [Yu2002a] \\
\hline
\end{tabular}

DEC 2002 
Table 1 (cont.): Best Defined HIV CTL Epitopes

\begin{tabular}{|c|c|c|c|c|}
\hline \multirow{2}{*}{$\frac{\text { HLA }}{A * 0301(\mathbf{A 3 )}}$} & \multirow[t]{2}{*}{ Protein } & \multirow[t]{2}{*}{ AA } & Sequence & \multirow{2}{*}{$\begin{array}{l}\text { Reference } \\
\text { DiBrino1993, Rammensee1995 }\end{array}$} \\
\hline & & & C & \\
\hline & & & $\mathrm{K}$ & \\
\hline & & & $\mathrm{v}$ & \\
\hline & & & $\mathbf{F}$ & \\
\hline A*0301 (A3) & p17 & $18-26$ & KIRLRPGGK & [Harrer1996b] \\
\hline A*0301 (A3) & p17 & $20-28$ & RLRPGGKKK & $\begin{array}{l}\text { Goulder1997a Culmann1999, Lewinsohn1999b } \\
\text { Wilkes1999b }\end{array}$ \\
\hline $\mathrm{A} * 0301(\mathrm{~A} 3)$ & p17 & $20-29$ & RLRPGGKKKY & Goulder2000b \\
\hline A*0301 (A3) & RT & $33-43$ & ALVEICTEMEK & [Haas1998, Haas1999] \\
\hline A*0301 (A3) & RT & 93-101 & GIPHPAGLK & [Yu2002a] \\
\hline A*0301 (A3) & RT & $158-166$ & AIFQSSMTK & [Threlkeld1997] \\
\hline A*0301 (A3) & RT & $269-277$ & QIYPGIKVR & [Yu2002a] \\
\hline A*0301 (A3) & Vif & $17-26$ & RIRTWKSLVK & [Altfeld2001a, Yu2002a] \\
\hline A*0301 (A3) & gp160 & $37-46$ & TVYYGVPVWK & [Johnson1994a] \\
\hline A*0301 (A3) & gp160 & $770-780$ & RLRDLLLIVTR & [Takahashi1991] \\
\hline A*0301 (A3) & Nef & $73-82$ & QVP LRPMTYK & [Koenig1990, Culmann1991] \\
\hline \multirow[t]{5}{*}{$A * 1101(\mathrm{A11})$} & & & C & [Zhang1993, Rammensee1995 \\
\hline & & & K & \\
\hline & & & I & \\
\hline & & & $\mathrm{F}$ & \\
\hline & & & $\mathrm{Y}$ & \\
\hline A*1101 (A11) & p17 & 84-92 & TLYCVHQRI & [Harrer1998] \\
\hline A*1101 (A11) & p24 & $217-227$ & ACQGVGGP GHK & [Sipsas1997] \\
\hline A*1101 (A11) & RT & $158-166$ & AIFQSSMTK & [Johnson1994b, Zhang 1993, Threlkeld1997] \\
\hline$A * 1101(\mathrm{~A} 11)$ & RT & $341-350$ & IYQEPFKNLK & Culmann1999 \\
\hline $\mathrm{A} * 1101(\mathrm{~A} 11)$ & RNase & $80-88$ & QIIEQLIKK & [Fukada1999] \\
\hline $\mathrm{A} * 1101(\mathrm{~A} 11)$ & Integrase & 179-188 & AVF I HNFKRK & [Fukada1999] \\
\hline $\mathrm{A} * 1101(\mathrm{~A} 11)$ & gp160 & 199-207 & SVITQACPK & [Fukada1999] \\
\hline A*1101 (A11) & Nef & $73-82$ & QVPLRPMTYK & [Buseyne1999] \\
\hline A*1101 (A11) & Nef & $75-82$ & PLRPMTYK & [Culmann1991] \\
\hline A*1101 (A11) & Nef & $84-92$ & AVDLSHFLK & Culmann1991 \\
\hline A*23 (A23) & gp41 & $74-82$ & RYLKDQQLL & [Cao2003 \\
\hline
\end{tabular}

DEC 2002 
Table 1 (cont.): Best Defined HIV CTL Epitopes

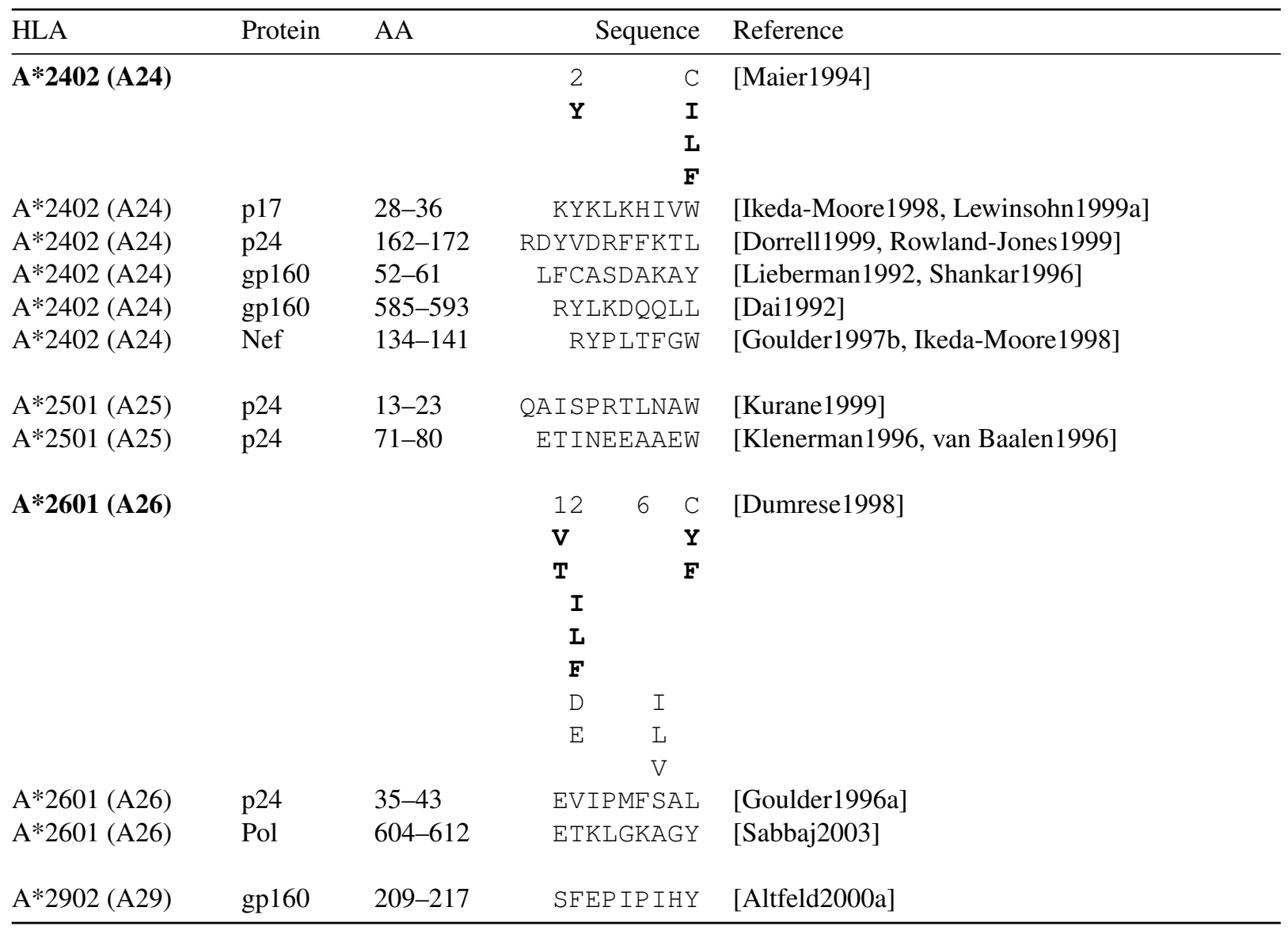


Table 1 (cont.): Best Defined HIV CTL Epitopes

\begin{tabular}{|c|c|c|c|c|}
\hline$\overline{\mathrm{HLA}}$ & Protein & AA & Sequence & Reference \\
\hline$A * 3002(\mathrm{A30})$ & & & $\begin{array}{rr}12 & \mathrm{C} \\
\mathbf{Y} & \mathbf{Y} \\
\mathbf{F} & \\
\mathrm{L} & \\
\mathrm{V} & \\
\mathrm{R} & \end{array}$ & [Rammensee1999] \\
\hline$A * 3002(\mathrm{~A} 30)$ & p17 & $76-86$ & RSLYNTVATLY & Goulder2001] \\
\hline$A * 3002(A 30)$ & RT & $173-181$ & KQNPDIVIY & [Goulder2001] \\
\hline$A * 3002(A 30)$ & RT & $263-271$ & KLNWASQIY & Goulder2001] \\
\hline$A * 3002(\mathrm{~A} 30)$ & RT & $356-365$ & RMRGAHTNDV & [Sabbaj2003] \\
\hline $\mathrm{A} * 3002(\mathrm{~A} 30)$ & Integrase & $219-227$ & KIQNFRVYY & [Sabbaj2003, Addo2002c \\
\hline$A * 3002(\mathrm{~A} 30)$ & gp160 & $704-712$ & IVNRNRQGY & [Goulder2001] \\
\hline$A * 3002(A 30)$ & gp120 & $310-318$ & HIGPGRAFY & [Sabbaj2003] \\
\hline$A * 3002(A 30)$ & gp41 & $283-291$ & KYCWNLLQY & Goulder2001] \\
\hline \multirow[t]{5}{*}{$A * 3101(\mathbf{A 3 1})$} & & & $\begin{array}{ll}2 & \mathbf{C} \\
& \mathbf{R}\end{array}$ & [Falk1994, Rammensee1999] \\
\hline & & & $\mathrm{L}$ & \\
\hline & & & V & \\
\hline & & & $\mathrm{Y}$ & \\
\hline & & & $\mathrm{F}$ & \\
\hline$A * 3101(\mathrm{~A} 31)$ & gp160 & $770-780$ & RLRDLLLIVTR & [Safrit1994a, Safrit1994b] \\
\hline $\mathrm{A} * 3201(\mathrm{~A} 32)$ & $\mathrm{RT}$ & $392-401$ & PIQKETWETW & [Harrer1996b] \\
\hline$A * 3201(\mathrm{~A} 32)$ & gp160 & $419-427$ & RIKQI INMW & Harrer1996b \\
\hline$A * 3303(\mathrm{~A} 33)$ & gp41 & $187-196$ & VFAVLS IVNR & [Hossain2001] \\
\hline A*3303 (A33) & gp41 & $320-327$ & EVAQRAYR & [Hossain2001] \\
\hline A*3303 (A33) & $\mathrm{Vpu}$ & $29-37$ & EYRKILRQR & Addo2002b \\
\hline A*3303 (A33) & Nef & $133-141$ & TRYPLTFGW & [Cao2002 \\
\hline$A * 6801(\mathrm{~A} 68)$ & Tat & $39-49$ & ITKGLGISYGR & Oxenius2002] \\
\hline$A * 6802(\mathrm{~A} 68)$ & Protease & $3-11$ & ITLWQRPLV & [Rowland-Jones1999] \\
\hline $\mathrm{A} * 6802(\mathrm{~A} 68)$ & Protease & $30-38$ & DTVLEEWNL & [Rowland-Jones1999] \\
\hline$A * 6802(\mathrm{~A} 68)$ & gp160 & $777-785$ & IVTRIVELL & Wilkes1999a \\
\hline A*7401 (A19) & Protease & $3-11$ & ITLWQRPLV & [Rowland-Jones1999] \\
\hline
\end{tabular}

DEC 2002 
Table 1 (cont.): Best Defined HIV CTL Epitopes

\begin{tabular}{|c|c|c|c|c|}
\hline HLA & Protein & AA & Sequence & Reference \\
\hline B*07 (B7) & $\mathrm{p} 24$ & $84-92$ & HPVHAGPIA & YYu2002a \\
\hline B*0702 (B7) & & & $\begin{array}{lll}123 & \text { C } \\
\text { P } & & \text { L } \\
A & R & \\
R & K & \end{array}$ & [Englehard1993, Rammensee1999] \\
\hline $\mathrm{B} * 0702$ (B7) & p24 & $16-24$ & SPRTLNAWV & [Lewinsohn1999a] \\
\hline B*0702 (B7) & p24 & $48-56$ & TPQDLNTML & $\begin{array}{l}\text { Wilson1999a, Wilkes1999c, Jin2000, } \\
\text { Wilson1997 }\end{array}$ \\
\hline $\mathrm{B} * 0702$ (B7) & p24 & $223-231$ & GPGHKARVL & Goulder1999] \\
\hline B*0702 (B7) & Vpr & $34-42$ & FPRIWLHGL & [Altfeld2001a \\
\hline B*0702 (B7) & Vif & $48-57$ & HPRVSSEVHI & [Altfeld2001a] \\
\hline B*0702 (B7) & gp160 & $298-307$ & RPNNNTRKSI & [Safrit1994b] \\
\hline B*0702 (B7) & gp160 & $843-851$ & IPRRIRQGL & [Wilkes1999b \\
\hline B*0702 (B7) & Nef & $68-77$ & FPVTPQVPLR & [Haas1996, Maier1999] \\
\hline B*0702 (B7) & Nef & $68-76$ & FPVTPQVPL & [Bauer1997, Frahm2002] \\
\hline B*0702 (B7) & Nef & $71-79$ & TPQVPLRPM & [Goulder1999] \\
\hline B*0702 (B7) & Nef & $77-85$ & RPMTYKAAL & [Bauer1997] \\
\hline B*0702 (B7) & Nef & $106-115$ & RQDILDLWIY & [Goulder1999] \\
\hline B*0702 (B7) & Nef & $128-137$ & TP GP GVRYPL & Culmann-Penciolelli1994, Haas1996 \\
\hline B*0801 (B8) & & & $\begin{array}{rrr}23 & 5 & \text { C } \\
\mathbf{K} \mathbf{K} & \mathbf{L} \\
\mathbf{R} & \\
\mathrm{PR} & \\
\mathrm{L} & \end{array}$ & Hill1992, Sutton1993, DiBrino1994a \\
\hline B*0801 (B8) & p17 & $24-32$ & GGKKKYKLK & [Rowland-Jones1993, Goulder1997d] \\
\hline B*0801 (B8) & p17 & $74-82$ & ELRSLYNTV & [Goulder1997d] \\
\hline B*0801 (B8) & p24 & $128-135$ & EIYKRWII & [Sutton1993, Goulder1997d] \\
\hline B*0801 (B8) & p24 & 197-205 & DCKTILKAL & [Sutton1993] \\
\hline B*0801 (B8) & RT & $18-26$ & GPKVKQWPL & Walker1989, Sutton1993 \\
\hline B*0801 (B8) & gp160 & $2-10$ & RVKEKYQHL & [Sipsas1997] \\
\hline B*0801 (B8) & gp160 & $586-593$ & YLKDQQLL & [Johnson1992, Shankar1996] \\
\hline B*0801 (B8) & Nef & $13-20$ & WP TVRERM & [Goulder1997d] \\
\hline B*0801 (B8) & Nef & $90-97$ & FLKEKGGL & Culmann-Penciolelli1994, Price1997 \\
\hline B*14 (B14) & p15 & $42-50$ & CRAPRKKGC & [Yu2002b] \\
\hline
\end{tabular}


Table 1 (cont.): Best Defined HIV CTL Epitopes

\begin{tabular}{|c|c|c|c|c|c|}
\hline$\frac{\text { HLA }}{B * 1402(B 14)}$ & Protein & AA & \multicolumn{2}{|c|}{ Sequence } & $\frac{\text { Reference }}{\mid \text { DiBrino1994b } \mid}$ \\
\hline B*1402 (B14) & & & $\begin{array}{ll}23 & 5 \\
\mathbf{R} & \mathbf{R} \\
\mathbf{K} & \mathbf{H} \\
\mathrm{L} & \\
\mathrm{Y} & \\
\mathrm{F} & \end{array}$ & $\begin{array}{l}\text { C } \\
\mathrm{L}\end{array}$ & [DiBrino1994b \\
\hline $\mathrm{B} * 1402(\mathrm{~B} 14)$ & p24 & $166-174$ & DRFYKTLF & & [Harrer1996b] \\
\hline $\mathrm{B} * 1402(\mathrm{~B} 14)$ & gp160 & 584-592 & ERYLKDQ & & [Johnson1992] \\
\hline $\mathrm{B} * 1501(\mathrm{~B} 62)$ & & & $\begin{array}{l}2 \\
\mathbf{Q} \\
\mathbf{L} \\
\mathbf{M}\end{array}$ & $\begin{array}{l}\mathrm{C} \\
\mathbf{Y} \\
\mathbf{F}\end{array}$ & $\begin{array}{l}\text { Barber1997] } \\
\text { Barber1997 } \\
\text { Barber1997 }\end{array}$ \\
\hline $\mathrm{B} * 1501(\mathrm{~B} 62)$ & p24 & $137-145$ & GLNKIVRI & & [Johnson1991, Goulder1999] \\
\hline $\mathrm{B} * 1501(\mathrm{~B} 62)$ & RT & $260-271$ & LVGKLNWASQ & & [Johnson1999] \\
\hline $\mathrm{B} * 1501(\mathrm{~B} 62)$ & RT & $309-318$ & ILKEPVHGY & & [Johnson1991, Johnson1999] \\
\hline $\mathrm{B} * 1501(\mathrm{~B} 62)$ & Nef & $19-27$ & RMRRAEP & & [Cao2002] \\
\hline $\mathrm{B} * 1501(\mathrm{~B} 62)$ & Nef & $117-127$ & TQGYFPDWQD & & Culmann1999 \\
\hline $\mathrm{B} * 1503(\mathrm{~B} 72)$ & Integrase & $263-271$ & RKAKIIRL & & Cao2003 \\
\hline B*1503 (B72) & Tat & $38-47$ & FQTKGLGIS & & [Novitsky2001] \\
\hline $\mathrm{B} * 1503(\mathrm{~B} 72)$ & Pol & $651-660$ & VTDSQYALC & & [Sabbaj2003] \\
\hline $\mathrm{B} * 1503(\mathrm{~B} 72)$ & Nef & $183-191$ & WREDSRLF & & [Cao2002] \\
\hline $\mathrm{B} * 1516(\mathrm{~B} 63)$ & & & $\begin{array}{l}2 \\
\mathbf{T} \\
\mathbf{S}\end{array}$ & $\begin{array}{l}9 \\
\mathbf{Y} \\
\mathrm{I} \\
\mathrm{V} \\
\mathbf{F}\end{array}$ & [Barber1997, Seeger1998] \\
\hline $\mathrm{B} * 1516(\mathrm{~B} 63)$ & gp160 & $375-383$ & SFNCGGEF & & Wilson1997, Wilson1999a \\
\hline $\mathrm{B} * 1801(\mathrm{~B} 18)$ & p24 & $161-170$ & FRDYVDRF & & [Ogg1998] \\
\hline B*1801 (B18) & Vif & $102-111$ & LADQLIHLF & & [Altfeld2001a \\
\hline B*1801 (B18) & Nef & $135-143$ & YPLTFGWC & & [Culmann1991, Culmann-Penciolelli1994] \\
\hline $\mathrm{B} * 2703(\mathrm{~B} 27)$ & p24 & $131-140$ & RRWIQLGL & & [Rowland-Jones1998, Rowland-Jones1999] \\
\hline
\end{tabular}


Table 1 (cont.): Best Defined HIV CTL Epitopes

\begin{tabular}{|c|c|c|c|c|}
\hline$\overline{\text { HLA }}$ & Protein & AA & Sequence & Reference \\
\hline \multirow[t]{7}{*}{ B*2705 (B27) } & & & 12 & [Jardetzky1991, Rammensee1995] \\
\hline & & & $\mathbf{R}$ & \\
\hline & & & $\mathbf{F}$ & \\
\hline & & & $\mathrm{K}$ & \\
\hline & & & $\mathrm{R}$ & \\
\hline & & & G & \\
\hline & & & A & \\
\hline B*2705 (B27) & p17 & $19-27$ & IRLRPGGKK & [McKinney1999, Lewinsohn1999a] \\
\hline $\mathrm{B} * 2705$ (B27) & p24 & $131-140$ & KRWI I LGLNK & [Nixon1988, Buseyne1993, Goulder1997c] \\
\hline B*2705 (B27) & gp160 & $786-795$ & GRRGWEALKY & [Lieberman1992, Lieberman1999] \\
\hline $\mathrm{B} * 2705$ (B27) & Nef & $105-114$ & RRQD ILDLWI & Goulder1997a \\
\hline \multirow[t]{6}{*}{$B * 3501(\mathrm{B35})$} & & & 2 & [Hill1992, Rammensee1999] \\
\hline & & & $\mathbf{P}$ & \\
\hline & & & A & \\
\hline & & & $\mathrm{V}$ & \\
\hline & & & $\mathrm{S}$ & \\
\hline & & & I & \\
\hline B*3501 (B35) & p17 & $36-44$ & WASRELERF & [Goulder1997b] \\
\hline $\mathrm{B} * 3501(\mathrm{~B} 35)$ & p17 & $124-132$ & NSSKVSQNY & Rowland-Jones1995] \\
\hline $\mathrm{B} * 3501(\mathrm{~B} 35)$ & p24 & $122-130$ & PPIPVGDIY & Rowland-Jones1995] \\
\hline $\mathrm{B} * 3501(\mathrm{~B} 35)$ & p24 & $122-130$ & NPVPVGNIY & Rowland-Jones1995 \\
\hline B*3501 (B35) & RT & $107-115$ & TVLDVGDAY & Wilkes1999b, Wilson1999b \\
\hline B*3501 (B35) & RT & $118-127$ & VPLDEDFRKY & [Sipsas1997, Shiga1996] \\
\hline B*3501 (B35) & RT & $175-183$ & NPDIVIYQY & [Sipsas1997, Shiga1996] \\
\hline $\mathrm{B} * 3501(\mathrm{~B} 35)$ & RT & $175-183$ & HPDIVIYQY & Rowland-Jones1995 \\
\hline B*3501 (B35) & gp160 & $42-52$ & VPVWKEATTTL & [Wilkes1999b] \\
\hline B*3501 (B35) & gp160 & $78-86$ & DPNPQEVVL & [Shiga1996] \\
\hline $\mathrm{B} * 3501(\mathrm{~B} 35)$ & gp160 & $606-614$ & TAVPWNASW & [Johnson1994a] \\
\hline B*3501 (B35) & Nef & $74-81$ & VPLRPMTY & [Culmann1991, Culmann-Penciolelli1994] \\
\hline \multirow[t]{5}{*}{ B*3701 (B37) } & & & 2 & [Falk1993] \\
\hline & & & D & \\
\hline & & & $\mathbf{E}$ & \\
\hline & & & $\mathrm{L}$ & \\
\hline & & & I & \\
\hline B*3701 (B37) & Nef & $120-128$ & YFPDWQNYT & Culmann1991, Culmann1999 \\
\hline
\end{tabular}


Table 1 (cont.): Best Defined HIV CTL Epitopes

\begin{tabular}{|c|c|c|c|c|}
\hline$\overline{\mathrm{HLA}}$ & Protein & AA & Sequence & Reference \\
\hline B*3801 (B38) & gp160 & $104-112$ & MHEDI ISLW & Cao2002 \\
\hline B*3901 (B39) & & & $\begin{array}{ll}2 & \text { C } \\
\text { R } & \text { L } \\
\text { H } & \end{array}$ & [Falk1995a] \\
\hline B*3901 (B39) & p24 & $61-69$ & GHQAAMQML & [Kurane1999] \\
\hline B*4001 (B60) & & & $\begin{array}{ll}2 & \text { C } \\
\mathbf{E} & \mathbf{L}\end{array}$ & [Falk1995b] \\
\hline $\mathrm{B} * 4001(\mathrm{~B} 60)$ & p17 & $92-101$ & IEIKDTKEAL & Altfeld2000b \\
\hline B*4001 (B60) & p24 & $44-52$ & SEGATPQDL & Altfeld2000b \\
\hline B*4001 (B60) & p6 & $33-41$ & KELYPLTSL & Yu2002b \\
\hline B*4001 (B60) & RT & $202-210$ & IEELRQHLL & Altfeld2000b \\
\hline B*4001 (B60) & gp160 & $805-814$ & QELKNSAVSL & Altfeld2000b \\
\hline $\mathrm{B} * 4001(\mathrm{~B} 60)$ & Nef & $92-100$ & KEKGGLEGL & Altfeld2000b \\
\hline $\mathrm{B} * 4002(\mathrm{~B} 61)$ & p17 & $11-19$ & GELDRWEKI & [Sabbaj2003] \\
\hline B*4002 (B61) & p24 & $70-78$ & KETINEEAA & [Sabbaj2003] \\
\hline B*4002 (B61) & p24 & $78-86$ & AEWDRVHPV & [Sabbaj2003] \\
\hline B*4002 (B61) & Nef & $92-100$ & KEKGGLEGL & [Sabbaj2003, Altfeld2000b \\
\hline $\mathrm{B} * 4002(\mathrm{~B} 61)$ & p15 & $64-71$ & TERQANFL & [Sabbaj2003] \\
\hline B*42 (B42) & Integrase & $260-268$ & VPRRKAKI I & [Kiepiela2002] \\
\hline$B * 4201(B 42)$ & p24 & $48-56$ & TPQDLNTML & Goulder2000a \\
\hline$B * 4201(B 42)$ & RT & $271-279$ & YPGIKVRQL & [Wilkes1999b \\
\hline $\mathrm{B} * 4201(\mathrm{~B} 42)$ & Nef & $128-137$ & TP GP GVRYPL & [Goulder1999] \\
\hline \multirow{3}{*}{$B * 4402(B 44)$} & & & $\mathrm{C}$ & [Rammensee1999] \\
\hline & & & $\mathbf{F}$ & \\
\hline & & & $\mathbf{Y}$ & \\
\hline B*4402 (B44) & p24 & $162-172$ & RDYVDRFYKTL & [Ogg1998] \\
\hline B*4402 (B44) & p24 & $174-184$ & AEQASQDVKNW & [Lewinsohn1999a] \\
\hline B*4402 (B44) & gp160 & $31-40$ & AENLWVTVYY & [Borrow1997] \\
\hline B*4415 (B12) & p24 & $28-36$ & EEKAFSPEV & [Bird2002] \\
\hline $\mathrm{B} * 51(\mathrm{~B} 51)$ & Vpr & $29-37$ & EAVRHFPRI & [Cao2003] \\
\hline
\end{tabular}


Table 1 (cont.): Best Defined HIV CTL Epitopes

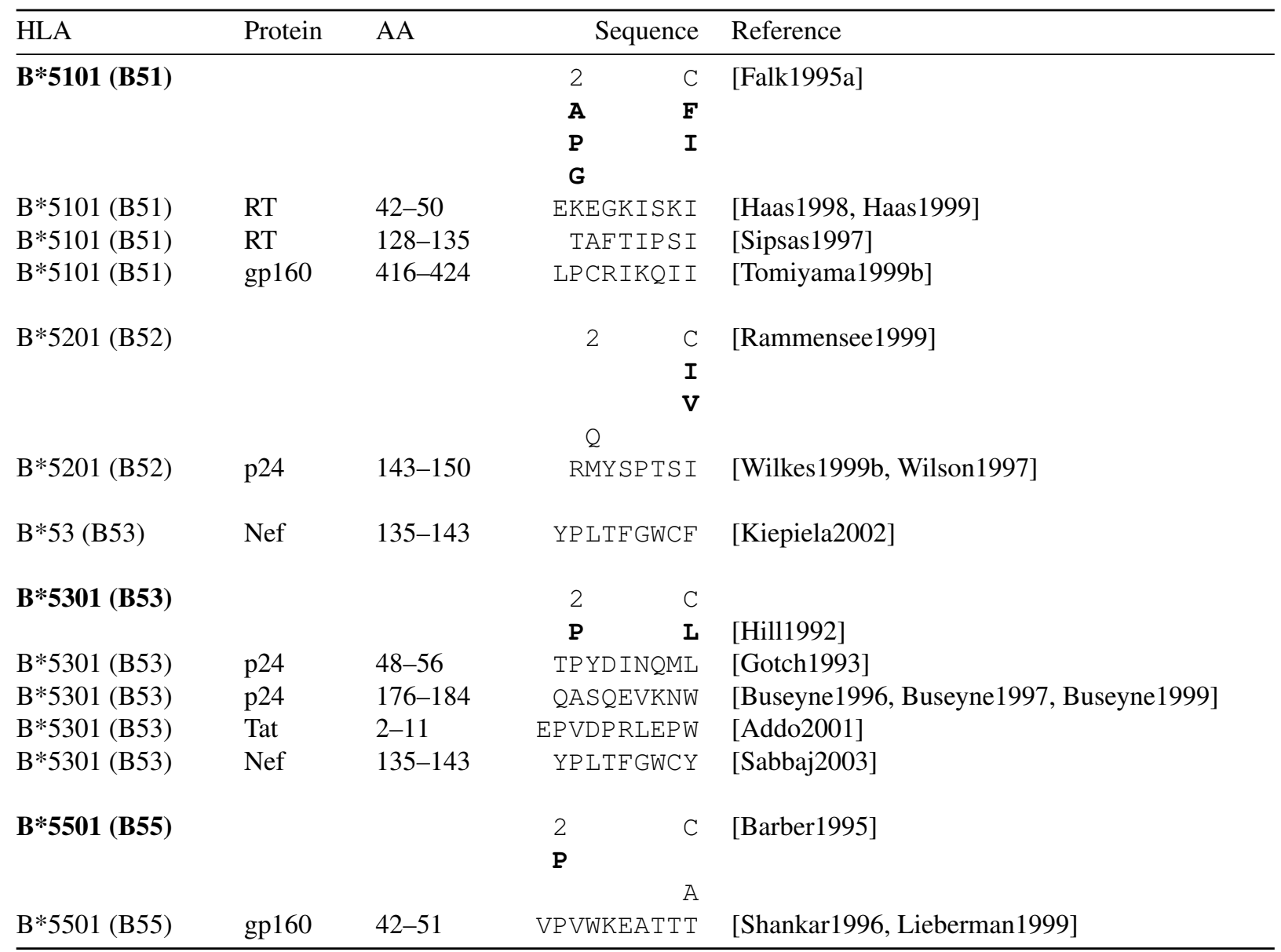


Table 1 (cont.): Best Defined HIV CTL Epitopes

\begin{tabular}{|c|c|c|c|c|}
\hline HLA & Protein & AA & Sequence & Reference \\
\hline \multirow{5}{*}{ B*5701 (B57) } & & & 12 & [Barber1997] \\
\hline & & & $\mathbf{A}$ & \\
\hline & & & $\mathbf{T}$ & \\
\hline & & & $\mathbf{s}$ & \\
\hline & & & $\mathrm{K}$ & \\
\hline B*5701 (B57) & p24 & $15-23$ & ISPRTLNAW & [Johnson1991, Goulder1996b] \\
\hline B*5701 (B57) & p24 & $30-40$ & KAFSPEVIPMF & [Goulder1996b] \\
\hline $\mathrm{B} * 5701(\mathrm{~B} 57)$ & p24 & $108-118$ & TSTLQEQIGWF & Goulder1996b \\
\hline B*5701 (B57) & p24 & $176-184$ & QASQEVKNW & [Goulder1996b] \\
\hline B*5701 (B57) & RT & $244-252$ & IVLPEKDSW & |van der Burg1997, Hay1999] \\
\hline B*5701 (B57) & Integrase & $173-181$ & KTAVQMAVF & [Goulder1996b, Hay 1999] \\
\hline B*5701 (B57) & Vpr & $30-38$ & AVRHFPRIW & Altfeld2001a \\
\hline B*5701 (B57) & Vif & $31-39$ & ISKKAKGWF & [Altfeld2001a] \\
\hline B*5701 (B57) & Rev & $14-23$ & KAVRLIKFLY & [Addo2001] \\
\hline $\mathrm{B} * 5701(\mathrm{~B} 57)$ & Nef & $116-125$ & HTQGYFPDWQ & [Culmann1991] \\
\hline B*5701 (B57) & Nef & $120-128$ & YFPDWQNYT & [Culmann1991 \\
\hline B57 (B57) & Nef & $116-124$ & HTQGYFPDW & [Draenert2002] \\
\hline B*5703 (B57) & p24 & $30-37$ & KAFSPEVI & Goulder2000b \\
\hline $\mathrm{B} * 5703(\mathrm{~B} 57)$ & p24 & $30-40$ & KAFSPEVIPMF & Goulder2000b \\
\hline \multirow[t]{7}{*}{ B*5801 (B58) } & & & 12 & [Barber1997, Falk1995b] \\
\hline & & & $\mathbf{F}$ & \\
\hline & & & $\mathbf{w}$ & \\
\hline & & & $\mathbf{S}$ & \\
\hline & & & $\mathrm{K}$ & \\
\hline & & & $\mathrm{V}$ & \\
\hline & & & I & \\
\hline B*5801 (B58) & p24 & $108-117$ & TSTVEEQQIW & [Bertoletti1998] \\
\hline $\mathrm{B} * 5801(\mathrm{~B} 58)$ & p24 & $108-117$ & TSTLQEQIGW & Goulder1996b \\
\hline B*5801 (B58) & RT & $375-383$ & IAMESIVIW & [Kiepiela2002] \\
\hline B*5801 (B58) & $\operatorname{Rev}$ & $14-23$ & KAVRLIKFLY & Addo2001] \\
\hline B*81 (B81) & Pol & $715-723$ & LFLDGIDKA & Addo2002a \\
\hline
\end{tabular}


Table 1 (cont.): Best Defined HIV CTL Epitopes

\begin{tabular}{|c|c|c|c|c|}
\hline$\overline{\mathrm{HLA}}$ & Protein & AA & Sequence & Reference \\
\hline $\mathrm{B} * 8101(\mathrm{~B} 81)$ & p24 & $48-56$ & TPQDLNTML & [Goulder2000a] \\
\hline $\mathrm{B} * 8101(\mathrm{~B} 81)$ & $\mathrm{Vpr}$ & $34-42$ & FPRIWLHGI & Altfeld2001a \\
\hline \multirow[t]{4}{*}{ Cw*0102 (Cw1) } & & & 23 & Barber1997 \\
\hline & & & A & \\
\hline & & & L & \\
\hline & & & $\mathrm{P}$ & \\
\hline $\mathrm{Cw}^{*} 0102(\mathrm{Cw} 1)$ & p24 & $36-43$ & VIPMFSAL & Goulder1997b \\
\hline $\mathrm{Cw}^{*} 0304(\mathrm{Cw} 10)$ & gp41 & $46-54$ & RAIEAQQHI & [Currier2002, Trocha2002] \\
\hline \multirow[t]{7}{*}{ Cw*0401 (Cw4) } & & & $6 \mathrm{c}$ & [Falk1994] \\
\hline & & & $\mathbf{Y}$ & \\
\hline & & & $P$ & \\
\hline & & & $\mathbf{F}$ & \\
\hline & & & V & \\
\hline & & & I & \\
\hline & & & L & \\
\hline $\mathrm{Cw}^{*} 0401(\mathrm{Cw} 4)$ & gp160 & $375-383$ & SFNCGGEFF & [Wilson1997, Johnson1993] \\
\hline Cw*0501 (Cw5) & Rev & $67-75$ & SAEPVPLQI & Addo2001] \\
\hline Cw*07 (Cw7) & Nef & $105-115$ & KRQEILDLWVY & [Kiepiela2002] \\
\hline $\mathrm{Cw}^{*} 07(\mathrm{Cw} 7)$ & Nef & $105-115$ & RRQD ILDLWIY & Yu2002a \\
\hline Cw*0802 (Cw8) & p24 & $48-56$ & TPQDLNTML & [Goulder2000a] \\
\hline Cw*0802 (Cw8) & Nef & $83-91$ & AAVDLSHFL & [Cao2003 \\
\hline $\mathrm{Cw}^{*} 12(\mathrm{Cw} 12)$ & Tat & $30-37$ & $\mathrm{CCFHCQVC}$ & [Cao2003, Nixon1999] \\
\hline $\mathrm{Cw}^{*} 15(\mathrm{Cw} 15)$ & gp41 & $46-54$ & RAIEAQQHL & [Trocha2002] \\
\hline
\end{tabular}




\section{References}

[Addo2001] M. Addo, M. Altfeld, E. Rosenberg, R. Eldridge, M. Philips, K. Habeeb, A. Khatri, C. Brander, G. Robbins, G. Mazzara, P. Goulder, \& B. Walker. The HIV-1 regulatory protein Tat and Rev are frequently targeted by cytotoxic T lymphocytes derived from HIV-1-infected individuals. Proc Natl Acad Sci U S A 98:1781-6, 2001. On p. 3] 14] 15$] 16$

[Addo2002a] M. Addo. pers. communication, 2002. On p.66 15

[Addo2002b] M. Addo, M. Altfeld, A. Rathod, M. Yu, X. Yu, P. Goulder, E. Rosenberg, \& B. Walker. HIV-1 Vpu represents a minor target for cytotoxic T lymphocytes in HIV-1-infection. AIDS 16:1071-3, 2002. On p.3 9

[Addo2002c] M. Addo, P. Kiepiela, \& P. Goulder. pers. communication, 2002. On p. 9

[Addo2003] M. Addo, X. Yu, A. Rathod, D. Cohen, R. Eldridge, D. Strick, M. Johnston, C. Corcoran, A. Wurcel, C. Fitzpatrick, M. Feeney, W. Rodriguez, N. Basgoz, R. Draenert, D. Stone, C. Brander, P. Goulder, E. Rosenberg, M. Altfeld, \& B. Walker. Comprehensive epitope analysis of human immunodeficiency virus type 1 (HIV-1)-specific T-cell responses directed against the entire expressed HIV-1 genome demonstrate broadly directed responses, but no correlation to viral load. J Virol 77:2081-92, 2003. On p. 3

[Alexander-Miller1996] M. A. Alexander-Miller, K. C. Parker, T. Tsukui, C. D. Pendleton, J. E. Coligan, \& J. A. Berzofsky. Molecular analysis of presentation by HLA-A2.1 of a promiscuously binding V3 loop peptide from the HIV-1 Envelope protein to human cytotoxic T lymphocytes. In Immunol 8:641-649, 1996. On p. 6

[Altfeld2000a] M. A. Altfeld. pers. communication, 2000. On p.8

[Altfeld2000b] M. A. Altfeld, A. Trocha, R. L. Eldridge, E. S. Rosenberg, M. N. Phillips, M. M. Addo, R. P. Sekaly, S. A. Kalams, S. A. Burchett, K. McIntosh, B. D. Walker, \& P. J. Goulde Identification of dominant optimal HLA-B60- and HLA-B61-restricted cytotoxic T-lymphocyte (CTL) epitopes: rapid characterization of CTL responses by enzyme-linked immunospot assay. $J$ Virol 74:8541-9, 2000. On p. 13

[Altfeld2001a] M. Altfeld, M. Addo, R. Eldridge, X. Yu, S. Thomas, A. Khatri, D. Strick M. Phillips, G. Cohen, S. Islam, S. Kalams, C. Brander, P. Goulder, E. Rosenberg, \& B. Walker Vpr is preferentially targeted by CTL during HIV-1 infection. J Immunol 167:2743-52, 2001. On p. 3 6 60 , $10,11,15,16$

[Altfeld2001b] M. A. Altfeld, B. Livingston, N. Reshamwala, P. T. Nguyen, M. M. Addo, A. Shea M. Newman, J. Fikes, J. Sidney, P. Wentworth, R. Chesnut, R. L. Eldridge, E. S. Rosenberg, G. K. Robbins, C. Brander, P. E. Sax, S. Boswell, T. Flynn, S. Buchbinder, P. J. Goulder, B. D. Walker, A. Sette, \& S. A. Kalams. Identification of Novel HLA-A2-Restricted Human Immunodeficiency Virus Type 1-Specific Cytotoxic T-Lymphocyte Epitopes Predicted by the HLA-A2 Supertype Peptide-Binding Motif. J Virol 75:1301-1311, 2001. On p.6

[Barber1995] L. D. Barber, B. Gillece-Castro, L. Percival, X. Li, C. Clayberger, \& P. Parham. Overlap in the repertoires of peptides bound in vivo by a group of related class I HLA-B allotypes. Curr Biol 5:179-90, 1995. On p.14.

[Barber1997] L. D. Barber, L. Percival, K. L. Arnett, J. E. Gumperz, L. Chen, \& P. Parham. Polymorphism in the $\alpha 1$ Helix of the HLA-B Heavy Chain Can Have an Overriding Influence on Peptide-Binding Specificity. J Immunol 158:1660-1669, 1997. On p. 11]15 16

[Barouch1995] D. Barouch, T. Friede, S. Stevanovic, L. Tussey, K. Smith, S. Rowland-Jones, V. Braud, A. McMichael, \& H. G. Rammensee. HLA-A2 subtypes are functionally distinct in peptide binding and presentation. J Exp Med 182:1847-56, 1995. On p.6

[Bauer1997] M. Bauer, M. Lucchiari-Hartz, R. Maier, G. Haas, B. Autran, K. Eichmann, R. Frank,

B. Maier, \& A. Meyerhans. Structural constraints of HIV-1 Nef may curtail escape from HLAB7-restricted CTL recognition. Immunol Lett 55:119-22, 1997. On p. 10

[Bertoletti1998] A. Bertoletti, F. Cham, S. McAdam, T. Rostron, S. Rowland-Jones, S. Sabally,
T. Corrah, K. Ariyoshi, \& H. Whittle. Cytotoxic T cells from human immunodeficiency virus type 2-infected patients frequently cross-react with different human immunodeficiency virus type 1 Clades. J Virol 72:2439-2448, 1998. On p. 15

[Betts2001] M. Betts, D. Ambrozak, D. Douek, S. Bonhoeffer, J. Brenchley, J. Casazza, R. Koup, \& L. Picker. Analysis of total human immunodeficiency virus (HIV)-specific CD4(+) and CD8(+) T-cell responses: relationship to viral load in untreated HIV infection. J Virol 75:11983-91, 2001 On p. 3

Bird2002] T. Bird, R. Kaul, T. Rostron, J. Kimani, J. Embree, P. Dunn, J. Bwayo, F. Plummer, S. Rowland-Jones, \& T. Dong. HLA typing in a Kenyan cohort identifies novel class I alleles that restrict cytotoxic T-cell responses to local HIV-1 clades. AIDS 16:1899-1904, 2002. On p.13

[Bond2001] K. Bond, B. Sriwanthana, T. Hodge, A. De Groot, T. Mastro, N. Young, N. Promadej, J. Altman, K. Limpakarnjanarat, \& J. McNicholl. An HLA-directed molecular and bioinformatics approach identifies new HLA-A11 HIV-1 subtype E cytotoxic T lymphocyte epitopes in HIV-1infected Thais. AIDS Res Hum Retroviruses 17:703-17, 2001. On p. 3

[Borrow1997] P. Borrow, H. Lewicki, X. Wei, M. S. Horwitz, N. Peffer, H. Meyers, J. A. Nelson, J. E. Gairin, B. H. Hahn, M. B. Oldstone, \& G. M. Shaw. Anti-viral pressure exerted by HIV-1-specific cytotoxic T lymphocytes (CTLs) during primary infection demonstrated by rapid selection of CTL escape virus. Nat Med 3:205-11, 1997. On p.13

[Brander1995] C. Brander \& B. Walker. The HLA class I restricted CTL response in HIV infection: identification of optimal epitopes. HIV Molecular Immunology Database 1995, Los Alamas National Laboratory 1995. On p. 3

[Brander1999] C. Brander, O. Yang, N. Jones, Y. Lee, P. Goulder, R. Johnson, A. Trocha, D. Colbert, C. Hay, S. Buchbinder, C. Bergmann, H. Zweerink, S. Wolinsky, W. Blattner, S. Kalams, $\&$ B. Walker. Efficient processing of the immunodominant, HLA-A*0201-restricted human immunodeficiency virus type 1 cytotoxic T-lymphocyte epitope despite multiple variations in the epitope flanking sequences. $\boldsymbol{J}$ Virol 73:10191-8, 1999. On p. 4

[Brander2002] C. Brander \& Y. Riviere. Early and late cytotoxic T lymphocyte responses in HIV infection. AIDS 16:S97, 2002. On p. 3 , 4

[Buseyne1993] F. Buseyne, M. McChesney, F. Porrot, S. Kovarik, B. Guy, \& Y. Riviere. Gagspecific cytotoxic T lymphocytes from human immunodeficiency virus type 1-infected individuals: Gag epitopes are clustered in three regions of the p24gag protein. $J$ Virol 67:694-702, 1993 On p.4 $4[12$

[Buseyne1996] F. Buseyne, M. Fevrier, S. Garcia, M. L. Gougeon, \& Y. Riviere. Dual function of a human immunodeficiency virus (HIV)-specific cytotoxic T-lymphocyte clone: inhibition of HIV replication by noncytolytic mechanisms and lysis of HIV-infected CD4+ cells. Virology 225:248-53, 1996. On p. 14

[Buseyne1997] F. Buseyne, S. Stevanovic, H. Rammensee, \& Y. Riviere. Characterization of an HIV-1 p24 gag epitope recognized by a CD8+ cytotoxic T-cell clone. Immunol Lett 55(3):145149, 1997. On p. 14

[Buseyne1999] F. Buseyne. pers. communication, 1999. On p.7 714

[Cao2002] J. Cao. pers. communication, 2002. On p.6 6 , 11,13

[Cao2003] J. Cao, J. McElrath, \& et al. Comprehensive analysis of HIV-1 specific IFN- $\gamma$ secreting CD8 T cells in primary HIV infection. submitted 2003. On p.7, $11,13,16$

[Culmann-Penciolelli1994] B. Culmann-Penciolelli, S. Lamhamedi-Cherradi, I. Couillin, N. Guegan, J. P. Levy, J. G. Guillet, \& E. Gomard. Identification of multirestricted immunodominant regions recognized by cytolytic $\mathrm{T}$ lymphocytes in the human immunodeficiency virus type $1 \mathrm{Nef}$ protein (See comments in J Virol 1995 Jan;69(1):618). J Virol 68:7336-43, 1994. On p.10]11 12

[Culmann1991] B. Culmann, E. Gomard, M.-P. Kieny, B. Guy, F. Dreyfus, A.-D. Saimot, D. Sereni, D. Sicard, \& J.-P. Levy. Six epitopes with human cytotoxic CD8+ cells in the central region of 
the HIV-1 Nef protein. J Immunol 146:1560-1565, 1991. On p.7 7 $11|12| 15$

[Culmann1999] B. Culmann. pers. communication, 1999. On p.7 $711 \mid 12$

[Currier2002] J. Currier, M. deSouza, P. Chanbancherd, W. Bernstein, D. Birx, \& J. Cox. Com-

prehensive screening for human immunodeficiency virus type 1 subtype-specific CD8 cytotoxic T lymphocytes and definition of degenerate epitopes restricted by HLA-A0207 and -C(W)0304 alleles. J Virology 76:4971-4986, 2002. On p.66 16

[Dai1992] L. C. Dai, K. West, R. Littaua, K. Takahashi, \& F. A. Ennis. Mutation of human immunodeficiency virus type 1 at amino acid 585 on gp 41 results in loss of killing by CD8+ A24-restricted cytotoxic T lymphocytes. J Virol 66:3151-3154, 1992. On p.8

[DiBrino1993] M. DiBrino, K. C. Parker, \& J. S. et al. Endogenous peptides bound to HLA-A3 possess a specific combination of anchor residues that permit identification of potential antigenic peptides. Proc Natl Acad Sci USA 90:1508-1512, 1993. On p.7

[DiBrino1994a] M. DiBrino, K. C. Parker, D. H. Margulies, J. Shiloach, R. V. Turner, M. Garfield, W. E. Biddison WE, \& J. E. Coligan. The HLA-B14 peptide binding site can accommodate peptides with different combinations of anchor residues. J Biol Chem 269, 1994. On p. 10

[DiBrino1994b] M. DiBrino, K. C. Parker, J. Shiloach, R. V. Turner, T. Tsuchida, M. Garfield, W. E Biddison, \& J. E. Coligan. Endogenous peptides with distinct amino acid anchor residue motif bind to HLA-A1 and HLA-B8. J Immunol 152:620-31, 1994. On p. 11

[Dorrell1999] L. Dorrell, T. Dong, G. S. Ogg, S. Lister, S. McAdam, T. Rostron, C. Conlon, A. J. McMichael, \& S. L. Rowland-Jones. Distinct recognition of non-clade B human immunodeficiency virus type 1 epitopes by cytotoxic T lymphocytes generated from donors infected in Africa J Virol 73:1708-14, 1999. On p.8

[Draenert2002] R. Draenert. pers. communication, 2002. On p. 15

[Dumrese1998] T. Dumrese, S. Stevanovic, F. H. Seeger, N. Yamada, Y. Ishikawa, K. Tokunaga, M. Takiguchi, \& H. Rammensee. HLA-A26 subtype A pockets accommodate acidic N-termin of ligands. Immunogenetics 48:350-3, 1998. On p. 8

[Dupuis1995] M. Dupuis, S. K. Kundu, \& T. C. Merigan. Characterization of HLA-A*0201 restricted cytotoxic T-cell epitopes in conserved regions of the HIV type 1 gp160 protein. $J$ Immunol 155:2232-2239, 1995. On p. 6

[Edwards2002] B. Edwards, A. Bansal, S. Sabbaj, J. Bakari, M. Mulligan, \& P. Goepfert. Magnitude of functional CD8+ T-cell responses to the gag protein of human immunodeficiency virus type correlates inversely with viral load in plasma. J Virol 76:2298-2305, 2002. On p. 3

[Englehard1993] V. H. Englehard, E. L. Huczko, \& W. Bodner et al. Peptides bound to HLA-B7 determined by mass spectrometry. J Cell Biochem Suppl 1993 17C:56, 1993. On p. 10

[Falk1991] K. Falk, O. Rotzschke, S. Stevanovic, G. Jung, \& H.-G. Rammensee. Allele-specific motifs revealed by sequencing of self-peptides eluted from MHC molecules. Nature 351:290 296, 1991. On p.6

[Falk1993] K. Falk, O. Rotzschke, B. Grahovac, D. Schendel, S. Stevanovic, G. Jung, \& H. G. Rammensee. Peptide motifs of HLA-B35 and -B37 molecules [published erratum appears in Immunogenetics 1994;39(5):379]. Immunogenetics 38:161-2, 1993. On p. 12

[Falk1994] K. Falk, O. Rotzschke, \& B. Grahavac. Allele-specific peptide motifs of HLA-C molecules. Proc Natl Acad Sci USA 90:12005-12009, 1994. On p.9 16

[Falk1995a] K. Falk, O. Rotzschke, M. Takiguchi, V. Gnau, S. Stevanovic, G. Jung, \& H. Rammensee. Peptide motifs of HLA-B38 and B39 molecules. Immunogenetics 41:162-164, 1995. On p. 13,14

[Falk1995b] K. Falk, O. Rotzschke, M. Takiguchi, V. Gnau, S. Stevanovic, G. Jung, \& H. Rammensee. Peptide motifs of HLA-B58, B60, B61, and B62 molecules. Immunogenetics 41:165 168, 1995. On $\mathrm{p} .1315$

[Frahm2002] N. Frahm \& P. Goulder. pers. communication, 2002. On p. 10

[Frahm2003] N. Frahm, C. Adams, R. Draenert, M. Feeney, K. Sango, N. Brown, D. SenGupta,
T. Simonis, F. Marincola, A. Wurcel, D. Stone, C. Russell, P. Adolf, D. Cohen, T. Roach, A. StJohn, B. Korber, J Szinger, K. Davies, J. Mullins, P. Goulder, B. Walker, \& C. Brander Identification of highly immunodominant regions in HIV by comprehensive CTL screening of ethnically diverse populations. Submitted 2003 . On p. 34

[Fukada1999] K. Fukada, Y. Chujoh, H. Tomiyama, K. Miwa, Y. Kaneko, S. Oka, \& M. Takiguchi. HLA-A*1101-restricted cytotoxic T lymphocyte recognition of HIV-1 Pol protein [letter]. AIDS 13:1413-4, 1999. On p.7

[Fukada2002] K. Fukada, H. Tomiyama, C. Wasi, T. Matsuda, S. Kusagawa, H. Sato, S. Oka, Y. Takebe, \& M. Takiguchi. Cytotoxic T-cell recognition of HIV-1 cross-clade and clade-specific epitopes in HIV-1-infected Thai and Japanese patients. AIDS 16:701-11, 2002. On p. 3

[Gaschen2002] B. Gaschen, J. Taylor, K. Yusim, B. Foley, F. Gao, D. Lang, V. Novitsky, B. Haynes, B. Hahn, T. Bhattacharya, \& B. Korber. Diversity considerations in HIV-1 vaccine selection Science 296:2354-60, 2002. On p.4

[Gileadi1999] U. Gileadi, A. Gallimore, P. Van der Bruggen, \& V. Cerundolo. Effect of epitope flanking residues on the presentation of N-terminal cytotoxic T lymphocyte epitopes. Eur J Immunol 29:2213-22, 1999. On p. 4

Gotch1993] F. Gotch, S. N. McAdam, \& C. E. Allsopp et al. Cytotoxic T-cells in HIV-2 seropositive Gambians. Identification of a virus specific MHC-restricted peptide epitope. J Immuno 151:3361-3369, 1993. On p. 14

Goulder1996a] P. Goulder, C. Conlon, K. Mclntyre, \& A. McMichael. Identification of a novel human leukocyte antigen A26-restricted epitope in a conserved region of Gag. AIDS 10(12):1441-3, 1996. On p. 8

[Goulder1996b] P. J. R. Goulder, M. Bunce, P. Krausa, K. McIntyre, S. Crowley, B. Morgan, A. Ed wards, P. Giangrande, R. E. Phillips, \& A. J. McMichael. Novel, cross-restricted, conserved and immunodominant cytotoxic T lymphocyte epitopes in slow HIV Type 1 infection. AIDS Res and Hum Retroviruses 12:1691-1698, 1996. On p. 15

[Goulder1997a] P. Goulder, A. Sewell, D. Lalloo, D. Price, J. Whelan, J. Evans, G. Taylor, G. Luzzi, P. Giangrande, R. Phillips, \& A. J. McMichael. Patterns of immunodominance in HIV-1-specific cytotoxic T lymphocyte responses in two human histocompatibility leukocyte antigens (HLA)identical siblings with HLA-A*0201 are influenced by epitope mutation. J Exp Med 8:1423-33, 1997. On p.7] 12

[Goulder1997b] P. J. Goulder, M. Bunce, G. Luzzi, R. E. Phillips, \& A. J. McMichael. Potential underestimation of HLA-C-restricted cytotoxic T-lymphocyte responses. AIDS 11(15): 1884-1886, 1997. On p. $8[12,16$

Goulder1997c] P. J. R. Goulder, R. E. Phillips, R. A. Colbert, S. McAdam, G. Ogg, M. A. Nowak, P. Giangrande, G. Luzzi, B. Morgan, A. Edwards, A. McMichael, \& S. Rowland-Jones. Late Escape from an immunodominant cytotoxic T-lymphocyte response associated with progression to AIDS. Nature Med 3:212-216, 1997. On p.12

[Goulder1997d] P. J. R. Goulder, S. W. Reid, D. A. Price, C. A. O’Callaghan, A. J. McMichael, R. E. Phillips, \& E. Y. Jones. Combined structural and immunological refinement of HIV-1 HLA-B8 restricted cytotoxic T lymphocyte epitopes. Eur J Immunol 27:1515-1521, 1997. On p.10

[Goulder1999] P. J. R. Goulder. pers. communication, 1999. On p. 6 10,11 13

[Goulder2000a] P. Goulder, C. Brander, K. Annamalai, N. Mngqundaniso, U. Govender, Y. Tang, S. He, K. Hartman, C. O’Callaghan, G. Ogg, M. Altfeld, E. Rosenberg, H. Cao, S. Kalams, M. Hammond, M. Bunce, S. Pelton, S. Burchett, K. McIntosh, H. Coovadia, \& B. Walker. Differential narrow focusing of immunodominant human immunodeficiency virus gag-specific cytotoxic T-lymphocyte responses in infected African and caucasoid adults and children. $J$ Viro 74:5679-90, 2000. On p. 4 13 [16

[Goulder2000b] P. Goulder, Y. Tang, S. Pelton, \& B. Walker. HLA-B57-restricted CTL activity in a single infected subject towards two optimal HIV epitopes, one of which is entirely contained 
within the other. $J$ Virol 74:5291-9, 2000. On p.7] 15

[Goulder2001] P. Goulder, M. Addo, M. Altfeld, E. Rosenberg, Y. Tang, U. Govender, N. Mngqundaniso, K. Annamalai, T. Vogel, M. Hammond, M. Bunce, H. Coovadia, \& B. Walker. Rapid definition of five novel HLA-A*3002-restricted human immunodeficiency virus-specific cytotoxic T-lymphocyte epitopes by elispot and intracellular cytokine staining assays. J Virol 75:1339-47, 2001. On p. 33

[Haas1996] G. Haas, U. Plikat, P. Debre, M. Lucchiari, C. Katlama, Y. Dudoit, O. Bonduelle, M. Bauer, H. Ihlenfeldt, G. Jung, B. Maier, A. Meyerhans, \& B. Autran. Dynamics of vira variants in HIV-1 Nef and specific cytotoxic T lymphocytes in vivo. J Immunol 157:4212-4221, 1996. On p.6610

[Haas 1998] G. Haas, A. Samri, E. Gomard, A. Hosmalin, J. Duntze, J. M. Bouley, H. G. Ihlenfeldt, C. Katlama, \& B. Autran. Cytotoxic T-cell responses to HIV-1 reverse transcriptase, integrase and protease. AIDS 12(12): 1427-36, 1998. On p. 677 14

[Haas1999] G. Haas. pers. communication, 1999. On p.6]7] 14

[Harrer1996a] E. Harrer, T. Harrer, P. Barbosa, M. Feinberg, R. P. Johnson, S. Buchbinder, \& B. D. Walker. Recognition of the highly conserved YMDD region in the human immunodeficiency virus type 1 reverse transcriptase by HLA-A2-restricted cytotoxic T lymphocytes from an asymptomatic long-term nonprogresser. J Inf Dis 173:476-479, 1996. On p.6

[Harrer1996b] T. Harrer, E. Harrer, S. A. Kalams, P. Barbosa, A. Trocha, R. P. Johnson, T. Elbeik, M. B. Feinberg, S. P. Buchbinder, \& B. D. Walker. Cytotoxic T lymphocytes in asymptomatic long-term nonprogressing HIV-1 infection. Breadth and specificity of the response and relation to in vivo viral quasispecies in a person with prolonged infection and low viral load. J Immunol 156:2616-2623, 1996. On p.7] 9 [1 11

[Harrer1998] T. Harrer, E. Harrer, P. Barbosa, F. Kaufmann, R. Wagner, S. Bruggemann, J. R Kalden, M. Feinberg, R. P. Johnson, S. Buchbinder, \& B. D. Walker. Recognition of two overlapping CTL epitopes in HIV-1 p17 by CTL from a long-term nonprogressing HIV-1-infected individual. J Immunol 161:4875-81, 1998. On p.7

[Hay1999] C. Hay. pers. communication, 1999. On p. 15

[Hill1992] A. V. Hill, J. Elvin, A. C. Willis, M. Aidoo, C. E. Allsopp, F. M. Gotch, X. M. Gao M. Takiguchi, B. M. Greenwood, \& A. R. Townsend et al. Molecular analysis of the association of HLA-B53 and resistance to severe malaria (see comments). Nature 360:434-9, 1992 . On p. 10 [1 12 14

[Hossain2001] M. Hossain, H. Tomiyama, T. Inagawa, B. Sriwanthana, S. Oka, \& M. Takiguchi. HLA-A*3303-restricted cytotoxic T lymphocyte recognition for novel epitopes derived from the highly variable region of the HIV-1 Env protein. AIDS 15:2199-2201, 2001. On p.9

[Ikeda-Moore1998] Y. Ikeda-Moore, H. Tomiyama, M. Ibe, S. Oka, K. Miwa, Y. Kaneko, \&

M. Takiguchi. Identification of a novel HLA-A24-restricted cytotoxic T-lymphocyte epitope derived from HIV-1 Gag protein. AIDS 12:2073-4, 1998. On p.8

[Jardetzky1991] T. S. Jardetzky, W. S. Lane, R. A. Robinson, D. R. Madden, \& D. C. Wiley. Identification of self peptides bound to purified HLA-B27. Nature 353:326-9, 1991. On p. 12

[Jin2000] X. Jin, C. G. Roberts, D. F. Nixon, J. T. Safrit, L. Q. Zhang, Y. X. Huang, N. Bhardwaj, B. Jesdale, A. S. DeGroot, \& R. A. Koup. Identification of subdominant cytotoxic T lymphocyte epitopes encoded by autologous HIV type 1 sequences, using dendritic cell stimulation an computer-driven algorithm. AIDS Res Hum Retroviruses 16:67-76, 2000. On p. 10

[Johnson1991] R. P. Johnson, A. Trocha, L. Yang, G. P. Mazzara, D. L. Panicali, T. M. Buchanan, \& B. D. Walker. HIV-1 gag-specific cytotoxic T lymphocytes recognize multiple highly conserved epitopes. Fine specificity of the gag-specific response defined by using unstimulated periphera blood mononuclear cells and cloned effector cells. J Immunol 147:1512-1521, 1991. On p.6 1115

[Johnson1992] R. P. Johnson, A. Trocha, T. M. Buchanan, \& B. D. Walker. Identification of overlap- ping HLA class I-restricted cytotoxic T-cell epitopes in a conserved region of the human immunodeficiency virus type 1 envelope glycoprotein: definition of minimum epitopes and analysis of the effects of sequence variation. J Exp Med 175:961-971, 1992. On p. 10. 11

[Johnson1993] R. P. Johnson, A. Trocha, T. M. Buchanan, \& B. D. Walker. Recognition of a highly conserved region of human immunodeficiency virus type 1 gp120 by an HLA-Cw4-restricted cytotoxic T-lymphocyte clone. J Virol 67:438-445, 1993. On p. 16

[Johnson1994a] R. P. Johnson, S. A. Hammond, A. Trocha, R. F. Siliciano, \& B. D. Walker. Induction of a major histocompatibility complex class I-restricted cytotoxic T-lymphocyte response to a highly conserved region of human immunodeficiency virus type 1 (HIV-1) gp120 in seronegative humans immunized with a candidate HIV-1 vaccine. J Virol 68:3145-3153, 1994. On p. 7] 12]

Johnson1994b] R. P. Johnson \& B. D. Walker. CTL in HIV-1 infection: Responses to structural proteins. Curr Topics Microbiol Immunol 189:35-63, 1994. On p.7

[Johnson1999] R. P. Johnson. pers. communication, 1999. On p.11

[Kiepiela2002] P. Kiepiela \& P. Goulder. pers. communication, 2002. On p.13 $14|15| 16$

[Klenerman1996] P. Klenerman, G. Luzzi, K. McIntyre, R. Phillips, \& A. McMichael. Identification of a novel HLA-A25 restricted epitope in a conserved region of p24 gag (positions 71-80). AIDS 10:348-350, 1996. On p. 8

[Koenig1990] S. Koenig, T. R. Fuerst, L. V. Wood, R. M. Woods, J. A. Suzich, G. M. Jones, V. F. de la Cruz, R. T. Davey, S. Venkatesan, B. Moss, W. E. Biddison, \& A. S. Fauci. Mapping the fine specificity of a cytotoxic T-cell response to HIV-1 Nef protein. J Immunol 145:127-135, 1990. On p.7

Kuckelkorn2002] U. Kuckelkorn, E. Ferreira, I. Drung, U. Liewer, P. Kloetzel, \& M. Theobald The effect of the interferon-gamma-inducible processing machinery on the generation of a naturally tumor-associated human cytotoxic T lymphocyte epitope within a wild-type and mutant p53 sequence context. Eur J Immunol 32:1368-75, 2002. On p. 4

[Kurane1999] I. Kurane \& K. West. pers. communication, 1999. On p. 8.13

\begin{tabular}{lll|l|l|l|} 
[Lewinsohn1999a] & D. Lewinsohn. pers. communication, 1999. On p. 8 |10 & 12 & 13 \\
\hline
\end{tabular}

[Lewinsohn1999b] D. Lewinsohn \& S. Riddell. pers. communication, 1999. On p.7

[Lieberman1992] J. Lieberman, J. A. Fabry, M.-C. Kuo, P. Earl, B. Moss, \& P. R. Skolnik. Cytotoxic T lymphocytes from HIV-1 seropositive individuals recognize immunodominant epitopes in gp160 and reverse transcriptase. J Immunol 148:2738-2747, 1992. On p. 8

[Lieberman1999] J. Lieberman. pers. communication, 1999. On p.12 14

[Lynch1998] J. Lynch, M. deSouza, M. Robb, L. Markowitz, S. Nitayaphan, C. Sapan, D. Mann, D. Birx, \& J. Cox. Cross-clade cytotoxic T cell response to human immunodeficiency virus type 1 proteins among HLA disparate North Americans and Thais. J Infect Dis 178:1040-6, 1998. On p. 3

Maier1994] R. Maier, K. Falk, O. Rotzschke, B. Maier, V. Gnau, S. Stevanovic, G. Jung, H. G. Rammensee, \& A. Meyerhans. Peptide motifs of HLA-A3, -A24, and -B7 molecules as determined by pool sequencing. Immunogenetics 40:306-8, 1994. On p.8

[Maier1999] B. Maier \& B. Autran. pers. communication, 1999. On p. 6

[McKinney 1999] D. McKinney, D. Lewinson, S. Riddell, P. Greenberg, \& D. Mosier. The Antiviral Activity of HIV-Specific CD8+ CTL clones is limited by elimination due to encounter with HIVinfected targets. J Imтиио 163:861-7, 1999. On p. 12

[Nixon1988] D. Nixon, A. Townsend, J. Elvin, C. Rizza, J. Gallway, \& A. McMichael. HIV-1 gagspecific cytotoxic T lymphocytes defined with recombinant vaccinia virus and synthetic peptides Nature 336:484-487, 1988. On p. 12

[Nixon1999] D. F. Nixon, D. Douek, P. J. Kuebler, X. Jin, M. Vesanen, S. Bonhoeffer, Y. Cao, R. A Koup, D. D. Ho, \& M. Markowitz. Molecular tracking of an Human Immunodeficiency Virus ne specific cytotoxic T-cell clone shows persistence of clone-specific T-cell receptor DNA but no mRNA following early combination antiretroviral therapy. Immunol Lett 66:219-28, 1999. On 
p. 16

[Novitsky2001] V. Novitsky, N. Rybak, M. McLane, \& et al. Identification of HIV type 1 subtype C Gag-,Tat-, Rev-, and Nef-specific Elispot based CTL responses to AIDS vaccine design. Virology 75:9210-9228, 2001. On p. 3] 11

[Novitsky2002] V. Novitsky, H. Cao, N. Rybak, P. Gilbert, M. McLane, S. Gaolekwe, T. Peter I. Thior, T. Ndung'u, R. Marlink, T. Lee, \& M. Essex. Magnitude and frequency of cytotoxic T-lymphocyte responses: identification of immunodominant regions of human immunodeficiency virus type 1 subtype C. $J$ Virol 76:10155-68, 2002. On p.33

[Novitsky2003] V. Novitsky, P. Gilbert, T. Peter, M. McLane, S. Gaolekwe, N. Rybak, I. Thior T. Ndung'u, R. Marlink, T. Lee, \& M. Essex. Association between virus-specific T-cell responses and plasma viral load in human immunodeficiency virus type 1 subtype $\mathrm{C}$ infection. $J$ Viro 77:882-90, 2003. On p. 3

[Ogg1998] G. S. Ogg, X. Jin, S. Bonhoeffer, P. R. Dunbar, M. A. Nowak, S. Monard, J. P. Segal, Y. Cao, S. L. Rowland-Jones, V. Cerundolo, A. Hurley, M. Markowitz, D. D. Ho, D. F. Nixon, \& A. J. McMichael. Quantitation of HIV-1-specific cytotoxic T lymphocytes and plasma load of viral RNA. Science 279:2103-6, 1998. On p. 11] 13

[Oxenius2002] A. Oxenius, B. Jakobsen, P. Easterbrook, J. Boulter, T. Tun, A. Waters, J. Agudelo, M. Barnardo, R. Phillips, \& D. Price. Complete mapping of a novel HLA A*6801-restricted HIV 1 Tat epitope directly with a rapid modified enzyme-linked immunospot assay. AIDS 16:12851287, 2002. On p.9

[Parker1992] K. C. Parker, M. A. Bednarek, L. K. Hull, U. Utz, B. C. H. J. Zweerink, W. E. Biddison, \& J. E. Coligan. Sequence motifs important for peptide binding to the human MHC class molecule, HLA-A2. J Immunol 149, 1992. On p.6

[Parker1994] K. C. Parker, M. A. Bednarek, \& J. E. Coligan. Scheme for ranking potential HLA-A2 binding peptides based on independent binding of individual peptide side-chains. J Immunol 152 1994. On p. 6

[Price1997] D. A. Price, P. J. Goulder, P. Klenerman, A. K. Sewell, P. J. Easterbrook, M. Troop C. R. Bangham, \& R. E. Phillips. Positive selection of HIV-1 cytotoxic T lymphocyte escape variants during primary infection. Proc Natl Acad Sci USA 94:1890-5, 1997. On p. 10

[Rammensee1995] H.-G. Rammensee, T. Friede, \& S. Stevanovic. MHC ligands and peptide motifs: first listing. Immunogenetics 41:178-228, 1995. On p.7] 12

[Rammensee1999] H. Rammensee, J. Bachmann, N. Emmerich, \& S. Stevanovic. SYFPEITHI: An Internet Database for MHC Ligands and Peptide Motifs. Immunogenetics 50:213-9, 1999. On p. $9\left[\begin{array}{l|l|l|l}10 & 12 & 13 & 14 \\ \hline\end{array}\right.$

[Rowland-Jones1993] S. L. Rowland-Jones, S. H. Powis, J. Sutton, I. Mockridge, F. M. Gotch

N. Murray, A. B. Hill, W. M. Rosenberg, J. Trowsdale, \& A. J. McMichael. An antigen processing polymorphism revealed by HLA-B8-restricted cytotoxic T lymphocytes which does not correlate with TAP gene polymorphism. Eur J Immunol 23:1999-2004, 1993. On p.10

[Rowland-Jones1995] S. L. Rowland-Jones, J. Sutton, K. Ariyoshi, T. Dong and , F. Gotch, S. McAdam, D. Whitby, S. Sabally, A. Gallimore, T. Corrah, M. Takiguchi, T. Schultz A. McMichael, \& H. Whittle. HIV-specific cytotoxic T-cells in HIV-exposed but uninfected Gambian women. Nature Medicine 1:59-64, 1995. On p. 12

[Rowland-Jones1998] S. L. Rowland-Jones, T. Dong, K. R. Fowke, J. Kimani, P. Krausa, H. Newell, T. Blanchard, K. Ariyoshi, J. Oyugi, E. Ngugi, J. Bwayo, K. S. MacDonald, A. J. McMichael, \& F. A. Plummer. Cytotoxic T cell responses to multiple conserved HIV epitopes in HIV- resistant prostitutes in Nairobi [see comments]. J Clin Invest 102:1758-65, 1998. On p. 11

Rowland-Jones1999] S. Rowland-Jones. pers. communication, 1999. On p.8 9

[Sabbaj2003] S. Sabbaj, A. Bansal, G.D.Ritter, C. Perkins, B. Edwards, E. Gough, J.Tang, J. Szinger, B. Korber, R. Kaslow, M. Mulligan, \& P. Goepfert. Cross-reactive CD8+ T cell epitopes identified in US adolescent minorities . submitted 2003. On p. 3 6 8 $9011,13,14$
[Safrit1994a] J. T. Safrit, C. A. Andrews, T. Zhu, D. D. Ho, \& R. A. Koup. Characterization of human immunodeficiency virus type 1-specific cytotoxic T lymphocyte clones isolated during acute seroconversion: recognition of autologous virus sequences within a conserved immunodominan epitope. J Exp Med 179:463-472, 1994. On p. 9

Safrit1994b] J. T. Safrit, A. Y. Lee, C. A. Andrews, \& R. A. Koup. A region of the Third Variable Loop of HIV-1 gp120 is recognized by HLA-B7-Restricted CTLs from two acute seroconversion patients. J Immunol 153:3822-3830, 1994. On p. 910

[Seeger1998] F. H. Seeger, D. Arnold, T. Dumrese, H. de la Salle, D. Fricker, H. Schild, H. G. Rammensee, \& S. Stevanovic. The HLA-B* 1516 motif demonstrates HLA-B-specific P2 pocket characteristics. Immunogenetics 48:156-60, 1998. On p. 11

Shankar1996] P. Shankar, J. A. Fabry, D. M. Fong, \& J. Lieberman. Three regions of HIV-1 gp160 contain clusters of immunodominant CTL epitopes. Immunol Lett 52:23-30, 1996. On p. 810

Shiga1996] H. Shiga, T. Shioda, H. Tomiyama, Y. Takamiya, S. Oka, S. Kimura, Y. Yamaguchi, T. Gojoubori, H. G. Rammensee, K. Miwa, \& M. Takiguchi. Identification of multiple HIV-1 cytotoxic T-cell epitopes presented by human leukocyte antigen B35 molecule. AIDS 10:10751083, 1996. On p. 12

[Sipsas 1997] N. V. Sipsas, S. A. Kalams, A. Trocha, S. He, W. A. Blattner, B. D. Walker, \& R. P. Johnson. Identification of type-specific cytotoxic $\mathrm{T}$ lymphocyte responses to homologous vira proteins in laboratory workers accidentally infected with HIV-1. J Clin Invest 99:752-62, 1997.

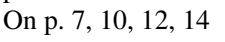

Sriwanthana2001] B. Sriwanthana, T. Hodge, T. Mastro, C. Dezzutti, K. Bond, H. Stephens, L. Kostrikis, K. Limpakarnjanarat, N. Young, S. Qari, R. Lal, D. Chandanayingyong, \& J. McNicholl. HIV-specific cytotoxic T lymphocytes, HLA-A11, and chemokine-related factors may act synergistically to determine HIV resistance in CCR5 delta32-negative female sex workers in Chiang Rai, northern Thailand. AIDS Res Hum Retroviruses 17:719-34, 2001. On p. 3

[Sutton1993] J. Sutton, S. Rowland-Jones, W. Rosenberg, D. Nixon, F. Gotch, X.-M. Gao, N. Murray, A. Spoonas, P. Driscoll, M. Smith, A. Willis, \& A. McMichael. A sequence pattern fo peptides presented to cytotoxic T lymphocytes by HLA B8 revealed by analysis of epitopes and eluted peptides. Eur J Immunol 23:447-453, 1993. On p. 10

[Takahashi1991] K. Takahashi, L.-C. Dai, T. R. Fuerst, W. E. Biddison, P. L. Earl, B. Moss, \& F. A. Ennis. Specific lysis of human immunodeficiency virus type 1-infected cells by a HLAA3.1-restricted CD8+ cytotoxic T-lymphocyte clone that recognizes a conserved peptide sequence within the gp41 subunit of the envelope protein. Proc Natl Acad Sci USA 88:10277-10281, 1991. On p. 7

[Threlkeld1997] S. C. Threlkeld, P. A. Wentworth, S. A. Kalams, B. M. Wilkes, D. J. Ruhl, E. Kepgh, J. Sidney, S. Southwood, B. D. Walker, \& A. Sette. Degenerate and promiscuous recognition by CTL of peptides presented by the MHC class I A3-like superfamily. J Immunol 159 (4): $1648-1657,1997$. On p.7]

Tomiyama1999a] H. Tomiyama, Y. Chujoh, T. Shioda, K. Miwa, S. Oka, Y. Kaneko, \& M. Takiguchi. Cytotoxic T lymphocyte recognition of HLA-B*5101-restricted HIV-1 Rev epitope which is naturally processed in HIV-1-infected cells [letter]. AIDS 13:861-3, 1999. On p. 3 .

Tomiyama1999b] H. Tomiyama, T. Sakaguchi, K. Miwa, S. Oka, A. Iwamoto, Y. Kaneko, \& M. Takiguchi. Identification of multiple HIV-1 CTL epitopes presented by HLA-B*5101 molecules. Hum Immunol 60:177-86, 1999. On p.14

[Trocha2002] A. Trocha. pers. communication, 2002. On p.16

[Tsomides1991] T. J. Tsomides, B. D. Walker, \& H. N. Eisen. An optimal viral peptide recognized by CD8+ T-cells binds very tightly to the restricting class I major histocompatibility complex protein on intact cells but not to the purified class I protein. Proc Natl Acad Sci USA 88:11276- 
11280, 1991. On p.6

[van Baalen1996] C. A. van Baalen, M. R. Klein, R. C. Huisman, M. E. Dings, S. R. Kerkhof Garde, A. M. Geretti, R. Gruters, C. A. van Els, F. Miedema, \& A. D. Osterhaus. Fine-specificity of cytotoxic T lymphocytes which recognize conserved epitopes of the Gag protein of human immunodeficiency virus type 1. J Gen Virol 77:1659-1665, 1996. On p.8

[van Baalen1997] C. van Baalen, O. Pontesilli, R. Huisman, A. Geretti, M. Klein, F. de Wolf, F. Miedema, R. Gruters, \& A. Osterhaus. Human immunodeficiency virus type 1 Rev- and Tatspecific cytotoxic T lymphocyte frequencies inversely correlate with rapid progression to AIDS. J Gen Virol 78 ( Pt 8): 1913-8, 1997. On p. 3

[van der Burg1997] S. H. van der Burg, M. R. Klein, O. Pontesilli, A. M. Holwerda, J. Drijfhout, W. M. Kast, F. Miedema, \& C. J. M. Melief. HIV-1 reverse transcriptase-specific CTL against conserved epitopes do not protect against progression to AIDS. J Immunol 159:3648-3654, 1997. On p.15

[Walker1989] B. D. Walker, C. Flexner, K. Birch-Limberger, L. Fisher, T. J. Paradis, A. Aldovini, R. Young, B. Moss, \& R. T. Schooley. Long-term culture and fine specificity of human cytotoxic T-lymphocyte clones reactive with human immunodeficiency virus type 1. Proc Natl Acad Sci USA 86:9514-9518, 1989. On p.6]10

[Wilkes1999a] B. Wilkes. pers. communication, 1999. On p.9

[Wilkes1999b] B. M. Wilkes \& D. J. Ruhl. pers. communication, 1999. On p.7

[Wilkes1999c] B. M. Wilkes, D. J. Ruhl, \& P. J. Goulder. pers. communication, 1999. On p.10

[Wilson1997] C. C. Wilson, S. A. Kalams, B. M. Wilkes, D. J. Ruhl, F. Gao, B. H. Hahn, I. C. Hanson, K. Luzuriaga, S. Wolinsky, R. Koup, S. P. Buchbinder, R. P. Johnson, \& B. D. Walker. Overlapping epitopes in human immunodeficiency virus type 1 gp120 presented by HLA A, B, and C molecules: effects of viral variation on cytotoxic T-lymphocyte recognition. J Virol 71:1256-64, 1997. On p. 10 $11,14,16$

[Wilson1999a] C. C. Wilson. pers. communication, 1999. On p.10]11

[Wilson1999b] C. C. Wilson, R. C. Brown, B. T. Korber, B. M. Wilkes, D. J. Ruhl, D. Sakamoto, K. Kunstman, K. Luzuriaga, I. C. Hanson, S. M. Widmayer, A. Wiznia, S. Clapp, A. J. Ammann, R. A. Koup, S. M. Wolinsky, \& B. D. Walker. Frequent detection of escape from cytotoxic T lymphocyte recognition in perinatal human immunodeficiency virus (HIV) type 1 transmission: the ariel project for the prevention of transmission of HIV from mother to infant. J Virol 73:397585, 1999. On p. 12

[Yellen-Shaw 1997] A. Yellen-Shaw, E. Wherry, G. Dubois, \& L. Eisenlohr. Point mutation flanking a CTL epitope ablates in vitro and in vivo recognition of a full-length viral protein. J Immuno 158:3227-34, 1997. On p. 4

[Yu2002a] X. Yu, M. Addo, E. Rosenberg, W. Rodriguez, P. Lee, C. Fitzpatrick, M. Johnston, D. Strick, P. Goulder, B. Walker, \& M. Altfeld. Consistent patterns in the development and immunodominance of human immunodeficiency virus type 1 (HIV-1)-specific CD8+ T-cell responses following acute HIV-1 infection. J Virology 76:8690-8701, 2002. On p.6 6] 10 [16

[Yu2002b] X. Yu, H. Shang, M. Addo, R. Eldridge, M. Phillips, M. Feeney, D. Strick, C. Brander, P. Goulder, E. Rosenberg, B. Walker, \& M. Altfeld. Important contribution of p15 Gag-specific responses to the total Gag-specific CTL responses. AIDS 16:321-328, 2002. On p. 610 13

[Yusim2002] K. Yusim, C. Kesmir, B. Gaschen, M. Addo, M. Altfeld, S. Brunak, A. Chigaev, V. Detours, \& B. Korber. Clustering patterns of cytotoxic T-lymphocyte epitopes in human immunodeficiency virus type 1 (HIV-1) proteins reveal imprints of immune evasion on HIV-1 global variation. J Virol 76:8757-68, 2002. On p. 34

[Zhang1993] Q. Zhang, R. Gavioli, G. Klein, \& M. G. Masucci. An HLA-All-specific motif in nonamer peptides derived from viral and cellular proteins. Proc Natl Acad Sci USA 90:22172221, 1993. On p.7 



\section{Mutational Analyses and Natural Variability of the gp41 Ectodomain}

Rogier W. Sanders ${ }^{1}$, Bette Korber ${ }^{2}$, Min Lu$^{3}$, Ben Berkhout ${ }^{1}$, and John P. Moore ${ }^{4}$

${ }^{1}$ Department of Human Retrovirology, Academic Medical Center, University of Amsterdam, 1105 AZ Amsterdam, The Netherlands; r.w.sanders@amc.uva.nl

${ }^{2}$ Theoretical Biology and Biophysics, MS K710, Los Alamos National Laboratory, Los Alamos, New Mexico 87545

${ }^{3}$ Department of Biochemistry and ${ }^{4}$ Department of Microbiology and Immunology, Weill Medical College, Cornell University, New York, New York 10021

The HIV-1 envelope glycoproteins mediate viral attachment and release of the viral core in susceptible target cells. A single gp160 precursor protein is processed intracellularly to yield the native form of the envelope complex, consisting of three gp120 and three gp41 molecules associated through non-covalent interactions. Upon receptor and co-receptor binding to the surface subunit gp120, conformational changes within the envelope glycoprotein complex enable the insertion of the hydrophobic fusion peptide of the transmembrane subunit gp41 into the target membrane. Subsequent rearrangements within gp41 allow fusion of viral and cellular membranes. These late structural alterations are targeted by the entry inhibitor T-20 (for reviews see 13, 20, 21, 24, 46, 75).

A considerable body of mutagenesis data on structure-function relationships within the HIV-1 gp41 ectodomain (gp41e) has been published over the years The value of this data-set has been increased considerably by the determination of the structure of the gp41e core, allowing some of the mutational effects to be interpreted and at least partially understood $(9,12,38,41,68,71)$. The native, pre-fusion structure of gp41e in the trimeric gp120-gp41 complex on the virion surface prior to receptor engagement is not known, however, and the various transitional structures of gp41 during the virus-cell fusion process are stil ill-defined. Consequently, the structural and functional consequences of many amino acid substitutions in gp41e remain unclear.

In HIV Molecular Immunology 2002. Bette T. M. Korber, Christian Brander, Barton F. Haynes, Richard Koup, Carla Kuiken, John P. Moore, Bruce D. Walker, and David I. Watkins, editors. Publisher: Los Alamos National Laboratory, Theoretical Biology and Biophysics, Los Alamos, New Mexico. LA-UR 03-5816. pp. 23.44
Here, we have summarized the results of published mutagenesis studies on gp41e (see the accompanying table). The HXB2 reference strain has been used as a basis for numbering individual amino acid residues (Figure 1). This information should facilitate the research of those who study the HIV-1 envelope glycoproteins as fusogens or vaccine antigens. In general, we have tabulated only data for single mutants, but several publications contain information on the effects of multiple amino acid substitutions $(25,43,44,49,56,57,62)$. The table does not include information on every naturally occurring gp4le sequence variant, as the variation is extensive. However, a summary of natural variability in clades B and $\mathrm{C}$ is presented in Figure 2. Also, the last two columns in the table present the entropy scores for gp41e positions that have a defined impact on Env function, for both the $\mathrm{B}$ clade and the $\mathrm{C}$ clade. Not surprisingly, positions identified through mutational analysis as those where substitutions can abrogate key functions, also tend to be highly conserved among the natural variants. The clearest example is provided by positions where substitutions essentially eliminate cell-cell fusion (i.e., where fusion efficiencies in syncytium assays or reporter gene assays have been reduced to less than $3 \%$ of the wild-type value). Sites at which substitutions can abrogate cell-cell fusion tended to be more invariant among 123 B clade sequences $(26 / 44,59 \%)$, compared to those sites where amino acid changes did not dramatically reduce fusion $(11 / 39,28 \%$, Fisher's exact test $p=0.004)$. Some unusual gp41e variants found in neutralization-resistant isolates are also included in the table, as are variants that arise in response to selection pressure, both in vitro and in vivo, from the entry inhibitor T-20, which targets gp41e.

The precision with which the available data could be analyzed was sometimes limited because different viral clones, isolates and assays were used to obtain the experimental data. We have therefore chosen to summarize quantitative parameters using the grading system,,-+++ and +++ , as indicated in the footnotes. In some cases these grades had to be deduced from the primary reports, so readers are encouraged to consult the original papers for quantitative details; we regret any errors of interpretation we may have made during this estimation process. Not surprisingly, perhaps, different studies sometimes yielded conflicting results. We have recorded the conflicting data sets but shall leave it to the readers to judge which are the more plausible.

The natural variability of residues in clade B and C isolates was analyzed and mapped on the structure of gp41 (see Figures 2 and 3). A focus of variable residues in clade B sequences is located in the upper part of the C-terminal helix 
centered around the highly variable leucine-glutamate-glutamine (LEQ) triplet, indicating that this region is under selective pressure. However, it is also possible that certain changes in residues in this region have little impact on Env function, particularly if there is some flexibility in Env structure(s) around this region. This relatively variable region also contains four glycosylation sites, which could be involved in immune evasion (30). Indeed, mutations that affect glycosylation in this region can modulate neutralization sensitivity (65). Of note is that no CTL or antibody epitopes have been mapped to this region despite the intense positive selection. One interpretation of this observation is that the selection pressure is exerted indirectly on distant antibody epitopes elsewhere in gp4le or even in gp120 (32). Another is that some neutralizing antibodies remain as yet undiscovered in this region of gp4le. In clade $C$ viruses the variability is somewhat shifted towards the 2F5 epitope, compared to clade B. Furthermore, certain residues are significantly more variable in clade $\mathrm{C}$ viruses compared to clade $\mathrm{B}$, and vice versa, suggesting that subtly different selection pressures may operate on viruses from the two clades.

Acknowledgments. We thank Brian Foley and Charles Calef for their help with graphical presentation of Figures and Tables. Financial support was obtained from the Dutch AIDS Fund, Amsterdam.

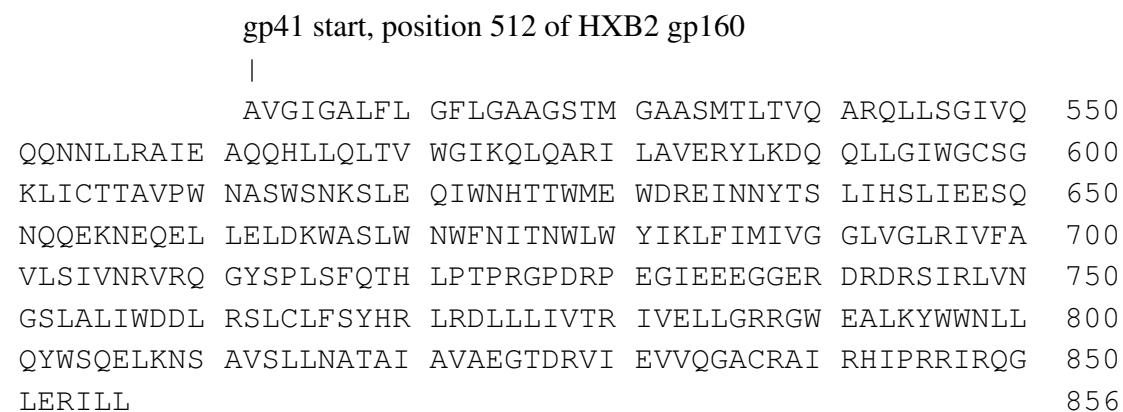

Figure 1: The HXB2 reference strain and the numbering of positions in the gp41 sequence. Only information on the ectodomain (residue 512-684) is incorporated in subsequent analyses. 

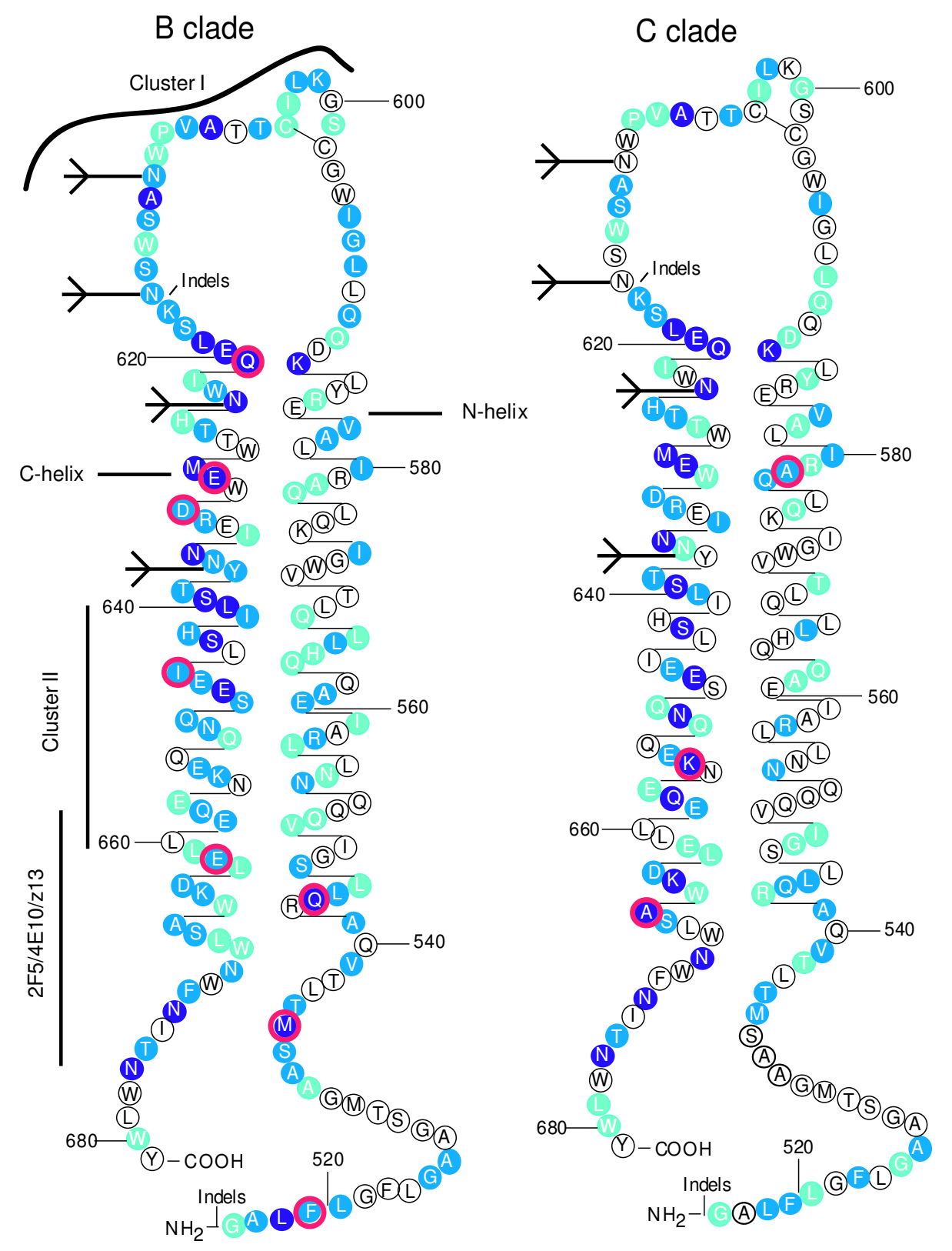

Invariant Highly conserved Moderately variable
Figure 2: Variability of gp41e. The relative entropies of residues were mapped onto a 2D representation of the HXB2 gp41e (adapted from 29, 61). The variability of residues in clade B isolates (left panel) and clade C isolates (right panel) is indicated according to their entropy values. The entropy is a simple measure of variation in each position based on a sequence alignment (33). Not surprisingly, entropy values for each amino acid were highly correlated with the ratio of the nonsynonymous/synonymous substitution rates, a measure which is indicative of selective pressure, calculated using PAML (76) (Spearman's rank correlation tests gave $z=7.3, p=2 \times 10^{-13}$ for the B clade, and $z=7.5, p=5 \times 10^{-14}$ for the $\mathrm{C}$ clade). We used the entropy scores as our measure of variability here because they lent themselves to testing for differences in variability between the $\mathrm{B}$ clade and $\mathrm{C}$ clade (33). The color coding for the sites is as follows: white, invariant (entropy score of zero); light blue, very conserved (entropy score below the median, corresponding to only one observed substitution); medium blue, variable (entropy score above the median: 2 or more observed substitutions); dark blue, highly variable (highest $10 \%$ of entropy scores: $>0.8$ for clade B and $>0.75$ for clade $\mathrm{C}$ ). Residues that are significantly more variable in clade $\mathrm{B}$ than in clade $\mathrm{C}$ or vice versa ( $p$ value $\leq 0.03$ after a Bonferroni correction for multiple tests, using a Monte Carlo scheme and randomizing the $\mathrm{B}$ and $\mathrm{C}$ clade data 10,000 times) are indicated by red circles. 123 clade $B$ sequences and 48 clade $C$ sequences were used for the analyses. The four glycans and the major antibody epitopes (non-neutralizing clusters I and II and the neutralizing 2F5/4E10/z13 cluster) are also indicated, as are regions labelled "indel" where insertions and deletions are frequently observed in natural variants. 


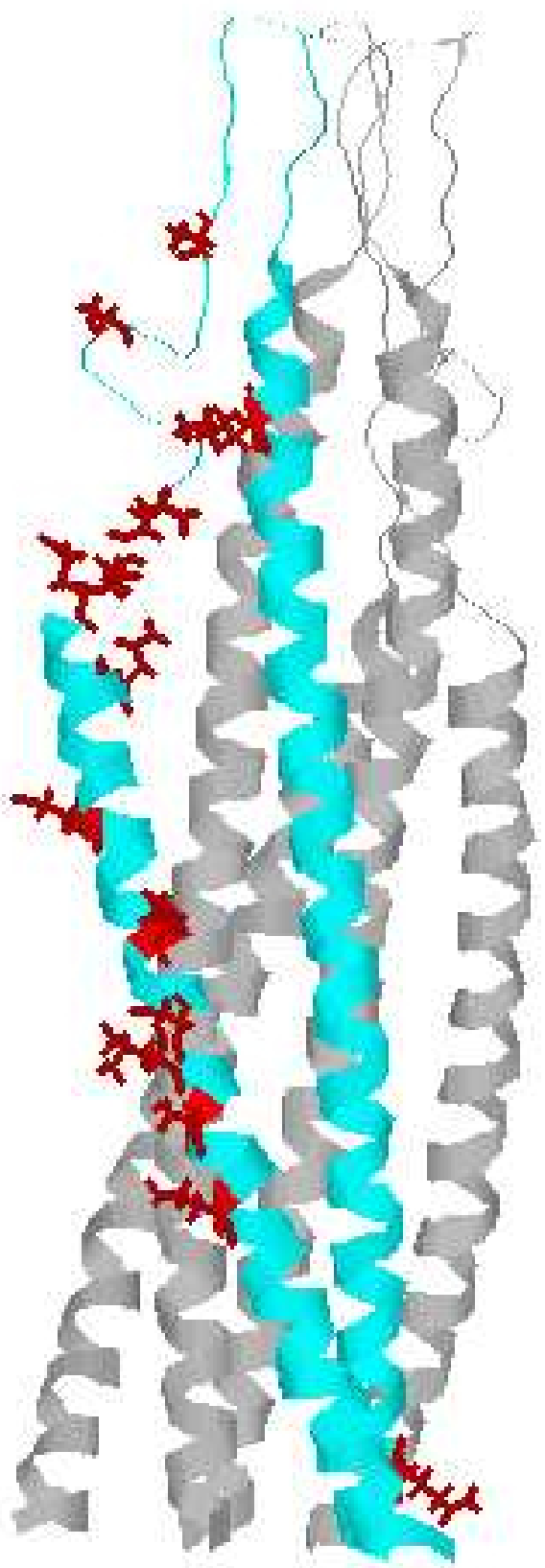

Figure 3: The residues with the highest $10 \%$ of entropy scores in clade B are indicated in red on the 3D structure model of Caffrey (pdb accession number 1IF3, (8)). These residues are only indicated in one monomer. The other two monomers are shown in grey for orientation purposes. 


\begin{tabular}{|c|c|c|c|c|}
\hline 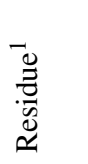 & 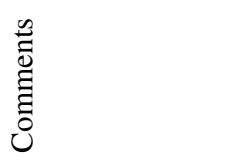 & 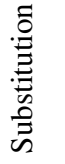 & 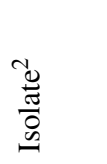 & 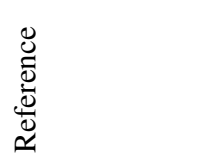 \\
\hline \multicolumn{5}{|l|}{ WT } \\
\hline \multirow[t]{2}{*}{ A512 } & & $\mathrm{V}^{16}$ & NL4-3 & Freed90 \\
\hline & & $\mathrm{E}$ & NL4-3 & Freed90 \\
\hline \multirow[t]{4}{*}{ V513 } & & $\mathrm{E}$ & NL4-3 & Freed90 \\
\hline & & A & NL4-3 & Buchschacher95 \\
\hline & & $\mathrm{G}$ & NL4-3 & \\
\hline & & $\mathrm{R}$ & NL4-3 & \\
\hline G514 & & V & NL4-3 & Delahunty96 \\
\hline G516 & & $\mathrm{V}$ & NL4-3 & Delahunty96 \\
\hline \multirow[t]{2}{*}{ A517 } & & 17 & HXB2 & Kowalski91 \\
\hline & & 18 & HXB2 & Kowalski91 \\
\hline M518 & & $\mathrm{V}^{19}$ & ELI1 & Kozak97 \\
\hline \multirow[t]{2}{*}{ F519 } & & $\mathrm{L}^{16}$ & NL4-3 & Freed90 \\
\hline & & V & NL4-3 & Delahunty96 \\
\hline L520 & & $\mathrm{R}$ & NL4-3 & Freed90 \\
\hline G521 & & $\mathrm{V}$ & NL4-3 & Delahunty96 \\
\hline \multirow[t]{2}{*}{ F522 } & & V & NL4-3 & Delahunty96 \\
\hline & & $\mathrm{G}$ & BH8 & Pritsker99 \\
\hline G524 & & V & NL4-3 & Delahunty96 \\
\hline A525 & & $\mathrm{T}^{20}$ & LAI & Bahbouhi01 \\
\hline A526 & & $\mathrm{E}$ & NL4-3 & Freed90 \\
\hline G527 & & $\mathrm{V}$ & NL4-3 & Delahunty96 \\
\hline S528 & & $\mathrm{T}$ & HXB2 & $\mathrm{Cao} 93$ \\
\hline M530 & & $\mathrm{S}$ & HXB2 & Cao93 \\
\hline G531 & & V & NL4-3 & Delahunty96 \\
\hline L537 & & $\mathrm{R}$ & NL4-3 & Freed90 \\
\hline V539 & & $\mathrm{E}$ & NL4-3 & Freed90 \\
\hline Q540 & & $\mathrm{L}$ & NL4-3 & Freed90 \\
\hline R542 & e in heptad-repeat & $\mathrm{G}$ & NL4-3 & Freed90 \\
\hline \multirow[t]{2}{*}{ Q543 } & $\mathrm{f}$ in heptad-repeat & $\mathrm{H}$ & PI & Wei02, Kilby02 \\
\hline & & $\mathrm{R}$ & & \\
\hline P543 & & $\mathrm{L}^{28}$ & $\mathrm{MN}$ & Park00 \\
\hline L544 & $\mathrm{g}$ in heptad-repeat & $S^{22}$ & PI & Fikkert02 \\
\hline
\end{tabular}

\begin{tabular}{|c|c|c|c|c|c|c|c|c|c|c|c|c|c|c|}
\hline 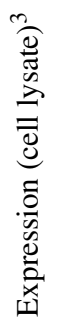 & 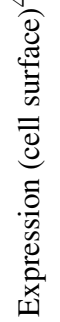 & 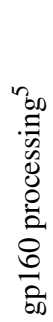 & 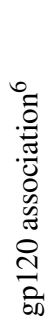 & 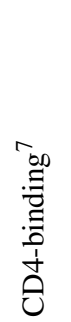 & 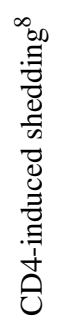 & 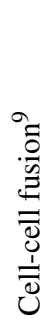 & 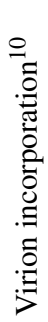 & 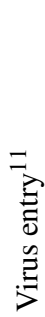 & $\begin{array}{l}0 \\
0 \\
0 \\
0 \\
0 \\
0 \\
0\end{array}$ & 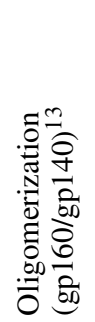 & 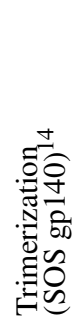 & 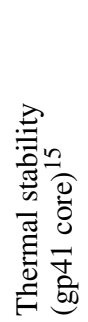 & 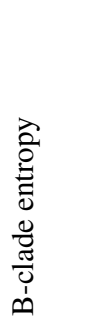 & 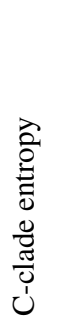 \\
\hline++ & ++ & ++ & ++ & ++ & ++ & & ++ & ++ & + & ++ & + & ++ & & \\
\hline++ & & ++ & ++ & ++ & & + & & & & & & & 0.136 & 0 \\
\hline++ & & ++ & ++ & ++ & & + & & & & & & & & \\
\hline \multirow[t]{3}{*}{++} & & ++ & ++ & ++ & & - & & & & & & & 0.326 & 0.44 \\
\hline & & & & & & ++ & & & & & & & & \\
\hline & & & & & & ++ & & & & & & & & \\
\hline++ & & ++ & & & & - & & & & & & & & \\
\hline++ & & ++ & ++ & & & ++ & & & & & & & 0.628 & 0.594 \\
\hline +++ & & ++ & ++ & & & ++ & & & & & & & 0.047 & 0.101 \\
\hline \multirow[t]{3}{*}{++} & ++ & ++ & ++ & ++ & & & & & + & & & & 0.115 & 0 \\
\hline & & & & & & & & & - & & & & & \\
\hline & & & & +++ & & & & & + & & & & 0.985 & 0.658 \\
\hline++ & & ++ & ++ & ++ & & + & & & & & & & 0.19 & 0.473 \\
\hline +++ & & ++ & ++ & & & ++ & & & +- & & & & & \\
\hline++ & & ++ & ++ & ++ & & - & & & & & & & 0.13 & 0.101 \\
\hline+ & ++ & ++ & ++ & & & - & & & - & & & & 0 & 0 \\
\hline+++ & & ++ & ++ & & & + & & & & & & & 0 & 0.302 \\
\hline++ & & ++ & & & & + & & & & & & & & \\
\hline +++ & ++ & ++ & ++ & & & + & & & + & & & & 0.083 & 0.101 \\
\hline++ & & ++ & & & & & ++ & & + & & & & 0.115 & 0.202 \\
\hline++ & & + & + & ++ & & - & & & & & & & 0 & 0 \\
\hline \multirow[t]{3}{*}{+++} & & ++ & - & & & - & & & & & & & 0 & 0 \\
\hline & + & + & - & & + & - & & + & & & & & 0 & 0 \\
\hline & ++ & - & - & & + & - & & - & & & & & 0 & 0 \\
\hline+++ & & ++ & ++ & & & ++ & & & & & & & 0 & 0 \\
\hline++ & & + & + & ++ & & - & & & & & & & 0 & 0 \\
\hline++ & & ++ & ++ & ++ & & + & & & & & & & 0.083 & 0.334 \\
\hline++ & & + & + & ++ & & - & & & & & & & 0 & 0 \\
\hline \multirow[t]{5}{*}{++} & & ++ & ++ & ++ & & + & & & & & & & 0 & 0.101 \\
\hline & & & & & & & & & + & & & & 0.811 & 0.202 \\
\hline & & & & & & & & & +- & & & & & \\
\hline & & & & & & & & & + & & & & & \\
\hline & & & & & & & & & +- & & & & 0.094 & 0.234 \\
\hline
\end{tabular}




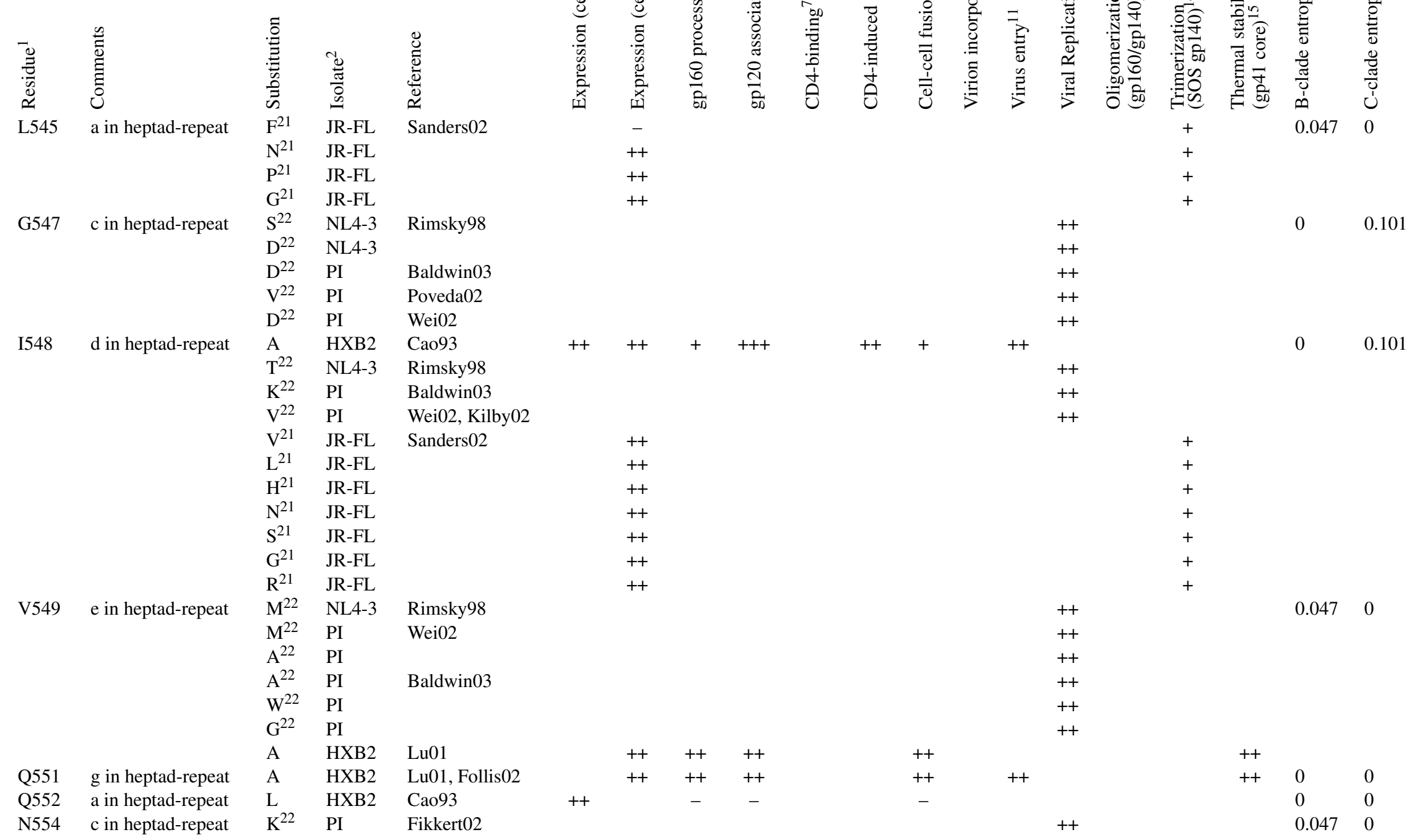




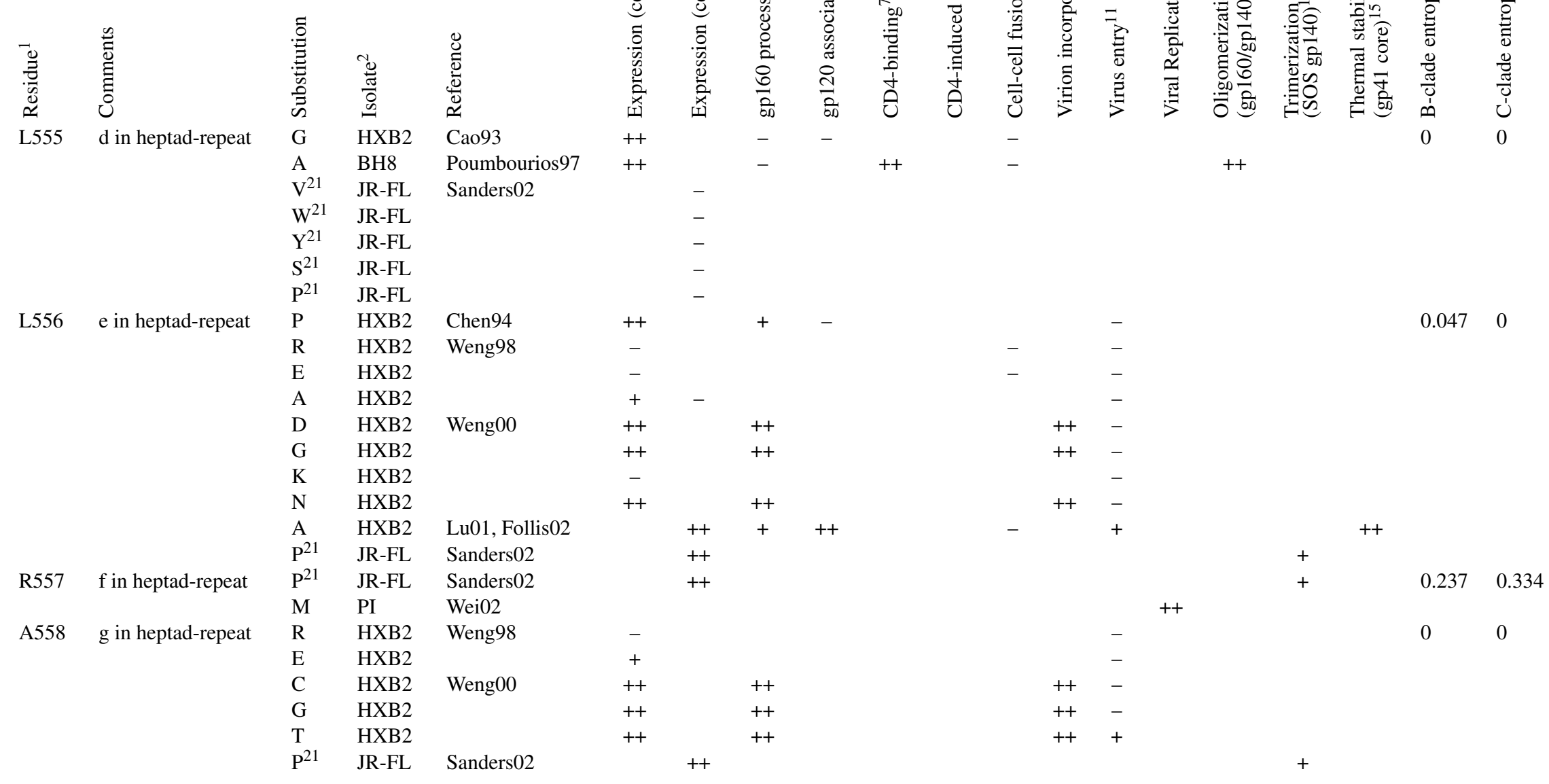




\begin{tabular}{|c|c|c|c|c|c|c|c|c|c|c|c|c|c|c|c|c|c|c|c|}
\hline 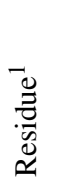 & 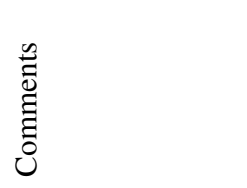 & 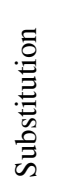 & 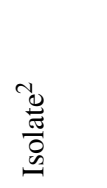 & 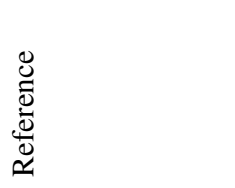 & 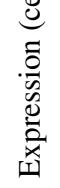 & 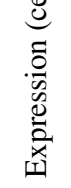 & 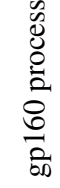 & 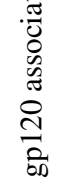 & 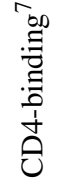 & 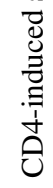 & $\begin{array}{l}\frac{0}{0} \\
\stackrel{2}{\Xi} \\
\overline{0} \\
\frac{0}{1} \\
\frac{0}{0}\end{array}$ & 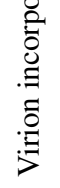 & 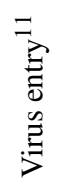 & 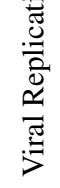 & 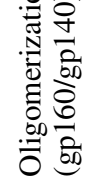 & 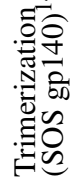 & 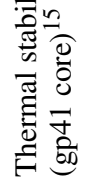 & 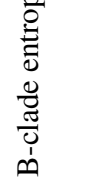 & 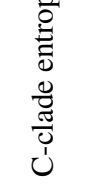 \\
\hline \multirow[t]{11}{*}{ I559 } & a in heptad-repeat & $\mathrm{P}$ & HXB2 & Chen93, Chen94 & ++ & ++ & - & - & ++ & & - & & - & & ++ & & & 0.047 & 0 \\
\hline & & A & BH8 & Poumbourios97 & ++ & & - & & ++ & & - & & & & ++ & & & & \\
\hline & & $\mathrm{V}^{21}$ & JR-FL & Sanders02 & & + & & & & & & & & & & + & & & \\
\hline & & $\mathrm{F}^{21}$ & JR-FL & & & - & & & & & & & & & & +++ & & & \\
\hline & & $\mathrm{N}^{21}$ & JR-FL & & & - & & & & & & & & & & +++ & & & \\
\hline & & $\mathrm{P}^{21}$ & JR-FL & & & ++ & ++ & & & & & & & & & +++ & - & & \\
\hline & & $\mathrm{G}^{21}$ & JR-FL & & & + & ++ & & & & & & & & & +++ & + & & \\
\hline & & $\mathrm{R}^{21}$ & JR-FL & & & + & & & & & & & & & & +++ & & & \\
\hline & & $\mathrm{P}$ & $\begin{array}{l}\text { LAI/ } \\
\text { JR-FL }\end{array}$ & Sanders $03 b$ & & & & & & & & & & - & & & - & & \\
\hline & & $\mathrm{G}$ & $\begin{array}{l}\text { LAI/ } \\
\text { JR-FL }\end{array}$ & & & & & & & & & & & - & & & + & & \\
\hline & & $\mathrm{L}$ & $\begin{array}{l}\text { LAI/ } \\
\text { JR-FL }\end{array}$ & & & & & & & & & & & ++ & & & ++ & & \\
\hline \multirow[t]{2}{*}{ E560 } & $\mathrm{b}$ in heptad-repeat & $\mathrm{P}^{21}$ & JR-FL & Sanders02 & & +++ & & & & & & & & & & + & & 0.217 & 0 \\
\hline & & $\mathrm{G}^{19}$ & ELI1 & Kozak97 & & & & & & & + & & & & & & & & \\
\hline A561 & $\mathrm{c}$ in heptad-repeat & $\mathrm{P}^{21}$ & JR-FL & Sanders02 & & +++ & & & & & & & & & & + & & 0.094 & 0.101 \\
\hline S561 & & $\mathrm{A}^{28}$ & $\mathrm{MN}$ & Park00 & & & & & & & & & & ++ & & & & & \\
\hline \multirow[t]{3}{*}{ Q562 } & $\mathrm{d}$ in heptad-repeat & $\mathrm{L}$ & HXB2 & $\mathrm{Ca0} 93$ & ++ & & + & - & & & - & & & & & & & 0 & 0.101 \\
\hline & & A & BH8 & Poumbourios97 & ++ & & ++ & + & ++ & & - & & & & ++ & & & & \\
\hline & & $\mathrm{P}^{21}$ & JR-FL & Sanders02 & & +++ & & & & & & & & & & + & & & \\
\hline \multirow[t]{7}{*}{ Q563 } & e in heptad-repeat & A & HXB2 & Weng00 & ++ & & ++ & & & & & ++ & ++ & & & & & 0.047 & 0 \\
\hline & & $\mathrm{E}$ & HXB2 & & ++ & & ++ & & & & & ++ & - & & & & & & \\
\hline & & M & HXB2 & & ++ & & ++ & & & & & ++ & - & & & & & & \\
\hline & & $\mathrm{G}$ & HXB2 & & ++ & & ++ & & & & & ++ & ++ & & & & & & \\
\hline & & $\mathrm{R}$ & HXB2 & & ++ & & ++ & & & & & ++ & + & & & & & & \\
\hline & & A & HXB2 & Lu01, Follis02 & & ++ & ++ & ++ & & & ++ & & ++ & & & & ++ & & \\
\hline & & $\mathrm{P}^{21}$ & JR-FL & Sanders02 & & +++ & & & & & & & & & & + & & & \\
\hline R564 & $\mathrm{f}$ in heptad-repeat & $\mathrm{P}^{21}$ & JR-FL & Sanders02 & & +++ & & & & & & & & & & + & & 0.047 & 0 \\
\hline \multirow[t]{2}{*}{ H564 } & & $\mathrm{N}^{28}$ & $\mathrm{MN}$ & Park00 & & & & & & & & & & ++ & & & & & \\
\hline & & $C^{26}$ & HXB2 & Rabenstein95 & & & & & & & & & & & ++ & & & & \\
\hline \multirow[t]{3}{*}{ L565 } & $\mathrm{g}$ in heptad-repeat & $\mathrm{P}$ & HXB2 & Chen94 & ++ & ++ & + & ++ & ++ & & - & & & & & & & 0.402 & 0.584 \\
\hline & & A & HXB2 & Lu01, Follis02 & & ++ & ++ & ++ & & & - & & - & & & & + & & \\
\hline & & $\mathrm{P}^{21}$ & JR-FL & Sanders02 & & ++ & & & & & & & & & & + & & & \\
\hline
\end{tabular}




\begin{tabular}{|c|c|c|c|c|c|c|c|c|c|c|c|c|c|c|c|c|c|c|c|}
\hline 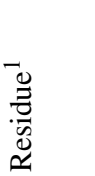 & 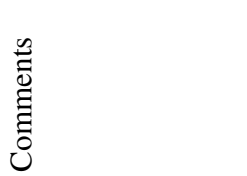 & 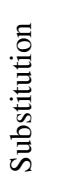 & 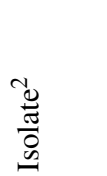 & 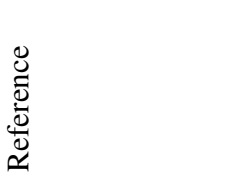 & 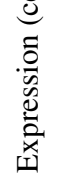 & 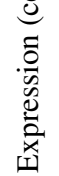 & $\begin{array}{l}0 \\
0 \\
0 \\
0 \\
0 \\
8 \\
0 \\
00\end{array}$ & 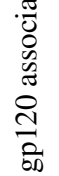 & 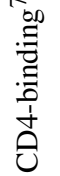 & 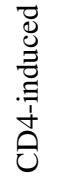 & 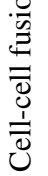 & 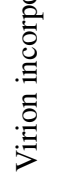 & 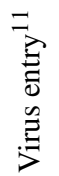 & 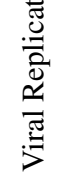 & 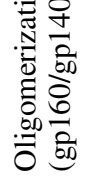 & 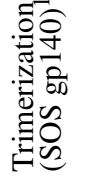 & 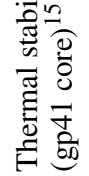 & 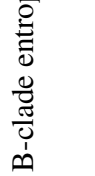 & 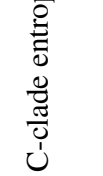 \\
\hline \multirow[t]{10}{*}{ L566 } & $\mathrm{a}$ in heptad-repeat & $\mathrm{G}$ & HXB2 & Cao93 & & ++ & + & + & & - & - & & + & & & & & 0.047 & 0 \\
\hline & & $\mathrm{P}$ & HXB2 & Chen93, Chen94 & ++ & ++ & + & + & ++ & & - & & - & & ++ & & & & \\
\hline & & A & BH8 & Poumbourios97 & ++ & & - & & ++ & & - & & & & ++ & & & & \\
\hline & & $\mathrm{V}^{23}$ & $\mathrm{BH} 8$ & Ear193 & & ++ & ++ & & ++ & & & & & & ++ & & & & \\
\hline & & $\mathrm{V}^{21}$ & JR-FL & Sanders02 & & + & ++ & & & & & & & & & ++ & ++ & & \\
\hline & & $\mathrm{I}^{21}$ & JR-FL & & & - & & & & & & & & & & + & & & \\
\hline & & $\mathrm{N}^{21}$ & JR-FL & & & + & & & & & & & & & & ++ & & & \\
\hline & & $\mathrm{T}^{21}$ & JR-FL & & & + & & & & & & & & & & ++ & & & \\
\hline & & $\mathrm{P}^{21}$ & JR-FL & & & + & ++ & & & & & & & & & + & & & \\
\hline & & $\mathrm{K}^{21}$ & JR-FL & & & - & & & & & & & & & & + & & & \\
\hline Q567 & $\mathrm{b}$ in heptad-repeat & $\mathrm{R}$ & LAI & Sanders03a & & & & & & & & & & ++ & & & ++ & 0.177 & 0 \\
\hline \multirow[t]{3}{*}{ L568 } & $\mathrm{c}$ in heptad-repeat & A & HXB2 & Cao93 & ++ & ++ & + & ++ & ++ & ++ & - & & + & & & & & 0 & 0 \\
\hline & & $\mathrm{P}$ & HXB2 & Chen94 & ++ & ++ & + & + & ++ & & - & & & & & & & & \\
\hline & & A & HXB2 & Ji00 & & & & & & & & & & & & & + & & \\
\hline \multirow[t]{6}{*}{ T569 } & $\mathrm{d}$ in heptad-repeat & A & BH8 & Poumbourios97 & ++ & & - & & ++ & & - & & & & ++ & & & 0 & 0.101 \\
\hline & & $\mathrm{C}$ & HXB2 & Farzan98 & & & - & & & & & & & & & & & & \\
\hline & & $S^{21}$ & JR-FL & Sanders02 & & + & & & & & & & & & & + & & & \\
\hline & & $\mathrm{P}^{21}$ & JR-FL & & & + & ++ & & & & & & & & & ++ & + & & \\
\hline & & $\mathrm{K}^{21}$ & JR-FL & & & + & & & & & & & & & & ++ & & & \\
\hline & & $\mathrm{E}^{21}$ & JR-FL & & & - & & & & & & & & & & & & & \\
\hline \multirow[t]{8}{*}{ V570 } & e in heptad-repeat & $\mathrm{R}$ & HXB2 & Weng98 & ++ & ++ & & & ++ & & - & ++ & - & & & & & 0 & 0 \\
\hline & & $E^{35}$ & HXB2 & & ++ & ++ & & & ++ & & & ++ & & & & & & & \\
\hline & & A & HXB2 & Weng00 & ++ & & ++ & & & & & ++ & - & & & & & & \\
\hline & & $\mathrm{D}$ & HXB2 & & ++ & & ++ & & & & & ++ & - & & & & & & \\
\hline & & $\mathrm{E}$ & HXB2 & & ++ & & ++ & & & & & ++ & - & & & & & & \\
\hline & & $\mathrm{G}$ & HXB2 & & ++ & & ++ & & & & & ++ & - & & & & & & \\
\hline & & I & HXB2 & & ++ & & ++ & & & & & ++ & ++ & & & & & & \\
\hline & & A & HXB2 & Lu01, Follis02 & & ++ & ++ & ++ & & & - & & - & & & & + & & \\
\hline \multirow[t]{3}{*}{ W571 } & $\mathrm{f}$ in heptad-repeat & $\mathrm{R}$ & HXB2 & Cao93 & ++ & ++ & + & ++ & ++ & - & - & & - & & & & & 0 & 0 \\
\hline & & $\mathrm{R}$ & HXB2 & $\mathrm{JiOO}$ & & & & & & & & & & & & & ++ & & \\
\hline & & $C^{26}$ & HXB2 & Rabenstein95 & & & & & & & & & & & ++ & & & & \\
\hline \multirow[t]{2}{*}{ G572 } & $\mathrm{g}$ in heptad-repeat & $\mathrm{G}$ & HXB2 & Weng98 & ++ & - & & & ++ & & - & - & - & & & & & 0 & 0 \\
\hline & & A & HXB2 & Lu01 & & ++ & ++ & ++ & & & - & & & & & & +++ & & \\
\hline
\end{tabular}




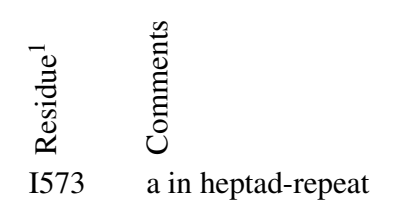

总 范

\section{HXB2 Dubay92}

HXB2

HXB2

HXB2

HXB2

HXB2

HXB2

HXB2 Bernstein95

HXB2

HXB2

HXB3 Shugars96

HXB3

$\begin{array}{ll}\text { HXB2 } & \text { Chen93, Chen94 } \\ \text { HXB2, Wild94 }\end{array}$

LAI

$\mathrm{A}^{26}$ HXB2,

LAI

HXB2,

LAI

HXB2 Rabenstein95

$\mathrm{HXB} 2$

HXB2

168P Liu01

LAI Sanders03a

BH8 Poumbourios97

HXB2 Markosyan02

HXB2

HXB2

HXB2

JR-FL Sanders02

JR-FL

JR-FL

JR-FL
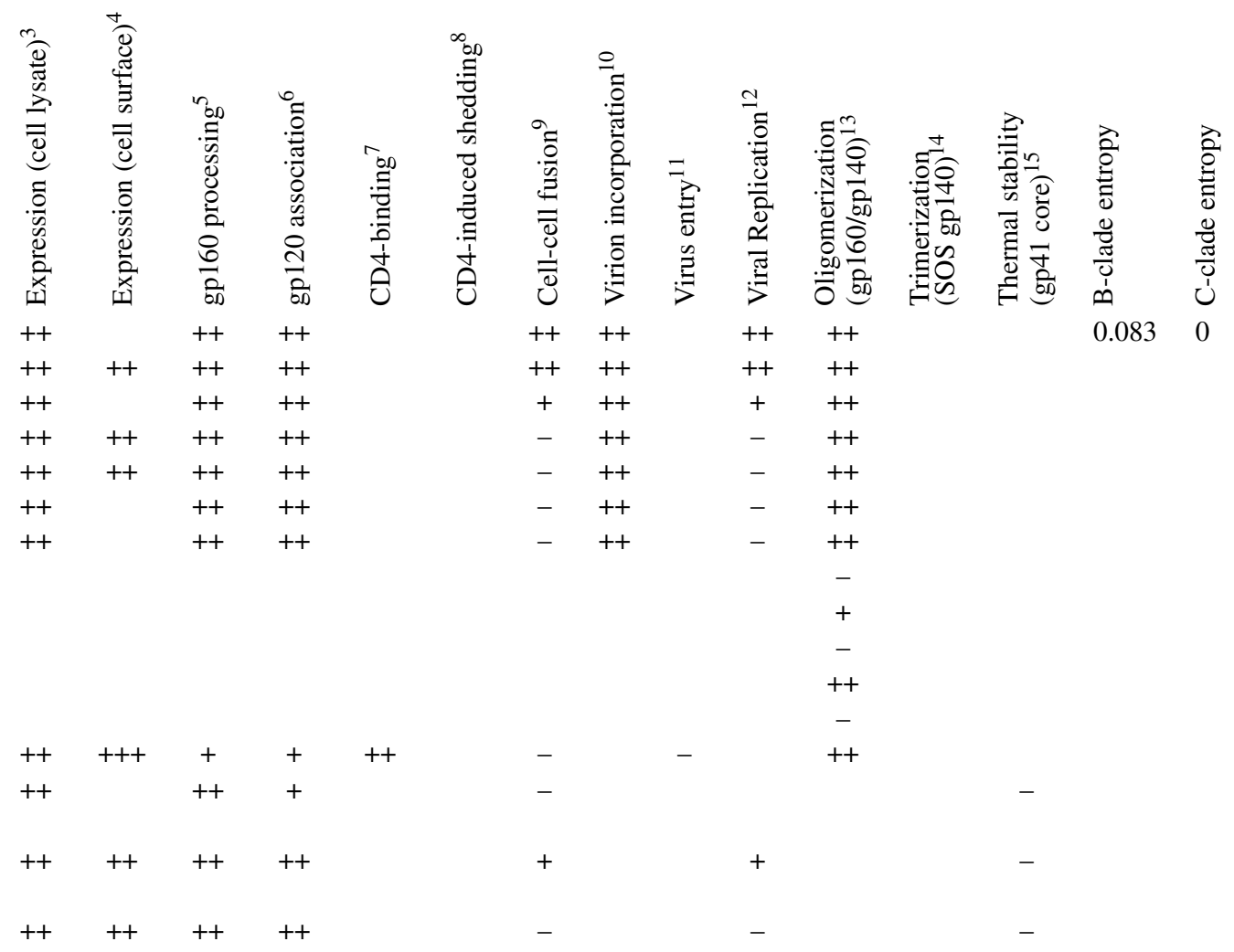

$++\quad++$

$+\quad+$

$\begin{array}{lll}+ & ++ \\ - & ++\end{array}$

$-\quad+$

$-\quad++$

$++$

$++$

$++$
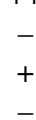

$-$

(2)

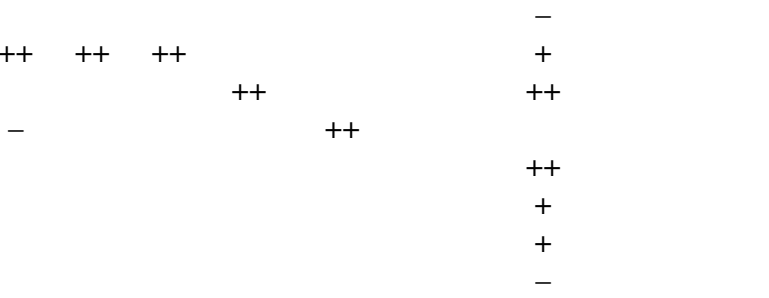




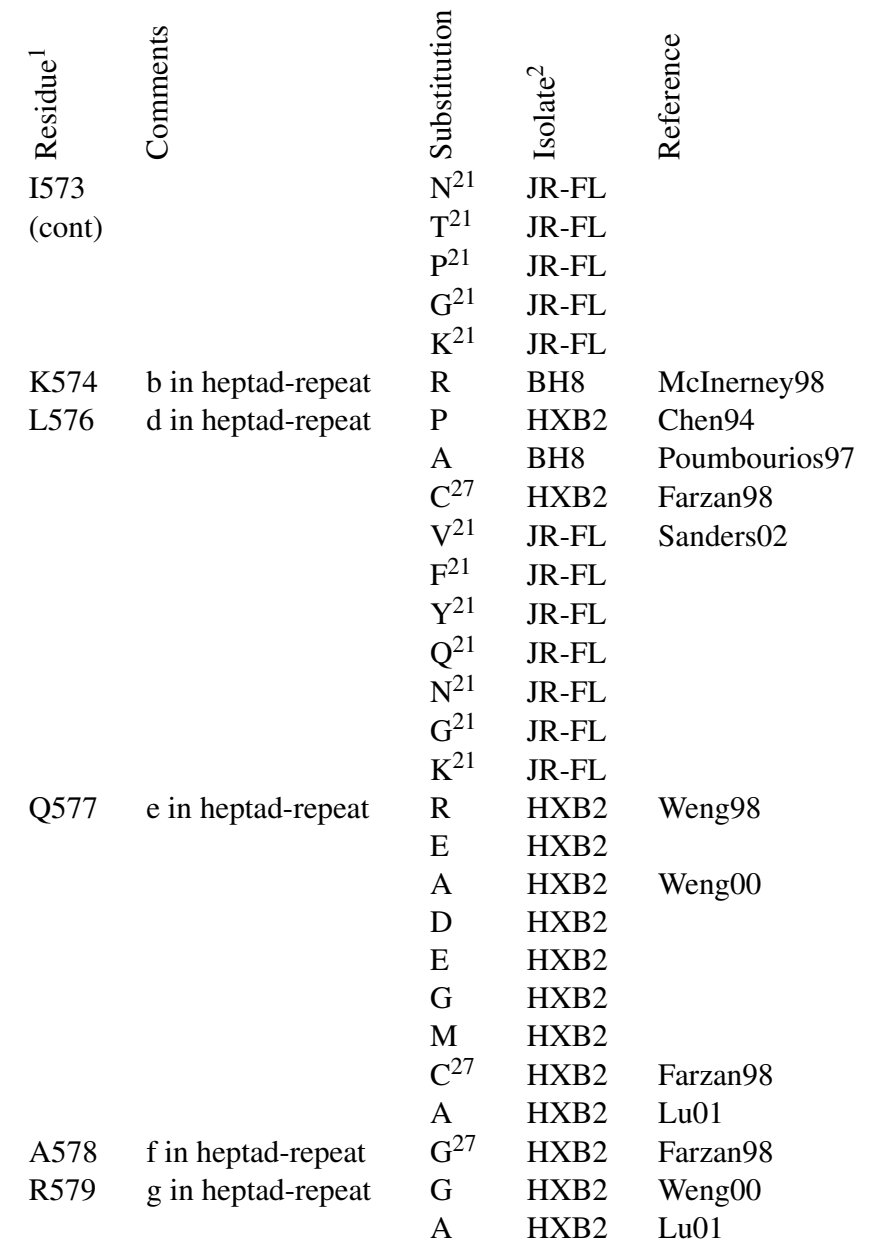

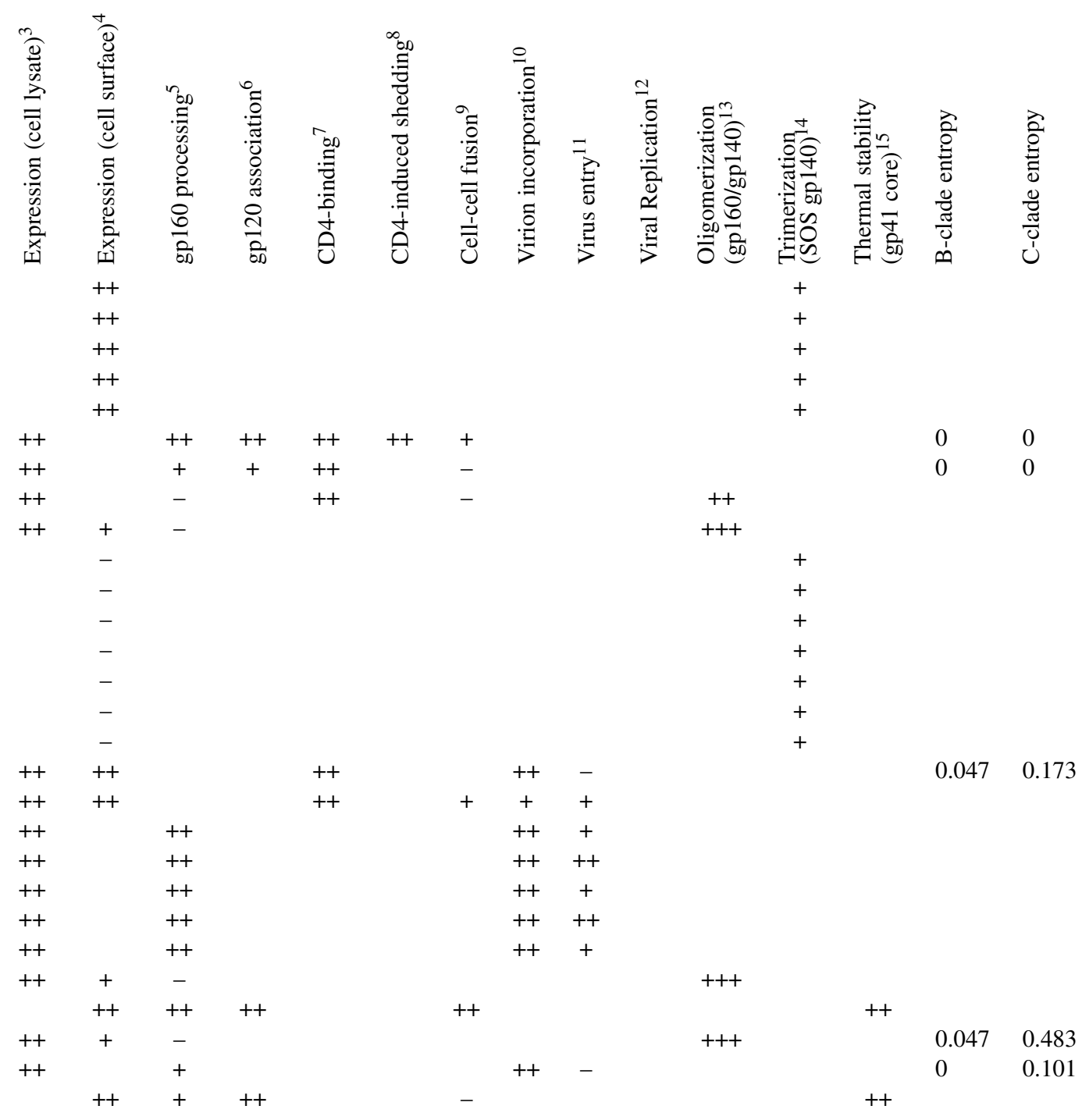




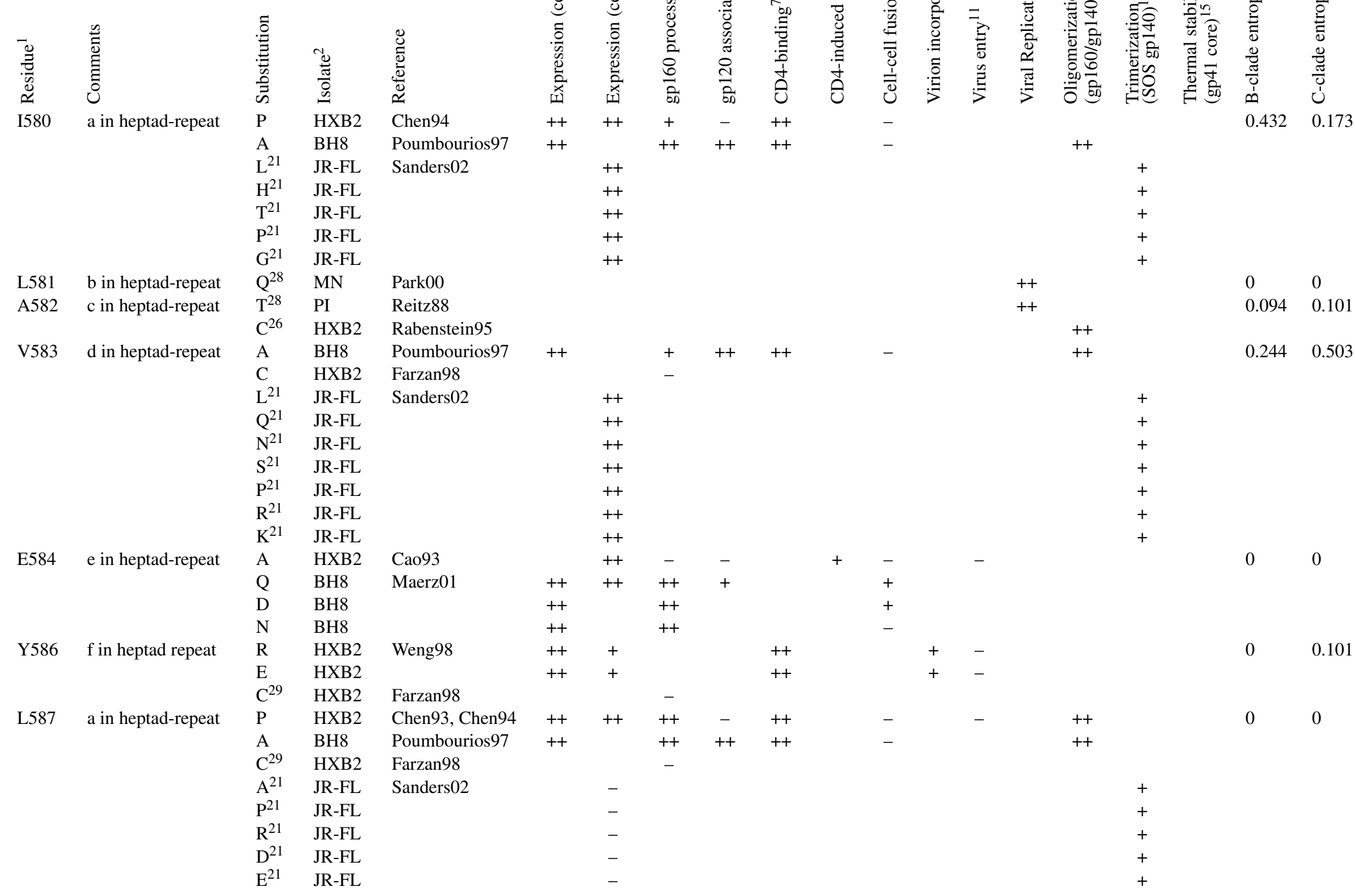




\begin{tabular}{|c|c|c|c|c|}
\hline 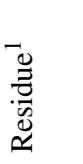 & 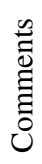 & 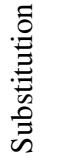 & 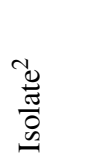 & 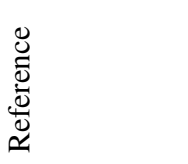 \\
\hline K588 & & $\mathrm{R}$ & BH8 & McInerney98 \\
\hline \multirow[t]{3}{*}{ D589 } & & $\mathrm{L}$ & HXB2 & Cao93 \\
\hline & & $C^{30}$ & JR-FL & Binley00 \\
\hline & & $\mathrm{K}$ & BH8 & Maerz01 \\
\hline \multirow[t]{3}{*}{ Q591 } & & A & BH8 & Maerz01 \\
\hline & & $\mathrm{K}$ & BH8 & \\
\hline & & $\mathrm{L}$ & LAI & Sanders $03 c$ \\
\hline \multirow[t]{2}{*}{ L592 } & & $\mathrm{V}$ & BH8 & Maerz01 \\
\hline & & A & BH8 & \\
\hline \multirow[t]{3}{*}{ L593 } & & V & BH8 & Maerz01 \\
\hline & & A & $\mathrm{BH} 8$ & \\
\hline & & Q & LAI & Sanders $03 \mathrm{c}$ \\
\hline I595 & & $\mathrm{F}^{31}$ & PI & Moore93 \\
\hline \multirow[t]{7}{*}{ W596 } & & M & HXB2 & Cao93, Cao94 \\
\hline & & $\mathrm{Y}$ & $\begin{array}{l}\text { LAI, } \\
\text { NL4-3 }\end{array}$ & Rovinski99 \\
\hline & & A & $\begin{array}{l}\text { LAI, } \\
\text { NL4-3 }\end{array}$ & \\
\hline & & $C^{30}$ & JR-FL & Binley00 \\
\hline & & $\mathrm{F}$ & BH8 & Maerz01 \\
\hline & & $\mathrm{H}$ & BH8 & \\
\hline & & $\mathrm{L}$ & BH8 & \\
\hline \multirow[t]{3}{*}{ G597 } & & $\mathrm{P}$ & BH8 & Maerz01 \\
\hline & & A & BH8 & \\
\hline & & $\mathrm{S}$ & BH8 & \\
\hline \multirow[t]{4}{*}{ C598 } & & $\mathrm{S}$ & HXB2 & Dedera92a \\
\hline & & $S^{23}$ & BH8 & Ear193 \\
\hline & & G & HXB2 & Syu91 \\
\hline & & A & LAI & Van Anken03 \\
\hline G600 & & A & $\begin{array}{l}\text { LAI, } \\
\text { NL4-3 }\end{array}$ & Rovinski99 \\
\hline
\end{tabular}

\begin{tabular}{|c|c|c|c|c|c|c|c|c|c|c|c|c|c|c|}
\hline 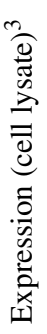 & 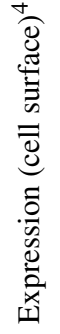 & 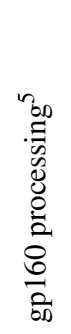 & 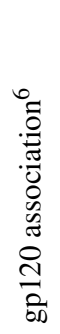 & 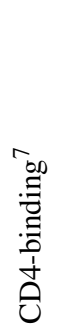 & 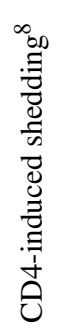 & 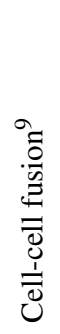 & 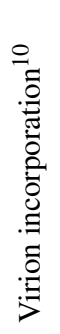 & 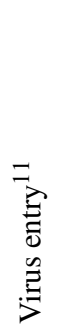 & 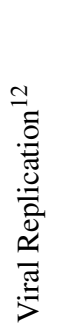 & 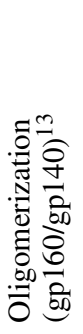 & 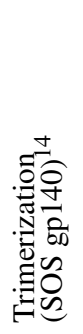 & 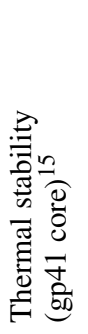 & $\begin{array}{l}\text { à } \\
0 \\
0 \\
0 \\
0 \\
0 \\
0 \\
0 \\
0 \\
0\end{array}$ & 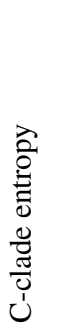 \\
\hline++ & & ++ & ++ & ++ & ++ & ++ & & & & & & & 1.112 & 0.775 \\
\hline ++ & $\begin{array}{l}++ \\
++\end{array}$ & +++ & + & ++ & + & - & & + & & & & & 0 & 0.101 \\
\hline++ & ++ & ++ & + & & & - & & & & & & & & \\
\hline++ & ++ & ++ & ++ & & & ++ & & & & & & & 0.083 & 0.101 \\
\hline \multirow[t]{2}{*}{++} & & ++ & & & & ++ & & & & & & & & \\
\hline & & & & & & & & & + & & & & & \\
\hline++ & & ++ & & & & ++ & & & & & & & 0 & 0.101 \\
\hline++ & & ++ & & & & ++ & & & & & & & & \\
\hline++ & & ++ & & & & + & & - & & & & & 0.143 & 0 \\
\hline \multirow[t]{3}{*}{ ++ } & ++ & ++ & - & & & + & & - & & & & & & \\
\hline & & & & & & & & & $+1-$ & & & & & \\
\hline & & & & & & & & & ++ & & & & 0.162 & 0.555 \\
\hline \multirow[t]{4}{*}{++} & ++ & ++ & + & ++ & & - & & ++ & ++ & ++ & & & 0 & 0 \\
\hline & & ++ & & & & & ++ & & & & & & & \\
\hline & & - & & & & & + & & & & & & & \\
\hline & ++ & & & & & & & & & & & & & \\
\hline++ & ++ & ++ & + & & & ++ & & ++ & & & & & & \\
\hline++ & & ++ & & & & + & & & & & & & & \\
\hline ++ & ++ & ++ & + & & & + & & & & & & & & \\
\hline++ & ++ & ++ & - & & & - & & & & & & & 0 & 0 \\
\hline++ & ++ & ++ & - & & & - & & & & & & & & \\
\hline++ & ++ & ++ & - & & & - & & & & & & & & \\
\hline \multirow[t]{2}{*}{++} & & - & & & & - & & & & & & & 0 & 0 \\
\hline & ++ & ++ & & ++ & & & & & & ++ & & & & \\
\hline \multirow[t]{3}{*}{++} & & - & & & & & & & - & & & & & \\
\hline & & & & & & & & & - & & & & & \\
\hline & & ++ & & & & & ++ & & & & & & 0 & 0.101 \\
\hline
\end{tabular}




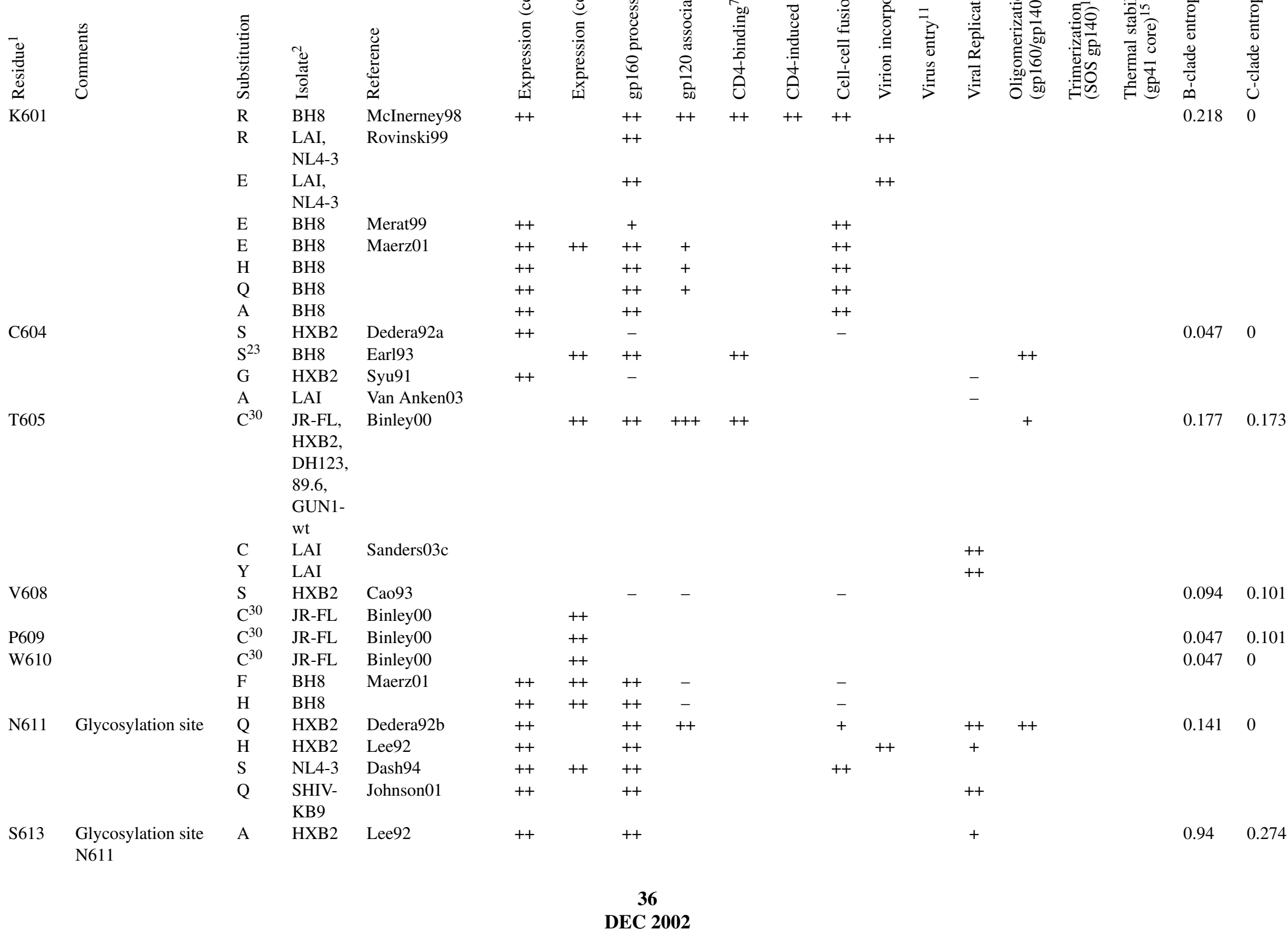




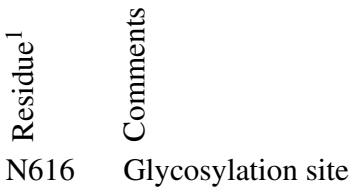

K617

S618 Glycosylation site N616

N624 d in heptad-repeat Glycosylation site (N625 in most isolates)

N625 e in heptad-repeat Glycosylation site

T626 f in heptad-repeat Glycosylation site N624

W628 a in heptad-repeat

W631 d in heptad-repea

D632 e in heptad-repea

R633 $\mathrm{f}$ in heptad-repeat

I635 a in heptad-repeat

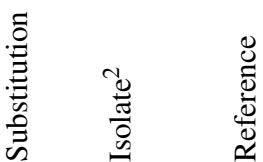

HXB2 Dedera92b

BH8 Ear193

HXB2 Lee92

NL4-3 Dash94

BH10 Perrin98

SHIV- Johnson01

KB9

BH8 McInerney98

HXB2 Lee92

HXB2 Lee92

BH10 Perrin98

SHIV- Johnson01

KB9

$\mathrm{Q}^{23} \quad \mathrm{BH} 8$

Ear193

M HXB2 Cao93

$\mathrm{M}^{28} \quad$ SHIV- Si01

$\mathrm{HXBc} 2 \mathrm{P}$

M HXB2 Cao93

A HXB2 Weng00

A $\quad$ HXB2 $\quad$ Wang02

HXB2 Wang02

BH10 Perrin98

PI Wei02

HXB2 Wang02

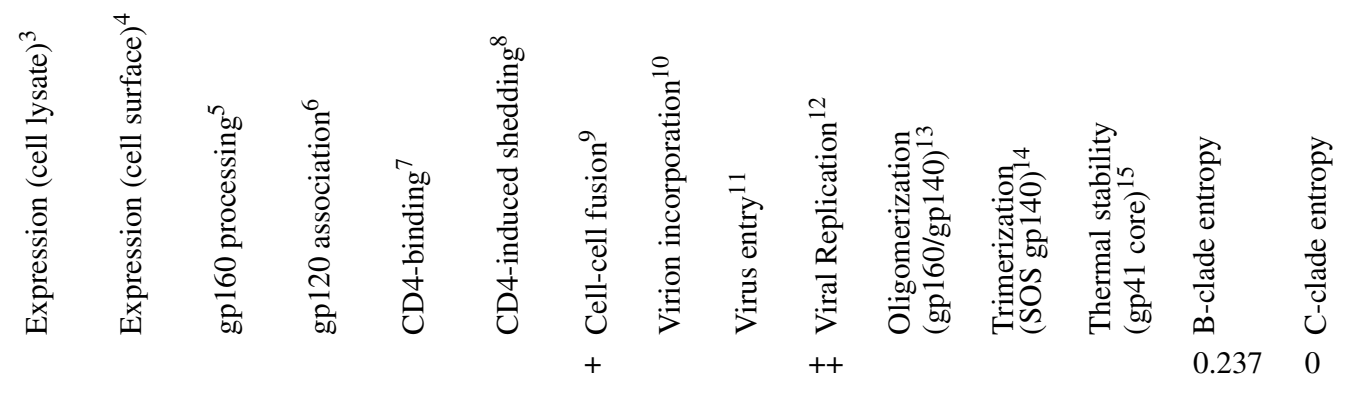

$++\quad++$

$++$

$++$

$\begin{array}{lll}++ & & ++ \\ ++ & ++ & ++\end{array}$

$\begin{array}{lll}++ & +2 \\ ++ & ++\end{array}$

$++$

++
++

$++$

$++$

++
++

$++$

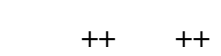

$++$

$-$

$++$

$0.047 \quad 0.274$

$0.244 \quad 0.444$

$0.348 \quad 0.658$

$0.495 \quad 0.483$

$1.153 \quad 1.305$

$++$

++
++

$\begin{array}{llll}++ & - & -1 & -1\end{array}$

$++$

$+$

$++\quad$ + 


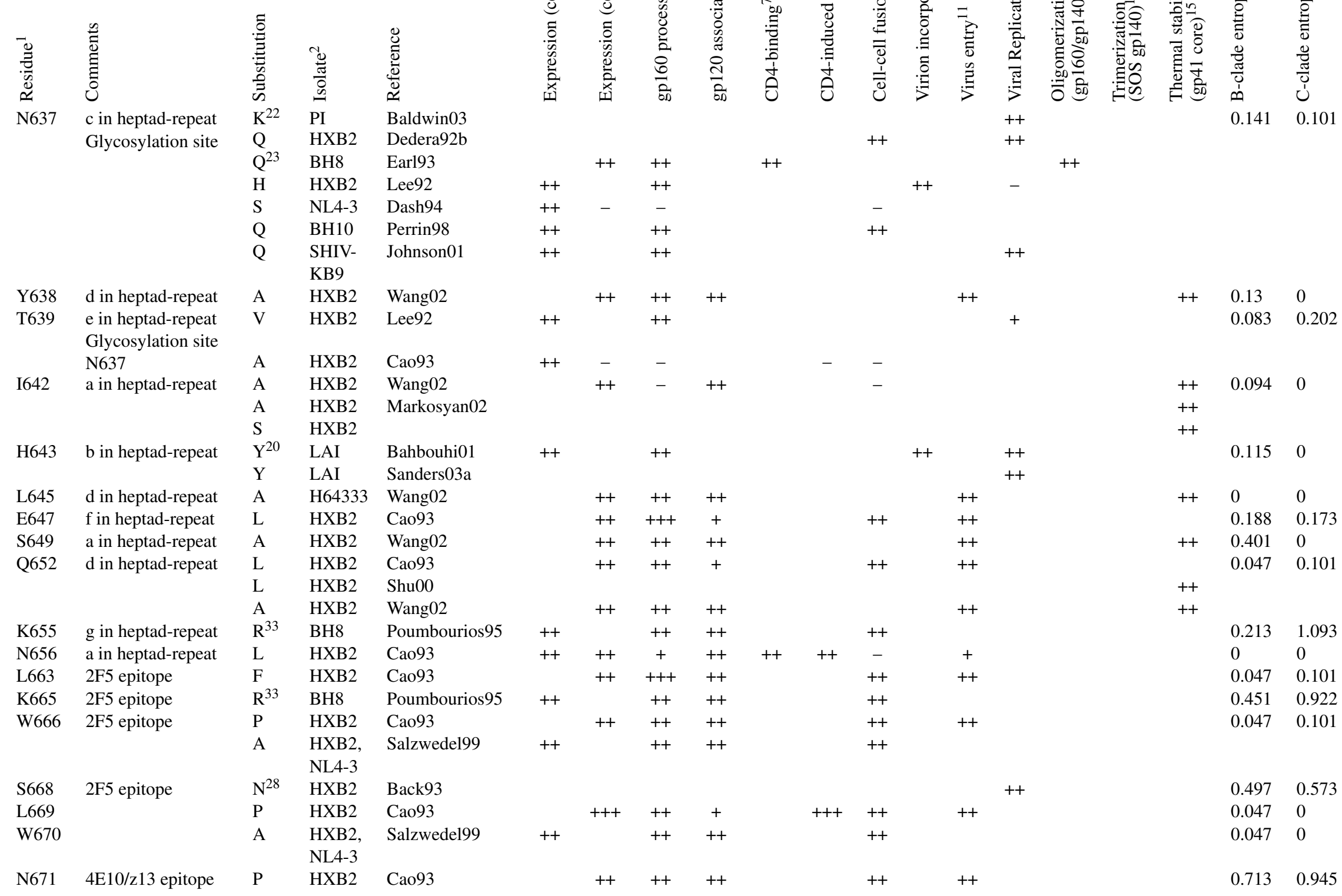




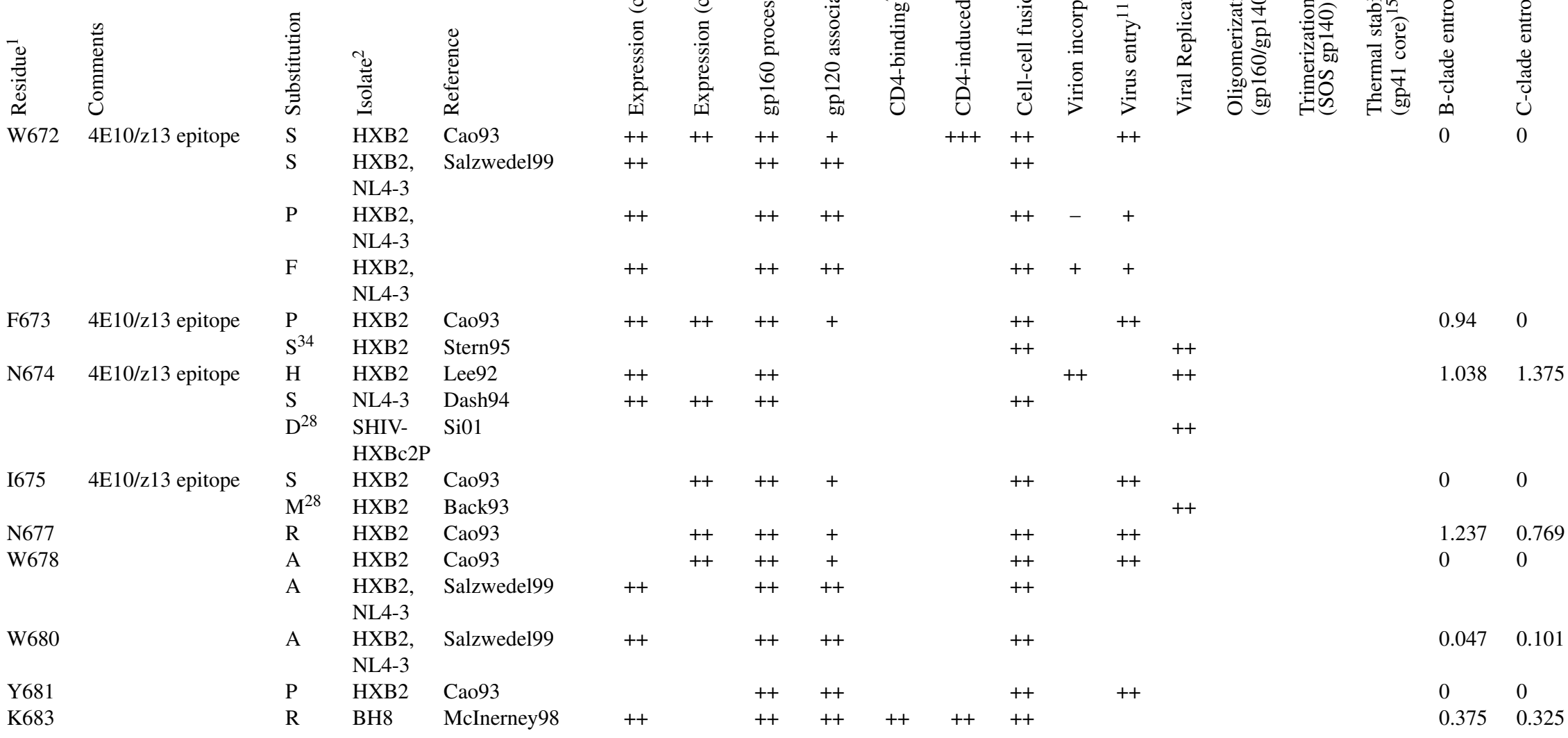

Table footnotes:

${ }^{1}$ Residue numbering is based on HXB2 gp160, although the amino-acids studied may be different in the isolate used. The one-letter code for amino acids is used ${ }^{2} \mathrm{PI}$ : primary isolate

${ }^{3}$ As assessed by western blot or immunoprecipitation. -, minimal or no expression; +, reduced expression; ++, expression similar to WT; +++, increased expression ${ }^{4}$ As assessed by surface biotinylation, iodination or FACS. When soluble gp140 constructs were used, the relative secretion levels (western blot or immunoprecipitation) are given. -, minimal or no expression; +, reduced expression; ++, expression similar to WT; +++, increased expression

${ }^{5}$ As assessed by western blot or immunoprecipitation in combination with densitometric measurements. - , minimal or no processing; + , reduced processing; ++, processing similar to WT; +++, increased processing 
${ }^{6}$ As assessed by western blot or immunoprecipitation in combination with densitometric measurements. - , minimal or no association; + , reduced association;,++ association similar to WT; +++, increased association

${ }^{7}$ As assessed by immunoprecipitation with CD4-based reagents. ++ , similar to WT; +++ , increased CD4 binding

${ }^{8}$ As assessed by immunoprecipitation. - , no shedding; +, reduced shedding; ++, shedding similar to WT; +++, increased shedding. Note that CD4-induced shedding and to a lesser extent gp120 association (i.e., the reverse of shedding), when measured in laboratory isolates, might be diminished in primary isolates that can retain gp120 more efficiently.

${ }^{9}$ As assessed by syncytium formation or reporter gene assays. -, fusion lower than $3 \%$ of WT; +, fusion between 3 and $30 \%$ of WT; ++, fusion greater than $30 \%$ of WT

${ }^{10}$ As assessed by western blot or immunoprecipitation. -, minimal or no incorporation; +, reduced incorporation; ++, incorporation similar to WT

${ }^{11}$ As assessed by various assays (replication complementation, use of reporter genes, p24 production). -, entry lower than $3 \%$ of WT; +, entry between 3 and $30 \%$ of WT; ++, entry greater that $30 \%$ of WT

12 , no apparent replication; +, replication with a delay of more than 2 days compared to WT; ++ replication similar to WT

${ }^{13}$ As assessed by sucrose gradient fractionation, immunoprecipitation, velocity sedimentation or FPLC, unless indicated otherwise. -, oligomerization below $25 \%$ of WT; + , oligomerization between $25 \%$ and $50 \%$ of WT; ++ , oligomerization similar to WT. No distinction between dimerization, trimerization or tetramerization is made.

${ }^{14}$ As assessed by Blue Native-PAGE. +, trimerization similar to WT SOS gp140 (occasional trimerization); ++, slightly more trimerization than in WT; +++, significantly more trimerization than in WT.

${ }^{15}$ As analyzed using the N34(L6)C28 or N36(L6)C34 peptide model, unless indicated otherwise. - , melting temperature $\left(T_{m}\right)$ below $40^{\circ} \mathrm{C}$;,$+ T_{m}$ between $40^{\circ} \mathrm{C}$ and $60^{\circ} \mathrm{C} ;++, T_{m}$ between $60^{\circ} \mathrm{C}$ and $80^{\circ} \mathrm{C} ;+++, T_{m}$ over $80^{\circ} \mathrm{C}$

${ }^{16}$ Analyzed in a double mutant, A512V + F519L

${ }^{17}$ Four amino-acid insertion GIPA

${ }^{18}$ Six amino-acid insertion IHRWIA

${ }^{19}$ Involved in cell line adaptation

${ }^{20}$ Identified in an isolate which is resistant to the furin inhibitor ( $\left.\alpha 1-\mathrm{PDX}\right)$

${ }^{21}$ Analyzed in soluble SOS gp140 constructs and so also contain the A501C and T605C substitutions

${ }^{22}$ Involved in T-20 resistance

${ }^{23}$ Analyzed in soluble gp140

${ }^{24}$ Analyzed in an N-peptide/Protein A fusion protein

${ }^{25}$ Analyzed in an N-peptide/maltose binding protein (MBP) fusion protein

${ }^{26}$ Thermal stability (74) or oligomerization (53) of N-peptides analyzed in the absence of C-peptides

${ }^{27}$ Analyzed in a triple mutant L576C + Q577C + A578G

${ }^{28}$ Involved in neutralization resistance

${ }^{29}$ Analyzed in a double mutant Y586C + L587C

${ }^{30}$ Analyzed in combination with gp120 cysteine substitutions in the context of soluble gp140

${ }^{31}$ Involved in resistance to soluble CD4 
${ }^{32}$ Generates a new glycosylation site

${ }^{33}$ Analyzed in a double mutant K655R + K665R

${ }^{34}$ Analyzed in a double mutant A582T + F673S

${ }^{35}$ Data on this mutant were corrected in reference 73 


\section{References}

[1] Van Anken, E., R. W. Sanders, B. Berkhout, and I. Braakman. 2003. unpublished results.

[2] Back, N. K., L. Smit, M. Schutten, P. L. Nara, M. Tersmette, and J. Goudsmit. 1993. Mutation in human immunodeficiency virus type 1 gp41 affect sensitivity to neutralization by gp120 antibodies. J. Virol. 67:6897-6902.

[3] Bahbouhi, B., N. G. Seidah, and E. Bahraoui. 2001. Replication of HIV-1 viruses in the presence of the Portland $\alpha 1$ - antitrypsin variant ( $\alpha 1$-PDX) inhibitor. Biochem. J. 360:127134.

[4] Baldwin, C. E., R. W. Sanders, S. Jurriaans, J. M. Lange, and B. Berkhout. 2003. Two step evolution of HIV-1 gp41 during therapy with the T20 entry inhibitor. Submitted.

[5] Bernstein, H. B., S. P. Tucker, S. R. Kar, S. A. McPherson, D. T. McPherson, J. W. Dubay, J Lebowitz, R. W. Compans, and E. Hunter. 1995. Oligomerization of the hydrophobic heptad repeat of gp41. J. Virol. 69:2745-2750.

[6] Binley, J. M., R. W. Sanders, B. Clas, N. Schuelke, A. Master, Y. Guo, F. Kajumo, D. J. Anselma, P. J. Maddon, W. C. Olson, and J. P. Moore. 2000. A recombinant HIV-1 envelope glycoprotein complex stabilized by an intermolecular disulfide bond between the gp120 and gp41 subunits is an antigenic mimic of the trimeric virion-associated structure. J. Virol. 74:627-643.

[7] Buchschacher, G. L., Jr., E. O. Freed, and A. T. Panganiban. 1995. Effects of second-site mutations on dominant interference by a human immunodeficiency virus type 1 envelope glycoprotein mutant. J. Virol. 69:1344-1348.

[8] Caffrey, M. 2001. Model for the structure of the HIV gp41 ectodomain: insight into the intermolecular interactions of the gp41 loop. Biochim. Biophys. Acta. 1536:116-122.

[9] Caffrey, M., M. Cai, J. Kaufman, S. J. Stahl, P. T. Wingfield, D. G. Covell, A. M. Gronenborn, and G. M. Clore. 1998. Three-dimensional solution structure of the $44 \mathrm{kDa}$ ectodomain of SIV gp41. ЕMBO J. 17:4572-4584.

[10] Cao, J., L. Bergeron, E. Helseth, M. Thali, H. Repke, and J. Sodroski. 1993. Effects of amino acid changes in the extracellular domain of the human immunodeficiency virus type $1 \mathrm{gp} 4$ envelope glycoprotein. J. Virol. 67:2747-2755.

[11] Cao, J., B. Vasir, and J. G. Sodroski. 1994. Changes in the cytopathic effects of human immunodeficiency virus type 1 associated with a single amino acid alteration in the ectodomain of the gp41 transmembrane glycoprotein. J. Virol. 68:4662-4668.

[12] Chan, D. C., D. Fass, J. M. Berger, and P. S. Kim. 1997. Core structure of gp41 from the HIV envelope glycoprotein. Cell 89:263-273.

[13] Chan, D. C. and P. S. Kim. 1998. HIV entry and its inhibition. Cell 93:681-684.

[14] Chen, S. S. 1994. Functional role of the zipper motif region of human immunodeficiency virus type 1 transmembrane protein gp41. J. Virol. 68:2002-2010.

[15] Chen, S. S., C. N. Lee, W. R. Lee, K. McIntosh, and T. H. Lee. 1993. Mutational analysis of the leucine zipper-like motif of the human immunodeficiency virus type 1 envelope transmembrane glycoprotein. J. Virol. 67:3615-3619.

[16] Dash, B., A. McIntosh, W. Barrett, and R. Daniels. 1994. Deletion of a single N-linked glycosylation site from the transmembrane envelope protein of human immunodeficiency virus type 1 stops cleavage and transport of gp160 preventing env-mediated fusion.J. Gen. Virol. 75( $\mathrm{Pt}$ 6):1389-1397.

[17] Dedera, D., R. L. Gu, and L. Ratner. 1992a. Conserved cysteine residues in the human immunodeficiency virus type 1 transmembrane envelope protein are essential for precurso envelope cleavage. J. Virol. 66:1207-1209.

[18] Dedera, D. A., R. L. Gu, and L. Ratner. 1992b. Role of asparagine-linked glycosylation in human immunodeficiency virus type 1 transmembrane envelope function. Virol. 187:377-382.
[19] Delahunty, M. D., I. Rhee, E. O. Freed, and J. S. Bonifacino. 1996. Mutational analysis of the fusion peptide of the human immunodeficiency virus type 1: identification of critical glycine residues. Virol. 218:94-102.

[20] Doms, R. W. 2000. Beyond receptor expression: the influence of receptor conformation, density, and affinity in HIV-1 infection. Virol. 276:229-237.

21] Doms, R. W. and J. P. Moore. 2000. HIV-1 membrane fusion: targets of opportunity. J. Cell Biol. 151:F9-14.

22] Dubay, J. W., S. J. Roberts, B. Brody, and E. Hunter. 1992. Mutations in the leucine zipper of the human immunodeficiency virus type 1 transmembrane glycoprotein affect fusion and infectivity. J. Virol. 66:4748-4756.

[23] Earl, P. L. and B. Moss. 1993. Mutational analysis of the assembly domain of the HIV-1 envelope glycoprotein. AIDS Res. Hum. Retroviruses 9:589-594.

[24] Eckert, D. M. and P. S. Kim. 2001. Mechanisms of viral membrane fusion and its inhibition. Annu. Rev. Biochem. 70:777-810.

[25] Farzan, M., H. Choe, E. Desjardins, Y. Sun, J. Kuhn, J. Cao, D. Archambault, P. Kolchinsky, M. Koch, R. Wyatt, and J. Sodroski. 1998. Stabilization of human immunodeficiency virus type 1 envelope glycoprotein trimers by disulfide bonds introduced into the gp 41 glycoprotein ectodomain. J. Virol. 72:7620-7625.

[26] Fikkert, V., P. Cherepanov, K. van Laethum, A. Hantson, B. van Remoortel, C. Pannecouque, E. de Clercq, Z. Debyser, A. Vandamme, and M. Witvrouw. 2002. Env chimeric virus technology for evaluating human immunodeficiency virus susceptility to entry inhibitors. Antimicrob. Agents Chemother. 46:3954-3962

[27] Follis, K. E., S. J. Larson, M. Lu, and J. H. Nunberg. 2002. Genetic evidence that interhelical packing interactions in the gp41 core are critical for transition of the human immunodeficiency virus type 1 envelope glycoprotein to the fusion-active state. J. Virol. 76:7356-7362.

[28] Freed, E. O., D. J. Myers, and R. Risser. 1990. Characterization of the fusion domain of the human immunodeficiency virus type 1 envelope glycoprotein gp41. Proc. Natl. Acad. Sci. U. S. A. 87:4650-4654.

29] Gallaher, W. R., J. M. Ball, R. F. Garry, A. M. Martin-Amedec, and R. C. Montelaro. 1989. A general model for the transmembrane proteins of HIV and other retroviruses. AIDS Res. Hum Retroviruses. 5:431-440.

[30] Johnson, W. E.. J. M. Sauvron, and R. C. Desrosiers. 2001. Conserved, N-linked carbohydrates of human immunodeficiency virus type $1 \mathrm{gp} 41$ are largely dispensable for viral replication. $J$. Virol. 75:11426-11436

31] Kilby, J. M., J. P. Lalezari, J. J. Eron, M. Carlson, C. Cohen, R. C. Arduino, J. C. Goodgame, J. E. Gallant, P. Volberding, R. L. Murphy, F. Valentine, M. S. Saag, E. L. Nelson, P. R. Sista, and A. Dusek. 2002. The Safety, Plasma Pharmacokinetics, and Antiviral Activity of Subcutaneou Enfuvirtide (T-20), a Peptide Inhibitor of gp41-Mediated Virus Fusion, in HIV-Infected Adults. AIDS Res. Hum. Retroviruses 18:685-693.

32] Klasse, P. J., J. A. McKeating, M. Schutten, M. S. Reitz Jr., and M. Robert-Guroff. 1993. An immune-selected point mutation in the transmembrane protein of human immunodeficiency virus type 1 (HXB2-Env:Ala $582(\rightarrow \mathrm{Thr})$ ) decreases viral neutralization by monoclonal antibodies to the CD4-binding site. Virol. 196:332-337.

[33] Korber BT, K Kunstman, BK Patterson, M Furtado, M McEvilly, R Levy, and SM Wolinsky. 1994. Genetic differences between blood- and brain-derived viral sequences from human immunodeficiency virus type 1-infected patients: evidence of conserved elements in the V3 region of the envelope protein of brain-derived sequences. J. Virol. 68(11):7467-81

[34] Kowalski, M., L. Bergeron, T. Dorfman, W. Haseltine, and J. Sodroski. 1991. Attenuation of human immunodeficiency virus type 1 cytopathic effect by a mutation affecting the transmembrane envelope glycoprotein. J. Virol. 65:281-291. 
[35] Kozak, S. L., E. J. Platt, N. Madani, F. E. Ferro, K. Peden, and D. Kabat. 1997. CD4, CXCR-4, and CCR-5 dependencies for infections by primary patient and laboratory-adapted isolates of human immunodeficiency virus type 1. J. Virol. 71:873-882.

[36] Lee, W. R., X. F. Yu, W. J. Syu, M. Essex, and T. H. Lee. 1992. Mutational analysis of conserved N-linked glycosylation sites of human immunodeficiency virus type $1 \mathrm{gp} 41$. J. Virol. 66:1799-1803.

[37] Liu, J., W. Shu, M. B. Fagan, J. H. Nunberg, and M. Lu. 2001. Structural and functional analysis of the HIV gp41 core containing an Ile573 to Thr substitution: implications for membrane fusion. Biochem. 40:2797-2807.

[38] Lu, M., H. Ji, and S. Shen. 1999. Subdomain folding and biological activity of the core structure from human immunodeficiency virus type $1 \mathrm{gp} 41$ : implications for viral membrane fusion. J. Virol. 73:4433-4438.

[39] Lu, M., M. O. Stoller, S. Wang, J. Liu, M. B. Fagan, and J. H. Nunberg. 2001. Structural and functional analysis of interhelical interactions in the human immunodeficiency virus type 1 gp41 envelope glycoprotein by alanine-scanning mutagenesis. J. Virol. 75:11146-11156.

[40] Maerz, A. L., H. E. Drummer, K. A. Wilson, and P. Poumbourios. 2001. Functional analysis of the disulfide-bonded loop/chain reversal region of human immunodeficiency virus type $1 \mathrm{gp} 4$ reveals a critical role in gp120-gp41 association. J. Virol. 75:6635-6644.

[41] Malashkevich, V. N., D. C. Chan, C. T. Chutkowski, and P. S. Kim. 1998. Crystal structure of the simian immunodeficiency virus (SIV) gp41 core: conserved helical interactions underlie the broad inhibitory activity of gp41 peptides. Proc. Natl. Acad. Sci. U.S.A. 95:9134-9139.

[42] Markosyan, R. B., X. Ma, M. Lu, F. Cohen, and G. Melikyan. 2002. The mechanism of HIVEnv-mediated cell-cell fusion by recombinant cores of gp41 ectodomain. Virol. 302:174-184.

[43] McInerney, T. L., W. El Ahmar, B. E. Kemp, and P. Poumbourios. 1998. Mutation-directed chemical cross-linking of human immunodeficiency virus type 1 gp41 oligomers. J. Virol. 72:1523-1533.

[44] Merat, R., H. Raoul, T. Leste-Lasserre, P. Sonigo, and G. Pancino. 1999. Variable constraints on the principal immunodominant domain of the transmembrane glycoprotein of human immunodeficiency virus type 1. J. Virol. 73:5698-5706.

[45] Moore, J. P., L. C. Burkly, R. I. Connor, Y. Cao, R. Tizard, D. D. Ho, and R. A. Fisher. 1993 Adaptation of two primary human immunodeficiency virus type 1 isolates to growth in transformed $\mathrm{T}$ cell lines correlates with alterations in the responses of their envelope glycoprotein to soluble CD4. AIDS Res. Hum. Retroviruses 9:529-539.

[46] Moore, J. P. and M. Stevenson. 2000. New targets for inhibitors of HIV-1 replication. Nat Rev. Mol. Cell Biol. 1:40-49.

[47] Park, E. J., M. K. Gorny, S. Zolla-Pazner, and G. V. Quinnan, Jr. 2000. A global neutralization resistance phenotype of human immunodeficiency virus type 1 is determined by distinct mechanisms mediating enhanced infectivity and conformational change of the envelope complex. $J$. Virol. 74:4183-4191.

[48] Perrin, C., E. Fenouillet, and I. M. Jones. 1998. Role of gp41 glycosylation sites in the biological activity of human immunodeficiency virus type 1 envelope glycoprotein. Virol. 242:338 345.

[49] Poumbourios, P., W. El Ahmar, D. A. McPhee, and B. E. Kemp. 1995. Determinants of human immunodeficiency virus type 1 envelope glycoprotein oligomeric structure. J. Virol. 69:12091218.

[50] Poumbourios, P., K. A. Wilson, R. J. Center, W. El Ahmar, and B. E. Kemp. 1997. Human immunodeficiency virus type 1 envelope glycoprotein oligomerization requires the gp41 amphipathic alpha-helical/leucine zipper-like sequence. J. Virol. 71:2041-2049.

[51] Poveda, E., B. Rodes, C. Toro, L. Martinez-Carbonero, J. Gonzalez-Lahoz, and V. Soriano. 2002. Evolution of the gp41 env region in HIV-infected patients receiving T-20, a fusion in- hibitor. AIDS 16:1959-1980.

52] Pritsker, M. J. Rucker, T. L. Hoffman, R. W. Doms, and Y. Shai. 1999. Effect of nonpolar substitutions of the conserved Phe11 in the fusion peptide of HIV-1 gp41 on its function, structure, and organization in membranes. Biochem. 38:11359-11371.

[53] Rabenstein, M. and Y. K. Shin. 1995. A peptide from the heptad repeat of human immunodeficiency virus gp41 shows both membrane binding and coiled-coil formation. Biochem. 34:13390-13397.

54] Reitz, M. S., Jr., C. Wilson, C. Naugle, R. C. Gallo, and M. Robert-Guroff. 1988. Generation of a neutralization-resistant variant of HIV-1 is due to selection for a point mutation in the envelope gene. Cell 54:57-63.

55] Rimsky, L. T., D. C. Shugars, and T. J. Matthews. 1998. Determinants of human immunodeficiency virus type 1 resistance to gp41- derived inhibitory peptides. J. Virol. 72:986-993.

[56] Rovinski, B., G. A. Dekaban, S. X. Cao, F. L. Yao, R. Persson, T. J. Matthews, and M. H. Klein 1999. Engineering of noninfectious HIV-1-like particles containing mutant gp41 glycoproteins as vaccine candidates that allow vaccinees to be distinguished from HIV-1 infectees. Virol. 257:438-448.

57] Salzwedel, K., J. T. West, and E. Hunter. 1999. A conserved tryptophan-rich motif in the membrane-proximal region of the human immunodeficiency virus type $1 \mathrm{gp} 41$ ectodomain is important for Env-mediated fusion and virus infectivity. J. Virol. 73:2469-2480.

[58] Sanders, R. W., E. Van Anken, M. Lu, I. Braakman, and B. Berkhout. 2003a. unpublished results.

59] Sanders, R. W., E. Busser, J. P. Moore, M. Lu, and B. Berkhout. 2003b. unpublished results.

[60] Sanders, R. W., M. Dankers, J. P. Moore, and B. Berkhout. 2003c. unpublished results.

[61] Sanders, R. W., M. Vesanen, N. Schuelke, A. Master, L. Schiffner, R. Kalyanaraman, M Paluch, B. Berkhout, P. J. Maddon, W. C. Olson, M. Lu, and J. P. Moore. 2002. Stabilization of the soluble, cleaved, trimeric form of the envelope glycoprotein complex of human immunodeficiency virus type 1. J. Virol. 76:8875-8889.

62] Schaal, H., M. Klein, P. Gehrmann, O. Adams, and A. Scheid. 1995. Requirement of N terminal amino acid residues of gp41 for human immunodeficiency virus type 1-mediated cell fusion. J. Virol. 69:3308-3314.

63] Shu, W., J. Liu, H. Ji, L. Radigen, S. Jiang, and M. Lu. 2000. Helical interactions in the HIV-1 gp41 core reveal structural basis for the inhibitory activity of gp41 peptides. Biochem. 39:1634-1642.

[64] Shugars, D. C., C. T. Wild, T. K. Greenwell, and T. J. Matthews. 1996. Biophysical characterization of recombinant proteins expressing the leucine zipper-like domain of the human immunodeficiency virus type 1 transmembrane protein gp41. J. Virol. 70:2982-2991.

[65] Si, Z., M. Cayabyab, and J. Sodroski. 2001. Envelope glycoprotein determinants of neutralization resistance in a simian-human immunodeficiency virus (SHIV-HXBc2P 3.2) derived by passage in monkeys. J. Virol. 75:4208-4218

[66] Stern, T. L., M. S. Reitz, Jr., and M. Robert-Guroff. 1995. Spontaneous reversion of human immunodeficiency virus type 1 neutralization-resistant variant HXB2thr582: in vitro selection against cytopathicity highlights gp120-gp41 interactive regions. J. Virol. 69:1860-1867.

[67] Syu, W. J., W. R. Lee, B. Du, Q. C. Yu, M. Essex, and T. H. Lee. 1991. Role of conserved gp41 cysteine residues in the processing of human immunodeficiency virus envelope precursor and viral infectivity. J. Virol. 65:6349-6352.

68] Tan, K., J. Liu, J. Wang, S. Shen, and M. Lu. 1997. Atomic structure of a thermostable subdomain of HIV-1 gp41. Proc. Natl. Acad. Sci. U.S.A. 94:12303-12308.

[69] Wang, S., J. York, W. Shu, M. O. Stoller, J. H. Nunberg, and M. Lu. 2002. Interhelica interactions in the gp41 core: implications for activation of HIV-1 membrane fusion. Biochem. 41:7283-7292. 
[70] Wei, X., J. M. Decker, H. Liu, Z. Zhang, R. B. Arani, J. M. Kilby, M. S. Saag, X. Wu, G. M. Shaw, and J. C. Kappes. 2002. Emergence of resistant human immunodeficiency virus type 1 in patients receiving fusion inhibitor (T-20) monotherapy. Antimicrob. Agents Chemother. 46:1896-1905.

[71] Weissenhorn, W., A. Dessen, S. C. Harrison, J. J. Skehel, and D. C. Wiley. 1997. Atomic structure of the ectodomain from HIV-1 gp41. Nature 387:426-430.

[72] Weng, Y. and C. D. Weiss. 1998. Mutational analysis of residues in the coiled-coil domain of human immunodeficiency virus type 1 transmembrane protein gp41. J. Virol. 72:9676-9682.

[73] Weng, Y., Z. Yang, and C. D. Weiss. 2000. Structure-function studies of the self-assembly domain of the human immunodeficiency virus type 1 transmembrane protein gp41. J. Virol. 74:5368-5372

[74] Wild, C., J. W. Dubay, T. Greenwell, T. Baird, Jr., T. G. Oas, C. McDanal, E. Hunter, an T. Matthews. 1994. Propensity for a leucine zipper-like domain of human immunodeficiency virus type $1 \mathrm{gp} 41$ to form oligomers correlates with a role in virus- induced fusion rather than assembly of the glycoprotein complex. Proc. Natl. Acad. Sci. U. S. A. 91:12676-12680.

[75] Wyatt, R. and J. Sodroski. 1998. The HIV-1 envelope glycoproteins: fusogens, antigens, and immunogens. Science 280: 1884-1888.

[76] Yang Z, Nielsen R, Goldman N, Pedersen AM. 2000. Codon-substitution models for heterogeneous selection pressure at amino acid sites. Genetics 155(1):431-49. 


\section{Web-based Tools for Vaccine Design}

Ole Lund ${ }^{1}$, Morten Nielsen ${ }^{1}$, Can Kesmir ${ }^{1,2}$, Jens K. Christensen ${ }^{1}$, Claus Lundegaard $^{1}$, Peder Worning ${ }^{1}$, and Søren Brunak ${ }^{1}$

${ }^{1}$ Center for Biological Sequence Analysis, BioCentrum, Technical University of Denmark.

${ }^{2}$ Theoretical Biology/Bioinformatics, Utrecht University, The Netherlands.

\section{Introduction}

Computational methods used in vaccine design have been changing drastically in recent years. In classical immunological research results could be recorded by pen and pencil or in a spreadsheet, but new experimental high-throughput methods such as sequencing, DNA arrays, and proteomics have generated a wealth of data that are not efficiently handled and mined by these approaches. This has fueled the rapid growth of the field of Immunological Bioinformatics (or Immunoinformatics) that addresses how to handle these large amounts of data in the field of immunology and vaccine design. Many of the methods have been made available on the Internet and can be used by experimental researchers without expert knowledge of bioinformatics. This review attempts to give an overview over the methods currently available and to point out the strengths and weaknesses of the different methods.

\section{Immunological processes described by prediction servers}

Only a small fraction of the possible peptides that can be generated from proteins of pathogenic organisms actually generate an immune response. In order to be presented to CD8+ T cells a precursor peptide must be generated by the proteasome. This peptide may be trimmed at the $\mathrm{N}$-terminal by other peptidases in the cytosol (Levy et al., 2002). It must then bind to the transporter associated with antigen processing (TAP) in order to be translocated to the endoplasmatic reticulum (ER). Here it can be trimmed N-terminally by the aminopeptidase associated

In HIV Molecular Immunology 2002. Bette T. M. Korber, Christian Brander, Barton F. Haynes, Richard Koup, Carla Kuiken, John P. Moore, Bruce D. Walker, and David I. Watkins, editors. Publisher: Los Alamos National Laboratory, Theoretical Biology and Biophysics, Los Alamos, New Mexico. LA-UR 03-5816. pp. 45 .51 with antigen processing (ERAAP) while it binds to the major histocompatibility complex class I (MHC I) molecule (Serwold, 2002). Hereafter it is transported to the cell surface. Only half the peptides presented on the cell surface are immunogenic probably due to the limited size of the $\mathrm{T}$ cell receptor (TCR) repertoire. The most selective step is binding to the MHC I molecule, since only 1/200 binds with an affinity strong enough to generate an immune response (Yewdell, 1999). For comparison the selectivity of TAP binding is reported to be $1 / 7$ (Uebel et al., 1997). This all happens in competition with other peptides so in order for a peptide to be immunogenic (immunodominant) it must go through the above described process more efficiently than other peptides produced in a given cell (Reviewed by Yewdell, 1999).

Whereas the MHC I molecule mainly samples peptides from the cytosol, the MHC II molecule presents peptides from endocytosed proteins. Unfolded polypeptides bind to MHC II in the endocytic organelles (Reviewed by Castllino, 1997). Both MHC I and MHC II are highly polymorphic, and the specificity of the alleles are often very different. Different individuals will thus typically react to a different set of peptides from a pathogen.

The specificity of some of the processes involved in antigen presentation can be predicted from the amino acid sequence. This can for example be used to select epitopes for use in a vaccine, and help to understand the role of the immune system in infectious diseases, autoimmune diseases and cancers. Below we describe a number of resources available on the web that can perform such predictions.

\section{Databases of MHC binding peptides}

Several databases of MHC binding peptides now exist on the web (Table 1).

SYFPEITHI: The SYFPEITHI database contains information on peptide sequences, anchor positions, MHC specificity, source proteins, source organisms, and publication references. The database comprise approximately 3500 peptide sequences known to bind class I and class II MHC molecules and is based on previous publications on T-cell epitopes and MHC ligands from many species (Rammensee, 1999).

MHCPEP: The other major database of MHC binding peptides, MHCPEP, (Brusic, 1997) comprises over 13,000 peptide sequences known to bind MHC 
molecules. Entries were compiled from published reports as well as from direct submissions of experimental data. Each entry contains the peptide sequence, its MHC specificity and, when available, experimental method, observed activity, binding affinity, source protein, anchor positions, and publication references Unfortunately the database has since June 1998 been static. The database can be downloaded as an ASCII file.

JenPep: The JenPep database is a newer database that contains quantitative binding data of peptides to MHC and TAP, as well as T cell epitopes (Blythe, 2001). The database contains more than 8000 entries .

FIMM: The database by Schoenbach \& Brusic is a functional database of molecular immunology. The database contains 571 antigens and 1591 peptides (Schonbach et al., 2002)

MHCBN: (Bhasin, 2002) is a database of MHC binding and non-binding peptides containing 14,816 binders, 1,782 non-binders and 5,456 T-cell epitope entries.

HLA Ligand/Motif database: This site's database can be searched by defining allele and specificity, amino acid pattern, ligand/motif in sequence of amino acids, author's last name, or advanced search with more criteria.

HIV Molecular Immunology database: The HIV Molecular Immunology Database is an annotated, searchable collection of HIV-1 cytotoxic and helper T-cell epitopes and antibody binding sites. The goal of the database is to provide a comprehensive listing of defined HIV epitopes (Korber et al., 2001).

EPIMHC: MHC ligand database that can be searched based on sequence, length, class, species, and on whether a ligand is an epitope or not.

NIH will over the next five to seven years fund an "Immune Epitope Database and Analysis Program' 1 to design, develop, populate, and maintain a publicly accessible, comprehensive Immune Epitope Database containing linear and conformational antibody epitopes and $\mathrm{T}$ cell epitopes. This database may eventually incorporate most of the data from the above described databases.

\section{Prediction of MHC binding}

Several peptide-MHC binding prediction servers exist on the web (Table 2). As indicated in the table some of the web based methods also allow prediction of binding to Class II molecules. Most methods available on the web for predicting

\footnotetext{
www.niaid.nih.gov/contract/archive/rfp0331.pdf
}

MHC-peptide binding are matrix methods. Parameters are often derived from pool sequencing of ligands. Matrices or hidden Markov models may however also be derived from a set of ligand sequences. In these methods the amino acid on each position in the motif gives an independent contribution to the prediction score. Neural networks are able to make more accurate predictions if correlations between positions exist, and there are enough data to model them. This has the potential advantage that it can take correlations between different positions in the binding motif into account.

BIMAS: The BIMAS method was developed by Parker et al., (1994). The method is based on coefficient tables deduced from the published literature. For HLA-A2, peptide binding data were combined together to generate a table containing 180 coefficients (20 amino acids x 9 positions), each of which represents the contribution of one particular amino acid residue at a specified position within the peptide (Parker et al., 1994).

SYFPEITHI: The SYFPEITHI prediction is based on published motifs (pool sequencing, natural ligands) and takes into consideration the amino acids in the anchor and auxiliary anchor positions, as well as other frequent amino acids. The score is calculated according to the following rules: The amino acids of a certain peptide are given a specific value depending on whether they are anchor, auxiliary anchor or preferred residue. Ideal anchors will be given 10 points, unusual anchors 6-8 points, auxiliary anchors $4-6$ and preferred residues $1-4$ points. Amino acids that are regarded as having a negative effect on the binding ability are given values between -1 and -3 (Rammensee, 1997; 1999). On the SYFPEITHI web site predictions can be made for 5 different MHC II alleles in addition to a number of Class I alleles.

PREDEPP: In this method the peptide structure in the MHC groove is used as a template upon which peptide candidates are threaded, and their compatibility to bind is evaluated by statistical pairwise potentials. This method has the advantage that it does not require experimental testing of peptide binding, and can thus be used for alleles where only limited data are available (Schueler-Furman et al., 2000).

Epipredict: Method using synthetic combinatorial peptide libraries to describe peptide-HLA class II interaction in a quantitative way. The binding contribution of every amino acid side chain in a class II-ligand is described by allele-specific two-dimensional databases (Jung et al., 2001).

Predict: The Predict method use neural networks to predict Class I, II and TAP binding (Yu et al., 2002). 
Propred: The Propred method (Singh, 2001) is based on the matrices published by Sturniolo (1999), and is an implementation and extension of the TEPITOPE program. (Hammer, 1995; Raddrizzani, 2000)). Besides differences that can be attributed to round off errors we have in our tests not seen any differences between the two implementations.

MHCPred: Prediction of binding to 11 different HLA class I alleles using a three-dimensional quantitative structure-activity relationship method (Doytchinova et al., 2002).

NetMHC: Prediction of HLA-A2 binding using neural networks. This method predicts quantitatively the binding affinity, and is different from methods performing classification only (binding versus non-binding according to a threshold). The method has been trained using quantitative binding data generated by the same assay (Buus et al., 2003), and some predicted binders have been tested for their ability to induce a CTL response in mice and be recognized by CD8+ T-cells from HLA-A2 HIV-1 positive patients (Corbet et al., 2003). Two well-known prediction methods, TEPITOPE and EpiMatrix (Meister 1995; De Groot, 1997) that are not available through the web are listed in Table 3. TEPITOPE is popular since it allows prediction of peptides to many different Class II molecules.

\section{Prediction of proteasomal cleavage sites}

The C terminal of MHC class I ligands must most likely be cleaved by the proteasome. The proteasome usually generates precursors of MHC ligands with an extension at the $\mathrm{N}$-termini. These precursors can be trimmed at the $\mathrm{N}$-terminal in the ER. The existence of proteasome cleavage sites within epitopes need not abrogate the immune response for such epitopes. They may, however, reduce the availability, and thereby the immunogenecity of a given peptide (Yewdell, 1999). The proteasome thus plays an important role in selecting which peptides are presented to $\mathrm{CD} 8+\mathrm{T}$ cells. In vertebrates stimulation with IFN- $\gamma$ leads to the replacement of three subunits of the constitutive proteasome to form the socalled immunoproteasome which has a different specificity (reviewed by Uebel, 1999). Different methods for predicting proteasomal cleavage sites exist on the web (Table 4).

PAProC: Prediction Algorithm for Proteasomal Cleavages is a prediction tool for cleavages by human and yeast proteasomes, based on experimental cleavage data. (Kuttler, 2000; Nussbaum, 2001). An updated version of the PAProC program based on in vitro immunoproteasome cleavage data (Toes, 2001) is also in the making according to the PAProC homepage.

FRAGPREDICT comprises two different algorithms. One that aims at predicting potential proteasomal cleavage, based on a statistical analysis of cleavagedetermining amino acid motifs present around the scissile bond (Holzhütter $e t$ al., 1999, 2000). The second algorithm, which uses the results of the cleavage site analysis as an input, provides predictions of major proteolytic fragments.

NetChop: (Kesmir, 2002) is a method based on neural networks that have been trained on different data sets. C Kesmir suggests to use the C-term 2.0 network which was trained on C-terminal cleavage sites of 1,110 publicly available $\mathrm{MHC}$ class I ligands for predicting the boundaries of CTL. The specificity of this network may resemble the specificity of the immunoproteasome.

Margalit's group have also recently made their proteasomal cleavage site propensities (Altuvia and Margalit, 2000) available on the net (bioinfo.md. huji.ac.il/marg/cleavage/index.html).

\section{Combined predictions}

A number of sites providing combined predictions have been developed recently. The MAPPP server (Table 2) allows the user to make an open reading frame (ORF) search combined with MHC binding and proteasomal cleavage site predictions, and Raghava have a prediction server ${ }^{2}$ which implements matrices for 47 MHC Class-I alleles and proteasomal and immunoproteasomal models. The NetMHC server allows combination of HLA-A2 and NetChop predictions.

\section{MHC sequence databases}

A number of databases containing sequences of proteins of immunological interest exist on the web (Table 5).

HIG: The HLA Sequence Database currently contains 1,596 allele sequences. To date (October 2002), some 263 HLA-A, 501 HLA-B, 125 HLA-C, 6 HLA-E, 1 HLA-F and 15 HLA-G class I alleles have been named. A total of 3 HLADRA, 397 HLA-DRB, 22 HLA-DQA1, 53 HLA-DQB1, 20 HLA-DPA1, 100 HLA-DPB1, 4 HLA-DMA, 6 HLA-DMB, 8 HLA-DOA and 8 HLA-DOB class II sequences have also been assigned. There are also 6 TAP1, 4 TAP2 and 54 $2 \longdiv { \text { www.imtech.res.in/raghava/propredl/index.html } }$ 
MICA sequences. The HLA Sequence Database also contains the comprehensive nomenclature for factors of the HLA system (listings for HLA class I and class II allele names) which is very helpful since the HLA nomenclature is very complicated and cumbersome.

IMGT: IMGT, the international ImMunoGeneTics project, is a collection of databases specializing in Immunoglobulins, T cell receptors and the Major Histocompatibility Complex (MHC) of all vertebrate species. The IMGT project was established in 1989 by the Université Montpellier II and the CNRS (Montpellier, France) and works in close collaboration with the EBI.

ASHI: The American Society for Histocompatibility and Immunogenetics (ASHI) hosts databases of gene and allele frequencies (www.ashi-hla.org/).

MHCDB: "Registered users only" database of MHC sequences. This is an ACeDB-style database holding the Human Major Histocompatibility Database. It is largely superseded by 6ace which is ACeDB-style database of human chromosome 6 from the Sanger Centre.

\section{Other sites}

A number of other databases relevant to immunology and vaccine design are listed in Table 6. Table 7 contains a compilation of lists of links. As stated in Table 7 we will also make an HTML version of this article available on the net.

\section{References}

Altuvia Y, Margalit H. Sequence signals for generation of antigenic peptides by the proteasome implications for proteasomal cleavage mechanism. J Mol Biol. 2000 295:879-90.

Bhasin M, Singh H, Raghava G. PS. (2002) MHCBN: A Comprehensive Database of MHC Binding and Non-Binding Peptides. Nucleic Acids Research, (online) www3.oup.co.uk/nar/ database/summary/180

Blythe MJ, Doytchinova IA, Flower DR. JenPep: a database of quantitative functional peptide data for immunology. Bioinformatics. 2002 18:434-9.

Brusic V, Rudy G, Harrison LC. MHCPEP, a database of MHC-binding peptides: update 1997. Nucleic Acids Res. 1998 26:368-71.

Buus S, Lauemøller SL, Worning P, Kesmir C, Frimurer T, Corbet S, Fomsgaard A, Hilden J, Holm A, and Brunak S. Sensitive quantitative predictions of peptide-MHC binding by a "Query by Committee" artificial neural network approach. Accepted for publication in Tissue Antigens, 2003.

Castellino F, Zhong G, Germain RN. Antigen presentation by MHC class II molecules: invarian chain function, protein trafficking, and the molecular basis of diverse determinant capture. Hum Immunol. 1997 54:159-69.

Corbet S, Nielsen HV, Vinner L, Lauemøller SL, Therrien D, Tang S, Kronborg G, Mathiesen L, Chaplin P, Brunak S, Buus S, and Fomsgaard A. Optimisation and immune recognition of multiple novel conserved HLA-A2, HIV-1-specific CTL epitopes. Accepted for publication in General Virology.

De Groot AS, Jesdale BM, Szu E, Schafer JR, Chicz RM, Deocampo G. An interactive Web site providing major histocompatibility ligand predictions: application to HIV research. AIDS Res. Hum. Retroviruses 1997 13:529-31.

Doytchinova IA, Flower DR. Physicochemical explanation of peptide binding to HLA-A*0201 major histocompatibility complex: a three-dimensional quantitative structure-activity relationship study. Proteins. 2002 48:505-18.

Hammer J. New methods to predict MHC-binding sequences within protein antigens. Curr Opin Immunol. 1995 7:263-9.

Holzhutter HG, Frommel C, Kloetzel PM. A theoretical approach towards the identification of cleavagedetermining amino acid motifs of the $20 \mathrm{~S}$ proteasome. J Mol Biol. 1999 286:1251-65.

Holzhutter HG, Kloetzel PM. A kinetic model of vertebrate 20S proteasome accounting for the generation of major proteolytic fragments from oligomeric peptide substrates. Biophys J. 2000 79:1196-205.

Johnson G, Wu TT. Kabat Database and its applications: future directions. Nucleic Acids Res. 2001 29:205-6.

Jung G, Fleckenstein B, von der Mulbe F, Wessels J, Niethammer D, Wiesmuller KH. From combinatorial libraries to MHC ligand motifs, T-cell superagonists and antagonists. Biologicals. 2001 29:179-81

HIV Molecular Immunology 2001, Editors: Bette T. M. Korber, Christian Brander, Barton F. Haynes, Richard Koup, Carla Kuiken, John P. Moore, Bruce D. Walker, and David I. Watkins. Publisher: Los Alamos National Laboratory, Theoretical Biology and Biophysics, Los Alamos, New Mexico. LA-UR 02-4663.

Kuttler C, Nussbaum AK, Dick TP, Rammensee HG, Schild H, Hadeler KP. An algorithm for the prediction of proteasomal cleavages. J Mol Biol. 2000 298:417-29.

Levy F, Burri L, Morel S, Peitrequin AL, Levy N, Bachi A, Hellman U, Van den Eynde BJ, Servis C. The final N-terminal trimming of a subaminoterminal proline-containing HLA class I-restricted antigenic peptide in the cytosol is mediated by two peptidases. J Immunol 2002 169:4161-71.

Meister GE, Roberts CG, Berzofsky JA, De Groot AS. Two novel T cell epitope prediction algorithms based on MHC-binding motifs; comparison of predicted and published epitopes from Mycobacterium tuberculosis and HIV protein sequences. Vaccine. 1995 13:581-91.

Nussbaum AK, Kuttler C, Hadeler KP, Rammensee HG, Schild H. PAProC: a prediction algorithm for proteasomal cleavages available on the WWW. Immunogenetics. 2001 53:87-94.

Parker KC, Bednarek MA, Coligan JE. Scheme for ranking potential HLA-A2 binding peptides based on independent binding of individual peptide side-chains. J Immunol. 1994 152:163-75.

Raddrizzani L, Hammer J. Epitope scanning using virtual matrix-based algorithms. Brief Bioinform. 2000 1:179-89.

Rammensee H, Bachmann J, Emmerich NP, Bachor OA, Stevanovic S. SYFPEITHI: database for MHC ligands and peptide motifs. Immunogenetics. 1999 50:213-9.

Rammensee H-G, Bachmann J, Stevanovic S. MHC ligands and peptide motifs. Landes Bioscience, 1997.

Serwold T, Gonzalez F, Kim J, Jacob R, Shastri N. ERAAP customizes peptides for MHC class I molecules in the endoplasmic reticulum. Nature. 2002 419:480-3.

Schonbach C, Koh JL, Flower DR, Wong L, Brusic V. FIMM, a database of functional molecular immunology: update 2002. Nucleic Acids Res. 2002 30:226-9.

Schueler-Furman O, Altuvia Y, Sette A, Margalit H. Structure-based prediction of binding peptides to MHC class I molecules: application to a broad range of MHC alleles. Protein Sci 2000 9:1838-46.

Singh H, Raghava GP. ProPred: prediction of HLA-DR binding sites. Bioinformatics. 2001 17:1236- 
Sturniolo T, Bono E, Ding J, Raddrizzani L, Tuereci O, Sahin U, Braxenthaler M, Gallazzi F, Protti MP, Sinigaglia F, Hammer J. Generation of tissue-specific and promiscuous HLA ligand databases using DNA microarrays and virtual HLA class II matrices. Nat Biotechnol. 1999 17:555-61.

Toes RE, Nussbaum AK, Degermann S, Schirle M, Emmerich NP, Kraft M, Laplace C, Zwinderman A, Dick TP, Muller J, Schonfisch B, Schmid C, Fehling HJ, Stevanovic S, Rammensee HG, Schild H. Discrete cleavage motifs of constitutive and immunoproteasomes revealed by quantitative analysis of cleavage products. J Exp Med. 2001 194:1-12.

Uebel S, Kraas W, Kienle S, Wiesmuller KH, Jung G, Tampe R. Recognition principle of the TAP transporter disclosed by combinatorial peptide libraries. Proc Natl Acad Sci U S A 1997 94:8976-81

Uebel S, Tampe R. Specificity of the proteasome and the TAP transporter. Curr Opin Immunol. 1999 11:203-8.

Yewdell JW, Bennink JR. Immunodominance in major histocompatibility complex class I-restricted T lymphocyte responses. Annu Rev Immunol. 1999 17:51-88.

Yu K, Petrovsky N, Schonbach C, Koh JY, Brusic V. Methods for prediction of peptide binding to MHC molecules: a comparative study. Mol Med. 2002 8:137-48 
Table 1: Databases of MHC binding peptides

\begin{tabular}{|c|c|c|c|}
\hline Name & Principal Investigator & URL & Description \\
\hline SYFPEITHI & Rammensee & syfpeithi.bmi-heidelberg.com/scripts/MHCServer.dll/home.htm & $\begin{array}{l}\text { Database and prediction server for } \\
\text { peptides that bind MHC molecules. }\end{array}$ \\
\hline МHCPEP & Brusic, Harrison & wehih.wehi.edu.au/mhcpep & Database of MHC binding peptides \\
\hline JenPep & Flower & www.jenner.ac.uk/JenPep & $\begin{array}{l}\text { Database of MHC and TAP binding } \\
\text { peptides }\end{array}$ \\
\hline FIMM & Schoenbach \& Brusic & sdmc.krdl.org.sg:8080/fimm & $\begin{array}{l}\text { Database of functional molecular } \\
\text { immunology/binding prediction }\end{array}$ \\
\hline MHCBN & Raghava & www.imtech.res.in/raghava/mhcbn & Tools for subunit vaccine design \\
\hline HLA Ligand/Motif Database & Hildebrand & hlaligand.ouhsc.edu & Ligand database/prediction \\
\hline HIV Molecular Immunology & Korber & hiv-web.lanl.gov/content/immunology/ & HIV CTL epitopes \\
\hline EPIMHC & Reinherz & mif.dfci.harvard.edu/Tools/db_query_epimhc.html & $\begin{array}{l}\text { Peptides that bind to MHC } \\
\text { molecules }\end{array}$ \\
\hline
\end{tabular}

Table 2: HLA Peptide Binding Predictions

\begin{tabular}{lll}
\hline Name & URL & Description \\
\hline BIMAS & bimas.dcrt.nih.gov/molbio/hla_bind & Prediction of MHC class I binding using matrices \\
SYFPEITHI & syfpeithi.bmi-heidelberg.com/Scripts/MHCServer.dll/EpPredict.htm & Prediction of Class I and II binding \\
PREDEPP & bioinfo.md.huji.ac.il/marg/Teppred/mhc-bind & MHC Class I epitope prediction \\
Epipredict & www.epipredict.de/index.html & Prediction of HLA class II restricted binding \\
Predict & http://sdmc.krdl.org.sg:8080/predict-demo & Prediction of Class I, II and TAP binding \\
Propred & www.imtech.res.in/raghava/propred & MHC class II prediction \\
MHCPred & www.jenner.ac.uk/MHCPred & HLA class I predictions \\
NetMHC & www.cbs.dtu.dk/services/NetMHC & Prediction of HLA-A2 binding using Neural \\
& & networks \\
MAPPP & www.mpiib-berlin.mpg.de/MAPPP/expertquery.html & Combined ORF, MHC binding and proteasomal \\
& & cleavage Registration needed for expert mode \\
\hline
\end{tabular}

Table 3: Non web MHC binding predictions

\begin{tabular}{lll}
\hline Name & URL & Description \\
\hline TEPITOPE & www.vaccinome.com & $\begin{array}{l}\text { PC Program for Class II predictions can be } \\
\text { downloaded }\end{array}$ \\
EpiMatrix & epivax.com/epimatrix.html & Commercial epitope prediction \\
\hline
\end{tabular}


Table 4: Prediction of proteasomal cleavage sites

\begin{tabular}{|c|c|c|}
\hline Name & URL & Description \\
\hline Paproc & paproc.de & $\begin{array}{l}\text { A matrix based method for prediction of } \\
\text { protasomal cleavage }\end{array}$ \\
\hline FRAGPREDICT & www.mpiib-berlin.mpg.de/MAPPP/cleavage.html & Proteolytic fragment predicter \\
\hline NetChop & www.cbs.dtu.dk/services/NetChop & $\begin{array}{l}\text { A neural network based method for prediction of } \\
\text { proteasomal cleavage }\end{array}$ \\
\hline
\end{tabular}

Table 5: MHC sequence databases

\begin{tabular}{|c|c|c|}
\hline Name & URL & Description \\
\hline HIG & www.anthonynolan.org.uk/HIG & HLA sequence database \\
\hline IMGT & www.ebi.ac.uk/imgt & $\begin{array}{l}\text { Sequences of MHC, TCR and immunoglobulin } \\
\text { molecules }\end{array}$ \\
\hline ASHI & www.ashi-hla.org & Sequences and Gene and Haplotype frequencies \\
\hline MHCDB & www.hgmp.mrc.ac.uk/Registered/Option/mhcdb.html & $\begin{array}{l}\text { Registered users only database of MHC } \\
\text { sequences }\end{array}$ \\
\hline
\end{tabular}

\section{Table 6: Other sites}

\begin{tabular}{llc}
\hline Name & URL & Description \\
\hline $\begin{array}{l}\text { HIV Molecular } \\
\text { Immunology database }\end{array}$ & hiv-web.lanl.gov/content/immunology & HIV immunology \\
$\begin{array}{l}\text { School of Crystallogra } \\
\text { phy, Birkbeck College, }\end{array}$ & www.cryst.bbk.ac.uk/pps97/assignments/projects/coadwell/MHCSTFU1.HTM & Structure and Function of the Major \\
$\begin{array}{l}\text { University of London } \\
\text { MHC-Peptide }\end{array}$ & surya.bic.nus.edu.sg/mpid/ & Histocompatibility Complex (MHC) Proteins \\
$\begin{array}{l}\text { Interaction Database } \\
\text { (MPID) }\end{array}$ & hiv-web.lanl.gov/content/hiv-db/ALABAMA/epitope_analyzer.html & Structural information and characterization of \\
ELF & www.ashi-hla.org & MHC peptide interaction \\
ASHI & & Epitope Location Finder \\
& & The American Society for Histocompatibility and \\
\hline
\end{tabular}

Table 7: Links to lists of links

\begin{tabular}{lll}
\hline Name & URL & Description \\
Syfpeithi & http://syfpeithi.bmi-heidelberg.com/Scripts/MHCServer.dll/Info.htm & Rammensee's links \\
FIMM & http://sdmc.krdl.org.sg:8080/fimm & Brusic's links \\
CBS & www.cbs.dtu.dk/courses/27485.imm/links.html & Our links \\
HLA-RELATED LINKS & home.att.net/dorak/hla/linkhla.html & Dorak's links \\
This article & www.cbs.dtu.dk/researchgroups/immunology/webreview.html & The present article in HTML format \\
\hline
\end{tabular}





\section{Part II}

\section{HIV CTL Epitopes}

53
DEC 2002 



\section{II-A Summary}

Part II includes tables, maps, and associated references of HIV-specific CTL epitopes arranged sequentially according to the location of the proteins in the HIV-1 genome. We attempted to make this section as comprehensive as possible, requiring that the epitope be contained within a defined region of a maximum of 30 amino acids, but not that the optimal boundaries be defined. Studies that were based on the analysis of whole proteins are described at the end of each protein section. The same epitope can have multiple entries, and each entry represents a single publication in this section of the database. For more recent updates and useful searching capabilities, please see our web site: http: //hiv-web.lanl.gov/immunology. For a concise listing of the best defined CTL epitopes, see the summary by Christian Brander and Philip Goulder on page 3 in Part 1 of this compendium. CTL protein reactions with no welldefined epitopes are listed at the end of each protein section.

Recent studies utilize multiple functions attributed to $\mathrm{T}$ cells to define responses, and the simple distinctions of cytotoxic T-cell and helper T-cells have become blurred as more is learned about the range of responses triggered in CD4 and CD8 positive T-cells responding to antigenic stimulus. When adding the most recent studies, we have tried to place $\mathrm{T}$ cell responses in a reasonable manner into our traditional helper $\mathrm{T}$ cell and CTL sections, and to specify the assay used to measure the response in each study.

\section{II-A-1 CTL Epitope Tables}

Each CTL reference has a six part basic entry:

HXB2 Location: The viral strain HXB2 (GenBank Accession Number K03455) is used as a reference strain throughout this publication. The position of the defined epitope location relative to the sequence of the HXB2 protein is indicated. The numbering in this table corresponds to the protein maps. Because of HIV-1 variation the epitope may not actually be present in HXB2, rather the position in HXB2 indicates the position aligned to the epitope. HXB2 was selected as the reference strain because so many studies use HXB2, and because crystal structures for HXB2-related proteins are often available. The precise positions of an epitope on the HXB2 reference strain can be readily obtained using the interactive position locator at our web site: http://hiv-web.lanl.gov/content/hiv-db/ LOCATE_SEQ/locate.html

Author Location: The amino acid positions of the epitope boundaries and the reference sequence are listed as given in the primary publication. Frequently, these positions as published are imprecise, and do not truly correspond to the numbering of the sequence, but they provide a reasonable guide to the peptide's approximate location in the protein. Also, in many cases the reference sequence identification was not provided, and in such cases it is not possible to use these numbers to specify precise locations.

Epitope Sequence: The amino acid sequence of the epitope of interest as defined in the reference, based on the reference strain used in the study defining the epitope. On occasions when only the position numbers and not the actual peptide sequence was specified in the original publication, we tried to fill in the peptide sequence based on the position numbers and reference strain. If the sequences were numbered inaccurately by the primary authors, or if we made a mistake in this process, we may have misrepresented the binding site's amino acid sequence. Because of this uncertainty, epitopes that were not explicitly written in the primary publication, that we determined by looking up the reference strain and the numbered location, are followed by a question mark in the table.

Immunogen: The original stimulus of the CTL response. Often this is an HIV-1 infection. If a vaccine was used as the original antigenic stimulation, not a natural infection, this is noted on a separate line, and additional information about the vaccine antigen is provided as available.

Species(HLA): The species responding and HLA or MHC specificity of the epitope.

Reference: The primary reference (sometimes two or more directly related studies are included). Details for some of the earlier references are in Part $\mathrm{V}$

Following the entry for a given CTL epitope are brief comments explaining the context in which the epitope was studied and what was learned about the epitope in a given study. 


\section{II-A-2 HIV Protein Epitope Maps}

All HIV CTL epitopes mapped to within a region of 21 amino acids or less are indicated on the HIV protein epitope maps. The location and HLA restriction elements of CTL epitopes are indicated on protein sequences of HXB2. These maps are meant to provide the relative location of defined epitopes on a given protein, but the HXB2 sequence may not actually carry the epitope of interest, as it may vary relative to the sequence for which the epitope was defined. Epitopes with identical boundaries and HLA fields are included in the maps only once. If one laboratory determines HLA presenting molecules at the serotype level (example: A2) and another at the genotype level (example: A*0201) both will be included in the map. MHC specificities are indicative of the host species; when no MHC presenting molecule is defined, the host species is noted.

\section{II-A-3 Alignments}

To conserve space, no epitope alignments are provided in this book, but they can be generated using the CTL epitope search tool at http: / / hiv-web. lanl. gov/immunology. All epitopes are aligned to the HXB2 sequence, with the sequence used to define the epitope indicated directly above it. Sequences are sorted by their subtype and country of origin.

The master alignment files from which the epitope alignments were created are available at our web site (http://hiv-web.lanl.gov/ALIGN CURRENT/ALIGN-INDEX.html). The alignments were modified in some cases to optimize the alignment relative to the defined epitope and minimize insertions and deletions; epitope alignments are generated by anchoring on the C-terminal residue. A dash indicates identity to the consensus sequence, and a period indicates an insertion made to maintain the alignment. Stop codons are indicated with a $\$$, and frameshifts by a \#, or ambiguous codons (nucleotide was $r, y$, or $n$ ) by an $x$; they are inserted to maintain the alignments. In consensus sequences an upper case letter indicates the amino acid was present in all sequences, a lower case letter indicates the amino acid was present in most sequences in a given position, and a question mark indicates two or more amino acids were represented with equal frequency. 


\section{II-B HIV CTL Epitope Tables}

All HIV CTL epitopes arranged by protein position. The table entries are sorted in a nested way-first by protein, then by HXB2 start location and finally by HLA. CTL reactions against proteins with undefined epitopes are listed at the end of the protein which stimulated the response.

\section{II-B-1 p17 CTL Epitopes}

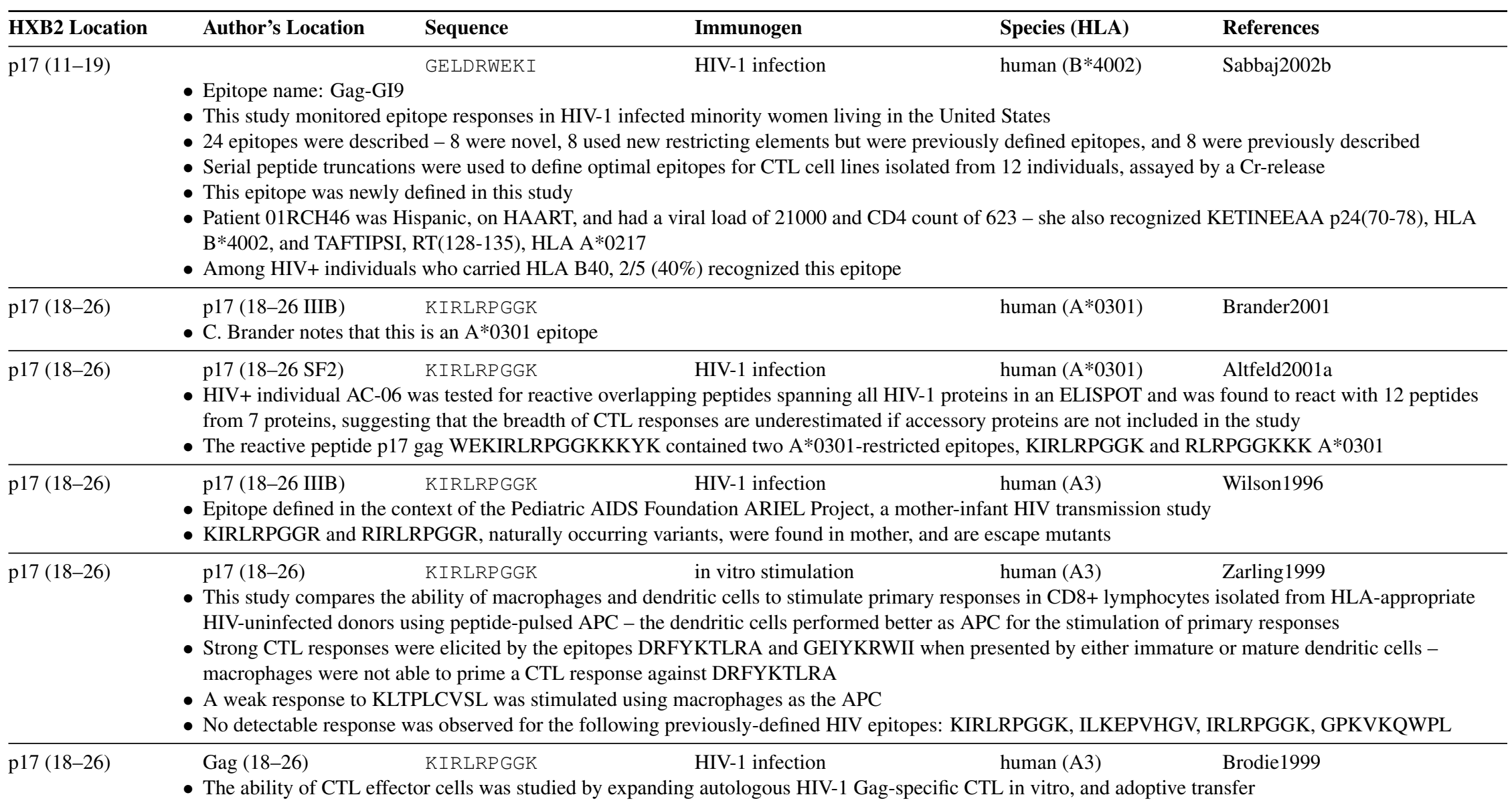




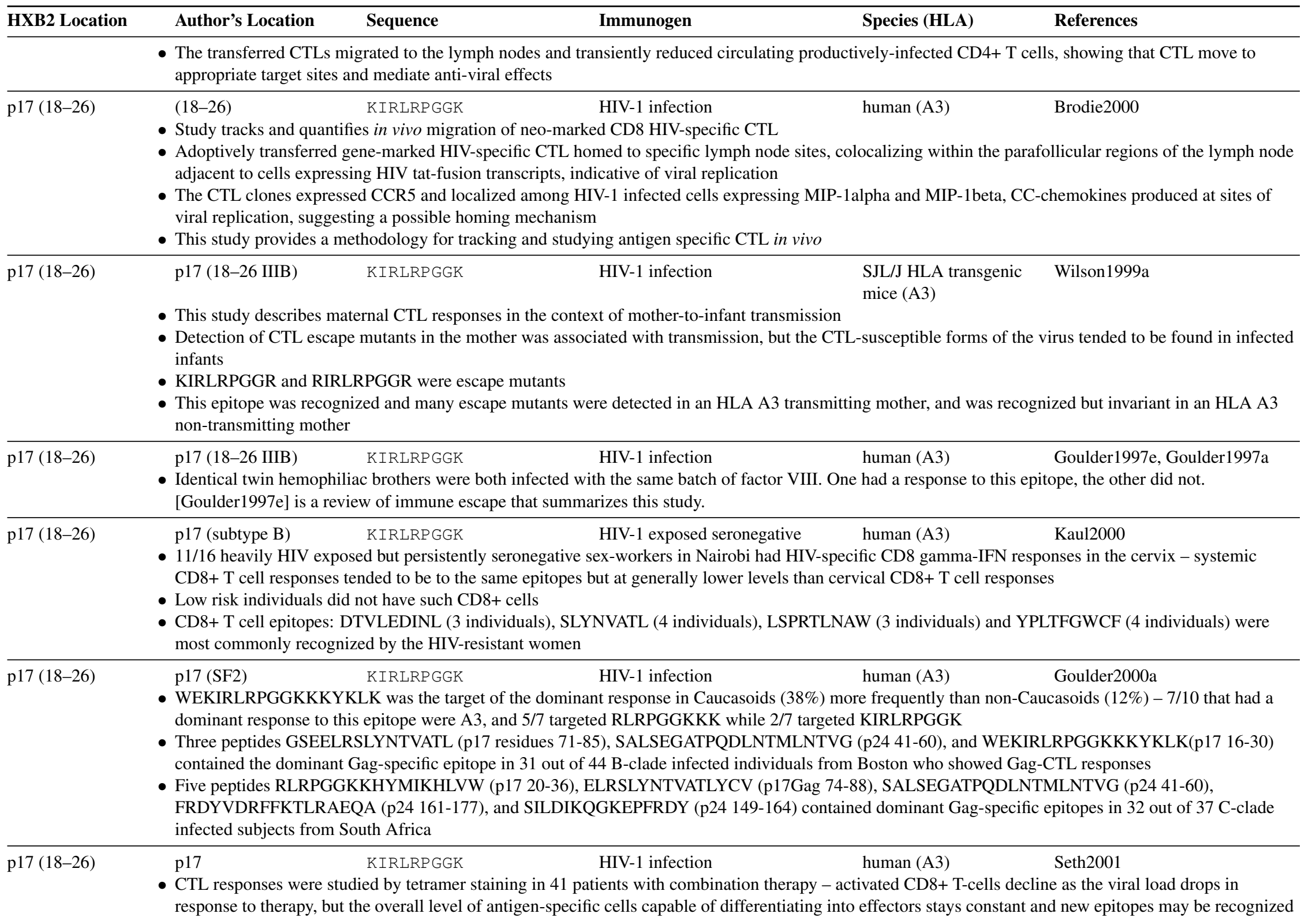




\begin{tabular}{lllll}
\hline HXB2 Location & Author's Location & Sequence & Immunogen & Species (HLA) \\
\hline p17 (18-26) & p17 (18-26 SF2) & KIRLRPGGK & HIV-1 infection & human (A3) \\
A
\end{tabular}

- Therapy provided during acute infection resulted in a narrower CTL response, stronger T help response, and a less diverse viral population than was seen in individuals treated during chronic infection

- The breadth and specificity of the response was determined using ELISPOT by studying 19 individuals with pre-seroconversion therapy (Group 1), 11 individuals with primary infection but post-seroconversion therapy (Group 2), and 10 individuals who responded to HAART given during chronic infection (Group 3), using 259 overlapping peptides spanning p17, p24, RT, gp41, gp120 and Nef

- Previously described and newly defined optimal epitopes were tested for CTL response

- Number of HLA-A3+ individuals that had a CTL response to this epitope broken down by group: 3/7 group 1, 0/4 group 2, and 2/2 group 3

p17 (18-26) p17 (18-26) KIRLRPGGK $\quad$ HIV-1 infection, HIV-1 exposed human (A3) Kaul2001a
seronegative

- KIRLRPGGK is cross-reactive for A, B, and D clades

- ELISPOT was used to study CTL responses to a panel of 54 predefined HIV-1 epitopes in 91 HIV-1-exposed, persistently seronegative (HEPS) and 87 HIV-1-infected female Nairobi sex workers

$\begin{array}{llcc}\text { p17 (18-26) } & \text { p17 (JRCSF) } & \text { KIRLRPGGK } & \text { HIV-1 infection }\end{array}$

- Primary HLA-A3+ CD4+ and HLA-mismatched lymphocytes from uninfected donors were infected with JRCSF after isolation then cocultured with the A3-restricted CTL clone 11504/A7 specific for KIRLRPGGK, and viral inhibition was MHC-restricted

- Primary monocytes and monocyte-derived DC were generated from the same donors, replication of HIV-1 in these cell types was less efficient than in lymphocytes and could also be inhibited by MHC-restricted CTL

- DC-lymphocyte cluster cultures allowed vigorous viral replication and MHC-restricted CTL viral inhibition was blunted or lost depending on the ratio of DC to CD4+ lymphocyte in the culture

p17 (18-26) p17 (18-26) KIRLRPGGK HIV-1 infection $\quad$ Day2001

- The CTL response to optimally defined CTL epitopes restricted by HLA class I A and B alleles in individuals who coexpressed HLA A2, A3, and B7 was studied in eight HIV-1-infected subjects, two with acute infection, five with chronic, and one long-term non-progressor (LTNP)

- 2 to 17 epitopes were recognized in a given individual, A2-restricted CTL response tended to be narrow and never dominated the response, and 25/27 epitopes were targeted by at least one person

- All patients recognized at least $1 \mathrm{~A} 3$ epitope, up to $8 \mathrm{~A} 3$ epitopes, but none was clearly dominant

p17 (18-26) p17 KIRLRPGGK HIV-1 infection $\quad$ Ostrowski2000

- The role of CD4+ T-cell help in expansion of virus-specific memory CTL was studied through co-culture ex vivo

- Optimal expansion of HIV-1-specific memory CTL depended on CD4+ T cell help in 9 of 10 patients - CD40 ligand trimer (CD40LT) could enhance CTL

in the absence of CD4+ T cell help to a variable degree in most of patients

- Those CTL that didn't respond to CD40LT could expand with IL2 present, and IL15 produced by dendritic cells also contributes

- The T-helper epitope used for CD4+ T cell stimulation was the universal tetanus helper epitope TET830-843 (QYIKANSKFIGITE)

\begin{tabular}{lllll}
\hline p17 (18-26) Gag (p17) (18-26) KIRLRPGGK & HIV-1 infection & human (A3)
\end{tabular}

- Epitope name: A3-KK9

- CTL responses in 18 acutely HIV-infected HLA-A3 $(n=7)$ or -B7 $(n=4)$ or both -A3 and B7 (n=7) positive individuals were studied.

- One individual, AC-06 was homozygous at all three class I alleles (A3, B7, Cw7), was treated during acute infection and had supervised treatment interruptions (STI). He had only two detectable CTL responses during acute infection, but after STI this broadened to 27 distinct epitopes including 15 restricted by HLA-A3, 11 by HLA-B7, and 1 by HLA-Cw7. 


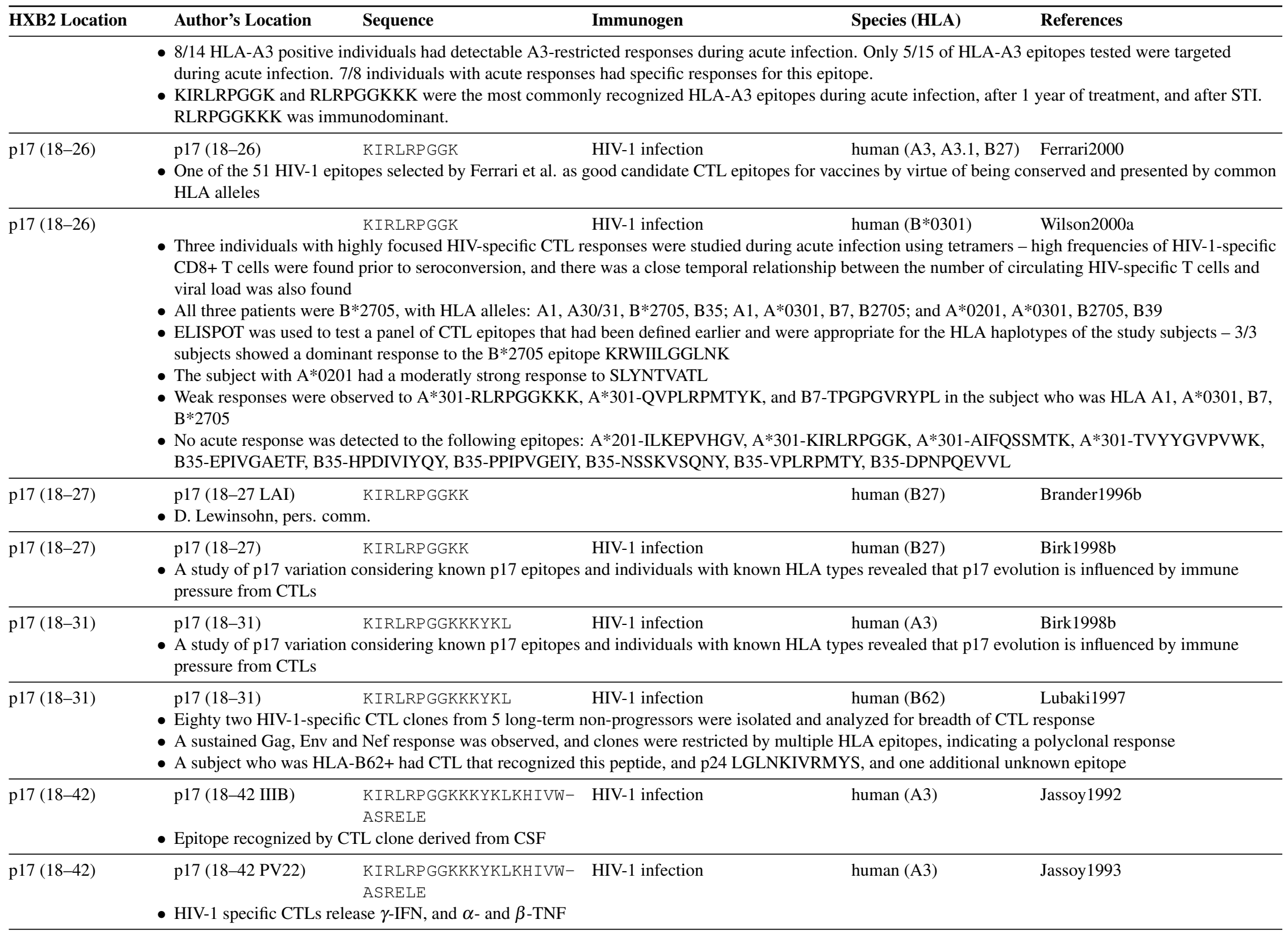




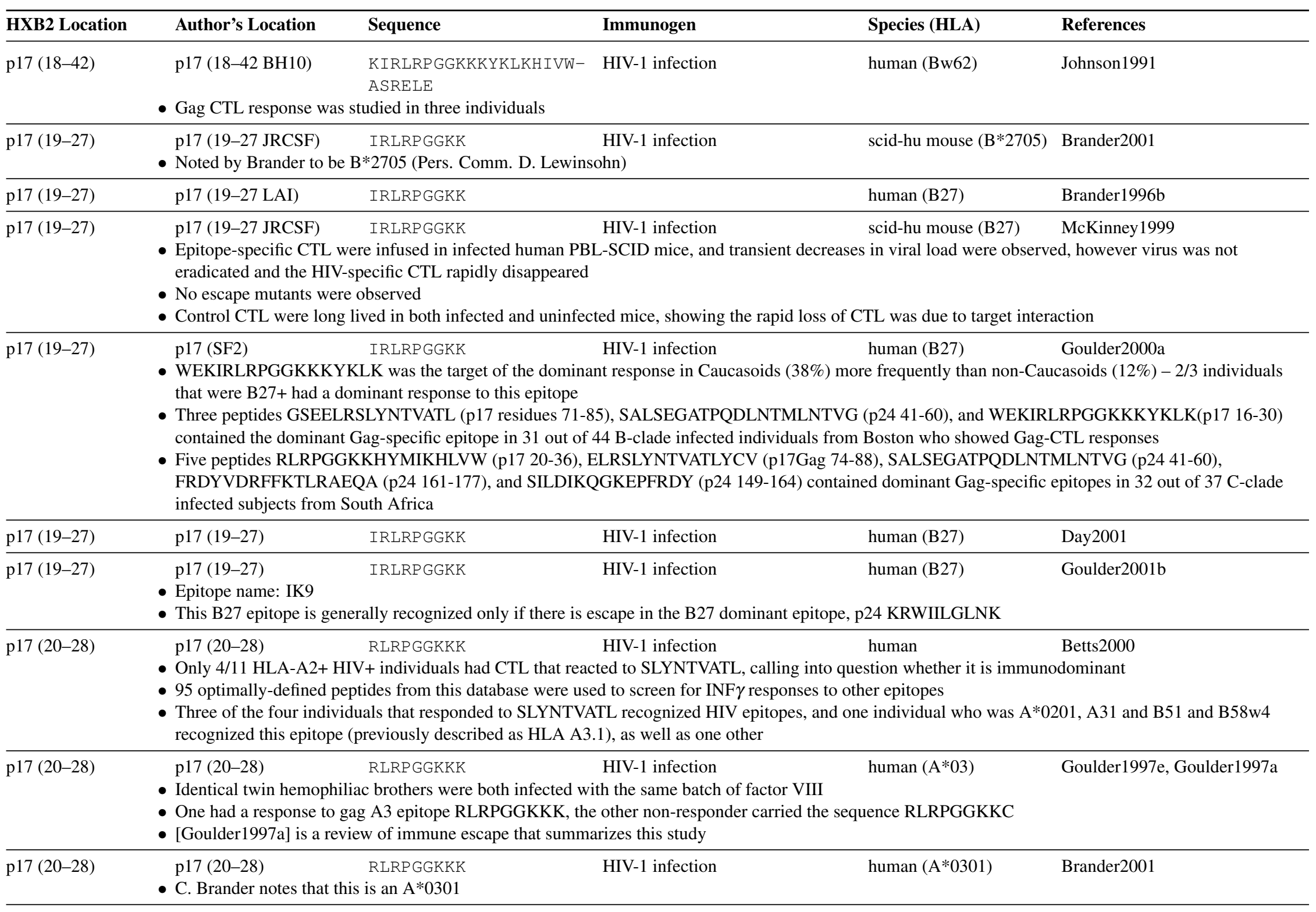




\begin{tabular}{lllcc}
\hline HXB2 Location & Author's Location & Sequence & Immunogen & Species (HLA) \\
\hline p17 (20-28) & p17 & RLRPGGKKK & HIV-1 infection & human (A*0301) \\
& - Three individuals with highly focused HIV-specific CTL responses were studied during acute infection using tetramers - high frequencies of HIV-1-specific
\end{tabular}

- Three individuals with highly focused HIV-specific CTL responses were studied during acute infection using tetramers - high frequencies of HIV-1-specific CD8+ T cells were found prior to seroconversion, and there was a close temporal relationship between the number of circulating HIV-specific T cells and viral load was also found

- All three patients were B*2705, with HLA alleles: A1, A30/31, B*2705, B35; A1, A*0301, B7, B2705; and A*0201, A*0301, B2705, B39

- ELISPOT was used to test a panel of CTL epitopes that had been defined earlier and were appropriate for the HLA haplotypes of the study subjects - 3/3 subjects showed a dominant response to the B*2705 epitope KRWIILGGLNK

- The subject with $\mathrm{A} * 0201$ had a moderatly strong response to SLYNTVATL

- Weak responses were observed to A*301-RLRPGGKKK, A*301-QVPLRPMTYK, and B7-TPGPGVRYPL in the subject who was HLA A1, A*0301, B7, B*2705

- No acute response was detected to the following epitopes: A*201-ILKEPVHGV, A*301-KIRLRPGGK, A*301-AIFQSSMTK, A*301-TVYYGVPVWK, B35-EPIVGAETF, B35-HPDIVIYQY, B35-PPIPVGEIY, B35-NSSKVSQNY, B35-VPLRPMTY, B35-DPNPQEVVL

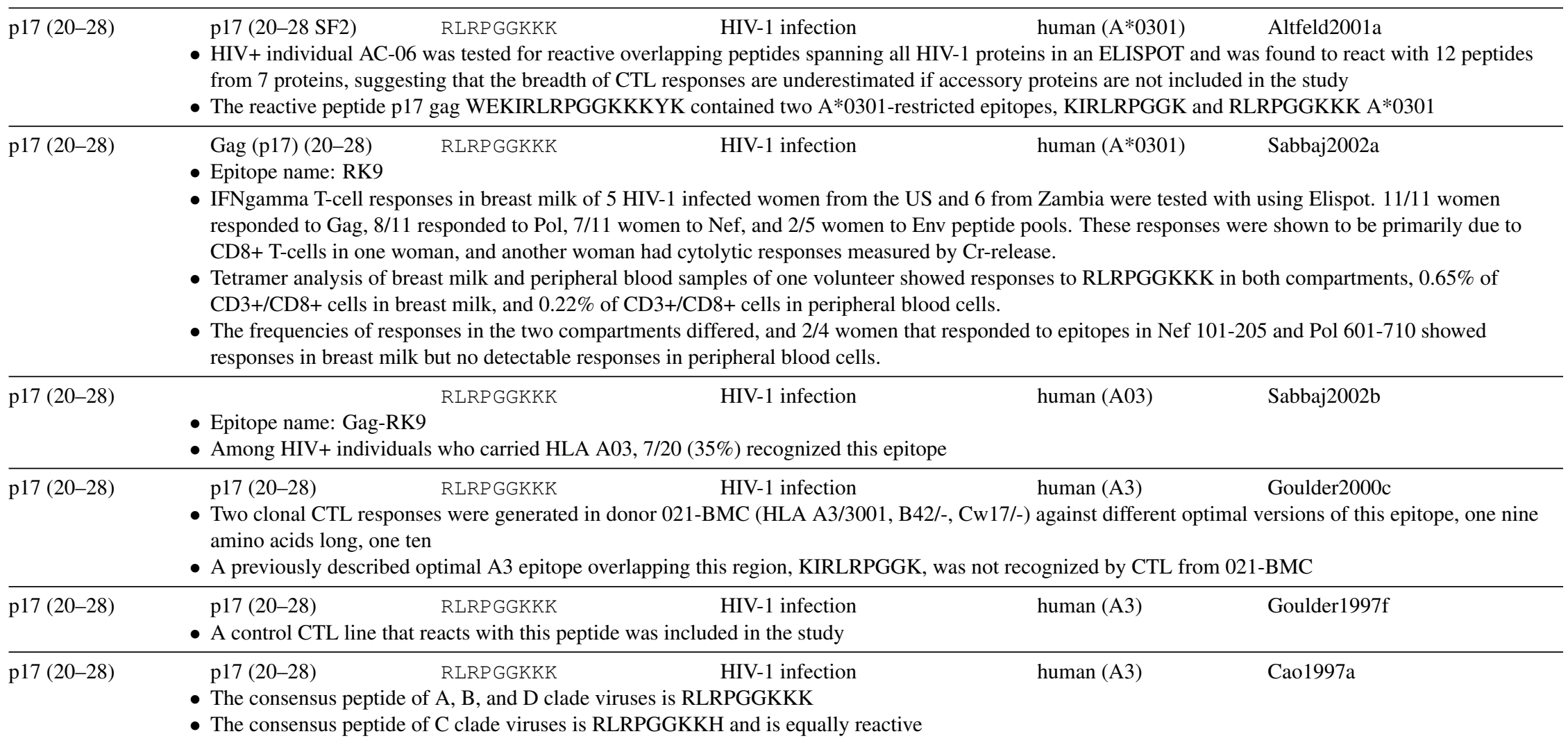




\begin{tabular}{|c|c|c|c|c|c|}
\hline HXB2 Location & Author's Location & Sequence & Immunogen & Species (HLA) & References \\
\hline p17 (20-28) & p17 (SF2) & RLRP GGKKK & HIV-1 infection & human (A3) & Goulder2000a \\
\hline
\end{tabular}

- WEKIRLRPGGKKKYKLK was the target of the dominant response in Caucasoids (38\%) more frequently than non-Caucasoids (12\%) - 7/10 that had a dominant response to this epitope were A3, and 5/7 targeted RLRPGGKKK while 2/7 targeted KIRLRPGGK

- Three peptides GSEELRSLYNTVATL (p17 residues 71-85), SALSEGATPQDLNTMLNTVG (p24 41-60), and WEKIRLRPGGKKKYKLK(p17 16-30) contained the dominant Gag-specific epitope in 31 out of 44 B-clade infected individuals from Boston who showed Gag-CTL responses

- Five peptides RLRPGGKKHYMIKHLVW (p17 20-36), ELRSLYNTVATLYCV (p17Gag 74-88), SALSEGATPQDLNTMLNTVG (p24 41-60), FRDYVDRFFKTLRAEQA (p24 161-177), and SILDIKQGKEPFRDY (p24 149-164) contained dominant Gag-specific epitopes in 32 out of 37 C-clade infected subjects from South Africa

p17 (20-28) p17 (20-28 SF2) RLRPGGKKK HIV-1 infection human (A3) Altfeld2001b

- Therapy provided during acute infection resulted in a narrower CTL response, stronger T help response, and a less diverse viral population than was seen in individuals treated during chronic infection

- The breadth and specificity of the response was determined using ELISPOT by studying 19 individuals with pre-seroconversion therapy (Group 1), 11 individuals with primary infection but post-seroconversion therapy (Group 2), and 10 individuals who responded to HAART given during chronic infection (Group 3), using 259 overlapping peptides spanning p17, p24, RT, gp41, gp120 and Nef

- Previously described and newly defined optimal epitopes were tested for CTL response

- Number of HLA-A3+ individuals that had a CTL response to this epitope broken down by group: 5/7 group 1, 2/4 group 2, and 2/2 group 3

p17 (20-28) p17 (20-28) RLRPGGKKK HIV-1 infection $\quad$ Day2001

- The CTL response to optimally defined CTL epitopes restricted by HLA class I A and B alleles in individuals who coexpressed HLA A2, A3, and B7 was studied in eight HIV-1-infected subjects, two with acute infection, five with chronic, and one long-term non-progressor (LTNP)

- 2 to 17 epitopes were recognized in a given individual, A2-restricted CTL response tended to be narrow and never dominated the response, and 25/27 epitopes were targeted by at least one person

- All patients recognized at least $1 \mathrm{~A} 3$ epitope, up to 8 A3 epitopes, but none was clearly dominant

p17 (20-28) p17 (20-28) RLRPGGKKK HIV-1 infection $\quad$ Goulder2001b

- Epitope name: RK9

- Responses to this dominant A3-restricted Gag epitope are present during the time of decreasing viral load in acute infection

- Mutations in this epitope were observed in autologous clones of subjects who were A3-positive with a higher frequency than those who were A3-negative $(\mathrm{P}=0.0002)$

- These mutations are being sexually transmitted in adult infections

p17 (20-28)

Gag (p17) (20-28) RLRPGGKKK HIV-1 infection
human (A3)

Yu2002a

- Epitope name: A3-RK9

- CTL responses in 18 acutely HIV-infected HLA-A3 (n=7) or -B7 (n=4) or both -A3 and B7 (n=7) positive individuals were studied.

- One individual, AC-06 was homozygous at all three class I alleles (A3, B7, Cw7), was treated during acute infection and had supervised treatment interruptions (STI). He had only two detectable CTL responses during acute infection, but after STI this broadened to 27 distinct epitopes including 15 restricted by HLA-A3, 11 by HLA-B7, and 1 by HLA-Cw7.

- 8/14 HLA-A3 positive individuals had detectable A3-restricted responses during acute infection. Only 5/15 of HLA-A3 epitopes tested were targeted during acute infection. 7/8 individuals with acute responses had specific responses for this epitope.

- KIRLRPGGK and RLRPGGKKK were the most commonly recognized HLA-A3 epitopes during acute infection, after 1 year of treatment, and after STI. RLRPGGKKK was immunodominant during acute infection and throughout the study period in the 5/6 individuals who targeted it. 


\begin{tabular}{llllll}
\hline HXB2 Location & Author's Location & Sequence & Immunogen & Species (HLA) & References \\
\hline p17 (20-28) & Gag (LAI) & RLRPGGKKK & HIV-1 infection & human (A3) & Lewinsohn2002 \\
& - CTL kill targets through releasing perforin, that forms pore in the plasma membrane, and granzymes, that induce apoptosis. &
\end{tabular}

- Vpr is capable of arresting infected cells in the G2 phase, and it was hypothesized that Vpr may inhibit CTL-mediated apoptosis because it interacts with the granzyme B molecular complex.

- Vpr expression in the target cell did not inhibit epitope specific lysis - neither perforin or granzyme mediated events were inhibited, as measured by a Chromium release assay and a TUNEL assay.

- In contrast, deletion of Nef, which is thought to protect primary HIV infected cells by down-regulating cell-surface expression of MHC class I complexes, increased the susceptibility of HIV-1 infected cells to CTL mediated killing 2-fold using the TUNEL assay

\begin{tabular}{llccc}
\hline p17 (20-28) & p17 & RLRPGGKKK & HIV-1 infection & human (A3)
\end{tabular}
responded to Gag, 8/11 responded to Pol, 7/11 women to Nef, and 2/5 women to Env peptide pools. These responses were shown to be primarily due to CD8+ T-cells in one woman, and another woman had cytolytic responses measured by Cr-release.

- T-cells in breast milk from a volunteer who was HLA A3, A11, B35, B51 induced IFNgamma after stimulation with a peptide that carries known A3 epitope RLRPGGKKK.

- The frequencies of responses in the two compartments differed, and 2/4 women that responded to epitopes in Nef 101-205 and Pol 601-710 showed responses in breast milk but no detectable responses in peripheral blood cells.

\begin{tabular}{llcccc}
\hline p17 (20-29) & p17 (20-29 LAI) & RLRPGGKKKY & HIV-1 infection & human (A*0301) & Brander2001 \\
& - C. Brander notes this is an A*0301 epitope & & & \\
\hline p17 (20-29) & p17 (20-29) & RLRPGGKKKY & HIV-1 infection & human (A3) & Goulder2000c \\
\hline
\end{tabular}

- Two clonal CTL responses were generated in donor 021-BMC (HLA A3/3001, B42/-, Cw17/-) against different optimal versions of this epitope, one nine amino acids long, one ten

- A previously described optimal A3 epitope overlapping this region, KIRLRPGGK, was not recognized by CTL from 021-BMC

\begin{tabular}{|c|c|c|c|c|}
\hline \multirow[t]{2}{*}{ p17 (20-29) } & RLRPGGKKKY & HIV-1 infection & human (A3.1) & Brander1995b \\
\hline & \multicolumn{4}{|c|}{ - Unpublished, C. Jassoy and Beatrice Culman, pers. comm. } \\
\hline p17 (20-29) & $\begin{array}{l}\text { p17 (20-29 LAI) RLRP GGKKKY } \\
\text { - Pers. comm., B. Wilkens and D. Ruhl }\end{array}$ & HIV-1 infection & human (A3.1) & Wilkens1999 \\
\hline p17 (20-29) & RLRPGGKKKY & HIV-1 infection & human (A30, A3.1) & Betts2000 \\
\hline
\end{tabular}

- Only 4/11 HLA-A2+ HIV+ individuals had CTL that reacted to SLYNTVATL, calling into question whether it is immunodominant

- 95 optimally-defined peptides from this database were used to screen for INF $\gamma$ responses to other epitopes

- 1/11 of the A2+ individuals was A30, and one was A3, and both responded to RLRPGGKKKY

- The A2+ A3 individual also reacted with two other A3.1 epitopes

p17 (20-29)

p17 (20-29 IIIB) RLRPGGKKKY HIV-1 infection

human (B42)

Wilson1996

- Epitope defined in the context of the Pediatric AIDS Foundation ARIEL Project, a mother-infant HIV transmission study

- RLRPGGKKRY, a naturally occurring variant, was found in non-transmitting mother and is recognized

- Binds HLA-A3 and Bw62 as well 


\begin{tabular}{lllll}
\hline HXB2 Location & Author's Location & Sequence & Immunogen & Species (HLA) \\
\hline p17 (20-29) & p17 (20-29) & RLRPGGKKKY & HIV-1 infection & human (B42, Bw62)
\end{tabular}

- One of the 51 HIV-1 epitopes selected by Ferrari et al. as good candidate CTL epitopes for vaccines by virtue of being conserved and presented by common HLA alleles

\begin{tabular}{lllll}
\hline p17 (20-29) p17 (20-29) & RLRPGGKKKY & human (B62) infection
\end{tabular}

- Study tracks and quantifies in vivo migration of neo-marked CD8 HIV-specific CTL

- Adoptively transferred gene-marked HIV-specific CTL homed to specific lymph node sites, colocalizing within the parafollicular regions of the lymph node adjacent to cells expressing HIV tat-fusion transcripts, indicative of viral replication

- The CTL clones expressed CCR5 and localized among HIV-1 infected cells expressing MIP-1alpha and MIP-1beta, CC-chemokines produced at sites of viral replication, suggesting a possible homing mechanism

- This study provides a methodology for tracking and studying antigen specific CTL in vivo

\begin{tabular}{|c|c|}
\hline p17 (20-29) & $\begin{array}{l}\text { p17 (20-29 LAI) RLRPGGKKKY } \\
\text { - Review of HIV CTL epitopes } \\
\text { - Also P. Johnson, pers. comm. }\end{array}$ \\
\hline p17 (20-30) & 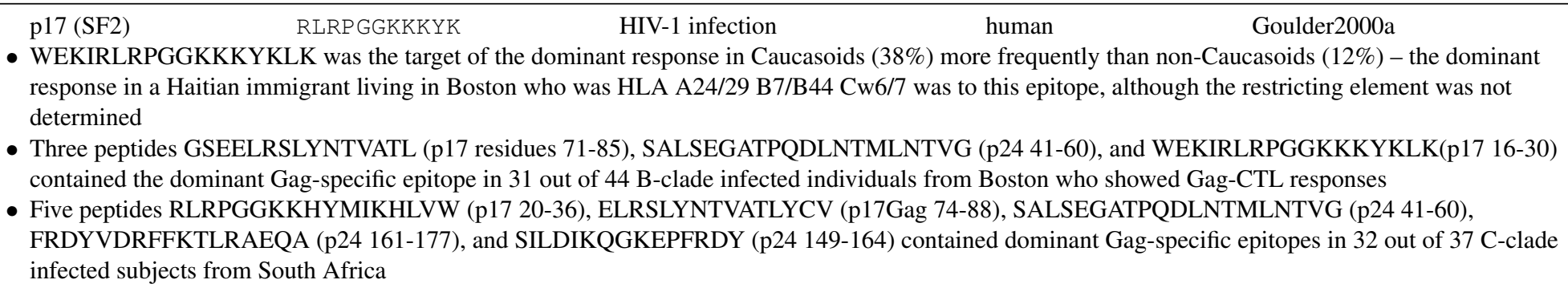 \\
\hline p17 (20-35) & $\begin{array}{l}\text { p17 (90-105 SF2) CLRPGGKKKYKLKHIV HIV-1 infection } \\
\text { - Of } 25 \text { patients, most had CTL specific for more than 1 HIV-1 protein } \\
\text { - Twelve subjects had CTL that could recognize vaccinia-expressed LAI gag } \\
\text { - One of these } 12 \text { had CTL response to this peptide } \\
\text { - The responding subject was HLA A-2, A-24, B-13, B-35 }\end{array}$ \\
\hline p17 (21-35) & 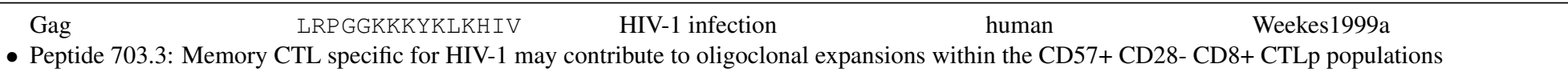 \\
\hline p17 (21-35) & $\begin{array}{l}\text { p17 (91-105 SF2) LRPGGKKKYKLKHIV HIV-1 infection } \\
\text { - Of } 25 \text { patients, most had CTL specific for more than 1 HIV-1 protein } \\
\text { - Twelve subjects had CTL that could recognize vaccinia-expressed LAI gag } \\
\text { - One of these } 12 \text { had CTL response to this peptide } \\
\text { - The responding subject was HLA-A1, A2, B50, B57 }\end{array}$ \\
\hline p17 (21-35) & $\begin{array}{l}\text { Gag LRPGGKKKYKLKHIV HIV-1 infection } \\
\text { - Peptide 703.3: Almost all CD8+ T cells are CD28+ at birth, and the proportion of CD28-CD8+ cells increases with age - this study examines the } \\
\text { contribution of CD8+CD28- cells to CTL memory pools for CTL clones specific for two persistent human viruses, CMV and HIV - clones were found to } \\
\text { be similarly distributed in the CD28 depleted cell population }\end{array}$ \\
\hline
\end{tabular}




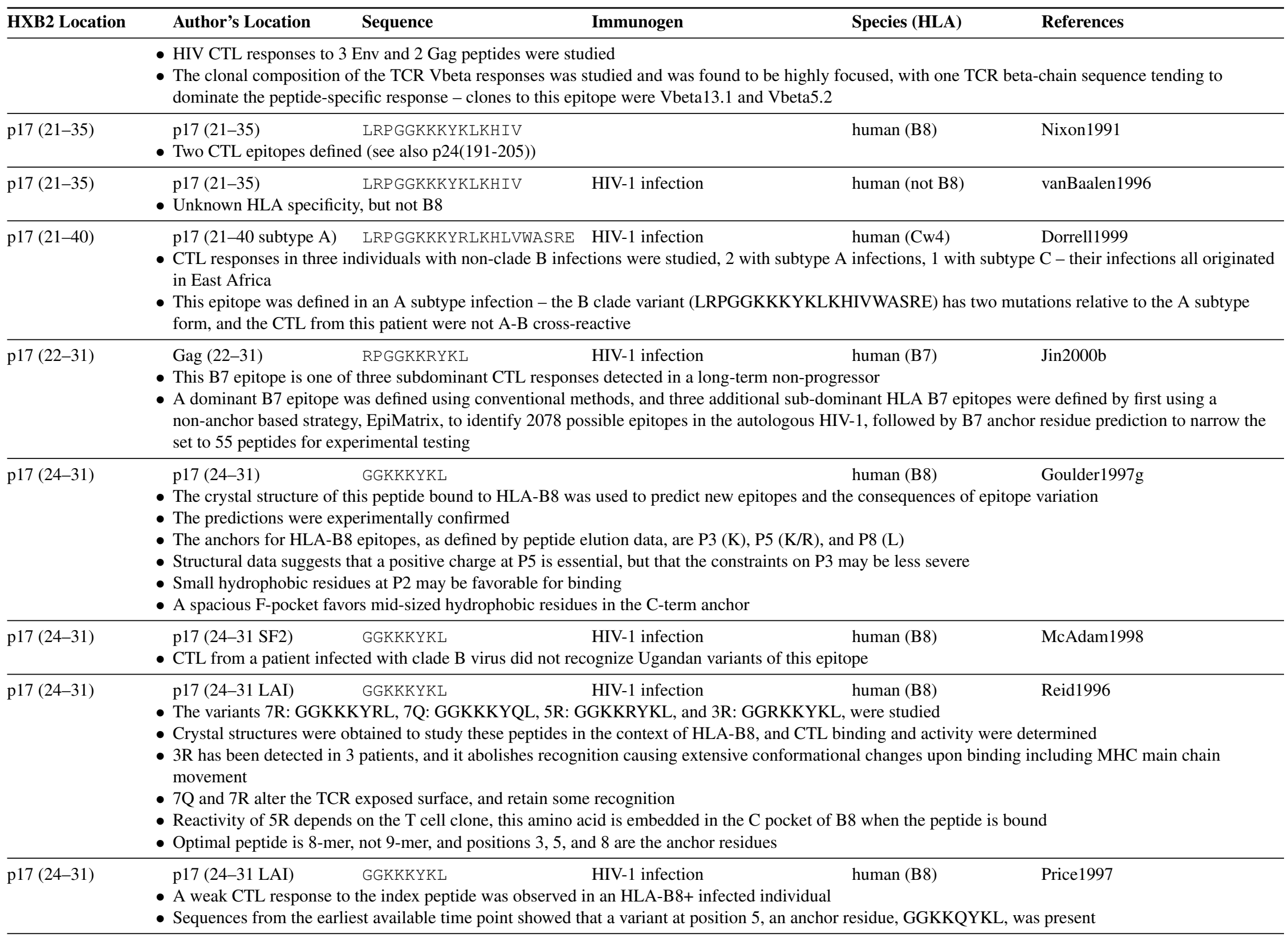




\begin{tabular}{lllll}
\hline HXB2 Location & Author's Location & Sequence & Immunogen & Species (HLA) \\
\hline p17 (24-31) & p17 (24-31 SF2) & GGKKKYKL & HIV-1 infection & human (B8) \\
( Therences & Altfeld2001b
\end{tabular}

- Therapy provided during acute infection resulted in a narrower CTL response, stronger T help response, and a less diverse viral population than was seen in individuals treated during chronic infection

- The breadth and specificity of the response was determined using ELISPOT by studying 19 individuals with pre-seroconversion therapy (Group 1), 11 individuals with primary infection but post-seroconversion therapy (Group 2), and 10 individuals who responded to HAART given during chronic infection (Group 3), using 259 overlapping peptides spanning p17, p24, RT, gp41, gp120 and Nef

- Previously described and newly defined optimal epitopes were tested for CTL response

- Number of HLA-B8+ individuals that had a CTL response to this epitope broken down by group: 0/3 group 1, 1/3 group 2, and 2/2 group 3

p17 (24-31) p17 (24-31) GGKKKYRL HIV-1 infection, HIV-1 exposed human (B8) Kaul2001a
seronegative

- ELISPOT was used to study CTL responses to a panel of 54 predefined HIV-1 epitopes in 91 HIV-1-exposed, persistently seronegative (HEPS) and 87 HIV-1-infected female Nairobi sex workers

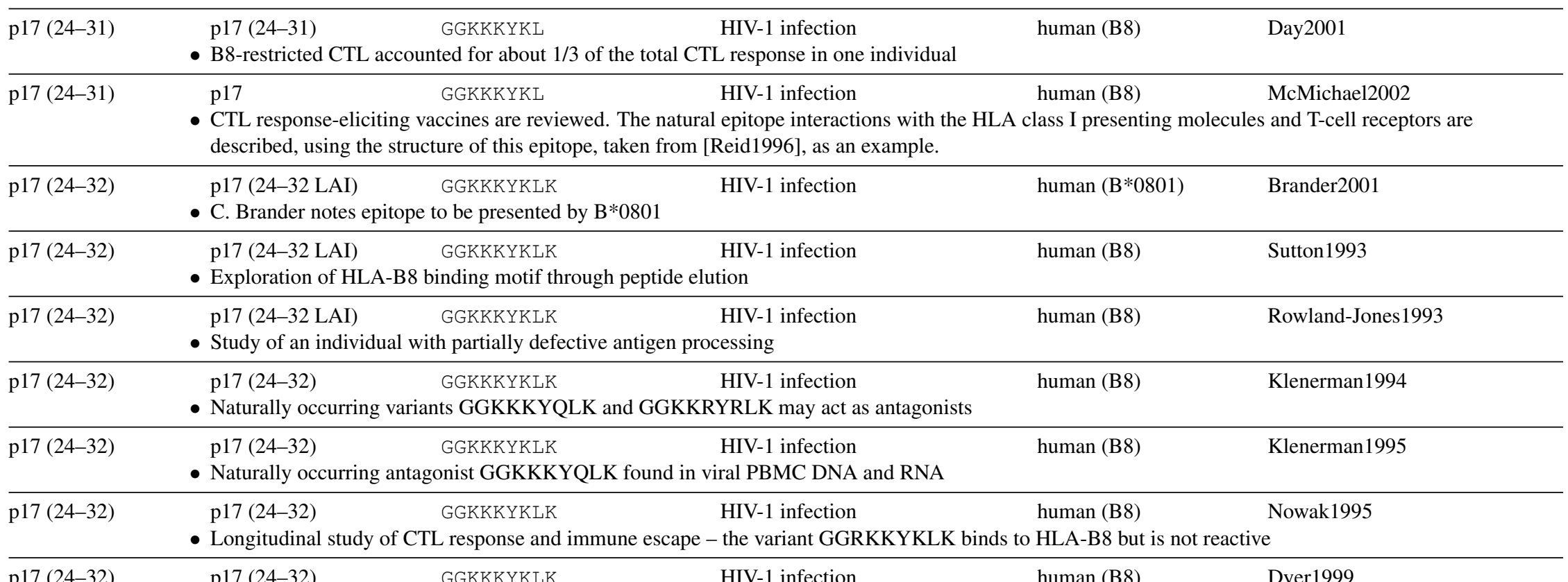

- CTL specific responses were measured over a 1.3 to 1.5 year period in members of the Sydney Blood Bank Cohort (SBBC) who had been infected with a natural attenuated strain of HIV-1 which was Nef-defective

- Some of these patients had prolonged high levels of CTL effector and memory cells despite low viral load

p17 (24-32) p17 GGKKKYKLK human (B8) Rowland-Jones1999

- CTL responses in seronegative highly HIV-exposed African female sex workers in Gambia and Nairobi were studied - these women had no delta 32 deletion in CCR5 


\begin{tabular}{|c|c|c|c|c|c|}
\hline HXB2 Location & Author's Location & Sequence & Immunogen & Species (HLA) & References \\
\hline & \multicolumn{5}{|c|}{$\begin{array}{l}\text { - In Gambia there is exposure to both HIV-1 and HIV-2, CTL responses to B35 epitopes in exposed, uninfected women are cross-reactive, and the B35 allele } \\
\text { seems to be protective } \\
\text { - HIV-2 sequence: GGKKKYKMK - no cross-reactivity [Phillips1991] }\end{array}$} \\
\hline $17(24$ & \multicolumn{5}{|c|}{$\begin{array}{l}\text { p17 (24-32) G HIV-1 infection } \\
\text { - Epitope name: GGK } \\
\text { - Patients who started therapy at acute HIV-1 infection (three with sustained therapy, two with limited therapy upon early infection) had strong HIV specific } \\
\text { CD4 proliferative responses and were able to maintain a CTL response even with undetectable viral load - three patients that had delayed initiation of } \\
\text { HAART had no HIV specific CD4 proliferative responses and lost their CTL responses when HAART was eventually given and their viral loads became } \\
\text { undetectable } \\
\text { - This epitope was recognized by } 1 / 7 \text { study subjects that were HLA-B8+ } \\
\text { - Patient SC12(HLA A1, B8/39, Cw0701/0702, DR2/3, DR51/52, DQ2/6) had sustained therapy started during acute infection and maintained an } \\
\text { immunodominant response to FLKEKGGL throughout and minor responses to GEIYKRWII, DCKTILKAL, GGKKKYKLK - GEIYKRWII and } \\
\text { GGKKKYKLK responses were stimulated by a brief period off therapy }\end{array}$} \\
\hline p17 (24-32) & \multicolumn{5}{|c|}{$\begin{array}{l}\text { - CTL responses were studied by tetramer staining in } 41 \text { patients with combination therapy - activated CD8+ T-cells decline as the viral load drops in } \\
\text { response to therapy, but the overall level of antigen-specific cells capable of differentiating into effectors stays constant and new epitopes may be recognizec }\end{array}$} \\
\hline p17 ( & $\begin{array}{l}\text { p17 } \\
\text { - Epitope name: GGK } \\
\text { - Using previously defi } \\
\text { period including ther: } \\
\text { - STIs induced increase } \\
\text { clearance rates. }\end{array}$ & $\begin{array}{l}\text { GGKKKKYKLK } \\
\text { epitopes Oxenius20 } \\
\text { vith standard treatm } \\
\text { cognition of CTL ep }\end{array}$ & HIV-1 infection & $\begin{array}{l}\text { human (B8) } \\
\text { Ly, } 13 \text { chronically } \\
\text { responses with } v\end{array}$ & $\begin{array}{l}\text { Oxenius } 2002 \mathrm{~b} \\
\text { fected patients were studied over a } \\
\text { ound rates, plateau viral loads, or }\end{array}$ \\
\hline p17 ( & \multicolumn{5}{|c|}{$\begin{array}{l}\text { - Longitudinal study of CTL escape mutants in people with the appropriate HLA types - little variation was observed in the immunodominant B27 epitope, } \\
\text { relative to B8 epitopes, which varied over time } \\
\text { - Goulder1997a is a review of immune escape that points out that there may be a protective effect associated with B27, and that HLA-B8 individuals tend to } \\
\text { progress more rapidly than HLA B27 patients }\end{array}$} \\
\hline p17 (24-35) & \multicolumn{5}{|c|}{$\begin{array}{l}\text { - A study of p17 variation considering known p17 epitopes and individuals with known HLA types revealed that p17 evolution is influenced by immune } \\
\text { pressure from CTLs }\end{array}$} \\
\hline p17 (28-36) & \multicolumn{5}{|c|}{$\begin{array}{l}\text { - This study examines CTL responses in HIV exposed, persistently seronegative individuals, HEPS, who eventually seroconverted - } 11 / 114 \text { HEPS Nairobi } \\
\text { sex workers eventually seroconverted, and for six of these HIV CTL reactive epitopes had been defined while seronegative } \\
\text { - The epidemiological factor associated with seroconversion was stopping sex work and HIV-specific CTL activity declines when HEPS sex workers stop } \\
\text { working for a period or retire } \\
\text { - This epitope was recognized in } 1 / 22 \text { HEPS sex worker controls (ML1573) }\end{array}$} \\
\hline $1 / 2$ & $\begin{array}{l}\text { p17 (28-36 LAI) } \\
\text { Ikeda-Moore }(1998)\end{array}$ & $\begin{array}{l}\text { IVW } \\
\text { ohn, p }\end{array}$ & & human $(A * 2402)$ & Brander2001 \\
\hline
\end{tabular}




\begin{tabular}{|c|c|c|c|c|c|}
\hline HXB2 Location & Author's Location & Sequence & Immunogen & Species (HLA) & References \\
\hline \multicolumn{6}{|c|}{ - C. Brander notes that this is an $A * 2402$ epitope } \\
\hline p17 (28-36) & p17 (28-36 SF2) & KYKLKHIVW & HIV-1 infection & human $(A * 2402)$ & Ikeda-Moore1998 \\
\hline
\end{tabular}

- Strong CTL activity to this peptide was detected in 2/3 HIV-infected individuals who were HLA A24+

- HLA A24 is very common in Japanese (70\% carry it) and is common globally

- This epitope was detected by looking for peptides with appropriate A24 anchor residues (Y at position 2, carb-term ILF or W) - 16/17 such peptides bound to A24 - KYKLKHIVW was found to be a naturally processed epitope that elicits a strong CTL response.

\begin{tabular}{llllc}
\hline p17 (28-36) & p17 (28-36 LAI) & KYKLKHIVW & human (A23) \\
& P. Goulder, pers. comm. & & Goulder1999b \\
\hline p17 (28-36) & p17 (28-36 LAI) & KYKLKHIVW & human (A24) \\
& D. Lewinsohn, pers. comm. & Brander1996b \\
\hline p17 (28-36) & p17 (28-36 SF2) & KYKLKHIVW & HIV-1 infection & human (A24)
\end{tabular}

- Therapy provided during acute infection resulted in a narrower CTL response, stronger T help response, and a less diverse viral population than was seen in individuals treated during chronic infection

- The breadth and specificity of the response was determined using ELISPOT by studying 19 individuals with pre-seroconversion therapy (Group 1), 11 individuals with primary infection but post-seroconversion therapy (Group 2), and 10 individuals who responded to HAART given during chronic infection (Group 3), using 259 overlapping peptides spanning p17, p24, RT, gp41, gp120 and Nef

- Previously described and newly defined optimal epitopes were tested for CTL response

- Number of HLA-A24+ individuals that had a CTL response to this epitope broken down by group: 0/0 group 1, 2/3 group 2, and 0/0 group 3

\begin{tabular}{lllll}
\hline p17 (28-36) & p17 (28-36 93TH253 KYKLKHIVW & HIV-1 infection & human (A24) Bond2001
\end{tabular}
subtype CRF01)

- More than half of a cohort of HIV+ female sex workers (FSW) from Northern Thailand were HLA-A11 positive, and this study concentrated on A11 epitopes in this group, although E clade versions of previously defined B-clade A2 and A24 epitopes were also tested.

- The only HLA-A24 FSWs tested did not recognized the E clade version of this epitope KYKMKHLVW, which differs from the previously defined B clade version by two amino acids, KYKLKHIVW

\begin{tabular}{llll}
\hline p17 (28-36) & p17 (728-736 subtype & KYRLKHLVW & $\begin{array}{l}\text { HIV-1 infection, HIV-1 exposed } \\
\text { seronegative }\end{array}$
\end{tabular}

- ELISPOT was used to study CTL responses to a panel of 54 predefined HIV-1 epitopes in 91 HIV-1-exposed, persistently seronegative (HEPS) and 87 HIV-1-infected female Nairobi sex workers

- Responses in HEPS women tended to be lower, and focused on different epitopes with HLA presenting molecules that have previously been associated with reduced risk of infection, and there was a shift in the response in the HEPS women upon late seroconversion to epitopes recognized by the HIV-1 infected women

- 43/91 HEPS women had CD8+ responses and detection of HIV-1-specific CTL in HEPS women increased with the duration of viral exposure

- Among HLA-Cw4 women, 2/2 HEPS and 7/11 HIV-1 infected women recognized this epitope

- The dominant response to this HLA allele was to this epitope in both of the $2 / 2$ HEPS cases and in 3 of the 7/11 HIV- 1 infected women

\begin{tabular}{lllll}
\hline p17 (28-36) & k17 (28-36) & KYRLKHLVW & HIV-1 infection & Appay2000
\end{tabular}

- This epitope is newly defined in this study

- Combined tetramer and intracellular cytokine staining was used to study the function of circulating CD8+ T cells specific for HIV and CMV 


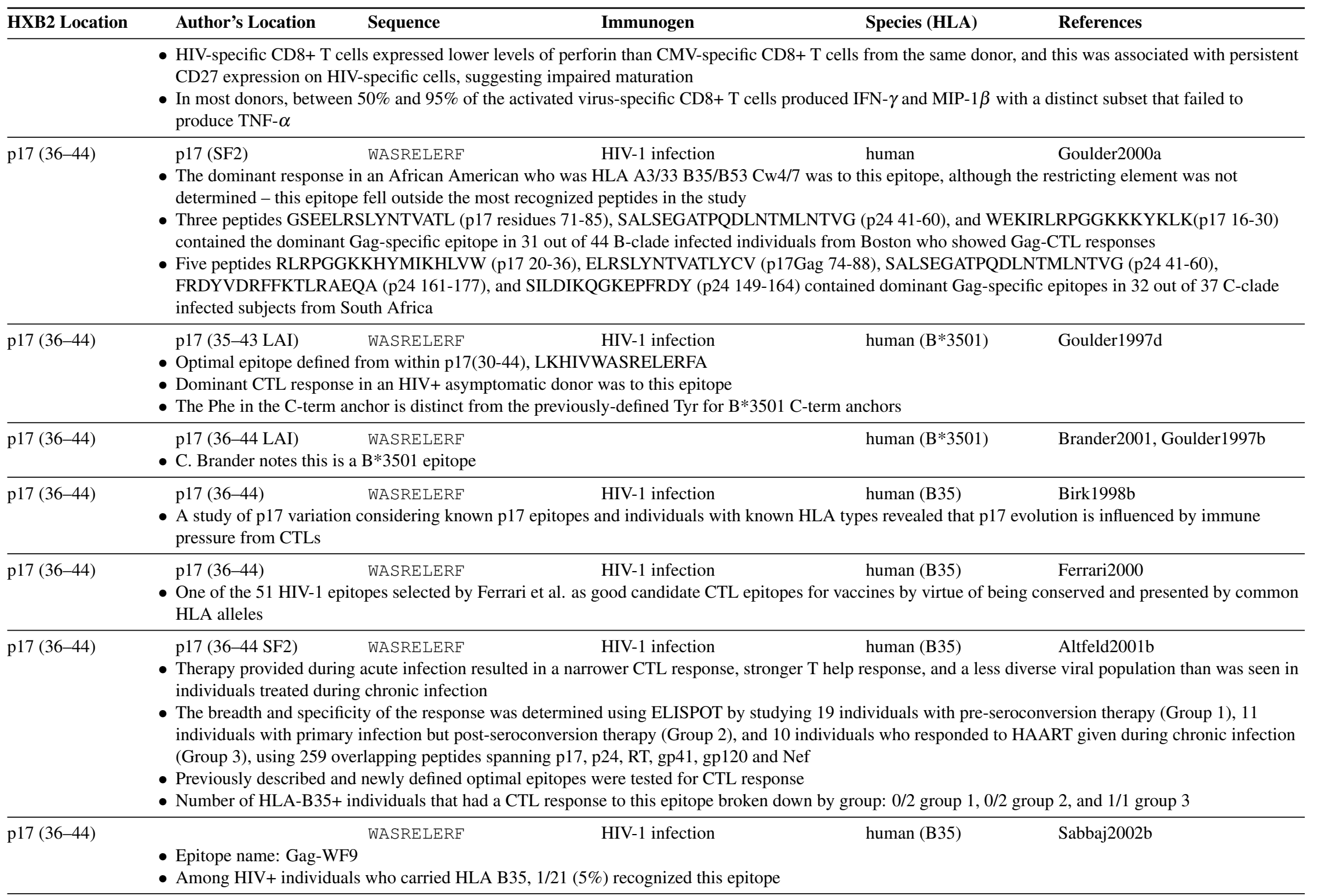




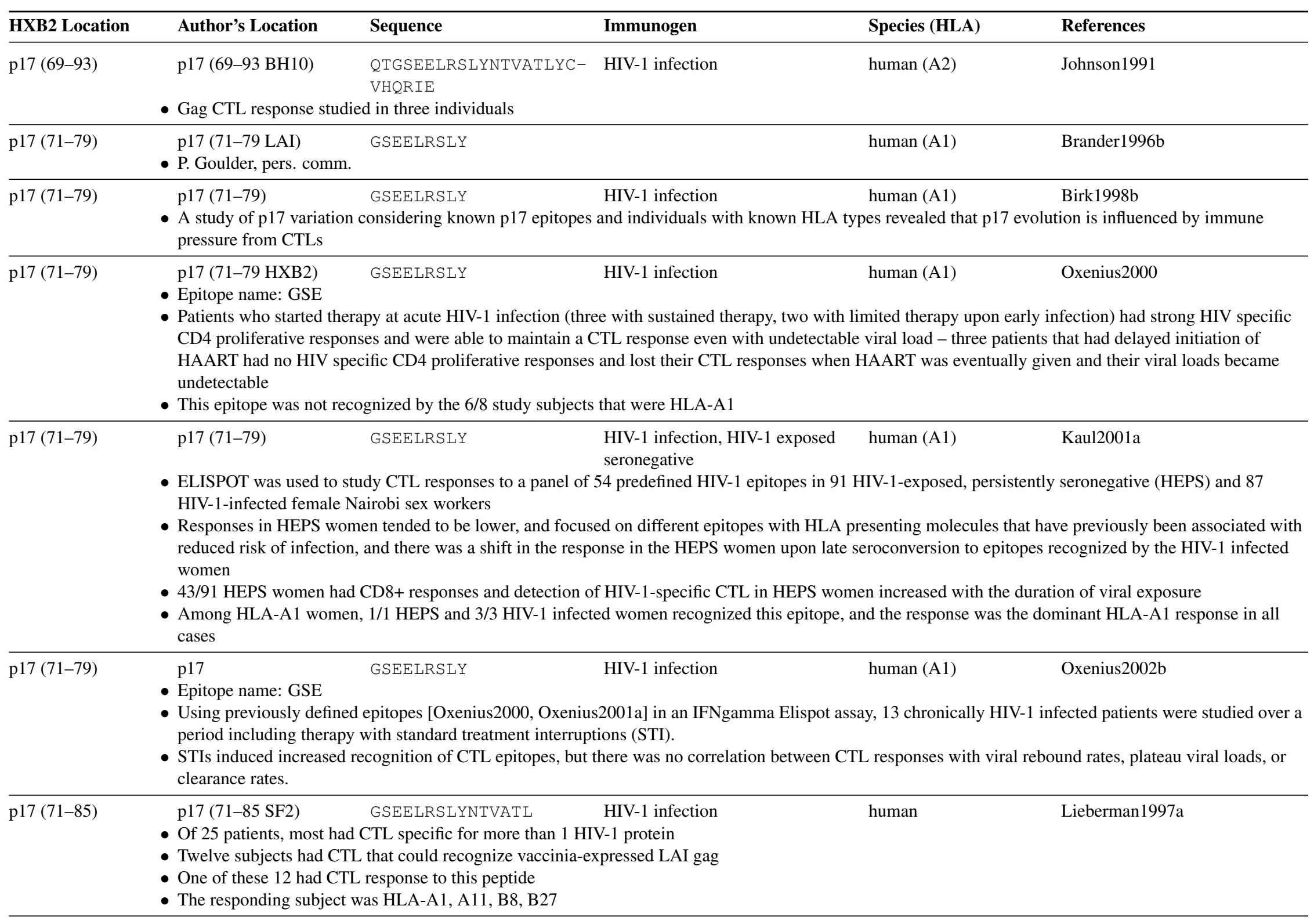




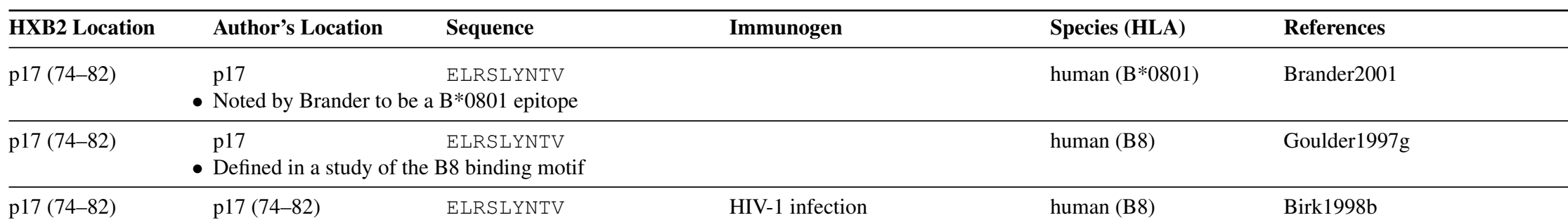

- A study of p17 variation considering known p17 epitopes and individuals with known HLA types revealed that p17 evolution is influenced by immune pressure from CTLs

p17 (74-82) p17 (74-82) ELRSLYNTV HIV-1 infection human (B8)

- One of the 51 HIV-1 epitopes selected by Ferrari et al. as good candidate CTL epitopes for vaccines by virtue of being conserved and presented by common HLA alleles

\begin{tabular}{|c|c|c|c|c|}
\hline p17 (74-82) & ELRSLYNTV & HIV-1 infection & human (B8) & Day2001 \\
\hline & - B8-restricted CTL accounted for about $1 / 3$ & L response in one & & \\
\hline p17 (76-86) & $\begin{array}{l}\text { p17 (74-86 LAI) RSLYNTVATLY } \\
\text { - C. Brander notes this is an A*3002 epitope }\end{array}$ & & human $(\mathrm{A} * 3002)$ & Brander2001 \\
\hline
\end{tabular}

- The CTL-dominant response was focused on this epitope in a single HIV+ individual from Boston - this epitope fell outside the most recognized peptides in the study

- Three peptides GSEELRSLYNTVATL (p17 residues 71-85), SALSEGATPQDLNTMLNTVG (p24 41-60), and WEKIRLRPGGKKKYKLK(p17 16-30) contained the dominant Gag-specific epitope in 31 out of 44 B-clade infected individuals from Boston who showed Gag-CTL responses

- Five peptides RLRPGGKKHYMIKHLVW (p17 20-36), ELRSLYNTVATLYCV (p17Gag 74-88), SALSEGATPQDLNTMLNTVG (p24 41-60),

FRDYVDRFFKTLRAEQA (p24 161-177), and SILDIKQGKEPFRDY (p24 149-164) contained dominant Gag-specific epitopes in 32 out of 37 C-clade infected subjects from South Africa

p17 (76-86) Gag (96ZM651.8) RLSYNTVATLY human (A*3002) Novitsky2001

- This study is provides a survey of CTL responses and full length HIV-1 genome sequences from a C subtype infected Botswanan cohort

- Only 3 of $13(23.1 \%)$ A*3002-positive subjects demonstrated moderate CTL responses to the peptide GTEELRSLYNTVATLYCVHE (residues 71 to 90), which contains the previously described A*3002 epitope RLSYNTVATLY

p17 (76-86) p17 (76-86) RSLYNTVATLY $\quad$ HIV-1 infection $\quad$ human (A*3002)

- Epitope name: RY11 (p17)

- HLA-A*3002 is very common in African populations, 50\% of Zimbabweans express HLA-A30, 44\% in African Zulu, so five new HIV epitopes were characterized that are presented by this HLA molecule

- A rapid method was developed combining ELISPOT with intracellular IFN- $\gamma$ staining of PBMCs to map optimal epitopes, then HLA presenting molecules were defined - this method was completed within 48 to 72 hours of receipt of blood

- Two individuals were studied: Subject 199 (HLA A*0201/*3002 B*4402/51 Cw2/5), a Caucasian, and Subject 6007 (HLA A*3002/ B53/*5801 Cw4/7) an African-Caribbean

- In both HLA-A*3002 individuals the response to RSLYNTVATLY was dominant

- Three quantitative assays, ELISPOT, precursor frequency and chromium release, confirmed a hierarchy of response: RY11 (p17) > KY9 (gp41) > KY9 (RT-53) > IY9 (gp41) 


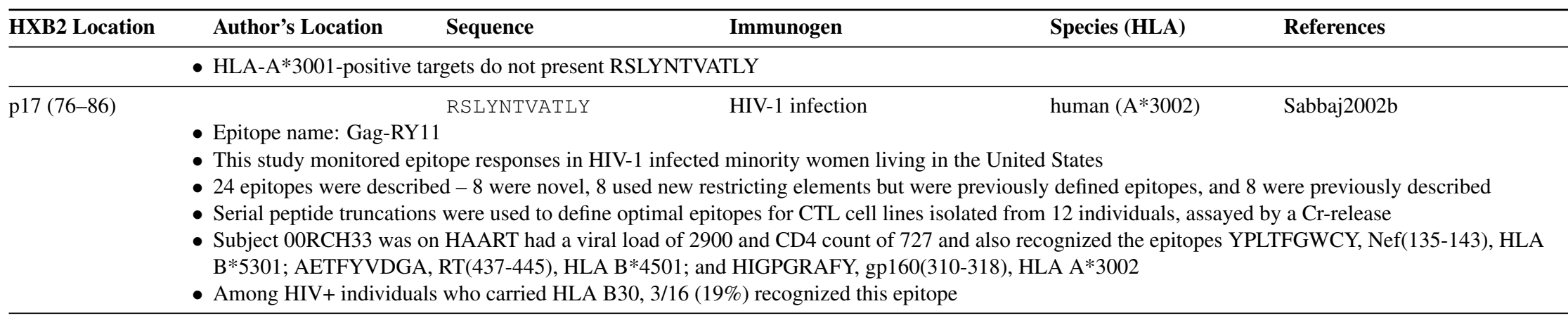

p17 (76-86) p17 (74-86 SF2) $\quad$ RSLYNTVATLY $\quad$ HIV-1 infection $\quad$ human (A30)

- Therapy provided during acute infection resulted in a narrower CTL response, stronger T help response, and a less diverse viral population than was seen in individuals treated during chronic infection

- The breadth and specificity of the response was determined using ELISPOT by studying 19 individuals with pre-seroconversion therapy (Group 1), 11 individuals with primary infection but post-seroconversion therapy (Group 2), and 10 individuals who responded to HAART given during chronic infection (Group 3), using 259 overlapping peptides spanning p17, p24, RT, gp41, gp120 and Nef

- Previously described and newly defined optimal epitopes were tested for CTL response

- Number of HLA-A30+ individuals that had a CTL response to this epitope broken down by group: 0/1 group 1, 0/0 group 2, and 1/1 group 3

\begin{tabular}{lllll}
\hline p17 (76-86) & RSLYNTVATLY & HIV-1 infection & human (A30)
\end{tabular}

- Epitope name: A30-RY11(p17)

- Peripheral blood (PB) and lymph node (LN) CD8+ T-cell responses were compared in 15 asymptomatic HIV-1 infected patients using all known optimal CTL epitopes (http://hiv-web.lanl.gov/content/hiv-db/REVIEWS/brander2001.html) for each person's class I HLA alleles.

- 60 epitope responses were detected in both PB and LN samples of the 15 patients, and an additional 8 responses were detected only in LN. The total magnitude of the response was similar in LN and PB, but the percentage of CD8+ T cells in the LN is lower so the number of HIV-specific cells per million CD8+ T-cells is higher in the LN.

- 1 year post-HAART treatment in five patients studied, the magnitude of the CD8 T-cell response was decreased in both LN and PB, but more dramatically in $\mathrm{PB}$, and 13/25 epitope responses in the PB became undetectable, in contrast to 5/26 in the LN.

- Treatment interruption following HAART induced resulted in increased viremia accompanied by the restoration of the detection of 13 epitopes that had become undetectable in the PB, and the addition of 9 novel epitope responses.

- Breakdowns of epitope responses were shown for 4 individuals. Patient D displayed the greatest response to B27-KK10 (p24), and also responded to A30-RY11(p17), A32-PW10(RT), A30-KY11(RT), A32-RW10(gp120), and B18-YY9(Nef).

$\begin{array}{llll}\text { p17 (77-85) } & \text { p17 } & \text { SLYNTVATL } & \text { HIV-1 infection }\end{array}$

- Review of the impact of CTL on viral immunity and escape that notes that SLYNTVATL-tetramer binding cells in individuals that react to this epitope inversely correlate with plasma viral load

\begin{tabular}{llll}
\hline p17 (77-85) & $\begin{array}{l}\text { (SF2, HXBc2/Bal } \\
\text { chimeric })\end{array}$ & SLYNTVATL & HIV-1 infection
\end{tabular}

- Epitope name: SL9

- Seven HIV-positive subjects tended to make their strongest CD8+ T-cell response against Gag; these responses had varying breadth and magnitude that were unrelated to disease progression.

- Patient TX7 primarily recognized SL9 during a three year study period and used six T-cell clonotypes for this recognition. 


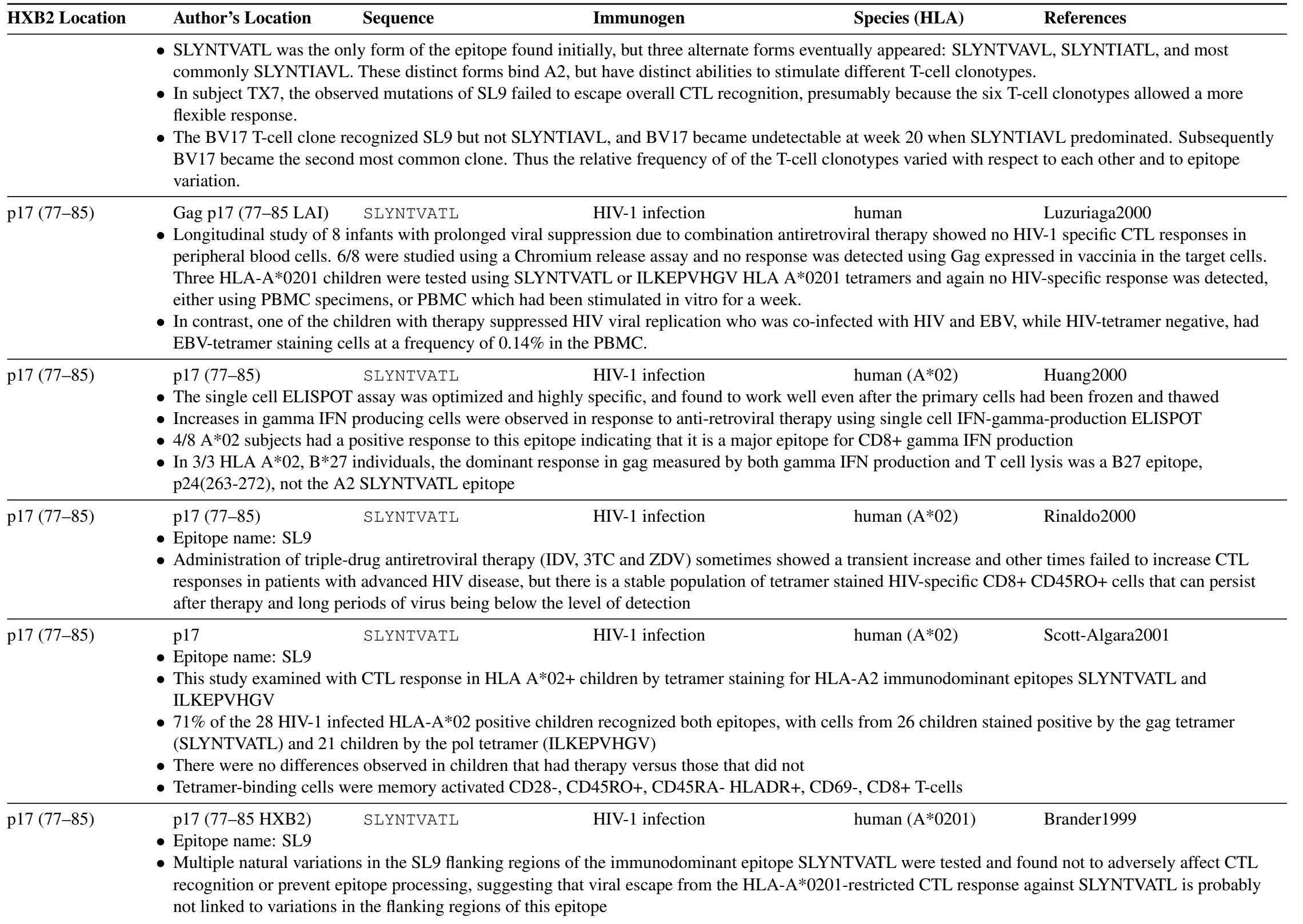




\begin{tabular}{|c|c|c|c|c|c|}
\hline HXB2 Location & Author's Location & Sequence & Immunogen & Species (HLA) & References \\
\hline & \multicolumn{5}{|c|}{$\begin{array}{l}\text { - The substitution Y79F was an escape mutation in that it interfered with CTL recognition by one CTL clone from an A*0201 infected individual, clone } \\
\text { 13010.B17, but it was still recognized by another CTL clone, 115.D4 }\end{array}$} \\
\hline $17(77-85)$ & \multicolumn{5}{|c|}{ 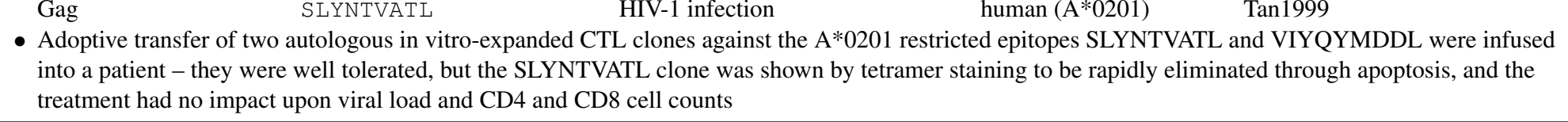 } \\
\hline $17(77-85)$ & \multicolumn{5}{|c|}{$\begin{array}{l}\text { p17 (77-85) SLYNTVATL HIV-1 infection } \\
\text { - Only 4/11 HLA-A2+ HIV+ individuals had CTL that reacted to SLYNTVATL, calling into question whether it is immunodominant } \\
\text { - } 95 \text { optimally-defined peptides from this database were used to screen for INF } \gamma \text { responses to other epitopes } \\
\text { - Individuals that did not respond to SLYNTVATL recognized other HIV epitopes, and 2/4 SLYNTVATL responders had stronger responses to epitopes } \\
\text { restricted by other class I alleles } \\
\text { - SLYNTVATL was the only response detected in a one individual that was HLA A*0201, B44, B70 }\end{array}$} \\
\hline p17 ( & \multicolumn{5}{|c|}{$\begin{array}{l}\text { - CTL effector levels were measured after potent ARV therapy using HLA-tetramer complexes for the A*0201 epitopes SLYNTVATL and ILKEPVHGV in } \\
\text { seven patients, and the } \mathrm{B} * 3501 \text { epitope DPNPQEVVL in one additional patient } \\
\text { - Levels of CTL effectors typically decline for 5-7 days and then rebound, fluctuating during the first two weeks of therapy } \\
\text { - After the early fluctuation, there was a steady exponential decay with a median half-life of } 45 \text { days }\end{array}$} \\
\hline 17( & $\begin{array}{l}\text { p17 (77-85) } \\
\text { - Epitope name: SL9 } \\
\text { - This paper introduce } \\
\text { were prepared that c } \\
\text { - Three patients only } \\
\text { Gag epitope }(0.28 \%\end{array}$ & $\begin{array}{l}\text { SLYNTVATL } \\
\text { tetramer metho } \\
\text { in CTL lines sp } \\
\text { d the Gag epito }\end{array}$ & $\begin{array}{l}\text { HIV-1 infection } \\
\text { mits quantificatio } \\
\text { VHGV and SLYN } \\
\text { one patient had tl }\end{array}$ & $\begin{array}{l}\text { human }\left(A^{*} 0201\right) \\
\text { ased on expressior } \\
\text { itate HIV-specific } \\
\text { y of tetramer stain }\end{array}$ & $\begin{array}{l}\text { Altman1996 } \\
\text { cific TCRs - HLA-A2 tetramers } \\
\text { ell lines in freshly isolated PBMCs } \\
\text { ne Pol epitope }(0.77 \%) \text {, less to the }\end{array}$ \\
\hline p17 & $\begin{array}{l}\text { Gag } \\
\text { - Administration of hi } \\
\text { to be memory cells, }\end{array}$ & $\begin{array}{l}\text { SLYNTVATL } \\
\text { active antiretrov } \\
\text { ating that persis }\end{array}$ & $\begin{array}{l}\text { HIV-1 infection } \\
\text { ART) reduced CD } \\
\text { viral populations }\end{array}$ & $\begin{array}{l}\text { human }(\mathrm{A} * 0201) \\
\text { and the } \mathrm{CD} 8+\text { cells } \\
\text { tain high frequenci }\end{array}$ & $\begin{array}{l}\text { Gray } 1999 \\
\text { ed by tetramer staining were likely } \\
\text { IIV-1 specific CTL }\end{array}$ \\
\hline p17 (77-85) & $\begin{array}{l}\text { p17 (77-85 SF2) } \\
\text { - Epitope name: SL9 } \\
\text { - CTL from a patient } \\
\end{array}$ & $\begin{array}{l}\text { SLYNTVATL } \\
\text { ed with clade B } \\
\end{array}$ & $\begin{array}{l}\text { HIV-1 infection } \\
\text { ognize the clade A } \\
\end{array}$ & $\begin{array}{l}\text { human }(\mathrm{A} * 0201) \\
\text { tope }\end{array}$ & McAdam1998 \\
\hline p17 (77-85) & $\begin{array}{l}\text { p17 (77-85) } \\
\text { - Epitope name: SL9 } \\
\text { - HIV+ individuals we } \\
\text { cells was followed ir } \\
\text { - Seven HIV+ people } \\
\text { - Three patients were } \\
\text { - An A2-Gag specific }\end{array}$ & $\begin{array}{l}\text { SLYNTVATL } \\
\text { llowed longitud } \\
\text { studied, and all } \\
\text { wed in detail, TC } \\
\text { from one patient }\end{array}$ & $\begin{array}{l}\text { HIV-1 infection } \\
\text { C tetramers in con } \\
\text { ons of particular T } \\
\text { as persisted for } 2 \mathrm{t} \\
\text { BV8, and at its hi }\end{array}$ & $\begin{array}{l}\text { human }(\mathrm{A} * 0201) \\
\text { nti-BV chain } \mathrm{MAb} \\
\text { en several, relative } \\
\text { asional transient in } \\
\text { nted } 17.5 \% \text { of the }\end{array}$ & $\begin{array}{l}\text { Wilson1998a } \\
\text { lonal expansion of HIV-specific T } \\
\text { CD8+ T cells }\end{array}$ \\
\hline
\end{tabular}




\begin{tabular}{|c|c|c|c|c|c|}
\hline HXB2 Location & Author's Location & Sequence & Immunogen & Species (HLA) & References \\
\hline p17 (77-85) & $\begin{array}{l}\text { p17 (77-85) } \\
\text { Epitope name: SL9 }\end{array}$ & SLYNTVATL & HIV-1 infection & human $\left(A^{*} 0201\right)$ & Ogg1998b \\
\hline
\end{tabular}

- HLA-tetrameric complexes were used in a cross-sectional study of 14 untreated HLA A*0201 positive individuals, revealing an inverse relationship

between HIV Gag and Pol specific CTL effector cells (CTLe) and viral load

- Inclusion of both the p17 SLYNTVATL and RT ILKEPVHGV epitopes gives a good representation of HLA A*0201-restricted activity

- No correlation was observed between the CTLe and CD4 count or clearance rate of productively infected cells

\begin{tabular}{lllll}
\hline p17 (77-85) & p17 (77-85) & in vitro stimulation & human (A*0201)
\end{tabular}

- Epitope name: SL9

- HLA-A2 heavy chain and $\beta 2$-microglobulin expressed in E. coli were refolded in the presence of this peptide

- The HLA-A2-peptide complex elicited HLA-A2 peptide-specific CTL response in cells lacking HLA-A2

- Suggests that preformed HLA-peptide complexes could provide an alternate to intracellular processing for immunogens

p17 (77-85) p17(77-85) SLYNTVATL HIV-1 infection $\quad$ Lalvani1997

- Epitope name: SL9

- A peptide-based protocol was optimized for restimulation of CTLp using optimized peptide and IL-7 concentrations - importantly this protocol does not stimulate a primary response, only secondary - peptide-specific CTLp counts could be obtained via staining with peptide-Class I tetramers

- This peptide was one of the test peptides for optimizing the protocol

\begin{tabular}{lll}
\hline p17 (77-85) & p17 (76-84) SLYNTVATL \\
& - Epitope name: SL9 \\
& - Slow dissociation rate is associated with immunogenicity \\
& CTL generated by in vitro stimulation of PBMC derived from uninfected individual
\end{tabular}

p17 (77-85) p17 (77-85) SLYNTVATL HIV-1 infection $\quad$ Goulder1997e Goulder1997a

- Epitope name: SL9

- Identical twin hemophiliac brothers were both infected with the same batch of factor VIII

- One had a response to gag A2 epitope SLYNTVATL, the other to pol A2 epitope ILKEPVHGV

- Viral sequencing from the twin that had no response to SLYNTVATL indicated his virus had the substituted form SLHNAVAVL

- $71 \%$ of an additional set of $22 \mathrm{HIV}-1$ infected HLA-A*0201 positive donors preferentially responded to gag SLYNTVATL

- Those individuals with a pol ILKEPVHGV response tended to have mutations in or around SLYNTVATL

- An additional subject went from SLYNTVATL responder to non-responder coincident with a switch to the variant SLFNTVATL

- [Goulder1997a] is a review of immune escape that summarizes this study

\begin{tabular}{lllll}
\hline p17 (77-85) Gag (77-85) & SLYNTVATL & HIV-1 infection & human (A*0201)
\end{tabular}

- Epitope name: SL9

- Peptide-tetramer complexes of $A * 0201$ and SLYNTVATL or ILKEPVHGV were used to study individuals receiving HAART to determine the frequency of Class I HLA-restricted anti-HIV CD8+ T cells

- 17/18 asymptomatic patients had a CTL response to one or both epitopes $-72 \%$ had a CTL response to SLYNTVATL

- After HAART, the majority of the epitope-specific CTL were apparently memory cells

p17 (77-85) p17 (77-85 subtype A) SLFNTVATL $\quad$ HIV-1 infection $\quad$ human (A*0201)

- Epitope name: SL9

- CTL responses in three individuals with non-clade B infections were studied, 2 with subtype A infections, 1 with subtype $\mathrm{C}$ - their infections all originated in East Africa 


\begin{tabular}{|c|c|c|c|c|}
\hline HXB2 Location & Author's Location & Sequence & Species (HLA) & References \\
\hline & \multicolumn{4}{|c|}{$\begin{array}{l}\text { - This epitope is most commonly SLYNTVATL in B subtype, and CTL from the C subtype infection did not recognize B clade gag or the } 3 \mathrm{Y} \text { form of the } \\
\text { epitope, but do recognize the predominant A and C clade form, SLFNTVATL }\end{array}$} \\
\hline p17 (77-85) & $\begin{array}{l}\text { p17 (77-85) } \\
\text { - Epitope name: SL9 } \\
\text { - Of } 17 \text { infected HLA } \\
\text { VIYQYMDDL, and } \\
\text { - Only one subject ha } \\
\text { - There was significar } \\
\text { - The overall variatiol } \\
\text { immune pressure } \\
\text { - Subjects were part o }\end{array}$ & $\begin{array}{l}\text { SLYNTVATL } \\
201 \text { subjects, } 13 \\
\text { e was no correla } \\
\text { L against all thre } \\
\text { erogeneity in the } \\
\text { his epitope amor } \\
\text { San Francisco C }\end{array}$ & $\begin{array}{l}\text { HIV-1 infection human (A*0201) } \\
\text { ses against the p17 SLYNTVATL epitope, six recognize } \\
1 \text { load and recognition of a specific epitope } \\
\text { this immunodominant epitope } \\
\text { a CTL response and } 11 \text { non-HLA A*0201 HIV-1+ ind } \\
\text { the ARIEL project and from the Boston area }\end{array}$ & $\begin{array}{l}\text { Brander1998a } \\
\text { EPVHGV and five recognized } \\
\text { Is was similar, suggesting a lack of }\end{array}$ \\
\hline p17 (77-85) & $\begin{array}{l}\text { p17 (77-85 HXB2) } \\
\text { - Epitope name: SL9 } \\
\text { - CTL response to IPI } \\
\text { interestingly, no res } \\
\text { - The individual show } \\
\text { - Despite the initial n } \\
\text { - No HIV-specific lyn } \\
\text { - A variant of this epi } \\
\text { could present the pe }\end{array}$ & $\begin{array}{l}\text { SLYNTVATL } \\
\text { QGL was the in } \\
\text { to commonly ir } \\
\text { trong initial CT } \\
\text { response to two } \\
\text { roliferative resp } \\
\text { vas observed in } \\
\text { to SLYNTVATI }\end{array}$ & $\begin{array}{l}\text { HIV-1 infection human }\left(\mathrm{A}^{* 0201)}\right. \\
\text { esponse in a rapid progressor - there was a subdominan } \\
\text { HLA A*0201 epitope SLYNTVATL, although this indiv } \\
\text { time of the initial drop in viremia, but it was quickly lo } \\
\text { er CTL responses developed } \\
\text { ted in this patient, and neutralizing antibody response w } \\
\text { out this mutation is recognized by SLYNTVATL-specific }\end{array}$ & $\begin{array}{l}\text { Hay1999b } \\
\text { nse to SPAIFQSSM in Pol, and } \\
\text { vas HLA A*0201 } \\
\text { ough memory cells persisted } \\
\text { and in this case the patient's cells } \\
\text { and }\end{array}$ \\
\hline p17 (7 & $\begin{array}{l}\text { p17 (77-85) } \\
\text { - Two patients were f } \\
\text { that by day } 260 \text { CTI } \\
\text { - ERYLKDQQL was } \\
\text { - Sporadic breakthrou } \\
\text { - Memory CTL frequ } \\
\text { the viral load }\end{array}$ & $\begin{array}{l}\text { SLYNTVATL } \\
\text { ed before and af } \\
\text { vities were unde } \\
\text { ominant respons } \\
\text { viremia resulte } \\
\text { directed against }\end{array}$ & $\begin{array}{l}\text { HIV-1 infection human (A*0201) } \\
\text { luced plasma HIV-1 RNA levels resulted in a decline in } \\
\text { dividuals, SLYNTVATL subdominant } \\
\text { eases in CTLp } \\
\text {, Vac-Env, and Vac-Nef initially increased with HAART }\end{array}$ & $\begin{array}{l}\text { Kalams1999b } \\
\text { ecific in-vivo activated CTL such } \\
\text { en decreased with the decline of }\end{array}$ \\
\hline p17 (77-85) & $\begin{array}{l}\text { Gag (77-85) } \\
\text { - High levels of CD8- } \\
\text { low CD4 counts, bu } \\
\text { - Thus HIV-1 specific } \\
\text { may be a useful ther }\end{array}$ & $\begin{array}{l}\text { SLYNTVATL } \\
-1 \text { specific and } \\
\text { T cell mediatec } \\
+ \text { cells may be } 1 \\
\text { ic strategy }\end{array}$ & $\begin{array}{l}\text { HIV-1 infection human }(\mathrm{A} * 0201) \\
\text { pecific CTL were detected by HLA-A*0201-peptide tet } \\
\text { was not seen } \\
\text { ck direct effector activity in late disease, suggesting tha }\end{array}$ & $\begin{array}{l}\text { Spiegel2000 } \\
\text { in } 3 \text { infected subjects with very } \\
\text { oming antigen unresponsiveness }\end{array}$ \\
\hline p17 (77-85) & $\begin{array}{l}\text { Gag (77-85) } \\
\text { - ELISPOT was used } \\
\text { - The highest CTL fre } \\
\text { - In A*0201 individu. } \\
\text { and ILKEPVHGV p }\end{array}$ & $\begin{array}{l}\text { SLYNTVATL } \\
\text { ay the CD8 T c } \\
\text { y was directed } \\
\text { gher numbers o } \\
\text { ted by A2 }\end{array}$ & $\begin{array}{l}\text { HIV-1 infection human }\left(\mathrm{A}^{*} 0201\right) \\
\text { e HIV-1 proteins Gag, Pol, Nef or Env expressed in vace } \\
\text { cells were directed against HIV-1 proteins expressed in }\end{array}$ & $\begin{array}{l}\text { Larsson1999 } \\
\text { ectors in } 19 \mathrm{HIV}+\text { people } \\
\text { iia than to peptides SLYNTVATL }\end{array}$ \\
\hline
\end{tabular}




\begin{tabular}{lllll}
\hline HXB2 Location & Author's Location & Sequence & Immunogen & Species (HLA) \\
\hline p17 (77-85) & p17 (SF2) & SLYNTVATL & HIV-1 infection & human $(\mathrm{A} * 0201)$
\end{tabular}

- The CTL-dominant response was focused on this epitope in 11/25 HLA A2 (A*0201 or A*0202) HIV+ individuals from Boston and in 1/8 HLA A2 HIV+ individuals from Durban

- Three peptides GSEELRSLYNTVATL (p17 residues 71-85), SALSEGATPQDLNTMLNTVG (p24 41-60), and WEKIRLRPGGKKKYKLK(p17 16-30) contained the dominant Gag-specific epitope in 31 out of 44 B-clade infected individuals from Boston who showed Gag-CTL responses

- Five peptides RLRPGGKKHYMIKHLVW (p17 20-36), ELRSLYNTVATLYCV (p17Gag 74-88), SALSEGATPQDLNTMLNTVG (p24 41-60), FRDYVDRFFKTLRAEQA (p24 161-177), and SILDIKQGKEPFRDY (p24 149-164) contained dominant Gag-specific epitopes in 32 out of 37 C-clade infected subjects from South Africa

p17 (77-85 LAI) SLYNTVATI

$\operatorname{human}(\mathrm{A} * 0201)$

Brander2001

- C. Brander notes this is an $A^{*} 0201$ epitope

p17 (77-85) p17 (77-85 SF2) SLYNTVATL HIV-1 infection human (A*0201) Goulder2001a

- Epitope name: SL9

- This epitope is targeted by $75 \%$ of HLA-A*0201, HIV+ adults, and the magnitude of the response is inversely correlated with viral load

- CTL responses to SL9 and autologous SL9 variants were not detected in 11 HLA-A*0201 positive subjects during acute infection

- Longitudinal studies of two individuals (AC13 and PI004) showed that the initial control of viremia was independent of the SL9 CTL response

- Low Gag expression levels did not correlate with the delayed CTL response to this epitope

- Autologous SL9 variants SLYNTIAVL, SLYNTVAVL, SLFNTVATL, SLFNTVATL, and SLFNTVATL are each capable of inducing a range of CTL responses, sometimes strong, sometimes diminished, and sometimes complete escape relative to the than the wild type variant SLYNTVATL in patients with chronic HIV-1 infection - the ability to cross-react with a particular variant was patient dependent

\begin{tabular}{lllll}
\hline p17 (77-85) & p17 & SLYNTVATL & HIV-1 infection & human (A*0201)
\end{tabular}

- Epitope name: p17 SL9

- HIV was scanned for all peptides which carried the A2-supermotif pattern conserved in more than $50 \%$ of B clade sequences - 233 peptides met this criteria, and 30 of these bound to HLA-A*0201 - 20/30 bound to at least 3/5 of HLA-A2 supertype alleles tested

- Three additional previously described HLA-A2 epitopes were added to the set of 20, including p17 SL9, and 18/22 chronically infected HLA-A2 individuals had CTL that recognized at least one of the 23 peptides (median of 2 and maximum of 6 ), while $6 / 12$ acute infected individuals recognized at least 1 (median of 1 and maximum of 2)

- p17 SL9 was recognized in 12/22 patients with chronic HIV-1 infection

- Only 1/13 patients with acute HIV-1 infection recognized p17 SL9

p17 (77-85) Gag SLYNTVATL HIV-1 infection human $\left(\mathrm{A}^{*} 0201\right)$

Goepfert2000

- Epitope name: (SL9)

- This paper describes a comparison of results of different CTL assays, a SL9 tetramer assay and IFN-gamma ELISPOT, using 7 HIV-positive patients

- The IFN-gamma ELISPOT assay was compared using the single SL9, a pool of overlapping 20 mers, and recombinant vaccinia encoding Gag as antigen pooled peptides gave the highest number of spot forming cells, vaccinia gave high background

- A correlation with results of the tetramer assay was found only for ELISPOT using the Gag epitope as antigen, but the tetramer assay detected a 10-fold higher number of cells than could produce IFN-gamma in the ELISPOT assay - the authors suggest not all tetramer-positive cells may produce IFN-gamma, some may be undergoing apoptosis, some may be producing other cytokines

- The tetramer assay could detect a reaction to SLYNTVATL in most of the HLA-A*0201 chronically HIV-1 infected study subjects 


\begin{tabular}{|c|c|c|c|c|c|}
\hline HXB2 Location & Author's Location & Sequence & Immunogen & Species (HLA) & References \\
\hline p17 (77-85) & Gag (LAI) & SLYNTVATL & in vitro stimulation & human $\left(\mathrm{A}^{* 0201)}\right.$ & Engelmayer2001 \\
\hline
\end{tabular}

- Recombinant canarypox virus vector containing HIV-1 sequences, upon infection of mature dendritic cells, can trigger specific lysis through in vitro by

T-cells from HIV-1 infected individuals at levels comparable to the response seen to HIV carried in vaccinia vectors

- Recombinant canarypox virus vector containing HIV-1 sequences can also stimulate HIV-specific CD4+ helper T-cell responses

\begin{tabular}{lllll}
\hline p17 (77-85) & p17 (77-85 LAI) & SLYNTVATL & HIV-1 infection & human (A*0201)
\end{tabular}

- A panel of 16 epitopes covering 15 class I alleles was tested in 14 HIV+ patients from an unselected Caucasian population treated with HAART, using tetramer staining or CD8+ cell IFNgamma production to measure responses

- In general, during the first month of treatment viral load decreased and frequencies of HIV-specific CTL tripled and broadened - eight new HIV specificities that were not previously detectable were newly detected, as were CMV specific CD8+ PBL - but with continued viral suppression, HIV-specific responses diminished

- Viral rebounds gave different patterns of response: increases or decreases in pre-existing response, new specificities, or no change

p17 (77-85) Gag SLYNTVATL HIV-1 infection $\quad$ Gea-Banacloche2000

- In a study including many long-term non-progressors, no correlation between plasma virus levels and number of HIV-specific CD8+ T-cells was found

- High frequencies of circulating CD8+ T-cells were HIV-1 specific, and the majority of these responses were to gag-pol gene products

- 4/21 subjects were HLA-(A*0201), and of these only 2 subjects (patient 3 and 19) tested positive to this epitope

p17 (77-85) p17 (77-85 SF2) SLYNTVATL $\quad$ HIV-1 infection $\quad$ Propato2001

- Long-term nonprogressors (LTNPs) had strong memory resting CD8+ T-cell responses against the majority of epitopes tested, (18 for the A2 supertype, 16 for the A3 supertype) while the effector cells of long-term nonprogressors recognized far fewer epitopes

- Progressors had memory resting CD8+ T-cells that recognized far fewer epitopes than LTNPs

- A positive correlation between effector CD8+ T-cells and plasma viremia and a negative correlation between CD8+ effector T-cells and CD4+ T-cells was observed, which may contribute to the inability of LTNPs to clear virus

- Tetramer staining with A2, beta2microglobulin, and either SLYNTVATL, KLVGKLNWA, or LTFGWCFKL revealed that tetramers detected more HIV-specific sells in LTNP than in progressors, activated effector cells were the minority population, and ELISPOT correlated better with the effector cell subpopulation than the total tetramer stained population

p17 (77-85) Gag (77-85) SLYNTVATL HIV-1 infection $\quad$ Jin2000a

- The CTL effector levels (CTLe) were compared in long term non-progressors (LTNP) with low viral load and in patients whose virus was well-suppressed by therapy, using a tetramer assay

- LTNPs have high memory CTLe numbers and low viral load, while HAART patients had low CTLe numbers and low viral load

p17 (77-85) p17(77-85) SLYNTVATL HIV-1 infection human (A*0201) Appay2000

- Combined tetramer and intracellular cytokine staining was used to study the function of circulating CD8+ T cells specific for HIV and CMV

- HIV-specific CD8+ T cells expressed lower levels of perforin than CMV-specific CD8+ T cells from the same donor, and this was associated with persistent CD27 expression on HIV-specific cells, suggesting impaired maturation

- In most donors, between $50 \%$ and $95 \%$ of the activated virus-specific CD8+ T cells produced IFN- $\gamma$ and MIP-1 $\beta$ with a distinct subset that failed to produce TNF- $\alpha$

p17 (77-85) p17 (77-85) SLYNTVATL HIV-1 infection $\quad$ Goulder2000b

- Tetramer assays were compared with three functional assays in 42 people with chronic HIV infection: ELISPOT, intracellular cytokine staining, and precursor frequency (limiting dilution assay [LDA]) 


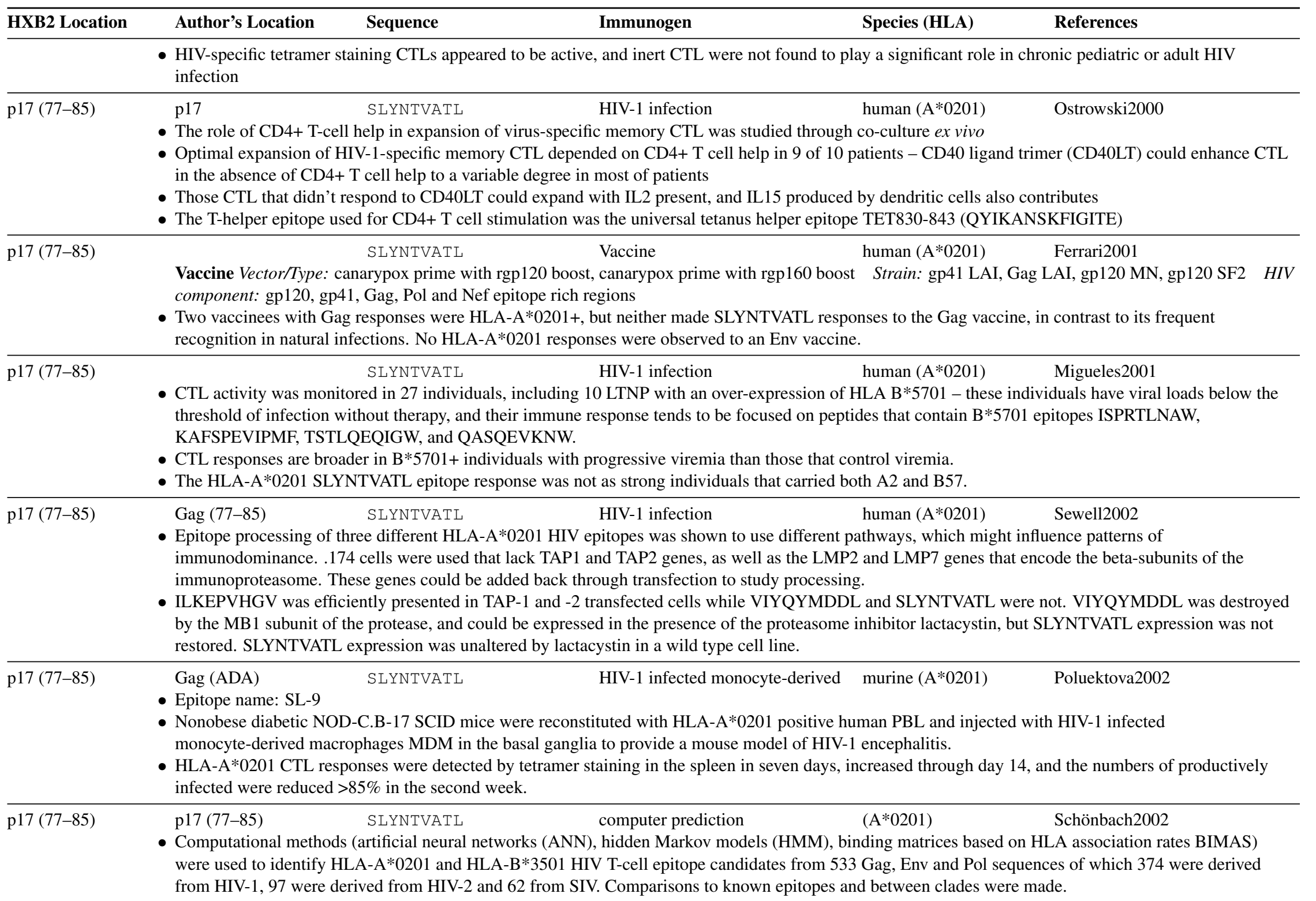




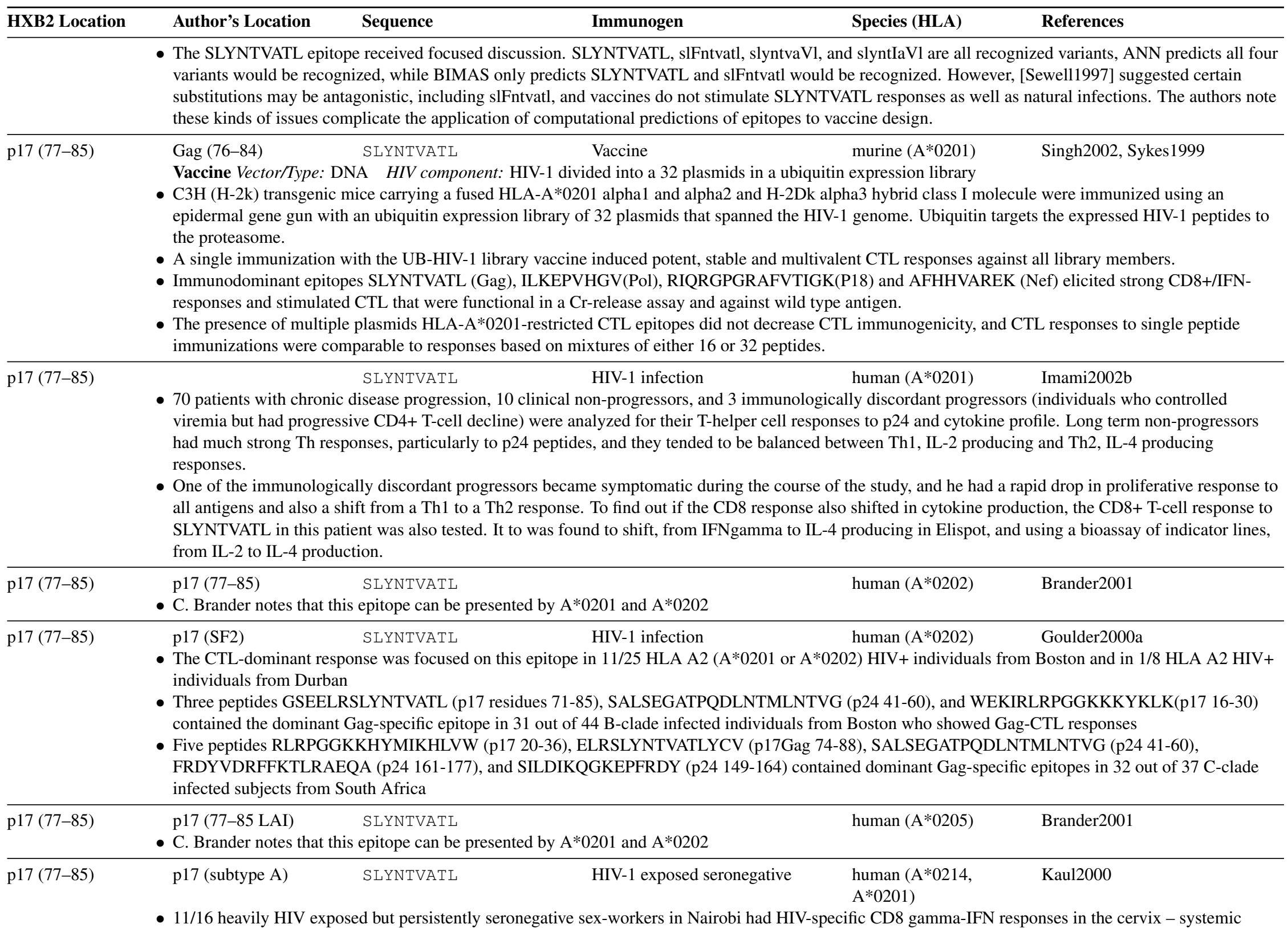

-11/16 heavily HIV exposed but persistently seronegative sex-workers in Nairobi had HIV-specific CD8 gamma-IFN responses in the cervix - systemic CD8+ T cell responses tended to be to the same epitopes but at generally lower levels than cervical CD8+ T cell responses 


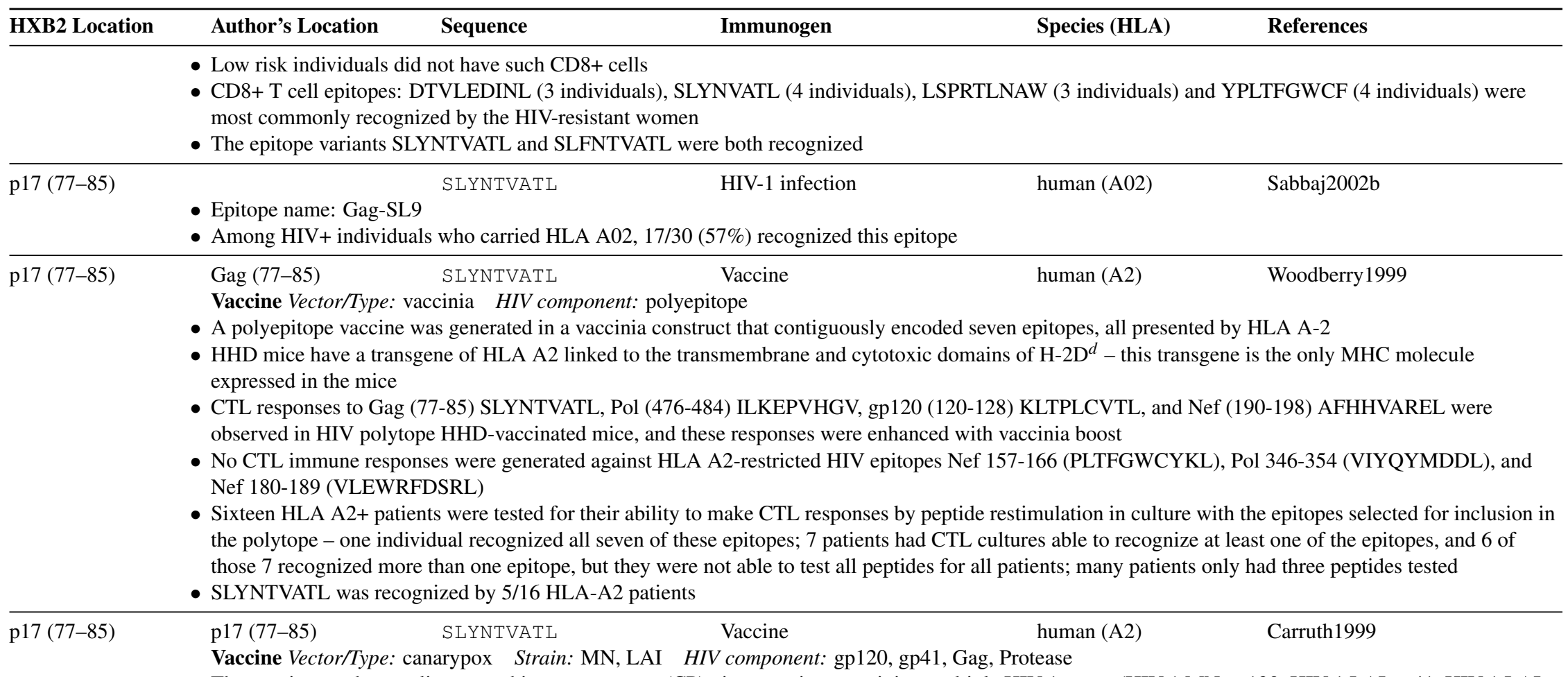

- The vaccine used was a live recombinant canarypox (CP) virus vaccine containing multiple HIV-1 genes (HIV-1 MN gp120, HIV-1 LAI gp41, HIV-1 LAI Gag, HIV-1 LAI protease)

- CD4+ and CD8+ Gag and Env specific CTL responses were detected in only 1/5 vaccinated volunteers, and were not detectable 1 year after vaccination

- CTL responses to epitopes SLYNTVATL and TVYYGVPVWK from HIV+ control patients were used as positive controls

- The study explored why vaccinees were non-responsive - non-response was not due to inherent defects or differences in the ability of these individuals to process and present antigen

- Lack of response to SLYNTVATL led the authors to speculate that the immunodominance of this epitope in natural infections may not be recapitulated by vaccine antigen

\begin{tabular}{lllll}
\hline p17 (77-85) p17 (77-85) & SLYNTVATL & Birk1998b
\end{tabular}

- Epitope name: SL9

- A study of p17 variation considering known p17 epitopes and individuals with known HLA types revealed that p17 evolution is influenced by immune pressure from CTLs

\begin{tabular}{lllll}
\hline p17 (77-85) & p17 (77-85) & SLYNTVATL & HIV-1 infection & human (A2)
\end{tabular}

- Epitope name: SL9

- Included as a negative control in a tetramer study of A2-EBV CTL response

\begin{tabular}{lllll}
\hline p17 (77-85) & p17 & SLYNTVATL & HIV-1 infection & human (A2)
\end{tabular}




\begin{tabular}{|c|c|c|c|c|}
\hline HXB2 Location & Author's Location & Sequence & Species (HLA) & References \\
\hline & \multicolumn{4}{|c|}{$\begin{array}{l}\text { - CTL specific for HIV epitopes were used to show that the mediators of both the cytolytic (granzyme A was used as the marker) and non-cytolytic (HIV-1 } \\
\text { inhibitory chemokines MIP-1 } \alpha \text { and RANTES were used as markers) anti-viral responses are localized within the CTL's cytotoxic granules }\end{array}$} \\
\hline p17 (77-85) & $\begin{array}{l}\text { p17 (77-85 HXB2) } \\
\text { - Epitope name: SL9 } \\
\text { - Two CTL clones rec } \\
\text { - Nef down-regulates I } \\
\text { soluble peptide }\end{array}$ & SLYNTVATL & \multicolumn{2}{|c|}{$\begin{array}{l}\text { HIV-1 infection human (A2) Collins1998 } \\
\text { form of the epitope SLYNTIAVL } \\
\text { s CTL killing, and this down-regulation can be partially compensated for by adding excess }\end{array}$} \\
\hline p17 (77-85) & $\begin{array}{l}\text { p17 }(77-\text { - } \\
\text { - Critope } n \\
\text { one A sul } \\
\text { - Pol reacti } \\
\text { - Gag react } \\
\text { - Nef react } \\
\text { - Env react }\end{array}$ & SLYNTVATL & HIV-1 infection & $\begin{array}{l}\text { Durali1998 } \\
1 \text { AG recombinant infections) and } \\
\text { LL }\end{array}$ \\
\hline p17 (77-85) & \multicolumn{4}{|c|}{$\begin{array}{l}\text { - Epitope name: SL9 } \\
\text { - Allogeneic dendritic cells (DCs) were obtained from HLA-identical siblings, pulsed with rgp160 MN or A2-restricted HIV-1 epitope peptides, and infused } \\
\text { monthly into six HIV-infected patients } \\
\text { - } 1 / 6 \text { showed increased env-specific CTL and increased lymphoproliferative responses, } 2 / 6 \text { showed increase only in proliferative responses, and } 3 / 6 \text { showed } \\
\text { no change - pulsed DCs were well tolerated } \\
\text { - SLYNTVATL is a conserved HLA-A2 epitope included in this study - } 3 / 6 \text { patients had this sequence as their HIV direct sequence, one had the form } \\
\text { SLYNTVAVL and all four of these had a detectable CTL response - the other two had either the sequence SLFSAVAVL or SLFSAVAAL and no detectable } \\
\text { CTL response }\end{array}$} \\
\hline p17 (77-85) & $\begin{array}{l}\text { p17 (77-85 IIIB) } \\
\text { - Epitope name: SL9 } \\
\text { - HIV IIIB proteins we } \\
\text { - SLYNTVAVL, a vari } \\
\text { - SLFNTVAVL, a vari }\end{array}$ & $\begin{array}{l}\text { SLYNTVATL } \\
\text { ed to define the } \\
\text { und in HIV-1 N } \\
\text { und in HIV-1 N }\end{array}$ & $\begin{array}{l}\text { HIV-1 infection human (A2) } \\
\text { bitopes recognized by } 3 \text { lab workers accidentally infec } \\
\text { recognized } \\
\text { recognized }\end{array}$ & Sipsas1997 \\
\hline p17 (77-85) & $\begin{array}{l}\text { p17 } \\
\text { - Epitope name: SL9 } \\
\text { - A CTL response was } \\
\text { and D clades - such } \\
\text { - The A subtype conse } \\
\text { - The D subtype conse }\end{array}$ & $\begin{array}{l}\text { SLYNTVATL } \\
\text { d in exposed bu } \\
\text { reactivity could } \\
\text { is SLfNtvatL } \\
\text { is SLyNTVATL }\end{array}$ & $\begin{array}{l}\text { HIV-1 infection human (A2) } \\
\text { titutes from Nairobi using previously-defined B clade } \\
\text { both A and D and confer protection in Nairobi where }\end{array}$ & $\begin{array}{l}\text { Rowland-Jones1998a } \\
\text { that tended to be conserved in A } \\
\text { ypes are circulating }\end{array}$ \\
\hline p17 (77-85) & $\begin{array}{l}\text { p17 } \\
\text { - Epitope name: SL9 } \\
\text { - Naturally occurring }\end{array}$ & $\begin{array}{l}\text { SLYNTVATL } \\
\text { ts of this epitop }\end{array}$ & $\begin{array}{l}\text { HIV-1 infection } \\
\text { and acted as antagonists }\end{array}$ & Sewell1997 \\
\hline
\end{tabular}




\begin{tabular}{|c|c|c|c|c|c|}
\hline HXB2 Location & Author's Location & Sequence & Immunogen & Species (HLA) & References \\
\hline & \multicolumn{5}{|c|}{$\begin{array}{l}\text { - The following variants were found in } \mathrm{HIV}-1 \text { infected patients who mounted a strong response against this epitope: }-\mathrm{F}-\mathrm{-},-\mathrm{F}-\mathrm{V}-,-\mathrm{S}-\mathrm{C},-\mathrm{SF}- \\
-\mathrm{L}-,-\mathrm{I}-,-\mathrm{I}-\mathrm{V}-,-\mathrm{F}-\mathrm{I}-,-\mathrm{F}-\mathrm{I}-\mathrm{V}-,-\mathrm{F}-\mathrm{A}-\text { - } \\
\text { - All variants bound to A2 with at least half the affinity of SLYNTVATL except the triple mutant: }-\mathrm{F}-\mathrm{I}-\mathrm{V}- \\
\text { - Antagonism could be observed at low concentrations, abrogating lysis at an antagonist:agonist ratio of } 1: 10-\text { the antagonism was observed in one } \\
\text { SLYNTVATL-specific CTL line but not another }\end{array}$} \\
\hline p17 (77-85) & \multicolumn{5}{|c|}{$\begin{array}{l}\text { - A chimeric universal } \mathrm{T} \text { cell receptor was created by linking CD4 or an HIV-specific anti-gp41 Ig sequence to the signaling domain of the T cell receptor } \\
\text { chain } \zeta \text {, and transduced into CD8+ cells } \\
\text { - The response using universal-receptor-bearing CD8+ cells to lyse infected cells in vitro was comparable to the natural occurring responses of CTL-clones } \\
\text { from HIV+ individuals in terms of kinetics and efficiency } \\
\text { - A CTL clone specific for this epitope was used for the comparison }\end{array}$} \\
\hline p17 (77-85) & \multicolumn{4}{|c|}{$\begin{array}{l}\text { - Keyhole limpit hemocyanin or tetanus toxoid Th epitope co-expression with peptide CTL epitopes on the same APC was required for induction of } \\
\text { peptide-specific CTL }\end{array}$} & Stuhler1997 \\
\hline p17 (77-85) & $\begin{array}{l}\text { p17 (77-85) } \\
\text { - Epitope name: SL9 } \\
\text { - CD4+ cell lines acut } \\
\text { - Clones specific for R } \\
\text { - The distinction was t } \\
\text { - CTL can lyse infecte }\end{array}$ & $\begin{array}{l}\text { SLYNTVATL } \\
\text { fected with HI } \\
\text { ed HIV-1 infect } \\
\text { ht to be due to } 1 \\
\text { ls early after inf }\end{array}$ & $\begin{array}{l}\text { HIV-1 infection } \\
\text { determine their susc } \\
\text { levels than Env or Ga } \\
\text { of RT relative to Env } \\
\text { prior to viral producti }\end{array}$ & $\begin{array}{l}\text { human (A2) } \\
\text { is by CTL } \\
\text { es }\end{array}$ & Yang1996 \\
\hline p17 (77-85) & $\begin{array}{l}\text { p17 (77-85) } \\
\text { - Epitope name: SL9 } \\
\text { - CTL inhibit HIV-1 re } \\
\text { - CTL produced HIV- } \\
\text { - CTL suppress HIV r }\end{array}$ & $\begin{array}{l}\text { SLYNTVATL } \\
\text { ation at effector } \\
\text { pressive soluble } \\
\text { ation more effic }\end{array}$ & $\begin{array}{l}\text { HIV-1 infection } \\
\text { ons comparable to tho } \\
\alpha, \text { MIP-1 } \beta, \text { RANTE } \\
\text { atched cells }\end{array}$ & $\begin{array}{l}\text { human (A2) } \\
\text { specific activation }\end{array}$ & Yang1997a \\
\hline p17 (77-85) & $\begin{array}{l}\text { p17 (77-85 LAI) } \\
\text { - Epitope name: SL9 } \\
\text { - Examined in the con }\end{array}$ & $\begin{array}{l}\text { SLYNTVATL } \\
\text { f motifs import }\end{array}$ & $\begin{array}{l}\text { HIV-1 infection } \\
\text { binding }\end{array}$ & human (A2) & Parker1992, Parker1994 \\
\hline p17 (77-85) & $\begin{array}{l}\text { p17 (77-85 LAI) } \\
\text { - Epitope name: SL9 } \\
\text { - Review of HIV CTL }\end{array}$ & $\begin{array}{l}\text { SLYNTVATL } \\
\text { spes }\end{array}$ & HIV-1 infection & human (A2) & McMichael1994 \\
\hline p17 (77-85) & $\begin{array}{l}\text { p17 (77-85) } \\
\text { - Epitope name: SL9 } \\
\text { - CTL clones recogniz }\end{array}$ & $\begin{array}{l}\text { SLYNTVATL } \\
\text { urally processec }\end{array}$ & HIV-1 infection & human (A2) & Tsomides1994 \\
\hline p17 (77-85) & $\begin{array}{l}\text { p17 (77-85) } \\
\text { Epitope name: SL9 }\end{array}$ & SLYNTVATL & in vitro stimulation & human (A2) & Stuhler1997 \\
\hline
\end{tabular}




\begin{tabular}{|c|c|c|c|c|}
\hline HXB2 Location & Author's Location & Sequence & Species (HLA) & References \\
\hline & \multicolumn{4}{|c|}{ - A three cell-type cluster consisting of APCs, Th, and CTLs is the minimal regulatory unit required for Th cell-dependent induction of CTLs } \\
\hline p17 (77-85) & \multicolumn{3}{|c|}{$\begin{array}{l}\text { p17 (77-85) SLYNTVATL HIV-1 infection } \\
\text { - Epitope name: SL9 } \\
\text { - The consensus peptides of B and D clade viruses and some Cs have the sequence SLYNTVATL } \\
\text { - The consensus peptide of A, and some C strains have SLFNTVATL, a form that is cross-reactive }\end{array}$} & Cao1997a \\
\hline p17 (77-85) & \multicolumn{2}{|c|}{$\begin{aligned} & \mathrm{C} \\
& \text { - } \mathrm{E} \\
& \text { - } \mathrm{C} \\
& \text { - } \mathrm{S} \\
&\end{aligned}$} & $\begin{array}{l}\text { HIV-1 infection human (A2) } \\
\text { ear period in members of the Sydney Blood Bank Coh } \\
\text { effector and memory cells despite low viral load }\end{array}$ & D) who had been infected with a \\
\hline p17 (77-85) & \multicolumn{2}{|l|}{$\begin{array}{l}\text { p17 (77-85) } \\
\text { - Epitope name: SL9 } \\
\text { - Two overlapping epi } \\
\text { (SLYNTVATL) } \\
\text { - Viral sequence subst } \\
\text { reduced recognition }\end{array}$} & $\begin{array}{l}\text { HIV-1 infection human (A2) } \\
\text { survivor, restricted by two different HLA molecules, } \\
\text { al which did not affect viral replication and did not alte } \\
\text { hune escape }\end{array}$ & $\begin{array}{l}\text { Harrer1998 } \\
\text { 1(TLYCVHQR) and -A2 } \\
\text { ecognition of the A2 epitope, but }\end{array}$ \\
\hline p17 (77-85) & \multicolumn{2}{|c|}{$\begin{array}{l}\text { p17 ( } 77-85 \mathrm{SF} 2) \\
\text { - The relative contribution } \\
\text { - Individuals with long-ter } \\
\text { - Vpr is a frequent target o } \\
\text { targeted proteins per uni } \\
\text { - The A2 eptiopes Vpr AI } \\
\text { individuals are excluded }\end{array}$} & $\begin{array}{l}\text { HIV-1 infection human (A2) } \\
\text { Vpr, Vpu, and Vif were analyzed in multiple HIV-1-in } \\
\text { onic HIV-1 infection targeted Vpr more frequently tha } \\
\text { response was detected in 45\% of individuals tested ar } \\
\text { do not account for the dominance of Vpr and p17, the }\end{array}$ & $\begin{array}{l}\text { Altfeld2001a } \\
\text { dividuals } \\
\text { luals with treated acute infection } \\
\text { ad p17 were the most preferentially } \\
\text { olds even when HLA-A2+ }\end{array}$ \\
\hline p17 (77-85) & $\begin{array}{l}\text { p17 (BRU) } \\
\text { - Epitope name: SL9 } \\
\text { - Exogenous presentat } \\
\text { epitope processing th } \\
\text { - Dendritic cells treate } \\
\text { without protein synth } \\
\text { - Exogenous Gag epitc }\end{array}$ & $\begin{array}{l}\text { SLYNTVATL } \\
\text { or cross-presenta } \\
\text { lay be important } \\
\text { th AZT to inhibi } \\
\text {, while macroph } \\
\text { presentation was }\end{array}$ & $\begin{array}{l}\text { in vitro stimulation human (A2) } \\
\text { by antigen presenting cells (APC) without protein syn } \\
\text { neration of viral specific CTL } \\
\text { sis were able to elicit a strong specific CTL response in } \\
\text { ed a decreased presentation efficiency } \\
\text { and required receptor-dependent fusion }\end{array}$ & $\begin{array}{l}\text { Buseyne2001 } \\
\text { VATL specific CTL line EM71-1 }\end{array}$ \\
\hline p17 (77-85) & $\begin{array}{l}\text { p17 } \\
\text { - HLA tetramers to si } \\
\text { with viral load in pa } \\
\text { - Most patients have h } \\
\text { - In } 15 \text { of the patients, } \\
\text { - In one patient with a } \\
\text { serum), suggesting } 1\end{array}$ & $\begin{array}{l}\text { SLYNVATL } \\
\text { opes were used } \\
\text { with high CD4, } \\
\text { vels of HIV-spe } \\
\text { roportion of IFI } \\
\text { NVATL respons } \\
\text { vivo efficacy of }\end{array}$ & $\begin{array}{l}\text { HIV-1 infection human (A2) } \\
\text { 2, B8 and B57 CTL in } 54 \text { patients - HIV-specific tetra } \\
\text { ith CD4 T-cells below } 400 \text { high tetramer frequencies } \\
\text { asions, but many of these cells aren't functional } \\
\text { ing tetramer cells correlated with AIDS-free survival } \\
\text { mutations were found among } 21 \text { clones despite high } \\
\text { response }\end{array}$ & $\begin{array}{l}\text { Kostense2001 } \\
\text { ive cells were inversely correlated } \\
\text { ad despite high viral load } \\
\text { d (260,000 RNA copies/ml }\end{array}$ \\
\hline
\end{tabular}




\begin{tabular}{llllll}
\hline HXB2 Location & Author's Location & Sequence & Immunogen & Species (HLA) \\
\hline p17 (77-85) & p17 (77-85) & SLYNTVATL & HIV-1 infection & human (A2) & Ferrari2000
\end{tabular}

- One of the 51 HIV-1 epitopes selected by Ferrari et al. as good candidate CTL epitopes for vaccines by virtue of being conserved and presented by common HLA alleles

\begin{tabular}{llccc}
\hline p17 (77-85) & p17 & SLYNVATL & HIV-1 infection & Suman (A2)
\end{tabular}

response to therapy, but the overall level of antigen-specific cells capable of differentiating into effectors stays constant and new epitopes may be recognized

- 6/10 A*0201+ individuals had HIV-specific tetramer staining cells, and 5 of these declined upon successful therapy

- 4/10 A*0201+ individuals with chronic HIV-1 infection recognized this epitope

- Prior to therapy, the mean percentage of CD8+ cells that recognized the immunodominant epitope SLYNVATL was six-fold greater than the percentage recognizing the epitope ILKEPVHGV

\begin{tabular}{lllll}
\hline p17 (77-85) & p17 (77-85) & SLYNTVATL & HIV-1 infection & human (A2)
\end{tabular}

- Epitope name: SL9

- Transcript frequencies were followed for four CTL clones from patient 115, with a chronic and stable HIV-1 infection, were tracked in a longitudinal study of samples collected 6-11 years post infection: clone M21 and E15 recognize ERYLKDQQL, and clone D87 recognizes variant ERYLQDQQL, and clone p175b recognizes the A2 epitope SLYNTVATL

- This epitope sequence from clone p175b uses the Vbeta5, CDR3 (FDS), Jbeta2.7 TCR beta gene

- Responses were stable even through HAART with undetectable viral loads, but frequencies varied over time by 100 -fold, ranging from $0.012 \%$ of the total population for SLYNTVATL at its lowest point to $3.78 \%$ for M21, with the relative frequencies of clones shifting over time

p17 (77-85) p17 (77-85 SF2) SLYNTVATL HIV-1 infection $\quad$ human (A2)

- Therapy provided during acute infection resulted in a narrower CTL response, stronger T help response, and a less diverse viral population than was seen in individuals treated during chronic infection

- The breadth and specificity of the response was determined using ELISPOT by studying 19 individuals with pre-seroconversion therapy (Group 1), 11 individuals with primary infection but post-seroconversion therapy (Group 2), and 10 individuals who responded to HAART given during chronic infection (Group 3), using 259 overlapping peptides spanning p17, p24, RT, gp41, gp120 and Nef

- Previously described and newly defined optimal epitopes were tested for CTL response

- Number of HLA-A2+ individuals that had a CTL response to this epitope broken down by group: 1/10 group 1, 2/6 group 2, and 2/4 group 3

p17 (77-85) p17(77-85) SLFNTVATL HIV-1 infection, HIV-1 exposed human (A2) Kaul2001a

- Variants SL(F/Y)NTVATL are A/B clade specific

- ELISPOT was used to study CTL responses to a panel of 54 predefined HIV-1 epitopes in 91 HIV-1-exposed, persistently seronegative (HEPS) and 87 HIV-1-infected female Nairobi sex workers

- Responses in HEPS women tended to be lower, and focused on different epitopes with HLA presenting molecules that have previously been associated with reduced risk of infection, and there was a shift in the response in the HEPS women upon late seroconversion to epitopes recognized by the HIV-1 infected women

- 43/91 HEPS women had CD8+ responses and detection of HIV-1-specific CTL in HEPS women increased with the duration of viral exposure

- Among HLA-A2 women, 1/10 HEPS and 22/26 HIV-1 infected women recognized this epitope, likelihood ratio 18.3, p value < 0.003, and ILK(D/E)PVHGV tended to be more reactive in HEPS women, SL(F/Y)NTVATL in infected women

- The dominant response to this HLA allele was to this epitope in the 1/10 HEPS case and in 18 of the 22/26 HIV-1 infected women that responded

- Differences in epitope specificity were only seen for responses restricted by class I HLA alleles A2, A24, A*6802, B14, and B18, previously shown to be associated with resistance to HIV-1 in this cohort 


\begin{tabular}{|c|c|c|c|c|}
\hline HXB2 Location & Author's Location & Sequence & Species (HLA) & References \\
\hline & \multicolumn{4}{|c|}{$\begin{array}{l}\text { - Subject ML } 1250 \text { had an A2 response to ILK(D/E)PVHGV prior to seroconversion, which switched to SL(F/Y)NTVATL post-seroconversion } \\
\text { - Subjects ML } 1575 \text { and ML } 1592 \text { had no response to SL(F/Y)NTVATL prior to seroconversion, but made responses post-seroconversion } \\
\text { - Subject ML } 1760 \text { had an A2 response to ILK(D/E)PVHGV prior to seroconversion, and gained responses to epitopes A2 SL(F/Y)NTVATL and B27 } \\
\text { KRWII(L/M)GLNK post-seroconversion }\end{array}$} \\
\hline p17 (77-85) & $\begin{array}{l}\text { p17 (77-85 93TH253 } \\
\text { subtype CRF01) } \\
\text { - Epitope name: G77-85 } \\
\text { - This was a study of HIV- } \\
\text { - HLA-A11 is very comm } \\
\text { and CTL responses were } \\
\text { - This epitope was reactive }\end{array}$ & $\begin{array}{l}\text { SLYNTIATL } \\
1 \text { exposed persis } \\
\text { n in this populat } \\
\text { found in } 8 / 8 \mathrm{HI} \\
\text { in HIV+ contro }\end{array}$ & $\begin{array}{l}\text { HIV-1 infection human (A2) } \\
\text { ve (HEPS) female sex workers in Chiang Mai, norther } \\
\text { iched among the HEPS sexworkers - weak CTL resp } \\
\text { 0/9 HIV- women that were not exposed } \\
25 \text { and } 144 \text { who carried HLA-A2 }\end{array}$ & $\begin{array}{l}\text { Sriwanthana2001 } \\
\text { nd } \\
\text { ee detected in 4/7 HEPS women, }\end{array}$ \\
\hline p17 (77-85) & $\begin{array}{l}\text { p17 (77-85 93TH253 } \\
\text { subtype CRF01) } \\
\text { - More than half of a coho } \\
\text { epitopes in this group, al } \\
\text { - } 2 / 4 \text { tested FSWs recogni } \\
\text { - This epitope was only co }\end{array}$ & $\begin{array}{l}\text { SLYNTIATL } \\
\text { th of HIV+ femal } \\
\text { hough E clade v } \\
\text { ed the E clade } \mathrm{v} \\
\text { nserved in CRFO }\end{array}$ & $\begin{array}{l}\text { HIV-1 infection human (A2) } \\
\text { FSW) from Northern Thailand were HLA-A11 positive } \\
\text { ously defined B-clade A2 and A24 epitopes were also } t \\
\text { itope, SLYNTIATL, the B clade version is SLYNTVAT } \\
\text { B and D, and exact matches were uncommon }\end{array}$ & Bond2001 \\
\hline p17 ( & $\begin{array}{l}\text { p17 (77-85) } \\
\text { - The CTL response to opt } \\
\text { studied in eight HIV-1-in } \\
\text { - } 2 \text { to } 17 \text { epitopes were rec } \\
\text { epitopes were targeted by } \\
\text { - SLYNTVATL was the do } \\
\text { - Three subjects had an A2 } \\
\text { - The two subjects with ac }\end{array}$ & $\begin{array}{l}\text { SLYNTVATL } \\
\text { imally defined C } \\
\text { fected subjects, } \\
\text { ognized in a give } \\
\text { at least one per } \\
\text { minant A2 epito } \\
\text { response only t } \\
\text { ute infection did }\end{array}$ & $\begin{array}{l}\text { HIV-1 infection human (A2) } \\
\text { tricted by HLA class I A and B alleles in individuals w } \\
\text { nfection, five with chronic, and one long-term non-pros } \\
\text { 2-restricted CTL response tended to be narrow and nev } \\
\text { patients with chronic infection, except for Subject } 118 \\
\text { LYNTVATL }\end{array}$ & $\begin{array}{l}\text { Day2001 } \\
\text { ressed HLA A2, A3, and B7 was } \\
\text { TNP) } \\
\text { ated the response, and } 25 / 27 \\
\text { recognized } 5 / 8 \text { epitopes }\end{array}$ \\
\hline p17 (77-85) & $\begin{array}{l}\text { p17 (77-85) } \\
\text { - Epitope name: SL9 } \\
\text { - Immune escape variants } \\
\text { - Eight transmitting mothe } \\
\text { but no link between varia }\end{array}$ & $\begin{array}{l}\text { SLYNTVATL } \\
\text { this epitope wl } \\
\text { and } 14 \text { non-tra } \\
\text { ion from the SL }\end{array}$ & $\begin{array}{l}\text { HIV-1 infection human (A2) } \\
\text { both horizontally and vertically in two families } \\
\text { ss were studied and variation within the SL9 epitope w } \\
\text { vertical transmission was established }\end{array}$ & $\begin{array}{l}\text { Goulder2001c } \\
\text { iated carrying HLA-A2 }(\mathrm{P}=0.04) \text {, }\end{array}$ \\
\hline p17 (77-85) & $\begin{array}{l}\text { - } 17 \text { (SF2) } \\
\text { an HLA-B60 individual } \\
\text { - The response to the pepti }\end{array}$ & $\begin{array}{l}\text { SLYNTVATL } \\
\text { by ELISPOT in } \\
\text { le was CD8 dep }\end{array}$ & $\begin{array}{l}\text { HIV-1 infection } \\
\text { ng new HLA-B60 epitopes, and was one of the epitop } \\
\text { LLA presenting molecule and optimal epitope were no }\end{array}$ & $\begin{array}{l}\text { Altfeld2000b } \\
\text { ted by another HLA molecule in }\end{array}$ \\
\hline p17 (77-85) & $\begin{array}{l}\text { p17 (77-85 LAI) } \\
\text { - Ritonavir (RTV) inhibi } \\
\text { there is concern protea } \\
\text { relevant concentrations }\end{array}$ & $\begin{array}{l}\text { SLYNTVATL } \\
\text { hymotryptic ac } \\
\text { nhibitors may a } \\
\text { RTV when the }\end{array}$ & $\begin{array}{l}\text { HIV-1 infection } \operatorname{human}(\mathrm{A} 2) \\
\text { roteasome in vitro, as does Saquinavir (SQV) to a le } \\
\text { TL epitope processing, but this paper indicates that } \mathrm{p} \\
\text { hctioning in in an intracellular context. }\end{array}$ & $\begin{array}{l}\text { Kelleher2001a } \\
\text { Indinavir (IDV) does not. Thus } \\
\text { not inhibited at therapeutically }\end{array}$ \\
\hline
\end{tabular}




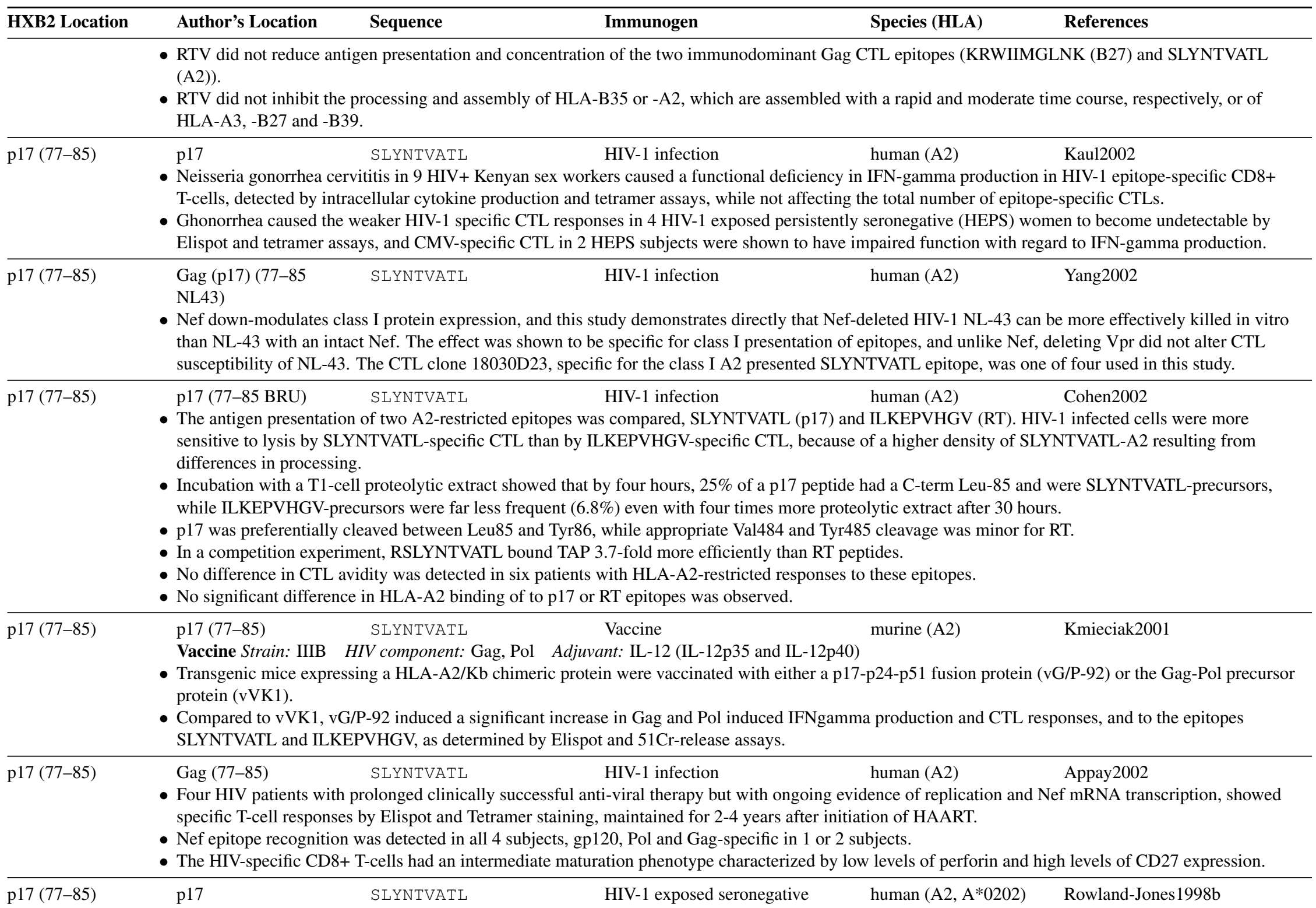

- Epitope name: SL9

- HIV-specific CTL were found in exposed seronegative prostitutes from Nairobi - these CTL may confer protection

- Seroprevalence in this cohort is $90-95 \%$ and their HIV-1 exposure is among the highest in the world 


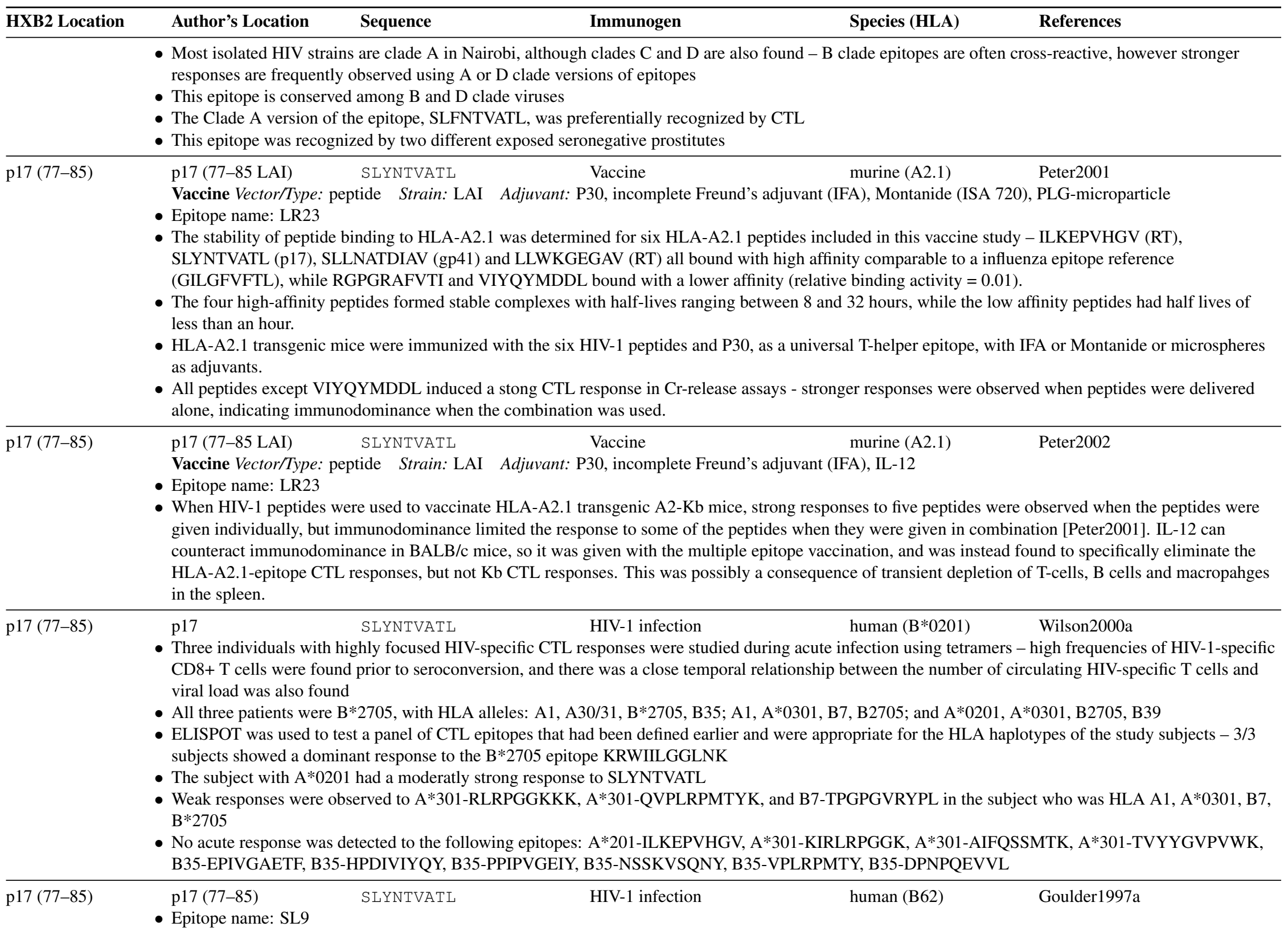




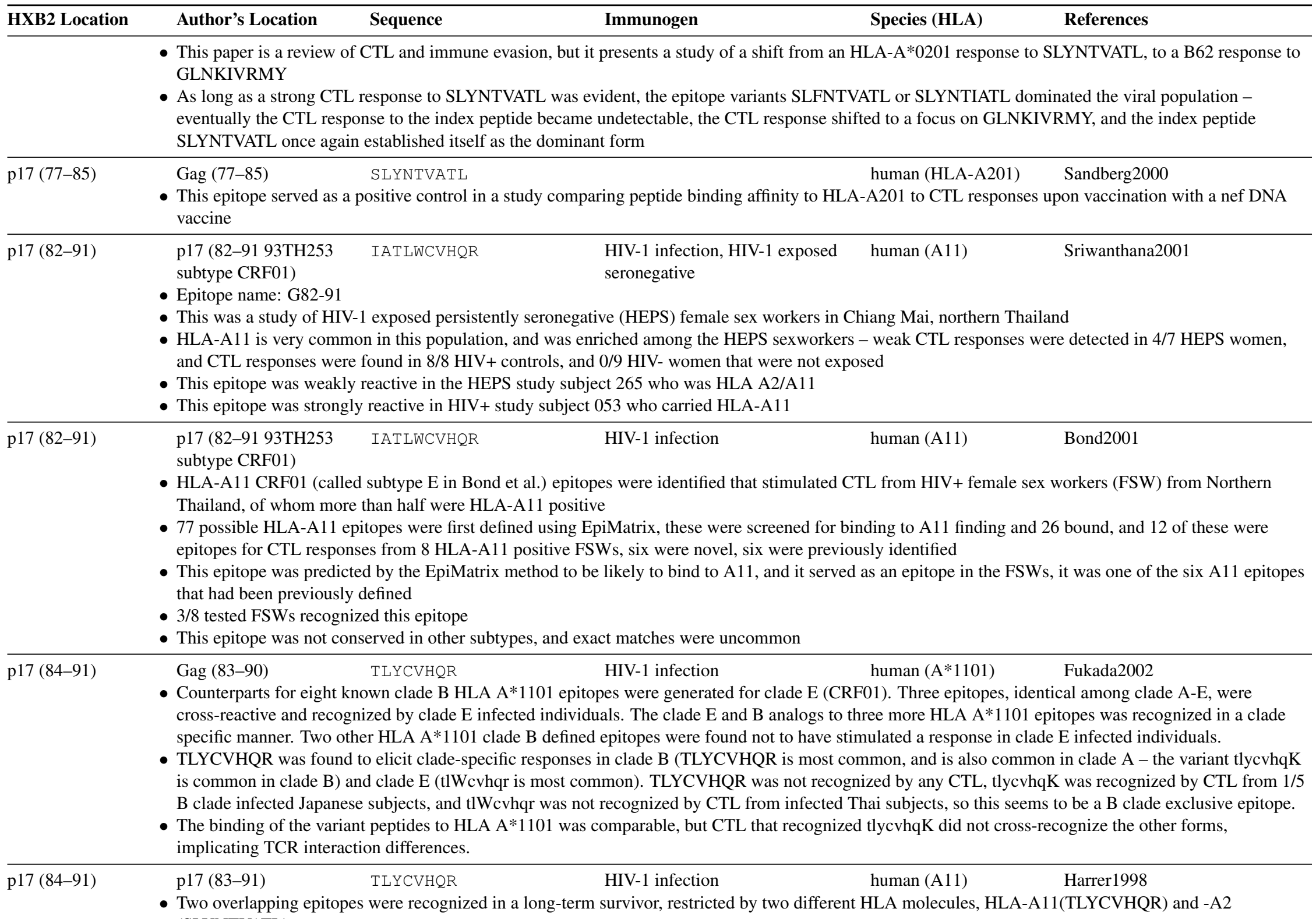
(SLYNTVATL) 


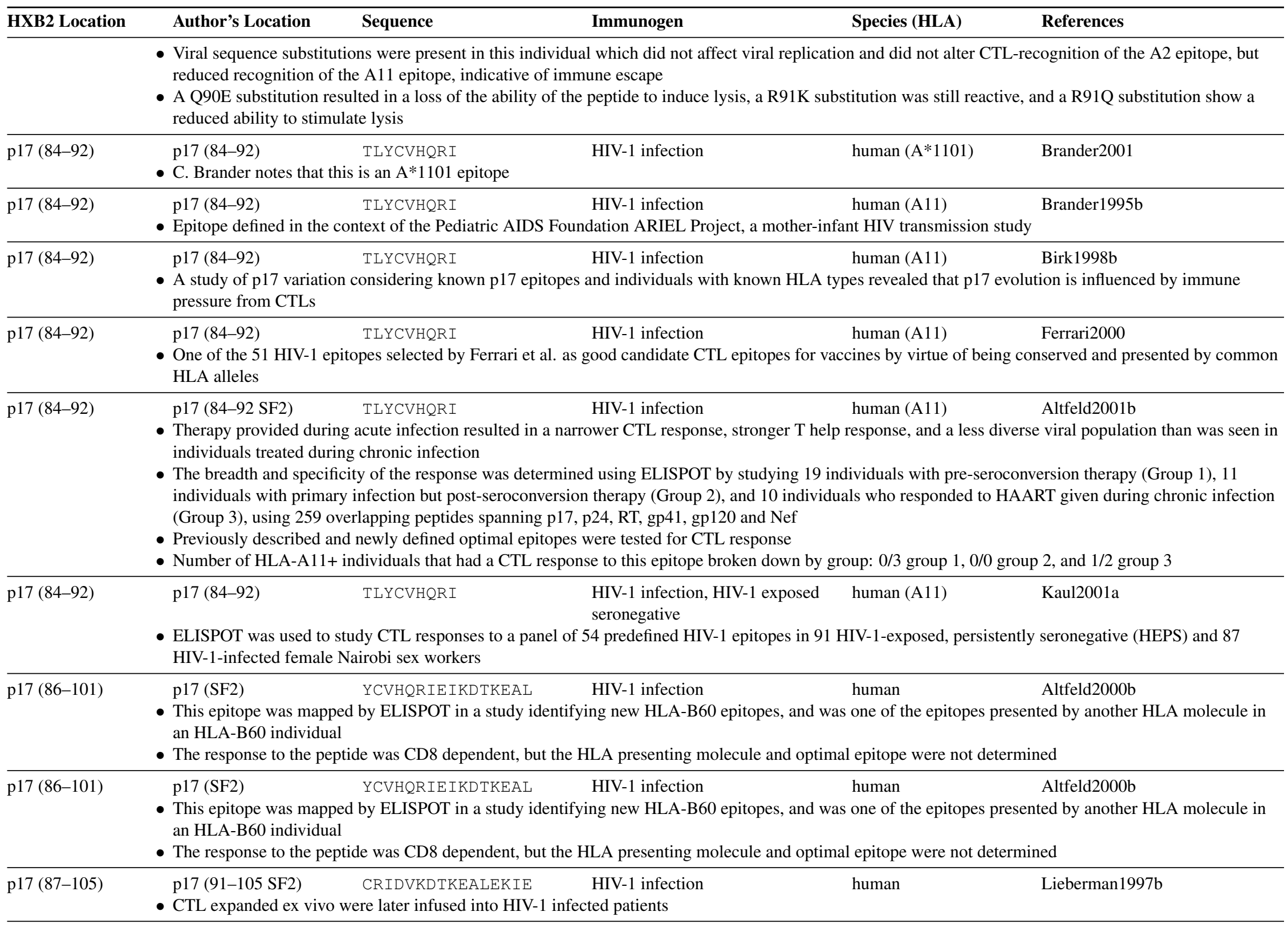




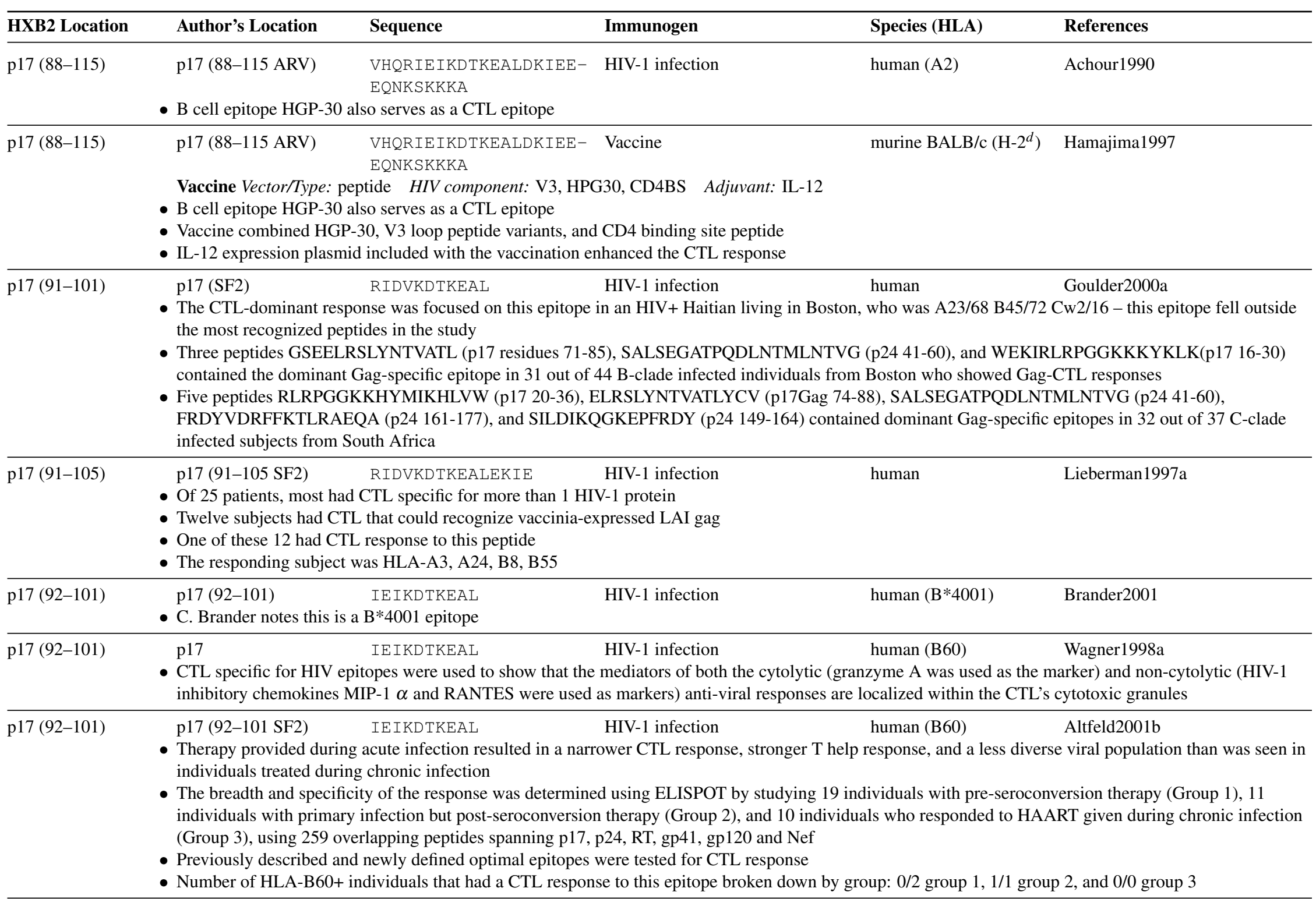




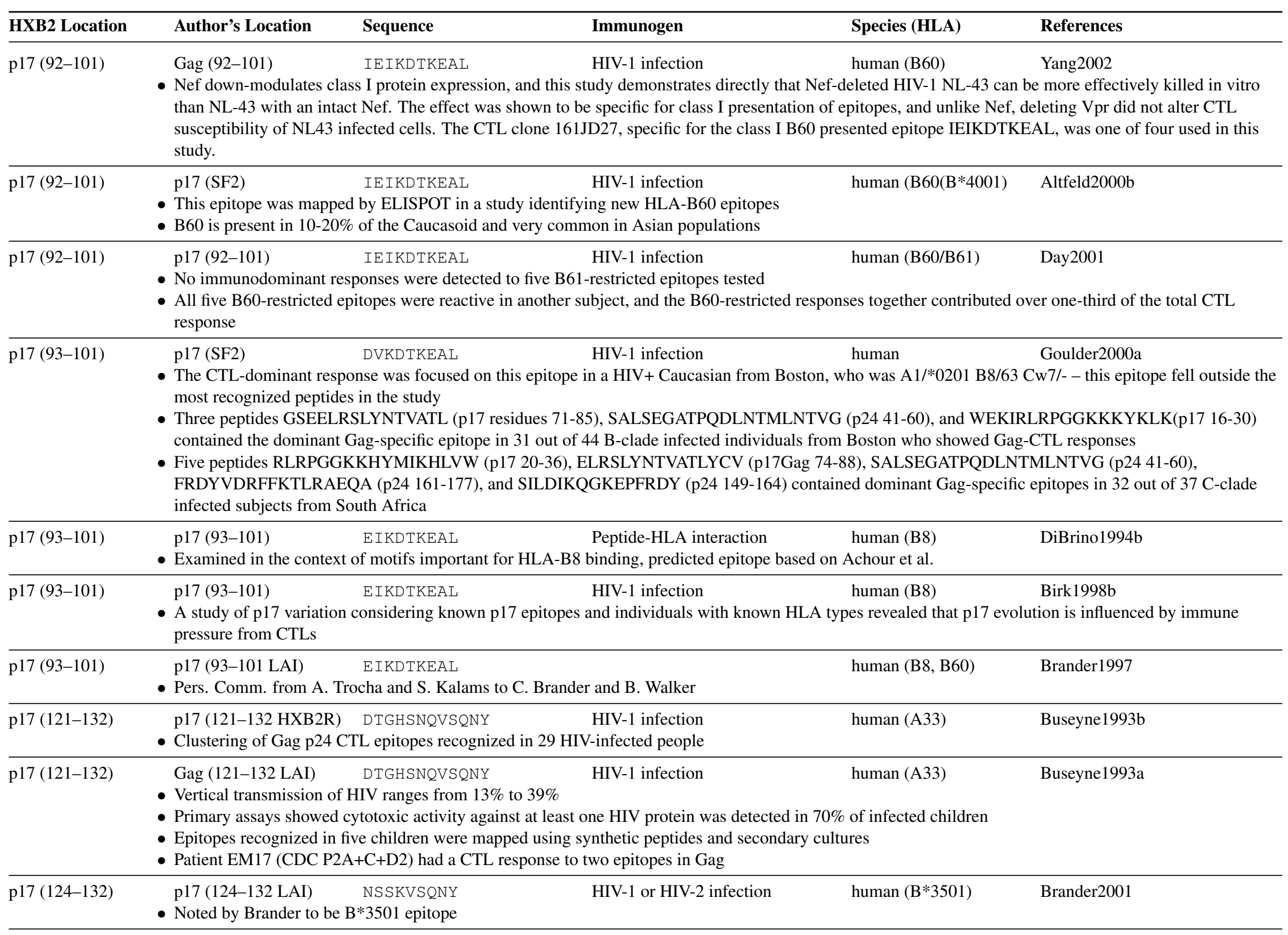




\begin{tabular}{lllll}
\hline HXB2 Location & Author's Location & Sequence & Immunogen & Species (HLA) \\
\hline p17 (124-132) & p17 & NSSQVSQNY & HIV-1 infection & human $(B * 3501)$
\end{tabular}

- The crystal structure of this epitope bound to HLA-B*3501 shows that a serine can fit into the B pocket, which is shared between B35 and B53, with the hydroxyl group of the $\mathrm{P} 2$ serine occupying a position almost identical to the $\mathrm{P} 2$ proline that was previously considered the anchor motif

- Novel B53 epitopes (DTINEEAAEW and QATQEVKNM) were defined in this study that showed that A and T can also serve as P2 anchor residues for the B pocket of HLA-B35 and B53 - while S, T, and P could all fit into the B pocket and form a hydrogen bond, A would not form a bond, so the authors propose compensatory interactions account for the high affinity of QATQEVKNM for B53

$\begin{array}{lll}\text { p17 (124-132 LAI) NSSKVSQNY } & \text { HIV-1 infection } & \text { human (B35) } \\ \text { Review of HIV CTL epitopes } & \end{array}$

p17 (124-132) NSSKVSQNY HIV-1 infection human (B35) Wilson2000a

- Three individuals with highly focused HIV-specific CTL responses were studied during acute infection using tetramers - high frequencies of HIV-1-specific CD8+ T cells were found prior to seroconversion, and there was a close temporal relationship between the number of circulating HIV-specific T cells and viral load was also found

- All three patients were B*2705, with HLA alleles: A1, A30/31, B*2705, B35; A1, A*0301, B7, B2705; and A*0201, A*0301, B2705, B39

- ELISPOT was used to test a panel of CTL epitopes that had been defined earlier and were appropriate for the HLA haplotypes of the study subjects - 3/3 subjects showed a dominant response to the B*2705 epitope KRWIILGGLNK

- The subject with $\mathrm{A}^{*} 0201$ had a moderatly strong response to SLYNTVATL

- Weak responses were observed to A*301-RLRPGGKKK, A*301-QVPLRPMTYK, and B7-TPGPGVRYPL in the subject who was HLA A1, A*0301, B7, $\mathrm{B} * 2705$

- No acute response was detected to the following epitopes: A*201-ILKEPVHGV, A*301-KIRLRPGGK, A*301-AIFQSSMTK, A*301-TVYYGVPVWK, B35-EPIVGAETF, B35-HPDIVIYQY, B35-PPIPVGEIY, B35-NSSKVSQNY, B35-VPLRPMTY, B35-DPNPQEVVL

p17 (124-132) p17 (124-132) NSSKVSQNY HIV-1 infection

- A study of p17 variation considering known p17 epitopes and individuals with known HLA types revealed that p17 evolution is influenced by immune pressure from CTLs

\begin{tabular}{llllll}
\hline p17 (124-132) & $\begin{array}{l}\text { p17 (124-132 LAI) } \\
\text { - Established by titration }\end{array}$ & NSSKVSQNY & HIV-1 or HIV-2 infection & human (B35) \\
\hline p17 (124-132) & p17 (124-132 LAI) & NSSKVSQNY & in vitro stimulation & human (B35)
\end{tabular}

- A peptide-based protocol was optimized for restimulation of CTLp using optimized peptide and IL-7 concentrations - importantly this protocol does not stimulate a primary response, only secondary - peptide-specific CTLp counts could be obtained via staining with peptide-Class I tetramers

- This peptide was one of the B35 presented test peptides used in control experiments showing that the assay gave no activity using lymphocytes from 21 healthy B35 seronegative donors

p17 (124-132) p17 NSSKVSONY human (B35) Rowland-Jones1999

- CTL responses in seronegative highly HIV-exposed African female sex workers in Gambia and Nairobi were studied - these women had no delta 32 deletion in CCR5

- In Gambia there is exposure to both HIV-1 and HIV-2, CTL responses to B35 epitopes in exposed, uninfected women are cross-reactive

- HIV-2 version of this epitope is not conserved: PPSGKGGNY, but the CTLs are cross-reactive - this is one of five B35 CTL epitopes that are cross-reactive, see also [Rowland-Jones1995b] 


\begin{tabular}{|c|c|c|c|c|c|}
\hline HXB2 Location & Author's Location & Sequence & Immunogen & Species (HLA) & References \\
\hline p17 (124-132) & \multicolumn{5}{|c|}{$\begin{array}{l}\text { - CTL responses were studied by tetramer staining in } 41 \text { patients with combination therapy - activated CD8+ T-cells decline as the viral load drops in } \\
\text { response to therapy, but the overall level of antigen-specific cells capable of differentiating into effectors stays constant and new epitopes may be recognized }\end{array}$} \\
\hline p17 (124-132) & \multicolumn{5}{|c|}{$\begin{array}{l}\text { - Therapy provided during acute infection resulted in a narrower CTL response, stronger T help response, and a less diverse viral population than was seen in } \\
\text { individuals treated during chronic infection } \\
\text { - The breadth and specificity of the response was determined using ELISPOT by studying } 19 \text { individuals with pre-seroconversion therapy (Group } 1 \text { ), } 11 \\
\text { individuals with primary infection but post-seroconversion therapy (Group 2), and } 10 \text { individuals who responded to HAART given during chronic infection } \\
\text { (Group 3), using } 259 \text { overlapping peptides spanning p17, p24, RT, gp41, gp120 and Nef } \\
\text { - Previously described and newly defined optimal epitopes were tested for CTL response } \\
\text { - Number of HLA-B35+ individuals that had a CTL response to this epitope broken down by group: } 0 / 2 \text { group } 1,0 / 2 \text { group } 2 \text {, and } 1 / 1 \text { group } 3\end{array}$} \\
\hline p17 (124-132) & - Epitope name: Gag & $\begin{array}{l}\text { NSSKVSQNY } \\
\text { who carried HI }\end{array}$ & $\begin{array}{l}\text { HIV-1 infection } \\
\text { o) recognized this }\end{array}$ & human (B35) & Sabbaj2002b \\
\hline
\end{tabular}




\section{II-B-2 p17-p24 CTL Epitopes}

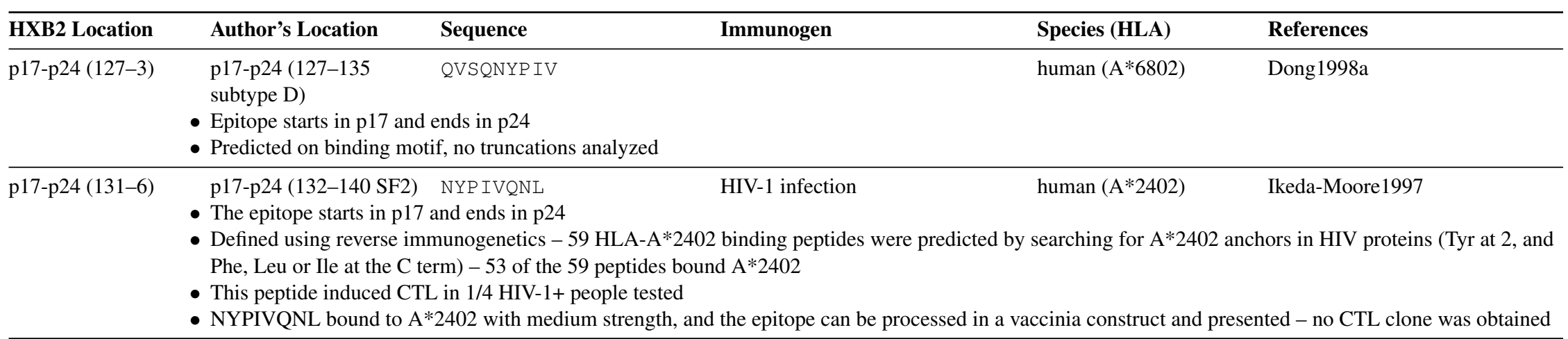




\section{II-B-3 p24 CTL Epitopes}

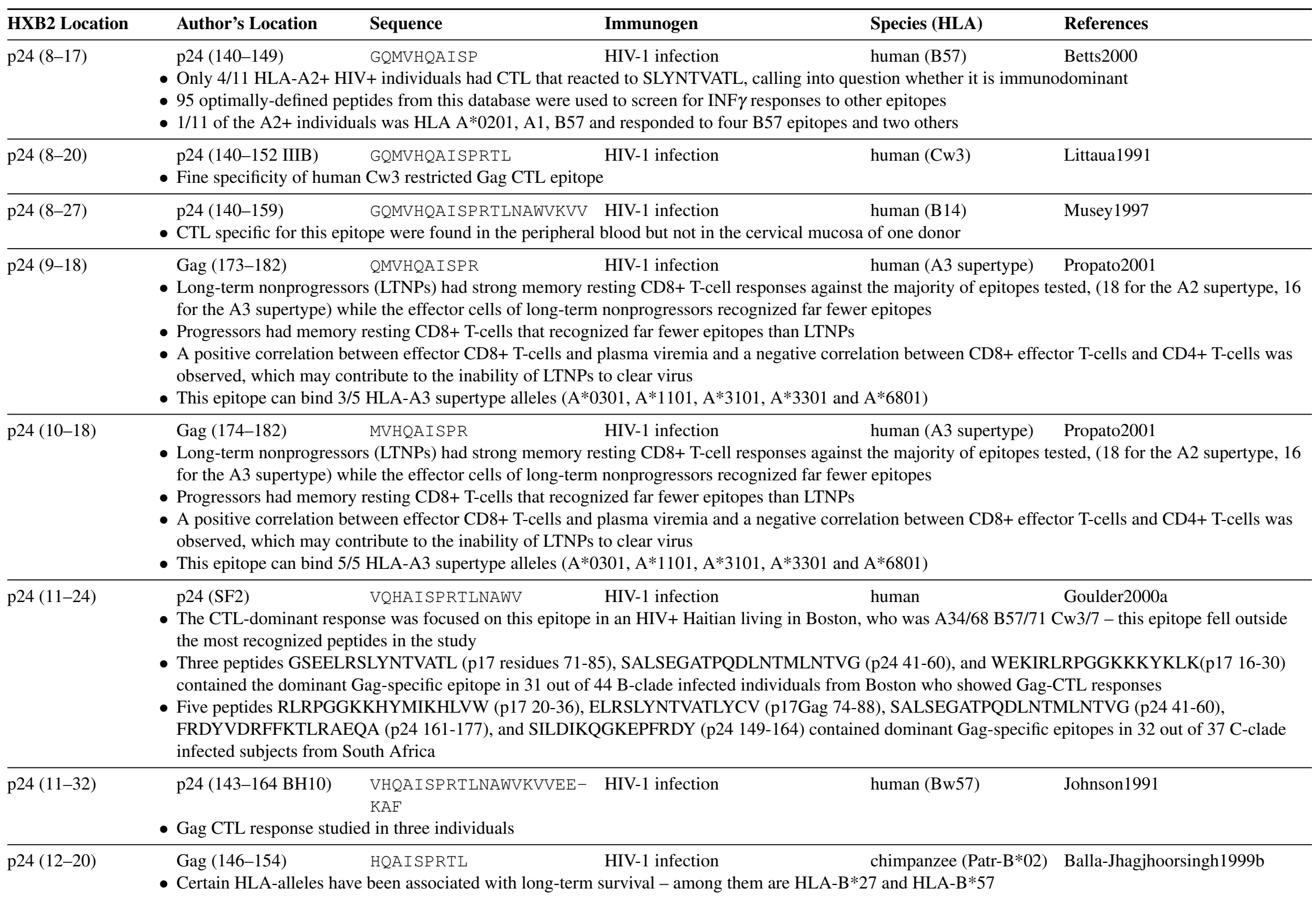




\begin{tabular}{|c|c|c|c|c|c|}
\hline HXB2 Location & Author's Location & Sequence & Immunogen & Species (HLA) & References \\
\hline & \multicolumn{5}{|c|}{$\begin{array}{l}\text { - Of more than } 150 \text { chimpanzees that have been reported to be infected with HIV-1, only one has developed AIDS } \\
\text { - CTL responses were studied in two HIV-1 infected chimpanzees that have strong CTL responses, and they were found to respond to highly conserved } \\
\text { epitopes that are recognized in humans in the context of HLA-B*27 and HLA-B*57 } \\
\text { - The human HLA protein which presents this Patr-B*02 epitope is HLA-B*5701 but the amino acid sequences in the binding pockets of HLA-B*5701 and } \\
\text { Patr-B*02 are distinctive }\end{array}$} \\
\hline p24 (13-20) & \multicolumn{5}{|c|}{$\begin{array}{l}\text { - ELISPOT was used to study CTL responses to a panel of } 54 \text { predefined HIV-1 epitopes in } 91 \text { HIV-1-exposed, persistently seronegative (HEPS) and } 87 \\
\text { HIV-1-infected female Nairobi sex workers }\end{array}$} \\
\hline $24(13-23)$ & \multicolumn{5}{|c|}{$\begin{array}{l}\text { - Only 4/11 HLA-A2+ HIV+ individuals had CTL that reacted to SLYNTVATL, calling into question whether it is immunodominant } \\
\text { - } 95 \text { optimally-defined peptides from this database were used to screen for INF } \gamma \text { responses to other epitopes } \\
\text { - } 1 / 11 \text { of the A2+ individuals was HLA A*0201, A1, B57 and responded to QAISPRTLNAW noted previously to be A25 }\end{array}$} \\
\hline p24 (13-23) & $\begin{array}{l}\text { p24 (145-155 LAI) } \\
\text { - C. Brander notes the }\end{array}$ & $\begin{array}{l}\text { QAISPRTLNAW } \\
\text { is an } \mathrm{A} * 2501 \text { epit }\end{array}$ & & human $\left(A^{*} 2501\right)$ & Brander2001 \\
\hline p24 (13-23) & \multicolumn{5}{|c|}{$\begin{array}{l}\text { - Therapy provided during acute infection resulted in a narrower CTL response, stronger T help response, and a less diverse viral population than was seen in } \\
\text { individuals treated during chronic infection } \\
\text { - The breadth and specificity of the response was determined using ELISPOT by studying } 19 \text { individuals with pre-seroconversion therapy (Group } 1 \text { ), } 11 \\
\text { individuals with primary infection but post-seroconversion therapy (Group 2), and } 10 \text { individuals who responded to HAART given during chronic infection } \\
\text { (Group 3), using } 259 \text { overlapping peptides spanning p17, p24, RT, gp41, gp120 and Nef } \\
\text { - Previously described and newly defined optimal epitopes were tested for CTL response } \\
\text { - Number of HLA-A25+ individuals that had a CTL response to this epitope broken down by group: } 0 / 0 \text { group } 1,0 / 2 \text { group } 2 \text {, and } 1 / 3 \text { group } 3\end{array}$} \\
\hline p24 (13-23) & p24 (145-155 LAI) & QAISPRTLNAW & & human (A5) & Kurane1998 \\
\hline p24 (15-23) & $\begin{array}{l}\text { - This study examine: } \\
\text { sex workers eventua } \\
\text { - The epidemiologica } \\
\text { working for a perioc } \\
\text { ISPRTLNAW was c } \\
\text { worker controls (MI }\end{array}$ & $\begin{array}{l}\text { LSPRTLNAW } \\
\text { responses in HIV } \\
\text { roconverted, and f } \\
\text { or associated with } \\
\text { etire } \\
\text { tently recognized b } \\
3 \text { and ML1589) }\end{array}$ & $\begin{array}{l}\text { HIV-1 infection, HIV-1 exposed } \\
\text { seronegative } \\
\text { istently seronegative individuals, } \\
\text { HIV CTL reactive epitopes had be } \\
\text { was stopping sex work and HIV-s } \\
\text { sex worker controls (ML1250), an }\end{array}$ & $\begin{array}{l}\text { human } \\
\text { S, who eventual } \\
\text { defined while se } \\
\text { ific CTL activity } \\
\text { SPRTLNAW wa }\end{array}$ & $\begin{array}{l}\text { Kaul2001c } \\
\text { nverted - 11/114 HEPS Nairobi } \\
\text { when HEPS sex workers stop } \\
\text { zed by } 2 \text { additional HEPS sex }\end{array}$ \\
\hline p24 (15-23) & $\begin{array}{l}\text { - N24 Neisseria gonorrhea } \\
\text { T-cells, detected by } \\
\text { - Ghonorrhea caused } \\
\text { Elispot and tetramer }\end{array}$ & $\begin{array}{l}\text { LSPRTLNAW } \\
\text { titis in } 9 \text { HIV+ Ke } \\
\text { ellular cytokine pr } \\
\text { eaker HIV-1 specif } \\
\text { S, and CMV-speci }\end{array}$ & $\begin{array}{l}\text { HIV-1 infection } \\
\text { ers caused a functional deficiency } \\
\text { etramer assays, while not affectir } \\
\text { ses in } 4 \text { HIV-1 exposed persisten } \\
\text { IEPS subjects were shown to hav }\end{array}$ & $\begin{array}{l}\text { human }(\mathrm{B} * 57) \\
\text { IFN-gamma pro } \\
\text { e total number o } \\
\text { eronegative }(\mathrm{HE} \\
\text { paired function }\end{array}$ & $\begin{array}{l}\text { Kaul2002 } \\
\text { HIV-1 epitope-specific CD8+ } \\
\text { specific CTLs. } \\
\text { en to become undetectable by } \\
\text { d to IFN-gamma production. }\end{array}$ \\
\hline
\end{tabular}




\begin{tabular}{|c|c|c|c|c|c|}
\hline HXB2 Location & Author's Location & Sequence & Immunogen & Species (HLA) & References \\
\hline p24 (15-23) & $\begin{array}{l}\text { p24 (147-155 IIIB) } \\
\text { - C. Brander notes thi }\end{array}$ & $\begin{array}{l}\text { ISPRTLNAW } \\
\text { B*5701 epitope }\end{array}$ & HIV-1 infection & human $(\mathrm{B} * 5701)$ & Brander2001 \\
\hline p24 (15-23) & & ISPRTLNAW & HIV-1 infection & human (B*5701) & Migueles2001 \\
\hline
\end{tabular}

- HLA B*5701 was found in a very high frequency in HIV-1 infected non-progressors, 11/13 (85\%) versus $19 / 200(9.5 \%)$ of progressors. Non-progressors tended to have an immune response that was highly focused on four p24 epitopes that were presented by B*5701, ISPRTLNAW, KAFSPEVIPMF, TSTLQEQIGW, and QASQEVKNW.

p24 (15-23) ISPRTLNAW $\quad$ HIV-1 infection $\quad$ Migueles2001

- CTL activity was monitored in 27 individuals, including 10 LTNP with an over-expression of HLA B*5701 - these individuals have viral loads below the threshold of infection without therapy, and their immune response tends to be focused on peptides that contain B*5701 epitopes ISPRTLNAW,

KAFSPEVIPMF, TSTLQEQIGW, and QASQEVKNW.

- CTL responses are broader in $\mathrm{B} * 5701+$ individuals with progressive viremia than those that control viremia.

- The HLA-A*0201 SLYNTVATL epitope response was not as strong individuals that carried both A2, B57.

\begin{tabular}{lllll}
\hline p24 (15-23) Gag (147-155 LAI) & ISPRTLNAW & HIV-1 infection & human (B*5701 \\
& & & B*5801)
\end{tabular}

- B57 has been associated with long-term non-progression in the Amsterdam cohort

- The most pronounced CTL responses in HLA B*5701 LTS were to RT and Gag

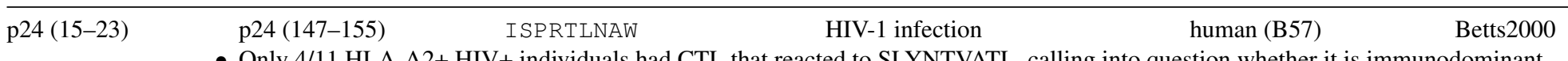

- Only 4/11 HLA-A2+ HIV+ individuals had CTL that reacted to SLYNTVATL, calling into question whether it is immunodominant

- 95 optimally-defined peptides from this database were used to screen for INF $\gamma$ responses to other epitopes

- $1 / 11$ of the A2+ individuals was HLA A*0201, A1, B57 and responded to four B57 epitopes and two others, but not SLYNTVATL

p24 (15-23) Gag (SF2) ISPRTLNAW HIV-1 infection human (B57) Goulder2001a

- Epitope name: IW9

- This epitope elicited the second strongest CTL response in patient PI004 during acute infection, and maintained the response

- Three CTL responses, to epitopes TSTLQEQIGW, ISPRTLNAW, and KAFSPEVIPMF, were evident early after infection; CTL responses to SLYNTVATL, QASQEVKNW, EIYKRWII, and FLKEKGGL were detectable at 5 months post-infection and beyond

p24 (15-23) p24(147-155) ISPRTLNAW $\quad$ HIV-1 infection

- Epitope name: ISP

- Patients who started therapy at acute HIV-1 infection (three with sustained therapy, two with limited therapy upon early infection) had strong HIV specific CD4 proliferative responses and were able to maintain a CTL response even with undetectable viral load - three patients that had delayed initiation of HAART had no HIV specific CD4 proliferative responses and lost their CTL responses when HAART was eventually given and their viral loads became undetectable

- None of the 8 study subjects recognized this epitope but none were HLA B57+

p24 (15-23) p24 (15-23) ISPRTLNAW HIV-1 infection human (B57) Ferrari2000

- One of the 51 HIV-1 epitopes selected by Ferrari et al. as good candidate CTL epitopes for vaccines by virtue of being conserved and presented by common HLA alleles

p24 (15-23) p24(147-155 SF2) ISPRTLNAW HIV-1 infection human (B57) Altfeld2001b

- Therapy provided during acute infection resulted in a narrower CTL response, stronger T help response, and a less diverse viral population than was seen in individuals treated during chronic infection 


\begin{tabular}{|c|c|c|c|c|c|}
\hline HXB2 Location & Author's Location & Sequence & nmunogen & Species (HLA) & References \\
\hline & \multicolumn{5}{|c|}{$\begin{array}{l}\text { - The breadth and specificity of the response was determined using ELISPOT by studying } 19 \text { individuals with pre-seroconversion therapy (Group } 1 \text { ), } 11 \\
\text { individuals with primary infection but post-seroconversion therapy (Group 2), and } 10 \text { individuals who responded to HAART given during chronic infection } \\
\text { (Group 3), using } 259 \text { overlapping peptides spanning p17, p24, RT, gp41, gp120 and Nef } \\
\text { - Previously described and newly defined optimal epitopes were tested for CTL response } \\
\text { - Number of HLA-B57+ individuals that had a CTL response to this epitope broken down by group: 0/0 group } 1,0 / 0 \text { group } 2 \text {, and } 2 / 2 \text { group } 3\end{array}$} \\
\hline $24(15-23)$ & \multicolumn{5}{|c|}{$\begin{array}{l}\text { - Epitope name: Gag-IW9 } \\
\text { - Among HIV+ individuals who carried HLA B57, } 2 / 5(40 \%) \text { recognized this epitope } \\
\text { - Among HIV+ individuals who carried HLA B58, 0/4 (0\%) recognized this epitope }\end{array}$} \\
\hline $24(15$ & \multicolumn{5}{|c|}{$\begin{array}{l}\text { - Epitope name: ISP } \\
\text { - Using previously defined epitopes [Oxenius2000, Oxenius2001a] in an IFNgamma Elispot assay, } 13 \text { chronically HIV-1 infected patients were studied over a } \\
\text { period including therapy with standard treatment interruptions (STI). } \\
\text { - STIs induced increased recognition of CTL epitopes, but there was no correlation between CTL responses with viral rebound rates, plateau viral loads, or } \\
\text { clearance rates. }\end{array}$} \\
\hline $24(15-23)$ & \multicolumn{5}{|c|}{$\begin{array}{l}\text { - Five slow progressors made a response to this epitope, and in two it was the dominant response } \\
\text { - Peptide defined on the basis of } \mathrm{B}^{*} 5801 \text { binding motif, yet not cross-restricted except at high concentrations }\end{array}$} \\
\hline $24(15-23)$ & \multicolumn{5}{|c|}{$\begin{array}{l}\text { - 11/16 heavily HIV exposed but persistently seronegative sex-workers in Nairobi had HIV-specific CD8 gamma-IFN responses in the cervix - systemic } \\
\text { CD8+ T cell responses tended to be to the same epitopes but at generally lower levels than cervical CD8+ T cell responses } \\
\text { - Low risk individuals did not have such CD8+ cells } \\
\text { - CD8+ T cell epitopes: DTVLEDINL ( } 3 \text { individuals), SLYNVATL (4 individuals), LSPRTLNAW ( } 3 \text { individuals) and YPLTFGWCF (4 individuals) were } \\
\text { most commonly recognized by the HIV-resistant women }\end{array}$} \\
\hline $24(15-23)$ & \multicolumn{5}{|c|}{$\begin{array}{l}\text { - Veronegative } \\
\text { - ELISPOT was used to study CTL responses to a panel of } 54 \text { predefined HIV-1 epitopes in } 91 \text { HIV-1-exposed, persistently seronegative (HEPS) and } 87 \\
\text { HIV-1-infected female Nairobi sex workers } \\
\text { - Responses in HEPS women tended to be lower, and focused on different epitopes with HLA presenting molecules that have previously been associated with } \\
\text { reduced risk of infection, and there was a shift in the response in the HEPS women upon late seroconversion to epitopes recognized by the HIV-1 infected } \\
\text { women } \\
\text { - } 43 / 91 \text { HEPS women had CD8+ responses and detection of HIV-1-specific CTL in HEPS women increased with the duration of viral exposure } \\
\text { - Among HLA-B57/B58 women, 4/6 HEPS and 14/17 HIV-1 infected women recognized this epitope } \\
\text { - The dominant response to this HLA allele was to this epitope in } 2 \text { of the } 4 / 6 \text { HEPS cases and in } 7 \text { of the } 14 / 17 \text { responsive HIV-1 infected women }\end{array}$} \\
\hline 24( & p24 & $\begin{array}{l}\text { PRTLNAWV } \\
\text { Gag-specific }\end{array}$ & IV-1 infection & himpanzee & \\
\hline
\end{tabular}




\begin{tabular}{|c|c|c|c|c|}
\hline HXB2 Location & Author's Location & Sequence & Species (HLA) & References \\
\hline & \multicolumn{4}{|c|}{$\begin{array}{l}\text { - No chimpanzee CTL were detected to the following human HIV-1 specific Gag epitopes, although they were embedded within 20mer peptides that } \\
\text { contained a reactive epitope: ISPRTLNAW, HLA-B57; KRWIILGLNK, HLA-B27; and DRFYKTLRA, HLA-B14 }\end{array}$} \\
\hline p24 (16-24) & \multicolumn{2}{|c|}{$\begin{array}{l}\text { p24 (148-156) SPRTLNAWV } \\
\text { - C. Brander notes this is a B*0702 epitope } \\
\text { - Optimal peptide mapped by titration, Pers }\end{array}$} & $\begin{array}{l}\text { human }(\mathrm{B} * 0702) \\
\text {. Lewinsohn to C. Brander and B. Walker }\end{array}$ & Brander2001 \\
\hline p24 (16-24) & \multicolumn{4}{|c|}{$\begin{array}{l}\text { - Epitope name: Gag-SW9 } \\
\text { - Among HIV+ individuals who carried HLA B07, 1/9 (11\%) recognized this epitope } \\
\text { - Among HIV+ individuals who carried HLA B81, 1/6 (17\%) recognized this epitope }\end{array}$} \\
\hline p24 (16-24) & - Optimal peptide mapped by titration, Pers. Comm. from D. Lewinsohn to C. Brander and B. Walker & $\begin{array}{l}\text { SPRTLNAWV } \\
\text { by titration, Pers }\end{array}$ & $\begin{array}{l}\text { human (B7) } \\
\text { Lewinsohn to C. Brander and B. Walker }\end{array}$ & Brander1997 \\
\hline p24 (16-24) & $\begin{array}{l}\text { p24 (148-156) } \\
\text { - Study tracks and qu } \\
\text { - Adoptively transferr } \\
\text { adjacent to cells exp } \\
\text { - The CTL clones exp } \\
\text { viral replication, sug } \\
\text { - This study provides }\end{array}$ & $\begin{array}{l}\text { SPRTLNAWV } \\
\text { es in vivo migrat } \\
\text { ne-marked HIV- } \\
\text { a HIV tat-fusio } \\
\text { d CCR5 and loc } \\
\text { ng a possible ho } \\
\text { thodology for tr. }\end{array}$ & $\begin{array}{l}\text { HIV-1 infection } \\
\text { ed CD8 HIV-specific CTL } \\
\text { med to specific lymph node sites, colocalizing within th } \\
\text { icative of viral replication } \\
\text { V-1 infected cells expressing MIP-1alpha and MIP-1be } \\
\text { ing antigen specific CTL in vivo }\end{array}$ & $\begin{array}{l}\text { Brodie2000 } \\
\text { hlicular regions of the lymph node }\end{array}$ \\
\hline p24 (16-24) & $\begin{array}{l}\text { p24 (148-156) } \\
\text { - ELISPOT was used } \\
\text { HIV-1-infected fem } \\
\text { - Responses in HEPS } \\
\text { reduced risk of infec } \\
\text { women } \\
\text { - } 43 / 91 \text { HEPS women } \\
\text { - Subject ML } 1203 \text { st } \\
\text { acquired additional }\end{array}$ & $\begin{array}{l}\text { SPRTLNAWV } \\
\text { dy CTL respons } \\
\text { airobi sex worke } \\
\text { en tended to be } \\
\text { and there was a } \\
\text { CD8+ responses } \\
\text { with CTL respo } \\
\text { nses to A*6802 }\end{array}$ & $\begin{array}{l}\text { HIV-1 infection, HIV-1 exposed human (B7) } \\
\text { seronegative } \\
54 \text { predefined HIV-1 epitopes in } 91 \text { HIV-1-exposed, per } \\
\text { ed on different epitopes with HLA presenting molecules } \\
\text { onse in the HEPS women upon late seroconversion to ep } \\
\text { f HIV-1-specific CTL in HEPS women increased with th } \\
\text { DTVLEDINL and to B7 FPVTPQVPLR prior to seroco } \\
\text { ich became dominant, B7 TPGPGVIRYPL, B7 IPRRIR }\end{array}$ & $\begin{array}{l}\text { Kaul2001a } \\
\text { seronegative (HEPS) and } 87 \\
\text { ve previously been associated with } \\
\text { ecognized by the HIV-1 infected } \\
\text { ion of viral exposure } \\
\text { h, and upon seroconversion } \\
\text { and B7 SPRTLNAWV }\end{array}$ \\
\hline p24 (16-24) & $\begin{array}{l}\text { p24 (16-24) } \\
\text { - The CTL response t } \\
\text { studied in eight HIV } \\
\text { - } 2 \text { to } 17 \text { epitopes wer } \\
\text { epitopes were target } \\
\text { - Subjects with chron } \\
\text { - An acute seroconver } \\
\text { - The other acute sero } \\
\text { - The B7-restricted C }\end{array}$ & $\begin{array}{l}\text { SPRTLNAWV } \\
\text { Imally defined C } \\
\text { fected subjects, } \\
\text { ognized in a give } \\
\text { at least one per } \\
\text { V-1 infection rec } \\
\text { omozygous for t } \\
\text { ertor failed to re } \\
\text { sponse was high }\end{array}$ & $\begin{array}{l}\text { HIV-1 infection human (B7) } \\
\text { ricted by HLA class I A and B alleles in individuals wh } \\
\text { fection, five with chronic, and one long-term non-progr } \\
\text {-restricted CTL response tended to be narrow and never } \\
\text { 2-8 out of 11 B7-restricted epitopes } \\
\text { gnized five B7-restricted epitopes } \\
\text { he } 11 \text { B7-restricted epitopes tested } \\
\text { here was no clearly dominant epitope }\end{array}$ & $\begin{array}{l}\text { Day2001 } \\
\text { ressed HLA A2, A3, and B7 was } \\
\text { TNP) } \\
\text { ated the response, and } 25 / 27\end{array}$ \\
\hline
\end{tabular}




\begin{tabular}{llllll}
\hline HXB2 Location & Author's Location & Sequence & Immunogen & Species (HLA) & References \\
\hline p24 (16-24) & p24 (16-24) & SPRTLNAWV & HIV-1 infection & human (B7) & Yu2002a
\end{tabular}

- CTL responses in 18 acutely HIV-infected HLA-A3 (n=7) or $-\mathrm{B} 7(\mathrm{n}=4)$ or both $-\mathrm{A} 3$ and $\mathrm{B} 7(\mathrm{n}=7)$ positive individuals were studied.

- One individual, AC-06, was homozygous at all three class I alleles (A3, B7, Cw7), was treated during acute infection and had supervised treatment interruptions (STI). He had only two detectable CTL responses during acute infection, but after STI this broadened to 27 distinct epitopes including 15 restricted by HLA-A3, 11 by HLA-B7, and 1 by HLA-Cw7.

- 1/11 HLA-B7 positive individuals had detectable B7-restricted responses to this epitope during acute infection - 10/15 of HLA-B7 epitopes tested were targeted by at least one person during acute infection. 1/4 individuals had detectable responses to this epitope after STI.

\begin{tabular}{llllll}
\hline p24 (16-24) p24 (subtype B) & SPRTLNAWV & HIV-1 exposed seronegative & human (B7, B*8101) & Kaul2000
\end{tabular}

- 11/16 heavily HIV exposed but persistently seronegative sex-workers in Nairobi had HIV-specific CD8 gamma-IFN responses in the cervix - systemic

$\mathrm{CD} 8+\mathrm{T}$ cell responses tended to be to the same epitopes but at generally lower levels than cervical CD8+ $\mathrm{T}$ cell responses

- Low risk individuals did not have such CD8+ cells

- CD8+ T cell epitopes: DTVLEDINL (3 individuals), SLYNVATL (4 individuals), LSPRTLNAW (3 individuals) and YPLTFGWCF (4 individuals) were most commonly recognized by the HIV-resistant women

\begin{tabular}{llllll}
\hline p24 (16-24) & Gag (subtype B) & SPRTLNAWV & HIV-1 exposed seronegative & human (B7, B*8101) & Rowland-Jones1998b
\end{tabular}

- HIV-specific CTL were found in exposed seronegative prostitutes from Nairobi - these CTL may confer protection

- Seroprevalence in this cohort is $90-95 \%$ and their HIV-1 exposure is among the highest in the world

- Most isolated HIV strains are clade A in Nairobi, although clades C and D are also found - B clade epitopes are often cross-reactive, however stronger responses are frequently observed using $\mathrm{A}$ or $\mathrm{D}$ clade versions of epitopes

- This epitope is conserved among A, B, and D clade viruses

p24 (19-27) p24 (151-159) $\quad$ TLNAWVKVV $\quad$ HIV-1 infection $\quad$ human (A*02)

- The single cell ELISPOT assay was optimized and highly specific, and found to work well even after the primary cells had been frozen and thawed

- Increases in gamma interferon producing cells were observed in response to anti-retroviral therapy using single cell IFN-gamma-production ELISPOT

- In 3/3 HLA-A*02, -B*27 subjects the immunodominant epitope was against HLA B*27 Gag p24 epitope KRWIILGL, not A2 Gag epitopes

p24 (19-27) p24 (151-159) TLNAWVKVV HIV-1 infection Ruman (A*02) Rinaldo2000

- Administration of triple-drug antiretroviral therapy (IDV, 3TC and ZDV) sometimes showed a transient increase and other times failed to increase CTL responses in patients with advanced HIV disease, but there is a stable population of tetramer stained HIV-specific CD8+ CD45RO+ cells that can persist after therapy and long periods of virus being below the level of detection

\begin{tabular}{llccc}
\hline p24 (19-27) & $\begin{array}{l}\text { p24 (151-159) } \\
\text { - Study of sequence motifs preferred for peptide binding to class I HLA-A2 }\end{array}$ & human (A2) \\
\hline p24 (19-27) & p24 (19-27) & TLNAWVKVV & HIV-1 infection & human (A2)
\end{tabular}

- One of the 51 HIV-1 epitopes selected by Ferrari et al. as good candidate CTL epitopes for vaccines by virtue of being conserved and presented by common HLA alleles

p24 (19-27) p24(150-159) TLNAWVKVI

HIV-1 infection, HIV-1 exposed human (A2)
seronegative

Kaul2001a

- Variants TLNAWVKV(I/V) are A/B clade specific 


\begin{tabular}{|c|c|c|c|c|}
\hline HXB2 Location & Author's Location & Immunogen & Species (HLA) & References \\
\hline & \multicolumn{4}{|c|}{$\begin{array}{l}\text { - ELISPOT was used to study CTL responses to a panel of } 54 \text { predefined HIV-1 epitopes in } 91 \text { HIV-1-exposed, persistently seronegative (HEPS) and } 87 \\
\text { HIV-1-infected female Nairobi sex workers }\end{array}$} \\
\hline p24 (19-27) & \multicolumn{4}{|c|}{$\begin{array}{l}\text { p24 (subtype B) TLNAWVKVV HIV-1 exposed seronegative human (A2, A*0202) Rowland-Jones1998b } \\
\text { - HIV-specific CTL were found in exposed seronegative prostitutes from Nairobi - these CTL may confer protection } \\
\text { - Seroprevalence in this cohort is 90-95\% and their HIV-1 exposure is among the highest in the world } \\
\text { - Most isolated HIV strains are clade A in Nairobi, although clades C and D are also found - B clade epitopes are often cross-reactive, however stronger } \\
\text { responses are frequently observed using A or D clade versions of epitopes } \\
\text { - This epitope is conserved among A, B and D clade viruses }\end{array}$} \\
\hline p24 (21-40) & \multicolumn{4}{|c|}{$\begin{array}{l}\text { - Of } 25 \text { patients, most had CTL specific for more than } 1 \text { HIV-1 protein } \\
\text { - Twelve subjects had CTL that could recognize vaccinia-expressed LAI gag } \\
\text { - One of these } 12 \text { had CTL response to this peptide } \\
\text { - The responding subject was HLA-A2, -B21 }\end{array}$} \\
\hline p24 (21-40) & \multicolumn{4}{|c|}{$\begin{array}{l}\text { Vaccine Vector/Type: virus-like particle HIV component: gag, gp120, V3, CD4BS } \\
\text { - A VLP is a non-infectious virus-like particle self-assembled from HIV Pr55 gag - macaques were immunized with VLPs bound to either gp120 or } \\
\text { V3+CD4 linear domains Gag and Env specific CTL were stimulated in each case, and Ab response to gag and gp120 was elicited, but the gp120 } \\
\text { neutralizing response occurred only with whole gp120, not V3+CD4 - despite the CTL and Ab response, immunized macaques were infected by } \\
\text { intervenous challenge with SHIV chimeric challenge stock [Wagner1998b] } \\
\text { - CTL specific for this epitope could be found both before and after SHIV challenge }\end{array}$} \\
\hline p24 (21-40) & \multicolumn{4}{|c|}{$\begin{array}{l}\text { - The ability of CTL effector cells was studied by expanding autologous HIV-1 Gag-specific CTL in vitro, and adoptively transferring them } \\
\text { - The transferred CTLs migrated to the lymph nodes and transiently reduced circulating productively infected CD4+ T cells, showing that CTL move to } \\
\text { appropriate target sites and mediate anti-viral effects }\end{array}$} \\
\hline p24 (21-40) & $\begin{array}{l}\text { p24 (153-172) } \\
\text { - Study tracks and quan } \\
\text { - Adoptively transferre } \\
\text { adjacent to cells expre } \\
\text { - The CTL clones expre } \\
\text { viral replication, sugg } \\
\text { - This study provides a }\end{array}$ & $\begin{array}{l}\text { NAWVKVVEEKAF SPEVIPMF HIV-1 infection } \\
\text { es in vivo migration of neo-marked CD8 HIV-specific CTL } \\
\text { ne-marked HIV-specific CTL homed to specific lymph node si } \\
\text { ng HIV tat-fusion transcripts, indicative of viral replication } \\
\text { d CCR5 and localized among HIV-1 infected cells expressing } \\
\text { ng a possible homing mechanism } \\
\text { thodology for tracking and studying antigen specific CTL in vil }\end{array}$ & $\begin{array}{l}\text { human (B57) } \\
\text { ocalizing within the pa } \\
\text { lpha and MIP-1beta, C }\end{array}$ & $\begin{array}{l}\text { Brodie2000 } \\
\text { llicular regions of the lymph node }\end{array}$ \\
\hline p24 (21-42) & $\begin{array}{l}\text { p24 (153-174 BH10) } \\
\text { Gag CTL response stı }\end{array}$ & $\begin{array}{l}\text { NAWVKVVEEKAFSPEVIPM- HIV-1 infection } \\
\text { FSA } \\
\text { din three individuals }\end{array}$ & human (Bw57) & Johnson1991 \\
\hline $24(28-36)$ & 24 & ction & 415) & Bird2002 \\
\hline
\end{tabular}

- Residues forming the B pocket of HLA B*4415 were identical to HLA B*4001, B*4402 and B*4403. These alleles preferred E, an acidic residue, at the P2 position. 


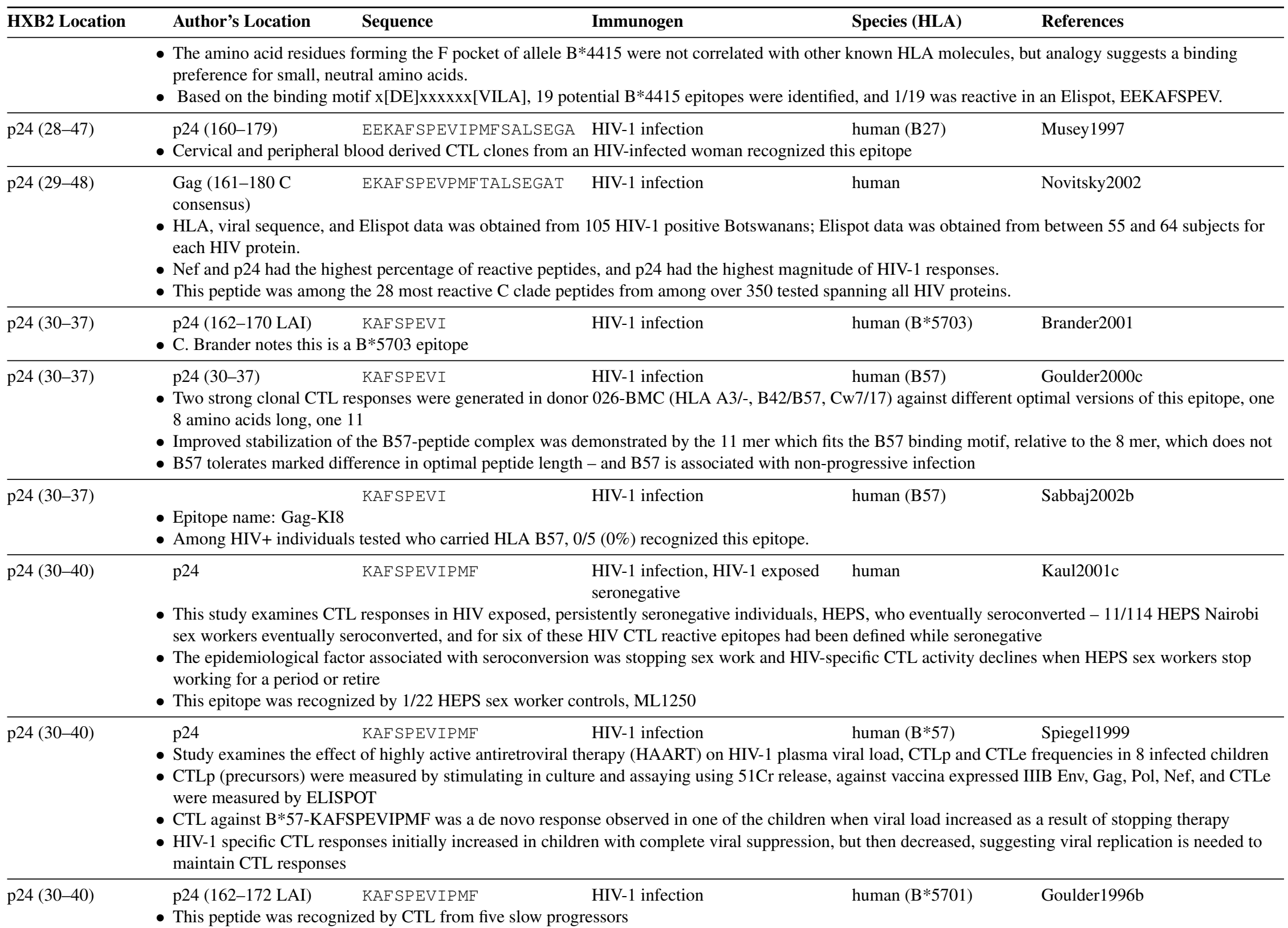

- Peptide defined on the basis of $\mathrm{B}^{*} 5801$ binding motif, yet not cross-restricted except at high concentrations 


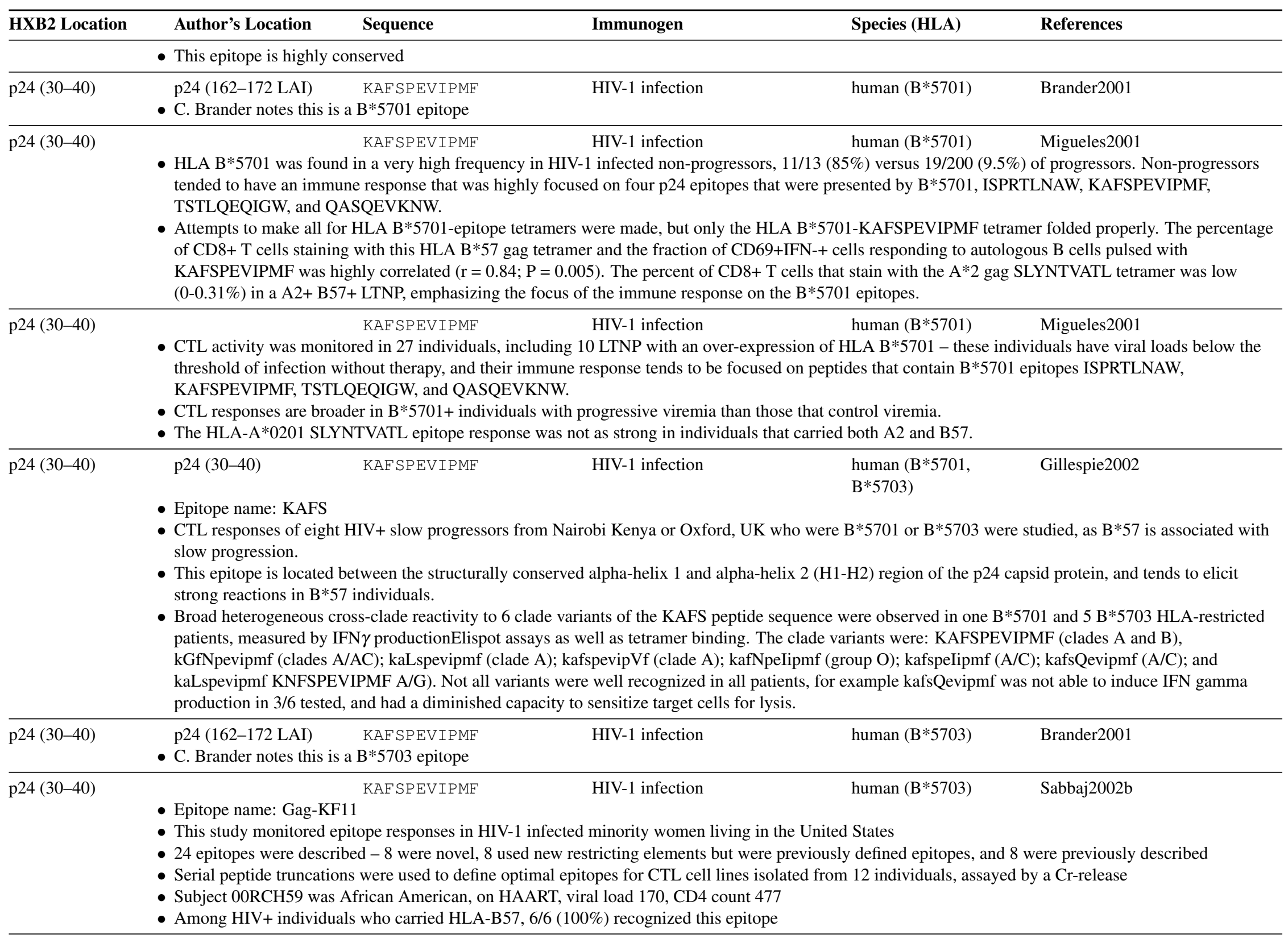




\begin{tabular}{lllll}
\hline HXB2 Location & Author's Location & Sequence & Immunogen & Species (HLA) \\
\hline p24 (30-40) & p24 (30-40) & KAF SPEVIPMF & HIV-1 infection & human (B57)
\end{tabular}

- Two strong clonal CTL responses were generated in donor 026-BMC (HLA A3/-, B42/B57, Cw7/17) against different optimal versions of this epitope, one 8 amino acids long, one 11

- Improved stabilization of the B57-peptide complex was demonstrated by the 11 mer which fits the B57 binding motif, relative to the 8 mer, which does not

- B57 tolerates marked difference in optimal peptide length - and B57 is associated with non-progressive infection

p24 (30-40) p24 (162-172) KAFSPEVIPMF $\quad$ HIV-1 infection $\quad$ Betts2000

- Only 4/11 HLA-A2+ HIV+ individuals had CTL that reacted to SLYNTVATL, calling into question whether it is immunodominant

- 95 optimally-defined peptides from this database were used to screen for INF $\gamma$ responses to other epitopes

- $1 / 11$ of the A2+ individuals was HLA A*0201, A1, B57 and responded to four B57 epitopes and two others

p24 (30-40) $\quad$ 24 (SF2) HAFSPEVIPMF HIV-1 infection

- The CTL-dominant response was focused on this epitope in a HIV+ Caucasian living in Boston - this epitope is not among the most recognized peptides in the study

- Three peptides GSEELRSLYNTVATL (p17 residues 71-85), SALSEGATPQDLNTMLNTVG (p24 41-60), and WEKIRLRPGGKKKYKLK(p17 16-30) contained the dominant Gag-specific epitope in 31 out of 44 B-clade infected individuals from Boston who showed Gag-CTL responses

- Five peptides RLRPGGKKHYMIKHLVW (p17 20-36), ELRSLYNTVATLYCV (p17Gag 74-88), SALSEGATPQDLNTMLNTVG (p24 41-60), FRDYVDRFFKTLRAEQA (p24 161-177), and SILDIKQGKEPFRDY (p24 149-164) contained dominant Gag-specific epitopes in 32 out of 37 C-clade infected subjects from South Africa

p24 (30-40)

Gag (SF2)

KAFSPEVIPMF

HIV-1 infection

human (B57)

Goulder2001a

- Epitope name: KF11

- Three CTL responses in patient PI004, to epitopes TSTLQEQIGW, ISPRTLNAW, and KAFSPEVIPMF, were evident early after infection; CTL responses to SLYNTVATL, QASQEVKNW, EIYKRWII, and FLKEKGGL were detectable at 5 months post-infection and beyond

\begin{tabular}{lllll}
\hline p24 (30-40) & p24 (162-172) & KIV-1 infection & Oxenius2000
\end{tabular}

- Epitope name: KAF

- Patients who started therapy at acute HIV-1 infection (three with sustained therapy, two with limited therapy upon early infection) had strong HIV specific CD4 proliferative responses and were able to maintain a CTL response even with undetectable viral load - three patients that had delayed initiation of HAART had no HIV specific CD4 proliferative responses and lost their CTL responses when HAART was eventually given and their viral loads became undetectable

- None of the 8 study subjects recognized this epitope but none were HLA B57+

p24 (30-40) p24 KAFSPEVIPMF HIV-1 infection $\quad$ Kostense2001

- HLA tetramers to six epitopes were used to study HLA-A2, B8 and B57 CTL in 54 patients - HIV-specific tetramer positive cells were inversely correlated with viral load in patients with high CD4, but in patients with CD4 T-cells below 400 high tetramer frequencies were found despite high viral load

- Most patients have high levels of HIV-specific T-cell expansions, but many of these cells aren't functional

- In 15 of the patients, the proportion of IFN gamma producing tetramer cells correlated with AIDS-free survival

p24 (30-40)

HIV-1 infection human (B57)

Altfeld2001b

- Therapy provided during acute infection resulted in a narrower CTL response, stronger T help response, and a less diverse viral population than was seen in individuals treated during chronic infection

- The breadth and specificity of the response was determined using ELISPOT by studying 19 individuals with pre-seroconversion therapy (Group 1 ), 11 individuals with primary infection but post-seroconversion therapy (Group 2), and 10 individuals who responded to HAART given during chronic infection (Group 3), using 259 overlapping peptides spanning p17, p24, RT, gp41, gp120 and Nef 


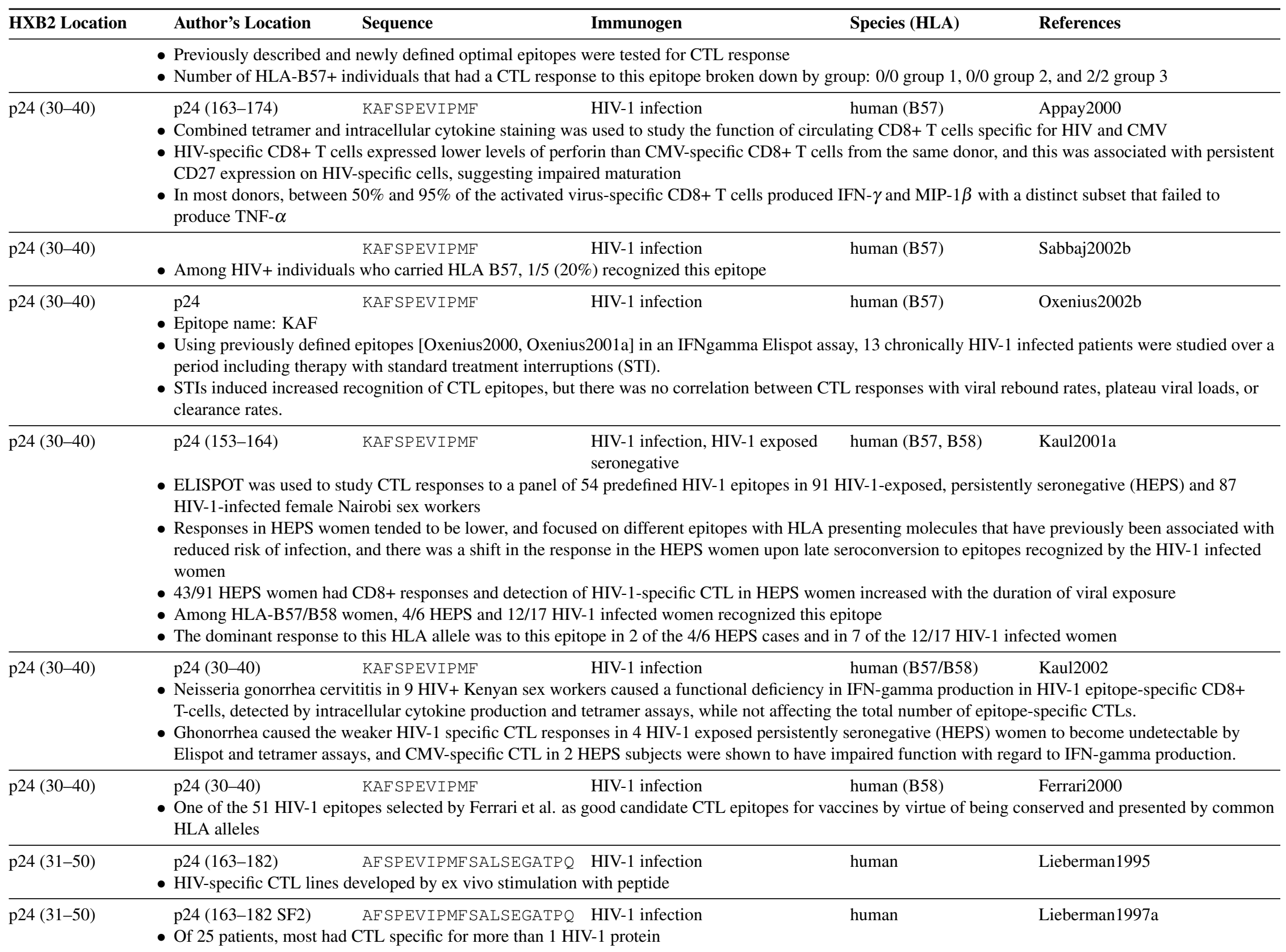




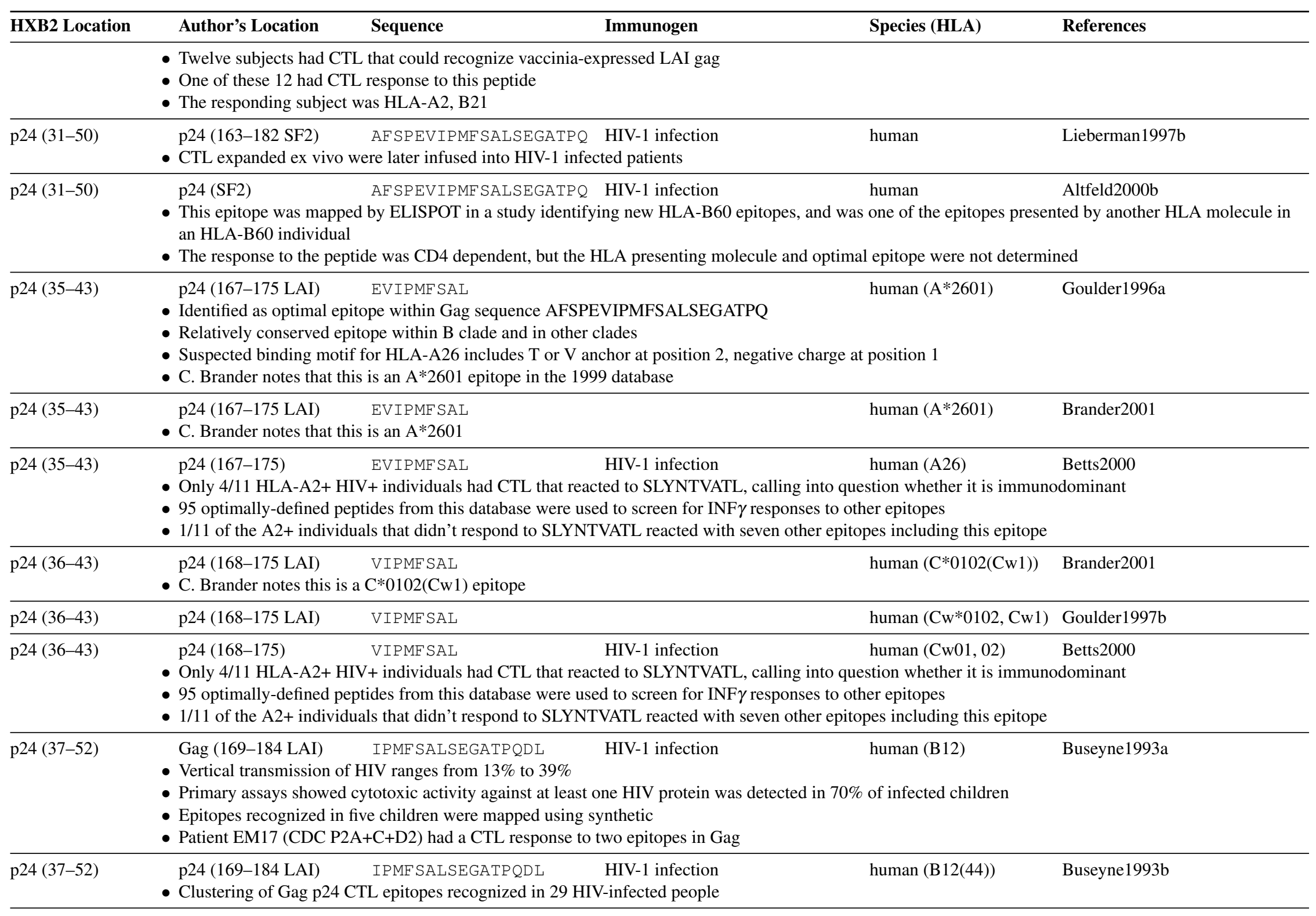




\begin{tabular}{lllll}
\hline HXB2 Location & Author's Location & Sequence & Immunogen & Species (HLA) \\
\hline p24 (37-52) & p24 (37-52) & IPMF SALSEGATPDQL & HIV-1 infection & human (B44)
\end{tabular}

- One of the $51 \mathrm{HIV}-1$ epitopes selected by Ferrari et al. as good candidate CTL epitopes for vaccines by virtue of being conserved and presented by common HLA alleles

\begin{tabular}{lll}
\hline p24 (39-58) & Gag (171-190) MF TALSEGTPQDLNTMLNT HIV-1 infection \\
& HLA, viral sequence, and Elispot data was obtained from 105 HIV-1 positive Botswanans; Elispot data was obtained from between 55 and 64 subjects for \\
& each HIV protein.
\end{tabular}

each HIV protein.

- Nef and p24 had the highest percentage of reactive peptides, and p24 had the highest magnitude of HIV-1 responses.

- This peptide was among the 8 most reactive C clade peptides from among over 350 tested spanning all HIV proteins.

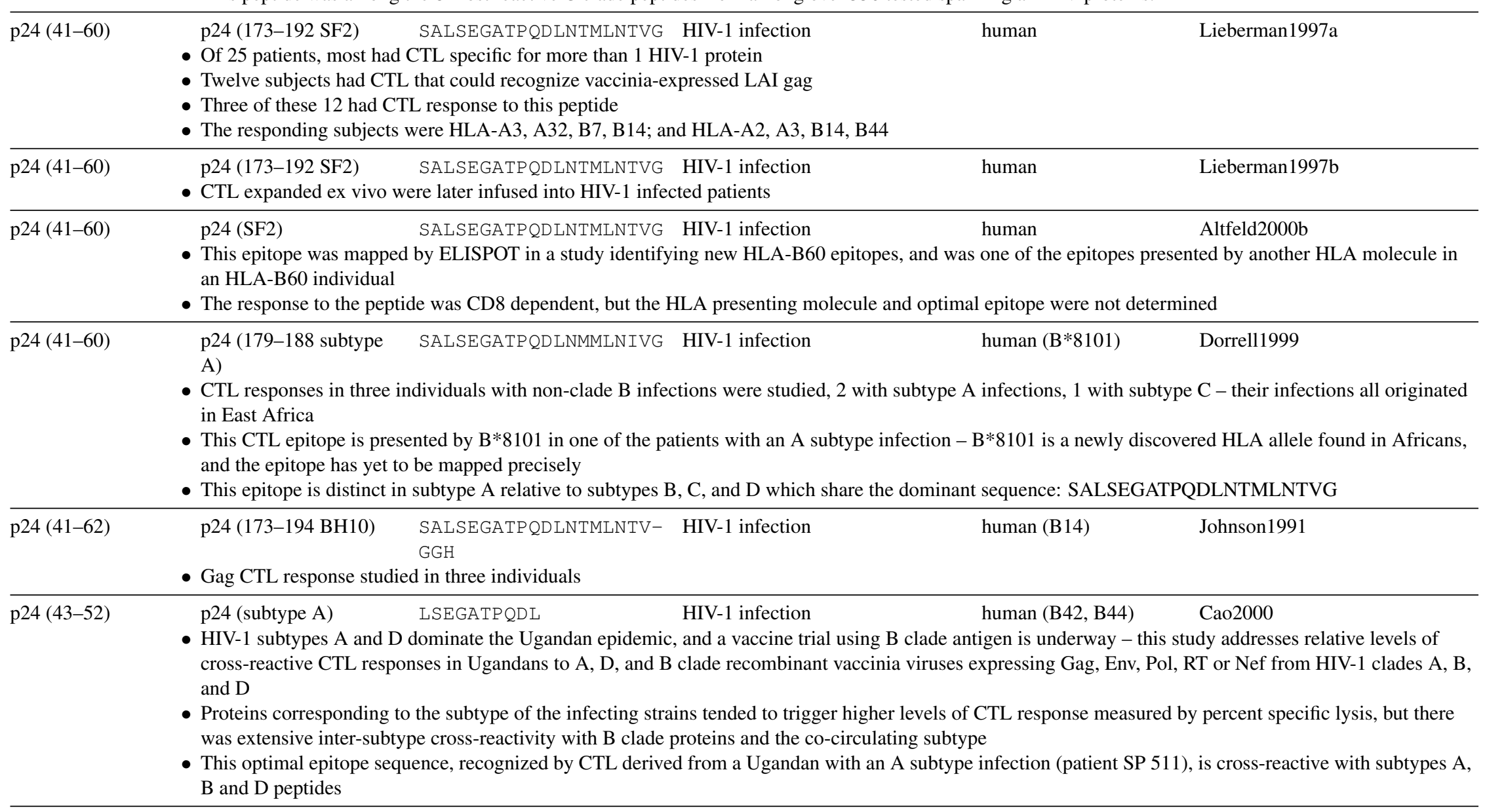




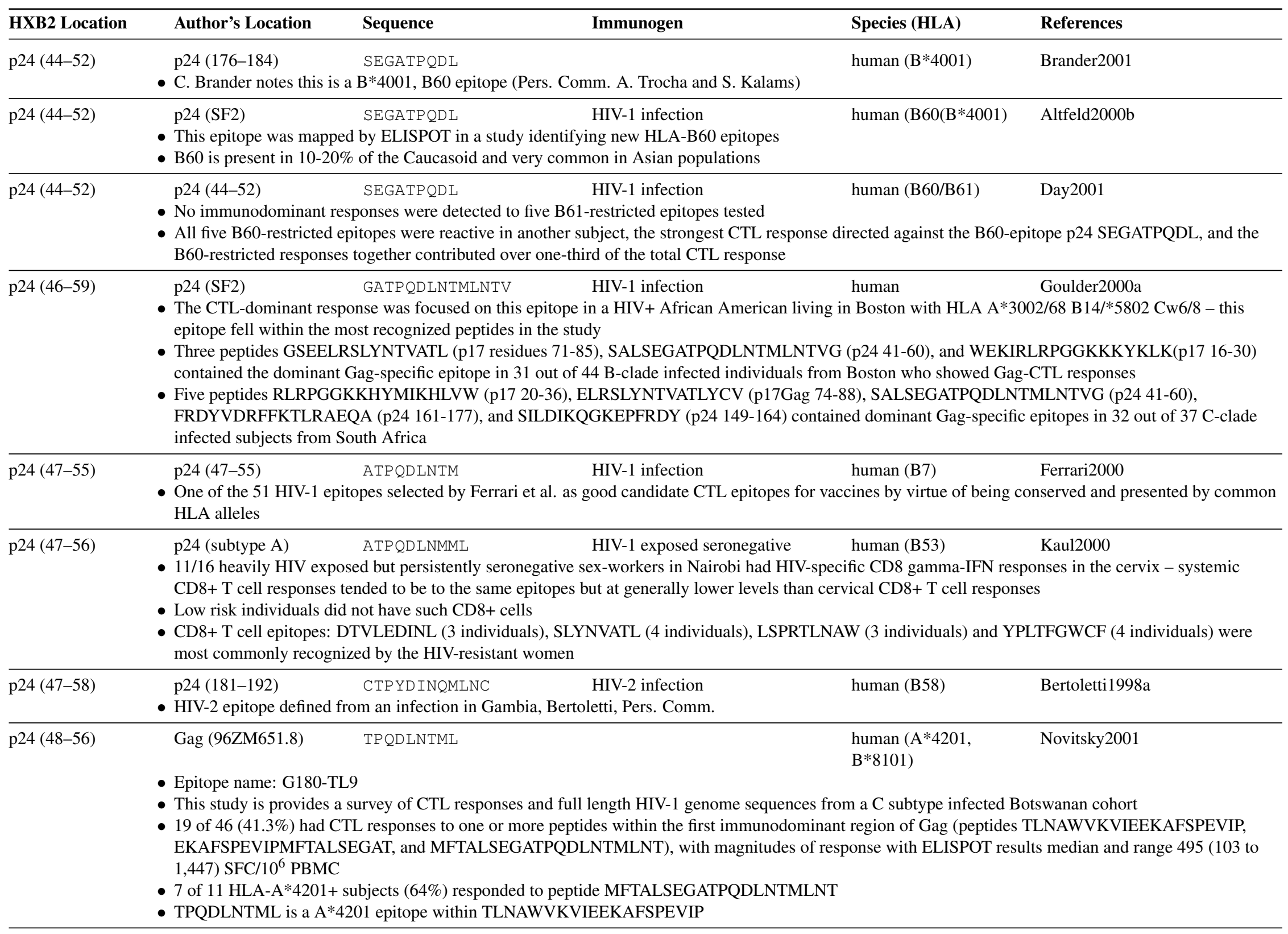




\begin{tabular}{|c|c|c|c|c|}
\hline HXB2 Location & Author's Location & Immunogen & Species (HLA) & References \\
\hline p24 (48-56) & $\begin{array}{l}\text { p24 (180-188 IIIB) } \\
\text { - C. Brander notes this is a B*0702 epitope }\end{array}$ & HIV-1 infection & human $(\mathrm{B} * 0702)$ & Brander2001 \\
\hline p24 (48-56) & $\begin{array}{l}\text { p24 (179-187 LAI) } \\
\text { - C. Brander notes this is a B*4201 epitope }\end{array}$ & & human $(B * 4201)$ & Brander2001 \\
\hline p24 (48-56) & $\begin{array}{l}\text { Gag (173-181 HIV-2) TPYD INQML } \\
\text { - C. Brander notes this is a B*5301 epitope }\end{array}$ & HIV-2 infection & human $(\mathrm{B} * 5301)$ & Brander2001 \\
\hline p24 (48-56) & $\begin{array}{l}\text { p24 (180-188 LAI) TPQDLNTML } \\
\text { C. Brander notes this is a B*8101 epitope }\end{array}$ & HIV-1 infection & human $(\mathrm{B} * 8101)$ & Brander2001 \\
\hline p24 (48-56) & TPQDLNTML & HIV-1 infection & $\begin{array}{l}\text { human }(\mathrm{B} * 8101 \\
\mathrm{B} * 5301, \mathrm{~B} 07)\end{array}$ & Sabbaj2002b \\
\hline
\end{tabular}

- Epitope name: Gag-TL9

- This study monitored epitope responses in HIV-1 infected minority women living in the United States

- 24 epitopes were described - 8 were novel, 8 used new restricting elements but were previously defined epitopes, and 8 were previously described

- Serial peptide truncations were used to define optimal epitopes for CTL cell lines isolated from 12 individuals, assayed by a Cr-release

- Subjects 00RCH86 and 03RCH59 both recognized this epitope, both restricted by HLA B*8101

- Subject 00RCH86 was African American, not on HAART, viral load 51000, CD4 count 520

- Subject 03RCH59 was African American, male, on HAART, viral load 22000, CD4 count 769

- Among HIV+ individuals who carried HLA B07, 2/9 (22\%) recognized this epitope

- Among HIV+ individuals who carried HLA B*5301,3/15 (20\%) recognized this epitope

- Among HIV+ individuals who carried HLA B81, 4/6 (67\%) recognized this epitope

p24 (48-56) p24 (C consensus) TPQDLNTML HIV-1 infection $\quad$ Goulder2000a

- B42 and B81 are very similar, and both can present this epitope to B42-positive effector cells - this epitope is almost certainly optimal for B81 as well B42 and or B81 are expressed in 40-45\% of Zulu and Xhosa infected individuals in South Africa, and in 14/18 B42 or B81+ individuals, the dominant gag response was to TPQDLNTML

- Three peptides GSEELRSLYNTVATL (p17 residues 71-85), SALSEGATPQDLNTMLNTVG (p24 41-60), and WEKIRLRPGGKKKYKLK(p17 16-30) contained the dominant Gag-specific epitope in 31 out of 44 B-clade infected individuals from Boston who showed Gag-CTL responses

- Five peptides RLRPGGKKHYMIKHLVW (p17 20-36), ELRSLYNTVATLYCV (p17Gag 74-88), SALSEGATPQDLNTMLNTVG (p24 41-60), FRDYVDRFFKTLRAEQA (p24 161-177), and SILDIKQGKEPFRDY (p24 149-164) contained dominant Gag-specific epitopes in 32 out of 37 C-clade infected subjects.

p24 (48-56) Gag $\quad$ TPQDLNTML $\quad$ HIV-1 infection $\quad$ Goulder2000b

- Tetramer assays were compared with three functional assays in 42 people with chronic HIV infection: ELISPOT, intracellular cytokine staining, and precursor frequency (limiting dilution assay [LDA])

- HIV-specific tetramer staining CTLs appeared to be active, and inert CTL were not found to play a significant role in chronic pediatric or adult HIV infection

$\mathrm{p} 24(48-56)$

TPQDLNQML

human (B53)

Rowland-Jones1999

- CTL responses in seronegative highly HIV-exposed African female sex workers in Gambia and Nairobi were studied - these women had no delta 32 deletion in CCR5 


\begin{tabular}{|c|c|c|c|c|c|}
\hline HXB2 Location & Author's Location & Sequence & Immunogen & Species (HLA) & References \\
\hline & \multicolumn{5}{|c|}{$\begin{array}{l}\text { - In Gambia there is exposure to both HIV-1 and HIV-2, CTL responses to B35 epitopes in exposed, uninfected women are cross-reactive, and the B35 allele } \\
\text { seems to be protective } \\
\text { - HIV-2 sequence: TPYDINQML, no cross-reactivity, Gotch1993 }\end{array}$} \\
\hline p24 (48-56) & Gag (173-181 HIV-2) & TPYD INQML & HIV-2 infection & human (B53) & Gotch1993 \\
\hline p24 (48-56) & $\begin{array}{l}\text { Gag (180-188 subtype } \\
\text { A) }\end{array}$ & TPQDLNMML & $\begin{array}{l}\text { HIV-1 infection, in vitro } \\
\text { stimulation }\end{array}$ & human (B53) & Dorrell2001 \\
\hline
\end{tabular}

- In vitro restimulation of CTL specific for dominant epitopes from infected individuals is possible using recombinant modified vaccinia virus Ankara (MVA) carrying A or D subtype HIV-1 Gag proteins

\begin{tabular}{lllll}
\hline p24 (48-56) p24 (180-188 subtype A TPQDLNMML & HIV-1 infection & human (B53) Dorrell2001
\end{tabular}

- In clade A infected Gambians, three HLA-B53 epitopes were defined in Gag p24 using ELISPOT, tetramer, and cytotoxicity assays

- This optimal epitope was identified within the 20 mer reactive peptide that carried it by homology with a B53 epitope from HIV-2, a B subtype B7 peptide that corresponds to it, as B53 is part of the B7 superfamily, and by the proline in the anchor at position 2

- TPQDLNMML was recognized in 6/7 HLA-B53 subjects and was immunodominant in most subjects

- TPQDLNMML was A subtype-specific with no cross-recognition of the subtype B, C, and D variant, TPQDLNTML, although the B/C/D variant bound more efficiently to B53 - position 7 show great positional variation in crystal structures of two HLA-B53 complexes, suggesting variation here might significantly alter the position of the peptide in the binding groove and thus affect TCR interactions

- Only one subject might have had a cross-reactive response with the HIV-2 and Mamu-A*01 variant CTPYDINQML, and this subject might have been dua infected with HIV-2

p24 (48-56) p24 (180-188 IIIB) TPQDLNTML HIV-1 infection

- This study describes maternal CTL responses in the context of mother-to-infant transmission

- Detection of CTL escape mutants in the mother was associated with transmission, but the CTL-susceptible forms of the virus tended to be found in infected infants

- No variants of this epitope were found in a non-transmitting mother that had a CTL response to this epitope

p24 (48-56) p24 (180-188) TPQDLNTML HIV-1 infection

- This is the optimal epitope for the immunodominant response defined using a conventional approach in an HLA B7+ long-term non-progressor

- Three additional sub-dominant HLA B7 epitopes were defined using EpiMatrix, a non-anchor based strategy for defining potential epitopes, which highlighted 2078 possible epitopes in the autologous HIV-1 derived from the study subject - this was followed by B7 anchor residue prediction which narrowed the set to 55 peptides, three of which could serve as functional CTL epitopes

p24 (48-56) p24 (SF2) TPQDLNTML HIV-1 infection

- Epitope name: TL9

- Recognized by patient 9354 during chronic infection, used as a positive control in a study of the SLYNTVATL epitope p24 (48-56)

TPQDLNTML

HIV-1 infection

human (B7)

Day2001

- The CTL response to optimally defined CTL epitopes restricted by HLA class I A and B alleles in individuals who coexpressed HLA A2, A3, and B7 was studied in eight HIV-1-infected subjects, two with acute infection, five with chronic, and one long-term non-progressor (LTNP)

- 2 to 17 epitopes were recognized in a given individual, A2-restricted CTL response tended to be narrow and never dominated the response, and 25/27 epitopes were targeted by at least one person

- Subjects with chronic HIV-1 infection recognized between 2-8 out of 11 B7-restricted epitopes 


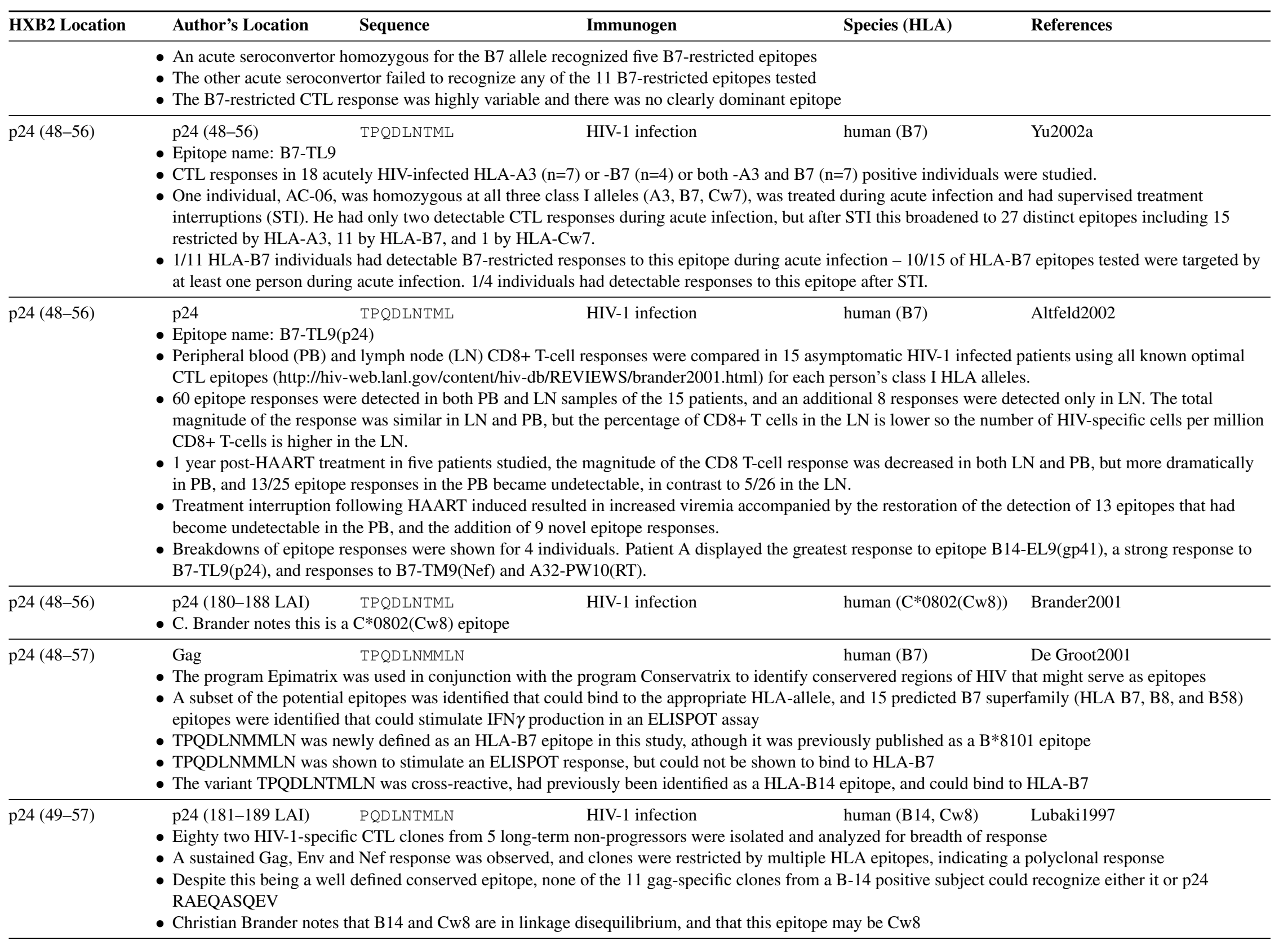




\begin{tabular}{lllll}
\hline HXB2 Location & Author's Location & Sequence & Immunogen & Species (HLA) \\
\hline p24 (51-59) & p24 & DLNTMLNTV & HIV-1 infection & chimpanzee
\end{tabular}

- Effector cells from two chimpanzees were able to recognize two epitopes also recognized by human HIV-1 Gag-specific CTL (SPRTLNAWV, HLA-B7, and DLNTMLNTV, HLA-B14)

- No chimpanzee CTL were detected to the following human HIV-1 specific Gag epitopes, although they were embedded within 20mer peptides that contained a reactive epitope: ISPRTLNAW, HLA-B57; KRWIILGLNK, HLA-B27; and DRFYKTLRA, HLA-B14

\begin{tabular}{ll}
\hline p24 (51-59) & p24 (subtype A) DLNMMLNIV HIV-1 exposed seronegative human (B14) \\
- & $11 / 16$ heavily HIV exposed but persistently seronegative sex-workers in Nairobi had HIV-specific CD8 gamma-IFN responses in the cervix - systemic \\
& CD8+ T cell responses tended to be to the same epitopes but at generally lower levels than cervical CD8+ T cell responses \\
& Low risk individuals did not have such CD8+ cells \\
- & CD8+ T cell epitopes: DTVLEDINL (3 individuals), SLYNVATL (4 individuals), LSPRTLNAW (3 individuals) and YPLTFGWCF (4 individuals) were \\
& most commonly recognized by the HIV-resistant women
\end{tabular}

p24 (51-59) p24 DLNMMLNIV HIV-1 infection human (B14) Kaul2001c

- This study examines CTL responses in HIV exposed, persistently seronegative individuals, HEPS, who eventually seroconverted - 11/114 HEPS Nairobi sex workers eventually seroconverted, and for six of these HIV CTL reactive epitopes had been defined while seronegative

- The epidemiological factor associated with seroconversion was stopping sex work and HIV-specific CTL activity declines when HEPS sex workers stop working for a period or retire

- This epitope was recognized in 1/22 HEPS sex worker controls, ML1792

\begin{tabular}{lllll}
\hline p24 (51-59) p24 (183-191 LAI) DLNTMLNTV & HIV-1 infection & human (B14)
\end{tabular}

- Epitope name: G5

- A panel of 16 epitopes covering 15 class I alleles was tested in 14 HIV+ patients from an unselected Caucasian population treated with HAART, using CD8+ cell IFNgamma production to measure responses

- In general, during the first month of treatment viral load decreased and frequencies of HIV-specific CTL tripled and broadened - eight new HIV specificities that were not previously detectable were newly detected, as were CMV specific CD8+ PBL - but with continued viral suppression, HIV-specific responses diminished

- Viral rebounds gave different patterns of response: increases or decreases in pre-existing response, new specificities, or no change

p24 (51-59) p24 (183-191) DLNMMLNIV HIV-1 infection, HIV-1 exposed human (B14) Kaul2001a

- Variants DLNMMLNIV/DLNTMLNVV are specific for clades A/B

- ELISPOT was used to study CTL responses to a panel of 54 predefined HIV-1 epitopes in 91 HIV-1-exposed, persistently seronegative (HEPS) and 87 HIV-1-infected female Nairobi sex workers

- Responses in HEPS women tended to be lower, and focused on different epitopes with HLA presenting molecules that have previously been associated with reduced risk of infection, and there was a shift in the response in the HEPS women upon late seroconversion to epitopes recognized by the HIV-1 infected women

- 43/91 HEPS women had CD8+ responses and detection of HIV-1-specific CTL in HEPS women increased with the duration of viral exposure

- Among HLA-B14 women, 4/4 HEPS and 3/7 HIV-1 infected women recognized this epitope, likelihood ratio 4.8, p value 0.1, and HEPS women tended to respond to DLNMMLNIV/DLNTMLNVV, while infected women tended to respond to DRF(F/W)KTLRA

- The dominant response to this HLA allele was to this epitope for all 4/4 HEPS cases and in only one of the 3/7 HIV-1 infected women

- Differences in epitope specificity were only seen for responses restricted by class I HLA alleles A2, A24, A*6802, B14, and B18, previously shown to be associated with resistance to HIV-1 in this cohort 


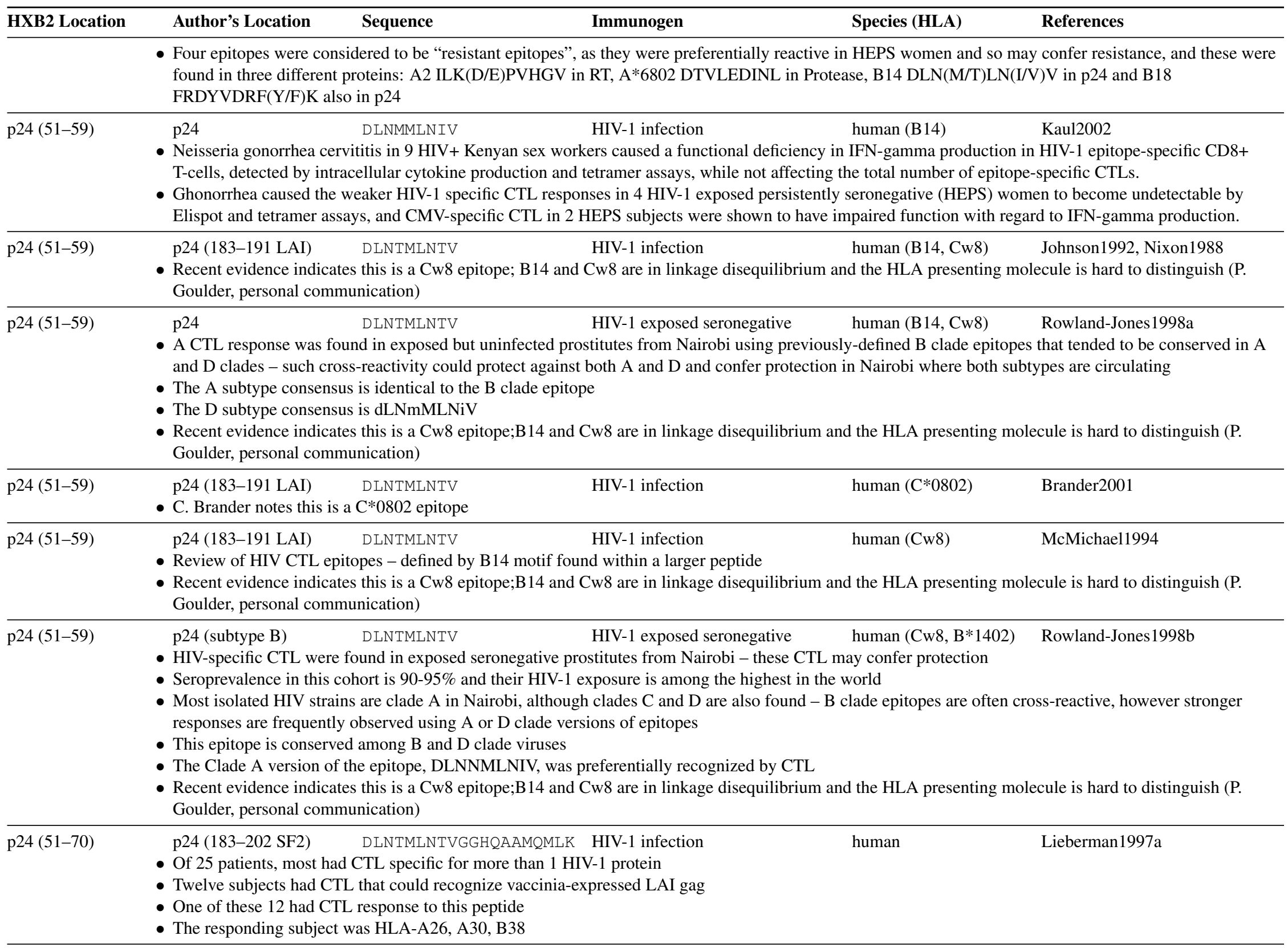




\begin{tabular}{|c|c|c|c|c|c|}
\hline HXB2 Location & Author's Location & Sequence & Immunogen & Species (HLA) & References \\
\hline p24 (51-82) & Gag (183-214 LAI) & $\begin{array}{l}\text { DLNTMLNTVGGHQAAMQML- } \\
\text { KETINEEAAEWDR }\end{array}$ & Vaccine & human & Gahery-Segard2000 \\
\hline
\end{tabular}

- Anti-HIV lipopeptide vaccine consisting of six long amino acid peptides derived from Nef, Gag and Env HIV-1 proteins modified by a palmitoyl chain was administered in a phase I trial

- A CD4+ T cell proliferative response to at least one of the six peptides was observed in 9/10 vaccinees $-2 / 10$ reacted to this peptide

- 9/12 tested mounted a CTL response to at least one of the six peptides; each of the six peptides elicited a CTL response in at least one individual

- None of the 12 tested had an IgG response to this peptide

\begin{tabular}{llccc}
\hline p24 (61-69) & p24 (193-201 LAI) & GHQAAMQML & human (B*3901) & Brander2001 \\
& C. Brander notes this is a B*3901 epitope & human (B39) \\
\hline p24 (61-69) & p24 (193-201 LAI) $\quad$ GHQAAMQML & Kurane1998 \\
\hline p24 (61-71) & Optimal peptide defined by titration & human (A2) & Claverie1988 \\
& - One of 4 epitopes first predicted, then shown to stimulate HLA-A2 restricted CTL line & & \\
\hline
\end{tabular}

p24 (61-80) p24 (193-212 SF2) GHQAAMQMKETINEEAAEW HIV-1 infection human Lieberman1997a

- Of 25 patients, most had CTL specific for more than $1 \mathrm{HIV}-1$ protein

- Twelve subjects had CTL that could recognize vaccinia-expressed LAI gag

- One of these 12 had CTL response to this peptide

- The responding subject was HLA-A26, A30, B38

\begin{tabular}{llll}
\hline p24 (61-82) p24 (193-214 BH10) & $\begin{array}{l}\text { GHQAAMQMLKETINEEAAE- HIV-1 infection } \\
\text { WDR }\end{array}$ & & Johnson1991 \\
& &
\end{tabular}

- Gag CTL response studied in three individuals

\begin{tabular}{lllll}
\hline p24 (62-70) & p24 (194-202 LAI) & HQAAMQMLK & human (B52) \\
& P. Goulder, pers. comm. & & Brander1996b \\
\hline p24 (65-73) & Gag (199-207 HXB2) & AMQMLKETI & Vaccine & murine (H-2 $\left.{ }^{d}\right)$
\end{tabular}

p24 (65-73)

Gag (199-207 HXB2) AMQMLKETI Vaccine

Vaccine Vector/Type: DNA Strain: HXB2 HIV component: gag

- Different expression vectors were tested to increase Gag expression in cell lines and create suitable vectors for DNA vaccines

- Stable Gag expression was achieved in murine p815 cells, using a Gag gene that had mutated silent base positions that disrupt inhibitory RNA sequences which promote RNA degradation

- Silent mutations were more effective than introduction of the D retrovirus cis-acting posttranscriptional control element (CTE) for enhancing Gag expression

- The gag vector with silent mutations given as a vaccine to BALB/c mice gave CTL responses in splenic mononuclear cells, using peptide pulsed cells as targets

\begin{tabular}{llccc}
\hline p24 (65-73) & p24 (199-207 SF2) & AMQMLKETI & Vaccine & murine $\left(\mathrm{H}-2^{d}\right)$ \\
& Vaccine Vector/Type: protein, vaccinia & Strain: SF2 & HIV component: soluble Gag, or GagPol expressing vaccinia & Adjuvant: heat-labile enterotoxin \\
& (LT) from E. coli & &
\end{tabular}

- Epitope name: p7g 


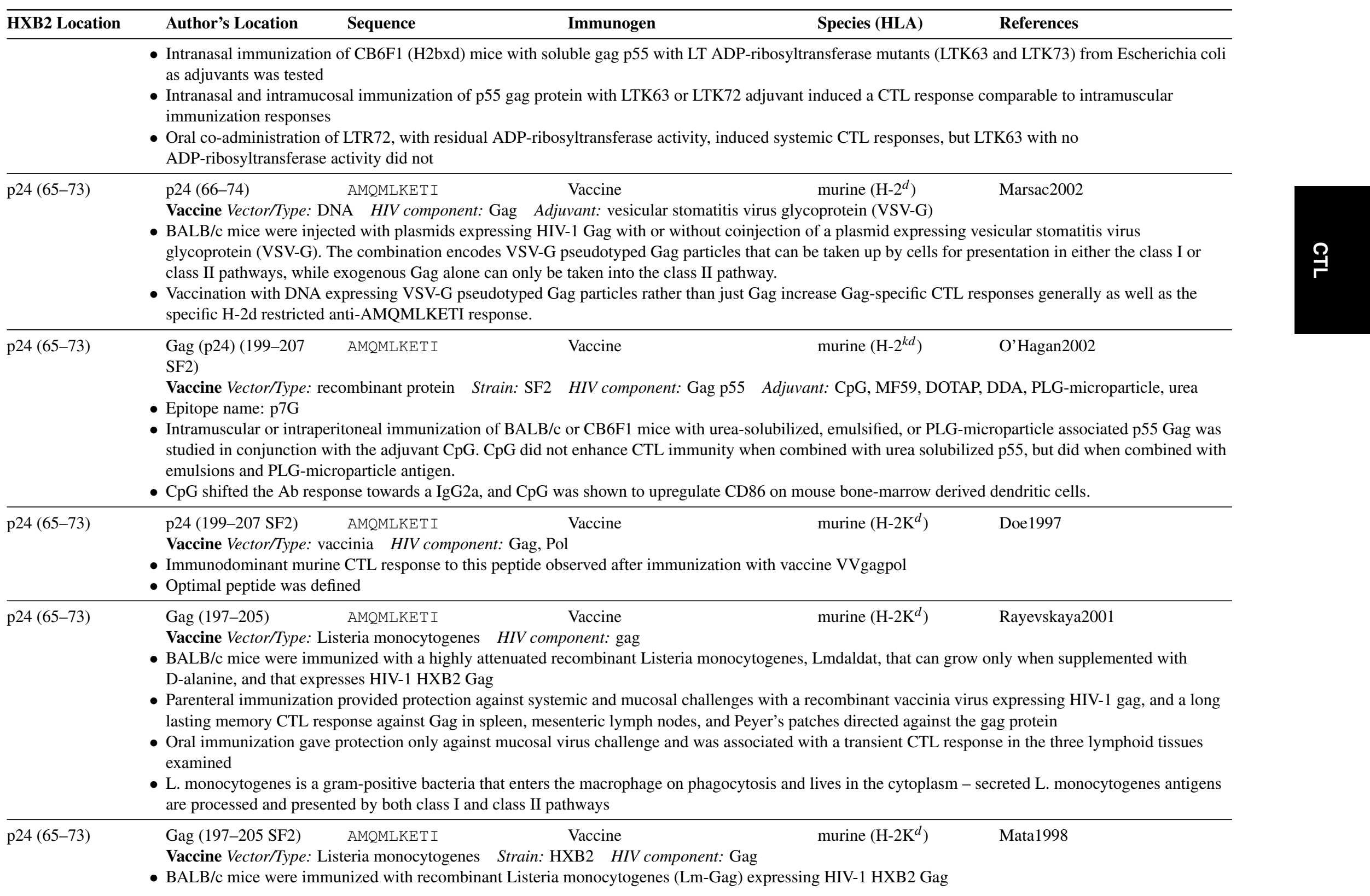




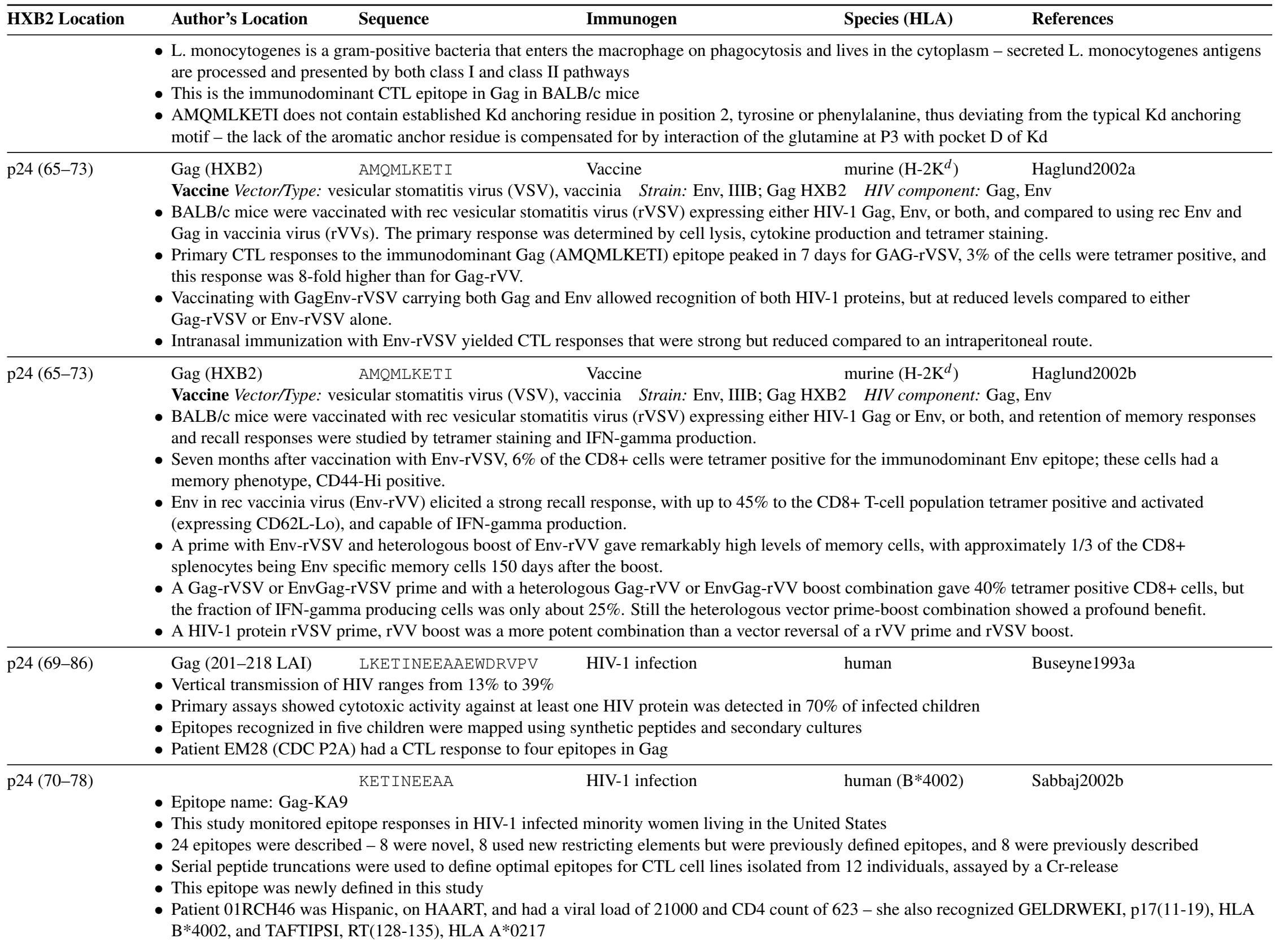




\begin{tabular}{|c|c|c|c|c|}
\hline HXB2 Location & Author's Location & Sequence & Species (HLA) & References \\
\hline & \multicolumn{4}{|c|}{ - Among HIV+ individuals who carried HLA B40, $3 / 5$ (60\%) recognized this epitope } \\
\hline p24 (71-80) & \multicolumn{4}{|c|}{$\begin{array}{l}\text { - The epitope was defined through direct stimulation of PBMC with 20-mer peptides } \\
\text { - It is in a conserved region, ETINEEAAEW is found in most B, D, and E subtype isolates } \\
\text { - DTINEEAAEW is found in A and some D subtype sequences }\end{array}$} \\
\hline p24 (71-80) & \multicolumn{3}{|c|}{ - C. Brander notes this is an $A^{*} 2501$ epitope } & Brander2001 \\
\hline p24 (71-80) & \multicolumn{4}{|c|}{$\begin{array}{l}\text { - Conserved between B and D subtypes, variable in other clades; a consensus of clades A,C, F, G, and H and a peptide of HIV-2ROD over this region were } \\
\text { not recognized by CTL recognizing the index peptide } \\
\text { - C. Brander notes that this is an A*2501 epitope in the } 1999 \text { database }\end{array}$} \\
\hline p24 (71-80) & $\begin{array}{l}\text { p24 } \\
\text { CTL responses in se } \\
\text { deletion in CCR5 } \\
\text { In Gambia there is e } \\
\text { seems to be protectiv } \\
\text { HIV-2 sequence: EII }\end{array}$ & $\begin{array}{l}\text { ETINEEAAEW } \\
\text { rative highly HIV } \\
\text { Ire to both HIV-1 } \\
\text { AAEW, no cross }\end{array}$ & $\begin{array}{l}\text { human (A25) } \\
\text { an female sex workers in Gambia and Nairobi were studi } \\
\text { L responses to B35 epitopes in exposed, uninfected won } \\
\text { Baalen1996 }\end{array}$ & $\begin{array}{l}\text { Rowland-Jones1999 } \\
\text { ese women had no delta } 32 \\
\text { cross-reactive, and the B35 allele }\end{array}$ \\
\hline p24 (71-80) & $\begin{array}{l}\text { p24 (203-212 SF2) } \\
\text { - Therapy provided du } \\
\text { individuals treated d } \\
\text { - The breadth and spe } \\
\text { individuals with prin } \\
\text { (Group 3), using } 259 \\
\text { - Previously described } \\
\text { - Number of HLA-A2 }\end{array}$ & $\begin{array}{l}\text { ETINEEAAEW } \\
\text { acute infection re } \\
\text { chronic infectior } \\
\text { ty of the response } \\
\text { infection but post } \\
\text { lapping peptides } \\
\text { newly defined op } \\
\text { dividuals that hac }\end{array}$ & $\begin{array}{l}\text { HIV-1 infection human (A25) } \\
\text { ower CTL response, stronger T help response, and a less } \\
\text { d using ELISPOT by studying } 19 \text { individuals with pre-se } \\
\text { n therapy (Group 2), and } 10 \text { individuals who responded t } \\
\text { p24, RT, gp41, gp } 120 \text { and Nef } \\
\text { were tested for CTL response } \\
\text { se to this epitope broken down by group: } 0 / 0 \text { group } 1,0 /\end{array}$ & $\begin{array}{l}\text { Altfeld } 2001 \mathrm{~b} \\
\text { e viral population than was seen in } \\
\text { zersion therapy (Group } 1), 11 \\
\text { RT given during chronic infection } \\
2 \text {, and } 1 / 3 \text { group } 3\end{array}$ \\
\hline p24 (71-80) & $\begin{array}{l}\text { - Epitope name: Gag-1 } \\
\text { - Among HIV+ indivi }\end{array}$ & $\begin{array}{l}\text { DTINEEAAEW } \\
0 \\
\text { who carried HL }\end{array}$ & $\begin{array}{l}\text { HIV-1 infection } \\
(13 \%) \text { recognized this epitope }\end{array}$ & Sabbaj2002b \\
\hline p24 (71-80) & $\begin{array}{l}\text { Epitope name: Gag-1 } \\
\text { - Among HIV+ indivi }\end{array}$ & $\begin{array}{l}\text { ETINEEAAEW } \\
0 \\
\text { who carried HL }\end{array}$ & $\begin{array}{l}\text { HIV-1 infection } \\
(13 \%) \text { recognized this epitope }\end{array}$ & Sabbaj2002b \\
\hline p24 (71-80) & $\begin{array}{l}\text { p24 (203-212) } \\
\text { ELISPOT was used } \\
\text { HIV-1-infected fema } \\
\text { Responses in HEPS } \\
\text { reduced risk of infec } \\
\text { women }\end{array}$ & $\begin{array}{l}\text { DT INEEAAEW } \\
\text { dy CTL response } \\
\text { airobi sex worker } \\
\text { en tended to be lo } \\
\text { and there was a s }\end{array}$ & $\begin{array}{l}\text { HIV-1 infection, HIV-1 exposed human (B53) } \\
\text { seronegative } \\
54 \text { predefined HIV-1 epitopes in } 91 \text { HIV-1-exposed, pers } \\
\text { ed on different epitopes with HLA presenting molecules } \\
\text { onse in the HEPS women upon late seroconversion to ep }\end{array}$ & $\begin{array}{l}\text { Kaul2001a } \\
\text { seronegative (HEPS) and } 87 \\
\text { ve previously been associated with } \\
\text { ecognized by the HIV-1 infected }\end{array}$ \\
\hline
\end{tabular}




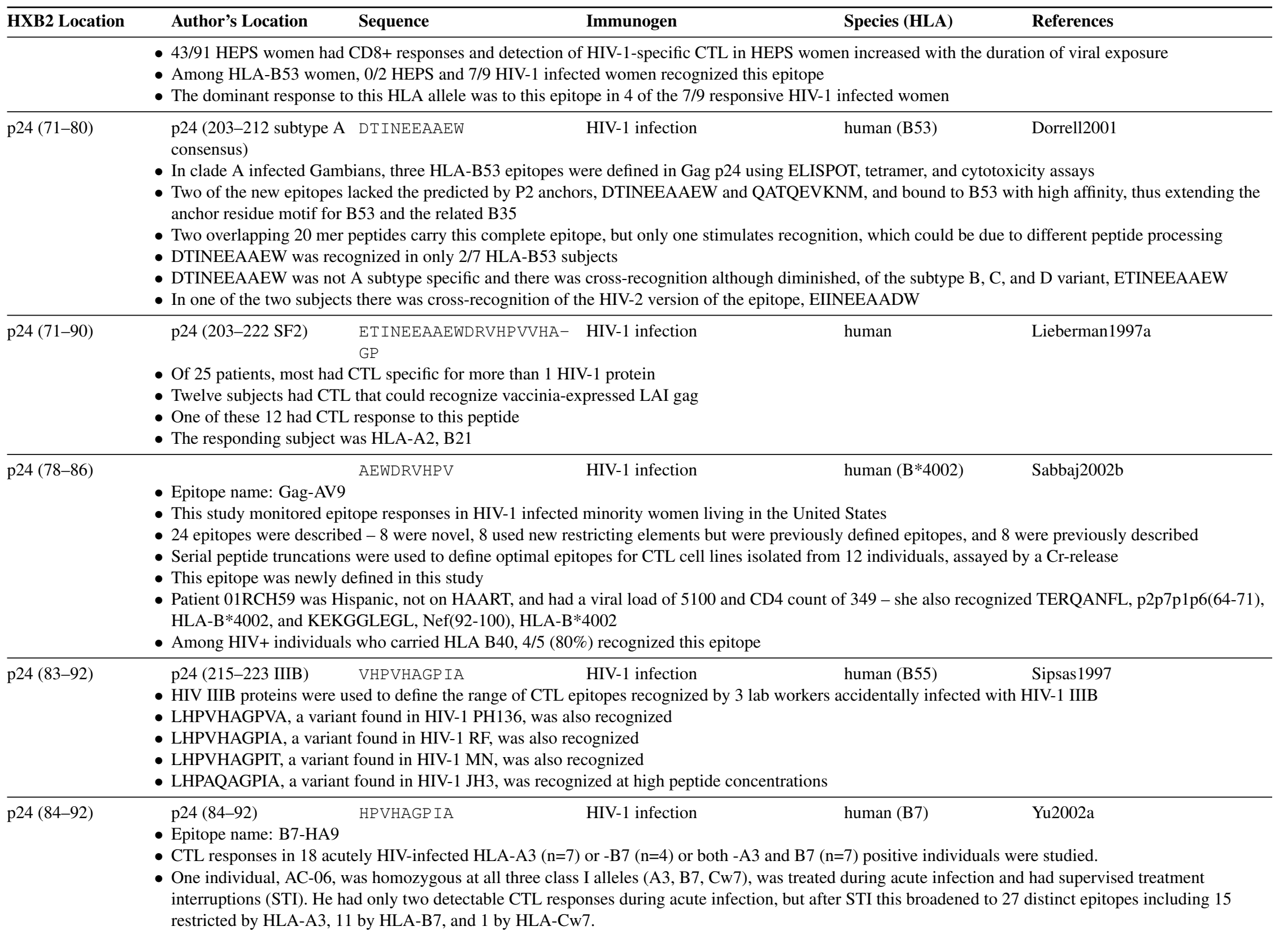




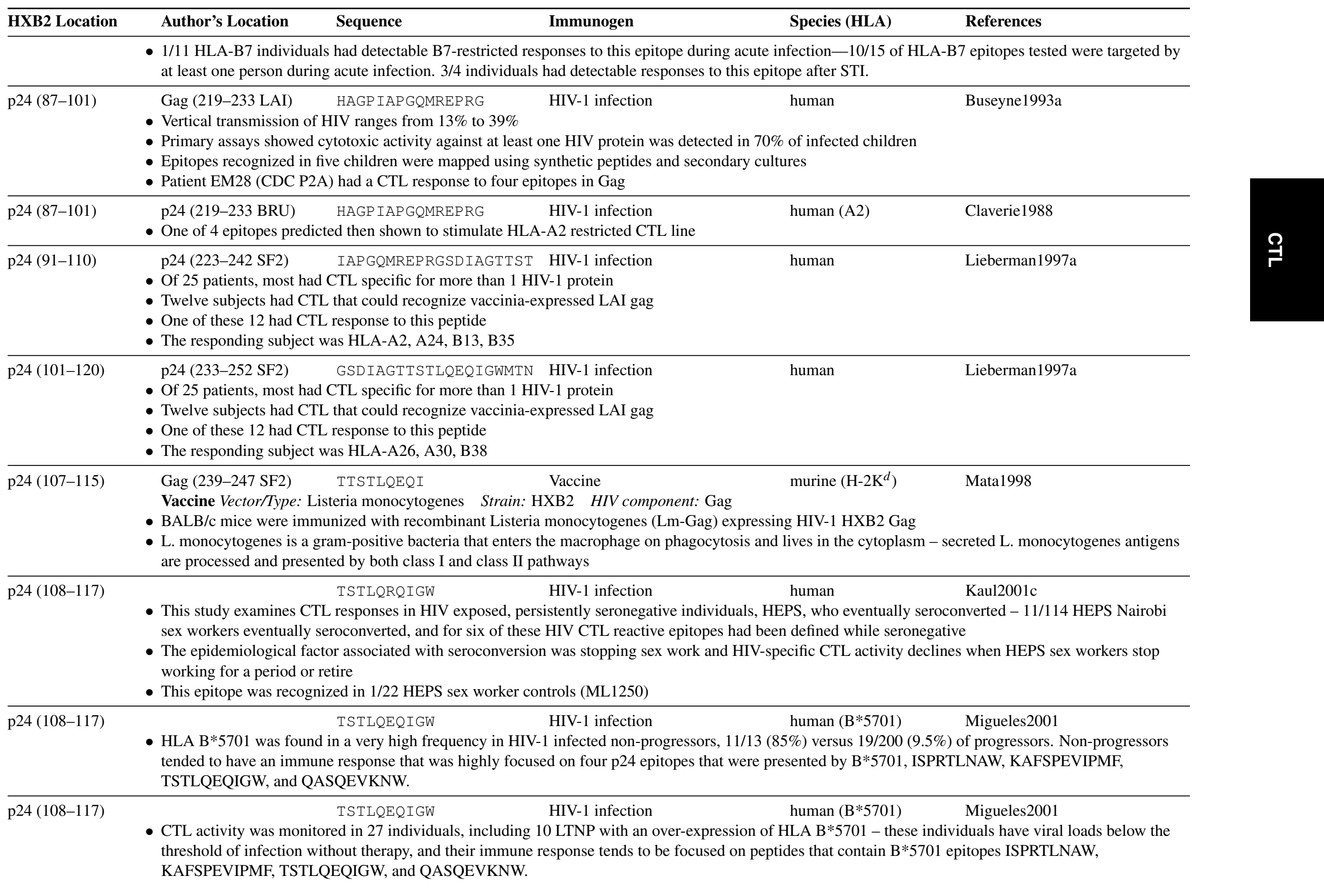




\begin{tabular}{|c|c|c|c|c|c|}
\hline HXB2 Location & Author's Location & Sequence & Immunogen & Species (HLA) & References \\
\hline & \multicolumn{5}{|c|}{$\begin{array}{l}\text { - CTL responses are broader in } B * 5701+\text { individuals with progressive viremia than those that control viremia. } \\
\text { - The HLA-A*0201 SLYNTVATL epitope response was not as strong individuals that carried both A2, B57. }\end{array}$} \\
\hline p24 (108-117) & \multicolumn{5}{|c|}{ - C. Brander notes this is a $\mathrm{B} * 5801$ epitope } \\
\hline p24 (108-117) & \multicolumn{4}{|c|}{ - C. Brander notes this is a B*5801 epitope } & Brander2001 \\
\hline p24 (108-117) & p24 (233-252) & TSTLQEQIGW & HIV-1 infection & human (B57) & Bernard1998 \\
\hline
\end{tabular}

- This study focuses on six rare long-term survivor HIV-infected people who were infected for many years without exhibiting immune dysregulation - such immunologically normal HIV-infected (INHI) cases occur at a frequency between 0.1 and $1 \%$ in the infected population

- No direct CTL were found in any of the six INHIs, but above background CTLp activity was founded in 3/6 INHIs

- Epitope sequences were deduced from larger reactive peptides based on HLA binding motifs - XSXXXXXXXW is a B57 binding motif, and CTL activity against TSTLQEQIGW has been found in two other B57 long-term non-progressors

p24 (108-117) Gag (SF2) TSTLQEQIGW $\quad$ HIV-1 infection

- Epitope name: TW10

- Dominant epitope in acute infection in patient PI004, who did not receive any antiviral therapy

- 1-2 months post seroconversion, subject PI004 displayed a significant decrease in TW10 peptide recognition, followed by an increased CTL response against epitope SL9, SLYNTVATL and other epitopes

- Three CTL responses, to epitopes TSTLQEQIGW, ISPRTLNAW, and KAFSPEVIPMF, were evident early after infection; CTL responses to SLYNTVATL, QASQEVKNW, EIYKRWII, and FLKEKGGL were detectable at 5 months post-infection and beyond

\begin{tabular}{lllll}
\hline p24 (108-117) p24 (108-117) & TSTLQEQIGW & Oxenius2000
\end{tabular}

- Epitope name: TST

- Patients who started therapy at acute HIV-1 infection (three with sustained therapy, two with limited therapy upon early infection) had strong HIV specific CD4 proliferative responses and were able to maintain a CTL response even with undetectable viral load - three patients that had delayed initiation of HAART had no HIV specific CD4 proliferative responses and lost their CTL responses when HAART was eventually given and their viral loads became undetectable

- None of the 8 study subjects recognized this epitope but none were HLA B57+

p24 (108-117) p24 (108-117) TSTLQEQIGW HIV-1 infection Fuman (B57) Ferrari2000

- One of the 51 HIV-1 epitopes selected by Ferrari et al. as good candidate CTL epitopes for vaccines by virtue of being conserved and presented by common HLA alleles

p24 (108-117) p24 TSTLQEQIGW HIV-1 infection $\quad$ Kaul2002

- Neisseria gonorrhea cervititis in 9 HIV+ Kenyan sex workers caused a functional deficiency in IFN-gamma production in HIV-1 epitope-specific CD8+ T-cells, detected by intracellular cytokine production and tetramer assays, while not affecting the total number of epitope-specific CTLs.

- Ghonorrhea caused the weaker HIV-1 specific CTL responses in 4 HIV-1 exposed persistently seronegative (HEPS) women to become undetectable by Elispot and tetramer assays, and CMV-specific CTL in 2 HEPS subjects were shown to have impaired function with regard to IFN-gamma production. p24

HIV-1 infection

human (B57)

Oxenius2002b

- Epitope name: TST

- Using previously defined epitopes [Oxenius2000 Oxenius2001a] in an IFNgamma Elispot assay, 13 chronically HIV-1 infected patients were studied over a period including therapy with standard treatment interruptions (STI). 


\begin{tabular}{|c|c|c|c|c|c|}
\hline HXB2 Location & Author's Location & Sequence & Immunogen & Species (HLA) & References \\
\hline & \multicolumn{5}{|c|}{$\begin{array}{l}\text { - STIs induced increased recognition of CTL epitopes, but there was no correlation between CTL responses with viral rebound rates, plateau viral loads, or } \\
\text { clearance rates. }\end{array}$} \\
\hline p24 (108-117) & \multicolumn{5}{|c|}{$\begin{array}{l}\text { - TSTLQEQIGW cross reacts with both for the A and B clades } \\
\text { - ELISPOT was used to study CTL responses to a panel of } 54 \text { predefined HIV-1 epitopes in } 91 \text { HIV-1-exposed, persistently seronegative (HEPS) and } 87 \\
\text { HIV-1-infected female Nairobi sex workers }\end{array}$} \\
\hline p24 (108-117) & $\begin{array}{l}\text { p24 (241-250) } \\
\text { - HIV-2 epitope define } \\
\text { - All HIV-2 sequences }\end{array}$ & $\begin{array}{l}\text { TSTVEEQQIW } \\
\mathrm{m} \text { an infection in } \\
\text { the database are }\end{array}$ & $\begin{array}{l}\text { HIV-2 infection } \\
\text { letti, Pers. Comm. } \\
\text { W in this region, not TSTVEEQQ }\end{array}$ & $\begin{array}{l}\text { human (B58) } \\
\text { as in the paper }\end{array}$ & Bertoletti1998a \\
\hline p24 (108-117) & $\begin{array}{l}\text { p24 } \\
\text { - CTL responses in ser } \\
\text { deletion in CCR5 } \\
\text { - In Gambia there is ex } \\
\text { seems to be protectiv } \\
\text { - HIV-2 sequence: TS }\end{array}$ & $\begin{array}{l}\text { TSTLQEQIGW } \\
\text { ative highly HIV- } \\
\text { Ire to both HIV-1 } \\
\text { EQIQW, CTL are }\end{array}$ & $\begin{array}{l}\text { HIV-1 exposed seronegative } \\
\text { an female sex workers in Gambia a } \\
\text { L responses to B35 epitopes in ex } \\
\text { Bertoletti1998b }\end{array}$ & $\begin{array}{l}\text { human (B58) } \\
\text { Nairobi were studic } \\
\text { sed, uninfected wom }\end{array}$ & $\begin{array}{l}\text { Rowland-Jones1999 } \\
\text { ese women had no delta } 32 \\
\text { cross-reactive, and the B35 allele }\end{array}$ \\
\hline p24 (108-117) & $\begin{array}{l}\text { p24 (240-249) } \\
\text { - CTL responses in HI } \\
\text { HLA-B*5801+ indiv } \\
\text { - This can be an immu } \\
\text { - HIV-2 sequence: HI } \\
\text { epitopes } \\
\text { - The epitope is TSTL } \\
\text { - HLA B*5801 and B? }\end{array}$ & $\begin{array}{l}\text { TSTLQEQIGW } \\
* 5801 \text { positive H } \\
\text { s may have an en } \\
\text { minant epitope ir } \\
\text { OD has the epito } \\
\text { IGW in HIV-1 B } \\
\text { ay preferentially }\end{array}$ & $\begin{array}{l}\text { HIV-2 infection } \\
\text { ndividuals have a dominant respon } \\
\text { al for cross-protection between HI } \\
\text { B*5801 infected individuals, and } \\
\text { STVEEQIQW, and the CTL from } \\
\text { TVEEQIQW in HIV-2 ROD } \\
\text { d HIV-2 cross-reactive epitopes }\end{array}$ & $\begin{array}{l}\text { human (B58) } \\
\text { to Gag and tolerate } \\
1 \text { and HIV-2 } \\
\text { associated with lons } \\
\text { erson infected with I }\end{array}$ & $\begin{array}{l}\text { Bertoletti1998b } \\
\text { ive substitution, thus } \\
\text { non-progression Goulder1996b } \\
\text { was cross-reactive with HIV-1 }\end{array}$ \\
\hline p24 (10 & $\begin{array}{l}\text { p24 (240-249 SF2) } \\
\text { - Therapy provided du } \\
\text { individuals treated di } \\
\text { - The breadth and spec } \\
\text { individuals with prin } \\
\text { (Group 3), using } 259 \\
\text { - Previously described } \\
\text { - Number of HLA-B5 }\end{array}$ & $\begin{array}{l}\text { TSTLQEQIGW } \\
\text { acute infection re } \\
\text { chronic infection } \\
\text { y of the response } \\
\text { nfection but post } \\
\text { lapping peptides } \\
\text { newly defined op } \\
\text { dividuals that had }\end{array}$ & $\begin{array}{l}\text { HIV-1 infection } \\
\text { ower CTL response, stronger T he } \\
\text { d using ELISPOT by studying } 19 \\
\text { therapy (Group 2), and } 10 \text { indivic } \\
\text { p24, RT, gp41, gp120 and Nef } \\
\text { were tested for CTL response } \\
\text { se to this epitope broken down by }\end{array}$ & $\begin{array}{l}\text { human (B58) } \\
\text { response, and a less } \\
\text { lividuals with pre-se } \\
\text { ls who responded to } \\
\text { oup: } 0 / 0 \text { group } 1,1 / 1\end{array}$ & $\begin{array}{l}\text { Altfeld2001b } \\
\text { e viral population than was seen in } \\
\text { ersion therapy (Group } 1), 11 \\
\text { RT given during chronic infection } \\
2 \text {, and } 0 / 0 \text { group } 3\end{array}$ \\
\hline p24 (108-117) & $\begin{array}{l}\text { p24 (108-117) } \\
\text { - Epitope name: TW1( } \\
\text { - Responses to this do } \\
\text { - Mutations in this epi } \\
(\mathrm{P}=0.02) \\
\text { - These mutations are }\end{array}$ & $\begin{array}{l}\text { TSTLQEQIGW } \\
\text { at A3-restricted C } \\
\text { were observed in } \\
\text { sexually transm }\end{array}$ & $\begin{array}{l}\text { HIV-1 infection } \\
\text { present during the time of decreas } \\
\text { nes of subjects who were B58-pos } \\
\text { fections }\end{array}$ & $\begin{array}{l}\text { human (B58) } \\
\text { g viral load in acute } \\
\text { je with a higher freq }\end{array}$ & $\begin{array}{l}\text { Goulder2001c } \\
\text { on } \\
\text { than those who were B58-negative }\end{array}$ \\
\hline
\end{tabular}




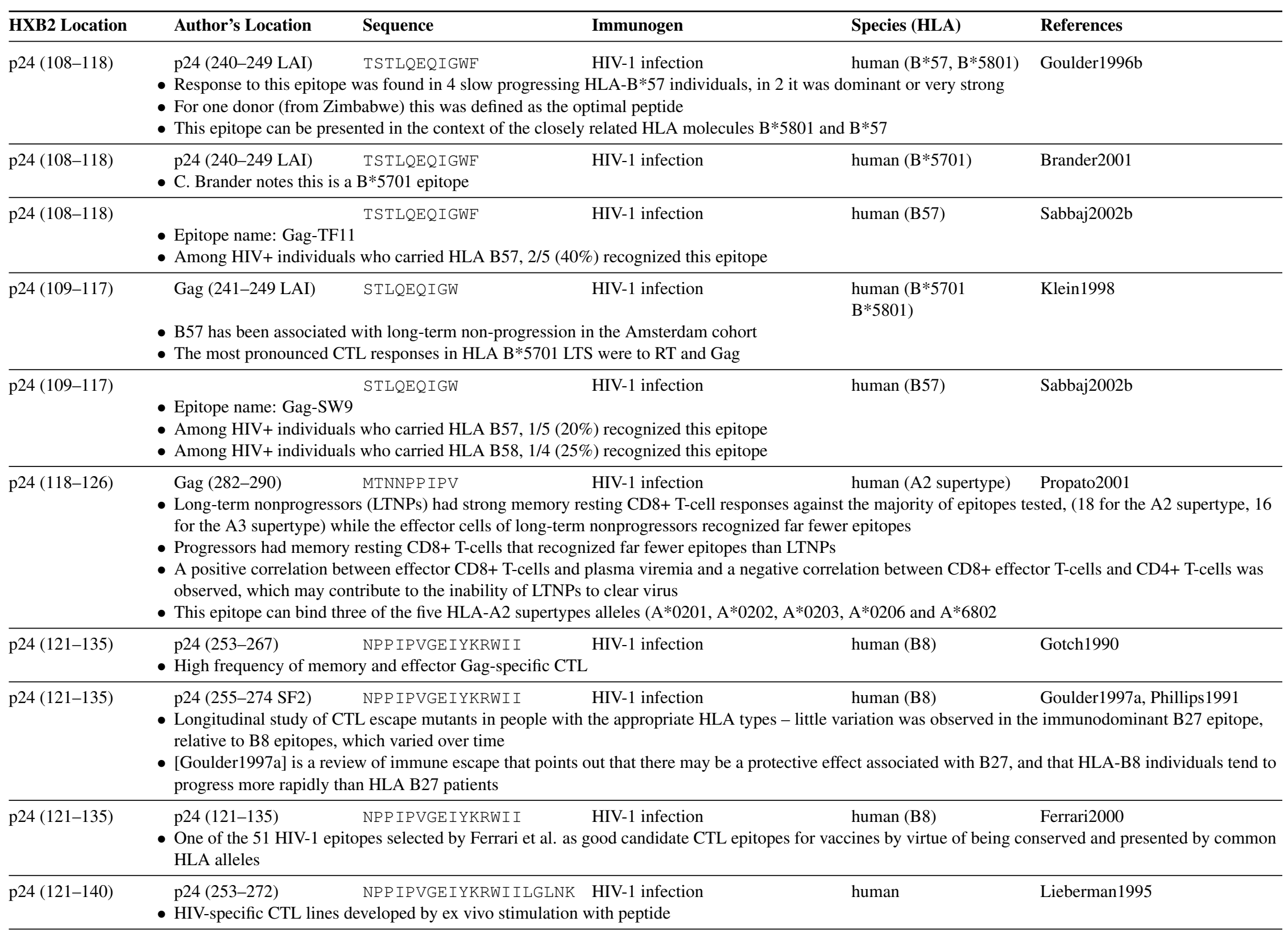




\begin{tabular}{|c|c|c|c|c|}
\hline HXB2 Location & Author's Location & Immunogen & Species (HLA) & References \\
\hline p24 (121-140) & $\begin{array}{l}\text { p24 (253-272 SF2) } \\
\text { - Of } 25 \text { patients, most h } \\
\text { - Twelve subjects had C } \\
\text { - Two of these } 12 \text { had C } \\
\text { - The responding subje }\end{array}$ & $\begin{array}{l}\text { NP P IPVGE I YKRWI ILGLNK HIV-1 infection } \\
\text { CTL specific for more than } 1 \text { HIV-1 protein } \\
\text { that could recognize vaccinia-expressed LAI gag } \\
\text { response to this peptide } \\
\text { vere HLA-A2, A3, B8, B62, and HLA-A1, B8, B18 }\end{array}$ & human & Lieberman1997a \\
\hline p24 (121-140) & $\begin{array}{l}\text { p24 (253-272 SF2) } \\
\text { - CTL expanded ex viv }\end{array}$ & $\begin{array}{l}\text { NPPIPGEIKRWIILGNIK HIV-1 infection } \\
\text { re later infused into HIV-1 infected patients }\end{array}$ & human & Lieberman1997b \\
\hline p24 (121-140) & $\begin{array}{l}\text { p24 (255-274 SF2) } \\
\text { - Gag CTL epitope prec }\end{array}$ & $\begin{array}{l}\text { NPP IPVGEIYKRWIILGLNK HIV-1 infection } \\
\text { or frequencies were estimated and peptide mapping }\end{array}$ & human & vanBaalen1993 \\
\hline p24 (121-142) & $\begin{array}{l}\text { p24 (253-274 BH10) } \\
\text { - Gag CTL response stı }\end{array}$ & $\begin{array}{l}\text { NPP IPVGEIYKRWIILGLN- HIV-1 infection } \\
\text { KIV } \\
\text { d in three individuals }\end{array}$ & human (B8) & Johnson1991 \\
\hline p24 (121-152) & Gag (183-214 LAI) & $\begin{array}{l}\text { NPP IPVGEIYKRWIILGLN }- \text { Vaccine } \\
\text { KIVRMYSPTSILD }\end{array}$ & human & Gahery-Segard2000 \\
\hline
\end{tabular}

- Anti-HIV lipopeptide vaccine consisting of six long amino acid peptides derived from Nef, Gag and Env HIV-1 proteins modified by a palmitoyl chain was administered in a phase I trial

- A CD4+ T cell proliferative response to at least one of the six peptides was observed in $9 / 10$ vaccinees $-9 / 10$ reacted to this peptide

- 9/12 tested mounted a CTL response to at least one of the six peptides; each of the six peptides elicited a CTL response in at least one individual - this peptide was particularly immunogenic, eliciting a CTL response in four vaccinees

- All of the 12 tested had an IgG response to this peptide

\begin{tabular}{llll}
\hline p24 (121-152) Gag & $\begin{array}{l}\text { NPPIPVGEIYKRWIILGLN- } \\
\text { KIVRMYSPTSILD }\end{array}$ & heth2000 infection, Vaccine \\
\hline
\end{tabular}

Vaccine Vector/Type: lipopeptide HIV component: gag peptide

- Immunization of 2/4 HIV seropositive HLA selected individuals with a 32 amino acid Gag lipopeptide that contains CTL epitopes restricted by HLA A33,

B8, B27, B35, and Bw62 gave a transient increase in peptide-specific bulk CTL response, but they did not decrease plasma viral load.

- Placebo and HLA mis-matched controls showed no change in CTL

- The responders carried HLA Bw62 and B35 - the two HLA-matched that did not respond carried B35 and B8

p24 (122-130) p24 PPIPVGDIH HIV-1 infection $\quad$ Kaul2001c

- This study examines CTL responses in HIV exposed, persistently seronegative individuals, HEPS, who eventually seroconverted - 11/114 HEPS Nairobi sex workers eventually seroconverted, and for six of these HIV CTL reactive epitopes had been defined while seronegative

- The epidemiological factor associated with seroconversion was stopping sex work and HIV-specific CTL activity declines when HEPS sex workers stop working for a period or retire

- This epitope was recognized in 1/22 HEPS sex worker controls, ML887

\begin{tabular}{lllll}
\hline p24 (122-130) & p24 (260-268 LAI) & PP IPVGDIY & HIV-1 or HIV-2 infection & human (B*3501) \\
\hline C24 (122-130) & p24 (245-253 HIV-2) & NPVPVGNIY & HIV-1 infection & human (B*3501) \\
\hline
\end{tabular}




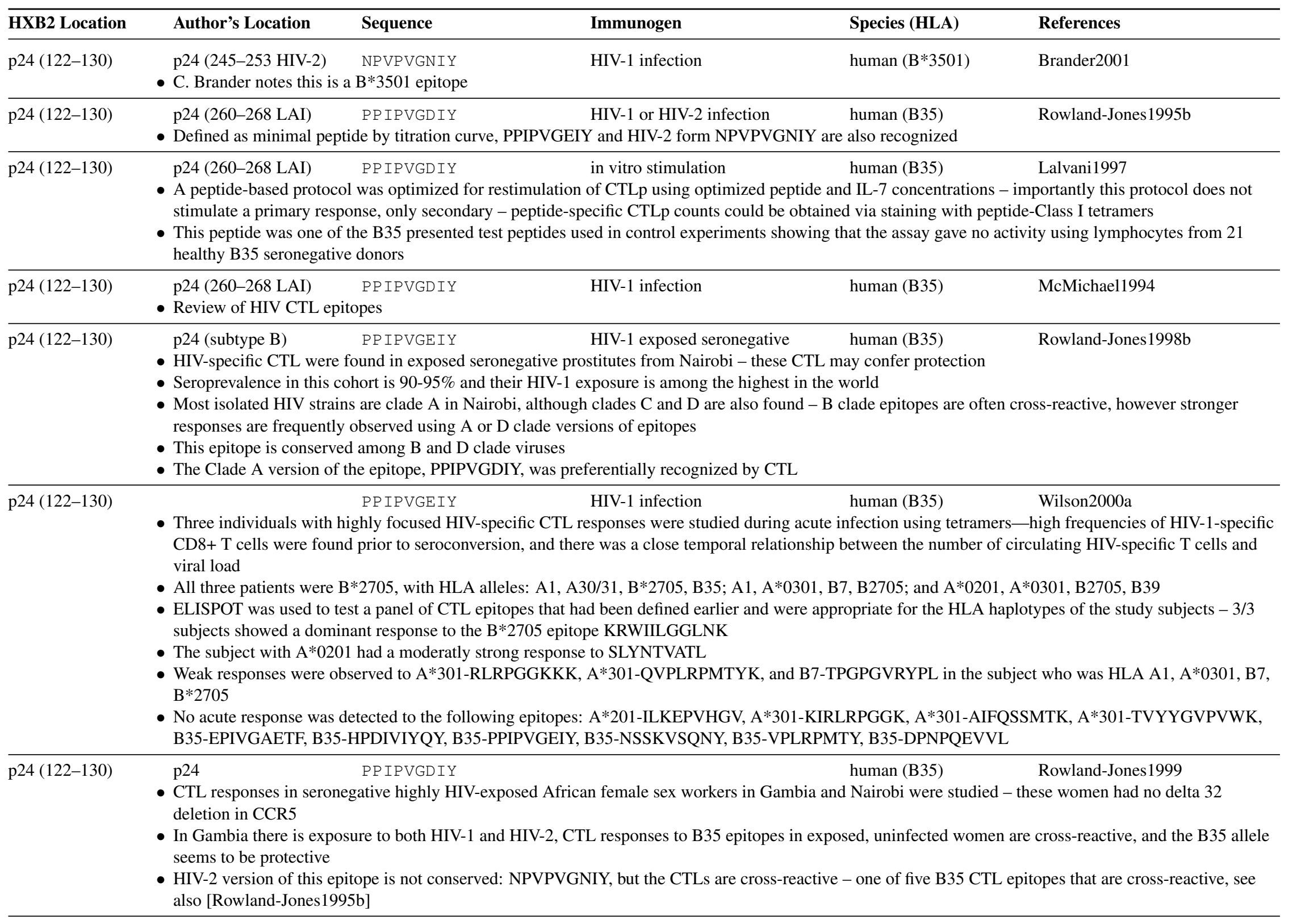




\begin{tabular}{lllll}
\hline HXB2 Location & Author's Location & Sequence & Immunogen & Species (HLA) \\
\hline p24 (122-130) & p24 (260-268) & PP IPVGDIY & HIV-1 infection & human (B35)
\end{tabular}

- Patients who started therapy at acute HIV-1 infection (three with sustained therapy, two with limited therapy upon early infection) had strong HIV specific CD4 proliferative responses and were able to maintain a CTL response even with undetectable viral load - three patients that had delayed initiation of HAART had no HIV specific CD4 proliferative responses and lost their CTL responses when HAART was eventually given and their viral loads became undetectable

- One of two HLA B35+ among the eight study subjects recognized this epitope

- Patient SC15 (HLA A1/68, B8/35, Bw4/6, Cw4/0704) was given acute and sustained therapy and recognized epitopes PPIPVGDIY and VPLRPMTY during 331 days of HAART treatment

\begin{tabular}{llccc}
\hline p24 (122-130) & p24 (122-130) & PPIPVGD IY & HIV-1 infection & herrari2000 \\
& One of the 51 HIV-1 epitopes selected by Ferrari et al. as good candidate CTL epitopes for vaccines by virtue of being conserved and presented by common \\
& HLA alleles
\end{tabular}

HLA alleles

p24 (122-130)

p24 (254-262 SF2) PP IPVGDIY
Therapy provided during acute infection rest

individuals treated during chronic infection

- The breadth and specificity of the response was determined using ELISPOT by studying 19 individuals with pre-seroconversion therapy (Group 1), 11

individuals with primary infection but post-seroconversion therapy (Group 2), and 10 individuals who responded to HAART given during chronic infection (Group 3), using 259 overlapping peptides spanning p17, p24, RT, gp41, gp120 and Nef

- Previously described and newly defined optimal epitopes were tested for CTL response

- Number of HLA-B35+ individuals that had a CTL response to this epitope broken down by group: 0/2 group 1,0/2 group 2, and 1/1 group 3

p24 (122-130) p24 (260-268) PPIPVGDIY $\quad \begin{aligned} & \text { HIV-1 infection, HIV-1 exposed human (B35) } \\ & \text { seronegative }\end{aligned}$

- ELISPOT was used to study CTL responses to a panel of 54 predefined HIV-1 epitopes in 91 HIV-1-exposed, persistently seronegative (HEPS) and 87 HIV-1-infected female Nairobi sex workers

- Responses in HEPS women tended to be lower, and focused on different epitopes with HLA presenting molecules that have previously been associated with reduced risk of infection, and there was a shift in the response in the HEPS women upon late seroconversion to epitopes recognized by the HIV-1 infected women

- 43/91 HEPS women had CD8+ responses and detection of HIV-1-specific CTL in HEPS women increased with the duration of viral exposure

- Among HLA-B35 women, 1/3 HEPS and 3/4 HIV-1 infected women recognized this epitope

- The dominant response to this HLA allele was to this epitope in the 1/3 HEPS case and in the all 3/4 responsive HIV-1 infected women

- Subject ML 857 shifted from a A*6802 DTVLEDINL and B35 (H/N)PDIVIYQY response prior to seroconversion to a B35 PPIPVGDIY and B35 VPLRPMTY response post-seroconversion

p24 (122-130) PPIPVGDIY HIV-1 infection $\quad$ Sabbaj2002b

- Epitope name: Gag-PY9

- Among HIV+ individuals who carried HLA B35, $2 / 21$ (10\%) recognized this epitope

- Among HIV+ individuals who carried HLA B*5301, 0/11 (0\%) recognized this epitope

p24 (122-130) p24 PPIPVGEIY HIV-1 infection, Vaccine human (B35) Wee2002
Vaccine Vector/Type: DNA prime with vaccinia MVA boost Strain: subtype A HIV component: p17, p24, polyepitope 


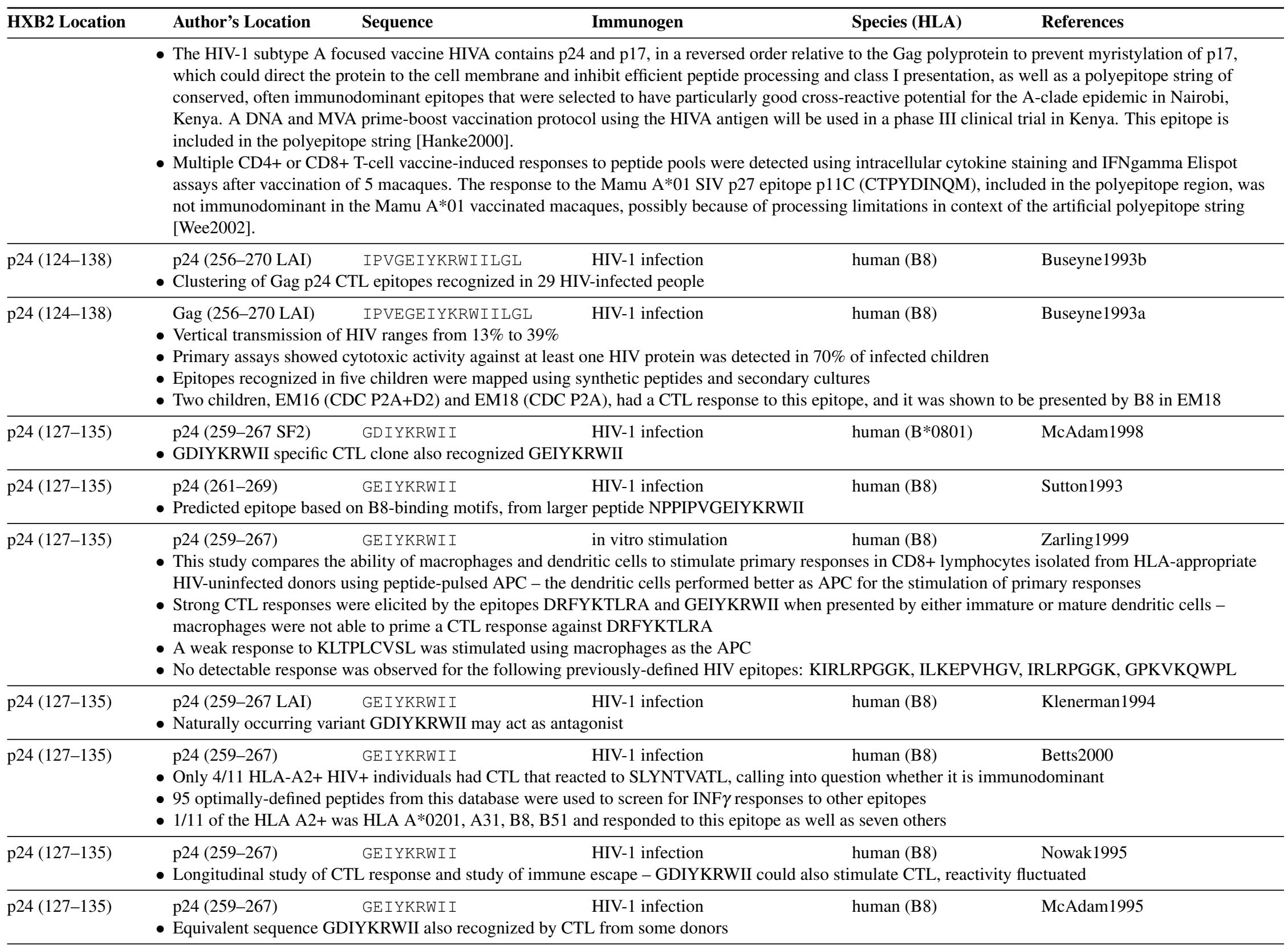




\begin{tabular}{lllll}
\hline HXB2 Location & Author's Location & Sequence & Immunogen & Species (HLA) \\
\hline p24 (127-135) & $\begin{array}{l}\text { p24 (259-267) } \\
\text { Epitope name: GEI }\end{array}$ & GEIYKRWII & HIV-1 infection & human (B8)
\end{tabular}

- Patients who started therapy at acute HIV-1 infection (three with sustained therapy, two with limited therapy upon early infection) had strong HIV specific CD4 proliferative responses and were able to maintain a CTL response even with undetectable viral load - three patients that had delayed initiation of HAART had no HIV specific CD4 proliferative responses and lost their CTL responses when HAART was eventually given and their viral loads became undetectable

- Six of the 7/8 study subjects that were HLA B8 recognized this epitope

- Patient SC2 (HLA A1, B7/8, Cw0701/0702, DR4/53, DQ7) had CTL responsiveness against epitopes FLKEKGGL, GPKVKQWPL, and GEIYKRWII peptides - FLKEKGGL tetramer staining steadily declined and at day 1340 the FLKEKGGL stained cells were no longer detected and the escape mutant FLKENGGI was found in $8 / 10$ clones

- Patient SC9 (HLA A1/2, B8/13, Cw0/0701, DR2/11, DQ6/7) had a CTL response against epitopes FLKEKGGL, ILKEPVHGV, SQRRQDILDLWIYHTQGYFPDWQNY, and GEIYKRWII and all responses declined during therapy initiated at day 390 but were restored when therapy become intermittent.

- Patient SC19(HLA A11/12, B8/44, Cw06/0701, DR3/7, DR52/53, DQ 2/8) had a CTL response to epitopes FLKEKGGL, GEIYKRWII, ACQGVGGPGHK, AVDLSHFLK, and FNCGGEFFY that declined during therapy initiated at day 197

- Patient SC10(HLA A1/3, B8/35, DR1/8, DQ 4/5) had sustained therapy started during acute infection and maintained an immunodominant response to FLKEKGGL and a response to GEIYKRWII through day 1088

- Patient SC12(HLA A1, B8/39, Cw0701/0702, DR2/3, DR51/52, DQ2/6) had sustained therapy started during acute infection and maintained an immunodominant response to FLKEKGGL throughout and minor responses to GEIYKRWII, DCKTILKAL, GGKKKYKLK - GEIYKRWII and GGKKKYKLK responses were stimulated by a brief period off therapy

- Patient SC11(HLA A1, B8, Cw0201, DR3/11, DR52, DQ2/7) started therapy early, remained on therapy for 40 days, then reinitiated HAART at day 640 had a CTL response to FLKEKGGL, GPKVKQWPL, and GEIYKRWII throughout and received a benefit from the early limited course therapy

p24 (127-135) p24(259-267 SF2) GEIYKRWII HIV-1 infection human (B8) Altfeld2001b

- Therapy provided during acute infection resulted in a narrower CTL response, stronger T help response, and a less diverse viral population than was seen in individuals treated during chronic infection

- The breadth and specificity of the response was determined using ELISPOT by studying 19 individuals with pre-seroconversion therapy (Group 1), 11 individuals with primary infection but post-seroconversion therapy (Group 2), and 10 individuals who responded to HAART given during chronic infection (Group 3), using 259 overlapping peptides spanning p17, p24, RT, gp41, gp120 and Nef

- Previously described and newly defined optimal epitopes were tested for CTL response

- Number of HLA-B8+ individuals that had a CTL response to this epitope broken down by group: 2/3 group 1,2/3 group 2, and 2/2 group 3

p24 (127-135) p24 GEIYKRWII HIV-1 infection human (B8) Oxenius2002b

- Epitope name: GEI

- Using previously defined epitopes [Oxenius2000 Oxenius2001a] in an IFNgamma Elispot assay, 13 chronically HIV-1 infected patients were studied over a period including therapy with standard treatment interruptions (STI).

- STIs induced increased recognition of CTL epitopes, but there was no correlation between CTL responses with viral rebound rates, plateau viral loads, or clearance rates.

\begin{tabular}{lllll}
\hline p24 (127-135) & p24 & GEIYKRWII & HIV-1 infection, Vaccine & human (B8) \\
& Vaccine Vector/Type: DNA prime with vaccinia MVA boost & Strain: subtype A & HIV component: p17, p24, polyepitope
\end{tabular}




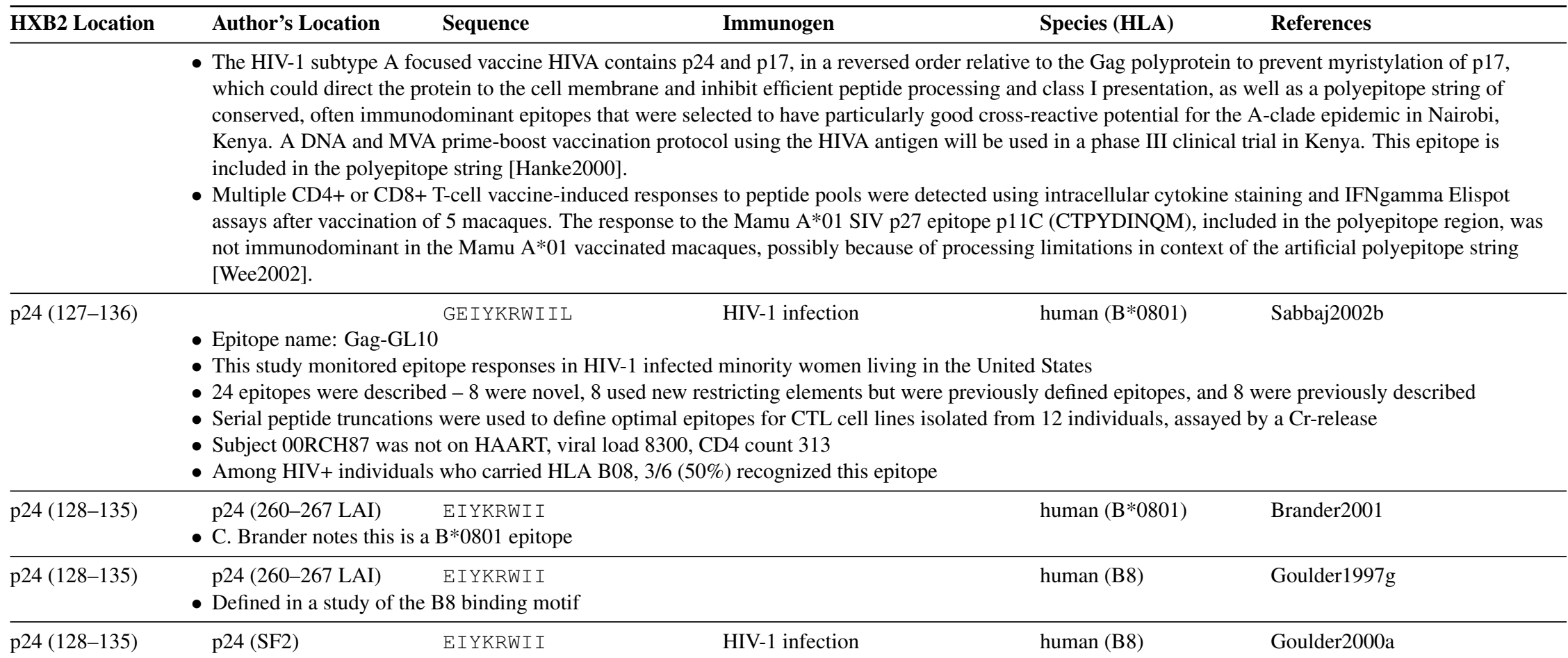

- The CTL-dominant response was focused on this epitope in a HIV+ Caucasian living in Boston - this epitope did not fall within the three most recognized peptides in the study

- Three peptides GSEELRSLYNTVATL (p17 residues 71-85), SALSEGATPQDLNTMLNTVG (p24 41-60), and WEKIRLRPGGKKKYKLK(p17 16-30) contained the dominant Gag-specific epitope in 31 out of 44 B-clade infected individuals from Boston who showed Gag-CTL responses

- Five peptides RLRPGGKKHYMIKHLVW (p17 20-36), ELRSLYNTVATLYCV (p17Gag 74-88), SALSEGATPQDLNTMLNTVG (p24 41-60), FRDYVDRFFKTLRAEQA (p24 161-177), and SILDIKQGKEPFRDY (p24 149-164) contained dominant Gag-specific epitopes in 32 out of 37 C-clade infected subjects from South Africa
p24 (128-135)
p24 (C consensus) DIYKRWII
HIV-1 infection
human (B8)
Goulder2000a

- The CTL-dominant response was focused on this epitope in a HIV+ South African - this epitope did not fall within the five most recognized peptides in the study

- Three peptides GSEELRSLYNTVATL (p17 residues 71-85), SALSEGATPQDLNTMLNTVG (p24 41-60), and WEKIRLRPGGKKKYKLK(p17 16-30) contained the dominant Gag-specific epitope in 31 out of 44 B-clade infected individuals from Boston who showed Gag-CTL responses

- Five peptides RLRPGGKKHYMIKHLVW (p17 20-36), ELRSLYNTVATLYCV (p17Gag 74-88), SALSEGATPQDLNTMLNTVG (p24 41-60),

FRDYVDRFFKTLRAEQA (p24 161-177), and SILDIKQGKEPFRDY (p24 149-164) contained dominant Gag-specific epitopes in 32 out of 37 C-clade infected subjects from South Africa

p24 (128-135) p24 (SF2) EIYKRWII

- This peptide elicited a weak CTL response during acute HIV-1 infection in patient PI004 


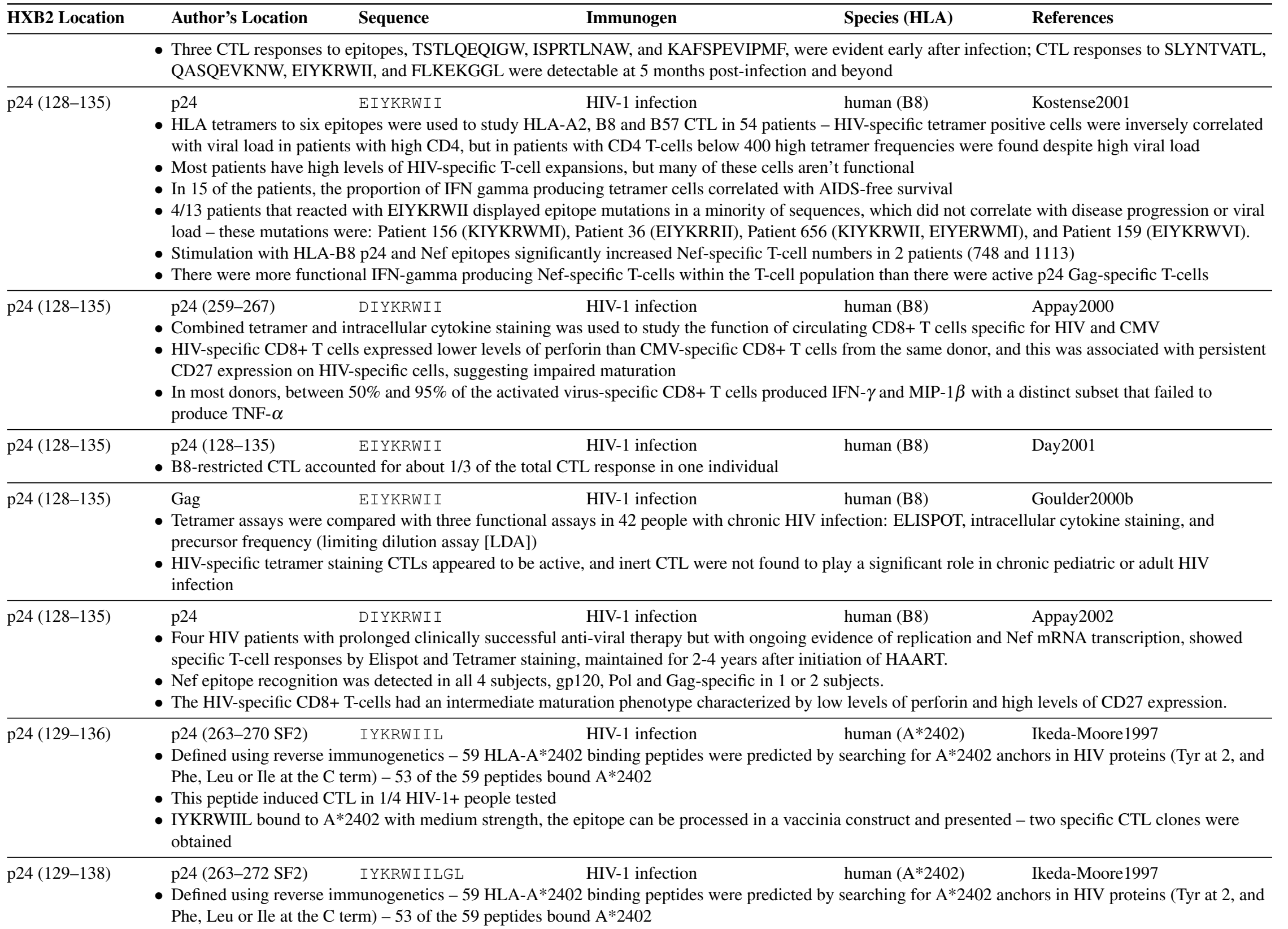




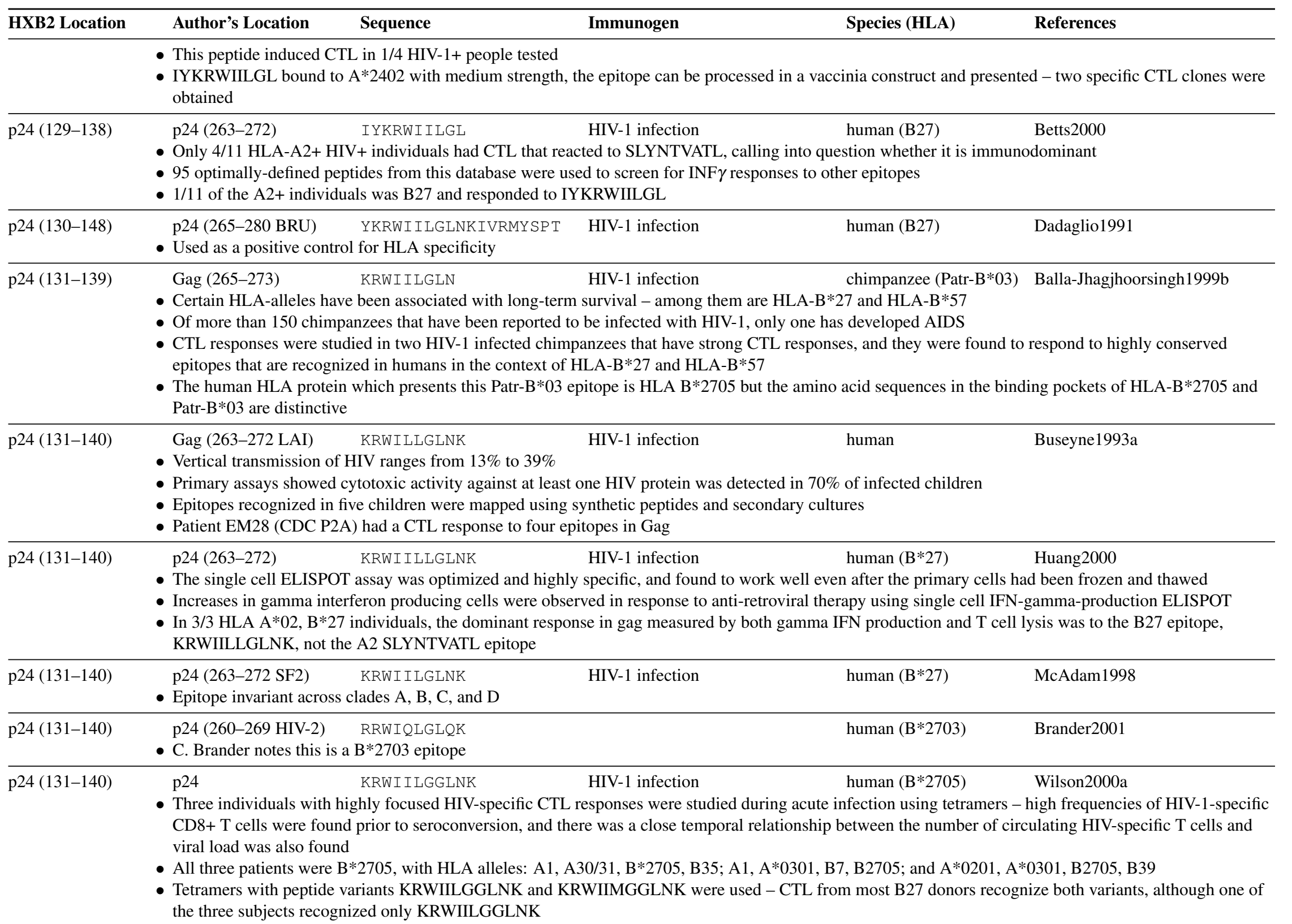




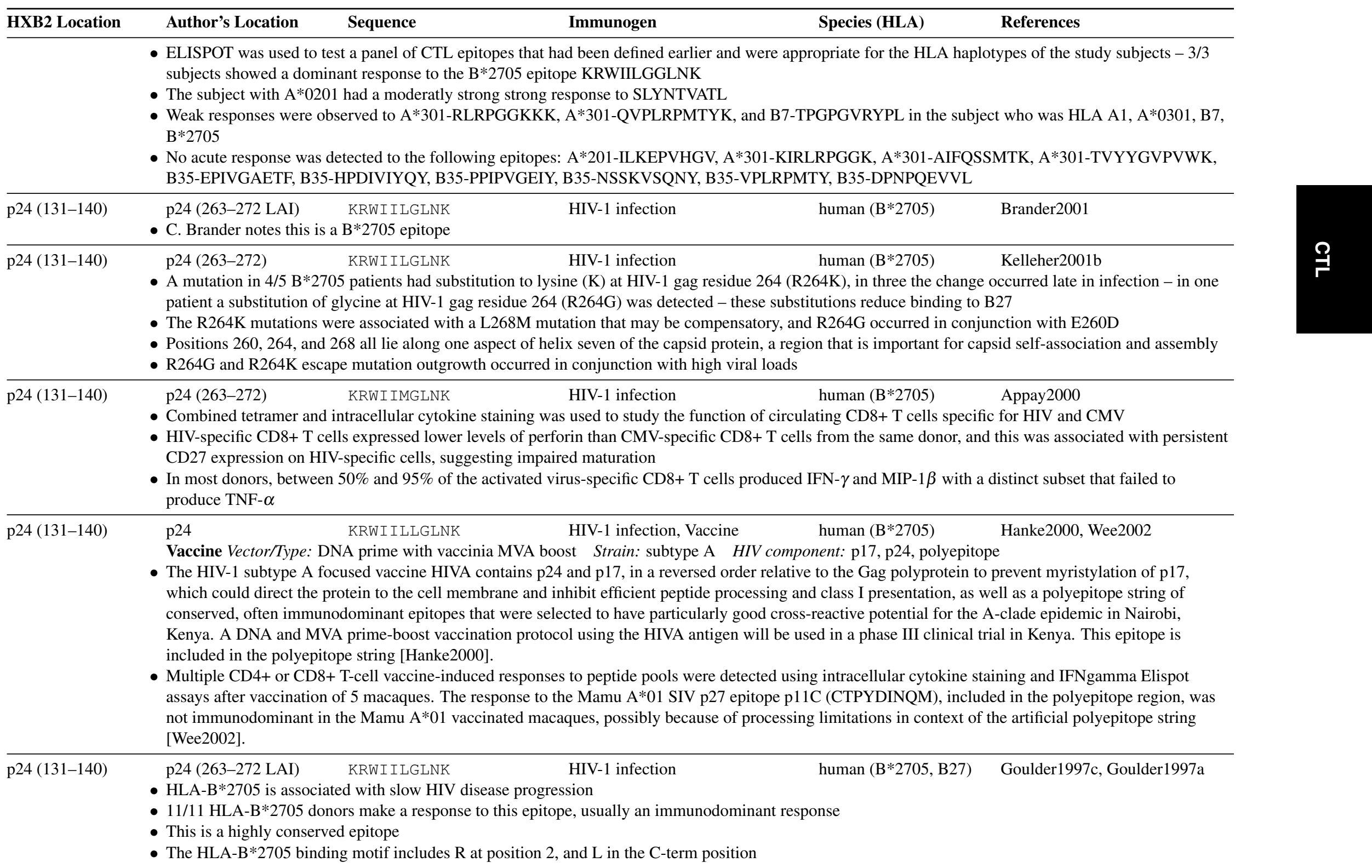




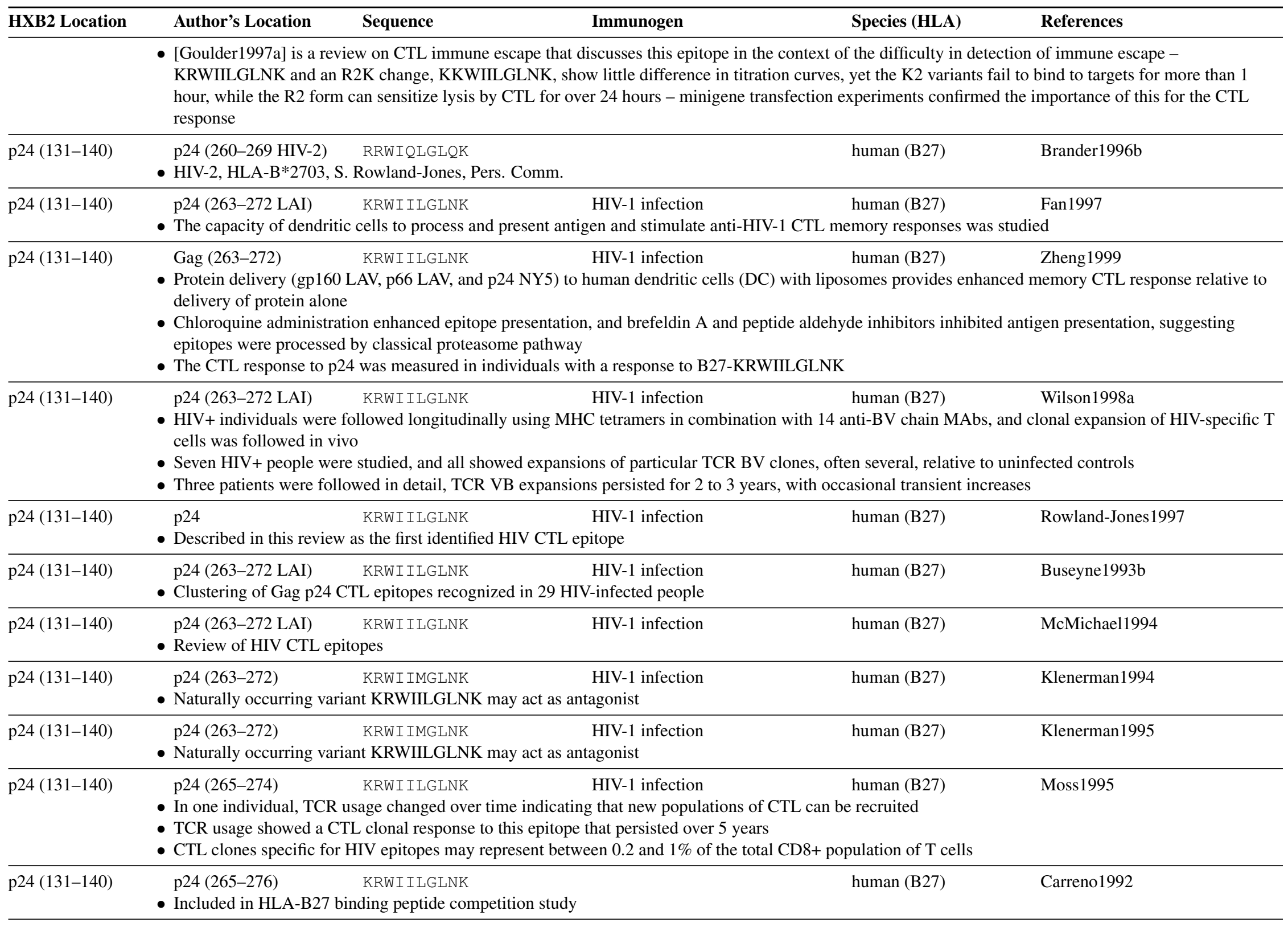




\begin{tabular}{|c|c|c|c|c|}
\hline HXB2 Location & Author's Location & Sequence & Species (HLA) & References \\
\hline 0) & \multicolumn{4}{|c|}{$\begin{array}{l}\text { - Longitudinal study of CTL escape mutants - little variation was observed in the immunodominant B27 epitope, relative to B8 epitope } \\
\text { - Goulder1997a is a review of immune escape that points out that there may be a protective effect associated with B27, and that HLA-B8 individuals tend to } \\
\text { progress more rapidly than HLA B27 patients }\end{array}$} \\
\hline 024 (131-140) & \multicolumn{4}{|c|}{$\begin{array}{l}\text { - Single point mutations were introduced and viral viability and CTL recognition tested - an Arg to Lys change at anchor position P2 abrogates binding to } \\
\text { B27, but doesn't change viral viability in vitro } \\
\text { - }[\text { Goulder1997a is a review of immune escape that summarizes this study }\end{array}$} \\
\hline $24(131-140)$ & \multicolumn{4}{|c|}{ - Longitudinal study of CTL response and immune escape - the form KRWIILGNK was also found, and both forms stimulate CTL } \\
\hline 40) & $\begin{array}{l}\text { p24 (263-272) } \\
\text { - Cross-clade CTL res } \\
\text { one A subtype infect } \\
\text { - Pol reactivity: 8/8 ha } \\
\text { - Gag reactivity: 7/8 re } \\
\text { - Nef reactivity: 7/8 re } \\
\text { - Env reactivity: } 3 / 8 \mathrm{re} \\
\text { - One of the patients w }\end{array}$ & $\begin{array}{l}\text { KRWI ILGNK } \\
\text { e was studied by } \\
\text { rom a person livir } \\
\text { TL to A subtype, a } \\
\text { d with A or B sub } \\
\text { d with A subtype, } \\
\text { d with A subtype, } \\
\text { hown to react to tl }\end{array}$ & $\begin{array}{l}\text { HIV-1 infection } \\
\text { e CTL activity in seven patients from Bangui, (6 A subt } \\
\text { iginally from Togo, to different antigens expressed in v } \\
\text { btype, and HIV-2 Pol was not tested } \\
\text { with HIV-2 Gag } \\
3 \text { subtype, none with HIV-2 Nef } \\
\text { btype, none with HIV-2 Env } \\
\text { RWIILGNK }\end{array}$ & $\begin{array}{l}\text { Durali1998 } \\
1 \text { AG recombinant infections) and }\end{array}$ \\
\hline 24( & $\begin{array}{l}\text { p24 (263-272) } \\
\text { - Six HLA-B27 donor } \\
\text { - In 4/6 cases, this was } \\
\text { - Two of the cases had } \\
\text { - The arginine to lysin } \\
\text { - Goulder1997a is a }\end{array}$ & $\begin{array}{l}\text { KRWI IMGLNK } \\
\text { ied make a stron } \\
\text { mmunodominant } \\
\text { oitope switch to t } \\
\text { tch is in an ancho } \\
N \text { of immune esce }\end{array}$ & $\begin{array}{l}\text { HIV-1 infection human (B27) } \\
\text { is epitope } \\
\text { esponse } \\
\text { IMGLNK during a period of rapid decline to AIDS, f } \\
\text { esults in immune escape due to severely diminished } \\
\text { rizes this study in the context of CTL escape to fixati }\end{array}$ & $\begin{array}{l}\text { Goulder1997f Goulder1997a } \\
\text { their asymptomatic period } \\
\text { the B27 molecule }\end{array}$ \\
\hline p24 (1 & $\begin{array}{l}\text { p24 } \\
\text { - } \text { CTL responses in ser } \\
\text { deletion in CCR5 } \\
\text { - In Gambia there is ex } \\
\text { seems to be protectiv } \\
\text { - HIV-2 sequence: RR }\end{array}$ & $\begin{array}{l}\text { KRWI ILGLNK } \\
\text { ative highly HIV- } \\
\text { re to both HIV-1 } \\
\text { LLLK - this ep }\end{array}$ & $\begin{array}{l}\text { human (B27) } \\
\text { In female sex workers in Gambia and Nairobi were stu } \\
\text { L responses to B35 epitopes in exposed, uninfected wo } \\
\text { IIV-1 and HIV-2 cross-reactive }\end{array}$ & $\begin{array}{l}\text { Rowland-Jones1999 } \\
\text { ese women had no delta } 32 \\
\text { cross-reactive, and the B35 allele }\end{array}$ \\
\hline p24 (131-140) & $\begin{array}{l}\text { Gag (263-) } \\
\text { - This study uses EpiN } \\
\text { - Based on EpiMatrix } \\
\text { were shown to bind t } \\
\text { - Two of these } 12 \text { pept } \\
\text { - This peptide sequenc }\end{array}$ & $\begin{array}{l}\text { KRWI LGLNK } \\
\text { for T cell epitop } \\
\text { ctions, } 28 \text { peptide } \\
\text { predicted HLA n } \\
\text { had been previou } \\
\text { hot conserved bet }\end{array}$ & $\begin{array}{l}\text { computer prediction } \\
\text { identify possible HLA-B27 and A-2 CTL epitopes in } \\
\text { ized and tested using T2 binding assays for potential } \\
\text { CTL epitopes: HLA-B27 KRWILGLNK and HLA-A } \\
\text { it is found in most B clade isolates }\end{array}$ & $\begin{array}{l}\text { Schafer1998 } \\
\text { VHGV } \\
\text { V } 27 \text { binding, and } 12 \text { of these }\end{array}$ \\
\hline
\end{tabular}




\begin{tabular}{lllll}
\hline HXB2 Location & Author's Location & Sequence & Immunogen & Species (HLA) \\
\hline p24 (131-140) & p24 (263-282) & KRWI I LGLNK & HIV-1 infection & human (B27)
\end{tabular}

- This study focuses on six rare long-term survivor HIV-infected people who were infected for many years without exhibiting immune dysregulation - such immunologically normal HIV-infected (INHI) cases occur at a frequency between 0.1 and $1 \%$ in the infected population

- No direct CTL were found in any of the six INHIs, but above background CTLp activity was founded in 3/6 INHIs

- Epitope sequences were deduced from larger reactive peptides based on HLA binding motifs - XRXXXXXXXK is a B*2705 binding motif

p24 (131-140) p24 (265-274 SF2) KRWIILGLNK HIV-1 infection human (B27) Altfeld2001b

- Therapy provided during acute infection resulted in a narrower CTL response, stronger T help response, and a less diverse viral population than was seen in individuals treated during chronic infection

- The breadth and specificity of the response was determined using ELISPOT by studying 19 individuals with pre-seroconversion therapy (Group 1), 11 individuals with primary infection but post-seroconversion therapy (Group 2), and 10 individuals who responded to HAART given during chronic infection (Group 3), using 259 overlapping peptides spanning p17, p24, RT, gp41, gp120 and Nef

- Previously described and newly defined optimal epitopes were tested for CTL response

- Number of HLA-B27+ individuals that had a CTL response to this epitope broken down by group: 0/0 group 1,0/0 group 2, and 1/1 group 3

p24 (131-140) p24 (263-272) KRWIILGLNK HIV-1 infection, HIV-1 exposed human (B27) Kaul2001a
seronegative

- ELISPOT was used to study CTL responses to a panel of 54 predefined HIV-1 epitopes in 91 HIV-1-exposed, persistently seronegative (HEPS) and 87

HIV-1-infected female Nairobi sex workers

- Responses in HEPS women tended to be lower, and focused on different epitopes with HLA presenting molecules that have previously been associated with reduced risk of infection, and there was a shift in the response in the HEPS women upon late seroconversion to epitopes recognized by the HIV-1 infected women

- 43/91 HEPS women had CD8+ responses and detection of HIV-1-specific CTL in HEPS women increased with the duration of viral exposure

- Subject ML 1760 had an A2 response to ILK(D/E)PVHGV prior to seroconversion, and gained responses to epitopes A2 SL(F/Y)NTVATL and B27 KRWII(L/M)GLNK post-seroconversion

\begin{tabular}{|c|c|c|c|c|c|}
\hline p24 (131-140) & p24 (131-140) & KRWI I LGLNK & HIV-1 infection & human (B27) & Day2001 \\
\hline p24 (131-140) & p24 (260-299) & RRWIQLGLQK & HIV-1 infection & human (B27) & Day2001 \\
\hline p24 (131-140) & p24 (131-140) & KRWI I LGLNK & HIV-1 infection & human (B27) & Goulder2001b \\
\hline
\end{tabular}

- Epitope name: KK10

- $85 \%$ of B27+ adults have CTL that recognize this epitope, but only $2 / 6$ children did

- Responses to this dominant B27-restricted Gag epitope are present during the time of decreasing viral load in acute infection

- Three children who shared B27 with their mothers did not respond to this epitope and inherited escape mutations from their mothers

- A transmitted R132T anchor residue mutation abrogated binding to B27

- In the three children infected with the non-binding KK10 variants, the dominant CTL specificity was still HLA-B27-restricted, but it was directed against an epitope in p17, IRLRPGGKK, only rarely recognized in adults when KRWIILGLNK is the dominant response

- Mutations in this epitope were observed in autologous clones of subjects who were B27-positive with a higher frequency than those who were B27-negative $(\mathrm{P}=0.0005)$

- These mutations are being sexually transmitted in adult infections 


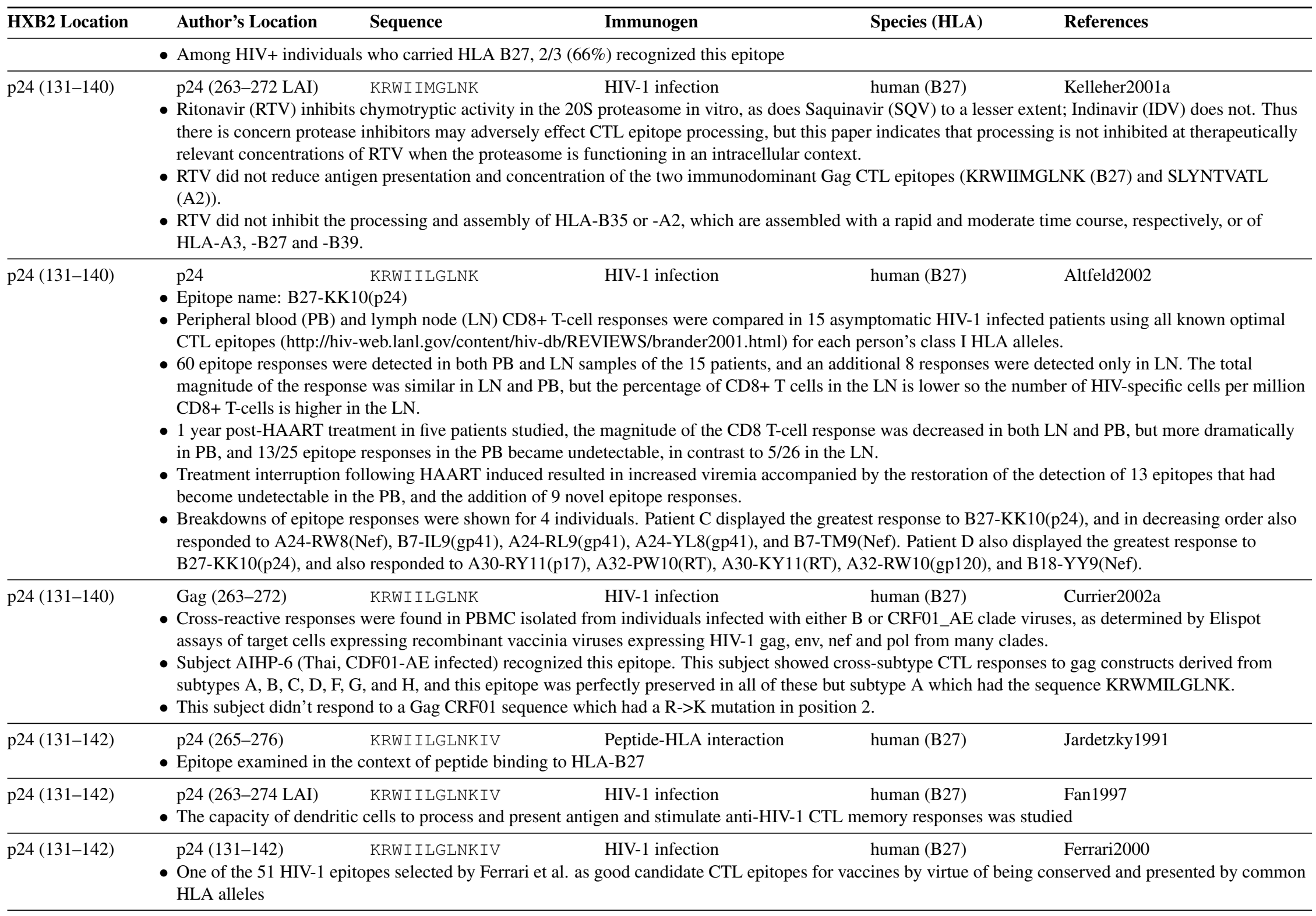




\begin{tabular}{|c|c|c|c|c|}
\hline HXB2 Location & Author's Location & Immunogen & Species (HLA) & References \\
\hline p24 (131-145) & \multicolumn{4}{|c|}{$\begin{array}{l}\text { - The CTL-dominant response was focused on this epitope in a HIV+ African American living in Boston with unknown HLA - this epitope did not fall } \\
\text { within the three most recognized peptides in the study } \\
\text { - Three peptides GSEELRSLYNTVATL (p17 residues 71-85), SALSEGATPQDLNTMLNTVG (p24 41-60), and WEKIRLRPGGKKKYKLK(p17 16-30) } \\
\text { contained the dominant Gag-specific epitope in } 31 \text { out of 44 B-clade infected individuals from Boston who showed Gag-CTL responses } \\
\text { - Five peptides RLRPGGKKHYMIKHLVW (p17 20-36), ELRSLYNTVATLYCV (p17Gag 74-88), SALSEGATPQDLNTMLNTVG (p24 41-60), } \\
\text { FRDYVDRFKTLRAEQA (p24 161-177), and SILDIKQGKEPFRDY (p24 149-164) contained dominant Gag-specific epitopes in } 32 \text { out of } 37 \text { C-clade } \\
\text { infected subjects from South Africa }\end{array}$} \\
\hline p24 (131-145) & \multicolumn{4}{|c|}{ - Clustering of Gag p24 CTL epitopes recognized in 29 HIV-infected people } \\
\hline p24 (131-145) & \multicolumn{4}{|c|}{$\begin{array}{l}\text { Vaccine Vector/Type: vaccinia HIV component: Gag } \\
\text { - Gag CTL epitope mapped with rec gag-vaccinia and synthetic peptides } \\
\text { - This was the first HIV-1 epitope to be mapped }\end{array}$} \\
\hline p24 (131-145) & $\begin{array}{l}\text { p24 (266-277 LAI) } \\
\text { - Longitudinal study show }\end{array}$ & $\begin{array}{ll}\text { KRWI ILGLNKIVMRY } & \text { HIV-1 infection } \\
\text { ng persistence of epitope despite CTL activity }\end{array}$ & human (B27) & Meyerhans1991 \\
\hline p24 (131-145) & \multicolumn{4}{|c|}{$\begin{array}{l}\text { - HIV-1 and HIV-2 cross-reactive CTL clone, highly conserved epitope } \\
\text { - Reviewed in Rowland-Jones99, notes that it did not appear cross-reactive with HIV-2 in Rowland-Jones98, HIV-2 form: RRWIQLGLQK }\end{array}$} \\
\hline p24 (131-146) & \multicolumn{4}{|c|}{ - HLA-B27 restricted epitope also binds to HLA-A2 and HLA-B37 in solid phase assay } \\
\hline p24 (131-150) & \multicolumn{4}{|c|}{$\begin{array}{l}\text { - Of } 25 \text { patients, most had CTL specific for more than } 1 \text { HIV-1 protein } \\
\text { - Twelve subjects had CTL that could recognize vaccinia-expressed LAI gag } \\
\text { - One of these } 12 \text { A-2 had CTL response to this peptide } \\
\text { - The responding subject was HLA-A3, A32, B51, B62 }\end{array}$} \\
\hline p24 (131-150) & \multicolumn{4}{|c|}{ - Gag CTL epitope precursor frequencies estimated } \\
\hline p24 (131-152) & $\begin{array}{l}\text { p24 (263-284 BH10) } \\
\text { - Gag CTL response studi }\end{array}$ & $\begin{array}{l}\text { KRWI ILGLNKIVRMYSPTS- HIV-1 infection } \\
\text { ILD } \\
\text { d in three individuals }\end{array}$ & human (Bw62) & Johnson1991 \\
\hline p24 (132-145) & \multicolumn{4}{|c|}{ - Peptide 728: Memory CTL specific for HIV-1 may contribute to oligoclonal expansions within the CD57+ CD28- CD8+ CTLp populations } \\
\hline p24 (132-145) & $\begin{array}{l}\text { - Gag } \\
\text { Peptide 728: Almost all } \\
\text { of } 8+C D 28-\text { cells to } ~ \\
\text { distributed the CD28 dep }\end{array}$ & $\begin{array}{l}\text { KWILGLNKIVRMY } \\
\text { ED8+ T cells are CD28+ at birth, and the proportion } \\
\text { TL memory pools for CTL clones specific for two } 1 \\
\text { leted cell population }\end{array}$ & $\begin{array}{l}\text { human (B27) } \\
\text { ells increases with } \\
\text { viruses, CMV and }\end{array}$ & $\begin{array}{l}\text { Weekes1999b } \\
\text { is study examines the contribution } \\
\text { lones were found to be similarly }\end{array}$ \\
\hline
\end{tabular}




\begin{tabular}{|c|c|c|c|c|}
\hline HXB2 Location & Author's Location & Immunogen & Species (HLA) & References \\
\hline & \multicolumn{4}{|c|}{$\begin{array}{l}\text { - HIV CTL responses to } 3 \text { Env and } 2 \text { Gag peptides were studied } \\
\text { - The clonal composition of the TCR Vbeta responses were studied and was found to be highly focused, with one TCR beta-chain sequence tending to } \\
\text { dominate the peptide-specific response - clones to this epitope were Vbeta22.1 }\end{array}$} \\
\hline p24 (134-143) & \multicolumn{4}{|c|}{$\begin{array}{l}\text { - HIV-specific CTL were found in exposed seronegative prostitutes from Nairobi - these CTL may confer protection } \\
\text { - Seroprevalence in this cohort is 90-95\% and their HIV-1 exposure is among the highest in the world } \\
\text { - Most isolated HIV strains are clade A in Nairobi, although clades C and D are also found - B clade epitopes are often cross-reactive, however stronger } \\
\text { responses are frequently observed using A or D clade versions of epitopes } \\
\text { - This epitope is conserved among A, B and D clade viruses }\end{array}$} \\
\hline p24 (136-145) & $\begin{array}{l}\text { p24 (268-277 LAI) LGLNKIVRMY } \\
\text { - Predicted from larger peptide } \\
\text { - Review of HIV CTL epitopes } \\
\text { - Also P. Johnson, Pers. Comm. }\end{array}$ & HIV-1 infection & human (Bw62) & McMichael1994 \\
\hline p24 (136-146) & $\begin{array}{l}\text { p24 (271-281) EGLNKIVRMYS } \\
\text { - Aghty two HIV-1-specific CTL clones fron } \\
\text { - A subject who was B62+ had CTL that rece } \\
\text { - The two clones that recognized this epitope }\end{array}$ & $\begin{array}{l}\text { HIV-1 infection } \\
\text { non-progressors were isolated } \\
\text { d clones were restricted by mu } \\
\text { ptide, p17 KIRLRPGGKKKY } \\
\text { rent V } \beta \text { genes, further demon }\end{array}$ & $\begin{array}{l}\text { human (B62) } \\
\text { lyzed for breadth } \\
\text { ILA epitopes, indi } \\
\text { d one additional u } \\
\text { a polyclonal resp }\end{array}$ & $\begin{array}{l}\text { Lubaki1997 } \\
\text { nse } \\
\text { polyclonal response } \\
\text { epitope }\end{array}$ \\
\hline p24 (136-146) & $\begin{array}{l}\text { p24 (136-146) One of the 51 HIV-1 epitopes selected by F } \\
\text { HLA alleles }\end{array}$ & $\begin{array}{l}\text { HIV-1 infection } \\
\text { good candidate CTL epitopes }\end{array}$ & $\begin{array}{l}\text { human (B62) } \\
\text { cines by virtue of }\end{array}$ & $\begin{array}{l}\text { Ferrari2000 } \\
\text { onserved and presented by common }\end{array}$ \\
\hline p24 (137-145) & $\begin{array}{l}\text { p24 (C consensus) GLNKIVRMY } \\
\text { - The CTL-dominant response was focused o } \\
\text { within the three most recognized peptides } \\
\text { - Three peptides GSEELRSLYNTVATL (p17 } \\
\text { contained the dominant Gag-specific epitop } \\
\text { - Five peptides RLRPGGKKHYMIKHLVW } \\
\text { FRDYVDRFFKTLRAEQA (p24 161-177) } \\
\text { infected subjects from South Africa }\end{array}$ & $\begin{array}{l}\text { HIV-1 infection } \\
\text { in a HIV+ South African livin } \\
\text { 5), SALSEGATPQDLNTMLI } \\
\text { 44 B-clade infected individual } \\
\text { ELRSLYNTVATLYCV (p17G } \\
\text { QGKEPFRDY (p24 149-164) }\end{array}$ & $\begin{array}{l}\text { human } \\
\text { arban, HLA A2/- I } \\
\text { (p24 41-60), and } \\
\text { Boston who show } \\
\text { 8), SALSEGATP } \\
\text { ed dominant Gag- }\end{array}$ & $\begin{array}{l}\text { Goulder2000a } \\
2 \text { Cw4/6 - this epitope did not fall } \\
\text { LRPGGKKKYKLK(p17 16-30) } \\
\text { CTL responses } \\
\text { MLNTVG (p24 41-60), } \\
\text { epitopes in } 32 \text { out of } 37 \text { C-clade }\end{array}$ \\
\hline p24 (137-145) & $\begin{array}{l}\text { p24 (272-280 LAI) GLNKIVRMY } \\
\text { C. Brander notes this is a B*1501 epitope }\end{array}$ & HIV-1 infection & human $(\mathrm{B} * 1501)$ & Brander2001 \\
\hline p24 (137-145) & $\begin{array}{l}\text { p24 (272-280 LAI) GLNKIVRMY } \\
\text { - This paper is a review of CTL and immune } \\
\text { GLNKIVRMY } \\
\text { - As long as a strong CTL response to SLYN } \\
\text { eventually the CTL response to the index p } \\
\text { SLYNTVATL once again established itself }\end{array}$ & $\begin{array}{l}\text { HIV-1 infection } \\
\text { presents a study of a shift fror } \\
\text { vident, the epitope variants SL } \\
\text { undetectable, the CTL respon } \\
\text { t form }\end{array}$ & $\begin{array}{l}\text { human (B62) } \\
\text { LA-A*0201 respo } \\
\text { TL or SLYNTIA } \\
\text { ed to a focus on C }\end{array}$ & $\begin{array}{l}\text { Goulder1997a } \\
\text { LYNTVATL, to a B62 response to } \\
\text { inated the viral population - } \\
\text { RMY, and the index peptide }\end{array}$ \\
\hline
\end{tabular}




\begin{tabular}{llllc}
\hline HXB2 Location & Author's Location & Sequence & Immunogen & Species (HLA) \\
\hline p24 (137-145) & p24 (SF2) & GLNKIVRMY & HIV-1 infection & human (B62) \\
& - The CTL-dominant response was focused on this epitope in a HIV+ African American living in Boston - this epitope did not fall within the three most & Goulder2000a
\end{tabular}

recognized peptides in the study

- Three peptides GSEELRSLYNTVATL (p17 residues 71-85), SALSEGATPQDLNTMLNTVG (p24 41-60), and WEKIRLRPGGKKKYKLK(p17 16-30) contained the dominant Gag-specific epitope in 31 out of 44 B-clade infected individuals from Boston who showed Gag-CTL responses

- Five peptides RLRPGGKKHYMIKHLVW (p17 20-36), ELRSLYNTVATLYCV (p17Gag 74-88), SALSEGATPQDLNTMLNTVG (p24 41-60), FRDYVDRFFKTLRAEQA (p24 161-177), and SILDIKQGKEPFRDY (p24 149-164) contained dominant Gag-specific epitopes in 32 out of 37 C-clade infected subjects from South Africa

p24 (137-145) p24(267-277 SF2) GLNKIVRMY HIV-1 infection

- Therapy provided during acute infection resulted in a narrower CTL response, stronger T help response, and a less diverse viral population than was seen in individuals treated during chronic infection

- The breadth and specificity of the response was determined using ELISPOT by studying 19 individuals with pre-seroconversion therapy (Group 1 ), 11 individuals with primary infection but post-seroconversion therapy (Group 2), and 10 individuals who responded to HAART given during chronic infection (Group 3), using 259 overlapping peptides spanning p17, p24, RT, gp41, gp120 and Nef

- Previously described and newly defined optimal epitopes were tested for CTL response

- Number of HLA-B62+ individuals that had a CTL response to this epitope broken down by group: 0/1 group 1, 0/1 group 2, and 1/1 group 3

\begin{tabular}{|c|c|c|c|c|}
\hline p24 (137-145) & $\begin{array}{l}\text { p24 (137-145) GLNKIVRMY } \\
\text { No immunodominant responses were dete }\end{array}$ & $\begin{array}{l}\text { HIV-1 infection } \\
\text {-restricted epitop }\end{array}$ & human (B62) & Day2001 \\
\hline p24 (143-150) & $\begin{array}{l}\text { p24 (273-283 IIIB) RMYSP TS I } \\
\text { C. Brander notes this is a B*5201 epitope }\end{array}$ & HIV-1 infection & human $(\mathrm{B} * 5201)$ & Brander2001 \\
\hline p24 (143-150) & p24 (273-283 IIIB) & HIV-1 infection & human (B52) & Brander1999 \\
\hline
\end{tabular}

- Epitope name: SL9

- Multiple natural variations in the SL9 flanking regions of the immunodominant epitope SLYNTVATL were tested and found not to adversely affect CTL recognition or prevent epitope processing, suggesting that viral escape from the HLA-A*0201-restricted CTL response against SLYNTVATL is probably not linked to variations in the flanking regions of this epitope

- The CTL response to RMYSPTSI was used as a control

p24 (143-150) p24(273-283 IIIB) RMYSPTSI HIV-1 infection human (B52) Wilson1999a

- This study describes maternal CTL responses in the context of mother-to-infant transmission

- Detection of CTL escape mutants in the mother was associated with transmission, but the CTL-susceptible forms of the virus tended to be found in infected infants

- No variants of this epitope were found in a non-transmitting mother that had a CTL response to this epitope

p24 (143-150) p24 (143-150) RMYSPTSI HIV-1 infection human (B52)

- One of the 51 HIV-1 epitopes selected by Ferrari et al. as good candidate CTL epitopes for vaccines by virtue of being conserved and presented by common HLA alleles

\begin{tabular}{lllll}
\hline p24 (151-170) & p24 (283-302 SF2) & LDIRQGPKEPFRDYVDRFYK & HIV-1 infection & human \\
\hline
\end{tabular}




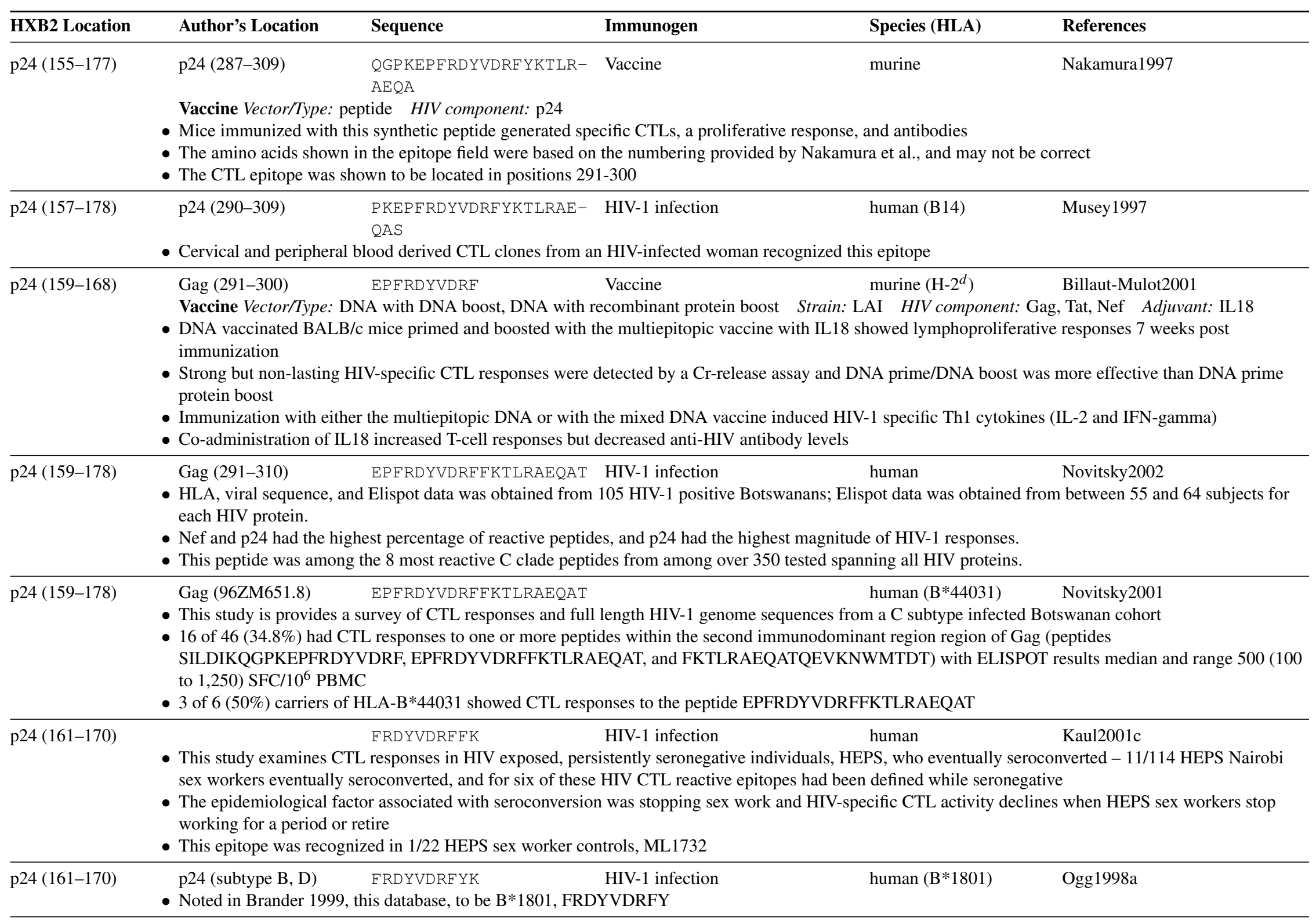




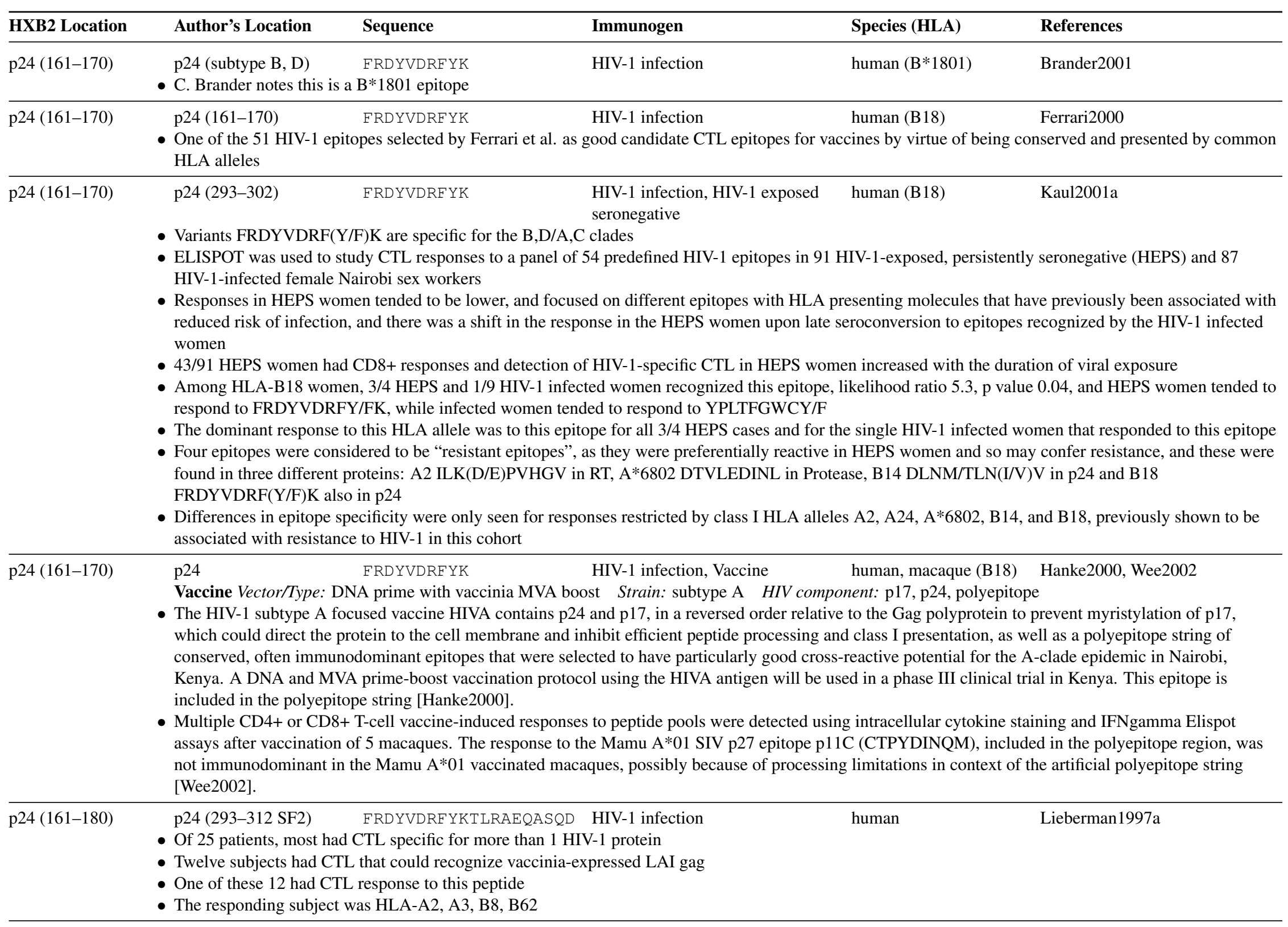




\begin{tabular}{|c|c|c|c|c|c|}
\hline HXB2 Location & Author's Location & Sequence & Immunogen & Species (HLA) & References \\
\hline p24 (161-180) & $\begin{array}{l}\text { p24 (293-312 SF2) } \\
\text { CTL expanded ex viv }\end{array}$ & $\begin{array}{l}\text { ERDYVDRFYKTLRAEQASQD } \\
\text { ere later infused into HIV-1 infec }\end{array}$ & $\begin{array}{l}\text { HIV-1 infection } \\
\text { ted patients }\end{array}$ & human & Lieberman1997b \\
\hline p24 (161-180) & p24 (293-312 SF2) & FRDYVDRFYKTLRAEQASQD & HIV-1 infection & human (B71) & McAdam1998 \\
\hline p24 (162-172) & p24 (296-306 subtyp & RDYVDRFFKTL & HIV-1 infection & human $(A * 2402)$ & Dorrell1999 \\
\hline
\end{tabular}

CTL responses in three individuals with non-clade B infections were studied, 2 with subtype $\mathrm{A}$ infections, 1 with subtype $\mathrm{C}$ - their infections all originated in East Africa

- This epitope is similar to the A24 DYVDRYFKT epitope found for B subtype, but CTL from this A subtype infection required the additional Arg - the B clade sequence change from $\mathrm{F}$ to $\mathrm{Y}$ diminished CTL reactivity

- C. Brander notes that this is an $A * 2402$ epitope in the 1999 database

\begin{tabular}{|c|c|c|c|c|c|}
\hline p24 (162-172) & $\begin{array}{l}\text { p24 (296-306 subtype } \\
\text { A) } \\
\text { - C. Brander notes this is ar }\end{array}$ & $\begin{array}{l}\text { RDYVDRFFKTL } \\
\text { A*2402 epitope }\end{array}$ & HIV-1 infection & human $(\mathrm{A} * 2402)$ & Brander2001 \\
\hline p24 (162-172) & p24 (296-306) & RDYVDRFFKTL & $\begin{array}{l}\text { HIV-1 infection, HIV-1 exposed } \\
\text { seronegative }\end{array}$ & human (A24) & Kaul2001a \\
\hline
\end{tabular}

- ELISPOT was used to study CTL responses to a panel of 54 predefined HIV-1 epitopes in 91 HIV-1-exposed, persistently seronegative (HEPS) and 87 HIV-1-infected female Nairobi sex workers

- Responses in HEPS women tended to be lower, and focused on different epitopes with HLA presenting molecules that have previously been associated with reduced risk of infection, and there was a shift in the response in the HEPS women upon late seroconversion to epitopes recognized by the HIV-1 infected women

- 43/91 HEPS women had CD8+ responses and detection of HIV-1-specific CTL in HEPS women increased with the duration of viral exposure

- Among HLA-A24 women, 0/4 HEPS and 6/10 HIV-1 infected women recognized this epitope, likelihood ratio 7.2, p value 0.03, and (R)YL(R/K)DQQLL tended to be reactive in HEPS and infected women, RDYVDRFFKTL in infected women only

- The dominant response to this HLA allele was to this epitope in all of the 6/10 HIV-1 infected women

- Differences in epitope specificity were only seen for responses restricted by class I HLA alleles A2, A24, A*6802, B14, and B18, previously shown to be associated with resistance to HIV-1 in this cohort

- Subject ML 1707 started with a CTL response to A*6802 DTVLEDINL prior to seroconversion, and switched to A*6802 ETAYFILKL and A24 RDYVDRFFKTL post-seroconversion

\begin{tabular}{llccc}
\hline p24 (162-172) & p24 (293-312 LAI) & RDYVDRFYKTL & HIV-1 infection & human (B*4402) \\
& C. Brander notes this is a B*4402 epitope & & Brander2001 \\
\hline p24 (162-172) & p24 (162-172) & RDYVDRFYKTL & HIV-1 infection & human (B44)
\end{tabular}

- One of the 51 HIV-1 epitopes selected by Ferrari et al. as good candidate CTL epitopes for vaccines by virtue of being conserved and presented by common HLA alleles

\begin{tabular}{|c|c|c|c|c|c|}
\hline p24 (162-172) & p24 (162-172) & RDYVDRFYKTL & HIV-1 infection & human (B44) & Day2001 \\
\hline p24 (162-172) & p24 & RDYVDRFYKTL & HIV-1 infection, Vaccine & human, macaque (B44) & Hanke2000 Wee2002 \\
\hline
\end{tabular}




\begin{tabular}{|c|c|c|c|c|c|}
\hline HXB2 Location & Author's Location & Sequence & Immunogen & Species (HLA) & References \\
\hline & \multicolumn{5}{|c|}{$\begin{array}{l}\text { - The HIV-1 subtype A focused vaccine HIVA contains p } 24 \text { and p17, in a reversed order relative to the Gag polyprotein to prevent myristylation of p17, } \\
\text { which could direct the protein to the cell membrane and inhibit efficient peptide processing and class I presentation, as well as a polyepitope string of } \\
\text { conserved, often immunodominant epitopes that were selected to have particularly good cross-reactive potential for the A-clade epidemic in Nairobi, } \\
\text { Kenya. A DNA and MVA prime-boost vaccination protocol using the HIVA antigen will be used in a phase III clinical trial in Kenya. This epitope is } \\
\text { included in the polyepitope string [Hanke2000]. } \\
\text { - Multiple CD4+ or CD8+ T-cell vaccine-induced responses to peptide pools were detected using intracellular cytokine staining and IFNgamma Elispot } \\
\text { assays after vaccination of } 5 \text { macaques. The response to the Mamu A*01 SIV p27 epitope p11C (CTPYDINQM), included in the polyepitope region, was } \\
\text { not immunodominant in the Mamu A*01 vaccinated macaques, possibly because of processing limitations in context of the artificial polyepitope string } \\
\text { Wee2002]. }\end{array}$} \\
\hline p24 (162-172) & p24 (293-312 LAI) & RDYVDRFYKTL & HIV-1 infection & $\begin{array}{l}\text { human (B44, A26 or } \\
\text { B70) }\end{array}$ & Ogg1998a \\
\hline p24 (163-172) & $\begin{array}{l}\text { p24 (163-172) } \\
\text { One of the } 51 \text { HIV-1 ep } \\
\text { HLA alleles }\end{array}$ & $\begin{array}{l}\text { DYVDRFYKTL } \\
\text { opes selected by Fe }\end{array}$ & $\begin{array}{l}\text { HIV-1 infection } \\
\text { good candidate CTL epitol }\end{array}$ & $\begin{array}{l}\text { human (A24) } \\
\text { cines by virtue of being }\end{array}$ & $\begin{array}{l}\text { Ferrari2000 } \\
\text { aserved and presented by common }\end{array}$ \\
\hline p24 (164-172) & $\begin{array}{l}\text { Gag (296-304) } \\
\text { - Cross-reactive response } \\
\text { assays of target cells ex } \\
\text { The Thai subject VAIP- } \\
\text { Sequence alignments or } \\
\text { H. YVDRFYKTL and }\end{array}$ & $\begin{array}{l}\text { YVDRFYKTL } \\
\text { were found in PBN } \\
\text { essing recombinan } \\
\text { demonstrated broa } \\
\text { his epitope showed } \\
\text { e variant epitope Y }\end{array}$ & $\begin{array}{l}\text { HIV-1 infection } \\
\text { om individuals infected wi } \\
\text { uses expressing HIV-1 gag } \\
\text { reactivity towards gag cons } \\
\text { for clades B and D, and Y } \\
\text { are recognized equally wel }\end{array}$ & $\begin{array}{l}\text { human }\left(A^{*} 0207\right) \\
\text { or CRF01_AE clade vir } \\
\text { hd pol from many clades } \\
\text { yed from subtypes A, B, } \\
\text { ations at position } 6 \text { for } s\end{array}$ & $\begin{array}{l}\text { Currier2002a } \\
\text { ses, as determined by Elispot } \\
\text { btypes A, F, CD, CD01-AE, F, G, and }\end{array}$ \\
\hline p24 (164-172) & $\begin{array}{l}\text { p24 (298-306 subtype } \\
\text { A) } \\
\text { - CTL responses in three } \\
\text { in East Africa } \\
\text { - This CTL epitope is co } \\
\text { CTL reactivity } \\
\text { CTL reacted with targe } \\
\text { terminus, and the B70 a }\end{array}$ & $\begin{array}{l}\text { YVDRFFKTL } \\
\text { dividuals with non } \\
\text { erved in A and C SI } \\
\text { presenting either in } \\
\text { chor residue motif }\end{array}$ & $\begin{array}{l}\text { HIV-1 infection } \\
\text { tions were studied, } 2 \text { with } \\
\text { clade sequences tend to h } \\
26 \text { or B70 - the epitope h }\end{array}$ & $\begin{array}{l}\text { human (A26 or B70) } \\
\text { infections, } 1 \text { with subty } \\
\text { ge from F to Y, YVDRF } \\
\text { A-26 motif of Val at posi }\end{array}$ & $\begin{array}{l}\text { Dorrell1999 } \\
\text { KTL - both variants showed strong } \\
\text { on } 2 \text { and Leu at the carboxy }\end{array}$ \\
\hline p24 (164-172) & $\begin{array}{l}\text { Gag (298-306 subtype } \\
\text { A) } \\
\text { In vitro restimulation o } \\
\text { carrying A or D subtyp }\end{array}$ & $\begin{array}{l}\text { YVDRFFKTL } \\
\text { HIL specific for do } \\
\text { HIV -1 Gag proteins }\end{array}$ & $\begin{array}{l}\text { HIV-1 infection, in vitro } \\
\text { stimulation } \\
\text { es from infected individua }\end{array}$ & $\begin{array}{l}\text { human (A26 or B70) } \\
\text { e using recombinant m }\end{array}$ & Dorrell2001 \\
\hline p24 (164-172) & $\begin{array}{l}\text { Gag (296-304 } \\
\text { 96ZM651.8) } \\
\text { - This study provides a s } \\
\text { - } 4 \text { subjects who respons } \\
\text { An HIV-1 B variant of } \\
\text { restriction of the epitop }\end{array}$ & $\begin{array}{l}\text { YVDRFFKRL } \\
\text { vey of CTL respon } \\
\text { to the CTL epitop } \\
\text { e epitope YVDRFY } \\
\text { was suggested to be }\end{array}$ & $\begin{array}{l}\text { gth HIV-1 genome seque } \\
\mathrm{L}-\text { all were HLA-B*151 } \\
\text { described, and was reco } \\
\text { - HLA-B*1510 is equival }\end{array}$ & $\begin{array}{l}\text { human }(\mathrm{B} * 1510, \mathrm{~B} 70) \\
\text { a C subtype infected Bot } \\
\text { shared HLA-Cw03, sug } \\
\text { CTL from an HIV-1 subty } \\
\text { serological specificity HI }\end{array}$ & $\begin{array}{l}\text { Novitsky2001 } \\
\text { wanan cohort. } \\
\text { sting linkage disequilibrium } \\
\text { A-infected patient, and the HLA } \\
\text { A B70 }\end{array}$ \\
\hline
\end{tabular}




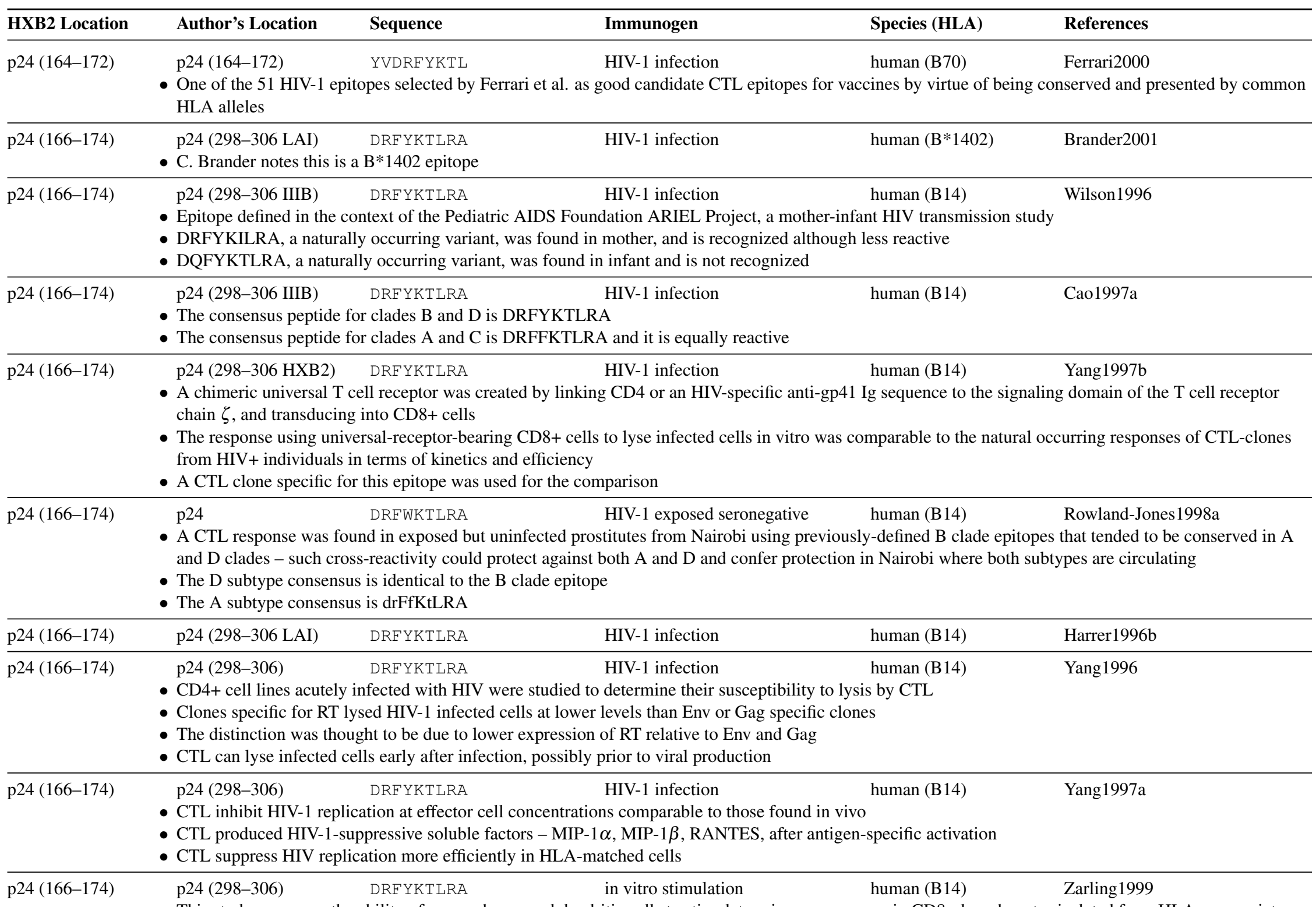

- This study compares the ability of macrophages and dendritic cells to stimulate primary responses in CD8+ lymphocytes isolated from HLA-appropriate HIV-uninfected donors using peptide-pulsed APC - the dendritic cells performed better as APC for the stimulation of primary responses 


\begin{tabular}{|c|c|c|c|c|}
\hline $\mathrm{HXB}$ & Author's Location & equence & Species (HLA) & References \\
\hline & \multicolumn{4}{|c|}{$\begin{array}{l}\text { - Strong CTL responses were elicited by the epitopes DRFYKTLRA and GEIYKRWII when presented by either immature or mature dendritic cells - } \\
\text { macrophages were not able to prime a CTL response against DRFYKTLRA } \\
\text { - A weak response to KLTPLCVSL was stimulated using macrophages as the APC } \\
\text { - No detectable response was observed for the following previously-defined HIV epitopes: KIRLRPGGK, ILKEPVHGV, IRLRPGGK, GPKVKQWPL }\end{array}$} \\
\hline $24(1$ & \multicolumn{4}{|c|}{$\begin{array}{l}\text { p24 DRFYKLTRA } \\
\text { - CTL responses in seronegative highly HIV-exposed African female sex workers in Gambia and Nairobi were studied - these women had no delta } 32 \\
\text { deletion in CCR5 } \\
\text { - In Gambia there is exposure to both HIV-1 and HIV-2, CTL responses to B35 epitopes in exposed, uninfected women are cross-reactive, and the B35 allele } \\
\text { seems to be protective } \\
\text { - HIV-2 sequence: DRFYKSLRA is cross-reactive, Harrer1993 }\end{array}$} \\
\hline $24(1$ & \multicolumn{4}{|c|}{$\begin{array}{l}\text { - This study describes maternal CTL responses in the context of mother-to-infant transmission } \\
\text { infants } \\
\text { - DRFYKILRA and DQFYKTLRA were escape mutants }\end{array}$} \\
\hline 24( & \multicolumn{4}{|c|}{$\begin{array}{l}\text { - The CTL-dominant response was focused on this epitope in } 2 / 5 \text { HIV+ individuals who were HLA B14 living in Boston - this epitope did not fall within the } \\
\text { three most recognized peptides in the study } \\
\text { - Three peptides GSEELRSLYNTVATL (p17 residues 71-85), SALSEGATPQDLNTMLNTVG (p24 41-60), and WEKIRLRPGGKKKYKLK(p17 16-30) } \\
\text { contained the dominant Gag-specific epitope in } 31 \text { out of 44 B-clade infected individuals from Boston who showed Gag-CTL responses } \\
\text { - Five peptides RLRPGGKKHYMIKHLVW (p17 20-36), ELRSLYNTVATLYCV (p17Gag 74-88), SALSEGATPQDLNTMLNTVG (p24 41-60), } \\
\text { FRDYVDRFFKTLRAEQA (p24 161-177), and SILDIKQGKEPFRDY (p24 149-164) contained dominant Gag-specific epitopes in } 32 \text { out of } 37 \text { C-clade } \\
\text { infected subjects from South Africa }\end{array}$} \\
\hline 24( & $\begin{array}{l}\text { p24 (SF2) } \\
\text { - Epitope name: DA9 } \\
\text { - Data from patient A } \\
\text { recognized during th } \\
\text { - A CTL response to }\end{array}$ & $\begin{array}{l}\text { DRFYKTLRA } \\
\text { uggest a role fo } \\
\text { tial decline in vi }\end{array}$ & $\begin{array}{l}\text { HIV-1 infection human (B14) } \\
\text { nitial control of viremia in acute infection, as it is on } \\
\text { til } 18 \text { months post-presentation }\end{array}$ & Goulder2001a \\
\hline 24( & $\begin{array}{l}\text { p24 (166-174) } \\
\text { - One of the } 51 \text { HIV } \\
\text { HLA alleles }\end{array}$ & $\begin{array}{l}\text { DRFYKTLRA } \\
\text { opes selected by }\end{array}$ & $\begin{array}{l}\text { hIV-1 infection } \\
\text { od candidate CTL epitopes for vaccines by virtue }\end{array}$ & Ferrari2000 \\
\hline $24(1$ & $\begin{array}{l}\text { p24 (298-306 SF2) } \\
\text { - Therapy provided du } \\
\text { individuals treated d } \\
\text { - The breadth and spe } \\
\text { individuals with prir } \\
\text { (Group 3), using 25s } \\
\text { - Previously describec }\end{array}$ & $\begin{array}{l}\text { DRFYKTLRA } \\
\text { acute infection } 1 \\
\text { chronic infectic } \\
\text { ty of the respons } \\
\text { infection but po } \\
\text { rlapping peptide } \\
\text { newly defined o }\end{array}$ & $\begin{array}{l}\text { HIV-1 infection human (B14) } \\
\text { wer CTL response, stronger T help response, and a } 1 \\
\text { using ELISPOT by studying } 19 \text { individuals with pre } \\
\text { therapy (Group 2), and } 10 \text { individuals who responde } \\
24, \text { RT, gp41, gp120 and Nef } \\
\text { vere tested for CTL response }\end{array}$ & $\begin{array}{l}\text { Altfeld } 2001 \mathrm{~b} \\
\text { viral population than was seen in } \\
\text { rsion therapy (Group 1), } 11 \\
\text { T given during chronic infection }\end{array}$ \\
\hline
\end{tabular}




\begin{tabular}{|c|c|c|c|}
\hline HXB2 Location & Author's Location & Immunogen & Species (HLA) \\
\hline & \multicolumn{3}{|c|}{ - Number of HLA-B14+ individuals that had a CTL response to this epitope broken down by group: $3 / 3$ group $1,1 / 2$ group 2 , and $0 / 0$ group 3} \\
\hline $24(166$ & \multicolumn{3}{|c|}{$\begin{array}{l}\text { - Variants DRF(F/W)KTLRA are specific for clades A/B } \\
\text { - ELISPOT was used to study CTL responses to a panel of } 54 \text { predefined HIV-1 epitopes in } 91 \text { HIV-1-exposed, persistently seronegative (HEPS) and } 87 \\
\text { HIV-1-infected female Nairobi sex workers } \\
\text { - Responses in HEPS women tended to be lower, and focused on different epitopes with HLA presenting molecules that have previously been associated with } \\
\text { reduced risk of infection, and there was a shift in the response in the HEPS women upon late seroconversion to epitopes recognized by the HIV-1 infected } \\
\text { women } \\
\text { - } 43 / 91 \text { HEPS women had CD8+ responses and detection of HIV-1-specific CTL in HEPS women increased with the duration of viral exposure } \\
\text { - Among HLA-B14 women, 0/4 HEPS and 6/7 HIV-1 infected women recognized this epitope, likelihood ratio } 14.4 \text {, p value } 0.004 \text { and HEPS women tended } \\
\text { to respond to DLNMMLNIV/DLNTMLNVV, while infected women tended to respond to DRF(F/W)KTLRA } \\
\text { - The dominant response to this HLA allele was to this epitope for all of the } 6 / 7 \text { HIV-1 infected women } \\
\text { - Differences in epitope specificity were only seen for responses restricted by class I HLA alleles A2, A24, A*6802, B14, and B18, previously shown to be } \\
\text { associated with resistance to HIV-1 in this cohort }\end{array}$} \\
\hline p24 (166-174) & \multicolumn{3}{|c|}{$\begin{array}{l}\text { - This epitope was mapped by ELISPOT in a study identifying new HLA-B60 epitopes, and was one of the epitopes presented by another HLA molecule in } \\
\text { an HLA-B60 individual }\end{array}$} \\
\hline 24( & $\begin{array}{l}\text { p24 } \\
\text { AC13 is a B14 restri } \\
\text { - CTL could be activa } \\
\text { exogenous protein a } \\
\text { generation of cells b }\end{array}$ & $\begin{array}{l}\text { DRFYKTLRA HIV-1 infection } \\
\text { CTL clone that recognizes DRFYKTLRA. } \\
\text { y a fusion protein of an HIV protein and anthrax lethal factor (LFn- } \\
\text { lows processing through the MHC class I pathway. This strategy for } \\
\text { standard methods of using live viral vectors carrying a protein, or b }\end{array}$ & $\begin{array}{l}\text { human (B14) Cao2002 } \\
\text { IV) that promotes antigen presenting cell uptake of } \\
\text { CTL detection could allow antigen presentation without } \\
\text { loading the cells with peptides and by-passing processing. }\end{array}$ \\
\hline 4( & $\begin{array}{l}\text { p24 } \\
\text { - Neisseria gonorrhea } \\
\text { T-cells, detected by } \\
\text { - Ghonorrhea caused } \\
\text { Elispot and tetramer }\end{array}$ & $\begin{array}{l}\text { DRFWKTLRA HIV-1 infection } \\
\text { ititis in } 9 \text { HIV+ Kenyan sex workers caused a functional deficienc } \\
\text { cellular cytokine production and tetramer assays, while not affecti } \\
\text { eaker HIV-1 specific CTL responses in } 4 \text { HIV-1 exposed persister } \\
\text { ys, and CMV-specific CTL in 2 HEPS subjects were shown to ha }\end{array}$ & $\begin{array}{l}\text { Kaul2002 } \\
\text { FN-gamma production in HIV-1 epitope-specific CD8+ } \\
\text { total number of epitope-specific CTLs. } \\
\text { ronegative (HEPS) women to become undetectable by } \\
\text { aired function with regard to IFN-gamma production. }\end{array}$ \\
\hline 24( & $\begin{array}{l}\text { p24 } \\
\text { Vaccine Vector/Type } \\
\text { - The HIV-1 subtype } \\
\text { which could direct } \mathrm{t} \\
\text { conserved, often im } \\
\text { Kenya. A DNA and } \\
\text { included in the poly } \\
\text { - Multiple CD4+ or C } \\
\text { assays after vaccina } \\
\text { not immunodominat } \\
\text { Wee2002. }\end{array}$ & $\begin{array}{l}\text { DRFYKTLRA HIV-1 infection, Vaccine } \\
\text { JA prime with vaccinia MVA boost Strain: subtype A HIV com } \\
\text { used vaccine HIVA contains p } 24 \text { and p17, in a reversed order relat } \\
\text { otein to the cell membrane and inhibit efficient peptide processing } \\
\text { dominant epitopes that were selected to have particularly good cro } \\
\text { prime-boost vaccination protocol using the HIVA antigen will be } \\
\text { pe string [Hanke2000]. } \\
\text { T-cell vaccine-induced responses to peptide pools were detected us } \\
\text { f } 5 \text { macaques. The response to the Mamu A*01 SIV p } 27 \text { epitope } p \\
\text { the Mamu A*01 vaccinated macaques, possibly because of process }\end{array}$ & $\begin{array}{l}\text { human (B14) Hanke2000 Wee2002 } \\
\text { nent: p17, p24, polyepitope } \\
\text { to the Gag polyprotein to prevent myristylation of p17, } \\
\text { d class I presentation, as well as a polyepitope string of } \\
\text { reactive potential for the A-clade epidemic in Nairobi, } \\
\text { ed in a phase III clinical trial in Kenya. This epitope is } \\
\text { g intracellular cytokine staining and IFNgamma Elispot } \\
\text { C (CTPYDINQM), included in the polyepitope region, was } \\
\text { limitations in context of the artificial polyepitope string }\end{array}$ \\
\hline
\end{tabular}




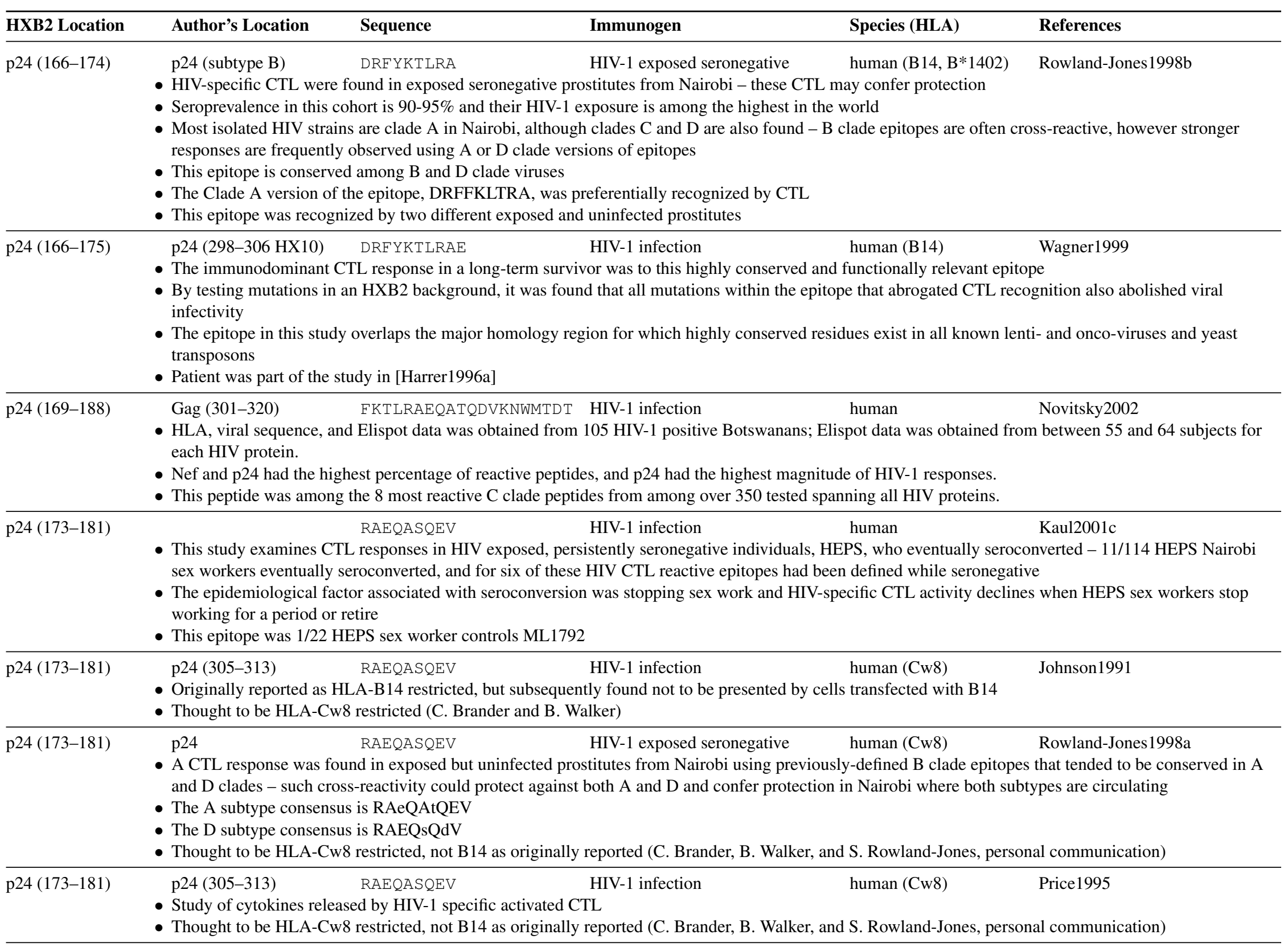




\begin{tabular}{|c|c|c|c|c|c|}
\hline HXB2 Location & Author's Location & Sequence & Immunogen & Species (HLA) & References \\
\hline p24 (173-181) & p24 (305-313) & RAEQASQEV & HIV-1 infection & human (Cw8) & Lubaki1997 \\
\hline
\end{tabular}

- Eighty two HIV-1-specific CTL clones from 5 long-term non-progressors were isolated and analyzed for breadth of response

- A sustained Gag, Env and Nef response was observed, and clones were restricted by multiple HLA epitopes, indicating a polyclonal response

- Despite this being a well defined conserved epitope, and thought to be presented by B14, none of the 11 gag-specific clones from a B-14 positive subject could recognize either it or p24 PQDLNTMLN

- Thought to be HLA-Cw8 restricted, not B14 as originally reported (C. Brander, B. Walker, and S. Rowland-Jones, personal communication)

p24 (173-181) p24 (305-313) RAEQASQEV $\quad \begin{aligned} & \text { HIV-1 infection, HIV-1 exposed human (Cw8) } \\ & \text { seronegative }\end{aligned}$

- ELISPOT was used to study CTL responses to a panel of 54 predefined HIV-1 epitopes in 91 HIV-1-exposed, persistently seronegative (HEPS) and 87 HIV-1-infected female Nairobi sex workers

\begin{tabular}{|c|c|c|c|}
\hline p24 (174-184) & $\begin{array}{l}\text { p24 (306-316 LAI) AEQASQDVKNW } \\
\text { - C. Brander notes this is a B*4402 epitope }\end{array}$ & human $(B * 4402)$ & Brander2001 \\
\hline p24 (174-184) & $\begin{array}{l}\text { p24 (306-316 LAI) AEQASQDVKNW } \\
\text { - Pers. Comm. from D. Lewinsohn to C. Brand }\end{array}$ & $\begin{array}{l}\text { human }(B * 4402, \mathrm{~B} 44) \\
1999\end{array}$ & Brander1997 \\
\hline p24 (174-184) & AEQASQEVKNW & human (B44) & Brodie1999 \\
\hline
\end{tabular}

- The ability of CTL effector cells was studied by expanding autologous HIV-1 Gag-specific CTL in vitro, and adoptively transferring them

- The transferred CTLs migrated to the lymph nodes and transiently reduced circulating productively infected CD4+ T cells, showing that CTL move to appropriate target sites and mediate anti-viral effects

p24 (174-184) p24 (306-316) AEQASQEVKNW $\quad$ HIV-1 infection $\quad$ human (B44) Brodie2000

- Study tracks and quantifies in vivo migration of neo-marked CD8 HIV-specific CTL

- Adoptively transferred gene-marked HIV-specific CTL homed to specific lymph node sites, colocalizing within the parafollicular regions of the lymph node adjacent to cells expressing HIV tat-fusion transcripts, indicative of viral replication

- The CTL clones expressed CCR5 and localized among HIV-1 infected cells expressing MIP-1alpha and MIP-1beta, CC-chemokines produced at sites of viral replication, suggesting a possible homing mechanism

- This study provides a methodology for tracking and studying antigen specific CTL in vivo

p24 (174-184) p24 (306-316 LAI) AEQASQDVKNW $\quad$ HIV-1 infection

- Epitope name: G3

- A panel of 16 epitopes covering 15 class I alleles was tested in 14 HIV+ patients from an unselected Caucasian population treated with HAART, using CD8+ cell IFNgamma production to measure responses

- In general, during the first month of treatment viral load decreased and frequencies of HIV-specific CTL tripled and broadened - eight new HIV specificities that were not previously detectable were newly detected, as were CMV specific CD8+ PBL - but with continued viral suppression, HIV-specific responses diminished

- Viral rebounds gave different patterns of response: increases or decreases in pre-existing response, new specificities, or no change

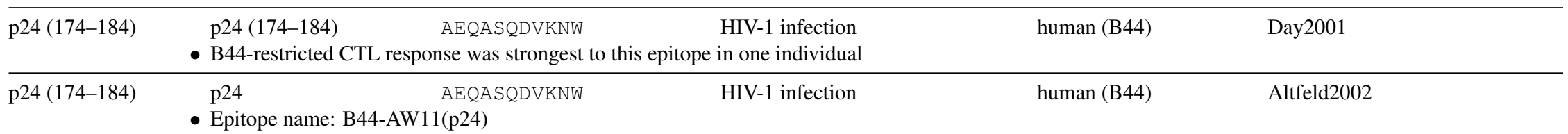




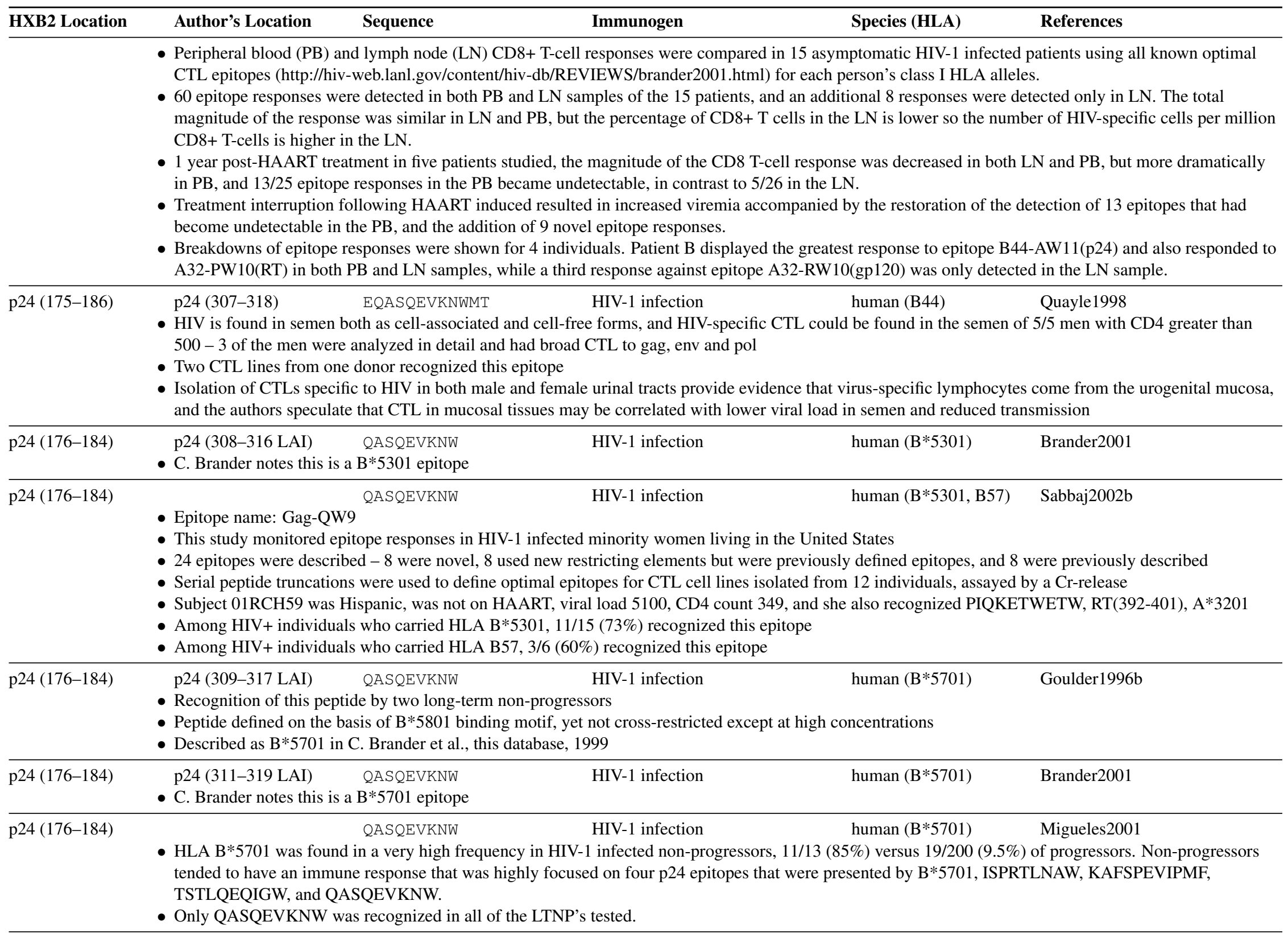




\begin{tabular}{lllcl}
\hline HXB2 Location & Author's Location & Sequence & Immunogen & Species (HLA) \\
\hline p24 (176-184) & & QASQEVKNW & HIV-1 infection & human (B*5701) \\
& - CTL activity was monitored in 27 individuals, including 10 LTNP with an over-expression of HLA B*5701 - these individuals have viral loads below the
\end{tabular}

threshold of infection without therapy, and their CD8+ T-cell response tends to be focused on peptides that contain B*5701 epitopes ISPRTLNAW, KAFSPEVIPMF, TSTLQEQIGW, or QASQEVKNW.

- CTL responses are broader in $B * 5701+$ individuals with progressive viremia than those that control viremia.

- The HLA-A*0201 SLYNTVATL epitope response was not as strong individuals that carried both A2, B57.

\begin{tabular}{|c|c|c|c|c|}
\hline p24 (176-184) & \multicolumn{4}{|c|}{$\begin{array}{l}\text { - Minimal sequence determined through epitope mapping } \\
\text { - This is a relatively conserved epitope } \\
\text { - HLA-Cw*0401 was defined as the restricting element, but cells that carry } \mathrm{Cw}^{*} 0401 \text { varied in their ability to present this epitope - this could be the result of } \\
\text { diminished cell-surface expression of } \mathrm{Cw}^{*} 0401 \text { in some cells } \\
\text { - The HLA presenting molecule for this epitope was originally described as } \mathrm{Cw} * 0401 \text {, but subsequent experiments with an HLA B53+ C4- cell line and with } \\
\text { C1R cells transfected with HLA-B53 have shown that the HLA restricting element is HLA-B53 (Pers. Comm., Dr. Florence Buseyne, 2000) }\end{array}$} \\
\hline p24 (176-184) & (LAI) & QASQEVKNW & human (B53) & Brander2001, Buseyne1999 \\
\hline $24(176-184)$ & p24 (NL43) & QASQEVKNW & In vitro stimulation & e2001 \\
\hline
\end{tabular}

- Epitope name: QW9

- Exogenous presentation or cross-presentation of epitopes by antigen presenting cells (APC) without protein synthesis is an alternative pathway for CTL epitope processing that may be important in the initial generation of viral specific CTL

- Dendritic cells treated with AZT to inhibit protein synthesis were able to elicit a strong specific CTL response in QASQEVKNW specific CTL clone 141 without protein synthesis, while macrophages demonstrated a decreased presentation efficiency

- Exogenous Gag epitope presentation was Env-dependent and required receptor-dependent fusion

p24 (176-184)

p24 (308-316) QATOEVKNG

HIV-1 infection, HIV-1 exposed

human (B53)

Kaul2001a

seronegative

- ELISPOT was used to study CTL responses to a panel of 54 predefined HIV-1 epitopes in $91 \mathrm{HIV}$-1-exposed, persistently seronegative (HEPS) and 87 HIV-1-infected female Nairobi sex workers

- Responses in HEPS women tended to be lower, and focused on different epitopes with HLA presenting molecules that have previously been associated with reduced risk of infection, and there was a shift in the response in the HEPS women upon late seroconversion to epitopes recognized by the HIV-1 infected women

- 43/91 HEPS women had CD8+ responses and detection of HIV-1-specific CTL in HEPS women increased with the duration of viral exposure

- Among HLA-B53 women, 1/2 HEPS and 7/9 HIV-1 infected women recognized this epitope p24 (308-316 subtype A QATQEVKNM HIV-1 infection consensus)

- In clade A infected Gambians, three HLA-B53 epitopes were defined in Gag p24 using ELISPOT, tetramer, and cytotoxicity assays

- Two of the new epitopes lacked the predicted by P2 anchors, DTINEEAAEW and QATQEVKNM, and bound to B53 with high affinity, thus extending the anchor residue motif for B53 and the related B35

- While S, T, and P could all fit into the HLA-B35 or HLA-B53 B pocket and form a hydrogen bond, A would not form a bond, so the authors propose compensatory interactions account for the high affinity of QATQEVKNM for B53

- QATQEVKNM was recognized in 6/7 HLA-B53 subjects 


\begin{tabular}{|c|c|c|c|c|}
\hline HXB2 Location & Author's Location & Sequence & Species (HLA) & References \\
\hline & \multicolumn{4}{|c|}{$\begin{array}{l}\text { - Cross-recognition of QATQEVKNM was not studied here, but it was noted that both the A, QATQEVKNM, and B, QASQDVKNW, subtype version of } \\
\text { this epitope, are also presented by HLA-B57 and B58, common HLA alleles in Africans }\end{array}$} \\
\hline p24 (176-184) & $\begin{array}{l}\text { Gag (SF2) } \\
\text { - Epitope name: QW9 } \\
\text { - This peptide elicited a we } \\
\text { Three CTL responses, to } \\
\text { QASQEVKNW, EIYKRV }\end{array}$ & $\begin{array}{l}\text { QASQEVKNW } \\
\text { eak CTL response } \\
\text { epitopes TSTLQ } \\
\text { WII, and FLKEK }\end{array}$ & $\begin{array}{l}\text { HIV-1 infection human (B57) } \\
\text { ffection of patient PI004 } \\
\text { LNAW, and KAFSPEVIPMF, were evident early after } \\
\text { ctable at } 5 \text { months post-infection and beyond }\end{array}$ & $\begin{array}{l}\text { Goulder2001a } \\
\text {; CTL responses to SLYNTVATL, }\end{array}$ \\
\hline p24 (176-184) & (LAI) & QASQEVKNW & human (Cw4) & Brander2001, Buseyne1999 \\
\hline p24 (176-184) & $\begin{array}{l}\text { p24 (176-184) } \\
\text { ELISPOT was used to stu } \\
\text { HIV-1-infected female Na }\end{array}$ & $\begin{array}{l}\text { QASGEVKNW } \\
\text { ady CTL respons } \\
\text { airobi sex worker }\end{array}$ & $\begin{array}{l}\text { HIV-1 infection, HIV-1 exposed human (Cw4) } \\
\text { seronegative } \\
54 \text { predefined HIV-1 epitopes in 91 HIV-1-exposed, pe }\end{array}$ & $\begin{array}{l}\text { Kaul2001a } \\
\text { seronegative (HEPS) and } 87\end{array}$ \\
\hline p24 (176-185) & $\begin{array}{l}\text { p24 (311-319 SF2) } \\
\text { - Therapy provided during } \\
\text { individuals treated during } \\
\text { - The breadth and specificit } \\
\text { individuals with primary i } \\
\text { (Group 3), using } 259 \text { over } \\
\text { - Previously described and } \\
\text { - Number of HLA-B57+ in }\end{array}$ & $\begin{array}{l}\text { QASKEVKNWV } \\
\text { acute infection re } \\
\text { chronic infectiol } \\
\text { ity of the response } \\
\text { infection but pos } \\
\text { rlapping peptides } \\
\text { newly defined op } \\
\text { adividuals that ha }\end{array}$ & $\begin{array}{l}\text { HIV-1 infection } \\
\text { ower CTL response, stronger T help response, and a le } \\
\text { d using ELISPOT by studying } 19 \text { individuals with pre- } \\
\text { n therapy (Group 2), and } 10 \text { individuals who responded } \\
\text { p24, RT, gp41, gp120 and Nef } \\
\text { were tested for CTL response } \\
\text { se to this epitope broken down by group: 0/0 group 1, }\end{array}$ & $\begin{array}{l}\text { Altfeld } 2001 \mathrm{~b} \\
\text { e viral population than was seen in } \\
\text { jersion therapy (Group } 1), 11 \\
\text { RT given during chronic infection } \\
2 \text {, and } 1 / 2 \text { group } 3\end{array}$ \\
\hline p24 (177-185) & $\begin{array}{l}\text { p24 (177-185) } \\
\text { - Variants A(T/S)QEVKNV } \\
\text { ELISPOT was used to stu } \\
\text { HIV-1-infected female Na } \\
\text { Responses in HEPS wom } \\
\text { reduced risk of infection, } \\
\text { women } \\
\text { - 43/91 HEPS women had } \\
\text { - The dominant response to }\end{array}$ & $\begin{array}{l}\text { ATQEVKNWM } \\
\text { WM are specific } f \\
\text { Idy CTL response } \\
\text { airobi sex worker } \\
\text { len tended to be } 1 \text { } \\
\text { and there was a } \\
\text { CD8+ responses } \\
\text { n, } 1 / 2 \text { HEPS and } \\
\text { this HLA allele }\end{array}$ & $\begin{array}{l}\text { HIV-1 infection, HIV-1 exposed human (B53) } \\
\text { seronegative } \\
\text { les } \\
54 \text { predefined HIV-1 epitopes in } 91 \text { HIV-1-exposed, p } \\
\text { ed on different epitopes with HLA presenting molecu } \\
\text { onse in the HEPS women upon late seroconversion to } \\
\text { f HIV-1-specific CTL in HEPS women increased with } \\
\text { ted women recognized this epitope } \\
\text { ope in the } 1 / 2 \text { HEPS case and in only one of the } 5 / 9 \mathrm{H}\end{array}$ & $\begin{array}{l}\text { Kaul2001a } \\
\text { seronegative (HEPS) and } 87 \\
\text { ve previously been associated with } \\
\text { ecognized by the HIV-1 infected } \\
\text { ion of viral exposure } \\
\text { cted women }\end{array}$ \\
\hline p24 (180-189) & $\begin{array}{l}\text { p24 (313-322) } \\
\text { ELISPOT was used to stu } \\
\text { HIV-1-infected female Na } \\
\end{array}$ & $\begin{array}{l}\text { EVKNWMTETL } \\
\text { ady CTL response } \\
\text { airobi sex worker }\end{array}$ & $\begin{array}{l}\text { HIV-1 infection, HIV-1 exposed human (B53) } \\
\text { seronegative } \\
54 \text { predefined HIV-1 epitopes in } 91 \text { HIV-1-exposed, pe }\end{array}$ & Kaul2001a \\
\hline p24 (181-190) & $\begin{array}{l}\text { p24 (313-322 LAI) } \\
\text { P. Johnson, pers. comm. }\end{array}$ & VKNWMTETLL & human (B8) & Brander1996b \\
\hline
\end{tabular}




\begin{tabular}{|c|c|c|c|c|c|}
\hline HXB2 Location & Author's Location & Sequence & Immunogen & Species (HLA) & References \\
\hline p24 (191-205) & \multicolumn{5}{|c|}{$\begin{array}{l}\text { - This study employs an antigenic similarity matrix to compare HIV-1 antigenic determinants with human proteins. } \\
\text { - This CTL epitope (the HIV-1 LAI fragment with high similarity to a human protein overlapping this epitope is TLLVQNANP) has similarity with growth } \\
\text { differentiation factor } 11 \text {, fragment THLVQQANP. }\end{array}$} \\
\hline p24 (191-205) & $\begin{array}{l}\text { p24 (191-205) } \\
\text { - One of the } 51 \text { HIV-1 el } \\
\text { HLA alleles }\end{array}$ & $\begin{array}{l}\text { VQNANPDCKTILKAL } \\
\text { opes selected by Ferrari e }\end{array}$ & $\begin{array}{l}\text { HIV-1 infection } \\
\text { good candidate CT }\end{array}$ & $\begin{array}{l}\text { human (B51) } \\
\text { cines by virtue }\end{array}$ & $\begin{array}{l}\text { Ferrari2000 } \\
\text { onserved and presented by common }\end{array}$ \\
\hline p24 (191-205) & $\begin{array}{l}\text { p24 (323-337) } \\
\text { - Two CTL epitopes defi }\end{array}$ & $\begin{array}{l}\text { VQNANPDCKTILKAL } \\
\text { (see also p17(21-35)) }\end{array}$ & HIV-1 infection & human (B8) & Nixon1991 \\
\hline
\end{tabular}

- Longitudinal study of CTL escape mutants in people with the appropriate HLA types - little variation was observed in the immunodominant B27 epitope, relative to the $\mathrm{B} 8$ epitopes, which varied over time

- Goulder1997a is a review of immune escape that points out that there may be a protective effect associated with B27, and that HLA-B8 individuals tend to progress more rapidly than HLA B27 patients

\begin{tabular}{|c|c|c|c|}
\hline p24 (191-210) & $\begin{array}{l}\text { p24 (323-342 SF2) VQNANPDCKTILKALGPAAT HIV-1 infection } \\
\text { - Of } 25 \text { patients, most had CTL specific for more than 1 HIV-1 protein } \\
\text { - Twelve subjects had CTL that could recognize vaccinia-expressed LAI gag } \\
\text { - Three of these } 12 \text { had CTL response to this peptide } \\
\text { - The responding subjects were HLA-A3, A24, B8, B55; HLA-A1, A11, B8, B27 }\end{array}$ & human & Lieberman1997a \\
\hline p24 (191-210) & $\begin{array}{l}\text { p24 (323-342 SF2) VQNANPDCKTILKALGPAAT HIV-1 infection } \\
\text { - CTL expanded ex vivo were later infused into HIV-1 infected patients }\end{array}$ & human & Lieberman1997b \\
\hline p24 (193-201) & Gag (327-335 SF2) & human $(\mathrm{B} * 5101)$ & Tomiyama1999 \\
\hline
\end{tabular}

- HLA-B27, -B51, and -B57 are associated with slow progression to AIDS, while HLA-B35, -B8, -B24 are associated with a rapid progression to AIDS (Nat. Med. 2:405, 1996;Lancet 22:1187, 1986;Hum Immunol 22:73, 1988;Hum Immunol 44:156, 1995)

- $15 \%$ of Japanese populations carry HLA-B51 while HLA-B27 and -B57 are detected in less than 0.3\%

- Of the 172 HIV-1 peptides with HLA-B*5101 anchor residues, 33 bound to HLA-B*5101, seven of these peptides were reactive with CTL from 3 B*5101 positive individuals, and six were properly processed

- Four of the six epitopes were highly conserved among B subtype sequences, NANPDCKTI is conserved

p24 (193-201) p24 (325-333) NANPDCKTI? HIV-1 infection

- Only 4/11 HLA-A2+ HIV+ individuals had CTL that reacted to SLYNTVATL, calling into question whether it is immunodominant

- 95 optimally-defined peptides from this database were used to screen for INF $\gamma$ responses to other epitopes

- 3/11 of the HLA A2+ individuals were HLA B51 and two of these responded to this epitope as well as to other epitopes

p24 (193-201) p24(324-335 IIIB) NANPDCKTI HIV-1 infection human (B51) Wilson1999a

- This study describes maternal CTL responses in the context of mother-to-infant transmission

- Detection of CTL escape mutants in the mother was associated with transmission, but the CTL-susceptible forms of the virus tended to be found in infected infants 


\begin{tabular}{llllll}
\hline HXB2 Location & \multicolumn{1}{c}{ Author's Location } & Sequence & Immunogen & Species (HLA) & References \\
\hline \multicolumn{7}{c}{ - No variants of this epitope were found in a non-transmitting mother that had a CTL response to this epitope } \\
\hline p24 (193-201) & p24 (323-333) & NANPDCKT I & HIV-1 infection & human (B51) & Oxenius2000 \\
\hline
\end{tabular}

- Epitope name: NAN

- Patients who started therapy at acute HIV-1 infection (three with sustained therapy, two with limited therapy upon early infection) had strong HIV specific CD4 proliferative responses and were able to maintain a CTL response even with undetectable viral load - three patients that had delayed initiation of HAART had no HIV specific CD4 proliferative responses and lost their CTL responses when HAART was eventually given and their viral loads became undetectable

- None of the 8 study subjects recognized this epitope but none were HLA B51+

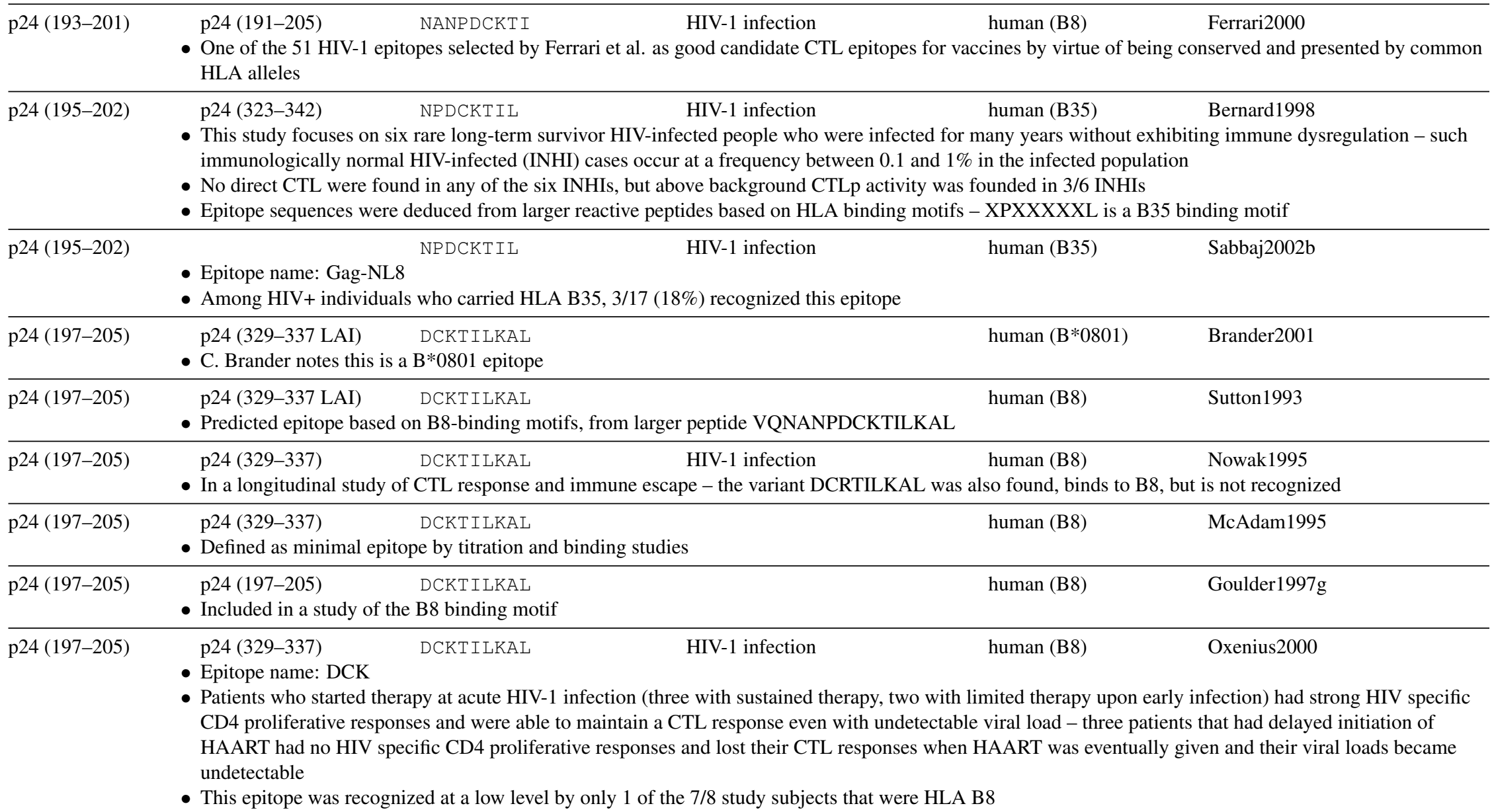




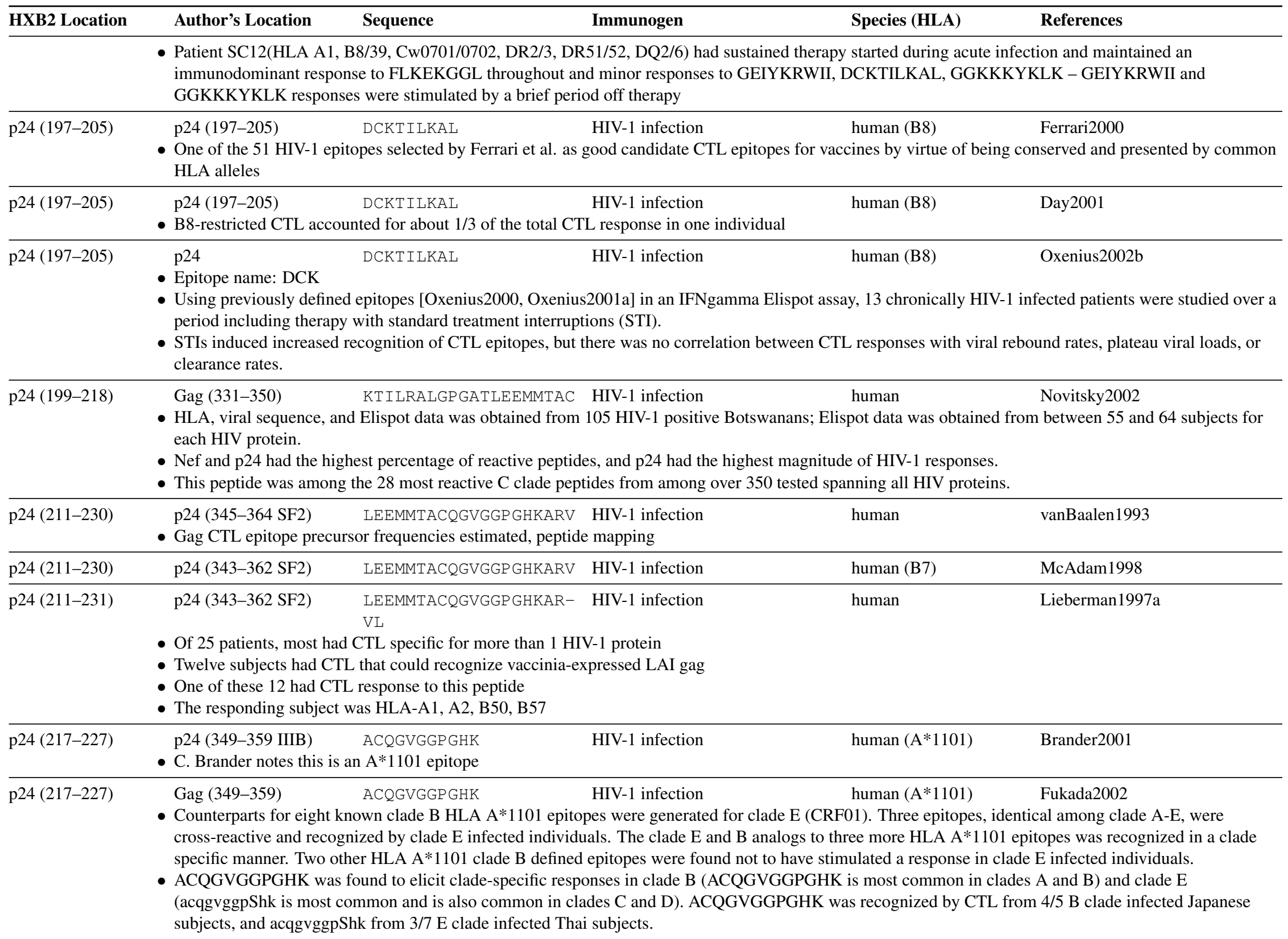




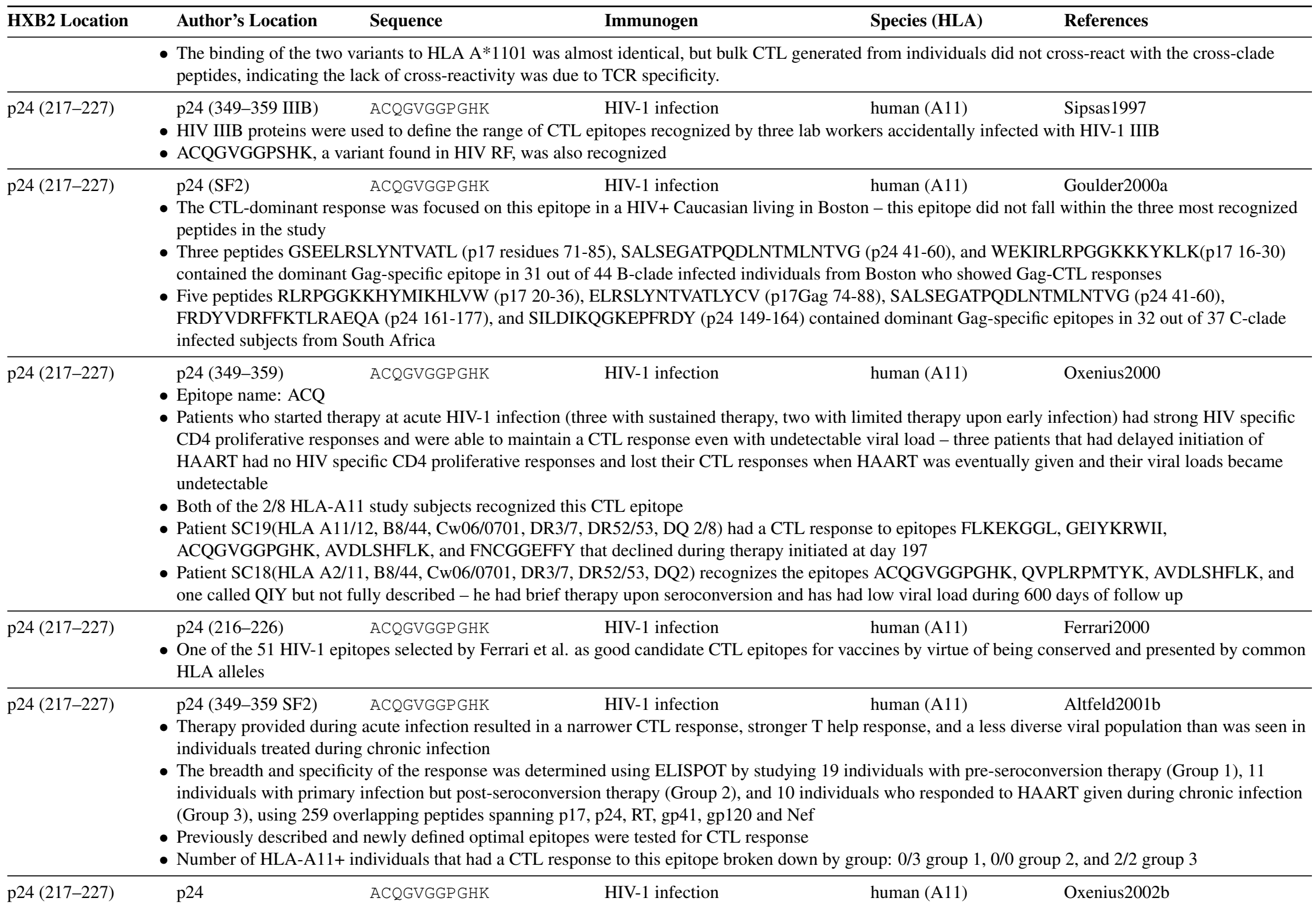

- Epitope name: ACQ

- Using previously defined epitopes [Oxenius2000 Oxenius2001a] in an IFNgamma Elispot assay, 13 chronically HIV-1 infected patients were studied over a period including therapy with standard treatment interruptions (STI). 


\begin{tabular}{|c|c|c|c|c|}
\hline HXB2 Location & Author's Location & Sequence & Species (HLA) & References \\
\hline & \multicolumn{4}{|c|}{$\begin{array}{l}\text { - STIs induced increased recognition of CTL epitopes, but there was no correlation between CTL responses with viral rebound rates, plateau viral loads, or } \\
\text { clearance rates. }\end{array}$} \\
\hline p24 (221-231) & $\begin{array}{l}\text { p24 (353-363 LAI) } \\
\text { - Epitope name: G1 } \\
\text { - A panel of } 16 \text { epitop } \\
\text { CD8+ cell IFNgamm } \\
\text { - In general, during th } \\
\text { specificities that wer } \\
\text { HIV-specific respons } \\
\text { - Viral rebounds gave }\end{array}$ & $\begin{array}{l}\text { VGGP GHKARVL } \\
\text { vering } 15 \text { class I a } \\
\text { oduction to measur } \\
\text { t month of treatme } \\
\text { previously detecta } \\
\text { iminished } \\
\text { rent patterns of res }\end{array}$ & $\begin{array}{l}\text { HIV-1 infection human (B7) } \\
\text { ed in } 14 \mathrm{HIV}+\text { patients from an unselected Caucasian } \\
\text { lecreased and frequencies of HIV-specific CTL tripled a } \\
\text { ly detected, as were CMV specific CD8+ PBL - but wit } \\
\text { ses or decreases in pre-existing response, new specificit }\end{array}$ & $\begin{array}{l}\text { Mollet } 2000 \\
\text { n treated with HAART, using } \\
\text { dened - eight new HIV } \\
\text { lued viral suppression, } \\
\text { o change }\end{array}$ \\
\hline p24 (223-231) & $\begin{array}{l}\text { p24 (223-231 SF2) } \\
\text { - Epitope name: GL9 } \\
\text { - HIV+ individual AC } \\
\text { from } 7 \text { proteins, sug } \\
\text { - The response to GPC }\end{array}$ & $\begin{array}{l}\text { GP GHKARVL } \\
\text { as tested for react } \\
\text { g that the breadth } \\
\text { ARVL was domina }\end{array}$ & $\begin{array}{l}\text { HIV-1 infection human (B*0702) } \\
\text { ag peptides spanning all HIV-1 proteins in an ELISPOT } \\
\text { nses are underestimated if accessory proteins are not in }\end{array}$ & $\begin{array}{l}\text { Altfeld2001a } \\
\text { found to react with } 12 \text { peptides } \\
\text { the study }\end{array}$ \\
\hline p24 (223-231) & $\begin{array}{l}\text { p24 (355-363 LAI) } \\
\text { - Identical twin hemor } \\
\text { - One had a strong res } \\
\text { - } \text { [Goulder1997a] is a }\end{array}$ & $\begin{array}{l}\text { GP GHKARVL } \\
c \text { brothers were bo } \\
\text { to this peptide, th } \\
w \text { of immune esca }\end{array}$ & $\begin{array}{l}\text { HIV-1 infection } \\
h \text { the same batch of factor VIII } \\
\text { response } \\
\text { rizes this study }\end{array}$ & Goulder1997e Goulder1997a \\
\hline p24 (223-231) & $\begin{array}{l}\text { p24 (SF2) } \\
\text { - The CTL-dominant } 1 \\
\text { peptides in the study } \\
\text { - Three peptides GSEl } \\
\text { contained the domin } \\
\text { - Five peptides RLRP } \\
\text { FRDYVDRFFKTLR } \\
\text { infected subjects fro }\end{array}$ & $\begin{array}{l}\text { GP SHKARVL } \\
\text { nse was focused or } \\
\text { SLYNTVATL (p17 } \\
\text { Jag-specific epitop } \\
\text { KHYMIKHLVW } \\
\text { QA (p24 161-177), } \\
\text { uth Africa }\end{array}$ & $\begin{array}{l}\text { HIV-1 infection human (B7) } \\
\text { n a HIV+ Caucasian living in Boston - this epitope did } \\
\text { 5), SALSEGATPQDLNTMLNTVG (p24 41-60), and } \\
\text { 4 B-clade infected individuals from Boston who show } \\
\text { LRSLYNTVATLYCV (p17Gag 74-88), SALSEGATPC } \\
\text { GKEPFRDY (p24 149-164) contained dominant Gag-s }\end{array}$ & $\begin{array}{l}\text { Goulder2000a } \\
\text { within the three most recognized } \\
\text { RPGGKKKYKLK(p17 16-30) } \\
\text { CTL responses } \\
\text { MLNTVG (p24 41-60), } \\
\text { epitopes in } 32 \text { out of } 37 \text { C-clade }\end{array}$ \\
\hline p24 (223-231) & $\begin{array}{l}\text { p24 (SF2) } \\
\text { - The CTL-dominant } 1 \\
\text { peptides in the study } \\
\text { - Three peptides GSEl } \\
\text { contained the domin } \\
\text { - Five peptides RLRP } \\
\text { FRDYVDRFFKTLR } \\
\text { infected subjects fro }\end{array}$ & $\begin{array}{l}\text { GP SHKARVL } \\
\text { ase was focused or } \\
\text { LYNTVATL (p17 } \\
\text { ag-specific epitope } \\
\text { KHYMIKHLVW } \\
\text { A (p24 161-177), } \\
\text { th Africa }\end{array}$ & $\begin{array}{l}\text { HIV-1 infection human (B7) } \\
\text { n a HIV+ Caucasian living in Boston - this epitope did } \\
\text { 5), SALSEGATPQDLNTMLNTVG (p24 41-60), and } \\
\text { 4 B-clade infected individuals from Boston who show } \\
\text { LRSLYNTVATLYCV (p17Gag 74-88), SALSEGATPC } \\
\text { GKEPFRDY (p24 149-164) contained dominant Gag-s }\end{array}$ & $\begin{array}{l}\text { Goulder2000a } \\
\text { within the three most recognized } \\
\text { RPGGKKKYKLK(p17 16-30) } \\
\text { CTL responses } \\
\text { MLNTVG (p24 41-60), } \\
\text { epitopes in } 32 \text { out of } 37 \text { C-clade }\end{array}$ \\
\hline p24 (223-231) & (LAI) & GP GHKARVL & (B7) & Brander2001, Goulder1999a \\
\hline
\end{tabular}




\begin{tabular}{llllll}
\hline HXB2 Location & Author's Location & Sequence & Immunogen & Species (HLA) \\
\hline p24 (223-231) & p24 (223-231 SF2) & GPGHKARVL & HIV-1 infection & human (B7)
\end{tabular}

- Therapy provided during acute infection resulted in a narrower CTL response, stronger T help response, and a less diverse viral population than was seen in individuals treated during chronic infection

- The breadth and specificity of the response was determined using ELISPOT by studying 19 individuals with pre-seroconversion therapy (Group 1), 11 individuals with primary infection but post-seroconversion therapy (Group 2), and 10 individuals who responded to HAART given during chronic infection (Group 3), using 259 overlapping peptides spanning p17, p24, RT, gp41, gp120 and Nef

- Previously described and newly defined optimal epitopes were tested for CTL response

- Number of HLA-B7+ individuals that had a CTL response to this epitope broken down by group: $1 / 4$ group $1,2 / 3$ group 2 , and $0 / 1$ group 3 p24 (223-231) GPGHKARVL HIV-1 infection human (B7)

Day2001

p24 (223-231)

- The CTL response to optimally defined CTL epitopes restricted by HLA class I A and B alleles in individuals who coexpressed HLA A2, A3, and B7 was studied in eight HIV-1-infected subjects, two with acute infection, five with chronic, and one long-term non-progressor (LTNP)

- 2 to 17 epitopes were recognized in a given individual, A2-restricted CTL response tended to be narrow and never dominated the response, and 25/27 epitopes were targeted by at least one person

- Subjects with chronic HIV-1 infection recognized between 2-8 out of 11 B7-restricted epitopes

- An acute seroconvertor homozygous for the B7 allele recognized five B7-restricted epitopes

- The other acute seroconvertor failed to recognize any of the 11 B7-restricted epitopes tested

- The B7-restricted CTL response was highly variable and there was no clearly dominant epitope

\begin{tabular}{lllll}
\hline p24 (223-231) p24(223-231) GPGHKARVL & HIV-1 infection & human (B7)
\end{tabular}

- Epitope name: B7-GL9

- CTL responses in 18 acutely HIV-infected HLA-A3 (n=7) or -B7 ( $n=4)$ or both -A3 and B7 (n=7) positive individuals were studied.

- One individual, AC-06, was homozygous at all three class I alleles (A3, B7, Cw7), was treated during acute infection and had supervised treatment interruptions (STI). He had only two detectable CTL responses during acute infection, but after STI this broadened to 27 distinct epitopes including 15 restricted by HLA-A3, 11 by HLA-B7, and 1 by HLA-Cw7.

- Only two epitopes were detected during acute infection in patient AC-06, B7 restricted gp41 epitope IPRRIRQGL and Gag GPGHKARVL. GPGHKARVL was the first targeted peptide, and remained immunodominant through the 34 month study period.

- 3/11 HLA-B7 individuals had detectable B7-restricted responses to this epitope during acute infection - 10/15 of HLA-B7 epitopes tested were targeted by at least one person during acute infection. 1/4 individuals had detectable responses to this epitope after STI. p24 (C consensus)

\section{HIV-1 infection}

human

Goulder2000a

- The CTL-dominant response was focused on this epitope in a HIV+ South African - this epitope did not fall within the five most recognized peptides in the study

- Three peptides GSEELRSLYNTVATL (p17 residues 71-85), SALSEGATPQDLNTMLNTVG (p24 41-60), and WEKIRLRPGGKKKYKLK(p17 16-30) contained the dominant Gag-specific epitope in 31 out of 44 B-clade infected individuals from Boston who showed Gag-CTL responses

- Five peptides RLRPGGKKHYMIKHLVW (p17 20-36), ELRSLYNTVATLYCV (p17Gag 74-88), SALSEGATPQDLNTMLNTVG (p24 41-60), FRDYVDRFFKTLRAEQA (p24 161-177), and SILDIKQGKEPFRDY (p24 149-164) contained dominant Gag-specific epitopes in 32 out of 37 C-clade infected subjects from South Africa 


\section{II-B-4 p24-p2p7p1p6 CTL Epitopes}

\begin{tabular}{|c|c|c|c|c|c|}
\hline HXB2 Location & Author's Location & Sequence & Immunogen & Species (HLA) & References \\
\hline $\begin{array}{l}\text { p24-p2p7p1p6 } \\
(223-1)\end{array}$ & \multicolumn{5}{|c|}{$\begin{array}{l}\text { - The program Epimatrix was used in conjunction with the program Conservatrix to identify conservered regions of HIV that might serve as epitopes } \\
\text { - A subset of the potential epitopes was identified that could bind to the appropriate HLA-allele, and } 15 \text { predicted B7 superfamily (HLA B7, B8, and B58) } \\
\text { epitopes were identified that could stimulate IFN } \gamma \text { production in an ELISPOT assay } \\
\text { - GPGHKARVLA was confirmed as an HLA-B7 epitope in this study, and had been previously published }\end{array}$} \\
\hline $\begin{array}{l}\text { p24-p2p7p1p6 } \\
(225-8)\end{array}$ & $\begin{array}{l}\text { Gag (357-372 LAI) } \\
\text { - Vertical transmission } \\
\text { - Primary assays show } \\
\text { - Epitopes recognized } \\
\text { - Patient EM28 (CDC }\end{array}$ & $\begin{array}{l}\text { GHKARVLAEATLSQVN } \\
\text { HIV ranges from } 13 \% \text { to } 3 \\
\text { ytotoxic activity against at } \\
\text { ve children were mapped } \\
\text { ) had a CTL response to } \mathrm{f}\end{array}$ & $\begin{array}{l}\text { HIV-1 infection } \\
\text { ne HIV protein w } \\
\text { ynthetic peptides a } \\
\text { topes in Gag }\end{array}$ & $\begin{array}{l}\text { human } \\
\text { of infected children } \\
\text { ares }\end{array}$ & Buseyne1993a \\
\hline $\begin{array}{l}\text { p24-p2p7p1p6 } \\
(230-7)\end{array}$ & $\begin{array}{l}\text { Gag (386-) } \\
\text { - Epitope name: Gag-- } \\
\text { - HIV was scanned for } \\
\text { criteria, and } 30 \text { of the } \\
\text { - Three additional pre } \\
\text { recognized at least o } \\
\text { maximum of 2) } \\
\text { - VLAEAMSQV bind } \\
\text { - } 4 / 22 \text { individuals with } \\
\text { - } 0 / 12 \text { acutely infected }\end{array}$ & $\begin{array}{l}\text { VLAEAMSQV } \\
\text { peptides which carried the } \\
\text { ound to HLA-A*0201-2 } \\
\text { ly described HLA-A2 epi } \\
\text { the } 23 \text { peptides (median } \\
\text { all five HLA-A2 supertype } \\
\text { onic HIV-1 infection reco } \\
\text { viduals recognized this ep }\end{array}$ & $\begin{array}{l}\text { HIV-1 infection } \\
\text { permotif pattern c } \\
\text { ound to at least } 3 / \text { s } \\
\text { vere added to the } \\
\text { d maximum of } 6 \text { ), } \\
\text { s tested: } A^{*} 0201 \text {, } \\
\text { this epitope, and i }\end{array}$ & $\begin{array}{l}\text { human }(\mathrm{A} * 0201) \\
\text { han } 50 \% \text { of B clade sequ } \\
\text { rtype alleles tested } \\
\text { chronically infected HL } \\
\text { hfected individuals recog } \\
\text { A*0206 and } \mathrm{A} * 6802 \text { (hi } \\
\text { inant in } 3 / 4 \text { by ELISPOT }\end{array}$ & $\begin{array}{l}\text { Altfeld2001c } \\
\text { nces }-233 \text { peptides met this } \\
\text { A-A2 individuals had CTL that } \\
\text { ized at least } 1 \text { (median of } 1 \text { and } \\
\text { hest affinity) }\end{array}$ \\
\hline $\begin{array}{l}\text { p24-p2p7p1p6 } \\
(230-7)\end{array}$ & $\begin{array}{l}\text { - Epitope name: Gag- } \\
\text { - Among HIV+ indivi }\end{array}$ & VLAEAMSQV & HIV-1 infection & human (A02) & Sabbaj2002b \\
\hline $\begin{array}{l}\text { p24-p2p7p1p6 } \\
(230-7)\end{array}$ & $\begin{array}{l}\text { Gag (397-405) } \\
\text { - Long-term nonprogr } \\
\text { for the A3 supertype } \\
\text { - Progressors had men } \\
\text { - A positive correlatior } \\
\text { observed, which may } \\
\text { - This epitope can bin }\end{array}$ & $\begin{array}{l}\text { VLAEAMSQV } \\
\text { s (LTNPs) had strong mer } \\
\text { ile the effector cells of lon } \\
\text { resting CD8+ T-cells that } \\
\text { ween effector CD8+ T-cel } \\
\text { tribute to the inability of I } \\
\text { five HLA-A2 supertypes a }\end{array}$ & $\begin{array}{l}\text { HIV-1 infection } \\
\text { esting CD8+ T-cel } \\
\text { nonprogressors re } \\
\text { ized far fewer epi } \\
\text { plasma viremia an } \\
\text { to clear virus } \\
(\mathrm{A} * 0201, \mathrm{~A} * 0202\end{array}$ & $\begin{array}{l}\text { human (A2 supertype) } \\
\text { the majority of epitopes } \\
\text { r epitopes } \\
\text { ation between } \mathrm{CD} 8+\text { eff } \\
\text { and } \mathrm{A} * 6802)\end{array}$ & $\begin{array}{l}\text { Propato2001 } \\
\text { ested, (18 for the A2 supertype, } 16 \\
\text { tor T-cells and CD4+ T-cells was }\end{array}$ \\
\hline
\end{tabular}




\section{II-B-5 p2p7p1p6 CTL Epitopes}

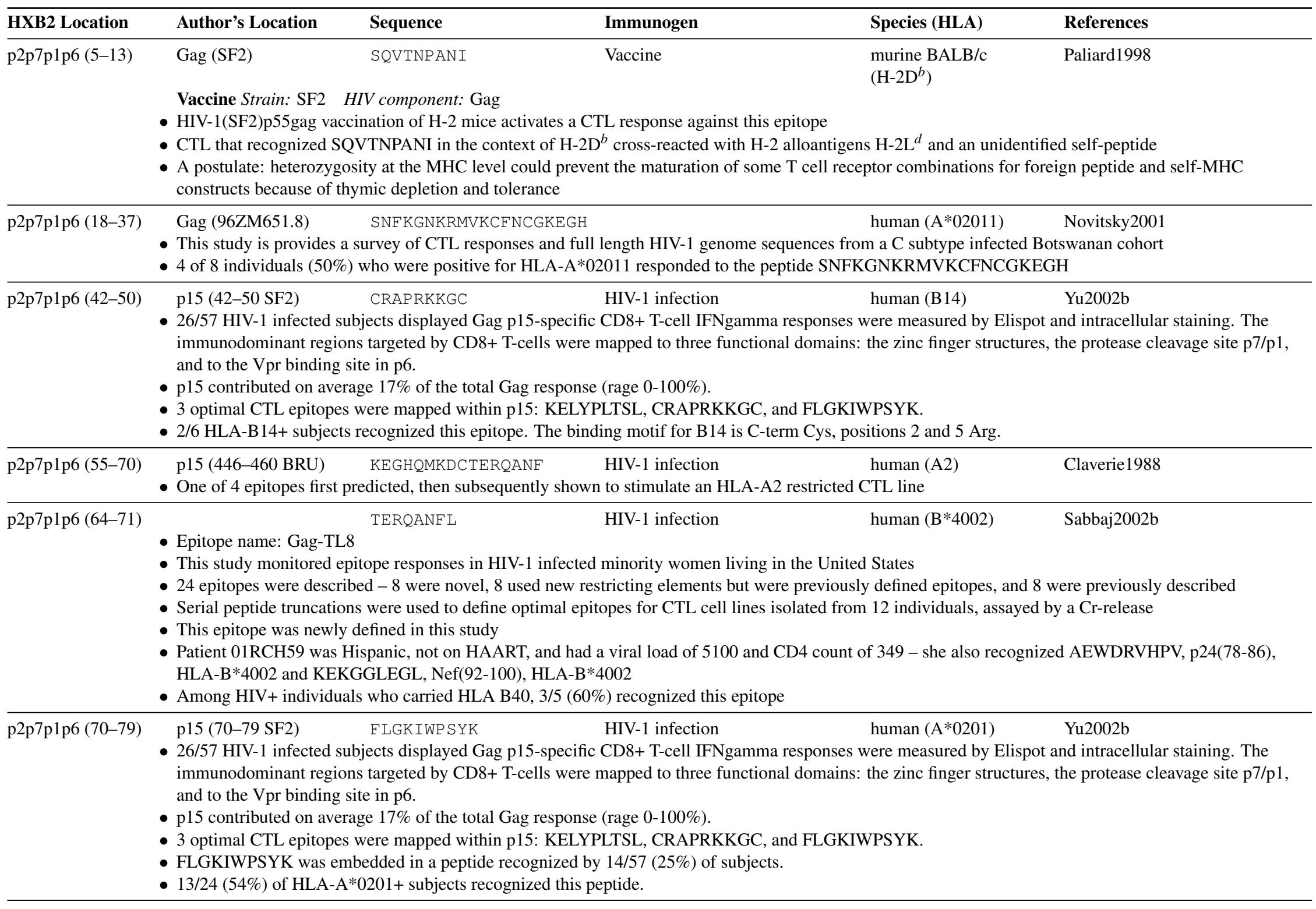




\begin{tabular}{|c|c|c|c|c|c|}
\hline HXB2 Location & Author's Location & Sequence & Immunogen & Species (HLA) & References \\
\hline p2p7p1p6 (83-97) & $\begin{array}{l}\text { Gag (453-462 BH10, } \\
\text { LAI) } \\
\text { - This study employs an a } \\
\text { - This CTL epitope (the F } \\
\text { surface glycoprotein CD }\end{array}$ & $\begin{array}{l}\text { GNFLQSRPEPTAPPF } \\
\text { tigenic similarity matrix } \\
\text { V-1 LAI fragment with } \mathrm{h} \\
\text {, fragment PEPTAPPRL }\end{array}$ & $\begin{array}{l}\text { HIV-1 infection } \\
\text { pare HIV-1 antigen } \\
\text { ilarity to a human }\end{array}$ & $\begin{array}{l}\text { human } \\
\text { ith human proteins. } \\
\mathrm{g} \text { this epitope is PEPTAP }\end{array}$ & $\begin{array}{l}\text { Maksiutov2002 } \\
\text { FLQ) has similarity with the T-cell }\end{array}$ \\
\hline p2p7p1p6 (83-97) & $\begin{array}{l}\text { p15 (418-433 BRU) } \\
\text { One of } 4 \text { epitopes first p }\end{array}$ & $\begin{array}{l}\text { GNFLQSRPEPTAPPF } \\
\text { edicted, then subsequentl }\end{array}$ & $\begin{array}{l}\text { HIV-1 infection } \\
\text { to stimulate an } \mathrm{H}\end{array}$ & $\begin{array}{l}\text { human (A2) } \\
\text { CTL line }\end{array}$ & Claverie1988 \\
\hline p2p7p1p6 (118-126) & $\begin{array}{l}\text { p2p7p1p6 (118-126) } \\
\text { C. Brander notes that th }\end{array}$ & $\begin{array}{l}\text { KELYPLTSL } \\
\text { is a } B * 4001 \text { epitope }\end{array}$ & & human $(\mathrm{B} * 4001(\mathrm{~B} 60))$ & Brander2001 \\
\hline p2p7p1p6 (118-126) & $\begin{array}{l}\text { p15 (118-126 SF2) } \\
\text { Epitope name: p15-24 } \\
\text { 26/57 HIV-1 infected su } \\
\text { immunodominant regior } \\
\text { and to the Vpr binding s } \\
\text { p15 contributed on aver } \\
\text { - } 3 \text { optimal CTL epitopes } \\
\text { - Four patients who were } \\
\text { - The binding motif for B } \\
\text { Four patients who did n } \\
\text { immediate region can be }\end{array}$ & $\begin{array}{l}\text { KELYPLTSL } \\
\text { jects displayed Gag p15- } \\
\text { targeted by CD } 8+\text { T cel } \\
\text { e in p6. } \\
\text { ge } 17 \% \text { of the total Gag r } \\
\text { vere mapped within p15: } \\
\text { ILA-B60+ recognized K } \\
0 \text { is C-term Leu and 2nd } \\
\text { carry HLA-B60 also rec } \\
\text { presented by other HLA }\end{array}$ & $\begin{array}{l}\text { HIV-1 infection } \\
\text { CD8+ T-cell IFNs } \\
\text { napped to three fu } \\
\text { (rage } 0-100 \% \text { ). } \\
\text { LTSL, CRAPRKF } \\
\text { SL. } \\
\text { Glu. } \\
\text { the } 15 \text { amino aci } \\
\text { olecules. }\end{array}$ & $\begin{array}{l}\text { human }(\mathrm{B} 60, \mathrm{~B} * 4001) \\
\text { vere measured by Elispot } \\
\text { the zinc finger structures } \\
\text { VPSYK. } \\
\text { ing KELYPLTSL, sugge }\end{array}$ & $\begin{array}{l}\text { Yu2002b } \\
\text { nd intracellular staining. The } \\
\text { he protease cleavage site } \mathrm{p} 7 / \mathrm{p} 1 \text {, } \\
\text { ing other epitopes in this }\end{array}$ \\
\hline p2p7p1p6 (121-130) & $\begin{array}{l}\text { Gag (484-493) } \\
\text { This B7 epitope is one } \\
\text { A dominant B7 epitope } \\
\text { non-anchor based strat } \\
\text { set to } 55 \text { peptides for ex }\end{array}$ & $\begin{array}{l}\text { YPLTSLRSLF } \\
\text { three subdominant CTL } \\
\text { as defined using conven } \\
\text { y, EpiMatrix, to identify } \\
\text { erimental testing }\end{array}$ & $\begin{array}{l}\text { HIV-1 infection } \\
\text { es detected in a lo } \\
\text { ethods, and three } \\
\text { sible epitopes in }\end{array}$ & $\begin{array}{l}\text { human (B7) } \\
\text { essor } \\
\text { inant HLA B7 epitopes } \\
\text {-1, followed by B7 anch }\end{array}$ & $\begin{array}{l}\text { Jin2000b } \\
\text { re defined by first using a } \\
\text { residue prediction to narrow the }\end{array}$ \\
\hline
\end{tabular}




\section{II-B-6 Gag CTL Epitopes}

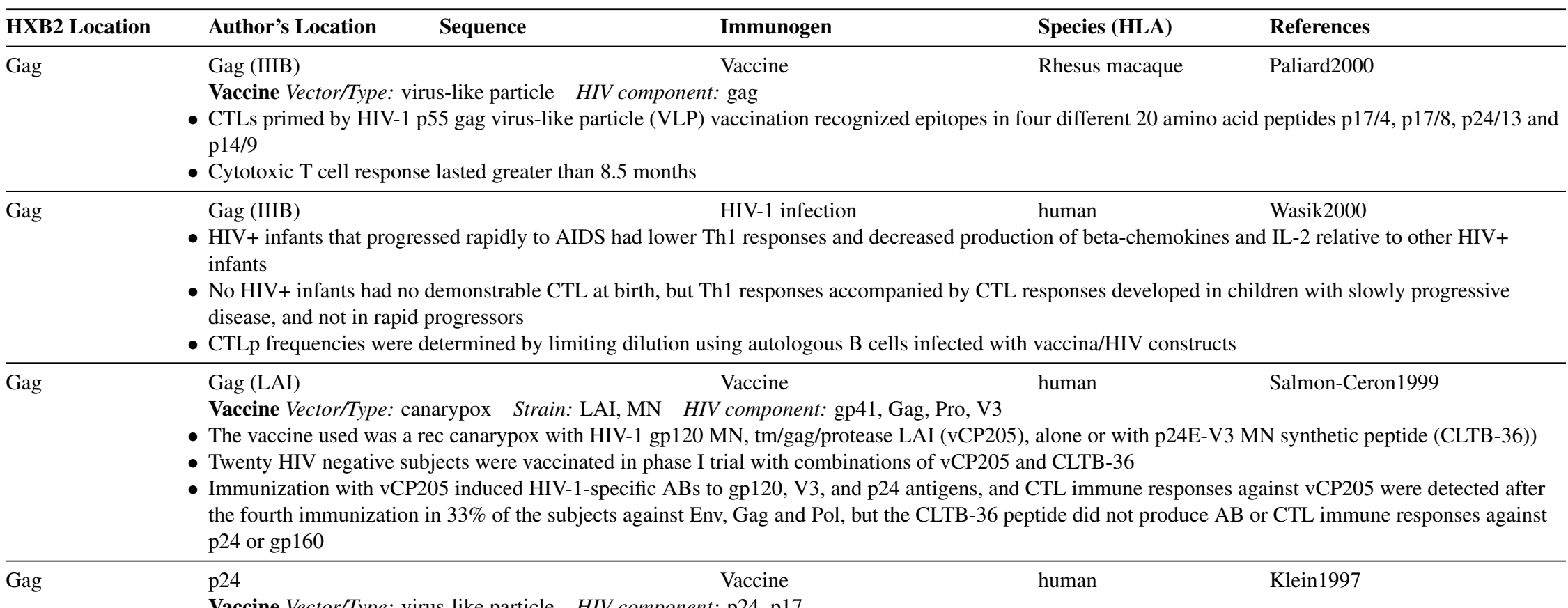

Vaccine Vector/Type: virus-like particle HIV component: p24, p17

- Immunization of HIV+ people with an HIV-1 p17/p24 Ty virus-like particle (p24-VLP) resulted in a marginal, short-lived increased proliferative response to 24 and p17 and a transient elevation in viral load

- Two of four subjects that received 500 or $1000 \mu \mathrm{g}$ of p24-VLP had an increase in gag-specific CTL

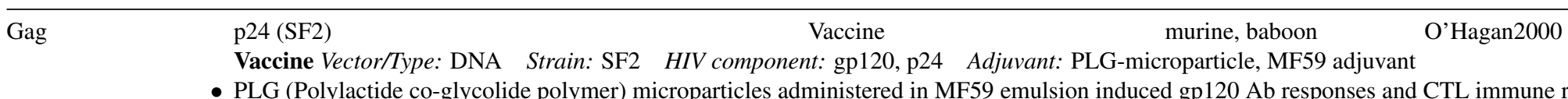

- PLG (Polylactide co-glycolide polymer) microparticles administered in MF59 emulsion induced gp120 Ab responses and CTL immune responses against p24 gag

\begin{tabular}{llll}
\hline Gag & Gag & HIV-1 infection & human \\
& Three strategies were used to analyze CTL activity: area under the net HIV-specific lysis curve (ACU), linear regression (LR) of net specific lysis, and the \\
& standard method, lytic units (LU20)
\end{tabular}

- A correlation between low HIV plasma viral load and increased levels of HIV-specific Gag and Nef CTL activity was observed using ACU and LR, but not LU20

$\begin{array}{ll}\text { Gag } & \text { Gag } \\ & \text { The presence of HIV-1 p24-specific proliferative responses was positively correlated with Gag-specific memory CTL and negatively correlated with viral } \\ & \text { load in untreated subjects }\end{array}$




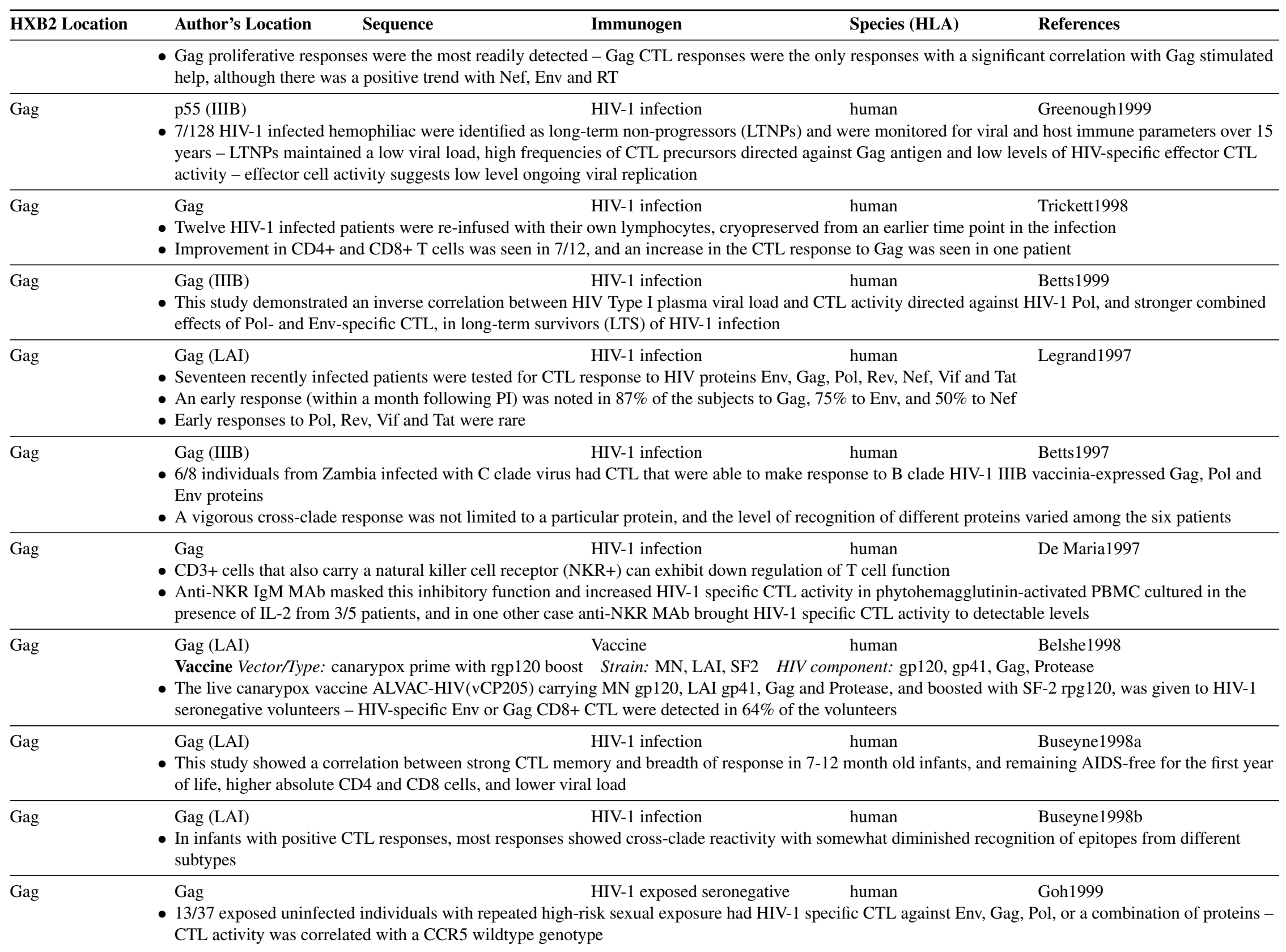




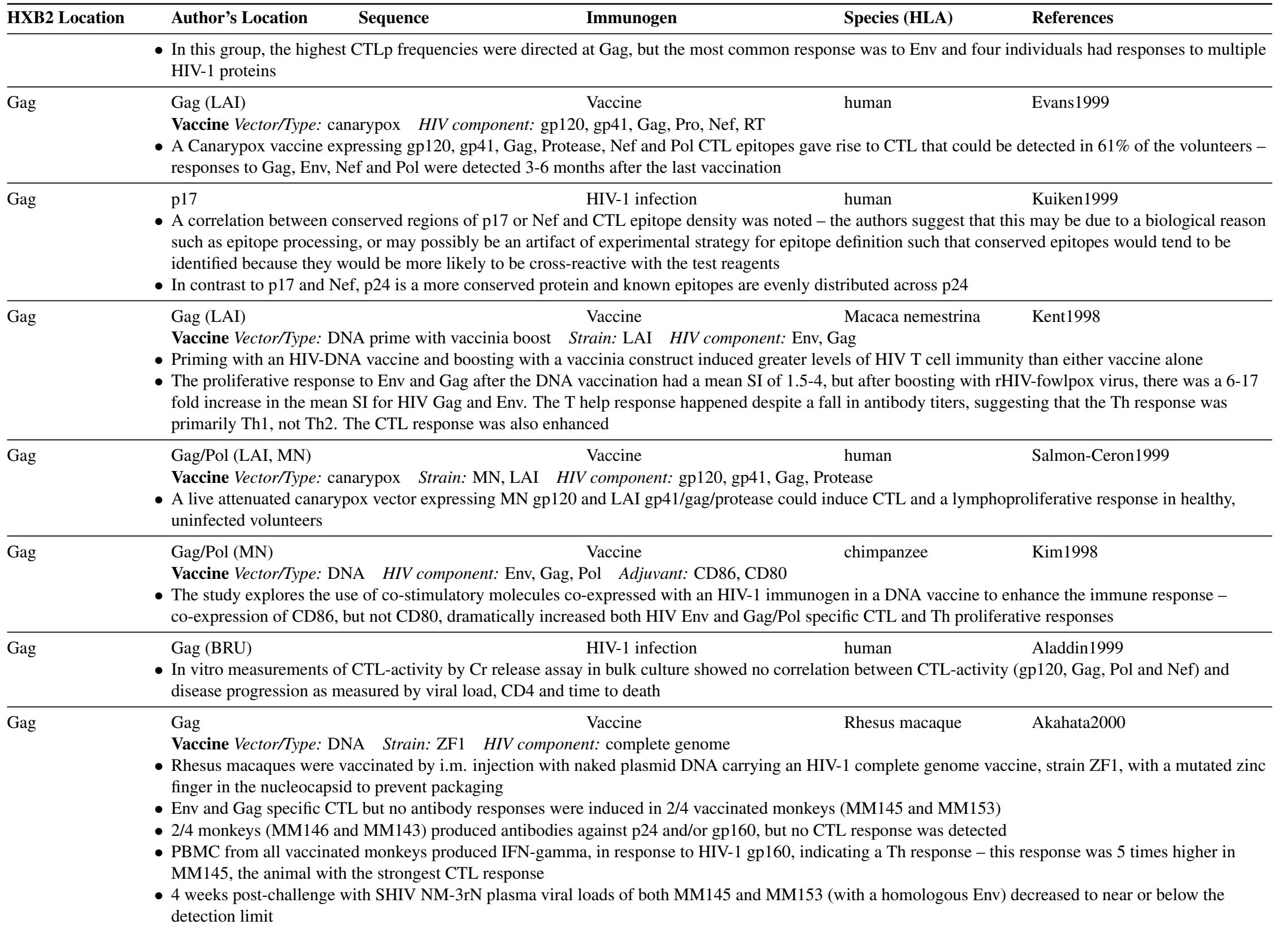




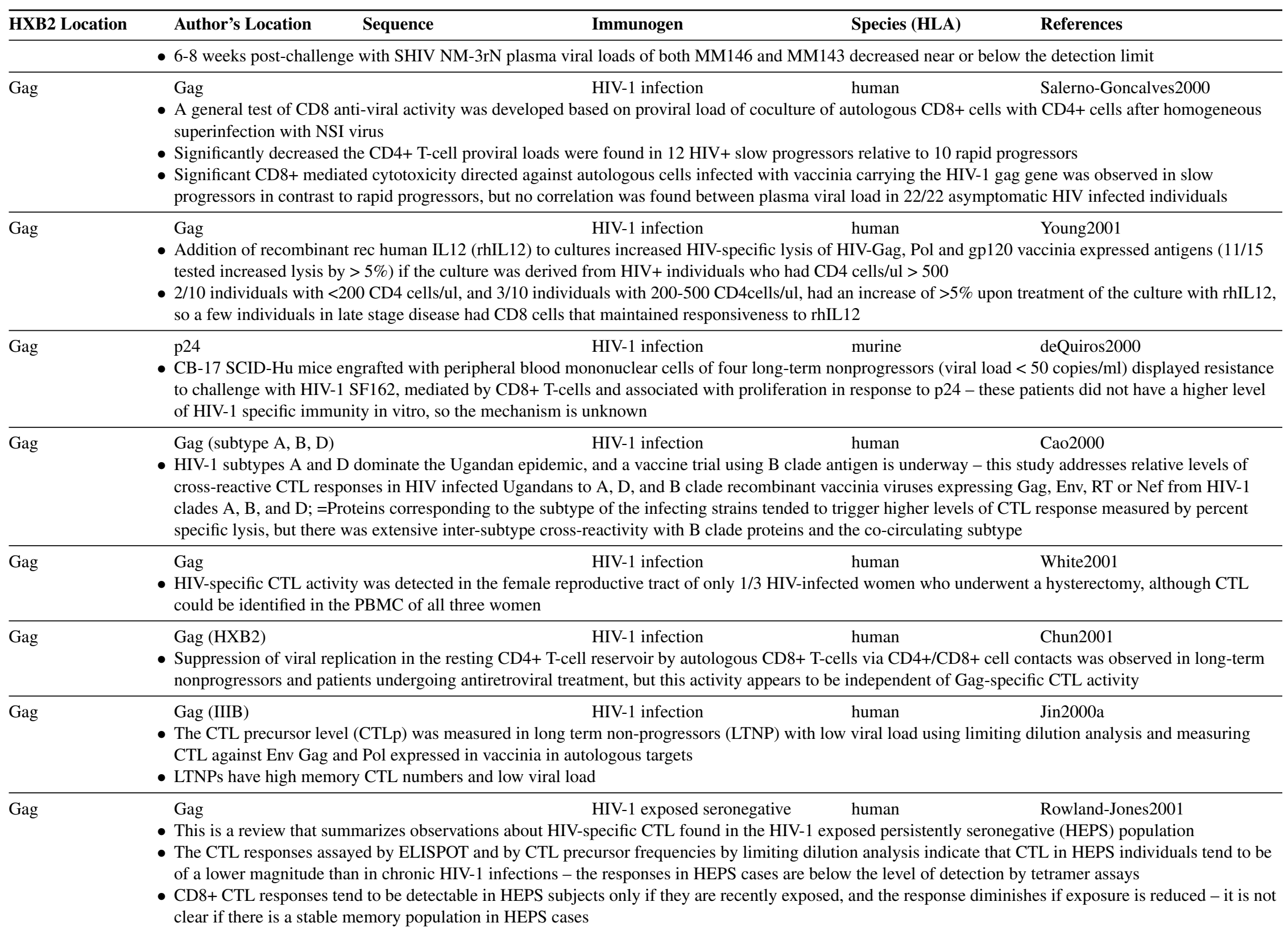




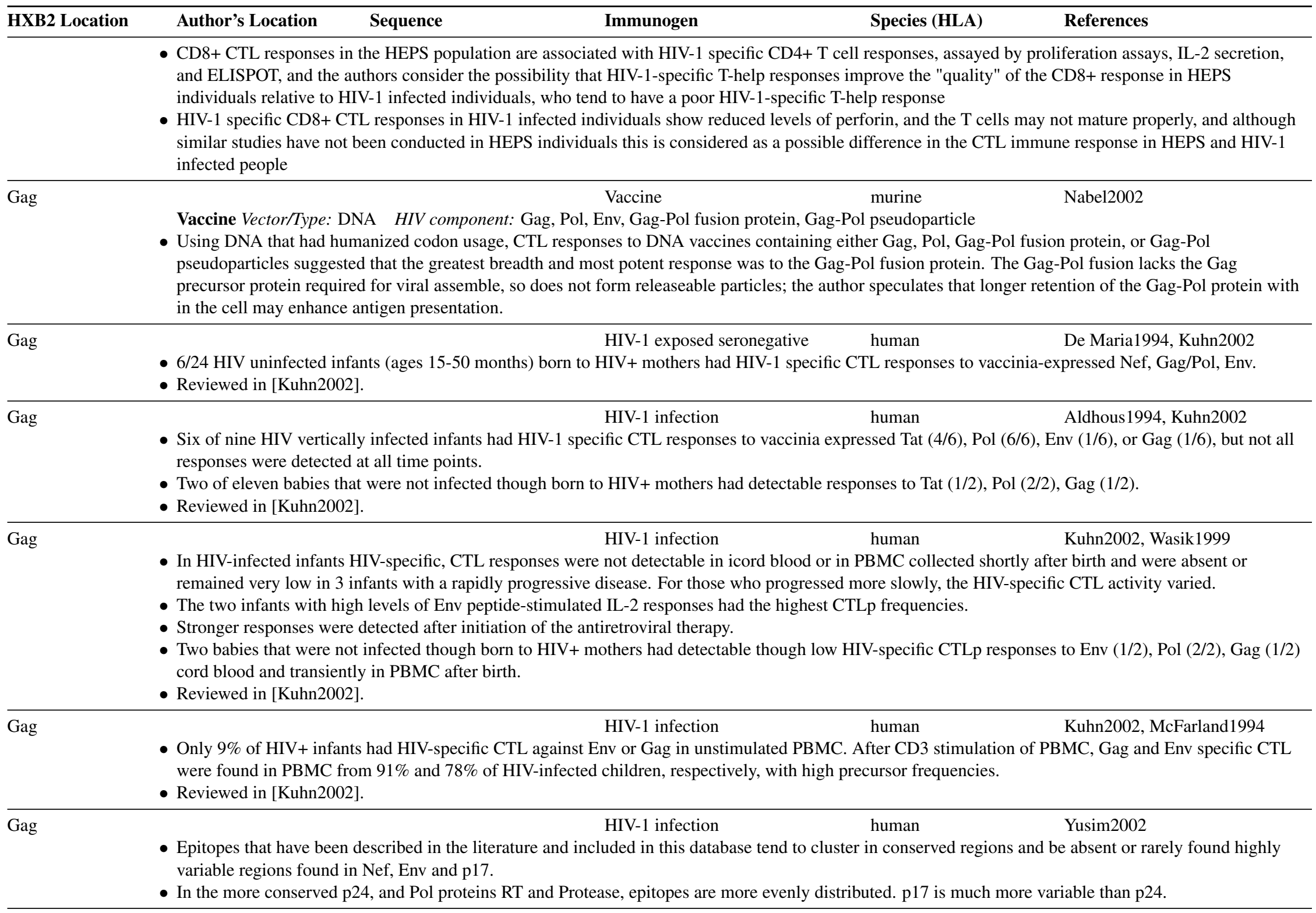




\begin{tabular}{lllll}
\hline HXB2 Location & Author's Location & Sequence & Immunogen & Species (HLA) \\
\hline Gag & p24 (HXB) & HIV-1 infection & heferences & Lu2000a
\end{tabular}

- Bacillus anthrax lethal toxin (LFn)-HIV fusion proteins are candidate HIV vaccines that are safe in mice, and LFn-V3 region fusion proteins induce CD8 T cells in BALBc mice. LFn causes exogenous protein to be taken up and processed in a class I pathway. Expressed proteins from Gag p24 and Nef fragments cloned into the LFn expression plasmid stimulate gag-specific CD4 proliferation and CTL responses in HIV-infected donor PBMCs in vitro.

Gag (HXB2) HIV-1 infection human Edwards2002

- $96 \%(26 / 27)$ chronically infected HIV-1 infected patients elicited gamma-IFN CD8+ T-cell responses against Gag

- Nef and/or Pol CTL responses were detected in $86 \%$ of the subjects

- The magnitude and breadth of Gag and p24 T-cell responses correlated with absolute CD4 counts, and inversely correlated with viral load

- Pol and Int CTL responses correlated positively with absolute CD4+ T-cell count

- Nef and Env responses did not correlate with either CD4 counts or viral load

Gag HIV-1 infection human Larsson2002b

- Autologous mature dendritic cells with rec vaccinia expressing Gag, Pol, Nef and Env could amplify CD8+ T-cell Elispot responses 4-38 fold in five HIV+ patients on successful HAART treatment, relative to autologous monocytes. Some weak responses could only be detected using mature dendritic cells as APCs, and this approach could be useful for detection of low frequency memory cells.

Gag (IIIB) HIV-1 infection Trickett2002

- Conditions were optimized for ex-vivo expansion of CD8+ and CD4+ T-cells with the goal of functional T-cell production for autologous immunotherapy. 10,000-fold expansions were obtained in 14 days with optimized concentrations of IL-2, anti-CD3 and anti-CD28 coated microspheres, and decreasing amounts of serum over the first 8 days.

Gag (IIIB) HIV-1 and HCV co-infection human Lauer2002

- HIV-1 and HCV immune responses were studied in 22 individuals who were co-infected with HIV-1 and hepatitis C virus (HCV). IFNgamma production was measured in an Elispot assay of CD8+ T-cells using targets expressing either Gag, RT, Env and Nef in a vaccinia construct, or one of seven HCV proteins.

- All 22 patients targeted at least one protein. 20/22 patients recognized RT, 17/22 patients recognized Gag, 13/22 subjects recognized Env and 11/22 patients recognized Nef. Robust CTL activity was independent of disease progression or viral load.

- Despite high HCV viral loads, very few HCV CD8+ T-cell Elispot responses were detected. In a control HCV infected person who did not have HIV-1, strong anti-HCV responses were mounted.

- HIV-specific CD4 proliferative responses were detected in 9/17 coinfected patients, but no HCV responses were detected.

Gag HIV-1 infection human Luzuriaga1995

- 2/3 infants infected in utero had detectable HIV-1 Gag and Env specific CTL responses, one by 4 months, one by 11 months of age. Levels of the responses varied at different time point. Pol responses were not detected.

- 2/4 infants infected intrapartum had detectable responses, one note until 11 months, one not until 42 months.

- HIV-specific CTL were not detected in ten HIV- infants that were born to HIV+ mothers.

Gag Vaccine Vector/Type: canarypox prime with rop120 boost Strain: Gag, LAI; gp120, MN; and gp41, LAI HIV component: gag, en

- A safety and immunogeniticity study of a vaccine dosing schedule was studied in a trial conducted in high and low risk study subjects. There was a $76 \%$ cumulative probability of detecting a Gag or Env CTL response by day 728 .

- CTL responses before and after initiation of ART were studied in 13 HIV-1 vertically infected infants $<6$ months of age, and 4 that were $>6$ months of age. 


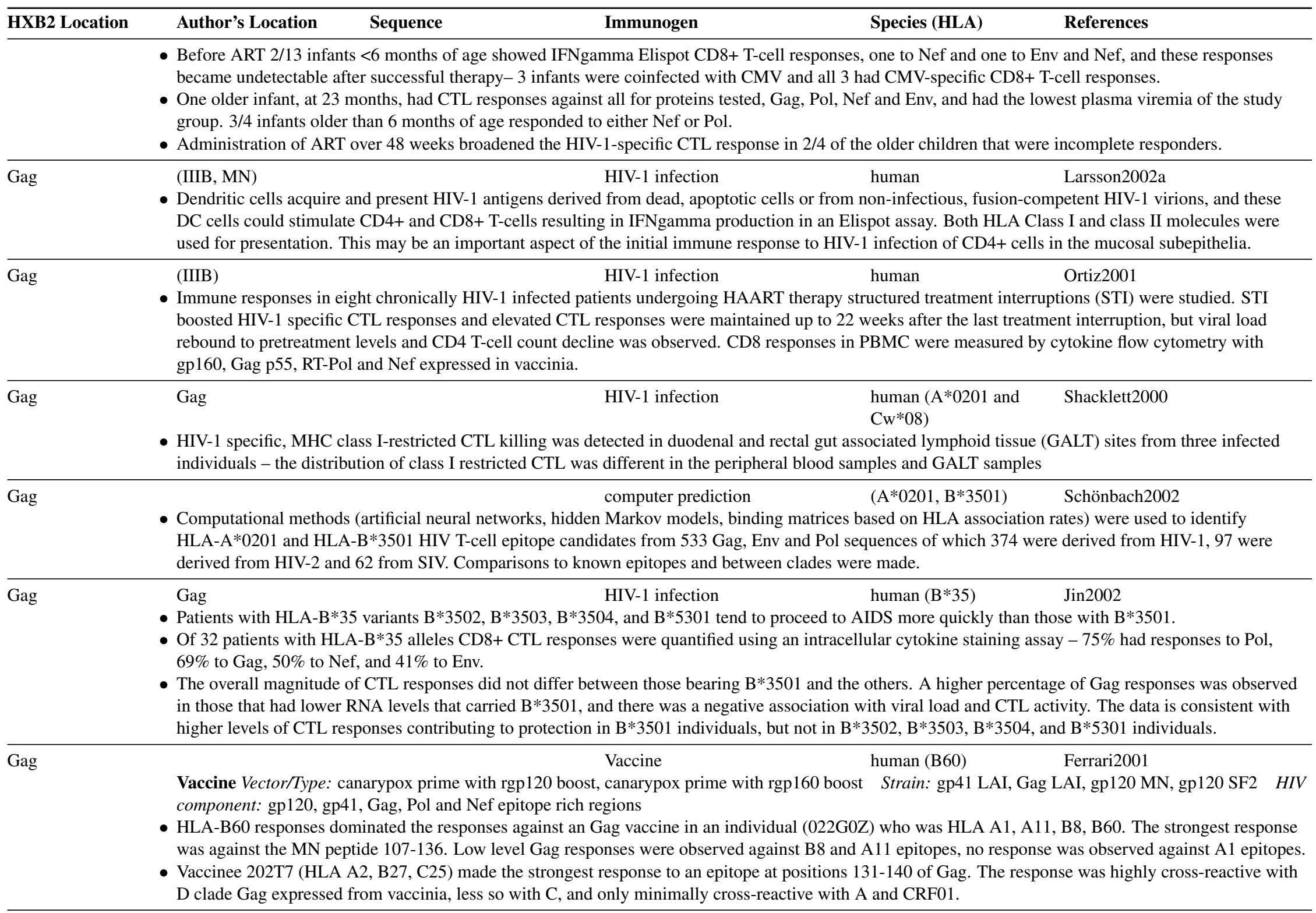




\begin{tabular}{|c|c|c|c|c|c|}
\hline HXB2 Location & Author's Location & Sequence & Immunogen & Species (HLA) & References \\
\hline Gag & $\mathrm{p} 24$ & & Vaccine & $\begin{array}{l}\text { murine }\left(\mathrm{H}-2^{b}, \mathrm{H}-2^{d},\right. \\
\left.\mathrm{H}-2^{k}\right)\end{array}$ & Iroegbu2000 \\
\hline
\end{tabular}

- The p24 sequence is more conserved than is p17 within patient, and nonsynonymous substitutions are spread evenly throughout its coding regions, not concentrated in CTL epitopes

- Minor changes in p24 did not alter the immunogenicity in H-2b,d, or k mice, while changes in p17 (92\% similarity) did alter immunogenicity

Gag p24 Vaccine murine (H-2 ${ }^{d}$ Q

Vaccine Vector/Type: DNA HIV component: gag

- Mice were injected with plasmid DNA at 0,2 and 4 weeks and lymphocyte proliferation was measured after 6 weeks with recombinant p24 protein

- Secreted HIV-1 Gag expression vectors generated a stronger response than standard Gag or cytoplasmic Gag expression vectors

- IFN-gamma levels were increased compared to an undetectable IL-4 response

- CTL levels were also increased in secreted Gag expression vaccination studies

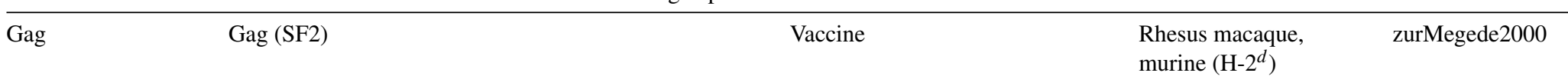

Vaccine Vector/Type: vaccinia Strain: SF2 HIV component: Gag, Protease, codon-optimized

- Sequence-modified Rev-independent gag and gag-protease gene constructs lead to increased expression levels and elevated CTL and antibody immunogenicity in BALB/c and CB6F1 mice

- A CTL response in mice could be detected after a single immunization with codon-optimized gag, using 2 ng of plasmid; wild type gag required 200 ng to detect a response

- Recognition of 3 different Gag peptide pools was observed, indicating a polyclonal CTL response

- Significant gag-specific CTL responses were detected in 4/4 rhesus monkeys, in contrast to $1 / 4$ using wildtype gag

\begin{tabular}{llcc}
\hline $\mathrm{Gag} 24$ & Vaccine & murine $\left(\mathrm{H}-2^{d}\right)$ & Halim2000
\end{tabular}

- An avirulent recombinant coxsackievirus (CB4-P) construct was generated that can express p24 Gag sequences - CB4-P is attenuated even in immunodeficient mice and T help responses can be elicited from peptides embedded in a surface loop of the VP1 capsid

- This paper describes the vaccine strategy and generation of constructs, and employs amino-terminal fusion of Gag sequences to the viral polyprotein with subsequent cleavage to elicit CTL responses via MHC class I presentation in BALB/c mice

\begin{tabular}{lllll}
\hline Gag & Gag & Vaccine & murine $\left(\mathrm{H}-2^{d}\right)$
\end{tabular}

- Mice were immunized with four humanized DNA constructs: GagPol, that would form a pseudoparticle carrying Gag and Pol, Gag, Pol or a GagPol fusion construct

- The GagPol pseudoparticle, Gag and GagPol fusion construct all elicited strong anti-Gag CTL, but only the GagPol fusion construct elicited strong anti-Pol CTL

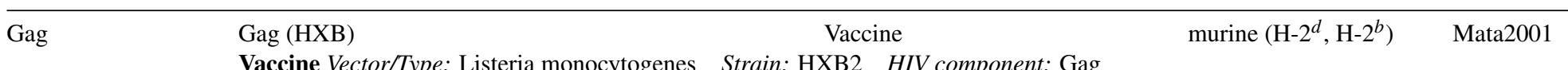

Vaccine Vector/Type: Listeria monocytogenes Strain: HXB2 HIV component: Gag

- BALB/c and C57BL/6 mice were immunized with recombinant Listeria monocytogenes (Lm-Gag) expressing HIV-1 HXB2 Gag and mice were challenged with vaccinia expressing Gag

- L. monocytogenes is a gram-positive bacteria that enters the macrophage on phocytosis and lives in the cytoplasm - secreted L. monocytogenes antigens are processed and presented by both class I and class II pathways 


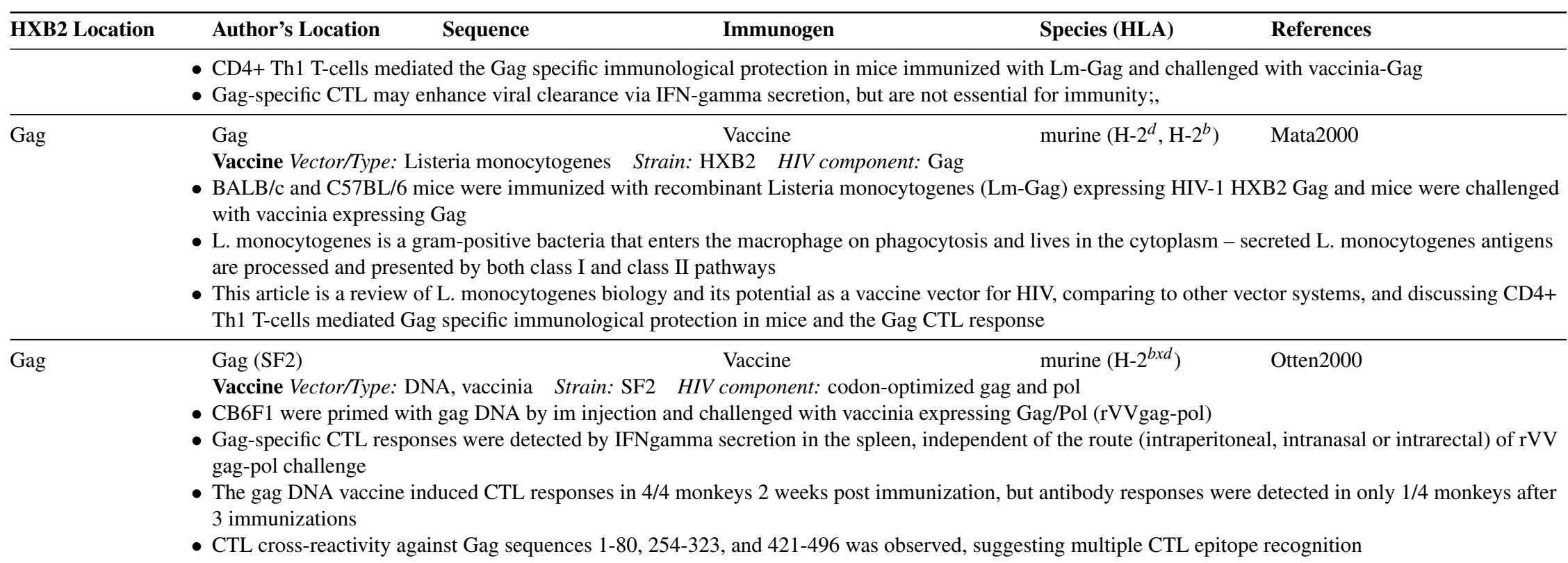




\section{II-B-7 Gag/Pol CTL Epitopes}

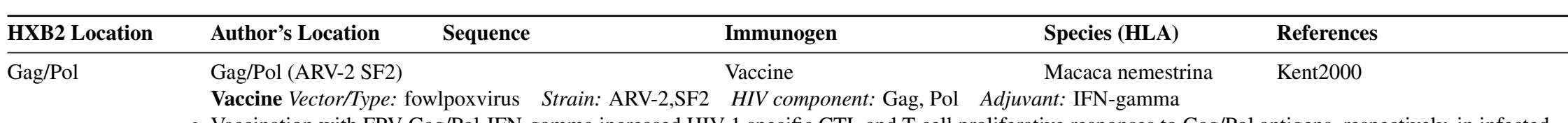

- Vaccination with FPV Gag/Pol-IFN-gamma increased HIV-1 specific CTL and T cell proliferative responses to Gag/Pol antigens, respectively, in infected

Macaca nemestrina

- HIV-1 viral loads remained low and unchanged following vaccinations

\begin{tabular}{llcc}
\hline Gag/Pol RT & Vaccine & murine & Kim1997d
\end{tabular}

- A Gag/Pol or Env DNA vaccine, when delivered in conjunction with the plasmid encoding the co-stimulatory molecules CD86, gave a dramatic increase in

both the cytotoxic and proliferative responses in mice

- When CD86 was present, CTL response could be detected even without in vitro stimulation

\begin{tabular}{lll}
\hline Gag/Pol & RT & HIV-1 infection \\
& & $13 / 13$ subjects with advanced HIV infections showed CD8 T cell proliferation and differentiation of CTL in vitro, and six individuals showed HIV-specific \\
& responses to Gag, Pol, Env or Nef antigens
\end{tabular}
responses to Gag, Pol, Env or Nef antigens

- Data suggests that the functional and genetic integrity of the CD8 T cell repertoire (TCR V beta gene intrafamily genetic diversity) remains intact through advanced HIV infection, although HIV-specific CTL activity decreases

\begin{tabular}{lccc}
\hline $\mathrm{Gag} / \mathrm{Pol}$ & Vaccine & murine
\end{tabular}

- Vpr can cause cells to go into G2 arrest, and it surpresses immune cell activation and inflammatory cytokine production, so co-immunization of BALB/c mice with recombinant adenovirus expressing Vpr and HIV-1 antigens Nef or Gag/Pol was tested to see if Vpr reduced the immune response to the other HIV antigens.

- Vpr compromised CD8+ T-cell lytic responses and T-helper proliferative responses in mice co-immunized with Vpr and Nef or Gag/Pol.

- In vitro, Vpr reduced T-cell cytokine production of IL-12 and TNFalpha, indicative of Vpr-mediated immune suppression. 


\section{II-B-8 Protease CTL Epitopes}

\begin{tabular}{lllll}
\hline HXB2 Location & Author's Location & Sequence & Immunogen & Species (HLA) \\
\hline Protease (3-11) & RT (71-79 subtype A, & ITLWQRPLV & human (A*6802) \\
& B, D) & & Brander2001 \\
& C. Brander notes this is an A*6802 epitope & & \\
\hline
\end{tabular}

\begin{tabular}{lllll}
\hline Protease (3-11) Pol & ITLWQRPLV HIV-1 infection, Vaccine & Hanke2000, Wee2002
\end{tabular}

Vaccine Vector/Type: DNA prime with vaccinia MVA boost Strain: subtype A HIV component: p17, p24, polyepitope

- The HIV-1 subtype A focused vaccine HIVA contains p24 and p17, in a reversed order relative to the Gag polyprotein to prevent myristylation of p17, which could direct the protein to the cell membrane and inhibit efficient peptide processing and class I presentation, as well as a polyepitope string of conserved, often immunodominant epitopes that were selected to have particularly good cross-reactive potential for the A-clade epidemic in Nairobi, Kenya. A DNA and MVA prime-boost vaccination protocol using the HIVA antigen will be used in a phase III clinical trial in Kenya. This epitope is included in the polyepitope string [Hanke2000].

- Multiple CD4+ or CD8+ T-cell vaccine-induced responses to peptide pools were detected using intracellular cytokine staining and IFNgamma Elispot assays after vaccination of 5 macaques. The response to the Mamu A*01 SIV p27 epitope p11C (CTPYDINQM), included in the polyepitope region, was not immunodominant in the Mamu $\mathrm{A} * 01$ vaccinated macaques, possibly because of processing limitations in context of the artificial polyepitope string [Wee2002].

\begin{tabular}{|c|c|c|c|}
\hline Protease (3-11) & $\begin{array}{l}\text { Protease (71-79 LAI) ITLWQRPLV } \\
\text { - Predicted on binding motif, no truncations analyzed } \\
\text { - Clade A/B/D consensus, S. Rowland-Jones, pers. comm. }\end{array}$ & $\begin{array}{l}\text { human }(A * 6802, \\
A * 7401, A 19)\end{array}$ & Dong1998a \\
\hline Protease (3-11) & $\begin{array}{l}\text { RT (71-79 subtype A, ITLWQRP LV } \\
\text { B, D) } \\
\text { - C. Brander notes this is an A*7401 epitope }\end{array}$ & human $(\mathrm{A} * 7401)$ & Brander2001 \\
\hline
\end{tabular}

ITLWORPLV HIV-1 infection human (A28) Ferrari2000

- One of the 51 HIV-1 epitopes selected by Ferrari et al. as good candidate CTL epitopes for vaccines by virtue of being conserved and presented by common HLA alleles

\begin{tabular}{lllll}
\hline Protease (3-11) & RT (71-79 LAI) & ITLWQRPLV & HIV-1 infection & human (A28supertype)
\end{tabular}

- Epitope name: P2

- A panel of 16 epitopes covering 15 class I alleles was tested in $14 \mathrm{HIV}+$ patients from an unselected Caucasian population treated with HAART, using CD8+ cell IFNgamma production to measure responses

- In general, during the first month of treatment viral load decreased and frequencies of HIV-specific CTL tripled and broadened - eight new HIV specificities that were not previously detectable were newly detected, as were CMV specific CD8+ PBL - but with continued viral suppression, HIV-specific responses diminished

- Viral rebounds gave different patterns of response: increases or decreases in pre-existing response, new specificities, or no change

Protease $(3-11)$

Pol ITLWQRPLV

HIV-1 infection, HIV-1 exposed human (A74)

Kaul2001a

- ITLWQRPLV cross-reacts with clades A, B and D

seronegative

DEC 2002 


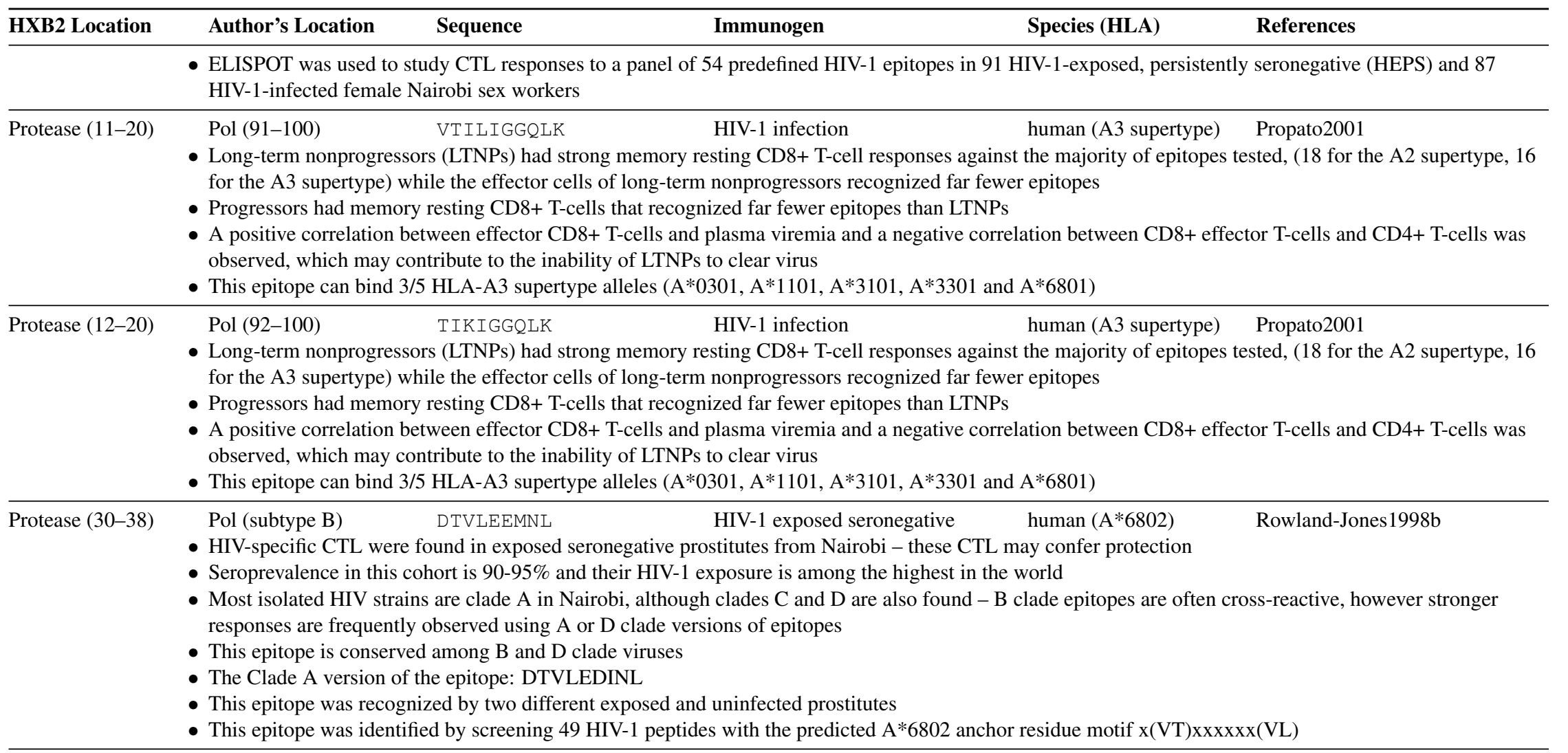

Protease (30-38)

Pol (subtype A)

HIV-1 exposed seronegative

human $\left(A^{*} 6802\right)$

Kaul2000

- 11/16 heavily HIV exposed but persistently seronegative sex-workers in Nairobi had HIV-specific CD8 gamma-IFN responses in the cervix - systemic

$\mathrm{CD} 8+\mathrm{T}$ cell responses tended to be to the same epitopes but at generally lower levels than cervical CD8+ T cell responses

- Low risk individuals did not have such CD8+ cells

- CD8+ T cell epitopes: DTVLEDINL (3 individuals), SLYNVATL (4 individuals), LSPRTLNAW (3 individuals) and YPLTFGWCF (4 individuals) were most commonly recognized by the HIV-resistant women

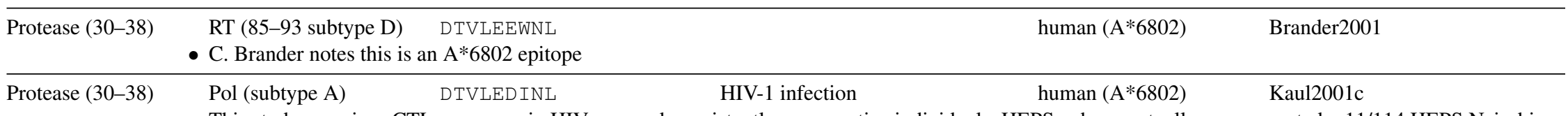

- This study examines CTL responses in HIV exposed, persistently seronegative individuals, HEPS, who eventually seroconverted - 11/114 HEPS Nairobi sex workers eventually seroconverted, and for six of these HIV CTL reactive epitopes had been defined while seronegative

- DTVLEDINL was recognized in 3 of the 6 women (ML857, ML1203, and ML1707), and the response was present in the last available sample prior to seroconversion, 3-7 months 


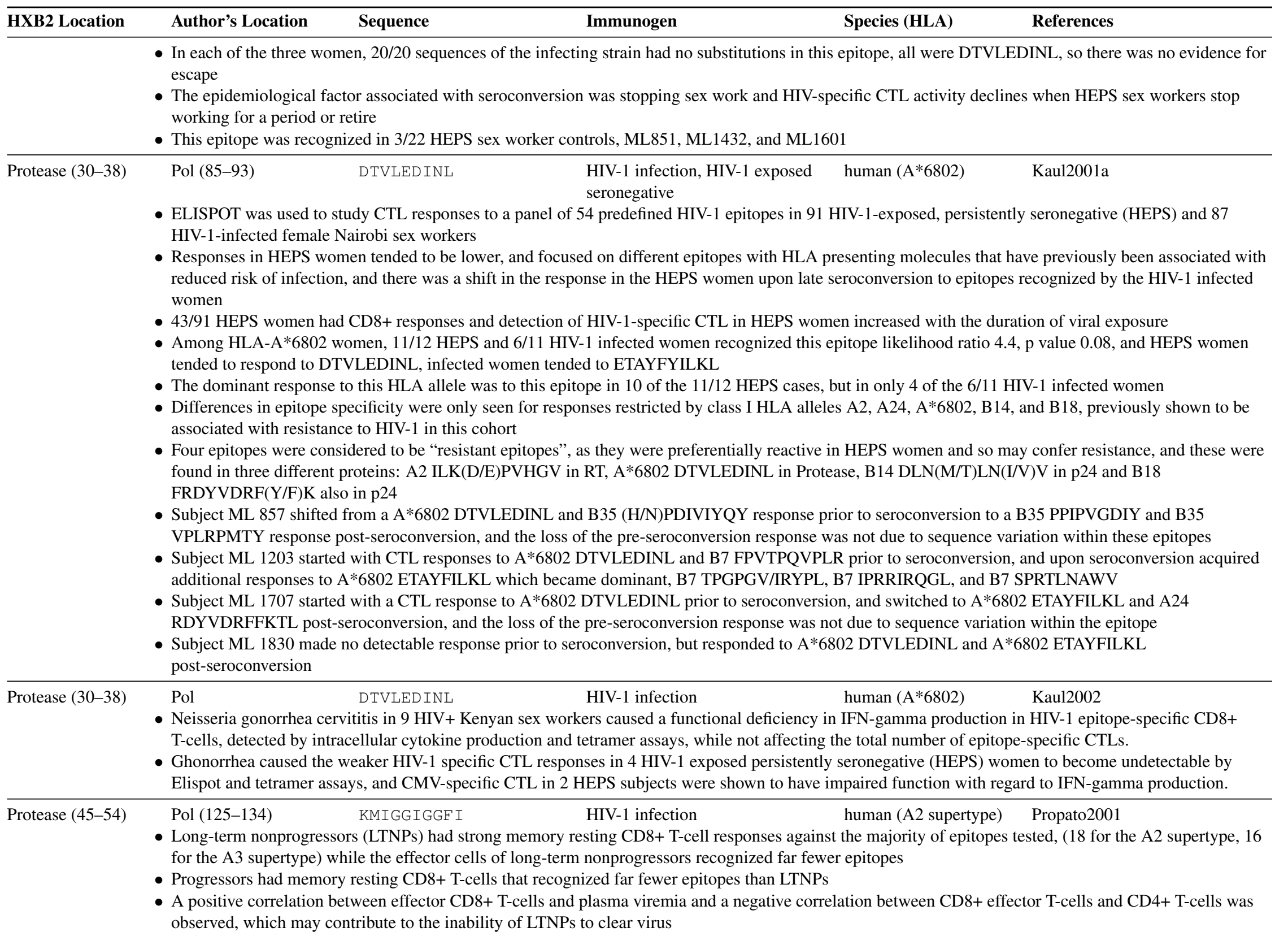




\begin{tabular}{|c|c|c|c|c|c|}
\hline HXB2 Location & Author's Location & Sequence & Immunogen & Species (HLA) & References \\
\hline & \multicolumn{5}{|c|}{ - This epitope can bind three of the five HLA-A2 supertypes alleles (A*0201, A*020 2, A*0203, A*0206 and A*6802) } \\
\hline Protease $(75-84)$ & $\begin{array}{l}\text { Protease (75-84 MN } \\
\text { - Peptide predicted to } \\
\text { - Peptide could stimula } \\
\text { - Peptide located in a } \\
\text { - Both 9-mer and } 10-\mathrm{m} \\
\text { - Binding affinity to A } \\
\text { - MAL variant of } \operatorname{Pr}(7\end{array}$ & $\begin{array}{l}\text { VLVGP TPVNI } \\
\text { active based on } \\
\text { TL in PBMC frc } \\
\text { y conserved regi } \\
\text { ould stimulate C } \\
1 \text { was measured } \\
\text { MN), with subst }\end{array}$ & $\begin{array}{l}\text { in vitro stimulation } \\
\text { inding motif } \\
\text { tive donors } \\
\text { VNI and LVGPTPVN } \\
=6 \text { for } 10-\text { mer, } 3 \text { for } \\
78 \text {, and P79, gave red }\end{array}$ & $\begin{array}{l}\text { human }(\mathrm{A} * 0201) \\
\text { nd CTL recognition }\end{array}$ & Konya1997 \\
\hline
\end{tabular}

Protease $(76-84)$

Pol (163-)

HIV-1 infection

human $(\mathrm{A} * 0201)$

Altfeld2001c

- Epitope name: Pol-163

- HIV was scanned for all peptides which carried the A2-supermotif pattern conserved in more than $50 \%$ of B clade sequences - 233 peptides met this criteria, and 30 of these bound to HLA-A*0201 - 20/30 bound to at least 3/5 of HLA-A2 supertype alleles tested

- Three additional previously described HLA-A2 epitopes were added to the set of 20, and 18/22 chronically infected HLA-A2 individuals had CTL that recognized at least one of the 23 peptides (median of 2 and maximum of 6), while 6/12 acute infected individuals recognized at least 1 (median of 1 and maximum of 2)

- LVGPTPVNI binds to 4/5 HLA-A2 supertype alleles: $\mathrm{A} * 0201, \mathrm{~A} * 0202, \mathrm{~A} * 0206$ (highest affinity) and $\mathrm{A} * 6802$, but not $\mathrm{A} * 0203$

- $1 / 22$ individuals with chronic HIV-1 infection recognized this epitope by ELISPOT

- 0/12 acutely infected individuals recognized this epitope
Protease (76-84)
Pol (156-164)
LVGPTPVNI
HIV-1 infection
human (A2 supertype) Propato2001

- Long-term nonprogressors (LTNPs) had strong memory resting CD8+ T-cell responses against the majority of epitopes tested, (18 for the A2 supertype, 16 for the A3 supertype) while the effector cells of long-term nonprogressors recognized far fewer epitopes

- Progressors had memory resting CD8+ T-cells that recognized far fewer epitopes than LTNPs

- A positive correlation between effector CD8+ T-cells and plasma viremia and a negative correlation between CD8+ effector T-cells and CD4+ T-cells was observed, which may contribute to the inability of LTNPs to clear virus

- This epitope can bind three of the five HLA-A2 supertypes alleles (A*0201, $A * 0202, \mathrm{~A} * 0203, \mathrm{~A} * 0206$ and $\mathrm{A} * 6802$ ) 


\section{II-B-9 Protease-RT CTL Epitopes}

\begin{tabular}{|c|c|c|c|c|c|}
\hline HXB2 Location & Author's Location & Sequence & Immunogen & Species (HLA) & References \\
\hline Protease-RT (95-5) & Gag (175-184) & CTLNFPISPI & HIV-1 infection & human (A2 supertype) & Propato2001 \\
\hline
\end{tabular}

- The epitope starts in Protease and ends in RT

- Long-term nonprogressors (LTNPs) had strong memory resting CD8+ T-cell responses against the majority of epitopes tested, (18 for the A2 supertype, 16 for the A3 supertype) while the effector cells of long-term nonprogressors recognized far fewer epitopes

- Progressors had memory resting CD8+ T-cells that recognized far fewer epitopes than LTNPs

- A positive correlation between effector CD8+ T-cells and plasma viremia and a negative correlation between CD8+ effector T-cells and CD4+ T-cells was observed, which may contribute to the inability of LTNPs to clear virus

- This epitope can bind all five HLA-A2 supertypes alleles $(A * 0201, A * 0202, A * 0203, A * 0206$ and $A * 6802)$

TLNFPISPI HIV-1 infection

human (A2 supertype) Propato2001

- Long-term nonprogressors (LTNPs) had strong memory resting CD8+ T-cell responses against the majority of epitopes tested, (18 for the A2 supertype, 16 for the A3 supertype) while the effector cells of long-term nonprogressors recognized far fewer epitopes

- Progressors had memory resting CD8+ T-cells that recognized far fewer epitopes than LTNPs

- A positive correlation between effector CD8+ T-cells and plasma viremia and a negative correlation between CD8+ effector T-cells and CD4+ T-cells was observed, which may contribute to the inability of LTNPs to clear virus

- This epitope can bind three of the five HLA-A2 supertypes alleles (A*0201, $A * 0202, \mathrm{~A} * 0203, \mathrm{~A} * 0206$ and $\mathrm{A} * 6802$ ) 


\section{II-B-10 RT CTL Epitopes}

\begin{tabular}{|c|c|c|c|c|}
\hline$\overline{\text { HXB2 Location }}$ & Author's Location & Immunogen & Species (HLA) & References \\
\hline RT (3-12) & \multicolumn{4}{|c|}{$\begin{array}{l}\text { - Recognized by CTL from a long-term survivor, EILKEPVGHGV was also recognized } \\
\text { - Highly conserved across clades }\end{array}$} \\
\hline RT (3-12) & \multicolumn{4}{|c|}{$\begin{array}{l}\text { - The program Epimatrix was used in conjunction with the program Conservatrix to identify conservered regions of HIV that might serve as epitopes } \\
\text { - A subset of the potential epitopes was identified that could bind to the appropriate HLA-allele, and } 15 \text { predicted B7 superfamily (HLA B7, B8, and B58) } \\
\text { epitopes could stimulate IFN } \gamma \text { production in an ELISPOT assay } \\
\text { - SPIETVPVKL was newly identified as HLA-B7 epitope in this study, it had been previously shown to be presented by HLA-A2 and B61 }\end{array}$} \\
\hline RT (5-29) & $\begin{array}{l}\text { RT (160-184 HXB2) } \\
\text { - One of five epitopes }\end{array}$ & $\begin{array}{l}\text { IETVPVKLKP GMDGPKVKQ- HIV-1 infection } \\
\text { WPLTEE } \\
\text { ed for RT-specific CTL clones in this study }\end{array}$ & human (B8) & Walker1989 \\
\hline RT (18-26) & $\begin{array}{l}\text { RT }(185-193 \text { LAI }) \\
\text { C. Brander notes this }\end{array}$ & $\begin{array}{l}\text { GPKVKQWPL } \\
\text { B*0801 epitope }\end{array}$ & human $(\mathrm{B} * 0801)$ & Brander2001 \\
\hline RT (18-26) & $\begin{array}{l}\text { RT (18-26) } \\
\text { - HIV proteins with m } \\
\text { - Article reviewed in [ }\end{array}$ & $\begin{array}{l}\text { GP KVKQWPL HIV-1 infection } \\
\text { ons in this epitope allowed transactive inhibition of sp } \\
\text { endez-Arias1998, with a discussion of antagonism }\end{array}$ & $\begin{array}{l}\text { human (B8) } \\
\text { diated lysis }\end{array}$ & $\begin{array}{l}\text { Meier1995 } \\
\text { Menendez-Arias1998 }\end{array}$ \\
\hline RT (18-26) & $\begin{aligned} & \text { RT (173-181) } \\
& \text { - Included in a study o } \\
& \text { - Article reviewed in I } \\
&\end{aligned}$ & $\begin{array}{l}\text { GPKVKQWPL } \\
\text { B8 binding motif } \\
\text { endez-Arias1998, with a discussion of antagonism }\end{array}$ & human (B8) & \begin{tabular}{|l|} 
Goulder1997g \\
Menendez-Arias1998 \\
\end{tabular} \\
\hline RT (18-26) & $\begin{array}{l}\text { RT (185-193 LAI) } \\
\text { - Predicted epitope ba }\end{array}$ & $\begin{array}{l}\text { GPKVKQWPL } \\
\text { n B8-binding motifs, from larger peptide IETVPVKL }\end{array}$ & $\begin{array}{c}\text { human (B8) } \\
\text { KQWPLTEE }\end{array}$ & Sutton1993 \\
\hline RT (18-26) & $\begin{array}{l}\text { RT (185-193 LAI) } \\
\text { - Naturally occurring } \\
\text { - Article reviewed in I }\end{array}$ & $\begin{array}{l}\text { GPKVKQWPL HIV-1 infection } \\
\text { onist GPRVKQWPL found in viral PBMC DNA and } \\
\text { endez-Arias1998| with a discussion of antagonism }\end{array}$ & human (B8) & \begin{tabular}{l|} 
Klenerman1995 \\
Menendez-Arias1998
\end{tabular} \\
\hline RT (18-26) & $\begin{array}{l}\text { RT (18-26) } \\
\text { - This study compares } \\
\text { HIV-uninfected donc } \\
\text { - Strong CTL respons } \\
\text { macrophages were n } \\
\text { - A weak response to } \\
\text { - No detectable respor }\end{array}$ & $\begin{array}{l}\text { GPKVKQWPL in vitro stimulation } \\
\text { bility of macrophages and dendritic cells to stimulate } \\
\text { ing peptide-pulsed APC - the dendritic cells perform } \\
\text { re elicited by the epitopes DRFYKTLRA and GEIYK } \\
\text { le to prime a CTL response against DRFYKTLRA } \\
\text { PCVSL was stimulated using macrophages as the AF } \\
\text { as observed for the following previously-defined HIV }\end{array}$ & $\begin{array}{l}\text { human (B8) } \\
\text { ases in CD8+ lympl } \\
\text { C for the stimulatio } \\
\text { esented by either in } \\
\text { LRPGGK, ILKEPV }\end{array}$ & $\begin{array}{l}\text { Zarling } 1999 \\
\text { isolated from HLA-appropriate } \\
\text { imary responses } \\
\text { e or mature dendritic cells - } \\
\text { IRLRPGGK, GPKVKQWPL }\end{array}$ \\
\hline
\end{tabular}




\begin{tabular}{|c|c|c|c|c|c|}
\hline HXB2 Location & Author's Location & Sequence & Immunogen & Species (HLA) & References \\
\hline RT (18-26) & $\begin{array}{l}\text { RT (185-193) } \\
\text { Epitope name: GPK }\end{array}$ & GPKVKQWP L & HIV-1 infection & human (B8) & Oxenius2000 \\
\hline
\end{tabular}

- Patients who started therapy at acute HIV-1 infection (three with sustained therapy, two with limited therapy upon early infection) had strong HIV specific CD4 proliferative responses and were able to maintain a CTL response even with undetectable viral load - three patients that had delayed initiation of HAART had no HIV specific CD4 proliferative responses and lost their CTL responses when HAART was eventually given and their viral loads became undetectable

- Two of the 7/8 study subjects that were HLA B8+ recognized this epitope

- Patient SC2 (HLA A1, B7/8, Cw0701/0702, DR4/53, DQ7) had CTL responsiveness against epitopes FLKEKGGL, GPKVKQWPL, and GEIYKRWII peptides - FLKEKGGL tetramer staining steadily declined and at day 1340 the FLKEKGGL stained cells were no longer detected and the escape mutant FLKENGGI was found in $8 / 10$ clones

- Patient SC11(HLA A1, B8, Cw0201, DR3/11, DR52, DQ2/7) started therapy early, remained on therapy for 40 days, then reinitiated HAART at day 640 had a CTL response to FLKEKGGL, GPKVKQWPL, and GEIYKRWII throughout and received a benefit from the early limited course therapy

\begin{tabular}{llccc}
\hline RT (18-26) & Pol & GPKVKQWPL & HIV-1 infection human (B8) & Seth2001
\end{tabular}

- CTL responses were studied by tetramer staining in 41 patients with combination therapy - activated CD8+ T-cells decline as the viral load drops in response to therapy, but the overall level of antigen-specific cells capable of differentiating into effectors stays constant and new epitopes may be recognized

RT (18-26) RT (185-193 SF2) GPKVKQWPL HIV-1 infection human (B8) Altfeld2001b

- Therapy provided during acute infection resulted in a narrower CTL response, stronger T help response, and a less diverse viral population than was seen in individuals treated during chronic infection

- The breadth and specificity of the response was determined using ELISPOT by studying 19 individuals with pre-seroconversion therapy (Group 1 ), 11 individuals with primary infection but post-seroconversion therapy (Group 2), and 10 individuals who responded to HAART given during chronic infection (Group 3), using 259 overlapping peptides spanning p17, p24, RT, gp41, gp120 and Nef

- Previously described and newly defined optimal epitopes were tested for CTL response

- Number of HLA-B8+ individuals that had a CTL response to this epitope broken down by group: 0/3 group 1, 0/3 group 2, and 2/2 group 3

\begin{tabular}{llll}
\hline RT (18-26) Pol (171-180) GPKVKQWPL & $\begin{array}{l}\text { HIV-1 infection, HIV-1 exposed human (B8) } \\
\text { seronegative }\end{array}$
\end{tabular}

- GPKVKQWPL is cross-reactive for clades A, B, C, and D

- ELISPOT was used to study CTL responses to a panel of 54 predefined HIV-1 epitopes in 91 HIV-1-exposed, persistently seronegative (HEPS) and 87 HIV-1-infected female Nairobi sex workers

\begin{tabular}{llcccc}
\hline RT (18-26) & RT (18-26) & GPKVKQWPL & HIV-1 infection & human (B8) & Day2001 \\
& $\bullet$ & B8-restricted CTL accounted for about 1/3 of the total CTL response in one individual & & \\
\hline RT (18-26) & RT & GPKVKQWPL & HIV-1 infection & human (B8) & Oxenius2002b \\
\hline
\end{tabular}

- Epitope name: GPK

- Using previously defined epitopes [Oxenius2000 Oxenius2001a] in an IFNgamma Elispot assay, 13 chronically HIV-1 infected patients were studied over a period including therapy with standard treatment interruptions (STI).

- STIs induced increased recognition of CTL epitopes, but there was no correlation between CTL responses with viral rebound rates, plateau viral loads, or clearance rates.

\begin{tabular}{llcc}
\hline RT (18-27) & Pol & GPKVKQWPLT & human (B7, B8) \\
& The program Epimatrix was used in conjunction with the program Conservatrix to identify conservered regions of HIV that might serve
\end{tabular}

- The program Epimatrix was used in conjunction with the program Conservatrix to identify conservered regions of HIV that might serve as epitopes 


\begin{tabular}{|c|c|c|c|c|c|}
\hline HXB2 Location & Author's Location & Sequence & Immunogen & Species (HLA) & References \\
\hline & \multicolumn{5}{|c|}{$\begin{array}{l}\text { - A subset of the potential epitopes was identified that could bind to the appropriate HLA-allele, and } 15 \text { predicted B7 superfamily (HLA B7, B8, and B58) } \\
\text { epitopes were identified that could stimulate IFN } \gamma \text { production in an ELISPOT assay } \\
\text { - GPKVKQWPLT was confirmed as a previously identified HLA-B8 epitope, and newly identified as an HLA-B7 epitope in this study }\end{array}$} \\
\hline $\mathrm{T}(33-41)$ & \multicolumn{5}{|c|}{ - C. Brander notes this is an $A^{*} 0201$ epitope } \\
\hline $\mathrm{T}(33-41)$ & \multicolumn{5}{|c|}{$\begin{array}{l}\text { - This epitope contains the mutation M41L, a mutation induced by nucleosidee reverse transcriptase inhibitors } \\
\text { - Patient 201\#5, (A*0201), was found by ELISPOT to recognize the mutated peptide after zidovudine treatment, but not the wild-type peptide - the mutation } \\
\text { M41L gave an increased A2 binding score (http://bimas.dcrt.nih.gov/molbio/hla_bind) compared to the wildtype RT sequence } \\
\text { - Three additional A*0201 individuals and one B27 individual did not respond to this epitope before or after treatment } \\
\text { - M41L occurred at anchor positions p2 and p9 in several computer predicted RT epitopes (33-41, 32-41, and 40-49) } \\
\text { (http//bimas.dcrt.nih.gov/molbio/hla_bind), and increased the predicted binding affinity for } 6 \text { HLA molecules (B2705, B5102, C3, A0201, B2705 and } \\
\text { B3901) }\end{array}$} \\
\hline RT (33-41) & \multicolumn{5}{|c|}{$\begin{array}{l}\text { - Of } 98 \text { patients in cross-sectional analysis, } 78 \% \text { had CTL against pol - RT was more immunogenic than Integrase and Protease }(81 \%, 51 \% \text {, and } 24 \% \text { of } 37 \\
\text { patients, respectively) } \\
\text { - New clusters of epitopes were defined utilizing different HLA molecules }\end{array}$} \\
\hline $\mathrm{T}(33-41)$ & \multicolumn{5}{|c|}{$\begin{array}{l}\text { - The CTL response to optimally defined CTL epitopes restricted by HLA class I A and B alleles in individuals who coexpressed HLA A2, A3, and B7 was } \\
\text { studied in eight HIV-1-infected subjects, two with acute infection, five with chronic, and one long-term non-progressor (LTNP) } \\
\text { - } 2 \text { to } 17 \text { epitopes were recognized in a given individual, A2-restricted CTL response tended to be narrow and never dominated the response, and } 25 / 27 \\
\text { epitopes were targeted by at least one person } \\
\text { - SLYNTVATL was the dominant A2 epitope recognized in patients with chronic infection, except for Subject } 11841 \text { who recognized } 5 / 8 \text { epitopes and who } \\
\text { had a dominant A-2 response to ALVEICTEM }\end{array}$} \\
\hline RT $(3$ & \multicolumn{5}{|c|}{$\begin{array}{l}\text { - Of } 98 \text { patients in cross-sectional analysis, } 78 \% \text { had CTL against pol - RT was more immunogenic than Integrase and Protease }(81 \%, 51 \% \text {, and } 24 \% \text { of } 37 \\
\text { patients, respectively) } \\
\text { - New clusters of epitopes were defined utilizing different HLA molecules } \\
\text { - C. Brander notes that this is an A*0301 epitope in the } 1999 \text { database, G. Haas pers. comm. }\end{array}$} \\
\hline RT (33-43) & \multicolumn{5}{|c|}{ - C. Brander notes this is an $A^{*} 0301$ epitope } \\
\hline RT (33-43) & $\begin{array}{l}\text { RT (33-43) } \\
\text { - The CTL response to } \\
\text { studied in eight HIV- } \\
\text { - } 2 \text { to } 17 \text { epitopes were } \\
\text { epitopes were targete } \\
\text { - All patients recogniz }\end{array}$ & $\begin{array}{l}\text { ALVE ICTEMEK } \\
\text { mally defined CTL } \\
\text { ected subjects, two } \\
\text { gnized in a given i } \\
\text { at least one person } \\
\text { least } 1 \mathrm{~A} 3 \text { epitope }\end{array}$ & $\begin{array}{l}\text { IIV-1 infection } \\
\text { ted by HLA cla } \\
\text { ction, five with } \\
\text { estricted CTL re } \\
\text { topes, but none }\end{array}$ & $\begin{array}{l}\text { human (A3) } \\
\text { in individuals wh } \\
\text { g-term non-progr } \\
\text { narrow and neve } \\
\text { it }\end{array}$ & $\begin{array}{l}\text { Day2001 } \\
\text { ressed HLA A2, A3, and B7 was } \\
\text { TNP) } \\
\text { ted the response, and 25/27 }\end{array}$ \\
\hline
\end{tabular}




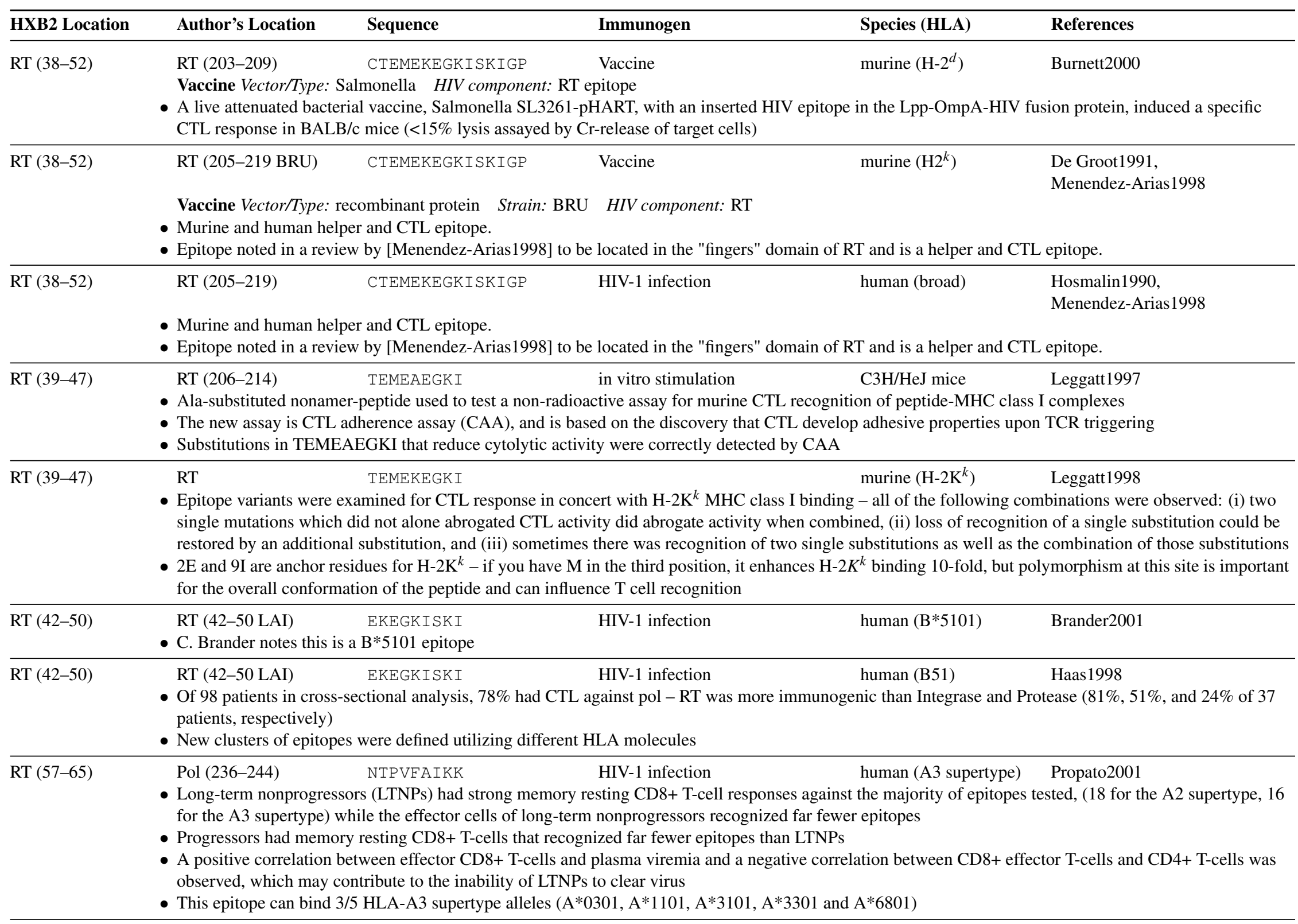




\begin{tabular}{lllll}
\hline HXB2 Location & Author's Location & Sequence & Immunogen & Species (HLA) \\
\hline RT (73-82) & RT (73-82 LAI) & KLVDFRELNK & HIV-1 infection & human (A3) \\
& - This epitope contains the mutation L74V, a frequent mutation induced by nucleoside reverse transcriptase inhibitors & Samri2000 &
\end{tabular}

- This epitope contains the mutation $\mathrm{L} 74 \mathrm{~V}$, a frequent mutation induced by nucleoside reverse transcriptase inhibitors

- The wild-type, but not the mutated peptide, was recognized before and after zidovudine treatment in A3-restricted patients $252 \# 0$ and 252\#4

- Mutation L74V affects the p2 anchor position in RT epitopes and was predicted to reduce binding to A3 (http://bimas.dcrt.nih.gov/molbio/hla_bind)

\begin{tabular}{lllll}
\hline RT (73-82) & RT (228-237) & KLVDFRELNK & HIV-1 infection & Yu2002a
\end{tabular}

- Epitope name: A3-KK10

- CTL responses in 18 acutely HIV-infected HLA-A3 $(n=7)$ or -B7 $(n=4)$ or both -A3 and B7 $(n=7)$ positive individuals were studied.

- One individual, AC-06, was homozygous at all three class I alleles (A3, B7, Cw7), was treated during acute infection and had supervised treatment interruptions (STI). He had only two detectable CTL responses during acute infection, but after STI this broadened to 27 distinct epitopes including 15 restricted by HLA-A3, 11 by HLA-B7, and 1 by HLA-Cw7.

- 0/14 HLA-A3 positive individuals had detectable A3-restricted responses to this epitope during acute infection, but only 5/15 of HLA-A3 epitopes tested were targeted during acute infection. 3/7 individuals began to have detectable responses to this epitope after STI.

\begin{tabular}{llllll}
\hline RT (93-101) & (LAI) & GIPHPAGLK & (A3) & Altfeld2000a, Brander2001 \\
\hline RT (93-101) & RT (248-257) & GIPHPAGLK & HIV-1 infection & human (A3) & Yu2002a
\end{tabular}

- Epitope name: A3-GK9

- CTL responses in 18 acutely HIV-infected HLA-A3 $(n=7)$ or -B7 $(n=4)$ or both -A3 and B7 $(n=7)$ positive individuals were studied.

- One individual, AC-06, was homozygous at all three class I alleles (A3, B7, Cw7), was treated during acute infection and had supervised treatment interruptions (STI). He had only two detectable CTL responses during acute infection, but after STI this broadened to 27 distinct epitopes including 15 restricted by HLA-A3, 11 by HLA-B7, and 1 by HLA-Cw7.

- 0/14 HLA-A3 positive individuals had detectable A3-restricted responses to this epitope during acute infection, but only 5/15 of HLA-A3 epitopes tested were targeted during acute infection. 1/7 individuals began to have detectable responses to this epitope after STI.

\begin{tabular}{lllll}
\hline RT (93-102) & $\begin{array}{l}\text { Pol (240-249 93TH253 } \\
\text { subtype CRF01) }\end{array}$ & GIPHPAGLKK & HIV-1 exposed seronegative & human (A11)
\end{tabular}

- Epitope name: P248-257

- This was a study of HIV-1 exposed persistently seronegative (HEPS) female sex workers in Chiang Mai, northern Thailand

- HLA-A11 is very common in this population, and was enriched among the HEPS sexworkers - weak CTL responses were detected in 4/7 HEPS women, and CTL responses were found in 8/8 HIV+ controls, and 0/9 HIV- women that were not exposed

- This epitope was weakly reactive in the HEPS study subject 265 who was HLA A2/A11 and after a second stimulation in vitro gave a strong response in HEPS study subject 128 who was HLA A11/A33

\begin{tabular}{|c|c|c|c|}
\hline RT (93-102) & $\begin{array}{l}\text { Pol (240-249 93TH253 } \\
\text { subtype CRF01) }\end{array}$ & GIPHPAGLKK & HIV-1 infection \\
\hline
\end{tabular}

- HLA-A11 CRF01 (called subtype E in Bond et al.) epitopes were identified that stimulated CTL from HIV+ female sex workers (FSW) from Northern Thailand, of whom more than half were HLA-A11 positive

- 77 possible HLA-A11 epitopes were first defined using EpiMatrix, these were screened for binding to A11 finding and 26 bound, and 12 of these were epitopes for CTL responses from 8 HLA-A11 positive FSWs, six were novel, six were previously identified

- This is one of the new A11 epitopes identified through the streamlined EpiMatrix method, and 2/8 tested FSWs recognized it

- This epitope was highly conserved in other subtypes, and exact matches were common 


\begin{tabular}{|c|c|c|c|c|c|}
\hline HXB2 Location & Author's Location & Sequence & Immunogen & Species (HLA) & References \\
\hline RT (98-113) & \multicolumn{5}{|c|}{$\begin{array}{l}\text { - This study employs an antigenic similarity matrix to compare HIV-1 antigenic determinants with human proteins. } \\
\text { - This CTL epitope (the HIV-1 LAI fragment with high similarity to a human protein overlapping this epitope is GLKKKKSVTVL) has similarity with the } \\
\text { CD166 antigen (activated leukocyte-cell adhesion molecule), fragment GLKKRESLTLI. }\end{array}$} \\
\hline RT (98-113) & \multicolumn{5}{|c|}{$\begin{array}{l}\text { - This study focuses on six rare long-term survivor HIV-infected people who were infected for many years without exhibiting immune dysregulation - such } \\
\text { immunologically normal HIV-infected (INHI) cases occur at a frequency between } 0.1 \text { and } 1 \% \text { in the infected population } \\
\text { - No direct CTL were found in any of the six INHIs, but above background CTLp activity was founded in } 3 / 6 \text { INHIs }\end{array}$} \\
\hline RT (103-117) & \multicolumn{5}{|c|}{$\begin{array}{l}\text { - This study focuses on six rare long-term survivor HIV-infected people who were infected for many years without exhibiting immune } \\
\text { - No direct CTL were found in any of the six INHIs, but above background CTLp activity was founded in 3/6 INHIs }\end{array}$} \\
\hline RT (107-115) & \multicolumn{5}{|c|}{ - C. Brander notes this is a B*3501 epitope } \\
\hline RT (107-115) & \multicolumn{5}{|c|}{$\begin{array}{l}\text { - Epitope defined in the context of the Pediatric AIDS Foundation ARIEL Project, a mother-infant HIV transmission study } \\
\text { - TVLDMGDAC is a naturally occurring variant that is less reactive } \\
\text { - [Menendez-Arias1998], in a review, notes that this epitope includes a catalytic residue (Asp-110) in the active site of RT }\end{array}$} \\
\hline
\end{tabular}

RT (107-115)
Pol (262-270 IIIB)
TVLDVGDAY
HIV-1 infection
- This study describes maternal CTL responses in the context of mother-to-infant transmission

human (B35)

Wilson1999a

- Detection of CTL escape mutants in the mother was associated with transmission, but the CTL-susceptible forms of the virus tended to be found in infected infants

- An additional variant that gave a positive CTL response: TVLDMGDAC

RT (107-115) Pol (262-270) TVLDVGDAY HIV-1 infection $\quad$ Ferrari2000

- One of the 51 HIV-1 epitopes selected by Ferrari et al. as good candidate CTL epitopes for vaccines by virtue of being conserved and presented by common HLA alleles

\begin{tabular}{lllll}
\hline RT (107-115) & RT (262-270 SF2) TVLDVGDAY & HIV-1 infection & human (B35)
\end{tabular}

- Therapy provided during acute infection resulted in a narrower CTL response, stronger T help response, and a less diverse viral population than was seen in individuals treated during chronic infection

- The breadth and specificity of the response was determined using ELISPOT by studying 19 individuals with pre-seroconversion therapy (Group 1), 11 individuals with primary infection but post-seroconversion therapy (Group 2), and 10 individuals who responded to HAART given during chronic infection (Group 3), using 259 overlapping peptides spanning p17, p24, RT, gp41, gp120 and Nef

- Previously described and newly defined optimal epitopes were tested for CTL response

- Number of HLA-B35+ individuals that had a CTL response to this epitope broken down by group: $1 / 2$ group 1,0/2 group 2, and 0/1 group 3

human (B35)

Sabbaj2002b

- Epitope name: Pol-TY9 


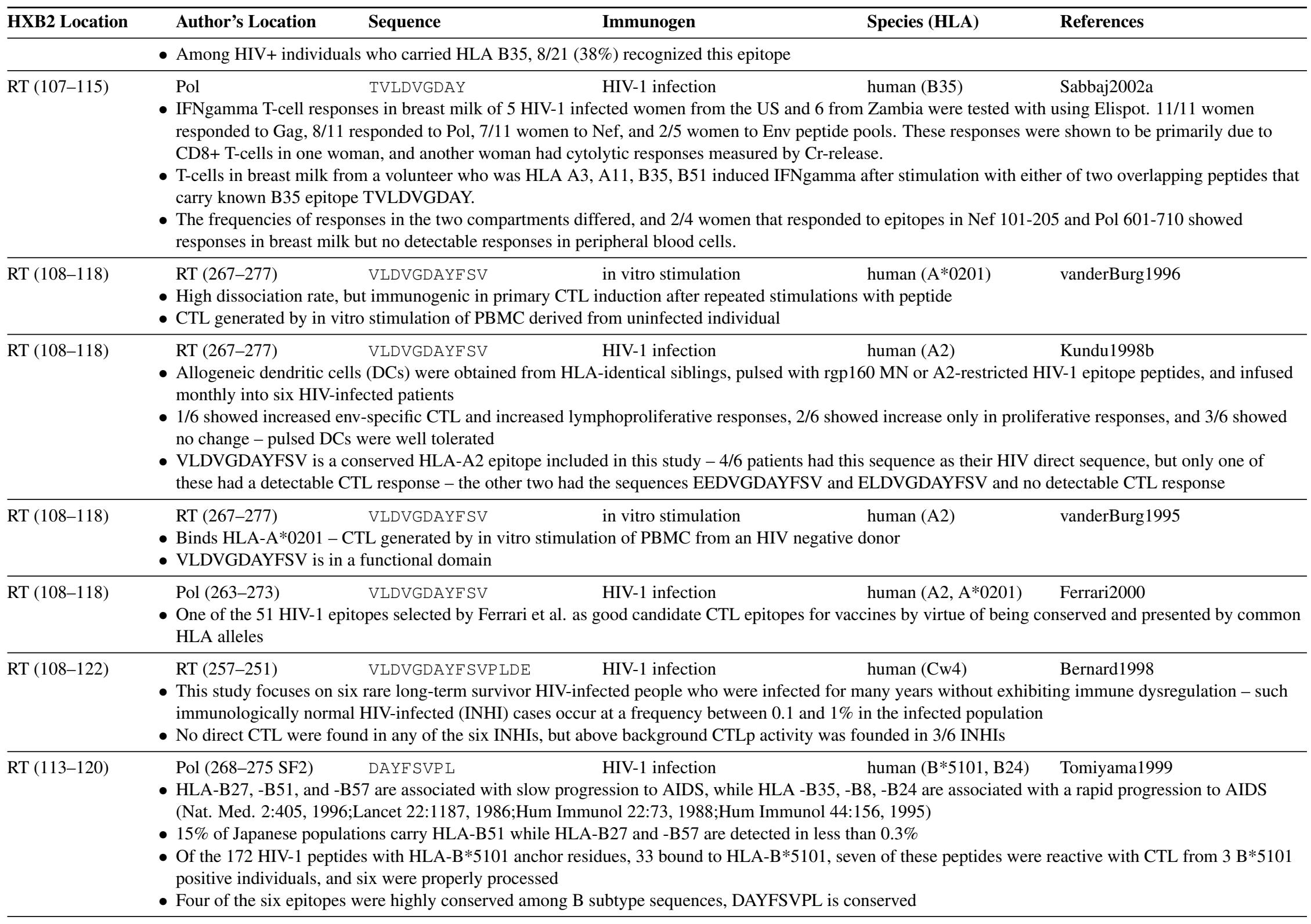




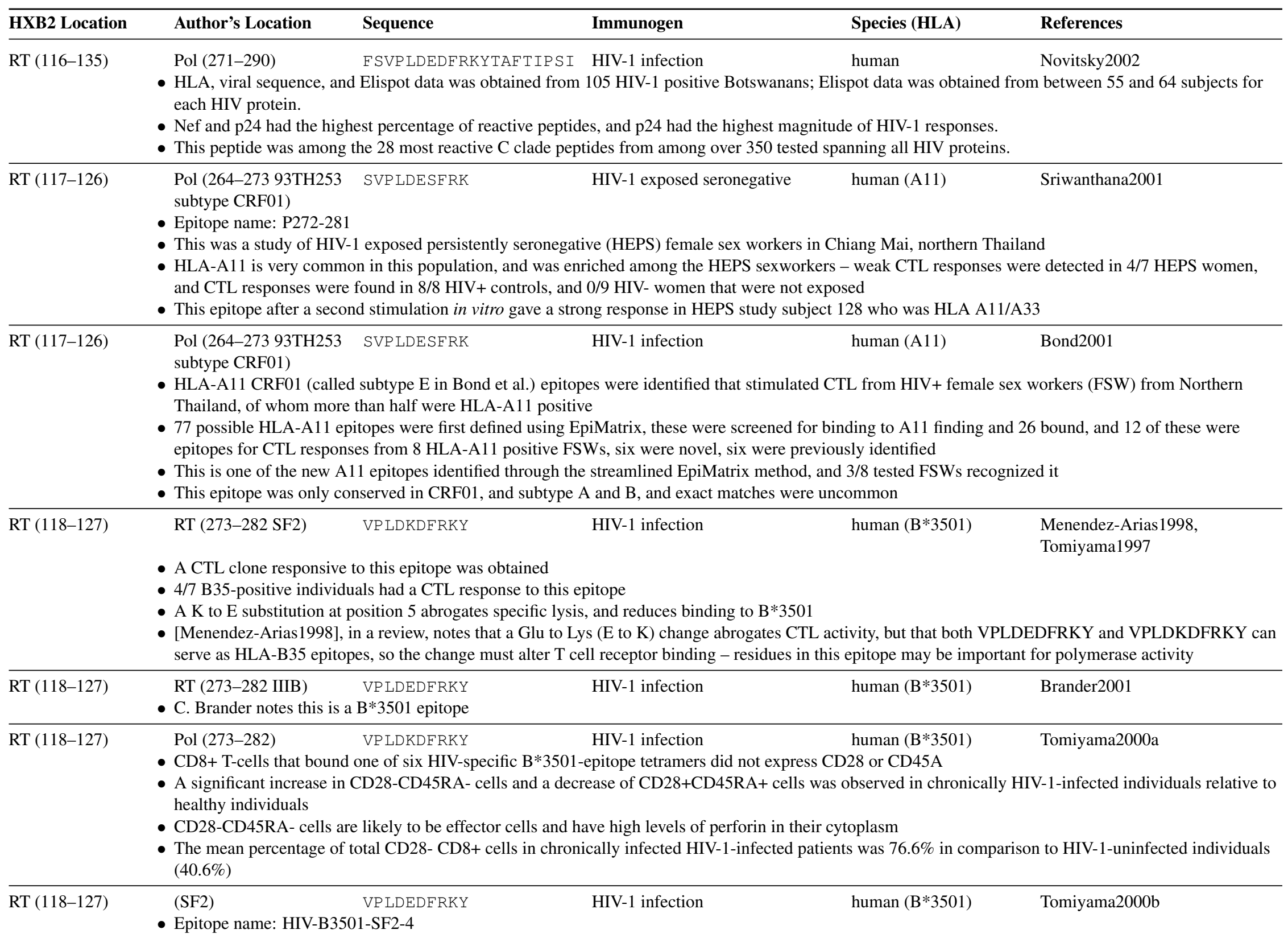




\begin{tabular}{|c|c|c|c|c|c|}
\hline HXB2 Location & Author's Location & Sequence & Immunogen & Species (HLA) & References \\
\hline & \multicolumn{5}{|c|}{$\begin{array}{l}\text { - B*3501 VPLDEDFRKY tetramer binding did not inhibit CTL activity of a clone that react with both HLA-B*3501 than HLA-B*5101 presentation of the } \\
\text { epitope IPLTEEAEL }\end{array}$} \\
\hline RT (118-127) & $\begin{array}{l}\text { RT (273-282 IIIB) } \\
\text { Binds HLA-B*3501 }\end{array}$ & VPLDEDFRKY & HIV-1 infection & human $(\mathrm{B} * 3501, \mathrm{~B} 35)$ & Shiga1996 \\
\hline RT (118-127) & $\begin{array}{l}\text { (SF2) } \\
\text { - HLA B35 is associat } \\
\text { - } \text { The sequences of } 9 \mathrm{p} \\
\text { 3/9 CTL epitopes hac } \\
\text { peptide to B35 and w }\end{array}$ & $\begin{array}{l}\text { VPLDKDFRKY } \\
\text { ith rapid disease } \\
\text { usly described H } \\
\text { stitutions that we } \\
\text { own to be an esc }\end{array}$ & $\begin{array}{l}\text { HIV-1 infection } \\
\text { epitopes were obt } \\
\text { on in B35+ individ }\end{array}$ & $\begin{array}{l}\text { human (B35) } \\
\text { 35+ and } 19 \text { HLA B35- i } \\
\text { individuals - only one of }\end{array}$ & $\begin{array}{l}\text { Kawana1999 } \\
\text { lividuals } \\
\text { nese reduced the binding of the }\end{array}$ \\
\hline
\end{tabular}

- - E- was found in 8/10 of the B35+ individuals, and three of the B35- individuals - the D $\rightarrow$ E substituted peptide had similar binding affinity to B35 and was equally susceptible to a CTL clone

\begin{tabular}{lllll}
\hline RT (118-127) RT (273-282 IIIB) & VPLDEDFRKY & HIV-1 infection & hipsas1997 (B35)
\end{tabular}

- HIV IIIB proteins were used to define the range of CTL epitopes recognized by three lab workers accidentally infected with HIV-1 IIIB

- VPLDKDFRKY, a variant found in HIV MN, was not recognized

- VPHDEDFRKY, a variant found in HIV YU2, was not recognized

- This epitope was type-specific and conserved in only one other B subtype sequence

RT (118-127) RT (273-282 SF2) VPLDEDFRKY HIV-1 infection human (B35) Altfeld2001b

- Therapy provided during acute infection resulted in a narrower CTL response, stronger T help response, and a less diverse viral population than was seen in individuals treated during chronic infection

- The breadth and specificity of the response was determined using ELISPOT by studying 19 individuals with pre-seroconversion therapy (Group 1), 11 individuals with primary infection but post-seroconversion therapy (Group 2), and 10 individuals who responded to HAART given during chronic infection (Group 3), using 259 overlapping peptides spanning p17, p24, RT, gp41, gp120 and Nef

- Previously described and newly defined optimal epitopes were tested for CTL response

- Number of HLA-B35+ individuals that had a CTL response to this epitope broken down by group: 1/2 group 1,0/2 group 2, and 1/1 group 3

RT (118-127)$$
\text { VPLDEDFRKY }
$$$$
\text { HIV-1 infection }
$$

human (B35)

Sabbaj2002b

- Epitope name: Pol-VY10

- Among HIV+ individuals who carried HLA B35, 5/21 (24\%) recognized this epitope

RT (126-135) RT (293-302 HXB) KYTAFTIPSI HIV-1 infection $\quad$ Shankar1998

- A novel CTL clone was defined with a panel of recombinant vaccinia-RT-infected B-LCL target cells using PBMCs donated by a patient who was HIV-seropositive for 6 years and had not received any antiretroviral therapy

- There is evidence that some CTL epitopes are poorly presented on the surface of infected cells, but this RT epitope was recognized as effectively on HIV-infected cells as on peptide-pulsed targets

\begin{tabular}{lllll}
\hline RT (127-135) & Pol (316-) & YTAFTIPSI & human (A2)
\end{tabular}

- Epitope name: Pol-316

- HIV was scanned for all peptides which carried the A2-supermotif pattern conserved in more than $50 \%$ of B clade sequences - 233 peptides met this criteria, and 30 of these bound to HLA-A $* 0201-20 / 30$ bound to at least $3 / 5$ of HLA-A2 supertype alleles tested 


\begin{tabular}{|c|c|c|c|c|c|}
\hline HXB2 Location & Author's Location & Sequence & Immunogen & Species (HLA) & References \\
\hline & \multicolumn{5}{|c|}{$\begin{array}{l}\text { - Three additional previously described HLA-A2 epitopes were added to the set of } 20 \text {, and } 18 / 22 \text { chronically infected HLA-A2 individuals had CTL that } \\
\text { recognized at least one of the } 23 \text { peptides (median of } 2 \text { and maximum of } 6 \text { ), while } 6 / 12 \text { acute infected individuals recognized at least } 1 \text { (median of } 1 \text { and } \\
\text { maximum of } 2 \text { ) } \\
\text { - } 2 / 22 \text { individuals with chronic HIV- } 1 \text { infection recognized this epitope in ELISPOT } \\
\text { - } 0 / 12 \text { acutely infected individuals recognized this epitope } \\
\text { - YTAFTIPSI binds to five HLA-A2 supertype alleles: A*0201, A*0202,A*0203, A*0206 and A*6802 (highest affinity) }\end{array}$} \\
\hline RT (127-135) & \multicolumn{5}{|c|}{$\begin{array}{l}\text { Pol (306-314) HIV-1 infection } \\
\text { - Long-term nonprogressors (LTNPs) had strong memory resting CD8+ T-cell responses against the majority of epitopes tested, (18 for the A2 supertype, } 16 \\
\text { for the A3 supertype) while the effector cells of long-term nonprogressors recognized far fewer epitopes } \\
\text { - Progressors had memory resting CD8+ T-cells that recognized far fewer epitopes than LTNPs } \\
\text { - A positive correlation between effector CD8+ T-cells and plasma viremia and a negative correlation between CD8+ effector T-cells and CD4+ T-cells was } \\
\text { observed, which may contribute to the inability of LTNPs to clear virus } \\
\text { - This epitope can bind all five HLA-A2 supertypes alleles (A*0201, A*0202, A*0203, A*0206 and A*6802) }\end{array}$} \\
\hline RT (128-135) & \multicolumn{5}{|c|}{$\begin{array}{l}\text { - Epitope name: Pol-TI8 } \\
\text { - This study monitored epitope responses in HIV-1 infected minority women living in the United States } \\
\text { - } 24 \text { epitopes were described - } 8 \text { were novel, } 8 \text { used new restricting elements but were previously defined epitopes, and } 8 \text { were previously described } \\
\text { - Serial peptide truncations were used to define optimal epitopes for CTL cell lines isolated from } 12 \text { individuals, assayed by a Cr-release } \\
\text { - Patient 01RCH46 was Hispanic, on HAART, and had a viral load of } 21000 \text { and CD4 count of } 623 \text { - she also recognized GELDRWEKI, p17(11-19), HLA } \\
\text { B*4002, and KETINEEAA p24(70-78), HLA B*4002 } \\
\text { - Among HIV+ individuals who carried HLA A*02,7/36 (19\%) recognized this epitope, two of which also carried B*5101 which can also restrict this } \\
\text { epitope }\end{array}$} \\
\hline RT (128-135) & \multicolumn{5}{|c|}{ - C. Brander notes this is a $\mathrm{B} * 5101$ epitope } \\
\hline RT (128-135) & $\begin{array}{l}\text { Pol (283-290 SF2) } \\
\text { - HLA-B27, -B51, an } \\
\text { Med. 2:405, 1996;L } \\
\text { - } 15 \% \text { of Japanese po } \\
\text { - Of the } 172 \text { HIV-1 pe } \\
\text { positive individuals, } \\
\text { - Four of the six epito }\end{array}$ & $\begin{array}{l}\text { TAFTIPS I } \\
7 \text { are associated } \\
\text { 22:1187, 1986; } \\
\text { ons carry HLA- } \\
\text { with HLA-B* } \\
\text { ix were properl } \\
\text { ere highly cons }\end{array}$ & $\begin{array}{l}\text { HIV-1 infection } \\
\text { ession to AIDS, w } \\
2: 73,1988 ; \text { Hum I } \\
\text {-B27 and -B57 are } \\
\text { dues, } 33 \text { bound to } \\
\text { subtype sequences }\end{array}$ & $\begin{array}{l}\text { human }(\mathrm{B} * 5101) \\
\text {, -B24 are associa } \\
95) \\
\text { an } 0.3 \% \\
\text { en of these peptide } \\
\text { omewhat variable }\end{array}$ & $\begin{array}{l}\text { Tomiyama1999 } \\
\text { a rapid progression to AIDS (Nat. } \\
\text { eactive with CTL from } 3 \mathrm{~B} * 5101\end{array}$ \\
\hline RT (128-135) & $\begin{array}{l}\text { RT (295-302) } \\
\text { - Epitope name: P5 } \\
\text { - The epitope TAFTIF }\end{array}$ & $\begin{array}{l}\text { TAFTIPS I } \\
\text { is recognized b }\end{array}$ & $\begin{array}{l}\text { HIV-1 infection } \\
\text { n a study of the ef }\end{array}$ & $\begin{array}{l}\text { human }(\mathrm{B} * 5101) \\
\text { ape mutations on }\end{array}$ & $\begin{array}{l}\text { Samri2000 } \\
\text { ognition }\end{array}$ \\
\hline
\end{tabular}




\begin{tabular}{|c|c|c|c|c|}
\hline HXB2 Location & Author's Location & Sequence & Species (HLA) & References \\
\hline Т (128-135) & \multicolumn{4}{|c|}{$\begin{array}{l}\text { - HIV polymorphisms in the RT protein were examined relation to HLA alleles found in a population of } 473 \text { participants in the Western Australian HIV } \\
\text { Cohort Study. } 64 \text { significant associations between polymorphisms at particular positions and HLA alleles were detected, for HLA-B7, -B12, -B35 and } \\
\text {-B15. Fifteen of these were in positions with known epitopes, } 4 \text { in anchor residues, } 11 \text { in other positions. Six additional polymorphic sites associated with } \\
\text { particular HLA molecules flanked known epitopes and may relate to processing. } \\
\text { - TAFTIPSI was one of two epitopes characterized in detail. C-terminal I135x substitutions were associated with people who carried HLA-B5 - 39/40 ( } 98 \% \text { ) } \\
\text { of HLA-B*5101 individuals had substitutions in this position, while only } 127 / 431(29 \%) \text { who did not have HLA-B*5101 did. The predominant substitution } \\
\text { was kytaftipsT, and this mutation is predicted to abrogate binding to HLA-B*5101. }\end{array}$} \\
\hline RT (128-135) & \multicolumn{4}{|c|}{$\begin{array}{l}\text { - HIV IIIB proteins were used to define the range of CTL epitopes recognized by } 3 \text { lab workers accidentally infected with HIV-1 IIIB } \\
\text { - TAFTIPST, a variant found in HIV-1 CAM1, was also recognized but } 100 \text {-fold more peptide was needed } \\
\text { - TAFTIPSV, a variant found in HIV-1 VE1RT, was also recognized, but } 10 \text {-fold more peptide was needed } \\
\text { - TVFTIPSI, a variant found in HIV-1 MANC, was also recognized } \\
\text { - Menendez-Arias1998, in a review, notes that this epitope includes a region near the active site of RT - the substitution of the position two conservative } \\
\text { change from A to V decreases CTL recognition }\end{array}$} \\
\hline RT (128-135) & $\begin{array}{l}\text { RT }(295-302) \\
\text { - Only } 4 / 11 \text { HLA-A2+ } \\
\text { - } 95 \text { optimally-defined } \\
\text { - Three of the four ind } \\
\text { epitope as well as otl }\end{array}$ & $\begin{array}{l}\text { TAFTIPSI } \\
+ \text { individuals } \\
\text { ides from this } \\
\text { als that respo } \\
\text { itopes }\end{array}$ & $\begin{array}{l}\text { HIV-1 infection } \\
\text { ted to SLYNTVATL, calling into question whether it is } \\
\text { d to screen for INF } \gamma \text { responses to other epitopes } \\
\text { TL recognized additional HIV epitopes, and all three we }\end{array}$ & $\begin{array}{l}\text { Betts2000 } \\
\text { odominant } \\
\text { HLA B51 and recognized this }\end{array}$ \\
\hline RT (128-135) & $\begin{array}{l}\text { RT (295-302) } \\
\text { - Epitope name: TAF } \\
\text { - Patients who started } \\
\text { CD4 proliferative res } \\
\text { HAART had no HIV } \\
\text { undetectable } \\
\text { - None of the } 8 \text { study }\end{array}$ & $\begin{array}{l}\text { TAFTIPSI } \\
\text { py at acute H } \\
\text { es and were a } \\
\text { ific CD4 prol } \\
\text { cts recognizec }\end{array}$ & $\begin{array}{l}\text { HIV-1 infection human (B51) } \\
\text { e with sustained therapy, two with limited therapy upo } \\
\text { TL response even with undetectable viral load - three } \\
\text { and lost their CTL responses when HAART was event } \\
\text { ne were HLA B51+ }\end{array}$ & $\begin{array}{l}\text { Oxenius } 2000 \\
\text { ffection) had strong HIV specific } \\
\text { that had delayed initiation of } \\
\text { en and their viral loads became }\end{array}$ \\
\hline RT (128-135) & $\begin{array}{l}\text { RT (295-302 LAI) } \\
\text { - Epitope name: P5 } \\
\text { - A panel of } 16 \text { epitop } \\
\text { CD8+ cell IFNgamm } \\
\text { - In general, during th } \\
\text { specificities that wer } \\
\text { HIV-specific respons } \\
\text { - Viral rebounds gave }\end{array}$ & $\begin{array}{l}\text { TAFT IP S I } \\
\text { vering } 15 \text { clas } \\
\text { duction to } \mathrm{m} \\
\text { month of tre } \\
\text { previously de } \\
\text { minished } \\
\text { ent patterns o }\end{array}$ & $\begin{array}{l}\text { HIV-1 infection } \\
\text { d in } 14 \mathrm{HIV}+\text { patients from an unselected Caucasian po } \\
\text { ecreased and frequencies of HIV-specific CTL tripled an } \\
\text { y detected, as were CMV specific CD8+ PBL - but witl } \\
\text { es or decreases in pre-existing response, new specificiti }\end{array}$ & $\begin{array}{l}\text { Mollet2000 } \\
\text { on treated with HAART, using } \\
\text { dened - eight new HIV } \\
\text { nued viral suppression, } \\
\text { lo change }\end{array}$ \\
\hline
\end{tabular}




\begin{tabular}{llllll}
\hline HXB2 Location & Author's Location & Sequence & Immunogen & Species (HLA) & References \\
\hline RT (128-135) & Pol & TAFT IP S & HIV-1 infection & human (B51) & Sabbaj2002a
\end{tabular}

- IFNgamma T-cell responses in breast milk of 5 HIV-1 infected women from the US and 6 from Zambia were tested with using Elispot. 11/11 women responded to Gag, 8/11 responded to Pol, 7/11 women to Nef, and 2/5 women to Env peptide pools. These responses were shown to be primarily due to CD8+ T-cells in one woman, and another woman had cytolytic responses measured by Cr-release.

- T-cells in breast milk from a volunteer who was HLA A3, A11, B35, B51 induced IFNgamma after stimulation with either of two overlapping peptides that carry known B51 epitope TAFTIPSI.

- The frequencies of responses in the two compartments differed, and 2/4 women that responded to epitopes in Nef 101-205 and Pol 601-710 showed responses in breast milk but no detectable responses in peripheral blood cells.

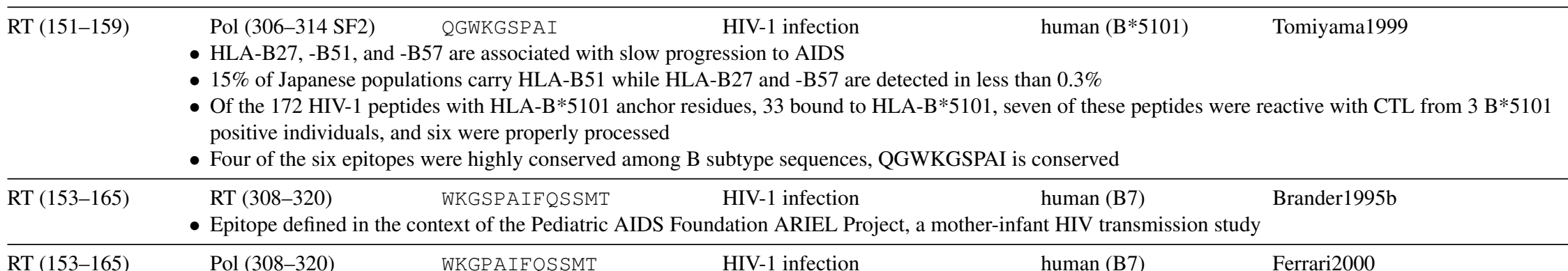

- One of the 51 HIV-1 epitopes selected by Ferrari et al. as good candidate CTL epitopes for vaccines by virtue of being conserved and presented by common HLA alleles

RT (153-167) RT (SF2) WKGSPAIFQSSMTKI HIV-1 infection human Altfeld2001a

- HIV+ individual AC-06 was tested for reactive overlapping peptides spanning all HIV-1 proteins in an ELISPOT and was found to react with 12 peptides from 7 proteins, suggesting that the breadth of CTL responses are underestimated if accessory proteins are not included in the study

- RT peptides SQIYPGIKVRQLCKL and WKGSPAIFQSSMTKI were recognized

RT (156-164) RT (311-319 SF2) SPAIFQSSM HIV-1 infection $\quad$ Menendez-Arias1998,

- A CTL clone responsive to this epitope was obtained

- Only 1/7 B35-positive individuals had a CTL response to this epitope

- [Menendez-Arias1998], in a review, notes that this epitope is near the active site of RT

\begin{tabular}{lllll}
\hline RT (156-164) RT (311-319 SF2) SPAIFQSSM & HIV-1 infection & human (B35) \\
\hline
\end{tabular}

- Binds HLA-B*3501

- [Menendez-Arias1998], in a review, notes that this epitope includes catalytic residues in the active site of RT

RT (156-164) Pol (311-319) SPAIFQSSM HIV-1 infection $\quad$ Ferrari2000

- One of the 51 HIV-1 epitopes selected by Ferrari et al. as good candidate CTL epitopes for vaccines by virtue of being conserved and presented by common HLA alleles

RT (156-164) Pol (156-164 HXB2) SPAIFQSSM $\quad$ HIV-1 infection human (B7) Hay 1999b

- CTL response to IPRRIRQGL was the immunodominant response in a rapid progressor - there was a subdominant response to SPAIFQSSM in Pol, and interestingly, no response to commonly immunodominant HLA A*0201 epitope SLYNTVATL, although this individual was HLA A*0201 


\begin{tabular}{|c|c|c|c|c|}
\hline HXB2 Location & Author's Location & Sequence & Species (HLA) & References \\
\hline & \multicolumn{4}{|c|}{$\begin{array}{l}\text { - The individual showed a strong initial CTL response at the time of the initial drop in viremia, but it was quickly lost, although memory cells persisted } \\
\text { - Despite the initial narrow response to two epitopes, no other CTL responses developed } \\
\text { - No HIV-specific lymphoproliferative responses were detected in this patient, and neutralizing antibody response was weak } \\
\text { - Variants of this epitopes were observed in vivo (spaifqCsm, spSifqssm), but the binding motifs for B7 were preserved (P2, and C-term aromatic or } \\
\text { hydrophobic) }\end{array}$} \\
\hline RT (156-164) & \multicolumn{4}{|c|}{$\begin{array}{l}\text { - Subject } 053 \mathrm{i} \text { was followed longitudinally from acute infection through death, and had rapid progression to AIDS } \\
\text { - This individual had a dominant response to IPRRIRQGL with strong in vivo activated responses and in vitro stimulated memory responses and a } \\
\text { subdominant response to SPAIFQSSM - during the course of disease progression (4 Years), the functional CTL responses were lost and no sequence } \\
\text { variation occurred with in both epitopes, but CTL clones specific for IPRRIRQGL persisted throughout }\end{array}$} \\
\hline RT (156-164) & \multicolumn{4}{|c|}{$\begin{array}{l}\text { - Therapy provided during acute infection resulted in a narrower CTL response, stronger T help response, and a less diverse viral population than was seen in } \\
\text { individuals treated during chronic infection } \\
\text { - The breadth and specificity of the response was determined using ELISPOT by studying } 19 \text { individuals with pre-seroconversion therapy (Group } 1 \text { ), } 11 \\
\text { individuals with primary infection but post-seroconversion therapy (Group 2), and } 10 \text { individuals who responded to HAART given during chronic infection } \\
\text { (Group 3), using } 259 \text { overlapping peptides spanning p17, p24, RT, gp41, gp } 120 \text { and Nef } \\
\text { - Previously described and newly defined optimal epitopes were tested for CTL response } \\
\text { - Number of HLA-B7+ individuals that had a CTL response to this epitope broken down by group: } 0 / 4 \text { group } 1,0 / 3 \text { group } 2 \text {, and } 1 / 1 \text { group } 3\end{array}$} \\
\hline RT (156-164) & $\begin{array}{l}\text { RT (156-164) } \\
\text { Epitope name: B7-S } \\
\text { CTL responses in } 18 \\
\text { One individual, AC- } \\
\text { interruptions (STI). } \\
\text { restricted by HLA-A } \\
1 / 11 \text { HLA-B7 indivi } \\
\text { at least one person d }\end{array}$ & $\begin{array}{l}\text { SPAIFQSSM } \\
\text { ely HIV-infected } \\
\text { as homozygous a } \\
\text { d only two detect } \\
\text { by HLA-B7, and } \\
\text { had detectable B } \\
\text { acute infection. }\end{array}$ & $\begin{array}{l}\text { HIV-1 infection human (B7) } \\
\text { or -B7 ( } \mathrm{n}=4) \text { or both -A3 and B7 }(\mathrm{n}=7) \text { positive indivi } \\
\text { I alleles (A3, B7, Cw7), was treated during acute infec } \\
\text { nses during acute infection, but after STI this broaden } \\
7 \text {. } \\
\text { ponses to this epitope during acute infection - 10/15 of } \\
\text { had detectable responses to this epitope after STI. }\end{array}$ & $\begin{array}{l}\text { Yu2002a } \\
\text { re studied. } \\
\text { had supervised treatment } \\
\text { distinct epitopes including } 15 \\
7 \text { epitopes tested were targeted by }\end{array}$ \\
\hline RT (156-165) & $\begin{array}{l}\text { RT (311-319 LAI) } \\
\text { - Epitope name: P4 } \\
\text { - This epitope contain } \\
\text { It was recognized by }\end{array}$ & $\begin{array}{l}\text { SPAIFQSSMT } \\
\text { mutation P157S } \\
\text { nt } 252 \# 0 \text { in a stu }\end{array}$ & $\begin{array}{l}\text { HIV-1 infection } \\
\text { duced by nucleosidee reverse transcriptase inhibitors } \\
\text { s of therapy escape mutations on CTL recognition }\end{array}$ & Samri2000 \\
\hline RT (156-165) & $\begin{array}{l}\text { RT (311-319 SF2) } \\
\text { - Pers. Comm. from } \\
\text { - Menendez-Arias19 }\end{array}$ & $\begin{array}{l}\text { SPAIFQSSMT } \\
\text { and D. Ruhl to } \\
\text { a review, notes }\end{array}$ & $\begin{array}{l}\text { human (B7) } \\
\text { B. Walker } \\
\text { includes catalytic residues in the active site of RT }\end{array}$ & \begin{tabular}{|l|} 
Brander1997, \\
Menendez-Arias1998 \\
\end{tabular} \\
\hline RT (156-165) & $\begin{array}{l}\text { RT (311-319 SF2) } \\
\text { - Epitope name: P4 } \\
\text { A panel of } 16 \text { epitop } \\
\text { CD8+ cell IFNgamr }\end{array}$ & $\begin{array}{l}\text { SPAIFQSSMT } \\
\text { vering } 15 \text { class I } \\
\text { duction to meast }\end{array}$ & $\begin{array}{l}\text { HIV-1 infection } \\
\text { human (B7) } 14 \text { HIV+ patients from an unselected Caucasian } p\end{array}$ & Mollet2000 \\
\hline
\end{tabular}




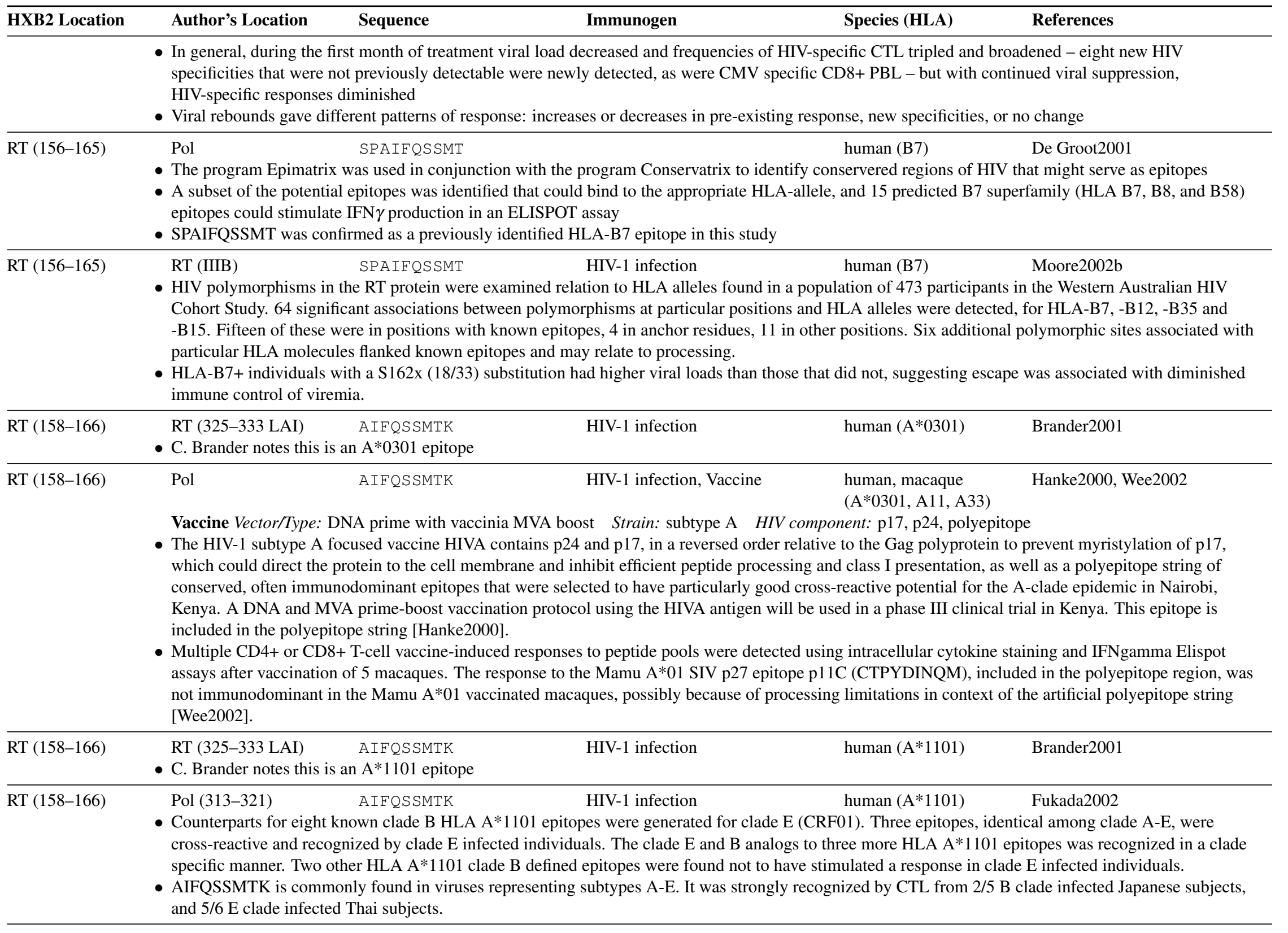




\begin{tabular}{|c|c|c|c|c|c|}
\hline HXB2 Location & Author's Location & Sequence & Immunogen & Species (HLA) & References \\
\hline RT (158-166) & RT (325-333) & AIFQSSMTK & HIV-1 infection & $\begin{array}{l}\text { human }(A * 1101, A 3, \\
A * 0301, A * 6801)\end{array}$ & \begin{tabular}{|l} 
Menendez-Arias1998, \\
Threlkeld1997
\end{tabular} \\
\hline
\end{tabular}

- Study of the fine specificity of an A3-like super-type epitope (the A3 super-type includes A*0301, A*1101, A*3101, A*3301, and A*6801)

- A3 super-type is characterized by a hydrophobic or hydroxyl containing anchor residue at position 2, and a positive charge in the C-term position

- While most lines were specific, promiscuous cloned CTL lines were also derived from HIV+ donors that could recognize epitope presented by either A3 or A11 or A*6801

- Alanine substitutions throughout the epitope and natural variants indicate that the same amino acid positions are critical for presentation by either MHC molecule, A3 or A11

- AIFQSSMTK is presented by three members of the A3 superfamily: $\mathrm{A} * 0301, \mathrm{~A} * 1101$, and $\mathrm{A} * 6801$, and the naturally occurring variants $\mathrm{A} 1 \mathrm{~S}$ and $\mathrm{K} 9 \mathrm{R}$ are recognized with similar efficiency to wild type epitope - AIFQRSMTR can also bind to two additional members of the A3 superfamily, A*3101 and A*3301

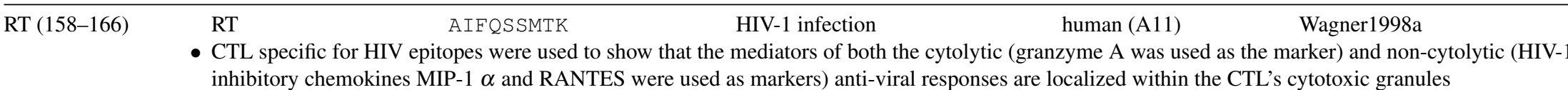
inhibitory chemokines MIP-1 $\alpha$ and RANTES were used as markers) anti-viral responses are localized within the CTL's cytotoxic granules

\begin{tabular}{lllll}
\hline RT (158-166) RT (325-333 LAI) AIFQSSMTK & Peptide-HLA interaction & human (A11) \\
\hline Zhang1993 &
\end{tabular}

- Exploration of A11 binding motif, based on Nixon et al. 1991

\begin{tabular}{lllll}
\hline RT (158-166) & $\begin{array}{l}\text { RT (325-333 LAI) } \\
\text { Review of HIV CTL epitopes }\end{array}$ & HIV-1 infection & human (A11) \\
\hline RT (158-166) & $\begin{array}{l}\text { Pol (305-313 93TH253 } \\
\text { subtype CRF01) }\end{array}$ & AIFQSSMTK & $\begin{array}{l}\text { HIV-1 infection, HIV-1 exposed } \\
\text { seronegative }\end{array}$ & human (A11)
\end{tabular}

pe CRF01)

- Epitope name: P313-321

- This was a study of HIV-1 exposed persistently seronegative (HEPS) female sex workers in Chiang Mai, northern Thailand

- HLA-A11 is very common in this population, and was enriched among the HEPS sexworkers - weak CTL responses were detected in 4/7 HEPS women, and CTL responses were found in 8/8 HIV+ controls, and 0/9 HIV- women that were not exposed

- This epitope was weakly reactive in the HEPS study subject 128 who was HLA A11/A33

- This epitope was strongly reactive in HIV+ study subjects 053 and 184 who carried HLA-A11 subtype CRF01)

- HLA-A11 CRF01 (called subtype E in Bond et al.) epitopes were identified that stimulated CTL from HIV+ female sex workers (FSW) from Northern Thailand, of whom more than half were HLA-A11 positive

- 77 possible HLA-A11 epitopes were first defined using EpiMatrix, these were screened for binding to A11 finding and 26 bound, and 12 of these were epitopes for CTL responses from 8 HLA-A11 positive FSWs, six were novel, six were previously identified

- This epitope was predicted by the EpiMatrix method to be likely to bind to A11, and it served as an epitope in the FSWs, it was one of the six A11 epitopes that had been previously defined

- 6/8 tested FSWs recognized this epitope

- An HLA-A11 tetramer was made for this epitope, which was recognized by two subjects - and both subjects had expanded tetramer staining T-cell populations after in vitro stimulation 


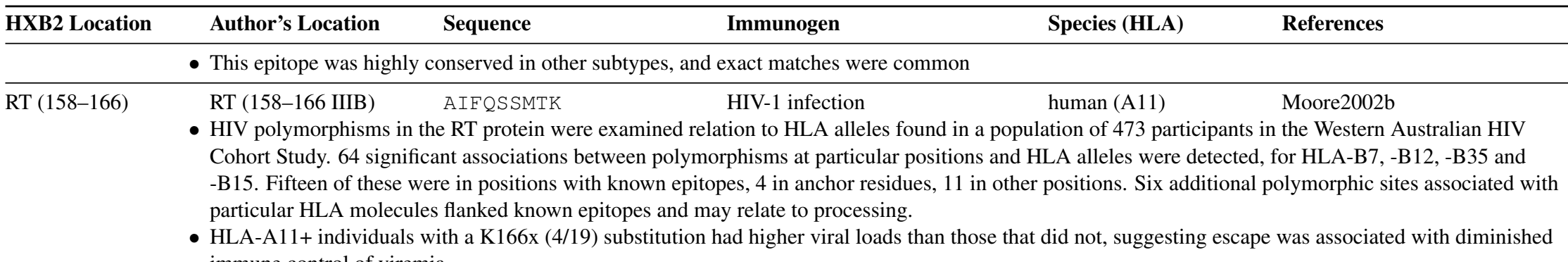

\begin{tabular}{llccc}
\hline RT (158-166) & Pol & SIFQSSMTK & HIV-1 infection human (A11) Appay2002 \\
& - Four HIV patients with prolonged clinically successful anti-viral therapy but with ongoing evidence of replication and Nef mRNA transcription, showed
\end{tabular}

- Four HIV patients with prolonged clinically successful anti-viral therapy but with ongoing evidence of replication
specific T-cell responses by Elispot and Tetramer staining, maintained for 2-4 years after initiation of HAART.

- Nef epitope recognition was detected in all 4 subjects, gp120, Pol and Gag-specific in 1 or 2 subjects.

- The HIV-specific CD8+ T-cells had an intermediate maturation phenotype characterized by low levels of perforin and high levels of CD27 expression.

RT (158-166) RT (325-333 IIIB) AIFQSSMTK $\quad$ HIV-1 infection

- Epitope defined in the context of the Pediatric AIDS Foundation ARIEL Project, a mother-infant HIV transmission study

- AIFQSSMTR and AILQSSMTK, naturally occurring variants, were found in infant, and are recognized

- TISQSSMTK, a naturally occurring variant, was found in infant and is not recognized

\begin{tabular}{llrll}
\hline RT (158-166) RT (325-333 LAI) AIFQSSMTK & HIV-1 infection & human (A3) Cao1997a
\end{tabular}

- The consensus peptide of B and D clade viruses is AIFQSSMTK

- The consensus peptide of a subset of As is AIFQASMTK and it is less able to stimulate the CTL clone

- The consensus peptide of a subset of As is SIFQSSMTK and is as reactive as the originally defined epitope

RT (158-166) Pol (325-333 IIIB) AIFQSSMTK HIV-1 infection $\quad$ Wilson1999a

- This study describes maternal CTL responses in the context of mother-to-infant transmission

- Detection of CTL escape mutants in the mother was associated with transmission, but the CTL-susceptible forms of the virus tended to be found in infected infants

- One variant found in an infant gave a positive CTL response: AIFQSSMTR

- AIFLSSMTK and TISQSSMTK were escape mutants

RT (158-166) RT (325-333 SF2) AIFQSSMTK HIV-1 infection human (A3) Altfeld2001b

- Therapy provided during acute infection resulted in a narrower CTL response, stronger T help response, and a less diverse viral population than was seen in individuals treated during chronic infection

- The breadth and specificity of the response was determined using ELISPOT by studying 19 individuals with pre-seroconversion therapy (Group 1), 11 individuals with primary infection but post-seroconversion therapy (Group 2), and 10 individuals who responded to HAART given during chronic infection (Group 3), using 259 overlapping peptides spanning p17, p24, RT, gp41, gp120 and Nef

- Previously described and newly defined optimal epitopes were tested for CTL response

- Number of HLA-A3+ individuals that had a CTL response to this epitope broken down by group: 0/7 group 1, 0/4 group 2, and 1/2 group 3

RT (158-166) AIFQSSMTK HIV-1 infection human (A3) Day2001

- The CTL response to optimally defined CTL epitopes restricted by HLA class I A and B alleles in individuals who coexpressed HLA A2, A3, and B7 was studied in eight HIV-1-infected subjects, two with acute infection, five with chronic, and one long-term non-progressor (LTNP) 


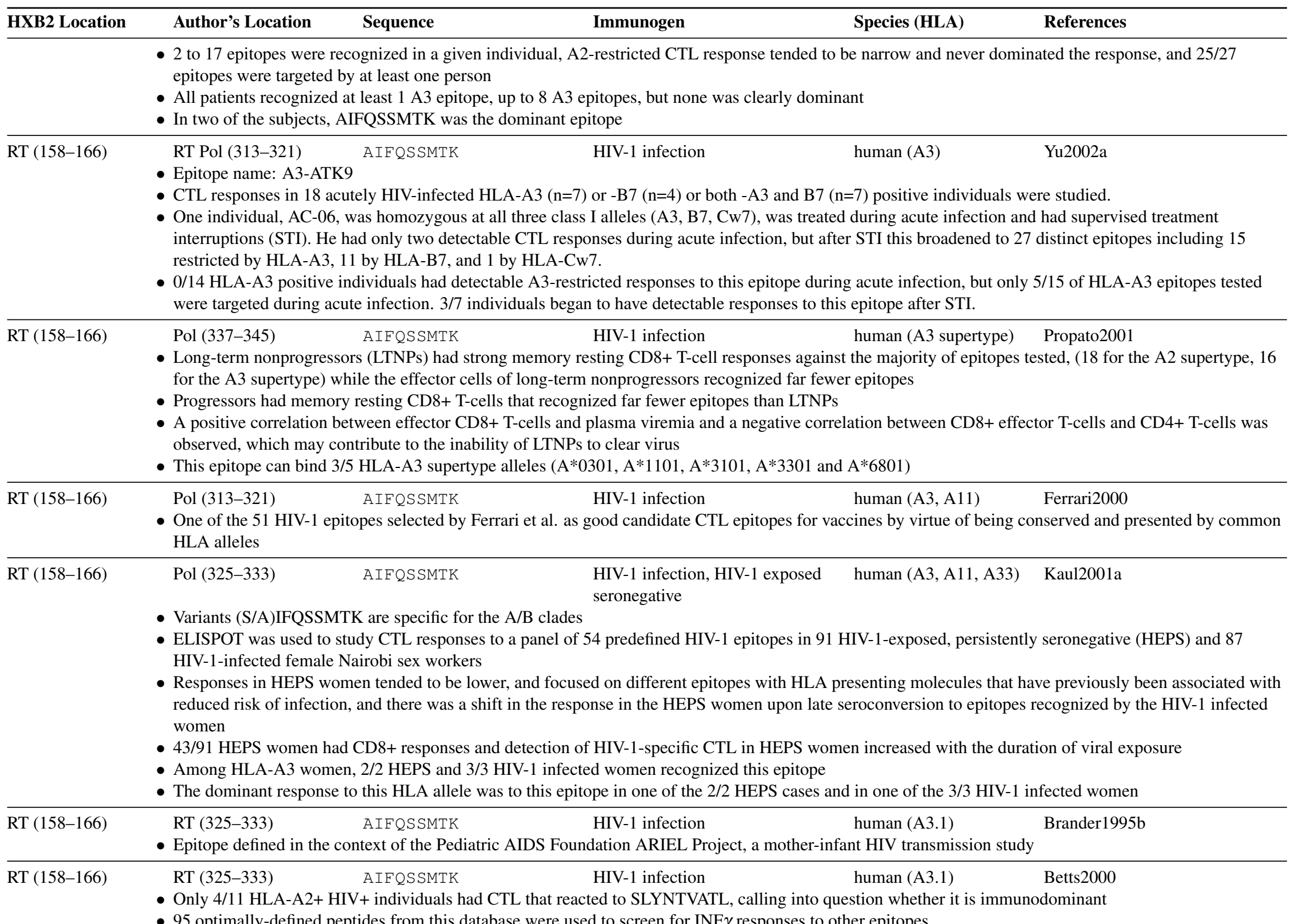




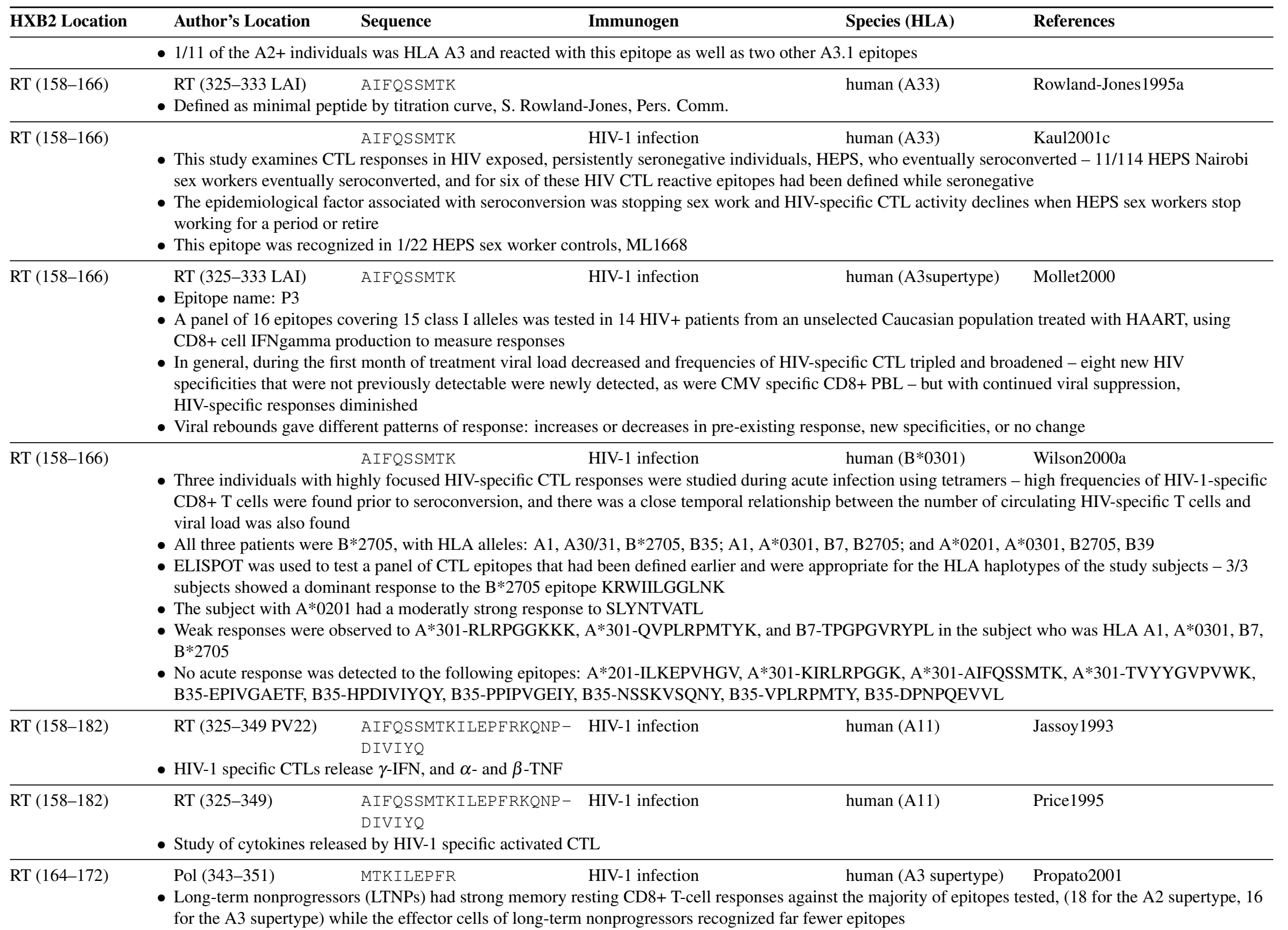




\begin{tabular}{|c|c|c|c|c|}
\hline HXB2 Location & Author's Location & Sequence & Species (HLA) & References \\
\hline & \multicolumn{4}{|c|}{$\begin{array}{l}\text { - Progressors had memory resting CD8+ T-cells that recognized far fewer epitopes than LTNPs } \\
\text { - A positive correlation between effector CD8+ T-cells and plasma viremia and a negative correlation between CD8+ effector T-cells and CD4+ T-cells was } \\
\text { observed, which may contribute to the inability of LTNPs to clear virus } \\
\text { - This epitope can bind 4/5 HLA-A3 supertype alleles }(A * 0301, A * 1101, A * 3101, A * 3301 \text { and } A * 6801)\end{array}$} \\
\hline RT (173-181) & \multicolumn{2}{|c|}{$\begin{array}{l}\text { RT }(173-181 \text { LAI }) \text { KQNPD IVIY } \\
\text { C. Brander notes this is an A*3002 epitope }\end{array}$} & human $(A * 3002)$ & Brander2001, Goulder2001a \\
\hline RT (173-181) & \multicolumn{4}{|c|}{$\begin{array}{l}\text { RT KQNPDIVIY } \\
\text { - Epitope name: KY9 (RT-53) } \\
\text { - HLA-A*3002 is very common in African populations, 50\% of Zimbabweans express HLA-A30, 44\% in African Zulu, so five new HIV epitopes were } \\
\text { characterized that are presented by this HLA molecule } \\
\text { - A rapid method was developed combining ELISPOT with intracellular IFN- } \gamma \text { staining of PBMCs to map optimal epitopes, then HLA presenting molecules } \\
\text { were defined - this method was completed within } 48 \text { to } 72 \text { hours of receipt of blood } \\
\text { - Two individuals were studied: Subject } 199 \text { (HLA A*0201/*3002 B*4402/51 Cw2/5), a Caucasian, and Subject 6007 (HLA A*3002/ B53/*5801 Cw4/7) an } \\
\text { African-Caribbean } \\
\text { - In both HLA-A*3002 individuals the response to RSLYNTVATLY was dominant } \\
\text { - In subject } 199 \text { four additional A*3002 epitopes were identified } \\
\text { - Three quantitative assays, ELISPOT, precursor frequency and chromium release, confirmed a hierarchy of response: RY11 (p17) > KY9 (gp41) > KY9 } \\
\text { (RT-53) > IY9 (gp41) }\end{array}$} \\
\hline$\Gamma(175-183)$ & \multicolumn{4}{|c|}{$\begin{array}{l}\text { - A CTL clone responsive to this epitope was obtained } \\
\text { - 3/7 B35-positive individuals had a CTL response to this epitope } \\
\text { - D to E, or V to I, substitutions at positions } 3 \text { or } 5 \text {, respectively, reduces CTL activity and binding to B*3501 }\end{array}$} \\
\hline RT (175-183) & \multicolumn{4}{|c|}{ - C. Brander notes this is a $\mathrm{B} * 3501$ epitope } \\
\hline RT (175-183) & \multicolumn{4}{|c|}{ - C. Brander notes this is a $\mathrm{B} * 3501$ epitope } \\
\hline RT (175-183) & \multicolumn{4}{|c|}{$\begin{array}{l}\text { - } \mathrm{CD} 8+\mathrm{T} \text {-cells that bound one of six HIV-specific } \mathrm{B} * 3501 \text {-epitope tetramers did not express CD28 or CD45A } \\
\text { - A significant increase in CD28-CD45RA- cells and a decrease of CD28+CD45RA+ cells was observed in chronically HIV-1-infected individuals relative to } \\
\text { healthy individuals } \\
\text { - CD28-CD45RA- cells are likely to be effector cells and have high levels of perforin in their cytoplasm } \\
\text { - The mean percentage of total CD28- CD8+ cells in chronically infected HIV-1-infected patients was } 76.6 \% \text { in comparison to HIV-1-uninfected individuals } \\
(40.6 \%)\end{array}$} \\
\hline $\mathrm{T}(175-183)$ & $\begin{array}{l}\text { RT (175-183 IIIB) } \\
\text { - HIV polymorphism } \\
\text { Cohort Study. } 64 \text { siq } \\
\text {-B15. Fifteen of the } \\
\text { particular HLA mol }\end{array}$ & $\begin{array}{l}\text { NPDIVIYQY } \\
\text { RT protein wer } \\
\text { ant associations b } \\
\text { re in positions wi } \\
\text { s flanked known }\end{array}$ & $\begin{array}{l}\text { HIV-1 infection } \\
\text { tion to HLA alleles found in a population of } 473 \text { partici } \\
\text { rphisms at particular positions and HLA alleles were de } \\
\text { es, } 4 \text { in anchor residues, } 11 \text { in other positions. Six addi }\end{array}$ & $\begin{array}{l}\text { Moore2002b } \\
\text { the Western Australian HIV } \\
\text { for HLA-B7, -B12, -B35 and } \\
\text { olymorphic sites associated with }\end{array}$ \\
\hline
\end{tabular}




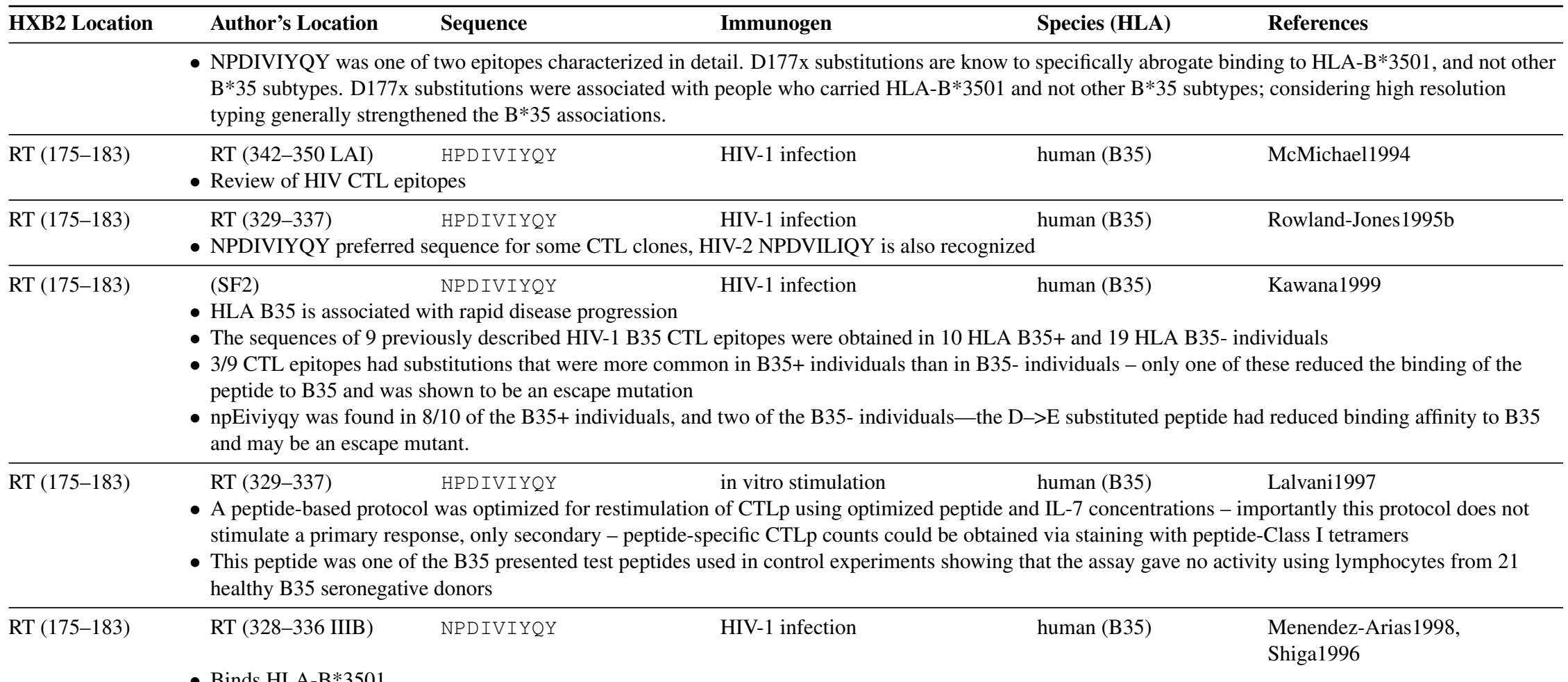

- Binds HLA-B*3501

- CTL activity to this epitope was originally detected in a long-term survivor, however it has since been found in normal progressors - it is cross-reactive with HIV-2 (HPDILIYQY), but D3E and V5I substitutions reduce binding [Menendez-Arias1998]

\begin{tabular}{lllll}
\hline RT (175-183) RT (328-336 IIIB) NPDIVIYQY & HIV-1 infection & human (B35)
\end{tabular}

- HIV IIIB proteins were used to define the range of CTL epitopes recognized by 3 lab workers accidentally infected with HIV-1 IIIB

- NPDIIIYQY, a variant found in HIV-1 JRCSF, was also recognized

- NPEIVIYQY, was also recognized

- NPDLVIYQY, was also recognized

- Menendez-Arias1998, in a review, notes that the YXDD motif, highly conserved among polymerases, overlaps this epitope - CTL activity to this epitope was originally detected in a long-term survivor, however it has since been found in normal progressors - it is cross-reactive with HIV-2 (HPDILIYQY), but D3E and V5I substitutions reduce binding

RT NPDIVIYQY

HIV-1 exposed seronegative

human (B35)

Menendez-Arias1998, Rowland-Jones1998a

- A CTL response was found in exposed but uninfected prostitutes from Nairobi using previously-defined B clade epitopes that tended to be conserved in A and D clades - such cross-reactivity could protect against both A and D and confer protection in Nairobi where both subtypes are circulating

- The A subtype consensus is HPDIVIYQY 


\begin{tabular}{|c|c|c|c|c|}
\hline HXB2 Location & Author's Location & Immunogen & Species (HLA) & References \\
\hline & \multicolumn{4}{|c|}{$\begin{array}{l}\text { - The D subtype consensus is NPEIVIYQY } \\
\text { - [Menendez-Arias1998], in a review, notes that the YXDD motif, highly conserved among polymerases, overlaps this epitope - CTL activity to this epitope } \\
\text { was originally detected in a long-term survivor, however it has since been found in normal progressors - it is cross-reactive with HIV-2 (HPDILIYQY), but } \\
\text { D3E and V5I substitutions reduce binding }\end{array}$} \\
\hline RT (175-183) & \multicolumn{4}{|c|}{$\begin{array}{l}\text { - HIV-specific CTL were found in exposed seronegative prostitutes from Nairobi - these CTL may confer protection } \\
\text { - Seroprevalence in this cohort is 90-95\% and their HIV-1 exposure is among the highest in the world } \\
\text { - Most isolated HIV strains are clade A in Nairobi, although clades C and D are also found - B clade epitopes are often cross-reactive, however stronger } \\
\text { responses are frequently observed using A or D clade versions of epitopes } \\
\text { - Clade A version of epitope HPDIVIYQY, Clade D NPEIVIYQY }\end{array}$} \\
\hline$\Gamma(175-183)$ & \multicolumn{4}{|c|}{$\begin{array}{l}\text { - CTL responses in seronegative highly HIV-exposed African female sex workers in Gambia and Nairobi were studied - these women had no delta } 32 \\
\text { deletion in CCR5 } \\
\text { seems to be protective } \\
\text { - HIV-2 version of this epitope is not conserved: NPDVILIQY, but the CTLs are cross-reactive - one of five B35 CTL epitopes that are cross-reactive, see } \\
\text { also Rowland-Jones1995b }\end{array}$} \\
\hline$(1$ & \multicolumn{4}{|c|}{$\begin{array}{l}\text { HPDIVIYQY } \\
\text { - Three individuals with highly focused HIV-specific CTL responses were studied during acute infection using tetramers - high frequencies of HIV-1-specific } \\
\text { CD8+ T cells were found prior to seroconversion, and there was a close temporal relationship between the number of circulating HIV-specific T cells and } \\
\text { viral load was also found } \\
\text { - All three patients were B*2705, with HLA alleles: A1, A30/31, B*2705, B35; A1, A*0301, B7, B2705; and A*0201, A*0301, B2705, B39 } \\
\text { - ELISPOT was used to test a panel of CTL epitopes that had been defined earlier and were appropriate for the HLA haplotypes of the study subjects - 3/3 } \\
\text { subjects showed a dominant response to the B*2705 epitope KRWIILGGLNK } \\
\text { - The subject with A*0201 had a moderately strong response to SLYNTVATL } \\
\text { - Weak responses were observed to A*301-RLRPGGKKK, A*301-QVPLRPMTYK, and B7-TPGPGVRYPL in the subject who was HLA A1, A*0301, B7, } \\
\text { B*2705 } \\
\text { - No acute response was detected to the following epitopes: A*201-ILKEPVHGV, A*301-KIRLRPGGK, A*301-AIFQSSMTK, A*301-TVYYGVPVWK, } \\
\text { B35-EPIVGAETF, B35-HPDIVIYQY, B35-PPIPVGEIY, B35-NSSKVSQNY, B35-VPLRPMTY, B35-DPNPQEVVL }\end{array}$} \\
\hline 3) & $\begin{array}{l}\text { Pol (subtype A) } \\
\text { - This study examines } \\
\text { sex workers eventua } \\
\text { - HPDIVIYQY or NP } \\
\text { seroconversion, } 7 \mathrm{~m} \\
\text { - } 20 / 20 \text { sequences of } \mathrm{t} \\
857 \text { - this was the or } \\
\text { - The epidemiological } \\
\text { working for a period } \\
\text { - NPDIVIYQY was re }\end{array}$ & $\begin{array}{l}\text { HPDIVIYQY HIV-1 infection } \\
\text { responses in HIV exposed, persistently seronegative individu } \\
\text { roconverted, and for six of these HIV CTL reactive epitopes } h \\
\text { YQY was recognized in } 1 \text { of the } 6 \text { women (ML857), and the } r \\
\text { fecting strain had three substitutions in this epitope, all } 20 \text { wer } \\
\text { se in the study where a virus carrying an unrecognized form o } \\
\text { or associated with seroconversion was stopping sex work and } \mathrm{t} \\
\text { tire } \\
\text { ized by } 1 / 22 \text { HEPS control sex workers, ML887 }\end{array}$ & $\begin{array}{l}\text { human (B35) } \\
\text { PS, who eventuall } \\
\text { defined while ser } \\
\text { was present in th } \\
\text { iyqy, and this for } \\
\text { itope broke throu } \\
\text { cific CTL activity }\end{array}$ & $\begin{array}{l}\text { Kaul2001c } \\
\text { nverted - 11/114 HEPS Nairobi } \\
\text { ailable sample prior to } \\
\text { when HEPS sex workers stop } \\
\text { whized by CTL from ML }\end{array}$ \\
\hline
\end{tabular}




\begin{tabular}{lllll}
\hline HXB2 Location & Author's Location & Sequence & Immunogen & Species (HLA) \\
\hline RT (175-183) & RT (175-183 SF2) & NPD IVIYQY & HIV-1 infection & human (B35)
\end{tabular}

- Therapy provided during acute infection resulted in a narrower CTL response, stronger $\mathrm{T}$ help response, and a less diverse viral population than was seen in individuals treated during chronic infection

- The breadth and specificity of the response was determined using ELISPOT by studying 19 individuals with pre-seroconversion therapy (Group 1), 11 individuals with primary infection but post-seroconversion therapy (Group 2), and 10 individuals who responded to HAART given during chronic infection (Group 3), using 259 overlapping peptides spanning p17, p24, RT, gp41, gp120 and Nef

- Previously described and newly defined optimal epitopes were tested for CTL response

- Number of HLA-B35+ individuals that had a CTL response to this epitope broken down by group: $1 / 2$ group 1, 0/2 group 2, and 1/1 group 3 Pol (342-350) HPDIVIYQY

HIV-1 infection, HIV-1 exposed human (B35) Kaul2001a

RT (175-183)

Variants (H/N)PDIVIYQY are specific for the A/B clades

- ELISPOT was used to study CTL responses to a panel of 54 predefined HIV-1 epitopes in 91 HIV-1-exposed, persistently seronegative (HEPS) and 87 HIV-1-infected female Nairobi sex workers

- Responses in HEPS women tended to be lower, and focused on different epitopes with HLA presenting molecules that have previously been associated with reduced risk of infection, and there was a shift in the response in the HEPS women upon late seroconversion to epitopes recognized by the HIV-1 infected women

- 43/91 HEPS women had CD8+ responses and detection of HIV-1-specific CTL in HEPS women increased with the duration of viral exposure

- Among HLA-B35 women, 2/3 HEPS and 1/4 HIV-1 infected women recognized this epitope

- The dominant response to this HLA allele was to this epitope in only one of the $2 / 3$ HEPS cases, and was not to this epitope in the one responsive HIV-1 infected women

- Subject ML 857 shifted from a A*6802 DTVLEDINL and B35 (H/N)PDIVIYQY response prior to seroconversion to a B35 PPIPVGDIY and B35 VPLRPMTY response post-seroconversion and the loss of the pre-seroconversion response was not due to sequence variation within these epitopes

\begin{tabular}{lllll}
\hline RT (175-183) HPDIVIYQY HIV-1 infection & Sabbaj2002b
\end{tabular}

- Epitope name: Pol-HY9

- Among HIV+ individuals who carried HLA B35, 4/21 (19\%) recognized this epitope

RT (175-183) Pol NPDIVIYQY HIV-1 infection habbaj2002a

- IFNgamma T-cell responses in breast milk of 5 HIV-1 infected women from the US and 6 from Zambia were tested with using Elispot. 11/11 women responded to Gag, 8/11 responded to Pol, 7/11 women to Nef, and 2/5 women to Env peptide pools. These responses were shown to be primarily due to CD8+ T-cells in one woman, and another woman had cytolytic responses measured by Cr-release.

- T-cells in breast milk from a volunteer who was HLA A3, A11, B35, B51 induced IFNgamma after stimulation with a peptide that carries known B35 epitope NPDIVIYQY.

- The frequencies of responses in the two compartments differed, and 2/4 women that responded to epitopes in Nef 101-205 and Pol 601-710 showed responses in breast milk but no detectable responses in peripheral blood cells.

\begin{tabular}{lllll}
\hline RT (175-183) Pol HPDIVIYQY & HIV-1 infection, Vaccine & human, macaque (B35) Hanke2000 Wee202
\end{tabular}

Vaccine Vector/Type: DNA prime with vaccinia MVA boost Strain: subtype A HIV component: p17, p24, polyepitope

- The HIV-1 subtype A focused vaccine HIVA contains p24 and p17, in a reversed order relative to the Gag polyprotein to prevent myristylation of p17, which could direct the protein to the cell membrane and inhibit efficient peptide processing and class I presentation, as well as a polyepitope string of conserved, often immunodominant epitopes that were selected to have particularly good cross-reactive potential for the A-clade epidemic in Nairobi, Kenya. A DNA and MVA prime-boost vaccination protocol using the HIVA antigen will be used in a phase III clinical trial in Kenya. This epitope is included in the polyepitope string [Hanke2000]. 


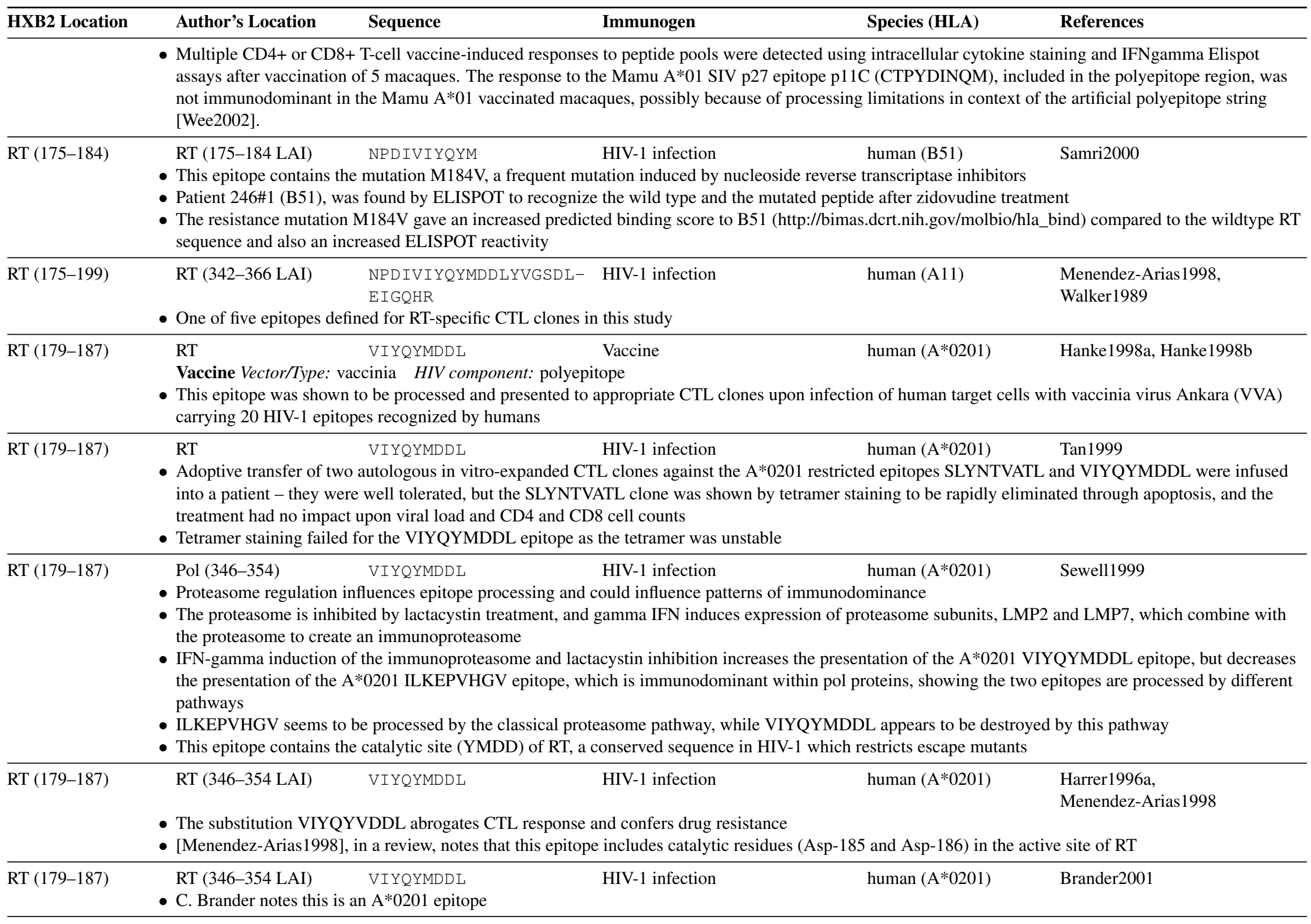




\begin{tabular}{|c|c|c|c|c|c|}
\hline HXB2 Location & Author's Location & Sequence & Immunogen & Species (HLA) & References \\
\hline RT (179-187) & RT (346-354) & VIYQYMDDL & HIV-1 infection & human $\left(A^{*} 0201\right)$ & $\frac{\mid \text { Brander1998a }}{\text { Menendez-Arias1998 }}$ \\
\hline
\end{tabular}

- Of 17 infected HLA A*0201 subjects, 13 had CTL responses against the p17 SLYNTVATL epitope, six recognized ILKEPVHGV and five recognized

VIYQYMDDL, and there was no correlation between viral load and recognition of a specific epitope or evidence of immune escape

- Only one subject had CTL against all three epitopes

- Subjects were part of the San Francisco City Clinic Cohort, the ARIEL project and from the Boston area

- In the review [Menendez-Arias1998] the authors note that substitution of three residues in this epitope can confer resistance to RT inhibitors (1, 3, and 6) substitutions V1E and M6V abolish CTL activity, and M6V confers resistance to 3TC - substitution Y3C reduces CTL activity and is associated with resistance to non-nucleoside RT inhibitors

\begin{tabular}{lllll}
\hline RT (179-187) & RT & VIYQYMDDL & HIV-1 infection & human (A*0201)
\end{tabular}

- Epitope name: RT VL9

- HIV was scanned for all peptides which carried the A2-supermotif pattern conserved in more than 50\% of B clade sequences - 233 peptides met this criteria, and 30 of these bound to HLA-A $* 0201-20 / 30$ bound to at least 3/5 of HLA-A2 supertype alleles tested

- Three additional previously described HLA-A2 epitopes were added to the set of 20, including RT VL9, and 18/22 chronically infected HLA-A2 individuals had CTL that recognized at least one of the 23 peptides (median of 2 and maximum of 6), while 6/12 acute infected individuals recognized at least 1 (median of 1 and maximum of 2)

- RT VL9 was not recognized by any of the 22 HLA-A2 patients with chronic HIV-1 infection or the 13 HLA-A2 patients with acute HIV-1 infection included in this study

\begin{tabular}{lllll}
\hline RT (179-187) & RT (346-354) & VIYQYMDDL & HIV-1 infection & human (A*0201)
\end{tabular}

- Epitope name: VL9

- Integration of HIV RT CTL epitopes into the N-terminus of the HLA-A2 heavy chain, or tethering the epitopes to the target chain, resulted in epitope-specific lysis by CD8+ CTL

- These antigens could also be used to stimulate primary responses in vitro

\begin{tabular}{llccc}
\hline RT (179-187) & Pol (346-354) & VIYQYMDDL & HIV-1 infection & human (A*0201)
\end{tabular}

- Epitope processing of three different HLA-A*0201 HIV epitopes was shown to use different pathways, which might influence patterns of immunodominance. .174 cells were used that lack TAP1 and TAP2 genes, as well as the LMP2 and LMP7 genes that encode the beta-subunits of the immunoproteasome. These genes could be added back through transfection to study processing.

- ILKEPVHGV was efficiently presented in TAP-1 and -2 transfected cells while VIYQYMDDL and SLYNTVATL were not. VIYQYMDDL was destroyed by the MB1 subunit of the protease, and could be expressed in the presence of the proteasome inhibitor lactacystin, but SLYNTVATL expression was not restored. SLYNTVATL expression was unaltered by lactacystin in a wild type cell line. Pol

VIYQYMDDL HIV-1 infection, Vaccine

\section{human, macaque} $(\mathrm{A} * 0201)$

Vaccine Vector/Type: DNA prime with vaccinia MVA boost Strain: subtype A HIV component: p17, p24, polyepitope

- The HIV-1 subtype A focused vaccine HIVA contains p24 and p17, in a reversed order relative to the Gag polyprotein to prevent myristylation of p17, which could direct the protein to the cell membrane and inhibit efficient peptide processing and class I presentation, as well as a polyepitope string of conserved, often immunodominant epitopes that were selected to have particularly good cross-reactive potential for the A-clade epidemic in Nairobi, Kenya. A DNA and MVA prime-boost vaccination protocol using the HIVA antigen will be used in a phase III clinical trial in Kenya. This epitope is included in the polyepitope string [Hanke2000]. 


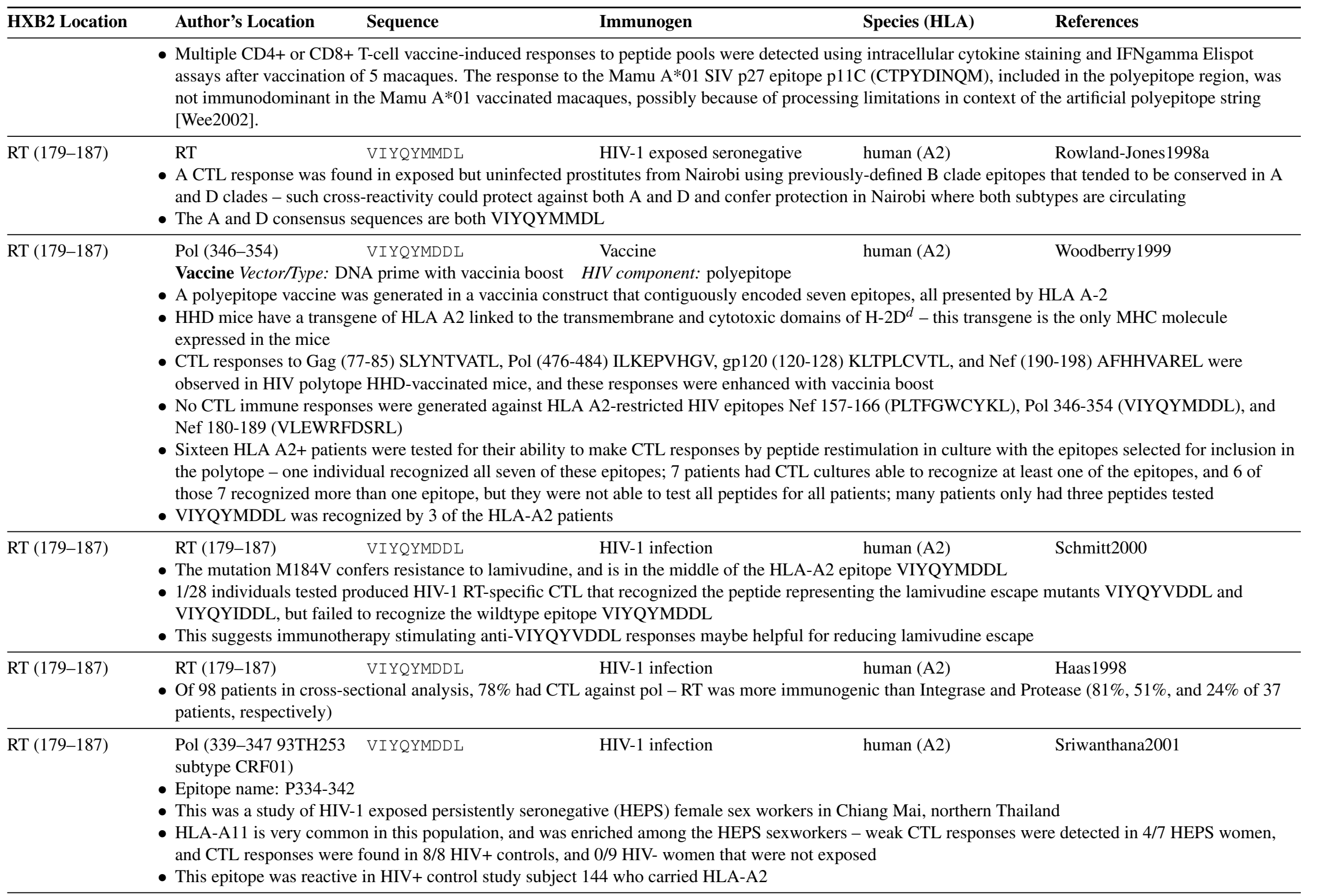




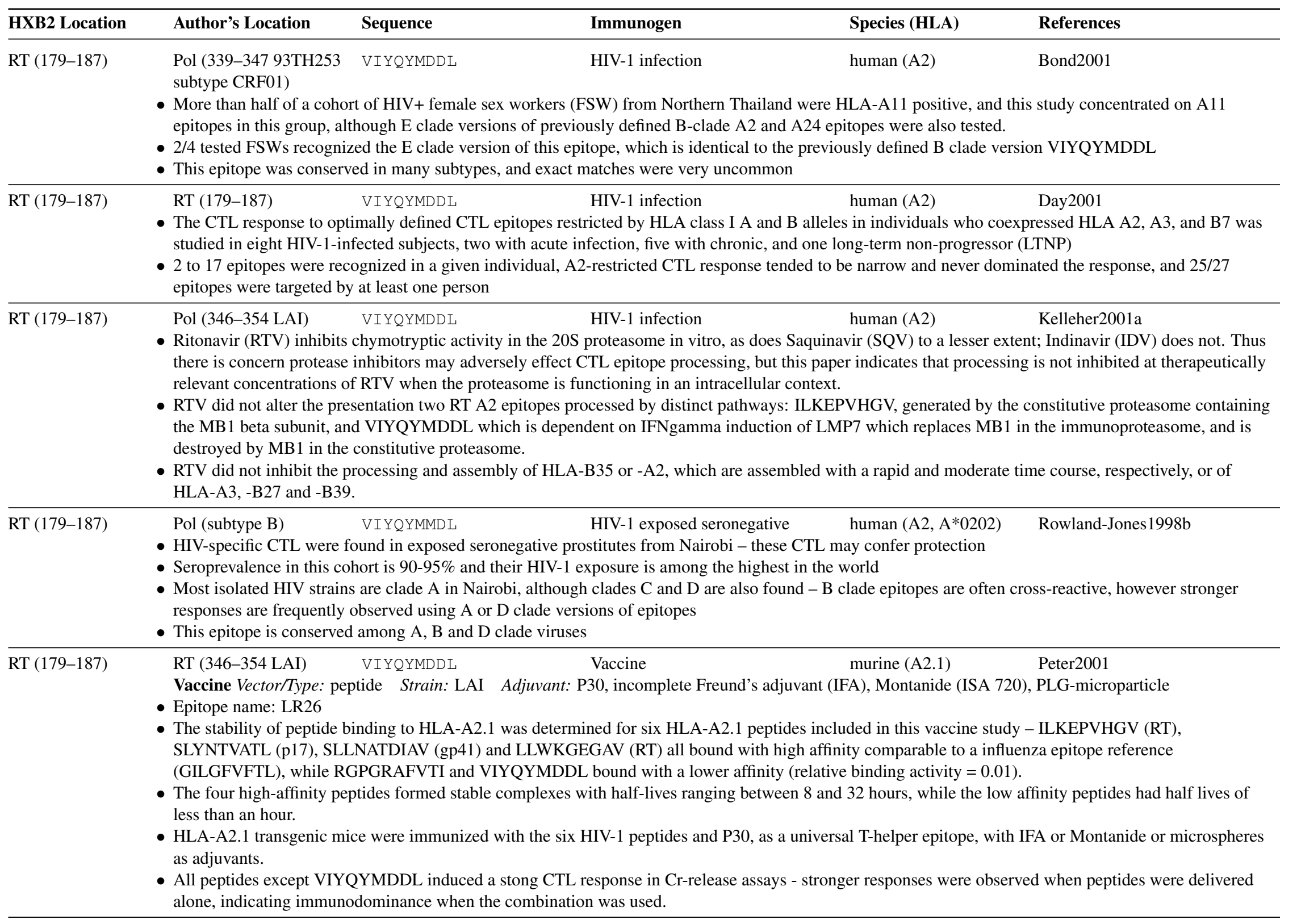




\begin{tabular}{|c|c|c|c|c|}
\hline HXB2 Location & Author's Location & Immunogen & Species (HLA) & References \\
\hline Г (179-187) & \multicolumn{4}{|c|}{$\begin{array}{l}\text { Vaccine Vector/Type: peptide Strain: LAI Adjuvant: P30, incomplete Freund's adjuvant (IFA), IL-12 } \\
\text { - Epitope name: LR26 } \\
\text { - When HIV-1 peptides were used to vaccinate HLA-A2.1 transgenic A2-Kb mice, strong responses to five peptides were observed when the peptides were } \\
\text { given individually, but immunodominance limited the response to some of the peptides when they were given in combination [Peter2001]. IL-12 can } \\
\text { counteract immunodominance in BALB/c mice, so it was given with the multiple epitope vaccination, and was instead found to specifically eliminate the } \\
\text { HLA-A2.1-epitope CTL responses, but not Kb CTL responses. This was possibly a consequence of transient depletion of T-cells, B cells and macropahges } \\
\text { in the spleen. }\end{array}$} \\
\hline RT (180-189) & \multicolumn{4}{|c|}{$\begin{array}{l}\text { - Recognized by CTL from a progressor, spans important RT functional domain } \\
\text { - A previous study determined that this was an epitope recognized by a long-term survivor }\end{array}$} \\
\hline RT (181-189) & \multicolumn{4}{|c|}{$\begin{array}{l}\text { - This epitope contains the mutation M184V, a frequent mutation induced by nucleoside reverse transcriptase inhibitors } \\
\text { - High levels of recognition by ELISPOT were observed for zidovudine induced mutation YQYVDDLYV and for the wildtype peptide YQYMDDLYV in } \\
\text { patient } 250 \# 0 \text { (HLA-A*0201), but neither were recognized by patient } 201 \# 5 \text { (also HLA-A*0201) } \\
\text { - Both the wild-type and the mutated peptide were computer predicted to have a high binding affinity for A2 (http//bimas.dcrt.nih.gov/molbio/hla_bind) }\end{array}$} \\
\hline RT (192-201) & $\begin{array}{l}\text { RT (192-201) } \\
\text { - Of } 98 \text { patients in cross- } \\
\text { patients, respectively) } \\
\text { - New clusters of epitope }\end{array}$ & $\begin{array}{l}\text { DLEIGQHRTK HIV-1 infection } \\
\text { tional analysis, 78\% had CTL against pol - RT w } \\
\text { vere defined utilizing different HLA molecules }\end{array}$ & $\begin{array}{l}\text { human (A3) } \\
\text { enic than Integrase }\end{array}$ & Haas 1998 tease $(81 \%, 51 \%$, and $24 \%$ of 37 \\
\hline RT (192-216) & $\begin{array}{l}\text { RT (359-383 HXB2) } \\
\text { - One of five epitopes def }\end{array}$ & $\begin{array}{l}\text { DLEIGQHRTKIEELRQHLL- HIV-1 infection } \\
\text { RWGLTT } \\
\text { ned for RT-specific CTL clones in this study }\end{array}$ & human (Bw60) & $\begin{array}{l}\text { Menendez-Arias1998, } \\
\text { Walker1989 }\end{array}$ \\
\hline RT (192-216) & $\begin{array}{l}\text { RT (191-215) } \\
\text { - Polyclonal CTL recogn } \\
\text { variant, RT T215Y }\end{array}$ & $\begin{array}{l}\text { DLEIGQHRTKIEELRQHLL- HIV-1 infection } \\
\text { RWGFTT } \\
\text { ion switched from RT 191-215 to RT 514-524 whe }\end{array}$ & $\begin{array}{l}\text { human (polyclona } \\
\text { cted for the resistar }\end{array}$ & $\begin{array}{l}\text { Haas1997, Menendez-Arias1998 } \\
\text { tation, and presumably the escape }\end{array}$ \\
\hline RT (198-212) & $\begin{array}{l}\text { RT (SF2) } \\
\text { - This epitope was mapp } \\
\text { an HLA-B60 individual } \\
\text { - The response to the pep }\end{array}$ & $\begin{array}{l}\text { HRTKIEELRQHLLRW HIV-1 infection } \\
\text { by ELISPOT in a study identifying new HLA-B6 } \\
\text { le was CD8 dependent, but the HLA presenting m }\end{array}$ & $\begin{array}{l}\text { human } \\
\text { one of the epitope } \\
\text { epitope were not }\end{array}$ & $\begin{array}{l}\text { Altfeld } 2000 \mathrm{~b} \\
\text { nted by another HLA molecule in } \\
\text { ined }\end{array}$ \\
\hline RT (201-209) & $\begin{array}{l}\text { RT (201-209) } \\
\text { - Of } 98 \text { patients in cross- } \\
\text { patients, respectively) } \\
\text { - New clusters of epitope }\end{array}$ & $\begin{array}{l}\text { KIEELRQHL } \\
\text { etional analysis, 78\% had CTL against pol - RT w } \\
\text { were defined utilizing different HLA molecules }\end{array}$ & $\begin{array}{l}\text { human (A2) } \\
\text { enic than Integrase }\end{array}$ & Haas 1998 \\
\hline
\end{tabular}




\begin{tabular}{llccc}
\hline HXB2 Location & Author's Location & Sequence & Immunogen & Species (HLA) \\
\hline RT (201-210) & Pol & KIEELRQHLL & References & human (B58) \\
& - The program Epimatrix was used in conjunction with the program Conservatrix to identify conservered regions of HIV that might serve as epitopes & De Groot2001
\end{tabular}

- A subset of the potential epitopes was identified that could bind to the appropriate HLA-allele, and 15 predicted B7 superfamily (HLA B7, B8, and B58) epitopes could stimulate IFN $\gamma$ production in an ELISPOT assay

- KIEELRQHLL was newly identified as a HLA-B58 epitope in this study, it had been previously shown to be presented by HLA-A2 and Bw60

- KIEELRQHLL did not bind detectably to B7

\begin{tabular}{llccc}
\hline RT (202-210) & $\begin{array}{l}\text { RT (202-210 LAI) } \\
\text { C. Brander notes this is a B*4001 epitope }\end{array}$ & human (B*4001) & Altfeld2000b Brander2001 \\
\hline RT (202-210) & RT (SF2) & IEELRQHLL & HIV-1 infection & human (B60)
\end{tabular}

- Therapy provided during acute infection resulted in a narrower CTL response, stronger T help response, and a less diverse viral population than was seen in individuals treated during chronic infection

- The breadth and specificity of the response was determined using ELISPOT by studying 19 individuals with pre-seroconversion therapy (Group 1 ), 11 individuals with primary infection but post-seroconversion therapy (Group 2), and 10 individuals who responded to HAART given during chronic infection (Group 3), using 259 overlapping peptides spanning p17, p24, RT, gp41, gp120 and Nef

- Previously described and newly defined optimal epitopes were tested for CTL response

- Number of HLA-B60+ individuals that had a CTL response to this epitope broken down by group: 0/2 group 1, 1/1 group 2, and 0/0 group 3

\begin{tabular}{lllll}
\hline RT (202-210) RT (SF2) IEELRQHLL & HIV-1 infection & human (B60(B*4001)
\end{tabular}

- This epitope was mapped by ELISPOT in a study identifying new HLA-B60 epitopes

- B60 is present in 10-20\% of the Caucasoid and very common in Asian populations

\begin{tabular}{lllll}
\hline RT (202-210) & RT (202-210) & IEELRQHLL & HIV-1 infection & human (B60/B61)
\end{tabular}

- All five B60-restricted epitopes were reactive in another subject, and the B60-restricted responses together contributed over one-third of the total CTL response

\begin{tabular}{lllll}
\hline RT (203-212) RT (LAI) EELRQHLLRW & HIV-1 infection & human (B44)
\end{tabular}

- The only epitope recognized by CTL from a long-term survivor in two samples taken six years apart

- Recognized by CTL from a progressor, EILKEPVGHGV and TWETWWTEYW were also recognized

\begin{tabular}{lllll}
\hline RT (209-220) & RT (209-220) & LLRWGLTTPDKK & HIV-1 infection & human (A2)
\end{tabular}

- Of 98 patients in cross-sectional analysis, $78 \%$ had CTL against pol - RT was more immunogenic than Integrase and Protease $(81 \%$, $51 \%$, and $24 \%$ of 37 patients, respectively)

- New clusters of epitopes were defined utilizing different HLA molecules

\begin{tabular}{lllll}
\hline RT (243-252) & RT (LAI) & HIV-1 infection & human (B*5701) \\
\hline vanderBurg1997
\end{tabular}

- Recognized by CTL from a progressor and a long-term survivor, KITTESIVIW was also recognized 


\begin{tabular}{lllll}
\hline HXB2 Location & Author's Location & Sequence & Immunogen & Species (HLA) \\
\hline RT (243-252) & RT (LAI) & P IVLPEKD SW & HIV-1 infection & human (B*5701) \\
& & & & Menendez-Arias1998, \\
\hline
\end{tabular}

- Recognized by CTL from long-term survivor, whose CTL response persisted for more than 10 years - the substitution V3M reduced affinity but was well recognized, on the other hand V3T and D8G did not reduce affinity, but abrogated CTL response

\begin{tabular}{lllll}
\hline RT (243-252) RT (410-419) & PIVLPEKDSW & Oxenius2000
\end{tabular}

- Epitope name: PIV

- Patients who started therapy at acute HIV-1 infection (three with sustained therapy, two with limited therapy upon early infection) had strong HIV specific CD4 proliferative responses and were able to maintain a CTL response even with undetectable viral load - three patients that had delayed initiation of HAART had no HIV specific CD4 proliferative responses and lost their CTL responses when HAART was eventually given and their viral loads became undetectable

- None of the 8 study subjects recognized this epitope but none were HLA B57+

\begin{tabular}{|c|c|c|c|c|}
\hline RT (243-252) & $\begin{array}{l}\text { RT } \\
\text { - Epitope name: PIV } \\
\text { - Using previously de } \\
\text { period including the } \\
\text { - STIs induced increa } \\
\text { clearance rates. }\end{array}$ & $\begin{array}{l}\text { P IVLPEKDSW } \\
\text { epitopes Oxeniu } \\
\text { vith standard tre } \\
\text { cognition of CTI }\end{array}$ & $\begin{array}{l}\text { HIV-1 infection human (B57) } \\
\text { 2001al in an IFNgamma Elispot assay, } 13 \text { chronically } \\
\text { tions (STI). } \\
\text { there was no correlation between CTL responses with v }\end{array}$ & $\begin{array}{l}\text { Oxenius } 2002 \mathrm{~b} \\
\text { fected patients were studied over a } \\
\text { und rates, plateau viral loads, or }\end{array}$ \\
\hline RT (244-252) & $\begin{array}{ll} & \text { RT (399-407) } \\
\text { - Subtype of B57 not } \\
\text { - C. Brander notes thi }\end{array}$ & $\begin{array}{l}\text { IVLPEKDSW } \\
\text { nined } \\
\text { B*5701 epitope }\end{array}$ & human $(B * 5701)$ & Brander2001 \\
\hline RT (244-252) & $\begin{array}{l}\text { RT (244-252 LAI) } \\
\text { - This peptide was de } \\
\text { - B57 has been associ } \\
\text { - The most pronounce } \\
\text { - B57 restricted CTL } \\
\text { were found in this L } \\
\text { by CTL, and IMLPI } \\
\text { - In an additional HIV } \\
\text { index peptide } \\
\text { - This epitope was rec }\end{array}$ & $\begin{array}{l}\text { IVLPEKDSW } \\
\text { as the optimal ep } \\
\text { vith long-term no } \\
\text { L responses in H } \\
\text { nses are targeted } \\
\text { CLPEKESW, wh } \\
\text { W, which bound } \\
\text { S, only the variar } \\
\text { ed in the context }\end{array}$ & $\begin{array}{l}\text { HIV-1 infection human }(\mathrm{B} * 5701 \text {, } \\
\text { in the Amsterdam cohort. } \\
\text { S were to RT and Gag } \\
\text { teins, but one LTS had a response that was dominated by } \\
5701 \text { with similar affinity as the index peptide but was } \\
\text { i reduced affinity but could still be recognized } \\
\text { was found, and this epitope was recognized by CTL b } \\
3 * 5701 \text { and } \mathrm{B} * 5801\end{array}$ & $\begin{array}{l}\text { Klein1998 } \\
\text { ity to the epitope }- \text { two variants } \\
\text { ee mutant that was not recognized } \\
\text { ess affinity for } B * 5701 \text { than the }\end{array}$ \\
\hline RT (244-252) & $\begin{array}{l}\text { Pol (244-252) } \\
\text { - Combined tetramer } \\
\text { - } \text { HIV-specific CD8+ } \\
\text { CD27 expression on } \\
\text { - In most donors, bet } \\
\text { produce TNF- } \alpha\end{array}$ & $\begin{array}{l}\text { IVLPEKDSW } \\
\text { tracellular cytok } \\
\text { s expressed lowe } \\
\text { specific cells, su } \\
50 \% \text { and } 95 \% \text { of }\end{array}$ & $\begin{array}{l}\text { HIV-1 infection } \\
\text { s used to study the function of circulating CD8+ T cells } \\
\text { orin than CMV-specific CD8+ T cells from the same dor } \\
\text { ed maturation } \\
\text { rus-specific CD8+ T cells produced IFN- } \gamma \text { and MIP-1 } \beta\end{array}$ & $\begin{array}{l}\text { Appay2000 } \\
\text { for HIV and CMV } \\
\text { this was associated with persistent } \\
\text { distinct subset that failed to }\end{array}$ \\
\hline RT (244-252) & RT (399-407) & IVLPEKDSW & human (B57) & vanderBurg1997 \\
\hline
\end{tabular}




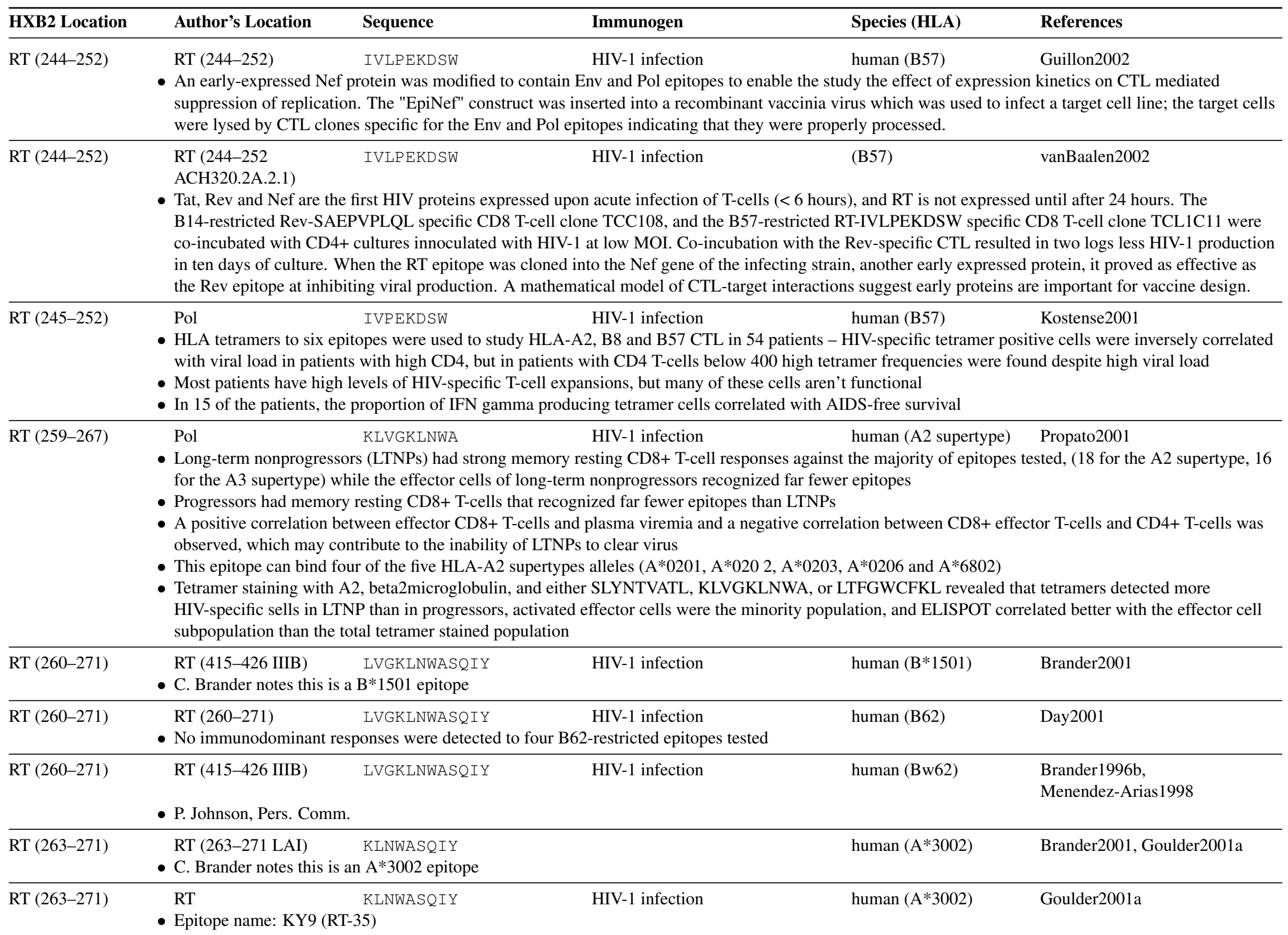




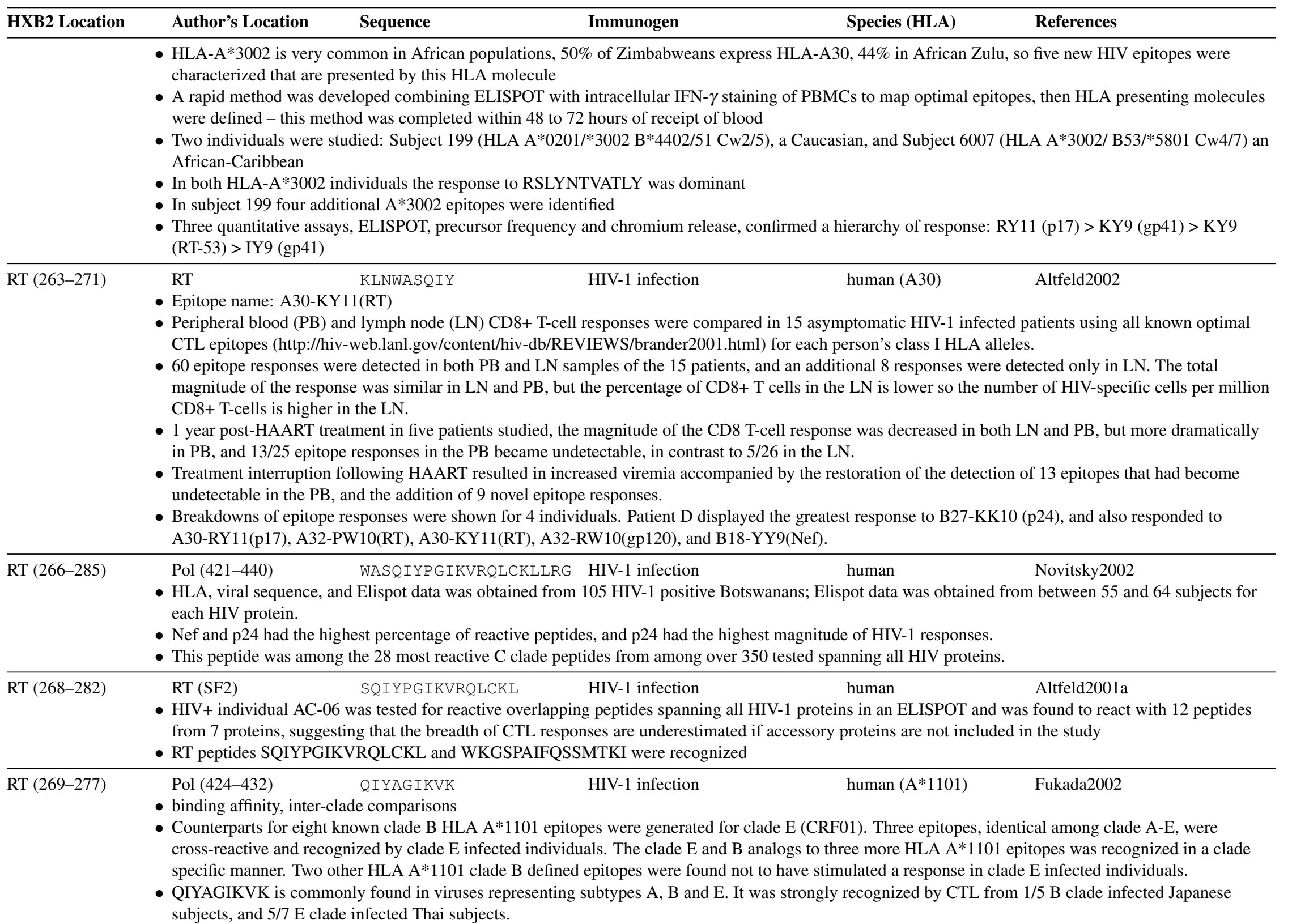




\begin{tabular}{|c|c|c|c|c|c|}
\hline HXB2 Location & Author's Location & Sequence & Immunogen & Species (HLA) & References \\
\hline & \multicolumn{5}{|c|}{$\begin{array}{l}\text { - QIYAGIKVK had the highest } A^{*} 1101 \text { binding affinity, but qiyagikvR and qiyPgikvR (the most common C and D clade variant both bound to A*1101). } \\
\text { QIYAGIKVK and qiyagikvR were both cross-presented by a clone from a B clade infection, but qiyPgikvR was not. }\end{array}$} \\
\hline RT (269-277) & (LAI) & QIYPGIKVR & & (A3) & Altfeld2000a, Brander2001 \\
\hline RT (269-277) & \multicolumn{5}{|c|}{$\begin{array}{l}\text { - The CTL response to optimally defined CTL epitopes restricted by HLA class I A and B alleles in individuals who coexpressed HLA A2, A3, and B7 was } \\
\text { studied in eight HIV-1-infected subjects, two with acute infection, five with chronic, and one long-term non-progressor (LTNP) } \\
\text { - } 2 \text { to } 17 \text { epitopes were recognized in a given individual, A2-restricted CTL response tended to be narrow and never dominated the response, and } 25 / 27 \\
\text { epitopes were targeted by at least one person } \\
\text { - All patients recognized at least } 1 \text { A3 epitope, up to } 8 \text { A3 epitopes, but none was clearly dominant }\end{array}$} \\
\hline RT (269-277) & \multicolumn{5}{|c|}{$\begin{array}{l}\text { - CTL responses in } 18 \text { acutely HIV-infected HLA-A3 }(n=7) \text { or }-\mathrm{B} 7(\mathrm{n}=4) \text { or both }-\mathrm{A} 3 \text { and } \mathrm{B} 7(\mathrm{n}=7) \text { positive individuals were studied. } \\
\text { - One individual, AC-06, was homozygous at all three class I alleles }(\mathrm{A} 3, \mathrm{~B} 7, \mathrm{Cw} 7) \text {, was treated during acute infection and had supervised treatment } \\
\text { interruptions (STI). He had only two detectable CTL responses during acute infection, but after STI this broadened to } 27 \text { distinct epitopes including } 15 \\
\text { restricted by HLA-A3, } 11 \text { by HLA-B7, and } 1 \text { by HLA-Cw7. } \\
\text { - } 1 / 14 \text { HLA-A3 positive individuals had detectable A3-restricted responses to this epitope during acute infection, but only } 5 / 15 \text { of HLA-A3 epitopes tested } \\
\text { were targeted during acute infection. } 4 / 7 \text { individuals began to have detectable responses to this epitope after STI. }\end{array}$} \\
\hline RT (271-279) & \multicolumn{5}{|c|}{ - C. Brander notes this is a B*4201 epitope } \\
\hline RT (271-279) & \multicolumn{5}{|c|}{$\begin{array}{l}\text { - YAGIKVRQL and YPGIKVKQL are naturally occurring variants that are both reactive } \\
\text { - YHKIKVRQL is a naturally occurring variant that has not been tested } \\
\text { - Epitope defined in the context of the Pediatric AIDS Foundation ARIEL Project, a mother-infant HIV transmission study }\end{array}$} \\
\hline RT (271-279) & $\begin{array}{l}\text { Pol (438-446 IIIB) } \\
\text { - This study describes } \\
\text { Detection of CTL es } \\
\text { infants } \\
\text { - An additional varian } \\
\text { YHGIKVRQL was }\end{array}$ & $\begin{array}{l}\text { YPGIKVRQL } \\
\text { rnal CTL respo } \\
\text { mutants in the } \mathrm{n} \\
\text { gave a positive } \\
\text { ape mutant }\end{array}$ & $\begin{array}{l}\text { HIV-1 infection } \\
\text { xt of mother-to-in } \\
\text { iated with transm } \\
\text { YPGIKVKQL, YA }\end{array}$ & $\begin{array}{l}\text { human (B42) } \\
\text { susceptible form }\end{array}$ & $\begin{array}{l}\text { Wilson1999a } \\
\text { virus tended to be found in infected }\end{array}$ \\
\hline RT (293-301) & $\begin{array}{l}\text { RT (448-456 SF2) } \\
\text { - A CTL clone respon } \\
\text { - Only 1/7 B35-positi } \\
\text { - An E to K substituti } \\
\text { - An I to V substitutic } \\
\text { - An I to V substitutic }\end{array}$ & $\begin{array}{l}\text { IPLTEEAEL } \\
\text { to this epitope } \mathrm{W} \\
\text { dividuals had a } \\
\text { position } 5 \text { abrog } \\
\text { position } 1, \mathrm{P} \text { to } \\
\text { position } 1 \text { did no } \\
\text { Arias1998], this }\end{array}$ & $\begin{array}{l}\text { HIV-1 infection } \\
\text { this epitope } \\
\text { is, but not binding } \\
\text { nd } E \text { to } K \text { at } 5 \text {, abi } \\
\text { ne thumb region o }\end{array}$ & $\begin{array}{l}\text { human }(\mathrm{B} * 3501) \\
\text { is and binding to } \mathrm{E}\end{array}$ & \begin{tabular}{|l|} 
Menendez-Arias1998 \\
Tomiyama1997
\end{tabular} \\
\hline
\end{tabular}




\begin{tabular}{|c|c|c|c|c|}
\hline HXB2 Location & Author's Location & Immunogen & Species (HLA) & References \\
\hline RT (293-301) & \multicolumn{4}{|c|}{$\begin{array}{l}\text { - Epitope name: HIV-B35-SF2-24 } \\
\text { - This epitope is naturally processed and presented by both HLA-B*3501 and HLA-B*5101 and is cross-recognized by a single CTL clone } \\
\text { - IPLTEEAEL binds approximately four times more tightly to HLA-B*3501 than HLA-B*5101. }\end{array}$} \\
\hline RT (293-301) & $\begin{array}{l}\text { Pol (489-456) } \\
\text { - The IPLTEEAEL epito } \\
\text { Valpha12.1 and Vbeta5 } \\
\text { presented by B*5301 ar } \\
\text { of HLA class I molecul }\end{array}$ & $\begin{array}{l}\text { IPLTEEAEL HIV-1 infection } \\
\text { was known to be presented by both HLA-B*3501 and -B*5101 to } \\
\text { was shown recognize the epitope in either HLA-B*3501 and -B*5 } \\
\mathrm{B}^{*} 0702 \text { in cytolytic CTL assays, demonstrating that this single TC }\end{array}$ & $\begin{array}{l}\text { human }(\mathrm{B} * 3501 \text {, } \\
\mathrm{B} * 5301, \mathrm{~B} * 5101 \text {, } \\
\mathrm{B} * 0702) \\
\text { dual specific CTL clone. } \\
1 \text {. Furthermore, this TCl } \\
\text { complex recognizes the }\end{array}$ & $\begin{array}{l}\text { Ueno2002 } \\
\text { A single TCR complex bearing } \\
\text { also recognized the peptide } \\
\text { ame peptide presented by a range }\end{array}$ \\
\hline RT (293-301) & $\begin{array}{l}\text { (SF2) } \\
\text { - HLA B35 is associated } \\
\text { - The sequences of } 9 \text { pre } \\
\text { - } 3 / 9 \text { CTL epitopes had } \mathrm{s} \\
\text { associated pattern of mı }\end{array}$ & $\begin{array}{l}\text { IPLTEEAEL HIV-1 infection } \\
\text { ith rapid disease progression } \\
\text { ously described HIV-1 B35 CTL epitopes were obtained in } 10 \text { HLA } \\
\text { ostitutions that were more common in B35+ individuals than in B3 } \\
\text { ation }\end{array}$ & $\begin{array}{l}\text { human (B35) } \\
335+\text { and } 19 \text { HLA B35- } \mathrm{i} \\
\text { individuals but this was }\end{array}$ & $\begin{array}{l}\text { Kawana1999 } \\
\text { dividuals } \\
\text { le of the six that had no B35 }\end{array}$ \\
\hline RT (293-301) & $\begin{aligned} & \text { RT (448-456 SF2) } \\
\text { - } & \text { Binds HLA-B*3501 an } \\
\text { - } & \text { Reviewed in Menende? }\end{aligned}$ & $\begin{array}{l}\text { IPLTEEAEL HIV-1 infection } \\
\mathrm{B} * 5101 \\
\text { Arias1998, this epitope lies in the thumb region of RT }\end{array}$ & human (B35, B51) & $\begin{array}{l}\text { Menendez-Arias 1998, } \\
\text { Shiga1996 }\end{array}$ \\
\hline RT (293-301) & $\begin{array}{l}\text { Pol (447-455) } \\
\text { - ELISPOT was used to s } \\
\text { HIV-1-infected female }\end{array}$ & $\begin{array}{l}\text { IPLTEEAEL } \begin{array}{l}\text { HIV-1 infection, HIV-1 exposed } \\
\text { seronegative }\end{array} \\
\text { ady CTL responses to a panel of } 54 \text { predefined HIV-1 epitopes in } 9 \\
\text { airobi sex workers }\end{array}$ & $\begin{array}{l}\text { human (B51) } \\
\text { HIV-1-exposed, persisten }\end{array}$ & Kaul2001a \\
\hline RT (294-318) & $\begin{array}{l}\text { RT (461-485 HXB2) } \\
\text { - One of five epitopes def }\end{array}$ & $\begin{array}{l}\text { PLTEEAELELAENREILKE- HIV-1 infection } \\
\text { PVHGVY } \\
\text { ned for RT-specific CTL clones in this study }\end{array}$ & human (A2) & $\begin{array}{l}\text { Menendez-Arias1998, } \\
\text { Walker1989 }\end{array}$ \\
\hline RT (308-317) & $\begin{array}{l}\text { RT (LAI) } \\
\text { - Recognized by CTL fro } \\
\text { - Recognized by CTL fro }\end{array}$ & $\begin{array}{l}\text { EILKEPVGHV HIV-1 infection } \\
\text { a long-term survivor, SPIETVPVKL was also recognized } \\
\text { a progressor, EELRQHLLRW and TWETWWTEYW were also r }\end{array}$ & $\begin{array}{l}\text { human }(\mathrm{A} * 0201) \\
\text { ognized }\end{array}$ & \begin{tabular}{|l|} 
Menendez-Arias1998, \\
vanderBurg1997
\end{tabular} \\
\hline RT (309-317) & $\begin{array}{l}\text { RT (476-484 LAI) } \\
\text { - Longitudinal study of } 8 \\
\text { peripheral blood cells. } \\
\text { Three HLA-A*0201 ch } \\
\text { either using PBMC spe }\end{array}$ & $\begin{array}{l}\text { ILKEPVHGV HIV-1 infection } \\
\text { nfants with prolonged viral suppression due to combination antire } \\
8 \text { were studied using a Chromium release assay and no response w } \\
\text { dren were tested using SLYNTVATL or ILKEPVHGV HLA A*02 } \\
\text { mens, or PBMC which had been stimulated in vitro for a week. }\end{array}$ & $\begin{array}{l}\text { human } \\
\text { iral therapy showed no } \mathrm{H} \\
\text { detected using Gag expre } \\
\text { tetramers and again no } \mathrm{H}\end{array}$ & $\begin{array}{l}\text { Luzuriaga2000 } \\
\mathrm{V} \text {-1 specific CTL responses in } \\
\text { sed in vaccinia in the target cells. } \\
\mathrm{V} \text {-specific response was detected, }\end{array}$ \\
\hline
\end{tabular}




\begin{tabular}{|c|c|c|c|c|c|}
\hline HXB2 Location & Author's Location & Sequence & Immunogen & Species (HLA) & References \\
\hline & \multicolumn{5}{|c|}{$\begin{array}{l}\text { - In contrast, one of the children with suppressed HIV viral replication who was co-infected with HIV and EBV, while HIV-tetramer negative, had } \\
\text { EBV-tetramer staining cells at a frequency of } 0.14 \% \text { in the PBMC. }\end{array}$} \\
\hline RT (309-317) & \multicolumn{5}{|c|}{$\begin{array}{l}\text { - The single cell ELISPOT assay was optimized and highly specific, and found to work well even after the primary cells had been frozen and thawed } \\
\text { - Increases in gamma interferon producing cells were observed in response to anti-retroviral therapy using single cell IFN-gamma-production ELISPOT }\end{array}$} \\
\hline RT (309-317) & \multicolumn{5}{|c|}{$\begin{array}{l}\text { - Administration of triple-drug antiretroviral therapy (IDV, 3TC and ZDV) sometimes showed a transient increase and other times failed to increase CTL } \\
\text { responses in patients with advanced HIV disease, but there is a stable population of tetramer stained HIV-specific CD8+ CD45RO+ cells that persist afte } \\
\text { therapy and long periods of virus being below the level of detection }\end{array}$} \\
\hline RT (309-317) & $\begin{array}{l}\text { RT } \\
\text { - Epitope name: IV9 } \\
\text { - This study examinec } \\
\text { ILKEPVHGV } \\
\text { - } 71 \% \text { of the } 28 \text { HIV- } \\
\text { (SLYNTVATL) and } \\
\text { - There were no differ } \\
\text { - Tetramer-binding ce }\end{array}$ & $\begin{array}{l}\text { ILKEPVHGV } \\
\text { CTL response } \\
\text { ted HLA-A*02 } \\
\text { ildren by the po } \\
\text { observed in ch } \\
\text { re memory acti }\end{array}$ & $\begin{array}{l}\text { HIV-1 infection } \\
\text { hildren by tetram } \\
\text { n recognized both } \\
\text { PVHGV) } \\
\text { erapy versus thos } \\
45 \mathrm{RO}+\text {, CD45R }\end{array}$ & $\begin{array}{l}\text { human }(\mathrm{A} * 02) \\
\text {-A2 immunodom } \\
\text { s from } 26 \text { childre } \\
\text { 9-, CD8+ T-cells }\end{array}$ & $\begin{array}{l}\text { Scott-Algara2001 } \\
\text { itopes SLYNTVATL and } \\
\text { positive by the gag tetramer }\end{array}$ \\
\hline RT (309-317) & & ILKEPVHGV & HIV-1 infection & human $(\mathrm{A} * 0201$ & Wilson2000a \\
\hline
\end{tabular}

- Three individuals with highly focused HIV-specific CTL responses were studied during acute infection using tetramers - high frequencies of HIV-1-specific CD8+ T cells were found prior to seroconversion, and there was a close temporal relationship between the number of circulating HIV-specific T cells and viral load was also found

- All three patients were B*2705, with HLA alleles: A1, A30/31, B*2705, B35; A1, A*0301, B7, B2705; and A*0201, A*0301, B2705, B39

- ELISPOT was used to test a panel of CTL epitopes that had been defined earlier and were appropriate for the HLA haplotypes of the study subjects - 3/3 subjects showed a dominant response to the B*2705 epitope KRWIILGGLNK

- The subject with $\mathrm{A}^{*} 0201$ had a moderatly strong response to SLYNTVATL

- Weak responses were observed to A*301-RLRPGGKKK, A*301-QVPLRPMTYK, and B7-TPGPGVRYPL in the subject who was HLA A1, A*0301, B7, $\mathrm{B} * 2705$

- No acute response was detected to the following epitopes: A*201-ILKEPVHGV, A*301-KIRLRPGGK, A*301-AIFQSSMTK, A*301-TVYYGVPVWK, B35-EPIVGAETF, B35-HPDIVIYQY, B35-PPIPVGEIY, B35-NSSKVSQNY, B35-VPLRPMTY, B35-DPNPQEVVL

RT (309-317) Pol (476-484) ILKEPVHGV HIV-1 infection

- High levels of CD8+ HIV-1 specific and cytomegalovirus specific CTL were detected by HLA-A*0201-peptide tetramers in 3 infected subjects with very low CD4 counts, but CD8 T cell mediated effector activity was not seen

- Thus HIV-1 specific CD8+ cells may be present but may lack direct effector activity in late disease, suggesting that overcoming antigen unresponsiveness may be a useful therapeutic strategy

RT (309-317)

Pol (476-484) ILKEPVHGV

HIV-1 infection

human $\left(\mathrm{A}^{*} 0201\right)$

Sewell1999

- Proteasome regulation influences epitope processing and could influence immunodominance

- The proteasome is inhibited by lactacystin treatment, and gamma IFN induces expression of proteasome subunits, LMP2 and LMP7, which combine with the proteasome to create an immunoproteasome 


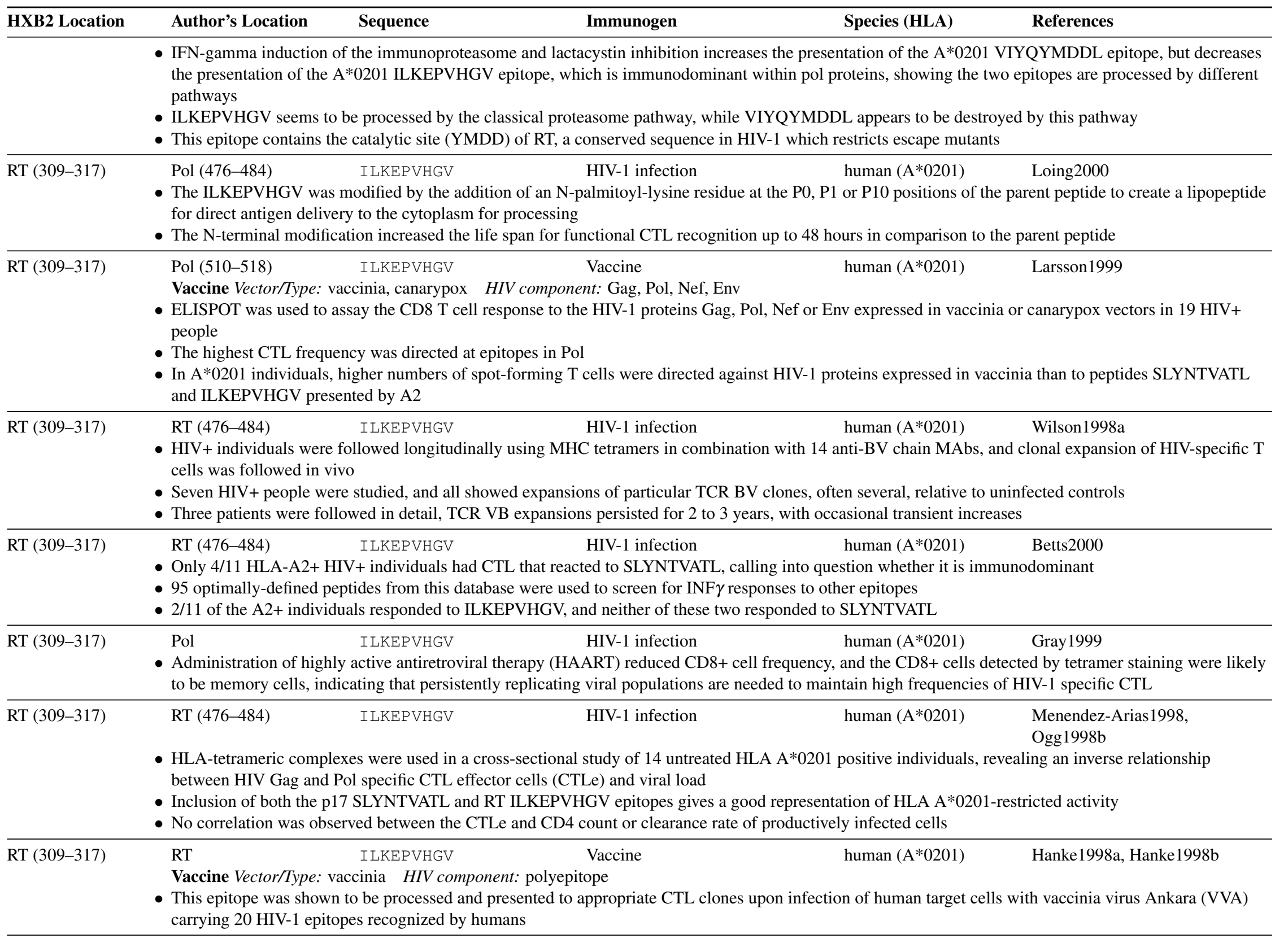




\begin{tabular}{|c|c|c|c|c|c|}
\hline HXB2 Location & Author's Location & Sequence & Immunogen & Species (HLA) & References \\
\hline RT (309-317) & \multicolumn{5}{|c|}{$\begin{array}{l}\text { - This epitope was included as a positive control } \\
\text { - Binding affinity to } \mathrm{A}^{*} 0201 \text { was measured, } C_{-} 1 / 2 \max \mu M=12\end{array}$} \\
\hline RT (309-317) & \multicolumn{5}{|c|}{$\begin{array}{l}\text { - Immunogenic in humans, slow dissociation rate, and associated with immunogenicity in transgenic HLA-A*0201/K } \mathrm{K}^{b} \text { mice } \\
\text { - CTL generated by in vitro stimulation of PBMC derived from uninfected individual }\end{array}$} \\
\hline RT (309-317) & \multicolumn{5}{|c|}{ - Binds HLA-A*0201 - CTL generated by in vitro stimulation of PBMC from an HIV negative donor } \\
\hline RT (309-317) & $\begin{array}{l}\text { RT (476-484) } \\
\text { - Mutational study: po }\end{array}$ & $\begin{array}{l}\text { ILKEPVHGV } \\
1 \text { I to Y incres }\end{array}$ & $\begin{array}{l}\text { HIV-1 infection } \\
\text { oility with HLA-A*02 }\end{array}$ & human $\left(\mathrm{A}^{*} 0201\right)$ & $\begin{array}{l}\text { Menendez-Arias1998, } \\
\text { Pogue1995 }\end{array}$ \\
\hline RT (309-317) & $\begin{array}{l}\text { RT (476-484) } \\
\text { - Identical twin hemor } \\
\text { - One had a response } \\
\text { - Viral sequencing fro } \\
\text { - } 71 \% \text { of an additional } \\
\text { - Those individuals wi } \\
\text { - Goulder1997a is a }\end{array}$ & $\begin{array}{l}\text { ILKEPVHGV } \\
\text { c brothers were } \\
\text { A2 epitope SL } \\
\text { twin that had r } \\
\text { f } 22 \text { HIV-1 infe } \\
\text { ol ILKEPVHC } \\
\text { w of immune es }\end{array}$ & $\begin{array}{l}\text { HIV-1 infection } \\
\text { th the same batch of } f \\
\text { other to pol A2 epitop } \\
\text { YNTVATL indicated } \\
01 \text { positive donors pre } \\
\text { ed to have mutations } i \\
\text { arizes this study }\end{array}$ & $\begin{array}{l}\text { human }\left(\mathrm{A}^{*} 0201\right) \\
\text { ee substituted form } \\
\text { YNTVATL } \\
\text { YNT gag SLYN }\end{array}$ & $\begin{array}{l}\text { Goulder1997e Goulder1997a } \\
\text { Menendez-Arias1998 } \\
\text { AVAVL } \\
\text { L }\end{array}$ \\
\hline RT (309-317) & $\begin{array}{l}\text { RT (309-317) } \\
\text { - This paper introduce } \\
\text { were prepared that c } \\
\text { PBMCs. } \\
\text { - Three patients only s } \\
\text { Gag epitope }(0.28 \%) \\
\text { - The A2-Pol CD8+c }\end{array}$ & $\begin{array}{l}\text { ILKEPVHGV } \\
\text { tetramer metho } \\
\text { in CTL lines sp } \\
\text { d the Gag epito } \\
\text { were CD45RO }\end{array}$ & $\begin{array}{l}\text { HIV-1 infection } \\
\text { rmits quantification o } \\
\text { VHGV and SLYNTV } \\
\text { one patient had the } 1 \\
\text { A-DR and CD38 nega }\end{array}$ & $\begin{array}{l}\text { human }\left(\mathrm{A}^{*} 0201\right) \\
\text { based on expression } \\
\text { fuantify HIV-specifi } \\
\text { cy of tetramer staini } \\
\text { g a memory rather } t\end{array}$ & $\begin{array}{l}\text { Altman1996 } \\
\text { +ific TCRs-HLA-A2 tetramers } \\
\text { he Pol epitope }(0.77 \%) \text {, less to the } \\
\text { ector phenotype }\end{array}$ \\
\hline RT (309-317) & $\begin{array}{l}\text { RT (476-484) } \\
\text { - HLA-A2 heavy chai } \\
\text { - The HLA-A2-peptid } \\
\text { - Suggests that preforr }\end{array}$ & $\begin{array}{l}\text { ILKEPVHGV } \\
\beta 2 \text {-microglobs } \\
\text { aplex elicited H } \\
\text { ILA-peptide co }\end{array}$ & $\begin{array}{l}\text { in vitro stimulation } \\
\text { E. coli were refolded } \\
\text { specific CTL response } \\
\text { rovide an alternate to }\end{array}$ & $\begin{array}{l}\text { human }\left(\mathrm{A}^{*} 0201\right) \\
\text { of this peptide } \\
\mathrm{g} \text { HLA-A2 } \\
\text { cessing for immun }\end{array}$ & $\begin{array}{l}\text { Menendez-Arias1998, } \\
\text { Walter1997 }\end{array}$ \\
\hline RT (309-317) & $\begin{array}{l}\text { RT (464-472) } \\
\text { - Peptide-tetramer con } \\
\text { Class I HLA-restrict } \\
\text { - } 17 / 18 \text { asymptomatic } \\
\text { - After HAART, the } \mathrm{m}\end{array}$ & $\begin{array}{l}\text { I LKEPVHGV } \\
\text { es of A*0201 a } \\
\text { ti-HIV CD8+ T } \\
\text { nts had a CTL } \\
\text { ty of the epitop }\end{array}$ & $\begin{array}{l}\text { HIV-1 infection } \\
\text { or ILKEPVHGV we } \\
\text { or both epitopes }-72 \% \\
\text { ere apparently memo }\end{array}$ & $\begin{array}{l}\text { human }(\mathrm{A} * 0201) \\
\text { individuals receivi } \\
\text { ponse to SLYNTVE }\end{array}$ & $\begin{array}{l}\text { Gray1999 } \\
\text { ART to determine the frequency of }\end{array}$ \\
\hline
\end{tabular}




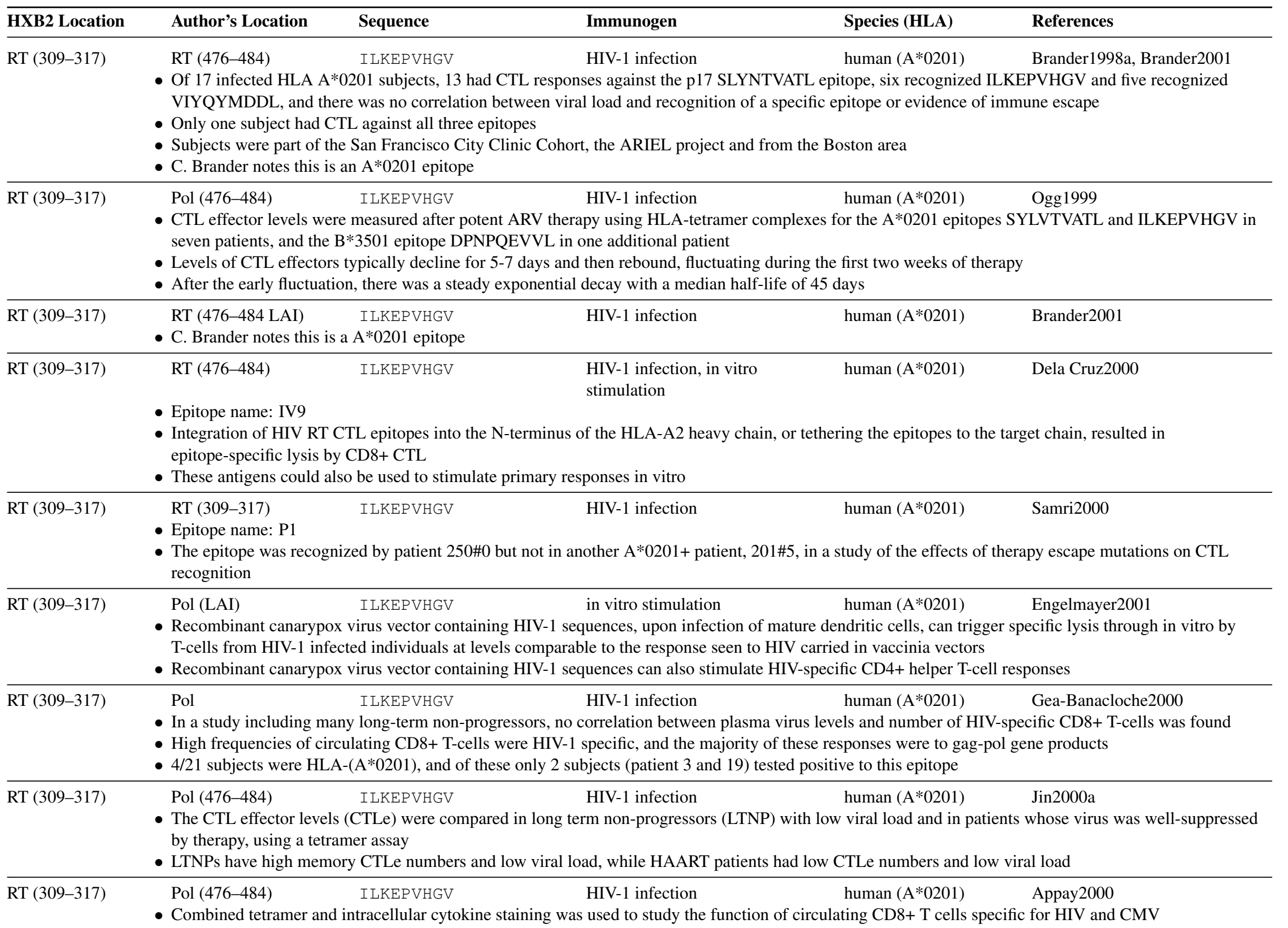




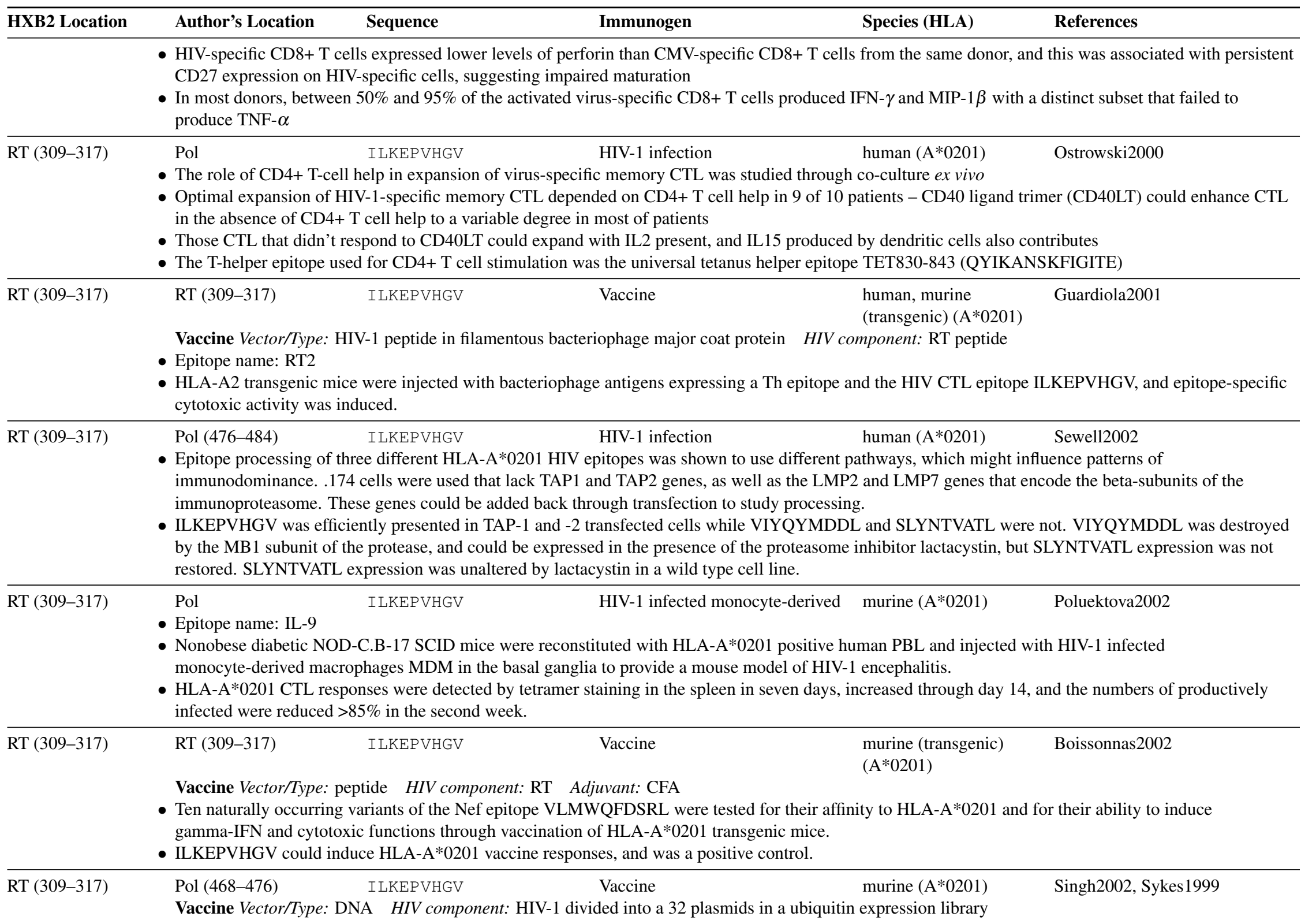




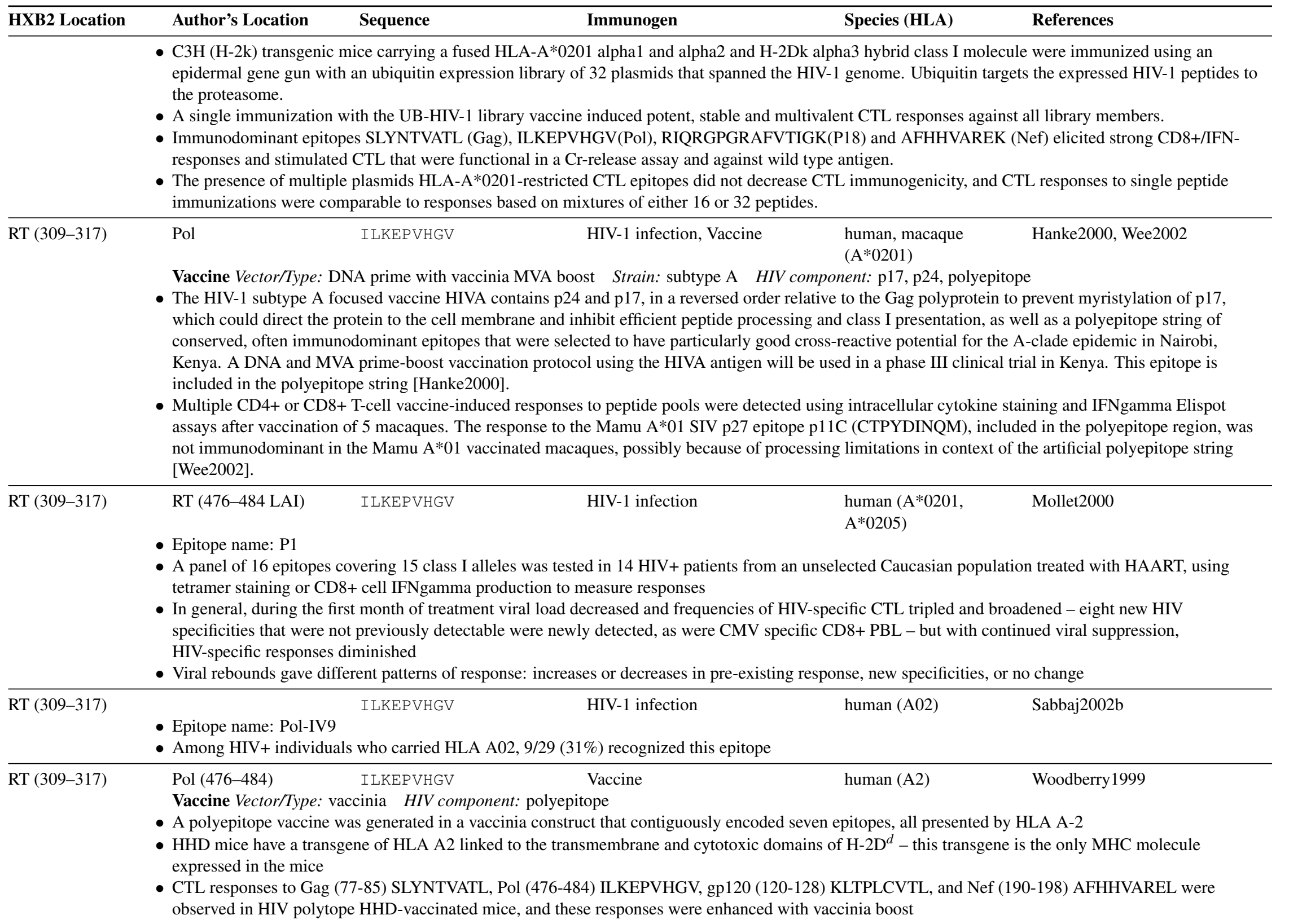




\begin{tabular}{|c|c|c|c|c|}
\hline HXB2 Location & Author's Location & Sequence & Species (HLA) & References \\
\hline & \multicolumn{4}{|c|}{$\begin{array}{l}\text { - No CTL immune responses were generated against HLA A2-restricted HIV epitopes Nef 157-166 (PLTFGWCYKL), Pol 346-354 (VIYQYMDDL), and } \\
\text { Nef 180-189 (VLEWRFDSRL) } \\
\text { - Sixteen HLA A2+ patients were tested for their ability to make CTL responses by peptide restimulation in culture with the epitopes selected for inclusion in } \\
\text { the polytope - one individual recognized all seven of these epitopes; } 7 \text { patients had CTL cultures able to recognize at least one of the epitopes, and } 6 \text { of } \\
\text { those } 7 \text { recognized more than one epitope, but they were not able to test all peptides for all patients; many patients only had three peptides tested } \\
\text { - ILKEPVHGV was recognized by } 2 \text { of the patients }\end{array}$} \\
\hline RT (309-317) & \multicolumn{4}{|c|}{$\begin{array}{l}\text { - TCR usage in CTL specific for this epitope was examined in three patients and identical Vbeta6.1 and Valpha2.5 gene segments were used and two of the } \\
\text { patients had very similar complementarity-determining regions - clonal expansion of RT-HIV-specific CTL can contribute to the skewed TCR repertoire in } \\
\text { HIV-1 infected patients } \\
\text { - CTL clones from all three patients showed similar sensitivity to mutation in the epitope, ilkepvhEv was well recognized (the sequence from SF2), } \\
\text { ilkDpvhgv was not (the common A clade form) }\end{array}$} \\
\hline RT (309-317) & \multicolumn{4}{|c|}{$\begin{array}{l}\text { - Nef down-regulates MHC class I molecules, which inhibits CTL killing of HIV-infected targets } \\
\text { - The anti-RT CTL clone killed Nef- cells less efficiently than anti-gag clones, correlated with the reduced expression of RT }\end{array}$} \\
\hline T (309-317) & \multicolumn{4}{|c|}{ - The capacity of dendritic cells to process and present antigen and stimulate anti-HIV-1 CTL memory responses was studied } \\
\hline$(309-317)$ & \multicolumn{4}{|c|}{$\begin{array}{l}\text { - Allogeneic dendritic cells (DCs) were obtained from HLA-identical siblings, pulsed with rgp } 160 \mathrm{MN} \text { or A2-restricted HIV-1 epitope peptides, and infused } \\
\text { monthly into six HIV-infected patients } \\
\text { - } 1 / 6 \text { showed increased env-specific CTL and increased lymphoproliferative responses, } 2 / 6 \text { showed increase only in proliferative responses, and } 3 / 6 \text { showed } \\
\text { no change - pulsed DCs were well tolerated } \\
\text { - ILKEPVHGV is a conserved HLA-A2 epitope included in this study - } 5 / 6 \text { patients had this sequence as their HIV direct sequence, and these had a } \\
\text { detectable CTL response- one person carried the form ILREPVHGV and had no detectable CTL }\end{array}$} \\
\hline RT (309-317) & $\begin{array}{l}\text { RT (476-484) } \\
\text { CTL clones recogni }\end{array}$ & $\begin{array}{l}\text { ILKEPVHGV } \\
\text { turally processec }\end{array}$ & $\begin{array}{l}\text { HIV-1 infection } \\
\text { de abundance corresponded to level of CTL killing }\end{array}$ & $\begin{array}{l}\text { Menendez-Arias1998 } \\
\text { Tsomides1994 }\end{array}$ \\
\hline RT (309-317) & \multicolumn{4}{|c|}{$\begin{array}{l}\text { - A CTL response was found in exposed but uninfected prostitutes from Nairobi using previously-defined B clade epitopes that tended to be conserved in A } \\
\text { and D clades - such cross-reactivity could protect against both A and D and confer protection in Nairobi where both subtypes are circulating } \\
\text { - The A subtype consensus is ILKDPVHGV } \\
\text { - The D subtype consensus is identical to the epitope ILKEPVHGV }\end{array}$} \\
\hline RT (309-317) & $\begin{array}{l}\text { RT (476-484) } \\
\text { - The consensus pepti } \\
\text { - The consensus pepti }\end{array}$ & $\begin{array}{l}\text { ILKEPVHGV } \\
\text { B and D clade } \\
\text { a subset of A cl }\end{array}$ & $\begin{array}{l}\text { HIV-1 infection human (A2) } \\
\text { e As have the sequence ILKEPVHGV } \\
\text { DPVHGV, is not cross-reactive }\end{array}$ & Cao1997a, Menendez-Arias1998 \\
\hline Т (309-317) & RT (476-484) & ILKEPVHGV & HIV-1 infection & Menendez-Arias1998, Yang1996 \\
\hline
\end{tabular}

- CD4+ cell lines acutely infected with HIV were studied to determine their susceptibility to lysis by CTL

- Clones specific for RT lysed HIV-1 infected cells at lower levels than Env or Gag specific clones 


\begin{tabular}{|c|c|c|c|c|c|}
\hline HXB2 Location & Author's Location & Sequence & Immunogen & Species (HLA) & References \\
\hline & \multicolumn{5}{|c|}{$\begin{array}{l}\text { - The distinction was thought to be due to lower expression of RT relative to Env and Gag } \\
\text { - CTL can lyse infected cells early after infection, possibly prior to viral production }\end{array}$} \\
\hline RT (309-317) & \multicolumn{5}{|c|}{$\begin{array}{l}\text { - CTL inhibit HIV-1 replication at effector cell concentrations comparable to those found in vivo } \\
\text { - CTL produced HIV-1-suppressive soluble factors - MIP-1 } \alpha \text {, MIP-1 } 1 \beta \text {, RANTES, after antigen-specific activation } \\
\text { - CTL suppress HIV replication more efficiently in HLA-matched cells }\end{array}$} \\
\hline RT (309-317) & $\begin{array}{l}\text { RT (309-317) } \\
\text { - Two clones were ob }\end{array}$ & \multicolumn{4}{|c|}{ - Two clones were obtained with different TCR usage, $\mathrm{V} \_\beta 1$ and $\mathrm{V} \_\beta 21$} \\
\hline RT (309-317) & $\begin{array}{l}\text { RT (476-484) } \\
\text { - Cervical CTL clone }\end{array}$ & $\begin{array}{l}\text { ILKEPVHGV } \\
\text { n an HIV-infecte }\end{array}$ & $\begin{array}{l}\text { HIV-1 infection } \\
\text { nized this epitope }\end{array}$ & human (A2) & \begin{tabular}{|l|} 
Menendez-Arias1998 \\
Musey1997 \\
\end{tabular} \\
\hline RT (309-317) & $\begin{array}{l}\text { RT (476-484 LAI) } \\
\text { - Precise identificatio }\end{array}$ & $\begin{array}{l}\text { ILKEPVHGV } \\
\text { he nonamer that }\end{array}$ & HIV-1 infection & human (A2) & $\begin{array}{l}\text { Menendez-Arias1998, } \\
\text { Tsomides1991 }\end{array}$ \\
\hline RT (309-317) & $\begin{array}{l}\text { RT (476-484 LAI) } \\
\text { - Promotes assembly }\end{array}$ & ILKEPVHGV & $\begin{array}{l}\text { Peptide-HLA interaction } \\
\text { tes }\end{array}$ & human (A2) & $\begin{array}{l}\text { Connan1994 } \\
\text { Menendez-Arias1998 } \\
\end{array}$ \\
\hline RT (309-317) & \multicolumn{4}{|c|}{ - Studied in the context of HLA-A2 peptide binding } & Parker1992 \\
\hline RT (309-317) & \multicolumn{5}{|c|}{$\begin{array}{l}\text { - CTL specific responses were measured over a } 1.3 \text { to } 1.5 \text { year period in members of the Sydney Blood Bank Cohort (SBBC) who had been infected with a } \\
\text { natural attenuated strain of HIV-1 which was Nef-defective } \\
\text { - Some of these patients had prolonged high levels of CTL effector and memory cells despite low viral load }\end{array}$} \\
\hline RT (309-317) & $\begin{array}{l}\text { RT (476-484) } \\
\text { - This study compare } \\
\text { HIV-uninfected don } \\
\text { - Strong CTL respons } \\
\text { macrophages were r } \\
\text { - A weak response to } \\
\text { - No detectable respo } \\
\end{array}$ & $\begin{array}{l}\text { ILKEPVHGV } \\
\text { ability of macro } \\
\text { sing peptide-pul } \\
\text { ere elicited by th } \\
\text { le to prime a CT } \\
\text { PLCVSL was st } \\
\text { jas observed for }\end{array}$ & $\begin{array}{l}\text { in vitro stimulation } \\
\text { itic cells to stimulate prim } \\
\text { ndritic cells performed bet } \\
\text { KTLRA and GEIYKRWI } \\
\text { ist DRFYKTLRA } \\
\text { acrophages as the APC } \\
\text { eviously-defined HIV epito }\end{array}$ & $\begin{array}{l}\text { human (A2) } \\
\text { ses in CD8+ lym } \\
\text { for the stimulat } \\
\text { sented by either } \\
\text { RPGGK, ILKEP }\end{array}$ & $\begin{array}{l}\text { Zarling1999 } \\
\text { isolated from HLA-appropriate } \\
\text { mary responses } \\
\text { or mature dendritic cells - } \\
\text { IRLRPGGK, GPKVKQWPL }\end{array}$ \\
\hline RT (309-317) & $\begin{array}{l}\text { RT (480-) } \\
\text { - This study uses Epil } \\
\text { - } \text { Based on EpiMatrix } \\
\text { were shown to bind } \\
\text { - Two of these } 12 \text { pep } \\
\text { - This sequence is no }\end{array}$ & $\begin{array}{l}\text { ILKEPVHGV } \\
\text { X for T cell epitc } \\
\text { ictions, } 28 \text { pepti } \\
\text { predicted HLA } \\
\text { had been previo } \\
\text { served between }\end{array}$ & $\begin{array}{l}\text { computer prediction } \\
\text { identify possible HLA-B2 } \\
\text { ized and tested using T2 b } \\
\text { CTL epitopes: HLA-B27 } \\
\text { d only in a small number }\end{array}$ & $\begin{array}{l}\text { (A2) } \\
\text { CTL epitopes in } \\
\text { says for potential I } \\
\text { SLNK and HLA-A } \\
\text { isolates }\end{array}$ & $\begin{array}{l}\text { Schafer1998 } \\
\text { or B27 binding, and } 12 \text { of these } \\
\text { PVHGV }\end{array}$ \\
\hline
\end{tabular}




\begin{tabular}{lllll}
\hline HXB2 Location & Author's Location & Sequence & Immunogen & Species (HLA) \\
\hline RT (309-317) & RT & ILKEPVHGV & HIV-1 infection & human (A2)
\end{tabular}

- HIV was scanned for all peptides which carried the A2-supermotif pattern conserved in more than $50 \%$ of B clade sequences - 233 peptides met this criteria, and 30 of these bound to HLA-A $* 0201-20 / 30$ bound to at least $3 / 5$ of HLA-A2 supertype alleles tested

- Three additional previously described HLA-A2 epitopes were added to the set of 20, and 18/22 chronically infected HLA-A2 individuals had CTL that recognized at least one of the 23 peptides (median of 2 and maximum of 6), while 6/12 acute infected individuals recognized at least 1 (median of 1 and maximum of 2)

- This peptide binds to four HLA-A2 supertype alleles: A*0201, A*0202, A*0206 (highest affinity) and A*6802

- RT IV9 was recognized in 7/22 patients with chronic HIV-1 infection

- $1 / 13$ patients with acute HIV-1 infection recognized RT IV9

RT (309-317) Pol (subtype A) ILKDPVHGV HIV-1 infection $\quad$ Kaul2001c

- This study examines CTL responses in HIV exposed, persistently seronegative individuals, HEPS, who eventually seroconverted - 11/114 HEPS Nairobi sex workers eventually seroconverted, and for six of these HIV CTL reactive epitopes had been defined while seronegative

- ILKDPVHGV or ILKEPVHGV was recognized in 1 of the 6 women (ML1760), and the response was present in the last available sample prior to seroconversion, 12 months

- 20/20 sequences of the infecting strain had no substitutions in this epitope, all were ILKDPVHGV, so there was no evidence for escape

- The epidemiological factor associated with seroconversion was stopping sex work and HIV-specific CTL activity declines when HEPS sex workers stop working for a period or retire

- This epitope was recognized by 4/22 HEPS control sex workers: ML887, ML1192, ML1250, and ML1749

$\begin{array}{lllll}\text { RT (309-317) RT (476-484) } & \text { ILKEPVHGV } & \text { Oxenius2000 }\end{array}$

- Epitope name: ILK

- Patients who started therapy at acute HIV-1 infection (three with sustained therapy, two with limited therapy upon early infection) had strong HIV specific CD4 proliferative responses and were able to maintain a CTL response even with undetectable viral load - three patients that had delayed initiation of HAART had no HIV specific CD4 proliferative responses and lost their CTL responses when HAART was eventually given and their viral loads became undetectable

- One of the 2/8 HLA-A2+ study subjects recognized this CTL epitope

- Patient SC9 (HLA A1/2, B8/13, Cw0/0701, DR2/11, DQ6/7) had a CTL response against epitopes FLKEKGGL, ILKEPVHGV,

SQRRQDILDLWIYHTQGYFPDWQNY, and GEIYKRWII peptides and all responses declined during therapy initiated at day 390 but were restored when therapy become intermittent

\begin{tabular}{llcl}
\hline RT (309-317) & Pol & ILKEPVHGV HIV-1 infection & human (A2) \\
& HLA tetramers to six epitopes were used to study HLA-A2, B8 and B57 CTL in 54 patients - HIV-specific tetramer positive cells were inversely correlated \\
& with viral load in patients with high CD4, but in patients with CD4 T-cells below 400 high tetramer frequencies were found despite high viral load
\end{tabular}

with viral load in patients with high CD4, but in patients with CD4 T-cells below 400 high tetramer frequencies were found despite high viral load

- Most patients have high levels of HIV-specific T-cell expansions, but many of these cells aren't functional

- In 15 of the patients, the proportion of IFN gamma producing tetramer cells correlated with AIDS-free survival

\begin{tabular}{lllll}
\hline RT (309-317) Pol & ILKEPVHGV & HIV-1 infection & human (A2)
\end{tabular}

- CTL responses were studied by tetramer staining in 41 patients with combination therapy - activated CD8+ T-cells decline as the viral load drops in response to therapy, but the overall level of antigen-specific cells capable of differentiating into effectors stays constant and new epitopes may be recognized

- 6/10 A*0201+ individuals had HIV-specific tetramer staining cells, and 5 of these declined upon successful therapy

- 3/10 A*0201+ individuals with chronic HIV-1 infection recognized this epitope 


\begin{tabular}{|c|c|c|c|c|}
\hline HXB2 Location & Author's Location & Sequence & Species (HLA) & References \\
\hline & \multicolumn{4}{|c|}{$\begin{array}{l}\text { - Prior to therapy, the mean percentage of CD8+ cells that recognized the immunodominant epitope SLYNVATL was six-fold greater than the percentage } \\
\text { recognizing the epitope ILKEPVHGV }\end{array}$} \\
\hline RT (309-317) & \multicolumn{4}{|c|}{$\begin{array}{l}\text { - Therapy provided during acute infection resulted in a narrower CTL response, stronger T help response, and a less diverse viral population than was seen in } \\
\text { individuals treated during chronic infection } \\
\text { individuals with primary infection but post-seroconversion therapy (Group 2), and } 10 \text { individuals who responded to HAART given during chronic infection } \\
\text { (Group 3), using } 259 \text { overlapping peptides spanning p17, p24, RT, gp41, gp120 and Nef } \\
\text { - Previously described and newly defined optimal epitopes were tested for CTL response } \\
\text { - Number of HLA-A2+ individuals that had a CTL response to this epitope broken down by group: } 1 / 10 \text { group 1, 0/6 group 2, and 3/4 group } 3\end{array}$} \\
\hline $\mathrm{T}(309-317)$ & $\begin{array}{l}\text { Pol (476-484) } \\
\text { - Variants ILK(D/E)PVHG } \\
\text { - ELISPOT was used to stu } \\
\text { HIV-1-infected female N } \\
\text { - Responses in HEPS wom } \\
\text { reduced risk of infection, } \\
\text { women } \\
\text { - } 43 / 91 \text { HEPS women had } \\
\text { - Among HLA-A2 women } \\
\text { women, SL(F/Y)NTVAT } \\
\text { - The dominant response to } \\
\text { - Four epitopes were consi } \\
\text { found in three different p } \\
\text { FRDYVDRF(Y/F)K also } \\
\text { - Differences in epitope sp } \\
\text { associated with resistance } \\
\text { - Subject ML } 1250 \text { had an } \\
\text { - Subject ML } 1760 \text { had an } \\
\text { KRWII(L/M)GLNK post }\end{array}$ & $\begin{array}{l}\text { ILKDPVHGV } \\
\text { V are A/B clade } \\
\text { Idy CTL respons } \\
\text { airobi sex worke } \\
\text { en tended to be } \\
\text { and there was a } \\
\text { CD8+ responses } \\
7 / 10 \text { HEPS and } \\
\text { in infected wo } \\
\text { this HLA allele } \\
\text { dered to be "resi } \\
\text { roteins: A2 ILK } \\
\text { in p24 } \\
\text { ecificity were on } \\
\text { to HIV-1 in thi } \\
\text { A2 response to } \\
\text { A2 response to I } \\
\text {-seroconversion }\end{array}$ & $\begin{array}{l}\text { HIV-1 infection, HIV-1 exposed human (A2) } \\
\text { seronegative } \\
54 \text { predefined HIV-1 epitopes in } 91 \text { HIV-1-exposed, pe } \\
\text { ed on different epitopes with HLA presenting molecul } \\
\text { onse in the HEPS women upon late seroconversion to } \\
\text { f HIV-1-specific CTL in HEPS women increased with } \\
\text { fected women recognized this epitope, and ILK(D/E)P } \\
\text { ope in all } 7 / 10 \text { HEPS cases but in only } 5 \text { of the } 14 / 26 \mathrm{H} \\
\text { as they were preferentially reactive in HEPS women ar } \\
\text { RT, A*6802 DTVLEDINL in Protease, B14 DLN(M/ } \\
\text { onses restricted by class I HLA alleles A2, A } 24, \mathrm{~A} * 680 \\
\text { V prior to seroconversion, which switched to SL(F/Y) } \\
\text { V prior to seroconversion, and gained responses to epi }\end{array}$ & $\begin{array}{l}\text { Kaul2001a } \\
\text { y seronegative (HEPS) and } 87 \\
\text { ve previously been associated with } \\
\text { recognized by the HIV-1 infected } \\
\text { tion of viral exposure } \\
\text { ended to be more reactive in HEPS } \\
\text { ected women } \\
\text { y)V in p24 and B18 } \\
\text { and B18, previously shown to be } \\
\text { post-seroconversion } \\
\text { SL(F/Y)NTVATL and B27 }\end{array}$ \\
\hline RT (309-317) & $\begin{array}{l}\text { Pol (93TH253 subtype } \\
\text { CRF01) } \\
\text { - Epitope name: P464-472 } \\
\text { - This was a study of HIV- } \\
\text { - HLA-A11 is very commo } \\
\text { and CTL responses were } \\
\text { - This epitope was reactive }\end{array}$ & $\begin{array}{l}\text { ILRIPVHGV } \\
1 \text { exposed persis } \\
\text { in this popula } \\
\text { found in } 8 / 8 \mathrm{HI} \\
\text { in HIV+ contro }\end{array}$ & $\begin{array}{l}\text { HIV-1 infection human (A2) } \\
\text { e (HEPS) female sex workers in Chiang Mai, norther } \\
\text { ched among the HEPS sexworkers - weak CTL resp } \\
4 \text { HIV- women that were not exposed } \\
4 \text { who carried HLA-A2 }\end{array}$ & $\begin{array}{l}\text { Sriwanthana2001 } \\
\text { nd } \\
\text { re detected in 4/7 HEPS women, }\end{array}$ \\
\hline
\end{tabular}




\begin{tabular}{|c|c|c|c|c|c|}
\hline HXB2 Location & Author's Location & Sequence & Immunogen & Species (HLA) & References \\
\hline RT (309-317) & $\begin{array}{l}\text { Pol (93TH253 subtype } \\
\text { CRF01) }\end{array}$ & ILRIPVHGV & HIV-1 infection & human (A2) & Bond2001 \\
\hline
\end{tabular}

- More than half of a cohort of HIV+ female sex workers (FSW) from Northern Thailand were HLA-A11 positive, and this study concentrated on A11 epitopes in this group, although E clade versions of previously defined B-clade A2 and A24 epitopes were also tested.

- 1/4 tested FSWs recognized the E clade version of this epitope, which differs from the previously defined B clade version by two amino acids: ILKEPVHGV

- This epitope was not conserved in many subtypes, and exact matches were very rare

- The CTL response to optimally defined CTL epitopes restricted by HLA class I A and B alleles in individuals who coexpressed HLA A2, A3, and B7 was studied in eight HIV-1-infected subjects, two with acute infection, five with chronic, and one long-term non-progressor (LTNP)

- 2 to 17 epitopes were recognized in a given individual, A2-restricted CTL response tended to be narrow and never dominated the response, and 25/27 epitopes were targeted by at least one person

RT (309-317) Pol (476-484 LAI) ILKEPVHGV HIV-1 infection $\quad$ Kelleher2001a

- Ritonavir (RTV) inhibits chymotryptic activity in the 20S proteasome in vitro, as does Saquinavir (SQV) to a lesser extent; Indinavir (IDV) does not. Thus there is concern protease inhibitors may adversely effect CTL epitope processing, but this paper indicates that processing is not inhibited at therapeutically relevant concentrations of RTV when the proteasome is functioning in in an intracellular context.

- RTV did not alter the presentation two RT A2 epitopes processed by distinct pathways: ILKEPVHGV, generated by the constitutive proteasome containing the MB1 beta subunit, and VIYQYMDDL which is dependent on IFNgamma induction of LMP7 which replaces MB1 in the immunoproteasome, and is destroyed by MB1 in the constitutive proteasome.

- RTV did not inhibit the processing and assembly of HLA-B35 or -A2, which are assembled with a rapid and moderate time course, respectively, or of HLA-A3, -B27 and -B39.

RT (309-317) Pol ILKDPVHGV HIV-1 infection human (A2) Kaul2002

- Neisseria gonorrhea cervititis in 9 HIV+ Kenyan sex workers caused a functional deficiency in IFN-gamma production in HIV-1 epitope-specific CD8+ T-cells, detected by intracellular cytokine production and tetramer assays, while not affecting the total number of epitope-specific CTLs.

- Ghonorrhea caused the weaker HIV-1 specific CTL responses in 4 HIV-1 exposed persistently seronegative (HEPS) women to become undetectable by Elispot and tetramer assays, and CMV-specific CTL in 2 HEPS subjects were shown to have impaired function with regard to IFN-gamma production.

RT (309-317)
RT (476-484 NL43)
ILKEPVHGV
HIV-1 infection
human (A2)
Yang2002

- Nef down-modulates class I protein expression, and this study demonstrates directly that Nef-deleted HIV-1 NL-43 can be more effectively killed in vitro than NL-43 with an intact Nef. The effect was shown to be specific for class I presentation of epitopes, and unlike Nef, deleting Vpr did not alter CTL susceptibility of NL-43 infected cells. The CTL clone 68A62, specific for the class I A2 presented ILKEPVHGV epitope, was one of four used in this study.

RT (476-484 BRU) ILKEPVHGV HIV-1 infection human (A2) Cohen2002

- The antigen presentation of two A2-restricted epitopes was compared, SLYNTVATL (p17) and ILKEPVHGV (RT). HIV-1 infected cells were more sensitive to lysis by SLYNTVATL-specific CTL than by ILKEPVHGV-specific CTL, because of a higher density of SLYNTVATL-A2 resulting from differences in processing.

- Incubation with a T1-cell proteolytic extract showed that by four hours, 25\% of a p17 peptide had a C-term Leu- 85 and were SLYNTVATL-precursors, while ILKEPVHGV-precursors were far less frequent (6.8\%) even with four times more proteolytic extract after 30 hours.

- p17 was preferentially cleaved between Leu85 and Tyr86, while appropriate Val484 and Tyr485 cleavage was minor for RT.

- In a competition experiment, RSLYNTVATL bound TAP 3.7-fold more efficiently than RT peptides.

- No difference in CTL avidity was detected in six patients with HLA-A2-restricted responses to these epitopes. 


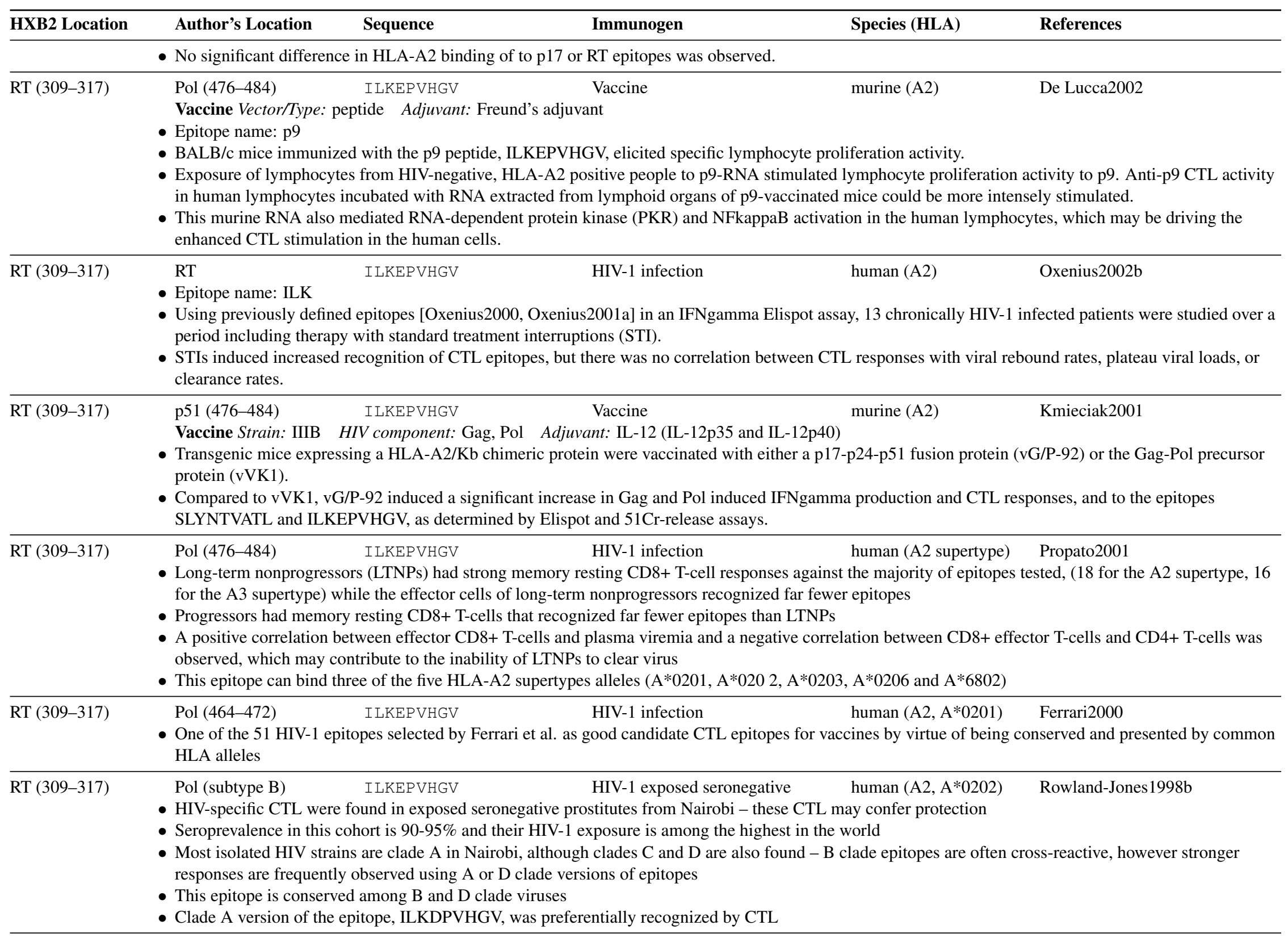




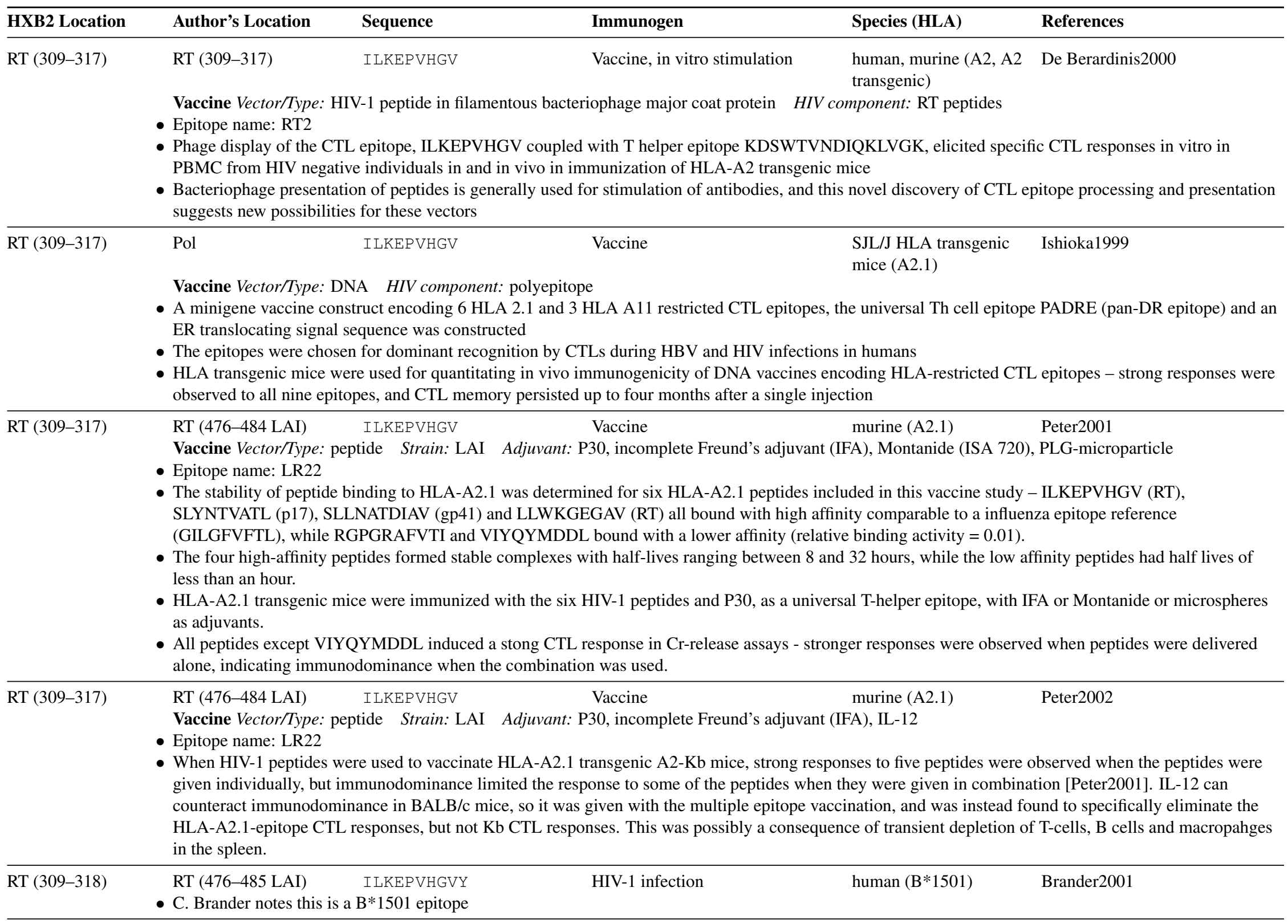




\begin{tabular}{|c|c|c|c|c|c|}
\hline HXB2 Location & Author's Location & Sequence & Immunogen & Species (HLA) & References \\
\hline RT (309-318) & $\begin{array}{l}\text { RT (309-318) } \\
\text { - No immunodominan }\end{array}$ & $\begin{array}{l}\text { IKLEPVHGVY } \\
\text { onses were detec }\end{array}$ & $\begin{array}{l}\text { HIV-1 infection } \\
\text {-restricted epitop }\end{array}$ & human (B62) & Day2001 \\
\hline RT (309-318) & RT (476-485 LAI) & ILKEPVHGVY & HIV-1 infection & human (Bw62) & \begin{tabular}{|l|} 
McMichael1994 \\
Menendez-Arias1998 \\
\end{tabular} \\
\hline
\end{tabular}

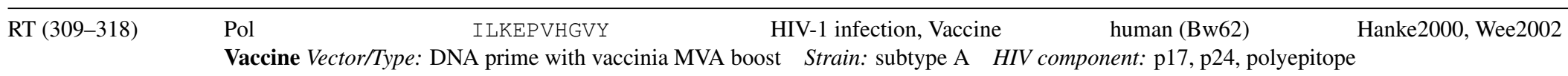

- The HIV-1 subtype A focused vaccine HIVA contains p24 and p17, in a reversed order relative to the Gag polyprotein to prevent myristylation of p17, which could direct the protein to the cell membrane and inhibit efficient peptide processing and class I presentation, as well as a polyepitope string of conserved, often immunodominant epitopes that were selected to have particularly good cross-reactive potential for the A-clade epidemic in Nairobi, Kenya. A DNA and MVA prime-boost vaccination protocol using the HIVA antigen will be used in a phase III clinical trial in Kenya. This epitope is included in the polyepitope string [Hanke2000].

- Multiple CD4+ or CD8+ T-cell vaccine-induced responses to peptide pools were detected using intracellular cytokine staining and IFNgamma Elispot assays after vaccination of 5 macaques. The response to the Mamu A*01 SIV p27 epitope p11C (CTPYDINQM), included in the polyepitope region, was not immunodominant in the Mamu $\mathrm{A}^{*} 01$ vaccinated macaques, possibly because of processing limitations in context of the artificial polyepitope string [Wee2002].

\begin{tabular}{|c|c|c|c|c|}
\hline RT (328-352) & $\begin{array}{cc}\text { RT (495-515 LAI) } & \text { EIQKQGQGQWTYQIYQEPF- } \\
\text { KNLKTG }\end{array}$ & $\begin{array}{l}\text { HIV-1 infection } \\
\text { this study }\end{array}$ & human (A11) & $\begin{array}{l}\text { Menendez-Arias1998, } \\
\text { Walker1989 }\end{array}$ \\
\hline RT (340-350) & $\begin{array}{l}\text { RT (507-516) QIYQEPEKNLK } \\
\text { - Study of cytokines released by HIV-1 specific activated C' }\end{array}$ & $\begin{array}{l}\text { HIV-1 infection } \\
\Gamma L\end{array}$ & human & Menendez-Arias1998, Price1995 \\
\hline RT (340-350) & $\begin{array}{l}\text { Pol (487-497 93TH253 QIYQEPFKNLK } \\
\text { subtype CRF01) }\end{array}$ & $\begin{array}{l}\text { HIV-1 infection, HIV-1 exposed } \\
\text { seronegative }\end{array}$ & human (A11) & Sriwanthana2001 \\
\hline
\end{tabular}

- Epitope name: P495-505

- This was a study of HIV-1 exposed persistently seronegative (HEPS) female sex workers in Chiang Mai, northern Thailand

- HLA-A11 is very common in this population, and was enriched among the HEPS sexworkers - weak CTL responses were detected in 4/7 HEPS women, and CTL responses were found in 8/8 HIV+ controls, and 0/9 HIV- women that were not exposed

- This epitope was weakly reactive in the HEPS study subject 128 who was HLA A11/A33

- This epitope was reactive in HIV+ study subjects 053 and 184 who carried HLA-A11

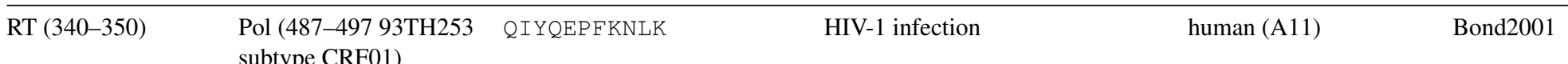

- HLA-A11 CRF01 (called subtype E in Bond et al.) epitopes were identified that stimulated CTL from HIV+ female sex workers (FSW) from Northern Thailand, of whom more than half were HLA-A11 positive

- 77 possible HLA-A11 epitopes were first defined using EpiMatrix, these were screened for binding to A11 finding and 26 bound, and 12 of these were epitopes for CTL responses from 8 HLA-A11 positive FSWs, six were novel, six were previously identified

- This epitope was not predicted by the EpiMatrix method to be likely to bind to A11, though it served as an epitope in the FSWs, and it was one of the six A11 epitopes that had been previously defined

- 5/8 tested FSWs recognized this epitope 


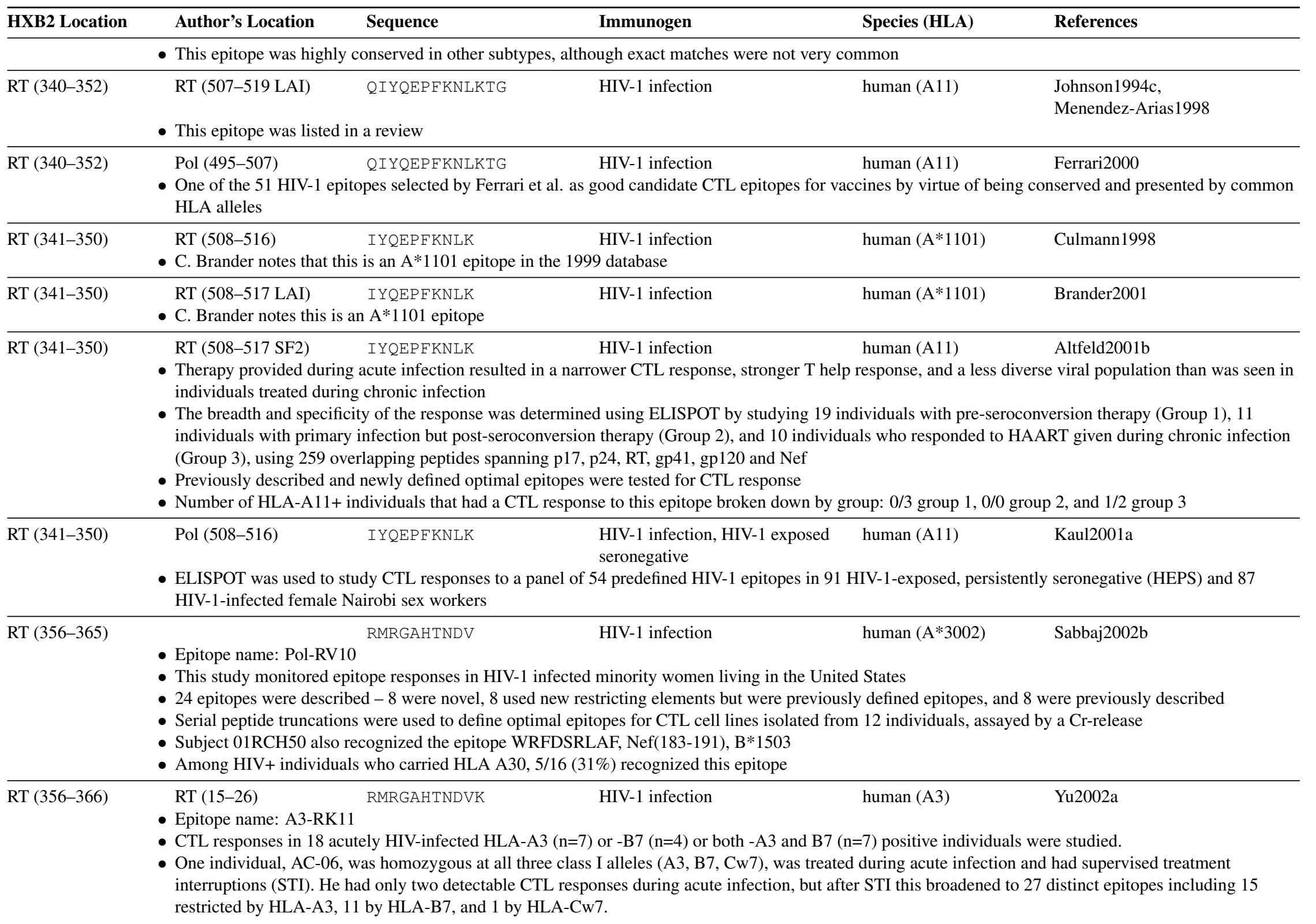




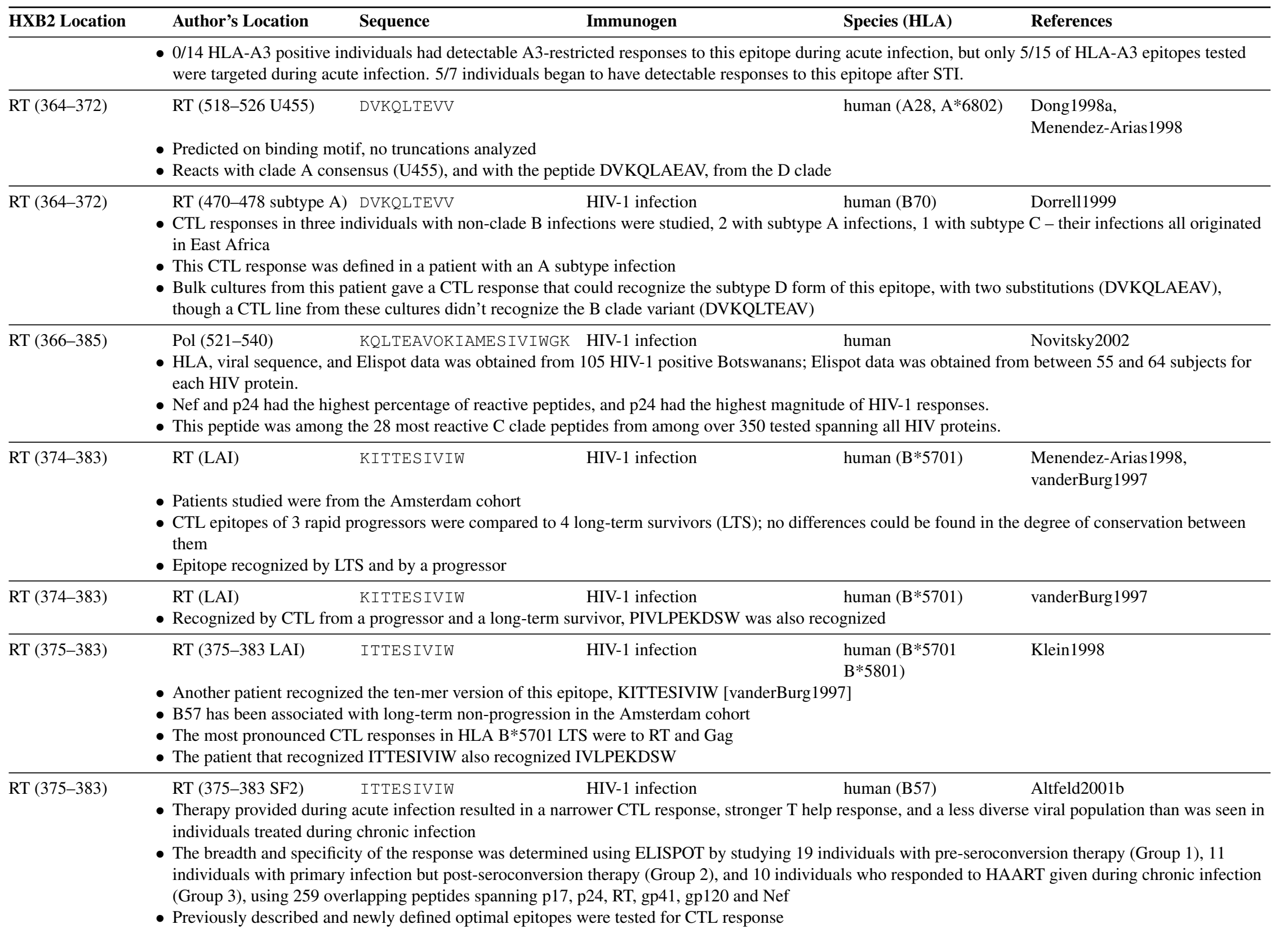




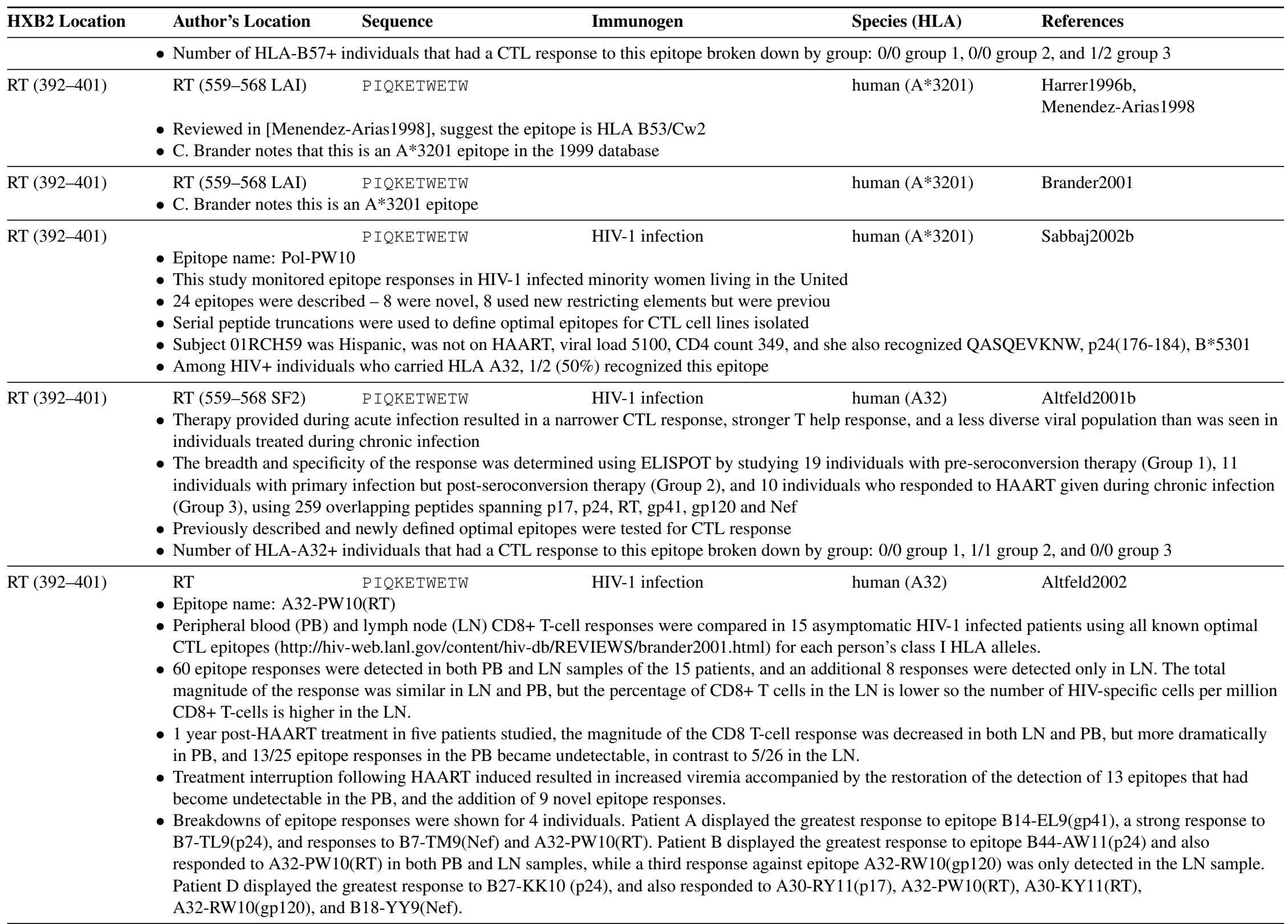




\begin{tabular}{|c|c|c|c|c|c|}
\hline HXB2 Location & Author's Location & Sequence & Immunogen & Species (HLA) & References \\
\hline RT (397-406) & RT (LAI) & TWETWWTEYW & HIV-1 infection & human (B44) & \begin{tabular}{|l|} 
Menendez-Arias1998 \\
vanderBurg1997
\end{tabular} \\
\hline
\end{tabular}

- Recognized by CTL from two progressors, EILKEPVGHGV and EELRQHLLRW were also recognized by one, and RETKLGKAGY was also recognized by the other

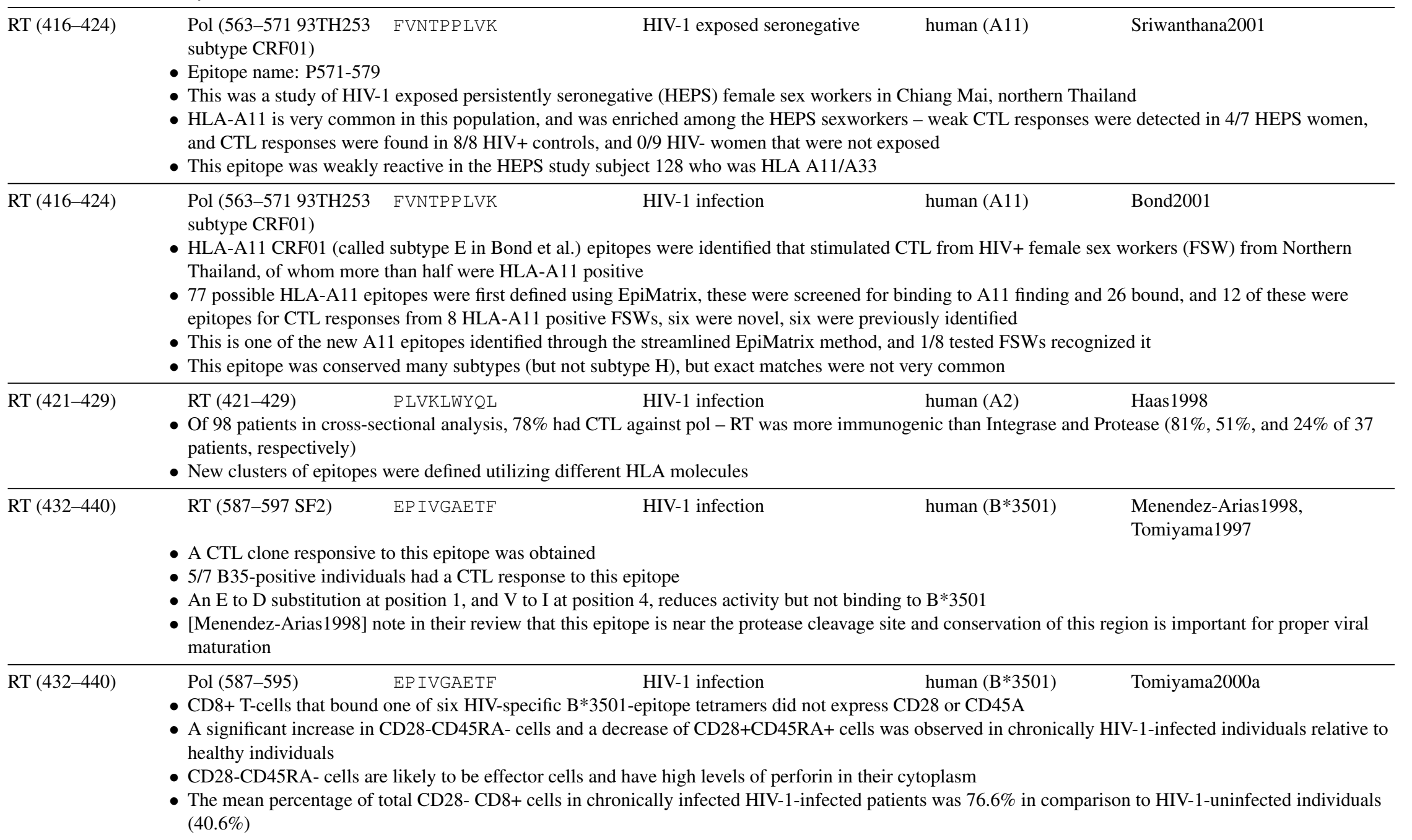




\begin{tabular}{llccc}
\hline HXB2 Location & Author's Location & Sequence & Immunogen & Species (HLA) \\
\hline RT (432-440) & & EPIVGAETF & HIV-1 infection & human (B35) \\
& - Three individuals with highly focused HIV-specific CTL responses were studied during acute infection using tetramers - high frequencies of HIV-1-specific
\end{tabular}

$\mathrm{CD} 8+\mathrm{T}$ cells were found prior to seroconversion, and there was a close temporal relationship between the number of circulating HIV-specific $\mathrm{T}$ cells and viral load was also found

- All three patients were B*2705, with HLA alleles: A1, A30/31, B*2705, B35; A1, A*0301, B7, B2705; and A*0201, A*0301, B2705, B39

- ELISPOT was used to test a panel of CTL epitopes that had been defined earlier and were appropriate for the HLA haplotypes of the study subjects - 3/3 subjects showed a dominant response to the $\mathrm{B} * 2705$ epitope KRWIILGGLNK

- The subject with $\mathrm{A}^{*} 0201$ had a moderatly strong response to SLYNTVATL

- Weak responses were observed to A*301-RLRPGGKKK, A*301-QVPLRPMTYK, and B7-TPGPGVRYPL in the subject who was HLA A1, A*0301, B7, $\mathrm{B} * 2705$

- No acute response was detected to the following epitopes: A*201-ILKEPVHGV, A*301-KIRLRPGGK, A*301-AIFQSSMTK, A*301-TVYYGVPVWK, B35-EPIVGAETF, B35-HPDIVIYQY, B35-PPIPVGEIY, B35-NSSKVSQNY, B35-VPLRPMTY, B35-DPNPQEVVL

$\begin{array}{llccc}\text { RT (432-440) } & \text { Pol (587-595) } & \text { EP IVGAETF } & \text { HIV-1 infection } & \text { human (B35) }\end{array}$

- CTL specific responses were measured over a 1.3 to 1.5 year period in members of the Sydney Blood Bank Cohort (SBBC) who had been infected with a natural attenuated strain of HIV-1 which was Nef-defective

- Some of these patients had prolonged high levels of CTL effector and memory cells despite low viral load

\begin{tabular}{llccc}
\hline RT (432-440) & RT (587-596 SF2) & EP IVGAETF & HIV-1 infection & $\begin{array}{c}\text { human (B35, B51) } \\
\end{array}$ \\
& Binds HLA-B*3501, and is also presented by B51 - but CTL could not kill RT-vaccinia virus infected cells that expressed B51 & Shig96 \\
\hline RT (432-440) & Pol (587-595) & EP IVGAETF & HIV-1 infection & human (B35, B51)
\end{tabular}

- One of the 51 HIV-1 epitopes selected by Ferrari et al. as good candidate CTL epitopes for vaccines by virtue of being conserved and presented by common HLA alleles

\begin{tabular}{llccc}
\hline RT (432-441) & Pol (587-596) & EP IVGAETFY & HIV-1 infection & human (B*3501)
\end{tabular}

- CD8+ T-cells that bound one of six HIV-specific B*3501-epitope tetramers did not express CD28 or CD45A

- A significant increase in CD28-CD45RA- cells and a decrease of CD28+CD45RA+ cells was observed in chronically HIV-1-infected individuals relative to healthy individuals

- CD28-CD45RA- cells are likely to be effector cells and have high levels of perforin in their cytoplasm

- The mean percentage of total CD28- CD8+ cells in chronically infected HIV-1-infected patients was 76.6\% in comparison to HIV-1-uninfected individuals (40.6\%)

RT (432-441) RT (587-597 SF2) EPIVGAETFY HIV-1 infection $\mathrm{C} 3 \mathrm{H} / \mathrm{HeJ}$ mice (B35)

\section{Menendez-Arias1998,} Shiga1996

- Binds HLA-B*3501, but not presented by B51, in contrast to the peptide EPIVGAETF

- [Menendez-Arias1998 note in their review that this epitope is located near the protease cleavage site and conservation of this region is important for viral maturation

- This epitope spans the Pol p66 RT - p15 (RNAse) domain

- HLA B35 is associated with rapid disease progression

- The sequences of 9 previously described HIV-1 B35 CTL epitopes were obtained in 10 HLA B35+ and 19 HLA B35- individuals 


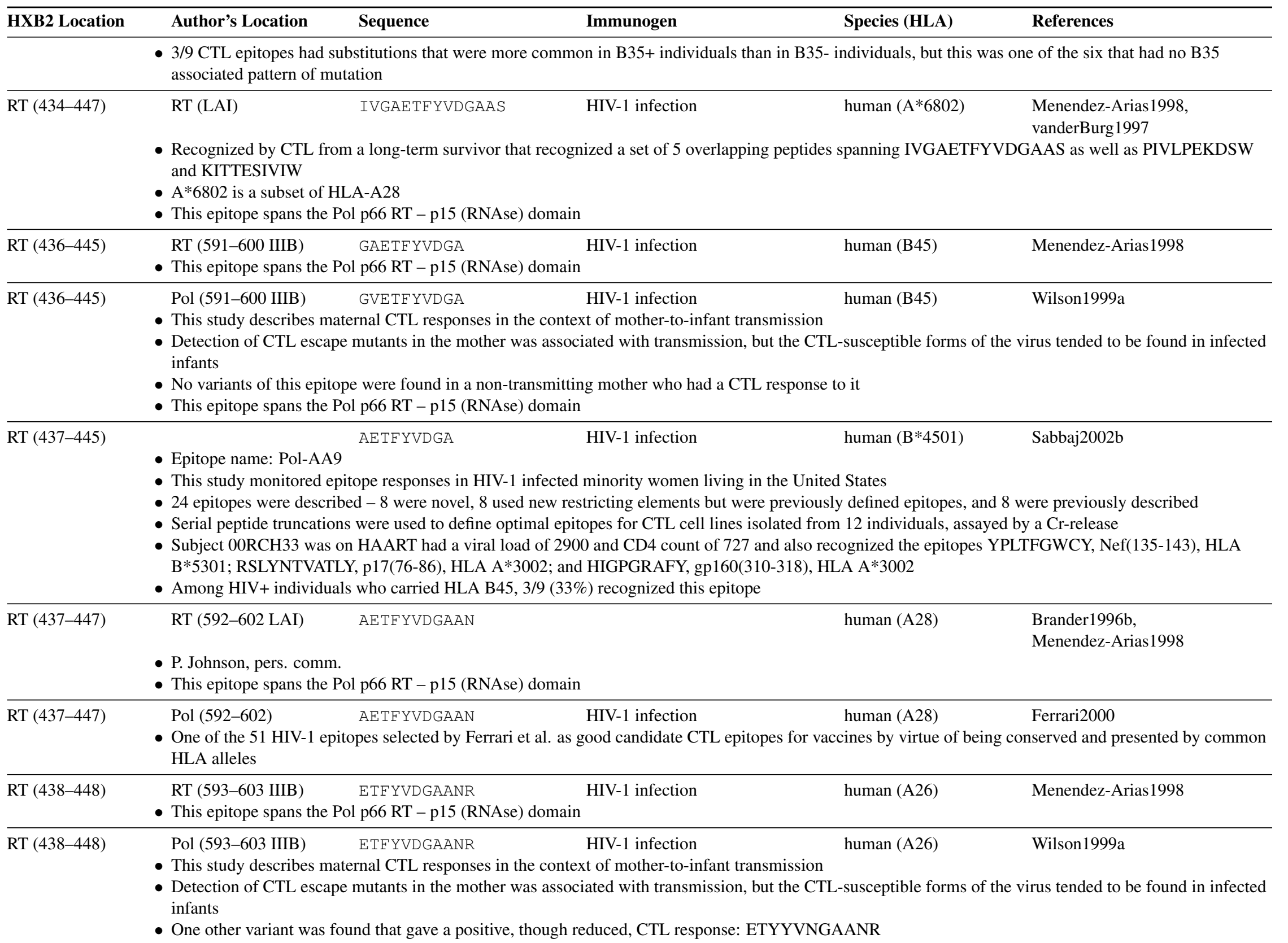




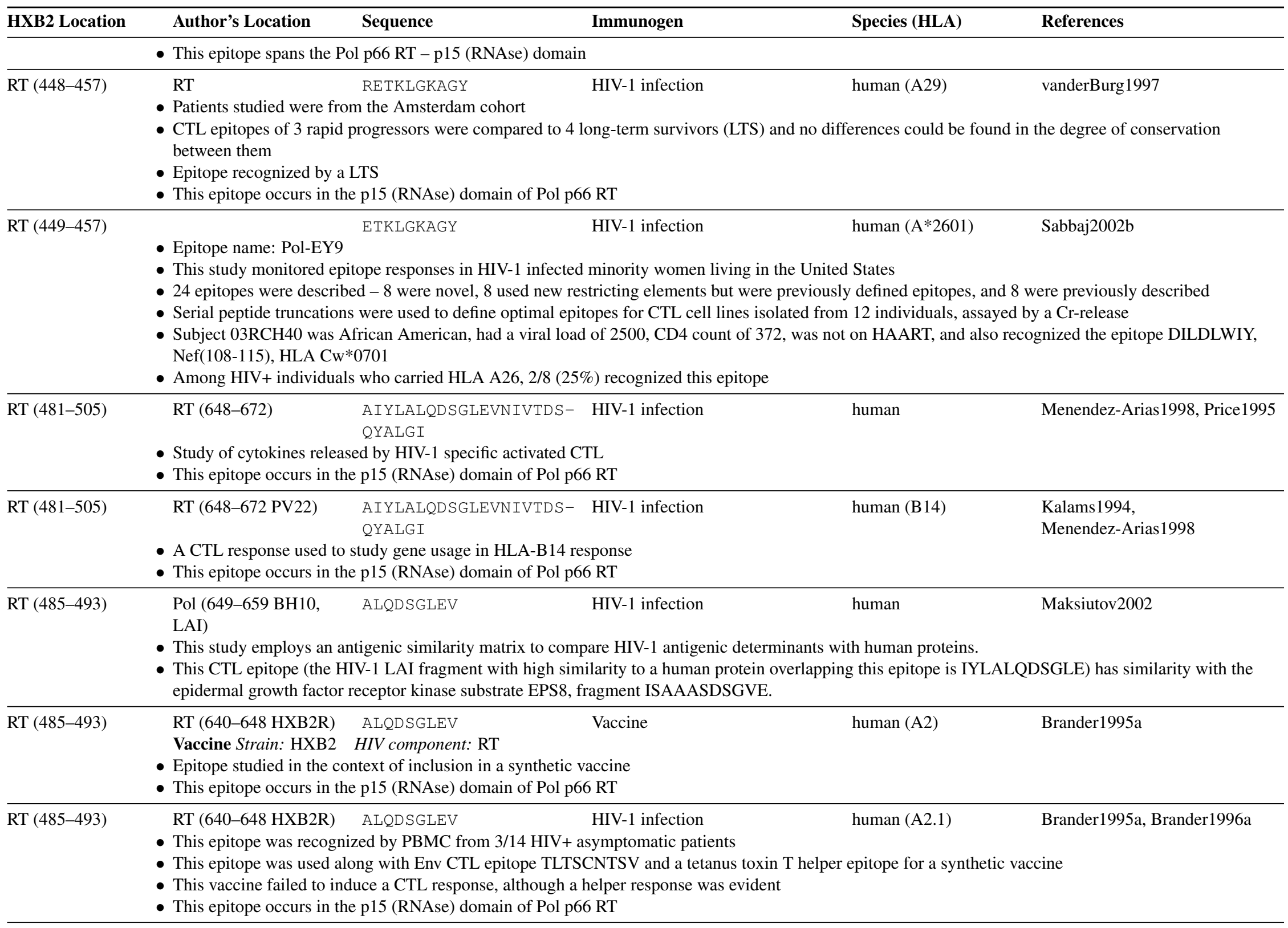




\begin{tabular}{|c|c|c|c|c|c|}
\hline HXB2 Location & Author's Location & Sequence & Immunogen & Species (HLA) & References \\
\hline RT (485-505) & $\begin{array}{l}\text { RT (648-672) } \\
\text { - Unpublished, S. Kalams } \\
\text { - This epitope occurs in the }\end{array}$ & $\begin{array}{l}\text { ALQD SGLEVVTDSQYALGI } \\
\text { p15 (RNAse) domain of Pol p }\end{array}$ & $\begin{array}{l}\text { HIV-1 infection } \\
6 \text { RT }\end{array}$ & human (B14) & Brander1995b \\
\hline RT (496-505) & & VTDSQYALGI & HIV-1 infection & human (B*1503) & Sabbaj2002b \\
\hline
\end{tabular}

- Epitope name: Pol-VI10

- This study monitored epitope responses in HIV-1 infected minority women living in the United States

- 24 epitopes were described - 8 were novel, 8 used new restricting elements but were previously defined epitopes, and 8 were previously described

- Serial peptide truncations were used to define optimal epitopes for CTL cell lines isolated from 12 individuals, assayed by a Cr-release

- Subject 01RCH51 was an African American on HAART, viral load 980, CD4 count 811

- Among HIV+ individuals who carried HLA B15, 1/17 (6\%) recognized this epitope

RT (496-505) Pol (subtype B) VTDSQYALGI HIV-1 exposed seronegative human (B14, B*1402) Rowland-Jones1998b

- HIV-specific CTL were found in exposed seronegative prostitutes from Nairobi - these CTL may confer protection

- Seroprevalence in this cohort is $90-95 \%$ and their HIV-1 exposure is among the highest in the world

- Most isolated HIV strains are clade A in Nairobi, although clades C and D are also found - B clade epitopes are often cross-reactive, however stronger responses are frequently observed using A or D clade versions of epitopes

- This epitope is conserved among A, B and D clade viruses

\begin{tabular}{lllll}
\hline RT (496-505) RT (663-672 IIIB) VTDSQYALGI & HIV-1 infection & human (Cw8)
\end{tabular}

- Unpublished, P. Johnson

- Published in this database in 1995 as B14, but B14 transfected cells did not present the peptide and it is thought to be presented by the genetically linked Cw8 molecule instead [Brander1996b]

- This epitope occurs in the p15 (RNAse) domain of Pol p66 RT

RT (496-505) RT VTDSQYALGI HIV-1 exposed seronegative human (Cw8) Rowland-Jones1998a

- A CTL response was found in exposed but uninfected prostitutes from Nairobi using previously-defined B clade epitopes that tended to be conserved in A and D clades - such cross-reactivity could protect against both A and D and confer protection in Nairobi where both subtypes are circulating

- The A and D subtype consensus are identical to the B clade epitope

- Thought to be HLA-Cw8 restricted, not B14 as originally reported (C. Brander, B. Walker, and S. Rowland-Jones, personal communication)

- This epitope occurs in the p15 (RNAse) domain of Pol p66 RT

RT (509-518) Pol QPDKSESELV De Groot2001

- The program Epimatrix was used in conjunction with the program Conservatrix to identify conservered regions of HIV that might serve as epitopes

- A subset of the potential epitopes was identified that could bind to the appropriate HLA-allele, and 15 predicted B7 superfamily (HLA B7, B8, and B58)

epitopes were identified that could stimulate IFN $\gamma$ production in an ELISPOT assay

- QPDKSESELV was newly identified as an HLA-B7 epitope in this study

RT (516-525) RT (516-525) ELVNQI IEQL $\quad$ HIV-1 infection

- Of 98 patients in cross-sectional analysis, $78 \%$ had CTL against pol - RT was more immunogenic than Integrase and Protease (81\%, 51\%, and $24 \%$ of 37

patients, respectively)

- New clusters of epitopes were defined utilizing different HLA molecules

- This epitope occurs in the p15 (RNAse) domain of Pol p66 RT 


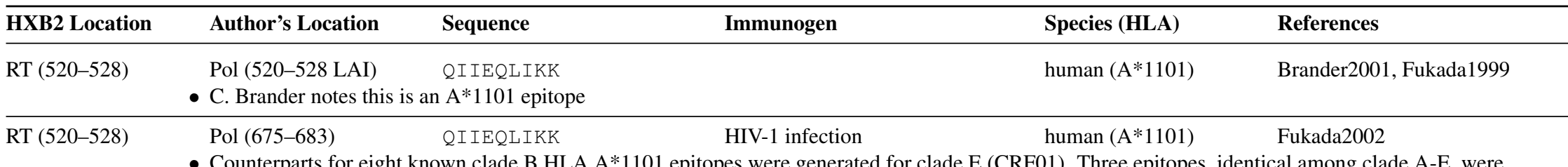

- Counterparts for eight known clade B HLA A*1101 epitopes were generated for clade E (CRF01). Three epitopes, identical among clade A-E, were cross-reactive and recognized by clade $\mathrm{E}$ infected individuals. The clade $\mathrm{E}$ and $\mathrm{B}$ analogs to three more HLA A*1101 epitopes was recognized in a clade specific manner. Two other HLA A*1101 clade B defined epitopes were found not to have stimulated a response in clade E infected individuals.

- QIIEQLIKK was found to elicit clade-specific responses in clade B (QIIEQLIKK is most common) and clade E (qiieElikk is most common). QIIEQLIKK was strongly recognized by CTL from $1 / 5$ B clade infected Japanese subjects, and qiieElikk from 3/7 E clade infected Thai subjects. The variant qiieKliEk, common in the A subtype, was also recognized in 2/7 E clade infected Thai subjects.

- The binding of QIIEQLIKK, qiieElikk and qiieKliEk to HLA A*1101 was similar, but CTL clones from individuals did not cross-react with the cross-clade peptides indicating that the substitutions inhibited TCR interaction.

\begin{tabular}{llll}
\hline RT (530-538) & Pol (680-691 BH10, KVYLAWVPA & HIV-1 infection & human
\end{tabular}

- This study employs an antigenic similarity matrix to compare HIV-1 antigenic determinants with human proteins.

- This CTL epitope (the HIV-1 LAI fragment with high similarity to a human protein overlapping this epitope is IKKEKVYLAWV) has similarity with B-cell growth factor precursor, fragment IKKERLWLGPV.

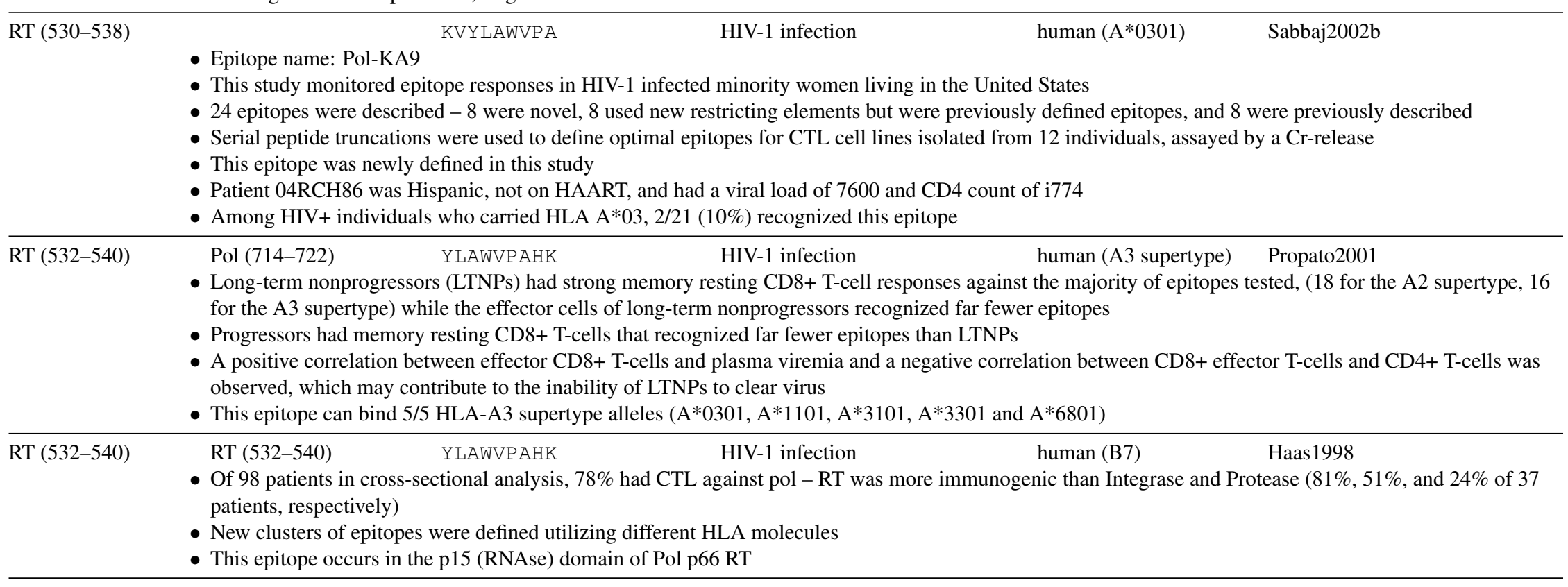




\section{II-B-11 Integrase CTL Epitopes}

\begin{tabular}{|c|c|c|c|c|}
\hline HXB2 Location & Author's Location & Sequence & Immunogen & Species (HLA) \\
\hline -28) & \multicolumn{4}{|c|}{$\begin{array}{l}\text { - Long-term nonprogressors (LTNPs) had strong memory resting CD8+ T-cell responses against the majority of epitopes tested, (18 for the A2 supertype, } 16 \\
\text { for the A3 supertype) while the effector cells of long-term nonprogressors recognized far fewer epitopes } \\
\text { - Progressors had memory resting CD8+ T-cells that recognized far fewer epitopes than LTNPs } \\
\text { - A positive correlation between effector CD8+ T-cells and plasma viremia and a negative correlation between CD8+ effector T-cells and CD4+ T-cells was } \\
\text { observed, which may contribute to the inability of LTNPs to clear virus } \\
\text { - This epitope can bind three of the five HLA-A2 supertypes alleles (A*0201, A*020 2, A*0203, A*0206 and A*6802) }\end{array}$} \\
\hline tegrase (22-31) & \multicolumn{4}{|c|}{$\begin{array}{l}\text { - Long-term nonprogressors (LTNPs) had strong memory resting CD8+ T-cell responses against the majority of epitopes tested, (18 for the A2 supertype, } 16 \\
\text { for the A3 supertype) while the effector cells of long-term nonprogressors recognized far fewer epitopes } \\
\text { - A positive correlation between effector CD8+ T-cells and plasma viremia and a negative correlation between CD8+ effector T-cells and CD4+ T-cells was } \\
\text { observed, which may contribute to the inability of LTNPs to clear virus } \\
\text { - This epitope can bind all five HLA-A2 supertypes alleles (A*0201, A*0202, A*0203, A*0206 and A*6802) }\end{array}$} \\
\hline -36) & \multicolumn{4}{|c|}{$\begin{array}{l}\text { - HLA-B27, -B51, and -B57 are associated with slow progression to AIDS, while HLA-B35, -B8, -B24 are associated with a rapid progression to AIDS (Nat. } \\
\text { Med. 2:405, 1996;Lancet 22:1187, 1986;Hum Immunol 22:73, 1988;Hum Immunol 44:156, 1995) } \\
\text { - } 15 \% \text { of Japanese populations carry HLA-B51 while HLA-B27 and -B57 are detected in less than 0.3\% } \\
\text { - Of the } 172 \text { HIV-1 peptides with HLA-B*5101 anchor residues, } 33 \text { bound to HLA-B*5101, seven of these peptides were reactive with CTL from } 3 \text { B*5101 } \\
\text { positive individuals, and six were properly processed } \\
\text { - Four of the six epitopes were highly conserved among B subtype sequences - LPPVVAKEI is highly conserved }\end{array}$} \\
\hline tegrase (82-89) & \multicolumn{4}{|c|}{$\begin{array}{l}\text { - Defined using reverse immunogenetics }-59 \text { HLA-A*2402 binding peptides were predicted by searching for A*2402 anchors in HIV proteins (Tyr at } 2 \text {, and } \\
\text { Phe, Leu or Ile at the C term) - } 53 \text { of the } 59 \text { peptides bound A*2402 } \\
\text { - This peptide induced CTL in } 1 / 4 \text { HIV-1+ people tested } \\
\text { - GYIEAEVI bound to A*2402 weakly, the epitope can be processed in a vaccinia construct and presented - two specific CTL clones were obtained }\end{array}$} \\
\hline tegrase (89-98) & $\begin{array}{l}\text { Pol (805-814 BH10, } \\
\text { LAI) } \\
\text { - This study employs a } \\
\text { - This CTL epitope (th } \\
\text { beta-4 precursor (GP }\end{array}$ & D104), fragı & rity to a human & $\begin{array}{l}\text { Maksiutov2002 } \\
\text { human proteins. } \\
\text { his epitope is PAETGQETAY) has similarity with Integrin }\end{array}$ \\
\hline ntegrase (89-98) & $\begin{array}{l}\text { Pol } \\
\text { - The program Epimat } \\
\text { - A subset of the poten } \\
\text { epitopes could stimu } \\
\text { - IPAETGQETA was } 1\end{array}$ & $\begin{array}{l}\text { IPAETGQETA } \\
\text { as used in conju } \\
\text { pitopes was ider } \\
\text { FN } \gamma \text { production } \\
\text { identified as an }\end{array}$ & $\begin{array}{l}\text { rogram Conserva } \\
\text { bind to the appro } \\
\text { ssay } \\
\text { e in this study }\end{array}$ & $\begin{array}{l}\text { Uman (B56) De Groot2001 } \\
\text { rvered regions of HIV that might serve as epitopes } \\
\text { d } 15 \text { predicted B7 superfamily (HLA B7, B8, and B58) }\end{array}$ \\
\hline
\end{tabular}




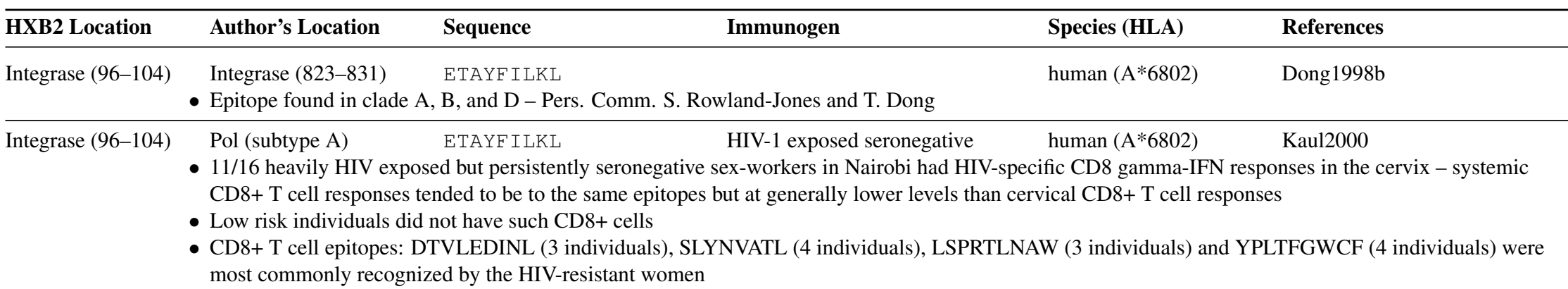

$\begin{array}{llcc}\text { ETAYF ILKL } & \text { HIV-1 infection } & \text { human }(A * 6802) & \text { Kaul2001c }\end{array}$ sex workers eventually seroconverted, and for six of these HIV CTL reactive epitopes had been defined while seronegative

- The epidemiological factor associated with seroconversion was stopping sex work and HIV-specific CTL activity declines when HEPS sex workers stop working for a period or retire

- This epitope was recognized in 1/22 HEPS sex worker controls (ML1671)

$\begin{array}{llll}\text { Integrase (96-104) Pol (744-752) } & \text { ETAYFYILKL } & \begin{array}{l}\text { HIV-1 infection, HIV-1 exposed } \\ \text { seronegative }\end{array} & \text { human (A*6802) }\end{array}$

- ETAYFYILKL cross-reacts with clades A, B and D

- ELISPOT was used to study CTL responses to a panel of 54 predefined HIV-1 epitopes in 91 HIV-1-exposed, persistently seronegative (HEPS) and 87 HIV-1-infected female Nairobi sex workers

- Responses in HEPS women tended to be lower, and focused on different epitopes with HLA presenting molecules that have previously been associated with reduced risk of infection, and there was a shift in the response in the HEPS women upon late seroconversion to epitopes recognized by the HIV-1 infected women

- 43/91 HEPS women had CD8+ responses and detection of HIV-1-specific CTL in HEPS women increased with the duration of viral exposure

- Among HLA-A*6802 women, 3/12 HEPS and 9/11 HIV-1 infected women recognized this epitope likelihood ratio 7.9, p value 0.01, and HEPS women tended to respond to DTVLEDINL, while infected women to ETAYFYILKL

- The dominant response to this HLA allele was to this epitope in 2 of the 3/12 HEPS cases and in all 9/11 HIV-1 infected women that responded to the epitope

- Differences in epitope specificity were only seen for responses restricted by class I HLA alleles A2, A24, A*6802, B14, and B18, previously shown to be associated with resistance to HIV-1 in this cohort

- Subject ML 1203 started with CTL responses to A*6802 DTVLEDINL and to B7 FPVTPQVPLR prior to seroconversion, and upon seroconversion acquired additional responses to A*6802 ETAYFILKL which became dominant, B7 TPGPG(V/I)RYPL, B7 IPRRIRQGL, and B7 SPRTLNAWV

- Subject ML 1707 started with a CTL response to A*6802 DTVLEDINL prior to seroconversion, and switched to A*6802 ETAYFILKL and A24 RDYVDRFFKTL post-seroconversion

- Subject ML 1830 made no detectable response prior to seroconversion, but responded to A*6802 DTVLEDINL and A*6802 ETAYFILKL post-seroconversion

$\begin{array}{llc}\text { Integrase (96-104) } & \text { Pol (744-752) } & \text { ETAYF ILKL } \\ & \text { - This epitope is newly defined in this study }\end{array}$

HIV-1 infection

human $(\mathrm{A} * 6802)$

Appay2000

- Combined tetramer and intracellular cytokine staining was used to study the function of circulating CD8+ T cells specific for HIV and CMV

- HIV-specific CD8+ T cells expressed lower levels of perforin than CMV-specific CD8+ T cells from the same donor, and this was associated with persistent CD27 expression on HIV-specific cells, suggesting impaired maturation 


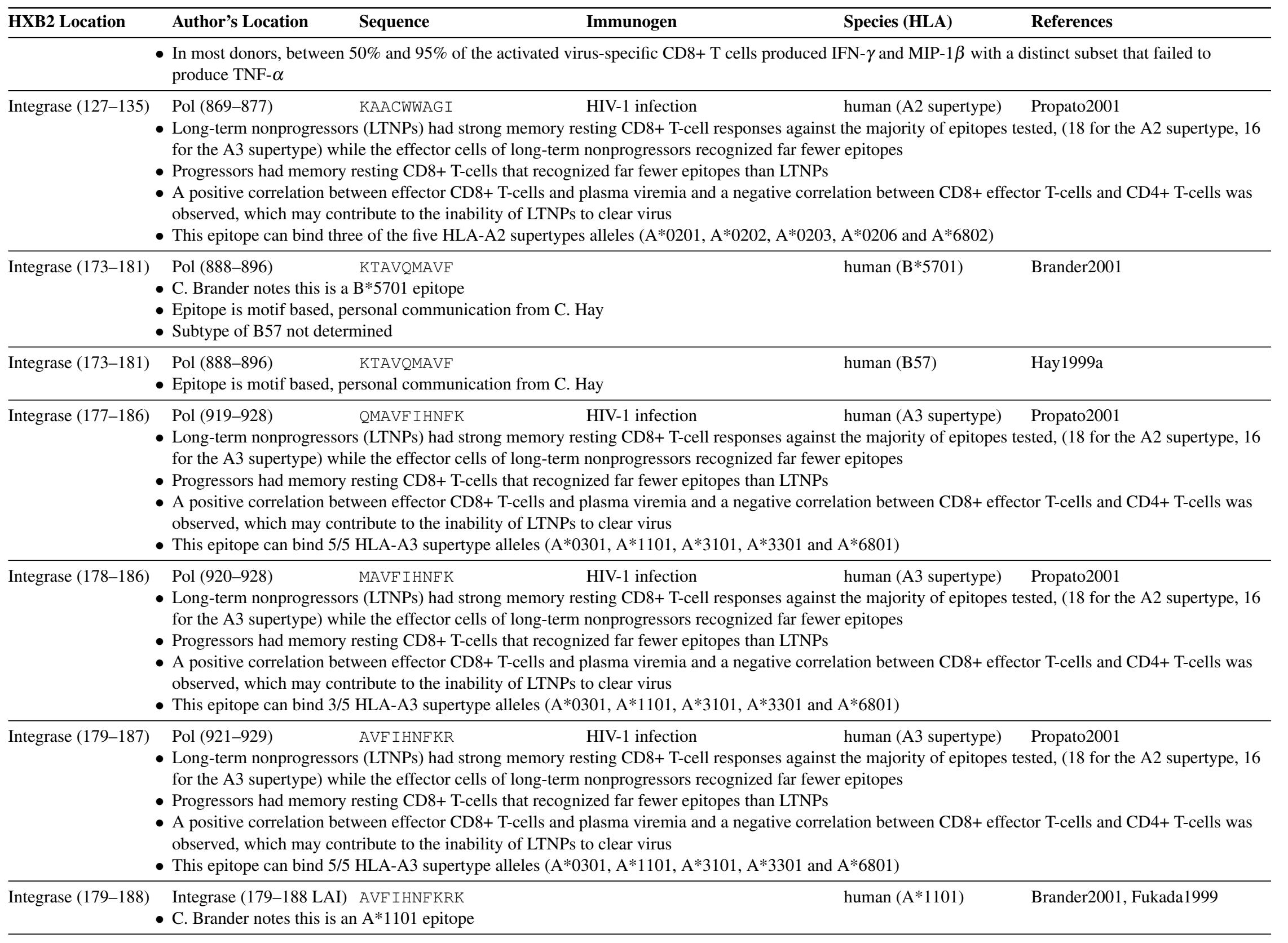




\begin{tabular}{llllc}
\hline HXB2 Location & Author's Location & Sequence & Immunogen & Species (HLA) \\
\hline Integrase (179-188) & $\begin{array}{l}\text { Pol (894-903) } \\
\text { - Counterparts for eight known clade B HLA A*1101 epitopes were generated for clade E (CRF01). Three epitopes, identical among clade A-E, were }\end{array}$ & AVF I HNFKR & HIV-1 infection
\end{tabular}

cross-reactive and recognized by clade $\mathrm{E}$ infected individuals. The clade $\mathrm{E}$ and $\mathrm{B}$ analogs to three more HLA A*1101 epitopes was recognized in a clade specific manner. Two other HLA A*1101 clade B defined epitopes were found not to have stimulated a response in clade E infected individuals.

- AVFIHNFKRK is commonly found in viruses representing subtypes A-E. It was strongly recognized by CTL from 4/7 E clade infected Thai subjects.

Pol (894-903 93TH253 AVF I HNFKRK

HIV-1 exposed seronegative

human (A11)

Bond2001

subtype CRF01)

- Epitope name: P894-903

- This was a study of HIV-1 exposed persistently seronegative (HEPS) female sex workers in Chiang Mai, northern Thailand

- HLA-A11 is very common in this population, and was enriched among the HEPS sexworkers - weak CTL responses were detected in 4/7 HEPS women, and CTL responses were found in 8/8 HIV+ controls, and 0/9 HIV- women that were not exposed

- This epitope was weakly reactive in the HEPS study subjects 265 who was HLA A2/A11 and 128 who was HLA A11/A33, and had been predicted to be a possible A11 epitope using Epimer in [Bond2001]

\begin{tabular}{lllll}
\hline Integrase (179-188) Int (894-904) AVFIHNFKRK HIV-1 infection & Yuman (A3)
\end{tabular}

- Epitope name: A3-AK10

- CTL responses in 18 acutely HIV-infected HLA-A3 $(n=7)$ or $-\mathrm{B} 7(\mathrm{n}=4)$ or both -A3 and B7 $(n=7)$ positive individuals were studied

- One individual, AC-06, was homozygous at all three class I alleles (A3, B7, Cw7), was treated during acute infection and had supervised treatment interruptions (STI). He had only two detectable CTL responses during acute infection, but after STI this broadened to 27 distinct epitopes including 15 restricted by HLA-A3, 11 by HLA-B7, and 1 by HLA-Cw7.

- 0/14 HLA-A3 positive individuals had detectable A3-restricted responses to this epitope during acute infection, but only 5/15 of HLA-A3 epitopes tested were targeted during acute infection. 1/7 individuals began to have detectable responses to this epitope after STI.

\begin{tabular}{lllll}
\hline Integrase (179-196) & Pol (894-911) AVF IHNFKRKGGIGGYSA & HIV-1 infection & hovitsky2002
\end{tabular}

- HLA, viral sequence, and Elispot data was obtained from 105 HIV-1 positive Botswanans; Elispot data was obtained from between 55 and 64 subjects for each HIV protein.

- Nef and p24 had the highest percentage of reactive peptides, and p24 had the highest magnitude of HIV-1 responses.

- This peptide was among the 28 most reactive $\mathrm{C}$ clade peptides from among over 350 tested spanning all HIV proteins.

Integrase (210-227) Pol (925-942) TKELQKQIIKIQNFRVYY HIV-1 infection human

- HLA, viral sequence, and Elispot data was obtained from 105 HIV-1 positive Botswanans; Elispot data was obtained from between 55 and 64 subjects for each HIV protein.

- Nef and p24 had the highest percentage of reactive peptides, and p24 had the highest magnitude of HIV-1 responses.

- This peptide was among the 28 most reactive $\mathrm{C}$ clade peptides from among over 350 tested spanning all HIV proteins.

Integrase (219-227) KIQNFRVYY HIV-1 infection human (A*3002) Sabbaj2002b

- Epitope name: Pol-KY9

- This study monitored epitope responses in HIV-1 infected minority women living in the United States

- 24 epitopes were described -8 were novel, 8 used new restricting elements but were previously defined epitopes, and 8 were previously described

- Serial peptide truncations were used to define optimal epitopes for CTL cell lines isolated from 12 individuals, assayed by a Cr-release

- Patient 00RCH28 was African American, not on HAART, had a viral load of 5900 and CD4 count of 889, and she also recognized RIRQGLERA, gp160(846-854), A*0205 


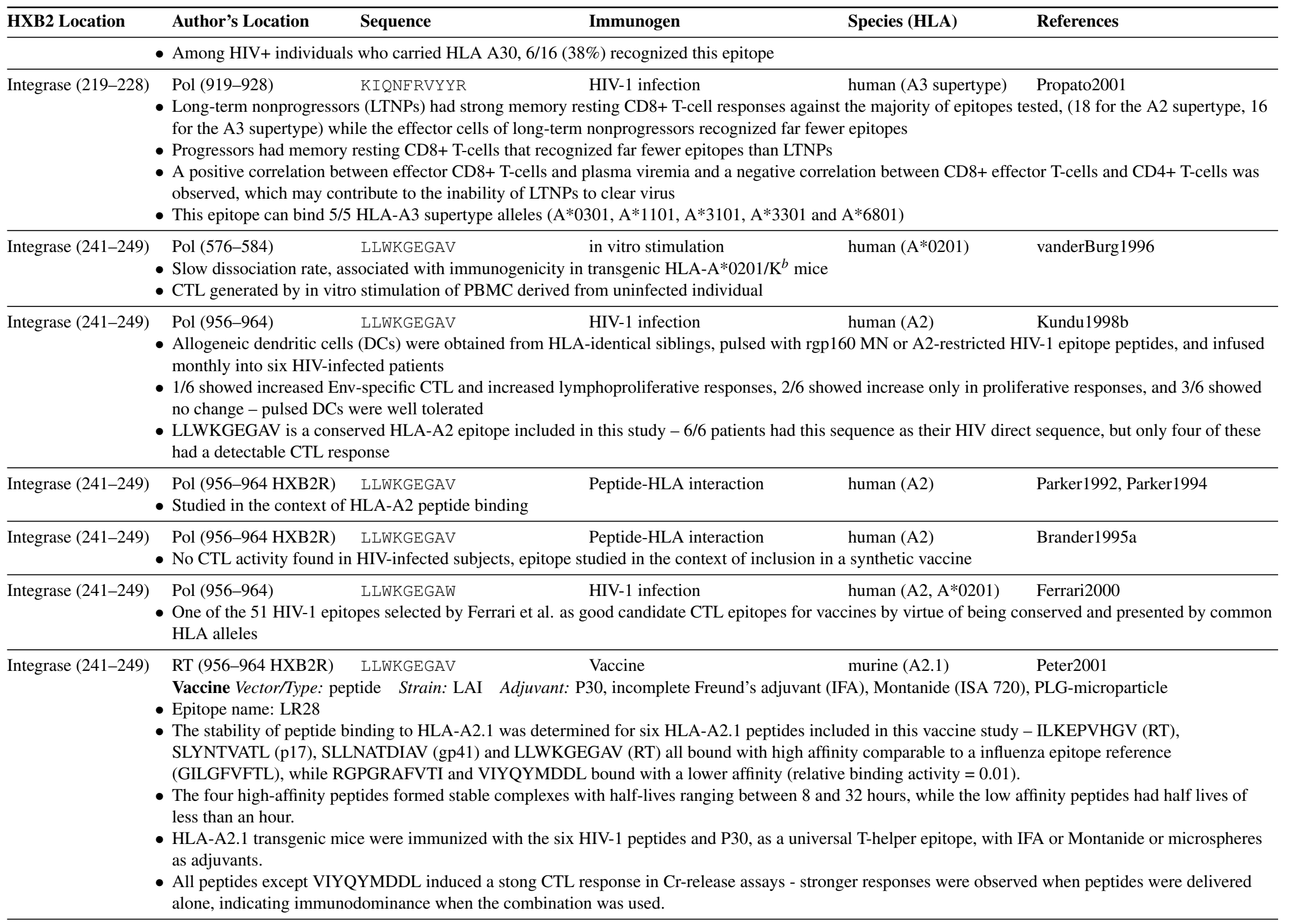




\begin{tabular}{|c|c|c|c|c|}
\hline HXB2 Location & Author's Location & Sequence & Species (HLA) & References \\
\hline \multirow[t]{2}{*}{ Integrase (241-249) } & RT (956-964 HXB2R) & LLWKGEGAV & murine (A2.1) & Peter2002 \\
\hline & \multicolumn{4}{|c|}{$\begin{array}{l}\text { Vaccine Vector/Type: peptide Strain: LAI Adjuvant: P30, incomplete Freund's adjuvant (IFA), IL-12 } \\
\text { - Epitope name: LR28 } \\
\text { - When HIV-1 peptides were used to vaccinate HLA-A2.1 transgenic A2-Kb mice, strong responses to five peptides were observed when the peptides were } \\
\text { given individually, but immunodominance limited the response to some of the peptides when they were given in combination [Peter2001]. IL-12 can } \\
\text { counteract immunodominance in BALB/c mice, so it was given with the multiple epitope vaccination, and was instead found to specifically eliminate the } \\
\text { HLA-A2.1-epitope CTL responses, but not Kb CTL responses. This was possibly a consequence of transient depletion of T-cells, B cells and macropahges } \\
\text { in the spleen. }\end{array}$} \\
\hline
\end{tabular}




\section{II-B-12 Pol CTL Epitopes}

\begin{tabular}{llccc}
\hline HXB2 Location & Author's Location & Sequence & Immunogen & Species (HLA) \\
\hline Pol & RT (LAI) & HIV-1 infection & human \\
& $\bullet$ & This study showed a correlation between strong CTL memory and breadth of response in 7-12 month old infants, and remaining AIDS-free for the first year
\end{tabular}

- This study showed a correlation between strong CTL memory and breadth of response in 7-12 month old infants, and remaining AIDS-free for the first year of life, higher absolute CD4 and CD8 cells, and lower viral load

Pol p66 (LAV) HIV-1 infection human

- Protein delivery (gp160 LAV, p66 LAV, and p24 NY5) to human dendritic cells (DC) with liposomes provides enhanced memory CTL response relative to delivery of protein alone

- Chloroquine administration enhanced epitope presentation, and brefeldin A and peptide aldehyde inhibitors inhibited antigen presentation, suggesting epitopes were processed by classical proteasome pathway
Pol
Pol (IIIB)
HIV-1 infection
human
Wasik2000

- HIV+ infants that progressed rapidly to AIDS had lower Th1 responses and decreased production of beta-chemokines and IL-2 relative to other HIV+ infants

- No HIV+ infants had no demonstrable CTL at birth, but Th1 responses accompanied by CTL responses developed in children with slowly progressive disease, and not in rapid progressors

- CTLp frequencies were determined by limiting dilution using autologous B cells infected with vaccina/HIV constructs

\begin{tabular}{lllc}
\hline Pol & Pol (LAI) & Vaccine & human
\end{tabular}

- The vaccine used was a rec canarypox with HIV-1 gp120 MN, tm/gag/protease LAI (vCP205), alone or with p24E-V3 MN synthetic peptide (CLTB-36))

- Twenty HIV negative subjects were vaccinated in phase I trial with combinations of vCP205 and CLTB-36

- Immunization with vCP205 induced HIV-1-specific ABs to gp120, V3, and p24 antigens, and CTL immune responses against vCP205 were detected after the fourth immunization in 33\% of the subjects against Env, Gag and Pol, but the CLTB-36 peptide did not produce AB or CTL immune responses against p24 or gp160

\begin{tabular}{llll}
\hline Pol & Pol (172-219 subtype B) & Vaccine & human \\
& Vaccine Vector/Type: canarypox prime with rgp120 boost & Strain: LAI and SF2 & HIV component: Env, Gag, Pro, Nef, Pro
\end{tabular}

- The vaccine used was rec canarypox expressing HIV-1 env, gag, pol, nef and protease (vCP300) with or without administration of HIV-1 SF-2 rgp120

- In vitro inducible CTL activity against HIV-1 Env, Gag, Pol, and Nef antigens was observed in 79\% (15 of 19) of vaccine recipients

- The combination of vCP300 and vP1291 together resulted in an overall increase in CTL induction and detection sensitivity

Pol Pol (IIIB) HIV-1 infection human

- This study demonstrated an inverse correlation between HIV Type I plasma viral load and CTL activity directed against HIV-1 Pol, and stronger combined effects of Pol- and Env-specific CTL, in long-term survivors (LTS) of HIV-1 infection

Pol Pol (BRU) HIV-1 infection human Aladdin1999

- In vitro measurements of CTL-activity by Cr release assay in bulk culture showed no correlation between CTL-activity (gp120, Gag, Pol and Nef) and disease progression as measured by viral load, CD4 and time to death
Pol
RT (LAI)
HIV-1 infection
human
Buseyne1998b
- In infants with positive CTL responses, most responses showed cross-clade reactivity with somewhat diminished recognition of epitopes from different subtypes 


\begin{tabular}{llccc}
\hline HXB2 Location & Author's Location & Sequence & Immunogen & Species (HLA) \\
\hline Pol & RT & References & murine & Kim1997c \\
& Vaccine Vector/Type: DNA & HIV component: Gag, Pol, Vif, Env & Adjuvant: B7, IL-12 &
\end{tabular}

A gag/pol, vif or gp160 DNA vaccine, when delivered in conjunction with the plasmid encoding the co-stimulatory molecules B7 and IL-12, gave a

dramatic increase in both the cytotoxic and proliferative responses in mice

- When IL-12 was present, CTL response could be detected even without in vitro stimulation

Pol RT $\quad$ HIV-1 infection human Trickett1998

- Twelve HIV-1 infected patients were re-infused with their own lymphocytes, cryopreserved from an earlier time point in the infection

- Improvement in CD4+ and CD8+ T cells were seen in 7/12, and an increase in the CTL response to Pol was seen in one patient

\begin{tabular}{llll}
\hline Pol & RT & HIV-1 infection & human
\end{tabular}

\begin{tabular}{llll}
\hline Pol & RT & HIV-1 infection & human
\end{tabular}

\begin{tabular}{llll}
\hline Pol & RT & HIV-1 infection & human
\end{tabular}

作 autologous EBV transformed B cells

- The child who progressed consistently had CTL against Pol and Tat

- The long-term non-progressing child had no detectable CTL, but was heterozygous for a mutation in the CCR5 receptor and for HLA-B49, which has been shown to be associated with slower progression

Pol Pol (IIIB) HIV-1 infection human Betts1997

- 6/8 individuals from Zambia infected with C clade virus had CTL that were able to make response to B clade HIV-1 IIIB vaccinia-expressed Gag, Pol and Env proteins

- A vigorous cross-clade response was not limited to a particular protein, and the level of recognition of different proteins varied among the six patients

Pol RT $\quad$ HIV-1 infection human Daria1997

- $\mathrm{CD} 3+$ cells that also carry a natural killer cell receptor (NKR+) can exhibit down regulation of $\mathrm{T}$ cell function

- Anti-NKR IgM MAb masked this inhibitory function and increased HIV-1 specific CTL activity in phytohemagglutinin-activated PBMC cultured in the presence of IL-2 from 3/5 patients, and in one other case anti-NKR MAb brought HIV-1 specific CTL activity to detectable levels

- 13/37 exposed uninfected individuals with repeated high-risk sexual exposure had HIV-1 specific CTL against Env, Gag, Pol, or a combination of proteins CTL activity was correlated with a CCR5 wildtype genotype

- In this group, the highest CTLp frequencies were directed at Gag, but the most common response was to Env and four individuals had responses to multiple HIV-1 proteins

Pol

Pol (LAI)

Vaccine

human

Evans1999

Vaccine Vector/Type: canarypox HIV component: gp120, gp41, Gag, Pro, Nef, RT

- A Canarypox vaccine expressing gp120, gp41, Gag, Protease, Nef and Pol CTL epitopes gave rise to CTL that could be detected in $61 \%$ of the volunteers responses to Gag, Env, Nef and Pol were detected 3-6 months after the last vaccination

Vaccine Vector/Type: DNA HIV component: Env, Gag, Pol Adjuvant: CD86, CD80

- The study explores the use of co-stimulatory molecules co-expressed with an HIV-1 immunogen in a DNA vaccine to enhance the immune response co-expression of CD86, but not CD80, dramatically increased both HIV Env and Gag/Pol specific CTL and Th proliferative responses 


\begin{tabular}{llllll}
\hline HXB2 Location & Author's Location & Sequence & Immunogen & Species (HLA) \\
\hline Pol & Pol (IIIB) & HIV-1 infection & human
\end{tabular}

- CTL precursor frequencies were determined in HIV-1 infected pregnant women, and significantly higher CTLp frequencies to Pol and Nef were found in non-transmitting mothers than in transmitting mothers; Pol

HIV-1 infection

human

Young2001

- Addition of recombinant rec human IL12 (rhIL12) to cultures increased HIV-specific lysis of HIV-Gag, Pol and gp120 vaccinia expressed antigens (11/15 tested increased lysis by $>5 \%$ ) if the culture was derived from HIV+ individuals who had CD4 cells/ul $>500$

- 2/10 individuals with $<200 \mathrm{CD} 4$ cells/ul, and 3/10 individuals with 200-500 CD4cells/ul, had an increase of $>5 \%$ upon treatment of the culture with rhIL12, so a few individuals in late stage disease had CD8 cells that maintained responsiveness to rhIL12

Pol RT (subtype A, B, D) HIV-1 infection human

- HIV-1 subtypes A and D dominate the Ugandan epidemic, and a vaccine trial using B clade antigen is underway - this study addresses relative levels of cross-reactive CTL responses in HIV infected Ugandans to A, D, and B clade recombinant vaccinia viruses expressing Gag, Env, Pol, RT or Nef from HIV-1 clades A, B, and D.

- Proteins corresponding to the subtype of the infecting strains tended to trigger higher levels of CTL response measured by percent specific lysis, but there was extensive inter-subtype cross-reactivity with B clade proteins and the co-circulating subtype.

\begin{tabular}{llcl}
\hline Pol & Pol & HIV-1 infection & human \\
& HIV-specific CTL activity was detected in the female reproductive tract of only $1 / 3$ HIV-infected women who underwent a hysterectomy,
\end{tabular}

- HIV-specific CTL activity was detected in the female reproductive tract of only $1 / 3$ HIV-infected women who underwent a hysterectomy, although CTL could be identified in the PBMC of all three women

Pol Pol (IIIB) HIV-1 infection human Jin2000a

- The CTL precursor level (CTLp) was measured in long term non-progressors (LTNP) with low viral load using limiting dilution analysis and measuring CTL against Env Gag and Pol expressed in vaccinia in autologous targets

- LTNPs have high memory CTL numbers and low viral load

Pol Pol HIV-1 exposed seronegative human Rowland-Jones2001

- This is a review that summarizes observations about HIV-specific CTL found in the HIV-1 exposed persistently seronegative (HEPS) population

- The CTL responses assayed by ELISPOT and by CTL precursor frequencies by limiting dilution analysis indicate that CTL in HEPS individuals tend to be of a lower magnitude than in chronic HIV-1 infections - the responses in HEPS cases are below the level of detection by tetramer assays

- CD8+ CTL responses tend to be detectable in HEPS subjects only if they are recently exposed, and the response diminishes if exposure is reduced - it is not clear if there is a stable memory population in HEPS cases

- CD8+ CTL responses in the HEPS population are associated with HIV-1 specific CD4+ T cell responses, assayed by proliferation assays, IL-2 secretion, and ELISPOT, and the authors consider the possibility that HIV-1-specific T-help responses improve the "quality" of the CD8+ response in HEPS individuals relative to HIV-1 infected individuals, who tend to have a poor HIV-1-specific T-help response

- HIV-1 specific CD8+ CTL responses in HIV-1 infected individuals show reduced levels of perforin, and the T cells may not mature properly, and although similar studies have not been conducted in HEPS individuals this is considered as a possible difference in the CTL immune response in HEPS and HIV-1 infected people

- 6/24 HIV uninfected infants (ages 15-50 months) born to HIV+ mothers had HIV-1 specific CTL responses to vaccinia-expressed Nef, Gag/Pol, Env. - Reviewed in [Kuhn2002]. 


\begin{tabular}{|c|c|c|c|c|c|}
\hline HXB2 Location & Author's Location & Sequence & Immunogen & Species (HLA) & References \\
\hline Pol & & & HIV-1 infection & human & \begin{tabular}{|l|l} 
Kuhn2002 Wasik1999 \\
\end{tabular} \\
\hline
\end{tabular}

- In HIV-infected infants HIV-specific, CTL responses were not detectable in icord blood or in PBMC collected shortly after birth and were absent or remained very low in 3 infants with a rapidly progressive disease. For those who progressed more slowly, the HIV-specific CTL activity varied.

- The two infants with high levels of Env peptide-stimulated IL-2 responses had the highest CTLp frequencies.

- Stronger responses were detected after initiation of the antiretroviral therapy.

- Two babies that were not infected though born to HIV+ mothers had detectable though low HIV-specific CTLp responses to Env (1/2), Pol (2/2), Gag (1/2) cord blood and transiently in PBMC after birth.

- Reviewed in [Kuhn2002].

- Six of nine HIV vertically infected infants had HIV-1 specific CTL responses to vaccinia expressed Tat (4/6), Pol (6/6), Env (1/6), or Gag (1/6), but not all responses were detected at all time points.

- Two of eleven babies that were not infected though born to HIV+ mothers had detectable responses Tat (1/2), Pol (2/2), Gag (1/2)

- Reviewed in [Kuhn2002].

- Epitopes that have been described in the literature and included in this database tend to cluster in conserved regions and be absent or rarely found highly variable regions found in Nef, Env and p17.

- In the more conserved p24, and Pol proteins RT and Protease, epitopes are more evenly distributed, however, epitopes were not found that span the invariant, most highly conserved regions of RT and Protease. This might be due to the virus evolving conserved features that disallow the CTL responses in these most conserved regions, as functional constraints for enzyme function would not tolerate change and normal capacity for immune escape by rapid evolution is lost in these domains.

- Therapeutic RT inhibitors were used to select in vitro for resistance mutations in subtype C viruses. Many of the resistance mutations were located within analogs to CTL epitopes that had been defined for the B subtype,

- Subjects treated with HAART early in HIV-infection showed a correlation between the number of viremic episodes and the total as well as the Pol-specific CD8 T-cell activity as measured by Elispot SFC per million PBMC summed across Pol, Env, Nef and Gag. The subjects treated early after infection had higher levels of $\mathrm{CD} 8+\mathrm{T}$-cell activity $(\mathrm{N}=31)$ than those treated later $(\mathrm{N}=23)$, and a greater capacity to enhance $\mathrm{CD} 8+\mathrm{T}$-cell responses to viremic episodes.

Pol (MN) HIV-1 infection $\quad$ human

- $96 \%$ (26/27) chronically infected HIV-1 infected patients elicited gamma-IFN CD8+ T-cell responses against Gag

- Nef and/or Pol CTL responses were detected in $86 \%$ of the subjects

- The magnitude and breadth of Gag and p24 T-cell responses correlated with absolute CD4 counts, and inversely correlated with viral load

- Pol and Int CTL responses correlated positively with absolute CD4+ T-cell count

- Nef and Env responses did not correlate with either CD4 counts or viral load

- Autologous mature dendritic cells with rec vaccinia expressing Gag, Pol, Nef and Env could amplify CD8+ T-cell Elispot responses 4-38 fold in five HIV+ patients on successful HAART treatment, relative to autologous monocytes. Some weak responses could only be detected using mature dendritic cells as APCs, and this approach could be useful for detection of low frequency memory cells. 


\begin{tabular}{lllll}
\hline HXB2 Location & Author's Location & Sequence & Immunogen & Species (HLA) \\
\hline Pol & (IIIB) & HIV-1 infection & human & Trickett2002
\end{tabular}

- Conditions were optimized for ex-vivo expansion of CD8+ and CD4+ T-cells with the goal of functional T-cell production for autologous immunotherapy. 10,000-fold expansions were obtained in 14 days with optimized concentrations of IL-2, anti-CD3 and anti-CD28 coated microspheres, and decreasing amounts of serum over the first 8 days.
Pol
(IIIB)
HIV-1 and HCV co-infection
human
Lauer2002

- HIV-1 and HCV immune responses were studied in 22 individuals who were co-infected with HIV-1 and hepatitis C virus (HCV). IFNgamma production was measured in an Elispot assay of CD8+ T-cells using targets expressing either Gag, RT, Env and Nef in a vaccinia construct, or one of seven HCV proteins.

- All 22 patients targeted at least one protein. 20/22 patients recognized RT, 17/22 patients recognized Gag, 13/22 subjects recognized Env and 11/22 patients recognized Nef. Robust CTL activity was independent of disease progression or viral load.

- Despite high HCV viral loads, very few HCV CD8+ T-cell Elispot responses were detected. In a control HCV infected person who did not have HIV-1, strong anti-HCV responses were mounted.

- HIV-specific CD4 proliferative responses were detected in 9/17 coinfected patients, but no HCV responses were detected.

$$
\text { HIV-1 infection human Scott2001 }
$$

- CTL responses before and after initiation of ART were studied in 13 HIV-1 vertically infected infants $<6$ months of age, and 4 that were $>6$ months of age.

- Before ART 2/13 infants $<6$ months of age showed IFNgamma Elispot CD8+ T-cell responses, one to Nef and one to Env and Nef, and these responses

became undetectable after successful therapy- 3 infants were coinfected with CMV and all 3 had CMV-specific CD8+ T-cell responses.

- One older infant, at 23 months, had CTL responses against all for proteins tested, Gag, Pol, Nef and Env, and had the lowest plasma viremia of the study group. 3/4 infants older than 6 months of age responded to either Nef or Pol.

- Administration of ART over 48 weeks broadened the HIV-1-specific CTL response in 2/4 of the older children that were incomplete responders.
Pol
(IIIB, MN)

\section{HIV-1 infection}
human
Larsson2002a

- Dendritic cells acquire and present HIV-1 antigens derived from dead, apoptotic cells or from non-infectious, fusion-competent HIV-1 virions, and these DC cells could stimulate CD4+ and CD8+ T-cells resulting in IFNgamma production in an Elispot assay. Both HLA Class I and class II molecules were used for presentation. This may be an important aspect of the initial immune response to HIV-1 infection of CD4+ cells in the mucosal subepithelia.

Pol (III) HIV-1 infection human Ortiz2001

- Immune responses in eight chronically HIV-1 infected patients undergoing HAART therapy structured treatment interruptions (STI) were studied. ST boosted HIV-1 specific CTL responses and elevated CTL responses were maintained up to 22 weeks after the last treatment interruption, but viral load rebound to pretreatment levels and CD4 T-cell count decline was observed. CD8 responses in PBMC were measured by cytokine flow cytometry with gp160, Gag p55, RT-Pol and Nef expressed in vaccinia.

\begin{tabular}{llll}
\hline Pol & Pol & HIV-1 infection & $\begin{array}{l}\text { human (A*0201 and } \\
\text { Cw*08) }\end{array}$
\end{tabular}

- HIV-1 specific, MHC class I-restricted CTL killing was detected in duodenal and rectal gut associated lymphoid tissue (GALT) sites from three infected individuals - the distribution of class I restricted CTL was different in the peripheral blood samples and GALT samples

computer prediction $\quad(\mathrm{A} * 0201, \mathrm{~B} * 3501) \quad$ Schönbach2002

- Computational methods (artificial neural networks, hidden Markov models, binding matrices based on HLA association rates) were used to identify HLA-A*0201 and HLA-B*3501 HIV T-cell epitope candidates from 533 Gag, Env and Pol sequences of which 374 were derived from HIV-1, 97 were derived from HIV-2 and 62 from SIV. Comparisons to known epitopes and between clades were made. 


\begin{tabular}{llllll}
\hline HXB2 Location & Author's Location & Sequence & Immunogen & Species (HLA) \\
\hline Pol & RT (IIIB) & HIV-1 infection & human (A2) & Moore2002b
\end{tabular}

- HIV polymorphisms in the RT protein were examined relation to HLA alleles found in a population of 473 participants in the Western Australian HIV Cohort Study. 64 significant associations between polymorphisms at particular positions and HLA alleles were detected, for HLA-B7, -B12, -B35 and

-B15. Fifteen of these were in positions with known epitopes, 4 in anchor residues, 11 in other positions. Six additional polymorphic sites associated with particular HLA molecules flanked known epitopes and may relate to processing.

- 25 negative associations were also found between polymorphism and HLA alleles. The authors propose this is due to escape mutations in epitopes presented by common HLA types dominating in the population, and give examples of five amino acids which are in the consensus and tend to be stable in those with the most common HLA allele, HLA-A2. Pol HIV-1 infection human $(\mathrm{B} * 35)$

Jin2002

- Patients with HLA-B*35 variants $\mathrm{B} * 3502, \mathrm{~B} * 3503, \mathrm{~B} * 3504$, and $\mathrm{B} * 5301$ tend to proceed to AIDS more quickly than those with $\mathrm{B} * 3501$.

- Of 32 patients with HLA-B*35 alleles CD8+CTL responses were quantified using an intracellular cytokine staining assay - 75\% had responses to Pol, $69 \%$ to Gag, $50 \%$ to Nef, and $41 \%$ to Env.

- The overall magnitude of CTL responses did not differ between those bearing B*3501 and the others. A higher percentage of Gag responses was observed in those that had lower RNA levels that carried $\mathrm{B} * 3501$, and there was a negative association with viral load and CTL activity. The data is consistent with higher levels of CTL responses contributing to protection in $\mathrm{B} * 3501$ individuals, but not in $\mathrm{B} * 3502$, $\mathrm{B} * 3503$, $\mathrm{B} * 3504$, and $\mathrm{B} * 5301$ individuals .

Pol Po Vaccine murine $\left(\mathrm{H}-2^{d}\right)$

Huang2001

Vaccine Vector/Type: DNA Strain: gag HxB2, pol NL43 HIV component: Gag, Pol

- Mice were immunized with four humanized DNA constructs: GagPol, that would form a pseudoparticle carrying Gag and Pol, Gag, Pol or a GagPol fusion construct

- The GagPol pseudoparticle, Gag and GagPol fusion construct all elicited strong anti-Gag CTL, but only the GagPol fusion construct elicited strong anti -Pol CTL 


\section{II-B-13 Vif CTL Epitopes}

\begin{tabular}{|c|c|c|c|c|}
\hline HXB2 Location & Author's Location & Sequence & Species (HLA) & References \\
\hline Vif (17-26) & \multicolumn{4}{|c|}{$\begin{array}{l}\text { - Epitopes that have been described in the literature and included in this database tend to cluster in conserved regions and be absent or rarely found highly } \\
\text { variable regions found in Nef, Env and p17. } \\
\text { - While the uneven distribution of epitopes may be in part due to a limited cross-recognition of specific responses because of differences between peptides } \\
\text { used to probe the immune response and autologous strains, regions with a paucity of defined epitopes also had higher frequencies of amino acids that tend } \\
\text { to not be found in C-terminal positions of epitopes, and had lower cleavage prediction scores for epitope processing. This suggests that in the regions of the } \\
\text { virus where variation is best tolerated traces of immune escape have left an imprint on the viral population. Epitopes also were concentrated in alpha-helix } \\
\text { and turn regions in the proteins. } \\
\text { - In the more conserved p24, and Pol proteins RT and Protease, epitopes are more evenly distributed. } \\
\text { - What was learned from proteins where many epitopes have been defined (Gag, Pol, Env and Nef) was used to develop an algorithm to predict where } \\
\text { epitopes would be localized in Rev, Tat, Vif, and Vpr. Predictions were made blinded, and then compared to the first } 15 \text { epitopes defined in these proteins; } \\
\text { the epitopes were concentrated in the predicted regions. }\end{array}$} \\
\hline $\operatorname{Vif}(17-26)$ & $\begin{array}{l}\text { Vif (17-26 SF2) } \\
\text { - Epitope name: RK10 } \\
\text { - CTL responses against H } \\
\text { - } 10 / 29(35 \%) \text { individuals t } \\
\text { - This epitope was recogniz } \\
\text { - HIV+ individual AC-06 y } \\
\text { from } 7 \text { proteins, suggestir } \\
\text { - Overlapping Vif peptides } \\
\text { RIRTWKSLVK }\end{array}$ & $\begin{array}{l}\text { RIRTWKSLVK } \\
\text { IV-1 Vpr, Vpu, at } \\
\text { ested responded } \\
\text { zed by } 3 / 15 \text { indiv } \\
\text { vas tested for rea } \\
\text { ag that the breadt } \\
\text { QVDRMRIRTW }\end{array}$ & $\begin{array}{l}\text { HIV-1 infection } \\
\text { lyzed in multiple HIV-1-infected individuals }\left(\mathrm{A}^{* 0301)}\right. \\
\text { ng A*0301 allele } \\
\text { ig peptides spanning all HIV-1 proteins in an ELISPOT } \\
\text { nses are underestimated if accessory proteins are not in } \\
\text { IRTWKSLVKHHMYI both reacted with T-cells from A }\end{array}$ & $\begin{array}{l}\text { Altfeld2001a } \\
\text { s found to react with } 12 \text { peptides } \\
\text { n the study } \\
\text { nd contained epitope }\end{array}$ \\
\hline Vif (17-26) & $\begin{array}{l}\text { Vif (17-26) } \\
\text { - CTL responses against re } \\
\text { - } 28 \% \text { targeted one or more } \\
\text { - All known optimally defi }\end{array}$ & $\begin{array}{l}\text { RIRTWKSLVK } \\
\text { gulatory and acce } \\
\text { overlapping Tat } \\
\text { ned epitopes wer }\end{array}$ & $\begin{array}{l}\text { HIV-1 infection human (A*0301) } \\
\text { n } 70 \text { HIV-1 infected patients were studied using overla } \\
\text { Rev peptides; 33\%, Vif peptides; 40\%, Vpr peptides; a } \\
\text { r the five proteins. }\end{array}$ & $\begin{array}{l}\text { Addo2002b } \\
\text { eptides and Elispot. } \\
\text { Vpu peptides. }\end{array}$ \\
\hline Vif (17-26) & $\begin{array}{l}\text { - Epitope name: Vif-RK10 } \\
\text { - Among HIV+ individuals }\end{array}$ & $\begin{array}{l}\text { RIRTWKSLVK } \\
\text { who carried HL }\end{array}$ & $\begin{array}{l}\text { HIV-1 infection } \\
4 \% \text { ) recognized this epitope }\end{array}$ & Sabbaj2002b \\
\hline Vif (17-26) & (LAI) & RIRTWKSLVK & (A3) & Altfeld2000a, Brander2001 \\
\hline $\operatorname{Vif}(17-26)$ & $\begin{array}{l}\text { Vif (17-26) } \\
\text { - Epitope name: A3-RK10 } \\
\text { - CTL responses in } 18 \text { acut } \\
\text { - One individual, AC-06, w } \\
\text { interruptions (STI). He ha } \\
\text { restricted by HLA-A3, } 11\end{array}$ & $\begin{array}{l}\text { RIRTWKSLVK } \\
\text { ely HIV-infected } \\
\text { as homozygous } \\
\text { ad only two detec } \\
\text { by HLA-B7, an }\end{array}$ & $\begin{array}{l}\text { HIV-1 infection } \\
\text { or -B7 }(n=4) \text { or both -A3 and B7 ( } \mathrm{n}=7 \text { ) positive indiv } \\
\text { I alleles (A3, B7, Cw7), was treated during acute infe } \\
\text { nses during acute infection, but after STI this broaden } \\
7 \text {. }\end{array}$ & $\begin{array}{l}\text { Yu2002a } \\
\text { ere studied. } \\
\text { had supervised treatment } \\
\text { distinct epitopes including } 15\end{array}$ \\
\hline
\end{tabular}




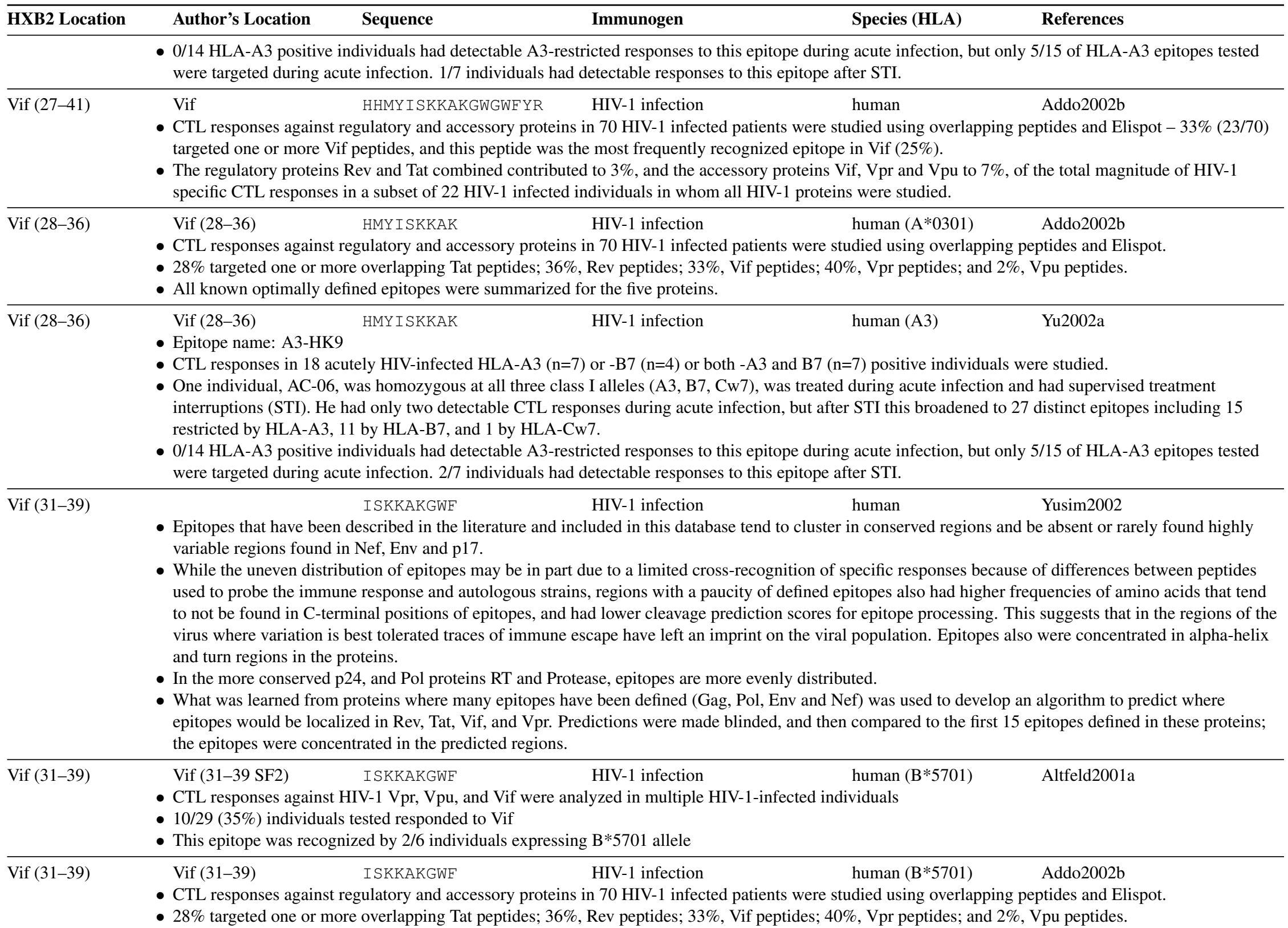




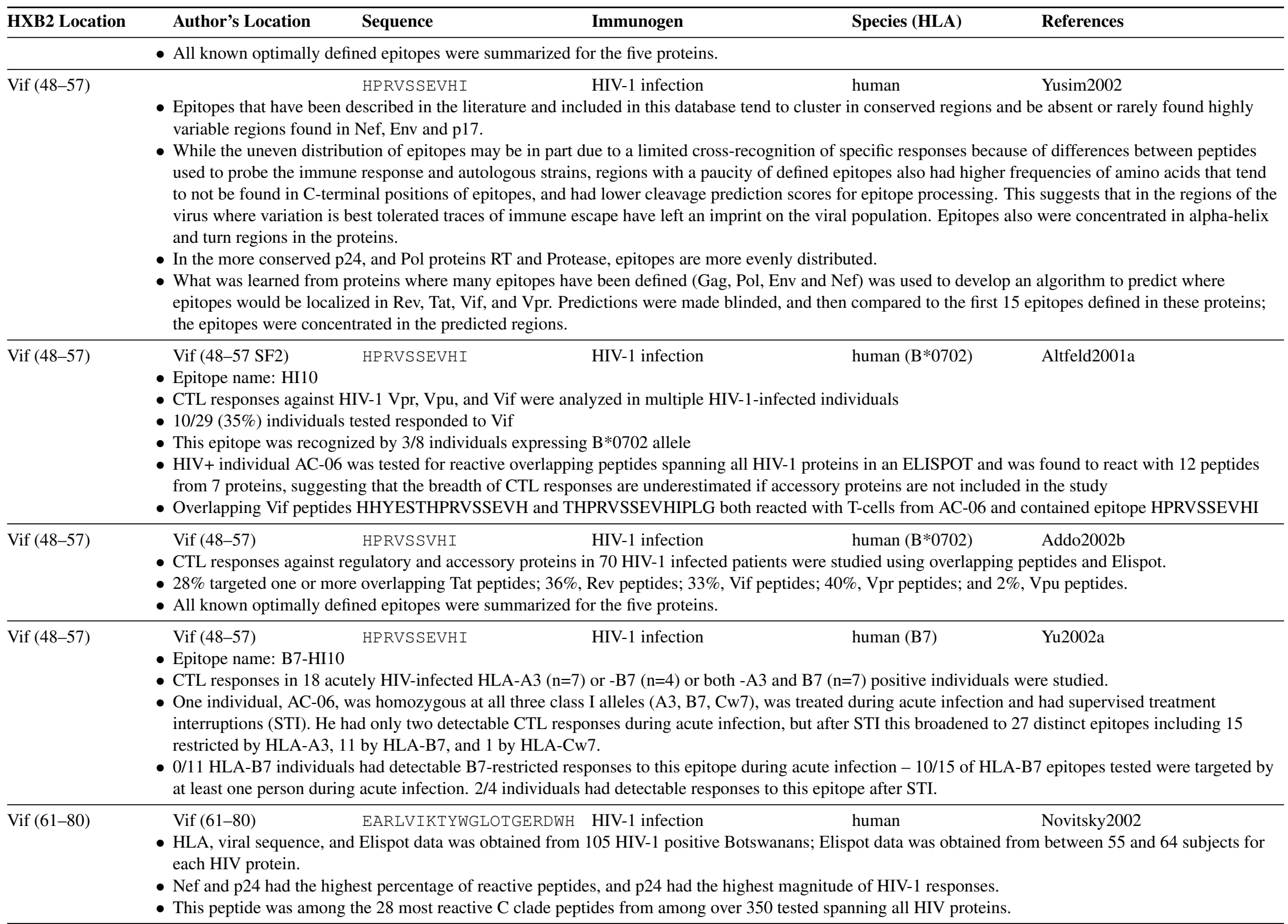




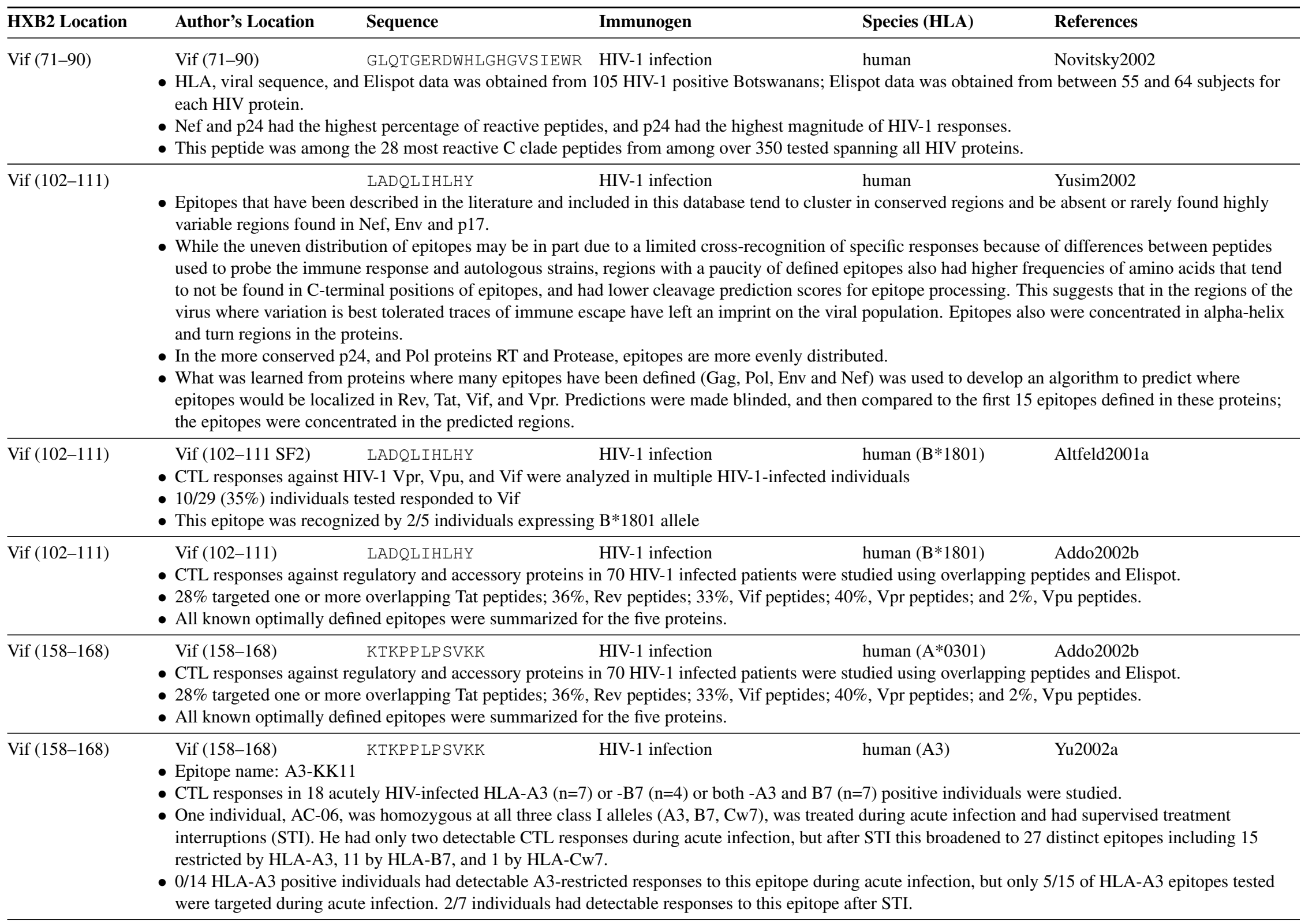




\begin{tabular}{lllcl}
\hline HXB2 Location & Author's Location & Sequence & Immunogen & Species (HLA) \\
\hline Vif (160-169) & Vif & KPP LP SVKKL & human (B7) \\
& - The program Epimatrix was used in conjunction with the program Conservatrix to identify conservered regions of HIV that might serve as epitopes
\end{tabular}

- A subset of the potential epitopes was identified that could bind to the appropriate HLA-allele, and 15 predicted B7 superfamily (HLA B7, B8, and B58) epitopes could stimulate IFN $\gamma$ production in an ELISPOT assay

- KPPLPSVKKL was newly identified as an HLA-B7 epitope in this study

\begin{tabular}{llll}
\hline Vif & Vif & Vaccine & murine
\end{tabular}

- A gag/pol, vif or env DNA vaccine, when delivered in conjunction with the plasmid encoding the co-stimulatory molecules B7 and IL-12, gave a dramatic increase in both the cytotoxic and proliferative responses in mice

- When IL-12 was present, CTL response could be detected even without in vitro stimulation

\begin{tabular}{|c|c|c|}
\hline Vif & $\begin{array}{l}\text { Vif } \\
\text { Vaccine Vector/Type: DNA HIV component: Vif, Vpu, Nef } \\
\text { - Splenocytes from BALB/c mice immunized with pVVN-P DNA were incubated with Vif, Vpu or Nef antigens } \\
\text { IFN-gamma levels } \\
\text { - Antigen stimulation increased IFN-gamma production in pVVN-P immunized mice, indicating a Th1 response } \\
\text { - IL-4 production was not significantly changed after antigen stimulation compared to control levels } \\
\text { - Cross-clade CTL activity was also observed: A, B clade, CRF01(AE) clade antigens could serve as targets for } \\
\text { an HIV-1 AC recombinant, however, did not stimulate a CTL response, but was expressed at lower levels on the }\end{array}$ & $\begin{array}{l}\text { Ayyavoo2000 } \\
\text { s and assayed for IL-4 and } \\
\text { e immunization-stimulated CTL - }\end{array}$ \\
\hline Vif & $\begin{array}{l}\text { Vif } \\
\text { Vaccine Vector/Type: DNA HIV component: Vif, Vpu, Nef } \\
\text { - Splenocytes from BALB/c mice immunized with pVVN-P DNA were incubated with Vif, Vpu or Nef antigens } \\
\text { IFN-gamma levels } \\
\text { - Antigen stimulation increased IFN-gamma production in pVVN-P immunized mice, indicating a Th1 response } \\
\text { - IL-4 production was not significantly changed after antigen stimulation compared to control levels } \\
\text { - Cross-clade CTL activity was also observed: A, B clade, CRF01(AE) clade antigens could serve as targets for } \\
\text { an HIV-1 AC recombinant, however, did not stimulate a CTL response, but was expressed at lower levels on the }\end{array}$ & $\begin{array}{l}\text { Ayyavoo2000 } \\
\text { s and assayed for IL-4 and } \\
\text { e immunization-stimulated CTL - } \\
\text { ll }\end{array}$ \\
\hline
\end{tabular}




\section{II-B-14 Vpr CTL Epitopes}

\begin{tabular}{llccc}
\hline HXB2 Location & Author's Location & Sequence & Immunogen & Species (HLA) \\
\hline $\operatorname{Vpr}(12-20)$ & & REP HNEWTL & HIV-1 infection & human \\
& - Epitopes that have been described in the literature and included in this database tend to cluster in conserved regions and be absent or rarely found highly
\end{tabular}

- Epitopes that have been described in the literature and included in this database tend to cluster in conserved regions and be absent or rarely found highly variable regions found in Nef, Env and p17.

- While the uneven distribution of epitopes may be in part due to a limited cross-recognition of specific responses because of differences between peptides used to probe the immune response and autologous strains, regions with a paucity of defined epitopes also had higher frequencies of amino acids that tend to not be found in C-terminal positions of epitopes, and had lower cleavage prediction scores for epitope processing. This suggests that in the regions of the virus where variation is best tolerated traces of immune escape have left an imprint on the viral population. Epitopes also were concentrated in alpha-helix and turn regions in the proteins.

- In the more conserved p24, and Pol proteins RT and Protease, epitopes are more evenly distributed.

- What was learned from proteins where many epitopes have been defined (Gag, Pol, Env and Nef) was used to develop an algorithm to predict where epitopes would be localized in Rev, Tat, Vif, and Vpr. Predictions were made blinded, and then compared to the first 15 epitopes defined in these proteins; the epitopes were concentrated in the predicted regions.

\begin{tabular}{llccc}
\hline $\operatorname{Vpr}(12-20)$ & Vpr $(12-20$ SF2) & REPHNEWTL & HIV-1 infection & human (B*4002) \\
& - & CTL responses against HIV-1 Vpr, Vpu, and Vif were analyzed in multiple HIV-1-infected individuals
\end{tabular}

- CTL responses against HIV-1 Vpr, Vpu, and Vif were analyzed in multiple HIV-1-infected individuals

- Individuals with long-term nonprogressive and treated chronic HIV-1 infection targeted Vpr more frequently than individuals with treated acute infection

- Only one B*4002+ individual was tested, and had a CTL response against REPHNEWTL

- Vpr is a frequent target of HIV-1 specific CD8+ T-cells - a response was detected in 45\% of individuals tested and Vpr and p17 were the most preferentially targeted proteins per unit length by CD8+ T-cells

Vpr (12-20) Vpr (12-20) REPHNEWTL HIV-1 infection $\quad$ Addo2002b

- CTL responses against regulatory and accessory proteins in $70 \mathrm{HIV}-1$ infected patients were studied using overlapping peptides and Elispot.

- $28 \%$ targeted one or more overlapping Tat peptides; 36\%, Rev peptides; 33\%, Vif peptides; 40\%, Vpr peptides; and 2\%, Vpu peptides.

- All known optimally defined epitopes were summarized for the five proteins.

Vpr (30-38) AVRHFRPIW HIV-1 infection human

- Epitopes that have been described in the literature and included in this database tend to cluster in conserved regions and be absent or rarely found highly variable regions found in Nef, Env and p17.

- While the uneven distribution of epitopes may be in part due to a limited cross-recognition of specific responses because of differences between peptides used to probe the immune response and autologous strains, regions with a paucity of defined epitopes also had higher frequencies of amino acids that tend to not be found in C-terminal positions of epitopes, and had lower cleavage prediction scores for epitope processing. This suggests that in the regions of the virus where variation is best tolerated traces of immune escape have left an imprint on the viral population. Epitopes also were concentrated in alpha-helix and turn regions in the proteins.

- In the more conserved p24, and Pol proteins RT and Protease, epitopes are more evenly distributed.

- What was learned from proteins where many epitopes have been defined (Gag, Pol, Env and Nef) was used to develop an algorithm to predict where epitopes would be localized in Rev, Tat, Vif, and Vpr. Predictions were made blinded, and then compared to the first 15 epitopes defined in these proteins; the epitopes were concentrated in the predicted regions.

Vpr (30-38) Vpr(29-38 SF2) AVRHFPRIW HIV-1 infection $\quad$ Altfeld2001a

- CTL responses against HIV-1 Vpr, Vpu, and Vif were analyzed in multiple HIV-1-infected individuals

- This epitope was recognized by $4 / 6$ individuals expressing $B * 5701$ allele 


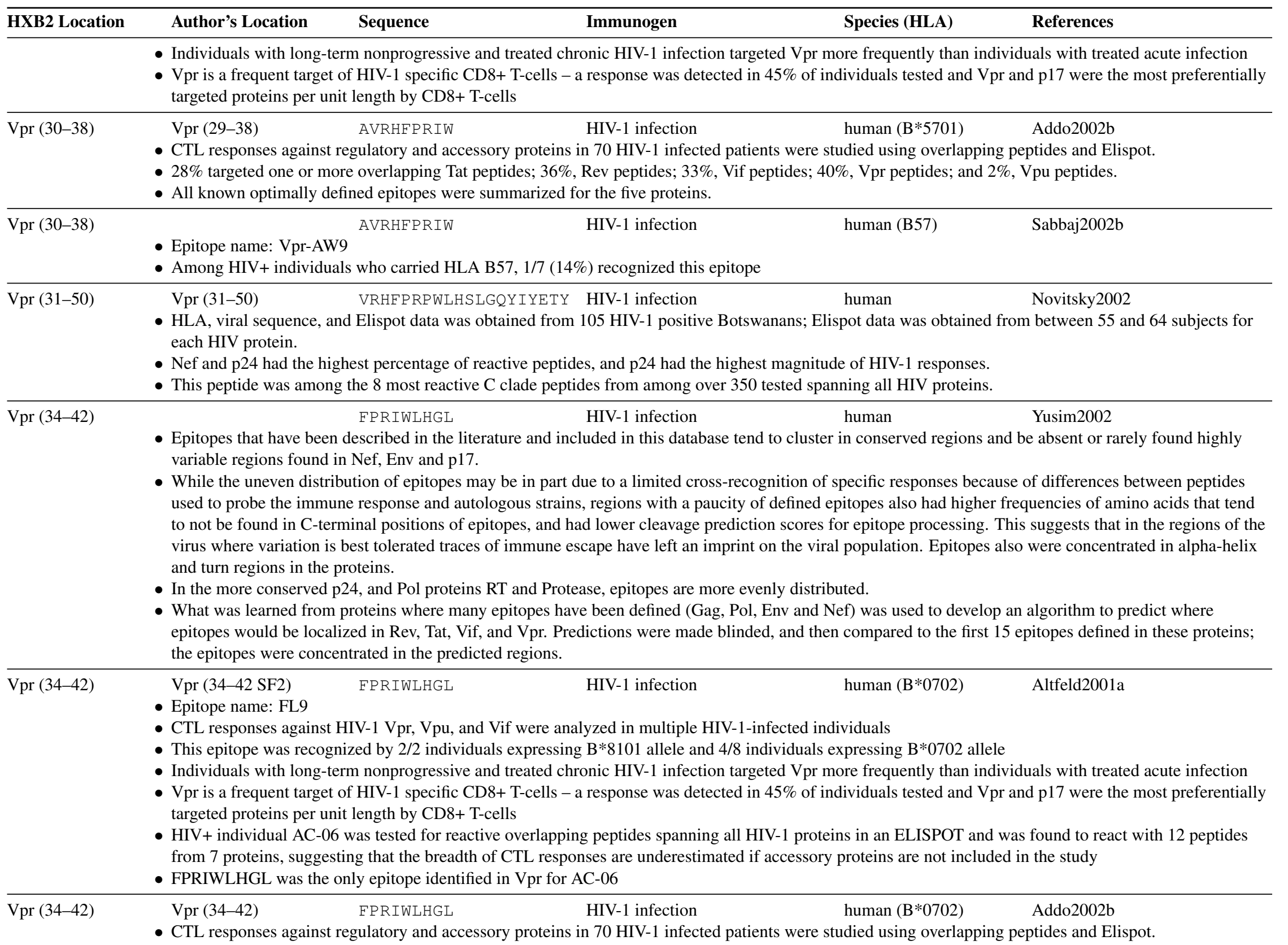




\begin{tabular}{|c|c|c|c|c|}
\hline HXB2 Location & Author's Location & Sequence & Species (HLA) & References \\
\hline & \multicolumn{4}{|c|}{$\begin{array}{l}\text { - } 28 \% \text { targeted one or more overlapping Tat peptides; } 36 \% \text {, Rev peptides; } 33 \% \text {, Vif peptides; } 40 \% \text {, Vpr peptides; and } 2 \% \text {, Vpu peptides. } \\
\text { - All known optimally defined epitopes were summarized for the five proteins. }\end{array}$} \\
\hline $\operatorname{Vpr}(34-42)$ & \multicolumn{4}{|c|}{$\begin{array}{l}\text { Vpr (34-42 SF2) FPRIWLHGL } \\
\text { - Epitope name: FL9 } \\
\text { - CTL responses against HIV-1 Vpr, Vpu, and Vif w } \\
\text { - This epitope was recognized by } 2 / 2 \text { individuals exp } \\
\text { - Individuals with long-term nonprogressive and trea } \\
\text { - Vpr is a frequent target of HIV-1 specific CD8+ T-c } \\
\text { targeted proteins per unit length by CD8+ T-cells }\end{array}$} \\
\hline $\operatorname{Vpr}(34-42)$ & \multicolumn{4}{|c|}{$\begin{array}{l}\text { - CTL responses against regulatory and accessory proteins in } 70 \mathrm{HIV}-1 \text { infected patients were studied using overlapping peptides and Elispot. } \\
\text { - } 28 \% \text { targeted one or more overlapping Tat peptides; } 36 \% \text {, Rev peptides; } 33 \% \text {, Vif peptides; } 40 \% \text {, Vpr peptides; and } 2 \% \text {, Vpu peptides. } \\
\text { - All known optimally defined epitopes were summarized for the five proteins. }\end{array}$} \\
\hline $\operatorname{Vpr}(34-42)$ & $\begin{array}{l}\text { Vpr (34-42) } \\
\text { - Epitope name: B7-F } \\
\text { - CTL responses in } 18 \\
\text { - One individual, AC- } \\
\text { interruptions (STI). } \\
\text { restricted by HLA-A } \\
\text { - } 1 / 11 \text { HLA-B7 indiv } \\
\text { at least one person }\end{array}$ & $\begin{array}{l}\text { FPRIWLHGL } \\
\text { tely HIV-infected HLA-A } \\
\text { vas homozygous at all thr } \\
\text { ad only two detectable CT } \\
1 \text { by HLA-B7, and } 1 \text { by H } \\
\text { had detectable B7-restric } \\
\text { acute infection. } 2 / 4 \text { indi }\end{array}$ & $\begin{array}{l}\text { HIV-1 infection human (B7) } \\
\text { or -B7 ( }=4) \text { or both -A3 and B7 }(n=7) \text { positive indivic } \\
\text { I alleles }(\mathrm{A} 3, \mathrm{~B} 7, \mathrm{Cw} 7) \text {, was treated during acute infec } \\
\text { onses during acute infection, but after STI this broadene } \\
\text { v7. } \\
\text { ponses to this epitope during acute infection - 10/15 of } \\
\text { had detectable responses to this epitope after STI. }\end{array}$ & $\begin{array}{l}\text { Yu2002a } \\
\text { ere studied. } \\
\text { d had supervised treatment } \\
\text { distinct epitopes including } 15 \\
37 \text { epitopes tested were targeted by }\end{array}$ \\
\hline $\operatorname{Vpr}(55-70)$ & $\begin{array}{l}\text { Vpr } \\
\text { - CTL responses agai } \\
\text { targeted one or mor } \\
\text { - The regulatory prote } \\
\text { specific CTL respon }\end{array}$ & $\begin{array}{l}\text { AGVEAIIRILQQLLFI } \\
\text { gulatory and accessory pr } \\
\text { peptides, and this peptide } \\
\text { ev and Tat combined con } \\
\text { a subset of } 22 \text { HIV-1 inf }\end{array}$ & $\begin{array}{l}\text { HIV-1 infection human } \\
70 \text { HIV-1 infected patients were studied using overla } \\
\text { most frequently recognized epitope in } \mathrm{Vpr}(41 \%) \text {. } \\
\text { to } 3 \% \text {, and the accessory proteins Vif, Vpr and Vpu t } \\
\text { dividuals in whom all HIV-1 proteins were studied. }\end{array}$ & $\begin{array}{l}\text { Addo2002b } \\
\text { ptides and Elispot - } 40 \%(28 / 70) \\
\text { he total magnitude of HIV-1 }\end{array}$ \\
\hline $\operatorname{Vpr}(59-67)$ & $\begin{array}{l}\text { - Epitopes that have b } \\
\text { variable regions fou } \\
\text { - While the uneven di } \\
\text { used to probe the im } \\
\text { to not be found in C } \\
\text { virus where variatio } \\
\text { and turn regions in t } \\
\text { - In the more conserv } \\
\text { - What was learned fr } \\
\text { epitopes would be l } \\
\text { the epitopes were cc }\end{array}$ & $\begin{array}{l}\text { AIIRILQQL } \\
\text { lescribed in the literature } \\
\text { Nef, Env and p17. } \\
\text { ation of epitopes may be i } \\
\text { e response and autologous } \\
\text { inal positions of epitopes } \\
\text { est tolerated traces of imr } \\
\text { oteins. } \\
4 \text {, and Pol proteins RT an } \\
\text { roteins where many epito } \\
\text { ted in Rev, Tat, Vif, and V } \\
\text { trated in the predicted reg }\end{array}$ & $\begin{array}{l}\text { HIV-1 infection human } \\
\text { uded in this database tend to cluster in conserved regio } \\
\text { ue to a limited cross-recognition of specific responses } \\
\text {, regions with a paucity of defined epitopes also had hi } \\
\text { d lower cleavage prediction scores for epitope processi } \\
\text { cape have left an imprint on the viral population. Epito } \\
\text { ase, epitopes are more evenly distributed. } \\
\text { been defined (Gag, Pol, Env and Nef) was used to der }\end{array}$ & $\begin{array}{l}\text { Yusim } 2002 \\
\text { be absent or rarely found highly } \\
\text { of differences between peptides } \\
\text { quencies of amino acids that tend } \\
\text { s suggests that in the regions of the } \\
\text { were concentrated in alpha-helix } \\
\text { algorithm to predict where } \\
\text { pitopes defined in these proteins; }\end{array}$ \\
\hline
\end{tabular}




\begin{tabular}{|c|c|c|c|c|}
\hline HXB2 Location & Author's Location & Sequence & Species (HLA) & References \\
\hline $\operatorname{Vpr}(59-67)$ & \multicolumn{2}{|c|}{$\begin{array}{l}\text { Vpr (58-66 LAI) AI IRILQQL } \\
\text { - C. Brander notes this is an A*0201 epitope }\end{array}$} & human $\left(A^{*} 0201\right)$ & Altfeld2001c, Brander2001 \\
\hline $\operatorname{Vpr}(59-67)$ & \multicolumn{2}{|c|}{$\begin{array}{l}\text { Vpr (58-66 SF2) } \\
\text { - Epitope name: AL9 } \\
\text { - CTL responses against H } \\
\text { - This epitope was recogni } \\
\text { - Epitope is located within } \\
\text { - Individuals with long-ter } \\
\text { - Vpr is a frequent target o } \\
\text { targeted proteins per unit } \\
\text { - The A2 eptiopes Vpr AII } \\
\text { individuals are excluded }\end{array}$} & $\begin{array}{l}\text { HIV-1 infection human }\left(\mathrm{A}^{*} 0201\right) \\
\text { lyzed in multiple HIV-1-infected individuals } \\
\text { ag A*0201 allele } \\
\mathrm{n} \mathrm{Vpr} \\
\text { onic HIV-1 infection targeted Vpr more frequently than } \\
\text { a response was detected in } 45 \% \text { of individuals tested and } \\
\text { do not account for the dominance of Vpr and p17, the }\end{array}$ & $\begin{array}{l}\text { Altfeld2001a } \\
\text { luals with treated acute infection } \\
\text { nd p17 were the most preferentially } \\
\text { olds even when HLA-A2+ }\end{array}$ \\
\hline $\operatorname{Vpr}(59-67)$ & $\begin{array}{l}\text { Vpr (59-) } \\
\text { - Epitope name: Vpr- } \\
\text { - HIV was scanned fo } \\
\text { criteria, and } 30 \text { of th } \\
\text { - Three additional pre } \\
\text { recognized at least o } \\
\text { maximum of 2) } \\
\text { - AIIRILQQL binds t } \\
\text { - } 5 / 22 \text { individuals witl } \\
\text { - } 2 / 12 \text { HLA-A2 patien } \\
\text { immunodominant w } \\
\text { - One of the the acute } \\
\text { - This peptide was sho }\end{array}$ & $\begin{array}{l}\text { AI IRILQQL } \\
\text { optides which car } \\
\text { ound to HLA-A*0 } \\
\text { ly described HLA } \\
\text { the } 23 \text { peptides ( } \mathrm{r} \\
\text { HLA-A2 superty } \\
\text { onic HIV-1 infecti } \\
\text { th acute HIV-1 inf } \\
\text { Jpr-59 was weak a } \\
\text { ected individuals, } \\
\text { o be properly proc }\end{array}$ & $\begin{array}{l}\text { HIV-1 infection human (A*0201) } \\
\text { permotif pattern conserved in more than } 50 \% \text { of B clade } \\
\text { ound to at least } 3 / 5 \text { of HLA-A } 2 \text { supertype alleles tested } \\
\text { vere added to the set of } 20 \text {, and } 18 / 22 \text { chronically infect } \\
\text { d maximum of } 6 \text { ), while } 6 / 12 \text { acute infected individuals } \\
0203, A^{*} 0201, A^{*} 0206 \text { and } A * 6802 \text { (highest affinity), b } \\
\text { this epitope, but with low magnitude responses in ELIS } \\
\text { ded strongly to this peptide, but during chronic infection } \\
\text { ant } \\
\text { LA A*0201/68 B } 44 / 14 \text { and also had a strong acute respo } \\
\text { sented in TAP-competent B-cell lines in vitro }\end{array}$ & $\begin{array}{l}\text { Altfeld2001c } \\
\text { nces }-233 \text { peptides met this } \\
\text { A-A2 individuals had CTL that } \\
\text { ized at least } 1 \text { (median of } 1 \text { and } \\
\text { A*0202 } \\
\text { nd Gag-386 tended to be } \\
\text { gp41 epitope SV10 SLLNATDIAV }\end{array}$ \\
\hline $\operatorname{Vpr}(59-67)$ & $\begin{array}{l}\text { Vpr (58-66) } \\
\text { - CTL responses agair } \\
\text { - } 28 \% \text { targeted one or } \\
\text { - All known optimally }\end{array}$ & $\begin{array}{l}\text { AIIRILQQL } \\
\text { gulatory and acces } \\
\text { overlapping Tat } \mathrm{p} \\
\text { ned epitopes were }\end{array}$ & $\begin{array}{l}\text { HIV-1 infection human (A*0201) } \\
\text { in } 70 \text { HIV-1 infected patients were studied using overlap } \\
\text { Rev peptides; 33\%, Vif peptides; 40\%, Vpr peptides; an } \\
\text { or the five proteins. }\end{array}$ & $\begin{array}{l}\text { Addo } 2002 \mathrm{~b} \\
\text { eptides and Elispot. } \\
\text { Vpu peptides. }\end{array}$ \\
\hline $\operatorname{Vpr}(59-67)$ & $\begin{array}{l}\text { - Epitope name: Vpr- } \\
\text { - Among HIV+ indivi }\end{array}$ & $\begin{array}{l}\text { AIIRILQQL } \\
\text { who carried HLA }\end{array}$ & $\begin{array}{l}\text { HIV-1 infection } \\
1 \% \text { ) recognized this epitope }\end{array}$ & Sabbaj2002b \\
\hline Vpr (59-67) & $\begin{array}{l}\text { Vpr (59-) } \\
\text { - Epitope name: AL9 } \\
\text { - Data from patient A } \\
\text { recognized during th } \\
\text { - A CTL response to }\end{array}$ & $\begin{array}{l}\text { AI IRILQQL } \\
\text { uggest a role for } \mathrm{t} \\
\text { ial decline in vire } \\
\text { SLYNTVATL, was }\end{array}$ & $\begin{array}{l}\text { HIV-1 infection human (A2) } \\
\text { initial control of viremia in acute infection, as it is one o } \\
\text { ntil } 18 \text { months post-presentation }\end{array}$ & Goulder2001a \\
\hline
\end{tabular}




\begin{tabular}{llllll}
\hline HXB2 Location & Author's Location & Sequence & Immunogen & Species (HLA) \\
\hline Vpr (59-67) & Vpr (59-67 SF2) & AIIRILQQL & HIV-1 infection & human (A2) & Altfeld2001b
\end{tabular}

- Therapy provided during acute infection resulted in a narrower CTL response, stronger T help response, and a less diverse viral population than was seen in individuals treated during chronic infection

- The breadth and specificity of the response was determined using ELISPOT by studying 19 individuals with pre-seroconversion therapy (Group 1), 11 individuals with primary infection but post-seroconversion therapy (Group 2), and 10 individuals who responded to HAART given during chronic infection (Group 3), using 259 overlapping peptides spanning p17, p24, RT, gp41, gp120 and Nef

- Previously described and newly defined optimal epitopes were tested for CTL response

- Number of HLA-A2+ individuals that had a CTL response to this epitope broken down by group: $1 / 10$ group 1, 0/6 group 2, and 0/4 group 3 Vpr (59-67) AIIRILQQL HIV-1 infection human (A2 supertype) Propato2001

$\operatorname{Vpr}(59-67)$

- Long-term nonprogressors (LTNPs) had strong memory resting CD8+ T-cell responses against the majority of epitopes tested, (18 for the A2 supertype, 16 for the A3 supertype) while the effector cells of long-term nonprogressors recognized far fewer epitopes

- Progressors had memory resting CD8+ T-cells that recognized far fewer epitopes than LTNPs

- A positive correlation between effector CD8+ T-cells and plasma viremia and a negative correlation between CD8+ effector T-cells and CD4+ T-cells was observed, which may contribute to the inability of LTNPs to clear virus

- This epitope can bind four of the five HLA-A2 supertypes alleles (A*0201, A*020 2, A*0203, A*0206 and A*6802)

\begin{tabular}{lllll}
\hline $\operatorname{Vpr}(62-70)$ & Vpr (62-) & HIV-1 infection & human (A*0201)
\end{tabular}

- Epitope name: Vpr-62

- HIV was scanned for all peptides which carried the A2-supermotif pattern conserved in more than $50 \%$ of B clade sequences - 233 peptides met this criteria, and 30 of these bound to HLA-A*0201 - 20/30 bound to at least 3/5 of HLA-A2 supertype alleles tested

- Three additional previously described HLA-A2 epitopes were added to the set of 20, and 18/22 chronically infected HLA-A2 individuals had CTL that recognized at least one of the 23 peptides (median of 2 and maximum of 6 ), while 6/12 acute infected individuals recognized at least 1 (median of 1 and maximum of 2)

- This epitope binds to three HLA-A2 supertype alleles: $A * 0202, \mathrm{~A} * 6802$ (strongest affinity) and A*0203

- 3/22 chronically infected patients had a weak ELISPOT response to this epitope

- 0/12 HLA-A2 patients with acute HIV-1 infection responded to this peptide

Vpr (62-70) Vpr (62-70) RILQQLLFI HIV-1 infection $\quad$ Addo2002b

- CTL responses against regulatory and accessory proteins in $70 \mathrm{HIV}-1$ infected patients were studied using overlapping peptides and Elispot.

- $28 \%$ targeted one or more overlapping Tat peptides; $36 \%$, Rev peptides; 33\%, Vif peptides; 40\%, Vpr peptides; and 2\%, Vpu peptides.

- All known optimally defined epitopes were summarized for the five proteins.

Vpr (62-70) Vpr (62-70) RILQQLLFI $\quad$ HIV-1 infection $\quad$ Propato2001

- Long-term nonprogressors (LTNPs) had strong memory resting CD8+ T-cell responses against the majority of epitopes tested, (18 for the A2 supertype, 16 for the A3 supertype) while the effector cells of long-term nonprogressors recognized far fewer epitopes

- Progressors had memory resting CD8+ T-cells that recognized far fewer epitopes than LTNPs

- A positive correlation between effector CD8+ T-cells and plasma viremia and a negative correlation between CD8+ effector T-cells and CD4+ T-cells was observed, which may contribute to the inability of LTNPs to clear virus

- This epitope can bind three of the five HLA-A2 supertypes alleles (A*0201, A*0202, A*0203, A*0206 and A*6802

Vaccine Vector/Type: adenovirus $\quad H I V$ component: $\mathrm{Vpr}$, Nef, Gag/Pol 
- Vpr can cause cells to go into G2 arrest, and it surpresses immune cell activation and inflammatory cytokine production, so co-immunization of BALB/c mice with recombinant adenovirus expressing Vpr and HIV-1 antigens Nef or Gag/Pol was tested to see if Vpr reduced the immune response to the other HIV antigens.

- Vpr compromised CD8+ T-cell lytic responses and T-helper proliferative responses in mice co-immunized with Vpr and Nef or Gag/Pol.

- In vitro, Vpr reduced T-cell cytokine production of IL-12 and TNFalpha, indicative of Vpr-mediated immune suppression. 


\section{II-B-15 Tat CTL Epitopes}

\begin{tabular}{|c|c|c|c|c|c|}
\hline HXB2 Location & Author's Location & Sequence & Immunogen & Species (HLA) & References \\
\hline Tat $(2-11)$ & \multicolumn{5}{|c|}{$\begin{array}{l}\text { - Epitopes that have been described in the literature and included in this database tend to cluster in conserved regions and be absent or rarely found highly } \\
\text { variable regions found in Nef, Env and p17. } \\
\text { - While the uneven distribution of epitopes may be in part due to a limited cross-recognition of specific responses because of differences between peptides } \\
\text { used to probe the immune response and autologous strains, regions with a paucity of defined epitopes also had higher frequencies of amino acids that tend } \\
\text { to not be found in C-terminal positions of epitopes, and had lower cleavage prediction scores for epitope processing. This suggests that in the regions of the } \\
\text { virus where variation is best tolerated traces of immune escape have left an imprint on the viral population. Epitopes also were concentrated in alpha-helix } \\
\text { and turn regions in the proteins. } \\
\text { - In the more conserved p24, and Pol proteins RT and Protease, epitopes are more evenly distributed. } \\
\text { - What was learned from proteins where many epitopes have been defined (Gag, Pol, Env and Nef) was used to develop an algorithm to predict where } \\
\text { epitopes would be localized in Rev, Tat, Vif, and Vpr. Predictions were made blinded, and then compared to the first } 15 \text { epitopes defined in these proteins; } \\
\text { the epitopes were concentrated in the predicted regions. }\end{array}$} \\
\hline Tat $(2-11)$ & $\begin{array}{l}\text { - Epitope name: Tat-E } \\
\text { - Among HIV+ indivi }\end{array}$ & $\begin{array}{l}\text { EPVDPRLEPW } \\
\text { who carried HL }\end{array}$ & $\begin{array}{l}\text { HIV-1 infection } \\
(20 \%) \text { recognize }\end{array}$ & human $(B * 5301)$ & Sabbaj2002b \\
\hline Tat $(2-11)$ & (LAI) & EPVDPRLEPW & & (B53) & Addo2001, Brander2001 \\
\hline Tat $(2-11)$ & Tat (2-11 BRU) & EPVDPRLEPW & HIV-1 infection & human (B53) & Addo2001 \\
\hline
\end{tabular}

- Epitope name: Tat 1

- Rev and Tat are expressed early in the virus life cycle and thus may be important targets for CTL against HIV early in infection and for vaccines - therefore CTL responses against Tat and Rev were screened using overlapping peptides

- 11/57 (19.3\%) HIV-1+ individuals recognized at least 1 Tat peptide, and 21/57 (37\%) responded to at least 1 Rev peptide

- EPVDPRLEPW was recognized by four individuals, but only two were B53, thus this epitope can probably be presented by other HLA alleles

Tat (2-11) Tat (2-11) EPVDPRLEPW $\quad$ HIV-1 infection $\quad$ Addo2002b

- CTL responses against regulatory and accessory proteins in $70 \mathrm{HIV}-1$ infected patients were studied using overlapping peptides and Elispot.

- $28 \%$ targeted one or more overlapping Tat peptides; $36 \%$, Rev peptides; $33 \%$, Vif peptides; $40 \%$, Vpr peptides; and $2 \%$, Vpu peptides.

- All known optimally defined epitopes were summarized for the five proteins.

Tat (16-30) Tat (16-30) SQPKTACNKCYCKRC HIV-1 infection $\quad$ Novitsky2002

- HLA, viral sequence, and Elispot data was obtained from 105 HIV-1 positive Botswanans; Elispot data was obtained from between 55 and 64 subjects for each HIV protein.

- Nef and p24 had the highest percentage of reactive peptides, and p24 had the highest magnitude of HIV-1 responses.

- This peptide was among the 28 most reactive $\mathrm{C}$ clade peptides from among over 350 tested spanning all HIV proteins.

Tat (17-26) $\quad$ EPKTACTTCY Epitopes that have been described in the literature and included in this database tend to cluster in conserved regions and be absent or rarely found highly
variable regions found in Nef, Env and p17. 


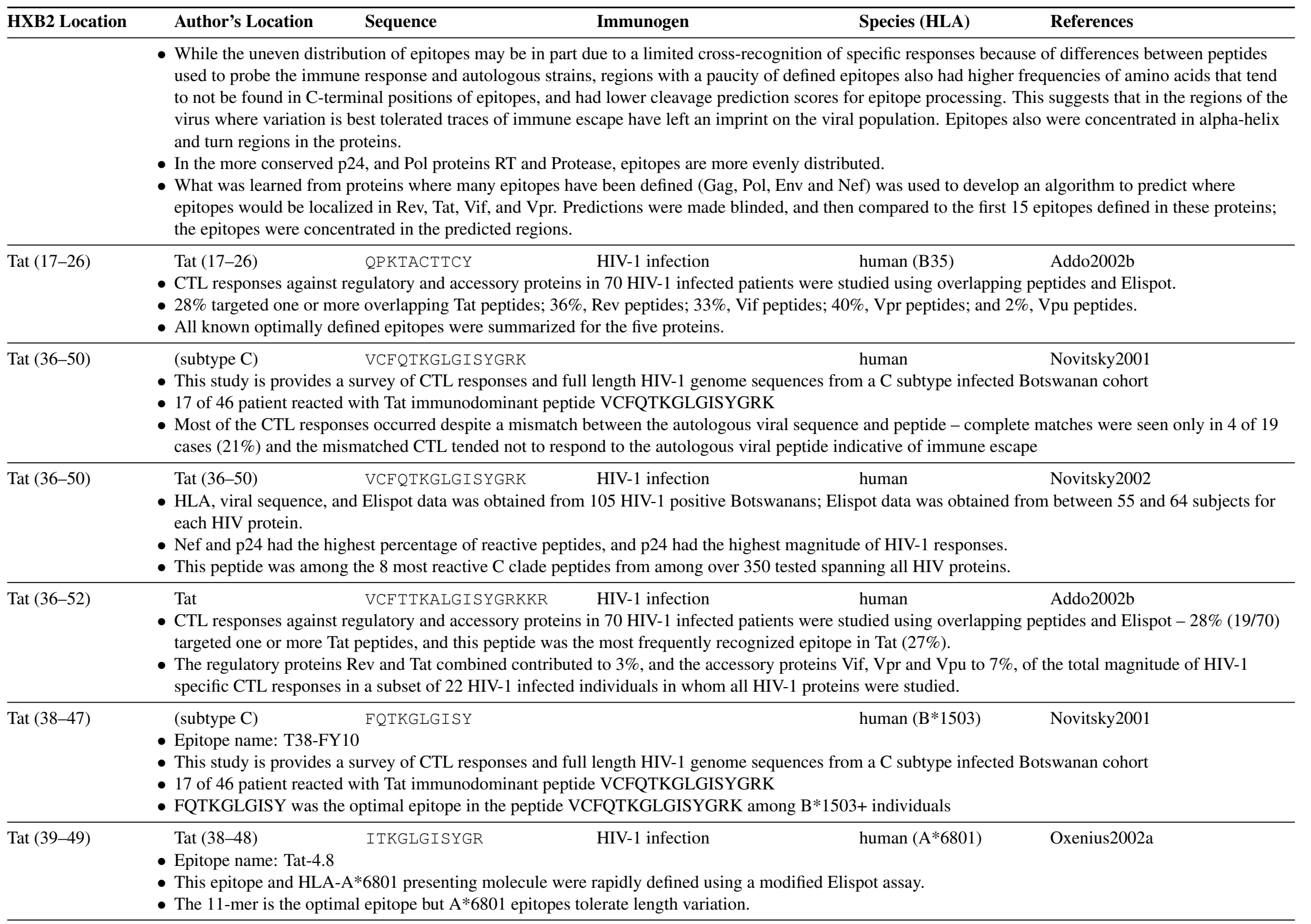




\begin{tabular}{|c|c|c|c|c|c|}
\hline HXB2 Location & Author's Location & Sequence & Immunogen & Species (HLA) & References \\
\hline Tat (39-49) & Tat $(38-48)$ & ITKGLGISYGR & HIV-1 infection & human (A68) & Addo2002b \\
\hline
\end{tabular}

- CTL responses against regulatory and accessory proteins in $70 \mathrm{HIV}-1$ infected patients were studied using overlapping peptides and Elispot.

- $28 \%$ targeted one or more overlapping Tat peptides; $36 \%$, Rev peptides; $33 \%$, Vif peptides; $40 \%$, Vpr peptides; and 2\%, Vpu peptides.

- All known optimally defined epitopes were summarized for the five proteins.

Tat (40-49) TKALGISYGR HIV-1 infection human

- Epitopes that have been described in the literature and included in this database tend to cluster in conserved regions and be absent or rarely found highly variable regions found in Nef, Env and p17.

- While the uneven distribution of epitopes may be in part due to a limited cross-recognition of specific responses because of differences between peptides used to probe the immune response and autologous strains, regions with a paucity of defined epitopes also had higher frequencies of amino acids that tend to not be found in C-terminal positions of epitopes, and had lower cleavage prediction scores for epitope processing. This suggests that in the regions of the virus where variation is best tolerated traces of immune escape have left an imprint on the viral population. Epitopes also were concentrated in alpha-helix and turn regions in the proteins.

- In the more conserved p24, and Pol proteins RT and Protease, epitopes are more evenly distributed.

- What was learned from proteins where many epitopes have been defined (Gag, Pol, Env and Nef) was used to develop an algorithm to predict where epitopes would be localized in Rev, Tat, Vif, and Vpr. Predictions were made blinded, and then compared to the first 15 epitopes defined in these proteins; the epitopes were concentrated in the predicted regions.
Tat (49-57)
Tat (49-57)
RKKRRQRRR
murine
Kim1997a

- The Tat peptide RKKRRQRRR when conjugated to a protein can cause that protein to be taken up by APCs and presented to CTL

- The system was demonstrated by vaccinating mice with an OVA-Tat peptide conjugate and immunizing $\mathrm{H}-2 \mathrm{~K}^{b}$ mice

- The CTL response to the $\mathrm{H}-2 \mathrm{~K}^{b}$ specific OVA peptide SIINFEKL was stimulated

Tat (49-57) Tat (49-57) RKKRRQRRR $\quad$ Baccine murine (H-2 ${ }^{d}$ )

Vaccine Vector/Type: DNA with DNA boost, DNA with recombinant protein boost Strain: LAI HIV component: Gag, Tat, Nef Adjuvant: IL18

- DNA vaccinated BALB/c mice primed and boosted with the multiepitopic vaccine with IL18 showed lymphoproliferative responses 7 weeks post immunization

- Strong but non-lasting HIV-specific CTL responses were detected by a Cr-release assay and DNA prime/DNA boost was more effective than DNA prime protein boost

- Immunization with either the multiepitopic DNA or with the mixed DNA vaccine induced HIV-1 specific Th1 cytokines (IL-2 and IFN-gamma)

- Co-administration of IL18 increased T-cell responses but decreased anti-HIV antibody levels

\begin{tabular}{llll}
\hline Tat (83-92) & Tat & GPKESKKKVE & human (B58)
\end{tabular}

- A subset of the potential epitopes was identified that could bind to the appropriate HLA-allele, and 15 predicted B7 superfamily (HLA B7, B8, and B58) epitopes could stimulate IFN $\gamma$ production in an ELISPOT assay

- GPKESKKKVE was newly identified as an HLA-B58 epitope in this study

Tat Tat Calarota1999

Vaccine Vector/Type: DNA HIV component: Nef, Rev Tat

- 9/9 HIV-1+ subjects were given one of three DNA vaccinations for nef, rev or tat, and novel proliferative and CTL responses were generated

- The nef DNA immunization induced the highest and most consistent CTLp activity, IFN-gamma production, and IL-6 and IgG responses 


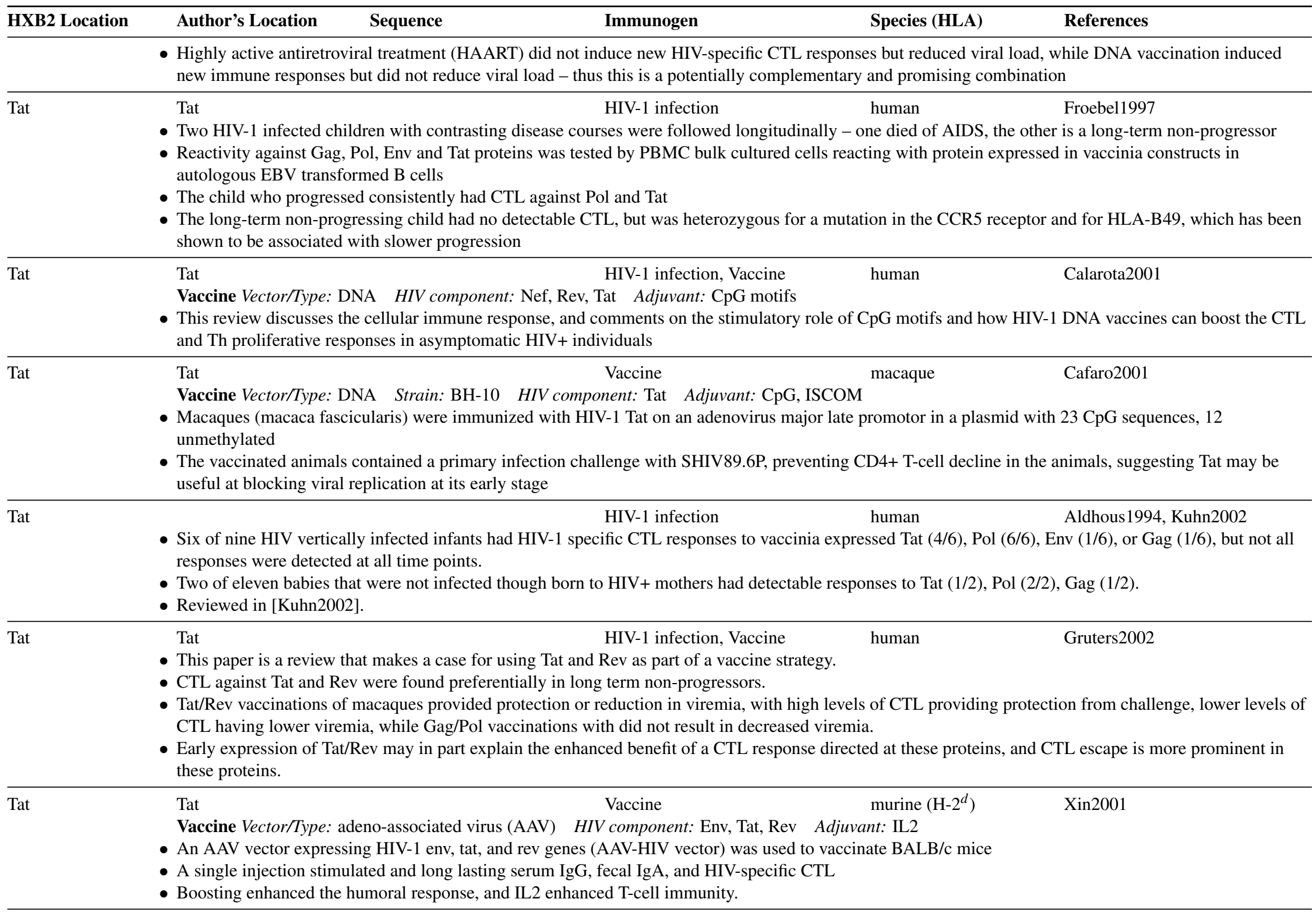




\section{II-B-16 Rev CTL Epitopes}

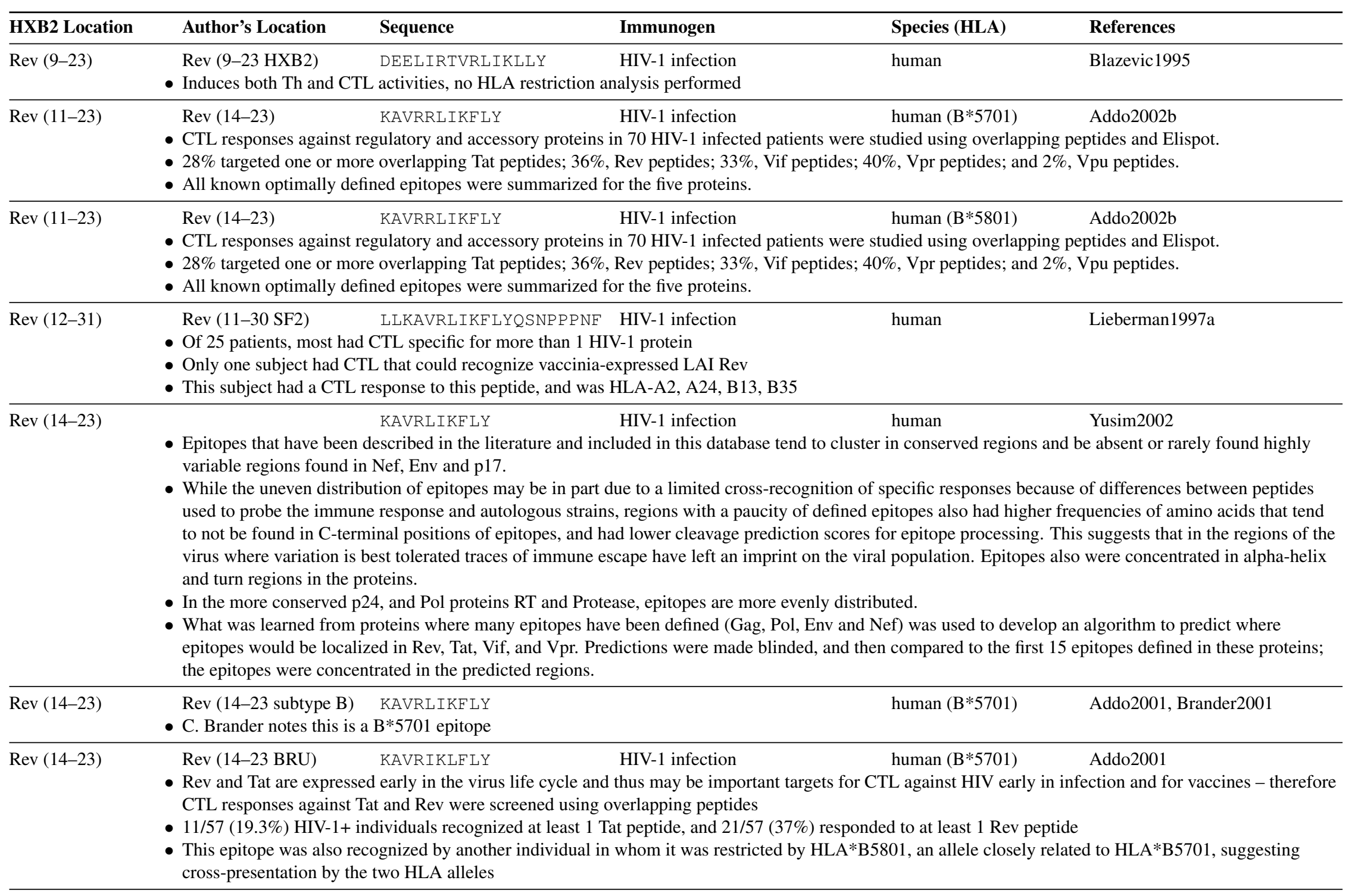




\begin{tabular}{|c|c|c|c|c|c|}
\hline HXB2 Location & Author's Location & Sequence & Immunogen & Species (HLA) & References \\
\hline $\operatorname{Rev}(14-23)$ & $\begin{array}{l}\text { Rev (14-23 subtype B) } \\
\text { - C. Brander notes this is a }\end{array}$ & $\begin{array}{l}\text { KAVRLIKFLY } \\
\text { B*5801 epitope }\end{array}$ & & human $(\mathrm{B} * 5801)$ & Addo2001, Brander2001 \\
\hline $\operatorname{Rev}(14-23)$ & Rev (14-23 BRU) & KAVRIKLFLY & HIV-1 infection & human $(\mathrm{B} * 5801)$ & Addo2001 \\
\hline
\end{tabular}

- Rev and Tat are expressed early in the virus life cycle and thus may be important targets for CTL against HIV early in infection and for vaccines - therefore

CTL responses against Tat and Rev were screened using overlapping peptides

- 11/57 (19.3\%) HIV-1+ individuals recognized at least 1 Tat peptide, and 21/57 (37\%) responded to at least 1 Rev peptide

- This epitope was also recognized by another individual in whom it was restricted by HLA*B5701, an allele closely related to HLA*B5801, suggesting cross-presentation by the two HLA alleles

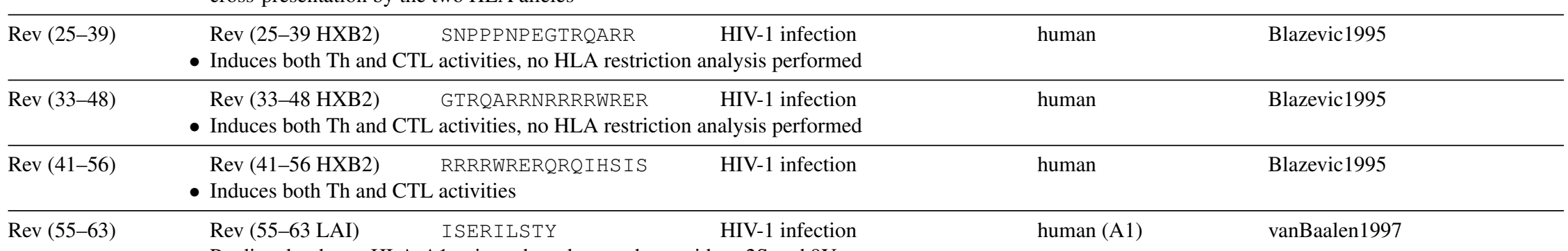

- Predicted to be an HLA-A1 epitope based on anchor residues $2 \mathrm{~S}$ and 9Y

- Both forms LSGWL(L or I)STY, with intact anchors, were found in an HLA-A1+ individual with Rev-responsive CTL

- An HLA-A1 individual who did not make a Rev response had lost the C-term anchor, ISGWILS(T or N)S

- 3/7 long-term non-progressors and 0/5 progressors were positive for HLA-B57 (associated with prolonged survival)

- CTLp frequencies to Rev and Tat were inversely correlated with rapid progression to AIDS, but not Gag, RT or Nef

$\operatorname{Rev}(55-63)$ $\operatorname{Rev}(55-63)$ ISERILSTY

$$
\begin{aligned}
& \text { HIV-1 infection, HIV-1 exposed human (A1) } \\
& \text { seronegative }
\end{aligned}
$$

- ELISPOT was used to study CTL responses to a panel of 54 predefined HIV-1 epitopes in 91 HIV-1-exposed, persistently seronegative (HEPS) and 87 HIV-1-infected female Nairobi sex workers

- Epitopes that have been described in the literature and included in this database tend to cluster in conserved regions and be absent or rarely found highly variable regions found in Nef, Env and p17.

- While the uneven distribution of epitopes may be in part due to a limited cross-recognition of specific responses because of differences between peptides used to probe the immune response and autologous strains, regions with a paucity of defined epitopes also had higher frequencies of amino acids that tend to not be found in C-terminal positions of epitopes, and had lower cleavage prediction scores for epitope processing. This suggests that in the regions of the virus where variation is best tolerated traces of immune escape have left an imprint on the viral population. Epitopes also were concentrated in alpha-helix and turn regions in the proteins.

- In the more conserved p24, and Pol proteins RT and Protease, epitopes are more evenly distributed.

- What was learned from proteins where many epitopes have been defined (Gag, Pol, Env and Nef) was used to develop an algorithm to predict where epitopes would be localized in Rev, Tat, Vif, and Vpr. Predictions were made blinded, and then compared to the first 15 epitopes defined in these proteins; the epitopes were concentrated in the predicted regions. 


\begin{tabular}{llllll}
\hline HXB2 Location & Author's Location & Sequence & Immunogen & Species (HLA) & References \\
\hline $\operatorname{Rev}(57-66)$ & Rev (57-66) & ERILSTYLGR & HIV-1 infection & human (A3)
\end{tabular}

- Epitope name: A3-ER10

- CTL responses in 18 acutely HIV-infected HLA-A3 $(n=7)$ or -B7 $(n=4)$ or both -A3 and B7 $(n=7)$ positive individuals were studied.

- One individual, AC-06, was homozygous at all three class I alleles (A3, B7, Cw7), was treated during acute infection and had supervised treatment interruptions (STI). He had only two detectable CTL responses during acute infection, but after STI this broadened to 27 distinct epitopes including 15 restricted by HLA-A3, 11 by HLA-B7, and 1 by HLA-Cw7.

- 0/14 HLA-A3 positive individuals had detectable A3-restricted responses to this epitope during acute infection, but only 5/15 of HLA-A3 epitopes tested were targeted during acute infection. 1/7 individuals had detectable responses to this epitope after STI.

Rev (58-66) Rev (58-66) RILSTYLGR HIV-1 infection $\quad$ Addo2002b

- CTL responses against regulatory and accessory proteins in $70 \mathrm{HIV}-1$ infected patients were studied using overlapping peptides and Elispot.

- $28 \%$ targeted one or more overlapping Tat peptides; 36\%, Rev peptides; 33\%, Vif peptides; $40 \%$, Vpr peptides; and 2\%, Vpu peptides.

- All known optimally defined epitopes were summarized for the five proteins.

Rev (66-81) Rev RAEPVPLQLPPLERL HIV-1 infection human Addo2002b

- CTL responses against regulatory and accessory proteins in $70 \mathrm{HIV}-1$ infected patients were studied using overlapping peptides and Elispot - 36\% (25/70) targeted one or more Rev peptides, and this peptide was the most frequently recognized epitope in Rev (32\%).

- The regulatory proteins Rev and Tat combined contributed to 3\%, and the accessory proteins Vif, Vpr and Vpu to 7\%, of the total magnitude of HIV-1 specific CTL responses in a subset of 22 HIV-1 infected individuals in whom all HIV-1 proteins were studied.
$\operatorname{Rev}(67-75)$ SAEPVPLQI
HIV-1 infection
human
Yusim2002

- Epitopes that have been described in the literature and included in this database tend to cluster in conserved regions and be absent or rarely found highly variable regions found in Nef, Env and p17.

- While the uneven distribution of epitopes may be in part due to a limited cross-recognition of specific responses because of differences between peptides used to probe the immune response and autologous strains, regions with a paucity of defined epitopes also had higher frequencies of amino acids that tend to not be found in C-terminal positions of epitopes, and had lower cleavage prediction scores for epitope processing. This suggests that in the regions of the virus where variation is best tolerated traces of immune escape have left an imprint on the viral population. Epitopes also were concentrated in alpha-helix and turn regions in the proteins.

- In the more conserved p24, and Pol proteins RT and Protease, epitopes are more evenly distributed.

- What was learned from proteins where many epitopes have been defined (Gag, Pol, Env and Nef) was used to develop an algorithm to predict where epitopes would be localized in Rev, Tat, Vif, and Vpr. Predictions were made blinded, and then compared to the first 15 epitopes defined in these proteins; the epitopes were concentrated in the predicted regions.

$\operatorname{Rev}(67-75)$ Rev (65-77 BH10, LAI) SAEPVPLQI

HIV-1 infection

human

Maksiutov2002

- This study employs an antigenic similarity matrix to compare HIV-1 antigenic determinants with human proteins.

- This CTL epitope (the HIV-1 LAI fragment with high similarity to a human protein overlapping this epitope is GRSAEPVPLQLPP) has similarity with transforming growth factor beta binding protein protein I, fragment ARSAEPEVATAPP.

- This CTL epitope (the HIV-1 LAI fragment with high similarity to a human protein overlapping this epitope is EPVPLQLPPL) also has similarity with the epidermal growth factor receptor substrate 15, fragment EPVPMSLPPA. 


\begin{tabular}{llllc}
\hline HXB2 Location & Author's Location & Sequence & Immunogen & Species (HLA) \\
\hline Rev (67-75) & Rev & SAEPVPLQL & HIV-1 infection & human (B14) \\
& - & Molecularly cloned primary NSI macrophage tropic strain 2.1 and SI non-macrophage tropic strain 1.2 were isolated from study participant ACH320 and
\end{tabular}

use to infect irradiated XID mice that had been reconstituted with human PBMC from B14+ seronegative donors - results indicate CTL may favor selective outgrowth of macrophage tropic strains

- The CTL clone TCC108 specific for SAEPVPLQL, previously described by van Baalen 1997, and van Baalen 1998, was stimulated in vitro and given to the mice to apply specific CTL pressure

- The macrophage-tropic HIV-1 strain \#2.1 escaped CTL pressure more efficiently (7/14 animals) than its non-macrophage-tropic counterpart \#1.2(SI) - the latter isolate was suppressed in 13/14 animals - macrophage may serve as a CTL sanctuary and reduced pressure on macrophage tropic HIV strains may allow additional replication to assist with acquisition of escape

- Specific HIV-1 variants selectively induced by TCC108 were for strain 1.2: SEEPVPLQL, and for strain 2.1: SAEHVPLQL, SAESVPLQL, SVEPVPLQL, SLEPVPLQL, SAEPVPFQL, and SAEPVPFQL

\begin{tabular}{llcc}
\hline Rev (67-75) & Rev (67-75) SAEPVPLQL $\quad$ HIV-1 infection $\quad$ human (B14) \\
& Tat, Rev and Nef are the first HIV proteins expressed upon acute infection of T-cells (<6 hours), and RT is not expressed until after 24 hours. The \\
& B14-restricted Rev-SAEPVPLQL specific CD8 T-cell clone TCC108, and the B57-restricted RT-IVLPEKDSW specific CD8 T-cell clone TCL1C11
\end{tabular}
B14-restricted Rev-SAEPVPLQL specific CD8 T-cell clone TCC108, and the B57-restricted RT-IVLPEKDSW specific CD8 T-cell clone TCL1C11 were co-incubated with CD4+ cultures innoculated with HIV-1 at low MOI. Co-incubation with the Rev-specific CTL resulted in two logs less HIV-1 production in ten days of culture. When the RT epitope was cloned into the Nef gene of the infecting strain, another early expressed protein, it proved as effective as the Rev epitope at inhibiting viral production. A mathematical model of CTL-target interactions suggest early proteins are important for vaccine design.

\begin{tabular}{lllll}
\hline $\operatorname{Rev}(67-75)$ & Rev (67-75) & SAEPVPLQL & HIV-1 infection & human (B14)
\end{tabular}

- CTL responses against regulatory and accessory proteins in $70 \mathrm{HIV}-1$ infected patients were studied using overlapping peptides and Elispot.

- $28 \%$ targeted one or more overlapping Tat peptides; 36\%, Rev peptides; 33\%, Vif peptides; 40\%, Vpr peptides; and 2\%, Vpu peptides.

- All known optimally defined epitopes were summarized for the five proteins.

Rev (67-75) Rev (67-75 IIIB) SAEPVPLQL HIV-1 infection $\quad$ vanBaalen1998

- The Rev-specific CTL response studied here was from an individual infected with HIV-1 for more than 12 years without developing symptoms - Rev and

Tat are expressed early and CTL activity against these proteins has been correlated with long-term survival

- The CTL clone TCC108 specific for this epitope was studied in vitro

- CTLs added immediately after infection suppressed viral production, indicative of CTL interference with viral production prior to lysis - CTL-mediated lysis occurred after the onset of progeny viral release, but prior to peak viral production

- Rapid selection of a E69K mutation, which abolished CTL, recognition was observed

- The epitope was originally listed as B14, but Cw8 and B14 are in linkage disequilibrium, and in this case were not distinguished (Pers. Comm., Christian Brander, 1999)

\begin{tabular}{lllll|l}
\hline $\operatorname{Rev}(67-75)$ & (LAI) & SAEPVPLQL & human (Cw5) & Addo2001, Brander2001 \\
\hline $\operatorname{Rev}(67-75)$ & Rev (SF2) & SAEPVPLQL & HIV-1 infection & human (Cw5) & Goulder2001a
\end{tabular}

- Epitope name: SL9

- Data from patient AC13 suggest a role for this epitope in initial control of viremia in acute infection, as it is one of several subdominant CTL epitopes recognized during the initial decline in viremia

- A CTL response to SL9, SLYNTVATL, was not evident until 18 months post-presentation

\begin{tabular}{llll}
\hline $\operatorname{Rev}(67-75)$ & Rev (67-75 SF2) SAEPVPLQL & HIV-1 infection & human (Cw5) \\
& Therapy provided during acute infection resulted in a narrower CTL response, stronger T help response, and a less diverse viral population than was seen in \\
& individuals treated during chronic infection
\end{tabular}




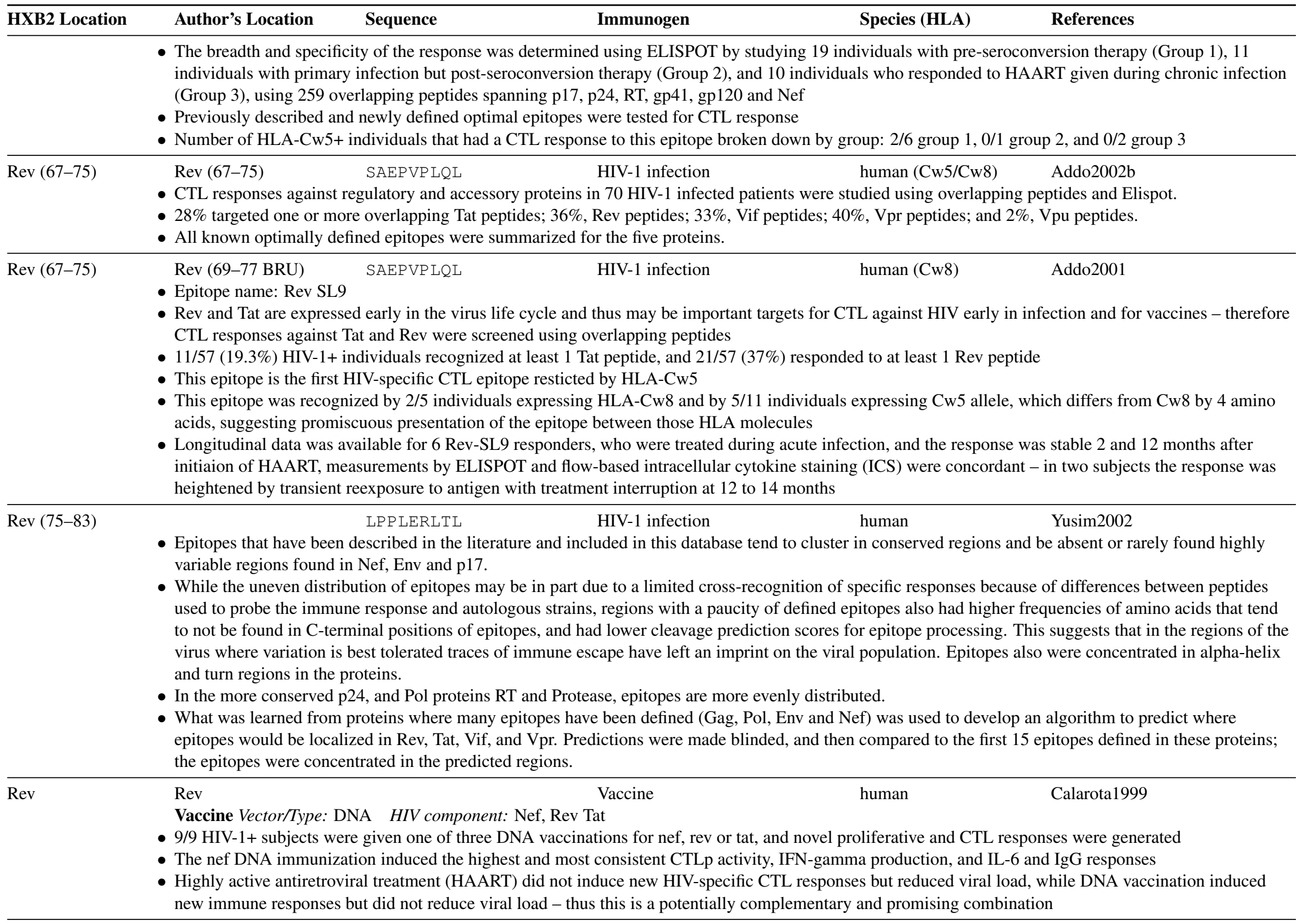




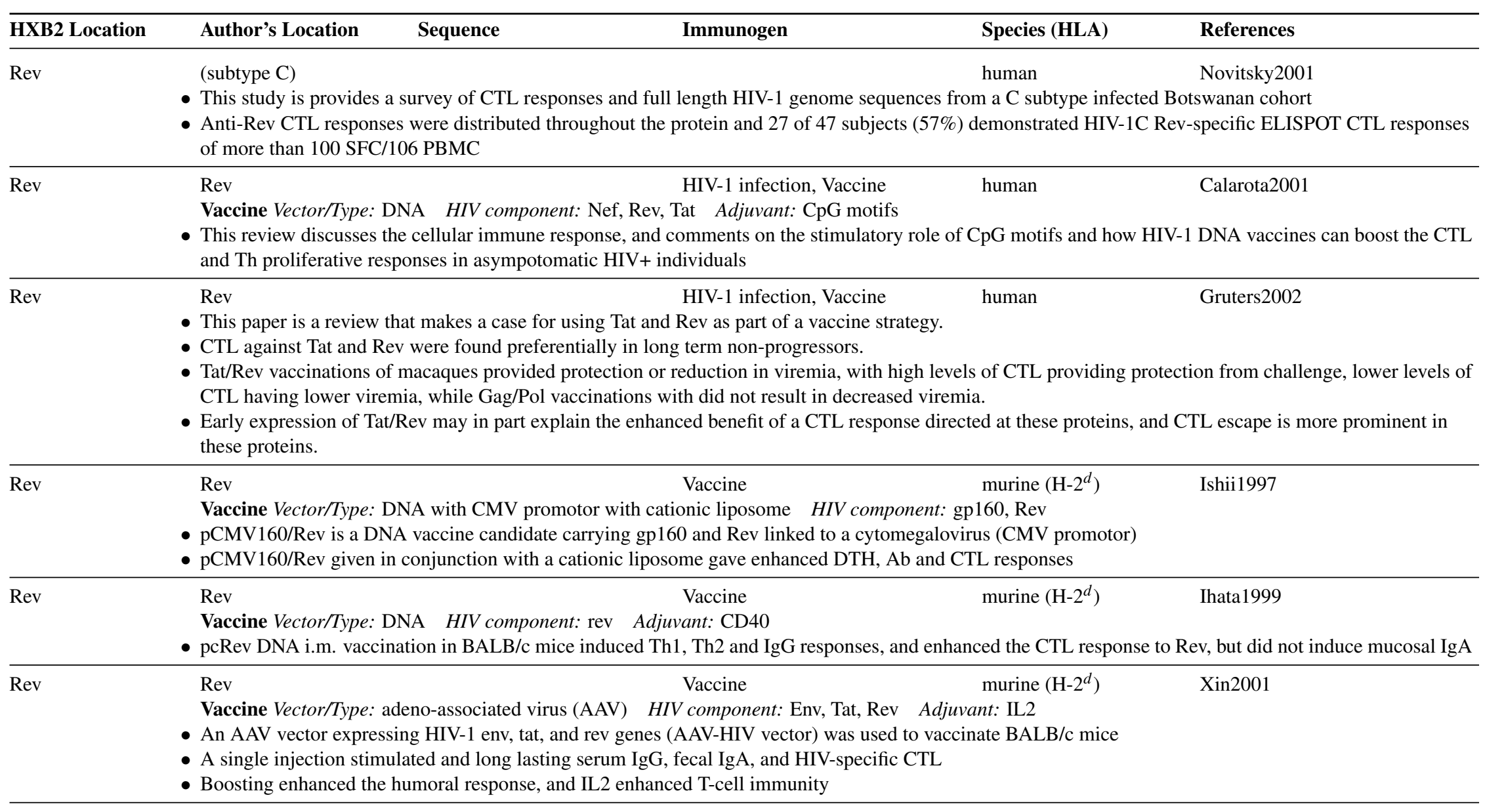




\section{II-B-17 Vpu CTL Epitopes}

\begin{tabular}{lllcl}
\hline HXB2 Location & Author's Location & Sequence & Immunogen & Species (HLA) \\
\hline Vpu (4-13) & Vpu & LVILAIVALV & human (B7) & De Groot2001 \\
& - The program Epimatrix was used in conjunction with the program Conservatrix to identify conservered regions of HIV that might serve as epitopes
\end{tabular}

- A subset of the potential epitopes was identified that could bind to the appropriate HLA-allele, and 15 predicted B7 superfamily (HLA B7, B8, and B58)

epitopes could stimulate IFN $\gamma$ production in an ELISPOT assay

- LVILAIVALV was newly identified as an HLA-B7 epitope in this study using ELISPOT, but could not be shown to bind to B7

- CTL responses against regulatory and accessory proteins in $70 \mathrm{HIV}-1$ infected patients were studied using overlapping peptides and Elispot - only $2 \%$ (2/70) targeted one or more Vpu peptides, including this peptide.

- The regulatory proteins Rev and Tat combined contributed to 3\%, and the accessory proteins Vif, Vpr and Vpu to 7\%, of the total magnitude of HIV-1 specific CTL responses in a subset of 22 HIV-1 infected individuals in whom all HIV-1 proteins were studied.

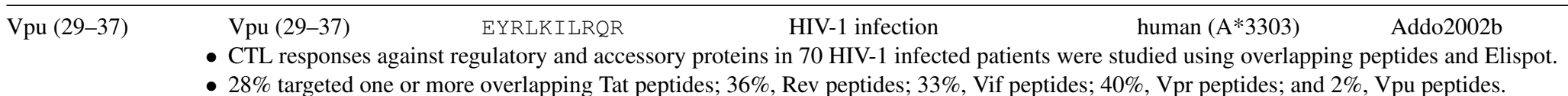

- All known optimally defined epitopes were summarized for the five proteins.

\begin{tabular}{lllll}
\hline Vpu (29-37) Vpu (29-37) & EYRLKILRQR & HIV-1 infection & human (A*3303)
\end{tabular}

- Detection of HIV CTL epitopes is rare in Vpu, and this is the first optimally defined Vpu epitope.

- This CTL response was first detected in a long term non-progressor, and 3/6 HLA A*3303 positive individuals were found to have a CTL response to this

epitope.

- HLA A*3303 is common in West Africa and Asia.

\begin{tabular}{llcc}
\hline $\mathrm{Vpu}$ & $\mathrm{Vpu}$ & Vaccine & murine $\left(\mathrm{H}-2^{d}\right)$
\end{tabular}

Vaccine Vector/Type: DNA HIV component: Vif, Vpu, Nef

- Splenocytes from BALB/c mice immunized with pVVN-P DNA were incubated with Vif, Vpu or Nef antigens for 3 days and assayed for IL-4 and IFN-gamma levels

- Antigen stimulation increased IFN-gamma production in pVVN-P immunized mice, indicating a Th1 response

- IL-4 production was not significantly changed after antigen stimulation compared to control levels

- Cross-clade CTL activity was also observed: A, B clade, CRF01(AE) clade antigens could serve as targets for the B clade immunization-stimulated CTL an HIV-1 AC recombinant, however, did not stimulate a CTL response, but was expressed at lower levels on the target cell 


\section{II-B-18 gp160 CTL Epitopes}

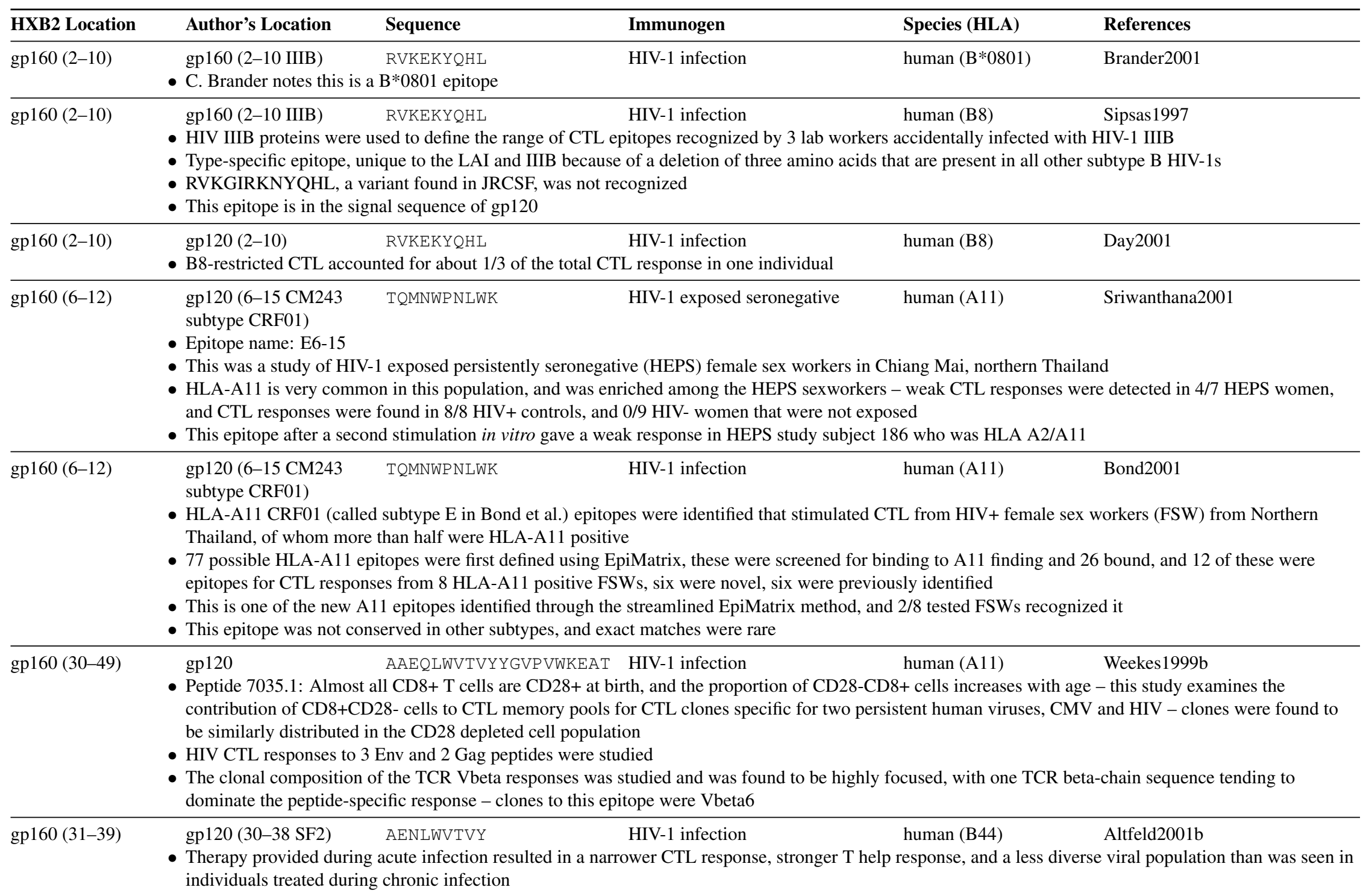




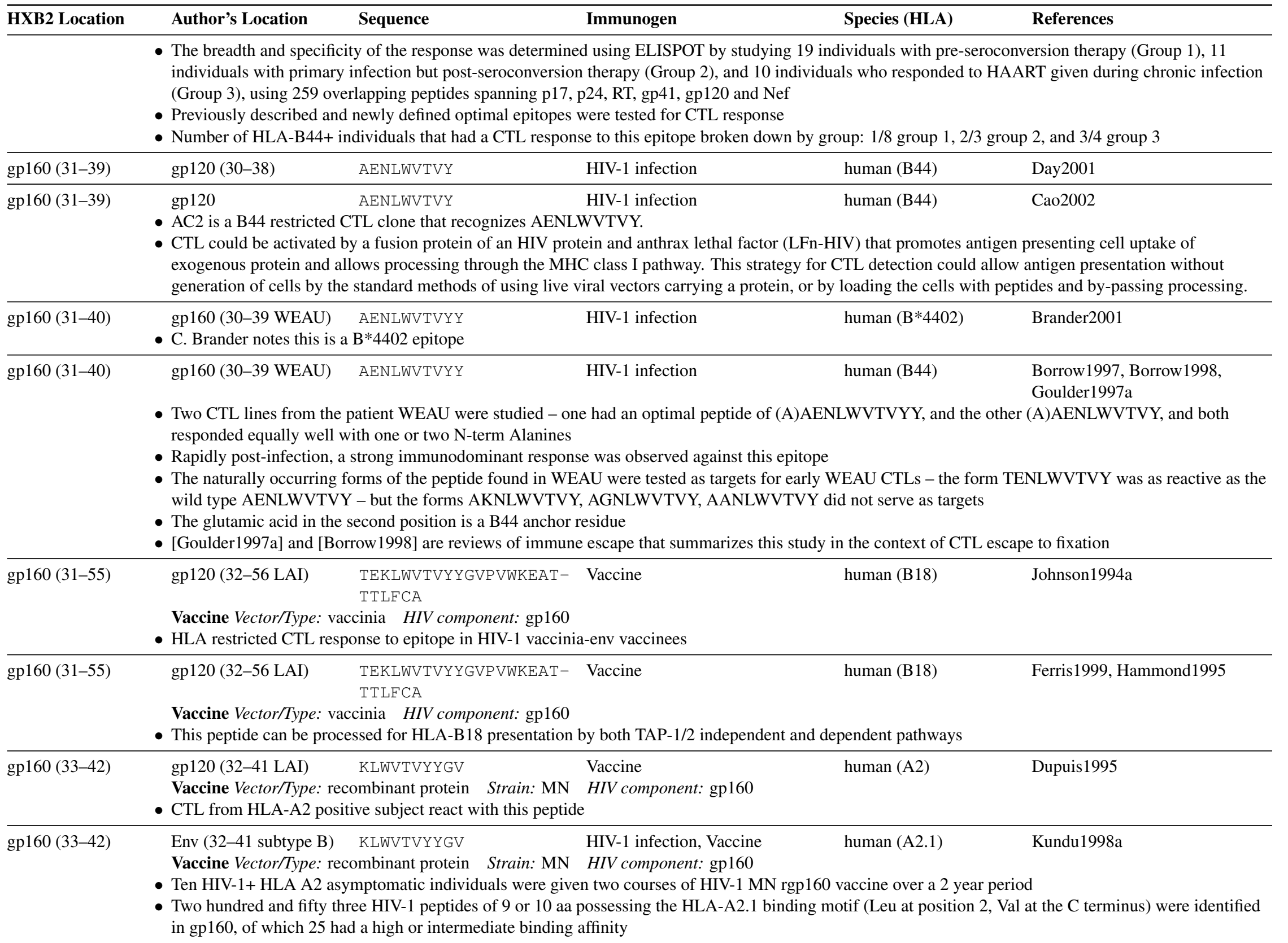




\begin{tabular}{|c|c|c|c|}
\hline HXB2 Location & Author's Location & Immunogen & Species (HLA) \\
\hline & \multicolumn{3}{|c|}{$\begin{array}{l}\text { - Eleven peptides were studied that had high HLA-A2 binding affinity - a CTL response was detected to 9/11 peptides in at least } 1 \text { individual } \\
\text { - CTL responses after reimmunization may include recall responses - only individuals with vaccine cross-reactive sequences prior to vaccination showed } \\
\text { detectable CTL responses }\end{array}$} \\
\hline gp160 (34-55) & $\begin{array}{l}\text { gp120 (25-46 BRU) } \\
\text { - Defined through peptide }\end{array}$ & $\begin{array}{l}\text { LWVTVYYGVPVWKEATTTL- HIV-1 infection } \\
\text { FCA } \\
\text { olocking of CTL activity, and Env deletions }\end{array}$ & Dadaglio1991 \\
\hline gp160 (36-46) & $\begin{array}{l}\text { gp120 (36-46 CM243 } \\
\text { subtype CRF01) } \\
\text { - Epitope name: E36-4 } \\
\text { - This was a study of HIV- } \\
\text { - HLA-A11 is very comm } \\
\text { and CTL responses were } \\
\text { - This epitope after a secol }\end{array}$ & $\begin{array}{l}\text { VTVYYGVPVWR HIV-1 exposed seronegative } \\
1 \text { exposed persistently seronegative (HEPS) female sex worker } \\
\text { n in this population, and was enriched among the HEPS sexwo } \\
\text { found in } 8 / 8 \text { HIV+ controls, and } 0 / 9 \text { HIV- women that were no } \\
\text { d stimulation in vitro gave a weak response in HEPS study sub }\end{array}$ & $\begin{array}{l}\text { human (A11) Sriwanthana2001 } \\
\text { hiang Mai, northern Thailand } \\
\text { - weak CTL responses were detected in 4/7 HEPS women, } \\
\text { sed } \\
86 \text { who was HLA A2/A11 }\end{array}$ \\
\hline gp160 (36-46) & $\begin{array}{l}\text { gp120 (36-46 CM243 } \\
\text { subtype CRF01) } \\
\text { - HLA-A11 CRF01 (callec } \\
\text { Thailand, of whom more } \\
\text { - } 77 \text { possible HLA-A11 ep } \\
\text { epitopes for CTL respon } \\
\text { - This epitope was not pre } \\
\text { A11 epitopes that had be } \\
\text { - } 1 / 8 \text { tested FSWs recogni } \\
\text { - This epitope was only co }\end{array}$ & $\begin{array}{l}\text { VTVYYGVPVWR HIV-1 infection } \\
\text { subtype E in Bond et al.) epitopes were identified that stimula } \\
\text { than half were HLA-A11 positive } \\
\text { itopes were first defined using EpiMatrix, these were screened } \\
\text { es from } 8 \text { HLA-A11 positive FSWs, six were novel, six were } \mathrm{p} \\
\text { licted by the EpiMatrix method to be likely to bind to A11, tho } \\
\text { en previously defined } \\
\text { ed this epitope } \\
\text { nserved in CRF01 and subtypes B and C, and exact matches we }\end{array}$ & $\begin{array}{l}\text { human (A11) Bond2001 } \\
\text { ГL from HIV+ female sex workers (FSW) from Northern } \\
\text { ding to A11 finding and } 26 \text { bound, and } 12 \text { of these were } \\
\text { sly identified } \\
\text { served as an epitope in the FSWs, and it was one of the six } \\
\text { common }\end{array}$ \\
\hline gp160 (36-46) & $\begin{array}{l}\text { gp120 } \\
\text { - Study of the fine specific } \\
\text { - The A3 super-type is cha } \\
\text { - While most lines were sp } \\
\text { or } A * 6801\end{array}$ & $\begin{array}{l}\text { VTVYYGVPVWK HIV-1 infection } \\
\text { ty of an A3-like-HLA-super-type epitope (the A3-super-type in } \\
\text { racterized as a hydrophobic or hydroxyl containing anchor resi } \\
\text { ecific, a promiscuous cloned CTL line was derived from an HI }\end{array}$ & $\begin{array}{l}\text { human (A11 and Threlkeld1997 } \\
\mathrm{A}^{* 6801)} \\
\left.\text { s A } 0301, \mathrm{~A}^{*} 1101, \mathrm{~A} * 3101, \mathrm{~A} * 3301, \text { and } \mathrm{A} * 6801\right) \\
\text { position 2, and a positive charge in the C-term position } \\
\text { nor that could recognize this epitope presented by either A11 }\end{array}$ \\
\hline gp160 (37-46) & $\begin{array}{l}\text { gp120 (37-46 LAI) } \\
\text { Vaccine Vector/Type: va } \\
\text { - Multiple CTL clones obt } \\
\text { - C. Brander notes that thi }\end{array}$ & $\begin{array}{l}\text { TVYYGVPVWK } \\
\text { cinia HIV component: gp } 160 \\
\text { ined from two vaccinees } \\
\text { is an } \mathrm{A} * 0301 \text { epitope in the } 1999 \text { database }\end{array}$ & human $\left(\mathrm{A}^{*} 0301\right)$ \\
\hline gp160 (37-46) & $\begin{array}{l}\text { gp120 (37-46 LAI) } \\
\text { Vaccine Vector/Type: va } \\
\text { - C. Brander notes this is a }\end{array}$ & $\begin{array}{l}\text { TVYYGVPVWK } \\
\text { cinia HIV component: gp160 } \\
\text { n A*0301 epitope }\end{array}$ & human $\left(\mathrm{A}^{* 0301)}\right.$ \\
\hline gp160 (37-46) & $\begin{array}{l}\text { gp120 } \\
\text { Vaccine Vector/Type: I }\end{array}$ & $\begin{array}{l}\text { TVYYGVPVWK } \\
\text { HIV-1 infection, Vaccine } \\
\text { A prime with vaccinia MVA boost } \quad \text { Strain: subtype A HIV }\end{array}$ & $\begin{array}{l}\text { human }\left(\mathrm{A}^{*} 0301\right) \text { Hanke2000 Wee2002 } \\
\text { lent: } \mathrm{p} 17, \mathrm{p} 24, \text { polyepitope }\end{array}$ \\
\hline
\end{tabular}




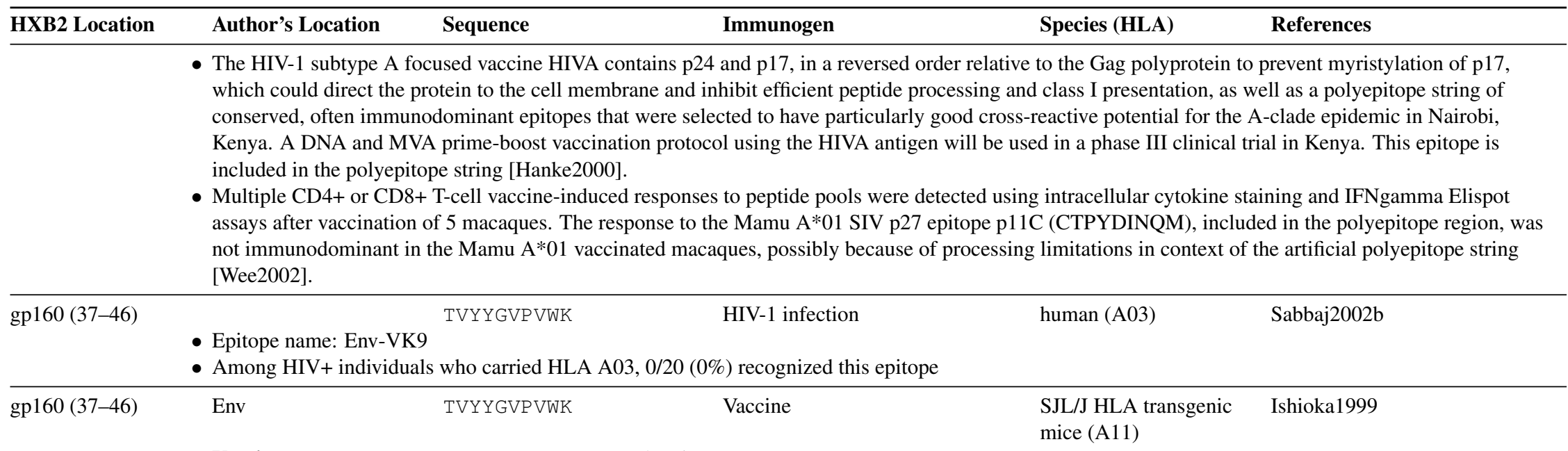

Vaccine Vector/Type: DNA HIV component: polyepitope

- A minigene vaccine construct encoding 6 HLA 2.1 and 3 HLA A11 restricted CTL epitopes, the universal Th cell epitope PADRE (pan-DR epitope) and an ER translocating signal sequence was constructed

- The epitopes were chosen for dominant recognition by CTLs during HBV and HIV infections in humans

- HLA transgenic mice were used for quantitating in vivo immunogenicity of DNA vaccines encoding HLA-restricted CTL epitopes.

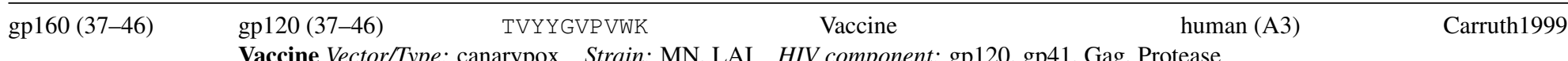

- The vaccine used was a live recombinant canarypox (CP) virus vaccine containing multiple HIV-1 genes (HIV-1 MN gp120, HIV-1 LAI gp41, HIV-1 LAI Gag, HIV-1 LAI protease)

- CD4+ and CD8+ Gag and Env specific CTL responses were detected in only 1/5 vaccinated volunteers, and were not detectable 1 year after vaccination

- CTL responses to epitopes SLYNTVATL and TVYYGVPVWK from HIV+ control patients were used as positive controls

- The study explored why vaccinees were non-responsive - non-response was not due to inherent defects or differences in the ability of these individuals to process and present antigen

gp160 (37-46) gp120 (37-46 LAI) TVYYGVPVWK $\quad$ HIV-1 infection $\quad$ human (A3)

- Identical twin hemophiliac brothers were both infected with the same batch of factor VIII

- One had a response to this epitope, the other did not

- [Goulder1997a] is a review of immune escape that summarizes this study

$\begin{array}{lllll}\text { gp160 (37-46) } & \text { gp120 (36-45) } & \text { TVYYGVPVWK } & \text { HIV-1 infection } & \text { human (A3) Ferrari2000 }\end{array}$

- One of the 51 HIV-1 epitopes selected by Ferrari et al. as good candidate CTL epitopes for vaccines by virtue of being conserved and presented by common HLA alleles

gp160 (37-46)

$\begin{array}{lllll}\text { gp120 (37-46) } & \text { TVYYGVPVWK } & \text { HIV-1 infection } & \text { Day2001 }\end{array}$

- The CTL response to optimally defined CTL epitopes restricted by HLA class I A and B alleles in individuals who coexpressed HLA A2, A3, and B7 was studied in eight HIV-1-infected subjects, two with acute infection, five with chronic, and one long-term non-progressor (LTNP)

- 2 to 17 epitopes were recognized in a given individual, A2-restricted CTL response tended to be narrow and never dominated the response, and 25/27 epitopes were targeted by at least one person 


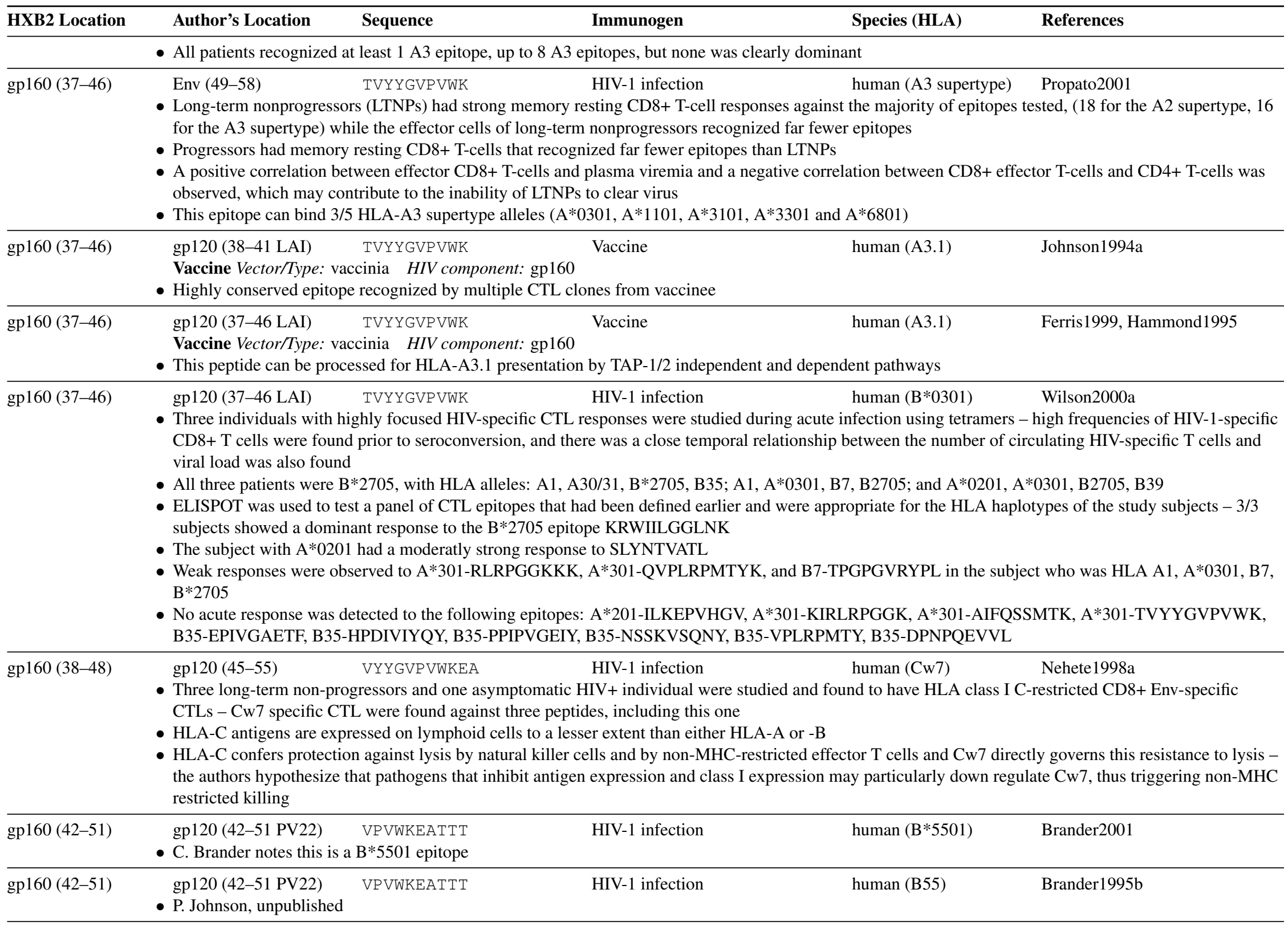




\begin{tabular}{llllll}
\hline HXB2 Location & Author's Location & Sequence & Immunogen & Species (HLA) \\
\hline gp160 (42-51) & gp120 (41-55) & VPVWKEATTT & HIV-1 infection & human (B55)
\end{tabular}

- One of the 51 HIV-1 epitopes selected by Ferrari et al. as good candidate CTL epitopes for vaccines by virtue of being conserved and presented by common HLA alleles

gp160 (42-52) Env (43-52 BH10, LAI) VPVWKEATTTL $\quad$ HIV-1 infection $\quad$ Maksiutov2002

- This study employs an antigenic similarity matrix to compare HIV-1 antigenic determinants with human proteins.

- This CTL epitope (the HIV-1 LAI fragment with high similarity to a human protein overlapping this peptide is PVWKEATTTL) has similarity with the platelet membrane glycoprotein IIIA precursor (GLIIIA) (integrin beta-3) (CD61): PLYKEATSTF.

\begin{tabular}{llllr}
\hline gp160 (42-52) & gp120 (42-52) & VPVWKEATTTL & HIV-1 infection & human (B*3501) \\
& - C. Brander notes this is a B*3501 epitope & Brander2001 \\
\hline gp160 (42-52) & gp120 (42-52 PV22) & VPVWKEATTTL & HIV-1 infection & human (B35)
\end{tabular}

- VPVWKEATTTL is the consensus sequence for clades B and D

- VPVWKDAETTL is the consensus sequence for clade A and it is cross-reactive

- VPVWKEADTTL is the consensus sequence for clade $\mathrm{C}$ and it is cross-reactive

- VPVWKEADTTL is the consensus sequence for clade $\mathrm{E}$ and even with three substitutions still retains some cross-reactivity

gp160 (42-52) gp120(41-51) VPVWKEATTTL HIV-1 infection human (B35)

- One of the 51 HIV-1 epitopes selected by Ferrari et al. as good candidate CTL epitopes for vaccines by virtue of being conserved and presented by common HLA alleles

\begin{tabular}{llrrr}
\hline gp160 (42-61) & gp120 (49-68) & VPVWKEATTTLFCASDAKAY & HIV-1 infection & human \\
& - HIV-specific CTL lines developed by ex vivo stimulation with peptide & Lieberman1995 & \\
\hline gp160 (42-61) & gp120 (49-68 SF2) & VPVWKEATTTLFCASDAKAY & HIV-1 infection & human
\end{tabular}

Of $120(49-68 \mathrm{SF} 2$

- Eleven subjects had CTL that could recognize vaccinia-expressed LAI gp160

- Three of these 11 had CTL response to this peptide

- The responding subjects were HLA-A2, A3, B8, B62; HLA-A3, A24, B7, B38

\begin{tabular}{llrl}
\hline gp160 (42-61) & gp120 (49-68 SF2) VPVWKEATTTLFCASDAKAY & HIV-1 infection & human \\
& - CTL expanded ex vivo were later infused into HIV-1 infected patients & Lieberman1997b
\end{tabular}

- CTL expanded ex vivo were later infused into HIV-1 infected patients

gp160 (50-59) Env (62-71) TTLFCASDAK HIV-1 infection human (A3 supertype) Propato2001

- Long-term nonprogressors (LTNPs) had strong memory resting CD8+ T-cell responses against the majority of epitopes tested, (18 for the A2 supertype, 16 for the A3 supertype) while the effector cells of long-term nonprogressors recognized far fewer epitopes

- Progressors had memory resting CD8+ T-cells that recognized far fewer epitopes than LTNPs

- A positive correlation between effector CD8+ T-cells and plasma viremia and a negative correlation between CD8+effector T-cells and CD4+ T-cells was observed, which may contribute to the inability of LTNPs to clear virus

- This epitope can bind 3/5 HLA-A3 supertype alleles $(A * 0301, A * 1101, A * 3101, A * 3301$ and $A * 6801)$

$\operatorname{gp} 160(51-59)$ TLFCASDAK HIV-1 infection

Long-term nonprogressors (LTNPs) had strong memory resting CD8+ T-cell responses against the majority of epitopes tested, (18 for the A2 supertype, 16 for the A3 supertype) while the effector cells of long-term nonprogressors recognized far fewer epitopes

- Progressors had memory resting CD8+ T-cells that recognized far fewer epitopes than LTNPs 


\begin{tabular}{|c|c|c|c|c|}
\hline HXB2 Location & Author's Location & Immunogen & Species (HLA) & References \\
\hline & \multicolumn{4}{|c|}{$\begin{array}{l}\text { - A positive correlation between effector CD8+ T-cells and plasma viremia and a negative correlation between CD8+ effector T-cells and CD4+ T-cells was } \\
\text { observed, which may contribute to the inability of LTNPs to clear virus } \\
\text { - This epitope can bind } 3 / 5 \text { HLA-A3 supertype alleles }\left(A * 0301, A^{*} 1101, A^{*} 3101, A^{*} 3301 \text { and } A^{*} 6801\right)\end{array}$} \\
\hline gp160 (52-61) & \multicolumn{4}{|c|}{$\begin{array}{l}\text { - CTL epitope defined by T cell line and peptide mapping } \\
\text { - C. Brander notes that this is an } \mathrm{A} * 2402 \text { epitope in the } 1999 \text { database }\end{array}$} \\
\hline gp160 (52-61) & $\begin{array}{l}\text { gp120 (53-62 LAI }) \\
\text { - C. Brander notes this is a }\end{array}$ & $\begin{array}{l}\text { LFCASDAKAY } \\
\text { n A*2402 epitope }\end{array}$ & human $(\mathrm{A} * 2402)$ & Brander2001 \\
\hline gp160 (52-61) & \multicolumn{4}{|c|}{$\begin{array}{l}\text { - ELISPOT was used to study CTL responses to a panel of } 54 \text { predefined HIV-1 epitopes in } 91 \text { HIV-1-exposed, persistently seronegative (HEPS) and } 87 \\
\text { HIV-1-infected female Nairobi sex workers }\end{array}$} \\
\hline gp160 (52-61) & $\begin{array}{l}\text { gp120 (53-62 LAI) } \\
\text { - Uncertain whether optim }\end{array}$ & $\begin{array}{l}\text { LFCASCAKAY } \\
\text { al, binds A24 as well }\end{array}$ & human (B38) & Shankar1996 \\
\hline gp160 (52-71) & $\begin{array}{l}\text { gp120 (59-78) } \\
\text { - HIV-specific CTL lines c }\end{array}$ & $\begin{array}{l}\text { LFCASDAKAYDTEVHINVW- HIV-1 infection } \\
\text { AT } \\
\text { eveloped by ex vivo stimulation with peptide }\end{array}$ & human & Lieberman1995 \\
\hline gp160 (52-71) & $\begin{array}{l}\text { gp120 (59-78 SF2) } \\
\text { - Of } 25 \text { patients, most had } \\
\text { - Eleven subjects had CTL } \\
\text { - One of these } 11 \text { had CTL } \\
\text { - The responding subject v }\end{array}$ & $\begin{array}{l}\text { LFCASDAKAYDTEVHINVW- HIV-1 infection } \\
\text { AT } \\
\text { CTL specific for more than } 1 \text { HIV-1 protein } \\
\text { that could recognize vaccinia-expressed LAI gp160 } \\
\text { response to this peptide } \\
\text { vas HLA-A2 and B-21 }\end{array}$ & human & Lieberman1997a \\
\hline gp160 (62-80) & $\begin{array}{l}\text { gp120 (69-88 SF2) } \\
\text { - Of } 25 \text { patients, most had } \\
\text { - Eleven subjects had CTL } \\
\text { - One of these } 11 \text { had CTL } \\
\text { - The responding subject }\end{array}$ & $\begin{array}{l}\text { DTEVHNVWATHACVPTDPN HIV-1 infection } \\
\text { CTL specific for more than } 1 \text { HIV-1 protein } \\
\text { that could recognize vaccinia-expressed LAI gp160 } \\
\text { response to this peptide } \\
\text { vas HLA-A2 and B-21 }\end{array}$ & human & Lieberman1997a \\
\hline gp160 (78-86) & $\begin{array}{l}\text { gp120 (77-85) } \\
\text { - This epitope was include } \\
\text { low viral load }\end{array}$ & $\begin{array}{l}\text { DPNPQEVVL HIV-1 infection } \\
\text { d to illustrate the specificity of HIV-tetrameric staining, in a cross-s }\end{array}$ & $\begin{array}{l}\text { human }(\mathrm{B} * 3501) \\
\text { tional study correla }\end{array}$ & $\begin{array}{l}\text { Ogg1998b } \\
\text { LA A*0201 CTL effector cells and }\end{array}$ \\
\hline gp160 (78-86) & $\begin{array}{l}\text { gp120 (77-85 SF2) } \\
\text { C. Brander notes this is a }\end{array}$ & $\begin{array}{c}\text { DPNPQEVVL } \\
\text { B*3501 epitope }\end{array}$ & human $(\mathrm{B} * 3501)$ & Brander2001 \\
\hline gp160 (78-86) & $\begin{array}{l}\text { gp120 (77-85 SF2) } \\
\text { - A CTL clone responsive } \\
\text { - } 2 / 7 \text { B35-positive individu } \\
\text { - This epitope is highly va }\end{array}$ & $\begin{array}{l}\text { DPNPQEVVL HIV-1 infection } \\
\text { to this epitope was obtained } \\
\text { als have a CTL response to this epitope } \\
\text { riable }\end{array}$ & human $(\mathrm{B} * 3501)$ & Tomiyama1997 \\
\hline
\end{tabular}




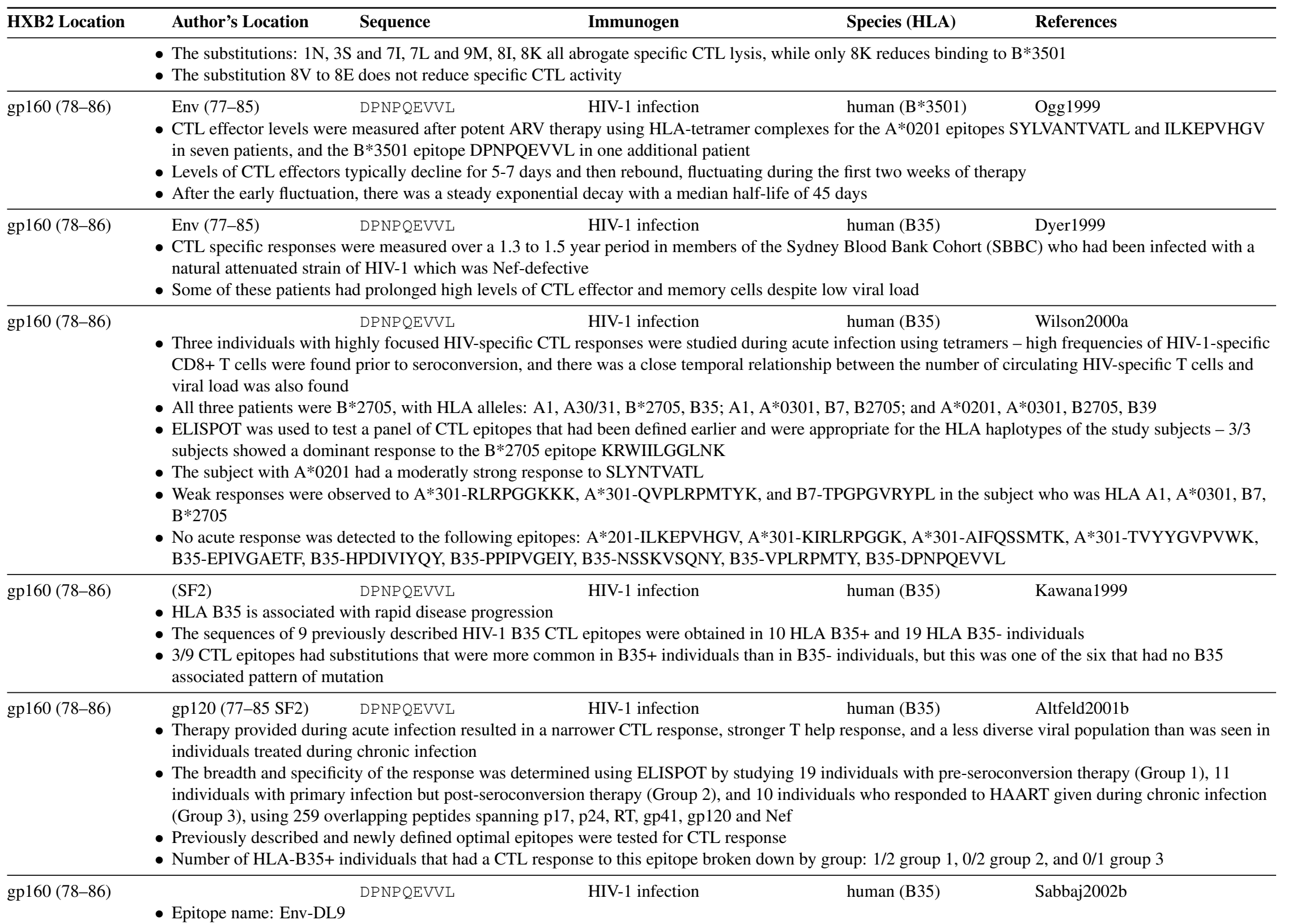




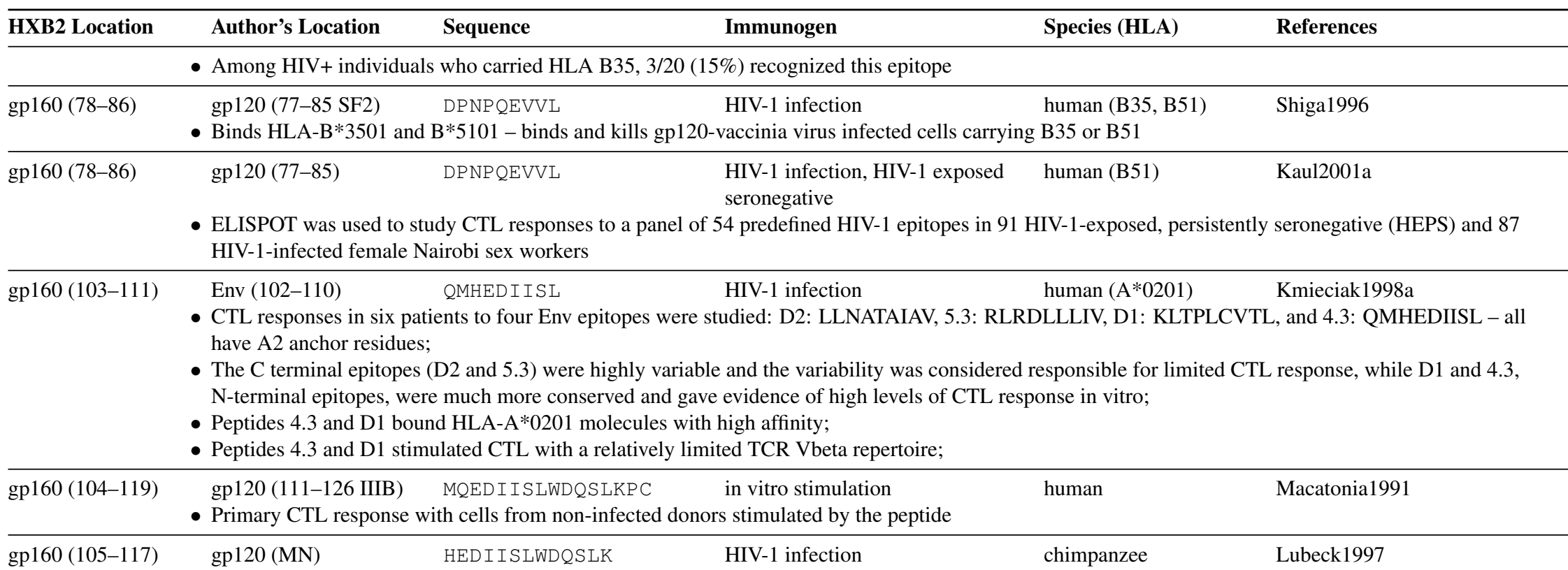

- No epitope-specific CTL were detected in chimpanzees immunized with adenovirus-HIV-1 MN gp160 recombinant despite a response to peptides P18 and $\mathrm{T} 1$

- Helper and cytotoxic $\mathrm{T}$ cells have been found to be stimulated by this peptide (T2)

\begin{tabular}{|c|c|c|c|c|}
\hline gp160 (105-117) & $\begin{array}{l}\text { gp120 (112-124 IIIB) HEDI ISLWDQSLK } \\
\text { - CTL and T helper cell reactivity in healthcare }\end{array}$ & $\begin{array}{l}\text { HIV-1 exposed seronegative } \\
\text { osed to HIV }\end{array}$ & human & Pinto1995 \\
\hline gp160 (105-117) & $\begin{array}{l}\text { gp120 (112-124 IIIB) HEDI ISLWDQSLK } \\
\text { - Helper and cytotoxic T cells can be stimulated }\end{array}$ & $\begin{array}{l}\text { HIV-1 infection } \\
\text { tide (T2) }\end{array}$ & human (A2) & Clerici1991a \\
\hline gp160 (108-116) & Env (107-115 subtype & Vaccine & human (A2.1) & Kundu1998a \\
\hline
\end{tabular}

B)

Vaccine Vector/Type: recombinant protein Strain: MN HIV component: gp160

- Ten HIV-1+ HLA A2 asymptomatic individuals were given two courses of HIV-1 MN rgp160 vaccine over a 2 year period

- Two hundred and fifty three HIV-1 peptides of 9 or 10 aa possessing the HLA-A2.1 binding motif (Leu at position 2, Val at the C terminus) were identified in gp160, of which 25 had a high or intermediate binding affinity

- Eleven peptides were studied that had high HLA-A2 binding affinity - a CTL response was detected to 9/11 peptides in at least 1 individual

- CTL responses after reimmunization may include recall responses - only individuals with vaccine cross-reactive sequences prior to vaccination showed detectable CTL responses

gp160 (109-117) Env(109-117 CM243 ISLWDQSLK $\quad$ HIV-1 exposed seronegative human (A11) Bond2001

subtype CRF01)

- Epitope name: E109-117

- This was a study of HIV-1 exposed persistently seronegative (HEPS) female sex workers in Chiang Mai, northern Thailand 


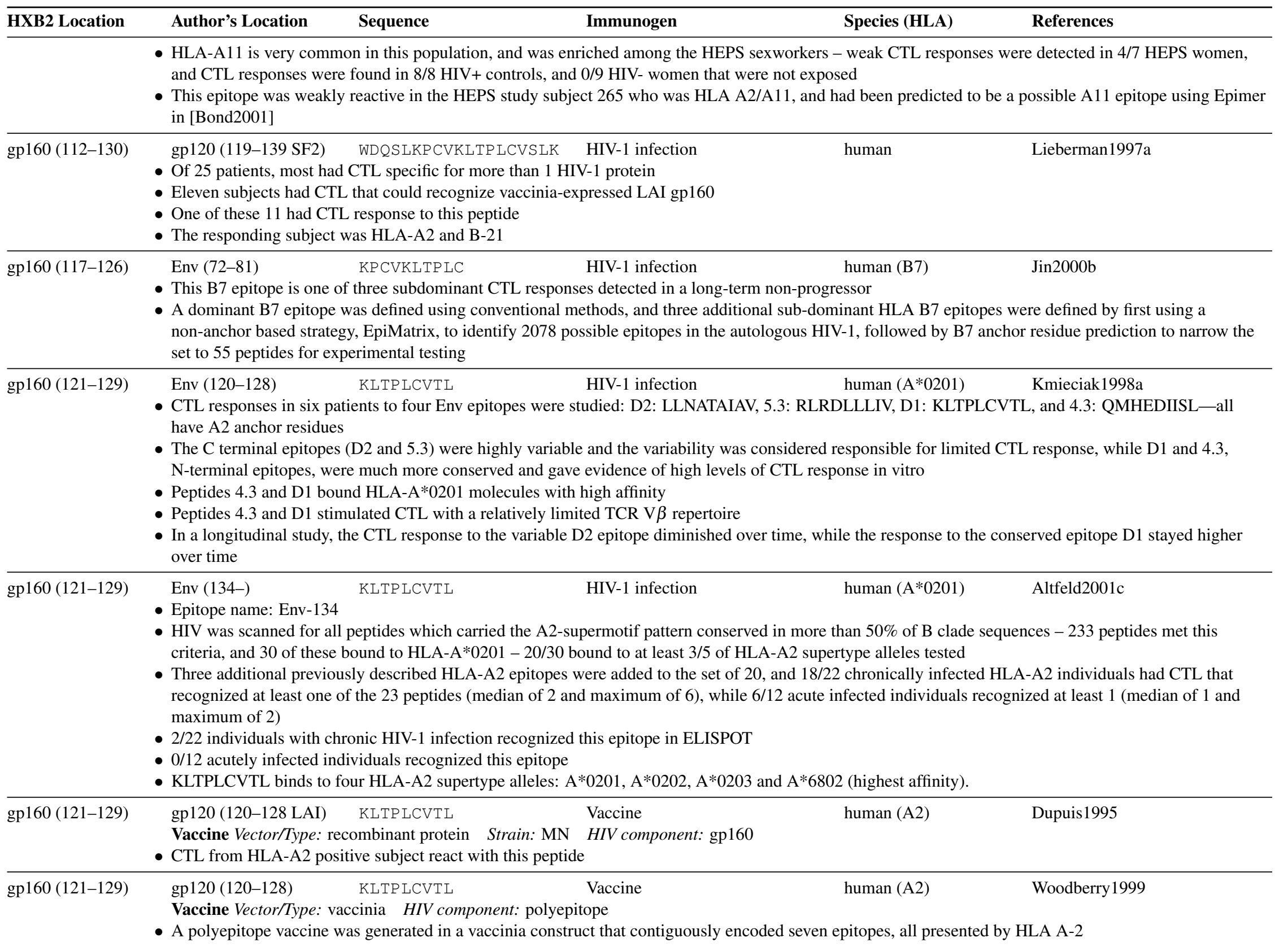




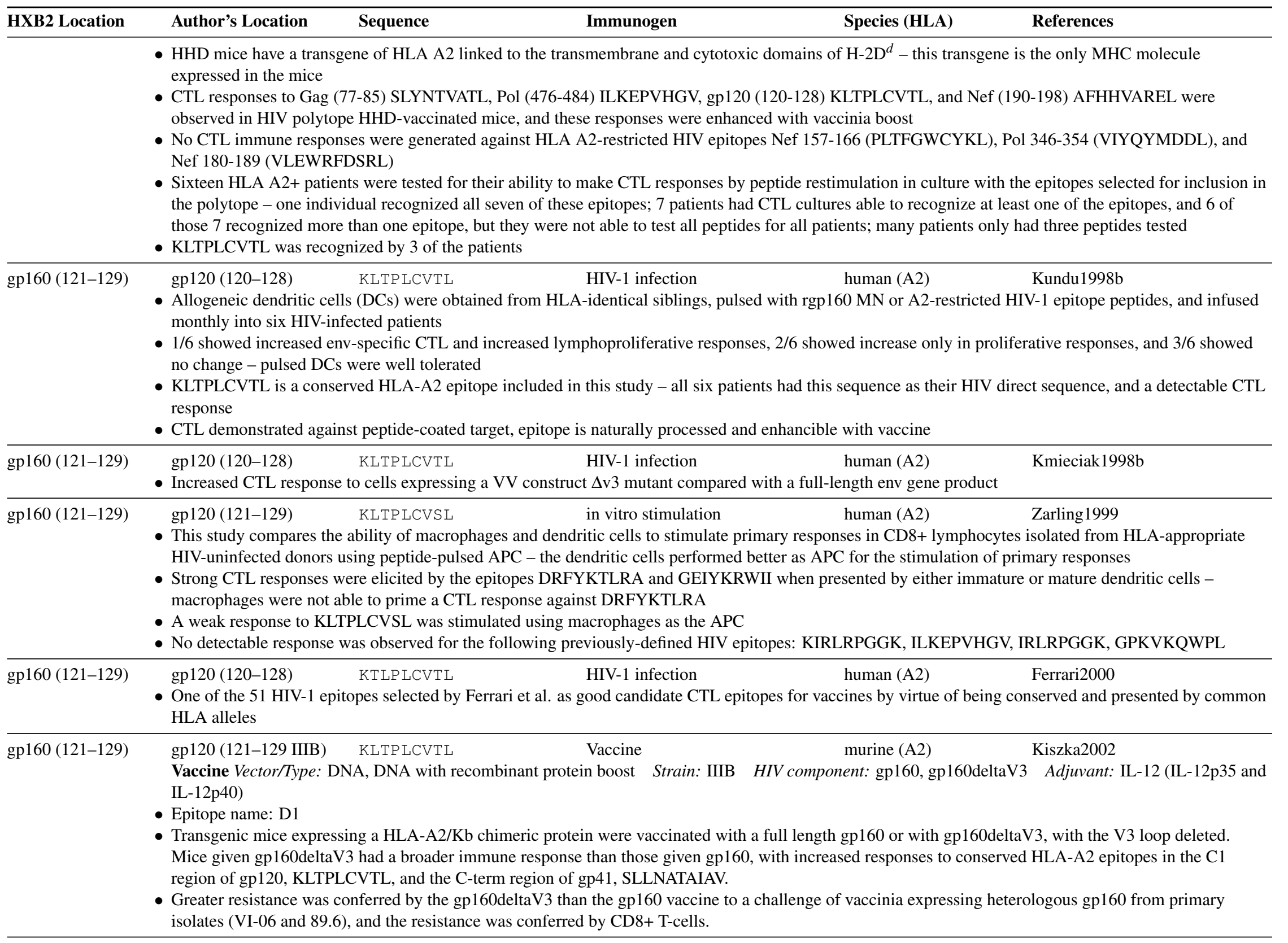




\begin{tabular}{llllc}
\hline HXB2 Location & Author's Location & Sequence & Immunogen & Species (HLA) \\
\hline gp160 (121-129) & Env (134-142) & $\begin{array}{c}\text { KLTPLCVTL } \\
\text { • }\end{array}$ & Rong-term nonprogressors (LTNPs) had strong memory resting CD8+ T-cell responses against the majority of epitopes tested, (18 for the A2 supertype, 16
\end{tabular}

- Long-term nonprogressors (LTNPs) had strong memory resting CD8+ T-cell responses against the majority of epitopes tested, (18 for the A2 supertype, 16 for the A3 supertype) while the effector cells of long-term nonprogressors recognized far fewer epitopes

- Progressors had memory resting CD8+ T-cells that recognized far fewer epitopes than LTNPs

- A positive correlation between effector CD8+ T-cells and plasma viremia and a negative correlation between CD8+ effector T-cells and CD4+ T-cells was observed, which may contribute to the inability of LTNPs to clear virus

- This epitope can bind four of the five HLA-A2 supertypes alleles (A*0201, A*020 2, A*0203, A*0206 and A*6802)

\begin{tabular}{llll}
\hline gp160 (121-129) Env & KLTPLCVTL & Vaccine & SJL/J HLA transgenic \\
mice (A2.1) & Ishioka1999
\end{tabular}

Vaccine Vector/Type: DNA HIV component: polyepitope

- A minigene vaccine construct encoding 6 HLA 2.1 and 3 HLA A11 restricted CTL epitopes, the universal Th cell epitope PADRE (pan-DR epitope) and an ER translocating signal sequence was constructed

- The epitopes were chosen for dominant recognition by CTLs during HBV and HIV infections in humans

- HLA transgenic mice were used for quantitating in vivo immunogenicity of DNA vaccines encoding HLA-restricted CTL epitopes - strong responses were observed to all nine epitopes, and CTL memory persisted up to four months after a single injection

\begin{tabular}{lllll}
\hline gp160 (121-129) & Env (120-128 subtype & KLTPLCVTL & Vaccine & human (A2.1)
\end{tabular}

Vaccine Vector/Type: recombinant protein Strain: MN HIV component: gp160

- Ten HIV-1+ HLA A2 asymptomatic individuals were given two courses of HIV-1 MN rgp160 vaccine over a 2 year period

- Two hundred and fifty three HIV-1 peptides of 9 or 10 aa possessing the HLA-A2.1 binding motif (Leu at position 2, Val at the C terminus) were identified in gp160, of which 25 had a high or intermediate binding affinity

- Eleven peptides were studied that had high HLA-A2 binding affinity - a CTL response was detected to 9/11 peptides in at least 1 individual

- CTL responses after reimmunization may include recall responses - only individuals with vaccine cross-reactive sequences prior to vaccination showed detectable CTL responses

\begin{tabular}{lllll}
\hline gp160 (156-165) Env (162-171 BH10, & NCSFNISTSI & HIV-1 infection & haman
\end{tabular}

LAI)

- This study employs an antigenic similarity matrix to compare HIV-1 antigenic determinants with human proteins.

- This CTL epitope (the HIV-1 LAI fragment with high similarity to a human protein overlapping this epitope is STSIRGKVQK) has similarity with the macrophage colony stimulating factor I receptor fragment SISIRLKVQK.

gp160 (156-165) gp120 (156-165) NCSFNISTSI HIV-1 infection

- Recognized by CTL clone LWF A5, isolated from a lab worker exposed to HIV-1 in 1985

- The processing of this epitope is TAP1/2-dependent, as are most Env epitopes, and it contains two N-linked glycosylation sites that are glycosylated in Env

- Only peptide that has been deglycosylated, a process that changes asparagine (N) to aspartic acid (D) was recognized: the aspartic acid at position 5 was critical, position 1 could be either $\mathrm{D}$ or $\mathrm{N}$

- This peptide also contains a Cys involved in a disulfide linkage but reducing conditions did not effect recognition by CTL clone LWF A5

- The HIV-1 Env epitopes are typically processed by a TAP1/2 dependent mechanism, which involves cotranslational translocation into the ER,

glycosylation, export back into the cytosol, and deglycosylation for processing, and retransport into the ER for the association with class I molecules

- The particular pathway of generating an epitope may have an impact on the presentation of that epitope, quantitatively as well as qualitatively 


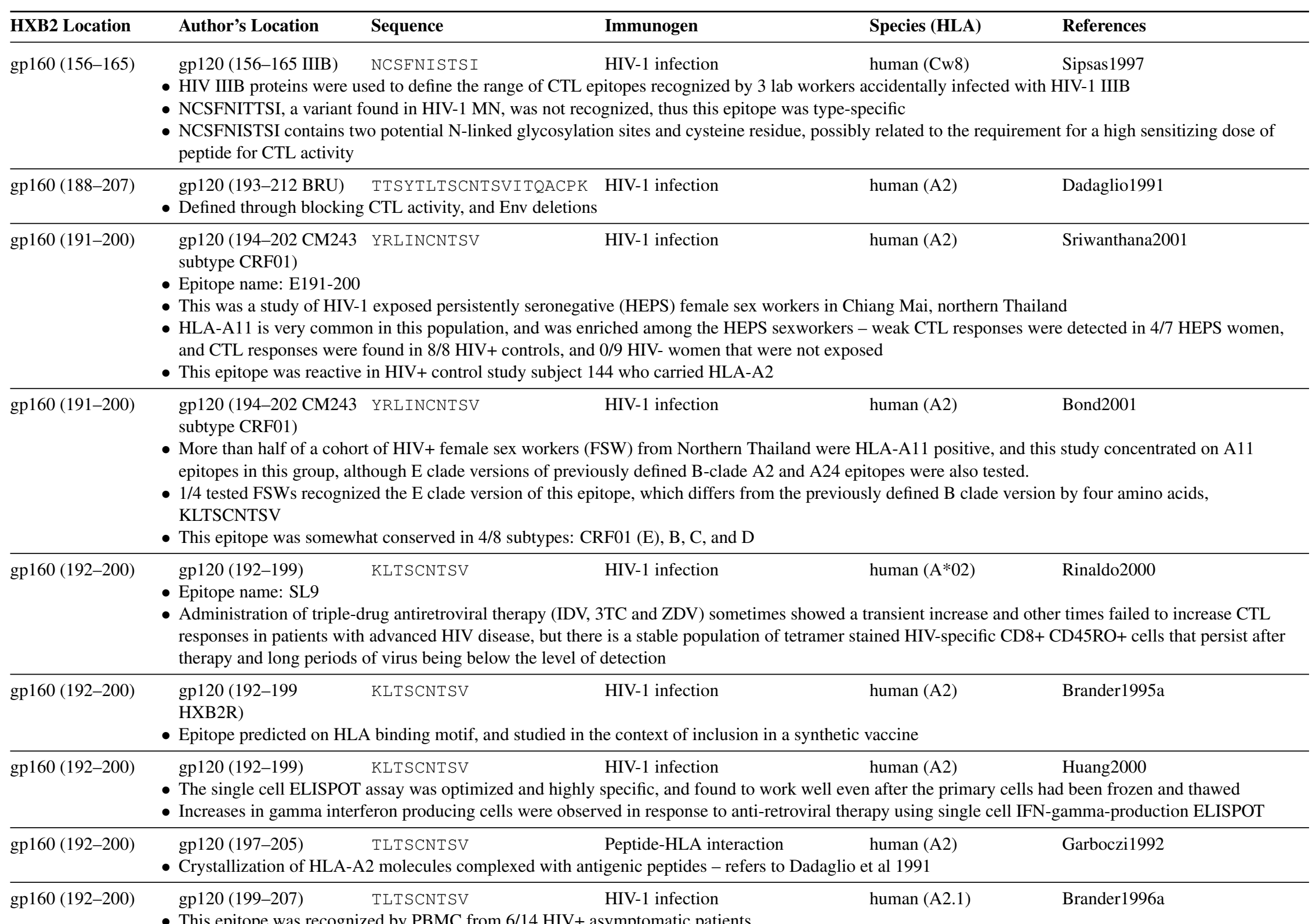

- This epitope was recognized by PBMC from 6/14 HIV+ asymptomatic patients

- This epitope was used along with pol CTL epitope ALQDSGLEV and a tetanus toxin T helper epitope for a synthetic vaccine 


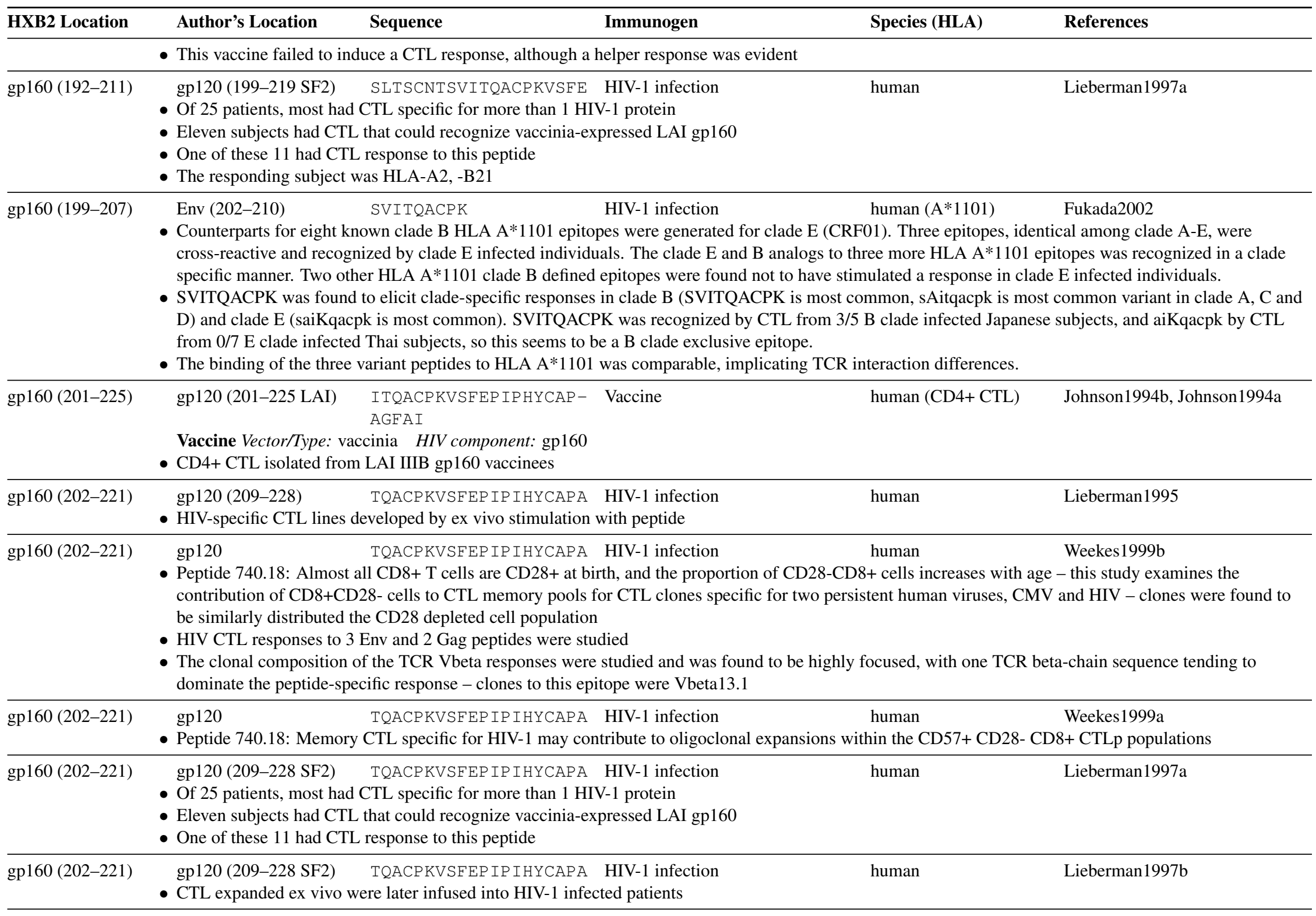




\begin{tabular}{|c|c|c|c|c|c|}
\hline HXB2 Location & Author's Location & Sequence & Immunogen & Species (HLA) & References \\
\hline gp160 (207-216) & gp120 (subtype A) & KMTFEPIPIH & HIV-1 infection & human (A29) & Cao2000 \\
\hline
\end{tabular}

- HIV-1 subtypes A and D dominate the Ugandan epidemic, and a vaccine trial using B clade antigen is underway - this study addresses relative levels of cross-reactive CTL responses in Ugandans to A, D, and B clade recombinant vaccinia viruses expressing Gag, Env, Pol, RT or Nef from HIV-1 clades A, B, and D

- Proteins corresponding to the subtype of the infecting strains tended to trigger higher levels of CTL response measured by percent specific lysis, but there was extensive inter-subtype cross-reactivity with B clade proteins and the co-circulating subtype

- CTL derived from subtype A clade infection (patient SP 528), recognized the subtype A version of the peptide (KMSFEPIPIH), had a slightly reduced specific lysis using the B clade version of the peptide (KVSFEPIPIH), and no lysis using the D clade version of the epitope (KVTFEPIPIH)

- Patient SP 528 is HLA A1, A29, B57, B81, Bw4, Bw6

gp160 (208-217) gp120 (subtype B) VSFEP IP IHY HIV-1 exposed seronegative human (A29)

- 11/16 heavily HIV exposed but persistently seronegative sex-workers in Nairobi had HIV-specific CD8 gamma-IFN responses in the cervix - systemic $\mathrm{CD} 8+\mathrm{T}$ cell responses tended to be to the same epitopes but at generally lower levels than cervical CD8+ T cell responses

- Low risk individuals did not have such CD8+ cells

- CD8+ T cell epitopes: DTVLEDINL (3 individuals), SLYNVATL (4 individuals), LSPRTLNAW (3 individuals) and YPLTFGWCF (4 individuals) were most commonly recognized by the HIV-resistant women

\begin{tabular}{lllll}
\hline gp160 (208-217) gp120(263-272) VSFEP IPHY & $\begin{array}{l}\text { HIV-1 infection, HIV-1 exposed human (A29) } \\
\text { seronegative }\end{array}$
\end{tabular}

- ELISPOT was used to study CTL responses to a panel of 54 predefined HIV-1 epitopes in 91 HIV-1-exposed, persistently seronegative (HEPS) and 87 HIV-1-infected female Nairobi sex workers

gp160 (208-219) Env $\quad$ VSFEPIPPHYCA HIV-1 infection $\quad$ Cao2002

- SP 511 is an A2 restricted CTL clone generated from a Ugandan subject that recognizes VSFEPIPPHYCA.

- CTL could be activated by a fusion protein of an HIV protein and anthrax lethal factor (LFn-HIV) that promotes antigen presenting cell uptake of exogenous protein and allows processing through the MHC class I pathway. This strategy for CTL detection could allow antigen presentation without generation of cells by the standard methods of using live viral vectors carrying a protein, or by loading the cells with peptides and by-passing processing

\begin{tabular}{|c|c|c|c|c|c|}
\hline gp160 (209-217) & (LAI) & SFEPIPIHY & & (A29) & Altfeld2000a Brander2001 \\
\hline $50(209-217)$ & gp120 (213-221 SF2) & SFEPIPIHY & HIV-1 infection & human (A29) & Altfeld2001b \\
\hline
\end{tabular}

- Therapy provided during acute infection resulted in a narrower CTL response, stronger T help response, and a less diverse viral population than was seen in individuals treated during chronic infection

- The breadth and specificity of the response was determined using ELISPOT by studying 19 individuals with pre-seroconversion therapy (Group 1), 11 individuals with primary infection but post-seroconversion therapy (Group 2), and 10 individuals who responded to HAART given during chronic infection (Group 3), using 259 overlapping peptides spanning p17, p24, RT, gp41, gp120 and Nef

- Previously described and newly defined optimal epitopes were tested for CTL response

- Number of HLA-A29+ individuals that had a CTL response to this epitope broken down by group: 1/3 group 1,0/0 group 2, and 0/0 group 3

gp160 (212-231) gp120 PIPIHYCAPAGFAILKCNNK HIV-1 infection human Weekes1999a

- Peptide 740.19: Memory CTL specific for HIV-1 may contribute to oligoclonal expansions within the CD57+ CD28- CD8+ CTLp populations

gp160 (212-231) gp120(219-238 HXB2) PIPIHYCAPAGFAILKCNNK HIV-1 infection human Lieberman1992

- CTL epitope defined by T cell line and peptide mapping 


\begin{tabular}{|c|c|c|c|c|c|}
\hline HXB2 Location & Author's Location & Sequence & Immunogen & Species (HLA) & References \\
\hline gp160 (212-231) & $\begin{array}{l}\text { gp120 (219-238) } \\
\text { - HIV-specific CTL li }\end{array}$ & $\begin{array}{l}\text { P IP I HYCAPAGE A I LKCNNK } \\
\text { eveloped by ex vivo stimulation }\end{array}$ & $\begin{array}{l}\text { HIV-1 infection } \\
\text { with peptide }\end{array}$ & human & Lieberman1995 \\
\hline gp160 (212-231) & gp120 & PIPIHYCAPAGFAILKCNNK & HIV-1 infection & human (A2) & Weekes1999b \\
\hline
\end{tabular}

- Peptide 740.19: Almost all CD8+ T cells are CD28+ at birth, and the proportion of CD28-CD8+ cells increases with age - this study examines the contribution of CD8+CD28- cells to CTL memory pools for CTL clones specific for two persistent human viruses, CMV and HIV - clones were found to be similarly distributed in the CD28 depleted cell population

- HIV CTL responses to 3 Env and 2 Gag peptides were studied

- The clonal composition of the TCR Vbeta responses was studied and was found to be highly focused, with one TCR beta-chain sequence tending to dominate the peptide-specific response - clones to this epitope were Vbeta13.6

gp160 (212-231) gp120 PIPIHYCAPAGFAILKCNNK HIV-1 infection human (B57) Jin1998b

- Progressive HIV-1 infection and CD4 decline was associated decreased the IL-2-expandable HIV-1 CTL response in 10 asymptomatic HIV-infected patients - this observation may be partially due to a reduction and impaired function of T helper cells, CTL exhaustion and APC dysfunction

- Continued presence of HIV-1 specific memory cells (CTLp) was observed in three patients, one to GIWGCSGKLICTTAV, one to ALIWEDLRSLCLFSY, and one to PIPIHYCAPAGFAILKCNNK

$\begin{array}{lll}\text { gp160 (237-246) } & \text { Env } \\ & \text { - The program Epimatrix was used in conjunction with the program Conservatrix to identify conservered regions of HIV that might serve as epitopes }\end{array}$

- A subset of the potential epitopes was identified that could bind to the appropriate HLA-allele, and 15 predicted B7 superfamily (HLA B7, B8, and B58) epitopes were identified that could stimulate IFN $\gamma$ production in an ELISPOT assay

- GPCKNVSTVQ was newly defined as an epitope in this study, was shown to stimulate an ELISPOT response, and to bind to HLA-B7

\begin{tabular}{|c|c|c|c|}
\hline gp160 (239-247) & $\begin{array}{l}\text { gp120 (241-249 LAI) CTNVSTVQC HIV-1 infection } \\
\text { - HIV IIIB proteins were used to define the range of CTL epitopes recognized by } 3 \text { lab v } \\
\text { - CTNVSTVQC contains a potential N-linked glycosylation site and cysteine residues, p } \\
\text { for CTL activity }\end{array}$ & $\begin{array}{l}\text { human }(\mathrm{Cw} 8) \\
\text { accidentally infecte } \\
\text { related to a requires }\end{array}$ & $\begin{array}{l}\text { Sipsas } 1997 \\
\text { HIV-1 IIIB } \\
\text { r a high sensitizing dose of peptide }\end{array}$ \\
\hline gp160 (242-261) & $\begin{array}{l}\text { gp120 (249-268) VSTVQCTHGIRPVVSTQLLL HIV-1 infection } \\
\text { - HIV-specific CTL lines developed by ex vivo stimulation with peptide }\end{array}$ & human & Lieberman1995 \\
\hline gp160 (242-261) & $\begin{array}{l}\text { gp120 (249-268 SF2) VSTVQCTHGIRPVVSTQLLL HIV-1 infection } \\
\text { - Of } 25 \text { patients, most had CTL specific for more than 1 HIV-1 protein } \\
\text { - Eleven subjects had CTL that could recognize vaccinia-expressed LAI gp160 } \\
\text { - One of these } 11 \text { had CTL response to this peptide } \\
\text { - The responding subject was HLA-2, -B21 }\end{array}$ & human & Lieberman1997a \\
\hline gp160 (242-261) & $\begin{array}{l}\text { gp120 (249-268) VSTVQCTHGIRPVVSTQLLL HIV-1 infection } \\
\text { - CTL expanded ex vivo were later infused into HIV-1 infected patients }\end{array}$ & human & Lieberman1997b \\
\hline gp160 (252-260) & $\begin{array}{l}\text { gp120 (255-263 SF2) RP IVSTQLL HIV-1 infection } \\
\text { - A CTL clone responsive to this epitope was obtained } \\
\text { - Only } 1 / 7 \text { B35-positive individuals had a CTL response to this epitope } \\
\text { - An I to V substitution at position } 3 \text { reduces specific lysis, but not binding to B*3501 } \\
\text { - A Q to H substitution at position } 7 \text { abrogates specific lysis, but not binding to B*3501 }\end{array}$ & human $(\mathrm{B} * 3501)$ & Tomiyama1997 \\
\hline
\end{tabular}




\begin{tabular}{lllll}
\hline HXB2 Location & Author's Location & Sequence & Immunogen & Species (HLA) \\
\hline gp160 (252-260) & $\begin{array}{l}\text { gp120 (255-263 SF2) } \\
\text { - Binds HLA-B*3501 }\end{array}$ & RP IVSTQLL & HIV-1 infection & human (B35) \\
\hline gp160 (252-260) & (SF2) & RP IVSTQLL & HIV-1 infection & Shiga1996 \\
\hline
\end{tabular}

- HLA B35 is associated with rapid disease progression

- The sequences of 9 previously described HIV-1 B35 CTL epitopes were obtained in 10 HLA B35+ and 19 HLA B35- individuals

- 3/9 CTL epitopes had substitutions that were more common in B35+ individuals than in B35- individuals, but this was one of the six that had no B35 associated pattern of mutation

\begin{tabular}{lll}
\hline gp160 (252-261) & Env & RPVVSTQLLL \\
& - The program Epimatrix was used in conjunction with the program Conservatrix to identify conservered regions of HIV that might serve as epitopes
\end{tabular}

- The program Epimatrix was used in conjunction with the program Conservatrix to identify conservered regions of HIV that might serve as epitopes
- A subset of the potential epitopes was identified that could bind to the appropriate HLA-allele, and 15 of the predicted B7 superfamily (HLA B7, B8, a

B58) epitopes were identified that could stimulate IFN $\gamma$ production in an ELISPOT assay

- RPVVSTQLLL was one of the 15, and had been previously identified as an HLA-B7 epitope, and was confirmed in this study

\begin{tabular}{lllll}
\hline gp160 (252-271) Env (256-268 BH10, RPVVSTQLLLNGSLAEEEVV & HIV-1 infection & haman
\end{tabular}

LAI)

- This study employs an antigenic similarity matrix to compare HIV-1 antigenic determinants with human proteins.

- This CTL epitope (the HIV-1 LAI fragment with high similarity to a human protein overlapping this epitope is STQLLLNGSLAEE) has similarity with the lymphatic endothelium-specific hyaluronan receptor LYVE-1 fragment TTRLLVQGSLRAEE.

\begin{tabular}{lllll}
\hline gp160 (252-271) & gp120(256-275 LAI) & RPVVSTQLLLNGSLAEEEVV & HIV-1 infection & human (B7) \\
\hline gp160 (291-307) & Env (292-301 BH10, & SVEINCTRPNNNTRKSI & HIV-1 infection & human \\
& LAI) & & Maksiutov2002
\end{tabular}

- This study employs an antigenic similarity matrix to compare HIV-1 antigenic determinants with human proteins.

- This CTL epitope (the HIV-1 LAI fragment with high similarity to a human protein overlapping this epitope is VEINCTRPNN) has similarity with the FasI receptor precursor (Apptosis-mediating surface antigen fas) (APO-1 antigen) (CD95 antigen) fragment VEINCTRQN.

\begin{tabular}{|c|c|c|c|c|}
\hline gp160 (291-307) & $\begin{array}{l}\text { gp120 (295-312 BRU) SVEINCTRPNNNTRKS I } \\
\text { - Defined through blocking CTL activity, and Env deletions }\end{array}$ & HIV-1 infection & human (A2) & Dadaglio1991 \\
\hline gp160 (291-307) & 91-307 IIIB) & Vaccine & murine (A2) & Kiszka2002 \\
\hline
\end{tabular}

Vaccine Vector/Type: DNA, DNA with recombinant protein boost Strain:

- Transgenic mice expressing a HLA-A2/Kb chimeric protein were vaccinated with a full length gp160 or with gp160deltaV3, with the V3 loop deleted. Mice given gp160deltaV3 had a broader immune response than those given gp160, with increased responses to conserved HLA-A2 epitopes in the C1 region of gp120, KLTPLCVTL, and the C-term region of gp41, SLLNATAIAV.

- Greater resistance was conferred by the gp160deltaV3 than the gp160 vaccine to a challenge of vaccinia expressing heterologous gp160 from primary isolates (VI-06 and 89.6), and the resistance was conferred by CD8+ T-cells.

- The most intense CTL responses to the intact gp160 vaccine were directed at three V3 peptides.

gp160 (297-322)

$\begin{array}{ll}\text { gp120 (297-322 IIIB) } & \text { TRPNNNTRKRIRIQRGPGR- Vaccine } \\ & \text { AFVTIGK }\end{array}$

Vaccine Vector/Type: peptide Strain: IIIB HIV component: V3 Adjuvant: liposome

- Induction of peptide-specific CTLs in BALB/c mice was dependent on immunization with peptide encapsulated liposomes containing MPL as adjuvant 


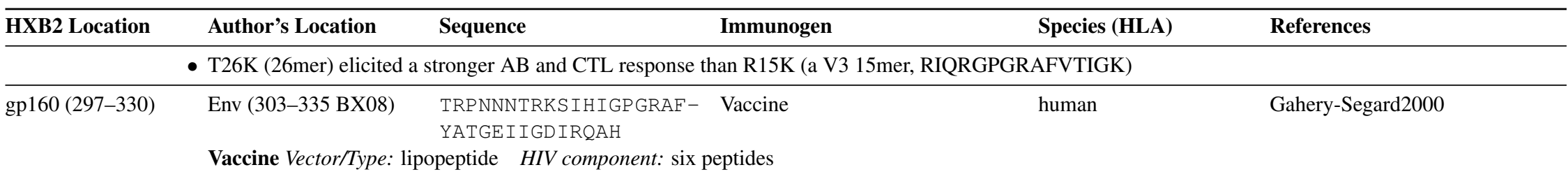

- Anti-HIV lipopeptide vaccine consisting of six long amino acid peptides derived from Nef, Gag and Env HIV-1 proteins modified by a palmitoyl chain was administered in a phase I trial

- A CD4+ T cell proliferative response to at least one of the six peptides was observed in $9 / 10$ vaccinees $-6 / 10$ reacted to this peptide

- 9/12 tested mounted a CTL response to at least one of the six peptides; each of the six peptides elicited a CTL response in at least one individual - this peptide was particularly immunogenic, eliciting a CTL response in five vaccinees

- None of the 12 tested had an IgG response to gp120 or gp160 and vaccinees could be differentiated from HIV-1 seropositive individuals with a commercial HIV detection kit - no neutralizing antibodies were observed

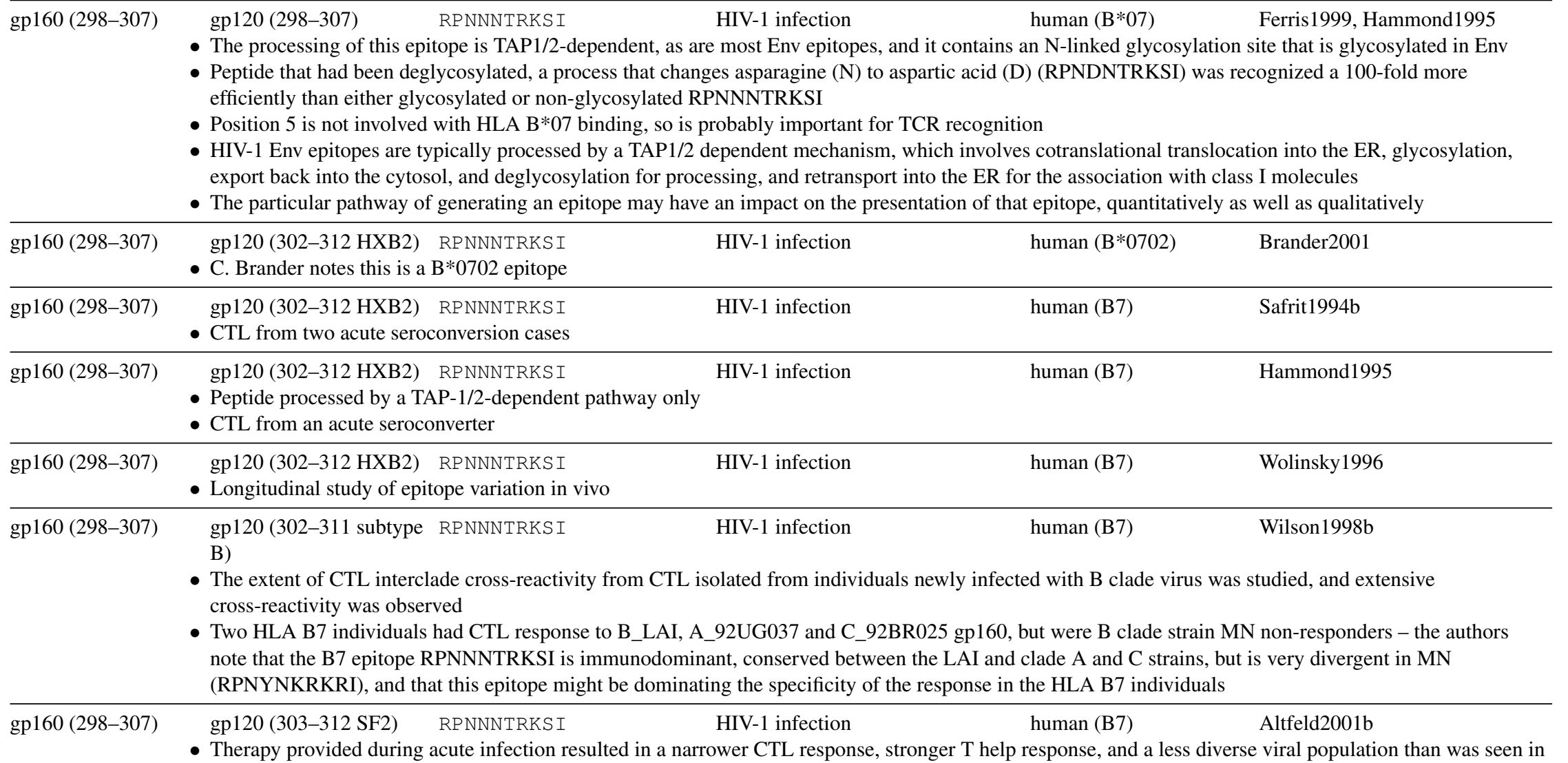

individuals treated during chronic infection 


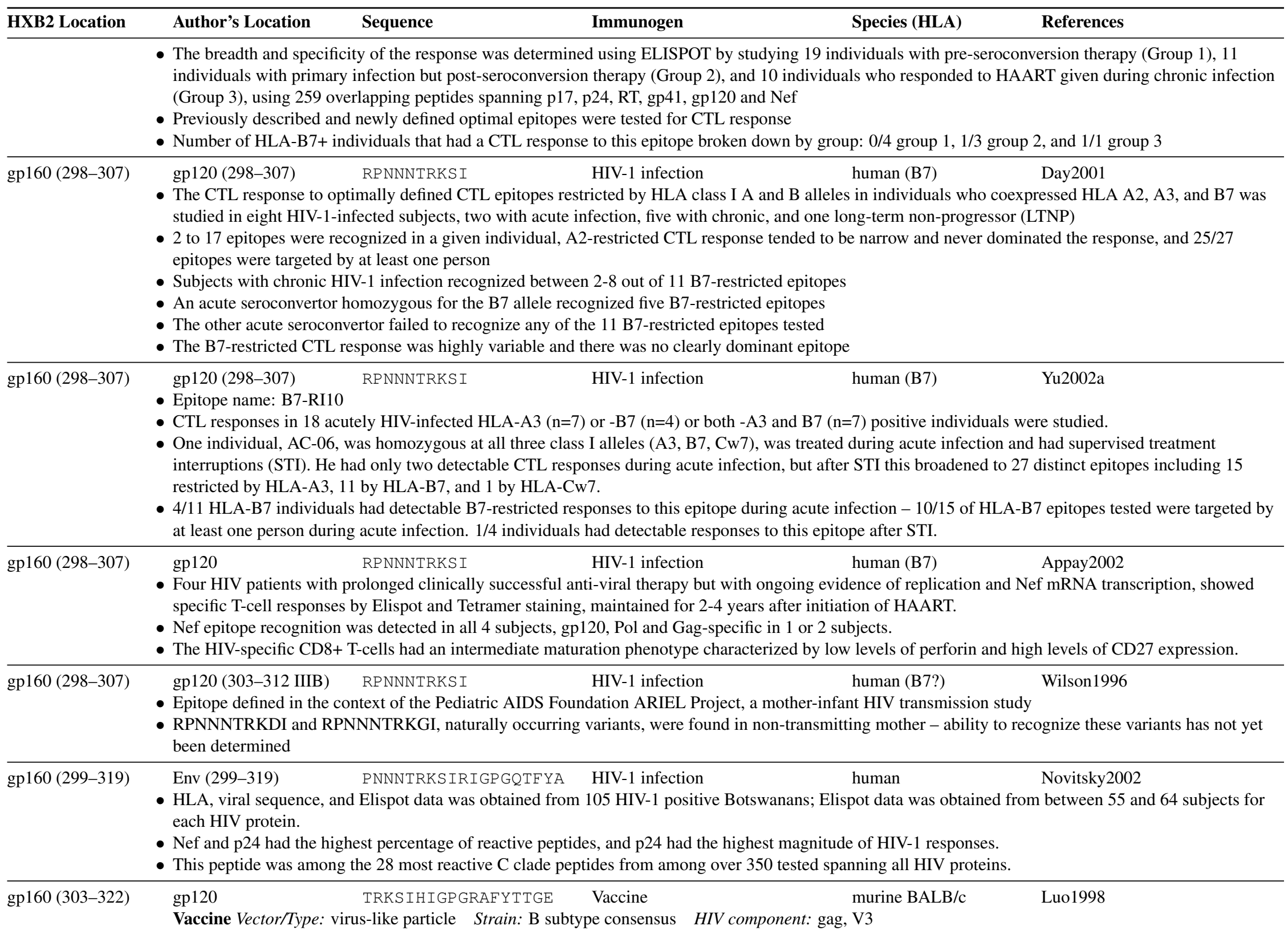




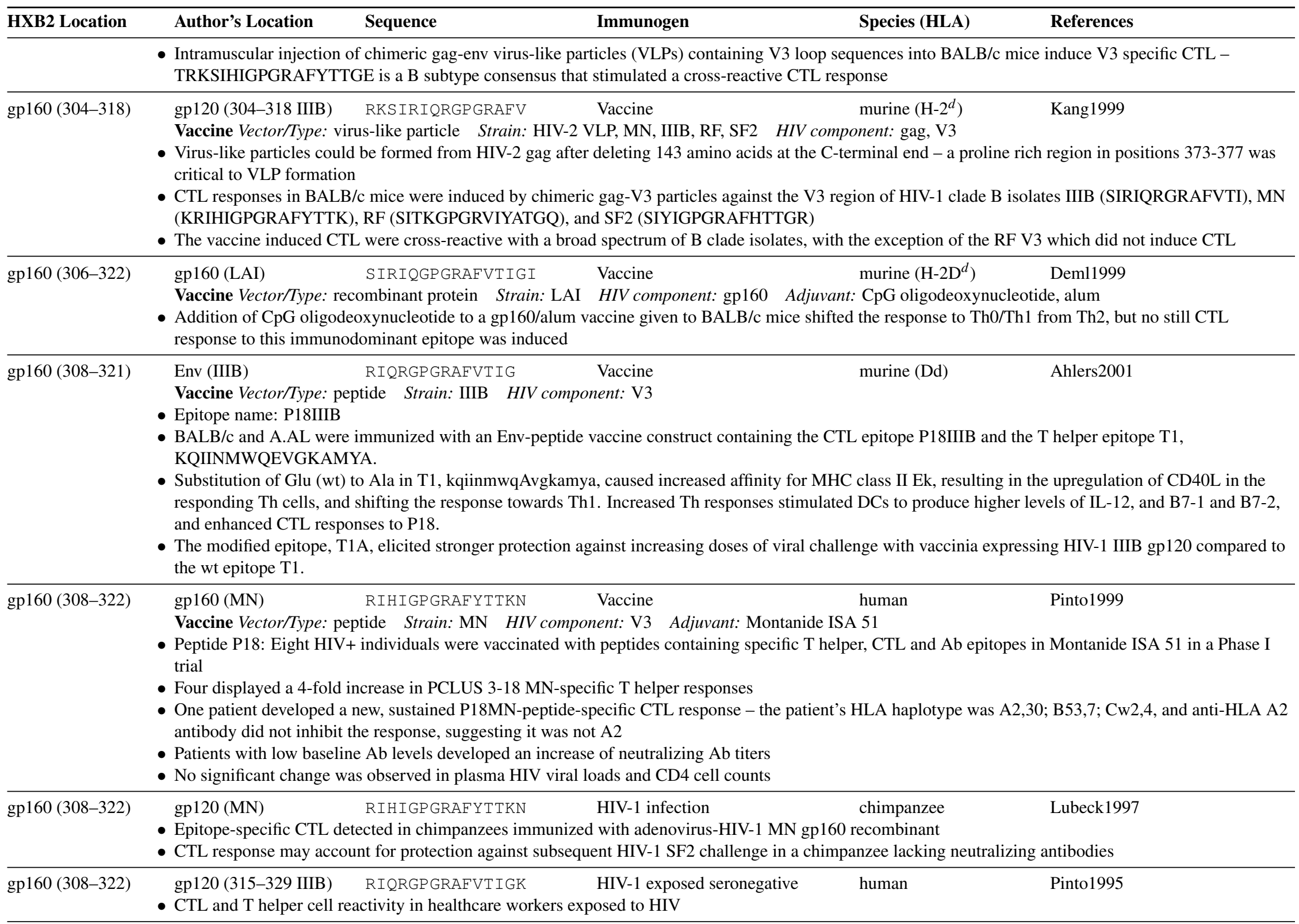




\begin{tabular}{|c|c|c|c|c|c|}
\hline HXB2 Location & Author's Location & Sequence & Immunogen & Species (HLA) & References \\
\hline gp160 (308-322) & $\begin{array}{l}\text { gp120 (313-327 MN) } \\
\text { - CTL and T helper cell }\end{array}$ & $\begin{array}{l}\text { RIHIGPGRAFYTTKN } \\
\text { ctivity in healthcare wor }\end{array}$ & $\begin{array}{l}\text { HIV-1 exposed seronegative } \\
\text { posed to HIV }\end{array}$ & human & Pinto1995 \\
\hline gp160 (308-322) & gp120 (110-122) & RIQRGPGRAFVTIGK & Vaccine & murine & Moore2002a \\
\hline
\end{tabular}

- Intramuscular immunization of BALB/c mice with DNA vaccines carrying either gp160 or Nef in the expression vector plasmid pNGVL gave different responses - gp160 induced strong gp160-specific CTL and IFN-responses and low-titer humoral responses, and Nef generated humoral (IgG1, IgG2a) responses and IFN-responses but little CTL activity.

- Co-injection of DNA plasmids encoding cytokines and/or hematopoietic growth factors, IL2, IL-12, IL-15, Flt3 ligand (FL), and GMCSF tended to give responses that were enhanced quantitatively, but not altered qualitatively.

- Co-administration of GMCSF most strongly enhanced CTL and IFN-responses against pNGVL-gp160.

- Repeated immunization with pNGVL-Nef failed to induce CTL responses. Co-administration of IL-12 most strongly enhanced humoral and IFNgamma responses.

- FL, which enhances innate immune responses, in combination with IL-2, IL-12 or IL-15 generated with most potent Nef responses.

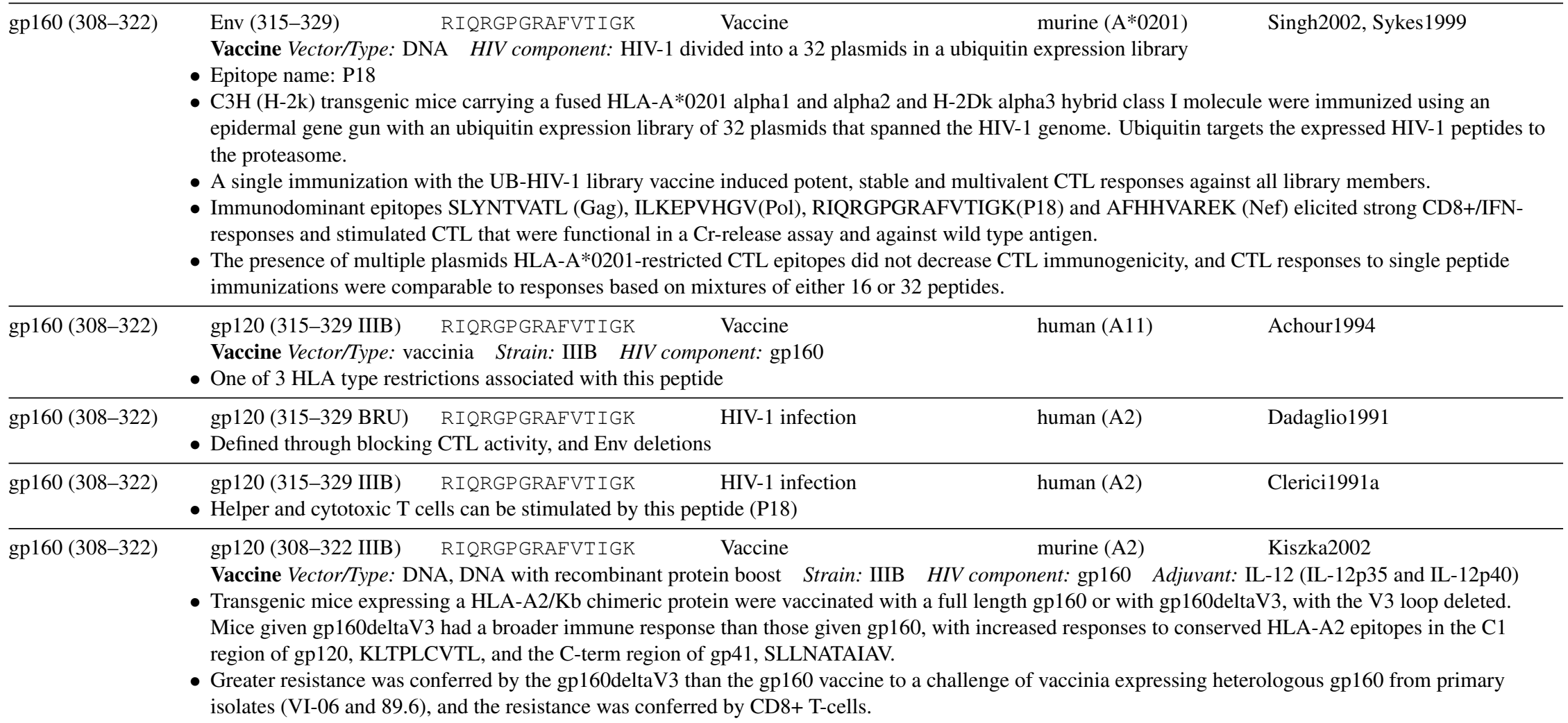




\begin{tabular}{|c|c|c|c|c|}
\hline HXB2 Location & Author's Location & Immunogen & Species (HLA) & References \\
\hline & \multicolumn{4}{|c|}{ - The most intense CTL responses to the intact gp160 vaccine were directed at three V3 peptides. } \\
\hline gp160 (308-322) & \multicolumn{4}{|c|}{$\begin{array}{l}\text { Vaccine Vector/Type: vaccinia HIV component: gp160 } \\
\text { - Two of } 3 \text { HLA type restrictions associated with this peptide }\end{array}$} \\
\hline gp160 (308-322) & \multicolumn{4}{|c|}{$\begin{array}{l}\text { Vaccine Vector/Type: peptide Strain: IIIB HIV component: V3 } \\
\text { - Positions } \mathrm{R}(8) \text { and } \mathrm{F}(10) \text { are important for MHC/peptide interaction. }\end{array}$} \\
\hline gp160 (308-322) & \multicolumn{4}{|c|}{$\begin{array}{l}\text { Vaccine Vector/Type: peptide Strain: IIIB HIV component: V3 } \\
\text { - Free peptide injected into the footpad of a mouse could stimulate specific CTL }\end{array}$} \\
\hline gp160 (308-322) & \multicolumn{4}{|c|}{$\begin{array}{l}\text { Vaccine Vector/Type: peptide Strain: MN HIV component: V3 } \\
\text { - PCLUS 3-18MN synthetic peptide vaccine construct contained T1 helper epitope covalently linked to truncated P18 CTL epitope } \\
\text { - A substitution in the T1 peptide stimulated an enhanced Th response and class II binding specificity, which in turn enhanced CTL induction by vaccine } \\
\text { - Construct PCLUS 3-18MN is currently in a phase I vaccine clinical trial }\end{array}$} \\
\hline gp160 (308-322) & \multicolumn{4}{|c|}{$\begin{array}{l}\text { Vaccine Vector/Type: vaccinia Strain: MN, IIIB HIV component: gp160 } \\
\text { - } \mathrm{Y}(11 \mathrm{MN}) \text { exchange with V(11 IIIB) interchanges specificities }\end{array}$} \\
\hline gp160 (308-322) & \multicolumn{4}{|c|}{$\begin{array}{l}\text { Vaccine Vector/Type: vaccinia Strain: RF HIV component: gp160 } \\
\text { Comparison of MN, IIIB, and RF specificities, position } 11 \text { is critical }\end{array}$} \\
\hline gp160 (308-322) & \multicolumn{4}{|c|}{$\begin{array}{l}\text { - The TCR repertoire and its specificity was studied through analyzing the spectrum of TCR-alpha and beta chains able to reconstitute a reaction to the H-2 } \\
\text { Dd-restricted P18 peptide. The RT-1 TCR alpha chain was able to react with } 1 / 3 \text { of the tested TCR beta chains to create a specific response. Experiments in } \\
\text { transgenic mice also supported the observation that a single TCR alpha chain would confer the specificity of the response and could interact with a large } \\
\text { variety of TCR beta chains. }\end{array}$} \\
\hline gp160 (308-322) & \multicolumn{4}{|c|}{$\begin{array}{l}\text { Vaccine Vector/Type: recombinant protein HIV component: Gag, V3 } \\
\text { - Gag-V3 fusion protein immunization elicited V3 CTL response in mice }\end{array}$} \\
\hline gp160 (308-322) & \multicolumn{4}{|c|}{$\begin{array}{l}\text { Vaccine Vector/Type: virus-like particle HIV component: Gag, Env } \\
\text { Env bound to virus-like particles (VLPs) can elicit a CTL response that is dependent on the amount of Env presented on the VLP }\end{array}$} \\
\hline gp160 (308-322) & $\begin{array}{l}\text { gp120 (313-327 MN) } \\
\text { Vaccine Vector/Type: D }\end{array}$ & $\begin{array}{l}\text { RIHIGPGRAFYTTKN } \quad \text { Vaccine } \\
\text { IA Strain: MN HIV component: gp160, V3 }\end{array}$ & murine $\mathrm{BALB} / \mathrm{c}$ & Fomsgaard1998a \\
\hline
\end{tabular}




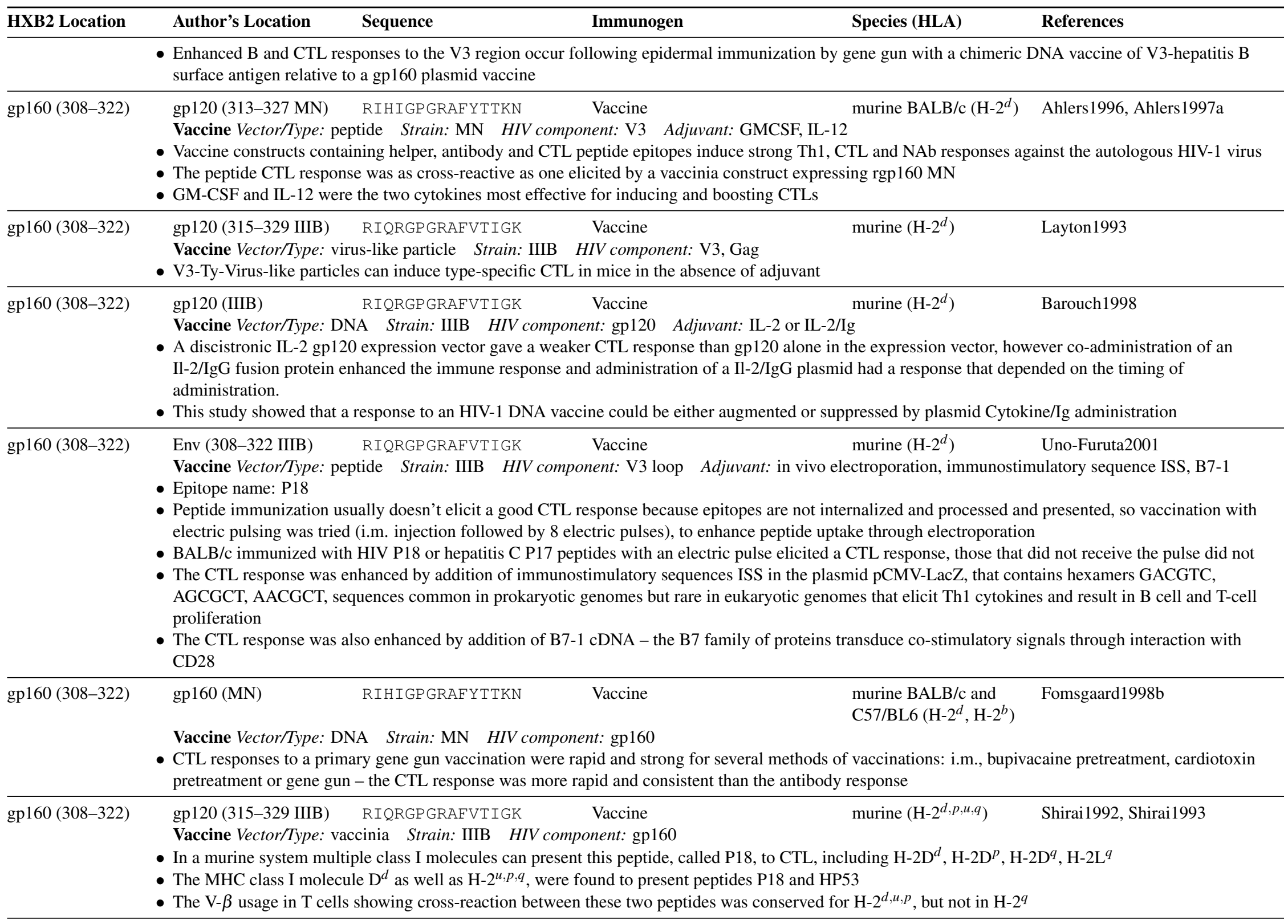




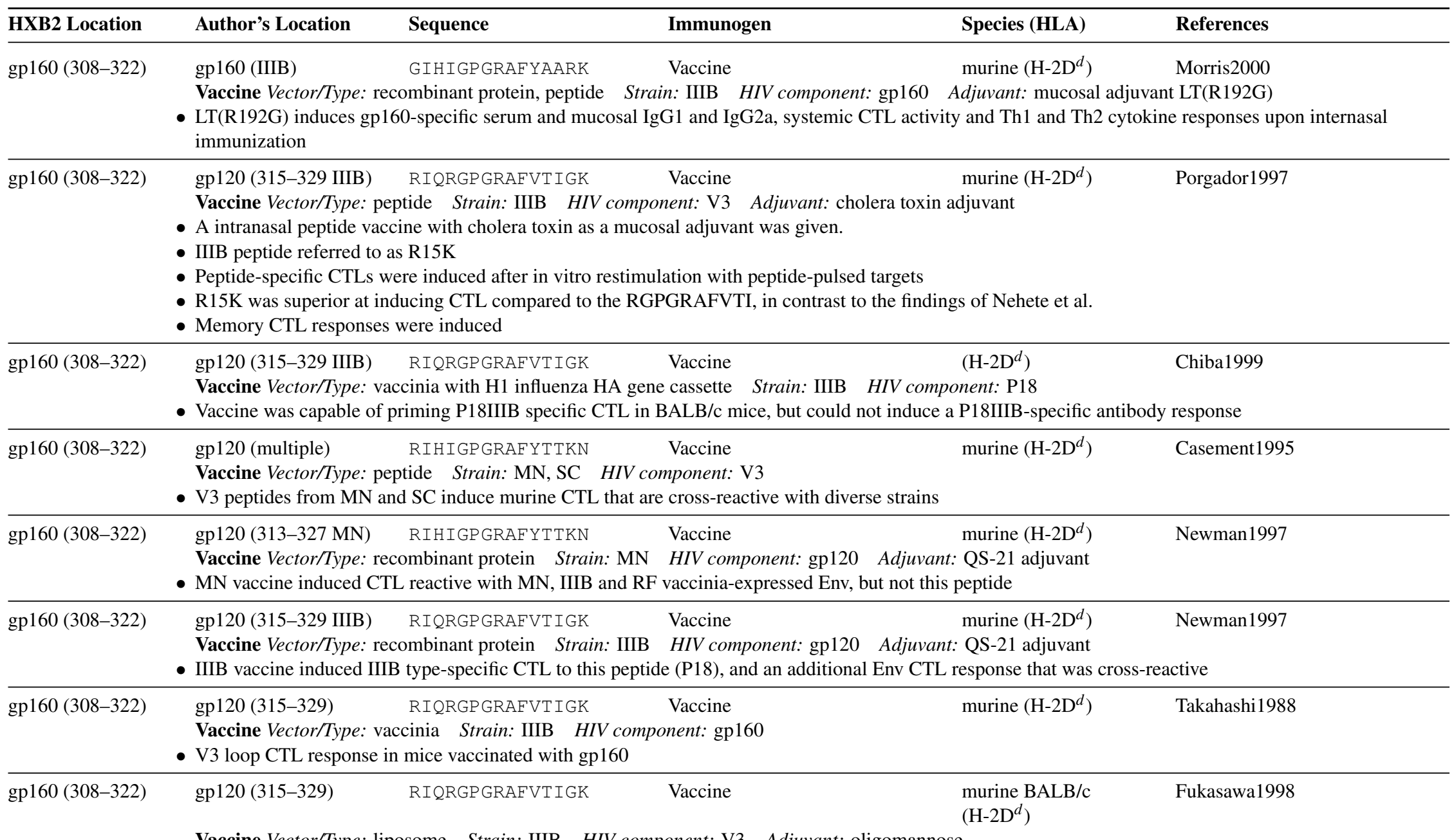

Vaccine Vector/Type: liposome Strain: IIIB HIV component: V3 Adjuvant: oligomannose

- The peptide RIQRGPGRAFVTIGK was incorporated into liposomes and given as a subcutaneous injection, which induces a MHC class I restricted CTL response in mice

- Liposomes coated with oligomannose show no toxicity and can elicit a potent CTL response upon a single subcutaneous infection, while non-coated liposomes do not, suggesting that oligomannose may be a good adjuvant for CTL responses

\begin{tabular}{cccc}
\hline gp160 (308-322) & RIQRGPGRAFVTIGK & Vaccine & murine $\left(\mathrm{H}-2 \mathrm{D}^{d}\right)$ \\
& Vaccine Vector/Type: fusion protein with anthrax delivery domain & HIV component: V3 & Adjuvant: B. anthracia lethal toxin LF component \\
& - Epitope name: P18
\end{tabular}




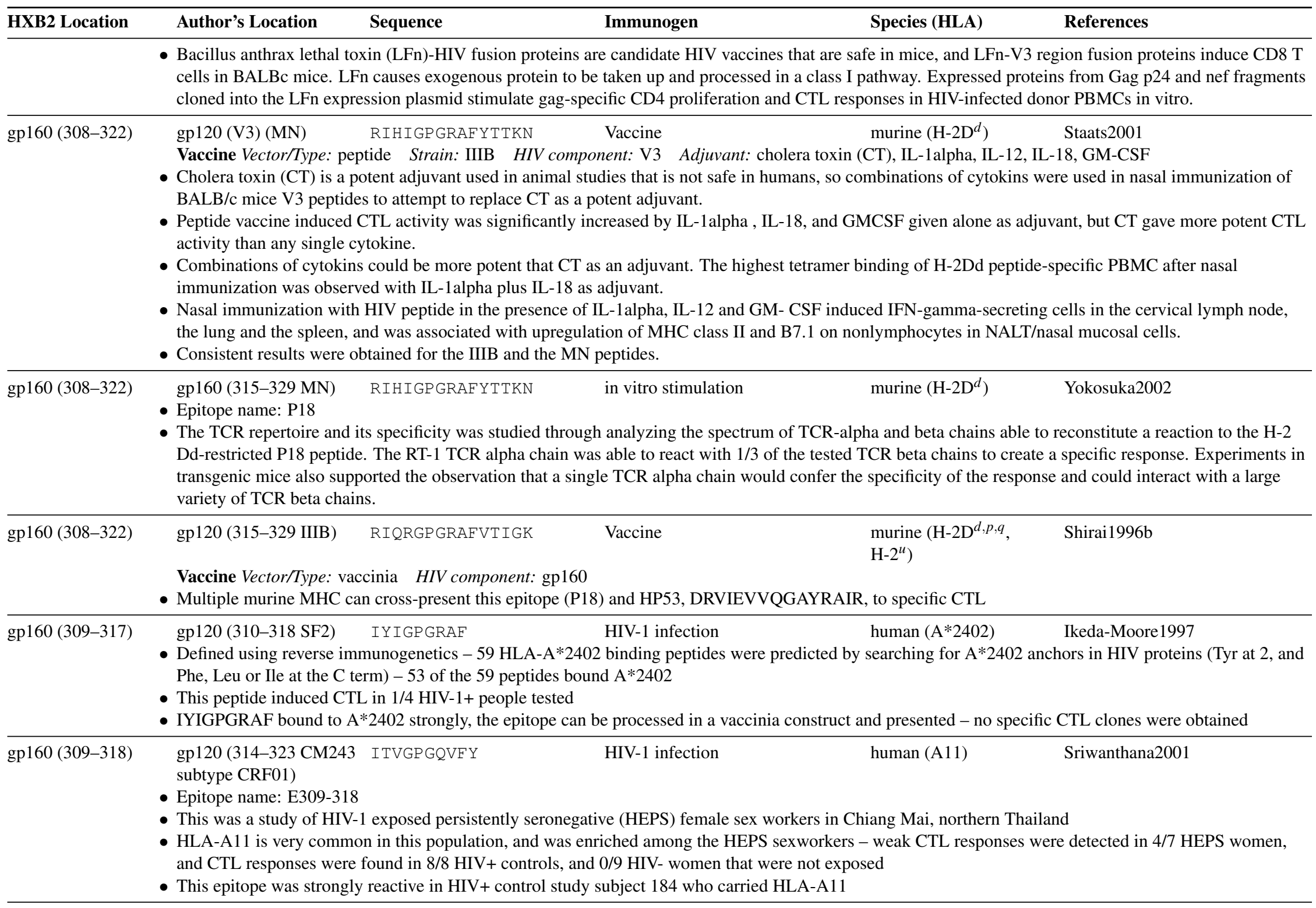




\begin{tabular}{lllll}
\hline HXB2 Location & Author's Location & Sequence & Immunogen & Species (HLA) \\
\hline gp160 (309-318) & $\begin{array}{l}\text { gp120 (314-323 CM243 } \\
\text { subtype CRF01) }\end{array}$ & ITVGPGQVFY & HIV-1 infection & human (A11)
\end{tabular}

- HLA-A11 CRF01 (called subtype E in Bond et al.) epitopes were identified that stimulated CTL from HIV+ female sex workers (FSW) from Northern Thailand, of whom more than half were HLA-A11 positive

- 77 possible HLA-A11 epitopes were first defined using EpiMatrix, these were screened for binding to A11 finding and 26 bound, and 12 of these were epitopes for CTL responses from 8 HLA-A11 positive FSWs, six were novel, six were previously identified

- This is one of the new A11 epitopes identified through the streamlined EpiMatrix method, and 2/8 tested FSWs recognized it

- This epitope was not conserved in other subtypes, and exact matches were rare

\begin{tabular}{|c|c|}
\hline gp160 (310-318) & $\begin{array}{l}\text { - Epitope name: Env-HY9 } \\
\text { - This study monitored epitope responses in HIV-1 infected minority women living in the United States } \\
\text { - } 24 \text { epitopes were described }-8 \text { were novel, } 8 \text { used new restricting elements but were previously defined epitopes, and } 8 \text { were previously described } \\
\text { - Serial peptide truncations were used to define optimal epitopes for CTL cell lines isolated from } 12 \text { individuals, assayed by a Cr-release } \\
\text { - This epitope was newly defined in this study } \\
\text { - Subject 00RCH33 was on HAART had a viral load of 2900 and CD4 count of 727 and also recognized the epitopes YPLTFGWCY, Nef(135-143), HLA } \\
\text { B*5301; AETFYVDGA, RT(437-445), HLA B*4501; and RSLYNTVATLY, p17(76-86), HLA A*3002 } \\
\text { - Among HIV+ individuals who carried HLA A30, 3/16 (19\%) recognized this epitope }\end{array}$ \\
\hline gp160 (310-318) & $\begin{array}{l}\text { - Epitope name: Env-HY9 } \\
\text { - Among HIV+ individuals who carried HLA A02, 6/29 (21\%) recognized this epitope }\end{array}$ \\
\hline gp160 (310-323) & $\begin{array}{l}\text { gp120 (315-328 MN) HIGP GRAFYTTKNI } \quad \text { Vaccine } \\
\text { Vaccine Vector/Type: canarypox prime with pseudovirion boost } \quad \text { Strain: MN, IIIB } \quad \text { HIV component: gp120, Gag, Pro } \\
\text { - Epitope name: } 97 \text { Arp1999 } \\
\text { - The vaccine vCP205, canarypox vector, MN gp120 + Gag/Pro IIIB, with a HIV-1 pseudovirion boost was given to mice; }) \\
\text { - HIV-1 pseudovirion boost enhanced the CTL to this epitope in immunized BALB/ c mice as measured by CTL lysis and IFN gamma production }\end{array}$ \\
\hline gp160 (311-318) & $\begin{array}{lccc}\text { (MN) } & \text { IGPGRAFY } & \text { Vaccine } & \text { murine }\left(\mathrm{H}_{-}-2 \mathrm{D}^{d}\right) \\
\text { Vaccine Vector/Type: } \text { B. abortus-peptide conjugate } & \text { Strain: } \mathrm{MN} \text { HIV component: V3 } & \\
\text { - Intranasal immunization of B. abortus conjugated to V3 peptides induces mucosal IFN-gamma producing T-cell responses in BALB/c mice }\end{array}$ \\
\hline gp160 (311-319) & $\begin{array}{l}\text { gp120 (311-320 IIIB) RGPGRAFVT } \quad \text { Vaccine } \\
\text { Vaccine Vector/Type: DNA, DNA with recombinant protein boost Strain: IIIB HIV component: gp160 Adjuvant: IL-12 (IL-12p35 and IL-12p40) } \\
\text { - Transgenic mice expressing a HLA-A2/Kb chimeric protein were vaccinated with a full length gp160 or with gp160deltaV3, with the V3 loop deleted. } \\
\text { Mice given gp160deltaV3 had a broader immune response than those given gp160, with increased responses to conserved HLA-A2 epitopes in the C1 } \\
\text { region of gp120, KLTPLCVTL, and the C-term region of gp41, SLLNATAIAV. } \\
\text { - Greater resistance was conferred by the gp160deltaV3 than the gp160 vaccine to a challenge of vaccinia expressing heterologous gp160 from primary } \\
\text { isolates (VI-06 and } 89.6 \text { ), and the resistance was conferred by CD8+ T-cells. } \\
\text { - The most intense CTL responses to the intact gp160 vaccine were directed at three V3 peptides. }\end{array}$ \\
\hline-319$)$ & $\begin{array}{lcc}\text { gp120 (312-320 SF2) } & \text { IGPGRAFHT } & \text { Vaccine } \\
\text { Vaccine Vector/Type: DNA } & \text { Strain: SF2 } & \text { HIV component: gp120 }\end{array}$ \\
\hline
\end{tabular}




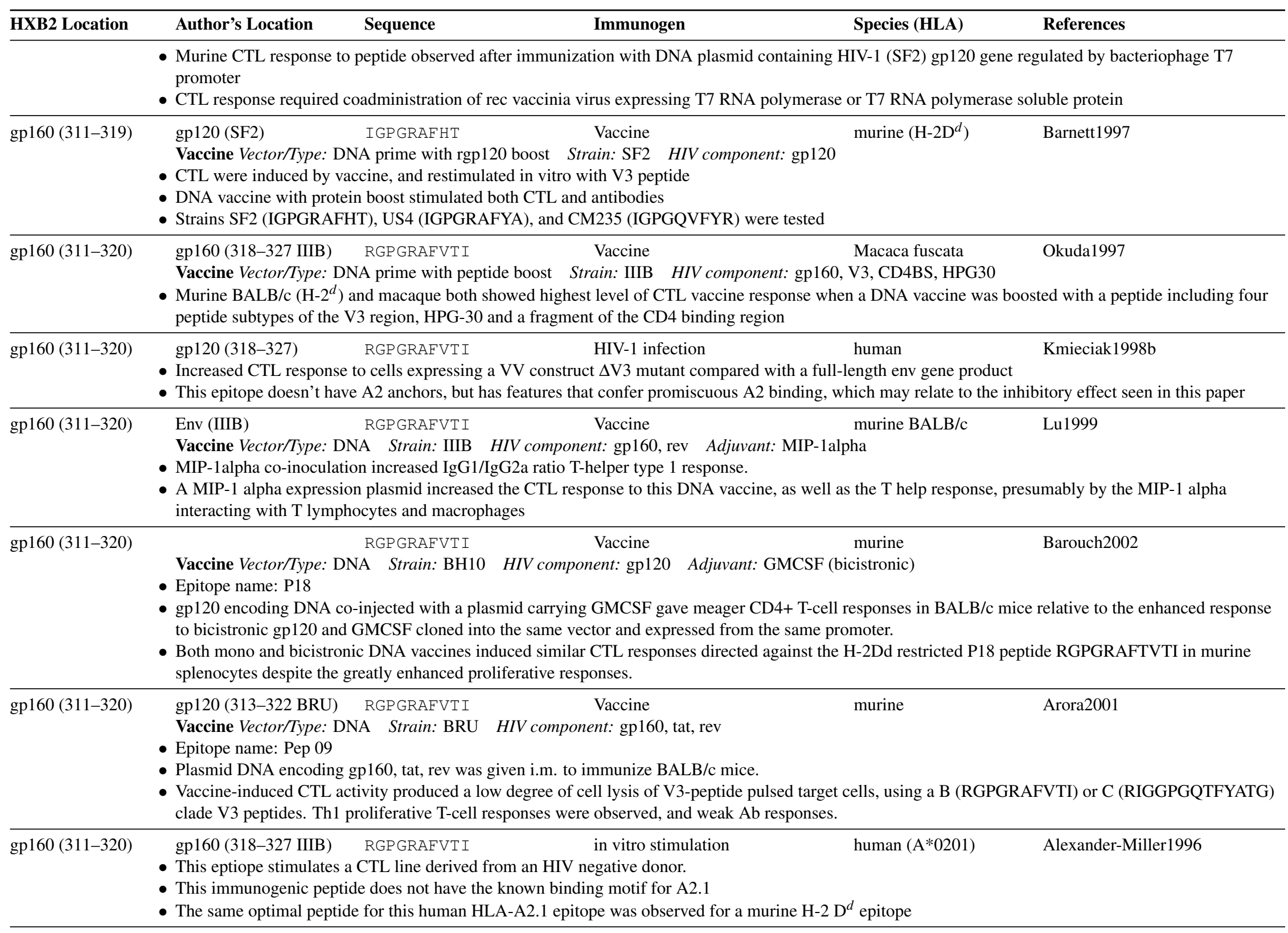




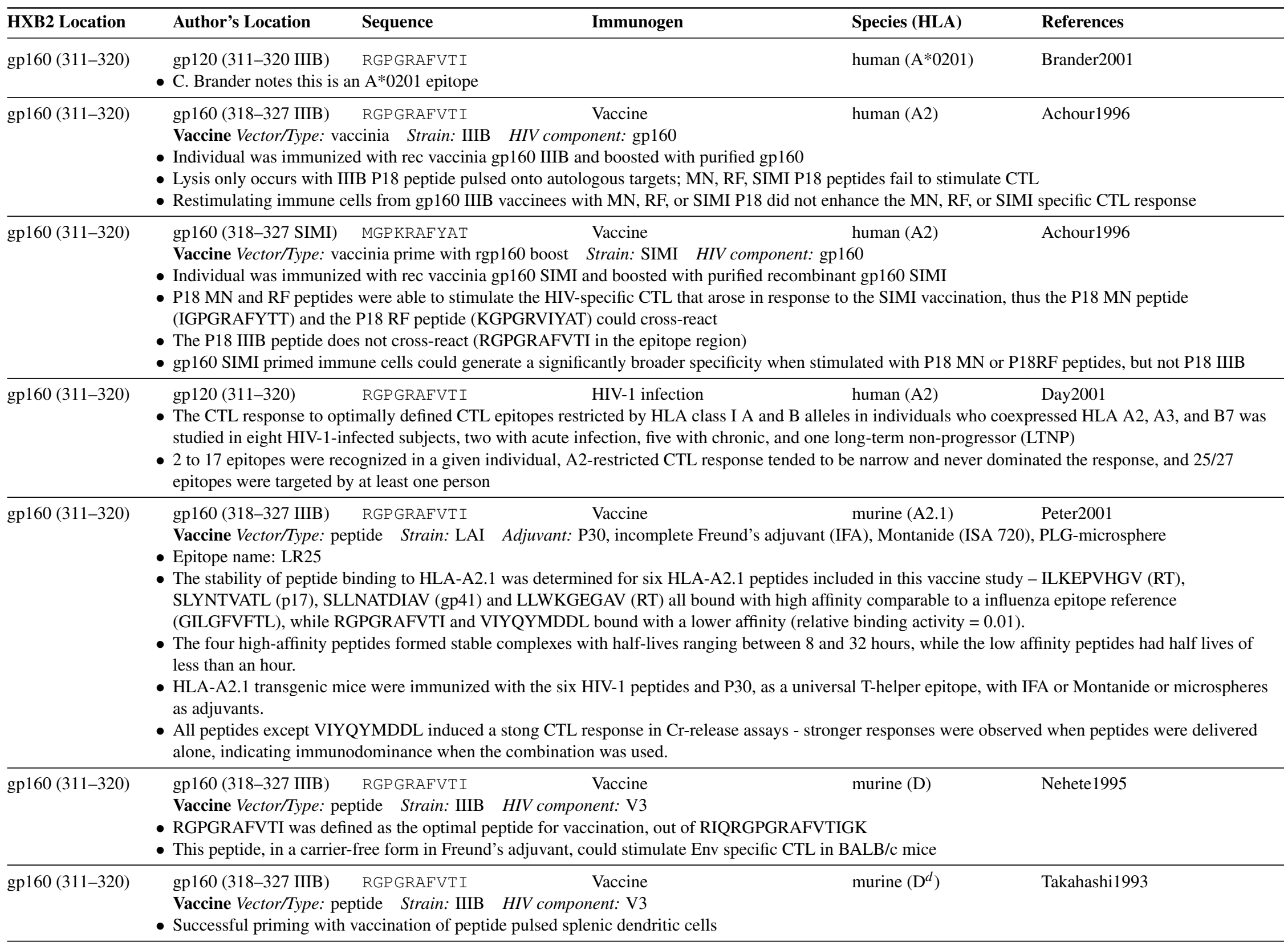




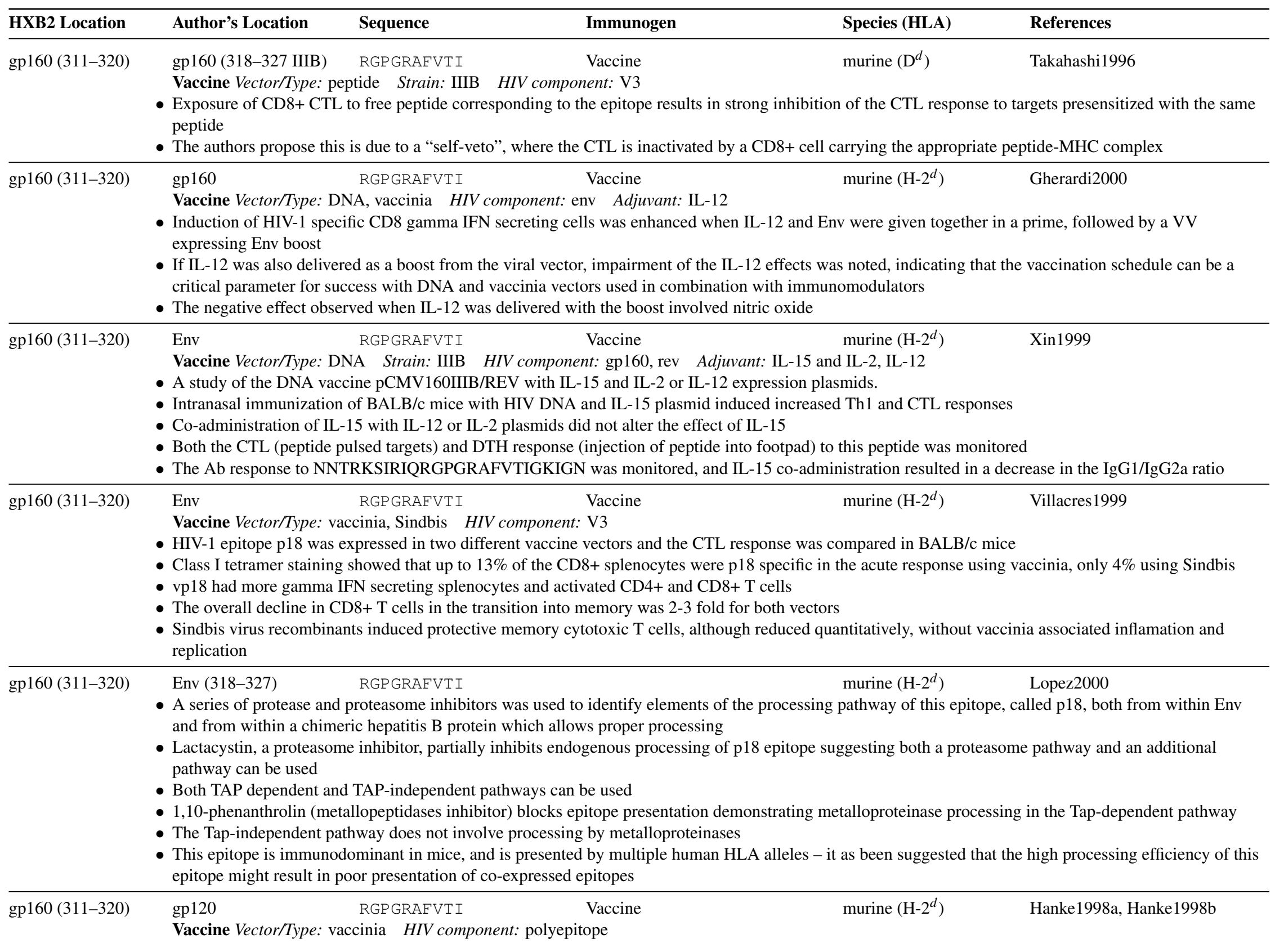




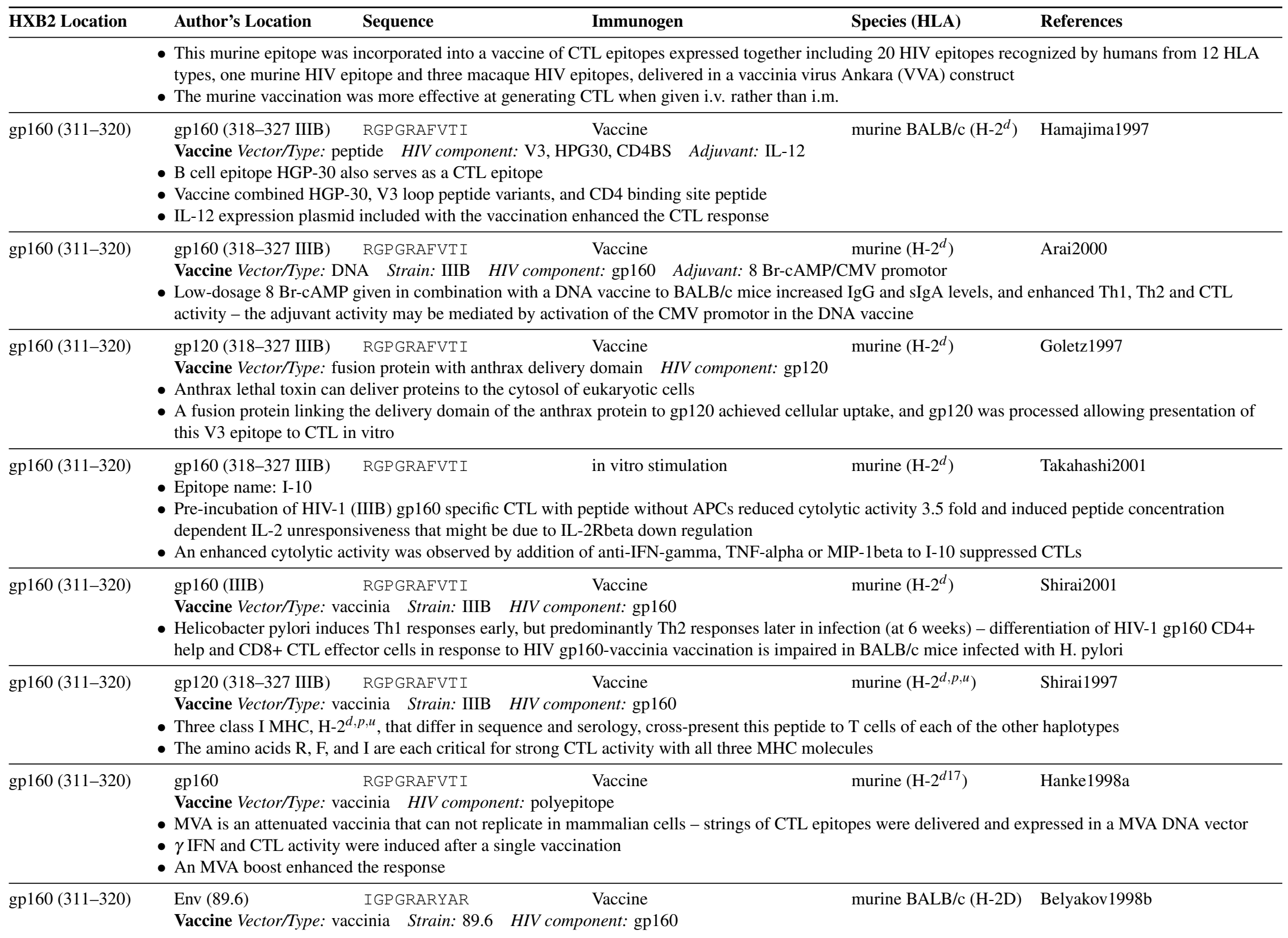




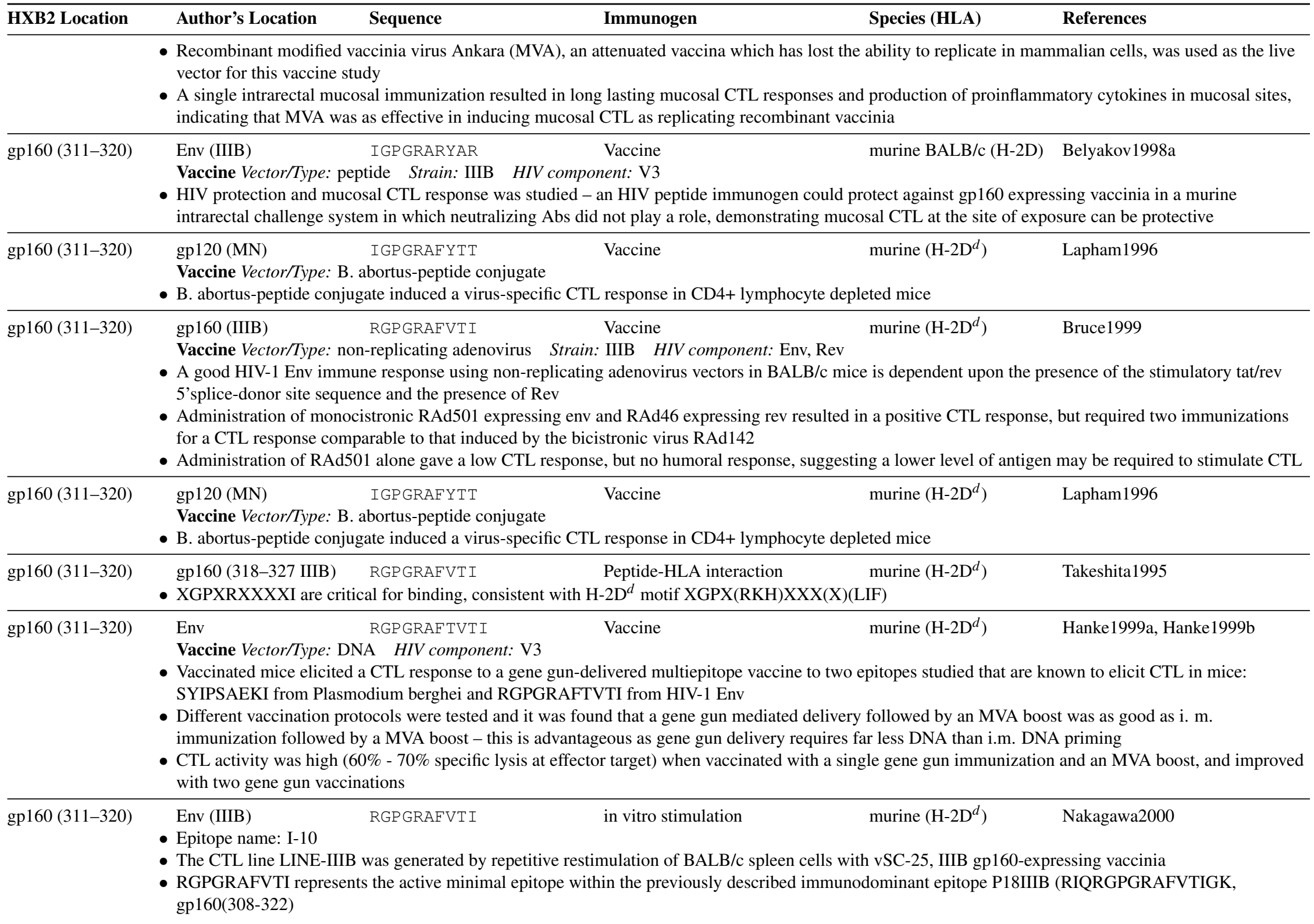




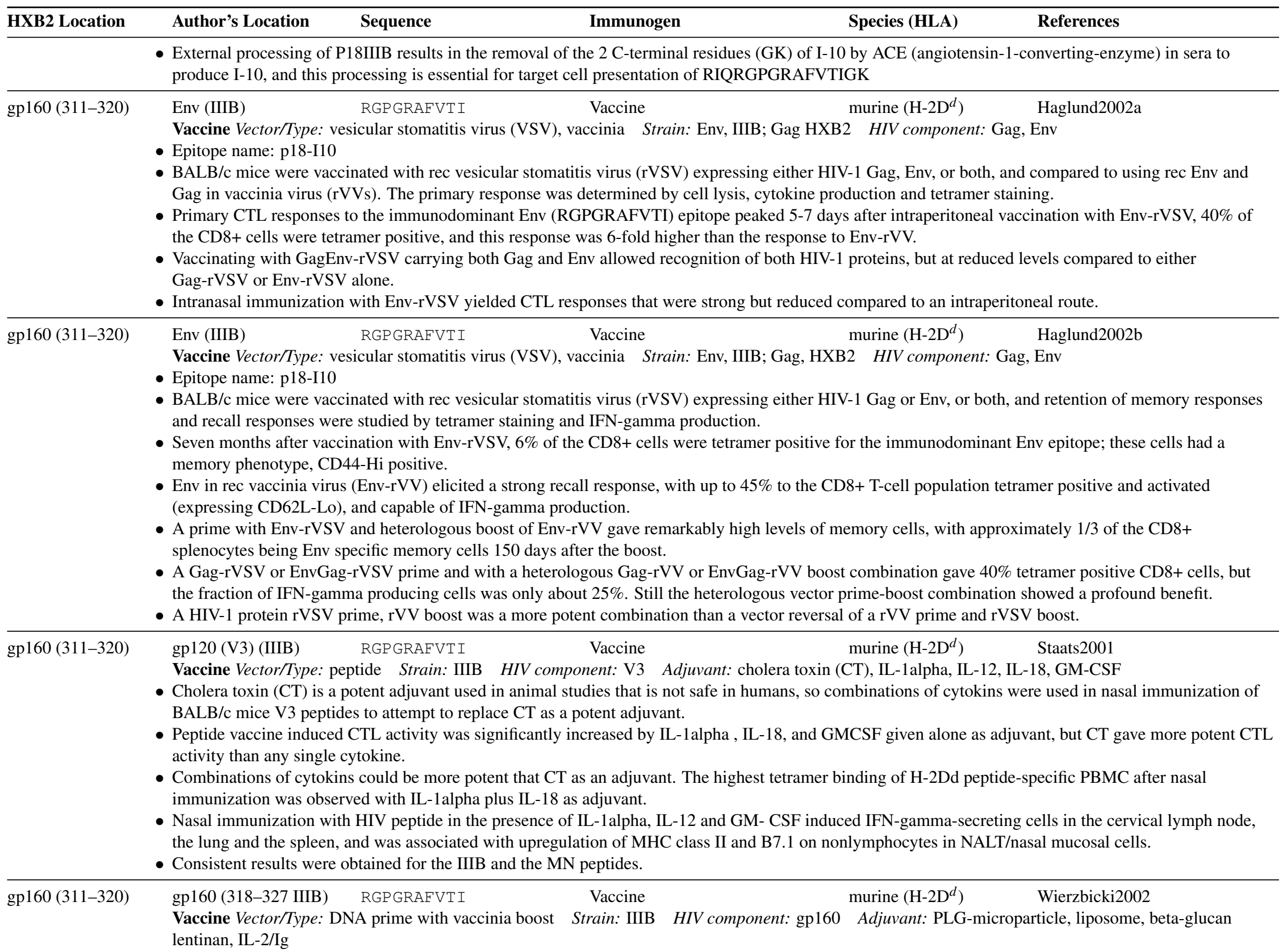




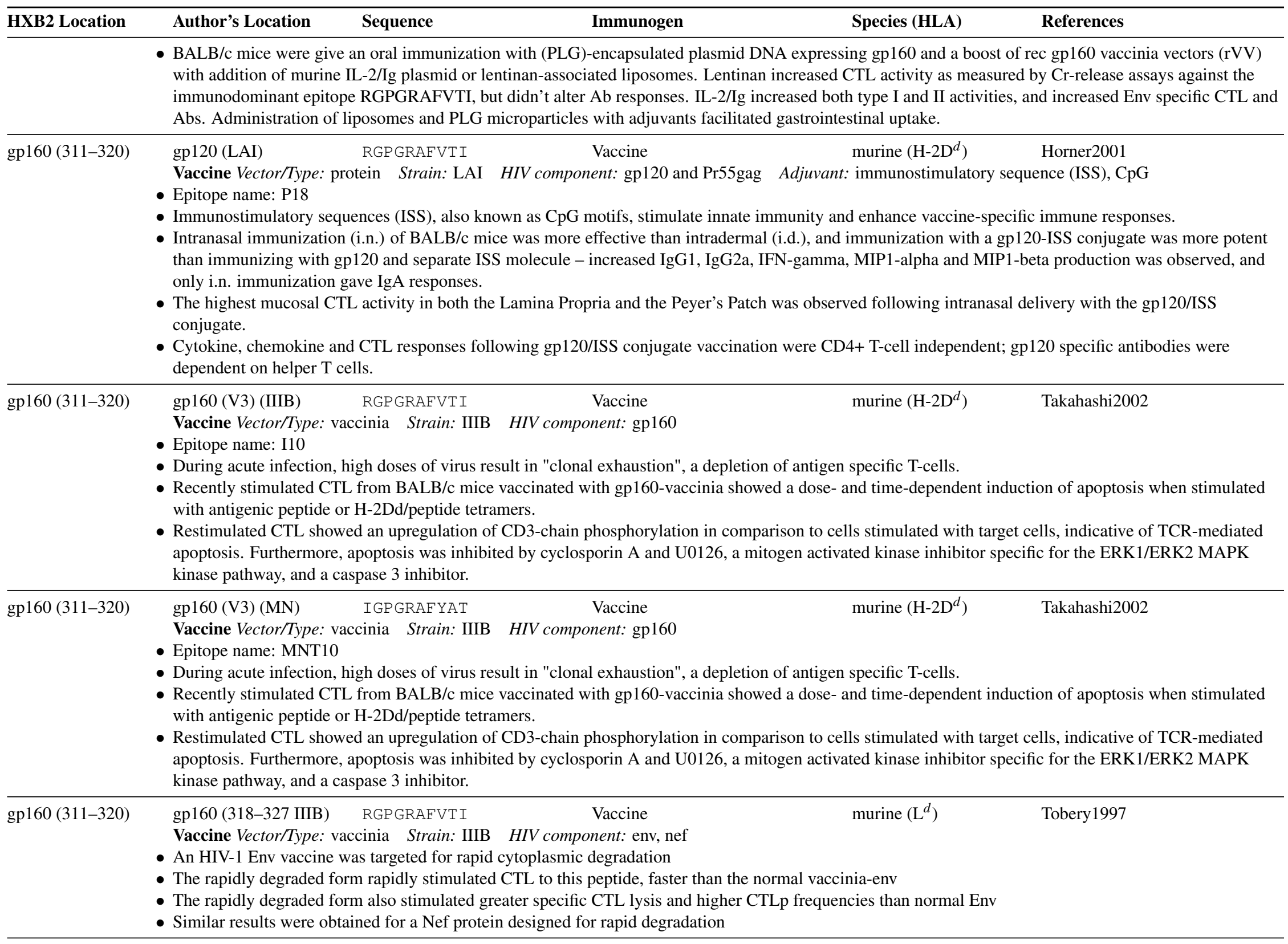




\begin{tabular}{llllc}
\hline HXB2 Location & Author's Location & Sequence & Immunogen & Species (HLA) \\
\hline gp160 (312-320) & $\begin{array}{l}\text { gp120 (V3 loop) (IIIB) } \\
\text { Vaccine Vector/Type: fowlpoxvirus }\end{array}$ & Strain: LR150, IIIB, JY1, RF, MN, BRVA & HIV component: concatonated 15 mer sections of six V3 loops & murine (H-2 ${ }^{d}$ )
\end{tabular}

- BALB/c mice were vaccinated with a polyepitope V3 vaccine in a fowlpoxvirus carrying concatonated 15 mer sections of the V3 loops of HIV-1 isolates

LR150, JY1, RF , MN, BRVA and IIIB with 5-aa linkers between, fused to the N-term of p64K protein from Neisseria meningitidis.

- Intraperitoneal immunization elicited the strongest V3-specific IFN-gamma response in splenocytes, compared to intravenous and subcutaneous immunization. Intraperitoneal immunization conferred protection in a recombinant vaccinia virus challenge model.

- The immunodominant response was directed against the IIIB peptide (the IIIB immunizing peptide was SIRIQRGPGRAFVTI, the peptide used to probe the response by Elispot was GPGRAFVTI).

- Low CTL responses were also detected to the LR150 (SRGIRIGPGRAILAT) and RF (RKRITMGPGRVYYTT) peptides, no responses were detected to the JY1 (RQSTPIGLGQALYTT), BRVA (RKSITKGPGRVIYAT), or MN (RKRIHIGPGRAFYTT) peptides.

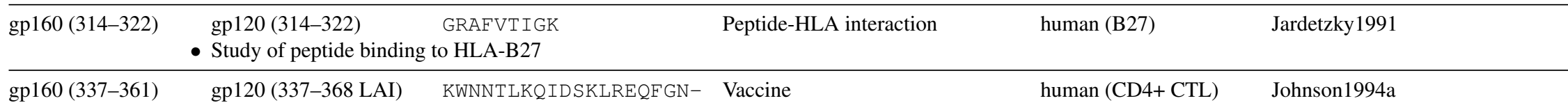
NKTIIF

Vaccine Vector/Type: vaccinia HIV component: gp160

- CD4+ CTL clones were obtained from an HIV-1 vaccinia-env vaccinee

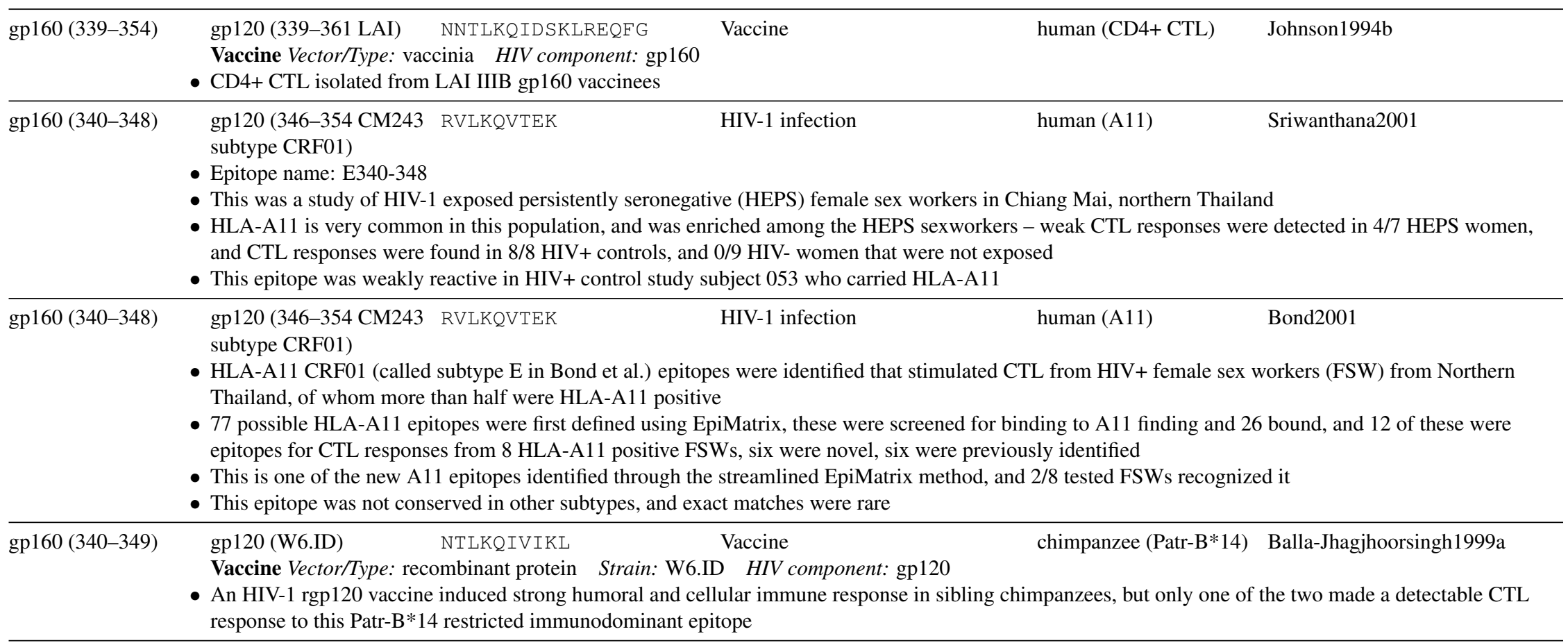




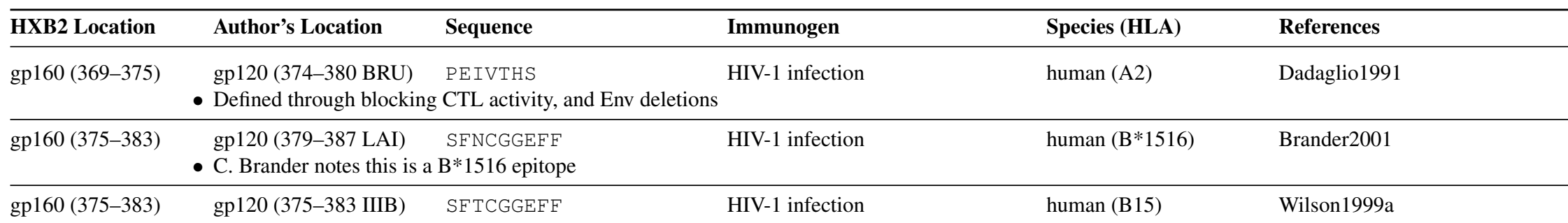

- This study describes maternal CTL responses in the context of mother-to-infant transmission

- Detection of CTL escape mutants in the mother was associated with transmission, but the CTL-susceptible forms of the virus tended to be found in infected infants

- An additional variant that gave a positive, though reduced, CTL response: SSTCGGEFF and SFTCGGGFF

- SFTCGGGVF was an escape mutant

gp160 (375-383) gp120(375-383 SF2) SFNCGGEFF HIV-1 infection human (B15) Altfeld2001b

- Therapy provided during acute infection resulted in a narrower CTL response, stronger T help response, and a less diverse viral population than was seen in individuals treated during chronic infection

- The breadth and specificity of the response was determined using ELISPOT by studying 19 individuals with pre-seroconversion therapy (Group 1 ), 11 individuals with primary infection but post-seroconversion therapy (Group 2), and 10 individuals who responded to HAART given during chronic infection (Group 3), using 259 overlapping peptides spanning p17, p24, RT, gp41, gp120 and Nef

- Previously described and newly defined optimal epitopes were tested for CTL response

- Number of HLA-B15+ individuals that had a CTL response to this epitope broken down by group: 0/0 group 1, 1/1 group 2, and 0/1 group 3

gp160 (375-383) gp120(375-383 IIIB) SFNCGGEFF HIV-1 infection human (B63, B15) Wilson1997a

- This is the optimal peptide for two CTL clones that recognize this epitope in the context of two different HLA molecules, Cw4 and B15

- Predominant form in proviral DNA of the individual with B15 restricted CTL was SFTCGGEFF and this was recognized

- Recognition of a minor autologous variant (SFNCRGEFF) from the B15 donor was greatly reduced

\begin{tabular}{|c|c|c|c|c|c|}
\hline gp160 (375-383) & $\begin{array}{l}\text { gp120 (376-383 PV22) } \\
\text { C. Brander notes this is a C }\end{array}$ & $\begin{array}{l}\text { SFNCGGEFF } \\
\text { C*0401 epitope }\end{array}$ & HIV-1 infection & human $(C * 0401)$ & Brander200 \\
\hline gp160 (375-383) & gp120 & SFNCGGEFF & HIV-1 infection & $\begin{array}{l}\text { human }\left(\mathrm{Cw}^{*} 0401,\right. \\
\mathrm{Cw} * 0407)\end{array}$ & Bird2002 \\
\hline
\end{tabular}

- 4/123 (2 HIV-1 positive, 2 HEPS) Kenyan female sex workers carried the novel allele HLA Cw*0407.

- HLA Cw*0407 did not differ from $\mathrm{Cw}^{*} 0401$ in the region associated with the binding pocket, and $\mathrm{Cw}^{*} 0407$ was shown to cross-present a previously defined $\mathrm{Cw}^{*} 0401$ epitope, SFNCGGEFF (gp120).

\begin{tabular}{llllll}
\hline gp160 (375-383) & $\begin{array}{l}\text { gp120 (376-383 PV22) } \\
\text { - Conserved epitope }\end{array}$ & SFNCGGEFF & HIV-1 infection & human (Cw4) \\
\hline gp160 (375-383) & $\begin{array}{lllll}\text { gp120 (376-383 PV22) } \\
\end{array}$ & $\begin{array}{l}\text { SFNCGGEFF } \\
\text { Longitudinal study of epitope variation in vivo }\end{array}$ & HIV-1 infection & human (Cw4) \\
\hline gp160 (375-383) & gp120 (376-383) & SFNCGGEFF & $\begin{array}{l}\text { HIV-1 infection, HIV-1 exposed } \\
\text { seronegative }\end{array}$ & human (Cw4) & Wolinsky1996 \\
\hline
\end{tabular}

- ELISPOT was used to study CTL responses to a panel of 54 predefined HIV-1 epitopes in 91 HIV-1-exposed, persistently seronegative (HEPS) and 87 HIV-1-infected female Nairobi sex workers 


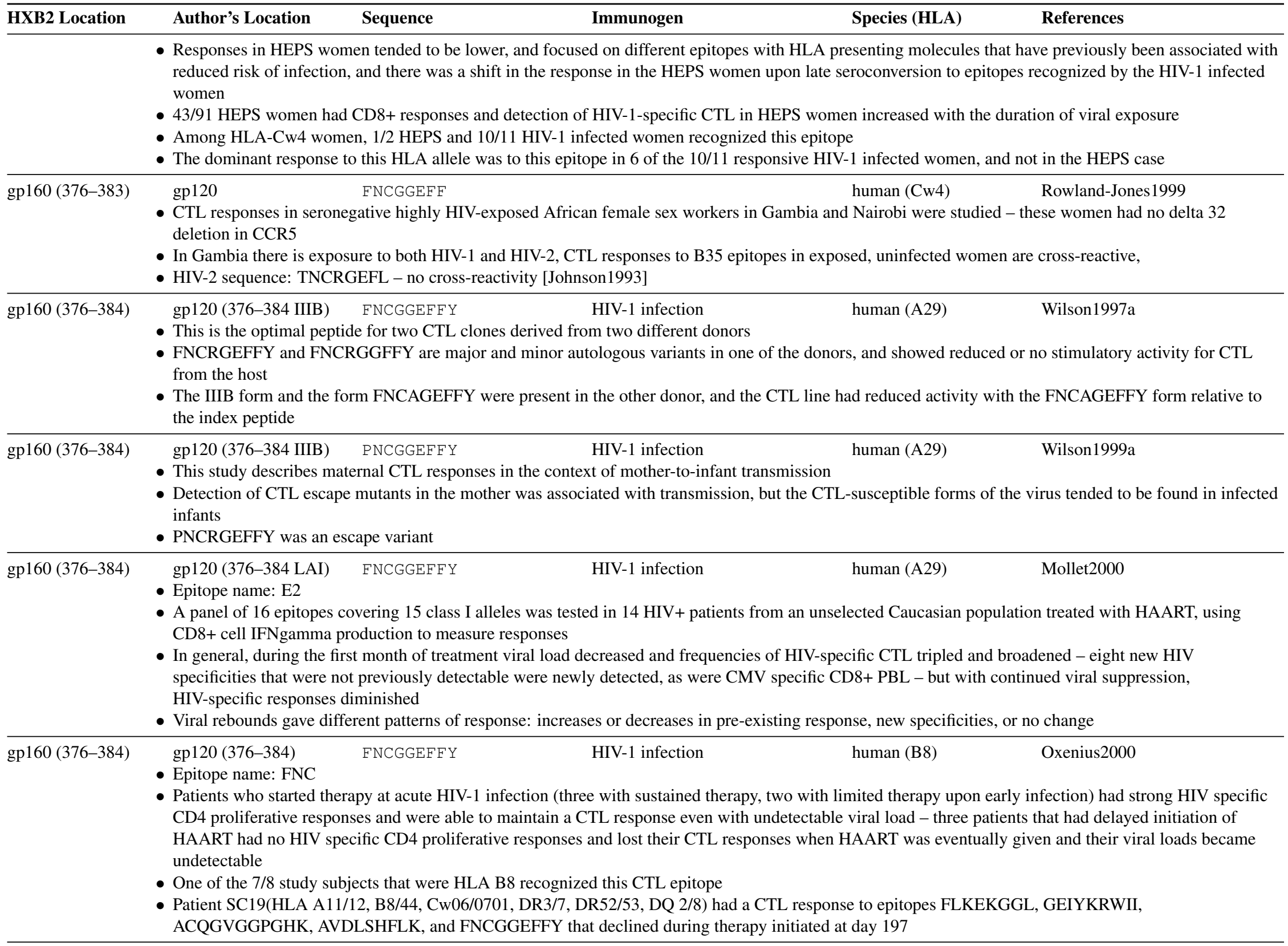




\begin{tabular}{lllll}
\hline HXB2 Location & Author's Location & Sequence & Immunogen & Species (HLA) \\
\hline gp160 (376-384) & gp160 & FNCGGEFFY & HIV-1 infection & human (B8)
\end{tabular}

- Using previously defined epitopes [Oxenius2000 Oxenius2001a] in an IFNgamma Elispot assay, 13 chronically HIV-1 infected patients were studied over a period including therapy with standard treatment interruptions (STI).

- STIs induced increased recognition of CTL epitopes, but there was no correlation between CTL responses with viral rebound rates, plateau viral loads, or clearance rates.

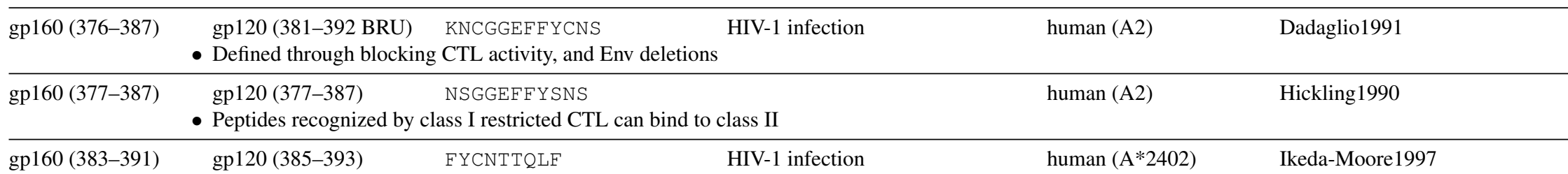

- Defined using reverse immunogenetics - 59 HLA-A $* 2402$ binding peptides were predicted by searching for A*2402 anchors in HIV proteins (Tyr at 2 , and Phe, Leu or Ile at the $\mathrm{C}$ term) -53 of the 59 peptides bound $\mathrm{A} * 2402$

- This peptide induced CTL in 1/4 HIV-1+ people tested

- FYCNTTQLF bound to A*2402 strongly, the epitope can be processed in a vaccinia construct and presented - two specific CTL clones were obtained

gp160 (410-429) gp120(410-429 PV22) GSDTITLPCRIKQF INMWQE in vitro stimulation human (CD4+DRA) Bouhdoud2000

- CTL were studied through PBMC stimulation in vitro by gp120 pulsed autologous monocytes.

- Human CD4+ CTL clone (Een217) is an MHC class II HLA-DRA restricted CTL clone that can lyse antigen presenting HLA-DRA-transfected murine L

cells - natural variants of the epitope resulted in an anergic response

- Low concentrations of the HXB2-derived variant (GSDTITLPCRIKQIINMWQK) induced T cell anergy - higher concentrations could induce proliferation and cytotoxic activity

- CDC42 (TGDIITLPCRIKQII-NRWQV), Eli (TNTNITLQCRIKQIIKMVAG) and Z3 (CTGNITLPCRIKQIIMNWQE) variants did not induce proliferation, cytotoxic or anergic responses

gp160 (416-424) Env (413-421 SF2) LPCRIKQII HIV-1 infection $\quad$ Tomiyama1999

- HLA-B27, -B51, and -B57 are associated with slow progression to AIDS, while HLA-B35, -B8, -B24 are associated with a rapid progression to AIDS (Nat. Med. 2:405, 1996;Lancet 22:1187, 1986;Hum Immunol 22:73, 1988;Hum Immunol 44:156, 1995)

- $15 \%$ of Japanese populations carry HLA-B51 while HLA-B27 and -B57 are detected in less than 0.3\%

- Of the 172 HIV-1 peptides with HLA-B*5101 anchor residues, 33 bound to HLA-B*5101, seven of these peptides were reactive with CTL from 3 B*5101 positive individuals, and six were properly processed

- Four of the six epitopes were highly conserved among B subtype sequences, LPCRIKQII is not conserved

\begin{tabular}{|c|c|c|c|c|}
\hline gp160 (416-424) & $\begin{array}{l}\text { gp160 (416-424 LAI) LPCRIKQI I } \\
\text { - C. Brander notes this is a B*5101 epitope }\end{array}$ & & human $(\mathrm{B} * 5101)$ & Brander2001 \\
\hline gp160 (416-424) & gp120 (378-385) & $\begin{array}{l}\text { HIV-1 infection, HIV-1 exposed } \\
\text { seronegative }\end{array}$ & human (B51) & Kaul2001a \\
\hline
\end{tabular}

- ELISPOT was used to study CTL responses to a panel of 54 predefined HIV-1 epitopes in 91 HIV-1-exposed, persistently seronegative (HEPS) and 87 HIV-1-infected female Nairobi sex workers 


\begin{tabular}{|c|c|c|c|c|c|}
\hline HXB2 Location & Author's Location & Sequence & Immunogen & Species (HLA) & References \\
\hline gp160 (416-429) & $\begin{array}{l}\text { gp120 (410-429 } \\
\text { H3DCG) } \\
\text { - CD4+ CTL restricted by }\end{array}$ & $\begin{array}{l}\text { LPCRIKQF INMWQE } \\
\text { class II HLA-DR4, targets prime }\end{array}$ & $\begin{array}{l}\text { HIV-1 infection } \\
\text { d by CD4 mediate }\end{array}$ & human (DR4 CD4+) & Siliciano1988 \\
\hline gp160 (416-435) & $\begin{array}{l}\text { gp120 (421-440 LAI) } \\
\text { - Defined through blocking }\end{array}$ & $\begin{array}{l}\text { LPCRIKQF INMWQEVGKAMY } \\
\text { CTL activity, and Env deletions }\end{array}$ & HIV-1 infection & human (A2) & Dadaglio1991 \\
\hline gp160 (419-427) & $\begin{array}{l}\text { gp120 (424-432 HXB2) } \\
\text { - C. Brander notes that this }\end{array}$ & $\begin{array}{l}\text { RIKQIINMW } \\
\text { is an A*3201 epitope in the } 199\end{array}$ & 9 database & human $(A * 3201)$ & Harrer1996b \\
\hline gp160 (419-427) & $\begin{array}{l}\text { gp120 (419-427 HXB2) } \\
\text { - C. Brander notes this is at }\end{array}$ & $\begin{array}{l}\text { RIKQI INMW } \\
\text { n A*3201 epitope }\end{array}$ & & human $(\mathrm{A} * 3201)$ & Brander2001 \\
\hline gp160 (419-427) & $\begin{array}{l}\text { gp120 (419-427) } \\
\text { - Only 4/11 HLA-A2+ HIV } \\
\text { - } 95 \text { optimally-defined pep } \\
\text { - } 1 / 11 \text { of the A2+ individua } \\
\text { - The sequence is unclear }\end{array}$ & $\begin{array}{l}\text { RIKQIINMW? } \\
\text { + individuals had CTL that react } \\
\text { tides from this database were use } \\
\text { als was A } 29 \text { and responded to RI } \\
\text { - Betts calls both peptide } 30 \text { and }\end{array}$ & $\begin{array}{l}\text { HIV-1 infection } \\
\text { ted to SLYNTVA' } \\
\text { ed to screen for IN } \\
\text { KQIINMW, and a } \\
\text { peptide } 32 \text { gp120 }\end{array}$ & $\begin{array}{l}\text { human (A29, A32) } \\
\text { estion whether it is imn } \\
\text { her epitopes } \\
\text { vas A32 and these are t } \\
\text { eptide sequences are n }\end{array}$ & $\begin{array}{l}\text { Betts2000 } \\
\text { odominant } \\
\text { ght to be presenting molecules } \\
\text { rovided }\end{array}$ \\
\hline gp160 (419-427) & $\begin{array}{l}\text { gp120 (424-432 LAI) } \\
\text { - Autologous virus was use } \\
\text { - The autologous epitope st } \\
\text { clones }\end{array}$ & $\begin{array}{l}\text { RIKQF INMW } \\
\text { d to detect CTL in two individua } \\
\text { equence was RIKQIINMW, MN }\end{array}$ & $\begin{array}{l}\text { HIV-1 infection } \\
\text { als, and in both ca } \\
\text { and RF were KIK }\end{array}$ & $\begin{array}{l}\text { human (A32) } \\
\text { autologous CTL were } \\
\text { KQFVNMW respecti }\end{array}$ & $\begin{array}{l}\text { Ray1998 } \\
\text { and all were reactive with CTL }\end{array}$ \\
\hline gp160 (419-427) & $\begin{array}{l}\text { gp120 (420-428) } \\
\text { - This epitope is processed }\end{array}$ & $\begin{array}{l}\text { RIKQIINMW } \\
\text { by a TAP } 1 / 2 \text { dependent mechani }\end{array}$ & $\begin{array}{l}\text { HIV-1 infection } \\
\text { ism }\end{array}$ & human (A32) & Ferris1999 \\
\hline gp160 (419-427) & gp120 & RIKQIINMW & HIV-1 infection & human (A32) & Altfeld2002 \\
\hline
\end{tabular}

- Epitope name: A32-RW10(gp120)

- Peripheral blood (PB) and lymph node (LN) CD8+ T-cell responses were compared in 15 asymptomatic HIV-1 infected patients using all known optimal CTL epitopes (http://hiv-web.lanl.gov/content/hiv-db/REVIEWS/brander2001.html) for each person's class I HLA alleles.

- 60 epitope responses were detected in both PB and LN samples of the 15 patients, and an additional 8 responses were detected only in LN. The total magnitude of the response was similar in LN and PB, but the percentage of CD8+ T cells in the LN is lower so the number of HIV-specific cells per million CD8+ T-cells is higher in the LN.

- 1 year post-HAART treatment in five patients studied, the magnitude of the CD8 T-cell response was decreased in both LN and PB, but more dramatically in PB, and 13/25 epitope responses in the PB became undetectable, in contrast to 5/26 in the LN.

- Treatment interruption following HAART induced resulted in increased viremia accompanied by the restoration of the detection of 13 epitopes that had become undetectable in the PB, and the addition of 9 novel epitope responses.

- Breakdowns of epitope responses were shown for 4 individuals. Patient B displayed the greatest response to epitope B44-AW11(p24) and also responded to A32-PW10(RT) in both PB and LN samples, while a third response against epitope A32-RW10(gp120) was only detected in the LN sample. Patient D displayed the greatest response to B27-KK10 (p24), and also responded to A30-RY11(p17), A32-PW10(RT), A30-KY11(RT), A32-RW10(gp120), and B18-YY9(Nef).

gp160 (421-435) gp120 (421-440 LAI) KQF INMWQEVGKAMY HIV-1 infection human (A2) Dadaglio1991

- Defined through blocking CTL activity, and Env deletions 


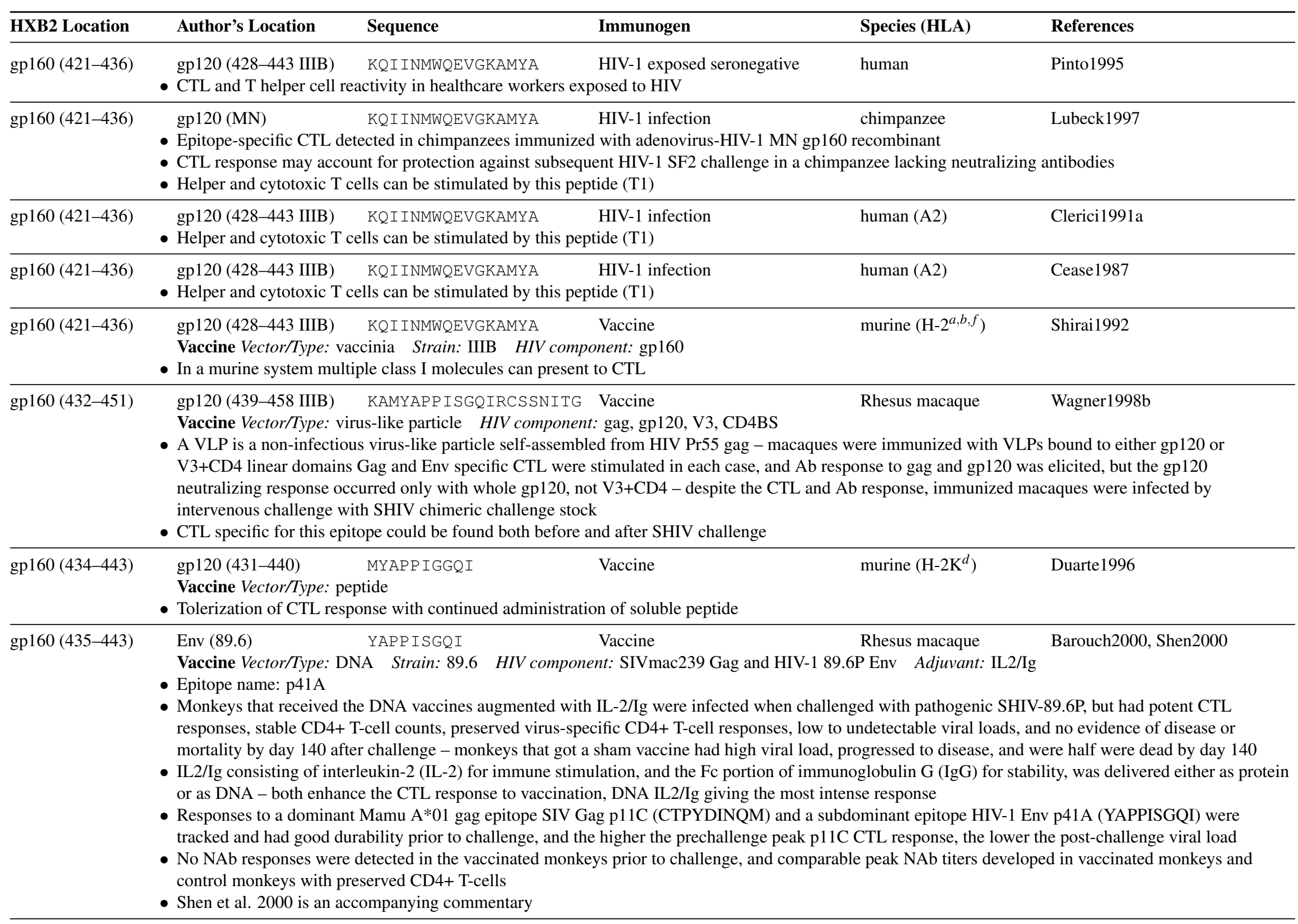




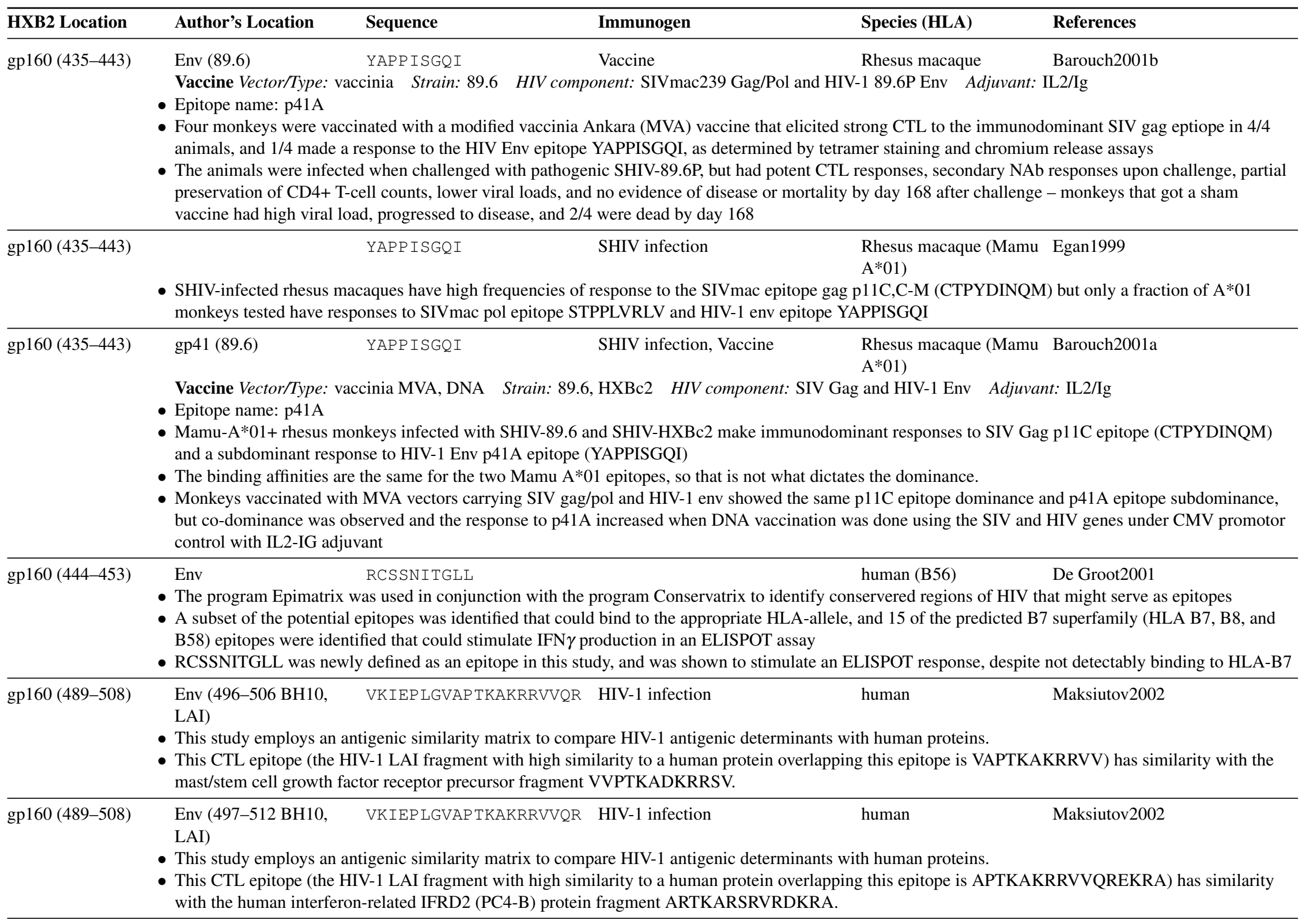




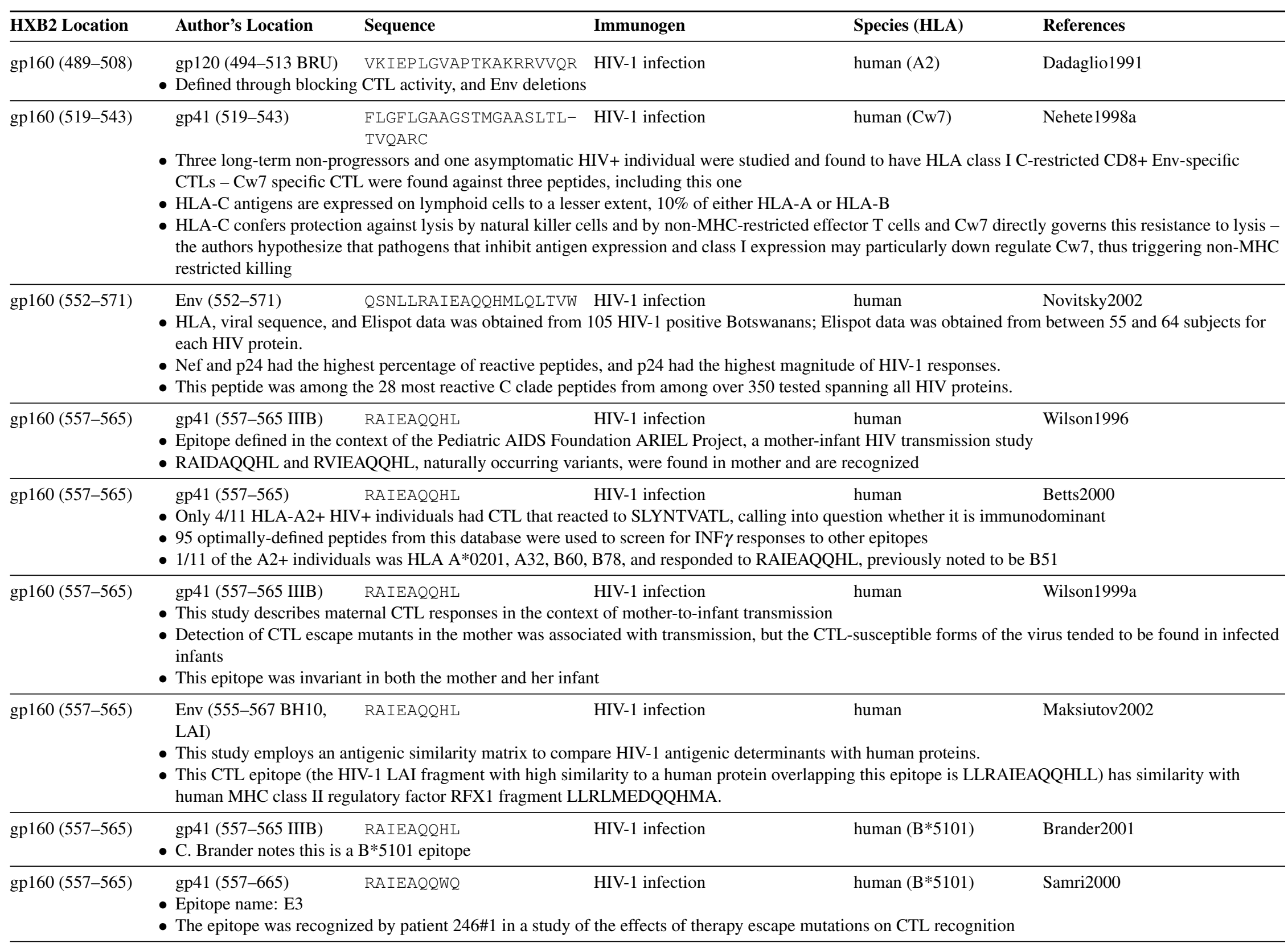




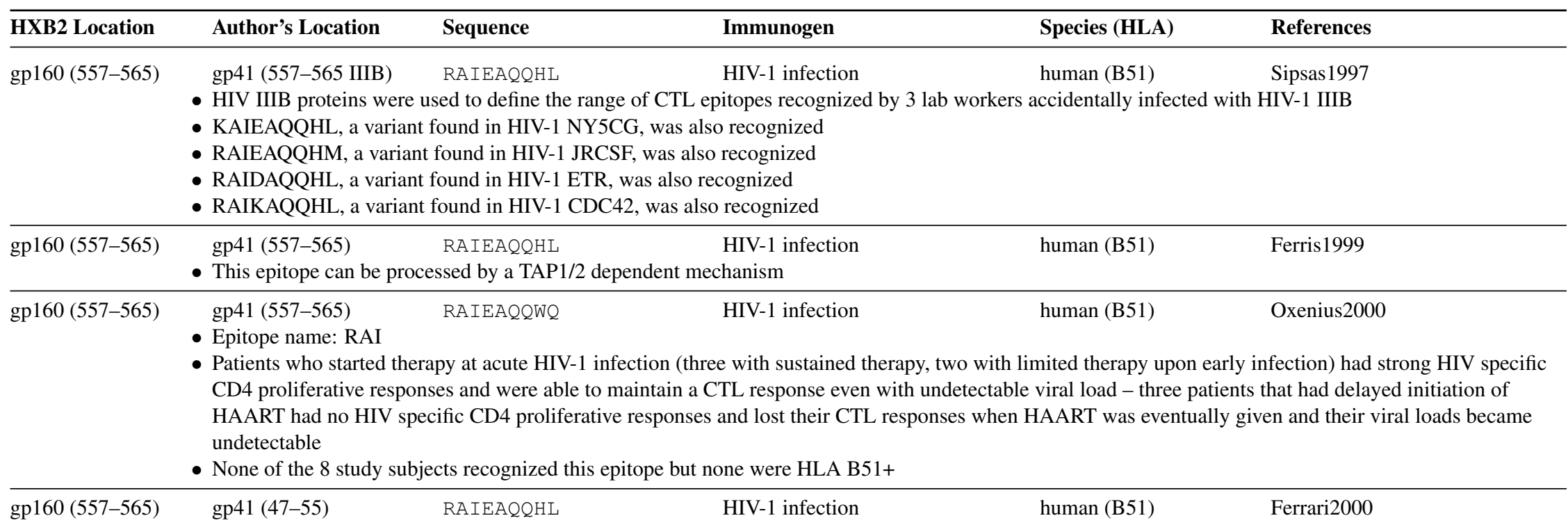

- One of the 51 HIV-1 epitopes selected by Ferrari et al. as good candidate CTL epitopes for vaccines by virtue of being conserved and presented by common HLA alleles

gp160 (557-565) gp41(557-565 LAI) RAIEAQQHL $\quad$ HIV-1 infection $\quad$ Mollet2000

- Epitope name: E3

- A panel of 16 epitopes covering 15 class I alleles was tested in $14 \mathrm{HIV}+$ patients from an unselected Caucasian population treated with HAART, using CD8+ cell IFNgamma production to measure responses

- In general, during the first month of treatment viral load decreased and frequencies of HIV-specific CTL tripled and broadened - eight new HIV specificities that were not previously detectable were newly detected, as were CMV specific CD8+ PBL - but with continued viral suppression, HIV-specific responses diminished

- Viral rebounds gave different patterns of response: increases or decreases in pre-existing response, new specificities, or no change

gp160 (557-565) Env (gp160) (557-565) RAIEAQQHL HIV-1 infection human (Cw*0304) Currier2002a

- Cross-reactive responses were found in PBMC isolated from individuals infected with either B or CRF01_AE clade viruses, as determined by Elispot assays of target cells expressing recombinant vaccinia viruses expressing HIV-1 gag, env, nef and pol from many clades.

- CTL from subject US101, infected with a clade B virus, displayed broad cross-reactivity to HIV-1 clade A, B, C, D, CRF01_AE, F G, recognized this epitope. Clade B and C had a L->M change in the $\mathrm{C}$-term position that was tolerated. The $\mathrm{H}$ clade Env was not cross-reactive, and had the sequence RAIAARQHM.

gp160 (565-573) Env (731-739) LLQLTVWGI $\quad$ HIV-1 infection $\quad$ human (A2 supertype) Propato2001

- Long-term nonprogressors (LTNPs) had strong memory resting CD8+ T-cell responses against the majority of epitopes tested, (18 for the A2 supertype, 16 for the A3 supertype) while the effector cells of long-term nonprogressors recognized far fewer epitopes

- Progressors had memory resting CD8+ T-cells that recognized far fewer epitopes than LTNPs

- A positive correlation between effector CD8+ T-cells and plasma viremia and a negative correlation between CD8+ effector T-cells and CD4+ T-cells was observed, which may contribute to the inability of LTNPs to clear virus 


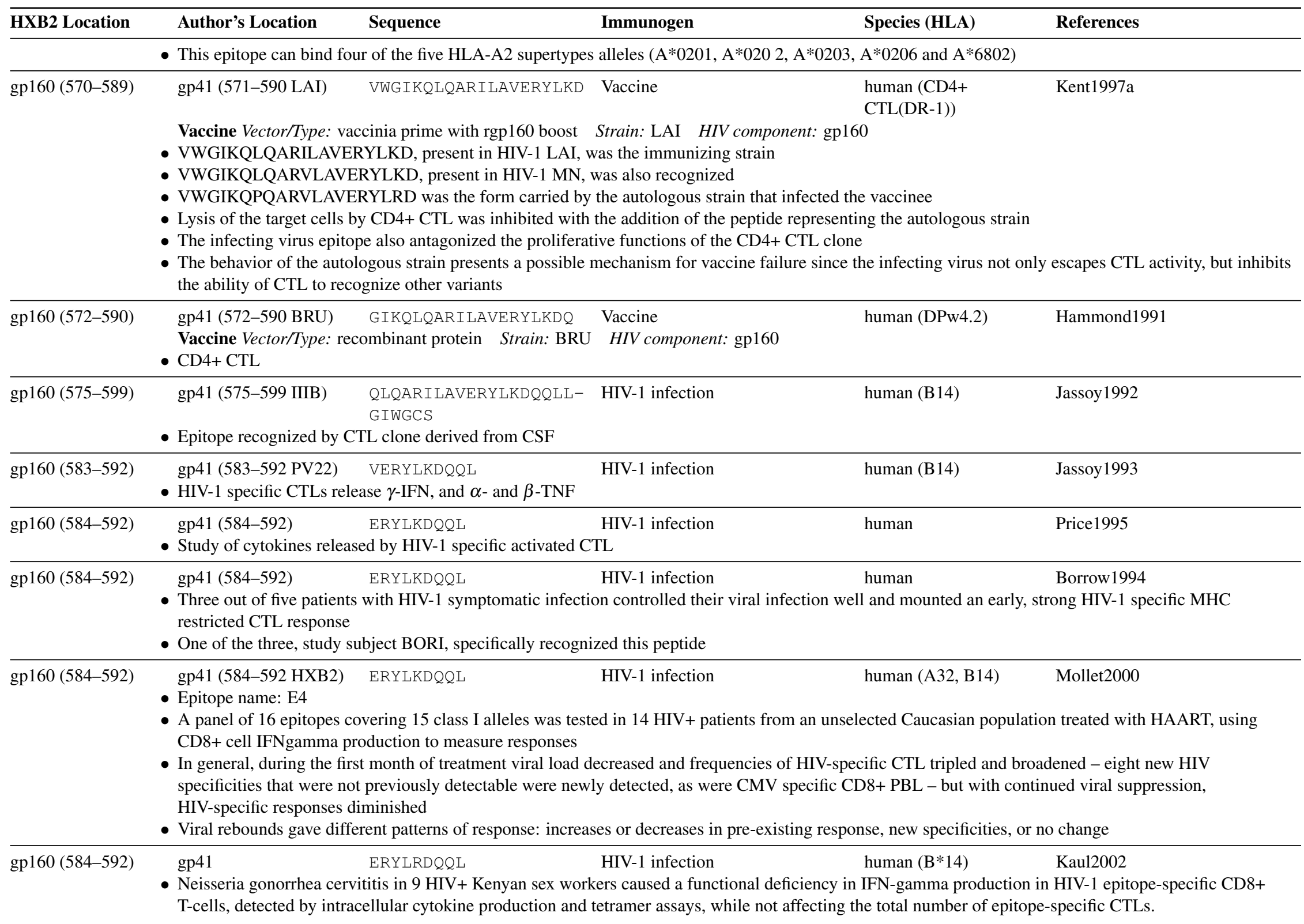




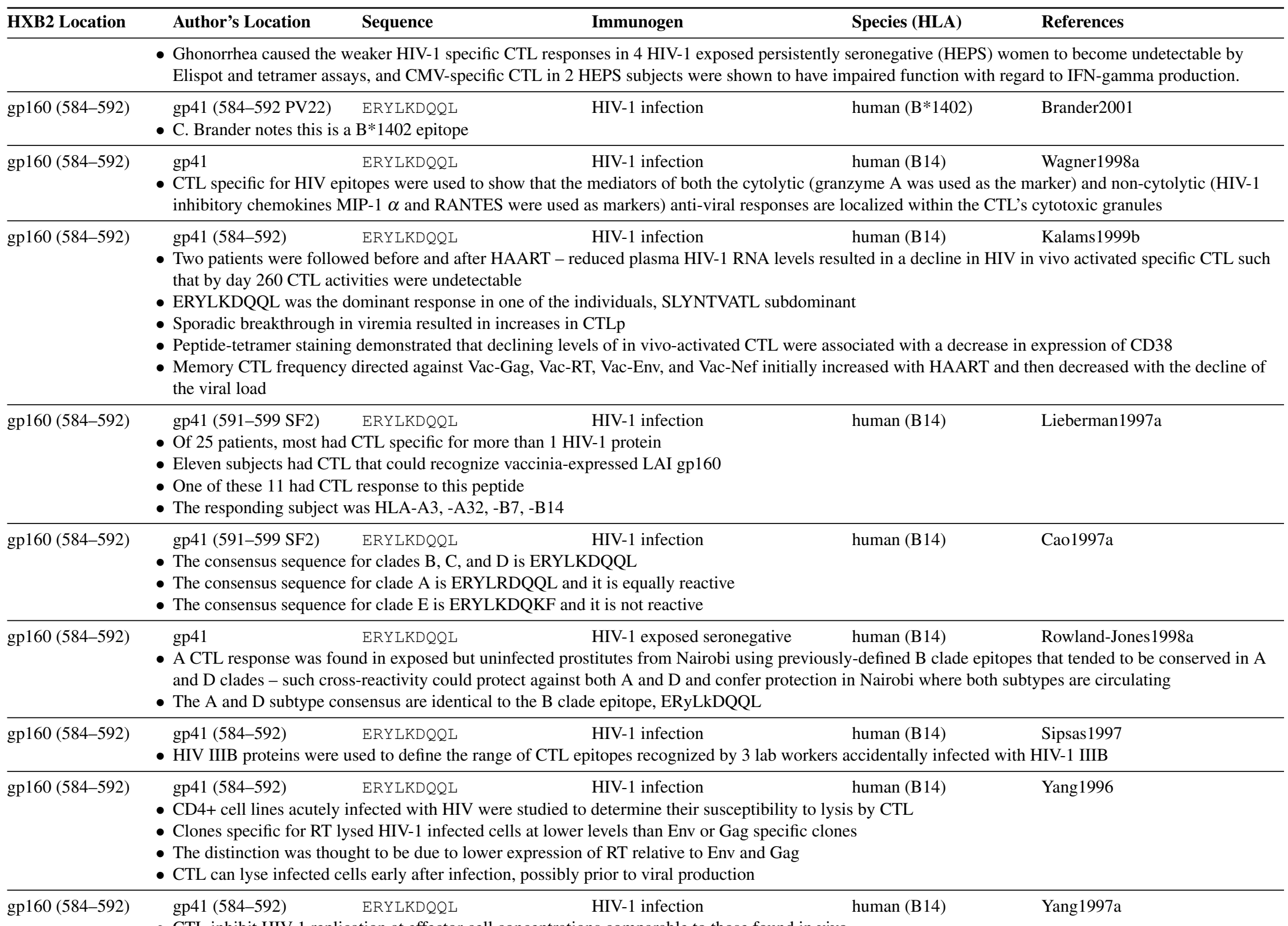




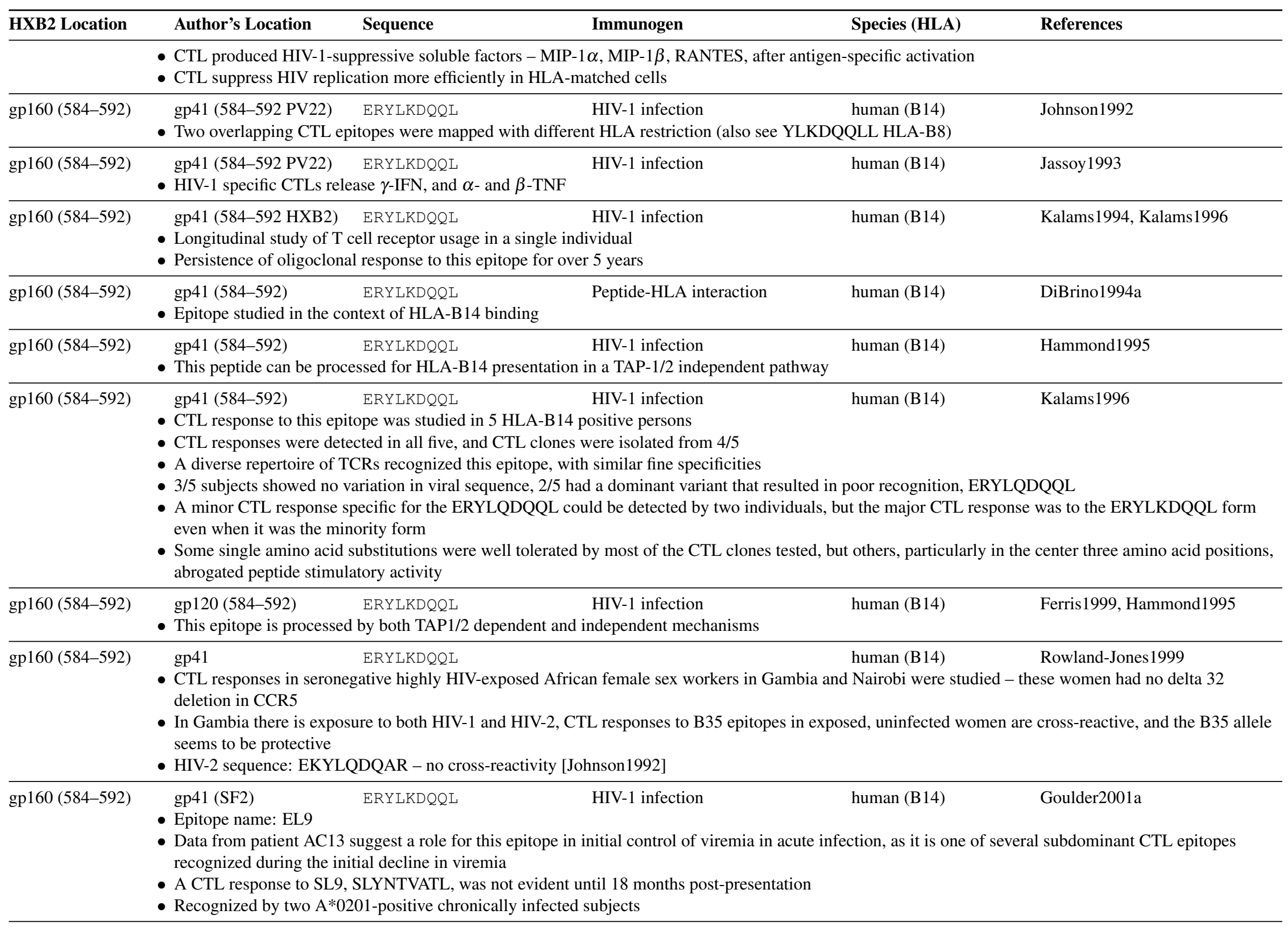




\begin{tabular}{lllll}
\hline HXB2 Location & Author's Location & Sequence & Immunogen & Species (HLA) \\
\hline gp160 (584-592) & $\begin{array}{c}\text { gp41 (584-592) } \\
\text { - Epitope name: 588K }\end{array}$ & ERYLKDQQL & HIV-1 infection & human (B14)
\end{tabular}

- Transcript frequencies of four CTL clones from patient 115, with a chronic and stable HIV-1 infection, were tracked in a longitudinal study of samples collected 6-11 years post infection: clone M21 and E15 recognize ERYLKDQQL, and clone D87 recognizes variant ERYLQDQQL, and clone p175b recognizes the A2 epitope SLYNTVATL

- CTL clone M21 uses the Vbeta 4, CDR3 VKDGA, Jbeta 1.2 TCR beta gene, and clone E15 uses the Vbeta 4, CDR3 VEDWGGAS Jbeta 2.1 TCR beta gene, and D87 uses Vbeta8, ALNRVD, Jbeta2.1

- Responses were stable even through HAART with undetectable viral loads but frequencies varied over time by 100 -fold, ranging from $0.012 \%$ of the total population for SLYNTVATL at its lowest point to $3.78 \%$ for M21, with the relative frequencies of clones shifting over time
gp160 (584-592) gp41 (589-597 SF2) ERYLKDQQL
HIV-1 infection
human (B14)
Altfeld2001b

- Therapy provided during acute infection resulted in a narrower CTL response, stronger T help response, and a less diverse viral population than was seen in individuals treated during chronic infection

- The breadth and specificity of the response was determined using ELISPOT by studying 19 individuals with pre-seroconversion therapy (Group 1), 11 individuals with primary infection but post-seroconversion therapy (Group 2), and 10 individuals who responded to HAART given during chronic infection (Group 3), using 259 overlapping peptides spanning p17, p24, RT, gp41, gp120 and Nef

- Previously described and newly defined optimal epitopes were tested for CTL response

- Number of HLA-B14+ individuals that had a CTL response to this epitope broken down by group: $3 / 3$ group 1, 1/2 group 2, and 0/0 group 3

\begin{tabular}{llll}
\hline gp160 (584-592) gp41 (589-597) ERYLRDQQL & $\begin{array}{l}\text { HIV-1 infection, HIV-1 exposed human (B14) } \\
\text { seronegative }\end{array}$
\end{tabular}

- ELISPOT was used to study CTL responses to a panel of 54 predefined HIV-1 epitopes in 91 HIV-1-exposed, persistently seronegative (HEPS) and 87 HIV-1-infected female Nairobi sex workers

gp160 (584-592) gp41(JRCSF) $\quad$ ERYLKDQQL $\quad$ HIV-1 infection $\quad$ Severino2000

- Primary HLA-B14+ CD4+ CD3+ and HLA-mismatched lymphocytes from uninfected donors were infected with JRCSF after isolation then cocultured with the B14-restricted CTL clone 15160/D75 specific for ERYLKDQQL, and viral inhibition was MHC-restricted

- Primary monocytes and monocyte-derived DC were generated from the same donors, replication of HIV-1 in these cell types was less efficient than in lymphocytes and could also be inhibited by MHC-restricted CTL

- DC-lymphocyte cluster cultures allowed vigorous viral replication and MHC-restricted CTL viral inhibition was blunted or lost depending on the ratio of $\mathrm{DC}$ to CD4+ lymphocyte in the culture

gp160 (584-592) gp41 (SF2) ERYLKQQL HIV-1 infection human (B14) Altfeld2000b

- This epitope was mapped by ELISPOT in a study identifying new HLA-B60 epitopes, and was one of the epitopes presented by another HLA molecule in an HLA-B60 individual

gp160 (584-592) Env (589-597) ERYLKDQQL HIV-1 infection $\quad$ Guillon2002

- An early-expressed Nef protein was modified to contain Env and Pol epitopes to enable the study the effect of expression kinetics on CTL mediated suppression of replication. The "EpiNef" construct was inserted into a recombinant vaccinia virus which was used to infect a target cell line; the target cells were lysed by CTL clones specific for the Env and Pol epitopes indicating that they were properly processed. 


\begin{tabular}{lllll}
\hline HXB2 Location & Author's Location & Sequence & Immunogen & Species (HLA) \\
\hline gp160 (584-592) & gp41 (584-592) & ERYLKDQQL & HIV-1 infection & human (B14)
\end{tabular}

- Nef down-modulates class I protein expression, and this study demonstrates directly that Nef-deleted HIV-1 NL-43 can be more effectively killed in vitro than NL-43 with an intact Nef. The effect was shown to be specific for class I presentation of epitopes, and unlike Nef, deleting Vpr did not alter CTL susceptibility of NL43 infected cells. The CTL clone 15160D75, specific for the class I B14 presented epitope ERYLKDQQL, was one of four used in this study.

\begin{tabular}{lllll}
\hline gp160 (584-592) & gp41 & ERYLKDQQL & HIV-1 infection & human (B14)
\end{tabular}

- Epitope name: B14-EL9(gp41)

- Peripheral blood (PB) and lymph node (LN) CD8+ T-cell responses were compared in 15 asymptomatic HIV-1 infected patients using all known optimal CTL epitopes (http://hiv-web.lanl.gov/content/hiv-db/REVIEWS/brander2001.html) for each person's class I HLA alleles.

- 60 epitope responses were detected in both PB and LN samples of the 15 patients, and an additional 8 responses were detected only in LN. The total magnitude of the response was similar in LN and PB, but the percentage of CD8+ T cells in the LN is lower so the number of HIV-specific cells per million $\mathrm{CD} 8+\mathrm{T}$-cells is higher in the LN.

- 1 year post-HAART treatment in five patients studied, the magnitude of the CD8 T-cell response was decreased in both LN and PB, but more dramatically in PB, and 13/25 epitope responses in the PB became undetectable, in contrast to 5/26 in the LN.

- Treatment interruption following HAART resulted in increased viremia accompanied by the restoration of the detection of 13 epitopes that had become undetectable in the $\mathrm{PB}$, and the addition of 9 novel epitope responses.

- Breakdowns of epitope responses were shown for 4 individuals. Patient A displayed the greatest response to epitope B14-EL9(gp41), a strong response to B7-TL9(p24), and responses to B7-TM9(Nef) and A32-PW10(RT).

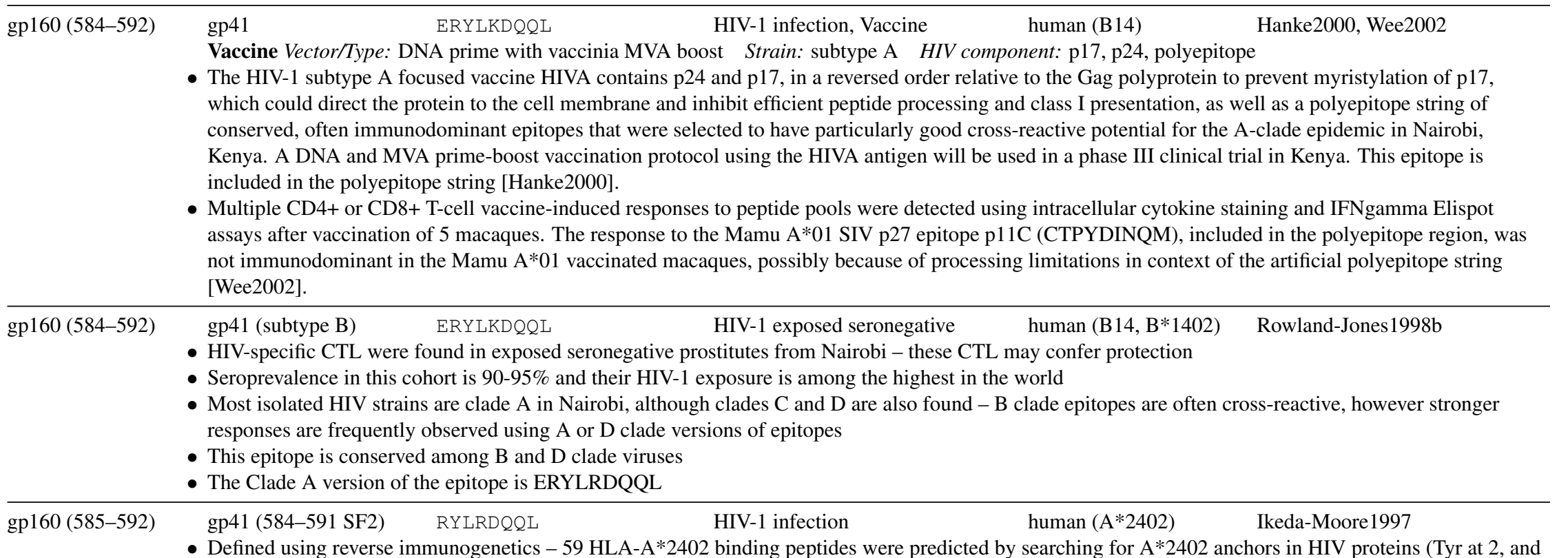

Defined using reverse immunogenetics -59 HLA-A*2402 binding pep 


\begin{tabular}{|c|c|c|c|c|c|}
\hline HXB2 Location & Author's Location & Sequence & Immunogen & Species (HLA) & References \\
\hline & \multicolumn{5}{|c|}{$\begin{array}{l}\text { - This peptide induced CTL in } 2 / 4 \mathrm{HIV}-1+\text { people tested } \\
\text { - RYLRDQQL bound to A*2402 weakly, the epitope can be processed in a vaccinia construct and presented - two specific CTL clones were obtained }\end{array}$} \\
\hline gp160 (585-592) & gp41 (590-597 LAI) & RYLKDQQL & HIV-1 infection & human (B27) & Shankar1996 \\
\hline gp160 (585-593) & \multicolumn{5}{|c|}{$\begin{array}{l}\text { - Defined using reverse immunogenetics }-59 \text { HLA-A } * 2402 \text { binding peptides were predicted by searching for A*2402 anchors in HIV proteins (Tyr at } 2 \text {, and } \\
\text { Phe, Leu or Ile at the C term) }-53 \text { of the } 59 \text { peptides bound } A * 2402 \\
\text { - This peptide induced CTL in } 4 / 4 \text { HIV- } 1+\text { people tested } \\
\text { - RYLRDQQLL bound to } A * 2402 \text { strongly, the epitope can be processed in a vaccinia construct and presented - two specific CTL clones were obtained }\end{array}$} \\
\hline gp160 (585-593) & \multicolumn{5}{|c|}{ - C. Brander notes this is an A*2402 epitope } \\
\hline gp160 (585-593) & $\begin{array}{l}\text { gp41 } \\
\text { - Epitope name: A24- } \\
\text { - Peripheral blood (PB } \\
\text { CTL epitopes (http:// } \\
\text { - } 60 \text { epitope responses } \\
\text { magnitude of the res } \\
\text { CD8+ T-cells is high } \\
\text { - } 1 \text { year post-HAART } \\
\text { in PB, and 13/25 epi } \\
\text { - Treatment interruptic } \\
\text { become undetectable } \\
\text { - Breakdowns of epito } \\
\text { responded to A24-R }\end{array}$ & $\begin{array}{l}\text { RYLKDQQLL } \\
\text { (gp41) } \\
\text { d lymph node (LN) } \\
\text { web.lanl.gov/conte } \\
\text { e detected in both I } \\
\text { e was similar in LI } \\
\text { the LN. } \\
\text { ment in five patien } \\
\text { responses in the PI } \\
\text { llowing HAART in } \\
\text { he PB, and the add } \\
\text { esponses were shov } \\
\text { Nef), B7-IL9(gp41) }\end{array}$ & $\begin{array}{l}\text { HIV-1 infection } \\
\text { esponses were co } \\
\text { IEWS/brander20 } \\
\text { nples of the } 15 \text { pa } \\
\text { he percentage of } \\
\text { magnitude of the } \\
\text { tectable, in contr } \\
\text { in increased vire } \\
\text { epitope response } \\
\text { luals. Patient C di } \\
41 \text { ), A24-YL8(gp }\end{array}$ & $\begin{array}{l}\text { human (A24) } \\
\text { nptomatic HIV-1 in } \\
\text { eerson's class I HL } \\
\text { tional } 8 \text { responses } \\
\text { e LN is lower so th } \\
\text { se was decreased ir } \\
\mathrm{N} \text {. } \\
\text { by the restoration c } \\
\text { st response to B27- } \\
\text { Nef). }\end{array}$ & $\begin{array}{l}\text { Altfeld2002 } \\
\text { patients using all known optimal } \\
\text { s. } \\
\text { tected only in LN. The total } \\
\text { er of HIV-specific cells per million } \\
\mathrm{N} \text { and PB, but more dramatically } \\
\text { tection of } 13 \text { epitopes that had } \\
\text { p24), and in decreasing order also }\end{array}$ \\
\hline gp160 (585-595) & $\begin{array}{l}\text { gp41 (584-591 SF2) } \\
\text { - Defined using revers } \\
\text { Phe, Leu or Ile at the } \\
\text { - This peptide induced } \\
\text { - RYLRDQQLLGI bo } \\
\text { were obtained }\end{array}$ & $\begin{array}{l}\text { RYLRDQQLLGI } \\
\text { nunogenetics }-59 \\
\mathrm{rm})-53 \text { of the } 59 \\
\text { in } 4 / 4 \text { HIV-1+ pe } \\
\text { o A*2402 with me }\end{array}$ & $\begin{array}{l}\text { HIV-1 infection } \\
\text { binding peptides } \\
d A * 2402 \\
\text { the epitope can } \mathrm{t}\end{array}$ & $\begin{array}{l}\text { human }(A * 2402) \\
\text { searching for } A * 2 \\
\text { ccinia construct at }\end{array}$ & $\begin{array}{l}\text { Ikeda-Moore1997 } \\
\text { hors in HIV proteins (Tyr at 2, and } \\
\text { nted - two specific CTL clones }\end{array}$ \\
\hline gp160 (585-595) & $\begin{array}{l}\text { Env (584-594) } \\
\text { Vaccine Vector/Type } \\
\text { - Epitope name: Env5 } \\
\text { - A Sendai virus vecto } \\
\text { responses and has th } \\
\text { - MHC class I/peptide } \\
\text { - Cells transfection wi }\end{array}$ & $\begin{array}{l}\text { RYLRDQQLLGI } \\
\text { ndai virus vector sy } \\
1 \\
\text { tem }(\mathrm{SeV}) \text { was des } \\
\text { ential to elicit imm } \\
\text { amers could be mac } \\
\text { eV modified to exp }\end{array}$ & $\begin{array}{l}\text { Vaccine } \\
\text { HIV component: } \\
\text { pressed HLA-A*2 } \\
\text { s. } \\
\text { ystem that bound } \\
\text { HIV epitope comp }\end{array}$ & $\begin{array}{l}\text { human }(\mathrm{A} * 2402) \\
\text { plexes } \\
\text { s I/peptide complex } \\
\text { CTLs in PBMCs. } \\
\text { mediated specific }\end{array}$ & $\begin{array}{l}\text { Kawana-Tachikawa2002 } \\
\text { system could be used to detect } \\
\text { is. }\end{array}$ \\
\hline
\end{tabular}




\begin{tabular}{lllll}
\hline HXB2 Location & Author's Location & Sequence & Immunogen & Species (HLA) \\
\hline gp160 (586-593) & gp160 & YLRDQQLL & HIV-1 infection & human
\end{tabular}

- This study examines CTL responses in HIV exposed, persistently seronegative individuals, HEPS, who eventually seroconverted - 11/114 HEPS Nairobi sex workers eventually seroconverted, and for six of these HIV CTL reactive epitopes had been defined while seronegative

- The epidemiological factor associated with seroconversion was stopping sex work and HIV-specific CTL activity declines when HEPS sex workers stop working for a period or retire

- This epitope was recognized in 1/22 HEPS sex worker controls, ML887

gp160(586-593) gp41(584-591 NL43) YLKDQQLL HIV-1 infection $\quad$ Dai1992

- The lysine $(\mathrm{K})$ is critical for eliciting a HLA-A24 CTL response

- C. Brander notes that this is an A*2402 epitope in the 1999 database, and suggested that the epitope is RYLKQQLL

\begin{tabular}{llll}
\hline gp160 (586-593) gp41 (591-598) YLRDQQLL & $\begin{array}{l}\text { HIV-1 infection, HIV-1 exposed human (A24) } \\
\text { seronegative }\end{array}$
\end{tabular}

- Variants $(\mathrm{R}) \mathrm{YL}(\mathrm{R} / \mathrm{K}) \mathrm{DQQLL}$ are specific for the A/B clade

- ELISPOT was used to study CTL responses to a panel of 54 predefined HIV-1 epitopes in 91 HIV-1-exposed, persistently seronegative (HEPS) and 87 HIV-1-infected female Nairobi sex workers

- Responses in HEPS women tended to be lower, and focused on different epitopes with HLA presenting molecules that have previously been associated with reduced risk of infection, and there was a shift in the response in the HEPS women upon late seroconversion to epitopes recognized by the HIV-1 infected women

- 43/91 HEPS women had CD8+ responses and detection of HIV-1-specific CTL in HEPS women increased with the duration of viral exposure

- Among HLA-A24 women, 3/4 HEPS and 10/10 HIV-1 infected women recognized this epitope, and (R)YL(R/K)DQQLL tended to be reactive in HEPS and infected women, RDYVDRFFKTL in infected women only

- The dominant response to this HLA allele was to this epitope in all 3/4 HEPS cases but in only 4 of the 10/10 HIV-1 infected women

- Differences in epitope specificity were only seen for responses restricted by class I HLA alleles A2, A24, A*6802, B14, and B18, previously shown to be associated with resistance to HIV-1 in this cohort

\begin{tabular}{lllll}
\hline gp160 (586-593) & $\begin{array}{l}\text { gp41 (580-587 CM243 } \\
\text { subtype CRF01) }\end{array}$ & YLKDQQLL & HIV-1 infection & human (A24) Bond2001
\end{tabular}

- More than half of a cohort of HIV+ female sex workers (FSW) from Northern Thailand were HLA-A11 positive, and this study concentrated on A11 epitopes in this group, although E clade versions of previously defined B-clade A2 and A24 epitopes were also tested.

- The only HLA-A24 FSW tested did not recognized the E clade version of this epitope RYLKDQKLL, which differs from the previously defined B clade version by one amino acid, YLKDQQLL, with an additional amino acid added on

gp160 (586-593) gp41 (subtype A) YLKDQQLL HIV-1 infection, Vaccine human, macaque (A24, Hanke2000 Wee2002 B8)

Vaccine Vector/Type: DNA prime with vaccinia MVA boost Strain: subtype A HIV component: p17, p24, polyepitope

- The HIV-1 subtype A focused vaccine HIVA contains p24 and p17, in a reversed order relative to the Gag polyprotein to prevent myristylation of p17, which could direct the protein to the cell membrane and inhibit efficient peptide processing and class I presentation, as well as a polyepitope string of conserved, often immunodominant epitopes that were selected to have particularly good cross-reactive potential for the A-clade epidemic in Nairobi, Kenya. A DNA and MVA prime-boost vaccination protocol using the HIVA antigen will be used in a phase III clinical trial in Kenya. This epitope is included in the polyepitope string [Hanke2000]. 


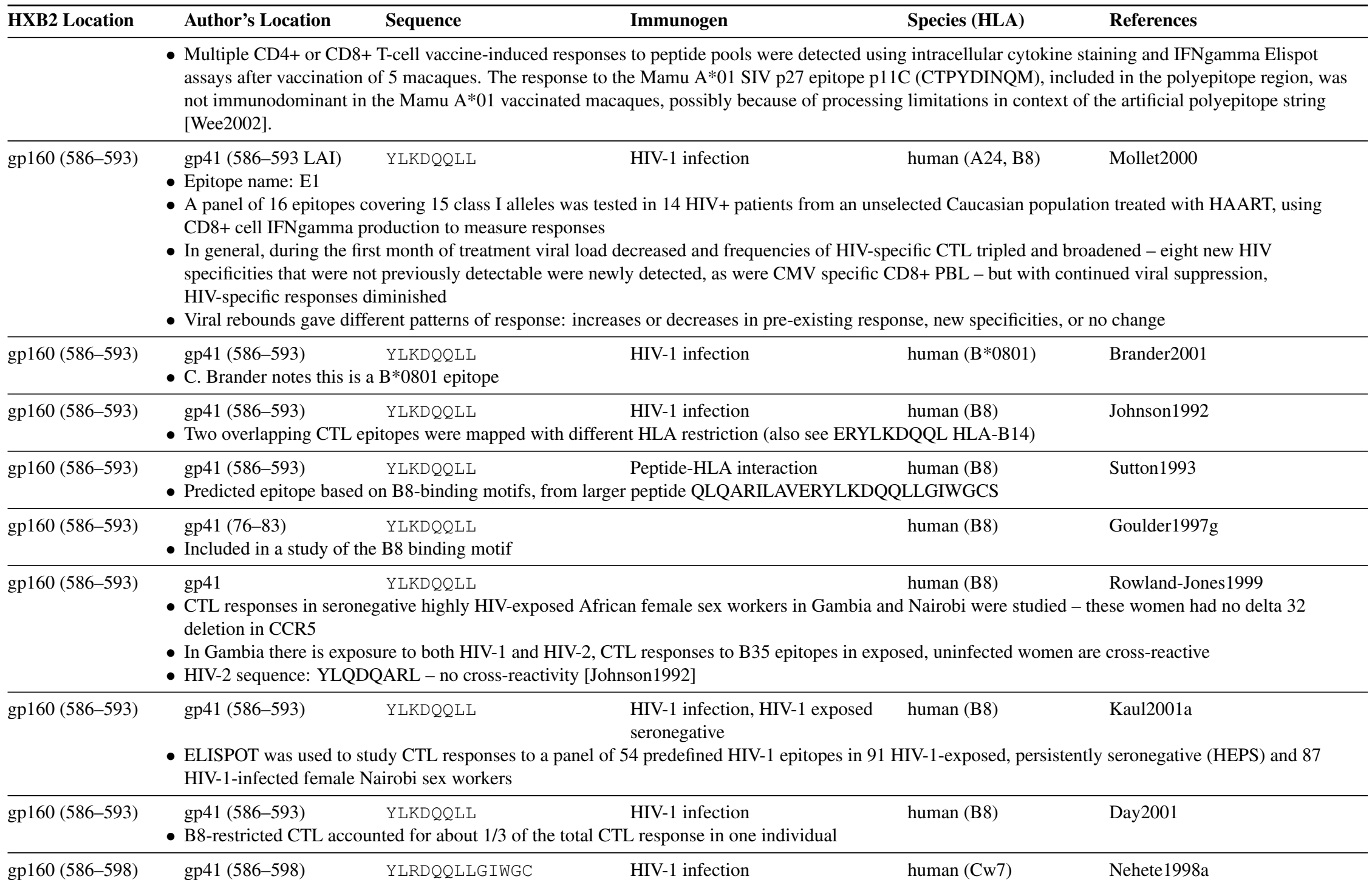

- Three long-term non-progressors and one asymptomatic HIV+ individual were studied and found to have HLA class I C-restricted CD8+ Env-specific

CTLs - Cw7 specific CTL were found against three peptides, including this one

- HLA-C antigens are expressed on lymphoid cells to a lesser extent, $10 \%$ of either HLA-A or HLA-B 


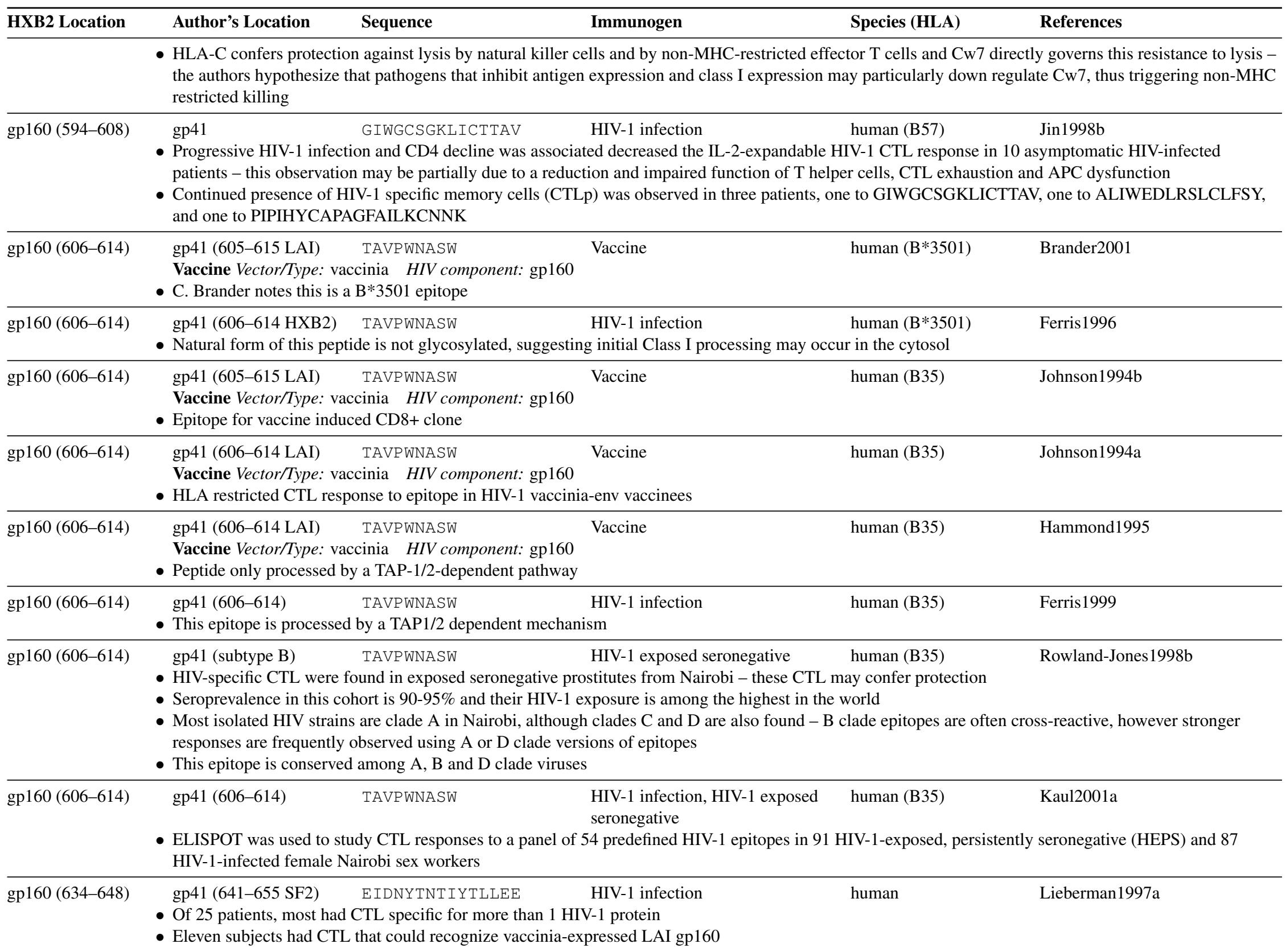




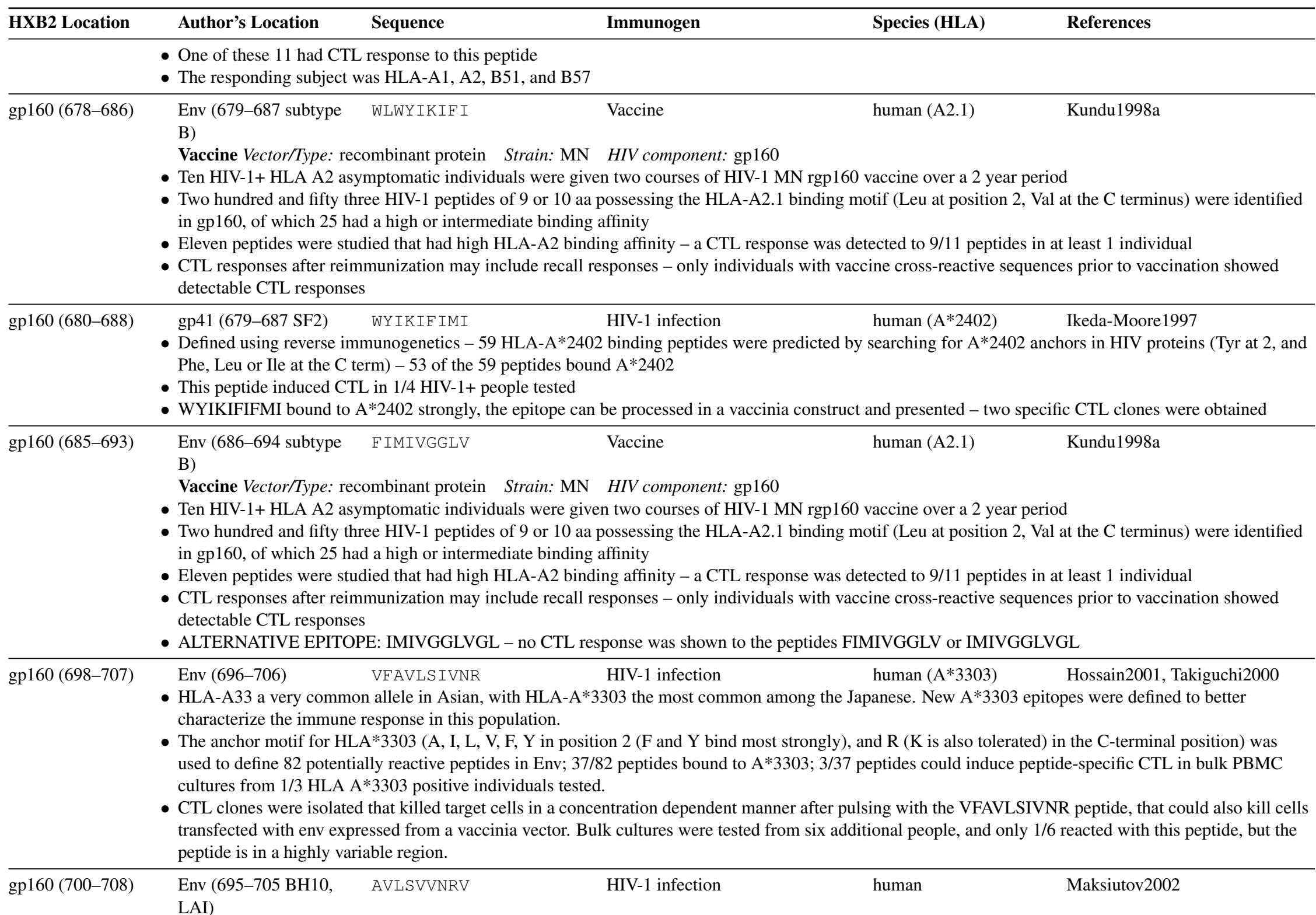

- This study employs an antigenic similarity matrix to compare HIV-1 antigenic determinants with human proteins. 


\begin{tabular}{|c|c|c|c|c|}
\hline HXB2 Location & Author's Location & Immunogen & Species (HLA) & References \\
\hline & \multicolumn{4}{|c|}{$\begin{array}{l}\text { - This CTL epitope (the HIV-1 LAI fragment with high similarity to a human protein overlapping this epitope is LRIVFAVLSVV) has similarity with the } \\
\text { human chemokine-factor } 3 \text { fragment LRLVFALVTAV. }\end{array}$} \\
\hline gp160 (700-708) & \multicolumn{3}{|c|}{ - This epitope is processed by a TAP1/2 dependent mechanism } & Ferris1999 \\
\hline gp160 (701-720) & \multicolumn{3}{|c|}{ - Recognized by CTL derived from acute seroconverter } & Safrit1994a \\
\hline gp160 (702-721) & \multicolumn{4}{|c|}{$\begin{array}{l}\text { - HLA, viral sequence, and Elispot data was obtained from } 105 \text { HIV-1 positive Botswanans; Elispot data was obtained from between } 55 \text { and } 64 \text { subjects for } \\
\text { each HIV protein. } \\
\text { - Nef and p24 had the highest percentage of reactive peptides, and p24 had the highest magnitude of HIV-1 responses. } \\
\text { - This peptide was among the } 28 \text { most reactive C clade peptides from among over } 350 \text { tested spanning all HIV proteins. }\end{array}$} \\
\hline gp160 (704-712) & \multicolumn{4}{|c|}{ - C. Brander notes this is an $\mathrm{A} * 3002$ epitope } \\
\hline gp160 (704-712) & $\begin{array}{l}\text { gp41 } \\
\text { - Epitope name: IY9 (gp41 } \\
\text { - HLA-A*3002 is very com } \\
\text { characterized that are pres } \\
\text { - A rapid method was deve } \\
\text { were defined - this metho } \\
\text { - Two individuals were stuc } \\
\text { African-Caribbean } \\
\text { - In both HLA-A*3002 ind } \\
\text { - In subject } 199 \text { four additi } \\
\text { - Three quantitative assays, } \\
\text { (RT-53) > IY9 (gp41) }\end{array}$ & $\begin{array}{l}\text { IVNRVRQGY HIV-1 infection } \\
\text { 1) } \\
\text { mmon in African populations, } 50 \% \text { of Zimbabwean } \\
\text { sented by this HLA molecule } \\
\text { eloped combining ELISPOT with intracellular IFN- } \\
\text { od was completed within } 48 \text { to } 72 \text { hours of receipt } \\
\text { idied: Subject } 199 \text { (HLA A*0201/*3002 B*4402/51 } \\
\text { dividuals the response to RSLYNTVATLY was dom } \\
\text { ional A*3002 epitopes were identified } \\
\text {, ELISPOT, precursor frequency and chromium rel }\end{array}$ & $\begin{array}{l}\text { human }(\mathrm{A} * 3002) \\
30,44 \% \text { in African } \\
\text { ICs to map optimal } \\
\text { sian, and Subject } 6 \\
\text { hierarchy of respol }\end{array}$ & $\begin{array}{l}\text { Goulder2001a } \\
\text { five new HIV epitopes were } \\
\text {, then HLA presenting molecules } \\
1(\mathrm{p} 17)>\mathrm{KY} 9(\mathrm{gp} 41)>\mathrm{KY} 9\end{array}$ \\
\hline gp160 (742-761) & $\begin{array}{l}\text { Env (742-761) } \\
\text { - HLA, viral sequence, and } \\
\text { each HIV protein. } \\
\text { - Nef and p24 had the high } \\
\text { - This peptide was among t }\end{array}$ & $\begin{array}{l}\text { RDRS IRLVSGF LALAWDDLR HIV-1 infection } \\
\text { Elispot data was obtained from } 105 \text { HIV-1 positive } \\
\text { test percentage of reactive peptides, and p } 24 \text { had the } \\
\text { the } 28 \text { most reactive } C \text { clade peptides from among }\end{array}$ & $\begin{array}{l}\text { human } \\
\text { pot data was obta } \\
\text { of HIV-1 respon } \\
\text { nning all HIV pro }\end{array}$ & $\begin{array}{l}\text { Novitsky2002 } \\
\text { between } 55 \text { and } 64 \text { subjects for }\end{array}$ \\
\hline gp160 (747-755) & $\begin{array}{l}\text { gp41 (747-755) } \\
\text { - Studied in the context of } 1\end{array}$ & $\begin{array}{l}\text { RLVNGSLAL } \\
\text { HLA-A2 peptide binding }\end{array}$ & human (A2) & Parker1992 \\
\hline gp160 (747-755) & $\begin{array}{l}\text { gp41 (741-749 CM243 } \\
\text { subtype CRF01) } \\
\text { - Epitope name: E747-755 } \\
\text { - This was a study of HIV-1 } \\
\text { - HLA-A11 is very commo } \\
\text { and CTL responses were }\end{array}$ & $\begin{array}{l}\text { RLVSGFLAL HIV-1 infection } \\
1 \text { exposed persistently seronegative (HEPS) female } \\
\text { on in this population, and was enriched among the } \mathrm{F} \\
\text { found in } 8 / 8 \mathrm{HIV}+\text { controls, and } 0 / 9 \mathrm{HIV} \text { - women } \mathrm{t}\end{array}$ & $\begin{array}{l}\text { human (A2) } \\
\text { iang Mai, northe } \\
\text { - weak CTL resp } \\
\text { ed }\end{array}$ & $\begin{array}{l}\text { Sriwanthana2001 } \\
\text { e detected in 4/7 HEPS women, }\end{array}$ \\
\hline
\end{tabular}




\begin{tabular}{|c|c|c|c|c|}
\hline HXB2 Location & Author's Location & Immunogen & Species (HLA) & References \\
\hline & \multicolumn{4}{|c|}{ - This epitope was reactive in HIV+ control study subject 144 who carried HLA-A2 } \\
\hline gp160 (747-755) & \multicolumn{2}{|c|}{$\begin{array}{l}\text { gp41 (741-749 CM243 RLVSGFLAL HIV-1 infection } \\
\text { subtype CRF01) } \\
\text { - More than half of a cohort of HIV+ female sex workers (FSW) from Northern } \\
\text { epitopes in this group, although E clade versions of previously defined B-clad } \\
\text { - 2/4 tested FSWs recognized the E clade version of this epitope, which differs } \\
\text { RLVNGSLAL } \\
\text { - This epitope was somewhat conserved 4/8 subtypes: CRF01 (E), B, C, and G }\end{array}$} & $\begin{array}{l}\text { human (A2) } \\
\text { HLA-A11 positive, } \\
\text { itopes were also te } \\
\text { sly defined B clade }\end{array}$ & $\begin{array}{l}\text { Bond2001 } \\
\text { study concentrated on A11 } \\
\text { by two amino acids, }\end{array}$ \\
\hline gp160 (754-768) & \multicolumn{4}{|c|}{$\begin{array}{l}\text { - } \text { Pp41 Progressive HIV-1 infection and CD4 decline was associated decreased the IL-2-expandable HIV-1 CTL response in 10 asymptomatic HIV-infected } \\
\text { patients - this observation may be partially due to a reduction and impaired function of T helper cells, CTL exhaustion and APC dysfunction } \\
\text { - Continued presence of HIV-1 specific memory cells (CTLp) was observed in three patients, one to GIWGCSGKLICTTAV, one to ALIWEDLRSLCLFSY, } \\
\text { and one to PIPIHYCAPAGFAILKCNNK }\end{array}$} \\
\hline gp160 (767-775) & \multicolumn{4}{|c|}{$\begin{array}{l}\text { - Defined using reverse immunogenetics }-59 \text { HLA-A } * 2402 \text { binding peptides were predicted by searching for A*2402 anchors in HIV proteins (Tyr at } 2 \text {, and } \\
\text { Phe, Leu or Ile at the C term) }-53 \text { of the } 59 \text { peptides bound A*2402 } \\
\text { - This peptide induced CTL in } 1 / 4 \text { HIV-1+ people tested } \\
\text { - SYRRLRDLL bound to A* } 2402 \text { moderately, the epitope can be processed in a vaccinia construct and presented - two specific CTL clones were obtained }\end{array}$} \\
\hline gp160 (767-780) & \multicolumn{4}{|c|}{$\begin{array}{l}\text { - Peptide only processed by a TAP-1/2-dependent pathway } \\
\text { - CTL from an acute seroconverter }\end{array}$} \\
\hline gp160 (769-777) & \multicolumn{4}{|c|}{ - Recognized by CTL derived from acute seroconverter } \\
\hline gp160 (770-778) & \multicolumn{4}{|c|}{$\begin{array}{l}\text { - CTL responses in six patients to four Env epitopes were studied: D2: LLNATAIAV, 5.3: RLRDLLLIV, D1: KLTPLCVTL, and 4.3: QMHEDIISL - all } \\
\text { have A2 anchor residues } \\
\text { - The C terminal epitopes (D2 and 5.3) were highly variable and the variability was considered responsible for limited CTL response, while D1 and 4.3, } \\
\text { N-terminal epitopes, were much more conserved and gave evidence of high levels of CTL response in vitro } \\
\text { - Peptides 5.3 and D2 bound to HLA A*0201 with low affinity. }\end{array}$} \\
\hline gp160 (770-780) & $\begin{array}{l}\text { gp41 }(775-785) \\
\text { - Only } 4 / 11 \text { HLA-A2+ HI } \\
\text { - } 1 / 11 \text { optimally-defined pep } \\
\text { others }\end{array}$ & $\begin{array}{l}\text { RLRDLLLIVTR HIV-1 infection } \\
\text { V+ individuals had CTL that reacted to SLYNTVAT } \\
\text { tides from this database were used to screen for INF } \\
\text { s HLA A*0201, A31, B8, B51 and responded to thi }\end{array}$ & $\begin{array}{l}\text { human } \\
\text { stion whether it } \\
\text { er epitopes } \\
\text { seen previously }\end{array}$ & $\begin{array}{l}\text { Betts2000 } \\
\text { odominant } \\
\text { HLA A3.1, as well as seven }\end{array}$ \\
\hline gp160 (770-780) & $\begin{array}{l}\text { gp41 }(768-778 \text { NL43) } \\
\text { CD8+ T cell clone }\end{array}$ & RLRDLLLIVTR & human $\left(\mathrm{A}^{* 0301)}\right.$ & Takahashi1991 \\
\hline
\end{tabular}




\begin{tabular}{|c|c|c|c|c|c|}
\hline HXB2 Location & Author's Location & Sequence & Immunogen & Species (HLA) & References \\
\hline gp160 (770-780) & \multicolumn{5}{|c|}{ - C. Brander notes this is an $\mathrm{A}^{*} 0301$ epitope } \\
\hline gp160 (770-780) & \multicolumn{5}{|c|}{$\begin{array}{l}\text { - Recognized by CTL derived from acute seroconverter } \\
\text { - C. Brander notes that this is an A*3101 epitope in the } 1999 \text { database }\end{array}$} \\
\hline gp160 (770-780) & \multicolumn{4}{|c|}{ - C. Brander notes this is an $\mathrm{A} * 3002$ epitope } & Brander2001 \\
\hline gp160 (770-780) & \multicolumn{5}{|c|}{$\begin{array}{l}\text { - The consensus peptide of clade B is RLRDLLLIVTR } \\
\text { - The consensus peptide of clades A, C and E is RLRDFILIVTR and it is less reactive } \\
\text { - The consensus peptide of clade D is SLRDLLLIVTR and it is less reactive }\end{array}$} \\
\hline gp160 (770-780) & \multicolumn{4}{|c|}{$\begin{array}{l}\text { - ELISPOT was used to study CTL responses to a panel of } 54 \text { predefined HIV-1 epitopes in } 91 \text { HIV-1-exposed, persistently seronegative (HEPS) and } 87 \\
\text { HIV-1-infected female Nairobi sex workers }\end{array}$} & $\begin{array}{l}\text { Kaul2001a } \\
\text { y seronegative (HEPS) and } 87\end{array}$ \\
\hline gp160 (770-780) & $\begin{array}{l}\text { gp41 (770-780) } \\
\text { - The CTL response to opti } \\
\text { studied in eight HIV-1-in } \\
\text { - } 2 \text { to } 17 \text { epitopes were rec } \\
\text { epitopes were targeted by } \\
\text { - All patients recognized at }\end{array}$ & $\begin{array}{l}\text { RLRDLLLIVTR } \\
\text { imally defined CTI } \\
\text { fected subjects, tw } \\
\text { ognized in a given } \\
\text { at least one persor } \\
\text { t least } 1 \text { A } 3 \text { epitope }\end{array}$ & $\begin{array}{l}\text { HIV-1 infection } \\
\text { ricted by HLA class I A and B all } \\
\text { afection, five with chronic, and on } \\
\text { 2-restricted CTL response tended } \\
\text { sitopes, but none was clearly dom }\end{array}$ & $\begin{array}{l}\text { human }(\mathrm{A} 3) \\
\text { es in individuals whe } \\
\text { ong-term non-progr } \\
\text { be narrow and never } \\
\text { ant }\end{array}$ & $\begin{array}{l}\text { Day2001 } \\
\text { pressed HLA A2, A3, and B7 was } \\
\text { LTNP) } \\
\text { nated the response, and } 25 / 27\end{array}$ \\
\hline gp160 (770-780) & $\begin{array}{l}\text { Nef (73-82) } \\
\text { - The CTL response to opti } \\
\text { studied in eight HIV-1-in } \\
\text { - } 2 \text { to } 17 \text { epitopes were rec } \\
\text { epitopes were targeted by } \\
\text { - All patients recognized at } \\
\text { - In two of the subjects, RL }\end{array}$ & $\begin{array}{l}\text { RLRDLLLIVTR } \\
\text { imally defined CTI } \\
\text { fected subjects, tw } \\
\text { ognized in a given } \\
\text { at least one persor } \\
\text { t least } 1 \text { A3 epitope } \\
\text { RDLLLIVTR was }\end{array}$ & $\begin{array}{l}\text { HIV-1 infection } \\
\text { ricted by HLA class I A and B all } \\
\text { ffection, five with chronic, and on } \\
\text { 2-restricted CTL response tended } \\
\text { pitopes, but none was clearly dom } \\
\text { tepitope }\end{array}$ & $\begin{array}{l}\text { human (A3) } \\
\text { in individuals who } \\
\text { ng-term non-progr } \\
\text { e narrow and never } \\
\text { nt }\end{array}$ & $\begin{array}{l}\text { Day2001 } \\
\text { ressed HLA A2, A3, and B7 was } \\
\text { TNP) } \\
\text { ated the response, and } 25 / 27\end{array}$ \\
\hline gp160 (770-780) & $\begin{array}{l}\text { gp41 (769-780) } \\
\text { - Epitope name: A3-RR11 } \\
\text { - CTL responses in } 18 \text { acut } \\
\text { - One individual, AC-06, w } \\
\text { interruptions (STI). He ha } \\
\text { restricted by HLA-A3, } 11 \\
\text { - } 0 / 14 \text { HLA-A3 positive in } \\
\text { were targeted during acut }\end{array}$ & $\begin{array}{l}\text { RLRDLLLIVTR } \\
\text { tely HIV-infected H } \\
\text { vas homozygous at } \\
\text { ad only two detecta } \\
1 \text { by HLA-B7, and } \\
\text { dividuals had detec } \\
\text { te infection. } 1 / 7 \text { ind }\end{array}$ & $\begin{array}{l}\text { HIV-1 infection } \\
\text { or -B7 ( } \mathrm{n}=4) \text { or both }-\mathrm{A} 3 \text { and } \mathrm{B} 7 \\
\text { I alleles (A3, B7, Cw7), was trea } \\
\text { onses during acute infection, but a } \\
\text { v. } \\
\text { ricted responses to this epitope du } \\
n \text { to have detectable responses to }\end{array}$ & $\begin{array}{l}\text { human (A3) } \\
=7 \text { ) positive individ } \\
\text { during acute infect } \\
\text { STI this broadene } \\
\text { g acute infection, bu } \\
\text { epitope after STI. }\end{array}$ & $\begin{array}{l}\text { Yu2002a } \\
\text { ere studied. } \\
\text { d had supervised treatment } \\
\text { distinct epitopes including } 15 \\
\text { 5/15 of HLA-A3 epitopes tested }\end{array}$ \\
\hline
\end{tabular}




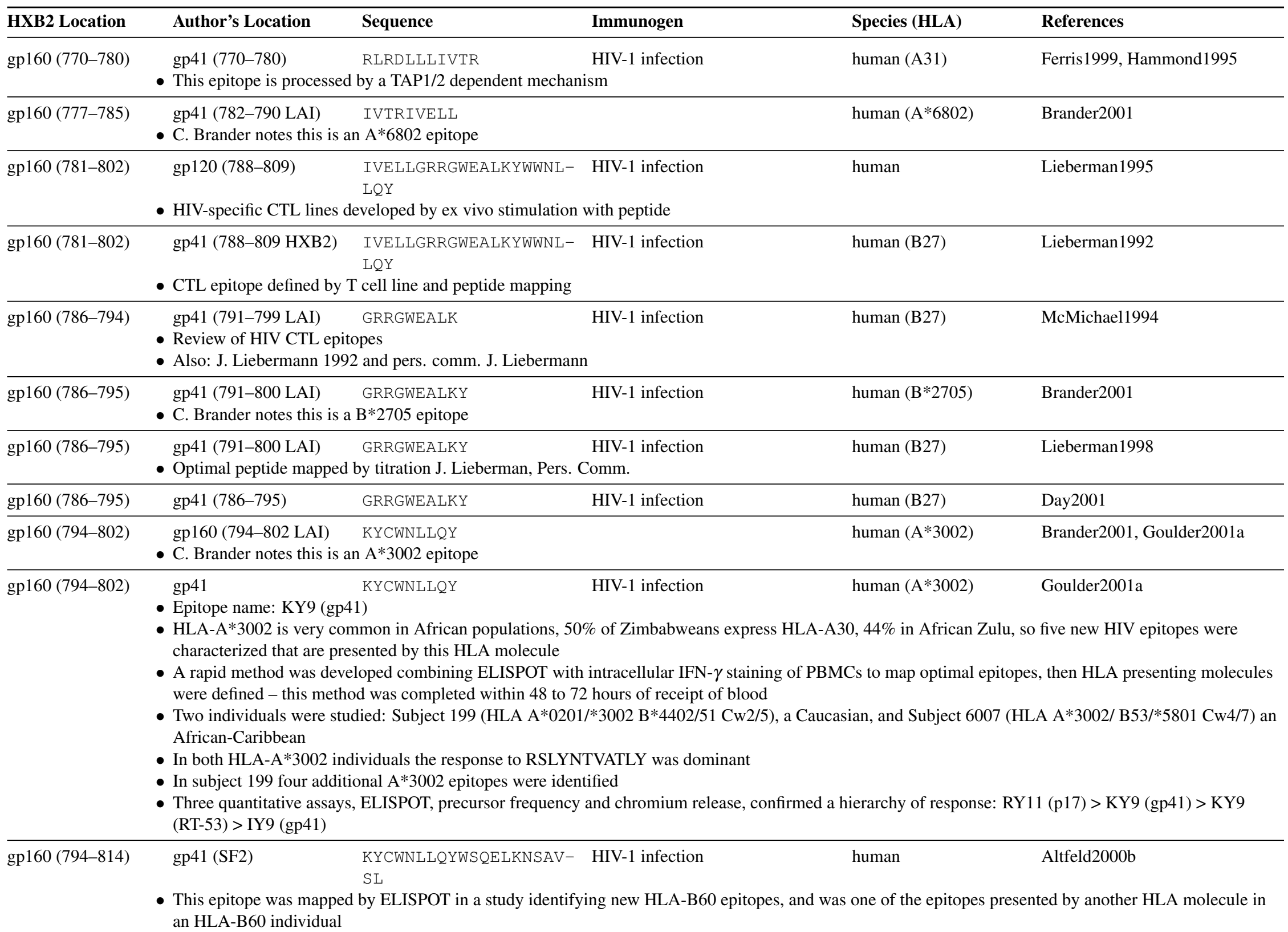




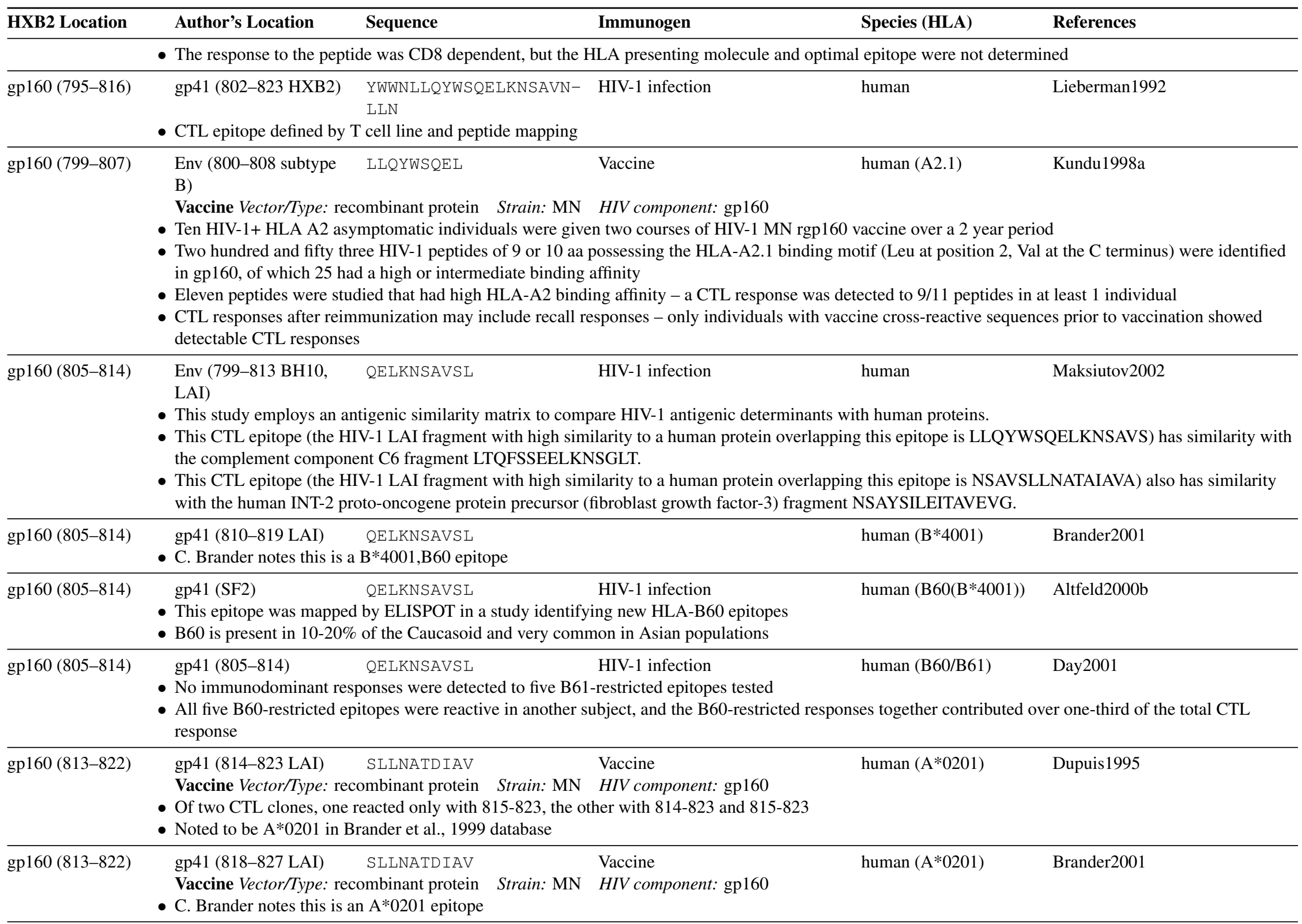




\begin{tabular}{llllc}
\hline HXB2 Location & Author's Location & Sequence & Immunogen & Species (HLA) \\
\hline gp160 (813-822) & gp41 (814-823) & SLLNATD IAV & HIV-1 infection & human (A2) \\
& - Allogeneic dendritic cells (DCs) were obtained from HLA-identical siblings, pulsed with rgp160 MN or A2-restricted HIV-1 epitope peptides, and infused
\end{tabular}

- Allogeneic dendritic cells (DCs) were obtained from HLA-identical siblings, pulsed with rgp160 MN or A2-restricted HIV-1 epitope peptides, and infused monthly into six HIV-infected patients

- 1/6 showed increased env-specific CTL and increased lymphoproliferative responses, 2/6 showed increase only in proliferative responses, and 3/6 showed no change - pulsed DCs were well tolerated

- SLLNATDIAV is a conserved HLA-A2 epitope included in this study - 4/6 patients had this sequence as their HIV direct sequence, and 3 of these had a

detectable CTL response - the other two had either the sequence SLFNAIDIAV or SLLNTTDIVV and no detectable CTL response

- CTL demonstrated against peptide-coated target, epitope is naturally processed and enhancible with vaccine

gp160 (813-822) gp41 (818-827) SLLNATDIAV HIV-1 infection

- Only 4/11 HLA-A2+ HIV+ individuals had CTL that reacted to SLYNTVATL, calling into question whether it is immunodominant

- 95 optimally-defined peptides from this database were used to screen for INF $\gamma$ responses to other epitopes

- $1 / 11$ of the A2+ individuals that didn't respond to SLYNTVATL reacted with seven other epitopes, including this epitope

gp160 (813-822) gp41 (SF2) SLLNATAIAV HIV-1 infection

- Epitope name: SV10

- Dominant CTL epitope in acute infection of patient AC13- response to this epitope corresponded to reduction of initial viremia

- Several other subdominant CTL epitopes were identified in the acute phase, but a response to SL9, SLYNTVATL, was not evident until 18 months post-presentation

gp160 (813-822) gp41 (77-85 SF2) SLLNATDIAV HIV-1 infection $\quad$ human (A2)

- Therapy provided during acute infection resulted in a narrower CTL response, stronger T help response, and a less diverse viral population than was seen in individuals treated during chronic infection

- The breadth and specificity of the response was determined using ELISPOT by studying 19 individuals with pre-seroconversion therapy (Group 1), 11 individuals with primary infection but post-seroconversion therapy (Group 2), and 10 individuals who responded to HAART given during chronic infection (Group 3), using 259 overlapping peptides spanning p17, p24, RT, gp41, gp120 and Nef

- Previously described and newly defined optimal epitopes were tested for CTL response

- Number of HLA-A2+ individuals that had a CTL response to this epitope broken down by group: 1/10 group 1, 2/6 group 2, and 1/4 group 3

gp160 (813-822) gp41 (814-823 CM243 SLLNATAIAV HIV-1 infection human (A2) Sriwanthana2001
subtype CRF01)

- Epitope name: E813-82

- This was a study of HIV-1 exposed persistently seronegative (HEPS) female sex workers in Chiang Mai, northern Thailand

- HLA-A11 is very common in this population, and was enriched among the HEPS sexworkers - weak CTL responses were detected in 4/7 HEPS women, and CTL responses were found in 8/8 HIV+ controls, and 0/9 HIV- women that were not exposed

- This epitope was reactive in HIV+ control study subjects 125 and 144 who carried HLA-A2

\begin{tabular}{llll}
\hline gp160 (813-822) & $\begin{array}{l}\text { gp41 (814-823 CM243 } \\
\text { subtype CRF01) }\end{array}$ & SLLNATAIAV & HIV-1 infection \\
\hline
\end{tabular}

- More than half of a cohort of HIV+ female sex workers (FSW) from Northern Thailand were HLA-A11 positive, and this study concentrated on A11 epitopes in this group, although E clade versions of previously defined B-clade A2 and A24 epitopes were also tested.

- 1/4 tested FSWs recognized the E clade version of this epitope, which differs from the previously defined B clade version by one amino acid, SLLNATDIAV

- This epitope was somewhat conserved 4/8 subtypes: CRF01 (E), B, D, and F 


\begin{tabular}{llllll}
\hline HXB2 Location & Author's Location & Sequence & Immunogen & Species (HLA) & References \\
\hline gp160 (813-822) & gp41 (813-822) & SLLNATDIAV & HIV-1 infection & human (A2)
\end{tabular}

- The CTL response to optimally defined CTL epitopes restricted by HLA class I A and B alleles in individuals who coexpressed HLA A2, A3, and B7 was studied in eight HIV-1-infected subjects, two with acute infection, five with chronic, and one long-term non-progressor (LTNP)

- 2 to 17 epitopes were recognized in a given individual, A2-restricted CTL response tended to be narrow and never dominated the response, and 25/27 epitopes were targeted by at least one person

\begin{tabular}{lllcc}
\hline gp160 (813-822) & gp41 (813-822 IIIB) & SLLNATAIAV & Vaccine & murine (A2) \\
& Vaccine Vector/Type: DNA, DNA with recombinant protein boost & Strain: $:$ IIIB & HIV component: gp160, gp160deltaV3 & Adjuvant: IL-12 (IL-12p35 and
\end{tabular}
IL-12p40)

- Epitope name: D2

- Transgenic mice expressing a HLA-A2/Kb chimeric protein were vaccinated with a full length gp160 or with gp160deltaV3, with the V3 loop deleted. Mice given gp160deltaV3 had a broader immune response than those given gp160, with increased responses to conserved HLA-A2 epitopes in the C1 region of gp120, KLTPLCVTL, and the C-term region of gp41, SLLNATAIAV.

- Greater resistance was conferred by the gp160deltaV3 than the gp160 vaccine to a challenge of vaccinia expressing heterologous gp160 from primary isolates (VI-06 and 89.6), and the resistance was conferred by CD8+ T-cells.

\begin{tabular}{lllll}
\hline gp160 (813-822) Env (814-823 subtype SLLNATDIAV & Vaccine & Kundu1998a \\
\hline
\end{tabular}

B)

Vaccine Vector/Type: recombinant protein Strain: MN HIV component: gp160

- Ten HIV-1+ HLA A2 asymptomatic individuals were given two courses of HIV-1 MN rgp160 vaccine over a 2 year period

- Two hundred and fifty three HIV-1 peptides of 9 or 10 aa possessing the HLA-A2.1 binding motif (Leu at position 2, Val at the C terminus) were identified in gp160, of which 25 had a high or intermediate binding affinity

- Eleven peptides were studied that had high HLA-A2 binding affinity - a CTL response was detected to 9/11 peptides in at least 1 individual

- CTL responses after reimmunization may include recall responses - only individuals with vaccine cross-reactive sequences prior to vaccination showed detectable CTL responses

- CTL to overlapping peptides in this region gave a positive response in the greatest number of patients

- ALTERNATIVE EPITOPES: LLNATDIAV and LLNATDIAVA - CTL were induced by vaccine in those that had the sequence SLLNATAIAVA in their own infection, but not in those with: NLLNTIAIAVA or NLFNTTAIAVA or SLLNATAITVA

gp160 (813-822) gp41 (814-823 LAI) SLLNATDIAV Paccine murine (A2.1) Peter2001

Vaccine Vector/Type: peptide Strain: LAI Adjuvant: P30, incomplete Freund's adjuvant (IFA), Montanide (ISA 720), PLG-microparticle

- Epitope name: LR27

- The stability of peptide binding to HLA-A2.1 was determined for six HLA-A2.1 peptides included in this vaccine study - ILKEPVHGV (RT), SLYNTVATL (p17), SLLNATDIAV (gp41) and LLWKGEGAV (RT) all bound with high affinity comparable to a influenza epitope reference (GILGFVFTL), while RGPGRAFVTI and VIYQYMDDL bound with a lower affinity (relative binding activity $=0.01$ ).

- The four high-affinity peptides formed stable complexes with half-lives ranging between 8 and 32 hours, while the low affinity peptides had half lives of less than an hour.

- HLA-A2.1 transgenic mice were immunized with the six HIV-1 peptides and P30, as a universal T-helper epitope, with IFA or Montanide or microspheres as adjuvants.

- All peptides except VIYQYMDDL induced a stong CTL response in Cr-release assays - stronger responses were observed when peptides were delivered alone, indicating immunodominance when the combination was used. 


\begin{tabular}{llllll}
\hline HXB2 Location & Author's Location & Sequence & Immunogen & Species (HLA) & References \\
\hline gp160 (813-822) & gp41 (814-823 LAI) & SLLNATDIAV & Vaccine & murine (A2.1) & Peter2002 \\
& $\begin{array}{l}\text { Vaccine Vector/Type: } \\
\text { - }\end{array}$ & Eeptide & Strain: LAI & Adjuvant: P30, incomplete Freund's adjuvant (IFA), IL-12 &
\end{tabular}

- Epitope name: LR27

- When HIV-1 peptides were used to vaccinate HLA-A2.1 transgenic A2-Kb mice, strong responses to five peptides were observed when the peptides were given individually, but immunodominance limited the response to some of the peptides when they were given in combination [Peter2001]. IL-12 can counteract immunodominance in BALB/c mice, so it was given with the multiple epitope vaccination, and was instead found to specifically eliminate the HLA-A2.1-epitope CTL responses, but not Kb CTL responses. This was possibly a consequence of transient depletion of T-cells, B cells and macropahges in the spleen.

\begin{tabular}{lllll}
\hline gp160 (813-822) gp41 & SLLNATDIAV & HIV-1 infection & human (A68) & Altfeld2001c
\end{tabular}

- Epitope name: gp41 SV10

- HIV was scanned for all peptides which carried the A2-supermotif pattern conserved in more than $50 \%$ of B clade sequences -233 peptides met this criteria, and 30 of these bound to HLA-A $* 0201-20 / 30$ bound to at least $3 / 5$ of HLA-A2 supertype alleles tested

- Three additional previously described HLA-A2 epitopes were added to the set of 20, and 18/22 chronically infected HLA-A2 individuals had CTL that recognized at least one of the 23 peptides (median of 2 and maximum of 6), while 6/12 acute infected individuals recognized at least 1 (median of 1 and maximum of 2)

- This epitope binds to three HLA-A2 supertype alleles: $A * 6802$ (highest affinity), $A * 0202$ and $A * 0203$ (but not $A * 0201$ and not $A * 0206$ )

- This epitope did not elicit an ELISPOT response in 22 chronic HIV HLA-A2 infections, but elicited a strong response in 1/12 acute HLA-A2 infections this individual, AC13, was HLA A*0201/68 B44/14 and also had a strong response to HLA-A2 vpr epitope AIIRILQQL

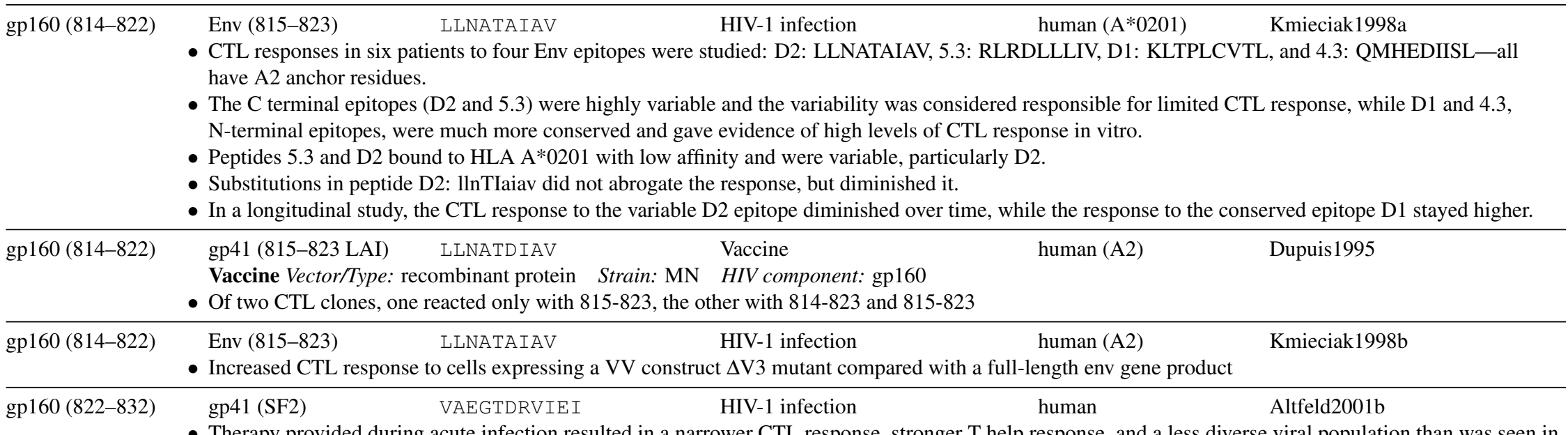

- Therapy provided during acute infection resulted in a narrower CTL response, stronger T help response, and a less diverse viral population than was seen in individuals treated during chronic infection

- The breadth and specificity of the response was determined using ELISPOT by studying 19 individuals with pre-seroconversion therapy (Group 1), 11 individuals with primary infection but post-seroconversion therapy (Group 2), and 10 individuals who responded to HAART given during chronic infection (Group 3), using 259 overlapping peptides spanning p17, p24, RT, gp41, gp120 and Nef

- Previously described and newly defined optimal epitopes were tested for CTL response 


\begin{tabular}{|c|c|c|c|c|}
\hline HXB2 Location & Author's Location & Immunogen & Species (HLA) & References \\
\hline & \multicolumn{4}{|c|}{$\begin{array}{l}\text { - Number of individuals that had a CTL response to this epitope (HLA presenting molecule uncertain) broken down by group: } 0 \text { group } 1,1 \text { group } 2 \text {, and } 0 \\
\text { group } 3\end{array}$} \\
\hline gp160 (827-841) & \multicolumn{4}{|c|}{ - $\mathrm{CTL}$ and T helper cell reactivity in healthcare workers exposed to HIV } \\
\hline gp160 (827-841) & \multicolumn{4}{|c|}{ - Helper and cytotoxic T cells can be stimulated by this peptide (Th4) } \\
\hline gp160 (827-841) & \multicolumn{4}{|c|}{$\begin{array}{l}\text { Vaccine Vector/Type: vaccinia Strain: IIIB HIV component: gp160 } \\
\text { - In a murine system multiple class I molecules can present to CTL }\end{array}$} \\
\hline gp160 (827-841) & \multicolumn{4}{|c|}{$\begin{array}{l}\text { Vaccine Vector/Type: vaccinia HIV component: gp160 } \\
\text { - Multiple murine MHC can cross-present this epitope (HP53), and P18 RIQRGPGRAFVTIGK, to specific CTL }\end{array}$} \\
\hline gp160 (828-836) & \multicolumn{4}{|c|}{$\begin{array}{l}\text { Vaccine Vector/Type: recombinant protein Strain: MN HIV component: gp160 } \\
\text { - CTL from HLA-A2 positive subject react with this peptide }\end{array}$} \\
\hline gp160 (828-836) & $\begin{array}{l}\text { gp41 (829-837 CM243 } \\
\text { subtype CRF01) } \\
\text { - More than half of a cohort } \\
\text { epitopes in this group, alth } \\
\text { - } 1 / 4 \text { tested FSWs recogniz } \\
\text { - This epitope was only con }\end{array}$ & $\begin{array}{l}\text { KVIEVAQGA HIV-1 infection } \\
\text { t of HIV+ female sex workers (FSW) from Northern Thailand } \\
\text { hough E clade versions of previously defined B-clade A2 and } \\
\text { sed the E clade version of this epitope, which differs from the p } \\
\text { nserved in CRF01 (subtype E), and identities were rare }\end{array}$ & $\begin{array}{l}\text { human (A2) } \\
\text { HLA-A11 positive, an } \\
\text { itopes were also teste } \\
\text { sly defined B clade ve }\end{array}$ & $\begin{array}{l}\text { Bond2001 } \\
\text { study concentrated on A11 } \\
\text { by three amino acids, RvievLqRa }\end{array}$ \\
\hline gp160 (828-836) & $\begin{array}{l}\text { Env (829-837 subtype } \\
\text { B) } \\
\text { Vaccine Vector/Type: reco } \\
\text { - Ten HIV-1+ HLA A2 asyr } \\
\text { - Two hundred and fifty thr } \\
\text { in gp160, of which } 25 \text { had } \\
\text { - Eleven peptides were stud } \\
\text { - CTL responses after reimr } \\
\text { detectable CTL responses }\end{array}$ & $\begin{array}{l}\text { RVIEVLQRA Vaccine } \\
\text { ombinant protein Strain: MN HIV component: gp160 } \\
\text { mptomatic individuals were given two courses of HIV-1 MN rg } \\
\text { ee HIV-1 peptides of } 9 \text { or } 10 \text { aa possessing the HLA-A2.1 bind } \\
\text { d a high or intermediate binding affinity } \\
\text { died that had high HLA-A2 binding affinity - a CTL response } \\
\text { munization may include recall responses - individuals with vac }\end{array}$ & $\begin{array}{l}\text { human (A2.1) } \\
\text { vaccine over a } 2 \text { year } \\
\text { otif (Leu at position } 2 \\
\text { tected to } 9 / 11 \text { peptide } \\
\text { ross-reactive sequenc }\end{array}$ & $\begin{array}{l}\text { Kundu1998a } \\
\text { at the } \mathrm{C} \text { terminus) were identified } \\
\text { tleast } 1 \text { individual } \\
\text { ior to vaccination showed }\end{array}$ \\
\hline gp160 (830-854) & $\begin{array}{l}\text { gp41 (831-853) } \\
\text { - Study of cytokines release }\end{array}$ & $\begin{array}{l}\text { IEVVQGAYRAIIRHIPRRI- HIV-1 infection } \\
\text { RQGLERI } \\
\text { ed by HIV-1 specific activated CTL }\end{array}$ & human & Price1995 \\
\hline gp160 (831-838) & $\begin{array}{l}\text { Env }(830-837) \\
\text { - HLA-A33 a very comm } \\
\text { characterize the immun }\end{array}$ & $\begin{array}{l}\text { EVAQRAYR HIV-1 infection } \\
n \text { allele in Asia, with HLA-A*3303 the most common amon } \\
\text { response in this population. }\end{array}$ & $\begin{array}{l}\text { human }(\mathrm{A} * 3303) \\
\text { anese. New } A * 3303\end{array}$ & $\begin{array}{l}\text { Hossain2001, Takiguchi2000 } \\
\text { es were defined to better }\end{array}$ \\
\hline
\end{tabular}




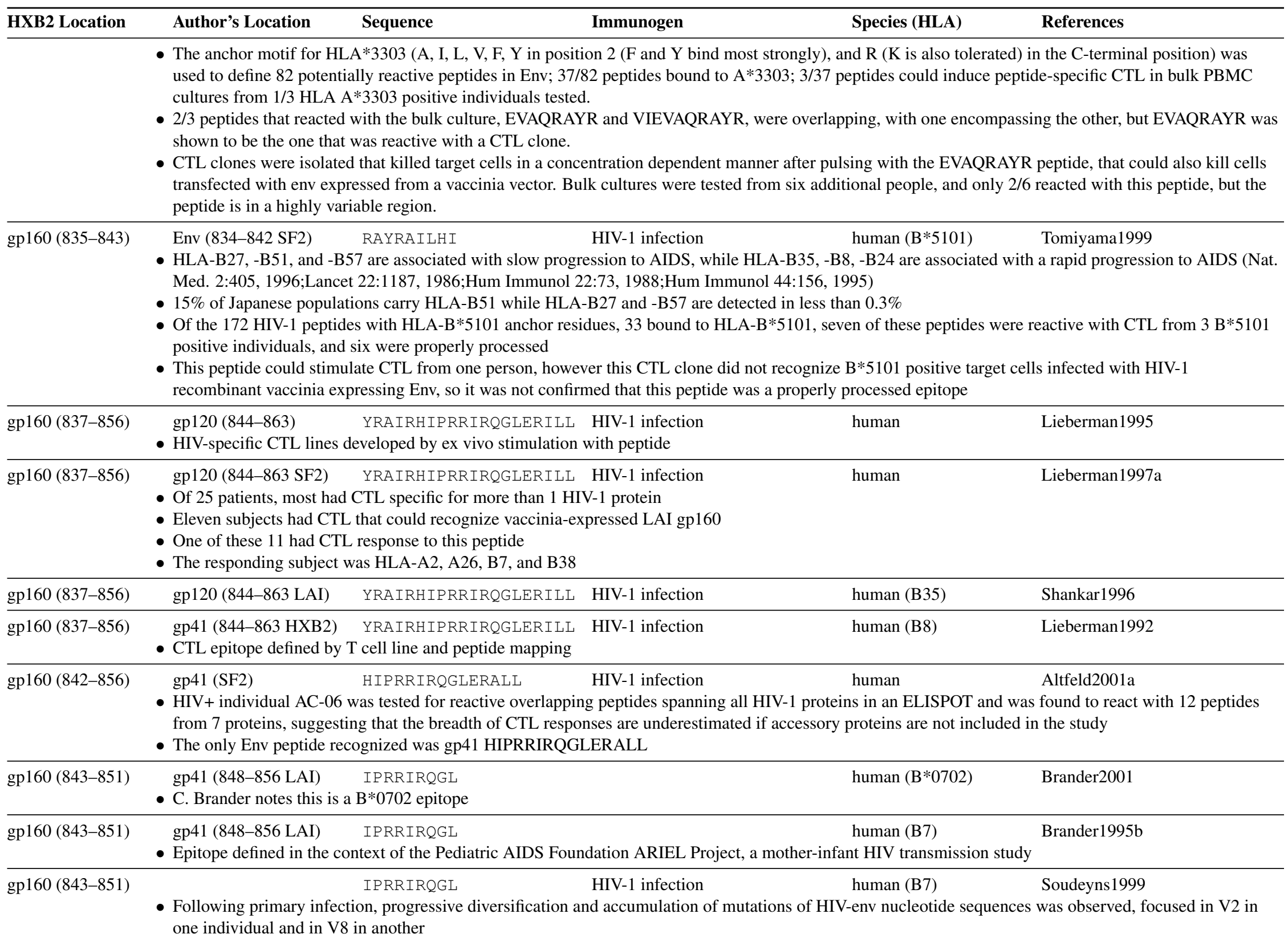




\begin{tabular}{|c|c|c|c|c|}
\hline HXB2 Location & Author's Location & Sequence & Species (HLA) & References \\
\hline & \multicolumn{4}{|c|}{$\begin{array}{l}\text { - The patient with the V2 diversification showed only transient CTL against Env and Nef } \\
\text { - The patient with the V8 diversification had an immunodominant CTL response to V8 epitope IPRRIRQGL, and multiple escape variants emerged within a } \\
\text { year: ipTrirqgl and ipTrirqgF, which abrogated the CT response in vitro, and also iprrLqgl and iprrirqDl which gave diminished responses. }\end{array}$} \\
\hline $160(843-851)$ & \multicolumn{4}{|c|}{$\begin{array}{l}\text { - The consensus peptide of clades } \mathrm{A}, \mathrm{B}, \mathrm{D} \text {, and } \mathrm{F} \text { is IPRRIRQGL } \\
\text { - The consensus peptide of clade } \mathrm{C} \text { is iprrirqgF, and it is equally reactive. }\end{array}$} \\
\hline 851) & \multicolumn{4}{|c|}{$\begin{array}{l}\text { - The extent of CTL interclade cross-reactivity from CTL isolated from individuals newly infected with B clade virus was studied, and extensive } \\
\text { cross-reactivity was observed } \\
\text { - Two HLA B7 individuals had CTL response to B_LAI, A_92UG037 and C_92BR025 gp160, but were B clade strain MN non-responders - the authors } \\
\text { note that the B7 epitope IPRRIRQGL is conserved between the LAI and clade A and C strains, but that MN has a non-conservative Arg to Thr substitution } \\
\text { at position three that may be contributing to the specificity of the response in the HLA B7 individuals }\end{array}$} \\
\hline 51) & \multicolumn{4}{|c|}{$\begin{array}{l}\text { - CTL response to IPRRIRQGL was the immunodominant response in a rapid progressor - there was a subdominant response to SPAIFQSSM in Pol, and } \\
\text { interestingly, no response to commonly immunodominant HLA A*0201 epitope SLYNTVATL, although this individual was HLA A*0201 } \\
\text { - The individual showed a strong initial CTL response at the time of the initial drop in viremia, but it was quickly lost, although memory cells persisted } \\
\text { - Despite the initial narrow response to two epitopes, no other CTL responses developed } \\
\text { - No HIV-specific lymphoproliferative responses were detected in this patient, and neutralizing antibody response was weak } \\
\text { - Variants were observed in vivo, the most common form of the viral epitope at presentation at } 3 \text { months was the only form that did not elicit a CTL response: } \\
\text { iprrTrqgl; the other forms detected were iprrirqgF, iprriLqgF, VprrirqgF and they could elicit a CTL response although the response to iprriLqgF was } \\
\text { reduced } \\
\text { - A second rapid progressor had a detectable CTL response exclusively to this epitope }\end{array}$} \\
\hline 1) & $\begin{array}{l}\text { gp41 (subtype A) } \\
\text { - HIV-1 subtypes A and D } \\
\text { cross-reactive CTL resp } \\
\text { and D } \\
\text { - Proteins corresponding t } \\
\text { was extensive inter-subt) } \\
\text { - This optimal epitope seq } \\
\text { subtype D }\end{array}$ & $\begin{array}{l}\text { IPRRIRQGF } \\
\text { lominate the U } \\
\text { ses in Ugandar } \\
\text { the subtype of } \\
\text { e cross-reactiv } \\
\text { ence, recognize }\end{array}$ & $\begin{array}{l}\text { HIV-1 infection human (B7) } \\
\text { and a vaccine trial using B clade antigen is underway } \\
\text { clade recombinant vaccinia viruses expressing Gag, E } \\
\text { ns tended to trigger higher levels of CTL response me } \\
\text { roteins and the co-circulating subtype } \\
\text { from a Ugandan with an A subtype infection, is cros }\end{array}$ & $\begin{array}{l}\text { Cao2000 } \\
\text { idy addresses relative levels of } \\
\text { T or Nef from HIV-1 clades A, B, } \\
\text { with subtypes A and B, but not in }\end{array}$ \\
\hline sp160 (843-851) & $\begin{array}{l}\text { gp41 } \\
\text { Subject } 053 \text { i was follow } \\
\text { This individual had a do } \\
\text { subdominant response to } \\
\text { variation occurred with } \\
\text { At } 3 \text { months post-presen } \\
\text { WAASS, two used Vbet } \\
\text { clones persisted over the }\end{array}$ & $\begin{array}{l}\text { IPRRIRQGL } \\
\text { d longitudinally } \\
\text { minant response } \\
\text { SPAIFQSSM - } \\
\text { n both epitopes } \\
\text { tation, seven IPR } \\
\text { 16S1, ERSPPG } \\
\text { course of the in }\end{array}$ & $\begin{array}{l}\text { HIV-1 infection human (B7) } \\
\text { tion through death, and had rapid progression to AIDS } \\
\text { with strong in vivo activated responses and in vitro stin } \\
\text { e of disease progression ( } 4 \text { Years), the functional CTL } \\
\text { lones were obtained, five used the T-cell receptor Vbet } \\
\text { one CTL clone isolated at } 39 \text { months was Vbeta 14S1, } \\
\text { ime of death, despite the loss of CTL functional respon }\end{array}$ & $\begin{array}{l}\text { Islam2001 } \\
\text { nemory responses and a } \\
\text { s were lost and no sequence } \\
\text { d Jbeta } 2.7 \text { and had the CDR3 } \\
\text { AAG, and Jbeta } 2.1 \text { - all of these } \\
\text { time }\end{array}$ \\
\hline
\end{tabular}




\begin{tabular}{lllll}
\hline HXB2 Location & Author's Location & Sequence & Immunogen & Species (HLA) \\
\hline gp160 (843-851) & gp41 (843-851 SF2) & IPRRIRQGL & HIV-1 infection & human (B7)
\end{tabular}

- Therapy provided during acute infection resulted in a narrower CTL response, stronger T help response, and a less diverse viral population than was seen in individuals treated during chronic infection

- The breadth and specificity of the response was determined using ELISPOT by studying 19 individuals with pre-seroconversion therapy (Group 1), 11 individuals with primary infection but post-seroconversion therapy (Group 2), and 10 individuals who responded to HAART given during chronic infection (Group 3), using 259 overlapping peptides spanning p17, p24, RT, gp41, gp120 and Nef

- Previously described and newly defined optimal epitopes were tested for CTL response

- Number of HLA-B7+ individuals that had a CTL response to this epitope broken down by group: $2 / 4$ group $1,1 / 3$ group 2 , and $1 / 1$ group 3

gp160 (843-851) gp41(848-856) IPRRIRQGL HIV-1 infection, HIV-1 exposed human (B7) Kaul2001a

- IPRRIRQGL cross-reacts with clades A, B and D

- ELISPOT was used to study CTL responses to a panel of 54 predefined HIV-1 epitopes in 91 HIV-1-exposed, persistently seronegative (HEPS) and 87 HIV-1-infected female Nairobi sex workers

- Responses in HEPS women tended to be lower, and focused on different epitopes with HLA presenting molecules that have previously been associated with reduced risk of infection, and there was a shift in the response in the HEPS women upon late seroconversion to epitopes recognized by the HIV-1 infected women

- 43/91 HEPS women had CD8+ responses and detection of HIV-1-specific CTL in HEPS women increased with the duration of viral exposure

- Among HLA-B7 women, 2/5 HEPS and 5/6 HIV-1 infected women recognized this epitope

- The dominant response to this HLA allele was to this epitope in 2 of the 5/6 HIV-1 infected women that responded to the epitope, but in neither of the 2/5 HEPS cases

- Subject ML 1203 started with CTL responses to A*6802 DTVLEDINL and to B7 FPVTPQVPLR prior to seroconversion, and upon seroconversion acquired additional responses to A*6802 ETAYFILKL which became dominant, B7 TPGPGV/IRYPL, B7 IPRRIRQGL, and B7 SPRTLNAWV

\begin{tabular}{llll}
\hline gp160 (843-851) gp41 (843-851) & IPRRIRQGL & HIV-1 infection & human (B7)
\end{tabular}

- The CTL response to optimally defined CTL epitopes restricted by HLA class I A and B alleles in individuals who coexpressed HLA A2, A3, and B7 was studied in eight HIV-1-infected subjects, two with acute infection, five with chronic, and one long-term non-progressor (LTNP)

- 2 to 17 epitopes were recognized in a given individual, A2-restricted CTL response tended to be narrow and never dominated the response, and 25/27 epitopes were targeted by at least one person

- Subjects with chronic HIV-1 infection recognized between 2-8 out of 11 B7-restricted epitopes

- An acute seroconvertor homozygous for the B7 allele recognized five B7-restricted epitopes

- The other acute seroconvertor failed to recognize any of the 11 B7-restricted epitopes tested

- The B7-restricted CTL response was highly variable and there was no clearly dominant epitope
gp160 (843-851)
gp41 (SF2)
IPRRIRQGL
HIV-1 infection
human (B7)
Altfeld2000b

- This epitope was mapped by ELISPOT in a study identifying new HLA-B60 epitopes, and was one of the epitopes presented by another HLA molecule in an HLA-B60 individual

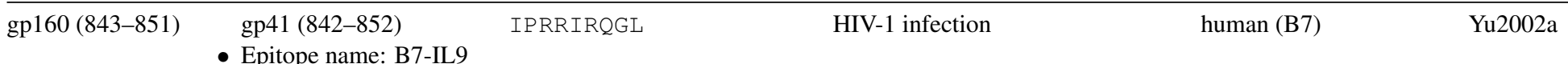

- Epitope name: B7-IL9

- CTL responses in 18 acutely HIV-infected HLA-A3 (n=7) or -B7 ( $=4)$ or both -A3 and B7 (n=7) positive individuals were studied.

- One individual, AC-06, was homozygous at all three class I alleles (A3, B7, Cw7), was treated during acute infection and had supervised treatment interruptions (STI). He had only two detectable CTL responses during acute infection, but after STI this broadened to 27 distinct epitopes including 15 restricted by HLA-A3, 11 by HLA-B7, and 1 by HLA-Cw7. 


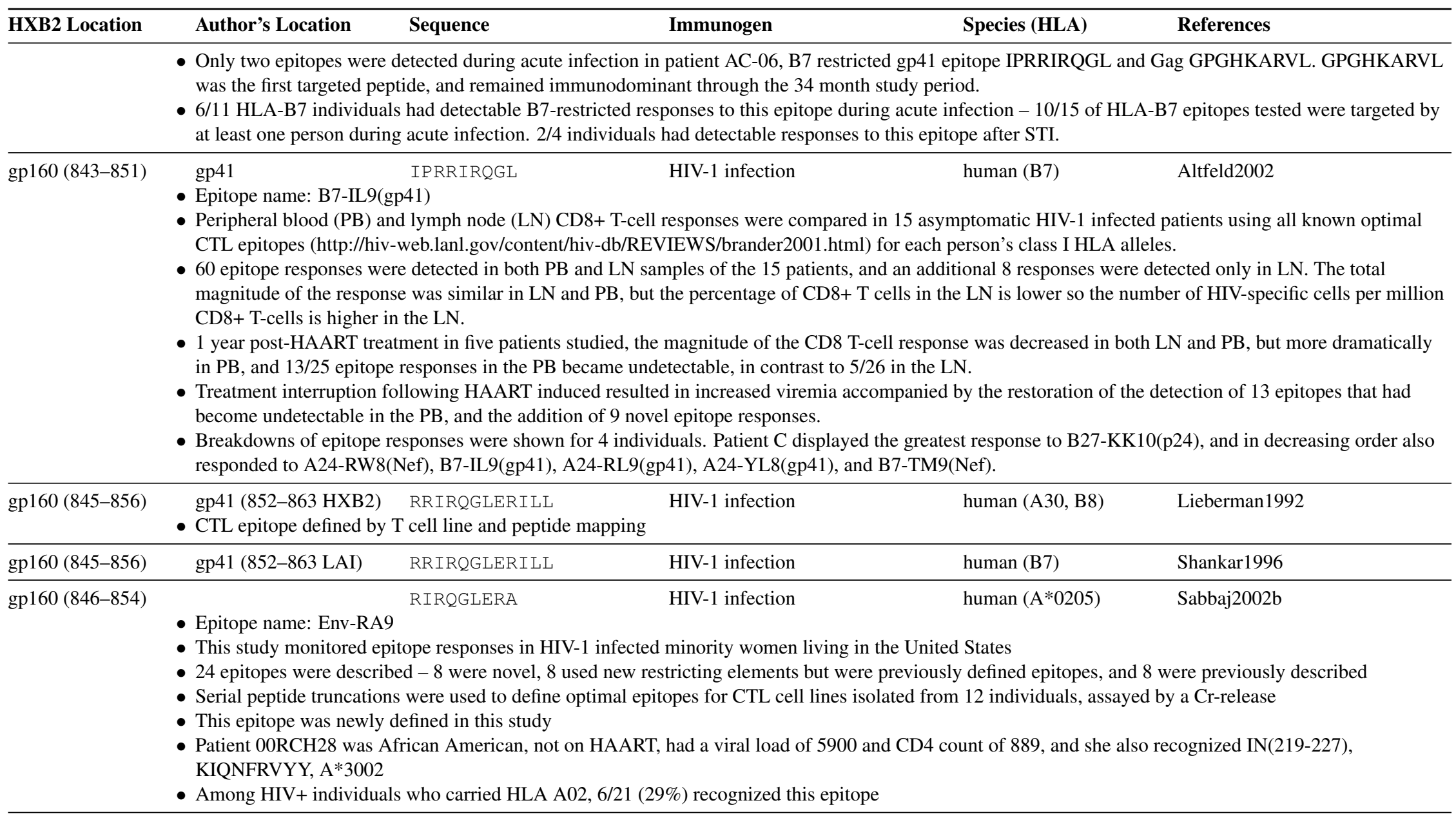




\section{II-B-19 Env CTL Epitopes}

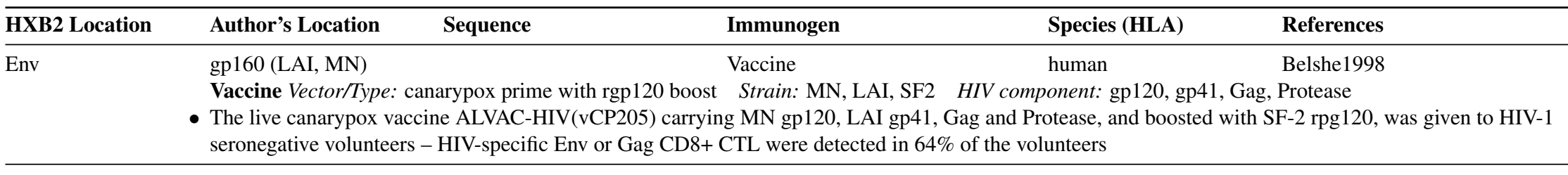

\begin{tabular}{llcc}
\hline Env & gp160 (LAV) & HIV-1 infection & human \\
& Protein delivery (gp160 LAV, p66 LAV, and p24 NY5) to human dendritic cells (DC) with liposomes provides enhanced memory CTL
\end{tabular}

- Protein delivery (gp160 LAV, p66 LAV, and p24 NY5) to human dendritic cells (DC) with liposomes provides enhanced memory CTL response relative to delivery of protein alone

- Chloroquine administration enhanced epitope presentation, and brefeldin A and peptide aldehyde inhibitors inhibited antigen presentation, suggesting epitopes were processed by classical proteasome pathway

Env Env (IIIB) HIV-1 infection human Wasik2000

- HIV+ infants that progressed rapidly to AIDS had lower Th1 responses and decreased production of IL-2, as well as beta-chemokines, relative to other HIV+ infants

- No HIV+ infants had no demonstrable CTL at birth, but Th1 responses accompanied by CTL responses developed in children with slowly progressive disease, and not in rapid progressors

- CTLp frequencies were determined by limiting dilution using autologous B cells infected with vaccina/HIV constructs

Env gp120 HIV-1 infection human Soudeyns2000

- Analysis of T cell receptor beta chain variable region repertoire indicates that antiretroviral therapy (ART) and highly active antiretroviral therapy

(HAART) decrease global CD8 T cell oligoclonality during primary HIV infection

- A sharp decline in HIV-1 gp120-specific CTL clones was observed in HAART-treated subjects

\begin{tabular}{llcc}
\hline Env & Env (LAI, MN) & Vaccine & human \\
& Vaccine Vector/Type: canarypox & Strain: LAI, MN & HIV component: gp41, Gag, Pro, V3
\end{tabular}

- The vaccine used was a rec canarypox with HIV-1 gp120 MN, tm/gag/protease LAI (vCP205), alone or with p24E-V3 MN synthetic peptide (CLTB-36))

- Twenty HIV negative subjects were vaccinated in phase I trial with combinations of vCP205 and CLTB-36

- Immunization with vCP205 induced HIV-1-specific ABs to gp120, V3, and p24 antigens, and CTL immune responses against vCP205 were detected after the fourth immunization in 33\% of the subjects against Env, Gag and Pol, but the CLTB-36 peptide did not produce AB or CTL immune responses against p24 or gp160

Env Env $\quad$ HIV-1 infection human

- 13/13 subjects with advanced HIV infections showed CD8 T cell proliferation and differentiation of CTL in vitro, and six individuals showed HIV-specific responses to Gag, Pol, Env or Nef antigens

- Data suggests that the functional and genetic integrity of the CD8 T cell repertoire (TCR betaV gene intrafamily genetic diversity) remains intact through advanced HIV infection, although HIV-specific CTL activity decreases

\begin{tabular}{lllc}
\hline Env & Env (LAI, MN) & Vaccine & human \\
& Vaccine Vector/Type: canarypox prime with rgp120 boost & Strain: LAI and SF2 & HIV component: Env, Gag, Pro, Nef, Pro
\end{tabular}

- The vaccine used was rec canarypox expressing HIV-1 env, gag, pol, nef and protease (vCP300) with or without administration of HIV-1 SF-2 rgp120

- In vitro inducible CTL activity against HIV-1 Env, Gag, Pol, and Nef antigens was observed in 79\% (15 of 19) of vaccine recipients 


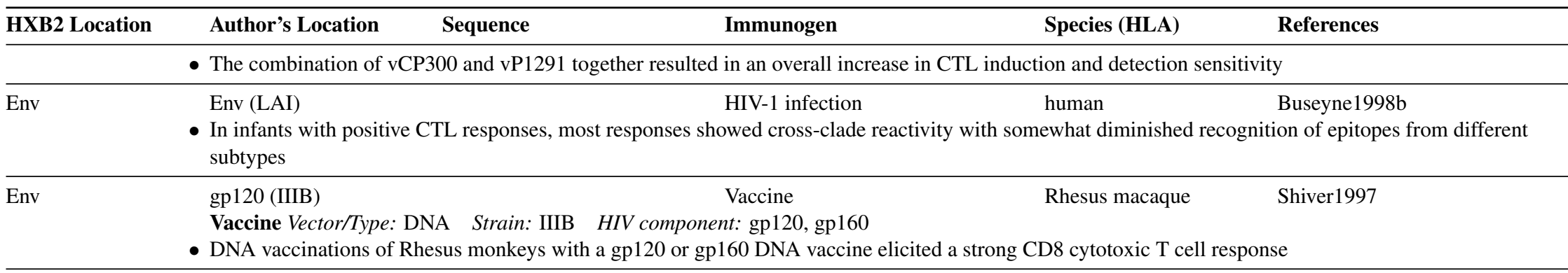

Env

$\begin{array}{lcc}\text { gp160 HIV-1 infection } & \text { Macaca nemestrina } \\ \text { Macaques can be infected with HIV, and clear the infection within } 6 \text { months, so it is of interest to examine their initial immune response }\end{array}$

- A strong CTL response against env, pol and gag antigens can be detected

- The CTL response peaked by 4 weeks and declined dramatically by 8 weeks

- The response in the lymph nodes and peripheral blood was comparable

Env gp160 Vaccine murine Kim1997c

Vaccine Vector/Type: DNA HIV component: Gag, Pol, Vif, Env Adjuvant: B7, IL-12

- A gag/pol, vif or env DNA vaccine, when delivered in conjunction with the plasmid encoding the co-stimulatory molecules B7 and IL-12, gave a dramatic increase in both the cytotoxic and proliferative responses in mice

- When IL-12 was present, CTL response could be detected even without in vitro stimulation

$\begin{array}{llll}\text { Env } & \text { Vp160 } & \text { Vaccine } & \text { murine } \\ & \text { Vaccine Vector/Type: DNA } & \text { HIV component: Gag, Pol, Vif, Env Adjuvant: B7, IL-12 }\end{array}$

- A gag/pol or env DNA vaccine, when delivered in conjunction with the plasmid encoding the co-stimulatory molecules CD86, gave a dramatic increase in both the cytotoxic and proliferative responses in mice

- When CD86 was present, CTL response could be detected even without in vitro stimulation

\begin{tabular}{llcc}
\hline Env & gp120 $(\mathrm{HXBc} 2)$ & Vaccine & Rhesus macaque
\end{tabular}

- Vaccination of Macaques mulatta (Rhesus monkeys) with an HXBc2 env DNA prime and a protein boost elicited a T cell proliferative response, a CTL response, and type-specific neutralizing antibodies

- Vaccinated animals challenged with SHIV-HXB2 were protected from infection

Env gp120 (MN) Vaccine human

Vaccine Vector/Type: DNA Strain: MN HIV component: Env, Rev

- An HIV DNA env and rev vaccine given to 15 asymptomatic HIV+ individuals at three different dosages, 30,100 or 300 ug, was safe

- The CTL response to gp120 was enhanced in 0/4 patients in the $30 \mu \mathrm{g}$ group, $2 / 3$ patients in the $100 \mu \mathrm{g}$ group, and $0 / 3$ in the $300 \mu \mathrm{g}$ group - but the non-responding patients in the $300 \mu \mathrm{g}$ group had a strong CTL response prior to vaccination, and the CTL results are inconclusive

\begin{tabular}{llcl}
\hline Env & gp120 (IIIB) HIV-1 infection & Trickett1998 \\
& - Twelve HIV-1 infected patients were re-infused with their own lymphocytes, cryopreserved from an earlier time point in the infection
\end{tabular}

- Improvement in CD4+ and CD8+ T cells was seen in 7/12, and an increase in the CTL response to Env was seen in one patient
Env
gp120 (LAI)
HIV-1 infection
human
Legrand1997
- Seventeen recently infected patients were tested for CTL response to HIV proteins Env, Gag, Pol, Rev, Nef, Vif and Tat 


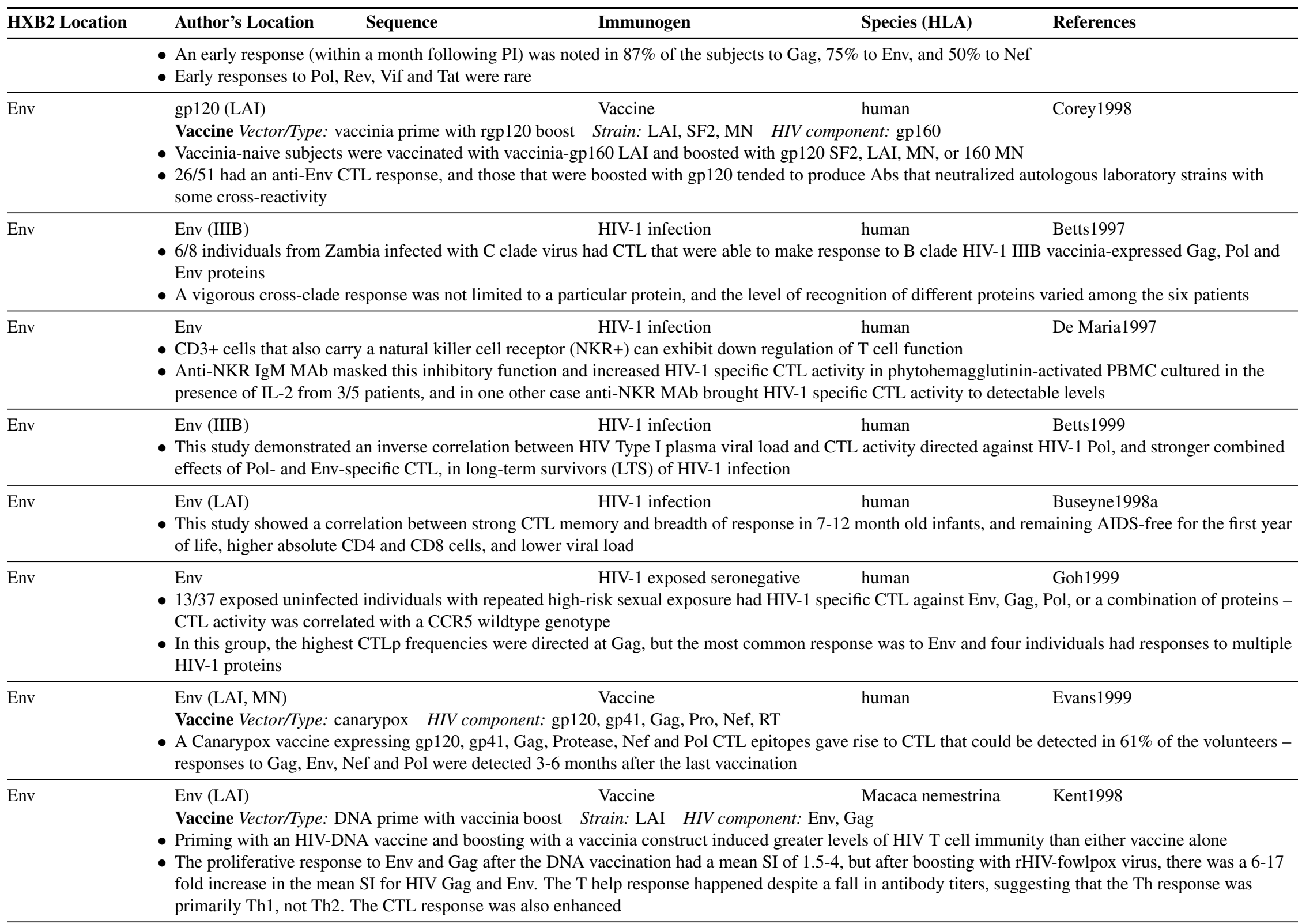




\begin{tabular}{llccc}
\hline HXB2 Location & Author's Location & Sequence & Immunogen & Species (HLA) \\
\hline Env & Env (LAI, MN) & & Vaccine & human \\
& Vaccine Vector/Type: canarypox & Strain: MN, LAI & HIV component: gp120, gp41, Gag, Protease
\end{tabular}

- A live attenuated canarypox vector expressing MN gp120 and LAI gp41/gag/protease could induce CTL and a lymphoproliferative response in healthy, uninfected volunteers

\begin{tabular}{ll}
\hline Env & Env (MN) Vaccine \\
& Vaccine Vector/Type: DNA HIV component: Env, Gag, Pol Adjuvant: CD86, CD80 \\
& The study explores the use of co-stimulatory molecules co-expressed with an HIV-1 immunogen in a DNA vaccine to enhance the immune response - \\
& co-expression of CD86, but not CD80, dramatically increased both HIV Env and Gag/Pol specific CTL and Th proliferative responses
\end{tabular}

Env gp120 (IIIB) Naccine Notka1999

Vaccine Vector/Type: Semliki-Forest Virus with virus-like particle boost Strain: IIIB HIV component: gag, gp120

- Immunization of SIV Pr56Gag-derived VLPs with HIV-1 gp120 anchored on their surface induced Abs, CTL and Th responses to HIV gp120; priming with the HIV antigens in Semliki-Forest Viruses enhanced the immunological outcome

- Immunized monkeys challenged with SHIV showed a more rapid reduction of plasma viremia

Env Env HIV-1 exposed seronegative human Akridge1999

- This study suggests that HIV-1-resistance in exposed and uninfected individuals is not only associated with the 32-bp deletion in the HIV-1 co-receptor CCR5, but can be related to HIV-1 specific CTL immunity

Env gp120 (BRU) HIV-1 infection human Aladdin1999

- In vitro measurements of CTL-activity by Cr release assay in bulk culture showed no correlation between CTL-activity (gp120, Gag, Pol and Nef) and disease progression as measured by viral load, CD4 and time to death

\begin{tabular}{llcl}
\hline Env & gp120 & HIV-1 infection & human \\
& $\bullet$ & The administration of IL-2 caused an initial enhancement of CD4 cell counts that was accompanied by a decrease in CTL activity - IL-2
\end{tabular}

- The administration of IL-2 caused an initial enhancement of CD4 cell counts that was accompanied by a decrease in CTL activity - IL-2 therapy did not reduce initial HIV viral load and viral replication was ultimately enhanced

Env Env HIV-1 infection

human

Jin1998a

- CTL precursor frequencies were determined in HIV-1 infected pregnant women, and higher CTLp frequencies to Pol and SF2 Nef, but not IIIB Nef, were found in non-transmitting mothers than in transmitting mothers - Nef CTL responses have been found in uninfected infants born to HIV+ women (Lazuriaga95);

- Very different CTLp frequencies were observed in env depending on whether IIIB, MN, RF, BK, or SF2 was used as antigen - no association between env specific CTL and transmission was observed

Env Env Vaccine Zavala2001

- This paper is a review of vaccinia in the context of vaccine vaccinia to very efficiently boost memory T-cell responses

- HIV is discussed in the context of Gonazalo et al. 1999, where a V3 CTL epitope expressed in reFlu was boosted most effectively by vaccinia expressing the full Env

\begin{tabular}{llcc}
\hline Env & Env & Vaccine & Rhesus macaque
\end{tabular}

Vaccine Vector/Type: DNA Strain: ZF1 HIV component: complete genome

- Rhesus macaques were vaccinated by i.m. injection with naked plasmid DNA carrying an HIV-1 complete genome vaccine, strain ZF1, with a mutated zinc finger in the nucleocapsid to prevent packaging 


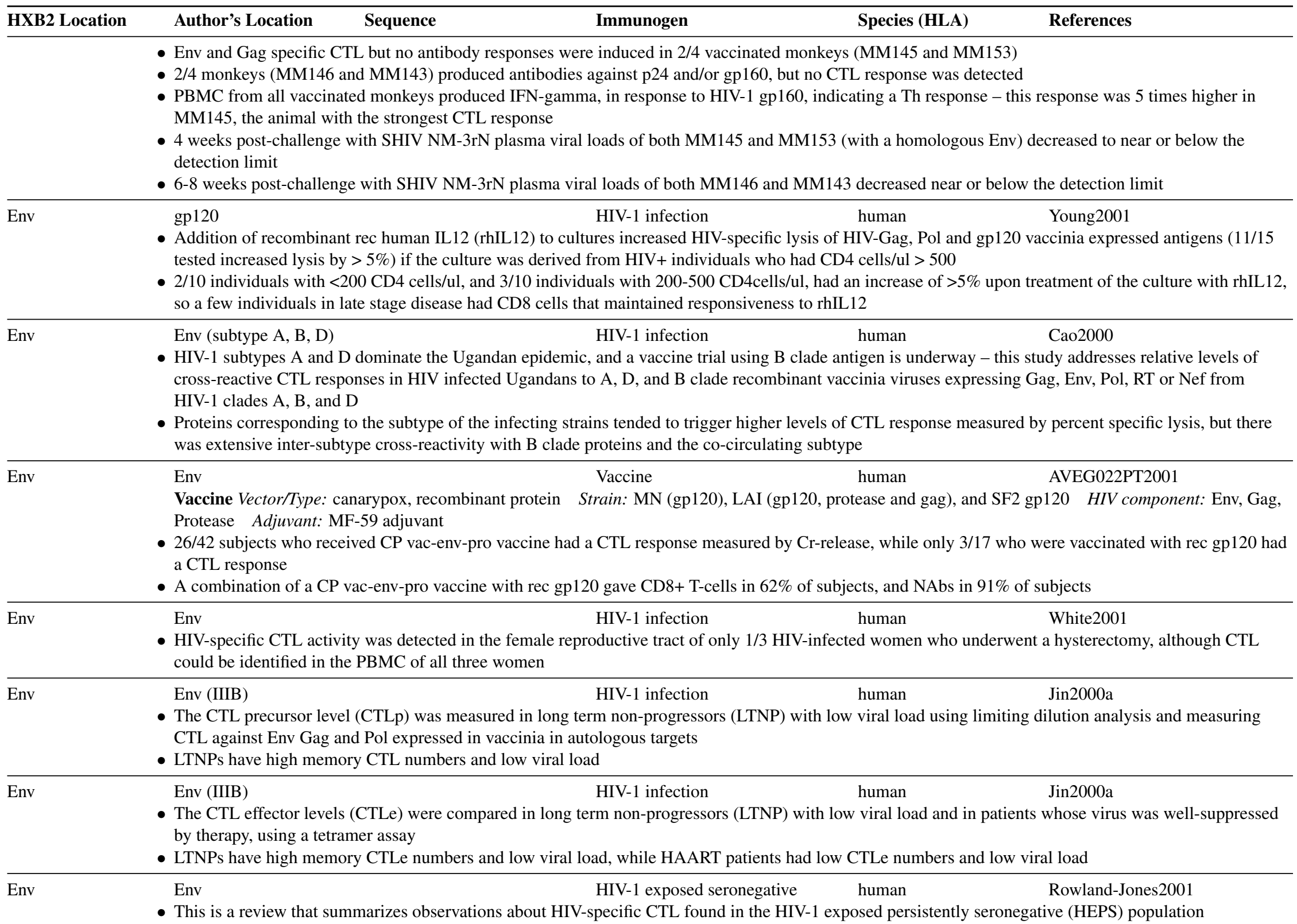




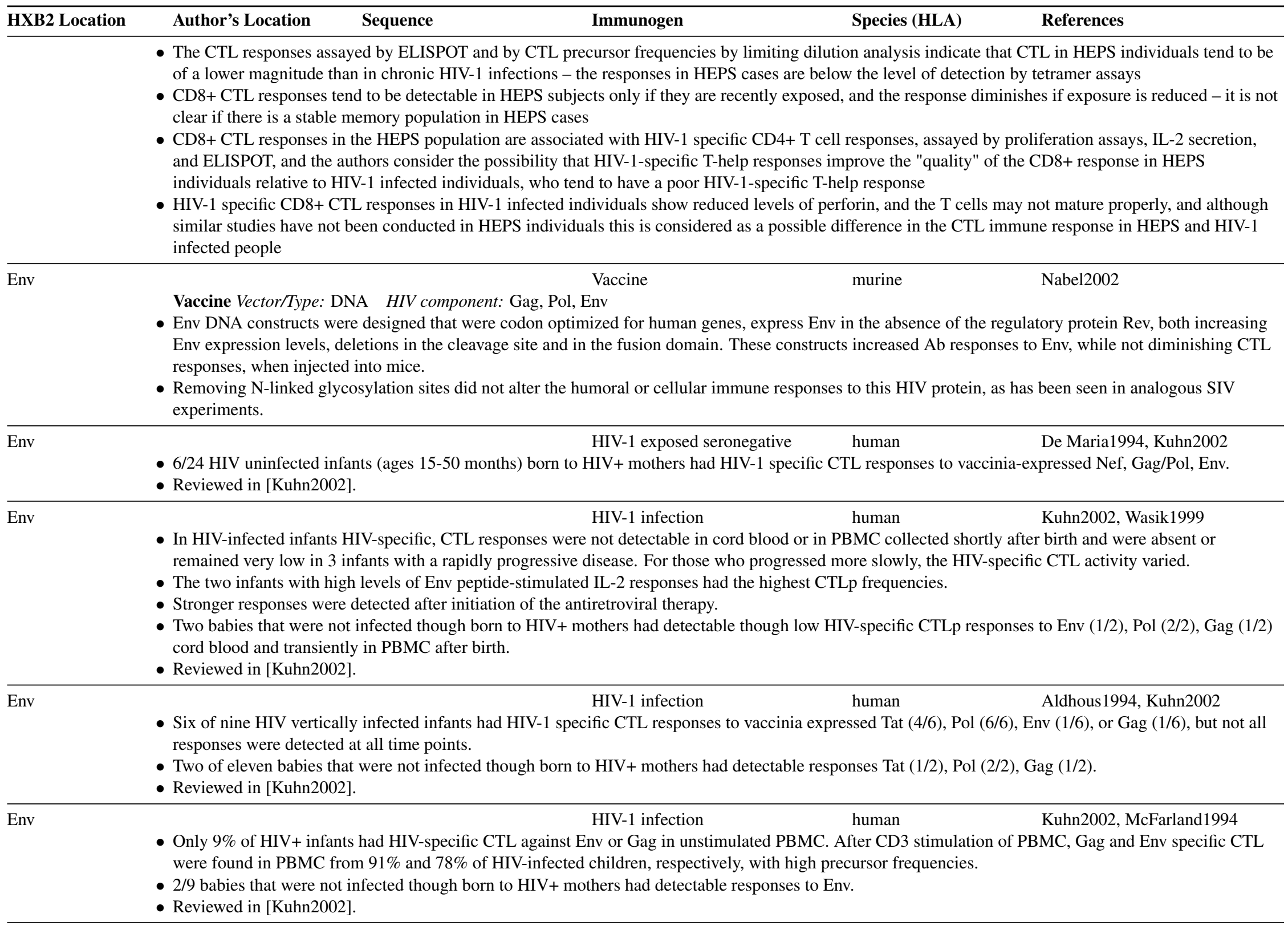




\begin{tabular}{lllll}
\hline HXB2 Location & Author's Location & Sequence & Immunogen & Species (HLA) \\
\hline Env & & HIV-1 infection & human
\end{tabular}

- Epitopes that have been described in the literature and included in this database tend to cluster in conserved regions and be absent or rarely found highly variable regions found in Nef, Env and p17.

- While the uneven distribution of epitopes may be in part due to a limited cross-recognition of specific responses because of differences between peptides used to probe the immune response and autologous strains, regions with a paucity of defined epitopes also had higher frequencies of amino acids that tend to not be found in C-terminal positions of epitopes, and had lower cleavage prediction scores for epitope processing. This suggests that in the regions of the virus where variation is best tolerated traces of immune escape have left an imprint on the viral population. Epitopes also were concentrated in alpha-helix and turn regions in the proteins.

- In the more conserved p24, and Pol proteins RT and Protease, epitopes are more evenly distributed.

$$
\text { HIV-1 infection human Trabattoni2002 }
$$

- CD8+ T-cells that were stimulated by HIV-1 Env expressing targets from $25 \mathrm{HIV}+$ patients receiving ART and 17 ART-naive patients were compared. CTL from the individuals receiving ART showed increased TNFalpha production and a reduction of perforin and granzyme expressing CTL, suggesting a functional defect in ART-treated individuals, and a potential benefit of immunomodulants during therapy.
Env (HXB2)
HIV-1 infection
human
Edwards2002

- $96 \%$ (26/27) chronically infected HIV-1 infected patients elicited gamma-IFN CD8+ T-cell responses against Gag

- Nef and/or Pol CTL responses were detected in $86 \%$ of the subjects

- The magnitude and breadth of Gag and p24 T-cell responses correlated with absolute CD4 counts, and inversely correlated with viral load

- Pol and Int CTL responses correlated positively with absolute CD4+ T-cell count

- Nef and Env responses did not correlate with either CD4 counts or viral load

\begin{tabular}{llccc}
\hline Env & Env & Vaccine & murine & Ishii2001 \\
& Vaccine Vector/Type: DNA & Strain: IIIB & HIV component: gp160, Rev & Adjuvant: cationic liposome, IL2, GMCSF
\end{tabular}

Vaccine Vector/Type: DNA Strain: IIIB HIV component: gp160, Rev Adjuvant: cationic liposome, IL2, GMCSF

- Vaccination route of HIV-1 DNA immunization with gp160 and Rev genes was compared including intranasal (i.n.), intramuscular (i.m.), and topical application of DNA directly on the skin after elimination of keratinocyte layers using a strong adhesive. Topical exposure resulted in high level CTL responses, IFN-gamma and IL-4 production, and delayed type hypersensitivity (DTH). Topical application favored Th2 responses.

- DNA delivered topically with adjuvant-like cationic liposomes gave a stronger response than DNA alone, and co-administration of the DNA vaccine with IL-12 and GM-CSF expression vectors enhanced cytotoxic activity and DTH.

- Autologous mature dendritic cells with rec vaccinia expressing Gag, Pol, Nef and Env could amplify CD8+ T-cell Elispot responses 4-38 fold in five HIV+ patients on successful HAART treatment, relative to autologous monocytes. Some weak responses could only be detected using mature dendritic cells as APCs, and this approach could be useful for detection of low frequency memory cells.

Env (IIIB) HIV-1 infection human Trickett2002

- Conditions were optimized for ex-vivo expansion of CD8+ and CD4+ T-cells with the goal of functional T-cell production for autologous immunotherapy. 10,000 -fold expansions were obtained in 14 days with optimized concentrations of IL-2, anti-CD3 and anti-CD28 coated microspheres, and decreasing amounts of serum over the first 8 days.

Env (IIIB)

HIV-1 and HCV co-infection

human

Lauer2002

- HIV-1 and HCV immune responses were studied in 22 individuals who were co-infected with HIV-1 and hepatitis C virus (HCV). IFNgamma production was measured in an Elispot assay of CD8+ T-cells using targets expressing either Gag, RT, Env and Nef in a vaccinia construct, or one of seven HCV proteins. 


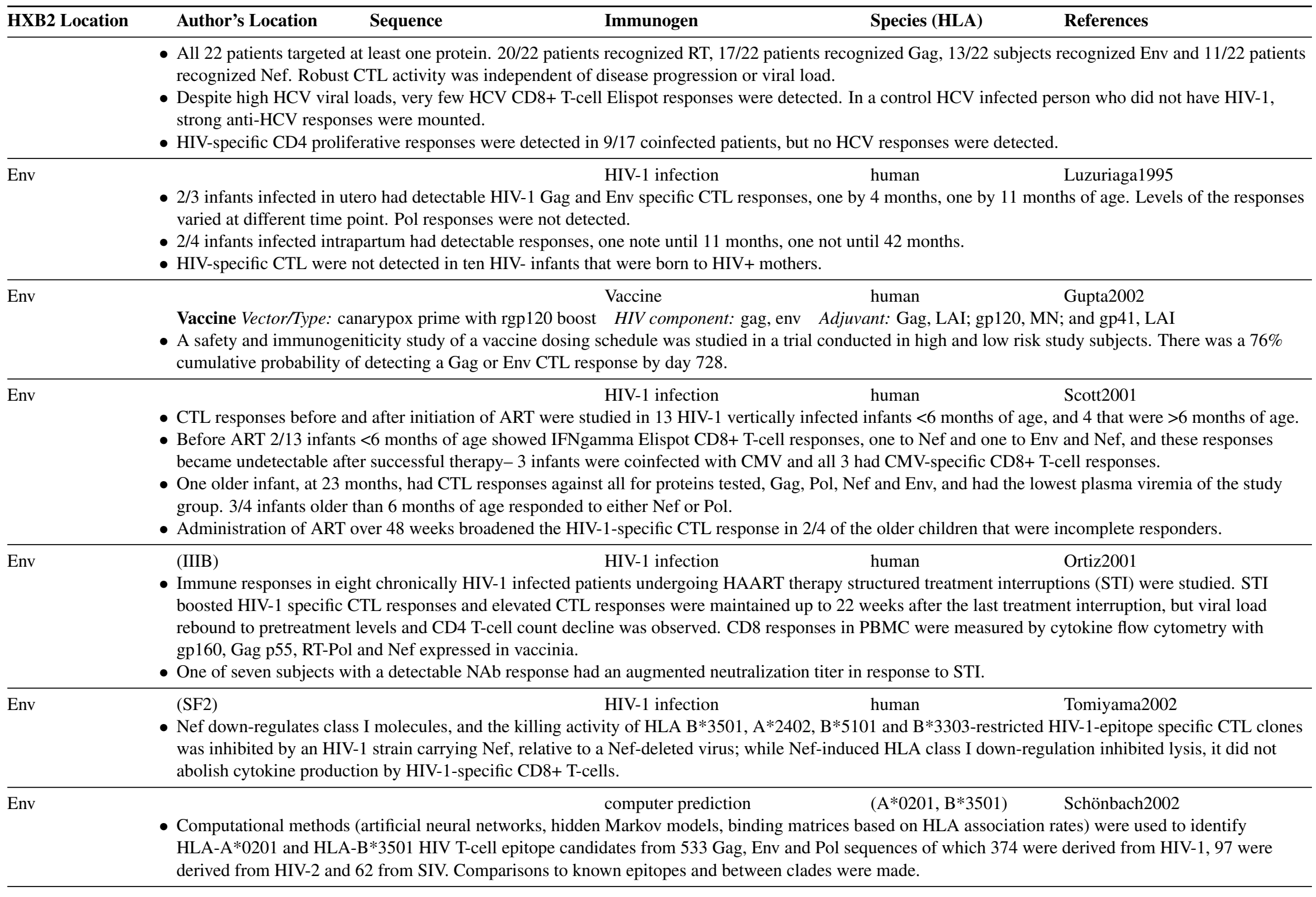




\begin{tabular}{|c|c|c|c|c|c|}
\hline HXB2 Location & Author's Location & Sequence & Immunogen & Species (HLA) & References \\
\hline Env & & & Vaccine & $\begin{array}{l}\text { human (A1, A2, A24, } \\
\text { B62, A25, A26, A30, } \\
\text { A31, B8, B17, B39, } \\
\text { B51, B57, B60, B62, } \\
\text { B70) }\end{array}$ & Ferrari2001 \\
\hline
\end{tabular}

component: gp120, gp41, Gag, Pol and Nef epitope rich regions

- HLA-B62 responses dominated the responses against an Env vaccine in an individual (022JAV) who was HLA A2, A26, B35, B62. The strongest response

was against the MN peptide 381-400; a response diminished by half was observed against vaccinia expressed clade $\mathrm{A}$ and clade $\mathrm{C}$ relative to clade $\mathrm{B}$.

- Class I presentation of Env CTL responses in vaccinee 022A12K: A25 > B39, A1 and B8 were undetectable.

- Class I presentation of Env CTL responses in vaccinee 022A12N: B57 » A2 > A26 and B60.

- Class I presentation of Env CTL responses in vaccinee 034GP3: A31 > A24 > B62 > B51.

- Class I presentation of Env CTL responses in vaccinee 0348PP: B17 > B70, A1 and A30 were undetectable.

Env gp120(303-327) HIV-1 infection $\quad$ Ferrari2000

- One of the 51 HIV-1 epitopes selected by Ferrari et al. as good candidate CTL epitopes for vaccines by virtue of being conserved and presented by common

HLA alleles

- For this cluster of epitopes spanning the tip of the V3 loop, they suggest including a sequence from each clade

Env

Vaccine Vector/Type: canarypox prime with rgp120 boost, canarypox prime with rgp160 boost Strain: gp41 LAI, Gag LAI, gp120 MN, gp120 SF2 HIV component: gp120, gp41, Gag, Pol and Nef epitope rich regions

- No HLA-A*0201 or B8 responses were made against the Env vaccine in individuals carrying these alleles, despite these being common presenting molecules for CTL responses to natural infections.

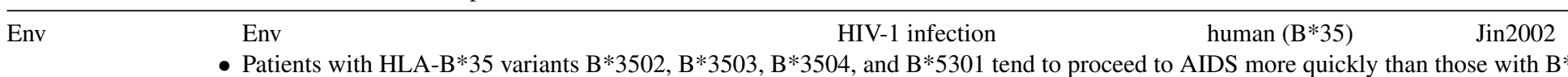

- Of 32 patients with HLA-B*35 alleles CD8+ CTL responses were quantified using an intracellular cytokine staining assay - 75\% had responses to Pol, $69 \%$ to Gag, $50 \%$ to Nef, and $41 \%$ to Env.

- The overall magnitude of CTL responses did not differ between those bearing B*3501 and the others. A higher percentage of Gag responses was observed in those that had lower RNA levels that carried $\mathrm{B} * 3501$, and there was a negative association with viral load and CTL activity. The data is consistent with higher levels of CTL responses contributing to protection in $\mathrm{B} * 3501$ individuals, but not in $\mathrm{B} * 3502, \mathrm{~B} * 3503$, $\mathrm{B} * 3504$, and $\mathrm{B} * 5301$ individuals.

\begin{tabular}{llll}
\hline Env & gp41 (842-850 IIIB, & HIV-1 infection & human (B7) Pantaleo1997, Soudeyns1997
\end{tabular}
BH8)

- Clonotype-specific PCR and analysis of in vivo HIV-specific CTL showed that in early infection HIV-specific CTL clones preferentially accumulate in blood rather than lymph nodes and that they accumulate prior to down-regulation of virus

\begin{tabular}{llcc}
\hline Env & gp160 (MN) & Vaccine & murine (H-2 $\left.{ }^{d}\right)$
\end{tabular}

- Mammalian codon optimization renders gp160 expression Rev independent, increases gp160 expression levels, and DNA vaccination of BALB/c mice yields a higher antibody response with an earlier onset than wild type

- Secreted gp120 gave higher antibody titers than membrane bound gp160 


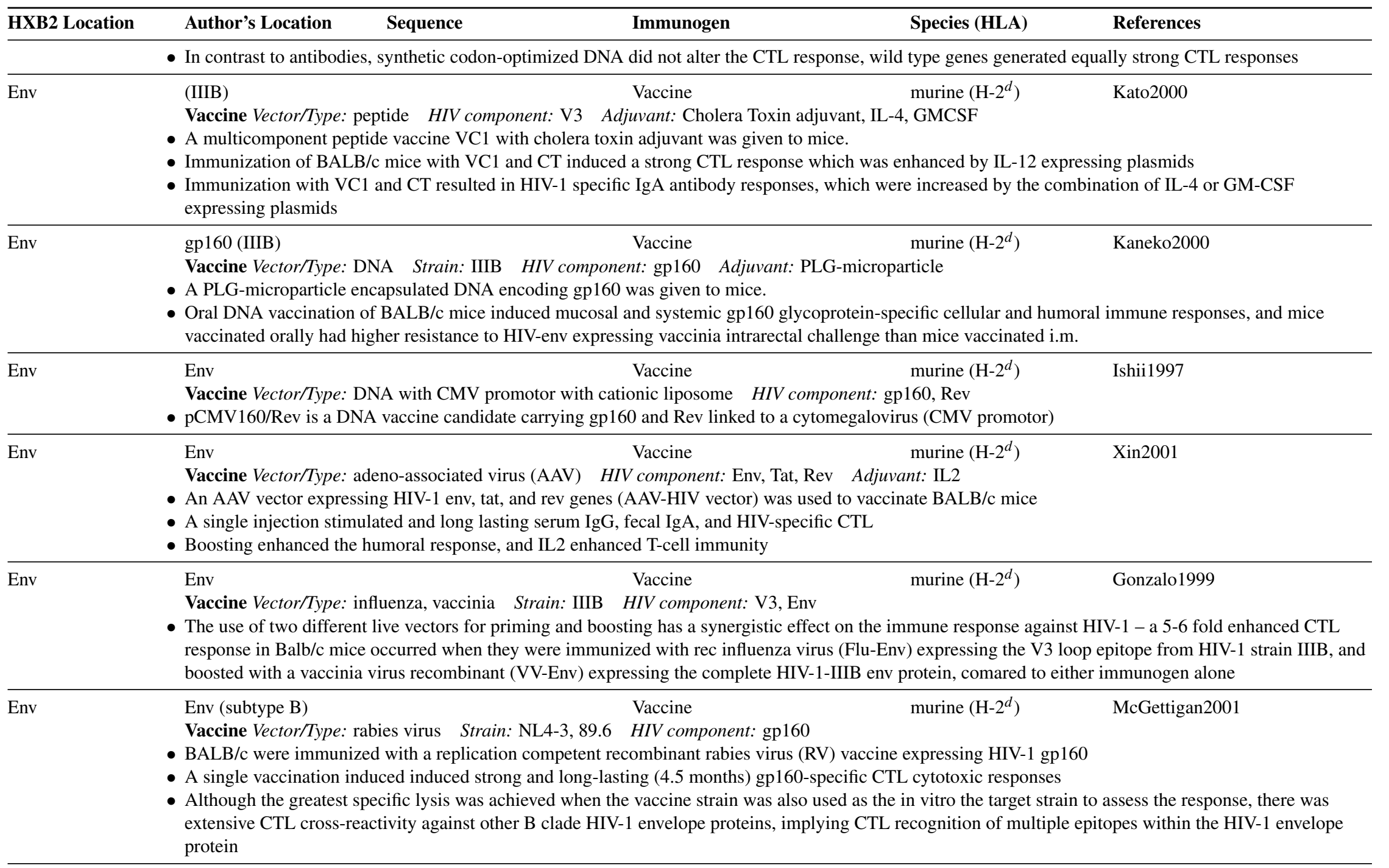




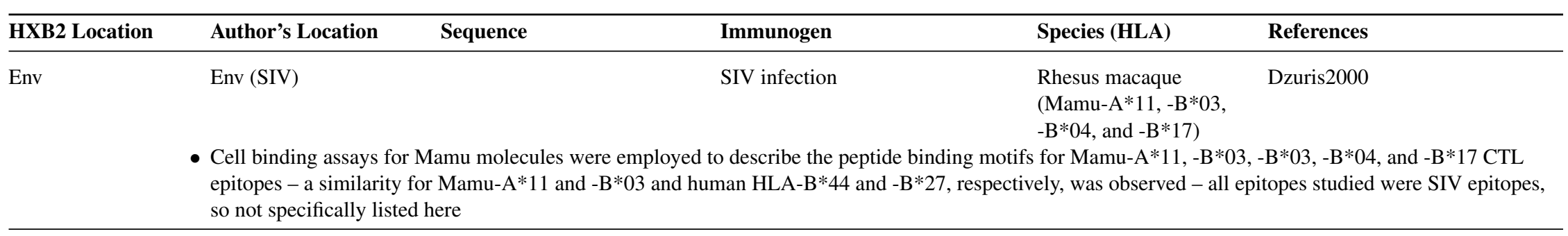




\section{II-B-20 Nef CTL Epitopes}

\begin{tabular}{llllc}
\hline HXB2 Location & Author's Location & Sequence & Immunogen & Species (HLA) \\
\hline Nef (1-16) & Nef (1-16) & MGGKWSKSS IVGWPAV & HIV-1 infection & human \\
& - HLA, viral sequence, and Elispot data was obtained from 105 HIV-1 positive Botswanans; Elispot data was obtained from between 55 and 64 subjects for
\end{tabular}
- HLA, viral sequence, and Elispot data was obtained from 105 HIV-1 positive Botswanans; Elispot data was obtained from between 55 and 64 subjects for
each HIV protein.

- Nef and p24 had the highest percentage of reactive peptides, and p24 had the highest magnitude of HIV-1 responses.

- This peptide was among the 28 most reactive $\mathrm{C}$ clade peptides from among over 350 tested spanning all HIV proteins.

\begin{tabular}{llcc}
\hline Nef (13-20) & Nef (13-20 LAI) $\begin{array}{c}\text { WP TVRERM } \\
\text { C. Brander notes this is a B*0801 epitope }\end{array}$ & HIV-1 infection & human (B*0801) \\
\hline Nef (13-20) & Nef (HXB2) $\quad$ WP TVRERM & HIV-1 infection & B $* 0801)$ \\
& $\begin{array}{l}\text { Deletion of the 19 N-terminal amino acids from Nef including the myristolation signal eliminates Nef-induced down-regulation of MHC class I and CD4 } \\
\text { molecules. Such a construct has the potential to serve as a more potent immunogen. The known T-cell epitopes that that would be disputed by this deletion }\end{array}$
\end{tabular}
are minimal, including the HLA-B8 CTL epitope WPTVRERM.

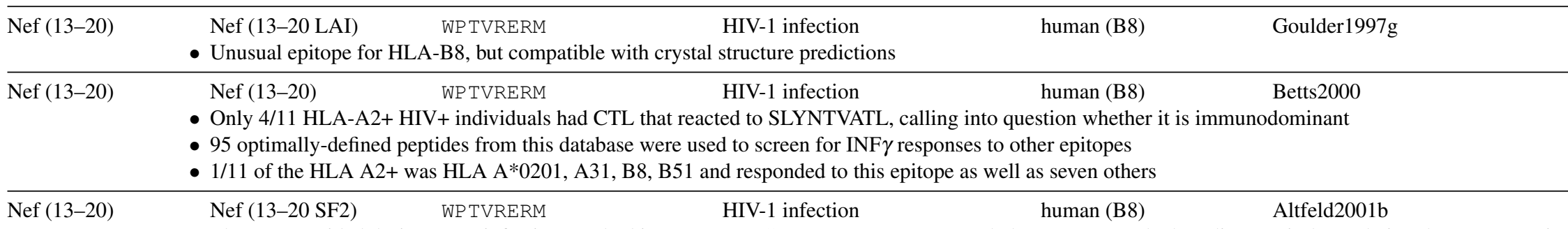

- Therapy provided during acute infection resulted in a narrower CTL response, stronger T help response, and a less diverse viral population than was seen in individuals treated during chronic infection

- The breadth and specificity of the response was determined using ELISPOT by studying 19 individuals with pre-seroconversion therapy (Group 1), 11 individuals with primary infection but post-seroconversion therapy (Group 2), and 10 individuals who responded to HAART given during chronic infection (Group 3), using 259 overlapping peptides spanning p17, p24, RT, gp41, gp120 and Nef

- Previously described and newly defined optimal epitopes were tested for CTL response

- Number of HLA-B8+ individuals that had a CTL response to this epitope broken down by group: 1/3 group 1,0/3 group 2, and 1/2 group 3

\begin{tabular}{|c|c|c|c|c|c|}
\hline \multirow[t]{2}{*}{ Nef (13-20) } & Nef (13-20) & WPTVRERM & HIV-1 infection & human (B8) & Day2001 \\
\hline & \multicolumn{5}{|c|}{ - B8-restricted CTL accounted for about $1 / 3$ of the total CTL response in one individual } \\
\hline Nef $(42-50)$ & Nef (44-52 HXB3) & ALTSSNTAA & Vaccine & $\begin{array}{l}\text { murine (HLA-A201 } \\
\text { transgenic) }\end{array}$ & Sandberg2000 \\
\hline
\end{tabular}

Vaccine Vector/Type: DNA, peptide Strain: HXB3 HIV component: Nef Adjuvant: Freund's adjuvant

- Ten Nef 9-mer peptides were predicted to have a strong binding affinity with HLA-A*0201 - of these, four did bind strongly by a T2 class I stabilization assay, several others bound weakly

- A CTL immune response to only 3/10 peptides was detected by a 51Cr-release assay after immunization of HLA-A201 transgenic mice with either nef

DNA under the control of a CMV promotor, coated on gold particles delivered to abdominal skin by gene gun

- ALTSSNTAA was also tested by subcutaneous injection of Nef peptides in Freund's adjuvant 


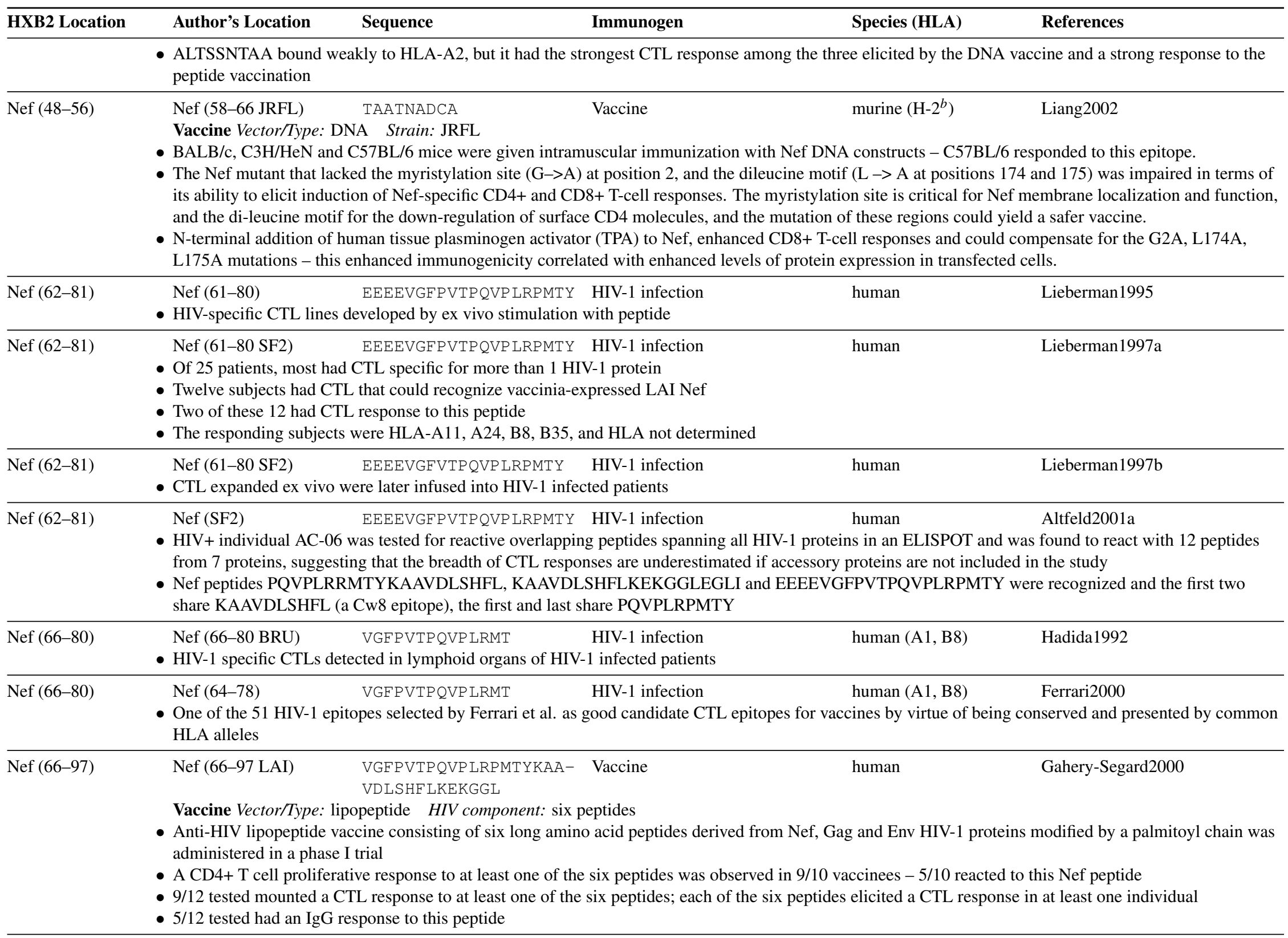




\begin{tabular}{lllll}
\hline HXB2 Location & Author's Location & Sequence & Immunogen & Species (HLA) \\
\hline Nef (67-81) & Nef (67-81) & GFPVRPQVP LRPMTY & HIV-1 infection & human
\end{tabular}

- HLA, viral sequence, and Elispot data was obtained from 105 HIV-1 positive Botswanans; Elispot data was obtained from between 55 and 64 subjects for each HIV protein.

- Nef and p24 had the highest percentage of reactive peptides, and p24 had the highest magnitude of HIV-1 responses.

- This peptide was among the 28 most reactive $\mathrm{C}$ clade peptides from among over 350 tested spanning all HIV proteins.

\begin{tabular}{|c|c|c|c|c|c|}
\hline Nef (68-76) & $\begin{array}{l}\text { Nef (72-80 SF2) } \\
\text { - } \text { A CTL clone resp } \\
\text { - } 3 / 7 \text { B35-positive } \\
\text { - An R to T substit }\end{array}$ & $\begin{array}{l}\text { FPVRPQVPL } \\
\text { o this epitope } \\
\text { als had a CTL } \\
\text { position } 4 \text { abro }\end{array}$ & $\begin{array}{l}\text { HIV-1 infection } \\
\text { pitope } \\
\text { s, but not binding }\end{array}$ & human $(\mathrm{B} * 3501)$ & Tomiyama1997 \\
\hline Nef (68-76) & Nef (72-80) & FPVRPQVPL & HIV-1 infection & human $(\mathrm{B} * 3501)$ & Tomiyama2000a \\
\hline
\end{tabular}

- CD8+ T-cells that bound one of six HIV-specific B*3501-epitope tetramers did not express CD28 or CD45A

- A significant increase in CD28-CD45RA- cells and a decrease of CD28+CD45RA+ cells was observed in chronically HIV-1-infected individuals relative to healthy individuals

- CD28-CD45RA- cells are likely to be effector cells and have high levels of perforin in their cytoplasm

- The mean percentage of total CD28- CD8+ cells in chronically infected HIV-1-infected patients was 76.6\% in comparison to HIV-1-uninfected individuals $(40.6 \%)$

\begin{tabular}{llllr}
\hline Nef (68-76) & $\begin{array}{l}\text { Nef (72-80 SF2) } \\
\text { Binds HLA-B*3501 }\end{array}$ & FPVRPQVPL & HIV-1 infection & human (B35) \\
\hline Nef (68-76) & (SF2) & FPVRPQVPL & HIV-1 infection & Shiga1996 \\
\hline
\end{tabular}

FPVRPQVPL
HLA B35 is associated with rapid disease progression

- The sequences of 9 previously described HIV-1 B35 CTL epitopes were obtained in 10 HLA B35+ and 19 HLA B35- individuals

- 3/9 CTL epitopes had substitutions that were more common in B35+ individuals than in B35- individuals, but this was one of the six that had no B35 associated pattern of mutation

Nef (68-76) Nef (66-74) FPVRPQVPL $\quad$ HIV-1 infection $\quad$ Ferrari2000

- One of the 51 HIV-1 epitopes selected by Ferrari et al. as good candidate CTL epitopes for vaccines by virtue of being conserved and presented by common HLA alleles

\begin{tabular}{lllcc}
\hline Nef (68-76) Nef (68-76 BRU) & FPVTPQVPL & HIV-1 infection & human (B35) \\
\hline
\end{tabular}

- Seventy-three 8-11 mer peptides were generated based on putative anchor motifs in the Nef BRU sequence for HLA A2, A3, A24, B7, B8, and B35; these 73 peptides had 81 motifs. 54\% bound to the predicted HLA molecule, particularly A2, B7/35, and B8. The strength of HLA-peptide binding was not directly related to the number of individuals that recognized a protein.

- 20 s proteasome cleavage of the Nef protein positions $66-100$ showed a large fraction of peptides were cleaved ending at: 87L, 83A, 81Y, 71P, 68F and 67G. The frequency of recognition may be in part dictated by the cleavage step in processing.

- FPVTPQVPL was recognized in 1/13 (8\%) of individuals with HLA B7, and 1/12 (8\%) of individuals with HLA B35. It was a high affinity HLA binder.

Nef (68-76) Nef (68-76) FPVTPQVPL $\quad$ in vitro stimulation human (B7) Wilson1999b

- Dendritic cells are the most potent for priming T cell responses - DCs can stimulate autologous CTL responses from T cells cultured from HIV negative donors

- Th1-biasing cytokines IL-12 or IFN alpha enhance CTL responses in vitro whether the epitope is delivered by pulsing from peptide, or expressed from within 


\begin{tabular}{llcccc}
\hline HXB2 Location & Author's Location & Sequence & Immunogen & Species (HLA) & References \\
\hline & $\bullet$ & B7 and A2 Nef epitopes were studied - FPVTPQVPL has a high affinity for B7 & & \\
\hline Nef (68-76) & Nef (68-76) & FPVTPQVPL & HIV-1 infection & human (B7)
\end{tabular}

- The CTL response to optimally defined CTL epitopes restricted by HLA class I A and B alleles in individuals who coexpressed HLA A2, A3, and B7 was studied in eight HIV-1-infected subjects, two with acute infection, five with chronic, and one long-term non-progressor (LTNP)

- 2 to 17 epitopes were recognized in a given individual, A2-restricted CTL response tended to be narrow and never dominated the response, and 25/27 epitopes were targeted by at least one person

- Subjects with chronic HIV-1 infection recognized between 2-8 out of 11 B7-restricted epitopes

- An acute seroconvertor homozygous for the B7 allele recognized five B7-restricted epitopes

- The other acute seroconvertor failed to recognize any of the 11 B7-restricted epitopes tested

- The B7-restricted CTL response was highly variable and there was no clearly dominant epitope

\begin{tabular}{|c|c|}
\hline Nef (68-76) & 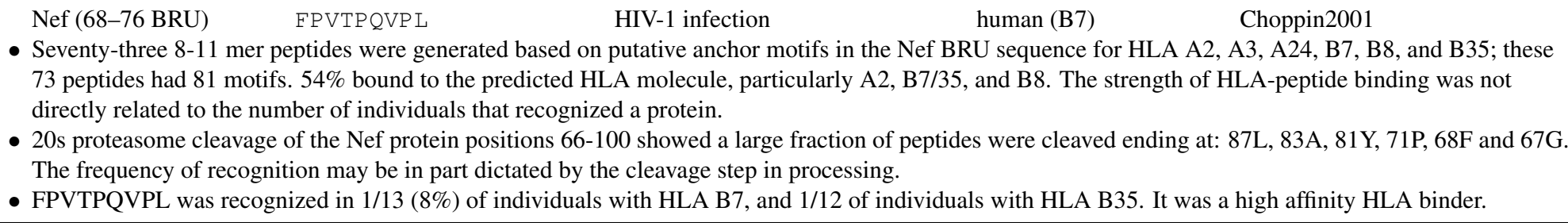 \\
\hline Nef (68-76) & $\begin{array}{l}\text { Nef (68-76) FPVTPQVPL } \\
\text { - CTL responses in 18 acutely HIV-infected HLA-A3 (n=7) or -B7 (n=4) or both -A3 and B7 (n=7) positive individuals were studied. } \\
\text { - One individual, AC-06, was homozygous at all three class I alleles (A3, B7, Cw7), was treated during acute infection and had supervised treatment } \\
\text { interruptions (STI). He had only two detectable CTL responses during acute infection, but after STI this broadened to } 27 \text { distinct epitopes including 15 } \\
\text { restricted by HLA-A3, } 11 \text { by HLA-B7, and 1 by HLA-Cw7. } \\
\text { - 0/11 HLA-B7 individuals had detectable B7-restricted responses to this epitope during acute infection - 10/15 of HLA-B7 epitopes tested were targeted by } \\
\text { at least one person during acute infection. Also, none of } 4 \text { individuals had detectable responses to this epitope after STI. }\end{array}$ \\
\hline Nef (68-77) & $\begin{array}{l}\text { Nef (68-77 LAI) EPVTPQVP LR } \\
\text { - C. Brander notes this is a B*0702 epitope }\end{array}$ \\
\hline Nef (68-77) & $\begin{array}{l}\text { Nef (68-77 LAI) FPVTPQVPLR } \\
\text { - There was a high degree of variation in three CTL epitopes in Nef in four slow and non-progressors, and variant specific CTLs arose over time to eliminate } \\
\text { variants, indicating immune selection }\end{array}$ \\
\hline Nef (68-77) & $\begin{array}{l}\text { Nef (subtype B) FPVTPQVPLR HIV-1 infection } \\
\text { - This study examines CTL responses in HIV exposed, persistently seronegative individuals, HEPS, who eventually seroconverted - 11/114 HEPS Nairobi } \\
\text { sex workers eventually seroconverted, and for six of these HIV CTL reactive epitopes had been defined while seronegative } \\
\text { - FPVTPQVPLR was recognized in } 1 \text { of the } 6 \text { women (ML1203), and the response was present in the last available sample prior to seroconversion, } 7 \text { months } \\
\text { - } 20 / 20 \text { sequences of the infecting strain had no substitutions in this epitope, all were FPVTPQVPLR, so there was no evidence for escape } \\
\text { - The epidemiological factor associated with seroconversion was stopping sex work and HIV-specific CTL activity declines when HEPS sex workers stop } \\
\text { working for a period or retire } \\
\text { - This epitope was recognized in 1/22 HEPS sex worker controls, ML851 }\end{array}$ \\
\hline
\end{tabular}




\begin{tabular}{lllll}
\hline HXB2 Location & Author's Location & Sequence & Immunogen & Species (HLA) \\
\hline Nef (68-77) & Nef (66-75) & FPVRP QVP LR & HIV-1 infection & human (B7)
\end{tabular}

- One of the 51 HIV-1 epitopes selected by Ferrari et al. as good candidate CTL epitopes for vaccines by virtue of being conserved and presented by common HLA alleles

\begin{tabular}{lllc}
\hline Nef (68-77) & Nef (68-77 SF2) F HIV-1 infection & human (B7) \\
& Therapy provided during acute infection resulted in a narrower CTL response, stronger Thelp response, and a less diverse viral population than was seen in \\
& individuals treated during chronic infection
\end{tabular}

- Therapy provided during acute infection resulted in a narrower CTL response, stronger T help response, and a less diverse viral population than was seen in
individuals treated during chronic infection

- The breadth and specificity of the response was determined using ELISPOT by studying 19 individuals with pre-seroconversion therapy (Group 1), 11 individuals with primary infection but post-seroconversion therapy (Group 2), and 10 individuals who responded to HAART given during chronic infection (Group 3), using 259 overlapping peptides spanning p17, p24, RT, gp41, gp120 and Nef

- Previously described and newly defined optimal epitopes were tested for CTL response

- Number of HLA-B7+ individuals that had a CTL response to this epitope broken down by group: 0/4 group 1, 0/3 group 2, and 1/1 group 3

\begin{tabular}{llll}
\hline Nef (68-77) Nef (68-77) FPVTPQVPLR & $\begin{array}{l}\text { HIV-1 infection, HIV-1 exposed human (B7) } \\
\text { seronegative }\end{array}$
\end{tabular}

- ELISPOT was used to study CTL responses to a panel of 54 predefined HIV-1 epitopes in 91 HIV-1-exposed, persistently seronegative (HEPS) and 87 HIV-1-infected female Nairobi sex workers

- Responses in HEPS women tended to be lower, and focused on different epitopes with HLA presenting molecules that have previously been associated with reduced risk of infection, and there was a shift in the response in the HEPS women upon late seroconversion to epitopes recognized by the HIV-1 infected women

- 43/91 HEPS women had CD8+ responses and detection of HIV-1-specific CTL in HEPS women increased with the duration of viral exposure

- Subject ML 1203 started with CTL responses to A*6802 DTVLEDINL and to B7 FPVTPQVPLR prior to seroconversion, and upon seroconversion acquired additional responses to A*6802 ETAYFILKL which became dominant, B7 TPGPG(V/I)RYPL, B7 IPRRIRQGL, and B7 SPRTLNAWV

\begin{tabular}{lllcl}
\hline Nef (68-77) & Nef (68-77) & FPVTPQVPLR & HIV-1 infection & human (B7) \\
& - & The CTL response to optimally defined CTL epitopes restricted by HLA class I A and B alleles in individuals who coexpressed HLA A2, A3, and B7 was
\end{tabular}

- 2 to 17 epitopes were recognized in a given individual, A2-restricted CTL response tended to be narrow and never dominated the response, and 25/27 epitopes were targeted by at least one person

- Subjects with chronic HIV-1 infection recognized between 2-8 out of 11 B7-restricted epitopes

- An acute seroconvertor homozygous for the B7 allele recognized five B7-restricted epitopes

- The other acute seroconvertor failed to recognize any of the $11 \mathrm{~B} 7$-restricted epitopes tested

- The B7-restricted CTL response was highly variable and there was no clearly dominant epitope

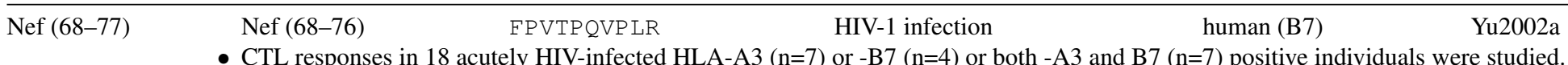

- One individual, AC-06, was homozygous at all three class I alleles (A3, B7, Cw7), was treated during acute infection and had supervised treatment interruptions (STI). He had only two detectable CTL responses during acute infection, but after STI this broadened to 27 distinct epitopes including 15 restricted by HLA-A3, 11 by HLA-B7, and 1 by HLA-Cw7.

- 0/11 HLA-B7 individuals had detectable B7-restricted responses to this epitope during acute infection - 10/15 of HLA-B7 epitopes tested were targeted by at least one person during acute infection. Also, none of 4 individuals had detectable responses to this epitope after STI. 


\begin{tabular}{lllll}
\hline HXB2 Location & Author's Location & Sequence & Immunogen & Species (HLA) \\
\hline Nef (68-81) & $\begin{array}{l}\text { Nef (82-95 HXB2) } \\
\text { - }\end{array}$ & $\begin{array}{c}\text { FPVTPQVP LRMTY } \\
\text { fprequences were obtained from Brazilians to study epitope diversity in this geographic region- the HXB2 sequence is FPVTPQVPLRMTY, but }\end{array}$ & HIV-1 infection & human \\
& &
\end{tabular}

Nef (68-84) Nef FPVRPQVP LRPMTYKGA human Jubier-Maurin1999

- 41 new HIV-1 strains describing envelope subtypes of HIV-1 A-H were genetically characterized in the nef region - 34 subtypes were classified in the same subtype in nef and env and 7 of the 41 strains were recombinants

- This region was defined as a CTL epitope region that is conserved among HIV-1 M group subtypes

\begin{tabular}{llllll}
\hline Nef (71-79) & \multicolumn{1}{l}{ Nef (71-79 LAI) } & TPQVP LRPM & HIV-1 infection & human (B*0702) & Brander2001 \\
& - C. Brander notes this is a B*0702 epitope & & & \\
\hline Nef (71-79) & Nef (71-79 BRU) & TPQVP LRPM & HIV-1 infection & human (B35) & Choppin2001 \\
\hline
\end{tabular}

- Seventy-three 8-11 mer peptides were generated based on putative anchor motifs in the Nef BRU sequence for HLA A2, A3, A24, B7, B8, and B35; these 73 peptides had 81 motifs. 54\% bound to the predicted HLA molecule, particularly A2, B7/35, and B8. The strength of HLA-peptide binding was not directly related to the number of individuals that recognized a protein.

- 20 s proteasome cleavage of the Nef protein positions $66-100$ showed a large fraction of peptides were cleaved ending at: 87L, 83A, 81Y, 71P, 68F and 67G. The frequency of recognition may be in part dictated by the cleavage step in processing.

- TPQVPLRPM was recognized in 1/10 (10\%) of individuals with HLA B7, and 1/10 (10\%) of individuals with HLA B35. It was a moderate affinity HLA binder.

\begin{tabular}{llccc}
\hline Nef (71-79) & Nef (71-79 SF2) & TPQVPLRPM & HIV-1 infection & human (B7) \\
& - Therapy provided during acute infection resulted in a narrower CTL response, stronger T help response, and a less diverse viral population than was seen in
\end{tabular}

- Therapy provided during acute infection resulted in a narrower CTL response, stronger T help response, and a less diverse viral population than was seen in individuals treated during chronic infection

- The breadth and specificity of the response was determined using ELISPOT by studying 19 individuals with pre-seroconversion therapy (Group 1), 11 individuals with primary infection but post-seroconversion therapy (Group 2), and 10 individuals who responded to HAART given during chronic infection (Group 3), using 259 overlapping peptides spanning p17, p24, RT, gp41, gp120 and Nef

- Previously described and newly defined optimal epitopes were tested for CTL response

- Number of HLA-B7+ individuals that had a CTL response to this epitope broken down by group: 0/4 group 1,0/3 group 2, and 1/1 group 3

Nef (71-79) Nef (71-79) TPQVPLRPM HIV-1 infection human (B7) Day2001

- The CTL response to optimally defined CTL epitopes restricted by HLA class I A and B alleles in individuals who coexpressed HLA A2, A3, and B7 was studied in eight HIV-1-infected subjects, two with acute infection, five with chronic, and one long-term non-progressor (LTNP)

- 2 to 17 epitopes were recognized in a given individual, A2-restricted CTL response tended to be narrow and never dominated the response, and 25/27 epitopes were targeted by at least one person

- Subjects with chronic HIV-1 infection recognized between 2-8 out of 11 B7-restricted epitopes

- An acute seroconvertor homozygous for the B7 allele recognized five B7-restricted epitopes

- The other acute seroconvertor failed to recognize any of the $11 \mathrm{~B} 7$-restricted epitopes tested

- The B7-restricted CTL response was highly variable and there was no clearly dominant epitope

Nef (71-79 BRU) TPOVPLRPM HIV-1 infection

human (B7)

Choppin2001

- Seventy-three 8-11 mer peptides were generated based on putative anchor motifs in the Nef BRU sequence for HLA A2, A3, A24, B7, B8, and B35; these 73 peptides had 81 motifs. 54\% bound to the predicted HLA molecule, particularly A2, B7/35, and B8. The strength of HLA-peptide binding was not directly related to the number of individuals that recognized a protein. 


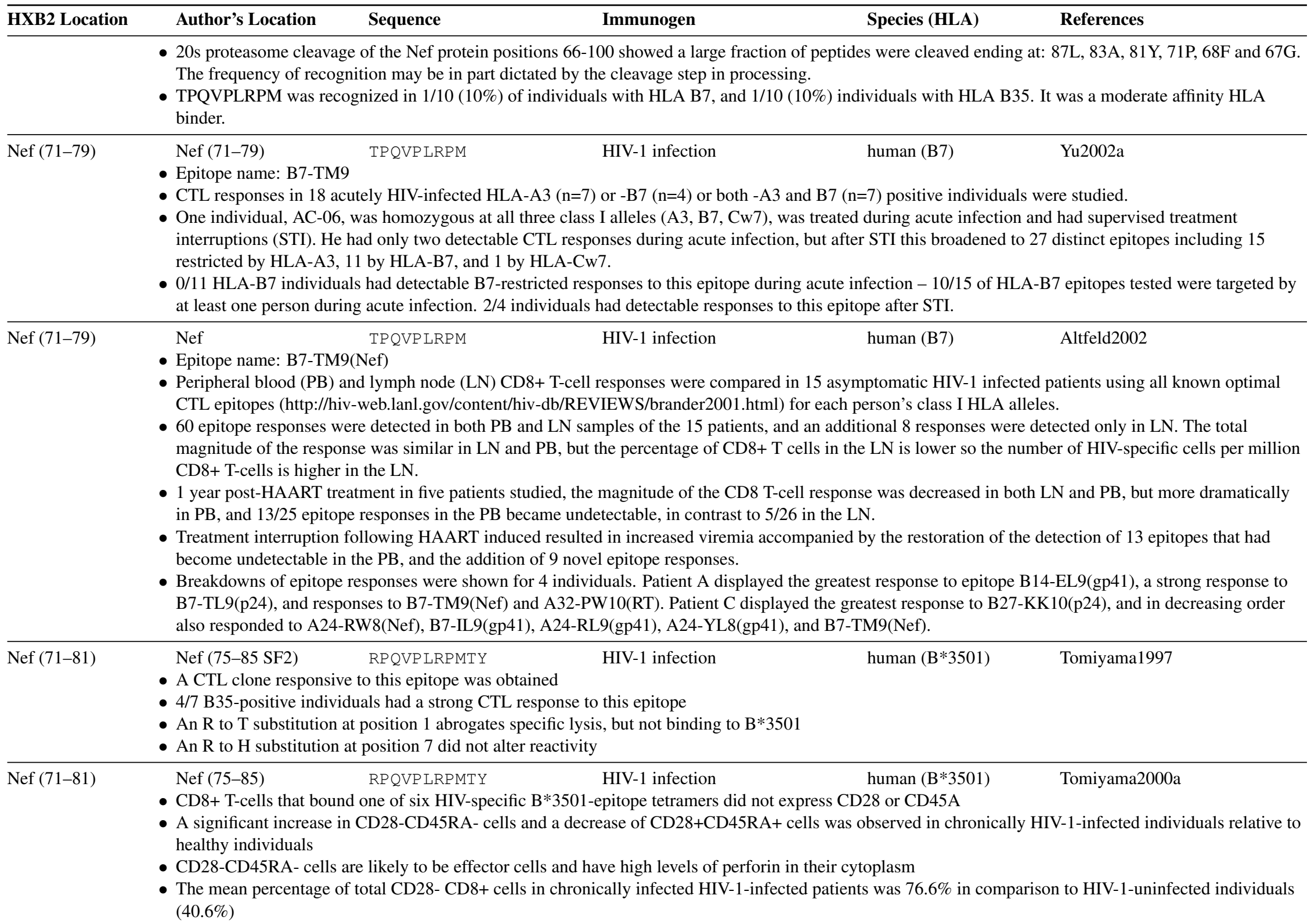




\begin{tabular}{llllll}
\hline HXB2 Location & Author's Location & Sequence & Immunogen & Species (HLA) & References \\
\hline Nef (71-81) & $\begin{array}{l}\text { Nef (75-85 SF2) } \\
\text { • Binds HLA-B*3501 }\end{array}$ & RPQVPLRPMTY & HIV-1 infection & human (B35) & Shiga1996 \\
\hline Nef (71-81) & (SF2) & RPQVP LRPMTY & HIV-1 infection & human (B35) & Kawana1999
\end{tabular}

- HLA B35 is associated with rapid disease progression

- The sequences of 9 previously described HIV-1 B35 CTL epitopes were obtained in 10 HLA B35+ and 19 HLA B35- individuals

- 3/9 CTL epitopes had substitutions that were more common in B35+ individuals than in B35- individuals - only one of these reduced the binding of the peptide to B35 and was shown to be an escape mutation

- rpqvplrpmtF was found in 9/10 of the B35+ individuals, none of the B35- individuals—-the $\mathrm{Y} \rightarrow \mathrm{F}$ substituted peptide had a similar binding affinity with B35 and was recognized by a CTL clone equally with wildtype.

\begin{tabular}{lllll}
\hline Nef (71-81) Nef (69-79) & RPQVPLRPMTY & HIV-1 infection & human (B35) & Ferrari2000 \\
\hline
\end{tabular}

- One of the 51 HIV-1 epitopes selected by Ferrari et al. as good candidate CTL epitopes for vaccines by virtue of being conserved and presented by common HLA alleles

\begin{tabular}{llccc}
\hline Nef (71-81) & Nef (71-81 BRU) & TPQVP LRPMTY & HIV-1 infection & human (B35) \\
\hline
\end{tabular}

- Seventy-three 8-11 mer peptides were generated based on putative anchor motifs in the Nef BRU sequence for HLA A2, A3, A24, B7, B8, and B35; these 73 peptides had 81 motifs. 54\% bound to the predicted HLA molecule, particularly A2, B7/35, and B8. The strength of HLA-peptide binding was not directly related to the number of individuals that recognized a protein.

- 20 s proteasome cleavage of the Nef protein positions $66-100$ showed a large fraction of peptides were cleaved ending at: $87 \mathrm{~L}, 83 \mathrm{~A}, 81 \mathrm{Y}, 71 \mathrm{P}, 68 \mathrm{~F}$ and $67 \mathrm{G}$.

The frequency of recognition may be in part dictated by the cleavage step in processing.

- TPQVPLRPMTY was recognized in 9/12 (75\%) of individuals with HLA B7, and 5/10 (50\%) of individuals with HLA B35. It was a moderate affinity HLA binder, and the C-term Y readily cleaved in vitro.

Nef (71-81) Nef RPQVPLRPMTY HIV-1 infection, Vaccine hanke2000, Wee2002

Vaccine Vector/Type: DNA prime with vaccinia MVA boost Strain: subtype A HIV component: p17, p24, polyepitope

- The HIV-1 subtype A focused vaccine HIVA contains p24 and p17, in a reversed order relative to the Gag polyprotein to prevent myristylation of p17, which could direct the protein to the cell membrane and inhibit efficient peptide processing and class I presentation, as well as a polyepitope string of conserved, often immunodominant epitopes that were selected to have particularly good cross-reactive potential for the A-clade epidemic in Nairobi, Kenya. A DNA and MVA prime-boost vaccination protocol using the HIVA antigen will be used in a phase III clinical trial in Kenya. This epitope is included in the polyepitope string [Hanke2000].

- Multiple CD4+ or CD8+ T-cell vaccine-induced responses to peptide pools were detected using intracellular cytokine staining and IFNgamma Elispot assays after vaccination of 5 macaques. The response to the Mamu A*01 SIV p27 epitope p11C (CTPYDINQM), included in the polyepitope region, was not immunodominant in the Mamu $\mathrm{A}^{*} 01$ vaccinated macaques, possibly because of processing limitations in context of the artificial polyepitope string Wee2002].

- Seventy-three 8-11 mer peptides were generated based on putative anchor motifs in the Nef BRU sequence for HLA A2, A3, A24, B7, B8, and B35; these 73 peptides had 81 motifs. 54\% bound to the predicted HLA molecule, particularly A2, B7/35, and B8. The strength of HLA-peptide binding was not directly related to the number of individuals that recognized a protein.

- 20 s proteasome cleavage of the Nef protein positions $66-100$ showed a large fraction of peptides were cleaved ending at: $87 \mathrm{~L}, 83 \mathrm{~A}, 81 \mathrm{Y}, 71 \mathrm{P}, 68 \mathrm{~F}$ and $67 \mathrm{G}$. The frequency of recognition may be in part dictated by the cleavage step in processing. 


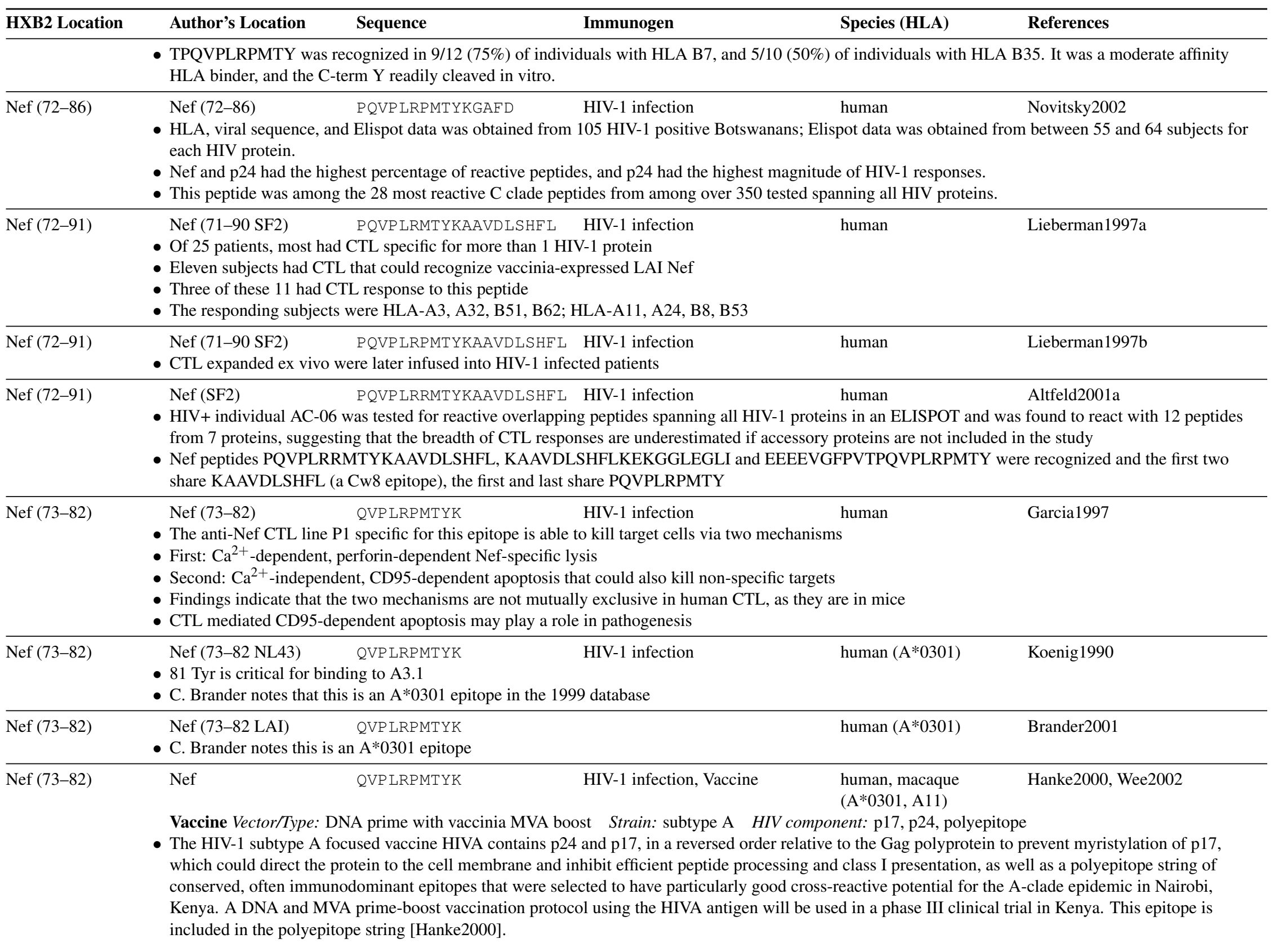




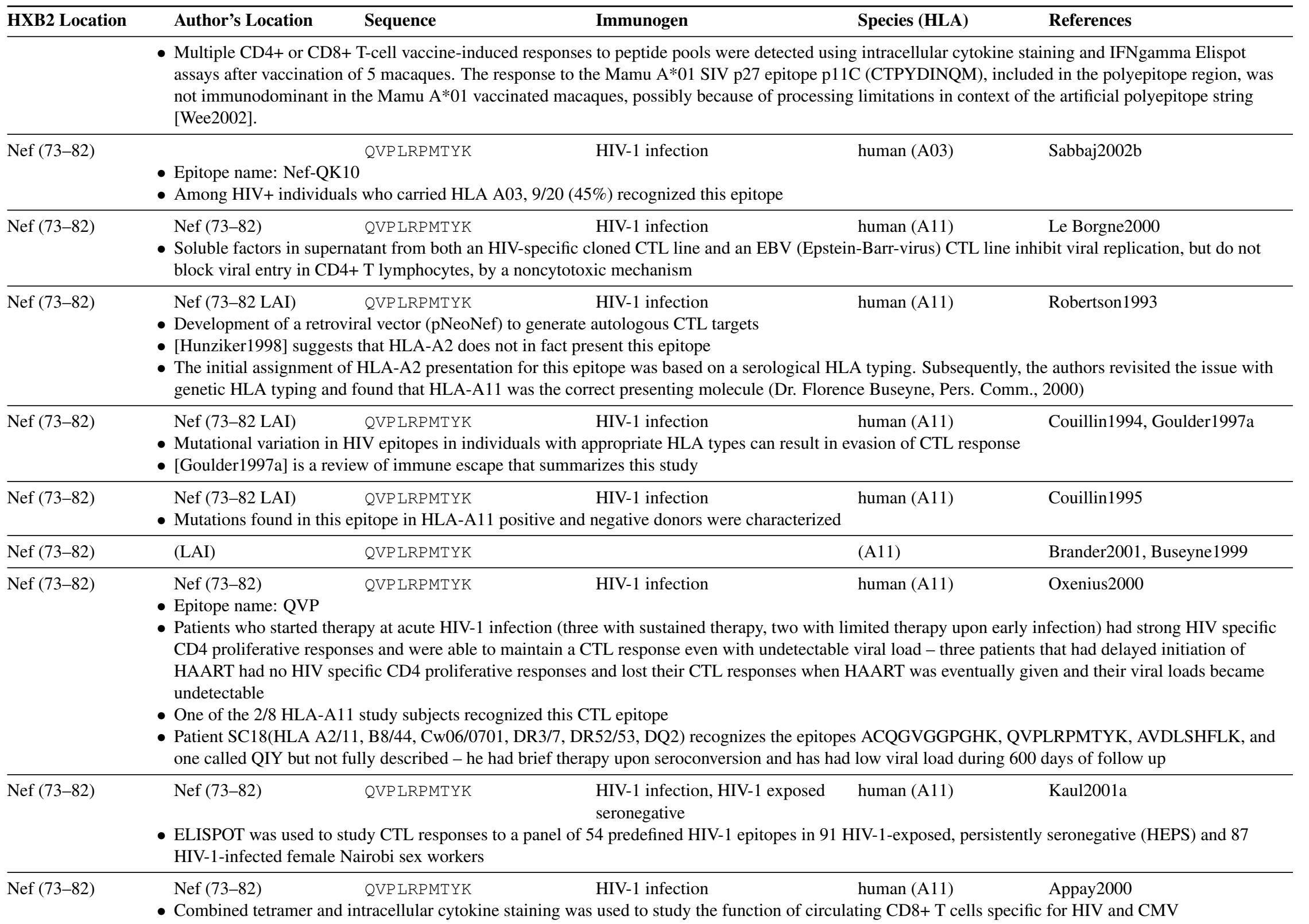




\begin{tabular}{|c|c|c|c|c|c|}
\hline HXB2 Location & Author's Location & Sequence & Immunogen & Species (HLA) & References \\
\hline & \multicolumn{5}{|c|}{$\begin{array}{l}\text { - HIV-specific CD8+ T cells expressed lower levels of perforin than CMV-specific CD8+ T cells from the same donor, and this was associated with persistent } \\
\text { CD27 expression on HIV-specific cells, suggesting impaired maturation } \\
\text { - In most donors, between } 50 \% \text { and } 95 \% \text { of the activated virus-specific CD8+ T cells produced IFN- } \gamma \text { and MIP- } 1 \beta \text { with a distinct subset that failed to } \\
\text { produce TNF- } \alpha\end{array}$} \\
\hline Nef (73-82) & $\begin{array}{l}\text { Nef (71-80 93TH253 } \\
\text { subtype CRF01) } \\
\text { - Epitope name: N73-82 } \\
\text { - This was a study of HIV- } \\
\text { - HLA-A11 is very comm } \\
\text { and CTL responses were } \\
\text { - This epitope was weakly } \\
\text { stimulation, in study sub } \\
\text { - This epitope was strongl }\end{array}$ & $\begin{array}{l}\text { QVPLRPMTYK } \\
1 \text { exposed persiste } \\
\text { on in this populatio } \\
\text { found in } 8 / 8 \text { HIV+ } \\
\text { reactive in HEPS } \\
\text { ect } 256 \text { who was } \mathrm{H} \\
\text { reactive in HIV+ }\end{array}$ & $\begin{array}{l}\text { HIV-1 infection, HIV-1 exposed } \\
\text { seronegative } \\
\text { ve (HEPS) female sex workers in } \\
\text { iched among the HEPS sexworker } \\
0 / 9 \text { HIV- women that were not exp } \\
265 \text { who was HLA A2/A11 and } 12 \\
\text { naking it the most reactive epitope } \\
533 \text { who carried HLA-A } 11\end{array}$ & $\begin{array}{l}\text { human (A11) } \\
\text { iang Mai, norther } \\
\text { weak CTL respo } \\
\text { ed } \\
\text { who was HLA A } \\
\text { sted in HLA-A11 }\end{array}$ & $\begin{array}{l}\text { Sriwanthana2001 } \\
\text { ad } \\
\text { e detected in } 4 / 7 \text { HEPS women, } \\
\text { omen, with } 3 / 4 \text { responding }\end{array}$ \\
\hline $\operatorname{Nef}(73-82)$ & $\begin{array}{l}\text { Nef (71-80 93TH253 } \\
\text { subtype CRF01) } \\
\text { - HLA-A11 CRF01 (calle } \\
\text { Thailand, of whom more } \\
\text { - } 77 \text { possible HLA-A11 er } \\
\text { epitopes for CTL respon } \\
\text { - This epitope was predict } \\
\text { that had been previously } \\
\text { - } 4 / 8 \text { tested FSWs recogni } \\
\text { - An HLA-A11 tetramer v } \\
\text { population after in vitro } \\
\text { - This epitope was highly }\end{array}$ & $\begin{array}{l}\text { QVPLRPMTYK } \\
\text { subtype E in Bon } \\
\text { than half were HL } \\
\text { itopes were first de } \\
\text { es from } 8 \text { HLA-A } \\
\text { d by the EpiMatri } \\
\text { defined } \\
\text { zed this epitope } \\
\text { as made for this ep } \\
\text { timulation } \\
\text { conserved in other }\end{array}$ & $\begin{array}{l}\text { HIV-1 infection } \\
\text { es were identified that stimulated } \\
\text { e } \\
\text { iMatrix, these were screened for } \\
\text { likely to bind to A11, and it serv } \\
\text { like novel, six were prev } \\
\text { vas recognized by two subjects - } \\
\text { exact matches were common }\end{array}$ & $\begin{array}{l}\text { human (A11) } \\
\text { CL from HIV+ fen } \\
\text { ading to A11 findi } \\
\text { sly identified } \\
\text { as an epitope in th } \\
\text { ly one subject had }\end{array}$ & $\begin{array}{l}\text { Bond2001 } \\
\text { borkers (FSW) from Northern } \\
\text { it was one of the six A11 epitopes } \\
\text { ded tetramer staining T-cell }\end{array}$ \\
\hline Nef (73-82) & $\begin{array}{l}\text { Nef } \\
\text { - Epitope name: QVP } \\
\text { - Using previously defined } \\
\text { period including therapy } \\
\text { - STIs induced increased r } \\
\text { clearance rates. }\end{array}$ & $\begin{array}{l}\text { QVPLRPMTYK } \\
\text { epitopes Oxenius } \\
\text { with standard treat } \\
\text { ecognition of CTL }\end{array}$ & $\begin{array}{l}\text { HIV-1 infection } \\
\text { 2001a] in an IFNgamma Elispot a } \\
\text { ions (STI). } \\
\text { here was no correlation between }\end{array}$ & $\begin{array}{l}\text { human (A11) } \\
\text { ay, } 13 \text { chronically } \\
\text { L responses with }\end{array}$ & $\begin{array}{l}\text { Oxenius } 2002 \mathrm{~b} \\
\text { fected patients were studied over a } \\
\text { und rates, plateau viral loads, or }\end{array}$ \\
\hline Nef (73-82) & $\begin{array}{l}\text { Nef } \\
\text { - Four HIV patients with } \mathrm{p} \\
\text { specific T-cell responses } \\
\text { - Nef epitope recognition } \\
\text { HLA-A3, one using HL } \\
\text { - The HIV-specific CD8+ }\end{array}$ & $\begin{array}{l}\text { QVPLRPMTYK } \\
\text { olonged clinically } \\
\text { y Elispot and Tet } \\
\text { as detected in all } \\
\text {-A11 } \\
\text {-cells had an inter }\end{array}$ & $\begin{array}{l}\text { HIV-1 infection } \\
\text { i-viral therapy but with ongoing } \\
\text { maintained for } 2-4 \text { years after ini } \\
20 \text {, Pol and Gag-specific in } 1 \text { or } 2 \\
\text { ation phenotype characterized by }\end{array}$ & $\begin{array}{l}\text { human (A11) } \\
\text { ence of replicatic } \\
\text { ion of HAART. } \\
\text { bjects - two patie } \\
\text { levels of perfori }\end{array}$ & $\begin{array}{l}\text { Appay2002 } \\
\text { f mRNA transcription, showed } \\
\text { nized this epitope, one using } \\
\text { h levels of CD27 expression. }\end{array}$ \\
\hline
\end{tabular}




\begin{tabular}{|c|c|c|c|c|c|}
\hline HXB2 Location & Author's Location & Sequence & Immunogen & Species (HLA) & References \\
\hline Nef (73-82) & Nef (73-81) & QVPLRPMTYK & HIV-1 infection & $\begin{array}{l}\text { human (A2, A3, A11, } \\
\text { B35) }\end{array}$ & Ferrari2000 \\
\hline
\end{tabular}

- One of the $51 \mathrm{HIV}-1$ epitopes selected by Ferrari et al. as good candidate CTL epitopes for vaccines by virtue of being conserved and presented by common HLA alleles

\begin{tabular}{|c|c|}
\hline Nef (73-82) & $\begin{array}{l}\text { Nef (73-82 LAI) QVPLRPMTYK } \\
\text { - Mutations in Nef that flank this epitope, Thr71Lys and Ala83Gly, may account for an observed loss of CTL reactivity, with escape due to the introduction } \\
\text { of proteasome processing defects }\end{array}$ \\
\hline Nef (73-82) & $\begin{array}{l}\text { Nef (73-82) CVPLRPMTYK HIV-1 infection } \\
\text { - Cross-clade CTL response was studied by determining the CTL activity in seven patients from Bangui, (6 A subtype, and } 1 \text { AG recombinant infections) and } \\
\text { one A subtype infection from a person living in France originally from Togo, to different antigens expressed in vaccinia } \\
\text { - Pol reactivity: } 8 / 8 \text { had CTL to A subtype, and 7/8 to B subtype, and HIV-2 Pol was not tested } \\
\text { - Gag reactivity: 7/8 reacted with A or B subtype gag, 3/8 with HIV-2 Gag } \\
\text { - Nef reactivity: 7/8 reacted with A subtype, and 5/8 with B subtype, none with HIV-2 Nef } \\
\text { - Env reactivity: 3/8 reacted with A subtype, 1/8 with B subtype, none with HIV-2 Env } \\
\text { - One of the patients was shown to react to this epitope: QVPLRPMTYK }\end{array}$ \\
\hline Nef (73-82) & $\begin{array}{l}\text { Nef (73-82 LAI) OVPLRPMTYK HIV-1 infection } \\
\text { - Identical twin hemophiliac brothers were both infected with the same batch of factor VIII } \\
\text { - Both had a response to this epitope } \\
\text { - } \text { Goulder1997a is a review of immune escape that summarizes this study }\end{array}$ \\
\hline Nef (73-82) & $\begin{array}{l}\text { Nef (73-82) } \quad \text { QVP LRPMTYK } \\
\text { - Eighty two HIV-1-specific CTL clones from } 5 \text { long-term non-progressors were isolated and analyzed for breadth of response } \\
\text { - A sustained Gag, Env and Nef response was observed, and clones were restricted by multiple HLA epitopes, indicating a polyclonal response } \\
\text { - An A3+ subject had a strong response to this epitope, with 10/11 CTL clones being specific for this epitope, isolated at two time points, } 1 \text { year apart }\end{array}$ \\
\hline Nef (73-82) & $\begin{array}{l}\text { Nef (73-82) QVPLRPMTYK } \\
\text { - Epitope name: N1 } \\
\text { - The epitope was recognized by patients } 252 \# 0 \text { and 252\#4 in a study of the effects of therapy escape mutations on CTL recognition }\end{array}$ \\
\hline $\operatorname{Nef}(73-82)$ & $\begin{array}{l}\text { Nef (73-82 SF2) PVPLRRMTYK HIV-1 infection } \\
\text { - Therapy provided during acute infection resulted in a narrower CTL response, stronger T help response, and a less diverse viral population than was seen in } \\
\text { individuals treated during chronic infection } \\
\text { - The breadth and specificity of the response was determined using ELISPOT by studying } 19 \text { individuals with pre-seroconversion therapy (Group 1), 11 } \\
\text { individuals with primary infection but post-seroconversion therapy (Group 2), and } 10 \text { individuals who responded to HAART given during chronic infection } \\
\text { (Group 3), using } 259 \text { overlapping peptides spanning p17, p24, RT, gp41, gp120 and Nef } \\
\text { - Previously described and newly defined optimal epitopes were tested for CTL response } \\
\text { - Number of HLA-A3+ individuals that had a CTL response to this epitope broken down by group: } 3 / 7 \text { group 1, 1/4 group 2, and 1/2 group 3 }\end{array}$ \\
\hline $\operatorname{Nef}(73-82)$ & $\begin{array}{l}\text { Nef (SF2) QVPLRPMTYK } \\
\text { - This epitope was mapped by ELISPOT in a study identifying new HLA-B60 epitopes, and was one of the epitopes presented by another HLA molecule in } \\
\text { an HLA-B60 individual }\end{array}$ \\
\hline
\end{tabular}




\begin{tabular}{|c|c|c|c|c|}
\hline HXB2 Location & Author's Location & Sequence & Immunogen & Species (HLA) \\
\hline Nef (73-82) & $\begin{array}{l}\text { Nef (73-82) } \\
\text { - Epitope name: A3-QK10 } \\
\text { - CTL responses in } 18 \text { acut } \\
\text { - One individual, AC-06, w } \\
\text { interruptions (STI). He ha } \\
\text { restricted by HLA-A3, } 11 \\
\text { - } 3 / 14 \text { HLA-A3 positive inc } \\
\text { were targeted during acut }\end{array}$ & $\begin{array}{l}\text { QVPLRPMTYK } \\
\text { tely HIV-infected } \\
\text { vas homozygous a } \\
\text { ad only two detec } \\
\text { l by HLA-B7, anc } \\
\text { dividuals had det } \\
\text { te infection. } 5 / 7 \text { ir }\end{array}$ & $\begin{array}{l}\text { HIV-1 infection } \\
\text { or -B7 ( } \mathrm{n}=4) \text { or b } \\
\text { I alleles }(\mathrm{A} 3, \mathrm{~B} 7 \text {, } \\
\text { onses during acute } \\
7 . \\
\text { icted responses to } \\
\text { etectable response }\end{array}$ & $\begin{array}{l}\text { human (A3) Yu2002a } \\
=7 \text { ) positive individuals were studied. } \\
\text { during acute infection and had supervised treatment } \\
\text { r STI this broadened to } 27 \text { distinct epitopes including } 15 \\
\text { g acute infection, but only } 5 / 15 \text { of HLA-A3 epitopes tested } \\
\text { fter STI. }\end{array}$ \\
\hline Nef (73-82) & $\begin{array}{l}\text { Nef } \\
\text { - Four HIV patients with pr } \\
\text { specific T-cell responses b } \\
\text { - Nef epitope recognition w } \\
\text { HLA-A3, one using HLA } \\
\text { - The HIV-specific CD8+ T }\end{array}$ & $\begin{array}{l}\text { QVPLRPMTYK } \\
\text { rolonged clinicall } \\
\text { by Elispot and Tet } \\
\text { vas detected in all } \\
\text {-A11. } \\
\text { T-cells had an inte }\end{array}$ & $\begin{array}{l}\text { HIV-1 infection } \\
\text { i-viral therapy but } \\
\text { maintained for 2- } \\
\text { 20, Pol and Gag-s } \\
\text { ation phenotype cl }\end{array}$ & $\begin{array}{l}\text { human (A3) Appay2002 } \\
\text { dence of replication and Nef mRNA transcription, showed } \\
\text { tion of HAART. } \\
\text { dbjects - two patients recognized this epitope, one using } \\
\text { w levels of perforin and high levels of CD27 expression. }\end{array}$ \\
\hline Nef (73-82) & $\begin{array}{l}\text { Nef (73-82 LAI) } \\
\text { - Epitope name: N1 } \\
\text { - A panel of } 16 \text { epitopes co } \\
\text { CD8+ cell IFNgamma pro } \\
\text { - In general, during the first } \\
\text { specificities that were not } \\
\text { HIV-specific responses di } \\
\text { - Viral rebounds gave differ }\end{array}$ & $\begin{array}{l}\text { QVPLRPMTYK } \\
\text { vering } 15 \text { class I } \\
\text { oduction to meast } \\
\text { t month of treatm } \\
\text { previously detect } \\
\text { minished } \\
\text { rent patterns of re }\end{array}$ & $\begin{array}{l}\text { HIV-1 infection } \\
\text { ed in } 14 \text { HIV+ pati } \\
\text { ecreased and frequ } \\
\text { y detected, as wer } \\
\text { es or decreases in }\end{array}$ & $\begin{array}{l}\text { human (A3 supertype) Mollet2000 } \\
\text { lected Caucasian population treated with HAART, using } \\
\text { ecific CTL tripled and broadened - eight new HIV } \\
\text { D8+ PBL - but with continued viral suppression, } \\
\text { nse, new specificities, or no change }\end{array}$ \\
\hline Nef (73-82) & $\begin{array}{l}\text { Nef (94-103) } \\
\text { - Long-term nonprogressor } \\
\text { for the A3 supertype) whi } \\
\text { - Progressors had memory } \\
\text { - A positive correlation bet } \\
\text { observed, which may con } \\
\text { - This epitope can bind } 3 / 5\end{array}$ & $\begin{array}{l}\text { QVPLRPMTYK } \\
\text { s (LTNPs) had str } \\
\text { ile the effector cel } \\
\text { resting CD8+ T-c } \\
\text { ween effector CD } \\
\text { tribute to the inab } \\
\text { HLA-A3 superty }\end{array}$ & $\begin{array}{l}\text { HIV-1 infection } \\
\text { sting CD8+ T-cell } \\
\text { nonprogressors re } \\
\text { ized far fewer epit } \\
\text { plasma viremia an } \\
\text { to clear virus } \\
301, A^{*} 1101, A^{* 3}\end{array}$ & $\begin{array}{l}\text { human (A3 supertype) Propato2001 } \\
\text { the majority of epitopes tested, (18 for the A2 supertype, } 16 \\
\text { epitopes } \\
\text { ation between CD8+ effector T-cells and CD4+ T-cells was } \\
\text { A*6801) }\end{array}$ \\
\hline Nef (73-82) & $\begin{array}{l}\text { Nef (73-82 BRU) } \\
\text { - Nef CTL clones from HIV }\end{array}$ & $\begin{array}{l}\text { QVPLRPMTYK } \\
\mathrm{V}+\text { donors }\end{array}$ & HIV-1 infection & human (A3, A11, B35) Culmann1991 \\
\hline Nef (73-82) & $\begin{array}{l}\text { Nef (73-82 LAI) } \\
\text { - Alanine substitutions L76 } \\
\text { - Nef CTL clones (4N225) } \\
\text { - Infusion led to outburst of }\end{array}$ & $\begin{array}{l}\text { QVPLRPMTYK } \\
\text { A, R77A, M79A, } \\
\text { were infused into } \\
\text { f escape variants }\end{array}$ & $\begin{array}{l}\text { HIV-1 infection } \\
\text { antly decreased im } \\
\text { ted volunteer to e } \\
\text { n higher viral loac }\end{array}$ & $\begin{array}{l}\text { human (A3.1) Koenig1995 } \\
\text { eptide } \\
\text { fusion on viral load/patient health } \\
\text { e progression }\end{array}$ \\
\hline $\operatorname{Nef}(73-82)$ & $\begin{array}{l}\text { Nef (73-82) } \\
\text { - Only 4/11 HLA-A2+ HIV } \\
\text { - } 95 \text { optimally-defined pept }\end{array}$ & $\begin{array}{l}\text { QVPLRPMTYK } \\
+ \text { individuals had } \\
\text { ides from this dat }\end{array}$ & $\begin{array}{l}\text { HIV-1 infection } \\
\text { ted to SLYNTVAT } \\
\text { d to screen for IN }\end{array}$ & $\begin{array}{l}\text { human (A3.1) Betts2000 } \\
\text { stion whether it is immunodominant } \\
\text { er epitopes }\end{array}$ \\
\hline
\end{tabular}




\begin{tabular}{|c|c|c|c|c|c|}
\hline HXB2 Location & Author's Location & Sequence & Immunogen & Species (HLA) & References \\
\hline Nef (73-82) & Nef (73-82) & RPMTYK & $\mathrm{H}$ & human $\left(\mathrm{B}^{*}\right.$ & Wilss \\
\hline
\end{tabular}

- Three individuals with highly focused HIV-specific CTL responses were studied during acute infection using tetramers - high frequencies of HIV-1-specific $\mathrm{CD} 8+\mathrm{T}$ cells were found prior to seroconversion, and there was a close temporal relationship between the number of circulating HIV-specific T cells and viral load was also found

- All three patients were B*2705, with HLA alleles: A1, A30/31, B*2705, B35; A1, A*0301, B7, B2705; and A*0201, A*0301, B2705, B39

- ELISPOT was used to test a panel of CTL epitopes that had been defined earlier and were appropriate for the HLA haplotypes of the study subjects - 3/3 subjects showed a dominant response to the $\mathrm{B} * 2705$ epitope KRWIILGGLNK

- The subject with $\mathrm{A}^{*} 0201$ had a moderatly strong response to SLYNTVATL

- Weak responses were observed to A*301-RLRPGGKKK, A*301-QVPLRPMTYK, and B7-TPGPGVRYPL in the subject who was HLA A1, A*0301, B7, $\mathrm{B} * 2705$

- No acute response was detected to the following epitopes: A*201-ILKEPVHGV, A*301-KIRLRPGGK, A*301-AIFQSSMTK, A*301-TVYYGVPVWK, B35-EPIVGAETF, B35-HPDIVIYQY, B35-PPIPVGEIY, B35-NSSKVSQNY, B35-VPLRPMTY, B35-DPNPQEVVL

\begin{tabular}{lllll}
\hline Nef (73-82) & $\begin{array}{l}\text { Nef (73-82 LAI) } \\
\text { Optimal epitope mapped by peptide titration }\end{array}$ & human (B27) & Culmann1998 \\
\hline Nef (73-82) & Nef (73-82 LAI) & SVPLRPMTYK & HIV-1 infection & human (B35 or C4)
\end{tabular}

- Vertical transmission of HIV ranges from $13 \%$ to $39 \%$

- Primary assays showed cytotoxic activity against at least one HIV protein was detected in $70 \%$ of infected children

- Epitopes recognized in five children were mapped using synthetic peptides and secondary cultures

- Patient EM13, who had a CTL response to three epitopes in Nef, was infected via blood transfusion after birth and went from CDC stage P2A to P2E during the study

\begin{tabular}{ll}
\hline Nef (73-83) & Nef (73-82 BRU) PVPLRPMTYKA $\quad$ HIV-1 infection $\quad$ human (A3) \\
& Seventy-three 8-11 mer peptides were generated based on putative anchor motifs in the Nef BRU sequence for HLA A2, A3, A24, B7, B8, and B35; these \\
& 73 peptides had 81 motifs. 54\% bound to the predicted HLA molecule, particularly A2, B7/35, and B8. The strength of HLA-peptide binding was not
\end{tabular}

- 20s proteasome cleavage of the Nef protein positions $66-100$ showed a large fraction of peptides were cleaved ending at: $87 \mathrm{~L}, 83 \mathrm{~A}, 81 \mathrm{Y}, 71 \mathrm{P}, 68 \mathrm{~F}$ and $67 \mathrm{G}$.

The frequency of recognition may be in part dictated by the cleavage step in processing.

- QVPLRPMTYKA was recognized in 9/15 (60\%) of individuals with HLA A3. It was a high affinity HLA-A3 binder.

\begin{tabular}{|c|c|c|c|c|}
\hline Nef (74-81) & $\begin{array}{lc}\text { Nef (74-82) } & \text { VP LRPMTY } \\
\text { - Included in HLA-A3 binding peptide com }\end{array}$ & & human (A3) & Carreno1992 \\
\hline Nef (74-81) & $\begin{array}{l}\text { Nef (73-82 LAI) VP LRPMTY } \\
\text { - C. Brander notes this is a B*3501 epitope }\end{array}$ & HIV-1 or HIV-2 infection & human $(\mathrm{B} * 3501)$ & Brander2001 \\
\hline Nef (74-81) & $\begin{array}{lc}\text { Nef (75-82) VPLRPMTY } \\
\text { - Crystal structure of VPLRPMTY-class I B }\end{array}$ & $\begin{array}{l}\text { Peptide-HLA interaction } \\
3501 \text { complex }\end{array}$ & human $(\mathrm{B} * 3501)$ & Smith1996 \\
\hline$\overline{\mathrm{Nef}}(74-81)$ & $\begin{array}{l}\text { Nef VPLRPMTY } \\
\text { - The role of CD4+ T-cell help in expansi } \\
\text { - Optimal expansion of HIV-1-specific me } \\
\text { in the absence of CD4+ T cell help to a }\end{array}$ & $\begin{array}{l}\text { HIV-1 infection } \\
\text { c memory CTL was studiec } \\
\text { ded on CD4+ T cell help in } \\
\text { most of patients }\end{array}$ & $\begin{array}{l}\text { human }(\mathrm{B} * 3501) \\
\text { co-culture ex vivo } \\
\text { patients - CD40 liga }\end{array}$ & $\begin{array}{l}\text { Ostrowski2000 } \\
\text { ner (CD40LT) could enhance CTL }\end{array}$ \\
\hline
\end{tabular}




\begin{tabular}{|c|c|c|c|c|}
\hline HXB2 Location & Author's Location & Sequence & Species (HLA) & References \\
\hline & \multicolumn{4}{|c|}{$\begin{array}{l}\text { - Those CTL that didn't respond to CD40LT could expand with IL2 present, and IL15 produced by dendritic cells also contributes } \\
\text { - The T-helper epitope used for CD4+ T cell stimulation was the universal tetanus helper epitope TET830-843 (QYIKANSKFIGITE) }\end{array}$} \\
\hline ef $(74-81)$ & \multicolumn{4}{|c|}{$\begin{array}{l}\text { - 11/16 heavily HIV exposed but persistently seronegative sex-workers in Nairobi had HIV-specific CD8 gamma-IFN responses in the cervix - systemic } \\
\text { CD8+ T cell responses tended to be to the same epitopes but at generally lower levels than cervical CD8+ T cell responses } \\
\text { - Low risk individuals did not have such CD8+ cells } \\
\text { - CD8+ T cell epitopes: DTVLEDINL ( } 3 \text { individuals), SLYNVATL ( } 4 \text { individuals), LSPRTLNAW ( } 3 \text { individuals) and YPLTFGWCF (4 individuals) were } \\
\text { most commonly recognized by the HIV-resistant women }\end{array}$} \\
\hline 81) & \multicolumn{4}{|c|}{$\begin{array}{l}\text { - Three individuals with highly focused HIV-specific CTL responses were studied during acute infection using tetramers - high frequencies of HIV-1-specific } \\
\text { CD8+ T cells were found prior to seroconversion, and there was a close temporal relationship between the number of circulating HIV-specific T cells and } \\
\text { viral load was also found } \\
\text { - All three patients were } \mathrm{B} * 2705 \text {, with HLA alleles: A1, A30/31, B*2705, B35; A1, A*0301, B7, B2705; and A*0201, A*0301, B2705, B39 } \\
\text { - ELISPOT was used to test a panel of CTL epitopes that had been defined earlier and were appropriate for the HLA haplotypes of the study subjects - 3/3 } \\
\text { subjects showed a dominant response to the B*2705 epitope KRWIILGGLNK } \\
\text { - The subject with A*0201 had a moderatly strong response to SLYNTVATL } \\
\text { - Weak responses were observed to A*301-RLRPGGKKK, A*301-QVPLRPMTYK, and B7-TPGPGVRYPL in the subject who was HLA A1, A*0301, B7, } \\
\text { B*2705 } \\
\text { - No acute response was detected to the following epitopes: A*201-ILKEPVHGV, A*301-KIRLRPGGK, A*301-AIFQSSMTK, A*301-TVYYGVPVWK, } \\
\text { B35-EPIVGAETF, B35-HPDIVIYQY, B35-PPIPVGEIY, B35-NSSKVSQNY, B35-VPLRPMTY, B35-DPNPQEVVL }\end{array}$} \\
\hline Nef (74-81) & \multicolumn{4}{|c|}{ - Review of HIV CTL epitopes - defined by B35 motif found within a larger peptide } \\
\hline Jef (74-81) & \multicolumn{4}{|c|}{ - VPLRPMTY also recognized by CTL from HIV-2 seropositives; epitope is conserved } \\
\hline lef (74-81) & \multicolumn{4}{|c|}{$\begin{array}{l}\text { - A CTL response was found in exposed but uninfected prostitutes from Nairobi using previously-defined B clade epitopes that tended to be conserved in A } \\
\text { and D clades - such cross-reactivity could protect against both A and D and confer protection in Nairobi where both subtypes are circulating } \\
\text { - The A and D subtype consensus are identical to the B clade epitope }\end{array}$} \\
\hline Nef (74-81) & $\begin{array}{l}\text { Nef }(75-82) \\
\text { - A peptide-based pro } \\
\text { stimulate a primary } 1 \\
\text { This peptide was one } \\
\text { healthy B35 seroneg }\end{array}$ & $\begin{array}{l}\text { VP LRPMTY } \\
\text { was optimizec } \\
\text { nse, only seco } \\
\text { he B35 presen } \\
\text { donors }\end{array}$ & $\begin{array}{l}\text { in vitro stimulation human (B35) } \\
\text { f CTLp using optimized peptide and IL-7 concentr } \\
\text { cific CTLp counts could be obtained via staining w } \\
\text { d in control experiments showing that the assay ga }\end{array}$ & $\begin{array}{l}\text { alvani1997 } \\
\text { ortantly this protocol does not } \\
\text { Class I tetramers } \\
\text { using lymphocytes from } 21\end{array}$ \\
\hline Nef $(74-81)$ & $\begin{array}{l}\text { Nef (subtype B) } \\
\text { - HIV-specific CTL w } \\
\text { - Seroprevalence in th } \\
\text { - Most isolated HIV st } \\
\text { responses are freque }\end{array}$ & $\begin{array}{l}\text { VP LRPMTY } \\
\text { ound in expose } \\
\text { hort is } 90-95 \% \\
\text { are clade A ir } \\
\text { observed using }\end{array}$ & $\begin{array}{l}\text { HIV-1 exposed seronegative human (B35) } \\
\text { stitutes from Nairobi - these CTL may confer protec } \\
\text { xposure is among the highest in the world } \\
\text { clades C and D are also found - B clade epitopes ar } \\
\text { ions of epitopes }\end{array}$ & Rowland-Jones1998b \\
\hline
\end{tabular}




\begin{tabular}{|c|c|c|c|c|}
\hline HXB2 Location & Author's Location & Sequence & Species (HLA) & References \\
\hline & \multicolumn{4}{|c|}{ - This epitope is conserved among A, B, and D clade viruses } \\
\hline Nef (74-81) & \multicolumn{2}{|c|}{$\begin{array}{l}\text { Nef } \\
\text { - CTL responses in seroneg } \\
\text { deletion in CCR5 } \\
\text { - In Gambia there is expost } \\
\text { - HIV-2 version of this epit } \\
\text { Rowland-Jones1995b }\end{array}$} & $\begin{array}{l}\text { human (B35) } \\
\text { female sex workers in Gambia and Nairobi were stu } \\
\text { responses to B35 epitopes in exposed, uninfected w } \\
\text { a CTLs are cross-reactive - one of five B35 CTL ep }\end{array}$ & $\begin{array}{l}\text { Rowland-Jones1999 } \\
\text { ese women had no delta } 32 \\
\text { cross-reactive, } \\
\text { at are cross-reactive, see also }\end{array}$ \\
\hline Nef (74-81) & \multicolumn{2}{|c|}{$\begin{array}{l}\text { Nef (74-81) VPPLRPMTY } \\
\text { - Epitope name: VPL } \\
\text { - Patients who started therapy at acute H } \\
\text { CD4 proliferative responses and were a } \\
\text { HAART had no HIV specific CD4 prol } \\
\text { undetectable } \\
\text { - One of two HLA B35+ among the eigh } \\
\text { - Patient SC15 (HLA A1/68, B8/35, Bw } \\
\text { during } 331 \text { days of HAART treatment }\end{array}$} & $\begin{array}{l}\text { HIV-1 infection human (B35) } \\
\text { e with sustained therapy, two with limited therapy up } \\
\text { TL response even with undetectable viral load - three } \\
\text { and lost their CTL responses when HAART was ever } \\
\text { ognized this epitope } \\
\text { given acute and sustained therapy and recognized ep }\end{array}$ & $\begin{array}{l}\text { Oxenius2000 } \\
\text { nfection) had strong HIV specific } \\
\text { that had delayed initiation of } \\
\text { ren and their viral loads became } \\
\text { IPVGDIY and VPLRPMTY }\end{array}$ \\
\hline Nef (74-81) & \multicolumn{4}{|c|}{$\begin{array}{l}\text { - ELISPOT was used to study CTL responses to a panel of } 54 \text { predefined HIV-1 epitopes in } 91 \text { HIV-1-exposed, persistently seronegative (HEPS) and } 87 \\
\text { HIV-1-infected female Nairobi sex workers } \\
\text { - Responses in HEPS women tended to be lower, and focused on different epitopes with HLA presenting molecules that have previously been associated with } \\
\text { reduced risk of infection, and there was a shift in the response in the HEPS women upon late seroconversion to epitopes recognized by the HIV-1 infected } \\
\text { women } \\
\text { - } 43 / 91 \text { HEPS women had CD } 8+\text { responses and detection of HIV-1-specific CTL in HEPS women increased with the duration of viral exposure } \\
\text { - Subject ML } 857 \text { shifted from a A*6802 DTVLEDINL and B35 (H/N)PDIVIYQY response prior to seroconversion to a B35 PPIPVGDIY and B35 } \\
\text { VPLRPMTY response post-seroconversion }\end{array}$} \\
\hline Nef (74-81) & \multicolumn{4}{|c|}{$\begin{array}{l}\text { - Epitope name: Nef-VY8 } \\
\text { - Among HIV+ individuals who carried HLA B35, 12/22 (55\%) recognized this epitope } \\
\text { - Among HIV+ individuals who carried HLA B*5301,0/11 (0\%) recognized this epitope }\end{array}$} \\
\hline Nef (74-81) & $\begin{array}{l}\text { Nef (74-81 BRU) } \\
73 \text { peptides had } 81 \mathrm{r} \\
\text { directly related to th } \\
\text { - 20s proteasome clea } \\
\text { The frequency of rec } \\
\text { - VPLRPMTY was re } \\
\text { frequent in vitro. }\end{array}$ & $\begin{array}{l}\text { VPLRPMTY } \\
\text { eptides were } \\
54 \% \text { bound } \\
\text { ber of indivi } \\
\text { of the Nef pre } \\
\text { ion may be in } \\
\text { zed in } 5 / 16\end{array}$ & $\begin{array}{l}\text { HIV-1 infection } \\
\text { putative anchor motifs in the Nef BRU sequence for } \\
\text { A molecule, particularly A2, B7/35, and B8. The stre } \\
\text { d a protein. } \\
00 \text { showed a large fraction of peptides were cleaved en } \\
\text { e cleavage step in processing. } \\
\text { with HLA B35, and it was a moderate affinity HLA b }\end{array}$ & $\begin{array}{l}\text { Choppin2001 } \\
\text { A3, A24, B7, B8, and B35; these } \\
\text { LA-peptide binding was not } \\
\text { eavage at the C-term Y was }\end{array}$ \\
\hline
\end{tabular}




\begin{tabular}{|c|c|c|c|c|}
\hline HXB2 Location & Author's Location & Sequence & Immunogen & Species (HLA) \\
\hline Nef $(74-81)$ & \multicolumn{4}{|c|}{$\begin{array}{l}\text { Vaccine Vector/Type: DNA prime with vaccinia MVA boost Strain: subtype A HIV component: p17, p24, polyepitope } \\
\text { - The HIV-1 subtype A focused vaccine HIVA contains p24 and p17, in a reversed order relative to the Gag polyprotein to prevent myristylation of p17, } \\
\text { which could direct the protein to the cell membrane and inhibit efficient peptide processing and class I presentation, as well as a polyepitope string of } \\
\text { conserved, often immunodominant epitopes that were selected to have particularly good cross-reactive potential for the A-clade epidemic in Nairobi, } \\
\text { Kenya. A DNA and MVA prime-boost vaccination protocol using the HIVA antigen will be used in a phase III clinical trial in Kenya. This epitope is } \\
\text { included in the polyepitope string [Hanke2000]. } \\
\text { - Multiple CD4+ or CD } 8+\text { T-cell vaccine-induced responses to peptide pools were detected using intracellular cytokine staining and IFNgamma Elispot } \\
\text { assays after vaccination of } 5 \text { macaques. The response to the Mamu A*01 SIV p27 epitope p11C (CTPYDINQM), included in the polyepitope region, was } \\
\text { not immunodominant in the Mamu A*01 vaccinated macaques, possibly because of processing limitations in context of the artificial polyepitope string } \\
\text { Wee2002]. }\end{array}$} \\
\hline Nef (74-82) & \multicolumn{4}{|c|}{ - Exploration of A11 binding motif } \\
\hline Nef $(75-82)$ & \multicolumn{4}{|c|}{$\begin{array}{l}\text { - Review of HIV CTL epitopes } \\
\text { - C. Brander notes that this is an A*1101 epitope in the } 1999 \text { database }\end{array}$} \\
\hline Nef (75-82) & \multicolumn{4}{|c|}{ - C. Brander notes this is an $\mathrm{A}^{*} 1101$ epitope } \\
\hline Nef (77-85) & \multicolumn{4}{|c|}{$\begin{array}{l}\text { - Structural constraints on the Nef protein may prevent escape } \\
\text { - Noted in Brander } 1999 \text {, this database, to be } \mathrm{B} * 0702\end{array}$} \\
\hline Nef (77-85) & \multicolumn{4}{|c|}{ - C. Brander notes this is a B*0702 epitope } \\
\hline Nef (77-85) & $\begin{array}{l}\text { Nef (75-83 IIIB) } \\
\text { - Study of tetramer sta } \\
\text { ELISPOT assay } \\
\text { - Autologous clones w } \\
\text { gave a more intense }\end{array}$ & $\begin{array}{l}\text { RPMTYKAAL } \\
\text { of B7 around I } \\
\text { hecked and 39/ } \\
\text { niform staining }\end{array}$ & $\begin{array}{l}\text { HIV-1 infection } \\
\text { ave quantitative results tha } \\
\text { wo time points had the vari } \\
\text { higher affinity to the RPM }\end{array}$ & $\begin{array}{l}\text { human (B7) Oxenius2001b } \\
\text { nce different than functional measurements based on an } \\
\text { neeta14 TCR }\end{array}$ \\
\hline Nef $(77-85)$ & $\begin{array}{l}\text { Nef (77-85 SF2) } \\
\text { - Therapy provided du } \\
\text { individuals treated du } \\
\text { - The breadth and spec } \\
\text { individuals with prim } \\
\text { (Group 3), using } 259 \\
\text { - Previously described } \\
\text { - Number of HLA-B7- }\end{array}$ & $\begin{array}{l}\text { RPMTYKAAL } \\
\text { acute infection } \\
\text { chronic infecti } \\
\text { y of the respon } \\
\text { infection but po } \\
\text { lapping peptide } \\
\text { newly defined } \\
\text { ividuals that ha }\end{array}$ & $\begin{array}{l}\text { HIV-1 infection } \\
\text { ower CTL response, strong } \\
\text { n using ELISPOT by study } \\
\text { p24, RT, gp41, gp120 and } \\
\text { were tested for CTL respor } \\
\text { e to this epitope broken dor }\end{array}$ & $\begin{array}{l}\text { human (B7) Altfeld2001b } \\
\text { response, and a less diverse viral population than was seen in } \\
\text { dividuals with pre-seroconversion therapy (Group } 1), 11 \\
\text { als who responded to HAART given during chronic infection } \\
\text { up: } 1 / 4 \text { group } 1,0 / 3 \text { group } 2 \text {, and } 1 / 1 \text { group } 3\end{array}$ \\
\hline
\end{tabular}




\begin{tabular}{llllll}
\hline HXB2 Location & Author's Location & Sequence & Immunogen & Species (HLA) & References \\
\hline Nef (77-85) & Nef (77-85) & RPMTYKAAL & HIV-1 infection & human (B7) & Day2001
\end{tabular}

- The CTL response to optimally defined CTL epitopes restricted by HLA class I A and B alleles in individuals who coexpressed HLA A2, A3, and B7 was studied in eight HIV-1-infected subjects, two with acute infection, five with chronic, and one long-term non-progressor (LTNP)

- 2 to 17 epitopes were recognized in a given individual, A2-restricted CTL response tended to be narrow and never dominated the response, and 25/27 epitopes were targeted by at least one person

- Subjects with chronic HIV-1 infection recognized between 2-8 out of 11 B7-restricted epitopes

- An acute seroconvertor homozygous for the B7 allele recognized five B7-restricted epitopes

- The other acute seroconvertor failed to recognize any of the 11 B7-restricted epitopes tested

- The B7-restricted CTL response was highly variable and there was no clearly dominant epitope

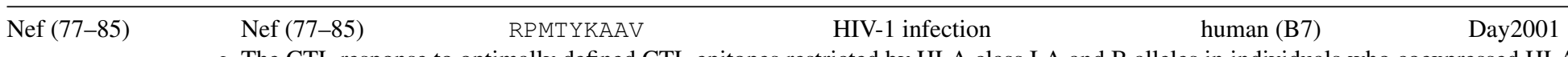

- The CTL response to optimally defined CTL epitopes restricted by HLA class I A and B alleles in individuals who coexpressed HLA A2, A3, and B7 was studied in eight HIV-1-infected subjects, two with acute infection, five with chronic, and one long-term non-progressor (LTNP)

- 2 to 17 epitopes were recognized in a given individual, A2-restricted CTL response tended to be narrow and never dominated the response, and 25/27 epitopes were targeted by at least one person

- Subjects with chronic HIV-1 infection recognized between 2-8 out of 11 B7-restricted epitopes

- An acute seroconvertor homozygous for the B7 allele recognized five B7-restricted epitopes

- The other acute seroconvertor failed to recognize any of the 11 B7-restricted epitopes tested

- The B7-restricted CTL response was highly variable and there was no clearly dominant epitope

\begin{tabular}{lllll}
\hline Nef (77-85) Nef (77-85 BRU) & RPMTYKAAV & HIV-1 infection & human (B7) & Choppin2001
\end{tabular}

- Seventy-three 8-11 mer peptides were generated based on putative anchor motifs in the Nef BRU sequence for HLA A2, A3, A24, B7, B8, and B35; these 73 peptides had 81 motifs. 54\% bound to the predicted HLA molecule, particularly A2, B7/35, and B8. The strength of HLA-peptide binding was not directly related to the number of individuals that recognized a protein.

- 20s proteasome cleavage of the Nef protein positions 66-100 showed a large fraction of peptides were cleaved ending at: 87L, 83A, 81Y, 71P, 68F and 67G. The frequency of recognition may be in part dictated by the cleavage step in processing.

- RPMTYKAAV was recognized in 7/10 (70\%) of individuals with HLA B7, and 0/3 (0\%) of individuals with HLA B35. It was a moderate affinity HLA binder.

\begin{tabular}{lllll}
\hline Nef (77-85) & Nef (77-85) & RPMTYKAAL & HIV-1 infection & human (B7) \\
& Epitope name: B7-RL9 & &
\end{tabular}

- CTL responses in 18 acutely HIV-infected HLA-A3 $(n=7)$ or -B7 $(n=4)$ or both -A3 and B7 $(n=7)$ positive individuals were studied.

- One individual, AC-06, was homozygous at all three class I alleles (A3, B7, Cw7), was treated during acute infection and had supervised treatment interruptions (STI). He had only two detectable CTL responses during acute infection, but after STI this broadened to 27 distinct epitopes including 15 restricted by HLA-A3, 11 by HLA-B7, and 1 by HLA-Cw7.

- 3/11 HLA-B7 individuals had detectable B7-restricted responses to this epitope during acute infection - 10/15 of HLA-B7 epitopes tested were targeted by at least one person during acute infection. 2/4 individuals had detectable responses to this epitope after STI.

\begin{tabular}{lllll}
\hline Nef (77-85) & Nef (77-85) & RPMTYKAAV & HIV-1 infection & human (B7)
\end{tabular}

- CTL responses in 18 acutely HIV-infected HLA-A3 $(n=7)$ or $-\mathrm{B} 7(\mathrm{n}=4)$ or both -A3 and B7 $(\mathrm{n}=7)$ positive individuals were studied.

- One individual, AC-06, was homozygous at all three class I alleles (A3, B7, Cw7), was treated during acute infection and had supervised treatment interruptions (STI). He had only two detectable CTL responses during acute infection, but after STI this broadened to 27 distinct epitopes including 15 restricted by HLA-A3, 11 by HLA-B7, and 1 by HLA-Cw7. 


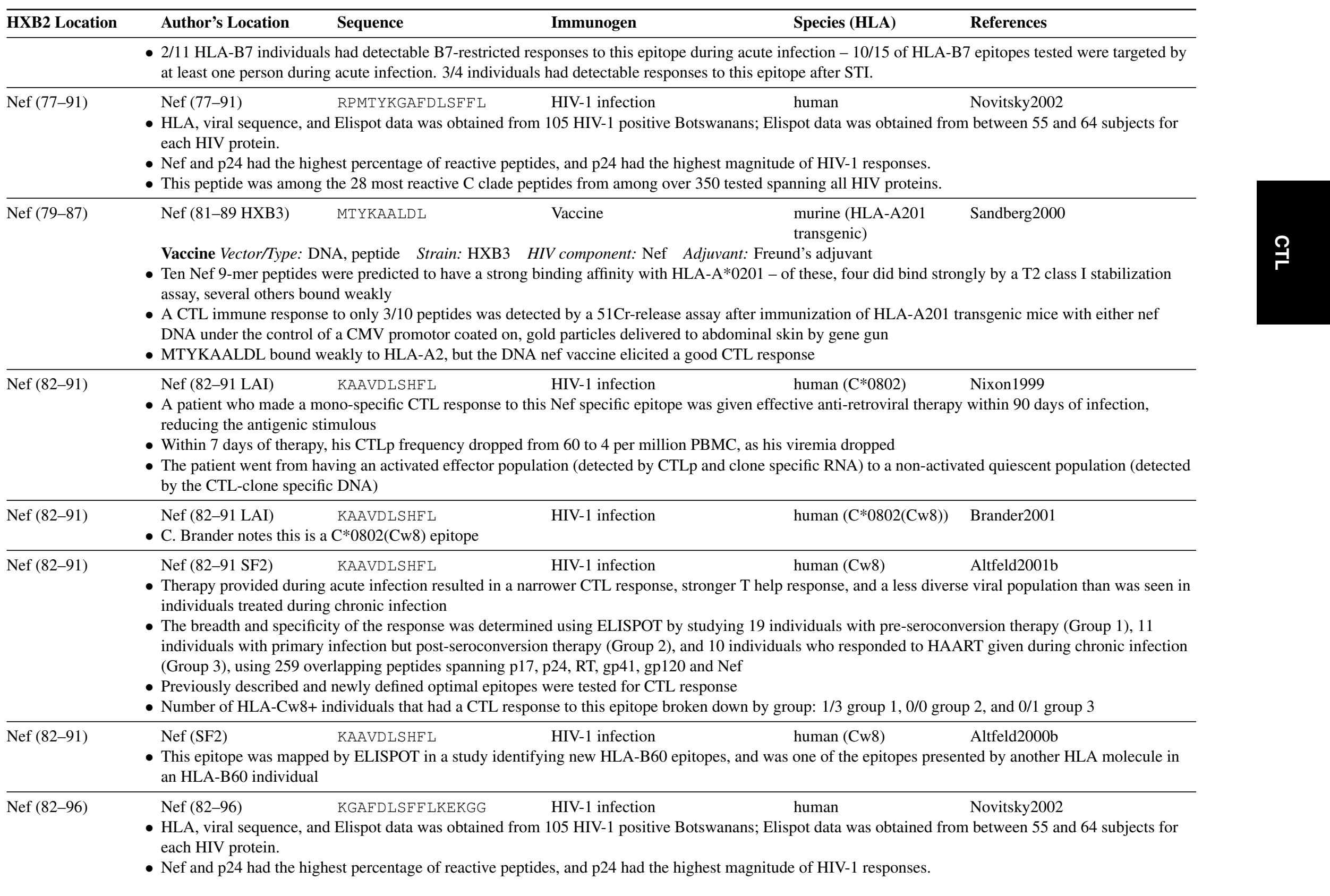




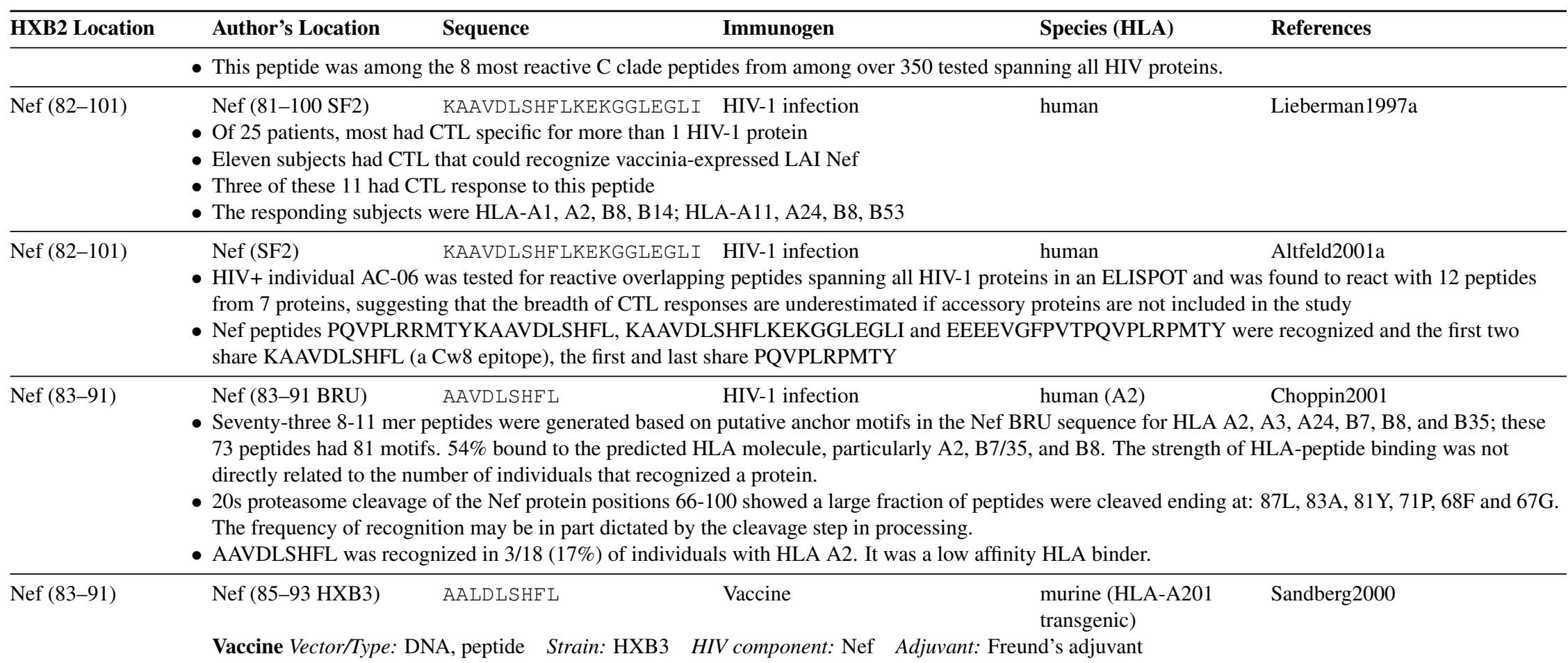

- Ten Nef 9-mer peptides were predicted to have strong binding affinity for HLA-A*0201 - of these, four did bind strongly by a T2 class I stabilization assay, several others bound weakly

- A CTL immune response to only 3/10 peptides was detected by a 51Cr-release assay after immunization of HLA-A201 transgenic mice with nef DNA under the control of a CMV promotor, coated on gold particles delivered to abdominal skin by gene gun

- AALDLSHFL was predicted to have a strong binding capacity for HLA-A2, and did, but it was the only one of the peptides recognized that was a strong binder, the other two recognized peptides were weak binders

- AALDLSHFL was also tested by subcutaneous injection of Nef peptides in Freund's adjuvant and gave a strong response to the peptide

Nef (83-92) Nef (81-90 93TH253 GAFDLSFFLK HIV-1 infection Sriwanthana2001
subtype CRF01)

- Epitope name: N83-92

- This was a study of HIV-1 exposed persistently seronegative (HEPS) female sex workers in Chiang Mai, northern Thailand

- HLA-A11 is very common in this population, and was enriched among the HEPS sexworkers - weak CTL responses were detected in 4/7 HEPS women, and CTL responses were found in 8/8 HIV+ controls, and 0/9 HIV- women that were not exposed

- This epitope was strongly reactive in HIV+ study subjects 053 and 184 who carried HLA-A11.

Nef $(83-92)$ Nef (81-90 93T
subtype CRF01)
HLA-A11 CRF01

HIV-1 infection

human (A11)

Bond2001

- HLA-A11 CRF01 (called subtype E in Bond et al.) epitopes were identified that stimulated CTL from HIV+ female sex workers (FSW) from Northern Thailand, of whom more than half were HLA-A11 positive 


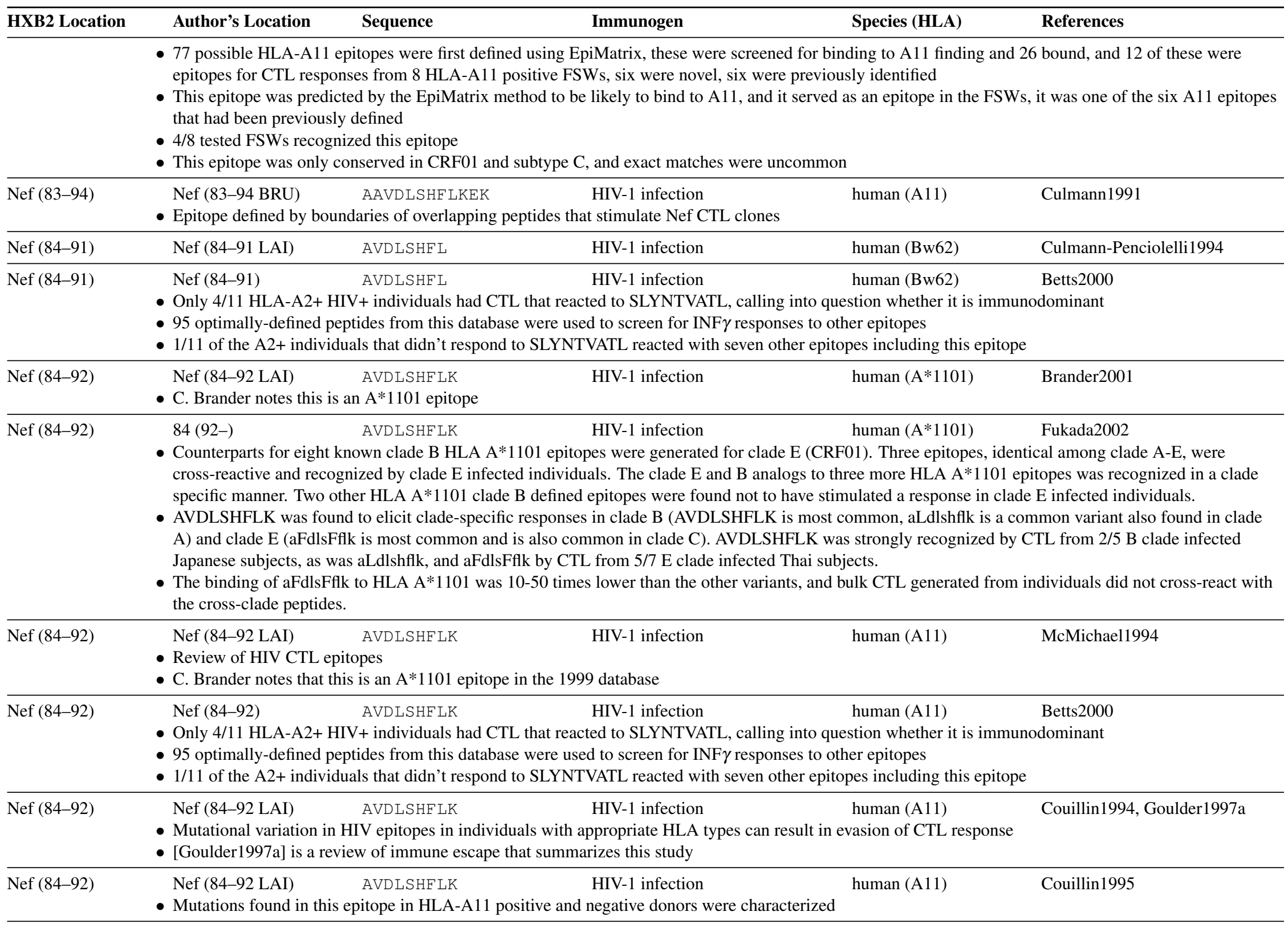




\begin{tabular}{lllll}
\hline HXB2 Location & Author's Location & Sequence & Immunogen & Species (HLA) \\
\hline Nef (84-92) & $\begin{array}{l}\text { Nef (84-92) } \\
\text { E Epitope name: AVD }\end{array}$ & AVDLSHFLK & HIV-1 infection & human (A11)
\end{tabular}

- Patients who started therapy at acute HIV-1 infection (three with sustained therapy, two with limited therapy upon early infection) had strong HIV specific CD4 proliferative responses and were able to maintain a CTL response even with undetectable viral load - three patients that had delayed initiation of HAART had no HIV specific CD4 proliferative responses and lost their CTL responses when HAART was eventually given and their viral loads became undetectable

- Both of the 2/8 HLA-A11 study subjects recognized this CTL epitope

- Patient SC19(HLA A11/12, B8/44, Cw06/0701, DR3/7, DR52/53, DQ 2/8) had a CTL response to epitopes FLKEKGGL, GEIYKRWII, ACQGVGGPGHK, AVDLSHFLK, and FNCGGEFFY that declined during therapy initiated at day 197

- Patient SC18(HLA A2/11, B8/44, Cw06/0701, DR3/7, DR52/53, DQ2) recognizes the epitopes ACQGVGGPGHK, QVPLRPMTYK, AVDLSHFLK, and one called QIY but not fully described - he had brief therapy upon seroconversion and has had low viral load during 600 days of follow up

Nef (84-92) Nef (82-90) AVDLSHFLK HIV-1 infection human (A11) Ferrari2000

- One of the 51 HIV-1 epitopes selected by Ferrari et al. as good candidate CTL epitopes for vaccines by virtue of being conserved and presented by common HLA alleles

Nef (84-92) Nef (84-92 SF2) AVDLSHFLK HIV-1 infection $\quad$ Altfeld2001b

- Therapy provided during acute infection resulted in a narrower CTL response, stronger T help response, and a less diverse viral population than was seen in individuals treated during chronic infection

- The breadth and specificity of the response was determined using ELISPOT by studying 19 individuals with pre-seroconversion therapy (Group 1), 11 individuals with primary infection but post-seroconversion therapy (Group 2), and 10 individuals who responded to HAART given during chronic infection (Group 3), using 259 overlapping peptides spanning p17, p24, RT, gp41, gp120 and Nef

- Previously described and newly defined optimal epitopes were tested for CTL response

- Number of HLA-A11+ individuals that had a CTL response to this epitope broken down by group: 0/3 group 1, 0/0 group 2, and 2/2 group 3

\begin{tabular}{llll}
\hline Nef (84-92) Nef (84-92) AVDLSHFLK & $\begin{array}{l}\text { HIV-1 infection, HIV-1 exposed human (A11) } \\
\text { seronegative }\end{array}$
\end{tabular}

- ELISPOT was used to study CTL responses to a panel of 54 predefined HIV-1 epitopes in 91 HIV-1-exposed, persistently seronegative (HEPS) and 87 HIV-1-infected female Nairobi sex workers

Nef (84-92) Nef AVDLSHFLK HIV-1 infection $\quad$ Oxenius2002b

- Epitope name: AVD

- Using previously defined epitopes [Oxenius2000, Oxenius2001a] in an IFNgamma Elispot assay, 13 chronically HIV-1 infected patients were studied over a period including therapy with standard treatment interruptions (STI).

- STIs induced increased recognition of CTL epitopes, but there was no correlation between CTL responses with viral rebound rates, plateau viral loads, or clearance rates.

Nef (84-92) Nef $\quad$ AVDLSHFLK $\quad$ HIV-1 infection, Vaccine human, macaque (A11) Hanke2000, Wee2002

Vaccine Vector/Type: DNA prime with vaccinia MVA boost Strain: subtype A HIV component: p17, p24, polyepitope

- The HIV-1 subtype A focused vaccine HIVA contains p24 and p17, in a reversed order relative to the Gag polyprotein to prevent myristylation of p17, which could direct the protein to the cell membrane and inhibit efficient peptide processing and class I presentation, as well as a polyepitope string of conserved, often immunodominant epitopes that were selected to have particularly good cross-reactive potential for the A-clade epidemic in Nairobi, Kenya. A DNA and MVA prime-boost vaccination protocol using the HIVA antigen will be used in a phase III clinical trial in Kenya. This epitope is included in the polyepitope string [Hanke2000]. 


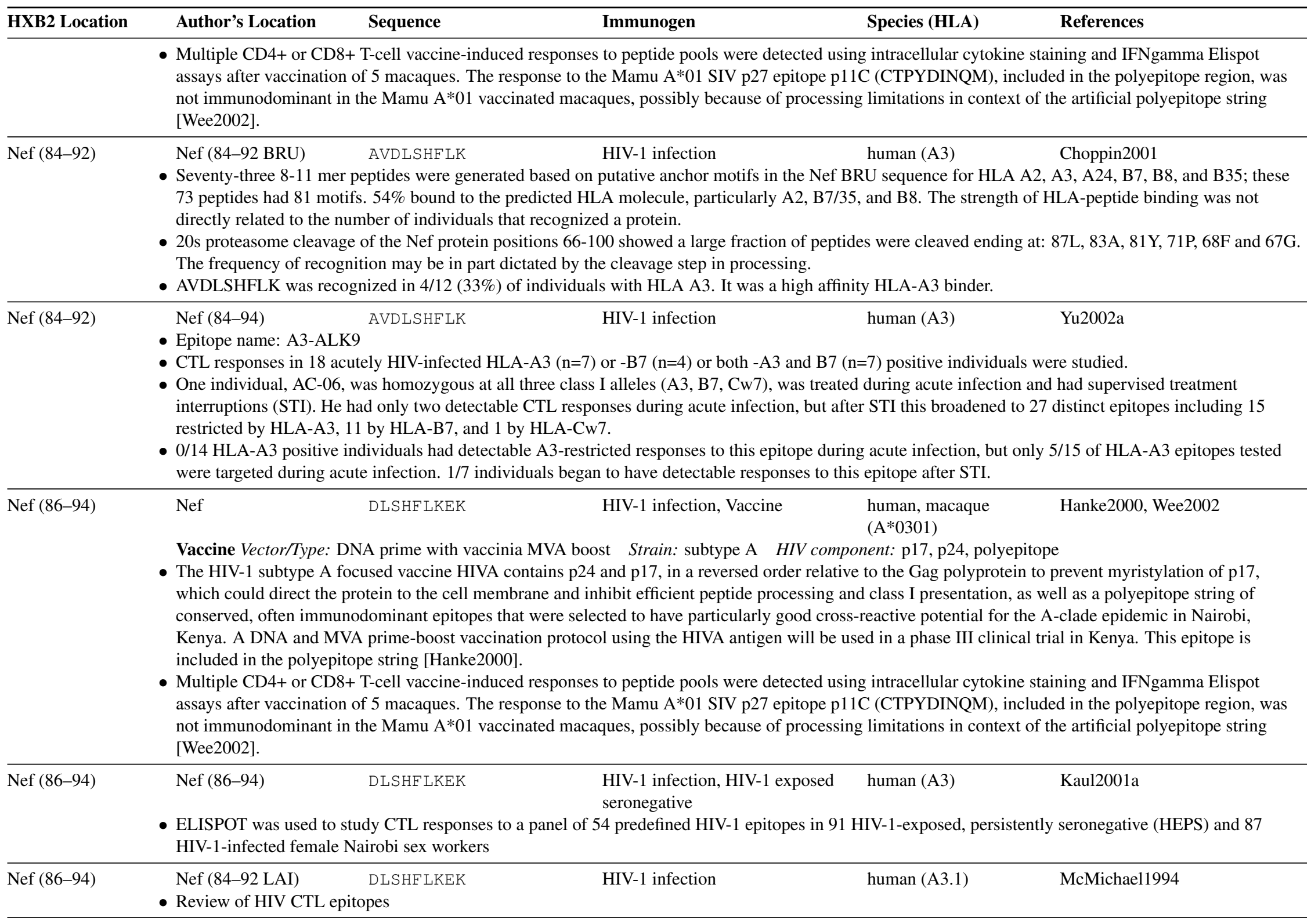




\begin{tabular}{|c|c|c|c|c|c|}
\hline HXB2 Location & Author's Location & Sequence & Immunogen & Species (HLA) & References \\
\hline Nef (86-100) & $\begin{array}{l}\text { Nef (86-100 LAI) } \\
\text { Development of a re }\end{array}$ & $\begin{array}{l}\text { DLSHF LKEKGGLEGL } \\
\text { al vector (pNeoNef) to }\end{array}$ & $\begin{array}{l}\text { HIV-1 infection } \\
\text { autologous targets }\end{array}$ & human (A2) & Robertson1993 \\
\hline Nef $(86-100)$ & Nef (86-100 LAI) & DLSHFLKEKGGLEGL & HIV-1 infection & human (B35) & Buseyne1993b \\
\hline Nef $(86-100)$ & Nef (86-100 LAI) & DLSHFLKEKGGLEGL & HIV-1 infection & human (B35 or C4) & Buseyne1993a \\
\hline
\end{tabular}

- Primary assays showed cytotoxic activity against at least one HIV protein was detected in $70 \%$ of infected children

- Epitopes recognized in five children were mapped using synthetic peptides and secondary cultures

- Patient EM13, who had a CTL response to three epitopes in Nef, was infected via blood transfusion after birth and went from CDC stage P2A to P2E during the study

\begin{tabular}{llcl}
\hline Nef (87-102) & Nef & F SHF LKEKGGLEGLIY & human
\end{tabular}

\begin{tabular}{llcl}
\hline Nef (87-102) & Nef & F SHF LKEKGGLEGLIY & human
\end{tabular}

\begin{tabular}{llcl}
\hline Nef (87-102) & Nef & F SHF LKEKGGLEGLIY & human
\end{tabular}

subtype in nef and env and 7 of the 41 strains were recombinants

- This region was defined as a CTL epitope region that is conserved among HIV-1 M group subtypes

Nef (88-100) Nef (103-116) SHFLKEKGGLEGL HIV-1 infection $\quad$ Guimaráes2002

- Nef sequences were obtained from Brazilians to study epitope diversity in this geographic region-most B subtype sequences are SHFLKEKGGLEGL, but sFflkekgglegl is found in most subtype $\mathrm{C}$ samples.

\begin{tabular}{llcc}
\hline Nef (90-97) & Nef (89-97) & FLKEKGGL & HIV-1 infection \\
& - Only 4/11 HLA-A2+ HIV+ individuals had CTL that reacted to SLYNTVATL, calling into question whether it is immunodominant \\
& - 95 optimally-defined peptides from this database were used to screen for INF $\gamma$ responses to other epitopes
\end{tabular}

- $1 / 11$ of the A2+ individuals that responded to SLYNTVATL reacted with seven other epitopes including this epitope previously described as presented by B8

Nef (90-97) Nef $\quad$ FLKEKGGL HIV-1 infection $\quad$ Ostrowski2000

- The role of CD4+ T-cell help in expansion of virus-specific memory CTL was studied through co-culture ex vivo

- Optimal expansion of HIV-1-specific memory CTL depended on CD4+ T cell help in 9 of 10 patients - CD40 ligand trimer (CD40LT) could enhance CTL

in the absence of CD4+ T cell help to a variable degree in most of patients

- Those CTL that didn't respond to CD40LT could expand with IL2 present, and IL15 produced by dendritic cells also contributes

- The T-helper epitope used for CD4+ T cell stimulation was the universal tetanus helper epitope TET830-843 (QYIKANSKFIGITE)

\begin{tabular}{|c|c|c|c|c|}
\hline Nef (90-97) & $\begin{array}{l}\text { FLKEKGGL } \\
\text { - Epitope name: Nef-FL8 } \\
\text { - Among HIV+ individuals who carried HLA }\end{array}$ & $\begin{array}{l}\text { HIV-1 infection } \\
\% \text { ) recognized this epitope. }\end{array}$ & human $(\mathrm{B} * 08)$ & Sabbaj2002b \\
\hline Nef (90-97) & $\begin{array}{l}\text { Nef }(89-97 \text { LAI }) \quad \text { FLKEKGGL } \\
\text { C. Brander notes this is a B*0801 epitope }\end{array}$ & HIV-1 infection & human $(\mathrm{B} * 0801)$ & Brander2001 \\
\hline Nef (90-97) & Nef (89-97 LAI) & HIV-1 infection & human (B8) & Price1997 \\
\hline
\end{tabular}

- CTL escape variants appeared over time in HLA-B8 HIV-1+ individual, providing evidence of immune escape

- Most variants appear at position 5, an anchor residue

- $\operatorname{FLKE}(\mathrm{E}, \mathrm{N}$ or Q)GGL showed reduced binding efficiency and recognition

- Double mutants (FIKENGGL, FLEENGGL, and FLKGNGGL) completely escaped recognition 


\begin{tabular}{lllcc}
\hline HXB2 Location & Author's Location & Sequence & Immunogen & Species (HLA) \\
\hline & $\bullet$ & Goulder1997a] is a review of immune escape that summarizes this study in the context of CTL escape to fixation & References & human (B8)
\end{tabular}

- Study examines the effect of highly active antiretroviral therapy (HAART) on HIV-1 plasma viral load, CTLp and CTLe frequencies in 8 infected children

- CTLp (precursors) were measured by stimulating in culture and assaying using 51Cr release, against vaccina expressed IIIB Env, Gag, Pol, Nef

- B7-FLKEKGGL tetramer complex was used for one of the children that was HLA-B7, and this infant showed a vigorous response ( $>4 \%$ of CD8+ T cells) at 9 months of age

- HIV-1 specific CTL responses initially increased in children with complete viral suppression, but then decreased, suggesting viral replication is needed to maintain CTL responses

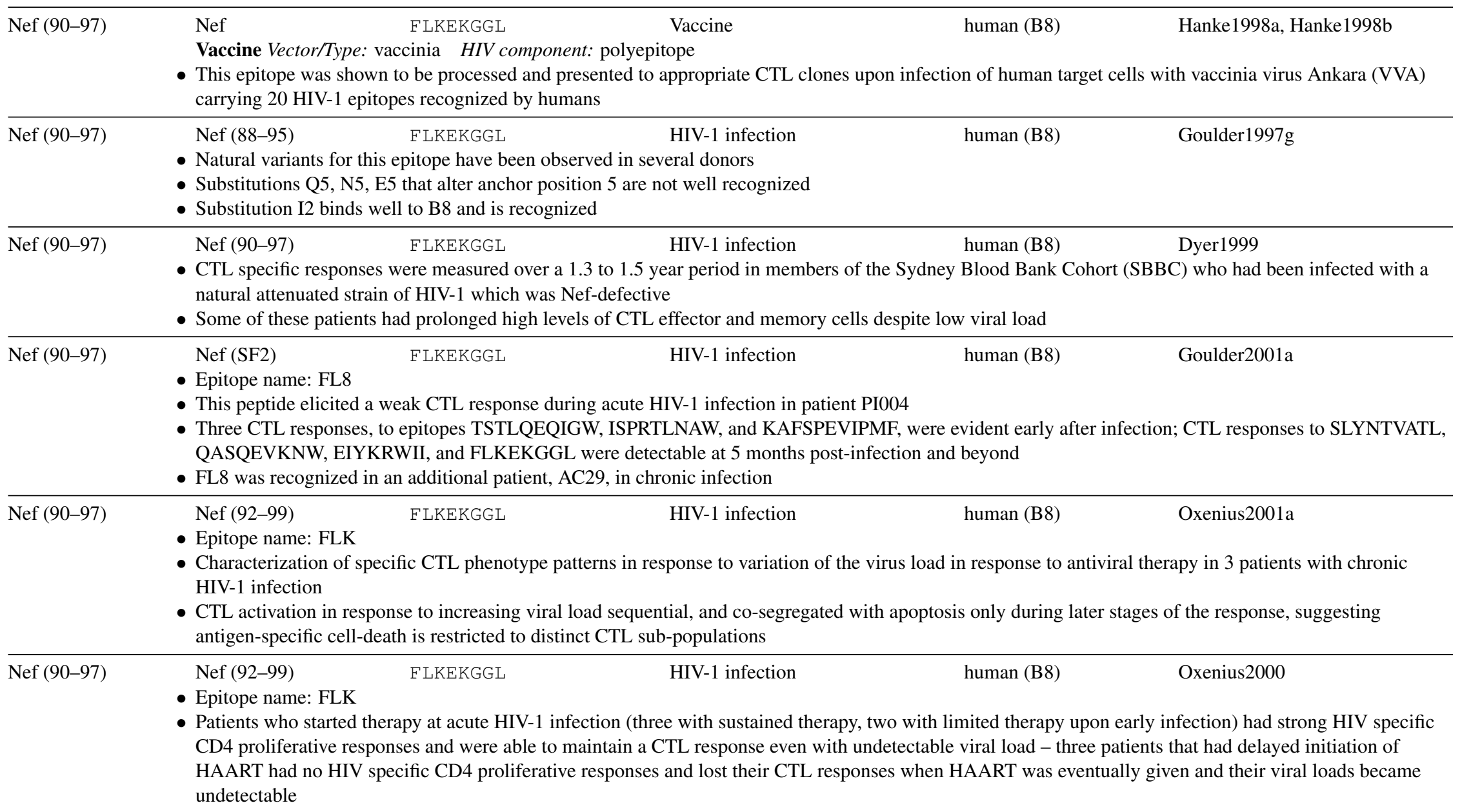




\begin{tabular}{|c|c|c|c|c|}
\hline HXB & 's Location & Sequence & Species (HLA) & ferences \\
\hline & \multicolumn{4}{|c|}{$\begin{array}{l}\text { - Six of the } 7 / 8 \text { study subjects that were HLA B8 recognized this early dominant CTL epitope } \\
\text { - Patient SC2 (HLA A1, B7/8, Cw0701/0702, DR4/53, DQ7) had CTL responsiveness against epitopes FLKEKGGL, GPKVKQWPL, and GEIYKRWII } \\
\text { peptides - FLKEKGGL tetramer staining steadily declined and at day } 1340 \text { the FLKEKGGL stained cells were no longer detected and the escape mutant } \\
\text { FLKENGGI was found in 8/10 clones } \\
\text { - Patient SC9 (HLA A1/2, B8/13, Cw0/0701, DR2/11, DQ6/7) had a CTL response against epitopes FLKEKGGL, ILKEPVHGV, } \\
\text { SQRRQDILDLWIYHTQGYFPWQNY, and GEIYKRWII peptides and all responses declined during therapy initiated at day } 390 \text { but were restored when } \\
\text { therapy become intermittent } \\
\text { - Patient SC19(HLA A11/12, B8/44, Cw06/0701, DR3/7, DR52/53, DQ 2/8) had a CTL response to epitopes FLKEKGGL, GEIYKRWII, } \\
\text { ACQGVGGPGHK, AVDLSHFLK, and FNCGGEFFY that declined during therapy initiated at day } 197 \\
\text { - Patient SC10(HLA A1/3, B8/35, DR1/8, DQ 4/5) had sustained therapy started during acute infection and maintained an immunodominant response to } \\
\text { FLKEKGGL and a response to GEIYKRWII through day 1088 } \\
\text { - Patient SC12(HLA A1, B8/39, Cw0701/0702, DR2/3, DR51/52, DQ2/6) had sustained therapy started during acute infection and maintained an } \\
\text { immunodominant response to FLKEKGGL throughout and minor responses to GEIYKRWII, DCKTILKAL, GGKKKYKLK - GEIYKRWII and } \\
\text { GGKKKYKLK responses were stimulated by a brief period off therapy } \\
\text { - Patient SC11(HLA A1, B8, Cw0201, DR3/11, DR52, DQ2/7) started therapy early, remained on therapy for } 40 \text { days, then reinitiated HAART at day } 640 \\
\text { had a CTL response to FLKEKGGL, GPKVKQWPL, and GEIYKRWII throughout and received a benefit from the early limited course therapy }\end{array}$} \\
\hline $\mathrm{f}(\mathrm{s}) \mathrm{s}$ & \multicolumn{4}{|c|}{$\begin{array}{l}\text { - HLA tetramers to six epitopes were used to study HLA-A2, B8 and B57 CTL in } 54 \text { patients - HIV-specific tetramer positive cells were inversely correlated } \\
\text { with viral load in patients with high CD4, but in patients with CD4 T-cells below } 400 \text { high tetramer frequencies were found despite high viral load } \\
\text { - Most patients have high levels of HIV-specific T-cell expansions, but many of these cells aren't functional } \\
\text { - In } 15 \text { of the patients, the proportion of IFN gamma producing tetramer cells correlated with AIDS-free survival } \\
\text { - Stimulation with HLA-B8 p24 and Nef epitopes significantly increased Nef-specific T-cell numbers in } 2 \text { patients (748 and } 1113 \text { ) } \\
\text { - There were more functional IFN-gamma producing Nef-specific T-cells within the T-cell population than there were active p24 Gag-specific T-cells } \\
\text { - No correlation between elevated numbers of Nef-specific CTL cells and plasma viral load was observed }\end{array}$} \\
\hline $\operatorname{Nef}(90-97)$ & $\begin{array}{l}\text { Nef }(88-95) \\
\text { - One of the } 51 \mathrm{HIV}-1 \\
\text { HLA alleles }\end{array}$ & $\begin{array}{l}\text { FLKEKGGI } \\
\text { pes selected }\end{array}$ & $\begin{array}{l}\text { HIV-1 infection } \\
\text { ood candidate CTL epitopes for vaccines by virtue }\end{array}$ & \\
\hline 10 & $\begin{array}{l}\text { Nef (88-95 SF2) } \\
\text { - Therapy provided du } \\
\text { individuals treated d } \\
\text { - The breadth and spec } \\
\text { individuals with prim } \\
\text { (Group 3), using } 259 \\
\text { - Previously described } \\
\text { - Number of HLA-B8 }\end{array}$ & $\begin{array}{l}\text { FLKEKGGL } \\
\text { acute infectio } \\
\text { chronic infec } \\
\text { y of the respo } \\
\text { nfection but } \\
\text { lapping pepti } \\
\text { newly definec } \\
\text { ividuals that } 1\end{array}$ & $\begin{array}{l}\text { IV-1 infection human (B8) } \\
\text { er CTL response, stronger T help response, and a le } \\
\text { sing ELISPOT by studying } 19 \text { individuals with pre } \\
\text { erapy (Group 2), and } 10 \text { individuals who respondec } \\
4, \text { RT, gp41, gp120 and Nef } \\
\text { re tested for CTL response } \\
\text { this epitope broken down by group: } 3 / 3 \text { group } 1,1\end{array}$ & $\begin{array}{l}\text { Altfeld } 2001 \mathrm{~b} \\
\text { viral population than was seen in } \\
\text { sion therapy (Group } 1), 11 \\
T \text { given during chronic infection } \\
\text { and } 1 / 2 \text { group } 3\end{array}$ \\
\hline $\operatorname{Nef}($ & $\begin{array}{l}\text { Nef (89-97) } \\
\text { Combined tetrame }\end{array}$ & FLKEKGG & $\begin{array}{l}\text { IV-1 infection human (B8) } \\
\text { sed to study the function of circulating CD8+ T } \\
\text { than CMV-specific CD8+ T cells from the sam } \\
\text { maturation }\end{array}$ & $\begin{array}{l}\text { ppay2000 } \\
\text { or HIV and CMV } \\
\text { is was associated with persistent }\end{array}$ \\
\hline
\end{tabular}




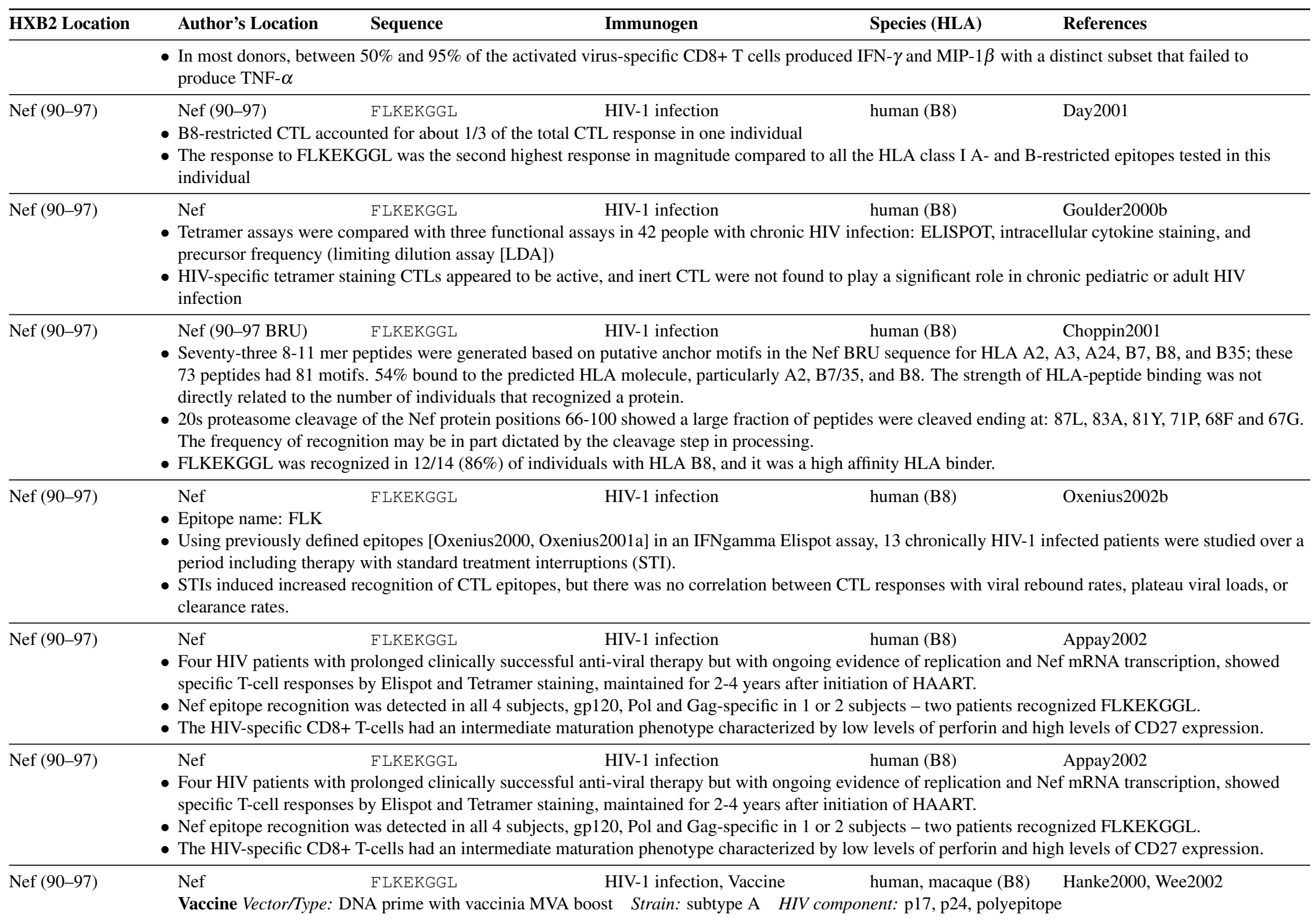




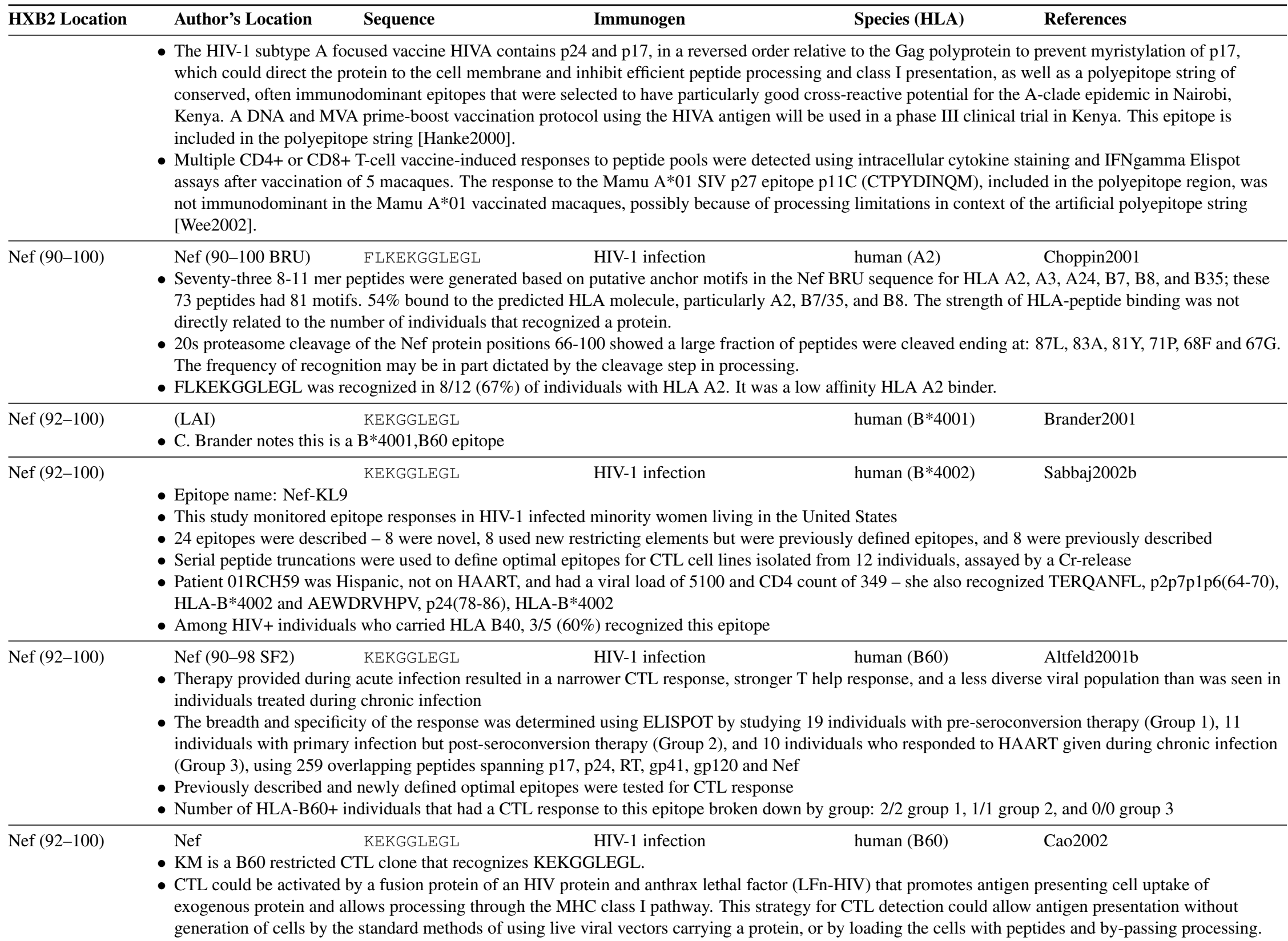




\begin{tabular}{|c|c|c|c|c|}
\hline HXB2 Location & Author's Location & Immunogen & Species (HLA) & References \\
\hline Nef $(92-100)$ & \multicolumn{4}{|c|}{$\begin{array}{l}\text { - This epitope was the dominant B60 (encoded by } \mathrm{B} * 4001 \text { ) response in } 6 / 8 \text { HLA-B60 individuals, and recognized in all eight } \\
\text { - This epitope was also recognized two expressing HLA-B61 individuals (B61 is usually encoded by B*4002, but this study did not distinguish between } \\
\mathrm{B} * 4002, \mathrm{~B} * 4003, \mathrm{~B} * 4004, \mathrm{~B} * 4006 \text {, and } \mathrm{B} * 4008 \text { ) } \\
\text { - ELISPOT was a rapid an effective method that was used to define five novel B60 epitopes } \\
\text { - HLA-B60 is present in } 10-20 \% \text { of the Caucasoid population and B60/B61 are very common in Asian populations }\end{array}$} \\
\hline Nef (92-100) & \multicolumn{4}{|c|}{$\begin{array}{l}\text { - No immunodominant responses were detected to five B61-restricted epitopes tested } \\
\text { - All five B60-restricted epitopes were reactive in another subject, and the B60-restricted responses together contributed over one-third of the total CTL } \\
\text { response }\end{array}$} \\
\hline Nef $(92-112)$ & \multicolumn{4}{|c|}{$\begin{array}{l}\text { - This epitope was mapped by ELISPOT in a study identifying new HLA-B60 epitopes, and was one of the epitopes presented by another HLA molecule in } \\
\text { an HLA-B60 individual } \\
\text { - The response to the peptide was CD8 dependent, but the HLA presenting molecule and optimal epitope were not determined }\end{array}$} \\
\hline Nef (92-112) & $\begin{array}{l}\text { Nef (SF2) } \\
\text { - This epitope was mi } \\
\text { an HLA-B60 indivic } \\
\text { - The response to the }\end{array}$ & $\begin{array}{l}\text { KEKGGLEGLIHSQRRQDIL- HIV-1 infection } \\
\text { DL } \\
\text { by ELISPOT in a study identifying new HLA-B60 } \\
\text { de was CD8 dependent, but the HLA presenting mo }\end{array}$ & $\begin{array}{l}\text { human } \\
\text { one of the epitopes pr }\end{array}$ & $\begin{array}{l}\text { Altfeld2000b } \\
\text { nted by another HLA molecule in } \\
\text { ined }\end{array}$ \\
\hline Nef (93-106) & $\begin{array}{l}\text { Nef (93-106 BRU) } \\
\text { - HIV-1 specific CTL }\end{array}$ & $\begin{array}{l}\text { EKGGLEGLIHSQRR } \\
\text { ected in lymphoid organs of HIV-1 infected patients }\end{array}$ & human (A1, B8) & Hadida1992 \\
\hline Nef $(97-111)$ & $\begin{array}{l}\text { Nef }(97-111) \\
\text { - HLA, viral sequenc } \\
\text { each HIV protein. } \\
\text { - Nef and p24 had the } \\
\text { - This peptide was an }\end{array}$ & $\begin{array}{l}\text { LEGLIYSKKRQEILD HIV-1 infection } \\
\text { Elispot data was obtained from } 105 \text { HIV-1 positiv } \\
\text { lest percentage of reactive peptides, and p } 24 \text { had the } \\
\text { the } 28 \text { most reactive } \mathrm{C} \text { clade peptides from among }\end{array}$ & $\begin{array}{l}\text { human } \\
\text { spot data was obtained } \mathrm{f} \\
\text { le of HIV-1 responses. } \\
\text { anning all HIV proteins. }\end{array}$ & Novitsky2002 \\
\hline Nef (102-115) & $\begin{array}{l}\text { Nef }(102-115 \text { LAI }) \\
\text { - Identical twin hemo } \\
\text { - One had a strong re } \\
\text { - } \text { Goulder1997a is a }\end{array}$ & $\begin{array}{l}\text { HSQRRQD ILDLWIY HIV-1 infection } \\
\text { ac brothers were both infected with the same batch } \\
\text { e to this peptide, the other did not } \\
\text { ew of immune escape that summarizes this study }\end{array}$ & human (B7) & Goulder1997e Goulder1997a \\
\hline Nef (102-121) & $\begin{array}{l}\text { Nef }(101-120 \mathrm{SF} 2) \\
\text { - Of } 25 \text { patients, most } \\
\text { - Eleven subjects had } \\
\text { - Two of these } 11 \mathrm{had} \\
\text { - The responding sub. }\end{array}$ & $\begin{array}{l}\text { HSQRRQD I LDLQIYHTQGYF HIV-1 infection } \\
\text { CTL specific for more than } 1 \mathrm{HIV}-1 \text { protein } \\
\text { that could recognize vaccinia-expressed LAI Nef } \\
\text { response to this peptide } \\
\text { were HLA-A2, A3, B8, B } 62 \text { and HLA-A2, B21 }\end{array}$ & human & Lieberman1997a \\
\hline
\end{tabular}




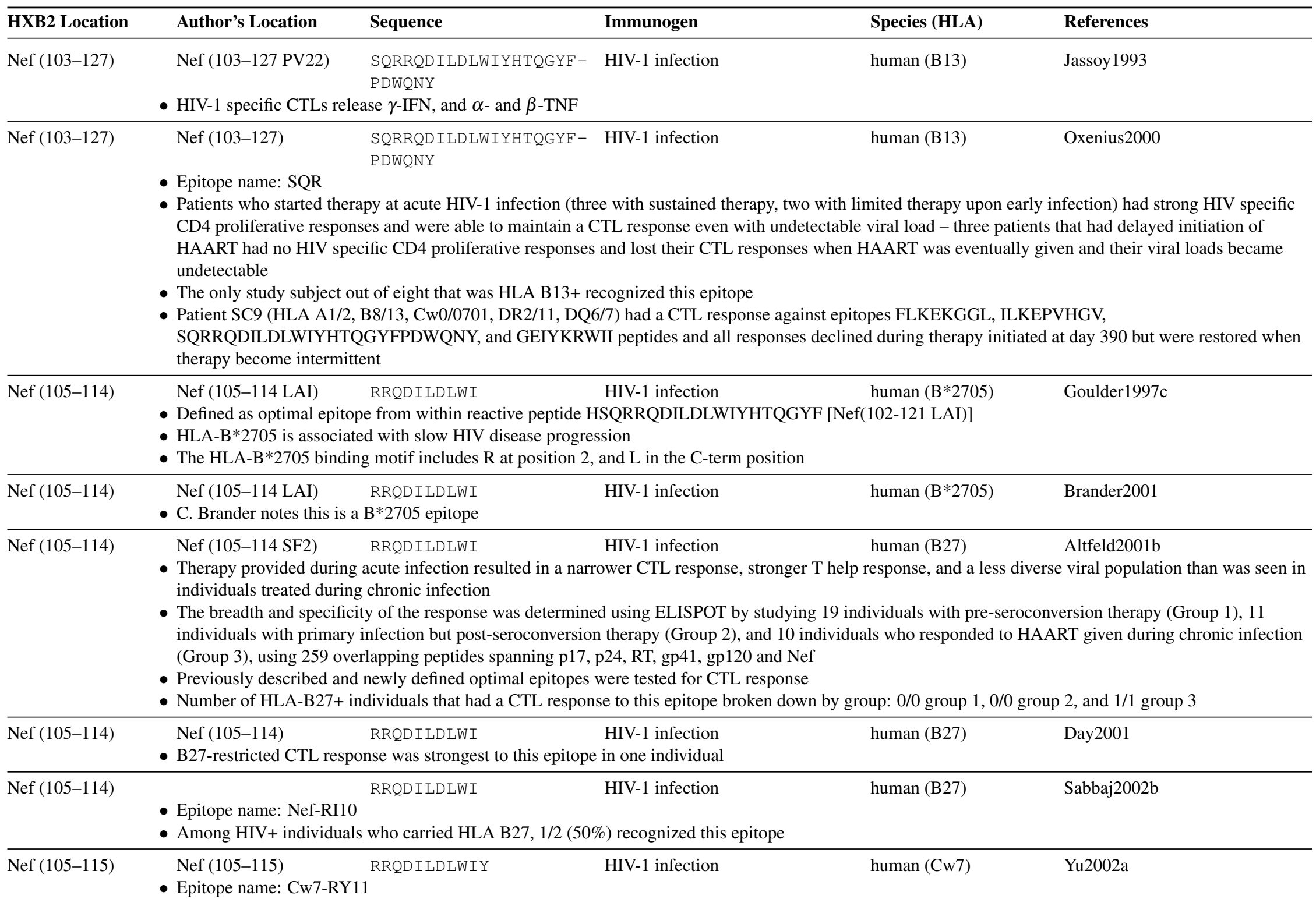




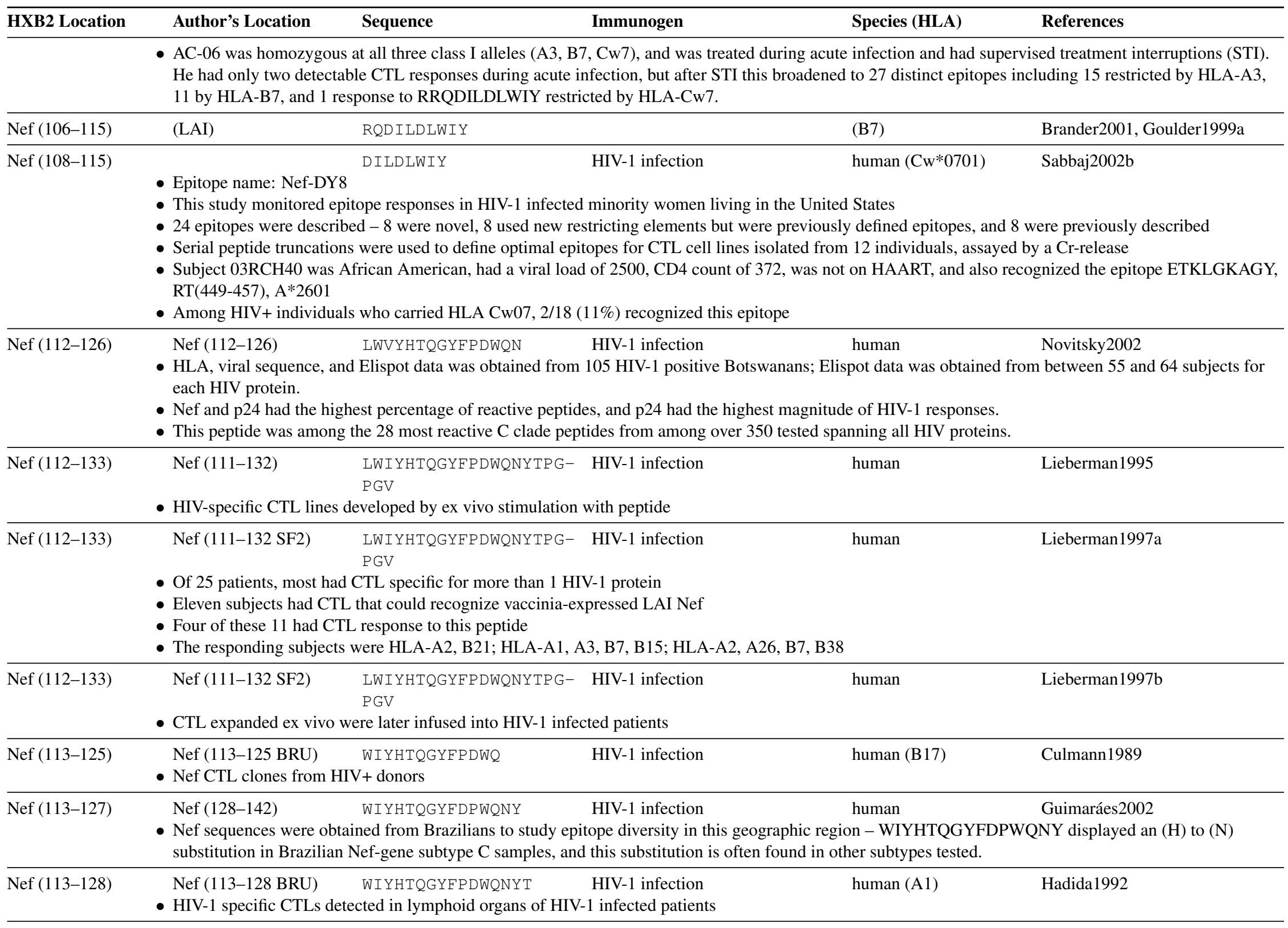




\begin{tabular}{|c|c|c|c|c|}
\hline HXB2 Location & Author's Location & Sequence & Species (HLA) & References \\
\hline $\operatorname{Nef}(113-128)$ & \multicolumn{4}{|c|}{$\begin{array}{l}\text { - Epitope name: N2 } \\
\text { - A panel of } 16 \text { epitopes covering } 15 \text { class I alleles was tested in } 14 \text { HIV+ patients from an unselected Caucasian population treated with HAART, using } \\
\text { CD8+ cell IFNgamma production to measure responses } \\
\text { - In general, during the first month of treatment viral load decreased and frequencies of HIV-specific CTL tripled and broadened - eight new HIV } \\
\text { specificities that were not previously detectable were newly detected, as were CMV specific CD8+ PBL - but with continued viral suppression, } \\
\text { HIV-specific responses diminished } \\
\text { - Viral rebounds gave different patterns of response: increases or decreases in pre-existing response, new specificities, or no change }\end{array}$} \\
\hline Nef (114-127) & Nef & VYHTQGYFPDWQNY & HIV-1 infection & Jubier-Maurin1999 \\
\hline Nef (115-125) & \multicolumn{4}{|c|}{ - Nef CTL clones from HIV+ donors } \\
\hline Nef (116-125) & \multicolumn{4}{|c|}{$\begin{array}{l}\text { - C. Brander notes this is a B*5701 epitope } \\
\text { - Subtype of B57 not determined }\end{array}$} \\
\hline $\operatorname{Nef}(116-125)$ & \multicolumn{4}{|c|}{$\begin{array}{l}\text { - Only 4/11 HLA-A2+ HIV+ individuals had CTL that reacted to SLYNTVATL, calling into question whether it is immunodominant } \\
\text { - } 95 \text { optimally-defined peptides from this database were used to screen for INF } \gamma \text { responses to other epitopes } \\
\text { - One of the A2+ individuals was HLA A*0201, A1, B57 and responded to four B57 epitopes and two others }\end{array}$} \\
\hline Nef (116-125) & \multicolumn{4}{|c|}{ - Nef CTL clones from HIV+ donors, optimal peptide mapped } \\
\hline Nef (116-125) & $\begin{array}{l}\text { Nef (116-125) } \\
\text { - Epitope name: HTQ } \\
\text { Patients who started ther: } \\
\text { CD4 proliferative respon } \\
\text { HAART had no HIV spe } \\
\text { undetectable } \\
\text { - None of the } 8 \text { study subje }\end{array}$ & $\begin{array}{l}\text { HTQGYFPDWQ } \\
\text { apy at acute HIV-1 infectio } \\
\text { ses and were able to maint } \\
\text { cific CD4 proliferative rest } \\
\text { ects recognized this epitop }\end{array}$ & $\begin{array}{l}\text { HIV-1 infection human (B57) } \\
\text { ee with sustained therapy, two with limited therapy upor } \\
\text { CTL response even with undetectable viral load - three } \\
\text { and lost their CTL responses when HAART was event } \\
\text { Ione were HLA B57+ }\end{array}$ & $\begin{array}{l}\text { Oxenius2000 } \\
\text { ffection) had strong HIV specific } \\
\text { that had delayed initiation of } \\
\text { en and their viral loads became }\end{array}$ \\
\hline $\operatorname{Nef}(116-125)$ & $\begin{array}{l}\text { - Epitope name: Nef-HQ1 } \\
\text { - Among HIV+ individual }\end{array}$ & $\begin{array}{l}\text { HTQGYFPDWQ } \\
\text { who carried HLA B57, 0/ }\end{array}$ & $\begin{array}{l}\text { HIV-1 infection } \\
\text { recognized this epitope }\end{array}$ & Sabbaj2002b \\
\hline $\operatorname{Nef}(117-127)$ & $\begin{array}{l}\text { Nef }(117-127) \\
\text { - Only } 4 / 11 \text { HLA-A2+ HI } \\
\text { - } 95 \text { optimally-defined pep } \\
\text { - } 1 / 11 \text { of the A2+ individu } \\
\text { individuals that was A2+ }\end{array}$ & $\begin{array}{l}\text { TQGYFPDWQNY } \\
V+\text { individuals had CTL th } \\
\text { tides from this database w } \\
\text { als was HLA A*0205/A*0 } \\
\text {, but otherwise of unknow }\end{array}$ & $\begin{array}{l}\text { HIV-1 infection human } \\
\text { ted to SLYNTVATL, calling into question whether it is } \\
\text { d to screen for INF } \gamma \text { responses to other epitopes } \\
30, B 27, \text { B44 but responded to HLA Bw62 epitope TQ } \\
\text { type, reacted with seven epitopes including this one }\end{array}$ & $\begin{array}{l}\text { Betts2000 } \\
\text { WQminant }\end{array}$ \\
\hline
\end{tabular}




\begin{tabular}{|c|c|c|c|c|c|}
\hline HXB2 Location & Author's Location & Sequence & Immunogen & Species (HLA) & References \\
\hline Nef $(117-127)$ & \multicolumn{5}{|c|}{ - C. Brander notes this is a B*1501 epitope } \\
\hline Nef (117-127) & \multicolumn{5}{|c|}{ - No immunodominant responses were detected to four B62-restricted epitopes tested } \\
\hline Nef (117-127) & \multicolumn{5}{|c|}{ - Optimal peptide defined by titration } \\
\hline Nef (117-128) & $\begin{array}{l}\text { Nef (117-128 BRU) } \\
\text { - Nef CTL clones from }\end{array}$ & $\begin{array}{l}\text { TQGYFPDWQNYT } \\
\text { + donors }\end{array}$ & HIV-1 infection & human (B17, B37) & Culmann1991 \\
\hline Nef (117-147) & $\operatorname{Nef}(117-147 \mathrm{LAI})$ & $\begin{array}{l}\text { TQGYFPDWQNYTP } \\
\text { PLTFGWCYKLVP }\end{array}$ & Vaccine & human & Gahery-Segard2000 \\
\hline
\end{tabular}

Vaccine Vector/Type: lipopeptide HIV component: six peptides

- Anti-HIV lipopeptide vaccine consisting of six long amino acid peptides derived from Nef, Gag and Env HIV-1 proteins modified by a palmitoyl chain was administered in a phase I trial

- A CD4+ T cell proliferative response to at least one of the six peptides was observed in $9 / 10$ vaccinees - $1 / 10$ reacted to this Nef peptide

- 9/12 tested mounted a CTL response to at least one of the six peptides; each of the six peptides elicited a CTL response in at least one individual

- 10/12 tested had an IgG response to this peptide

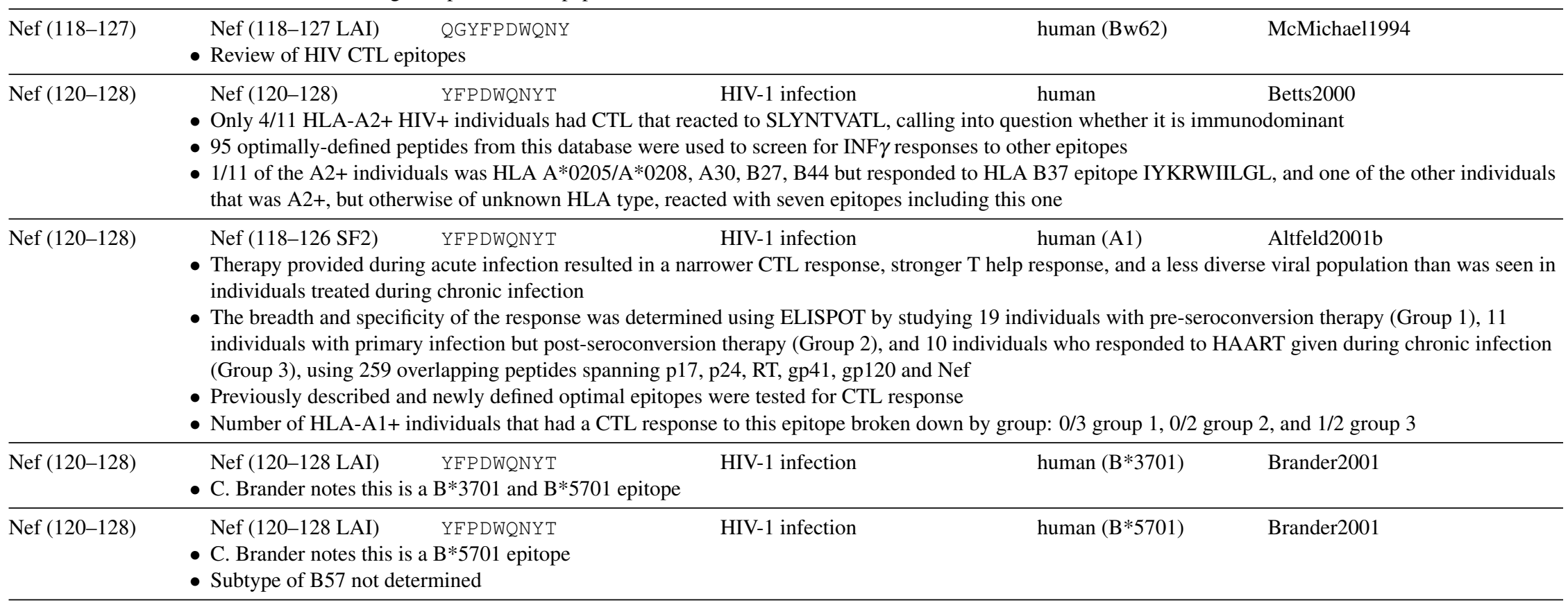




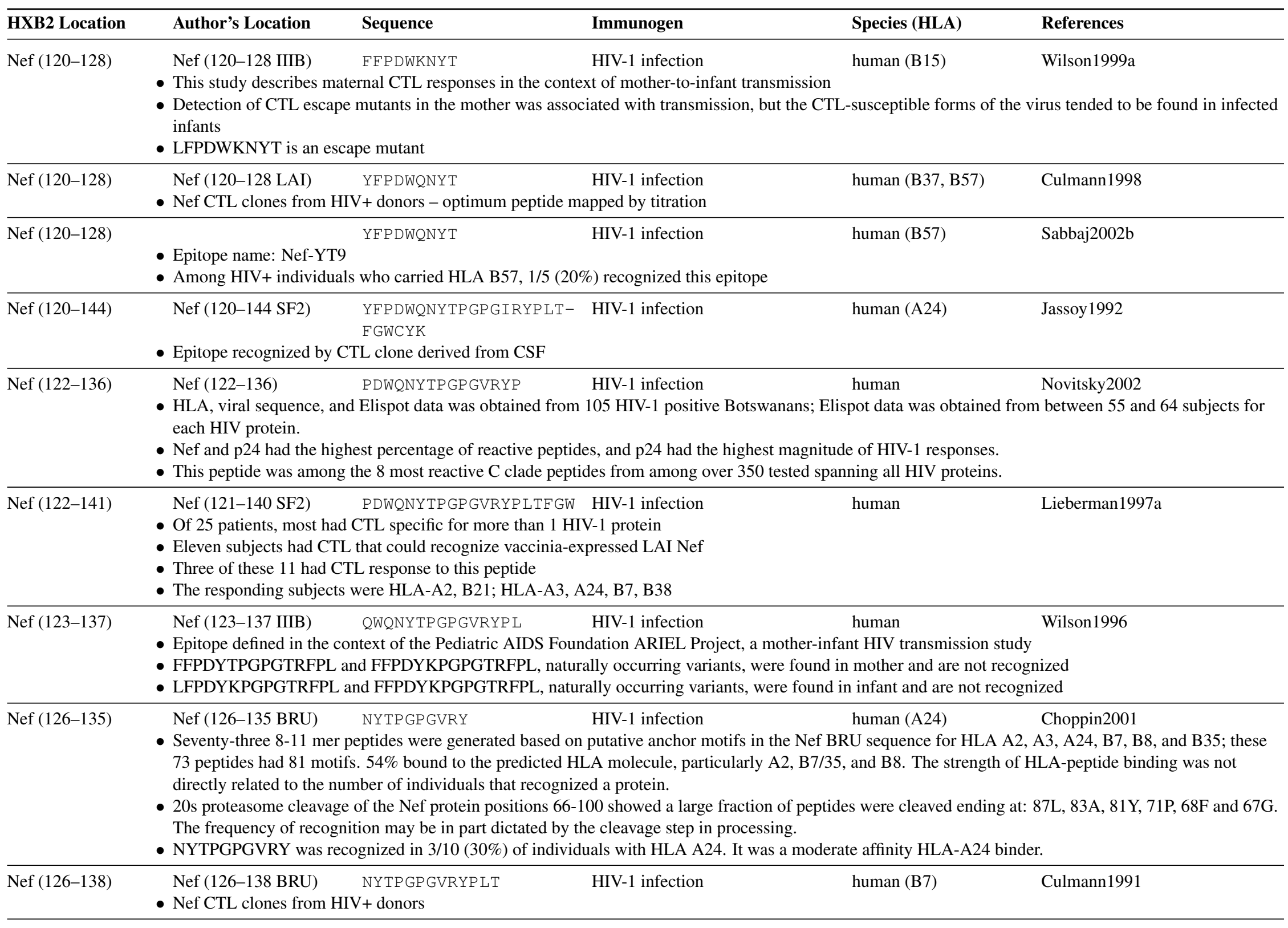




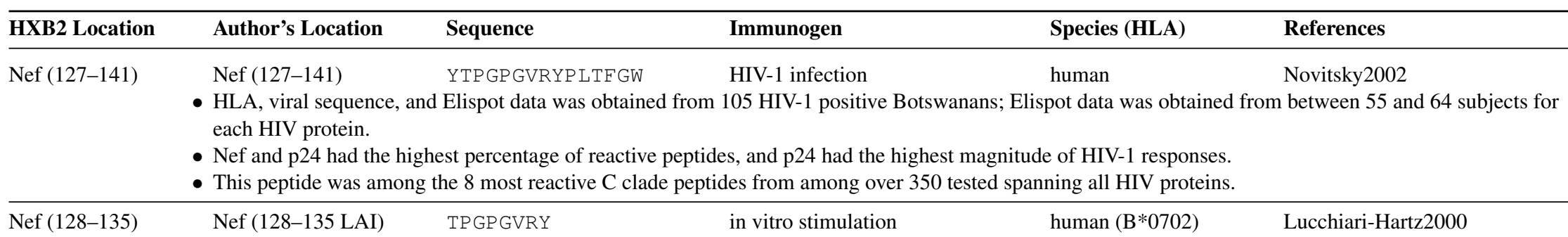

- Five naturally processed MHC class I ligands were identified in Nef in the conserved immunogenic region Nef between 123-152

- All five could be transported by TAP, and 4/5 had N-termini that were major cleavage points for the proteasome, only one had extended precursor fragments

- Both TPGPGVRYPL and TPGPGVRY are naturally processed ligands that can be eluted from HLA-B7 molecules, both are recognized by the same CTL, and both peptides seem to be the direct product of a proteasomal digest

- The peptide TPGPGVRY is present in a high copy number, TPGPGVRYPL at a more moderate level, possibly due to a major cleavage site between the Y and $\mathrm{P}$

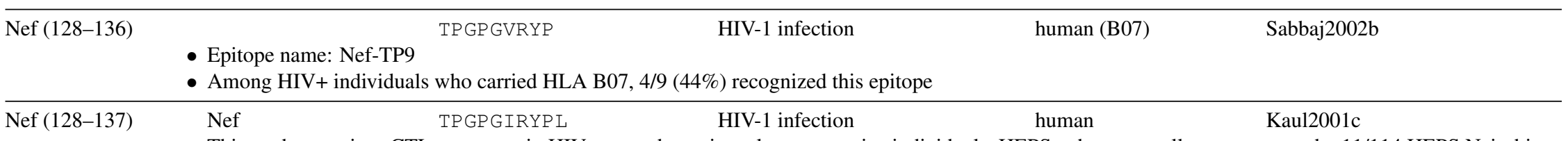

- This study examines CTL responses in HIV exposed, persistently seronegative individuals, HEPS, who eventually seroconverted - 11/114 HEPS Nairobi sex workers eventually seroconverted, and for six of these HIV CTL reactive epitopes had been defined while seronegative

- The epidemiological factor associated with seroconversion was stopping sex work and HIV-specific CTL activity declines when HEPS sex workers stop working for a period or retire

- This epitope was recognized by $1 / 22$ HEPS control sex workers, ML851

\begin{tabular}{llrrr}
\hline Nef (128-137) & $\begin{array}{l}\text { Nef (128-137 LAI) } \\
\text { C. Brander notes this is a B*0702 epitope }\end{array}$ & HIV-1 infection & human (B*0702) & Brander2001 \\
\hline Nef (128-137) & Nef (128-137 LAI) & TPGP GVRYPL & in vitro stimulation & human (B*0702)
\end{tabular}

- Five naturally processed MHC class I ligands were identified in Nef in the conserved immunogenic region Nef between 123-152

- All five could be transported by TAP, and 4/5 had N-termini that were major cleavage points for the proteasome, only one had extended precursor fragments

- Both TPGPGVRYPL and TPGPGVRY are naturally processed ligands that can be eluted from HLA-B7 molecules, both are recognized by the same CTL, and both peptides seem to be the direct product of a proteasomal digest

- The peptide TPGPGVRY is present in a high copy number, TPGPGVRYPL at a more moderate level, possibly due to a major cleavage site between the Y and $\mathrm{P}$

\begin{tabular}{llrrr}
\hline Nef (128-137) & Nef (128-137 LAI) & TPGPGVRYPL & human (B*4201) \\
& C. Brander notes this is a B*4201 epitope & Brander2001 \\
\hline Nef (128-137) & Nef (128-137 BRU) & TPGPGVRYPL & HIV-1 infection & human (B35)
\end{tabular}

- Seventy-three 8-11 mer peptides were generated based on putative anchor motifs in the Nef BRU sequence for HLA A2, A3, A24, B7, B8, and B35; these 73 peptides had 81 motifs. 54\% bound to the predicted HLA molecule, particularly A2, B7/35, and B8. The strength of HLA-peptide binding was not directly related to the number of individuals that recognized a protein.

- 20 s proteasome cleavage of the Nef protein positions $66-100$ showed a large fraction of peptides were cleaved ending at: $87 \mathrm{~L}, 83 \mathrm{~A}, 81 \mathrm{Y}, 71 \mathrm{P}, 68 \mathrm{~F}$ and $67 \mathrm{G}$. The frequency of recognition may be in part dictated by the cleavage step in processing. 


\begin{tabular}{llccc}
\hline HXB2 Location & Author's Location & Sequence & Immunogen & Species (HLA) \\
\hline & - TPGPGVRYPL was recognized in 8/16 (50\%) of individuals with HLA B7, and 1/9 (11\%) of individuals with HLA B35. It was a high affinity HLA binder. & TPGP GVRYPL & HIV-1 infection & human (B7) \\
\hline Nef (128-137) & & Wilson2000a & Three individuals with highly focused HIV-specific CTL responses were studied during acute infection using tetramers - high frequencies of HIV-1-specific \\
& CD8+ T cells were found prior to seroconversion, and there was a close temporal relationship between the number of circulating HIV-specific T cells and
\end{tabular}
viral load was also found

- All three patients were $\mathrm{B} * 2705$, with HLA alleles: A1, A30/31, B*2705, B35; A1, A*0301, B7, B*2705; and A*0201, A*0301, B*2705, B39

- ELISPOT was used to test a panel of CTL epitopes that had been defined earlier and were appropriate for the HLA haplotypes of the study subjects $-3 / 3$ subjects showed a dominant response to the B*2705 epitope KRWIILGGLNK

- The subject with $\mathrm{A}^{*} 0201$ had a moderatly strong response to SLYNTVATL

- Weak responses were observed to A*301-RLRPGGKKK, A*301-QVPLRPMTYK, and B7-TPGPGVRYPL in the subject who was HLA A1, A*0301, B7, $\mathrm{B} * 2705$

- No acute response was detected to the following epitopes: A*201-ILKEPVHGV, A*301-KIRLRPGGK, A*301-AIFQSSMTK, A*301-TVYYGVPVWK, B35-EPIVGAETF, B35-HPDIVIYQY, B35-PPIPVGEIY, B35-NSSKVSQNY, B35-VPLRPMTY, B35-DPNPQEVVL

Nef (128-137) Nef (128-137 LAI) TPGPGVRYPL HIV-1 infection Haas1996, Haas

- There was a high degree of variation in three CTL epitopes in Nef in four slow and non-progressors, and variant specific CTLs arose over time to eliminate variants, indicating immune selection

- The epitope position was taken from [Haas1997]

Nef (128-137) Nef TPGPGVRYPL $\quad$ HIV-1 exposed seronegative human (B7) Rowland-Jones1998a

- A CTL response was found in exposed but uninfected prostitutes from Nairobi using previously-defined B clade epitopes that tended to be conserved in A and D clades - such cross-reactivity could protect against both A and D and confer protection in Nairobi where both subtypes are circulating

- The D subtype consensus is identical to the B clade epitope

- The A subtype consensus is TPGPGIRYPL

Nef (128-137) Nef (subtype B) TPGPGVRYPL $\quad$ HIV-1 exposed seronegative human (B7)

- HIV-specific CTL were found in exposed seronegative prostitutes from Nairobi - these CTL may confer protection

- Seroprevalence in this cohort is $90-95 \%$ and their HIV-1 exposure is among the highest in the world

- Most isolated HIV strains are clade A in Nairobi, although clades C and D are also found - B clade epitopes are often cross-reactive, however stronger responses are frequently observed using A or D clade versions of epitopes

- This epitope is conserved among B and D clade viruses

- The Clade A version of the epitope: TPGPGIRYPL

Nef (128-137) Nef (128-137) TPGPGVRYPL in vitro stimulation human (B7) Wilson1999b

- Dendritic cells are the most potent for priming T cell responses - DCs can stimulate autologous CTL responses from T cells cultured from HIV negative donors

- Th1-biasing cytokines IL-12 or IFN alpha enhance CTL responses in vitro whether the epitope is delivered by pulsing from peptide, or expressed from within

- CTL from a B7 donor displayed no reactivity to this epitope, although it had been immunodominant in another study [Haas1996]

Nef(128-137 SF2) TPGPGVRYPL HIV-1 infection human (B7) Altfeld2001b

- Therapy provided during acute infection resulted in a narrower CTL response, stronger T help response, and a less diverse viral population than was seen in individuals treated during chronic infection 


\begin{tabular}{|c|c|c|c|c|}
\hline HXB2 Location & Author's Location & Sequence & Species (HLA) & References \\
\hline & \multicolumn{4}{|c|}{$\begin{array}{l}\text { - The breadth and specificity of the response was determined using ELISPOT by studying } 19 \text { individuals with pre-seroconversion therapy (Group } 1 \text { ), } 11 \\
\text { individuals with primary infection but post-seroconversion therapy (Group 2), and } 10 \text { individuals who responded to HAART given during chronic infection } \\
\text { (Group 3), using } 259 \text { overlapping peptides spanning p17, p24, RT, gp41, gp120 and Nef } \\
\text { - Previously described and newly defined optimal epitopes were tested for CTL response } \\
\text { - Number of HLA-B7+ individuals that had a CTL response to this epitope broken down by group: } 0 / 4 \text { group } 1,0 / 3 \text { group } 2 \text {, and } 1 / 1 \text { group } 3\end{array}$} \\
\hline Nef (128-137) & $\begin{array}{l}\text { Nef (128-137) } \\
\text { - ELISPOT was used } \\
\text { HIV-1-infected fema } \\
\text { - Responses in HEPS } \\
\text { reduced risk of infec } \\
\text { women } \\
\text { - } 43 / 91 \text { HEPS women } \\
\text { - Among HLA-B7 wo } \\
\text { - The dominant respor } \\
\text { - Subject ML } 1203 \text { sta } \\
\text { acquired additional r }\end{array}$ & $\begin{array}{l}\text { TPGPGVRYPL } \\
\text { dy CTL response } \\
\text { airobi sex worker } \\
\text { len tended to be lo } \\
\text { and there was a s } \\
\text { CD8+ responses } \\
4 / 5 \text { HEPS and } 5 \text { / } \\
\text { this HLA allele } \\
\text { with CTL respon } \\
\text { nses to A*6802 E }\end{array}$ & $\begin{array}{l}\text { HIV-1 infection, HIV-1 exposed human (B7) } \\
\text { seronegative } \\
54 \text { predefined HIV-1 epitopes in } 91 \text { HIV-1-exposed, pe } \\
\text { ed on different epitopes with HLA presenting molecule } \\
\text { onse in the HEPS women upon late seroconversion to e } \\
\text { f HIV-1-specific CTL in HEPS women increased with } \\
\text { d women recognized this epitope } \\
\text { ope in } 3 \text { of the } 4 / 5 \text { HEPS cases and in } 2 \text { of the } 5 / 6 \text { HIV } \\
\text { DTVLEDINL and to B7 FPVTPQVPLR prior to seroc } \\
\text { lich became dominant, B7 TPGPG(V/I)RYPL, B7 IPR }\end{array}$ & $\begin{array}{l}\text { Kaul2001a } \\
\text { y seronegative (HEPS) and } 87 \\
\text { ve previously been associated with } \\
\text { recognized by the HIV-1 infected } \\
\text { tion of viral exposure } \\
\text { ed women } \\
\text { n, and upon seroconversion } \\
\text { L, and B7 SPRTLNAWV }\end{array}$ \\
\hline Nef (128-137) & $\begin{array}{l}\text { Nef }(128-137) \\
\text { - } \text { Combined tetramer a } \\
\text { HIV-specific CD8+ } \\
\text { CD27 expression on } \\
\text { - In most donors, betw } \\
\text { produce TNF- } \alpha\end{array}$ & $\begin{array}{l}\text { TP GP GVRYP L } \\
\text { tracellular cytok } \\
\text { expressed lowe } \\
\text { specific cells, su } \\
0 \% \text { and } 95 \% \text { of }\end{array}$ & $\begin{array}{l}\text { HIV-1 infection } \\
\text { used to study the function of circulating CD8+ T cell } \\
\text { rin than CMV-specific CD8+ T cells from the same do } \\
\text { d maturation } \\
\text { us-specific CD8+ T cells produced IFN- } \gamma \text { and MIP-1f }\end{array}$ & $\begin{array}{l}\text { Appay2000 } \\
\text { for HIV and CMV } \\
\text { this was associated with persistent } \\
\text { distinct subset that failed to }\end{array}$ \\
\hline Nef (128-137) & $\begin{array}{l}\text { Nef (128-137) } \\
\text { - The CTL response tc } \\
\text { studied in eight HIV- } \\
\text { - } 2 \text { to } 17 \text { epitopes wer } \\
\text { epitopes were target } \\
\text { - Subjects with chroni } \\
\text { - An acute seroconver } \\
\text { - The other acute sero } \\
\text { - The B7-restricted C }\end{array}$ & $\begin{array}{l}\text { TPGP GVRYPL } \\
\text { mally defined CT } \\
\text { ected subjects, tv } \\
\text { gnized in a giver } \\
\text { at least one pers } \\
\text { I-1 infection reco } \\
\text { mozygous for th } \\
\text { rtor failed to rec } \\
\text { ponse was highl }\end{array}$ & $\begin{array}{l}\text { HIV-1 infection human (B7) } \\
\text { ricted by HLA class I A and B alleles in individuals } \\
\text { fection, five with chronic, and one long-term non-pro } \\
\text { 2-restricted CTL response tended to be narrow and ne } \\
\text { 2-8 out of } 11 \text { B7-restricted epitopes } \\
\text { gnized five B7-restricted epitopes } \\
\text { he } 11 \text { B7-restricted epitopes tested } \\
\text { here was no clearly dominant epitope }\end{array}$ & $\begin{array}{l}\text { Day2001 } \\
\text { essed HLA A2, A3, and B7 was } \\
\text { TNP) } \\
\text { ted the response, and } 25 / 27\end{array}$ \\
\hline Nef (128-137) & $\begin{array}{l}\text { Nef (128-137 BRU) } \\
\text { - Seventy-three 8-11 n } \\
73 \text { peptides had } 81 \mathrm{n} \\
\text { directly related to th } \\
\text { - 20s proteasome clea } \\
\text { The frequency of rec } \\
\text { - TPGPGVRYPL was }\end{array}$ & $\begin{array}{l}\text { TPGP GVRYPL } \\
\text { ptides were gen } \\
54 \% \text { bound to } t \\
\text { ber of individual } \\
\text { f the Nef proteir } \\
\text { ion may be in pa } \\
\text { nized in } 8 / 16(5\end{array}$ & $\begin{array}{l}\text { HIV-1 infection human (B7) } \\
\text { putative anchor motifs in the Nef BRU sequence for } \\
\text { A molecule, particularly A2, B7/35, and B8. The stre } \\
\text { d a protein. } \\
00 \text { showed a large fraction of peptides were cleaved en } \\
\text { e cleavage step in processing. } \\
\text { ls with HLA B7, and } 1 / 9(11 \%) \text { of individuals with } \mathrm{H}\end{array}$ & $\begin{array}{l}\text { Choppin2001 } \\
\text { A3, A24, B7, B8, and B35; these } \\
\text { LA-peptide binding was not } \\
\text { 87L, 83A, } 81 \mathrm{Y}, 71 \mathrm{P}, 68 \mathrm{~F} \text { and } 67 \mathrm{G} \text {. } \\
\text { It was a high affinity HLA binder. }\end{array}$ \\
\hline
\end{tabular}




\begin{tabular}{llllll}
\hline HXB2 Location & Author's Location & Sequence & Immunogen & Species (HLA) & References \\
\hline Nef (128-137) & $\begin{array}{l}\text { Nef } \\
\text { • Epitope name: B7-TL10 }\end{array}$ & TPGPGVRYPL & HIV-1 infection & human (B7) & Yu2002a
\end{tabular}

- Epitope name: B7-TL10

- CTL responses in 18 acutely HIV-infected HLA-A3 $(n=7)$ or -B7 $(n=4)$ or both -A3 and B7 $(n=7)$ positive individuals were studied.

- One individual, AC-06, was homozygous at all three class I alleles (A3, B7, Cw7), was treated during acute infection and had supervised treatment interruptions (STI). He had only two detectable CTL responses during acute infection, but after STI this broadened to 27 distinct epitopes including 15 restricted by HLA-A3, 11 by HLA-B7, and 1 by HLA-Cw7.

- 0/11 HLA-B7 individuals had detectable B7-restricted responses to this epitope during acute infection - 10/15 of HLA-B7 epitopes tested were targeted by at least one person during acute infection. 2/4 individuals had detectable responses to this epitope after STI.

\begin{tabular}{lllll}
\hline Nef (128-137) Nef & TPGPGVRYPL & HIV-1 infection & human (B7)
\end{tabular}

- Four HIV patients with prolonged clinically successful anti-viral therapy but with ongoing evidence of replication and Nef mRNA transcription, showed specific T-cell responses by Elispot and Tetramer staining, maintained for 2-4 years after initiation of HAART.

- Nef epitope recognition was detected in all 4 subjects, gp120, Pol and Gag-specific in 1 or 2 subjects.

- The HIV-specific CD8+ T-cells had an intermediate maturation phenotype characterized by low levels of perforin and high levels of CD27 expression.

Nef (128-137) Nef TPGPGVRYL HIV-1 infection, Vaccine Hanke2000, Wee2002

Vaccine Vector/Type: DNA prime with vaccinia MVA boost Strain: subtype A HIV component: p17, p24, polyepitope

- The HIV-1 subtype A focused vaccine HIVA contains p24 and p17, in a reversed order relative to the Gag polyprotein to prevent myristylation of p17, which could direct the protein to the cell membrane and inhibit efficient peptide processing and class I presentation, as well as a polyepitope string of conserved, often immunodominant epitopes that were selected to have particularly good cross-reactive potential for the A-clade epidemic in Nairobi, Kenya. A DNA and MVA prime-boost vaccination protocol using the HIVA antigen will be used in a phase III clinical trial in Kenya. This epitope is included in the polyepitope string [Hanke2000].

- Multiple CD4+ or CD8+ T-cell vaccine-induced responses to peptide pools were detected using intracellular cytokine staining and IFNgamma Elispot assays after vaccination of 5 macaques. The response to the Mamu A*01 SIV p27 epitope p11C (CTPYDINQM), included in the polyepitope region, was not immunodominant in the Mamu $\mathrm{A}^{*} 01$ vaccinated macaques, possibly because of processing limitations in context of the artificial polyepitope string [Wee2002].

Nef (128-137) Nef (subtype B) TPGPGVRYPL $\quad$ HIV-1 exposed seronegative $\quad$ human (B7(*8101))

- HIV-specific CTL were found in exposed seronegative prostitutes from Nairobi - these CTL may confer protection

- Seroprevalence in this cohort is $90-95 \%$ and their HIV-1 exposure is among the highest in the world

- Most isolated HIV strains are clade A in Nairobi, although clades C and D are also found - B clade epitopes are often cross-reactive, however stronger responses are frequently observed using A or D clade versions of epitopes

- Clade A version of the epitope: TPGPGIRYPL, clade D version: TPGPGIRYPL

\begin{tabular}{lllll}
\hline Nef (128-137) Nef (128-137 subtype TPGPGVRYPL & HIV-1 exposed seronegative & human (B7, B*8101)
\end{tabular}
B)

- 11/16 heavily HIV exposed but persistently seronegative sex-workers in Nairobi had HIV-specific CD8 gamma-IFN responses in the cervix - systemic $\mathrm{CD} 8+\mathrm{T}$ cell responses tended to be to the same epitopes but at generally lower levels than cervical CD8+ T cell responses

- Low risk individuals did not have such CD8+ cells

- CD8+ T cell epitopes: DTVLEDINL (3 individuals), SLYNVATL (4 individuals), LSPRTLNAW (3 individuals) and YPLTFGWCF (4 individuals) were most commonly recognized by the HIV-resistant women 


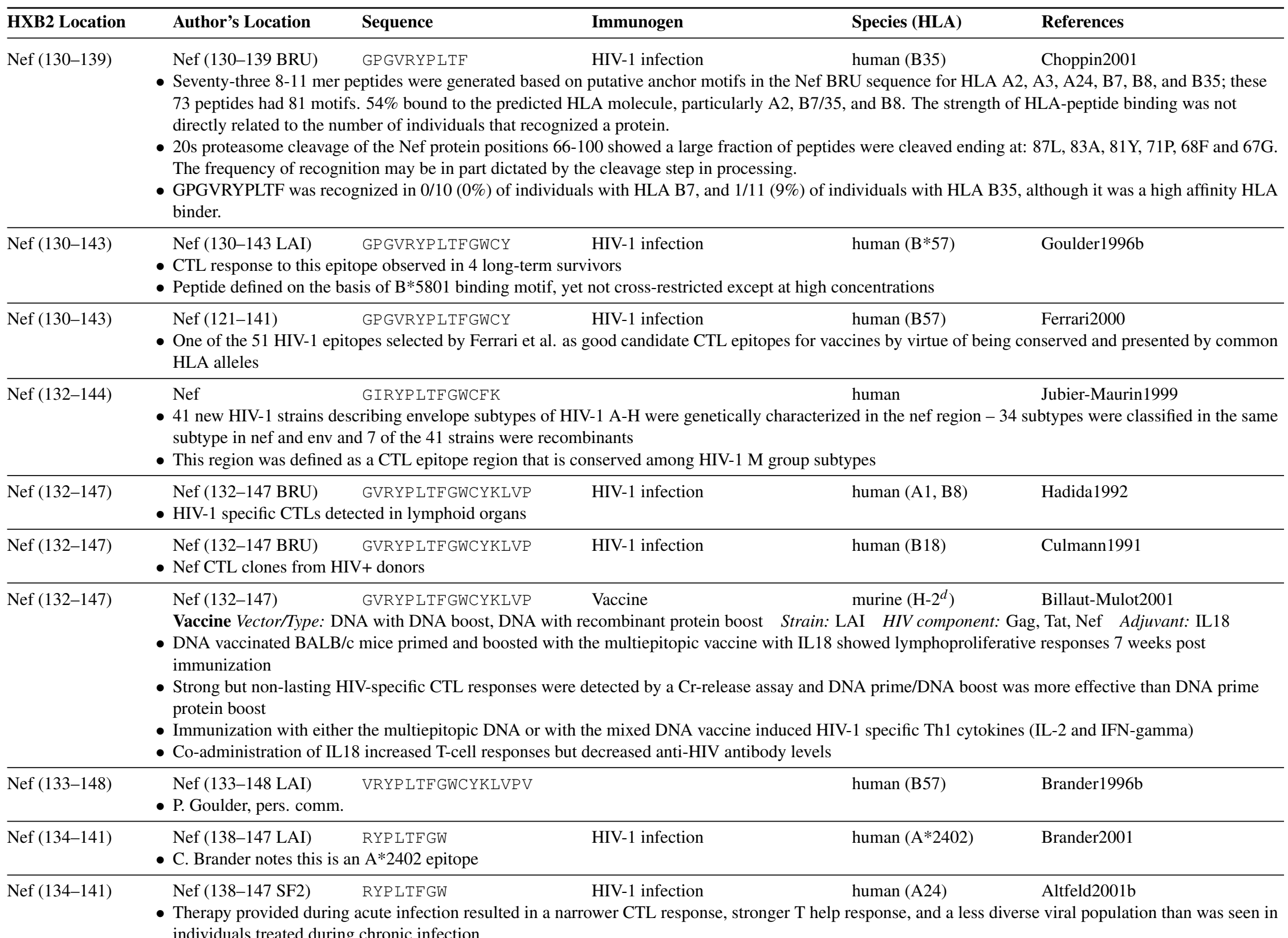




\begin{tabular}{|c|c|c|c|c|}
\hline HXB2 Location & Author's Location & Sequence & Species (HLA) & References \\
\hline & \multicolumn{4}{|c|}{$\begin{array}{l}\text { - The breadth and specificity of the response was determined using ELISPOT by studying } 19 \text { individuals with pre-seroconversion therapy (Group } 1 \text { ), } 11 \\
\text { individuals with primary infection but post-seroconversion therapy (Group 2), and } 10 \text { individuals who responded to HAART given during chronic infection } \\
\text { (Group 3), using } 259 \text { overlapping peptides spanning p17, p24, RT, gp41, gp120 and Nef } \\
\text { - Previously described and newly defined optimal epitopes were tested for CTL response } \\
\text { - Number of HLA-A24+ individuals that had a CTL response to this epitope broken down by group: } 0 / 0 \text { group } 1,2 / 3 \text { group } 2 \text {, and } 0 / 0 \text { group } 3\end{array}$} \\
\hline ef (134-141) & \multicolumn{4}{|c|}{$\begin{array}{l}\text { Nef } \\
\text { - Epitope name: A24-RW8(Nef) } \\
\text { - Peripheral blood (PB) and lymph node (LN) CD8+ T-cell responses were compared in } 15 \text { asymptomatic HIV-1 infected patients using all known optimal } \\
\text { CTL epitopes (http://hiv-web.lanl.gov/content/hiv-db/REVIEWS/brander2001.html) for each person's class I HLA alleles. } \\
\text { - } 60 \text { epitope responses were detected in both PB and LN samples of the } 15 \text { patients, and an additional } 8 \text { responses were detected only in LN. The total } \\
\text { magnitude of the response was similar in LN and PB, but the percentage of CD8+ T cells in the LN is lower so the number of HIV-specific cells per million } \\
\text { CD8+ T-cells is higher in the LN. } \\
\text { - } 1 \text { year post-HAART treatment in five patients studied, the magnitude of the CD8 T-cell response was decreased in both LN and PB, but more dramatically } \\
\text { in PB, and 13/25 epitope responses in the PB became undetectable, in contrast to 5/26 in the LN. } \\
\text { - Treatment interruption following HAART induced resulted in increased viremia accompanied by the restoration of the detection of } 13 \text { epitopes that had } \\
\text { become undetectable in the PB, and the addition of 9 novel epitope responses. } \\
\text { - Breakdowns of epitope responses were shown for } 4 \text { individuals. Patient C displayed the greatest response to B27-KK10(p24), and in decreasing order also } \\
\text { responded to A24-RW8(Nef), B7-IL9(gp41), A24-RL9(gp41), A24-YL8(gp41), and B7-TM9(Nef). }\end{array}$} \\
\hline ef ( & \multicolumn{4}{|c|}{ - Optimal peptide defined by titration } \\
\hline ef ( & $\begin{array}{l}\text { Nef (138-147 SF2) } \\
\text { - Defined using revers } \\
\text { Phe, Leu or Ile at th } \\
\text { - This peptide induce } \\
\text { RYPLTFGWCF bou }\end{array}$ & $\begin{array}{l}\text { RYPLTFGWCF } \\
\text { munogenetics }-59 \\
\text { rm) }-53 \text { of the } 59 \\
\text { in } 3 / 4 \text { HIV-1+ pc } \\
\text { A*2402 strongly, }\end{array}$ & $\begin{array}{l}\text { HIV-1 infection human }(A * 2402) \\
\text { binding peptides were predicted by searching for } A * 2 \\
d A * 2402 \\
\text { be processed in a vaccinia construct and presented - }\end{array}$ & $\begin{array}{l}\text { Ikeda-Moore1997 } \\
\text { hors in HIV proteins (Tyr at 2, and } \\
\text { cific CTL clones were obtained }\end{array}$ \\
\hline ef ( & $\begin{array}{l}\text { Nef (138-147) } \\
\text { Vaccine Vector/Type } \\
\text { - Epitope name: Nef1 } \\
\text { - A Sendai virus vect } \\
\text { responses and has th } \\
\text { - MHC class I/peptide } \\
\text { - Cells transfection w }\end{array}$ & $\begin{array}{l}\text { RYP LTFGWCF } \\
\text { dai virus vector } \mathrm{s} \\
\text { tem (SeV) was de } \\
\text { ential to elicit imn } \\
\text { amers could be ma } \\
\text { eV modified to exp }\end{array}$ & $\begin{array}{l}\text { Vaccine human }\left(\mathrm{A}^{* 2402)}\right. \\
\text { HIV component: class I/peptide complexes } \\
\text { oressed HLA-A*2402-restricted class I/peptide compl } \\
\text { stem that bound to epitope-specific CTLs in PBMCs. } \\
\text { IV epitope complexes induced CTL mediated specific }\end{array}$ & Kawana-Tachikawa2002 \\
\hline $\operatorname{Nef}(13$ & $\begin{array}{l}\text { Nef (134-143 BRU) } \\
\text { - Seventy-three 8-11 } \\
73 \text { peptides had } 81 \mathrm{r} \\
\text { directly related to th } \\
\text { - 20s proteasome clea } \\
\text { The frequency of re } \\
\text { - RYPLTFGWCY wa }\end{array}$ & $\begin{array}{l}\text { RYPLTFGWCY } \\
\text { eptides were gene } \\
54 \% \text { bound to th } \\
\text { nber of individual } \\
\text { of the Nef protein } \\
\text { tion may be in par } \\
\text { gnized in } 5 / 12 \text { ( } 4\end{array}$ & $\begin{array}{l}\text { HIV-1 infection human (A24) } \\
\text { putative anchor motifs in the Nef BRU sequence for } \mathrm{H} \\
\text { A molecule, particularly A2, B7/35, and B8. The strer } \\
\text { d a protein. } \\
00 \text { showed a large fraction of peptides were cleaved en } \\
\text { e cleavage step in processing. } \\
\text { als with HLA A24. It was a moderate affinity HLA-A }\end{array}$ & $\begin{array}{l}\text { Choppin2001 } \\
\text { A3, A24, B7, B8, and B35; these } \\
\text { ILA-peptide binding was not } \\
\text { 87L, 83A, 81Y, 71P, 68F and 67G. } \\
\text { r. }\end{array}$ \\
\hline
\end{tabular}




\begin{tabular}{|c|c|c|c|c|c|}
\hline HXB2 Location & Author's Location & Sequence & Immunogen & Species (HLA) & References \\
\hline Nef (134-144) & Nef (134-144 LAI) & RYPLTEGWCYK & HIV-1 infection & human (B18) & Couillin1994 Goulder1997a \\
\hline
\end{tabular}

- Goulder1997a is a review of immune escape that summarizes this study

\begin{tabular}{lllll}
\hline Nef (134-144) Nef(134-144) & RYPLTFGWCYK & Oxenius2000
\end{tabular}

- Epitope name: RYP

- Patients who started therapy at acute HIV-1 infection (three with sustained therapy, two with limited therapy upon early infection) had strong HIV specific CD4 proliferative responses and were able to maintain a CTL response even with undetectable viral load - three patients that had delayed initiation of HAART had no HIV specific CD4 proliferative responses and lost their CTL responses when HAART was eventually given and their viral loads became undetectable

- None of the 8 study subjects recognized this epitope but none were HLA B18+

Nef (135-143) Nef(135-143 LAI) YPLTFGWCY in vitro stimulation human (B*0702) Lucchiari-Hartz2000

- Five naturally processed MHC class I ligands were identified in Nef in the conserved immunogenic region Nef between 123-152

- All five could be transported by TAP, and 4/5 had N-termini that were major cleavage points for the proteasome, only one had extended precursor fragments

- YPLTFGWCY is the naturally processed ligand for $\mathrm{B} 7$, and this epitope is the only one of the five that may require trimming at the N-termini

- YPLTFGWCY is present in low copy number in the cell, possibly due to a predominant proteasomal cleavage site between $\mathrm{Y}$ and $\mathrm{P}$

\begin{tabular}{llccc}
\hline Nef (135-143) & $\begin{array}{l}\text { Nef (135-143 LAI) } \\
\text { - C. Brander notes this is a B*1801 epitope }\end{array}$ & HIV-1 exposed seronegative & human (B*1801) \\
\hline Nef (135-143) & & YPLTFGWCY & HIV-1 infection & human (B*5301, B35) \\
Sabbaj2002b
\end{tabular}

- Epitope name: Nef-YY9

- This study monitored epitope responses in HIV-1 infected minority women living in the United States

- 24 epitopes were described - 8 were novel, 8 used new restricting elements but were previously defined epitopes, and 8 were previously described

- Serial peptide truncations were used to define optimal epitopes for CTL cell lines isolated from 12 individuals, assayed by a Cr-release

- Subject 00RCH33 was on HAART had a viral load of 2900 and CD4 count of 727 and also recognized the epitopes HIGPGRAFY, gp160(310-318), HLA A*3002; AETFYVDGA, RT(437-445), HLA B*4501; and RSLYNTVATLY, p17(76-86), HLA A*3002

- Among HIV+ individuals who carried HLA B53, 8/15 (53\%) recognized this epitope - one subject also carried B7, previously shown to restrict this epitope

- Among HIV+ individuals who carried HLA B35, 13/19 (68\%) recognized this epitope

Nef (135-143) Nef (subtype D) YPLTFGWCF HIV-1 exposed seronegative human (B18)

- 11/16 heavily HIV exposed but persistently seronegative sex-workers in Nairobi had HIV-specific CD8 gamma-IFN responses in the cervix - systemic

$\mathrm{CD} 8+\mathrm{T}$ cell responses tended to be to the same epitopes but at generally lower levels than cervical CD8+ T cell responses

- Low risk individuals did not have such CD8+ cells

- CD8+ T cell epitopes: DTVLEDINL (3 individuals), SLYNVATL (4 individuals), LSPRTLNAW (3 individuals) and YPLTFGWCF (4 individuals) were most commonly recognized by the HIV-resistant women

\begin{tabular}{lllll}
\hline Nef (135-143) & Nef (135-143 LAI) YPLTFGWCY HIV-1 exposed seronegative & human (B18) Culmann1991 \\
& - Nef CTL clones from HIV+ donors & &
\end{tabular}

Nef (135-143) Nef(135-143 SF2) YPLTFGWCY HIV-1 infection $\quad$ Altfeld2001b

- Therapy provided during acute infection resulted in a narrower CTL response, stronger T help response, and a less diverse viral population than was seen in individuals treated during chronic infection 


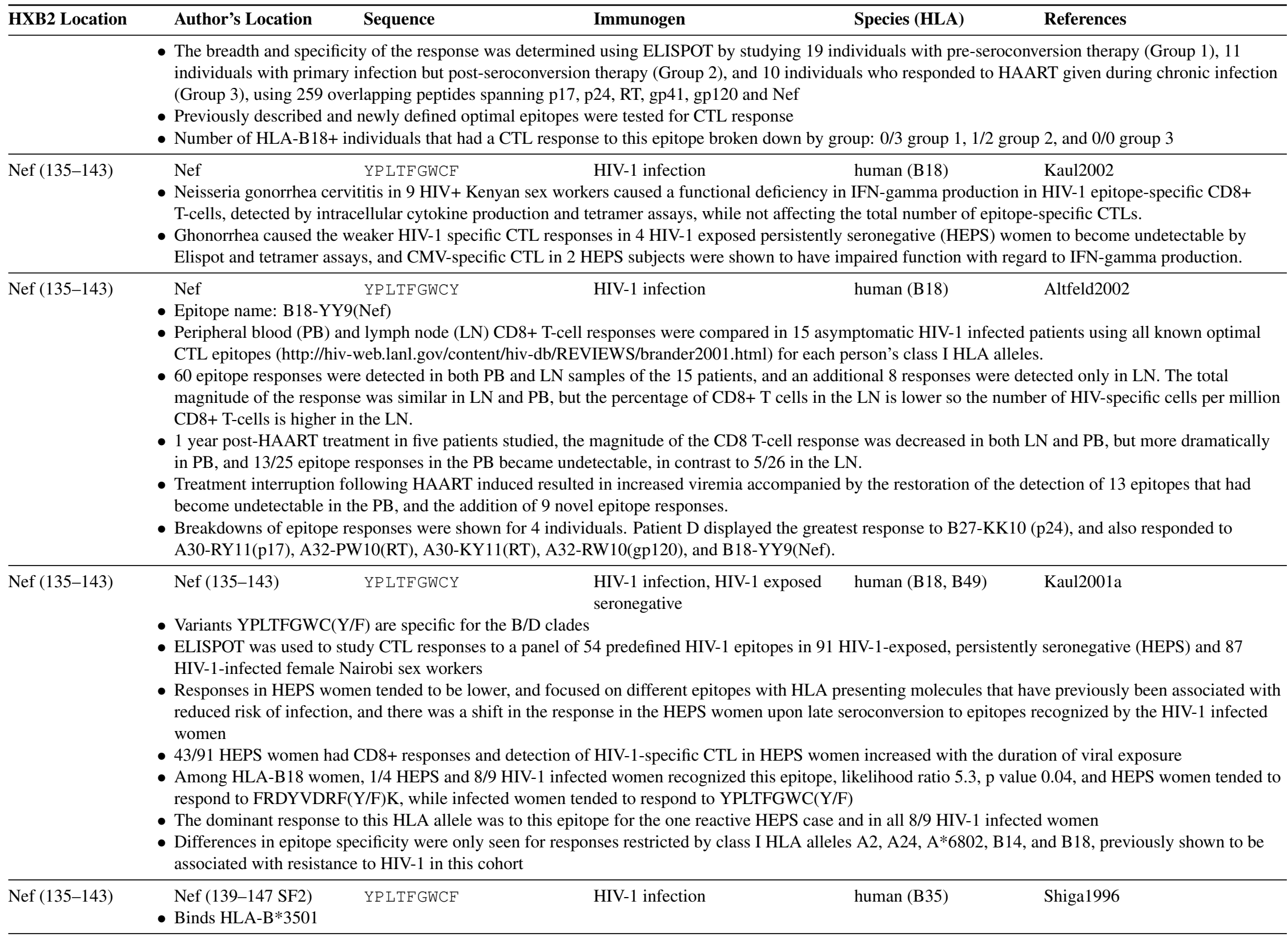




\begin{tabular}{lllll}
\hline HXB2 Location & Author's Location & Sequence & Immunogen & Species (HLA) \\
\hline Nef (135-143) & Nef (135-143 BRU) & YP LTFGWCY & HIV-1 infection & human (B35)
\end{tabular}

- Seventy-three 8-11 mer peptides were generated based on putative anchor motifs in the Nef BRU sequence for HLA A2, A3, A24, B7, B8, and B35; these 73 peptides had 81 motifs. 54\% bound to the predicted HLA molecule, particularly A2, B7/35, and B8. The strength of HLA-peptide binding was not directly related to the number of individuals that recognized a protein.

- 20 s proteasome cleavage of the Nef protein positions $66-100$ showed a large fraction of peptides were cleaved ending at: $87 \mathrm{~L}, 83 \mathrm{~A}, 81 \mathrm{Y}, 71 \mathrm{P}, 68 \mathrm{~F}$ and $67 \mathrm{G}$. The frequency of recognition may be in part dictated by the cleavage step in processing.

- YPLTFGWCY was recognized in 2/13 (15\%) of individuals with HLA B7, and 11/14 (79\%) of individuals with HLA B35, and it was a moderate affinity HLA binder.

Nef (135-143) Nef YPLTFGWCY HIV-1 infection Sabbaj2002a

- IFNgamma T-cell responses in breast milk of 5 HIV-1 infected women from the US and 6 from Zambia were tested with using Elispot. 11/11 women responded to Gag, 8/11 responded to Pol, 7/11 women to Nef, and 2/5 women to Env peptide pools. These responses were shown to be primarily due to CD8+ T-cells in one woman, and another woman had cytolytic responses measured by Cr-release.

- T-cells in breast milk from a volunteer who was HLA A3, A11, B35, B51 induced IFNgamma after stimulation with a peptide that carries known B35 epitope YPLTFGWCY.

- The frequencies of responses in the two compartments differed, and 2/4 women that responded to epitopes in Nef 101-205 and Pol 601-710 showed responses in breast milk but no detectable responses in peripheral blood cells.

Nef (135-143) Nef YPLTFGWCY HIV-1 exposed seronegative human (B49) Rowland-Jones1998a

- A CTL response was found in exposed but uninfected prostitutes from Nairobi using previously-defined B clade epitopes that tended to be conserved in A and D clades - such cross-reactivity could protect against both A and D and confer protection in Nairobi where both subtypes are circulating

- The A subtype consensus is identical to the B clade epitope

- The D subtype consensus is ypltfgwcF.

Nef (135-143) Nef (subtype B) YLPTFGWCY HIV-1 exposed seronegative human (B49)

- HIV-specific CTL were found in exposed seronegative prostitutes from Nairobi - these CTL may confer protection

- Seroprevalence in this cohort is $90-95 \%$ and their HIV-1 exposure is among the highest in the world

- Most isolated HIV strains are clade A in Nairobi, although clades C and D are also found - B clade epitopes are often cross-reactive, however stronger responses are frequently observed using $\mathrm{A}$ or $\mathrm{D}$ clade versions of epitopes

- This epitope is conserved among A and B clade viruses

- The Clade D version of the epitope, YPLTFGWCF, was preferentially recognized by CTL

Nef $(135-143)$

$$
\begin{array}{llll}
\text { YPLTFGWCY HIV-1 infection human (B49) } & \text { Kaulc }
\end{array}
$$

- This study examines CTL responses in HIV exposed, persistently seronegative individuals, HEPS, who eventually seroconverted - 11/114 HEPS Nairobi sex workers eventually seroconverted, and for six of these HIV CTL reactive epitopes had been defined while seronegative

- The epidemiological factor associated with seroconversion was stopping sex work and HIV-specific CTL activity declines when HEPS sex workers stop working for a period or retire

- This epitope, YPLTFGWC(Y/F), was recognized in 1/22 HEPS sex worker controls (ML1668)

Nef (135-143) Nef(135-143 BRU) YPLTFGWCY HIV-1 infection $\quad$ Choppin2001

- Seventy-three 8-11 mer peptides were generated based on putative anchor motifs in the Nef BRU sequence for HLA A2, A3, A24, B7, B8, and B35; these 73 peptides had 81 motifs. $54 \%$ bound to the predicted HLA molecule, particularly A2, B7/35, and B8. The strength of HLA-peptide binding was not directly related to the number of individuals that recognized a protein. 


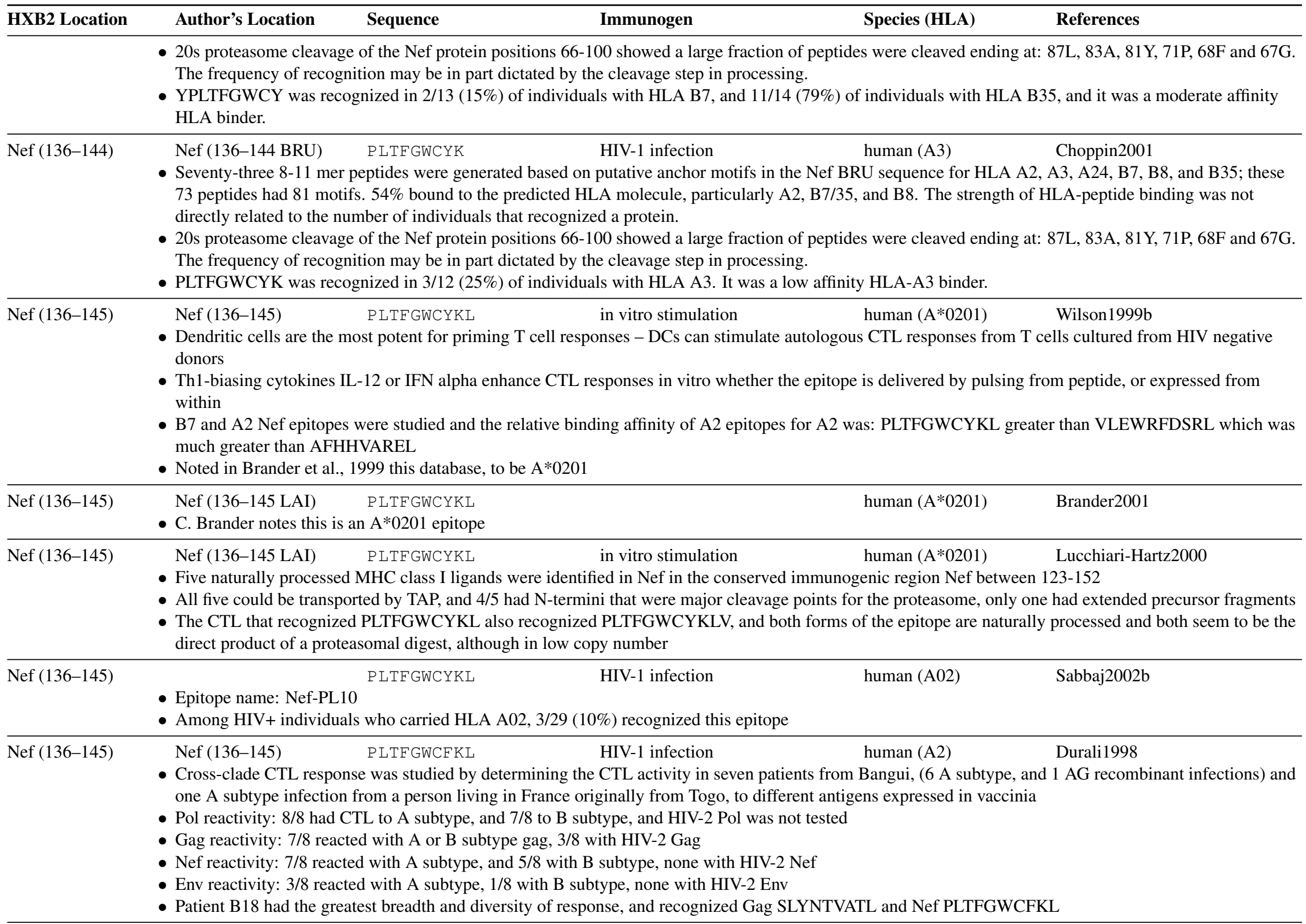




\begin{tabular}{|c|c|c|c|c|}
\hline HXB2 Location & Author's Location & Sequence & Species (HLA) & References \\
\hline 51 & \multicolumn{4}{|c|}{$\begin{array}{l}\text { Vaccine Vector/Type: DNA prime with vaccinia boost HIV component: polyepitope } \\
\text { - A polyepitope vaccine was generated in a vaccinia construct that contiguously encoded seven epitopes, all presented by HLA A-2 } \\
\text { - HHD mice have a transgene of HLA A2 linked to the transmembrane and cytotoxic domains of H-2D }{ }^{d}-\text { this transgene is the only MHC molecule } \\
\text { expressed in the mice } \\
\text { - CTL responses to Gag (77-85) SLYNTVATL, Pol (476-484) ILKEPVHGV, gp120 (120-128) KLTPLCVTL, and Nef (190-198) AFHHVAREL were } \\
\text { observed in HIV polytope HHD-vaccinated mice, and these responses were enhanced with vaccinia boost } \\
\text { - No CTL immune responses were generated against HLA A2-restricted HIV epitopes Nef 157-166 (PLTFGWCYKL), Pol 346-354 (VIYQYMDDL), and } \\
\text { Nef } 180-189 \text { (VLEWRFDSRL) } \\
\text { - Sixteen HLA A2+ patients were tested for their ability to make CTL responses by peptide restimulation in culture with the epitopes selected for inclusion in } \\
\text { the polytope - one individual recognized all seven of these epitopes; } 7 \text { patients had CTL cultures able to recognize at least one of the epitopes, and } 6 \text { of } \\
\text { those } 7 \text { recognized more than one epitope, but they were not able to test all peptides for all patients; many patients only had three peptides tested } \\
\text { - PLTFGWCFKL was recognized by } 1 \text { of the HLA-A2 patients }\end{array}$} \\
\hline Jef $(13 \mathrm{C}$ & $\begin{array}{l}\text { Nef (135-144 93TH253 } \\
\text { subtype CRF01) } \\
\text { - More than half of a cohor } \\
\text { epitopes in this group, altl } \\
\text { - } 0 / 4 \text { tested FSWs recogniz } \\
\text { acids, PLTFGWCYKL } \\
\text { - This epitope was only con }\end{array}$ & $\begin{array}{l}\text { PLTFGWCYKL } \\
\text { t of HIV+ female s } \\
\text { hough E clade vers } \\
\text { ed the E clade vers } \\
\text { hserved in CRF01 }\end{array}$ & $\begin{array}{l}\text { HIV-1 infection } \\
\text { SW) from Northern Thailand were HLA-A11 positive } \\
\text { usly defined B-clade A2 and A24 epitopes were also te } \\
\text { itope PLCFGWCFKL, which differs from the previous } \\
\text { d subtype B }\end{array}$ & $\begin{array}{l}\text { Bond2001 } \\
\text { s study concentrated on A11 } \\
\text { B clade version by two amino }\end{array}$ \\
\hline $\operatorname{Nef}(1$ & $\begin{array}{l}\text { Nef (136-145) } \\
\text { - The CTL response to opti } \\
\text { studied in eight HIV-1-inf } \\
\text { - } 2 \text { to } 17 \text { epitopes were recc } \\
\text { epitopes were targeted by }\end{array}$ & $\begin{array}{l}\text { PLTFGWCYKL } \\
\text { imally defined CTL } \\
\text { fected subjects, two } \\
\text { ognized in a given } \\
\text { at least one person }\end{array}$ & $\begin{array}{l}\text { hIV-1 infection } \\
\text { ted by HLA class I A and B alleles in individuals v } \\
\text { ction, five with chronic, and one long-term non-pro } \\
\text { estricted CTL response tended to be narrow and ne }\end{array}$ & $\begin{array}{l}\text { Day2001 } \\
\text { essed HLA A2, A3, and B7 was } \\
\text { TNP) } \\
\text { ited the response, and 25/27 }\end{array}$ \\
\hline $\operatorname{Nef}($ & $\begin{array}{l}\text { Nef (136-145 BRU) } \\
\text { - Seventy-three 8-11 mer p } \\
73 \text { peptides had } 81 \text { motifs } \\
\text { directly related to the num } \\
\text { - 20s proteasome cleavage } \\
\text { The frequency of recognit } \\
\text { - PLTFGWCYKL was reco }\end{array}$ & $\begin{array}{l}\text { PLTFGWCYKL } \\
\text { eptides were gener } \\
54 \% \text { bound to the } \\
\text { nber of individuals } \\
\text { of the Nef protein } \\
\text { tion may be in part } \\
\text { gnized in } 9 / 28 \text { ( } 32\end{array}$ & $\begin{array}{l}\text { HIV-1 infection human (A2) } \\
\text { utative anchor motifs in the Nef BRU sequence for } \\
\text { A molecule, particularly A2, B7/35, and B8. The str } \\
\text { a protein. } \\
0 \text { showed a large fraction of peptides were cleaved e } \\
\text { cleavage step in processing. } \\
\text { als with HLA A2. It was a low affinity HLA-A2 bine }\end{array}$ & $\begin{array}{l}\text { Choppin2001 } \\
\text { 3, A24, B7, B8, and B35; these } \\
\text { A-peptide binding was not } \\
7 \mathrm{~L}, 83 \mathrm{~A}, 81 \mathrm{Y}, 71 \mathrm{P}, 68 \mathrm{~F} \text { and } 67 \mathrm{G} \text {. }\end{array}$ \\
\hline Nef (136-146) & $\begin{array}{l}\text { Nef (136-146 LAI) } \\
\text { - Five naturally processed } \\
\text { - All five could be transport } \\
\text { - The CTL that recognized } \\
\text { direct product of a proteas }\end{array}$ & $\begin{array}{l}\text { PLTFGWCYKLV } \\
\text { MHC class I ligand } \\
\text { ted by TAP, and } 4 / 5 \\
\text { PLTFGWCYKL a } \\
\text { somal digest, altho }\end{array}$ & $\begin{array}{l}\text { in vitro stimulation } \\
\text { ed in Nef in the conserved immunogenic region Nef } b \\
\text { i that were major cleavage points for the proteasome, } \\
\text { PLTFGWCYKLV, and both forms of the epitope are } \\
\text { y number }\end{array}$ & $\begin{array}{l}\text { Lucchiari-Hartz2000 } \\
23-152 \\
\text { had extended precursor fragments } \\
\text { processed and both seem to be the }\end{array}$ \\
\hline
\end{tabular}




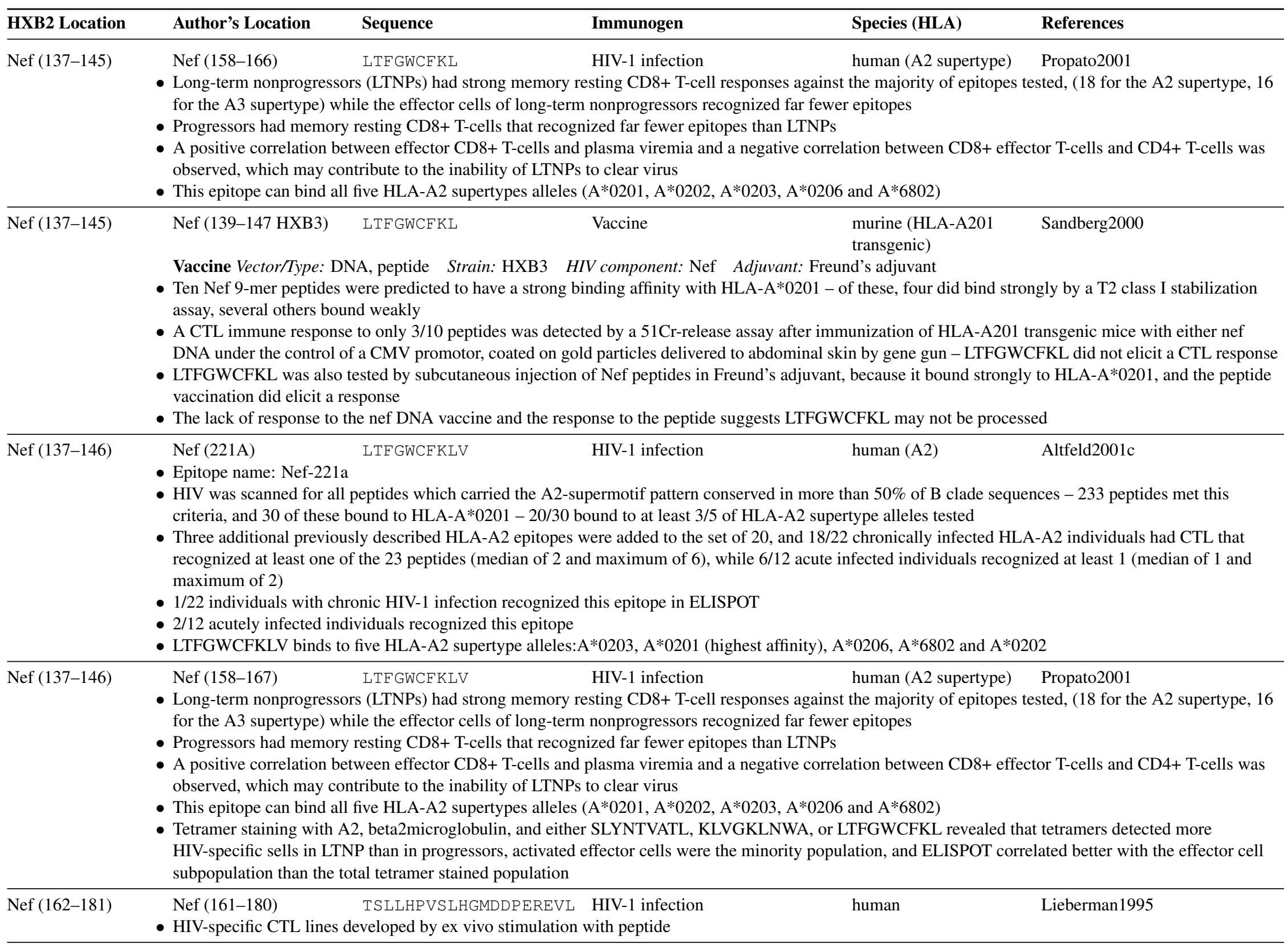




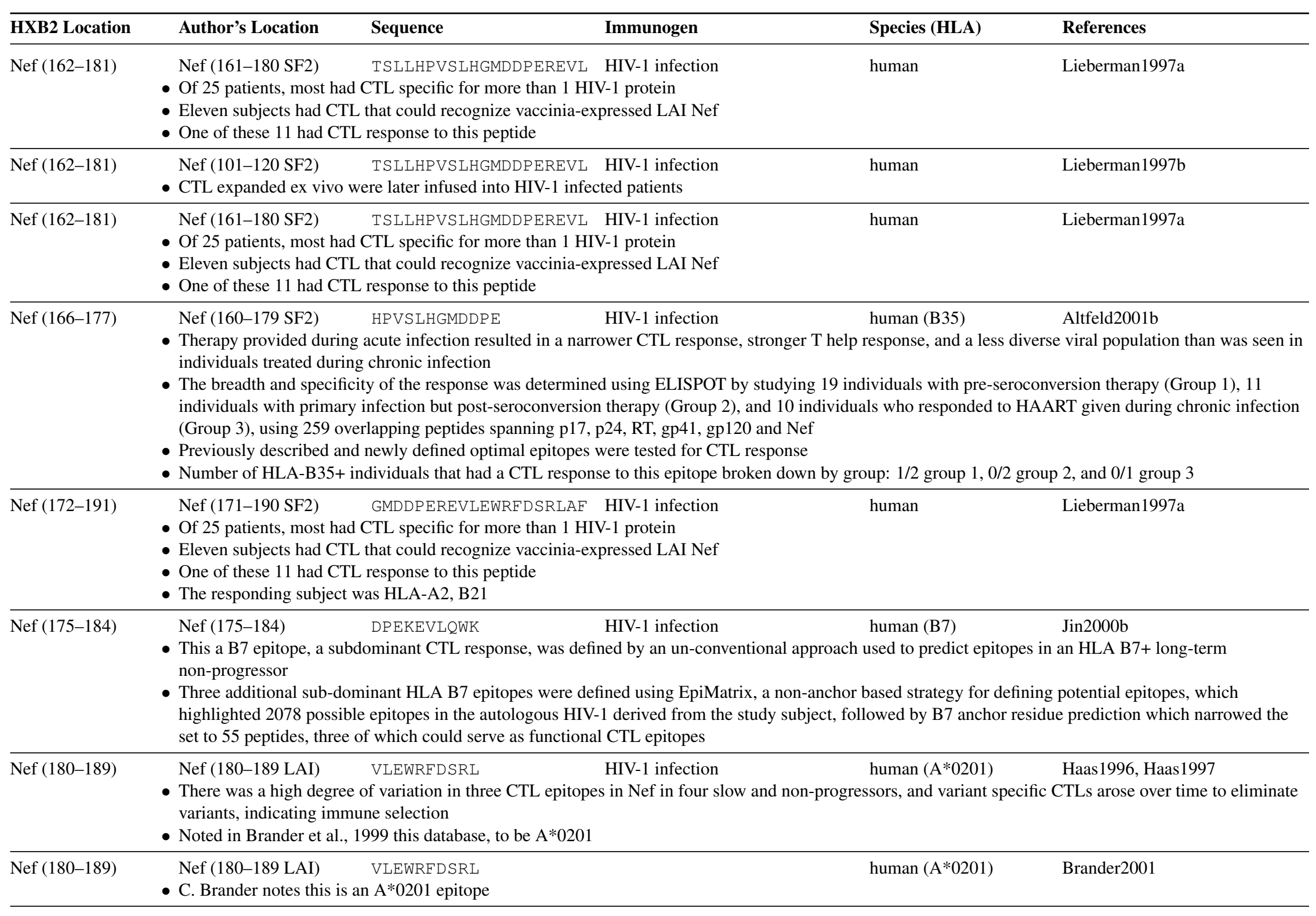




\begin{tabular}{lllll}
\hline HXB2 Location & Author's Location & Sequence & Immunogen & Species (HLA) \\
\hline Nef (180-189) & Nef (180-189 LAI) & VLMWQFD SRL & Vaccine & murine (transgenic) \\
& Vefences & Boissonnas2002 & (A*0201)
\end{tabular}

- Ten naturally occurring variants of this epitope were tested for their affinity to HLA-A*0201 and for their ability to induce gamma-IFN and cytotoxic functions through vaccination of HLA-A $* 0201$ transgenic mice.

- Only two variants could induce vaccine responses: VLMWQFDSRL, a high affinity binder, and VLQWRFDSRL a medium affinity binder to A*0201.

- In vivo priming with Nef peptide VLMWQFDSRL induced cross-reactive CTL to 6/7 peptides tested (AlmwKfdsKl, vlmwKfdsrl, vlmwKfdsKl,

vlQwRfdsKl, vlVwrfdTrl, and vlAwKLdsrl but not the LAI peptide vlEwrfdsrl)

- In vivo priming with Nef peptide VLQWRFDTRL induced cross-reactive CTL to 3/6 variant Nef peptides (vlMwQfdsrl, vlqwrfdSrl and vlEwrfdsrl).

\begin{tabular}{lllcl}
\hline Nef (180-189) & Nef (190-198) & VLEWRFDSRL & Vaccine & murine (A*0201) \\
& Vaccine Vector/Type: DNA & HIV component: HIV-1 divided into a 32 plasmids in a ubiquitin expression library & Sykes1999
\end{tabular}

- $\mathrm{C} 3 \mathrm{H}(\mathrm{H}-2 \mathrm{k})$ transgenic mice carrying a fused HLA-A*0201 alpha1 and alpha2 and H-2Dk alpha3 hybrid class I molecule were immunized using an epidermal gene gun with an ubiquitin expression library of 32 plasmids that spanned the HIV-1 genome. Ubiquitin targets the expressed HIV-1 peptides to the proteasome.

- A single immunization with the UB-HIV-1 library vaccine induced potent, stable and multivalent CTL responses against all library members.

- Immunodominant epitopes SLYNTVATL (Gag), ILKEPVHGV(Pol), RIQRGPGRAFVTIGK(P18) and AFHHVAREK (Nef) elicited strong CD8+/IFNresponses and stimulated CTL that were functional in a Cr-release assay and against wild type antigen.

- The presence of multiple plasmids HLA-A*0201-restricted CTL epitopes did not decrease CTL immunogenicity, and CTL responses to single peptide immunizations were comparable to responses based on mixtures of either 16 or 32 peptides.

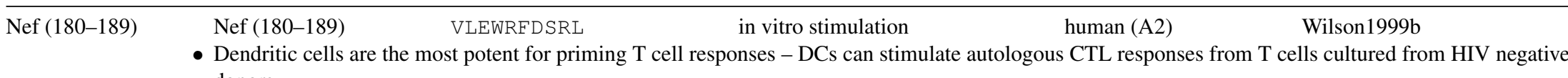

- Dendritic cells are the most potent for priming T cell responses - DCs can stimulate autologous CTL responses from T cells cultured from HIV negative

Th1-biasing cytokines IL-12 or IFN alpha enhance CTL responses in vitro whether the epitope is delivered by pulsing from peptide, or expressed from within

- B7 and A2 Nef epitopes were studied and the relative binding affinity of A2 epitopes for A2 was: PLTFGWCYKL greater than VLEWRFDSRL which was much greater than AFHHVAREL

\begin{tabular}{llccc}
\hline Nef (180-189) & Nef (180-189) & VLEWRFDSRL & Vaccine & human (A2) \\
& Vaccine Vector/Type: DNA prime with vaccinia boost & HIV component: polyepitope & Woodberry1999
\end{tabular}

- A polyepitope vaccine was generated in a vaccinia construct that contiguously encoded seven epitopes, all presented by HLA A-2

- HHD mice have a transgene of HLA A2 linked to the transmembrane and cytotoxic domains of $\mathrm{H}-2 \mathrm{D}^{d}-$ this transgene is the only MHC molecule expressed in the mice

- CTL responses to Gag (77-85) SLYNTVATL, Pol (476-484) ILKEPVHGV, gp120 (120-128) KLTPLCVTL, and Nef (190-198) AFHHVAREL were observed in HIV polytope HHD-vaccinated mice, and these responses were enhanced with vaccinia boost

- No CTL immune responses were generated against HLA A2-restricted HIV epitopes Nef 157-166 (PLTFGWCYKL), Pol 346-354 (VIYQYMDDL), and Nef 180-189 (VLEWRFDSRL)

- Sixteen HLA A2+ patients were tested for their ability to make CTL responses by peptide restimulation in culture with the epitopes selected for inclusion in the polytope - one individual recognized all seven of these epitopes; 7 patients had CTL cultures able to recognize at least one of the epitopes, and 6 of those 7 recognized more than one epitope, but they were not able to test all peptides for all patients; many patients only had three peptides tested

- VLEWRFDSRL was recognized by 2 of the HLA-A2 patients 


\begin{tabular}{|c|c|c|c|c|}
\hline HXB2 Location & Author's Location & Immunogen & Species (HLA) & References \\
\hline Nef (180-189) & $\begin{array}{l}\text { Nef (180-189 LAI) } \\
\text { - Epitope name: N3 } \\
\text { - A panel of } 16 \text { epitopes co } \\
\text { CD8+ cell IFNgamma pro } \\
\text { - In general, during the first } \\
\text { specificities that were not } \\
\text { HIV-specific responses dil } \\
\text { - Viral rebounds gave differ }\end{array}$ & $\begin{array}{l}\text { VLEWRFDSRL HIV-1 infection } \\
\text { vering } 15 \text { class I alleles was tested in } 14 \text { HIV+ pat } \\
\text { oduction to measure responses } \\
\text { t month of treatment viral load decreased and frequ } \\
\text { previously detectable were newly detected, as wer } \\
\text { minished } \\
\text { rent patterns of response: increases or decreases in }\end{array}$ & $\begin{array}{l}\text { human (A2) } \\
\text { lected Caucasian } \\
\text { cific CTL tripled } \\
\text { D8+ PBL - but wi } \\
\text { nse, new specifici }\end{array}$ & $\begin{array}{l}\text { Mollet2000 } \\
\text { n treated with HAART, using } \\
\text { dened - eight new HIV } \\
\text { ued viral suppression, } \\
\text { o change }\end{array}$ \\
\hline Nef (180-189) & $\begin{array}{l}\text { Nef (179-188 93TH253 } \\
\text { subtype CRF01) } \\
\text { - More than half of a cohort } \\
\text { epitopes in this group, alth } \\
\text { - } 0 / 4 \text { tested FSWs recogniz } \\
\text { acids, VLEWRFDSRL }\end{array}$ & $\begin{array}{l}\text { VLEWRFDSRL HIV-1 infection } \\
\text { t of HIV+ female sex workers (FSW) from Northe } \\
\text { hough E clade versions of previously defined B-cla } \\
\text { the E clade version of this epitope VLIWKFDS }\end{array}$ & $\begin{array}{l}\text { human (A2) } \\
\text { HLA-A11 positive } \\
\text { itopes were also t } \\
\text { from the previous }\end{array}$ & $\begin{array}{l}\text { Bond2001 } \\
\text { study concentrated on A11 } \\
\text { d B clade version by three amino }\end{array}$ \\
\hline Nef (180-189) & $\begin{array}{l}\text { Nef (180-189) } \\
\text { - The CTL response to opti } \\
\text { studied in eight HIV-1-inf } \\
\text { - } 2 \text { to } 17 \text { epitopes were reco } \\
\text { epitopes were targeted by }\end{array}$ & $\begin{array}{l}\text { VLEWRFDSRL HIV-1 infection } \\
\text { Imally defined CTL epitopes restricted by HLA cla } \\
\text { fected subjects, two with acute infection, five with } \\
\text { ognized in a given individual, A2-restricted CTL re } \\
\text { at least one person }\end{array}$ & $\begin{array}{l}\text { human (A2) } \\
\text { s in individuals wl } \\
\text { ong-term non-prog } \\
\text { e narrow and nev }\end{array}$ & $\begin{array}{l}\text { Day2001 } \\
\text { ressed HLA A2, A3, and B7 was } \\
\text { TNP) } \\
\text { ated the response, and 25/27 }\end{array}$ \\
\hline Nef (182-198) & $\begin{array}{l}\text { Nef (182-198 BRU) } \\
\text { - HIV-1 specific CTLs dete }\end{array}$ & $\begin{array}{l}\text { EWREDSRLAFHHVAREL HIV-1 infection } \\
\text { cted in lymphoid organs of HIV-1 infected patients }\end{array}$ & human (A1, B8) & Hadida1992 \\
\hline Nef (182-198) & $\begin{array}{l}\text { Nef (182-198 LAI) } \\
\text { - The C-terminal region of }\end{array}$ & $\begin{array}{cc}\text { EWRFDSRLAFHHVAREL } & \text { HIV-1 infection } \\
\text { Nef (182-205) contains multiple CTL epitopes wit }\end{array}$ & $\begin{array}{l}\text { human }(\mathrm{A} 2, \mathrm{~A} 25 \\
\text { estrictions }\end{array}$ & Hadida1995 \\
\hline Nef (182-198) & $\begin{array}{l}\text { Nef (182-198 BRU) } \\
\text { - CTL isolated in children b }\end{array}$ & $\begin{array}{ll}\text { EWRFDSRLAFHHVAREL } & \text { HIV-1 infection } \\
\text { born to HIV-1 positive mothers }\end{array}$ & human (A25) & Cheynier1992 \\
\hline Nef (182-198) & $\begin{array}{l}\text { Nef (182-198 LAI) } \\
\text { - The C-terminal region of }\end{array}$ & $\begin{array}{l}\text { EWRFDSRLAFHHVAREL } \\
\text { Nef (182-205) contains multiple } \text { CTL epitopes wit }\end{array}$ & $\begin{array}{l}\text { human (B35) } \\
\text { estrictions }\end{array}$ & Hadida1995 \\
\hline Nef (182-198) & $\begin{array}{l}\text { Nef (182-198 LAI) } \\
\text { Vaccine Vector/Type: Mer } \\
\text { - Macaca mulatta did not ha } \\
\text { - BALB/c mice had a weak } \\
\text { presented in a vaccinia ba }\end{array}$ & $\begin{array}{l}\text { EWREDSRLAFHHVAREL Vaccine } \\
\text { ngo virus, vaccinia Strain: LAI HIV componen } \\
\text { ave a detectable response to Rec Mengo virus-HIV } \\
\text { response to this epitope in the Mengo virus constr } \\
\text { ckground }\end{array}$ & $\begin{array}{l}\text { murine }\left(\mathrm{H}-2^{d}\right) \\
\text { cine } \\
\text { HIV-1 Nef induces }\end{array}$ & $\begin{array}{l}\text { VanderRyst1998 } \\
\text { CTL response in mice when }\end{array}$ \\
\hline Nef (182-201) & $\begin{array}{l}\text { Nef (191-205 SF2) } \\
\text { - Of } 25 \text { patients, most had C } \\
\text { - Eleven subjects had CTL } \\
\text { - One of these } 11 \text { had CTL } \\
\text { - The responding subject w }\end{array}$ & $\begin{array}{l}\text { EWREDSRLAFHHVARELHPE HIV-1 infection } \\
\text { CTL specific for more than } 1 \text { HIV-1 protein } \\
\text { that could recognize vaccinia-expressed LAI Nef } \\
\text { response to this peptide } \\
\text { as HLA-A2, B21 }\end{array}$ & human & Lieberman1997a \\
\hline
\end{tabular}




\begin{tabular}{|c|c|c|c|c|c|}
\hline HXB2 Location & Author's Location & Sequence & Immunogen & Species (HLA) & References \\
\hline Nef (182-205) & Nef (182-205 LAI) & $\begin{array}{l}\text { EWRFDSRLAFHHVARELHP- } \\
\text { EYFKN }\end{array}$ & Vaccine & human & Gahery-Segard2000 \\
\hline
\end{tabular}

Vaccine Vector/Type: lipopeptide HIV component: six peptides

- Anti-HIV lipopeptide vaccine consisting of six long amino acid peptides derived from Nef, Gag and Env HIV-1 proteins modified by a palmitoyl chain was administered in a phase I trial

- A CD4+ T cell proliferative response to at least one of the six peptides was observed in 9/10 vaccinees - 4/10 reacted to this Nef peptide

- 9/12 tested mounted a CTL response to at least one of the six peptides; each of the six peptides elicited a CTL response in at least one individual

- None of the 12 tested had an $\operatorname{IgG}$ response to this peptide

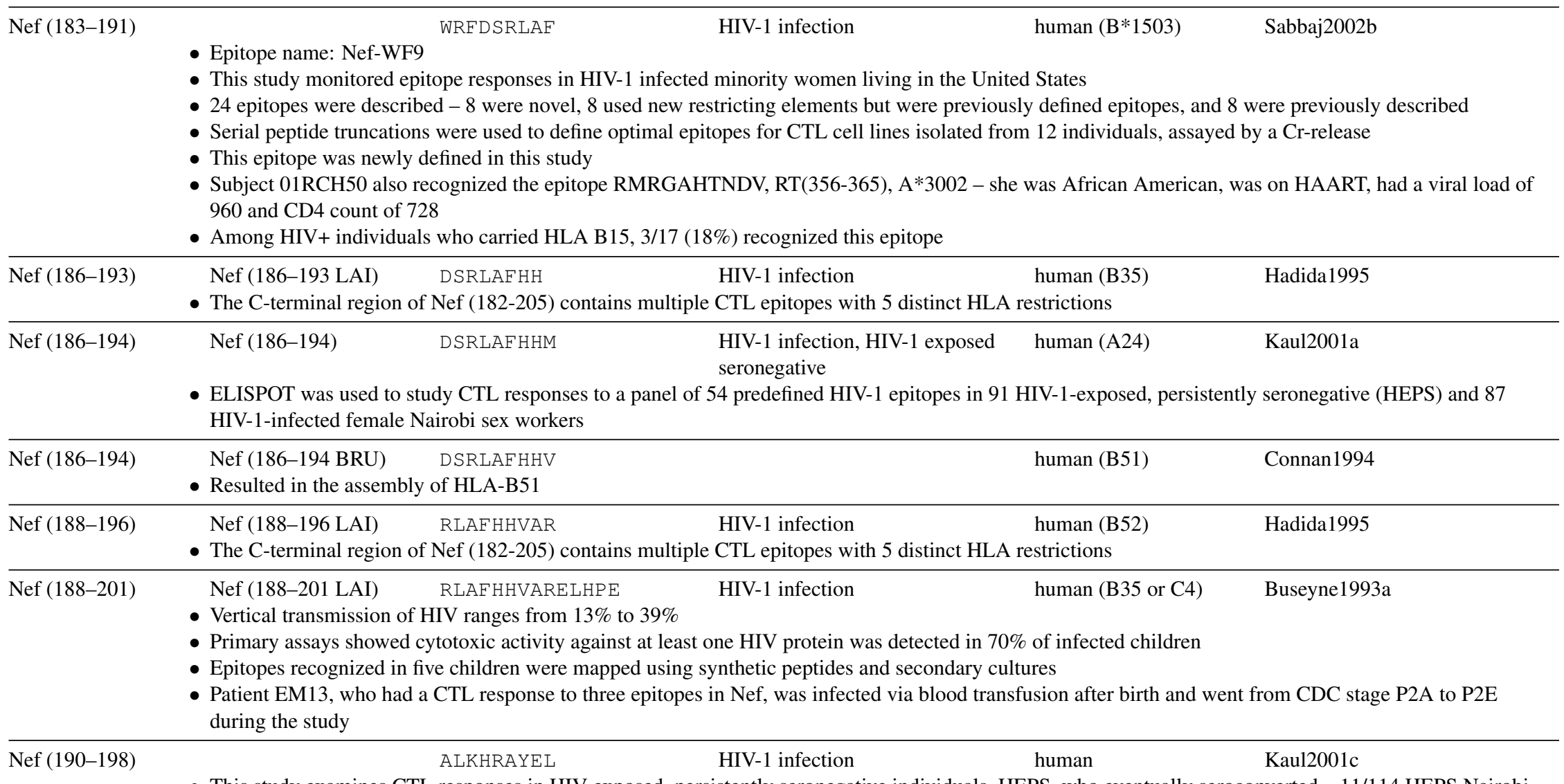

- This study examines CTL responses in HIV exposed, persistently seronegative individuals, HEPS, who eventually seroconverted - 11/114 HEPS Nairob sex workers eventually seroconverted, and for six of these HIV CTL reactive epitopes had been defined while seronegative

- The epidemiological factor associated with seroconversion was stopping sex work and HIV-specific CTL activity declines when HEPS sex workers stop working for a period or retire 


\begin{tabular}{lllll}
\hline HXB2 Location & Author's Location & Sequence & Immunogen & Species (HLA) \\
\hline & - This epitope was in 1/22 HEPS controls, ML1749 & & References \\
\hline Nef (190-198) & Nef & AFHHVAREL & HIV-1 infection & human (A*0201)
\end{tabular}

- Epitope name: Nef AL9

- HIV was scanned for all peptides which carried the A2-supermotif pattern conserved in more than 50\% of B clade sequences - 233 peptides met this criteria, and 30 of these bound to HLA-A*0201 - 20/30 bound to at least 3/5 of HLA-A2 supertype alleles tested

- Three additional previously described HLA-A2 epitopes were added to the set of 20, including Nef AL9, and 18/22 chronically infected HLA-A2 individuals had CTL that recognized at least one of the 23 peptides (median of 2 and maximum of 6), while $6 / 12$ acute infected individuals recognized at least 1 (median of 1 and maximum of 2)

- RT VL9 was not recognized by any of the 22 HLA-A2 patients with chronic HIV-1 infection or the 13 HLA-A2 patients with acute HIV-1 infection included in this study

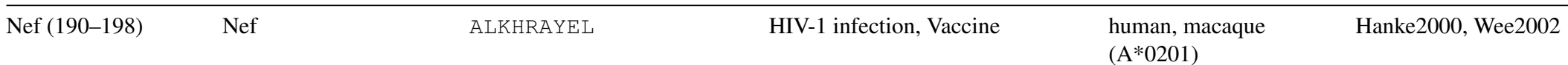

Vaccine Vector/Type: DNA prime with vaccinia MVA boost Strain: subtype A HIV component: p17, p24, polyepitope

- The HIV-1 subtype A focused vaccine HIVA contains p24 and p17, in a reversed order relative to the Gag polyprotein to prevent myristylation of p17, which could direct the protein to the cell membrane and inhibit efficient peptide processing and class I presentation, as well as a polyepitope string of conserved, often immunodominant epitopes that were selected to have particularly good cross-reactive potential for the A-clade epidemic in Nairobi, Kenya. A DNA and MVA prime-boost vaccination protocol using the HIVA antigen will be used in a phase III clinical trial in Kenya. This epitope is included in the polyepitope string [Hanke2000].

- Multiple CD4+ or CD8+ T-cell vaccine-induced responses to peptide pools were detected using intracellular cytokine staining and IFNgamma Elispot assays after vaccination of 5 macaques. The response to the Mamu A*01 SIV p27 epitope p11C (CTPYDINQM), included in the polyepitope region, was not immunodominant in the Mamu $\mathrm{A} * 01$ vaccinated macaques, possibly because of processing limitations in context of the artificial polyepitope string [Wee2002].

Nef (190-198) Nef (190-198 LAI) AFHHVAREL $\quad$ HIV-1 exposed seronegative human (A2) Rowland-Jones1998a

- CTL recognition reported in the context of HLA-B52 and A2.1, A2.2 and A2.4

- A CTL response was found in exposed but uninfected prostitutes from Nairobi using previously-defined B clade epitopes that tended to be conserved in A and D clades - such cross-reactivity could protect against both A and D and confer protection in Nairobi where both subtypes are circulating

- The A subtype consensus is ALKHRAYEL

- The D subtype consensus is AfEHKAREm

- [Hunziker1998] suggests that HLA-A2 does not in fact present this epitope, and notes that it does not promote A2 assembly [Connan1994 - also see |Brander1998b

- [Hunziker1998] maintains that HLA-A2 does not present this epitope contrary to an earlier report [Hadida1995], (also see [Brander1998a])—despite the position of Hunziker et al., Rowland-Jones and colleagues are confident that this epitope in its A clade form is presented by HLA-A*0201 and A*0202, and it is one of the most common responses seen in both seropositive and exposed-uninfected donors from Nairobi (Rupert Kaul, Pers. Comm.)

Nef (190-198) Nef (190-198) AFHHVAREL in vitro stimulation human (A2) Wilson1999b

- Dendritic cells are the most potent for priming T cell responses - DCs can stimulate autologous CTL responses from T cells cultured from HIV negative donors

- Th1-biasing cytokines IL-12 or IFN alpha enhance CTL responses in vitro whether the epitope is delivered by pulsing from peptide, or expressed from within 


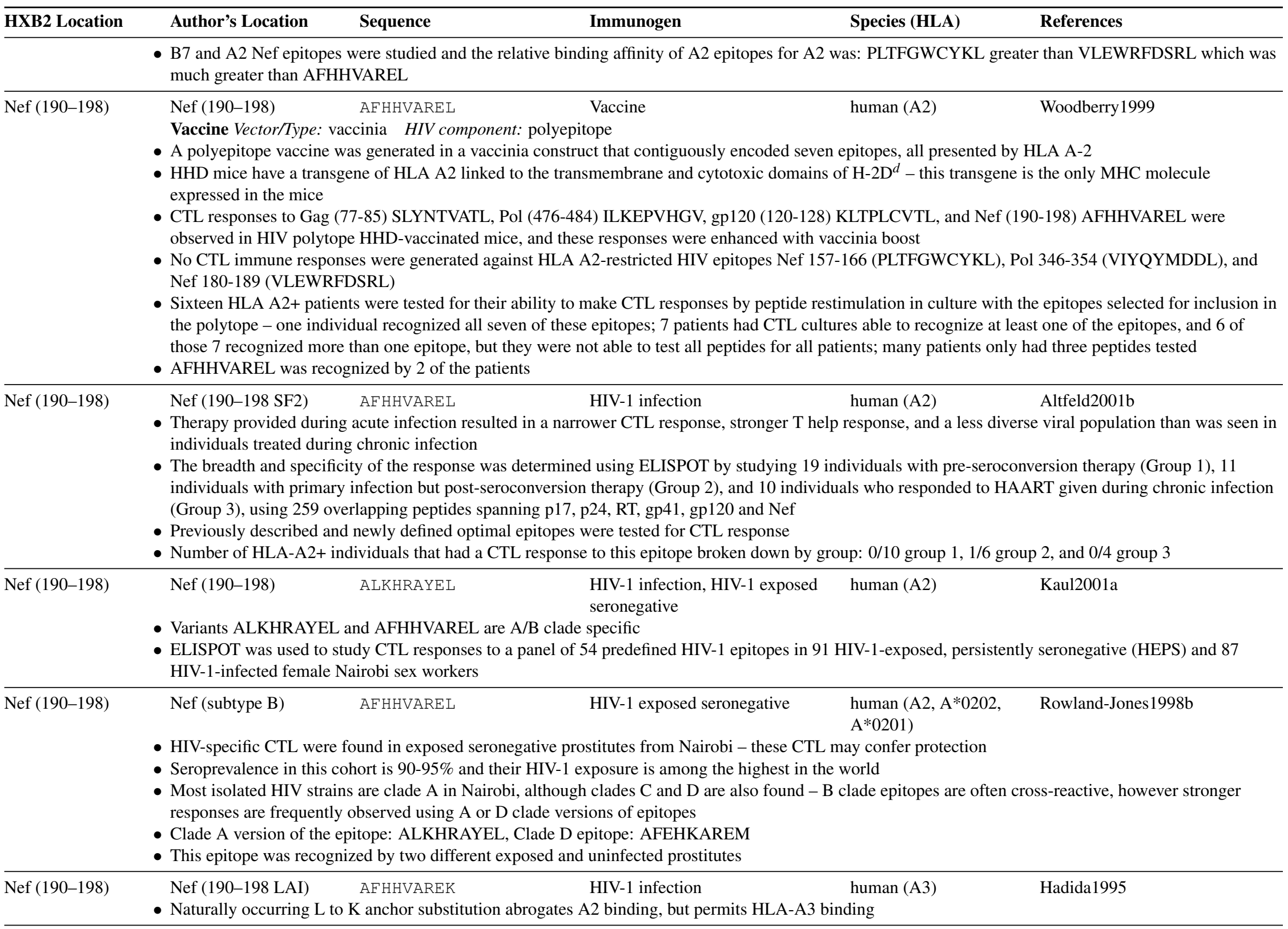




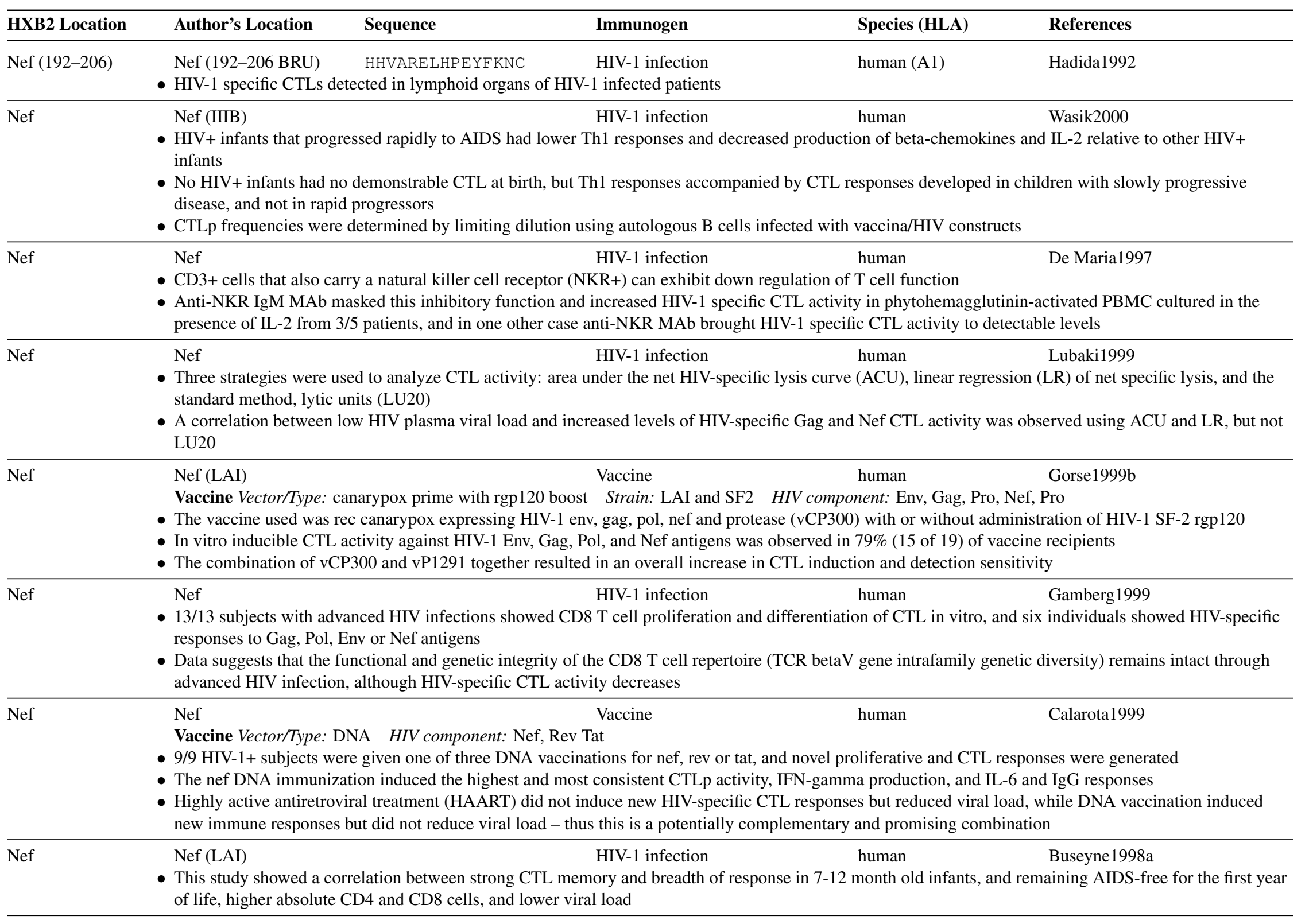




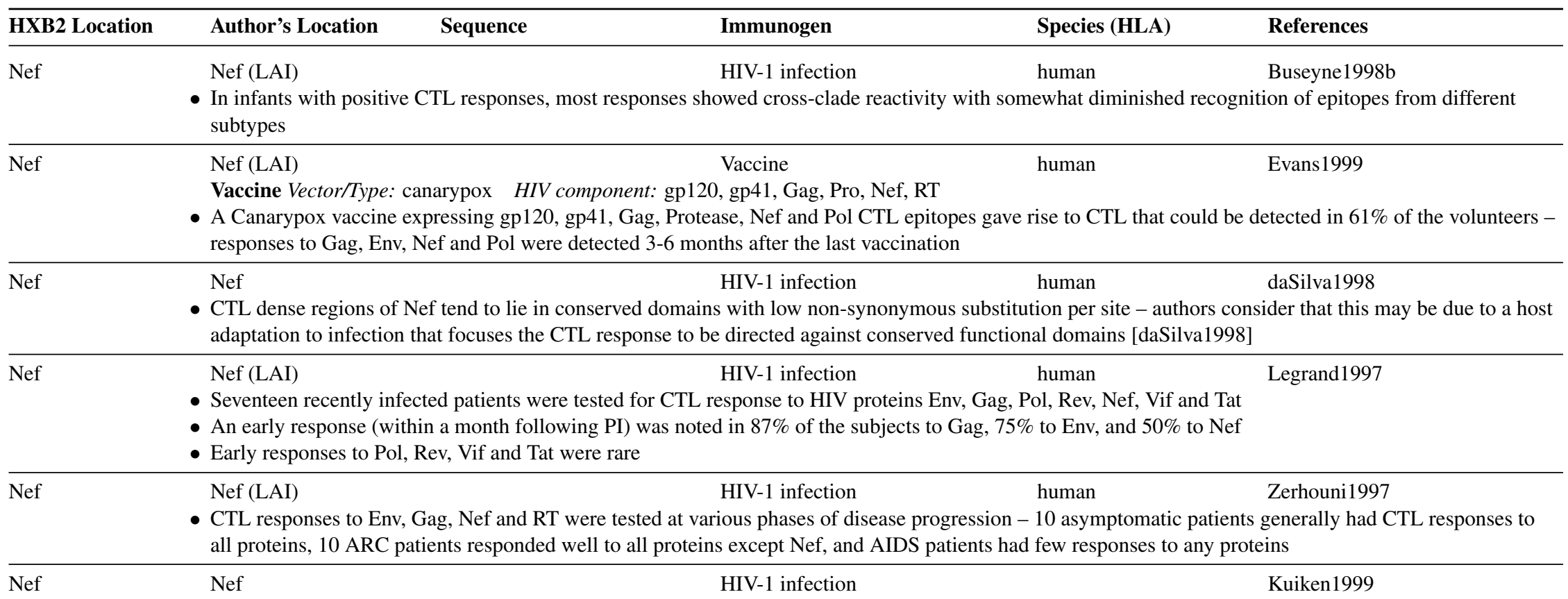

- A correlation between conserved regions of Nef and CTL epitope density was also noted in [Kuiken1999]. The authors suggest that this may be due to biological reasons such as the one described above daSilva1998, or due to epitope processing, or may possibly be an artifact of experimental strategy for epitope definition such that conserved epitopes would tend to be identified because they would be more likely to be cross-reactive with the test reagents

- Both p17 and Nef show a correlation between epitope density and conserved regions in the protein - in contrast, p24 is a more conserved protein and known epitopes are evenly distributed across p24

Nef Nef (BRU) HIV-1 infection $\quad$ human

- In vitro measurements of CTL-activity by $\mathrm{Cr}$ release assay in bulk culture showed no correlation between CTL-activity (gp120, Gag, Pol and Nef) and disease progression as measured by viral load, CD4 and time to death

\begin{tabular}{lll}
\hline Nef & Nef (SF2) HIV-1 infection & human \\
& CTL precursor frequencies were determined in HIV-1 infected pregnant women, and higher CTLp frequencies to Pol and SF2 Nef, but not IIIB Nef, were \\
& found in non-transmitting mothers than in transmitting mothers - Nef CTL responses have been found in uninfected infants born to HIV+ women
\end{tabular}

found in non-transmitting mothers than in transmitting mothers - Nef CTL responses have been found in uninfected infants born to HIV+ women (Lazuriaga95);

- This study is provides a survey of CTL responses and full length HIV-1 genome sequences from a C subtype infected Botswanan cohort

- 37 of 45 subjects (82\%) demonstrated Nef specific ELISPOT CTL responses of more than 100 SFC/106 PBMC

- Two Nef-immunodominant regions were identified, one spanned amino acid positions 67 to 96 using HXB2 numbering system while the second corresponded to amino acid positions 122 to 141 


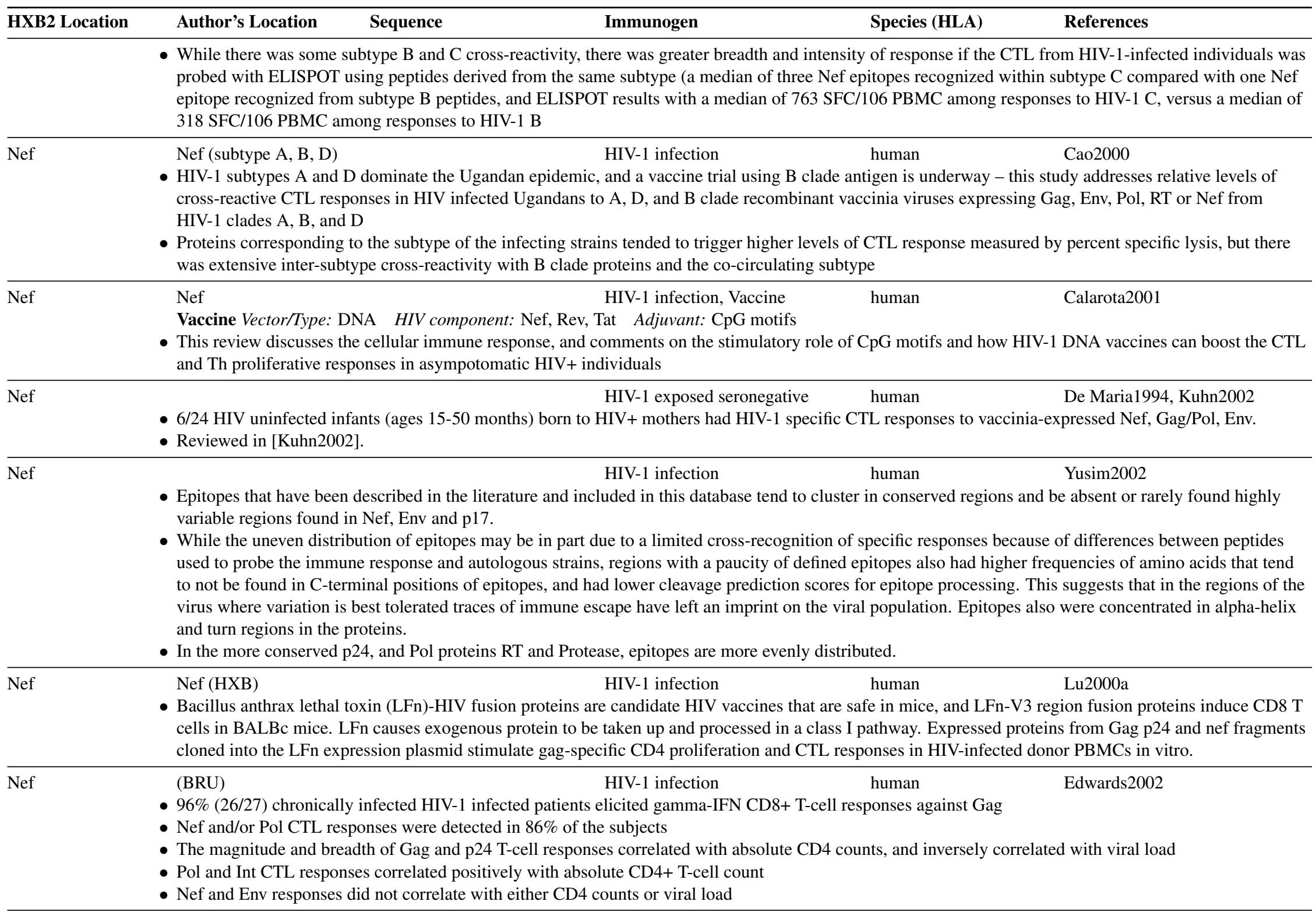




\begin{tabular}{|c|c|c|c|c|c|}
\hline HXB2 Location & Author's Location & Sequence & Immunogen & Species (HLA) & References \\
\hline Nef & & & HIV-1 infection & human & Larsson $2002 b$ \\
\hline
\end{tabular}

patients on successful HAART treatment, relative to autologous monocytes. Some weak responses could only be detected using mature dendritic cells as APCs, and this approach could be useful for detection of low frequency memory cells.

Nef $\quad$ (SF2) $\quad$ HIV-1 and HCV immune responses were studied in 22 individuals who were co-infected with HIV-1 and hepatitis C virus (HCV). IFN

- HIV-1 and HCV immune responses were studied in 22 individuals who were co-infected with HIV-1 and hepatitis C virus (HCV). IFNgamma production was measured in an Elispot assay of CD8+ T-cells using targets expressing either Gag, RT, Env and Nef in a vaccinia construct, or one of seven HCV proteins.

- All 22 patients targeted at least one protein. 20/22 patients recognized RT, 17/22 patients recognized Gag, 13/22 subjects recognized Env and 11/22 patients recognized Nef. Robust CTL activity was independent of disease progression or viral load.

- Despite high HCV viral loads, very few HCV CD8+ T-cell Elispot responses were detected. In a control HCV infected person who did not have HIV-1, strong anti-HCV responses were mounted.

- HIV-specific CD4 proliferative responses were detected in 9/17 coinfected patients, but no HCV responses were detected.

Nef HIV-1 infection human Scott2001

- CTL responses before and after initiation of ART were studied in 13 HIV-1 vertically infected infants $<6$ months of age, and 4 that were $>6$ months of age.

- Before ART 2/13 infants $<6$ months of age showed IFNgamma Elispot CD8+ T-cell responses, one to Nef and one to Env and Nef, and these responses

became undetectable after successful therapy- 3 infants were coinfected with CMV and all 3 had CMV-specific CD8+ T-cell responses.

- One older infant, at 23 months, had CTL responses against all for proteins tested, Gag, Pol, Nef and Env, and had the lowest plasma viremia of the study group. 3/4 infants older than 6 months of age responded to either Nef or Pol.

- Administration of ART over 48 weeks broadened the HIV-1-specific CTL response in 2/4 of the older children that were incomplete responders.

Nef (IIIB) HIV-1 infection human Ortiz2001

- Immune responses in eight chronically HIV-1 infected patients undergoing HAART therapy structured treatment interruptions (STI) were studied. STI boosted HIV-1 specific CTL responses and elevated CTL responses were maintained up to 22 weeks after the last treatment interruption, but viral load rebound to pretreatment levels and CD4 T-cell count decline was observed. CD8 responses in PBMC were measured by cytokine flow cytometry with gp160, Gag p55, RT-Pol and Nef expressed in vaccinia.

Vaccine Vector/Type: adenovirus HIV component: Vpr, Nef, Gag/Pol

- Vpr can cause cells to go into G2 arrest, and it surpresses immune cell activation and inflammatory cytokine production, so co-immunization of BALB/c mice with recombinant adenovirus expressing Vpr and HIV-1 antigens Nef or Gag/Pol was tested to see if Vpr reduced the immune response to the other HIV antigens.

- Vpr compromised CD8+ T-cell lytic responses and T-helper proliferative responses in mice co-immunized with Vpr and Nef or Gag/Pol.

- In vitro, Vpr reduced T-cell cytokine production of IL-12 and TNFalpha, indicative of Vpr-mediated immune suppression.

\begin{tabular}{llll}
\hline Nef Nef & HIV-1 infection & $\begin{array}{l}\text { human (A*0201 and } \\
\text { Cw*08) }\end{array}$
\end{tabular}

- HIV-1 specific, MHC class I-restricted CTL killing was detected in duodenal and rectal gut associated lymphoid tissue (GALT) sites from three infected individuals - the distribution of class I restricted CTL was different in the peripheral blood samples and GALT samples

Nef Nef HIV-1 infection human (B*35) Jin2002

- Patients with HLA-B*35 variants $B * 3502, B * 3503, B * 3504$, and $B * 5301$ tend to proceed to AIDS more quickly than those with $B * 3501$. 


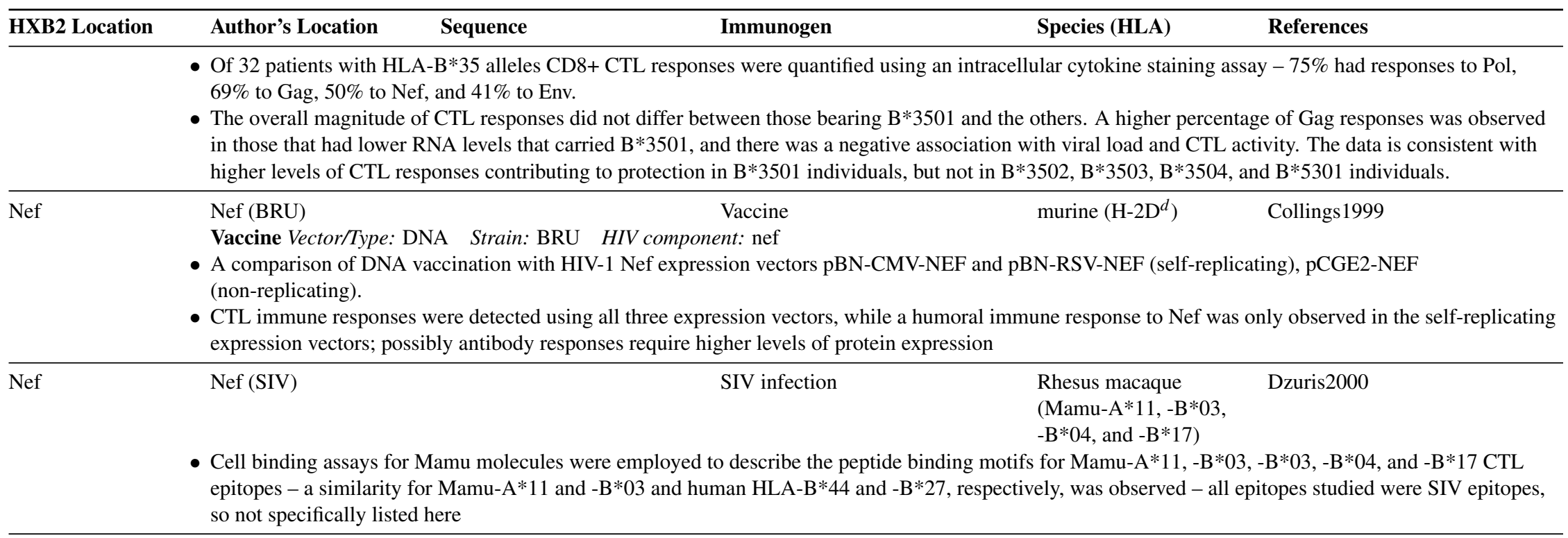




\section{II-B-21 HIV-1 CTL Epitopes}

\begin{tabular}{lllll}
\hline HXB2 Location & Author's Location & Sequence & Immunogen & Species (HLA) \\
\hline HIV-1 & & HIV-1 infection & human
\end{tabular}

- Longitudinal analysis (72 weeks) of 15 patients with acute or recent HIV-1 infection implies that HAART treatment alone can not completely conserve $\mathrm{CD} 8+$ cell homeostasis and preserve the original T-cell receptor repertoire

HIV-1 HIV-1 infection human

- Non-cytotoxic anti-HIV responses of CD8+ T cells cultured with CD4 infected HIV cells are mediated be blocking expression of viral RNA, and do not influence viral replication steps through integration of provirus

- This study employs a mathematical model to study the consequences of increasing the T-helper response through a vaccine, which would have counter-balancing effects in a new infection: a more intense response provides more help but also more target cells. The model indicates that if the infecting virus had a low replication rate, then CTLp and CD4 helper cells could control an infection. Only a vaccine that could increase CTL responsiveness could reduce viral set point with observed replication rates.

- A CD4+ T-cell response without maintained CTL response was deleterious in this model.

HIV-1 HIV-1 infection human Currier2002b

- Elispot standardization was sought using a reference peptide pool of 23, 8-11 mer epitopes from Influenza, cytomegalovirus (CMV), and Epstein Bar Virus (EBV) presented by 11 common HLA class I molecules.

- $15 / 17$ (88\%) HIV- and 14/20 (70\%) HIV+ individuals reacted with this test set and in vitro simulation of the PBMC from these individuals were capable of killing cells expressing the target antigen.

HIV-1 HIV-1 infection human, macaque

Wodarz2002

- Mathematical modeling is used to support the idea that T-helper cell dysfunction results in a compromised ability to maintain an anti-HIV CTL memory response. Models suggest strategies to restore CTL memory through therapy and improve long-term immunological control of the virus.

HIV-1 HIV-1 infection, Vaccine human Zinkernagel2002

- HIV immunity and vaccine strategies are compared with to other pathogens. We do not have a successful vaccine agains TB leprosy, HIV, HCV and most parasites, and the author suggests this is associated with the need for a strong T-cell response to these diseases. Vaccine strategies that achieve a physiological low does infection that is well controlled but persists may be required to alter the immunopathological consequences of infection with HIV.

- The concept of using an artificial consensus sequence for vaccine design is discussed, comparing the concepts of a model ancestor sequence or a consensus sequence, with illustrations of the potential advantages of the strategy based on C-clade comparisons.

- See also a comment [Nickle2003], and reply [Gao2003]

- Reviews evidence for CTL escape in HIV epitopes in natural human infections, and in SIV infections of macaque where viral clones with a known time of infection and multiple animals with the same HLA molecules can be tracked.

- Vigorous CTL responses are made despite class I down-regulation by the Nef protein, but it may delay cytolysis of infected cells. Too great a loss of MHC proteins may enhance NK cell killing so the fitness advantage of this function of Nef may be in balance. 


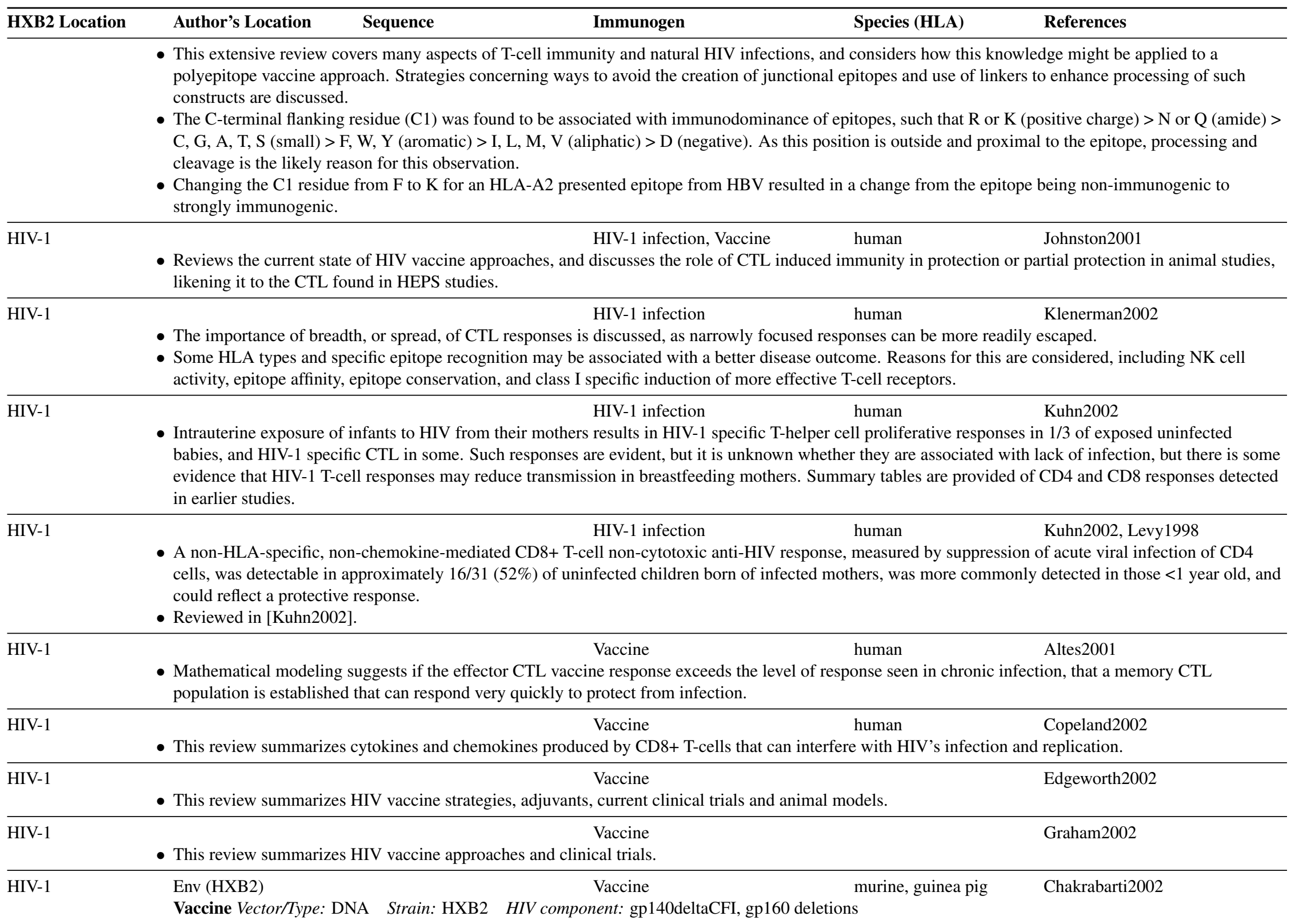




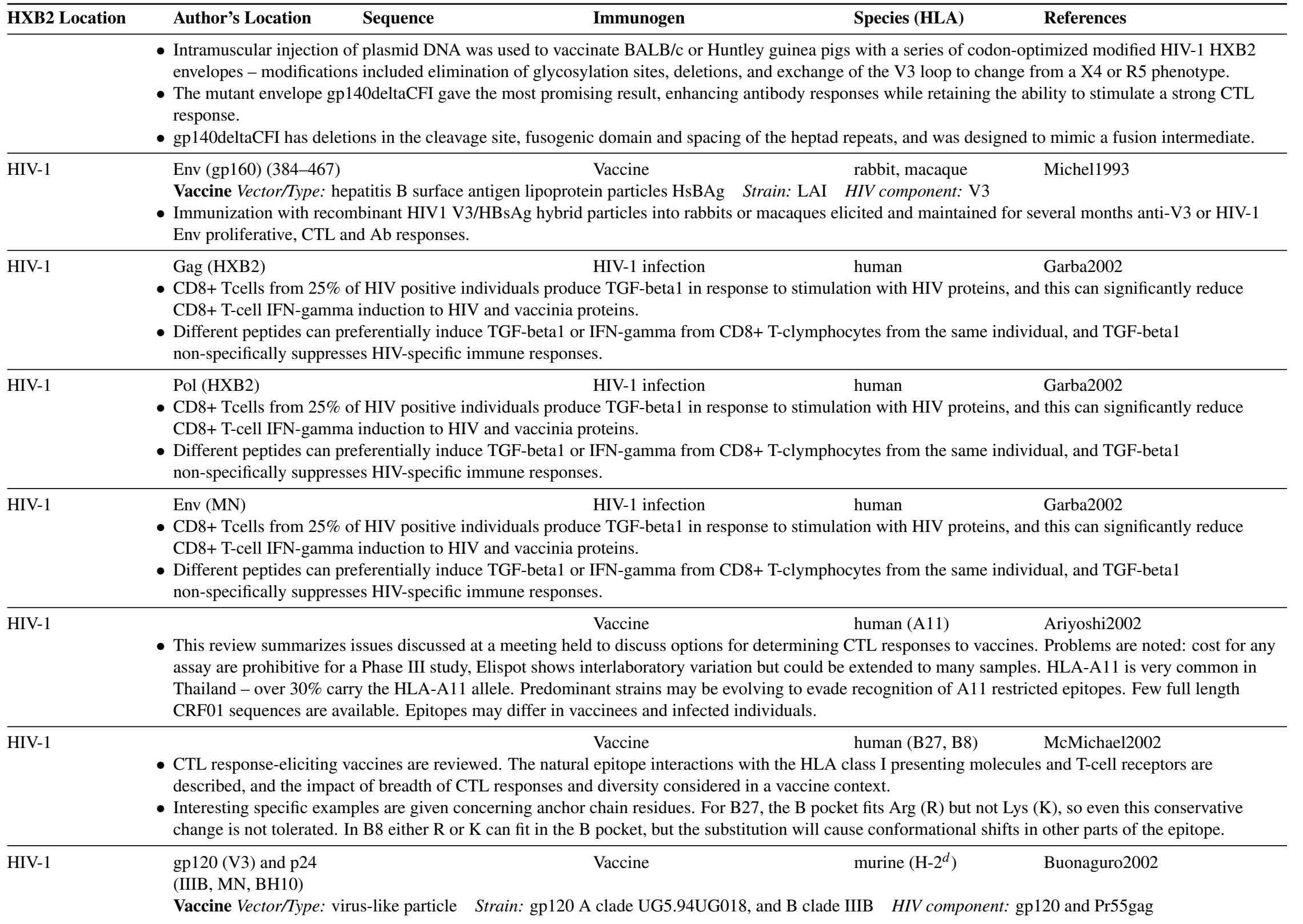




\begin{tabular}{|c|c|c|c|c|c|}
\hline HXB2 Location & Author's Location & Sequence & Immunogen & Species (HLA) & References \\
\hline & \multicolumn{5}{|c|}{$\begin{array}{l}\text { - BALB/c mice were given intraperitoneal immunization with virus-like particle (VLPs) expressing recombinant subtype A gp120 and Pr55gag in the } \\
\text { absence of adjuvants. } \\
\text { - High dose-independent humoral responses against both gp120 and p24 peptides were detected. Antibodies able to elicit 50\% neutralization against A clade } \\
\text { IIIB and the autologous clade a virus were obtained. } \\
\text { - Recombinant rgp120 (clade B, MN) induced T-cell proliferative responses in vitro from vaccinated animals. } \\
\text { - CTL activity was observed against splenocytes expressing Env (clade A) and Gag (clade B, BH10) from a vaccinia construct. }\end{array}$} \\
\hline HIV-1 & $\begin{array}{l}\text { Vaccine Vector/Type } \\
\text { - Attenuated Listeria } \\
\text { vaccinia-gag challen }\end{array}$ & $\begin{array}{l}\text { teria monoc } \\
\text { cytogenes } \mathrm{v}\end{array}$ & $\begin{array}{l}\text { Vaccine } \\
\text { ponent: Gag } \\
\text { ersistent CTL r }\end{array}$ & $\begin{array}{l}\text { murine }(\mathrm{MHC} \mathrm{H} 2 \mathrm{~d}) \\
\text { ns of } \mathrm{BALB} / \mathrm{c} \text { mice a }\end{array}$ & $\begin{array}{l}\text { Lieberman2002 } \\
\text { an protect mice from a }\end{array}$ \\
\hline
\end{tabular}





\section{II-C Maps of CTL Epitope Locations Plotted by Protein}

II-C-1 p17 CTL Epitope Map

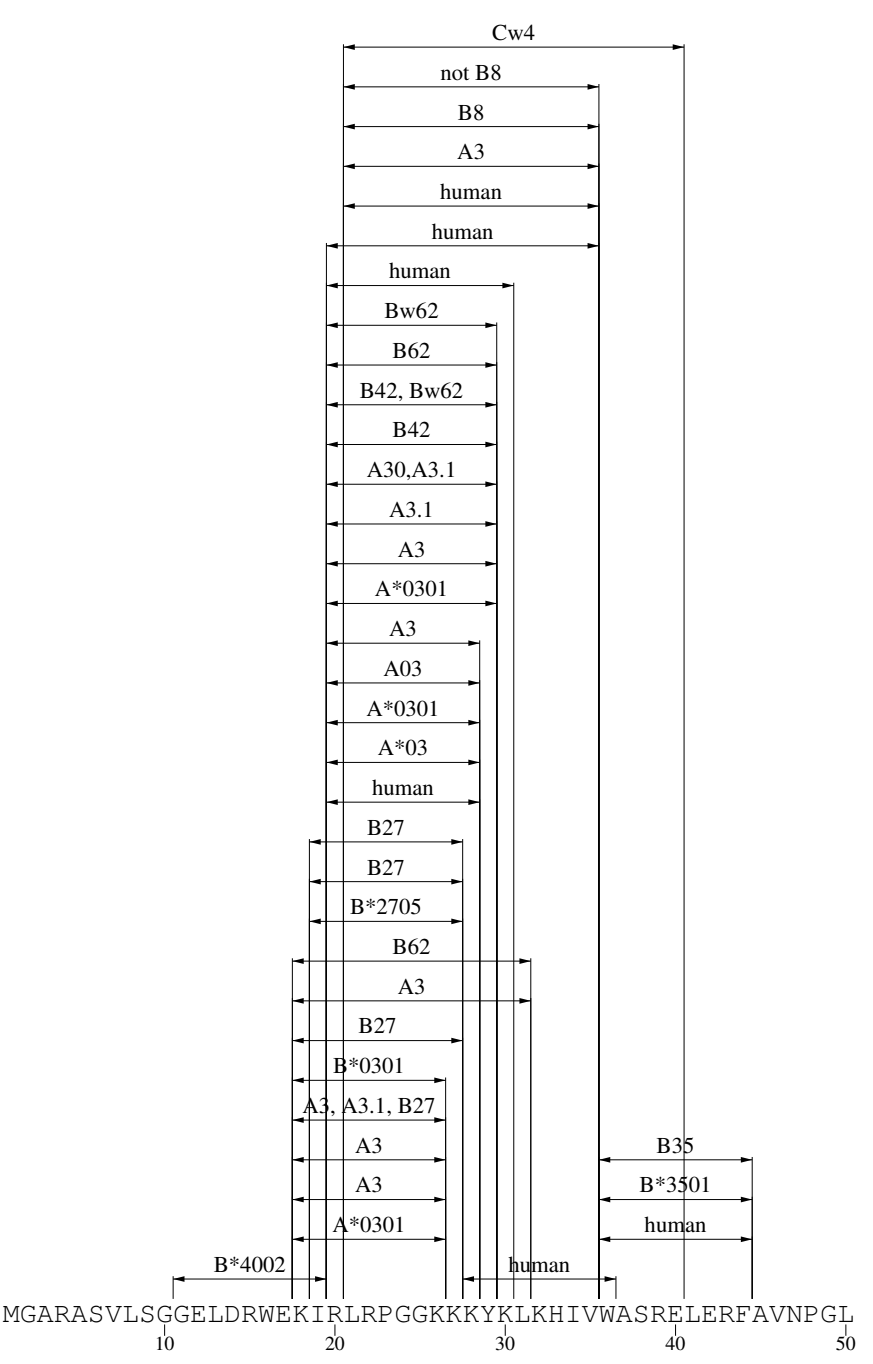




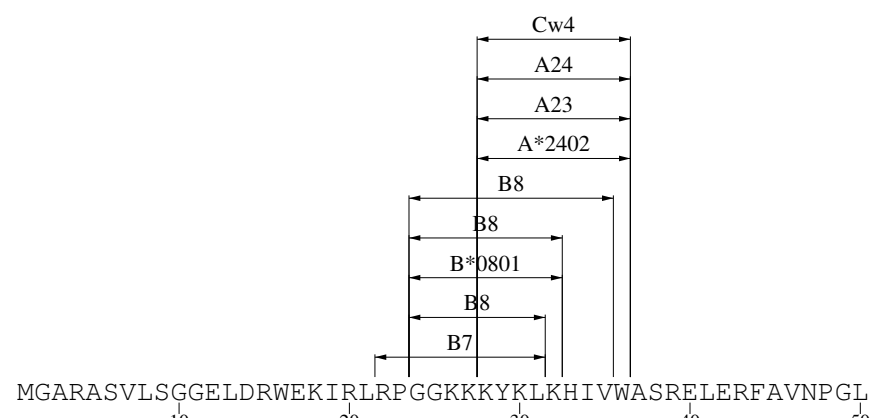

20

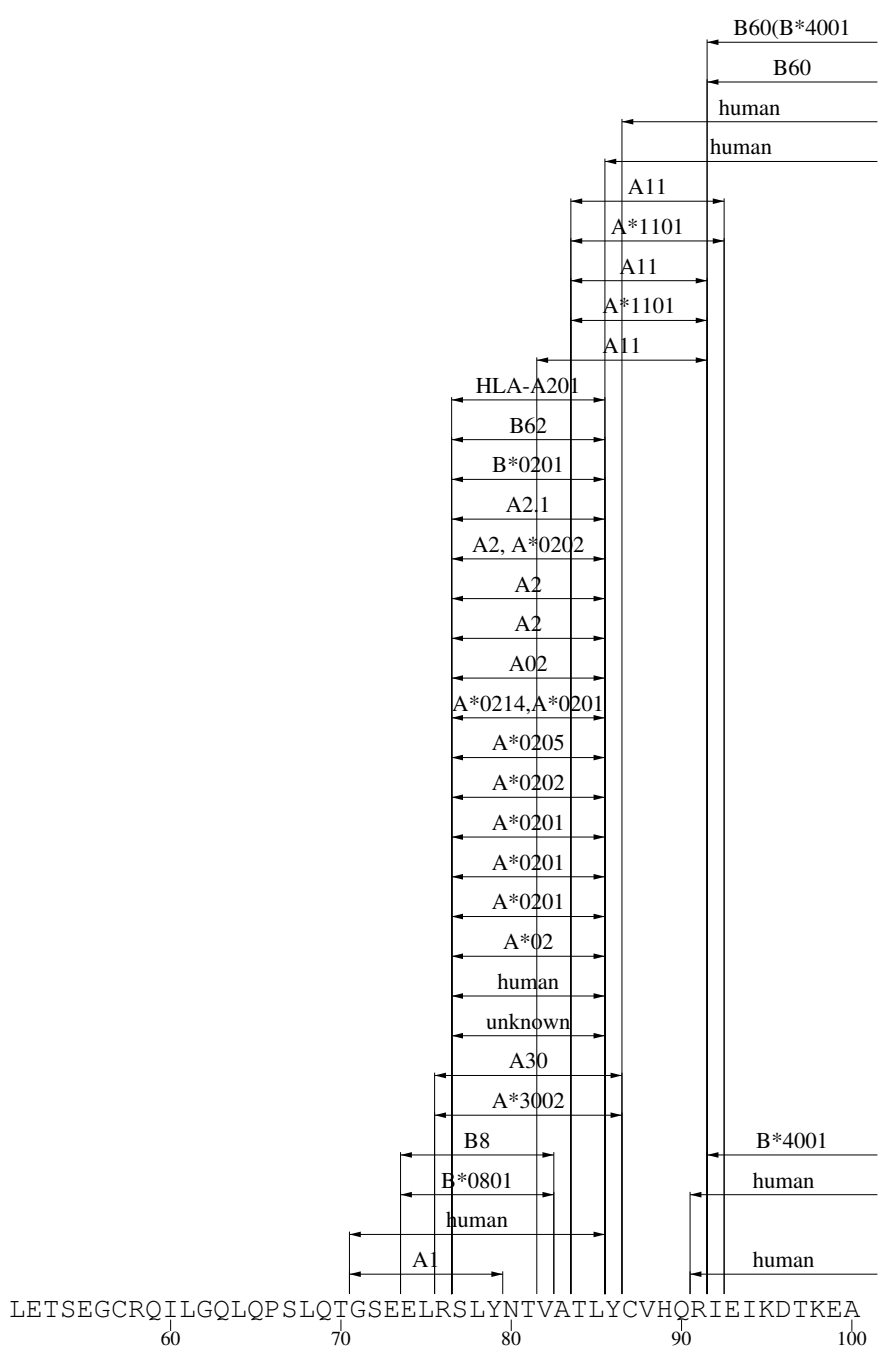




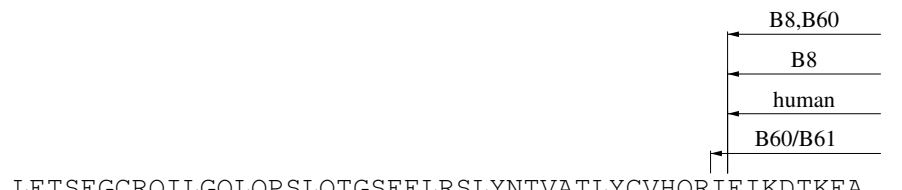

LETSEGCRQILGQLOP SLOTGSEELRSLYNTVATLYCVHORTEIKDTKEA

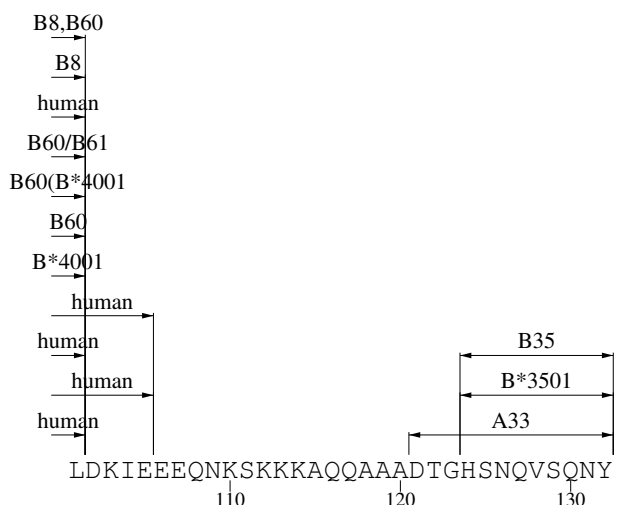

\section{II-C-2 p24 CTL Epitope Map}

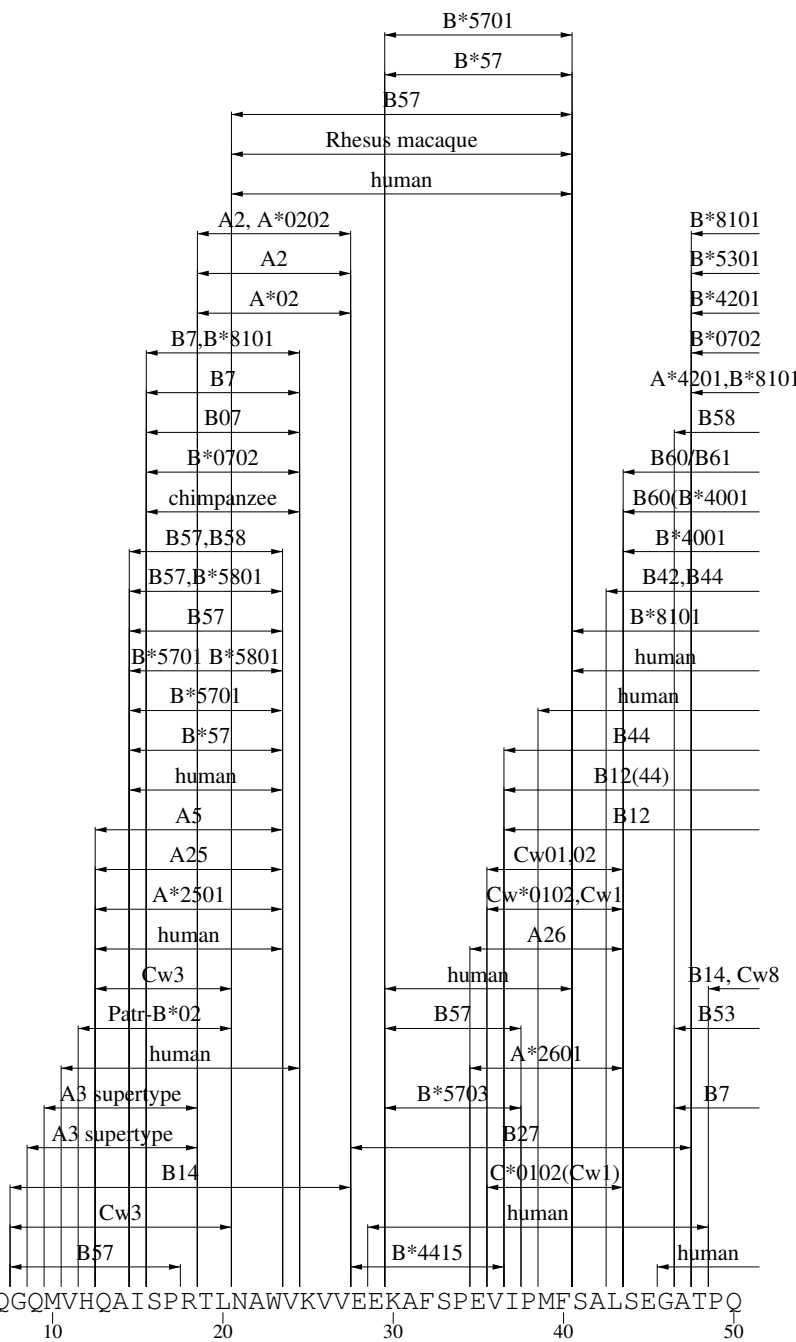



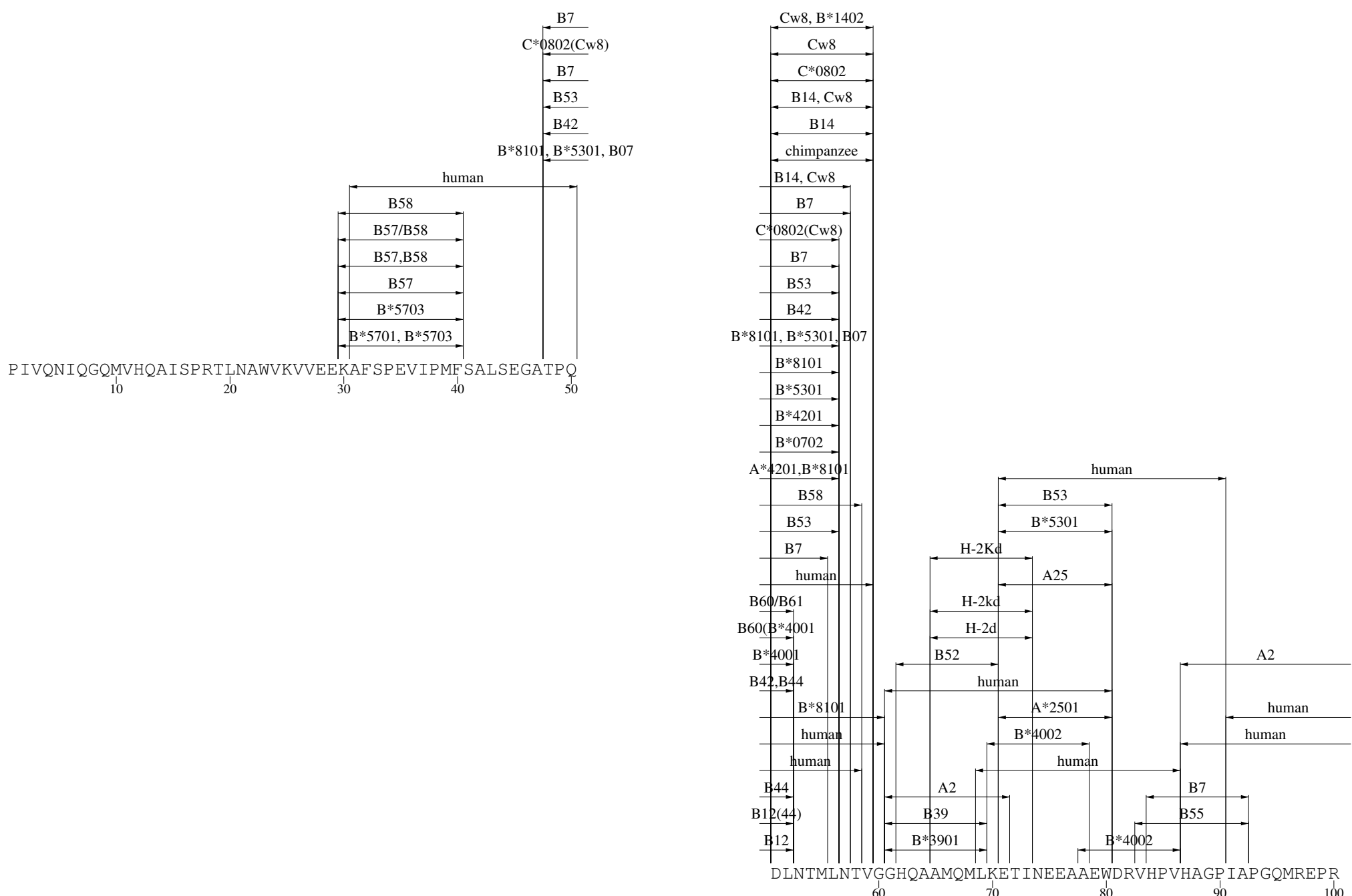
human

DLNTMLNTVGGHQAAMQMLKET INEEAAEWDRVHPVHAGP IAP GQMREPR

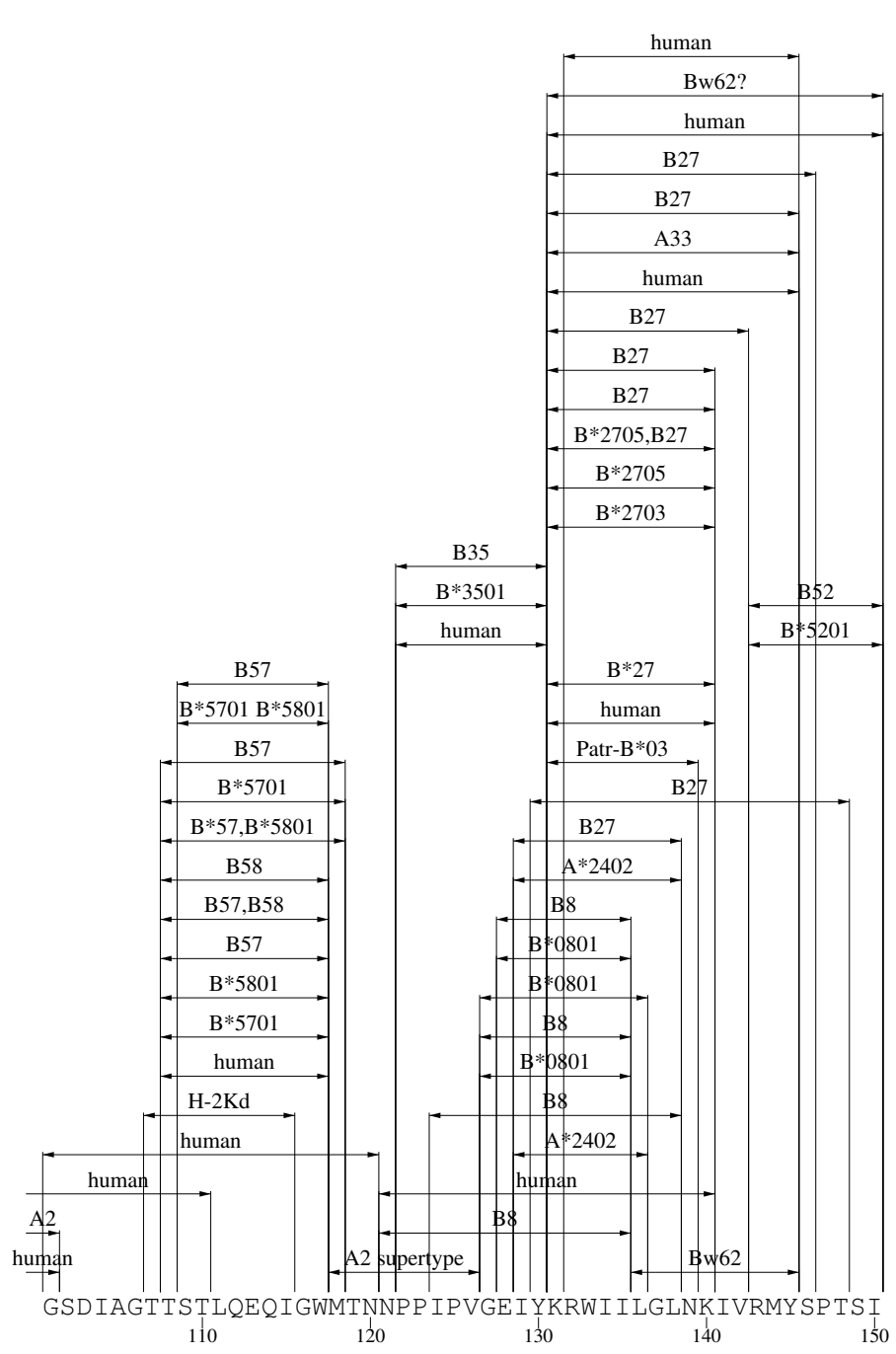

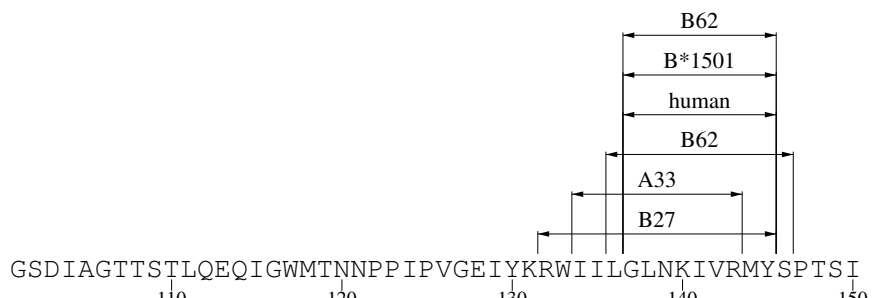

120
130

140
150 


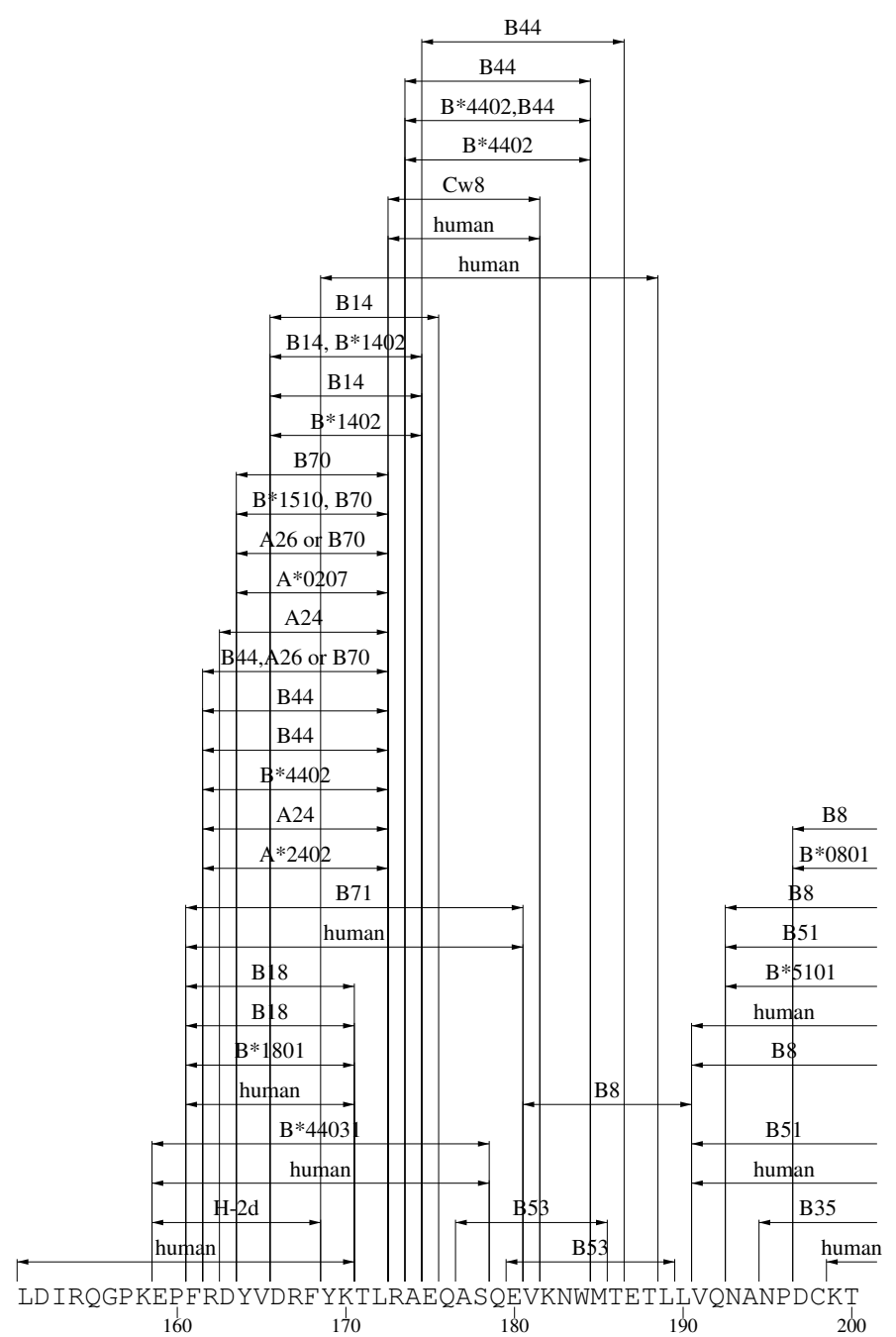

\begin{tabular}{|c|}
\hline $\mathrm{B} 57$ \\
\hline $\mathrm{C} 45$ \\
\hline $\mathrm{B} 57$ \\
\hline $\mathrm{B} 53$ \\
\hline $\mathrm{B} * 5701$ \\
\hline $\mathrm{B} * 5301, \mathrm{~B} 57$ \\
\hline $\mathrm{B} * 5301$ \\
\hline
\end{tabular}

LDIRQGPKEPFRDYVDRFYKTLRAEQASQEVKNWMTETLLVQNANPDCKT

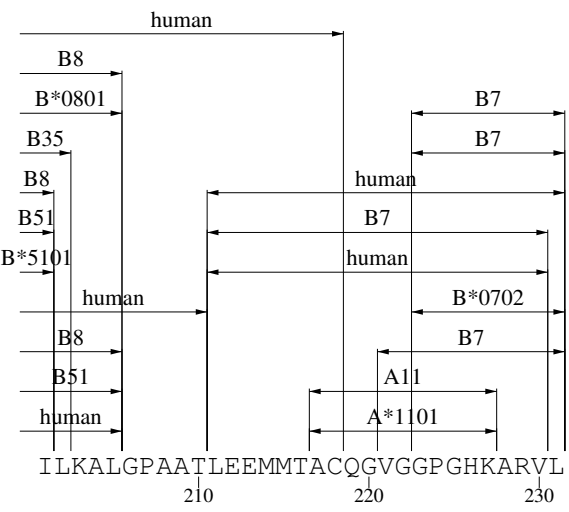




\section{II-C-3 p2p7p1p6 CTL Epitope Map}
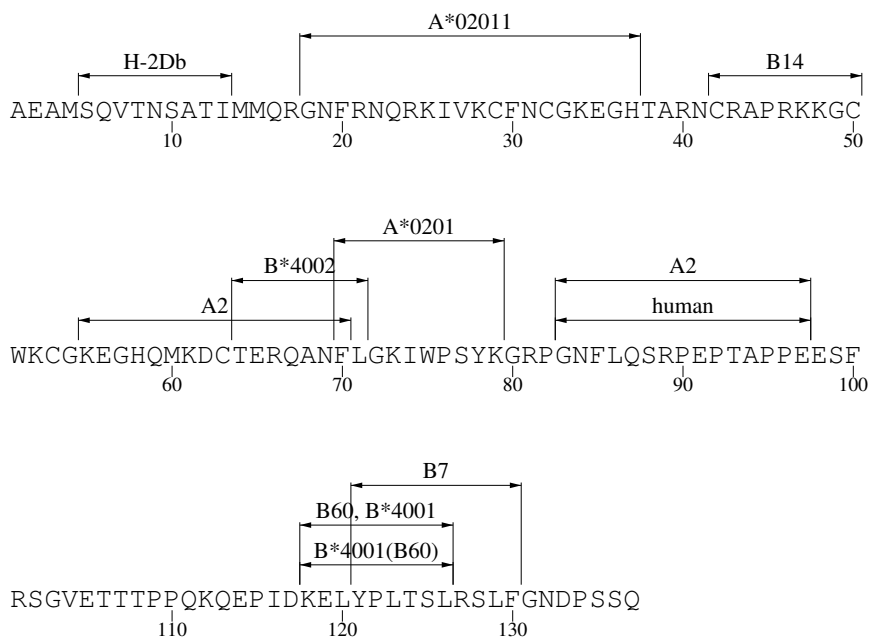

\section{II-C-4 Protease CTL Epitope Map}
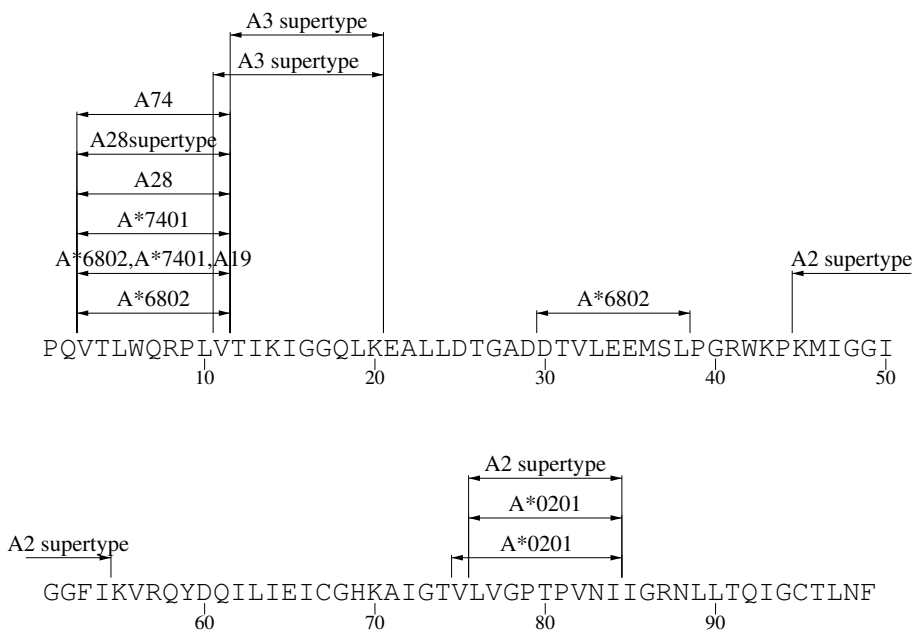


\section{II-C-5 RT CTL Epitope Map}
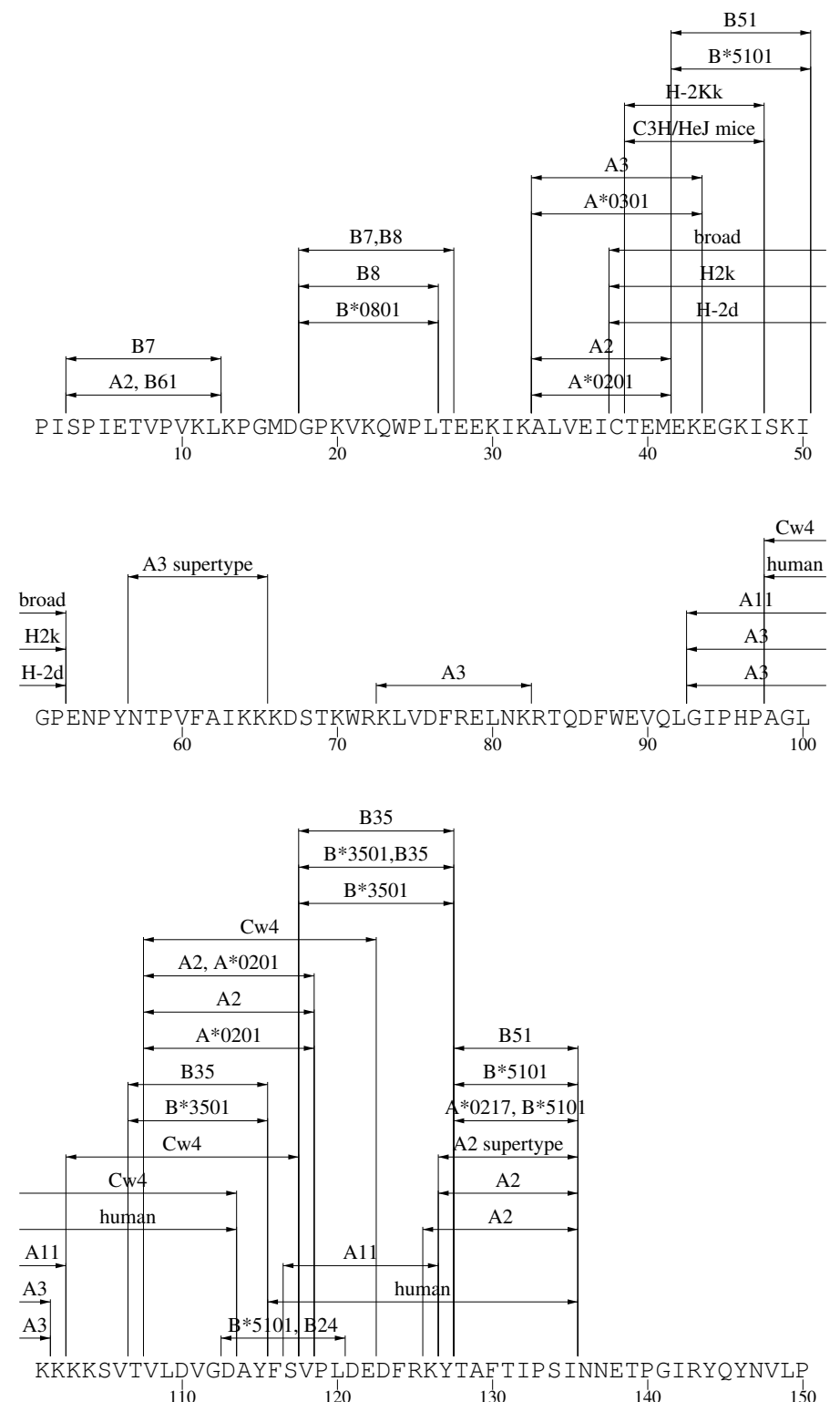
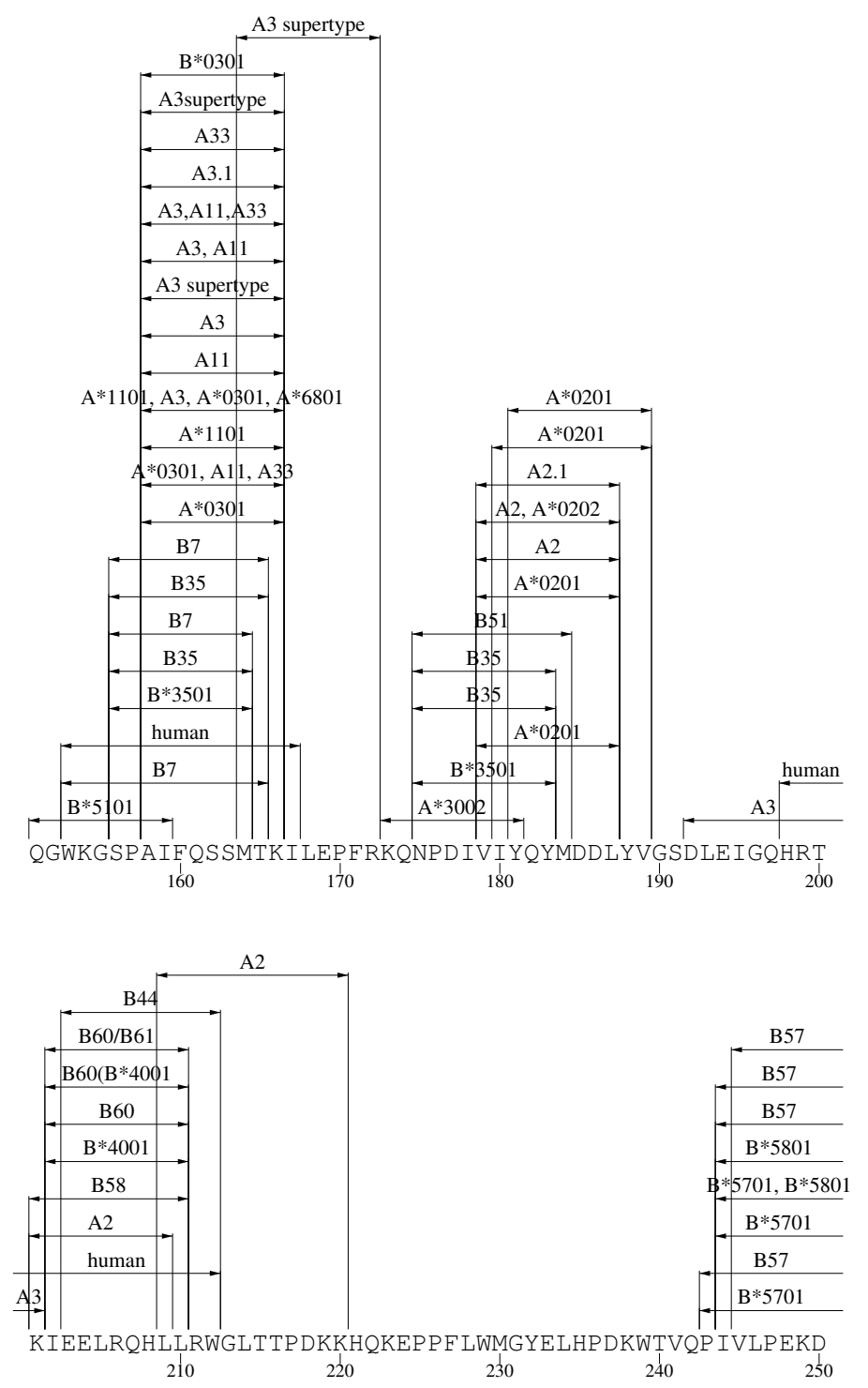

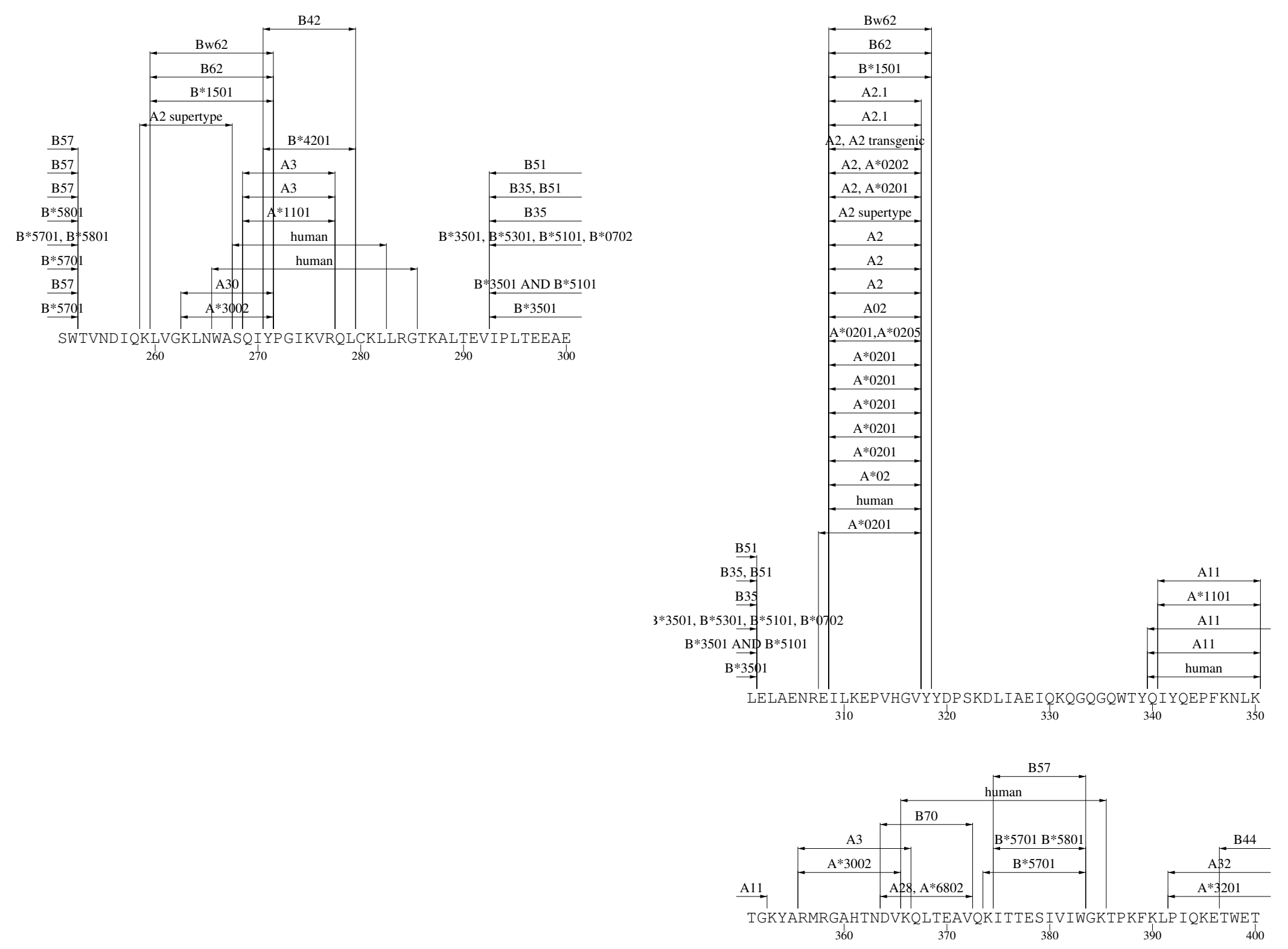

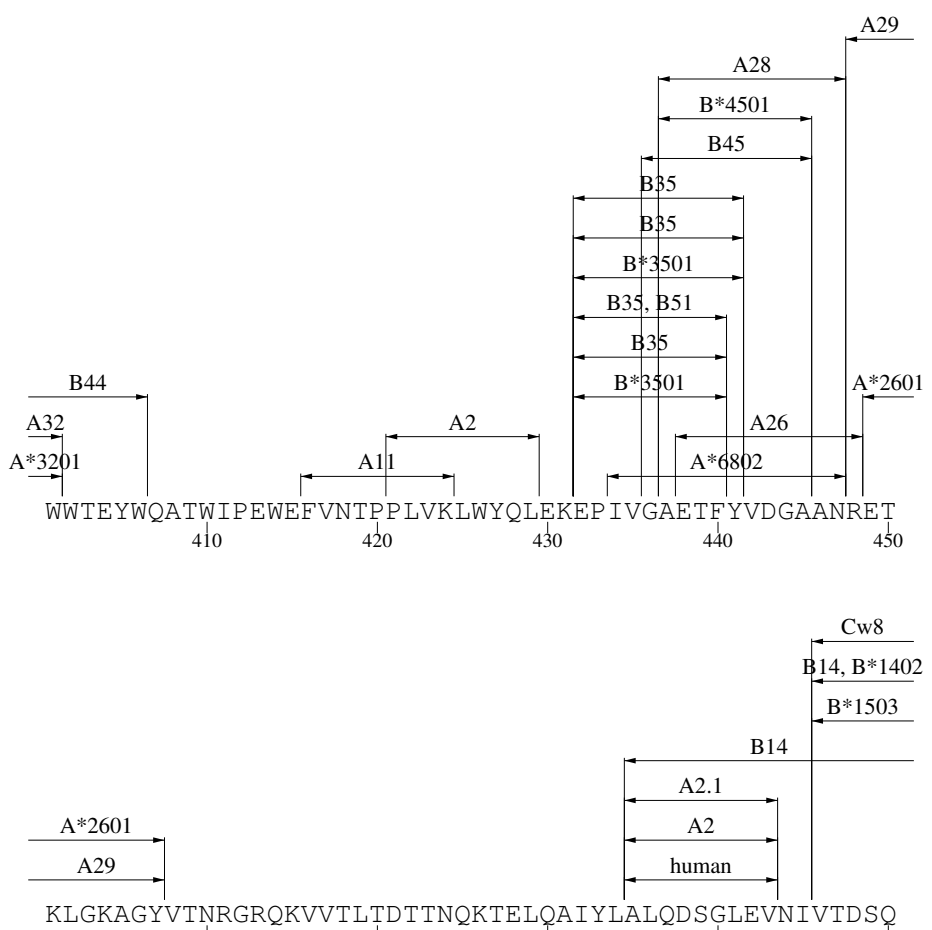

$$
470
$$

480

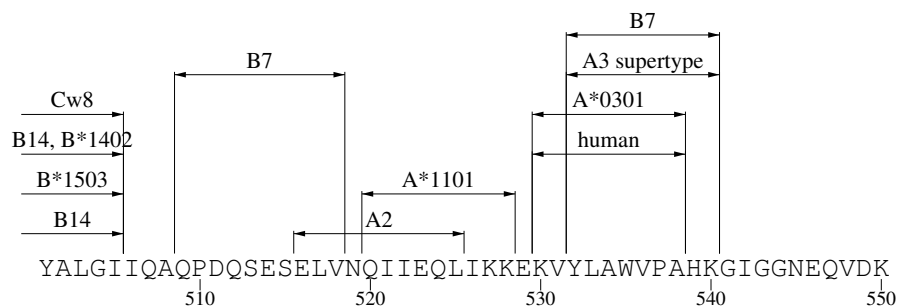

LVSAGIRKVI

\section{II-C-6 Integrase CTL Epitope Map}

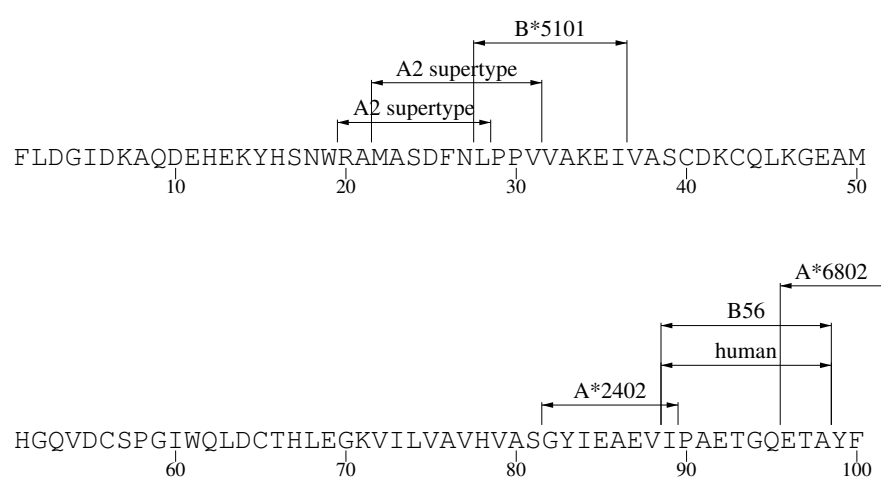

$\stackrel{\text { A*6802 }}{\rightarrow}$ A2 supertype

LLKLAGRWPVKT IHTDNGSNFTGATVRAACWWAGIKQEFGIPYNPQSQGV

120

130

$140 \quad 150$

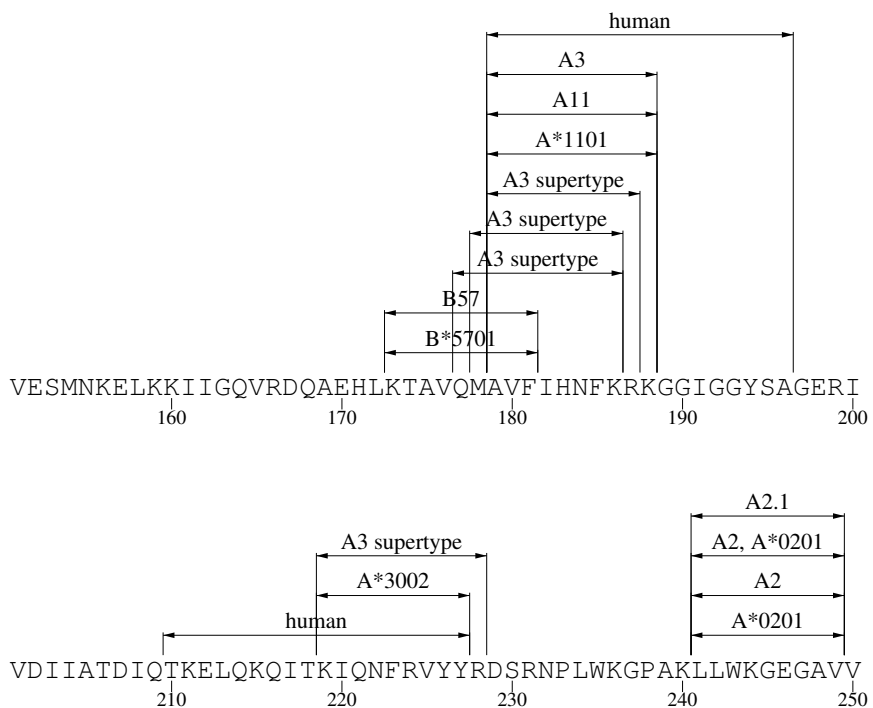

220

230

IQDNSD I KVVP RRKAKI I RDYGKQMAGDDCVASRODED
270 


\section{II-C-7 Rev CTL Epitope Map}
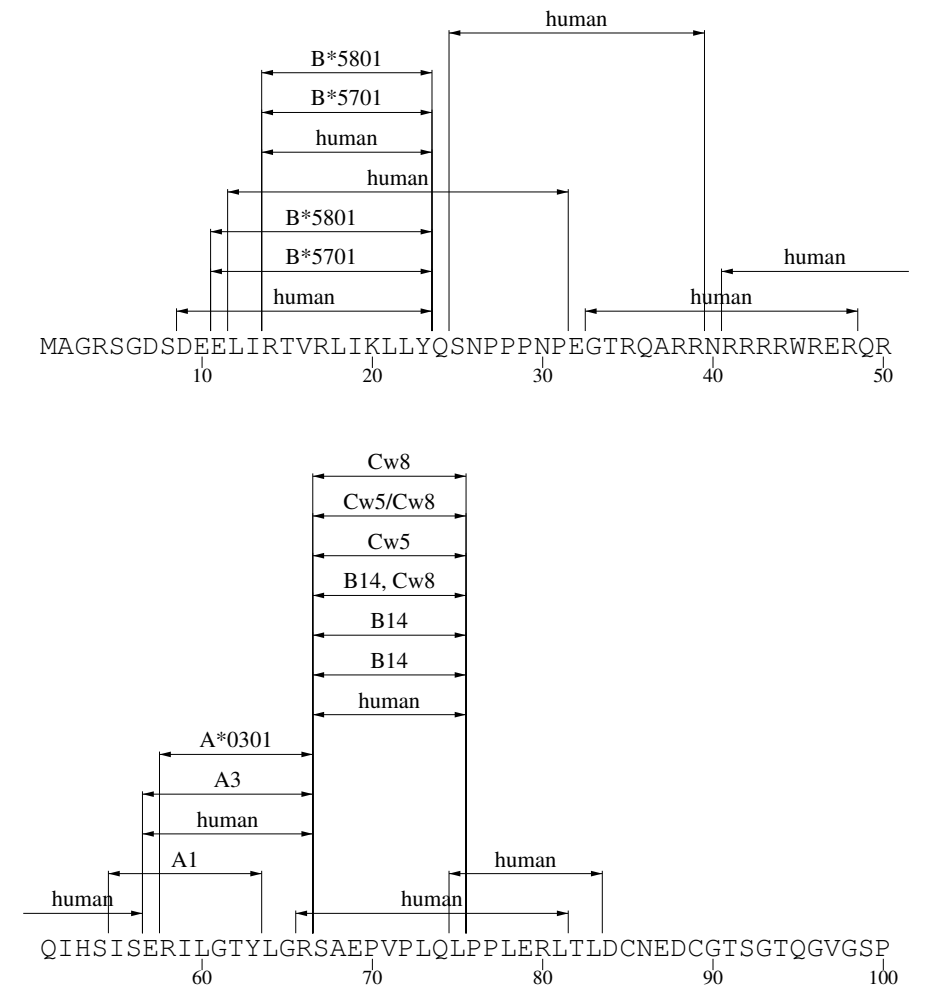

QILVESPTVLESGTKE

\section{II-C-8 Tat CTL Epitope Map}
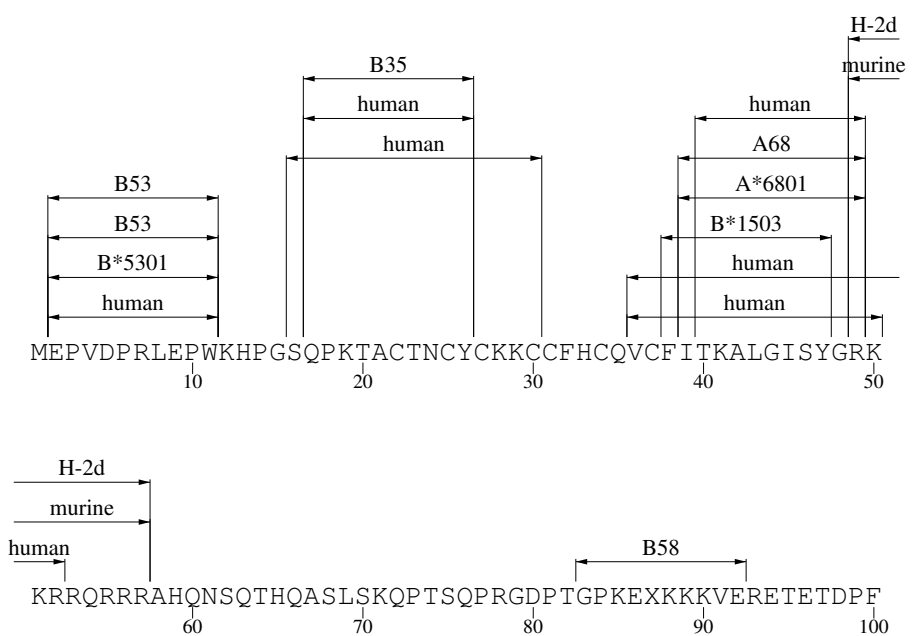

$\underset{1}{D}$ 


\section{II-C-9 Vif CTL Epitope Map}
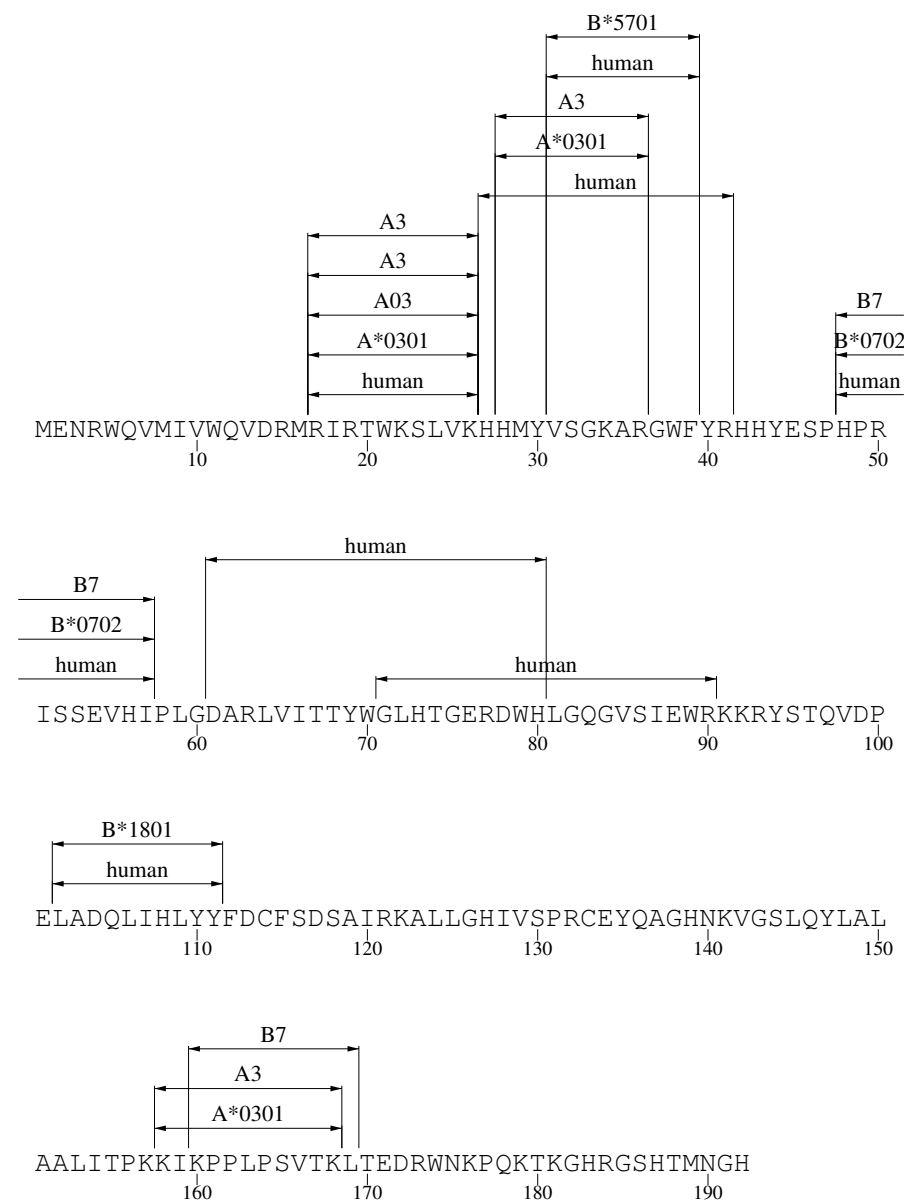

$170 \quad 18$

\section{II-C-10 Vpr CTL Epitope Map}
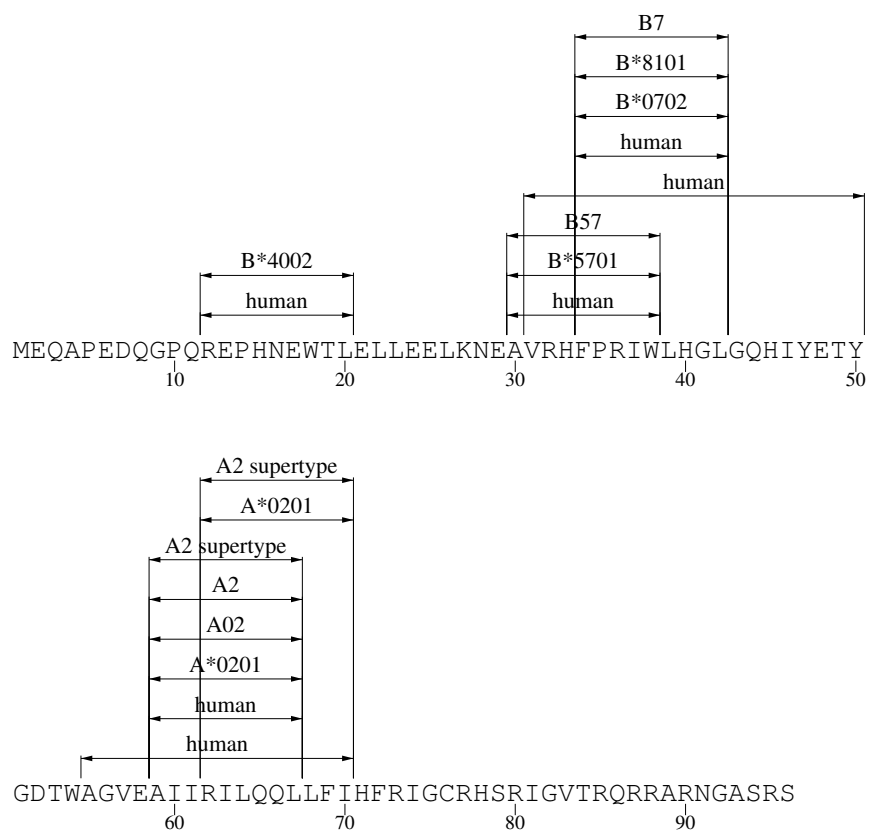


\section{II-C-11 Vpu CTL Epitope Map}

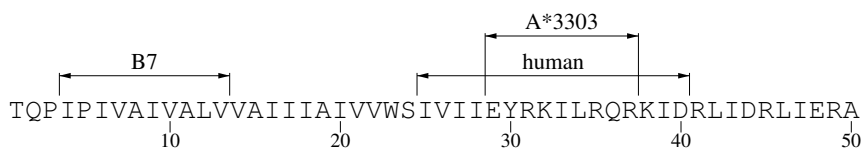

EDSGNESEGE ISALVEMGVEMGHHAPWDVDDI

\section{II-C-12 gp160 CTL Epitope Map}
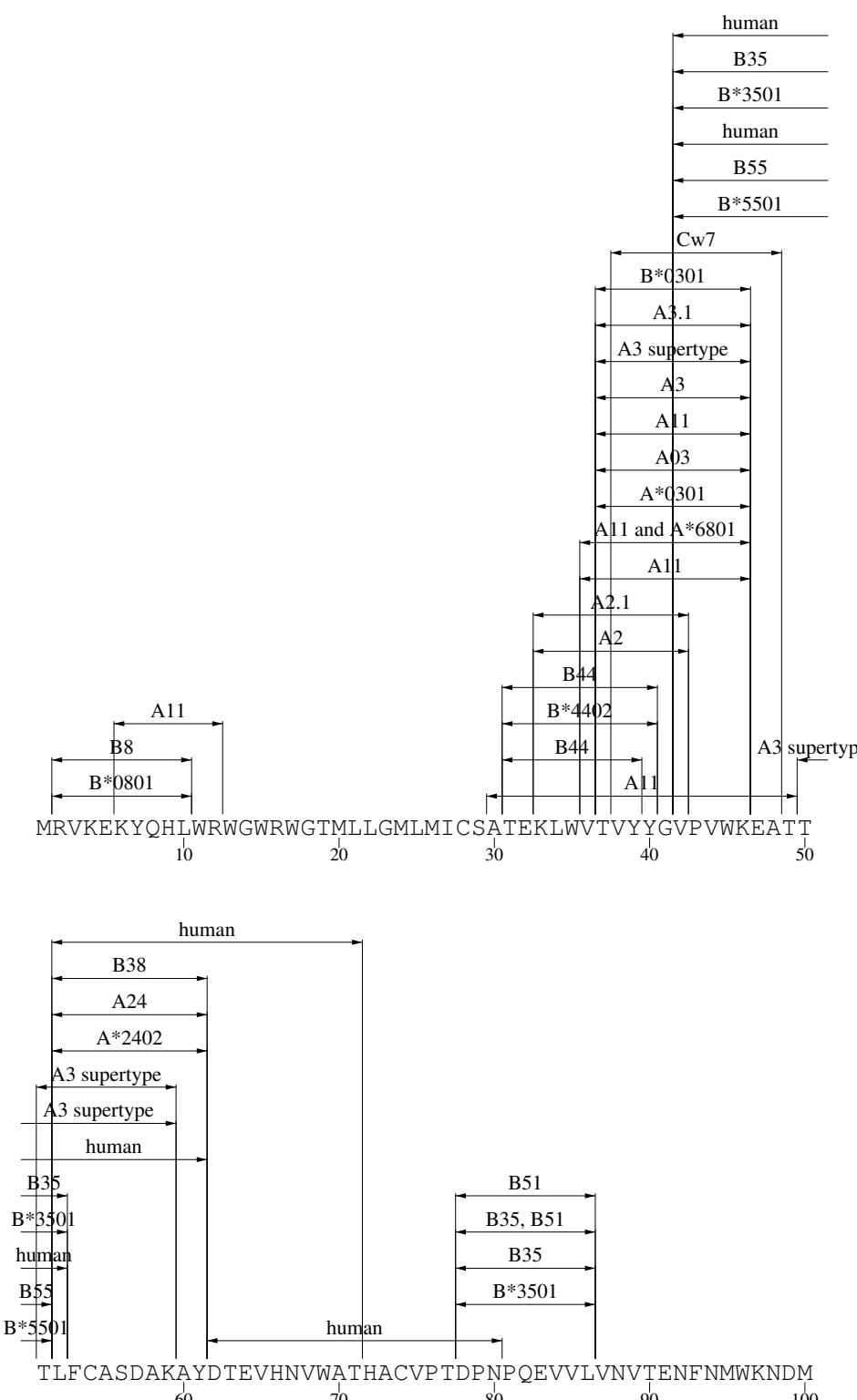

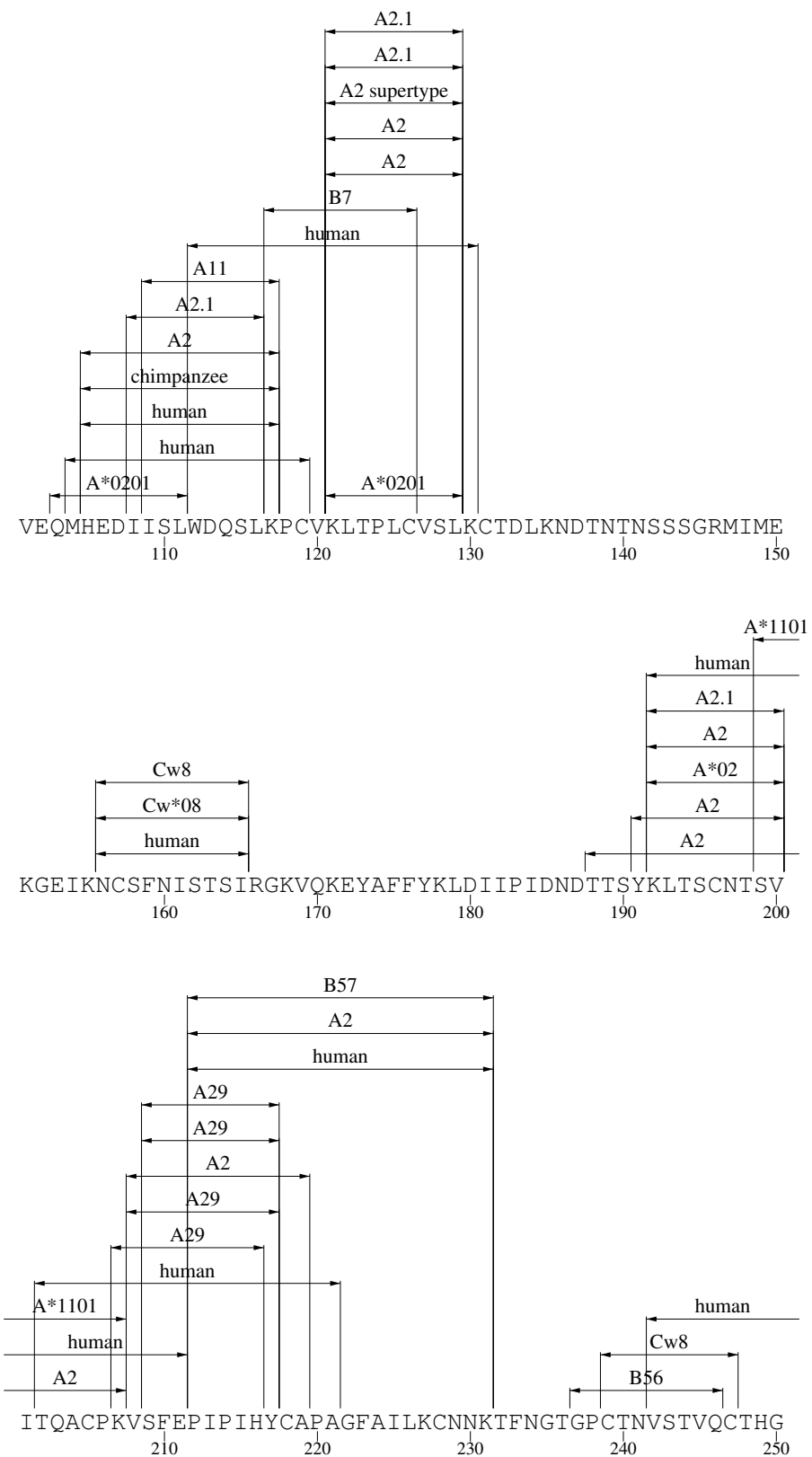

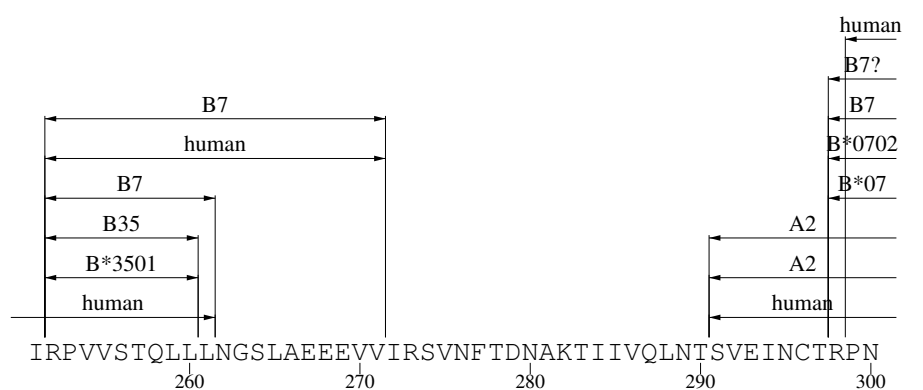

280 

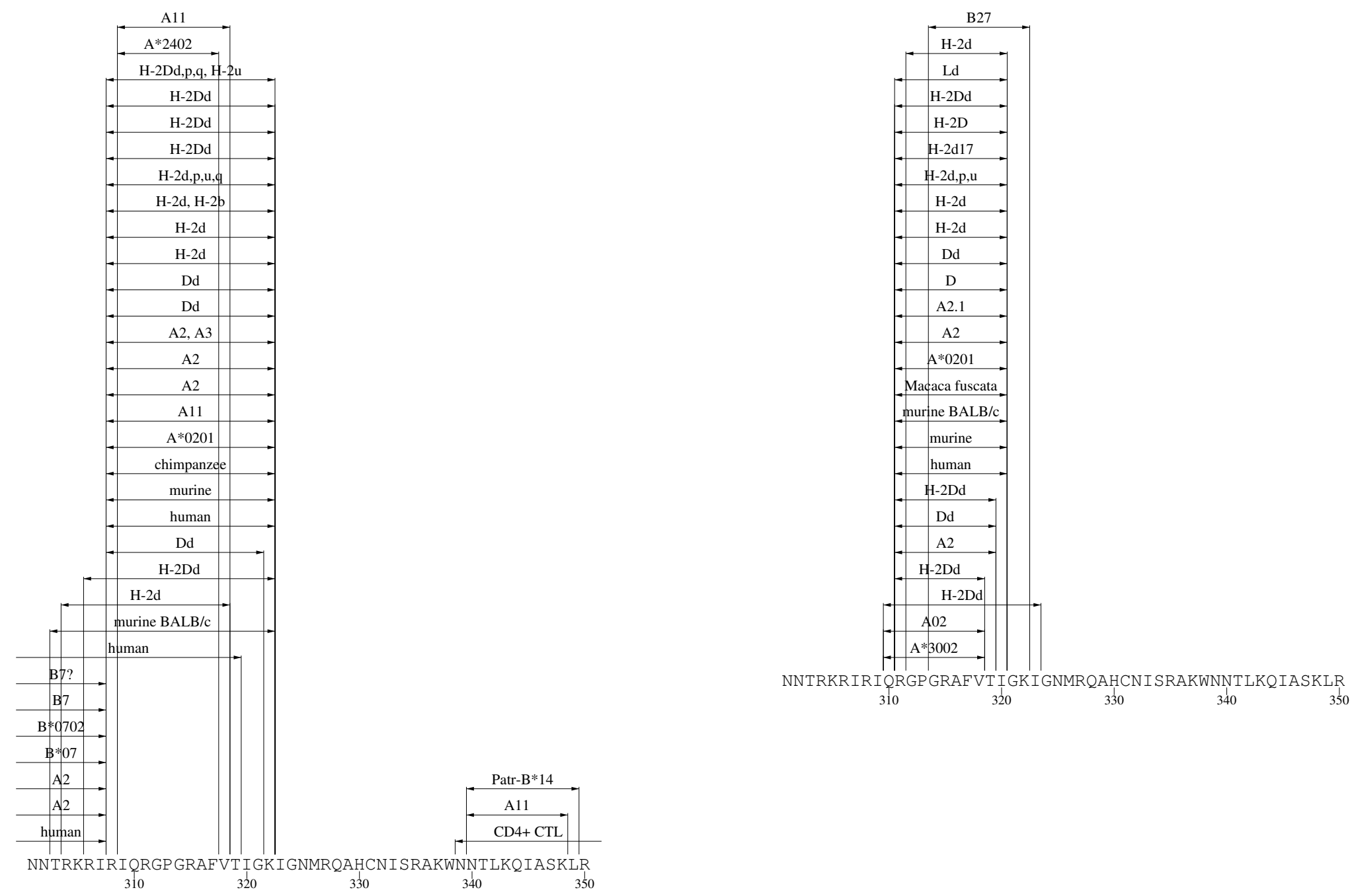

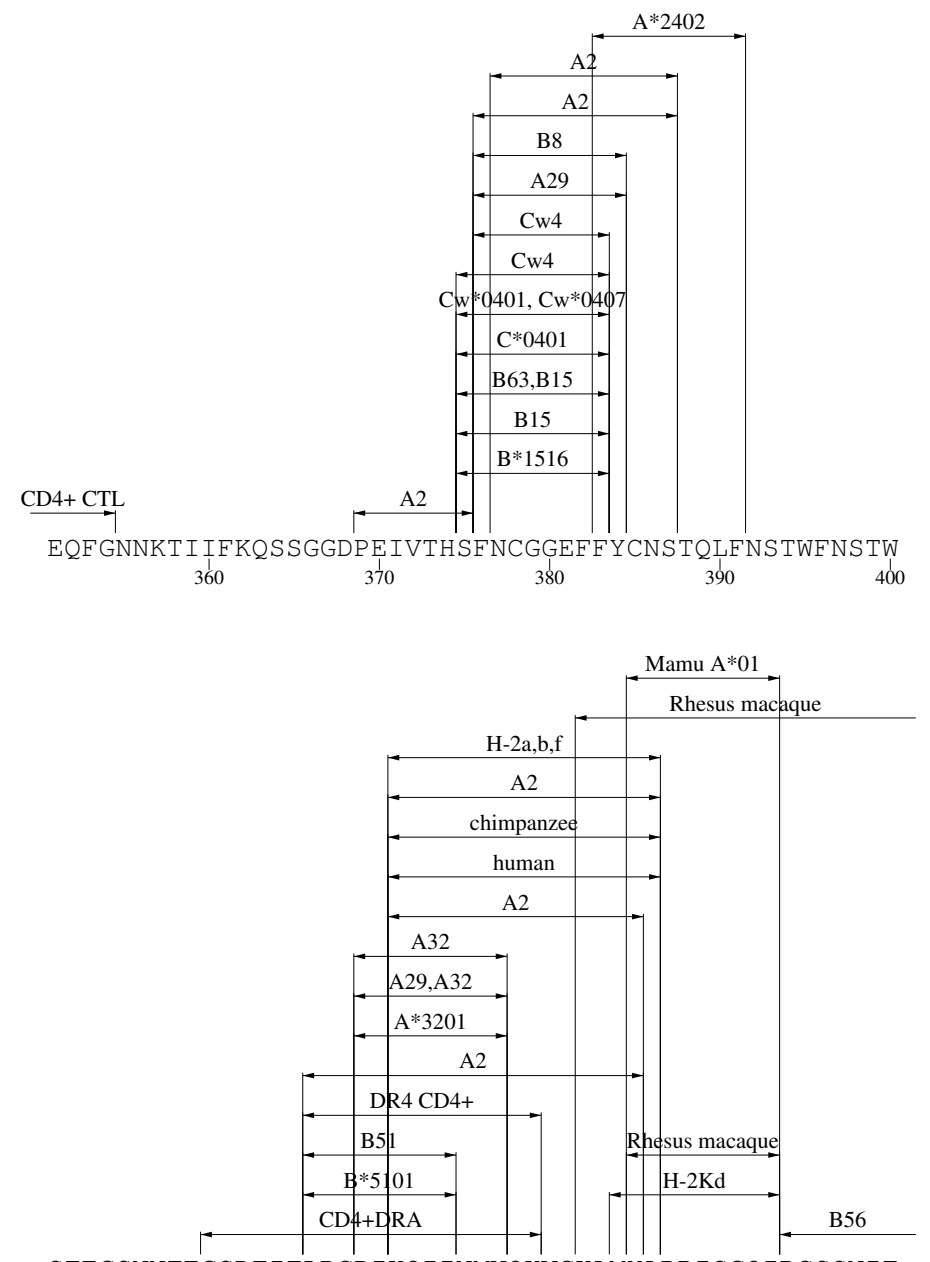

$\begin{array}{cccc}\text { STEGSNNTEGSDDT ITLPCRIKQI INMWQKVGKAMYAPP I SGQ IRCSSNIT } \\ 410 & 420 & 430 & 440\end{array}$$$
420
$$

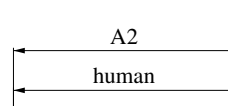

Rhesus macaque

GLLLTRDGGNSNNESEIFRPGGGDMRDNWRSELYKYKVVKIEPLGVAPTK

$$
470
$$$$
480
$$

$$
\underset{\text { AKRRVVQRE }}{\stackrel{\mathrm{A} 2}{\stackrel{\text { human }}{\longrightarrow}}}
$$

AKRRVVQREKRAVG I GALF LGF LGAAGSTMGAASMTLTVQARQLLSGIVQ

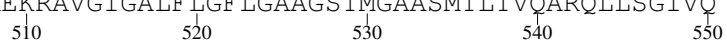
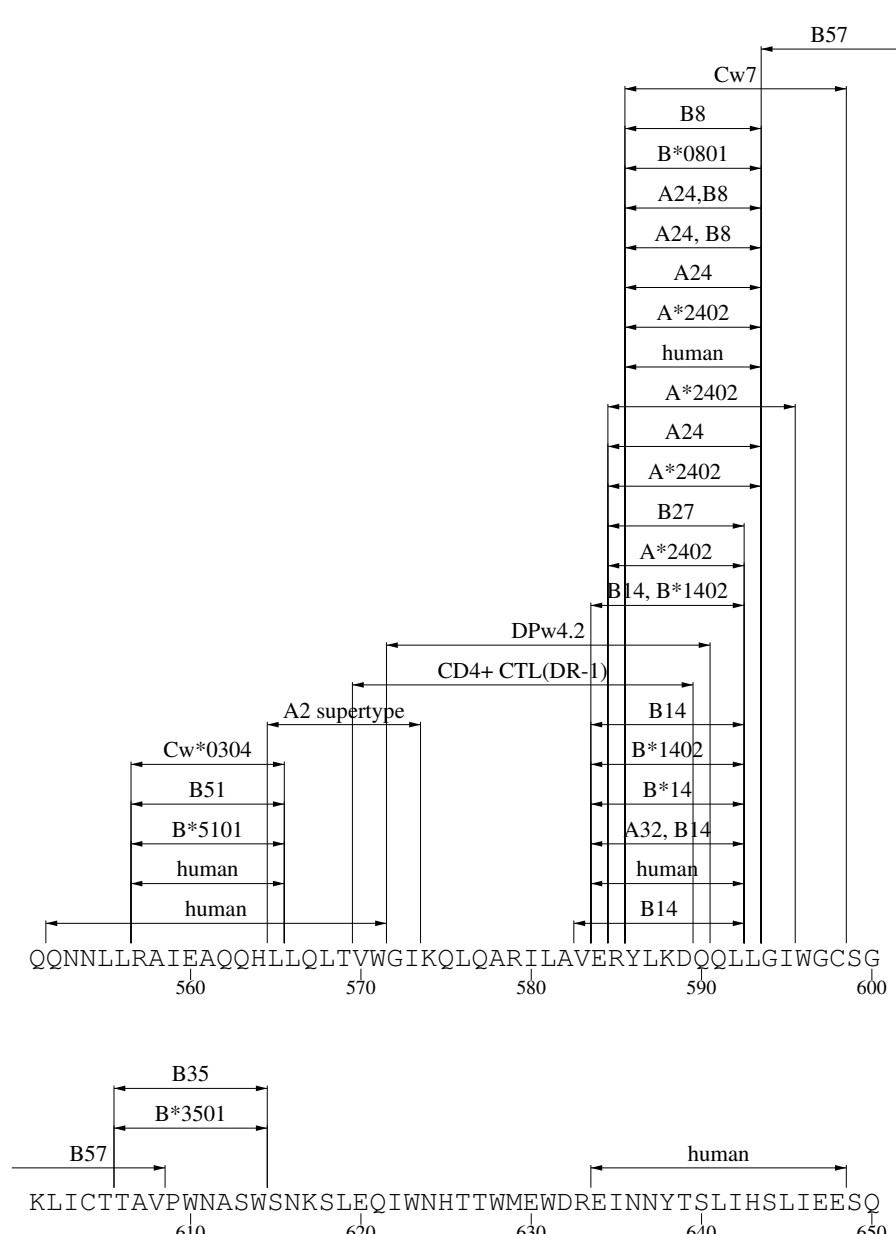

610 

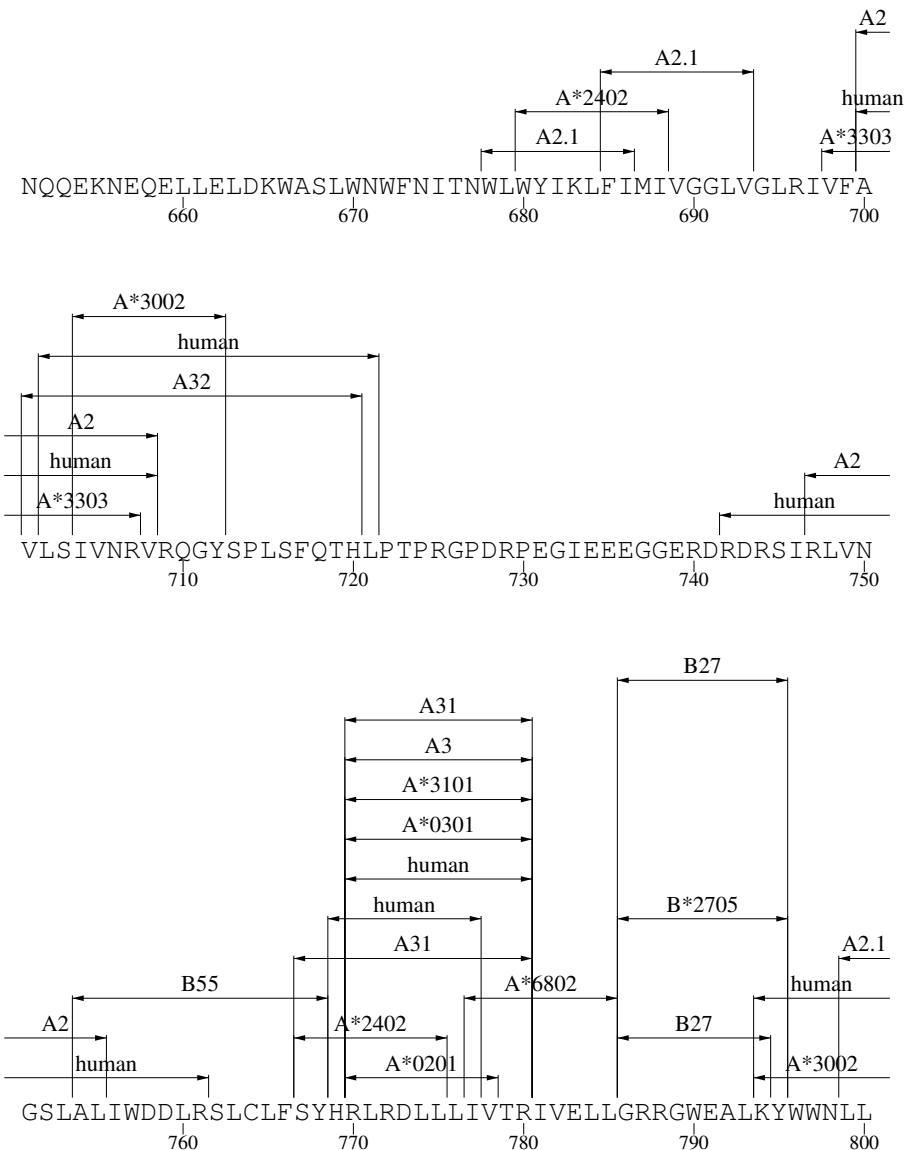

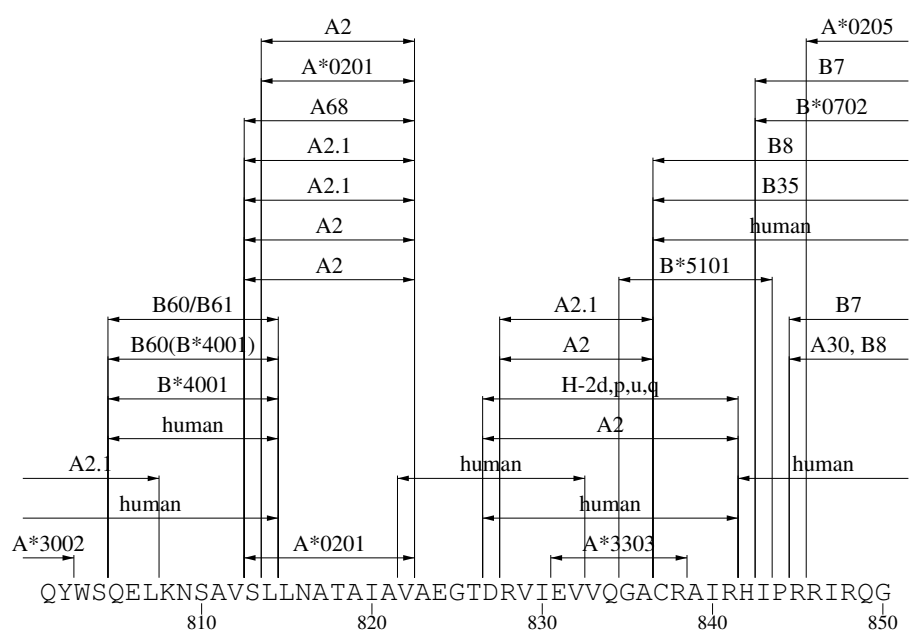

$$
\begin{aligned}
& \stackrel{\mathrm{A} * 0205}{\mathrm{~B} 7} \\
& \text { A30, B } 8 \\
& \stackrel{\mathrm{B} 7}{\rightarrow} \\
& \begin{array}{c}
\text { human } \\
\text { B8 }
\end{array} \\
& \text { B35 } \\
& \text { human } \\
& \begin{array}{r}
\text { LERILL } \\
856
\end{array}
\end{aligned}
$$




\section{II-C-13 Nef CTL Epitope Map}
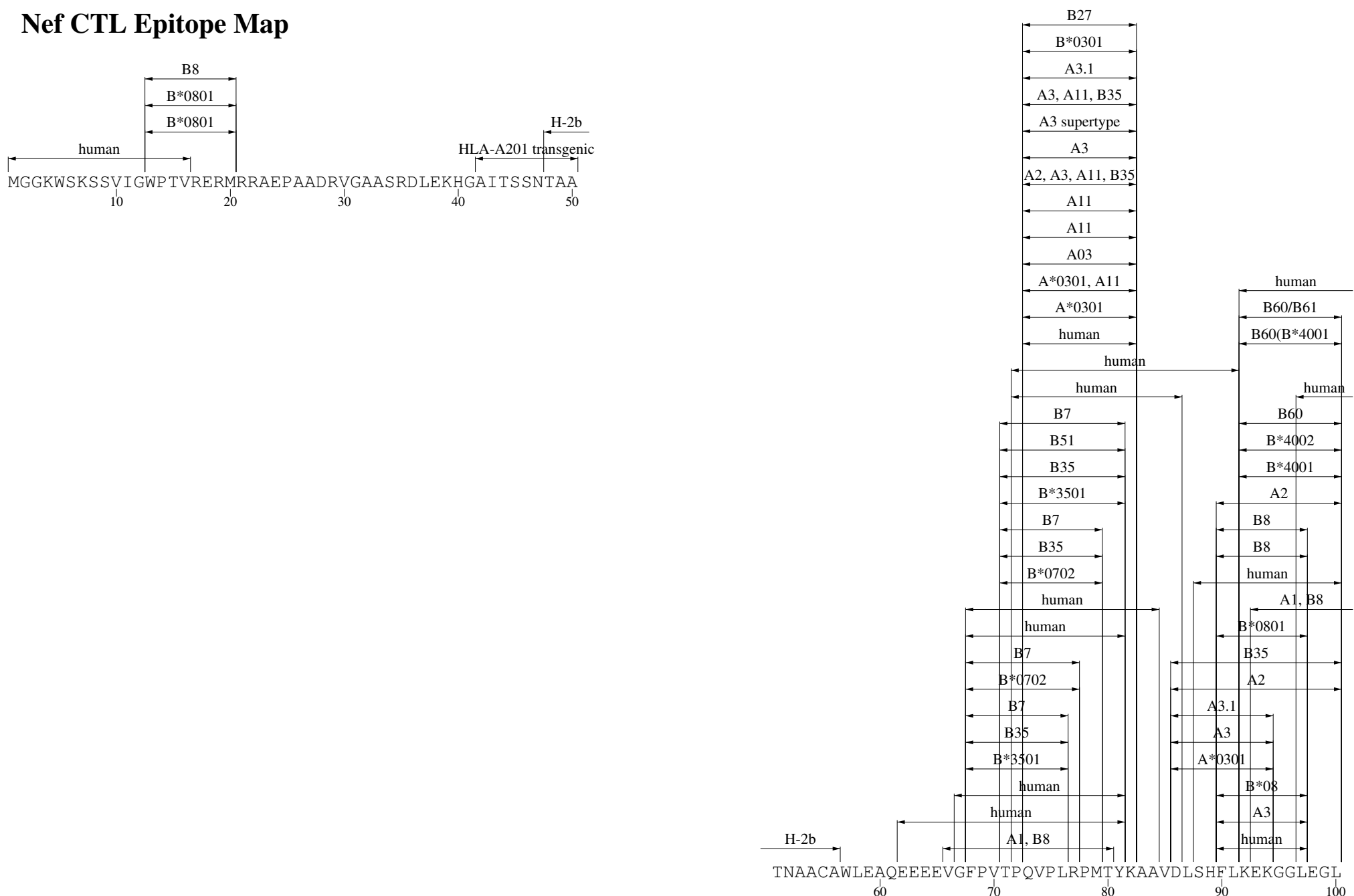

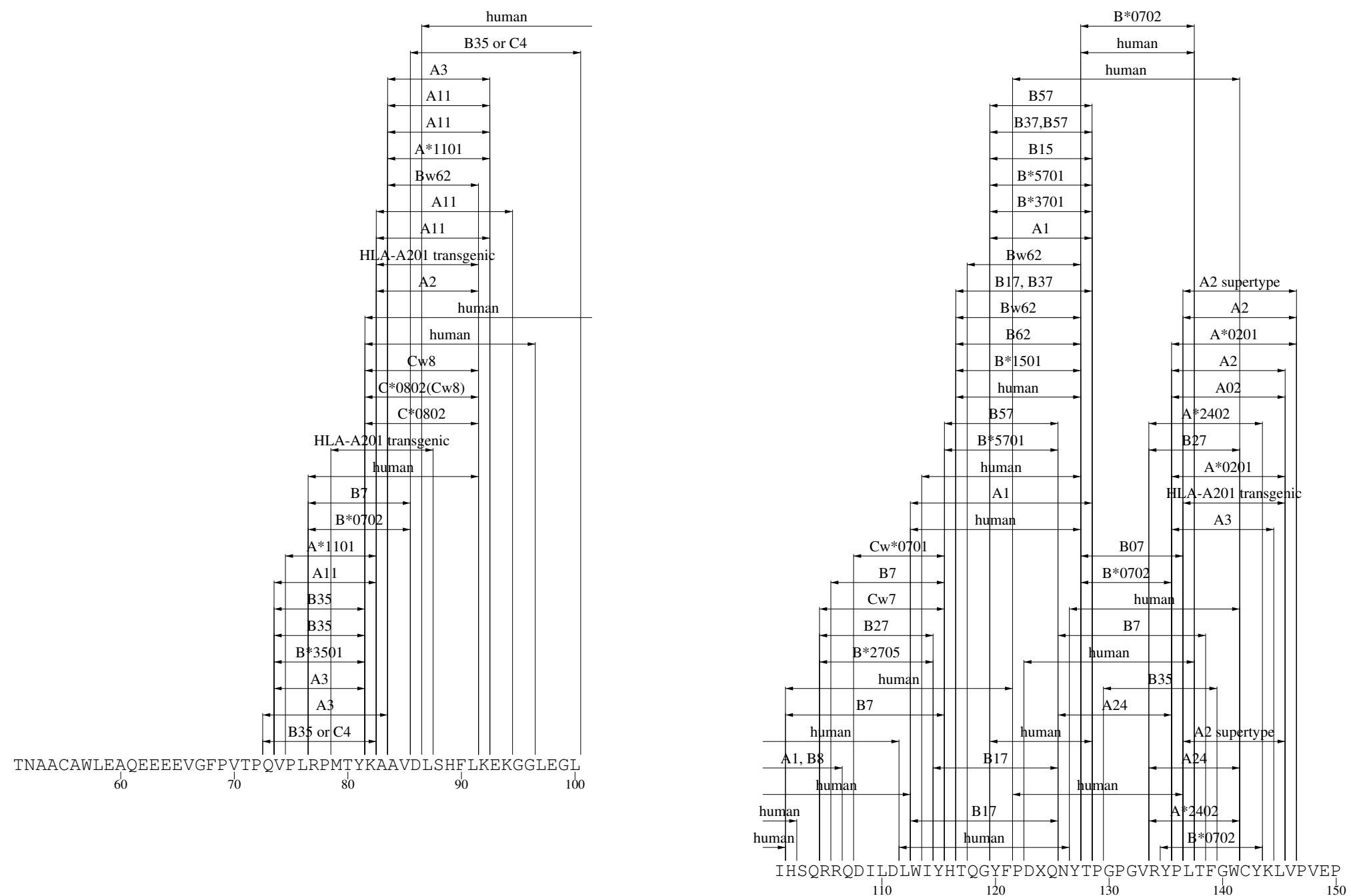


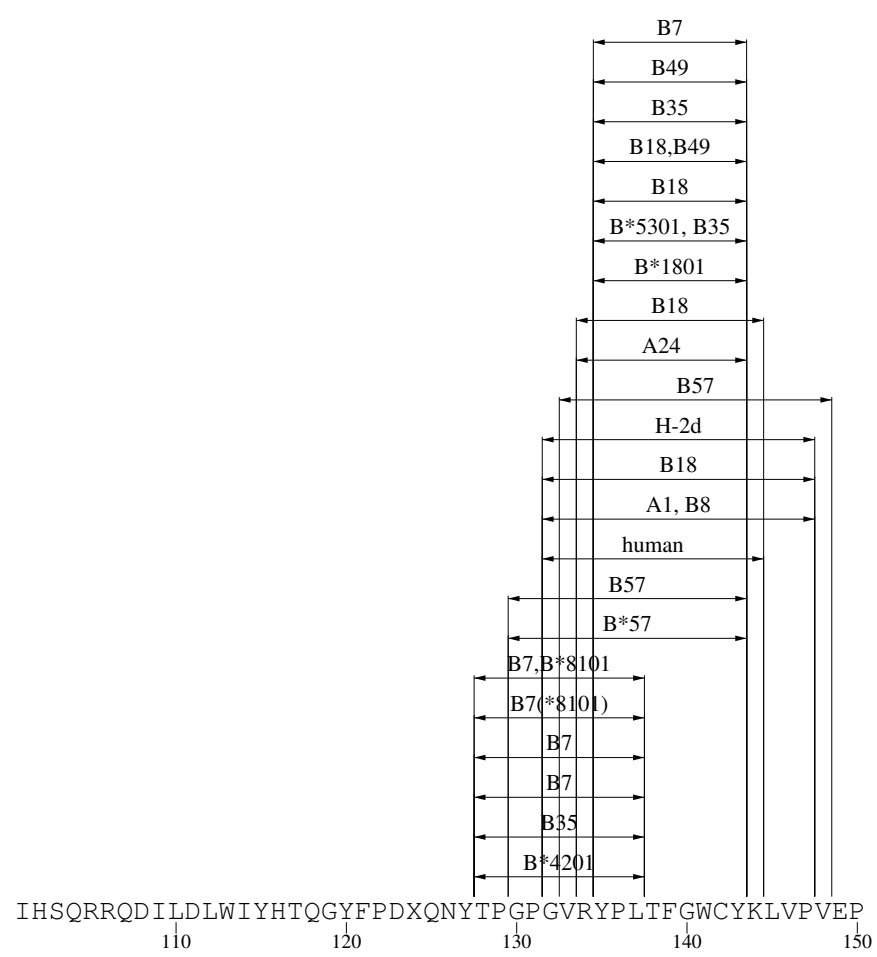




\section{Part III}

\section{HIV Helper T-Cell Epitopes}





\section{III-A Summary}

Part III includes tables and maps of HIV-specific helper T-cell (Th) epitopes arranged sequentially according to the location of the proteins in the HIV-1 genome. This section parallels the organization of the CTL section. We attempted to make this section as comprehensive as possible, requiring that the epitope be contained within a region of 30 amino acids maximum, but not that the precise boundaries be defined. The HLA specificity is usually not determined for Th epitopes. For more recent updates, epitope sequence alignments, and useful searching capabilities, please see our web site: http:/ / hiv-web. lanl . gov/immunology. The same epitope can have multiple entries, as each en try represents a single publication. Helper T-cell responses to proteins with no defined epitope are described at the end of each protein section.

Recent studies utilize multiple functions attributed to $\mathrm{T}$ cells to define responses, and the simple distinctions of cytotoxic T-cell and helper T-cells have become blurred as more is learned about the range of responses triggered in CD4 and CD8 positive T-cells responding to antigenic stimulus. When adding the most recent studies to the database, we have tried to place $\mathrm{T}$ cell responses in a reasonable manner into our traditional helper T cell and CTL sections, and to specify the assay used to measure the response in each study.

\section{III-A-1 Tables}

Each Th epitope has a six-part basic entry:

HXB2 Location: The viral strain HXB2 (GenBank Accession Number K03455) is used as a reference strain throughout this publication. The position of the defined epitope location relative to the sequence of the HXB2 protein is indicated. The numbering in this table corresponds to the protein maps. Because of HIV-1 variation the epitope may not actually be present in HXB2, rather the position in HXB2 indicates the position aligned to the epitope. HXB2 was selected as the reference strain because so many stud ies use HXB2, and because crystal structures for HXB2-related protein are often available. The precise positions of an epitope on the HXB2 reference strain can be readily obtained using the interactive position locator a our web site: http://hiv-web.lanl.gov/content/hiv-db/ LOCATE_SEQ/locate.html.
Author Location: The amino acid positions of the epitope boundaries and the reference sequence are listed as given in the primary publication. Frequently, these positions as published are imprecise, and do not truly correspond to the numbering of the sequence, but they provide a reasonable guide to the peptide's approximate location in the protein. Also, in many cases the reference sequence identification was not provided, and in such cases it is not possible to use these numbers to specify precise locations.

Epitope Sequence: The amino acid sequence of the epitope of interest as defined in the reference, based on the reference strain used in the study defining the epitope. On occasions when only the position numbers and not the actual peptide sequence was specified in the original publication, we tried to fill in the peptide sequence based on the position numbers and reference strain. If the sequences were numbered inaccurately by the primary authors, or if we made a mistake in this process, we may have misrepresented the binding site's amino acid sequence. Because of this uncertainty, epitopes that were not explicitly written in the primary publication, that we determined by looking up the reference strain and the numbered location, are followed by a question mark in the table.

Immunogen: The antigenic stimulus of the Th response to the defined epitope. Often this is an HIV-1 infection. If a vaccine was used as the original antigenic stimulation, not a natural infection, this is noted on a separate line, and additional information about the vaccine antigen is provided as available.

Species(HLA): The species responding and HLA specificity of the epitope, when known.

Reference: The primary reference (sometimes two or more directly related studies are included). Details for some of the earlier references are in Part $\mathrm{V}$

Following the entry for a given Th epitope are brief comments explaining the context in which the epitope was studied and what was learned about the epitope in a given study. 


\section{III-A-2 HIV Protein Epitope Maps}

All HIV Th epitopes mapped to within a region of 21 amino acids or less are indicated on the HIV protein epitope maps. The location and HLA restriction elements of Th epitopes are indicated on protein sequences of HXB2. These maps are meant to provide the relative location of defined epitopes on a given protein, but the HXB2 sequence may not actually carry the epitope of interest, as it may vary relative to the sequence for which the epitope was defined. Epitopes with identical boundaries and HLA fields are included in the maps only once. If one laboratory determines HLA presenting molecules at the serotype level (example: A2) and another at the genotype level (example: A*0201) both will be included in the map. MHC specificities are indicative of the host species; when no MHC presenting molecule is defined, the host species is noted.

\section{III-A-3 Alignments}

To conserve space, no epitope alignments are provided in this book, but they can be generated using the Thelper epitope search tool at http://hiv-web. lanl.gov/immunology. All epitopes are aligned to the HXB2 sequence, with the sequence used to define the epitope indicated directly above it. Sequences are sorted by their subtype and country of origin.

The master alignment files from which the epitope alignments were created are available at our web site (http://hiv-web.lanl.gov/ALIGN_ CURRENT/ALIGN-INDEX.html). The alignments were modified in some cases to optimize the alignment relative to the defined epitope and minimize insertions and deletions; epitope alignments are generated by anchoring on the C-terminal residue. A dash indicates identity to the consensus sequence, and a period indicates an insertion made to maintain the alignment. Stop codons are indicated with a $\$$, and frameshifts by a \#, or ambiguous codons (nucleotide was $r, y$, or $n$ ) by an $x$; they are inserted to maintain the alignments. In consensus sequences an upper case letter indicates the amino acid was present in all sequences, a lower case letter indicates the amino acid was present in most sequences in a given position, and a question mark indicates two or more amino acids were represented with equal frequency. 


\section{III-B HIV Helper T-Cell Epitope Tables}

All HIV Helper T-Cell epitopes arranged by protein position. The table entries are sorted in a nested way-first by protein, then by HXB2 start location within the protein and finally by HLA presenting molecule. Epitopes for which the HXB2 location is unknown appear at the end of the listing of the protein in which they are located.

\section{III-B-1 p17 Helper T-Cell Epitopes}

\begin{tabular}{lllll}
\hline HXB2 Location & Author's Location & Sequence & Immunogen & Species (HLA) \\
\hline p17 (18-42) & p17 (18-42 PV22) & KIRLRPGGKKKYKLKHIVW- & HIV-1 infection & human (DRB1*13) \\
& & ASRELE & Lotti2002
\end{tabular}

10/49 chronically HIV-1 infected patients had low p55-Gag-specific CD4+ T cell responses prior to therapy, and these responses remained unchanged 3 and 6 months after initiation of HAART. There was no difference in level of response in those with or without a detectable p55 response.

- For one individual, patient F45 CDC stage A2, CD4+ p55 responding clones were generated. Her response was consistently strong and heterogeneous in terms of HLA restriction and Vbeta usage, and some clones had a Th1 cytokine secretion profile (high IFNgamma production) while some had a Th2 profile (high IL-4 and IL-5 production). 5/10 CD4+ clones could also induce cytotoxicity.

- 4/10 clones from patient F45 had their epitopes mapped using overlapping peptides. Clone 6 recognized this peptide sequence restricted by DRB $1 * 13$. This clone had a high SI (27.1 to p55, 90.6 to peptide) secreted IFNgamma, indicative of a Th1 response, as well as TNFalpha. Clone 6 was highly cytotoxic, through a perforin-mediated pathway.

p17 (21-35) p17(21-35 SF2) LRPGGKKKYKLKHIV HIV-1 infection human (DR13.02) Harcourt1998

- 43 asymptomatic HIV+ individuals were screened for proliferative responses to HIV - 12 showed a response, and dominant epitopes were mapped for two individuals, one in p24 and one in p17

- Patient 024's naturally occurring variant LRPGGKKKYQLKHIV also elicited a strong proliferative response.

- Naturally occurring variants of this epitope were found within the individual who made this response - several did not stimulate the CD4+ T-cell line that recognized the index peptide, suggestive of immune escape

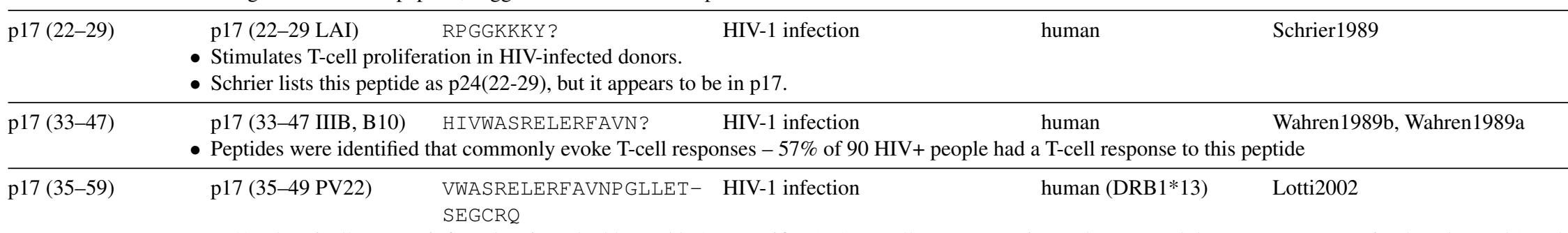

- 10/49 chronically HIV-1 infected patients had low p55-Gag-specific CD4+ T cell responses prior to therapy, and these responses remained unchanged 3 and 6 months after initiation of HAART. There was no difference in level of response in those with or without a detectable p55 response.

- For one individual, patient F45 CDC stage A2, CD4+ p55 responding clones were generated. Her response was consistently strong and heterogeneous in terms of HLA restriction and Vbeta usage, and some clones had a Th1 cytokine secretion profile (high IFNgamma production) while some had a Th2 profile (high IL-4 and IL-5 production). 5/10 CD4+ clones could also induce cytotoxicity. 


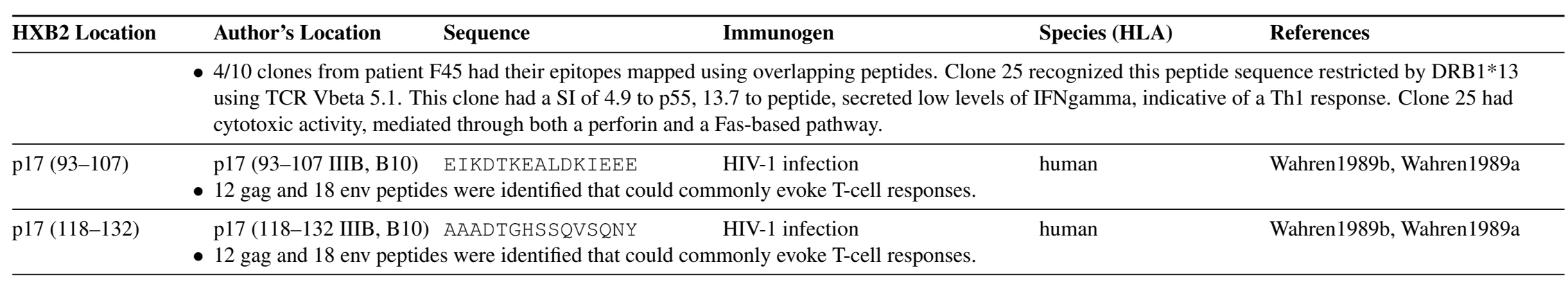




\section{III-B-2 p24 Helper T-Cell Epitopes}

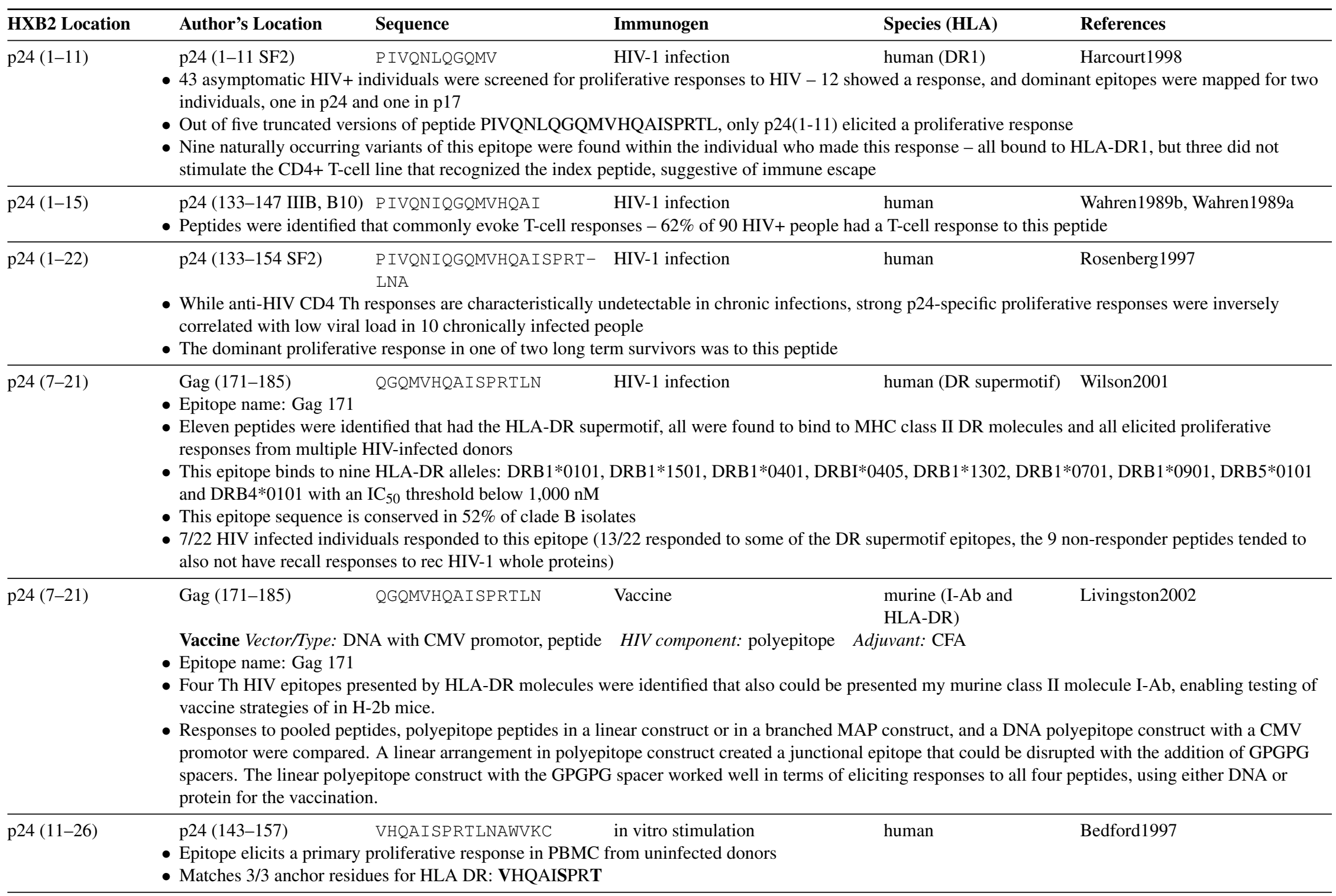




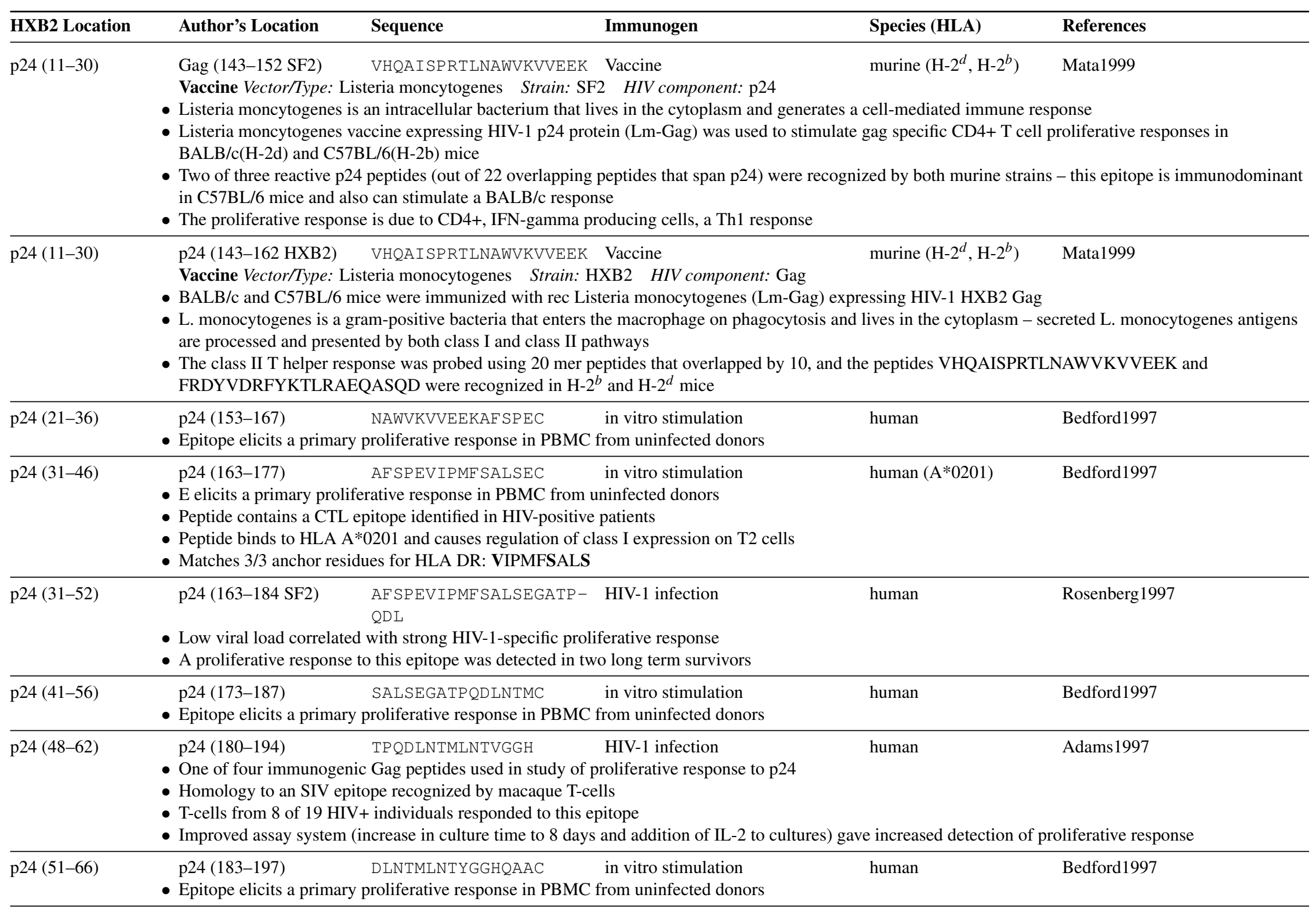




\begin{tabular}{|c|c|c|c|c|}
\hline HXB2 Location & Author's Location & Immunogen & Species (HLA) & References \\
\hline p24 (51-82) & $\begin{array}{l}\text { Gag (183-214 LAI) } \\
\text { Vaccine Vector/Type: lip } \\
\text { - Anti-HIV lipopeptide vac } \\
\text { administered in a phase I } \\
\text { - A CD4+ T cell proliferati } \\
\text { - } 9 / 12 \text { tested mounted a CT } \\
\text { - None of the } 12 \text { tested had }\end{array}$ & $\begin{array}{l}\text { DLNTMLNTVGGHQAAMQML- Vaccine } \\
\text { KETINEEAAEWDR } \\
\text { opeptide } \\
\text { cine consisting of six long peptides derived from Nef } \\
\text { trial } \\
\text { ve response to at least one of the six peptides was obs } \\
\text { L responses to at least one of the six peptides, each o } \\
\text { an IgG response to this peptide }\end{array}$ & $\begin{array}{l}\text { human } \\
\text { IIV-1 proteins modifi } \\
\text { scinees - } 2 / 10 \text { reacte } \\
\text { s elicited a CTL resp }\end{array}$ & $\begin{array}{l}\text { Gahery-Segard2000 } \\
\text { a palmitoyl chain was } \\
\text { his peptide } \\
\mathrm{n} \text { at least one individual }\end{array}$ \\
\hline p24 (69-88) & $\begin{array}{l}\text { Gag (p24) (201-220 } \\
\text { IIIB) } \\
\text { - Epitope name: P21 } \\
\text { - PBMC from a seronegati } \\
\text { Gag-specific CD4+ T-cel } \\
\text { dilution. All reacted with } \\
\text { - Clone } 85 \text { recognized this } \\
\text { clone was only activated }\end{array}$ & $\begin{array}{l}\text { LKETINEEAAEWDRVHPVHA in vitro stimulation } \\
\text { ve donor, the healthy brother of a pair of monozygotic } \\
\text { clones by in vitro immunization with HIV-1 overlapp } \\
\text { p24 except one which recognized a p } 24 \text { peptide and a } \\
\text { peptide using TCR Vbeta } 8 \text { and } 18 \text {; the two TCR rece } \\
\text { by peptide, not by processed protein from vaccinia vir }\end{array}$ & $\begin{array}{l}\text { human (DR) } \\
\text { t for HIV-1 infection } \\
\text { ides spanning p55. S } \\
\text { CD4+ T cell clones } \\
\text { his limiting dilution } \\
\text { B-LCL. }\end{array}$ & $\begin{array}{l}\text { Venturini2002 } \\
\text { re used to generate HIV-1 } \\
\text { ones were generated by limiting } \\
\text { HLA clas II DR restricted. } \\
\text { sents a mixed population. This }\end{array}$ \\
\hline p24 (71-86) & $\begin{array}{l}\text { p24 (203-217) } \\
\text { - Epitope elicits a primary }\end{array}$ & $\begin{array}{l}\text { ETINEEAAEWDRVHPC } \\
\text { proliferative response in PBMC from uninfected dono }\end{array}$ & human & Bedford1997 \\
\hline p24 (73-97) & $\begin{array}{l}\text { p24 (205-229 PV22) } \\
\text { - } 10 / 49 \text { chronically HIV-1 } \\
6 \text { months after initiation } \\
\text { - For one individual, patien } \\
\text { terms of HLA restriction } \\
\text { profile (high IL-4 and IL- } \\
\text { - 4/10 clones from patient } \\
\text { using TCR Vbeta } 22 \text {. Thi } \\
\text { cytotoxic activity, mediat }\end{array}$ & $\begin{array}{l}\text { INEEAAEWDRVHPVHAGP I- HIV-1 infection } \\
\text { APGQMR } \\
\text { infected patients had low p55-Gag-specific CD4+ T ce } \\
\text { f HAART. There was no difference in level of respon } \\
\text { it F } 45 \text { CDC stage A2, CD4+ p55 responding clones w } \\
\text { and Vbeta usage, and some clones had a Th1 cytokine } \\
5 \text { production). 5/10 CD4+ clones could also induce cy } \\
\text { F45 had their epitopes mapped using overlapping pept } \\
\text { s clone had a SI of } 12.4 \text { to p55, } 49.6 \text { to peptide, secret } \\
\text { ed through both a perforin and a Fas-based pathway. }\end{array}$ & $\begin{array}{l}\text { human }(\mathrm{DRB} 1 * 03) \\
\text { or to therapy, and the } \\
\text { or without a detectal } \\
\text { fer response was con } \\
\text { le (high IFNgamma } \\
\text { recognized this pepti } \\
\text { IFNgamma, indicati }\end{array}$ & $\begin{array}{l}\text { Lotti2002 } \\
\text { sponses remained unchanged } 3 \text { and } \\
55 \text { response. } \\
\text { tlly strong and heterogeneous in } \\
\text { ction) while some had a Th2 } \\
\text { quence restricted by DRB } 1 * 03 \\
\text { a Th1 response. Clone } 12 \text { had }\end{array}$ \\
\hline p24 (76-85) & $\begin{array}{l}\text { p24 (208-217) } \\
\text { - One of four immunogeni } \\
\text { - T-cells from } 11 \text { of } 24 \mathrm{HI} \\
\text { - Improved assay system (i }\end{array}$ & $\begin{array}{l}\text { EAAEWDRVHP HIV-1 infection } \\
\text { Gag peptides used in study of the proliferative resp } \\
+ \text { individuals responded to this epitope } \\
\text { ncrease in culture time to } 8 \text { days and addition of IL- } 2\end{array}$ & $\begin{array}{l}\text { human } \\
\text { e increased detection }\end{array}$ & $\begin{array}{l}\text { Adams1997 } \\
\text { oliferative response }\end{array}$ \\
\hline p24 (76-90) & $\begin{array}{l}\text { p24 (208-222 IIIB, B10) } \\
\text { - } 12 \text { gag and } 18 \text { env peptid }\end{array}$ & $\begin{array}{l}\text { EAAEWDRVHPVHAGP HIV-1 infection } \\
\text { es were identified that could commonly evoke T-cell } r\end{array}$ & human & Wahren1989b Wahren1989a \\
\hline p24 (81-95) & $\begin{array}{l}\text { p24 (215-229 SF2) } \\
\text { Vaccine Vector/Type: vir } \\
\text { - Responses to } 3 \text { T-cell and }\end{array}$ & 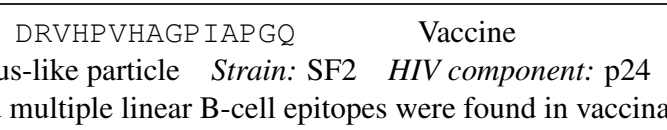 & macaque & Mills1990 \\
\hline
\end{tabular}




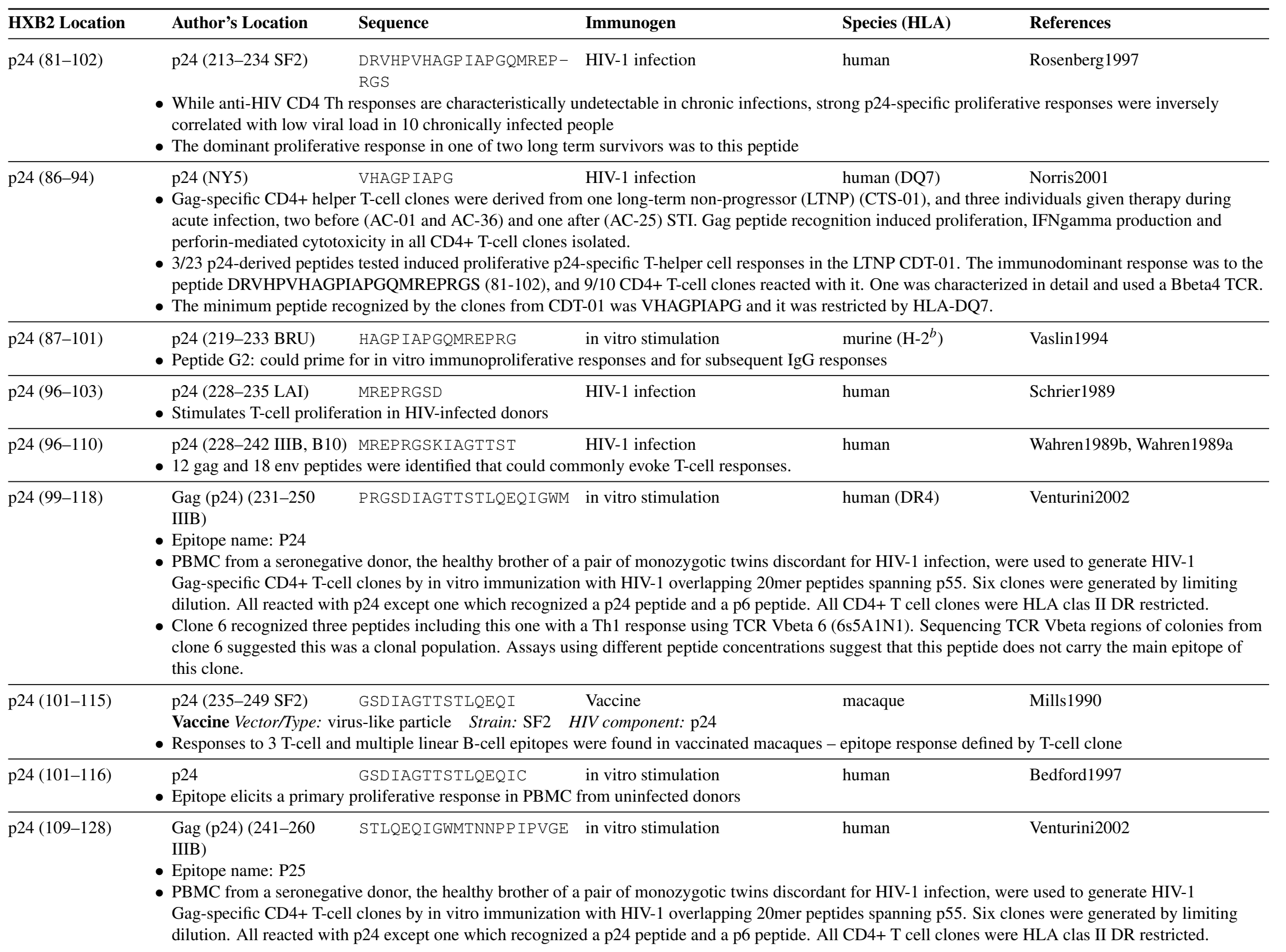




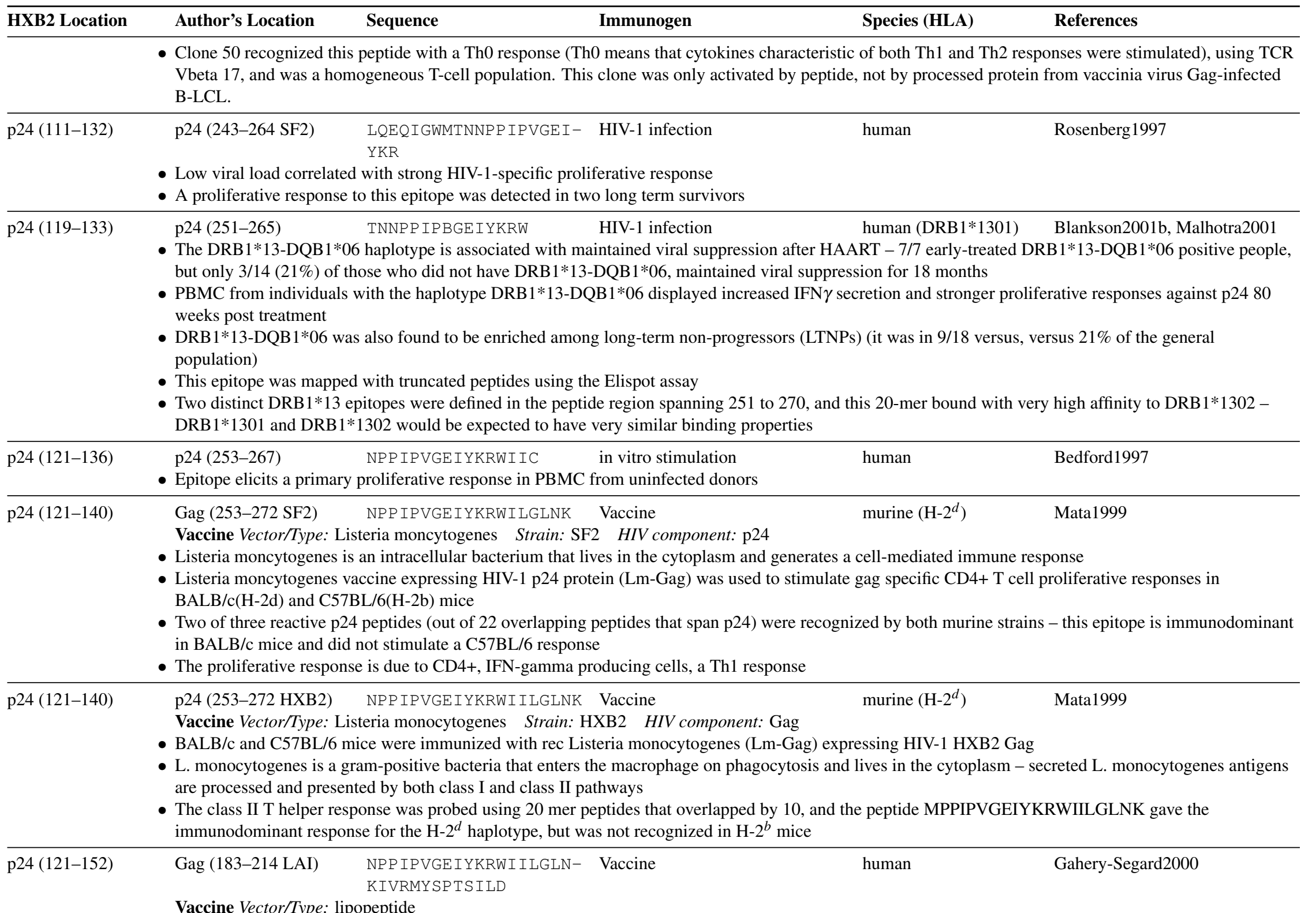

- Anti-HIV lipopeptide vaccine consisting of six long peptides derived from Nef, Gag and Env HIV-1 proteins modified by a palmitoyl chain was administered in a phase I trial 


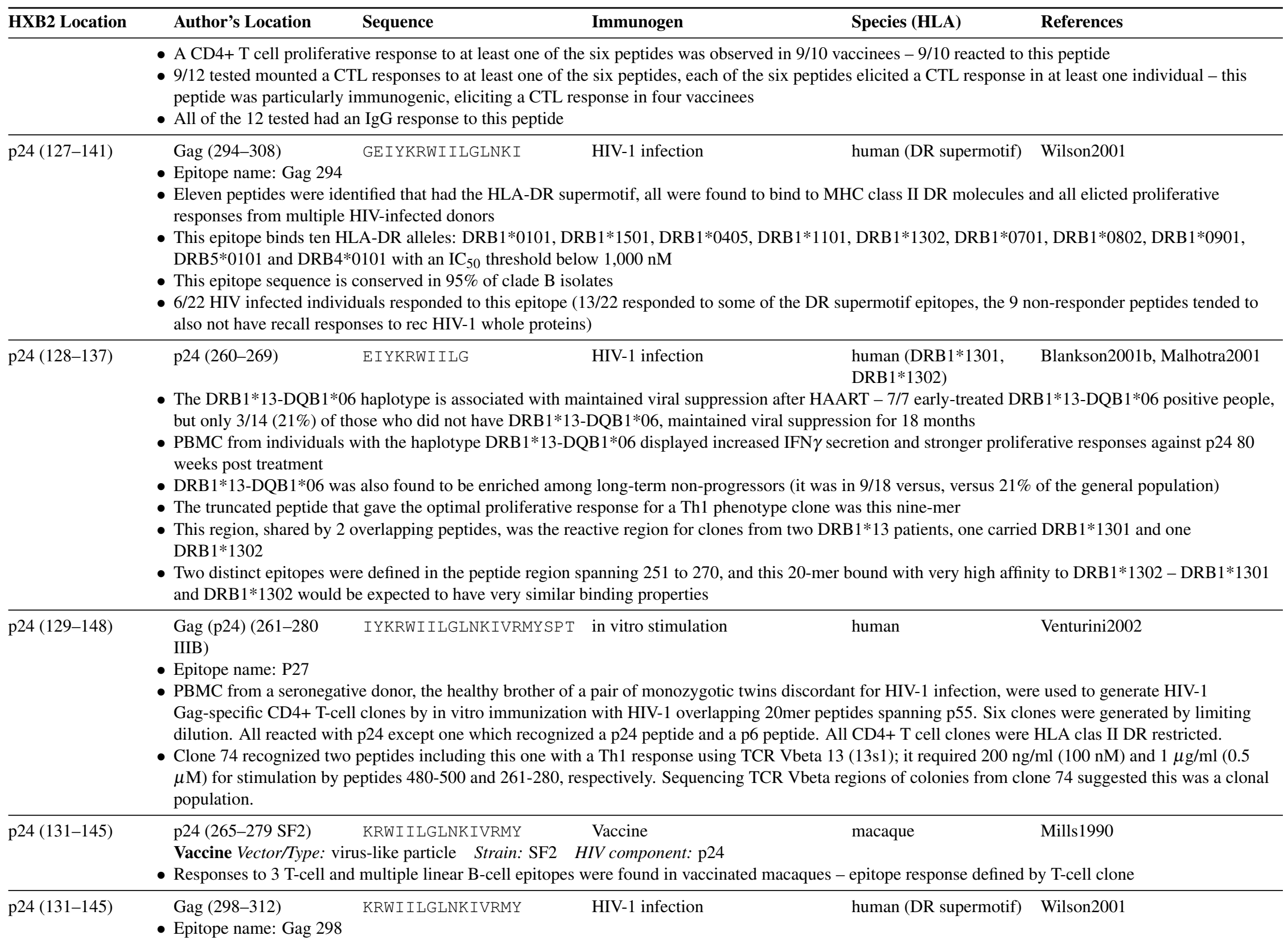




\begin{tabular}{|c|c|c|c|c|}
\hline HXB2 Location & Author's Location & Immunogen & Species (HLA) & References \\
\hline & \multicolumn{4}{|c|}{$\begin{array}{l}\text { - Eleven peptides were identified that had the HLA-DR supermotif, all were found to bind to MHC class II DR molecules and all elicited proliferative } \\
\text { responses from multiple HIV-infected donors } \\
\text { - This epitope binds thirteen HLA-DR alleles: DRB } 4 * 0101, \mathrm{DRB} 5 * 0101, \mathrm{DRB} 1 * 0901 \text {, DRB } 1 * 0802, \mathrm{DRB} 1 * 0701, \mathrm{DRB} 1 * 1302, \mathrm{DRB} 1 * 1201, \mathrm{DRB} 1 * 1101 \text {, } \\
\text { DRB } 1 * 0405, \mathrm{DRB} 1 * 0401, \mathrm{DRB} * 0301, \mathrm{DRB} 1 * 1501 \text { and DRB } 1 * 0101 \text {, with an } \mathrm{IC}_{50} \text { threshold below } 1,000 \mathrm{nM} \\
\text { - This epitope sequence is conserved in } 94 \% \text { of clade B isolate } \\
\text { - } 8 / 22 \mathrm{HIV} \text { infected individuals responded to this epitope }(13 / 22 \text { responded to some of the DR supermotif epitopes, the } 9 \text { non-responder peptides tended to } \\
\text { also not have recall responses to rec HIV- } 1 \text { whole proteins) }\end{array}$} \\
\hline $\mathrm{p} 24(131-152)$ & $\begin{array}{l}\text { p24 (263-284 SF2) } \\
\text { Low viral load correlated } \\
\text { A proliferative response t }\end{array}$ & $\begin{array}{l}\text { KRWI ILGLNKIVRMYSPTS- HIV-1 infection } \\
\text { ILD } \\
\text { with strong HIV-1-specific proliferative response } \\
\text { o this epitope was detected in two long term survivors }\end{array}$ & human & Rosenberg1997 \\
\hline p24 (135-154) & $\begin{array}{l}\text { p24 (267-286) } \\
\text { One of four immunogenic } \\
8 \text { of } 24 \text { HIV+ individuals } \\
\text { Improved assay system (i }\end{array}$ & $\begin{array}{l}\text { ILGLNKIVRMYSP TSILDIR HIV-1 infection } \\
\text { Gag peptides used in study of the proliferative respon } \\
\text { responded to this epitope } \\
\text { ncrease in culture time to } 8 \text { days and addition of IL- } 2 \mathrm{t}\end{array}$ & $\begin{array}{l}\text { human } \\
\text { re increased detect }\end{array}$ & $\begin{array}{l}\text { Adams1997 } \\
\text { oliferative response }\end{array}$ \\
\hline p24 (139-157) & $\begin{array}{l}\text { Gag (p24) (271-290 } \\
\text { IIIB) } \\
\text { Epitope name: P28 } \\
\text { PBMC from a seronegati } \\
\text { Gag-specific CD4+ T-cell } \\
\text { dilution. All reacted with } \\
\text { Clone } 6 \text { recognized three } \\
\text { clone } 6 \text { suggested this wa } \\
\text { of this clone. Upon activa } \\
\text { Clone } 6 \text { was activated in } 1 \\
\text { Clone } 37 \text { recognized this } \\
\text { activated by peptide, not } 1 \\
\text { Clone } 97 \text { recognized this } \\
\text { population. This clone wa }\end{array}$ & $\begin{array}{l}\text { NKIVRMYSP TS ILD IRQGP in vitro stimulation } \\
\text { ve donor, the healthy brother of a pair of monozygotic } \\
\text { clones by in vitro immunization with HIV-1 overlapp } \\
\text { p24 except one which recognized a p24 peptide and a } \\
\text { peptides including this one with a Th1 response using } \\
\text { s a clonal population. Assays using different peptide c } \\
\text { tion, clone } 6 \text { was observed to induce a cytopathic effec } \\
\text { response to vaccinia virus Gag-infected B-LCL, so cou } \\
\text { peptide sequence with a Th2 response using TCR Vbe } \\
\text { by processed protein from vaccinia virus Gag-infected } \\
\text { peptide sequence with a using TCR Vbeta } 9 \text { and } 14 \text {; th } \\
\text { as only activated by peptide, not by processed protein } f\end{array}$ & $\begin{array}{l}\text { human (DR4) } \\
\text { nt for HIV-1 infec } \\
\text { tides spanning p55 } \\
\text { 1 CD4+ T cell clor } \\
\text { (6s5A1N1). Seque } \\
\text { uggest that this pe } \\
\text { nt layer of fibrobl } \\
\text { aturally processed } \\
\text { homogeneous T-c } \\
\text { eptors used indica } \\
\text { irus Gag-infected }\end{array}$ & $\begin{array}{l}\text { Venturini2002 } \\
\text { e used to generate HIV-1 } \\
\text { nes were generated by limiting } \\
\text { HLA clas II DR restricted. } \\
\text { CR Vbeta regions of colonies from } \\
71-290 \text {, contains the main epitope } \\
\text { essing HLA DR4W14 and -W15. } \\
\text { lation. This clone was only } \\
\text { imiting dilution represents a mixed }\end{array}$ \\
\hline p24 (141-156) & $\begin{array}{l}\text { p24 (273-287) } \\
\text { Epitope elicits a primary } \\
\text { Matches } 3 / 3 \text { anchor resid }\end{array}$ & $\begin{array}{l}\text { IVRMYSP TS ILDIRQC in vitro stimulation } \\
\text { proliferative response in PBMC from uninfected donor } \\
\text { ues for HLA DR: IVRMYSPTS }\end{array}$ & human & Bedford1997 \\
\hline p24 (146-160) & $\begin{array}{l}\text { p24 (278-292 IIIB, B10) } \\
12 \text { gag and } 18 \text { env peptide }\end{array}$ & $\begin{array}{l}\text { SPTS ILDIRQGPKEP HIV-1 infection } \\
\text { es were identified that could commonly evoke T-cell }\end{array}$ & human & Wahren1989b Wahren1989a \\
\hline p24 (149-168) & $\begin{array}{l}\text { Gag (p24) (281-300 } \\
\text { IIIB) } \\
\text { Epitope name: P29 }\end{array}$ & SILDIRQGPKEPERDYVDRF in vitro stimulation & uman (DR4) & Venturini2002 \\
\hline
\end{tabular}




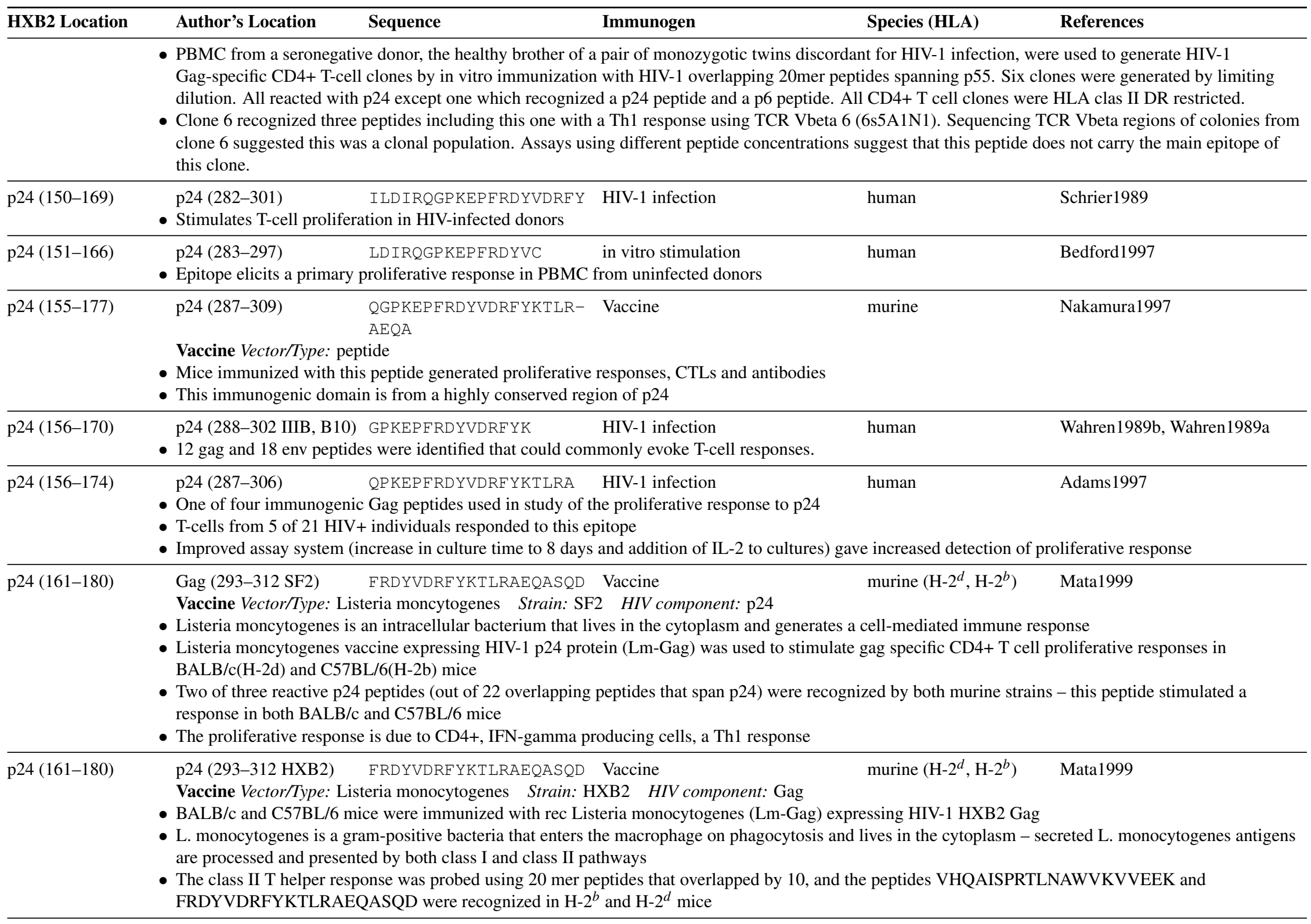




\begin{tabular}{|c|c|c|c|c|}
\hline HXB2 Location & Author's Location & Immunogen & Species (HLA) & References \\
\hline p24 (163-177) & \multicolumn{4}{|c|}{$\begin{array}{l}\text { - The DRB } 1 * 13-\mathrm{DQB} 1 * 06 \text { haplotype is associated with maintained viral suppression after HAART }-7 / 7 \text { early-treated DRB } 1 * 13-\mathrm{DQB} 1 * 06 \text { positive people, } \\
\text { but only } 3 / 14(21 \%) \text { of those who did not have DRB } 1 * 13-\mathrm{DQB} 1 * 06 \text {, maintained viral suppression for } 18 \text { months } \\
\text { - PBMC from individuals with the haplotype DRB } 1 * 13-\mathrm{DQB} 1 * 06 \text { displayed increased IFN } \gamma \text { secretion and stronger proliferative responses against p24 } 80 \\
\text { weeks post treatment } \\
\text { - DRB } 1 * 13-\mathrm{DQB} 1 * 06 \text { was also found to be enriched among long-term non-progressors (it was in } 9 / 18 \text { versus, versus } 21 \% \text { of the general population) } \\
\text { - This epitope was mapped with truncated peptides using the Elispot assay, and is highly conserved }\end{array}$} \\
\hline p24 (175-199) & $\begin{array}{l}\text { p17 (307-331 PV22) } \\
\text { - } 10 / 49 \text { chronically HI } \\
6 \text { months after initiat } \\
\text { - For one individual, } \mathrm{p} \\
\text { terms of HLA restric } \\
\text { profile (high IL-4 an } \\
\text { - } 4 / 10 \text { clones from pat } \\
\text { This clone had a SI o } \\
\text { cytotoxic activity. }\end{array}$ & $\begin{array}{l}\text { EQASQEVKNWMTETLLVQN- HIV-1 infection } \\
\text { ANPDCK } \\
\text { infected patients had low p55-Gag-specific CD4+ } \\
\text { of HAART. There was no difference in level of resp } \\
\text { t F45 CDC stage A2, CD4+ p55 responding clone } \\
\text { and Vbeta usage, and some clones had a Th1 cytok } \\
-5 \text { production). } 5 / 10 \text { CD4+ clones could also induc } \\
\text { F45 had their epitopes mapped using overlapping p } \\
\text { Ito p55, } 5.3 \text { to peptide, secreted high levels of IFNg }\end{array}$ & $\begin{array}{l}\text { human }(\mathrm{DRB} 1 * 03) \\
\text { ior to therapy, and these } \\
\text { or without a detectable } \\
\text { Her response was consi } \\
\text { le (high IFNgamma pro } \\
\text { recognized this peptide } \\
\text { of a Th1 response, but a }\end{array}$ & $\begin{array}{l}\text { Lotti2002 } \\
\text { sponses remained unchanged } 3 \text { and } \\
55 \text { response. } \\
\text { ttly strong and heterogeneous in } \\
\text { ction) while some had a Th2 } \\
\text { quence restricted by DRB } 1 * 03 \text {. } \\
\text { IL- } 4 \text { and IL- } 5 \text {. Clone } 26 \text { had no }\end{array}$ \\
\hline p24 (181-196) & $\begin{aligned} & \text { p24 (313-327) } \\
& \text { - Epitope elicits a pri } \\
& \text { - Matches 3/3 anchor }\end{aligned}$ & $\begin{array}{l}\text { VKNWMTETLLVQNANC in vitro stimulati } \\
\text { oroliferative response in PBMC from uninfected d } \\
\text { les for HLA DR: VKNWMTETL }\end{array}$ & human & Bedford1997 \\
\hline
\end{tabular}




\section{III-B-3 p2p7p1p6 Helper T-Cell Epitopes}

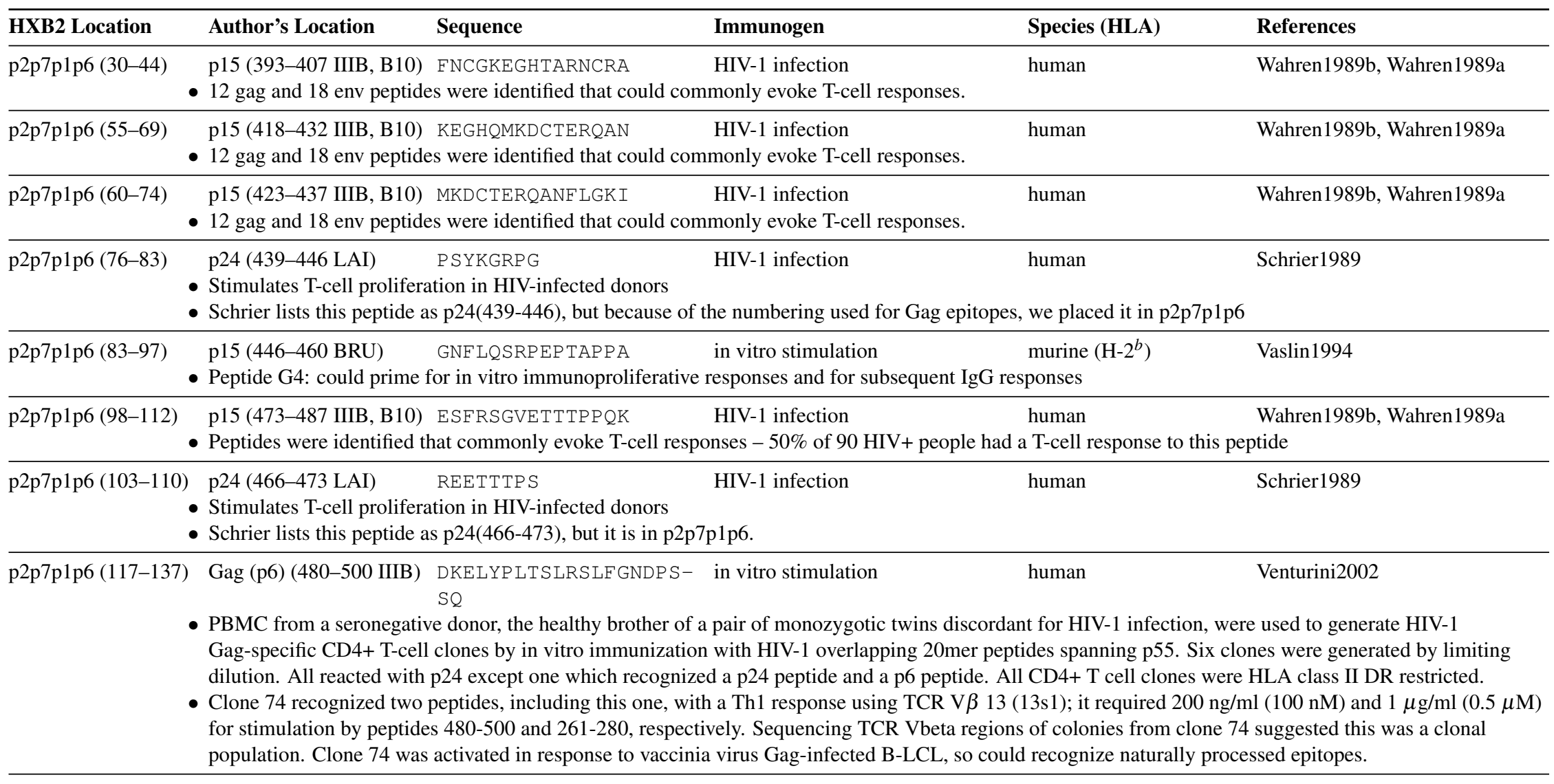




\section{III-B-4 Gag Helper T-Cell Epitopes}

\begin{tabular}{lllll}
\hline HXB2 Location & Author's Location & Sequence & Immunogen & Species (HLA) \\
\hline Gag & p24 & HIV-1 infection, Vaccine & human & Kelleher1998b \\
& Vaccine Vector/Type: virus-like particle & HIV component: $\mathrm{p} 24, \mathrm{p} 17$ &
\end{tabular}

- Immunization of HIV+ people with a p24-VLP virus-like particle did not significantly impact CD4+ lymphocyte count, viral load, or p24 antibody titre

- Immunization with p24-VLP showed a modest, short-lived increased proliferative response to p24

\begin{tabular}{llcll}
\hline Gag & p24 & HIV-1 infection, Vaccine & human & Maino2000
\end{tabular}

- 18 HIV-1-seropositive patients with a low frequency or no detectable CD4+ T cell response to HIV-1 antigen received an HIV-1 immunogen consisting of

10 units of native p24 and 100 ug of HZ321, a gp120 depleted antigen

- Using flow-cytometric methods, HIV-1 specific CD4+ T cells were shown to increase in response to immunization - in many patients significant enhancement was observed after a single immunization

- The frequency of CD4+ T cells expressing cytokines in response to antigen by FACS was correlated with a lymphoproliferation assay

Gag p24 HIV-1 infection human Ruiz2000

- Structured treatment interruption in chronically infected patients allowed recovery of p24 Th proliferative responses after HAART therapy discontinuation in $2 / 12$ patients

- The Th response to p24 was identified during peak viremia in one patient, while in the second it was noted when viremia was controlled after restarting antiviral therapy

Gag p24 HIV-1 infection human

- Ten patients with acute, pre-seroconversion HIV-1 infections were treated with didanosine, indinavir and hydroxyurea - this treatment is associated with normalization of immune parameters

- A vigorous HIV-specific Th response (stimulation index greater than 8) was observed in 7/8 patients treated before complete WB seroconversion, but in only $1 / 5$ controls treated after seroconversion

- Vigorous Th responses were detected as early as 34 days after treatment begin

- Patients treated prior to seroconversion had no loss of naive CD4 T lymphocytes, recovery of up to 35\% of the naive CD8 cells in several weeks, and a reduced latent viral reservoir

\begin{tabular}{llcc}
\hline Gag & p24 & HIV-1 infection & human \\
- $11 / 22$ adult patients on HAART showed strong CD4+ T-cell IFN-gamma producing Th1 responses to HIV p24
\end{tabular}

- The magnitude of the Th1 response correlated with previous interruptions in HAART, suggesting the interruptions primed or boosted the response

- In contrast, the magnitude of the CD8+ CTL response did not correlate with interruptions in therapy, although a greater breadth in response was associated with interruptions in HAART

Gag p24 HIV-1 infection, Vaccine human

Vaccine Vector/Type: virus-like particle HIV component: p24, p17

- Immunization of HIV+ people with a HIV-1 p17/p24 Ty virus-like particle (p24-VLP) resulted in a marginal, short-lived increased proliferative response to p24 and p17 and a transient elevation in viral load

- Two of four subjects that received 500 or $1000 \mathrm{ug}$ of p24-VLP had an increase in gag-specific CTL

\begin{tabular}{llccc}
\hline Gag & p24 & Vaccine & human & Moss1998 \\
& Vaccine Vector/Type: gp120 depleted virus HZ321 (REMUNE(TM)) & Strain: Z321 & HIV component: gp120 depleted virus
\end{tabular}




\begin{tabular}{|c|c|c|c|c|c|}
\hline HXB2 Location & Author's Location & Sequence & Immunogen & Species (HLA) & References \\
\hline & \multicolumn{5}{|c|}{$\begin{array}{l}\text { - Immunization with gp120 depleted HZ321 virus (REMUNE }{ }^{T M} \text { ) triggered an increase in lymphocyte proliferative response to native p24, a clade B virus } \\
\text { and clade E viral antigens - Z321 is clade A in env and clade G in gag. [Moss1998 }\end{array}$} \\
\hline Gag & \multicolumn{5}{|c|}{$\begin{array}{l}\text { p24 HIV-1 infection } \\
\text { - This paper reviews the role of T-cells in viral control and HIV disease outcome } \\
\text { - Strong anti-p24 lymphoproliferative responses were found in seven persons who were treated with potent anti-viral therapy during acute HIV-1 infection } \\
\text { syndrome } \\
\text { - This suggests that Th cells are part of the normal response to HIV-1 infection, but their numbers are rapidly diminished by either being infected during the } \\
\text { peak viremia or by activation-induced cell death - if peak viremia can be controlled, a robust anti-p24 Th response can be maintained }\end{array}$} \\
\hline$\overline{\mathrm{Gag}}$ & \multicolumn{5}{|c|}{$\begin{array}{l}\text { - Strong Th responses have been found in rare individuals who effectively maintain low viral loads } \\
\text { - If aggressive anti-retroviral therapy is given prior to sero-conversion, strong helper responses can be maintained. }\end{array}$} \\
\hline Gag & \multicolumn{5}{|c|}{$\begin{array}{l}\text { Vaccine Vector/Type: recombinant protein HIV component: p17 } \\
\text { - Different p17 genes derived from the same quasispecies and expressed and purified in E. coli primed different Th } 1 \text { and Th } 2 \text { subsets in mice, depending on } \\
\text { their H-2 type. }\end{array}$} \\
\hline Gag & $\begin{array}{l}\text { - Gag } \\
\text { - Study of parameters } \\
\text { - HIV-1 replication in } \\
\text { - Gag proteins includ } \\
\text { - Frozen samples can }\end{array}$ & $\begin{array}{l}\text { might influes } \\
\text { is unlikely t } \\
17 \text { and possil } \\
\text { sed in T-proli }\end{array}$ & $\begin{array}{l}\text { HIV-1 infection } \\
\text { or reproducibility } \\
\text { y } \\
4 \text { perform better th } \\
\text { with lower radiolal }\end{array}$ & $\begin{array}{l}\text { human } \\
\text { liferative assays } \\
\text { orporation }\end{array}$ & Schiller2000 \\
\hline Gag & $\begin{array}{l}\text { Gag } \\
\text { - In contrast to earlier } \\
\text { paper shows using f } \\
\text { subjects } \\
\text { - Effective anti-viral t }\end{array}$ & $\begin{array}{l}\text { suggestin } \\
\text { tometric de }\end{array}$ & $\begin{array}{l}\text { HIV-1 infection } \\
\text { c Th responses we } \\
\text { duced cytokines th } \\
\text { cells, presumably }\end{array}$ & $\begin{array}{l}\text { human } \\
\text { e early stages of in } \\
\text { mory gag-specific } \\
\text { tigenic stimulus }\end{array}$ & $\begin{array}{l}\text { Pitcher1999 } \\
\text { in most HIV+ individuals, this } \\
\text { are detectable in most HIV+ }\end{array}$ \\
\hline Gag & $\begin{array}{l}\text { Gag } \\
\text { Patients from later s }\end{array}$ & of infection & $\begin{array}{l}\text { HIV-1 infection } \\
\text { ot show restoration }\end{array}$ & $\begin{array}{l}\text { human } \\
\text { Th proliferative } 1\end{array}$ & Plana1998 \\
\hline Gag & $\begin{array}{l}\text { Gag } \\
\text { Env and gag Th epit } \\
\text { lymphocyte count, i }\end{array}$ & ere pooled & $\begin{array}{l}\text { HIV-1 infection } \\
\text { proliferative respo } \\
\text { liferative response }\end{array}$ & $\begin{array}{l}\text { human } \\
\text { apy - while IL2 th }\end{array}$ & $\begin{array}{l}\text { Kelleher1998a } \\
\text { auses an increase in CD4+ }\end{array}$ \\
\hline Gag & $\begin{array}{l}\text { Gag (LAI) } \\
\text { Vaccine Vector/Typ } \\
\text { - Priming with an HI } \\
\text { - The proliferative res } \\
\text { fold increase in the } \\
\text { Th1, not Th2. The } C\end{array}$ & $\begin{array}{l}\text { A prime wit } \\
\text { A vaccine ar } \\
\text { to Env and } \\
\text { SI for HIV } \\
\text { sponse was }\end{array}$ & $\begin{array}{l}\text { Vaccine } \\
\text { rain: LAI HIV } \\
\text { ccinia construct ir } \\
\text { accination had a } \\
\text { h response happe }\end{array}$ & $\begin{array}{l}\text { Macaca nemestri } \\
\text { GAG } \\
\text { els of HIV T-cell ir } \\
\text { out after boosting v } \\
\text { n Ab titers, sugges }\end{array}$ & $\begin{array}{l}\text { Kent1998 } \\
\text { than either vaccine alone } \\
\text { J-fowlpox virus, there was a 6-17 } \\
\text { the Th response was primarily }\end{array}$ \\
\hline
\end{tabular}




\begin{tabular}{|c|c|c|c|c|c|}
\hline HXB2 Location & Author's Location & Sequence & Immunogen & Species (HLA) & References \\
\hline Gag & & & Vaccine & Rhesus macaque & Heeney1999 \\
\hline
\end{tabular}

- Ten different vaccine strategies were evaluated for their ability to protect from infection in a rhesus macaque model using a non-pathogenic SHIV challenge

- Protection correlated with the magnitude of NAb responses, beta-chemokines, and a balanced Th response

- DNA, protein+adjuvant, VLP and ISCOM vaccines were tested

- HIV-1/ISCOMS gave the highest NAb titers, Th1 and Th2 responses, was the only vaccine formulation tested with a detectable CTL response, and gave enhanced beta-chemokine production

\begin{tabular}{lllcr}
\hline Gag & Gag/Pol (MN) & Vaccine & chimpanzee & Kim1998 \\
& Vaccine Vector/Type: DNA & Strain: MN & HIV component: GAG, POL, ENV & Adjuvant: CD80 and CD86 expression vectors
\end{tabular}

- Co-stimulatory molecules co-expressed with an HIV-1 immunogen in a DNA vaccine used to enhance the immune response - co-expression of CD86, but not CD80, dramatically increased both HIV Env and Gag/Pol specific CTL and Th proliferative responses

\begin{tabular}{lllc}
\hline Gag & Gag/Pol (LAI, MN) & Vaccine & human \\
& Vaccine Vector/Type: canarypox & Strain: MN, LAI & HIV component: gp120, gp41, Gag, Protease
\end{tabular}

- A live attenuated canarypox vector expressing MN gp120 and LAI gp41/gag/protease could induce CTL and a lymphoproliferative response in healthy uninfected volunteers

Gag p55 (IIIB) HIV-1 infection human Zhang

- T-helper cell proliferative responses to HIV p24, p55 and gp120 were tested in 27 patients with HIV infection - vigorous responses directed at Gag were detected in ten patients, but an Env specific response was detected in only one patient

- Untreated patients showed a negative correlation between plasma viral load and HIV p24-specific T-cell responses, and the responses could be detected after extended HAART therapy with viremia below the detection limit

\begin{tabular}{ll}
\hline Gag & p24 \\
& Repeated structured HAART therapy interruptions (STI) in 3 chronically HIV infected patients induced rapid but transient (<3 weeks) HIV-1 specific \\
& CD4+ Th1 responses concurrently with viral rebound, as measured by proliferation assays and by IFN $\gamma$ production by CD8-depleted PBMC
\end{tabular}

CD4+ Th1 responses concurrently with viral rebound, as measured by proliferation assays and by IFN $\gamma$ production by CD8-depleted PBMC

- Kinetics suggest that viral replication leads to rapid destruction of the HIV-specific Th1 cell response

- HIV-specific CD8+ T-cell responses were delayed relative to the Th1 responses and were not sustained

Gag Gag HIV-1 infection human Bankson2001a

- 5/10 chronically HIV infected patients with low CD4+ counts who received HAART therapy and experienced immune reconstitution displayed p24, p17 and p66 T-helper CD4 proliferative responses, in contrast to 0/8 chronically HIV infected patients with high CD4+ counts at the initiation of antiretroviral treatment

- This surprising result could be due to the low CD4 nadir patients being more likely to have thymic regeneration or a peripheral expansion of T cells p24

HIV-1 infection

human

Angel2001

- Prolonged viral suppression resulting from potent anti-retroviral therapy allowed a T helper response to Gag p24 and PHA to develop in many HIV+ patients

- At baseline, $2 / 41$ (4.9\%) subjects had a proliferative response to Gag p24, and 7/41 (17.1\%) had a response to PHA, but by week 72 of therapy, $53 \%$ had a detectable response to p24 and 94\% to PHA 


\begin{tabular}{lllcl}
\hline HXB2 Location & Author's Location & Sequence & Immunogen & Species (HLA) \\
\hline Gag & p24 & HIV-1 infection & human & Blazevic2000 \\
& - Prolonged viral suppression resulting from potent anti-retroviral therapy did not allow an HIV T helper response to p24 or gp160, but Th proliferative
\end{tabular}

responses to influenza, alloantigen, and PHA did develop in many HIV+ patients, and asymptomatic patients had stronger and more frequent Th response recovery than AIDS patients

\begin{tabular}{llcc}
\hline Gag & Gag (SF2) HIV-1 infection & human \\
- Therapy provided during acute infection resulted in a narrower CTL response, stronger T help response, and a less diverse viral population
\end{tabular}

- Therapy provided during acute infection resulted in a narrower CTL response, stronger T help response, and a less diverse viral population than was seen in individuals treated during chronic infection

- The breadth and specificity of the CTL response was determined using Elispot by studying 19 individuals with pre-seroconversion therapy (Group 1), 11 individuals with primary infection but post-seroconversion therapy (Group 2), and 10 individuals who responded to HAART given during chronic infection (Group 3), using 259 overlapping peptides spanning p17, p24, RT, gp41, gp120 and Nef

- Individuals who were given HAART during acute or early in infection had significantly stronger proliferative responses than individuals who were chronically infected

Gag

p24 HIV-1 infection

human

Oxenius2000

- Patients who started therapy at acute HIV infection (three with sustained therapy, two with limited therapy upon early infection) had strong HIV-specific CD4 proliferative responses and were able to maintain a CTL response even with undetectable viral load - three patients that had delayed initiation of HAART had no HIV-specific CD4 proliferative responses and lost their CTL responses when HAART was eventually given and their viral loads became undetectable

- In 3/4 responders tested p24 gave the strongest T helper response

\begin{tabular}{|c|c|c|}
\hline Gag & $\begin{array}{l}\mathrm{p} 24 \\
\text { Vaccine Vector/Type: gp120 depleted whole killed virus } \\
\text { Freund's adjuvant } \\
\text { - Lewis rats simultaneously immunized with HIV-1 antigen } \\
\text { CpG was given as a prime prior to the injection of HIV-1 }\end{array}$ & $\begin{array}{l}\text { Vaccine } \quad \text { rat } \\
\text { Strain: HZ321 (subtype A env, subtype G gag) }\end{array}$ \\
\hline Gag & $\begin{array}{l}\mathrm{p} 24 \\
\text { Vaccine Vector/Type: gp120 depleted whole killed virus } \\
\text { Freund's adjuvant } \\
\text { Lewis rats co-immunized with HIV-1 antigen in Freund's } \\
\text { CD8+ T cells and anti-p24 antibodies relative to antigen i }\end{array}$ & $\begin{array}{l}\text { Vaccine } \quad \text { rat } \\
\text { Strain: HZ321 (subtype A env, subtype G gag) }\end{array}$ \\
\hline Gag & $\begin{array}{l}\text { p } 24 \\
\text { The strength of p24 specific Gag proliferative responses ( } \\
\text { IFN } \gamma \text { producing. Proliferative responses against gp160 we } \\
\text { Gag specific CTL levels were correlated with Gag prolife } \\
\text { proliferative responses, and } 4 / 8 \text { had no CTLp to any HIV- }\end{array}$ & $\begin{array}{l}\text { HIV-1 infection human } \\
\text { SIs) were inversely correlated with viral load in } 21 \text { ARV naive patients. The responses were Th1, } \\
\text { re rarely observed (only } 4 \text { cases). } \\
\text { ative responses but were not correlated with viral load. } 8 \text { subjects lacked p24 specific Gag } \\
1 \text { antigen tested. }\end{array}$ \\
\hline Gag & $\begin{array}{l}\text { p24 } \\
\text { - This paper reviews the role of specific T cell help in many } \\
\text { advantages of HAART during acute HIV infection to prev }\end{array}$ & $\begin{array}{l}\text { HIV-1 infection human } \\
\text { viral infections, and covers the interplay between Th, CTL and survival, and discusses briefly } \\
\text { ent the early decimation of the Th response in HIV infections. }\end{array}$ \\
\hline Gag & $\begin{array}{l}\text { p24 } \\
\text { Dysfunction of HIV-1 specific proliferative responses, }\end{array}$ & 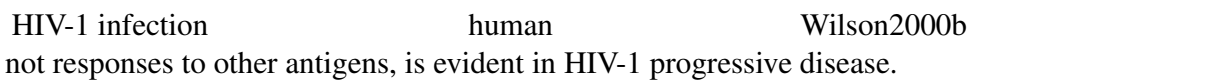 \\
\hline
\end{tabular}




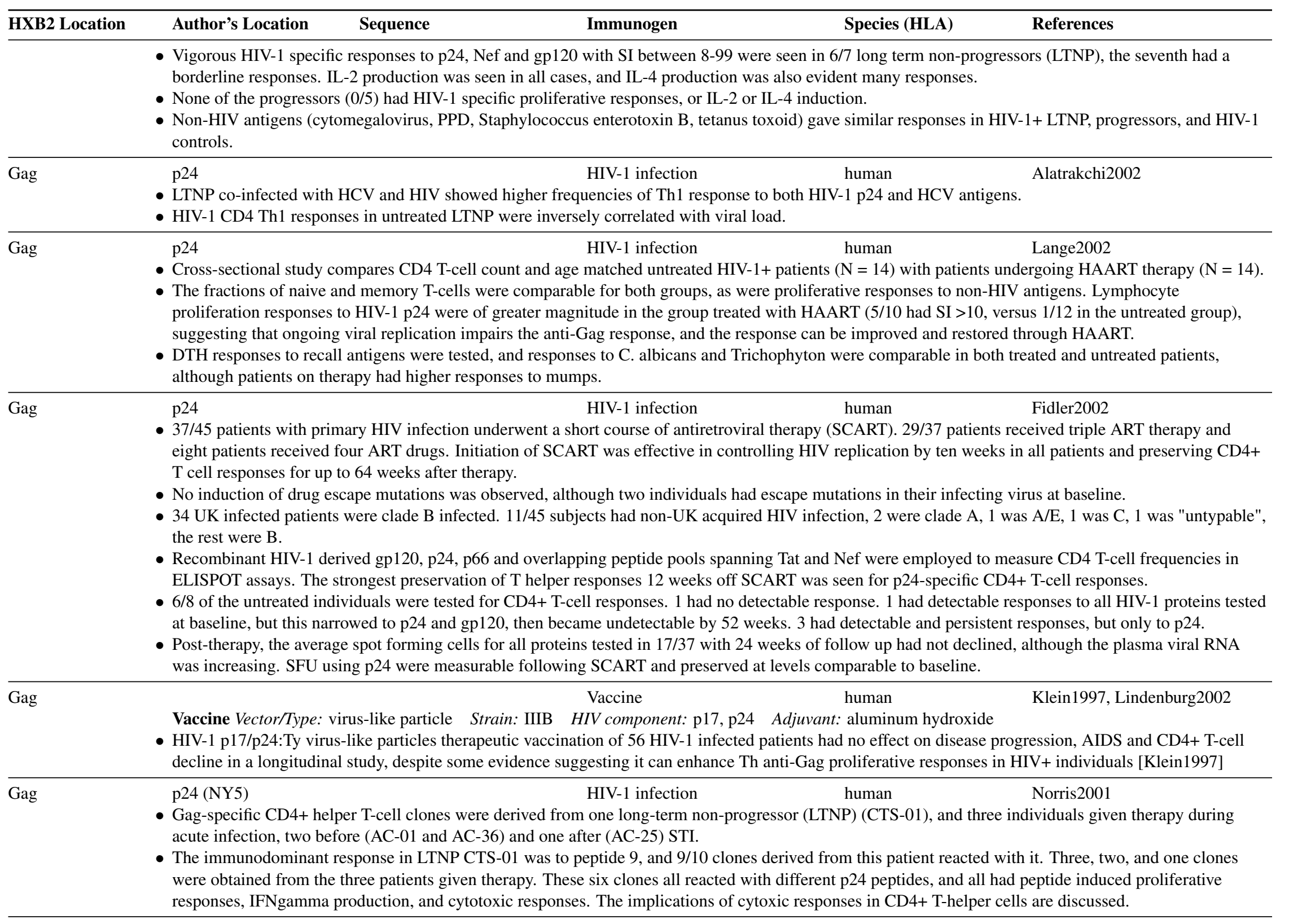




\begin{tabular}{|c|c|c|c|c|c|}
\hline HXB2 Location & Author's Location & Sequence & Immunogen & Species (HLA) & References \\
\hline Gag & p24 & & HIV-1 infection & human & Palmer2002 \\
\hline
\end{tabular}

(HAART failures and HAART naive). Patients with HAART suppression showed stronger p24- and p66-specific proliferative responses compared to patient groups with active HIV-1 replication, suggesting active viral replication in vivo specifically reduces proliferation responses.

- No differences in the frequency of HIV-specific CD4+ T-cells that were positive for cytokine secretion in a flow cytometry assay were found in the HAART suppressed group versus the group with active viral replication.

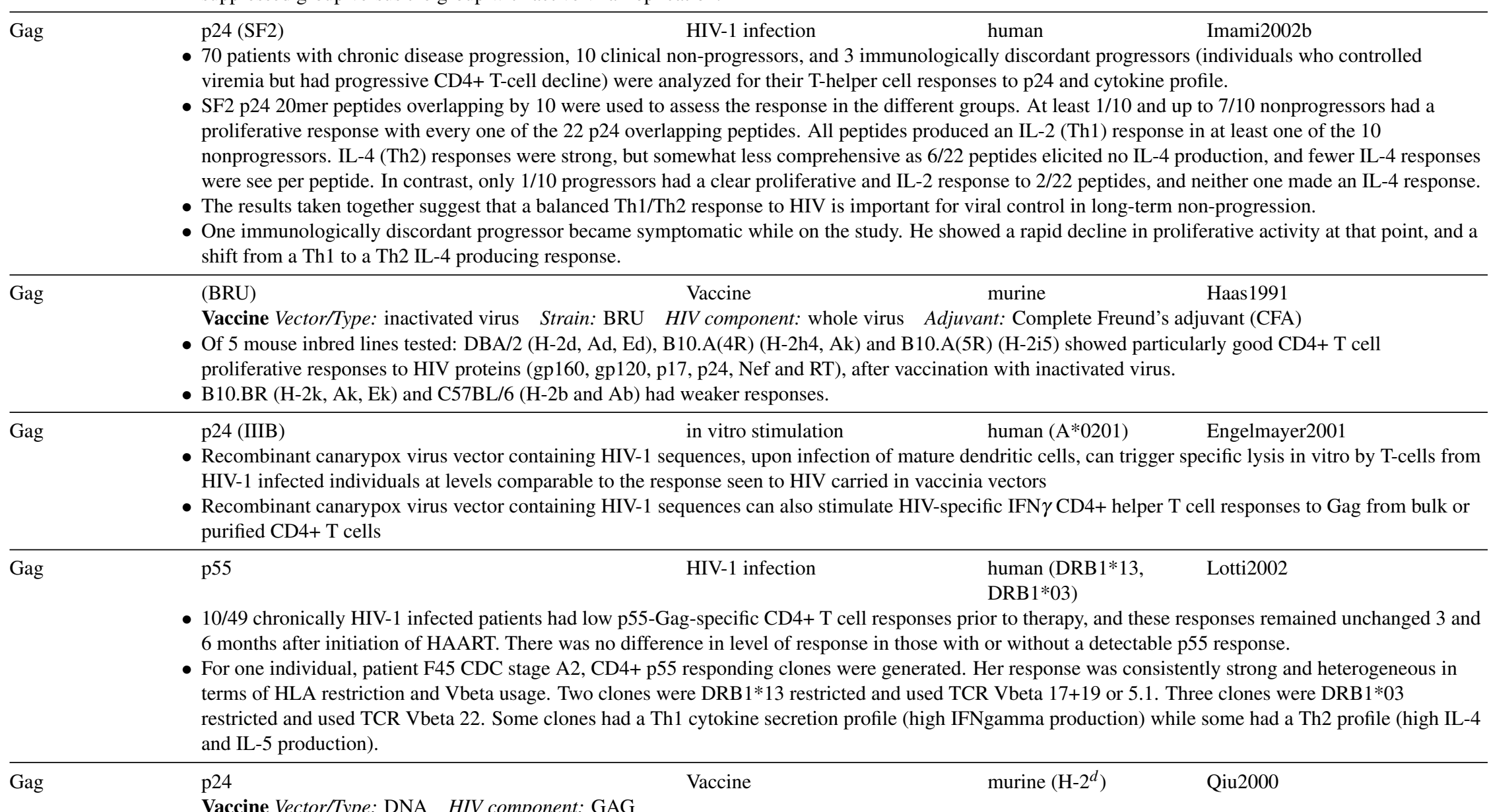

- Mice were injected with plasmid DNA at 0,2 and 4 weeks and lymphocyte proliferation was measured after 6 weeks with recombinant p24 protein

- Secreted HIV-1 Gag expression vectors generated a stronger response than standard Gag or cytoplasmic Gag expression vectors

- IFN-gamma levels were increased compared to an undetectable IL-4 response 


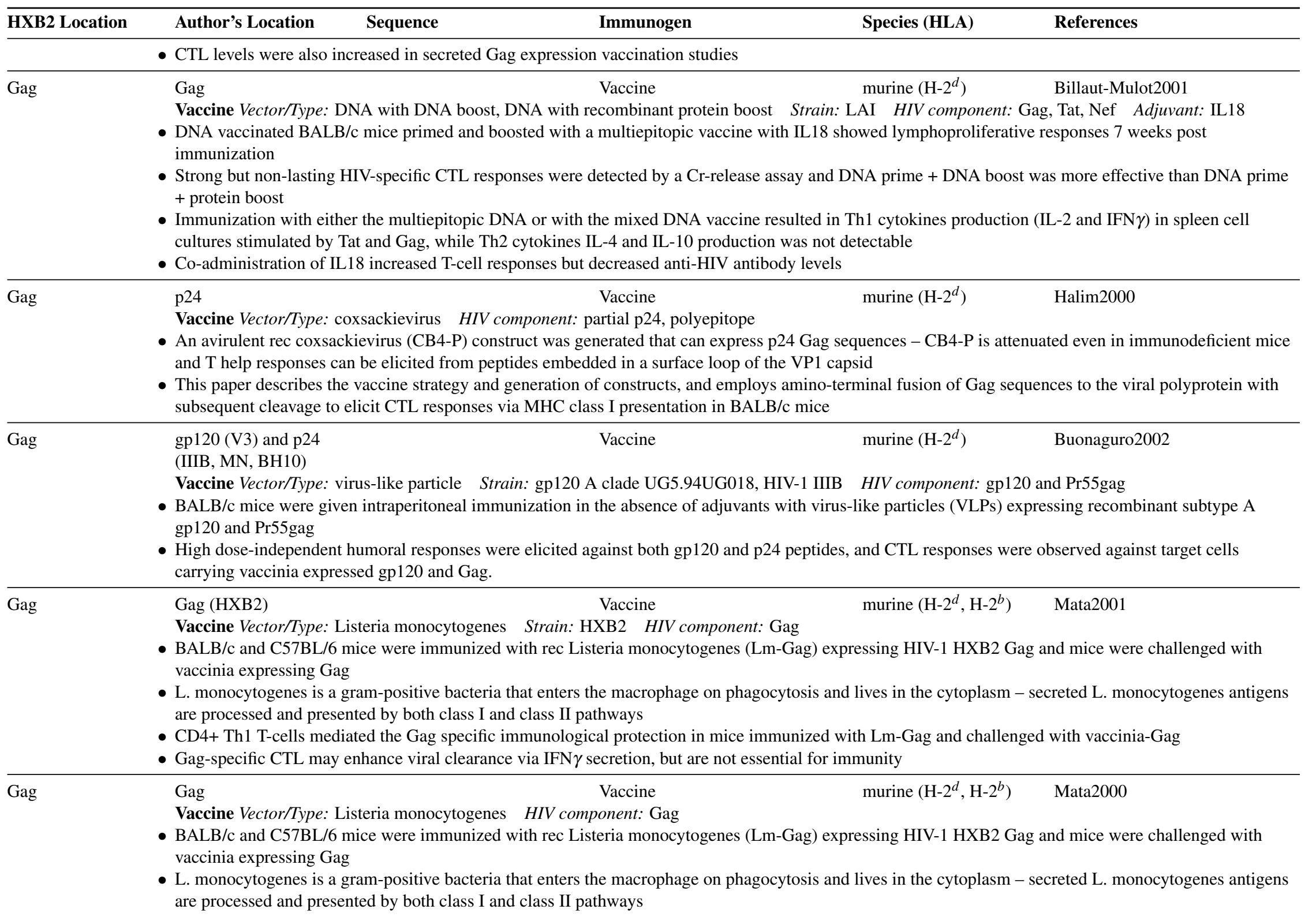


- This article is a review of L. monocytogenes biology and its potential as a vaccine vector for HIV, comparing to other vector systems, and discussing CD4+ Th1 T-cells mediated Gag specific immunological protection in mice and the Gag CTL response 


\section{III-B-5 RT Helper T-Cell Epitopes}

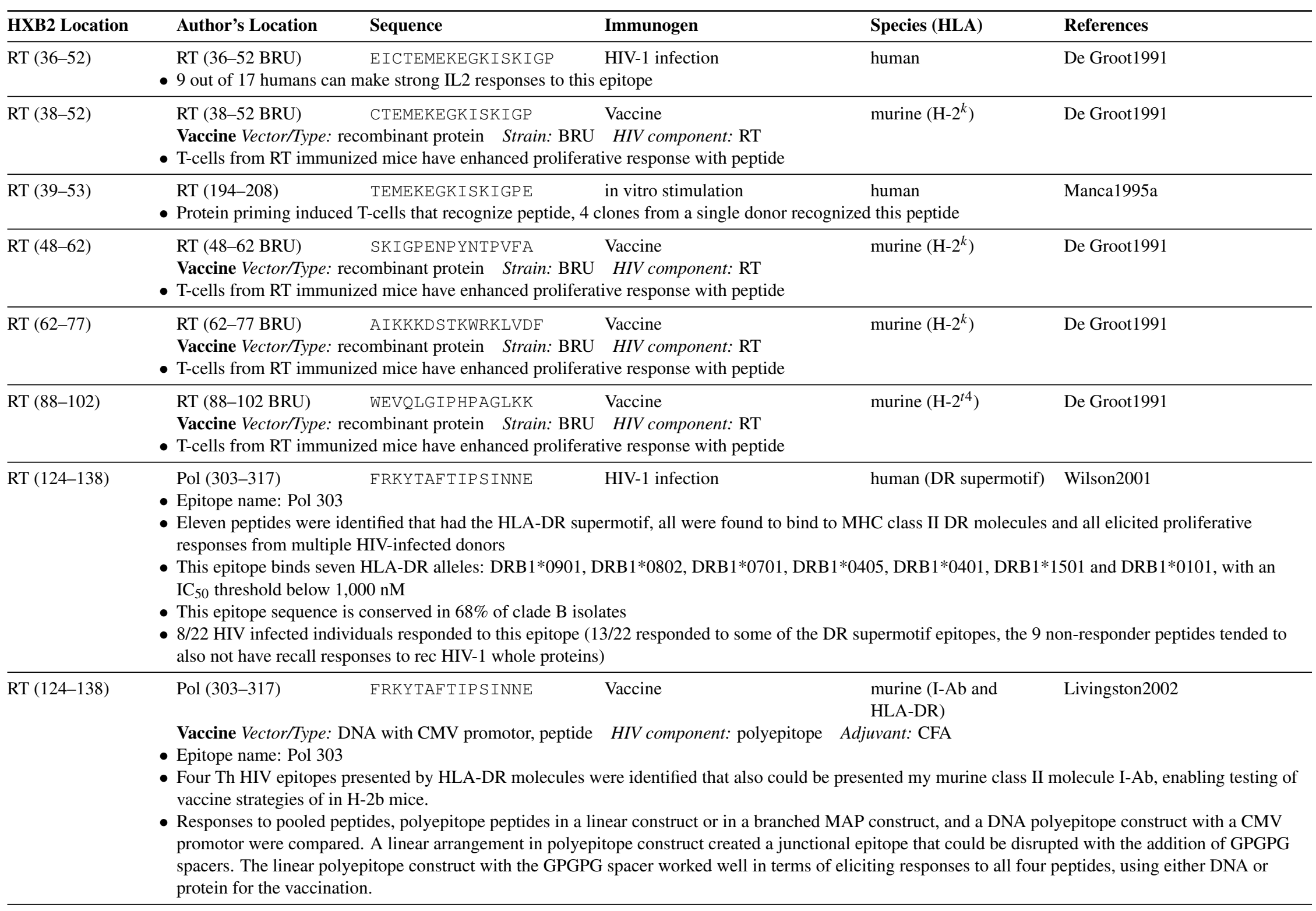




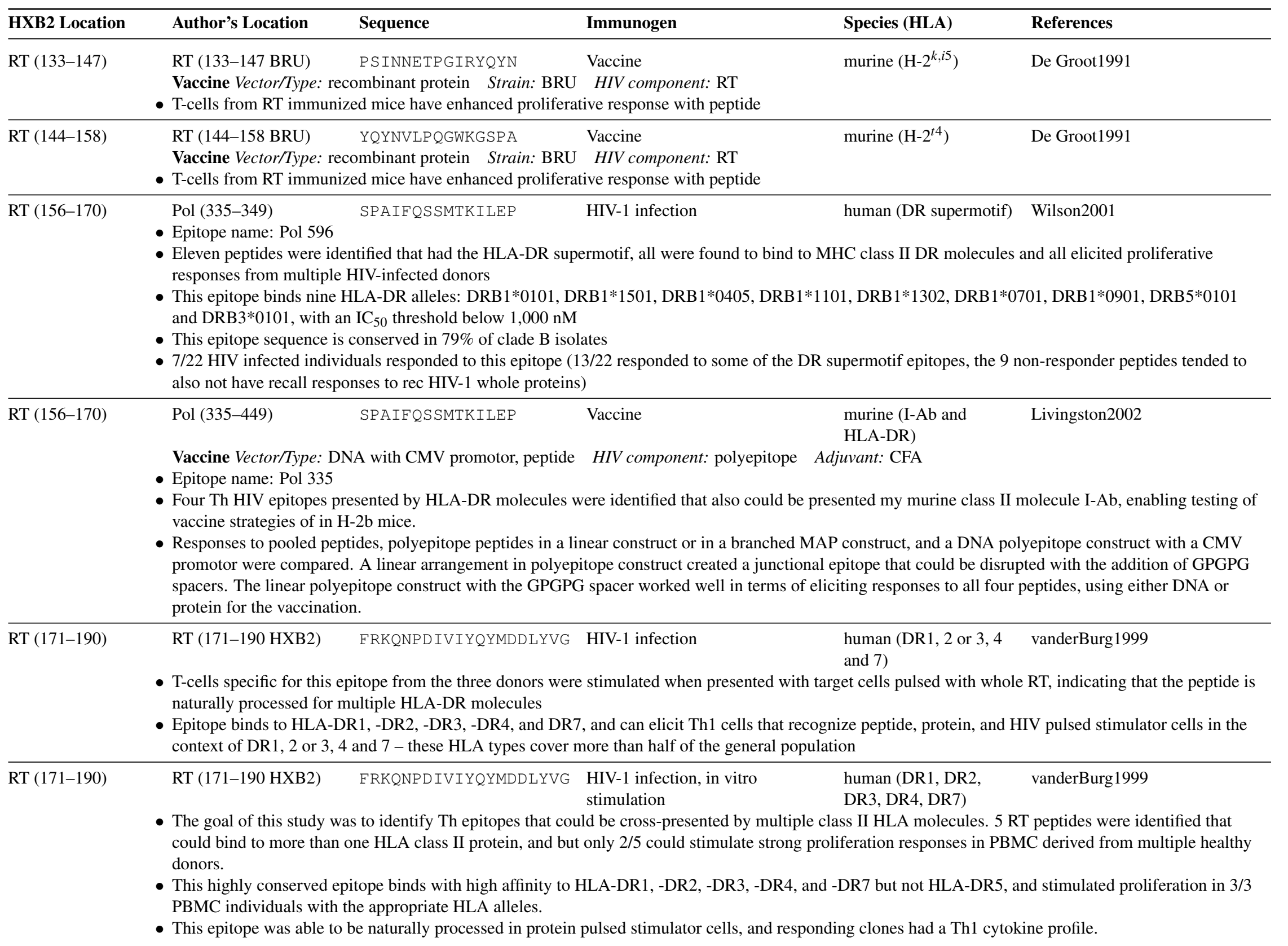




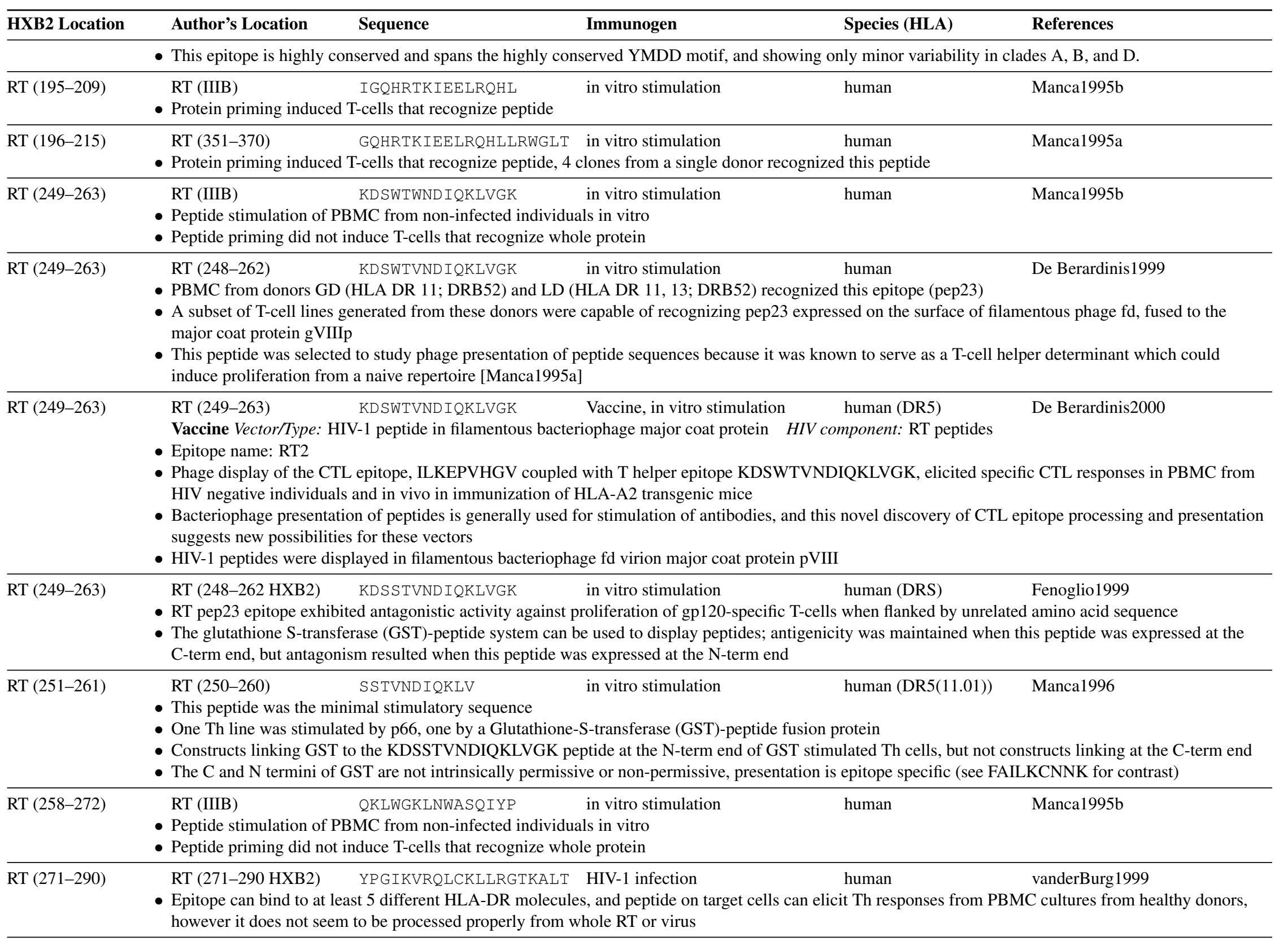




\begin{tabular}{|c|c|c|c|c|c|}
\hline HXB2 Location & Author's Location & Sequence & Immunogen & Species (HLA) & References \\
\hline RT (271-290) & \multicolumn{5}{|c|}{$\begin{array}{l}\text { - The goal of this study was to identify Th epitopes that could be cross-presented by multiple class II HLA molecules. } 5 \text { RT peptides were identified that } \\
\text { could bind to more than one HLA class II protein, but only } 2 / 5 \text { could stimulate strong proliferation responses in PBMC derived from multiple healthy } \\
\text { donors. } \\
\text { - This epitope binds with high affinity to HLA-DR1, -DR2, -DR3, -DR5, and -DR7 but not HLA-DR4, and stimulated proliferation in } 3 / 4 \text { individuals with } \\
\text { the appropriate HLA alleles. } \\
\text { - This epitope was not able to be naturally processed in protein-pulsed stimulator cells. }\end{array}$} \\
\hline RT (276-290) & $\begin{array}{l}\text { RT (IIIB) } \\
\text { - Protein priming induced }\end{array}$ & $\begin{array}{l}\text { WRQLCKLLRGTKALT } \\
\text { T-cells that recognize peptide }\end{array}$ & in vitro stimulation & human & Manca1995b \\
\hline RT (285-299) & $\begin{array}{l}\text { RT (IIIB) } \\
\text { • Protein priming induced }\end{array}$ & $\begin{array}{l}\text { GTKALTEVIPLTEEA } \\
\text { I-cells that recognize peptide }\end{array}$ & in vitro stimulation & human & Manca1995b \\
\hline RT (294-308) & $\begin{array}{l}\text { RT (IIIB) } \\
\text { - Protein priming induced }\end{array}$ & $\begin{array}{l}\text { PLTEEAELELAENRE } \\
\text { T-cells that recognize peptide }\end{array}$ & in vitro stimulation & human & Manca1995b \\
\hline RT (303-317) & $\begin{array}{l}\text { RT (IIIB) } \\
\text { - Protein priming induced }\end{array}$ & $\begin{array}{l}\text { LAENRE I LKEPVHGV } \\
\text { T-cells that recognize peptide }\end{array}$ & in vitro stimulation & human & Manca1995b \\
\hline RT (384-398) & $\begin{array}{l}\text { RT (IIIB) } \\
\text { - Protein priming induced }\end{array}$ & $\begin{array}{l}\text { GKTPKFKLPIQKETW } \\
\text { I-cells that recognize peptide }\end{array}$ & in vitro stimulation & human & Manca1995b \\
\hline RT (414-428) & $\begin{array}{l}\text { Pol (596-610) } \\
\text { - Epitope name: Pol } 596 \\
\text { - Eleven peptides were ide } \\
\text { responses from multiple } \\
\text { - This epitope binds eleve } \\
\text { DRB } 1^{*} 0901, \text { DRB5*01 } \\
\text { - This epitope sequence is } \\
\text { - } 6 / 22 \text { HIV infected indivi } \\
\text { also not have recall resp }\end{array}$ & $\begin{array}{l}\text { WEFVNTP P LVKLWYQ } \\
\text { atified that had the HLA-DR sup } \\
\text { HIV-infected donors } \\
\text { HLA-DR alleles: DRB } 1 * 0101 \text {, } \\
1 \text { and DRB } 4 * 0101 \text {, with an IC } 50 \\
\text { conserved in } 84 \% \text { of clade B iso } \\
\text { luals responded to this epitope } \\
\text { nses to rec HIV- } 1 \text { whole protein }\end{array}$ & $\begin{array}{l}\text { HIV-1 infection } \\
\text { ermotif, all were found to } \\
\text { DRB } 1 * 1501 \text {, DRB } 1 * 0401 \\
\text { threshold below } 1,000 \mathrm{nM} \\
\text { lates } \\
\text { 3/22 responded to some of } \\
\text { s) }\end{array}$ & $\begin{array}{l}\text { human (DR superm } \\
\text { IC class II DR molec } \\
405, \text { DRB } 1 * 1101, \text { D } \\
\text { permotif epitopes, th }\end{array}$ & $\begin{array}{l}\text { Wilson2001 } \\
\text { nd all elicited proliferative } \\
302 \text {, DRB } 1 * 0701 \text {, DRB } 1 * 0802 \text {, } \\
\text { n-responder peptides tended to }\end{array}$ \\
\hline RT (429-443) & $\begin{array}{l}\text { RT (IIIB) } \\
\text { - Protein priming induce }\end{array}$ & $\begin{array}{l}\text { LEKEP IVGAETFYVD } \\
\text { T-cells that recognize peptide }\end{array}$ & in vitro stimulation & human & Manca1995b \\
\hline RT (432-450) & $\begin{array}{l}\text { RT (431-450 HXB2) } \\
\text { The goal of this study w } \\
\text { could bind to more than } \\
\text { donors. } \\
\text { This epitope binds with } \\
\text { was not considered broa }\end{array}$ & $\begin{array}{l}\text { EP IVGAETFYVDGAANRET } \\
\text { s to identify Th epitopes that col } \\
\text { ne HLA class II protein, and bu } \\
\text { igh affinity to HLA-DR1, -DR2, } \\
\text { ly cross-presented. }\end{array}$ & $\begin{array}{l}\text { HIV-1 infection, in vitro } \\
\text { stimulation } \\
\text { ld be cross-presented by } \mathrm{m} \\
\text { tonly } 2 / 5 \text { could stimulate } \mathrm{s} \\
\text {-DR3, and -DR4, but stim }\end{array}$ & $\begin{array}{l}\text { human (DR1, DR2, } \\
\text { DR3, DR4) } \\
\text { ss II HLA molecules } \\
\text { feration responses in } \\
\text { ong proliferation res }\end{array}$ & $\begin{array}{l}\text { vanderBurg } 1999 \\
\text { T peptides were identified that } \\
\text { AC derived from multiple healthy } \\
\text { e in only } 1 / 4 \text { individuals tested so }\end{array}$ \\
\hline
\end{tabular}




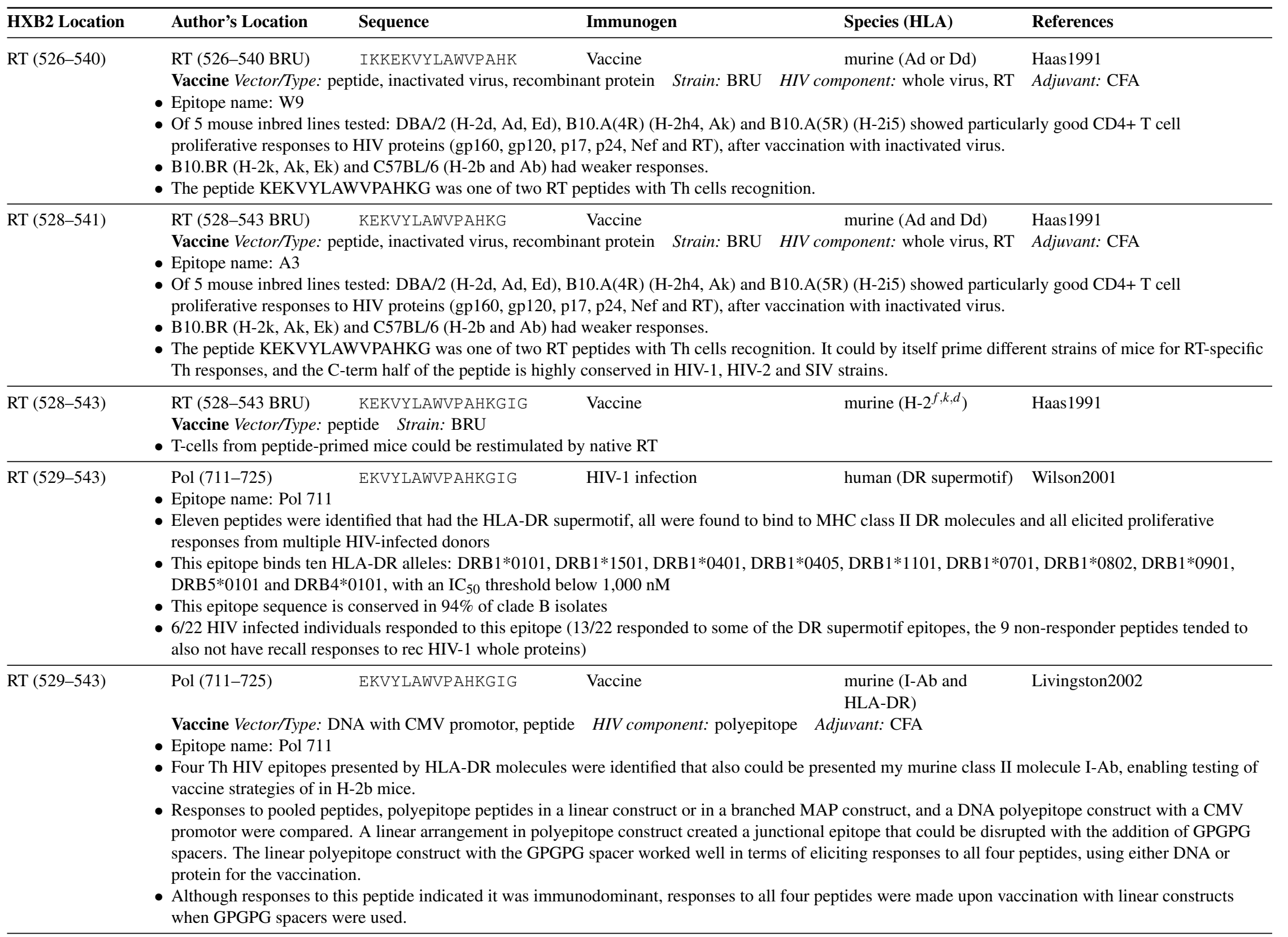




\begin{tabular}{|c|c|c|c|c|c|}
\hline HXB2 Location & Author's Location & Sequence & Immunogen & Species (HLA) & References \\
\hline RT (530-544) & $\begin{array}{l}\text { Pol (712-726) } \\
\text { - Epitope name: Pol } 712 \\
\text { - Eleven peptides were ide } \\
\text { responses from multiple } \\
\text { - This epitope binds ten H } \\
\text { DRB5*0101 and DRB4 } \\
\text { - This epitope sequence is } \\
\text { - } 6 / 22 \text { HIV infected indivi } \\
\text { also not have recall resp }\end{array}$ & $\begin{array}{l}\text { KVYLAWVPAHKGIGG } \\
\text { tified that had the HLA- } \\
\text { HIV-infected donors } \\
\text { A-DR alleles: DRB } 1 * 0 \\
0101 \text {, with an } \text { IC }_{50} \text { thresl } \\
\text { conserved in } 89 \% \text { of clac } \\
\text { luals responded to this ep } \\
\text { nses to rec HIV-1 whole }\end{array}$ & $\begin{array}{l}\text { HIV-1 infection } \\
\text { ermotif, all were } \\
\text { B } 1 * 1501, \text { DRB1 } \\
\text { ates } 1,000 \mathrm{nM} \\
3 / 22 \text { responded t }\end{array}$ & $\begin{array}{l}\text { human (DR supermotif) } \\
\text { IC class II DR molecules a } \\
\text { DRB } 1 * 1101, \mathrm{DRB} 1 * 070 \\
\text { permotif epitopes, the } 9 \mathrm{n}\end{array}$ & $\begin{array}{l}\text { Wilson2001 } \\
\text { and all elicited proliferative } \\
\text { on-responder peptides tended to }\end{array}$ \\
\hline
\end{tabular}




\section{III-B-6 RT-Integrase Helper T-Cell Epitopes}

\begin{tabular}{lllll}
\hline HXB2 Location & Author's Location & Sequence & Immunogen & Species (HLA) \\
\hline RT-Integrase (553-3) & RT (720-730 LAI) & SAGIRKVLFLD & HIV-1 infection & human \\
& Stimulates T-cell proliferation in HIV-infected donors & & Schrier1989 \\
\hline
\end{tabular}




\section{III-B-7 Integrase Helper T-Cell Epitopes}

\begin{tabular}{|c|c|c|c|c|}
\hline HXB2 Location & Author's Location & Sequence & Immunogen & Species (HLA) \\
\hline ntegrase (16-30) & $\begin{array}{l}\text { Pol (758-772) } \\
\text { - Epitope name: Pol } 758 \\
\text { - Eleven peptides were ide } \\
\text { responses from multiple } \\
\text { - This epitope binds eight } \\
\text { DRB } 1^{*} 0101 \text {, with an } \mathrm{IC}_{5} \\
\text { - This epitope sequence is } \\
\text { - } 8 / 22 \text { HIV infected indivic } \\
\text { also not have recall respo }\end{array}$ & $\begin{array}{l}\text { HSNWRAMASDFNLPP } \\
\text { ntified that had the HLA-I } \\
\text { HIV-infected donors } \\
\text { HLA-DR alleles: DRB4*( } \\
0 \text { threshold below } 1,000 \mathrm{n} \\
\text { conserved in } 68 \% \text { of clad } \\
\text { luals responded to this ep } \\
\text { nses to rec HIV-1 whole } \mathrm{p}\end{array}$ & $\begin{array}{l}\text { HIV-1 infection } \\
\text { ermotif, all were } \mathrm{f} \\
\text { RB } 5 * 0101, \mathrm{DRB} \\
\text { lates } \\
3 / 22 \text { responded to } \\
\text { ) }\end{array}$ & $\begin{array}{l}\text { human (DR supermotif) Wilson2001 } \\
\text { HC class II DR molecules and all elicited proliferative } \\
01 \text {, DRB } 1 * 1101, \text { DRB } 1 * 0405, \text { DRB } 1 * 0401 \text { and } \\
\text { upermotif epitopes, the } 9 \text { non-responder peptides tended to }\end{array}$ \\
\hline Integrase (172-186) & $\begin{array}{l}\text { RT (899-913 LAI) } \\
\text { - Stimulates T-cell prolifer }\end{array}$ & $\begin{array}{l}\text { LKTAVQMAVF I HNFK } \\
\text { ation in HIV-infected don }\end{array}$ & HIV-1 infection & Schrier1989 \\
\hline Integrase (173-187) & $\begin{array}{l}\text { Pol (915-929) } \\
\text { - Epitope name: Pol } 915 \\
\text { - Eleven peptides were ide } \\
\text { responses from multiple } \\
\text { - This epitope binds seven } \\
\text { IC } 50 \text { threshold below } 1,0 \\
\text { - This epitope sequence is } \\
\text { - } 6 / 22 \text { HIV infected indivic } \\
\text { also not have recall respo }\end{array}$ & $\begin{array}{l}\text { KTAVQMAVFF I HNFKR } \\
\text { ntified that had the HLA-I } \\
\text { HIV-infected donors } \\
\text { HLA-DR alleles: DRB5* } \\
00 \mathrm{nM} \\
\text { conserved in } 94 \% \text { of clad } \\
\text { luals responded to this ep } \\
\text { nses to rec HIV-1 whole } \mathrm{p}\end{array}$ & $\begin{array}{l}\text { HIV-1 infection } \\
\text { ermotif, all were } \mathrm{f} \\
\text { DRB } 1 * 1302 \text {, DRB } \\
\text { lates } \\
\text { 3/22 responded to } \\
\text { ) }\end{array}$ & $\begin{array}{l}\text { human (DR supermotif) Wilson2001 } \\
\text { HC class II DR molecules and all elicited proliferative } \\
405 \text {, DRB } 1 * 0401 \text {, DRB } 1 * 1501 \text { and DRB } 1 * 0101 \text {, with an } \\
\text { upermotif epitopes, the } 9 \text { non-responder peptides tended to }\end{array}$ \\
\hline Integrase (196-210) & $\begin{array}{l}\text { RT (923-937 LAI) } \\
\text { - Stimulates T-cell prolifer }\end{array}$ & $\begin{array}{l}\text { AGERIVDI IATD IQT } \\
\text { ation in HIV-infected don }\end{array}$ & HIV-1 infection & Schrier1989 \\
\hline Integrase (214-228) & $\begin{array}{l}\text { Pol }(956-970) \\
\text { - Epitope name: Pol } 956 \\
\text { - Eleven peptides were ide } \\
\text { responses from multiple } \\
\text { - This epitope binds twelve } \\
\text { DRB } 1 * 0405, \text { DRB } 1 * 040 \\
\text { - This epitope sequence is } \\
\text { - } 8 / 22 \text { HIV infected indivic } \\
\text { also not have recall respo }\end{array}$ & $\begin{array}{l}\text { QKQITKIQNFRVYYR } \\
\text { ntified that had the HLA-I } \\
\text { HIV-infected donors } \\
\text { HLA-DR alleles: DRB4 } \\
\text { 1, DRB1*1501 and DRB } \\
\text { conserved in } 95 \% \text { of clad } \\
\text { luals responded to this ep } \\
\text { nses to rec HIV-1 whole } \mathrm{p}\end{array}$ & $\begin{array}{l}\text { HIV-1 infection } \\
\text { ermotif, all were f } \\
\text { DRB }{ }^{*} 0101 \text {, DRF } \\
\text {, with an } \mathrm{IC}_{50} \text { thre } \\
\text { lates } \\
3 / 22 \text { responded to } \\
\text { ) }\end{array}$ & $\begin{array}{l}\text { human (DR supermotif) Wilson2001 } \\
\text { HC class II DR molecules and all elicited proliferative } \\
802 \text {, DRB } 1 * 0701, \text { DRB } 1 * 1302 \text {, DRB } 1 * 1201 \text {, DRB } 1 * 1101 \text {, } \\
\text { nM } \\
\text { upermotif epitopes, the } 9 \text { non-responder peptides tended to }\end{array}$ \\
\hline Integrase (215-227) & $\begin{array}{l}\text { RT (942-954 LAI) } \\
\text { - Stimulates T-cell prolifer }\end{array}$ & $\begin{array}{l}\text { KQITKIQNFRVYY } \\
\text { ation in HIV-infected do }\end{array}$ & HIV-1 infection & Schrier1989 \\
\hline
\end{tabular}




\section{III-B-8 Pol Helper T-Cell Epitopes}

\begin{tabular}{lllll}
\hline HXB2 Location & Author's Location & Sequence & Immunogen & Species (HLA) \\
\hline Pol & Gag/Pol & References & murine & Kim1997b \\
& Vaccine Vector/Type: DNA & HIV component: GAG, POL, VIF Adjuvant: B7 and IL-12 expression vector & A A
\end{tabular}

- A gag/pol DNA vaccine delivered in conjunction with the plasmid encoding the co-stimulatory molecules B7 and IL-12 gives a dramatic increase in both the cytotoxic and proliferative responses in mice

\begin{tabular}{lllc}
\hline Pol Gag/Pol & Vaccine & murine & Kim1997d \\
& Vaccine Vector/Type: DNA & HIV component: gp160, GAG, POL Adjuvant: CD86 expression vectors
\end{tabular}

- A gag/pol DNA vaccine delivered in conjunction with the plasmid encoding the co-stimulatory molecule CD86 gives an increase in proliferative responses to $\operatorname{Pr} 55$ in mice

\begin{tabular}{lllc}
\hline Pol & Gag/Pol (MN) & Vaccine & chimpanzee
\end{tabular}

- Co-stimulatory molecules co-expressed with an HIV-1 immunogen in a DNA vaccine used to enhance the immune response - co-expression of CD86, but not CD80, dramatically increased both HIV Env and Gag/Pol specific CTL and Th proliferative responses

Pol Pol HIV-1 infection human

- 5/10 chronically HIV infected patients with low CD4+ counts who received HAART therapy and experienced immune reconstitution displayed p24, p17 and p66 T-helper CD4 proliferative responses, in contrast to 0/8 chronically HIV infected patients with high CD4+ counts at the initiation of antiretroviral treatment

- This surprising result could be due to the low CD4 nadir patients being more likely to have thymic regeneration or a peripheral expansion of T cells

Pol p66 HIV-1 infection human Oxenius2000

- Patients who started therapy at acute HIV infection (three with sustained therapy, two with limited therapy upon early infection) had strong HIV specific CD4 proliferative responses and were able to maintain a CTL response even with undetectable viral load - three patients that had delayed initiation of HAART had no HIV specific CD4 proliferative responses and lost their CTL responses when HAART was eventually given and their viral loads became undetectable

Pol p66 HIV-1 infection $\quad$ Palmer2002

- CD4+ T cell proliferative responses from 33 HIV-1 infected patients with HAART suppression were compared to 19 patients with active viral replication (HAART failures and HAART naive). Patients with HAART suppression showed stronger p24- and p66-specific proliferative responses compared to patient groups with active HIV-1 replication, suggesting active viral replication in vivo specifically reduces proliferation responses.

- No differences in the frequency of HIV-specific CD4+ T-cells that were positive for cytokine secretion in a flow cytometry assay were found in the HAART suppressed group versus the group with active viral replication.

\begin{tabular}{llcc}
\hline Pol & (BRU) & Vaccine & murine
\end{tabular}

- Of 5 mouse inbred lines tested DBA/2 (H-2d, Ad, Ed), B10.A(4R) (H-2h4, Ak) and B10.A(5R) (H-2i5) showed particularly good CD4+ T cell proliferative responses to HIV proteins (gp160, gp120, p17, p24, Nef and RT), after vaccination with inactivated virus.

- B10.BR (H-2k, Ak, Ek) and C57BL/6 (H-2b and Ab) had weaker responses.

\begin{tabular}{llll}
\hline Pol & RT (248-256 HXB2) & in vitro stimulation & human (DR5) \\
& CD4+ T-cell lines from uninfected individuals by stimulation with p66-pulsed APC & Manca1995b
\end{tabular}




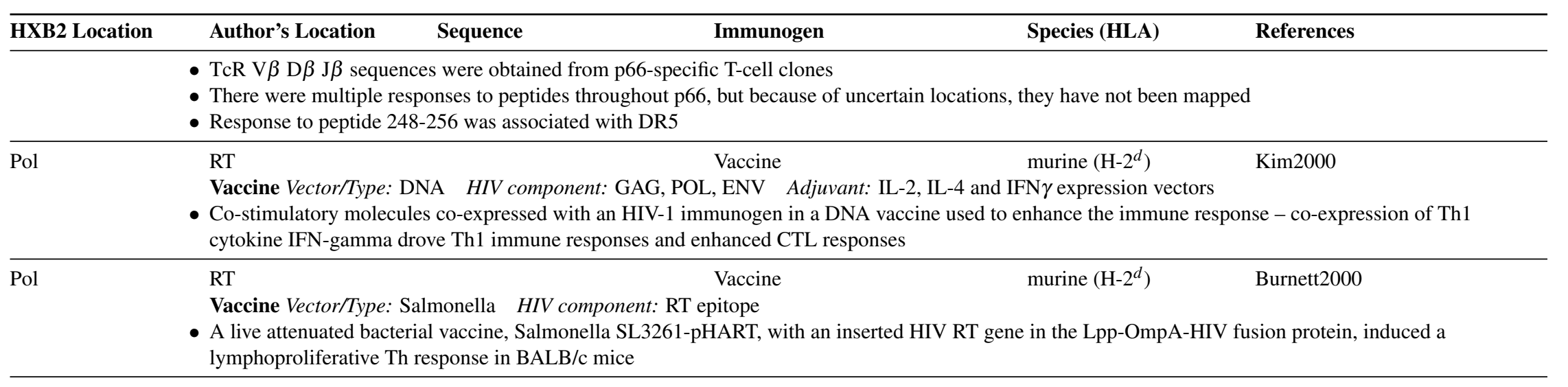




\section{III-B-9 Vif Helper T-Cell Epitopes}

\begin{tabular}{|c|c|c|c|c|c|}
\hline HXB2 Location & Author's Location & Sequence & Immunogen & Species (HLA) & References \\
\hline Vif $(65-76)$ & \multicolumn{5}{|c|}{ - T-cell response to this epitope persisted after seroreversion } \\
\hline Vif (81-96) & \multicolumn{4}{|c|}{ - T-cell response to this epitope persisted after seroreversion } & Ranki1997 \\
\hline Vif & \multicolumn{5}{|c|}{$\begin{array}{l}\text { Vaccine Vector/Type: DNA HIV component: Vif, Vpu, Nef } \\
\text { - Splenocytes from BALB/c mice immunized with pVVN-P DNA were incubated with Vif, Vpu or Nef antigens for } 3 \text { days and assayed for IL-4 and } \\
\text { IFN-gamma levels } \\
\text { - Antigen stimulation increased IFN-gamma production in pVVN-P immunized mice, indicating a Th1 response } \\
\text { - IL-4 production was not significantly changed after antigen stimulation compared to control levels } \\
\text { - Cross-clade CTL activity was also observed: A, B clade, CRF01(AE) clade antigens could serve as targets for the B clade immunization stimulated CTL - } \\
\text { an HIV-1 AC recombinant, however, did not stimulate a CTL response, but was expressed at lower levels on the target cell }\end{array}$} \\
\hline
\end{tabular}




\section{III-B-10 Vpr Helper T-Cell Epitopes}

\begin{tabular}{|c|c|c|c|c|c|}
\hline$\overline{\text { HXB2 Location }}$ & Author's Location & Sequence & Immunogen & Species (HLA) & References \\
\hline \multirow[t]{2}{*}{ Vpr (66-80) } & Vpr (66-80 IIIB) & QLLF IHFRIGCRHSR & HIV-1 infection & human & Sarobe1994 \\
\hline & - This peptide was for & stimulate proliferative $r$ & $\mathrm{~s}$ in $37.5 \%$ of $\mathrm{HI}$ & uals & \\
\hline \multirow[t]{2}{*}{$\operatorname{Vpr}(66-80)$} & Vpr (66-80 IIIB) & QLLFIHFRIGCRHSR & Vaccine & murine $\left(\mathrm{H}-2^{d}\right)$ & Sarobe1994 \\
\hline & $\begin{array}{l}\text { Vaccine Vector/Type } \\
\text { - Included as a Th stir }\end{array}$ & $\begin{array}{l}\text { ory component of peptid } \\
\text { orde }\end{array}$ & es that also inco & & \\
\hline
\end{tabular}




\section{III-B-11 Tat Helper T-Cell Epitopes}

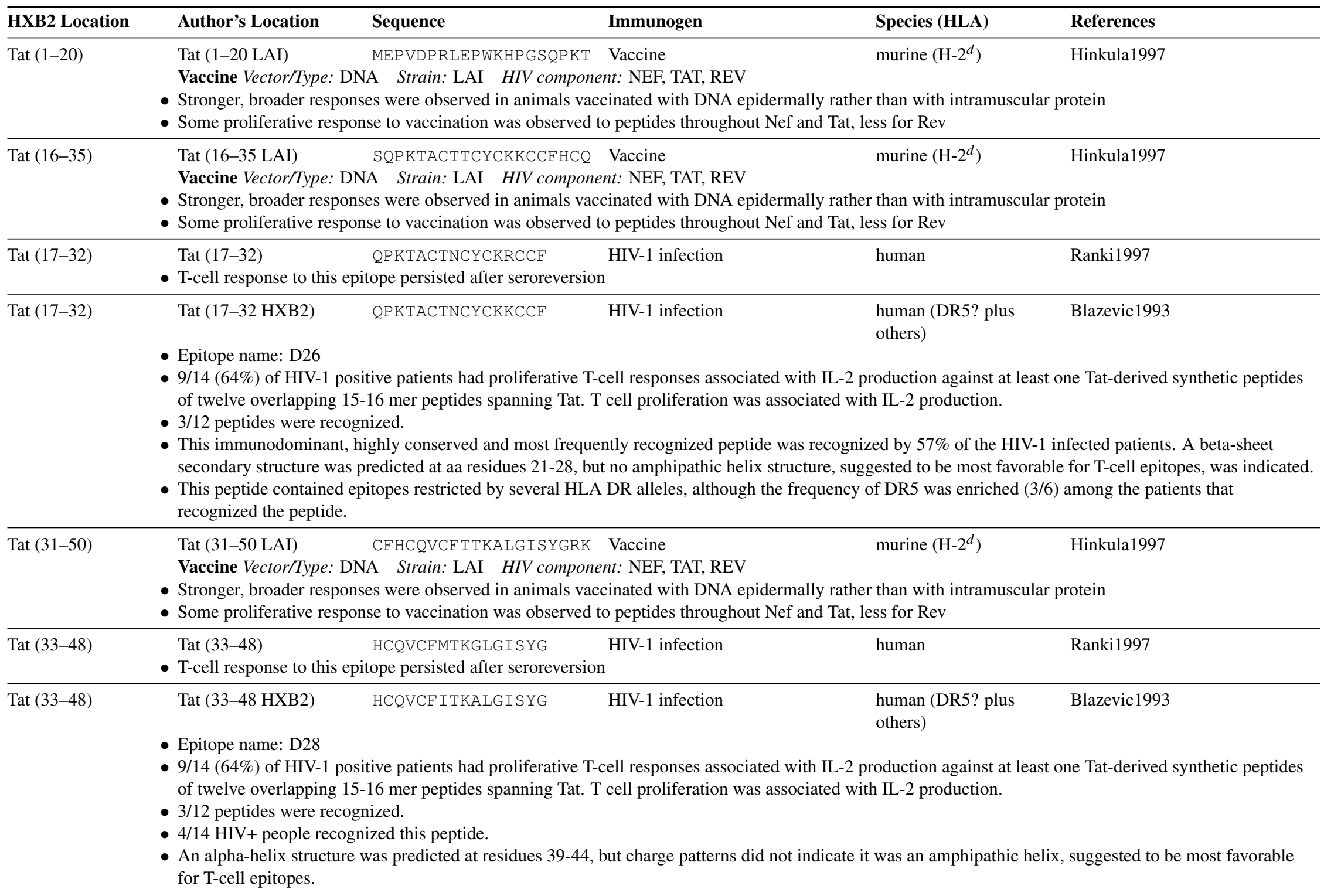




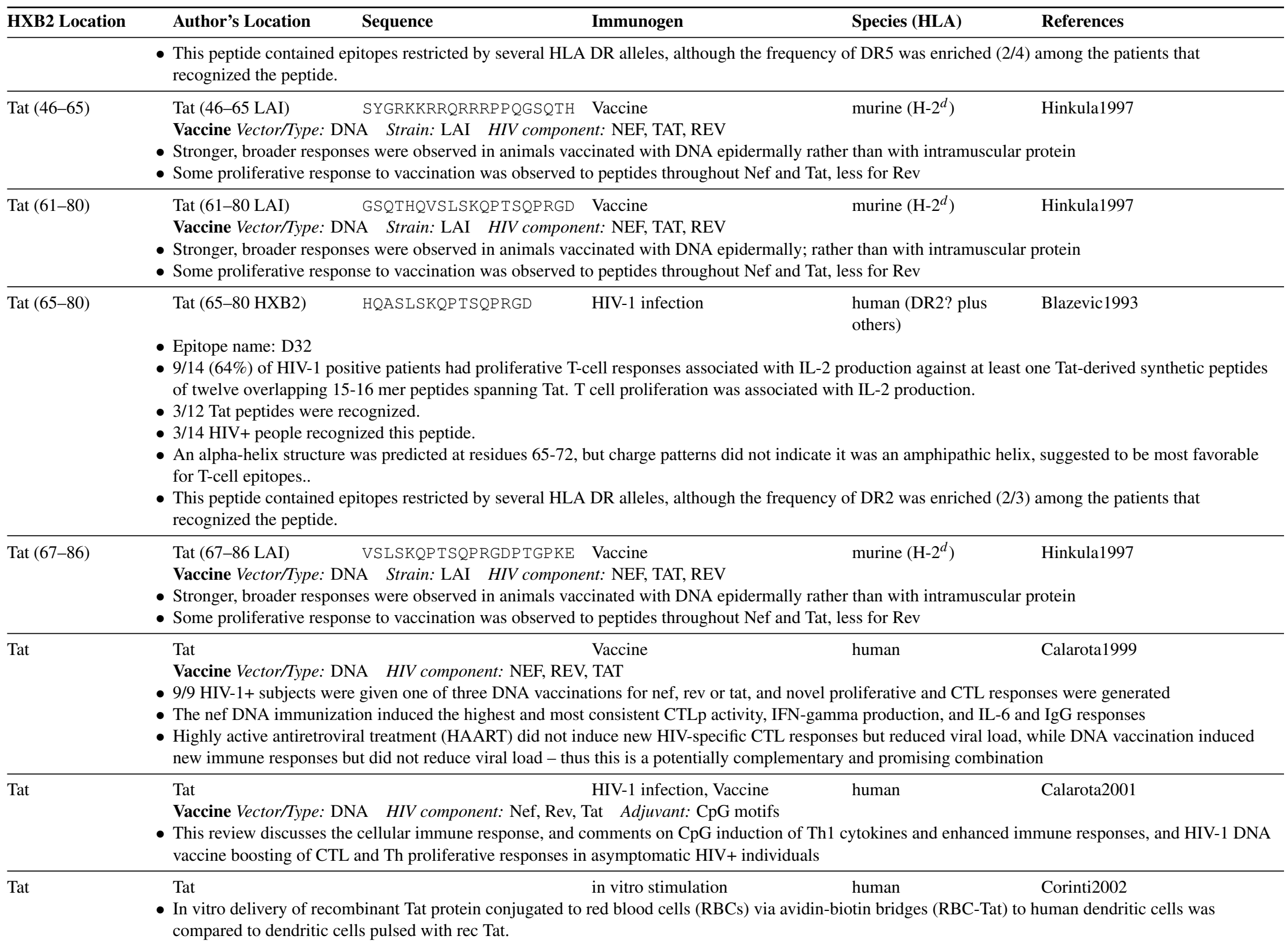




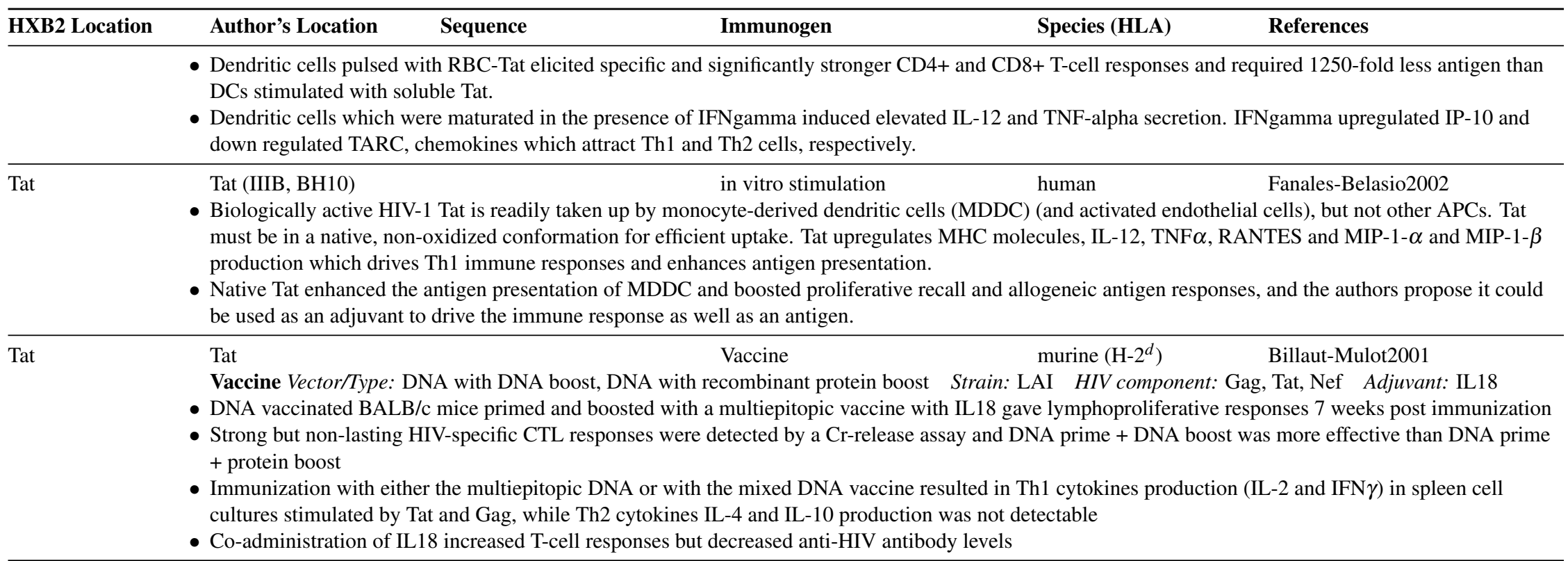




\section{III-B-12 Rev Helper T-Cell Epitopes}

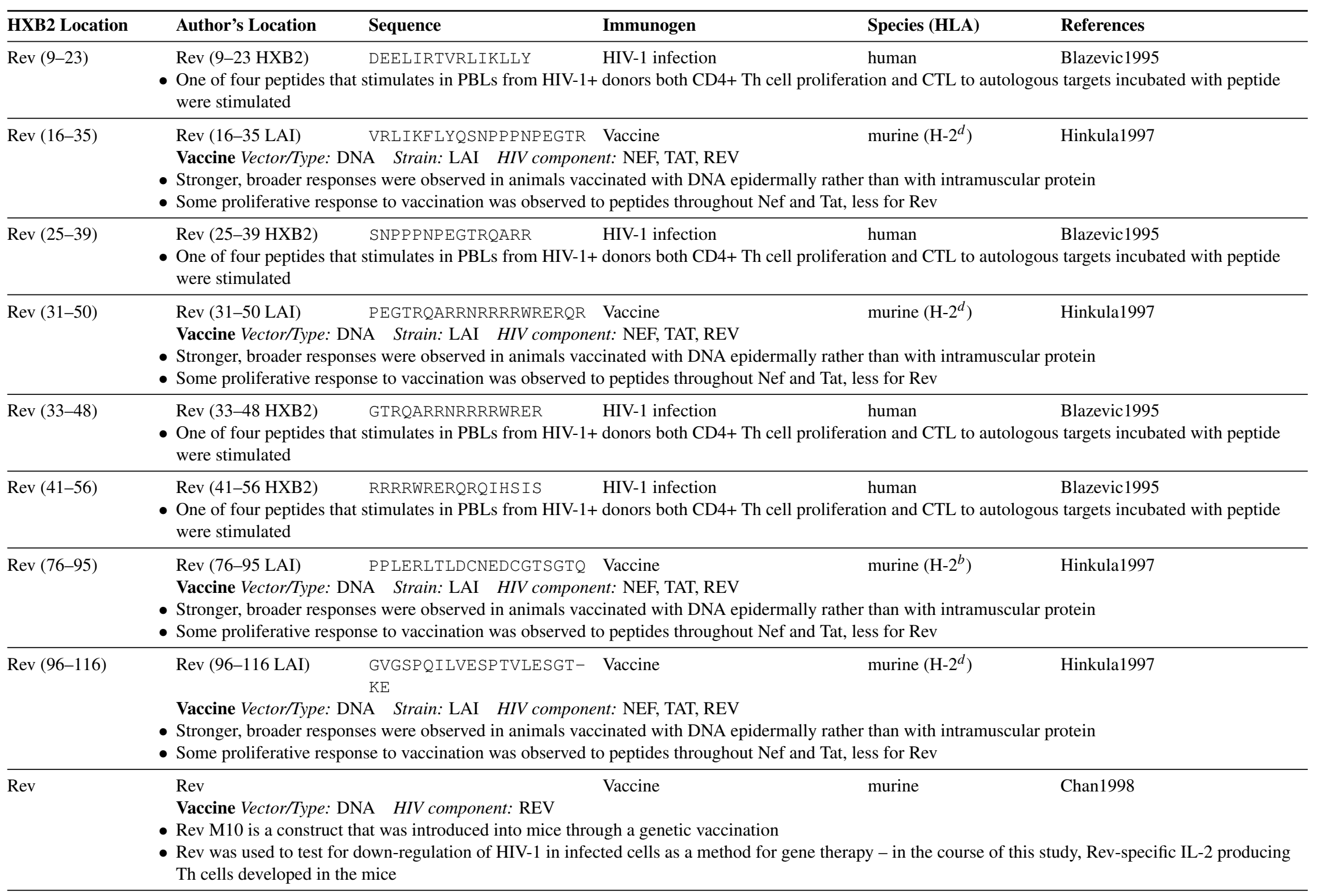




\begin{tabular}{lllccc}
\hline HXB2 Location & Author's Location & Sequence & Immunogen & Species (HLA) & References \\
\hline Rev & Rev & Vaccine & human & Calarota1999
\end{tabular}

Vaccine Vector/Type: DNA HIV component: Nef, Rev Tat

- 9/9 HIV-1 + subjects were given one of three DNA vaccinations for nef, rev or tat, and novel proliferative and CTL responses were generated

- The nef DNA immunization induced the highest and most consistent CTLp activity, IFN-gamma production, and IL-6 and IgG responses

- Highly active antiretroviral treatment (HAART) did not induce new HIV-specific CTL responses but reduced viral load, while DNA vaccination induced new immune responses but did not reduce viral load - thus this is a potentially complementary and promising combination

\begin{tabular}{llcc}
\hline Rev & Rev & HIV-1 infection, Vaccine & human \\
& Vaccine Vector/Type: DNA & HIV component: Nef, Rev, Tat Adjuvant: CpG motifs &
\end{tabular}

Vaccine Vector/Type: DNA HIV component: Nef, Rev, Tat Adjuvant: CpG motifs

- This review discusses the cellular immune response, and comments on CpG induction of Th1 cytokines and enhanced immune responses, and HIV-1 DNA vaccine boosting of CTL and Th proliferative responses in asymptomatic HIV+ individuals

Rev

Vaccine human

Vaccine Vector/Type: DNA with CMV promotor Strain: MN HIV component: Env, Rev Adjuvant: bupivacaine

MacGregor2002

- A phase I clinical trial of a HIV-1 Env and Rev DNA vaccine with a CMV promoter was conducted and Th proliferative, CTL and Elispot responses monitored. The construct was modified for safety and included no LTRs or packaging signals. The vaccine strategy was safe, and elicited strong CD4-T cell responses, but not CD8 T-cell responses. Rev elicited strong Th responses, and is a early produced protein so may confer advantages.

- With a 300 ug dose, 4/6 individuals had a lymphocyte proliferation (LP) responses to gp120, 3/6 to Rev.

- With a 1000 ug dose, 4/6 individuals had a LP and 2/6 had IFNgamma Elispot responses to gp160; 3/6 had LP, and 4/6 had IFNgamma Elispot responses to Rev.

- No responses to three specific CTL epitopes were observed by Elispot in individuals with appropriate HLA. Some cytoxic activity against whole protein was observed that was CD4+ T-cell mediated. 


\section{III-B-13 Vpu Helper T-Cell Epitopes}

\begin{tabular}{|c|c|c|c|c|c|}
\hline HXB2 Location & Author's Location & Sequence & Immunogen & Species (HLA) & References \\
\hline Vpu (19-34) & \multicolumn{5}{|c|}{ - T-cell response to this epitope persisted after seroreversion } \\
\hline Vpu & \multicolumn{5}{|c|}{$\begin{array}{l}\text { Vaccine Vector/Type: DNA HIV component: Vif, Vpu, Nef } \\
\text { - Splenocytes from BALB/c mice immunized with pVVN-P DNA were incubated with Vif, Vpu or Nef antigens for } 3 \text { days and assayed for IL-4 and } \\
\text { IFN-gamma levels } \\
\text { - Antigen stimulation increased IFN-gamma production in pVVN-P immunized mice, indicating a Th1 response } \\
\text { - IL-4 production was not significantly changed after antigen stimulation compared to control levels } \\
\text { - Cross-clade CTL activity was also observed: A, B clade, CRF01(AE) clade antigens could serve as targets for the B clade immunization stimulated CTL - } \\
\text { an HIV-1 AC recombinant, however, did not stimulate a CTL response, but was expressed at lower levels on the target cell }\end{array}$} \\
\hline
\end{tabular}




\section{III-B-14 gp160 Helper T-Cell Epitopes}

\begin{tabular}{|c|c|c|c|c|c|}
\hline HXB2 Location & Author's Location & Sequence & Immunogen & Species (HLA) & References \\
\hline gp160 (30-51) & \multicolumn{5}{|c|}{$\begin{array}{l}\text { - Epitope name: A1 } \\
\text { - Th proliferative responses were studied in } 36 \text { asymptomatic HIV-1+ patients. PBMC from } 15 / 36 \text { patients responded to stimulation with gp120 synthetic } \\
\text { overlapping peptide pools. } 10 \text { of the responding patients recognized three or more peptide pools. } \\
\text { - After } 12 \text { months, most responses were lost or diminished, and specific responses fluctuated. } 4 / 10 \text { of the responders at baseline had new responses at } 12 \\
\text { months, as did } 5 / 15 \text { non-responders. } \\
\text { - IL-2 production was a more sensitive and well-preserved measure of Th function than proliferation. } \\
\text { - } 2 / 15 \text { responders recognized this peptide, mean SI }=4.6 \text {. }\end{array}$} \\
\hline gp160 (32-44) & \multicolumn{5}{|c|}{$\begin{array}{l}\text { Vaccine Vector/Type: peptide } \\
\text { - Peptides induced T-cell proliferative response to immunizing peptide and to gp160 }\end{array}$} \\
\hline gp160 (38-48) & \multicolumn{5}{|c|}{$\begin{array}{l}\text { Vaccine Vector/Type: peptide } \\
\text { - Synthetic peptide derived from conserved region of the HIV-1 envelope that stimulates a proliferative response in mice } \\
\text { - Proliferative response to this peptide was observed in } 3 / 3 \text { immunized rhesus monkeys }\end{array}$} \\
\hline gp160 (38-48) & $\begin{array}{l}\text { Env (45-55) } \\
\text { - Seven out of nine HI } \\
\text { peptide (peptide 104 } \\
\text { - This peptide, along v } \\
\text { - Peptide } 104 \text { elicited }\end{array}$ & $\begin{array}{l}\text { VYYGVPVWKEA } \\
\text { ected chimpanzees and eight ou } \\
\text { HIV negative individuals show } \\
\text { other peptides from conserved } \\
\text { erative responses in inbred mou }\end{array}$ & $\begin{array}{l}\text { HIV-1 infection } \\
\text { t of seventeen HIV-positive } \\
\text { ed a response } \\
\text { regions of envelope, can in } \\
\text { se strains and outbred rhes }\end{array}$ & $\begin{array}{l}\text { human, chimpanzee } \\
\text { humans exhibited positive prol } \\
\text { duce proliferative responses to } \mathrm{F} \\
\text { us monkeys in previous study by }\end{array}$ & $\begin{array}{l}\text { Nehete } 1998 \mathrm{~b} \\
\text { tive responses to this conserved } \\
\text { and may be useful for vaccines } \\
\text { ne group }\end{array}$ \\
\hline gp160 (38-48) & $\begin{array}{l}\text { gp120 (45-55) } \\
\text { Vaccine Vector/Type } \\
\text { - Peptides induced T-c }\end{array}$ & $\begin{array}{l}\text { VYYGVPVWKEA } \\
\text { tide } \\
\text { oliferative response to immuni }\end{array}$ & $\begin{array}{l}\text { Vaccine } \\
\text { ing peptide and to gp160 }\end{array}$ & murine $\left(\mathrm{H}-2^{b x k, s x d}\right)$ & Sastry1991 \\
\hline gp160 (41-54) & $\begin{array}{l}\text { Env }(48-60) \\
\text { Vaccine Vector/Type } \\
\text { - Synthetic peptide des } \\
\text { - Despite the prolifera }\end{array}$ & $\begin{array}{l}\text { GVPVWKEATTLEC } \\
\text { tide } \\
\text { from conserved region of the HI } \\
\text { esponse to this peptide in mice, }\end{array}$ & $\begin{array}{l}\text { Vaccine } \\
\text { IV-1 envelope that stimulat } \\
\text { no response was observed i }\end{array}$ & $\begin{array}{l}\text { Rhesus macaque } \\
\text { a proliferative response in mi } \\
3 \text { rhesus monkeys }\end{array}$ & Nehete1993 \\
\hline gp160 (41-54) & $\begin{array}{l}\text { gp120 (48-61) } \\
\text { Vaccine Vector/Type } \\
\text { - Peptides induced T-c }\end{array}$ & $\begin{array}{l}\text { GVPVWKEATTLFC } \\
\text { tide } \\
\text { oliferative response to immuni }\end{array}$ & $\begin{array}{l}\text { Vaccine } \\
\text { ing peptide and to gp160 }\end{array}$ & murine $\left(\mathrm{H}-2^{s x d}\right)$ & Sastry1991 \\
\hline gp160 (41-60) & $\begin{array}{l}\text { gp120 (40-59 89.6) } \\
\text { Vaccine Vector/Type } \\
\text { coli (mLT) } \\
\text { - Epitope name: Pepti }\end{array}$ & $\begin{array}{l}\text { GVPVWREATTTLFCASDAKA } \\
\text { ombinant protein } \quad \text { Strain: } 89.6\end{array}$ & $\begin{array}{l}\text { Vaccine } \\
\text { HIV component: gp120 }\end{array}$ & $\begin{array}{l}\text { murine } \\
\text { djuvant: } \mathrm{R} 192 \mathrm{G} \text { mutant heat- }\end{array}$ & $\begin{array}{l}\text { Dai2001 } \\
\text { le toxin from enterotoxigenic } \mathrm{E} \text {. }\end{array}$ \\
\hline
\end{tabular}




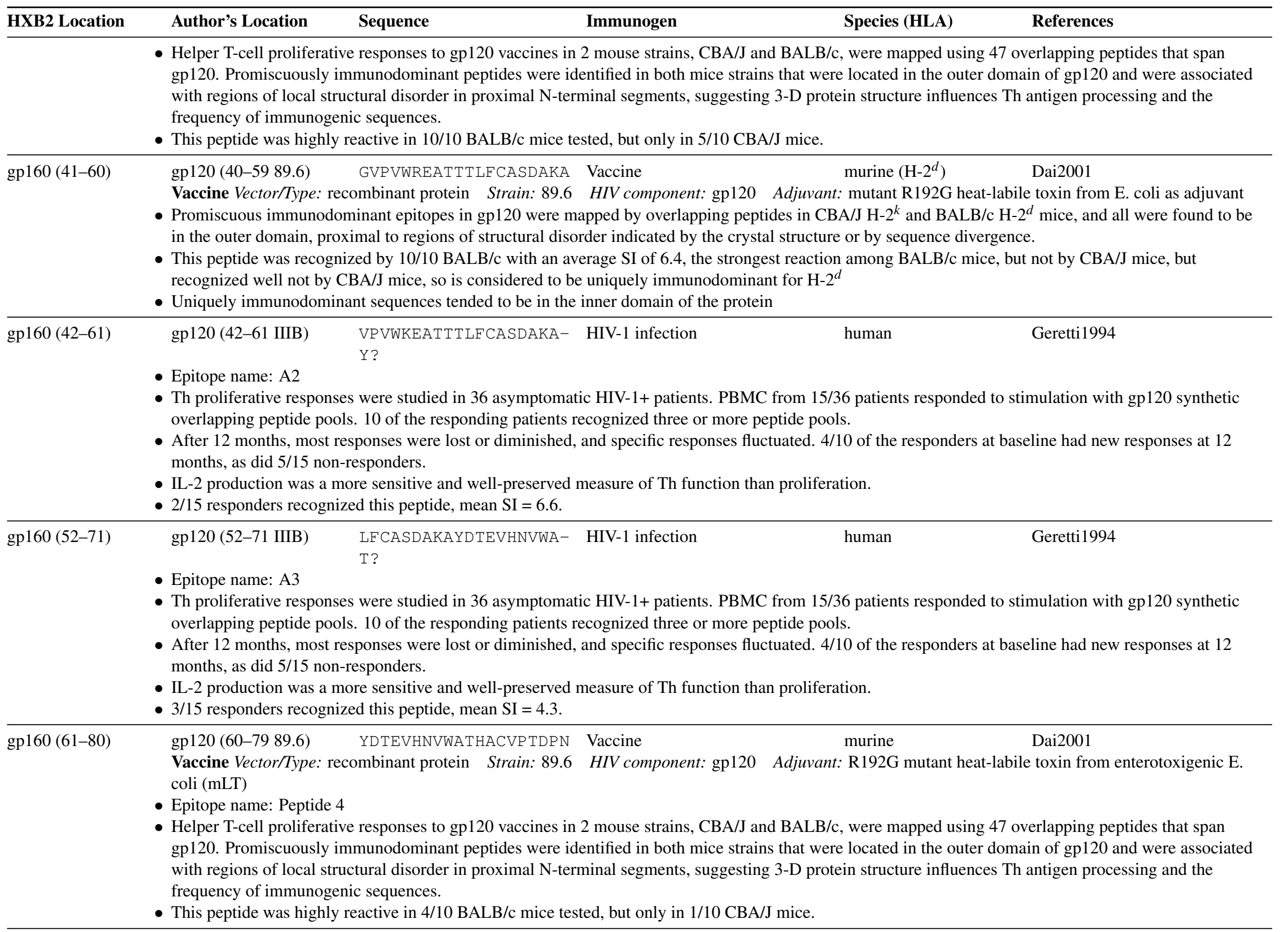




\begin{tabular}{|c|c|c|c|c|c|}
\hline HXB2 Location & Author's Location & Sequence & Immunogen & Species (HLA) & References \\
\hline gp160 (62-80) & $\begin{array}{l}\text { gp120 (62-80 IIIB) } \\
\text { Epitope name: A4 }\end{array}$ & DTEVHNVWATHACVPTDPN? & HIV-1 infection & human & Geretti1994 \\
\hline
\end{tabular}

- Epitope name: A4

- Th proliferative responses were studied in 36 asymptomatic HIV-1+ patients. PBMC from 15/36 patients responded to stimulation with gp120 synthetic overlapping peptide pools. 10 of the responding patients recognized three or more peptide pools.

- After 12 months, most responses were lost or diminished, and specific responses fluctuated. 4/10 of the responders at baseline had new responses at 12 months, as did 5/15 non-responders.

- IL-2 production was a more sensitive and well-preserved measure of Th function than proliferation.

- $1 / 15$ responders recognized this peptide, $\mathrm{SI}=3.5$.

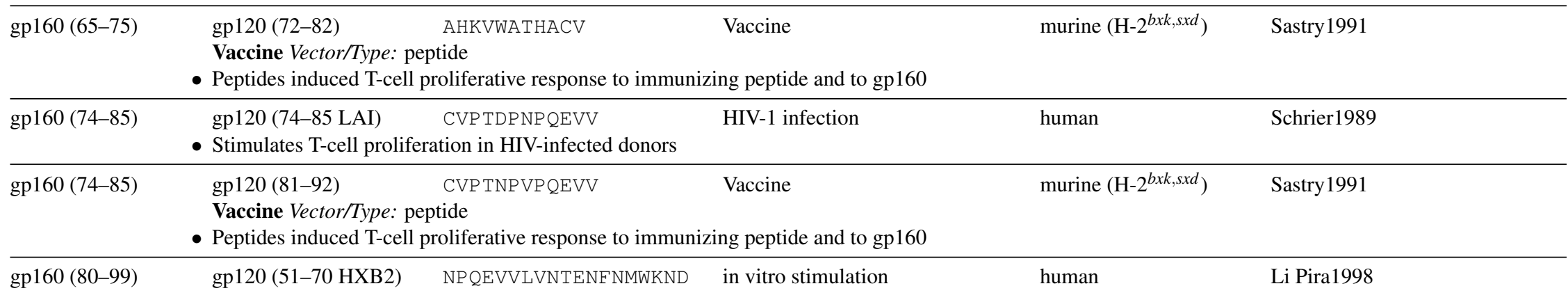

- Clonal heterogeneity was broad for a recall response to tetanus toxoid or PPD, but oligoclonal to primary HIV antigens, dominated in this case by TCR V $\beta$ 13 usage

- Donor of PBMC that recognized this epitope had HLA-DR alleles 2 and 7

\begin{tabular}{|c|c|}
\hline gp160 (81-100) & $\begin{array}{l}\text { gp120 (80-99 89.6) PQEVVLGNVTENFNMWKNNM Vaccine } \\
\text { Vaccine Vector/Type: recombinant protein Strain: } 89.6 \text { HIV component: gp120 Adjuvant: R192G mutant heat-labile toxin from enterotoxigenic E. } \\
\text { coli (mLT) } \\
\text { - Epitope name: Peptide } 6 \\
\text { - Helper T-cell proliferative responses to gp120 vaccines in } 2 \text { mouse strains, CBA/J and BALB/c, were mapped using } 47 \text { overlapping peptides that span } \\
\text { gp120. Promiscuously immunodominant peptides were identified in both mice strains that were located in the outer domain of gp120 and were associated } \\
\text { with regions of local structural disorder in proximal N-terminal segments, suggesting 3-D protein structure influences Th antigen processing and the } \\
\text { frequency of immunogenic sequences. } \\
\text { - This peptide was not reactive in any BALB/c mice tested (0/10), but was highly reactive in all }(10 / 10) \text { CBA/J mice. }\end{array}$ \\
\hline gp160 (81-100) & $\begin{array}{l}\text { gp120 (80-99 89.6) PQEVVLGNVTENFNMWKNNM Vaccine murine }\left(\mathrm{H}-2^{k}\right) \\
\text { Vaccine Vector/Type: recombinant protein Strain: } 89.6 \text { HIV component: gp120 Adjuvant: mutant R192G heat-labile toxin from E. coli as adjuvant } \\
\text { - Promiscuous immunodominant epitopes in gp120 were mapped by overlapping peptides in CBA/J H-2 }{ }^{k} \text { and BALB/c H-2 } 2^{d} \text { mice, and all were found to be } \\
\text { in the outer domain, proximal to regions of structural disorder indicated by the crystal structure or by sequence divergence. } \\
\text { - This peptide was recognized by 10/10 CBA/J with an average SI of 8.2, and not by BALB/c mice, so is considered to be uniquely immunodominant for H- } 2^{k} \\
\text { - Uniquely immunodominant sequences tended to be in the inner domain of the protein }\end{array}$ \\
\hline gp160 (81-101) & $\begin{array}{l}\text { PQEVVLVNVTENFNMWKND - HIV-1 infection } \\
\text { MV? }\end{array}$ \\
\hline
\end{tabular}

- Epitope name: B1 


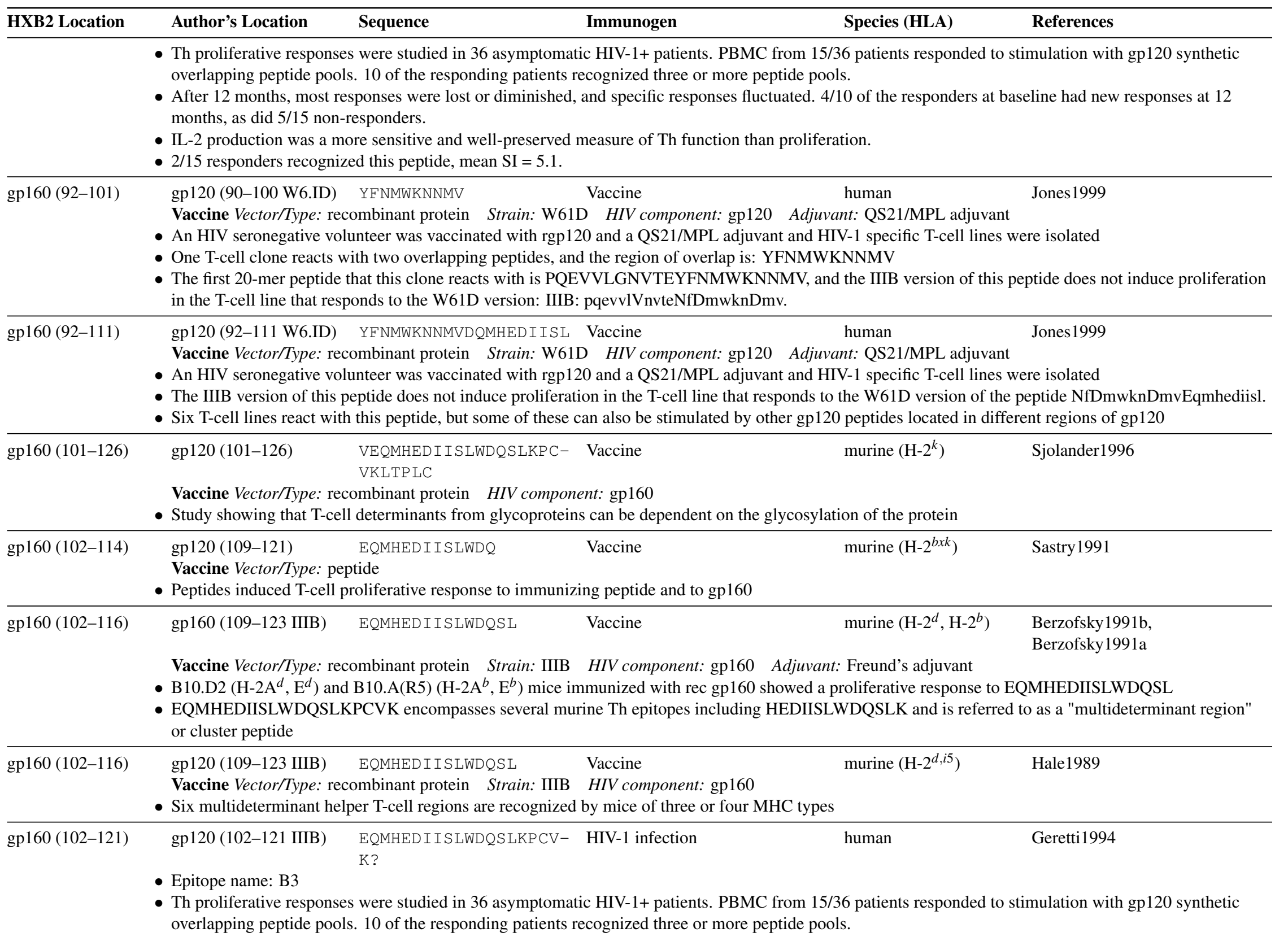




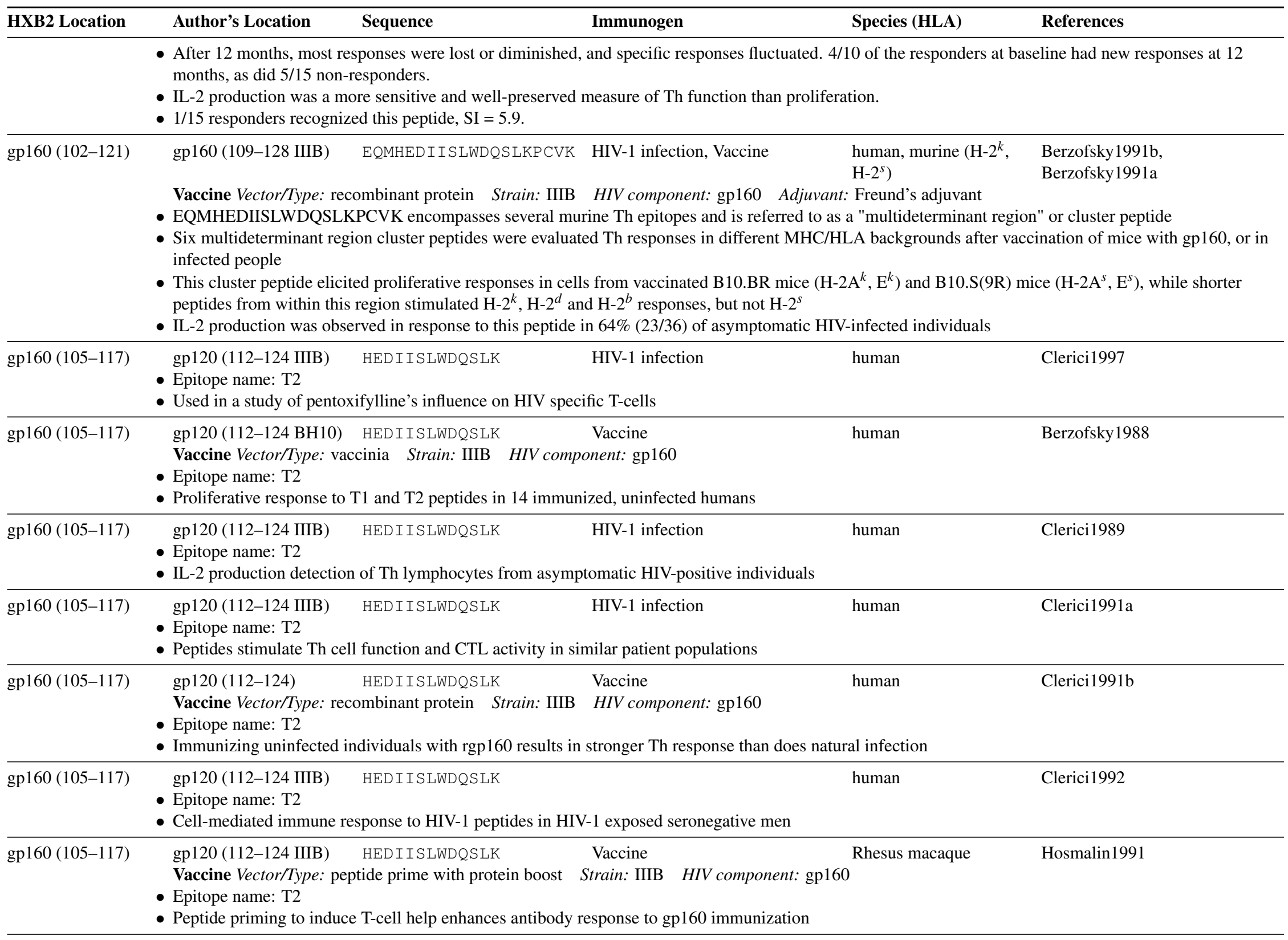




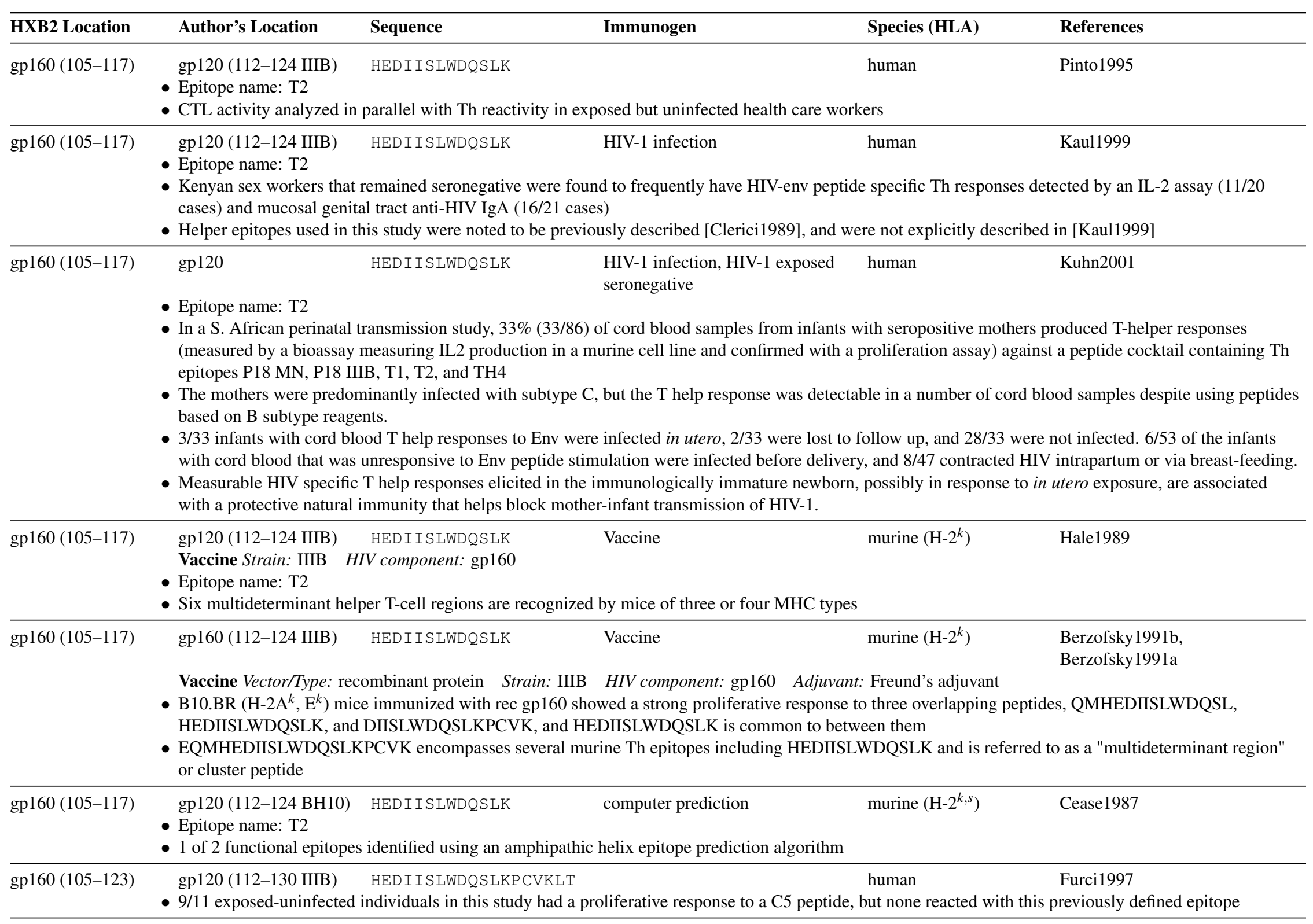




\begin{tabular}{|c|c|c|c|c|c|}
\hline HXB2 Location & Author's Location & Sequence & Immunogen & Species (HLA) & References \\
\hline gp160 (108-119) & $\begin{array}{l}\text { gp120 (108-119 LAI) } \\
\text { - Stimulates T-cell proli }\end{array}$ & $\begin{array}{l}\text { I ISLWDQSLKPC } \\
\text { ation in HIV-infectec }\end{array}$ & HIV-1 infection & human & Schrier1989 \\
\hline gp160 (110-125) & gp120 (110-125) & SLWDQSLKPCVKI & HIV-1 infection & human & Caruso1997 \\
\hline
\end{tabular}

- As HIV-1-infected individuals progress to disease, T-cells show reduced ability to proliferate in response to HIV antigen, but retain the ability to express the activation antigens CD25 and CD71

- The ability to express activation markers in response to HIV is retained, but the response to tetanus toxoid recall antigen is lost

- This study investigated CD25 and CD71 expression in PBMC from patients at various stages of progression, measuring the response to in vitro stimulation by peptide cocktail containing four antigenic Env peptides, or p17 and p24

\begin{tabular}{lllc}
\hline gp160 (111-123) & gp120 (118-130) LWDQSLKPCVKLT & Vaccine & Rhesus macaque \\
Vaccine Vector/Type: peptide & Nehete1993
\end{tabular}

- Synthetic peptide derived from conserved region of the HIV-1 envelope that stimulates a proliferative response in mice

- Proliferative response to this peptide was observed in $3 / 3$ immunized rhesus monkeys

gp160(112-130) gp120(112-130 IIIB) WDQSLKPCVKLTPLCVSLK? HIV-1 infection $\quad$ Geretti1994

- Epitope name: B4

- Th proliferative responses were studied in 36 asymptomatic HIV-1+ patients. PBMC from 15/36 patients responded to stimulation with gp120 synthetic overlapping peptide pools. 10 of the responding patients recognized three or more peptide pools.

- After 12 months, most responses were lost or diminished, and specific responses fluctuated. 4/10 of the responders at baseline had new responses at 12 months, as did 5/15 non-responders.

- IL-2 production was a more sensitive and well-preserved measure of Th function than proliferation.

- 3/15 responders recognized this peptide, average $\mathrm{SI}=4.4$.

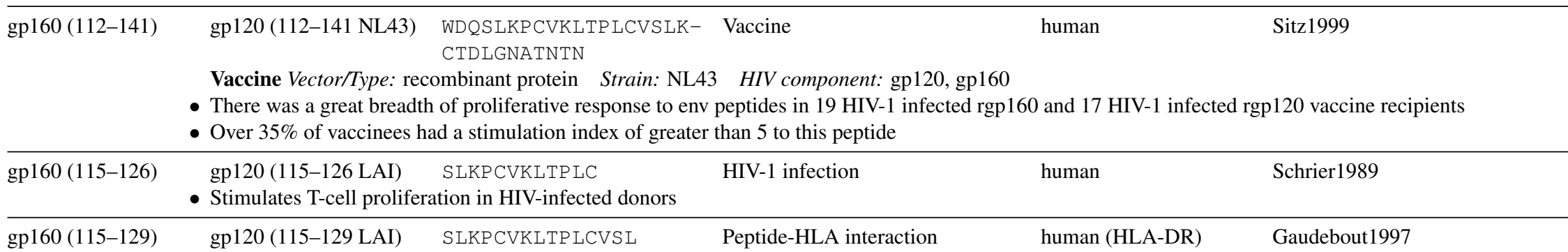

- Peptide bound to both HLA-DR*1101 and HLA-DR*0401 with high affinity

- Because of the distinctive binding pockets of HLA-DR*1101 and HLA-DR*0401, peptides that bound both were considered candidates for promiscuous HLA-DR binding

gp160 (121-140)

gp120 (120-139 89.6) KLTPLCVTLNCTNLNITKNT Vaccine

Vaccine Vector/Type: recombinant protein Strain: 89.6 HIV component: gp120 Adjuvant: R192G mutant heat-labile toxin from enterotoxigenic E. coli (mLT)

- Epitope name: Peptide 10 


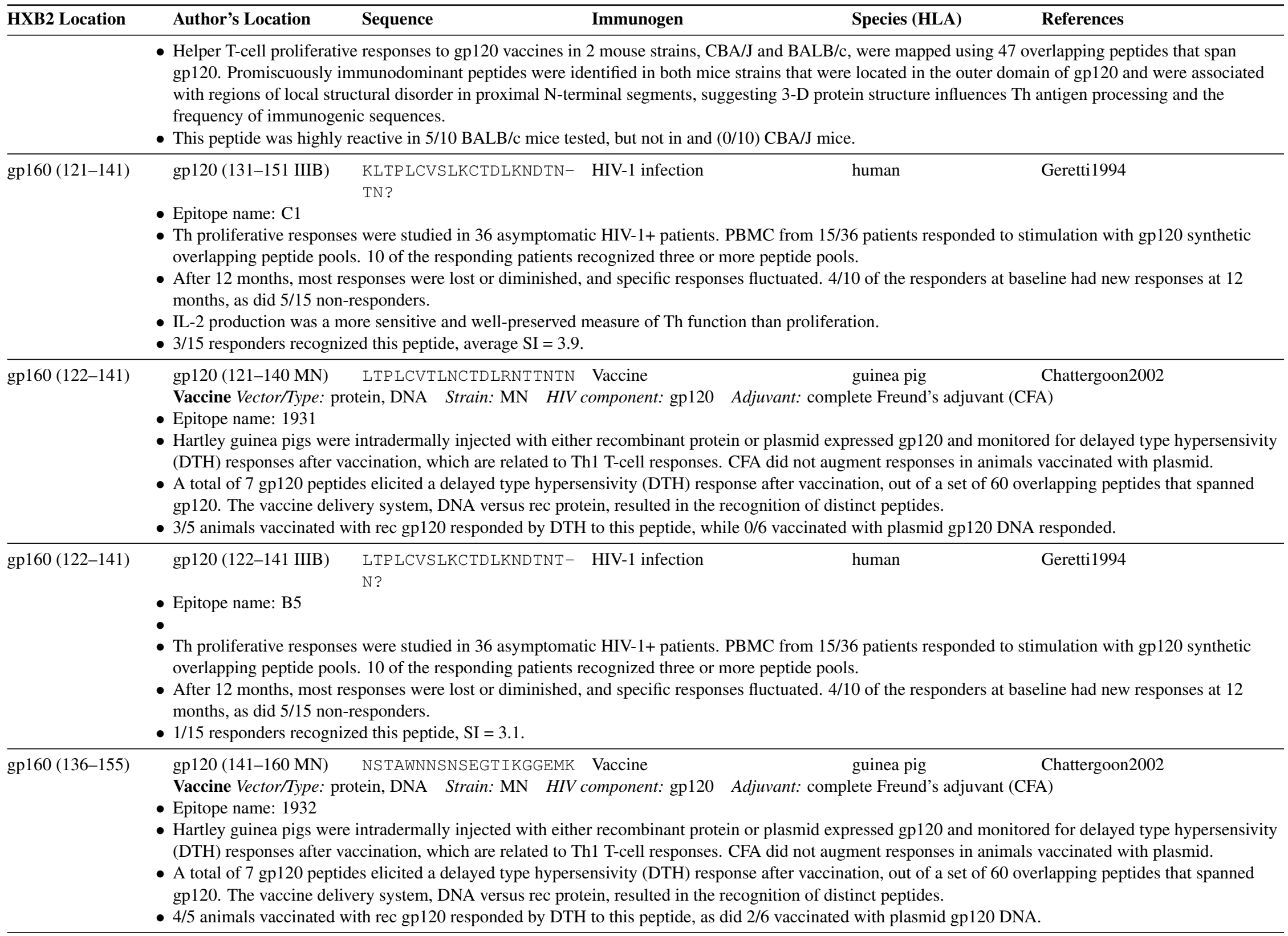




\begin{tabular}{|c|c|c|c|c|c|}
\hline HXB2 Location & Author's Location & Sequence & Immunogen & Species (HLA) & Reference: \\
\hline p160 (138-159) & 160 W6.ID) & TTSNGWTGEIRKGEIKNCSF & Vaccine & human & Jones1999 \\
\hline
\end{tabular}

Vaccine Vector/Type: recombinant protein Strain: W61D HIV component: gp120 Adjuvant: QS21/MPL adjuvant

- An HIV seronegative volunteer was vaccinated with rgp120 and a QS21/MPL adjuvant and HIV-1 specific T-cell lines were isolated

- The IIIB version of this peptide does not induce proliferation in the T-cell line that responds to the W61D version of the peptide: IIIB: ttsnSSGRMIMEgeikncsf.

\begin{tabular}{lllll}
\hline gp160 (142-161) gp120(142-161 IIIB) & $\begin{array}{l}\text { SSSGRMIMEKGEIKNCSFN- HIV-1 infection } \\
\text { I? }\end{array}$ & Geretti1994 \\
\hline
\end{tabular}

- Epitope name: $\mathrm{C} 2$

- Th proliferative responses were studied in 36 asymptomatic HIV-1+ patients. PBMC from 15/36 patients responded to stimulation with gp120 synthetic overlapping peptide pools. 10 of the responding patients recognized three or more peptide pools.

- After 12 months, most responses were lost or diminished, and specific responses fluctuated. 4/10 of the responders at baseline had new responses at 12 months, as did 5/15 non-responders.

- IL-2 production was a more sensitive and well-preserved measure of Th function than proliferation.

- Five peptides were recognized most frequently: C2 (aa 142-161), C3 (aa 152-171), C5 (aa 172-191), E5 (aa 272-291) and G4 (aa 380-393). The first three were in or near V2, the other two were proximal to the V3 and V4 loops.

- $4 / 15$ responders recognized this immunodominant peptide, average $\mathrm{SI}=4.3$.

gp160 (147-168) gp120 (152-173 NL43) MMMEKGEIKNCSFNISTSI- Vaccine RGK

human

Sitz1999

Vaccine Vector/Type: recombinant protein Strain: NL43 HIV component: gp120, gp160

- There was a great breadth of proliferative response to env peptides in 19 HIV-1 infected rgp160 and 17 HIV-1 infected rgp120 vaccine recipients

- Over $50 \%$ of vaccinees had a stimulation index of greater than 5 to this peptide

gp160 (152-171) gp120 (152-171 IIIB) GEIKNCSFNISTSIRGKVQ- HIV-1 infection $K$ ?

human

Geretti1994

- Epitope name: C3

- Th proliferative responses were studied in 36 asymptomatic HIV-1+ patients. PBMC from 15/36 patients responded to stimulation with gp120 synthetic overlapping peptide pools. 10 of the responding patients recognized three or more peptide pools.

- After 12 months, most responses were lost or diminished, and specific responses fluctuated. 4/10 of the responders at baseline had new responses at 12 months, as did 5/15 non-responders.

- IL-2 production was a more sensitive and well-preserved measure of Th function than proliferation.

- Five peptides were recognized most frequently: C2 (aa 142-161), C3 (aa 152-171), C5 (aa 172-191), E5 (aa 272-291) and G4 (aa 380-393). The first three were in or near V2, the other two were proximal to the V3 and V4 loops.

- $4 / 15$ responders recognized this immunodominant peptide, average $\mathrm{SI}=4.4$.

$\begin{array}{llccc}\text { gp160 (155-169) Env (UG92005) } & \text { KNCSFNITTELIDKK } & \text { Vaccine } & \left.\text { murine (H-2 IA }{ }^{b}\right) & \text { Surman2001 }\end{array}$
Vaccine Vector/Type: DNA, vaccinia, recombinant protein Strain: 1007 (clade B), UG92005 (clade D) HIV component: gp140 Adjuvant: Freund's adjuvant

- This epitope is located in the V2 region of UG92005 (UG, clade D) and the hybridoma that recognized it used V $\beta 5$

- C57BL/6 mice were immunized with a prime-boost strategy involving three HIV-1 Env antigens: Mice were primed with DNA given i.m., 3-4 weeks later boosted with VV, and 3-4 weeks later boosted again with purified protein in Freund's adjuvant 


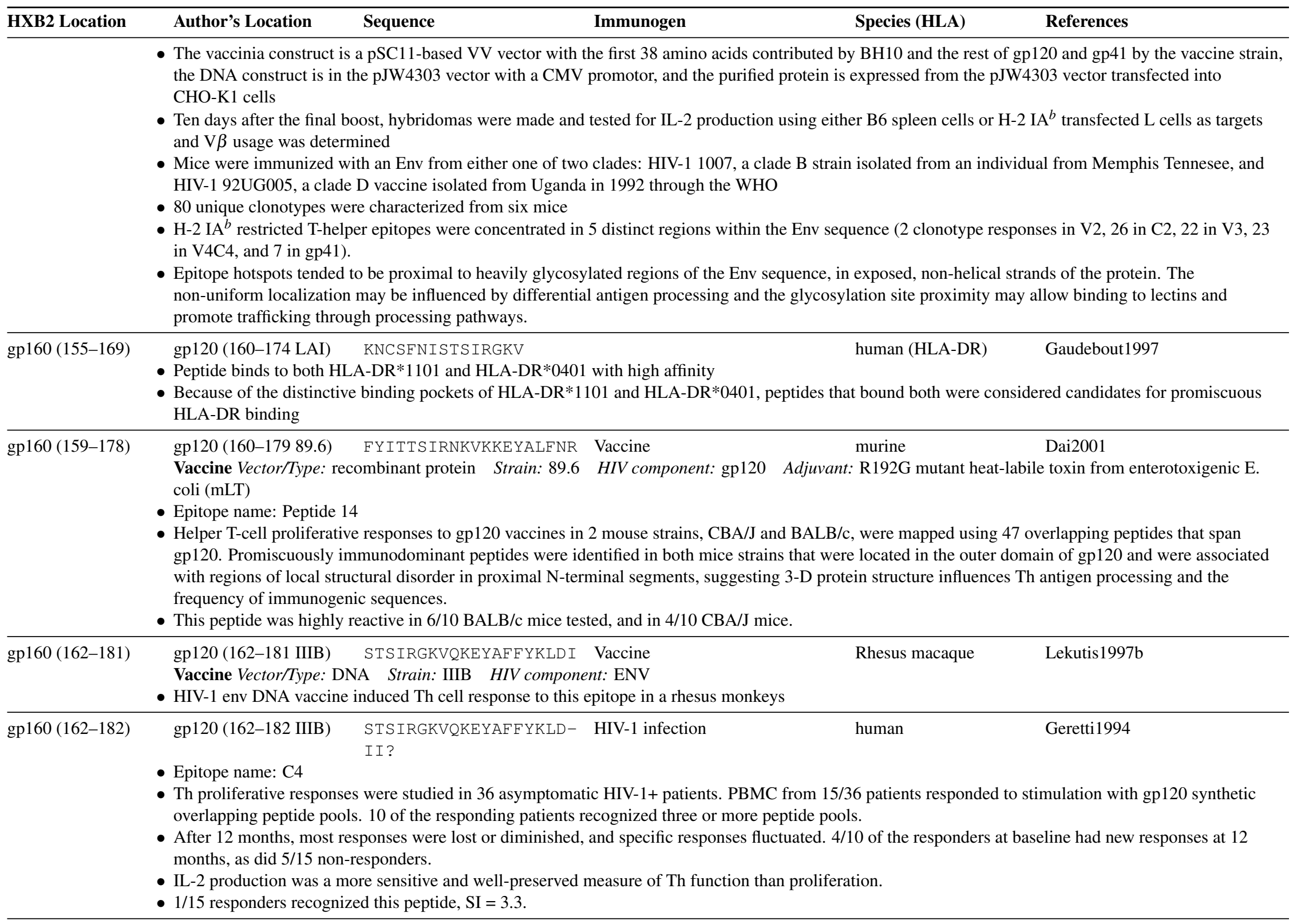




\begin{tabular}{|c|c|c|c|c|c|}
\hline HXB2 Location & Author's Location & Sequence & Immunogen & Species (HLA) & References \\
\hline gp160 (169-189) & gp120 (141-160 W6.ID) & $\begin{array}{l}\text { VQKEYALFYNLDVVP IDDD- } \\
\text { NA }\end{array}$ & Vaccine & human & Jones1999 \\
\hline
\end{tabular}

Vaccine Vector/Type: recombinant protein Strain: W61D HIV component: gp120 Adjuvant: QS21/MPL adjuvant

- An HIV seronegative volunteer was vaccinated with rgp120 and a QS21/MPL adjuvant and HIV-1 specific T-cell lines were isolated

- The IIIB version of this peptide does not induce proliferation in the T-cell line that responds to the W61D version of the peptide - - F-K-II- N-TT vqkeyaFfyKldIIpidNdTT.

- Two T-cell lines react specifically with this peptide

\begin{tabular}{llrl}
\hline gp160 (172-191) & gp120(172-191 IIIB) EYAFFYKLDIIPIDNDTTSY Vaccine & Rhesus macaque \\
& Vaccine Vector/Type: DNA Strain: IIIB HIV component: ENV & Lekutis1997b
\end{tabular}

- HIV-1 env DNA vaccine induced Th cell response to this epitope in a rhesus monkey

\begin{tabular}{llll}
\hline gp160 (172-191) gp120(172-191 IIIB) & $\begin{array}{l}\text { EYAFFYKLDIIPIDNDTTS- HIV-1 infection } \\
\text { Y? }\end{array}$ & Geretti1994 \\
\hline
\end{tabular}

- Epitope name: C5

- Th proliferative responses were studied in 36 asymptomatic HIV-1+ patients. PBMC from 15/36 patients responded to stimulation with gp120 synthetic overlapping peptide pools. 10 of the responding patients recognized three or more peptide pools.

- After 12 months, most responses were lost or diminished, and specific responses fluctuated. 4/10 of the responders at baseline had new responses at 12 months, as did 5/15 non-responders.

- IL-2 production was a more sensitive and well-preserved measure of Th function than proliferation.

- Five peptides were recognized most frequently: C2 (aa 142-161), C3 (aa 152-171), C5 (aa 172-191), E5 (aa 272-291) and G4 (aa 380-393). The first three were in or near V2, the other two were proximal to the V3 and V4 loops.

- $4 / 15$ responders recognized this immunodominant peptide, average $\mathrm{SI}=7.4$.

gp160 (175-189) Env (UG92005) LFYKLDVVQIDNSTN $\quad$ Vaccine $\quad$ Surman2001

Vaccine Vector/Type: DNA, vaccinia, recombinant protein Strain: 1007 (clade B), UG92005 (clade D) HIV component: gp140 Adjuvant: Freund's adjuvant

- This epitope is located in the V2 region of UG92005 (UG, clade D) and the V $\beta$ usage of the TCR was not determined

- C57BL/6 mice were immunized with a prime-boost strategy involving three HIV-1 Env antigens: Mice were primed with DNA given i.m., 3-4 weeks later boosted with VV, and 3-4 weeks later boosted again with purified protein in Freund's adjuvant

- The vaccinia construct is a pSC11-based VV vector with the first 38 amino acids contributed by BH10 and the rest of gp120 and gp41 by the vaccine strain, the DNA construct is in the pJW4303 vector with a CMV promotor, and the purified protein is expressed from the pJW4303 vector transfected into CHO-K1 cells

- Ten days after the final boost, hybridomas were made and tested for IL-2 production using either B6 spleen cells or H-2 IA ${ }^{b}$ transfected L cells as targets and $\mathrm{V} \beta$ usage was determined

- Mice were immunized with an Env from either one of two clades: HIV-1 1007, a clade B strain isolated from an individual from Memphis Tennesee, and HIV-1 92UG005, a clade D vaccine isolated from Uganda in 1992 through the WHO

- 80 unique clonotypes were characterized from six mice

- $\mathrm{H}-2 \mathrm{IA}^{b}$ restricted T-helper epitopes were concentrated in 5 distinct regions within the Env sequence (2 clonotype responses in V2, 26 in C2, 22 in V3, 23 in $\mathrm{V} 4 \mathrm{C} 4$, and 7 in gp41). 


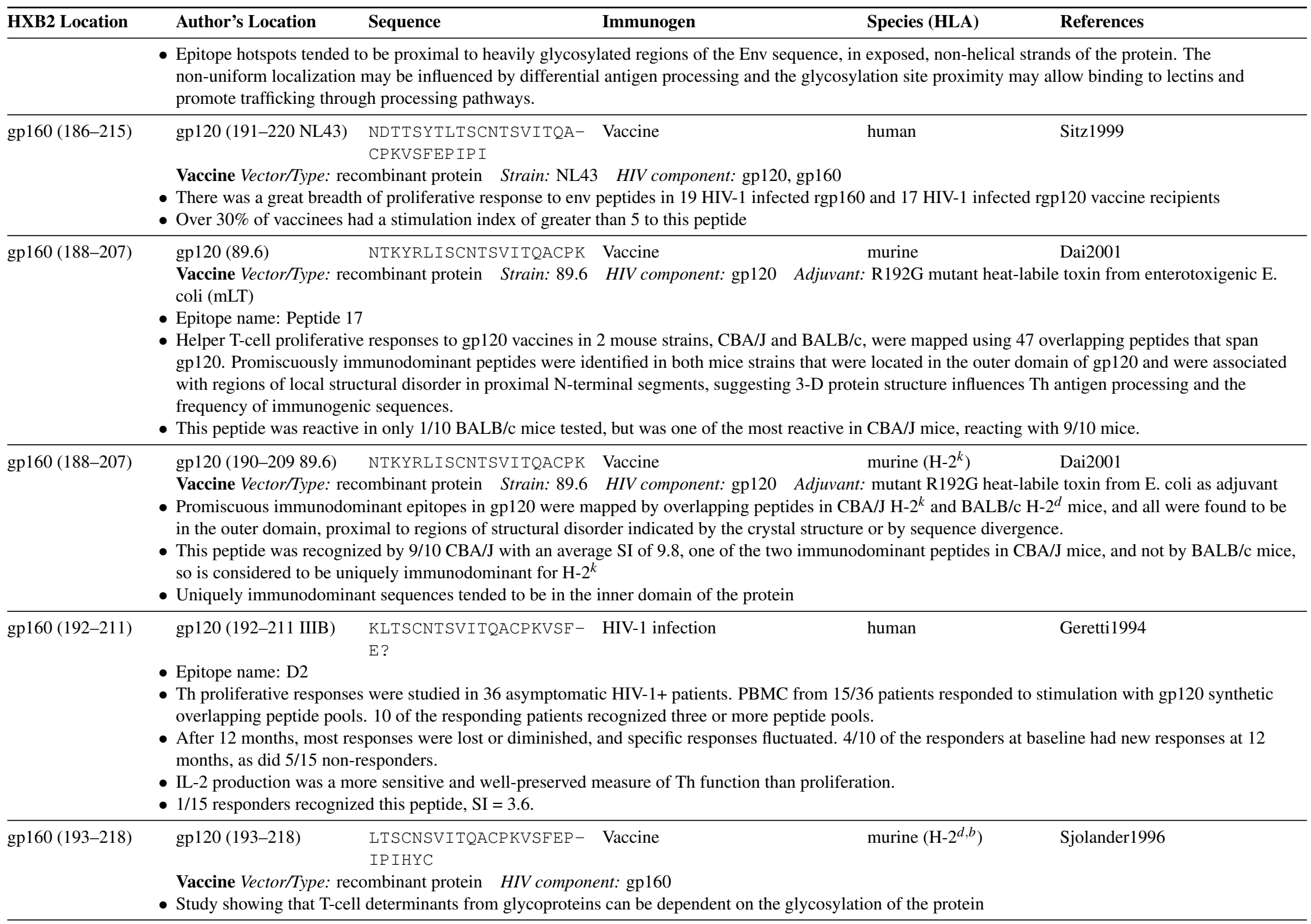




\begin{tabular}{|c|c|c|c|}
\hline HXB2 Location & Author's Location & Immunogen & Species (HLA) \\
\hline gp160 (198-212) & $\begin{array}{l}\text { Env (1007) } \\
\text { Vaccine Vector/Type } \\
\text { adjuvant } \\
\text { - This epitope is locat } \\
\text { - C57BL/6 mice were } \\
\text { boosted with VV, an } \\
\text { - The vaccinia constru } \\
\text { the DNA construct i } \\
\text { CHO-K1 cells } \\
\text { - Ten days after the fir } \\
\text { and V } \beta \text { usage was d } \\
\text { - Mice were immuniz } \\
\text { HIV-1 92UG005, a } \\
\text { - } 80 \text { unique clonotype } \\
\text { - H-2 IA }{ }^{b} \text { restricted T } \\
\text { in V4C4, and } 7 \text { in g } \\
\text { - Epitope hotspots ten } \\
\text { non-uniform localiz: } \\
\text { promote trafficking }\end{array}$ & $\begin{array}{l}\text { TSVITQACPKVSFEP Vaccine } \\
\text { A, vaccinia, recombinant protein Strain: } 1007 \\
\text { the C2 region of } 1007 \text { (US, clade B) and the V } \beta \\
\text { unized with a prime-boost strategy involving thr } \\
\text { weeks later boosted again with purified protein } \\
\text { a pSC11-based VV vector with the first } 38 \text { amin } \\
\text { he pJW4303 vector with a CMV promotor, and t } \\
\text { ost, hybridomas were made and tested for IL-2 } \\
\text { hined } \\
\text { th an Env from either one of two clades: HIV-1 } \\
\text { D vaccine isolated from Uganda in } 1992 \text { throug } \\
\text { e characterized from six mice } \\
\text { er epitopes were concentrated in } 5 \text { distinct regio } \\
\text { o be proximal to heavily glycosylated regions of } \\
\text { may be influenced by differential antigen proces } \\
\text { gh processing pathways. }\end{array}$ & $\begin{array}{l}\text { murine }\left(\mathrm{H}-2 \mathrm{IA}^{b}\right) \\
\text { (clade D) HIV component: gp140 Adjuvant: Freund's } \\
\text { or two clonotypes was } \mathrm{V} \beta 3 \text { and } \mathrm{V} \beta 8.1-2 \\
\text { ns: Mice were primed with DNA given i.m., 3-4 weeks later } \\
\text { y BH10 and the rest of gp120 and gp41 by the vaccine strain, } \\
\text { expressed from the pJW4303 vector transfected into } \\
\text { er B6 spleen cells or H-2 IA }{ }^{b} \text { transfected L cells as targets } \\
\text { isolated from an individual from Memphis Tennesee, and } \\
\text { uence ( } 2 \text { clonotype responses in V2, } 26 \text { in C2, } 22 \text { in V3, } 23 \\
\text { exposed, non-helical strands of the protein. The } \\
\text { lation site proximity may allow binding to lectins and }\end{array}$ \\
\hline gp160 (198-215) & $\begin{array}{l}\text { Env (1007) } \\
\text { Vaccine Vector/Type } \\
\text { adjuvant } \\
\text { - This epitope is locat } \\
\text { - C57BL/6 mice were } \\
\text { boosted with VV, an } \\
\text { - The vaccinia constru } \\
\text { the DNA construct i } \\
\text { CHO-K1 cells } \\
\text { - Ten days after the fir } \\
\text { and V } \beta \text { usage was d } \\
\text { - Mice were immuniz } \\
\text { HIV-1 92UG005, a } \\
\text { - } 80 \text { unique clonotype } \\
\text { - H-2 IA }{ }^{b} \text { restricted T } \\
\text { in V4C4, and } 7 \text { in gr } \\
\text { - Epitope hotspots ten } \\
\text { non-uniform localiz: } \\
\text { promote trafficking }\end{array}$ & $\begin{array}{l}\text { TSVITQACPKVSFEP IP I Vaccine } \\
\text { A, vaccinia, recombinant protein Strain: } 1007 \\
\text { the C2 region of } 1007 \text { (US, clade B) and the V } \beta \\
\text { unized with a prime-boost strategy involving thr } \\
\text { weeks later boosted again with purified protein } \\
\text { a pSC11-based VV vector with the first } 38 \text { amin } \\
\text { he pJW4303 vector with a CMV promotor, and t } \\
\text { oost, hybridomas were made and tested for IL-2 } \\
\text { ined } \\
\text { th an Env from either one of two clades: HIV-1 } \\
\text { D vaccine isolated from Uganda in } 1992 \text { throug } \\
\text { e characterized from six mice } \\
\text { er epitopes were concentrated in } 5 \text { distinct regio } \\
\text { o be proximal to heavily glycosylated regions of } \\
\text { may be influenced by differential antigen proces } \\
\text { gh processing pathways. }\end{array}$ & $\begin{array}{l}\text { murine }\left(\mathrm{H}-2 \mathrm{IA}^{b}\right) \\
\text { (clade D) HIV component: gp140 Adjuvant: Freund's } \\
\text { as V } \beta 6 \\
\text { ns: Mice were primed with DNA given i.m., 3-4 weeks later } \\
\text { y BH10 and the rest of gp } 120 \text { and gp } 41 \text { by the vaccine strain, } \\
\text { expressed from the pJW4303 vector transfected into } \\
\text { ler B6 spleen cells or H-2 IA }{ }^{b} \text { transfected L cells as targets } \\
\text { isolated from an individual from Memphis Tennesee, and } \\
\text { uence (2 clonotype responses in V2, } 26 \text { in C2, } 22 \text { in V3, } 23 \\
\text { lation site proximity may allow binding to lectins and }\end{array}$ \\
\hline 11) & $\begin{array}{l}\text { Env (204-216) } \\
\text { Vaccine Vector/Typ }\end{array}$ & $\begin{array}{l}\text { SVITQACSKVSFE } \\
\text { ide }\end{array}$ & hesus macaque \\
\hline
\end{tabular}




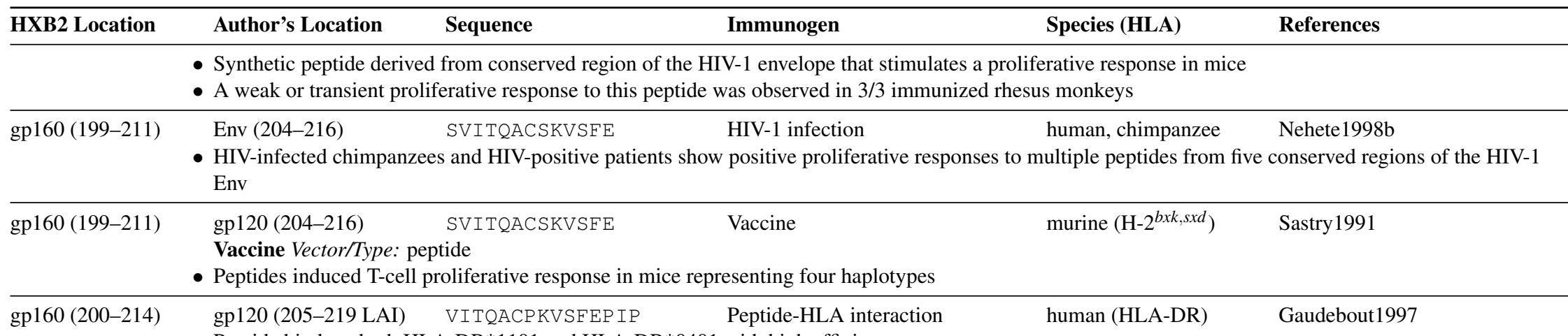

- Peptide binds to both HLA-DR*1101 and HLA-DR*0401 with high affinity

- Because of the distinctive binding pockets of HLA-DR*1101 and HLA-DR*0401, peptides that bound both were considered candidates for promiscuous HLA-DR binding

gp160 (201-212) Env (1007) ITQACPKVSFEP $\quad$ Vaccine $\quad$ Surman2001]

Vaccine Vector/Type: DNA, vaccinia, recombinant protein Strain: 1007 (clade B), UG92005 (clade D) HIV component: gp140 Adjuvant: Freund's adjuvant

- This epitope is located in the $\mathrm{C} 2$ region of 1007 (US, clade $\mathrm{B}$ ) and the V $\beta$ usage of the TCR was V $\beta 3$

- The epitope described here is the region of overlap of two 15 mers that were both able to stimulate IL-2 production from the hybridoma (TSVITQACPKVSFEP and ITQACPKVSFEPIPI)

- C57BL/6 mice were immunized with a prime-boost strategy involving three HIV-1 Env antigens: Mice were primed with DNA given i.m., 3-4 weeks later boosted with VV, and 3-4 weeks later boosted again with purified protein in Freund's adjuvant

- The vaccinia construct is a pSC11-based VV vector with the first 38 amino acids contributed by BH10 and the rest of gp120 and gp41 by the vaccine strain, the DNA construct is in the pJW4303 vector with a CMV promotor, and the purified protein is expressed from the pJW4303 vector transfected into CHO-K1 cells

- Ten days after the final boost, hybridomas were made and tested for IL-2 production using either B6 spleen cells or H-2 IA ${ }^{b}$ transfected L cells as targets and $\mathrm{V} \beta$ usage was determined

- Mice were immunized with an Env from either one of two clades: HIV-1 1007, a clade B strain isolated from an individual from Memphis Tennesee, and HIV-1 92UG005, a clade D vaccine isolated from Uganda in 1992 through the WHO

- 80 unique clonotypes were characterized from six mice

- $\mathrm{H}-2 \mathrm{IA}^{b}$ restricted T-helper epitopes were concentrated in 5 distinct regions within the Env sequence (2 clonotype responses in V2, 26 in C2, 22 in V3, 23 in $\mathrm{V} 4 \mathrm{C} 4$, and 7 in gp41).

- Epitope hotspots tended to be proximal to heavily glycosylated regions of the Env sequence, in exposed, non-helical strands of the protein. The non-uniform localization may be influenced by differential antigen processing and the glycosylation site proximity may allow binding to lectins and promote trafficking through processing pathways.

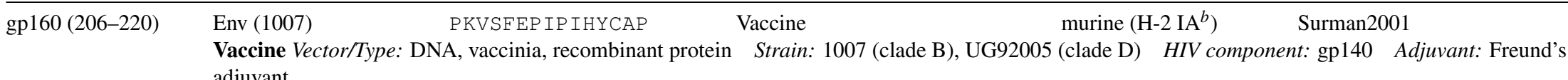
adjuvant

- This epitope is located in the C2 region of 1007 (US, clade B) and 12 hybridomas recognized the peptide with V $\beta$ usage of V $\beta 4,6,7,8.1-2,8.3,11,12$ and others not determined 


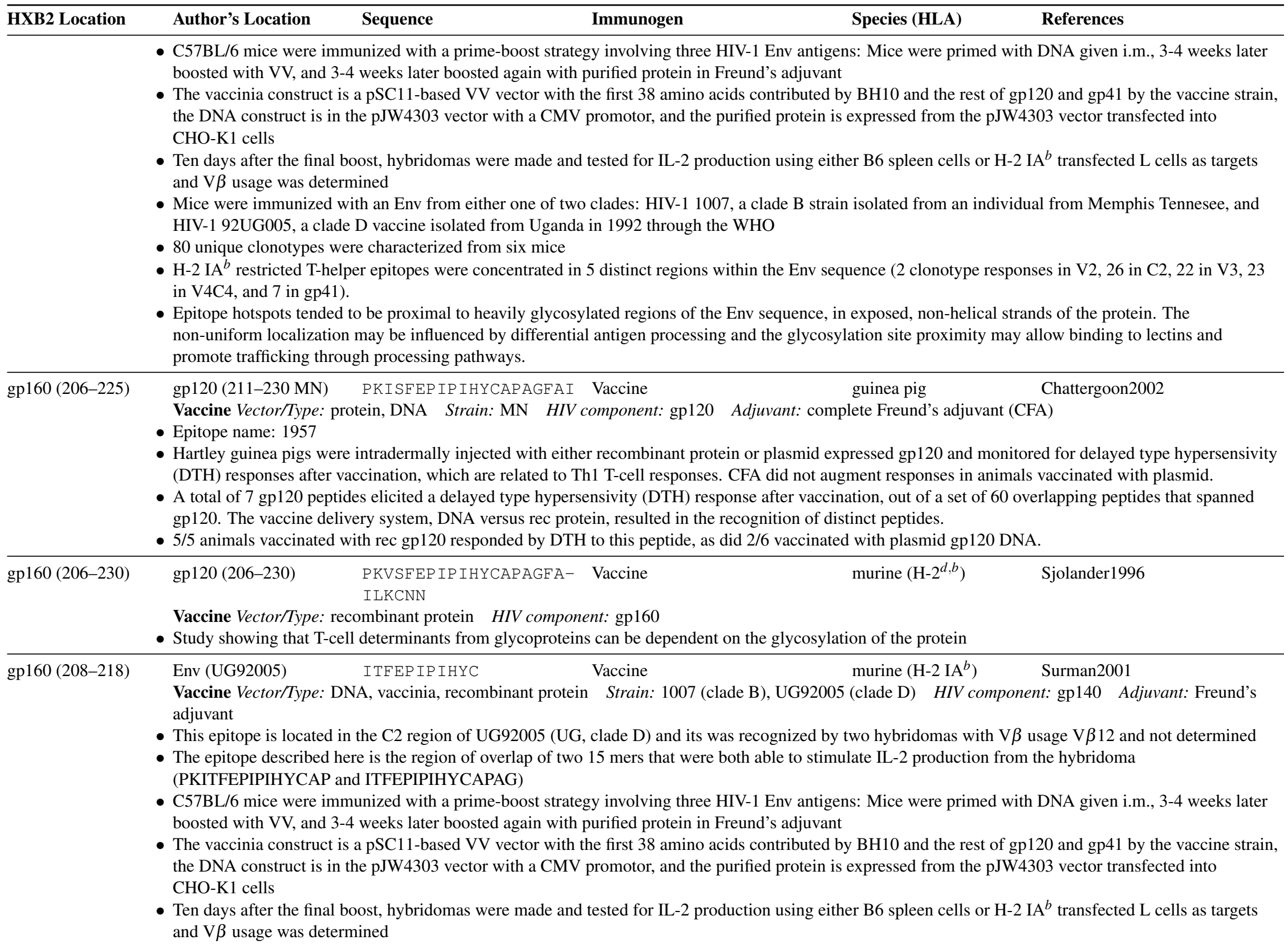




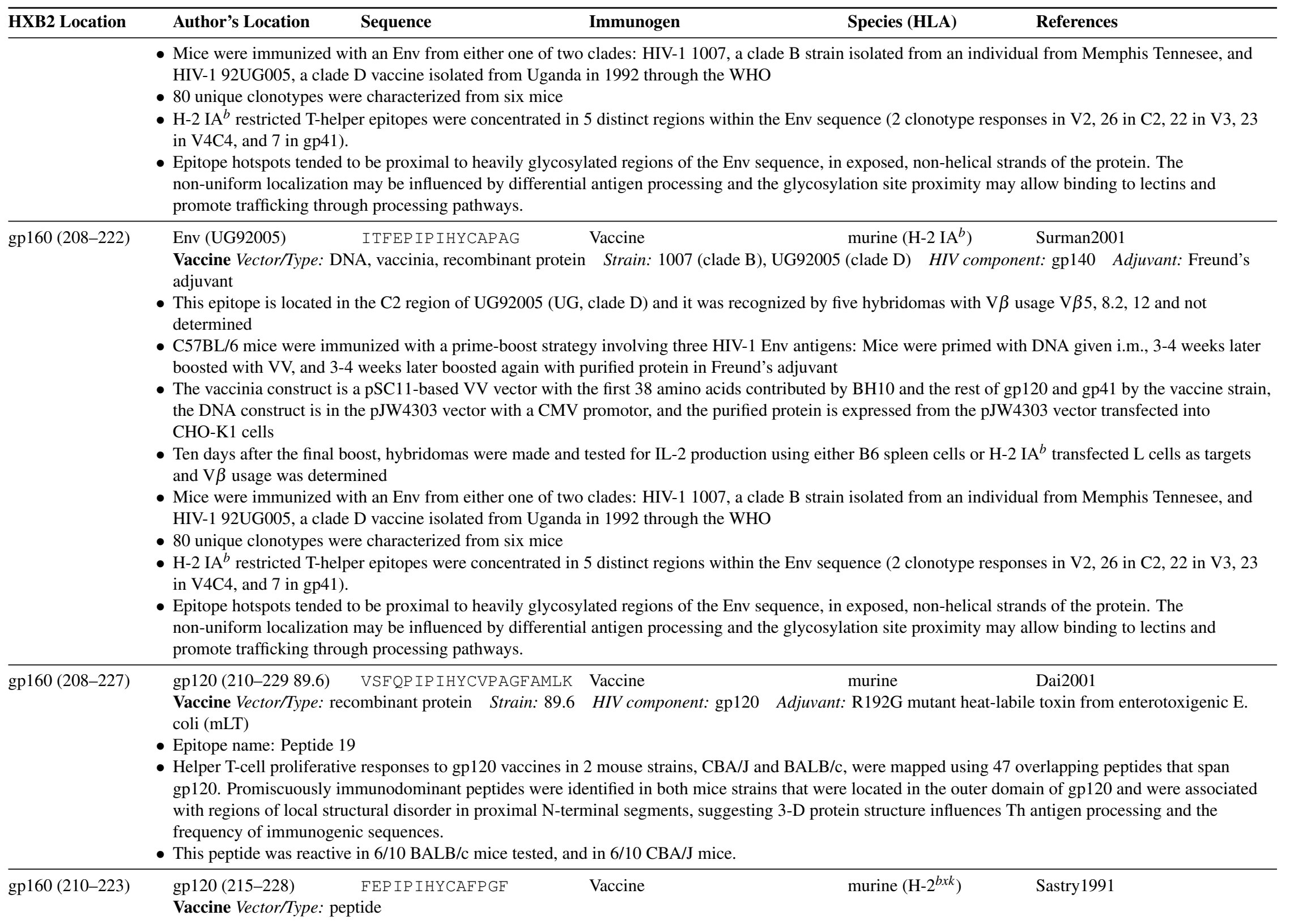




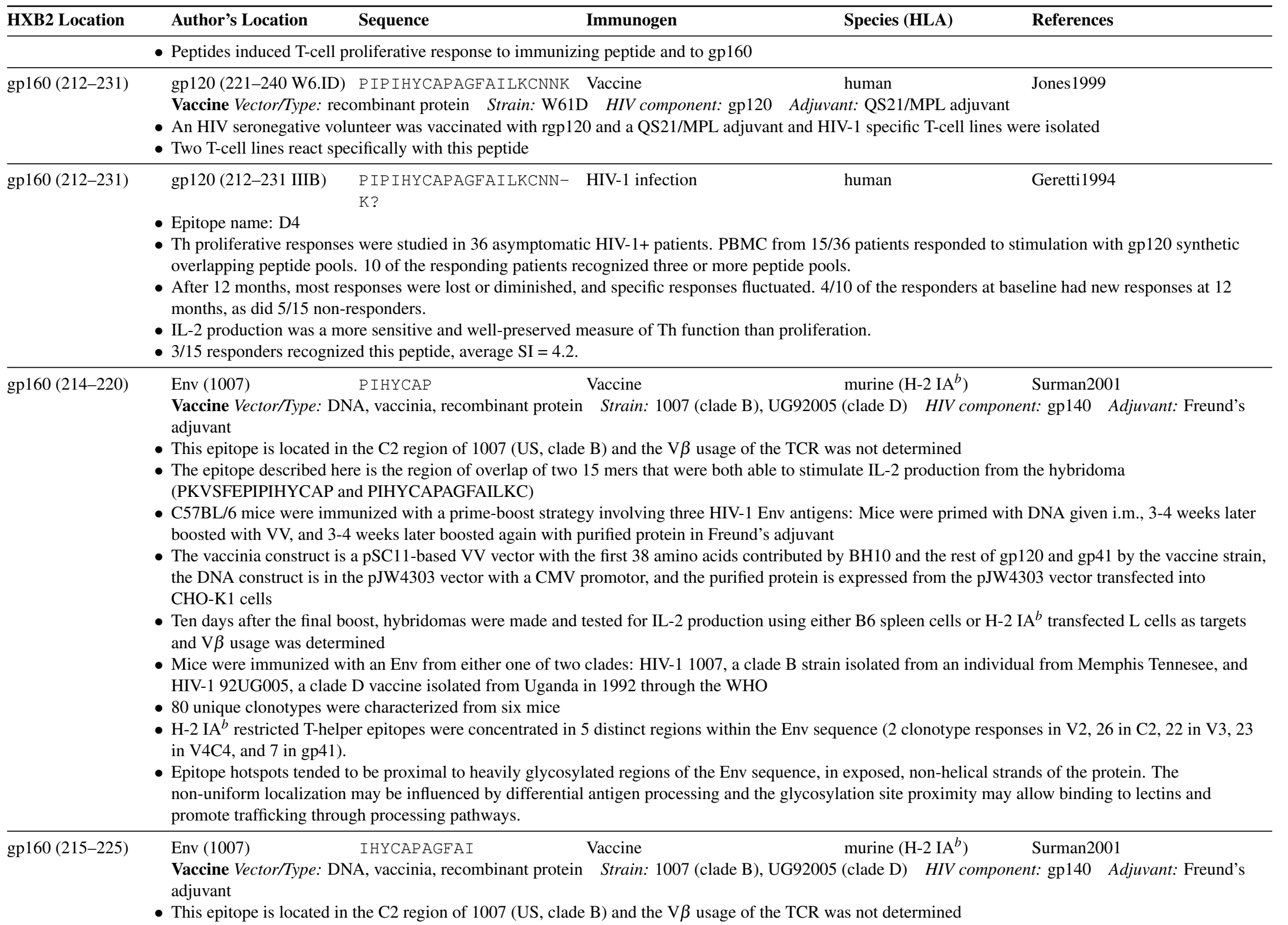




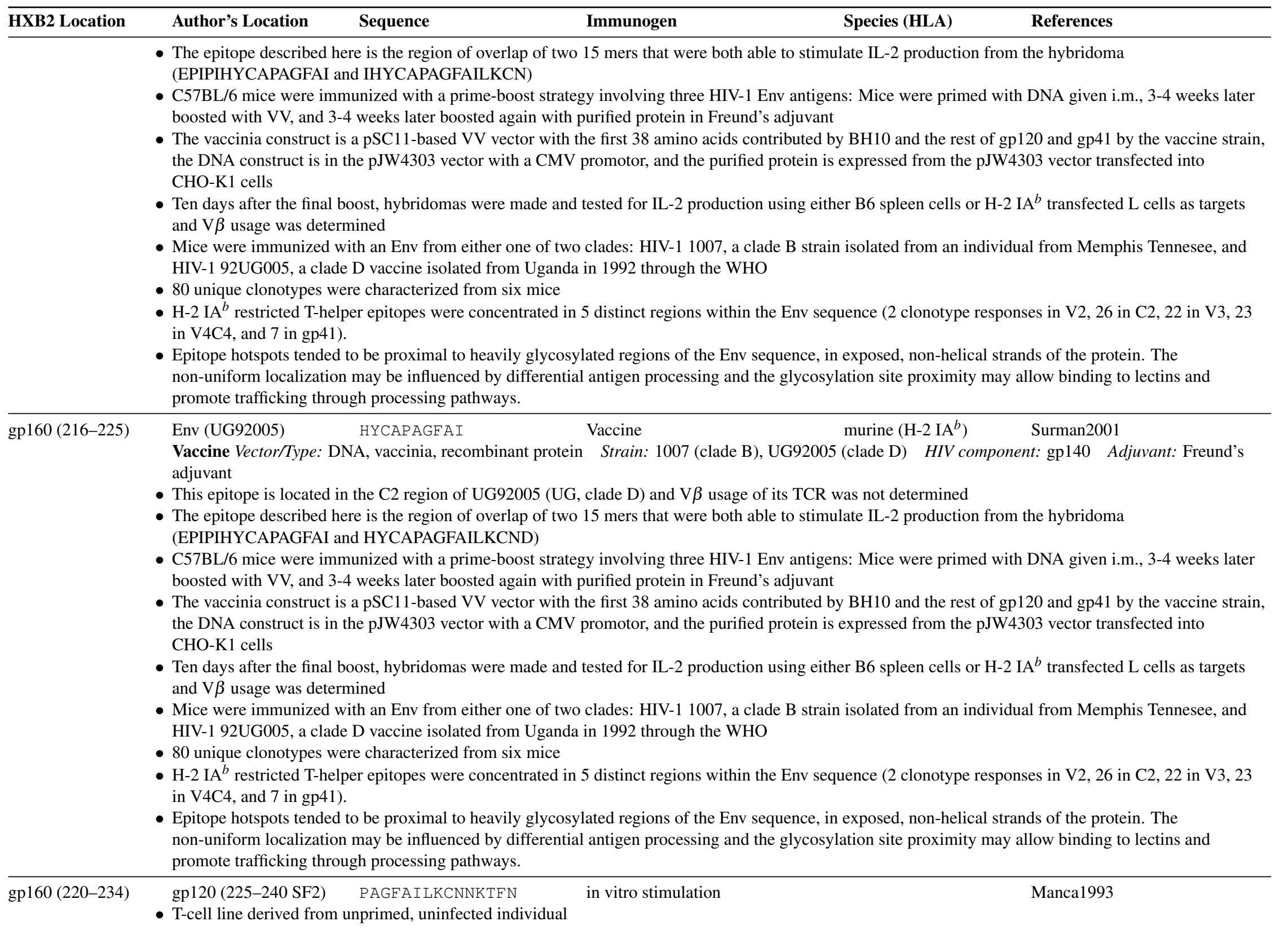




\begin{tabular}{|c|c|c|c|c|}
\hline HXB2 Location & Author's Location & Immunogen & Species (HLA) & References \\
\hline & \multicolumn{4}{|c|}{$\begin{array}{l}\text { - Responds to APC pulsed with either synthetic peptide or gp120 } \\
\text { - Human MAbs 448-D and 450-D enhance APC gp120 uptake and presentation }\end{array}$} \\
\hline gp160 (220-234) & \multicolumn{4}{|c|}{$\begin{array}{l}\text { Vaccine Vector/Type: Streptococcus gordonii HIV component: gp120 } \\
\text { - Epitope name: pep24 } \\
\text { - This previously described immunodominant Th cell epitope was fused to the streptococcal surface protein M6 (emm-6.1), for expression on the surface of } \\
\text { the bacterium Streptococcus gordonii. } \\
\text { - Recombinant bacteria showed efficient MHC class II mediated presentation of gp120 to T-cells by stimulation of a proliferative response in a human T cell } \\
\text { clone specific for pep24. }\end{array}$} \\
\hline gp160 (220-235) & \multicolumn{4}{|c|}{$\begin{array}{l}\text { - Peptide stimulation of PBMC from non-infected individuals in vitro } \\
\text { - Peptide priming does not always induce T-cells that recognize whole protein } \\
\text { - gp120 priming induced T-cells that recognize this peptide }\end{array}$} \\
\hline gp160 (220-235) & \multicolumn{4}{|c|}{$\begin{array}{l}\text { - Listeria monocytogenes, an intracellular pathogen which is ingested by macrophages and can escape from the phagosome to replicate in the cytoplasm, was } \\
\text { used successfully as carrier to deliver this gp120 epitope to CD4+ T-cells }\end{array}$} \\
\hline gp160 (220-235) & \multicolumn{4}{|c|}{$\begin{array}{l}\text { - gp120 pep24 epitope exhibited antagonistic activity against proliferation of gp120-specific T-cells when flanked by unrelated amino acid sequence } \\
\text { - The glutathione S-transferase (GST)-peptide system can be used to display peptides; antigenicity was maintained when this peptide was expressed at the } \\
\text { C-term end, but antagonism resulted when this peptide was expressed at the N-term end }\end{array}$} \\
\hline gp160 (222-241) & $\begin{array}{l}\text { gp120 (222-241 IIIB) } \\
\text { - Epitope name: D5 } \\
\text { - Th proliferative responses } \\
\text { overlapping peptide pools } \\
\text { - After } 12 \text { months, most res } \\
\text { months, as did 5/15 non-r } \\
\text { - IL-2 production was a mo } \\
\text { - } 2 / 15 \text { responders recognize }\end{array}$ & $\begin{array}{l}\text { GFAILKCNNKTFNGTGPCT- HIV-1 infection } \\
\text { N? } \\
\text { were studied in } 36 \text { asymptomatic HIV-1+ patients. P } \\
10 \text { of the responding patients recognized three or m } \\
\text { sponses were lost or diminished, and specific response } \\
\text { esponders. } \\
\text { re sensitive and well-preserved measure of Th functic } \\
\text { ed this peptide, average } \mathrm{SI}=4.8 \text {. }\end{array}$ & $\begin{array}{l}\text { human } \\
36 \text { patients responc } \\
\text { ls. } \\
10 \text { of the responde } \\
\text { ation. }\end{array}$ & $\begin{array}{l}\text { Geretti1994 } \\
\text { mulation with gp120 synthetic } \\
\text { eline had new responses at } 12\end{array}$ \\
\hline gp160 (223-231) & $\begin{array}{l}\text { gp120 (238-246 HXB2) } \\
\text { - Clonal heterogeneity was } \\
22 \text { usage } \\
\text { - Donor of PBMC that reco } \\
\text { - The only (detected) immu }\end{array}$ & $\begin{array}{l}\text { FAILKCNNK in vitro stimulation } \\
\text { broad for a recall response to tetanus toxoid or PPD, } \\
\text { gnized this epitope had HLA-DR alleles } 2 \text { and } 6 \\
\text { anogenic variant of this epitope was derived from strai }\end{array}$ & $\begin{array}{l}\text { human } \\
\text { primary HIV } \\
\text { CNNK) }\end{array}$ & $\begin{array}{l}\text { Li Pira1998 } \\
\text { ominated in this case by TCR V } \beta\end{array}$ \\
\hline gp160 (223-231) & $\begin{array}{l}\text { gp120 (194-202 HXB2) } \\
\text { - Epitope was the minimal } \\
\text { - One Th line was stimulate } \\
\text { - Alanine substitutions at p }\end{array}$ & $\begin{array}{l}\text { FAILKCNNK in vitro stimulation } \\
\text { stimulatory sequence defined for two Th lines stimula } \\
\text { ed by gp120, one by a Glutathione-S-transferase (GST } \\
\text { osition 914, 196, and } 202 \text { abrogated activity for the G }\end{array}$ & $\begin{array}{l}\text { human (DR2, 6) } \\
\text { llated line, but no }\end{array}$ & Manca1996 \\
\hline
\end{tabular}




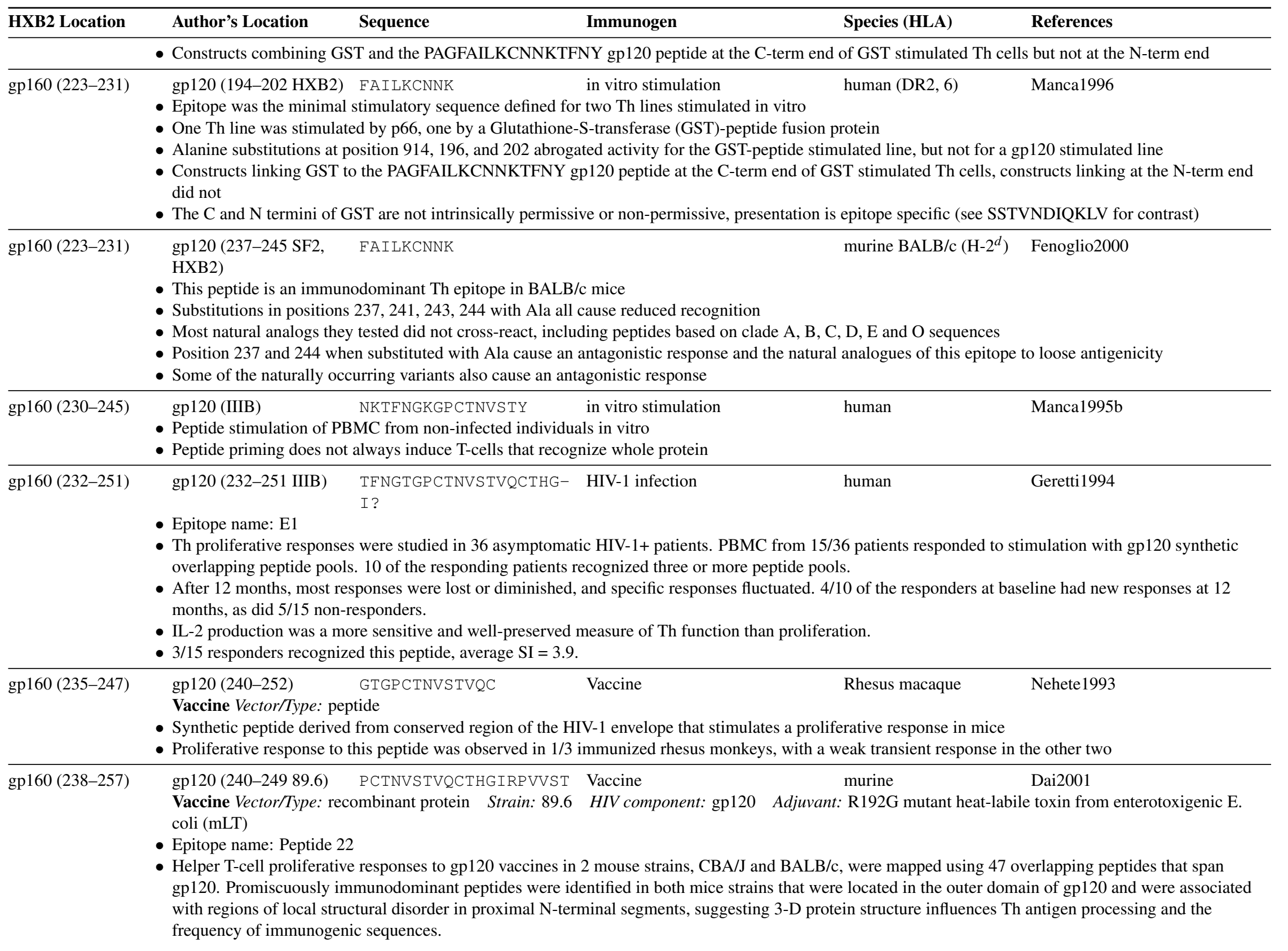




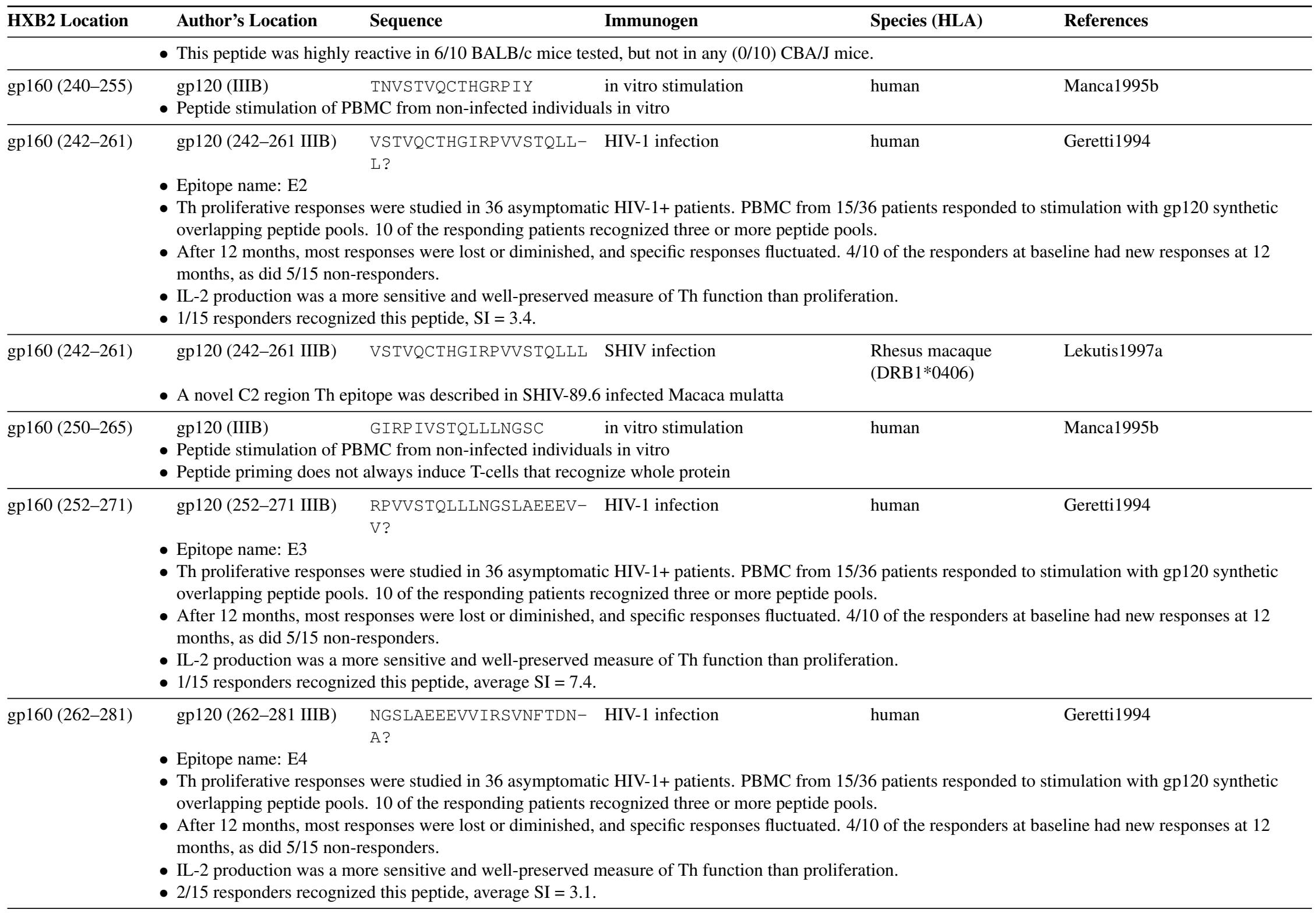




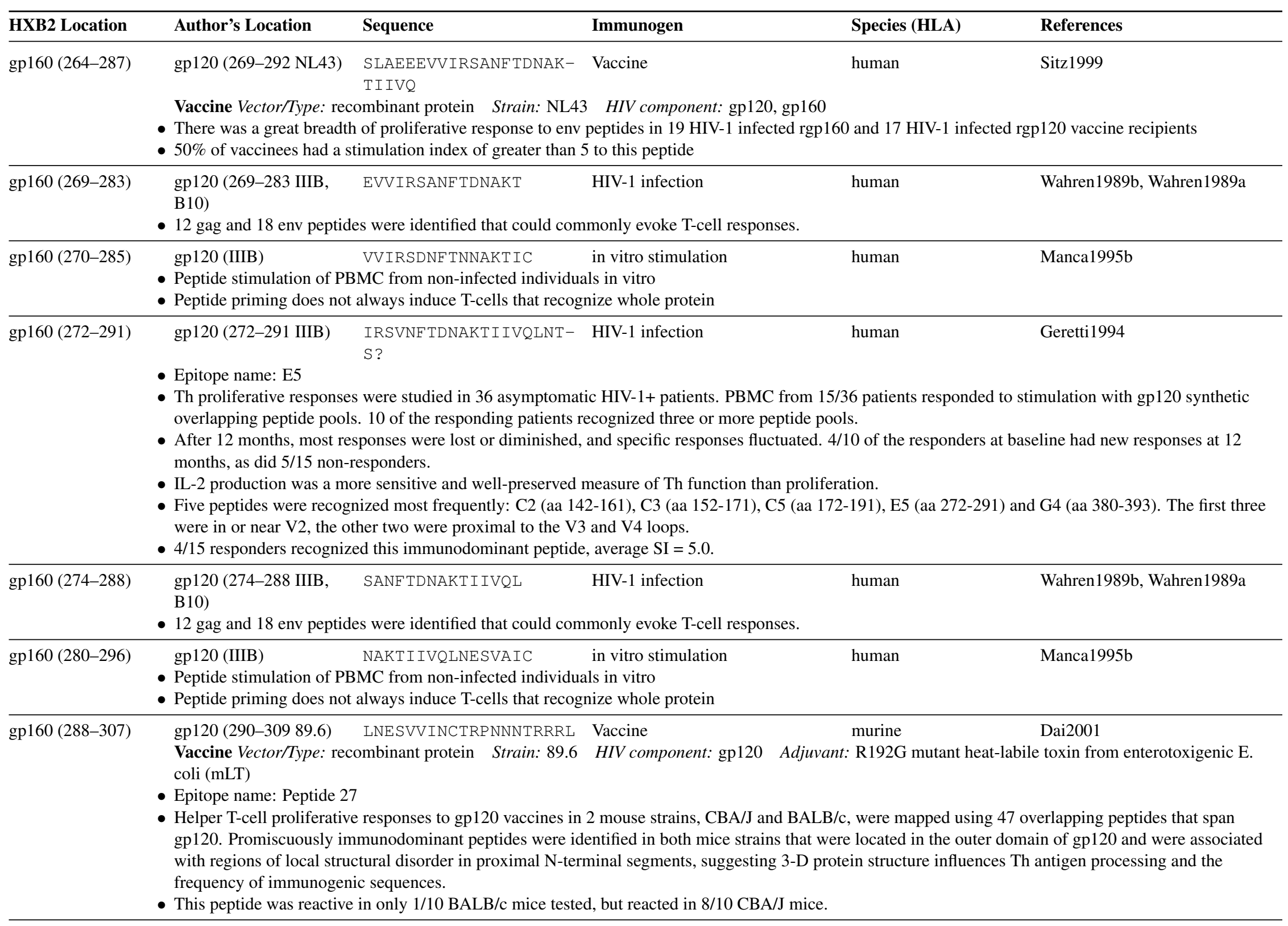




\begin{tabular}{lllll}
\hline HXB2 Location & Author's Location & Sequence & Immunogen & Species (HLA) \\
\hline gp160 (289-297) & $\begin{array}{l}\text { gp120 (292-300 SF2) } \\
\text { Vaccine Vector/Type: } \text { recombinant protein }\end{array}$ & Strain: SF2 & VIV component: gp120 & human
\end{tabular}

Vaccine Vector/Type: recombinant protein Strain: SF2 HIV component: gp120

- A non-glycosylated form of SF2 gp120, env 2-3, was used as an immunogen - 20\% of T-cell clones do not recognize the glycosylated form

\begin{tabular}{lllll}
\hline gp160 (290-306) & $\begin{array}{l}\text { gp120 (296-312 LAI) } \\
\text { - Stimulates T-cell proliferation in HIV-infected donors }\end{array}$ & HIV-1 infection & human \\
\hline gp160 (292-310) & gp120 (292-310 IIIB) & VEINCTRPNNNTRKRIRIQ? & HIV-1 infection & human
\end{tabular}

- Epitope name: F1

- Th proliferative responses were studied in 36 asymptomatic HIV-1+ patients. PBMC from 15/36 patients responded to stimulation with gp120 synthetic overlapping peptide pools. 10 of the responding patients recognized three or more peptide pools.

- After 12 months, most responses were lost or diminished, and specific responses fluctuated. 4/10 of the responders at baseline had new responses at 12 months, as did 5/15 non-responders.

- IL-2 production was a more sensitive and well-preserved measure of Th function than proliferation.

- Only 1/15 responders recognized this peptide, but it had the highest SI in the study of 9.9.

gp160 (296-307) gp120(301-324 RF) CTRPNNNTRKSI HIV-1 infection deLorimier1994

- Proton NMR spectroscopy was employed to analyze the solution conformation of a hybrid peptide, T1-SP10RF, in order to better understand the immunogenicity of its' T helper (KQIINMWQEVGKAMYA, CTRPNNNTRKSI), CTL (SITKGPGRVIYATG) and B-cell epitopes (RKSITKGPGRVIYATG).

- This epitope embedded in the T1-SP10RF peptide does not form a helical amphipathic conformation. It lacks random-coil conformations, and this may make a peptide less susceptible to complete proteolytic degredation and be favored within epitopes.

\begin{tabular}{lllll}
\hline gp160 (296-314) gp120(303-321 IIIB) & $\begin{array}{l}\text { CTRPNNNTRKSIRIQRGPG- } \\
\text { (Y) }\end{array}$ & Vaccine & goal
\end{tabular}

Vaccine Vector/Type: peptide Strain: IIIB

- Goats were immunized with peptides containing V3 type-specific neutralizing determinants coupled to T1

gp160 (297-321) gp120(302-324 MN) TRPNYNKRKRIHIGPGRAF - Vaccine murine BALB/c (H-2 $\left.{ }^{d}\right) \quad$ Oscherwitz1999b
YTTK

Vaccine Vector/Type: peptide Strain: MN HIV component: V3

- Epitope presented as a tandem repeat (eight copies) elicits stronger B-cell and T-cell responses than the epitope presented as a single copy

- This study indicates that the increased response was not due to neodeterminants created at the junction of the peptides, but rather due to an epitope density effect, increased immunogenicity through a high ratio of epitope to protein

\begin{tabular}{llll}
\hline gp160 (297-330) Env (303-335 BX08) & $\begin{array}{l}\text { TRPNNNTRKSIHIGPGRAF - Vaccine } \\
\text { YATGEIIGDIRQAH }\end{array}$ & human
\end{tabular}

Vaccine Vector/Type: lipopeptide

- Anti-HIV lipopeptide vaccine consisting of six long peptides derived from Nef, Gag and Env HIV-1 proteins modified by a palmitoyl chain was administered in a phase I trial

- A CD4+ T cell proliferative response to at least one of the six peptides was observed in $9 / 10$ vaccinees $-6 / 10$ reacted to this peptide

- 9/12 tested mounted a CTL responses to at least one of the six peptides, each of the six peptides elicited a CTL response in at least one individual - this peptide was particularly immunogenic, eliciting a CTL response in five vaccinees 


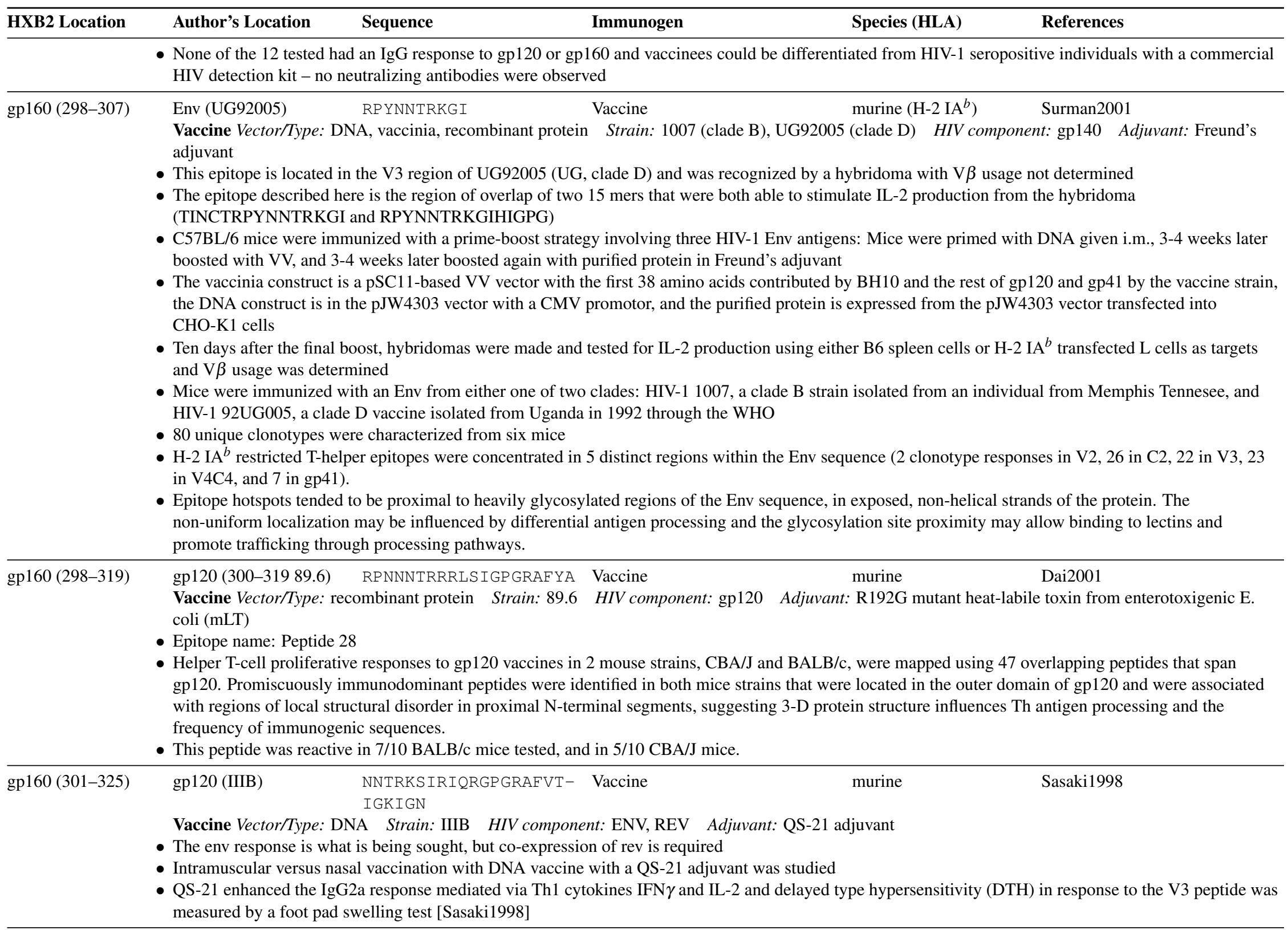




\begin{tabular}{|c|c|c|c|c|}
\hline HXB2 Location & Author's Location & Immunogen & Species (HLA) & References \\
\hline gp160 (302-315) & $\begin{array}{l}\text { gp120 (307-322 IIIB) } \\
\text { Vaccine Vector/Type: } \\
\text { - Identification of putati }\end{array}$ & $\begin{array}{l}\text { NTRKSIRIQRGPGR } \\
\text { tide Strain: IIIB HIV component: V3 } \\
\text { Th epitopes that can stimulate an antibody respons }\end{array}$ & $\begin{array}{l}\text { murine } \\
\text { aized mice }\end{array}$ & Goodman-Snitkoff1990 \\
\hline gp160 (302-321) & $\begin{array}{l}\text { gp120 (302-321 IIIB) } \\
\text { - Epitope name: F2 } \\
\text { - Th proliferative respon } \\
\text { overlapping peptide po } \\
\text { - After } 12 \text { months, most } \\
\text { months, as did } 5 / 15 \text { no } \\
\text { - IL-2 production was a } \\
\text { - } 1 / 15 \text { responders recogr }\end{array}$ & $\begin{array}{l}\text { NTRKRIRIQRGPGRAFVTI- HIV-1 infection } \\
\text { G? } \\
\text { were studied in } 36 \text { asymptomatic HIV-1+ patient } \\
10 \text { of the responding patients recognized three o } \\
\text { ponses were lost or diminished, and specific respc } \\
\text { esponders. } \\
\text { re sensitive and well-preserved measure of Th fun } \\
\text { ed this peptide, SI = 5.6. }\end{array}$ & $\begin{array}{l}\text { human } \\
36 \text { patients respor } \\
\text { Is. } \\
10 \text { of the respond } \\
\text { tion. }\end{array}$ & $\begin{array}{l}\text { Geretti1994 } \\
\text { seline had new responses at } 12\end{array}$ \\
\hline gp160 (302-327) & $\begin{array}{l}\text { gp120 (307-332 MN) } \\
\text { Vaccine Vector/Type: } \\
\text { - Hypervariable epitope } \\
\text { degenerative peptide ce } \\
\text { more durable Th respo } \\
\text { transient MN-specific }\end{array}$ & $\begin{array}{l}\text { NKRKRIHIGPGRAFYTTKN- Vaccine } \\
\text { IIGTIR } \\
\text { tide Strain: MN HIV component: V3 Adjuve } \\
\text { nstructs (HECs) are degenerative peptide cocktails } \\
\text { tail containing } 64 \text { distinct peptides, NTRK-[SR]-I } \\
\text { s than the MN V3 peptide alone in BALB/c mice } \\
\text { response. }\end{array}$ & $\begin{array}{l}\text { murine } \\
\text {-51 } \\
\text { single peptide sy } \\
\text {-AFY-[AT]-TG-[1 } \\
\text { osted with V3 pe] }\end{array}$ & $\begin{array}{l}\text { Anderson2001 } \\
\text { eaction. Vaccination with a V3 } \\
\text { DN]-IRQ, elicited broader and } \\
\text { though the MN peptide elicited a }\end{array}$ \\
\hline gp160 (305-321) & $\begin{array}{l}\text { gp120 (312-329) } \\
\text { - Used as positive contro }\end{array}$ & $\begin{array}{l}\text { (CG) KSIRIQRGPGRAFVT- HIV-1 infection } \\
\text { IG } \\
\text { n study examining T-cell response to four p } 24 \text { Gag }\end{array}$ & human & Adams1997 \\
\hline gp160 (308-319) & gp120 (subtype C) & (CKR) KIHIGPGQAFYT & murine $\left(\mathrm{H}-2^{b, c}\right.$ & Ahluwalia1997 \\
\hline
\end{tabular}

- A V3 loop peptide modified to resemble an Indian form (GPGQ) was incorporated into ISCOMS (immune stimulating complexes) or liposomes, and used to immunize mice - the IgG2a/IgG2b Ab response was enhanced by the presentation in the ISCOM suggestive of a Th1 response

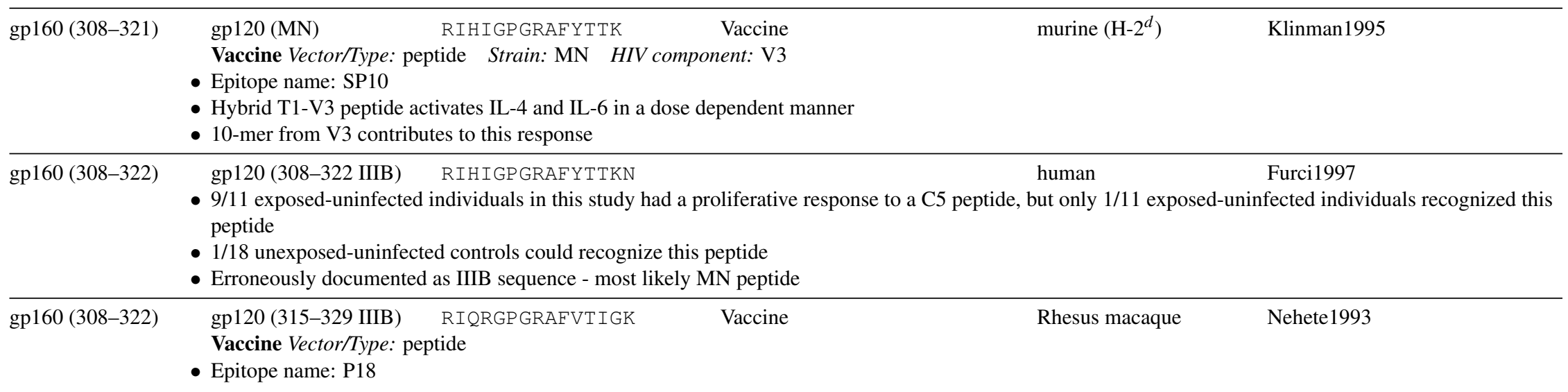




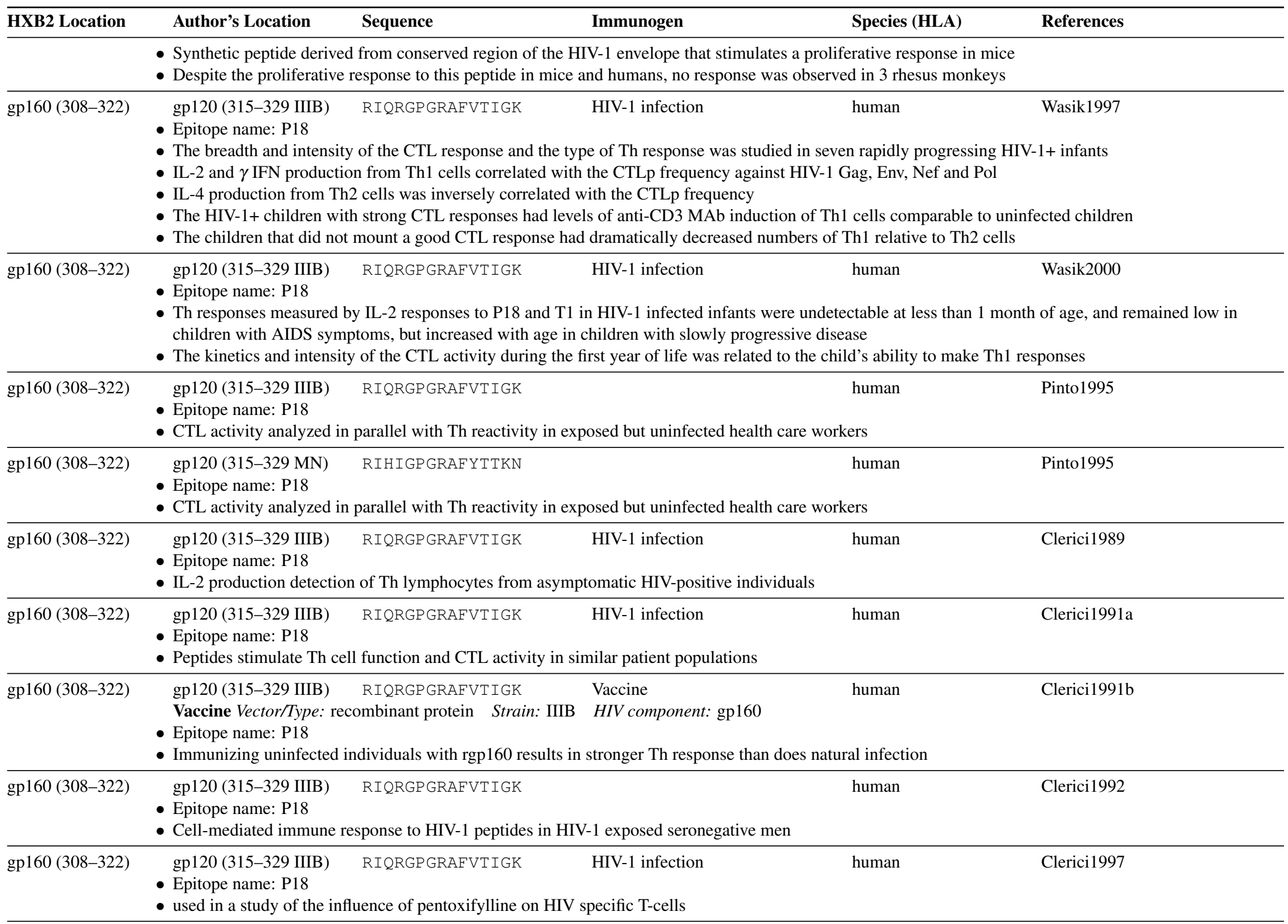




\begin{tabular}{|c|c|c|c|c|c|}
\hline HXB2 Location & Author's Location & Sequence & Immunogen & Species (HLA) & References \\
\hline \multirow[t]{2}{*}{ gp160 (308-322) } & gp120 (MN) & RIHIGPGRAFYTTKN & & human & Clerici1992 \\
\hline & - Epitope P18 MN: Cel & diated immune response & -1 peptides in HIV-1 exposed sero & gative men & \\
\hline gp160 (308-322) & gp160 (315-329 IIIB) & RIQRGP GRAFVTIGK & $\begin{array}{l}\text { HIV-1 infection, HIV-1 exposed } \\
\text { seronegative }\end{array}$ & human & Wasik1999 \\
\hline
\end{tabular}

- Epitope name: P18

- IL-2 responses associated with beta-chemokine expression were detectable at birth in the majority of uninfected infants born to HIV+ mothers, declining by age 6 months

- In both uninfected and infected infants of HIV-positive mothers, responses to the T1 peptide (KQIINMWQEVGKAMYA) were more frequent than responses to P18

- T1 is a highly conserved epitope, whereas P18 has a higher mutation rate due to its location in the immunodominant V3 loop region

gp160 (308-322) gp120(315-329 IIIB) RIQRGPGRAFVTIGK HIV-1 infection haul1999

- Epitope name: P18

- Kenyan sex workers that remained seronegative were found to frequently have HIV-env peptide specific Th responses detected by an IL-2 assay (11/20 cases) and mucosal genital tract anti-HIV $\operatorname{IgA}(16 / 21$ cases)

- Helper epitopes used in this study were noted to be previously described [Clerici1989], and were not explicitly described in [Kaul1999]

\begin{tabular}{llll}
\hline gp160 (308-322) gp120(315-329 IIIB) & RIQRGPGRAFVTIGK & $\begin{array}{l}\text { HIV-1 infection, HIV-1 exposed human } \\
\text { seronegative }\end{array}$
\end{tabular}

- Epitope name: P18

- In a S. African perinatal transmission study, 33\% (33/86) of cord blood samples from infants with seropositive mothers produced T-helper responses (measured by a bioassay measuring IL2 production in a murine cell line and confirmed with a proliferation assay) against a peptide cocktail containing Th epitopes P18 MN, P18 IIIB, T1, T2, and TH4

- The mothers were predominantly infected with subtype $\mathrm{C}$, but the $\mathrm{T}$ help response was detectable in a number of cord blood samples despite using peptides based on B subtype reagents.

- 3/33 infants with cord blood T help responses to Env were infected in utero, 2/33 were lost to follow up, and 28/33 were not infected. 6/53 of the infants with cord blood that was unresponsive to Env peptide stimulation were infected before delivery, and 8/47 contracted HIV intrapartum or via breast-feeding.

- Measurable HIV specific T help responses elicited in the immunologically immature newborn, possibly in response to in utero exposure, are associated with a protective natural immunity that helps block mother-infant transmission of HIV-1.

\begin{tabular}{llll}
\hline gp160 (308-322) gp120 (315-329 MN) RIHIGPGRAFYTTKN & $\begin{array}{l}\text { HIV-1 infection, HIV-1 exposed human } \\
\text { seronegative }\end{array}$
\end{tabular}

- Epitope name: P18

- In a S. African perinatal transmission study, 33\% (33/86) of cord blood samples from infants with seropositive mothers produced T-helper responses (measured by a bioassay measuring IL2 production in a murine cell line and confirmed with a proliferation assay) against a peptide cocktail containing Th epitopes P18 MN, P18 IIIB, T1, T2, and TH4

- The mothers were predominantly infected with subtype $\mathrm{C}$, but the $\mathrm{T}$ help response was detectable in a number of cord blood samples despite using peptides based on B subtype reagents.

- 3/33 infants with cord blood T help responses to Env were infected in utero, 2/33 were lost to follow up, and 28/33 were not infected. 6/53 of the infants with cord blood that was unresponsive to Env peptide stimulation were infected before delivery, and 8/47 contracted HIV intrapartum or via breast-feeding.

- Measurable HIV specific T help responses elicited in the immunologically immature newborn, possibly in response to in utero exposure, are associated with a protective natural immunity that helps block mother-infant transmission of HIV-1. 


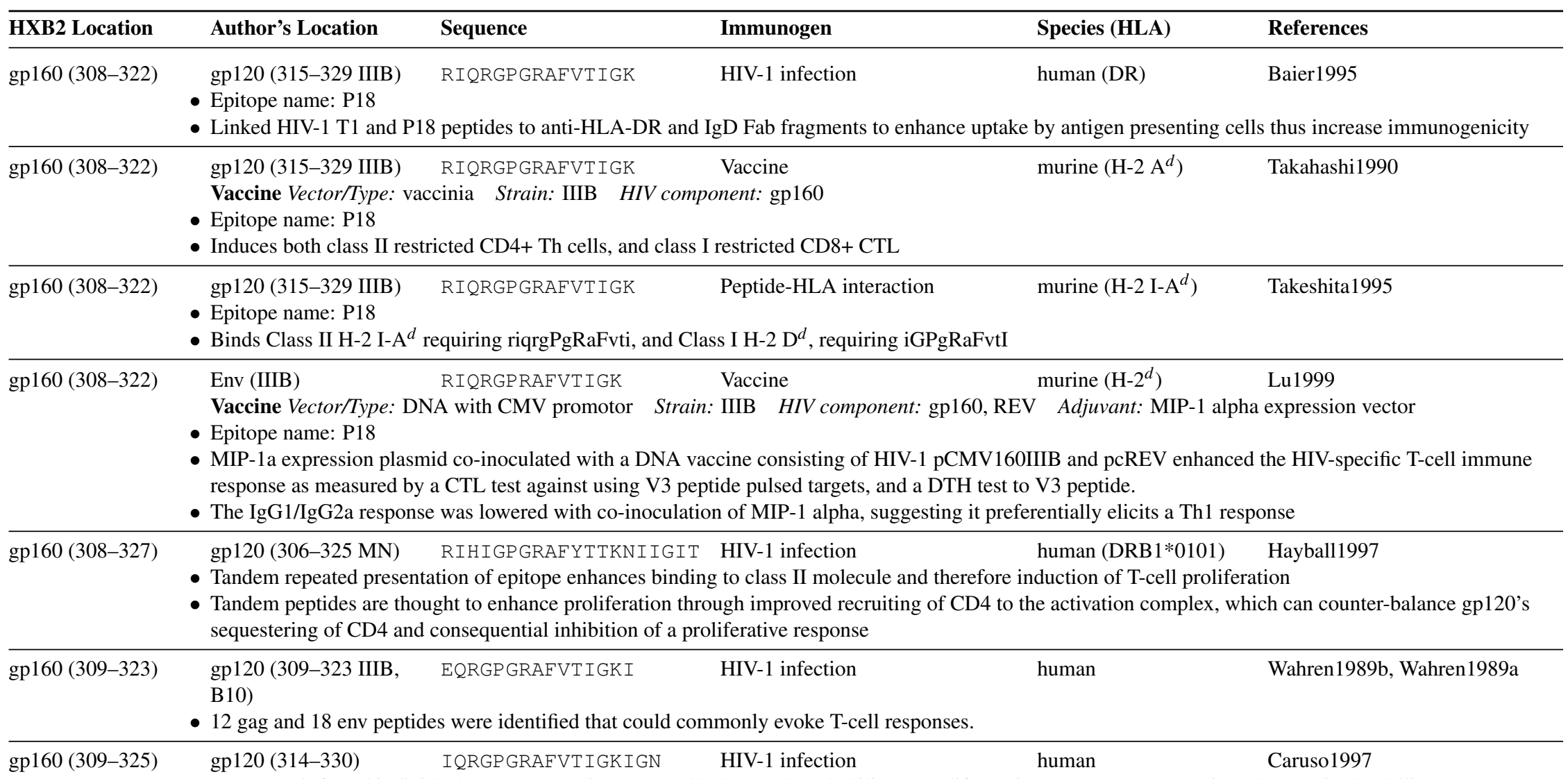

- As HIV-1-infected individuals progress to disease, T-cells show reduced ability to proliferate in response to HIV antigen, but retain the ability to express the activation antigens CD25 and CD71

- The ability to express activation markers in response to HIV is retained, but the response to tetanus toxoid recall antigen is lost

- This study investigated CD25 and CD71 expression in PBMC from patients at various stages of progression, measuring the response to in vitro stimulation by peptide cocktail containing four antigenic Env peptides, or p17 and p24

gp160 (310-328) gp120(310-329 89.6) S IGPGRAFYARRNI IGDIRQ Vaccine murine Dai2001 Vaccine Vector/Type: recombinant protein Strain: 89.6 HIV component: gp120 Adjuvant: R192G mutant heat-labile toxin from enterotoxigenic E. coli (mLT)

- Epitope name: Peptide 29

- Helper T-cell proliferative responses to gp120 vaccines in 2 mouse strains, CBA/J and BALB/c, were mapped using 47 overlapping peptides that span gp120. Promiscuously immunodominant peptides were identified in both mice strains that were located in the outer domain of gp120 and were associated with regions of local structural disorder in proximal N-terminal segments, suggesting 3-D protein structure influences Th antigen processing and the frequency of immunogenic sequences. 


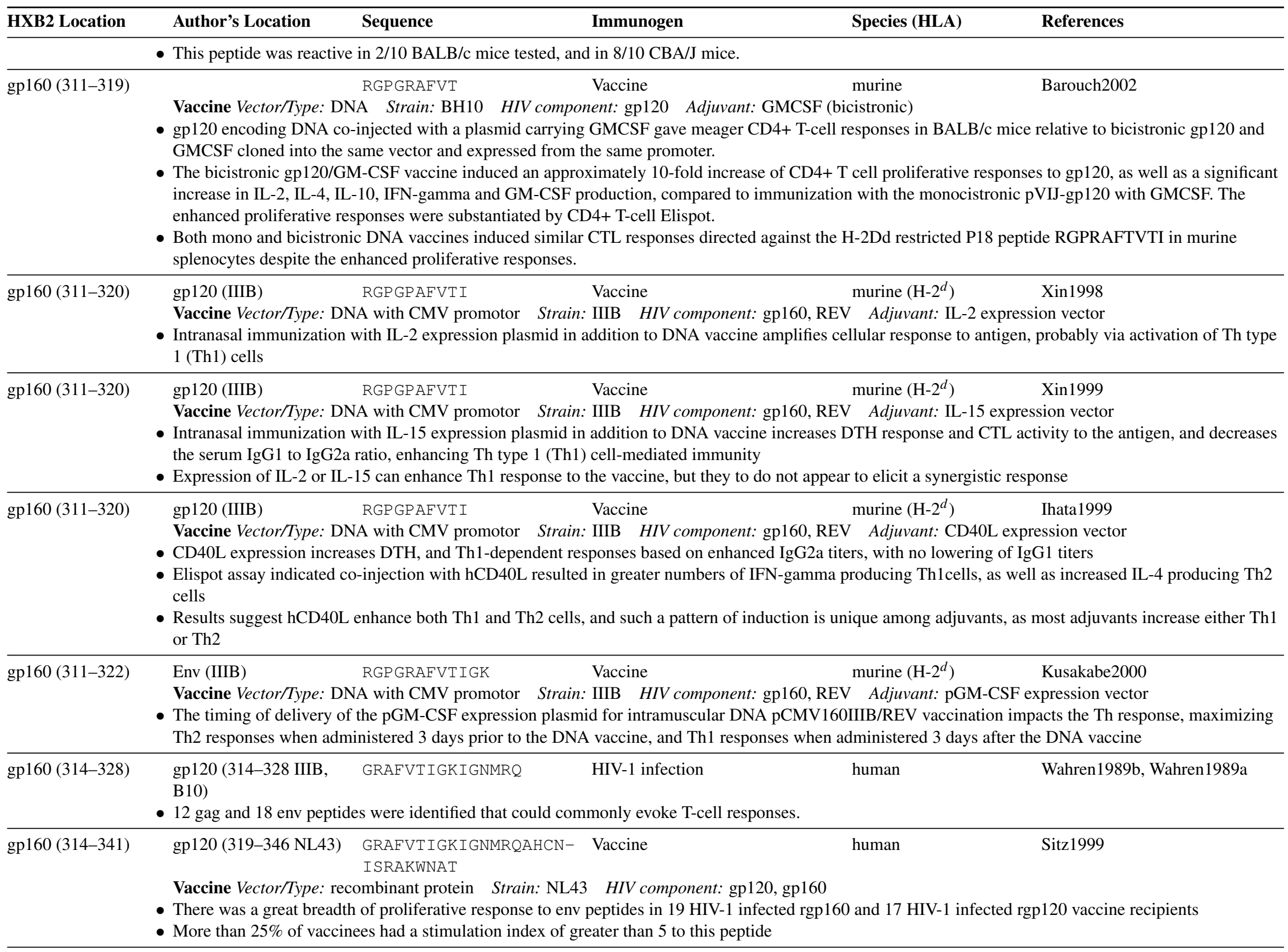




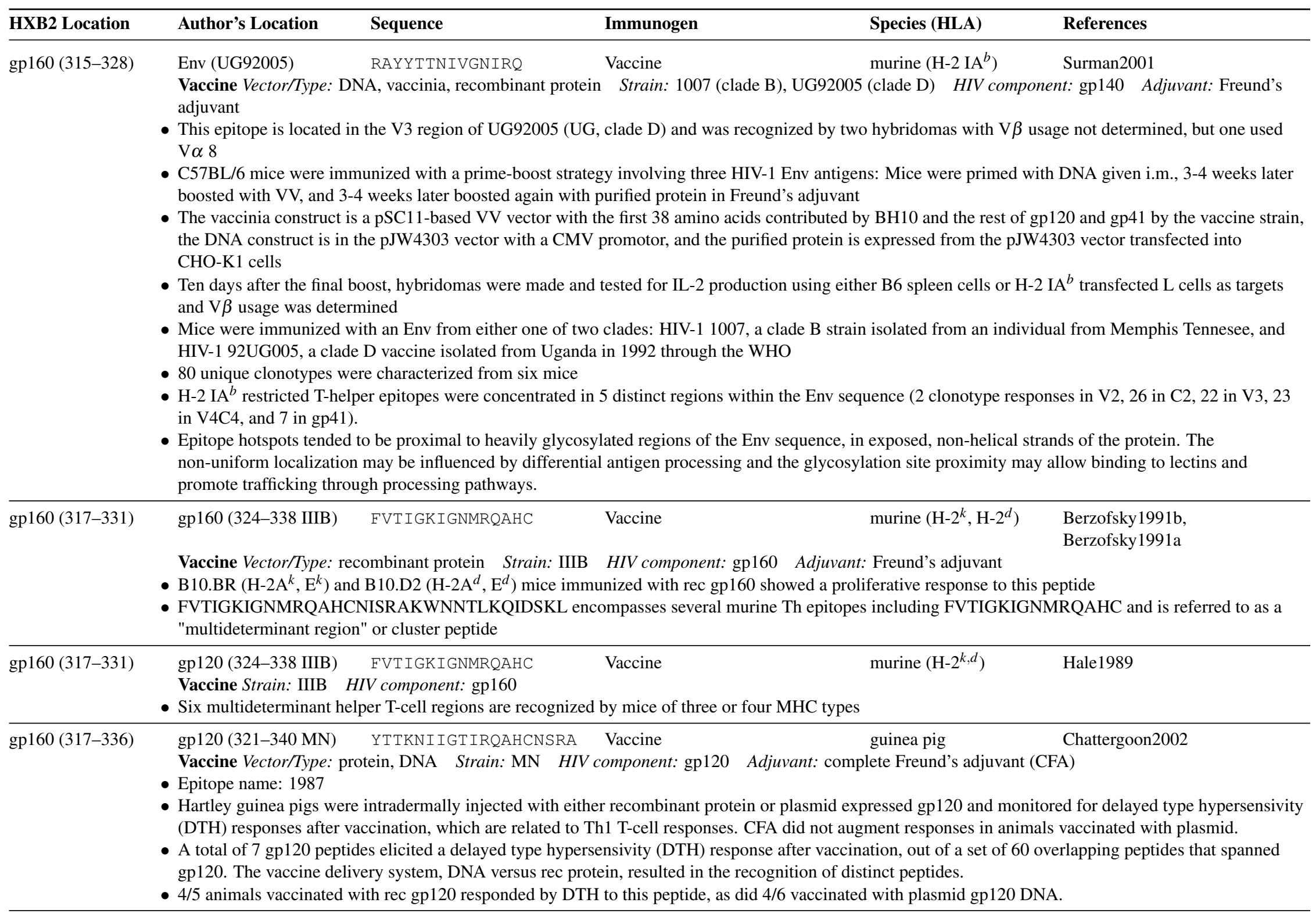




\begin{tabular}{|c|c|c|c|c|c|}
\hline HXB2 Location & Author's Location & Sequence & Immunogen & Species (HLA) & References \\
\hline gp160 (317-349) & gp160 (324-356 IIIB) & $\begin{array}{l}\text { FVTIGKIGNMRQAHCNISR- } \\
\text { AKWNNTLKQIDSKL }\end{array}$ & HIV-1 infection, Vaccine & $\begin{array}{l}\text { human, murine }\left(\mathrm{H}-2^{k} \text {, }\right. \\
\left.\mathrm{H}-2^{d}\right)\end{array}$ & \begin{tabular}{|l|} 
Berzofsky1991b \\
Berzofsky1991a
\end{tabular} \\
\hline
\end{tabular}

Vaccine Vector/Type: recombinant protein Strain:

- FVTIGKIGNMRQAHCNISRAKWNNTLKQIDSKL encompasses several murine Th epitopes and is referred to as a "multideterminant region" or cluster peptide

- Six multideterminant region cluster peptides were evaluated Th responses in different MHC/HLA backgrounds after vaccination of mice with gp160, or in infected people

- This cluster peptide elicited proliferative responses in cells from B10.BR mice $\left(\mathrm{H}-2 \mathrm{~A}^{k}, \mathrm{E}^{k}\right)$ and B10.D2 mice $\left(\mathrm{H}-2 \mathrm{~A}^{d}, \mathrm{E}^{d}\right)$, but shorter peptides from within this region stimulated $\mathrm{H}-2^{k}, \mathrm{H}-2^{d}, \mathrm{H}-2^{b}$ and $\mathrm{H}-2^{s}$ responses

- IL-2 production in response to this peptide was observed in 58\% (21/36) of asymptomatic HIV-infected individuals

\begin{tabular}{lllll}
\hline gp160 (319-338) gp120 (320-339 89.6) RRNIIGDIRQAHCNISRAKW Vaccine & Dai2001
\end{tabular}

Vaccine Vector/Type: recombinant protein Strain: 89.6 HIV component: gp120 Adjuvant: R192G mutant heat-labile toxin from enterotoxigenic E coli (mLT)

- Epitope name: Peptide 30

- Helper T-cell proliferative responses to gp120 vaccines in 2 mouse strains, CBA/J and BALB/c, were mapped using 47 overlapping peptides that span gp120. Promiscuously immunodominant peptides were identified in both mice strains that were located in the outer domain of gp120 and were associated with regions of local structural disorder in proximal N-terminal segments, suggesting 3-D protein structure influences Th antigen processing and the frequency of immunogenic sequences.

- This peptide was highly reactive in 7/10 BALB/c mice tested, and in 7/10 CBA/J mice and was consider one of the 3 immunodominant peptides identified that were shared in both mouse strains: RRNIIGDIRQAHCNISRAKW, NNTLQQIVIKLREKFRNKTI, GTNGTEGNDIITLQCRIKQI.

gp160 (319-338) gp120(320-339 89.6) RRNIIGDIRQAHCNISRAKW Vaccine murine (H-2 ${ }^{k}$, H-2 $\left.^{d}\right)$ Dai2001

Vaccine Vector/Type: recombinant protein Strain: 89.6 HIV component: gp120 Adjuvant: mutant R192G heat-labile toxin from E. coli as adjuvant

- Promiscuous immunodominant epitopes in gp120 were mapped by overlapping peptides in CBA/J H-2 ${ }^{k}$ and BALB/c H-2 ${ }^{d}$ mice, and all were found to be in the outer domain, proximal to regions of structural disorder indicated by the crystal structure or by sequence divergence.

- This peptide was recognized by $7 / 10 \mathrm{CBA} / \mathrm{J}$ and $7 / 10 \mathrm{BALB} / \mathrm{c}$ mice with $\mathrm{SI}>4$, averaging 6.3 and 4.8 , and is considered to be promiscuously immunodominant

- Uniquely immunodominant sequences tended to be in the inner domain of the protein

\begin{tabular}{|c|c|c|c|c|}
\hline gp160 (321-336) & $\begin{array}{l}\text { gp120 (IIIB) RIIGDIRKAHCNISRY } \\
\text { - Peptide stimulation of PBMC from non-infected in } \\
\text { - Peptide priming does not always induce T-cells that }\end{array}$ & $\begin{array}{l}\text { in vitro stimulation } \\
\text { ls in vitro } \\
\text { iize whole protein }\end{array}$ & human & Manca1995b \\
\hline gp160 (322-336) & Env (1007) & Vaccine & murine $\left(\mathrm{H}-2 \mathrm{IA}^{b}\right)$ & Surman2001 \\
\hline
\end{tabular}

Vaccine Vector/Type: DNA, vaccinia, recombinant protein Strain: 1007 (clade B), UG92005 (clade D) HIV component: gp140 Adjuvant: Freund's adjuvant

- This epitope is located in the V3 region of 1007 (US, clade B) and was recognized by three hybridomas with $\mathrm{V} \beta$ usage $\mathrm{V} \beta 6$ and not determined

- C57BL/6 mice were immunized with a prime-boost strategy involving three HIV-1 Env antigens: Mice were primed with DNA given i.m., 3-4 weeks later boosted with VV, and 3-4 weeks later boosted again with purified protein in Freund's adjuvant

- The vaccinia construct is a pSC11-based VV vector with the first 38 amino acids contributed by BH10 and the rest of gp120 and gp41 by the vaccine strain, the DNA construct is in the pJW4303 vector with a CMV promotor, and the purified protein is expressed from the pJW4303 vector transfected into CHO-K1 cells 


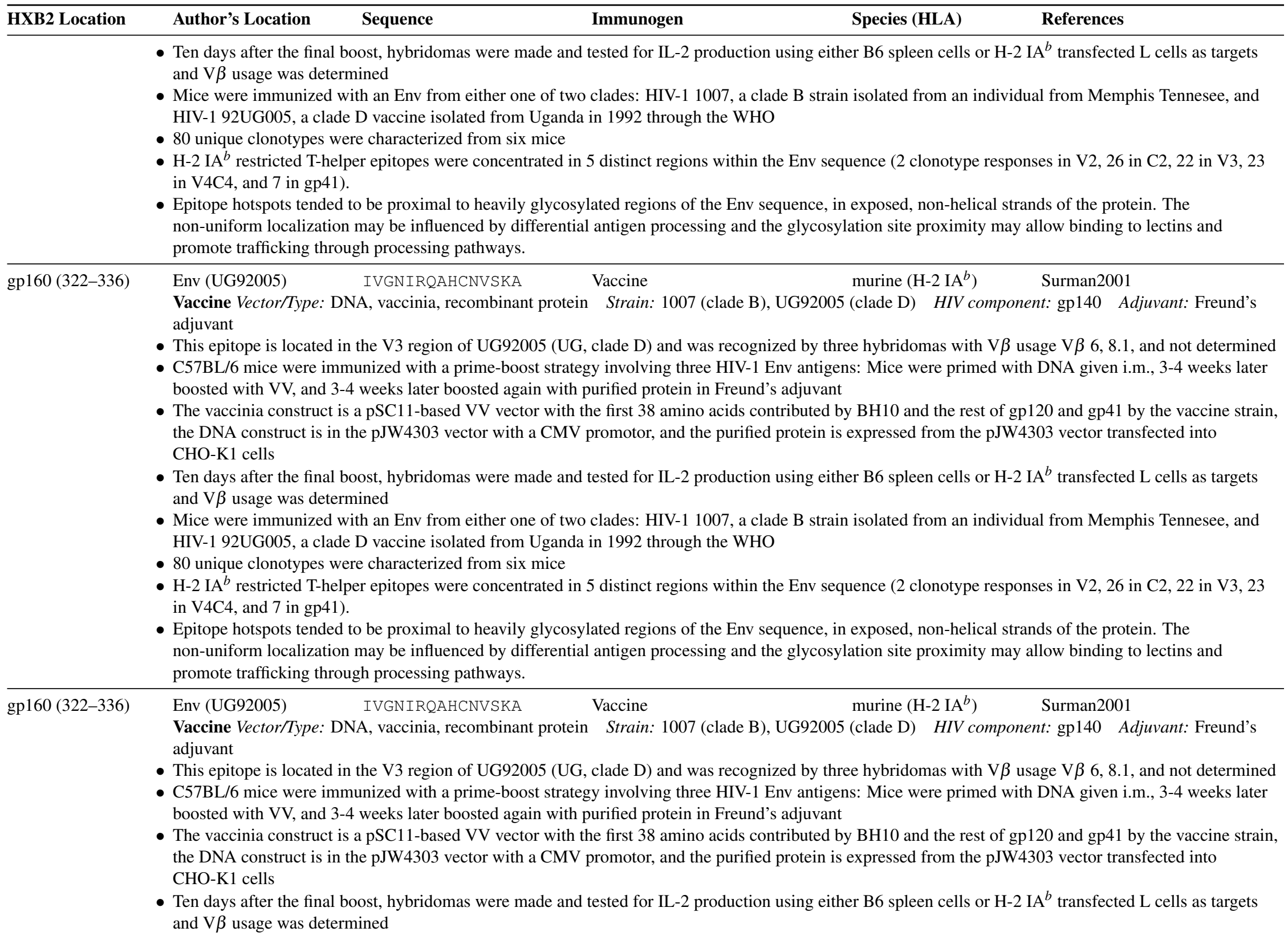




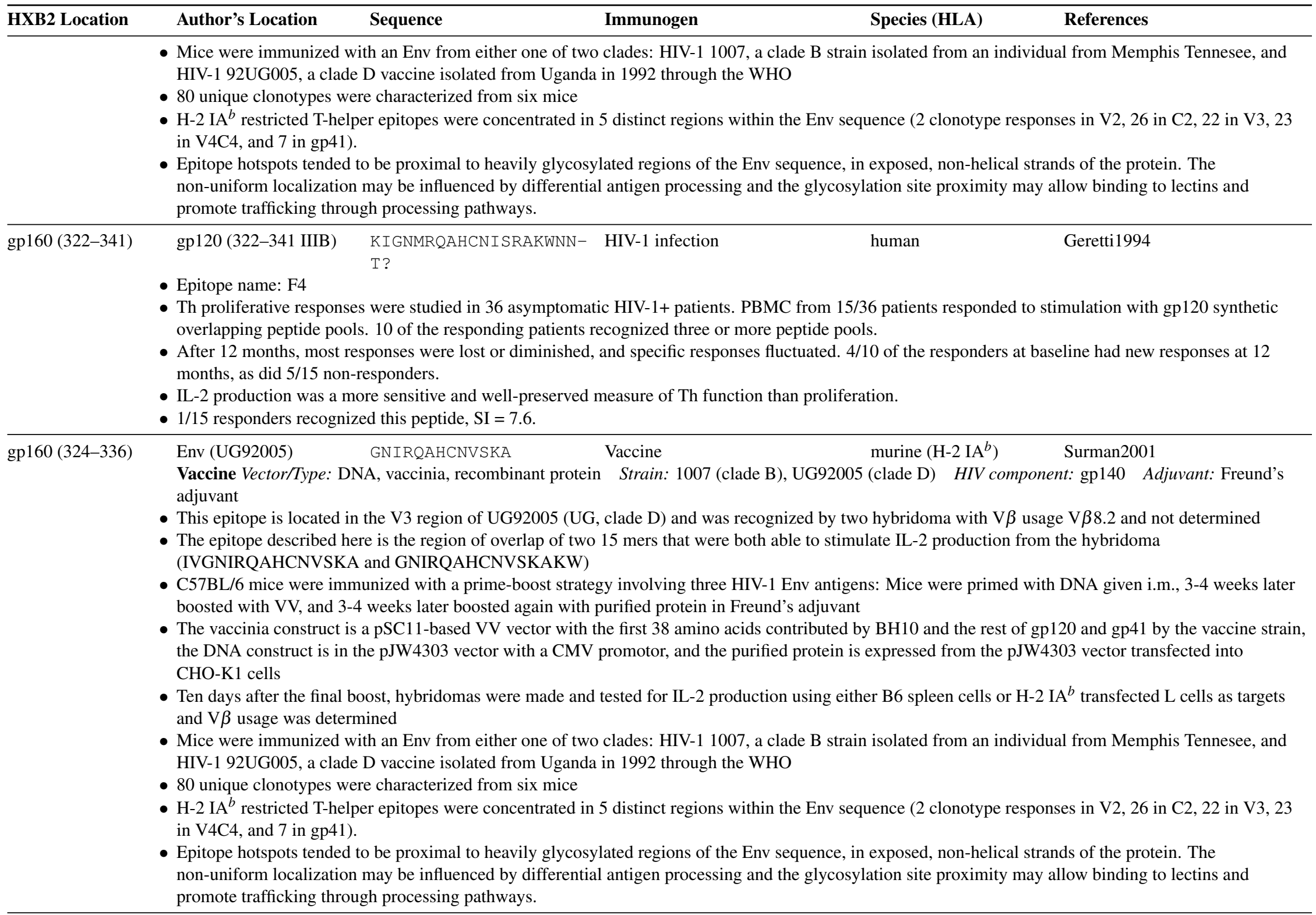




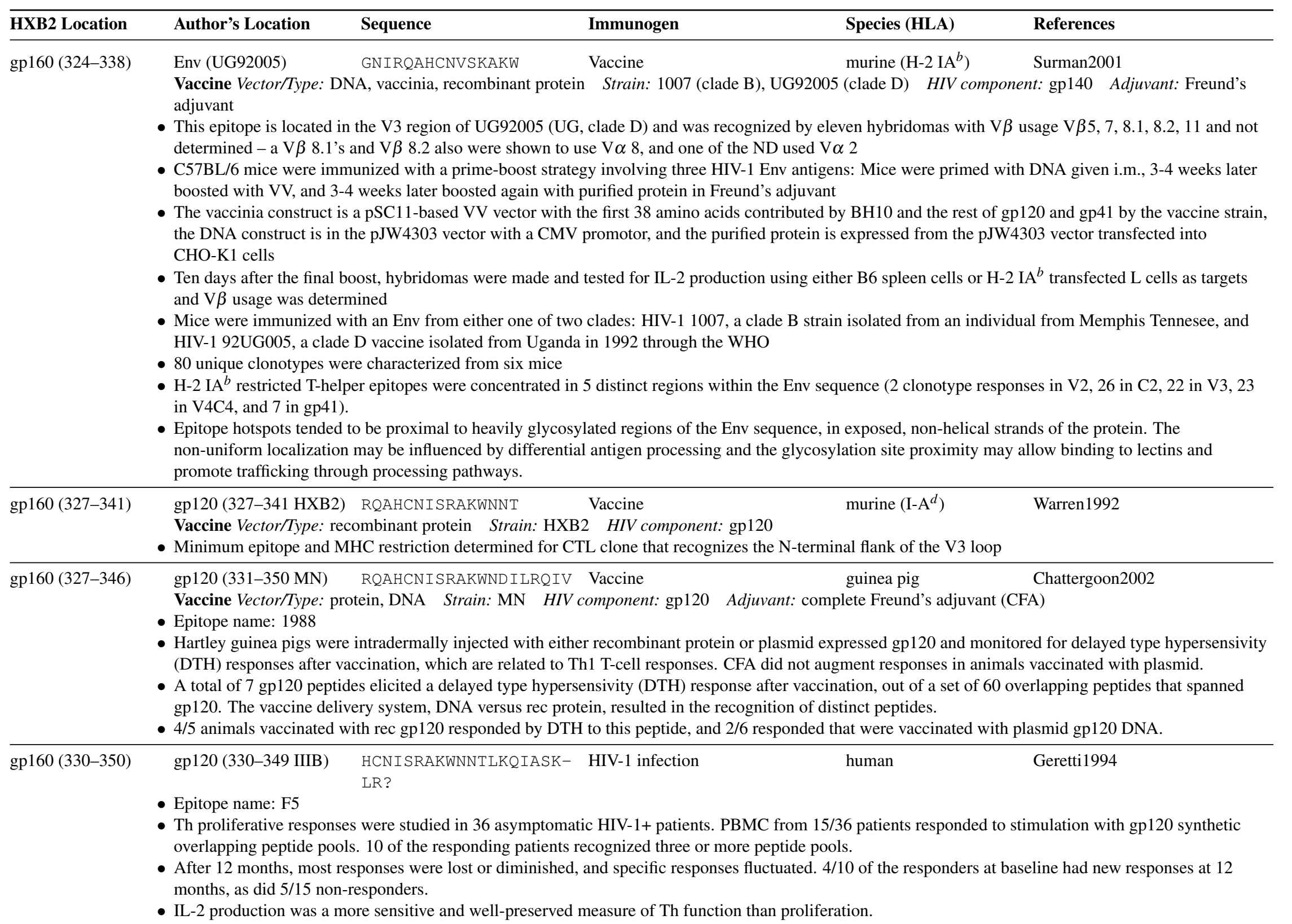




\begin{tabular}{|c|c|c|c|c|}
\hline HXB2 Location & Author's Location & Immunogen & Species (HLA) & References \\
\hline & \multicolumn{4}{|c|}{ - $3 / 15$ responders recognized this peptide, average $\mathrm{SI}=5.5$} \\
\hline gp160 (331-345) & $\begin{array}{l}\text { gp120 (IIIB) } \\
\text { Peptide stimulation of PI } \\
\text { Peptide priming does no }\end{array}$ & $\begin{array}{l}\text { CNISRAQWNNTLEQI in vitro stimulation } \\
\text { MC from non-infected individuals in vitro } \\
\text { always induce T-cells that recognize whole protein }\end{array}$ & human & Manca1995b \\
\hline gp160 (332-354) & gp120 (337-359 NL43) & NISRAKWNATLKQIASKLR- Vaccine & human & Sitz1999 \\
\hline
\end{tabular}

Vaccine Vector/Type: recombinant protein Strain: NL43 HIV component: gp120, gp160

- There was a great breadth of proliferative response to env peptides in 19 HIV-1 infected rgp160 and 17 HIV-1 infected rgp120 vaccine recipients

- More than $30 \%$ of vaccinees had a stimulation index of greater than 5 to this peptide

\begin{tabular}{|c|c|c|c|c|c|}
\hline gp160 (335-349) & gp160 (342-356 IIIB) & RAKWNNTLKQIDSKL & Vaccine & $\begin{array}{l}\text { murine }\left(\mathrm{H}-2^{k}, \mathrm{H}-2^{b},\right. \\
\left.\mathrm{H}-2^{s}\right)\end{array}$ & \begin{tabular}{|l|} 
Berzofsky1991b \\
Berzofsky1991a \\
\end{tabular} \\
\hline
\end{tabular}

B10.BR $\left(\mathrm{H}-2 \mathrm{~A}^{k}, \mathrm{E}^{k}\right), \mathrm{B} 10 . \mathrm{A}(5 \mathrm{R})\left(\mathrm{H}-2 \mathrm{~A}^{b}, \mathrm{E}^{b}\right)$ and $\mathrm{B} 10 . \mathrm{S}(9 \mathrm{R})\left(\mathrm{H}-2 \mathrm{~A}^{s}, \mathrm{E}^{s}\right)$ mice immunized with rec gp160 showed a proliferative response to this peptide

- FVTIGKIGNMRQAHCNISRAKWNNTLKQIDSKL encompasses several murine Th epitopes including RAKWNNTLKQIDSKL and is referred to as a "multideterminant region" or cluster peptide

gp160 (335-349) gp120(342-356 IIIB) RAKWNNTLKQICSKL $\quad$ Vaccine Hurine (H-2 $\left.{ }^{k, t 4, i 5}\right)$

Vaccine Strain: IIIB HIV component: gp160

- Six multideterminant helper T-cell regions are recognized by mice of three or four MHC types

gp160(337-356) gp120(341-360 MN) KWNDTLRQIVSKLKEQFKNK Vaccine guinea pig Chattergoon2002

Vaccine Vector/Type: protein, DNA Strain: MN HIV component: gp120 Adjuvant: complete Freund's adjuvant (CFA)

- Epitope name: 1989

- Hartley guinea pig were intradermally injected with either recombinant protein or plasmid expressed gp120 and monitored for delayed type hypersensivity (DTH) responses after vaccination, which are related to Th1 T-cell responses. CFA did not augment responses in animals vaccinated with plasmid.

- A total of 7 gp120 peptides elicited a delayed type hypersensivity (DTH) response after vaccination, out of a set of 60 overlapping peptides that spanned gp120. The vaccine delivery system, DNA versus rec protein, resulted in the recognition of distinct peptides.

- 3/5 animals vaccinated with rec gp120 responded by DTH to this peptide, and 2/6 responded that were vaccinated with plasmid gp120 DNA.

gp160 (339-359) gp120(340-359 89.6) NNTLQQIVIKLREKFRNKTI Vaccine murine Dai2001

Vaccine Vector/Type: recombinant protein Strain: 89.6 HIV component: gp120 Adjuvant: R192G mutant heat-labile toxin from enterotoxigenic E. coli (mLT)

- Epitope name: Peptide 32

- Helper T-cell proliferative responses to gp120 vaccines in 2 mouse strains, CBA/J and BALB/c, were mapped using 47 overlapping peptides that span gp120. Promiscuously immunodominant peptides were identified in both mice strains that were located in the outer domain of gp120 and were associated with regions of local structural disorder in proximal N-terminal segments, suggesting 3-D protein structure influences Th antigen processing and the frequency of immunogenic sequences.

- This peptide was reactive in 6/10 BALB/c mice tested, and in 4/10 CBA/J mice and was consider one of the 3 immunodominant peptides identified that were shared in both mouse strains: RRNIIGDIRQAHCNISRAKW, NNTLQQIVIKLREKFRNKTI, GTNGTEGNDIITLQCRIKQI.

gp160 (339-359) gp120(340-359 89.6) NNTLQQIVIKLREKFRNKT I Vaccine murine (H-2 ${ }^{k}$, H-2 $\left.^{d}\right) \quad$ Dai2001 Vaccine Vector/Type: recombinant protein Strain: 89.6 HIV component: gp120 Adjuvant: mutant R192G heat-labile toxin from E. coli as adjuvant 


\begin{tabular}{|c|c|c|c|c|}
\hline HXB2 Location & Author's Location & Immunogen & Species (HLA) & References \\
\hline & \multicolumn{4}{|c|}{$\begin{array}{l}\text { - Promiscuous immunodominant epitopes in gp } 120 \text { were mapped by overlapping peptides in } \mathrm{CBA} / \mathrm{J} \mathrm{H}-2^{k} \text { and BALB/c } \mathrm{H}-2^{d} \text { mice, and all were found to be } \\
\text { in the outer domain, proximal to regions of structural disorder indicated by the crystal structure or by sequence divergence. } \\
\text { - This peptide was recognized by } 4 / 10 \mathrm{CBA} / \mathrm{J} \text { and } 6 / 10 \mathrm{BALB} / \mathrm{c} \text { mice with } \mathrm{SI}>4 \text {, averaging } 4.9 \text { and } 5.5 \text { and is considered to be promiscuously } \\
\text { immunodominant } \\
\text { - Uniquely immunodominant sequences tended to be in the inner domain of the protein }\end{array}$} \\
\hline gp160 (341-356) & \multicolumn{4}{|c|}{$\begin{array}{l}\text { - Peptide stimulation of PBMC from non-infected individuals in vitro } \\
\text { - Peptide priming does not always induce T-cells that recognize whole protein }\end{array}$} \\
\hline gp160 (342-361) & \multicolumn{4}{|c|}{$\begin{array}{l}\text { - Epitope name: G1 } \\
\text { - Th proliferative responses were studied in } 36 \text { asymptomatic HIV-1+ patients. PBMC from } 15 / 36 \text { patients responded to stimulation with gp120 synthetic } \\
\text { overlapping peptide pools. } 10 \text { of the responding patients recognized three or more peptide pools. } \\
\text { - After } 12 \text { months, most responses were lost or diminished, and specific responses fluctuated. } 4 / 10 \text { of the responders at baseline had new responses at } 12 \\
\text { months, as did } 5 / 15 \text { non-responders. } \\
\text { - IL-2 production was a more sensitive and well-preserved measure of Th function than proliferation. } \\
\text { - } 2 / 15 \text { responders recognized this peptide, average } \mathrm{SI}=6.0 \text {. }\end{array}$} \\
\hline gp160 (344-357) & \multicolumn{4}{|c|}{ - Conjugation of HIV peptides to liposomes and rIL-2 stimulation may enhance cell-mediated responses } \\
\hline gp160 ( & $\begin{array}{l}\text { gp120 (350-369 89.6) } \\
\text { Vaccine Vector/Type: rec } \\
\text { coli (mLT) } \\
\text { - Epitope name: Peptide 3. } \\
\text { - Helper T-cell proliferativ } \\
\text { gp120. Promiscuously in } \\
\text { with regions of local stru } \\
\text { frequency of immunogen } \\
\text { - This peptide was reactive }\end{array}$ & $\begin{array}{l}\text { LREKFRNKTIAFNQSSGGD Vaccine } \\
\text { ombinant protein Strain: } 89.6 \quad \text { HIV component: gp120 } \\
\text { esponses to gp120 vaccines in } 2 \text { mouse strains, CBA/J an } \\
\text { munodominant peptides were identified in both mice strain } \\
\text { ctural disorder in proximal N-terminal segments, suggesting } \\
\text { ic sequences. } \\
\text { in } 3 / 10 \mathrm{BALB} / \mathrm{c} \text { mice tested, and in } 5 / 10 \mathrm{CBA} / \mathrm{J} \text { mice. }\end{array}$ & $\begin{array}{l}\text { BALB/c, were mappec } \\
\text { that were located in the } \\
\text { 3-D protein structure in }\end{array}$ & $\begin{array}{l}\text { Dai2001 } \\
\text { le toxin from enterotoxigenic E. } \\
\text { verlapping peptides that span } \\
\text { ain of gp120 and were associated } \\
\text { antigen processing and the }\end{array}$ \\
\hline gp160 (350-370) & $\begin{array}{l}\text { gp120 (350-370 IIIB) } \\
\text { - Epitope name: G2 } \\
\text { - Th proliferative response } \\
\text { overlapping peptide pool } \\
\text { - After } 12 \text { months, most re } \\
\text { months, as did } 5 / 15 \text { non-1 } \\
\text { - IL-2 production was a m } \\
\text { - } 1 / 15 \text { responders recogniz }\end{array}$ & $\begin{array}{l}\text { REQFGNNKT I IFKQSSGGD- HIV-1 infection } \\
\text { PE? } \\
\text { were studied in } 36 \text { asymptomatic HIV-1+ patients. PBMC } \\
\text { sponses were lost or diminished, and specific responses fluc } \\
\text { esponders. } \\
\text { re sensitive and well-preserved measure of Th function tha } \\
\text { ed this peptide, average } \mathrm{SI}=3.2 \text {. }\end{array}$ & $\begin{array}{l}\text { human } \\
\text { eptide pools. } \\
\text { tuated. } 4 / 10 \text { of the respor } \\
\text { n proliferation. }\end{array}$ & $\begin{array}{l}\text { Geretti1994 } \\
\text { imulation with gp120 synthetic } \\
\text { eline had new responses at } 12\end{array}$ \\
\hline gp160 (353-360) & $\begin{array}{l}\text { gp120 }(355-362 \text { IIIB }) \\
\text { C3 region minimal epitc }\end{array}$ & $\begin{array}{l}\text { FGNNKTI I SHIV infection } \\
\text { e determined through fine epitope mapping }\end{array}$ & Rhesus macac & Lekutis1997a \\
\hline
\end{tabular}




\begin{tabular}{|c|c|c|c|c|}
\hline HXB2 Location & Author's Location & Immunogen & Species (HLA) & References \\
\hline & \multicolumn{4}{|c|}{ - Cell line was lost prior to confirmation of MHC requirements } \\
\hline gp160 (363-372) & \multicolumn{4}{|c|}{ - Stimulates T-cell proliferation in HIV-infected donors } \\
\hline gp160 (364-378) & $\begin{array}{l}\text { gp120 (364-378 IIIB, } \\
\text { B10) } \\
\text { - } 12 \text { gag and } 18 \text { env pep }\end{array}$ & $\begin{array}{l}\text { SSGGKPEIVTHSENC HIV-1 infection } \\
\text { les were identified that could commonly evoke T-cell } \mathrm{r}\end{array}$ & human & Wahren1989b Wahren1989a \\
\hline gp160 (369-383) & $\begin{array}{l}\text { gp120 (369-383 IIIB, } \\
\text { B10) } \\
\text { - } 12 \text { gag and } 18 \text { env pep }\end{array}$ & $\begin{array}{l}\text { PEIVTHSFNCGGEFF HIV-1 infection } \\
\text { es were identified that could commonly evoke T-cell r }\end{array}$ & human & Wahren1989b Wahren1989a \\
\hline gp160 (380-393) & $\begin{array}{l}\text { gp120 (380-393 IIIB) } \\
\text { - Epitope name: G4 } \\
\text { - Th proliferative respon } \\
\text { overlapping peptide pc } \\
\text { - After } 12 \text { months, most } \\
\text { months, as did } 5 / 15 \text { no } \\
\text { - IL-2 production was a } \\
\text { - Five peptides were rec } \\
\text { were in or near V2, the } \\
\text { - } 4 / 15 \text { responders recogi }\end{array}$ & $\begin{array}{l}\text { GEFFYCNSTQLFNS? HIV-1 infection } \\
\text { S were studied in } 36 \text { asymptomatic HIV-1+ patients. P } \\
\text { s. } 10 \text { of the responding patients recognized three or m } \\
\text { sponses were lost or diminished, and specific response } \\
\text { responders. } \\
\text { ore sensitive and well-preserved measure of Th functio } \\
\text { nized most frequently: C2 (aa 142-161), C3 (aa 152-1 } \\
\text { ther two were proximal to the V3 and V4 loops. } \\
\text { ed this immunodominant peptide, average SI }=4.4 \text {. }\end{array}$ & $\begin{array}{l}\text { human } \\
6 \text { patients respon } \\
\text { s. } \\
10 \text { of the respond } \\
\text { tion. } \\
\text { 191), E5 (aa } 272\end{array}$ & $\begin{array}{l}\text { Geretti1994 } \\
\text { imulation with gp120 synthetic } \\
\text { eline had new responses at } 12 \\
\text { G4 (aa 380-393). The first three }\end{array}$ \\
\hline gp160 (381-395) & $\begin{array}{l}\text { gp120 (IIIB) } \\
\text { - Peptide stimulation of } \\
\text { - Peptide priming does } r\end{array}$ & $\begin{array}{l}\text { EFFYCNTTQLFNNTW in vitro stimulation } \\
\text { MC from non-infected individuals in vitro } \\
\text { always induce T-cells that recognize whole protein }\end{array}$ & human & Manca1995b \\
\hline gp160 (392-411) & $\begin{array}{l}\text { gp120 (392-411 IIIB) } \\
\text { - Epitope name: G5 } \\
\text { - Th proliferative respon } \\
\text { overlapping peptide po } \\
\text { - After } 12 \text { months, most } \\
\text { months, as did } 5 / 15 \text { no } \\
\text { - IL-2 production was a } \\
\text { - } 1 / 15 \text { responders recogi }\end{array}$ & $\begin{array}{l}\text { NSTWFNSTWSTEGSNNTEG- HIV-1 infection } \\
\text { S? } \\
\text { S were studied in } 36 \text { asymptomatic HIV-1+ patients. P } \\
\text { s. } 10 \text { of the responding patients recognized three or mo } \\
\text { sponses were lost or diminished, and specific response } \\
\text { responders. } \\
\text { ore sensitive and well-preserved measure of Th functio } \\
\text { ed this peptide, SI }=9.3 \text {. }\end{array}$ & $\begin{array}{l}\text { human } \\
6 \text { patients respon } \\
\text { s. } \\
10 \text { of the respond } \\
\text { tion. }\end{array}$ & $\begin{array}{l}\text { Geretti1994 } \\
\text { elinulation with gp120 synthetic } \\
\text { hew responses at } 12\end{array}$ \\
\hline gp160 (394-408) & $\begin{array}{l}\text { gp120 (394-408 IIIB, } \\
\text { B10) } \\
\text { - } 12 \text { gag and } 18 \text { env pep }\end{array}$ & $\begin{array}{l}\text { TWFNSTWSTKGSNNT HIV-1 infection } \\
\text { es were identified that could commonly evoke T-cell re }\end{array}$ & human & Wahren1989b Wahren1989a \\
\hline gp160 (396-411) & $\begin{array}{l}\text { gp120 (IIIB) } \\
\text { - Peptide stimulation of } \\
\text { - Peptide priming does } \mathrm{r}\end{array}$ & $\begin{array}{l}\text { FNNTWRLNHTEGTKGC in vitro stimulation } \\
\text { MC from non-infected individuals in vitro } \\
\text { always induce T-cells that recognize whole protein }\end{array}$ & human & Manca1995b \\
\hline
\end{tabular}




\begin{tabular}{lllll}
\hline HXB2 Location & Author's Location & Sequence & Immunogen & Species (HLA) \\
\hline gp160 (399-413) & gp120 (399-413 IIIB, & TWSTKGSNNTEGSDT & HIV-1 infection & human \\
& $\begin{array}{ll}\text { B10) } \\
\text { 12 gag and 18 env peptides were identified that could commonly evoke T-cell responses. }\end{array}$ &
\end{tabular}

$\begin{array}{lllll}\text { gp160 (404-423) } & \text { gp120 (400-419 89.6) } & \text { GTNGTEGNDIITQCRIKQI } & \text { Vaccine } & \text { murine } \\ & \text { Vaccine Vector/Type: recombinant protein } & \text { Strain: } 89.6 & \text { HIV component: gp120 } & \text { Adjuvant: R192G mutant heat-labile toxin from enterotoxigenic E. } \\ & \text { coli (mLT) } & & \end{array}$

12 gag and 18 env peptides were identified that could commonly evoke T-cell responses. coli (mLT)

- Epitope name: Peptide 38

- Helper T-cell proliferative responses to gp120 vaccines in 2 mouse strains, CBA/J and BALB/c, were mapped using 47 overlapping peptides that span gp120. Promiscuously immunodominant peptides were identified in both mice strains that were located in the outer domain of gp120 and were associated with regions of local structural disorder in proximal N-terminal segments, suggesting 3-D protein structure influences Th antigen processing and the frequency of immunogenic sequences.

- This peptide was reactive in 8/10 BALB/c mice tested, and in 6/10 CBA/J mice, and was consider one of the 3 immunodominant peptides identified that were shared in both mouse strains: RRNIIGDIRQAHCNISRAKW, NNTLQQIVIKLREKFRNKTI, GTNGTEGNDIITLQCRIKQI.

gp160 (404-423) gp120(400-419 89.6) GTNGTEGNDIITLQCRIKQI Vaccine murine (H-2 $2^{k}, \mathrm{H}_{-2}{ }^{d}$ Dai2001

Vaccine Vector/Type: recombinant protein Strain: 89.6 HIV component: gp120 Adjuvant: mutant R192G heat-labile toxin from E. coli as adjuvant

- Promiscuous immunodominant epitopes in gp120 were mapped by overlapping peptides in CBA/J H-2 ${ }^{k}$ and BALB/c H-2 ${ }^{d}$ mice, and all were found to be in the outer domain, proximal to regions of structural disorder indicated by the crystal structure or by sequence divergence.

- This peptide was recognized by 4/10 CBA/J and 6/10 BALB/c mice with SI > 4, averaging 4.9 and 5.5 and is considered to be promiscuously immunodominant

- Uniquely immunodominant sequences tended to be in the inner domain of the protein

gp160 (405-420) Env (1007) SNNTVGNP I ILPCRI $\quad$ Vaccine $\quad$ Surman2001
Vaccine Vector/Type: DNA, vaccinia, recombinant protein Strain: 1007 (clade B), UG92005 (clade D) HIV component: gp140 Adjuvant: Freund's adjuvant

- This epitope is located in the V4C4 region of 1007 (US, clade B) and was recognized by 13 hybridomas with $\mathrm{V} \beta$ usage $\mathrm{V} \beta 4,7,8.1,8.2,10,12$ and not determined - one of the $\mathrm{V} \beta 8.2$ was shown to utilize $\mathrm{V} \alpha 2$

- C57BL/6 mice were immunized with a prime-boost strategy involving three HIV-1 Env antigens: Mice were primed with DNA given i.m., 3-4 weeks later boosted with VV, and 3-4 weeks later boosted again with purified protein in Freund's adjuvant

- The vaccinia construct is a pSC11-based VV vector with the first 38 amino acids contributed by BH10 and the rest of gp120 and gp41 by the vaccine strain, the DNA construct is in the pJW4303 vector with a CMV promotor, and the purified protein is expressed from the pJW4303 vector transfected into CHO-K1 cells

- Ten days after the final boost, hybridomas were made and tested for IL-2 production using either B6 spleen cells or H-2 IA ${ }^{b}$ transfected L cells as targets and $\mathrm{V} \beta$ usage was determined

- Mice were immunized with an Env from either one of two clades: HIV-1 1007, a clade B strain isolated from an individual from Memphis Tennesee, and HIV-1 92UG005, a clade D vaccine isolated from Uganda in 1992 through the WHO

- 80 unique clonotypes were characterized from six mice

- $\mathrm{H}-2 \mathrm{IA}^{b}$ restricted T-helper epitopes were concentrated in 5 distinct regions within the Env sequence (2 clonotype responses in V2, 26 in C2, 22 in V3, 23 in $\mathrm{V} 4 \mathrm{C} 4$, and 7 in gp41). 


\begin{tabular}{|c|c|c|c|c|}
\hline HXB2 Location & Author's Location & Immunogen & Species (HLA) & References \\
\hline & \multicolumn{4}{|c|}{$\begin{array}{l}\text { - Epitope hotspots tended to be proximal to heavily glycosylated regions of the Env sequence, in exposed, non-helical strands of the protein. The } \\
\text { non-uniform localization may be influenced by differential antigen processing and the glycosylation site proximity may allow binding to lectins and } \\
\text { promote trafficking through processing pathways. }\end{array}$} \\
\hline gp160 (410-429) & \multicolumn{4}{|c|}{ - Synthetic peptides representing natural variants were used to test for recognition in the context DR4 } \\
\hline gp160 (410-429) & \multicolumn{4}{|c|}{ - Human CD4+ T-cell clones lyse recombinant vaccinia virus-infected cells that synthesize envelope gp160 } \\
\hline gp160 (412-431) & $\begin{array}{l}\text { gp120 (412-431 IIIB) } \\
\text { Epitope name: H2 } \\
\text { Th proliferative response } \\
\text { overlapping peptide pool } \\
\text { After } 12 \text { months, most re } \\
\text { months, as did } 5 / 15 \text { non-1 } \\
\text { IL-2 production was a mo } \\
1 / 15 \text { responders recogniz }\end{array}$ & $\begin{array}{l}\text { DTITLPCRIKQI INMWQKV- HIV-1 infection } \\
\text { G? } \\
\text { s were studied in } 36 \text { asymptomatic HIV-1+ patients. PI } \\
\text { s. } 10 \text { of the responding patients recognized three or mo } \\
\text { sponses were lost or diminished, and specific response } \\
\text { esponders. } \\
\text { ore sensitive and well-preserved measure of Th functio } \\
\text { ed this peptide, SI }=5.7 \text {. }\end{array}$ & $\begin{array}{l}\text { human } \\
36 \text { patients responded to } \\
1 \mathrm{~s} \text {. } \\
10 \text { of the responders at } \\
\text { ation. }\end{array}$ & $\begin{array}{l}\text { Geretti1994 } \\
\text { imulation with gp120 synthetic } \\
\text { seline had new responses at } 12\end{array}$ \\
\hline gp160 (416-431) & $\begin{array}{l}\text { gp120 (IIIB) } \\
\text { - Peptide stimulation of PB } \\
\text { Peptide priming does not }\end{array}$ & $\begin{array}{l}\text { LPCRIKQ I INMWQEVY in vitro stimulation } \\
\text { MC from non-infected individuals in vitro } \\
\text { always induce T-cells that recognize whole protein }\end{array}$ & human & Manca1995b \\
\hline gp160 (418-436) & $\begin{array}{l}\text { Env (417-435) } \\
\text { EnV-infected chimpanzee }\end{array}$ & $\begin{array}{l}\text { CRIKQI INMWQGVGKAMYA HIV-1 infection } \\
\text { es and HIV-positive patients show positive proliferative }\end{array}$ & $\begin{array}{l}\text { human, chimpanzee } \\
\text { ultiple peptides from } \mathrm{f}\end{array}$ & $\begin{array}{l}\text { Nehete1998b } \\
\text { conserved regions of the HIV-1 }\end{array}$ \\
\hline gp160 (421-436) & $\begin{array}{l}\text { gp120 (426-441 IIIB) } \\
\text { Epitope T1 variant: } 9 / 11 \\
\text { defined epitope } \\
\text { IIIB position } 435 \text { listed a }\end{array}$ & $\begin{array}{l}\text { KQF INMWQEWGKAMYA } \\
\text { exposed-uninfected individuals in this study had a prol } \\
\mathrm{S} W \text { in this epitope as opposed to } \mathrm{V} \text { in the sequence. }\end{array}$ & $\begin{array}{l}\text { human } \\
\text { nse to a C5 peptide, but }\end{array}$ & $\begin{array}{l}\text { Furci1997 } \\
\text { one reacted with this previously }\end{array}$ \\
\hline gp160 (421-436) & $\begin{array}{l}\text { gp120 (428-433 IIIB) } \\
\text { - Epitope name: T1 } \\
\text { Th responses measured b } \\
\text { children with AIDS symp } \\
\text { - The kinetics and intensity }\end{array}$ & $\begin{array}{l}\text { KQI INMWQEVGKAMYA HIV-1 infection } \\
\text { y IL-2 responses to P18 and T1 in HIV-1 infected infar } \\
\text { toms, but increased with age in children with slowly p } \\
\text { of the CTL activity during the first year of life was re }\end{array}$ & $\begin{array}{l}\text { human } \\
\text { ectable at less than } 1 \mathrm{mc} \\
\text { ld's ability to make Th1 }\end{array}$ & $\begin{array}{l}\text { Wasik2000 } \\
\text { of age, and remained low in } \\
\text { ponses }\end{array}$ \\
\hline gp160 (421-436) & $\begin{array}{l}\text { gp120 (428-433 IIIB) } \\
\text { - Epitope name: T1 } \\
\text { - The breadth and intensity } \\
\text { IL-2 and } \gamma \text { IFN productio } \\
\text { - IL-4 production from Th2 } \\
\text { - The HIV-1+ children witl }\end{array}$ & $\begin{array}{l}\text { KQI INMWQEVGKAMYA HIV-1 infection } \\
\text { of the CTL response and the type of Th response was } \\
\text { in from Th1 cells correlated with the CTLp frequency a } \\
\text { h strong CTL responses had levels of anti-CD3 MAb in }\end{array}$ & $\begin{array}{l}\text { human } \\
\text { en rapidly progressing } \mathrm{H} \\
\text { Gag, Env, Nef and Pol } \\
1 \text { cells comparable to th }\end{array}$ & $\begin{array}{l}\text { Wasik1997 } \\
\text { I-1+ infants } \\
\text { of uninfected children }\end{array}$ \\
\hline
\end{tabular}




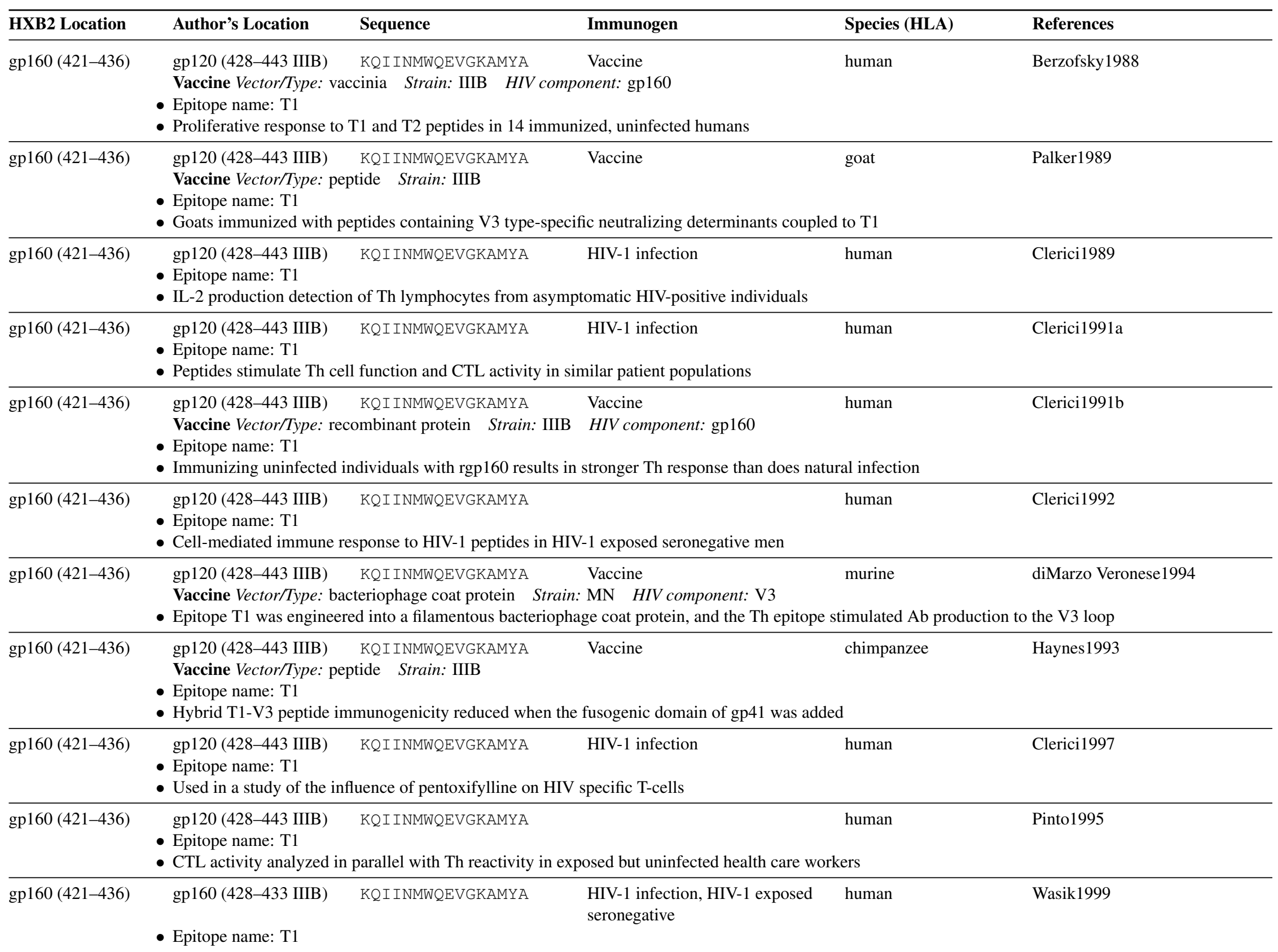




\begin{tabular}{|c|c|c|c|c|}
\hline HXB2 Location & Author's Location & Sequence & Species (HLA) & References \\
\hline & \multicolumn{4}{|c|}{$\begin{array}{l}\text { - IL-2 responses associated with beta-chemokine expression were detectable at birth in the majority of uninfected infants born to HIV+ mothers, declining by } \\
\text { age } 6 \text { months } \\
\text { - T1 peptide: In both uninfected and infected infants of HIV-positive mothers, responses to the T1 peptide were more frequent than responses to P18 } \\
\text { (RIQRGPGRAFVTIGK) } \\
\text { - T1 is a highly conserved epitope, whereas P18 has a higher mutation rate due to its location in the immunodominant V3 loop region }\end{array}$} \\
\hline gp160 (421-436) & $\begin{array}{l}\text { gp120 (428-443 IIIB) } \\
\text { - Epitope name: T1 } \\
\text { - Kenyan sex workers that } \\
\text { cases) and mucosal geni } \\
\text { - Helper epitopes used in }\end{array}$ & $\begin{array}{l}\text { KQI INMWQEVGKAMYA } \\
\text { remained seronegative wer } \\
\text { al tract anti-HIV IgA (16/2 } \\
\text { is study were noted to be }\end{array}$ & $\begin{array}{l}\text { HIV-1 infection human } \\
\text { ad to frequently have HIV-env peptide specific Th resp } \\
\text { s) } \\
\text { usly described [Clerici1989], and were not explicitly d }\end{array}$ & $\begin{array}{l}\text { Kaul1999 } \\
\text { ected by an IL-2 assay (11/20 } \\
\text { in Kaul1999 }\end{array}$ \\
\hline gp160 (421-436) & $\begin{array}{l}\text { gp120 (MN) } \\
\text { Vaccine Vector/Type: pe } \\
\text { - Epitope name: T1 } \\
\text { - C4-V3 PV (polyvalent } \mathrm{H} \\
\text { one of four different Nol } \\
\text { - This was a pilot phase I } \\
\text { - Enhanced lymphoprolife }\end{array}$ & $\begin{array}{l}\text { KQI INMWQEVGKAMYA } \\
\text { tide Strain: MN HIV C } \\
\text { IV envelope synthetic pept } \\
\text { h American strains } \\
\text { tudy involving vaccination } \\
\text { ative response to peptide }\end{array}$ & $\begin{array}{l}\text { HIV-1 infection, Vaccine human } \\
\text { nent: polyepitope } \\
\text { Imunogen) consisted of T1 helper epitope presented in } \\
\text { HIV-infected subjects who were HLA-B7-positive } \\
\text { served in } 5 / 8 \text { vaccinees - increase in neutralizing antib }\end{array}$ & $\begin{array}{l}\text { Bartlett1998 } \\
\text { with a V3 loop CTL epitope from } \\
\text { onses in 4/8 vacinees }\end{array}$ \\
\hline gp160 (421-436) & $\begin{array}{l}\text { gp120 } \\
\text { - Epitope name: T1 } \\
\text { - In a S. African perinatal } \\
\text { (measured by a bioassay } \\
\text { epitopes P18 MN, P18 I } \\
\text { - The mothers were predo } \\
\text { based on B subtype reag } \\
\text { - } 3 / 33 \text { infants with cord bl } \\
\text { with cord blood that was } \\
\text { - Measurable HIV specific } \\
\text { with a protective natural }\end{array}$ & $\begin{array}{l}\text { KQI INMWQEVGKAMYA } \\
\text { ransmission study, 33\% (3 } \\
\text { measuring IL2 production } \\
\text { IB, T1, T2, and TH4 } \\
\text { ninantly infected with subt } \\
\text { nts. } \\
\text { od T help responses to En } \\
\text { unresponsive to Env peptic } \\
\text { T help responses elicited i } \\
\text { mmunity that helps block }\end{array}$ & $\begin{array}{l}\text { HIV-1 infection, HIV-1 exposed human } \\
\text { seronegative } \\
\text { f cord blood samples from infants with seropositive } \\
\text { arine cell line and confirmed with a proliferation assa } \\
\text { but the T help response was detectable in a number c } \\
\text { infected in utero, } 2 / 33 \text { were lost to follow up, and } 28 \\
\text { ulation were infected before delivery, and } 8 / 47 \text { contra } \\
\text { nmunologically immature newborn, possibly in respc } \\
\text {-infant transmission of HIV-1. }\end{array}$ & $\begin{array}{l}\text { Kuhn2001 } \\
\text { roduced T-helper responses } \\
\text { a peptide cocktail containing Th } \\
\text { od samples despite using peptides } \\
\text { not infected. } 6 / 53 \text { of the infants } \\
\text { intrapartum or via breast-feeding. } \\
\text { utero exposure, are associated }\end{array}$ \\
\hline gp160 (421-436) & $\begin{array}{l}\text { gp120 (428-443 RF) } \\
\text { - Epitope name: T1 } \\
\text { - Proton NMR spectrosco } \\
\text { immunogenicity of its' } \\
\text { (RKSITKGPGRVIYATC } \\
\text { - As a free peptide, the T1 } \\
\text { gp120, and a nonnative } \\
\text { gp120. It lacks random- } \\
\text { favored within epitopes. }\end{array}$ & $\begin{array}{l}\text { KQI INMWQEVGKAMYA } \\
\text { y was employed to analyze } \\
\text { helper (KQIINMWQEVG } \\
\text { ). } \\
\text { segment, a T-helper epitop } \\
\text { onformation may account } f \\
\text { oil conformations, and it is }\end{array}$ & $\begin{array}{l}\text { HIV-1 infection } \\
\text { lution conformation of a hybrid peptide, T1-SP10RF } \\
\text { YA, CTRPNNNTRKSI), CTL (SITKGPGRVIYATG) } \\
\text { an extended conformation with nascent helical confo } \\
\text { inability of free T1 peptide to elicit antibody respons } \\
\text { sted that this may make the peptide less susceptible } t\end{array}$ & $\begin{array}{l}\text { deLorimier1994 } \\
\text { to better understand the } \\
\text { ll epitopes } \\
\text { t may form a beta strand in native } \\
\text { trast to the T1 segment in native } \\
\text { e proteolytic degredation, and be }\end{array}$ \\
\hline
\end{tabular}




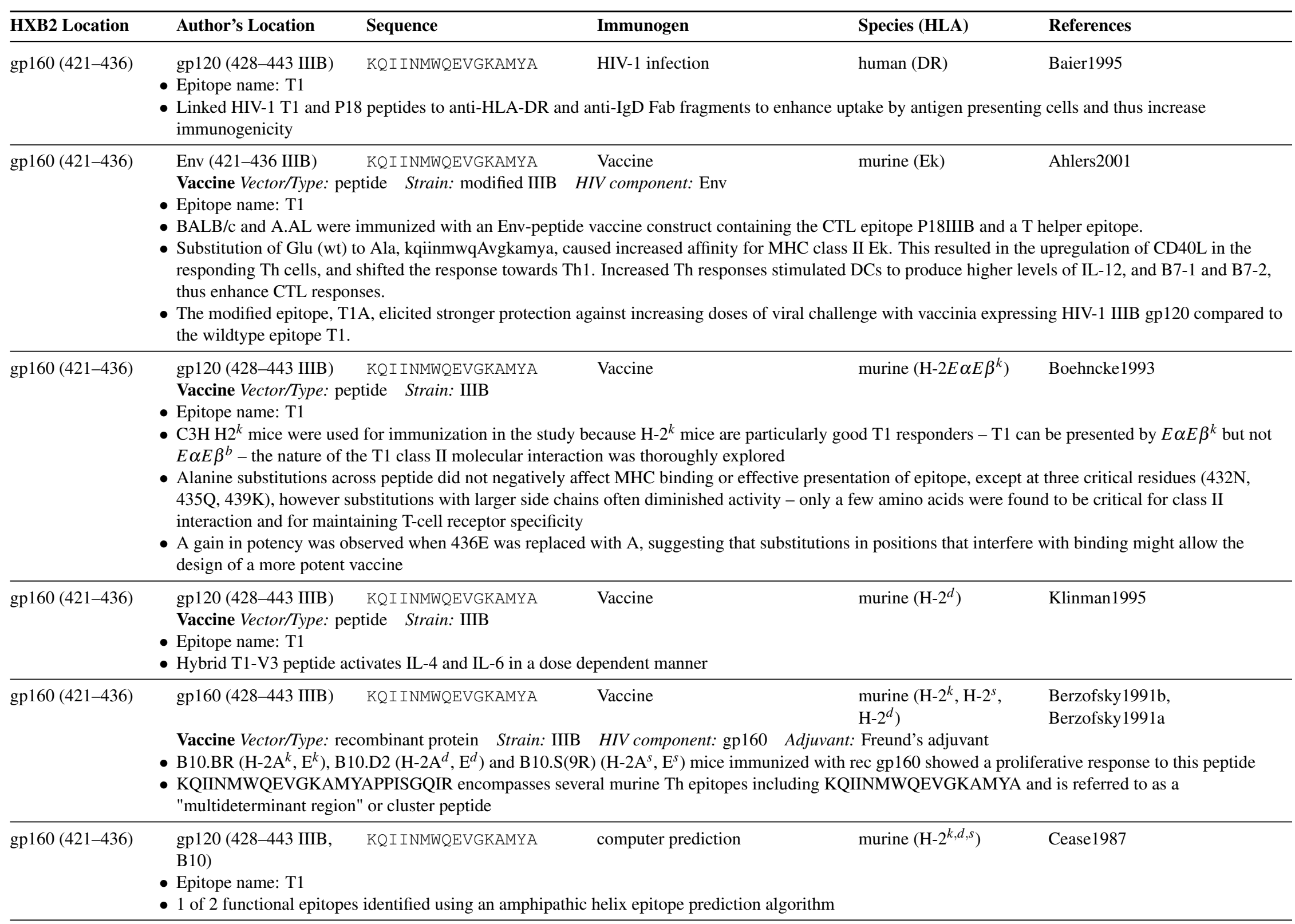




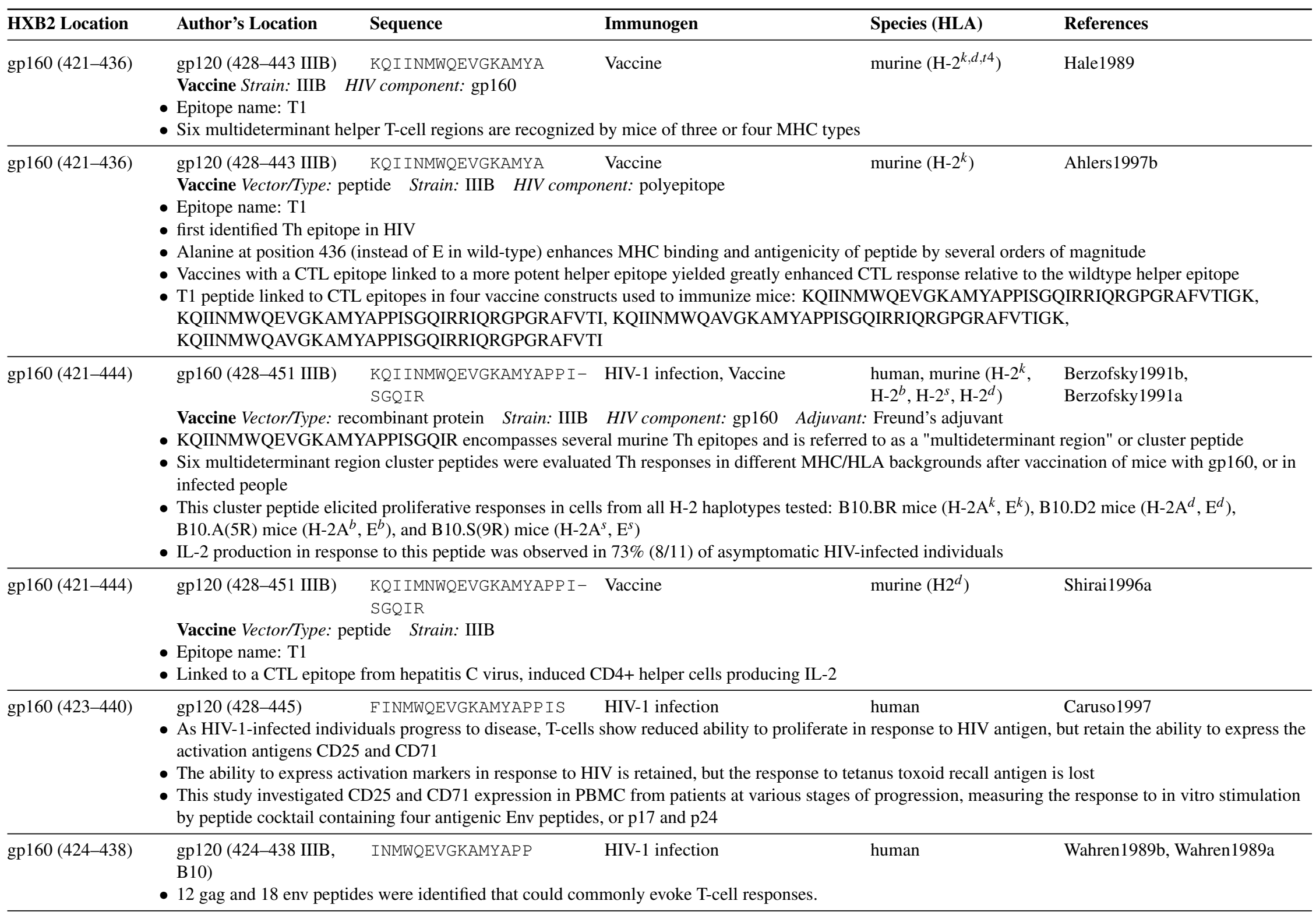




\begin{tabular}{|c|c|c|c|c|c|}
\hline HXB2 Location & Author's Location & Sequence & Immunogen & Species (HLA) & References \\
\hline gp160 (425-439) & \multicolumn{5}{|c|}{$\begin{array}{l}\text { Vaccine Vector/Type: recombinant protein Strain: IIIB HIV component: gp160 Adjuvant: Freund's adjuvant } \\
\text { - B10.S(9R) }\left(\mathrm{H}_{-}-\mathrm{A}^{s}, \mathrm{E}^{s}\right) \text { mice immunized with rec gp160 showed a proliferative response to this peptide } \\
\text { - KQIINMWQEVGKAMYAPPISGQIR encompasses several murine Th epitopes including NMWQEVGKAMYAPPI and is referred to as a } \\
\text { "multideterminant region" or cluster peptide }\end{array}$} \\
\hline gp160 (425-439) & $\begin{array}{l}\text { gp120 (432-446 IIIB) } \\
\text { Vaccine Strain: IIIB } \\
\text { - Six multideterminant he }\end{array}$ & $\begin{array}{l}\text { NMWQEVGKAMYAPP I } \\
\text { V component: gp160 } \\
\text { ger T-cell regions are recognized }\end{array}$ & $\begin{array}{l}\text { Vaccine } \\
\text { by mice of three or fo }\end{array}$ & murine $\left(\mathrm{H}-2^{t 4}\right)$ & Hale1989 \\
\hline gp160 (426-441) & $\begin{array}{l}\text { - Pp120 (IIIB) } \\
\text { - Peptide stimulation of } \mathrm{F} \\
\text { Peptide priming does nc }\end{array}$ & $\begin{array}{l}\text { MWQEVGKAMYAPP IGC } \\
\text { MC from non-infected individua } \\
\text { always induce T-cells that recogn }\end{array}$ & $\begin{array}{l}\text { in vitro stimulation } \\
\text { ls in vitro } \\
\text { nize whole protein }\end{array}$ & human & Manca1995b \\
\hline gp160 (430-444) & $\begin{array}{l}\text { gp160 (437-451 IIIB) } \\
\text { Vaccine Vector/Type: re } \\
\text { - This peptide elicited pro } \\
\left(\mathrm{H}^{-} 2 \mathrm{~A}^{b}, \mathrm{E}^{b}\right) \text {, and B10.S } \\
\text { KQIINMWQEVGKAN } \\
\text { region" or cluster peptic }\end{array}$ & $\begin{array}{l}\text { VGKAMYAPP ISGQIR } \\
\text { ombinant protein Strain: IIIB } \\
\text { ferative responses in cells from a } \\
\text { R) mice }\left(\mathrm{H}^{-} 2 \mathrm{~A}^{s}, \mathrm{E}^{s}\right) \\
\text { APPISGQIR encompasses sever }\end{array}$ & $\begin{array}{l}\text { Vaccine } \\
\text { HIV component: gp } \\
\text { ll H-2 haplotypes test } \\
\text { al murine Th epitopes }\end{array}$ & $\begin{array}{l}\text { murine }\left(\mathrm{H}-2^{k}, \mathrm{H}-2^{b},\right. \\
\left.\mathrm{H}-2^{s}, \mathrm{H}-2^{d}\right) \\
: \text { Freund's adjuvant } \\
\text { ice }\left(\mathrm{H}-2 \mathrm{~A}^{k}, \mathrm{E}^{k}\right), \mathrm{B} 10.1 \\
\text { KAMYAPPISGQIR a }\end{array}$ & $\begin{array}{l}\text { Berzofsky1991b } \\
\text { Berzofsky1991a } \\
\text { hice }\left(\mathrm{H}-2 \mathrm{~A}^{d}, \mathrm{E}^{d}\right), \mathrm{B} 10 . \mathrm{A}(5 \mathrm{R}) \text { mice } \\
\text { referred to as a "multideterminant }\end{array}$ \\
\hline gp160 (430-444) & $\begin{array}{l}\text { gp120 (437-451 IIIB) } \\
\text { Vaccine Strain: IIIB } \\
\text { - Six multideterminant he }\end{array}$ & $\begin{array}{l}\text { VGKAMYAPP ISGQIR } \\
\text { V component: gp } 160 \\
\text { ger T-cell regions are recognized }\end{array}$ & $\begin{array}{l}\text { Vaccine } \\
\text { by mice of three or fo }\end{array}$ & murine $\left(\mathrm{H}-2^{k, d, i 5, t 4}\right)$ & Hale1989 \\
\hline gp160 (430-453) & $\begin{array}{l}\text { gp120 (430-453) } \\
\text { Vaccine Vector/Type: re } \\
\text { - Study demonstrates that } \\
\text { - Peptide stimulation of a } \\
\text { - Local glycosylation site }\end{array}$ & $\begin{array}{l}\text { VGKAMYAPP ISGQIRCSSN- } \\
\text { ITGLL } \\
\text { ombinant protein HIV compone } \\
\text {-cell determinants from glycopro } \\
\text { in vitro proliferative response rec } \\
\text { thought not to be part of the epito }\end{array}$ & $\begin{array}{l}\text { Vaccine } \\
\text { ent: gp160 } \\
\text { oteins can depend on } \\
\text { quired in vivo priming } \\
\text { ope, but may be impo }\end{array}$ & $\begin{array}{l}\text { murine }\left(\mathrm{H}-2^{b}\right) \\
\text { on of the protein } \\
\text { ated protein } \\
\text { e processing }\end{array}$ & Sjolander1996 \\
\hline gp160 (432-451) & $\begin{array}{l}\text { gp120 (432-451 IIIB) } \\
\text { - Epitope name: H4 } \\
\text { - Th proliferative respons } \\
\text { overlapping peptide poc } \\
\text { - After } 12 \text { months, most } 1 \\
\text { months, as did } 5 / 15 \text { non } \\
\text { - IL-2 production was a n } \\
\text { 1/15 responders recogn }\end{array}$ & $\begin{array}{l}\text { KAMYAPP ISGQIRCSSNIT- } \\
\text { G? } \\
\text { were studied in } 36 \text { asymptomati } \\
10 \text { of the responding patients re } \\
\text { ponses were lost or diminished, } \\
\text { esponders. } \\
\text { re sensitive and well-preserved n } \\
\text { ed this peptide, SI }=6.3 \text {. }\end{array}$ & $\begin{array}{l}\text { HIV-1 infection } \\
\text { ic HIV-1+ patients. PI } \\
\text { ecognized three or mo } \\
\text { and specific response } \\
\text { neasure of Th functio }\end{array}$ & $\begin{array}{l}\text { human } \\
36 \text { patients responded } \\
\text { ls. } \\
10 \text { of the responders a } \\
\text { ation. }\end{array}$ & $\begin{array}{l}\text { Geretti1994 } \\
\text { eline had new responses at } 12\end{array}$ \\
\hline
\end{tabular}




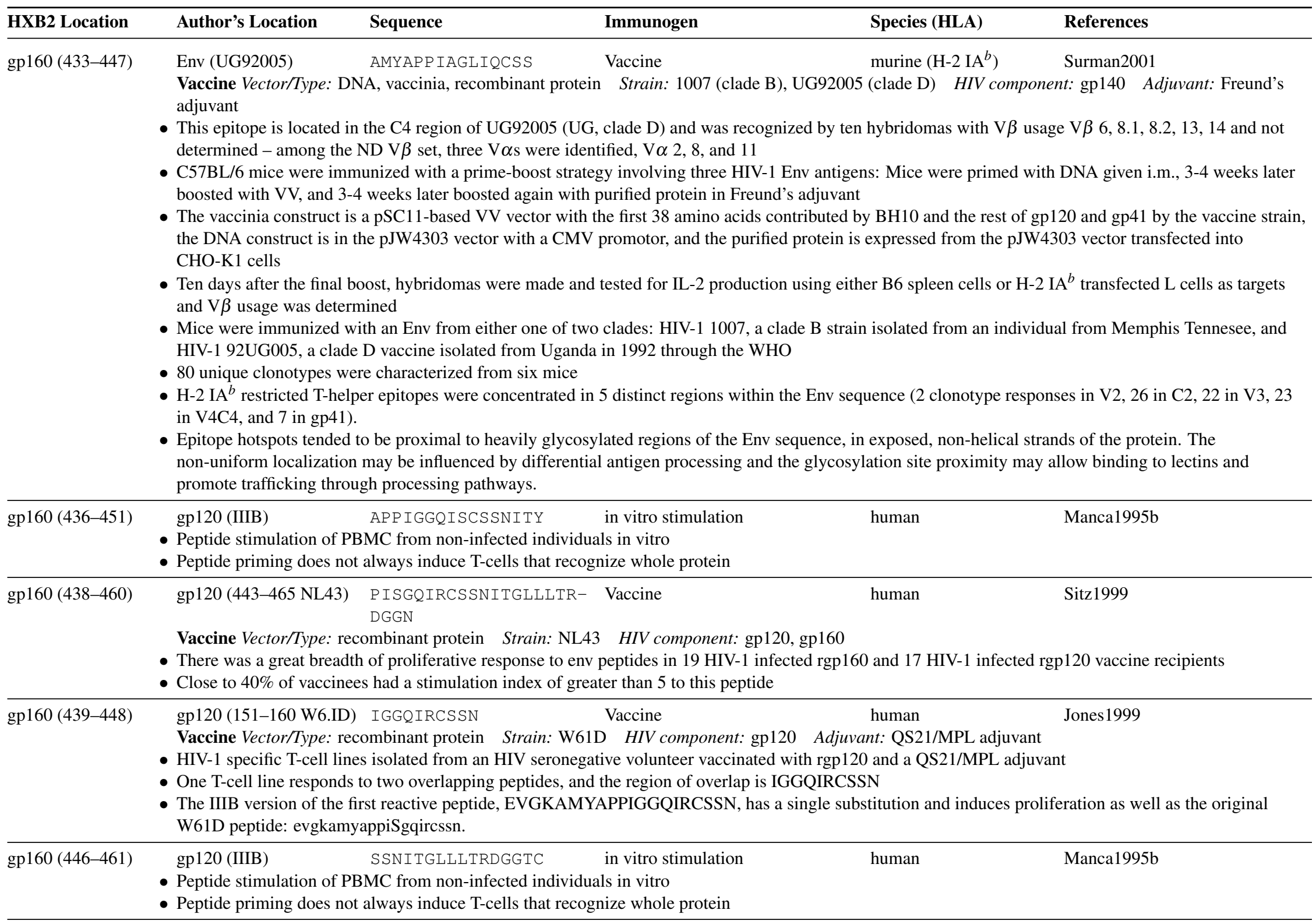




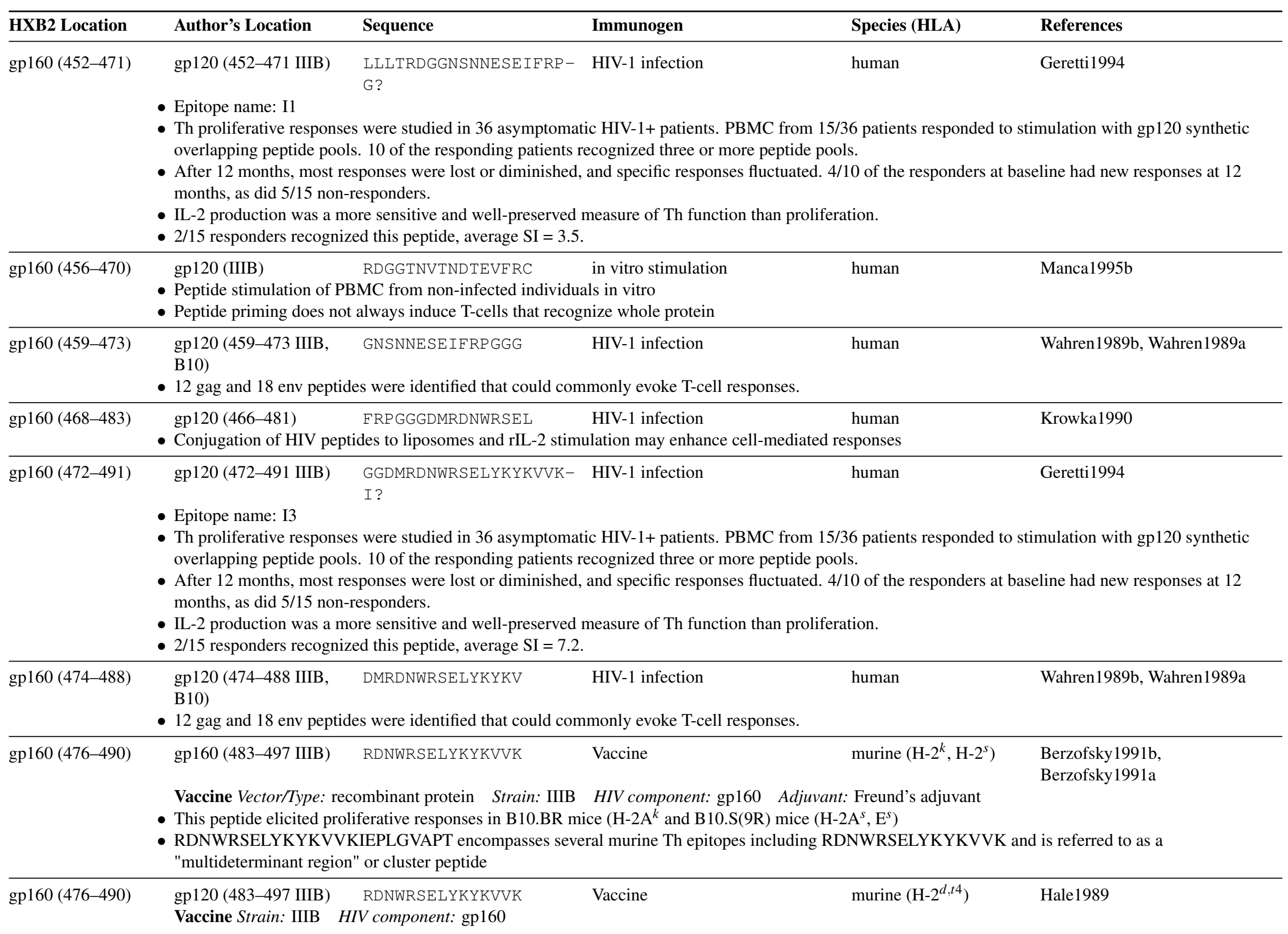




\begin{tabular}{|c|c|c|c|c|c|}
\hline HXB2 Location & Author's Location & Sequence & Immunogen & Species (HLA) & References \\
\hline \multicolumn{6}{|c|}{ - Six multideterminant helper T-cell regions are recognized by mice of three or four MHC types } \\
\hline gp160 (476-499) & gp160 (483-506 IIIB) & $\begin{array}{l}\text { RDNWRSELYKYKVVKIEPL- } \\
\text { GVAPT }\end{array}$ & HIV-1 infection, Vaccine & $\begin{array}{l}\text { human, murine }\left(\mathrm{H}-2^{k},\right. \\
\left.\mathrm{H}-2^{b}, \mathrm{H}-2^{s}, \mathrm{H}-2^{d}\right)\end{array}$ & \begin{tabular}{|l|} 
Berzofsky 1991b \\
Berzofsky 1991a \\
\end{tabular} \\
\hline
\end{tabular}

Vaccine Vector/Type: recombinant protein Strain: IIIB HIV component: gp160 Adjuvant: Freund's adjuvant

- RDNWRSELYKYKVVKIEPLGVAPT encompasses several murine Th epitopes and is referred to as a "multideterminant region" or cluster peptide

- Six multideterminant region cluster peptides were evaluated Th responses in different MHC/HLA backgrounds after vaccination of mice with gp160, or in infected people

- This cluster peptide elicited proliferative responses in cells from all H-2 haplotypes tested: B10.BR mice $\left(\mathrm{H}-2 \mathrm{~A}^{k}, \mathrm{E}^{k}\right), \mathrm{B} 10 . \mathrm{D} 2$ mice $\left(\mathrm{H}-2 \mathrm{~A}^{d}, \mathrm{E}^{d}\right)$,

B10.A (5R) mice $\left(\mathrm{H}-2 \mathrm{~A}^{b}, \mathrm{E}^{b}\right)$, and B10.S(9R) mice $\left(\mathrm{H}-2 \mathrm{~A}^{s}, \mathrm{E}^{s}\right)$

- IL-2 production in response to this peptide was observed in 52\% (14/27) of asymptomatic HIV-infected individuals

gp160 (479-498) gp120(481-500 MN) WRSELYKYKVVTIEPLGVAP Vaccine guinea pig Chattergoon2002

Vaccine Vector/Type: protein, DNA Strain: MN HIV component: gp120 Adjuvant: complete Freund's adjuvant (CFA)

- Epitope name: 2013

- Hartley guinea pigs were intradermally injected with either recombinant protein or plasmid expressed gp120 and monitored for delayed type hypersensivity (DTH) responses after vaccination, which are related to Th1 T-cell responses. CFA did not augment responses in animals vaccinated with plasmid.

- A total of 7 gp120 peptides elicited a delayed type hypersensivity (DTH) response after vaccination, out of a set of 60 overlapping peptides that spanned gp120. The vaccine delivery system, DNA versus rec protein, resulted in the recognition of distinct peptides.

- 0/5 animals vaccinated with rec gp120 responded by DTH to this peptide, while 6/6 vaccinated with plasmid gp120 DNA responded.

gp160 (482-501) gp120(482-501 IIIB) ELYKYKVVKIEPLGVAPTKA Vaccine Rhesus macaque Lekutis1997b

Vaccine Vector/Type: DNA Strain: IIIB HIV component: ENV

- HIV-1 env DNA vaccine induced Th cell response to this epitope in a rhesus monkey

- Epitope was recognized by both monkeys used in this study

\begin{tabular}{lllll}
\hline gp160 (482-501) gp120 (482-501 IIIB) & $\begin{array}{l}\text { ELYKYKVVKIEPLGVAPTK- HIV-1 infection } \\
\text { A? }\end{array}$ & Geretti1994 \\
\end{tabular}

- Epitope name: I4

- Th proliferative responses were studied in 36 asymptomatic HIV-1+ patients. PBMC from 15/36 patients responded to stimulation with gp120 synthetic overlapping peptide pools. 10 of the responding patients recognized three or more peptide pools.

- After 12 months, most responses were lost or diminished, and specific responses fluctuated. 4/10 of the responders at baseline had new responses at 12 months, as did 5/15 non-responders.

- IL-2 production was a more sensitive and well-preserved measure of Th function than proliferation.

- $3 / 15$ responders recognized this peptide, average $\mathrm{SI}=6.0$.

gp160 (483-502)

gp120 (480-499 89.6) LYKYKVVRIEPIGVAPTRAK Vaccine

murine

Dai2001

Vaccine Vector/Type: recombinant protein Strain: 89.6 HIV component: gp120 Adjuvant: R192G mutant heat-labile toxin from enterotoxigenic E. coli $(\mathrm{mLT})$

- Epitope name: Peptide 46

- Helper T-cell proliferative responses to gp120 vaccines in 2 mouse strains, CBA/J and BALB/c, were mapped using 47 overlapping peptides that span gp120. Promiscuously immunodominant peptides were identified in both mice strains that were located in the outer domain of gp120 and were associated with regions of local structural disorder in proximal N-terminal segments, suggesting 3-D protein structure influences Th antigen processing and the frequency of immunogenic sequences. 


\begin{tabular}{lllcl}
\hline HXB2 Location & Author's Location & Sequence & Immunogen & Species (HLA) \\
\hline & - This peptide was reactive in 7/10 BALB/c mice tested, and in only 1/10 CBA/J mice. & \\
\hline gp160 (484-496) & gp120 (484-496 HXB2) & YKYKVVKIEPLGV & Vaccine & Rhesus macaque \\
& Vaccine Vector/Type: DNA & Strain: HXB2 & HIV component: ENV & Lekutis1998 \\
& (DR*W201) &
\end{tabular}

- Variants of this epitope with substitutions at position 490(K) retained ability to bind to MHC class II, but failed to induce proliferation/cytokine secretion in HIV-1 env-specific CD4+ Th cells

- The modified peptide antagonized the wildtype peptide-induced proliferative response

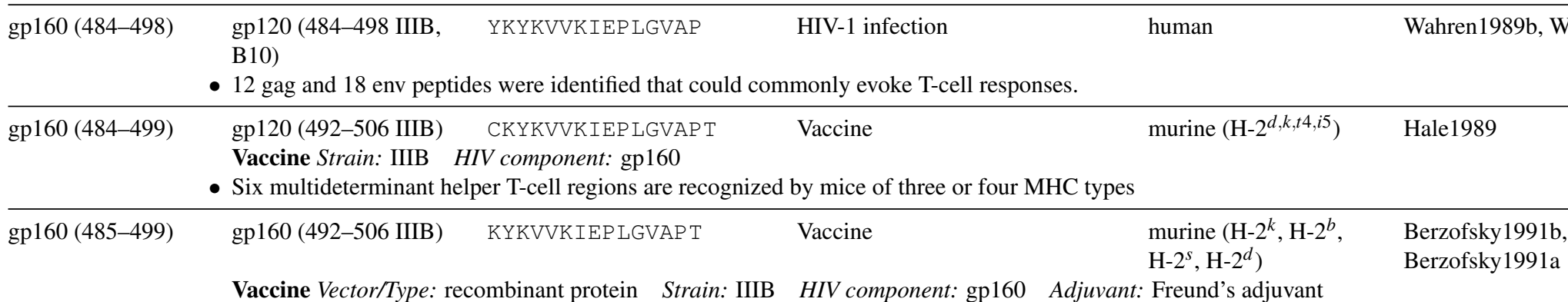

- This peptide elicited proliferative responses in cells from all H-2 haplotypes tested: B10.BR mice $\left(\mathrm{H}-2 \mathrm{~A}^{k}, \mathrm{E}^{k}\right), \mathrm{B} 10 . \mathrm{D} 2$ mice $\left(\mathrm{H}-2 \mathrm{~A}^{d}, \mathrm{E}^{d}\right), \mathrm{B} 10 . \mathrm{A}(5 \mathrm{R})$ mice $\left(\mathrm{H}-2 \mathrm{~A}^{b}, \mathrm{E}^{b}\right)$, and B10.S(9R) mice $\left(\mathrm{H}-2 \mathrm{~A}^{s}, \mathrm{E}^{s}\right)$

- RDNWRSELYKYKVVKIEPLGVAPT encompasses several murine Th epitopes including KYKVVKIEPLGVAPT and is referred to as a "multideterminant region" or cluster peptide

gp160 (485-500) gp120(IIIB) KYKVIKIEPLGIAPTC in vitro stimulation $\quad$ Manca1995b

- Peptide stimulation of PBMC from non-infected individuals in vitro

- Peptide priming does not always induce T-cells that recognize whole protein

\begin{tabular}{|c|c|c|c|c|c|}
\hline gp160 (486-494) & gp120 (486-494 IIIB) & YKVVKIEPL & SHIV infection & $\begin{array}{l}\text { Rhesus macaque } \\
(\mathrm{DRB} * \mathrm{~W} 201)\end{array}$ & Lekutis1997a \\
\hline
\end{tabular}

\begin{tabular}{llrl}
\hline gp160 (487-512) & gp120(494-518 IIIB) & KVVKIEPLGVAPTKAKRRV- Vaccine & GQREKRC \\
& Vaccine Vector/Type: peptide Strain: IIIB & Goodman-Snitkoff1990
\end{tabular}

- Identification of putative Th epitopes that stimulate an antibody response in peptide immunized mice

\begin{tabular}{lllll}
\hline gp160 (492-512) gp120 (492-512 IIIB) & EPLGVAPTKAKRRVVQREK- HIV-1 infection \\
RA?
\end{tabular}

- Epitope name: I5

- Th proliferative responses were studied in 36 asymptomatic HIV-1+ patients. PBMC from 15/36 patients responded to stimulation with gp120 synthetic overlapping peptide pools. 10 of the responding patients recognized three or more peptide pools.

- After 12 months, most responses were lost or diminished, and specific responses fluctuated. 4/10 of the responders at baseline had new responses at 12 months, as did 5/15 non-responders.

- IL-2 production was a more sensitive and well-preserved measure of Th function than proliferation. 


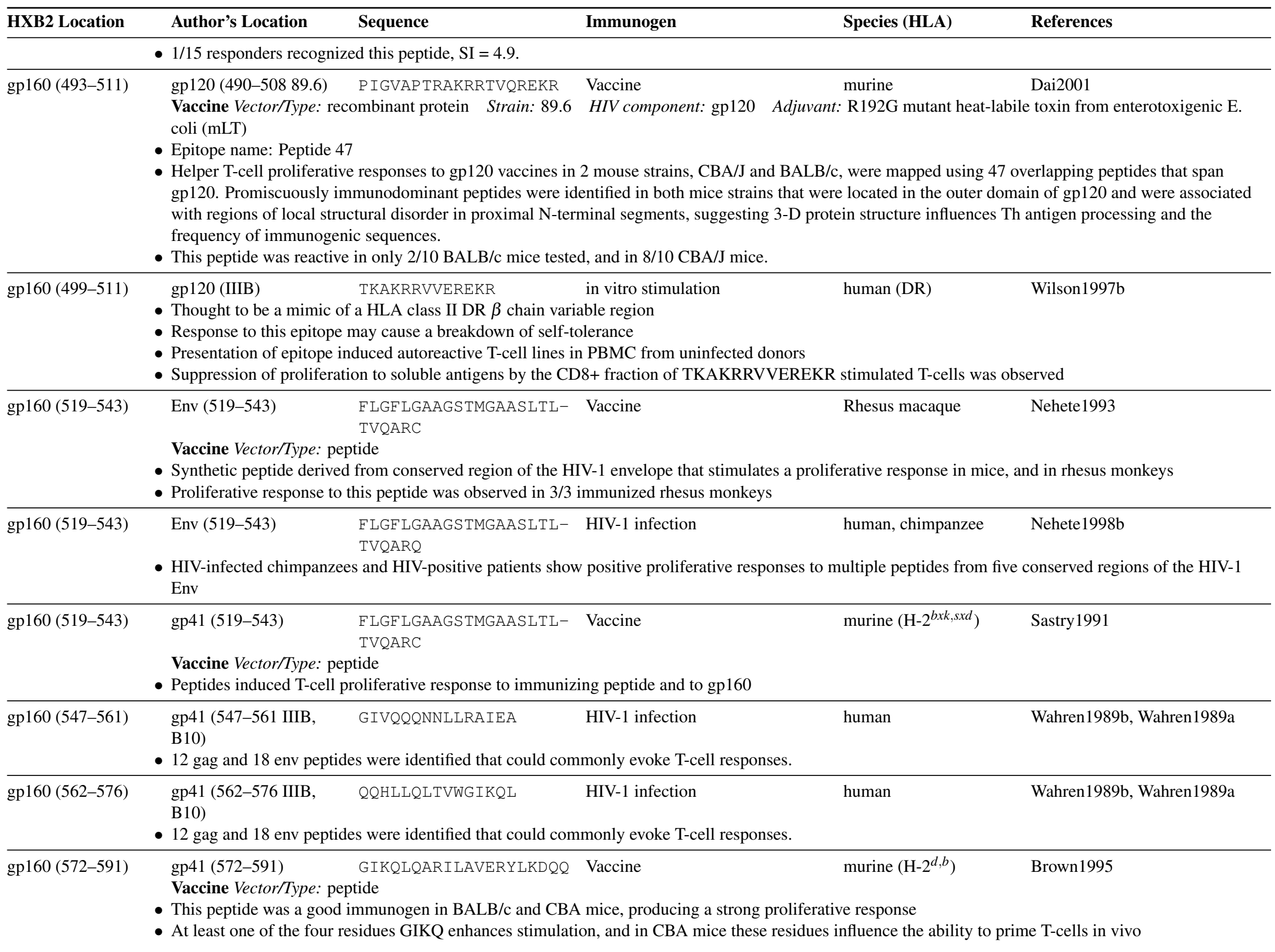




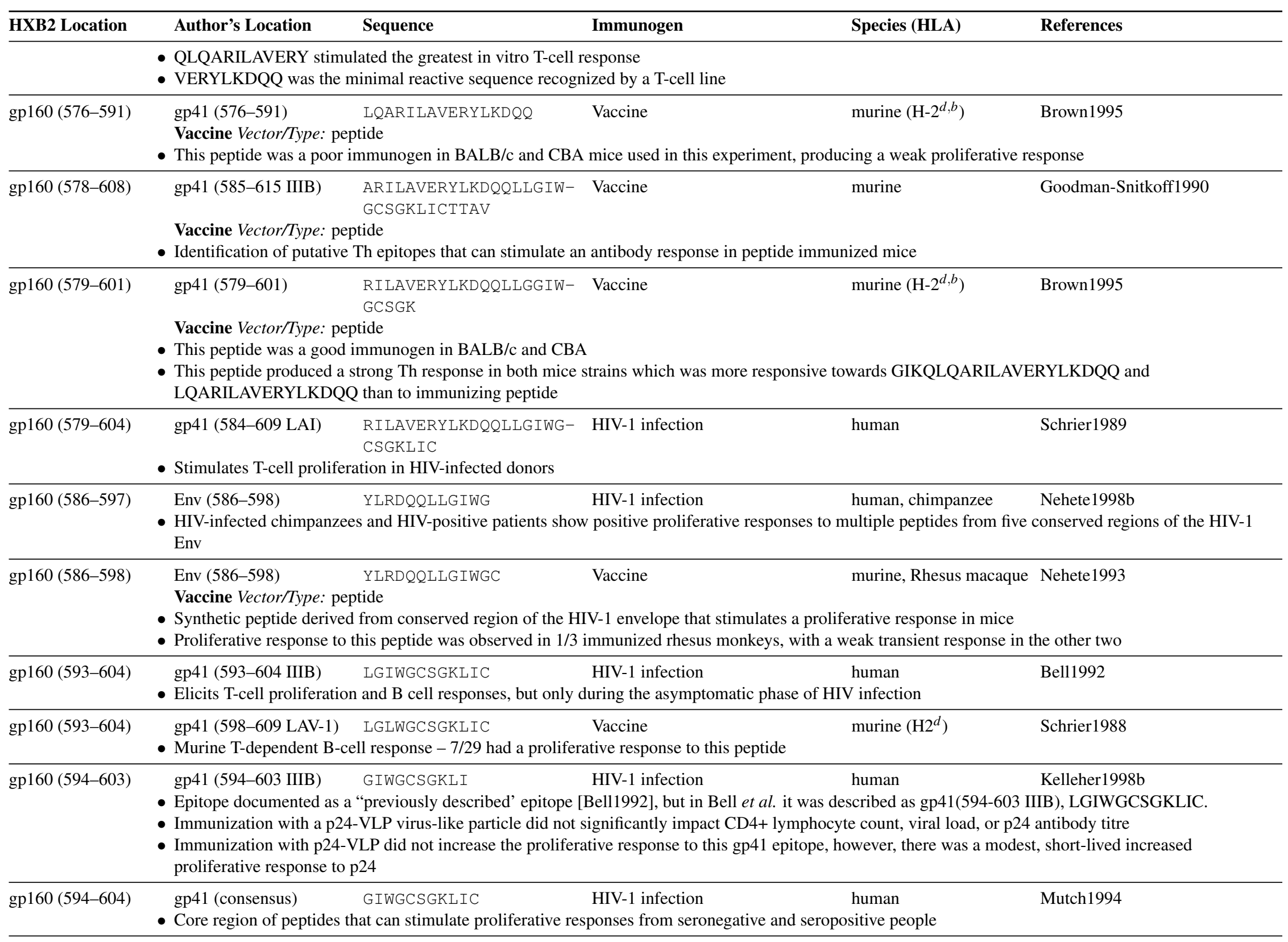




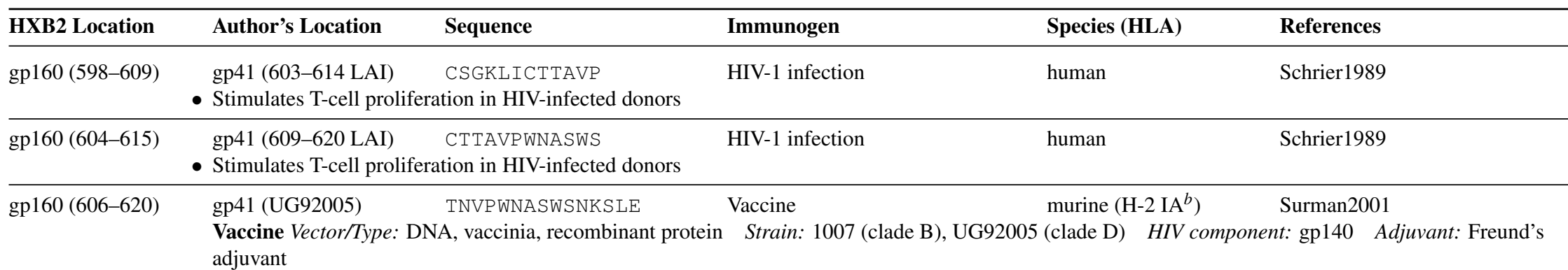

- This gp140 epitope of UG92005 (UG, clade D) was recognized by five hybridomas with V $\beta$ usage V $\beta$ 8.1, 14 and not determined - one of the V $\beta$ 8.1 was shown to utilize $\mathrm{V} \alpha 8$

- C57BL/6 mice were immunized with a prime-boost strategy involving three HIV-1 Env antigens: Mice were primed with DNA given i.m., 3-4 weeks later boosted with VV, and 3-4 weeks later boosted again with purified protein in Freund's adjuvant

- The vaccinia construct is a pSC11-based VV vector with the first 38 amino acids contributed by BH10 and the rest of gp120 and gp41 by the vaccine strain, the DNA construct is in the pJW4303 vector with a CMV promotor, and the purified protein is expressed from the pJW4303 vector transfected into CHO-K1 cells

- Ten days after the final boost, hybridomas were made and tested for IL-2 production using either B6 spleen cells or H-2 IA ${ }^{b}$ transfected L cells as targets and $\mathrm{V} \beta$ usage was determined

- Mice were immunized with an Env from either one of two clades: HIV-1 1007, a clade B strain isolated from an individual from Memphis Tennesee, and HIV-1 92UG005, a clade D vaccine isolated from Uganda in 1992 through the WHO

- 80 unique clonotypes were characterized from six mice

- H-2 IA ${ }^{b}$ restricted T-helper epitopes were concentrated in 5 distinct regions within the Env sequence (2 clonotype responses in V2, 26 in C2, 22 in V3, 23 in $\mathrm{V} 4 \mathrm{C} 4$, and 7 in gp41).

- Epitope hotspots tended to be proximal to heavily glycosylated regions of the Env sequence, in exposed, non-helical strands of the protein. The non-uniform localization may be influenced by differential antigen processing and the glycosylation site proximity may allow binding to lectins and promote trafficking through processing pathways.

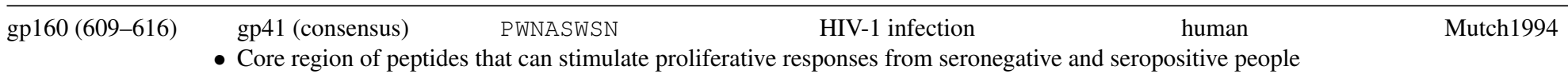

gp160 (611-620) gp41 (1007, UG92005) NASWSNKSLE $\quad$ Vaccine $\quad$ Surman2001 Vaccine Vector/Type: DNA, vaccinia, recombinant protein Strain: 1007 (clade B), UG92005 (clade D) HIV component: gp140 Adjuvant: Freund's adjuvant

- This gp41 epitope is conserved in 1007 (US, clade B) and UG92005 (UG, clade D) and was recognized by two hybridomas from two different mice that were vaccinated with different clades - the $\mathrm{V} \beta$ usage was $\mathrm{V} \beta 4$ and 14

- The epitope described here is the region of overlap of two 15 mers that were both able to stimulate IL-2 production from the hybridoma (T[TN]VPWNASWSNKSLE and NASWSNKSLEQIWNN) - the only difference between 1007 and UG92005 for these two proteins is that 1007 has a T and UG92005 has an $\mathrm{N}$ in the second position of the first peptide

- C57BL/6 mice were immunized with a prime-boost strategy involving three HIV-1 Env antigens: Mice were primed with DNA given i.m., 3-4 weeks later boosted with VV, and 3-4 weeks later boosted again with purified protein in Freund's adjuvant

- The vaccinia construct is a pSC11-based VV vector with the first 38 amino acids contributed by BH10 and the rest of gp120 and gp41 by the vaccine strain, the DNA construct is in the pJW4303 vector with a CMV promotor, and the purified protein is expressed from the pJW4303 vector transfected into CHO-K1 cells 


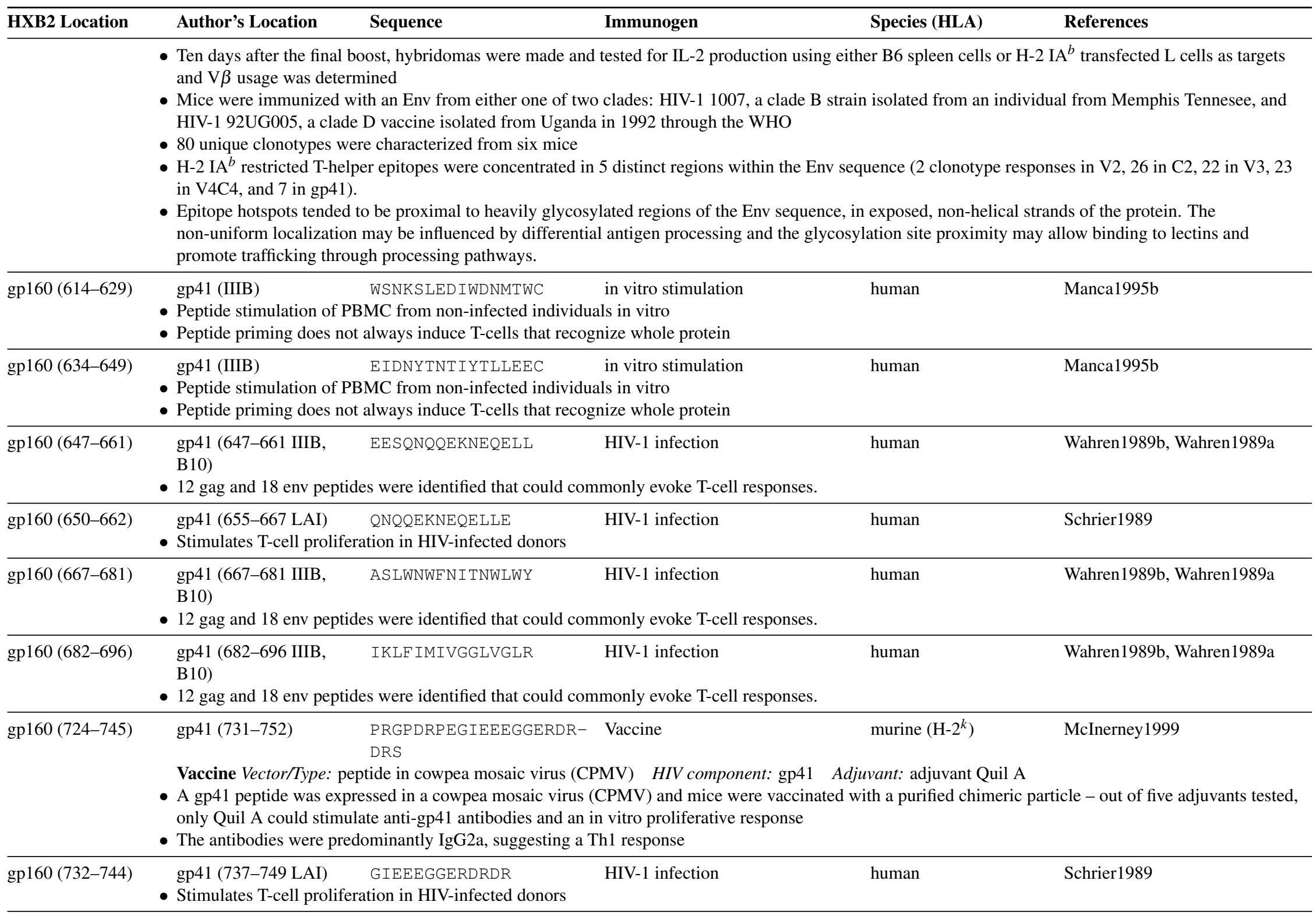




\begin{tabular}{|c|c|c|c|c|c|}
\hline HXB2 Location & Author's Location & Sequence & Immunogen & Species (HLA) & References \\
\hline gp160 (780-794) & gp160 (787-801 IIIB) & RIVELLGRRGWEALK & Vaccine & $\begin{array}{l}\text { murine }\left(\mathrm{H}-2^{k}, \mathrm{H}-2^{d},\right. \\
\left.\mathrm{H}-2^{s}\right)\end{array}$ & \begin{tabular}{|l|} 
Berzofsky1991b \\
Berzofsky1991a \\
\end{tabular} \\
\hline
\end{tabular}

- This peptide elicited proliferative responses in cells from B10.BR mice $\left(\mathrm{H}-2 \mathrm{~A}^{k}, \mathrm{E}^{k}\right), \mathrm{B} 10 . \mathrm{D} 2$ mice $\left(\mathrm{H}_{-}-2 \mathrm{~A}^{d}, \mathrm{E}^{d}\right)$, and B10.S $(9 \mathrm{R})$ mice $\left(\mathrm{H}-2 \mathrm{~A}^{s}, \mathrm{E}^{s}\right)$

- RIVELLGRRGWEALKYWWNLLQYWSQELKNSAVS encompasses several murine Th epitopes including RIVELLGRRGWEALK and is referred to as a "multideterminant region" or cluster peptide, but the longer peptide only stimulates cells from $\mathrm{H}-2^{k}$ mice

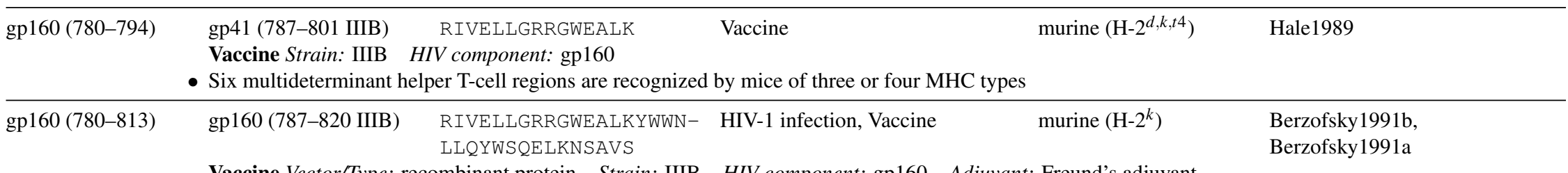

Vaccine Vector/Type: recombinant protein Strain: IIIB HIV component: gp160 Adjuvant: Freund's adjuvant

- RIVELLGRRGWEALKYWWNLLQYWSQELKNSAVS encompasses several murine Th epitopes and is referred to as a "multideterminant region" or cluster peptide

- Six multideterminant region cluster peptides were evaluated Th responses in different MHC/HLA backgrounds after vaccination of mice with gp160, or in infected people

- This cluster peptide elicited proliferative responses in cells from only B10.BR mice $\left(\mathrm{H}-2 \mathrm{~A}^{k}, \mathrm{E}^{k}\right)$, and not from B10.D2 mice $\left(\mathrm{H}-2 \mathrm{~A}^{d}, \mathrm{E}^{d}\right), \mathrm{B} 10 . \mathrm{A}(5 \mathrm{R}) \mathrm{mice}$ $\left(\mathrm{H}-2 \mathrm{~A}^{b}, \mathrm{E}^{b}\right)$, or B10.S(9R) mice $\left(\mathrm{H}-2 \mathrm{~A}^{s}, \mathrm{E}^{s}\right)$

- IL-2 production in response to this peptide was observed in 59\% (17/29) of asymptomatic HIV-infected individuals

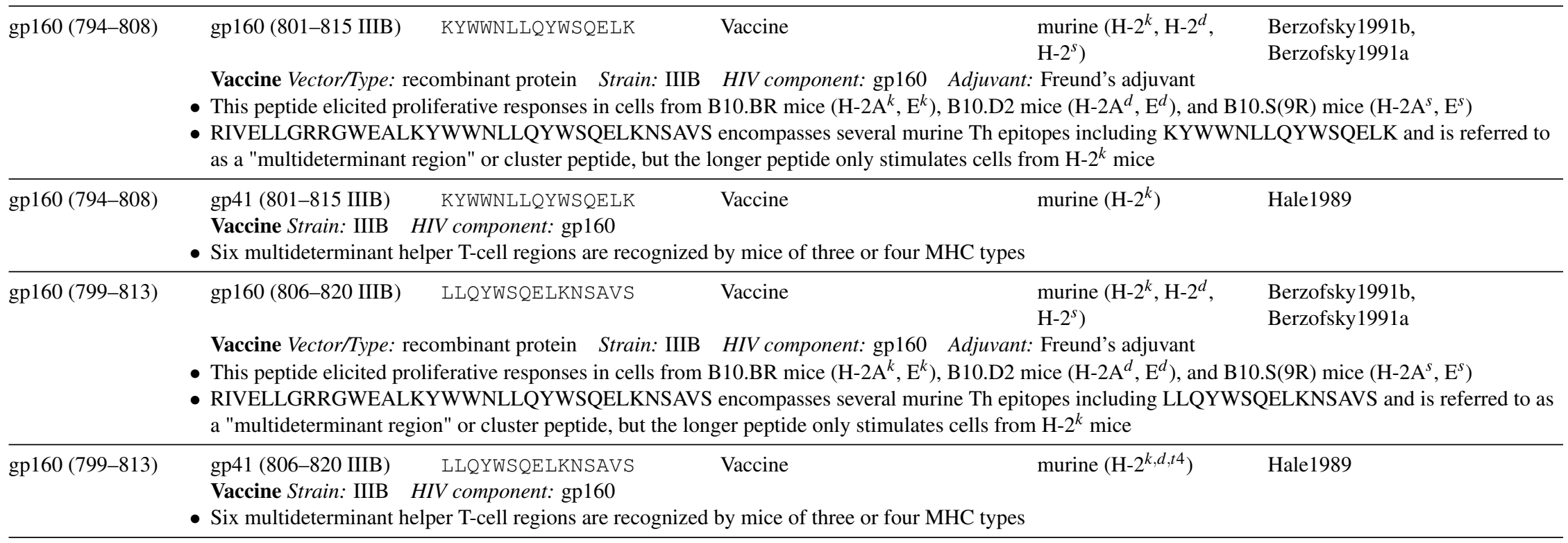




\begin{tabular}{|c|c|c|c|c|c|}
\hline HXB2 Location & Author's Location & Sequence & Immunogen & Species (HLA) & References \\
\hline gp160 (799-813) & $\begin{array}{l}\text { gp41 (806-820 IIIB) } \\
\text { Vaccine Strain: IIIB } \\
\text { - Six multideterminant }\end{array}$ & $\begin{array}{l}\text { LLQYWSQELKNSAVS } \\
\text { IV component: gp160 } \\
\text { per T-cell regions are reco }\end{array}$ & $\begin{array}{l}\text { Vaccine } \\
\text { by mice of three or } f\end{array}$ & murine $\left(\mathrm{H}-2^{k, d, t 4}\right)$ & Hale1989 \\
\hline gp160 (814-829) & $\begin{array}{l}\text { gp41 (IIIB) } \\
\text { - Peptide stimulation of } \\
\text { - Peptide priming does }\end{array}$ & $\begin{array}{l}\text { WLNATAIAVTEGTDRC } \\
\text { 3C from non-infected in } \\
\text { always induce T-cells tha }\end{array}$ & $\begin{array}{l}\text { in vitro stimulation } \\
\text { ls in vitro } \\
\text { aize whole protein }\end{array}$ & human & Manca1995b \\
\hline gp160 (821-835) & gp160 (828-842 IIIB) & AVAEGTDRVIEVVQG & Vaccine & $\begin{array}{l}\text { murine }\left(\mathrm{H}-2^{k}, \mathrm{H}-2^{b},\right. \\
\left.\mathrm{H}-2^{s}\right)\end{array}$ & \begin{tabular}{|l|} 
Berzofsky1991b \\
Berzofsky1991a
\end{tabular} \\
\hline
\end{tabular}

Vaccine Vector/Type: recombinant protein Strain: IIIB HIV component: gp160 Adjuvant: Freund's adjuvant

- This peptide elicited proliferative responses in cells from B10.BR mice $\left(\mathrm{H}-2 \mathrm{~A}^{k}, \mathrm{E}^{k}\right), \mathrm{B} 10 . \mathrm{A}(5 \mathrm{R})$ mice $\left(\mathrm{H}-2 \mathrm{~A}^{b}, \mathrm{E}^{b}\right)$, and $\mathrm{B} 10 . \mathrm{S}(9 \mathrm{R}) \mathrm{mice}\left(\mathrm{H}-2 \mathrm{~A}^{s}, \mathrm{E}^{s}\right)$

- AVAEGTDRVIEVVQGAYRAIRHIPRRIRQGLER encompasses several murine Th epitopes including AVAEGTDRVIEVVQG and is referred to as a "multideterminant region" or cluster peptide

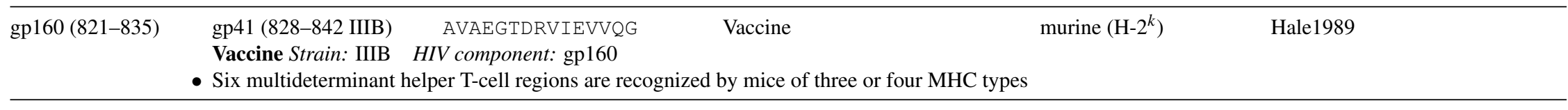

gp160 (821-838) gp41(827-843) YVAEGTDRVIEVVQGACR HIV-1 infection human

- As HIV-1-infected individuals progress to disease, T-cells show reduced ability to proliferate in response to HIV antigen, but retain the ability to express the activation antigens CD25 and CD71

- The ability to express activation markers in response to HIV is retained, but the response to tetanus toxoid recall antigen is lost

- This study investigated CD25 and CD71 expression in PBMC from patients at various stages of progression, measuring the response to in vitro stimulation by peptide cocktail containing four antigenic Env peptides, or p17 and p24

\begin{tabular}{|c|c|c|c|c|c|}
\hline gp160 (821-853) & gp160 (828-860 IIIB) & $\begin{array}{l}\text { AVAEGTDRVIEVVQGAYRA- } \\
\text { IRHIPRRIRQGLER }\end{array}$ & HIV-1 infection, Vaccine & $\begin{array}{l}\text { human, murine }\left(\mathrm{H}-2^{k}\right. \\
\left.\mathrm{H}-2^{b}, \mathrm{H}-2^{s}, \mathrm{H}-2^{d}\right)\end{array}$ & \begin{tabular}{|l|} 
Berzofsky1991b \\
Berzofsky1991a \\
\end{tabular} \\
\hline
\end{tabular}

Vaccine Vector/Type: recombinant protein Strain: IIIB HIV component: gp160 Adjuvant: Freund's adjuvant

- AVAEGTDRVIEVVQGAYRAIRHIPRRIRQGLER encompasses several murine Th epitopes and is referred to as a "multideterminant region" or cluster peptide

- Six multideterminant region cluster peptides were evaluated for Th responses in different MHC/HLA backgrounds after vaccination of mice with gp160, or in infected people

- This cluster peptide elicited proliferative responses in cells from all four MHC types tested: B10.BR mice $\left(\mathrm{H}-2 \mathrm{~A}^{k}, \mathrm{E}^{k}\right), \mathrm{B} 10 . \mathrm{D} 2$ mice $\left(\mathrm{H}-2 \mathrm{~A}^{d}, \mathrm{E}^{d}\right)$, B10.A (5R) mice $\left(\mathrm{H}-2 \mathrm{~A}^{b}, \mathrm{E}^{b}\right)$, and B10.S $(9 \mathrm{R})$ mice $\left(\mathrm{H}-2 \mathrm{~A}^{s}, \mathrm{E}^{s}\right)$

- IL-2 production in response to this peptide was observed in only $8 \%(1 / 12)$ of asymptomatic HIV-infected individuals

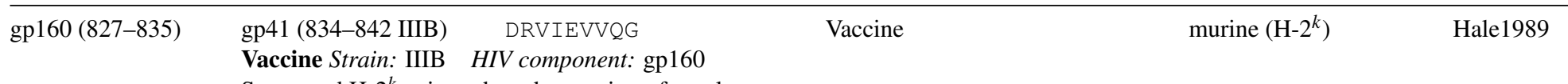

- Suggested H-2 ${ }^{k}$ epitope based on region of overlap

gp160 (827-841) gp41 (834-848 IIIB) DRVIEVVQGAYRAIR Vaccine Hosmalin1991

Vaccine Vector/Type: peptide prime with protein boost Strain: IIIB HIV component: gp160

- Epitope name: TH4

- Peptide priming to induce T-cell help enhances antibody response to gp160 immunization 


\begin{tabular}{|c|c|c|c|c|c|}
\hline HXB2 Location & Author's Location & Sequence & Immunogen & Species (HLA) & References \\
\hline & \multicolumn{5}{|l|}{ - Called Th4.1 and TH4 } \\
\hline gp160 (827-841) & $\begin{array}{l}\text { gp41 (834-848 IIIB) } \\
\text { - Epitope name: TH4 } \\
\text { - used in a study of the in }\end{array}$ & $\begin{array}{l}\text { DRVIEVVQGAYRAIR } \\
\text { luence of pentoxifylline on HIV } \mathrm{s}\end{array}$ & $\begin{array}{l}\text { HIV-1 infection } \\
\text { specific T-cells }\end{array}$ & human & Clerici1997 \\
\hline gp160 (827-841) & \multicolumn{5}{|c|}{$\begin{array}{l}\text { - Epitope name: TH4 } \\
\text { - CTL activity analyzed in parallel with Th reactivity in exposed but uninfected health care workers } \\
\text { - Called Th4.1 and TH4 }\end{array}$} \\
\hline gp160 (827-841) & \multicolumn{5}{|c|}{$\begin{array}{l}\text { - Epitope name: TH4 } \\
\text { - Peptides stimulate Th cell function and CTL activity in similar patient populations } \\
\text { - Called Th4.1 and TH4 }\end{array}$} \\
\hline gp160 (827-841) & \multicolumn{5}{|c|}{$\begin{array}{l}\text { Vaccine Vector/Type: recombinant protein Strain: IIIB HIV component: gp160 } \\
\text { - Epitope name: TH4 } \\
\text { - Immunizing uninfected individuals with rgp160 results in stronger Th response than does natural infection } \\
\text { - Called Th4.1 and TH4 }\end{array}$} \\
\hline gp160 (827-841) & $\begin{array}{l}\text { gp41 (834-848 IIIB) } \\
\text { - Epitope name: TH4 } \\
\text { - Cell-mediated immune } 1 \\
\text { - Called Th4.1 and TH4 } \\
\end{array}$ & \multicolumn{2}{|l|}{ DRVIEVVQGAYRAIR } & human & Clerici1992 \\
\hline gp160 (827-841) & $\begin{array}{l}\text { gp41 (834-848 IIIB) } \\
\text { - Epitope name: TH4 } \\
\text { - IL-2 production detectio } \\
\text { - Called Th4.1 and TH4 }\end{array}$ & $\begin{array}{l}\text { DRVIEVVQGAYRAIR } \\
\text { of Th lymphocytes from asympt }\end{array}$ & $\begin{array}{l}\text { HIV-1 infection } \\
\text { tomatic HIV-positive individuals }\end{array}$ & human & Clerici1989 \\
\hline gp160 (827-841) & $\begin{array}{l}\text { gp41 (834-848 IIIB) } \\
\text { - Epitope name: TH4 } \\
\text { - Kenyan sex workers tha } \\
\text { cases) and mucosal geni } \\
\text { - Helper epitopes used in }\end{array}$ & $\begin{array}{l}\text { DRVIEVVQGAYRAIR } \\
\text { emained seronegative were foun } \\
1 \text { tract anti-HIV IgA ( } 16 / 21 \text { case } \\
\text { is study were noted to be previou }\end{array}$ & $\begin{array}{l}\text { HIV-1 infection } \\
\text { ad to frequently have HIV-env pept } \\
\text { s) } \\
\text { usly described Clerici1989, and }\end{array}$ & $\begin{array}{l}\text { human } \\
\text { e specific Th resp } \\
\text { ere not explicitly d }\end{array}$ & $\begin{array}{l}\text { Kaul1999 } \\
\text { tected by an IL-2 assay }(11 / 20 \\
\text { in Kaul1999 }\end{array}$ \\
\hline gp160 (827-841) & $\begin{array}{l}\text { gp41 } \\
\text { - Epitope name: TH4, Th } \\
\text { - In a S. African perinatal } \\
\text { (measured by a bioassay } \\
\text { epitopes P18 MN, P18 I } \\
\text { - The mothers were predo } \\
\text { based on B subtype reag }\end{array}$ & $\begin{array}{l}\text { DRVIEVVQGAYRAIR } \\
1 \\
\text { ansmission study, } 33 \%(33 / 86) \text { c } \\
\text { neasuring IL2 production in a m } \\
\mathrm{B}, \mathrm{T} 1, \mathrm{~T} 2 \text {, and TH4 } \\
\text { inantly infected with subtype C, } \\
\text { nts. }\end{array}$ & $\begin{array}{l}\text { HIV-1 infection, HIV-1 exposed } \\
\text { seronegative } \\
\text { of cord blood samples from infants } \\
\text { urine cell line and confirmed with } \\
\text {, but the T help response was detec }\end{array}$ & $\begin{array}{l}\text { human } \\
\text { th seropositive } \mathrm{m} \\
\text { roliferation assay } \\
\text { le in a number of }\end{array}$ & $\begin{array}{l}\text { Kuhn2001 } \\
\text { roduced T-helper responses } \\
\text { t a peptide cocktail containing Th } \\
\text { ood samples despite using peptides }\end{array}$ \\
\hline
\end{tabular}




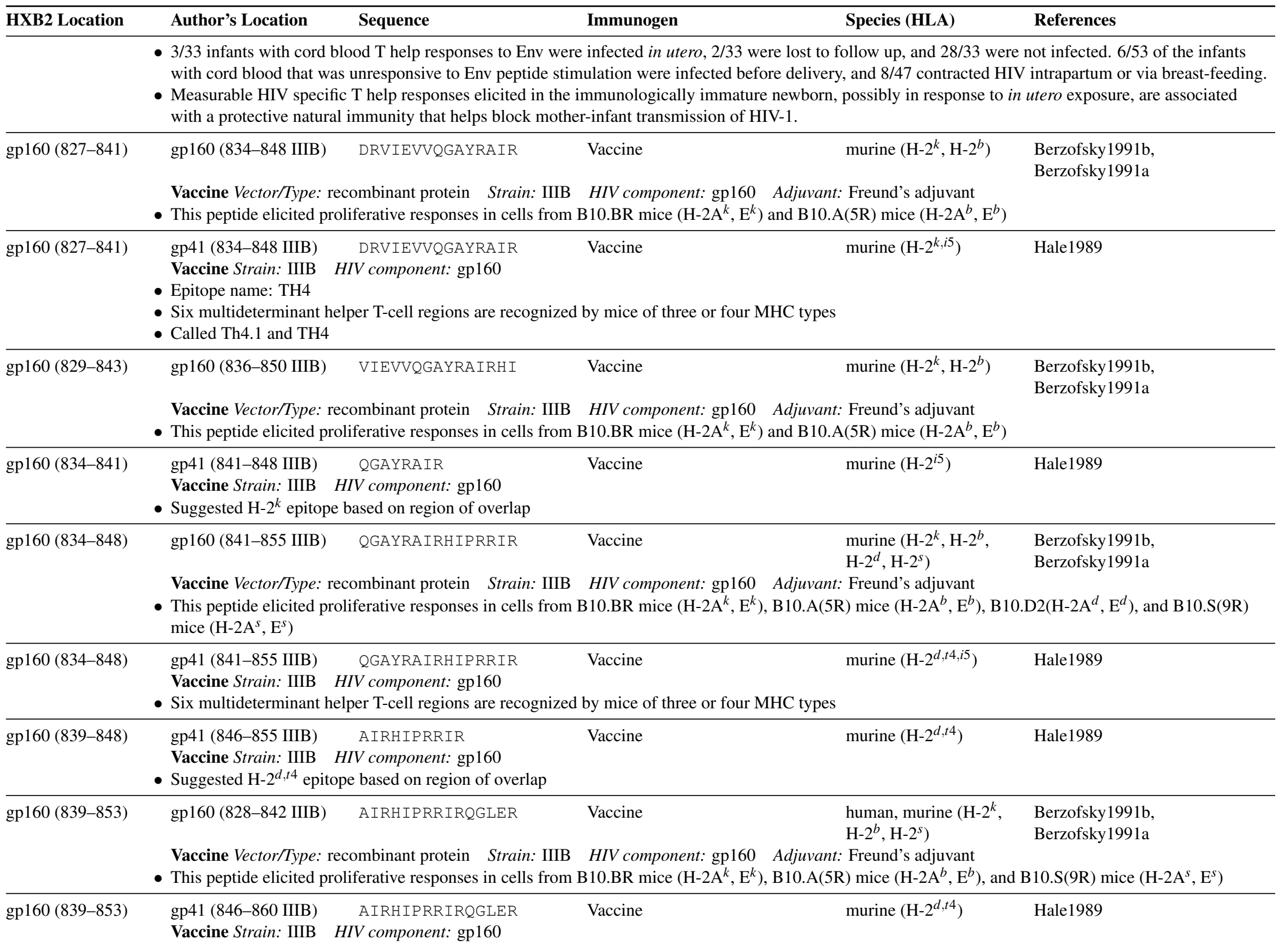




\begin{tabular}{|c|c|c|c|c|c|}
\hline HXB2 Location & Author's Location & Sequence & Immunogen & Species (HLA) & References \\
\hline & \multicolumn{5}{|c|}{ - Six multideterminant helper T-cell regions are recognized by mice of three or four MHC types } \\
\hline gp160 (842-856) & $\begin{array}{l}\text { gp41 (842-856 IIIB, } \\
\text { B10) } \\
12 \text { gag and } 18 \text { env pe }\end{array}$ & $\begin{array}{l}\text { HIPRRIRQGLERILL } \\
\text { es were identified that ce }\end{array}$ & $\begin{array}{l}\text { HIV-1 infection } \\
\text { amonly evoke T-ce }\end{array}$ & human & Wahren1989b Wahren1989a \\
\hline gp160 & gp120 (IIIB) & & HIV-1 infection & human & Geretti1994 \\
\hline
\end{tabular}

- Th proliferative responses were studied in 36 asymptomatic HIV-1+ patients. PBMC from 15/36 patients responded to stimulation with gp120 synthetic overlapping peptide pools. 10 of the responding patients recognized three or more peptide pools.

- After 12 months, most responses were lost or diminished, and specific responses fluctuated. 4/10 of the responders at baseline had new responses at 12 months, as did 5/15 non-responders.

- IL-2 production was a more sensitive and well-preserved measure of Th function than proliferation.

- $4 / 15$ responders recognized this peptide, average $\mathrm{SI}=4.4$.

- Th proliferative responses were studied in 36 asymptomatic HIV-1+ patients. PBMC from 15/36 patients responded to stimulation with gp120 synthetic overlapping peptide pools. 10 of the responding patients recognized three or more peptide pools.

- After 12 months, most responses were lost or diminished, and specific responses fluctuated. 4/10 of the responders at baseline had new responses at 12 months, as did 5/15 non-responders.

- IL-2 production was a more sensitive and well-preserved measure of Th function than proliferation.

- $4 / 15$ responders recognized this peptide, average $\mathrm{SI}=4.4$ 


\section{III-B-15 Env Helper T-Cell Epitopes}

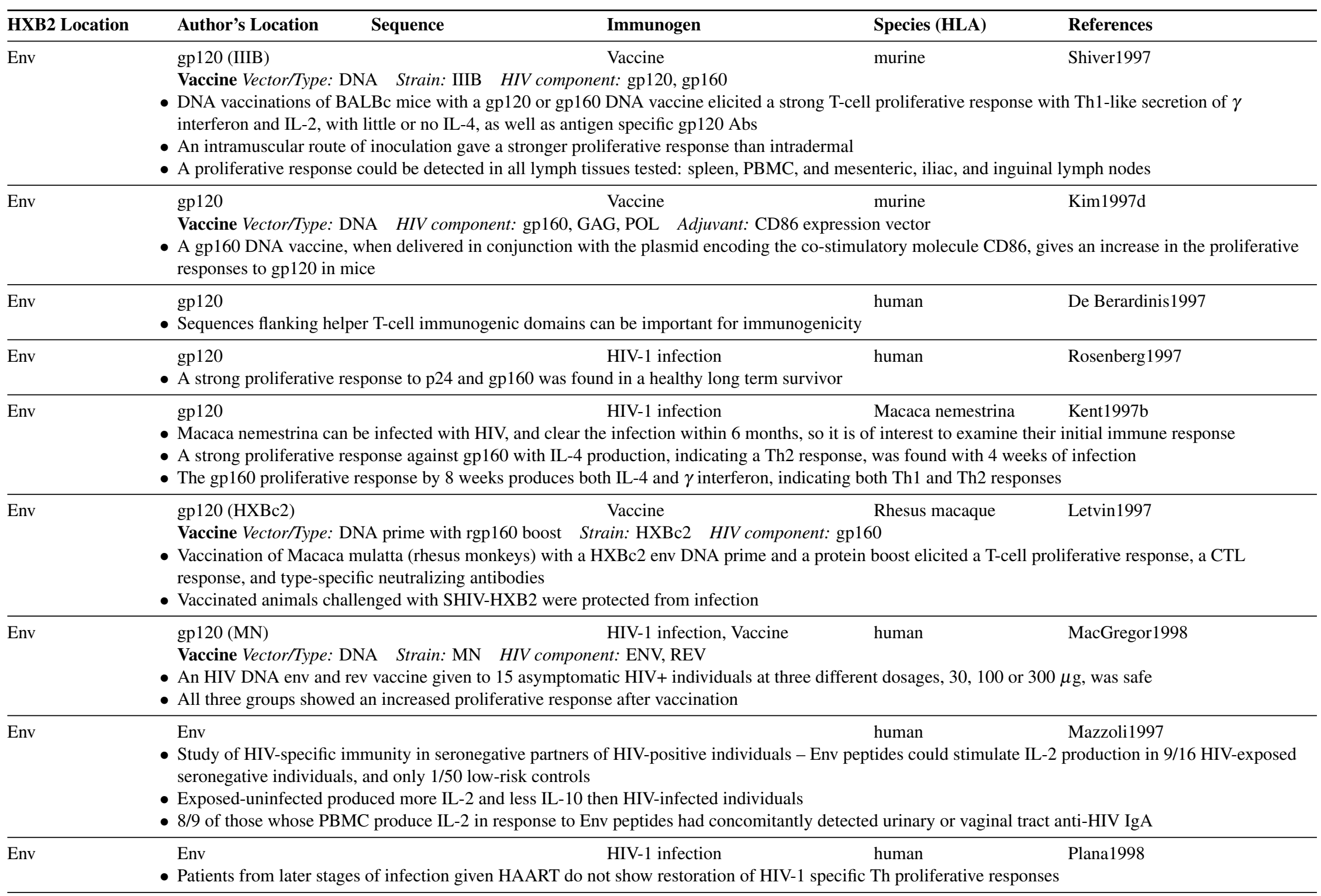




\begin{tabular}{llllll}
\hline HXB2 Location & Author's Location & Sequence & Immunogen & Species (HLA) & References \\
\hline Env & Env & HIV-1 infection & human & Kelleher1998a
\end{tabular}

- Env and gag Th epitopes were pooled and used to test Th proliferative responses after IL2 therapy - while IL2 therapy causes an increase in CD4+ lymphocyte count, it does not increase HIV-1 specific proliferative responses

\begin{tabular}{llll}
\hline Env & gp160 & HIV-1 infection, Vaccine human \\
& Vaccine Vector/Type: recombinant protein & HIV component: gp160
\end{tabular}

- Vaccinations with rgp160 did not enhance Th immunoproliferative responses in individuals who were immunized every 2 months for 5 years starting early in infection

\begin{tabular}{llll}
\hline Env & gp160 HIV-1 infection, Vaccine human \\
& Vaccine Vector/Type: recombinant protein & HIV component: gp160
\end{tabular}

- 27 HIV subtype B, 4 subtype C, 2 D and one of each subtype E, F, G infected individuals were either given rgp160 B clade immunizations or placebo. All rgp160 immunized individuals showed increased proliferation responses to the B clade immunizing antigen rgp160.

- gp120 was prepared from A, B, C, D, and E subtype virions and used as antigenic stimulus - 7 of 10 tested individuals responded to native gp120 from at least one additional subtype in addition to B subtype, while a placebo recipient did not respond to any gp120

- This study shows that cross-subtype HIV-specific T-cell proliferative responses can be stimulated in patients already infected with another HIV-1 subtype all immunized subjects could respond to the subtype B immunogen, but many developed responses to at least one more subtype

\begin{tabular}{llcc}
\hline Env & gp160 (MN) & Vaccine & human \\
& Vaccine Vector/Type: gp160 prime with gp120 boost & Strain: MN & HIV component: gp160, gp120
\end{tabular}

- Helper T-cell memory responses were induced by MN rgp160 as measured by proliferation and Th1 and Th2 cytokine release - this response could be boosted by MN rgp120

\begin{tabular}{llcl}
\hline Env & gp120 & Vaccine & Rhesus macaque \\
& Vaccine Vector/Type: ISCOM or fowlpoxvirus & Strain: SF2 HIV component: gp120 & Heeney9b
\end{tabular}

- Vaccinated monkeys with the highest level of Th1 and Th2 responses and the highest levels of NAbs were protected against a SHIV SF13 challenge - the ISCOM strategy gave more potent anti -gp120 responses than the Fowl pox strategy

- When animals were challenged 4 months after boost, those that maintained high levels of HIV-1 specific IFN-gamma responses, indicative of a Th response, were still protected

Env (IIIB) $\quad$ HIV-1 infection, Vaccine $\quad$ human

Vaccine Vector/Type: DNA Strain: IIIB HIV component: ENV, REV

- A DNA vaccine containing env and rev was tested for safety and immune response in $15 \mathrm{HIV+}$ asymptomatic individuals

- Enhanced proliferative activity and higher levels of MIP-1 alpha were detected in multiple study subjects

\begin{tabular}{llccc}
\hline Env & Env & Vaccine & murine BALB/c
\end{tabular}

Vaccine Vector/Type: vaccinia Strain: IIIB HIV component: gp160 Adjuvant: GM-CSF-ENV chimera

- A chimeric GM-CSF-env antigen expressed in a vaccinia vector elicits a higher HIV-specific env cellular immune response than when native env is used

\begin{tabular}{llcc}
\hline Env & Env (LAI) & Vaccine & Macaca nemestrina \\
& Vaccine Vector/Type: DNA prime with vaccinia boost & Strain: LAI & HIV component: ENV, GAG
\end{tabular}

- Priming with an HIV-DNA vaccine and boosting with a vaccinia construct induced greater levels of HIV T-cell immunity than either vaccine alone 


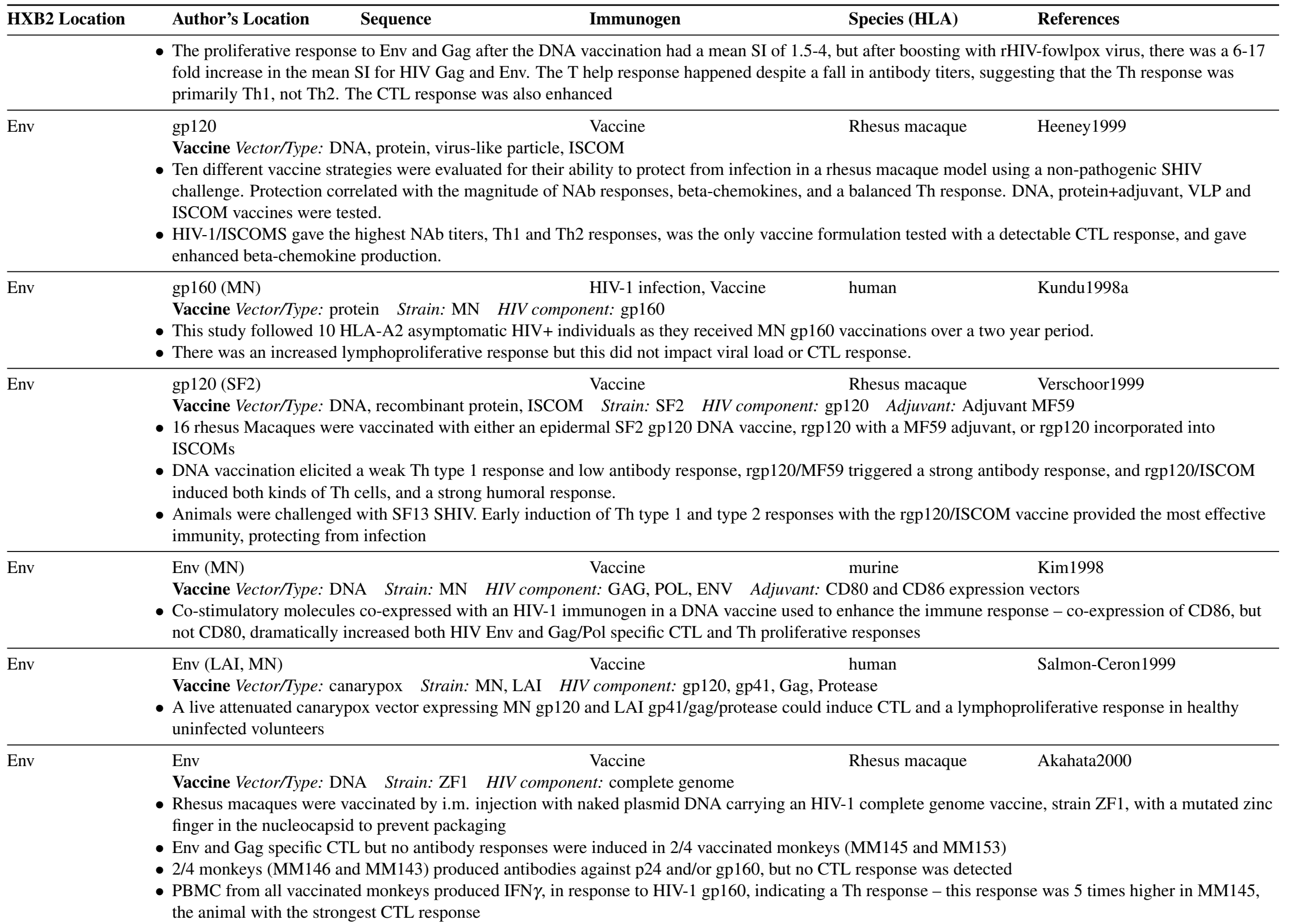




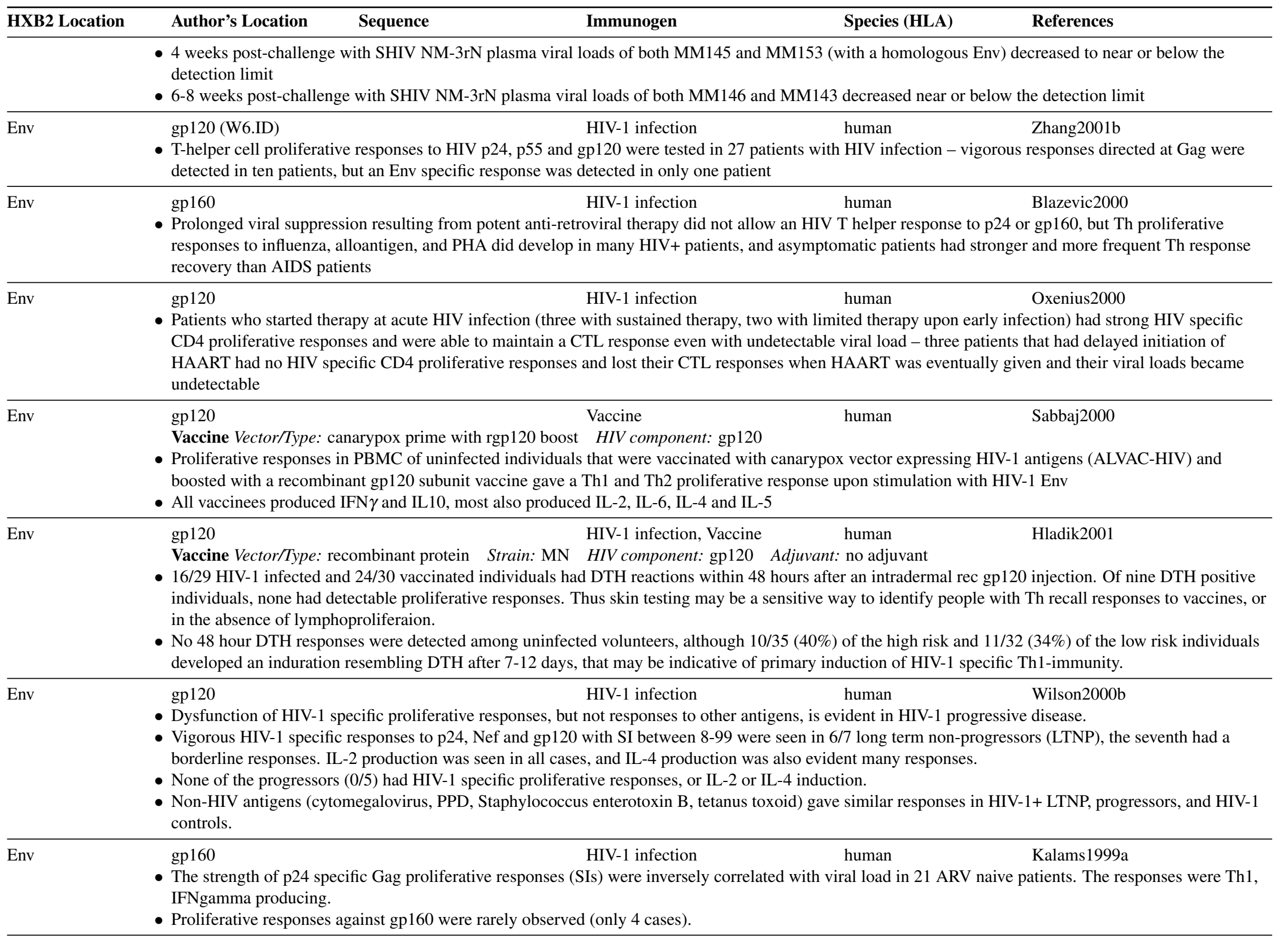




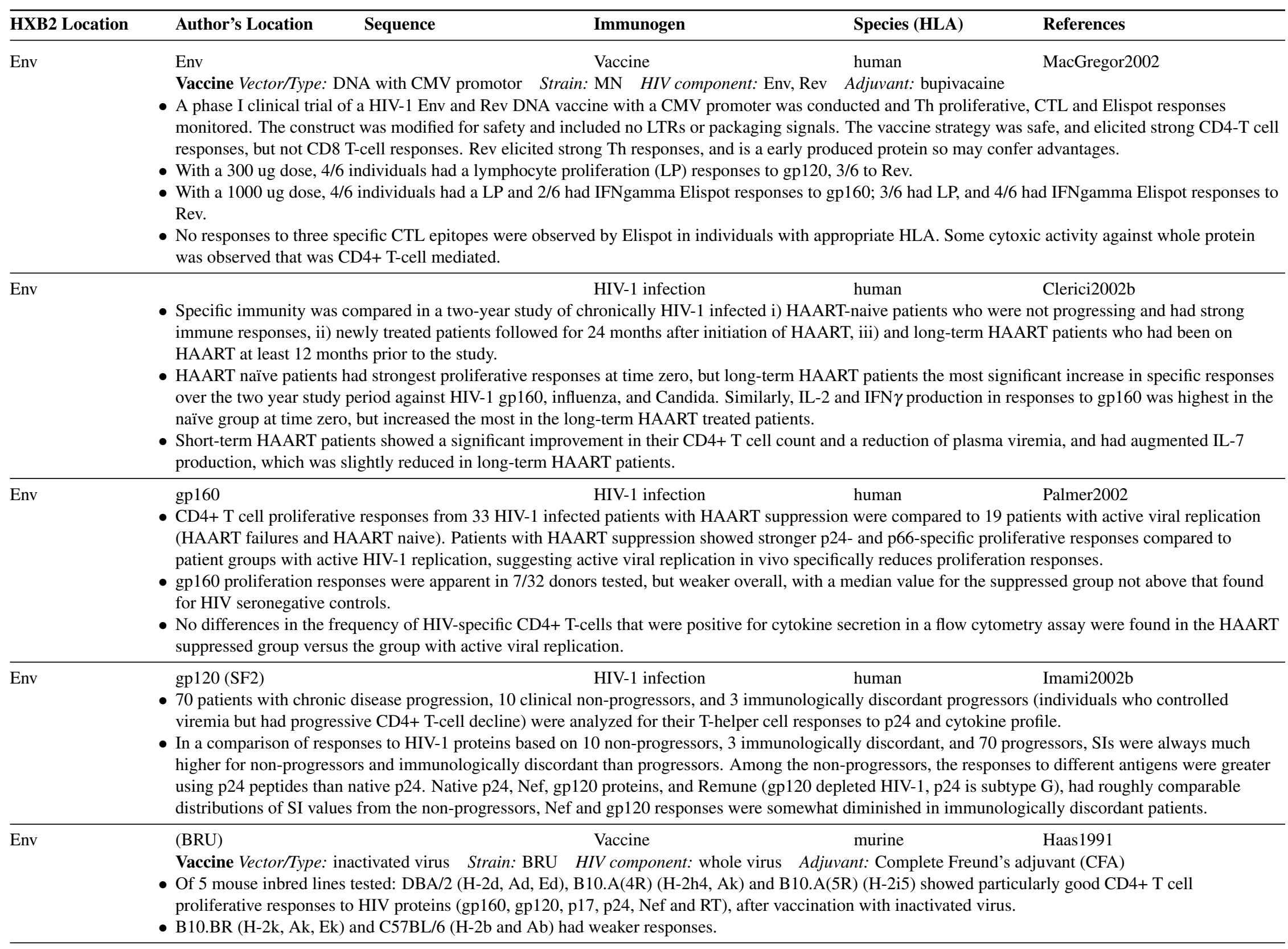




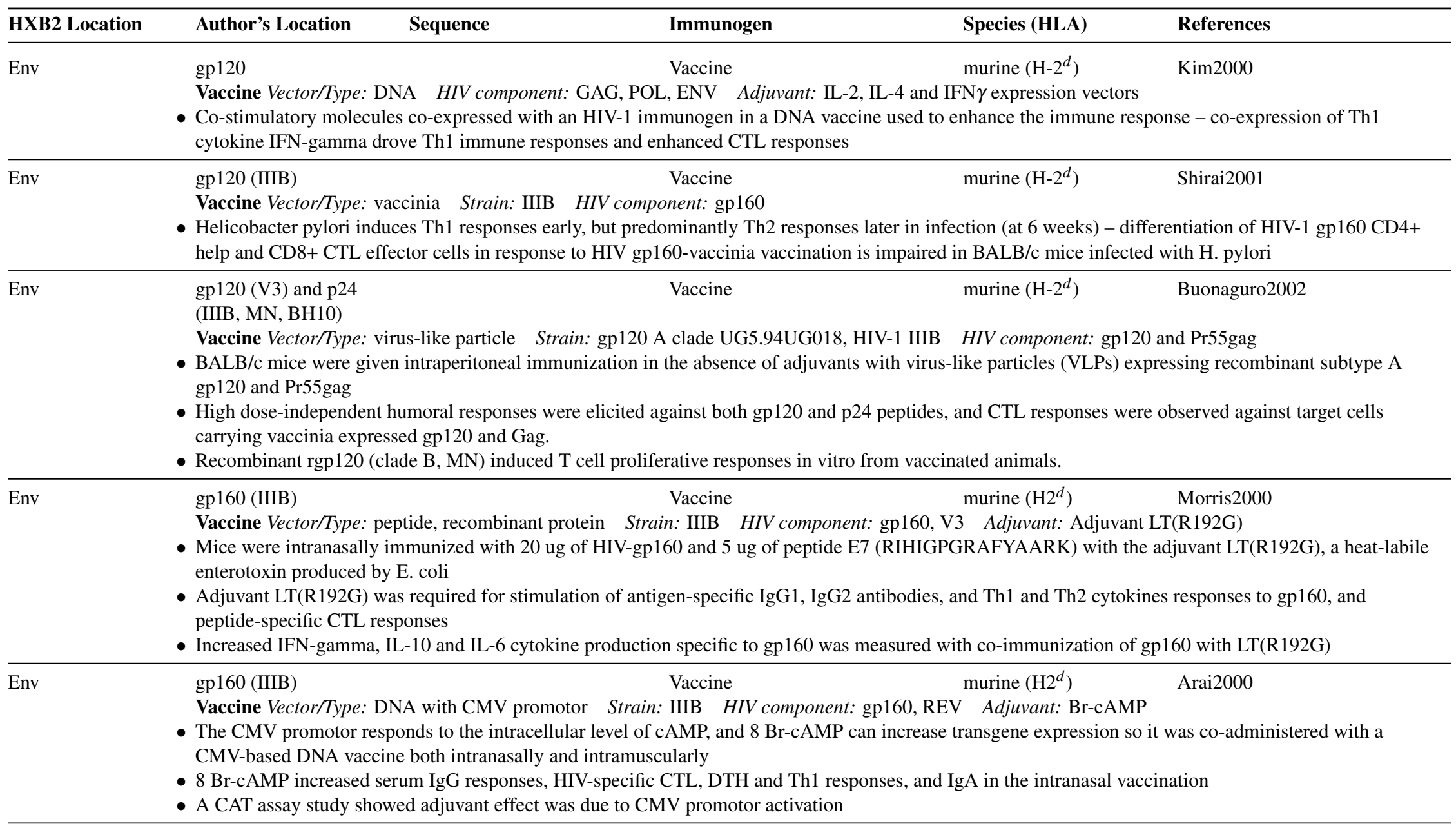




\section{III-B-16 Nef Helper T-Cell Epitopes}

\begin{tabular}{|c|c|c|c|c|}
\hline HXB2 Location & Author's Location & Immunogen & Species (HLA) & References \\
\hline Nef (1-20) & \multicolumn{4}{|c|}{$\begin{array}{l}\text { Vaccine Vector/Type: DNA Strain: LAI HIV component: NEF, TAT, REV } \\
\text { - Stronger, broader responses were observed in animals vaccinated with DNA epidermally rather than with intramuscular protein } \\
\text { - Proliferative response to vaccination was observed to peptides throughout Nef and Tat, less for Rev }\end{array}$} \\
\hline Nef (1-20) & $\begin{array}{l}\text { Nef }(1-20 \text { HXB2) } \\
\text { Deletion of the } 19 \mathrm{~N} \\
\text { molecules. Such a c } \\
\text { are minimal, a muri }\end{array}$ & $\begin{array}{l}\text { MGGKWSKSSVIGWPTVRERM HIV-1 infectio } \\
\text { ninal amino acids from Nef including the myrist } \\
\text { uct has the potential to serve as a more potent in } \\
\text { 2d Th epitope in the peptide MGGKWSKSSVI }\end{array}$ & $\begin{array}{l}\left(\mathrm{H}-2^{d}\right) \\
\text { tes Nef-induced d } \\
\text { n T-cell epitopes } \\
\text { HLA-B8 CTL e }\end{array}$ & $\begin{array}{l}\text { Peng2001 } \\
\text { ulation of MHC class I and CD4 } \\
\text { would be disputed by this deletion } \\
\text { VPTVRERM. }\end{array}$ \\
\hline Nef (16-35) & $\begin{array}{l}\text { Nef (16-35 LAI) } \\
\text { Vaccine Vector/Type } \\
\text { - Stronger, broader re } \\
\text { - Some proliferative } \mathrm{r}\end{array}$ & $\begin{array}{l}\text { VRERMRRAEPAADGVGAASR Vaccine } \\
\text { JA Strain: LAI HIV component: NEF, TAT, } \\
\text { ses were observed in animals vaccinated with DI } \\
\text { ase to vaccination was observed to peptides thro }\end{array}$ & $\begin{array}{l}\text { murine }\left(\mathrm{H}-2^{d}\right) \\
\text { than with intram } \\
\text { ess for Rev }\end{array}$ & $\begin{array}{l}\text { Hinkula1997 } \\
\text { rotein }\end{array}$ \\
\hline Nef (31-50) & $\begin{array}{l}\text { Nef (31-50 LAI) } \\
\text { Vaccine Vector/Type } \\
\text { - Stronger, broader re } \\
\text { - Some proliferative } \mathrm{r}\end{array}$ & $\begin{array}{l}\text { GAASRDLEKHGAITSSNTAA Vaccine } \\
\text { JA Strain: LAI HIV component: NEF, TAT, } \\
\text { ses were observed in animals vaccinated with DI } \\
\text { nse to vaccination was observed to peptides thro }\end{array}$ & $\begin{array}{l}\text { murine }\left(\mathrm{H}-2^{d}\right) \\
\text { than with intram } \\
\text { ess for Rev }\end{array}$ & $\begin{array}{l}\text { Hinkula1997 } \\
\text { rotein }\end{array}$ \\
\hline Nef (45-69) & $\begin{array}{l}\text { Nef (45-69 BRU) } \\
\text { Vaccine Vector/Type } \\
\text { - Antigenic domain: }\end{array}$ & $\begin{array}{l}\text { SSNTAATNAACAWLEAQEE- Vaccine } \\
\text { EEVGFP } \\
\text { tide prime with protein boost Strain: BRU } \\
\text { AACAWL, priming with peptide enhanced subse }\end{array}$ & $\begin{array}{l}\text { chimpanzee, rat } \\
\text { Nef protein imm }\end{array}$ & Estaquier1992 \\
\hline Nef (45-69) & $\begin{array}{r}\text { Nef (45-69) } \\
\text { Vaccine Vector/Type } \\
\text { - Covalently linking t } \\
\text { epitope from Schist }\end{array}$ & $\begin{array}{l}\text { SSNTAATNAACAWLEAQEE- Vaccine } \\
\text { EEVGFP } \\
\text { otide Adjuvant: no adjuvant, aluminum hydrox } \\
\text { tent Th epitope Nef } 45-69 \text {, which can induce Th } \\
\text { a mansoni allows the induction of detectable Th }\end{array}$ & $\begin{array}{l}\text { rat } \\
\text { es at low doses w } \\
\text { stosoma epitope. }\end{array}$ & $\begin{array}{l}\text { Rouaix1994 } \\
\text { ljuvant in Lou/M rats, to a weaker }\end{array}$ \\
\hline Nef (46-65) & $\begin{array}{l}\text { Nef (46-65 LAI) } \\
\text { Vaccine Vector/Type } \\
\text { - Stronger, broader re } \\
\text { - Some proliferative } \mathrm{r}\end{array}$ & $\begin{array}{l}\text { SNTAATNAACAWLEAQEEEE Vaccine } \\
\text { JA Strain: LAI HIV component: NEF, TAT, } \\
\text { ses were observed in animals vaccinated with DI } \\
\text { ase to vaccination was observed to peptides thro }\end{array}$ & $\begin{array}{l}\text { murine }\left(\mathrm{H}-2^{d}\right) \\
\text { than with intram } \\
\text { ess for Rev }\end{array}$ & $\begin{array}{l}\text { Hinkula1997 } \\
\text { protein }\end{array}$ \\
\hline Nef (56-68) & Nef (56-68 HXB2) & AWLEAQEEEEVGF & $\begin{array}{l}\text { murine (DQ2, } \\
\text { DQ5, DQ6, DC }\end{array}$ & Pancré2002 \\
\hline
\end{tabular}

- This highly conserved Nef epitope has promiscuous HLA-DQ class II binding potential. It has a can bind to 6 different HLA-DQ alleles, but did not bind to any HLA-DR alleles tested. It bound to DQ2 and DQ8 with particularly high affinity, and with DQ7 with low affinity.

- DQ transgenic mice (in particular DQ8) mounted strong cellular and humoral responses after immunization with this peptide. 


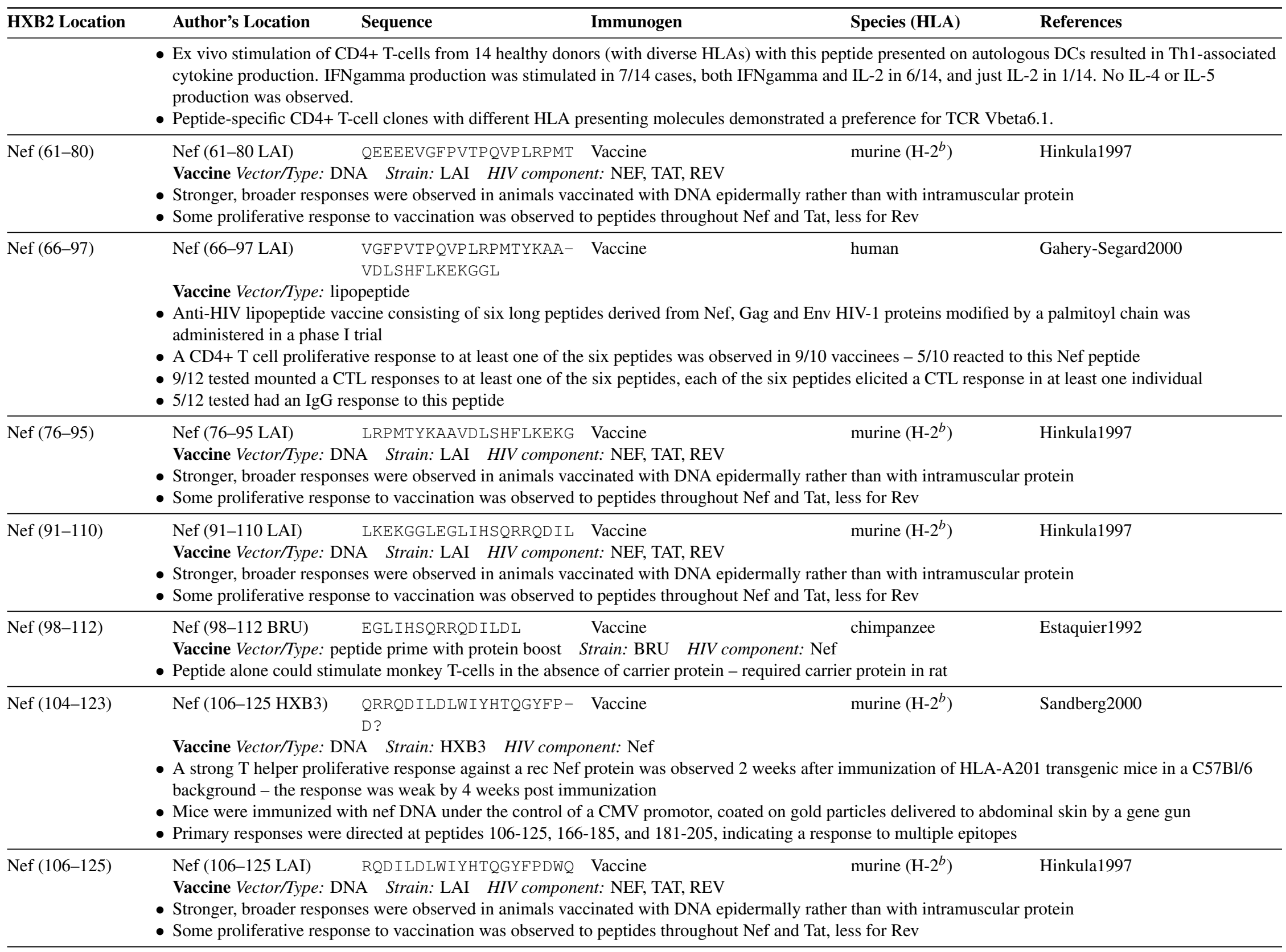




\begin{tabular}{|c|c|c|c|c|}
\hline HXB2 Location & Author's Location & Immunogen & Species (HLA) & References \\
\hline Nef (117-147) & \multicolumn{4}{|c|}{$\begin{array}{l}\text { Vaccine Vector/Type: lipopeptide } \\
\text { - Anti-HIV lipopeptide vaccine consisting of six long peptides derived from Nef, Gag and Env HIV-1 proteins modified by a palmitoyl chain was } \\
\text { administered in a phase I trial } \\
\text { - A CD4+ T cell proliferative response to at least one of the six peptides was observed in } 9 / 10 \text { vaccinees }-1 / 10 \text { reacted to this Nef peptide } \\
\text { - } 9 / 12 \text { tested mounted a CTL responses to at least one of the six peptides, each of the six peptides elicited a CTL response in at least one individual } \\
\text { - } 10 / 12 \text { tested had an IgG response to this peptide }\end{array}$} \\
\hline Nef (121-140) & $\begin{array}{l}\text { Nef (121-140 LAI) } \\
\text { Vaccine Vector/Type: D } \\
\text { - Stronger, broader respo } \\
\text { - Some proliferative resp } \\
\end{array}$ & $\begin{array}{l}\text { FPDWQNYTPGPGVRYPLTFG Vaccine } \\
\text { JA Strain: LAI HIV component: NEF, TAT, } \\
\text { ses were observed in animals vaccinated with DI } \\
\text { ase to vaccination was observed to peptides thro }\end{array}$ & $\begin{array}{l}\text { murine }\left(\mathrm{H}-2^{b}\right) \\
\text { than with intram } \\
\text { ss for Rev }\end{array}$ & $\begin{array}{l}\text { Hinkula1997 } \\
\text { protein }\end{array}$ \\
\hline Nef (136-155) & $\begin{array}{l}\text { Nef (136-155 LAI) } \\
\text { Vaccine Vector/Type: D } \\
\text { - Stronger, broader respo } \\
\text { - Some proliferative resp }\end{array}$ & $\begin{array}{l}\text { PLTFGWCYKLVPVEPDKVEE Vaccine } \\
\text { JA Strain: LAI HIV component: NEF, TAT, } \\
\text { ses were observed in animals vaccinated with DI } \\
\text { nse to vaccination was observed to peptides thro }\end{array}$ & $\begin{array}{l}\text { murine }\left(\mathrm{H}-2^{d}\right) \\
\text { than with intram } \\
\text { ss for Rev }\end{array}$ & $\begin{array}{l}\text { Hinkula1997 } \\
\text { protein }\end{array}$ \\
\hline Nef (151-170) & $\begin{array}{l}\text { Nef }(151-170 \text { LAI }) \\
\text { Vaccine Vector/Type: } \mathrm{D} \\
\text { - Stronger, broader respo } \\
\text { - Some proliferative resp }\end{array}$ & $\begin{array}{l}\text { DKVEEANKGENTSLLHPVSL Vaccine } \\
\text { JA Strain: LAI HIV component: NEF, TAT, } \\
\text { ses were observed in animals vaccinated with DI } \\
\text { ase to vaccination was observed to peptides thro }\end{array}$ & $\begin{array}{l}\text { murine }\left(\mathrm{H}-2^{d}\right) \\
\text { than with intram } \\
\text { ss for Rev }\end{array}$ & $\begin{array}{l}\text { Hinkula1997 } \\
\text { protein }\end{array}$ \\
\hline Nef (164-183) & $\begin{array}{l}\text { Nef (166-185 HXB3) } \\
\text { Vaccine Vector/Type: } \\
\text { - A strong T helper proli } \\
\text { background - the respo } \\
\text { - Mice were immunized } \\
\text { - Primary responses were }\end{array}$ & $\begin{array}{l}\text { LLHPVSLHGMDDPEREVLE- Vaccine } \\
\text { W? } \\
\text { JA Strain: HXB3 HIV component: Nef } \\
\text { rative response against a rec Nef protein was ob } \\
\text { se was weak by } 4 \text { weeks post immunization } \\
\text { ith nef DNA under the control of a CMV promo } \\
\text { directed at peptides } 106-125,166-185 \text {, and } 181-2\end{array}$ & $\begin{array}{l}\text { murine }\left(\mathrm{H}-2^{b}\right) \\
\text { mmunization of } \mathrm{H} \\
\text { ticles delivered to } \\
\text { nse to multiple e }\end{array}$ & $\begin{array}{l}\text { Sandberg2000 } \\
1 \text { transgenic mice in a C57B1/6 } \\
\text { nal skin by a gene gun }\end{array}$ \\
\hline Nef (166-185) & $\begin{array}{l}\text { Nef (166-185 LAI) } \\
\text { Vaccine Vector/Type: I } \\
\text { - Stronger, broader respo } \\
\text { - Some proliferative resp }\end{array}$ & $\begin{array}{l}\text { HPVSLHGMDDPEREVLEWRF Vaccine } \\
\text { JA Strain: LAI HIV component: NEF, TAT, } \\
\text { ses were observed in animals vaccinated with DI } \\
\text { ase to vaccination was observed to peptides thro }\end{array}$ & $\begin{array}{l}\text { murine }\left(\mathrm{H}-2^{b, d}\right) \\
\text { than with intram } \\
\text { ss for } \mathrm{Rev}\end{array}$ & $\begin{array}{l}\text { Hinkula1997 } \\
\text { protein }\end{array}$ \\
\hline Nef (179-198) & $\begin{array}{l}\text { Nef (181-205 HXB3) } \\
\text { Vaccine Vector/Type: I } \\
\text { - A strong T helper proli } \\
\text { background - the respo } \\
\text { - Mice were immunized } \\
\text { - Primary responses were }\end{array}$ & $\begin{array}{l}\text { EVLEWREDSRLAFHHVARE- Vaccine } \\
\text { L? } \\
\text { JA Strain: HXB3 HIV component: Nef } \\
\text { rative response against a rec Nef protein was ob } \\
\text { ee was weak by } 4 \text { weeks post immunization } \\
\text { ith nef DNA under the control of a CMV promo } \\
\text { directed at peptides } 106-125,166-185 \text {, and } 181-2\end{array}$ & $\begin{array}{l}\text { murine }\left(\mathrm{H}-2^{b}\right) \\
\text { mmunization of } \mathrm{H} \\
\text { rticles delivered } \mathrm{t} \\
\text { onse to multiple e }\end{array}$ & $\begin{array}{l}\text { Sandberg2000 } \\
01 \text { transgenic mice in a C57B1/6 } \\
\text { inal skin by a gene gun }\end{array}$ \\
\hline
\end{tabular}




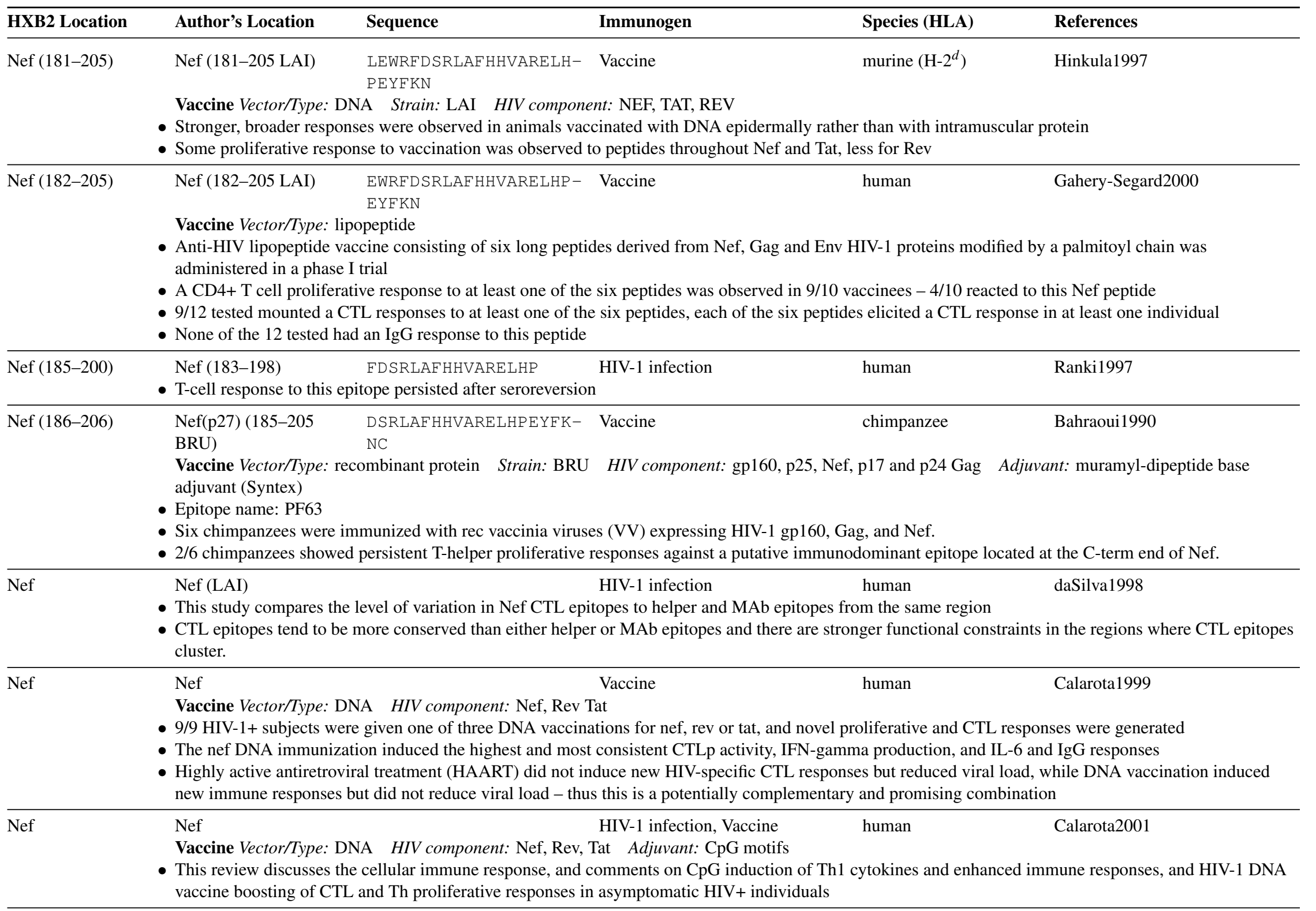




\begin{tabular}{|c|c|c|c|c|}
\hline HXB2 Location & Author's Location & Sequence & Species (HLA) & References \\
\hline Nef & $\begin{array}{l}\text { Nef } \\
\text { Patients who started } \\
\text { CD4 proliferative re } \\
\text { HAART had no HIV } \\
\text { undetectable }\end{array}$ & $\begin{array}{l}\text { py at acute } \mathrm{H} \\
\text { es and were } \\
\text { ific CD4 pro }\end{array}$ & $\begin{array}{l}\text { HIV-1 infection human } \\
\text { with sustained therapy, two with limited therapy upon } \\
\text { TTL response even with undetectable viral load - three } \\
\text { and lost their CTL responses when HAART was even }\end{array}$ & $\begin{array}{l}\text { Oxenius } 2000 \\
\text { that had delayed initiation of } \\
\text { ren and their viral loads became }\end{array}$ \\
\hline $\mathrm{Nef}$ & $\begin{array}{l}\text { Nef } \\
\text { - Dysfunction of HIV } \\
\text { Vigorous HIV-1 spe } \\
\text { borderline responses } \\
\text { - None of the progres } \\
\text { Non-HIV antigens ( } \\
\text { controls. }\end{array}$ & $\begin{array}{l}\text { ecific prolife } \\
\text { responses to } \\
2 \text { production } \\
\text { (0/5) had HIV } \\
\text { megalovirus, }\end{array}$ & $\begin{array}{l}\text { HIV-1 infection } \\
\text { not responses to other antigens, is evident in HIV-1 pr } \\
\text { with SI between } 8-99 \text { were seen in } 6 / 7 \text { long term non- } \\
\text { es, and IL-4 production was also evident many response } \\
\text { ative responses, or IL-2 or IL-4 induction. } \\
\text { as enterotoxin B, tetanus toxoid) gave similar response }\end{array}$ & $\begin{array}{l}\text { Wilson2000b } \\
\text { e disease. } \\
\text { ors (LTNP), the seventh had a } \\
\text {-1+ LTNP, progressors, and HIV-1 }\end{array}$ \\
\hline $\mathrm{Nef}$ & $\begin{array}{l}\text { Nef (BRU) } \\
\text { Vaccine Vector/Type } \\
\text { BALB/c mice were } \\
\text { - High Ab titers (pred } \\
\text { 5-fold higher than N } \\
\text { CD4+ T-cell lymph }\end{array}$ & $\begin{array}{l}\text { combinant pr } \\
\text { unized with I } \\
\text { lantly IgG1) } \\
\text { one. } \\
\text { iferative wer }\end{array}$ & $\begin{array}{l}\text { Vaccine murine } \\
\text { HIV component: Nef Adjuvant: poly(DL-lactide-c } \\
\text { Freund's adjuvant, or Nef encapsulated in poly(DL-lac } \\
\text { tained for seven months in the mice infected with Nef-1 } \\
\text { kine profiles indicated this was primarily a Th2 respon }\end{array}$ & $\begin{array}{l}\text { Moureau2002 } \\
\text { ide) (PLG), Freund's adjuvant } \\
\text { lycolide) PLG microparticles. } \\
\text { old higher than Nef in Freund's, }\end{array}$ \\
\hline Nef & $\begin{array}{l}\text { Nef (SF2) } \\
70 \text { patients with chr } \\
\text { viremia but had pros } \\
\text { In a comparison of } 1 \\
\text { higher for non-prog } \\
\text { using p24 peptides t } \\
\text { distributions of SI v }\end{array}$ & $\begin{array}{l}\text { isease progr } \\
\text { e CD4+ T- } \\
\text { ses to HIV- } \\
\text { and immu } \\
\text { ative p24. N } \\
\text { from the nor }\end{array}$ & $\begin{array}{l}\text { HIV-1 infection human } \\
\text { n-progressors, and } 3 \text { immunologically discordant pro } \\
\text { lyzed for their T-helper cell responses to p24 and cyt } \\
0 \text { non-progressors, } 3 \text { immunologically discordant, anc } \\
\text { t than progressors. Among the non-progressors, the r } \\
0 \text { proteins, and Remune (gp120 depleted HIV-1, p24 i } \\
\text { d gp120 responses were somewhat diminished in imn }\end{array}$ & $\begin{array}{l}\text { Imami2002b } \\
\text { (individuals who controlled } \\
\text { file. } \\
\text { ressors, SIs were always much } \\
\text { to different antigens were greater } \\
\text { e), had roughly comparable } \\
\text { cally discordant patients. }\end{array}$ \\
\hline $\mathrm{Nef}$ & $\begin{array}{l}\text { (BRU) } \\
\text { Vaccine Vector/Type } \\
\text { - Of } 5 \text { mouse inbred } 1 \\
\text { proliferative respons } \\
\text { B10.BR }(\mathrm{H}-2 \mathrm{k}, \mathrm{Ak} \text {, }\end{array}$ & $\begin{array}{l}\text { ctivated viru } \\
\text { ested: DBA/ } \\
\text { HIV protein } \\
\text { nd C57BL/6 }\end{array}$ & $\begin{array}{l}\text { Vaccine } \\
\text { IV component: whole virus, } \mathrm{RT} \quad \text { Adjuvant: } \text { Complete } \\
\text { 10.A(4R) }(\mathrm{H}-2 \mathrm{~h} 4, \mathrm{Ak}) \text { and } \mathrm{B} 10 . \mathrm{A}(5 \mathrm{R})(\mathrm{H}-2 \mathrm{i} 5) \text { showec } \\
\text { 7, p24, Nef and } \mathrm{RT}) \text {, after vaccination with inactivated } \\
\text { weaker responses. }\end{array}$ & $\begin{array}{l}\text { Haas1991 } \\
\text { larly adjuvant }(\mathrm{CFA})\end{array}$ \\
\hline $\mathrm{Nef}$ & $\begin{array}{l}\text { Nef } \\
\text { Vaccine Vector/Type } \\
\text { - Splenocytes from B } \\
\text { IFN-gamma levels } \\
\text { - Antigen stimulation } \\
\text { - IL-4 production was } \\
\text { - Cross-clade CTL ac } \\
\text { an HIV-1 AC recom }\end{array}$ & $\begin{array}{l}\text { A HIV co } \\
\text { c mice imm } \\
\text { ased IFN-ga } \\
\text { ignificantly } \\
\text { was also ob } \\
\text { t, however, }\end{array}$ & $\begin{array}{l}\text { Vaccine murine }\left(\mathrm{H}-2^{d}\right) \\
\text { Nef } \\
\text { P DNA were incubated with Vif, Vpu or Nef antigens } \mathrm{f} \\
\text { pVVN-P immunized mice, indicating a Th1 response } \\
\text { en stimulation compared to control levels } \\
\text { CRF01(AE) clade antigens could serve as targets for th } \\
\text { CTL response, but was expressed at lower levels on the }\end{array}$ & $\begin{array}{l}\text { Ayyavoo2000 } \\
\text { s and assayed for IL-4 and } \\
\text { le immunization stimulated CTL - } \\
\text { ll }\end{array}$ \\
\hline
\end{tabular}




\section{III-B-17 HIV-1 Helper T-Cell Epitopes}

\begin{tabular}{|c|c|c|c|c|c|}
\hline HXB2 Location & Author's Location & Sequence & Immunogen & Species (HLA) & References \\
\hline HIV-1 & & & HIV-1 infection & human & Kuhn2002 \\
\hline
\end{tabular}

and HIV-1 specific CTL in some. It is unknown whether these responses are associated with lack of infection, but there is some evidence that HIV-1 T-cell responses may reduce transmission in breastfeeding mothers. Summary tables are provided of CD4 and CD8 T-cell responses detected in earlier studies.

HIV-1 HIV-1 infection, Vaccine

human Kahn2000

Vaccine Vector/Type: gp120 depleted virus HZ321 (REMUNE(TM)) Strain: Z321 HIV component: gp120-depleted HIV-1 antigen Adjuvant: Incomplete Freund adjuvant (IFA)

- No benefit was observed in terms of progression free survival for HIV-1 patients on ART given vaccinations with HIV-1 antigen (N=1,262) versus those vaccinated with placebo $(\mathrm{N}=1,265)$. There was no statistically different outcome in HIV RNA, CD4 percentage, or body weight. HIV-1 ART patients that were vaccinated did have higher absolute CD4 counts.

HIV-1

$$
\text { HIV-1 infection, Vaccine }
$$

human

Moss1999

Vaccine Vector/Type: gp120 depleted virus HZ321 (REMUNE(TM)) Strain: Z321 HIV component: gp120-depleted HIV-1 antigen Adjuvant: Incomplete Freund adjuvant (IFA)

- 15 HIV-1+ patients on ARV given vaccinations with HIV-1 antigen versus vaccinated with placebo. Lymphocyte proliferation of CD4+, CD8+ memory cells and NK cells to p24 and Remune HIV-1 antigen increased in HAART treated patients after vaccination.

\begin{tabular}{|c|c|}
\hline HIV-1 & HIV-1 infection, Vaccine \\
\hline
\end{tabular}
Incomplete Freund adjuvant (IFA)

- HIV-1 specific stimulation of T-cell proliferation, and beta-chemokines (RANTES) and Th1-type cytokine (IFNgamma) production are found after immunization of HIV-1+ individuals with HIV-1 immunogen.

\begin{tabular}{llc}
\hline HIV-1 & HIV-1 infection, Vaccine & human \\
& Vaccine Vector/Type: gp120 depleted virus HZ321 (REMUNE(TM)) & Strain: Z321 HIV component: gp120-depleted HIV-1 antigen \\
& Incomplete Freund adjuvant (IFA)
\end{tabular}
Incomplete Freund adjuvant (IFA)

- Long-term follow up of HIV-1+ individuals given HIV-1 immunogen, suggesting those patients who became HIV-DTH-responsive in response to the HIV-1 immunogen had a better clinical outcome. Of twelve who developed DTH-responsiveness, one got an opportunistic infection and died, and one developed KS. Of the 13 patients who remained HIV-DTH-nonresponsive, 9 (69\%) progressed to AIDS and 7 of these had died.

HIV-1 $\quad$ Vaccine $\quad$ human

Vaccine Vector/Type: HIV-1 immunogen Adjuvant: Incomplete Freund adjuvant (IFA)

- A dose response study of HIV immunogen in IFA was conducted. Doses of 50, 100, 200, or 400 micrograms (total protein) were tested by DTH skin testing to the inactivated HIV-1 antigen. The HIV-1 immunogen was well tolerated, and the minimum dose required to induce HIV-1 DTH was 100 micrograms.

- Mathematical modeling is used to support the idea that T-helper cell dysfunction results in a compromised ability to maintain an anti-HIV CTL memory response. Models suggest strategies to restore CTL memory through therapy and improve long-term immunological control of the virus.

HIV-1 infection, Vaccine human Imami2002a

Vaccine Vector/Type: gp120 depleted virus HZ321 (REMUNE(TM)), recombinant protein, virus-like particle, canarypox, adenovirus, DNA Adjuvant: CpG, IL-2, GMCSF, IL-7, IL-12, Growth Hormone, Thymosin alpha-1. 


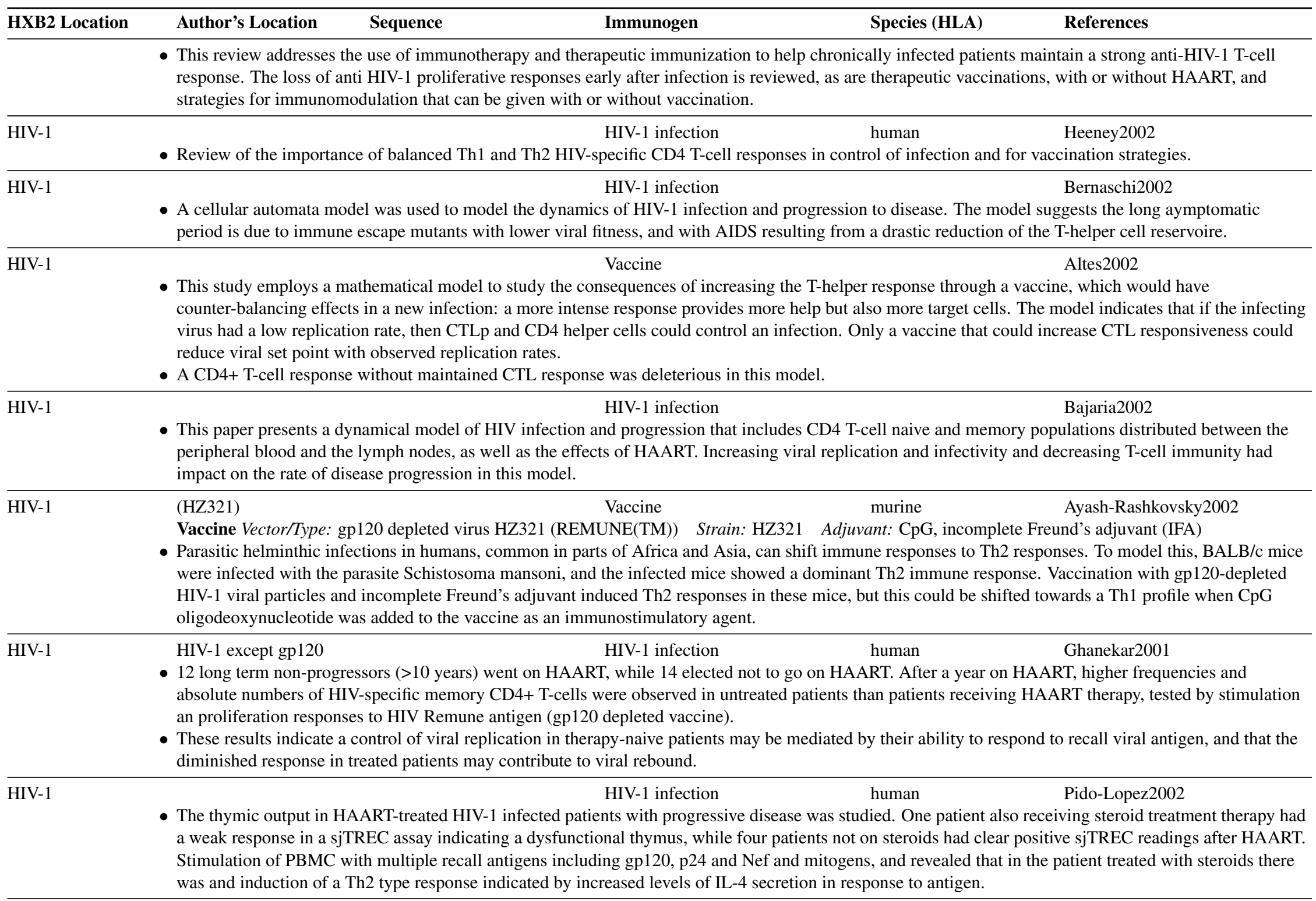




\section{III-C Maps of T-Helper Epitope Locations Plotted by Protein}

Linear helper $\mathrm{T}$ cell epitopes less than twenty-two amino acids long are III-C-1 p17 T-Helper Epitope Map shown.

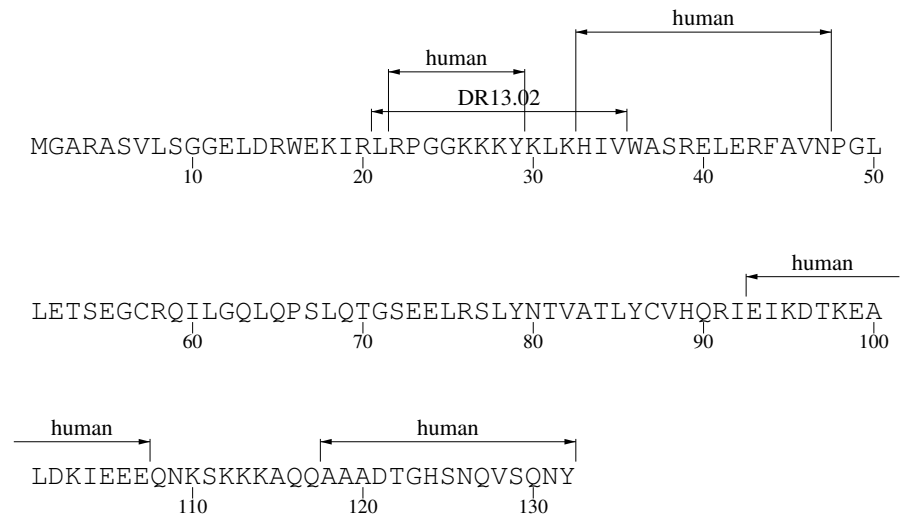




\section{III-C-2 p24 T-Helper Epitope Map}
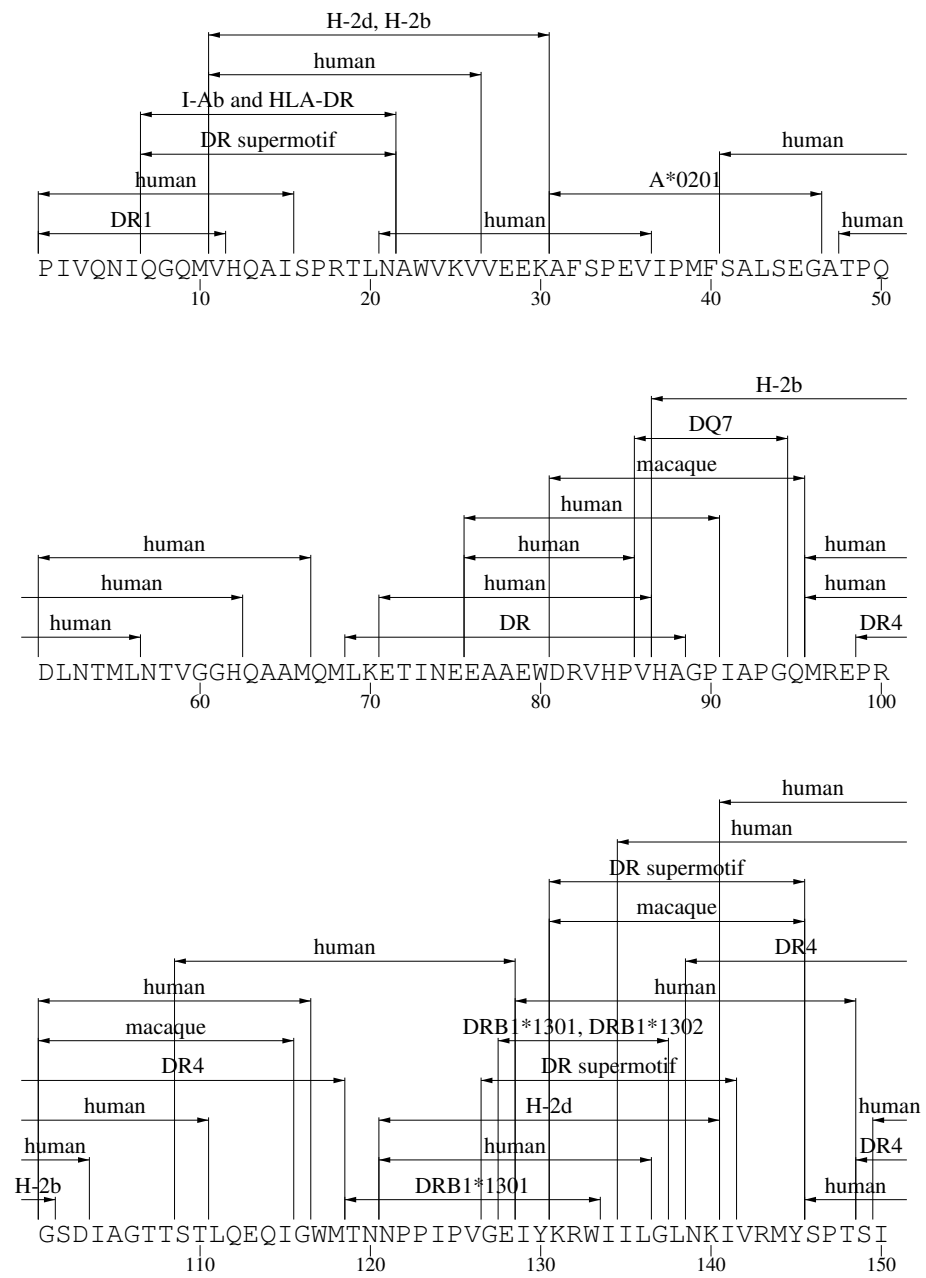

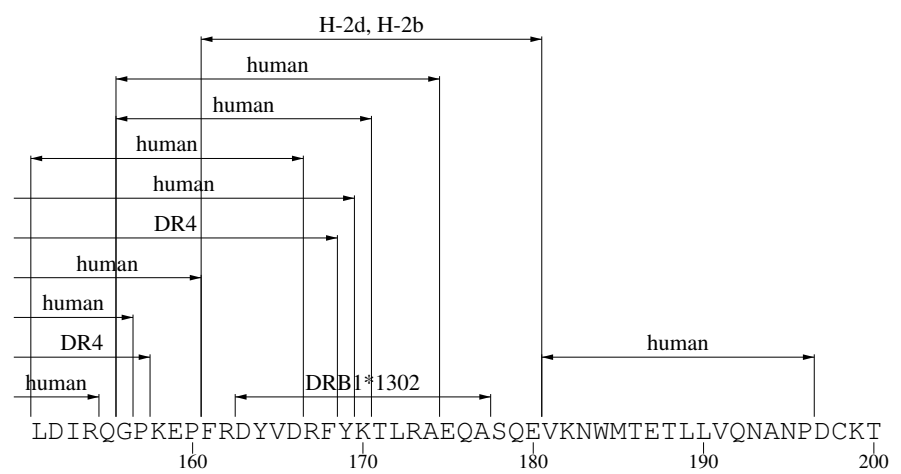

ILKALGPAATLEEMMTACQGVGGP GHKARVI

$$
\begin{array}{lll}
210 & 220 & 230
\end{array}
$$




\section{III-C-3 p2p7p1p6 T-Helper Epitope Map}

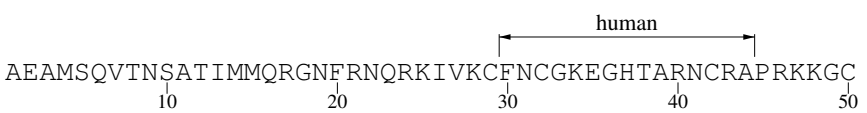
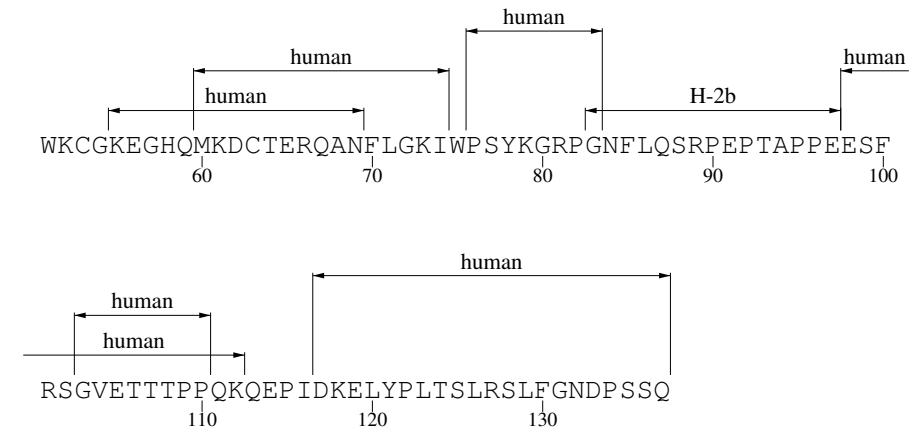

\section{III-C-4 Protease T-Helper Epitope Map}

PQVTLWQRP LVT IKIGGQLKEALLDTGADDTVLEEMSLP GRWKPKMIGG I

GGF IKVRQYDQI L IE ICGHKA I GTVLVGP TPVNI I GRNLLTQI GCT LNF

$60 \quad 70$ 


\section{III-C-5 RT T-Helper Epitope Map}
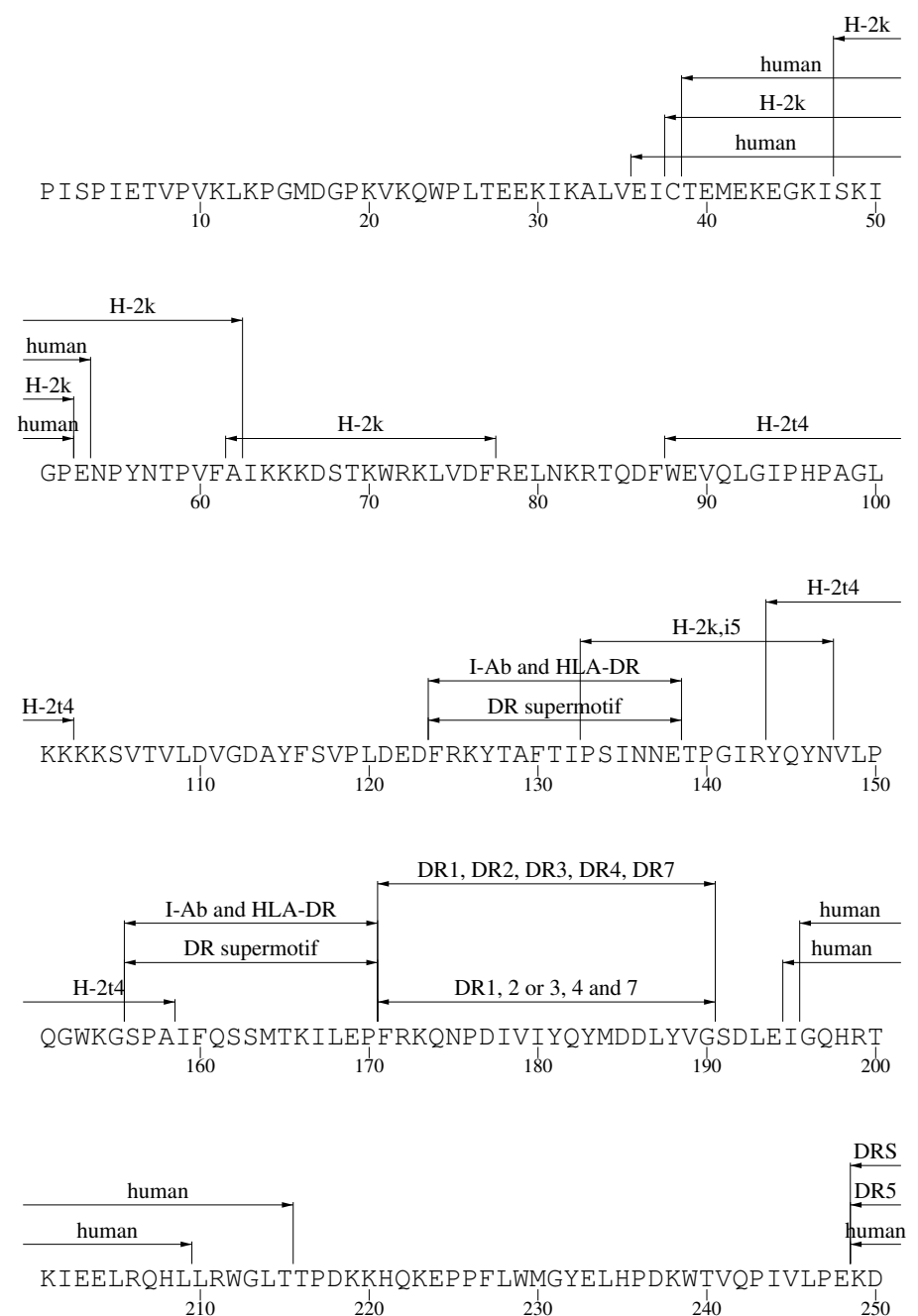
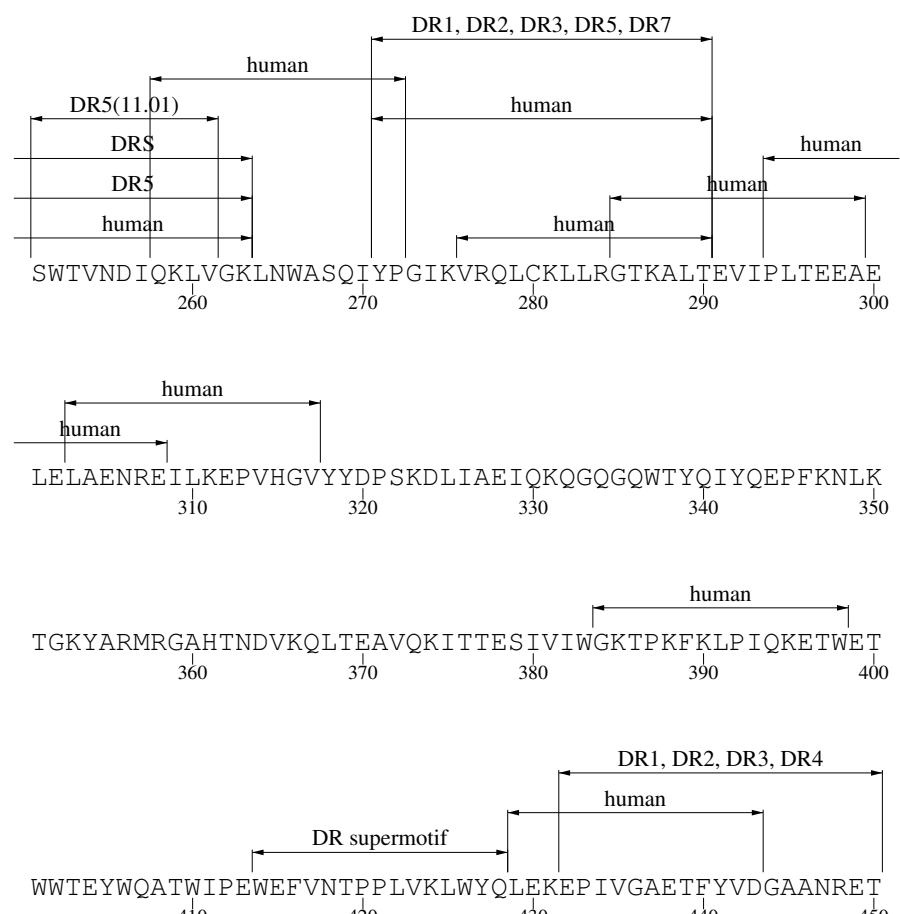

$$
410
$$
KLGKAGYVTNRGRQKVVTLTDTTNQKTELQA IYLALQD SGLEVNIVTD SO
460

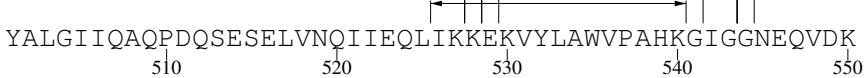

LVSAGIRKVL 


\section{III-C-6 Integrase T-Helper Epitope Map}

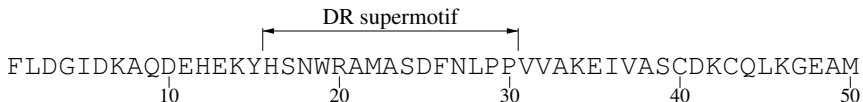
HGQVDCSP GIWQLDCTHLEGKVILVAVHVASGYIEAEVIPAETGQETAYF T0 $\begin{array}{cccc}\text { LLKLAGRWPVKT I HTDNGSNF TGA TVRAACWWAG IKQEF G IPYNP QSQGV } \\ 110 & 120 & 130 & 140\end{array}$ VESMNKELKKI IGQVRDOAEELKTAVOMAVF I hNAFKRKGGIGGYSAGERI $\begin{array}{llll}160 & 170 & 180 & 190\end{array}$

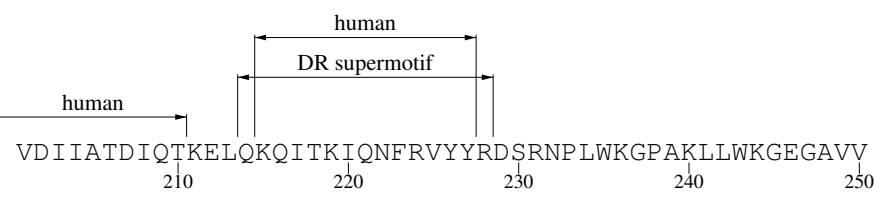
IQDNSD IKVVPRRKAKI IRDYGKQMAGDDCVASRQDED 260

\section{III-C-7 Rev T-Helper Epitope Map}
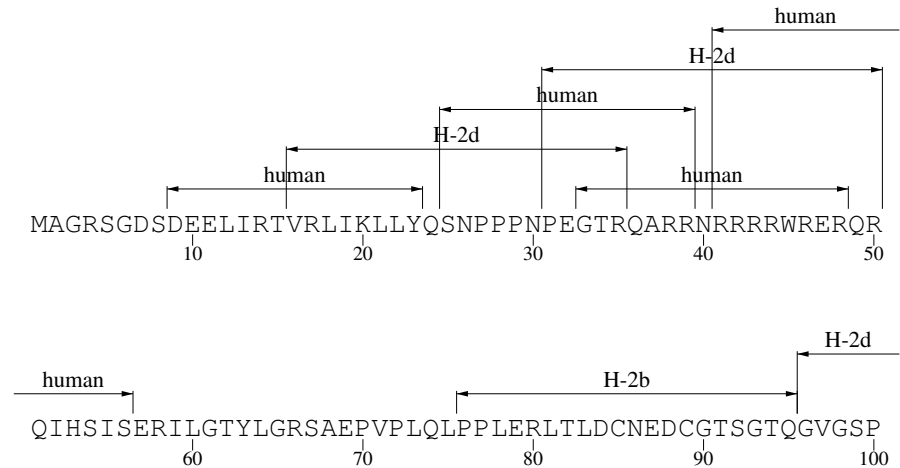

$\frac{\mathrm{H}-2 \mathrm{~d}}{\substack{\text { QILVESPTVLESGTKE } \\ 11_{0}}}$ 


\section{III-C-8 Tat T-Helper Epitope Map}
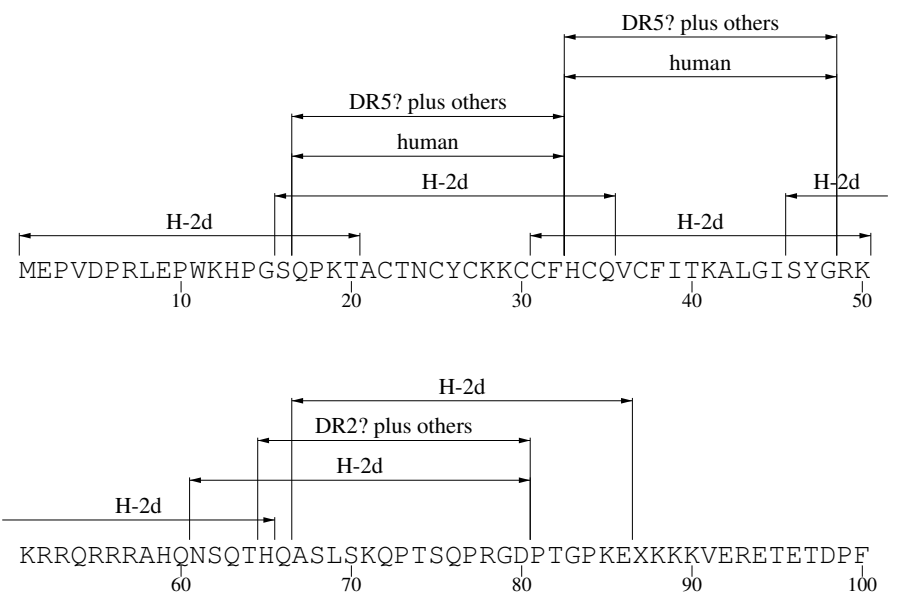

$\underset{\substack{D \\ 101}}{\mathrm{D}}$

\section{III-C-9 Vif T-Helper Epitope Map}

MENRWQVMIVWQVDRMRIRTWKS LVKHHMYVSGKARGWFYRHHYESPHPR

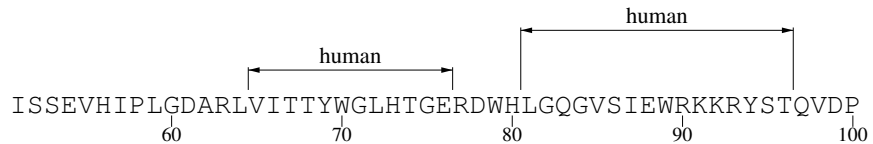

ELADQLIHLYYFDCF SDSAIRKALLGH IVSPRCEYQAGHNKVGSLQYLAL
110
130
140

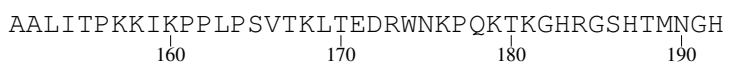




\section{III-C-10 Vpr T-Helper Epitope Map}

MEQAPEDQGP QREP HNEWT LELLEELKNEAVRHFPR R WLHGLGQH I YETY

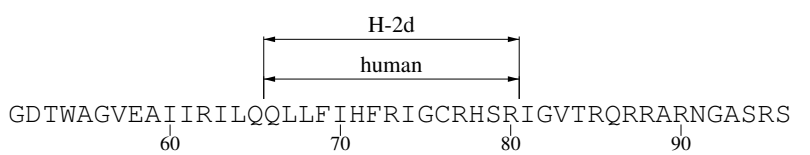

\section{III-C-11 Vpu T-Helper Epitope Map}

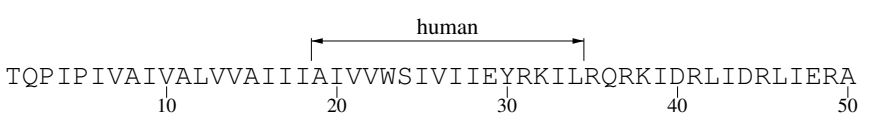

EDSGNESEGEISALVEMGVEMGHHAPWDVDDL

$\begin{array}{lll}1 \\ 60 & 10 & 1\end{array}$ 


\section{III-C-12 gp160 T-Helper Epitope Map}

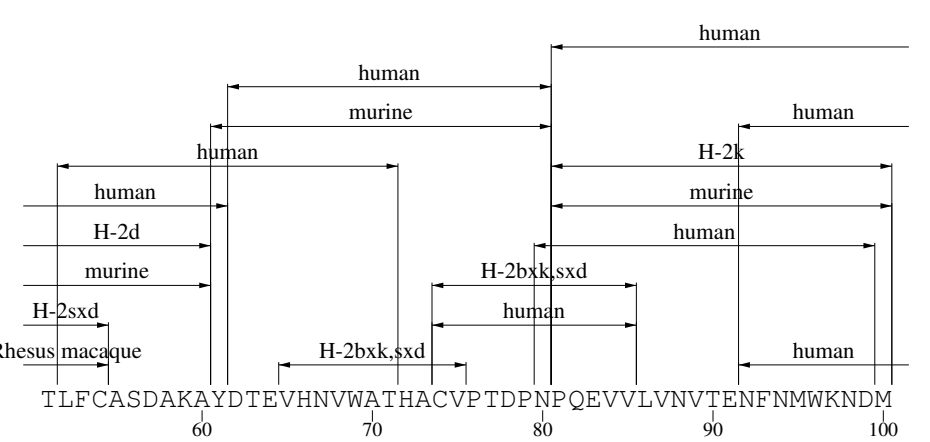

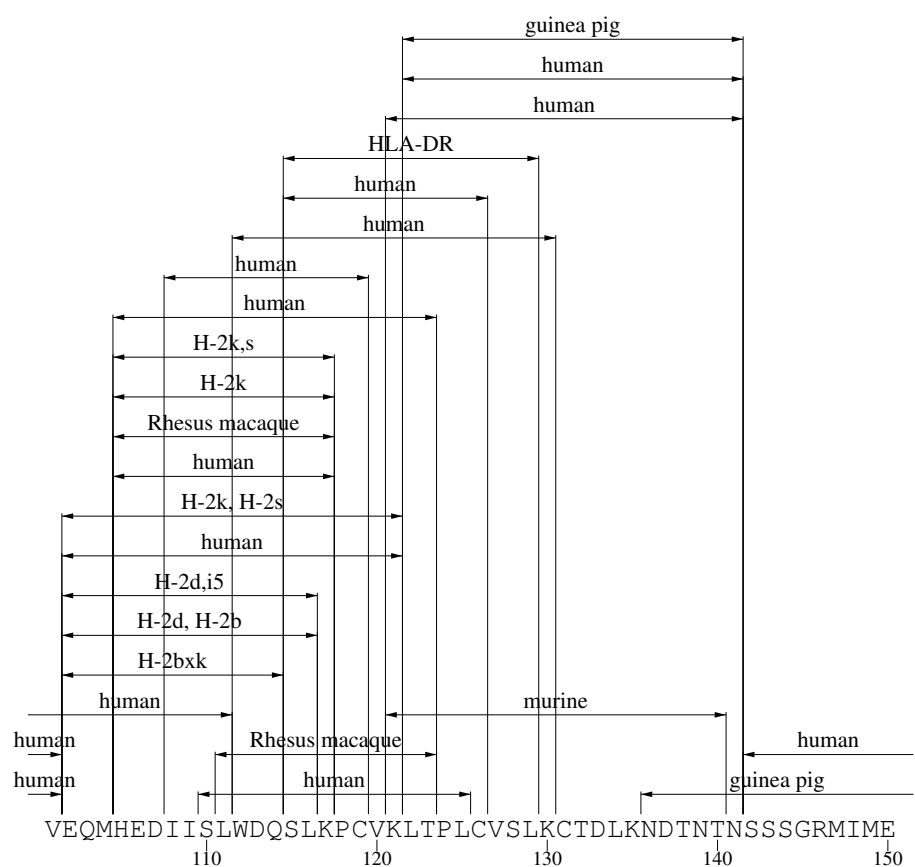

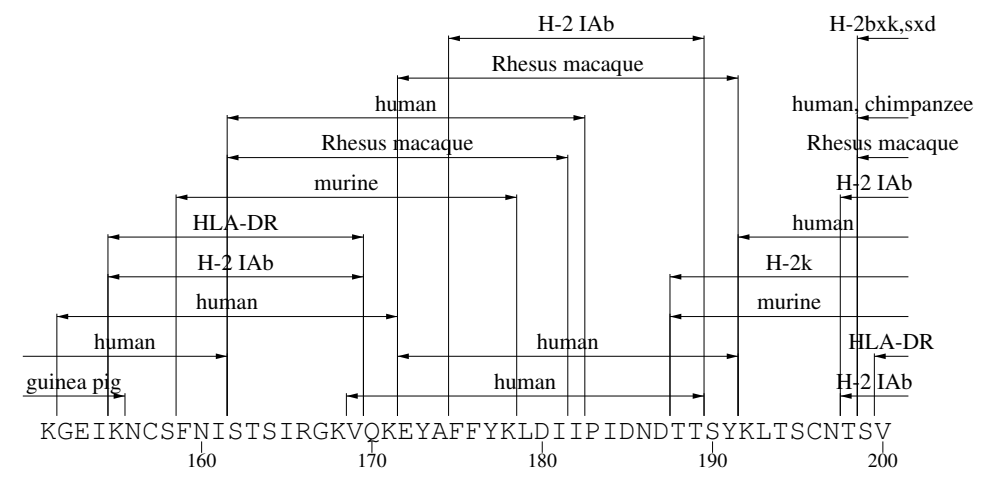



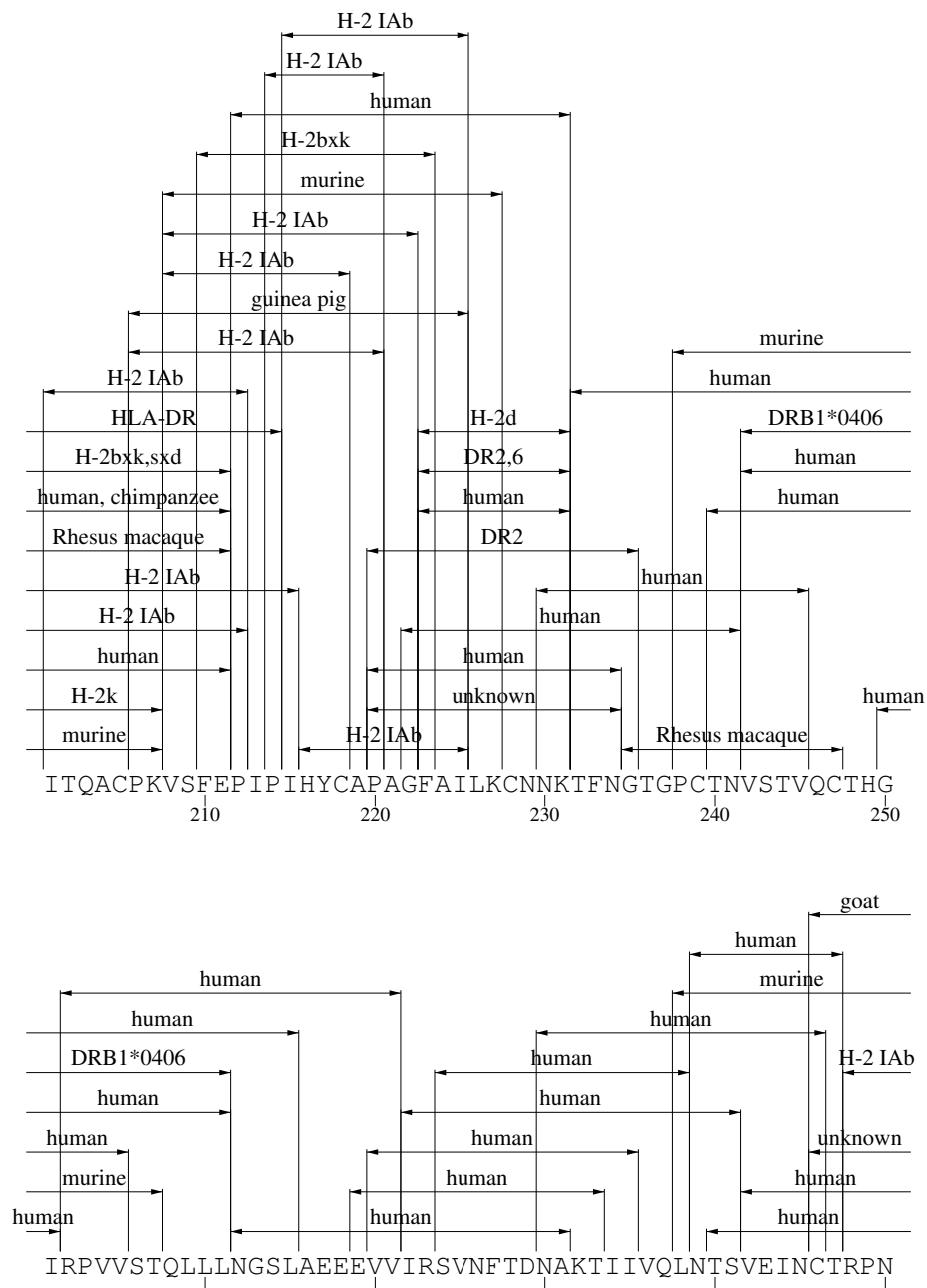

260

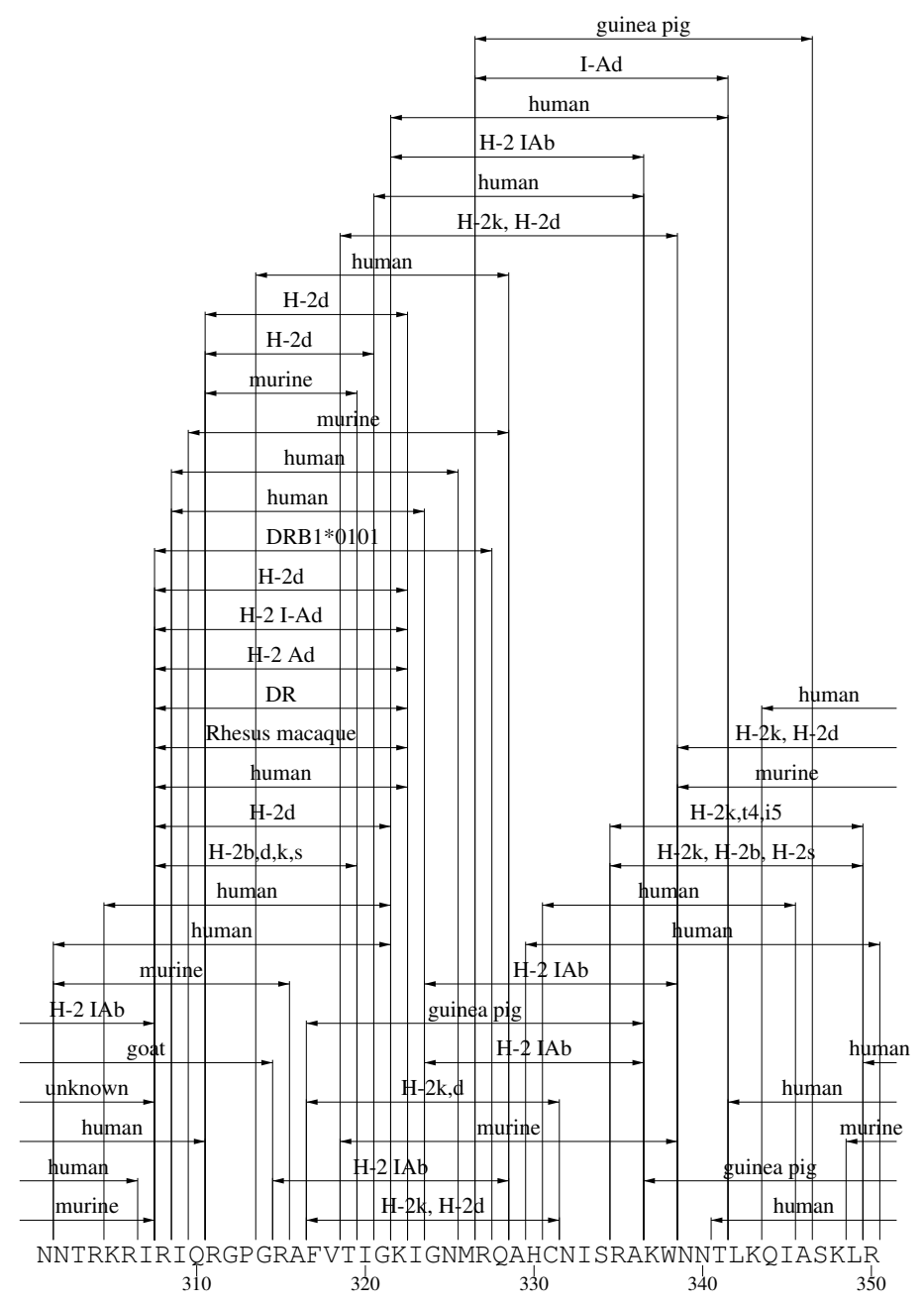



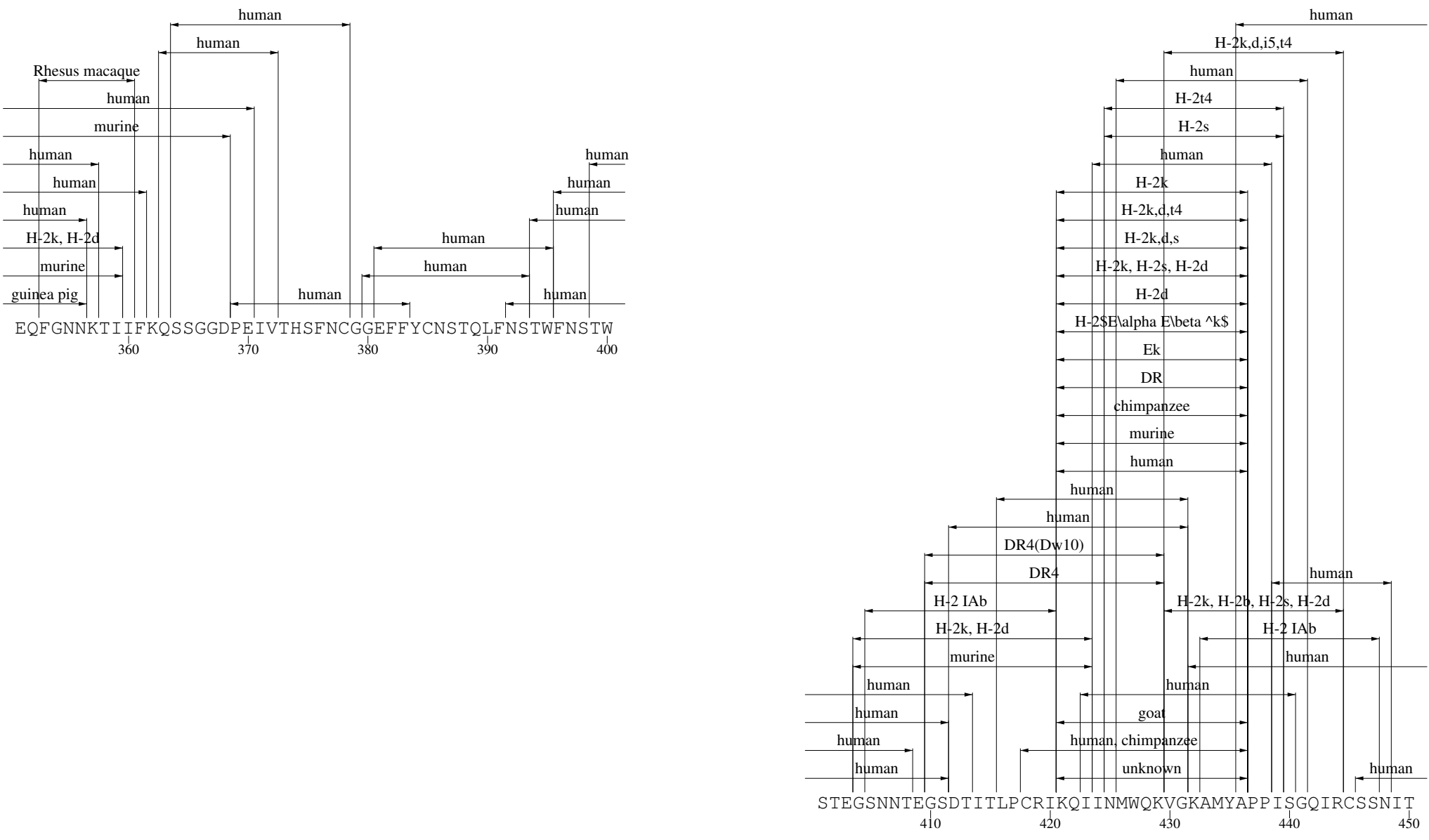

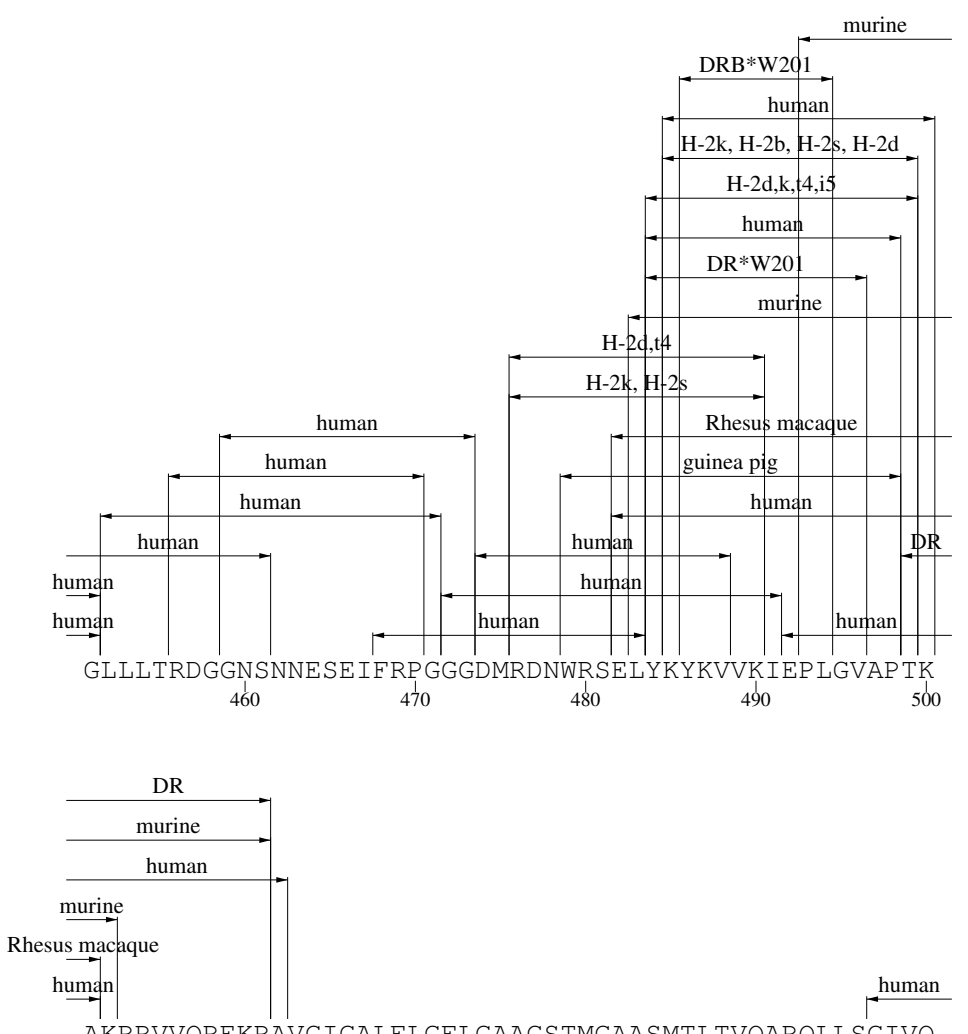

AKRRVVQREKRAVGIGAIFIGEIGAAGSTMGAASMTI TVQARQLISC

$$
520 \quad 530
$$

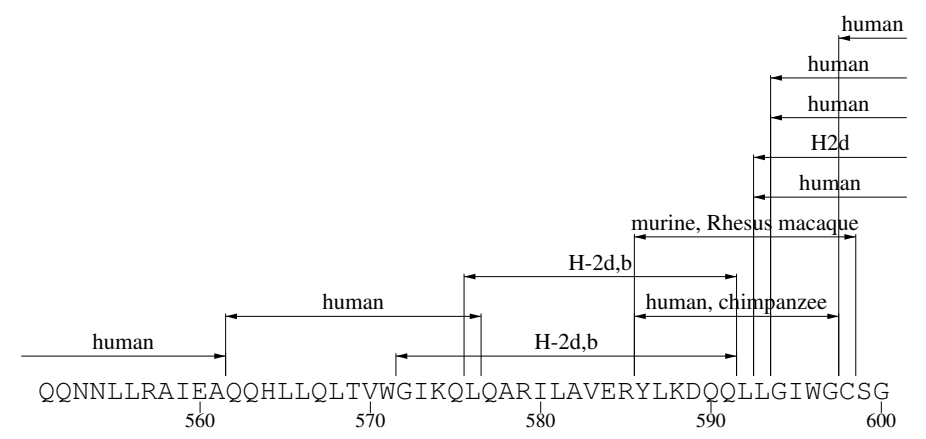

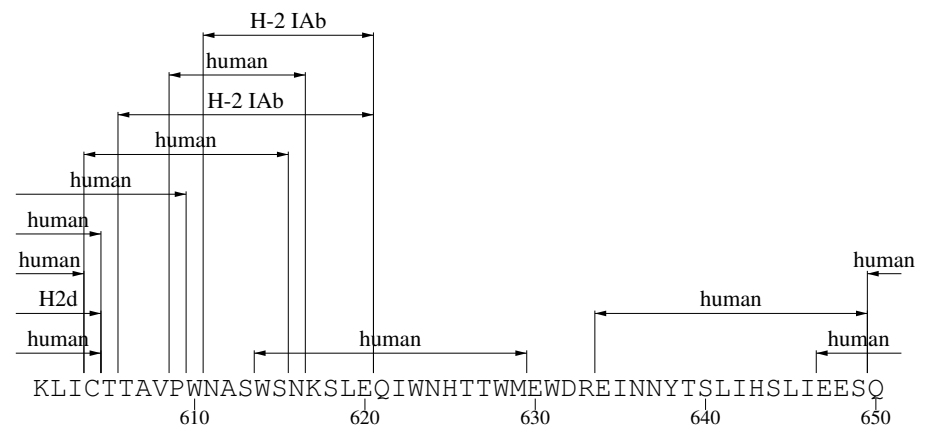

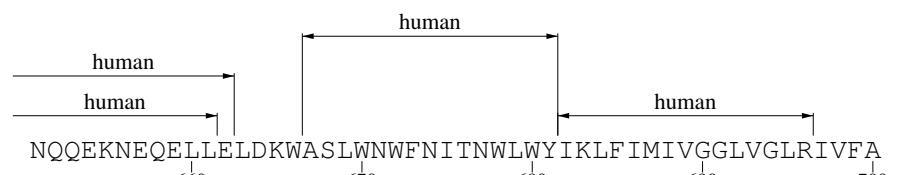

$$
\begin{array}{ccccc}
660 & 670 & 680 & 690 & 700
\end{array}
$$

$$
\text { human }
$$

VLSIVNRVRQGYSPLSFQTHLPTPRGPDRPEGIEEEGGERDRDRS IRLVN

$$
720
$$




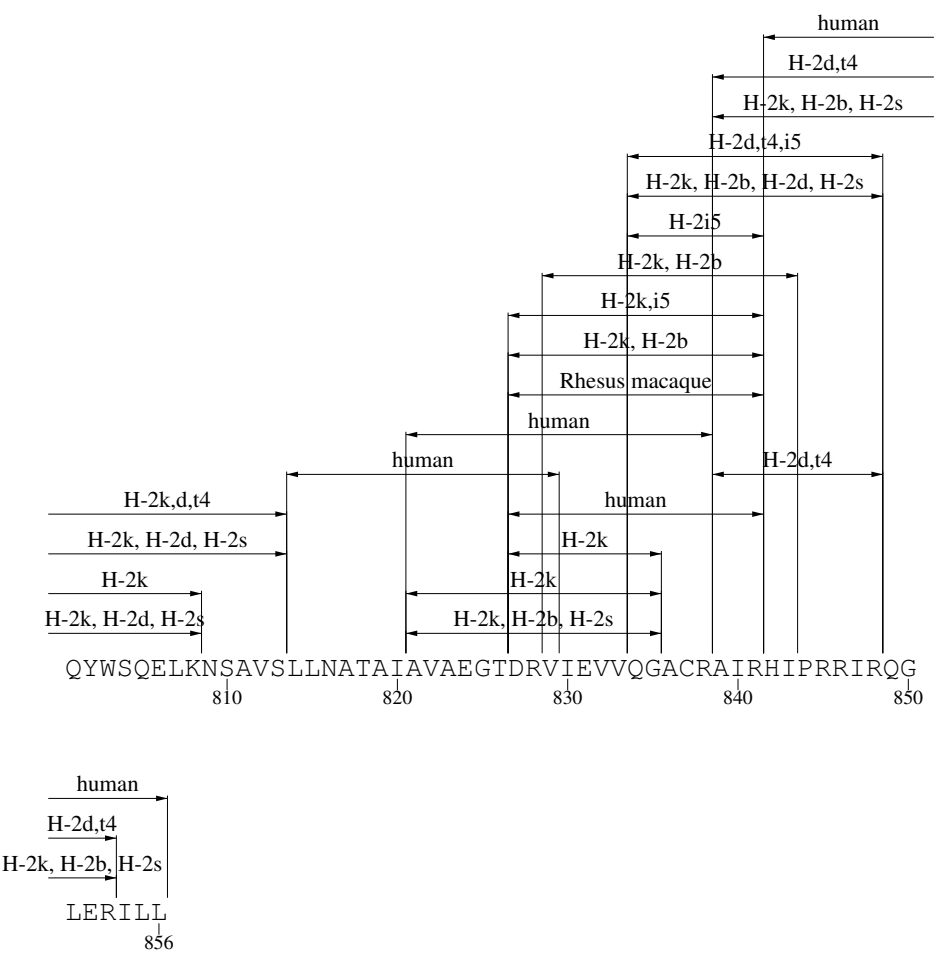

\section{III-C-13 Nef T-Helper Epitope Map}
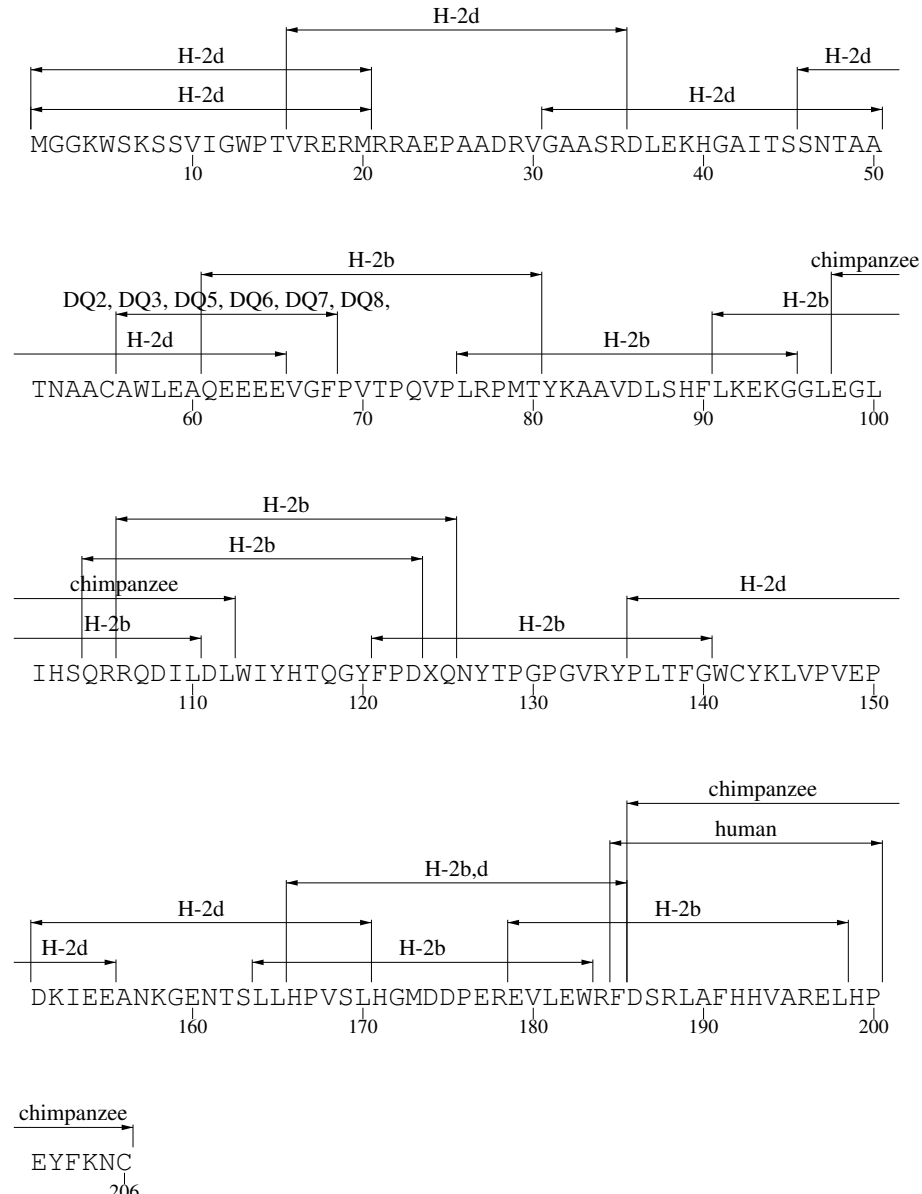


\section{Part IV \\ HIV Antibody Binding Sites}

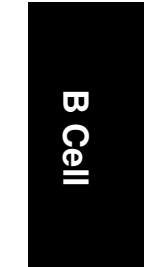





\section{IV-A Summary}

Part [V] section summarizes HIV-specific antibodies (Abs) arranged sequentially according to the location of their binding domain, organized by protein. We attempted to make this section as comprehensive as possible. For the monoclonal (MAbs) capable of binding to linear peptides, we require that the binding site be contained within a region of 30 or so amino acids to define the epitope, but not that the precise boundaries be defined. MAbs that do not bind to defined linear peptides are grouped by category at the end of each protein. Antibody categories, for example CD4 binding site (CD4BS) antibodies, are also noted in the index at the beginning of this section. Studies of polyclonal Ab responses are also included. Responses that are just characterized by binding to a protein, with no known specific binding site, are listed at the end of each protein. For more recent updates, epitope sequence alignments, and search capabilities, please see our web site: http://hiv-web.lanl.gov/immunology

\section{IV-A-1 Indices}

Three indices are provided. The first provides a concise list of anti-HIV-1 MAbs by cross-competition category, with both discontinuous epitopes (for example, CD4BS) and some well known linear epitopes (for example, cluster I) summarized. The second lists the MAb's IDs in alphabetical order so one can find their location in the table. The third is a listing by order of appearance in the tables.

\section{IV-A-2 Tables}

Each MAb has an ten-part basic entry:

Number: Order of appearance in this table.

MAb ID: The name of the monoclonal antibody with synonyms in parentheses. MAbs often have several names. For example, punctuation can be lost and names are often shortened (M-70 in one paper can be M70 in another) Polyclonal responses are listed as "polyclonal" in this field.

HXB2 Location: Position of the Ab binding site relative to the viral strain HXB2 (GenBank Accession Number K03455), which is used as a reference strain throughout this publication. The numbering in this table corresponds to the protein maps. Because of HIV-1 variation the epitope may not actually be present in $\mathrm{HXB} 2$, rather the position in HXB2 indicates the position aligned to the epitope. HXB2 was selected as the reference strain because so many studies use HXB2, and because crystal structures for HXB2-related proteins are often available. The precise positions of an epitope on the HXB2 reference strain can be readily obtained using the interactive position locator at our web site: http://hiv-web. lanl. gov/content/hiv-db/LOCATE_SEQ/locate.html.

Author Location: The amino acid positions of the epitope boundaries and the reference sequence used to define the epitope are listed as given in the primary publication. Frequently, these positions as published are imprecise, and do not truly correspond to the numbering of the sequence, but they provide a reasonable guide to the peptide's approximate location in the protein. Also, in many cases, position numbers were provided but the reference sequence identification was not. Because of HIV-1's variability, position numbers require a reference strain to be meaningful. Binding sites that cannot be defined through peptide binding or interference studies are labeled as discontinuous. The approximate location on the protein, sequence number, and reference sequence are listed.

Sequence: The amino acid sequence of the binding region of interest, based on the reference strain used in the study defining the binding site. On occasions when only the position numbers and not the actual peptide sequence was specified in the original publication, we tried to fill in the peptide sequence based on the position numbers and reference strain. If the sequences were numbered inaccurately by the primary authors, or if we made a mistake in this process, we may have misrepresented the binding site's amino acid sequence. Because of this uncertainty, epitopes that were not explicitly written in the primary publication, that we determined by looking up the reference strain and the numbered location, are followed by a question mark in the table.

Neutralizing: L: neutralizes lab strains. P: neutralizes at least some primary isolates. no: does not neutralize. No information in this field means that neutralization was either not discussed or unresolved in the primary publications referring to the MAb. 
Immunogen: The antigenic stimulus of the original B cell response. Often this is an HIV-1 infection. If a vaccine was used as the original antigenic stimulation, not a natural infection, this is noted on a separate line, and additional information about the vaccine antigen is provided as available.

Species(Isotype): The host that the antibody was generated in, and the isotype of the antibody.

References: All publications that we could find that refer to the use of a specific monoclonal antibody. First is a list of all references. Additional details for some of older references can be found in Part $\mathrm{V}$, although we have tried to keep the entries self-contained since 1997. The "donor" field is meant to serve as a potential guide to a source of information about an antibody or how to obtain it, as well as to provide credit.

Notes: Describe the context of each study, and what was learned about the antibody in the study.

\section{IV-A-3 HIV Protein Binding Site Maps}

The names of MAbs and the location of well characterized linear binding sites of 21 amino acids or less are indicated relative to the protein sequences of the HXB2 clone. This map is meant to provide the relative location of epitopes on a given protein, but the HXB2 sequence may not actually bind to the MAb of interest, as it may vary relative to the sequence for which the epitope was defined. Above each linear binding site, the MAb name is given followed by the species in parentheses. Human is represented by ' $h$ ', non-human primate by 'p', mouse by ' $m$ ', and others by ' $\mathrm{o}$ '. More precise species designations for any given MAb can be found using the web search interface or in the tables in this section.

\section{IV-A-4 Alignments}

To conserve space, no epitope alignments are provided in this book, but they can be generated using the MAb search tool at http://hiv-web.lanl. gov/immunology, All epitopes are aligned to the HXB2 sequence, with the sequence used to define the epitope indicated directly above it. Sequences are sorted by their subtype and country of origin.

The master alignment files from which the epitope alignments were created are available at our web site (http://hiv-web.lanl.gov/ALIGN CURRENT/ALIGN-INDEX.html). The alignments were modified in some cases to optimize the alignment relative to the defined epitope and minimize insertions and deletions; epitope alignments are generated by anchoring on the C-terminal residue. A dash indicates identity to the consensus sequence, and a period indicates an insertion made to maintain the alignment. Stop codons are indicated with a $\$$, and frameshifts by a \#, or ambiguous codons (nucleotide was $r, y$, or $n$ ) by an $x$; they are inserted to maintain the alignments. In consensus sequences an upper case letter indicates the amino acid was present in all sequences, a lower case letter indicates the amino acid was present in most sequences in a given position, and a question mark indicates two or more amino acids were represented with equal frequency. 


\section{IV-B Cross Reference Listing of MAbs}

\section{IV-B-1 MAbs by binding type}

Cross reference by protein and binding type of MAb names and their order of appearance in the tables.

\begin{tabular}{|c|c|}
\hline Binding type & MAb ID (No.) \\
\hline \multicolumn{2}{|l|}{ p17 } \\
\hline C-term & sc-FV p17 33 \\
\hline \multicolumn{2}{|l|}{ p24 } \\
\hline C-term & $13 \mathrm{~B} 5 \sqrt{115}$ \\
\hline \multicolumn{2}{|l|}{ Protease } \\
\hline N-term & $1 6 9 6 \longdiv { 1 7 0 }$ \\
\hline flap region & F11.2.32 172 \\
\hline \multicolumn{2}{|l|}{ RT } \\
\hline palm domain & $6 \mathrm{~B} 9 \sqrt{193}$ \\
\hline thumb domain & 5F (194), 5G $(195), 7 C 4(196)$ \\
\hline \multicolumn{2}{|l|}{ Integrase } \\
\hline $\begin{array}{l}\text { Integrase DNA binding } \\
\text { domain }\end{array}$ & 5D9 213, 2-19 216, 8-22 217, 4-20 218, 6-19 219 \\
\hline Integrase catalytic core & $7-16 \sqrt[210]{ }, 4 \mathrm{~F} 6$ 211 \\
\hline N-term & 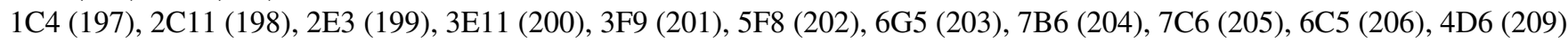 \\
\hline \multicolumn{2}{|r|}{ 型 } \\
\hline C-term & $33(244)$ \\
\hline \multicolumn{2}{|l|}{ Vif } \\
\hline C-term & TG001 246 \\
\hline \multicolumn{2}{|l|}{ Tat } \\
\hline C-term & 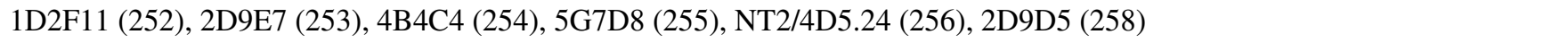 \\
\hline N-term & 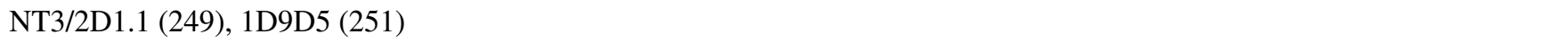 \\
\hline \multicolumn{2}{|l|}{ Env (gp160) } \\
\hline C-domain & $\begin{array}{l}\text { polyclonal 601, 5B2 669, 9G11 670, TH-Ab1 671), polyclonal 672, polyclonal 673, polyclonal 674, polyclonal } \\
675 \text {, }\end{array}$ \\
\hline C-term & $\begin{array}{l}\text { 105-306 (574), 750-D 576), 722-D (580), polyclonal (581), 1131-A (582), 858-D (583), 989-D (584), Z13 (677), } 1575 \text { (698), } \\
\text { polyclonal (702), polyclonal }(703), 1577 \text { (705), polyclonal }(706), 101-342(853), 101-451(854), 120-1 \text { (855) }\end{array}$ \\
\hline
\end{tabular}




\begin{tabular}{|c|c|}
\hline Binding type & MAb ID (No.) \\
\hline $\mathrm{C} 1$ & 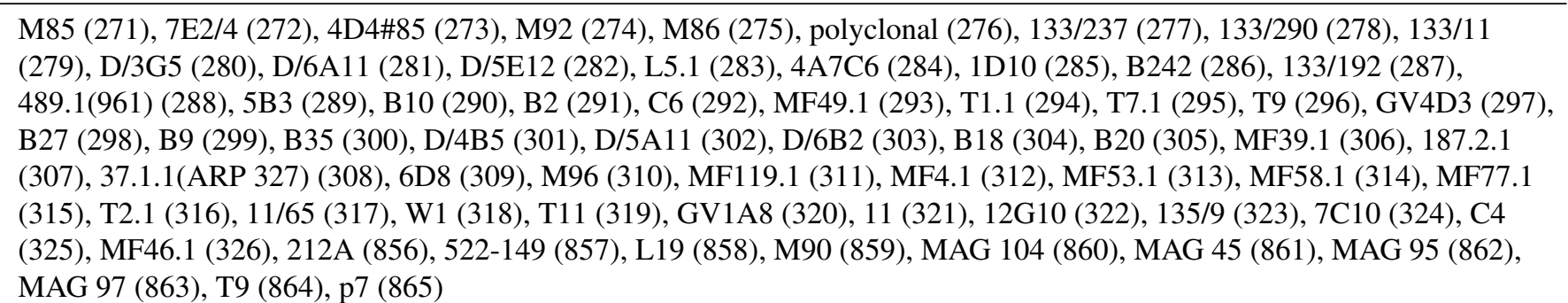 \\
\hline $\mathrm{C} 1-\mathrm{C} 2$ & L100 866 \\
\hline $\mathrm{C} 1-\mathrm{C} 4$ & $2 / 11 \mathrm{c} \sqrt[867]{86}, \mathrm{~A} 32 \sqrt{868}$ \\
\hline $\mathrm{C} 1-\mathrm{C} 5$ & C11 $\sqrt{8690, \text { L81 }} 8$ \\
\hline $\mathrm{C} 2$ & 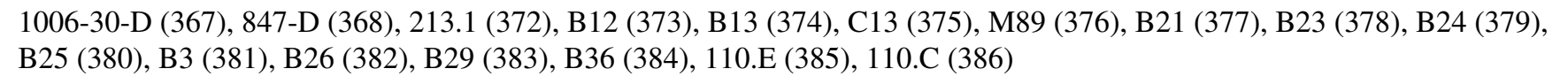 \\
\hline $\mathrm{C} 3$ & 2H1B $340,110 . \mathrm{D} 522$, B32 523, 2F19C 871, B2C 872, polyclonal 873, \\
\hline $\mathrm{C} 3, \mathrm{C} 4$ & ICR38.1a 533) \\
\hline $\mathrm{C} 4$ & 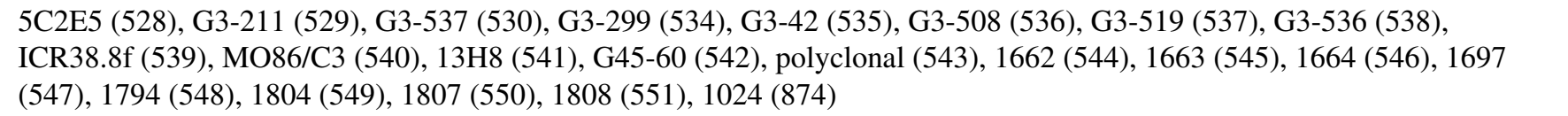 \\
\hline $\mathrm{C} 5$ & 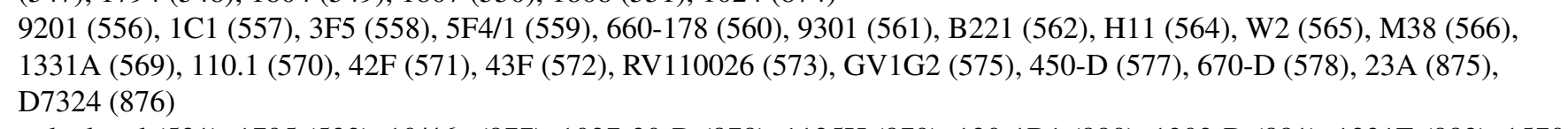 \\
\hline CD4BS & 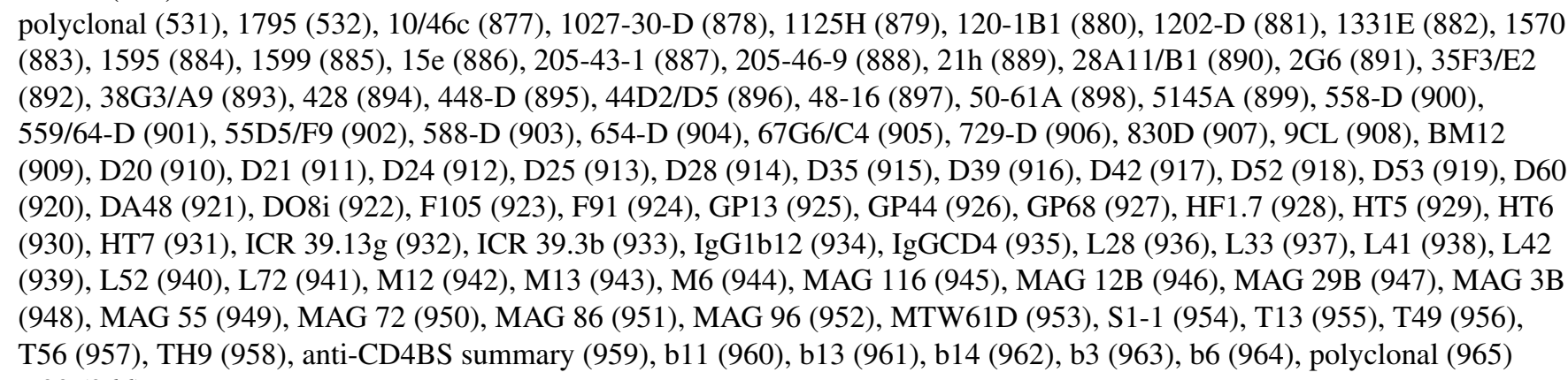 \\
\hline CD4BS, C-term, N-term & D33 966 \\
\hline CD4BS, CD4i, V3, V2 & 967 \\
\hline CD4i & 17b 968, 21c 969, 23e 970, 48d 971, 49e 972, X5 973, \\
\hline Env oligomer & $\mathrm{T} 22(\overrightarrow{974}$ \\
\hline HIV-2 V3 & anti-HIV-2 polyclonal 516 \\
\hline Leucine zipper motif & 593,594 \\
\hline
\end{tabular}




\begin{tabular}{|c|c|}
\hline Binding type & MAb ID (No.) \\
\hline $\begin{array}{l}\text { N-HR, C-HR, and six-helix } \\
\text { bundle }\end{array}$ & polyclonal 975 \\
\hline N-term & polyclonal 602, 2A2 976, , AC4 977, , AD3 978, AD3 979, ID6 980, ID6 981) \\
\hline V1 & $\begin{array}{l}\text { 35D10/D2 (330), 40H2/C7 331, 43A3/E4 332), 43C7/B9 333), 45D1/B7 334], 46E3/E6 335, 58E1/B3 [336, 64B9/A6 } \\
\text { (337), 69D2/A1 (338), 82D3/C3 (339) }\end{array}$ \\
\hline $\mathrm{V} 1, \mathrm{~V} 2, \mathrm{~V} 3, \mathrm{~V} 4, \mathrm{~V} 5$ & polyclonal 552$)$ \\
\hline V1-V2 & 11/68b (982), 62c 983, CRA-6 (984), L15 (985), T52 (986, T54 987) \\
\hline $\mathrm{V} 1-\mathrm{V} 2$ and $\mathrm{V} 3-\mathrm{V} 5$ & polyclonal 988 \\
\hline $\mathrm{V} 2$ & 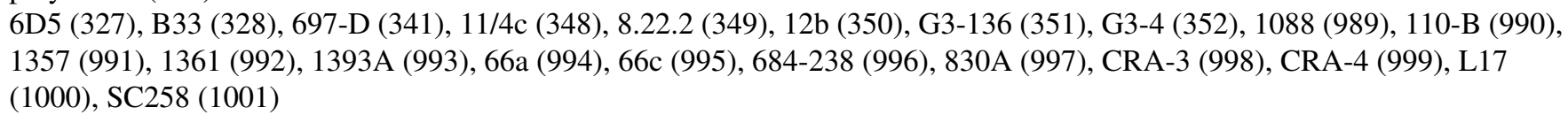 \\
\hline V2-CD4BS & L25 (1002, L39 (1003), L40 (1004), L78 (1005) \\
\hline $\mathrm{V} 3$ & 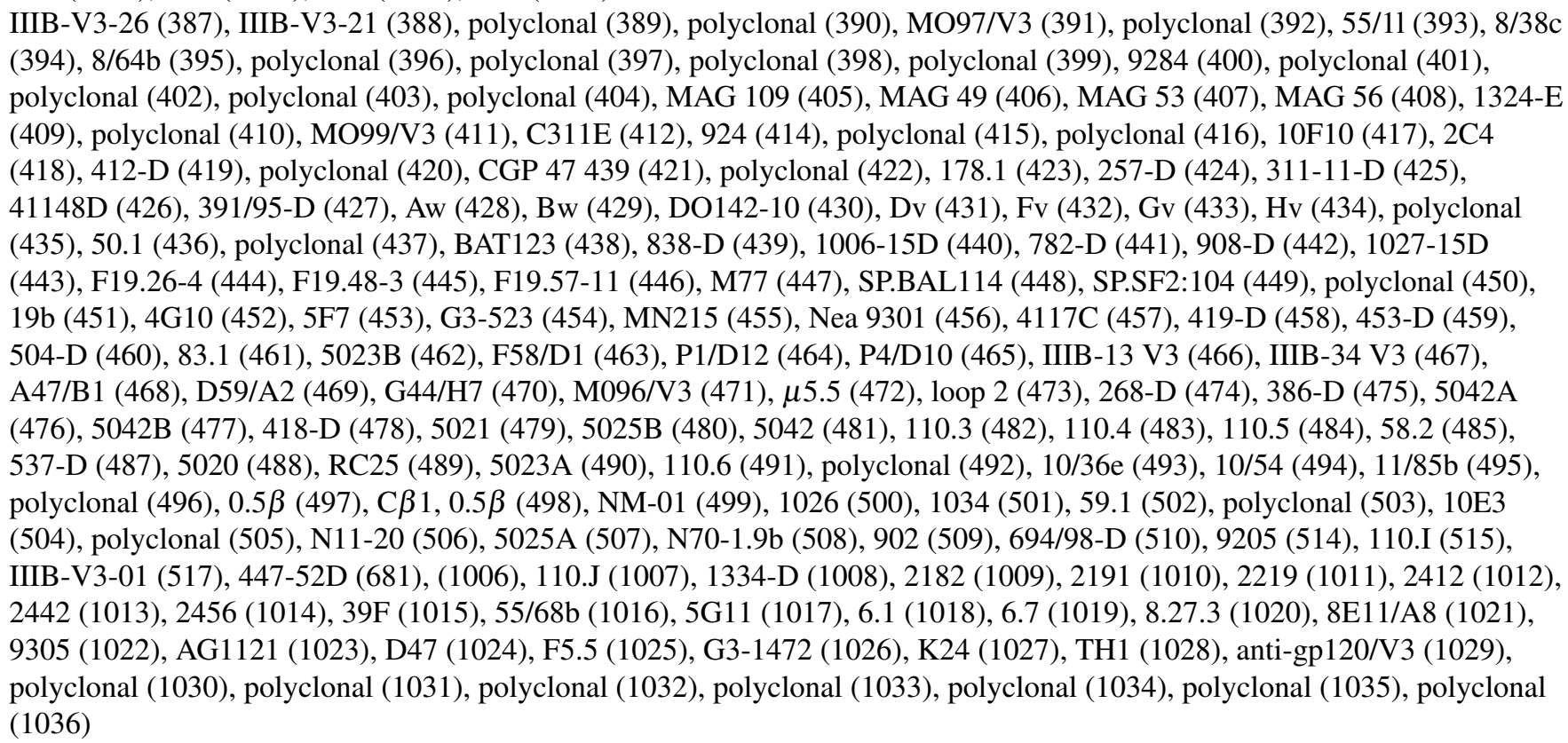 \\
\hline V3 discontinuous & $11 / 75 \mathrm{a} / 21 / 41(1037), 41.1[1038,, 55 / 45 \mathrm{a} / 11$ 1039) \\
\hline V3 mimotope & $1 1 0 8 \longdiv { 1 0 4 0 ) }$ \\
\hline $\mathrm{V} 3, \mathrm{~V} 4$ & polyclonal (1041) \\
\hline $\mathrm{V} 3-\mathrm{C} 4$ & MO101/V3,C4 (511), polyclonal 1042 \\
\hline $\mathrm{V} 3-\mathrm{C} 5$ & MO101/V3,C4 (512), MO101/V3,C4 (513) \\
\hline V3-CD4BS & D27 1043, D56(1044) \\
\hline
\end{tabular}




\begin{tabular}{|c|c|}
\hline Binding type & MAb ID (No.) \\
\hline V4 & D/6D1 $\sqrt{518}), 4 \mathrm{D} 7 / 4 \sqrt{519}), 36.1(\mathrm{ARP} 329) \sqrt{520},, \mathrm{C} 12 \sqrt{521})$, polyclonal $\sqrt{524}), \mathrm{B} 15 \sqrt{525}), \mathrm{B} 34 \sqrt{526})$ \\
\hline V5 & polyclonal (553) \\
\hline V5-C5 & 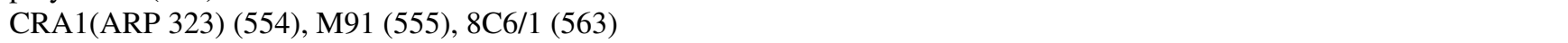 \\
\hline adjacent to cluster II & $2 \mathrm{~F} 5667$ \\
\hline $\begin{array}{l}\text { alpha-helical C-HR, hairpin } \\
\text { intermediate }\end{array}$ & $98-6$ 663 \\
\hline $\begin{array}{l}\text { carbohydrates at glycosylation } \\
\text { residues in } \mathrm{C} 2, \mathrm{C} 3, \mathrm{C} 4 \text {, and } \\
\mathrm{V} 4\end{array}$ & $2 \mathrm{G} 12$ \\
\hline cluster I & 50-69 (605), 246-D (623), 181-D (625), 240-D (627), F240 (628), D49 (629), D61 (630), T32 (631), T34 (632), 1367 (1046) \\
\hline cluster II & $\begin{array}{l}\text { D50 (660), 167-7 (664), ND-15G1 (665), 126-6 (1047), 1342 (1048), } 1379(1049), \text { Fab D11 (1050), Fab D5 (1051), Fab G1 } \\
(1052), \text { Fab M10 (1053), Fab M12 (1054), Fab M15 (1055), Fab S10 (1056), Fab S6 (1057), Fab S8 (1058), Fab S9 (1059), } \\
\text { Fab T3 (1060), Md-1 (1061) }\end{array}$ \\
\hline cluster II, six-helix bundle & 167-D (666), $1 2 8 1 \longdiv { 1 0 6 2 ) }$ \\
\hline cluster III & Fab A9 $(\overrightarrow{1063}$, Fab G15 1064$)$, Fab G5 $(1065)$, Fab L1 (1066), Fab L11 (1067), Fab L2 $\sqrt{1068}$ \\
\hline cytoplasmic domain & Chessie $8 \longdiv { 1 0 6 9 ) }$ \\
\hline gp120-CD4 complex & 8F101 $1070,, 8 F 102 \sqrt{1071}), C G-10 \sqrt{1072})$, CG-25 (1073), CG-4 1074$),$ CG-76 (1075), CG-9 (1076) \\
\hline immunodominant region & $3 \mathrm{D} 6(658), 105-518$ (1077) \\
\hline p24+gp41 & 31A1 1078, 39A64 1079), 39B86 (1080, $9303 \sqrt{1081)}$ \\
\hline six helix bundle & NC-1 (1082) \\
\hline Nef & \\
\hline C-term & AE6 1116, AG11 1117, EH1 (1118), AE6 1123) \\
\hline
\end{tabular}




\section{IV-B-2 Alphabetical listing of MAbs}

Cross reference of MAb 1025 names and their order of ap- 1026 pearance in the tables. Al- 1027-15D phanumeric sorting is sym- 1027-30-D bols, digits, uppercase letters 1034 and lowercase letters.

MAb ID

$\alpha(566-586)$

$\mu 5.5$

$0.5 \beta$

$1-\mathrm{B}-7$

$1-\mathrm{E}-4$

$1-\mathrm{E}-9$

$1.152 \mathrm{~B} 3$

$1.153 \mathrm{G} 10$

$1.158 \mathrm{E} 2$

$160 \mathrm{~B} 3$

1.17 .3

1.2

$10-\mathrm{E}-7$

$10-\mathrm{G}-9$

10.1

$10 / 36 \mathrm{e}$

$10 / 46 \mathrm{c}$

$10 / 76 b$

1006-15D

1006-30-D

101-342

101-451

102-135

1024

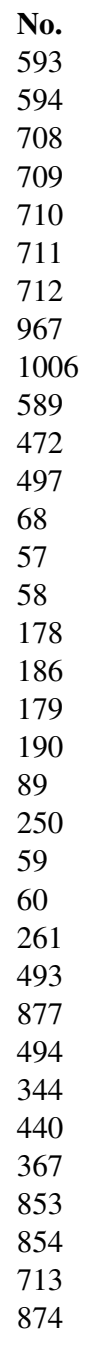

\begin{tabular}{|c|c|}
\hline 714 & $111 / 073$ \\
\hline 500 & $111 / 182$ \\
\hline 443 & $112 / 021$ \\
\hline 878 & $112 / 047$ \\
\hline 501 & $1125 \mathrm{H}$ \\
\hline 715 & $113 / 038$ \\
\hline 574 & $113 / 072$ \\
\hline 1077 & $1131-\mathrm{A}$ \\
\hline 657 & 115.8 \\
\hline 116 & $11 \mathrm{C} 10 \mathrm{~B} 10$ \\
\hline 110 & 11D11F2 \\
\hline 989 & $11 \mathrm{H} 9$ \\
\hline 504 & 12 \\
\hline \begin{tabular}{|l|}
171 \\
\end{tabular} & $12-\mathrm{B}-4$ \\
\hline 716 & $120-1$ \\
\hline 417 & $120-16$ \\
\hline 321 & 120-1B1 \\
\hline 61 & 1202-D \\
\hline 345 & $126-50$ \\
\hline 346 & $126-6$ \\
\hline 348 & 1281 \\
\hline 317 & 12G-A8g2 \\
\hline 982 & 12G-D7h11 \\
\hline 1037 & 12G-H1c7 \\
\hline 495 & $12 \mathrm{G} 10$ \\
\hline $9 \overline{990}$ & 12H-D3b3 \\
\hline 363 & $12 \mathrm{H} 2$ \\
\hline 570 & 12I-D12g2 \\
\hline 482 & $12 b$ \\
\hline $4 \overline{483}$ & 13 \\
\hline $4 \overline{484}$ & $13-102-100$ \\
\hline 491 & 13.10 \\
\hline 386 & $13 / 035$ \\
\hline 522 & $13 / 042$ \\
\hline $3 \overline{385}$ & $13 / 058$ \\
\hline 515 & 1324-E \\
\hline 1007 & $133 / 11$ \\
\hline 111 & $133 / 192$ \\
\hline 1040 & $133 / 237$ \\
\hline 49 & $133 / 290$ \\
\hline 46 & $1331 \mathrm{~A}$ \\
\hline
\end{tabular}

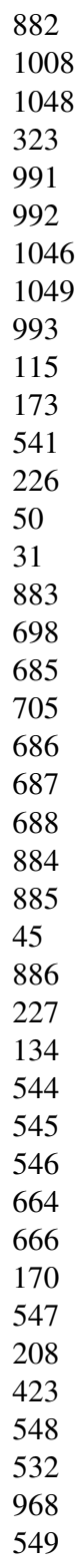

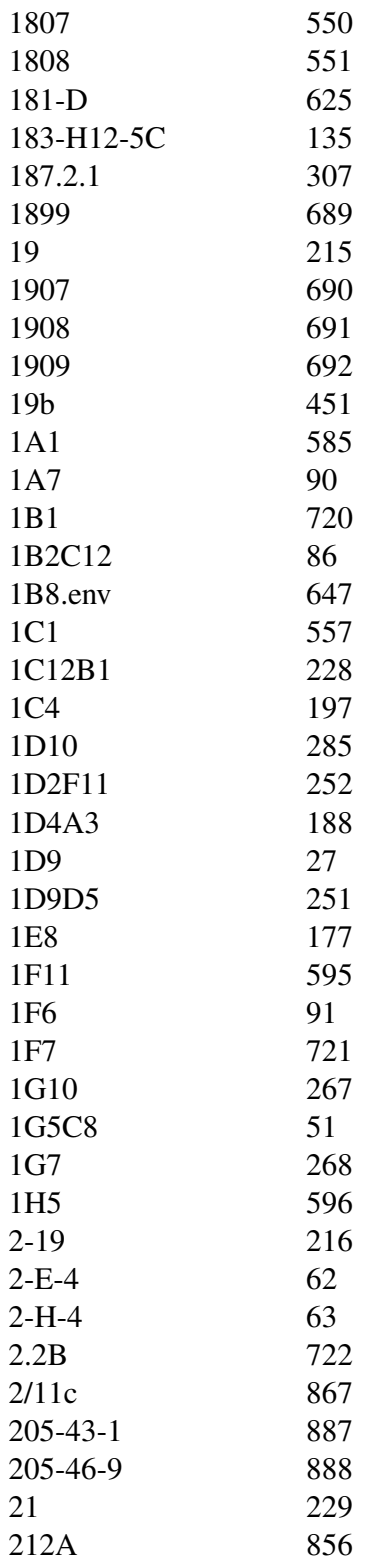

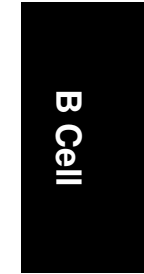




\begin{tabular}{|c|c|c|c|c|c|c|c|c|c|}
\hline 213.1 & 372 & $2 \mathrm{H} 12$ & 1111 & $3 \mathrm{D} 12$ & 1092 & 448-D & 895 & $5145 \mathrm{~A}$ & 899 \\
\hline 2182 & \begin{tabular}{|l|l|}
1009 \\
\end{tabular} & $2 \mathrm{H} 1 \mathrm{~B}$ & 340 & $3 \mathrm{D} 3$ & 43 & 44D2/D5 & 896 & $522-149$ & 857 \\
\hline 2191 & $\begin{array}{l}1010 \\
\end{array}$ & $3-B-7$ & 69 & 3D3.B8 & $3 \overrightarrow{360}$ & $450-\mathrm{D}$ & 577 & $52 \mathrm{G} 5 / \mathrm{B} 9$ & 731 \\
\hline $21 \mathrm{c}$ & 969 & $3-\mathrm{H}-7$ & 25 & 3D5 & 727 & 453-D & 459 & 537-D & 487 \\
\hline $21 \mathrm{~h}$ & 889 & $30: 3 \mathrm{E} 5$ & 83 & 3D6 & 658 & 45D1/B7 & 334 & $55 / 11$ & 393 \\
\hline 2219 & |011 & $30 \mathrm{D}$ & 723 & 3D9 & 597 & 46E3/E6 & 335 & $55 / 45 \mathrm{a} / 11$ & 1039 \\
\hline $23 \mathrm{~A}$ & 875 & $31-11$ & 32 & $3 \mathrm{E} 11$ & 13 & $47-2$ & 52 & $55 / 68 b$ & 1016 \\
\hline $23 \mathrm{~A} 5 \mathrm{G} 4$ & 92 & $31 / 03$ & 1101 & 3E11 & 200 & $48-16$ & 897 & 558-D & 900 \\
\hline 23A5G5 & 93 & 311-11-D & 425 & 3E6 & $\overrightarrow{1108}$ & 489.1(961) & 288 & 559/64-D & 901 \\
\hline $23 \mathrm{e}$ & 970 & $31710 \mathrm{~B}$ & 724 & $3 \mathrm{~F} 10$ & 233 & $48 \mathrm{~d}$ & 971 & 55D5/F9 & 902 \\
\hline $240-D$ & 627 & $31 \mathrm{~A} 1$ & 1078 & $3 \mathrm{~F} 2$ & $\overrightarrow{1091}$ & 493-156 & 362 & $55 \mathrm{E} 4 / \mathrm{H} 1$ & 732 \\
\hline 241-D & 136 & 31D6 & 180 & $3 \mathrm{~F} 5$ & 558 & 49B11/A1 & 730 & $56 \mathrm{C} 4 / \mathrm{C} 8$ & 733 \\
\hline 2412 & 1012 & $31 \mathrm{G} 8$ & 181 & $3 \mathrm{~F} 9$ & 201 & $49 \mathrm{e}$ & 972 & 57B6/F1 & 734 \\
\hline 2442 & 1013 & 32 & 230 & $3 \mathrm{G} 12$ & 1095 & $4 \mathrm{~A} 7 \mathrm{C} 6$ & 284 & $57 \mathrm{H} 5 / \mathrm{D} 7$ & 735 \\
\hline 2456 & \begin{tabular}{|l|}
1014 \\
\end{tabular} & $32 / 1.24 .89$ & 11 & $3 \mathrm{G} 4$ & 266 & $4 \mathrm{~B} 3$ & 598 & 58.2 & 485 \\
\hline 246-D & 623 & $32 / 5.8 .42$ & 3 & $3 \mathrm{H} 6$ & 262 & $4 \mathrm{~B} 4 \mathrm{C} 4$ & 254 & 588-D & 903 \\
\hline $24 \mathrm{G} 3$ & 586 & $32 / 5.8 .42$ & 4 & $3 \mathrm{H} 6$ & 728 & 4C11.D8 & 361 & 58E1/B3 & 336 \\
\hline 25.3 & 75 & $322-151$ & 359 & 4 & 234 & $4 C 9$ & 28 & 59.1 & 502 \\
\hline $25 / 03$ & 1089 & $32: 32 \mathrm{~K}$ & 112 & 4 & 650 & $4 \mathrm{D} 4$ & 599 & $5 \mathrm{~B} 2$ & \begin{tabular}{|l|l|}
184 \\
\end{tabular} \\
\hline 257-D & 424 & $32 \mathrm{E} 7$ & 182 & $4-20$ & 218 & 4D4\#85 & 273 & $5 \mathrm{~B} 2$ & 669 \\
\hline $25 \mathrm{C} 2$ & 587 & 33 & 244 & $406 / 01$ & 78 & 4D6 & 209 & $5 \mathrm{~B} 3$ & 289 \\
\hline $26 / 028$ & 1097 & 33D5 & 183 & 40D3/C11 & 729 & $4 \mathrm{D} 7 / 4$ & 519 & $5 \mathrm{C} 2 \mathrm{E} 5$ & 528 \\
\hline $26 / 76$ & 1090 & 35 & 231 & $40 \mathrm{H} 2 / \mathrm{C} 7$ & 331 & $4 \mathrm{E} 10$ & 676 & 5D9 & 213 \\
\hline 268-D & 474 & $35 \mathrm{D} 10 / \mathrm{D} 2$ & 330 & $41-1$ & 608 & $4 \mathrm{~F} 6$ & 211 & 5E2.A3k & $1 \overline{138}$ \\
\hline 28A11/B1 & 890 & $35 \mathrm{~F} 3 / \mathrm{E} 2$ & 892 & $41-1$ & 693 & $4 \mathrm{G} 10$ & 452 & $5 \mathrm{~F}$ & 194 \\
\hline $2 \mathrm{~A} 2$ & 976 & 36.1(ARP 329) & 520 & $41-2$ & 694 & $4 \mathrm{G} 2$ & 600 & $5 \mathrm{~F} 3$ & 588 \\
\hline $2 \mathrm{~A} 2 / 26$ & 604 & 37.1.1(ARP 327) & 308 & $41-3$ & 695 & $4 \mathrm{G} 9$ & 259 & $5 \mathrm{~F} 4 / 1$ & 559 \\
\hline $2 \mathrm{~A} 3$ & 1109 & $38 / 12 b$ & 356 & $41-6$ & 651 & $4 \mathrm{H} 2 \mathrm{~B} 1$ & 29 & $5 \mathrm{~F} 7$ & 453 \\
\hline $2 \mathrm{~A} 6$ & 137 & $38 / 60 b$ & 357 & $41-7$ & 652 & $4 \mathrm{H} 4$ & 1083 & $5 \mathrm{~F} 8$ & 202 \\
\hline $2 \mathrm{C} 11$ & 198 & $386-\mathrm{D}$ & 475 & 41.1 & 1038 & $5-21-3$ & 661 & $5 \mathrm{G}$ & 195 \\
\hline $2 \mathrm{C} 4$ & 418 & $38: 9.6 \mathrm{~K}$ & 80 & 41.4 & 609 & $50-61 \mathrm{~A}$ & 898 & $5 \mathrm{G} 11$ & 1017 \\
\hline 2D9D5 & 258 & $38 \mathrm{~B} 5 / \mathrm{C} 9$ & 725 & 41148D & 426 & $50-69$ & 605 & 5G7D8 & 255 \\
\hline 2D9E7 & 253 & 38G3/A9 & 893 & $4117 \mathrm{C}$ & 457 & 50.1 & 436 & $6-19$ & 219 \\
\hline $2 \mathrm{E} 3$ & 199 & 391/95-D & 427 & 412-D & 419 & 5020 & 488 & 6-D-12 & 70 \\
\hline $2 \mathrm{E} 3$ & 1098 & 39A64 & $\overrightarrow{1079}$ & 418-D & 478 & 5021 & 479 & $6-E-7$ & 71 \\
\hline $2 \mathrm{E} 4$ & 1110 & $39 \mathrm{~B} 86$ & $\overline{1080}$ & 419-D & 458 & $5023 \mathrm{~A}$ & 490 & 6.1 & $\overrightarrow{1018}$ \\
\hline $2 \mathrm{~F} 11$ & 622 & $39 \mathrm{~F}$ & $\overline{1015}$ & $41 \mathrm{~S}-2$ & 680 & $5023 \mathrm{~B}$ & 462 & 6.1 & $\overline{1119}$ \\
\hline $2 \mathrm{~F} 19 \mathrm{C}$ & 871 & 39H10/A11 & 726 & 428 & 894 & $5025 \mathrm{~A}$ & 507 & 6.7 & $\overline{1019}$ \\
\hline $2 \mathrm{~F} 2$ & 1106 & $3 \mathrm{~A} 2$ & $\overline{1112}$ & $42 \mathrm{~F}$ & 571 & $5025 \mathrm{~B}$ & 480 & $60 \mathrm{~b}$ & 354 \\
\hline $2 \mathrm{~F} 5$ & 667 & $3 \mathrm{~A} 6$ & 35 & $43 \mathrm{~A} 3 / \mathrm{E} 4$ & 332 & 504-D & 460 & $62 c$ & 983 \\
\hline $2 \mathrm{G} 12$ & 1045 & $3 \mathrm{~B} 10$ & 12 & $43 \mathrm{C} 7 / \mathrm{B} 9$ & 333 & 5042 & 481 & $63 \mathrm{G} 4 / \mathrm{E} 2$ & 736 \\
\hline $2 \mathrm{G} 2$ & 270 & 3D10G6 & 94 & $43 \mathrm{~F}$ & 572 & $5042 \mathrm{~A}$ & 476 & 64B9/A6 & 337 \\
\hline 2G6 & 891 & 3D12 & 232 & $447-52 \mathrm{D}$ & 681 & $5042 B$ & 477 & 654-D & 904 \\
\hline
\end{tabular}




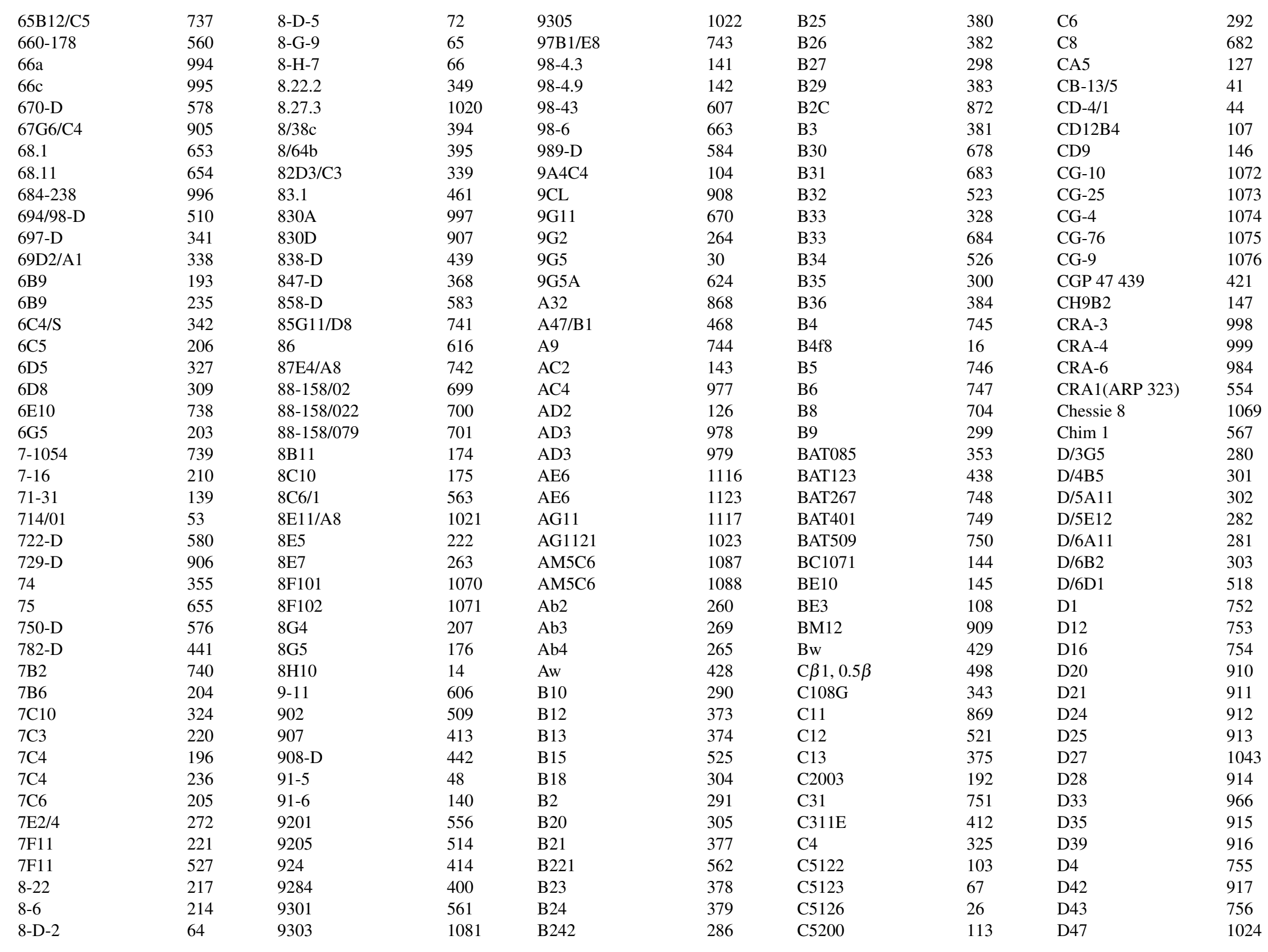




\begin{tabular}{|c|c|c|c|c|c|c|c|c|c|}
\hline D49 & 629 & F91 & 924 & G3-523 & 454 & IIIB-13 V3 & 466 & M-11 & 635 \\
\hline D50 & 60 & FC12 & $\overline{130}$ & G3-536 & 538 & IIIB-34 V3 & 467 & M-13 & 636 \\
\hline D52 & 918 & $\mathrm{FF} 1$ & 73 & G3-537 & 530 & IIIB-V3-01 & 517 & M-2 & 637 \\
\hline D53 & 919 & FH2 & 114 & $\mathrm{G} 44 / \mathrm{H} 7$ & 470 & IIIB-V3-21 & 388 & M-22 & 638 \\
\hline D56 & 1044 & Fab A1 & 610 & G45-60 & 542 & IIIB-V3-26 & 387 & M-24 & 639 \\
\hline D59/A2 & 469 & Fab A12 & $7 \overrightarrow{760}$ & GE4 & 131 & IVI-4G6 & 769 & M-25 & 640 \\
\hline D60 & 920 & Fab A2 & 761 & GP13 & 925 & IgG1b12 & 934 & M-28 & 641 \\
\hline D61 & 630 & Fab A4 & 611 & GP44 & 926 & IgGCD4 & 935 & M-29 & 642 \\
\hline D7324 & 876 & Fab A9 & 1063 & GP68 & 927 & $\mathrm{~J} 1$ & 365 & M-36 & 643 \\
\hline DA48 & 921 & Fab D11 & 1050 & GV1A8 & 320 & $\mathrm{~J} 3$ & 366 & M-4 & 644 \\
\hline DF3 & 128 & Fab D5 & $\overline{1051}$ & GV1G2 & 575 & $\mathrm{~J} 4$ & 247 & M-6 & 645 \\
\hline DG8 & 123 & Fab G1 & 1052 & GV4D3 & 297 & JB7 & 132 & M096/V3 & 471 \\
\hline DO142-10 & 430 & Fab G15 & $\overline{1064}$ & GV4H3 & 364 & JF11 & 133 & M12 & 122 \\
\hline DO8i & 922 & Fab G5 & 1065 & $\mathrm{Gv}$ & 433 & K14 & 770 & M12 & 942 \\
\hline $\mathrm{DZ}$ & 707 & Fab L1 & 1066 & H11 & 564 & $\mathrm{~K} 24$ & 1027 & M13 & 943 \\
\hline Dv & 431 & Fab L11 & 1067 & $\mathrm{H} 2$ & 765 & L-anti-Tat & 257 & M25 & 771 \\
\hline E7 & 1115 & Fab L2 & $\overline{1068}$ & $\mathrm{H} 8$ & $\overline{766}$ & L100 & 866 & M38 & 566 \\
\hline E9 & 1107 & Fab L9 & 762 & HBW4 & 767 & L14 & 109 & M6 & 944 \\
\hline EB1A9 & 81 & Fab M10 & 1053 & HF 1.7 & 928 & L14.17 & 1 & M77 & 447 \\
\hline EB5 & 124 & Fab M12 & 1054 & HH3 & 125 & L15 & 985 & M85 & 271 \\
\hline EC3 & 129 & Fab M12B & 612 & HIVIG & 7768 & L17 & 1000 & M86 & 275 \\
\hline EC6 & 121 & Fab M15 & $\overline{1055}$ & HT5 & 92 & L19 & 858 & M89 & 376 \\
\hline ED6 & 696 & Fab M26B & 613 & HT6 & 930 & L25 & 1002 & M90 & 859 \\
\hline ED8 & 148 & Fab M8B & 614 & HT7 & 931 & L28 & 936 & M91 & 555 \\
\hline EF7 & 84 & Fab S10 & 1056 & $\mathrm{Hv}$ & 434 & L33 & 937 & M92 & 274 \\
\hline EH1 & 1118 & Fab S6 & 1057 & HyHIV-1 & 5 & L39 & 1003 & M96 & 310 \\
\hline EH12E1 & 149 & Fab S8 & 1058 & HyHIV-15 & 34 & L40 & 1004 & MAG 104 & 860 \\
\hline $\mathrm{F} 1$ & 1105 & Fab S9 & 1059 & HyHIV-19 & 152 & L41 & 938 & MAG 109 & 405 \\
\hline F105 & 923 & Fab T2 & 615 & HyHIV-2 & 6 & $\mathrm{~L} 42$ & 939 & MAG 116 & 945 \\
\hline F11.2.32 & 172 & Fab T3 & 1060 & HyHIV-21 & 15 & L5.1 & 283 & MAG 12B & 946 \\
\hline F14.11 & 1100 & Fv & 432 & HyHIV-22 & 17 & L52 & 940 & MAG 29B & 947 \\
\hline F172-D8 & 659 & G11G1 & 150 & HyHIV-3 & 7 & L72 & 941 & MAG 3B & 948 \\
\hline F19.26-4 & 444 & G11H3 & 151 & HyHIV-4 & 8 & $\mathrm{~L} 78$ & 1005 & MAG 45 & 861 \\
\hline F19.48-3 & 445 & G12 & 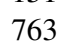 & HyHIV-5 & 9 & L81 & 870 & MAG 49 & 406 \\
\hline F19.57-11 & 446 & G2 & 764 & HyHIV-6 & 10 & LA9 (121-134) & 697 & MAG 53 & 407 \\
\hline F223 & 757 & G3-136 & 351 & ICR $39.13 \mathrm{~g}$ & 932 & LH-104-A & 88 & MAG 55 & 949 \\
\hline F240 & 628 & G3-1472 & $\overline{1026}$ & ICR $39.3 b$ & 933 & LH-104-B & 117 & MAG 56 & 408 \\
\hline F285 & 758 & G3-211 & 529 & ICR38.1a & 533 & LH-104-C & 101 & MAG 6B & 772 \\
\hline F5-2 & 40 & G3-299 & 534 & ICR38.8f & 539 & LH-104-E & 85 & MAG 72 & 950 \\
\hline F5-4 & 96 & G3-4 & 352 & ID6 & 980 & LH-104-G & 119 & MAG 86 & 951 \\
\hline F5.5 & 1025 & G3-42 & 535 & ID6 & 981 & LH-104-I & 118 & MAG 95 & 862 \\
\hline F58/D1 & 463 & G3-508 & 536 & ID8F6 & 39 & LH-104-K & 87 & MAG 96 & 952 \\
\hline F7 & 759 & G3-519 & 537 & IE8G2 & 153 & M-1 & 634 & MAG 97 & 863 \\
\hline
\end{tabular}




\begin{tabular}{|c|c|c|c|c|c|c|c|c|c|}
\hline MAb 35 & 223 & P5-3 & 779 & V7-8 & 154 & polyclonal & 167 & polyclonal & 592 \\
\hline MF119.1 & 311 & PC5009 & $\overline{590}$ & W1 & $3 \overline{318}$ & polyclonal & 168 & polyclonal & 601 \\
\hline MF169.1 & 369 & $\mathrm{RC} 25$ & $4 \overline{489}$ & W2 & 565 & polyclonal & 185 & polyclonal & $\overline{602}$ \\
\hline MF170.1 & 370 & RL4.72.1 & 77 & $\mathrm{X} 5$ & 973 & polyclonal & 191 & polyclonal & 603 \\
\hline MF39.1 & 306 & RSD-33 & 347 & $\mathrm{Z} 13$ & 677 & polyclonal & 241 & polyclonal & 617 \\
\hline MF4.1 & 312 & RT-4 & 237 & anti-CD4BS summary & 959 & polyclonal & 242 & polyclonal & 619 \\
\hline MF46.1 & 326 & RT6H & 189 & anti-HIV-1 RT & 240 & polyclonal & 243 & polyclonal & 620 \\
\hline MF49.1 & 293 & RT7O & 238 & anti-HIV-2 polyclonal & 516 & polyclonal & 248 & polyclonal & 621 \\
\hline MF53.1 & 313 & RT7U & 239 & anti-K159 & 212 & polyclonal & 276 & polyclonal & 626 \\
\hline MF58.1 & 314 & RTMAb8 & $\overline{187}$ & anti-gp120/V3 & $\overline{1029}$ & polyclonal & 329 & polyclonal & 648 \\
\hline MF77.1 & 315 & RV110026 & 573 & anti-p24 & 155 & polyclonal & 358 & polyclonal & 656 \\
\hline MF87.1 & 371 & $\mathrm{~S} 1-1$ & 954 & b11 & 960 & polyclonal & 389 & polyclonal & 668 \\
\hline MN215 & 455 & SC 258 & 1001 & b13 & 961 & polyclonal & 390 & polyclonal & 672 \\
\hline MO101/V3,C4 & 511 & SP.BAL114 & 448 & b14 & 962 & polyclonal & 392 & polyclonal & 673 \\
\hline MO101/V3,C4 & 512 & SP.SF2:104 & $4 \overline{449}$ & b3 & 963 & polyclonal & 396 & polyclonal & 674 \\
\hline MO101/V3,C4 & 513 & $\mathrm{~T} 1.1$ & 294 & b6 & 964 & polyclonal & 397 & polyclonal & 675 \\
\hline $\mathrm{MO} 28$ & 7773 & $\mathrm{~T} 11$ & 319 & clone 3 & 6 & polyclonal & 398 & polyclonal & 679 \\
\hline MO30 & $\overline{7774}$ & $\mathrm{~T} 13$ & 955 & human sera & $\overline{156}$ & polyclonal & 399 & polyclonal & 702 \\
\hline MO43 & 775 & T15G1 & $\overline{780}$ & i5B11 & $\overline{120}$ & polyclonal & 401 & polyclonal & 703 \\
\hline MO86/C3 & 540 & $\mathrm{~T} 2.1$ & 316 & loop 2 & \begin{tabular}{|l|}
473 \\
\end{tabular} & polyclonal & 402 & polyclonal & 706 \\
\hline MO9.42.2 & 97 & $\mathrm{~T} 20$ & 781 & multiple Fabs & $7 \overline{786}$ & polyclonal & 403 & polyclonal & $\overline{790}$ \\
\hline MO9.50.2 & 9 & $\mathrm{~T} 22$ & 97 & multiple MAbs & $\overline{787}$ & polyclonal & 404 & polyclonal & $\overline{91}$ \\
\hline MO97/V3 & $\overrightarrow{391}$ & $\mathrm{~T} 27$ & $7 \overline{782}$ & multiple MAbs & $\overline{788}$ & polyclonal & 410 & polyclonal & $7 \overline{292}$ \\
\hline MO99/V3 & 411 & $\mathrm{~T} 3$ & 783 & multiple MAbs & 789 & polyclonal & 415 & polyclonal & 793 \\
\hline MTW61D & 953 & T30 & $7 \overline{784}$ & p7 & 865 & polyclonal & 416 & polyclonal & 794 \\
\hline Md-1 & \begin{tabular}{|l|l|}
1061 \\
\end{tabular} & $\mathrm{~T} 32$ & 631 & polyclonal & 2 & polyclonal & 420 & polyclonal & 795 \\
\hline N11-20 & 506 & T34 & 632 & polyclonal & 23 & polyclonal & 422 & polyclonal & 796 \\
\hline N2-4 & 776 & $\mathrm{~T} 4$ & 785 & polyclonal & 42 & polyclonal & 435 & polyclonal & 97 \\
\hline $\mathrm{N} 70-1.9 \mathrm{~b}$ & 508 & $\mathrm{~T} 49$ & 956 & polyclonal & 47 & polyclonal & 437 & polyclonal & $\overline{98}$ \\
\hline N70-2.3a & \begin{tabular}{|l|}
777 \\
\end{tabular} & $\mathrm{~T} 52$ & 986 & polyclonal & 54 & polyclonal & 450 & polyclonal & $\overline{99}$ \\
\hline $\mathrm{NC}-1$ & 1082 & $\mathrm{~T} 54$ & 987 & polyclonal & 79 & polyclonal & 486 & polyclonal & 00 \\
\hline ND-15G1 & 665 & $\mathrm{~T} 56$ & 957 & polyclonal & 82 & polyclonal & 492 & polyclonal & 01 \\
\hline NF1A1 & 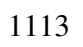 & $\mathrm{T} 7.1$ & 295 & polyclonal & 95 & polyclonal & 496 & polyclonal & $\overline{02}$ \\
\hline NF2B2 & 1120 & T9 & 296 & polyclonal & 157 & polyclonal & 503 & polyclonal & $\overline{03}$ \\
\hline NF3A3 & $\overline{1121}$ & T9 & 864 & polyclonal & $\overline{158}$ & polyclonal & 505 & polyclonal & $\overline{04}$ \\
\hline NF8B4 & 11122 & TG001 & 246 & polyclonal & 159 & polyclonal & 524 & polyclonal & $\overline{05}$ \\
\hline NM-01 & 499 & TG002 & 245 & polyclonal & $\overline{160}$ & polyclonal & 531 & polyclonal & $\overline{06}$ \\
\hline NT2/4D5.24 & 256 & $\mathrm{TH}-\mathrm{Ab} 1$ & 671 & polyclonal & 161 & polyclonal & 543 & polyclonal & 07 \\
\hline NT3/2D1.1 & 249 & TH1 & $\overline{1028}$ & polyclonal & $1 \overline{162}$ & polyclonal & 552 & polyclonal & $\overline{08}$ \\
\hline Nea 9301 & 456 & TH9 & 958 & polyclonal & 163 & polyclonal & 553 & polyclonal & $\overline{09}$ \\
\hline P1/D12 & $4 \overline{464}$ & V10 & 99 & polyclonal & 164 & polyclonal & 568 & polyclonal & 810 \\
\hline $\mathrm{P} 4 / \mathrm{D} 10$ & 465 & V10-9 & 618 & polyclonal & 165 & polyclonal & 579 & polyclonal & 811 \\
\hline P43110 & $\overline{778}$ & V107 & $\overline{100}$ & polyclonal & $\overline{166}$ & polyclonal & 581 & polyclonal & 812 \\
\hline
\end{tabular}




\begin{tabular}{|c|c|c|c|}
\hline polyclonal & 813 & polyclonal & 988 \\
\hline polyclonal & 814 & polyclonal & 1030 \\
\hline polyclonal & 815 & polyclonal & $\overline{1031}$ \\
\hline polyclonal & 816 & polyclonal & 1032 \\
\hline polyclonal & 817 & polyclonal & 1033 \\
\hline polyclonal & 818 & polyclonal & $\overline{1034}$ \\
\hline polyclonal & 819 & polyclonal & 1035 \\
\hline polyclonal & 820 & polyclonal & 1036 \\
\hline polyclonal & 821 & polyclonal & 1041 \\
\hline polyclonal & 822 & polyclonal & $\overline{1042}$ \\
\hline polyclonal & 823 & polyclonal & $\overline{084}$ \\
\hline polyclonal & 824 & polyclonal & 1093 \\
\hline polyclonal & 825 & polyclonal & $\overline{094}$ \\
\hline polyclonal & 826 & polyclonal & 1099 \\
\hline polyclonal & 827 & polyclonal & 11102 \\
\hline polyclonal & 828 & polyclonal & 1103 \\
\hline polyclonal & 829 & polyclonal & 11104 \\
\hline polyclonal & 830 & polyclonal & $\overline{1114}$ \\
\hline polyclonal & 831 & polyclonal & $\overline{124}$ \\
\hline polyclonal & 832 & polyclonal & 11125 \\
\hline polyclonal & 833 & polyclonal & $\overline{1126}$ \\
\hline polyclonal & 834 & polyclonal & 11127 \\
\hline polyclonal & 835 & polyclonal & $\overline{128}$ \\
\hline polyclonal & 836 & polyclonal & 11129 \\
\hline polyclonal & 837 & polyclonal & 11130 \\
\hline polyclonal & 838 & polyclonal & 1131 \\
\hline polyclonal & 839 & polyclonal $\alpha 577-596$ & 591 \\
\hline polyclonal & 840 & polyclonal $\alpha 598-609$ & 646 \\
\hline polyclonal & 841 & polyclonal HIVIG & 169 \\
\hline polyclonal & 842 & sc-FV p17 & 33 \\
\hline polyclonal & 843 & & \\
\hline polyclonal & 844 & & \\
\hline polyclonal & 845 & & \\
\hline polyclonal & 846 & & \\
\hline polyclonal & 847 & & \\
\hline polyclonal & 848 & & \\
\hline polyclonal & 849 & & \\
\hline polyclonal & 850 & & \\
\hline polyclonal & 851 & & \\
\hline polyclonal & 852 & & \\
\hline polyclonal & 873 & & \\
\hline polyclonal & 965 & & \\
\hline polyclonal & 975 & & \\
\hline
\end{tabular}


IV-B-3 MAbs by order of appearance in tables

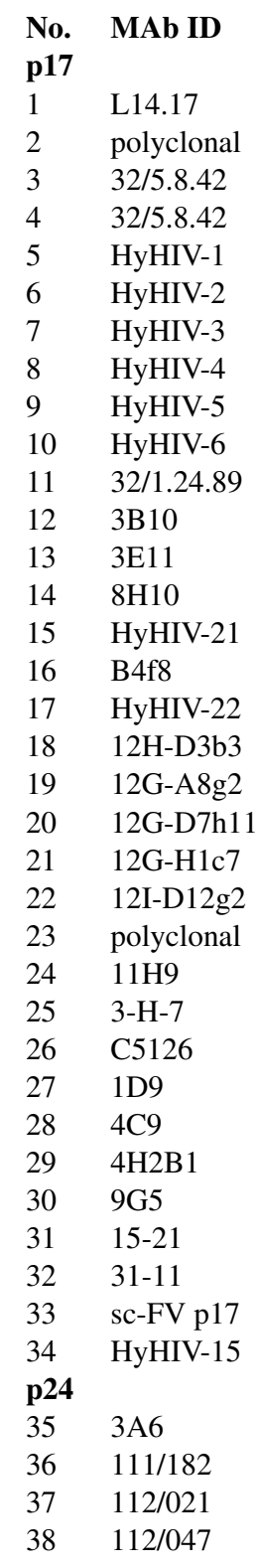

\begin{tabular}{|c|c|}
\hline 39 & ID8F6 \\
\hline 40 & F5-2 \\
\hline 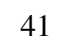 & CB-13/5 \\
\hline 42 & polyclonal \\
\hline 43 & $3 \mathrm{D} 3$ \\
\hline 4 & $\mathrm{CD}-4 / 1$ \\
\hline & $15 \mathrm{~F} 8 \mathrm{C} 7$ \\
\hline & $111 / 052$ \\
\hline & polyclonal \\
\hline & $91-5$ \\
\hline & $1109 / 01$ \\
\hline 0 & 14D4E11 \\
\hline 1. & $1 \mathrm{G} 5 \mathrm{C} 8$ \\
\hline$\overline{\overline{5}}$ & $47-2$ \\
\hline 5 & $714 / 01$ \\
\hline 5 & polyclonal \\
\hline & $111 / 073$ \\
\hline & $113 / 038$ \\
\hline & $1-E-4$ \\
\hline & $1-E-9$ \\
\hline 5 & $10-E-7$ \\
\hline 6 & $10-\mathrm{G}-9$ \\
\hline 6 & $11-\mathrm{C}-5$ \\
\hline 6 & $2-\mathrm{E}-4$ \\
\hline$\overline{6}$ & $2-\mathrm{H}-4$ \\
\hline & 8-D-2 \\
\hline 6 & 8-G-9 \\
\hline 6 & 8-H-7 \\
\hline & C5123 \\
\hline & $1-B-7$ \\
\hline$\overline{6}$ & $3-B-7$ \\
\hline & 6-D-12 \\
\hline$\overline{7}$ & $6-E-7$ \\
\hline & 8-D-5 \\
\hline & FF1 \\
\hline & $113 / 072$ \\
\hline & 25.3 \\
\hline & $13-102-100$ \\
\hline & RL4.72.1 \\
\hline & $406 / 01$ \\
\hline & polyclonal \\
\hline
\end{tabular}

\begin{tabular}{|c|c|}
\hline 80 & $38: 9.6 \mathrm{~K}$ \\
\hline 8 & EB1A9 \\
\hline 8 & polyclonal \\
\hline 83 & $30: 3 E 5$ \\
\hline$\overline{84}$ & EF7 \\
\hline 8 & LH-104-E \\
\hline $8 \overline{86}$ & $1 \mathrm{~B} 2 \mathrm{C} 12$ \\
\hline 87 & LH-104-K \\
\hline & LH-104-A \\
\hline & 1.17 .3 \\
\hline$\overline{9}$ & $1 \mathrm{~A} 7$ \\
\hline$\overline{9}$ & 1F6 \\
\hline & 23A5G4 \\
\hline 9 & 23A5G5 \\
\hline$\overline{94}$ & 3D10G6 \\
\hline$\overline{9}$ & polyclonal \\
\hline & F5-4 \\
\hline & MO9.42.2 \\
\hline 9 & MO9.50.2 \\
\hline$\overline{9}$ & V10 \\
\hline $\overrightarrow{100}$ & V107 \\
\hline 101 & LH-104-C \\
\hline$\overline{2}$ & $12-\mathrm{B}-4$ \\
\hline 103 & C5122 \\
\hline$\overline{\overline{104}}$ & $9 \mathrm{~A} 4 \mathrm{C} 4$ \\
\hline 1 & $11 \mathrm{C} 10 \mathrm{~B} 10$ \\
\hline 6 & 11D11F2 \\
\hline 107 & CD12B4 \\
\hline$=$ & BE3 \\
\hline$\overline{109}$ & L14 \\
\hline$\overline{\overline{0}}$ & $108 / 03$ \\
\hline$\overline{111}$ & $110 / 015$ \\
\hline$\overline{112}$ & $32: 32 \mathrm{~K}$ \\
\hline$\overline{113}$ & C5200 \\
\hline$=$ & FH2 \\
\hline & 13B5 \\
\hline$\overline{1}$ & $106 / 01$ \\
\hline$\overline{117}$ & LH-104-B \\
\hline & LH-104-I \\
\hline p24 & 2p7p1p6 \\
\hline & LH-104-G \\
\hline
\end{tabular}

\begin{tabular}{|c|c|}
\hline \multicolumn{2}{|c|}{ p2p7p1p6 } \\
\hline 120 & i5B11 \\
\hline 121 & EC6 \\
\hline 122 & M12 \\
\hline 123 & DG8 \\
\hline 124 & EB5 \\
\hline 125 & HH3 \\
\hline 126 & $\mathrm{AD} 2$ \\
\hline 127 & CA5 \\
\hline 128 & DF3 \\
\hline$\overline{129}$ & EC3 \\
\hline 130 & FC12 \\
\hline 131 & GE4 \\
\hline 132 & JB7 \\
\hline$\overline{133}$ & $\mathrm{JF} 11$ \\
\hline Gag & \\
\hline 134 & $16 / 4 / 2$ \\
\hline 135 & $183-\mathrm{H} 12-5 \mathrm{C}$ \\
\hline$\overline{136}$ & $241-D$ \\
\hline 137 & $2 \mathrm{~A} 6$ \\
\hline 138 & 5E2.A3k \\
\hline 139 & $71-31$ \\
\hline 140 & $91-6$ \\
\hline 141 & $98-4.3$ \\
\hline 142 & $98-4.9$ \\
\hline 143 & $\mathrm{AC} 2$ \\
\hline 144 & BC 1071 \\
\hline 145 & BE10 \\
\hline$\overline{146}$ & CD9 \\
\hline 147 & CH9B2 \\
\hline$\overline{148}$ & ED8 \\
\hline$\overline{149}$ & EH12E1 \\
\hline 150 & G11G1 \\
\hline$\overline{151}$ & G11H3 \\
\hline$\overline{152}$ & HyHIV-19 \\
\hline$\overline{153}$ & IE8G2 \\
\hline 154 & V7-8 \\
\hline$\overline{155}$ & anti-p24 \\
\hline 156 & human sera \\
\hline 157 & polyclonal \\
\hline 15 & polyclonal \\
\hline
\end{tabular}

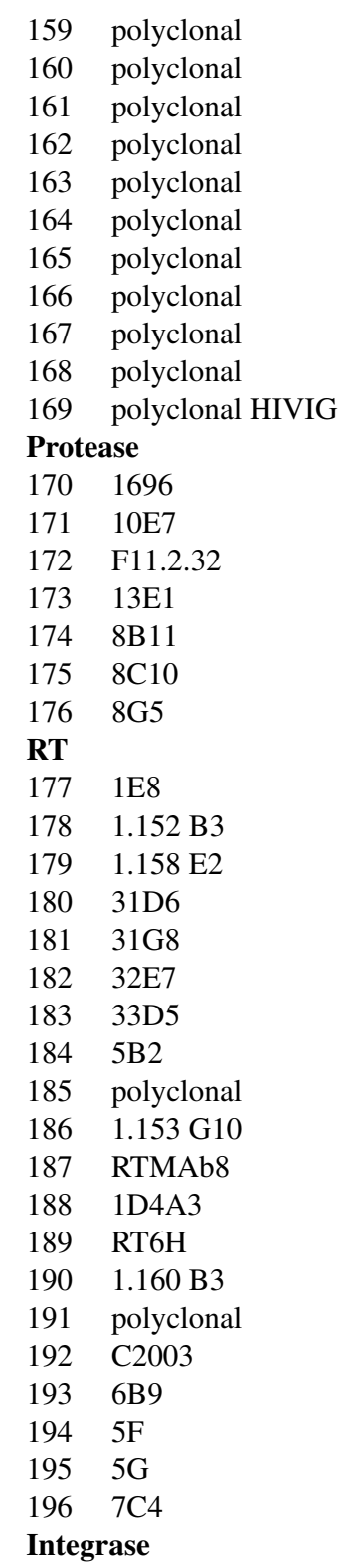




\begin{tabular}{|c|c|}
\hline 197 & $1 \mathrm{C} 4$ \\
\hline 198 & $2 \mathrm{C} 11$ \\
\hline 199 & 2E3 \\
\hline 200 & 3E11 \\
\hline 201 & $3 \mathrm{~F} 9$ \\
\hline 202 & $5 \mathrm{~F} 8$ \\
\hline 203 & 6G5 \\
\hline 204 & 7B6 \\
\hline 205 & 7C6 \\
\hline 206 & $6 \mathrm{C} 5$ \\
\hline 207 & 8G4 \\
\hline 208 & 17 \\
\hline 209 & 4D6 \\
\hline 210 & $7-16$ \\
\hline 211 & 4F6 \\
\hline$\overline{212}$ & anti-K159 \\
\hline$\overline{213}$ & 5D9 \\
\hline 214 & $8-6$ \\
\hline 215 & 19 \\
\hline$\overline{216}$ & $2-19$ \\
\hline$\overline{217}$ & $8-22$ \\
\hline $2 \overline{218}$ & $4-20$ \\
\hline$\overline{219}$ & $6-19$ \\
\hline$\overline{220}$ & $7 \mathrm{C} 3$ \\
\hline$\overline{221}$ & $7 F 11$ \\
\hline 222 & $8 \mathrm{E} 5$ \\
\hline$\overline{223}$ & MAb 35 \\
\hline Pol & \\
\hline 224 & 12 \\
\hline 225 & 13 \\
\hline$\overline{226}$ & 14 \\
\hline 227 & 16 \\
\hline 228 & 1C12B1 \\
\hline$\overline{229}$ & 21 \\
\hline$\overline{230}$ & 32 \\
\hline$\overline{231}$ & 35 \\
\hline$\overline{232}$ & 3D12 \\
\hline$\overline{233}$ & $3 F 10$ \\
\hline$\overline{234}$ & 4 \\
\hline$\overline{235}$ & $6 \mathrm{~B} 9$ \\
\hline$\overline{236}$ & $7 \mathrm{C} 4$ \\
\hline$\overline{237}$ & RT-4 \\
\hline 238 & RT7O \\
\hline
\end{tabular}

\begin{tabular}{|c|c|}
\hline 239 & RT7U \\
\hline$\overline{240}$ & anti-HIV-1 RT \\
\hline$\overline{241}$ & polyclonal \\
\hline$\overline{242}$ & polyclonal \\
\hline$\overline{243}$ & polyclonal \\
\hline$\overline{244}$ & 33 \\
\hline & \\
\hline 245 & TG002 \\
\hline$\overline{246}$ & TG001 \\
\hline 247 & $\mathrm{~J} 4$ \\
\hline$\overline{248}$ & polyclonal \\
\hline Tat & \\
\hline 249 & NT3/2D1.1 \\
\hline 250 & 1.2 \\
\hline 251 & 1D9D5 \\
\hline 252 & 1D2F11 \\
\hline 253 & 2D9E7 \\
\hline 254 & $4 \mathrm{~B} 4 \mathrm{C} 4$ \\
\hline$\overline{255}$ & 5G7D8 \\
\hline $2 \overline{256}$ & NT2/4D5.24 \\
\hline 257 & L-anti-Tat \\
\hline$\overline{258}$ & 2D9D5 \\
\hline $\operatorname{Rev}$ & \\
\hline 259 & 4G9 \\
\hline 260 & $\mathrm{Ab} 2$ \\
\hline 261 & 10.1 \\
\hline$\overline{262}$ & $3 \mathrm{H} 6$ \\
\hline $2 \overline{263}$ & $8 \mathrm{E} 7$ \\
\hline 264 & 9G2 \\
\hline 265 & $\mathrm{Ab} 4$ \\
\hline 266 & $3 \mathrm{G} 4$ \\
\hline 267 & $1 \mathrm{G} 10$ \\
\hline 268 & $1 \mathrm{G} 7$ \\
\hline 269 & Ab3 \\
\hline$\overline{270}$ & $2 \mathrm{G} 2$ \\
\hline gp1 & \\
\hline 271 & M85 \\
\hline$\overline{272}$ & $7 \mathrm{E} 2 / 4$ \\
\hline$\overline{273}$ & 4D4\#85 \\
\hline$\overline{274}$ & M92 \\
\hline$\overline{275}$ & M86 \\
\hline$\overline{276}$ & polyclonal \\
\hline$\overline{277}$ & $133 / 237$ \\
\hline
\end{tabular}

\begin{tabular}{|c|c|}
\hline 278 & $133 / 290$ \\
\hline 279 & $133 / 11$ \\
\hline 280 & D/3G5 \\
\hline 281 & $\mathrm{D} / 6 \mathrm{~A} 11$ \\
\hline 282 & $\mathrm{D} / 5 \mathrm{E} 12$ \\
\hline 283 & L5.1 \\
\hline 284 & 4A7C6 \\
\hline 285 & $1 \mathrm{D} 10$ \\
\hline 286 & B242 \\
\hline 287 & $133 / 192$ \\
\hline 288 & 489.1(961) \\
\hline 289 & $5 B 3$ \\
\hline 290 & B10 \\
\hline 291 & $\mathrm{~B} 2$ \\
\hline 292 & C6 \\
\hline $2 \overline{293}$ & MF49.1 \\
\hline 294 & $\mathrm{~T} 1.1$ \\
\hline 295 & $\mathrm{~T} 7.1$ \\
\hline 296 & T9 \\
\hline$\overline{297}$ & GV4D3 \\
\hline 298 & B27 \\
\hline 299 & B9 \\
\hline 300 & B35 \\
\hline 301 & $\mathrm{D} / 4 \mathrm{~B} 5$ \\
\hline 302 & $\mathrm{D} / 5 \mathrm{~A} 11$ \\
\hline 303 & $\mathrm{D} / 6 \mathrm{~B} 2$ \\
\hline 304 & B18 \\
\hline$\overline{305}$ & B20 \\
\hline 306 & MF39.1 \\
\hline 307 & 187.2.1 \\
\hline 308 & 37.1.1(ARP 327) \\
\hline$\overline{304}$ & $6 \mathrm{D} 8$ \\
\hline 310 & M96 \\
\hline 311 & MF119.1 \\
\hline 312 & MF4.1 \\
\hline 313 & MF53.1 \\
\hline 314 & MF58.1 \\
\hline 315 & MF77.1 \\
\hline $3 \overline{316}$ & $\mathrm{~T} 2.1$ \\
\hline 317 & $11 / 65$ \\
\hline 318 & W1 \\
\hline 319 & $\mathrm{~T} 11$ \\
\hline 230 & GV1A8 \\
\hline
\end{tabular}

\begin{tabular}{|c|c|}
\hline 321 & 11 \\
\hline 322 & $12 \mathrm{G} 10$ \\
\hline$\overline{323}$ & $135 / 9$ \\
\hline 324 & $7 \mathrm{C} 10$ \\
\hline 325 & $\mathrm{C} 4$ \\
\hline 326 & MF46.1 \\
\hline 327 & $6 \mathrm{D} 5$ \\
\hline 328 & B33 \\
\hline 329 & polyclonal \\
\hline 330 & $35 \mathrm{D} 10 / \mathrm{D} 2$ \\
\hline 331 & $40 \mathrm{H} 2 / \mathrm{C} 7$ \\
\hline 332 & 43A3/E4 \\
\hline 333 & 43C7/B9 \\
\hline 334 & $45 \mathrm{D} 1 / \mathrm{B} 7$ \\
\hline 335 & 46E3/E6 \\
\hline 336 & 58E1/B3 \\
\hline 337 & 64B9/A6 \\
\hline 338 & 69D2/A1 \\
\hline 339 & $82 \mathrm{D} 3 / \mathrm{C} 3$ \\
\hline 340 & $2 \mathrm{H} 1 \mathrm{~B}$ \\
\hline 341 & 697-D \\
\hline $3 \overline{342}$ & $6 \mathrm{C} 4 / \mathrm{S}$ \\
\hline $3 \overline{343}$ & C108G \\
\hline 344 & $10 / 76 b$ \\
\hline 345 & $11 / 41 \mathrm{e}$ \\
\hline 346 & $11 / 4 b$ \\
\hline 347 & RSD-33 \\
\hline 348 & $11 / 4 c$ \\
\hline 349 & 8.22 .2 \\
\hline 350 & $12 b$ \\
\hline 351 & G3-136 \\
\hline 352 & G3-4 \\
\hline 353 & BAT085 \\
\hline 354 & $60 \mathrm{~b}$ \\
\hline 355 & 74 \\
\hline 356 & $38 / 12 b$ \\
\hline 357 & $38 / 60 \mathrm{~b}$ \\
\hline 358 & polyclonal \\
\hline$\overline{355}$ & $322-151$ \\
\hline $3 \overline{360}$ & 3D3.B8 \\
\hline 361 & 4C11.D8 \\
\hline 362 & 493-156 \\
\hline$\overline{363}$ & 110.1 \\
\hline
\end{tabular}

\begin{tabular}{|c|c|}
\hline 64 & GV4H3 \\
\hline 365 & $\mathrm{~J} 1$ \\
\hline 7 & $\mathrm{~J} 3$ \\
\hline & 1006-30-D \\
\hline 0 & 847-D \\
\hline & MF169.1 \\
\hline (0) & MF170.1 \\
\hline 1 & MF87.1 \\
\hline 2 & 213.1 \\
\hline 3 & B12 \\
\hline 4 & B13 \\
\hline & $\mathrm{C} 13$ \\
\hline 6 & M89 \\
\hline 7 & B21 \\
\hline & B23 \\
\hline (9) & B24 \\
\hline 30 & B25 \\
\hline & B3 \\
\hline 8 & B26 \\
\hline 33 & B29 \\
\hline & B36 \\
\hline & 110.E \\
\hline 6 & 110.C \\
\hline & IIIB-V3-26 \\
\hline & IIIB-V3-21 \\
\hline & polyclonal \\
\hline & polyclonal \\
\hline & MO97/V3 \\
\hline & polyclonal \\
\hline & $55 / 11$ \\
\hline & $8 / 38 c$ \\
\hline & $8 / 64 b$ \\
\hline & polyclonal \\
\hline & polyclonal \\
\hline & polyclonal \\
\hline & polyclonal \\
\hline & 9284 \\
\hline & polyclonal \\
\hline & polyclonal \\
\hline & polyclonal \\
\hline & polyclonal \\
\hline & MAG 109 \\
\hline & MAG 49 \\
\hline
\end{tabular}




\begin{tabular}{|c|c|}
\hline 407 & MAG 53 \\
\hline 408 & MAG 56 \\
\hline$\overline{409}$ & 1324-E \\
\hline $4 \overline{410}$ & polyclonal \\
\hline 11 & MO99/V3 \\
\hline$\overline{412}$ & C311E \\
\hline 413 & 907 \\
\hline 414 & 924 \\
\hline 415 & polyclonal \\
\hline 416 & polyclonal \\
\hline 417 & $10 \mathrm{~F} 10$ \\
\hline 418 & $2 \mathrm{C} 4$ \\
\hline 419 & 412-D \\
\hline 420 & polyclonal \\
\hline 421 & CGP 47439 \\
\hline$\overline{22}$ & polyclonal \\
\hline $4 \overline{423}$ & 178.1 \\
\hline$\overline{424}$ & 257-D \\
\hline$\overline{425}$ & 311-11-D \\
\hline 26 & 41148D \\
\hline$\overline{427}$ & 391/95-D \\
\hline$\overline{42}$ & Aw \\
\hline$\overline{429}$ & $\mathrm{Bw}$ \\
\hline$\overline{4}$ & DO142-10 \\
\hline $4 \overline{431}$ & Dv \\
\hline$\overline{4}$ & $\mathrm{Fv}$ \\
\hline 433 & $\mathrm{Gv}$ \\
\hline 43 & $\mathrm{Hv}$ \\
\hline $4 \overline{435}$ & polyclonal \\
\hline$\overline{43}$ & 50.1 \\
\hline$\overline{43}$ & polyclonal \\
\hline$\overline{433}$ & BAT123 \\
\hline & 838-D \\
\hline$\overline{440}$ & 1006-15D \\
\hline T & 782-D \\
\hline$=$ & 908-D \\
\hline$=$ & 1027-15D \\
\hline 4 & F19.26-4 \\
\hline & F19.48-3 \\
\hline & F19.57-11 \\
\hline & M77 \\
\hline & SP.BAL114 \\
\hline & SP.SF2:104 \\
\hline
\end{tabular}

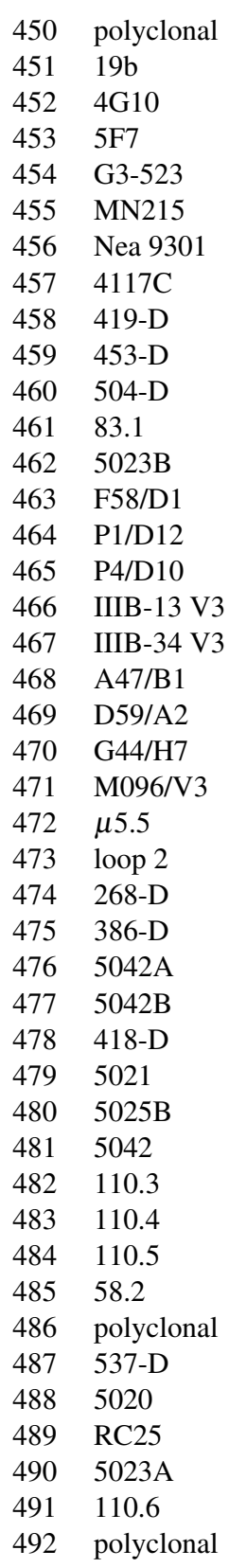

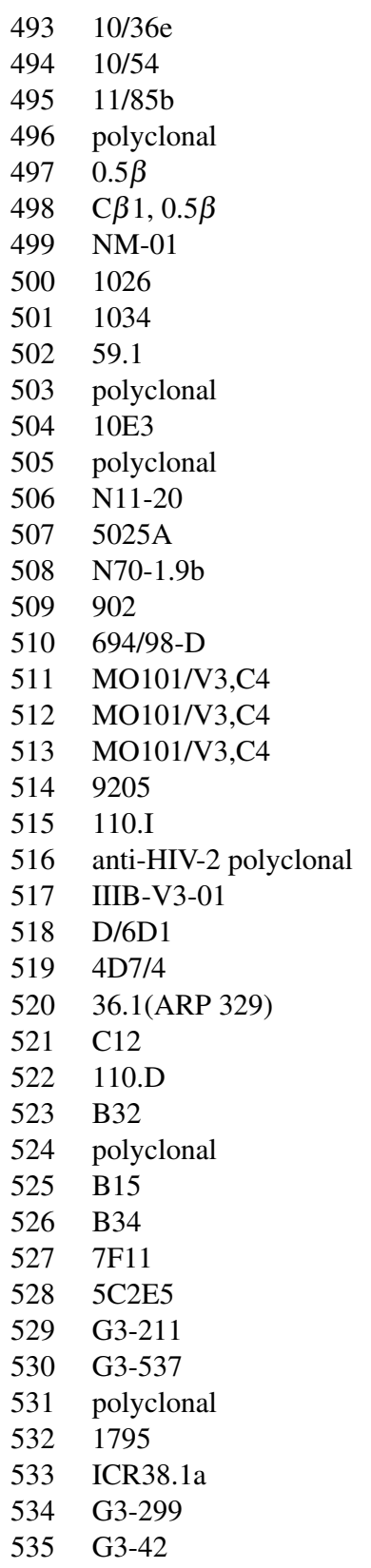

\begin{tabular}{|c|c|}
\hline 536 & G3-508 \\
\hline 537 & G3-519 \\
\hline 538 & G3-536 \\
\hline 539 & ICR38.8f \\
\hline 540 & MO86/C3 \\
\hline 541 & $13 \mathrm{H} 8$ \\
\hline$\overline{542}$ & G45-60 \\
\hline 543 & polyclonal \\
\hline 544 & 1662 \\
\hline 545 & 1663 \\
\hline 546 & 1664 \\
\hline 547 & 1697 \\
\hline 548 & 1794 \\
\hline 549 & 1804 \\
\hline 550 & 1807 \\
\hline 551 & 1808 \\
\hline 552 & polyclonal \\
\hline 553 & polyclonal \\
\hline$\overline{554}$ & CRA1(ARP 323) \\
\hline 555 & M91 \\
\hline 556 & 9201 \\
\hline 557 & $1 \mathrm{C} 1$ \\
\hline 558 & $3 \mathrm{~F} 5$ \\
\hline$\overline{559}$ & $5 \mathrm{~F} 4 / 1$ \\
\hline 560 & $660-178$ \\
\hline 561 & 9301 \\
\hline 562 & B221 \\
\hline $5 \overline{563}$ & $8 C 6 / 1$ \\
\hline 564 & H11 \\
\hline 565 & W2 \\
\hline 566 & M38 \\
\hline 567 & Chim 1 \\
\hline 568 & polyclonal \\
\hline 569 & $1331 \mathrm{~A}$ \\
\hline 570 & 110.1 \\
\hline 571 & $42 \mathrm{~F}$ \\
\hline 572 & $43 \mathrm{~F}$ \\
\hline 573 & RV110026 \\
\hline 574 & $105-306$ \\
\hline 575 & GV1G2 \\
\hline 576 & $750-\mathrm{D}$ \\
\hline 57 & $450-\mathrm{D}$ \\
\hline 57 & $670-\mathrm{D}$ \\
\hline
\end{tabular}

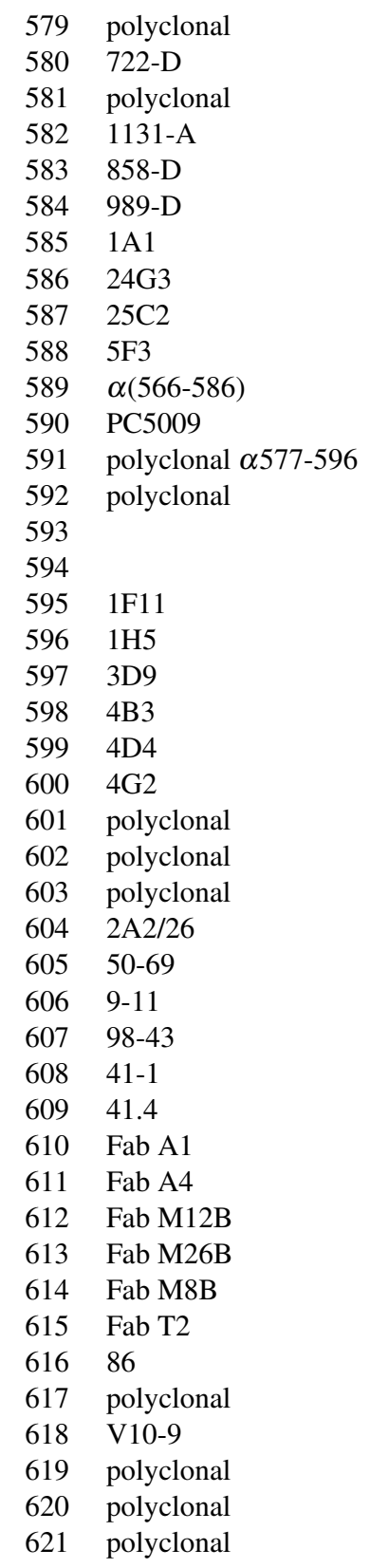




\begin{tabular}{|c|c|}
\hline 622 & $2 \mathrm{~F} 11$ \\
\hline 623 & 246-D \\
\hline 624 & 9G5A \\
\hline 625 & 181-D \\
\hline 626 & polyclonal \\
\hline 627 & $240-\mathrm{D}$ \\
\hline 628 & F240 \\
\hline 629 & D49 \\
\hline 630 & D61 \\
\hline 631 & $\mathrm{~T} 32$ \\
\hline 632 & T34 \\
\hline 633 & 115.8 \\
\hline 634 & M-1 \\
\hline 635 & M-11 \\
\hline 636 & M-13 \\
\hline 637 & M-2 \\
\hline 638 & M-22 \\
\hline 639 & M-24 \\
\hline 640 & M-25 \\
\hline 641 & M-28 \\
\hline 642 & M-29 \\
\hline 643 & M-36 \\
\hline 644 & M-4 \\
\hline 645 & M-6 \\
\hline 646 & polyclonal $\alpha 598-609$ \\
\hline 647 & 1B8.env \\
\hline 648 & polyclonal \\
\hline 649 & clone 3 \\
\hline 650 & 4 \\
\hline 651 & $41-6$ \\
\hline 652 & $41-7$ \\
\hline 653 & 68.1 \\
\hline 654 & 68.11 \\
\hline 655 & 75 \\
\hline 656 & polyclonal \\
\hline 657 & $105-732$ \\
\hline 658 & 3D6 \\
\hline 65 & F172-D8 \\
\hline 660 & D50 \\
\hline 661 & $5-21-3$ \\
\hline 662 & $120-16$ \\
\hline 663 & $98-6$ \\
\hline 664 & $167-7$ \\
\hline
\end{tabular}

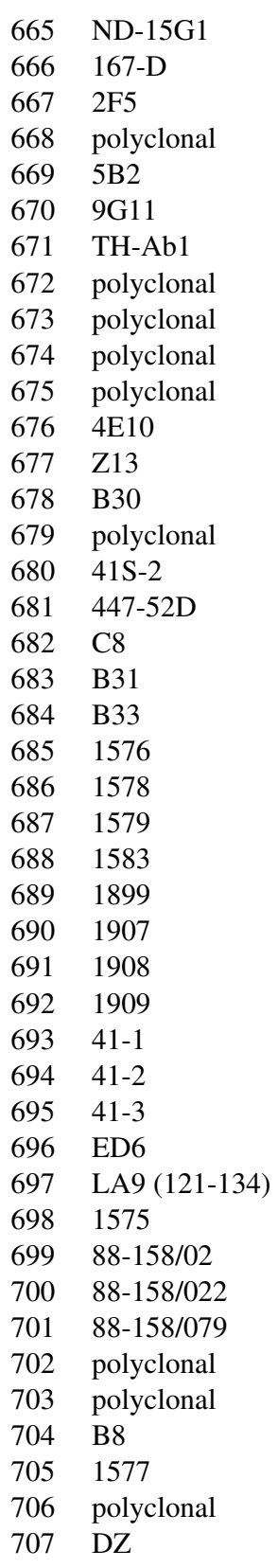

\begin{tabular}{|c|c|}
\hline \multirow{2}{*}{\multicolumn{2}{|c|}{$\begin{array}{l}\text { Env } \\
708\end{array}$}} \\
\hline & \\
\hline 709 & \\
\hline 710 & \\
\hline 711 & \\
\hline $7 \overline{712}$ & \\
\hline 713 & $102-135$ \\
\hline 714 & 1025 \\
\hline 715 & $105-134$ \\
\hline 716 & 10E9 \\
\hline 717 & $126-50$ \\
\hline 718 & $12 \mathrm{H} 2$ \\
\hline 719 & 13.10 \\
\hline 720 & 1B1 \\
\hline 721 & 1F7 \\
\hline$\overline{722}$ & $2.2 \mathrm{~B}$ \\
\hline 723 & $30 \mathrm{D}$ \\
\hline 724 & $31710 \mathrm{~B}$ \\
\hline 725 & 38B5/C9 \\
\hline 726 & 39H10/A11 \\
\hline 727 & 3D5 \\
\hline 728 & $3 \mathrm{H} 6$ \\
\hline $7 \overline{729}$ & 40D3/C11 \\
\hline 730 & 49B11/A1 \\
\hline 731 & $52 \mathrm{G} 5 / \mathrm{B} 9$ \\
\hline 732 & 55E4/H1 \\
\hline 733 & $56 \mathrm{C} 4 / \mathrm{C} 8$ \\
\hline 734 & $57 \mathrm{~B} 6 / \mathrm{F} 1$ \\
\hline 735 & 57H5/D7 \\
\hline 736 & $63 \mathrm{G} 4 / \mathrm{E} 2$ \\
\hline 737 & 65B12/C5 \\
\hline 738 & $6 \mathrm{E} 10$ \\
\hline 739 & $7-1054$ \\
\hline 740 & $7 \mathrm{~B} 2$ \\
\hline 741 & 85G11/D8 \\
\hline$\overline{742}$ & 87E4/A8 \\
\hline 743 & 97B1/E8 \\
\hline 744 & A9 \\
\hline 745 & B4 \\
\hline 746 & B5 \\
\hline 747 & B6 \\
\hline 748 & BAT267 \\
\hline 749 & BAT401 \\
\hline
\end{tabular}

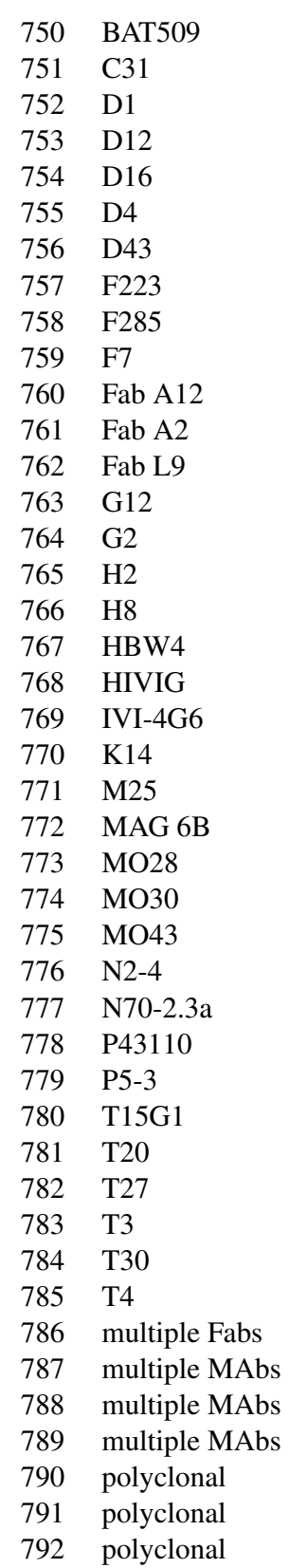

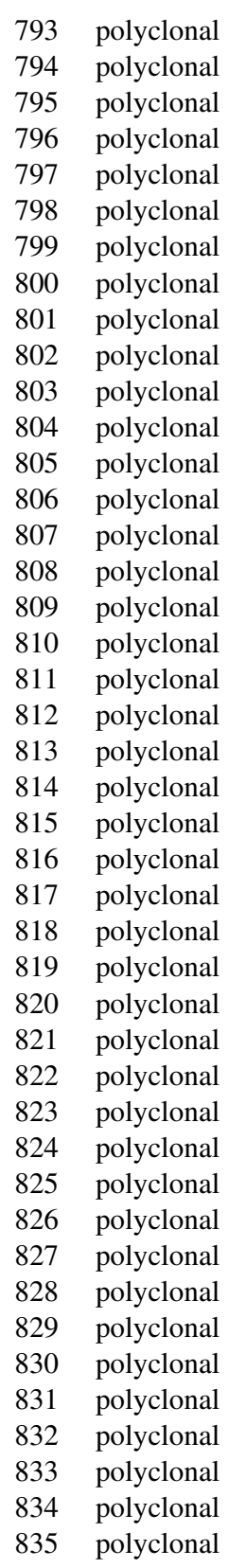




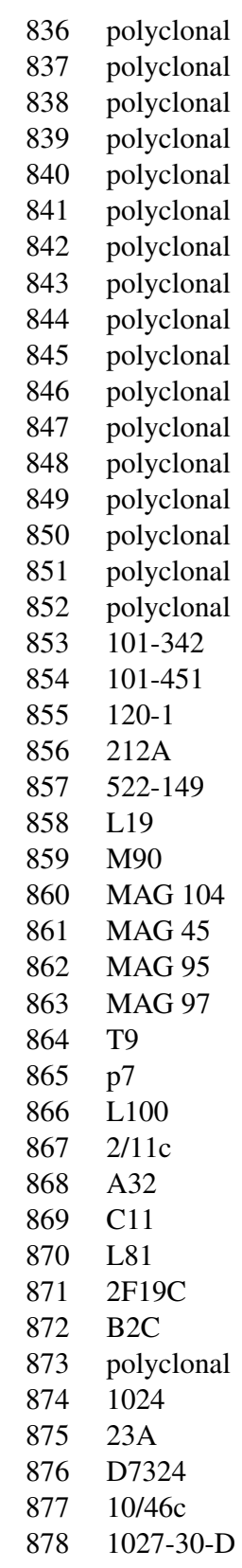

\begin{tabular}{|c|c|}
\hline & $1125 \mathrm{H}$ \\
\hline & $120-1 \mathrm{~B} 1$ \\
\hline & 1202-D \\
\hline & $1331 \mathrm{E}$ \\
\hline & 1570 \\
\hline & 1595 \\
\hline 5 & 1599 \\
\hline & $15 \mathrm{e}$ \\
\hline & $205-43-1$ \\
\hline & $205-46-9$ \\
\hline & $21 \mathrm{~h}$ \\
\hline & 28A11/B1 \\
\hline & 2G6 \\
\hline 2 & $35 \mathrm{~F} 3 / \mathrm{E} 2$ \\
\hline & 38G3/A9 \\
\hline & 428 \\
\hline 95 & 448-D \\
\hline 6 & 44D2/D5 \\
\hline & $48-16$ \\
\hline 9 & $50-61 \mathrm{~A}$ \\
\hline 9 & $5145 \mathrm{~A}$ \\
\hline$\overline{0}$ & 558-D \\
\hline 1 & 559/64-D \\
\hline$\overline{90}$ & 55D5/F9 \\
\hline$\overline{3}$ & 588-D \\
\hline$\overline{4}$ & 654-D \\
\hline$\overline{9}$ & $67 \mathrm{G} 6 / \mathrm{C} 4$ \\
\hline 96 & 729-D \\
\hline$\overline{907}$ & 830D \\
\hline & 9CL \\
\hline$\overline{9}$ & BM12 \\
\hline$\overline{91}$ & D20 \\
\hline 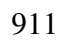 & D21 \\
\hline$\overline{9}$ & D24 \\
\hline$\overline{9}$ & D25 \\
\hline & D28 \\
\hline & D35 \\
\hline$\overline{91}$ & D39 \\
\hline & D42 \\
\hline & D52 \\
\hline & D53 \\
\hline & D60 \\
\hline & DA48 \\
\hline
\end{tabular}

\begin{tabular}{|c|c|}
\hline 922 & DO8i \\
\hline$\overline{923}$ & F105 \\
\hline$\overline{924}$ & F91 \\
\hline$\overline{925}$ & GP13 \\
\hline 926 & GP44 \\
\hline 927 & GP68 \\
\hline 928 & HF1.7 \\
\hline$\overline{929}$ & HT5 \\
\hline $9 \overline{930}$ & HT6 \\
\hline 931 & HT7 \\
\hline 932 & ICR $39.13 \mathrm{~g}$ \\
\hline 933 & ICR $39.3 b$ \\
\hline 934 & $\operatorname{IgG1b} 12$ \\
\hline 935 & IgGCD4 \\
\hline 936 & L28 \\
\hline 937 & L33 \\
\hline 938 & L41 \\
\hline$\overline{939}$ & L42 \\
\hline$\overline{940}$ & L52 \\
\hline$\overline{941}$ & L72 \\
\hline$\overline{942}$ & M12 \\
\hline$\overline{943}$ & M13 \\
\hline$\overline{944}$ & M6 \\
\hline$\overline{945}$ & MAG 116 \\
\hline$\overline{946}$ & MAG 12B \\
\hline 947 & MAG 29B \\
\hline$\overline{948}$ & MAG 3B \\
\hline$\overline{949}$ & MAG 55 \\
\hline$\overline{950}$ & MAG 72 \\
\hline 951 & MAG 86 \\
\hline 952 & MAG 96 \\
\hline$\overline{953}$ & MTW61D \\
\hline$\overline{954}$ & S1-1 \\
\hline 955 & $\mathrm{~T} 13$ \\
\hline$\overline{956}$ & $\mathrm{~T} 49$ \\
\hline 957 & T56 \\
\hline$\overline{95}$ & TH9 \\
\hline$\overline{959}$ & anti-CD4BS summary \\
\hline 960 & b11 \\
\hline 961 & b13 \\
\hline$\overline{962}$ & b14 \\
\hline$\overline{9 x}$ & b3 \\
\hline & b6 \\
\hline
\end{tabular}

\begin{tabular}{|c|c|}
\hline \begin{tabular}{|l|}
965 \\
966
\end{tabular} & $\begin{array}{l}\text { polyclonal } \\
\text { D33 }\end{array}$ \\
\hline 967 & \\
\hline$\overline{968}$ & $17 \mathrm{~b}$ \\
\hline 969 & $21 \mathrm{c}$ \\
\hline$\overline{970}$ & $23 e$ \\
\hline 971 & $48 d$ \\
\hline 972 & $49 \mathrm{e}$ \\
\hline 973 & $\mathrm{X} 5$ \\
\hline 974 & $\mathrm{~T} 22$ \\
\hline$\overline{975}$ & polyclonal \\
\hline 976 & 2A2 \\
\hline 977 & $\mathrm{AC} 4$ \\
\hline$\overline{978}$ & AD3 \\
\hline$\overline{979}$ & AD3 \\
\hline$\overline{980}$ & ID6 \\
\hline 981 & ID6 \\
\hline$\overline{982}$ & $11 / 68 b$ \\
\hline$\overline{983}$ & $62 c$ \\
\hline$\overline{984}$ & CRA-6 \\
\hline 985 & L15 \\
\hline$\overline{986}$ & T52 \\
\hline$\overline{987}$ & T54 \\
\hline$\overline{988}$ & polyclonal \\
\hline$\overline{989}$ & 1088 \\
\hline 990 & $110-\mathrm{B}$ \\
\hline$\overline{991}$ & 1357 \\
\hline 992 & 1361 \\
\hline$\overline{993}$ & $1393 \mathrm{~A}$ \\
\hline$\overline{994}$ & $66 a$ \\
\hline$\overline{995}$ & $66 c$ \\
\hline$\overline{996}$ & $684-238$ \\
\hline$\overline{997}$ & $830 \mathrm{~A}$ \\
\hline$\overline{998}$ & CRA-3 \\
\hline 999 & CRA-4 \\
\hline$\overline{1000}$ & L17 \\
\hline$\overline{1001}$ & SC258 \\
\hline$\overline{1002}$ & L25 \\
\hline$\overline{1003}$ & L39 \\
\hline$\overline{1004}$ & L40 \\
\hline 1005 & L78 \\
\hline 1006 & \\
\hline 1007 & 110.J \\
\hline
\end{tabular}

\begin{tabular}{|c|c|}
\hline 1008 & 1334-D \\
\hline$\overline{1009}$ & 2182 \\
\hline$\overline{1010}$ & 2191 \\
\hline 1011 & 2219 \\
\hline$\overline{1012}$ & 2412 \\
\hline 1013 & 2442 \\
\hline 1014 & 2456 \\
\hline 1015 & $39 \mathrm{~F}$ \\
\hline 1016 & $55 / 68 b$ \\
\hline 1017 & $5 \mathrm{G} 11$ \\
\hline 1018 & 6.1 \\
\hline 1019 & 6.7 \\
\hline 1020 & 8.27 .3 \\
\hline 1021 & $8 \mathrm{E} 11 / \mathrm{A} 8$ \\
\hline 1022 & 9305 \\
\hline 1023 & AG1121 \\
\hline 1024 & D47 \\
\hline 1025 & F5.5 \\
\hline 1026 & G3-1472 \\
\hline 1027 & K24 \\
\hline 1028 & TH1 \\
\hline 1029 & anti-gp120/V3 \\
\hline 1030 & polyclonal \\
\hline 1031 & polyclonal \\
\hline 1032 & polyclonal \\
\hline 1033 & polyclonal \\
\hline 1034 & polyclonal \\
\hline 1035 & polyclonal \\
\hline 1036 & polyclonal \\
\hline 1037 & $11 / 75 a / 21 / 41$ \\
\hline 1038 & 41.1 \\
\hline 1039 & $55 / 45 a / 11$ \\
\hline 1040 & 1108 \\
\hline 1041 & polyclonal \\
\hline 1042 & polyclonal \\
\hline 1043 & D27 \\
\hline 1044 & D56 \\
\hline 1045 & $2 \mathrm{G} 12$ \\
\hline 1046 & 1367 \\
\hline 1047 & $126-6$ \\
\hline 1048 & 1342 \\
\hline$\overline{1044}$ & 1379 \\
\hline$\overline{105}$ & Fab D11 \\
\hline
\end{tabular}




\begin{tabular}{|c|c|}
\hline 1051 & Fab D5 \\
\hline 1052 & Fab G1 \\
\hline 1053 & Fab M10 \\
\hline 1054 & Fab M12 \\
\hline 1055 & Fab M15 \\
\hline 1056 & Fab S10 \\
\hline 1057 & Fab S6 \\
\hline 1058 & Fab S8 \\
\hline 1059 & Fab S9 \\
\hline 1060 & Fab T3 \\
\hline 1061 & Md-1 \\
\hline 1062 & 1281 \\
\hline 1063 & Fab A9 \\
\hline 1064 & Fab G15 \\
\hline 1065 & Fab G5 \\
\hline 1066 & Fab L1 \\
\hline 1067 & Fab L11 \\
\hline 1068 & Fab L2 \\
\hline 1069 & Chessie 8 \\
\hline 1070 & $8 \mathrm{~F} 101$ \\
\hline 1071 & $8 \mathrm{~F} 102$ \\
\hline 1072 & CG-10 \\
\hline 1073 & CG-25 \\
\hline 1074 & CG-4 \\
\hline 1075 & CG-76 \\
\hline 1076 & CG-9 \\
\hline 1077 & $105-518$ \\
\hline 1078 & $31 \mathrm{~A} 1$ \\
\hline 1079 & 39A64 \\
\hline 1080 & 39B86 \\
\hline 1081 & 9303 \\
\hline 1082 & NC-1 \\
\hline \multicolumn{2}{|l|}{ Nef } \\
\hline 1083 & $4 \mathrm{H} 4$ \\
\hline 1084 & polyclonal \\
\hline$\overline{108}$ & $13 / 042$ \\
\hline 1086 & $13 / 035$ \\
\hline 1087 & AM5C6 \\
\hline$\overline{108}$ & AM5C6 \\
\hline 1089 & $25 / 03$ \\
\hline 1090 & $26 / 76$ \\
\hline 1091 & $3 \mathrm{~F} 2$ \\
\hline 1092 & 3D12 \\
\hline
\end{tabular}

\begin{tabular}{|c|c|}
\hline 1093 & polyclonal \\
\hline 1094 & polyclonal \\
\hline 1095 & $3 \mathrm{G} 12$ \\
\hline 1096 & $13 / 058$ \\
\hline 1097 & $26 / 028$ \\
\hline 1098 & $2 \mathrm{E} 3$ \\
\hline 1099 & polyclonal \\
\hline 1100 & F14.11 \\
\hline \begin{tabular}{|l|l|}
1101 \\
\end{tabular} & $31 / 03$ \\
\hline 1102 & polyclonal \\
\hline$\overline{110}$ & polyclonal \\
\hline 1104 & polyclonal \\
\hline 1105 & F1 \\
\hline 1106 & $2 \mathrm{~F} 2$ \\
\hline$\overline{110}$ & E9 \\
\hline$\overline{110}$ & 3E6 \\
\hline \begin{tabular}{|l|l|}
1109 \\
\end{tabular} & $2 \mathrm{~A} 3$ \\
\hline$\overline{111}$ & $2 \mathrm{E} 4$ \\
\hline$\overline{111}$ & $2 \mathrm{H} 12$ \\
\hline 1112 & $3 \mathrm{~A} 2$ \\
\hline 1113 & NF1A1 \\
\hline 11 & polyclonal \\
\hline 111. & E7 \\
\hline 1116 & AE6 \\
\hline$\overline{111}$ & AG11 \\
\hline 11 & EH1 \\
\hline 1119 & 6.1 \\
\hline 1120 & NF2B2 \\
\hline 112 & NF3A3 \\
\hline 1122 & NF8B4 \\
\hline 1123 & AE6 \\
\hline HIV- & \\
\hline 112 & polyclonal \\
\hline 112 & polyclonal \\
\hline 1126 & polyclonal \\
\hline$\overline{112}$ & polyclonal \\
\hline 1128 & polyclonal \\
\hline 11 & polyclonal \\
\hline $\begin{array}{l}1130 \\
1131\end{array}$ & $\begin{array}{l}\text { polyclonal } \\
\text { polyclonal }\end{array}$ \\
\hline
\end{tabular}




\section{IV-C HIV Antibodies Tables}

All HIV MAbs and polyclonal Abs that bind to linear epitopes 30 amino acids or less in length arranged by protein position. The table entries are sorted in a nested way - first by protein, then by HXB2 start location, then by antibody type and finally by antibody name. Abs that bind to conformational epitopes or with unknown epitopes are listed at the end of each protein section.

\section{IV-C-1 p17 Antibodies}

\begin{tabular}{|c|c|c|c|c|c|c|}
\hline No. & MAb ID & Author's Location & Sequence & Neutralizing & Immunogen & Species(Isotype) \\
\hline 1 & L14.17 & \multicolumn{5}{|c|}{$\begin{array}{l}\text { Vaccine Vector/Type: viral lysate Strain: BRU HIV component: virus } \\
\text { References Tatsumi1990, Robert-Hebmann1992b, Robert-Hebmann1992a }\end{array}$} \\
\hline 2 & polyclonal & $\begin{array}{l}\mathrm{p} 17(11-25) \quad \mathrm{p} 17 \text { (11-25 LAI) } \\
\text { Vaccine Vector/Type: recombinant protein, } \\
\text { References Truong1997 } \\
\text { An ELISA assay was used to study a panel } \\
\text { recognized by antibodies elicited by rp24CA } \\
\text { antibodies raised against anti-p55 virus-like } \\
\text { they are produced against the mature soluble }\end{array}$ & $\begin{array}{l}\text { GELDRWEKIRLRPGG } \\
\text {-like particle Strain: LAI } \\
\text { g peptides - mature p24 CA } \\
\text { ne p17MA epitope, residues } \\
\text { cles, suggesting a differentan } \\
\text { tein or the immature assembl }\end{array}$ & $\begin{array}{l}\mathrm{N} \\
\text { nent: } \mathrm{p} 24, \mathrm{p} 17, \mathrm{f} \\
\text { pped to residues } \\
\text { ne p24CA epito } \\
\text { erties for p24CA } \\
\text { he gag proteins }\end{array}$ & $\begin{array}{l}\text { Vaccine } \\
\text { p55 Adjuvan } \\
\text { 176-192, } 201 \\
\text { pe, residues } 17 \\
\text { A and p17MA } \\
\text { [Truong1997] }\end{array}$ & $\begin{array}{l}\text { mouse } \\
\text { ljuvant } \\
\text { ecognized by } \\
\text { pending on whether }\end{array}$ \\
\hline 3 & $32 / 5.8 .42$ & $\begin{array}{l}\text { p17 (12-19+ p17 (12-19 IIIB) } \\
\text { 100-105) } \\
\text { Vaccine Vector/Type: viral lysate } \\
\text { References Papsidero1989 } \\
\text { 32/5.8.42: Binds to two discontinuous region } \\
\text { |Papsidero1989] }\end{array}$ & $\begin{array}{l}\text { ELDRWEKI+ALDKIE } \\
\text { ositions } 12-19 \text { and 100-105, }\end{array}$ & no & $\begin{array}{l}\text { Vaccine } \\
\text { LDKIE - inhit }\end{array}$ & $\begin{array}{l}\text { murine }(\operatorname{IgG}) \\
\text { ty of cell free virus }\end{array}$ \\
\hline 4 & $32 / 5.8 .42$ & $\begin{array}{l}\text { p17 (12-19+ p17 (IIIB) } \\
\text { 100-105) } \\
\text { Vaccine Vector/Type: viral lysate HIV con } \\
\text { References Papsidero1989 } \\
\text { - 32/5.8.42: Inhibited infectivity of cell free v }\end{array}$ & $\begin{array}{l}\text { ELDRWEKI+ALDKIE } \\
\text { ent: virus } \\
\text { - bound to two peptides, ELI }\end{array}$ & no & Vaccine & osidero1989| \\
\hline 5 & HyHIV-1 & $\begin{array}{l}\text { p17 (12-29) p17 (12-29 JMH1) } \\
\text { Vaccine Vector/Type: recombinant protein } \\
\text { References Liu1995, Ota1998b } \\
\text { - HyHIV-1: This paper compares the results o } \\
\text { isotope-free BIAcore system, and results we } \\
\text { for both membrane binding and nuclear loca }\end{array}$ & $\begin{array}{l}\text { ELDKWEKIRLRPGGKTLY } \\
\text { component: } 17 \\
\text { inity constant }(\mathrm{Ka}) \text { measurem } \\
\text { und to be similar for HyHIV. } \\
\text { ion Ota1998b }\end{array}$ & $\begin{array}{l}\text { no } \\
17 \text { MAbs using } \\
\text { lAbs all bind to }\end{array}$ & $\begin{array}{l}\text { Vaccine } \\
\text { g double Ab m } \\
\text { the first alpha }\end{array}$ & $\begin{array}{l}\text { murine (IgG1) } \\
\text { the faster, } \\
\text { a functional domain }\end{array}$ \\
\hline
\end{tabular}




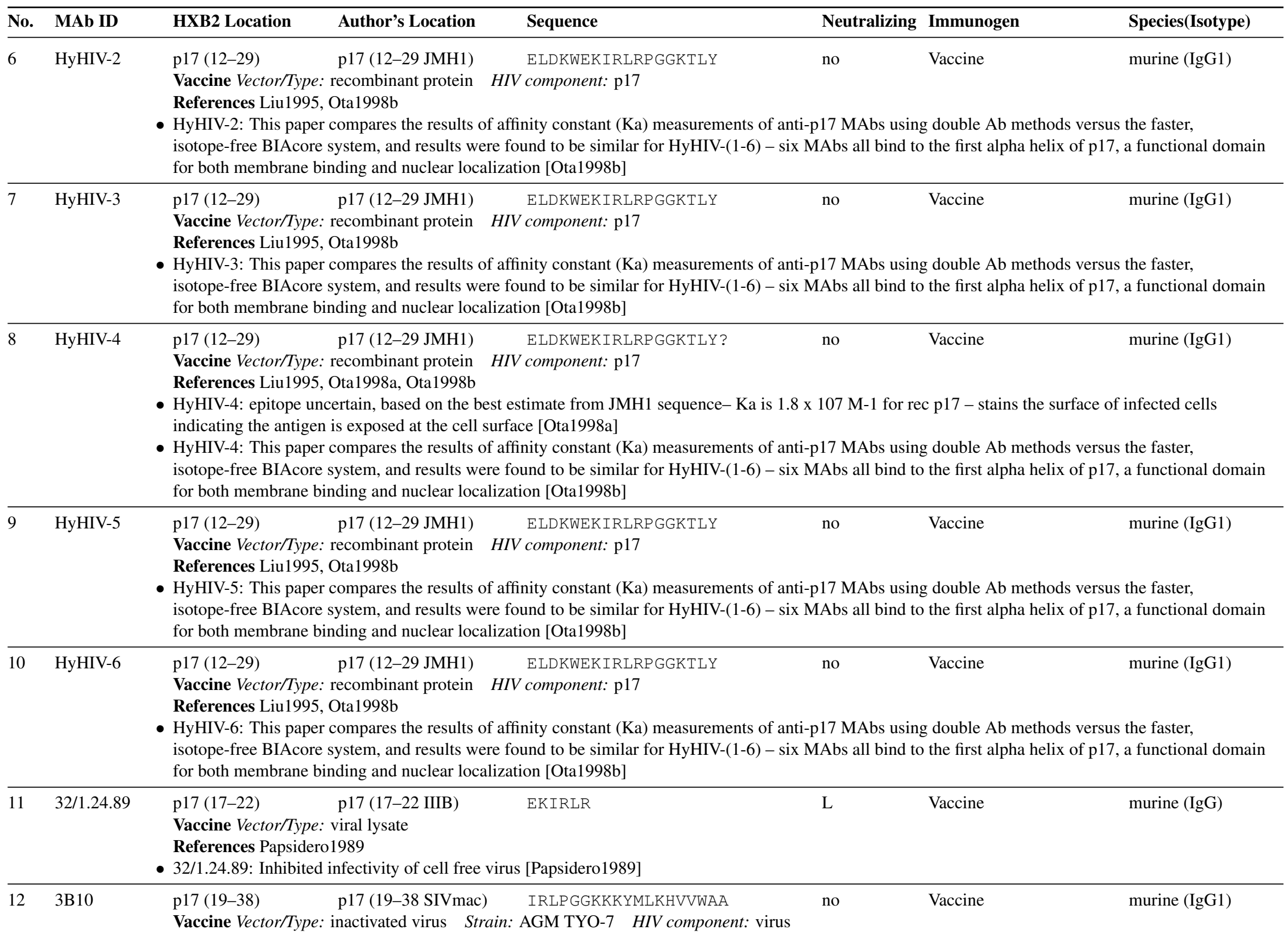




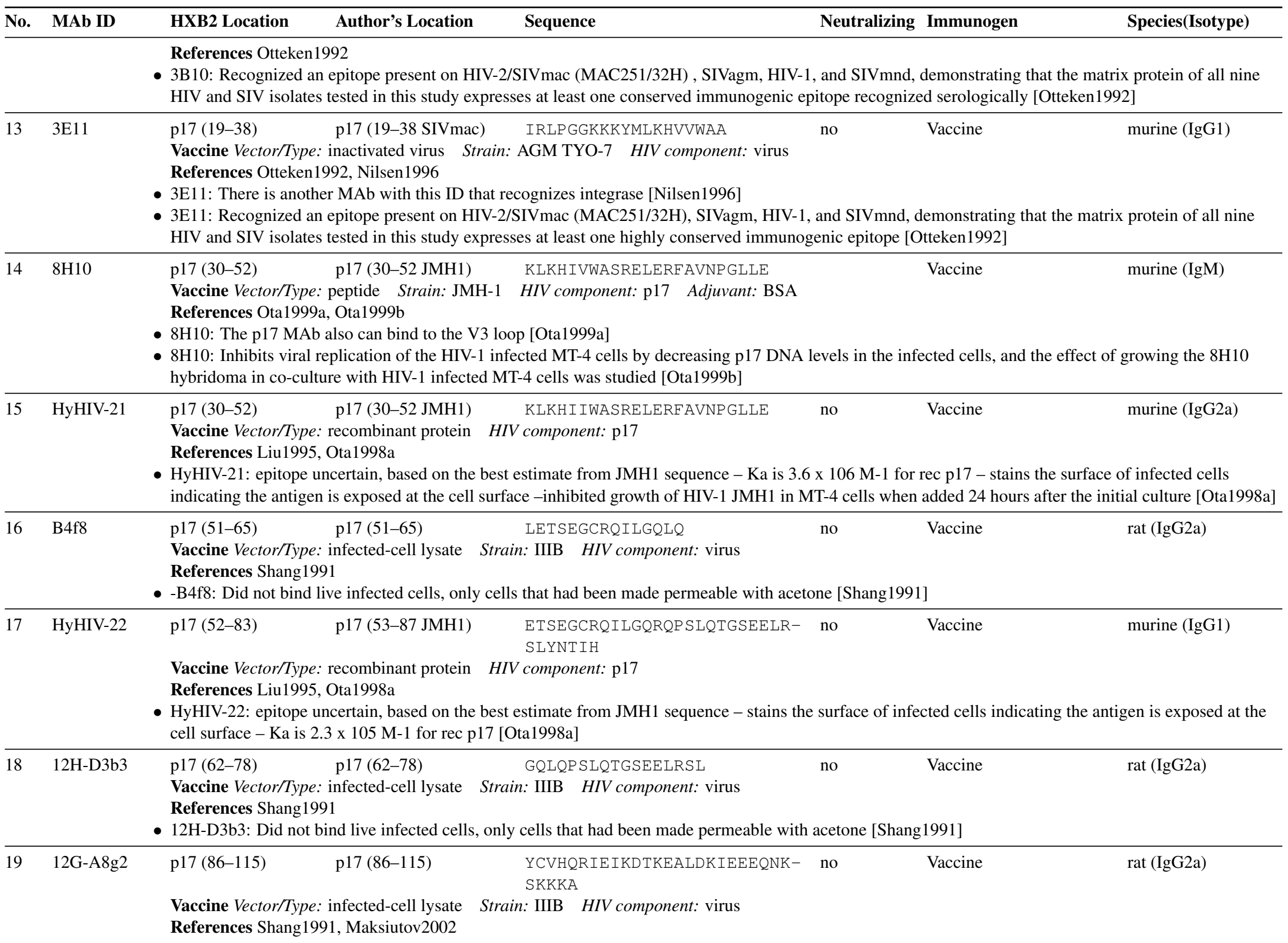




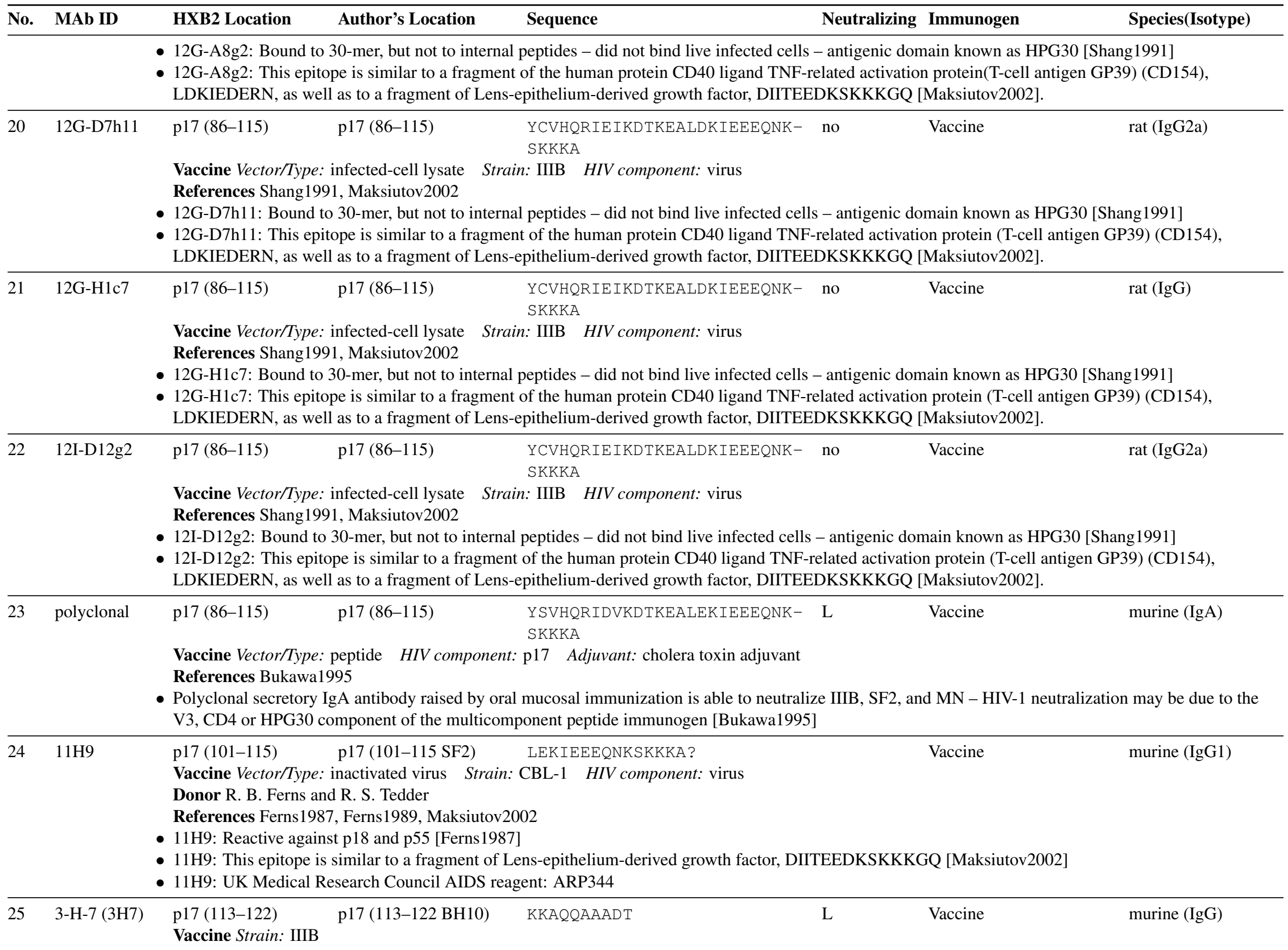




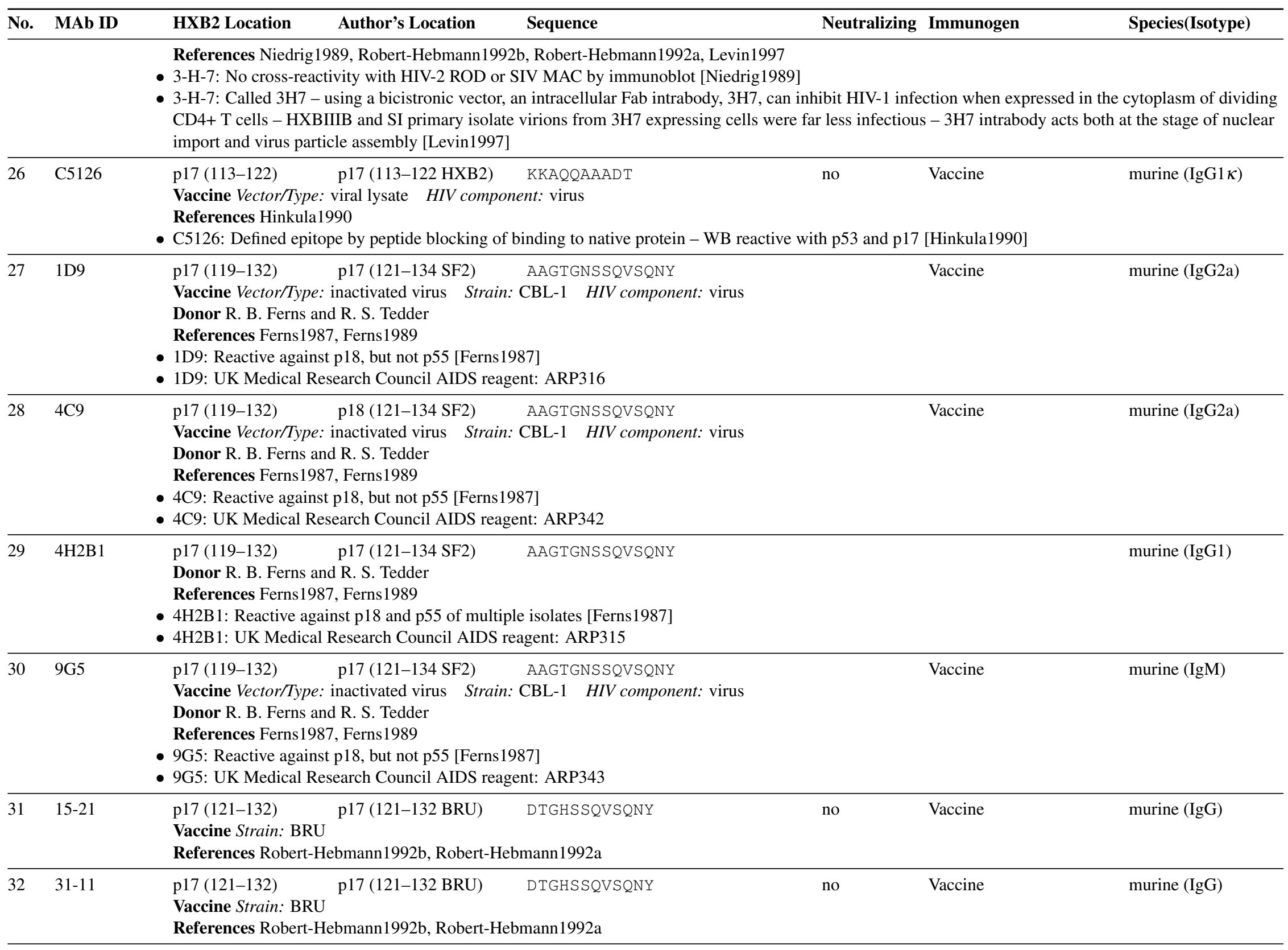




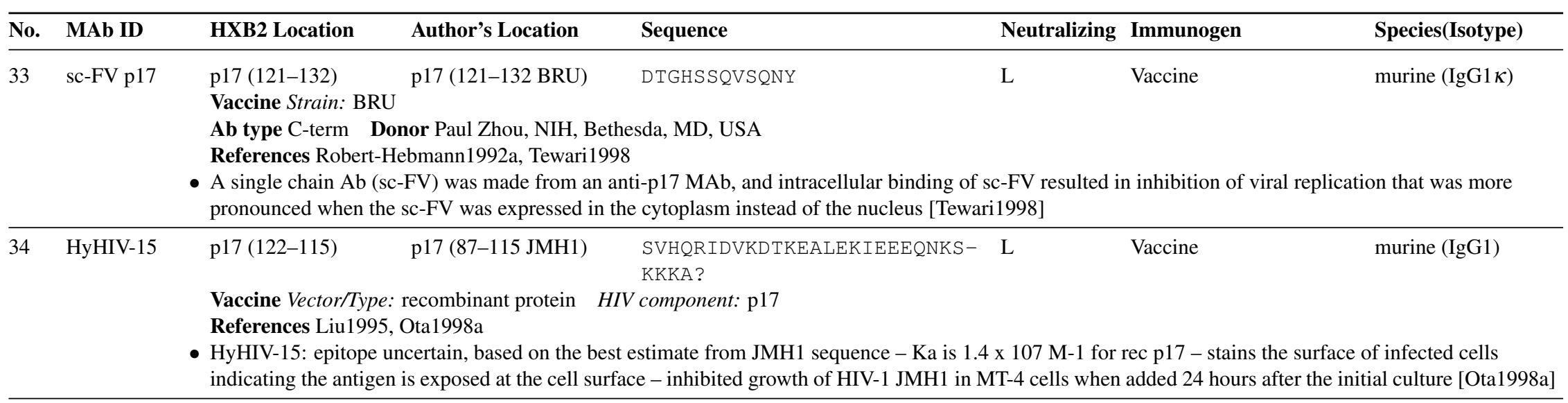




\section{IV-C-2 p24 Antibodies}

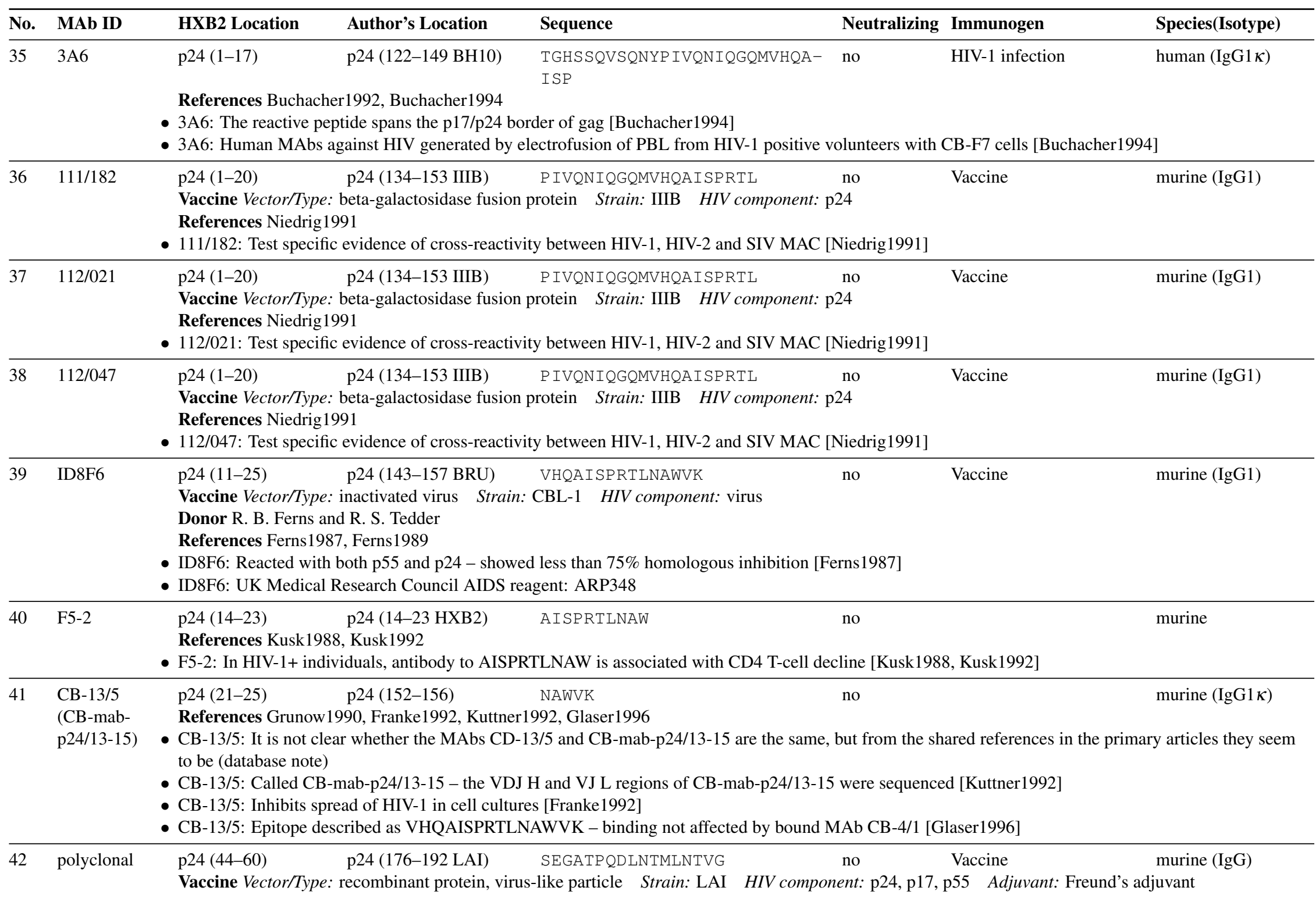




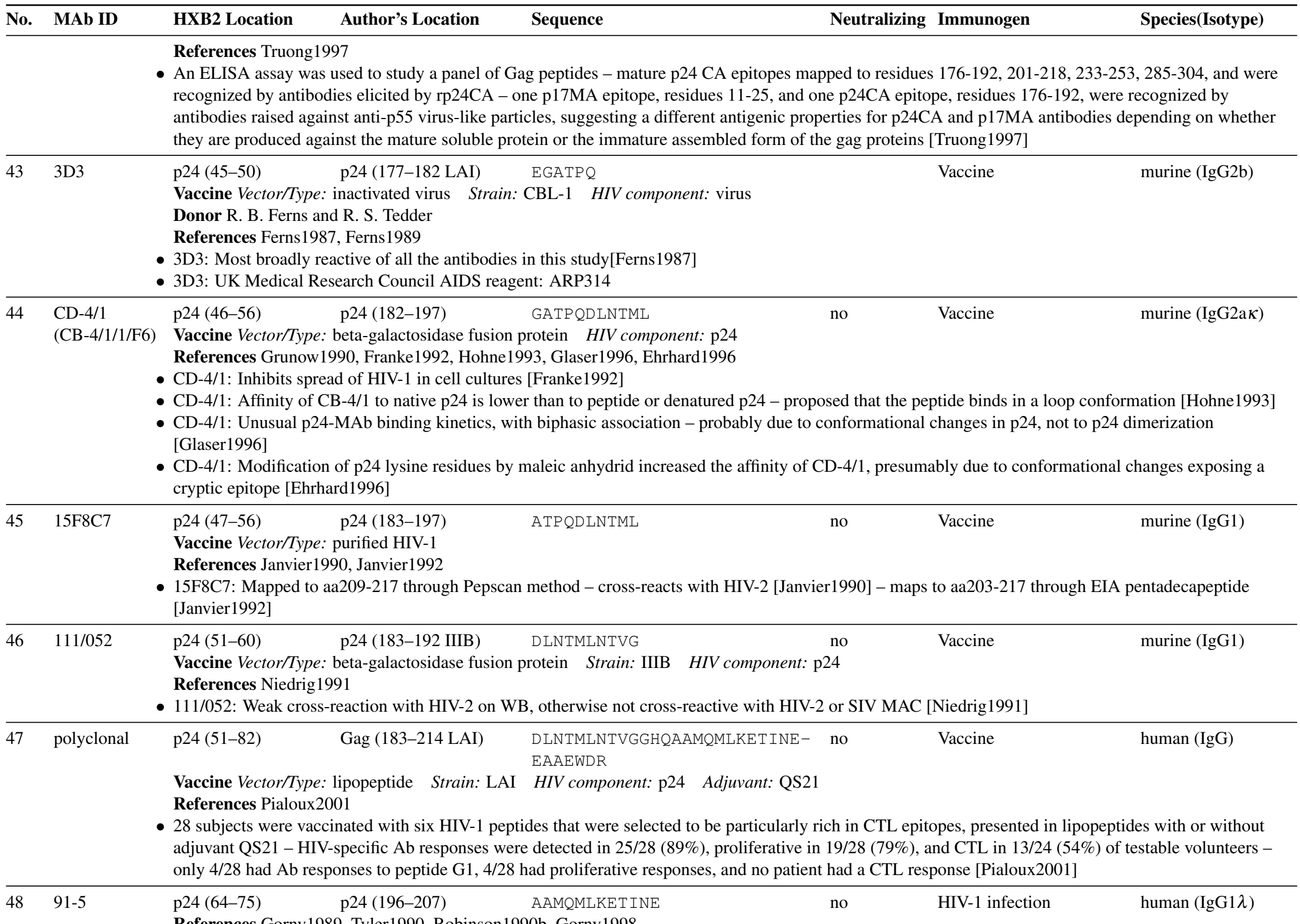

References Gorny1989, Tyler1990 Robinson1990b Gorny1998

- 91-5: Synthesized by immortalization of peripheral blood cells with Epstein-Barr virus [Gorny1989] 


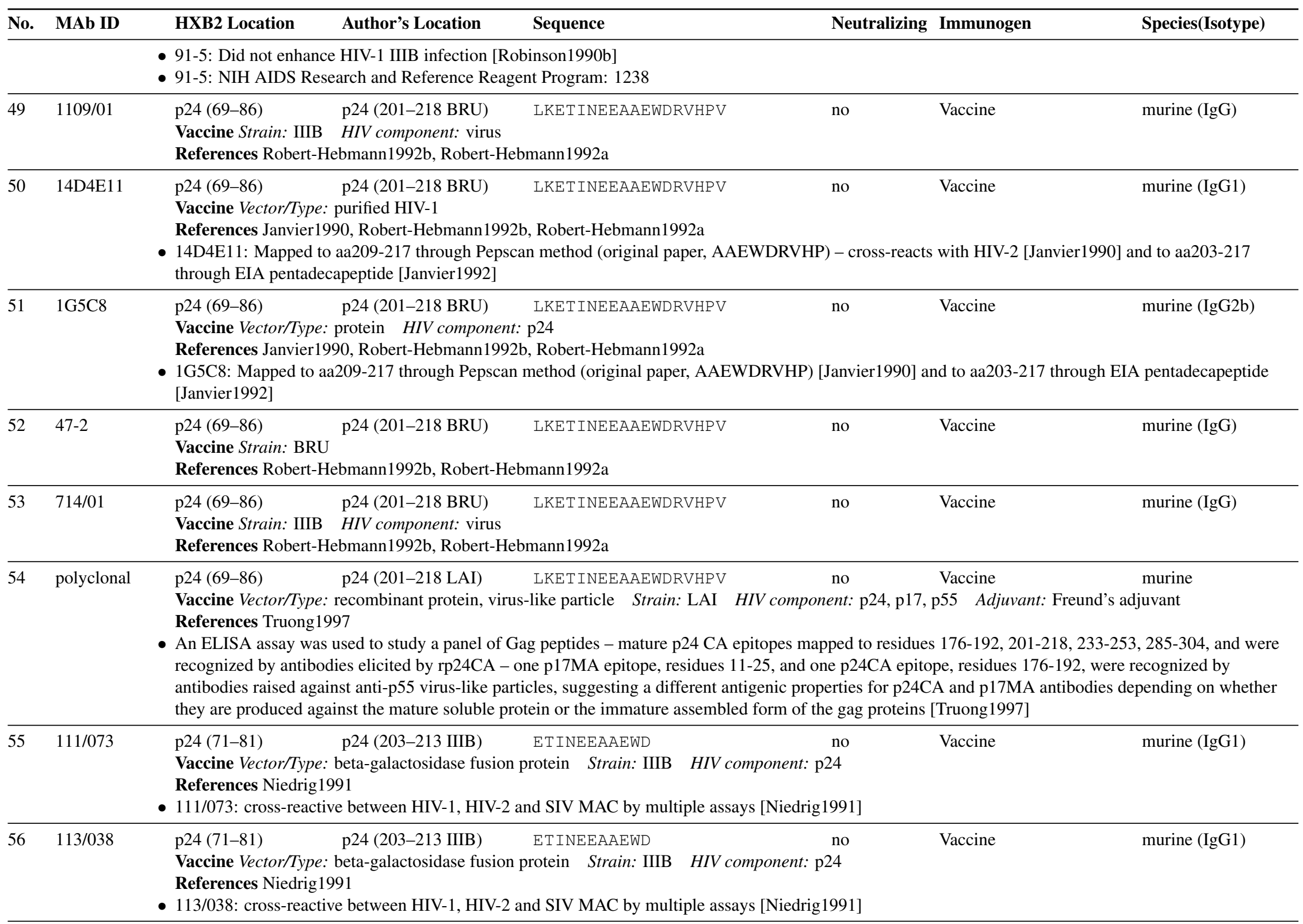




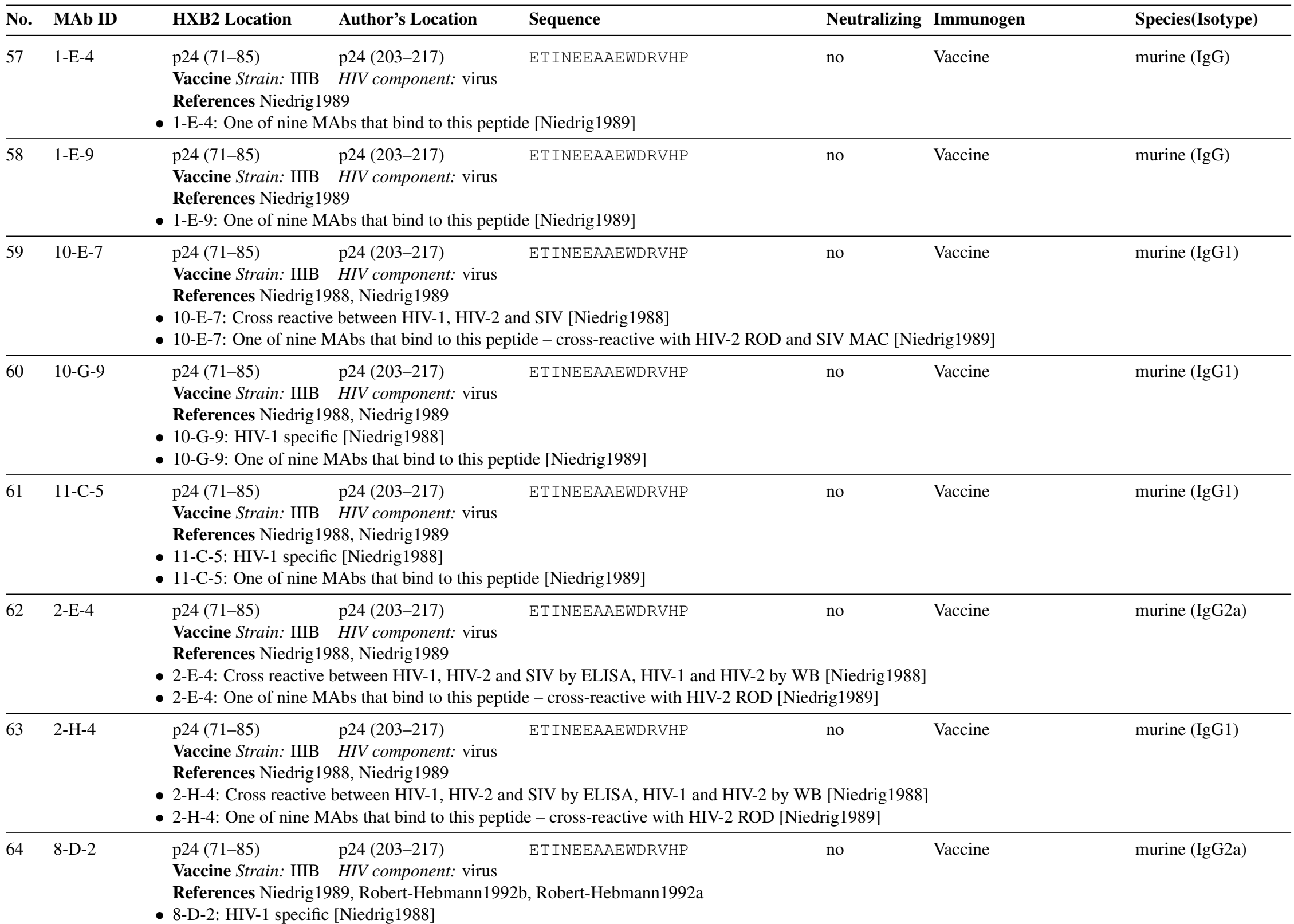




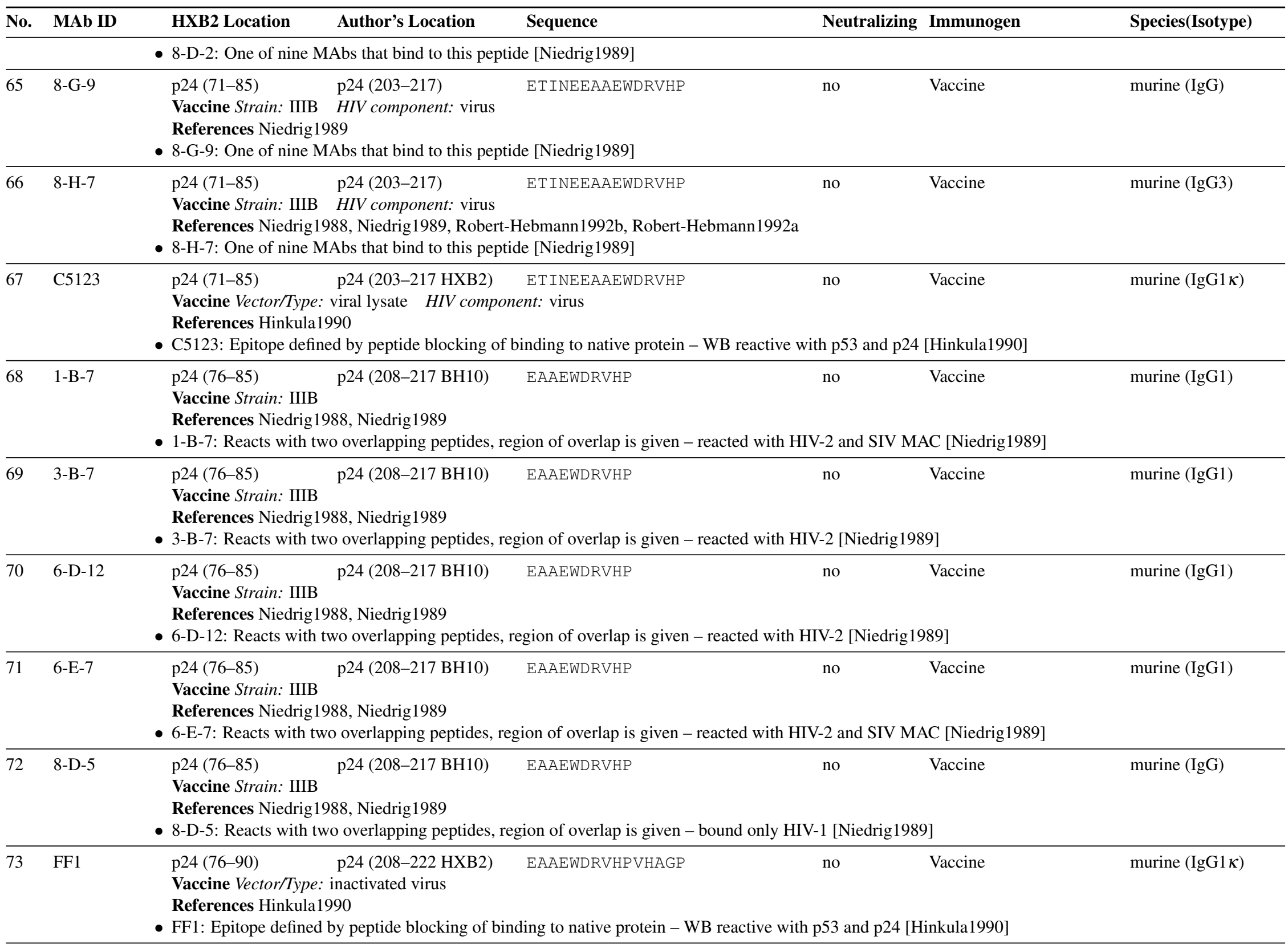




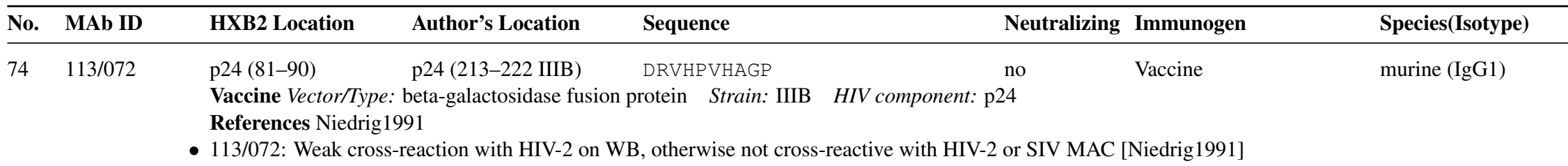

\begin{tabular}{llllll}
\hline 75 & 25.3 & p24 (82-102) & p24 (82-102) no & murine (IgG1 $\kappa)$
\end{tabular}

References Momany1996

- 25.3: Crystal structure of the CA protein bound to Fab 25.3 was solved - monomers form 7 alpha-helices arranged in a coiled-coil - Fab binds to a long antigenic peptide that separates the longest helices, with a salt bridge at CA 82 R, and interactions as far away as positions 100 and 102 [Momany1996]

\begin{tabular}{lllcc}
\hline 76 & $13-102-100$ & p24 (84-94) m24 (102-112 IIIB) HPVHAGP IAPG \\
& & Donor Advanced Technologies, Inc., Columbia, MD
\end{tabular}

References Parker1996, Qian1998

- 13-102-100: Binding site (HPVHAGPIAPG) defined by epitope footprinting - first binding p24 to MAb, then allowing proteolytic cleavage to take place to cleave unprotected residues, then performing mass spectrometry to identify protected residues of epitope [Parker1996]

- 13-102-100: Affinity capillary electrophoresis was used to fine map this epitope, and the optimal peptide was defined as VHAGPIAPGIAP - this method uses migration time shifts to probe relative affinities of Abs - the antibody binds to the cyclophilin A binding domain [Qian1998]

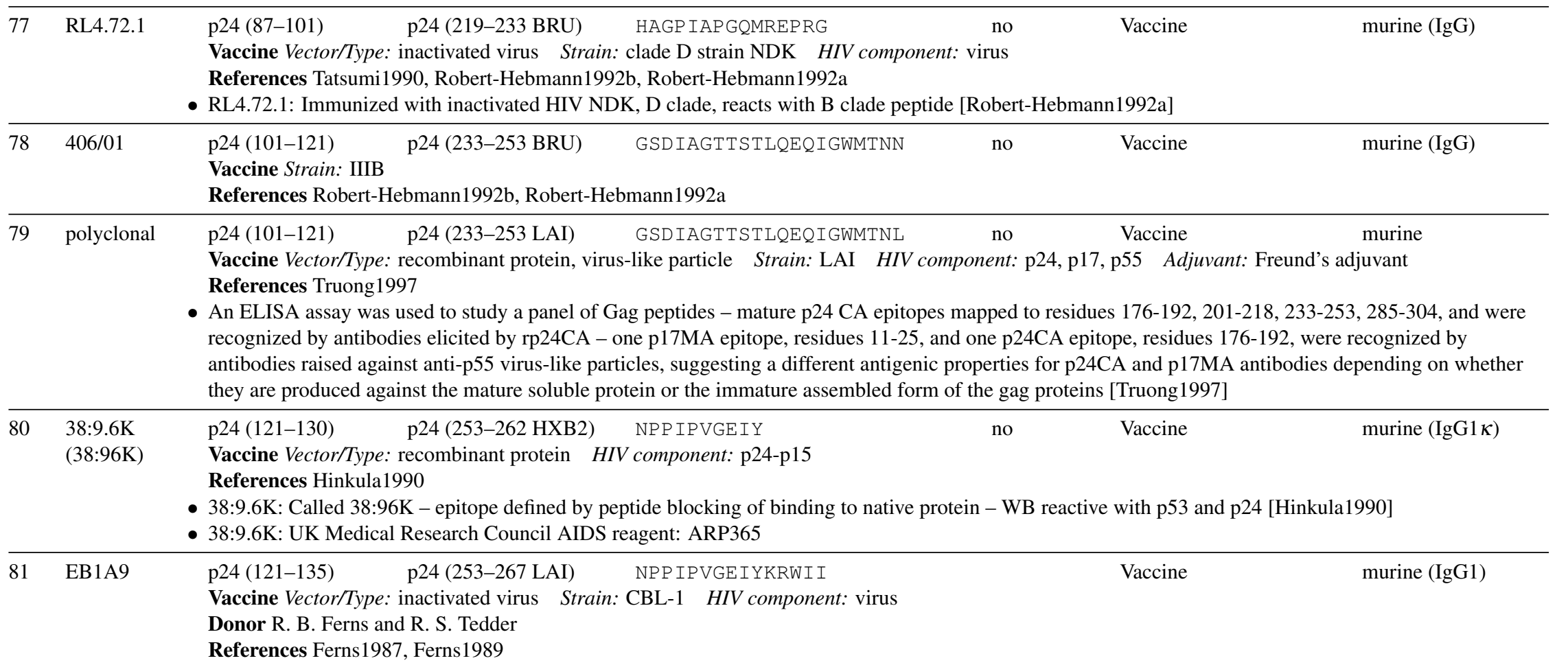




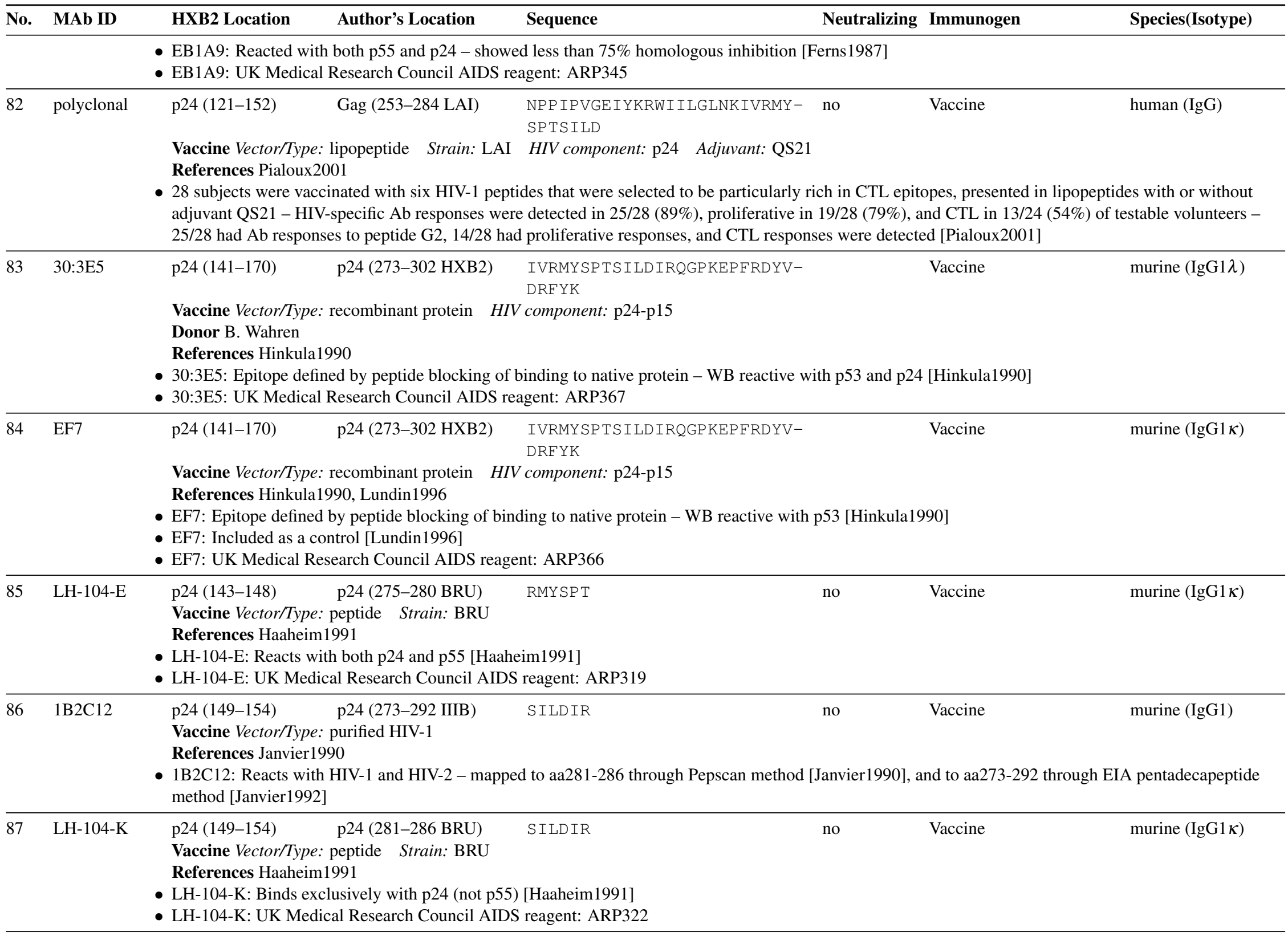




\begin{tabular}{|c|c|c|c|c|c|}
\hline No. & MAb ID & Author's Location & Neutralizing & Immunogen & Species(Isotype) \\
\hline 88 & LH-104-A & $\begin{array}{l}\text { p24 }(152-157+\quad \text { p24 (BRU) } \\
\text { 219-224) } \\
\text { Vaccine Vector/Type: peptide HIV component. } \\
\text { References Haaheim1991 } \\
\text { LF-104-A: A } 104 \text { amino acid peptide was used t } \\
\text { indicated the region 270-286 [Haaheim1991] } \\
\text { LH-104-A: UK Medical Research Council AID }\end{array}$ & $\begin{array}{l}\text { DIRQGP +QGVGGP } \\
\text { p24 } \\
\text { o immunize mice - hexapeptide scans revealed two reacti } \\
\text { reagent: ARP307 }\end{array}$ & $\begin{array}{l}\text { Vaccine } \\
\text { ve p24 peptide }\end{array}$ & $\begin{array}{l}\text { murine }(\operatorname{IgG} 1 \kappa) \\
\text { petition studies }\end{array}$ \\
\hline 89 & 1.17 .3 & $\begin{array}{l}\text { p24 (152-172) p24 (152-172 SIVmac) } \\
\text { Vaccine Vector/Type: inactivated virus Strain: } \\
\text { References Otteken1992 } \\
\text { 1.17.3: Recognized an epitope present on HIV-2 }\end{array}$ & $\begin{array}{l}\text { CVKQGPKEPFQSYVDRFYKSL } \\
\text { AGM TYO-7 HIV component: virus } \\
\text { /SIVmac (MAC251/32H) and HIV-2smmH4, but not SIVa }\end{array}$ & $\begin{array}{l}\text { Vaccine } \\
\text { agmTYO-1, H }\end{array}$ & $\begin{array}{l}\text { murine (IgG1) } \\
\text { IVmnd Otteken1992 }\end{array}$ \\
\hline 90 & $1 \mathrm{~A} 7$ & $\begin{array}{l}\text { p24 (152-172) p24 (152-172 SIVmac) } \\
\text { Vaccine Vector/Type: inactivated virus Strain: } \\
\text { References Otteken1992 } \\
\text { - 1A7: Recognized an epitope present on HIV-2/S }\end{array}$ & $\begin{array}{l}\text { CVKQGPKEPFQSYVDRFYKSL } \\
\text { AGM TYO-7 HIV component: virus } \\
\text { IVmac (MAC251/32H) and HIV-2smmH4, but not SIVag }\end{array}$ & $\begin{array}{l}\text { Vaccine } \\
\text { mTYO-1, HIV }\end{array}$ & $\begin{array}{l}\text { murine (IgG1) } \\
\text { Imnd Otteken1992] }\end{array}$ \\
\hline 91 & $1 \mathrm{~F} 6$ & $\begin{array}{l}\text { p24 (152-172) p24 (152-172 SIVmac) } \\
\text { Vaccine Vector/Type: inactivated virus Strain: } \\
\text { References Otteken1992 } \\
\text { - 1F6: Recognized an epitope present on HIV-2/S }\end{array}$ & $\begin{array}{l}\text { CVKQGPKEPFQSYVDRFYKSL } \\
\text { AGM TYO-7 HIV component: virus } \\
\text { IVmac (MAC251/32H) and HIV-2smmH4, but not SIVag, }\end{array}$ & $\begin{array}{l}\text { Vaccine } \\
\text { mTYO-1, HIV }\end{array}$ & $\begin{array}{l}\text { murine (IgG1) } \\
\text { mnd [Otteken1992] }\end{array}$ \\
\hline 92 & 23A5G4 & $\begin{array}{l}\text { p24 (153-172) p24 (285-304 IIIB) } \\
\text { Vaccine Vector/Type: protein HIV component: } \\
\text { References|Janvier1990 Janvier1996 } \\
\text { 23A5G4: Mapped to aa209-217 through Pepsca } \\
\text { 23A5G4: A few sera which were able to bind th } \\
\text { 23A5G4 to the native p24 [Janvier1996] }\end{array}$ & $\begin{array}{l}\text { IRQGP KEPFRDYVDRFYKTL no } \\
\text { p24 } \\
\text { method [Janvier1990] and to aa285-304 through EIA pe } \\
\text { linear sequence 178-192, but not sequence 288-302 in a }\end{array}$ & $\begin{array}{l}\text { Vaccine } \\
\text { ntadecapeptid } \\
\text { n indirect pept }\end{array}$ & $\begin{array}{l}\text { murine (IgG1) } \\
\text { vier1992] } \\
\text { iibited the binding of }\end{array}$ \\
\hline 93 & 23A5G5 & $\begin{array}{l}\text { p24 (153-172) p24 (285-304 BRU) } \\
\text { Vaccine Vector/Type: protein Strain: IIIB H } \\
\text { References Robert-Hebmann1992b Robert-Het }\end{array}$ & $\begin{array}{l}\text { IRQGP KEPFRDYVDRFYKTL } \\
\text { IV component: } \mathrm{p} 24 \\
\text { mann1992a }\end{array}$ & Vaccine & murine (IgG) \\
\hline 94 & 3D10G6 & $\begin{array}{l}\text { p24 (153-172) p24 (285-304 IIIB) } \\
\text { Vaccine Vector/Type: purified HIV-1 } \\
\text { References|Janvier1990 } \\
\text { 3D10G6: Epitope cross-reacts with HIV-1 and H } \\
\text { pentadecapeptide method [Janvier1992] }\end{array}$ & $\begin{array}{l}\text { IRQGP KEPFRDYVDRFYKTL no } \\
\text { IIV-2 - mapped to aa260-267 through Pepscan method } \mathrm{Ja}\end{array}$ & Vaccine & $\begin{array}{l}\text { murine }(\mathrm{IgG} 1) \\
4 \text { through EIA }\end{array}$ \\
\hline 95 & polyclonal & $\begin{array}{l}\mathrm{p} 24(153-172) \quad \mathrm{p} 24(285-304 \mathrm{LAI}) \\
\text { Vaccine Vector/Type: recombinant protein, virt } \\
\text { References Truong1997 }\end{array}$ & $\begin{array}{l}\text { IRQGPKEPERDYVDRFYKTL } \\
\text {-like particle Strain: LAI HIV component: } 24, \text { p17 }\end{array}$ & $\begin{array}{l}\text { Vaccine } \\
\text { p55 Adjuva }\end{array}$ & $\begin{array}{l}\text { murine } \\
\text { juvant }\end{array}$ \\
\hline
\end{tabular}




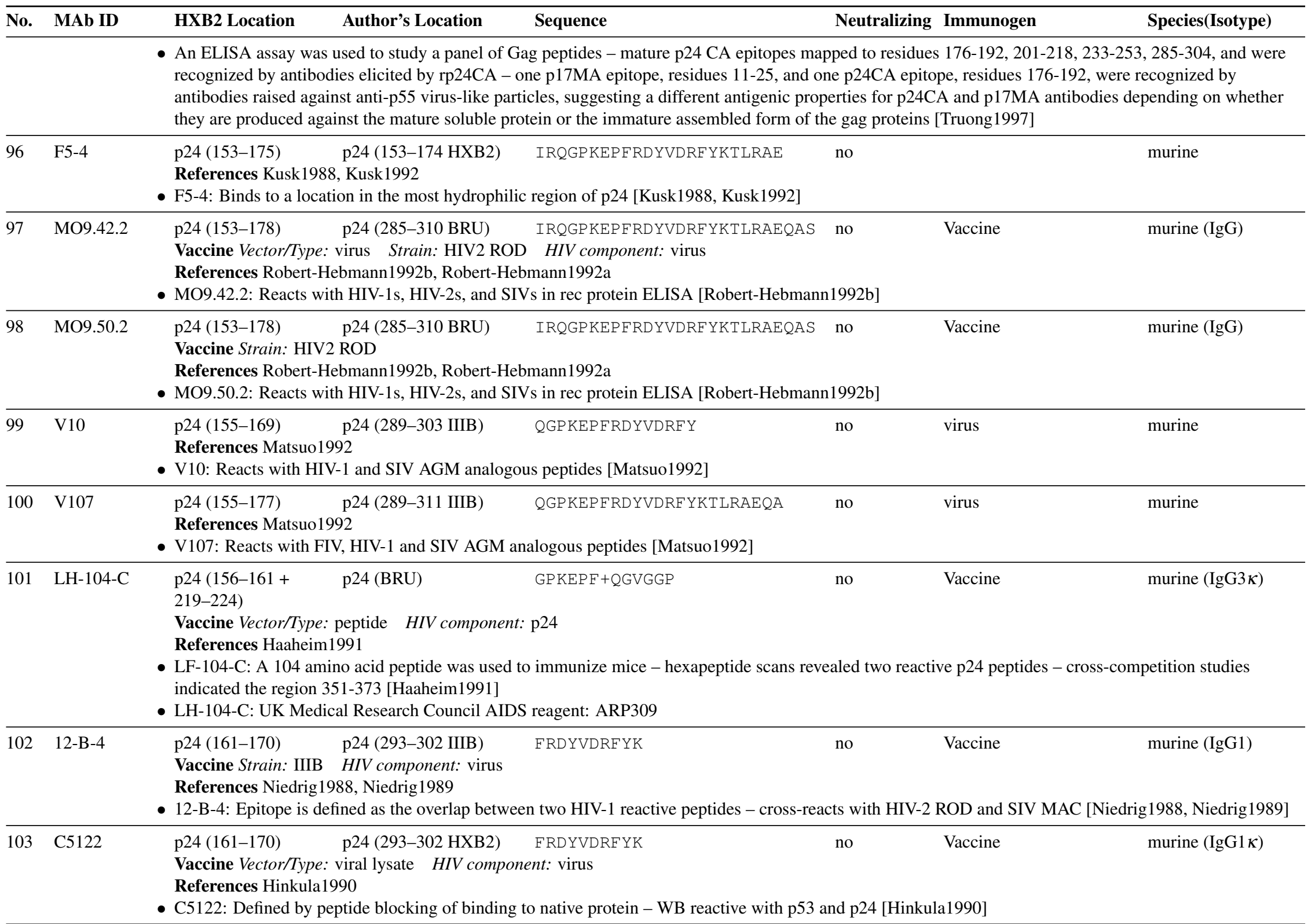




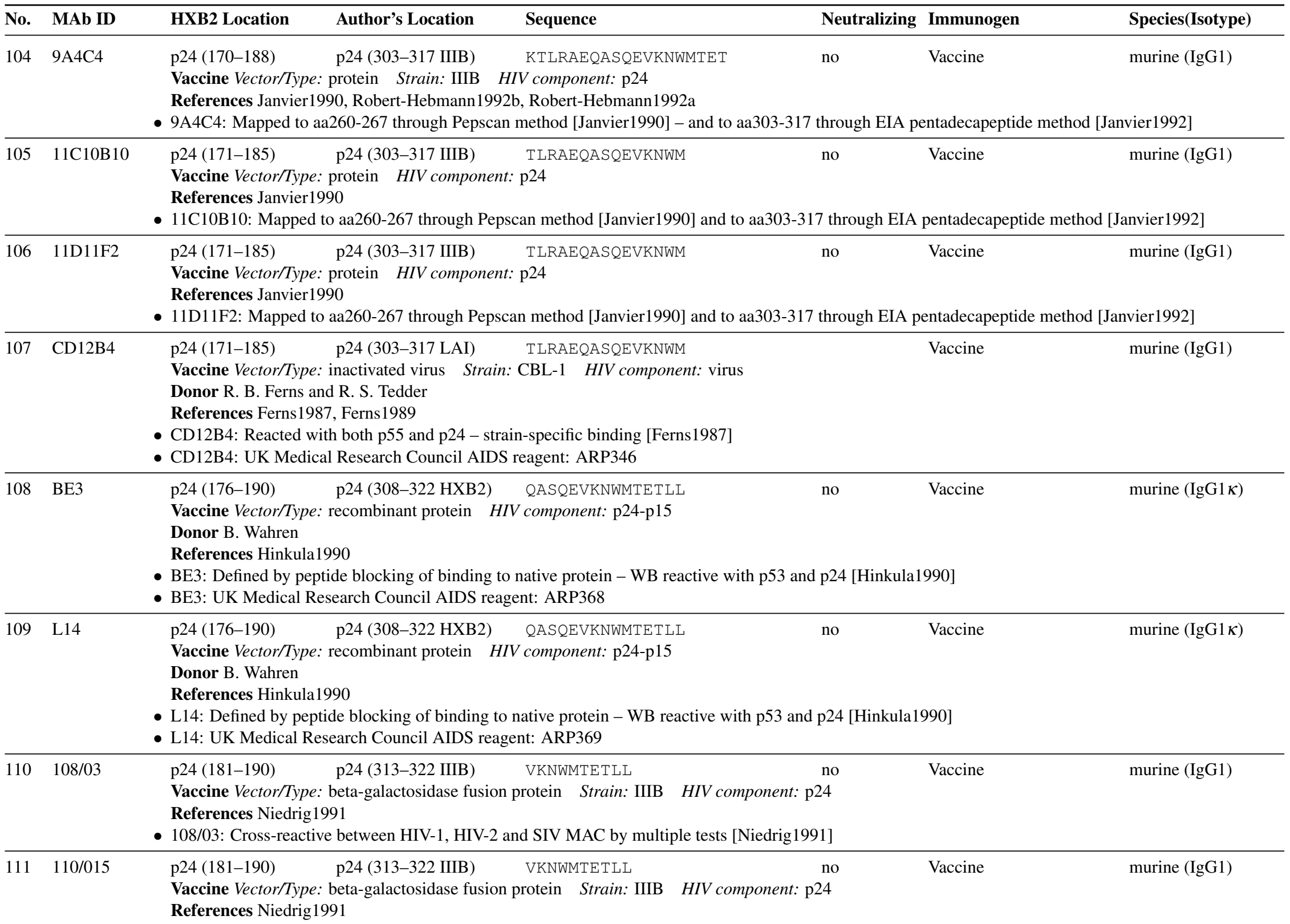




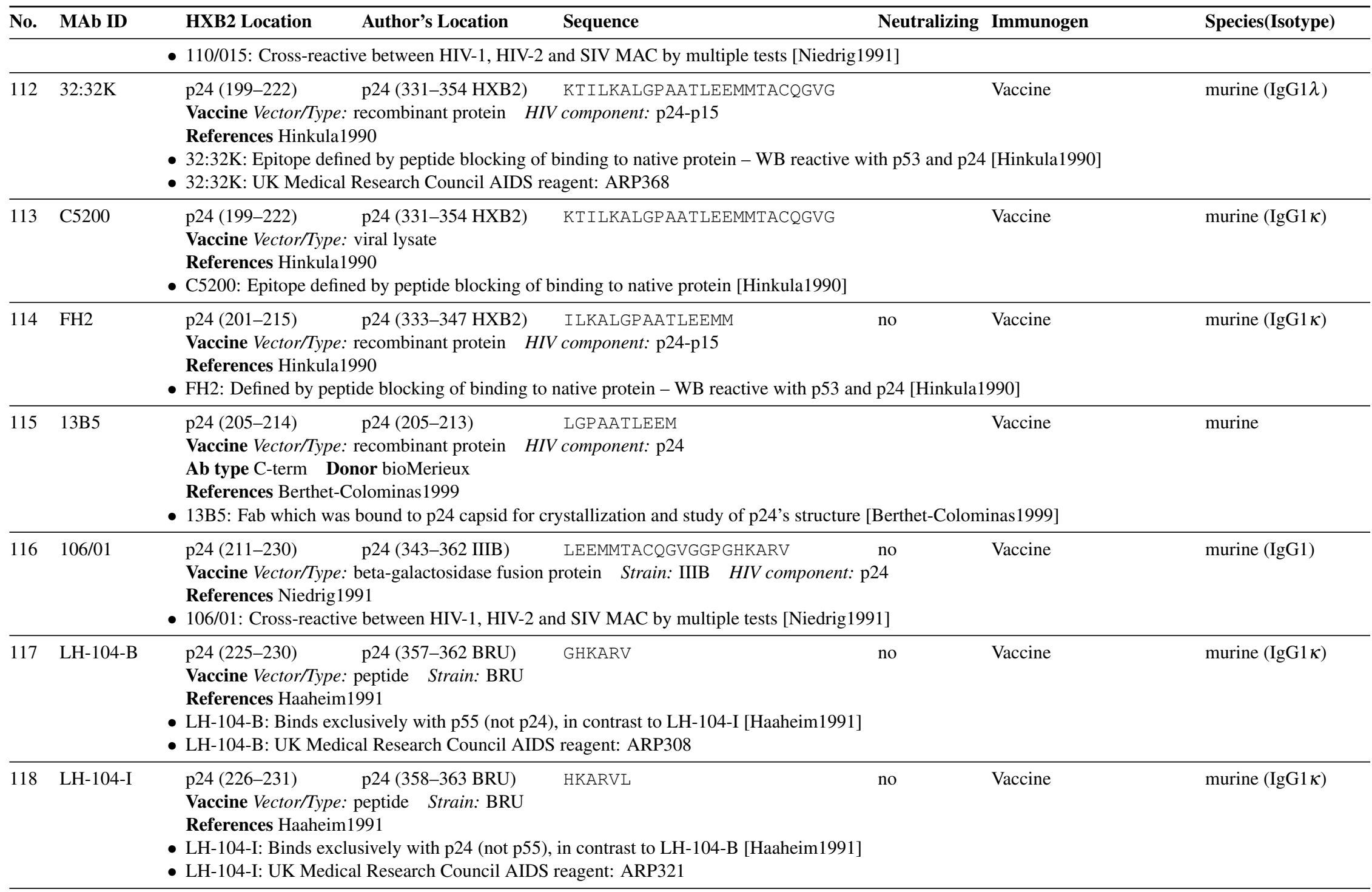




\section{IV-C-3 p24-p2p7p1p6 Antibodies}

\begin{tabular}{|c|c|c|c|c|c|c|c|}
\hline No. & MAb ID & HXB2 Location & Author's Location & Sequence & Neutralizing & Immunogen & Species(Isotype) \\
\hline 119 & LH-104-G & $\begin{array}{ll} & \text { p24-p2p7p1p6 } \\
& (231-5) \\
& \text { Vaccine Vector/Ty } \\
& \text { References Haah } \\
\text { - } & \text { LH-104-G: React } \\
\text { - } & \text { LH-104-G: This e } \\
\text { - } & \text { LH-104-G: UK N }\end{array}$ & $\begin{array}{l}\text { p24 (363-368 BRU) } \\
\text { peptide Strain: BRU } \\
991 \\
\text { th both p24 and p55, in } \\
\text { pe overlaps the p24-p2 } \\
\text { al Research Council Al }\end{array}$ & $\begin{array}{l}\text { LAEAMS } \\
\text { trast to LH-104-I [Haaheim1991] } \\
\text { vage site, database note } \\
\text { reagent: ARP320 }\end{array}$ & no & Vaccine & murine $(\operatorname{IgG} 1 \kappa)$ \\
\hline
\end{tabular}




\section{IV-C-4 p2p7p1p6 Antibodies}

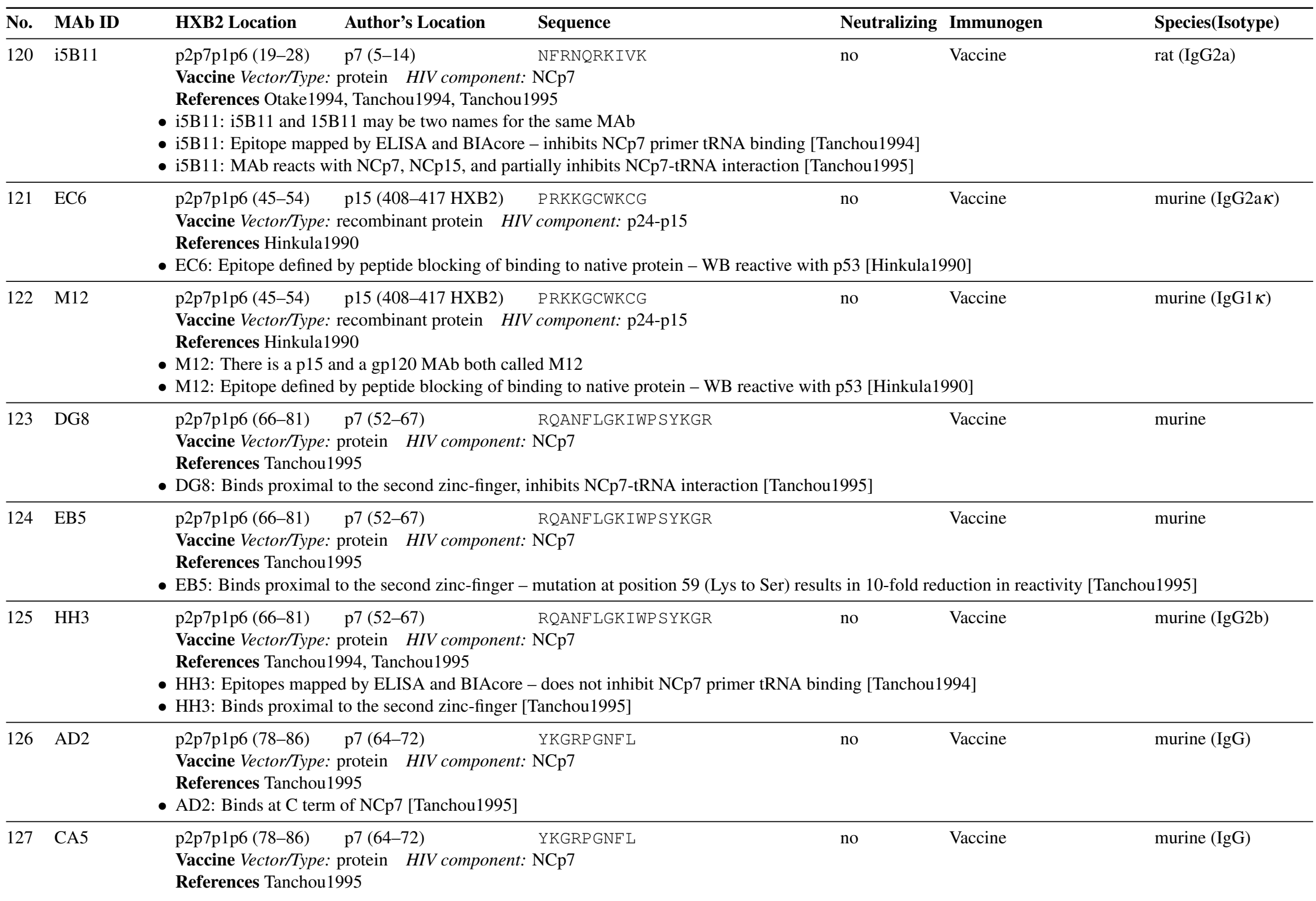




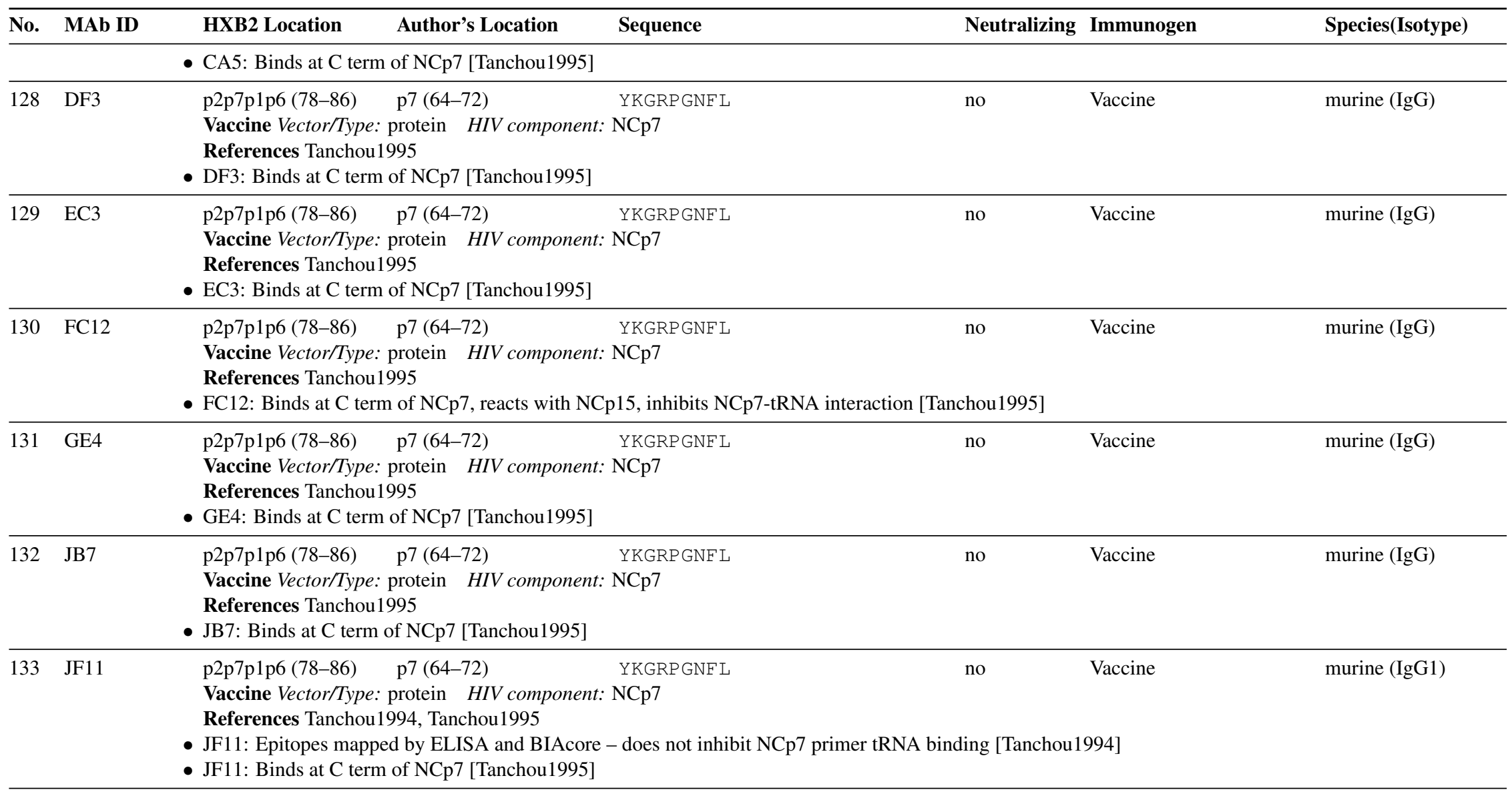




\section{IV-C-5 Gag Antibodies}

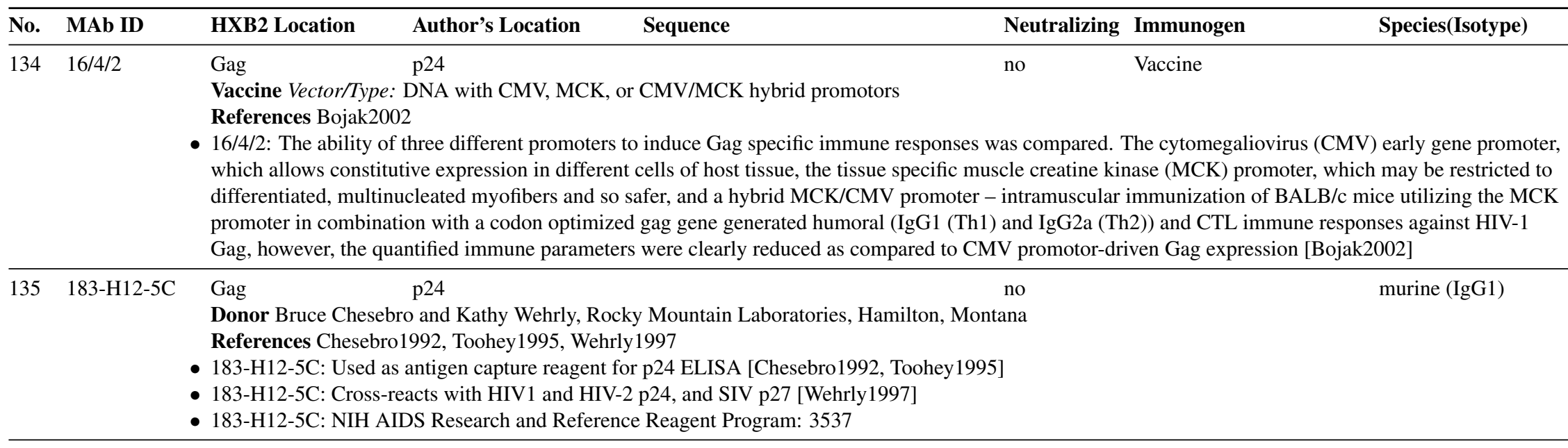

Donor Susan Zolla-Pazner (Zollas01@ mcrcr6.med.nyu) (NYU Med. Center)

References Gorny1989, Tyler1990 Robinson1991

- 241-D: An antibody by this name is available in the NIH AIDS Research and Reference Reagent Program, and they refer to the papers |Gorny1989 Tyler1990 Robinson1991], but no p24 MAb by this name is discussed in these papers

- 241-D: MH AIDS Research and Reference Reagent program: 1244

$137 \quad 2 \mathrm{~A} 6$

Gag p17

Donor A. O. Arthur, Frederick Cancer Research and Development Center, Frederick, MD

References Pincus1998

- 2A6: Part of a panel of 17 MAbs used as controls testing for the dual specificity of MAb G11H3 for both p17 and mycoplasma [Pincus1998]

\section{Gag p24 (1-158 SF2)}

murine $(\mathrm{IgG} 1)$

Donor Biodesign International, Kennebunk, Maine, USA

References Hochleitner2000a

- 5E2.A3k: The Ab binding site was studied with epitope excision (protein is bound in native conformation to immobilized MAb, then digested with proteolytic enzymes) and extraction (protein is digested then allowed to react with Ab), followed by mass spectroscopy, as well as lysine modification - the epitope is discontinuous, but involves the highly conserved N-term proline, and the antibody recognizes SIVs and HIV-2 as well as HIV-1 p24 Hochleitner2000a

References Gorny 1989, Robinson1990b, Robinson1991, Spear1993 Gorny1997 Gorny 1998, Bandres1998

- 71-31: Did not enhance HIV-1 IIIB infection Robinson1990b

- 71-31: No enhancing or neutralizing activity Robinson1991]

- 71-31: Did not mediate deposition of complement component C3 on HIV infected cells [Spear1993] 


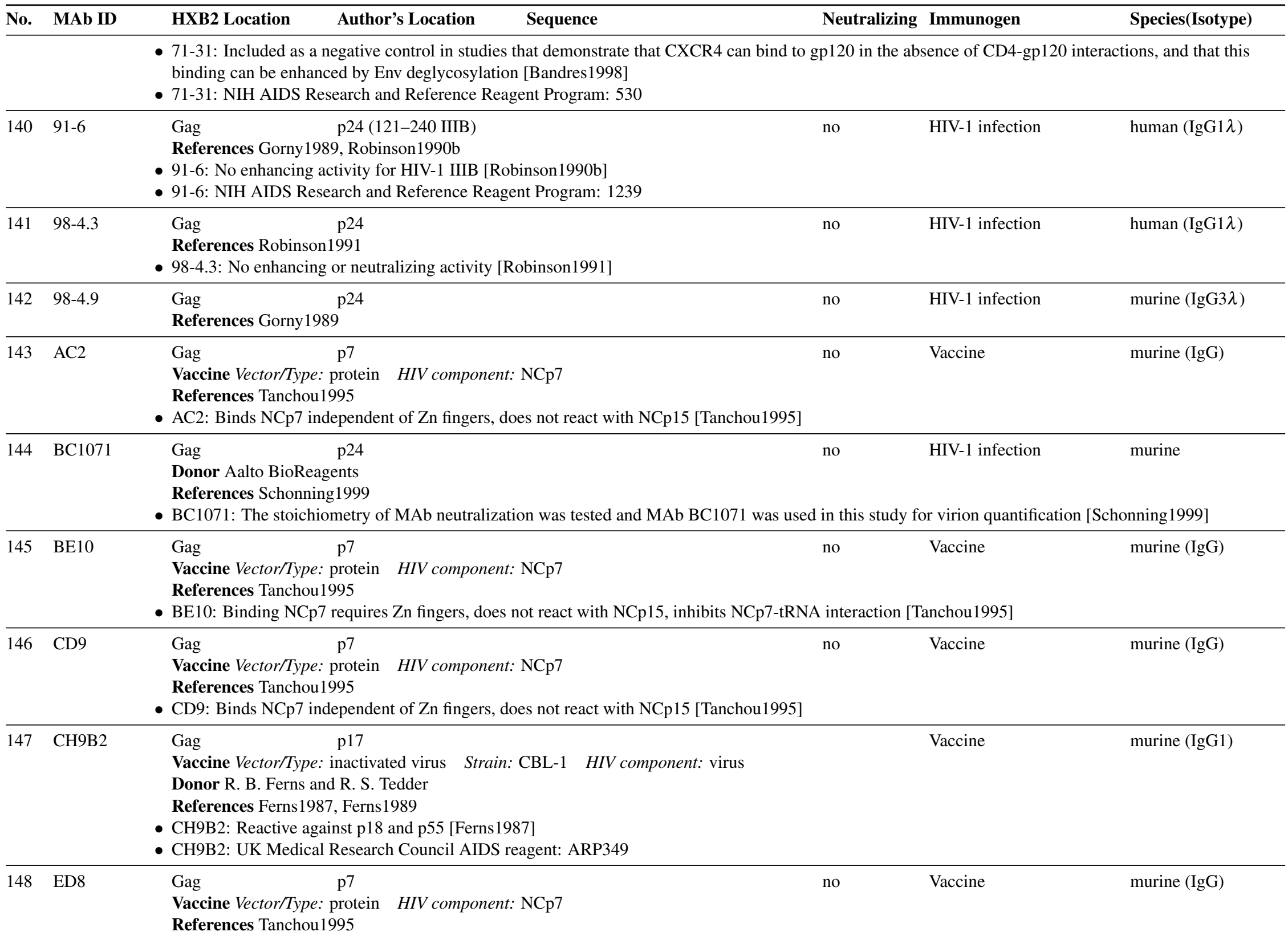




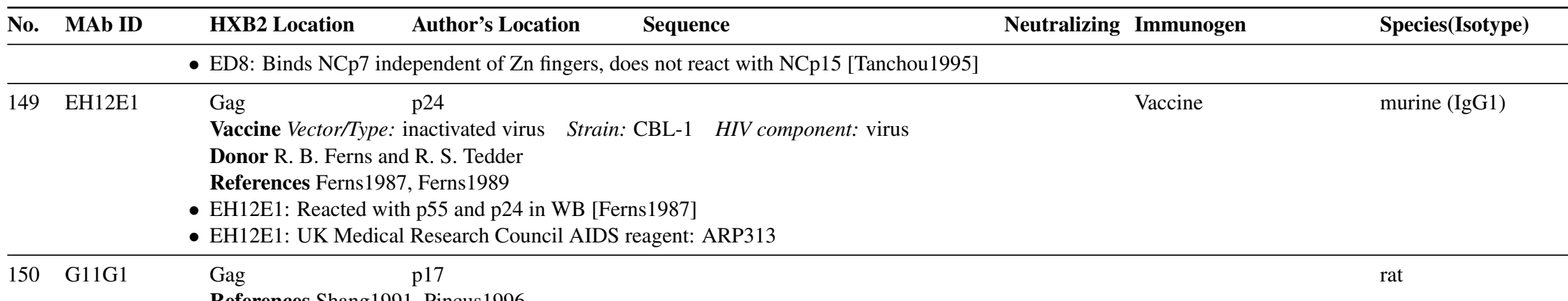

G11G1: Immunotoxins were generated by linking Env MAbs to ricin A - immunotoxins mediated cell killing, but only if the antigen was expressed at the cell surface - ricin-G11G1 did not mediate cell killing [Pincus1996]

\begin{tabular}{lll}
\hline 51 & G11H3 & Gag \\
& References & Shang1991, Pincus1998
\end{tabular}

- G11H3: This MAb is cross-reactive between p17 and mycoplasma - this antibody binds strain specifically to the variable lipoprotein (Vlp) F of M.

hyorhinis, in the region of the carboxy-terminal repeat CGGSTPTPEQGNNQGGSTPTPEQGNSQVSK - the p17 epitope is discontinuous, but p17 and Vlp F share the tetrapeptide SQVS [Pincus1998]

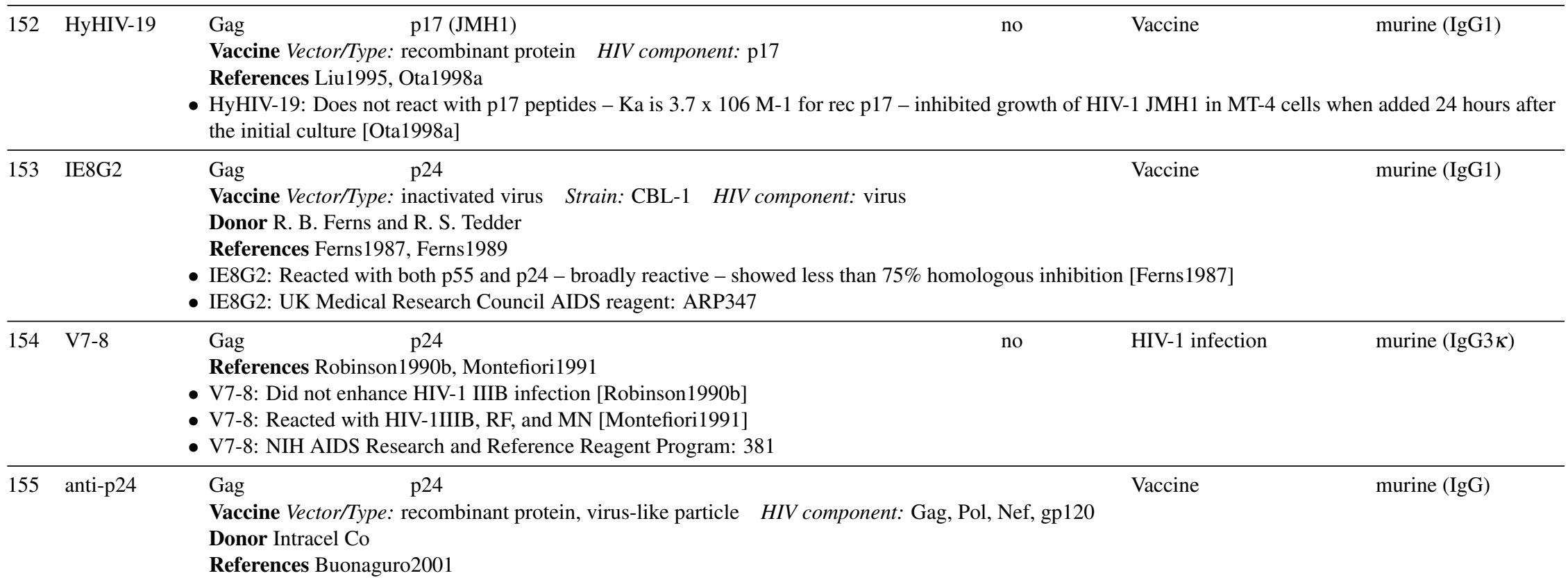




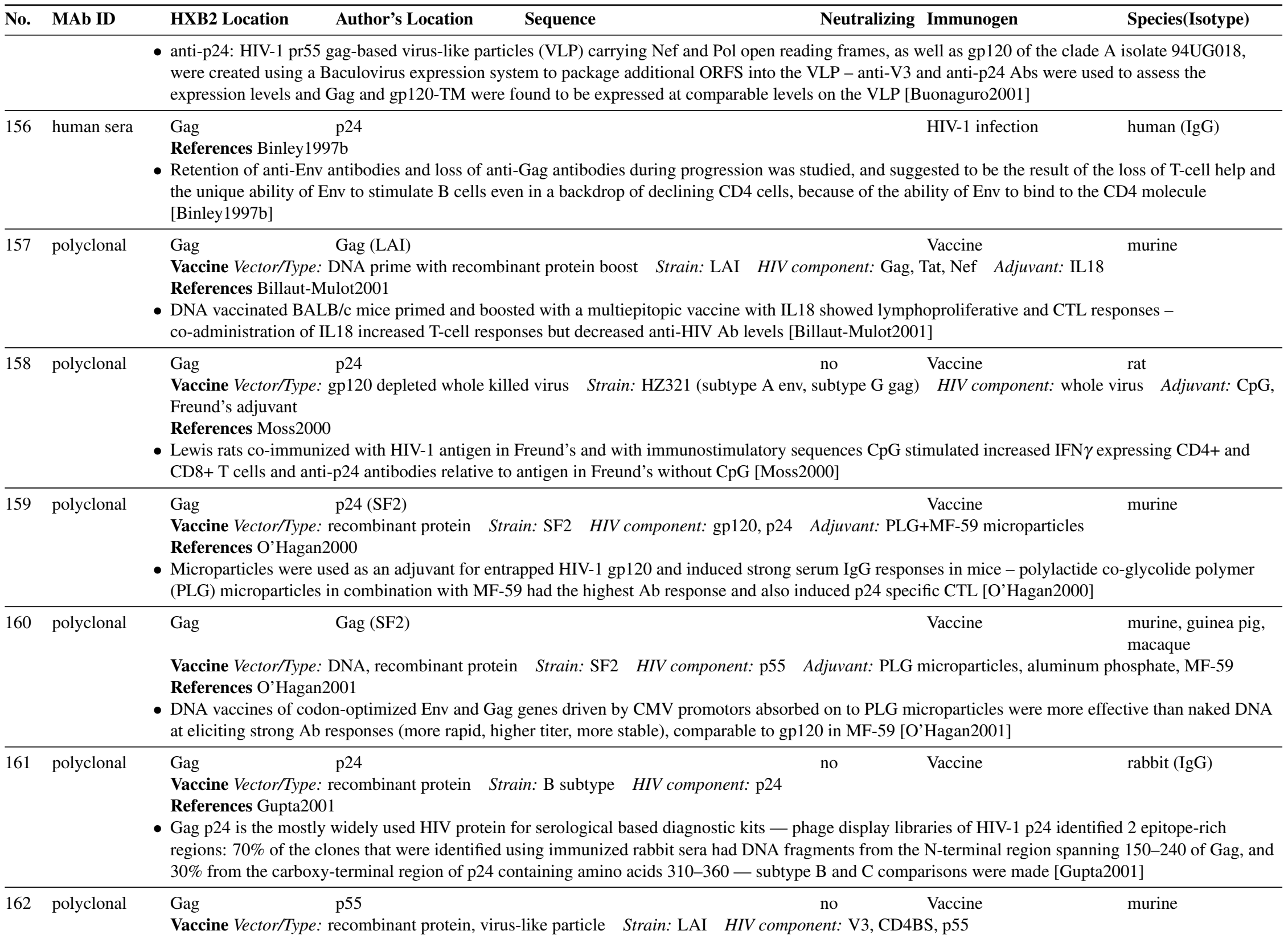




\begin{tabular}{|c|c|c|c|c|c|}
\hline No. & MAb ID & HXB2 Location & Author's Location & Neutralizing Immunogen & Species(Isotype) \\
\hline & & \multicolumn{4}{|c|}{$\begin{array}{l}\text { References Truong1996 } \\
\text { Antibodies raised against recombinant anti-p55 virus-like particles with the p24 region 196-226 deleted, bearing inserts of either the V3 or the CD4BS } \\
\text { regions of gp120 were studied - no neutralizing responses, weak Env and strong Gag responses were elicited - the major homology region (MHR) and } \\
\text { proximal sequences was found to be required for capsid assembly [Truong1996] }\end{array}$} \\
\hline 163 & polyclonal & $\begin{array}{l}\text { Gag } \\
\text { Vaccine Vector/Ty } \\
\text { and incomplete ad } \\
\text { References Devit } \\
\text { To compare vacci } \\
\text { peptides - the rab } \\
\text { shown to capture } \\
\text { IgG that was capa }\end{array}$ & $\begin{array}{l}\text { p24 (LAI) } \\
\text { virion, baculovirus and E. coli recombinant protein, peptides } \\
\text { ant, } \\
\text { Oc } \\
\text { rategies, rabbits were immunized with virion HIV-1/Lai, bacul } \\
\text { mmunized with peptides had the broadest linear epitope respon } \\
\text { tes from HIV-1 subtypes or clades A to G - only immunization } \\
\text { of efficiently capturing HIV-1 p24 in ELISA producing Abs ab }\end{array}$ & $\begin{array}{l}\text { Vaccine } \\
\text { Strain: LAI HIV component: } \mathrm{p} 24 \\
\text { ovirus recombinant } \mathrm{p} 24, \text { E. coli recom } \\
\text { ses - the capture ELISA method usin } \\
\text { with virion HIV-1/Lai and baculovir } \\
\text { le to recognise native configurations [1] }\end{array}$ & $\begin{array}{l}\text { rabbit }(\mathrm{IgG}) \\
\text { Adjuvant: Freund's complete } \\
\text { Ibinant p24-15, and p24-derived } \\
\text { g anti-p24 IgG preparations was } \\
\text { us recombinant p24 developed } \\
\text { Devito2000c }\end{array}$ \\
\hline
\end{tabular}
Gag

Vaccine Vector/Type: DNA Adjuvant: CpG, phosphorothioate oligodeoxynucleotides (ODNs)

Vaccine

murine

References Deml2001

- Immunization mice with a codon-optimized Gag was compared with a non-optimized Rev dependent Gag expression vector - Gag expression was at higher levels and Rev independent with the codon-optimized Gag, and i.m. immunization gave a stronger Th1-driven humoral and cellular immune response intradermal immunization with either Gag DNA induced a Th2 response and no CTL [Deml2001]

165 polyclonal Gag References Montefiori2001

human

- In 7/9 patients in whom HAART was initiated during early seroconversion, NAbs to autologous strains were not found immediately following treatment interuption after 1-3 years, and Env and Gag Abs were low or undetected by ELISA indicating, that early HAART suppresses the normal antibody response to HIV-1, presumably by limiting the concentration of viral antigens needed to drive B-cell maturation - in 3 patients with a viral rebound autologous NAbs rapidly appeared and correlated with spontaneous down-regulation of viremia - prolonged control of viremia after stopping treatment persisted in the absence of detectable NAbs, suggesting that cellular immune responses alone can control viremia under certain circumstances - these results support the notion that virus-specific B-cell priming, combined with CD8+ CTL induction, may be beneficial for HIV-1 vaccines that aim to suppress viremia in the absence of complete protection to prevent disease and reduce the rate of virus transmission [Montefiori2001]

166 polyclonal Gag

Vaccine Vector/Type: virus-like particle HIV component: Env, Gag Adjuvant: Freund's adjuvant

Vaccine

murine $(\operatorname{IgG})$

References Lebedev2000

- Virus-like particles (VLPs) in the form of spherical particles with yeast dsRNA enveloped in a polysaccharide matrix carrying the protein TBI, that contains fragments of HIV Env and Gag, were used to immunize BALB/c mice and induced specific Abs against HIV-1 as measured by ELISA with TBI |Lebedev2000

167 polyclonal Gag

Vaccine Vector/Type: DNA with CMV, MCK, or CMV/MCK hybrid promotors References Bojak2002
Vaccine

murine (IgG1,

IgG2a) 


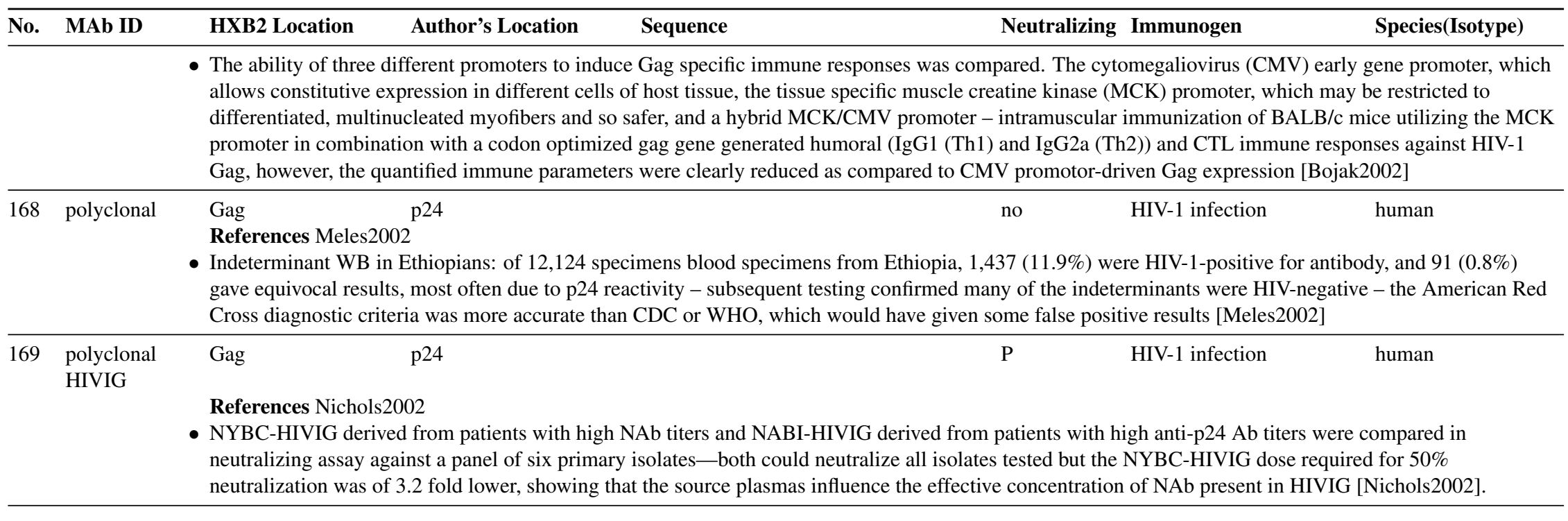




\section{IV-C-6 Protease Antibodies}

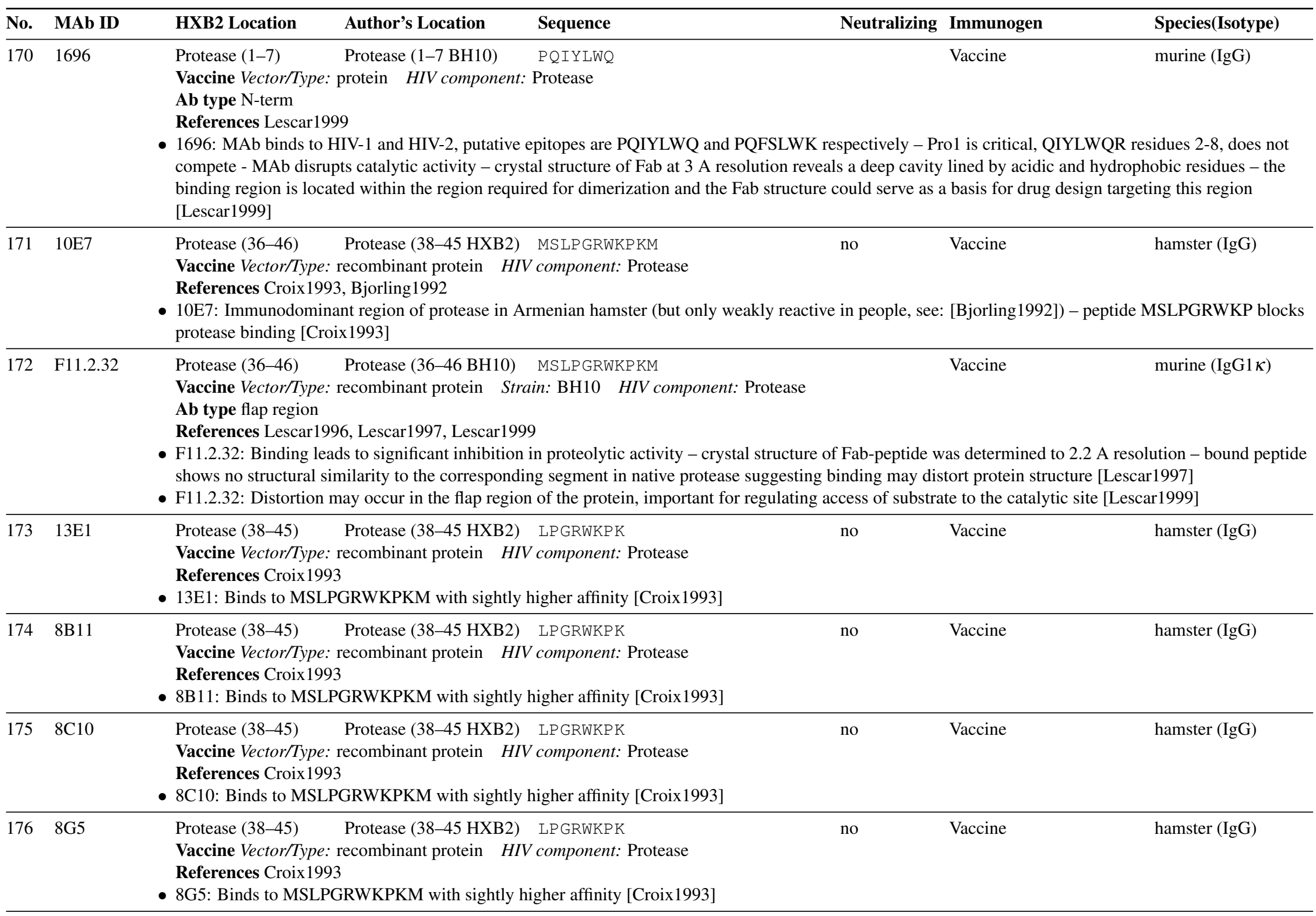


No. MAb ID HXB2 Location

Author's Location

Sequence

Neutralizing Immunogen

Species(Isotype) 


\section{IV-C-7 RT Antibodies}

\begin{tabular}{|c|c|c|c|c|c|c|}
\hline No. & MAb ID & Author's Location & Sequence & Neutralizing & Immunogen & Species(Isotype) \\
\hline 177 & $1 \mathrm{E} 8$ & \multicolumn{5}{|c|}{$\begin{array}{l}\text { Vaccine Vector/Type: recombinant protein HIV component: RT Adjuvant: nitrocellulose } \\
\text { References Wu1993, Gu1996 } \\
\text { - 1E8: Inhibits RT activity, binding site overlaps with two AZT resistance mutations [Wu1993 } \\
\text { - 1E8: Significantly inhibits DNA polymerase activity of RT by hindering binding of dNTPs - additive or synergistic RT inhibition with nevirapine and } \\
\text { delavirdine [Gu1996] }\end{array}$} \\
\hline 178 & $1.152 \mathrm{~B} 3$ & $\begin{array}{l}\text { RT (294-302) RT (294-302) } \\
\text { Vaccine Vector/Type: recombinant protein } \\
\text { References Orvell1991 } \\
\text { - } 1.152 \text { B3: Weakly positive by immunofluore }\end{array}$ & $\begin{array}{l}\text { PLTEEAELE } \\
\text { V component: } \mathrm{RT} \\
\text { nce - binding inhibits RT enzymatic acti }\end{array}$ & $\begin{array}{l}\text { no } \\
\text { ty Orvell1991 }\end{array}$ & Vaccine & murine (IgG1) \\
\hline 179 & $1.158 \mathrm{E} 2$ & $\begin{array}{l}\text { RT (294-302) RT (294-302) } \\
\text { Vaccine Vector/Type: recombinant protein } \\
\text { References Orvell1991 } \\
\text { - } 1.158 \text { E2: Negative by immunofluorescence }\end{array}$ & $\begin{array}{l}\text { PLTEEAELE } \\
\text { V component: } \mathrm{RT} \\
\text { inding inhibits RT enzymatic activity } \overline{\mathrm{O}}\end{array}$ & $\begin{array}{l}\text { no } \\
\text { ell1991] }\end{array}$ & Vaccine & murine (IgG1) \\
\hline 180 & 31D6 & $\begin{array}{l}\text { RT (294-318) RT (294-319) } \\
\text { Vaccine Vector/Type: E. coli Trp fusion prot } \\
\text { References Szilvay1992 } \\
\text { - 31D6: Strong inhibitor of RT, > 50\% inhibit }\end{array}$ & $\begin{array}{l}\text { PLTEEAELELAENRE I LKEPVHGVY } \\
\text { HIV component: RT } \\
\text { [Szilvay1992] }\end{array}$ & no & Vaccine & murine (IgG1) \\
\hline 181 & $31 \mathrm{G} 8$ & $\begin{array}{l}\text { RT (294-318) RT (294-319) } \\
\text { Vaccine Vector/Type: E. coli Trp fusion pro } \\
\text { References Szilvay1992 } \\
\text { - 31G8: Weak inhibitor of RT, reactive by im }\end{array}$ & $\begin{array}{l}\text { PLTEEAELELAENRE I LKEPVHGVY } \\
\text { HIV component: RT } \\
\text { ofluorescence [Szilvay1992] }\end{array}$ & no & Vaccine & murine (IgG1) \\
\hline 182 & $32 \mathrm{E} 7$ & $\begin{array}{l}\text { RT (294-318) RT (294-319) } \\
\text { Vaccine Vector/Type: E. coli Trp fusion pro } \\
\text { References Szilvay1992 } \\
\text { - 32E7: Weak inhibitor of RT, reactive by imr }\end{array}$ & $\begin{array}{l}\text { PLTEEAELELAENRE I LKEPVHGVY } \\
\text { HIV component: RT } \\
\text { fluorescence [Szilvay1992] }\end{array}$ & no & Vaccine & murine (IgG1) \\
\hline 183 & 33D5 & $\begin{array}{l}\text { RT (294-318) RT (294-319) } \\
\text { Vaccine Vector/Type: E. coli Trp fusion pro } \\
\text { References Szilvay1992 } \\
\text { - 33D5: Weak inhibitor of RT, reactive by im }\end{array}$ & $\begin{array}{l}\text { PLTEEAELELAENRE ILKEPVHGVY } \\
\text { HIV component: RT } \\
\text { hofluorescence [Szilvay1992] }\end{array}$ & no & Vaccine & murine (IgG1) \\
\hline 184 & $5 \mathrm{~B} 2$ & $\begin{array}{l}\text { RT (294-318) RT (294-319) } \\
\text { Vaccine Vector/Type: E. coli Trp fusion pro } \\
\text { References Szilvay1992 } \\
\text { - 5B2: There is an RT specific Ab [Szilvay19 } \\
\text { - 5B2: Weak inhibitor of RT, reactive by imm }\end{array}$ & $\begin{array}{l}\text { PLTEEAELELAENREILKEPVHGVY } \\
\text { HIV component: RT } \\
\text { and a gp41 specific Ab [Tian2001] both } \\
\text { fluorescence [Szilvay1992] }\end{array}$ & $\begin{array}{l}\text { no } \\
\text { led 5B2 }\end{array}$ & Vaccine & murine (IgG1) \\
\hline
\end{tabular}




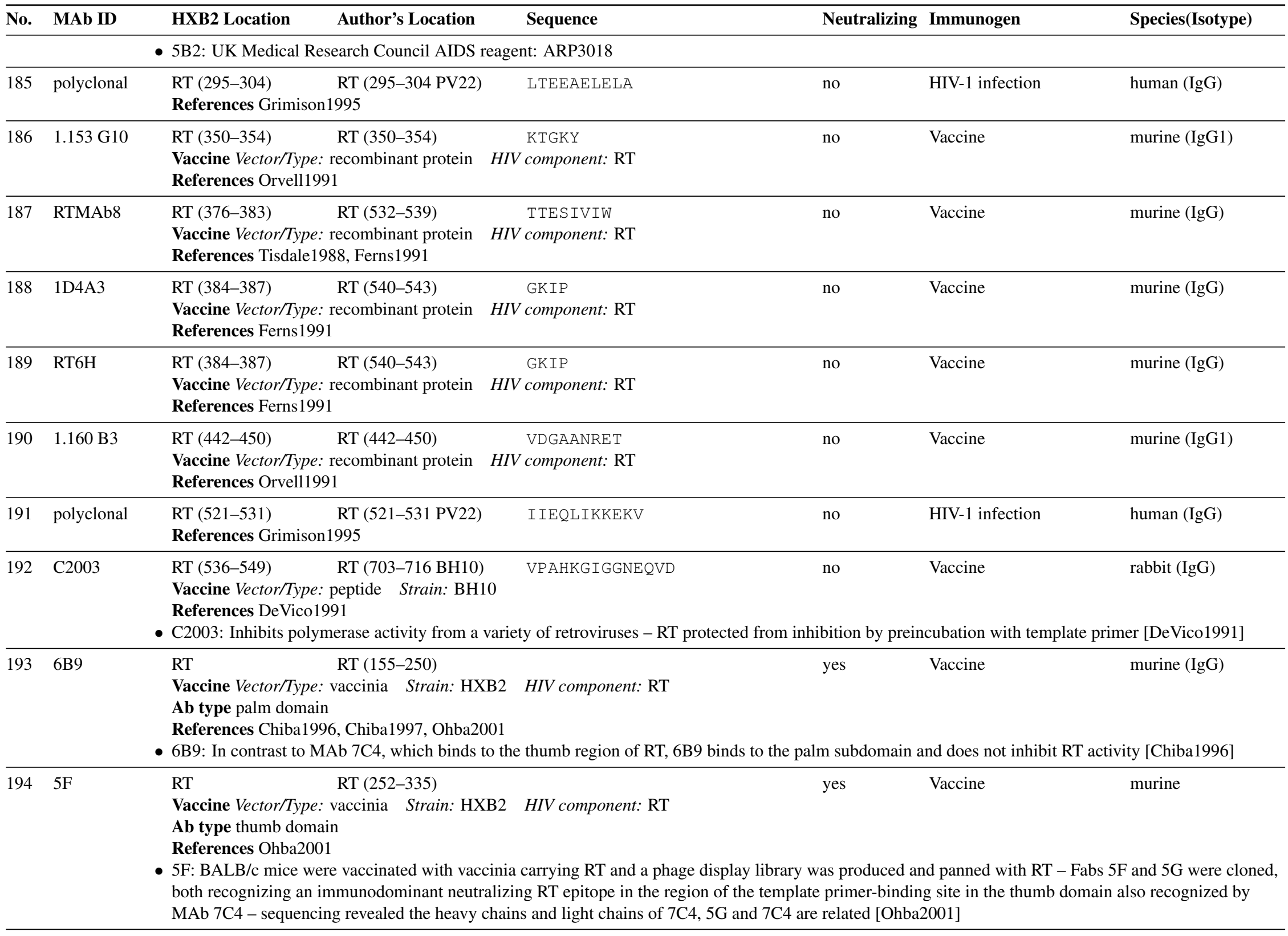




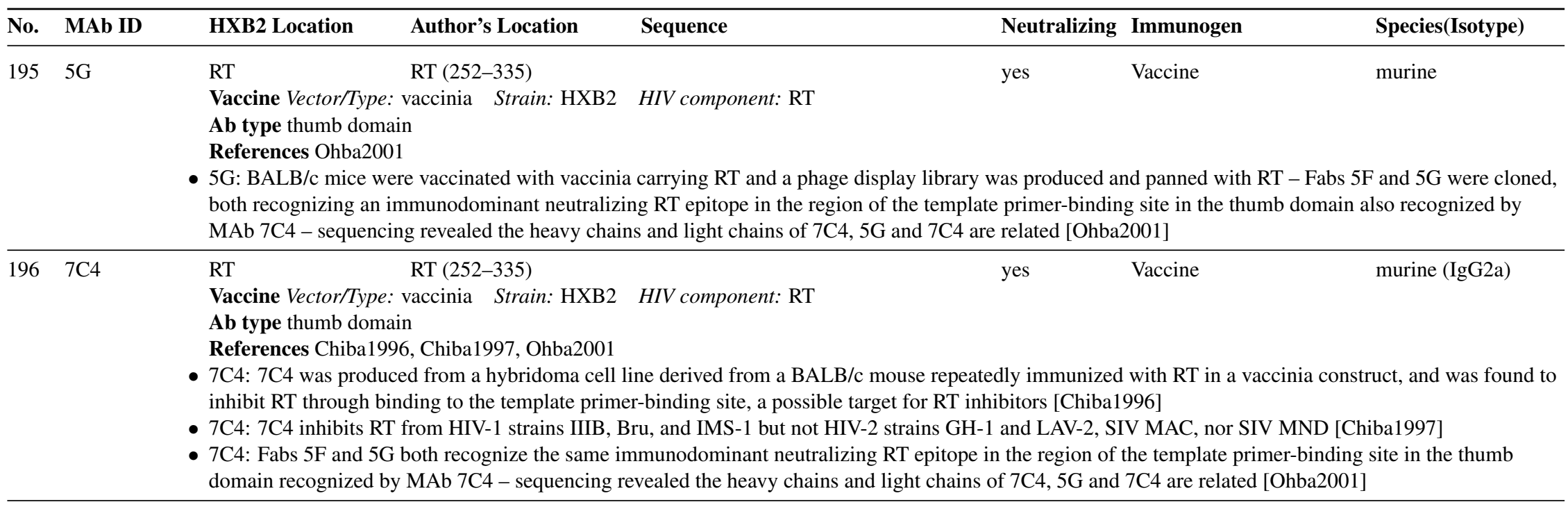




\section{IV-C-8 Integrase Antibodies}

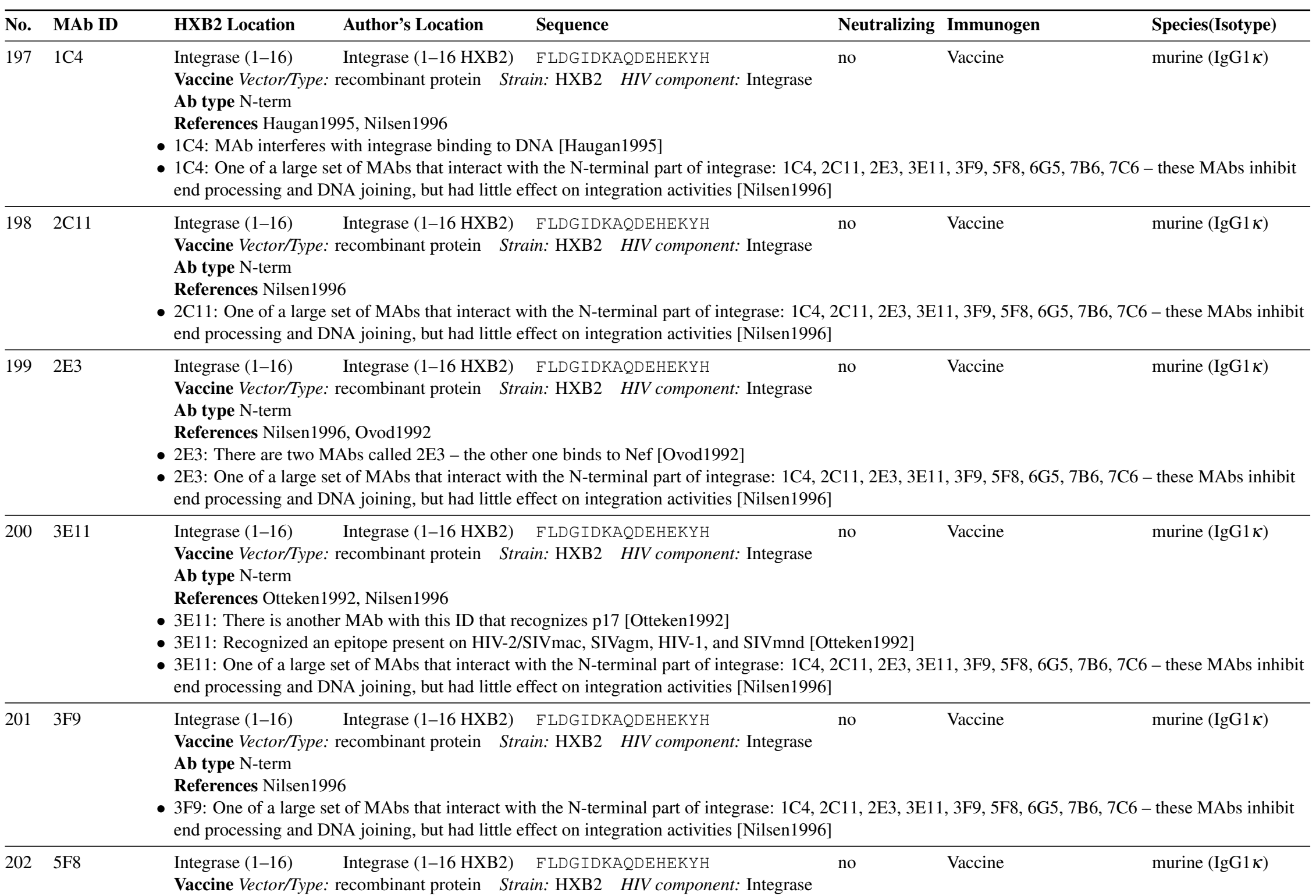




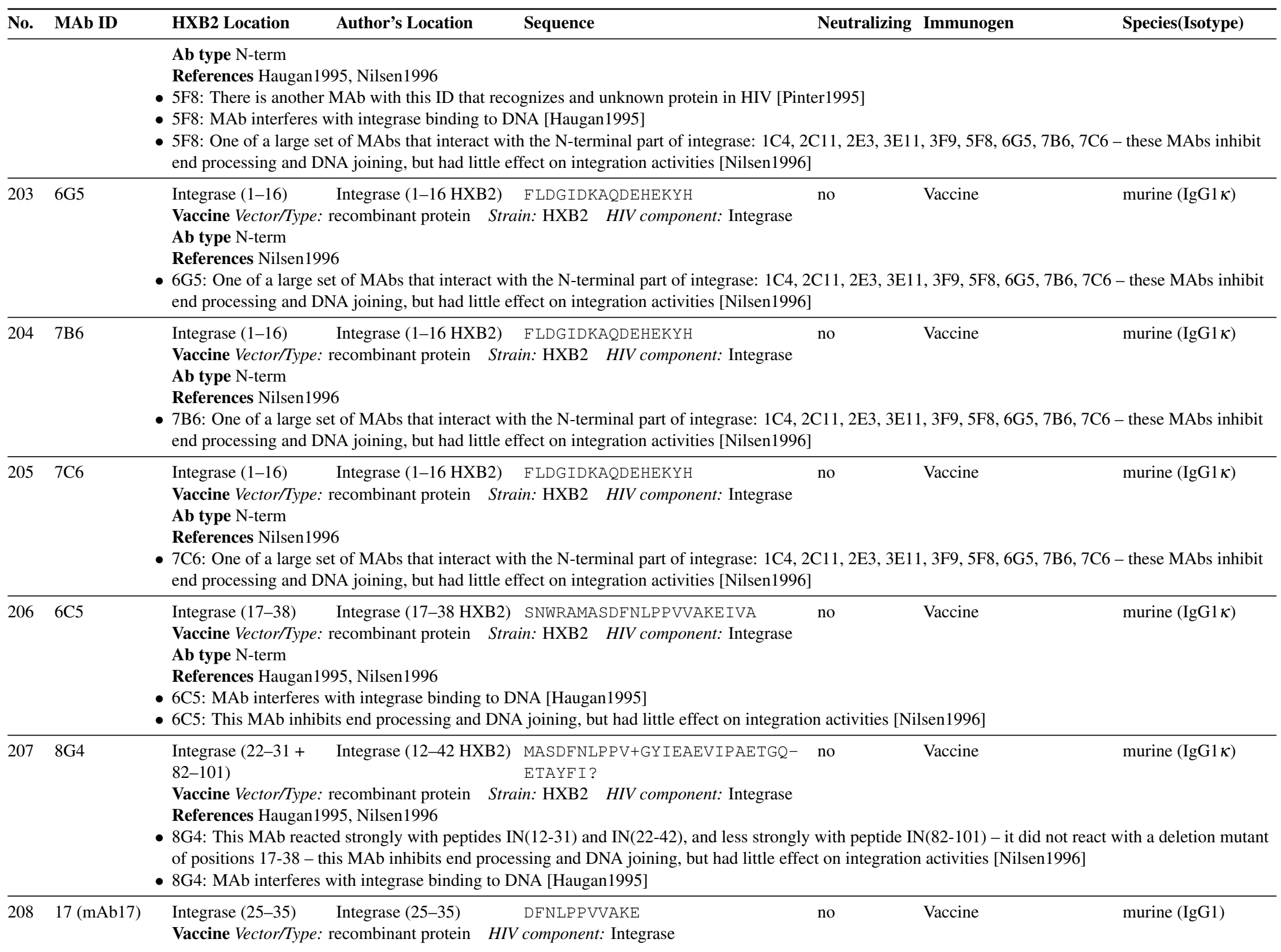




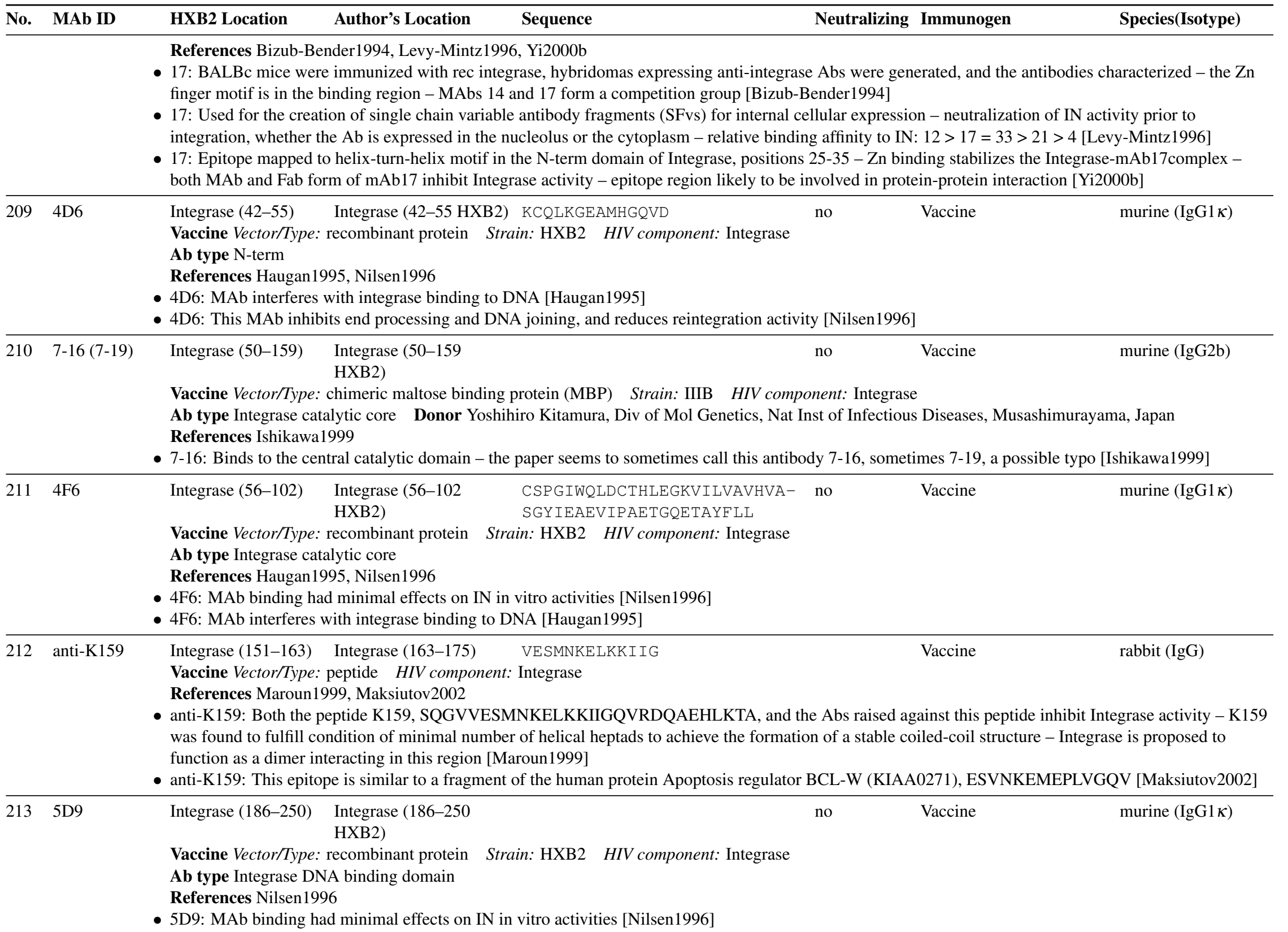




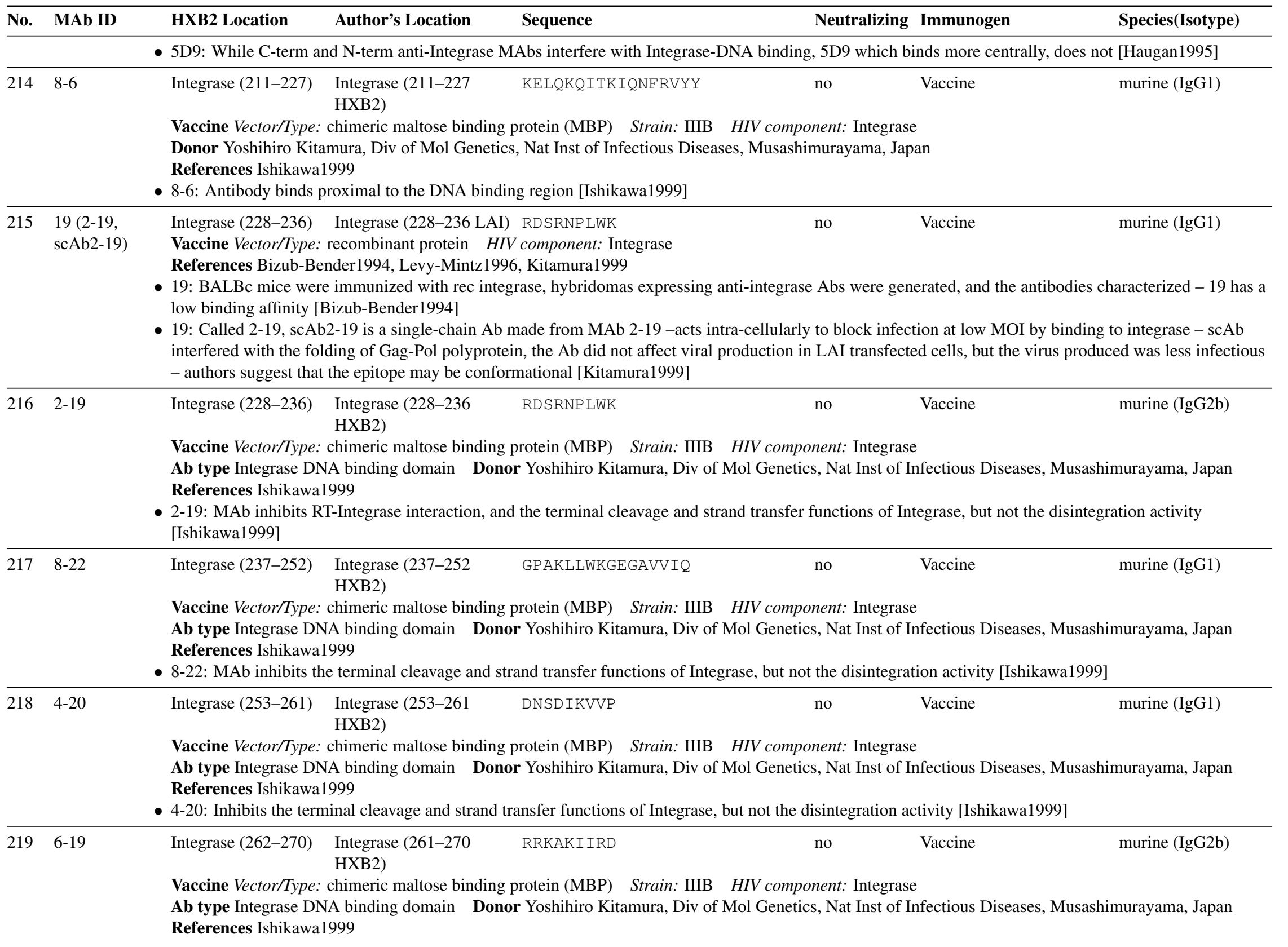




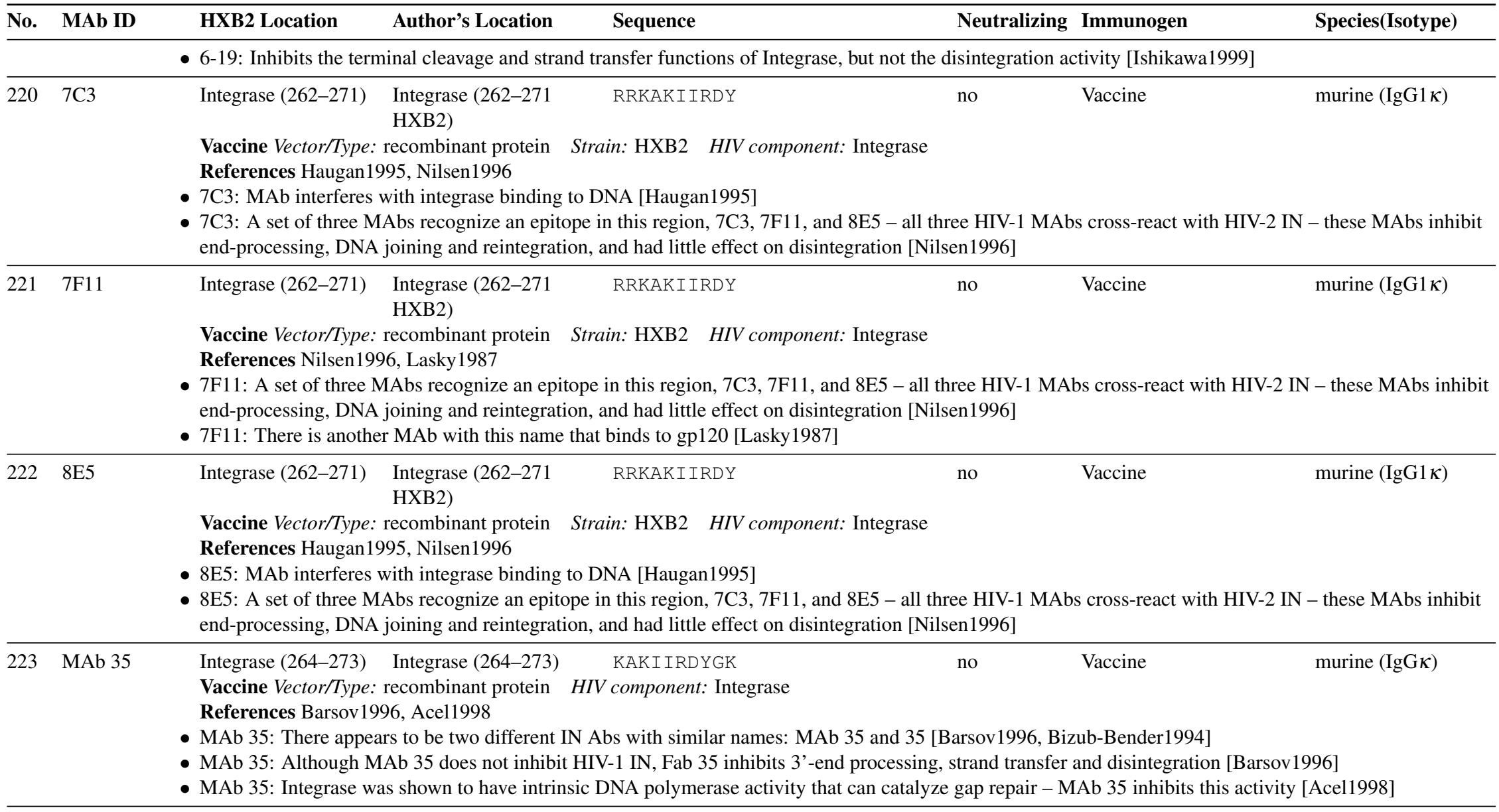




\section{IV-C-9 Pol Antibodies}

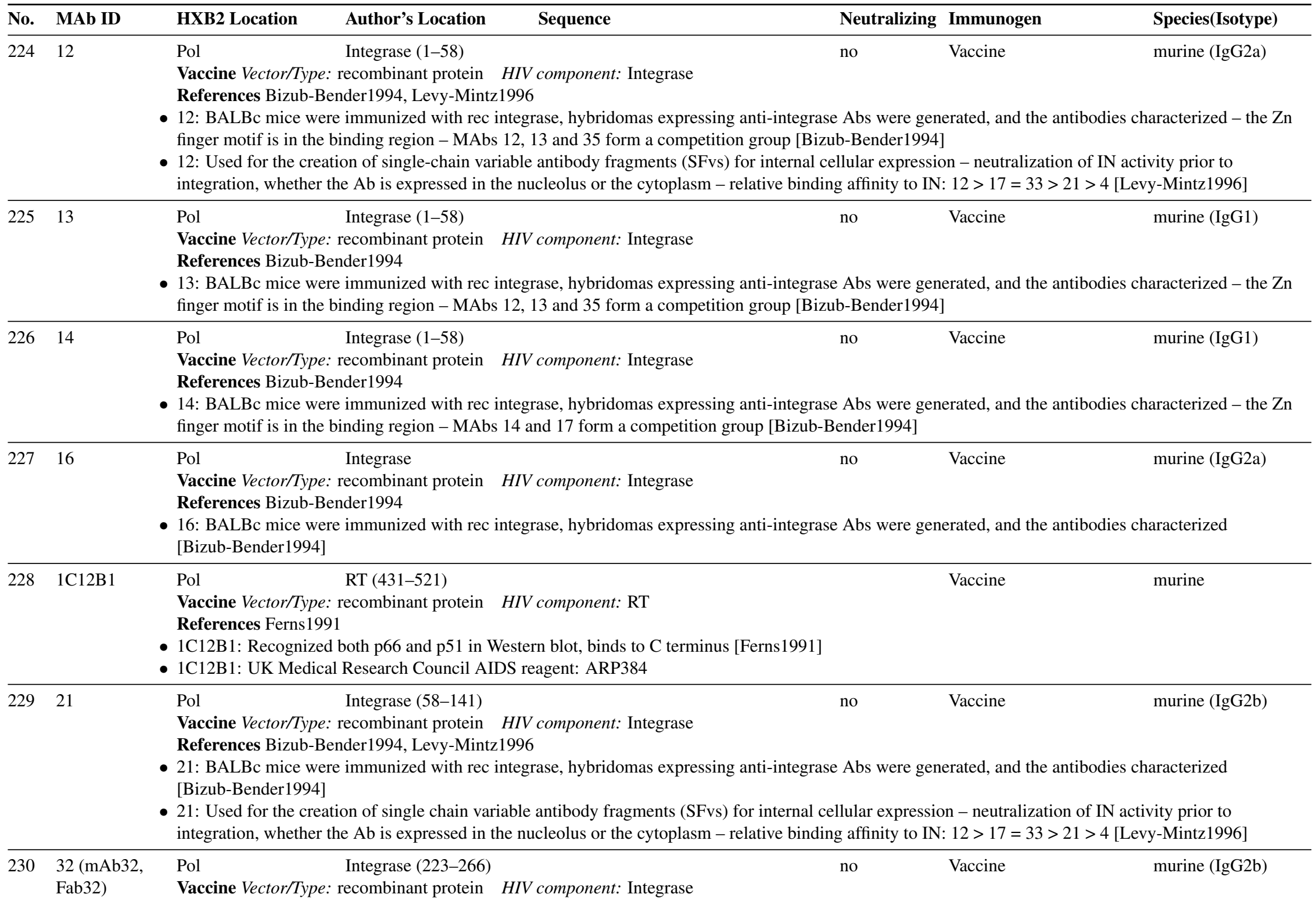




\begin{tabular}{|c|c|c|c|c|}
\hline No. & MAb ID & Author's Location & Neutralizing Immunogen & Species(Isotype) \\
\hline & & \multicolumn{3}{|c|}{$\begin{array}{l}\text { References Bizub-Bender1994 Yi2000a Yi2002 } \\
\text { 32: BALBc mice were immunized with rec integrase, hybridomas expressing anti-integrase Abs were generated, and the antibodies characterized - MAbs } \\
\text { - 32: Limited proteolysis combined with mass spectrometric analysis indicates Fab32 binds to two strands of the beta sheet, beta1 223F, 224R, 226Y, and } \\
\text { 228R and beta5 } 264 \mathrm{~K} \text { and } 266 \mathrm{~K} \text { [Yi2000a] } \\
\text { - 32: Called mAb32 - mAb33 and mAb32 compete for binding to the C-term domain of Integrase - while mAb32 only weakly inhibits IN activity, mAb33 } \\
\text { inhibits strongly, mAb32 has a lower affinity than mAb33, and Fab32 does not inhibit at all while Fab33 inhibits DNA binding a catalytic activity [Yi2002] }\end{array}$} \\
\hline 231 & 35 & \multicolumn{3}{|c|}{$\begin{array}{l}\text { Vaccine Vector/Type: recombinant protein HIV component: Integrase } \\
\text { References Bizub-Bender1994] } \\
\text { - 35: There appears to be two IN Abs with similar names: MAb } 35 \text { and } 35 \text { [Barsov1996 Bizub-Bender1994] } \\
\text { 35: BALBc mice were immunized with rec integrase, hybridomas expressing anti-integrase Abs were generated, and the antibodies characterized - the Zn } \\
\text { finger motif is in the binding region - MAbs 12, } 13 \text { and } 35 \text { form a competition group [Bizub-Bender1994] }\end{array}$} \\
\hline 232 & 3D12 & $\begin{array}{l}\text { Pol RT } \\
\text { Vaccine Vector/Type: vaccinia HIV compc } \\
\text { References Chiba1997 } \\
\text { - 3D12: There is an anti-Nef MAb that also } \mathrm{h}\end{array}$ & $\begin{array}{l}\text { RT } \\
\text { s name (see Chiba1997) }\end{array}$ & murine (IgG2a) \\
\hline 233 & $3 F 10$ & $\begin{array}{l}\text { Pol RT } \\
\text { Vaccine Vector/Type: vaccinia HIV comp } \\
\text { References Chiba1997 }\end{array}$ & Vaccine & murine (IgG2a) \\
\hline 234 & 4 & $\begin{array}{l}\text { Pol Integrase (141-172) } \\
\text { Vaccine Vector/Type: recombinant protein } \\
\text { References Bizub-Bender1994 Levy-Mint } \\
\text { - 4: There is another MAb with this ID that re } \\
\text { - 4: BALBc mice were immunized with rec ir } \\
\text { low binding affinity [Bizub-Bender1994] } \\
\text { - 4: Used for the creation of single chain vari } \\
\text { integration, whether the Ab is expressed in }\end{array}$ & $\begin{array}{l}\text { component: Integrase } \\
\text { with gp41 Oldstone1991, Bizub-Bender1994 } \\
\text { ase, hybridomas expressing anti-integrase Abs were generated, and the } \\
\text { antibody fragments (SFvs) for internal cellular expression - neutralizati } \\
\text { ucleolus or the cytoplasm - relative binding affinity to IN: } 12>17=33\end{array}$ & $\begin{array}{l}\text { murine (IgG2b) } \\
\text { racterized - } 4 \text { has a } \\
\text { ity prior to } \\
\text { y-Mintz1996 }\end{array}$ \\
\hline 235 & $6 \mathrm{~B} 9$ & $\begin{array}{l}\text { Pol RT } \\
\text { Vaccine Vector/Type: vaccinia HIV compe } \\
\text { References Chiba1997 }\end{array}$ & Vaccine & murine (IgG2a) \\
\hline 236 & $7 \mathrm{C} 4$ & $\begin{array}{l}\text { Pol RT } \\
\text { Vaccine Vector/Type: vaccinia HIV comp } \\
\text { References Chiba1997] } \\
\text { 7C4: Dose-dependent inhibition of polymer } \\
\text { MND [Chiba1997] }\end{array}$ & $\begin{array}{l}\text { Vaccine } \\
\text { RT } \\
\text { tivity of RT of strains IIIB, Bru and IMS-1, but not HIV-2 strains GH- }\end{array}$ & $\begin{array}{l}\text { murine (IgG1) } \\
\text { SIV strains MAC or }\end{array}$ \\
\hline 237 & RT-4 & \begin{tabular}{l|l|l} 
Pol & \multicolumn{1}{c}{ RT } \\
References & Li1993 & Gu1996 \\
\end{tabular} & no & murine (IgG2b) \\
\hline
\end{tabular}




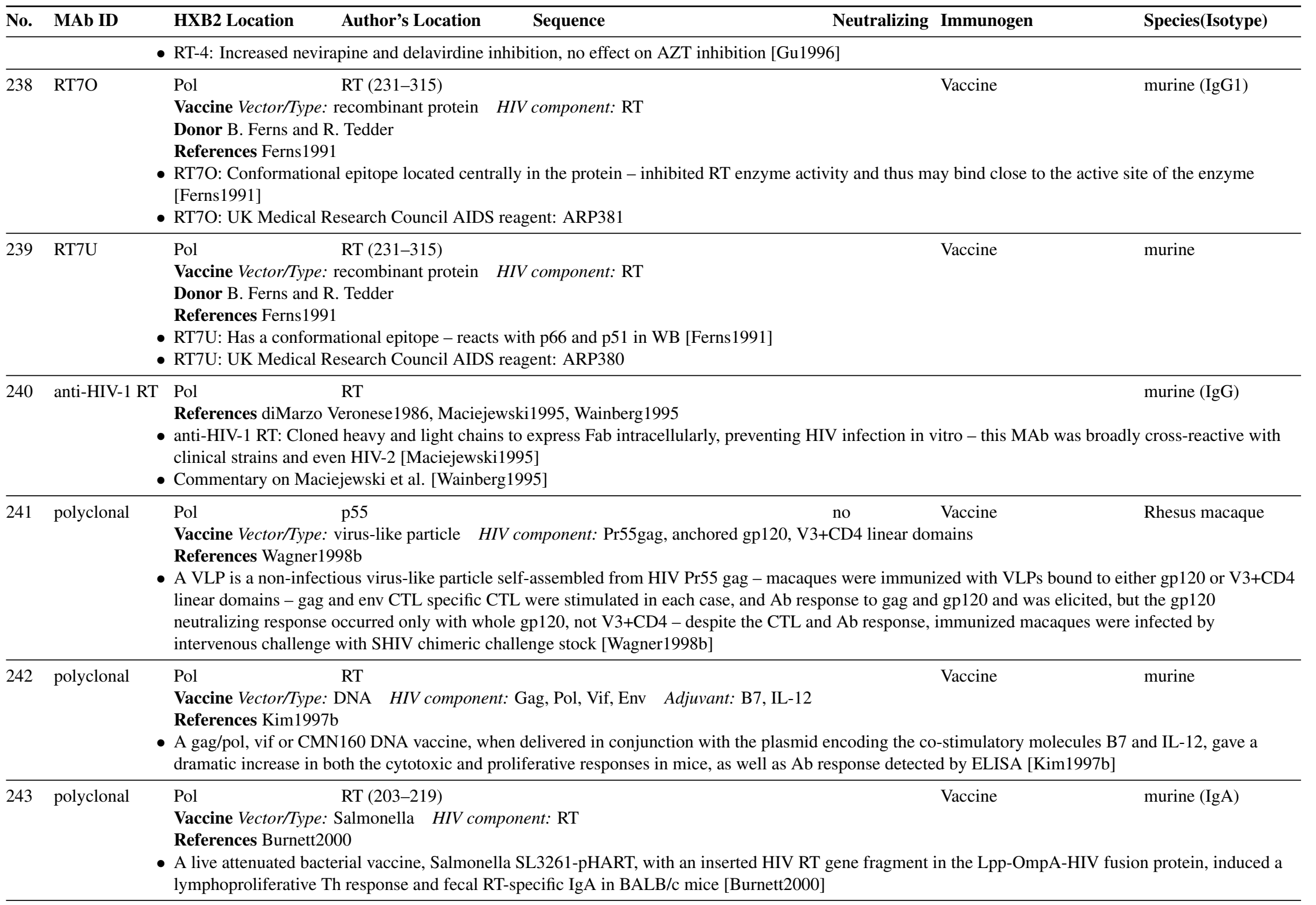




\begin{tabular}{|c|c|c|c|c|c|c|}
\hline No. & MAb ID & HXB2 Location & Author's Location & Neutralizing & Immunogen & Species(Isotype) \\
\hline 244 & $\begin{array}{l}33 \text { (mAb33, } \\
\text { Fab33, 33D5, } \\
\text { mab 33) }\end{array}$ & $\begin{array}{l}\text { Pol } \\
\text { Vaccine Vector/Type: } \\
\text { Ab type C-term } \\
\text { References Bizub-Be } \\
\text { 33: BALBc mice wer } \\
\text { 32 and } 33 \text { form a com } \\
\text { 33: Used for the creat } \\
\text { integration, whether t } \\
\text { 33: Limited proteolys } \\
\text { C-term domain left it } \\
\text { 33: Called mAb33 - } \\
\text { inhibits strongly, mA } \\
\text { heteronuclear NMR i } \\
\text { form a contiguous pa } \\
\text { Fab33-IN complex is }\end{array}$ & $\begin{array}{l}\text { Integrase (223-268 } \\
\text { HXB2) } \\
\text { recombinant protein } \\
\text { nder1994 Levy-Mintz } \\
\text { e immunized with rec i } \\
\text { petition group [Bizub- } \\
\text { ion of single chain vari } \\
\text { he Ab is expressed in th } \\
\text { is combined with mass } \\
\text { resistant to proteolytic } \\
\text { nAb33 and mAb32 cor } \\
32 \text { has a lower affinity } \\
\text { adicated eight residues } \\
\text { ch likely to contain the } \\
\text { far more soluble than I }\end{array}$ & $\begin{array}{l}\text { HIV component: Integrase } \\
\text { 1996. Yi2000a, Yi2002 } \\
\text { integrase, hybridomas expressing anti-integrase Abs were gene } \\
\text {-Bender1994] } \\
\text { iable antibody fragments (SFvs) for internal cellular expressio } \\
\text { he nucleolus or the cytoplasm - relative binding affinity to IN: } \\
\text { s spectrometric analysis were used to define the binding site fo } \\
\text { digestion [Yi2000a] } \\
\text { mpete for binding to the C-term domain of Integrase - while } \\
\text { y than mAb33, and Fab32 does not inhibit at all while Fab33 in } \\
\text { of Integrase are immobilized upon Fab33 binding, two in the } \\
\text { e epitope - 223F, 224R, 226Y, 244K, 267I, and 268I, which ma } \\
\text { IN alone and may be useful for crystallization [Yi2002] }\end{array}$ & $\begin{array}{l}\text { Vaccine } \\
\text { erated, and the } \\
\text { n - neutralizat } \\
\text { : } 12>17=33 \\
\text { r Fab32, but F } \\
\text { nAb32 only we } \\
\text { nhibits catalyti } \\
\text { core of the pro } \\
\text { ay be a useful }\end{array}$ & $\begin{array}{l}\text { aracterized - MAbs } \\
\text { vity prior to } \\
\text { y-Mintz1996 } \\
\text { to the Intergrase } \\
\text { IN activity, mAb33 } \\
\text { DNA binding - } \\
\text { the outer face that } \\
\text { g design - the }\end{array}$ \\
\hline
\end{tabular}




\section{IV-C-10 Vif Antibodies}

\begin{tabular}{|c|c|c|c|c|c|c|}
\hline No. & MAb ID & Author's Location & Sequence & Neutralizing & Immunogen & Species(Isotype) \\
\hline 245 & TG002 & $\begin{array}{l}\text { Vif (34-47) Vif (34-47) } \\
\text { Vaccine Vector/Type: recombinant protein } \\
\text { Donor Transgene } \\
\text { - TG002: This MAb was raised in response to } \\
\text { - TG002: NIH AIDS Research and Reference }\end{array}$ & $\begin{array}{l}\text { KARGWEYRHHYESP? } \\
\text { HIV component: Vif } \\
\text { a rec Vif protein derived from E. coli } \\
\text { Reagent Program: } 2746\end{array}$ & no & Vaccine & murine \\
\hline 246 & TG001 & $\begin{array}{l}\text { Vif (176-192) Vif (176-192) } \\
\text { Vaccine Vector/Type: recombinant protein } \\
\text { Ab type C-term Donor Transgene } \\
\text { - TG001: This antibody was raised in respons } \\
\text { - TG001: NIH AIDS Research and Reference }\end{array}$ & $\begin{array}{l}\quad \text { KPQKTKGHRGSHTMNGH? } \\
\text { HIV component: Vif } \\
\text { se to a rec Vif protein derived from E. coli } \\
\text { Reagent Program: } 2745\end{array}$ & no & Vaccine & murine \\
\hline 247 & $\mathrm{~J} 4$ & Vif & & & & $\begin{array}{l}\text { chimeric } \\
\text { rabbit/human FAb }\end{array}$ \\
\hline
\end{tabular}

- J4: The authors developed a Vif-specific intrabody single-chain FAb fragment of J4 called 14BL - when expressed intracellularly in the cytoplasm this intrabody efficiently bound Vif protein and neutralized its infectivity enhancing function - intrabody-expressing transduced cells were shown to be highly refractory to challenge with the laboratory strain NL43 and with primary isolates strains of HIV-1 [Goncalves2002]

\begin{tabular}{ll}
\hline 248 polyclonal & Vif Vif \\
& Vaccine Vector/Type: DNA HIV component: Gag, Pol, Vif, Env Adjuvant: B7, IL-12 \\
& References Kim1997b \\
& A gag/pol, vif or CMN160 DNA vaccine, when delivered in conjunction with the plasmid encoding the co-stimulatory molecules B7 and IL-12, gave a \\
& dramatic increase in both the cytotoxic and proliferative responses in mice, as well as an Ab response detected by ELISA [Kim1997b] \\
\hline
\end{tabular}




\section{IV-C-11 Tat Antibodies}

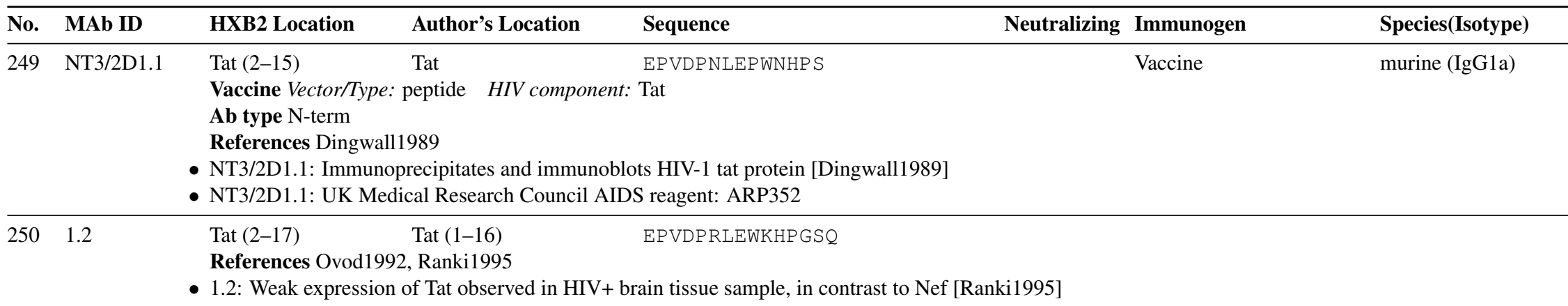

$\begin{array}{lrr}\text { Tat }(2-21) & \text { Tat } & \text { EPVDPRLEWKHPGSQPKTA } \\ \text { Vaccine Vector/Type: } & \text { recombinant protein } & \text { HIV component: Tat }\end{array}$

Vaccine murine $(\operatorname{IgG} 1)$

Vaccine Vector/Type: recombinant protein HIV component: Tat

Ab type N-term

References Mhashilkar1995 Valvatne1996

- 1D9D5: Single chain antibodies, intrabodies, were engineered that can be stably expressed in the cytoplasm of mammalian cells - co-expression of an $\mathrm{N}$-term intrabody can inhibit transactivation of an HIV LTR-CAT construct and block import into nucleus, but intrabody specific for exon 2 did not inhibit activity Mhashilkar1995

- 1D9D5: Exogenously delivered Tat can efficiently transactivate an HIV-LTR-CAT construct in HeLa cells in the presence of 1D9D5, suggesting when considered with the results of [Mhashilkar1995], that free Tat and not Ab bound is taken up by cells [Valvatne1996]

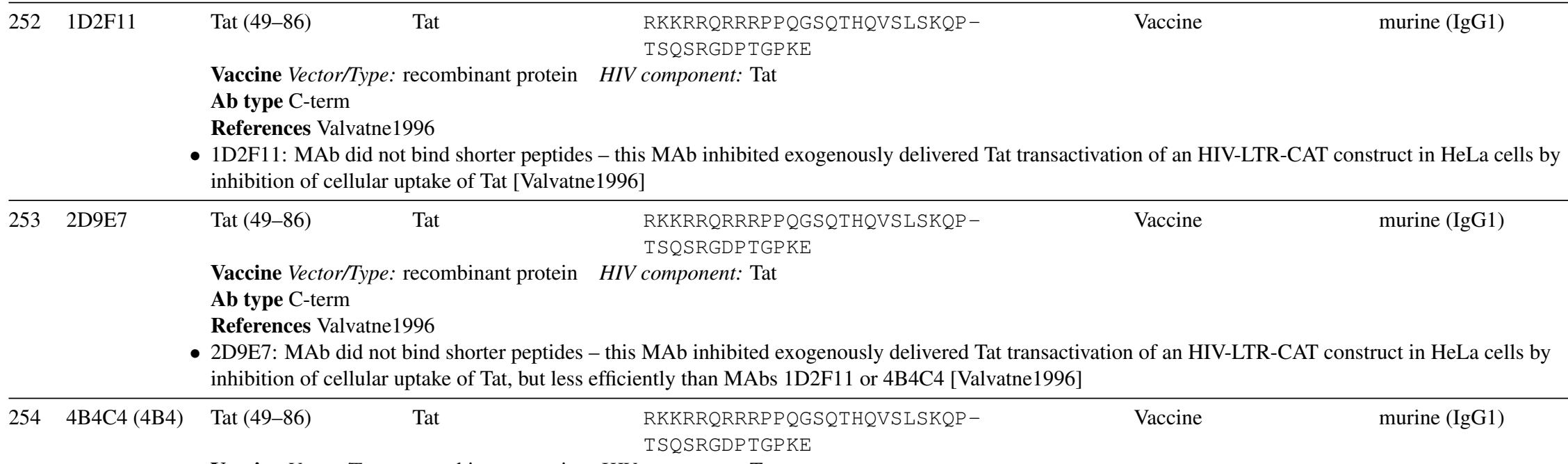

Vaccine Vector/Type: recombinant protein HIV component: Tat

Ab type C-term

References Valvatne1996, Jensen1997 


\begin{tabular}{|c|c|c|c|c|c|c|}
\hline No. & MAb ID & Author's Location & Sequence & Neutralizing & Immunogen & Species(Isotype) \\
\hline & & \multicolumn{5}{|c|}{$\begin{array}{l}\text { - 4B4C4: MAb did not bind shorter peptides - this MAb inhibited exogenously delivered Tat transactivation of an HIV-LTR-CAT construct in HeLa cells by } \\
\text { inhibition of cellular uptake of Tat [Valvatne1996] }\end{array}$} \\
\hline 255 & 5G7D8 & $\begin{array}{l}\text { Tat (49-86) Tat } \\
\text { Vaccine Vector/Type: recombinant protein } \\
\text { Ab type C-term } \\
\text { References Valvatne1996 } \\
\text { 5G7D8: MAb did not bind shorter peptides } \\
\text { inhibition of cellular uptake of Tat, but less }\end{array}$ & $\begin{array}{l}\text { RKKRRQRRRPPQGSQTHQVSLSKQP- } \\
\text { TSQSRGDPTGPKE } \\
\text { component: Tat } \\
\text { MAb inhibited exogenously delivered T } \\
\text { ently than 1D2F11 or 4B4C4 IValvatne19 }\end{array}$ & transactivatio & in of an HIV-L & uct in HeLa cells by \\
\hline 256 & NT2/4D5.24 & $\begin{array}{l}\text { Tat (73-86) Tat } \\
\text { Vaccine Vector/Type: peptide HIV compo } \\
\text { Ab type C-term } \\
\text { References Dingwall1989 } \\
\text { - NT2/4D5.24: Immunoprecipitates and imm }\end{array}$ & $\begin{array}{l}\text { PTSQPRGDPTGPKE } \\
\text { Tat } \\
\text { lots HIV-1 tat protein Dingwall1989] }\end{array}$ & & Vaccine & murine \\
\hline \multirow[t]{2}{*}{257} & L-anti-Tat & Tat & & $\begin{array}{l}\text { L P (when } \\
\text { lipidated) }\end{array}$ & Vaccine & murine (IgG1) \\
\hline & & $\begin{array}{l}\text { Vaccine Vector/Type: recombinant protein } \\
\text { Donor AGMED, Inc., Bedford, MA USA } \\
\text { References Cruikshank1997 } \\
\text { - L-anti-Tat: Lipidated antibody can be taken } \\
\text { [Cruikshank1997] }\end{array}$ & $\begin{array}{l}\text { component: Tat } \\
\text { cells and effectively block IIIB and prin }\end{array}$ & ary virus HIV- & 1 replication & atently infected cells \\
\hline 258 & 2D9D5 & $\begin{array}{l}\text { Tat Tat } \\
\text { Vaccine Vector/Type: recombinant protein } \\
\text { Ab type C-term } \\
\text { References Mhashilkar1995 } \\
\text { 2D9D5: Single chain antibodies, intrabodie } \\
\text { intrabody did not inhibit transactivation of a }\end{array}$ & $\begin{array}{l}\text { component: Tat } \\
\text { engineered that can be stably express } \\
\text { LTR-CAT construct, in contrast to M }\end{array}$ & $\begin{array}{l}\text { n the cytoplas } \\
\text { ID9D5 [Mhas }\end{array}$ & $\begin{array}{l}\text { Vaccine } \\
\text { m of mamma } \\
\text { shilkar1995 }\end{array}$ & $\begin{array}{l}\text { murine }(\mathrm{IgG}) \\
\text { expression of C-term }\end{array}$ \\
\hline
\end{tabular}




\section{IV-C-12 Rev Antibodies}

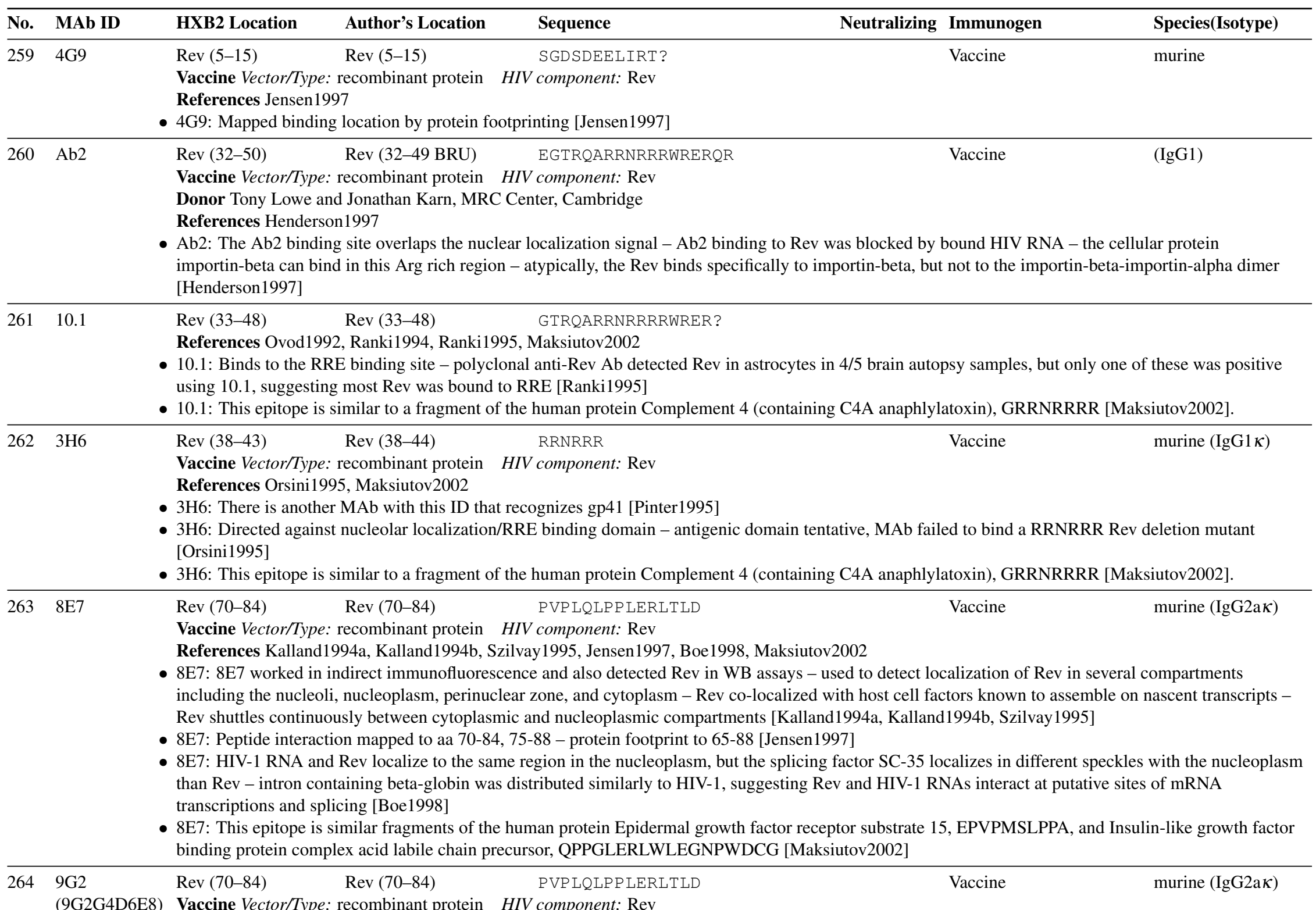




\begin{tabular}{|c|c|c|c|c|}
\hline No. & MAb ID & Author's Location & Neutralizing Immunogen & Species(Isotype) \\
\hline & & \multicolumn{3}{|c|}{$\begin{array}{l}\text { Donor Anne Marie Szilvay } \\
\text { References Kalland1994a, Jensen1997, Maksiutov2002] } \\
\text { - 9G2: Worked in indirect immunofluorescence and also detected Rev in WB assays - used to detect localization of Rev throughout the cell [Kalland1994a] } \\
\text { - 9G2: Peptide interaction mapped to aa 70-84, 75-88 - protein footprint to 65-88 [Jensen1997] } \\
\text { binding protein complex acid labile chain precursor, QPPGLERLWLEGNPWDCG [Maksiutov2002] } \\
\text { - 9G2: Called 9G2G4D6E8: UK Medical Research Council AIDS reagent: ARP3058 }\end{array}$} \\
\hline 265 & $\mathrm{Ab} 4$ & $\begin{array}{l}\text { Rev (72-91) Rev (72-91 BRU) } \\
\text { Vaccine Vector/Type: recombinant protein } \\
\text { Donor Tony Lowe and Jonathan Karn, MRC } \\
\text { References Henderson1997, Maksiutov2002 } \\
\text { - Ab4: The binding site overlaps the nuclear e } \\
\text { - Ab4: This epitope is similar fragments of th } \\
\text { binding protein complex acid labile chain pr }\end{array}$ & $\begin{array}{l}\text { PLQLPPLERLTLDCNEDCGT } \\
\text { component: Rev } \\
\text { nter, Cambridge } \\
\text { t signal - binding was not blocked by bound HIV RNA and may be ac } \\
\text { man protein Epidermal growth factor receptor substrate 15, EPVPMSL } \\
\text { sor, QPPGLERLWLEGNPWDCG [Maksiutov2002] }\end{array}$ & $\begin{array}{l}\text { (IgG1) } \\
\text { tein interaction } \\
\text { in-like growth factor }\end{array}$ \\
\hline 266 & $3 \mathrm{G} 4$ & $\begin{array}{l}\text { Rev (90-116) } \quad \operatorname{Rev}(90-116) \\
\text { Vaccine Vector/Type: recombinant protein } \\
\text { References Orsini1995 } \\
\text { - 3G4: Binds to a region that can be dispensed }\end{array}$ & $\begin{array}{l}\text { GTSGTQGVGSPQILVESPTVLESGT- } \\
\text { KE? } \\
\text { component: Rev } \\
\text { h and still retain Rev function Orsini1995 }\end{array}$ & murine $(\operatorname{IgG} 1 \kappa)$ \\
\hline 267 & $\begin{array}{l}1 \mathrm{G} 10 \\
(\mathrm{IG} 10 \mathrm{~F} 4)\end{array}$ & $\begin{array}{l}\text { Rev }(96-105) \quad \operatorname{Rev}(95-105) \\
\text { Vaccine Vector/Type: recombinant protein } \\
\text { Donor Anne Marie Szilvay } \\
\text { References Kalland1994a } \\
\text { - 1G10: Bound Rev in indirect immunofluore } \\
\text { - 1G10: Peptide interaction mapped to aa } 91-1 \\
\text { 1G10: Called IG10F4: UK Medical Researc }\end{array}$ & $\begin{array}{l}\text { GVGSPQILVE } \\
\text { component: Rev } \\
\text { ce and also detected Rev in WB - used to detect localization of Rev thr } \\
96-110 \text { - protein footprint to aa 10-20, and 95-105 [Jensen1997] } \\
\text { uncil AIDS reagent: ARP3060 }\end{array}$ & murine $(\operatorname{IgG} 2 \mathrm{~b} \kappa)$ \\
\hline 268 & $1 \mathrm{G} 7$ & $\begin{array}{l}\text { Rev (96-105) Rev (95-105) } \\
\text { Vaccine Vector/Type: recombinant protein } \\
\text { References Kalland1994a, Jensen1997 } \\
\text { - 1G7: Worked in indirect immunofluorescenc } \\
\text { - 1G7: Peptide interaction mapped to aa 91-10 }\end{array}$ & $\begin{array}{l}\text { GVGSPQILVE } \\
\text { component: Rev } \\
\text { d also detected Rev in WB - used to detect localization of Rev throug } \\
6-110 \text { - protein footprint to aa 95-105 [Jensen1997] }\end{array}$ & $\begin{array}{l}\text { murine }(\operatorname{IgG} 2 \mathrm{~b} \kappa) \\
\text { alland1994a }\end{array}$ \\
\hline 269 & Ab3 & $\begin{array}{l}\text { Rev (102-116) Rev (102-116 BRU) } \\
\text { Vaccine Vector/Type: recombinant protein } \\
\text { Donor Tony Lowe and Jonathan Karn, MRC } \\
\text { References Henderson1997 } \\
\text { Ab3: This binding site is at the carboxy end }\end{array}$ & $\begin{array}{l}\text { ILVESP TVLESDKTE } \\
\text { component: Rev } \\
\text { mbridge } \\
\text { ev - Ab3 binding was not blocked by bound HIV RNA Henderson } 199\end{array}$ & $\operatorname{IgG1})$ \\
\hline 70 & $2 \mathrm{G} 2$ & $\begin{array}{l}\text { Rev } \quad \text { Rev } \\
\text { Vaccine Vector/Type: recombinant protein }\end{array}$ & component: Rev & $(\operatorname{IgG} 1 \kappa)$ \\
\hline
\end{tabular}




\begin{tabular}{lll}
\hline No. MAb ID & HXB2 Location Author's Location & Sequence \\
\hline & References Orsini1995 \\
& 2G2: Does not bind to any of a set of glutathione S-transferase (GST) Rev fusion proteins, or to Rev in a RIPA buffer, suggesting a conformational epitope \\
& Orsini1995]
\end{tabular}




\section{IV-C-13 gp160 Antibodies}

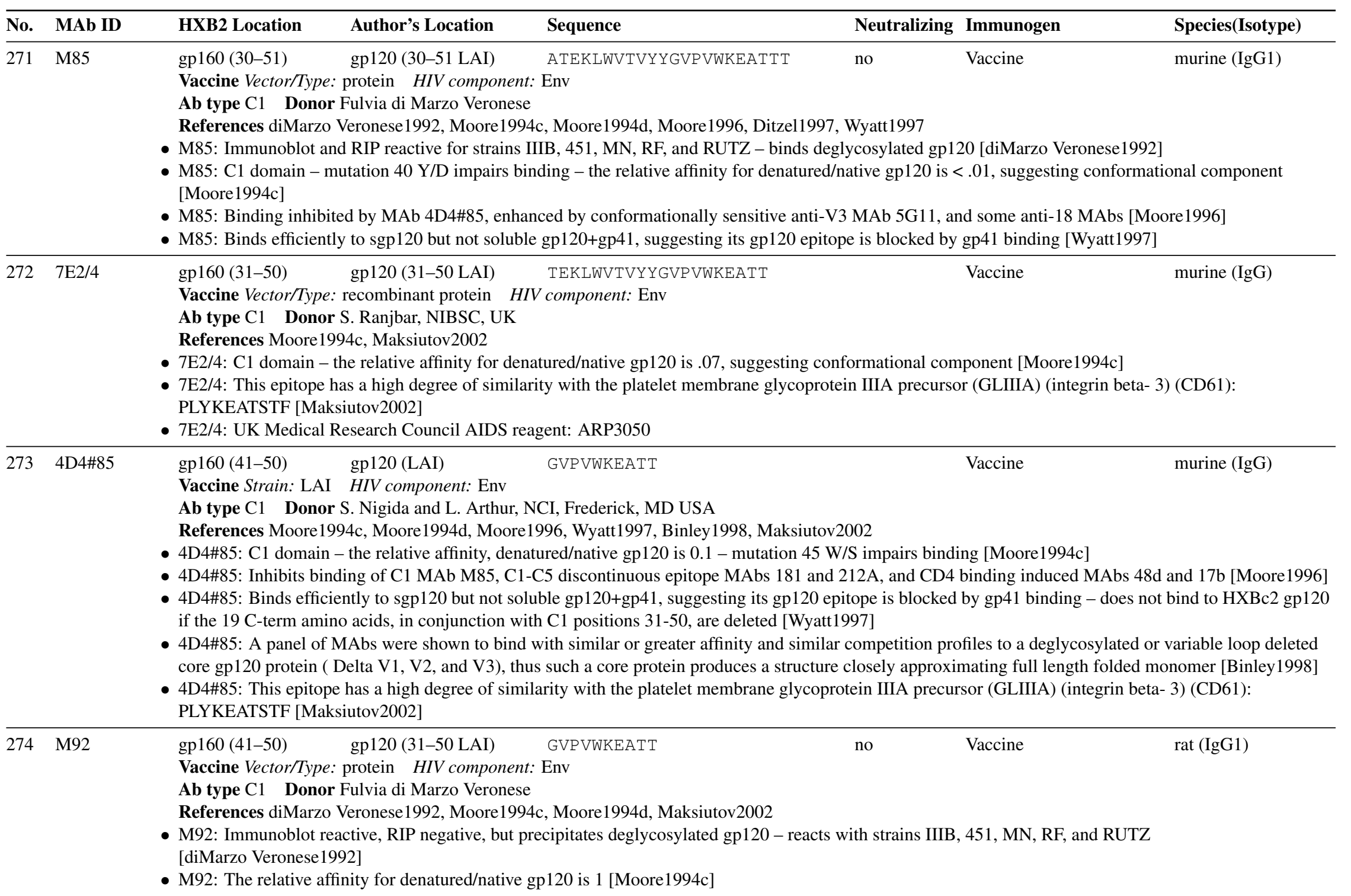




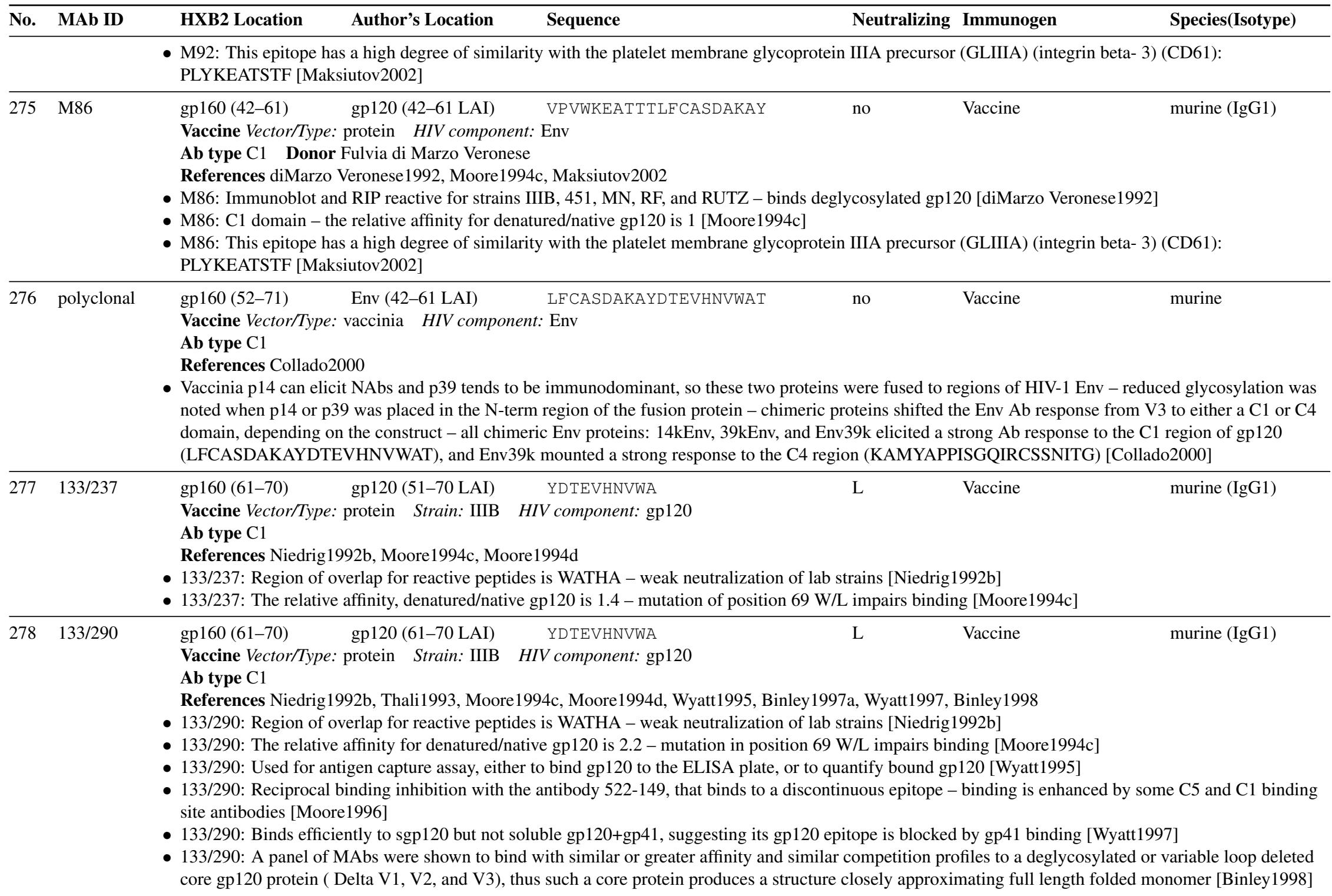




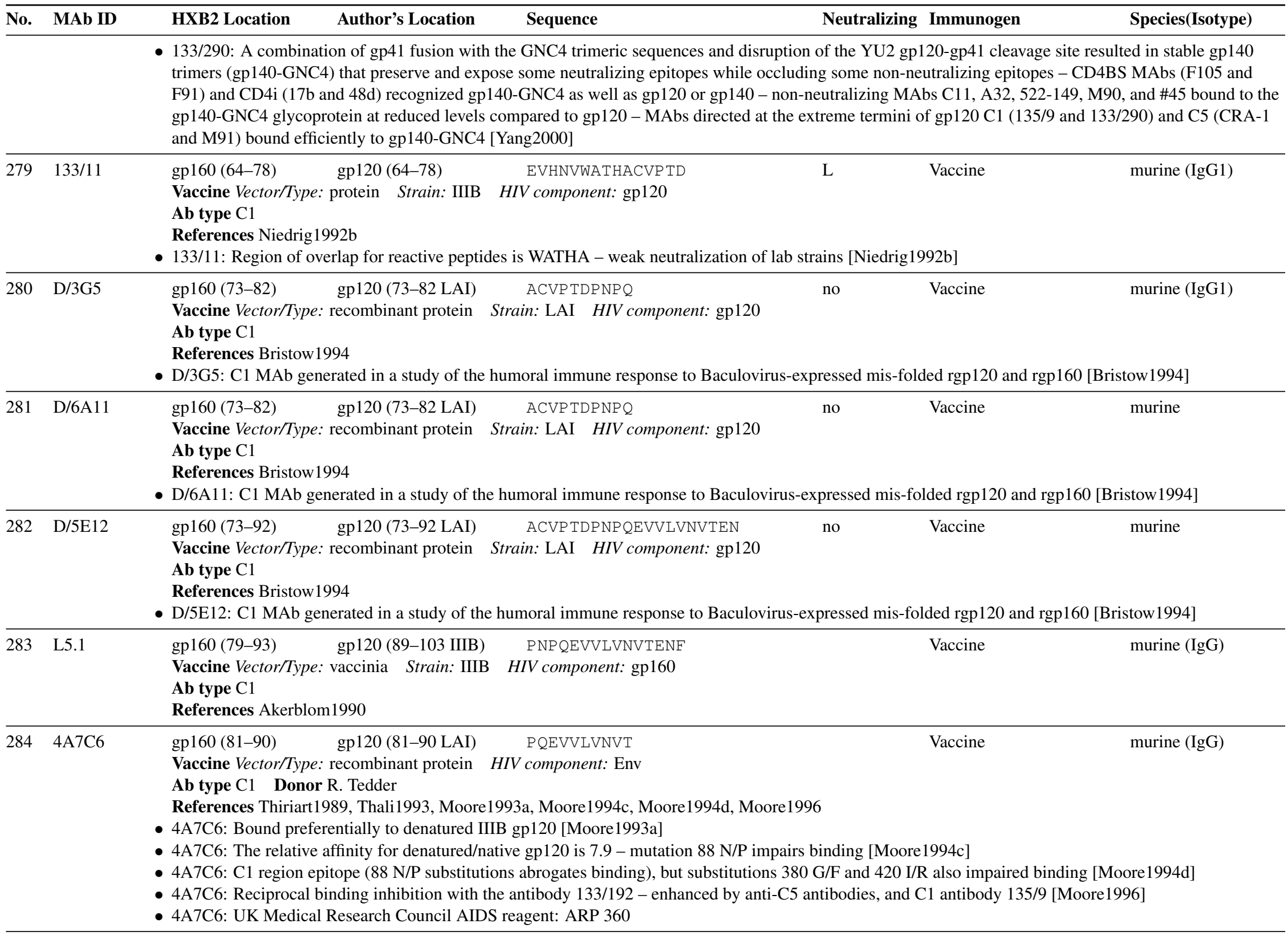




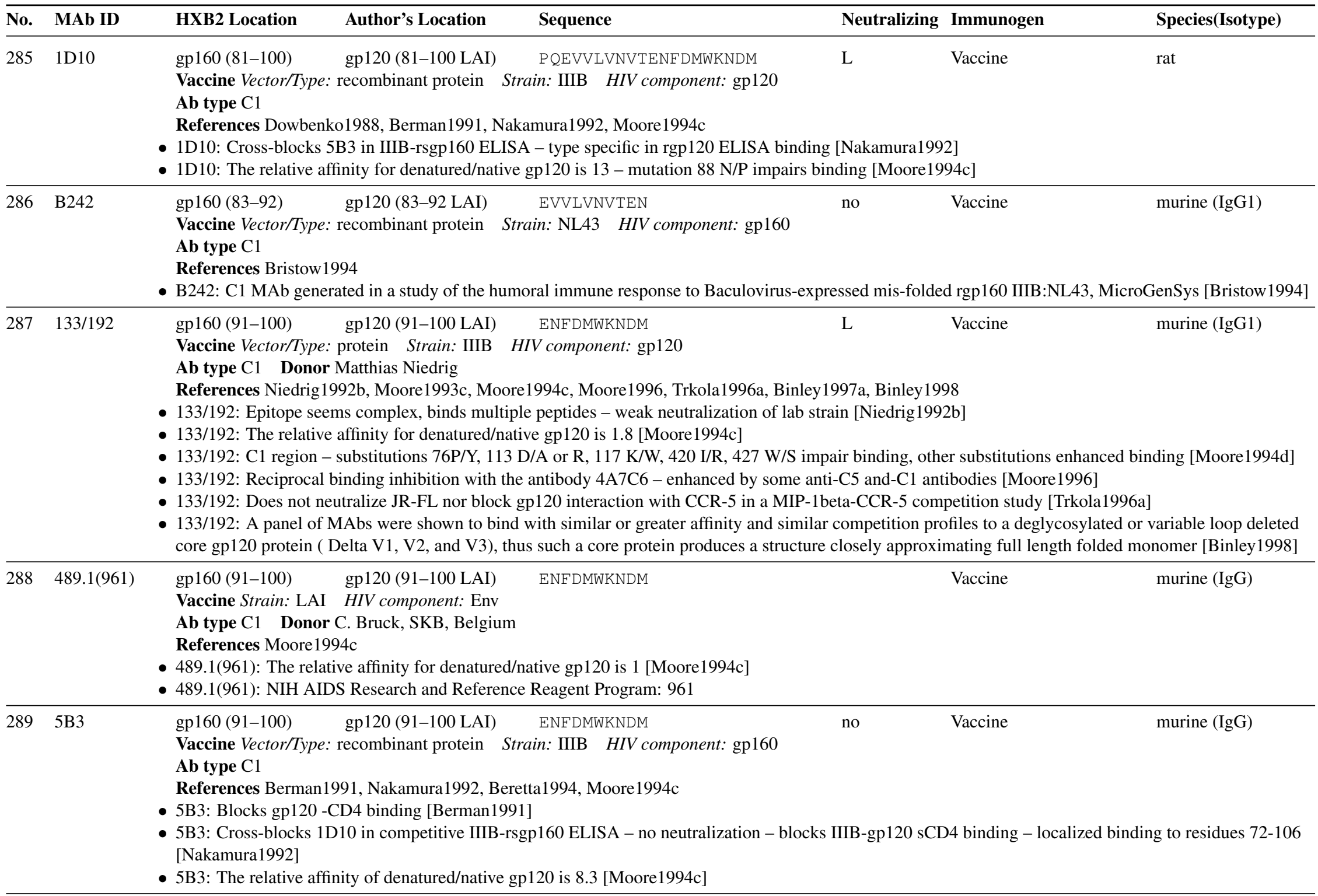




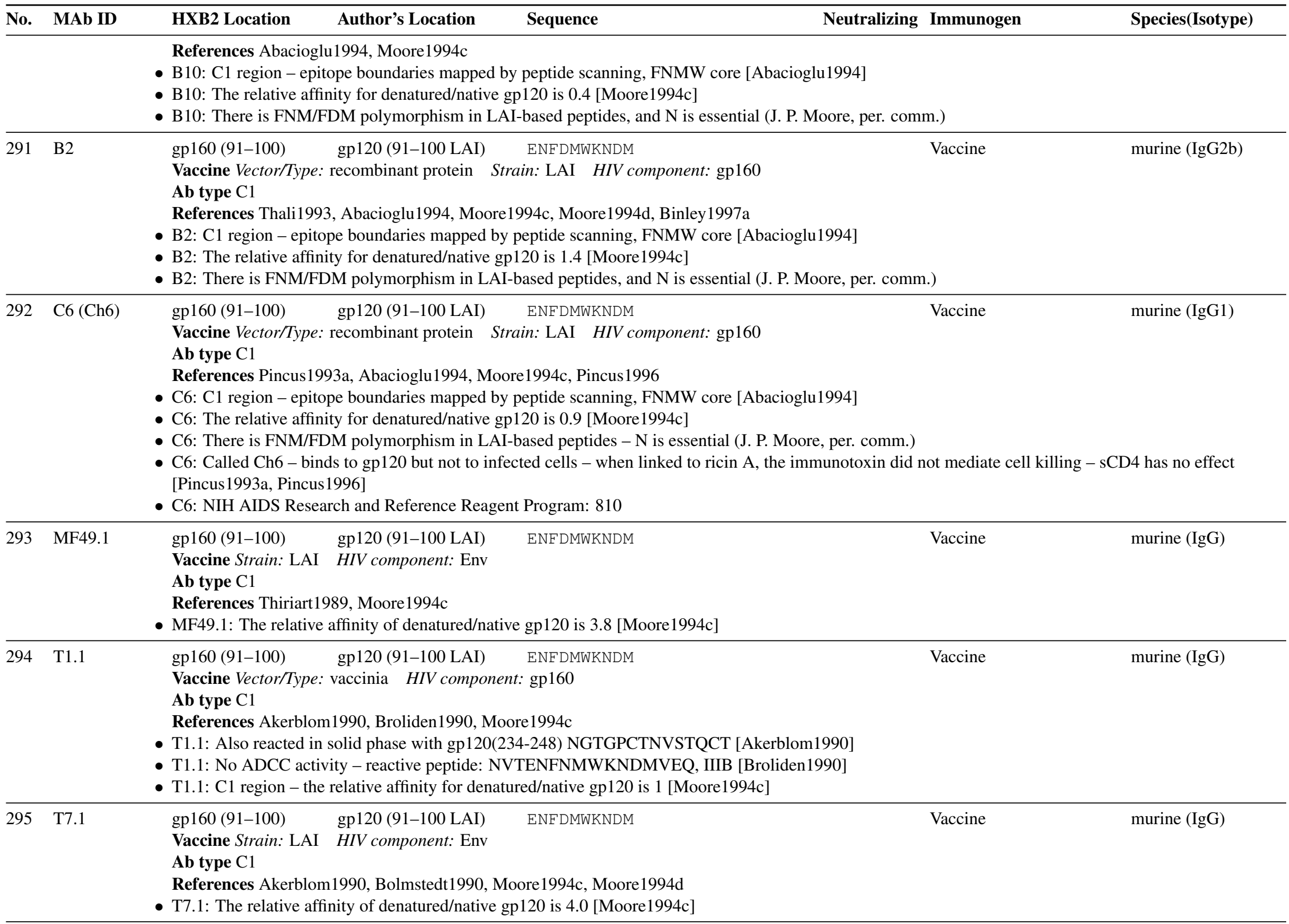




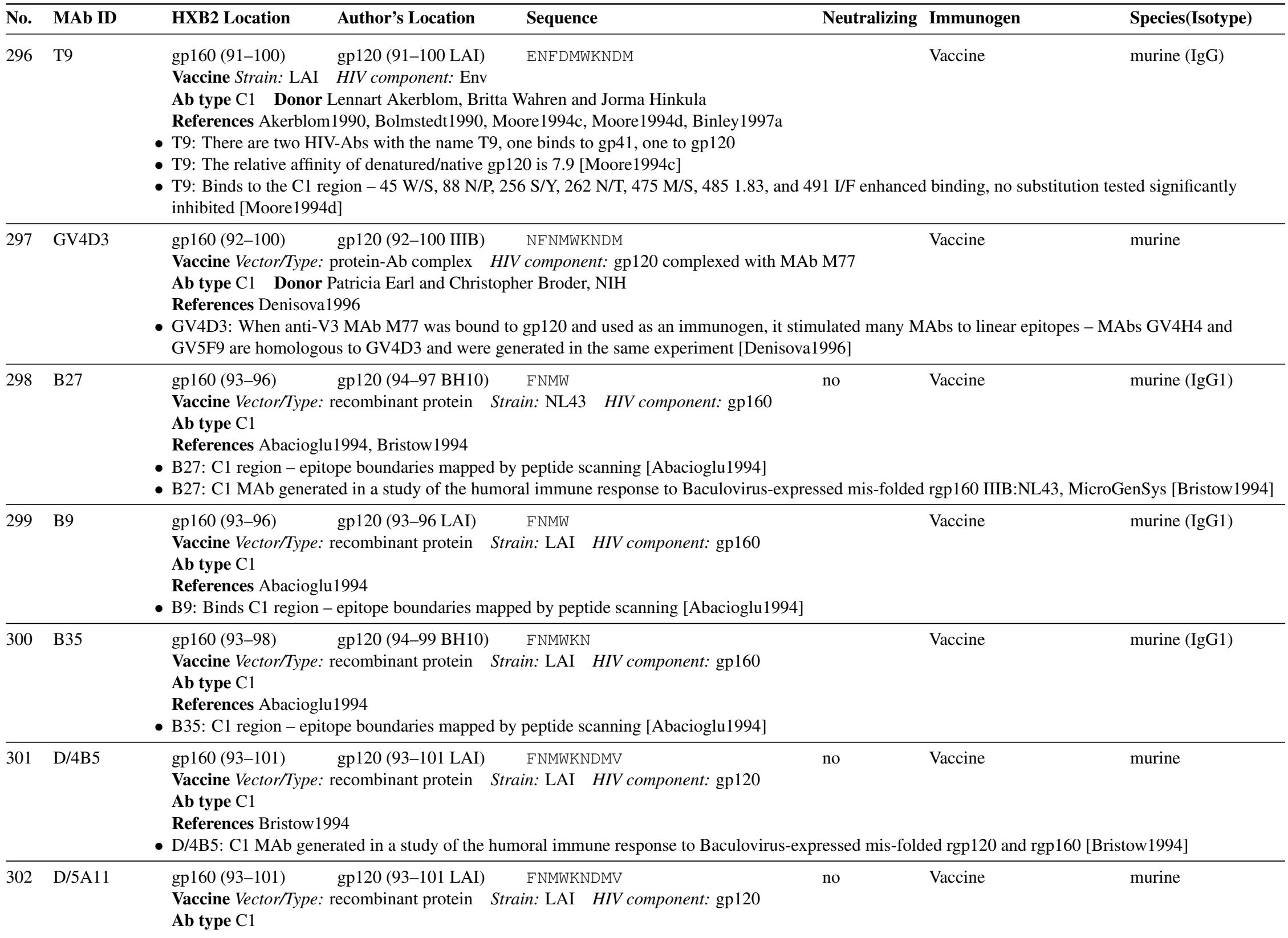




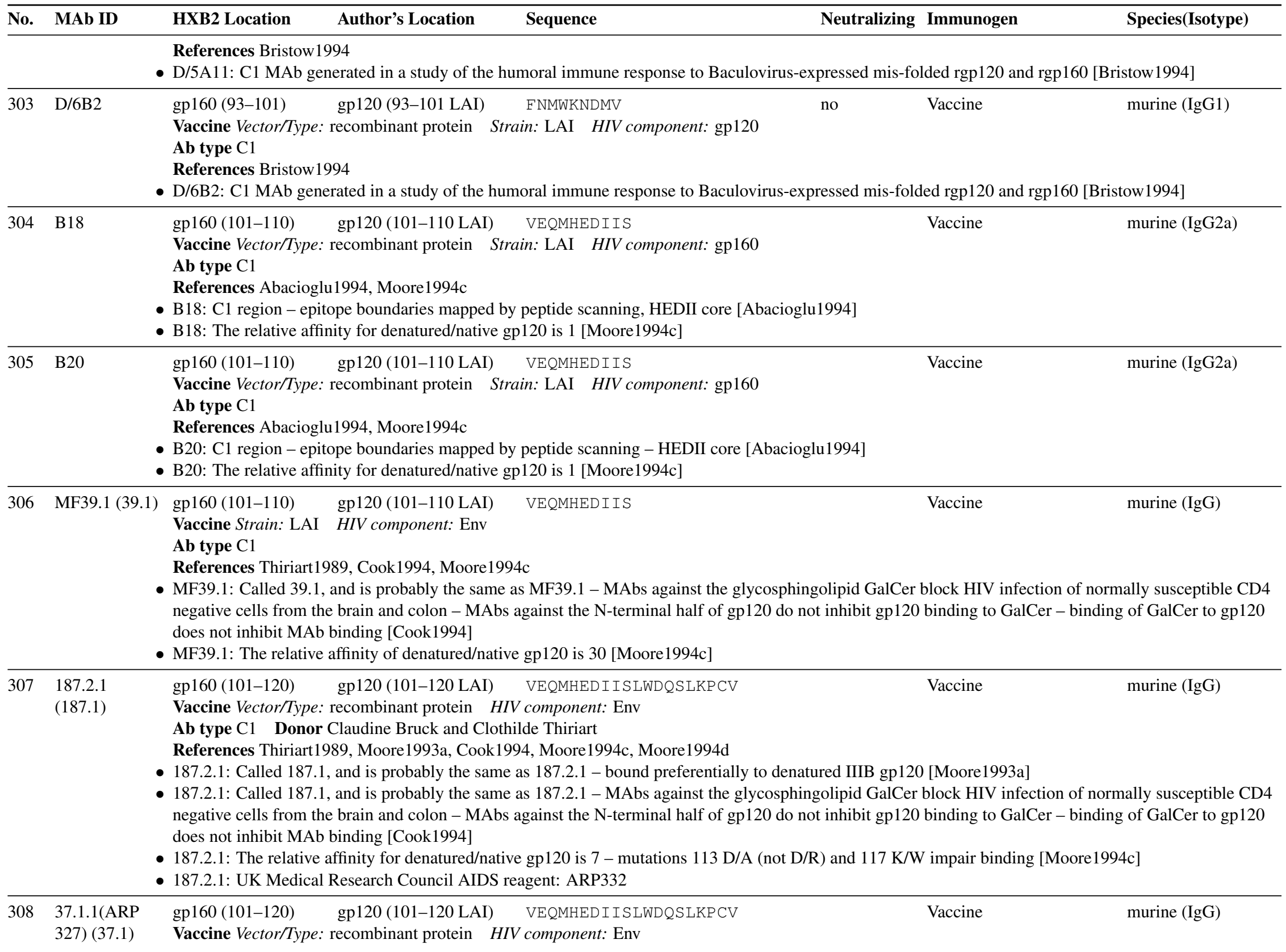




\begin{tabular}{|c|c|c|c|c|}
\hline No. & MAb ID & Author's Location & Neutralizing Immunogen & Species(Isotype) \\
\hline & & \multicolumn{3}{|c|}{$\begin{array}{l}\text { Ab type C1 Donor Claudine Bruck } \\
\text { References|Thiriart1989, Moore1993a |Moore1994c } \\
\text { - 37.1.1: Called 37.1 - bound preferentially to denatured IIIB gp120 [Moore1993a] } \\
\text { - 37.1.1: The relative affinity for denatured/native gp120 is } 8.6 \text { - mutations } 113 \text { D/R (not D/A) and } 117 \text { K/W impair binding [Moore1994c] } \\
\text { - 37.1.1: UK Medical Research Council AIDS reagent: ARP327 }\end{array}$} \\
\hline 309 & $6 \mathrm{D} 8$ & \multicolumn{3}{|c|}{$\begin{array}{l}\text { Vaccine Vector/Type: recombinant protein Strain: IIIB HIV component: gp120 } \\
\text { Ab type C1 } \\
\text { References Dowbenko1988, Nakamura1992, Moore1994c } \\
\text { - 6D8: Highly cross reactive with multiple stains by rgp120 ELISA [Nakamura1992] } \\
\text { - 6D8: The relative affinity for denatured/native gp120 is } 15 \text { - mutations } 113 \text { D/R and 113 D/A impair binding [Moore1994c] }\end{array}$} \\
\hline 310 & M96 & \multicolumn{3}{|c|}{$\begin{array}{l}\text { Vaccine Vector/Type: protein HIV component: Env } \\
\text { Ab type C1 Donor Fulvia di Marzo Veronese } \\
\text { References diMarzo Veronese1992, Moore1994c, Moore1994d } \\
\text { - M96: Immunoblot reactive for strains IIIB, 451, MN, RF, and RUTZ [diMarzo Veronese1992] } \\
\text { - M96: C1 region - the relative affinity for denatured/native gp120 is } 6 \text { [Moore1994c] }\end{array}$} \\
\hline 311 & MF119.1 & $\begin{array}{l}\text { gp160 (101-120) } \quad \text { gp120 (101-120 LAI) } \\
\text { Vaccine Strain: LAI HIV component: Env } \\
\text { Ab type C1 } \\
\text { References Thiriart1989, Moore1994c } \\
\text { MF119.1: The relative affinity for denatured/n }\end{array}$ & $\begin{array}{l}\text { VEQMHED I I LLWDQSLKPCV } \\
\text { ve gp120 is } 30 \text { - mutations } 113 \mathrm{D} / \mathrm{A}, 113 \mathrm{D} / \mathrm{R} \text {, and } 117 \mathrm{~K} / \mathrm{W} \text { impair bin }\end{array}$ & $\begin{array}{l}\text { murine }(\operatorname{IgG}) \\
994 \mathrm{c}\end{array}$ \\
\hline 312 & MF4.1 & $\begin{array}{l}\text { gp160 (101-120) gp120 (101-120 LAI) } \\
\text { Vaccine Strain: LAI } \quad \text { HIV component: Env } \\
\text { Ab type C1 } \\
\text { References Thiriart1989, Moore1994c } \\
\text { MF4.1: The relative affinity for denatured/nati }\end{array}$ & $\begin{array}{l}\text { VEQMHED I I SLWDQSLKPCV } \\
\text { gp120 is } 8 \text { Moore1994c }\end{array}$ & murine (IgG) \\
\hline 313 & MF53.1 & $\begin{array}{l}\text { gp160 (101-120) } \quad \text { gp120 (101-120 LAI) } \\
\text { Vaccine Strain: LAI } \text { HIV component: Env } \\
\text { Ab type C1 } \\
\text { References Thiriart1989, Moore1994c } \\
\text { MF53.1: The relative affinity for denatured/na }\end{array}$ & $\begin{array}{l}\text { VEQMHED I I SLWDQSLKP CV } \\
\text { e gp120 is } 10 \text { Moore1994c }\end{array}$ & murine (IgG) \\
\hline 314 & MF58.1 & $\begin{array}{ll}\text { gp160 }(101-120) & \text { gp120 }(101-120 \text { LAI }) \\
\text { Vaccine Strain: LAI } & \text { HIV component: Env } \\
\text { Ab type C1 } \\
\text { References Thiriart } 1989, \text { Moore } 1994 \mathrm{c}\end{array}$ & VEQMHED I I SLWDQSLKPCV & murine (IgG) \\
\hline 315 & MF77.1 & $\begin{array}{ll}\text { gp160 }(101-120) & \text { gp120 (101-120 LAI) } \\
\text { Vaccine Strain: LAI } & \text { HIV component: } \text { Env }\end{array}$ & VEQMHED I I SLWDQSLKPCV & murine (IgG) \\
\hline
\end{tabular}




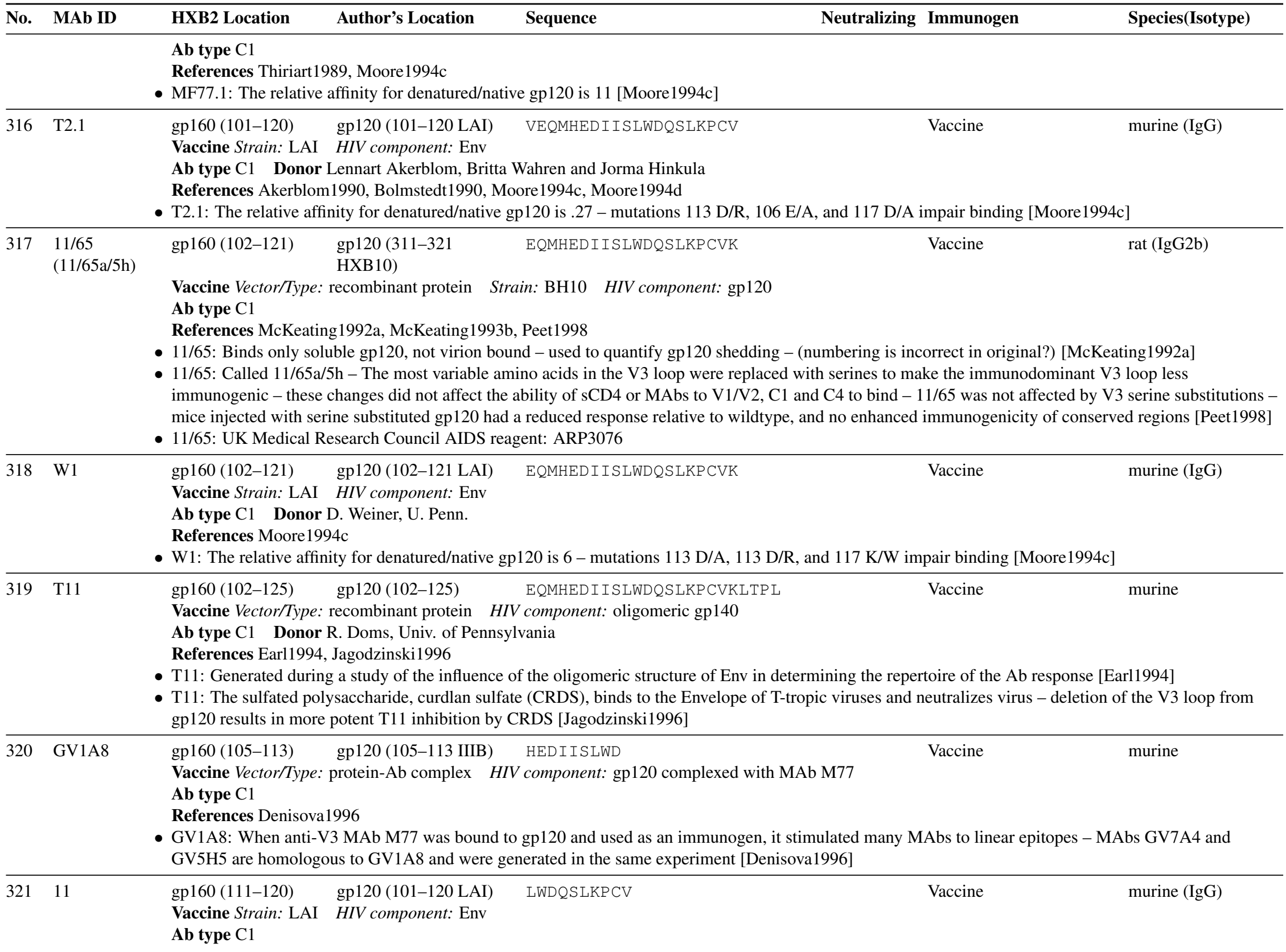




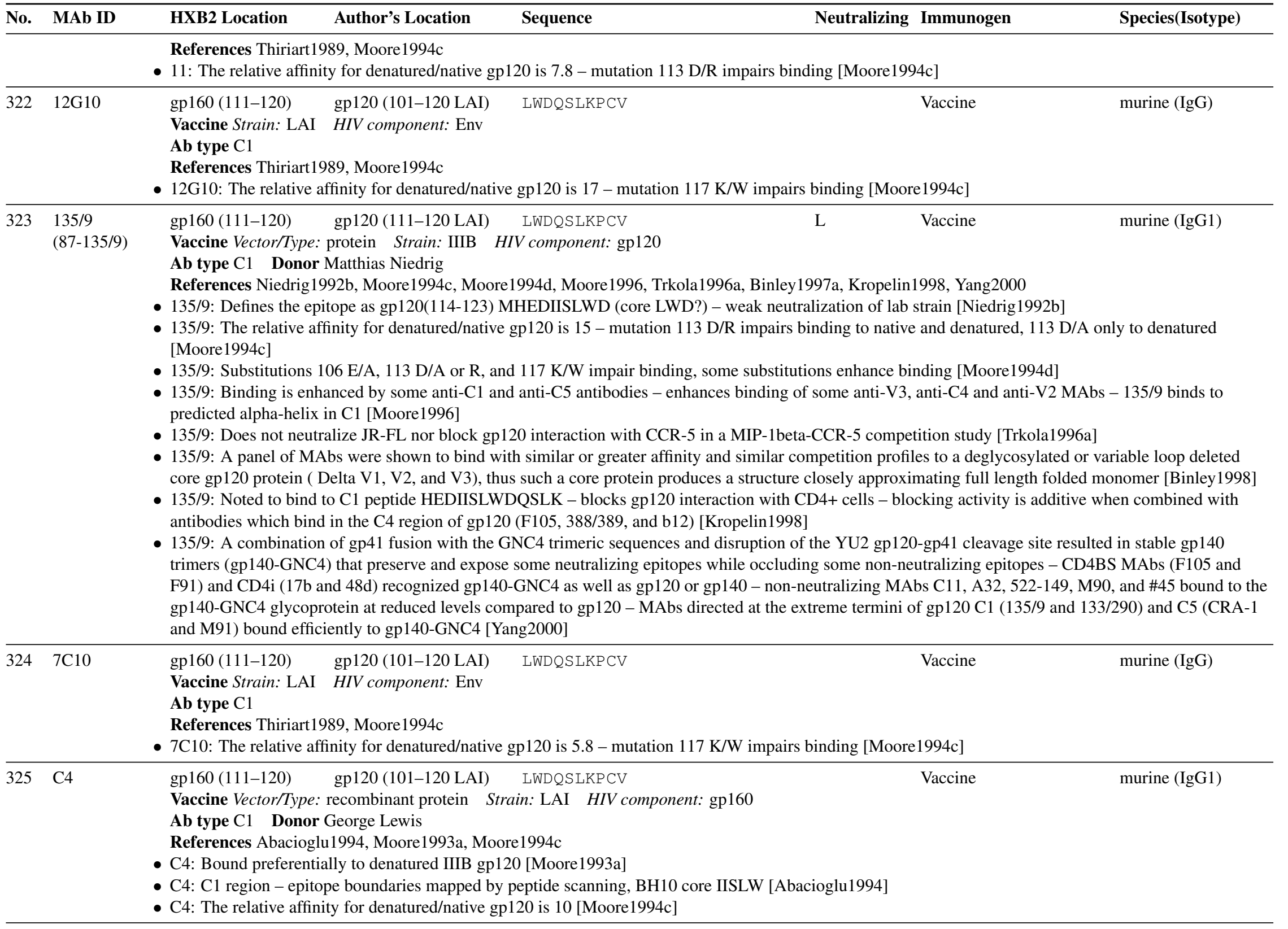




\begin{tabular}{|c|c|c|c|c|c|c|}
\hline No. & MAb ID & HXB2 Location & Author's Location & Neutralizing & Immunogen & Species(Isotype) \\
\hline 326 & MF46.1 & $\begin{array}{l}\text { gp160 }(111-120) \\
\text { Vaccine Strain: LAI } \\
\text { Ab type C1 } \\
\text { References Thiriart19 } \\
\text { MF46.1: The relative }\end{array}$ & $\begin{array}{l}\text { gp120 (101-120 LAI) } \\
\text { HIV component: Env } \\
\text { 89. Moore } 1994 \mathrm{c} \\
\text { affinity for denatured/na }\end{array}$ & $\begin{array}{l}\text { LWDQSLKPCV } \\
\text { e gp120 is } 8.5 \text { Moore1994c }\end{array}$ & Vaccine & murine (IgG) \\
\hline 327 & $6 \mathrm{D} 5$ & $\begin{array}{l}\text { gp160 (122-141) } \\
\text { Vaccine Strain: LAI } \\
\text { Ab type V2 Donor } \\
\text { References Moore196 } \\
\text { 6D5: The relative affil }\end{array}$ & $\begin{array}{l}\text { gp120 (122-141 LAI) } \\
\text { HIV component: Env } \\
\text { S. Nigida and L. Arthur } \\
\text { 4c Moore1994d } \\
\text { iity for denatured/native }\end{array}$ & $\begin{array}{l}\text { LTPLCVSLKCTDLKNDTNTN } \\
\text { CI, Frederick, MD USA } \\
120 \text { is } 15 \text { - mutations Delta119-205 and } 125 \text { L/G impair }\end{array}$ & $\begin{array}{l}\text { Vaccine } \\
\text { binding Moor }\end{array}$ & murine (IgG) \\
\hline
\end{tabular}

References Abacioglu1994 Bristow1994

- B33: There are two MAbs in the literature named B33, see also gp160(727-734) [Abacioglu1994]

- B33: Epitope boundaries mapped by peptide scanning [Abacioglu1994

- B27: C1 MAb generated in a study of the humoral immune response to Baculovirus-expressed mis-folded rgp160 IIIB:NL43, MicroGenSys [Bristow1994|

- B33: UK Medical Research Council AIDS reagent: ARP304, gp160/41 binding

\begin{tabular}{lllll}
\hline polyclonal & gp160 (131-151) Env (131-151) & CTDLKNDTNTNSSSGRMMMEK & hIV-1 infection \\
(VEI1) & References Carlos1999 &
\end{tabular}

- Antibody response to the epitopes in a vaccine construct (VEI) containing peptides from 5 hypervariable regions of gp120 was detected in the sera of HIV-1 positive subjects, including sera from 6 non-subtype B infections - serum samples from San Francisco, Canada and Puerto Rico cohort showed presence of antibodies against all five VEI hypervariable regions, but most consistently against the V3 region peptide NNNTRKSIRIGPGRAFYTTGDIGNIRQ Carlos1999

\begin{tabular}{|c|c|c|c|c|c|c|c|}
\hline \multirow[t]{2}{*}{330} & \multirow[t]{2}{*}{$35 \mathrm{D} 10 / \mathrm{D} 2$} & gp160 (139-155) & gp120 & NTKSSNWKEMDGEIK & $\mathrm{L}$ & Vaccine & $\begin{array}{l}\text { human from } \\
\text { transgenic mice } \\
(\operatorname{IgG} 2 \kappa)\end{array}$ \\
\hline & & $\begin{array}{l}\text { Vaccine Vector/Type } \\
\text { Ab type V1 Dono } \\
\text { References He2002 } \\
\text { 35D10/D2: Transge } \\
\text { create a panel of ant } \\
\text { neutralizing the autc } \\
\text { autologous strain SF }\end{array}$ & $\begin{array}{l}\text { recombi } \\
\text { Dr. Abre } \\
\text { c mice }( \\
\text { HIV gp1 } \\
\text { gous str } \\
62 \text { but } w\end{array}$ & $\begin{array}{l}\text { lin: SF162 HIV compor } \\
\text { c Health Research Institu } \\
\text { G2) carrying human gen } \\
\text { g hybridomas by immuni } \\
\text { region was immunodomi } \\
\text { ecific He2002] }\end{array}$ & inte & $\begin{array}{l}\text { ljuvant }(\mathrm{M} \\
\text { human IgC } \\
\text { - several o } \\
\text { 1-specific }\end{array}$ & $\begin{array}{l}\text { re used to rapidly } \\
\text { ed were effective at } \\
\text { tly neutralize }\end{array}$ \\
\hline 331 & $40 \mathrm{H} 2 / \mathrm{C} 7$ & gp160 (139-155) & gp120 & NTKSSNWKEMDGEIK & $\mathrm{L}$ & Vaccine & $\begin{array}{l}\text { human from } \\
\text { transgenic mice } \\
(\operatorname{IgG} 2 \kappa)\end{array}$ \\
\hline
\end{tabular}

Vaccine Vector/Type: recombinant protein Strain: SF162 HIV component: gp120 Adjuvant: Ribi adjuvant (MPL+TDM) $(\operatorname{IgG} 2 \kappa)$

Ab type V1Ｄonor Dr. Abraham Pinter, Public Health Research Institute, Newark, NJ, pinter@phri.org 


\begin{tabular}{|c|c|c|c|c|c|c|}
\hline No. & MAb ID & HXB2 Location & Author's Location & Sequence & Neutralizing Immunogen & Species(Isotype) \\
\hline & & \multicolumn{5}{|c|}{$\begin{array}{l}\text { References } \mathrm{He} 2002 \\
40 \mathrm{H} 2 / \mathrm{C} 7: \text { Transgenic mice (strain XenoMouse G2) carrying human genes allowing production of fully human IgG2kappa MAbs were used to rapidly } \\
\text { create a panel of anti-HIV gp120 MAb-producing hybridomas by immunization with HIV SF162 gp120 - several of the MAbs obtained were effective at } \\
\text { neutralizing the autologous strain - C-term-V1- region was immunodominant in these mice and the ten V1-specific MAbs could potently neutralize } \\
\text { autologous strain SF162 but were highly type specific [He2002] }\end{array}$} \\
\hline \multirow[t]{2}{*}{332} & $43 \mathrm{~A} 3 / \mathrm{E} 4$ & gp160 (139-155) & gp120 & NTKSSNWKEMDGEIK & Vaccine & $\begin{array}{l}\text { human from } \\
\text { transgenic mice } \\
(\operatorname{IgG} 2 \kappa)\end{array}$ \\
\hline & & \multicolumn{5}{|c|}{$\begin{array}{l}\text { Vaccine Vector/Type: recombinant protein Strain: SF162 HIV component: gp120 Adjuvant: Ribi adjuvant (MPL+TDM) } \\
\text { Ab type V1 Donor Dr. Abraham Pinter, Public Health Research Institute, Newark, NJ, pinter@phri.org } \\
\text { References He2002] } \\
\text { 43A3/E4: Transgenic mice (strain XenoMouse G2) carrying human genes allowing production of fully human IgG2kappa MAbs were used to rapidly create } \\
\text { a panel of anti-HIV gp120 MAb-producing hybridomas by immunization with HIV SF162 gp120 - several of the MAbs obtained were effective at } \\
\text { neutralizing the autologous strain - C-term-V1- region was immunodominant in these mice and the ten V1-specific MAbs could potently neutralize } \\
\text { autologous strain SF162 but were highly type specific [He2002] }\end{array}$} \\
\hline \multirow[t]{2}{*}{333} & 43C7/B9 & gp160 (139-155) & gp120 & NTKSSNWKEMDGEIK & Vaccine & $\begin{array}{l}\text { human from } \\
\text { transgenic mice } \\
(\operatorname{IgG} 2 \kappa)\end{array}$ \\
\hline & & \multicolumn{5}{|c|}{$\begin{array}{l}\text { Vaccine Vector/Type: recombinant protein Strain: SF162 HIV component: gp120 Adjuvant: Ribi adjuvant (MPL+TDM) } \\
\text { Ab type V1 Donor Dr. Abraham Pinter, Public Health Research Institute, Newark, NJ, pinter@ phri.org } \\
\text { References He2002] } \\
\text { 43C7/B9: Transgenic mice (strain XenoMouse G2) carrying human genes allowing production of fully human IgG2kappa MAbs were used to rapidly create } \\
\text { a panel of anti-HIV gp120 MAb-producing hybridomas by immunization with HIV SF162 gp120 - several of the MAbs obtained were effective at } \\
\text { neutralizing the autologous strain - C-term-V1- region was immunodominant in these mice and the ten V1-specific MAbs could potently neutralize } \\
\text { autologous strain SF162 but were highly type specific [He2002] }\end{array}$} \\
\hline \multirow[t]{2}{*}{334} & 45D1/B7 & gp160 (139-155) & gp120 & NTKSSNWKEMDGEIK & Vaccine & $\begin{array}{l}\text { human from } \\
\text { transgenic mice } \\
(\operatorname{IgG} 2 \kappa)\end{array}$ \\
\hline & & \multicolumn{5}{|c|}{$\begin{array}{l}\text { Vaccine Vector/Type: recombinant protein Strain: SF162 HIV component: gp120 Adjuvant: Ribi adjuvant (MPL+TDM) } \\
\text { Ab type V1 Donor Dr. Abraham Pinter, Public Health Research Institute, Newark, NJ, pinter@phri.org } \\
\text { References He2002 } \\
\text { 45D1/B7: Transgenic mice (strain XenoMouse G2) carrying human genes allowing production of fully human IgG2kappa MAbs were used to rapidly } \\
\text { create a panel of anti-HIV gp120 MAb-producing hybridomas by immunization with HIV SF162 gp120 - several of the MAbs obtained were effective at } \\
\text { neutralizing the autologous strain - C-term-V1- region was immunodominant in these mice and the ten V1-specific MAbs could potently neutralize } \\
\text { autologous strain SF162 but were highly type specific [He2002] }\end{array}$} \\
\hline 35 & 46E3/E6 & gp160 (139-155) & gp120 & NTKSSNWKEMDGEIK & Vaccine & $\begin{array}{l}\text { human from } \\
\text { transgenic mice } \\
(\mathrm{IgG} 2 \kappa)\end{array}$ \\
\hline
\end{tabular}

Vaccine Vector/Type: recombinant protein Strain: SF162 HIV component: gp120 Adjuvant: Ribi adjuvant (MPL+TDM) $(\operatorname{IgG} 2 \kappa)$

Ab type V1 Donor Dr. Abraham Pinter, Public Health Research Institute, Newark, NJ, pinter@phri.org 


\begin{tabular}{|c|c|c|c|c|c|c|}
\hline No. & MAb ID & HXB2 Location & Author's Location & Sequence & Neutralizing Immunogen & Species(Isotype) \\
\hline & & \multicolumn{5}{|c|}{$\begin{array}{l}\text { References He2002 } \\
\text { 46E3/E6: Transgenic mice (strain XenoMouse G2) carrying human genes allowing production of fully human IgG2kappa MAbs were used to rapidly create } \\
\text { a panel of anti-HIV gp120 MAb-producing hybridomas by immunization with HIV SF162 gp120 - several of the MAbs obtained were effective at } \\
\text { neutralizing the autologous strain - C-term-V1- region was immunodominant in these mice and the ten V1-specific MAbs could potently neutralize } \\
\text { autologous strain SF162 but were highly type specific [He2002] }\end{array}$} \\
\hline \multirow[t]{2}{*}{336} & 58E1/B3 & gp160 (139-155) & gp120 & NTKSSNWKEMDGEIK & Vaccine & $\begin{array}{l}\text { human from } \\
\text { transgenic mice } \\
(\operatorname{IgG} 2 \kappa)\end{array}$ \\
\hline & & \multicolumn{5}{|c|}{$\begin{array}{l}\text { Vaccine Vector/Type: recombinant protein Strain: SF162 HIV component: gp120 Adjuvant: Ribi adjuvant (MPL+TDM) } \\
\text { Ab type V1 Donor Dr. Abraham Pinter, Public Health Research Institute, Newark, NJ, pinter@phri.org } \\
\text { References He2002] } \\
\text { 58E1/B3: Transgenic mice (strain XenoMouse G2) carrying human genes allowing production of fully human IgG2kappa MAbs were used to rapidly create } \\
\text { a panel of anti-HIV gp120 MAb-producing hybridomas by immunization with HIV SF162 gp120 - several of the MAbs obtained were effective at } \\
\text { neutralizing the autologous strain - C-term-V1- region was immunodominant in these mice and the ten V1-specific MAbs could potently neutralize } \\
\text { autologous strain SF162 but were highly type specific [He2002] }\end{array}$} \\
\hline \multirow[t]{2}{*}{337} & 64B9/A6 & gp160 (139-155) & gp120 & NTKSSNWKEMDGEIK & Vaccine & $\begin{array}{l}\text { human from } \\
\text { transgenic mice } \\
(\operatorname{IgG} 2 \kappa)\end{array}$ \\
\hline & & \multicolumn{5}{|c|}{$\begin{array}{l}\text { Vaccine Vector/Type: recombinant protein Strain: SF162 HIV component: gp120 Adjuvant: Ribi adjuvant (MPL+TDM) } \\
\text { Ab type V1 Donor Dr. Abraham Pinter, Public Health Research Institute, Newark, NJ, pinter@ phri.org } \\
\text { References He2002 } \\
\text { - 64B9/A6: Transgenic mice (strain XenoMouse G2) carrying human genes allowing production of fully human IgG2kappa MAbs were used to rapidly } \\
\text { create a panel of anti-HIV gp120 MAb-producing hybridomas by immunization with HIV SF162 gp120 - several of the MAbs obtained were effective at } \\
\text { neutralizing the autologous strain - C-term-V1- region was immunodominant in these mice and the ten V1-specific MAbs could potently neutralize } \\
\text { autologous strain SF162 but were highly type specific [He2002] }\end{array}$} \\
\hline \multirow[t]{2}{*}{338} & 69D2/A1 & gp160 (139-155) & gp120 & NTKSSNWKEMDGEIK & Vaccine & $\begin{array}{l}\text { human from } \\
\text { transgenic mice } \\
(\operatorname{IgG} 2 \kappa)\end{array}$ \\
\hline & & \multicolumn{5}{|c|}{$\begin{array}{l}\text { Vaccine Vector/Type: recombinant protein Strain: SF162 HIV component: gp120 Adjuvant: Ribi adjuvant (MPL+TDM) } \\
\text { Ab type V1 Donor Dr. Abraham Pinter, Public Health Research Institute, Newark, NJ, pinter@phri.org } \\
\text { References He2002 } \\
\text { 69D2/A1: Transgenic mice (strain XenoMouse G2) carrying human genes allowing production of fully human IgG2kappa MAbs were used to rapidly } \\
\text { create a panel of anti-HIV gp120 MAb-producing hybridomas by immunization with HIV SF162 gp120 - several of the MAbs obtained were effective at } \\
\text { neutralizing the autologous strain - C-term-V1- region was immunodominant in these mice and the ten V1-specific MAbs could potently neutralize } \\
\text { autologous strain SF162 but were highly type specific He2002] }\end{array}$} \\
\hline 339 & $82 \mathrm{D} 3 / \mathrm{C} 3$ & gp160 (139-155) & gp120 & NTKSSNWKEMDGEIK & Vaccine & $\begin{array}{l}\text { human from } \\
\text { transgenic mice } \\
(\operatorname{IgG} 2 \kappa)\end{array}$ \\
\hline
\end{tabular}

Vaccine Vector/Type: recombinant protein Strain: SF162 HIV component: gp120 Adjuvant: Ribi adjuvant (MPL+TDM) $(\operatorname{IgG} 2 \kappa)$

Ab type V1 Donor Dr. Abraham Pinter, Public Health Research Institute, Newark, NJ, pinter@ phri.org 


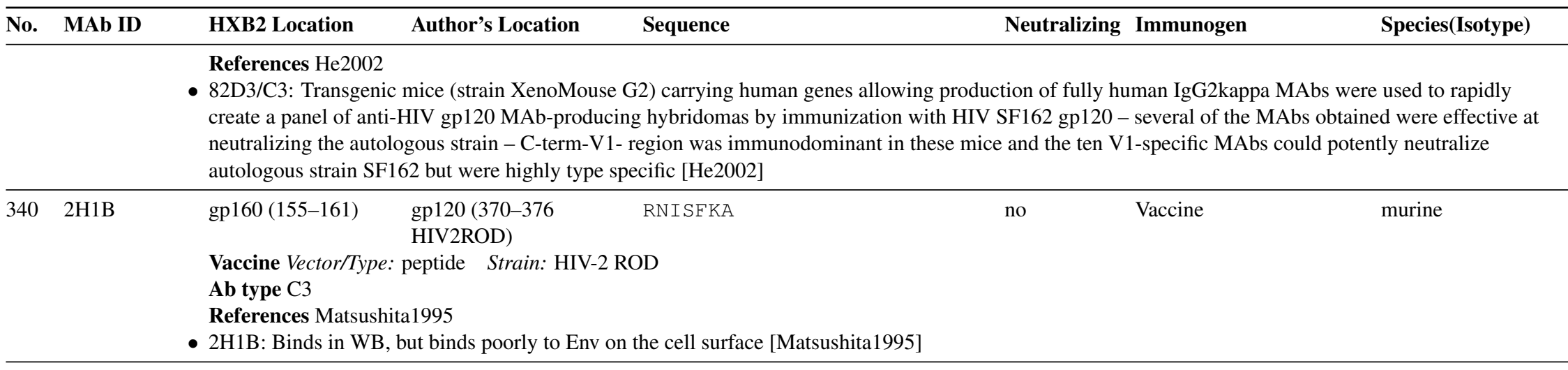

697-30D) Ab type V2 Donor Susan Zolla-Pazner (Zollas01@ mcrcr6.med.nyu) (NYU Med. Center) or Cellular Products Inc, Buffalo NY References Gorny1994, Forthal1995, Moore1995b Trkola1996a, Binley1997a, Fouts 1997, Parren1997c, Nyambi1998, Stamatatos1998, Gorny2000a Hioe2000, Nyambi2000, Edwards2002 Maksiutov2002

- 697-D: Conformational with weak reactivity to V2 peptide ISTSIRGKVQKEYAFFYKLD - neutralized 3/4 primary isolates, but none of 4 lab strains - V2 substitutions 176/177 FY/AT, 179/180 LD/DL, 183/184 PI/SG, and 192-194 YSL/GSS abrogate binding - anti-C4 MAbs G3-536 and G45-60 enhance binding - mild oxidation of carbohydrate moieties inhibits binding [Gorny1994]

- 697-D: Not neutralizing, no ADCC activity, and no viral enhancing activity [Forthal1995

- 697-D: Review: called 697/30D - neutralizes some primary, but not lab adapted strains [Moore1995b]

- 697-D: Partial inhibition of gp120 interaction with CCR-5 in a MIP-1beta-CCR-5 competition study [Trkola1996a]

- 697-D: Study shows neutralization is not predicted by MAb binding to JRFL monomeric gp120, but is associated with oligomeric Env binding - 697-D bound monomer, did not bind oligomer or neutralize JRFL [Fouts1997]

- 697-D: Does not neutralize TCLA strains but neutralizes some primary isolates weakly [Parren1997c]

- 697-D: Using a whole virion-ELISA method, 18 human MAbs were tested for their ability to bind to a panel of 9 viruses from clades A, B, D, F, G, and H V2 Abs 697-D, 1361, and 1357 tended to bind weakly with a similar pattern of specificity to virions, and bound well to soluble gp120: weak binding to 1/4 B clade viruses (CA5), and weak binding to viruses from subtype A and D [Nyambi1998]

- 697-D: Called 697-30D - deleting the V2 loop of neutralization-resistant HIV-1 isolate SF162 does not abrogate its replication in PBMC or macrophages, but it enhances its neutralization sensitivity to sera from patients with B clade infection up to 170-fold, and also enhances sensitivity to sera from clades A through F - deletion of V1 or V2 did not enable neutralization by V2 MAbs G3.4, G3.136, or 687-30D [Stamatatos1998]

- 697-D: Binding of panel of 21 MAbs to soluble oligomeric gp140 versus gp41 or gp120 monomers was compared - no MAb was oligomer specific, though anti-V3 and CD4BS MAbs reacted better with the oligomer and V2 and C5 tended to favor the monomer - V2 MAbs 697-D, 1357 and 1361 favored the monomer by approximately 2 fold|Gorny2000a

- 697-D: Ab responses, because of their capacity to alter antigen uptake and processing, can influence helper T cell responses - CD4BS MAbs or serum Ig from HIV+ individuals inhibited proliferative responses of gp120 specific T cells - V2 MAb 697-D did not effect proliferation [Hioe2000]

- 697-D: 26 HIV-1 group M isolates (clades A to H) were tested for binding to 47 MAbs, including 5 anti-V2 MAbs, which showed weak and sporadic binding, with the most frequent binding to $\mathrm{C}$ and D clades [Nyambi2000] 


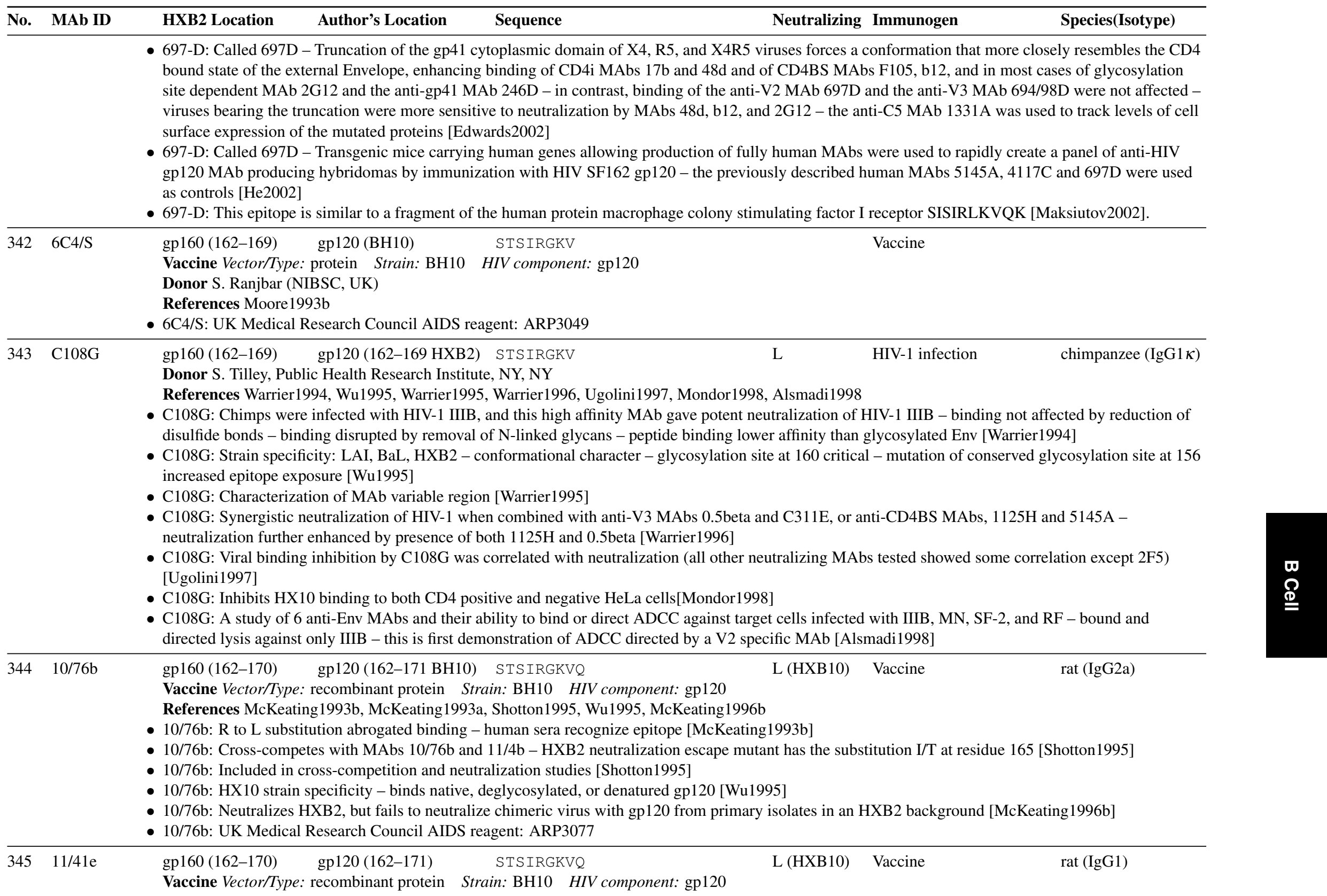




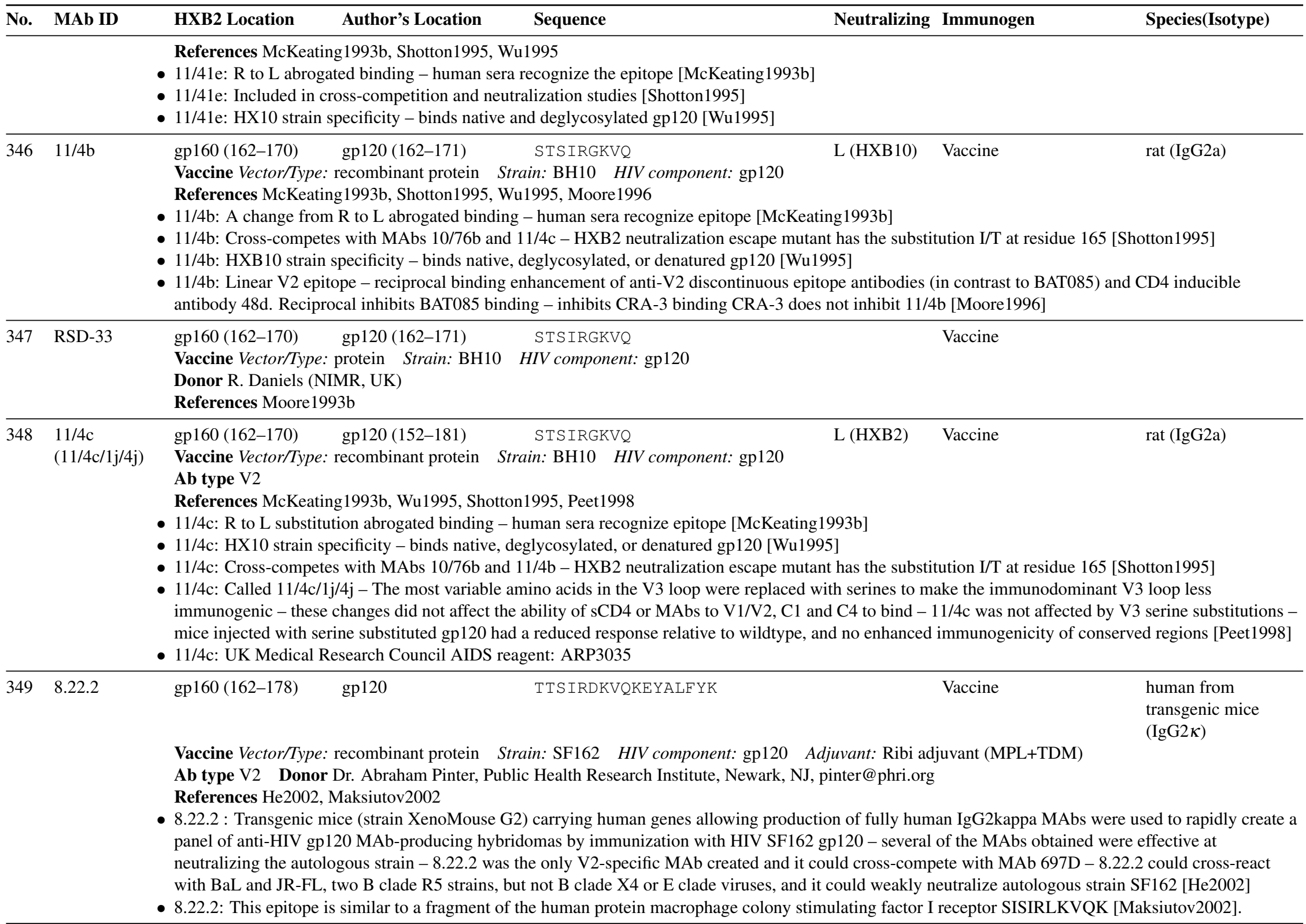




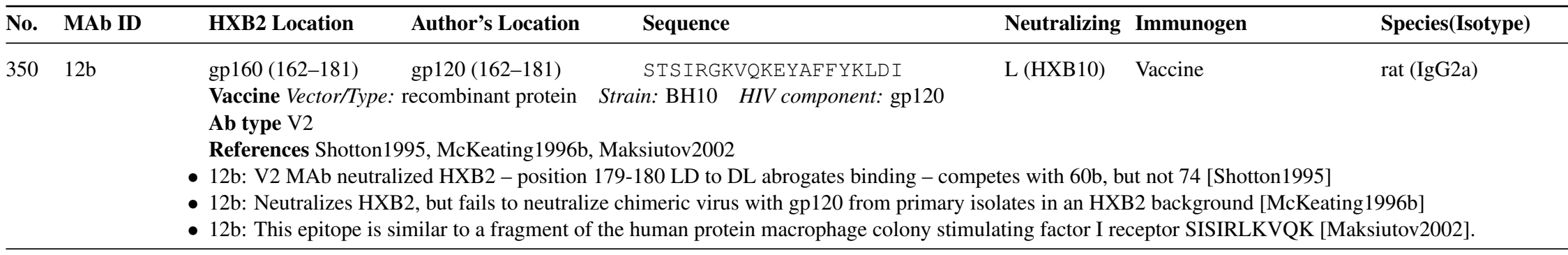

- G3-136: V2 region - binds and neutralizes IIIB and RF in CEM-SS cells, but not MN - neutralization activity against a few primary isolates in PBMC sCD4 binding inhibits binding (contrast with BAT085) - deglycosylation or reduction of gp120 by DTT diminishes reactivity [Fung1992]

- G3-136: Conformational, does not bind well to denatured gp120 - not reactive with SF-2 gp120, and does not inhibit HIV-1 sera from binding to IIIB gp120 [Moore1993a]

- G3-136: Marginal binding to peptide, binding inhibited by 183/184 PI/SG substitution [Moore1993b]

- G3-136: Binding enhanced by selected antibodies to C1, C4, C5, V3 and anti-CD4 binding site MAbs - enhances binding of selected V3, C4 and anti-CD4 binding site MAbs [Moore1993b]

- G3-136: HIV-1 RF V2 substitutions $177 \mathrm{Y} / \mathrm{H}$ and 179 L/P in the V2 loop of RF reduce affinity [Yoshiyama1994]

- G3-136: The binding of conformation-dependent anti-V2, anti-V3, and anti-CD4BS MAbs to monomeric and virion-associated gp120 from HIV-1 isolates with differences in cell tropism was studied - V2 loop epitopes are somewhat occluded in the oligomeric gp120 epitopes on the virion surface relative to the gp120 monomer as indicated by an increase in the half-maximal binding values to macrophage-tropic isolates SF162 and SF128a- anti-V2 MAbs G3-4 and G3.136 don't bind to T-cell tropic SF2 [Stamatatos1995]

- G3-136: Bound preferentially to the monomeric rather than oligomeric form of LAI gp120 - neutralizes cell free Hx10 [Sattentau1995b

- G3-136: Described epitope as STSIRGKVKEYAFFYKLDI - binds oligomer - binding of V2 MAbs G3-136, G3-4 or BAT123 did not significantly alter gp120 dissociation from virus or expose the gp41 epitope of MAb 50-69, in contrast to anti-V3 MAbs [Poignard1996a

- G3-136: Called G3.136 - does not mediate gp120 virion dissociation in contrast to anti-V2 MAb G3-4 - not neutralizing for SF162 or SF128A in either primary macrophages or PBMC [Stamatatos1997]

- G3-136: Binds both gp120 and soluble gp120+gp41 complex efficiently, suggesting its gp120 epitope is not blocked by gp41 binding [Wyatt1997]

- G3-136: The MAb and Fab binding to the oligomeric form of gp120 and neutralization were highly correlated - authors suggest that neutralization is determined by the fraction of Ab sites occupied on a virion irrespective of the epitope [Parren1998a

- G3-136: Called G3.136 - deleting the V2 loop of neutralization-resistant HIV-1 isolate SF162 does not abrogate its replication in PBMC or macrophages, but it enhances its neutralization sensitivity to sera from patients with B clade infection up to 170 -fold, and also enhances sensitivity to sera from clades A through F - deletion of V1 or V2 did not enable neutralization by V2 MAbs G3.4, G3.136, or 687-30D [Stamatatos1998]

- G3-136: Called G3.136 - SF162 is a neutralization-resistant HIV-1 isolate - N-linked glycosylation modifications in the V2 loop of the SF162 gp120 revealed that these sites prevent neutralization by CD4BS MAbs (IgG1b12 and IgGCD4), and protect against neutralization by V3 MAbs (447-D and 391-95D) - V2-region glycosylation site mutations did not alter neutralization resistance to V2 MAbs (G3.4 and G3.136) or CD4i MAbs (17b and 48d) V2 glycosylation site modification allows infection of macrophages, probably due to glycosylated forms requiring fewer CCR5 molecules for viral entry [Ly2000] 


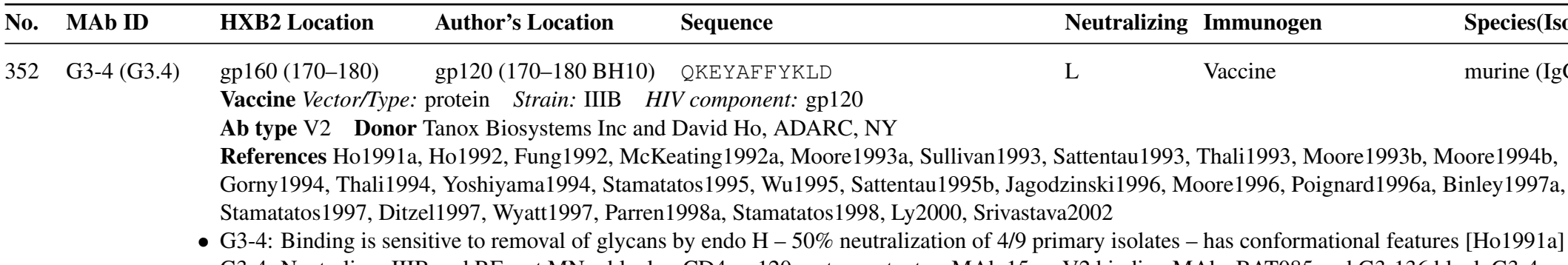

- G3-4: Binding is sensitive to removal of glycans by endo H - 50\% neutralization of 4/9 primary isolates - has conformational features [Ho1991a
- G3-4: Neutralizes IIIB and RF, not MN - blocks sCD4-gp120, not as potent as MAb 15e - V2 binding MAbs BAT085 and G3-136 block G3-4 gp120

binding - sensitive to reduction of gp120 by DTT [Ho1992]

- G3-4: Substitutions in residues 176 to 184 affect MAb recognition - substitutions in V2 can result in gp120-gp41 dissociation [Sullivan1993

- G3-4: Increased binding in the presence of sCD4 [Sattentau1993]

- G3-4: Conformational, does not bind well to denatured gp120 - not reactive with SF-2 gp120, and does not inhibit HIV-1 sera from binding to IIIB gp120 |Moore1993a

- G3-4: V2 region, marginal binding to peptide, binding inhibited by 183/184 PI/SG substitution [Moore1993b]

- G3-4: Conformationally sensitive - sporadic cross-reactivity among, and outside, B clade gp120s [Moore1994b

- G3-4: Weakly neutralizing, IC $50=53 \mathrm{mug} / \mathrm{ml}$ [Gorny1994]

- G3-4: gp41 mutation (582 A/T) that reduces neutralization of anti-CD4 binding site MAbs does not alter G3-4s ability to neutralize [Thali1994]

- G3-4: Neutralizes RF - substitutions 177 Y/H and 179 L/P in the V2 loop of RF reduce affinity and result in neutralization escape [Yoshiyama1994

- G3-4: The binding of conformation-dependent anti-V2, anti-V3, and anti-CD4BS MAbs to monomeric and virion-associated gp120 from HIV-1 isolates with differences in cell tropism was studied - V2 loop epitopes are somewhat occluded in the oligomeric gp120 epitopes on the virion surface relative to the gp120 monomer as indicated by an increase in the half-maximal binding values to macrophage-tropic isolates SF162 and SF128a - anti-V2 MAbs G3-4 and G3.136 don't bind to T-cell tropic SF2 [Stamatatos1995]

- G3-4: Reactive with BH10, RF, and MN - binds native, but not denatured or deglycosylated gp120, binds to deglycosylated V1V2 fusion protein, suggesting importance of glycans outside the V1V2 region Wu1995

- G3-4: Bound preferentially to the monomeric rather than oligomeric form of LAI gp120 - neutralizes Hx10 cell-free virus [Sattentau1995b]

- G3-4: The sulfated polysaccharide curdlan sulfate (CRDS) binds to the Envelope of T-tropic viruses and neutralizes virus - deletion of the V3 loop from gp120 results in more potent G3-4 binding inhibition by CRDS - G3-4 epitope described as 176-184 FYKLDIIPI and 191-193 YSL [Jagodzinski1996]

- G3-4: Binding enhanced by selected antibodies to C1, C4, C5, V3 and anti-CD4 binding site MAbs - enhances binding of selected V3, C4 and anti-CD4 binding site MAbs [Moore1996]

- G3-4: Described epitope as STSIRGKVKEYAFFYKLDI - binds oligomer - binding of V2 MAbs G3-136, G3-4 or BAT085 did not significantly alter gp120 dissociation from virus or expose the gp41 epitope of MAb 50-69, in contrast to anti-V3 MAbs [Poignard1996a]

- G3-4: Called G3.4 - mediates gp120 virion dissociation in contrast to anti-V2 MAb G3-136 - not neutralizing for SF162 or SF128A in either primary macrophages or PBMC [Stamatatos1997]

- G3-4: Binds both gp120 and soluble gp120+gp41 complex efficiently, suggesting its gp120 epitope is not blocked by gp41 binding |Wyatt1997

- G3-4: The MAb and Fab binding to the oligomeric form of gp120 and neutralization were highly correlated - authors suggest that neutralization is determined by the fraction of Ab sites occupied on a virion irrespective of the epitope [Parren1998a]

- G3-4: Called G3.4 - Deleting the V2 loop of neutralization-resistant HIV-1 isolate SF162 does not abrogate its replication in PBMC or macrophages, but it enhances its neutralization sensitivity to sera from patients with B clade infection up to 170-fold, and also enhances sensitivity to sera from clades A through F - deletion of V1 or V2 did not enable neutralization by V2 MAbs G3.4, G3.136, or 687-30D [Stamatatos1998 


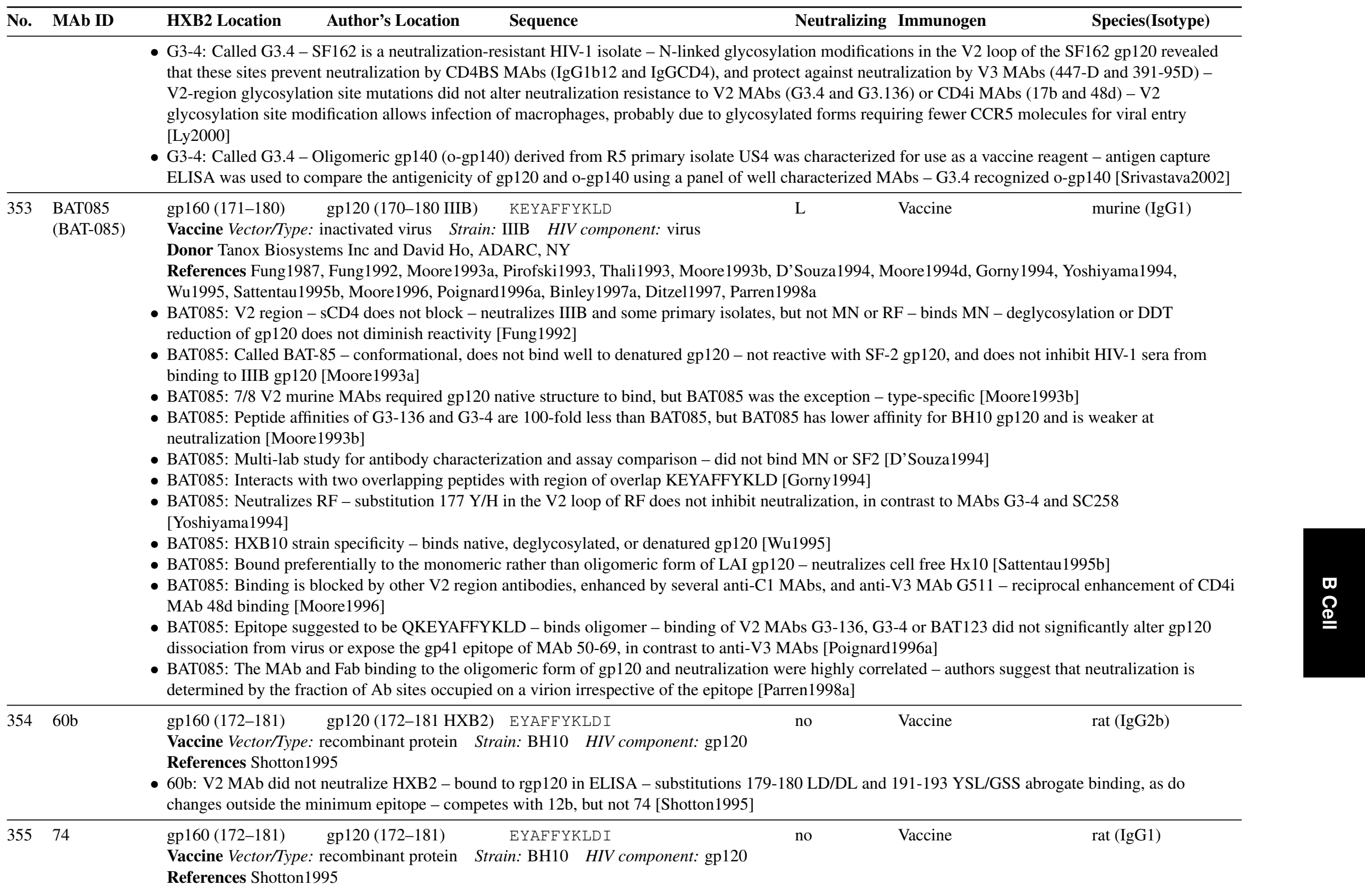




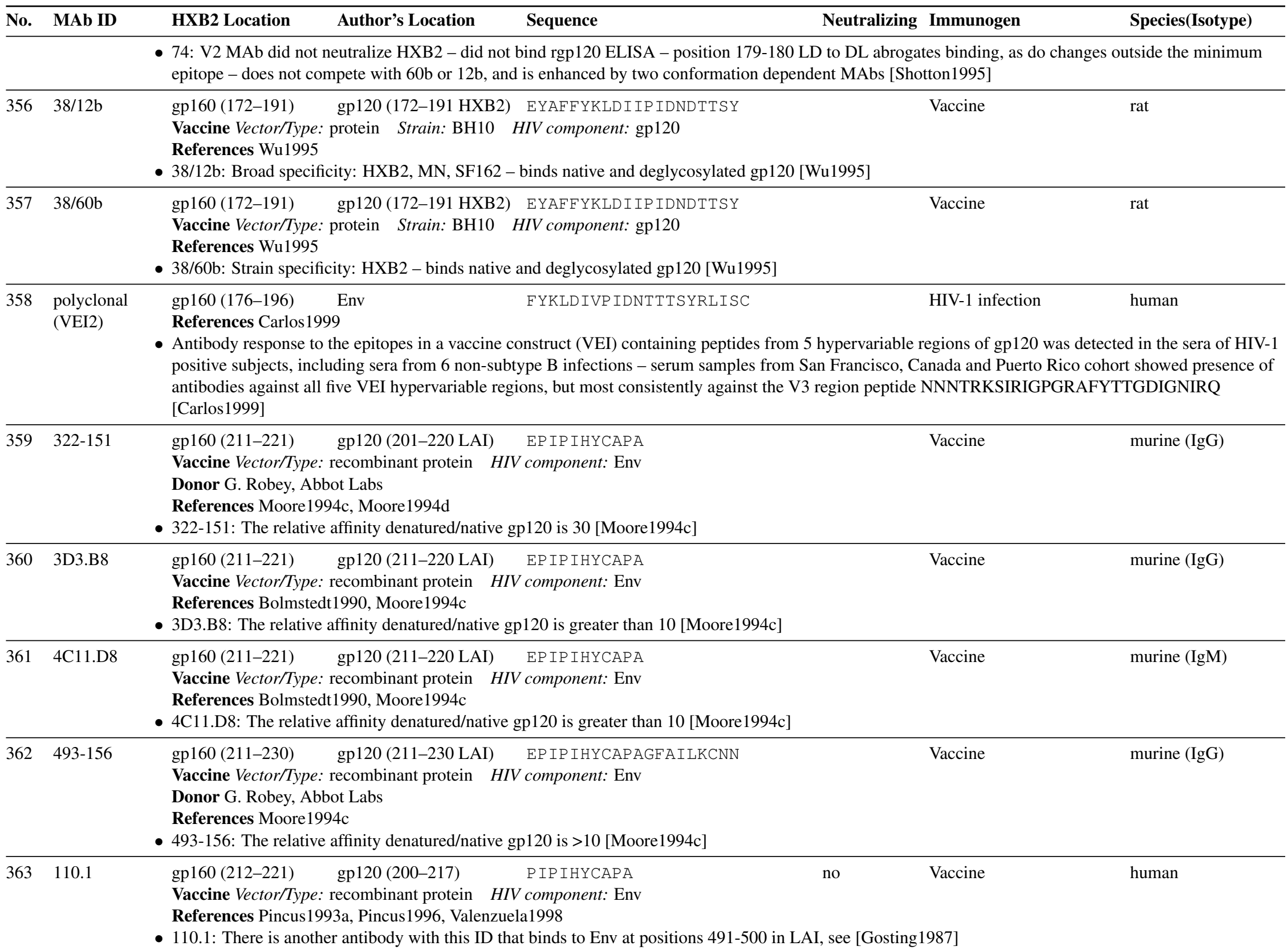




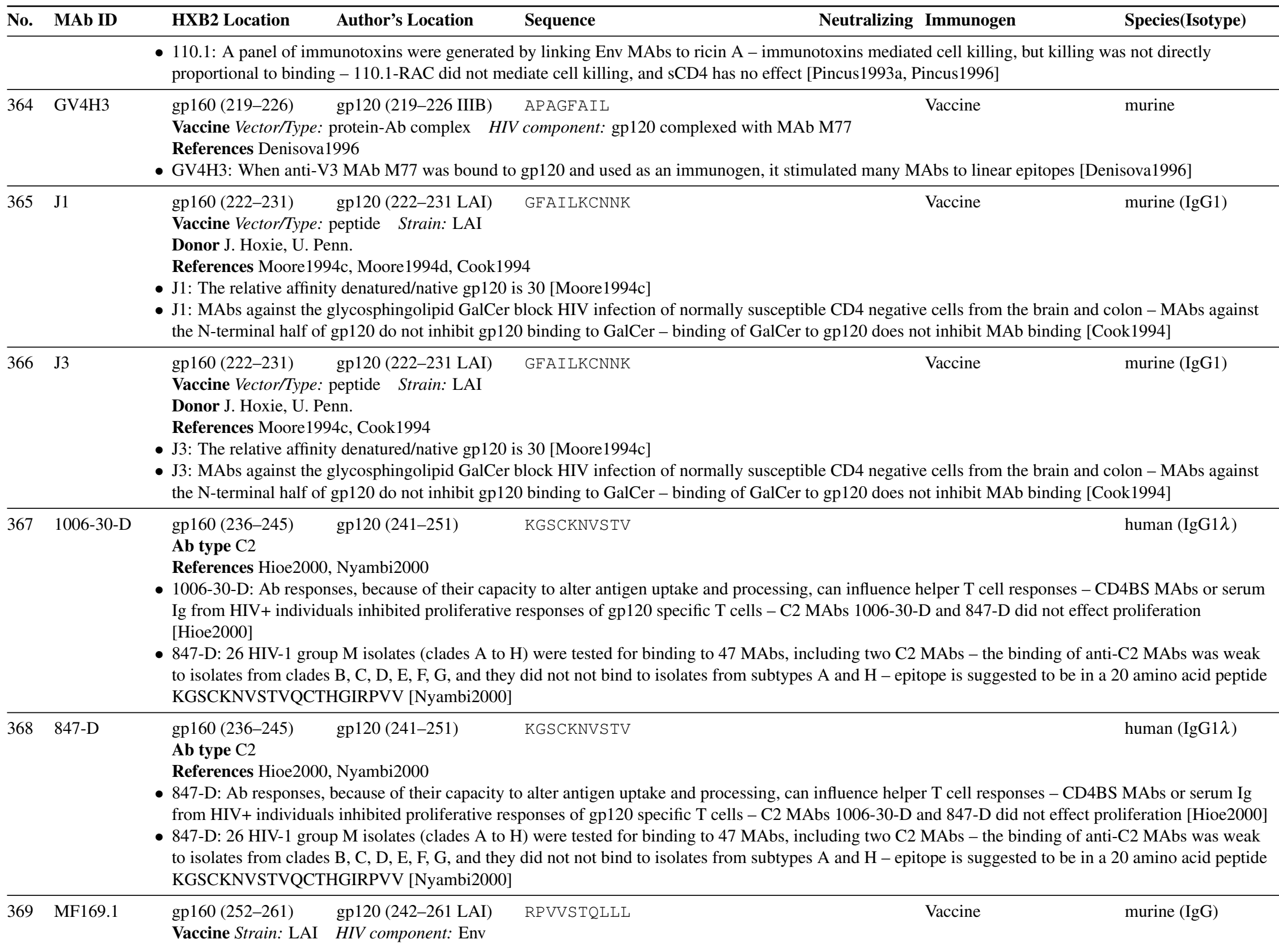




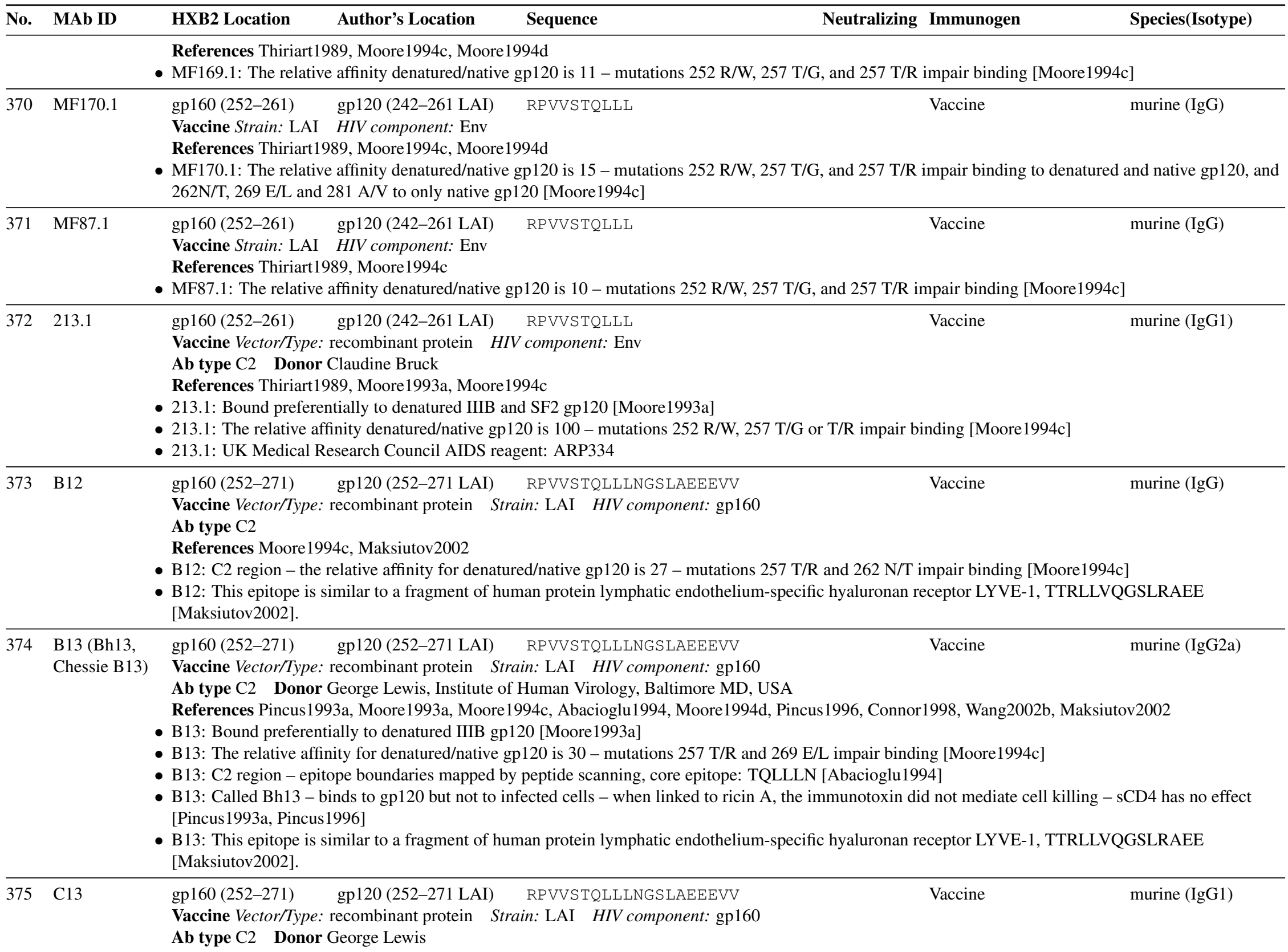




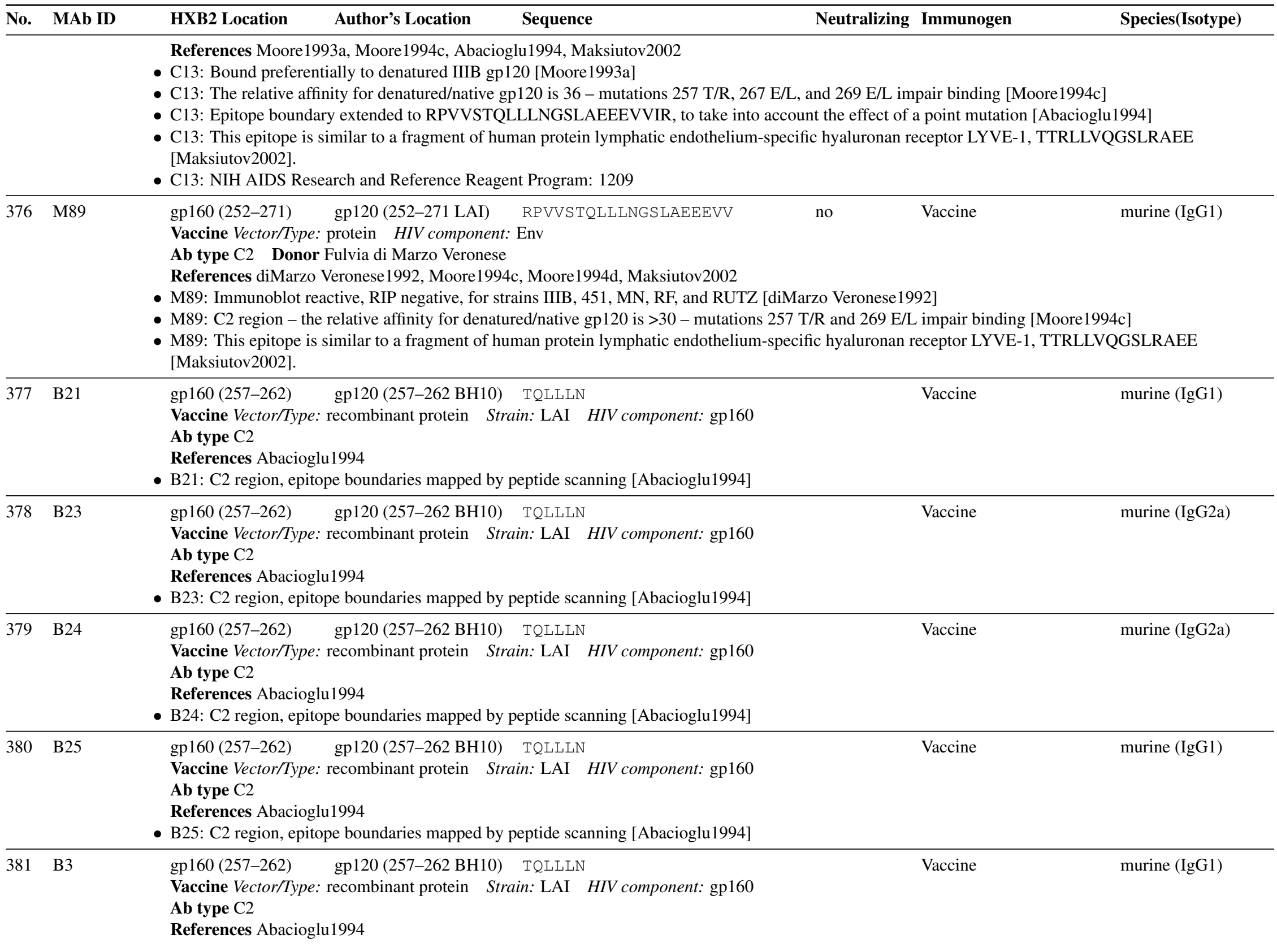




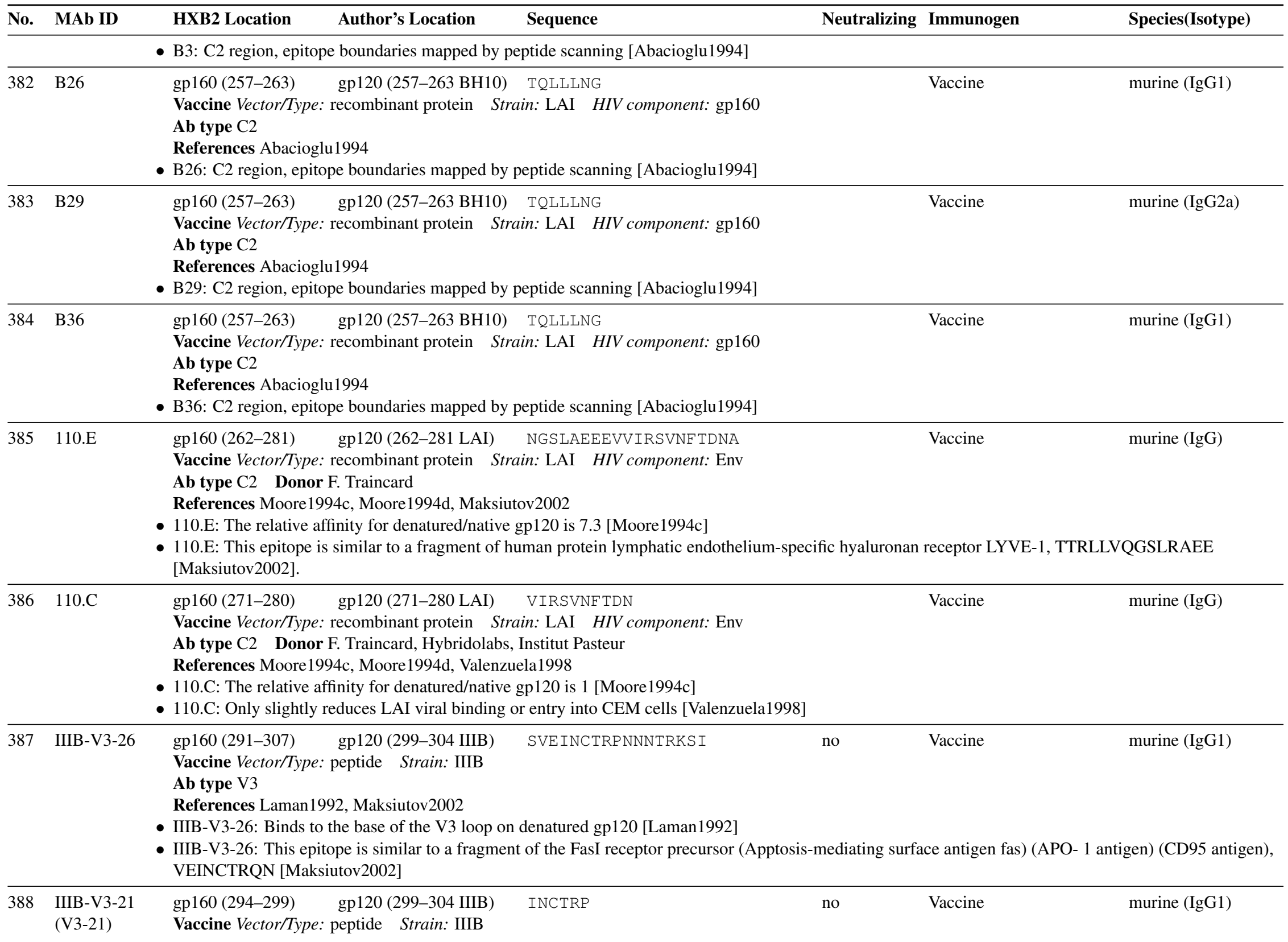




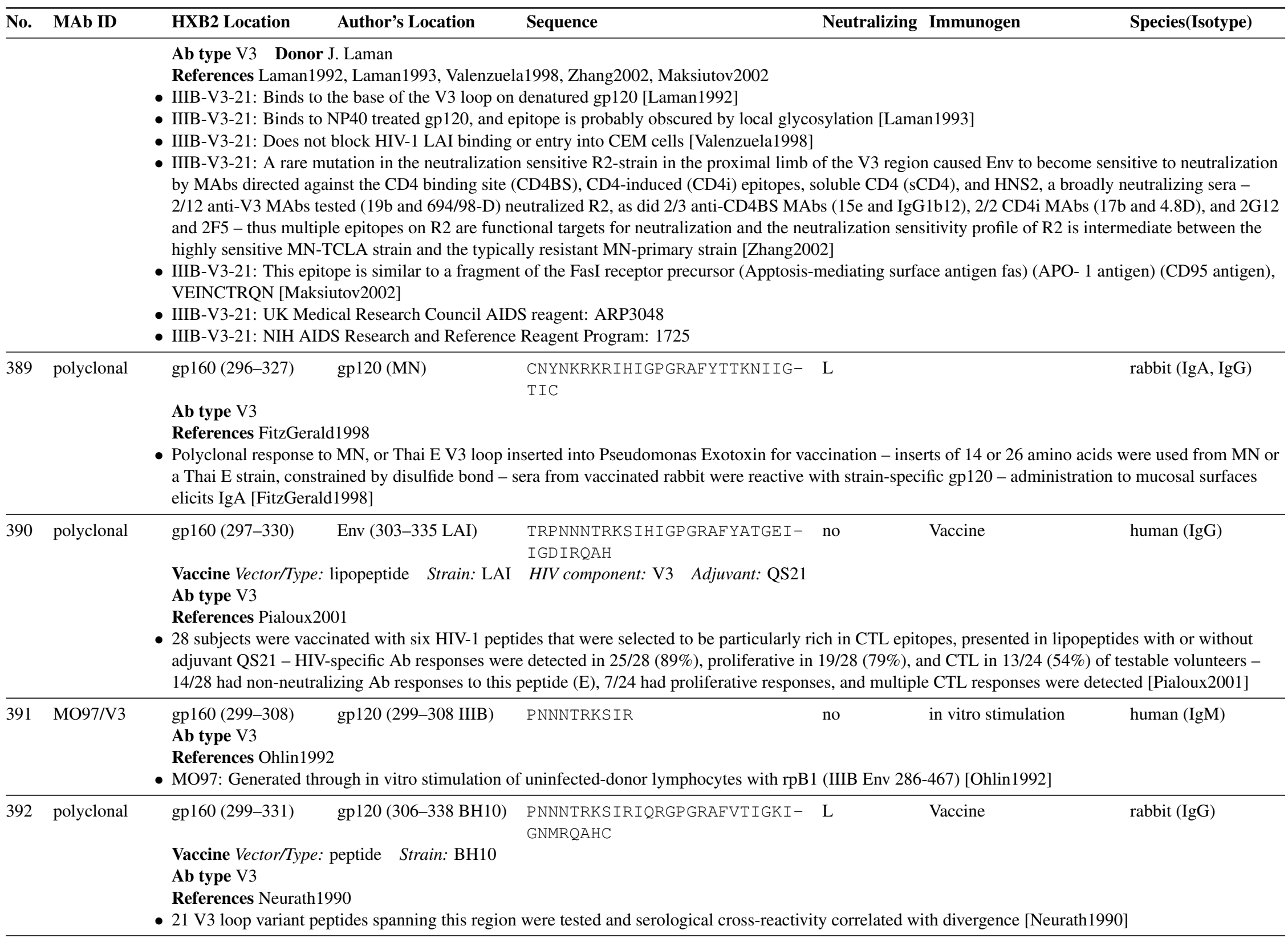




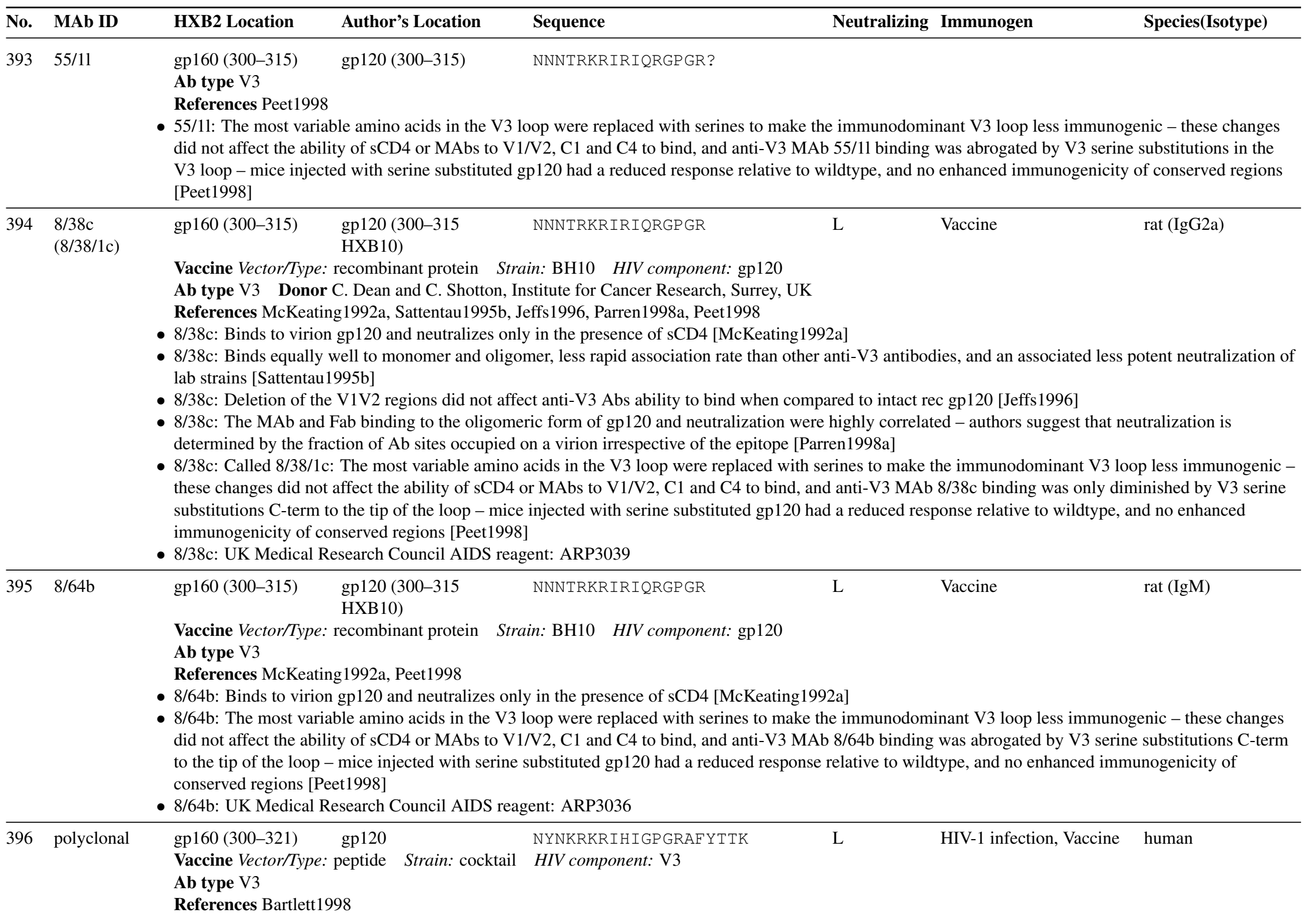




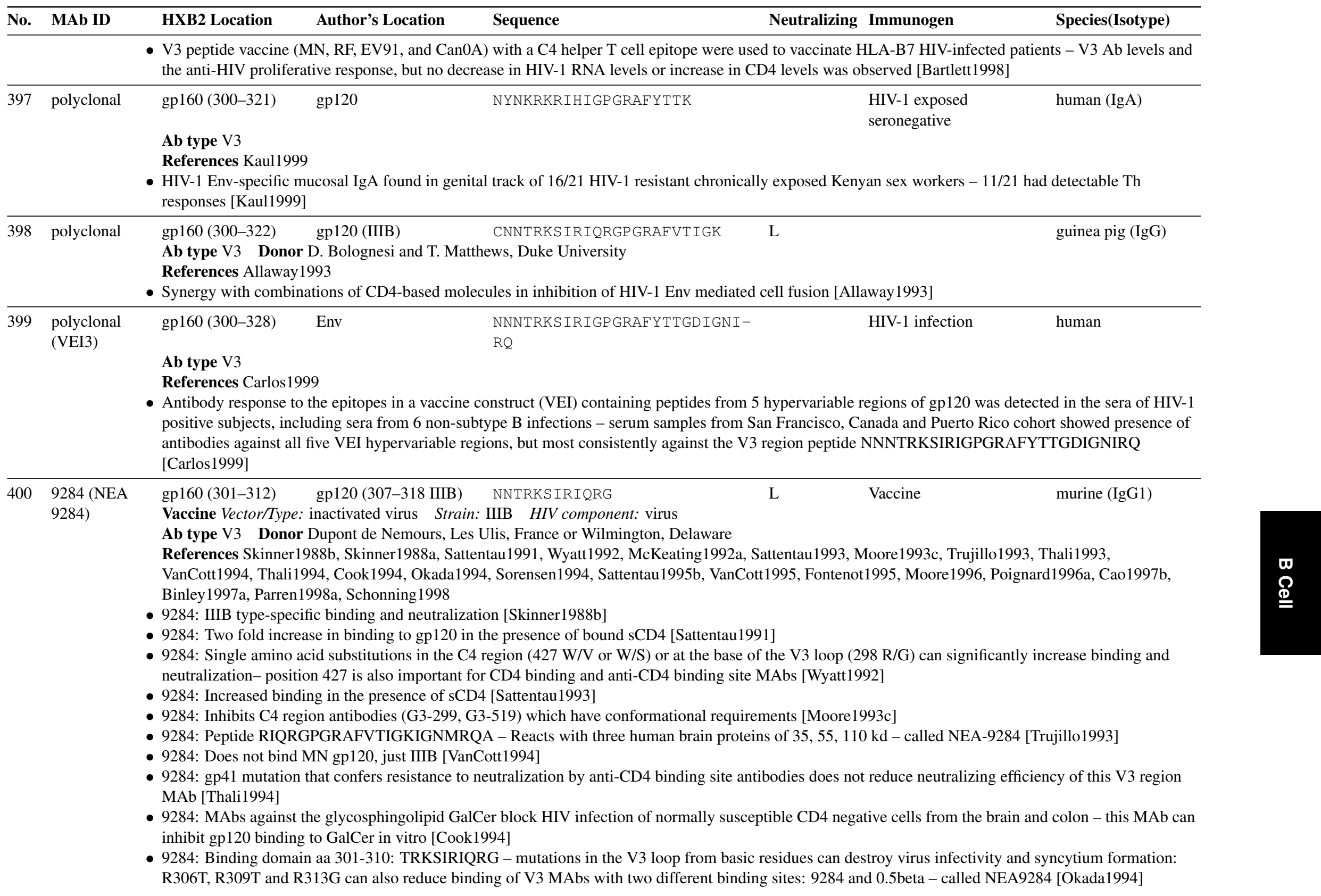




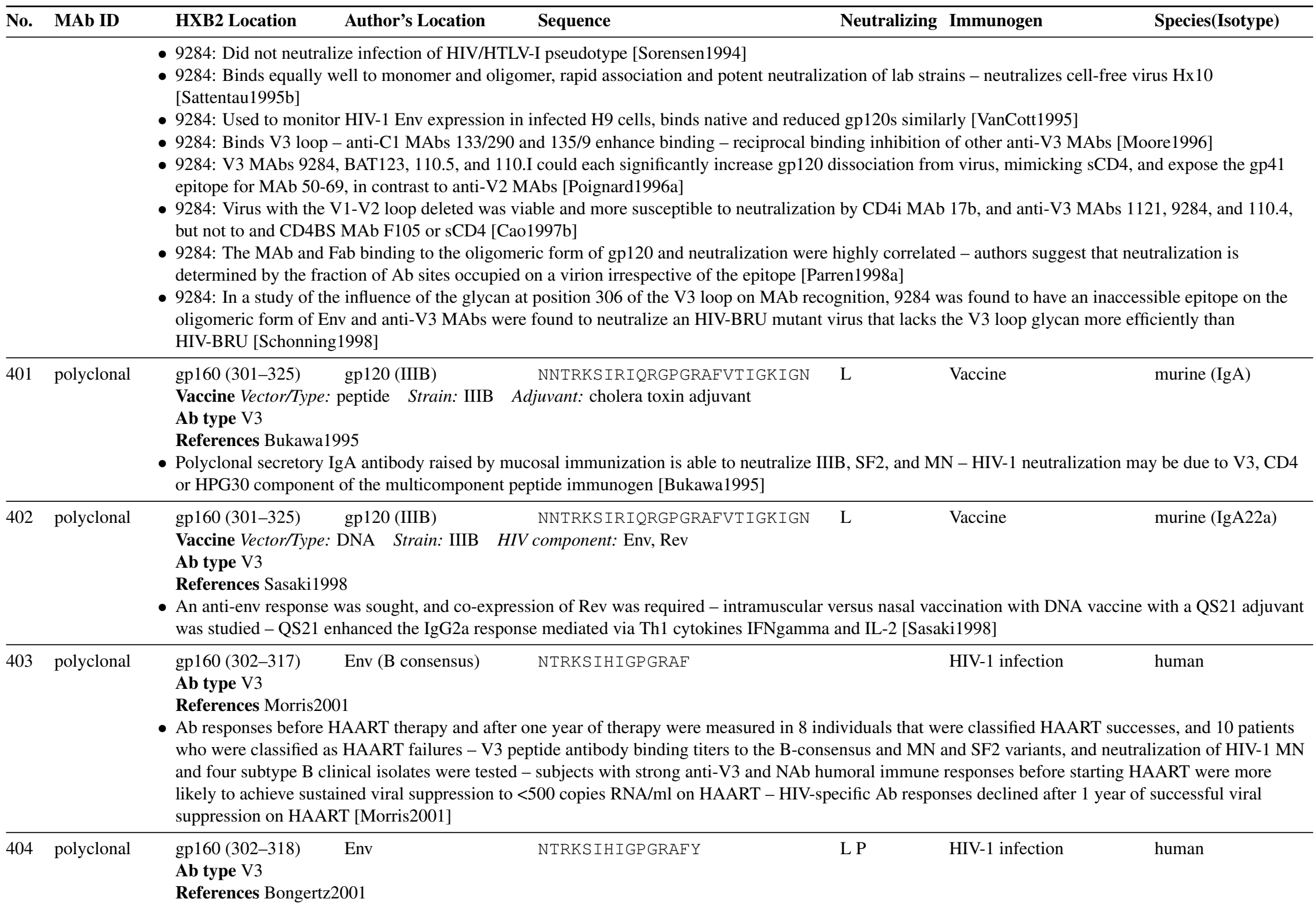




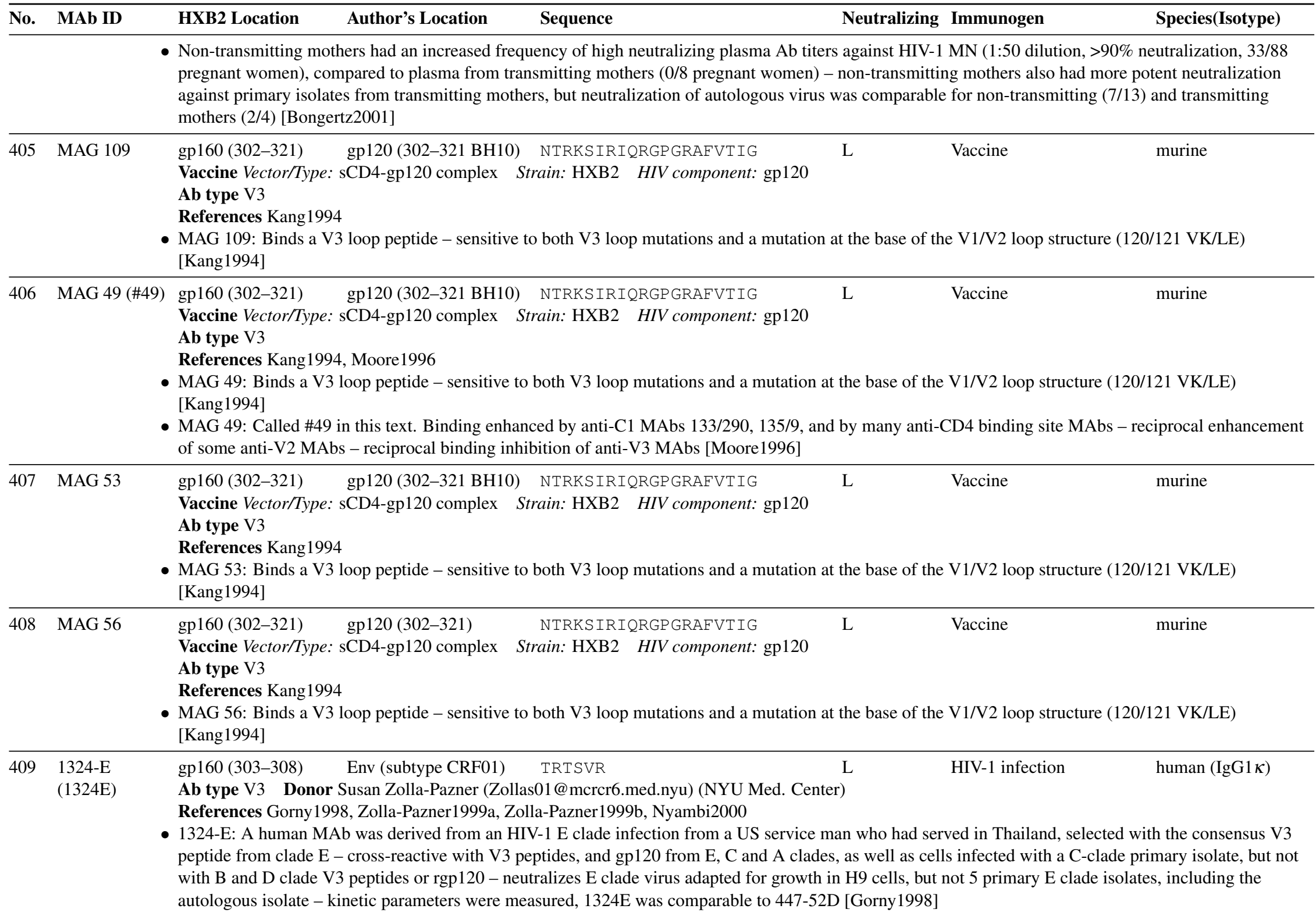




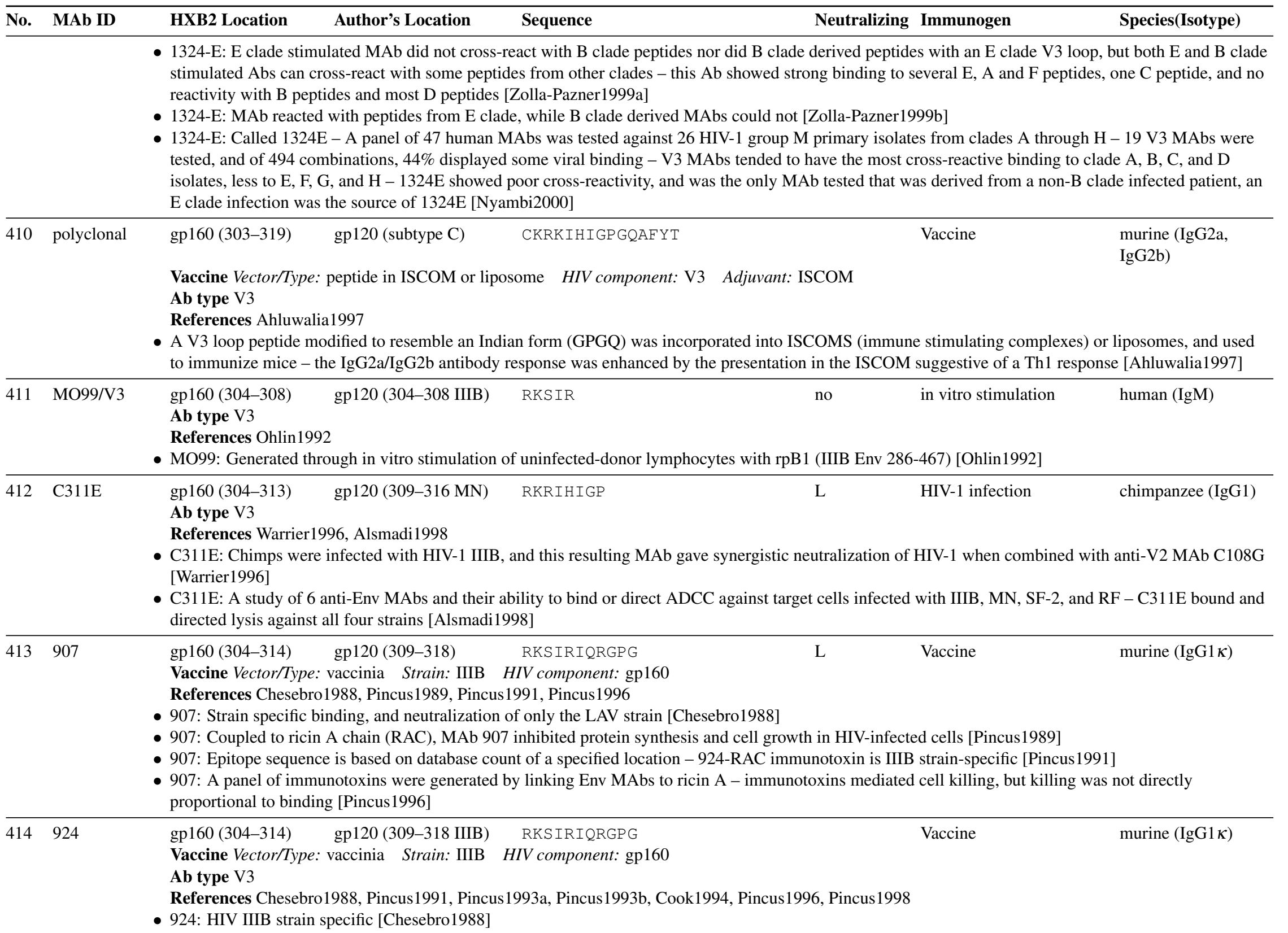




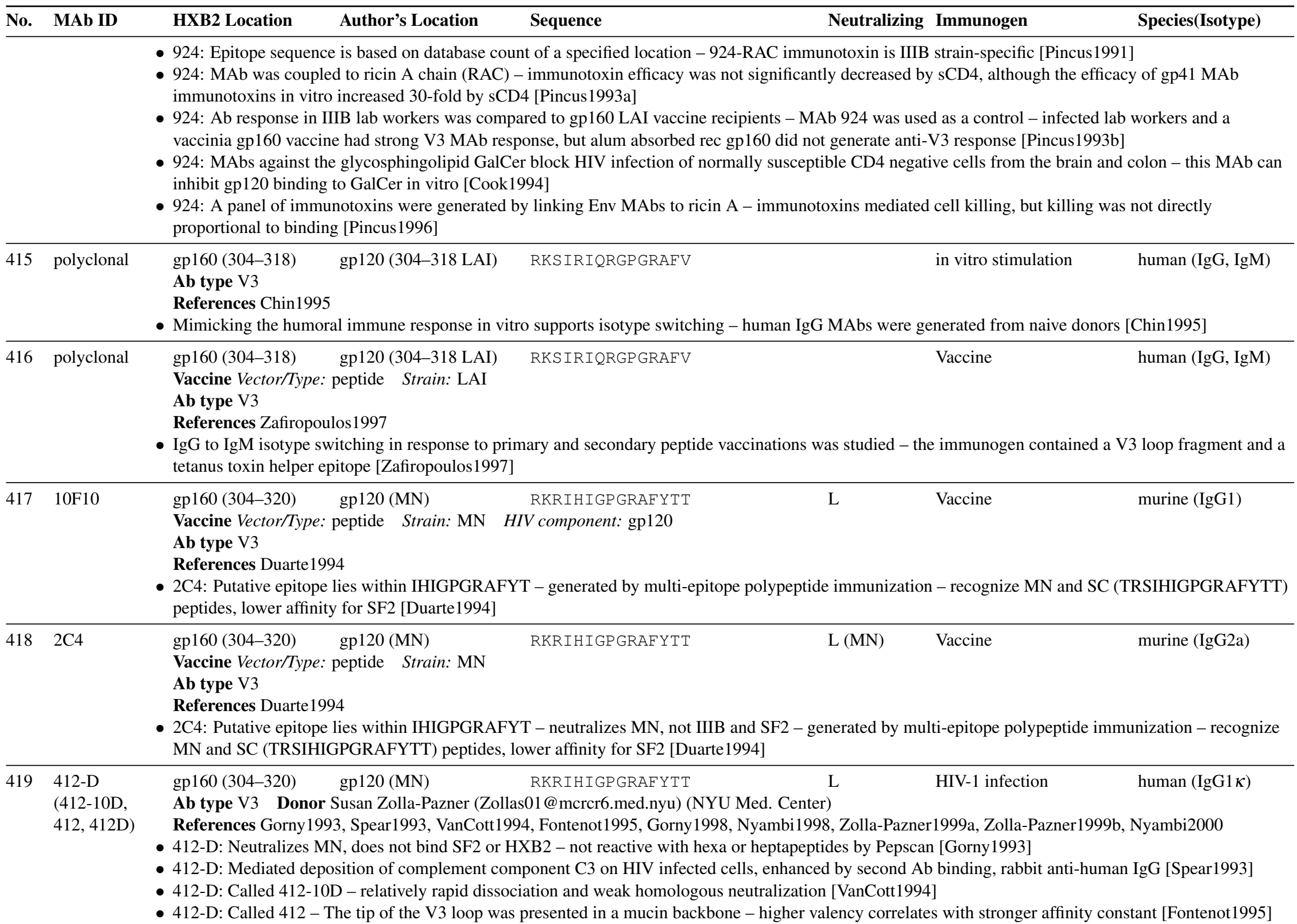




\begin{tabular}{|c|c|c|c|c|}
\hline No. MAb ID & HXB2 Location & Author's Location & Sequence & Neutralizing Immunogen \\
\hline
\end{tabular}

- 412-D: Kinetic parameters were measured, and the association rates were similar, but dissociation rate constants were quite variable for V3 MAbs, 412-D

has a relatively fast dissociation, thus low affinity among V3 MAbs [Gorny1998]

- 412-D: Using a whole virion-ELISA method, 18 human MAbs were tested for their ability to bind to a panel of 9 viruses from clades A, B, D, F, G, and H 412-D was bound only to B clade virions and to D clade MAL [Nyambi1998]

- 412-D: Review of clade specificity and anti-V3 HIV-1-Abs [Zolla-Pazner1999a]

- 412-D: MAb peptide-reactivity pattern clustered with immunological related MAbs: 391.5, 412 and 418, all selected with MN V3 peptide - the core amino acids HIGPGR tended to be critical for reactivity in this group [Zolla-Pazner1999b]

- 412-D: A panel of 47 human MAbs was tested against 26 HIV-1 group M primary isolates from clades A through H - 19 V3 MAbs were tested, and of 494 combinations, 44\% displayed some viral binding - V3 MAbs tended to have the most cross-reactive binding to clade A, B, C, and D isolates, less to E, F, G, and $\mathrm{H}$ - 412-D showed limited reactivity [Nyambi2000

\begin{tabular}{|c|c|c|c|c|c|}
\hline 420 polyclonal & gp160 (304-320) & gp120 (MN) & RKRIHIGPGRAFYTT & $\begin{array}{l}\text { L (MN } \\
\text { ALA-1) }\end{array}$ & HIV-1 infection \\
\hline
\end{tabular}

Ab type V3

References Spear1994

- $40 \%$ of antibody in serum that can bind to native viral proteins on MN-infected cells can be blocked by the peptide RKRIHIGPGRAFYTT, which can also block 75-95\% of the complement activation on HIV infected cells [Spear1994]

421 CGP $47439 \quad$ gp160 (304-322) gp120 RKRIRIQRGPGRAFVTIGK? $\quad$ L human (Ig)

Vaccine Vector/Type: protein Strain: IIIB HIV component: gp120

Ab type V3

References Liou1989 Safrit1993, Gunthard1994, Gauduin1998, Jacobson1998

- CGP 47 439: passive transfer to Hu-PBS-SCID mice confers protection against challenge with homologous cell-free virus - CGP 47439 is a BAT123-human Ig chimera [Safrit1993]

- CGP 47 439: Phase I/IIA clinical trial studying multidose tolerability, immunogenicity and pharmacokinetic responses - GP 47439 was well tolerated, serum t_1/2 was 8-16 days, and a virus burden reduction was noted in some patients [Gunthard1994]

- CGP 47 439: Post-exposure passive transfer of murine BAT123 can confer protection to hu-PBL-SCID mice challenged with HIV-1 LAI - this protection is not elicited by CGP 47439 , suggesting that the protection is mediated by complement - the protective ability of BAT123 is lost when mice were treated with cobra venom factor, which inactivates serum complement - in this circumstance complement activation provided a protective advantage [Gauduin1998]

- CGP 47 439: Review of passive immunotherapy, summarizing [Gunthard1994] in relation to other studies [Jacobson1998]

422 polyclonal gp160(304-322) (MN) RKRIHIGPGRAFYTTKN HIV-1 infection

\section{Ab type V3}

- The Ab response of 829 HIV-1 infected subjects from eight geographic areas to a set of different V3 peptides was determined by ELISA and cross-inhibition studies - the Ab binding pattern was highly variable, depended on the geographic origin of the sample - 297 sera were tested in a neutralization assay - there was a correlation between $\mathrm{Ab}$ binding to the MN V3 loop and MN neutralizing titer, but with neutralization of IIIB or CBL-4 [Cheingsong-Popov1992]

423 178.1 gp160(305-309) gp120(305-309 BH10) $\quad$ KSiRI $\quad$ L $\quad$ Vaccine $\quad$ IgG2a)

(178.1.1) Vaccine Vector/Type: recombinant protein Strain: IIIB HIV component: yeast derived gp160

Ab type V3 Donor C. Thiriart, Smith Kline and MRC AIDS reagent project

References Thiriart1989, Back1993, Moore1993a, Cook1994,

- 178.1: Reacts to gp120 and gp160 in RIPA EIA and immunoblot [Thiriart1989]

- 178.1: Called 178.1.1 - conformational, does not bind well to denatured gp120 [Moore1993a 


\begin{tabular}{llll}
\hline No. MAb ID & HXB2 Location Author's Location & Sequence & Neutralizing Immunogen \\
\hline & & 178.1: gp41 amino acid substitutions $668(\mathrm{~N} / \mathrm{S})$ and $675(\mathrm{I} / \mathrm{M})$ in gp41 interfere with 5023s neutralization potency, region $662-675$ is \\
& ELDKWANLWNWFNI [Back1993] \\
& - $178.1:$ MAbs against the glycosphingolipid GalCer block HIV infection of normally susceptible CD4 negative cells from the brain and colon - this MAb \\
& can inhibit gp120 binding to GalCer in vitro - binding of GalCer to gp120 inhibited but did not completely block MAb binding[Cook1994] \\
& - $178.1:$ UK Medical Research Council AIDS reagent: ARP331
\end{tabular}

424 257-D (257, gp160(305-309)

257-2-D-IV, Ab type V3 Donor Susan Zolla-Pazner (Zollas01@ mcrcr6.med.nyu) (NYU Med. Center)

257-D-IV, References Gorny1991. D’Souza1991, Karwowska1992b, Gorny1993 Cavacini1993a Spear1993, D’Souza1994, VanCott1994, Stamatatos1995.

257, 257-2D, D’Souza1995, Zolla-Pazner1995a, Schutten1995a, Schutten1995b, Fontenot1995, Wisnewski1996, Schutten1996, Schutten1997, Stamatatos1997

257D, $\quad$ Hill1997, Hioe1997b LaCasse1998, Yang1998, Gorny 1998, Stamatatos 1998 Zolla-Pazner1999a Zolla-Pazner1999b, Beddows1999, Oggioni1999.

ARP3023) Nyambi2000, Park2000, York2001, Zhang2002

- 257-D: Called 257-2-D-IV - potent neutralizing MAb [D'Souza1991]

- 257-D: Reacts with MN, NY5, CDC4 and SF2, does not cross-react with RF, WM52, or HXB2 [Karwowska1992b]

- 257-D: Neutralizes MN - binds SF2: KSIYI - specificity: MN, SF2, NY5, RF. [Gorny1993]

- 257-D: Additive MN or SF2 neutralization when combined with CD4 binding site MAb F105 - does not neutralize RF [Cavacini1993a]

- 257-D: Mediated deposition of complement component C3 on HIV infected cells, enhanced by second Ab binding, rabbit anti-human IgG - complement

mediated virolysis of MN, but not in the presence of sCD4 [Spear1993]

- 257-D: Included a multi-lab study for antibody characterization and assay comparison - best NAb against MN, but not IIIB [D’Souza1994

- 257-D: Potent MN neutralization, slow dissociation constant [VanCott1994]

- 257-D: The binding of conformation-dependent anti-V2, anti-V3, and anti-CD4BS MAbs to monomeric and virion-associated gp120 from HIV-1 isolates with differences in cell tropism was studied - V3 loop epitopes were less accessible to Ab binding on the virion surface than in the gp120 monomer, particularly for macrophage-tropic isolates SF162 and SF128a, relative to T-cell tropic SF2 - sCD4 association with gp120 better revealed this V3 epitope on TCLA SF2 and dual tropic (MU3) viruses than on macrophage tropic isolates [Stamatatos1995]

- 257-D: Called 257-D-IV - could neutralize MN and closely related JRCSF, but not 2 B subtype and 1 D subtype primary isolates in a multi-laboratory study involving 11 labs [D'Souza1995]

- 257-D: In serotyping study using flow-cytometry, bound only to virus with KRIHI Zolla-Pazner1995a

- 257-D: Only inhibition of SI phenotype virus, and strong enhancement of NSI phenotype chimeric viruses, that incorporated different envs from the same donor [Schutten1995a]

- 257-D: Comparable affinity for SI and NSI viruses, in contrast to MAb MN215 [Schutten1995b

- 257-D: 257-D is V H5 - V-region heavy chain usage was examined and a bias of enhanced V H1 and V H4, and reduced V H3, was noted among HIV infected individuals [Wisnewski1996]

- 257-D: IIIB neutralizing MAbs in vitro fail to neutralize in a mouse model it in vivo [Schutten1996]

- 257-D: Neutralized (>90\%) an SI-env chimeric virus and enhanced (>200\%) an NSI-env chimeric virus [Schutten1997]

- 257-D: Binds less extensively than MAb 391-95D on the surface of HIV-1 isolates SF162 and SF128A - neutralizes less potently than 391-95D - stronger neutralization of primary macrophage targets than PBMC [Stamatatos1997]

- 257-D: Called 257 - gp120 can inhibit MIP-1alpha from binding to CCR5, but this inhibitory effect is blocked by pre-incubation of gp120 with three anti-V3 MAbs: 447, 257, 1027 - MAb 670 which binds in the C5 region had no effect [Hill1997]

- 257-D: A T-cell line-adapted (TCLA) derivative of SI primary isolate 168P acquired the ability to be neutralized by anti-V3 MAbs - the primary isolate could use either CCR5 or CXCR4, and was not neutralized when infection was directed via either pathway, however the TCLA derivative uses CXCR4 only and is neutralized [LaCasse1998] 


\begin{tabular}{lllll}
\hline No. MAb ID & HXB2 Location & Author's Location & Sequence & Neutralizing Immunogen
\end{tabular}

- 257-D: A neutralization assay was developed based on hemi-nested PCR amplification of the LTR (HNPCR) - LTR-HNPCR consistently revealed HIV

DNA and was shown to be a rapid, specific and reliable neutralization assay based on tests with 6 MAbs and 5 isolates [Yang1998]

- 257-D: Kinetic parameters were measured, and the association rates were similar, but dissociation rate constants were quite variable for V3 MAbs, 257-D has a slow dissociation, thus the highest affinity among V3 MAbs [Gorny1998]

- 257-D: Called 257D - deleting the V2 loop of neutralization-resistant HIV-1 isolate SF162 does not abrogate its replication in PBMC or macrophages, but it enhances its neutralization sensitivity to sera from patients with B clade infection up to 170-fold, and also enhances sensitivity to sera from clades A through F - deletion of V1 or V2 did not enable neutralization by V3 MAbs 391-95D or 257D [Stamatatos1998]

- 257-D: Review of clade specificity and anti-V3 HIV-1-Abs [Zolla-Pazner1999a]

- 257-D: MAb peptide reactivity pattern clustered with immunological related MAbs: $1108,386,268,311,257,694.8$ - the amino acids HI tended to be critical for reactivity in this group [Zolla-Pazner1999b]

- 257-D: rgp120 derived from a R5X4 subtype B virus, HIV-1 W61D, was used to vaccinate healthy volunteers and the resulting sera were compared with sera from HIV-1 positive subjects and neutralizing MAbs - 257-D bound rgp120 W61D but could only neutralize the W61D isolate following T-cell line adaptation [Beddows1999]

- 257-D: Study of a live-vector mucosal vaccine that expresses HIV-1 V3 domains using the bacterium Streptococcus gordonii which can express heterologous Ag and can colonize the oral cavity and vagina of mice - 268-D and 257-D recognized S. gordonii expressing the V3 domain of MN - the vaccine stimulated V3-specific IgG2a in mice [Oggioni1999]

- 257-D: A panel of 47 human MAbs was tested against 26 HIV-1 group M primary isolates from clades A through H - 19 V3 MAbs were tested, and of 494 combinations, $44 \%$ displayed some viral binding - V3 MAbs tended to have the most cross-reactive binding to clade A, B, C, and D isolates, less to E, F, G, and $\mathrm{H}$ - 257-D showed intermediate reactivity [Nyambi2000]

- 257-D: Called 257D - six mutations in MN change the virus from a high-infectivity neutralization resistant phenotype to low-infectivity neutralization sensitive - V3, CD4BS, and CD4i MAbs are 20-100 fold more efficient at neutralizing the sensitive form - the mutation L544P reduced binding of all MAbs against gp120 by causing conformational changes [Park2000]

- 257-D: Abs against the V3 loop (50.1, 58.2, 59.1, 257-D, 268-D, 447-52D), CD4BS (IgG1b12, 559-64D, F105), CD4i (17b), and to gp41 (2F5, F240) each showed similar binding efficiency to Env derived from related pairs of primary and TCLA lines (primary: 168P and 320SI, and TCLA: 168C and

320SI-C3.3), but the TCLA lines were much more susceptible to neutralization suggesting that the change in TCLA lines that make them more susceptible to NAbs alters some step after binding [York2001]

- 257-D: Called ARP3023: Herpesvirus saimiri-immortalized CD4+ T lymphocytes (HVS T cells) were used to isolate virus and perform HIV-1 neutralization assays, and compared with a standard PBMC protocol - neutralization sensitivities to a panel of MAbs and to homologous or heterologous plasma/sera were similar for HVS T cells (CN-2 cells) and PBMCs [Vella2002]

- 257-D: A rare mutation in the neutralization sensitive R2-strain in the proximal limb of the V3 region caused Env to become sensitive to neutralization by MAbs directed against the CD4 binding site (CD4BS), CD4-induced (CD4i) epitopes, soluble CD4 (sCD4), and HNS2, a broadly neutralizing sera - 2/12 anti-V3 MAbs tested (19b and 694/98-D) neutralized R2, as did 2/3 anti-CD4BS MAbs (15e and IgG1b12), 2/2 CD4i MAbs (17b and 4.8D), and 2G12 and $2 \mathrm{~F} 5$ - thus multiple epitopes on R2 are functional targets for neutralization and the neutralization sensitivity profile of R2 is intermediate between the highly sensitive MN-TCLA strain and the typically resistant MN-primary strain [Zhang2002]

- 257-D: UK Medical Research Council AIDS reagent: ARP3023

- 257-D: NIH AIDS Research and Reference Reagent Program: 1510

$425 \begin{array}{lll}311-11-D & \text { gp160 (305-313) gp120 KRIHIGP }\end{array}$

311, 311D, References Gorny1991, Gorny1993. Spear1993 Gorny1998 Zolla-Pazner1999a Zolla-Pazner1999b, Nyambi2000

311-D) - 311-11-D: Neutralizes MN - binds SF2: KSIYIGP [Gorny1993]

- 311-11-D: Mediated deposition of complement component C3 on HIV infected cells, enhanced by second Ab binding, rabbit anti-human IgG [Spear1993] 


\begin{tabular}{lll}
\hline No. MAb ID & HXB2 Location Author's Location $\quad$ Sequence & Neutralizing Immunogen \\
\hline & 311-11-D: Review of clade specificity and anti-V3 HIV-1-Abs [Zolla-Pazner1999a] \\
& 311-11-D: MAb peptide reactivity pattern clustered with immunological related MAbs: 1108, 386, 268, 311, 257, 694.8 - the amino acids HI tended to be \\
& critical for reactivity in this group [Zolla-Pazner1999b] \\
& 311-11-D: A panel of 47 human MAbs was tested against 26 HIV-1 group M primary isolates from clades A through H - 19 V3 MAbs were tested, and of \\
& 494 combinations, 44\% displayed some viral binding - V3 MAbs tended to have the most cross-reactive binding to clade A, B, C, and D isolates, less to E, \\
& F, G, and H - 311-11D showed weak reactivity [Nyambi2000]
\end{tabular}

Ab type V3

References Pinter1993b Alsmadi1998

- 41148D: Neutralizes less potently than 4117C, reacts with MN, IIIB, SF2 [Pinter1993b]

- 41148D: A study of 6 anti-Env MAbs and their ability to bind or direct ADCC against target cells infected with IIIB, MN, SF-2, and RF - bound and directed lysis against strains IIIB, MN, SF-2, comparable to 4117C, however 41148D is 10x less efficient at neutralization, showing ADCC and neutralization don't always correlate [Alsmadi1998]

gp120 (MN)
KRIHIGPGRAFY $\mathrm{L}$

HIV-1 infection
human $(\operatorname{IgG} 1 \kappa)$

(391-95D,

Ab type V3 Donor Susan Zolla-Pazner (Zollas01@ mcrcr6.med.nyu) (NYU Med. Center)

391.5 References Gorny1991, Gorny1993, Fontenot1995, Stamatatos1995, Seligman1996, Stamatatos1997, Stamatatos1998, Zolla-Pazner1999a

391/95D) Zolla-Pazner1999b, Ly2000, Park2000 Guillon2002, Lawson2002, Zhang2002

- 391/95-D: Neutralizes MN - binds to SF2, not IIIB [Gorny1993

- 391/95-D: The binding of conformation-dependent anti-V2, anti-V3, and anti-CD4BS MAbs to monomeric and virion-associated gp120 from HIV-1 isolates with differences in cell tropism was studied - V3 loop epitopes were less accessible to Ab binding on the virion surface than in the gp120 monomer, particularly for macrophage-tropic isolates SF162 and SF128a, relative to T-cell tropic SF2 - sCD4 association with gp120 better revealed this V3 epitope on macrophage tropic and dual tropic (MU3) viruses, but not in TCLA SF2 [Stamatatos1995]

- 391/95-D: Competition ELISAs with serial deletions estimated the epitope to be KRIHIGPGRAFY - unconstrained peptide had higher affinity than cyclic [Seligman1996]

- 391/95-D: Called 391-95D - binds more extensively than MAb 257-D on the surface of HIV-1 isolates SF162 and SF128A - neutralizes more potently than 257-D - stronger neutralization of primary macrophage targets than PBMC - binding post-gp120-sCD4 association related to anti-V3 Abs neutralizing capacity [Stamatatos1997]

- 391/95-D: Called 391-95D - deleting the V2 loop of neutralization-resistant HIV-1 isolate SF162 does not abrogate its replication in PBMC or macrophages, but it enhances its neutralization sensitivity to sera from patients with B clade infection up to 170-fold, and also enhances sensitivity to sera from clades A through F - deletion of V1 or V2 did not enable neutralization by V3 MAbs 391-95D or 257D [Stamatatos1998]

- 391/95-D: Review of clade specificity and anti-V3 HIV-1-Abs [Zolla-Pazner1999a]

- 391/95-D: Called 391.5 - MAb peptide-reactivity pattern clustered with immunological related MAbs: 391.5, 412 and 418 , all selected with MN V3 peptide - the core amino acids HIGPGR tended to be critical for reactivity in this group [Zolla-Pazner1999b]

- 391/95-D: Called 391-95D - SF162 is a neutralization-resistant HIV-1 isolate - N-linked glycosylation modifications in the V2 loop of the SF162 gp120 revealed that these sites prevent neutralization by CD4BS MAbs (IgG1b12 and IgGCD4), and protect against neutralization by V3 MAbs (447-D and 391-95D) - V2-region glycosylation site mutations did not alter neutralization resistance to V2 MAbs (G3.4 and G3.136) or CD4i MAbs (17b and 48d) V2 glycosylation site modification allows infection of macrophages, probably due to glycosylated forms requiring fewer CCR5 molecules for viral entry Ly2000

- 391/95-D: Called 391/95D - six mutations in MN change the virus from a high-infectivity neutralization resistant phenotype to low-infectivity neutralization sensitive - V3, CD4BS, and CD4i MAbs are 20-100 fold more efficient at neutralizing the sensitive form - the mutation L544P reduced binding of all MAbs against gp120 by causing conformational changes [Park2000 


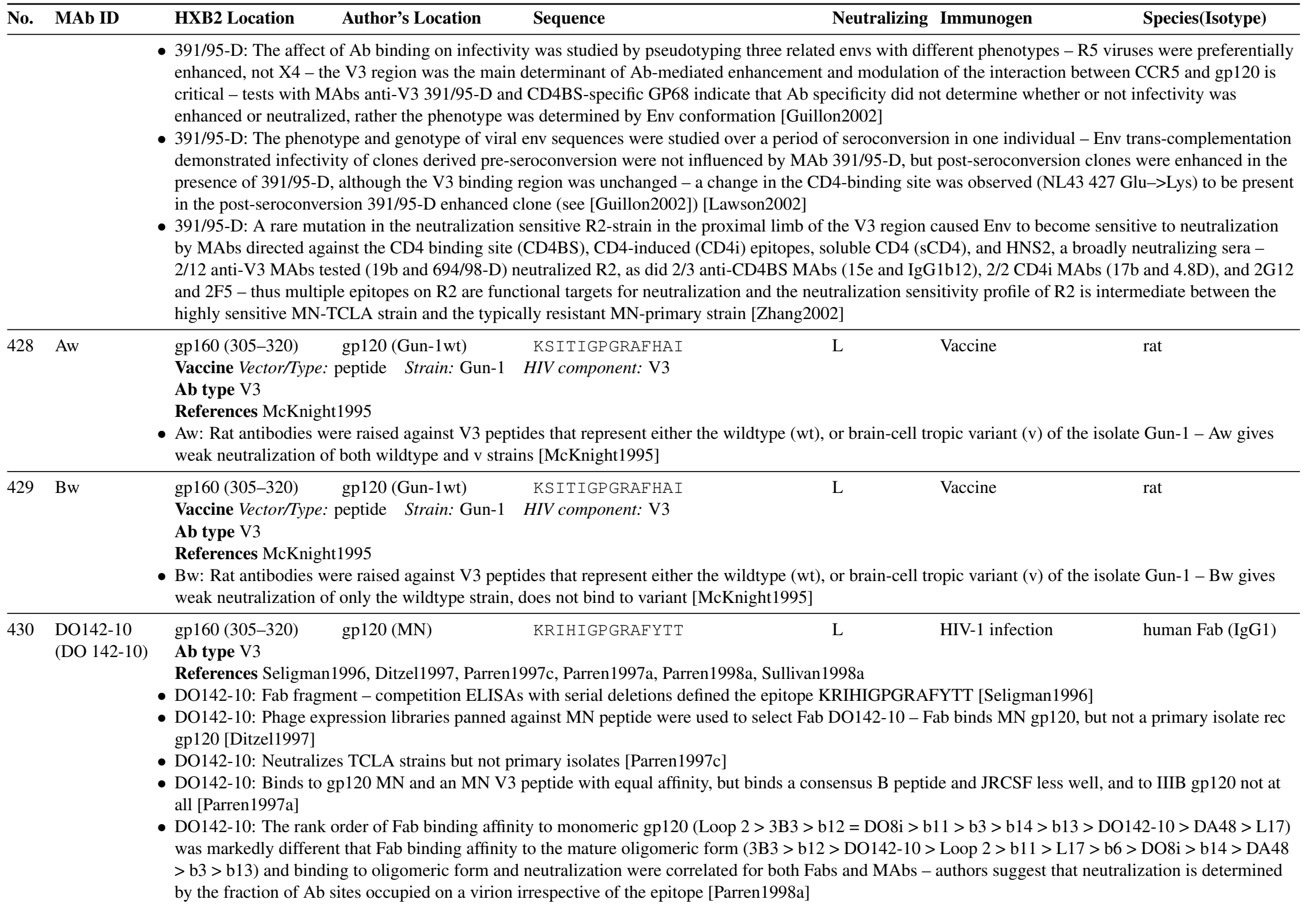




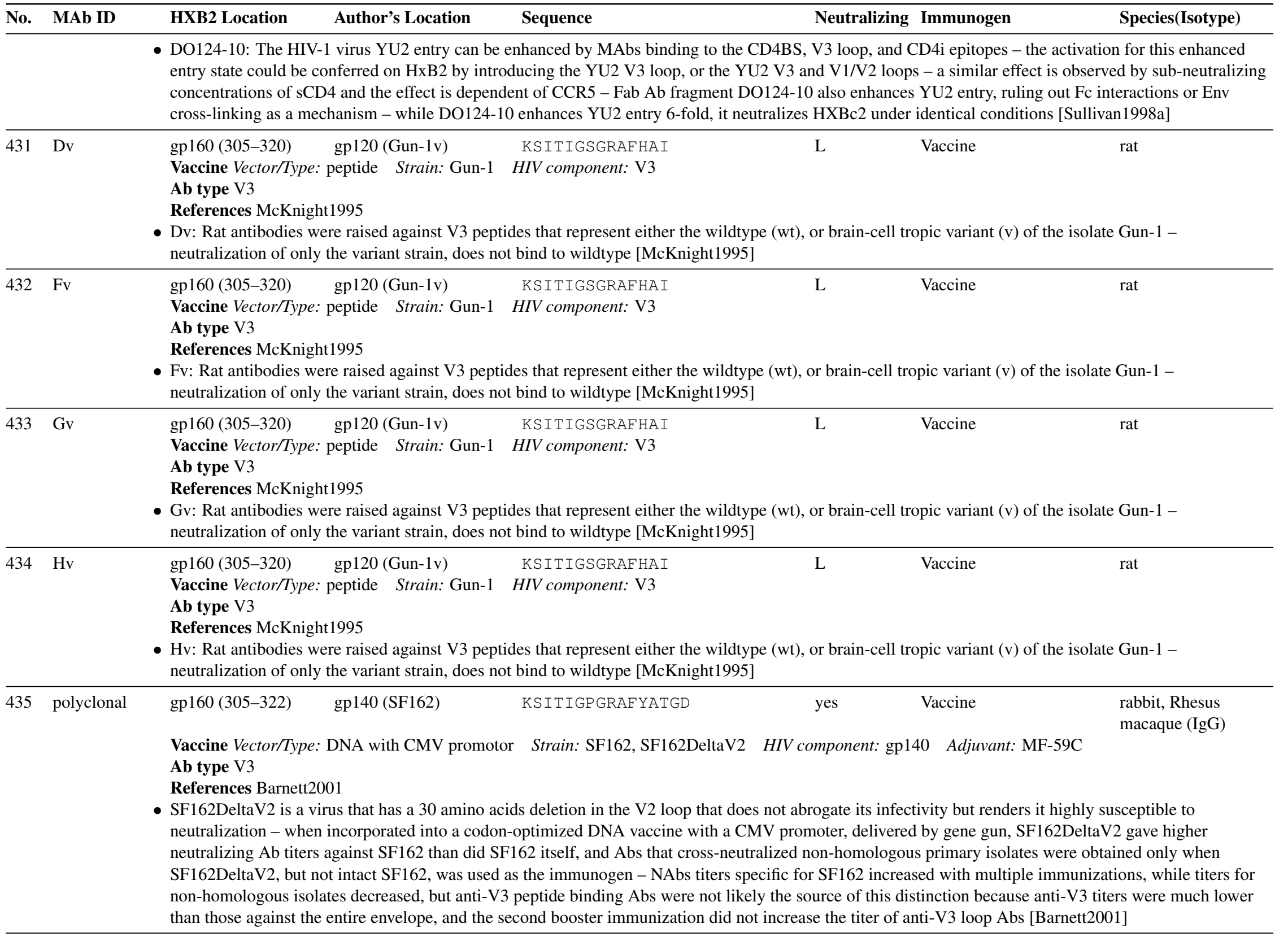




\begin{tabular}{|c|c|c|c|c|c|c|c|}
\hline No. & MAb ID & HXB2 Location & Author's Location & Sequence & Neutralizing & Immunogen & Species(Isotype) \\
\hline 436 & 50.1 & $60(306-310)$ & gp120 (MN) & RIHIG & $\mathrm{L}$ & Vaccine & murine $(\operatorname{IgG} 1 \kappa)$ \\
\hline
\end{tabular}

(R/V3-50.1, Vaccine Vector/Type: peptide Strain: MN HIV component: V3

Fab 50.1) Ab type V3 Donor Mary White-Scharf, Repligen Corporation, Cambridge, MA

References D'Souza1991, White-Scharf1993, Potts1993, Ghiara1993, Rini1993, Bou-Habib1994, VanCott1994, Robert-Guroff1994, Moore1994b

VanCott1995 Fontenot1995 Seligman1996, Berman1997, LaCasse1998, Stanfield1999, Hoffman1999, Park2000, York2001, Zhang2002

- 50.1: Called R/V3-50.1 - potent neutralizing of lab strains|D’Souza1991]

- 50.1: Epitope defined by peptide reactivity and changes affinity with amino acid substitutions - epitope RIHIGP [White-Scharf1993]

- 50.1: No synergistic neutralization of MN when combined with CD4BS MAb F105 - isotype stated to be IgG2a [Potts1993]

- 50.1: Crystal structure of a 24 amino acid peptide from the V3 loop bound to 59.1 and 50.1 Fab fragments - epitope KRIHIGP [Ghiara1993]

- 50.1: Crystal structure of V3 loop bound to 50.1 - light chain binds just to the left of GPG, heavy chain binds further to the left [Rini1993]

- 50.1: No neutralization of primary isolate JR-CSF - greater affinity for and neutralization of T cell tropic strain T-CSF, derived from JR-CSF

[Bou-Habib1994]

- 50.1: Potent MN neutralization, slow dissociation rate [VanCott1994]

- 50.1: Chimeric MN V3 loop in an HXB2 background allows increased FACS signal, Ab affinity, and viral neutralization [Robert-Guroff1994]

- 50.1: Shows modest cross-reactivity among B clade gp120s, little outside B clade [Moore1994b

- 50.1: Used to monitor HIV-1 Env expression in infected H9 cells [VanCott1995]

- 50.1: Competition ELISAs with serial deletions produced comparable estimate of epitope length to crystal structure and alanine substitution - KRIHIGP [Seligman1996]

- 50.1: Binds to 6/7 isolates from breakthrough cases from a MN gp120 vaccine trial [Berman1997]

- 50.1: A T-cell line-adapted (TCLA) derivative of SI primary isolate 168P acquired the ability to be neutralized by anti-V3 MAbs - the primary isolate could use either CCR5 or CXCR4, and was not neutralized when infection was directed via either pathway, however the TCLA derivative uses CXCR4 only and is neutralized [LaCasse1998]

- 50.1: The crystal structure of V3 loop peptides bound to Fabs was obtained - conformational changes in the tip of the V3 loop (GPGR) were observed when different Fabs were bound [Stanfield1999]

- 50.1: Called R/V3-50.1 - six mutations in MN change the virus from a high-infectivity neutralization resistant phenotype to low-infectivity neutralization sensitive - V3, CD4BS, and CD4i MAbs are 20-100 fold more efficient at neutralizing the sensitive form - the mutation L544P reduced binding of all MAbs against gp120 by causing conformational changes -50.1 could only neutralize the sensitive form [Park2000]

- 50.1: Abs against the V3 loop (50.1, 58.2, 59.1, 257-D, 268-D, 447-52D), CD4BS (IgG1b12, 559-64D, F105), CD4i (17b), and to gp41 (2F5, F240) each showed similar binding efficiency to Env derived from related pairs of primary and TCLA lines (primary: 168P and 320SI, and TCLA: 168C and 320SI-C3.3), but the TCLA lines were much more susceptible to neutralization suggesting that the change in TCLA lines that make them more susceptible to NAbs alters some step after binding - the dissociation constant, Kd of 50.1 for the cell associated primary and TCLA Envs was equal, 7nM [York2001]

- 50.1: Called R/V3-50.1 - A rare mutation in the neutralization sensitive R2-strain in the proximal limb of the V3 region caused Env to become sensitive to neutralization by MAbs directed against the CD4 binding site (CD4BS), CD4-induced (CD4i) epitopes, soluble CD4 (sCD4), and HNS2, a broadly neutralizing sera - 2/12 anti-V3 MAbs tested (19b and 694/98-D) neutralized R2, as did 2/3 anti-CD4BS MAbs (15e and IgG1b12), 2/2 CD4i MAbs (17b and 4.8D), and 2G12 and 2F5 - thus multiple epitopes on R2 are functional targets for neutralization and the neutralization sensitivity profile of R2 is intermediate between the highly sensitive MN-TCLA strain and the typically resistant MN-primary strain [Zhang2002]

- 50.1: NIH AIDS Research and Reference Reagent Program: 1289

437 polyclonal gp160(306-318) gp120(NY5) KKGIAIGPGRTLY Ab type V3

References Metlas1999b, Metlas 1999a 


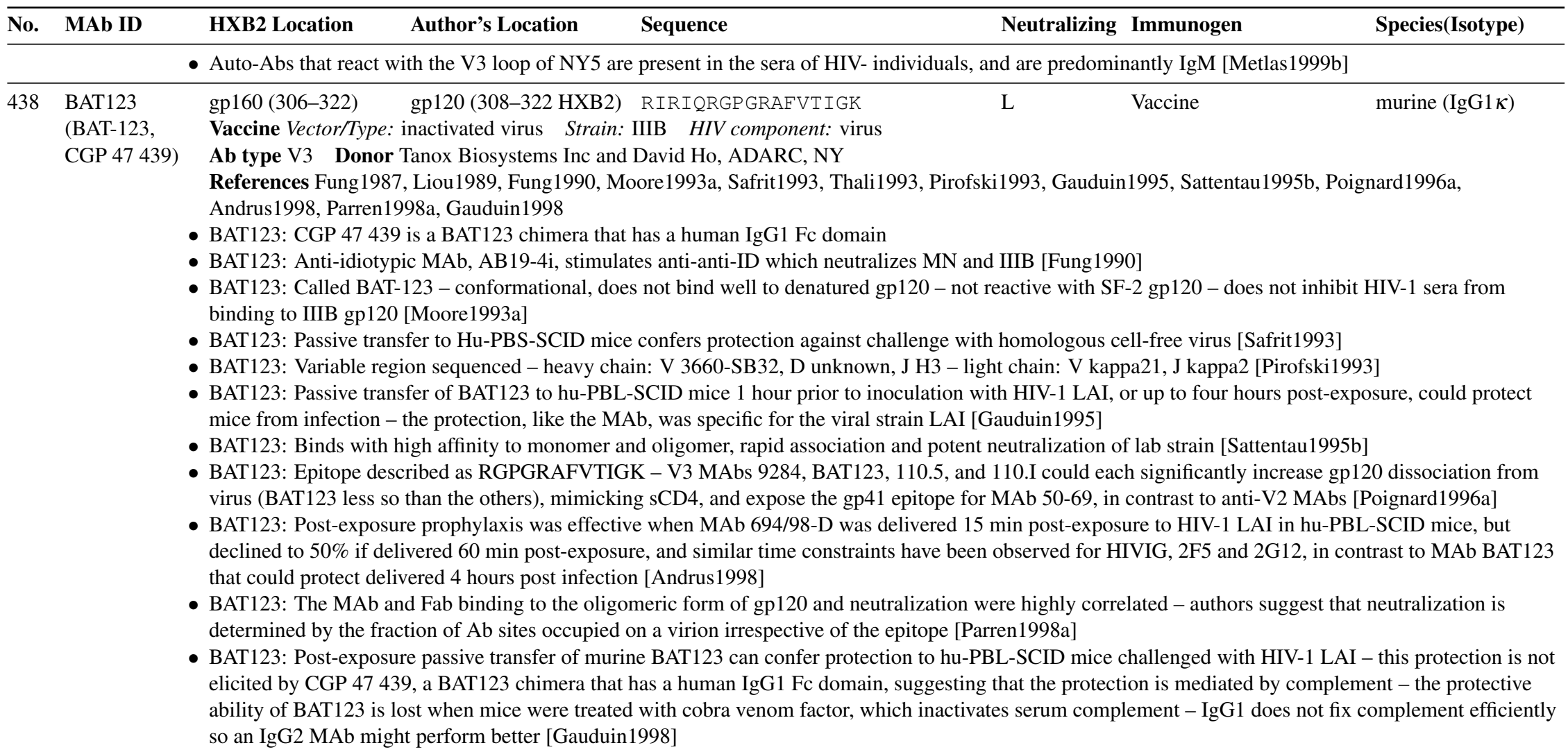

- 838-D: Five human MAbs against were derived from HIV-infected North American subjects after selection by the V3 RF peptide - 838-D was cross-reactive with V3 peptides from clade A and C, and could bind to 5/8 B clade V3 peptides - 50\% neutralization of RF was obtained [Gorny1997]

- 838-D: Four primary isolates showed distinct patterns of sensitivity to neutralization by polyclonal sera or plasma and MAbs - BZ167 was the only isolate inhibited by all polyclonal sera and plasma tested, and was also neutralized by 8/17 MAbs, in particular anti-V3 loop (419-D, 447-52D, 782-D, and 838-D), anti-CD4bd (559/64-D, 654-D and 830-D and a cluster II of gp41 directed MAb (98-6) - isolates 92HT593 and 91 US056 were neutralized by V3 loop (419-D, and 447-52D)and cluster II gp41 (98-6) MAbs at higher concentrations - US4 was neutralized by some of the polyclonal sera/plasma tested and not at all by MAbs individually or by a cocktail of ten MAbs consisting of 419-D, 447-52D, 782-D, 838-D, 559/64-D, 654-D, 450-D, 670-D, 1281-D and 98-6 Hioe1997b

- 838-D: Using a whole virion-ELISA method, 18 human MAbs were tested for their ability to bind to a panel of 9 viruses from clades A, B, D, F, G, and H 838-D bound B clade virions but had limited cross-reactivity with other clades, with low levels of binding to A and D virions [Nyambi1998]

- 838-D: Review of clade specificity and anti-V3 HIV-1-Abs - this Ab showed strong binding to many A, B, C and F peptides, poor binding to D and E [Zolla-Pazner1999a] 


\begin{tabular}{lllll}
\hline No. MAb ID & HXB2 Location & Author's Location & Sequence & Neutralizing Immunogen
\end{tabular}

- 838-D: MAb peptide-reactivity pattern clustered with immunological related MAbs: 838, 782, 1027, 908, and 1006, all selected with RF V3 peptide - the core amino acids KSITK tended to be critical for reactivity in this group [Zolla-Pazner1999b]

- 838-D: Binding of panel of 21 MAbs to soluble oligomeric gp140 versus gp41 or gp120 monomers was compared - no MAb was oligomer specific, though anti-V3 and CD4BS MAbs reacted better with the oligomer and V2 and C5 tended to favor the monomer - V3 MAbs 447-52D, 838-D, and 1334 bound with a 7-10 fold preference for the oligomer [Gorny2000a]

- 838-D: A panel of 47 human MAbs was tested against 26 HIV-1 group M primary isolates from clades A through H - 19 V3 MAbs were tested, and of 494 combinations, 44\% displayed some viral binding - V3 MAbs tended to have the most cross-reactive binding to clade A, B, C, and D isolates, less to E, F, G, and $\mathrm{H}$ - 838-D showed intermediate reactivity [Nyambi2000]

- 838-D: Called 838 - Transgenic mice carrying human genes allowing production of fully human MAbs were used to rapidly create a panel of anti-HIV gp120 MAb producing hybridomas by immunization with HIV SF162 gp120 - the previously described human MAbs 5145A(CD4BS) , 4117C (plus others, V3) and 697D (and SC258, V2) were used as controls [He2002]

- 838-D: A rare mutation in the neutralization sensitive R2-strain in the proximal limb of the $\mathrm{V} 3$ region caused Env to become sensitive to neutralization by MAbs directed against the CD4 binding site (CD4BS), CD4-induced (CD4i) epitopes, soluble CD4 (sCD4), and HNS2, a broadly neutralizing sera - 2/12 anti-V3 MAbs tested (19b and 694/98-D) neutralized R2, as did 2/3 anti-CD4BS MAbs (15e and IgG1b12), 2/2 CD4i MAbs (17b and 4.8D), and 2G12 and 2F5 - thus multiple epitopes on R2 are functional targets for neutralization and the neutralization sensitivity profile of R2 is intermediate between the highly sensitive MN-TCLA strain and the typically resistant MN-primary strain [Zhang2002]

440 1006-15D $\quad$ gp160(307-312) $\quad$ gp120 (RF) $\quad$ KSITKG $\quad$ no HIV-1 infection

(1006) Ab type V3 Donor Susan Zolla-Pazner (Zollas01@ mcrcr6.med.nyu) (NYU Med. Center)

References Gorny 1997, Zolla-Pazner1999a, Zolla-Pazner1999b, Nyambi2000, He2002

- 1006-15D: Five human MAbs against were derived from HIV-infected North American subjects after selection by the V3 RF peptide - was somewhat cross-reactive with V3 peptides from clade A, C and other B clade V3 peptides, but not E clade [Gorny1997]

- 1006-15D: Review of clade specificity and anti-V3 HIV-1-Abs - this Ab showed strong binding to several B and F peptides, one C peptide, and some reactivity with A peptides - no binding was observed with D and E peptides [Zolla-Pazner1999a]

- 1006-15D: MAb peptide-reactivity pattern clustered with immunological related MAbs: 838, 782, 1027, 908, and 1006, all selected with RF V3 peptide the core amino acids KSITK tended to be critical for reactivity in this group [Zolla-Pazner1999b]

- 1006-15D: A panel of 47 human MAbs was tested against 26 HIV-1 group M primary isolates from clades A through H - 19 V3 MAbs were tested, and of 494 combinations, 44\% displayed some viral binding - V3 MAbs tended to have the most cross-reactive binding to clade A, B, C, and D isolates, less to E, F, G, and $\mathrm{H}-1006-15 \mathrm{D}$ showed strong cross-reactivity [Nyambi2000]

- 1006-15D: Called 1006 - Transgenic mice carrying human genes allowing production of fully human MAbs were used to rapidly create a panel of anti-HIV gp120 MAb producing hybridomas by immunization with HIV SF162 gp120 - the previously described human MAbs 5145A(CD4BS), 4117C (plus others, V3) and 697D (and SC258, V2) were used as controls [He2002]

$\begin{array}{llll}441 & \text { 782-D (782) } & \text { gp160 (307-312) Env (RF) } & \text { KS ITKG } \\ & \text { Ab type V3 Donor Susan Zolla-Pazner (Zollas01@ @ mcrcr6.med.nyu) (NYU Med. Center) }\end{array}$ Ab type V3 Donor Susan Zolla-Pazner (Zollas01@ @ mcrcr6.med.nyu) (NYU Med. Center)
References Gorny1997, Hioe1997b, Zolla-Pazner1999a, Zolla-Pazner1999b Nyambi2000

- 782-D: Five human MAbs against were derived from HIV-infected North American subjects after selection by the V3 RF peptide - 782-D was not cross-reactive with V3 peptides from clade A or E, but could bind to 3/8 B clade V3 peptides, and 1/2 C clade V3 peptides - 50\% neutralization of RF was obtained Gorny1997] 


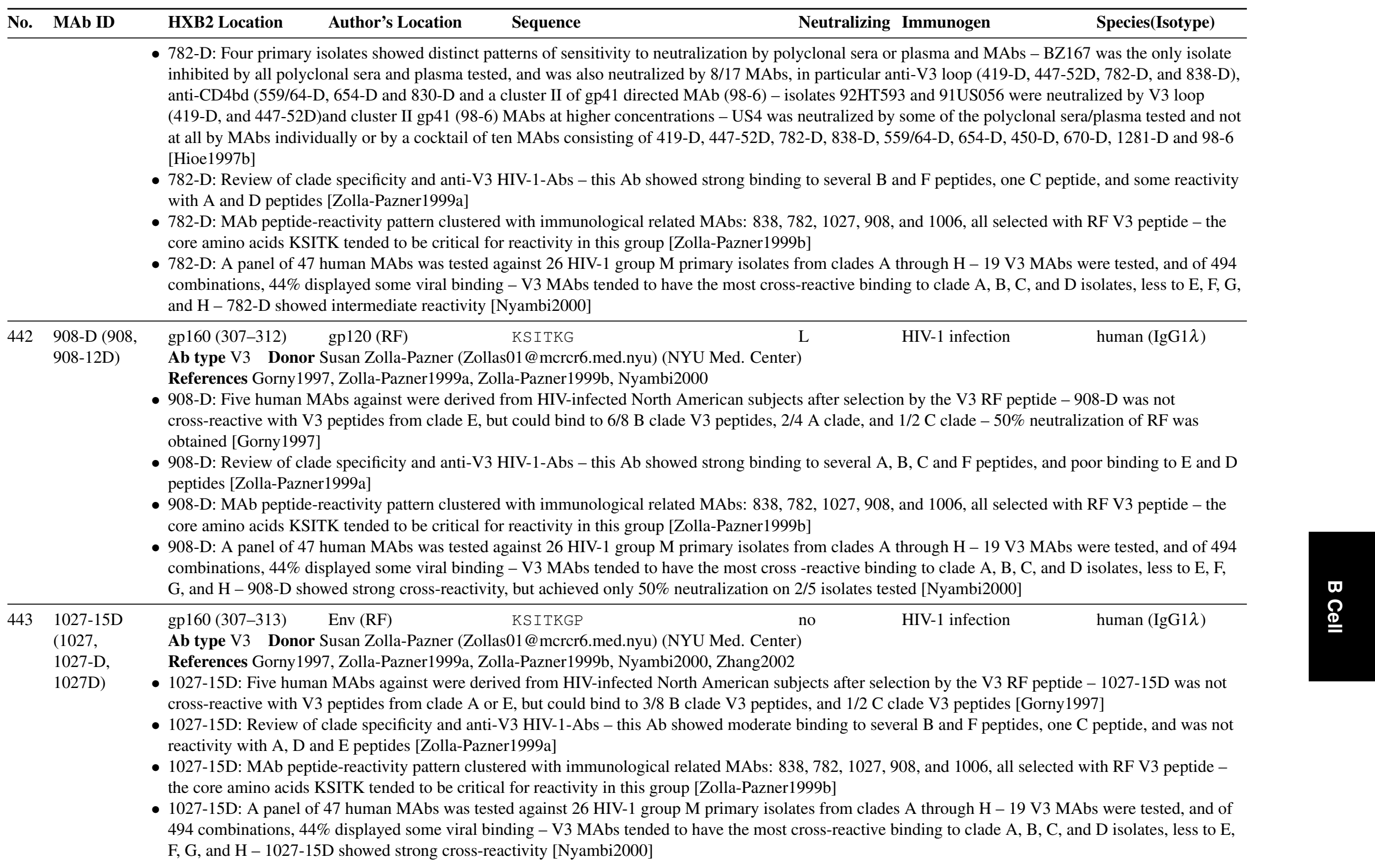




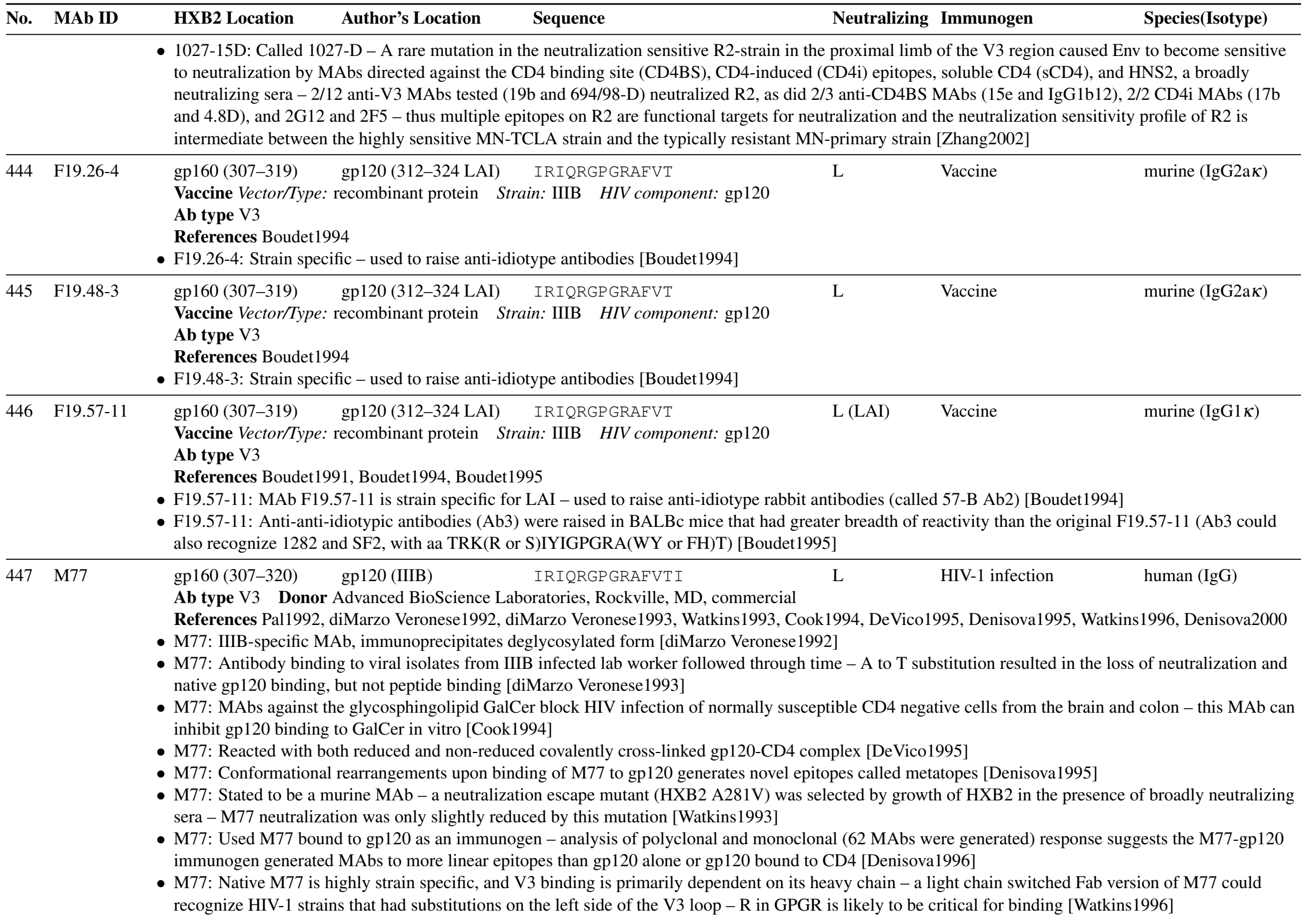




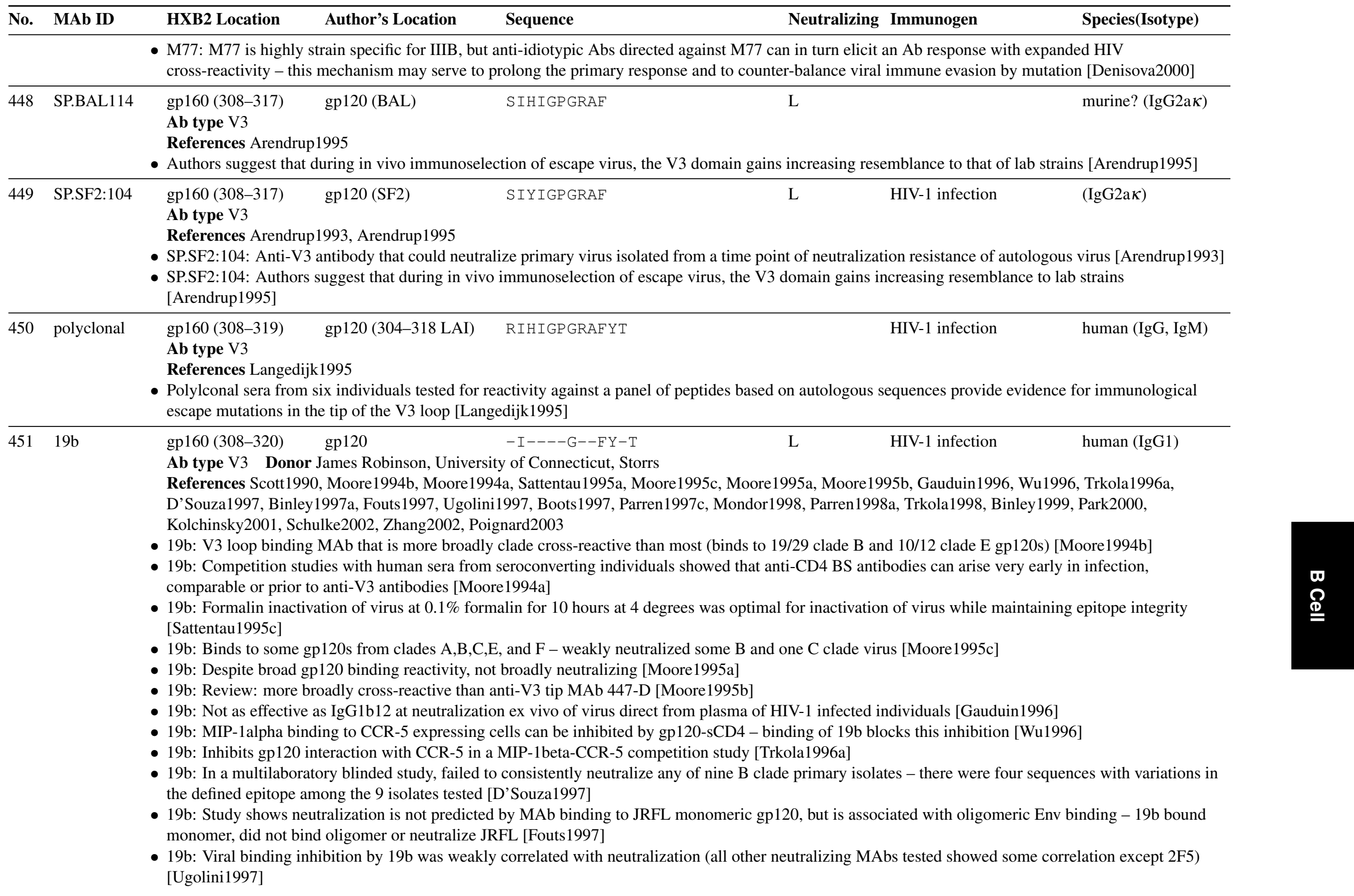




No. MAb ID HXB2 Location $\quad$ Author's Location $\quad$ Sequence $\quad$ Neutralizing Immunogen

- 19b: Abs that recognize discontinuous epitopes can identify mimotopes from a phage peptide display library - 19b has an epitope involving the tip of the V3 loop, with 5 or 6 essential amino acids distributed within a 12 amino acid stretch - the previously determined binding site was confirmed -I--G-FY-T and some tolerated variants described, the I can be I, V, or L, the Y can be Y, F, or W - probably a beta-turn is required for FY or FF binding, but WY in can bind with out the context of the turn [Boots1997]

- 19b: Neutralizes TCLA strains but not primary isolates [Parren1997c]

- 19b: Used as a control in this Hx10 binding and neutralizing MAb study because 19b does not bind to Hx10 [Mondor1998

- 19b: The MAb and Fab binding to the oligomeric form of gp120 and neutralization were highly correlated - authors suggest that neutralization is determined by the fraction of Ab sites occupied on a virion irrespective of the epitope [Parren1998a

- 19b: No detectable neutralizing activity among primary isolates with different co-receptor usage - some neutralization of TCLA strains [Trkola1998]

- 19b: The MAbs with the broadest neutralizing activity, IgG1b12, 2G12 and 2F5, all have high affinity for the native trimer, indicating that they were raised in an immune response to the oligomer on the virion surface rather than dissociated subunits - a disulfide linked gp120-gp41 (SOS gp140) was created to mimic the native conformation of Env and explore its potential as an immunogen - SOS gp140 is recognized by NAbs IgG1b12, 2G12, and CD4-IgG2, and also by anti-V3 MAbs 19b and 83.1 - SOSgp140 is not recognized by C4 region MAbs that neutralize only TCLA strains, G3-42 and G3-519 - nor did it bind C11, 23A, and M90, MAbs that bind to gp120 C1 and C5, where it interacts with gp41 - MAbs that bind CD4 inducible epitopes, 17b and A32 were very strongly induced by CD4 in SOS gp140 - anti-gp41 MAbs that bind in the region that interacts with gp120, 7B2, 2.2B, T4, T15G1 and 4D4, did not bind to SOSgp140, in contrast to 2F5, which binds to the only gp41 epitope that is well exposed in native gp120-gp41 complexes |Binley1999|

- 19b: Six mutations in MN change the virus from a high-infectivity neutralization resistant phenotype to low-infectivity neutralization sensitive - V3, CD4BS, and CD4i MAbs are 20-100 fold more efficient at neutralizing the sensitive form but 19b was an exception and required around 950 ng/ml to neutralize either form [Park2000]

- 19b: Mutations in two glycosylation sites in the V2 region of HIV-1 ADA at positions 190 and 197 (187 DNTSYRLINCNTS 199) cause the virus to become CD4-independent and able to enter cells through CCR5 alone - these same mutations tended to increase the neutralization sensitivity of the virus, including to $19 \mathrm{~b}$ |Kolchinsky2001].

- 19b: Ab binding characteristics of SOS gp140 were tested using SPR and RIPA - SOS gp140 is gp120-gp41 bound by a disulfide bond - NAbs 2G12, 2F5, IgG1b12, CD4 inducible 17b, and 19b bound to SOS gp140 better than uncleaved gp140 (gp140unc) and gp120 - non-neutralizing MAbs 2.2B (binds to gp41 in gp140unc) and 23A (binds gp120) did not bind SOS gp140 [Schulke2002]

- 19b: A rare mutation in the neutralization sensitive R2-strain in the proximal limb of the V3 region caused Env to become sensitive to neutralization by MAbs directed against the CD4 binding site (CD4BS), CD4-induced (CD4i) epitopes, soluble CD4 (sCD4), and HNS2, a broadly neutralizing sera - 2/12 anti-V3 MAbs tested (19b and 694/98-D) neutralized R2, as did 2/3 anti-CD4BS MAbs (15e and IgG1b12), 2/2 CD4i MAbs (17b and 4.8D), and 2G12 and $2 \mathrm{~F} 5$ - thus multiple epitopes on R2 are functional targets for neutralization and the neutralization sensitivity profile of R2 is intermediate between the highly sensitive MN-TCLA strain and the typically resistant MN-primary strain [Zhang2002]

- 19b: Virion capture assays are not a good preditor of neutralization, and the presentation of epitopes using this assay seems to be different from that of functional Envelope spikes on primary isolates - F105 and b6 could efficiently block the b12-mediated capture of infectious virions in a virus capture, but did not inhibit b12 neutralization - while b12 was potent at neutralizing the three primary virions JR-CSF, A DA, and 89.6, the Abs F105, 19b, and Fab b6 were overall very poor neutralizers [Poignard2003] gp160 (308-322) gp120(308-322 LAI) RIQRGPGRAFVTGK

Ab type V3 Donor Dr. Albrecht von Brunn, Max-von-Pettenkofer-Institut, Ludwig-Maximilians-Universitat Munchen, Germany

References vonBrunn1993

- 4G10: A 25 amino acid V3-loop sequence fused to HBcAg enhanced V3 immunogenicity |vonBrunn1993

- 4G10: NIH AIDS Research and Reference Reagent Program: 2534 


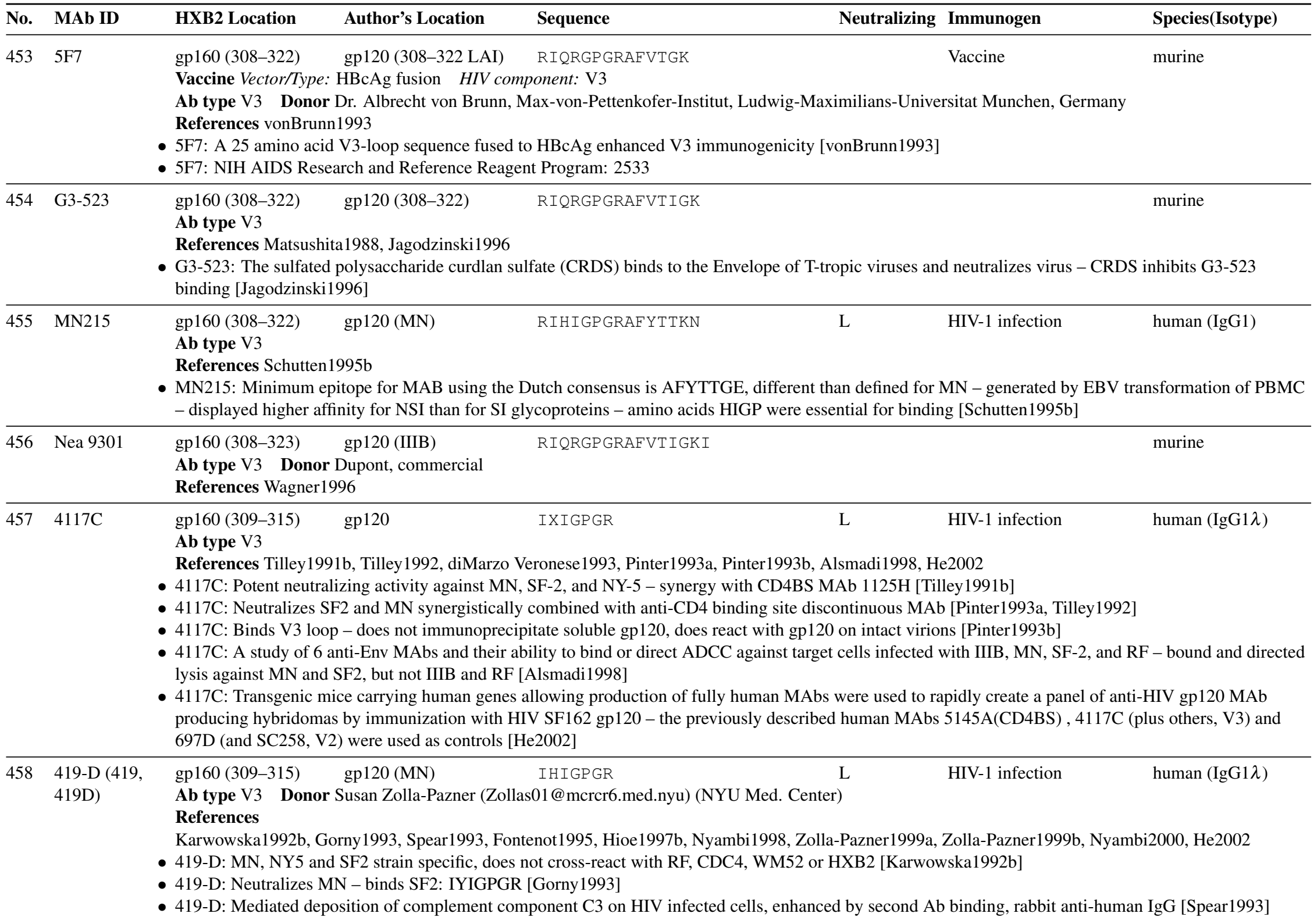




No. MAb ID HXB2 Location $\quad$ Author's Location $\quad$ Sequence $\quad$ Neutralizing Immunogen

- 419-D: Four primary isolates showed distinct patterns of sensitivity to neutralization by polyclonal sera or plasma and MAbs - BZ167 was the only isolate inhibited by all polyclonal sera and plasma tested, and was also neutralized by 8/17 MAbs, in particular anti-V3 loop (419-D, 447-52D, 782-D, and 838-D), anti-CD4bd (559/64-D, 654-D and 830-D and a cluster II of gp41 directed MAb (98-6) - isolates 92HT593 and 91 US056 were neutralized by V3 loop (419-D, and 447-52D)and cluster II gp41 (98-6) MAbs at higher concentrations - US4 was neutralized by some of the polyclonal sera/plasma tested and not at all by MAbs individually or by a cocktail of ten MAbs consisting of 419-D, 447-52D, 782-D, 838-D, 559/64-D, 654-D, 450-D, 670-D, 1281-D and 98-6 Hioe1997b

- 419-D: Using a whole virion-ELISA method, 18 human MAbs were tested for their ability to bind to a panel of 9 viruses from clades A, B, D, F, G, and H 419-D bound to 3/4 B clade virions, and to D clade MAL [Nyambi1998]

- 419-D: Review of clade specificity and anti-V3 HIV-1-Abs - epitope is described as KRIHIGP [Zolla-Pazner1999a]

- 419-D: MAb peptide-reactivity pattern clustered with immunological related MAbs: 1334, 419, 504, 447, 453 and 537 - the core amino acids GP tended to be critical for reactivity in this group [Zolla-Pazner1999b]

- 419-D: A panel of 47 human MAbs was tested against 26 HIV-1 group M primary isolates from clades A through H - 19 V3 MAbs were tested, and of 494 combinations, 44\% displayed some viral binding - V3 MAbs tended to have the most cross-reactive binding to clade A, B, C, and D isolates, less to E, F, G, and $\mathrm{H}-419-\mathrm{D}$ showed intermediate reactivity, and no neutralization when tested against five strains - discrepancy between the epitope as described in earlier papers and as described here, KRIHIGP [Nyambi2000]

- 419-D: Called 419 - Transgenic mice carrying human genes allowing production of fully human MAbs were used to rapidly create a panel of anti-HIV gp120 MAb producing hybridomas by immunization with HIV SF162 gp120 - the previously described human MAbs 5145A(CD4BS) , 4117C (plus others, V3) and 697D (and SC258, V2) were used as controls [He2002]

$459 \quad 453-\mathrm{D}(453)$

$\begin{array}{lccc}\text { gp160 (309-315) gp120 (MN) } & \text { IHIGPGR } & \text { L } \\ \text { Ab type V3 Donor Susan Zolla-Pazner (Zollas01@ mcrcr6.med.nyu) (NYU Med. Center) }\end{array}$

L HIV-1 infection

human $(\operatorname{IgG} 1 \lambda)$

References Gorny1991. Gorny1993. VanCott1994, Fontenot1995, Zolla-Pazner1999a, Zolla-Pazner1999b Nyambi2000

- 453-D: Neutralizes MN - binds SF2: IYIGPGR - specificity: MN, SF2, NY5, RF [Gorny1993]

- 453-D: Moderate homologous neutralization, moderately slow dissociation rate [VanCott1994]

- 453-D : Called 453, epitope described as KRIHIGPGR - the tip of the V3 loop was presented in a mucin backbone - higher valency correlates with stronger affinity constant [Fontenot1995]

- 453-D: Review of clade specificity and anti-V3 HIV-1-Abs [Zolla-Pazner1999a]

- 453-D : MAb peptide-reactivity pattern clustered with immunological related MAbs: 1334, 419, 504, 447, 453 and 537 - the core amino acids GP tended to be critical for reactivity in this group - MAb 268, with a previously defined core epitope identical to 453 (HIGPGR), was not part of this reactivity group, illustrating that context can be critical [Zolla-Pazner1999b]

- 453-D: A panel of 47 human MAbs was tested against 26 HIV-1 group M primary isolates from clades A through H - 19 V3 MAbs were tested, and of 494 combinations, 44\% displayed some viral binding - V3 MAbs tended to have the most cross-reactive binding to clade A, B, C, and D isolates, less to E, F, G, and $\mathrm{H}-453-\mathrm{D}$ showed intermediate reactivity [Nyambi2000]

504-10D) Ab type V3 Donor Susan Zolla-Pazner (Zollas01@ mcrcr6.med.nyu) (NYU Med. Center)

References Gorny1993, Zolla-Pazner1999a, Zolla-Pazner1999b, Nyambi2000

- 504-D - Neutralizes MN - binds SF2: IYIGPGR [Gorny1993]

- 504-D: Review of clade specificity and anti-V3 HIV-1-Abs [Zolla-Pazner1999a]

- 504-D: MAb peptide-reactivity pattern clustered with immunological related MAbs: 1334, 419, 504, 447, 453 and 537 - the core amino acids GP tended to be critical for reactivity in this group [Zolla-Pazner1999b 


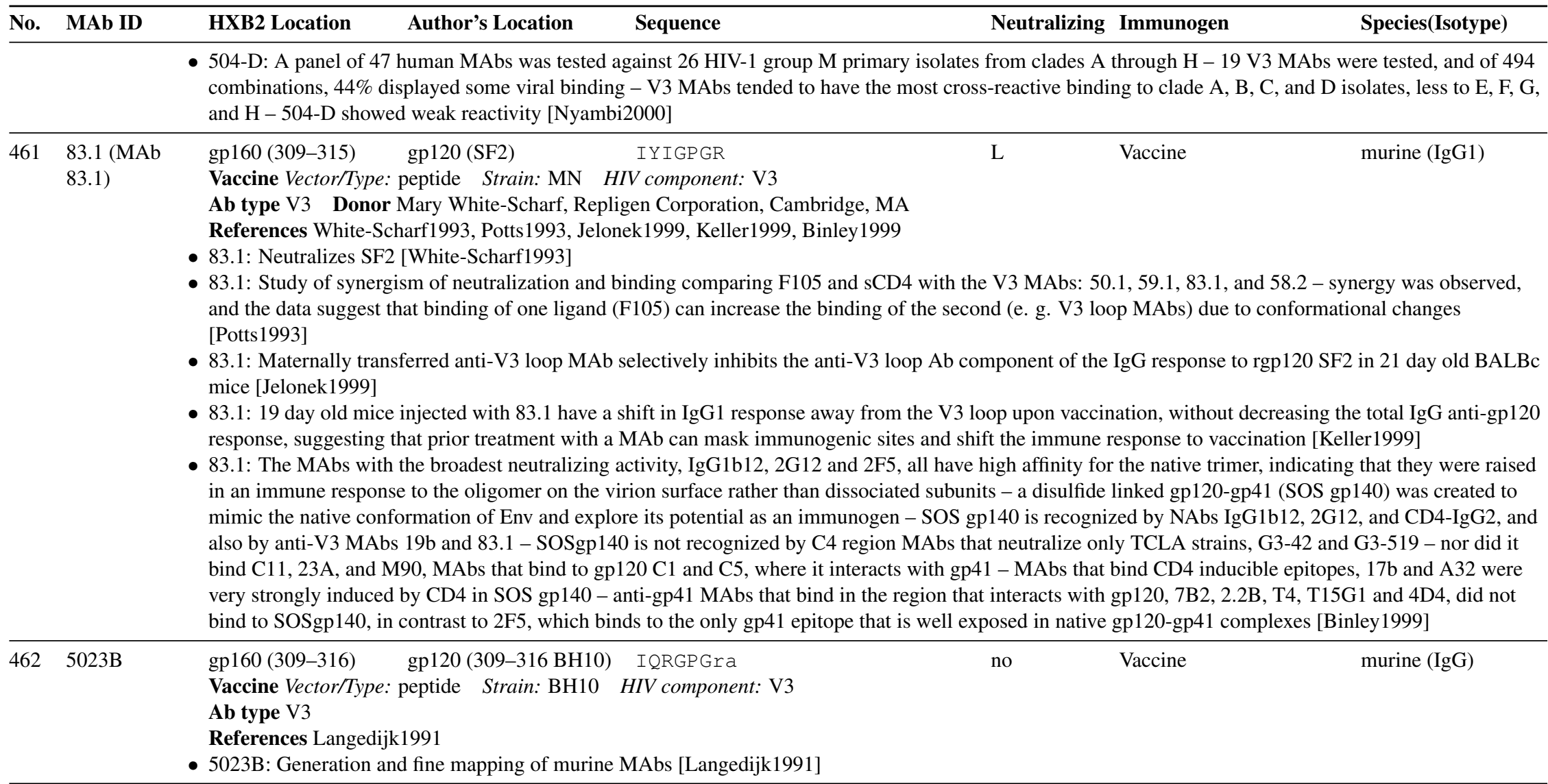

Vaccine Vector/Type: virus derived protein HIV component: gp120

$\mathrm{L}$

Vaccine

murine $(\operatorname{IgG} 1)$

Ab type V3

References Akerblom1990, Broliden1991 Moore1993c, Millar1998, Jackson1999

- F58/D1: Binding to native gp120 1-3 fold greater than to denatured - 314G/W substitution abolishes binding, changes outside the loop have little effect Moore1993c

- F58/D1: The interaction of a 17-amino-acid neutralizing microantibody (MicroAB) based on F58 and HIV-1 env was studied by electrospray ionization mass spectrometry [Millar1998]

- F58/D1: A 17 amino acid MicroAB was made from the third complementarity-determining region of the heavy chain of MAb - F58 neutralized 5x's more efficiently in terms of mass than the original MAb, 32-fold less on a molar basis - neutralization does not involve initial attachment, but fusion and events in early infection [Jackson1999]

Vaccine

murine $(\mathrm{IgG})$

Vaccine Vector/Type: virus derived protein Strain: IIIB HIV component: gp120 


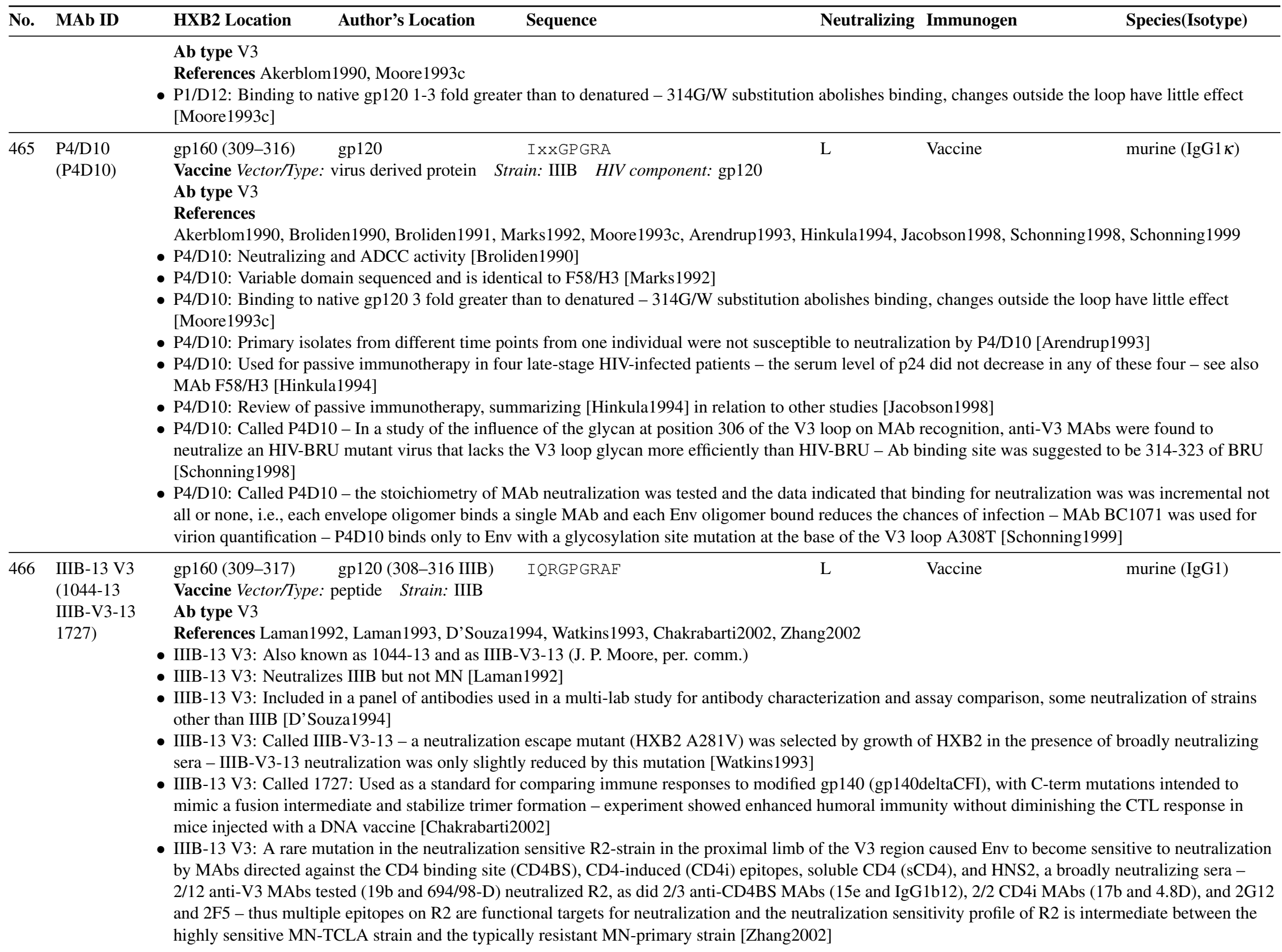




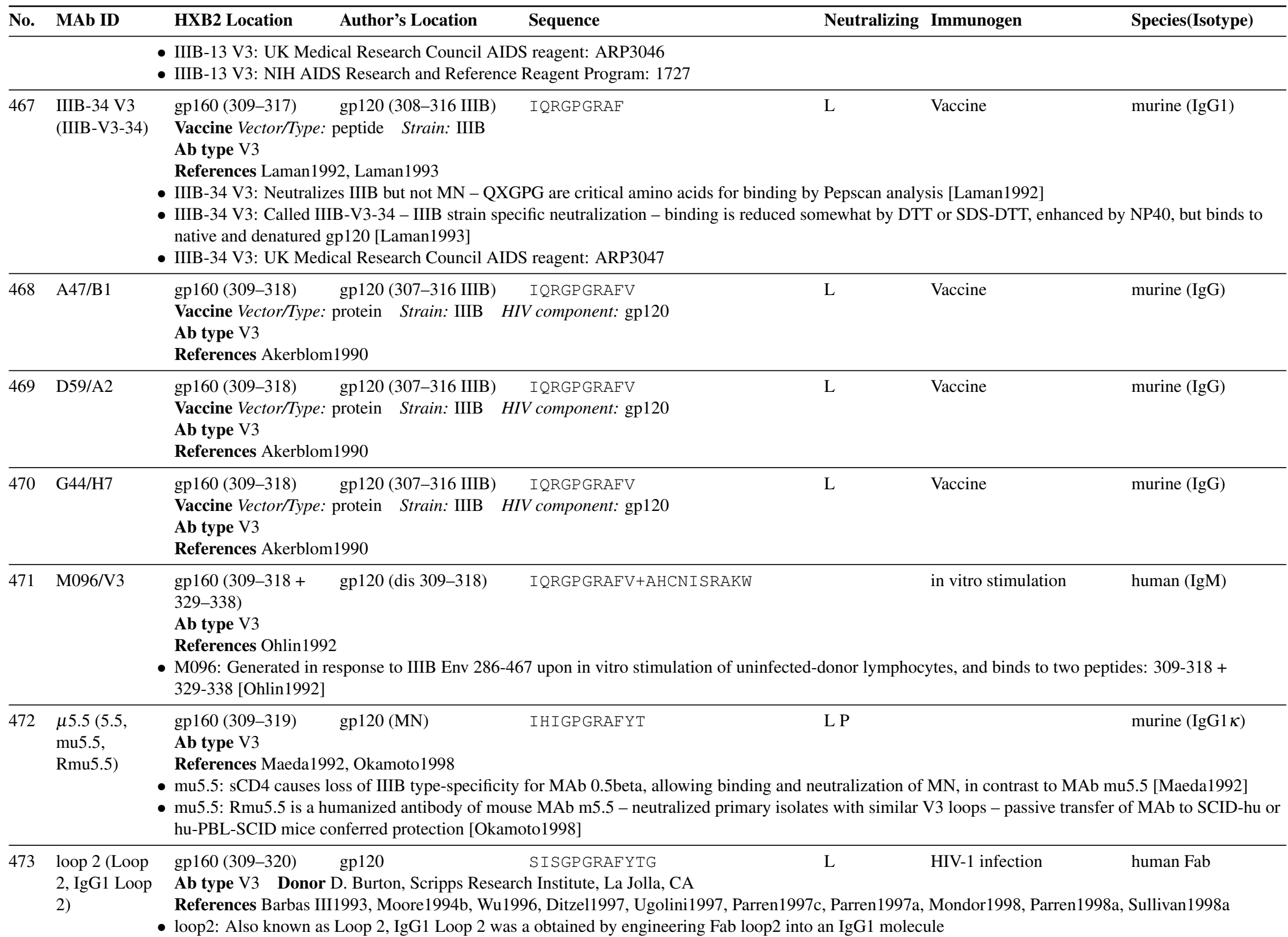




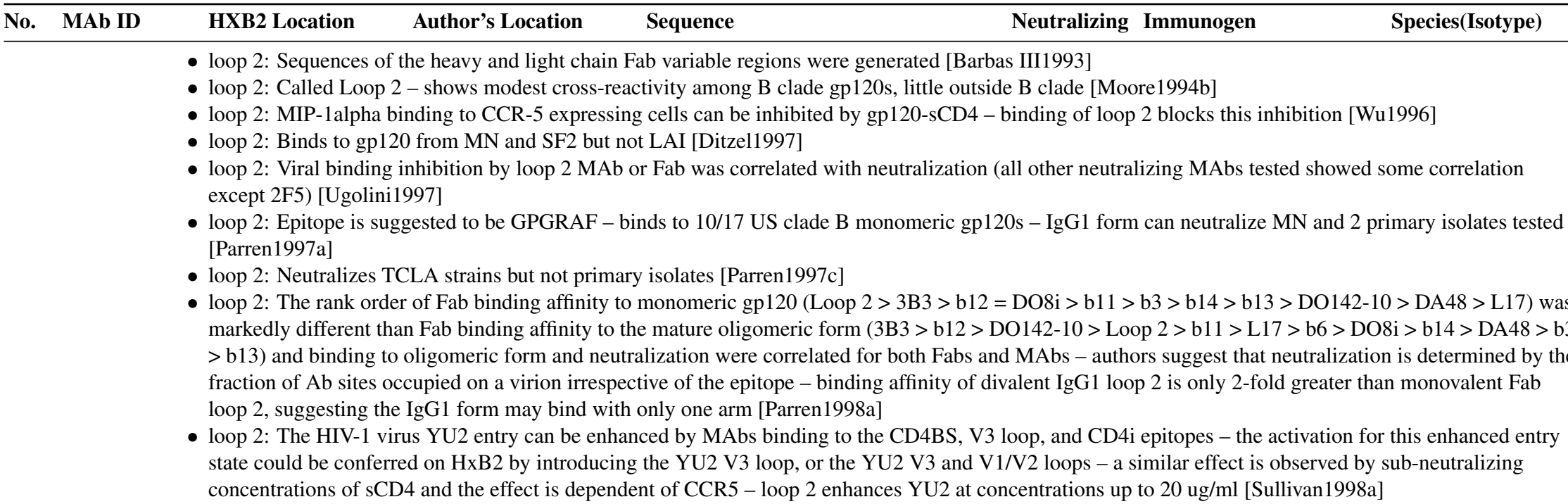
gp160 (310-315) gp120 (MN) HIGPGR $\mathrm{L}$ HIV-1 infection human $(\operatorname{IgG} 1 \lambda)$

(268-11-D-IV, Ab type V3 Donor Susan Zolla-Pazner (Zollas01@ @crcr6.med.nyu) (NYU Med. Center)

268D, 268, References Gorny1991, D’Souza1991, Karwowska1992b, Gorny1993 | Spear1993. VanCott1994, Stamatatos1995, Zolla-Pazner1995a, Fontenot1995 268-11D, McKeating1996b, Wisnewski1996, Hioe1997b, Stamatatos1997, LaCasse1998, Zolla-Pazner1999a, Zolla-Pazner1999b, Beddows1999, Oggioni1999 268-10D,

MAb 268, 268-10-D, ARP3024) Lioe2000 Nyambi2000 Park2000 York2001 Vella2002 Zhang2002

- 268-D: Called 268-11-D-IV - strain specific weakly neutralizing [D’Souza1991

- 268-D: Reacts with MN, NY5, CDC4, RF and SF2, does not cross-react with WM52 or HXB2 [Karwowska1992b]

- 268-D: Neutralizes MN - binds SF2: YIGPGR - specificity: MN, SF2, NY5, RF, CDC4 Gorny1993

- 268-D: Mediated deposition of complement component C3 on HIV infected cells, but not in the presence of sCD4 [Spear1993]

- 268-D: Moderate dissociation rate and homologous neutralization titer [VanCott1994]

- 268-D: Serotyping study using flow-cytometry, if H of HIGPGR was substituted in virus, 268-D did not bind [Zolla-Pazner1995a

- 268-D: The binding of conformation-dependent anti-V2, anti-V3, and anti-CD4BS MAbs to monomeric and virion-associated gp120 from HIV-1 isolates with differences in cell tropism was studied - V3 loop epitopes were less accessible to Ab binding on the virion surface than in the gp120 monomer, particularly for macrophage-tropic isolates SF162 and SF128a, relative to T-cell tropic SF2 - sCD4 association with gp120 did not influence the binding of 268-D to virion-associated gp120, although sCD4 binding did alter epitope exposure for other anti-V3 MAbs [Stamatatos1995]

- 268-D: Failed to neutralize HXB2 and chimeric virus with gp120 from primary isolates in an HXB2 background [McKeating1996b

- 268-D: 268-D is V H4 - V-region heavy chain usage was examined and a bias of enhanced V H1 and V H4, and reduced V H3, was noted among HIV infected individuals [Wisnewski1996]

- 268-D: Poor reactivity against HIV-1 isolates SF162 and SF128A and no neutralization, in contrast to MAbs 391/95-D and 257-D [Stamatatos1997]

- 268-D: A T-cell line-adapted (TCLA) derivative of SI primary isolate 168P acquired the ability to be neutralized by anti-V3 MAbs - the primary isolate could use either CCR5 or CXCR4, and was not neutralized when infection was directed via either pathway, however the TCLA derivative uses CXCR4 only and is neutralized [LaCasse1998

- 268-D: Review of clade specificity and anti-V3 HIV-1-Abs [Zolla-Pazner1999a] 


No. MAb ID $\quad$ HXB2 Location $\quad$ Author's Location $\quad$ Sequence $\quad$ Neutralizing Immunogen

- 268-D: Peptide reactivity pattern clustered with immunological related MAbs: $1108,386,268,311,257,694.8$ - the amino acids HI tended to be critical for reactivity in this group - MAb 453, with an identical core epitope to 268 based on prior experiments (HIGPGR), was not part of this reactivity group, illustrating that context can be critical [Zolla-Pazner1999b]

- 268-D: rgp120 derived from a R5X4 subtype B virus, HIV-1 W61D, was used to vaccinate healthy volunteers and the resulting sera were compared with sera from HIV-1 positive subjects and neutralizing MAbs - 268-D bound rgp120 W61D but could only neutralize the W61D isolate following T-cell line adaptation [Beddows1999]

- 268-D: Called 268-11D - Study of a live-vector mucosal vaccine that expresses HIV-1 V3 domains using the bacterium Streptococcus gordonii which can express heterologous Ag and can colonize the oral cavity and vagina of mice - 268-D and 257-D recognized S. gordonii expressing the V3 domain of MN the vaccine stimulated V3-specific IgG2a in mice [Oggioni1999]

- 268-D: Called MAb 268 - To identify potential mimotopes of V3, a hexapeptide phage library was screened with MAb 268 - two hexamers were identified, HLGPGR or KAIHRI that bind to 268 with the same binding site as the V3 loop and inhibit 268 MN gp120 - KLH conjugated hexamer KAIHRI stimulates Abs in rabbits that cross-react with ML gp120 [Laisney1999]

- 268-D: Ab responses, because of their capacity to alter antigen uptake and processing, can influence helper T cell responses - CD4BS MAbs or serum Ig from HIV+ individuals inhibited proliferative responses of gp120 specific T cells - V3 MAbs 447-52-D and 268-10-D did not effect proliferation [Hioe2000]

- 268-D: A panel of 47 human MAbs was tested against 26 HIV-1 group M primary isolates from clades A through H - 19 V3 MAbs were tested, and of 494 combinations, 44\% displayed some viral binding - V3 MAbs tended to have the most cross-reactive binding to clade A, B, C, and D isolates, less to E, F, G, and $\mathrm{H}$ - 268-D showed weak reactivity [Nyambi2000]

- 268-D: Called 268D - six mutations in MN change the virus from a high-infectivity neutralization resistant phenotype to low-infectivity neutralization sensitive - V3, CD4BS, and CD4i MAbs are 20-100 fold more efficient at neutralizing the sensitive form - the mutation L544P reduced binding of all MAbs against gp120 by causing conformational changes [Park2000]

- 268-D: Abs against the V3 loop (50.1, 58.2, 59.1, 257-D, 268-D, 447-52D), CD4BS (IgG1b12, 559-64D, F105), CD4i (17b), and to gp41 (2F5, F240) each showed similar binding efficiency to Env derived from related pairs of primary and TCLA lines (primary: 168P and 320SI, and TCLA: 168C and

320SI-C3.3), but the TCLA lines were much more susceptible to neutralization suggesting that the change in TCLA lines that make them more susceptible to NAbs alters some step after binding - one of the TCLA V3 viruses 320SI-C3.3 shows reduced binding with this MAb, the sequence of the epitope in 320SI is HIGPGR and in 320SI-C3.3 is RIGPGR [York2001]

- 268-D: Called ARP3024: Herpesvirus saimiri-immortalized CD4+ T lymphocytes (HVS T cells) were used to isolate virus and perform HIV-1 neutralization assays, and compared with a standard PBMC protocol - neutralization sensitivities to a panel of MAbs and to homologous or heterologous plasma/sera were similar for HVS T cells (CN-2 cells) and PBMCs [Vella2002].

- 268-D: A rare mutation in the neutralization sensitive R2-strain in the proximal limb of the $\mathrm{V} 3$ region caused Env to become sensitive to neutralization by MAbs directed against the CD4 binding site (CD4BS), CD4-induced (CD4i) epitopes, soluble CD4 (sCD4), and HNS2, a broadly neutralizing sera-2/12 anti-V3 MAbs tested (19b and 694/98-D) neutralized R2, as did 2/3 anti-CD4BS MAbs (15e and IgG1b12), 2/2 CD4i MAbs (17b and 4.8D), and 2G12 and 2F5-thus multiple epitopes on R2 are functional targets for neutralization and the neutralization sensitivity profile of R2 is intermediate between the highly sensitive MN-TCLA strain and the typically resistant MN-primary strain [Zhang2002].

- 268-D: UK Medical Research Council AIDS reagent: ARP3024

- 268-D: NIH AIDS Research and Reference Reagent Program: 1511

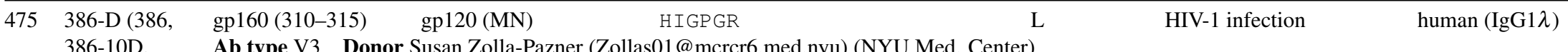

386-10D, $\quad$ Ab type V3 Donor Susan Zolla-Pazner (Zollas01@ @ mcrcr6.med.nyu) (NYU Med. Center)

386D) References Karwowska1992b, Gorny1993, VanCott1994, Fontenot1995, Zolla-Pazner1999a, Zolla-Pazner1999b Nyambi2000

- 386-D: Neutralizes MN - binds SF2: YIGPGR - specificity: MN, SF2, NY5, RF, CDC4 [Gorny1993]

- 386-D: Slow dissociation rate, potent homologous neutralization [VanCott1994] 


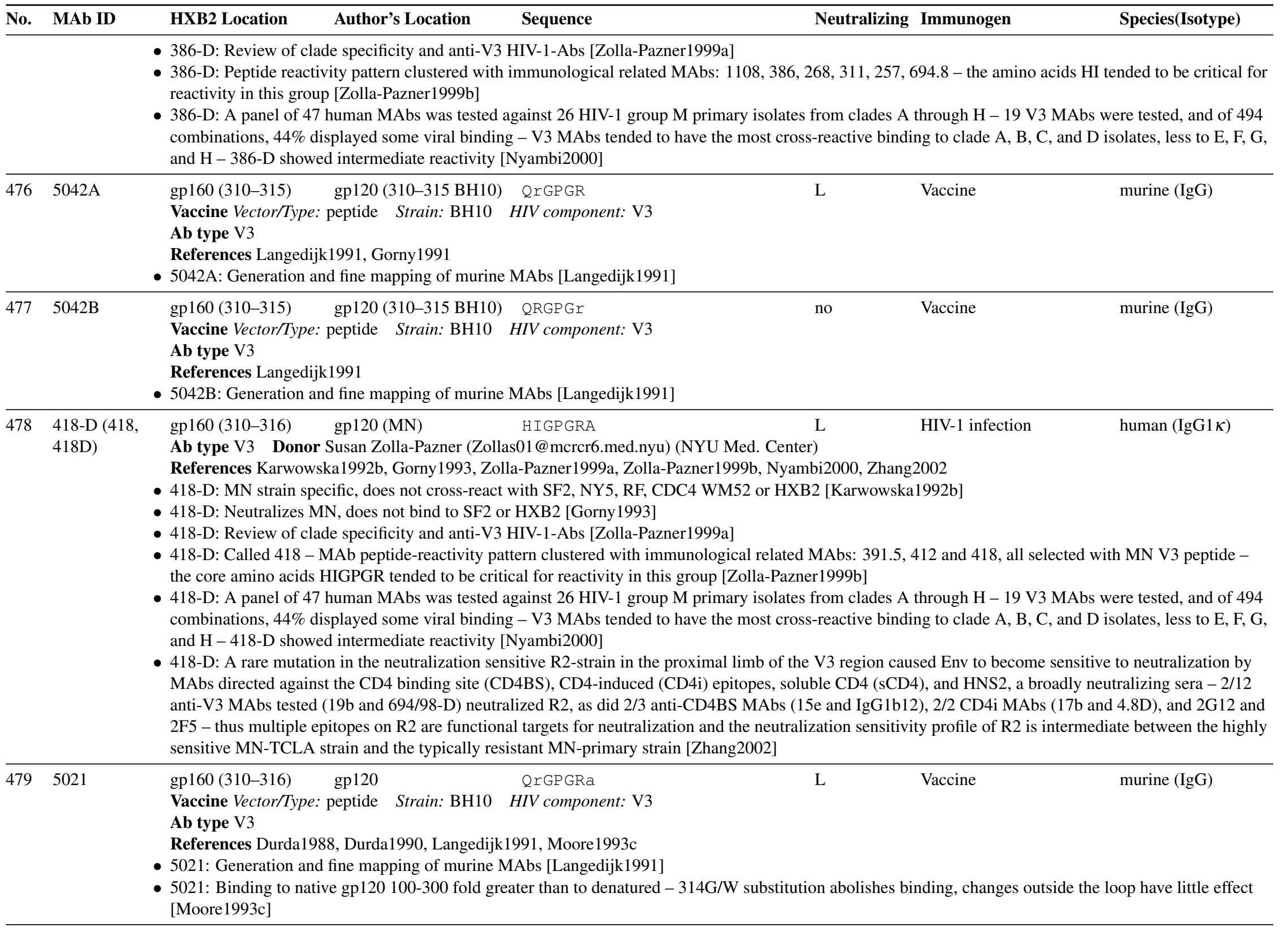




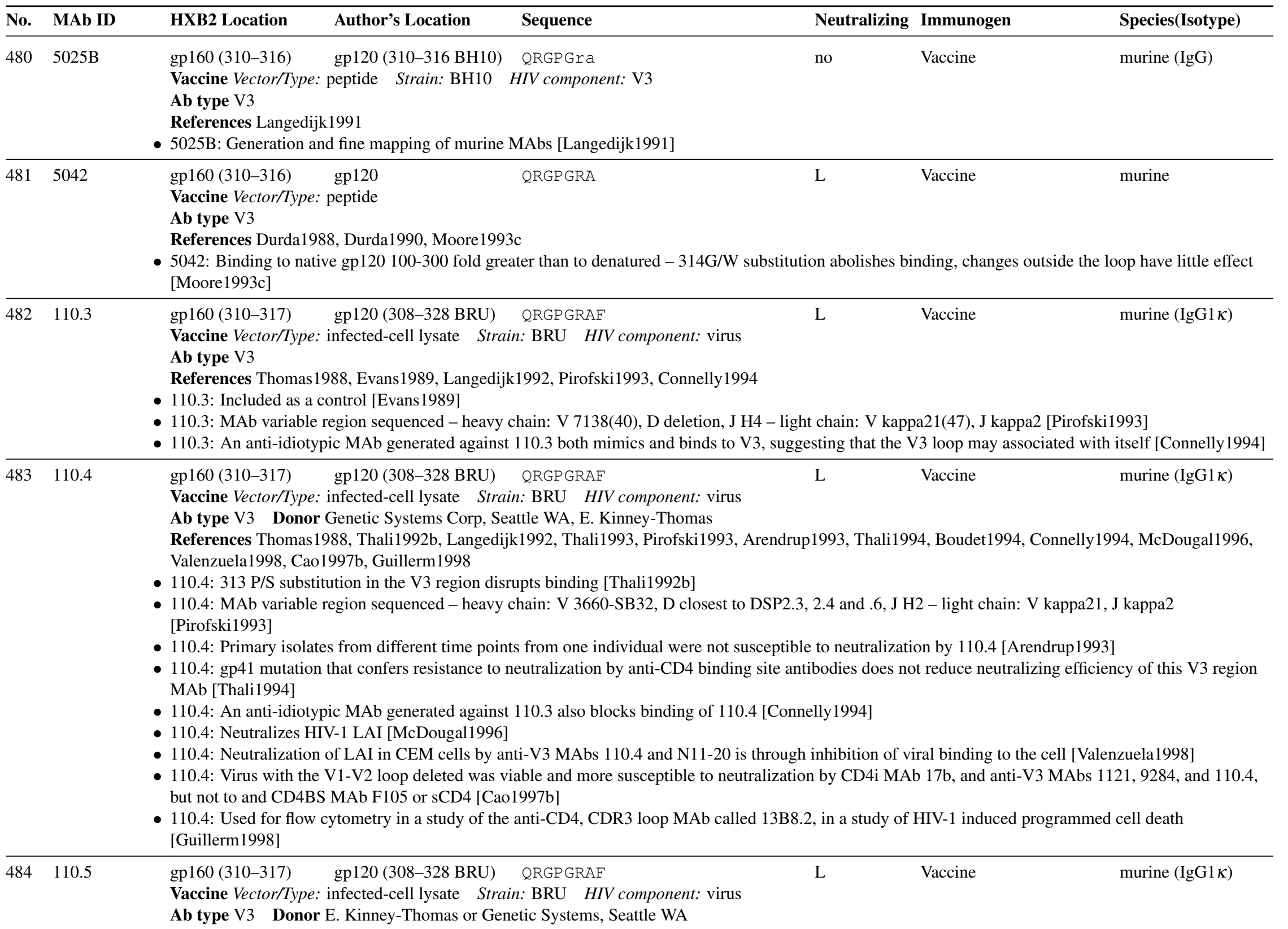




\begin{tabular}{|c|c|c|c|c|c|}
\hline \multirow[t]{2}{*}{ No. } & \multirow[t]{2}{*}{ MAb ID } & HXB2 Location & Author's Location & Neutralizing Immunogen & Species(Isotype) \\
\hline & & \multicolumn{4}{|c|}{ 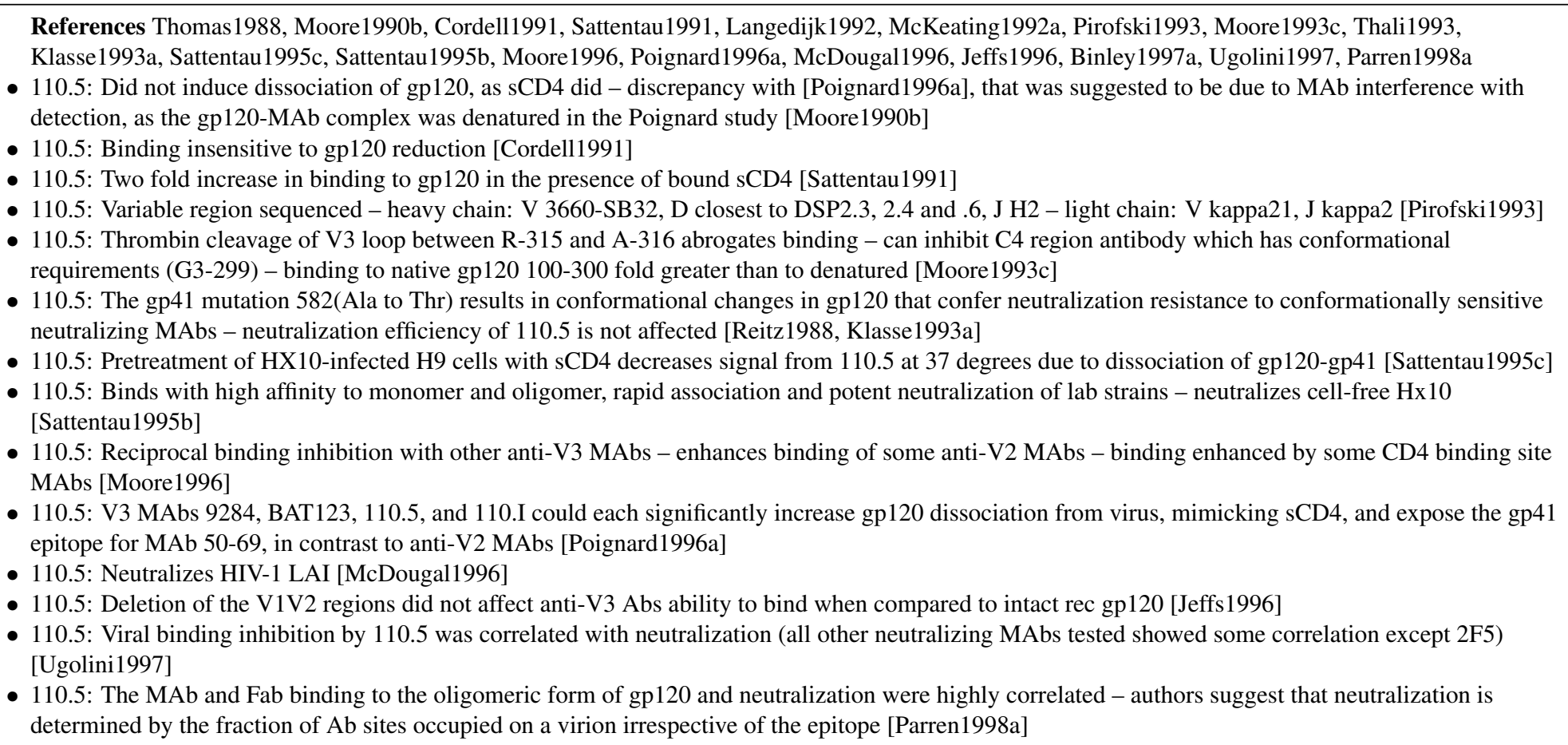 } \\
\hline 485 & 58.2 & $\begin{array}{l}\text { gp160 (310-317) } \\
\text { Vaccine Vector/T } \\
\text { Ab type V3 Do } \\
\text { References Whit } \\
\text { - 58.2: Epitope def } \\
\text { White-Scharf19 } \\
\text { - 58.2: Did not syn } \\
\text { - 58.2: Modest cro } \\
\text { - 58.2: Competitio } \\
\text { significance of nc } \\
\text { - 58.2: The crystal } \\
\text { when different M } \\
\text { - 58.2: } 58.2 \text { 's epito } \\
\text { CD4i (17b), and } \\
\text { and 320SI, and T } \\
\text { that make them } \mathrm{n}\end{array}$ & $\begin{array}{l}\text { llly neutralize MN } \\
\text { vity among B clad } \\
\text { s with serial delet } \\
\text { ct residues [Selign } \\
\text { e of Fab } 58.2 \text { boun } \\
\text { e bound - 58.2's } \\
\text { oted to be IGPGR }\end{array}$ & $\begin{array}{l}\text { H IGPGRAF } \\
\text { IV component: V3 } \\
\text { e1994b, Seligman1996 Stanfield1999, York2001] } \\
\text { hanges in affinity with amino acid substitutions - 4/7 primarily isolates } \\
\text { ombination with MAb F105 - there was synergistic neutralization when } \\
\text { 120s, little outside B clade - core epitope as I-IHIG [Moore1994b] } \\
\text { produced longer estimates of epitope length, RIHIGPGRAFY, than Alan } \\
\text { 996] } \\
\text { V3 loop peptides was obtained - conformational changes in the tip of the } \\
\text { - Abs defined as KRKRIHIGPGRAFY [Stanfield1999] } \\
\text { ed similar binding efficiency to Env derived from related pairs of primary } \\
\text { ut the TCLA lines were much more susceptible to neutralization suggest } \\
\text { some step after binding [York2001] }\end{array}$ & $\begin{array}{l}\text { murine (IgG1 } \kappa) \\
\text { zed } \\
\text { th sCD4 [Potts1993] } \\
\text { ion, suggesting } \\
\text { PGR) were observed } \\
\text { lines (primary: 168P } \\
\text { change in TCLA lines }\end{array}$ \\
\hline
\end{tabular}




\begin{tabular}{lllllll}
\hline No. & MAb ID & HXB2 Location & Author's Location & Sequence & Neutralizing & Immunogen \\
\hline 486 & polyclonal & gp160 (310-318) & gp120 & QRGPGRAFV? & L & Vaccine \\
& & & Vaccine Vector/Type: & peptide Brucella abortus $(\mathrm{Ba})$ conjugate, peptide keyhole limpet hemocyanin (KLH) conjugate, peptide lipopolysaccharide (LPS)
\end{tabular}

conjugate Strain: MN HIV component: V3

References Golding2002a

- Internasal (i.n.) immunization with V3-Ba induced mucosal anti-V3 NAbs and IFN-gamma secreting T cells - V3-Ba, V3-KLH and V3-LPS could each induce serum and mucosal IgA and IgG in BALB/c mice - i.n. plus i.p. immunizations gave higher titers than i.n. alone - the response to V3-KLH was mainly restricted to IgG1, and to V3-Ba, IgG2a - class II KO mice (CD4+-deficient) did not respond to V3-KLH, but did respond to V3-Ba, suggesting that V3-Ba may be effective in eliciting Ab responses in HIV-1 infected individuals that have impaired CD4+ T cell function [Golding2002a]

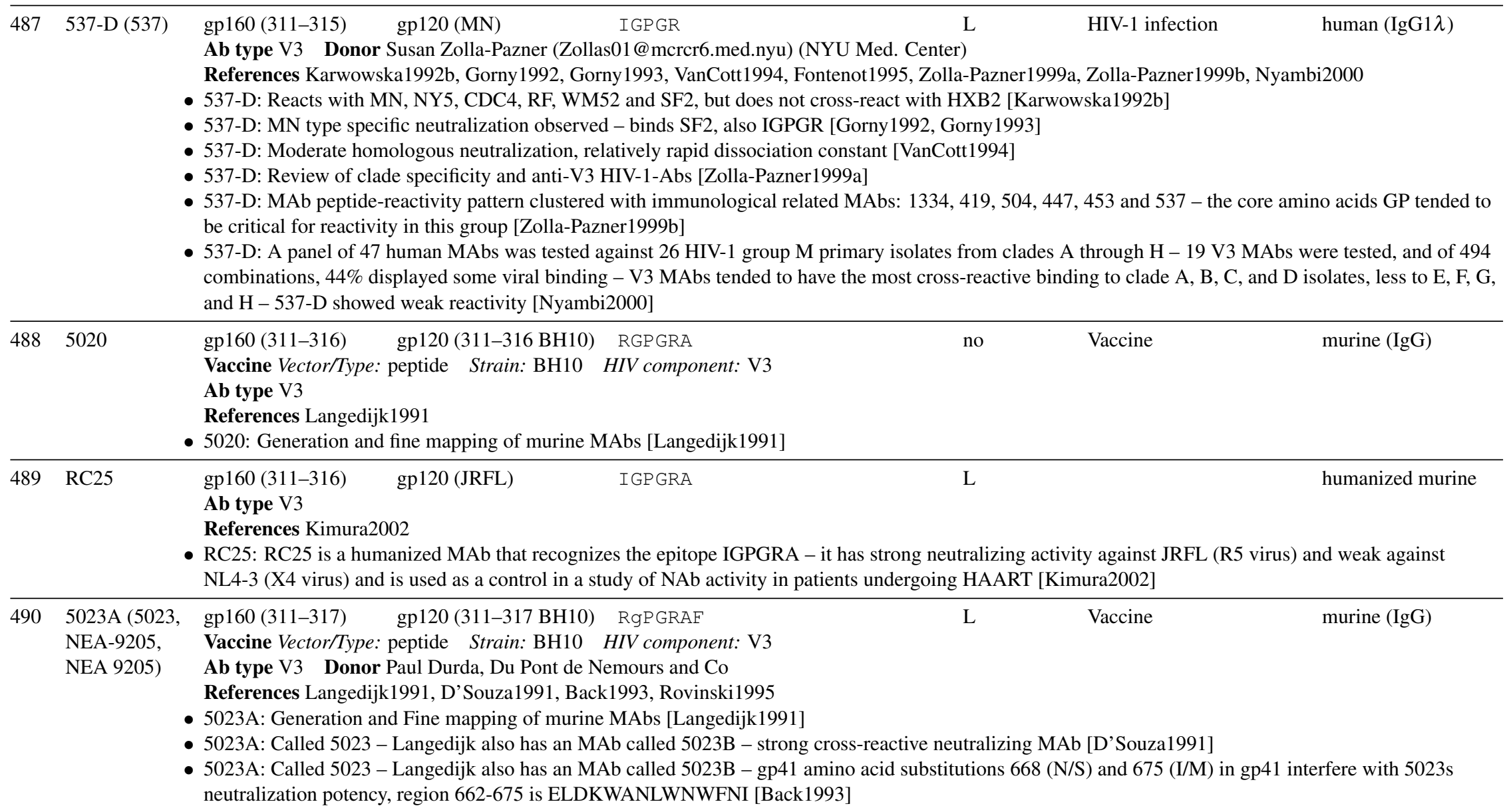




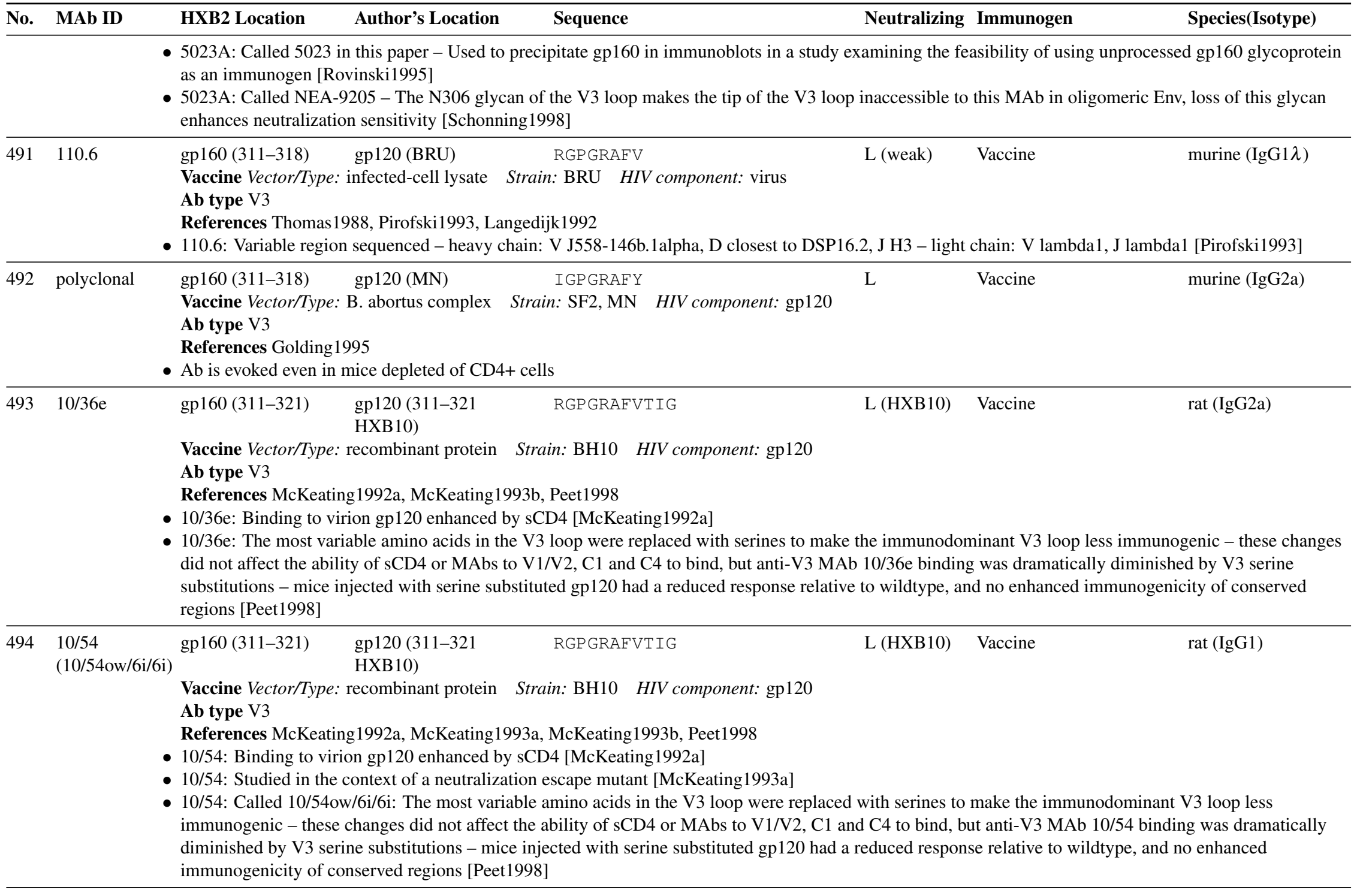

$(11 / 85 \mathrm{~b} / 14 \mathrm{I} / 14 \mathrm{I})$

HXB10)

Vaccine Vector/Type: recombinant protein Strain: BH10 HIV component: gp120
Ab type V3 Ab type V3 


\begin{tabular}{|c|c|c|c|c|c|c|}
\hline No. & MAb ID & Author's Location & Sequence & Neutralizing & Immunogen & Species(Isotype) \\
\hline & & \multicolumn{5}{|c|}{$\begin{array}{l}\text { References McKeating1992a } \\
\text { - } 11 / 85 \text { b: Binding to virion gp120 enhanced by sCD4 [McKeating1992a }\end{array}$} \\
\hline \multirow[t]{2}{*}{496} & \multirow[t]{2}{*}{ polyclonal } & gp160 (311-322) gp120 (MN) & IGPGRAFYTTKN & $\begin{array}{l}\mathrm{L}(\mathrm{MN} \\
\text { ALA-1) }\end{array}$ & Vaccine & guinea pig \\
\hline & & $\begin{array}{l}\text { Vaccine Vector/Type: human rhinovirus } 14 \\
\text { Ab type V3 } \\
\text { References Smith1998 } \\
\text { - The tip of the MN V3 loop (IGPGRAFYTT } \\
\text { chimeric viruses were neutralized by anti-V }\end{array}$ & $\begin{array}{l}\text { Strain: MN HIV component: } \mathrm{V} 3 \\
\mathrm{KN} \text { ) was inserted into cold-causing } \mathrm{I} \\
\text { loop antibodies - chimeric viruses }\end{array}$ & $\begin{array}{l}\text { novirus } 14 \text { (HR } \\
\text { otent NAbs aga }\end{array}$ & $\begin{array}{l}\text { V14) }- \text { chim } \\
\text { inst ALA-1 }\end{array}$ & $\begin{array}{l}\text { noselected, and } \\
998 \mid\end{array}$ \\
\hline
\end{tabular}

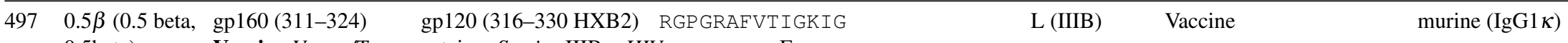

0.5beta) Vaccine Vector/Type: protein Strain: IIIB HIV component: Env

Ab type V3 Donor Shuzo Matsushita or Toshio Hattori of Kumamoto University

References Matsushita1988 Skinner1988b, Skinner1988a, Reitz1988, Nara1990, D’Souza1991, Matsushita1992, Emini1992, Maeda1992,

McKeating1992a |Sperlagh1993, diMarzo Veronese1993, Moore1993c |Klasse1993a| Watkins1993 |Cook1994, Thali1994| Okada1994| Boudet1994,

Broder1994|Zvi1995b Zvi1995a, Jagodzinski1996, Warrier1996, McDougal1996, Jeffs1996, Huang1997, Zvi1997, Wyatt1997| Faiman1997.

Tugarinov1999, Fortin2000, Jagodzinski2000, Tugarinov2000, Zvi2000

- 0.5beta: Type-specific neutralization of IIIB - does not neutralize MN or RF [Matsushita1988, Skinner1988b]

- 0.5beta: Emergence of virus resistant to MAb 0.5beta and autologous sera neutralization in IIIB infected chimps [Nara1990]

- 0.5beta: Potent neutralizing activity [D'Souza1991]

- 0.5beta: Chimeric mouse-human MAb Cbeta1 was constructed by combining the human Cgamma1 and Ckappa constant regions with the 0.5beta murine

$\mathrm{MAb}-\mathrm{ADCC}$ and neutralizing activity[Matsushita1992]

- 0.5beta: sCD4 causes loss of IIIB type-specificity, allowing binding and neutralization of MN, in contrast to MAb mu5.5 [Maeda1992]

- 0.5beta: Monoclonal anti-idiotype antibodies that mimic the 0.5beta epitope were generated [Sperlagh1993]

- 0.5beta: Neutralization of virus carrying an A to T substitution (contrast with MAb M77) diMarzo Veronese1993

- 0.5beta: Binding to native gp120 100-300 fold greater than to denatured [Moore1993c]

- 0.5beta: The gp41 mutation 582(Ala to Thr) results in conformational changes in gp120 that confer neutralization resistance to some antiserum and conformationally sensitive neutralizing MAbs - neutralization efficiency of 0.5beta is not affected [Reitz1988 Klasse1993a]

- 0.5beta: A neutralization escape mutant (HXB2 A281V) was selected by growth of HXB2 in the presence of broadly neutralizing sera - of the MAbs tested , 0.5beta neutralization was the most profoundly affected by this mutation [Watkins1993]

- 0.5beta: MAbs against the glycosphingolipid GalCer block HIV infection of normally susceptible CD4 negative cells from the brain and colon - this MAb can inhibit gp120 binding to GalCer in vitro [Cook1994]

- 0.5beta: gp41 mutation that confers resistance to neutralization by anti-CD4 binding site antibodies does not reduce neutralizing efficiency of this $\mathrm{V} 3$ region MAb [Thali1994]

- 0.5beta: Binding domain aa 310-319: RGPGRAFVTIGKIG - mutations in the V3 loop from basic residues can destroy virus infectivity and syncytium

formation: R306T, R309T and R313G can also reduce binding of V3 MAbs with two different binding sites: 9284 and 0.5beta [Okada1994]

- 0.5beta: Type-specific neutralization of IIIB - does not neutralize SF2 [Broder1994]

- 0.5beta: The interactions of the peptide RKSIRIQRGPGRAFVT 0.5beta were studied by NMR, and hydrophobic interactions between the two Is and the V form the base of a 12 amino acid loop with GPGR at the apex[Zvi1995b]

- 0.5beta: NMR of 0.5beta bound NNTRKSIRIQRGPGRAFVTIGKIG suggests that the bound amino acids are in the region SIRIQRGPGRAFVT [Zvi1995a] 


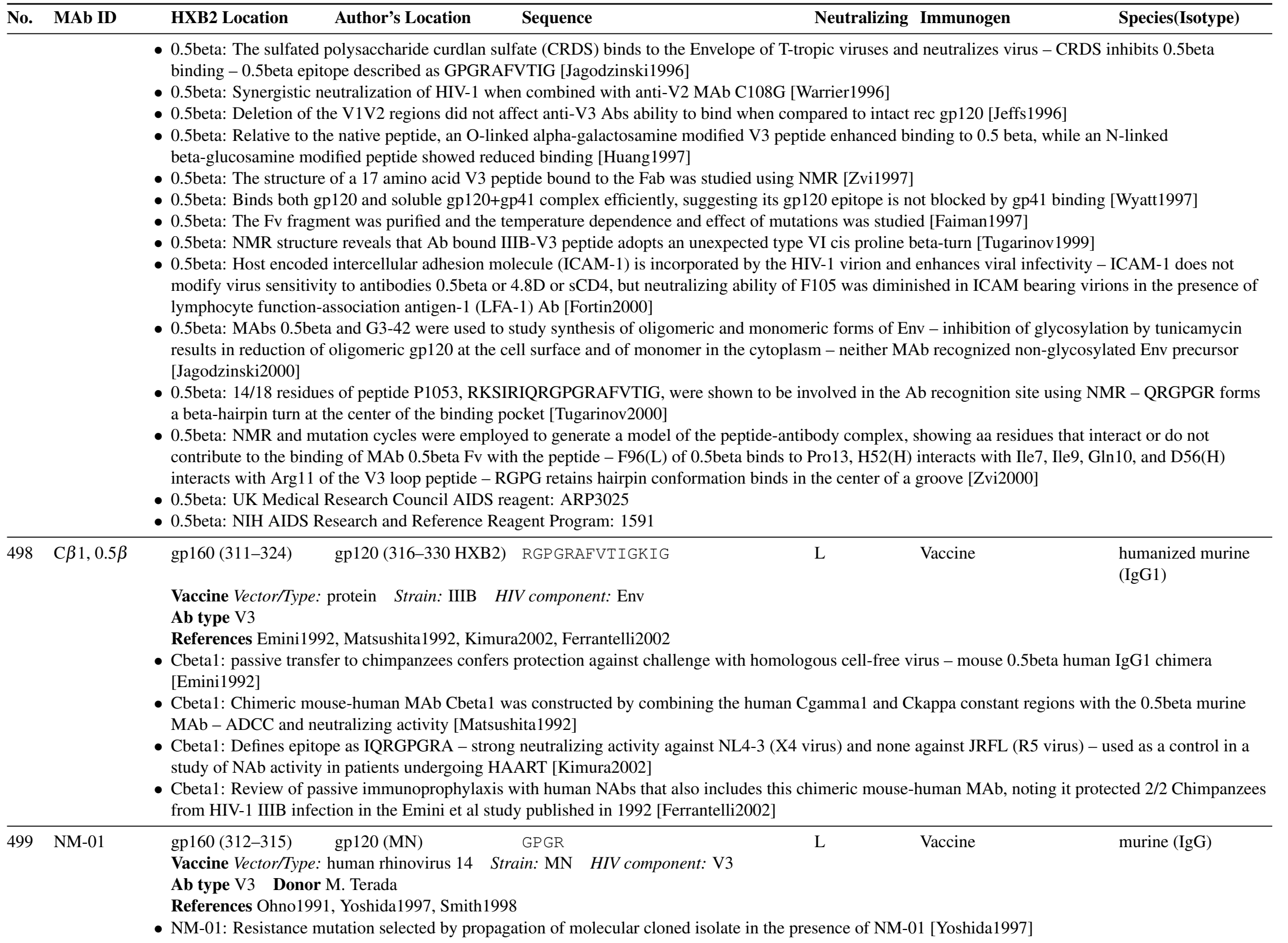




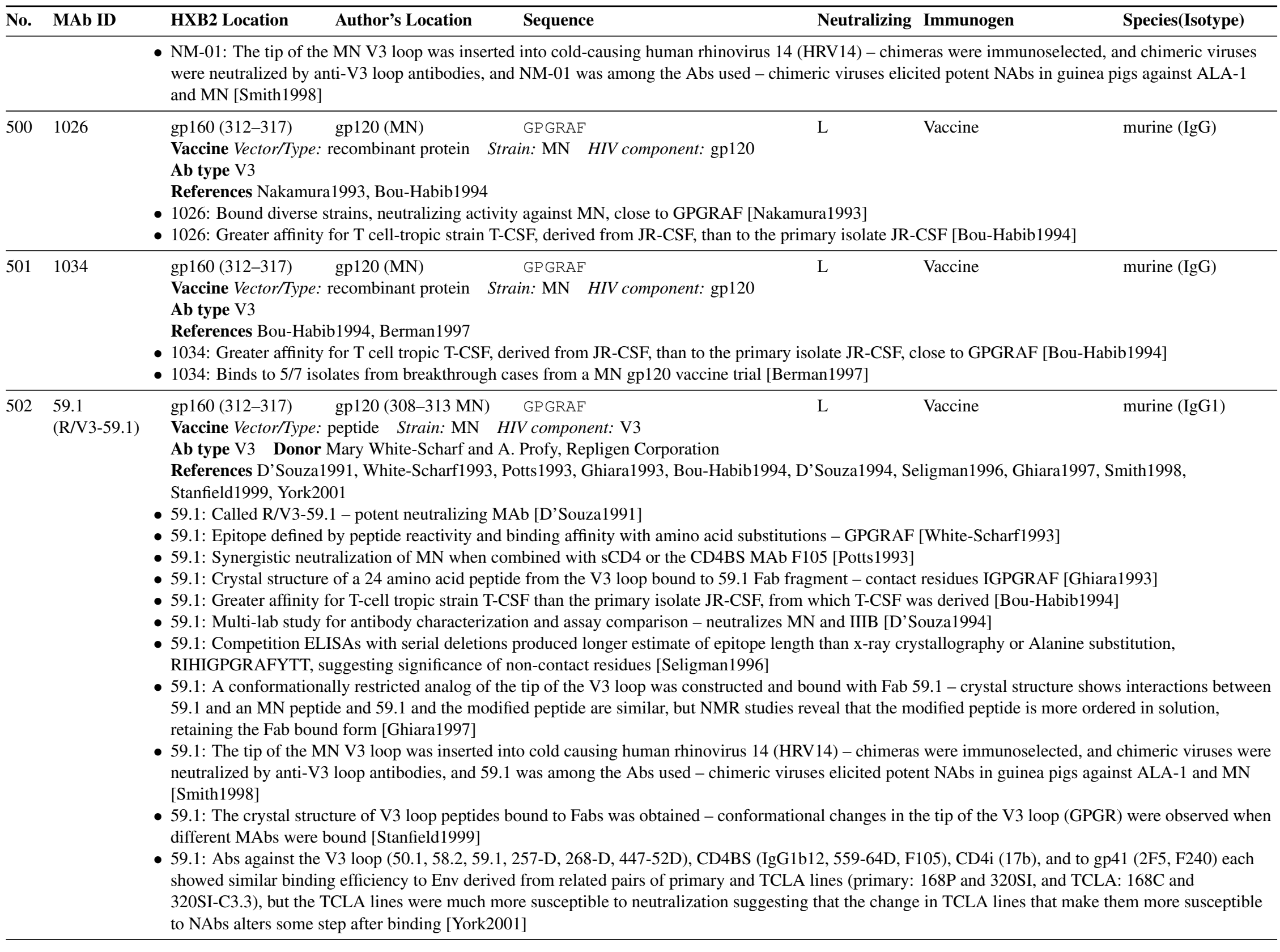




\begin{tabular}{|c|c|c|c|c|}
\hline No. & MAb ID & Author's Location & Immunogen & Species(Isotype) \\
\hline 503 & polyclonal & $\begin{array}{l}\text { gp160 (312-317) gp120 (316-321) } \quad \text { GPGRAF } \\
\text { Vaccine Vector/Type: polyepitope, protein } \quad \text { HIV component: gp160 Adjuvant: BSA } \\
\text { Ab type V3 } \\
\text { References Lu2000c, Lu2000b } \\
\text { High titer response to ELDKWA and RILAVERYLKD was observed upon vaccination with multiple-epit } \\
\text { CG-GPGRAFY-G-ELDKWA-G-RILAVERYLKD conjugated to BSA, a weak response to GPGRAFY - } \\
\text { CG-(ELDKWA-GPGRAFY)_2-K was also tried, yielding a strong Ab response to ELDKWA, weak to GF } \\
\text { response but not to any of the peptides studied here [Lu2000c, Lu2000b] }\end{array}$ & $\begin{array}{l}\text { Vaccine } \\
\text { ope vaccine } \\
\text { immunization w } \\
\text { PGRAF - gp160 }\end{array}$ & yielded strong $\mathrm{Ab}$ \\
\hline 504 & $10 \mathrm{E} 3$ & $\begin{array}{l}\text { gp160 (312-318) gp120 (317-323 IIIB) GPGRAFY } \\
\text { Vaccine Vector/Type: peptide keyhole limpet hemocyanin (KLH) conjugate Strain: IIIB HIV compone } \\
\text { Ab type V3 } \\
\text { ReferencesTian2001 } \\
\text { 10E3: Peptides GPGRAFY and ELDKWAG were conjugated to KLH and used to raise mouse monoclona } \\
\text { defined specificity - 10E3 binds to the peptide GPGRAFY and to rgp160 [Tian2001] }\end{array}$ & $\begin{array}{l}\text { Vaccine } \\
\text { ent: } \mathrm{V3} \\
\mathrm{al} \mathrm{Ab}-\mathrm{MAb} \text { hy }\end{array}$ & $\begin{array}{l}\text { murine (IgG) } \\
\text { ere generated with }\end{array}$ \\
\hline 505 & polyclonal & $\begin{array}{l}\text { gp160 (312-318) gp120 (317-323) GPGRAFY } \\
\text { Vaccine Vector/Type: peptide HIV component: V3 Adjuvant: BSA } \\
\text { Ab type V3 } \\
\text { References Yu2000 } \\
\text { High levels of epitope-specific Abs were induced by the peptide-BSA conjugates C-(GPGRAF)_4-BSA o } \\
\text { KI)-BSA but not by rgp160 vaccine [Yu2000] }\end{array}$ & Vaccine & $\begin{array}{l}\text { murine, rabbit } \\
\text { GPGRAFYTIG }\end{array}$ \\
\hline 506 & $\begin{array}{l}\text { N11-20 } \\
(110-\mathrm{H})\end{array}$ & $\begin{array}{l}\text { gp160 (312-320) gp120 }(317-325) \quad \text { GPGRAFVTI } \\
\text { Ab type V3 Donor J. C. Mazie, Hybridolab, Institut Pasteur } \\
\text { References Valenzuela1998 } \\
\text { N11-20: Neutralization of LAI in CEM cells by anti-V3 MAbs 110.4 and N11-20 is through inhibition of }\end{array}$ & ind & murine $(\operatorname{IgG1} \kappa)$ \\
\hline 507 & $5025 \mathrm{~A}(5025)$ & $\begin{array}{l}\text { gp160 (313-317) gp120 (313-317 BH10) pgRAF } \\
\text { Vaccine Vector/Type: peptide Strain: BH10 HIV component: V3 } \\
\text { Ab type V3 Donor Paul Durda, Du Pont de Nemours and Co } \\
\text { References Langedijk1991, D’Souza1991. } \\
\text { 5025A: Generation and fine mapping of murine MAbs [Langedijk1991] } \\
\text { 5025: Called 5025 - strain specific weakly neutralizing [D’Souza1991] }\end{array}$ & Vaccine & murine $(\operatorname{IgG})$ \\
\hline 508 & N70-1.9b & $\begin{array}{l}\text { gp160 (313-318) gp120 (316-322) PGRAFY } \\
\text { Ab type V3 } \\
\text { References Robinson1990a, Scott1990 } \\
\text { N70-1.9b: Type specificity Robinson1990a] } \\
\text { N70-1.9b: Type specific neutralization, ADCC directed against MN infected cells [Scott1990] }\end{array}$ & HIV-1 infectio & human (IgG1) \\
\hline 509 & 902 & $\begin{array}{llc}\text { gp160 (313-324) gp120 (IIIB) } & \text { PGRAFVTIGKIG } \\
\text { Vaccine Vector/Type: vaccinia Strain: IIIB } & \text { HIV component: gp160 } \\
\text { Ab type V3 } & \text { Donor Bruce Chesebro, Rocky Mountain National Laboratory, Montana }\end{array}$ & Vaccine & murine $(\operatorname{IgG} 1 \kappa)$ \\
\hline
\end{tabular}




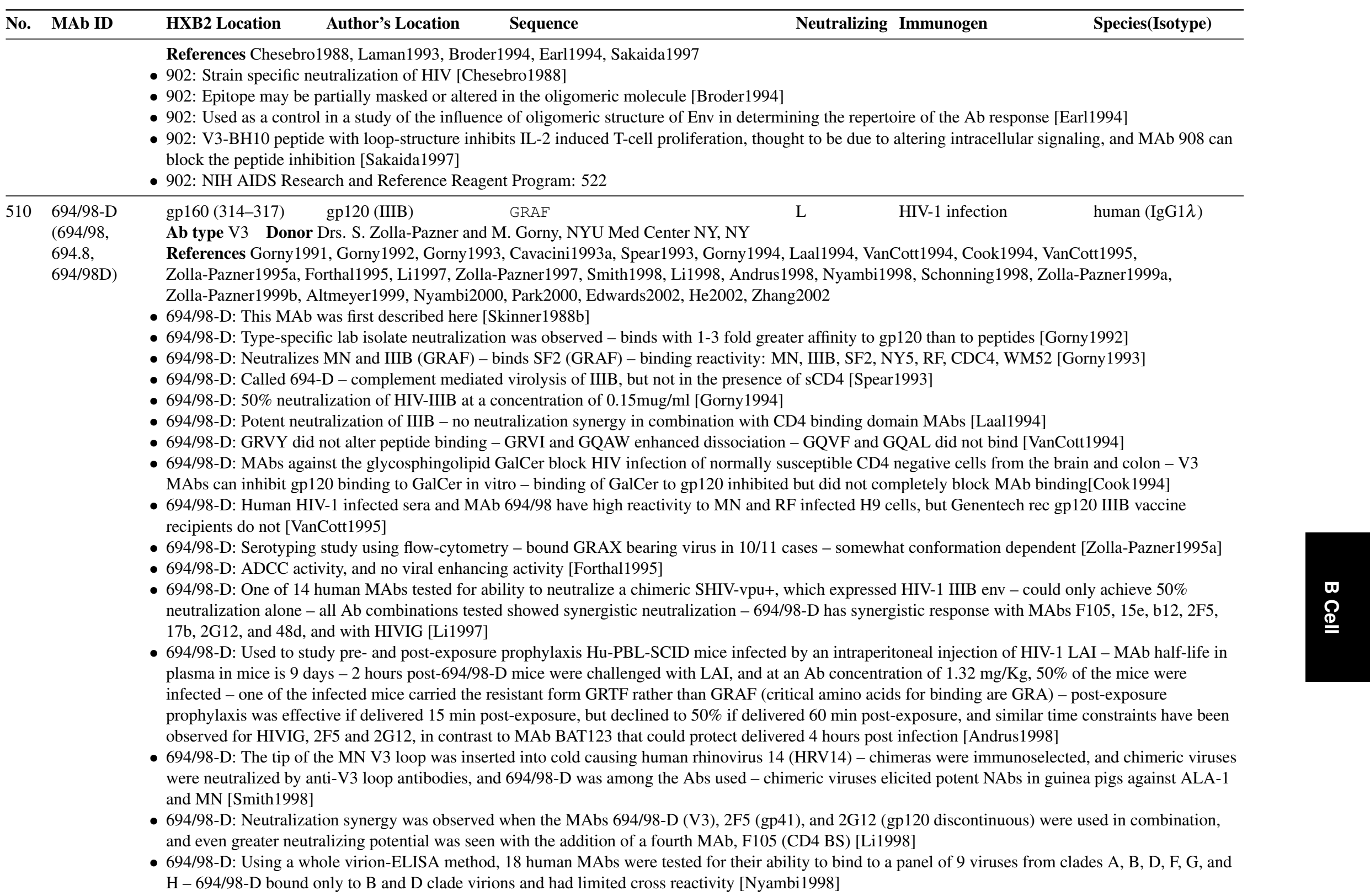




\begin{tabular}{lllll}
\hline No. MAb ID & HXB2 Location & Author's Location & Sequence & Neutralizing Immunogen
\end{tabular}

- 694/98-D: In a study of the influence of the glycan at position 306 of the V3 loop on MAb recognition, anti-V3 MAbs were found to neutralize an

HIV-BRU mutant virus that lacks the V3 loop glycan more efficiently than HIV-BRU [Schonning1998]

- 694/98-D: Review of clade specificity and anti-V3 HIV-1-Abs [Zolla-Pazner1999a]

- 694/98-D: MAb peptide reactivity pattern clustered with immunological related MAbs: 1108, 386, 268, 311, 257, 694.8 - the amino acids HI tended to be critical for reactivity in this group [Zolla-Pazner1999b]

- 694/98-D: A Semliki Forest virus (SFV) expression system carrying BX08 env was used to study the conformation of gp120 - intracytoplasmic gp120 was recognized by the anti-V3 MAbs K24 and F5.5, while gp120 at the plasma membrane was detected only by conformation dependent MAbs 2G12, 670-D and 694/98D and not linear V3 MAbs - expression in rat brain also showed that surface-expressed Env was recognized only by the conformation-dependent antibodies and not by anti-V3 antibodies [Altmeyer1999]

- 694/98-D: A panel of 47 human MAbs was tested against $26 \mathrm{HIV}-1$ group M primary isolates from clades A through $\mathrm{H}-19 \mathrm{~V} 3 \mathrm{MAbs}$ were tested, and of 494 combinations, $44 \%$ displayed some viral binding - V3 MAbs tended to have the most cross-reactive binding to clade A, B, C, and D isolates, less to E, F, G, and H - 694/98-D showed intermediate reactivity [Nyambi2000]

- 694/98-D: Called 694/98D - six mutations in MN change the virus from a high-infectivity neutralization resistant phenotype to low-infectivity neutralization sensitive - V3, CD4BS, and CD4i MAbs are 20-100 fold more efficient at neutralizing the sensitive form - the mutation L544P reduced binding of all MAbs against gp120 by causing conformational changes [Park2000]

- 694/98-D: Called 694/98D - Truncation of the gp41 cytoplasmic domain of X4, R5, and X4R5 viruses forces a conformation that more closely resembles the CD4 bound state of the external Envelope, enhancing binding of CD4i MAbs 17b and 48d and of CD4BS MAbs F105, b12, and in most cases of glycosylation site dependent MAb 2G12 and the anti-gp41 MAb 246D - in contrast, binding of the anti-V2 MAb 697D and the anti-V3 MAb 694/98D were not affected - viruses bearing the truncation were more sensitive to neutralization by MAbs 48d, b12, and 2G12 - the anti-C5 MAb 1331A was used to track levels of cell surface expression of the mutated proteins [Edwards2002]

- 694/98-D: Called 694 - Transgenic mice carrying human genes allowing production of fully human MAbs were used to rapidly create a panel of anti-HIV gp120 MAb producing hybridomas by immunization with HIV SF162 gp120 - the previously described human MAbs 5145A(CD4BS), 4117C (plus others, V3) and 697D (and SC258, V2) were used as controls [He2002]

511 MO101/V3,C4 gp160(314-323 + gp120 (dis 314-323) GRAFVTIGKI+LGVAPTKAKR in vitro stimulation $\quad$ human (IgM)
494-503)

Ab type V3-C4

References Ohlin1992

- MO101: Generated in response to IIIB Env 286-467 upon in vitro stimulation of uninfected-donor lymphocytes - reacts with peptides 314-323 + 494-503 from the V3 and C4 regions Ohlin1992]

512 MO101/V3,C4 gp160(314-323+ gp120(314-323)

GRAFVTIGKI+LGVAPTKAKR

in vitro stimulation

human $(\operatorname{IgM})$ 494-503)

Ab type V3-C5

References Ohlin1992

- MO101: Generated through in vitro stimulation of uninfected-donor lymphocytes with pB1 containing IIIB Env 286-467 - reacts with peptides from the V3 and C4 regions, positions 314-323 + 494-503, peptides GRAFVTIGKI + LGVAPTKAKR [Ohlin1992]

513 MO101/V3,C4 gp160(314-323 + gp120(494-503) GRAFVTIGKI+LGVAPTKAKR 494-503)

in vitro stimulation

human $(\operatorname{IgM})$

Ab type V3-C5

References Ohlin1992 


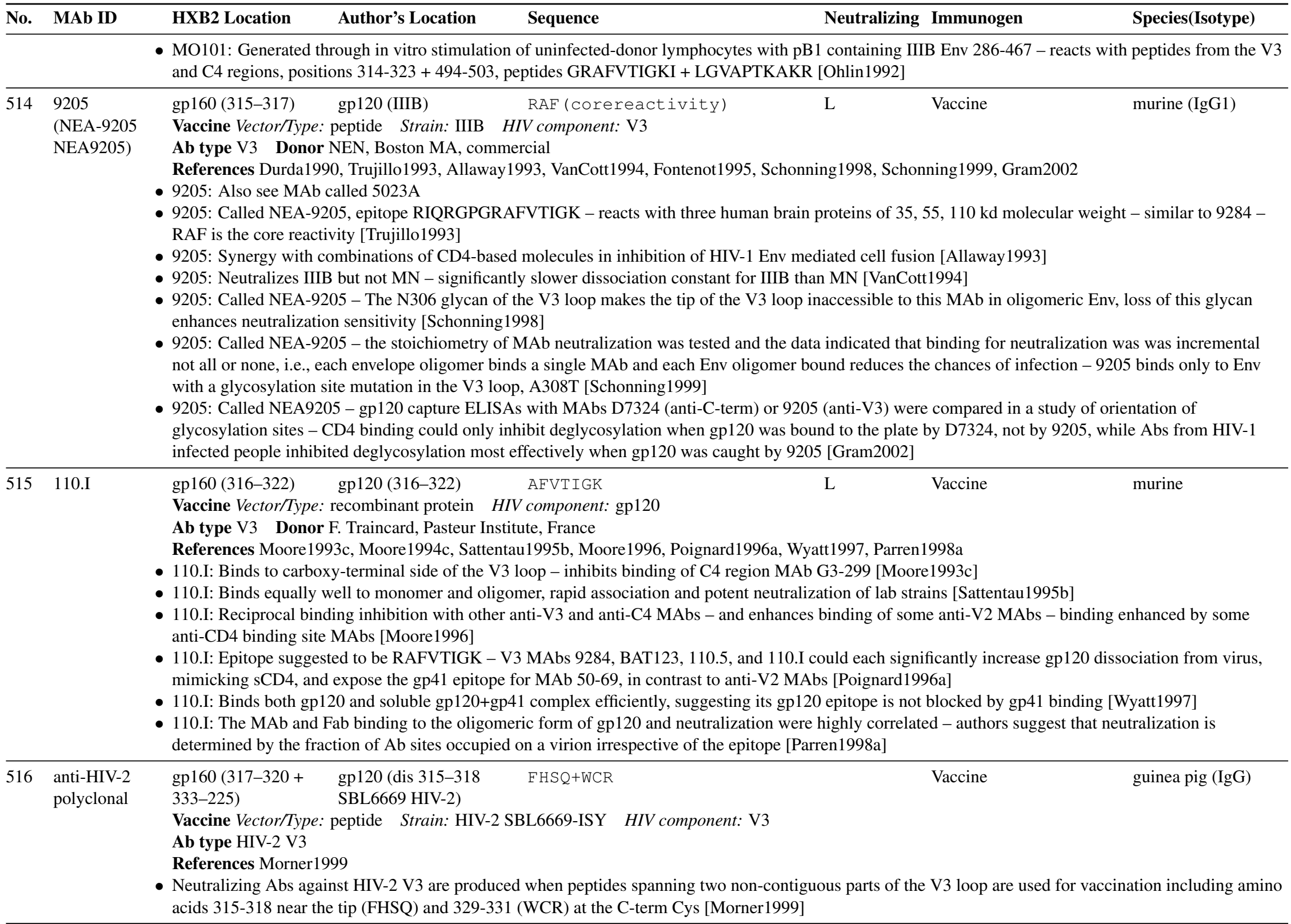




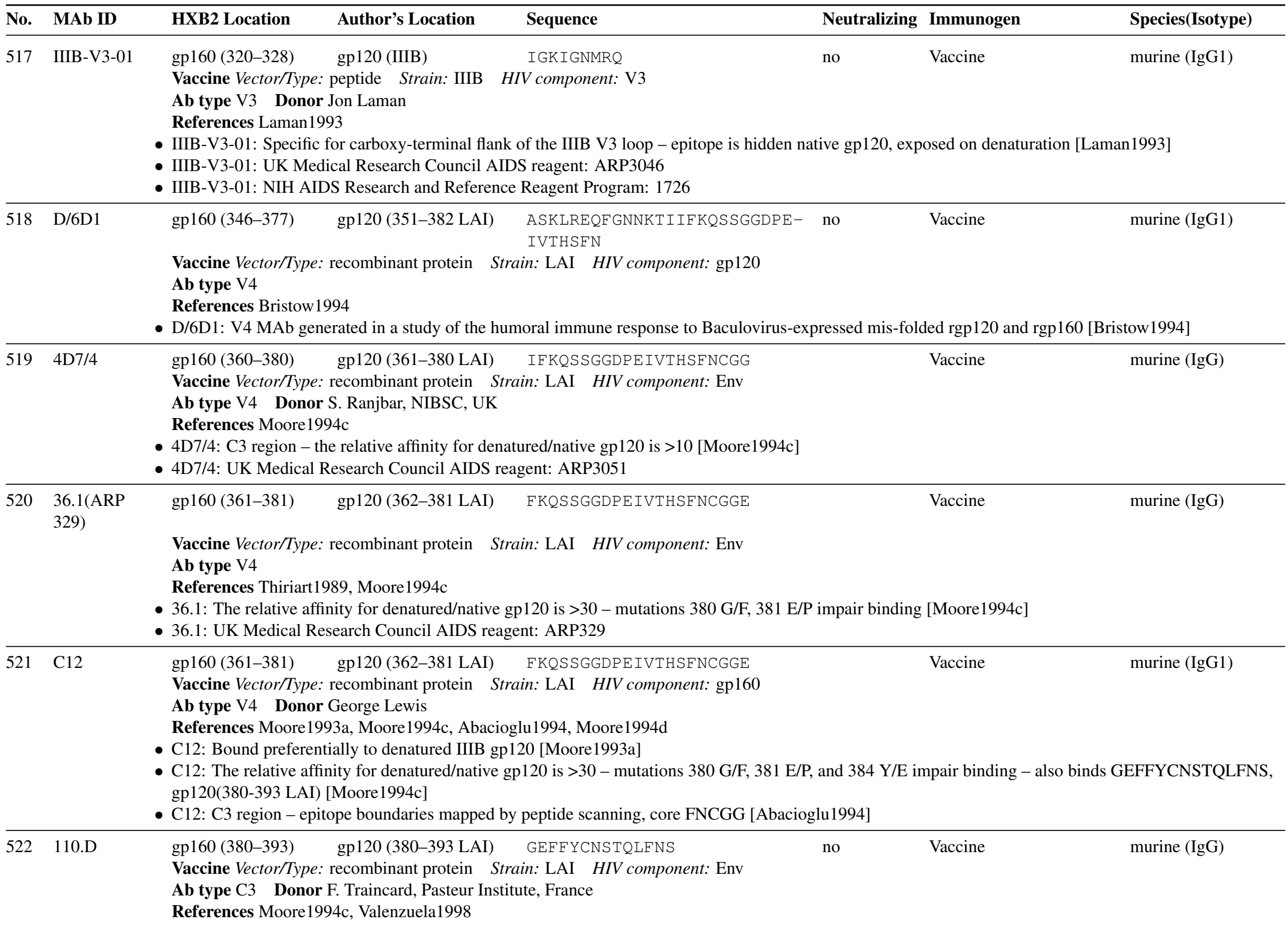




\begin{tabular}{|c|c|c|c|c|}
\hline No. & MAb ID & Author's Location & Immunogen & Species(Isotype) \\
\hline & & \multicolumn{3}{|l|}{ - 110.D: The relative affinity for denatured/native gp120 is $>50$ |Moore1994c } \\
\hline 523 & B32 & \multicolumn{3}{|c|}{$\begin{array}{l}\text { Vaccine Vector/Type: recombinant protein Strain: LAI HIV component: gp160 } \\
\text { Ab type C3 } \\
\text { References Moore1994c Abacioglu1994 } \\
\text { - B32: The relative affinity for denatured/native gp120 is >100 - mutations } 380 \text { G/F, } 381 \text { G/P, } 382 \text { F/L, } 384 \text { Y/E, and } 386 \text { N/R impair binding [Moore1994c] } \\
\text { - B32: C3 region - epitope boundaries mapped by peptide scanning - FFY(core) [Abacioglu1994] }\end{array}$} \\
\hline 524 & $\begin{array}{l}\text { polyclonal } \\
\text { (VEI4) }\end{array}$ & \multicolumn{3}{|c|}{$\begin{array}{l}\text { References Carlos1999 } \\
\text { postibody response to the epitopes in a vaccine construct (VEI) containing peptides from } 5 \text { hypervariable regions of gp120 was detected in the sera of HIV-1 } \\
\text { antibodies against all five VEI hypervariable regions, but most consistently against the V3 region peptide NNNTRKSIRIGPGRAFYTTGDIGNIRQ } \\
\text { Carlos1999 }\end{array}$} \\
\hline 525 & B15 & $\begin{array}{l}\text { gp160 (395-400) gp120 (395-400 BH10) WFNSTW } \\
\text { Vaccine Vector/Type: recombinant protein Strain: LAI HIV component: gp160 } \\
\text { Ab type V4 Donor George Lewis } \\
\text { References Moore1993a Moore1993c, Abacioglu1994 } \\
\text { B15: Bound preferentially to denatured IIIB gp120 [Moore1993a] } \\
\text { B15: Binds native BH10 gp120 with } 5 \text { fold less affinity than denatured - does not bind native or denatured } \\
\text { B15: V4 region - epitope boundaries mapped by peptide scanning [Abacioglu1994] }\end{array}$ & $\begin{array}{l}\text { Vaccine } \\
\text { MN gp120 Moore1993c }\end{array}$ & murine (IgG2b) \\
\hline 526 & B34 & $\begin{array}{l}\text { gp160 (395-400) gp120 (395-400 BH10) WFNSTW } \\
\text { Vaccine Vector/Type: recombinant protein Strain: LAI HIV component: gp160 } \\
\text { Ab type V4 } \\
\text { References Abacioglu1994 } \\
\text { B34: V4 region - epitope boundaries mapped by peptide scanning Abacioglu1994 }\end{array}$ & Vaccine & murine (IgG2b) \\
\hline 527 & $7 F 11$ & $\begin{array}{l}\text { gp160 (397-439) gp120 (IIIB) } \\
\text { Vaccine Vector/Type: protein HIV component: gp120 } \\
\text { References Lasky1987 Nilsen1996 } \\
\text { 7F11: There is another MAb with this name that binds to integrase [Nilsen1996] }\end{array}$ & Vaccine & murine \\
\hline 528 & $5 \mathrm{C} 2 \mathrm{E} 5$ & $\begin{array}{l}\text { gp160 (422-431) gp120 (406-415 IIIB) QF INMWQEVK } \\
\text { Vaccine Vector/Type: protein HIV component: gp120 } \\
\text { Ab type C4 Donor T. Gregory and R. Ward, Genentech, San Francisco } \\
\text { References Lasky1987] Cordell1991] } \\
\text { 5C2E5: Blocks the gp120-CD4 interaction [Lasky1987] } \\
\text { 5C2E5: Cross-competition with MAbs 5C2E5, ICR38.8f and ICR38.1a [Cordell1991] }\end{array}$ & Vaccine & murine \\
\hline 529 & G3-211 & $\begin{array}{lll}\text { gp160 (423-437) gp120 (423-437 IIIB) } & \text { I INMWQKVGKAMYAP } \\
\text { Vaccine Vector/Type: virus derived protein } & \text { Strain: IIIB } & \text { HIV component: gp120 }\end{array}$ & Vaccine & gG1) \\
\hline
\end{tabular}




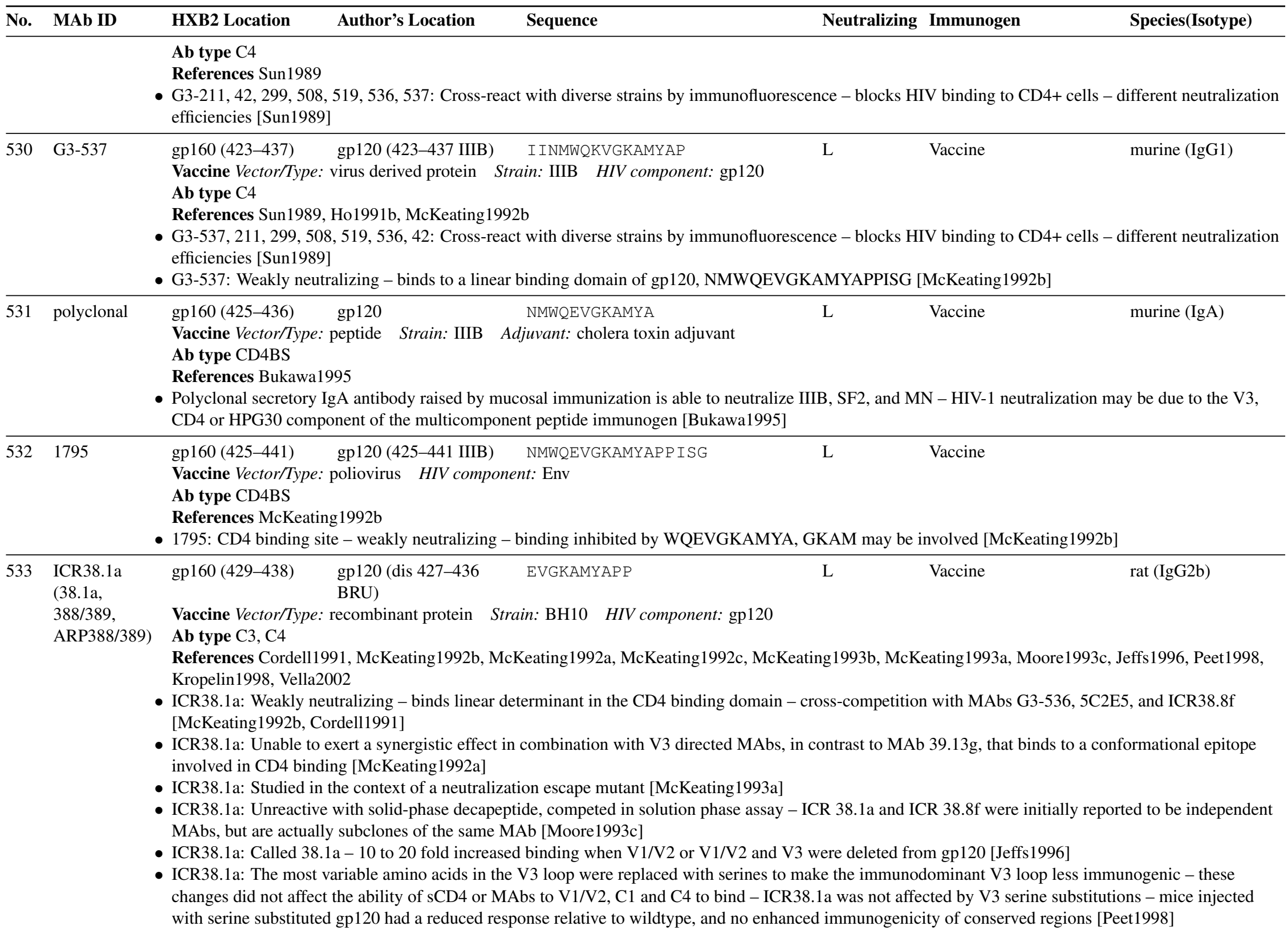




\begin{tabular}{|c|c|c|c|c|c|}
\hline No. & MAb ID & HXB2 Location & Author's Location & Neutralizing Immunogen & Species(Isotype) \\
\hline & & \multicolumn{4}{|c|}{$\begin{array}{l}\text { - ICR38.1a: Called 388/389 - anti-C1 region MAb 87-135/9 blocks gp120 interaction with CD4+ cells - blocking activity is additive when combined with } \\
\text { antibodies which bind in the C4 region of gp120 (F105, 388/389, and b12) |Kropelin1998] } \\
\text { - ICR38.1a: Called ARP388/ARP389: Herpesvirus saimiri-immortalized CD4+ T lymphocytes (HVS T cells) were used to isolate virus and perform HIV-1 } \\
\text { neutralization assays, and compared with a standard PBMC protocol - neutralization sensitivities to a panel of MAbs and to homologous or heterologous } \\
\text { plasma/sera were similar for HVS T cells (CN-2 cells) and PBMCs - lists epitope as WQEVGKAMYA [Vella2002] } \\
\text { - ICR38.1a: UK Medical Research Council AIDS reagent: ARP388/ARP389 }\end{array}$} \\
\hline 534 & G3-299 & $\begin{array}{l}\text { gp160 (429-438) } \\
\text { Vaccine Vector/Ty } \\
\text { Ab type C4 Dol } \\
\text { References Sun1 } \\
\text { - G3-299: Best neu } \\
\text { - G3-299: C4 regio } \\
\text { and G3-536- bou } \\
\text { impaired binding, } \\
\text { - G3-299: Binds wi } \\
\text { neutralization of } 1 \\
\text { - G3-299: Disconti } \\
\text { anti-V3 MAbs - C } \\
\text { - G3-299: Epitope } \\
\text { for MAb 50-69 |P } \\
\text { - G3-299: Binds bo } \\
\text { - G3-299: The MA } \\
\text { determined by the }\end{array}$ & $\begin{array}{l}\text { gp120 (429-438 BRU) } \\
\text { virus derived protein } \\
\text { M. Fung and Tanox Bios } \\
\text { Moore1993c. Sattentau1 } \\
\text { zation of IIIB in panel of } \\
\text { sinds HXB2 20mer KQII } \\
\text { ative gp120, not denatur } \\
\text { loop cleavage or insertio } \\
\text { igher affinity to monome } \\
\text { train [Sattentau1995b] } \\
\text { ls V3-C4 epitope, bindin } \\
29 \text { enhances the binding } \\
\text { ribed as KQIINMWQK } \\
\text { lard1996a] } \\
\text { p120 and soluble gp120- } \\
\text { d Fab binding to the olig } \\
\text { tion of Ab sites occupie }\end{array}$ & $\begin{array}{l}\text { EVGKAMYAPP } \\
\text { V component: gp120 } \\
\text { tems Inc and David Ho, ADARC, NY } \\
\text { 55b, Moore1996, Poignard1996a. Binley1997a, Ditzel1997, Wyatt1997 } \\
\text { MAbs that bind overlapping epitope [Sun1989] } \\
\text { MWQKVGKAMYAPPIS, and SF-2 and MN gp120s - G3-42, G3-299 } \\
\text { - poor peptide binding, epitope spans V3-C4 regions - 433A/L, 435Y/ } \\
\text { abolished binding [Moore1993c] } \\
\text { han to oligomer, slow association rate, although faster than other C4 M } \\
\text { enhanced by a few anti-C1, anti-CD4 binding site, and V2 MAbs - bind } \\
\text { some anti-V2 MAbs [Moore1996] } \\
\text { KAMYAPPIS - binding resulted in slight gp120 dissociation from viru } \\
\text { p41 complex efficiently, suggesting its gp120 epitope is not blocked by } \\
\text { neric form of gp120 and neutralization were highly correlated - authors } \\
\text { on a virion irrespective of the epitope [Parren1998a] }\end{array}$ & $\begin{array}{l}\text { murine (IgG1) } \\
\text { han G3-508, G3-519, } \\
\text { substitutions } \\
\text { h more potent } \\
\text { ly inhibited by } \\
\text { Wyatt1997) } \\
\text { eutralization is }\end{array}$ \\
\hline
\end{tabular}

535 G3-42 (G3 42) gp160 (429-438) gp120 (429-438 BRU) EVGKAMYAPP $\quad$ L $\quad$ Vaccine $\quad$ (IgG1) Vaccine Vector/Type: virus derived protein Strain: IIIB HIV component: gp120

Ab type C4 Donor Tanox Biosystems Inc and David Ho, ADARC, NY

References Sun1989, Moore1993c, Thali1993, Sattentau1995b, Jagodzinski1996 Moore1996, Poignard1996a, Trkola1996a, Binley 1997a, Binley1999. Jagodzinski2000

- G3-42: Neutralization of IIIB but not RF [Sun1989]

- G3-42: C4 region - binds HXB2 20mer KQIINMWQKVGKAMYAPPIS, and SF-2 and MN gp120s - G3-42, G3-299 have lower affinity than G3-508, G3-519, and G3-536 - bound native gp120, not denatured - poor peptide binding, epitope spans V3-C4 regions - 433A/L, 435Y/H and 430V/S substitutions impaired binding, V3 loop insertion abolished binding |Moore1993c

- G3-42: Inhibits binding of CD4 inducible MAb 48d [Thali1993]

- G3-42: Binds with higher affinity to monomer than to oligomer, slow association rate [Sattentau1995b]

- G3-42: The sulfated polysaccharide curdlan sulfate (CRDS) binds to the Envelope of T-tropic viruses and neutralizes virus - CRDS potently inhibits G3-42 binding - G3-42 epitope described as KVGKAMYAPP [Jagodzinski1996]

- G3-42: Inhibits binding of many anti-V3, -CD4 binding site, and -C4 region MAbs - enhances binding of some anti-V2 region MAbs [Moore1996]

- G3-42: Epitope described as KQIINMWQKVGKAMYAPPIS - binding resulted in slight gp120 dissociation from virus and exposure of the gp41 epitope for MAb 50-69 [Poignard1996a]

- G3-42: Called G3 42 - Does not inhibit gp120 interaction with CCR-5 in a MIP-1beta-CCR-5 competition study - described as V3-C4 discontinuous epitope [Trkola1996a] 


No. MAb ID HXB2 Location $\quad$ Author's Location $\quad$ Sequence $\quad$ Neutralizing Immunogen

- G3-42: The MAbs with the broadest neutralizing activity, IgG1b12, 2G12 and 2F5, all have high affinity for the native trimer, indicating that they were raised in an immune response to the oligomer on the virion surface rather than dissociated subunits - a disulfide linked gp120-gp41 (SOS gp140) was created to mimic the native conformation of Env and explore its potential as an immunogen - SOS gp140 is recognized by NAbs IgG1b12, 2G12, and CD4-IgG2, and also by anti-V3 MAbs 19b and 83.1 - SOSgp140 is not recognized by C4 region MAbs that neutralize only TCLA strains, G3-42 and G3-519 - nor did it bind C11, 23A, and M90, MAbs that bind to gp120 C1 and C5, where it interacts with gp41 - MAbs that bind CD4 inducible epitopes, $17 \mathrm{~b}$ and A32 were very strongly induced by CD4 in SOS gp140 - anti-gp41 MAbs that bind in the region that interacts with gp120, 7B2, 2.2B, T4, T15G1 and 4D4, did not bind to SOSgp140, in contrast to 2F5, which binds to the only gp41 epitope that is well exposed in native gp120-gp41 complexes Binley1999

- 0.5beta: MAbs 0.5beta and G3-42 were used to study synthesis of oligomeric and monomeric forms of Env - inhibition of glycosylation by tunicamycin results in reduction of oligomeric gp120 at the cell surface and of monomer in the cytoplasm - neither MAb recognized non-glycosylated Env precursor |Jagodzinski2000|

$536 \quad \mathrm{G} 3-508(\mathrm{G} 3-\mathrm{gp} 160(429-438)$ gp120 (429-438 BRU) EVGKAMYAPP $\mathrm{L}$

Vaccine murine $(\operatorname{IgG} 1)$

508) Vaccine Vector/Type: virus derived protein Strain: IIIB HIV component: gp120

Ab type C4 Donor M. Fung and Tanox Biosystems Inc and David Ho, ADARC, NY

References Sun1989, Thali1993, Moore1993c |Sattentau1995b, Moore1996| Poignard1996a| Trkola1996a, Binley1997a, Parren1998a, Binley1998

- G3-508: Neutralization of IIIB and RF [Sun1989]

- G3-508: Inhibits binding of CD4 inducible MAb 48d [Thali1993]

- G3-508: C4 region - binds HXB2 20mer KQIINMWQKVGKAMYAPPIS, and SF-2 and MN gp120s - bound denatured with 10 fold greater affinity than native $-433 \mathrm{~A} / \mathrm{L}, 435 \mathrm{Y} / \mathrm{H}$ and 430V/S substitutions impaired binding [Moore1993c]

- G3-508: Binds with higher affinity to monomer than to oligomer, slow association rate [Sattentau1995b]

- G3-508: Inhibits binding of some V3, C4 and CD4 binding site MAbs, enhances binding of V2 region MAbs [Moore1996]

- G3-508: Binding resulted in slight gp120 dissociation from virus and exposure of the gp41 epitope for MAb 50-69 |Poignard1996a|

- G3-508: Called G3 508 - inhibits gp120 interaction with CCR-5 in a MIP-1beta-CCR-5 competition study [Trkola1996a]

- G3-508: The MAb and Fab binding to the oligomeric form of gp120 and neutralization were highly correlated - authors suggest that neutralization is

determined by the fraction of Ab sites occupied on a virion irrespective of the epitope [Parren1998a

- G3-508: A panel of MAbs were shown to bind with similar or greater affinity and similar competition profiles to a deglycosylated or variable loop deleted core gp120 protein ( Delta V1, V2, and V3), thus such a core protein produces a structure closely approximating full length folded monomer [Binley1998] gp160 (429-438) gp120 (429-438 BRU) EVGKAMYAPP

Vaccine Vector/Type: virus derived protein Strain: IIIB HIV component: gp120

Ab type C4 Donor Tanox Biosystems Inc and David Ho, ADARC, NY

References

Sun1989, Moore1993a Moore1993c, D’Souza1994, Sattentau1995b, Moore1996, Poignard1996a, Binley1997a, Wyatt1997, Parren1998a, Binley1999

- G3-519: Best neutralization of RF in panel of 7 MAbs that bind overlapping epitope [Sun1989]

- G3-519: Neutralizes IIIB, is reactive with SF-2 gp120, mild inhibition of HIV-1+ sera binding to IIIB gp120 [Moore1993a

- G3-519: C4 region - binds HXB2 20mer KQIINMWQKVGKAMYAPPIS, and SF-2 and MN gp120s - bound denatured with 5 fold greater affinity than native - 433A/L, 435Y/H, 438P/R and 430V/S substitutions impaired binding [Moore1993c]

- G3-519: Included in a multi-lab study for antibody characterization, and binding and neutralization assay comparison, also binds IIIB: IINMWQKVGKAMYAPP [D’Souza1994]

- G3-519: Binds with higher affinity to monomer than to oligomer, slow association rate [Sattentau1995b]

- G3-519: Non-reciprocal enhanced binding in the presence of the C5 MAb 1C1 and the C1 MAb 135/9 - reciprocal enhanced binding with some V2 MAbs Inhibited binding the presence of some C4, V3 and CD4 binding site MAbs [Moore1996] 


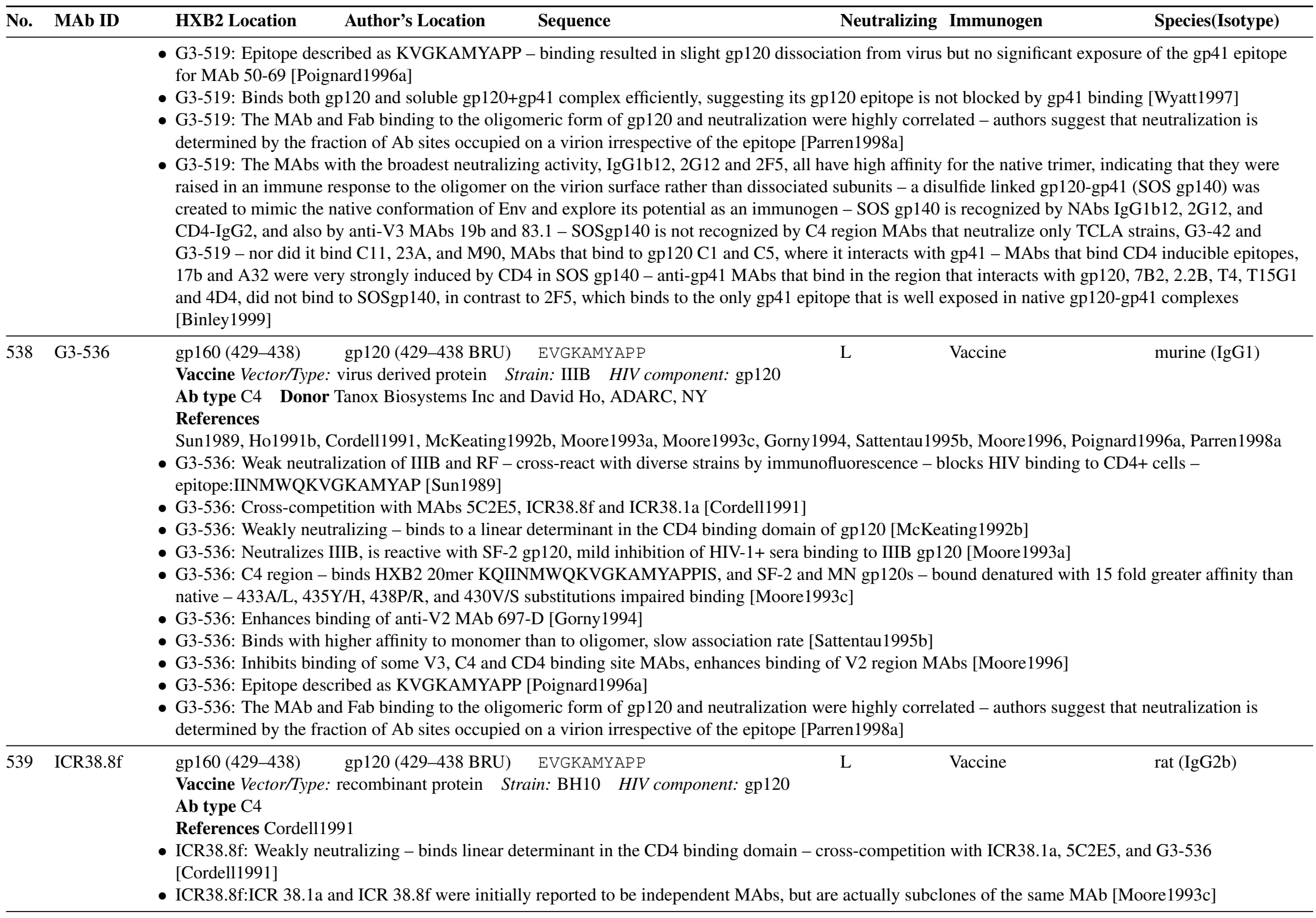
gp160 (429-443) gp120 (429-443) EVGKAMYAPP I SGQ in vitro stimulation human $(\operatorname{IgM})$ Ab type C4 


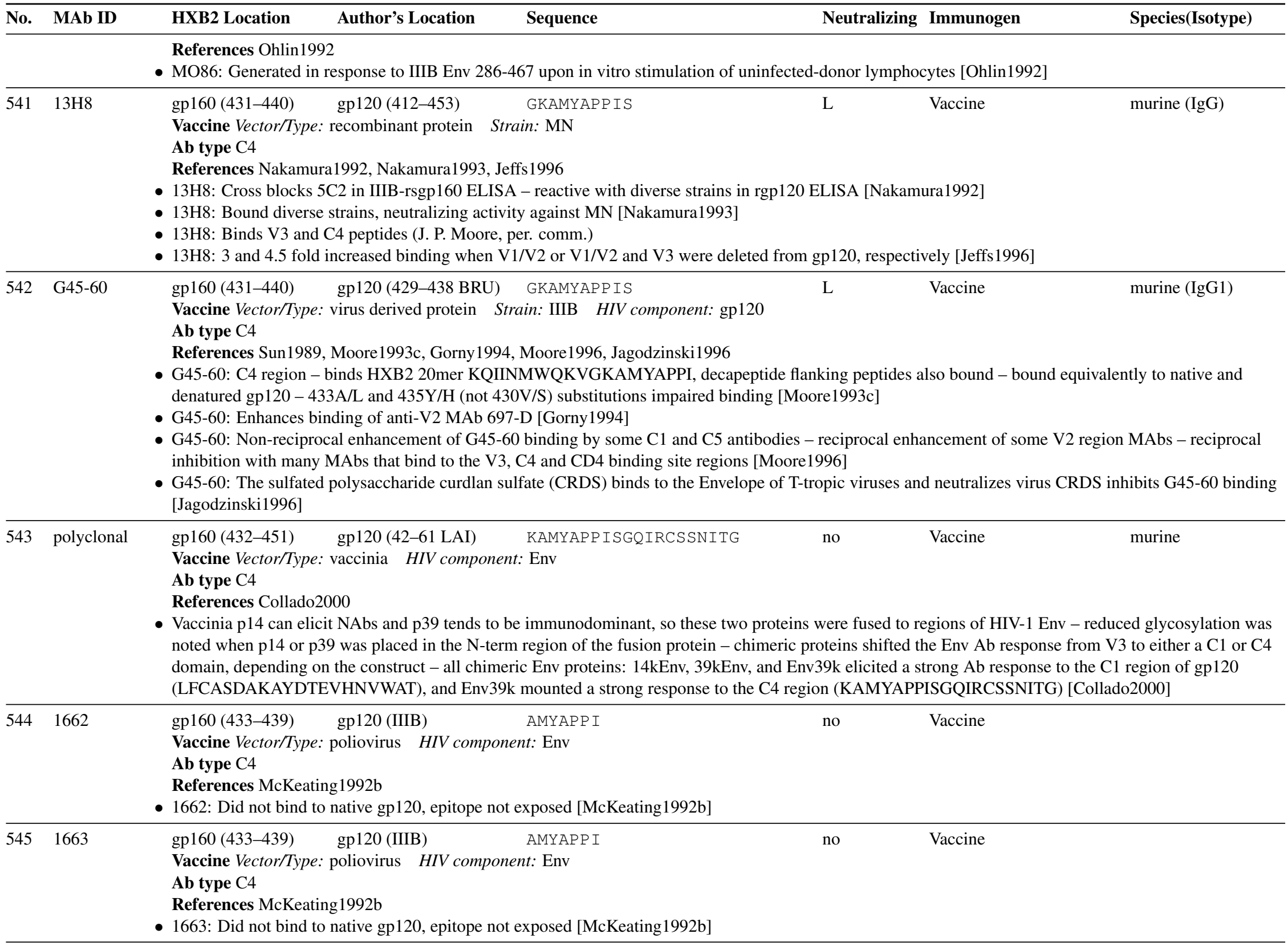




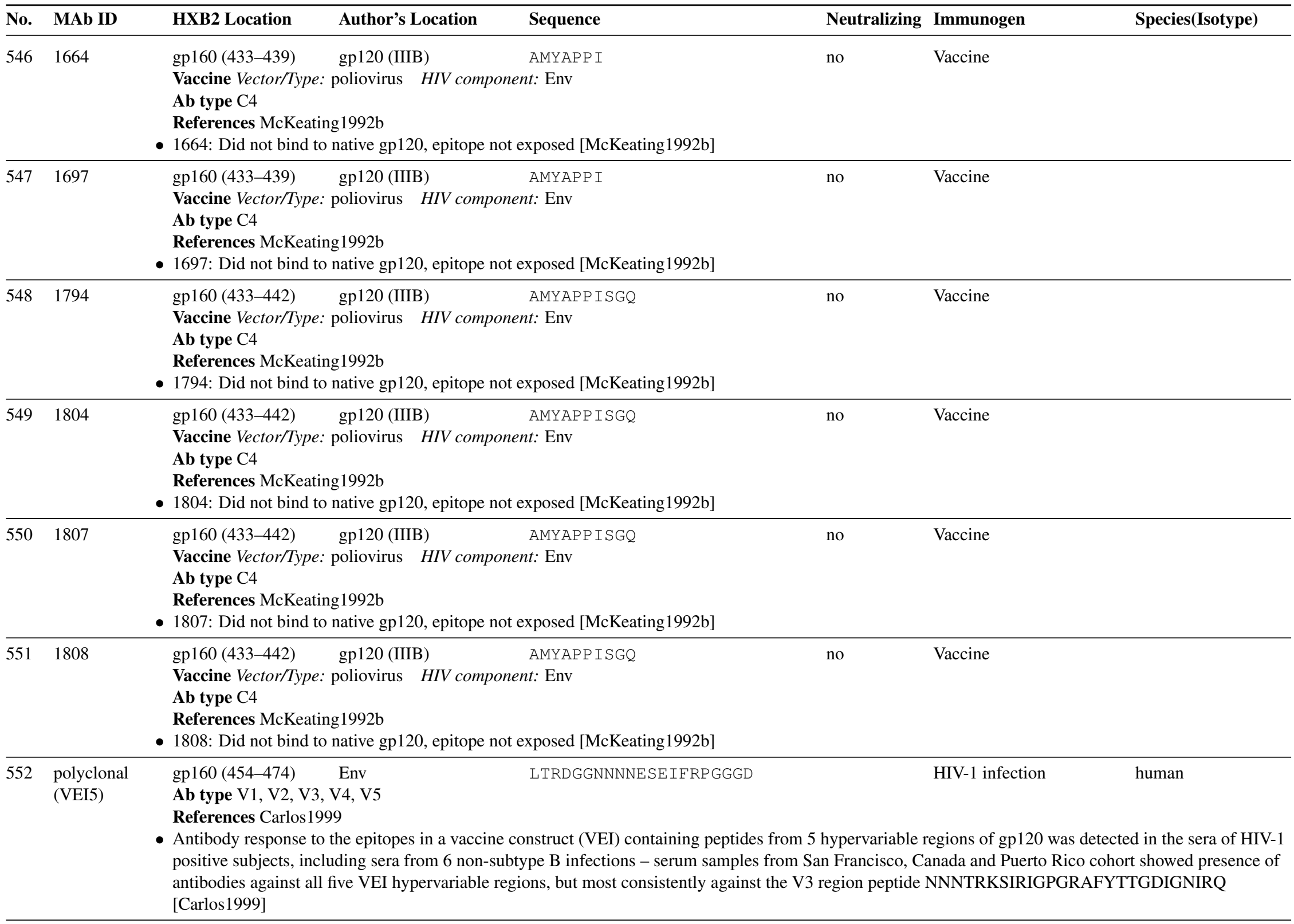




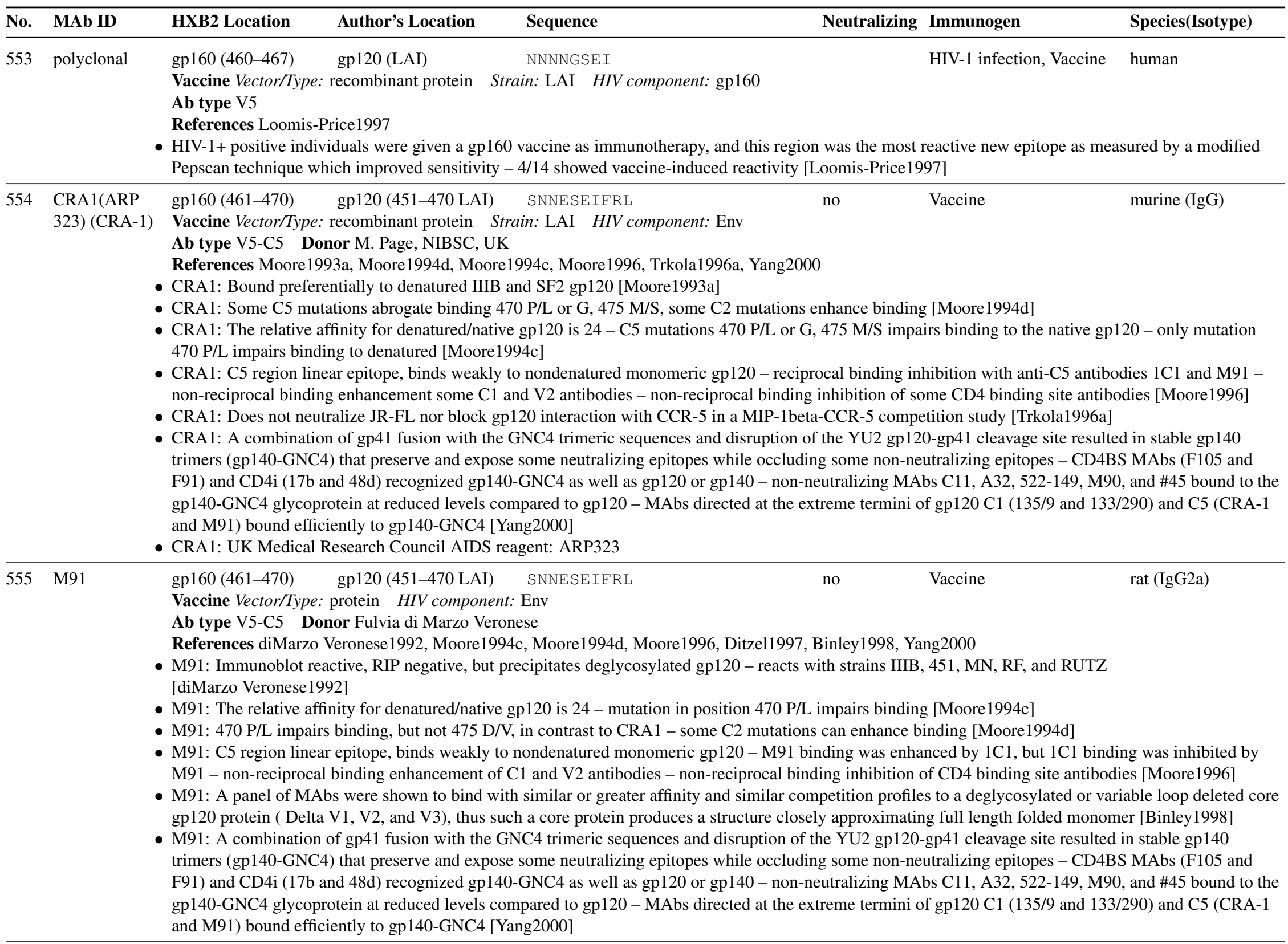




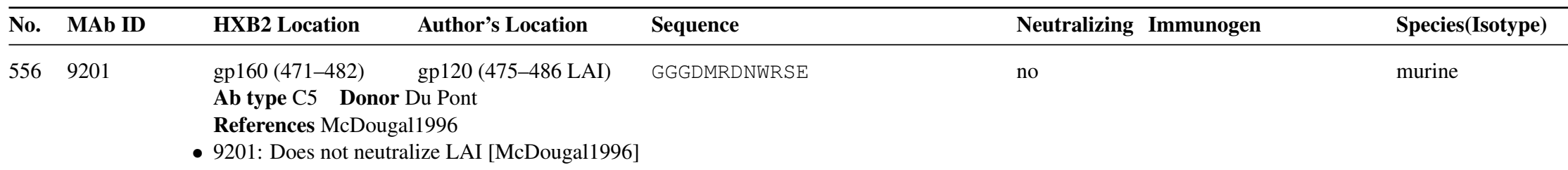

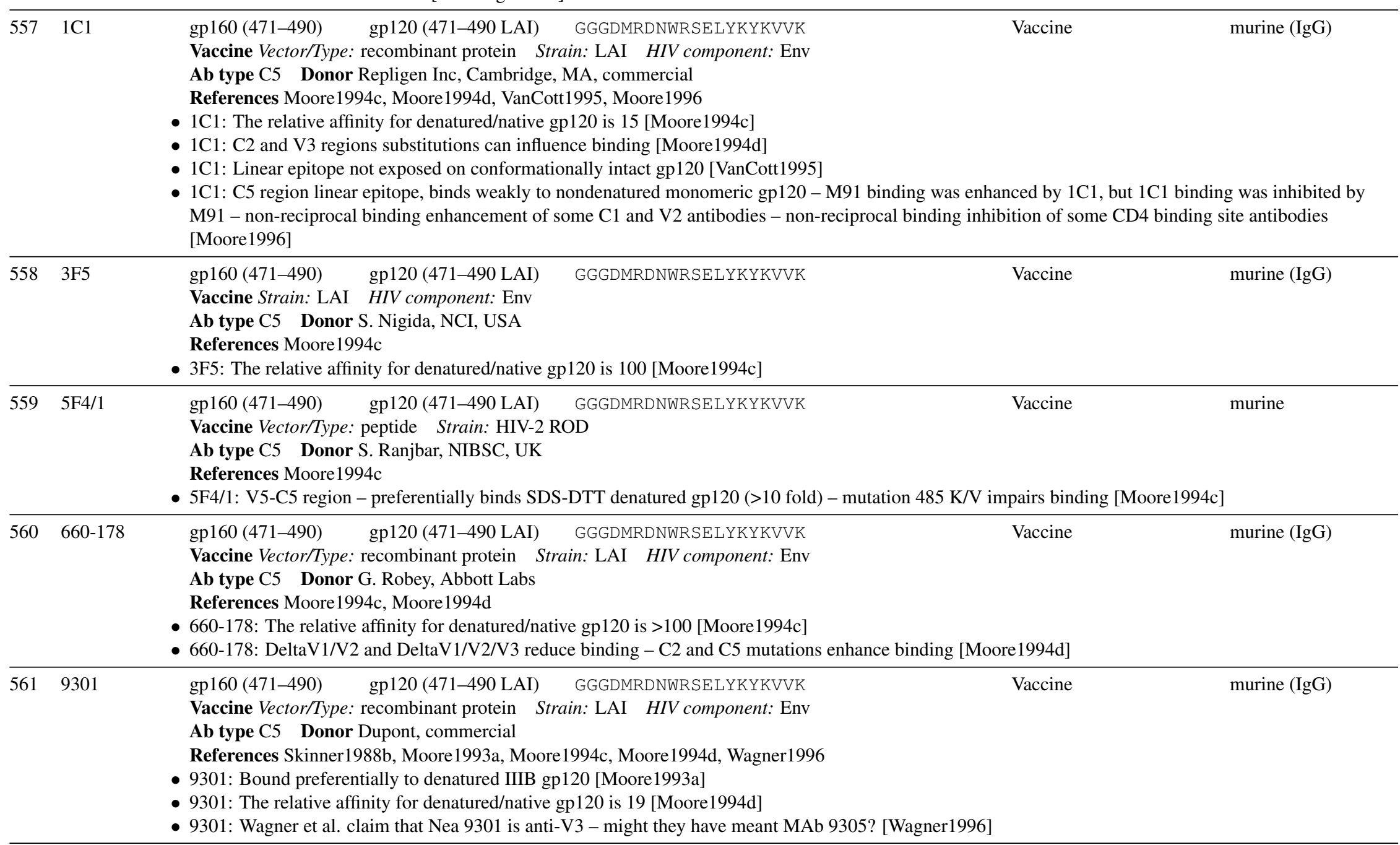




\begin{tabular}{|c|c|c|c|c|c|}
\hline No. & MAb ID & Author's Location & Neutralizing & Immunogen & Species(Isotype) \\
\hline 562 & B221 (221) & $\begin{array}{l}\text { gp160 (471-490) gp120 (471-490 LAI) } \\
\text { Vaccine Vector/Type: recombinant protein Strc } \\
\text { Ab type C5 Donor Rod Daniels } \\
\text { References Moore1993a, Bristow1994, Moore1 } \\
\text { B221: Called } 221 \text { - bound preferentially to dena } \\
\text { B221: MAb generated in a study of the humoral } \\
\text { B221: The relative affinity for denatured/native } \\
\text { B221: Called 221 - C2 and V3 substitutions infl } \\
\text { B221: UK Medical Research Council AIDS reas }\end{array}$ & $\begin{array}{l}\text { GGGDMRDNWRSELYKYKVVK } \\
\text { in: NL43 HIV component: gp160 } \\
994 \mathrm{c} \\
\text { tured IIIB gp120 [Moore1993a] } \\
\text { immune response to Baculovirus-expressed mis-folded rg } \\
\text { sp120 is } 12 \text { - mutation } 477 \text { D/V impairs binding [Moore1 } \\
\text { uence binding [Moore1994d] } \\
\text { sent: ARP301 }\end{array}$ & Vaccine & $\begin{array}{l}\text { murine }(\operatorname{IgG} 1 \kappa) \\
\text { Sys } \text { Bristow1994 }\end{array}$ \\
\hline 563 & $8 \mathrm{C} 6 / 1$ & $\begin{array}{l}\text { gp160 (471-490) gp120 (471-490 LAI) } \\
\text { Vaccine Strain: LAI } \\
\text { Ab type V5-C5 Donor S. Ranjbar, NIBSC, Ul } \\
\text { References Moore1994c } \\
\text { 8C6/1: V5-C5 region - preferentially binds SDS } \\
\text { 8C6/1: UK Medical Research Council AIDS rea }\end{array}$ & $\begin{array}{l}\text { GGGDMRDNWRSELYKYKVVK } \\
\text {-DTT denatured gp120 (>30 fold })- \text { mutation } 485 \mathrm{~K} / \mathrm{V} \text { im } \\
\text { gent: ARP3052 }\end{array}$ & $\begin{array}{l}\text { Vaccine } \\
\text { apairs binding }\end{array}$ & murine $(\operatorname{IgG})$ \\
\hline 564 & H11 & $\begin{array}{l}\text { gp160 (472-477) gp120 (472-477 HXB2) } \\
\text { Ab type C5 } \\
\text { References Pincus1993a, Pincus1996 } \\
\text { H11: Binds to gp120 but not to infected cells - v } \\
\text { [Pincus1993a Pincus1996] }\end{array}$ & $\begin{array}{l}\text { GGDMRD } \\
\text { vhen linked to ricin A, the immunotoxin did not mediate } \mathrm{c}\end{array}$ & cell killing - sc & murine \\
\hline 565 & W2 & $\begin{array}{l}\text { gp160 (472-491) gp120 (472-491 LAI) } \\
\text { Vaccine Strain: LAI HIV component: Env } \\
\text { Ab type C5 Donor D. Weiner, U. Penn., USA } \\
\text { References Moore1994c } \\
\text { W2: The relative affinity for denatured/native gp }\end{array}$ & $\begin{array}{l}\text { GGDMRDNWRSELYKYKVVKI } \\
120 \text { is } 30 \text { - mutation } 485 \mathrm{~K} / \mathrm{V} \text { impairs binding } \text { Moore199 }\end{array}$ & Vaccine & murine $(\operatorname{IgG})$ \\
\hline 566 & M38 & $\begin{array}{l}\text { gp160 (485-504) gp120 (490-508) } \\
\text { Vaccine Vector/Type: virus Strain: IIIB HIV } \\
\text { Ab type C5 } \\
\text { References Beretta1987, Grassi1991, Lopalco1s } \\
\text { M38: Binds to gp120 and to a } 80 \mathrm{kd} \text { human prot } \\
\text { M38: Binds to the carboxy terminus of gp120, ir } \\
\text { M38: Infected individuals have HLA class I-gp1 } \\
\text { M38: This epitope is similar to a fragment of the } \\
\text { fragment of IFN-related IFRD2 (PC4-B) protein }\end{array}$ & $\begin{array}{l}\text { KYKVVKEIPLGVAPTKAKRR no } \\
\text { component: virus } \\
\text { 93. DeSantis1994, Beretta1994, Maksiutov2002 } \\
\text { ein expressed on a small fraction of mononuclear cells in } \\
\text { a gp41 binding region, and also to denatured human HL } \\
20 \text { cross-reactive antibodies [DeSantis1994] } \\
\text { human protein mast/stem cell growth factor receptor prec } \\
\text { ARTKARSRVRDKRA [Maksiutov2002]. }\end{array}$ & $\begin{array}{l}\text { Vaccine } \\
\text { the lymph nod } \\
\text { As (antigenic } 1 \\
\text { cursor, VVPTI }\end{array}$ & $\begin{array}{l}\text { murine } \\
\text { ap well as to a }\end{array}$ \\
\hline 567 & Chim $1(\mathrm{C}-1)$ & gp120 (492-498 HXB2) & KVVKEIP & & $\begin{array}{l}\text { humanized } \\
\text { chimpanzee }\end{array}$ \\
\hline
\end{tabular}




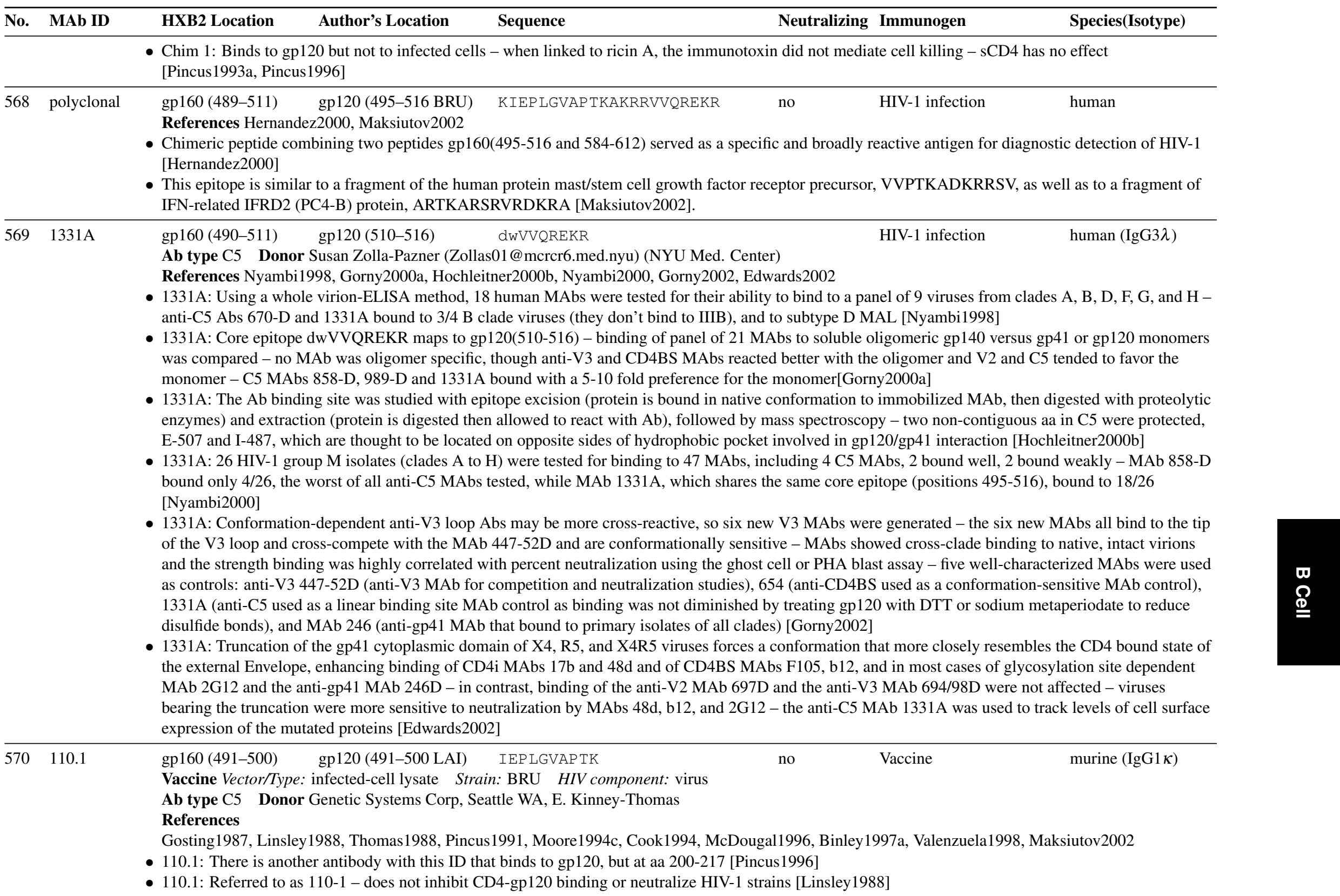




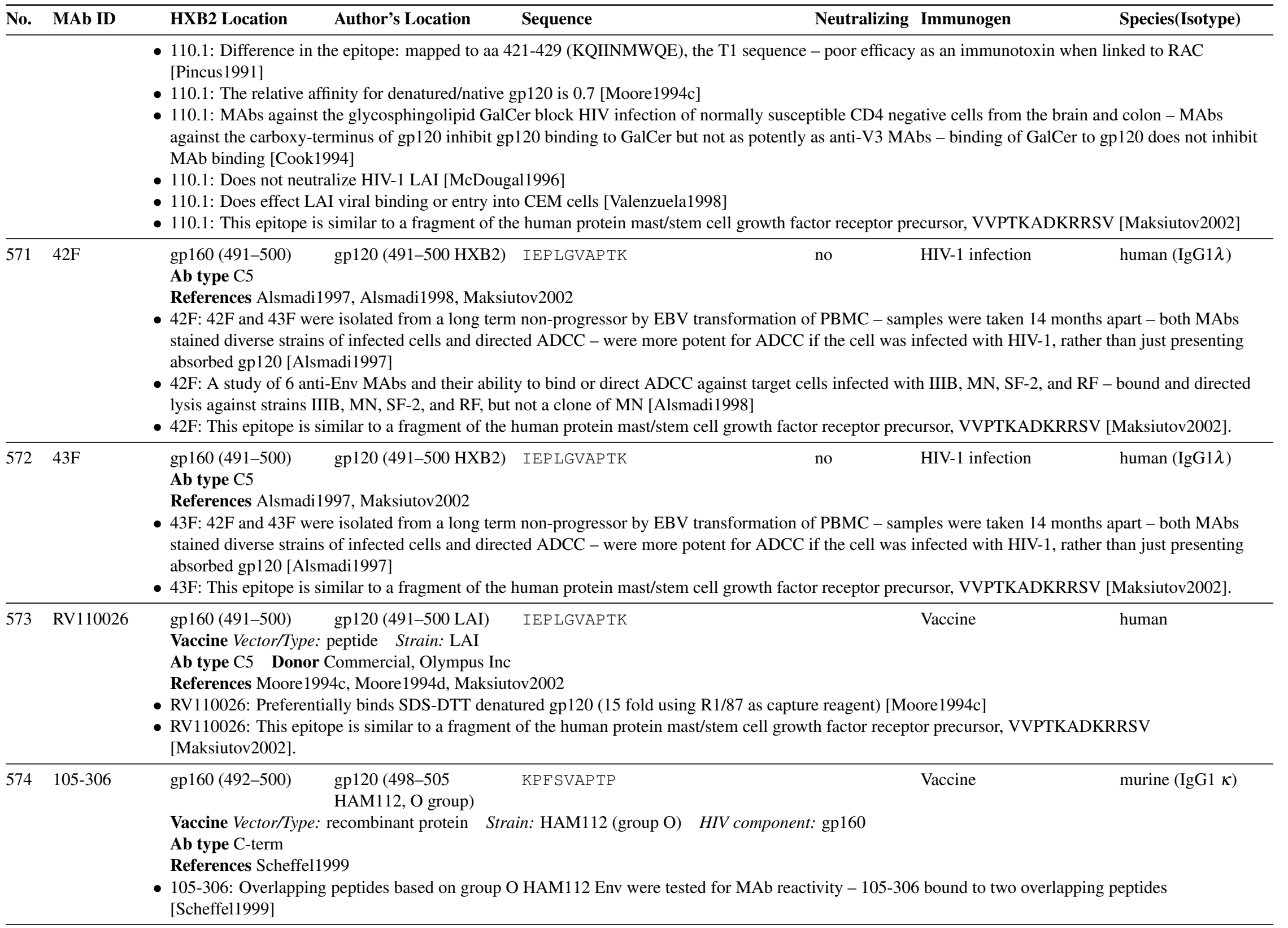




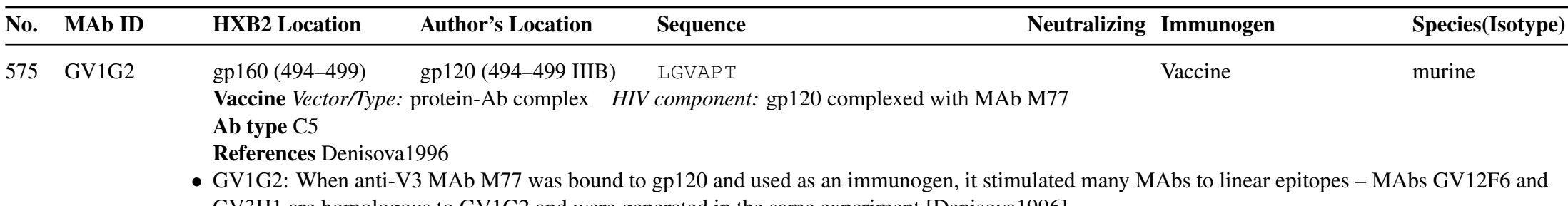

GV3H1 are homologous to GV1G2 and were generated in the same experiment [Denisova1996]

\begin{tabular}{|c|c|c|c|c|c|}
\hline 576 & $750-\mathrm{D}$ & $\begin{array}{l}\text { gp160 (498-504) gp120 (503-509) } \\
\text { Ab type C-term } \\
\text { References Forthal1995, Hioe2000 } \\
\text { - 750-D: Not neutralizing, positive ADCC activity } \\
\text { - 750-D: Ab responses, because of their capacity t } \\
\text { from HIV+ individuals inhibited proliferative res }\end{array}$ & $\begin{array}{l}\text { PTKAKRR } \\
\text { and no viral enhancing activity [Forthal1995] } \\
\text { alter antigen uptake and processing, can influ } \\
\text { ponses of gp120 specific T cells - C5 MAbs } 4 .\end{array}$ & $\begin{array}{l}\text { HIV-1 infection } \\
\text { r T cell responses } \\
\text { 750-D did not effe }\end{array}$ & $\begin{array}{l}\text { human }(\operatorname{IgG} 3 \lambda) \\
\text { MAbs or serum Ig } \\
\text { ation [Hioe2000] }\end{array}$ \\
\hline 577 & $\begin{array}{l}450-\mathrm{D} \\
(450-\mathrm{D}-3 \\
450 \mathrm{D})\end{array}$ & gp120 (475-486 BH10) & $\begin{array}{l}\text { PTKAKRR (orRRVVQRE, orMRDNW- no } \\
\text { RSELYKYdependingonreferen- } \\
\text { ce) }\end{array}$ & HIV-1 infection & human $(\operatorname{IgG} 1 \lambda)$ \\
\hline
\end{tabular}

Ab type C5 Donor Susan Zolla-Pazner (Zollas01 @mcrcr6.med.nyu), NYU Med Center, NY, NY

References Durda1988, Karwowska1992a, Karwowska1992b, Spear1993, Laal1994, Gorny1994, Cook1994, Forthal1995, Manca1995a, Li1997,

Hioe1997b Hioe2000, Hioe2001, Verrier2001

- 450-D: Bound to MN, SF-2 and IIIB, but was not neutralizing |Karwowska1992a

- 450-D: Did not mediate deposition of complement component C3 on HIV infected cells [Spear1993]

- 450-D: Not neutralizing alone, could synergize anti-CD4 binding site antibody neutralization [Laal1994]

- 450-D: Epitope is defined as PTKAKRR [Gorny1994]

- 450-D: MAbs against the glycosphingolipid GalCer block HIV infection of normally susceptible CD4 negative cells from the brain and colon - MAbs

against the carboxy-terminus of gp120 do not inhibit gp120 binding to GalCer - binding of GalCer to gp120 does not inhibit MAb binding [Cook1994]

- 450-D: No neutralizing activity, no ADCC activity, and no viral enhancing activity [Forthal1995]

- 450-D: Virions complexed to gp120 Ab facilitate presentation of p66 RT epitopes to Th cells [Manca1995a

- 450-D: One of 14 human MAbs tested for ability to neutralize a chimeric SHIV-vpu+, which expressed HIV-1 IIIB env - 50\% neutralization could not be achieved at a maximal concentration of $6 \mathrm{mug} / \mathrm{ml}$ [Li1997]

- 450-D: Four primary isolates showed distinct patterns of sensitivity to neutralization by polyclonal sera or plasma and MAbs - BZ167 was the only isolate inhibited by all polyclonal sera and plasma tested, and was also neutralized by 8/17 MAbs, in particular anti-V3 loop (419-D, 447-52D, 782-D, and 838-D), anti-CD4bd (559/64-D, 654-D and 830-D and a cluster II of gp41 directed MAb (98-6) - isolates 92HT593 and 91 US056 were neutralized by V3 loop (419-D, and 447-52D)and cluster II gp41 (98-6) MAbs at higher concentrations - US4 was neutralized by some of the polyclonal sera/plasma tested and not at all by MAbs individually or by a cocktail of ten MAbs consisting of 419-D, 447-52D, 782-D, 838-D, 559/64-D, 654-D, 450-D, 670-D, 1281-D and 98-6 Hioe1997b

- 450-D: Ab responses, because of their capacity to alter antigen uptake and processing, can influence helper T cell responses - CD4BS MAbs or serum Ig from HIV+ individuals inhibited proliferative responses of gp120 specific T cells - C5 MAbs 450-D and 750-D did not effect proliferation [Hioe2000]

- 450-D: CD4BS MAbs when complexed with gp120, inhibit proliferation of gp120-specific CD4 T-cells and IFN gamma production - 450-D does not have this effect and was used as a control in this study [Hioe2001] 


\begin{tabular}{|c|c|c|c|c|}
\hline No. MAb ID & HXB2 Location & Author's Location & Sequence & Neutralizing Immunogen \\
\hline
\end{tabular}

- 450-D: A panel of 12 MAbs was used to identify those that could neutralize the dual-tropic primary isolate HIV-1 89.6 - six gave significant neutralization at 2 to $10 \mathrm{ug} / \mathrm{ml}$ : 2F5, 50-69, IgG1b12, 447-52D, 2G12, and 670-D six did not have neutralizing activity: 654-D, 4.8D, 450-D, 246-D, 98-6, and 1281 - no synergy, only additive effects were seen for pairwise combinations of MAbs, and antagonism was noted between gp41 MAbs 50-69 and 98-6, as well as 98-6 and 2F5 [Verrier2001]

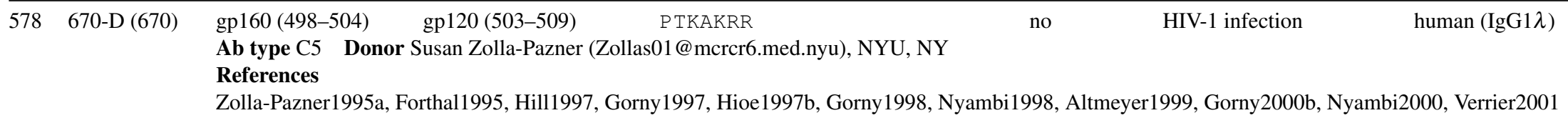

Zolla-Pazner1995a Fortha11995, Hill1997, Gorny1997, Hioe1997b, Gorny1998, Nyambi1998, Altmeyer1999, Gorny2000b Nyambi2000 Verrier2001

- 670-D: Group specific cross-clade binding in serotyping study using flow-cytometry [Zolla-Pazner1995a]

- 670-D: Not neutralizing, positive ADCC activity, and no viral enhancing activity, numbering provided suggests epitope is RRVVQRE [Forthal1995]

- 670-D: gp120 can inhibit MIP-1alpha from binding to CCR5, but this inhibitory effect is blocked by pre-incubation of gp120 with three anti-V3 MAbs: 447, 257, 1027 - MAb 670 which binds in the C5 region had no effect [Hill1997]

- 670-D: Four primary isolates showed distinct patterns of sensitivity to neutralization by polyclonal sera or plasma and MAbs - BZ167 was the only isolate inhibited by all polyclonal sera and plasma tested, and was also neutralized by 8/17 MAbs, in particular anti-V3 loop (419-D, 447-52D, 782-D, and 838-D), anti-CD4bd (559/64-D, 654-D and 830-D and a cluster II of gp41 directed MAb (98-6) - isolates 92HT593 and 91 US056 were neutralized by V3 loop (419-D, and 447-52D)and cluster II gp41 (98-6) MAbs at higher concentrations - US4 was neutralized by some of the polyclonal sera/plasma tested and not at all by MAbs individually or by a cocktail of ten MAbs consisting of 419-D, 447-52D, 782-D, 838-D, 559/64-D, 654-D, 450-D, 670-D, 1281-D and 98-6 Hioe1997b

- 670-D: Using a whole virion-ELISA method, 18 human MAbs were tested for their ability to bind to a panel of 9 viruses from clades A, B, D, F, G, and H anti-C5 Abs 670-D and 1331A bound to 3/4 B clade viruses (they didn't bind to IIIB), and to subtype D MAL - 670-D also reacted with subtype A[Nyambi1998]

- 670-D: A Semliki Forest virus (SFV) expression system carrying BX08 env was used to study the conformation of gp120 - intracytoplasmic gp120 was recognized by the anti-V3 MAbs K24 and F5.5, while gp120 at the plasma membrane was detected only by conformation dependent MAbs 2G12, 670-D and 694/98D and not V3 MAbs - expression in rat brain also showed that surface-expressed Env was recognized only by the conformation-dependent antibodies and not by anti-V3 antibodies [Altmeyer1999]

- 670-D: A gp120 C5 MAb used as a negative control in a study of anti-gp41 MAbs [Gorny2000b]

- 670-D: 26 HIV-1 group M isolates (clades A to H) were tested for binding to 47 MAbs, including 4 C5 MAbs, 2 bound well, 2 bound weakly - MAb 670-D bound 21/26, and was the most cross-reactive C5 MAb [Nyambi2000]

- 670-D: A panel of 12 MAbs was used to identify those that could neutralize the dual-tropic primary isolate HIV-1 89.6 - six gave significant neutralization at 2 to $10 \mathrm{ug} / \mathrm{ml}$ : 2F5, 50-69, IgG1b12, 447-52D, 2G12, and 670-D six did not have neutralizing activity: 654-D, 4.8D, 450-D, 246-D, 98-6, and 1281 - no synergy, only additive effects were seen for pairwise combinations of MAbs, and antagonism was noted between gp41 MAbs 50-69 and 98-6, as well as 98-6 and 2F5 [Verrier2001]

Vaccine Vector/Type: peptide HIV component: gp120

References Jeyarajah1998

- Mice were immunized with peptide APTKAKRRVVQREKR - epitope excision and extraction combined with mass spectrometry was used to map the fine structure of epitopes recognized by polyclonal Ab to HIV-1 Env - a major epitope was identified between positions 472 and 478 [Jeyarajah1998] 


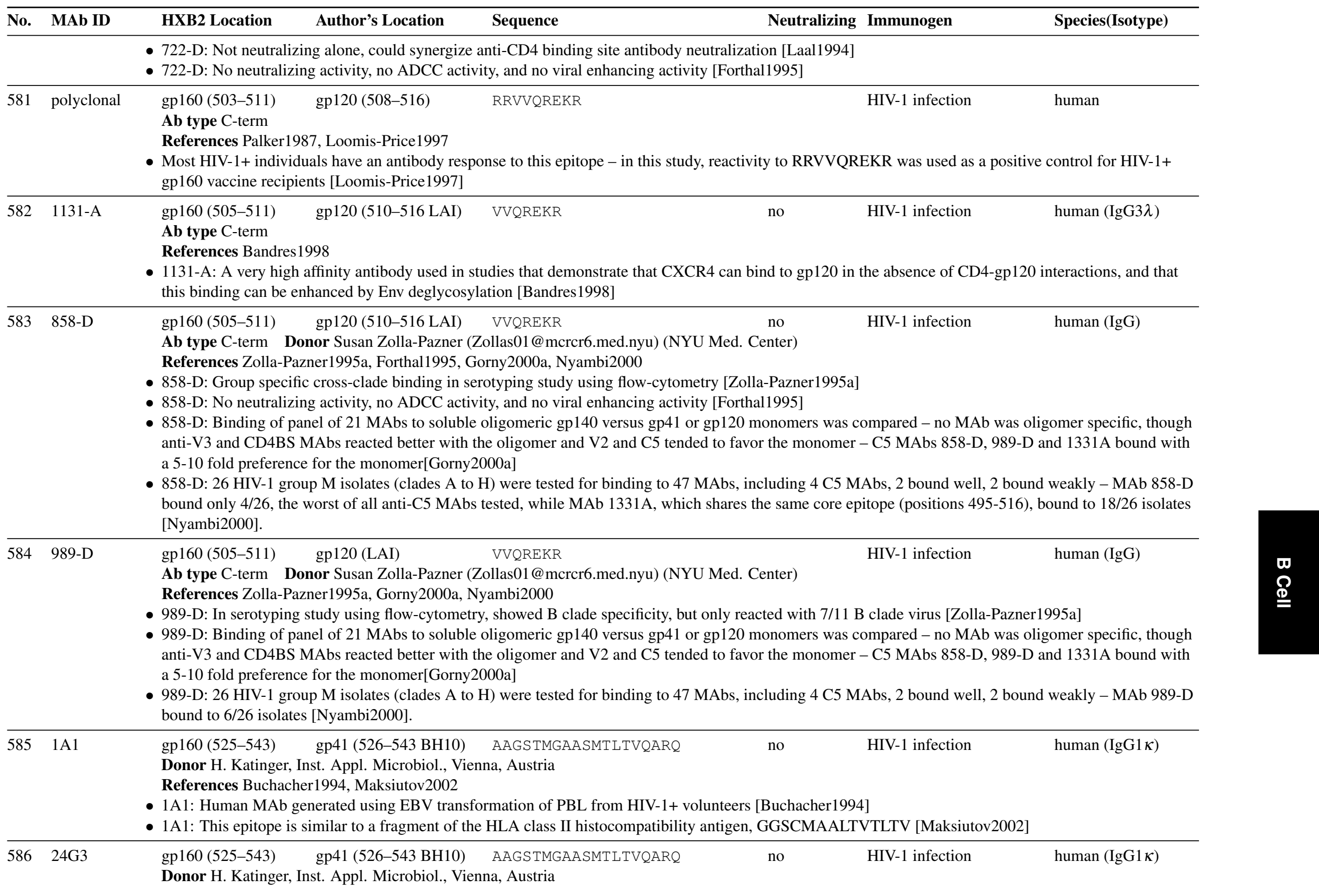




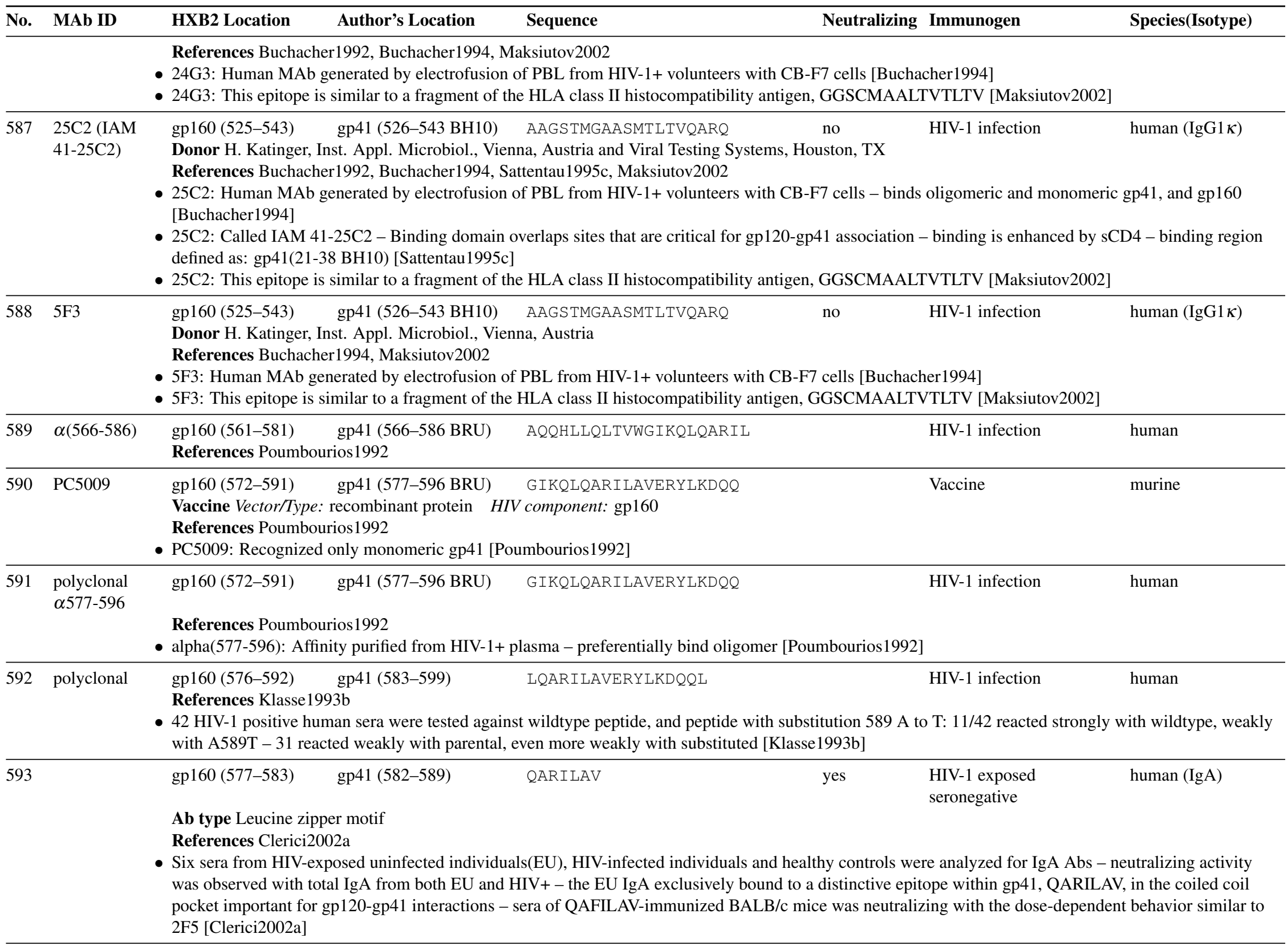




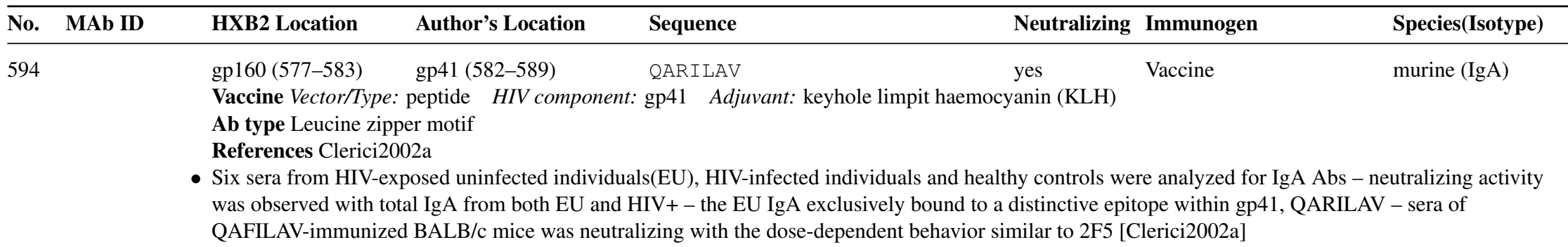

Donor H. Katinger, Inst. Appl. Microbiol., Vienna, Austria

References Buchacher1992, Buchacher1994

- 1F11: Generated by electrofusion of PBL from HIV-1 positive volunteers with CB-F7 cells [Buchacher1994]

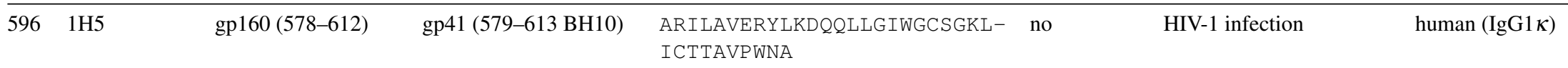

References Buchacher1992, Buchacher1994

- 1H5: Generated by electrofusion of PBL from HIV-1 positive volunteers with CB-F7 cells [Buchacher1994]

\begin{tabular}{llll}
\hline 597 3D9 gp160(578-612) gp41 (579-613 BH10) & $\begin{array}{l}\text { ARILAVERYLKDQQLLGIWGCSGKL- no } \\
\text { ICTTAVPWNA }\end{array}$ & homan (IgG1 $)$
\end{tabular}

Donor H. Katinger, Inst. Appl. Microbiol., Vienna, Austria

References Buchacher1992, Buchacher1994

- 3D9: Generated by electrofusion of PBL from HIV-1 positive volunteers with CB-F7 cells [Buchacher1994]

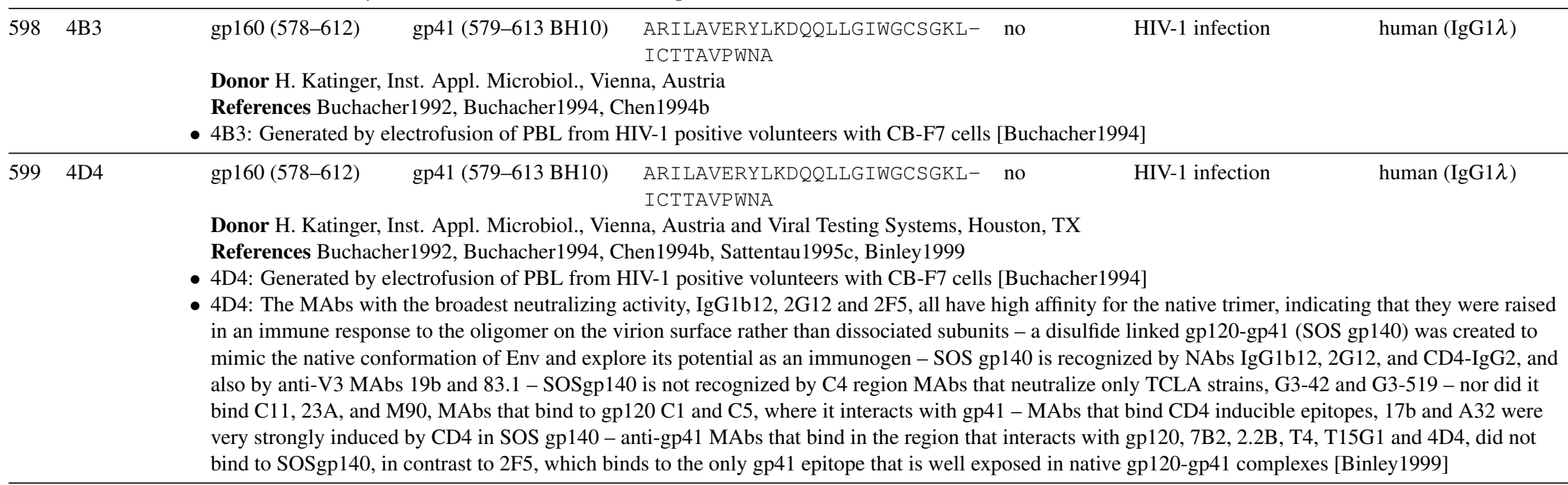




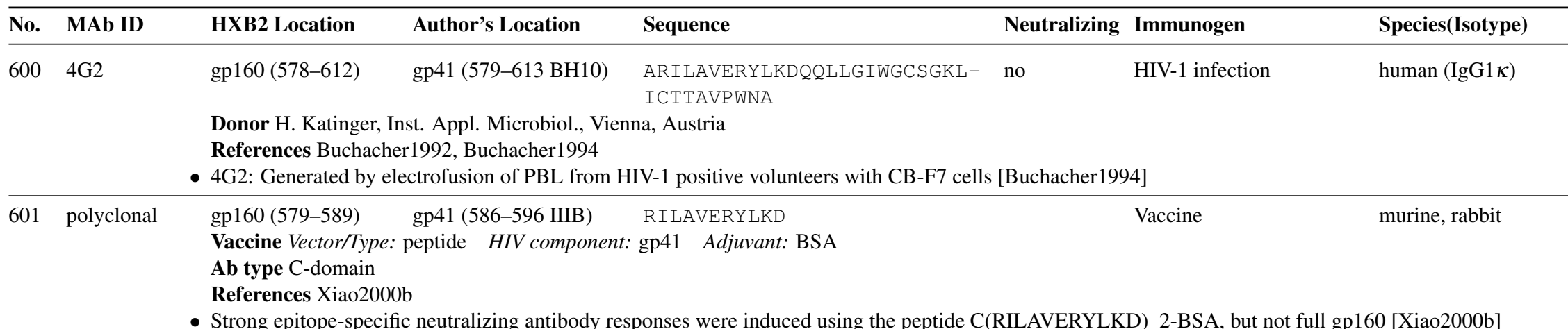

\begin{tabular}{|c|c|c|}
\hline 602 & polyclonal & $\begin{array}{l}\text { gp160 (579-589) gp41 (586-596) } \\
\text { Vaccine Vector/Type: } \text { polyepitope, protein } \\
\text { Ab type N-term }\end{array}$ \\
\hline 603 & polyclonal & 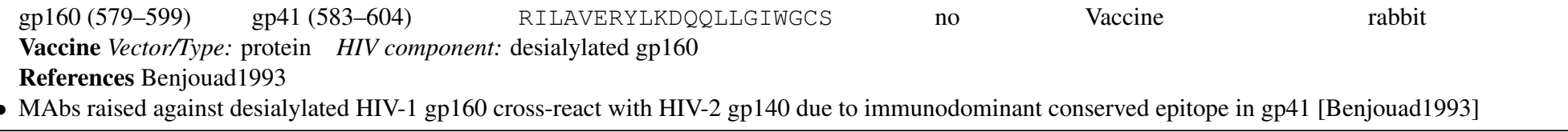 \\
\hline 604 & $2 \mathrm{~A} 2 / 26$ & $\begin{array}{l}\text { gp160 (579-601) gp41 (584-606 BRU) RILAVERYLKDQQLLGIWGCSGK } \\
\text { Vaccine Vector/Type: protein HIV component: gp41 } \\
\text { References Poumbourios1992] Poumbourios1995] } \\
\text { 2A2/26: Immunodominant region, binds both oligomer and monomer [Poumbourios1992] } \\
\text { 2A2/26: Delta 550-561 (Delta LLRAIEAQQHLL), a region important for oligomer formation diminishes binding, Delta (550-561 +571-581) abrogates } \\
\text { binding [Poumbourios1995] }\end{array}$ \\
\hline 605 & $\begin{array}{l}50-69 \\
(S Z-50.69)\end{array}$ & 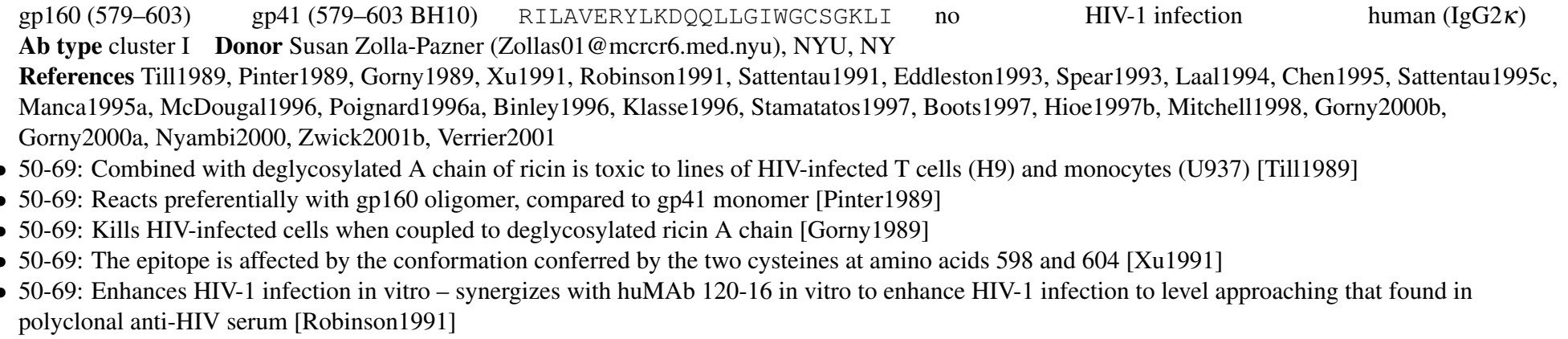 \\
\hline
\end{tabular}




No. MAb ID $\quad$ HXB2 Location $\quad$ Author's Location $\quad$ Sequence $\quad$ Neutralizing Immunogen

- 50-69: Two fold increase in binding to gp120 in the presence of bound SCD4 [Sattentau1991]

- 50-69: Called SZ-50.69 - binds to an epitope within aa 579-613 [Eddleston1993]

- 50-69: Did not mediate deposition of complement component C3 on HIV infected cells unless cells were pre-incubated with sCD4 - complement mediated virolysis of MN and IIIB in the presence of SCD4 [Spear1993]

- 50-69: Epitope described as cluster I, 601-604, conformational - does not neutralize IIIB or synergize neutralization by anti-V3 MAb 447-52D or by CD4 BS MAbs [Laal1994]

- 50-69: One of several anti-gp41 MAbs that bind to a gp41-maltose binding fusion protein designed to study the leucine zipper domain of gp41, showing that the construct has retained aspects of normal gp41 conformation [Chen1995]

- 50-69: Preferentially binds oligomer - binding increased after pretreatment of infected cells with sCD4 - binding domain overlaps site that is critical for gp120-gp41 association [Sattentau1995c]

- 50-69: Virions complexed to gp41 Ab facilitate presentation of p66 RT epitopes to Th cells [Manca1995a]

- 50-69: Does not neutralize HIV-1 LAI [McDougal1996]

- 50-69: Prebinding of anti-V3, and CD4i MAbs 48d and 17b, but not anti-V2 neutralizing MAbs, expose the 50-69 epitope [Poignard1996a

- 50-69: Binds to a linear epitope located in the cluster I region - binding of 50-69 and 240-D inhibited by Fabs A1, A4, M8B, M26B, M12B and T2 [Binley1996

- 50-69: Used to test exposure of gp41 upon sCD4 binding [Klasse1996

- 50-69: Binding of anti-gp120 MAbs IgG1b12 or 654-30D does not mediate significant exposure of the gp41 epitopes for MAbs 2F5 and 50-69 [Stamatatos1997]

- 50-69: Abs that recognize discontinuous epitopes can identify mimotopes from a phage peptide display library - 50-69 maps to an immunodominant domain in gp41 - three groups of peptides were selected, one which seems most closely related to gp41 sequence peptide consensus is WGCxx(RK)(x n)LxC - the analogous gp41 sequence WGCSGKLIC is present in most M group clades, except D with a common L to H substitution [Boots1997]

- 50-69: Mutations in BH10 gp160, W596Y and T605A, as well as deletions of 605-609 (TTAVP) and 597-609 (GCSGKLICTTAVP), abrogate binding of enhancing MAbs 86, 240D, 50-69, and 246-D - 5/6 enhancing MAbs identified to date bind to the immunodominant region 579-613 - identifies non-contiguous W596-G597-C598 and C604-T605 as minimal epitope [Mitchell1998

- 50-69: A cluster I epitope that binds to rgp41 567-647, recognizes a peptide N51-C43 complex trimer of heterodimers that approximates the core of the fusogenic form of gp41 - this MAb doesn't react with either of the peptides N51 or C43 individually - MAbs 50-69 and 1367 had similar properties - MAb $50-69$ bound the fusogenic form of the protein in liquid phase [Gorny2000b]

- 50-69: Binding of panel of 21 MAbs to soluble oligomeric gp140 versus gp41 or gp120 monomers was compared - no MAb was oligomer specific, but gp41 MAb 50-69 bound with a 5 fold preference for the oligomer, while other gp41 MAbs (1367, 98-6, 167-D, 1281, 1342, and 1379) did not show a preference |Gorny2000a

- 50-69: $26 \mathrm{HIV}-1$ group M isolates (clades A to H) were tested for binding to 47 MAbs, including 5 cluster I anti-gp41 MAbs which showed good cross clade reactivity - 50-69 bound the majority of isolates although binding was moderate to weak - specifies discontinuous binding site range as aa $579-613$ [Nyambi2000]

- 50-69: This paper primarily concerns 4E10 and Z13, MAbs that both bind proximally to the 2F5 binding site to a conserved epitope, and that neutralize some primary isolates from clades B, C, and E - MAb 50-69 binding to infected cells is enhanced by sCD4, while 4E10 and Z13 binding is essentially unaltered [Zwick2001b]

- 50-69: A panel of 12 MAbs was used to identify those that could neutralize the dual-tropic primary isolate HIV-1 89.6 - six gave significant neutralization at 2 to $10 \mathrm{ug} / \mathrm{ml}$ : 2F5, 50-69, IgG1b12, 447-52D, 2G12, and 670-D six did not have neutralizing activity: 654-D, 4.8D, 450-D, 246-D, 98-6, and 1281 - no synergy, only additive effects were seen for pairwise combinations of MAbs, and antagonism was noted between gp41 MAbs 50-69 and 98-6, as well as 98-6 and 2F5 [Verrier2001]

- 50-69: NIH AIDS Research and Reference Reagent Program: 531 


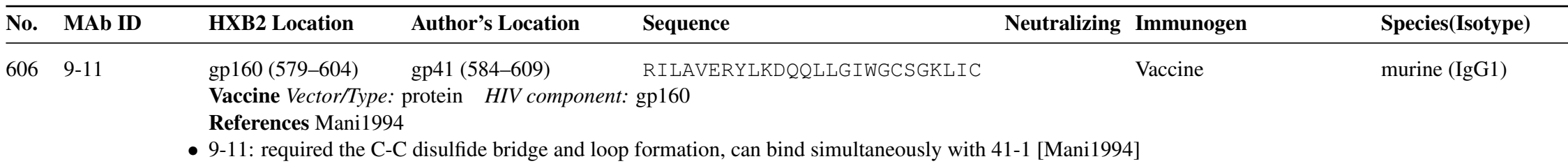

$607 \quad 98-43$ gp160 (579-604) gp41 (579-604 HXB2) RILAVERYLKDQQLLGIWGCSGKLIC no

References Pinter1989, Gorny1989, Tyler1990, Xu1991

- 98-43: Reacts equally well with oligomer and monomer [Pinter1989

- 98-43: Poor ADCC (in contrast to MAb 120-16, gp41(644-663)) [Tyler1990]

- 98-43: 579-604 binds in the immunodominant region [Xu1991]

- 98-43: NIH AIDS Research and Reference Reagent Program: 1241

Vaccine Vector/Type: protein HIV component: gp160

References Gosting 1987, Dalgleish1988 Pincus1991| Pincus1993a, Mani1994, Pincus1996 Pincus1998

- 41-1: This antibody to gp41(584-609) [Mani1994] seems to have been named the same as a different MAb to gp41(735-752 IIIB) [Dalgleish1988]

- 41-1: Also called 41.1, although possibly not, the literature is confusing because two gp41 MAbs that bind to this region with similar names (dash versus period) are listed as murine and human

- 41-1: Broadly reactive [Gosting1987]

- 41-1: This antibody seems to have been named the same as a different MAb to gp41(735-752) [Dalgleish1988]

- 41-1: Efficacious as an immunotoxin when coupled to RAC - gave linear epitope as gp160 579-603 [Pincus1991]

- 41-1: Called 41.1, and described as a human MAb - cross-competes with 41.4 - sCD4 enhances the efficacy of immunotoxins in vitro 30-fold - MAb was coupled to ricin A chain (RAC) [Pincus1993a]

- 41-1: Did not require the C-C disulfide bridge and loop formation, can bind simultaneously with 9-11 [Mani1994]

- 41-1: Called 41.1, and described as a human MAb, binding 579-604 - a panel of immunotoxins was generated by linking Env MAbs to ricin A immunotoxins mediated cell killing, but killing was not directly proportional to binding [Pincus1996
$609 \quad 41.4$ gp160 (579-608)

$$
\text { gp41 (584-609) }
$$

$$
\text { RILAVERYLKDQQLLGIWGCSGKLI- }
$$

$$
\text { CTTAV }
$$

Donor Jan McClure, Bristol-Myers Squibb Pharmaceutical Res Inst, Seattle, WA

References Pincus1993a

- 41.4: Binds to peptide weakly, but to gp160 with higher affinity than 41.1, and cross-competes with 41.1 - probably conformational - MAb was coupled to ricin A chain (RAC) - sCD4 enhances the efficacy of immunotoxins in vitro 30-fold [Pincus1993a]

610 Fab A

gp160 (579-608)

gp41 (584-609 LAI)

RILAVERYLKDQQLLGIWGCSGKLI- no
CTTAV

HIV-1 infection

human $(\operatorname{IgG} 1 \kappa)$

References Binley1996

- Fab A1: Binds to cluster I region - competes with MAbs 240-D and 50-69 - conformation sensitive - variable regions sequenced |Binley1996

gp160 (579-608)

gp41 (584-609 LAI)

\begin{abstract}
RILAVERYLKDQQLLGIWGCSGKLI- no CTTAV
\end{abstract}

HIV-1 infection

human $(\operatorname{IgG} 1 \kappa)$

\title{
References Binley1996
}




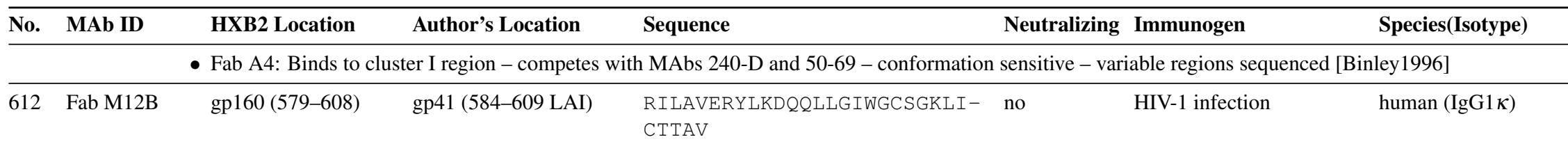

References Binley1996

- Fab M12B: Binds to cluster I region - competes with MAbs 240-D and 50-69 - conformation sensitive - variable regions sequenced [Binley1996]

613 Fab M26B

gp160 (579-608)

RILAVERYLKDQQLLGIWGCSGKLI- no

HIV-1 infection

human $(\operatorname{IgG} 1 \kappa)$

References Binley1996

- Fab M26B: Binds to cluster I region - competes with MAbs 240-D and 50-69 - conformation sensitive - variable regions sequenced [Binley1996]

614 Fab M8B

gp160 (579-608)

gp41 (584-609 LAI)

RILAVERYLKDQQLLGIWGCSGKLI- no

HIV-1 infection

human $(\operatorname{IgG} 1 \kappa)$

References Binley1996

- Fab M8B: Binds to cluster I region - competes with MAbs 240-D and 50-69 - conformation sensitive - variable regions sequenced [Binley1996]

$615 \quad$ Fab T2 gp160 (579-608) gp41 (584-609 LAI)

RILAVERYLKDQQLLGIWGCSGKLI- no

HIV-1 infection

human $(\operatorname{IgG} 1 \kappa)$

References Binley1996

- Fab T2: Binds to cluster I region - competes with MAbs 240-D and 50-69 - conformation sensitive - variable regions sequenced [Binley1996]

\begin{tabular}{lllll}
\hline 616 (No. 86) & gp160 (579-613) & gp41 (586-620 IIIB) & $\begin{array}{l}\text { RILAVERYLKDQQLLGIWGCSGKLI- no } \\
\text { CTTAVPWNAS }\end{array}$ & hIV-1 infection
\end{tabular}

Donor Evan Hersh and Yoh-Ichi Matsumoto

References Sugano1988, Robinson1990b, Robinson1990c Pincus1991, Moran1993 Wisnewski1996, Mitchell1998

- 86: Reacts with gp41 and also reacted weakly with gp120 [Sugano1988]

- 86: Antibody dependent enhancement (ADE) of HIV-1 IIIB infectivity in the presence of complement [Robinson1990b

- 86: Peptide 586-620 blocks complement mediated ADE [Robinson1990c

- 86: Poor immunotoxin activity when coupled to RAC - peptide binding stated to be aa 579-603 [Pincus1991]

- 86: Heavy (V HI) and light (V kappaI) chain sequenced - enhancing activity - similar germline sequence to MAb S1-1, but very different activity Moran1993

- 86: 86 is V H1 - V-region heavy chain usage was examined and a bias of enhanced V H1 and V H4, and reduced V H3, was noted among HIV infected individuals [Wisnewski1996]

- 86: Mutations in BH10 gp160, W596Y and T605A, as well as deletions of 605-609 (TTAVP) and 597-609 (GCSGKLICTTAVP), abrogate binding of enhancing MAbs 86, 240D, 50-69, and 246-D - 5/6 enhancing MAbs identified to date bind to the immunodominant region 579-613 [Mitchell1998]

- 86: NIH AIDS Research and Reference Reagent Program: 380

\begin{tabular}{|c|c|c|c|c|c|c|}
\hline 617 & polyclonal & $\begin{array}{l}\text { gp160 (580-597) gp41 (584-602) } \\
\text { References Petrov1990 } \\
\text { Immunodominant and broadly reactive pepti }\end{array}$ & $\begin{array}{l}\text { ILAVERYLKDQQLLGIWG } \\
\text { Petrov1990 }\end{array}$ & no & HIV-1 infection & human \\
\hline 618 & V10-9 & gp41 (586-620 IIIB) & $\begin{array}{l}\text { ILAVERYLKDQQLLGIWGCSGKLIC- } \\
\text { TTAVPWNAS }\end{array}$ & no & HIV-1 infection & human $(\operatorname{IgG} 1 \kappa)$ \\
\hline
\end{tabular}




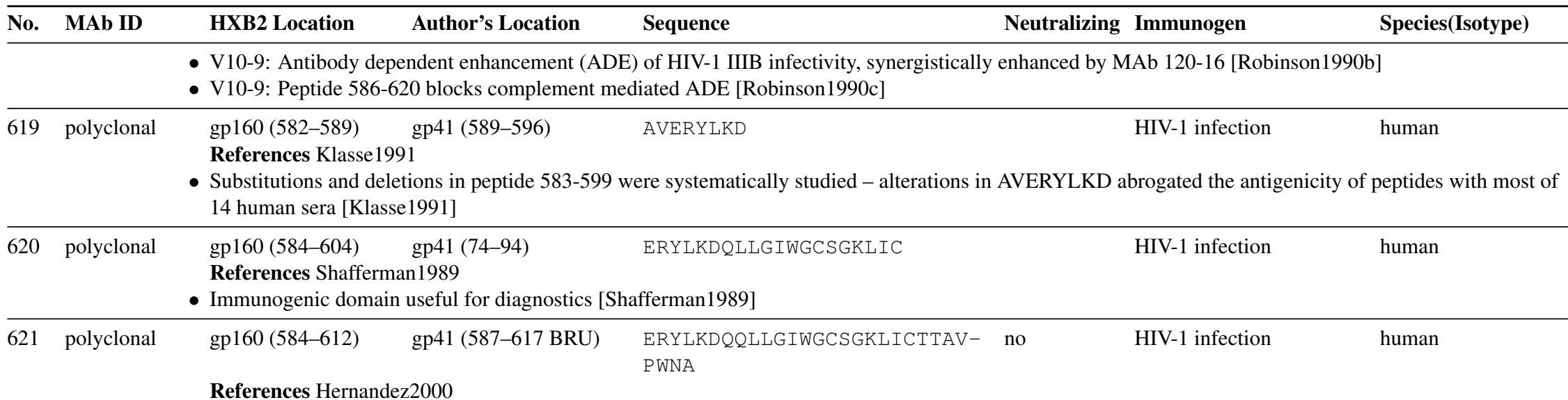

- Chimeric peptide combining two peptides gp160(495-516 and 584-612) served as a specific and broadly reactive antigen for diagnostic detection of HIV-1 [Hernandez2000]

\begin{tabular}{|c|c|c|}
\hline 622 & $2 \mathrm{~F} 11$ & $\begin{array}{l}\text { gp160 (589-600) gp41 (589-600 HXB2) DQQLLGIWGCSG } \\
\text { References Eaton1994 Enshell-Seijffers2001] } \\
\text { - 2F11: Enhances infectivity even in the absence of complement - does not mediate ADCC or neutralize virus [Eaton1994] } \\
\text { - 2F11: Monoclonal antibodies to this epitope have distinct phenotypes-41-7 and 1B8.env were found to be not neutralizing, 2F11 possibly enhancing, and } \\
\text { clone } 3 \text { beneficial [Enshell-Seijffers2001]. }\end{array}$ \\
\hline 623 & $\begin{array}{l}246-D \\
(S Z-246 . D, \\
246,246 D)\end{array}$ & 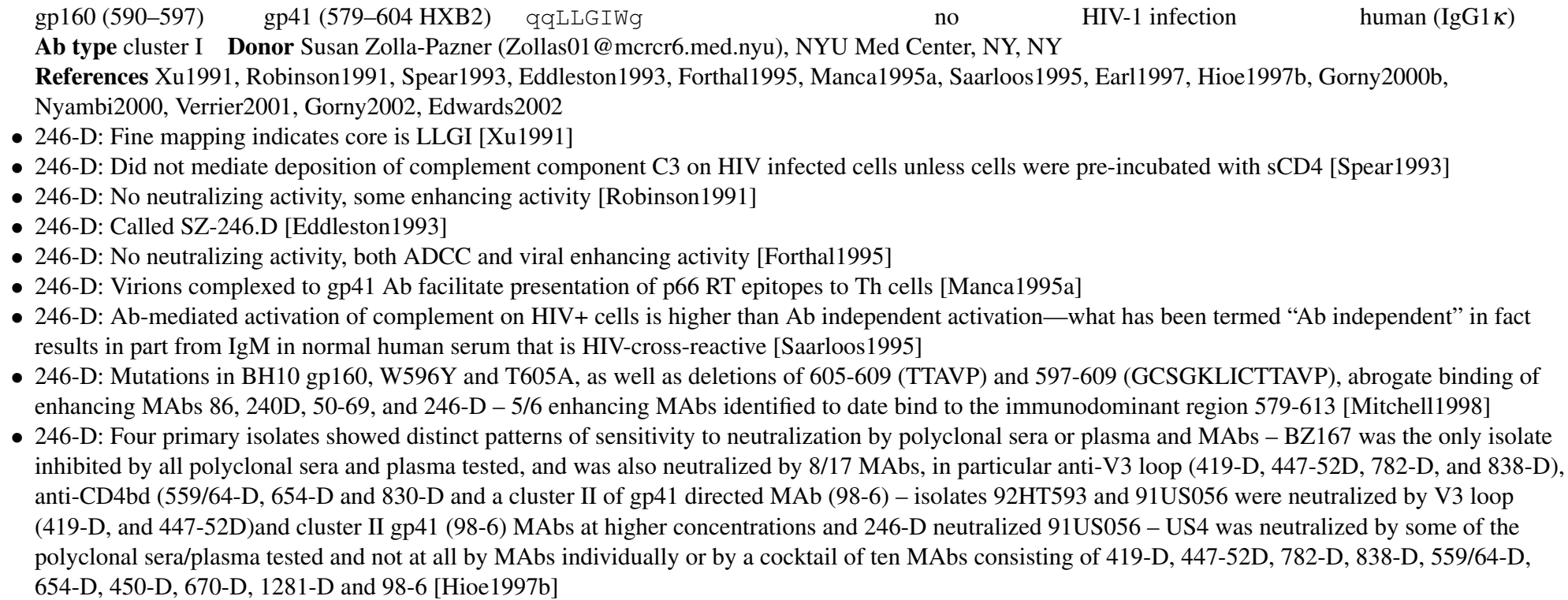 \\
\hline
\end{tabular}




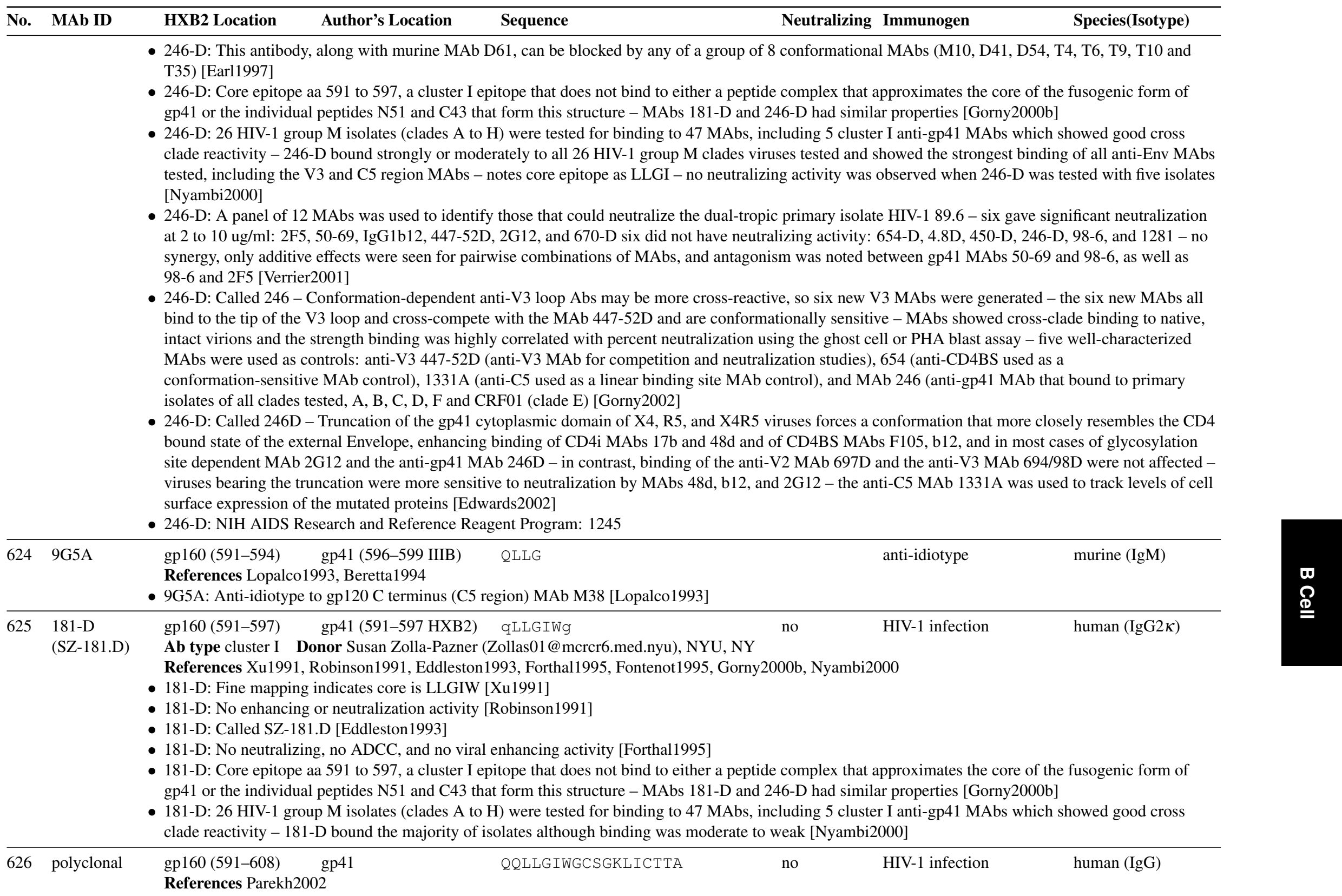




No. MAb ID HXB2 Location Author's Location $\quad$ Sequence Npecies(Isotype)

- A simple enzyme immunoassay (EIA) that detects increasing levels of anti-HIV IgG after seroconversion can be used for detecting recent HIV-1 infection longitudinal specimens from 139 incident infections in the US and Thailand were used in the study - the method was generally applicable for HIV-1 subtypes A, B, C, D and E(CRF01) [Parekh2002]

\begin{tabular}{|c|c|c|}
\hline 627 & 40-D (F240) & 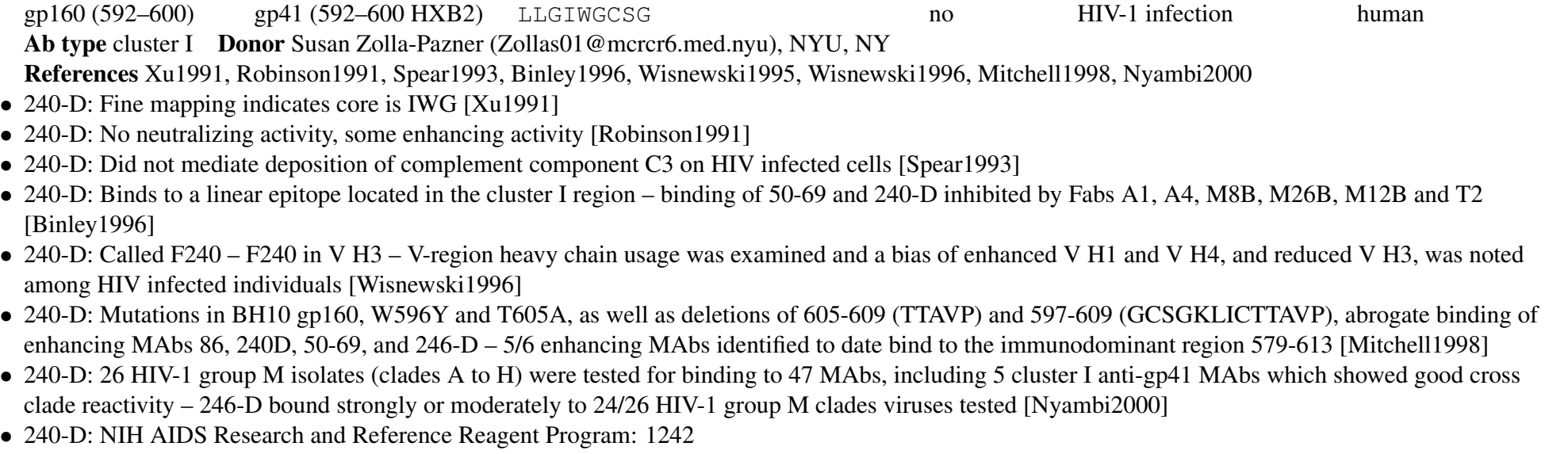 \\
\hline 628 & 40 & $\begin{array}{l}\text { gp160 (592-606) gp41 (592-606 BH10) LLGIWGCSGKLICTT ho Human (IgG1 } \kappa) \\
\text { Ab type cluster I Donor L. Cavacina or M. Posner, Dept. of Med. Harvard Med. School, Boston MA, USA } \\
\text { References Cavacini1998a, York2001 } \\
\text { - F240: Seems to be distinct from MAb 240-D, an antibody with a similar epitope in the immunodominant region of gp41 - dose-dependent reactivity with } \\
\text { HIV isolates RF, SF2, IIIB, and MN was observed - F240 had no neutralizing activity and enhances infection in the presence of complement - reactivity of } \\
\text { F240 is enhanced by preincubation of cells with sCD4 or anti-CD4BS MAb F105 - heavy and light chain variable domains were sequenced, and a strong } \\
\text { homology to hu MAb 3D6 was observed, as 3D6 binds to the same epitope, these MAbs may define a human Ab clonotype [Cavacini1998a] } \\
\text { - F240: Abs against the V3 loop (50.1, 58.2, 59.1, 257-D, 268-D, 447-52D), CD4BS (IgG1b12, 559-64D, F105), CD4i (17b), and to gp41 (2F5, F240) each } \\
\text { showed similar binding efficiency to Env derived from related pairs of primary and TCLA lines (primary: 168P and 320SI, and TCLA: 168C and } \\
\text { 320SI-C3.3), but the TCLA lines were much more susceptible to neutralization suggesting that the change in TCLA lines that make them more susceptible } \\
\text { to NAbs alters some step after binding [York2001] }\end{array}$ \\
\hline 629 & D49 & $\begin{array}{l}\text { gp160 (592-608) gp41 (597-613) LLGIWGCSGKLICTTAV } \\
\text { Vaccine Vector/Type: protein HIV component: dimeric Env } \\
\text { Ab type cluster I } \\
\text { References|Earl1994, Earl1997] } \\
\text { - D49: Generated during a study of the influence of the oligomeric structure of Env in determining the repertoire of the Ab response [Earl1994] } \\
\text { - D49: Binding maps to region 597-613: WGCSGKLICTTAVPWNA - immunodominant region containing two Cys residues [Earl1997] }\end{array}$ \\
\hline 630 & D61 & $\begin{array}{l}\text { gp160 (592-608) gp41 (592-608 HXB2) LLGIWGCSGKLICTTAV } \\
\text { Vaccine Vector/Type: protein HIV component: dimeric Env } \\
\text { Ab type cluster I Donor Patricia Earl and Christopher Broder, NIH } \\
\text { References Earl1994, Richardson1996, Weissenhorn1996, Earl1997, Golding2002b } \\
\text { D61: Generated during a study of the influence of the oligomeric structure of Env in c }\end{array}$ \\
\hline
\end{tabular}




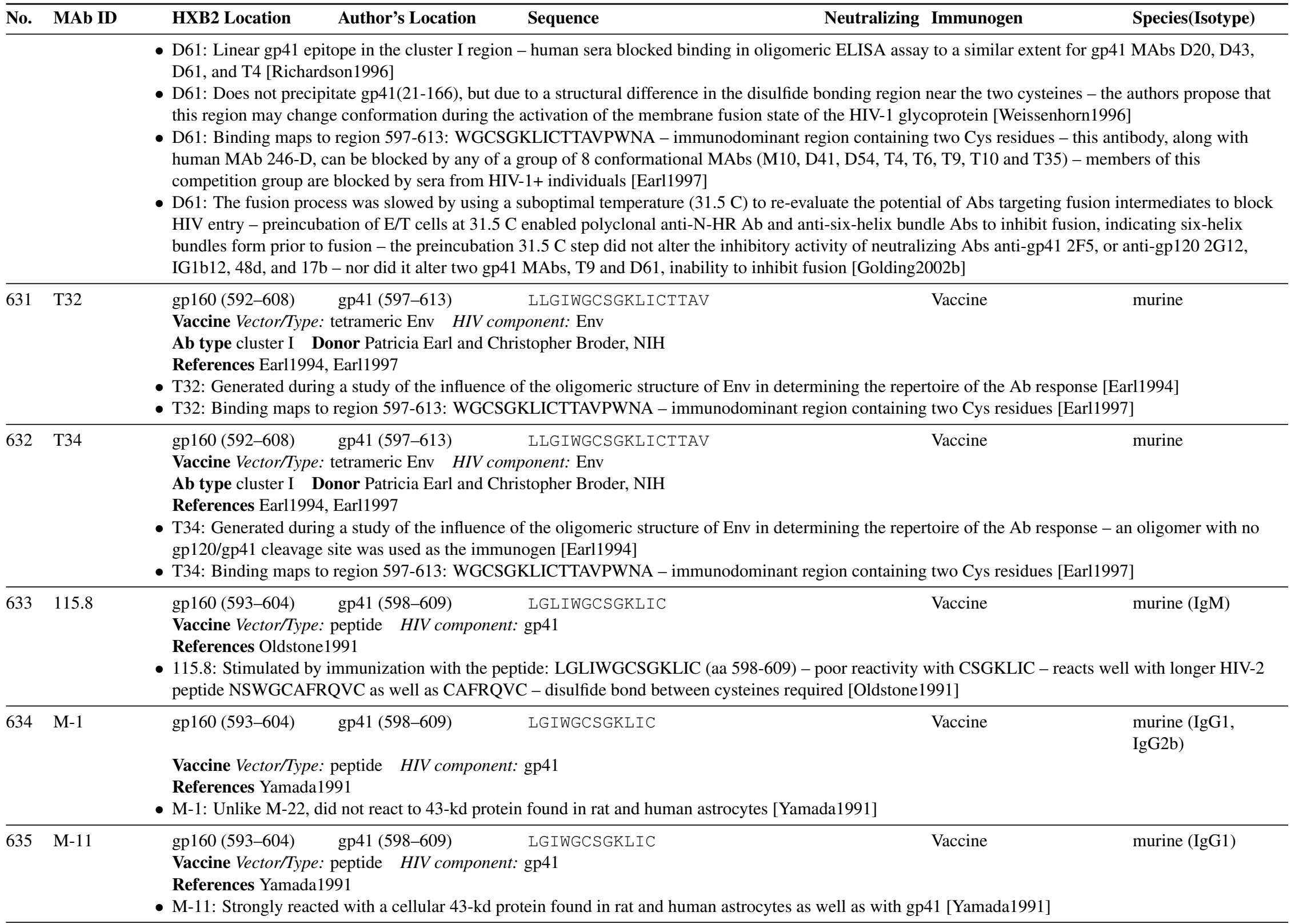




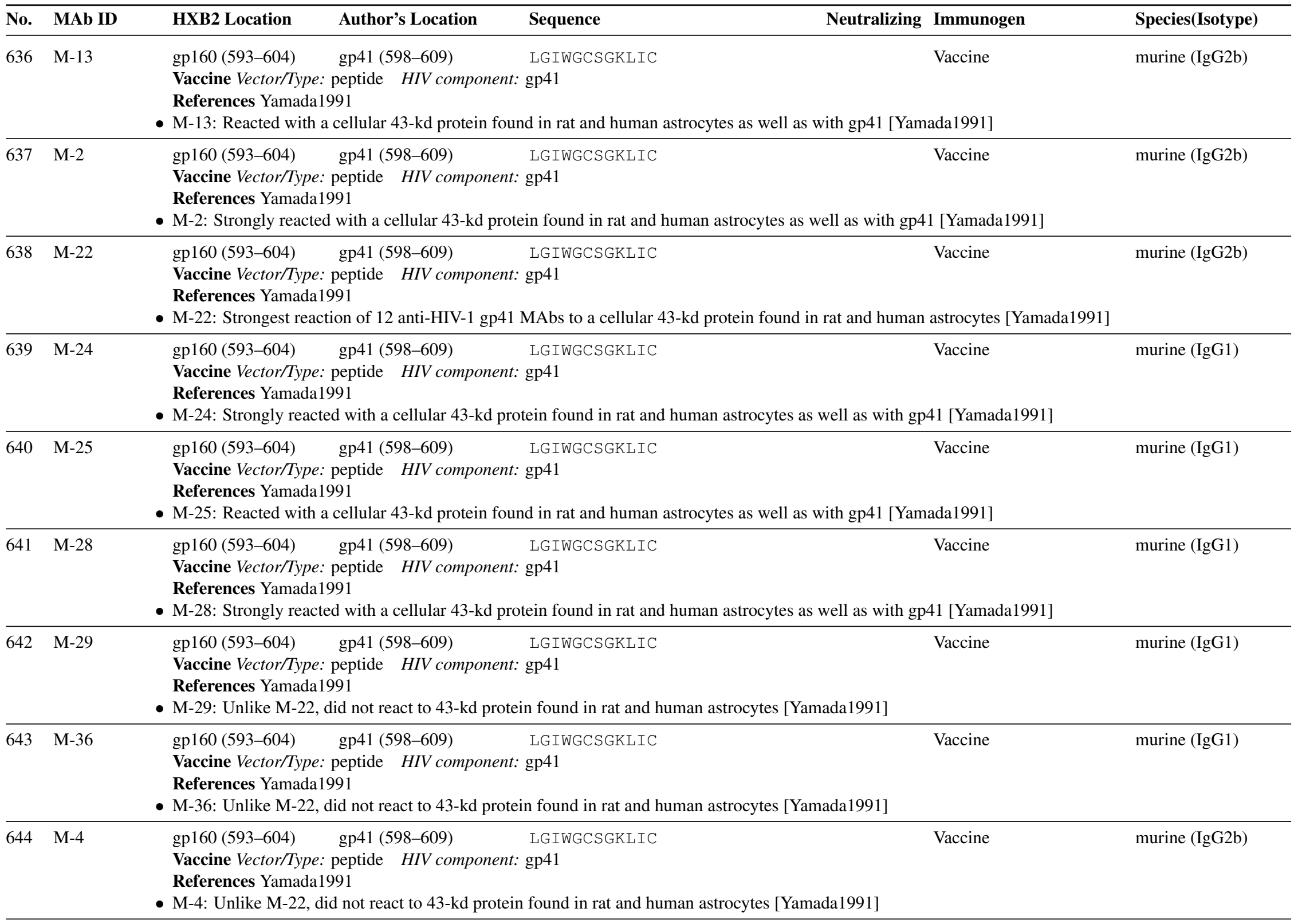




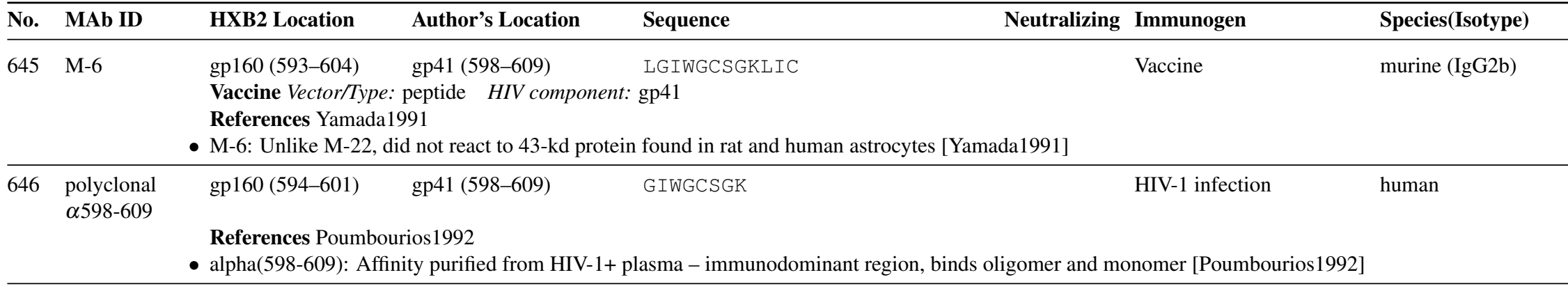
gp160 (594-604)
gp41 (594-605 HXB2) GIWGCSGKLIC
no
HIV-1 infection
human $(\operatorname{IgG} 2 \lambda)$

References Banapour1987. Enshell-Seijffers2001

- 1B8.env: Highly conserved epitope recognized by the majority of HIV-1 infected people - MAb does not neutralize [Banapour1987.

- 1B8.env: Monoclonal antibodies to this epitope have distinct phenotypes-41-7 and 1B8.env were found to be not neutralizing, 2F11 possibly enhancing, and clone 3 beneficial [Enshell-Seijffers2001].

\begin{tabular}{|c|c|c|c|c|c|c|}
\hline 648 & polyclonal & $\begin{array}{l}\text { gp160 (594-609) gp41 (601-616) } \\
\text { References Petrov1990 } \\
\text { Immunodominant and broadly reactive }\end{array}$ & $\begin{array}{l}\text { GIWGCSGKLICTTAVP } \\
\text { Petrov1990 }\end{array}$ & no & HIV-1 infection & human \\
\hline 649 & clone 3 & gp160 (597-606) gp41 (597-606) & GCSGKLICTT & $\mathrm{L}$ & HIV-1 infection & human (IgG1) \\
\hline
\end{tabular}

clone 3: Core binding domain gcsgkLIC - lack of serological activity to this region correlates with rapid progression in infants ([Broliden1989]) Cotropia1992

- clone 3: Inhibits replication of three diverse HIV-1 laboratory strains, as well as an AZT-resistant isolate |Cotropia1996

- clone 3: Monoclonal antibodies to this epitope have distinct phenotypes-41-7 and 1B8.env were found to be not neutralizing, 2F11 possibly enhancing, and clone 3 beneficial [Enshell-Seijffers2001].

\begin{tabular}{lllcc}
\hline 6504 & gp160 (598-604) & gp41 (598-609) & CSGKLIC & Vaccine \\
& Vaccine Vector/Type: & peptide & HIV component: gp41
\end{tabular}

References Oldstone1991, Bizub-Bender1994

- There is another MAb with this ID that reacts with integrase [Oldstone1991, Bizub-Bender1994]

- 4: Stimulated by immunization with the peptide: LGLIWGCSGKLIC (aa 598-609) - poor cross-reactivity with HIV-2 peptide CAFRQVC - slightly more reactive with longer HIV-2 peptide NSWGCAFRQVC [Oldstone1991]

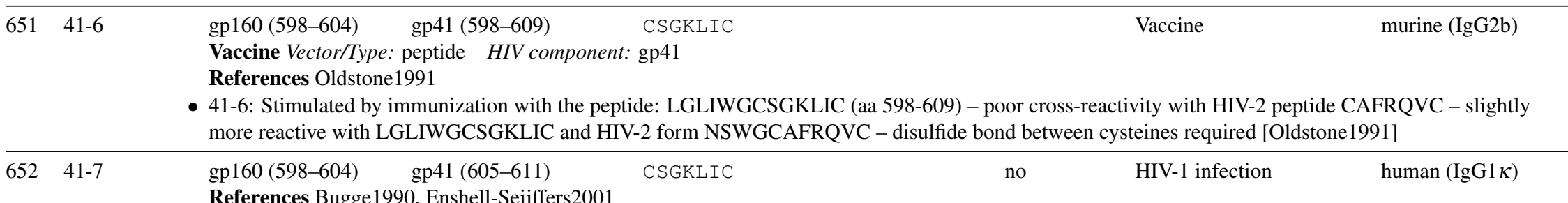

- 41-7: Sera from 6/6 HIV-1 positive, but no HIV-2 positive individuals, interfered with 41-7 binding - Ab does not neutralize [Bugge1990] 


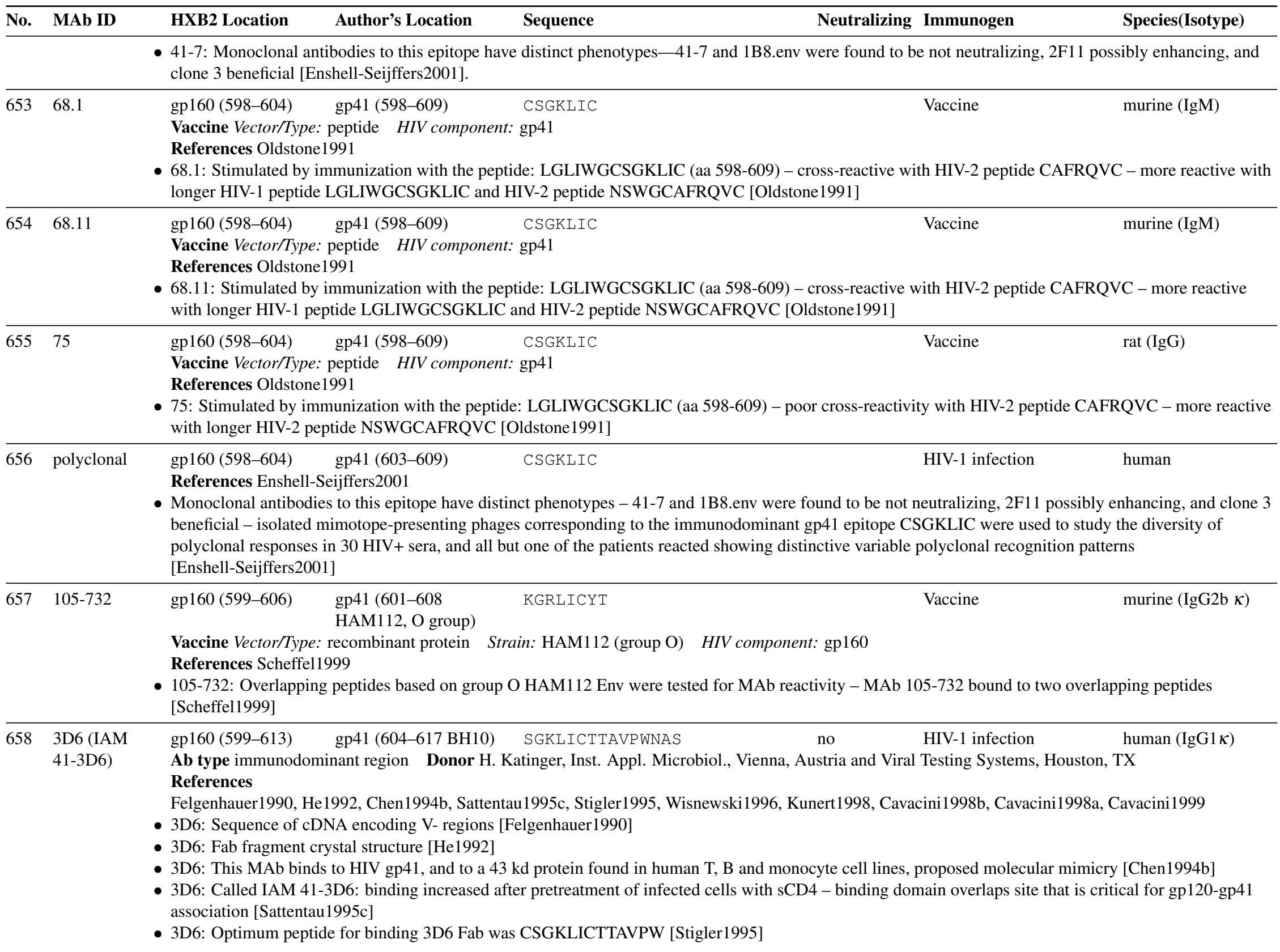




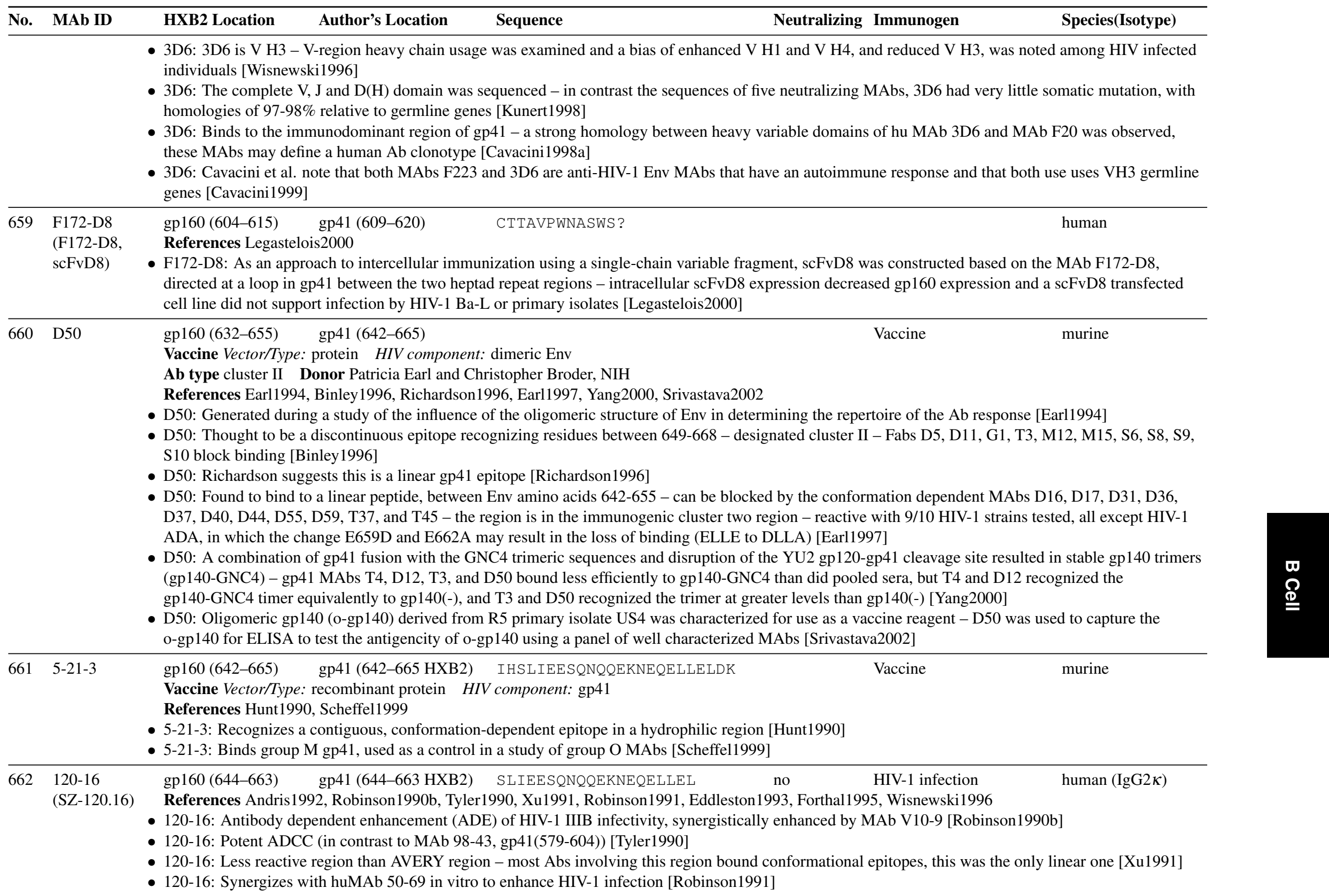




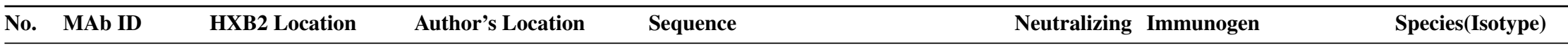

- 120-16: Called SZ-120.16 [Eddleston1993]

- 120-16: No neutralizing activity, both ADCC and viral enhancing activity [Forthal1995]

- 120-16: 120-16 is V H4 - V-region heavy chain usage was examined and a bias of enhanced V H1 and V H4, and reduced V H3, was noted among HIV infected individuals [Wisnewski1996]

- 98-6: Reacts preferentially with gp160 oligomer, compared to gp41 monomer [Pinter1989]

- 98-6: Kills HIV-infected cells when coupled to deglycosylated ricin A chain [Gorny1989]

- 98-6: Toxic to HIV-infected T cells (H9) and monocytes (U937) when coupled to deglycosylated A chain of ricin [Till1989]

- 98-6: No neutralizing or enhancing activity for HIV-1 IIIB [Robinson1990b]

- 98-6: Serves as target for antibody-dependent cellular cytotoxicity, ADCC [Tyler1990]

- 98-6: Two fold increase in binding to gp120 in the presence of bound sCD4 [Sattentau1991]

- 98-6: No neutralizing or enhancing activity [Robinson1991]

- 98-6: Appeared to be specific for a conformational or discontinuous epitope [Xu1991]

- 98-6: Called SZ-98.6 - binds to a conformational domain within aa 644-663 of gp41, and reacts with astrocytes, as do 167-7 and ND-15G1 [Eddleston1993]

- 98-6: Did not mediate deposition of complement component C3 on HIV infected cells, binding enhanced by sCD4 [Spear1993]

- 98-6: This MAb was expressed as a surface anti-gp41 monoclonal antibody receptor for gp41 on a CD4-negative B-cell line. Transfected cells could bind HIV Envelope, but could not be infected by HIV-1. When CD4 delivered by retroviral constructs was expressed on these cells, they acquired the ability to replicate HIV-1, and sIg/gp41 specifically enhanced viral replication [Tani1994]

- 98-6: Epitope described as cluster II, 644-663, conformational - does not neutralize IIIB or synergize neutralization by anti-V3 MAb 447-52D or by CD4 BS MAbs [Laal1994]

- 98-6: One of several anti-gp41 MAbs that bind to a gp41-maltose binding fusion protein designed to study the leucine zipper domain of gp41, showing that the construct has retained aspects of normal gp41 conformation Chen1995

- 98-6: No neutralizing activity, positive ADCC activity, and no viral enhancing activity [Forthal1995]

- 98-6: Virions complexed to gp41 Ab facilitate presentation of p66 RT epitopes to Th cells [Manca1995a]

- 98-6: Preferentially recognizes oligomeric form of gp41 - enhanced binding to HIV-1 infected cells at 37 degrees relative to 4 degrees - addition of sCD4 enhances binding [Sattentau1995c]

- 98-6: 98-6 is V H4 - V-region heavy chain usage was examined and a bias of enhanced V H1 and V H4, and reduced V H3, was noted among HIV infected individuals Wisnewski1996

- 98-6: Four primary isolates showed distinct patterns of sensitivity to neutralization by polyclonal sera or plasma and MAbs - BZ167 was the only isolate inhibited by all polyclonal sera and plasma tested, and was also neutralized by 8/17 MAbs, in particular anti-V3 loop (419-D, 447-52D, 782-D, and 838-D), anti-CD4bd (559/64-D, 654-D and 830-D and a cluster II of gp41 directed MAb (98-6) - isolates 92HT593 and 91 US056 were neutralized by V3 loop (419-D, and 447-52D)and cluster II gp41 (98-6) MAbs at higher concentrations - US4 was neutralized by some of the polyclonal sera/plasma tested and not at all by MAbs individually or by a cocktail of ten MAbs consisting of 419-D, 447-52D, 782-D, 838-D, 559/64-D, 654-D, 450-D, 670-D, 1281-D and 98-6 Hioe1997b

- 98-6: Using a whole virion-ELISA method, 18 human MAbs were tested for their ability to bind to a panel of 9 viruses from clades A, B, D, F, G, and H anti-gp41 Abs 98-6, 1367 and 1342 were not able to bind detectably with any of the viruses from any clade [Nyambi1998] 


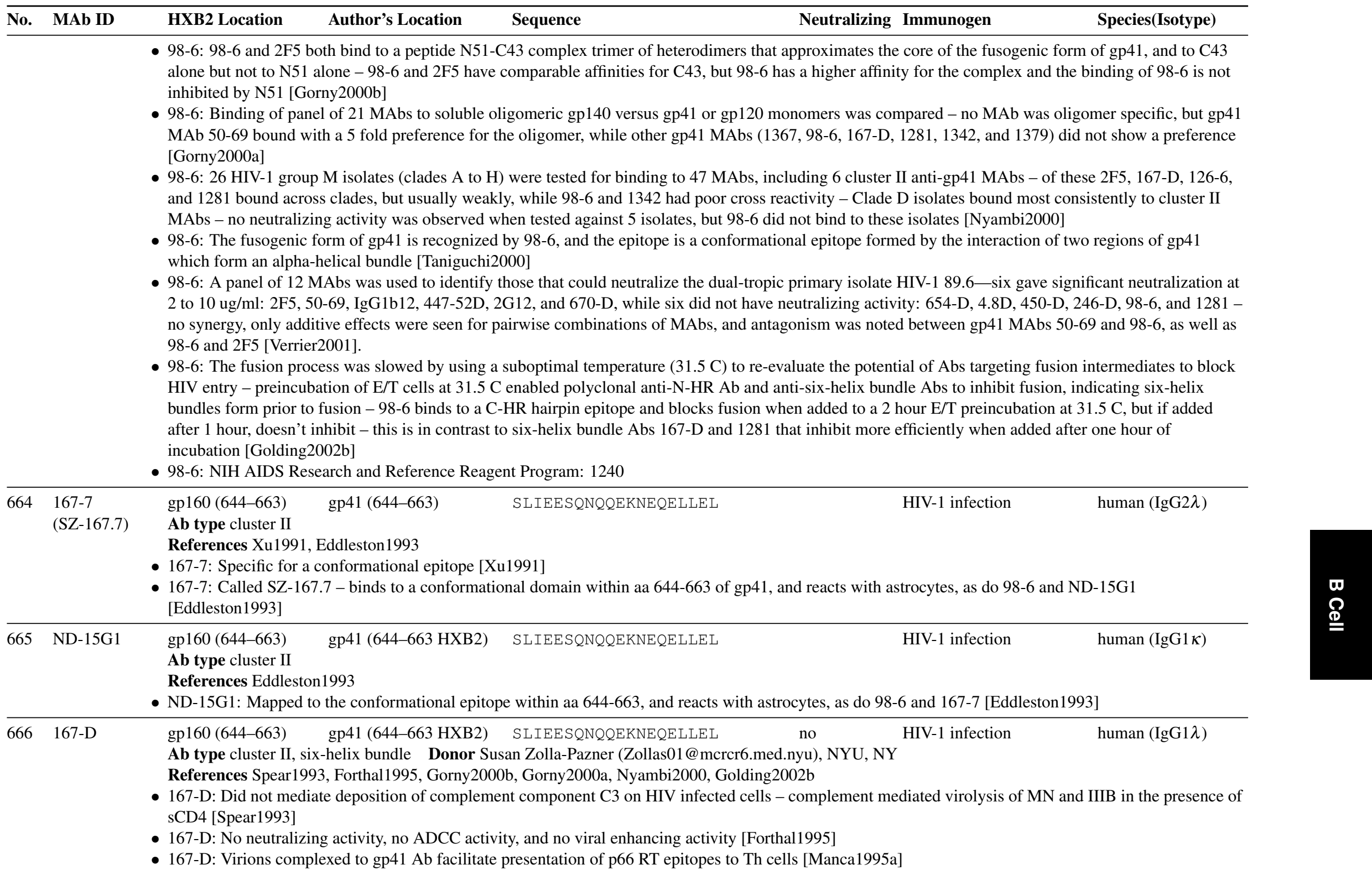




No. MAb ID HXB2 Location Author's Location $\quad$ Sequence $\quad$ Neutralizing Immunogen

- 167-D: This cluster II MAb binds to a conformational epitope in the region 644-663 - like most cluster II MAbs (126-6, 167-D, 1281, 1342, and 1379 all reacted similarly) it binds to a peptide N51-C43 complex trimer of heterodimers that approximates the core of the fusogenic form of gp41, but not to C43 nor to N51 alone [Gorny2000b]

- 167-D: Binding of panel of 21 MAbs to soluble oligomeric gp140 versus gp41 or gp120 monomers was compared - no MAb was oligomer specific, but gp41 MAb 50-69 bound with a 5 fold preference for the oligomer, while other gp41 MAbs (1367, 98-6, 167-D, 1281, 1342, and 1379) did not show a preference [Gorny2000a]

- 167-D: 26 HIV-1 group M isolates (clades A to H) were tested for binding to 47 MAbs, including 6 cluster II anti-gp41 MAbs - of these 2F5, 167-D, 126-6, and 1281 bound across clades, but usually weakly, while 98-6 and 1342 had poor cross reactivity - Clade D isolates bound most consistently to cluster II MAbs [Nyambi2000]

- 167-D: The fusion process was slowed by using a suboptimal temperature (31.5 C) to re-evaluate the potential of Abs targeting fusion intermediates to block HIV entry - preincubation of E/T cells at 31.5 C enabled polyclonal anti-N-HR Ab and anti-six-helix bundle Abs to inhibit fusion, indicating six-helix bundles form prior to fusion - 98-6 binds to a C-HR hairpin epitope and blocks fusion when added to a 2 hour E/T preincubation at $31.5 \mathrm{C}$, but if added after 1 hour, doesn't inhibit - this is in contrast to six-helix bundle Abs 167-D and 1281 that inhibit more efficiently when added after one hour of incubation [Golding2002b

667 2F5 (IAM gp160 (656-671) gp41 (662-667 BH10) $\quad$ NEQELLELDKWASLWN $\quad$ L P $\quad$ HIV-1 infection $\quad$ human (IgG3א) 2F5, Ab type adjacent to cluster II Donor Hermann Katinger, Institute of Applied Microbiology, Vienna, or Polymun Scientific Inc., Vienna, Austria, or Viral IAM-41-2F5, Testing Systems Corp., Houson TX

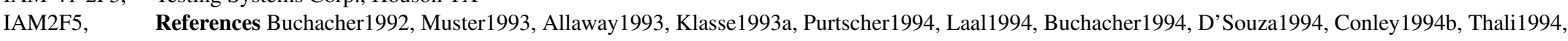
c2F5) Chen1994b, Muster1994, Beretta1994||D'Souza1995, Trkola1995, Sattentau1995c, Moore1995b Neurath1995, Kessler1995, Calarota1996

McKeating1996a Poignard1996b, Sattentau1996, Conley1996, Pincus1996, McKeating1996b, Stoiber1996, Purtscher1996, Schutten1997, D’Souza1997, Mo1997, Li1997, Kessler II1997, Moore1997, Mascola1997, Stamatatos1997| Turbica1997, Ugolini1997, Burton1997, Earl1997, Gorny 1997| Andrus1998, Mondor 1998 Connor1998, Parren1998a, Yang1998 Trkola1998, Fouts1998 Ernst1998, Takefman1998, Li1998, Jiang1998, Parren1998b Geffin1998, Kunert1998, Frankel1998, Montefiori1999, Poignard1999, Beddows1999, Muhlbacher1999 Parren1999, Mascola1999, Mascola2000, Baba2000 Robert-Guroff2000 Gorny2000b, Kunert2000, Liao2000, Lu2000c, Lu2000b, Nyambi2000, Park2000, Pai2002 Sanhadji2000, Coeffier2000, Xiao2000c Yang2000 Si2001 Dong2001 Kolchinsky2001, Tumanova2001, York2001, Zwick2001b, Zwick2001c, Mascola2001||Barnett2001, Moore2001, Zeder-Lutz2001, Parker2001, Spenlehauer2001, Verrier2001, Stiegler2001, Hofmann-Lehmann2001, Xu2001, Root2001, Armbruster2002, Srivastava2002, Golding2002b, Schulke2002, Tian2002, Ho2002, Xu2002, Chakrabarti2002, Joyce2002, Clerici2002a, Xiang2002b Grundner2002, Mascola2002, Kunert2002, Zhang2002, Ferrantelli2002, Liu2002

- 2F5: DKWA defined as the core sequence - highly conserved epitope neutralizing MAb [Buchacher1992, Muster1993

- 2F5: Synergy with combinations of CD4-based molecules in inhibition of HIV-1 Env mediated cell fusion [Allaway1993]

- 2F5: Called IAM-41-2F5 - reports MAb to be IgG1 - the gp41 mutation 582(Ala to Thr) results in conformational changes in gp120 that confer neutralization resistance to conformationally sensitive neutralizing MAbs - neutralization efficiency of 2F5 is not affected [Klasse1993a

- 2F5: Broadly reactive neutralizing activity, ELDKWA is relatively conserved - neutralized 2 primary isolates [Purtscher1994]

- 2F5: Failed to show synergy with anti-CD4 binding site IIIB neutralizing antibodies [Laal1994]

- 2F5: MAb generated by electrofusion of PBL from HIV-1 positive volunteers with CB-F7 cells [Buchacher1994]

- 2F5: Included in a multi-lab study for antibody characterization binding and neutralization assay comparison [D'Souza1994]

- 2F5: Called IAM-41-2F5 - neutralized lab and primary isolates - $\mathrm{t}$ 1/2 dissociation $122 \mathrm{~min}$ for the peptide, and $156 \mathrm{~min}$ for gp41 - core $\mathrm{D}(\mathrm{K} / \mathrm{R}) \mathrm{W}-\mathrm{Ab}$ resistant isolate had the sequence KLDNWA Conley1994b

- 2F5: gp41 mutation (582 A/T) that reduces neutralization of anti-CD4 binding site MAbs does not alter 2F5's ability to neutralize |Thali1994|

- 2F5: 2F5 epitope ELDKWA inserted into an immunogenic loop in influenza virus hemagglutinin can elicit IIIB, MN and RF neutralizing sera in immunized mice [Muster1994] 


No. MAb ID

HXB2 Location Author's Location Sequence

Neutralizing Immunogen

Species(Isotype)

- 2F5: Found to neutralize MN, JRCSF, and two B subtype primary isolates, but not a D subtype primary isolate, by most labs in a multi-laboratory study involving 11 labs [D'Souza1995]

- 2F5: Cross-clade primary virus neutralizing activity - LDKW defined as the core epitope [Trkola1995]

- 2F5: Called IAM 41-2F5 - exposed in the presence of gp120 on the cell surface, while most of gp41 is masked - binds proximal to transmembrane region Sattentau1995c

- 2F5: Review: binds to the only generally accepted strong neutralizing epitope outside of gp120, one of only 3 MAbs with strong broad activity against primary viruses, the others are 2G12 and IgG1b12 - unique member of epitope cluster [Moore1995b] and John Moore, per comm 1996

- 2F5: MAb binding decreases the accessibility or alters the conformation of the gp41 fusion domain and of gp120 domains, including the binding site for the CD4 cell receptor [Neurath1995]

- 2F5: Broad cross-clade neutralization of primary isolates - additive neutralization in combination with anti-CD4BS MAb IgG1b12 (Called BM12) [Kessler1995

- 2F5: Only 4/20 Argentinian and 3/43 Swedish HIV+ sera reacted with LLELDKWASL - sera reacting with peptides that contained ELDKWA tended to have high neutralization titers - the region carboxyl terminal to EDLKWA was found to be more important for polyclonal sera AB binding, 670-675 WNWFDI - 2F5 bound most strongly to the peptide QELLELDKWA Calarota1996

- 2F5: ELDKWAS is in a gp41 binding region for the negative regulator of complement factor H (CFH) - Abs to HIV generally do not cause efficient complement-mediated lysis, but binding of 2F5 can interfere with CHF binding, facilitating HIV destruction by complement [Stoiber1996]

- 2F5: Primary isolates from clade A, B, and E are neutralized by 2F5 - neutralization requires the LDKW motif - neutralization resistant isolates or 2F5 selected variants all had substitutions in the D or K [Purtscher1996]

- 2F5: Neutralizes HXB2, primary isolates, and chimeric virus with gp120 from primary isolates in an HXB2 background [McKeating1996b]

- 2F5: Review: one of three MAbs (IgG1b12, 2G12, and 2F5) generally accepted as having significant potency against primary isolates [Poignard1996b]

- 2F5: Review: only four epitopes have been described which can stimulate a useful neutralizing response to a broad spectrum of primary isolates, represented by the binding sites of MAbs: 447-52-D, 2G12, Fab b12, and 2F5 [Sattentau1996]

- 2F5: 2F5 was infused into two chimpanzees which were then given an intravenous challenge with a primary HIV-1 isolate - both became infected, but with delayed detection and prolonged decrease in viral load relative to controls, indicating that preexisting, neutralizing antibodies (passively administered or actively elicited) affect the course of acute-phase virus replication and can be influential after the Ab can no longer be detected in the peripheral circulation [Conley1996]

- 2F5: A panel of immunotoxins were generated by linking Env MAbs to ricin A - immunotoxins mediated cell killing, but killing was not directly proportional to binding [Pincus1996]

- 2F5: Called IAM 2F5 - antibody mediated enhancement or inhibition seemed to be determined by isolate rather than antibody specificity - in this study, only 2F5 inhibited the entry of all the viruses studied, irrespective of their phenotype, and directly proportional to its affinity to monomeric HIV-1 gp160 [Schutten1997]

- 2F5: Of three neutralizing MAbs (257-D, IgG1b12, and 2F5), 2F5 was the only one to inhibit the entry of all viruses studied, both SI and NSI, with a potency proportional to its affinity for monomeric gp126 [Schutten1997]

- 2F5: In a multilab evaluation of monoclonal antibodies, only IgG1b12, 2G12, and 2F5 could neutralize at least half of the 9 primary test isolates at a concentration of $<25$ mug per ml for 90\% viral inhibition - the isolates with no 2F5 neutralizing susceptibility had the sequences ALGQWA or ELDTWA instead of EDLKWA - 7/9 primary isolates were neutralized, and ALDKWQ and ALDKWA were susceptible to neutralization [D'Souza1997]

- 2F5: A JRCSF variant that was selected for IgG1b12 resistance remained sensitive to MAbs 2G12 and 2F5, for combination therapy [Mo1997]

- 2F5: One of 14 human MAbs tested for ability to neutralize a chimeric SHIV-vpu+, which expressed HIV-1 IIIB Env - strong neutralizer of SHIV-vpu+ all Ab combinations tested showed synergistic neutralization - 2F5 has synergistic response with MAbs 694/98-D (anti-V3), 2G12, b12, and F105 [Li1997]

- 2F5: IgG1b12 was more potent with greater breadth than MAb 2F5 in an infection reduction assay including 35 primary isolates [Kessler II1997] 


\begin{tabular}{lllll}
\hline No. MAb ID & HXB2 Location & Author's Location & Sequence & Neutralizing Immunogen
\end{tabular}

- 2F5: Review: MAbs 2F5, 2G12 and IgG1b12 have potential for use in combination with CD4-IgG2 as an immunotherapeutic or immunoprophylactic homologous MAbs to these are rare in humans and vaccine strategies should consider including constructs that may enhance exposure of these MAbs' epitopes [Moore1997]

- 2F5: Binding of anti-gp120 MAbs IgG1b12 or 654-30D does not mediate significant exposure of the gp41 epitopes for MAbs 2F5 and 50-69 [Stamatatos1997]

- 2F5: Using concentrations of Abs achievable in vivo, the triple combination of 2F5, 2G12 and HIVIG was found to be synergistic to have the greatest breadth and magnitude of response against 15 clade B primary isolates [Mascola1997]

- 2F5: Used to standardize polyclonal response to CD4 BS [Turbica1997]

- 2F5: The only MAb out of a large panel to show no correlation between Viral binding inhibition and neutralization [Ugolini1997]

- 2F5: This review summarizes results about 2F5: it binds extracellularly, near the transmembrane domain, it is the only gp41 MAb that is neutralizing, it reacts with many non-B clade viruses and has a paradoxically weak binding to virus, given the neutralizing titers [Burton1997]

- 2F5: Post-exposure prophylaxis was effective when MAb 694/98-D was delivered 15 min post-exposure to HIV-1 LAI in hu-PBL-SCID mice, but declined to $50 \%$ if delivered 60 min post-exposure, and similar time constraints have been observed for HIVIG, 2F5 and 2G12, in contrast to MAb BAT123 that could protect delivered 4 hours post infection Andrus1998

- 2F5: This MAb and the results of [Ugolini1997] are discussed - the authors propose that an Ab bound to gp41 would typically project less from the surface of the virion and so be unable to interfere with attachment [Parren1998a]

- 2F5: Ab from gp120 vaccinated individuals prior to infection, who subsequently became HIV infected, could not achieve $90 \%$ neutralization of the primary virus by which the individuals were ultimately infected - these viruses were not particularly refractive to neutralization, as determined by their susceptibility to neutralization by MAbs 2G12, IgG1b12, 2F5 and 447-52D [Connor1998]

- 2F5: A neutralization assay was developed based on hemi-nested PCR amplification of the LTR (HNPCR) - LTR-HNPCR consistently revealed HIV DNA and was shown to be a rapid, specific and reliable neutralization assay based on tests with 6 MAbs and 5 isolates [Yang1998]

- 2F5: A wide range of neutralizing titers was observed that was independent of co-receptor usage - 2F5 was the most potent of the MAbs tested [Trkola1998]

- 2F5: Points out that 2G12 and 2F5, potent neutralizing antibodies, were identified by screening for cell surface (oligomeric Envelope) reactivity [Fouts1998]

- 2F5: The ELDKWA epitope was inserted into the antigenic site B of influenza hemagglutinin and expressed on baculovirus infected insect cells, flanked by 3 additional random amino acids, xELDKWAxx - FACS was used to isolate the clone that displayed the epitope with the most markedly increased binding capacity for 2F5, to identify particularly specific immunogenic constructs - PELDKWAPP was a high affinity form selected by FACS [Ernst1998]

- 2F5: Induces complement-mediated lysis in MN but not primary isolates - primary isolates are refractive to CML [Takefman1998]

- 2F5: Neutralization synergy was observed when the MAbs 694/98-D (V3), 2F5 (gp41), and 2G12 (gp120 discontinuous) were used in combination, and even greater neutralizing potential was seen with the addition of a fourth MAb, F105 (CD4 BS) [Li1998]

- 2F5: Used as a control in the study of anti-gp41 MAb NC-1 - 2F5 does not react with HIV-2 gp41 or gp160 [Jiang1998]

- 2F5: MAbs 2G12, 2F5 and b12 are broadly neutralizing, as are some human polyconal sera, but this paper describes a set of primary isolates that are resistant to all three MAbs and 2 broadly neutralizing sera - results indicate that resistance levels of pediatric isolates might be higher than adult isolates resistance in general did not seem to be conferred by a loss of binding affinity for gp120 or gp41, rather by a more global perturbation of oligomeric Envelope [Parren1998b]

- 2F5: The natural immune response to the epitope of 2F5, ELDKWA, was studied in perinatally infected children and levels of reactivity to this epitope were correlated with absolute CD4 numbers over time and health status - 3/10 children who had no antibody reactivity to ELDKWA had substitutions in the epitope (ALDKWA, ELDQWA, and KLDKWA) - 2F5 competed with the ELDKWA-reactive sera depending on the serum titer [Geffin1998] 


No. MAb ID HXB2 Location Author's Location $\quad$ Sequence $\quad$ Neutralizing Immunogen

- 2F5: The complete $\mathrm{V}$, J and $\mathrm{D}(\mathrm{H})$ domain was sequenced - unlike non-neutralizing anti-gp41 MAb 3D6, five neutralizing MAbs (2F5, 2G12, 1B1, 1F7, and 3D5) showed extensive somatic mutations giving evidence of persistent antigenic pressure over long periods - in contrast to Geffin98, where multiple pediatric sera were found to compete with $2 \mathrm{~F} 5$, cross-competition was noted to be very rare in sera from HIV+ adults - Kunert et al. propose that because there is a binding site of human complement factor $\mathrm{H}$ which overlaps the $2 \mathrm{~F} 5$ binding site, it may generally be masked from the immune system - $2 \mathrm{~F} 5$ also has a remarkably long CDR3 loop of 22 amino acids, and this region could not be readily assigned to any described $\mathrm{D}(\mathrm{H})$ fragment, leading to the suggestion of recombination of two fragments from novel regions [Kunert1998]

- 2F5: Infection of dendritic cells cultured from CD14+ blood cells or from cadaveric human skin was blocked by neutralizing MAbs IgG1b12, or 2F5 and $2 \mathrm{G} 12$ delivered together, but not by control non-neutralizing anti-gp120 MAb 4.8D, indicating that NAbs could interrupt early mucosal transmission events [Frankel1998]

- 2F5: rgp120 derived from a R5X4 subtype B virus was used to vaccinate healthy volunteers and the resulting sera were compared with sera from HIV-1 positive subjects and neutralizing MAbs [Beddows1999]

- 2F5: A meeting summary presented results regarding neutralization-MAbs $2 \mathrm{G} 12$ and 2F5 tested for their ability to neutralize primary isolate infection of genetically engineered cell lines (cMAGI and others, presented by T. Matthews, A. Trkola, J. Bradac) - an advantage of such cells lines over PBMCs is that markers (X-Gal) can be added for staining to simplify the assay - the consensus of the meeting was that these engineered cell lines did not improve the sensitivity of detection of primary isolate neutralization - D. Burton and J. Mascola presented results concerning passive immunization and protection of hu-PBL-SCID mice and macaques, respectively, and both found combinations of MAbs that were able to achieve $99 \%$ neutralization in vitro corresponded to efficacy in vivo [Montefiori1999]

- 2F5: Hu-PBL-SCID mice were infected with HIV-1s JRCSF and SF162 to study the effect of NAbs on an established infection - no significant differences in the initial rate of decrease in viral load or the plateau levels of viral RNA between the b12 treated and control mice were seen - in most of the Ab treated mice b12 escape mutants were observed with varying patterns of mutations - a combination of b12, 2G12 and 2F5 protected 1/3 mice, and an isolate from one of the other two was resistant to neutralization by all three MAbs |Poignard1999

- 2F5: In a study of 116 HIV-1+ individuals, Ab reactivity to a peptide encompassing the ELDKWA peptide decreased in CDC stage C patients compared with stage A patients, and longitudinal studies showed a decline in $6 / 8$ patients, while overall Ab reactivity to rec soluble gp160 stayed constant [Muhlbacher1999]

- 2F5: Review of the neutralizing Ab response to HIV-1 [Parren1999]

- 2F5: Combinations of HIVIG, 2F5, 2 G12 were administered in passive-transfer experiments 24 hours prior to challenge with pathogenic SHIV 89.6PD $3 / 6$ animals given HIVIG/2F5/2G12 were completely protected, the others had reduced viremia and normal CD4 counts - 1/3 monkeys given 2F5/2G12 showed transient infection, the other two had reduced viral load - all monkeys that received HIVIG, 2F5, or 2G12 alone became infected and developed high-level plasma viremia, although animals that got HIVIG or 2G12 had a less profound CD4 T cell decline [Mascola1999]

- 2F5: Because HIV-1 is most often transmitted across mucosal surfaces, the ability of passive transfer of infused HIVIG/2F5/2G12 to protect against mucosal exposure of macaques to pathogenic SHIV 89.6PD was studied - HIVIG/2F5/2G12 protected 4/5 animals against vaginal challenge, 2F5/2G12 combined protected 2/5 animals, and 2G12 alone protected 2/4 animals - in contrast, Mascola and co-workers had previously shown single MAbs could not protect against intervenous challenge - Ab treated animals that got infected through vaginal innoculation had low viral loads and only modest declines in CD4 counts - the infused Abs were detected in the nasal, vaginal, and oral mucosa [Mascola2000]

- 2F5: Paper uses IgG1 form of 2F5 - a triple combination of 2F5, F105 and 2G12 effectively neutralized perinatal infection of macaque infants when challenged with SHIV-vpu+ - the plasma half-life was $4.2+/-0.8$ days [Baba2000]

- 2F5: A mini-review of observations of passive administration of IgG NAbs conferring protection against intervenous or vaginal SHIV challenge, that considers why IgG MAbs might protect against mucosal challenge [Robert-Guroff2000]

- 2F5: MAbs 98-6 and 2F5 both bind to a peptide N51-C43 complex trimer of heterodimers that approximates the core of the fusogenic form of gp41, and to C43 alone but not to N51 alone - 98-6 and 2F5 have comparable affinities for C43, but 98-6 has a higher affinity for the complex and 2F5 may bind to an epitope of $\mathrm{C} 43$ that is directly involved with complex formation -and IgG1 rec form of the Ab was used in this study [Gorny2000b] 


No. MAb ID HXB2 Location $\quad$ Author's Location $\quad$ Sequence $\quad$ Neutralizing Immunogen

- 2F5: 2F5 is a candidate for immunotherapy, but generally IgG1 has a longer half life in humans than IgG3, so the isotype was switched - rec CHO-derived

MAb 2F5 IgG1kappa and hybridoma-derived MAb 2F5 IgG3kappa displayed identical specificity, in vitro function, and epitope (ELDKWA) - it remains to be determined if isotype switching will prolongs beta-clearance [Kunert2000]

- 2F5: Low levels of anti-ELDKWA antibodies are observed in HIV-1+ individuals, so a C-domain P2 peptide linked to a carrier was used to immunize mice and rabbits, and stimulated a high-level anti-ELDKWA response [Liao2000]

- 2F5: A rare mutation in the neutralization sensitive R2-strain in the proximal limb of the V3 region caused Env to become sensitive to neutralization by MAbs directed against the CD4 binding site (CD4BS), CD4-induced (CD4i) epitopes, soluble CD4 (sCD4), and HNS2, a broadly neutralizing sera - 2/12 anti-V3 MAbs tested (19b and 694/98-D) neutralized R2, as did 2/3 anti-CD4BS MAbs (15e and IgG1b12), 2/2 CD4i MAbs (17b and 4.8D), and 2G12 and $2 \mathrm{~F} 5$ - thus multiple epitopes on R2 are functional targets for neutralization and the neutralization sensitivity profile of R2 is intermediate between the highly sensitive MN-TCLA strain and the typically resistant MN-primary strain [Zhang2002]

- 2F5: ELDKWA peptide vaccine study [Lu2000c]

- 2F5: ELDKWA peptide vaccine study [Lu2000b]

- 2F5: 26 HIV-1 group M isolates (clades A to H) were tested for binding to 47 MAbs, including 6 cluster II anti-gp41 MAbs - of these 2F5, 167-D, 126-6, and 1281 bound across clades, but usually weakly, while 98-6 and 1342 had poor cross reactivity - Clade D isolates bound most consistently to cluster II MAbs [Nyambi2000]

- 2F5: Six mutations in MN change the virus from a high-infectivity neutralization resistant phenotype to low-infectivity neutralization sensitive - V3, CD4BS, and CD4i gp120 specific MAbs are 20-100 fold more efficient at neutralizing the sensitive form - gp41 MAbs bind less, and 2F5 behaves the opposite of gp120 MAbs in that it neutralizes the "sensitive" form less efficiently [Park2000]

- 2F5: ELDKWAS co-crystallized bound to the Fab' 2F5 fragment showed the epitope peptide in a type I beta-turn conformation [Pai2002]

- 2F5: 2F5 or sCD4-IgG chimeric immunoadhesin were transferred into 3T3 cells, incorporated into a collagen structure called the neo-organ, and transplanted into SCIDhu mice that were then challenged with MN or LAI - the continuous production of the therapeutic molecules in this context resulted in dramatic reduction of viral load [Sanhadji2000]

- 2F5: A combination of gp41 fusion with the GNC4 trimeric sequences and disruption of the YU2 gp120-gp41 cleavage site resulted in stable gp140 trimers (gp140-GNC4) - gp41 MAbs T4, D12, T3, and D50 bound less efficiently to gp140-GNC4 than did pooled sera, but T4 and D12 recognized the gp140-GNC4 timer equivalently to gp140(-), and T3 and D50 recognized the trimer at greater levels than gp140(-) - 2F5 did not bind efficiently to these constructs, presumably because of the YU2 strain has a substitution in the 2F5 epitope (ALDKWA instead of ELDKWA) [Yang2000]

- 2F5: SHIV-HXBc2 is a neutralization sensitive non-pathogenic virus, and several in vivo passages through monkey's yielded highly pathogenic SHIV KU-1 $-\mathrm{HXBc} 2$ and the KU-1 clone $\mathrm{HXBc} 2 \mathrm{P} 3.2$ differ in 12 amino acids in gp160 - substitutions in both gp120 and gp41 reduced the ability of sCD4, IgG1b12, F105 and AG1121 to Env achieve saturation and full occupancy, and neutralize KU-1 - 17b and 2F5 also bound less efficiently to HXBc2P3.2, although 2G12 was able to bind both comparably [Si2001]

- 2F5: ELNKWA is an escape variant not recognized by the broadly neutralizing MAb 2F5, which recognizes the core epitope ELDKWA - Abs were raised against the peptide escape variant CGELNKWAGELNKWA linked to KLH carrier - these polyclonal antibodies, like the monoclonal antibody TH-Ab1 also raised to ELNKWA, could recognize ELDKWA and escape mutant peptide epitopes ELEKWA and ELDEWA [Dong2001]

- 2F5: Mutations in two glcosylation sites in the V2 region of HIV-1 ADA at positions 190 and 197 (187 DNTSYRLINCNTS 199) cause the virus to become CD4-independent and able to enter cells through CCR5 alone - these same mutations tended to increase the neutralization sensitivity of the virus, including to antibody 2F5 [Kolchinsky2001]

- 2F5: A peptide called 5-Helix was designed that binds to the C-peptide region of gp41 - 5-Helix is a potent inhibitor of HIV-1 entry that binds immediately $\mathrm{COOH}$-terminal to the C-peptide region targeted by 5-Helix - the conformation of the bound 2F5 epitope is a hairpin turn [Root2001]

- 2F5: A phage peptide library was screened with MAb 2F5, and from the peptides that bound the amino acids DKW were found to be most critical for binding - the mimetic peptide RDWSFDRWSLSEFWL elicited a cross-reactive Ab response to gp41 when used to immunize rabbits [Tumanova2001] 


No. MAb ID HXB2 Location Author's Location $\quad$ Sequence $\quad$ Neutralizing Immunogen

- 2F5: Abs against the V3 loop (50.1, 58.2, 59.1, 257-D, 268-D, 447-52D), CD4BS (IgG1b12, 559-64D, F105), CD4i (17b), and to gp41 (2F5, F240) each showed similar binding efficiency to Env derived from related pairs of primary and TCLA lines (primary: 168P and 320SI, and TCLA: 168C and 320SI-C3.3), but the TCLA lines were much more susceptible to neutralization suggesting that the change in TCLA lines that make them more susceptible to NAbs alters some step after binding [York2001]

- 2F5: This paper primarily concerns 4E10 and Z13, MAbs that both bind proximally to the 2F5 binding site to a conserved epitope, and that neutralize some primary isolates from clades B, C, and E - the minimal 2F5 epitope is determined to be EQELLELDKWASLW, based on screening a gp160 fragment expression library, longer than previous studies - broadly neutralizing MAbs 2F5, IgG1b12, and 4E10 and Z13 fail to neutralize different subsets of viruses [Zwick2001b]

- 2F5: Neutralization synergy between anti-HIV NAbs b12, 2G12, 2F5, and 4E10 was studied - a classic fixed-ratio method was used, as well as a method where one $\mathrm{Ab}$ was fixed at a low neutralization titer and the other was varied - using primary isolates, a two-four fold enhancement of neutralization was observed with MAb pairs, and a ten-fold enhancement with a quadruple Ab combination - no synergy was observed with any MAb pair in the neutralization of TCLA strain HXB2 [Zwick2001c]

- 2F5: Review of studies in macaques that have shown immune control of pathogenic SHIV viremia, improved clinical outcome, and protection, and the implications of the observations for HIV vaccines [Mascola2001]

- 2F5: SF162DeltaV2 is a virus that has a 30 amino acids deletion in the V2 loop that does not abrogate its infectivity but renders it highly susceptible to neutralization - when incorporated into a codon-optimized DNA vaccine with a CMV promoter and delivered by gene gun, SF162DeltaV2 gave higher neutralizing Ab titers against SF162 than did SF162 itself, and Abs that cross-neutralized non-homologous primary isolates were obtained only when SF162DeltaV2, but not intact SF162, was used as the immunogen - Control MAbs 2F5 and 2G12 could neutralize all of the following primary isolates: 91US056(R5), 92US714(R5), 92US660(R5), 92HT593(R5X4), and BZ167(R5X4), while after the first protein boost, the sera from two SF162DeltaV2 immunized macaques could neutralize 91US056(R5), 92US714(R5), 92US660(R5) and ADA(R5), but not 92HT593(R5X4) or 92US657(R5) - the pattern of cross-recognition shifted after the second boost [Barnett2001]

- 2F5: Moore and colleagues review the data concerning the lack of a clear relationship between genetic subtype and serotype - 2F5 is considered in some detail, as it represents a rare vulnerability from the neutralizing antibody perspective, although while it is apparently linear, attempts to present the peptide to the immune system have failed to elicit neutralizing Abs [Moore2001]

- 2F5: Neutralizing synergy between MAbs 1b12, 2G12 and 2F5 was studied using surface plasmon resonance to determine the binding kinetics for these three mAbs with respect to monomeric and oligomeric env protein gp160 IIIB - the 2G12 epitope is highly accessible on both monomeric and oligomeric Envs, $1 \mathrm{~b} 12$ is highly accessible on monomers but not oligomers, and 2F5 on neither form - binding of 2G12 exposes the 2F5 epitope on gp160 oligomers [Zeder-Lutz2001]

- 2F5: Matrix-assisted laser desorption ionization mass spectrometry (MALDI-MS) in combination with proteolytic protection was used to identify the functional epitope for MAb 2F5, NEQELLELDKWASLWN, in the disulfide bond associated gp120/gp41 protein SOS-gp140 (JRFL) - this minimal epitope is much larger than the ELDKWA core epitope previously defined by peptide ELISA, and this could help explain why ELDKWA-peptides are poor immunogens in terms of eliciting a 2F5-like antibody response [Parker2001]

- 2F5: A luciferase-reporter gene-expressing T-cell line was developed to facilitate neutralization and drug-sensitivity assays - luciferase and p24 antigen neutralization titer end points were found comparable using NAb from sera from HIV+ donors, and MAbs 2F5, 2G12 and IgG1b12 [Spenlehauer2001]

- 2F5: A panel of 12 MAbs was used to identify those that could neutralize the dual-tropic primary isolate HIV-1 89.6 - six gave significant neutralization a 2 to $10 \mathrm{ug} / \mathrm{ml}$ : 2F5, 50-69, IgG1b12, 447-52D, 2G12, and 670-D six did not have neutralizing activity: 654-D, 4.8D, 450-D, 246-D, 98-6, and 1281 - no synergy, only additive effects were seen for pairwise combinations of MAbs, and antagonism was noted between gp41 MAbs 50-69 and 98-6, as well as 98-6 and 2F5 [Verrier2001]

- 2F5: $4 \mathrm{E} 10$ binds proximal to $2 \mathrm{~F} 5$ and neutralizes primary isolates of clades $\mathrm{A}, \mathrm{B}, \mathrm{C}, \mathrm{D}$, and $\mathrm{E}$ - viruses that were resistant to $2 \mathrm{~F} 5$ were neutralized by $4 \mathrm{E} 10$ and vice versa [Stiegler2001 


\begin{tabular}{lllll}
\hline No. MAb ID & HXB2 Location & Author's Location & Sequence & Neutralizing Immunogen
\end{tabular}

- 2F5: A combination of MAbs IgG1b12, 2F5, and 2G12 was given postnatally to four neonates macaques that were then challenged with highly pathogenic SHIV89.6P - one of the four infants remained uninfected after oral challenge, two infants had no or a delayed CD4(+) T-cell decline Hofmann-Lehmann2001

- 2F5: Twenty HIV clade C isolates from five different countries were susceptible to neutralization by anti-clade B MAbs in a synergistic quadruple combination of mAbs IgG1b12, 2G12, 2F5, and 4E10 [Xu2001]

- 2F5: Oligomeric gp140 (o-gp140) derived from R5 primary isolate US4 was characterized for use as a vaccine reagent - antigen capture ELISA was used to compare the antigenicity of gp120 and o-gp140 using a panel of well characterized MAbs - 2F5 recognized o-gp140 [Srivastava2002]

- 2F5: The fusion process was slowed by using a suboptimal temperature $(31.5 \mathrm{C})$ to re-evaluate the potential of Abs targeting fusion intermediates to block HIV entry - preincubation of E/T cells at 31.5 C enabled polyclonal anti-N-HR Ab and anti-six-helix bundle Abs to inhibit fusion, indicating six-helix bundles form prior to fusion - the preincubation $31.5 \mathrm{C}$ step did not alter the inhibitory activity of neutralizing Abs anti-gp41 2F5, or anti-gp120 2G12, IG1b12, 48d, and 17b [Golding2002b]

- 2F5: Ab binding characteristics of SOS gp140 were tested using SPR and RIPA - SOS gp140 is gp120-gp41 bound by a disulfide bond - NAbs 2G12, 2F5, IgG1b12, CD4 inducible 17b, and 19b bound to SOS gp140 better than uncleaved gp140 (gp140unc) and gp120 - non-neutralizing MAbs 2.2B (binds to gp41 in gp140unc) and 23A (binds gp120) did not bind SOS gp140 - SOS gp140-2F5-IgG1b12 formed multiple ring structures composed of two SOS gp140 proteins bridged by two Ab molecules, while 2F5 and 2G12 formed extended chains rather than closed rings [Schulke2002]

- 2F5: Expanding the minimal epitope ELDKWA to an end-capped, linear nonapeptide, Ac-LELDKWASL-amide attained maximal affinity within a set of native gp41-sequence peptides - scanning single residue substitutions confirmed that essential recognition requirements were the central DKW core sequence and the importance of the terminal Leu residues for high-affinity binding - high specificity binding pockets at central Lys and Trp side-chains and an absolute requirement for the carboxylate group of the Asp side chain were found - the nine residue fragment flanked by pairs of Ser and constrained by a disulfide bridge had high affinity for 2F5 [Tian2002]

- 2F5: ELDKWAS was embedded into a beta-turn-like conformational site on a framework of an antibody specific for human leukocyte antigen HLA-DR this construct was recognized by $2 \mathrm{~F} 5$, and is suggested as an adjuvant-independent vaccine candidate [Ho2002]

- 2F5: Passive immunization of neonate macaques with a combination of F105+2G12+2F5 conferred complete protection against oral challenge with SHIV-vpu+ or - the combination b12+2G12+2F5 conferred partial protection against SHIV89.6 - such combinations may be useful for prophylaxis at birth and against milk born transmission - the synergistic combination of IgG1b12, 2G12, 2F5, and $4 \mathrm{E} 10$ neutralized a collection of HIV clade C primary isolates [Xu2002]

- 2F5: A modified gp140 (gp140deltaCFI), with C-term mutations intended to mimic a fusion intermediate and stabilize trimer formation, retained antigenic conformational determinants as defined by binding to CD4 and to MAbs $2 \mathrm{~F} 5,2 \mathrm{G} 12, \mathrm{~F} 105$, and b12, and enhanced humoral immunity without diminishing the CTL response in mice injected with a DNA vaccine [Chakrabarti2002]

- 2F5: DP178 is a peptide derived from the C-term heptad repeat of gp41 that is a potent inhibitor of viral-mediated fusion—it contains the 2F5 epitope but fails to stimulate 2F5-like NAbs upon immunization - the peptide was extended to force an increase in helicity, and the modified peptide had a increase in affinity for 2F5, but upon guinea pig immunization although high peptide-specific $\mathrm{Ab}$ titers were achieved the sera were incapable of viral neutralization - the authors propose that 2F5 may bind with low affinity to a maturation intermediate, which may account for its breadth and why it is hard to recreate the epitope, but also suggests that the high concentrations required for neutralization are not relevant in vivo [Joyce2002].

- 2F5: Six sera from HIV-exposed uninfected individuals(EU) had IgA neutralizing activity dominated by recognition of a distinctive epitope within gp41, QARILAV - sera of QAFILAV-immunized BALB/c mice was neutralizing with the dose-dependent behavior similar to 2F5 [Clerici2002a]

- 2F5: A combination of MAbs $2 \mathrm{~F} 5$ and 2G12 given in multiple infusions was found to be safe and well tolerated even in high doses in a phase I study of seven HIV-1 infected healthy volunteers-the median elimination half-life was 7.94 days for $2 \mathrm{~F} 5$, and 16.48 for 2G12 —no anti-2F5 or anti-2G12 IgM or IgG responses were detected - although there was some transient increases, overall plasma viral RNA levels decreased in 6/7 volunteers, by a median of 0.62 log_10 Armbruster2002]. 


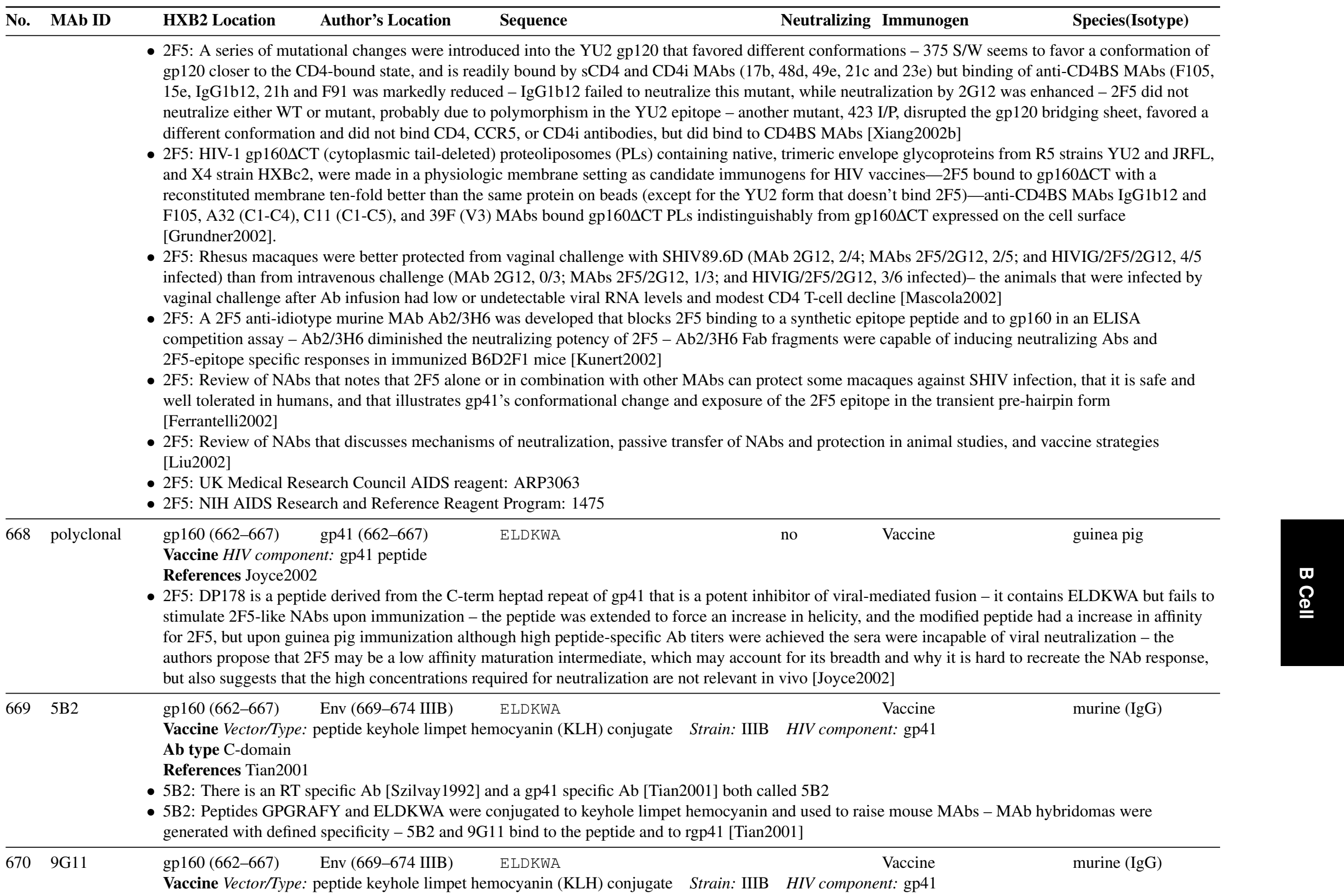




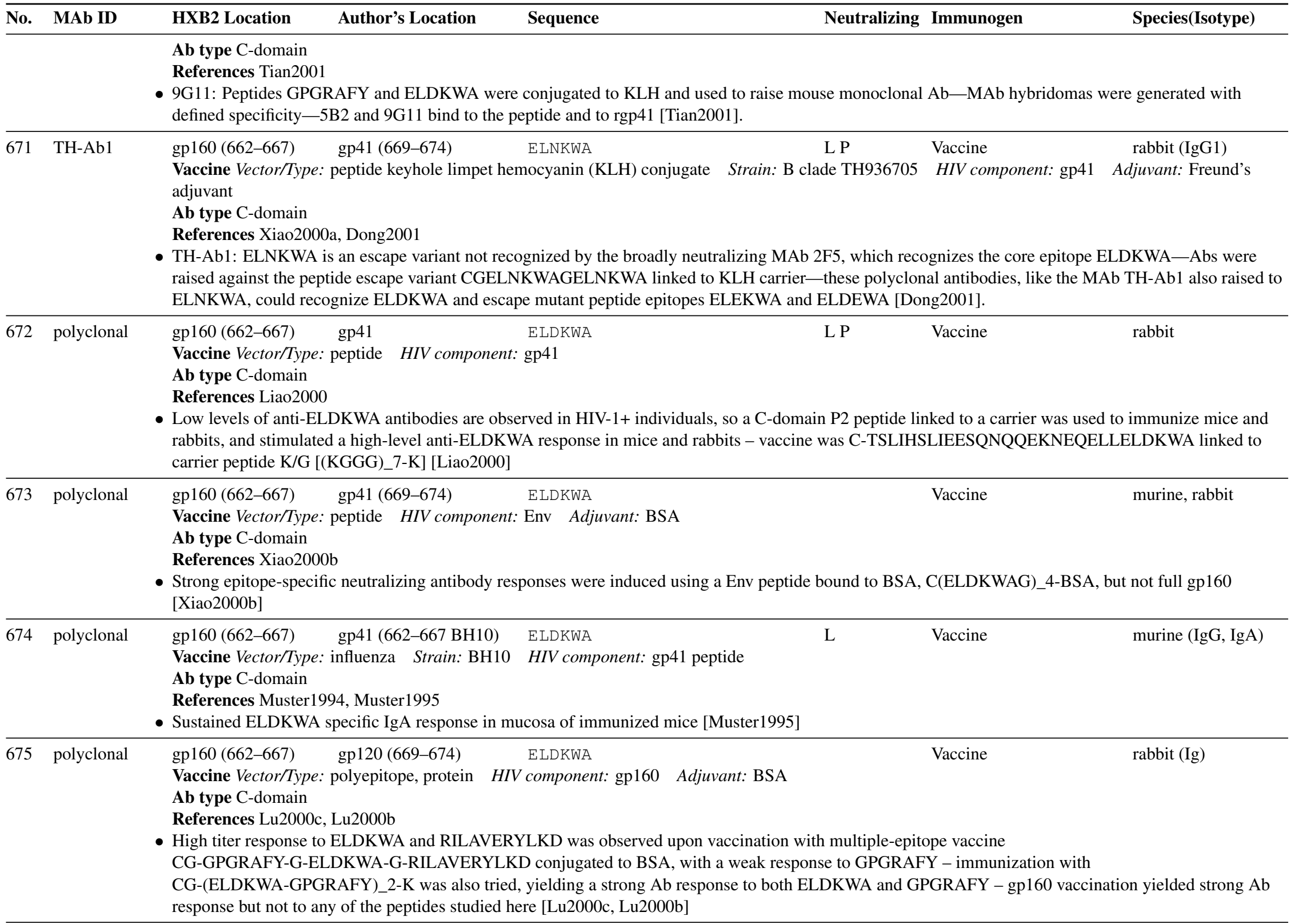




\begin{tabular}{|c|c|c|c|c|c|c|c|}
\hline No. & MAb ID & HXB2 Location & Author's Location & Sequence & Neutralizing & Immunogen & Species(Isotype) \\
\hline 676 & 4E10 & gp160 (671-676) & gp160 (671-676 MN) & NWFDIT & $\mathrm{P}$ & HIV-1 infection & human $(\operatorname{IgG} 3 \kappa)$ \\
\hline
\end{tabular}

References Buchacher1992, Buchacher1994, D’Souza1994, Stiegler2001, Zwick2001b, Zwick2001c, Xu2001, Xu2002, Ferrantelli2002

- 4E10: MAbs generated by hybridoma, electrofusion of PBL from HIV-1+ volunteers with CB-F7 heteromyeloma cells - also binds to MHC class II proteins - anti-class II Abs are only found in HIV-1 positive people - this paper maps 4E10's binding site to AEGTDRV, gp160(823-829), but the later Zwick et al. study in 2001 revised the epitope location [Buchacher1994]

- 4E10: Included in a multi-lab study for antibody characterization, binding and neutralization assay comparison [D’Souza1994]

- 4E10: 4E10 binds proximal to $2 \mathrm{~F} 5$ and neutralizes primary isolates of clades A, B, C, D, and E - viruses that were resistant to $2 \mathrm{~F} 5$ were neutralized by 4E10 and vice versa [Stiegler2001]

- 4E10: MAbs 4E10 and Z13 both bind proximally to 2F5 to a conserved linear epitope that has some conformational aspects - both bind to MN virions, bind weakly to infected cells in a manner that is not disrupted by sCD4 and neutralize some primary isolates from clades B, C, and E - maps minimal 4E10 epitope to NWFDIT, contrary to an earlier report - different strains were refractive to neutralization by broadly neutralizing Abs IgG1b12, 2F5, Z13 and 4E10 [Zwick2001b]

- 4E10: Neutralization synergy between anti-HIV NAbs b12, 2G12, 2F5, and 4E10 was studied - a classic fixed-ratio method was used, as well as a method where one $\mathrm{Ab}$ was fixed at a low neutralization titer and the other was varied - using primary isolates, a two-four fold enhancement of neutralization was observed with MAb pairs, and a ten-fold enhancement with a quadruple Ab combination - no synergy was observed with any MAb pair in the neutralization of TCLA strain HXB2 [Zwick2001c]

- 4E10: Twenty HIV clade C isolates from five different countries were susceptible to neutralization by anti-clade B MAbs in a synergistic quadruple combination of mAbs IgG1b12, 2G12, 2F5, and 4E10 [Xu2001]

- 4E10: Passive immunization of neonate macaques with a combination of F105+2G12+2F5 conferred complete protection against oral challenge with SHIV-vpu+ - the combination b12+2G12+2F5 conferred partial protection against SHIV89.6 - such combinations may be useful for prophylaxis at birth and against milk born transmission - the synergistic combination of IgG1b12, 2G12, 2F5, and 4E10 neutralized a collection of HIV clade C primary isolates [Xu2002].

- 4E10: Review of NAbs illustrating gp41's conformational change and exposure of the 4E10/Z13 epitope in the transient pre-hairpin form [Ferrantelli2002]

gp160 (671-676) gp41 (671-676 MN) NWFDIT $\quad$ P $\quad$ HIV-1 infection

Ab type C-term

References Zwick2001b, Ferrantelli2002

- Z13: MAb 4E10 and FAb Z13 both bind proximally to 2F5 to a relatively conserved linear epitope that has some conformational aspects - both bind to MN virions, bind weakly to infected cells in a manner that is not disrupted by sCD4 and can neutralize some primary isolates from clades B, C, and E - Z13 was selected using a phage display library with the MN gp41 peptide LLELDKWASLWNWFDITNWSW from an HIV infected donor who had an exceptionally broad NAb response - different strains were refractive to neutralization by broadly neutralizing Abs IgG1b12, 2F5, Z13 and 4E10 - epitope location noted here is by analogy to MAb 4E10 [Zwick2001b]

- Z13: Review of NAbs that notes Z13 is a phage display generated FAb fragment from a B clade infected individual and that illustrates gp41's conformational change and exposure of the 4E10/Z13 epitope in the transient pre-hairpin form [Ferrantelli2002]

Vaccine Vector/Type: recombinant protein Strain: LAI HIV component: gp160

Vaccine

murine (IgG1)

Donor George Lewis

References Abacioglu1994

- B30: Epitope boundaries mapped by peptide scanning [Abacioglu1994] 


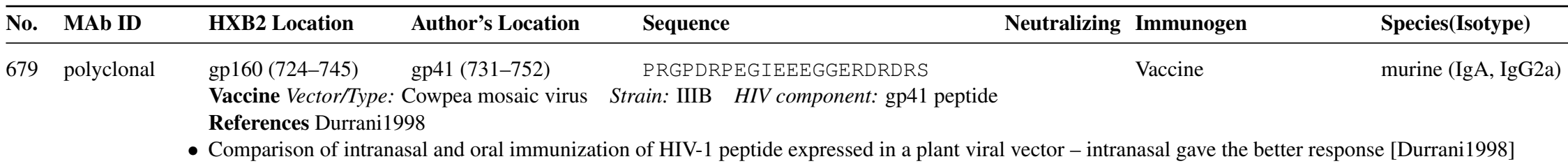

$680 \quad 41 S-2$

gp160 (725-745) gp160 (732-750) RGPDRPEGIEEEGGERDRDRS yes

Vaccine Vector/Type: peptide keyhole limpet hemocyanin (KLH) conjugate HIV component: gp41

References Hifumi2000

- 41S-2: BALBc mice were immunized with gp41 peptide and a MAb specific for the peptide was generated - isolated MAb light chains displayed proteolytic activity toward the peptide epitope which may be due to a catalytic triad on light chain (Asp73, Ser76, and His79) - no catalytic activity was observed for the whole antibody [Hifumi2000]

$681 \quad 447-52 \mathrm{D}$ gp160 (726-729) gp120 (MN) GPXR

(447/52-DII, Ab type V3 Donor Dr. Susan Zolla-Pazner, NYU Med Center NY, NY, or Cellular Products Inc, Buffalo, NY, USA

447-52-D, References Gorny1992, Buchbinder1992, Karwowska1992b, Gorny1993, Keller1993, Cavacini1993a, Spear1993, Conley1994a Laal1994, VanCott1994

447-52-D,

447-D, 447,

Gorny1994 Moore1994a, Sattentau1995a Fontenot1995, Saarloos1995, Zolla-Pazner1995a |Zolla-Pazner1995b |Moore1995a, Moore1995b |Forthal1995 Jagodzinski1996, Trkola1996a, Sattentau1996, D’Souza1997, Binley1997a, Fouts 1997, Hioe1997a, Hioe 1997b, Boots1997, Parren1997c, Hill1997.

Gorny 1997 Inouye1998 |Mondor1998, Smith1998, Parren1998a Zolla-Pazner1999a Zolla-Pazner1999b, Connor1998 Gorny1998 |Nyambi1998

Hioe1999., Beddows1999 Sharon2002 Gorny2002 He2002, Ferrantelli2002, Poignard2003

- 447-52D: Requires GPXR at the tip of the V3 loop - neutralizes a broad array of B clade lab isolates [Gorny1992]

- 447-52D: 60-fold increase in neutralization potency when combined 1:1 with human MAb 588-D [Buchbinder1992]

- 447-52D: Reacts with MN, NY5, CDC4, SF2, RF, WM52, and HXB2 [Karwowska1992b]

- 447-52D: Neutralizes MN and IIIB: GPGR, and binds SF2: GPGR [Gorny1993

- 447-52D: Peptide phage library showed that any of the residues ADGLMNQRS in the X position tolerated in peptides that react well with the antibody |Keller1993|

- 447-52D: Additive neutralization of MN and SF2 when combined with CD4 binding site MAb F105 - supra-additive neutralization of RF [Cavacini1993a]

- 447-52D: Complement mediated virolysis of IIIB, but not in the presence of SCD4 [Spear1993]

- 447-52D: Requires GPxR at the tip of the V3 loop, common in B clade - neutralized primary isolates [Conley1994a

- 447-52D: Neutralization synergy in combination with CD4 binding domain MAbs [Laal1994]

- 447-52D: GPGQ in MAL resulted in enhanced dissociation - GPGQ in CM234 or K14T did not bind - binding affected by identity of amino acids flanking GPGR core [VanCott1994

- 447-52D: Mild oxidation of carbohydrate moieties does not alter binding [Gorny1994]

- 447-52D: Competition studies with human sera from seroconverting individuals showed that anti-CD4 BS antibodies can arise very early in infection, comparable or prior to anti-V3 antibodies [Moore1994a]

- 447-52D: Called 447d - Formalin inactivation of virus at $0.1 \%$ formalin for 10 hours at 4 degrees was optimal for inactivation of virus while maintaining epitope integrity [Sattentau1995c]

- 447-52D: Called 447 - The tip of the V3 loop was presented in a mucin backbone - higher valency correlates with stronger affinity constant [Fontenot1995

- 447-52D: Ab-mediated activation of complement on HIV+ cells is higher than Ab independent activation-what has been termed "Ab independent" in fact results in part from IgM in normal human serum that is HIV-cross-reactive [Saarloos1995]

- 447-52D: Serotyping study using flow-cytometry - bound only to GPGR V3 loop tips [Zolla-Pazner1995a]

- 447-52D: Neutralization of primary and prototype laboratory HIV-1 isolates using a resting cell assay enhances sensitivity [Zolla-Pazner1995b 


No. MAb ID HXB2 Location Author's Location $\quad$ Sequence $\quad$ Neutralizing Immunogen

- 447-52D: Binding affected by identity of amino acids flanking GPGR core - poor breadth of primary virus neutralization |Moore1995a

- 447-52D: Review: the V3 loop motif GPGR is not common outside subtype B isolates, MAb 19b is more cross-reactive [Moore1995b]

- 447-52D: Neutralizing (- complement), no ADCC activity, and no viral enhancing activity [Forthal1995]

- 447-52D: Called 447-52-D - The sulfated polysaccharide curdlan sulfate (CRDS) binds to the Envelope of T-tropic viruses and neutralizes virus - CRDS inhibits binding [Jagodzinski1996]

- 447-52D: Neutralizes JR-FL - strongly inhibits gp120 interaction with CCR-5 in a MIP-1beta-CCR-5 competition study [Trkola1996a]

- 447-52D: Review: called 447-52-D - only four epitopes have been described which can stimulate a useful neutralizing response to a broad spectrum of primary isolates, represented by the binding sites of MAbs: 447-52-D, 2G12, Fab b12, and 2F5 [Sattentau1996]

- 447-52D: In a multilaboratory blinded study, failed to consistently neutralize any of nine B clade primary isolates - many of these isolates had the GPGR motif at the apex of the V3 loop [D'Souza1997]

- 447-52D: Study shows neutralization is not predicted by MAb binding to JRFL monomeric gp120, but is associated with oligomeric Env binding - 447-52D bound monomer, oligomer, and neutralized JRFL [Fouts1997]

- 447-52D: Tested using a resting cell neutralization assay [Hioe1997a]

- 447-52D: Four primary isolates showed distinct patterns of sensitivity to neutralization by polyclonal sera or plasma and MAbs - BZ167 was the only isolate inhibited by all polyclonal sera and plasma tested, and was also neutralized by 8/17 MAbs, in particular anti-V3 loop (419-D, 447-52D, 782-D, and 838-D), anti-CD4bd (559/64-D, 654-D and 830-D and a cluster II of gp41 directed MAb (98-6) - isolates $92 \mathrm{HT} 593$ and 91 US056 were neutralized by V3 loop (419-D, and 447-52D)and cluster II gp41 (98-6) MAbs at higher concentrations - US4 was neutralized by some of the polyclonal sera/plasma tested and not at all by MAbs individually or by a cocktail of ten MAbs consisting of 419-D, 447-52D, 782-D, 838-D, 559/64-D, 654-D, 450-D, 670-D, 1281-D and 98-6 [Hioe1997b

- 447-52D: Viral binding inhibition by 447-D was correlated with neutralization (all other neutralizing MAbs tested showed some correlation except 2F5) |Ugolini1997|

- 447-52D: Neutralizes TCLA strains but not primary isolates [Parren1997c]

- 447-52D: Called 447 - gp120 can inhibit MIP-1alpha from binding to CCR5, but this inhibitory effect is blocked by pre-incubation of gp120 with three anti-V3 MAbs: 447, 257, 1027 - MAb 670 which binds in the C5 region had no effect [Hill1997]

- 447-52D: Abs that recognize discontinuous epitopes can identify mimotopes from a phage peptide display library - 447-52D has an epitope involving the tip of the V3 loop, that was previously studied with this method [Keller1993] - in Keller et al., with no competition, LxGPxR was the most common six-mer, 38\% of the peptides - after competition with a gp120 IIIB ligand (QRGPGR)i, RGPxR was the most common and one peptide had the sequence QRGPGR, showing type specific mimotopes can be enriched by strain specific ligand competition protocols [Boots1997]

- 447-52D: Used as a control for comparison to five V3 RF selected antibodies - 447-52D was reactive with A, B, and C clade peptides, but not E |Gorny1997|

- 447-52D: Called 447-D - 447-D resistance took longer to acquire in virus with the M184V substituted RT, and had the form (AAC N to TAC Y) at position 5 of the V3 loop, rather than the GPGR to GPGR resistance found with wildtype RT [Inouye1998]

- 447-52D: Inhibits binding of Hx10 to both CD4 positive and negative HeLa cells [Mondor1998]

- 447-52D: Called 447-52-D - The tip of the MN V3 loop was inserted into cold causing human rhinovirus 14 (HRV14) - chimeras were immunoselected, and chimeric viruses were neutralized by anti-V3 loop antibodies, and 447-52D was among the Abs used - chimeric viruses elicited potent NAbs in guinea pigs against ALA-1 and MN [Smith1998]

- 447-52D: The MAb and Fab binding to the oligomeric form of gp120 and neutralization were highly correlated - authors suggest that neutralization is determined by the fraction of Ab sites occupied on a virion irrespective of the epitope [Parren1998a]

- 447-52D: Ab from gp120 vaccinated individuals prior to infection, who subsequently became HIV infected, could not achieve $90 \%$ neutralization of the primary virus by which the individuals were ultimately infected - these viruses were not particularly refractive to neutralization, as determined by their susceptibility to neutralization by MAbs 2G12, IgG1b12, 2F5 and 447-52D [Connor1998] 


No. MAb ID $\quad$ HXB2 Location $\quad$ Author's Location $\quad$ Sequence $\quad$ Neutralizing Immunogen

- 447-52D: Kinetic parameters were measured, and the association rates were similar, but dissociation rate constants were quite variable for V3 MAbs, 1324E was comparable to 447-52D [Gorny1998]

- 447-52D: Using a whole virion-ELISA method, 18 human MAbs were tested for their ability to bind to a panel of 9 viruses from clades A, B, D, F, G, and $\mathrm{H}-447-52 \mathrm{D}$ was the most potent and cross-reactive of 18 human MAbs tested and was the only MAb which bound to virions from isolates CA20 (subtype F), CA13 (subtype H), and VI526 (subtype G) [Nyambi1998]

- 447-52D: Review of clade specificity and anti-V3 HIV-1-Abs [Zolla-Pazner1999a]

- 447-52D: MAb peptide-reactivity pattern clustered with the immunological related MAbs: 1334, 419, 504, 447, 453 and 537 - the core amino acids GP tended to be critical for reactivity in this group - 447 reacted with peptides containing GPGR, but also with many lacking this sequence (GPGQ, for example), and it failed to react with 2/14 peptides containing GPGR, illustrating the importance of context [Zolla-Pazner1999b]

- 447-52D: The presence of leukocyte function-associated molecule 1 (LFA-1) promotes virus infectivity and hinders neutralization, and anti-LFA-1 MAbs can enhance the neutralizing effect of anti-HIV V3 MAb 447-52D and anti-HIV CD4BS MAb IgG1b12 - non-neutralizing anti-HIV CD4BS MAb 654-D did not become neutralizing in the presence of anti-LFA-1 MAbs [Hioe1999]

- 447-52D: rgp120 derived from a R5X4 subtype B virus, HIV-1 W61D, was used to vaccinate healthy volunteers and the resulting sera were compared with sera from HIV-1 positive subjects and neutralizing MAbs - TCLA strains showed enhanced 447-52D neutralization sensitivity relative to PBMC-adapted lines (32X increase between HIV-1(M2424/PBMC(p0)) and HIV-1(M2424/H9(p9)) and a >128X increase between HIV-1(W61D/PBMC) and HIV-1(W61D/SupT1) isolates) [Beddows1999]

- 447-52D: Binding of panel of 21 MAbs to soluble oligomeric gp140 versus gp41 or gp120 monomers was compared - no MAb was oligomer specific, though anti-V3 and CD4BS MAbs reacted better with the oligomer and V2 and C5 tended to favor the monomer - V3 MAbs 447-52D, 838-D, and 1334 bound with a 7-10 fold preference for the oligomer [Gorny2000a]

- 447-52D: To determine the antigenicity of virus killed by thermal and chemical inactivation, retention of conformation-dependent neutralization epitopes was examined, and exposure of CD4BS epitopes was found to be enhanced (MAbs IgG1b12, 205-46-9, and 205-43-1) - binding to 2G12 and 447-52D epitopes was essentially unaltered - the 17b CD4i epitope was also exposed [Grovit-Ferbas2000]

- 447-52D: Ab responses, because of their capacity to alter antigen uptake and processing, can influence helper T cell responses - CD4BS MAbs or serum Ig from HIV+ individuals inhibited proliferative responses of gp120 specific T cells - V3 MAbs 447-52-D and 268-10-D did not effect proliferation [Hioe2000]

- 447-52D: Called 447D - SF162 is a neutralization-resistant HIV-1 isolate - N-linked glycosylation modifications in the V2 loop of the SF162 gp120 revealed that these sites prevent neutralization by CD4BS MAbs (IgG1b12 and IgGCD4), and protect against neutralization by V3 MAbs (447D and 391-95D) - V2-region glycosylation site mutations did not alter neutralization resistance to V2 MAbs (G3.4 and G3.136) or CD4i MAbs (17b and 48d) V2 glycosylation site modification allows infection of macrophages, probably due to glycosylated forms requiring fewer CCR5 molecules for viral entry [Ly2000]

- 447-52D: A panel of 47 human MAbs was tested against 26 HIV-1 group M primary isolates from clades A through H - 19 V3 MAbs were tested, and of 494 combinations, $44 \%$ displayed some viral binding - V3 MAbs tended to have the most cross-reactive binding to clade A, B, C, and D isolates, less to E, F, G, and $\mathrm{H}-447-52 \mathrm{D}$ showed the highest cross-reactivity, bound to 24/26 viruses tested, but achieved $90 \%$ neutralization only against MN, 50\% against CA5, and no neutralization was observed for 3 other isolates tested [Nyambi2000]

- 447-52D: Six mutations in MN change the virus from a high-infectivity neutralization resistant phenotype to low-infectivity neutralization sensitive - V3, CD4BS, and CD4i MAbs are 20-100 fold more efficient at neutralizing the sensitive form - the mutation L544P reduced binding of all MAbs against gp120 by causing conformational changes [Park2000]

- 447-52D: Abs against the V3 loop (50.1, 58.2, 59.1, 257-D, 268-D, 447-52D), CD4BS (IgG1b12, 559-64D, F105), CD4i (17b), and to gp41 (2F5, F240) each showed similar binding efficiency to Env derived from related pairs of primary and TCLA lines (primary: 168P and 320SI, and TCLA: 168C and 320SI-C3.3), but the TCLA lines were much more susceptible to neutralization suggesting that the change in TCLA lines that make them more susceptible to NAbs alters some step after binding - the dissociation constant, $\mathrm{Kd}$ of 447-52D for the cell associated primary and TCLA Envs was equal, 3nM York2001] 


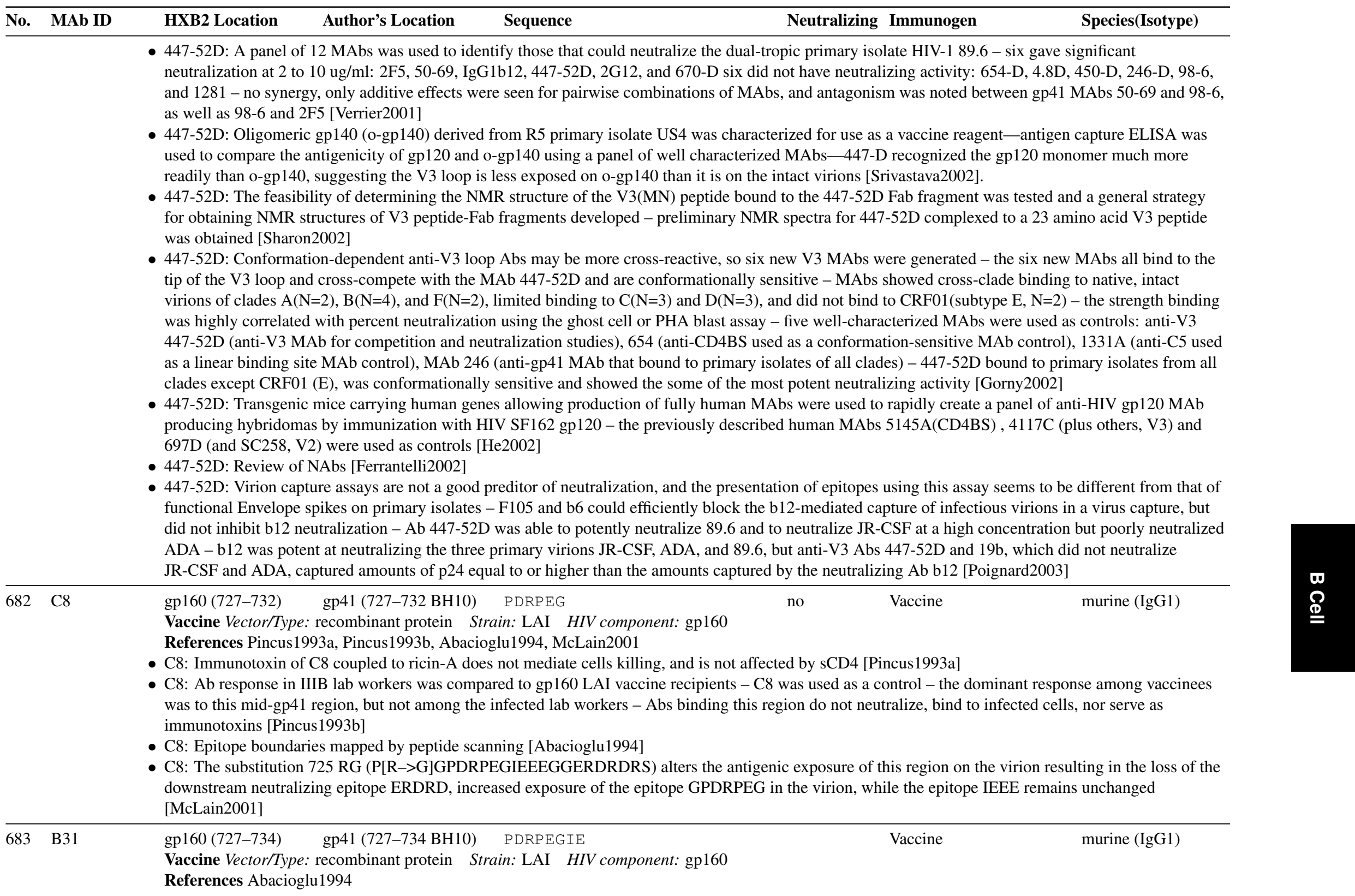




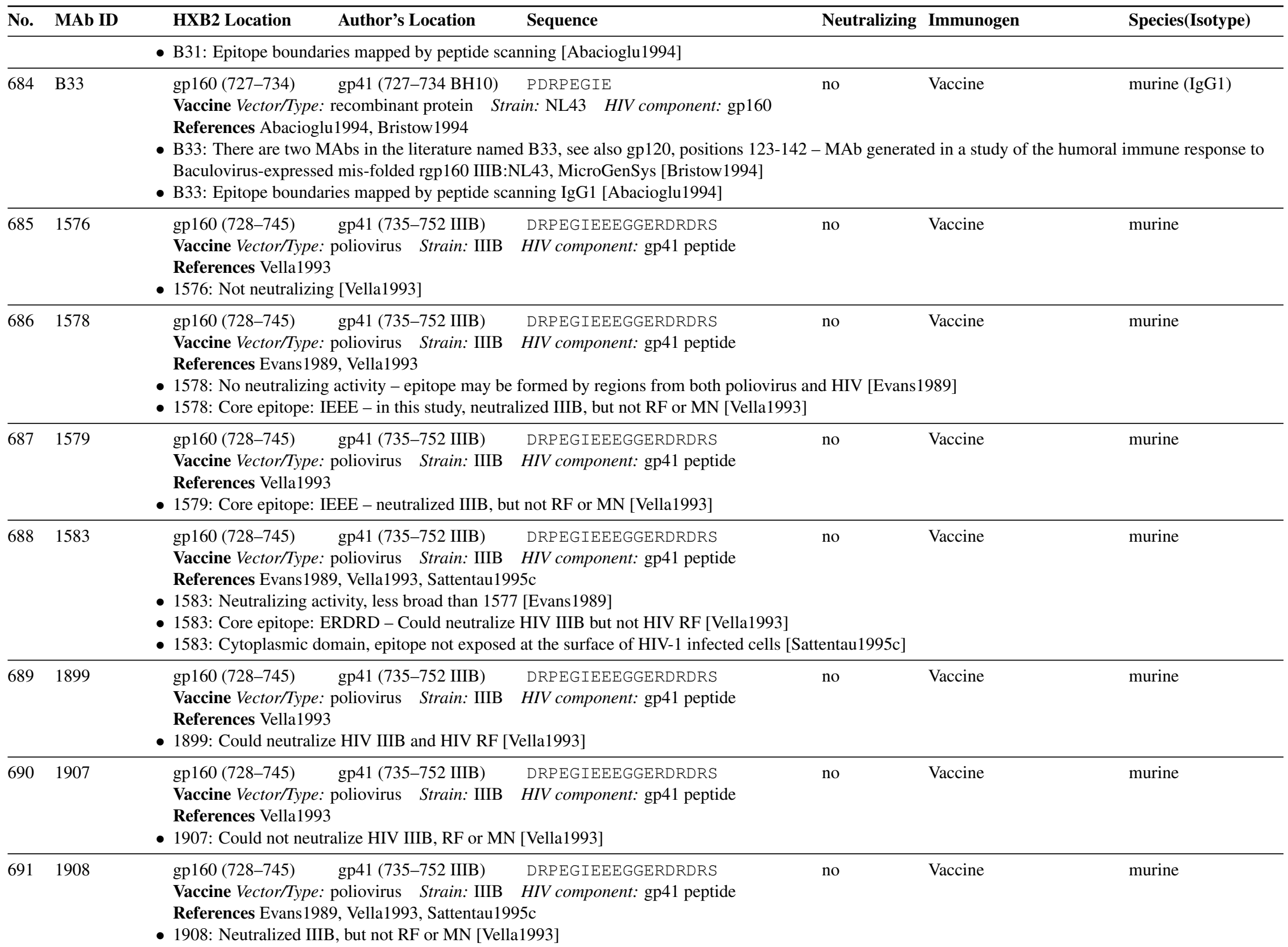




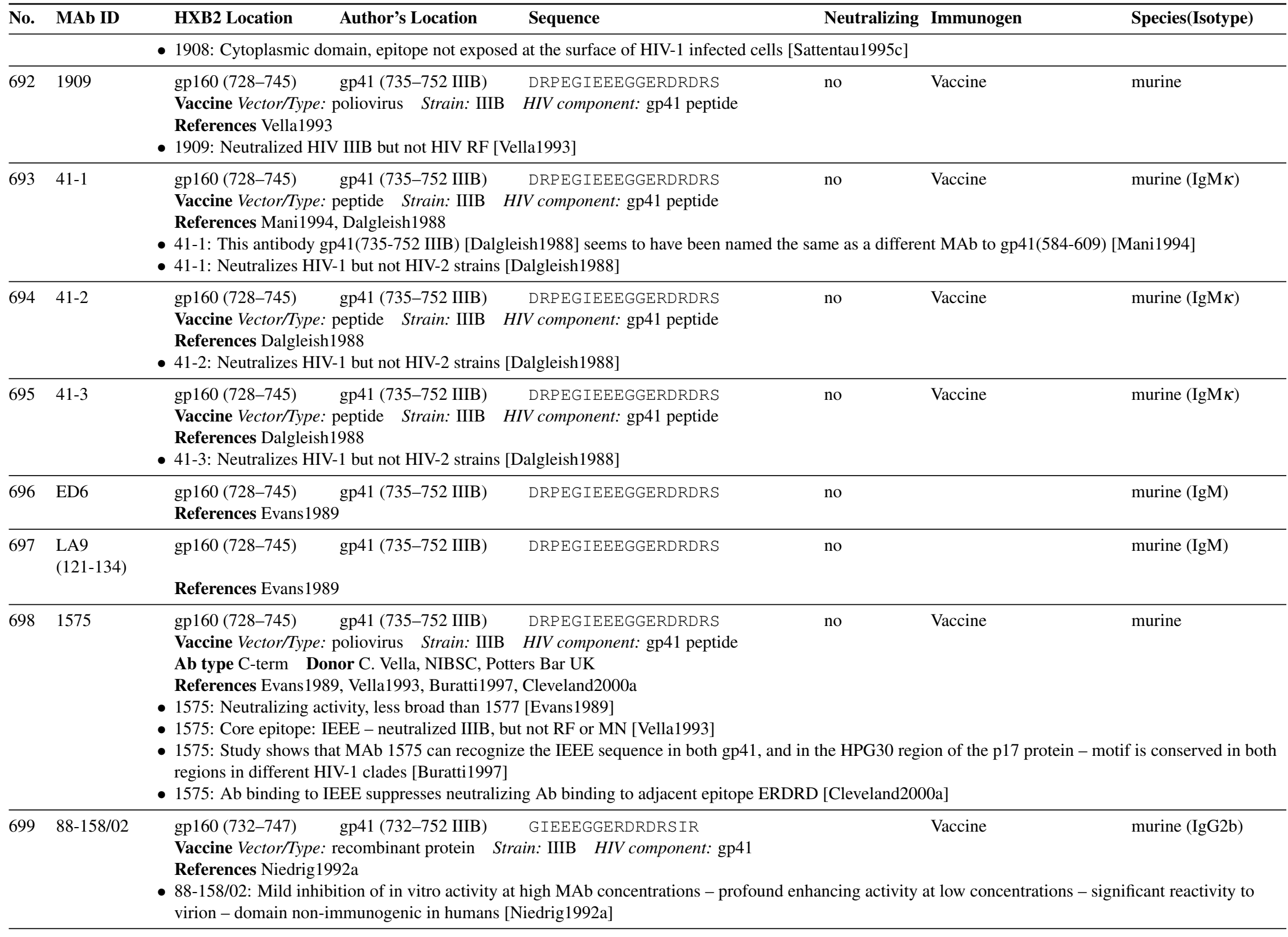




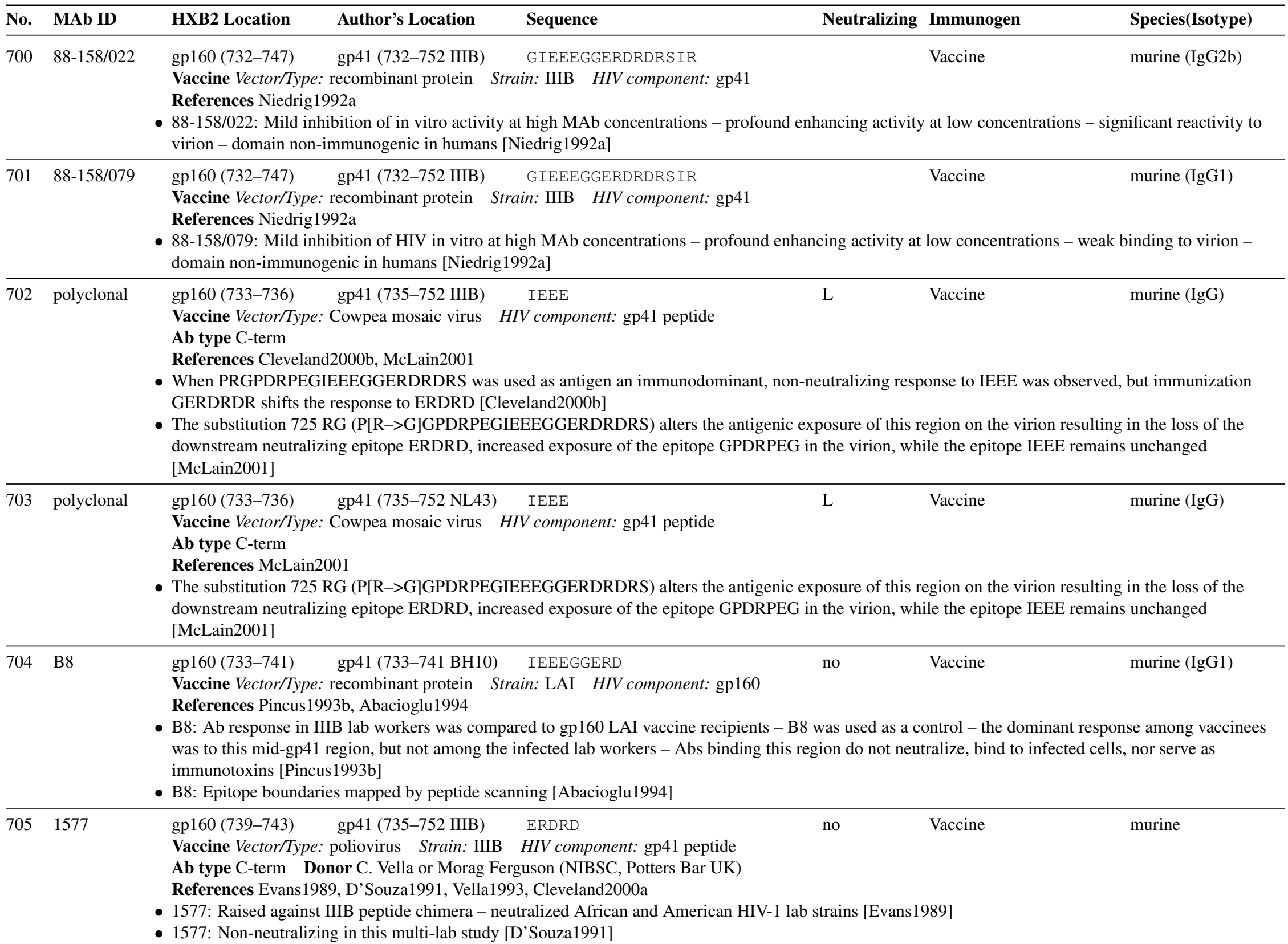




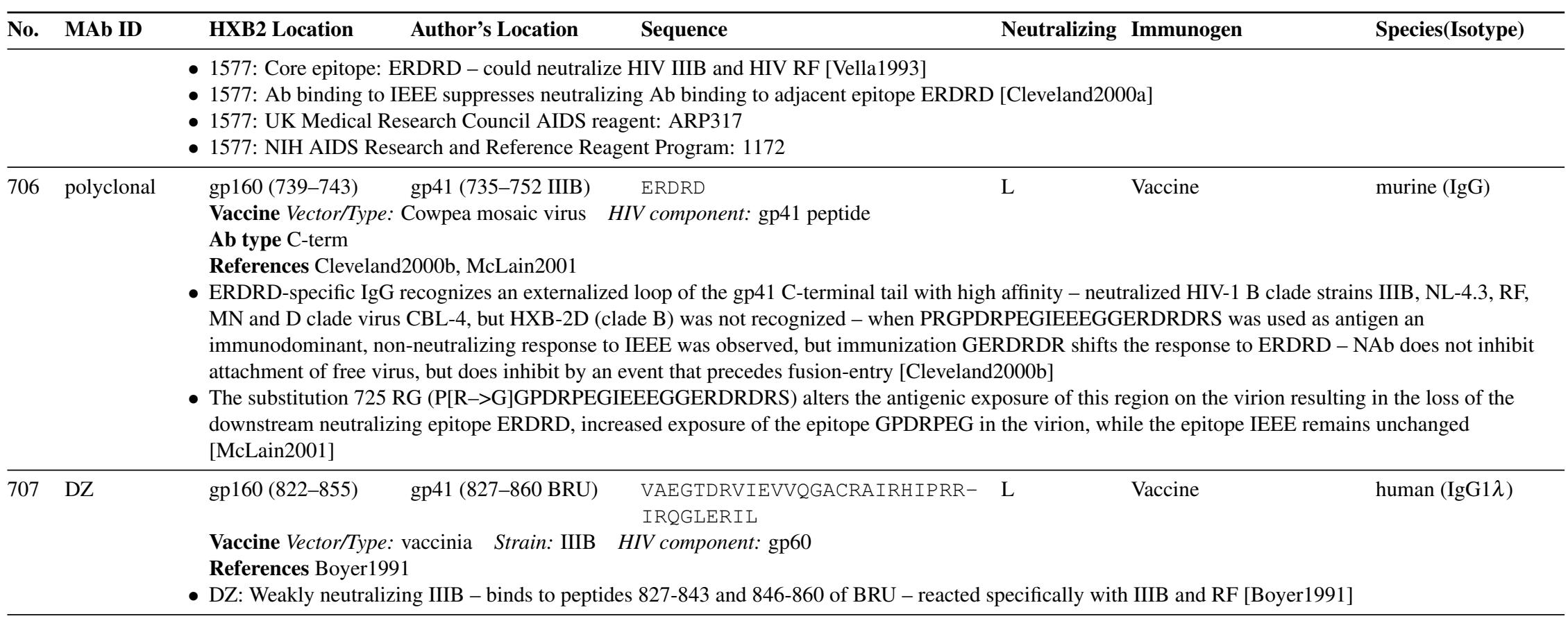




\section{IV-C-14 Env Antibodies}

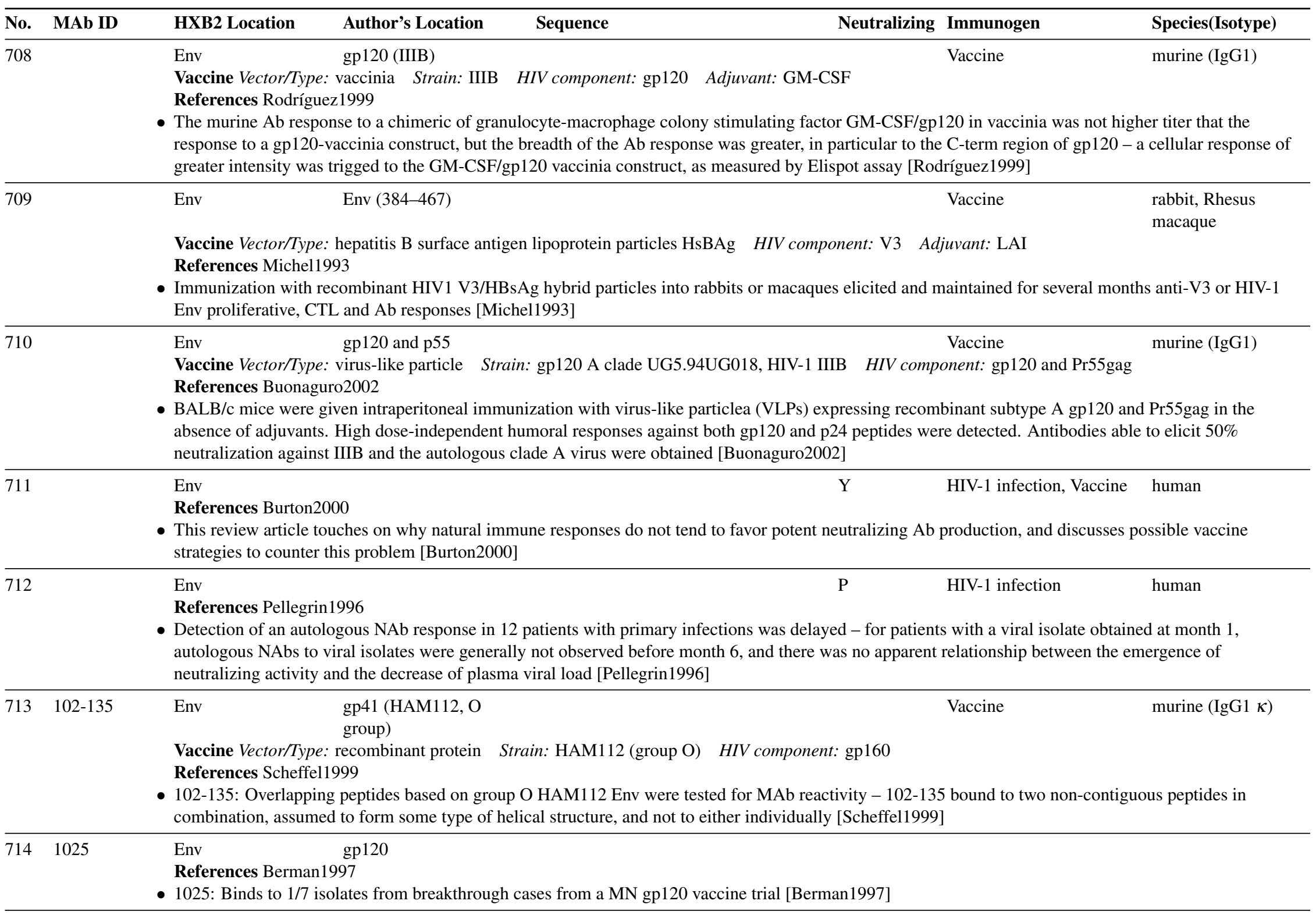




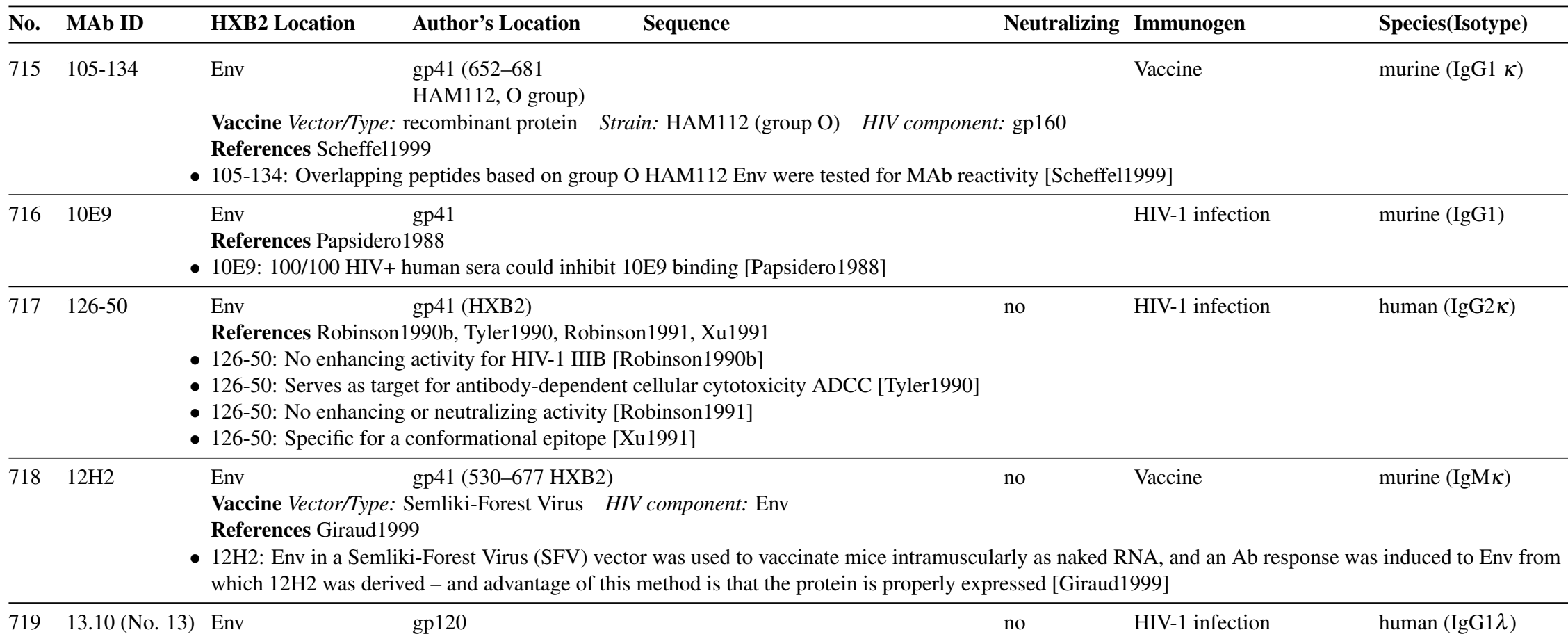

Donor Evan Hersh and Yoh-Ichi Matsumoto

References Lake1989, Moran1993 Wisnewski1996

- 13.10: First HIV-1 specific human-mouse hybridoma that produces a MAb that binds to gp120 and gp160 [Lake1989]

- 13.10: Heavy (V HI) and light (V lambdaII) chain sequenced - no enhancing or neutralizing activity - called No. 13 [Moran1993

- 13.10: 13.10 is V H1 - V-region heavy chain usage was examined and a bias of enhanced V H1 and V H4, and reduced V H3, was noted among HIV infected individuals [Wisnewski1996]

- 13.10: NIH AIDS Research and Reference Reagent Program: 377

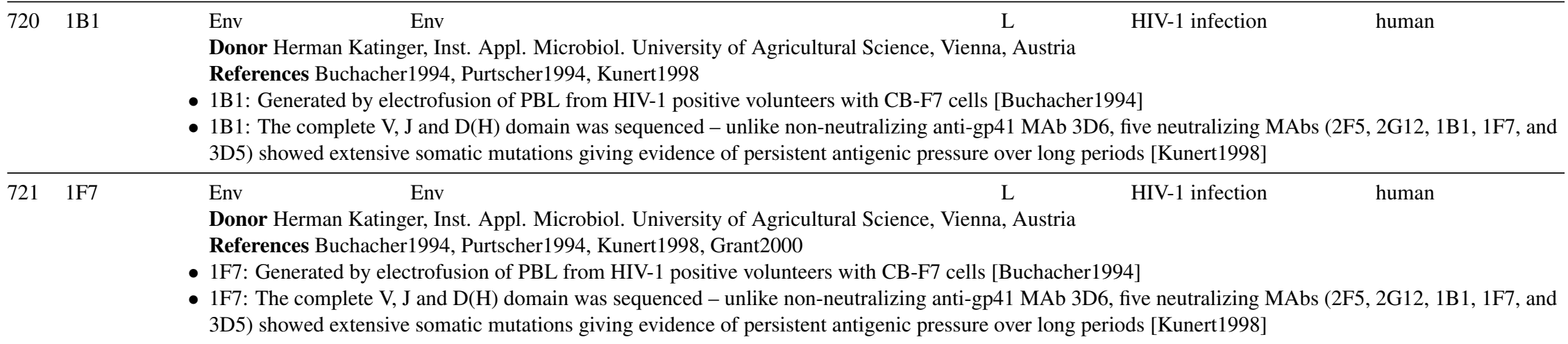




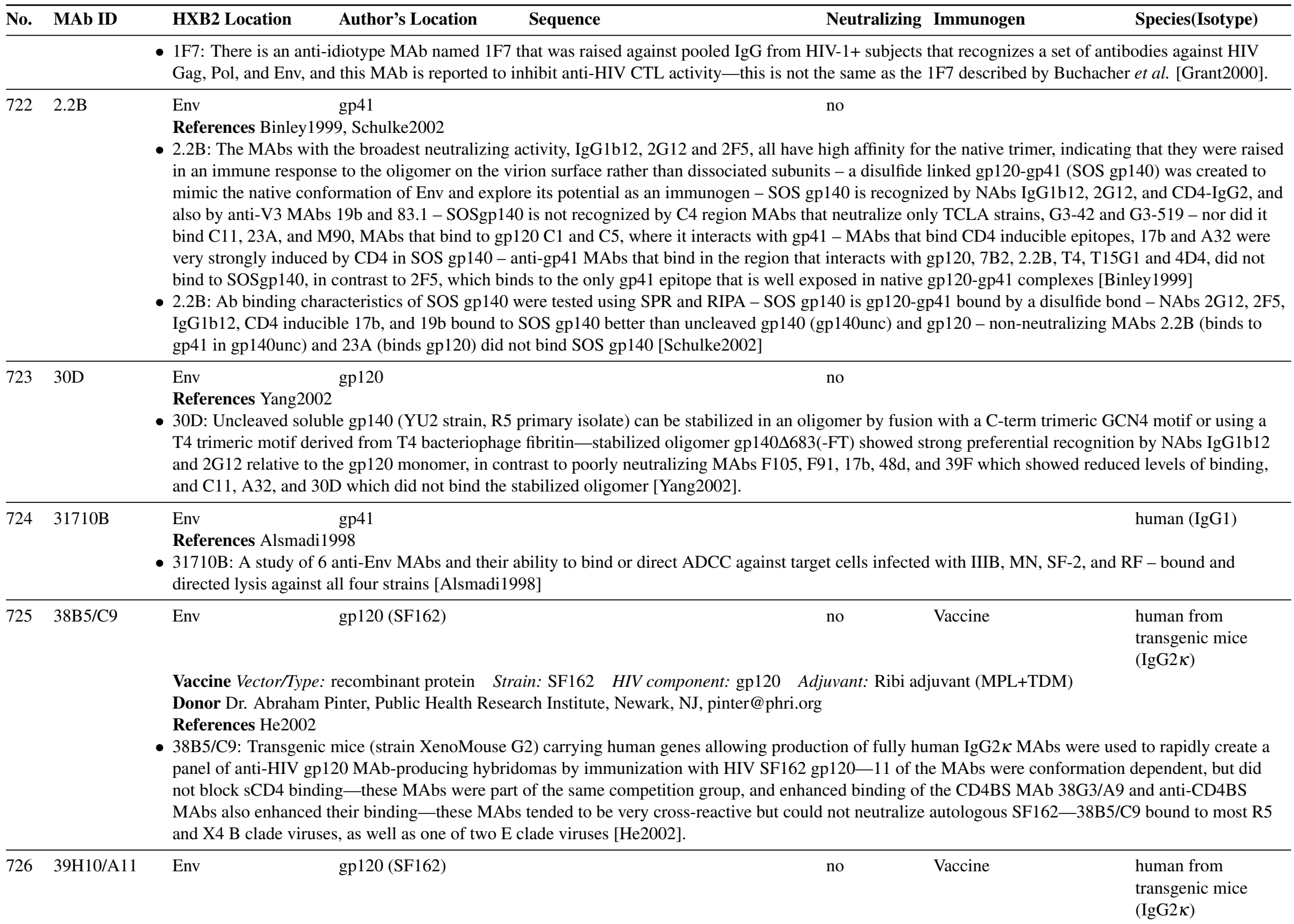

Vaccine Vector/Type: recombinant protein Strain: SF162 HIV component: gp120 Adjuvant: Ribi adjuvant (MPL+TDM) $(\operatorname{IgG} 2 \kappa)$ 


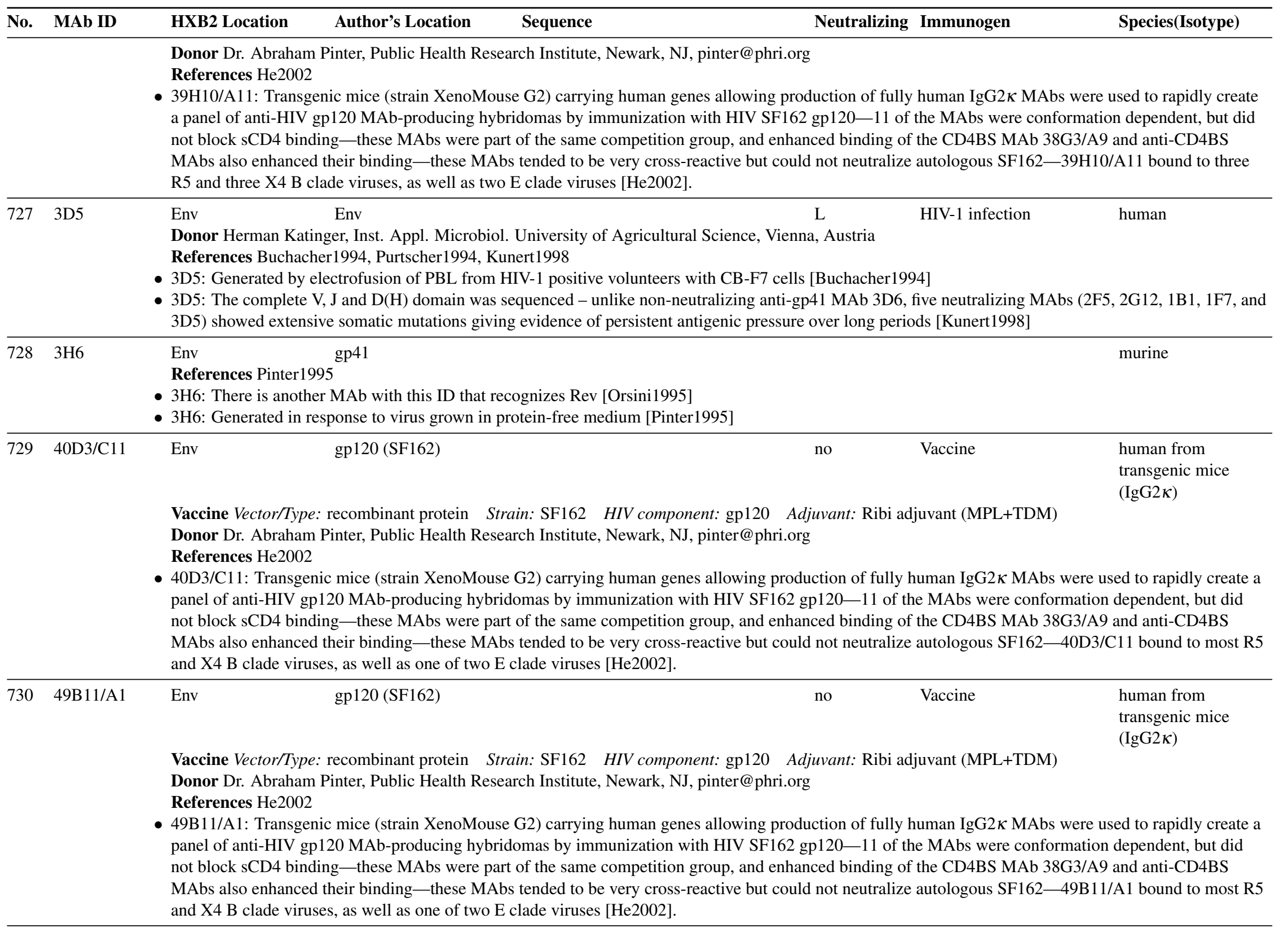




\begin{tabular}{|c|c|c|c|c|c|c|}
\hline No. & MAb ID & HXB2 Location & Author's Location & Sequence & Neutralizing Immunogen & Species(Isotype) \\
\hline \multirow[t]{2}{*}{731} & $52 \mathrm{G} 5 / \mathrm{B} 9$ & Env & gp120 (SF162) & & Vaccine & $\begin{array}{l}\text { human from } \\
\text { transgenic mice } \\
(\mathrm{IgG} 2 \kappa)\end{array}$ \\
\hline & & \multicolumn{5}{|c|}{$\begin{array}{l}\text { Vaccine Vector/Type: recombinant protein Strain: SF162 HIV component: gp120 Adjuvant: Ribi adjuvant (MPL+TDM) } \\
\text { Donor Dr. Abraham Pinter, Public Health Research Institute, Newark, NJ, pinter@ phri.org } \\
\text { References He2002] } \\
\text { 52G5/B9: Transgenic mice (strain XenoMouse G2) carrying human genes allowing production of fully human IgG2 } \kappa \text { MAbs were used to rapidly create a } \\
\text { panel of anti-HIV gp120 MAb-producing hybridomas by immunization with HIV SF162 gp120-11 of the MAbs were conformation dependent, but did } \\
\text { not block sCD4 binding —-these MAbs were part of the same competition group, and enhanced binding of the CD4BS MAb 38G3/A9 and anti-CD4BS } \\
\text { MAbs also enhanced their binding—-these MAbs tended to be very cross-reactive but could not neutralize autologous SF162-52G5/B9 bound to most R5 } \\
\text { and X4 B clade viruses, as well as one of two E clade viruses [He2002]. }\end{array}$} \\
\hline \multirow[t]{2}{*}{732} & $55 \mathrm{E} 4 / \mathrm{H} 1$ & Env & gp120 (SF162) & - & Vaccine & $\begin{array}{l}\text { human from } \\
\text { transgenic mice } \\
(\mathrm{IgG} 2 \kappa)\end{array}$ \\
\hline & & \multicolumn{5}{|c|}{ 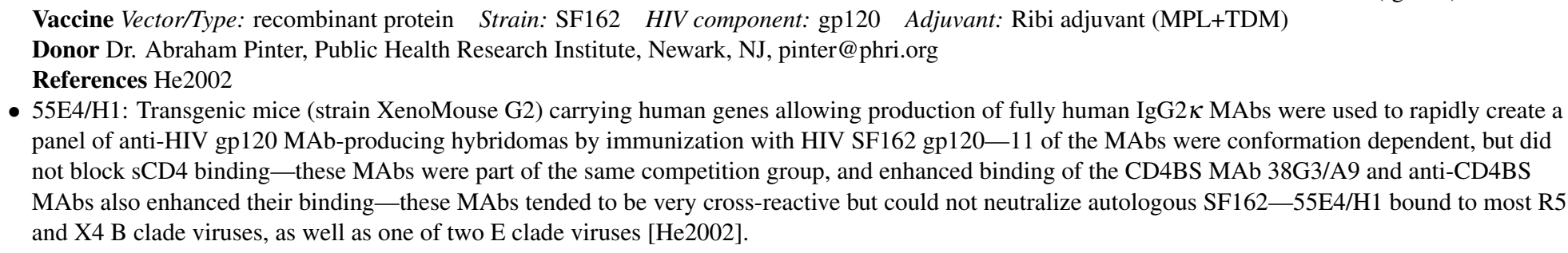 } \\
\hline \multirow[t]{2}{*}{733} & $56 \mathrm{C} 4 / \mathrm{C} 8$ & Env & gp120 (SF162) & & Vaccine & $\begin{array}{l}\text { human from } \\
\text { transgenic mice } \\
(\operatorname{IgG} 2 \kappa)\end{array}$ \\
\hline & & \multicolumn{5}{|c|}{ 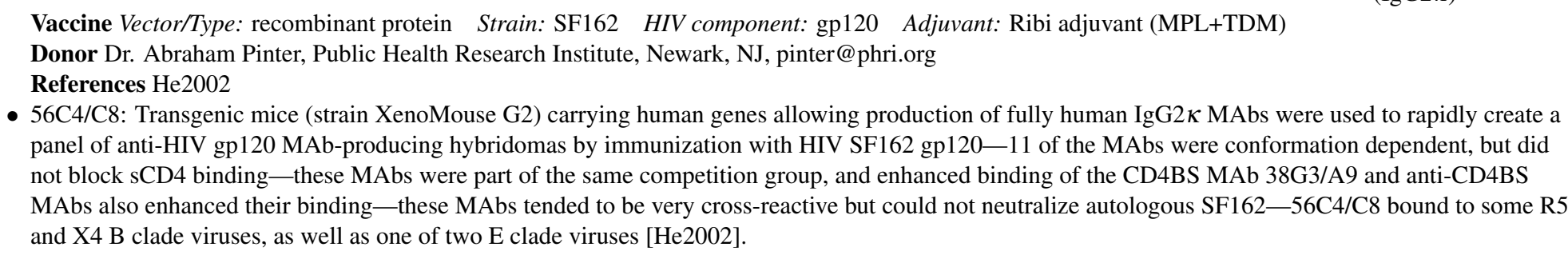 } \\
\hline 734 & $57 \mathrm{~B} 6 / \mathrm{F} 1$ & Env & gp120 (SF162) & & Vaccine & $\begin{array}{l}\text { human from } \\
\text { transgenic mice } \\
(\operatorname{IgG} 2 \kappa)\end{array}$ \\
\hline
\end{tabular}

Vaccine Vector/Type: recombinant protein Strain: SF162 HIV component: gp120 Adjuvant: Ribi adjuvant (MPL+TDM) $(\operatorname{IgG} 2 \kappa)$

Donor Dr. Abraham Pinter, Public Health Research Institute, Newark, NJ, pinter@phri.org

References $\mathrm{He} 2002$ 


\begin{tabular}{|c|c|c|c|c|c|c|}
\hline No. & MAb ID & HXB2 Location & Author's Location & Sequence & Neutralizing Immunogen & Species(Isotype) \\
\hline & & \multicolumn{5}{|c|}{$\begin{array}{l}\text { 57B6/F1: Transgenic mice (strain XenoMouse G2) carrying human genes allowing production of fully human IgG2 } \kappa \text { MAbs were used to rapidly create a } \\
\text { panel of anti-HIV gp120 MAb-producing hybridomas by immunization with HIV SF162 gp120-11 of the MAbs were conformation dependent, but did } \\
\text { not block sCD4 binding-these MAbs were part of the same competition group, and enhanced binding of the CD4BS MAb 38G3/A9 and anti-CD4BS } \\
\text { MAbs also enhanced their binding-these MAbs tended to be very cross-reactive but could not neutralize autologous SF162-57B6/F1 bound some R5 and } \\
\text { X4 B clade viruses, and no E clade viruses [He2002]. }\end{array}$} \\
\hline \multirow[t]{2}{*}{735} & 57H5/D7 & Env & gp120 (SF162) & & no Vaccine & $\begin{array}{l}\text { human from } \\
\text { transgenic mice } \\
(\operatorname{IgG} 2 \kappa)\end{array}$ \\
\hline & & \multicolumn{5}{|c|}{$\begin{array}{l}\text { Vaccine Vector/Type: recombinant protein Strain: SF162 HIV component: gp120 Adjuvant: Ribi adjuvant (MPL+TDM) } \\
\text { Donor Dr. Abraham Pinter, Public Health Research Institute, Newark, NJ, pinter@phri.org } \\
\text { References He2002] } \\
\text { 57H5/D7: Transgenic mice (strain XenoMouse G2) carrying human genes allowing production of fully human IgG2 } \kappa \text { MAbs were used to rapidly create a } \\
\text { panel of anti-HIV gp120 MAb-producing hybridomas by immunization with HIV SF162 gp120-11 of the MAbs were conformation dependent, but did } \\
\text { not block sCD4 binding - these MAbs were part of the same competition group, and enhanced binding of the CD4BS MAb 38G3/A9 and anti-CD4BS } \\
\text { MAbs also enhanced their binding—-these MAbs tended to be very cross-reactive but could not neutralize autologous SF162-57H5/D7 bound to most R5 } \\
\text { and X4 B clade viruses, as well as one of two E clade viruses [He2002]. }\end{array}$} \\
\hline \multirow[t]{2}{*}{736} & 63G4/E2 & 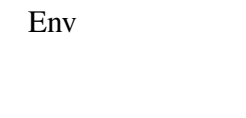 & gp120 (SF162) & & no Vaccine & $\begin{array}{l}\text { human from } \\
\text { transgenic mice } \\
(\operatorname{IgG} 2 \kappa)\end{array}$ \\
\hline & & \multicolumn{5}{|c|}{$\begin{array}{l}\text { Vaccine Vector/Type: recombinant protein Strain: SF162 HIV component: gp120 Adjuvant: Ribi adjuvant (MPL+TDM) } \\
\text { Donor Dr. Abraham Pinter, Public Health Research Institute, Newark, NJ, pinter@phri.org } \\
\text { References He2002] } \\
\text { 63G4/E2: Transgenic mice (strain XenoMouse G2) carrying human genes allowing production of fully human IgG2 } \kappa \text { MAbs were used to rapidly create a } \\
\text { panel of anti-HIV gp120 MAb-producing hybridomas by immunization with HIV SF162 gp120-11 of the MAbs were conformation dependent, but did } \\
\text { not block sCD4 binding - these MAbs were part of the same competition group, and enhanced binding of the CD4BS MAb 38G3/A9 and anti-CD4BS } \\
\text { MAbs also enhanced their binding—-these MAbs tended to be very cross-reactive but could not neutralize autologous SF162-63G4/E2 bound to three R5 } \\
\text { and three X4 B clade viruses, as well as two E clade viruses [He2002]. }\end{array}$} \\
\hline \multirow[t]{2}{*}{737} & $65 \mathrm{~B} 12 / \mathrm{C} 5$ & 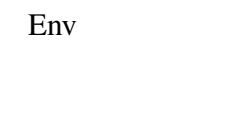 & gp120 (SF162) & & no Vaccine & $\begin{array}{l}\text { human from } \\
\text { transgenic mice } \\
(\operatorname{IgG} 2 \kappa)\end{array}$ \\
\hline & & \multicolumn{5}{|c|}{$\begin{array}{l}\text { Vaccine Vector/Type: recombinant protein Strain: SF162 HIV component: gp120 Adjuvant: Ribi adjuvant (MPL+TDM) } \\
\text { Donor Dr. Abraham Pinter, Public Health Research Institute, Newark, NJ, pinter@ phri.org } \\
\text { References He2002 } \\
\text { 65B12/C5: Transgenic mice (strain XenoMouse G2) carrying human genes allowing production of fully human IgG2 } \% \text { MAbs were used to rapidly create a } \\
\text { panel of anti-HIV gp120 MAb-producing hybridomas by immunization with HIV SF162 gp120-11 of the MAbs were conformation dependent, but did } \\
\text { not block sCD4 binding-these MAbs were part of the same competition group, and enhanced binding of the CD4BS MAb 38G3/A9 and anti-CD4BS } \\
\text { MAbs also enhanced their binding - these MAbs tended to be very cross-reactive but could not neutralize autologous SF162-65B12/C5 bound some R5 } \\
\text { and X4 B clade viruses, and no E clade viruses [He2002]. }\end{array}$} \\
\hline 738 & $6 \mathrm{E} 10$ & $\begin{array}{l}\text { Env } \\
\text { Vaccine Vector/T }\end{array}$ & $\begin{array}{l}\text { gp120 } \\
\text { recombinant protein }\end{array}$ & component & Vaccine & \\
\hline
\end{tabular}




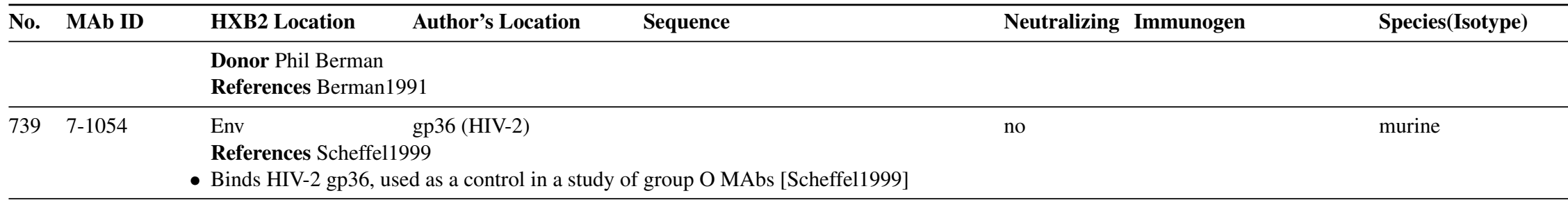

\begin{tabular}{llll}
\hline 740 & $7 \mathrm{~B} 2$ & Env & np41 \\
& & References & Binley1999
\end{tabular}

References Binley1999

- 7B2: The MAbs with the broadest neutralizing activity, IgG1b12, 2G12 and 2F5, all have high affinity for the native trimer, indicating that they were raised in an immune response to the oligomer on the virion surface rather than dissociated subunits - a disulfide linked gp120-gp41 (SOS gp140) was created to mimic the native conformation of Env and explore its potential as an immunogen - SOS gp140 is recognized by NAbs IgG1b12, 2G12, and CD4-IgG2, and also by anti-V3 MAbs 19b and 83.1 - SOSgp140 is not recognized by C4 region MAbs that neutralize only TCLA strains, G3-42 and G3-519 - nor did it bind C11,23A, and M90, MAbs that bind to gp120 C1 and C5, where it interacts with gp41 - MAbs that bind CD4 inducible epitopes, $17 \mathrm{~b}$ and A32 were very strongly induced by CD4 in SOS gp140 - anti-gp41 MAbs that bind in the region that interacts with gp120, 7B2, 2.2B, T4, T15G1 and 4D4, did not bind to SOSgp140, in contrast to 2F5, which binds to the only gp41 epitope that is well exposed in native gp120-gp41 complexes [Binley1999]

\begin{tabular}{llllll}
\hline 741 & 85G11/D8 & Env $120(\mathrm{SF} 162)$ & no & Vaccine & tramsgenic mice \\
& & & $(\mathrm{IgG} 2 \kappa)$
\end{tabular}

Vaccine Vector/Type: recombinant protein Strain: SF162 HIV component: deglycosylated gp120 Adjuvant: Ribi adjuvant (MPL+TDM)

Donor Dr. Abraham Pinter, Public Health Research Institute, Newark, NJ, pinter@phri.org

References $\mathrm{He} 2002$

- 85G11/D8: Transgenic mice (strain XenoMouse G2) carrying human genes allowing production of fully human IgG2 $\kappa$ MAbs were used to rapidly create a panel of anti-HIV gp120 MAb-producing hybridomas by immunization with HIV SF162 gp120 — three of the MAbs were conformation dependent, but did not block sCD4 binding and were part of the same competition group - these MAbs were all raised against a deglycosylated form of gp120 - they could not neutralize autologous SF162 and bound some R5 and X4 B clade viruses, and no E clade viruses [He2002].

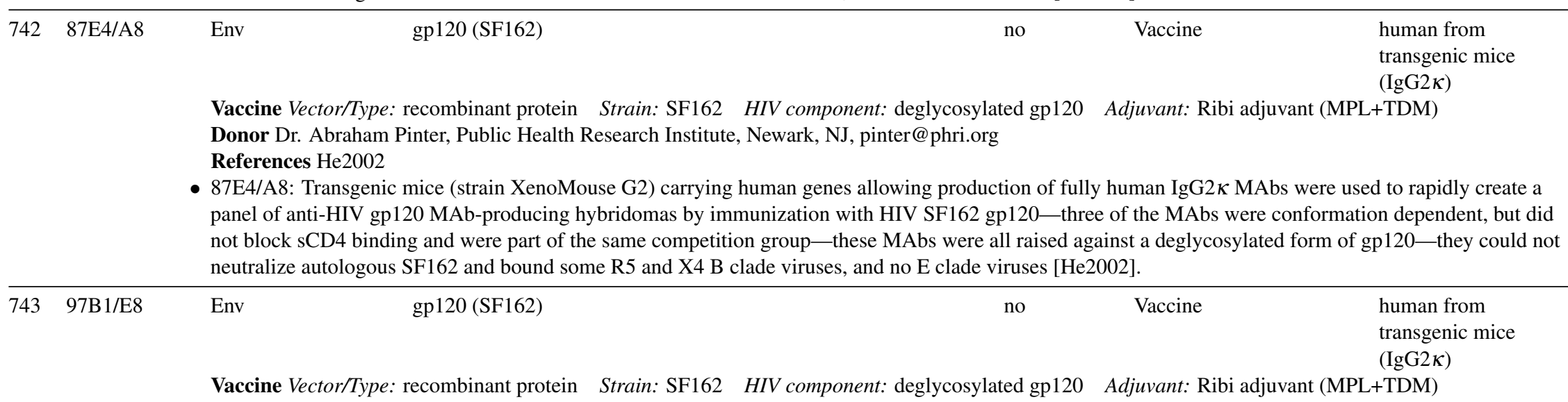

Donor Dr. Abraham Pinter, Public Health Research Institute, Newark, NJ, pinter@phri.org 


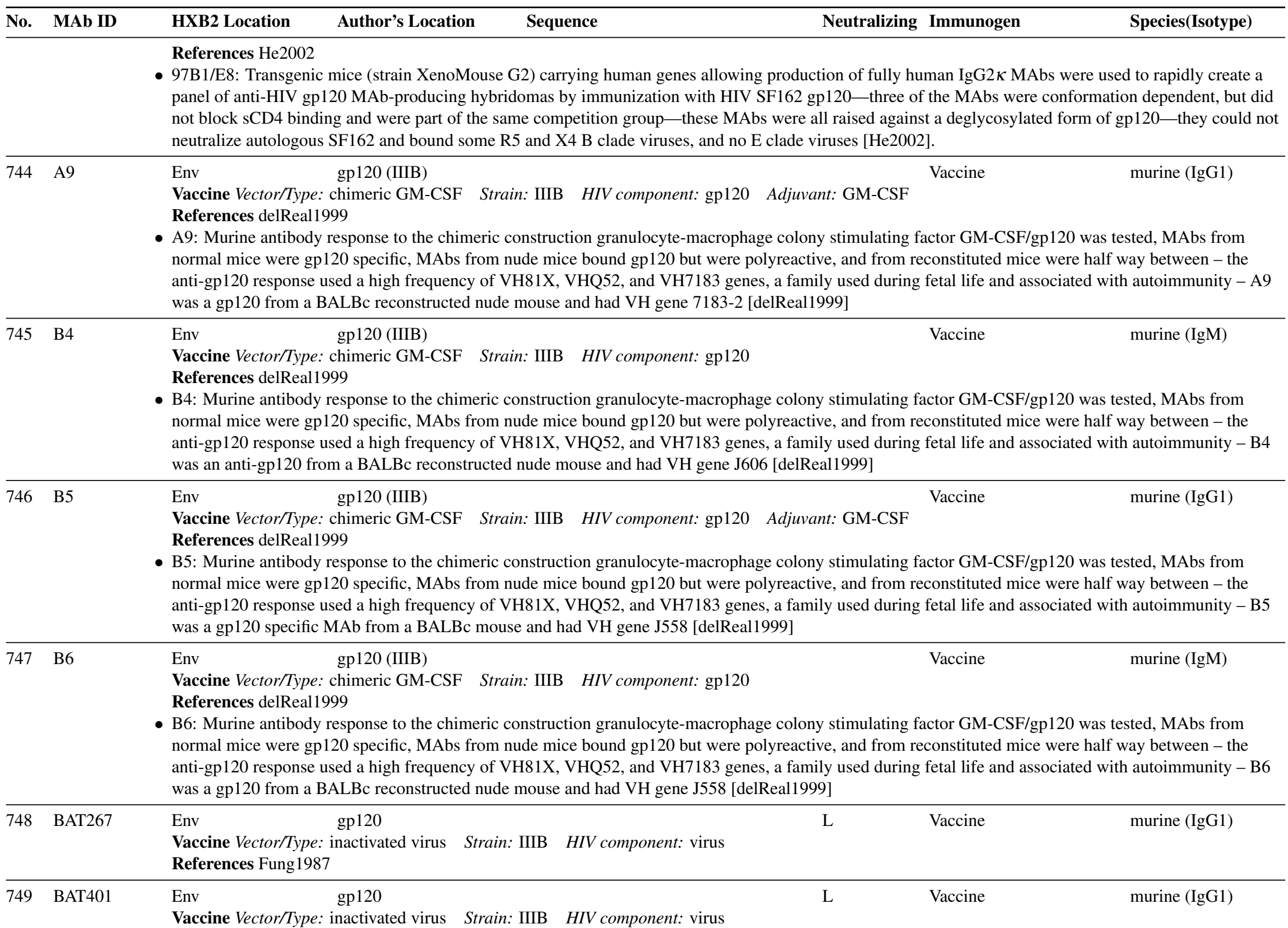




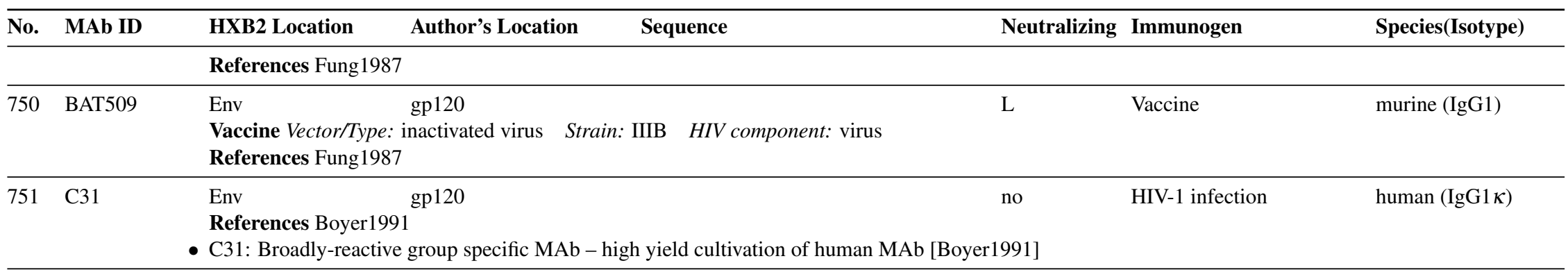

752 D1 Env gp41 (IIIB) murine (IgG)

Vaccine Vector/Type: vaccinia Strain: IIIB HIV component: oligomeric gp140

References Otteken1996

- D1: MAbs D1, D16, had T37 bind to oligomeric gp160 equally well - pulse label experiments of MAb binding to noncleavable gp160 revealed that these MAbs bound with a delay, epitopes forming with a half life of 30 min [Otteken1996]

\begin{tabular}{lllllll}
\hline 753 & D12 Env & gp41 (IIIB) & L & Vaccine
\end{tabular}

Vaccine Vector/Type: vaccinia Strain: IIIB HIV component: oligomeric gp140

Donor Patricia Earl and Christopher Broder, NIH

References Ear11994, Broder1994, Richardson1996, Ear11997, Otteken1996, LaBranche1999

- D12: Generated during a study of the influence of the oligomeric structure of Env in determining the repertoire of the Ab response [Earl1994]

- D12: One of 18 MAbs (e. g. D4 and D40) that bind to a conformation-dependent epitope in gp41 that bind preferentially, but not exclusively, to oligomers neutralizes IIIB and SF2 [Broder1994]

- D12: This antibody was blocked more strongly by human sera than other anti-gp41 MAbs (D20, D43, D61, and T4) in a oligomeric ELISA assay Richardson1996]

- D12: MAbs D10 and D12 are very easily blocked by human sera from HIV+ individuals [Earl1997]

- D12: MAbs D4, D10, D11, D12, and D41 all bind only to complete oligomer - pulse label experiments of MAb binding to noncleavable gp160 revealed that these MAbs bound with a delay, epitopes forming with a half life of 30 min [Otteken1996]

- D12: D12 was used in WB of HIV-1 transmembrane proteins in a study which showed that determinants of HIV-1 CD4 independence map outside regions required for coreceptor specificity - IIIBx, a CD4-independent variant of IIIB, has a truncated gp41 [LaBranche1999]

- D12: A combination of gp41 fusion with the GNC4 trimeric sequences and disruption of the YU2 gp120-gp41 cleavage site resulted in stable gp140 trimers (gp140-GNC4) - gp41 MAbs T4, D12, T3, and D50 bound less efficiently to gp140-GNC4 than did pooled sera, but T4 and D12 recognized the gp140-GNC4 timer equivalently to gp140(-), and T3 and D50 recognized the trimer at greater levels than gp140(-) [Yang2000] Env gp41 (IIIB)

L Vaccine murine (IgG)

Vaccine Vector/Type: protein HIV component: dimeric Env

Donor Patricia Earl and Christopher Broder, NIH

References Earl1994, Weissenhorn1996, Earl1997

- D16: Generated during a study of the influence of the oligomeric structure of Env in determining the repertoire of the Ab response [Earl1994]

- D16: Precipitates both oligomeric gp140 and soluble monomeric gp41(21-166)that lacks the fusion peptide and membrane anchor, along with MAbs D16, D38, D40, D41, and D54 [Weissenhorn1996]

- D16: One of eleven MAbs (D16, D17, D31, D36, D37, D40, D44, D55, D59, T37, and T45) that are conformation dependent and that can block the binding of the MAb D50 that binds to the linear peptide gp41(642-665) - reactive with 9/10 HIV-1 strains all except HIV-1 ADA, which has the change E659D and E662A that may result in the loss of binding (ELLE to DLLA) [Earl1997] 


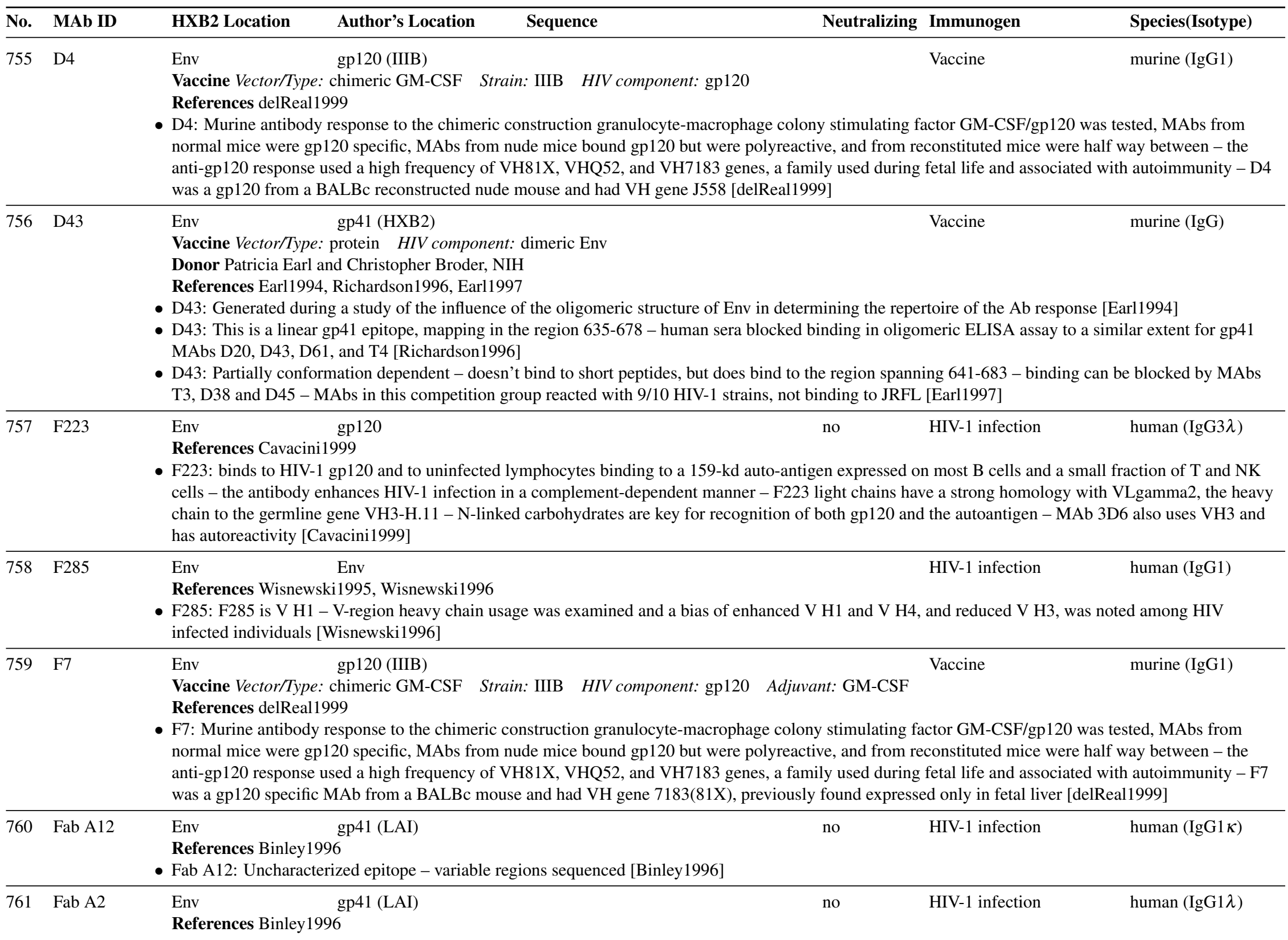




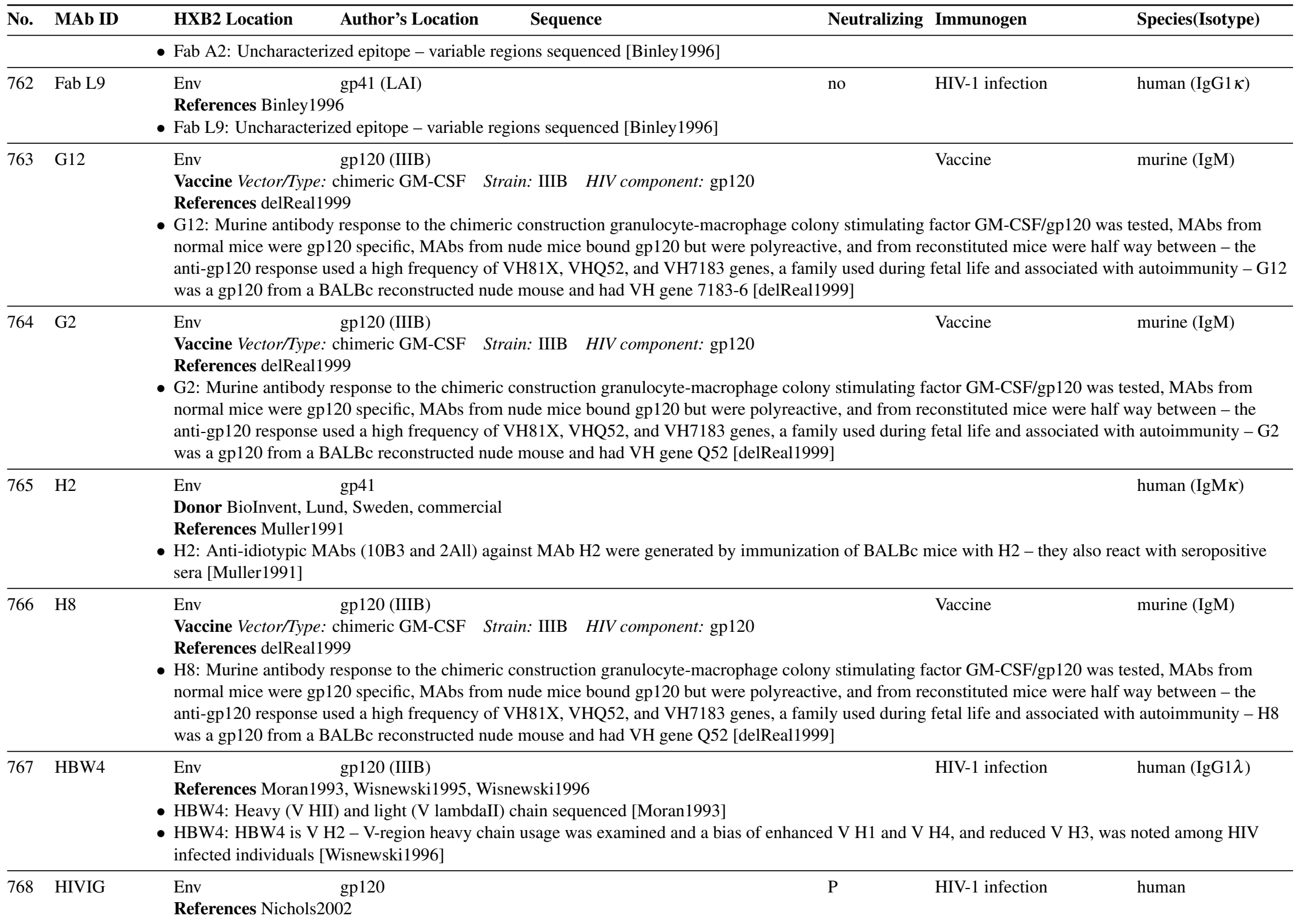




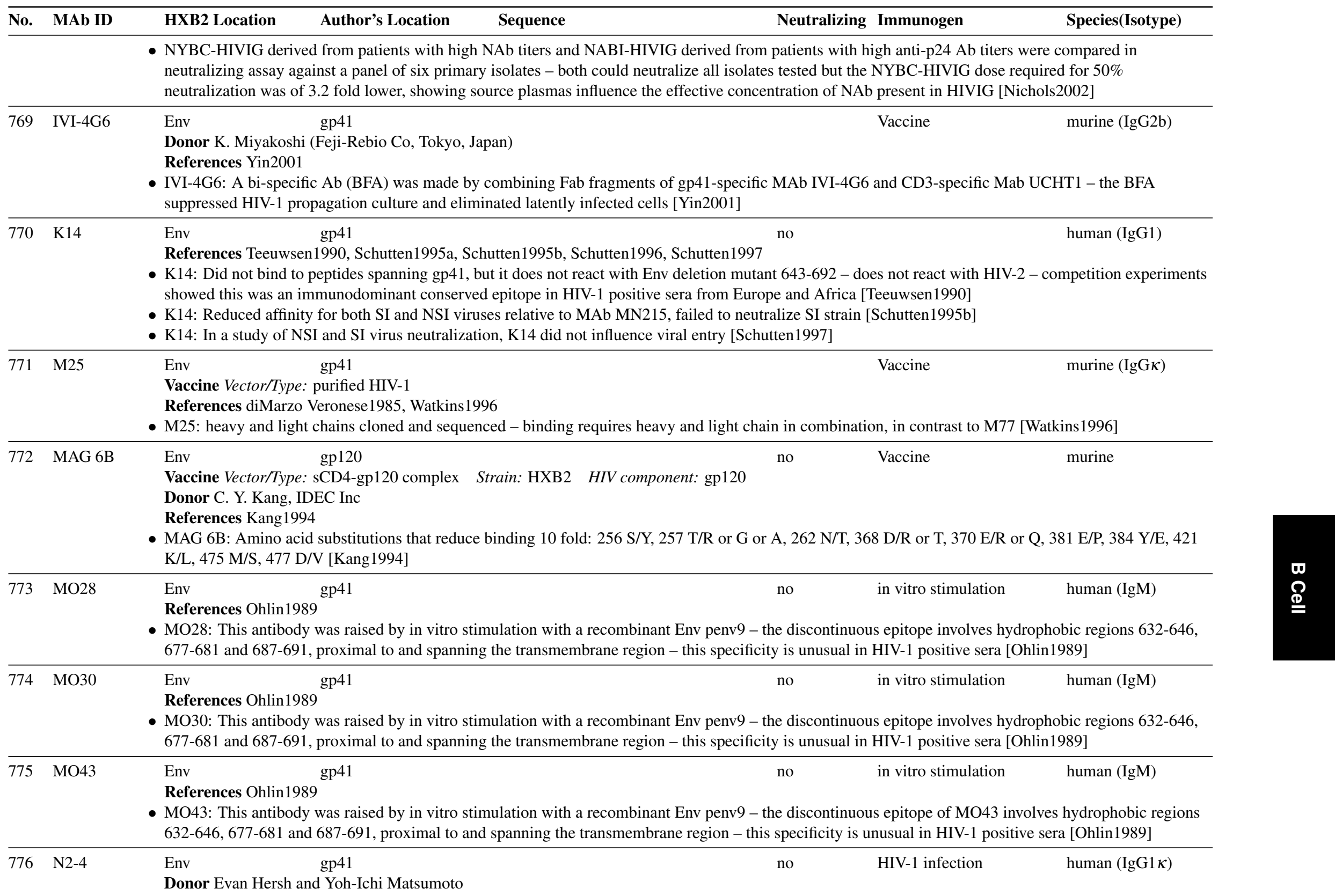




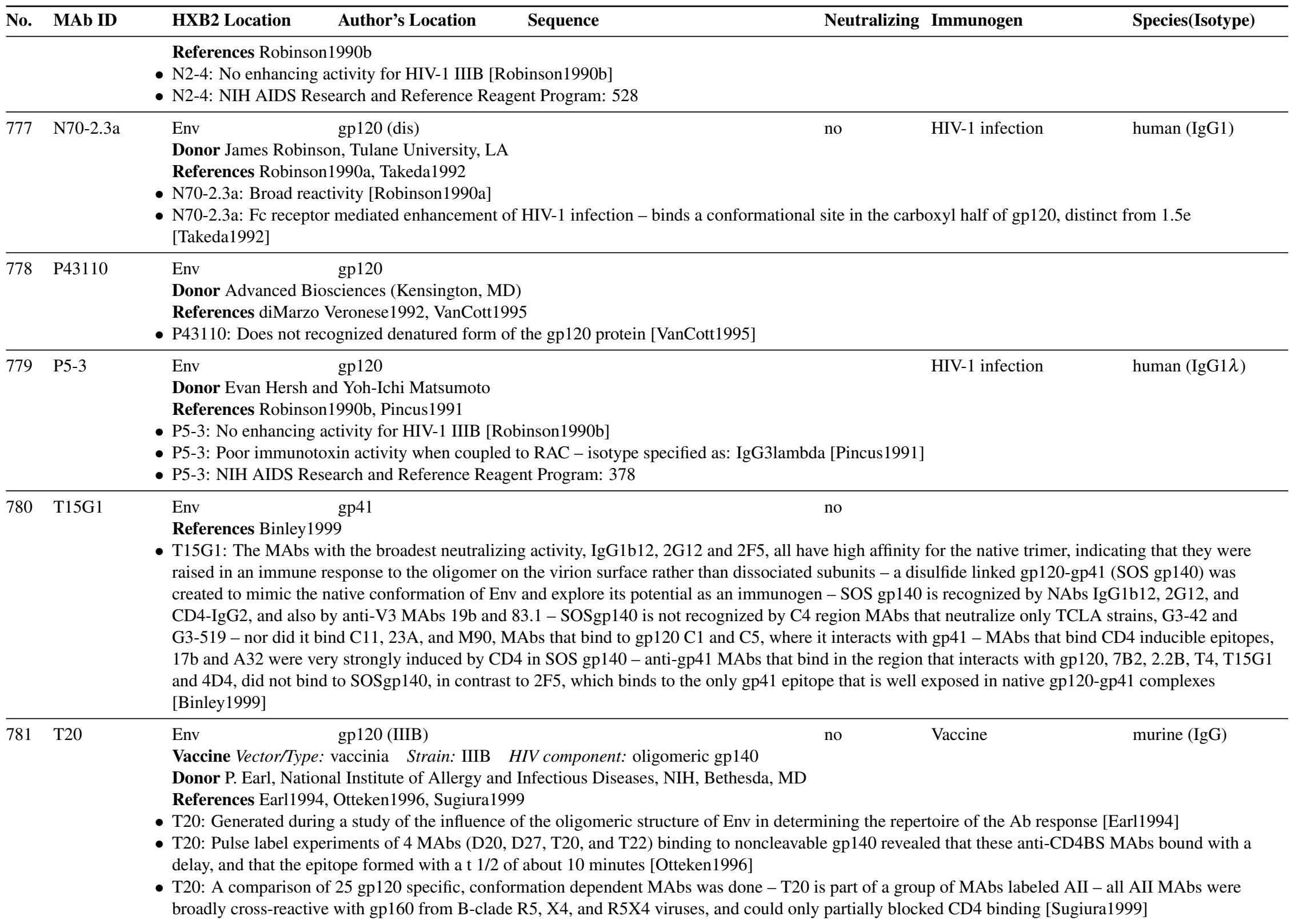




\begin{tabular}{|c|c|c|c|c|c|c|c|}
\hline No. & MAb ID & HXB2 Location & Author's Location & Sequence & Neutralizing & Immunogen & Species(Isotype) \\
\hline 782 & $\mathrm{~T} 27$ & Env & gp120 (IIIB) & & no & Vaccine & murine $(\operatorname{IgG})$ \\
\hline
\end{tabular}

Vaccine Vector/Type: vaccinia Strain: IIIB HIV component: oligomeric gp140

Donor P. Earl, National Institute of Allergy and Infectious Diseases, NIH, Bethesda, MD

References Earl1994, Sugiura1999

- T27: Generated during a study of the influence of the oligomeric structure of Env in determining the repertoire of the Ab response [Earl1994]

- T27: A comparison of 25 gp120 specific, conformation dependent MAbs was done - T27 is part of a group of MAbs labeled AII - all AII MAbs were broadly cross-reactive with gp160 from B-clade R5, X4, and R5X4 viruses, and could only partially blocked CD4 binding [Sugiura1999]

\begin{tabular}{lllll}
\hline 783 & T3 Env & gp41 (HXB2) & Vaccine \\
& & Vaccine Vector/Type: tetrameric Env HIV component: Env &
\end{tabular}

References Earl1994 Ear11997 Zwick2001b Yang2000

- T3: Generated during a study of the influence of the oligomeric structure of Env in determining the repertoire of the Ab response [Earl1994]

- T3: Partially conformation dependent - doesn't bind to short peptides, but does bind to the region spanning 641-683 - binding can be blocked by MAbs D43, D38 and D45 - MAbs in this competition group reacted with 9/10 HIV-1 strains, not binding to JRFL [Earl1997]

- T3: T3 partially competes with MAb Z13, but not MAb 4E10, both of which bind to gp41 proximally to the 2F5 epitope and have a broad neutralizing potential [Zwick2001b

- T3: A combination of gp41 fusion with the GNC4 trimeric sequences and disruption of the YU2 gp120-gp41 cleavage site resulted in stable gp140 trimers (gp140-GNC4) - gp41 MAbs T4, D12, T3, and D50 bound less efficiently to gp140-GNC4 than did pooled sera, but T4 and D12 recognized the gp140-GNC4 timer equivalently to gp140(-), and T3 and D50 recognized the trimer at greater levels than gp140(-) [Yang2000]

\begin{tabular}{llllll}
\hline 784 & T30 & Env & gp41 & no & Vaccine
\end{tabular}

References Earl1994, Earl1997

- T30: Generated during a study of the influence of the oligomeric structure of Env in determining the repertoire of the Ab response [Earl1994]

- T30: Binds in the region 580 to 640, but does not bind to peptides spanning this region - binding depends on N-linked glycosylation of Asn 616 - no other antibody tested inhibited binding, but binding could be inhibited by sera from HIV+ individuals [Earl1997]

\begin{tabular}{lllllll}
\hline 785 & T4 & Env & gp4 (IIIB) & L & Vaccine
\end{tabular}

Vaccine Vector/Type: vaccinia Strain: IIIB HIV component: oligomeric gp140

References Ear11994, Broder1994, Richardson1996, Weissenhorn1996, Otteken1996, Ear11997, Binley1999, Stamatatos2000, Yang2000, Srivastava2002

- T4: Generated during a study of the influence of the oligomeric structure of Env in determining the repertoire of the Ab response [Earl1994]

- T4: one of five MAbs (T4, T6, T9, T10 and T35) in a competition group that bind to a conformation-dependent epitope in gp41 and is oligomer specific neutralizes IIIB and SF2 [Broder1994]

- T4: Does not bind to soluble monomeric gp41(21-166) that lacks the fusion peptide and membrane anchor, only to the oligomer gp140, as does T6 [Weissenhorn1996]

- T4: MAbs T4 and T6 bind only to oligomer, and pulse chase experiments indicate that the epitope is very slow to form, requiring one to two hours [Otteken1996]

- T4: This antibody, along with 7 others (M10, D41, D54, T6, T9, T10 and T35), can block the linear murine MAb D61, and the human MAb 246-D, which both bind to the immunodominant region near the two Cys in gp41 - most of these antibodies are oligomer dependent - all of the MAbs are reactive with ten different HIV-1 strains - members of this competition group are blocked by sera from HIV-1+ individuals [Earl1997] 


\begin{tabular}{ll}
\hline No. MAb ID & HXB2 Location Author's Location $\quad$ Sequence \\
\hline & T4: The MAbs with the broadest neutralizing activity, IgG1b12, 2G12 and 2F5, all have high affinity for the native trimer, indicating that they were raised in \\
an immune response to the oligomer on the virion surface rather than dissociated subunits - a disulfide linked gp120-gp41 (SOS gp140) was created to \\
mimic the native conformation of Env and explore its potential as an immunogen - SOS gp140 is recognized by NAbs IgG1b12, 2G12, and CD4-IgG2, and \\
also by anti-V3 MAbs 19b and 83.1 - SOSgp140 is not recognized by C4 region MAbs that neutralize only TCLA strains, G3-42 and G3-519- nor did it \\
bind C11, 23A, and M90, MAbs that bind to gp120 C1 and C5, where it interacts with gp41 - MAbs that bind CD4 inducible epitopes, 17b and A32 were \\
very strongly induced by CD4 in SOS gp140 - anti-gp41 MAbs that bind in the region that interacts with gp120, 7B2, 2.2B, T4, T15G1 and 4D4, did not \\
bind to SOSgp140, in contrast to 2F5, which binds to the only gp41 epitope that is well exposed in native gp120-gp41 complexes [Binley1999] \\
- T4: Soluble gp140 derived from SF162, a neutralization-resistant primary isolate, and SF162AV2 a neutralization-susceptible isolate with 30 amino acids \\
deleted from the V2 loop, were generated with or without the gp120-gp41 cleavage site intact - all forms are recognized by oligomer-specific MAb T4 and \\
show enhanced binding of CD4i MAb 17b when sCD4 is bound - the fused forms are less efficiently recognized than the cleaved forms by polyclonal \\
neutralizing sera from HIV-infected patients - the V3 loop is more exposed on the fused form [Stamatatos2000] \\
- T4: A combination of gp41 fusion with the GNC4 trimeric sequences and disruption of the YU2 gp120-gp41 cleavage site resulted in stable gp140 trimers \\
(gp140-GNC4) - gp41 MAbs T4, D12, T3, and D50 bound less efficiently to gp140-GNC4 than did pooled sera, but T4 and D12 recognized the \\
gp140-GNC4 timer equivalently to gp140(-), and T3 and D50 recognized the trimer at greater levels than gp140(-) [Yang2000] \\
- T4: Oligomeric gp140 (o-gp140) derived from R5 primary isolate US4 was characterized for use as a vaccine reagent - antigen capture ELISA was used to \\
compare the antigenicity of gp120 and o-gp140 using a panel of well characterized MAbs - T4 recognized o-gp140 [Srivastava2002]
\end{tabular}
786 multiple Fabs Env gp120
HIV-1 infection
human
References Burton1991

- A panel of anti-gp120 Fabs was generated by antigen selection from a random combinatorial library prepared from bone marrow from an asymptomatic individual [Burton1991]

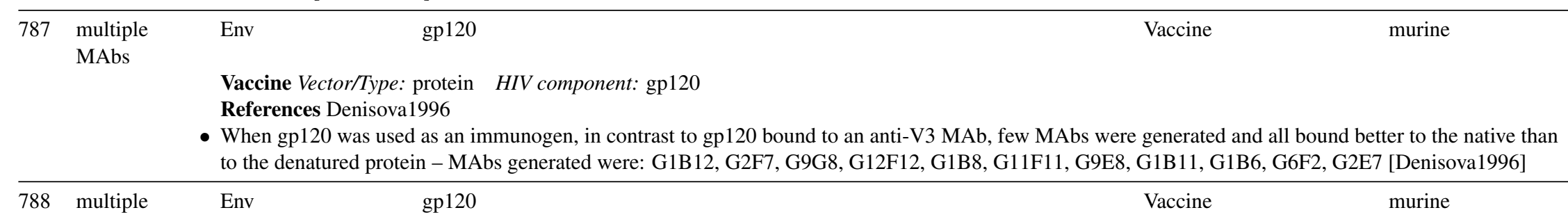
MAbs
Vaccine Vector/Type: gp120-CD4 complex HIV component: gp120

References Denisova1996

- When gp120-CD4 was used as an immunogen, in contrast to gp120 bound to an anti-V3 MAb, few MAbs were generated and all bound better to the native than to the denatured protein - MAbs generated were: CG43, CG41, CG49, CG53, CG42, CG4, CG46, CG40, CG52, CG51, CG48, CG50, CG125, CG124, CG121 [Denisova1996]

\begin{tabular}{llll}
\hline 789 & multiple & Env & gp120 \\
MAbs & & \\
& Vaccine Vector/Type: protein-Ab complex & HIV component: gp120 complexed with MAb M77 \\
& References Denisova1996
\end{tabular}

References Denisova1996 


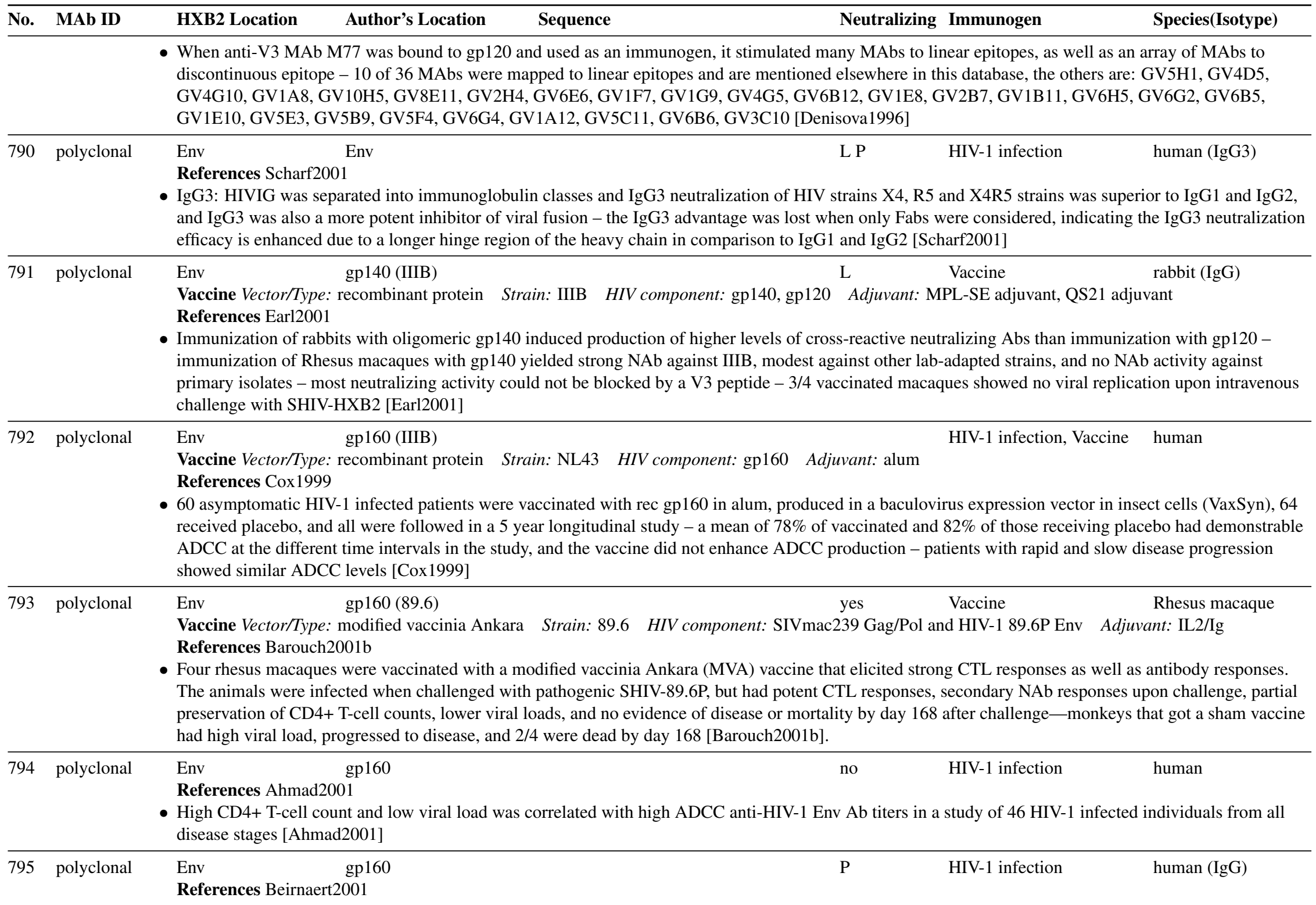




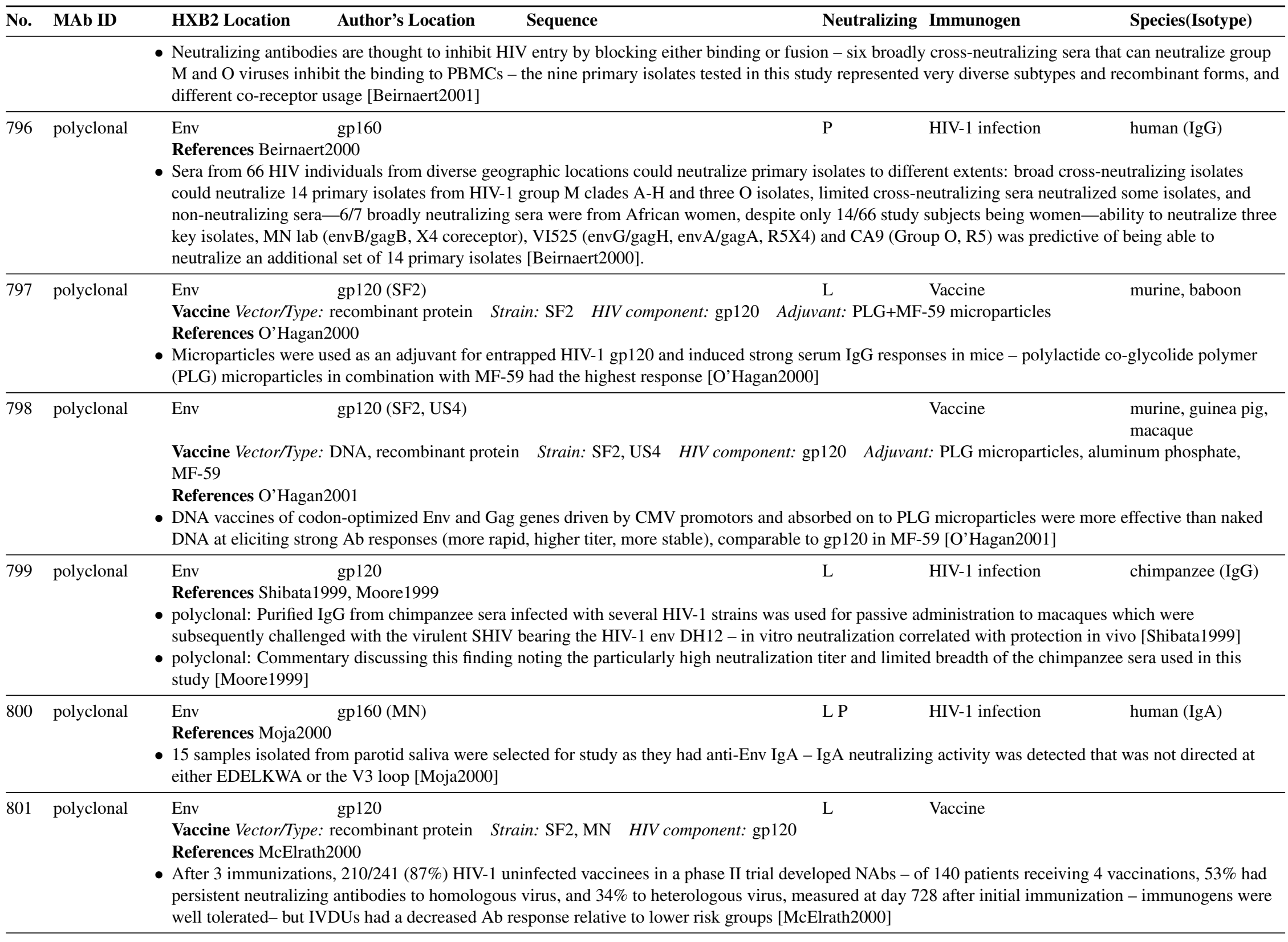




\begin{tabular}{lllllll}
\hline No. & MAb ID & HXB2 Location & Author's Location & Sequence & Neutralizing & Immunogen \\
\hline 802 & polyclonal & Env & gp120 & & Species(Isotype)
\end{tabular}

Vaccine Vector/Type: vaccinia Strain: IIIB HIV component: gp120 Adjuvant: GM-CSF/gp120 chimera

References Rodríguez1999

- The murine $\mathrm{Ab}$ response to a chimeric of granulocyte-macrophage colony stimulating factor GM-CSF/gp120 in vaccinia was not higher titer that the response to a gp120-vaccinia construct, but the breadth of the Ab response was greater - a cellular response of greater intensity was trigged to the GM-CSF/gp120 vaccinia construct, as measured by proliferation and Elispot [Rodríguez1999]

803 polyclonal

\begin{tabular}{llll}
\hline polyclonal & Env & gp120 (YU2) & \\
& Vaccine Vector/Type: stabilized Env trimer Strain: YU2, HXBc2 & HIV component: Env \\
& Donor Joseph Sodroski, Harvard Medical School
\end{tabular}

Donor Joseph Sodroski, Harvard Medical School

References Yang2001

- Soluble Env trimers were created that were designed to mimic functional Env oligomers - stabilized timers could induce neutralizing antibodies more effectively than gp120, and Abs to the YU2 trimer were cross-reactive within clade B and could neutralize several primary and TCLA reactive strains - the stabilized primers did not neutralize primary isolates outside the B clade, from clades $\mathrm{C}, \mathrm{D}$, and $\mathrm{E}-\mathrm{HXBc} 2$ stabilized trimer antigen elicited strong neutralizing Abs against the homologous isolate HXBc2 TCLA strain, but not against primary isolates [Yang2001]

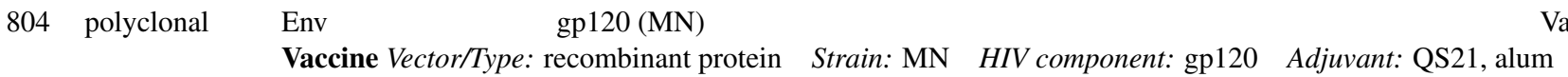

Vaccine human

References Evans2001

- Vaccination with QS21 adjuvant and rsgp120 elicited stronger and more sustained neutralizing antibody responses and lymphocyte proliferation with lower doses of rsgp120 than alum formulations, suggesting QS21 may be a means to reduce the does of soluble protein [Evans2001]

\begin{tabular}{|c|c|c|c|}
\hline 805 & polyclonal & $\begin{array}{l}\text { Env gp120 yes } \\
\text { References Binley2000 } \\
\text { HAART inhibited the development of anti-gp120 Ab when initiated during primary infection at } \\
\text { infection - HAART during primary infection usually did not inhibit the development of weak } \mathrm{N} \\
\text { intermittently adherent developed high titers of autologous NAbs, largely coincident with brief }\end{array}$ & $\begin{array}{l}\text { HIV-1 infection human } \\
\text { les in patients treated within } 2 \text { years of HIV-1 } \\
\text { ses against autologous virus }-3 / 4 \text { patients } \\
\text { iods [Binley2000] }\end{array}$ \\
\hline 806 & polyclonal & $\begin{array}{l}\text { Env gp120 (SIV) yes } \\
\text { References Reitter1998 } \\
\text { This study concerned an SIV mutated strain that lacked 4th, 5th and 6th sites for N-linked glyce } \\
\text { increased neutralizing activity in their sera relative to monkeys infected with the parental strain }\end{array}$ & $\begin{array}{l}\text { HIV-1 infection macaque } \\
\text { monkeys infected with the mutant viruses had }\end{array}$ \\
\hline 807 & polyclonal & $\begin{array}{l}\text { Env Env } \\
\text { References Kim2001 } \\
\text { After HAART reduction of viral load to }<400 \text { for three visits over a } 12 \text { month interval, } 2 / 11 \text { pat } \\
\text { titers, and neutralizing Abs titers increased against primary isolates US1, and CM237 - no NAP } \\
\text { BZ167 - this suggests that in certain individuals the control of HIV-1 by HAART may augmen }\end{array}$ & $\begin{array}{l}\text { HIV-1 infection human } \\
\text { found to have increased anti-Env Ab binding } \\
\text { ase was seen to more readily neutralized isolate } \\
\text { ontrol of HIV [Kim2001 }\end{array}$ \\
\hline 808 & polyclonal & Env & $\begin{array}{l}\text { HIV-1 exposed } \\
\text { seronegative }\end{array}$ \\
\hline
\end{tabular}

References Kaul2001b

- Kaul et al. provide a concise summary of the findings concerning the presence of Mucosal IgA in highly exposed, uninfected subjects, arguing for a role in protection [Kaul2001b 


\begin{tabular}{|c|c|c|c|c|c|}
\hline No. & MAb ID & Author's Location & Neutralizing & Immunogen & Species(Isotype) \\
\hline 809 & polyclonal & $\begin{array}{l}\text { Env gp120 } \\
\text { Vaccine Vector/Type: recombinant protein } \\
\text { References Nitayaphan2000 } \\
\text { A phase I/II trial was conducted in } 52 \text { serone } \\
\text { autologous SF2, while 22/40 were able to cr }\end{array}$ & $\begin{array}{l}\text { Strain: SF2 HIV component: gp120 Adjuvant: MF-59 } \\
\text { egative Thais immunizing with rgp120 SF2 - the vaccine was } \\
\text { oss-neutralize the heterologous strain MN [Nitayaphan2000] }\end{array}$ & Vaccine & $\begin{array}{l}\text { human } \\
\text { Ab responses to the }\end{array}$ \\
\hline 810 & polyclonal & $\begin{array}{l}\text { Env gp120 (SF2) } \\
\text { Vaccine Vector/Type: recombinant protein } \\
\text { References Heeney1998a }\end{array}$ & $\begin{array}{ll} & \text { yes } \\
\text { train: SF2 HIV component: gp120, p24 } & \text { Adjuvant: ISCO }\end{array}$ & $\begin{array}{l}\text { Vaccine } \\
\mathrm{M}\end{array}$ & macaque \\
\hline
\end{tabular}

- The immune responses induced in Rhesus monkeys using two different immunization strategies was studied - one vaccine group was completely protected from challenge infection, the other vaccinees and controls became infected - protected animals had high titers of heterologous NAbs, and HIV-1-specific T helper responses - increases in RANTES, MIP 1 alpha and MIP 1 beta produced by circulating CD8+ T cells were also associated with protection [Heeney1998a]

811 polyclon Env gp120

Vaccine

macaque

Vaccine Vector/Type: peptide, recombinant protein Strain: SF2, SF33 HIV component: gp120 Adjuvant: ISCOM, MF-59

References Verschoor1999

- Attempts were made to broaden immune responses induced in Rhesus monkeys by immunization of animals previously immunized that had resisted homologous challenge, with a second immunization with ISCOM-peptides or a boost with gp120 from SF33 - animals didn't survive a second challenge heterologous challenge virus SHIV(SF33) raising concerns about early antigenic sin [Verschoor1999]

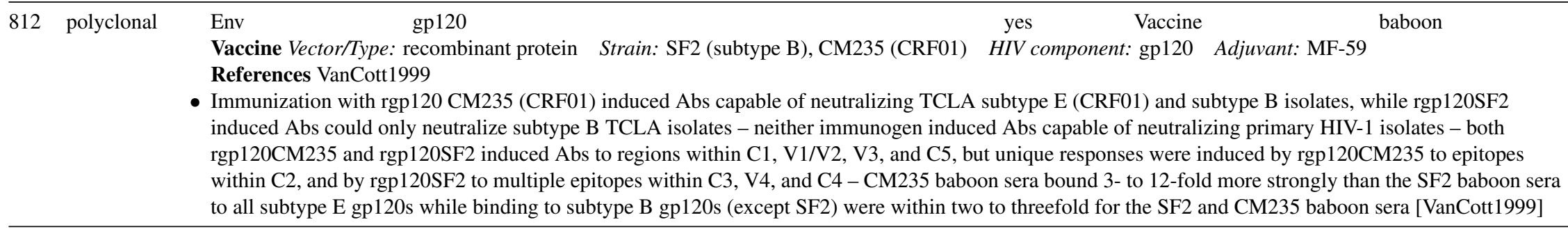

813 polyclonal Env gp140 (SF162DeltaV2) rabbit, Rhesus

Vaccine Vector/Type: DNA with CMV promotor Strain: SF162, SF162 2 V2 HIV component: gp140 Adjuvant: MF-59C macaque $(\operatorname{IgG})$ References Barnett2001

- SF162 $\Delta$ V2 is a virus that has a 30 amino acids deletion in the V2 loop that does not abrogate its infectivity but renders it highly susceptible to neutralization - when incorporated into a codon-optimized DNA vaccine with a CMV promoter and delivered by gene gun, SF162 $\Delta$ V2 gave higher neutralizing Ab titers against SF162 than did SF162 itself, and Abs that cross-neutralized non-homologous primary isolates were obtained only when SF162 $\Delta$ V2, but not intact SF162, was used as the immunogen - Control MAbs 2F5 and 2G12 could neutralize all of the following primary isolates: 91US056(R5), 92US714(R5), 92US660(R5), 92HT593(R5X4), and BZ167(R5X4), while after the first protein boost, the sera from two SF162 $\Delta$ V2 immunized macaques could neutralize 91US056(R5), 92US714(R5), 92US660(R5) and ADA(R5), but not 92HT593(R5X4) or 92US657(R5)-the pattern of cross-recognition shifted after the second boost [Barnett2001].

814 polyclonal Env gp120

References Binley 1997b

HIV-1 infection

human (IgG) 


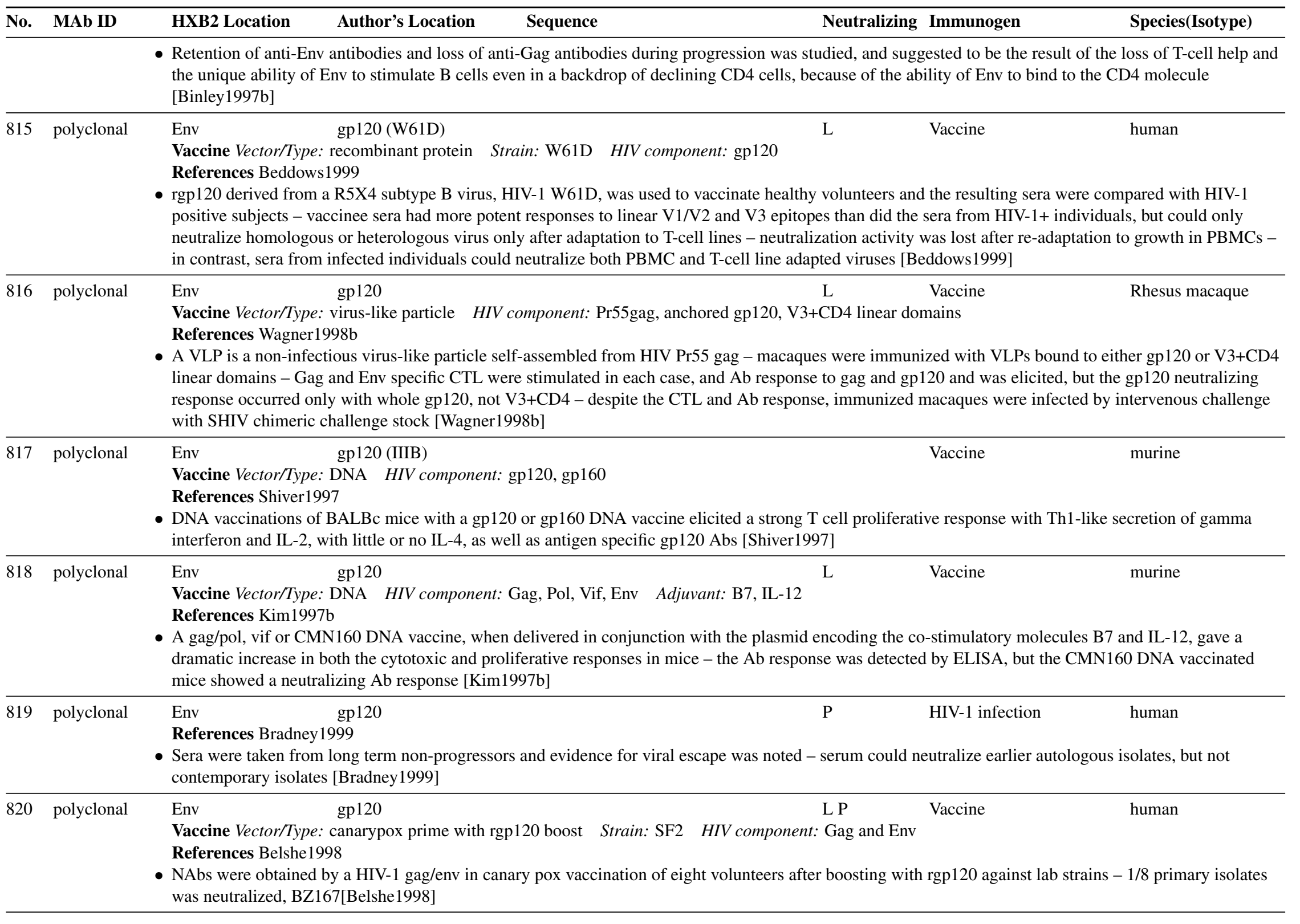




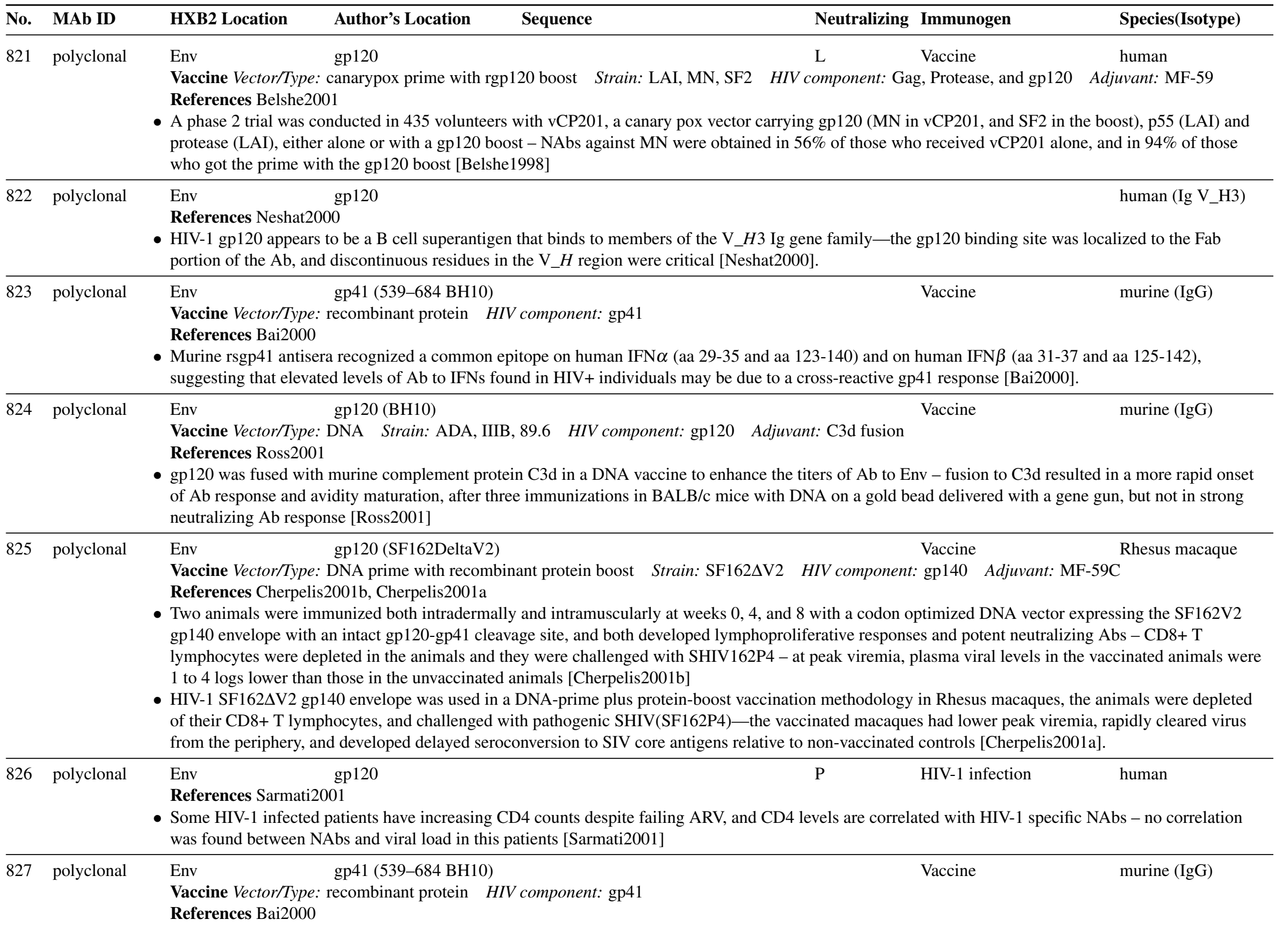




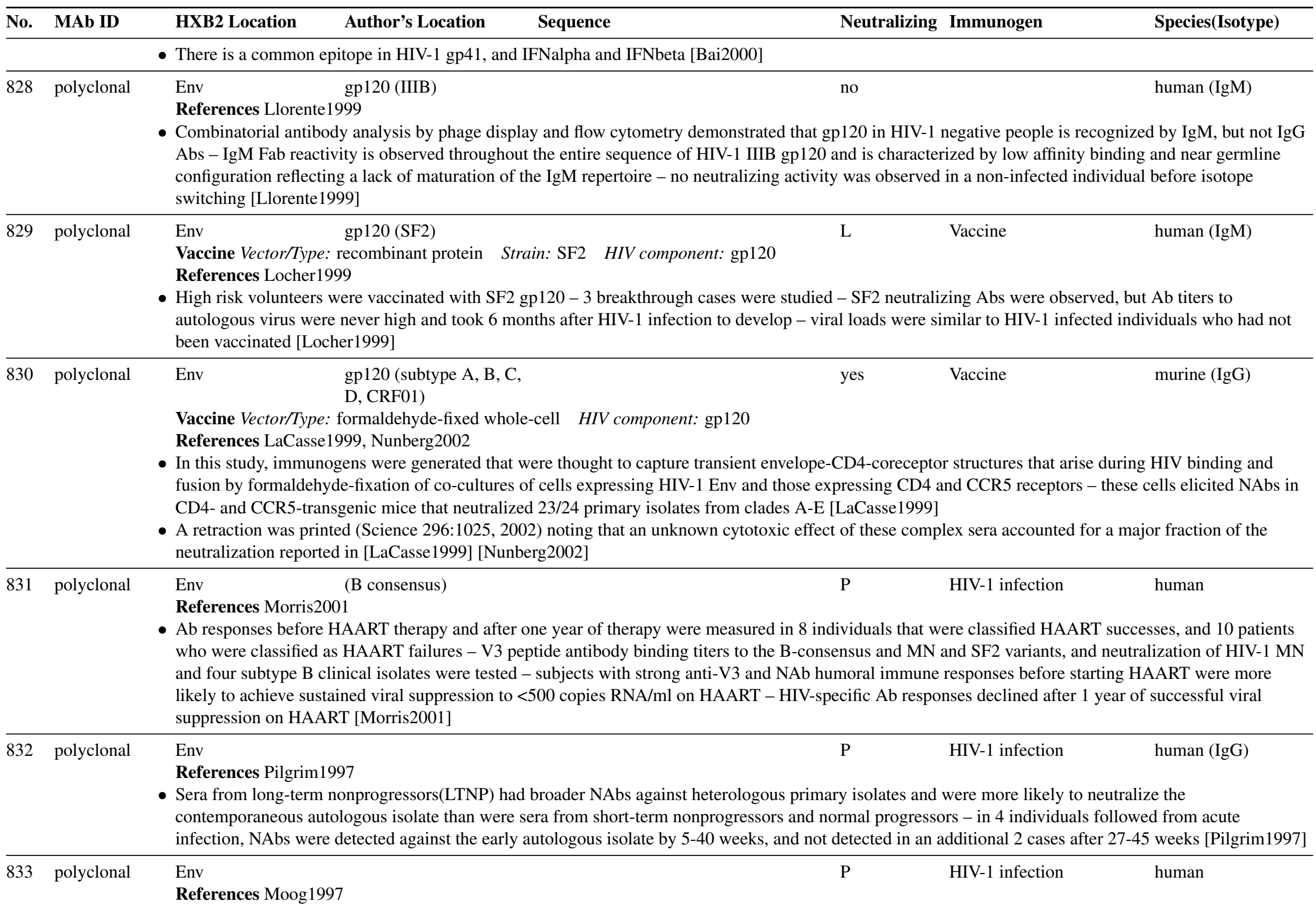




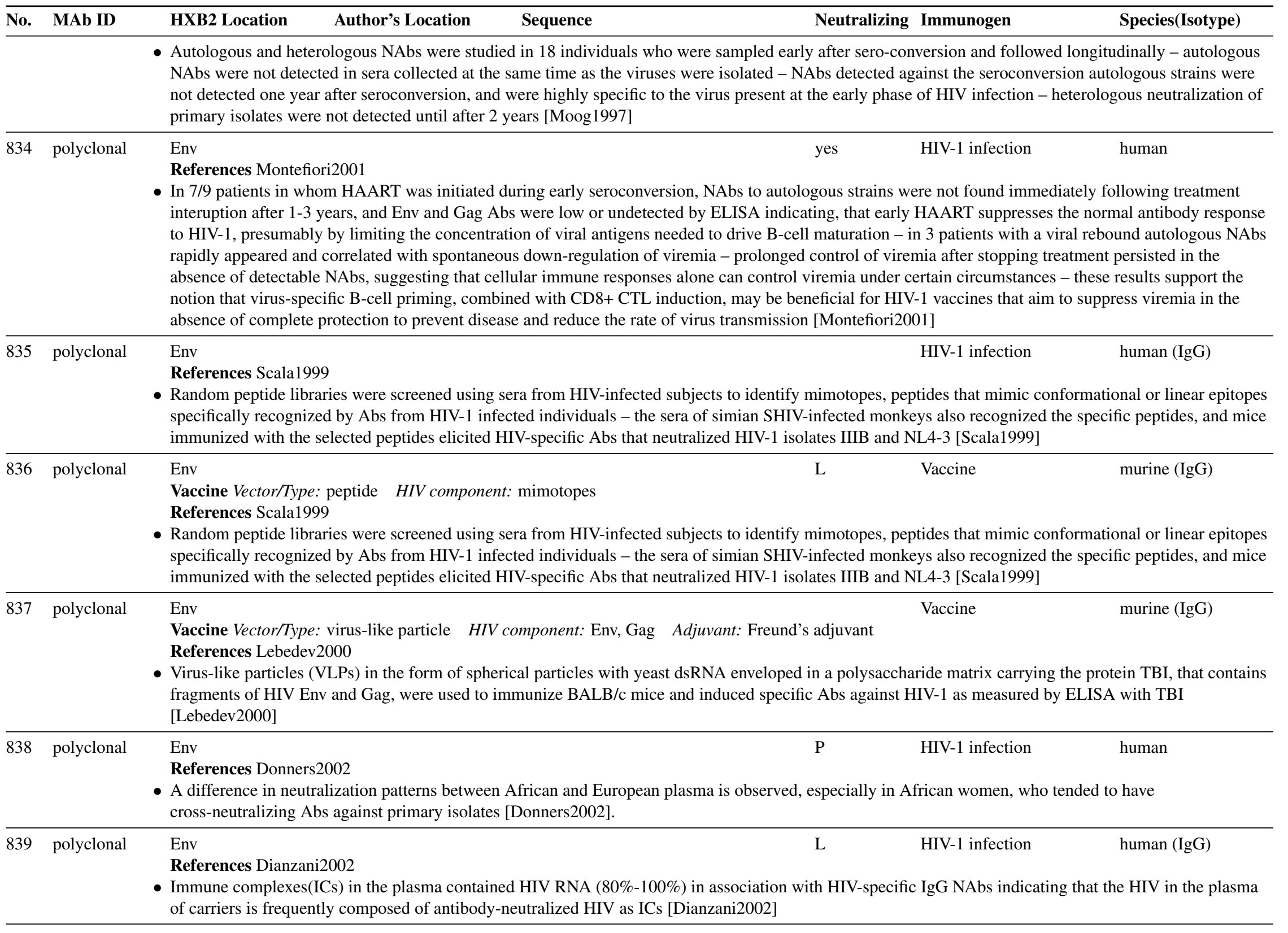




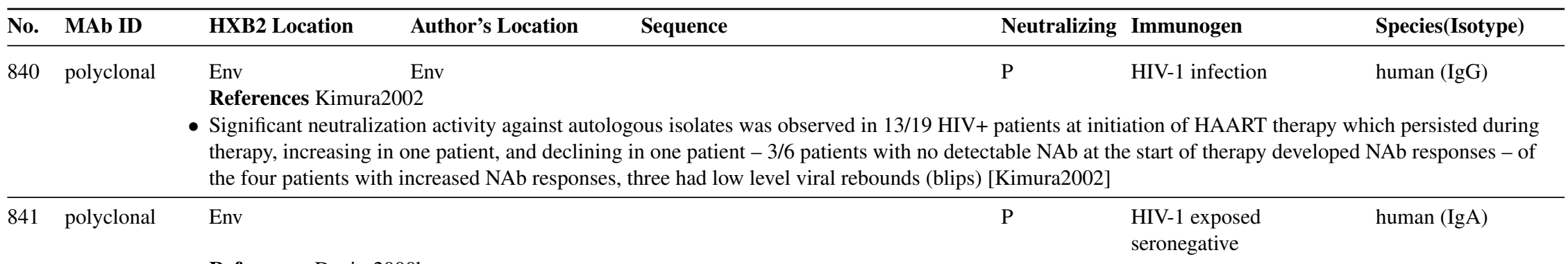

References Devito2000b

seronegative

- Mucosal and plasma HIV-specific IgA that can neutralize primary isolates is present saliva (11/15 tested) and plasma (11/15) and cervicovaginal fluid (11/14) from highly exposed persistently seronegative (HEPS) individuals [Devito2000b]

842 polyclonal Env $\quad \begin{array}{lll}\text { HIV-1 exposed } & \text { human (IgA) } \\ \text { seronegative }\end{array}$

References Devito2000a

- IgA from the genital tract, saliva and plasma from highly exposed persistently seronegative (HEPS) individuals can inhibit transcytosis of HIV-1 across a transwall system that provides a tight epithelial cell layer- $50 \%$ of the $\operatorname{IgA}$ samples studied were able to inhibit transcytosis of at least one of two primary isolates tested, indicating this may be an important mechanism against sexual acquisition of HIV-1 [Devito2000a].

\begin{tabular}{lllll}
\hline 843 polyclonal Env & P & $\begin{array}{l}\text { HIV-1 exposed } \\
\text { seronegative }\end{array}$ & human (IgA) &
\end{tabular}

References Broliden2001

- IgA isolated from the saliva, genital tract, and plasma of most highly exposed persistently seronegative (HEPS) sex workers in a Kenyan cohort could neutralize a B, A and D clade primary isolates and could inhibit transcytosis of HIV across a transwall model of the human mucosal epithelium [Broliden2001]

\begin{tabular}{lllll}
\hline 844 polyclonal & Env & P & HIV-1 exposed & human (IgA) \\
& seronegative
\end{tabular}

- IgA isolated from the saliva, genital tract, and plasma of most highly exposed persistently seronegative (HEPS) Kenyan sex workers mediated broad cross-clade neutralization of primary isolates (A, B, C, D, and CRF01) - 6/10 HEPS individuals that were persistently exposed to a stable HIV+ B clade infected partner showed less breadth of neutralization, and were able to neutralize clade A and B primary isolates, but not clades C, D, or CRF01 [Devito2002]

845 polyclonal Env

$\mathrm{P}$

HIV-1 exposed $\quad$ human (IgA)
seronegative

References Mazzoli1999

- Serum HIV-specific IgA is present in highly exposed persistently seronegative individuals (HEPS) in the absence of serum IgG - serum IgA can be found in productively infected individuals and exposed seronegatives at similar titers - 5/15 sera from HEPS had neutralizing activity, 2 of these in purified IgA HIV-1 specific serum IgA concentrations declined after one year of interruption of at-risk sex [Mazzoli1999]

\begin{tabular}{lllll}
\hline 846 polyclonal & Env & P & HIV-1 exposed \\
seronegative
\end{tabular}




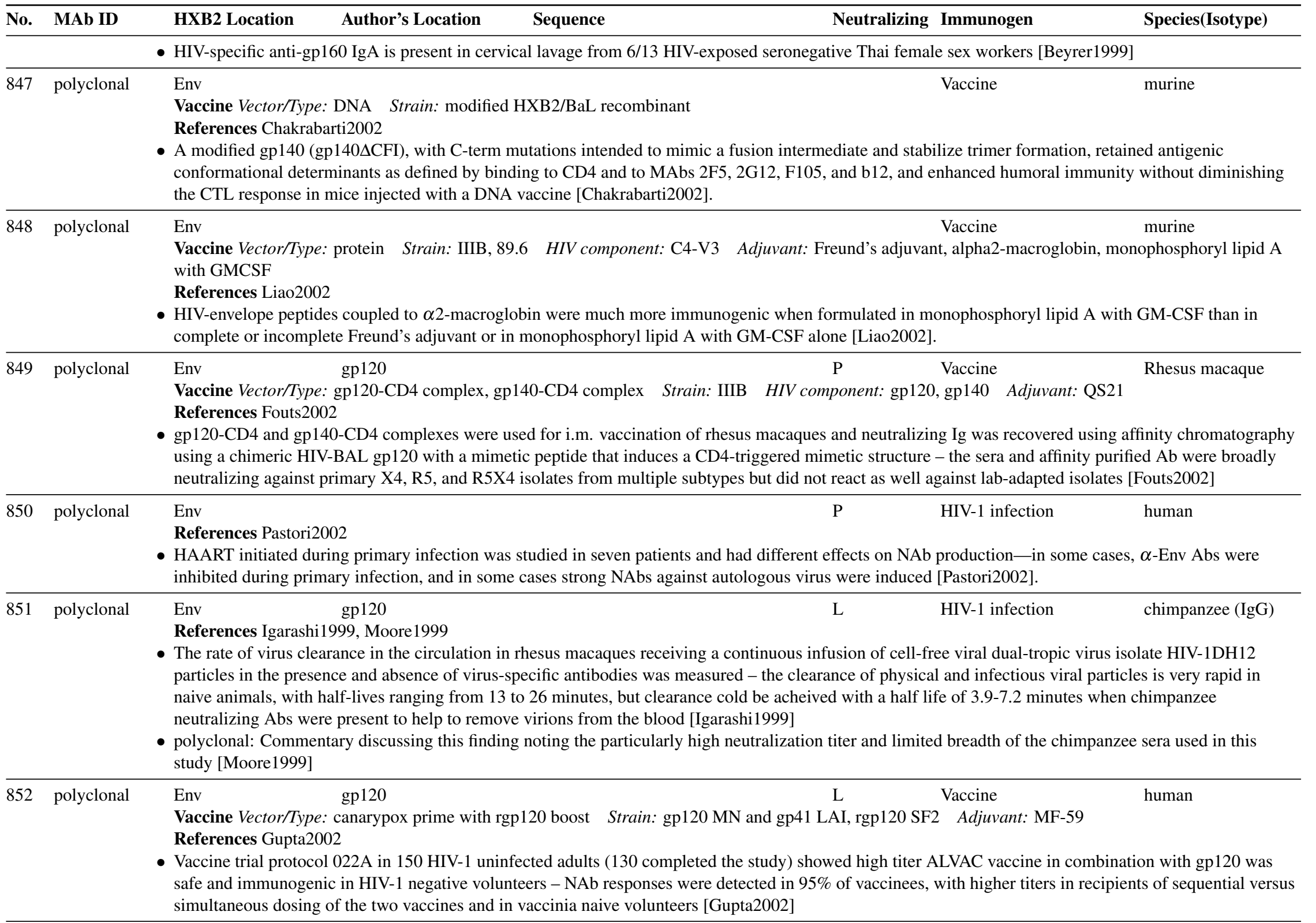




\begin{tabular}{|c|c|c|c|c|c|c|c|}
\hline No. & MAb ID & HXB2 Location & Author's Location & Sequence & Neutralizing & Immunogen & Species(Isotype) \\
\hline \multirow[t]{2}{*}{853} & $101-342$ & Env & $\begin{array}{l}\text { gp120 (476-505 } \\
\text { HAM112, O group) }\end{array}$ & & & Vaccine & murine $(\operatorname{IgG} 2 \mathrm{a} \kappa)$ \\
\hline & & $\begin{array}{l}\text { Vaccine Vector/T } \\
\text { Ab type C-term } \\
\text { References Sche }\end{array}$ & $\begin{array}{l}\text { recombinant protein } \\
999\end{array}$ & Strain: HAM112 (group O) & HIV component: gp160 & & \\
\hline
\end{tabular}

\begin{tabular}{|c|c|c|}
\hline 854 & $101-451$ & $\begin{array}{l}\text { Env gp120 (498-527 } \\
\text { HAM112, O group) }\end{array}$ \\
\hline 855 & $120-1$ & $\begin{array}{l}\text { Env gp120 (503-532) } \\
\text { Vaccine Vector/Type: peptide } \\
\text { Ab type C-term } \\
\text { References Chanh1986, Dalgleish1988 }\end{array}$ \\
\hline 856 & $212 \mathrm{~A}$ & $\begin{array}{l}\text { Env } \\
\text { Ab type C1 Donor James Robinson, Tulane University, LA } \\
\text { References Robinson1992, Moore1994d Moore1996, Binley1997a Fouts1997, Ditzel1997, Wyatt1997], Parren1997c, Sullivan1998b, Binley1998 } \\
\text { - 212A: Mutations that inhibit binding: C1 (45 W/S) and V5 (463 N/D) - and enhance binding: V2 (179/180 LD/DL) and C5 (495 G/K) [Moore1994d] } \\
\text { - 212A: Binding enhanced by anti-V3 MAb 5G11 - reciprocal inhibition with anti-C1 MAbs [Moore1996] } \\
\text { - 212A: Study shows neutralization is not predicted by MAb binding to JRFL monomeric gp120, but is associated with oligomeric Env binding - 212A } \\
\text { bound monomer, did not bind oligomer or neutralize JRFL [Fouts1997] } \\
\text { - 212A: Binds efficiently to sgp120 but not soluble gp120+gp41, suggesting its gp120 epitope is blocked by gp41 binding - does not bind to HXBc2 gp120 if } \\
\text { the 19 C-term amino acids are deleted [Wyatt1997] } \\
\text { - 212A: Does not neutralize TCLA strains or primary isolates [Parren1997c] } \\
\text { - 212A: Does not compete with binding of MAb generated in response to gp120-CD4 complex, CG10 [Sullivan1998b] } \\
\text { - 212A: A panel of MAbs were shown to bind with similar or greater affinity and similar competition profiles to a deglycosylated or variable loop deleted } \\
\text { core gp120 protein ( Delta V1, V2, and V3), thus such a core protein produces a structure closely approximating full length folded monomer [Binley1998] }\end{array}$ \\
\hline 857 & $522-149$ & $\begin{array}{l}\text { Env gp120 no } \\
\text { Vaccine Vector/Type: recombinant protein HIV component: Env } \\
\text { Ab type C1 Donor G. Robey, Abbott Inc. } \\
\text { References Moore1996, Trkola1996a, Binley1998 Yang2000 } \\
\text { - 522-149: Binding is enhanced by C5 antibodies M91 and 1C1 - mutual binding-inhibition with anti-C1 antibody 133/290 - binding is destroyed by a W/L } \\
\text { (position 61, LAI) gp120 amino acid substitution - other C1 antibodies enhance binding to gp120 [Moore1996] } \\
\text { - 522-149: Does not neutralize JR-FL nor block gp120 interaction with CCR-5 in a MIP-1beta-CCR-5 competition study [Trkola1996a] } \\
\text { - 522-149: A panel of MAbs were shown to bind with similar or greater affinity and similar competition profiles to a deglycosylated or variable loop deleted } \\
\text { core gp120 protein ( Delta V1, V2, and V3), thus such a core protein produces a structure closely approximating full length folded monomer [Binley1998] }\end{array}$ \\
\hline
\end{tabular}




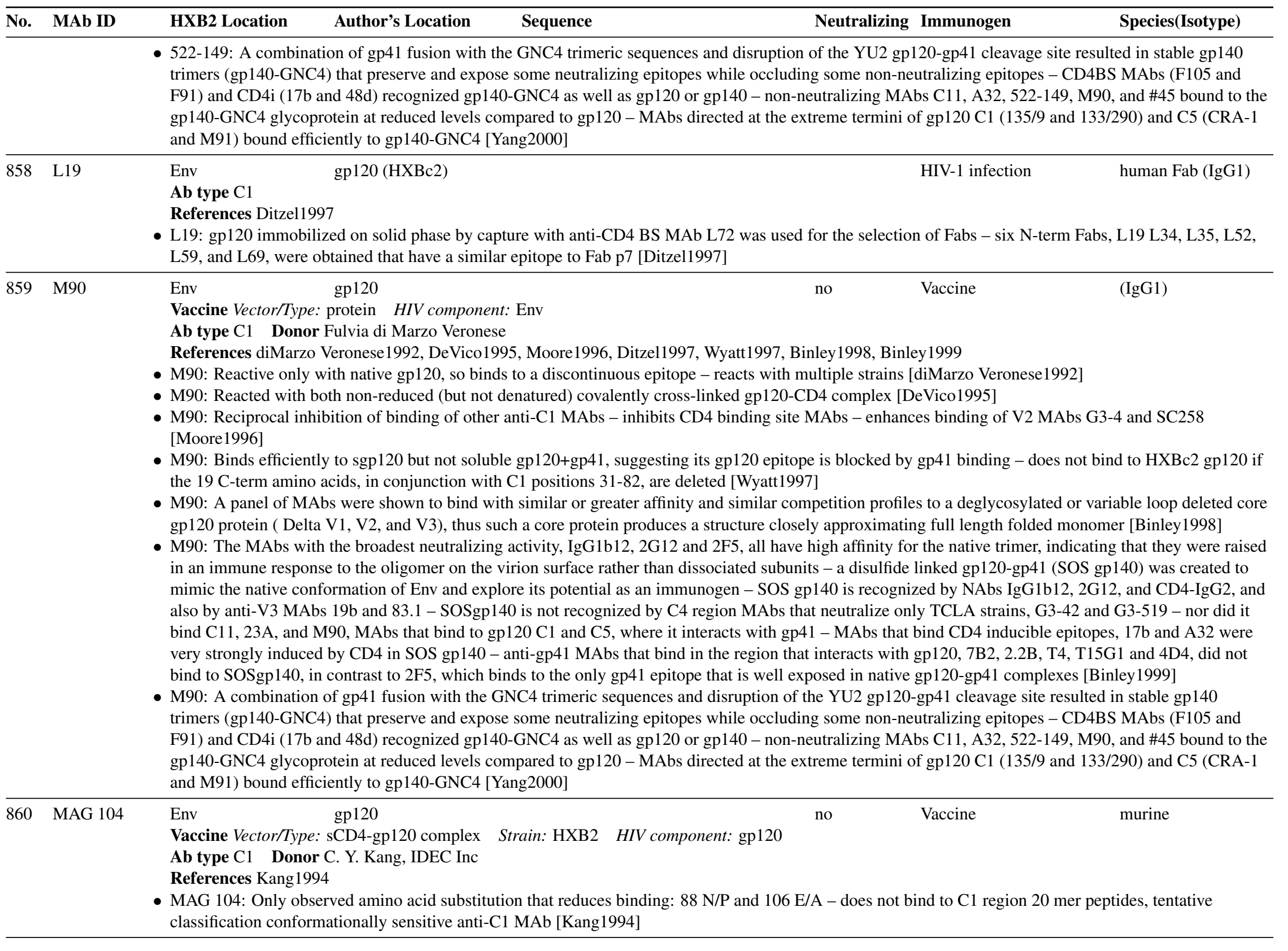




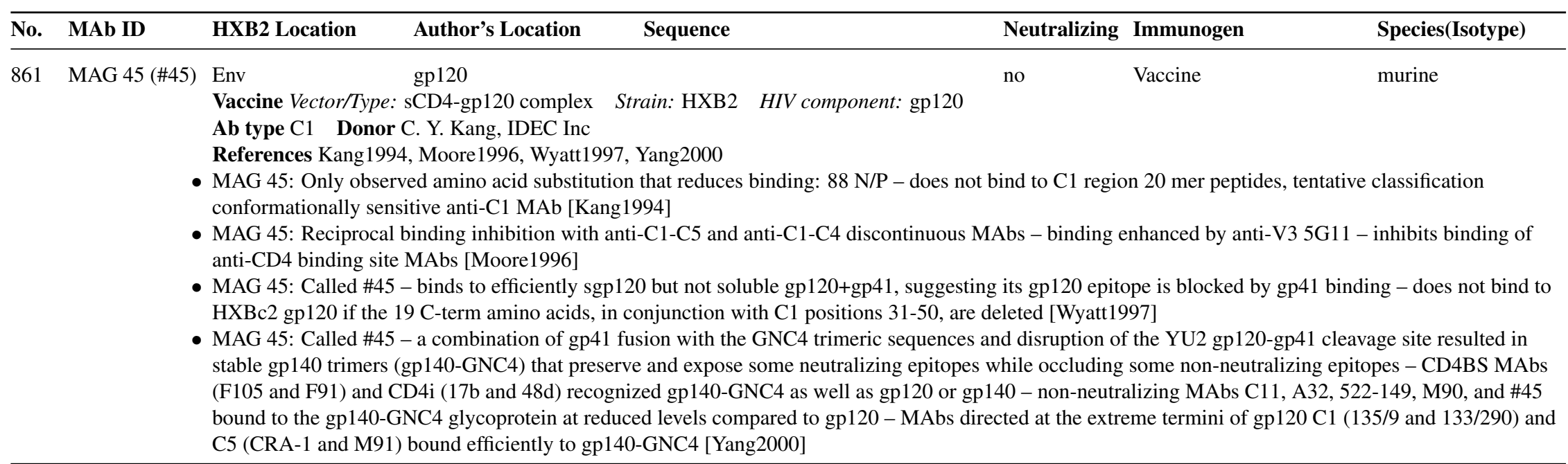

862 MAG 95

\begin{tabular}{|c|c|}
\hline gp120 & \\
\hline Vaccine Vector/Type: sCD4-gp120 complex & Strain: HXB2 HIV component: gp120 \\
\hline
\end{tabular}

Ab type C1 Donor C. Y. Kang, IDEC Inc

References Kang1994

- MAG 95: Only observed amino acid substitution that reduces binding: $88 \mathrm{~N} / \mathrm{P}$ - does not bind to $\mathrm{C} 1$ region 20 mer peptides, tentative classification conformationally sensitive anti-C1 MAb [Kang1994]

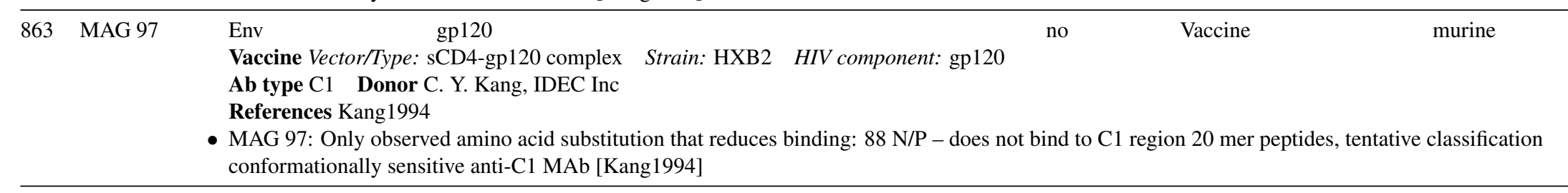

Env gp41

Ab type C1 Donor Patricia Earl and Christopher Broder, NIH

Vaccine murine (IgG)

References Broder1994, Ear11997, Golding2002b

- There are two HIV-Abs with the name T9, one binds to gp41, one to gp120

- T9: One of five MAbs (T4, T6, T9, T10 and T35) in a competition group that bind to a conformation-dependent epitope in gp41 and is oligomer specific neutralizes IIIB and SF2 [Broder1994]

- T9: This antibody, along with 7 others (M10, D41, D54, T6, T4, T10 and T35), can block the linear murine MAb D61, and the human MAb 246-D, which both bind to the immunodominant region near the two Cys in gp41 - most of these antibodies are oligomer dependent - all of the MAbs are reactive with ten different HIV-1 strains - members of this competition group are blocked by sera from HIV-1+ individuals [Earl1997] 


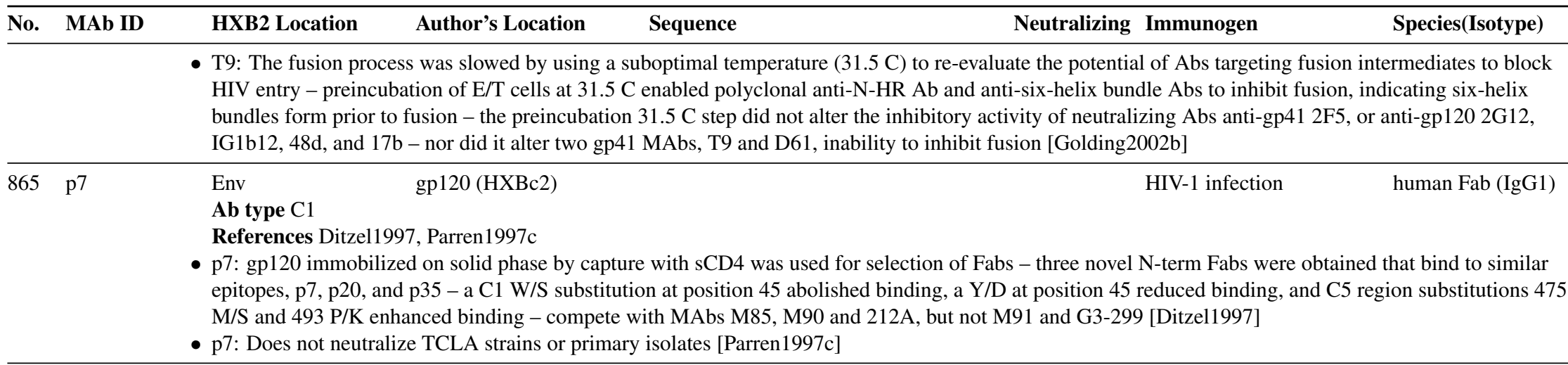

$$
866 \quad \text { L100 }
$$$$
\text { Env }
$$$$
\text { gp120 (HXBc2) }
$$$$
\text { HIV-1 infection }
$$$$
\text { human Fab (IgG1) }
$$

Ab type C1-C2

References Ditzel1997, Parren1997c, Parren1997a

- L100: Does not neutralize TCLA strains or primary isolates [Parren1997c|

- L100: gp120 immobilized on solid phase by capture with sCD4 and then masked with Fab p7 allowed selection of a new Fab, L100, with a novel specificity for $\mathrm{C} 1$ and $\mathrm{C} 2-$ gp120 C1 substitutions $69 \mathrm{~W} / \mathrm{L}$ and 76 P/Y abolish L100 binding, and C2 substitutions 252 R/W, 256 S/Y, 262 N/T and 267 E/L abolish or strongly inhibit L100 binding - inhibits binding of MAbs M90 and G3-299, but not M85, 212A, and M91 [Ditzel1997, Parren1997a]

$867 \quad 2 / 11 \mathrm{c}(211 \mathrm{c}, \quad$ Env gp120

$\mathrm{L}$ (weak)

HIV-1 infection

human

$2.11 \mathrm{c}, 211 / \mathrm{c}, \quad$ Ab type C1-C4 Donor James Robinson, Tulane University, LA

2-11c) References Moore1996, Trkola1996a, Binley1997a, Fouts1997, Li1997, Wyatt1997, Binley1998, Xiang2002a

- 2/11c: Inhibits binding of anti-C1, -C5, -C4, -V3 and anti-CD4 binding site MAbs - induces binding of some anti-V2 and CD4i MAbs (48d and 17b) -

similar reactivity pattern to A32, but less cross-reactive and lower affinity - A32 and 211/c are unique among known human and rodent MAbs [Moore1996]

- 2/11c: Called 211c - does not neutralize JR-FL nor block gp120 interaction with CCR-5 in a MIP-1beta-CCR-5 competition study [Trkola1996a]

- 2/11c: Study shows neutralization is not predicted by MAb binding to JRFL monomeric gp120, but is associated with oligomeric env binding - 2/11c bound monomer, did not bind oligomer or neutralize JRFL [Fouts1997]

- 2/11c: Called 2.11c - One of 14 human MAbs tested for ability to neutralize a chimeric SHIV-vpu+, which expressed HIV-1 IIIB env - 50\% neutralization could not be achieved at a maximal concentration of $67 \mathrm{mug} / \mathrm{ml}$ [Li1997]

- 2/11c: Binds efficiently to sgp120 but not soluble gp120+gp41, suggesting its gp120 epitope is blocked by gp41 binding - does not bind to HXBc2 gp120 if the 19 C-term amino acids, in conjunction with $\mathrm{C} 1$ positions 31-74, are deleted [Wyatt1997]

- 2/11c: Called 211/c - a panel of MAbs were shown to bind with similar or greater affinity and similar competition profiles to a deglycosylated or variable loop deleted core gp120 protein ( Delta V1, V2, and V3), thus such a core protein produces a structure closely approximating full length folded monomer Binley1998

- 2/11c: Used as a negative control in a study of CD4i MAbs [Xiang2002a]

\begin{tabular}{llll}
\hline 868 & A32 & $\begin{array}{l}\text { Env } \\
\text { Ab type C1-C4 }\end{array}$ & $\begin{array}{c}\text { gp120 } \\
\text { Donor James Robinson, Tulane University, New Orleans, LA, USA }\end{array}$
\end{tabular}

References Moore1994b Wyatt1995 Moore1995b Moore1996 Wu1996 Trkola1996 Binley1997a Fouts1997 Burton1997 Wyatt1997 Boots1997

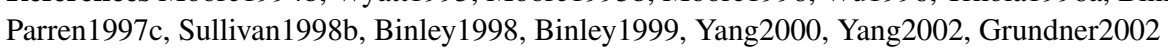

- A32: Reacted with virtually every gp120 monomer of every clade tested, most conserved gp120 monomer epitope known [Moore1994b] 


\begin{tabular}{lllll}
\hline No. MAb ID & HXB2 Location & Author's Location & Sequence & Neutralizing Immunogen
\end{tabular}

- A32: Epitope is better exposed upon CD4 binding to gp120 - binding of A32 enhances binding of 48d and 17b - studies using a V1/V2 deletion mutant demonstrated that enhanced binding of 48d in the presence sCD4 involves the V1/V2 loops, with more significant involvement of V2 [Wyatt1995]

- A32: Review: epitope is distinct from CD4BS MAbs, 48d and 17b, and 2G12 [Moore1995b]

- A32: Reciprocal inhibition of binding of anti-C1, - C5, -C4, -V3 and anti-CD4 binding site MAbs - induces binding of some anti-V2 and sCD4 inducible MAbs (48d and 17b) - very similar competition pattern to 2/11c, A32 and 211/c are unique among known human and rodent MAbs [Moore1996]

- A32: Not neutralizing - binds domains that interact with gp41 - MIP-1alpha binding to CCR-5 expressing cells can be inhibited by gp120-sCD4 and binding of A32 does not block this inhibition [Wu1996

- A32: Does not neutralize JR-FL, or any strain strongly - partial inhibition of gp120 interaction with CCR-5 in a MIP-1beta-CCR-5 competition study [Trkola1996a]

- A32: Study shows neutralization is not predicted by MAb binding to JRFL monomeric gp120, but is associated with oligomeric env binding - A32 bound monomer, did not bind oligomer or neutralize JRFL [Fouts1997]

- A32: Review Burton1997

- A32: Binds efficiently to sgp120 but not soluble gp120+gp41, suggesting its gp120 epitope is blocked by gp41 binding [Wyatt1997]

- A32: Does not neutralize TCLA strains or primary isolates [Parren1997c

- A32: Abs that recognize discontinuous epitopes can identify mimotopes from a phage peptide display library - A32 has a unique epitope involving mostly $\mathrm{C} 2$ but $\mathrm{C} 1$ and $\mathrm{C} 4$ contribute - six quite variable phage inserts were recognized, with a consensus of LPWYN - a central Trp was the most conserved element, consistent with W427 being an important residue for binding gp120 [Boots1997]

- A32: Enhances binding of CD4i MAbs 17b and 48d, and a MAb generated in response to gp120-CD4 complex, CG10 [Sullivan1998b]

- A32: A panel of MAbs were shown to bind with similar or greater affinity and similar competition profiles to a deglycosylated or variable loop deleted core gp120 protein ( Delta V1, V2, and V3), thus such a core protein produces a structure closely approximating full length folded monomer [Binley1998]

- A32: The MAbs with the broadest neutralizing activity, IgG1b12, 2G12 and 2F5, all have high affinity for the native trimer, indicating that they were raised in an immune response to the oligomer on the virion surface rather than dissociated subunits - a disulfide linked gp120-gp41 (SOS gp140) was created to mimic the native conformation of Env and explore its potential as an immunogen - SOS gp140 is recognized by NAbs IgG1b12, 2G12, and CD4-IgG2, and also by anti-V3 MAbs 19b and 83.1 - SOSgp140 is not recognized by C4 region MAbs that neutralize only TCLA strains, G3-42 and G3-519 - nor did it bind $\mathrm{C} 11,23 \mathrm{~A}$, and M90, MAbs that bind to gp120 C1 and C5, where it interacts with gp41 - MAbs that bind CD4 inducible epitopes, $17 \mathrm{~b}$ and A32 were very strongly induced by CD4 in SOS gp140 - anti-gp41 MAbs that bind in the region that interacts with gp120, 7B2, 2.2B, T4, T15G1 and 4D4, did not bind to SOSgp140, in contrast to 2F5, which binds to the only gp41 epitope that is well exposed in native gp120-gp41 complexes |Binley1999|

- A32: A combination of gp41 fusion with the GNC4 trimeric sequences and disruption of the YU2 gp120-gp41 cleavage site resulted in stable gp140 trimers (gp140-GNC4) that preserve and expose some neutralizing epitopes while occluding some non-neutralizing epitopes - CD4BS MAbs (F105 and F91) and CD4i (17b and 48d) recognized gp140-GNC4 as well as gp120 or gp140 - non-neutralizing MAbs C11, A32, 522-149, M90, and \#45 bound to the gp140-GNC4 glycoprotein at reduced levels compared to gp120 - MAbs directed at the extreme termini of gp120 C1 (135/9 and 133/290) and C5 (CRA-1 and M91) bound efficiently to gp140-GNC4 [Yang2000]

- A32: Uncleaved soluble gp140 (YU2 strain, R5 primary isolate) can be stabilized in an oligomer by fusion with a C-term trimeric GCN4 motif or using a T4 trimeric motif derived from T4 bacteriophage fibritin - stabilized oligomer gp140 delta683(-FT) showed strong preferential recognition by NAbs IgG1b12 and 2G12 relative to the gp120 monomer, in contrast to poorly neutralizing MAbs F105, F91, 17b, 48d, and 39F which showed reduced levels of binding, and C11, A32, and 30D which did not bind the stabilized oligomer [Yang2002]

- A32: HIV-1 gp160deltaCT (cytoplasmic tail-deleted) proteoliposomes (PLs) containing native, trimeric envelope glycoproteins from R5 strains YU2 and JRFL, and X4 strain $\mathrm{HXBc} 2$, were made in a physiologic membrane setting as candidate immunogens for HIV vaccines - 2F5 bound to gp160deltaCT with a reconstituted membrane ten-fold better than the same protein on beads - anti-CD4BS MAbs IgG1b12 and F105, A32 (C1-C4), C11 (C1-C5), and 39F (V3) MAbs bound gp160deltaCT PLs indistinguishably from gp160deltaCT expressed on the cell surface - non-neutralizing MAbs C11 and A32 bound with lower affinity than NAb IgG1b12 - the MAb 17b was sCD4 inducible on gp160deltaCT PL [Grundner2002] 


\begin{tabular}{|c|c|c|c|c|c|c|c|}
\hline No. & MAb ID & HXB2 Location & Author's Location & Sequence & Neutralizing & Immunogen & Species(Isotype) \\
\hline 369 & C11 (c11) & Env & gp120 & & no & HIV-1 infection & human \\
\hline
\end{tabular}

Ab type C1-C5 Donor James Robinson, Tulane University, LA

References Robinson1992, Moore1994d, Moore1996, Trkola1996a, Wu1996, Binley1997a, Fouts1997, Wyatt1997, Parren1997c, Sullivan1998b

Binley1999, Yang2002, Grundner2002, Basmaciogullari2002

- C11: Mutations that inhibit binding: C1 (45 W/S, 88 N/P) - V5 (463 N/D) - and C5 (491 I/F, 493 P/K and 495 G/K) and enhance binding: C1 (36 V/L) V1-V2 (152/153 GE/SM) - and DeltaV1/V2/V3 [Moore1994d]

- C11: Binding enhanced by anti-V3 MAb 5G11 - reciprocal inhibition with anti-C1 MAbs [Moore1996]

- C11: Did not block ability of gp120-sCD4 complexes to inhibit MIP-1alpha binding - binds to gp41-binding domain [Wu1996]

- C11: Does not neutralize JR-FL nor block gp120 interaction with CCR-5 in a MIP-1beta-CCR-5 competition study [Trkola1996a

- C11: Study shows neutralization is not predicted by MAb binding to JRFL monomeric gp120, but is associated with oligomeric Env binding - C11 bound monomer, did not bind oligomer or neutralize JRFL [Fouts1997]

- C11: Binds efficiently to sgp120 but not soluble gp120+gp41, suggesting its gp120 epitope is blocked by gp41 binding - partial re-exposure if sCD4 was bound - does not bind to HXBc2 gp120 if the 19 C-term amino acids are deleted [Wyatt1997]

- C11: Does not neutralize TCLA strains or primary isolates [Parren1997c]

- C11: Does not compete with binding of MAb generated in response to gp120-CD4 complex, CG10 [Sullivan1998b]

- C11: The MAbs with the broadest neutralizing activity, IgG1b12, 2G12 and 2F5, all have high affinity for the native trimer, indicating that they were raised in an immune response to the oligomer on the virion surface rather than dissociated subunits - a disulfide linked gp120-gp41 (SOS gp140) was created to mimic the native conformation of Env and explore its potential as an immunogen - SOS gp140 is recognized by NAbs IgG1b12, 2G12, and CD4-IgG2, and also by anti-V3 MAbs 19b and 83.1 - SOSgp140 is not recognized by C4 region MAbs that neutralize only TCLA strains, G3-42 and G3-519- nor did it bind $\mathrm{C} 11,23 \mathrm{~A}$, and M90, MAbs that bind to gp120 C1 and C5, where it interacts with gp41 - MAbs that bind CD4 inducible epitopes, 17b and A32 were very strongly induced by CD4 in SOS gp140 - anti-gp41 MAbs that bind in the region that interacts with gp120, 7B2, 2.2B, T4, T15G1 and 4D4, did not bind to SOSgp140, in contrast to 2F5, which binds to the only gp41 epitope that is well exposed in native gp120-gp41 complexes [Binley1999]

- C11: Uncleaved soluble gp140 (YU2 strain, R5 primary isolate) can be stabilized in an oligomer by fusion with a C-term trimeric GCN4 motif or using a T4 trimeric motif derived from T4 bacteriophage fibritin - stabilized oligomer gp140 delta683(-FT) showed strong preferential recognition by NAbs IgG1b12 and 2G12 relative to the gp120 monomer, in contrast to poorly neutralizing MAbs F105, F91, 17b, 48d, and 39F which showed reduced levels of binding, and C11, A32, and 30D which did not bind the stabilized oligomer [Yang2002]

- C11: HIV-1 gp160deltaCT (cytoplasmic tail-deleted) proteoliposomes (PLs) containing native, trimeric envelope glycoproteins from R5 strains YU2 and JRFL, and X4 strain $\mathrm{HXBc} 2$, were made in a physiologic membrane setting as candidate immunogens for HIV vaccines - 2F5 bound to gp160deltaCT with a reconstituted membrane ten-fold better than the same protein on beads - anti-CD4BS MAbs IgG1b12 and F105, A32 (C1-C4), C11 (C1-C5), and 39F (V3) MAbs bound gp160deltaCT PLs indistinguishably from gp160deltaCT expressed on the cell surface - non-neutralizing MAbs C11 and A32 bound with lower affinity than NAb IgG1b12 - the MAb 17b was sCD4 inducible on gp160deltaCT PL [Grundner2002]

- C11: gp120 mutants were used to define the CXCR4 binding site using CXCR4 displayed on paramagnetic proteoliposomes (PMPLs) to reduce non-specific gp120 binding - basic residues in the V3 loop and the beta19 strand (RIKQ, positions 419-422) were involved, and deletion of the V1-V2 loops allowed CD4-independent CXCR4 binding - MAbs 17b (CD4i) and F105 (CD4BS) were used to study conformational changes in the mutants - C11 was used to detect gp120 binding to CXCR4 or CCR5 on PMPLs [Basmaciogullari2002]

References Ditzel1997, Parren1997c

- L81: gp120 immobilized on solid phase by capture with anti-CD4 BS MAb L72 was used for selection of Fabs - L81 binding is abolished by C1 substitution 45 W/S, C5 substitution 491 I/F, and C3 substitution L/A [Ditzel1997] 


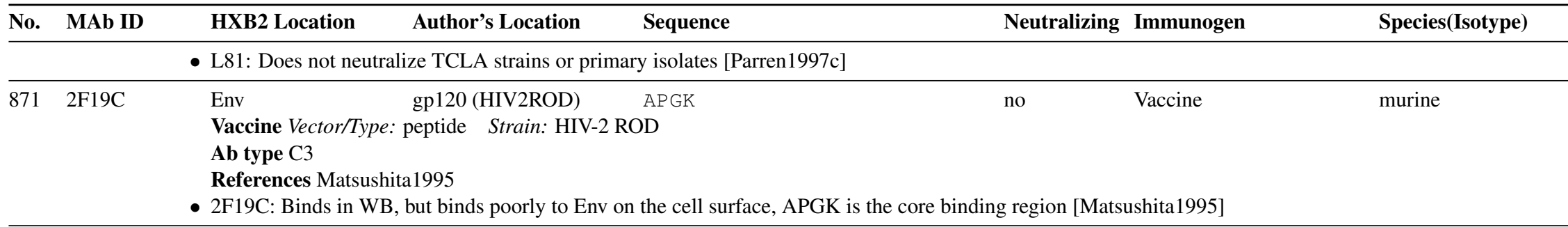

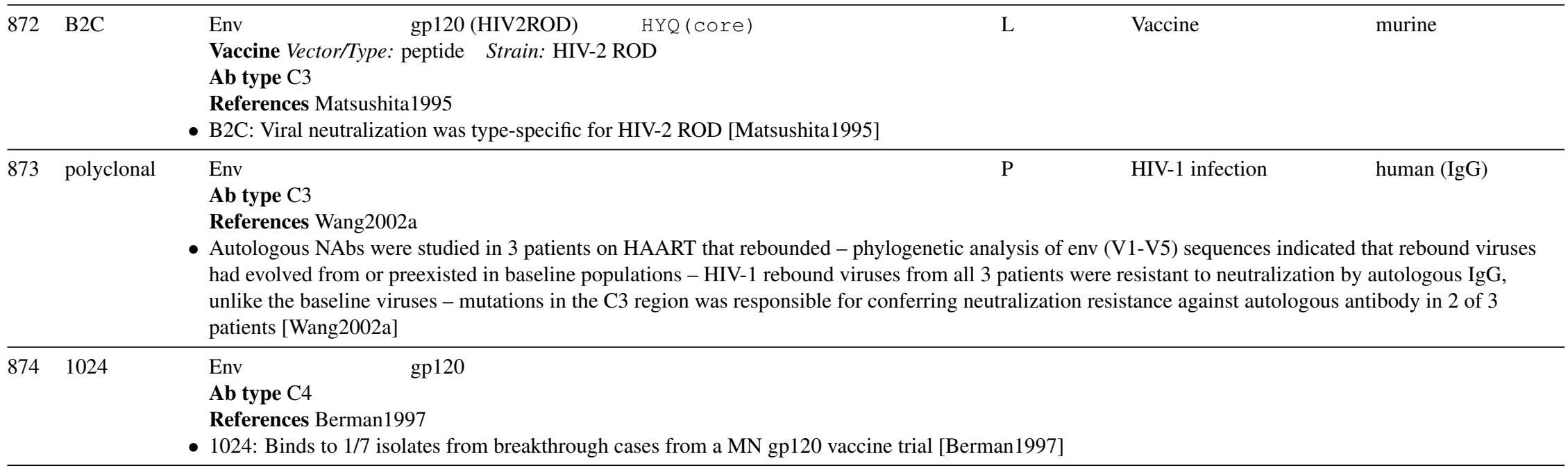

Ab type C5 Donor James Robinson, Tulane University, LA

References Thali1992a, Thali1993, Wu1996, Trkola1996a, Fouts1997, Binley1999, Schulke2002,

- 23A: Called 2.3A - Did not block ability of gp120-sCD4 complexes to inhibit MIP-1alpha binding - binds to gp41-binding domain of gp120 |Wu1996

- 23A: C5 binding MAb - does not inhibit gp120 interaction with CCR-5 in a MIP-1beta-CCR-5 competition study [Trkola1996a]

- 23A: Study shows neutralization is not predicted by MAb binding to JRFL monomeric gp120, but is associated with oligomeric env binding - 23A bound monomer, did not bind oligomer or neutralize JRFL [Fouts1997]

- 23A: The MAbs with the broadest neutralizing activity, IgG1b12, 2G12 and 2F5, all have high affinity for the native trimer, indicating that they were raised in an immune response to the oligomer on the virion surface rather than dissociated subunits - a disulfide linked gp120-gp41 (SOS gp140) was created to mimic the native conformation of Env and explore its potential as an immunogen - SOS gp140 is recognized by NAbs IgG1b12, 2G12, and CD4-IgG2, and also by anti-V3 MAbs 19b and 83.1 - SOSgp140 is not recognized by C4 region MAbs that neutralize only TCLA strains, G3-42 and G3-519- nor did it bind $\mathrm{C} 11,23 \mathrm{~A}$, and M90, MAbs that bind to gp120 C1 and C5, where it interacts with gp41 - MAbs that bind CD4 inducible epitopes, 17b and A32 were very strongly induced by CD4 in SOS gp140 - anti-gp41 MAbs that bind in the region that interacts with gp120, 7B2, 2.2B, T4, T15G1 and 4D4, did not bind to SOSgp140, in contrast to 2F5, which binds to the only gp41 epitope that is well exposed in native gp120-gp41 complexes [Binley1999]

- 23A: Ab binding characteristics of SOS gp140 were tested using SPR and RIPA - SOS gp140 is gp120-gp41 bound by a disulfide bond - NAbs 2G12, 2F5, IgG1b12, CD4 inducible 17b, and 19b bound to SOS gp140 better than uncleaved gp140 (gp140unc) and gp120 - non-neutralizing MAbs 2.2B (binds to gp41 in gp140unc) and 23A (binds gp120) did not bind SOS gp140 [Schulke2002] 


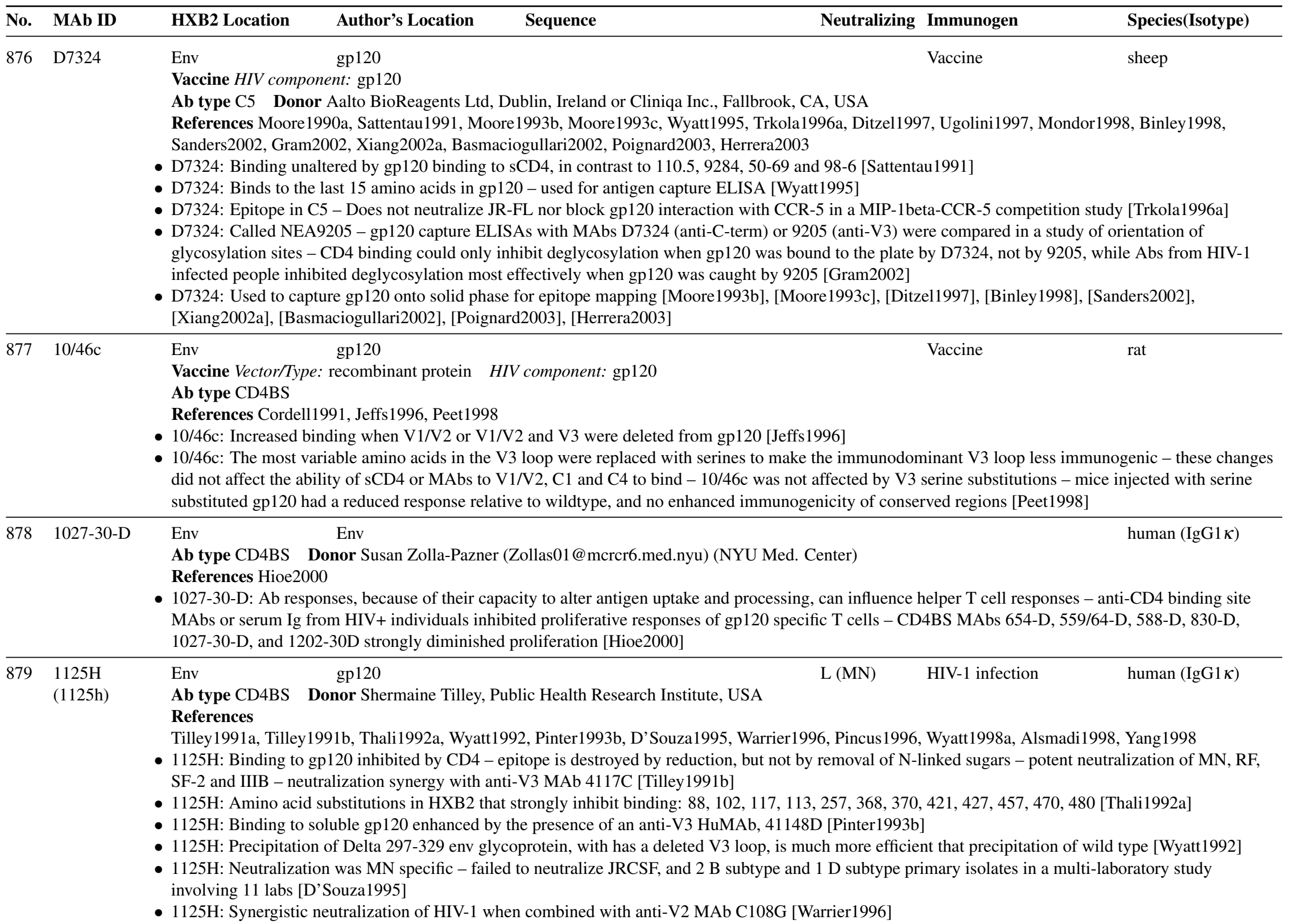




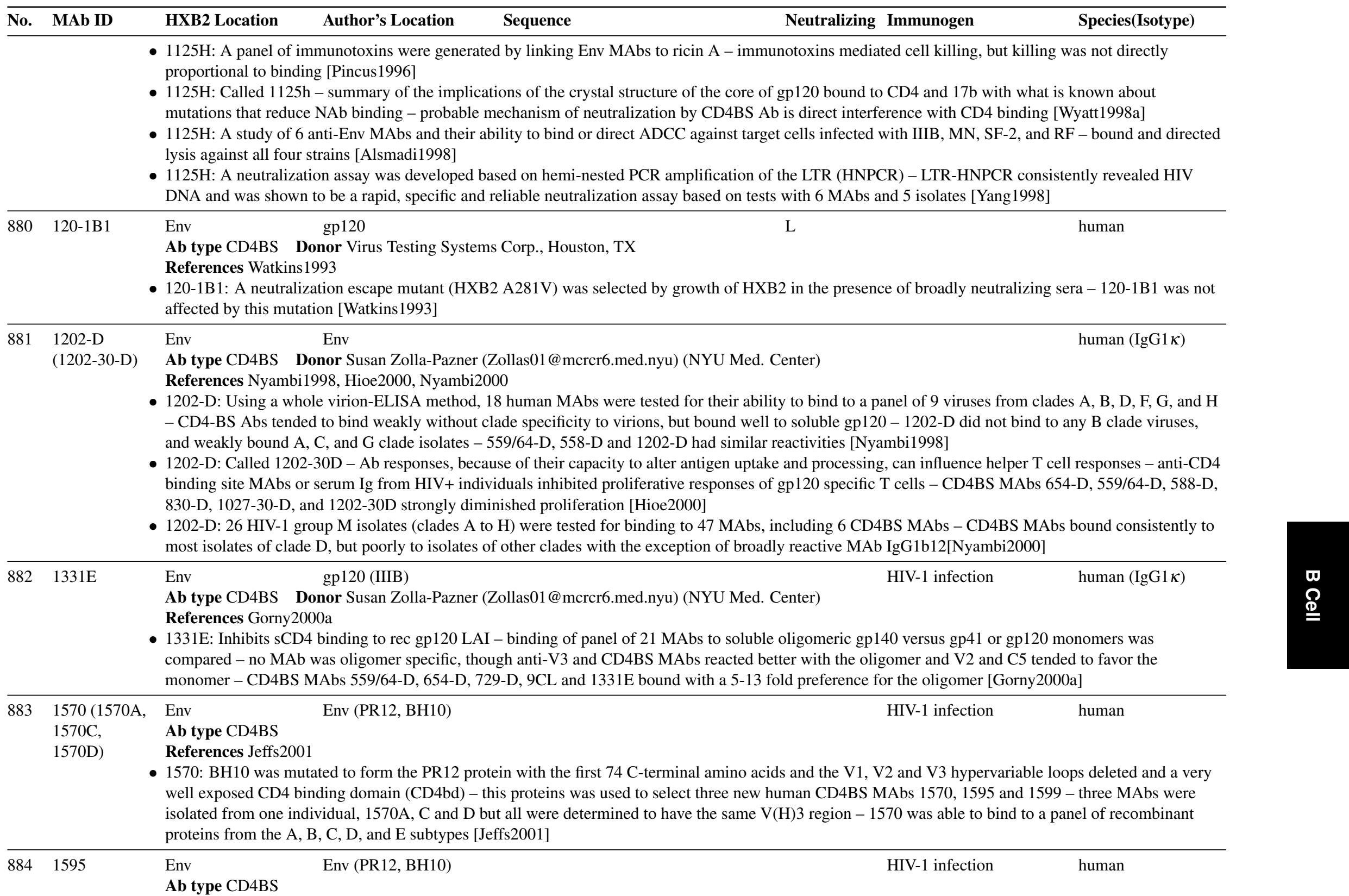




\begin{tabular}{|c|c|c|c|c|c|}
\hline No. & MAb ID & HXB2 Location & Author's Location & Neutralizing Immunogen & Species(Isotype) \\
\hline & & \multicolumn{4}{|c|}{$\begin{array}{l}\text { References Jeffs2001 } \\
\text { 1595: BH10 was mutated to form the PR12 protein with the first } 74 \text { C-terminal amino acids and the V1, V2 and V3 hypervariable loops deleted and a very } \\
\text { well exposed CD4 binding domain (CD4bd) - this proteins was used to select three new human CD4BS MAbs } 1570,1595 \text { and } 1599-1595 \text { was able to } \\
\text { bind gp120 from the A, B, and D clades from a panel of recombinant proteins from the A, B, C, D, and E subtypes [Jeffs } 2001]\end{array}$} \\
\hline 885 & 1599 & $\begin{array}{l}\text { Env } \\
\text { Ab type CD4BS } \\
\text { References Jeffs } 2001\end{array}$ & Env (PR12, BH10) & HIV-1 infectio & human \\
\hline
\end{tabular}

- 1599: BH10 was mutated to form the PR12 protein with the first 74 C-terminal amino acids and the V1, V2 and V3 hypervariable loops deleted and a very well exposed CD4 binding domain (CD4bd) - this proteins was used to select three new human CD4BS MAbs 1570, 1595 and 1599 - 1599 was able to bind gp120 only from the B clade from a panel of recombinant proteins from the A, B, C, D, and E subtypes [Jeffs2001]

$88615 \mathrm{e}(1.5 \mathrm{e}, \quad$ Env $\quad$ gp120 human (IgG1 $\kappa)$

1.5E, 15E) Ab type CD4BS Donor James Robinson, Tulane University, LA, and David Ho, ADARC, NY, NY

References Robinson1990a, Thali1991 Cordell1991, Ho1991b, Koup1991, Ho1992, Wyatt1992, Thali1992a, Takeda1992, Moore1993a, Thali1993, Wyatt1993, Bagley1994, Thali1994, Cook1994, Moore1994b, Moore1994a Sattentau1995b, Lee1995, McKeating1996b, Moore1996, Poignard1996a. Trkola1996a McDougal1996 Wisnewski1996, Binley1997a. Fouts1997, Li1997, Wyatt1997, Berman1997, Parren1997c Wyatt1998a. Parren1998a Sullivan1998b, Binley1998 Trkola1998, Fouts1998 Sullivan1998a Park2000, Kolchinsky2001, Xiang2002b, Zhang2002, Pantophlet2003

- 15e: Broadly neutralizing, binds multiple strains, competes with CD4 for gp120 binding, DTT reduction of env abrogates binding - more potent blocking of gp120-sCD4 binding than MAbs G3-536 and G3-537 [Ho1991b]

- 15e: Cross-competes with MAbs ICR 39.13g and ICR 39.3b [Cordell1991]

- 15e: Binds to gp120 of HIV-1 IIIB, but not RF - mediates ADCC - deletion of the V3 loop from gp120 does not alter ADCC activity |Koup1991|

- 15e: gp120 mutants that affect 15e epitope binding: 113, 257, 368, 370, 421, 427, 475 - four of these coincide with amino acids important for the CD4 binding domain [Ho1992]

- 15e: Precipitation of Delta 297-329 env glycoprotein, with a deleted V3 loop, is much more efficient that precipitation of wild type [Wyatt1992

- 15e: Amino acid substitutions in HXB2 that strongly inhibit binding, similar to [Ho1992], some additional, 88, 102, 117, 113, 257, 368, 370, 421, 427, 457, 470, 480 [Thali1992a]

- 15e: Called N70-1.5e - does not enhance infection of HIV-1 IIIB and MN [Thali1992a

- 15e: Conformational, does not bind denatured gp120 - neutralizes IIIB - reactive with SF-2 gp120 - strong inhibition of HIV+ human sera binding to IIIB gp120 Moore1993a

- 15e: Binding to Delta V1/2 and Delta V1/2/3 mutant glycoproteins is greater than binding to wildtype gp120 [Wyatt1993]

- 15e: Called 15E - a neutralization escape mutant (HXB2 A281V) was selected by growth of HXB2 in the presence of broadly neutralizing sera - 15E neutralization was not affected by this mutation [Watkins1993]

- 15e: Heavy chain is V HIV, V2-1 - light chain is V_kappaI, Hum01/012. Compared to 21h and F105 [Bagley1994]

- 15e: A mutation in gp41, $582 \mathrm{~A} / \mathrm{T}$, confers resistance to neutralization (also confers resistance to MAbs F105, 48d, 21h and 17b) [Thali1994]

- 15e: MAbs against the glycosphingolipid GalCer block HIV infection of normally susceptible CD4 negative cells from the brain and colon - anti-CD4

MAbs moderately inhibit gp120 binding to GalCer, possibly through steric hindrance - binding of GalCer to gp120 inhibited but did not completely block 15e binding [Cook1994]

- 15e: Cross-reactive with gp120 proteins from clades B and D, less so with A and C, and not reactive with clade E and F [Moore1994b

- 15e: Binds with higher affinity to monomer than to oligomer, moderate association rate [Sattentau1995b]

- 15e: The V4 and V5 domains are essential for 1.5e binding, in contrast to the V1, V2, and V3 loops [Lee1995]

- 15e: Called 1.5e - Neutralizes HXB2, but fails to neutralize chimeric virus with gp120 from primary isolates in an HXB2 background [McKeating1996b] 
- 15e: gp120 binding enhanced by anti-V3 MAb 5G11 and anti-V2 MAb G3-136 - binding inhibited by other CD4 binding site MAbs, antibodies that bind to gp120 only when CD4 is bound, and CD4-IgG [Moore1996]

- 15e: Anti-CD4BS MAbs 15e, 21h, and IgG1b12 did not cause gp120 dissociation from virus, or exposure of the gp41 epitope of MAb 50-69, in contrast to CD4i MAb 48d and anti-V3 neutralizing MAbs |Poignard1996a|

- 15e: Inhibits gp120 interaction with CCR-5 in a MIP-1beta-CCR-5 competition study [Trkola1996a]

- 15e: Neutralizes HIV-1 LAI less potently than V3 specific MAbs [McDougal1996]

- 15e: 15e is V H4 - V-region heavy chain usage was examined and a bias of enhanced V H1 and V H4, and reduced V H3, was noted among HIV infected individuals [Wisnewski1996

- 15e: Study shows neutralization is not predicted by MAb binding to JRFL monomeric gp120, but is associated with oligomeric Env binding - 15e bound monomer, did not bind oligomer or neutralize JRFL [Fouts1997]

- 15e: One of 14 human MAbs tested for ability to neutralize a chimeric SHIV-vpu+, which expressed HIV-1 IIIB env - 15e could only achieve 50\% neutralization, but could act synergistically with anti-V3 MAb 694/98-D to achieve 90\% [Li1997]

- 15e: Does not bind to HXBc2 gp120 if the 19 C-term amino acids, in conjunction with C1 positions 31-93, are deleted [Wyatt1997]

- 15e: Called 1.5E - Binds to 7/7 isolates from breakthrough cases from a MN gp120 vaccine trial [Berman1997]

- 15e: Neutralizes TCLA strains, but not primary isolates [Parren1997c]

- 15e: Summary of the implications of the crystal structure of the core of gp120 bound to CD4 and 17b with what is known about mutations that reduce NAb binding - probable mechanism of neutralization by CD4BS Ab is direct interference with CD4 binding [Wyatt1998a]

- 15e: The MAb and Fab binding to the oligomeric form of gp120 and neutralization were highly correlated - authors suggest that neutralization is determined by the fraction of Ab sites occupied on a virion irrespective of the epitope [Parren1998a]

- 15e: A panel of MAbs were shown to bind with similar or greater affinity and similar competition profiles to a deglycosylated or variable loop deleted core gp120 protein ( Delta V1, V2, and V3), thus such a core protein produces a structure closely approximating full length folded monomer - CD4BS MAbs $15 \mathrm{e}, \mathrm{F} 91$ and IgG1b12 bound better to the deleted protein than to wild type [Binley1998]

- 15e: Competes with CG-10 binding, a MAb raised against a gp120 CD4 complex, this was probably due to the disruption of CD4-gp120 by $15 \mathrm{e}$ [Sullivan1998b]

- 15e: No detectable neutralizing activity among primary isolates with different co-receptor usage - some neutralization of TCLA strains [Trkola1998]

- 15e: CD4BS MAbs 15e, 21h, and F91 bind with even lower affinity than 205-43-1 and 205-42-15 to JRFL oligomer [Fouts1998]

- 15e: Called 1.5e - the HIV-1 virus YU2 entry can be enhanced by MAbs binding to the CD4BS, V3 loop, and CD4i epitopes - the activation for this enhanced entry state could be conferred on HxB2 by introducing the YU2 V3 loop, or the YU2 V3 and V1/V2 loops - a similar effect is observed by sub-neutralizing concentrations of sCD4 and the effect is dependent of CCR5 - 1.5e enhances and does not neutralize YU2 env even at 50 ug/ml [Sullivan1998a]

- 15e: Six mutations in MN change the virus from a high-infectivity neutralization resistant phenotype to low-infectivity neutralization sensitive - V3, CD4BS, and CD4i MAbs are 20-100 fold more efficient at neutralizing the sensitive form - the mutation L544P reduced binding of all MAbs against gp120 by causing conformational changes [Park2000]

- 15e: Mutations in two glcosylation sites in the V2 region of HIV-1 ADA at positions 190 and 197 (187 DNTSYRLINCNTS 199) cause the virus to become CD4-independent and able to enter cells through CCR5 alone - these same mutations tended to increase the neutralization sensitivity of the virus, including to $15 \mathrm{e}$ Kolchinsky2001]

- 15e: A series of mutational changes were introduced into the YU2 gp120 that favored different conformations - $375 \mathrm{~S} / \mathrm{W}$ seems to favor a conformation of gp120 closer to the CD4-bound state, and is readily bound by sCD4 and CD4i MAbs (17b, 48d, 49e, 21c and 23e) but binding of anti-CD4BS MAbs (F105, $15 \mathrm{e}, \mathrm{IgG1b} 12,21 \mathrm{~h}$ and F91 was markedly reduced - IgG1b12 failed to neutralize this mutant, while neutralization by $2 \mathrm{G} 12$ was enhanced - 2F5 did not neutralize either WT or mutant, probably due to polymorphism in the YU2 epitope - another mutant, 423 I/P, disrupted the gp120 bridging sheet, favored a different conformation and did not bind CD4, CCR5, or CD4i antibodies, but did bind to CD4BS MAbs [Xiang2002b] 


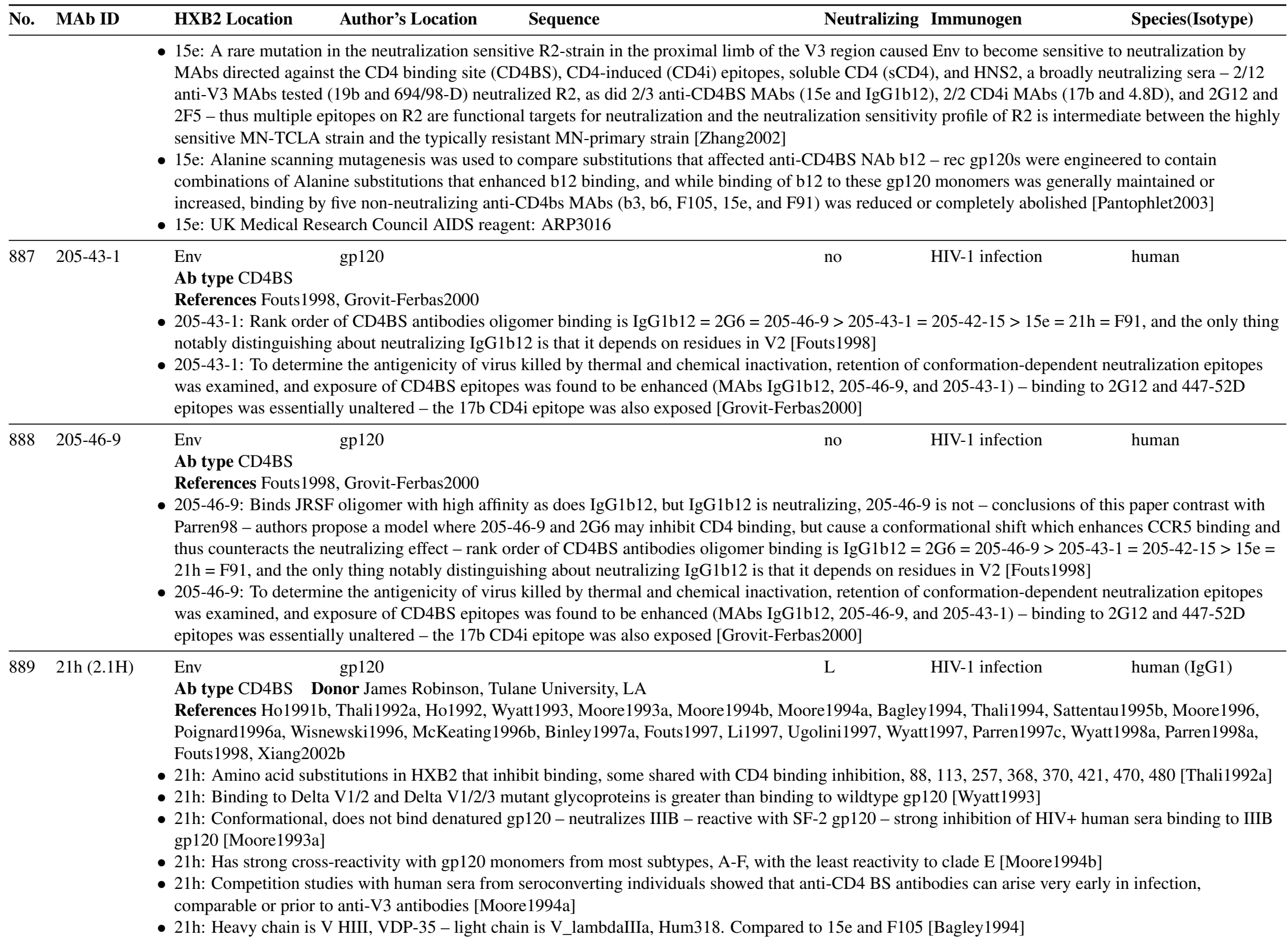




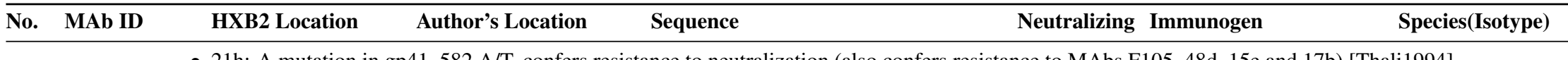

- 21h: A mutation in gp41, $582 \mathrm{~A} / \mathrm{T}$, confers resistance to neutralization (also confers resistance to MAbs F105, 48d, 15e and 17b) [Thali1994]

- 21h: Binds with higher affinity to monomer than to oligomer, moderate association rate [Sattentau1995b]

- 21h: Anti-CD4 binding site MAb - reciprocal inhibition by anti-C1, -C4 and other anti-CD4 binding site antibodies - enhanced by some anti-V2 MAbs and anti-V3 MAb 5G11 - enhances binding of some anti-V3 and -V2 MAbs [Moore1996]

- 21h: Anti-CD4BS MAbs 15e, 21h, and IgG1b12 did not cause gp120 dissociation from virus, or exposure of the gp41 epitope of MAb 50-69, in contrast to CD4i MAb 48d and anti-V3 neutralizing MAbs |Poignard1996a|

- 21h: $21 \mathrm{~h}$ is V H3 - V-region heavy chain usage was examined and a bias of enhanced V H1 and V H4, and reduced V H3, was noted among HIV infected individuals Wisnewski1996

- 21h: Called 2.1H - Neutralizes HXB2, but fails to neutralize chimeric virus with gp120 from primary isolates in an HXB2 background |McKeating1996b

- 21h: Study shows neutralization is not predicted by MAb binding to JRFL monomeric gp120, but is associated with oligomeric Env binding - 21h bound monomer, did not bind oligomer or neutralize JRFL [Fouts1997]

- 21h: One of 14 human MAbs tested for ability to neutralize a chimeric SHIV-vpu+, which expressed HIV-1 IIIB env - 50\% neutralization could not be achieved at a maximal concentration of $67 \mathrm{mug} / \mathrm{ml}$ [Li1997]

- $21 \mathrm{~h}$ : Viral binding inhibition by $21 \mathrm{~h}$ strongly correlated with neutralization (all other neutralizing MAbs tested showed some correlation except 2F5) |Ugolini1997|

- 21h: Binds both gp120 and soluble gp120+gp41 complex efficiently, suggesting its gp120 epitope is not blocked by gp41 binding - major deletions in C1 and C5 and deletions of the V1V2 and V3 loops do not diminish binding [Wyatt1997]

- 21h: Neutralizes TCLA strains, but not primary isolates [Parren1997c]

- $21 \mathrm{~h}$ : Summary of the implications of the crystal structure of the core of gp120 bound to CD4 and 17b with what is known about mutations that reduce NAb binding - probable mechanism of neutralization by CD4BS Ab is direct interference with CD4 binding [Wyatt1998a]

- 21h: The MAb and Fab binding to the oligomeric form of gp120 and neutralization were highly correlated - authors suggest that neutralization is determined by the fraction of Ab sites occupied on a virion irrespective of the epitope [Parren1998a]

- 21h: CD4BS MAbs 15e, 21h, and F91 bind with even lower affinity than 205-43-1 and 205-42-15 to JRFL oligomer - conclusions of this paper contrast with [Parren1998a] [Fouts1998]

- 21h: A series of mutational changes were introduced into the YU2 gp120 that favored different conformations-375 S/W seems to favor a conformation of gp120 closer to the CD4-bound state, and is readily bound by sCD4 and CD4i MAbs (17b, 48d, 49e, 21c and 23e) but binding of anti-CD4BS MAbs (F105, $15 \mathrm{e}, \mathrm{IgG} 1 \mathrm{~b} 12,21 \mathrm{~h}$ and F91) was markedly reduced-IgG1b12 failed to neutralize this mutant, while neutralization by $2 \mathrm{G} 12$ was enhanced-2F5 did not neutralize either WT or mutant, probably due to polymorphism in the YU2 epitope-another mutant, $423 \mathrm{I} / \mathrm{P}$, disrupted the gp120 bridging sheet, favored a different conformation and did not bind CD4, CCR5, or CD4i antibodies, but did bind to CD4BS MAbs [Xiang2002b].

- 21h: UK Medical Research Council AIDS reagent: ARP3017

Vaccine

human from transgenic mice

Vaccine Vector/Type: recombinant protein Strain: SF162 HIV component: gp120 Adjuvant: Ribi adjuvant (MPL+TDM) $(\operatorname{IgG} 2 \kappa)$

Ab type CD4BS Donor Dr. Abraham Pinter, Public Health Research Institute, Newark, NJ, pinter@phri.org

References $\mathrm{He} 2002$

- 28A11/B1: Transgenic mice (strain XenoMouse G2) carrying human genes allowing production of fully human IgG2 $\kappa$ MAbs were used to rapidly create a panel of anti-HIV gp120 MAb-producing hybridomas by immunization with HIV SF162 gp120—6 anti-CD4BS MAbs competed with anti-CD4BS MAb 5145A, blocked sCD4 binding and were conformationally sensitive-4/6 could neutralize the autologous strain SF162, and were broadly cross-reactive with B clade R5 and X4 strains (not E clade)-28A11/B1 was one of these four MAbs [He2002]. 


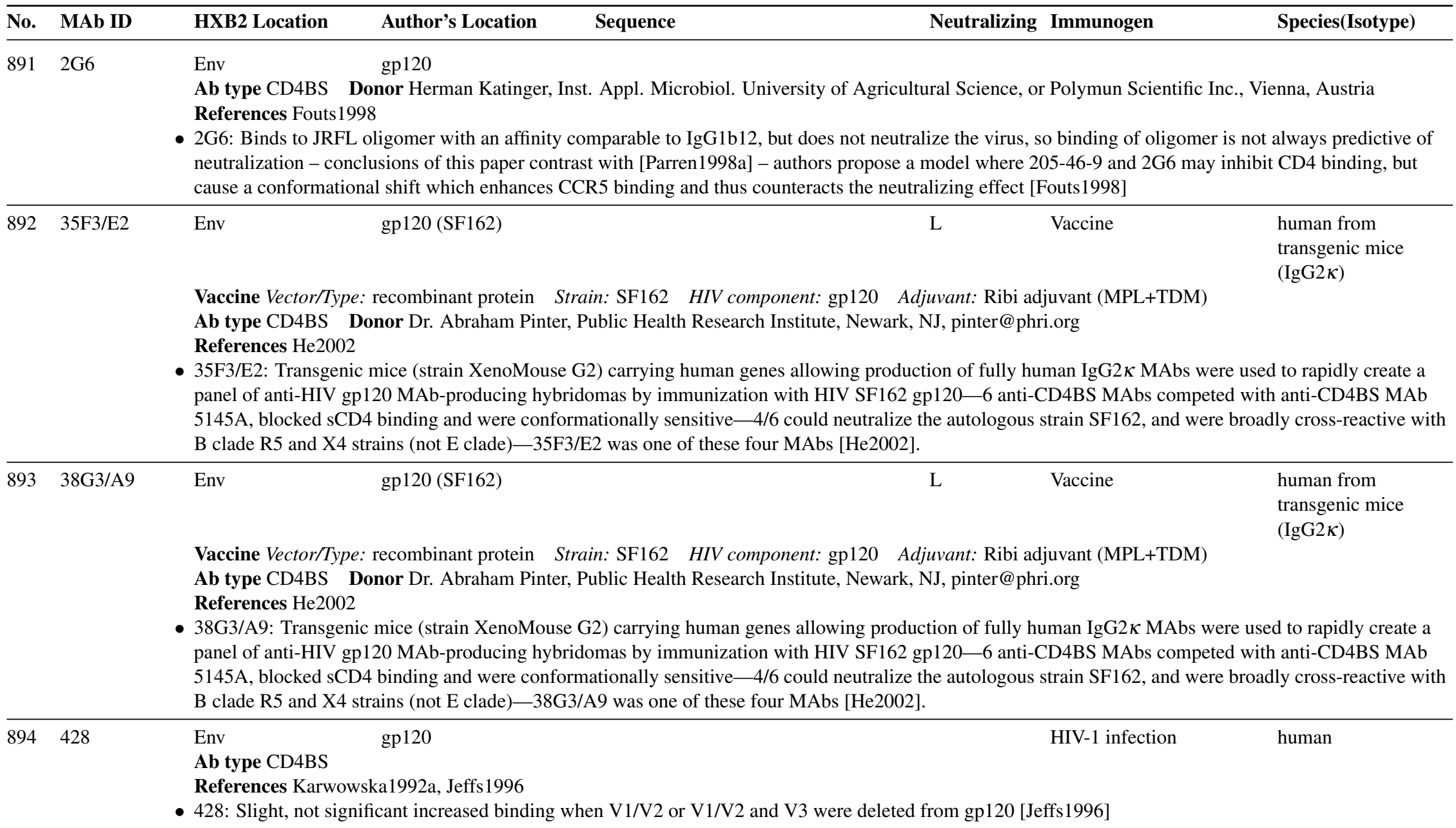

Ab type CD4BS Donor Susan Zolla-Pazner (Zollas01 @ mcrcr6.med.nyu), NYU Med Center, NY, NY

References Karwowska1992a, McKeating1992c, Spear1993, Laal1994, Forthal1995, Manca1995a, Li1997, Wyatt1998a. Nyambi2000

- 448-D: Conformational - reactive with IIIB gp120 in RIP, but not WB assay [Karwowska1992a

- 448-D: Called 448D - blocks gp120-CD4 binding - substitutions at gp120 residues 88, 113, 117, 257, 368 and 370 reduce binding - epitope similar to rat

MAbs 39.13g and 39.3b [McKeating1992c

- 448-D: Did not mediate deposition of complement component C3 on HIV infected cells [Spear1993]

- 448-D: Dissociation constant gp120 IIIB 0.029 - neutralizes IIIB, acts synergistically with anti-V3 MAb 447-52D [Laal1994]

- 448-D: Neutralizing activity, positive ADCC activity, and no viral enhancing activity [Forthal1995] 


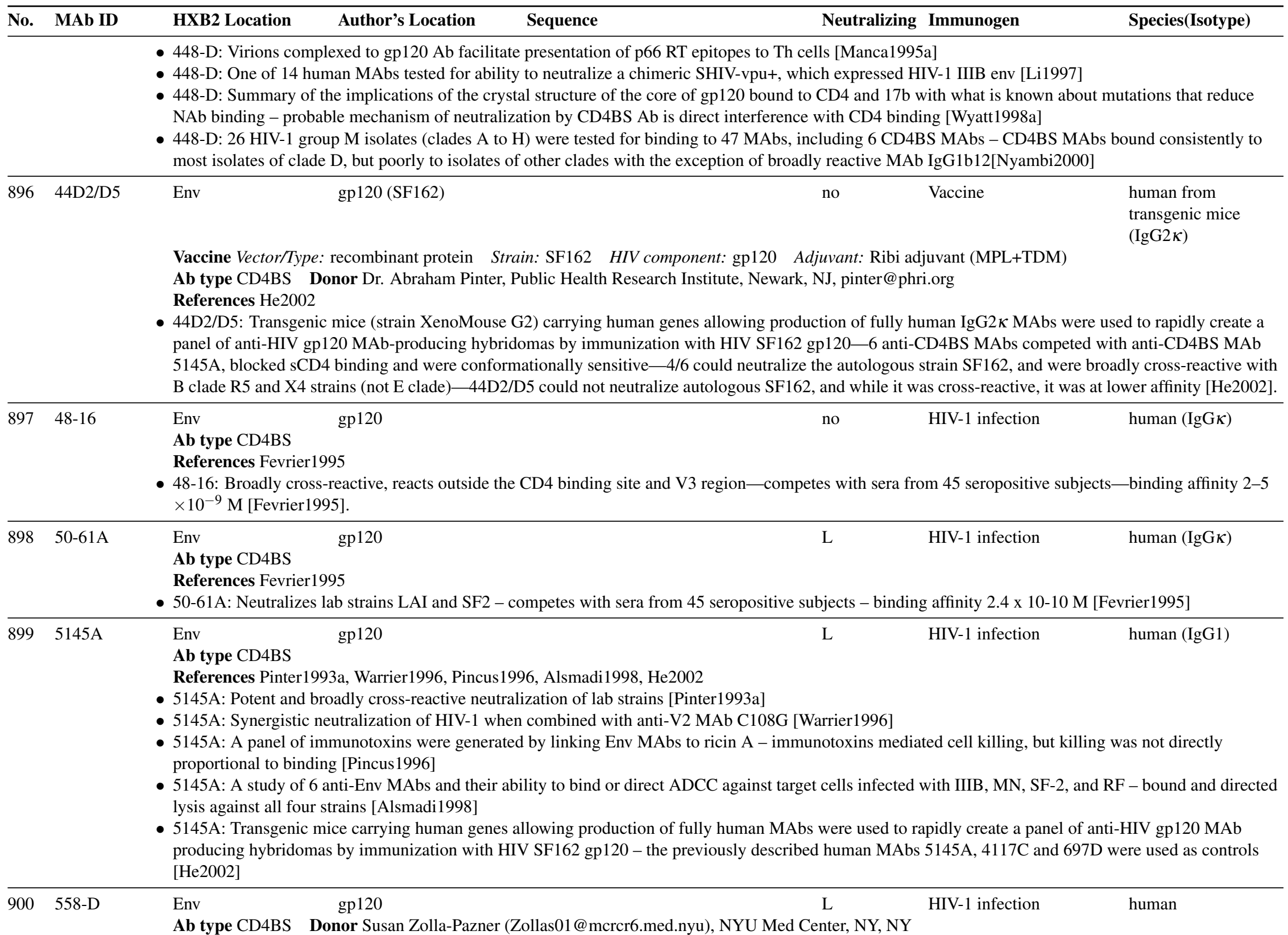




\begin{tabular}{|c|c|c|c|c|c|c|}
\hline No. & MAb ID & HXB2 Location & Author's Location & Sequence & Neutralizing Immunogen & Species(Isotype) \\
\hline
\end{tabular}

- 558-D: Blocks gp120-CD4 binding - binds a panel of mutants all except for $256 \mathrm{~S} / \mathrm{Y}$ and $262 \mathrm{~N} / \mathrm{T}$, which are probably conformationally disruptive |McKeating1992c |

- 558-D: Using a whole virion-ELISA method, 18 human MAbs were tested for their ability to bind to a panel of 9 viruses from clades A, B, D, F, G, and H CD4BS Abs tended to bind weakly without clade specificity to virions, but bound well to soluble gp120 - 558-D did not bind to any B clade viruses, and weakly bound to clade A, C, and G isolates - 559/64-D, 558-D and 1202-D had similar reactivities [Nyambi1998]

\begin{tabular}{llll}
\hline 901 & $559 / 64-D$ & Env & gp120 (LAI) \\
& (559, & Ab type CD4BS & Donor Susan Zolla-Pazner (Zollas01@ @mcrcr6.med.nyu), NYU Med Center, NY, NY
\end{tabular}

559-64D) ～References Karwowska1992a, McKeating1992c, Spear1993, Stamatatos1995, Forthal1995, Jeffs 1996, Hioe1997a, Hioe1997b, Nyambi1998, Gorny2000a Hioe2000, Nyambi2000, Hioe2001, York2001

- 559/64-D: Conformational - reactive with IIIB gp120 in RIP, but not WB assay [Karwowska1992a]

- 559/64-D: Did not mediate deposition of complement component C3 on HIV infected cells [Spear1993]

- 559/64-D: Called 559-64D - The binding of conformation-dependent anti-V2, anti-V3, and anti-CD4BS MAbs to monomeric and virion-associated gp120 from HIV-1 isolates with differences in cell tropism was studied - CD4BS loop epitopes are somewhat occluded in the oligomeric gp120 epitopes on the virion surface relative to the gp120 monomer as indicated by an increase in the half-maximal binding values to macrophage-tropic isolates SF162 and SF128a and to T-cell tropic SF2 - binding of anti-CD4BS MAbs to SF2 resulted in a significant amount of dissociation of gp120 from virion surface [Stamatatos1995]

- 559/64-D: Neutralizing activity, no ADCC activity, and no viral enhancing activity [Forthal1995]

- 559/64-D: Called 559 - slight, not significant increased binding when V1/V2 or V1/V2 and V3 were deleted from gp120 [Jeffs1996]

- 559/64-D: Used in the development of resting cell neutralization assay [Hioe1997a]

- 559/64-D: Four primary isolates showed distinct patterns of sensitivity to neutralization by polyclonal sera or plasma and MAbs - BZ167 was the only isolate inhibited by all polyclonal sera and plasma tested, and was also neutralized by 8/17 MAbs, in particular anti-V3 loop (419-D, 447-52D, 782-D, and 838-D), anti-CD4bd (559/64-D, 654-D and 830-D and a cluster II of gp41 directed MAb (98-6) - isolates 92HT593 and 91 US056 were neutralized by V3 loop (419-D, and 447-52D)and cluster II gp41 (98-6) MAbs at higher concentrations - US4 was neutralized by some of the polyclonal sera/plasma tested and not at all by MAbs individually or by a cocktail of ten MAbs consisting of 419-D, 447-52D, 782-D, 838-D, 559/64-D, 654-D, 450-D, 670-D, 1281-D and 98-6 Hioe1997b

- 559/64-D: Using a whole virion-ELISA method, 18 human MAbs were tested for their ability to bind to a panel of 9 viruses from clades A, B, D, F, G, and $\mathrm{H}$ - CD4BS Abs tended to bind weakly without clade specificity to virions, but bound well to soluble gp120 - 559/64-D did not bind to any B clade viruses, and weakly bound clade A, C, and G isolates - 559/64-D, 558-D and 1202-D had similar reactivities [Nyambi1998]

- 559/64-D: Binding of panel of 21 MAbs to soluble oligomeric gp140 versus gp41 or gp120 monomers was compared - no MAb was oligomer specific, though anti-V3 and CD4BS MAbs reacted better with the oligomer and V2 and C5 tended to favor the monomer - CD4BS MAbs 559/64-D, 654-D, 729-D, 9CL and 1331E bound with a 5-13 fold preference for the oligomer [Gorny2000a]

- 559/64-D: Ab responses, because of their capacity to alter antigen uptake and processing, can influence helper T cell responses - anti-CD4 binding site MAbs or serum Ig from HIV+ individuals inhibited proliferative responses of gp120 specific T cells - CD4BS MAbs 654-D, 559/64-D, 588-D, 830-D, 1027-30-D, and 1202-30D strongly diminished proliferation [Hioe2000]

- 559/64-D: 26 HIV-1 group M isolates (clades A to H) were tested for binding to 47 MAbs, including 6 CD4BS MAbs - CD4BS MAbs bound consistently to most isolates of clade D, but poorly to isolates of other clades with the exception of broadly reactive MAb IgG1b12]Nyambi2000]

- 559/64-D: CD4BS MAbs when complexed with gp120, inhibit proliferation of gp120-specific CD4 T-cells and IFN $\gamma$ production-anti-CD4BS MAbs inhibit gp120 presentation by altering the uptake and/or processing of gp120 by the APCs, not by blocking of gp120 attachment to CD4 on the surface of APCs [Hioe2001]. 


\begin{tabular}{|c|c|c|c|c|c|}
\hline No. & MAb ID & HXB2 Location & Author's Location & Neutralizing Immunogen & Species(Isotype) \\
\hline & & \multicolumn{4}{|c|}{$\begin{array}{l}\text {-559/64-D: Abs against the V3 loop (50.1, 58.2, 59.1, 257-D, 268-D, 447-52D), CD4BS (IgG1b12, 559-64D, F105), CD4 induced or CD4i (17b), and to } \\
\text { gp41 (2F5, F240) each showed similar binding efficiency to Env derived from related pairs of primary and TCLA lines (primary: } 168 \mathrm{P} \text { and 320SI, and } \\
\text { TCLA: } 168 \mathrm{C} \text { and 320SI-C3.3), but the TCLA lines were much more susceptible to neutralization suggesting that the change in TCLA lines that make them } \\
\text { more susceptible to NAbs alters some step after binding [York2001] }\end{array}$} \\
\hline \multirow[t]{2}{*}{902} & 55D5/F9 & Env & gp120 (SF162) & Vaccine & $\begin{array}{l}\text { human from } \\
\text { transgenic mice } \\
(\operatorname{IgG} 2 \kappa)\end{array}$ \\
\hline & & \multicolumn{4}{|c|}{$\begin{array}{l}\text { Vaccine Vector/Type: recombinant protein Strain: SF162 HIV component: gp120 Adjuvant: Ribi adjuvant (MPL+TDM) } \\
\text { Ab type CD4BS Donor Dr. Abraham Pinter, Public Health Research Institute, Newark, NJ, pinter@ phri.org } \\
\text { References He2002] } \\
\text { 55D5/F9: Transgenic mice (strain XenoMouse G2) carrying human genes allowing production of fully human IgG2 } \kappa \text { MAbs were used to rapidly create a } \\
\text { panel of anti-HIV gp120 MAb-producing hybridomas by immunization with HIV SF162 gp120—6 anti-CD4BS MAbs competed with anti-CD4BS MAb } \\
\text { 5145A, blocked sCD4 binding and were conformationally sensitive-4/6 could neutralize the autologous strain SF162, and were broadly cross-reactive with } \\
\text { B clade R5 and X4 strains (not E clade)—55D5/F9 was one of these four MAbs [He2002]. }\end{array}$} \\
\hline
\end{tabular}

903 588-D (588) Env gp120

Ab type CD4BS Donor Susan Zolla-Pazner (Zollas01@mcrcr6.med.nyu), NYU Med Center, NY, NY

References Karwowska1992a, Buchbinder1992, Moore1993a, Jeffs1996, Nyambi1998, Hioe2000, Nyambi2000

- 588-D: Conformational - reactive with IIIB gp120 in RIP, but not WB assay [Karwowska1992a]

- 588-D: 4-fold increase in neutralization potency for 588-D when combined 1:1 with human MAb 447-D [Buchbinder1992]

- 588-D: Weak neutralization of IIIB - strong inhibition of HIV+ human sera binding to IIIB gp120 [Moore1993a]

- 588-D: Called 588 - slight, not significant increased binding when V1/V2 or V1/V2 and V3 were deleted from gp120 [Jeffs1996]

- 588-D: Using a whole virion-ELISA method, 18 human MAbs were tested for their ability to bind to a panel of 9 viruses from clades A, B, D, F, G, and H CD4-BS Abs tended to bind weakly without clade specificity to virions, but bound well to soluble gp120 - 588-D did not bind to any B clade viruses, and weakly bound a clade A, C, and G clade isolate - 559/64-D, 558-D and 1202-D reacted had similar reactivities [Nyambi1998]

- 588-D: Ab responses, because of their capacity to alter antigen uptake and processing, can influence helper T cell responses - anti-CD4 binding site MAbs or serum Ig from HIV+ individuals inhibited proliferative responses of gp120 specific T cells - CD4BS MAbs 654-D, 559/64-D, 588-D, 830-D, 1027-30-D, and 1202-30D strongly diminished proliferation [Hioe2000

- 588-D: 26 HIV-1 group M isolates (clades A to H) were tested for binding to 47 MAbs, including 6 CD4BS MAbs - CD4BS MAbs bound consistently to most isolates of clade $\mathrm{D}$, but poorly to isolates of other clades with the exception of broadly reactive MAb IgG1b12[Nyambi2000]
904 654-D Env gp120 (LAI) L
(654-30D, $\quad$ Ab type CD4BS Donor Susan Zolla-Pazner (Zollas01@ mcrcr6.med.nyu), NYU Med Center, NY, NY HIV-1 infection

human $(\operatorname{IgG} \kappa)$

654/30D, $\quad$ References Karwowska1993, Laal1994 Gorny1994| Stamatatos1995, Li1997| Stamatatos1997, Gorny 1997, Hioe1997b Gorny1998, Schonning1998

654-D100, Nyambi1998, Stamatatos1998, Hioe1999, Gorny2000a. Hioe2000, Hioe2001, Nyambi2000, Verrier2001, Gorny2002

654.30D, 654)• 654-D: Dissociation constant gp120 IIIB 0.008 - neutralizes IIIB, acts synergistically with anti-V3 MAb 447-52D - reported to be human(IgG1lambda) [Laal1994]

- 654-D: Mild oxidation of carbohydrate moieties inhibits binding |Gorny1994 


\begin{tabular}{lllll}
\hline No. MAb ID & HXB2 Location & Author's Location & Sequence & Neutralizing Immunogen
\end{tabular}

- 654-D: Called 654-30D - The binding of conformation-dependent anti-V2, anti-V3, and anti-CD4BS MAbs to monomeric and virion-associated gp120 from HIV-1 isolates with differences in cell tropism was studied - CD4BS loop epitopes are somewhat occluded in the oligomeric gp120 epitopes on the virion surface relative to the gp120 monomer as indicated by an increase in the half-maximal binding values to macrophage-tropic isolates SF162 and SF128a and to T-cell tropic SF2 - binding of anti-CD4BS MAbs to SF2 resulted in a significant amount of dissociation of gp120 from virion surface [Stamatatos1995]

- 654-D: Called 654-30D - One of 14 human MAbs tested for ability to neutralize a chimeric SHIV-vpu+, which expressed HIV-1 IIIB env [Li1997]

- 654-D: Anti-CD4 BS MAb 654-30D and IgG1b12 have comparable binding affinities, neither mediates gp120-virion dissociation, but IgG1b12 can neutralize SF128A and SF162 and 654-D cannot - 654-D actually enhances infection by both viruses in primary macrophages [Stamatatos1997]

- 654-D: Four primary isolates showed distinct patterns of sensitivity to neutralization by polyclonal sera or plasma and MAbs - BZ167 was the only isolate inhibited by all polyclonal sera and plasma tested, and was also neutralized by 8/17 MAbs, in particular anti-V3 loop (419-D, 447-52D, 782-D, and 838-D), anti-CD4bd (559/64-D, 654-D and 830-D and a cluster II of gp41 directed MAb (98-6) - isolates 92HT593 and 91 US056 were neutralized by V3 loop (419-D, and 447-52D)and cluster II gp41 (98-6) MAbs at higher concentrations - US4 was neutralized by some of the polyclonal sera/plasma tested and not at all by MAbs individually or by a cocktail of ten MAbs consisting of 419-D, 447-52D, 782-D, 838-D, 559/64-D, 654-D, 450-D, 670-D, 1281-D and 98-6 Hioe1997b

- 654-D: Called 654-D100 - 654-D100 and IgG1b12 neutralized viruses HIV-BRU and a mutated virus that lacks the V3 loop glycan equally effectively - in contrast, sera from guinea pigs immunized with BRU gp120 neutralize viruses more effectively that lack the V3 glycan[Schonning1998]

- 654-D: Using a whole virion-ELISA method, 18 human MAbs were tested for their ability to bind to a panel of 9 viruses from clades A, B, D, F, G, and H CD4-BS Abs tended to bind very weakly without clade specificity to virions, but bound well to soluble gp120 - 654-D bound only to JRFL [Nyambi1998]

- 654-D: Called 654.30D - deleting the V2 loop of neutralization-resistant HIV-1 isolate SF162 does not abrogate its replication in PBMC or macrophages, but it enhances its neutralization sensitivity to sera from patients with B clade infection up to 170-fold, and also enhances sensitivity to sera from clades A through $\mathrm{F}$ - deletion of V2 but not V1 slightly allowed neutralization by CD4BS MAb 654.30D [Stamatatos1998]

- 654-D: The presence of leukocyte function-associated molecule 1 (LFA-1) promotes virus infectivity and hinders neutralization, and anti-LFA-1 MAbs can enhance the neutralizing effect of anti-HIV V3 MAb 447-52D and anti-HIV CD4BS MAb IgG1b12 - non-neutralizing anti-HIV CD4BS MAb 654-D did not become neutralizing in the presence of anti-LFA-1 MAbs [Hioe1999]

- 654-D: Binding of panel of 21 MAbs to soluble oligomeric gp140 versus gp41 or gp120 monomers was compared - no MAb was oligomer specific, though anti-V3 and CD4BS MAbs reacted better with the oligomer and V2 and C5 tended to favor the monomer - CD4BS MAbs 559/64-D, 654-D, 729-D, 9CL and 1331E bound with a 5-13 fold preference for the oligomer [Gorny2000a]

- 654-D: Ab responses, because of their capacity to alter antigen uptake and processing, can influence helper T cell responses - CD4BS MAbs or serum Ig from HIV+ individuals inhibited proliferative responses of gp120 specific T cells - MAb 654-D strongly diminished proliferation - there is a discrepancy in isotyping this antibody, previous reports indicated IgG1kappa, while Hioe suggests it is IgG1lambda [Hioe2000]

- 654-D: 26 HIV-1 group M isolates (clades A to H) were tested for binding to 47 MAbs, including 6 CD4BS MAbs - CD4BS MAbs bound consistently to most isolates of clade D, but poorly to isolates of other clades with the exception of broadly reactive MAb IgG1b12 - 654-D had the weakest binding among CD4BS MAbs, binding to only 4/26 isolates [Nyambi2000]

- 654-D: CD4BS MAbs when complexed with gp120, inhibit proliferation of gp120-specific CD4 T-cells and IFN gamma production - anti-CD4BS MAbs inhibit gp120 presentation by altering the uptake and/or processing of gp120 by the APCs, not by blocking of gp120 attachment to CD4 on the surface of APCs [Hioe2001]

- 654-D: A panel of 12 MAbs was used to identify those that could neutralize the dual-tropic primary isolate HIV-1 89.6-six gave significant neutralization at 2 to $10 \mathrm{ug} / \mathrm{ml}:$ 2F5, 50-69, IgG1b12, 447-52D, 2G12, and 670-D, while six did not have neutralizing activity: 654-D, 4.8D, 450-D, 246-D, 98-6, and 1281 - no synergy, only additive effects were seen for pairwise combinations of MAbs, and antagonism was noted between gp41 MAbs 50-69 and 98-6, as well as 98-6 and 2F5 [Verrier2001]. 


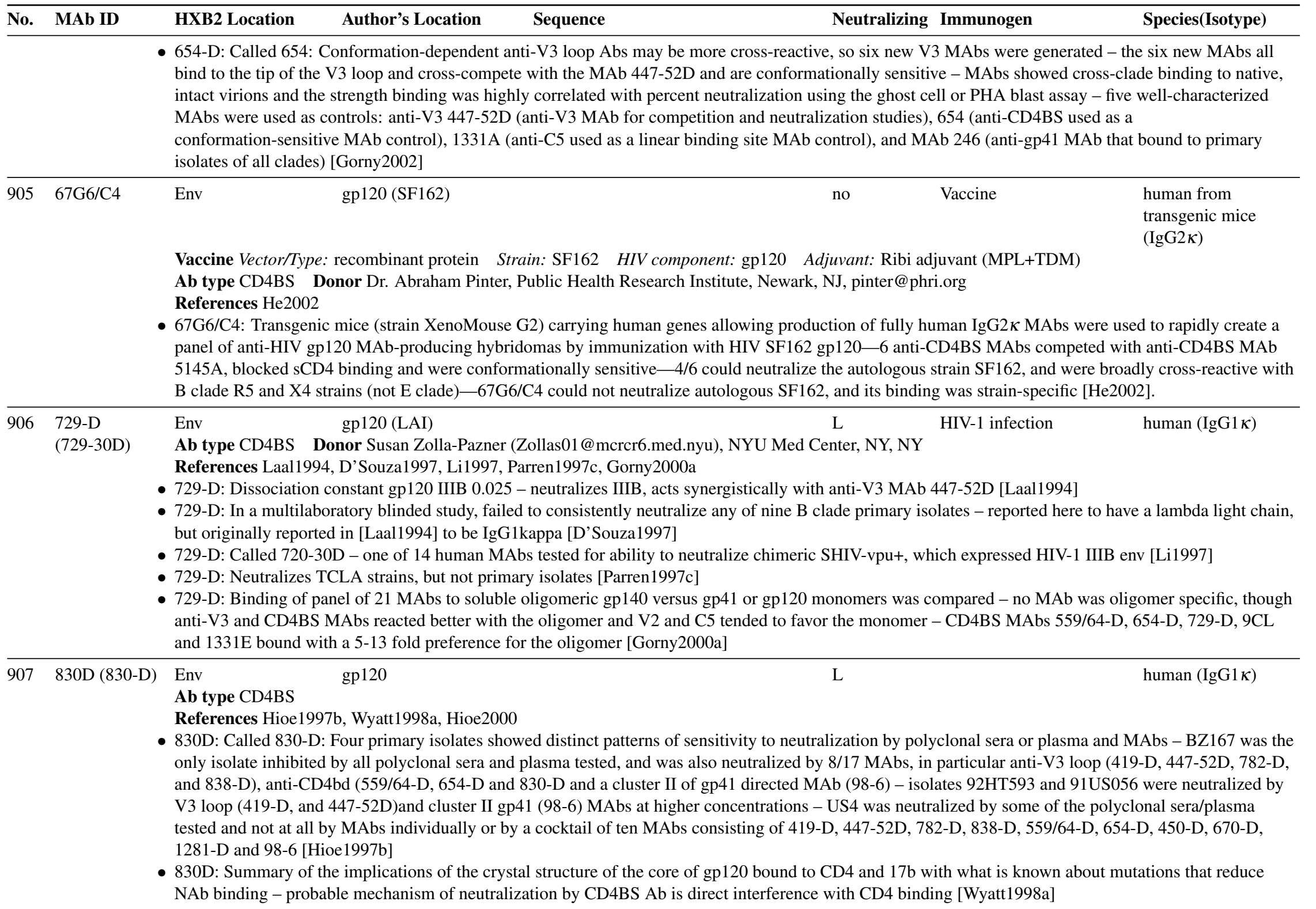




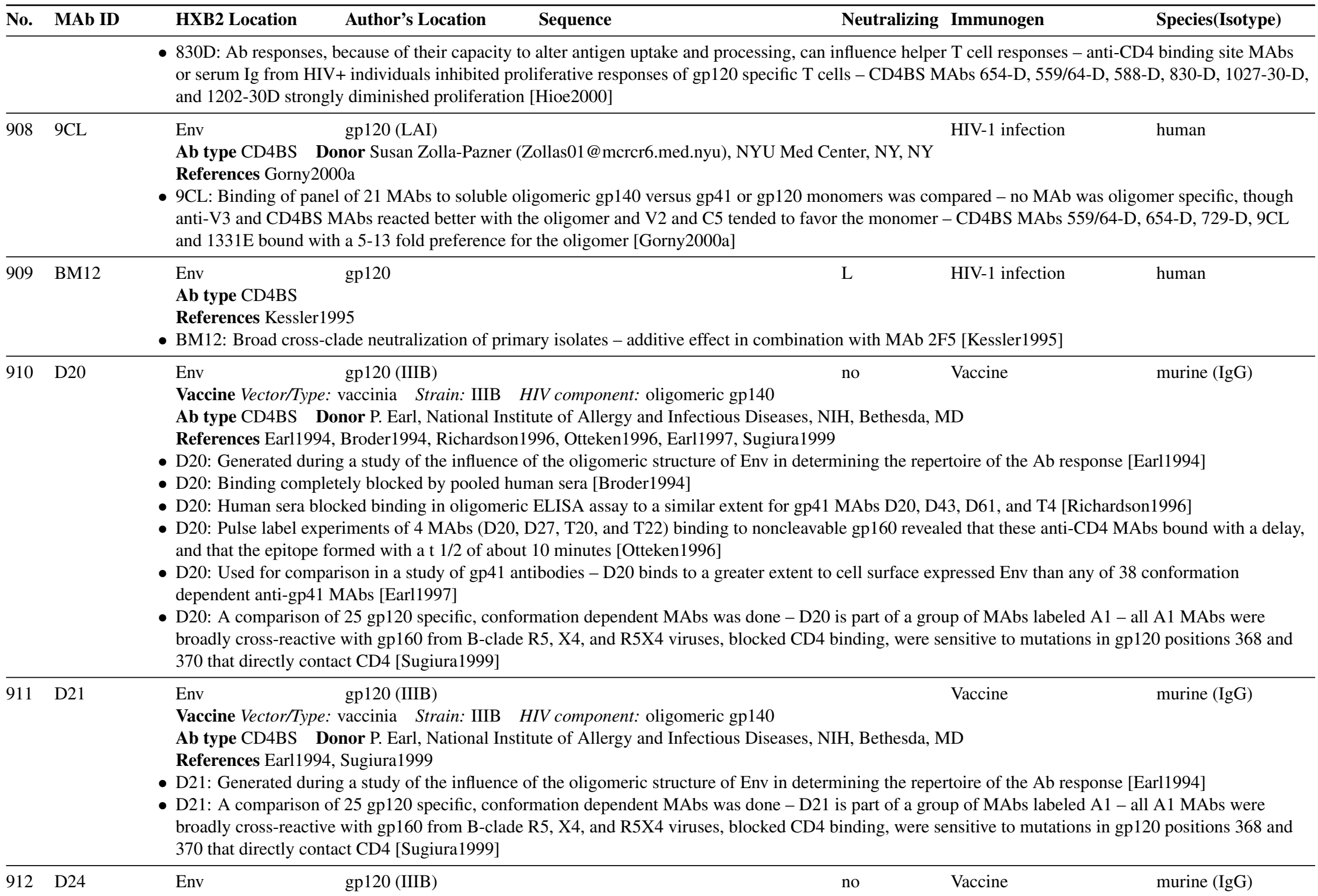

Vaccine Vector/Type: vaccinia Strain: IIIB HIV component: oligomeric gp 140

Ab type CD4BS Donor P. Earl, National Institute of Allergy and Infectious Diseases, NIH, Bethesda, MD

References Earl1994, Sugiura1999 


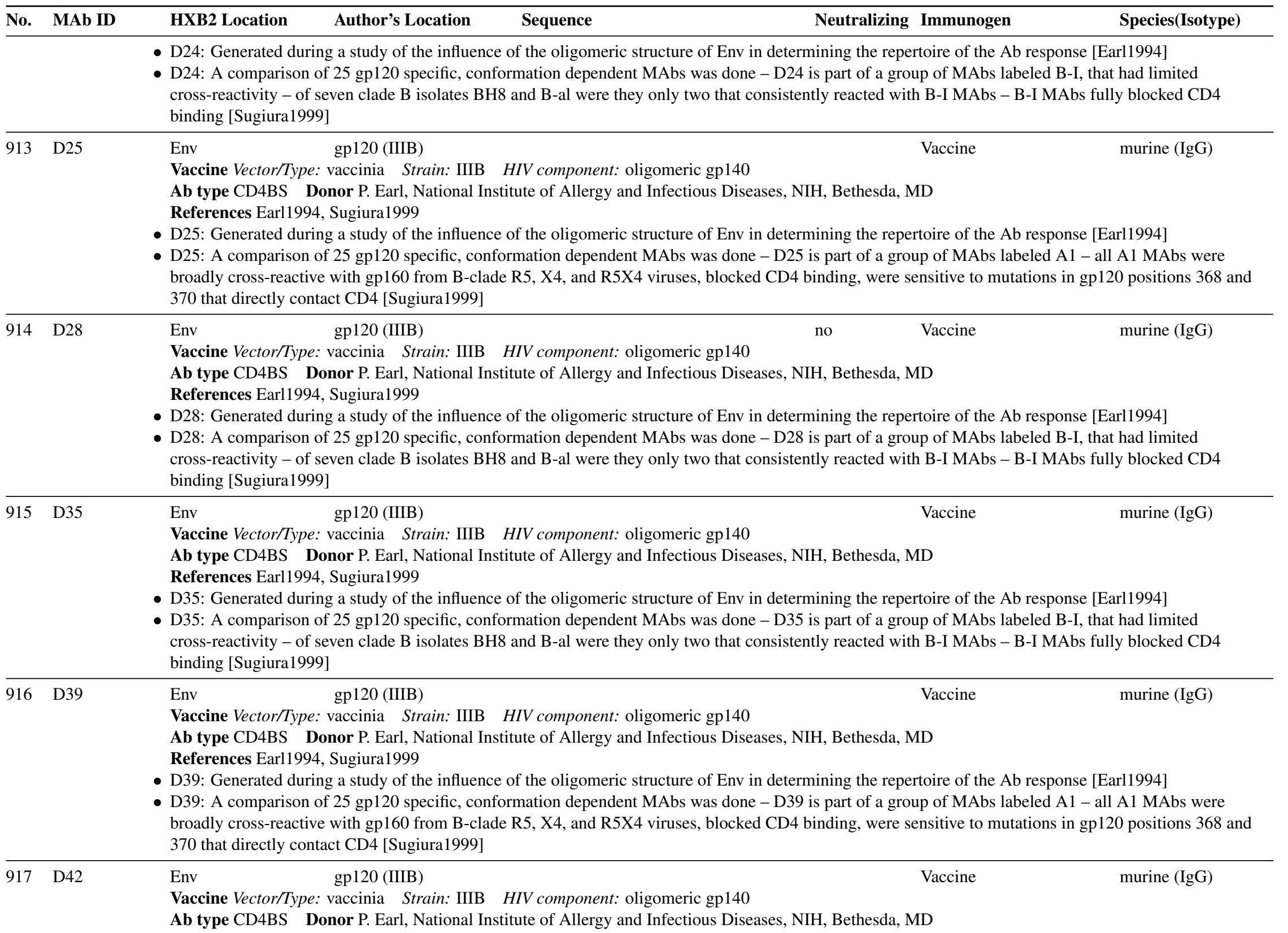




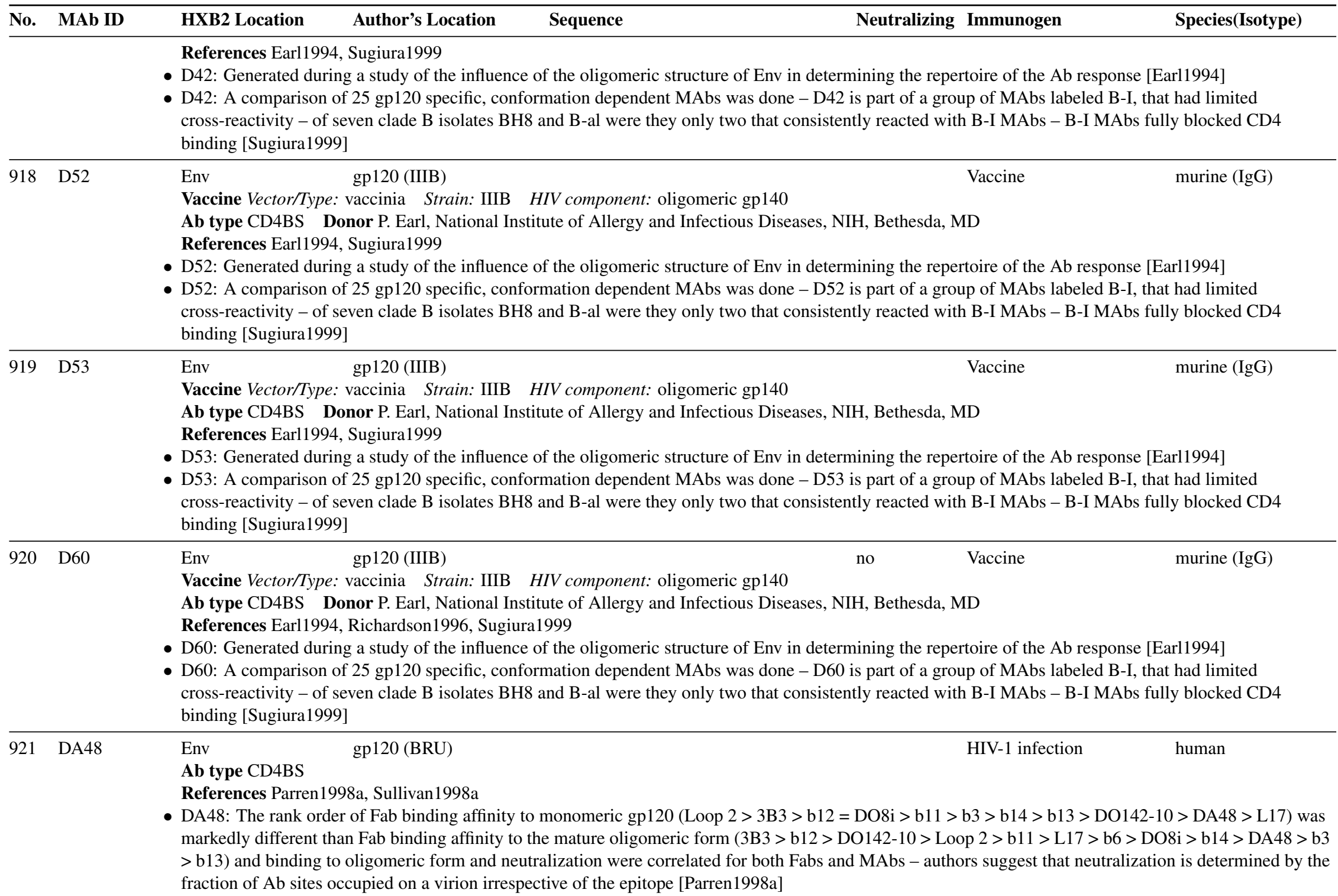




\begin{tabular}{lllll}
\hline No. MAb ID & HXB2 Location & Author's Location & Sequence & Neutralizing Immunogen
\end{tabular}

- DA48: The HIV-1 virus YU2 entry can be enhanced by MAbs binding to the CD4BS, V3 loop, and CD4i epitopes - the activation for this enhanced entry state could be conferred on HxB2 by introducing the YU2 V3 loop, or the YU2 V3 and V1/V2 loops - a similar effect is observed by sub-neutralizing concentrations of sCD4 and the effect is dependent of CCR5 - Fab Ab fragment DA48 also enhances YU2 entry, ruling out Fc interactions or Env cross-linking as a mechanism - while DA48 enhances YU2, it neutralizes HXBc2 - DA48 was obtained by panning libraries derived from bone marrow from a >15 year long term non-progressor against BRU gp120 [Sullivan1998a]

\begin{tabular}{lllll}
\hline 922 DO8i & Env & gp120 (BRU) HIV-1 infection & human Fab \\
& & Ab type CD4BS
\end{tabular}

\section{References Parren1998a}

- DO8i: The rank order of Fab binding affinity to monomeric gp120 (Loop $2>3$ B3 $>$ b12 = DO8i $>$ b11 $>$ b3 $>$ b14 $>$ b13 $>$ DO142-10 $>$ DA48 $>$ L17) was markedly different than Fab binding affinity to the mature oligomeric form $(3 \mathrm{~B} 3>$ b12 $>$ DO142-10 $>$ Loop $2>$ b1 $>$ L17 $>$ b6 $>$ DO8i $>$ b14 $>$ DA $48>$ b3 $>$ b13) and binding to oligomeric form and neutralization were correlated for both Fabs and MAbs - authors suggest that neutralization is determined by the fraction of Ab sites occupied on a virion irrespective of the epitope [Parren1998a

- DO8i - the HIV-1 virus YU2 entry can be enhanced by MAbs binding to the CD4BS, V3 loop, and CD4i epitopes - the activation for this enhanced entry state could be conferred on HxB2 by introducing the YU2 V3 loop, or the YU2 V3 and V1/V2 loops - a similar effect is observed by sub-neutralizing concentrations of sCD4 and the effect is dependent of CCR5 - Fab fragment DO8i also enhances YU2 entry, ruling out Fc interactions or Env cross-linking as a mechanism, while neutralizing $\mathrm{HXBc} 2$ - DO8i was obtained by panning libraries derived from bone marrow from a long term asymptomatic donor against BRU gp120 [Sullivan1998a]

Ab type CD4BS Donor Marshall Posner, Boston MA

References Posner1991 Thali1991, Thali1992a Marasco1992 Wyatt1992, Posner1992b, Posner1992a, Moore1993a, Posner1993, Cavacini1993a

Cavacini1993b, Wyatt1993, Montefiori1993, Potts1993, Klasse1993a, Pincus1993b, Watkins1993, Marasco1993, Bagley1994, Thali1994, Cook1994 Cavacini1994b, Cavacini1994a, Ear11994, Chen1994a, Turbica1995 Posner1995, Cavacini1995, Sullivan1995, Khouri1995, Jagodzinski1996, Wolfe1996, McDougal1996, Wisnewski1996 |Pincus1996, Litwin1996, Chen1996, Parren1997c, D’Souza1997, Li1997| Cao1997b, Wyatt1997. Wyatt1998a. Cavacini1998b, Li1998, Cavacini1998a Brand1998 Sullivan1998a Kropelin1998, Sugiura1999, Giraud1999, Cavacini1999, Oscherwitz1999a Robert-Guroff2000 Baba2000, Park2000, Yang2000, Si2001, Kolchinsky2001 York2001, Yang2002, Xu2002, Chakrabarti2002, Xiang2002b Edwards2002, Grundner2002, Basmaciogullari2002, Zhang2002 |Ferrantelli2002, Liu2002, Pantophlet2003

- F105: First description of F105, binds topographically near the CD4-binding site - inhibits binding of free, infectious virions to uninfected HT-H9 cells, but does not react with virus adsorbed to uninfected HT-H9 cells - soluble rCD4 pre-bound to infected cells inhibits F105 binding - F105 inhibits infection of HT-H9 cells in standard neutralization assays with HIV-1 and MN strains [Posner1991]

- F105: F105 neutralization escape mutants result from changes in amino acids in discontinuous regions: C2, 256-262 and C3, 386-370 [Thali1991]

- F105: Amino acid substitutions that impair F105 neutralization inhibit gp120-CD4 interaction [Thali1992a]

- F105: MAb cDNA sequence - V H4 V71-4 rearranged with a D H D-D fusion product of dlr4 and da4, and with J H5 - V kappa is from the Humvk325 germline gene joined with Jkappa 2 [Marasco1992]

- F105: Precipitation of Delta 297-329 env glycoprotein, which has a deleted V3 loop, is much more efficient than precipitation of wild type [Wyatt1992]

- F105: F105 mediates ADCC against SF2 through the CD16+ population of PBMC - does not mediate complement-dependent cytotoxicity [Posner1992b]

- F105: Significant enhancement of F105 binding to RF infected cells preincubated with V3-specific MAbs V3-2 and V3-1 [Posner1992a]

- F105: Called F-105 - neutralizes IIIB - strong inhibition of HIV+ human sera binding to IIIB gp120 [Moore1993a]

- F105: F105 binds to and neutralizes selected lab strains and 3/9 HIV-1 primary isolates - synergistic enhancement of neutralization by seropositive sera Posner1993

- F105: No neutralization of primary isolates observed (John Moore, pers comm)

- F105: Additive MN or SF2 neutralization when combined with anti-V3 MAbs 447-52D and 257-D [Cavacini1993a] 


No. MAb ID HXB2 Location Author's Location $\quad$ Sequence $\quad$ Neutralizing Immunogen

- F105: Serum from all asymptomatic HIV-1 positive people tested block F105 binding, but only from 27\% of symptomatic individuals [Cavacini1993b]

- F105: Binding to Delta V1/2 and Delta V1/2/3 mutant glycoproteins is 2.4- and 13-fold greater, respectively, than binding to wildtype gp120 [Wyatt1993]

- F105: Study of synergism between F105 and sera from vaccinated volunteers with V3-loop specific neutralization activity - 2/3 sera demonstrated neutralization synergy, and 3/3 binding/fusion-inhibition synergy [Montefiori1993]

- F105: Study of synergism of neutralization and binding comparing F105 and sCD4 with the V3 MAbs: 50.1, 59.1, 83.1, and 58.2 - synergy was observed, and the data suggest that binding of one ligand (F105) can increase the binding of the second (e. g. V3 loop MAbs) due to conformational changes [Potts1993]

- F105: The gp41 mutation 582(Ala to Thr) results in conformational changes in gp120 that confer neutralization resistance to a class of conformation sensitive neutralizing MAbs - required >81 fold higher concentrations to neutralize the mutant than wild type [Klasse1993a]

- F105: Ab response in IIIB lab workers was compared to gp160 LAI vaccine recipients - F105 was used as a control - infected lab workers and some of the gp160 vaccinees had a MAb response that could inhibit gp120-CD4 binding, at lower titers than the infected lab workers [Pincus1993b]

- F105: A neutralization escape mutant (HXB2 A281V) was selected by growth of HXB2 in the presence of broadly neutralizing sera - F105 neutralization was not affected by this mutation [Watkins1993]

- F105: Comparison of MAb F105 sequences with those of MAbs 21h and 15e [Bagley1994]

- F105: A mutation in gp41, $582 \mathrm{~A} / \mathrm{T}$, confers resistance to neutralization (also confers resistance to MAbs 48d, 21h, 15e and 17b) [Thali1994]

- F105: MAbs against the glycosphingolipid GalCer block HIV infection of normally susceptible CD4 negative cells from the brain and colon - anti-CD4 MAbs moderately inhibit gp120 binding to GalCer, possibly through steric hindrance - binding of GalCer to gp120 inhibited but did not completely block F105 binding[Cook1994]

- F105: Administered intravenously to four cynomologus monkeys, plasma pharmacokinetics and biological activity tested [Cavacini1994b]

- F105: Fab fragments show reduced capacity to neutralize IIIB, MN, and RF compared to intact IgG1, suggesting bivalent interaction may be important in binding and neutralization [Cavacini1994a]

- F105: Used as a positive control for CD4 BS antibodies in a study of the influence of oligomeric structure of Env in determining the repertoire of the Ab response [Earl1994]

- F105: A human CD4+ T lymphocyte line was transduced to express Fab fragments of F105 - heavy and light chains are joined by an inter-chain linker - in the transduced cells infected with HIV-1, the Fab binds intracellularly to the envelope protein and inhibits HIV-1 production - secreted Fab fragments neutralize cell-free HIV-1 - combined intra- and extracellular binding activities of the expressed Fab make transduced cells resistant to HIV-1 infection and also can protect surrounding lymphocytes by secreting neutralizing antibodies [Marasco1993, Chen1994a]

- F105: An immunoassay for titrating CD4BS serum antibody was developed using a gp120-coated solid phase and competition with MAb F105 - 109/110 French HIV-1+ sera and 51/56 HIV-1+ African sera had detectable CD4BS Abs using this assay, demonstrating CD4 binding site conservation among diverse subtypes - CD4BS Abs were detected soon after seroconversion and persisted - 0/21 HIV-2+ sera reacted, indicating that the HIV-1 and HIV-2 CD4BS Abs are not cross-reactive [Turbica1995]

- F105: Eight patient phase Ia trial for use as an immunotherapeutic - no clinical or biochemical side effects observed, plasma levels of 10 ug/ml maintained for 21 days [Posner1995]

- F105: Efficient neutralization of T-cell adapted lines HXBc2 and MN, no neutralization of primary isolates 89.6, ADA and YU2 - even some enhancement of infection of ADA and YU2 was observed [Sullivan1995]

- F105: Biotinylated F105 was used for competition studies with Ab derived from pregnant HIV-1+ women - a correlation between maternal anti-CD4 BS Abs overlapping the F105 binding site and lack of HIV-1 transmission to infants was noted [Khouri1995]

- F105: Changing heavy chain from IgG1 to IgG3 increased neutralization efficiency [Cavacini1995]

- F105: The sulfated polysaccharide curdlan sulfate (CRDS) binds to the Envelope of T-tropic viruses and neutralizes virus - deletion of the V3 loop results in less potent inhibition of F105 binding by CRDS - binding site of F105 described as 256-257 ST, 368-370 DPE, $421 \mathrm{~K}$, and 470-484 PGGGDMRDNWRSELY [Jagodzinski1996]

- F105: Phase I study - MAb clearance in plasma has a 13 day half-life [Wolfe1996 


\begin{tabular}{lllll}
\hline No. MAb ID & HXB2 Location & Author's Location & Sequence & Neutralizing Immunogen
\end{tabular}

- F105: Neutralizes HIV-1 LAI less potently than V3 specific MAbs [McDougal1996]

- F105: F105 is V H4 - V-region heavy chain usage was examined and a bias of enhanced V H1 and V H4, and reduced V H3, was noted among HIV infected individuals [Wisnewski1996]

- F105: A panel of immunotoxins were generated by linking Env MAbs to ricin A - immunotoxins mediated cell killing, but killing was not directly proportional to binding [Pincus1996]

- F105: Binding of F105 to oligomeric gp120 occurs despite the fact it cannot neutralize primary isolates [Litwin1996]

- F105: Intracellular co-expression of heavy and light chains of the Fab105 fragment MAb F105 was enhanced by inclusion of an internal ribosome entry site (IRES) sequence - the Fab105 IRES expression cassette was cloned into an adeno-associated virus (AAV) shuttle vector, and transduced into human lymphocytes which were able to produce and secrete the Fab105 fragments while maintaining normal growth - several primary HIV-1 patient isolates were effectively blocked [Chen1996]

- F105: Neutralizes TCLA strains, but not primary isolates [Parren1997c]

- F105: In a multilaboratory blinded study, failed to neutralize any of nine B clade primary isolates [D'Souza1997]

- F105: One of 14 human MAbs tested for ability to neutralize a chimeric SHIV-vpu+, which expressed HIV-1 IIIB env - F105 could only achieve 50\% neutralization alone - all Ab combinations tested showed synergistic neutralization - F105 has synergistic response with MAbs 694/98-D (anti-V3), 48d, 2F5, and 2G12, and also with HIVIG [Li1997]

- F105: Virus with the V1-V2 loop deleted was viable and more susceptible to neutralization by CD4i MAb 17b, and anti-V3 MAbs 1121, 9284, and 110.4, but not to a CD4BS MAb, F105 or SCD4 [Cao1997b]

- F105: Binds both gp120 and soluble gp120+gp41 complex efficiently, suggesting its gp120 epitope is not blocked by gp41 binding - does not bind to $\mathrm{HXBc} 2 \mathrm{gp} 120$ if the $19 \mathrm{C}$-term amino acids, in conjunction with C1 positions 31-93, are deleted [Wyatt1997]

- F105: Summary of the implications of the crystal structure of the core of gp120 bound to CD4 and 17b with what is known about mutations that reduce $\mathrm{NAb}$ binding - probable mechanism of neutralization by CD4BS Ab is direct interference with CD4 binding [Wyatt1998a

- F105: Phase I dose escalation study, single dose of 100 or $500 \mathrm{mg} / \mathrm{m} 2$ was given to 4 HIV+ patients - sustained levels, no immune response against F105, no toxicity, infused $\mathrm{Ab}$ retained function - there was no evidence of anti-HIV-1 activity and virus was not diminished at day 1 or 7, by culture or plasma RNA [Cavacini1998b]

- F105: Neutralization synergy was observed when the MAbs 694/98-D (V3), 2F5 (gp41), and 2G12 (gp120 discontinuous) were used in combination, and even greater neutralizing potential was seen with the addition of a fourth MAb, F105 (CD4 BS) [Li1998]

- F105: The MAb F240 binds to the immunodominant region of gp41 and enhances infection in the presence of complement - reactivity of F240 is enhanced by preincubation of cells with sCD4 or anti-CD4BS MAb F105 [Cavacini1998a]

- F105: Immunoprecipitation of gp120 and gp160 expressed from a rec Semliki Forest virus by F105 and IgG1b12 indicated that the SFV expressed HIV-1 Env was folded appropriately - and SVF-HIV-1 Env vaccine gave the strongest anti-HIV-1 Env response in mice, when compared to an HIV-1 Env DNA vaccine and a rgp160 protein Brand1998

- F105: A comparison of 25 gp120 specific, conformation dependent MAbs was done and F105 was used for competition studies - F105 did cross-compete with multiple CD4BS specific MAbs, however most could not neutralize even the autologous NL4-3 strains [Sugiura1999]

- F105: F105 enhances viral entry of viruses carrying the YU2 envelope glycoproteins, but neutralizes HXBc2 [Sullivan1998a]

- F105: Anti-C1 region MAb 87-135/9 blocks gp120 interaction with CD4+ cells - blocking activity is additive when combined with antibodies which bind in the C4 region of gp120 (F105, 388/389, and b12) [Kropelin1998]

- F105: A mini-review of observations of passive administration of IgG NAbs conferring protection against intervenous or vaginal SHIV challenge, that considers why IgG MAbs might protect against mucosal challenge [Robert-Guroff2000]

- F105: A triple combination of 2F5, F105 and 2G12 effectively neutralized perinatal infection of macaque infants when challenged with SHIV-vpu+ - the plasma half-life was $7.2+/-2.2$ days [Baba2000] 


No. MAb ID HXB2 Location Author's Location $\quad$ Sequence $\quad$ Neutralizing Immunogen

- F105: Host encoded intercellular adhesion molecule (ICAM-1) is incorporated by the HIV-1 virion and enhances viral infectivity - ICAM-1 does not modify virus sensitivity to antibodies 0.5 beta or $4.8 \mathrm{D}$ or sCD4, but neutralizing ability of F105 was diminished in ICAM bearing virions in the presence of lymphocyte function-association antigen-1 (LFA-1) Ab [Fortin2000]

- F105: Six mutations in MN change the virus from a high-infectivity neutralization resistant phenotype to low-infectivity neutralization sensitive - V3, CD4BS, and CD4i MAbs are 20-100 fold more efficient at neutralizing the sensitive form, although F105 was an exception and cannot neutralize either form of MN - the mutation L544P reduced binding of all MAbs against gp120 by causing conformational changes [Park2000]

- F105: A combination of gp41 fusion with the GNC4 trimeric sequences and disruption of the YU2 gp120-gp41 cleavage site resulted in stable gp140 trimers (gp140-GNC4) that preserve and expose some neutralizing epitopes while occluding some non-neutralizing epitopes - CD4BS MAbs (F105 and F91) and CD4i (17b and 48d) recognized gp140-GNC4 as well as gp120 or gp140 - non-neutralizing MAbs C11, A32, 522-149, M90, and \#45 bound to the gp140-GNC4 glycoprotein at reduced levels compared to gp120 - MAbs directed at the extreme termini of gp120 C1 (135/9 and 133/290) and C5 (CRA-1 and M91) bound efficiently to gp140-GNC4 [Yang2000]

- F105: SHIV-HXBc2 is a neutralization sensitive non-pathogenic virus, and several in vivo passages through monkies yielded highly pathogenic SHIV KU-1- HXBc2 and the KU-1 clone HXBc2P3.2 differ in 12 amino acids in gp160 - substitutions in both gp120 and gp41 reduced the ability of sCD4, IgG1b12, F105 and AG1121 to Env achieve saturation and full occupancy, and neutralize KU-1-17b and 2F5 also bound less efficiently to HXBc2P3.2, although 2G12 was able to bind both comparably [Si2001].

- F105: Mutations in two glcosylation sites in the V2 region of HIV-1 ADA at positions 190 and 197 (187 DNTSYRLINCNTS 199) cause the virus to become CD4-independent and able to enter cells through CCR5 alone - these same mutations tended to increase the neutralization sensitivity of the virus, including to F105 [Kolchinsky2001]

- F105: Abs against the V3 loop (50.1, 58.2, 59.1, 257-D, 268-D, 447-52D), CD4BS (IgG1b12, 559-64D, F105), CD4i (17b), and to gp41 (2F5, F240) each showed similar binding efficiency to Env derived from related pairs of primary and TCLA lines (primary: 168P and 320SI, and TCLA: 168C and 320SI-C3.3), but the TCLA lines were much more susceptible to neutralization suggesting that the change in TCLA lines that make them more susceptible to NAbs alters some step after binding [York2001]

- F105: Uncleaved soluble gp140 (YU2 strain, R5 primary isolate) can be stabilized in an oligomer by fusion with a C-term trimeric GCN4 motif or using a

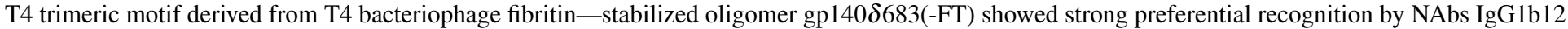
and 2G12 relative to the gp120 monomer, in contrast to poorly neutralizing MAbs F105, F91, 17b, 48d, and 39F which showed reduced levels of binding, and C11, A32, and 30D which did not bind the stabilized oligomer [Yang2002].

- F105: Passive immunization of neonate macaques with a combination of F105+2G12+2F5 conferred complete protection against oral challenge with SHIV-vpu+ or - the combination b12+2G12+2F5 conferred partial protection against SHIV89.6 - such combinations may be useful for prophylaxis at birth and against milk born transmission - the synergistic combination of IgG1b12, 2G12, 2F5, and 4E10 neutralized a collection of HIV clade C primary isolates [Xu2002]

- F105: A modified gp140 (gp140deltaCFI), with C-term mutations intended to mimic a fusion intermediate and stabilize trimer formation, retained antigenic conformational determinants as defined by binding to CD4 and to MAbs 2F5, 2G12, F105, and b12, and enhanced humoral immunity without diminishing the CTL response in mice injected with a DNA vaccine [Chakrabarti2002]

- F105: A series of mutational changes were introduced into the YU2 gp120 that favored different conformations - 375 S/W seems to favor a conformation of gp120 closer to the CD4-bound state, and is readily bound by sCD4 and CD4i MAbs (17b, 48d, 49e, 21c and 23e) but binding of anti-CD4BS MAbs (F105, $15 \mathrm{e}, \mathrm{IgG1b} 12,21 \mathrm{~h}$ and F91 was markedly reduced - IgG1b12 failed to neutralize this mutant, while neutralization by $2 \mathrm{G} 12$ was enhanced - 2F5 did not neutralize either WT or mutant, probably due to polymorphism in the YU2 epitope - another mutant, 423 I/P, disrupted the gp120 bridging sheet, favored a different conformation and did not bind CD4, CCR5, or CD4i antibodies, but did bind to CD4BS MAbs [Xiang2002b] 


\begin{tabular}{lllll}
\hline No. MAb ID & HXB2 Location & Author's Location & Sequence & Neutralizing Immunogen
\end{tabular}

- F105: Truncation of the gp41 cytoplasmic domain of X4, R5, and X4R5 viruses forces a conformation that more closely resembles the CD4 bound state of the external Envelope, enhancing binding of CD4i MAbs 17b and 48d and of CD4BS MAbs F105, b12, and in most cases of glycosylation site dependent MAb 2G12 and the anti-gp41 MAb 246D - in contrast, binding of the anti-V2 MAb 697D and the anti-V3 MAb 694/98D were not affected - viruses bearing the truncation were more sensitive to neutralization by MAbs 48d, b12, and 2G12 - the anti-C5 MAb 1331A was used to track levels of cell surface expression of the mutated proteins [Edwards2002]

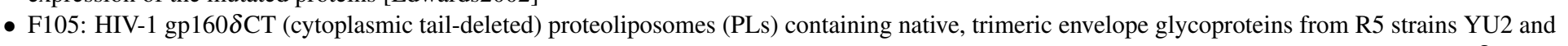
JRFL, and X4 strain HXBc2, were made in a physiologic membrane setting as candidate immunogens for HIV vaccines-2F5 bound to gp160 $\delta$ CT with a reconstituted membrane ten-fold better than the same protein on beads, while such an affinity difference was not seen with F105 and 2G12-anti-CD4BS MAbs IgG1b12 and F105, A32 (C1-C4), C11 (C1-C5), and 39F (V3) MAbs bound gp160 $\delta$ CT PLs indistinguishably from gp160 $\delta$ CT expressed on the cell surface [Grundner2002].

- F105: gp120 mutants were used to define the CXCR4 binding site using CXCR4 displayed on paramagnetic proteoliposomes (PMPLs) to reduce non-specific gp120 binding - basic residues in the V3 loop and the $\beta 19$ strand (RIKQ, positions 419-422) were involved, and deletion of the V1-V2 loops allowed CD4-independent CXCR4 binding-MAbs 17b (CD4i) and F105 (CD4BS) were used to study conformational changes in the mutants-the affinity of $\Delta \mathrm{V} 1$ and $\Delta \mathrm{V} 1-\mathrm{V} 2$ mutants for F105 was comparable to the wildtype-V3 mutants did not affect F105 binding - the K421A mutation in the $\beta 19$ strand dramatically reduced F105 affinity, consistent with what is known about the F105 epitope [Basmaciogullari2002].

- F105: A rare mutation in the neutralization sensitive R2-strain in the proximal limb of the V3 region caused Env to become sensitive to neutralization by MAbs directed against the CD4 binding site (CD4BS), CD4-induced (CD4i) epitopes, soluble CD4 (sCD4), and HNS2, a broadly neutralizing sera - 2/12 anti-V3 MAbs tested (19b and 694/98-D) neutralized R2, as did 2/3 anti-CD4BS MAbs (15e and IgG1b12), 2/2 CD4i MAbs (17b and 4.8D), and 2G12 and $2 \mathrm{~F} 5$ - thus multiple epitopes on R2 are functional targets for neutralization and the neutralization sensitivity profile of R2 is intermediate between the highly sensitive MN-TCLA strain and the typically resistant MN-primary strain [Zhang2002]

- F105: Review of NAbs that notes that F105 binds the CD4BS, in combination with other MAbs it can protect some macaques against SHIV infection, and that it has strong ADCC activity [Ferrantelli2002]

- F105: Review of NAbs that discusses mechanisms of neutralization, passive transfer of NAbs and protection in animal studies, and vaccine strategies [Liu2002]

- F105: Virion capture assays are not a good preditor of neutralization, and the presentation of epitopes using this assay seems to be different from that of functional Envelope spikes on primary isolates - F105 and b6 could efficiently block the b12-mediated capture of infectious virions in a virus capture, but did not inhibit b12 neutralization - while b12 was potent at neutralizing the three primary virions JR-CSF, ADA, and 89.6, the Abs F105, 19b, and Fab b6 were overall very poor neutralizers [Poignard2003]

- F105: Alanine scanning mutagenesis was used to compare substitutions that affected anti-CD4BS NAb b12 - rec gp120s were engineered to contain combinations of Alanine substitutions that enhanced b12 binding, and while binding of b12 to these gp120 monomers was generally maintained or increased, binding by five non-neutralizing anti-CD4bs MAbs (b3, b6, F105, 15e, and F91) was reduced or completely abolished [Pantophlet2003]

- F105: NIH AIDS Research and Reference Reagent Program: 857

924 F91 (F-91)

$$
\text { gp120 }
$$

Ab type CD4BS Donor James Robinson, University of Connecticut, Storrs

References Moore1993a, Moore1994b, Moore1996, Fouts1997, Mondor1998 Parren1998a, Binley1998 |Fouts 1998, Yang2000, Yang2002, Xiang2002b Pantophlet2003

- F91: Called F-91 - neutralizes IIIB - reactive with SF-2 gp120 - strong inhibition of HIV+ human sera binding to IIIB gp120 [Moore1993a]

- F91: Has strong cross-reactivity with gp120 monomers from most subtypes, A-F [Moore1994b]

- F91: Unusual pattern of reciprocal enhancement with several anti-V2 and V3 directed MAbs - reciprocal inhibition of other CD4BS MAbs [Moore1996]

- F91: Study shows neutralization is not predicted by MAb binding to JRFL monomeric gp120, but is associated with oligomeric Env binding - F91 bound monomer, did not bind oligomer or neutralize JRFL [Fouts1997] 


\begin{tabular}{lllll}
\hline No. MAb ID & HXB2 Location & Author's Location & Sequence & Neutralizing Immunogen
\end{tabular}

- F91: Weak inhibition of binding of Hx10 to CD4 positive or negative cells, weakly neutralizing [Mondor1998]

- F91: The MAb and Fab binding to the oligomeric form of gp120 and neutralization were highly correlated - authors suggest that neutralization is determined by the fraction of Ab sites occupied on a virion irrespective of the epitope [Parren1998a]

- F91: A panel of MAbs were shown to bind with similar or greater affinity and similar competition profiles to a deglycosylated or variable loop deleted core gp120 protein ( Delta V1, V2, and V3), thus such a core protein produces a structure closely approximating full length folded monomer - CD4BS MAbs $15 \mathrm{e}, \mathrm{F} 91$ and IgG1b12 bound better to the deleted protein than to wild type [Binley1998]

- F91: CD4BS MAbs 15e, 21h, and F91 bind with even lower affinity than 205-43-1 and 205-42-15 to JRFL oligomer - conclusions of this paper contrast with [Parren1998a] [Fouts1998]

- F91: A combination of gp41 fusion with the GNC4 trimeric sequences and disruption of the YU2 gp120-gp41 cleavage site resulted in stable gp140 trimers (gp140-GNC4) that preserve and expose some neutralizing epitopes while occluding some non-neutralizing epitopes - CD4BS MAbs (F105 and F91) and CD4i (17b and 48d) recognized gp140-GNC4 as well as gp120 or gp140 - non-neutralizing MAbs C11, A32, 522-149, M90, and \#45 bound to the gp140-GNC4 glycoprotein at reduced levels compared to gp120 - MAbs directed at the extreme termini of gp120 C1 (135/9 and 133/290) and C5 (CRA-1 and M91) bound efficiently to gp140-GNC4 [Yang2000]

- F91: Uncleaved soluble gp140 (YU2 strain, R5 primary isolate) can be stabilized in an oligomer by fusion with a C-term trimeric GCN4 motif or using a T4 trimeric motif derived from T4 bacteriophage fibritin - stabilized oligomer gp140 delta683(-FT) showed strong preferential recognition by NAbs IgG1b12 and 2G12 relative to the gp120 monomer, in contrast to poorly neutralizing MAbs F105, F91, 17b, 48d, and 39F which showed reduced levels of binding, and C11, A32, and 30D which did not bind the stabilized oligomer [Yang2002]

- F91: A series of mutational changes were introduced into the YU2 gp120 that favored different conformations - $375 \mathrm{~S} / \mathrm{W}$ seems to favor a conformation of gp120 closer to the CD4-bound state, and is readily bound by sCD4 and CD4i MAbs (17b, 48d, 49e, 21c and 23e) but binding of anti-CD4BS MAbs (F105, $15 \mathrm{e}, \mathrm{IgG1b} 12,21 \mathrm{~h}$ and F91 was markedly reduced - IgG1b12 failed to neutralize this mutant, while neutralization by $2 \mathrm{G} 12$ was enhanced - 2F5 did not neutralize either WT or mutant, probably due to polymorphism in the YU2 epitope - another mutant, 423 I/P, disrupted the gp120 bridging sheet, favored a different conformation and did not bind CD4, CCR5, or CD4i antibodies, but did bind to CD4BS MAbs [Xiang2002b]

- F91: Alanine scanning mutagenesis was used to compare substitutions that affected anti-CD4BS NAb b12 - rec gp120s were engineered to contain combinations of Alanine substitutions that enhanced b12 binding, and while binding of b12 to these gp120 monomers was generally maintained or increased, binding by five non-neutralizing anti-CD4bs MAbs (b3, b6, F105, 15e, and F91) was reduced or completely abolished [Pantophlet2003] Env gp120

\section{References}

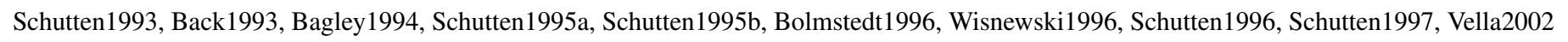

- GP13: Neutralized a broad range of HIV-1 strains from phylogenetically different subfamilies - the following gp120 amino acid substitutions strongly inhibit binding: 256(S/Y), 257(T/G), 262(N/T), 368(D/R or K), 370(E/R or Q or D), 384(Y/E) [Schutten1993]

- GP13: Mutations in a neutralization resistant isolate obtained by passage of the IIIB isolate in chimpanzees reduced neutralization, but the escape was not as clear as seen with anti-V3 MAbs [Back1993]

- GP13: Neutralizes IIIB - only slight inhibition of SI phenotype, and strong enhancement of NSI phenotype chimeric viruses, that incorporated different envs from the same donor [Schutten1995a]

- GP13: Neutralizes T-cell adapted viruses but not the SI strain 16.2, despite high binding affinity [Schutten1995b]

- GP13: Sera were obtained from guinea pigs vaccinated either with gp160, or with gp160 lacking N-linked glycans at N406, N448, and N463 - these sera could block equally well both the CD4 BS MAb GP13 and the V3 MAb F58/H3 [Bolmstedt1996]

- GP13: GP13 is V H5 - V-region heavy chain usage was examined and a bias of enhanced V H1 and V H4, and reduced V H3, was noted among HIV infected individuals [Wisnewski1996]

- GP13: IIIB neutralizing MAbs in vitro fail to neutralize in a mouse model it in vivo [Schutten1996] 


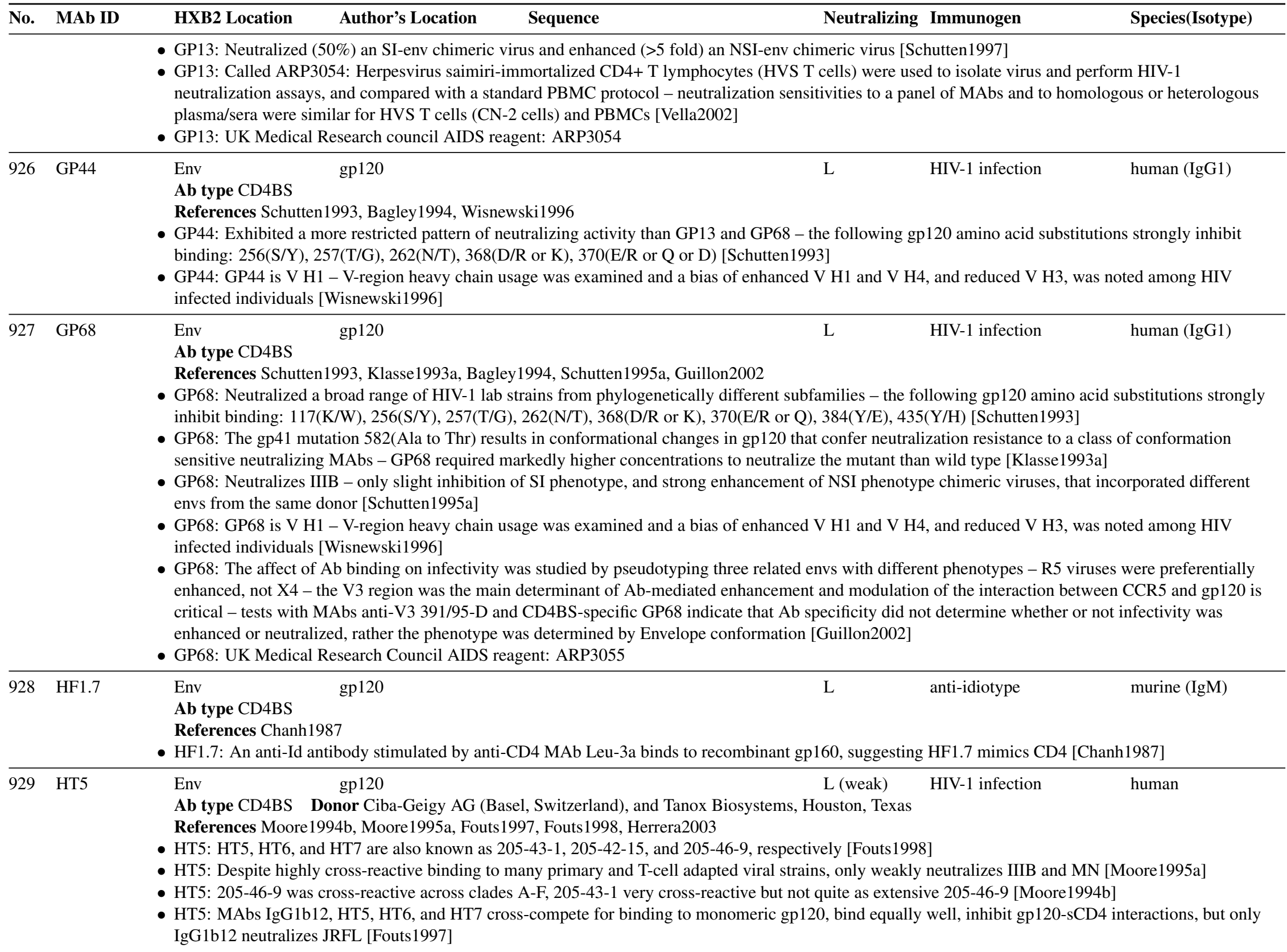




No. MAb ID HXB2 Location Author's Location $\quad$ Sequence $\quad$ Neutralizing Immunogen

- HT5: HT5 and HT6 bind JRSF oligomer but with low affinity, and are not neutralizing - conclusions of this paper contrast with [Parren1998a] [Fouts1998]

- HT5: Called 205-43-1 - CD4BS MAbs b12 (neutralizing) and 205-42-15, 204-43-1, 205-46-9 (nonneutralizing) all cross-competed for binding to monomeric gp120, indicating the topological proximity of their epitopes, however, the nonneutralizing CD4BS MAbs did not interfere with the neutralization activity of MAb b12 - the nonneutralizing MAbs partially competed with b12 for Env binding of the surface of Env-transfected cells - this suggests Env has two categories of binding site for CD4BS MAbs, one recognized by both b12 and nonneutralizing CD4BS MAbs, the other is recognized by only b12 - Ab-gp120 interactions based on the use of monomeric gp120 or Env-transfected cells do not predict the outcome of HIV-1 neutralization assays, and they should be interpreted with caution [Herrera2003]

\begin{tabular}{lllcc}
\hline 930 & HT6 & Env & gp120 & L (weak) HIV-1 infection \\
& & Ab type CD4BS & Donor Ciba-Geigy AG Basel, Switzerland, and Tanox Biosystems, Houston, Texas
\end{tabular}

Ab type CD4BS Donor Ciba-Geigy AG Basel, Switzerland, and Tanox Biosystems, Houston, Texas

References Moore1994b, Moore1995a, Fouts1997, Fouts1998, Herrera2003

- HT6: HT5, HT6, and HT7 are also known as 205-43-1 , 205-42-15, and 205-46-9, respectively [Fouts1998]

- HT6: Despite highly cross-reactive binding to many primary and T-cell adapted viral strains, only weakly neutralizes IIIB and MN [Moore1995a

- HT6: 205-46-9 was cross-reactive across clades A-F, 205-43-1 was not quite as extensively cross-reactive [Moore1994b]

- HT6: MAbs IgG1b12, HT5, HT6, and HT7 cross-compete for binding to monomeric gp120, bind equally well, inhibit gp120-sCD4 interactions, but only IgG1b12 neutralizes JRFL [Fouts1997

- HT6: HT5 and HT6 bind JRSF oligomer but with low affinity, and are not neutralizing - conclusions of this paper contrast with [Parren1998a] [Fouts1998]

- HT6: Called 205-42-15 - CD4BS MAbs b12 (neutralizing) and 205-42-15, 204-43-1, 205-46-9 (nonneutralizing) all cross-competed for binding to monomeric gp120, indicating the topological proximity of their epitopes, however, the nonneutralizing CD4BS MAbs did not interfere with the neutralization activity of MAb b12 - the nonneutralizing MAbs partially competed with b12 for Env binding of the surface of Env-transfected cells - this suggests Env has two categories of binding site for CD4BS MAbs, one recognized by both b12 and nonneutralizing CD4BS MAbs, the other is recognized by only b12 - Ab-gp120 interactions based on the use of monomeric gp120 or Env-transfected cells do not predict the outcome of HIV-1 neutralization assays, and they should be interpreted with caution [Herrera2003]

$\begin{array}{lccc}\text { Env } & \text { gp120 } & \text { L (IIIB) } & \text { HIV-1 infection } \\ \text { Ab type CD4BS } & \text { Donor Ciba-Geigy AG (Basel, Switzerland), and Tanox Biosystems, Houston, Texas } & \end{array}$

References Moore1994b, Moore1995a, Fouts1997, Fouts1998, Herrera2003.

- HT7: HT5, HT6, and HT7 are also known as 205-43-1, 205-42-15, and 205-46-9, respectively [Fouts1998]

- HT7: Despite highly cross-reactive binding to many primary and T-cell adapted viral strains, only neutralizes IIIB well, with sporadic weak neutralization of other isolates [Moore1995a]

- HT7: 205-46-9 was cross-reactive across clades A-F, 205-43-1 was cross-reactive, but not quite as extensive [Moore1994b

- HT7: MAbs IgG1b12, HT5, HT6, and HT7 cross-compete for binding to monomeric gp120, bind equally well, inhibit gp120-sCD4 interactions, but only IgG1b12 neutralizes JRFL [Fouts1997]

- HT7: Binds JRSF oligomer with high affinity, at least as high as IgG1b12, but IgG1b12 is neutralizing, H7 is not - conclusions of this paper contrast with [Parren1998a] - authors propose a model where H7 may inhibit CD4 binding, but cause a conformational shift which enhances CCR5 binding and thus counteracts the neutralizing effect [Fouts1998]

- HT7: Called 205-46-9 - CD4BS MAbs b12 (neutralizing) and 205-42-15, 204-43-1, 205-46-9 (nonneutralizing) all cross-competed for binding to monomeric gp120, indicating the topological proximity of their epitopes, however, the nonneutralizing CD4BS MAbs did not interfere with the neutralization activity of MAb b12 - the nonneutralizing MAbs partially competed with b12 for Env binding of the surface of Env-transfected cells - this suggests Env has two categories of binding site for CD4BS MAbs, one recognized by both b12 and nonneutralizing CD4BS MAbs, the other is recognized by only b12 - Ab-gp120 interactions based on the use of monomeric gp120 or Env-transfected cells do not predict the outcome of HIV-1 neutralization assays, and they should be interpreted with caution [Herrera2003] 


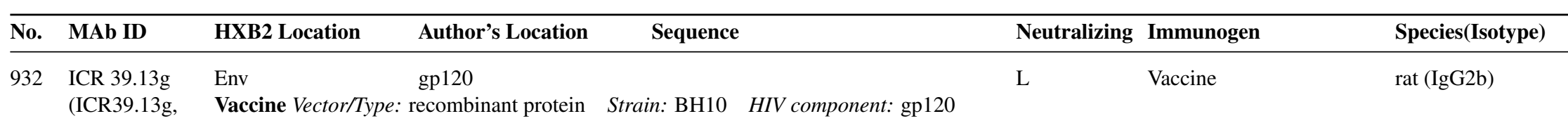

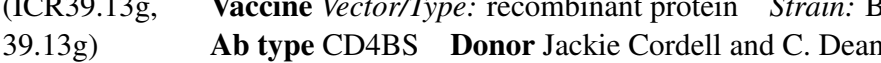

References Cordell1991, McKeating1992a, McKeating1992c, McKeating1993b, Moore1993a Thali1993, Klasse1993a, McLain1994, Beretta1994 McKeating1996b, Armstrong1996a Klasse1996, Peet1998, Vella2002

- ICR 39.13g: Cross-competes with MAbs ICR 39.3b and 15e [Cordell1991]

- ICR 39.13g: Binds to a conformational epitope involved in CD4 binding - exerts a synergistic effect in combination with V3 directed MAbs [McKeating1992a

- ICR 39.13g: Neutralization activity against HXB10, RF, SF-2 and MN strains of HIV-1 [McKeating1993b]

- ICR 39.13g: Conformational, does not bind denatured gp120 - weak neutralization of IIIB - strong inhibition of HIV+ human sera binding to IIIB gp120 |Moore1993a|

- ICR 39.13g: Strongly inhibits CD4 inducible MAb 48d [Thali1993]

- ICR 39.13g: Kinetics of neutralization studied - no lag for 39.3b, while ICR 39.13g and ICR 41.1i have lags of 5 and 15 min respectively - mediates neutralization with 2.3 molecules of IgG [McLain1994]

- ICR 39.13g: The gp41 mutation 582(Ala to Thr) results in conformational changes in gp120 that confer neutralization resistance to a class of conformation sensitive neutralizing MAbs - ICR 39.13g required moderately higher concentrations to neutralize the mutant than wild type [Klasse1993a]

- ICR 39.13g: Called 39.13g Neutralizes HXB2, but fails to neutralize chimeric virus with gp120 from primary isolates in an HXB2 background [McKeating1996b]

- ICR 39.13g: Post-attachment neutralization mechanism, in contrast to MAb 39.3b |Armstrong1996a

- ICR 39.13g: Variants of LAI have differing neutralization susceptibility to 39.13g [Klasse1996]

- ICR 39.13g: The most variable amino acids in the V3 loop were replaced with serines to make the immunodominant V3 loop less immunogenic - these changes did not affect the ability of SCD4 or MAbs to V1/V2, C1 and C4 to bind - ICR 39.13g was not affected by V3 serine substitutions - mice injected with serine substituted gp120 had a reduced response relative to wildtype, and no enhanced immunogenicity of conserved regions [Peet1998]

- ICR 39.13g: Called ARP390/391, but no such entry was found at the UK Medical Research Council AIDS reagent web site: Herpesvirus

saimiri-immortalized CD4+ T lymphocytes (HVS T cells) were used to isolate virus and perform HIV-1 neutralization assays, and compared with a standard PBMC protocol - neutralization sensitivities to a panel of MAbs and to homologous or heterologous plasma/sera were similar for HVS T cells (CN-2 cells) and PBMCs Vella2002

- ICR 39.13g: UK Medical Research Council AIDS reagent: ARP390

933 ICR $39.3 b$ $(39.3,39.3 \mathrm{~b}$, ICR39.3b)

Env gp120

Vaccine Vector/Type: recombinant protein Strain: BH10 HIV component: gp120

Ab type CD4BS Donor J. Cordell and C. Dean

References Cordell1991, McKeating1992c, Moore1993c, McLain1994, Armstrong1996a Jeffs 1996 Wyatt1998a

- ICR 39.3b: also known as 39.3, 39.3b and ICR39.3b

- ICR 39.3b: Cross-competes with MAbs ICR 39.13g and 15e [Cordell1991]

- ICR 39.3b: Conformational, does not bind to denatured IIIB [Moore1993a]

- ICR 39.3b: Kinetics of neutralization studied - no lag for 39.3b, while ICR 39.13g and ICR 41.1i have lags of 5 and 15 min respectively [McLain1994]

- ICR 39.3b: Neutralizes only if the antibody is added prior to the attachment of the virus to the cell, in contrast to 39.13g |Armstrong1996a]

- ICR 39.3b: Called 39.3b - increased binding when V1/V2 or V1/V2 and V3 were deleted from gp120 [Jeffs 1996]

- ICR 39.3b: Called 39.3 - summary of the implications of the crystal structure of the core of gp120 bound to CD4 and 17b with what is known about mutations that reduce NAb binding - probable mechanism of neutralization by CD4BS Ab is direct interference with CD4 binding [Wyatt1998a] 


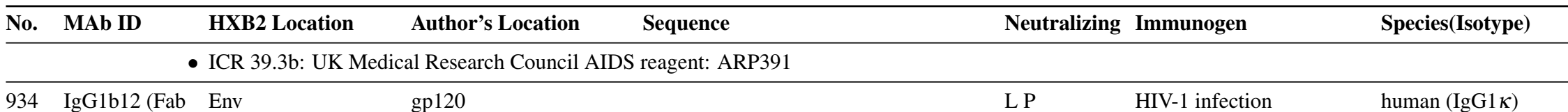

b12, Fab 3B3, Ab type CD4BS Donor D. Burton, Scripps Research Institute, La Jolla, CA, also J. Geltowsky and J. Pyati, R. W. Johnson Pharmaceutical Research Inst. $\mathrm{MAb} \quad$ La Jolla, CA

IgG1b12, References Burton1991, Barbas III1992 Roben1994 Burton1994, Moore1994b, Sattentau1995a, Moore1995a, Moore1995b, Parren1995 Trkola1995.

IgG1-b12, Ditzel1995 Sullivan1995 Yang1997c Moore1996, Gauduin1996, Poignard1996b, Poignard1996a, Trkola1996a Sattentau1996, McKeating1996a.

IgG1 b12, D’Souza1997, Schutten1997, Mo1997, Fouts1997, Li1997, Kessler II1997, Moore1997, Stamatatos1997, Ditzel1997, Ugolini1997 |Wyatt1997.

IgGB12, $\quad$ Burton1997, Boots1997, Parren1997c Parren1997b, Parren1997a Valenzuela1998, Wyatt1998a, Mondor1998 Parren1998a, Connor1998, Binley1998

b4/12, b12, $\quad$ Fouts1998, Takefman1998, Parren1998b Brand1998 Schonning1998 Sullivan1998a, Frankel1998, Kropelin1998 Stamatatos1998, Poignard1999

1b12, im- Jackson1999, Hioe1999, Montefiori1999, Giraud1999 Beddows1999 Binley 1999. Grovit-Ferbas2000, Ly2000. Nyambi2000. Park2000, Si2001,

munoglobulin Kolchinsky2001, Saphire2001a, Saphire2001b Yang2001, York2001, Zwick2001a, Zwick2001b, Zwick2001c||Parren2001, Poignard2001

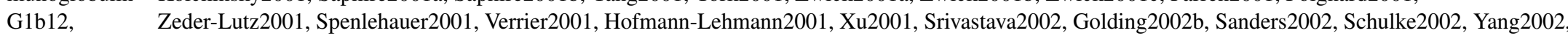

ARP3065, Saphire2002 Scanlan2002, Xu2002, Chakrabarti2002, Vella2002, Xiang2002b, Edwards2002, Grundner2002, Zhang2002, Klasse2002, Ferrantelli2002,

IgG1 b12) Liu2002, Poignard2003, Pantophlet2003 Herrera2003

- IgG1b12: Fab b12 was derived from IgG1b12, Fab 3B3 was derived from Fab b12 by random mutagenesis and selected for increased affinity to sgp120 database note

- IgG1b12: The original Fab fragment was derived from a combinatorial phage library from bone marrow of an HIV-1 positive individual who had been asymptomatic for six years [Burton1991]

- IgG1b12: Anti-CD4 binding site Fab, potent neutralizing activity, greater affinity for a subpopulation of gp120 molecules suggested to be in a mature confirmation - mutations in gp120 that abrogate binding: 368 D/R or D/T, $370 \mathrm{E} / \mathrm{R}$, and $477 \mathrm{D} / \mathrm{V}$, of clone HXBc2 of LAI - sensitive to V1 and V2 substitutions [Roben1994]

- IgG1b12: Very potent neutralization, of primary and lab strains, at concentrations that could be achieved by passive immunization - reduced binding with A,C, and D clade viruses relative to B clade, poor reactivity with E clade - isolates that were refractive to neutralization by sera from HIV-1+ donors could be neutralized by IgG1 b12 [Burton1994]

- IgG1b12: Cross-reactive with some gp120s, (but not all), from clades A-D - not reactive with gp120 from clades E or F [Moore1994b]

- IgG1b12: Formalin inactivation of virus at $0.1 \%$ formalin for 10 hours at 4 degrees was optimal for inactivation of virus while maintaining epitope integrity [Sattentau1995c]

- IgG1b12: Anti-CD4 binding site MAb - very potent neutralization of a number of primary isolates [Moore1995a]

- IgG1b12: Complete protection against HIV-1 infection was achieved in hu-PBL-SCID mice by passive immunization with physiologically relevant doses pharmacokinetics showed serum half-life of 30.2 +/- 1.3 hours for Fab b12 and 7.4 +/- 0.7 days for IgG1 b12 in mice, but IgG1 half-lives in human are generally between 21-23 days [Parren1995, Parren1997a]

- IgG1b12: Called BM12 - broad cross-clade neutralization of primary isolates - additive neutralization in combination with MAb 2F5 [Kessler1995]

- IgG1b12: Review: unusual properties for anti-CD4 BS MAb: sensitive to V2 substitutions, preferential recognition of the oligomer on the cell surface Moore1995b

- IgG1b12: Could potently neutralize primary isolates from within clade B, but showed a slight reduction in efficacy outside of clade B [Trkola1995]

- IgG1b12: Because Fab b12 shows reduction in binding when the V2 loop is deleted and when aa 183/184 PI/SG substitutions are made competition studies were done with Fab L78 and anti-V2 MAbs SC258 and 684-238 and they do not compete with IgG1b12 [Ditzel1995]

- IgG1b12: Fab b12 showed potent neutralization of T-cell-line-adapted strains, but much reduced neutralization of 3 primary isolates -2 of the 3 primary isolates also had reduced binding affinity, but the third was as efficiently immunoprecipitated as HXBc2 [Sullivan1995]

- IgG1b12: Saturation mutagenesis of the complementarity-determining region and optimization strategies were used to create very high affinity versions of this Fab - increased affinity was dominated by a slowing of the off rate |Yang1997c| 


No. MAb ID $\quad$ HXB2 Location $\quad$ Author's Location $\quad$ Sequence $\quad$ Neutralizing Immunogen

- IgG1b12: Potent neutralizing ex vivo of virus taken directly from plasma of HIV-1 infected individuals - little correlation between neutralization sensitivity of passaged virus and plasma derived virus - more effective than MAb 19b [Gauduin1996]

- IgG1b12: Review: Unique among anti-CD4BS MAbs in terms of being potent against both lab adapted virus and primary isolates - one of three MAbs (IgG1b12, 2G12, and 2F5) generally accepted as having significant potency against primary isolates [Poignard1996b]

- IgG1b12: Anti-CD4BS MAbs 15e, 21h, and IgG1b12 did not cause gp120 dissociation from virus, or exposure of the gp41 epitope of MAb 50-69, in contrast to CD4i MAb 48d and anti-V3 neutralizing MAbs [Poignard1996a]

- IgG1b12: Neutralizes JR-FL - inhibits gp120 interaction with CCR-5 in a MIP-1beta-CCR-5 competition study [Trkola1996a]

- IgG1b12: Review: Only four epitopes have been described which can stimulate a useful neutralizing response to a broad spectrum of primary isolates, represented by the binding sites of MAbs: 447-52-D, 2G12, Fab b12, and 2F5 [Sattentau1996]

- IgG1b12: In a multilab evaluation of monoclonal antibodies, only IgG1b12, 2G12, and 2F5 could neutralize at least half of the 9 primary test isolates at a concentration of $<25 \mathrm{mug}$ per $\mathrm{ml}$ for $90 \%$ viral inhibition $-\operatorname{IgG1b} 12$ failed to neutralize only $1 / 9$ primary isolates, although there was some variation between test sites [D’Souza1997]

- IgG1b12: Inhibited some SI- and NSI-env chimeric viruses but enhanced one NSI-env chimeric virus 3 fold [Schutten1997]

- IgG1b12: JRCSF was cultured in the presence of IgG1b12 until a 100-fold resistance to neutralization was selected - resistance was due to three changes: V2 substitution D182N and C3 substitution P365L conferred resistance, and V2 D164N was also required for a viable virus - IgG1b12 resistant virus remained sensitive to MAbs 2G12 and 2F5 [Mo1997]

- IgG1b12: Study shows neutralization is not predicted by MAb binding to JRFL monomeric gp120, but is associated with oligomeric Env binding IgG1b12 bound monomer, oligomer, and neutralized JRFL [Fouts1997]

- IgG1b12: b12 was used in its IgG1 form - of 14 human MAbs, the most potent neutralizer of SHIV-vpu+, which expressed HIV-1 IIIB env - all Ab combinations tested showed synergistic neutralization - b12 has a synergistic response with MAbs 694/98-D (anti-V3), 2F5, and 2G12 [Li1997]

- IgG1b12: 35 primary isolates were tested and all were neutralized by IgG1b12 (including 4, UG270, RW92/026, ZB20, and 301727 which been had reported as not neutralized by IgG1b12 [Trkola1995]) - IgG1b12 could neutralize even when added after the virus to the culture - selection for 400-fold increased affinity did not enhance neutralization by antibody - IgG1b12 was more potent with greater breadth than MAb 2F5 [Kessler II1997]

- IgG1b12: Review: MAbs 2F5, 2G12 and IgG1b12 have potential for use in combination with CD4-IgG2 as an immunotherapeutic or immunoprophylactic - homologous MAbs to these are rare in humans and vaccine strategies should consider including constructs that may enhance exposure of these MAbs' epitopes [Moore1997]

- IgG1b12: Viral binding inhibition by IgG1b12 strongly correlated with neutralization (all other neutralizing MAbs tested showed some correlation except 2F5) [Ugolini1997]

- IgG1b12: Major deletions in C1 and C5 and deletions of the V1V2 and V3 loops do not diminish binding [Wyatt1997]

- IgG1b12: This is a review that includes a description of IgG1b12, noting approximately equivalent affinities for sgp120 and unprocessed gp160, and somewhat enhanced affinity for the native oligomer on TCLA viruses - primary viruses have reduced affinity, but still in the useful range for neutralization - there can be complete protection in hu-PBL-SCID mice with Ab even when administered several hours after viral challenge - competes with sCD4, but unlike other CD4BS antibodies, it is sensitive to mutations in V2 [Burton1997]

- IgG1b12: In this review, the technique and potential application of Fab expression and selection in phage display libraries, and subsequent production of IgG molecules is discussed - b12 is exceptionally potent at neutralization and can successfully neutralize most B clade primary isolates, and many isolates from other subtypes as well - 3B3 was derived from b12 by selection for higher affinity using the CDR walking strategy - 3B3 has 8-fold enhancement of binding, a linear correlation was found between neutralization and affinity, and 3B3 can neutralize strains b12 cannot [Parren1997a]

- IgG1b12: Abs that recognize discontinuous epitopes can identify mimotopes from a phage peptide display library - IgG1b12 blocks CD4 binding and is the most potent neutralizing $\mathrm{Ab}$ - many 15 and 21-mer phage inserts were recognized, but it was not possible to derive a consensus - common features were a $\mathrm{W}$ and at least one acidic residue, and one sequence was found multiple times: NWPRWWEEFVDKHSS, and this peptide could compete with gp120 - two short stretches found in the phage peptides might mimic gp120 components of the epitope: positions 382-384, FFY(I), and 423-426 I(FV)I(V)NM [Boots1997] 


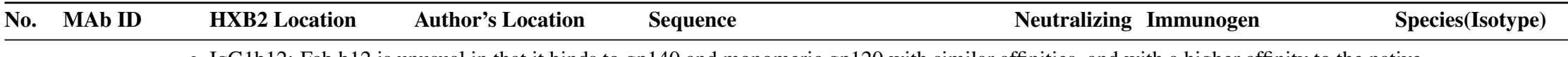

- IgG1b12: Fab b12 is unusual in that it binds to gp140 and monomeric gp120 with similar affinities, and with a higher affinity to the native

oligomer-authors propose this antibody may be exceptional because it binds the virus rather than viral debris-IgG1b12 can protect against infection prior to or shortly after challenge of hu-PBL-SCID mice with TCLA strains and primary strains, but the serum concentrations required in vivo were higher than for in vitro neutralization [Parren1997c Parren1997b].

- IgG1b12: MAb was slightly more efficient at neutralization than Fab - inhibits viral binding to cells and viral entry - doesn't affect CD4-independent binding to T-cells [Valenzuela1998]

- IgG1b12: Summary of the implications of the crystal structure of the core of gp120 bound to CD4 and 17b with what is known about mutations that reduce NAb binding - probable mechanism of neutralization by CD4BS Ab is direct interference with CD4 binding - IgG1b12 is an unusual CD4BS antibody because it is particularly potent as a neutralizing antibody and it is susceptible to changes in the V1-V2 stem loop structure, and so it may disrupt an interaction between CD4 and conserved amino acids on the V1-V2 stem [Wyatt1998a]

- IgG1b12: Enhances binding of Hx10 to CD4 positive or negative HeLa cells, inhibits binding to CD4+ T-cell line A3.01 - neutralizes HeLa and A3.01 cell Hx10 infection [Mondor1998]

- IgG1b12: IgG1b12, Fab b12 and 3B3 derived from b12 were all included in this study - the rank order of Fab binding affinity to monomeric gp120 (Loop 2 $>$ 3B3 $>$ b12 $=$ DO8i $>$ b11 $>$ b3 $>$ b14 $>$ b13 $>$ DO142-10 $>$ DA48 $>$ L17) was markedly different than Fab binding affinity to the mature oligomeric form $(3 \mathrm{~B} 3>\mathrm{b} 12>$ DO142-10 $>$ Loop $2>$ b1 $>$ L17 $>$ b6 $>$ DO8i $>$ b14 $>$ DA48 $>$ b3 $>$ b13) and binding to oligomeric form and neutralization were correlated for both Fabs and MAbs - authors suggest that neutralization is determined by the fraction of Ab sites occupied on a virion irrespective of the epitope binding affinity of divalent IgG1b12 is 17-fold greater than monovalent Fab b12 [Parren1998a]

- IgG1b12: Ab from gp120 vaccinated individuals prior to infection, who subsequently became HIV infected, could not achieve $90 \%$ neutralization of the primary virus by which the individuals were ultimately infected - these viruses were not particularly refractive to neutralization, as determined by their susceptibility to neutralization by MAbs 2G12, IgG1b12, 2F5 and 447-52D [Connor1998]

- IgG1b12: A panel of MAbs were shown to bind with similar or greater affinity and similar competition profiles to a deglycosylated or variable loop deleted core gp120 protein ( Delta V1, V2, and V3), thus such a core protein produces a structure closely approximating full length folded monomer - CD4BS MAbs 15e, F91 and IgG1b12 bound better to the deleted protein than to wild type [Binley1998]

- IgG1b12: Binds JRSF oligomer with high affinity, as do 205-46-9 and 2G6, but IgG1b12 is neutralizing, the other two are not - conclusions of this paper contrast with Parren98 - authors propose a model where 205-46-9 and 2G6 may inhibit CD4 binding, but cause a conformational shift which enhances CCR5 binding and thus counteracts the neutralizing effect - rank order of CD4BS antibodies oligomer binding is IgG1b12 = 2G6 $=205-46-9>205-43-1=$ 205-42-15 $>15 \mathrm{e}=21 \mathrm{~h}=\mathrm{F} 91$, and the only thing notably distinguishing about neutralizing IgG1b12 is that it depends on residues in V2 [Fouts1998]

- IgG1b12: Induces Complement-mediated lysis in MN but not primary isolates - primary isolates are refractive to CML [Takefman1998]

- IgG1b12: MAbs 2G12, 2F5 and b12 are broadly neutralizing, as are some human polyconal sera, but this paper describes a set of primary isolates that are resistant to all three MAbs and 2 broadly neutralizing sera - results indicate that resistance levels of pediatric isolates might be higher than adult isolates resistance in general did not seem to be conferred by a loss of binding affinity for gp120 or gp41, rather by a more global perturbation of oligomeric Envelope [Parren1998b]

- IgG1b12: Immunoprecipitation of gp120 and gp160 expressed from a rec Semliki Forest virus by F105 and IgG1b12 indicated that the SFV expressed HIV-1 Env was folded appropriately - and SVF-HIV-1 Env vaccine gave the strongest anti-HIV-1 Env response in mice, when compared to an HIV-1 Env DNA vaccine and a rgp160 protein |Brand1998|

- IgG1b12: MAbs 654-D100 and IgG1b12 neutralized viruses HIV-BRU and a mutated virus that lacks the V3 loop glycan equally effectively - in contrast, sera from guinea pigs immunized with BRU gp120 neutralize viruses more effectively that lack the V3 glycan[Schonning1998]

- IgG1b12: Fab b12 - the HIV-1 virus YU2 entry can be enhanced by MAbs binding to the CD4BS, V3 loop, and CD4i epitopes - the activation for this enhanced entry state could be conferred on HxB2 by introducing the YU2 V3 loop, or the YU2 V3 and V1/V2 loops - a similar effect is observed by sub-neutralizing concentrations of sCD4 and the effect is dependent of CCR5 - Fab fragment b12 also enhances YU2 entry, ruling out Fc interactions or Env cross-linking as a mechanism, while neutralizing HXBc2 [Sullivan1998a] 


No. MAb ID HXB2 Location Author's Location $\quad$ Sequence $\quad$ Neutralizing Immunogen

- IgG1b12: anti-C1 region MAb 87-135/9 blocks gp120 interaction with CD4+ cells - blocking activity is additive when combined with antibodies which bind in the C4 region of gp120 (F105, 388/389, and b12) [Kropelin1998]

- IgG1b12: Deleting the V2 loop of neutralization-resistant HIV-1 isolate SF162 does not abrogate its replication in PBMC or macrophages, but it enhances its neutralization sensitivity to sera from patients with B clade infection up to 170-fold, and also enhances sensitivity to sera from clades A through F deletion of V2, but not V1, diminished neutralization by CD4BS MAb IgG1b12, in contrast to 654.30D and IgGCD4 [Stamatatos1998]

- IgG1b12: Infection of dendritic cells cultured from CD14+ blood cells or from cadaveric human skin was blocked by neutralizing MAbs IgG1b12, or 2F5 and 2G12 delivered together, but not by control non-neutralizing anti-gp120 MAb 4.8D, indicating that NAbs could interrupt early mucosal transmission events [Frankel1998]

- IgG1b12: The presence of leukocyte function-associated molecule 1 (LFA-1) promotes virus infectivity and hinders neutralization, and anti-LFA-1 MAbs can enhance the neutralizing effect of anti-HIV V3 MAb 447-52D and anti-HIV CD4BS MAb IgG1b12 - non-neutralizing anti-HIV CD4BS MAb 654-D did not become neutralizing in the presence of anti-LFA-1 MAbs [Hioe1999]

- IgG1b12: rgp120 derived from a R5X4 subtype B virus was used to vaccinate healthy volunteers and the resulting sera were compared with sera from HIV-1 positive subjects and neutralizing MAbs - TCLA strains showed enhanced IgG1b12 neutralization sensitivity relative to PBMC-adapted lines IgG1b12 was able to bind, with low affinity, to the rgp120 monomer HIV-1 W61D [Beddows1999]

- IgG1b12: A meeting summary presented results regarding neutralization - D. Burton and J. Mascola presented results concerning passive immunization and protection of hu-PBL-SCID mice and macaques, respectively, and both found combinations of MAbs that were able to achieve $99 \%$ neutralization in vitro corresponded to efficacy in vivo [Montefiori1999]

- IgG1b12: does not inhibit attachment of virus to cells and was used as a control of a study of neutralization by a MAb F58 based micro antibody [Jackson1999]

- IgG1b12: Hu-PBL-SCID mice were infected with HIV-1s JRCSF and SF162 to study the effect of NAbs on an established infection - at day 6 post infection, mice were given $50 \mathrm{mg} / \mathrm{kg}$ of b12, an amount that would have been protective if given up to 8 hours post-infection, and 100-fold higher than the amount required for $90 \%$ neutralization in vitro - no significant differences in the initial rate of decrease in viral load or the plateau levels of viral RNA between the b12 treated and control mice were seen - in most of the Ab treated mice escape mutants were observed with varying patterns of mutations - a combination of b12, 2G12 and 2F5 protected 1/3 mice, and an isolate from one of the other two was resistant to neutralization by all three MAbs Poignard1999

- IgG1b12: The MAbs with the broadest neutralizing activity, IgG1b12, $2 \mathrm{G} 12$ and 2F5, all have high affinity for the native trimer, indicating that they were raised in an immune response to the oligomer on the virion surface rather than dissociated subunits - a disulfide linked gp120-gp41 (SOS gp140) was created to mimic the native conformation of Env and explore its potential as an immunogen - SOS gp140 is recognized by NAbs IgG1b12, 2G12, and CD4-IgG2, and also by anti-V3 MAbs $19 \mathrm{~b}$ and 83.1 - SOSgp140 is not recognized by C4 region MAbs that neutralize only TCLA strains, G3-42 and G3-519 - nor did it bind C11, 23A, and M90, MAbs that bind to gp120 C1 and C5, where it interacts with gp41 - MAbs that bind CD4 inducible epitopes, $17 \mathrm{~b}$ and A32 were very strongly induced by CD4 in SOS gp140 - anti-gp41 MAbs that bind in the region that interacts with gp120, 7B2, 2.2B, T4, T15G1 and 4D4, did not bind to SOSgp140, in contrast to 2F5, which binds to the only gp41 epitope that is well exposed in native gp120-gp41 complexes [Binley1999

- IgG1b12: To determine the antigenicity of virus killed by thermal and chemical inactivation, retention of conformation-dependent neutralization epitopes was examined, and exposure of CD4BS epitopes was found to be enhanced (MAbs IgG1b12, 205-46-9, and 205-43-1) - binding to 2G12 and 447-52D epitopes was essentially unaltered - the 17b CD4i epitope was also exposed [Grovit-Ferbas2000]

- IgG1b12: SF162 is a neutralization-resistant HIV-1 isolate - N-linked glycosylation modifications in the V2 loop of the SF162 gp120 revealed that these sites prevent neutralization by CD4BS MAbs (IgG1b12 and IgGCD4), and protect against neutralization by V3 MAbs (447-D and 391-95D) - V2-region glycosylation site mutations did not enhance neutralization resistance to V2 MAbs (G3.4 and G3.136) or CD4i MAbs (17b and 48d) - V2 glycosylation site modification allows increased infection of macrophages, probably due to glycosylated forms requiring fewer CCR5 molecules for viral entry [Ly2000] 


No. MAb ID $\quad$ HXB2 Location $\quad$ Author's Location $\quad$ Sequence $\quad$ Neutralizing Immunogen

- IgG1b12: 26 HIV-1 group M isolates (clades A to H) were tested for binding to 47 MAbs, including 6 CD4BS MAbs - CD4BS MAbs bound consistently to most isolates of clade D, but poorly to isolates of other clades with the exception of broadly reactive MAb IgG1b12, binding to 22 of 26 isolates tested - 8 MAbs were tested for neutralization and MAb IgG1b12 was most potent, with 90\% neutralization of 3/5 isolates tested [Nyambi2000]

- IgG1b12: Fab b12 was used - six mutations in MN change the virus from a high-infectivity neutralization resistant phenotype to low-infectivity neutralization sensitive - V3, CD4BS, and CD4i MAbs are 20-100 fold more efficient at neutralizing the sensitive form - the mutation L544P reduced binding of all MAbs against gp120 by causing conformational changes [Park2000]

- IgG1b12: SHIV-HXBc2 is a neutralization sensitive non-pathogenic virus, and several in vivo passages through monkey's yielded highly pathogenic SHIV $\mathrm{KU}-1$ - HXBc2 and the KU-1 clone HXBc2P3.2 differ in 12 amino acids in gp160 - substitutions in both gp120 and gp41 reduced the ability of sCD4, IgG1b12, F105 and AG1121 to Env achieve saturation and full occupancy, and neutralize KU-1 - 17b and 2F5 also bound less efficiently to HXBc2P3.2, although 2G12 was able to bind both comparably [Si2001]

- IgG1b12: Mutations in two glcosylation sites in the V2 region of HIV-1 ADA at positions 190 and 197 (187 DNTSYRLINCNTS) cause the virus to become CD4-independent and able to enter cells through CCR5 alone - these same mutations tended to increase the neutralization sensitivity of the virus, except the mutation $197 \mathrm{~S} / \mathrm{R}$ which resulted in a carbohydrate addition to $195 \mathrm{~N}$ that disrupts the IgG1b12 binding site [Kolchinsky2001]

- IgG1b12: This paper describes the technical aspects of the crystallization of b12 at a resolution of 2.7 angstroms with all 12 Ig domains resolved [Saphire2001a|

- IgG1b12: This paper describes the biological implications of the crystal structure of b12 - a remarkable feature of this antibody is a long protruding finger-like CDR H3 that can dock in the recessed CD4-binding site - a contact residues in gp120 are modeled, with numbering based on the variable loop-deleted crystal structure of gp120 [Saphire2001b]

- IgG1b12: Primary isolates YU2 and ADA are more resistant to IgG1b12 neutralization than HXBc2: 90\% Neutralization of HXBc2 is observed with 1.25 ug of IgG1b12, while ADA and YU2 require 2.5 and 5 ug respectively to achieve 50\% neutralization, and $90 \%$ neutralization could not be achieved with 10 or 20 ug of IgG1b12, respectively [Yang2001]

- IgG1b12: Abs against the V3 loop (50.1, 58.2, 59.1, 257-D, 268-D, 447-52D), CD4BS (IgG1b12, 559-64D, F105), CD4i (17b), and to gp41 (2F5, F240) each showed similar binding efficiency to Env derived from related pairs of primary and TCLA lines (primary: 168P and 320SI, and TCLA: 168C and 320SI-C3.3), but the TCLA lines were much more susceptible to neutralization suggesting that the change in TCLA lines that make them more susceptible to NAbs alters some step after binding [York2001]

- IgG1b12: b12 recognizes a conformational epitope that overlaps with the CD4 binding site - a phage displayed peptide library was used to identify a peptide which bound b12, called B2.1, which competes with b12 in competition assays - B2.1 has significant homology to the D loop of gp120: upper case letters indicate residues B2.1 shares with gp120, heRsymFSDlenrcI - one of the goals of defining peptide mimics to the b12 epitope is to develop an immunogen that can stimulate b12-like antibodies, but B2.1 cross-linked to phage and ovalbumin bound IgG1b12 did not elicit cross-reactive gp120 Abs in mice or rabbits [Zwick2001a]

- IgG1b12: This paper primarily concerns 4E10 and Z13, MAbs that both bind proximally to the 2F5 binding site to a conserved epitope, and that neutralize some primary isolates from clades B, C, and E - broadly neutralizing MAbs 2F5, IgG1b12, and 4E10 and Z13 fail to neutralize different subsets of viruses [Zwick2001b]

- IgG1b12: Neutralization synergy between anti-HIV NAbs b12, 2G12, 2F5, and 4E10 was studied - a classic fixed-ratio method was used, as well as a method where one $\mathrm{Ab}$ was fixed at a low neutralization titer and the other was varied - using primary isolates, a two-four fold enhancement of neutralization was observed with MAb pairs, and a ten-fold enhancement with a quadruple Ab combination - no synergy was observed with any MAb pair in the neutralization of TCLA strain HXB2 - whole IgG1b12 and b12 Fab fragments behaved similarly in the neutralization assays - there was no evidence for cooperativity of binding between b12 and 2G12 to envelope spikes expressed on the cell surface of TCLA or primary isolates [Zwick2001c] 


No. MAb ID $\quad$ HXB2 Location $\quad$ Author's Location $\quad$ Sequence $\quad$ Neutralizing Immunogen

- IgG1b12: Intravenous passive transfer of MAb b12 provides dose-dependent protection from infection to macaques vaginally challenged with the R5 virus SHIV(162P4) - the primary isolate HIV-1SF162 is neutralized 90\% (IC90) by b12 at $2 \mu \mathrm{g} / \mathrm{ml}$, and SHIV162P4, derived from HIV-1SF162, was neutralized by $90 \%$ at $2 \mu \mathrm{g} / \mathrm{ml}$ in PHA-activated PBMC from rhesus macaques - the 90\% neutralization titers achieved in three groups of animals that were given $25-$, $5-$, and 1-mg/kg doses were approximately 1:400, 1:80, and 1:16, respectively - the half-life of IgG1 b12 in plasma was about 1 week, but while the peak b12 plasma concentration was immediately after the infusion, the peak vaginal fluid concentration was 7-14 days later [Parren2001]

- IgG1b12: Structural aspects of the interaction of neutralizing Abs with HIV-1 Env are reviewed - Env essentially has three faces, one is largely inaccessible on the native trimer, and two that exposed but have low immunogenicity on primary viruses - neutralization is suggested to occur by inhibition of the interaction between gp120 and the target cell membrane receptors as a result of steric hindrance and it is noted that the attachment of approximately 70 IgG molecules per virion is required for neutralization, which is equivalent to about one $\mathrm{IgG}$ molecule per spike - the $2 \mathrm{G} 12,17 \mathrm{~b}$ and b12 epitopes are discussed in detail - the structure of CD4-bound gp120 reveals features that HIV has evolved to escape anti-CD4BS Abs like IgG1b12 despite profound functional constraints - CD4BS Abs must first access the CD4 binding site, deeply recessed within the gp120 core, and the Fab of an Ab molecule is "wider" than $\mathrm{CD} 4$, and in addition the binding site is flanked by variable and glycosylated regions [Poignard2001]

- IgG1b12: Neutralizing synergy between MAbs 1b12, 2G12 and 2F5 was studied using surface plasmon resonance to determine the binding kinetics for these three MAbs with respect to monomeric and oligomeric env protein gp160 IIIB - the 2G12 epitope is highly accessible on both monomeric and oligomeric Envs, 1 b12 is highly accessible on monomers but not oligomers, and 2F5 on neither form - binding of 2G12 exposes the 2F5 epitope on gp160 oligomers [Zeder-Lutz2001]

- IgG1b12: A luciferase-reporter gene-expressing T-cell line was developed to facilitate neutralization and drug-sensitivity assays - luciferase and p24 antigen neutralization titer end points were found comparable using NAb from sera from HIV+ donors, and MAbs 2F5, 2G12 and IgG1b12 [Spenlehauer2001]

- IgG1b12: A panel of 12 MAbs was used to identify those that could neutralize the dual-tropic primary isolate HIV-1 89.6 - six gave significant neutralization at 2 to $10 \mathrm{ug} / \mathrm{ml}$ : 2F5, 50-69, IgG1b12, 447-52D, 2G12, and 670-D six did not have neutralizing activity: 654-D, 4.8D, 450-D, 246-D, 98-6, and 1281 - no synergy, only additive effects were seen for pairwise combinations of MAbs, and antagonism was noted between gp41 M Abs 50-69 and 98-6, as well as 98-6 and 2F5 [Verrier2001]

- IgG1b12: A combination of MAbs IgG1b12, 2F5, and 2G12 was given postnatally to four neonates macaques that were then challenged with highly pathogenic SHIV89.6P - one of the four infants remained uninfected after oral challenge, two infants had no or a delayed CD4(+) T-cell decline - the most potent combination included IgG1b12, which alone does not alone neutralize SHIV89.6P [Hofmann-Lehmann2001]

- IgG1b12: Twenty HIV clade C isolates from five different countries were susceptible to neutralization by anti-clade B MAbs in a synergistic quadruple combination of mAbs IgG1b12, 2G12, 2F5, and 4E10 [Xu2001]

- IgG1b12: Oligomeric gp140 (o-gp140) derived from R5 primary isolate US4 was characterized for use as a vaccine reagent - antigen capture ELISA was used to compare the antigenicity of gp120 and o-gp140 using a panel of well characterized MAbs - Abs directed against the CD4 binding site (IgGCD4 and IgG1b12) reacted slightly more strongly with the gp120 monomer than with the oligomer, as did sCD4 [Srivastava2002]

- IgG1b12: The fusion process was slowed by using a suboptimal temperature $(31.5 \mathrm{C})$ to re-evaluate the potential of Abs targeting fusion intermediates to block HIV entry - preincubation of E/T cells at 31.5 C enabled polyclonal anti-N-HR Ab and anti-six-helix bundle Abs to inhibit fusion, indicating six-helix bundles form prior to fusion - the preincubation $31.5 \mathrm{C}$ step did not alter the inhibitory activity of neutralizing Abs anti-gp41 2F5, or anti-gp120 2G12, IG1b12, 48d, and 17b [Golding2002b]

- IgG1b12: Deglycosylation of gp120 does not significantly affect IG1b12 binding, in contrast to MAB 2G12 [Sanders2002]

- IgG1b12: Ab binding characteristics of SOS gp140 were tested using SPR and RIPA - SOS gp140 is gp120-gp41 bound by a disulfide bond - NAbs 2G12, 2F5, IgG1b12, CD4 inducible 17b, and 19b bound to SOS gp140 better than uncleaved gp140 (gp140unc) and gp120 - non-neutralizing MAbs 2.2B (binds to gp41 in gp140unc) and 23A (binds gp120) did not bind SOS gp140 - SOS gp140-2F5-IgG1b12 formed multiple ring structures composed of two SOS gp140 proteins bridged by two Ab molecules, while 2F5 and 2G12 formed extended chains rather than closed rings [Schulke2002] 


\begin{tabular}{lllll}
\hline No. MAb ID & HXB2 Location & Author's Location & Sequence & Neutralizing Immunogen
\end{tabular}

- IgG1b12: Uncleaved soluble gp140 (YU2 strain, R5 primary isolate) can be stabilized in an oligomer by fusion with a C-term trimeric GCN4 motif or using a T4 trimeric motif derived from T4 bacteriophage fibritin—-stabilized oligomer gp140 683 (-FT) showed strong preferential recognition by NAbs IgG1b12 and 2G12 relative to the gp120 monomer, in contrast to poorly neutralizing MAbs F105, F91, 17b, 48d, and 39F which showed reduced levels of binding, and C11, A32, and 30D which did not bind the stabilized oligomer [Yang2002].

- IgG1b12: The crystal structure of IgG1b12 is resolved and is the first structure of an intact human Ab with an ordered, full length hinge - the structure is extremely asymmetric and flexible with an antigen-binding site that has an unusually long CDR H3 region with a ten residue insertion that projects above the rest of the antigen-binding site - this loop may be required for recognition of the recessed CD4 binding site of gp120 [Saphire2002] ${ }^{\circ}$

- IgG1b12: Alanine scanning mutagenesis used in conjunction with competition and replacement studies of N-linked carbohydrates and sugars suggest that the $2 \mathrm{G} 12$ epitope is formed from mannose residues contributed by the glycans attached to N295 and N332, with the other N-linked carbohydrates in positions N339, N386, and N392 playing a role in maintaining conformation relevant to 2G12 binding - N295A and N332A mutants showed essentially unchanged anti-CD4BS NAb b12 binding affinities, while N339A, N386A and N392A mutants displayed significantly lowered b12 affinity, presumably due to conformational changes [Scanlan2002]

- IgG1b12: Passive immunization of neonate macaques with a combination of F105+2G12+2F5 conferred complete protection against oral challenge with SHIV-vpu+ or - the combination b12+2G12+2F5 conferred partial protection against SHIV89.6 - such combinations may be useful for prophylaxis at birth and against milk born transmission - the synergistic combination of IgG1b12, 2G12, 2F5, and 4E10 neutralized a collection of HIV clade C primary isolates [Xu2002]

- IgG1b12: A modified gp140 (gp140 $\mathrm{CFI}$ ), with C-term mutations intended to mimic a fusion intermediate and stabilize trimer formation, retained antigenic conformational determinants as defined by binding to CD4 and to MAbs 2F5, 2G12, F105, and b12, and enhanced humoral immunity without diminishing the CTL response in mice injected with a DNA vaccine [Chakrabarti2002.

- IgG1b12: Called ARP3065: Herpesvirus saimiri-immortalized CD4+ T lymphocytes (HVS T cells) were used to isolate virus and perform HIV-1 neutralization assays, and compared with a standard PBMC protocol - neutralization sensitivities to a panel of MAbs and to homologous or heterologous plasma/sera were similar for HVS T cells (CN-2 cells) and PBMCs [Vella2002]

- IgG1b12: A series of mutational changes were introduced into the YU2 gp120 that favored different conformations - $375 \mathrm{~S} / \mathrm{W}$ seems to favor a conformation of gp120 closer to the CD4-bound state, and is readily bound by sCD4 and CD4i MAbs (17b, 48d, 49e, 21c and 23e) but binding of anti-CD4BS MAbs (F105, 15e, IgG1b12, 21h and F91 was markedly reduced - IgG1b12 failed to neutralize this mutant, while neutralization by 2 G12 was enhanced - 2F5 did not neutralize either WT or mutant, probably due to polymorphism in the YU2 epitope - another mutant, $423 \mathrm{I} / \mathrm{P}$, disrupted the gp120 bridging sheet, favored a different conformation and did not bind CD4, CCR5, or CD4i antibodies, but did bind to CD4BS MAbs [Xiang2002b]

- IgG1b12: Truncation of the gp41 cytoplasmic domain of X4, R5, and X4R5 viruses forces a conformation that more closely resembles the CD4 bound state of the external Envelope, enhancing binding of CD4i MAbs 17b and 48d and of CD4BS MAbs F105, b12, and in most cases of glycosylation site dependent MAb 2G12 and the anti-gp41 MAb 246D - in contrast, binding of the anti-V2 MAb 697D and the anti-V3 MAb 694/98D were not affected - viruses bearing the truncation were more sensitive to neutralization by MAbs 48d, b12, and 2G12 - the anti-C5 MAb 1331A was used to track levels of cell surface expression of the mutated proteins [Edwards2002]

- IgG1b12: Truncation of the gp41 cytoplasmic domain of X4, R5, and X4R5 viruses forces a conformation that more closely resembles the CD4 bound state of the external Envelope, enhancing binding of CD4i MAbs 17b and 48d and of CD4BS MAbs F105, b12, and in most cases of glycosylation site dependent MAb 2G12 and the anti-gp41 MAb 246D - in contrast, binding of the anti-V2 MAb 697D and the anti-V3 MAb 694/98D were not affected - viruses bearing the truncation were more sensitive to neutralization by MAbs 48d, b12, and 2G12 - the anti-C5 MAb 1331A was used to track levels of cell surface expression of the mutated proteins [Edwards2002]

- IgG1b12: HIV-1 gp160 $\mathrm{CT}$ (cytoplasmic tail-deleted) proteoliposomes (PLs) containing native, trimeric envelope glycoproteins from R5 strains YU2 and JRFL, and X4 strain HXBc2, were made in a physiologic membrane setting as candidate immunogens for HIV vaccines-2F5 bound to gp160 $\Delta \mathrm{CT}$ with a reconstituted membrane ten-fold better than the same protein on beads-anti-CD4BS MAbs IgG1b12 and F105, A32 (C1-C4), C11 (C1-C5), and 39F (V3)

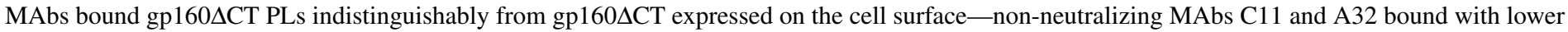
affinity than NAb IgG1b12 - the MAb 17b was sCD4 inducible on gp160 $\Delta$ CT PL [Grundner2002]. 


No. MAb ID HXB2 Location $\quad$ Author's Location $\quad$ Sequence $\quad$ Neutralizing Immunogen

- IgG1b12: A rare mutation in the neutralization sensitive R2-strain in the proximal limb of the $\mathrm{V} 3$ region caused Env to become sensitive to neutralization by MAbs directed against the CD4 binding site (CD4BS), CD4-induced (CD4i) epitopes, soluble CD4 (sCD4), and HNS2, a broadly neutralizing sera - 2/12 anti-V3 MAbs tested (19b and 694/98-D) neutralized R2, as did 2/3 anti-CD4BS MAbs (15e and IgG1b12), 2/2 CD4i MAbs (17b and 4.8D), and 2G12 and $2 \mathrm{~F} 5$ - thus multiple epitopes on R2 are functional targets for neutralization and the neutralization sensitivity profile of R2 is intermediate between the highly sensitive MN-TCLA strain and the typically resistant MN-primary strain [Zhang2002]

- IgG1b12: A broad review of NAbs that mentions IgG1b12 as an example of a NAb that does not alter the conformation of gp120, but interferes with CD4 binding [Klasse2002]

- IgG1b12: Review of NAbs that notes IgG1b12 is a recombinant IgG1 from a phage displayed Fab generated against gp120 from a B clade infected individual, that it binds the CD4BS, that alone or in combination with other MAbs it can protect some macaques against SHIV infection, and that it has strong ADCC activity [Ferrantelli2002]

- IgG1b12: Review of NAbs that discusses mechanisms of neutralization, passive transfer of NAbs and protection in animal studies, and vaccine strategies [Liu2002]

- IgG1b12: Virion capture assays are not a good preditor of neutralization, and the presentation of epitopes using this assay seems to be different from that of functional Envelope spikes on primary isolates - F105 and b6 could efficiently block the b12-mediated capture of infectious virions in a virus capture, but did not inhibit b12 neutralization - b12 was potent at neutralizing the three primary virions JR-CSF, ADA, and 89.6, but anti-V3 Abs 447-52D and 19b, which did not neutralize JR-CSF and ADA captured amounts of p24 equal to or higher than the amounts captured by the neutralizing Ab b12 |Poignard2003|

- IgG1b12: Called b12 - Alanine scanning mutagenesis was used to compare substitutions that affected anti-CD4BS NAb b12 binding to those that affect binding of sCD4 and two non-neutralizing anti-CD4BS Abs b3 and b6 - while the epitope maps overlapped, there were some differences observed binding of CD4 was never in enhanced, indicating it had evolved to be optimal - rec gp120s were engineered to contain combinations of Alanine substitutions that enhanced b12 binding, and while binding of b12 to these gp120 monomers was generally maintained or increased, binding by five non-neutralizing anti-CD4bs MAbs (b3, b6, F105, 15e, and F91) was reduced or completely abolished - 2G12 binding was largely unperturbed, indicating these proteins were not grossly misfolded - for twelve mutants, b12 neutralization sensitivity and affinity correlated, but for five mutants neutralization efficiency was maintained or increased despite a decrease in affinity [Pantophlet2003]

- IgG1b12: Called b12 - CD4BS MAbs b12 (neutralizing) and 205-42-15, 204-43-1, 205-46-9 (nonneutralizing) all cross-competed for binding to monomeric gp120, indicating the topological proximity of their epitopes, however, the nonneutralizing CD4BS MAbs did not interfere with the neutralization activity of MAb b12 - the nonneutralizing MAbs partially competed with b12 for Env binding of the surface of Env-transfected cells - this suggests Env has two categories of binding site for CD4BS MAbs, one recognized by both b12 and nonneutralizing CD4BS MAbs, the other is recognized by only b12 - Ab-gp120 interactions based on the use of monomeric gp120 or Env-transfected cells do not predict the outcome of HIV-1 neutralization assays, and they should be interpreted with caution [Herrera2003]

- IgG1b12: UK Medical Research Council AIDS reagent: ARP3065

- IgG1b12: NIH AIDS Research and Reference Reagent Program: 2640

935 IgGCD4 (IgG-CD4)

Env gp120

Ab type CD4BS

References Capon1989, Stamatatos1998, Ly2000, Srivastava2002

- IgGCD4: An antibody-like immunoadhesins molecule was constructed incorporating the gp120-binding domain of CD4 [Capon1989]

- IgGCD4: Deleting the V2 loop of neutralization-resistant HIV-1 isolate SF162 does not abrogate its replication in PBMC or macrophages, but it enhances its neutralization sensitivity to sera from patients with B clade infection up to 170-fold, and also enhances sensitivity to sera from clades A through F deletion of V2 but not V1 slightly enhanced neutralization by CD4BS MAb IgGCD4 [Stamatatos1998] 


\begin{tabular}{|c|c|c|c|c|c|}
\hline No. & MAb ID & Author's Location & Sequence & Neutralizing Immunogen & Species(Isotype) \\
\hline & & \multicolumn{4}{|c|}{$\begin{array}{l}\text { - IgGCD4: SF162 is a neutralization-resistant HIV-1 isolate - N-linked glycosylation modifications in the V2 loop of the SF162 gp120 revealed that these } \\
\text { sites prevent neutralization by CD4BS MAbs (IgG1b12 and IgGCD4), and protect against neutralization by V3 MAbs (447-D and 391-95D) - V2-region } \\
\text { glycosylation site mutations did not alter neutralization resistance to V2 MAbs (G3.4 and G3.136) or CD4i MAbs (17b and 48d) - V2 glycosylation site } \\
\text { modification allows infection of macrophages, probably due to glycosylated forms requiring fewer CCR5 molecules for viral entry [Ly2000] } \\
\text { - IgGCD4: Oligomeric gp140 (o-gp140) derived from R5 primary isolate US4 was characterized for use as a vaccine reagent - antigen capture ELISA was } \\
\text { used to compare the antigenicity of gp120 and o-gp140 using a panel of well characterized MAbs - Abs directed against the CD4 binding site (IgGCD4 and } \\
\text { IgG1b12) reacted slightly more strongly with the gp120 monomer than with the oligomer, as did sCD4 [Srivastava2002] }\end{array}$} \\
\hline 936 & $\mathrm{~L} 28$ & $\begin{array}{l}\text { Env } \\
\text { Ab type CD4BS } \\
\text { References Ditzel1995 } \\
\text { L28: Substitutions at 257 T/R, 368 D/R, 37 } \\
\text { V3 loop and by substitutions 45 W/S, 298 F } \\
\text { variable region sequence is available [Ditze }\end{array}$ & $\begin{array}{l}\text { and } 370 \mathrm{E} / \\
81 \mathrm{E} / \mathrm{P}, 382\end{array}$ & $\begin{array}{l}\text { L HIV-1 infection } \\
\text { N/D reduce binding - binding wa } \\
- \text { binding is sensitive to deglyco }\end{array}$ & $\begin{array}{l}\text { human }(\operatorname{IgG1} \kappa) \\
\text { d by removal of the } \\
\text { heavy and light chain }\end{array}$ \\
\hline 937 & L33 & $\begin{array}{l}\text { Env } \\
\text { Ab type CD4BS } \\
\text { References Ditzel1995 } \\
\text { L33: binding is sensitive to deglycosylation }\end{array}$ & y and ligh & $\begin{array}{l}\text { L HIV-1 infection } \\
\text { ce is available [Ditzel1995] }\end{array}$ & human $(\operatorname{IgG} 1 \kappa)$ \\
\hline 938 & L41 & $\begin{array}{l}\text { Env gp120 } \\
\text { Ab type CD4BS } \\
\text { References Ditzel1995 } \\
\text { L41: Substitutions at } 133 \text { D/R, 256 S/Y, 25 } \\
\text { retrieved from the library after masking wit } \\
\text { sequence is available [Ditzel1995] }\end{array}$ & $\begin{array}{l}368 \mathrm{D} / \mathrm{R} \text { o } \\
\text { in anti-CD }\end{array}$ & $\begin{array}{l}\text { L HIV-1 infection } \\
\text { E, and } 421 \mathrm{~K} / \mathrm{L} \text { reduce binding - } \\
\text { ive to deglycosylation - heavy an }\end{array}$ & $\begin{array}{l}\text { human }(\operatorname{IgG} 1 \kappa) \\
\text { ally, this Fab was } \\
\text { ain variable region }\end{array}$ \\
\hline 939 & L42 & $\begin{array}{l}\text { Env gp120 } \\
\text { Ab type CD4BS } \\
\text { References Ditzel1995 } \\
\text { L42: Substitutions at 257 T/R, 368 D/R, } 37 \\
\text { binding is sensitive to deglycosylation - hea }\end{array}$ & $\begin{array}{l}266 \mathrm{~A} / \mathrm{E} \text { a } \\
\text { dight chai }\end{array}$ & $\begin{array}{l}\text { L HIV-1 infection } \\
\text { binding was significantly enhance } \\
\text { available [Ditzel1995] }\end{array}$ & $\begin{array}{l}\text { human }(\operatorname{IgG} 1 \kappa) \\
\text { E/P and } 382 \mathrm{~F} / \mathrm{L}-\end{array}$ \\
\hline 940 & L52 & $\begin{array}{l}\text { Env gp120 } \\
\text { Ab type CD4BS } \\
\text { References Ditzel1995 } \\
\text { - L52: Binding is sensitive to deglycosylation }\end{array}$ & vy and ligl & $\begin{array}{l}\text { L HIV-1 infection } \\
\text { cee is available Ditzel1995 }\end{array}$ & human $(\operatorname{IgG} 1 \kappa)$ \\
\hline 941 & L72 & $\begin{array}{l}\text { Env } \quad \text { gp120 } \\
\text { Ab type CD4BS } \quad \text { Donor Dr. Hariharam, I } \\
\text { References Ditzel1997 } \\
\text { - L72: Used to bind gp120 to solid phase to s }\end{array}$ & $\begin{array}{l}\text { Pharmaceut } \\
\text { MAbs from }\end{array}$ & zel1997] & murine \\
\hline 942 & M12 & $\begin{array}{l}\text { Env } \quad \mathrm{gp} 120(\mathrm{IIIB}) \\
\text { Vaccine Vector/Type: vaccinia Strain: II }\end{array}$ & compon & Vaccine & (IgG) \\
\hline
\end{tabular}




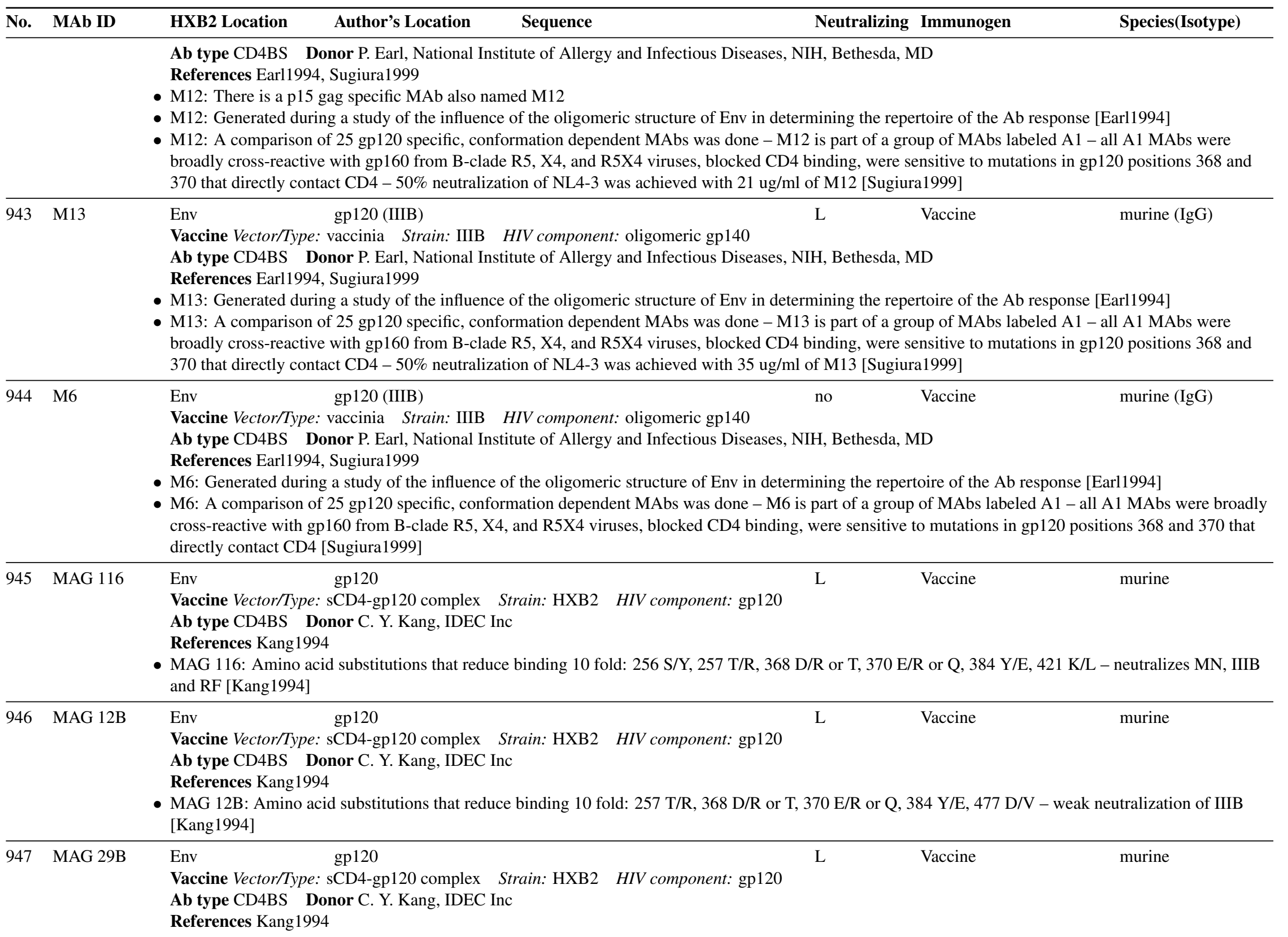




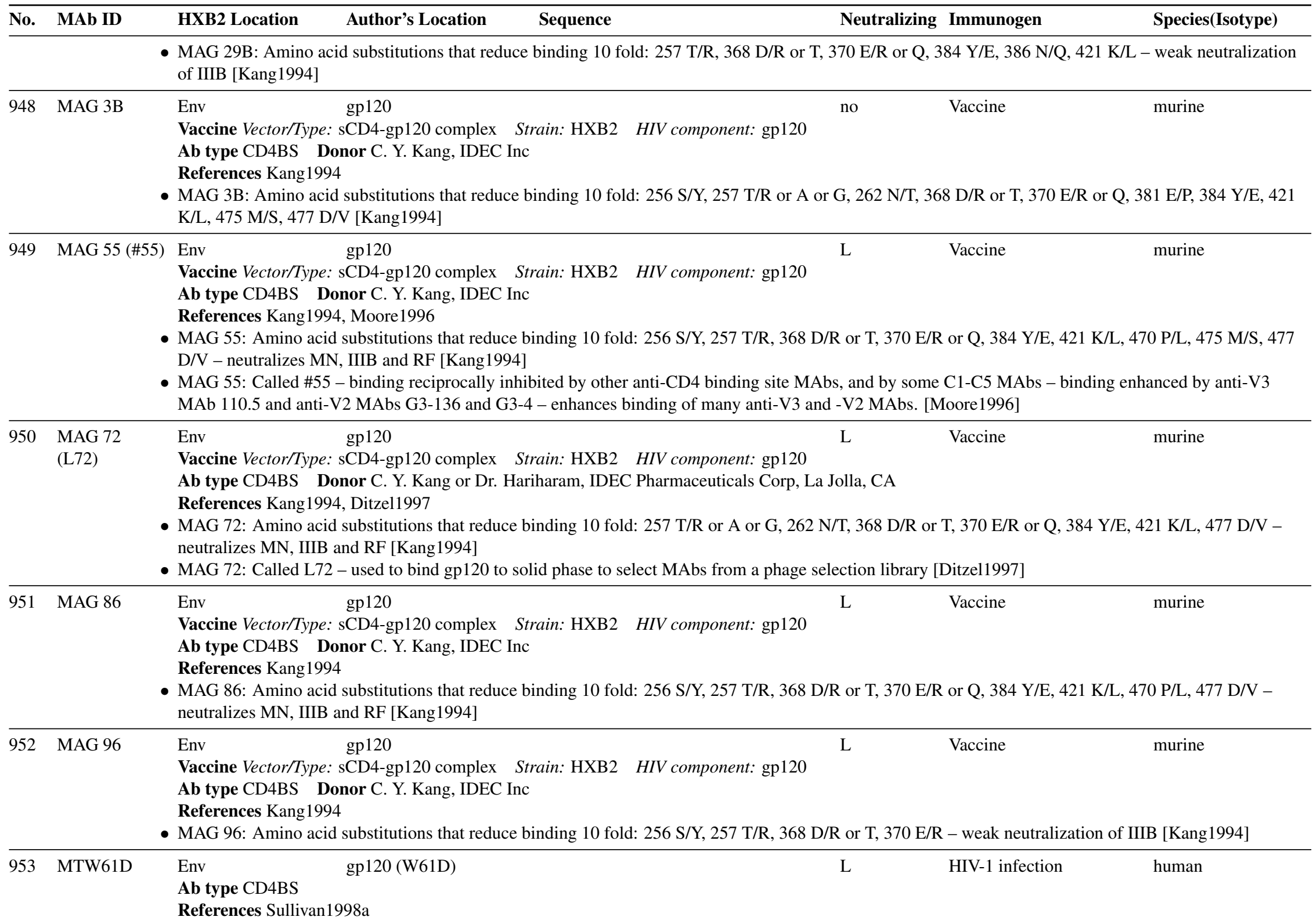




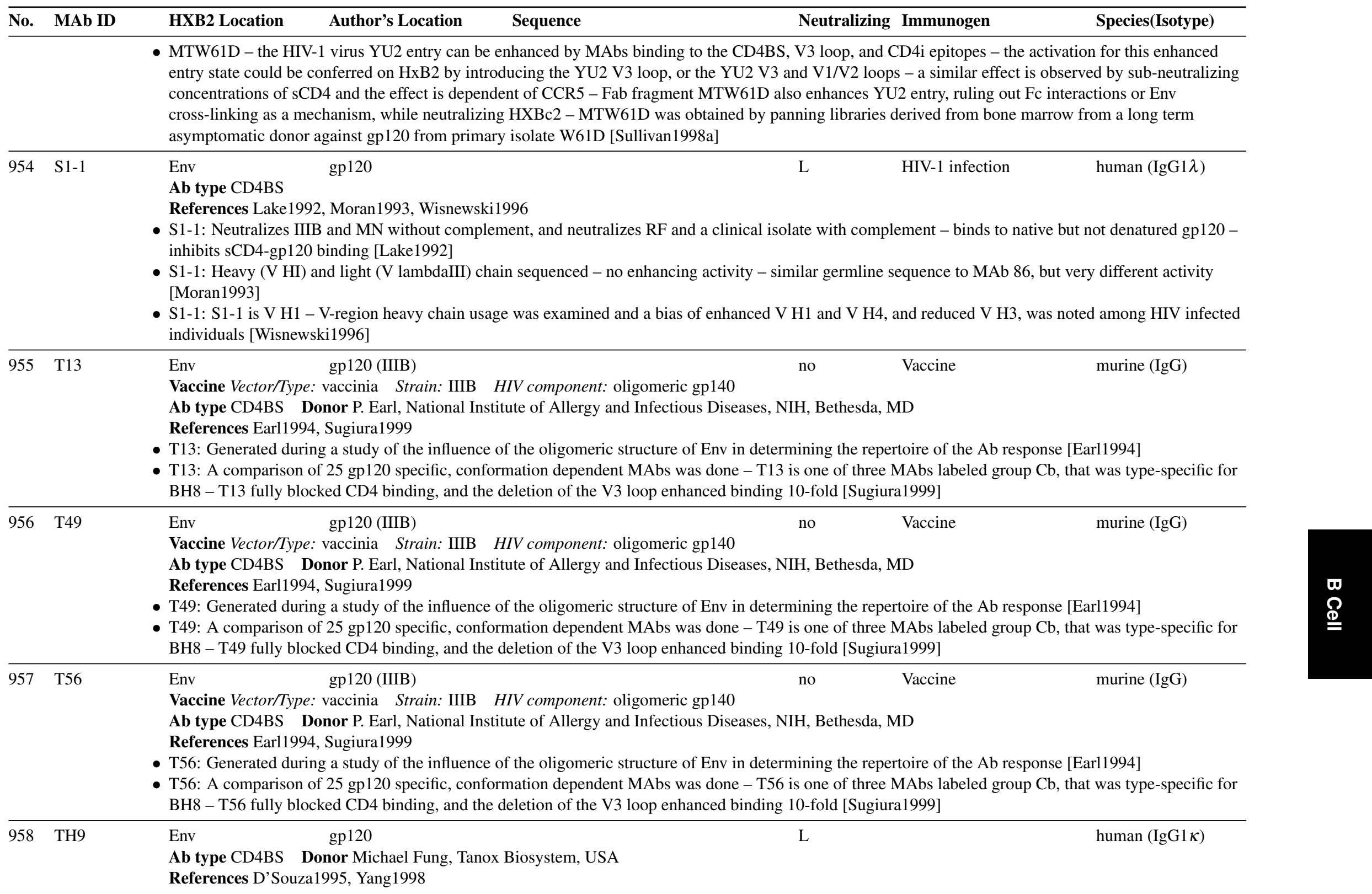




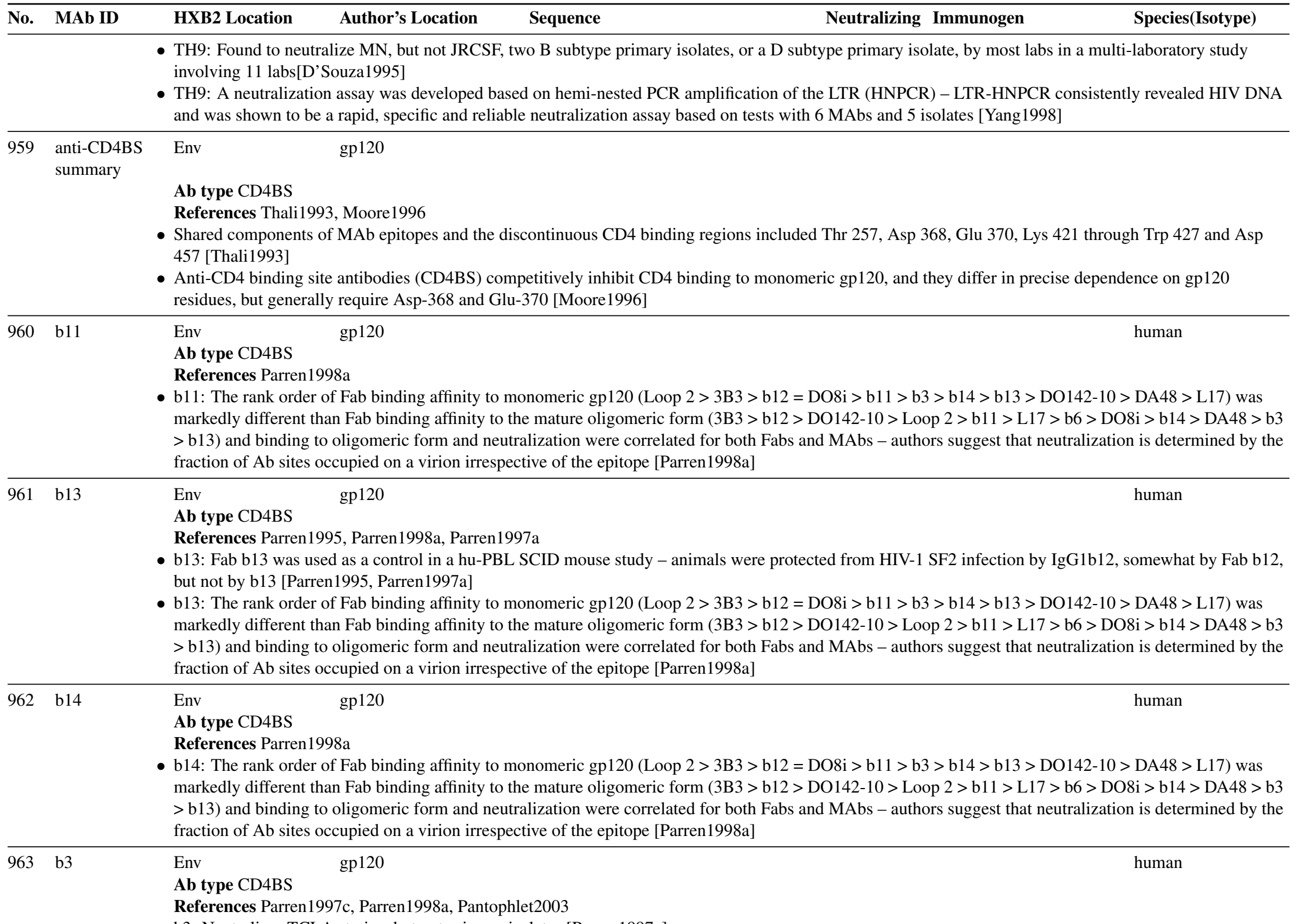

- b3: Neutralizes TCLA strains, but not primary isolates $\mid$ Parren1997c 


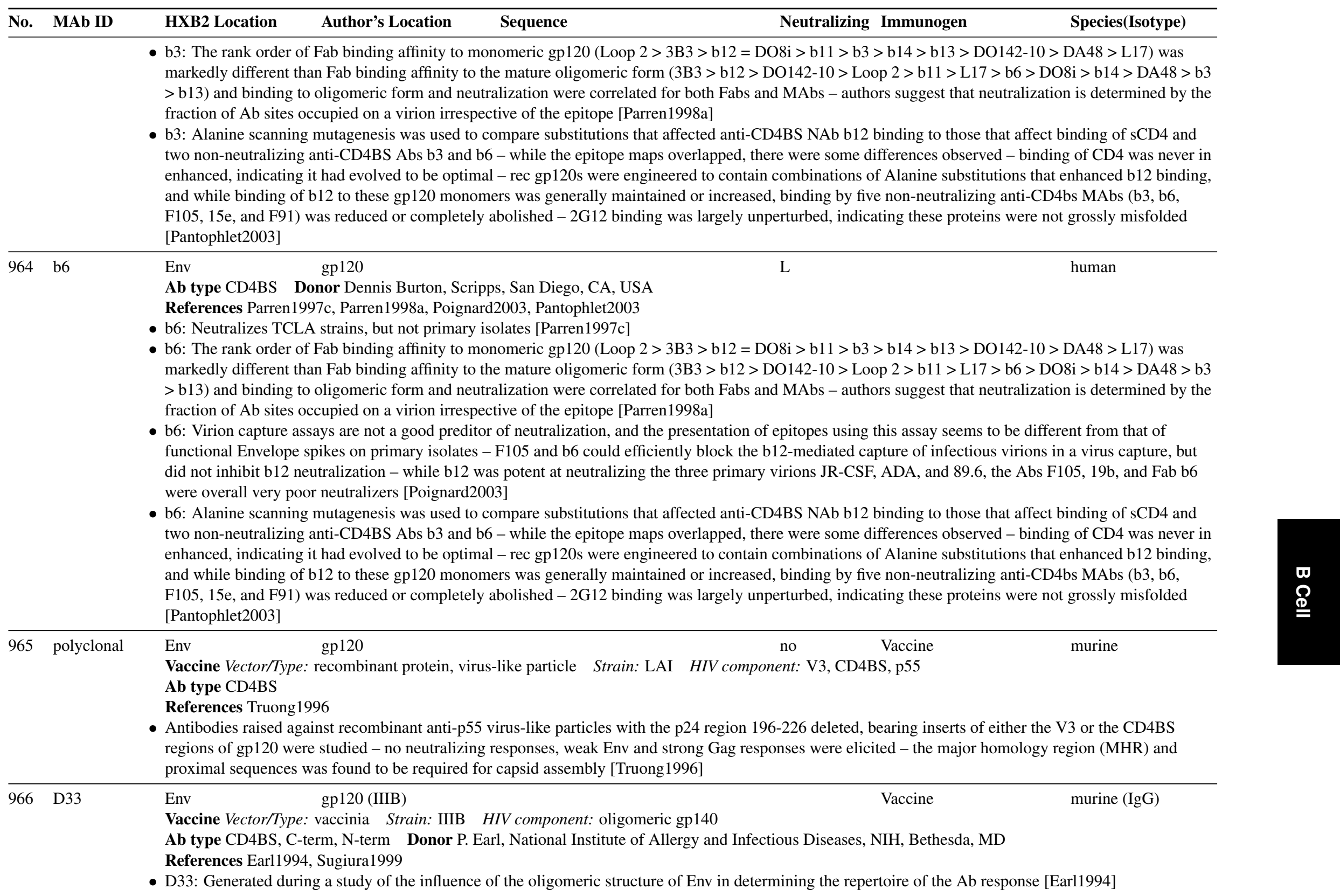




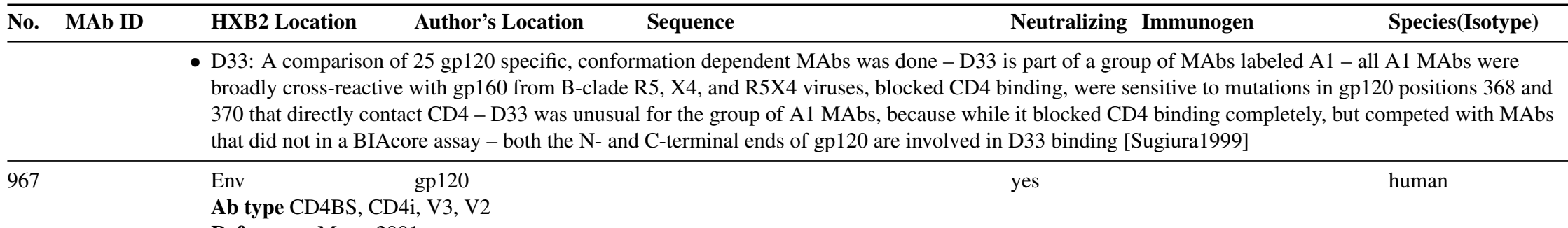

References Moore2001

- Moore and colleagues review structural aspects of gp120 and how they relate to antigenic domains, and review the data concerning the lack of a clear relationship between genetic subtype and serotype - they suggest the primary goal in vaccine efforts should be to design an immunogen that can be shown to elicit neutralizing antibodies against a significant proportion of primary isolates - assay artifacts that can result in confused interpretations are also discussed, such as Ab binding to defective spikes, which does not affect HIV-1 infectivity, but can dominant an assay signal [Moore2001] Env gp120

L P (weak) HIV-1 infection

human

Ab type CD4i Donor James Robinson, Tulane University, New Orleans, LA, USA

References

Thali1993, Moore1993d Thali1994, Beretta1994, Wyatt1995 Sattentau1995b, Moore1996 Poignard1996a Wu1996, Trkola1996a, Binley1997a,

Fouts1997, Li1997, Weinberg1997, Ditzel1997, Cao1997b, Wyatt1997, Parren1997c, Kwong1998, Wyatt1998a, Moore1998, Rizzuto1998, Sullivan1998b

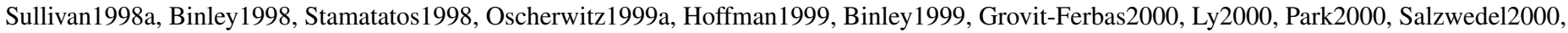

Stamatatos2000, Yang2000, Rizzuto2000, Si2001, Kolchinsky2001 York2001, Zhang2001a Poignard2001, Srivastava2002, Golding2002b, Schulke2002, Yang2002, Dowd2002, Xiang2002b, Xiang2002a, Edwards2002 Grundner2002, Basmaciogullari2002 Zhang2002, Arthos2002

- 17b: 48d and 17b have similar epitopes, and the pair are unique among human and rodent MAbs

- 17b: Epitope is better exposed upon CD4 binding to gp120 - competes with 15e and 21h, anti-CD4 binding site MAbs - 113 D/R, 252 R/W, 257 T/A or G, $370 \mathrm{E} / \mathrm{D}, 382 \mathrm{~F} / \mathrm{L}, 420 \mathrm{I} / \mathrm{R}, 433 \mathrm{~A} / \mathrm{L}, 438 \mathrm{P} / \mathrm{R}$ and $475 \mathrm{M} / \mathrm{S}$ confer decreased sensitivity to neutralization [Thali1993]

- 17b: Binding of 48d is much more influenced by sequence variation among molecular clones of LAI than is binding of 17b [Moore1993d

- 17b: A mutation in gp41, $582 \mathrm{~A} / \mathrm{T}$, confers resistance to neutralization (also confers resistance to MAbs F105, 48d, 21h and 15e) [Thali1994]

- 17b: Studies using a V1/V2 deletion mutant demonstrated that enhanced binding of 17b in the presence sCD4 involves the V1/V2 loops, with more significant involvement of V2 - similar effect observed for 48d and A32 [Wyatt1995]

- 17b: Binds with higher affinity to monomer and oligomer, slow association rate, poor neutralization of lab strain - this is in contrast to 48d, which has very different kinetics [Sattentau1995b]

- 17b: Many MAbs inhibit binding (anti-C1, -C5, -C4, -CD4BS) - anti-V3 MAb 5G11 enhances binding, as do C1-C4 discontinuous epitopes A32 and 2/11c - enhances binding of some anti-V2 MAbs [Moore1996]

- 17b: Binding did not result in significant gp120 dissociation from virion, in contrast to 48d, although the gp41 epitope of MAb 50-69 was exposed Poignard1996a

- 17b: MIP-1 $\alpha$ binding to CCR-5 expressing cells can be inhibited by gp120-sCD4 - binding of 17b blocks this inhibition [Wu1996].

- 17b: Neutralizes JR-FL - inhibits gp120 interaction with CCR-5 in a MIP-1beta-CCR-5 competition study [Trkola1996a]

- 17b: Study shows neutralization is not predicted by MAb binding to JRFL monomeric gp120, but is associated with oligomeric Env binding - 17b bound monomer, oligomer, and neutralized JRFL in the presence of sCD4, but if sCD4 was not present, 17b only bound monomer [Fouts1997]

- 17b: One of 14 human MAbs tested for ability to neutralize a chimeric SHIV-vpu+, which expressed HIV-1 IIIB env - 17b has synergistic response in combination with anti-V3 MAb 694/98-D [Li1997]

- 17b: $48 \mathrm{~d}$ binds to the IIIB protein and not IIIB V3 peptide, while binding to the Can0A V3 peptide, suggesting Can0A V3 is a conformer that mimics the $48 \mathrm{~d}$ - it does not bind to 17b, distinguishing the epitopes [Weinberg1997] 


No. MAb ID

- 17b: Virus with the V1-V2 loop deleted was viable and more susceptible to neutralization by CD4i MAb 17b, and anti-V3 MAbs 1121, 9284, and 110.4, but not to a CD4BS MAb, F105, or sCD4 [Cao1997b]

- 17b: Binds to sgp120 efficiently, but not soluble gp120+gp41, suggesting its gp120 epitope is blocked by gp41 binding - partial re-exposure if sCD4 was bound - could not bind to HXBc2 gp120 if the 19 C-term amino acids were deleted in conjunction with amino acids 31-93 in C1, but binding was restored in the presence of sCD4 |Wyatt1997|

- 17b: Neutralizes TCLA strains, but not primary isolates [Parren1997c]

- 17b: 17b Fab was co-crystallized with a gp120 core and CD4, and it's binding site can be directly visualized-17b binds to the "bridging sheet" of gp120, an antiparallel beta sheet region, contacting residues from the $\mathrm{C} 4$ region and the V1/V2 stem-the contact area is small for an Ab-antigen interactive surface, and dominated in the Ab by the heavy chain-the center of the binding region has hydrophobic interactions, and the periphery charge interactions, acidic on $17 \mathrm{~b}$ and basic on gp120 [Kwong1998]

- 17b: Summary of the implications of the crystal structure of a gp120 core bound to CD4 and 17b, combined with what is known about mutations that reduce NAb binding to gp 120 - probable mechanism of neutralization is interference with chemokine receptor binding - mutations in $88 \mathrm{~N}, 117 \mathrm{~K}, 121 \mathrm{~K}$, 256S, 257T, N262, Delta V3, E370, E381, F 382, R 419, I 420, K 421, Q 422, I 423, W 427, Y 435, P 438, M 475 of HXBc2 (IIIB) reduce binding - the only variable residues in gp120 that contact $17 \mathrm{~b}$ are $202 \mathrm{~T}$ and $434 \mathrm{M}$ - the contact points for $17 \mathrm{~b}$ with the crystallized incomplete gp 120 are mostly in the heavy chain of the Ab, and there is a gap between 17b's light chain and the partial gp120 which may be occupied by the V3 loop in a complete gp120 molecule - the authors propose that the V2 and V3 loops may mask the CD4i Ab binding site, and that the V2 loop may be repositioned upon CD4 binding [Wyatt1998a]

- 17b: Moore and Binley provide a commentary on the papers by [Rizzuto1998], [Wyatt1998a] and [Kwong1998] - they point out 17b shares binding elements in gp120 with chemokine receptor molecules, and that CD4 needs to bind to gp120 first to make the 17b epitope accessible and it may be sterically blocked in the CD4 bound virus, thus making it a poor NAb for primary isolates [Moore1998]

- 17b: Site directed mutagenesis of a WU2 protein with the V1-V2 loops deleted revealed key residues for 17b-gp120 interaction and interaction of gp120 and CCR5 - mutations in residues that reduced $17 \mathrm{~b}$ by $70 \%$ were R/D 419, I/R 420, Q/L 422, Y/S 435, I/S 423, K/D 121 and K/D 421- 17b can neutralize HIV-1 strains that use different chemokine receptors, supporting a common region in gp120 in chemokine-receptor interaction [Rizzuto1998]

- 17b: sCD4 induces 17b binding in primary isolates and TCLA strains - amino acids that reduce the efficiency of binding were determined and found also to compromise syncytia formation and viral entry - V1V2 deletion or sCD4 binding can expose the 17b epitope for both HXBc2 and macrophage tropic YU2 - neutralizing potency of $17 \mathrm{~b}$ is probably weak due to poor exposure of the epitope - 17b epitope exposure upon sCD4 binding can occur over a wide range of temperatures, consistent with the energy of CD4 binding being sufficient to drive the V1/V2 loop into a new conformation [Sullivan1998b]

- 17b: The HIV-1 virus YU2 entry can be enhanced by MAbs binding to the CD4BS, V3 loop, and CD4i epitopes - the activation for this enhanced entry state could be conferred on HxB2 by introducing the YU2 V3 loop, or the YU2 V3 and V1/V2 loops, and the presence of V1/V2 increased the enhancement - a similar effect is observed by sub-neutralizing concentrations of sCD4 and the effect is dependent of CCR5 - 17b enhances YU2 enhanced viral entry 10-fold, whereas HXBc2 was neutralized [Sullivan1998a]

- 17b: A panel of MAbs was shown to bind with similar or greater affinity and similar competition profiles to a deglycosylated or variable loop deleted core gp120 protein ( Delta V1, V2, and V3), thus such a core protein produces a structure closely approximating full length folded monomer - CD4i MAbs 17b and $48 \mathrm{~d}$ bound better to the deleted protein than to wild type [Binley1998]

- 17b: Deleting the V2 loop of neutralization-resistant HIV-1 isolate SF162 does not abrogate its replication in PBMC or macrophages, but it enhances its neutralization sensitivity to sera from patients with B clade infection up to 170-fold, and also enhances sensitivity to sera from clades A through F - deletion of V2 but not V1 enabled neutralization by CD4i MAbs 17b and 48d [Stamatatos1998]

- 17b: A CD4-independent viral variant of IIIB, IIIBx, was generated on CXCR4-expressing cells - IIIBx exhibited greater exposure of the 17b and 48d epitopes and enhanced neutralization by CD4i MAbs and by polyclonal human sera - the 17b epitope has significant overlap with the CCR5 coreceptor binding site [Hoffman1999] 


No. MAb ID HXB2 Location Author's Location $\quad$ Sequence $\quad$ Neutralizing Immunogen

- 17b: The MAbs with the broadest neutralizing activity, IgG1b12, 2G12 and 2F5, all have high affinity for the native trimer, indicating that they were raised in an immune response to the oligomer on the virion surface rather than dissociated subunits - a disulfide linked gp120-gp41 (SOS gp140) was created to mimic the native conformation of Env and explore its potential as an immunogen - SOS gp140 is recognized by NAbs IgG1b12, 2G12, and CD4-IgG2, and also by anti-V3 MAbs 19b and 83.1 - SOSgp140 is not recognized by C4 region MAbs that neutralize only TCLA strains, G3-42 and G3-519 - nor did it bind C11, 23A, and M90, MAbs that bind to gp120 C1 and C5, where it interacts with gp41 - MAbs that bind CD4 inducible epitopes, 17b and A32 were very strongly induced by CD4 in SOS gp140 - anti-gp41 MAbs that bind in the region that interacts with gp120, 7B2, 2.2B, T4, T15G1 and 4D4, did not bind to SOSgp140, in contrast to 2F5, which binds to the only gp41 epitope that is well exposed in native gp120-gp41 complexes [Binley1999]

- 17b: To determine the antigenicity of virus killed by thermal and chemical inactivation, retention of conformation-dependent neutralization epitopes was examined, and exposure of CD4BS epitopes was found to be enhanced (MAbs IgG1b12, 205-46-9, and 205-43-1) - binding to 2G12 and 447-52D epitopes was essentially unaltered - the $17 \mathrm{~b}$ CD4i epitope was also exposed [Grovit-Ferbas2000]

- 17b: SF162 is a neutralization-resistant HIV-1 isolate - N-linked glycosylation modifications in the V2 loop of the SF162 gp120 revealed that these sites prevent neutralization by CD4BS MAbs (IgG1b12 and IgGCD4), and protect against neutralization by V3 MAbs (447-D and 391-95D) - V2-region glycosylation site mutations did not alter neutralization resistance to V2 MAbs (G3.4 and G3.136) or CD4i MAbs (17b and 48d) - V2 glycosylation site modification allows infection of macrophages, probably due to glycosylated forms requiring fewer CCR5 molecules for viral entry [Ly2000]

- 17b: Six mutations in MN change the virus from a high-infectivity neutralization resistant phenotype to low-infectivity neutralization sensitive - V3, CD4BS, and CD4i MAbs are 20-100 fold more efficient at neutralizing the sensitive form - the mutation L544P reduced binding of all MAbs against gp120 by causing conformational changes [Park2000]

- 17b: sCD4 can activate fusion between effector cells expressing Env and target cells expressing coreceptor (CCR5 or CXCR4) alone without CD4 - CD4i MAbs 17b and 48d have little effect on a standard cell fusion assay but potently block sCD4 activated fusion - 17b was broadly cross-reactive inhibiting sCD4 activated fusion with Env from clades A, B, C, D, E, F, and F/B [Salzwedel2000]

- 17b: Soluble gp140 derived from SF162, a neutralization-resistant primary isolate, and SF162AV2 a neutralization-susceptible isolate with 30 amino acids deleted from the V2 loop, were generated with or without the gp120-gp41 cleavage site intact - all forms are recognized by oligomer-specific MAb T4 and show enhanced binding of CD4i MAb 17b when SCD4 is bound - the fused forms are less efficiently recognized than the cleaved forms by polyclonal neutralizing sera from HIV-infected patients - the V3 loop is more exposed on the fused form [Stamatatos2000]

- 17b: A combination of gp41 fusion with the GNC4 trimeric sequences and disruption of the YU2 gp120-gp41 cleavage site resulted in stable gp140 trimers (gp140-GNC4) that preserve and expose some neutralizing epitopes while occluding some non-neutralizing epitopes - CD4BS MAbs (F105 and F91) and CD4i (17b and 48d) recognized gp140-GNC4 as well as gp120 or gp140 - non-neutralizing MAbs C11, A32, 522-149, M90, and \#45 bound to the gp140-GNC4 glycoprotein at reduced levels compared to gp120 - MAbs directed at the extreme termini of gp120 C1 (135/9 and 133/290) and C5 (CRA-1 and M91) bound efficiently to gp140-GNC4 [Yang2000]

- 17b: Mutagenesis defines Ile-420, Lys-421, Gln-422, Pro-438, and Gly-441 to be important residues for CCR5 binding - these positions are located on two strands that connect the gp120 bridging sheet and outer domain, suggesting a mechanism for conformational shifts induced by CD4 binding to facilitate CCR5 binding [Rizzuto2000]

- SHIV-HXBc2 is a neutralization sensitive non-pathogenic virus, and several in vivo passages through monkey's yielded highly pathogenic SHIV KU-1 HXBc2 and the KU-1 clone HXBc2P3.2 differ in 12 amino acids in gp160 - substitutions in both gp120 and gp41 reduced the ability of sCD4, IgG1b12, F105 and AG1121 to Env achieve saturation and full occupancy, and neutralize KU-1 - 17b and 2F5 also bound less efficiently to HXBc2P3.2, although 2G12 was able to bind both comparably [Si2001]

- 17b: Mutations in two glycosylation sites in the V2 region of HIV-1 ADA at positions 190 and 197 (187 DNTSYRLINCNTS 199) cause the virus to become CD4-independent and able to enter cells through CCR5 alone-these same mutations tended to increase the neutralization sensitivity of the virus, including to 17b—only the CD4i antibodies 17b and 48d showed an increased affinity of the CD4 independent viruses relative to wild-type [Kolchinsky2001]. 


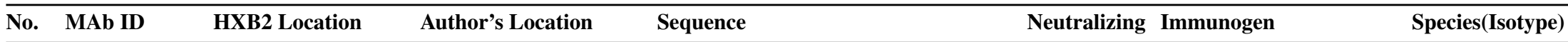

- 17b: Abs against the V3 loop (50.1, 58.2, 59.1, 257-D, 268-D, 447-52D), CD4BS (IgG1b12, 559-64D, F105), CD4i (17b), and to gp41 (2F5, F240) each showed similar binding efficiency to Env derived from related pairs of primary and TCLA lines (primary: 168P and 320SI, and TCLA: 168C and 320SI-C3.3), but the TCLA lines were much more susceptible to neutralization suggesting that the change in TCLA lines that make them more susceptible to NAbs alters some step after binding $-17 \mathrm{~b}$ bound at somewhat greater levels to $168 \mathrm{C}$ than to $168 \mathrm{P}$, but this is not a general feature of $17 \mathrm{~b}$ binding to primary versus TCLA strains [York2001]

- 17b: 17b binds to a CD4 inducible epitope which partially overlaps the CCR5 binding site - JRFL, YU2, 89.6, and HXB2 and their C1-, V1/V2-, C5 -deletion mutants were used to study how 17b binding affects gp120-CD4 interactions - 17b reduced CD4-gp120 interactions by decreasing the on-rate and increasing the off-rate of sCD4, while enhanced binding of sCD4 binding was observed for the 17b-bound, V1/V2 deleted gp120s - 17b was considered to be a surrogate for CCR5, and the authors suggest that $17 \mathrm{~b}$ binding may shift V1/V2 into a position that interferes with CD4 binding, forcing a release |Zhang2001a|

- 17b: Structural aspects of the interaction of neutralizing Abs with HIV-1 Env are reviewed - Env essentially has three faces, one is largely inaccessible on the native trimer, and two that exposed but have low immunogenicity on primary viruses - neutralization is suggested to occur by inhibition of the interaction between gp120 and the target cell membrane receptors as a result of steric hindrance and it is noted that the attachment of approximately 70 IgG molecules per virion is required for neutralization, which is equivalent to about one IgG molecule per spike - the 2G12, 17b and b12 epitopes are discussed in detail - the 17b epitope is masked prior to CD4 binding by the V1-V2 loop and in contrast to sCD4, the binding of cell surface CD4 to virus does not appear to make the epitope accessible to binding by $17 \mathrm{~b}$ to allow neutralization [Poignard2001]

- 17b: Oligomeric gp140 (o-gp140) derived from R5 primary isolate US4 was characterized for use as a vaccine reagent - antigen capture ELISA was used to compare the antigenicity of gp120 and o-gp140 using a panel of well characterized MAbs - 17b recognized both gp120 monomer and o-gp140 [Srivastava2002

- 17b: The fusion process was slowed by using a suboptimal temperature (31.5 C) to re-evaluate the potential of Abs targeting fusion intermediates to block HIV entry - preincubation of E/T cells at $31.5 \mathrm{C}$ enabled polyclonal anti-N-HR Ab and anti-six-helix bundle Abs to inhibit fusion, indicating six-helix bundles form prior to fusion - the preincubation $31.5 \mathrm{C}$ step did not alter the inhibitory activity of neutralizing Abs anti-gp41 2F5, or anti-gp120 2G12, IG1b12, 48d, and 17b [Golding2002b]

- 17b: Ab binding characteristics of SOS gp140 were tested using SPR and RIPA - SOS gp140 is gp120-gp41 bound by a disulfide bond - NAbs 2G12, 2F5, IgG1b12, CD4 inducible 17b, and 19b bound to SOS gp140 better than uncleaved gp140 (gp140unc) and gp120 - non-neutralizing MAbs 2.2B (binds to gp41 in gp140unc) and 23A (binds gp120) did not bind SOS gp140 [Schulke2002]

- 17b: Uncleaved soluble gp140 (YU2 strain, R5 primary isolate) can be stabilized in an oligomer by fusion with a C-term trimeric GCN4 motif or using a T4 trimeric motif derived from T4 bacteriophage fibritin—stabilized oligomer gp140 683 (-FT) showed strong preferential recognition by NAbs IgG1b12 and 2 G12 relative to the gp120 monomer, in contrast to poorly neutralizing MAbs F105, F91, 17b, 48d, and 39F which showed reduced levels of binding, and C11, A32, and 30D which did not bind the stabilized oligomer [Yang2002].

- 17b: CD4 residue Phe43 significantly contributes to the affinity of CD4-gp120 interactions - despite decreased affinities for gp120, CD4 proteins and CD4-mimetic peptides lacking a Phe side-chain enhance binding of gp120 to $17 \mathrm{~b}$ in a manner similar to Phe-bearing ligands indicating the Phe42 interaction is not critical for CD4-induced conformational changes in gp120 [Dowd2002]

- 17b: A series of mutational changes were introduced into the YU2 gp120 that favored different conformations - $375 \mathrm{~S} / \mathrm{W}$ seems to favor a conformation of gp120 closer to the CD4-bound state, and is readily bound by sCD4 and CD4i MAbs (17b, 48d, 49e, 21c and 23e) but binding of anti-CD4BS MAbs (F105, 15e, IgG1b12, 21h and F91 was markedly reduced - IgG1b12 failed to neutralize this mutant, while neutralization by $2 \mathrm{G} 12$ was enhanced - 2F5 did not neutralize either WT or mutant, probably due to polymorphism in the YU2 epitope - another mutant, 423 I/P, disrupted the gp120 bridging sheet, favored a different conformation and did not bind CD4, CCR5, or CD4i antibodies, but did bind to CD4BS MAbs [Xiang2002b] 


\begin{tabular}{lllll}
\hline No. MAb ID & HXB2 Location & Author's Location & Sequence & Neutralizing Immunogen
\end{tabular}

- 17b: Five CD4i MAbs were studied, 17b, 48d and three new MAbs derived by Epstein-Barr virus transformation of PBMC from an HIV+ long term non-progressor - 23e and 21c were converted to hybridomas to increase Ab production - all compete with the well-characterized 17b CD4i MAb in an ELISA antigen capture assay - critical binding residues are mapped and the CD4i MAb epitopes were distinct but share a common element near isoleucine 420, also important for CCR5 binding, and all five can block CCR5 binding to a sCD4-gp120 complex - the MAb 48d has the epitope most similar to the CCR5 binding site [Xiang2002a]

- 17b: Truncation of the gp41 cytoplasmic domain of X4, R5, and X4R5 viruses forces a conformation that more closely resembles the CD4 bound state of the external Envelope, enhancing binding of CD4i MAbs 17b and 48d and of CD4BS MAbs F105, b12, and in most cases of glycosylation site dependent MAb 2G12 and the anti-gp41 MAb 246D - in contrast, binding of the anti-V2 MAb 697D and the anti-V3 MAb 694/98D were not affected - viruses bearing the truncation were more sensitive to neutralization by MAbs 48d, b12, and 2G12 - the anti-C5 MAb 1331A was used to track levels of cell surface expression of the mutated proteins [Edwards2002]

- 17b: HIV-1 gp160 $\mathrm{CT}$ (cytoplasmic tail-deleted) proteoliposomes (PLs) containing native, trimeric envelope glycoproteins from R5 strains YU2 and JRFL, and $\mathrm{X} 4$ strain $\mathrm{HXBc} 2$, were made in a physiologic membrane setting as candidate immunogens for HIV vaccines-2F5 bound to gp160 $\Delta \mathrm{CT}$ with a reconstituted membrane ten-fold better than the same protein on beads-anti-CD4BS MAbs IgG1b12 and F105, A32 (C1-C4), C11 (C1-C5), and 39F (V3) MAbs bound gp160 $\Delta$ CT PLs indistinguishably from gp160 $\Delta$ CT expressed on the cell surface-non-neutralizing MAbs C11 and A32 bound with lower affinity than NAb IgG1b12 - the MAb 17b was sCD4 inducible on gp160 $\Delta$ CT PL [Grundner2002].

- 17b: gp120 mutants were used to define the CXCR4 binding site using CXCR4 displayed on paramagnetic proteoliposomes (PMPLs) to reduce non-specific gp120 binding — basic residues in the V3 loop and the $\beta 19$ strand (RIKQ, positions 419-422) were involved, and deletion of the V1-V2 loops allowed CD4-independent CXCR4 binding-MAbs 17b (CD4i) and F105 (CD4BS) were used to study conformational changes in the mutants-the affinity of $\Delta \mathrm{V} 1$ and $\Delta \mathrm{V} 1-\mathrm{V} 2$ for $17 \mathrm{~b}$ was dramatically increased and no longer inducible in the presence of sCD4-V3 mutants R298A and R327A were not recognized by $17 \mathrm{~b}$ except in the presence of sCD4-mutations in the $\beta 19$ strand dramatically reduced $17 \mathrm{~b}$ affinity in the presence or absence of sCD4, consistent with known $17 \mathrm{~b}$ contact residues in this region [Basmaciogullari2002].

- 17b: A rare mutation in the neutralization sensitive R2-strain in the proximal limb of the V3 region caused Env to become sensitive to neutralization by MAbs directed against the CD4 binding site (CD4BS), CD4-induced (CD4i) epitopes, soluble CD4 (sCD4), and HNS2, a broadly neutralizing sera - 2/12 anti-V3 MAbs tested (19b and 694/98-D) neutralized R2, as did 2/3 anti-CD4BS MAbs (15e and IgG1b12), 2/2 CD4i MAbs (17b and 4.8D), and 2G12 and $2 \mathrm{~F} 5$ - thus multiple epitopes on R2 are functional targets for neutralization and the neutralization sensitivity profile of R2 is intermediate between the highly sensitive MN-TCLA strain and the typically resistant MN-primary strain [Zhang2002]

- 17b: The two N-terminal domains of CD4, termed D1 and D2, when expressed in the absence of the remaining domains of CD4 retain the capacity to bind to gp120 — coding sequences of D1D2 and Ig $\alpha$ tp were fused to create a large, multivalent rec protein D1D2Ig $\alpha$ tp, which, unlike CD4, does not enhance infection at sub-optimal concentrations-the MAb 17b can also enhance viral replication at sub-optimal concentrations, but D1D2-Ig $\alpha$ inhibited the 17b enhancement of two primary isolates [Arthos2002].

- 17b: NIH AIDS Research and Reference Reagent Program: 4091

Ab type CD4i Donor James Robinson, Tulane University, New Orleans, LA, USA

L

HIV-1 infection

human $(\operatorname{IgG})$

References Xiang2002a Xiang2002b

- 21c: Five CD4i MAbs were studied, 17b, 48d and three new MAbs derived by Epstein-Barr virus transformation of PBMC from an HIV+ long term non-progressor - 23e and 21c were converted to hybridomas to increase Ab production - all compete with the well-characterized 17b CD4i MAb in an ELISA antigen capture assay - critical binding residues are mapped and the CD4i MAb epitopes were distinct but share a common element near isoleucine 420, also important for CCR5 binding, and all five can block CCR5 binding to a sCD4-gp120 complex - the MAb 48d has the epitope most similar to the CCR5 binding site [Xiang2002a] 


\begin{tabular}{lllll}
\hline No. MAb ID & HXB2 Location & Author's Location & Sequence & Neutralizing Immunogen
\end{tabular}

- 21c: A series of mutational changes were introduced into the YU2 gp120 that favored different conformations - $375 \mathrm{~S} / \mathrm{W}$ seems to favor a conformation of gp120 closer to the CD4-bound state, and is readily bound by sCD4 and CD4i MAbs (17b, 48d, 49e, 21c and 23e) but binding of anti-CD4BS MAbs (F105, 15e, IgG1b12, 21h and F91 was markedly reduced - IgG1b12 failed to neutralize this mutant, while neutralization by 2 G12 was enhanced - 2F5 did not neutralize either WT or mutant, probably due to polymorphism in the YU2 epitope - another mutant, $423 \mathrm{I} / \mathrm{P}$, disrupted the gp120 bridging sheet, favored a different conformation and did not bind CD4, CCR5, or CD4i antibodies, but did bind to CD4BS MAbs [Xiang2002b]

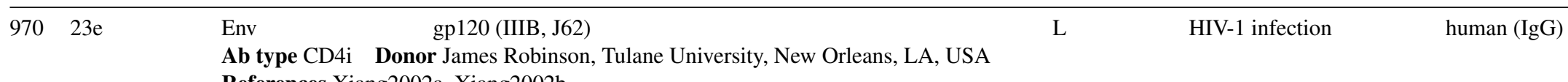
References Xiang2002a Xiang2002b

- 23e: Five CD4i MAbs were studied, 17b, 48d and three new MAbs derived by Epstein-Barr virus transformation of PBMC from an HIV+ long term non-progressor - 23e and 21c were converted to hybridomas to increase Ab production - all compete with the well-characterized 17b CD4i MAb in an ELISA antigen capture assay - critical binding residues are mapped and the CD4i MAb epitopes were distinct but share a common element near isoleucine 420, also important for CCR5 binding, and all five can block CCR5 binding to a sCD4-gp120 complex - the MAb 48d has the epitope most similar to the CCR5 binding site [Xiang2002a]

- 23e: A series of mutational changes were introduced into the YU2 gp120 that favored different conformations - 375 S/W seems to favor a conformation of gp120 closer to the CD4-bound state, and is readily bound by sCD4 and CD4i MAbs (17b, 48d, 49e, 21c and 23e) but binding of anti-CD4BS MAbs (F105, $15 \mathrm{e}, \mathrm{IgG} 1 \mathrm{~b} 12,21 \mathrm{~h}$ and F91 was markedly reduced - IgG1b12 failed to neutralize this mutant, while neutralization by $2 \mathrm{G} 12$ was enhanced - 2F5 did not neutralize either WT or mutant, probably due to polymorphism in the YU2 epitope - another mutant, 423 I/P, disrupted the gp120 bridging sheet, favored a different conformation and did not bind CD4, CCR5, or CD4i antibodies, but did bind to CD4BS MAbs [Xiang2002b]

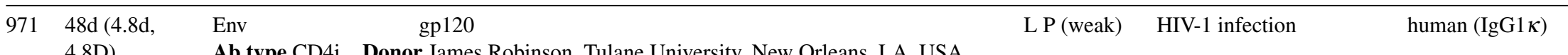

4.8D) Ab type CD4i Donor James Robinson, Tulane University, New Orleans, LA, USA

References Thali1993 Moore1993a, Moore1993d Thali1994, Moore1994b D'Souza1995, Sattentau1995a, Wyatt1995 |Sattentau1995b | Moore1996 Poignard1996a Trkola1996a, Binley1997a, Li1997, Weinberg 1997, Lee1997, Ugolini1997, Wyatt1997, Parren1997c. Frankel1998, Wyatt1998a Mondor1998 |Parren1998a, Sullivan1998b, Yang1998. Binley1998, Stamatatos1998 Oscherwitz1999a, Hoffman1999, Fortin2000, Ly2000, Park2000 Yang2000, Salzwedel2000 Kolchinsky2001, Verrier2001 Golding2002b, Yang2002, Xiang2002b, Xiang2002a Edwards2002, Zhang2002

- 48d: 48d and 17b have similar epitopes, and the pair are unique among human and rodent MAbs

- 48d: Epitope is better exposed upon CD4 binding to gp120 - competes with ICR 39.13, 15e and 21h, anti-CD4 binding site MAbs - inhibited by anti-CD4BS MAb ICR 39.13g and linear anti-C4 MAbs G3-42 and G3-508 - 113 D/R, 252 R/W, 257 T/A or G, 370 E/D, 382 F/L, 420 I/R, 421 K/L, 433A/L, $438 \mathrm{P} / \mathrm{R}$ and $475 \mathrm{M} / \mathrm{S}$ confer decreased sensitivity to neutralization [Thali1993]

- 48d: Called 4.8d - Neutralizes IIIB - reactive with SF-2 gp120 - does not inhibit HIV-1 sera from binding to IIIB gp120 [Moore1993a]

- 48d: Binding of 48d is much more influenced by sequence variation among molecular clones of LAI than is binding of 17b [Moore1993d

- 48d: A mutation in gp41, $582 \mathrm{~A} / \mathrm{T}$, confers resistance to neutralization (also confers resistance to MAbs F105, 21h, 15e and 17b) [Thali1994]

- 48d: Poor cross-reactivity with gp120 from most clades [Moore1994b]

- 48d: Called 4.8D - Found to neutralize MN, but not JRCSF, two B subtype primary isolates, or a D subtype primary isolate, by most labs in a multi-laboratory study involving 11 labs[D'Souza1995]

- 48d: Studies using a V1/V2 deletion mutant demonstrated that enhanced binding of 48d in the presence of sCD4 involves the V1/V2 loops, with more significant involvement of V2 - similar effect observed for 17b and A32 [Wyatt1995

- 48d: Formalin inactivation of virus at $0.1 \%$ formalin for 10 hours at 4 degrees was optimal for inactivation of virus while maintaining epitope integrity [Sattentau1995c]

- 48d: Binds with similar affinity to monomer and oligomer, moderate association rate, potent neutralization - this is in contrast to $17 \mathrm{~b}$, which has very different kinetics [Sattentau1995b] 


No. MAb ID HXB2 Location Author's Location $\quad$ Sequence $\quad$ Neutralizing Immunogen

- 48d: Many MAbs inhibit binding (anti-C1, -C5, -C4, -CD4BS) - anti-C1-C4 discontinuous epitope MAbs A32 and 2/11c enhance binding - reciprocal enhanced binding with some anti-V2 MAbs [Moore1996]

- 48d: Binding resulted in gp120 dissociation from virion, mimicking sCD4, and exposure of the gp41 epitope of MAb 50-69, in contrast to CD4BS MAbs [Poignard1996a]

- 48d: Neutralizes JR-FL - slightly inhibits gp120 interaction with CCR-5 in a MIP-1beta-CCR-5 competition study [Trkola1996a]

- 48d: One of 14 human MAbs tested for ability to neutralize a chimeric SHIV-vpu+, which expressed HIV-1 IIIB env - all Ab combinations tested showed synergistic neutralization - 48d has synergistic response with MAbs 694/98-D (anti-V3) and F105 [Li1997]

- 48d: 48d binds to the IIIB protein and not IIIB V3 peptide, while binding to the Can0A V3 peptide, suggesting Can0A V3 is a conformer that mimics the 48d, (but not 17b), epitope [Weinberg1997]

- 48d: Prefers CD4-gp120 complex to gp120 alone, but does not enhance fusion, in contrast to MAb CG10, in fact it inhibits syncytium formation [Lee1997

- 48d: Viral binding inhibition by 48d was strongly correlated with neutralization (all other neutralizing MAbs tested showed some correlation except 2F5) |Ugolini1997]

- 48d: Binds efficiently to sgp120 but not soluble gp120+gp41, suggesting its gp120 epitope is blocked by gp41 binding [Wyatt1997]

- 48d: Neutralizes TCLA strains, but not primary isolates [Parren1997c]

- 48d: Summary of the implications of the crystal structure of the core of gp120 bound to CD4 and 17b with what is known about mutations that reduce NAb binding - probable mechanism of neutralization of 48d is interference with chemokine receptor binding - CD4 binding increases exposure of epitope due to V2 loop movement - 88N, 117K, 121K, 256S, 257T, N262, delta V3, E370, E381, F 382, R 419, I 420, K 421, Q 422, I 423, W 427, Y 435, P 438, M 475 mutations in HXBc2 (IIIB) decrease binding [Wyatt1998a]

- 48d: Inhibits binding of Hx10 to both CD4 positive and CD4 negative HeLa cells [Mondor1998]

- 48d: The MAb and Fab binding to the oligomeric form of gp120 and neutralization were highly correlated - authors suggest that neutralization is determined by the fraction of Ab sites occupied on a virion irrespective of the epitope [Parren1998a]

- 48d: CD4i MAbs 17b and 48d compete with MAb CG10, and the binding sites may overlap - MAb A32 enhances binding of 17b, 48d and CG10 [Sullivan1998b]

- 48d: A neutralization assay was developed based on hemi-nested PCR amplification of the LTR (HNPCR) - LTR-HNPCR consistently revealed HIV DNA and was shown to be a rapid, specific and reliable neutralization assay based on tests with 6 MAbs and 5 isolates [Yang1998]

- 48d: A panel of MAbs were shown to bind with similar or greater affinity and similar competition profiles to a deglycosylated or variable loop deleted core gp120 protein ( Delta V1, V2, and V3), thus such a core protein produces a structure closely approximating full length folded monomer - CD4i MAbs 17b and $48 \mathrm{~d}$ bound better to the deleted protein than to wild type [Binley1998]

- 48d: Deleting the V2 loop of neutralization-resistant HIV-1 isolate SF162 does not abrogate its replication in PBMC or macrophages, but it enhances its neutralization sensitivity to sera from patients with B clade infection up to 170-fold, and also enhances sensitivity to sera from clades A through $\mathrm{F}$ - deletion of V2 but not V1 enabled neutralization by CD4i MAbs 17b and 48d [Stamatatos1998]

- 48d: Infection of dendritic cells cultured from CD14+ blood cells or from cadaveric human skin was blocked by neutralizing MAbs IgG1b12, or 2F5 and $2 \mathrm{G} 12$ delivered together, but not by control non-neutralizing anti-gp120 MAb 4.8D, indicating that NAbs could interrupt early mucosal transmission events [Frankel1998

- 48d: A CD4-independent viral variant of IIIB, IIIBx, was generated on CXCR4-expressing cells - IIIBx exhibited greater exposure of the 17b and 48d epitopes and enhanced neutralization by CD4i MAbs and by polyclonal human sera [Hoffman1999]

- 48d: Called 4.8D - host encoded intercellular adhesion molecule (ICAM-1) is incorporated by the HIV-1 virion and enhances viral infectivity - ICAM-1 does not modify virus sensitivity to antibodies 0.5 beta or $4.8 \mathrm{D}$ or sCD4, but neutralizing ability of F105 was diminished in ICAM bearing virions in the presence of lymphocyte function-association antigen-1 (LFA-1) Ab [Fortin2000] 


No. MAb ID HXB2 Location Author's Location Sequence $\quad$ Neutralizing Immunogen

- 48d: SF162 is a neutralization-resistant HIV-1 isolate - N-linked glycosylation modifications in the V2 loop of the SF162 gp120 revealed that these sites prevent neutralization by CD4BS MAbs (IgG1b12 and IgGCD4), and protect against neutralization by V3 MAbs (447-D and 391-95D) - V2-region glycosylation site mutations did not alter neutralization resistance to V2 MAbs (G3.4 and G3.136) or CD4i MAbs (17b and 48d) - V2 glycosylation site modification allows infection of macrophages, probably due to glycosylated forms requiring fewer CCR5 molecules for viral entry [Ly2000]

- 48d: Six mutations in MN change the virus from a high-infectivity neutralization resistant phenotype to low-infectivity neutralization sensitive - V3, CD4BS, and CD4i MAbs are 20-100 fold more efficient at neutralizing the sensitive form - the mutation L544P reduced binding of all MAbs against gp120 by causing conformational changes [Park2000]

- 48d: SCD4 can activate fusion between effector cells expressing Env and target cells expressing coreceptor (CCR5 or CXCR4) alone without CD4 - CD4i MAbs 17b and 48d have little effect on a standard cell fusion assay but potently block SCD4 activated fusion [Salzwedel2000]

- 48d: A combination of gp41 fusion with the GNC4 trimeric sequences and disruption of the YU2 gp120-gp41 cleavage site resulted in stable gp140 trimers (gp140-GNC4) that preserve and expose some neutralizing epitopes while occluding some non-neutralizing epitopes - CD4BS MAbs (F105 and F91) and CD4i (17b and 48d) recognized gp140-GNC4 as well as gp120 or gp140 - non-neutralizing MAbs C11, A32, 522-149, M90, and \#45 bound to the gp140-GNC4 glycoprotein at reduced levels compared to gp120 - MAbs directed at the extreme termini of gp120 C1 (135/9 and 133/290) and C5 (CRA-1 and M91) bound efficiently to gp140-GNC4 [Yang2000]

- 48d: Mutations in two glcosylation sites in the V2 region of HIV-1 ADA at positions 190 and 197 (187 DNTSYRLINCNTS 199) cause the virus to become CD4-independent and able to enter cells through CCR5 alone - these same mutations tended to increase the neutralization sensitivity of the virus, including to $48 \mathrm{~d}$ - only the CD4i antibodies 17b and 48d showed an increased affinity of the CD4 independent viruses relative to wild-type [Kolchinsky2001]

- 48d: Called 4.8d - A panel of 12 MAbs was used to identify those that could neutralize the dual-tropic primary isolate HIV-1 89.6 - six gave significant neutralization at 2 to $10 \mathrm{ug} / \mathrm{ml}$ : 2F5, 50-69, IgG1b12, 447-52D, 2G12, and 670-D six did not have neutralizing activity: 654-D, 4.8D, 450-D, 246-D, 98-6, and 1281 - no synergy, only additive effects were seen for pairwise combinations of MAbs, and antagonism was noted between gp41 MAbs 50-69 and 98-6, as well as 98-6 and 2F5 [Verrier2001

- 48d: The fusion process was slowed by using a suboptimal temperature $(31.5 \mathrm{C})$ to re-evaluate the potential of Abs targeting fusion intermediates to block HIV entry - preincubation of E/T cells at $31.5 \mathrm{C}$ enabled polyclonal anti-N-HR Ab and anti-six-helix bundle Abs to inhibit fusion, indicating six-helix bundles form prior to fusion - the preincubation $31.5 \mathrm{C}$ step did not alter the inhibitory activity of neutralizing Abs anti-gp41 2F5, or anti-gp120 2G12, IG1b12, 48d, and 17b [Golding2002b]

- 48d: Uncleaved soluble gp140 (YU2 strain, R5 primary isolate) can be stabilized in an oligomer by fusion with a C-term trimeric GCN4 motif or using a T4 trimeric motif derived from T4 bacteriophage fibritin - stabilized oligomer gp140 delta683(-FT) showed strong preferential recognition by NAbs IgG1b12 and 2G12 relative to the gp120 monomer, in contrast to poorly neutralizing MAbs F105, F91, 17b, 48d, and 39F which showed reduced levels of binding, and C11, A32, and 30D which did not bind the stabilized oligomer [Yang2002]

- 48d: A series of mutational changes were introduced into the YU2 gp120 that favored different conformations $-375 \mathrm{~S} / \mathrm{W}$ seems to favor a conformation of gp120 closer to the CD4-bound state, and is readily bound by sCD4 and CD4i MAbs (17b, 48d, 49e, 21c and 23e) but binding of anti-CD4BS MAbs (F105, 15e, IgG1b12, 21h and F91 was markedly reduced - IgG1b12 failed to neutralize this mutant, while neutralization by 2G12 was enhanced - 2F5 did not neutralize either WT or mutant, probably due to polymorphism in the YU2 epitope - another mutant, $423 \mathrm{I} / \mathrm{P}$, disrupted the gp120 bridging sheet, favored a different conformation and did not bind CD4, CCR5, or CD4i antibodies, but did bind to CD4BS MAbs [Xiang2002b]

- 48d: Five CD4i MAbs were studied, 17b, 48d and three new MAbs derived by Epstein-Barr virus transformation of PBMC from an HIV+ long term non-progressor - 23e and 21c were converted to hybridomas to increase Ab production - all compete with the well-characterized 17b CD4i MAb in an ELISA antigen capture assay - critical binding residues are mapped and the CD4i MAb epitopes were distinct but share a common element near isoleucine 420, also important for CCR5 binding, and all five can block CCR5 binding to a sCD4-gp120 complex - the MAb 48d has the epitope most similar to the CCR5 binding site Xiang2002a 


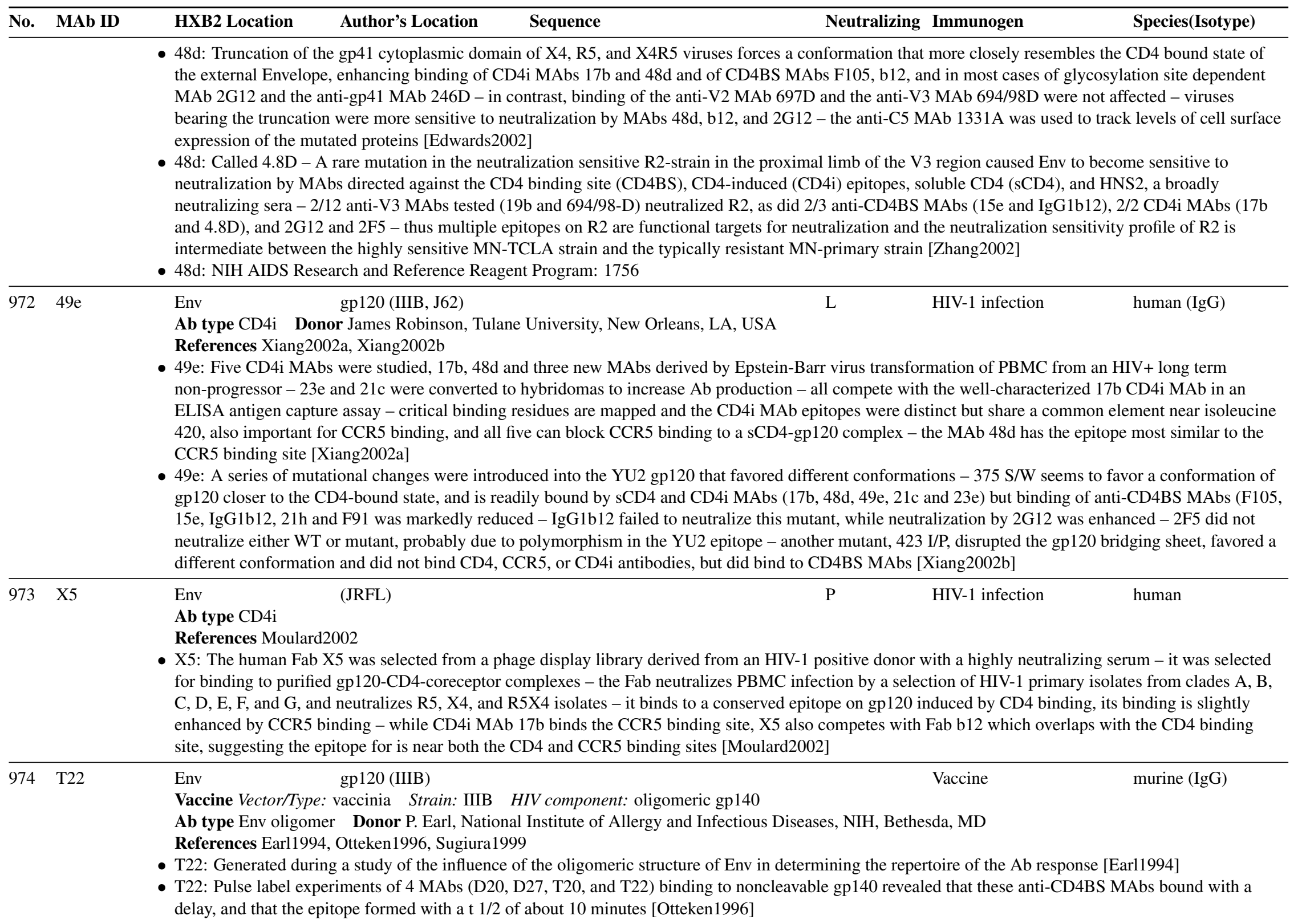




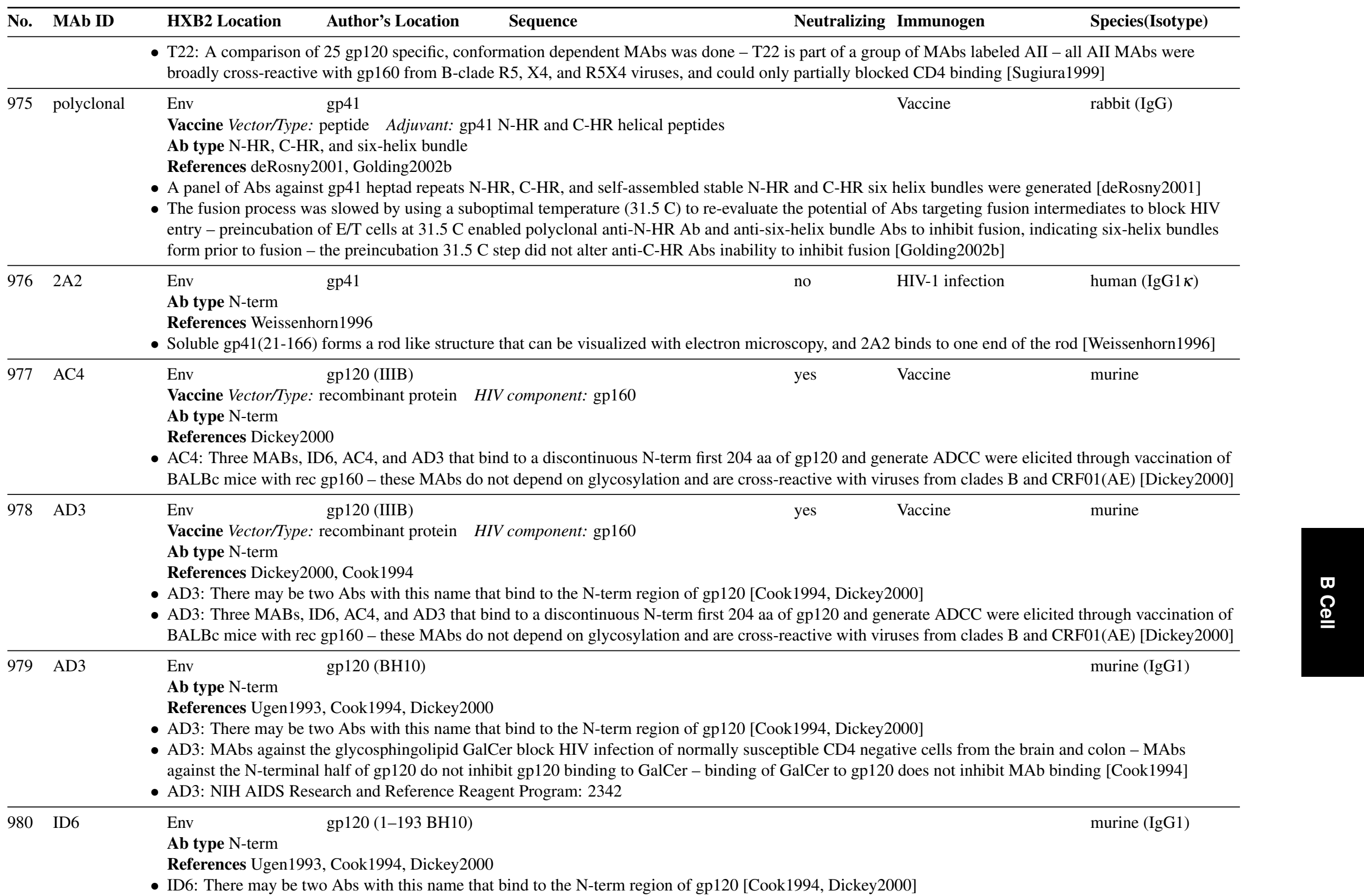




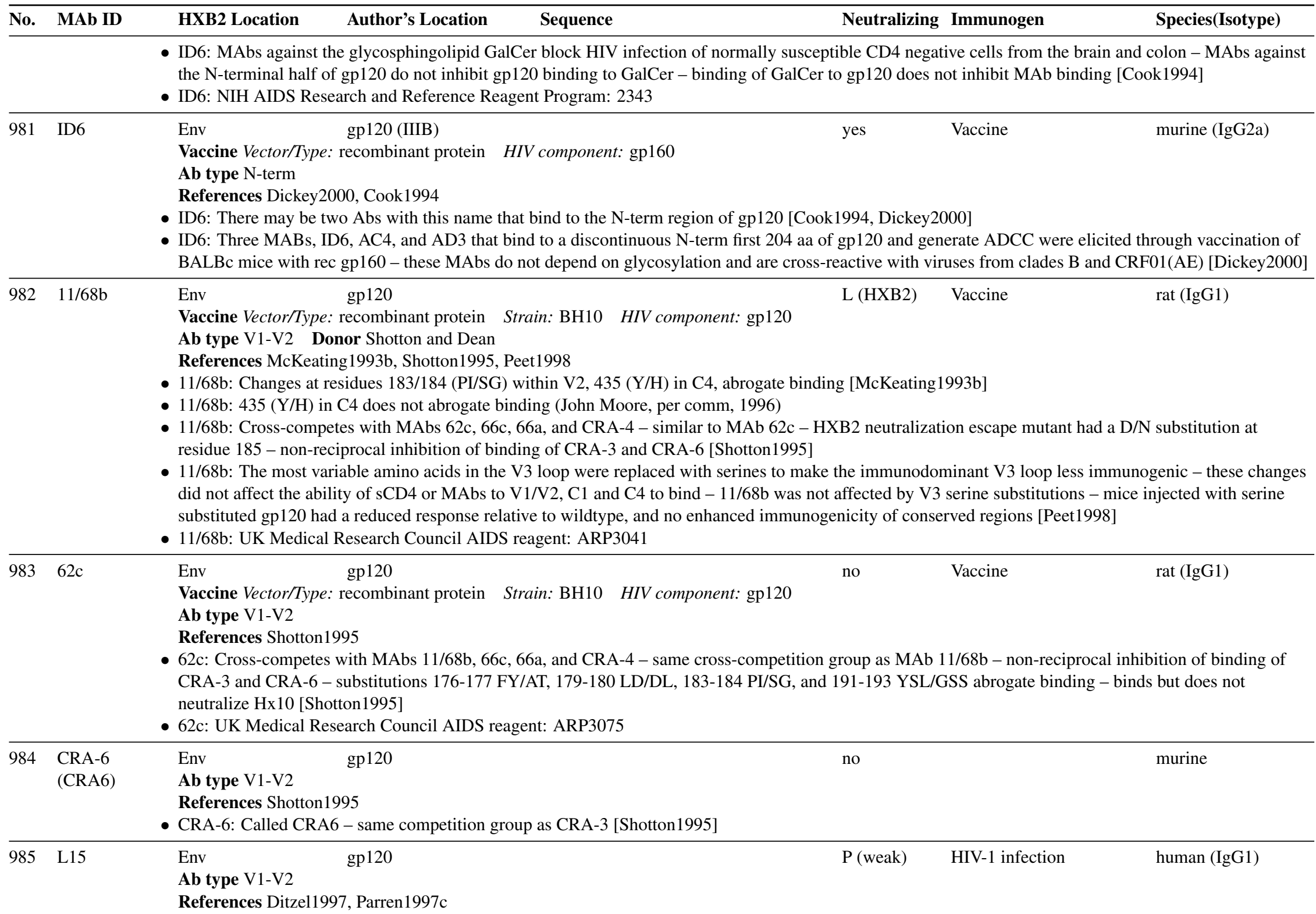




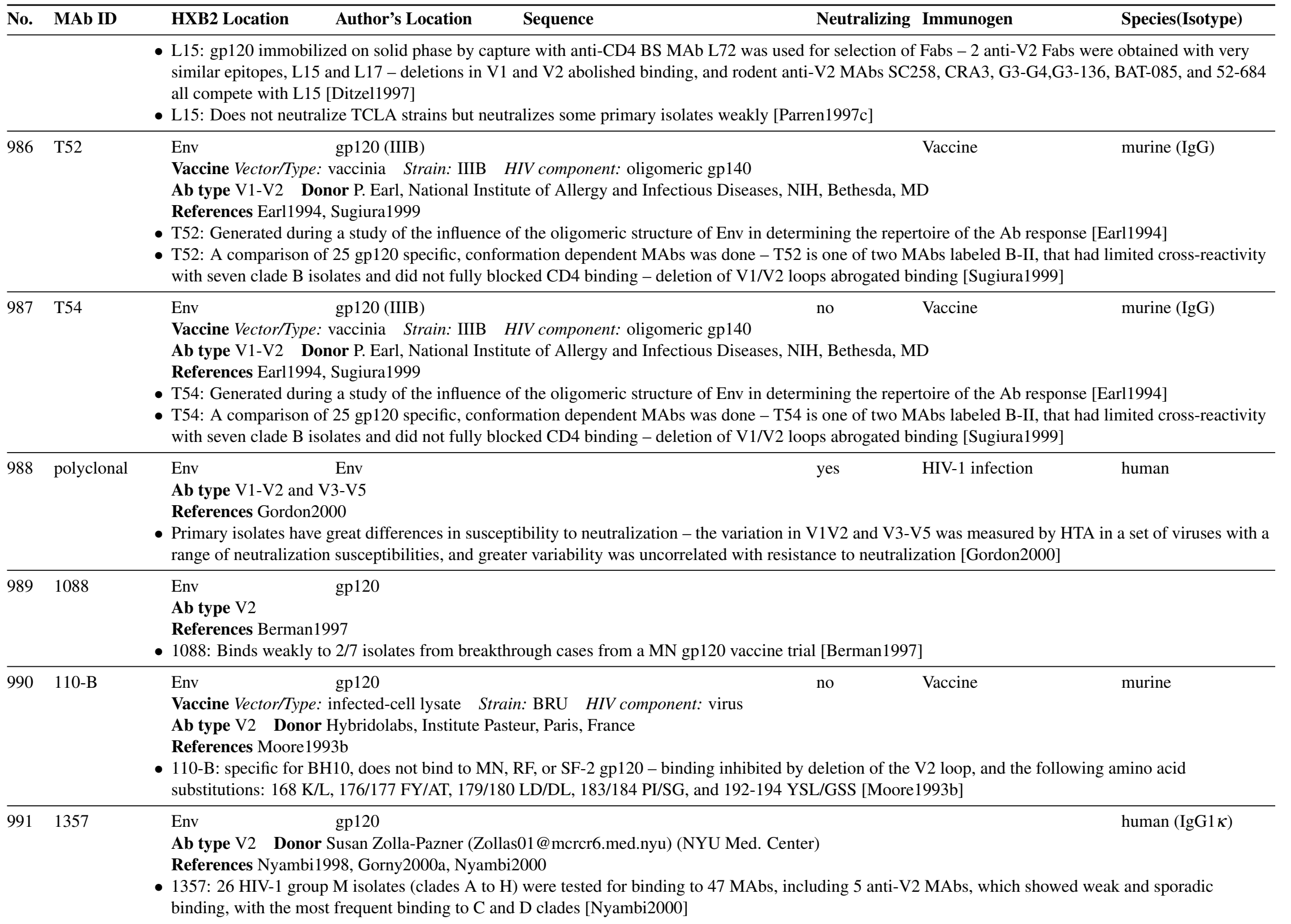




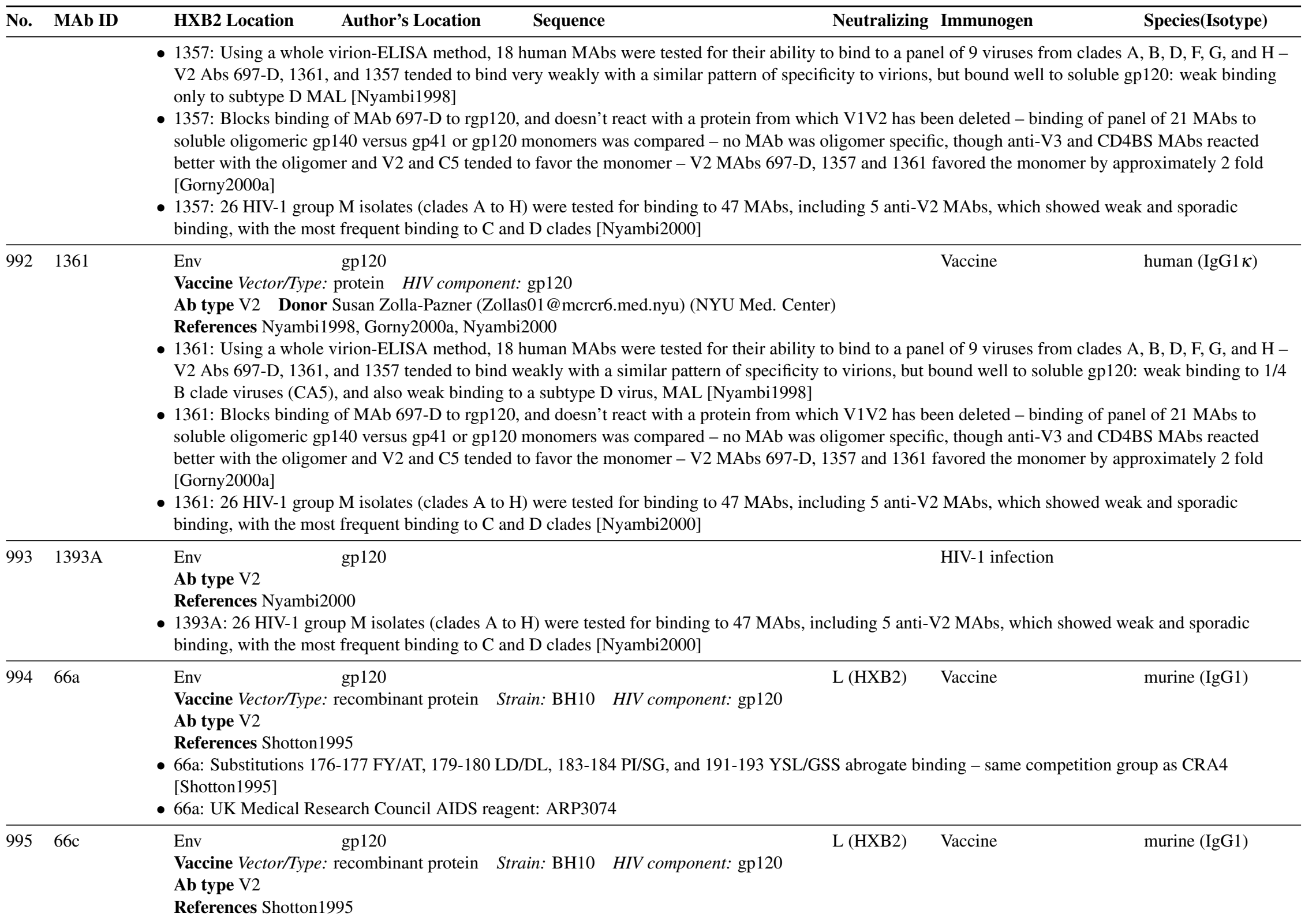




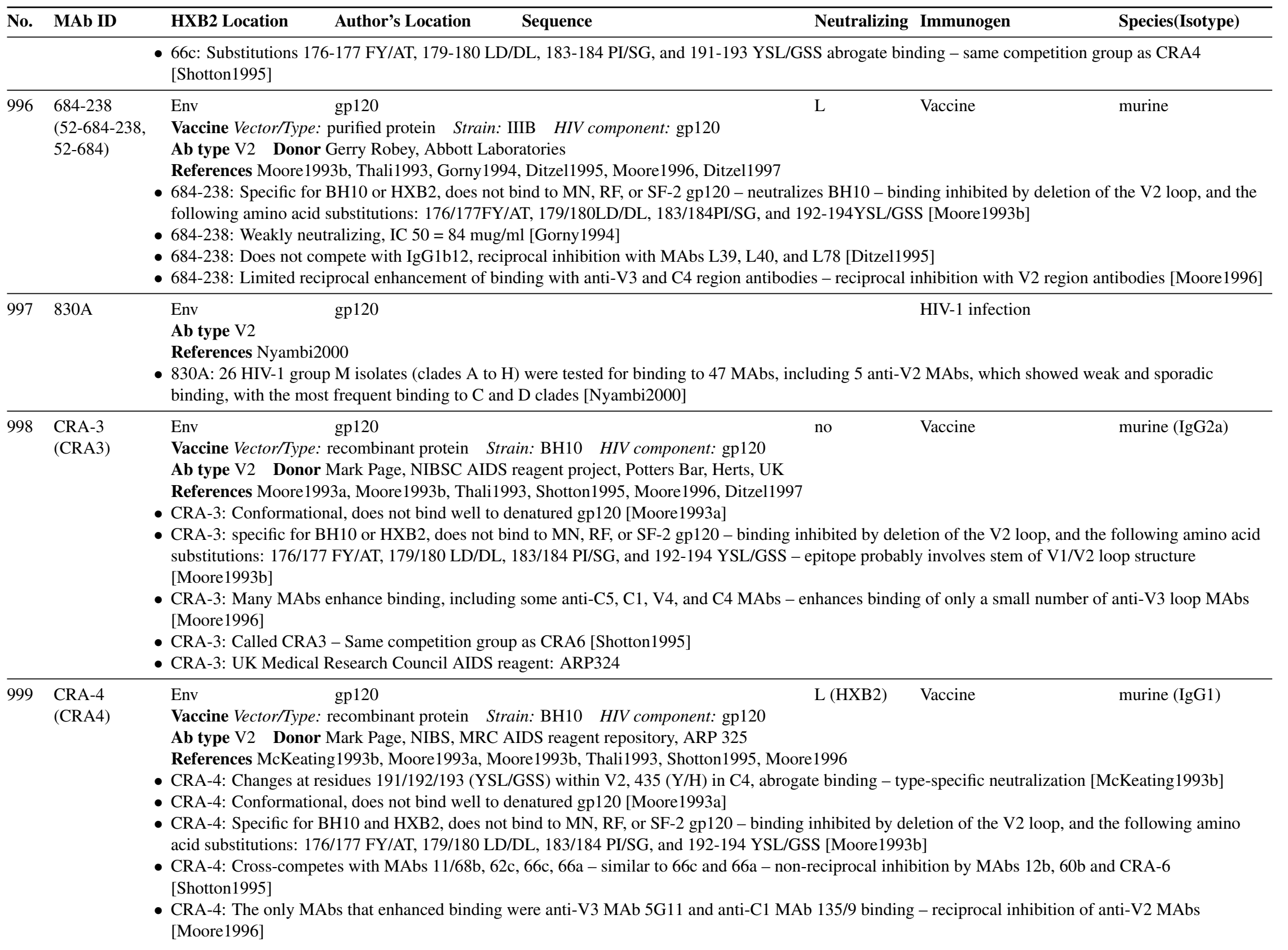




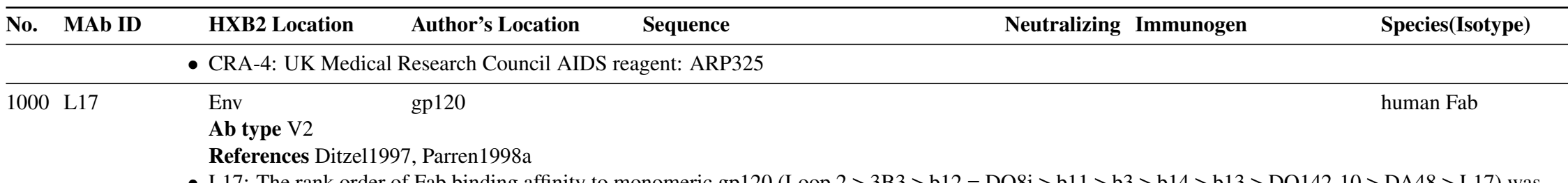

- L17: The rank order of Fab binding affinity to monomeric gp120 (Loop $2>3$ B3 $>$ b12 $=$ DO8i $>$ b11 $>$ b3 $>$ b14 $>$ b13 $>$ DO142-10 $>$ DA48 $>$ L17) was markedly different than Fab binding affinity to the mature oligomeric form $(3 \mathrm{~B} 3>$ b12 $>$ DO142-10 $>$ Loop $2>$ b11 $>$ L17 $>$ b6 $>$ DO8i $>$ b14 $>$ DA48 $>$ b3 $>$ b13) and binding to oligomeric form and neutralization were correlated for both Fabs and MAbs - authors suggest that neutralization is determined by the fraction of Ab sites occupied on a virion irrespective of the epitope [Parren1998a]

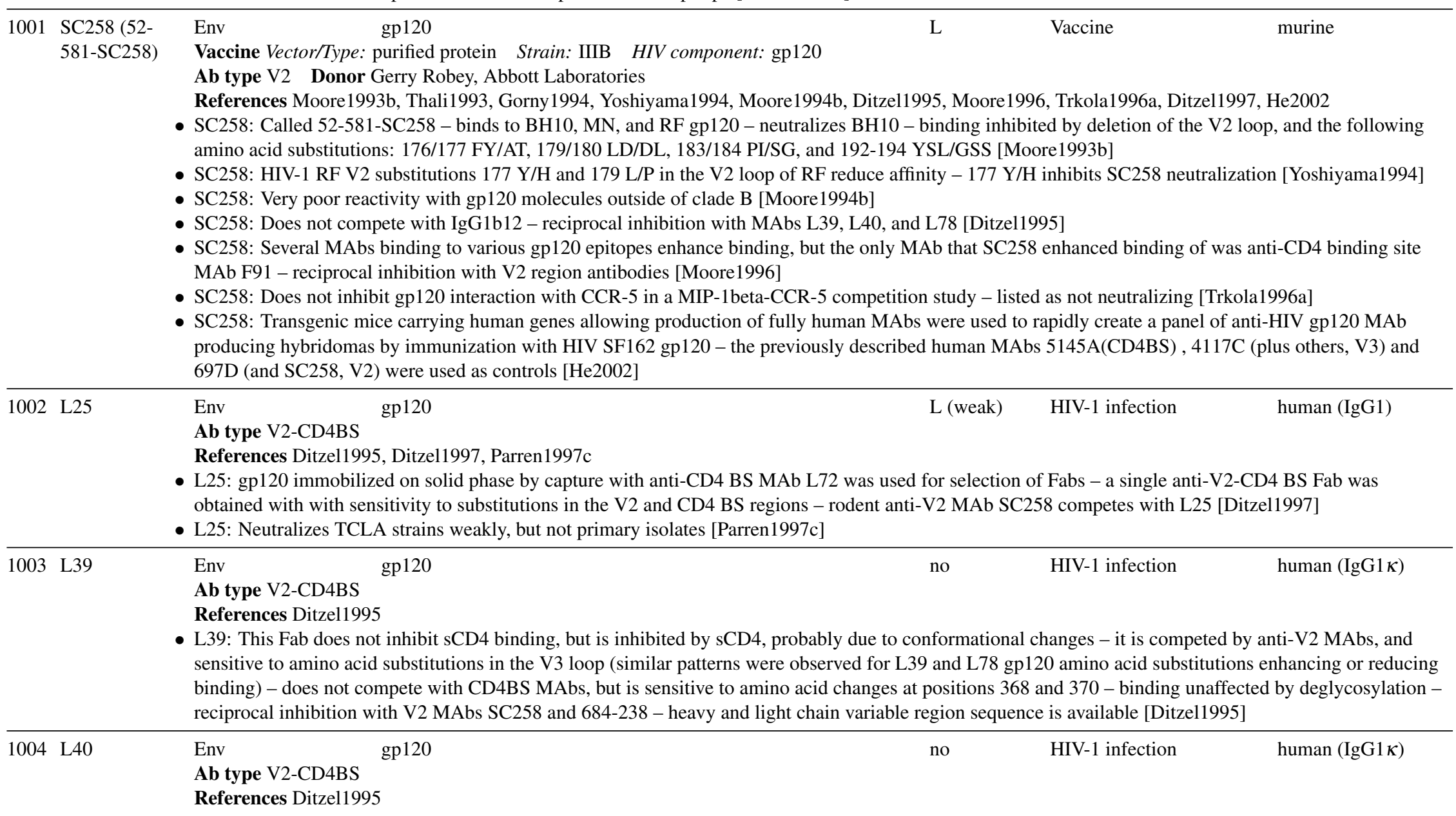




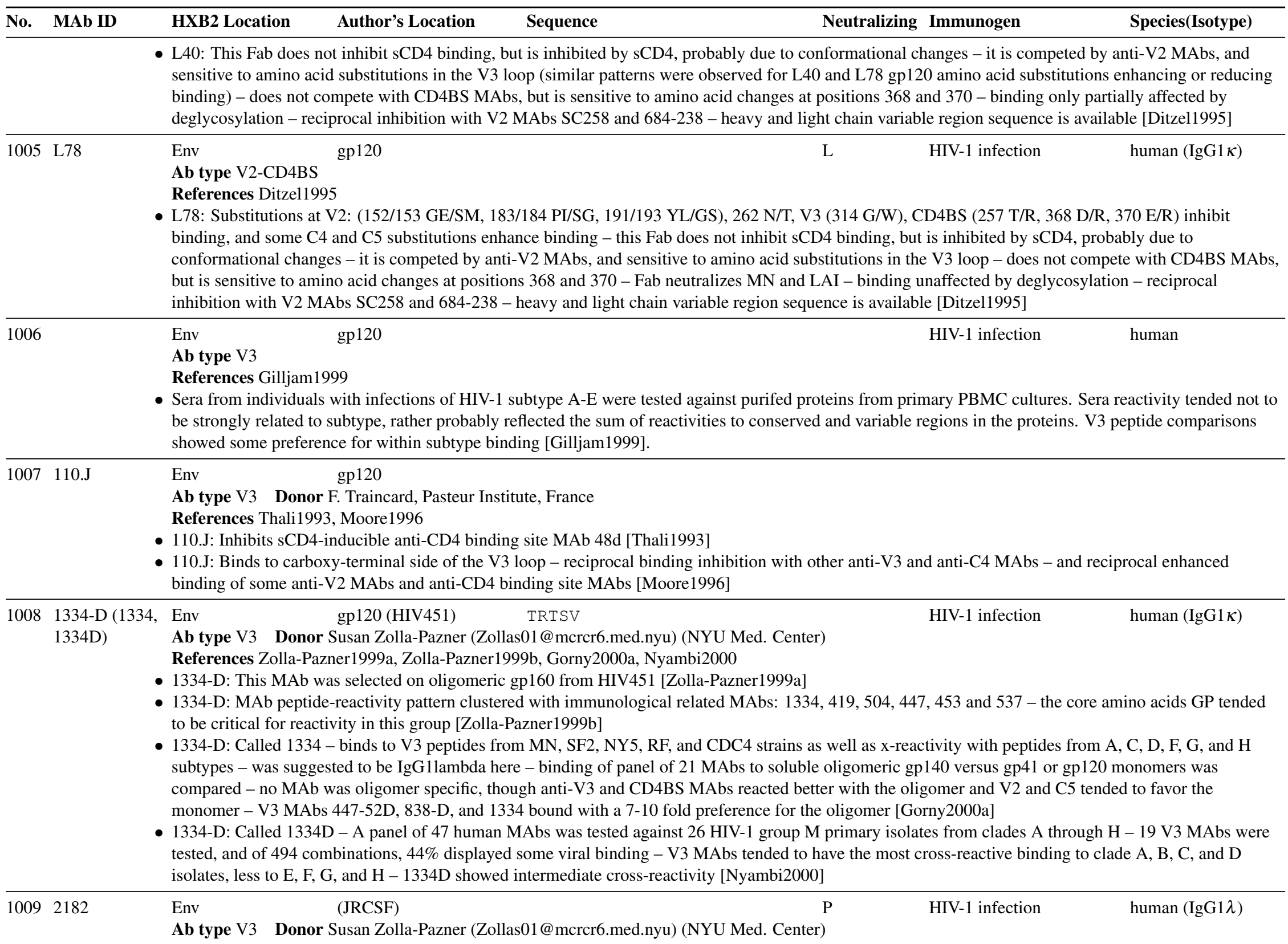




\begin{tabular}{ll}
\hline No. MAb ID & HXB2 Location Author's Location $\quad$ Sequence \\
\hline & References Gorny2002 \\
& 2182: Conformation-dependent anti-V3 loop Abs may be more cross-reactive, so six new V3 MAbs were generated from cells of asymptomatic \\
& HIV-1-infected individuals by selection of heterhybridomas using a V3-fusion protein (V3-fp), the HIV-1 JRCSF V3 loop inserted into a truncated murine \\
& leukemia virus gp70 - the six new MAbs all bind to the tip of the V3 loop and cross-compete with the MAb 447-52D and are conformationally sensitive - \\
& MAbs showed cross-clade binding to native, intact virions of clades A(N=2), B(N=4), and F(N=2), limited binding to C(N=3) and D(N=3), and did not bind \\
& to CRF01(subtype E, N=2) - the strength binding was highly correlated with percent neutralization using the ghost cell or PHA blast assay - five \\
& well-characterized MAbs were used as controls: anti-V3 447-52D (anti-V3 MAb for competition and neutralization studies), 654 (anti-CD4BS used as a \\
& conformation-sensitive MAb control), 1331A (anti-C5 used as a linear binding site MAb control), MAb 246 (anti-gp41 MAb that bound to primary isolates \\
& of all clades) - 5/6 MAbs were derived from individuals infected in the US, presumably with clade B, and one, 2182, was derived from an individual who \\
& was infected abroad with clade A who is presently living in New York city - 2412 and 2456 were produced from cells obtained from the same individual, \\
& while the other MAbs were each generated from different subjects - 2182 bound to 8/16 of the diverse isolates, not to any clade C or CRF01 [Gorny2002]
\end{tabular}

$\begin{array}{llllll}1010 & 2191 & \text { Env } & \text { (JRCSF) } & \text { HIV-1 infection }\end{array}$

Ab type V3 Donor Susan Zolla-Pazner (Zollas01@mcrcr6.med.nyu) (NYU Med. Center)

References Gorny2002

- 2191: Conformation-dependent anti-V3 loop Abs may be more cross-reactive, so six new V3 MAbs were generated from cells of asymptomatic HIV-1-infected individuals by selection of heterhybridomas using a V3-fusion protein (V3-fp), the HIV-1 JRCSF V3 loop inserted into a truncated murine leukemia virus gp70 - the six new MAbs all bind to the tip of the V3 loop and cross-compete with the MAb 447-52D and are conformationally sensitive MAbs showed cross-clade binding to native, intact virions of clades $\mathrm{A}(\mathrm{N}=2), \mathrm{B}(\mathrm{N}=4)$, and $\mathrm{F}(\mathrm{N}=2)$, limited binding to $\mathrm{C}(\mathrm{N}=3)$ and $\mathrm{D}(\mathrm{N}=3)$, and did not bind to $\mathrm{CRF} 01$ (subtype $\mathrm{E}, \mathrm{N}=2$ ) - the strength binding was highly correlated with percent neutralization using the ghost cell or PHA blast assay - five well-characterized MAbs were used as controls: anti-V3 447-52D (anti-V3 MAb for competition and neutralization studies), 654 (anti-CD4BS used as a conformation-sensitive MAb control), 1331A (anti-C5 used as a linear binding site MAb control), MAb 246 (anti-gp41 MAb that bound to primary isolates of all clades) - 5/6 MAbs were derived from individuals infected in the US, presumably with clade B, and one, 2182, was derived from an individual who was infected abroad with clade A who is presently living in New York city - 2412 and 2456 were produced from cells obtained from the same individual, while the other MAbs were each generated from different subjects - 2191 bound to 10/16 of the diverse isolates, not to any clade D or CRF01 [Gorny2002]

Ab type V3 Donor Susan Zolla-Pazner (Zollas01@ mcrcr6.med.nyu) (NYU Med. Center)

References Gorny2002

- 2219: Conformation-dependent anti-V3 loop Abs may be more cross-reactive, so six new V3 MAbs were generated from cells of asymptomatic HIV-1-infected individuals by selection of heterhybridomas using a V3-fusion protein (V3-fp), the HIV-1 JRCSF V3 loop inserted into a truncated murine leukemia virus gp70 - the six new MAbs all bind to the tip of the V3 loop and cross-compete with the MAb 447-52D and are conformationally sensitive MAbs showed cross-clade binding to native, intact virions of clades $\mathrm{A}(\mathrm{N}=2), \mathrm{B}(\mathrm{N}=4)$, and $\mathrm{F}(\mathrm{N}=2)$, limited binding to $\mathrm{C}(\mathrm{N}=3)$ and $\mathrm{D}(\mathrm{N}=3)$, and did not bind to CRF01(subtype $\mathrm{E}, \mathrm{N}=2$ ) - the strength binding was highly correlated with percent neutralization using the ghost cell or PHA blast assay - five well-characterized MAbs were used as controls: anti-V3 447-52D (anti-V3 MAb for competition and neutralization studies), 654 (anti-CD4BS used as a conformation-sensitive MAb control), 1331A (anti-C5 used as a linear binding site MAb control), MAb 246 (anti-gp41 MAb that bound to primary isolates of all clades) - 5/6 MAbs were derived from individuals infected in the US, presumably with clade B, and one, 2182, was derived from an individual who was infected abroad with clade A who is presently living in New York city - 2412 and 2456 were produced from cells obtained from the same individual, while the other MAbs were each generated from different subjects - 2219 bound to 13/16 of the diverse isolates [Gorny2002] 


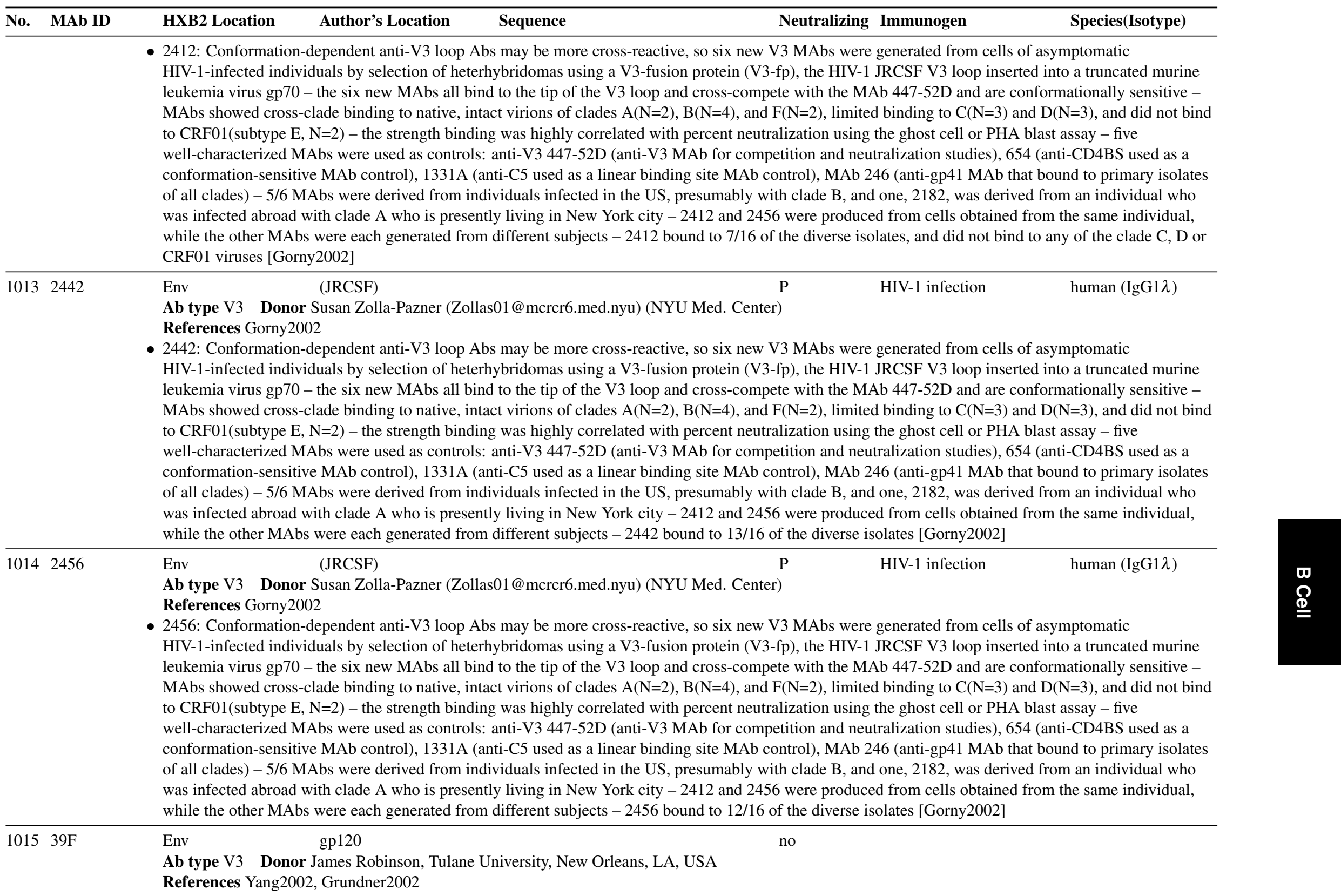




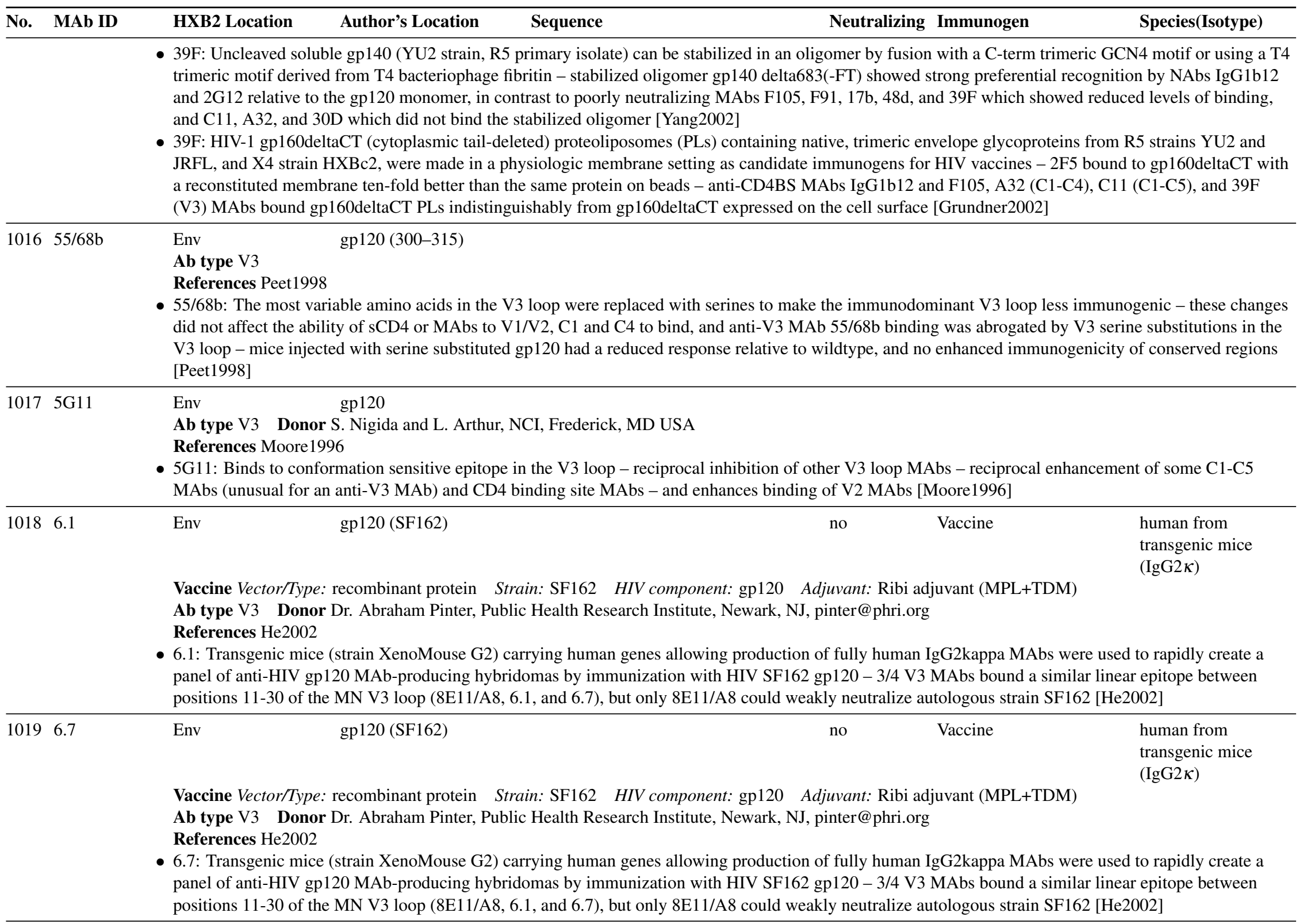




\begin{tabular}{lllllll}
\hline No. & MAb ID & HXB2 Location & Author's Location & Sequence & Neutralizing & Immunogen \\
\hline 1020 & 8.27 .3 & Env & gp120 (SF162) & Species(Isotype) & L & Vaccine \\
& & & & $\begin{array}{l}\text { human from } \\
\text { (IgG2 } \kappa)\end{array}$
\end{tabular}

Vaccine Vector/Type: recombinant protein Strain: SF162 HIV component: gp120 Adjuvant: Ribi adjuvant (MPL+TDM)

$(\operatorname{IgG} 2 \kappa)$

Ab type V3 Donor Dr. Abraham Pinter, Public Health Research Institute, Newark, NJ, pinter@phri.org

References $\mathrm{He} 2002$

- 8.27.3: Transgenic mice (strain XenoMouse G2) carrying human genes allowing production of fully human IgG2kappa MAbs were used to rapidly create a panel of anti-HIV gp120 MAb-producing hybridomas by immunization with HIV SF162 gp120 - 1/4 V3 MAbs, 8.27.3, bound a discontinuous epitope that was broadly cross-reactive with B clade R5 and X4 strains (not E clade) and could neutralize autologous strain SF162 [He2002]

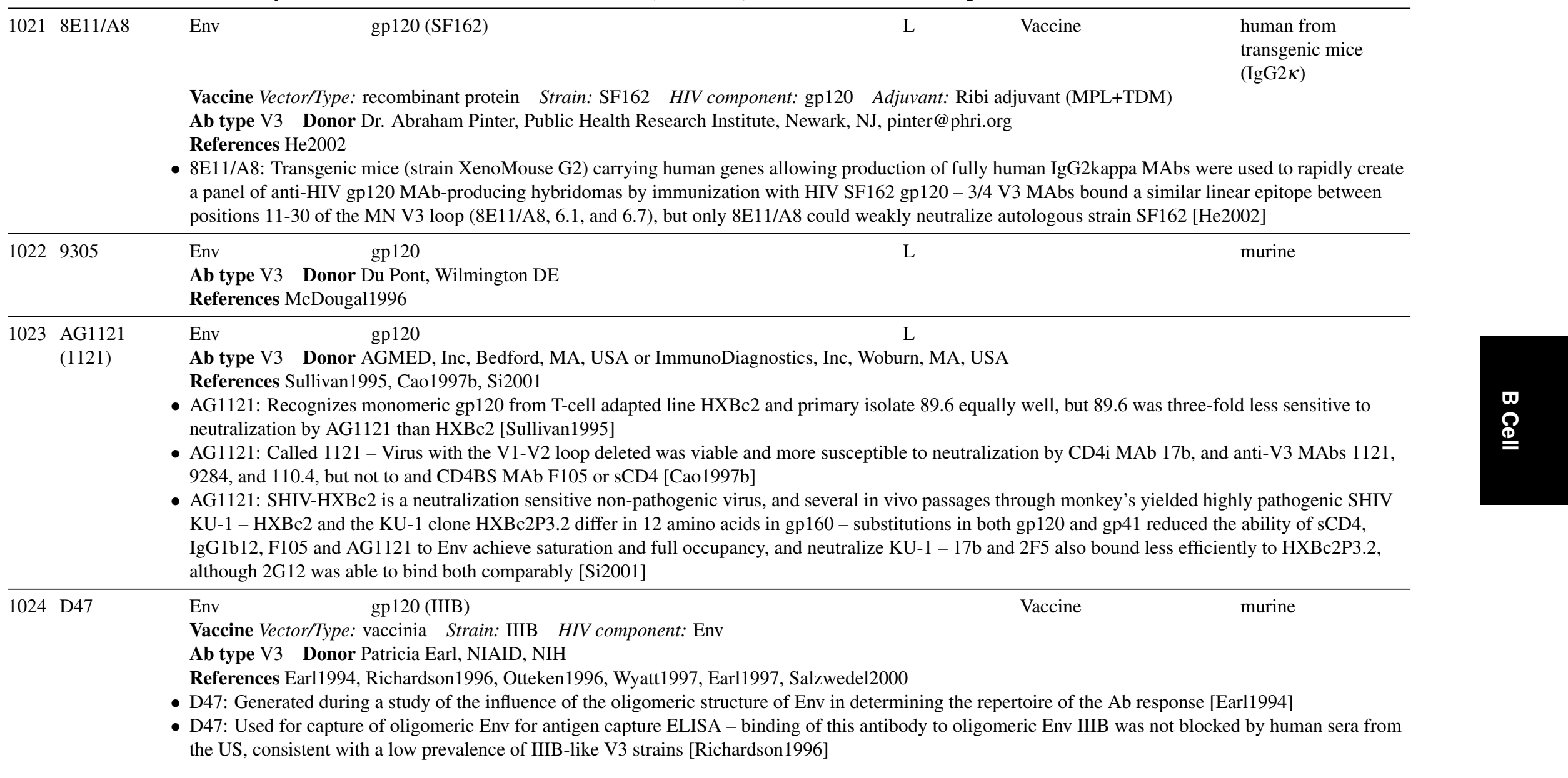




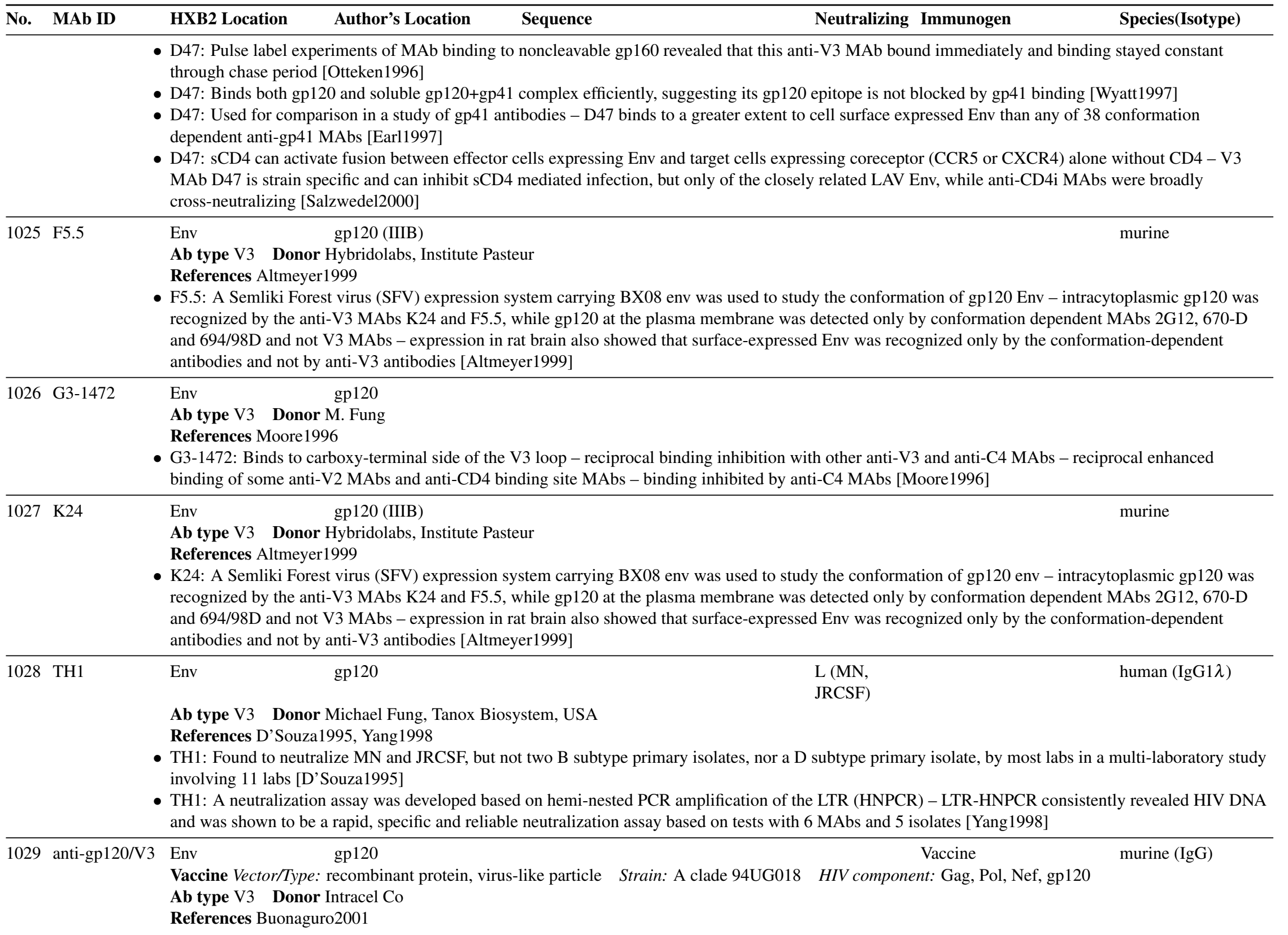




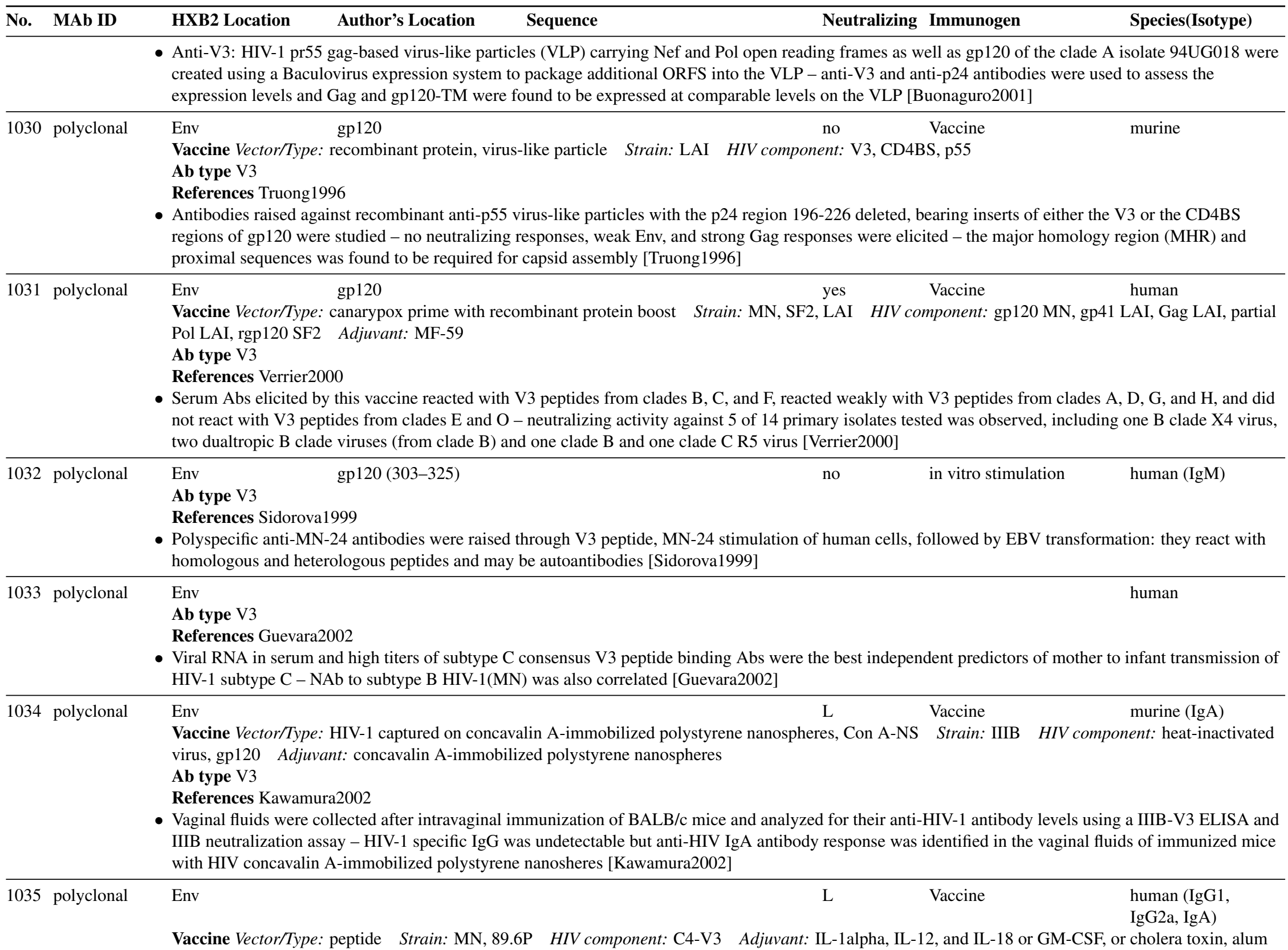




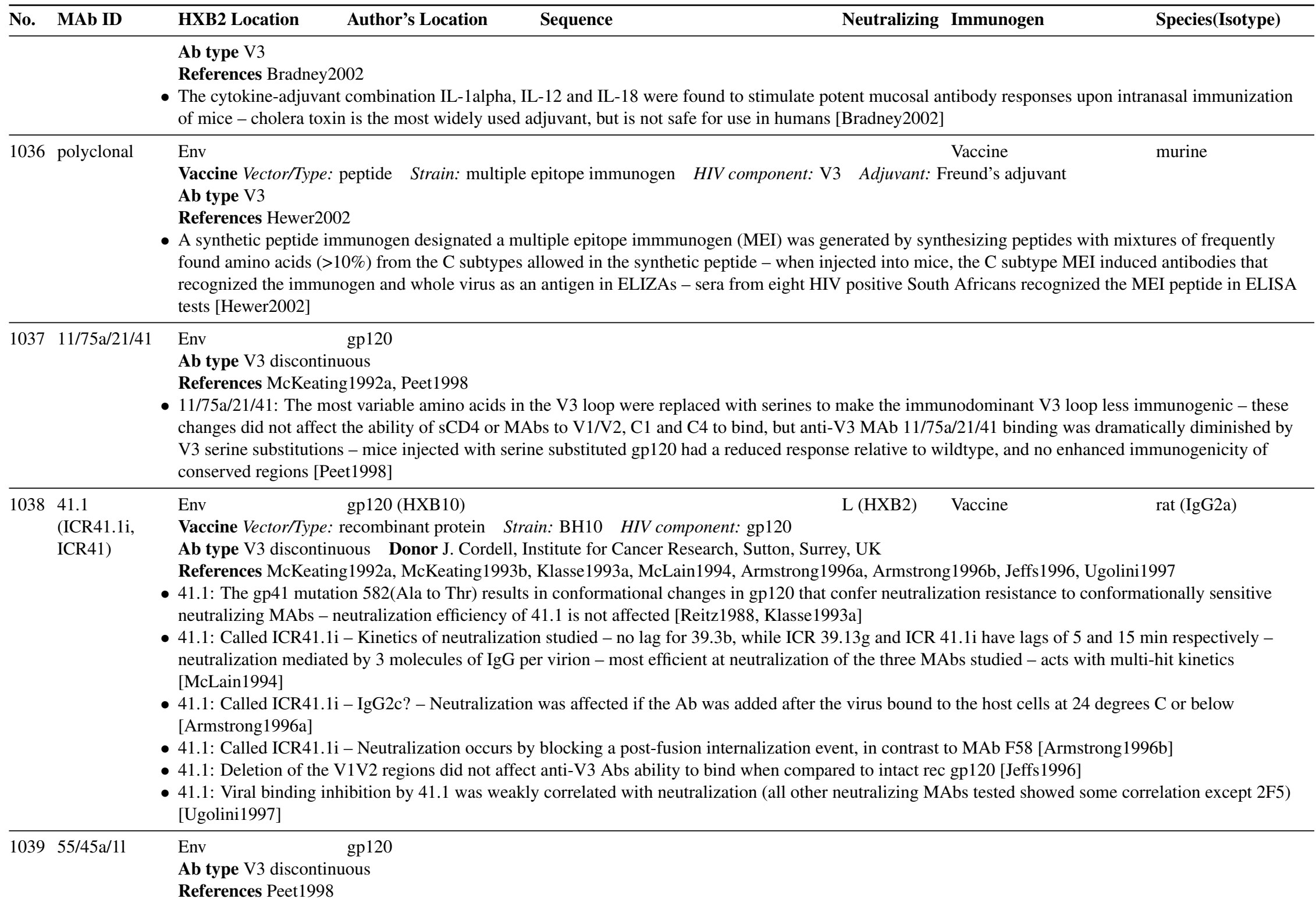




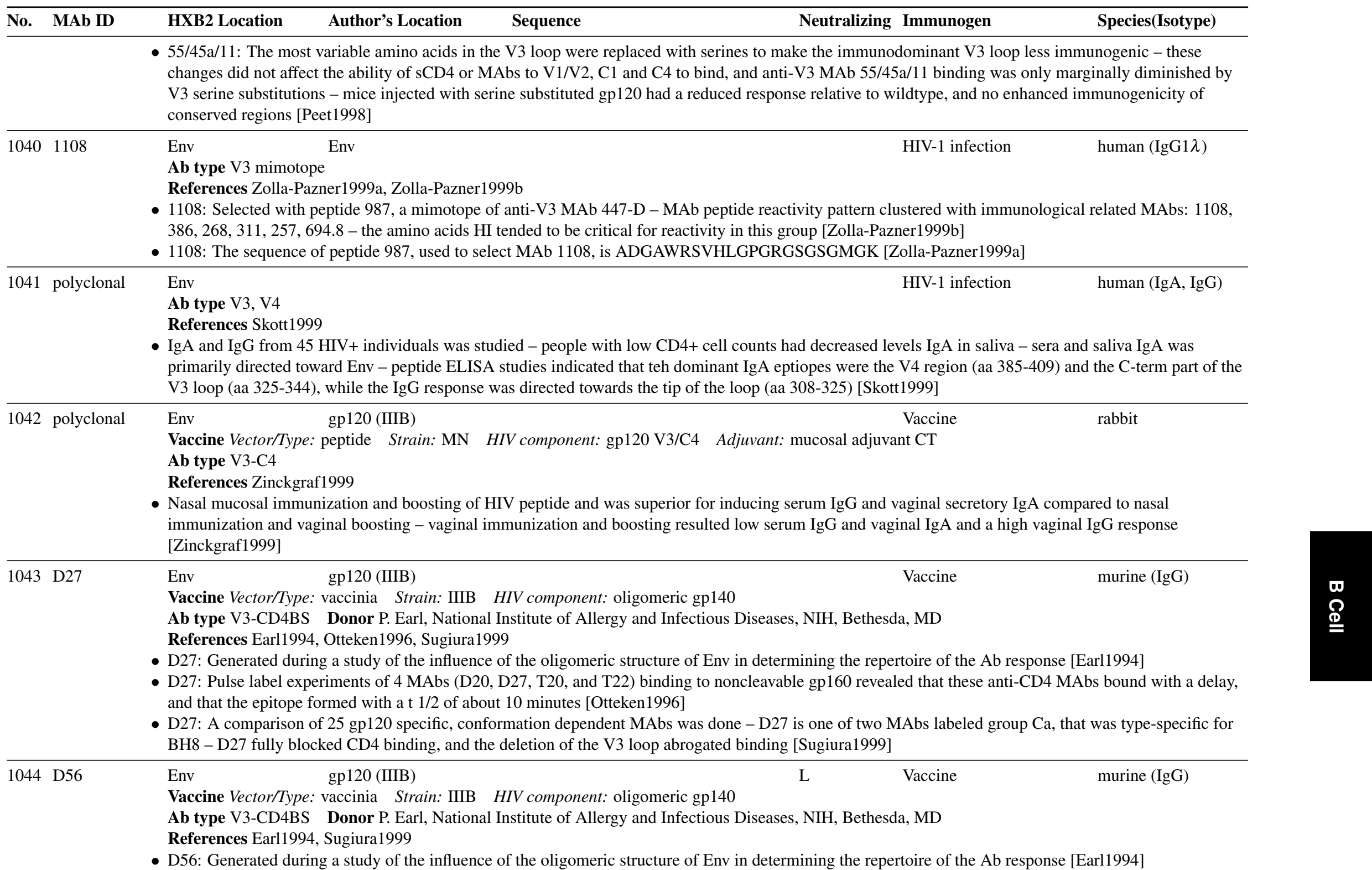




\begin{tabular}{lllll}
\hline No. MAb ID & HXB2 Location & Author's Location & Sequence & Neutralizing Immunogen
\end{tabular}

- D56: A comparison of 25 gp120 specific, conformation dependent MAbs was done - D56 is one of two MAbs labeled group Ca, that was type-specific for BH8 - D56 fully blocked CD4 binding, and the deletion of the V3 loop abrogated binding $-12.5 \mathrm{ug} / \mathrm{ml}$ of D56 was required to achieve $50 \%$ neutralization of HIV-1 NL4-3 [Sugiura1999]

1045 2G12 (c2G12) Env gp120 L P HIV-1 infection human $(\operatorname{IgG1} \kappa)$ Ab type carbohydrates at glycosylation residues in C2, C3, C4, and V4 Donor Herman Katinger, Inst. Appl. Microbiol. or Polymun Scientific Inc., Vienna, Austria, MRC AIDS reagent project

References Buchacher1994, Trkola1995, Moore1995b, McKeating1996b | McKeating1996a, Trkola1996b, Moore1996, Poignard1996b, Trkola1996a, Sattentau1996, D’Souza1997, Mo1997. Binley1997a. Fouts1997, Li1997, Moore1997, Mascola1997, Ugolini1997, Burton1997, Parren1997c Andrus1998. Wyatt1998a, Mondor1998, Parren1998a , Sullivan1998b, Connor1998, Binley1998, Trkola1998, Fouts1998, Takefman1998, Parren1998b, Li1998. Wyatt1998b Frankel1998, Kunert1998, Schonning1998, Montefiori1999, Beddows1999, Altmeyer1999, Poignard1999, Parren1999, Mascola1999. Mascola2000, Binley1999, Robert-Guroff2000, Baba2000, Grovit-Ferbas2000, Park2000, Si2001, Mascola2001 |Zwick2001c |Barnett2001, Moore2001, Poignard2001, Zeder-Lutz2001, Verrier2001, Stiegler2001 Spenlehauer2001, Hofmann-Lehmann2001, Xu2001, Savarino2001, Golding2002b Sanders2002, Scanlan2002, Schulke2002, Yang2002, Xu2002, Chakrabarti2002, Armbruster2002, Edwards2002, Grundner2002, Mascola2002, Zhang2002, Ferrantelli2002 Liu2002 Pantophlet2003, Herrera2003

- 2G12: Human MAb generated by electrofusion of PBL from HIV-1+ volunteers with CB-F7 cells [Buchacher1994]

- 2G12: Highly potent Cross-clade neutralizing activity [Trkola1995]

- 2G12: Conformationally sensitive epitope destroyed by mutations altering the N-linked glycosylation sites near the base of the V3 loop and the amino-terminal flank of the V4 loop [Trkola1996b]

- 2G12: Binding weakly enhanced by some anti-C1, -C4, -V3, and CD4 binding site MAbs - unusual in that $2 \mathrm{G} 12$ binding neither enhanced or inhibited the binding of other MAbs included in the study |Moore1996

- 2G12: Review: binding site is distinct from CD4BS MAbs epitope and is unique among known gp120 MAbs, human or rodent [Moore1995b]

- 2G12: Review: exceptional capacity to neutralize primary isolates in terms of both breadth and potency - one of three MAbs (IgG1b12, 2G12, and 2F5) generally accepted as having significant potency against primary isolates [Poignard1996b

- 2G12: Neutralizes JR-FL - inhibits gp120 interaction with CCR-5 in a MIP-1beta-CCR-5 competition study [Trkola1996a]

- 2G12: Neutralizes primary isolates, HXB2, and chimeric virus with gp120 from primary isolates in an HXB2 background |McKeating1996b

- 2G12: Review: Only four epitopes have been described which can stimulate a useful neutralizing response to a broad spectrum of primary isolates, represented by the binding sites of MAbs: 447-52-D, 2G12, Fab b12, and 2F5 [Sattentau1996]

- 2G12: In a multilab evaluation of monoclonal antibodies, only IgG1b12, 2G12, and 2F5 could neutralize at least half of the 9 primary test isolates at a concentration of < 25 mug per $\mathrm{ml}$ for $90 \%$ viral inhibition - neutralized 6 of 9 primary isolates [D'Souza1997]

- 2G12: A JRCSF variant that was selected for IgG1b12 resistance remained sensitive to MAbs 2G12 and 2F5, for combination therapy [Mo1997]

- 2G12: Study shows neutralization is not predicted by MAb binding to JRFL monomeric gp120, but is associated with oligomeric Env binding - 2 G12 bound monomer, and weakly bound oligomer and neutralized JRFL [Fouts1997]

- 2G12: One of 14 human MAbs tested for ability to neutralize a chimeric SHIV-vpu+, which expressed HIV-1 IIIB Env - 2G12 was a strong neutralizer of SHIV-vpu+ - all Ab combinations tested showed synergistic neutralization - 2G12 has synergistic response with MAbs 694/98-D (anti-V3), 2F5, F105, and b12 Li1997

- 2G12: Review: MAbs 2F5, 2G12 and IgG1b12 have potential for use in combination with CD4-IgG2 as an immunotherapeutic or immunoprophylactic homologous MAbs to these are rare in humans and vaccine strategies should consider including constructs that may enhance exposure of these MAbs' epitopes [Moore1997]

- 2G12: Using concentrations of Abs achievable in vivo, the triple combination of 2F5, 2G12 and HIVIG was found to be synergistic to have the greatest breadth and magnitude of response against 15 clade B primary isolates [Mascola1997] 


No. MAb ID HXB2 Location $\quad$ Author's Location $\quad$ Sequence $\quad$ Neutralizing Immunogen

- 2G12: Viral binding inhibition by 2G12 was strongly correlated with neutralization (all other neutralizing MAbs tested showed some correlation except 2F5) [Ugolini1997]

- 2G12: Review that discusses this MAb - reacts with residues at the base of the V3 loop and V4, and most of the changes that reduce binding are glycosylation sites - it is not clear whether the binding site is peptidic or direct carbohydrate [Burton1997]

- 2G12: Neutralizes TCLA strains and primary isolates [Parren1997c]

- 2G12: Post-exposure prophylaxis was effective when MAb 694/98-D was delivered 15 min post-exposure to HIV-1 LAI in hu-PBL-SCID mice, but declined to $50 \%$ if delivered 60 min post-exposure, and similar time constraints have been observed for HIVIG, 2F5 and 2G12, in contrast to MAb BAT123 that could protect delivered 4 hours post infection [Andrus1998]

- 2G12: The MAb and Fab binding to the oligomeric form of gp120 and neutralization were highly correlated - authors suggest that neutralization is determined by the fraction of Ab sites occupied on a virion irrespective of the epitope [Parren1998a]

- 2G12: Summary of the implications of the crystal structure of gp120 combined with what is known about mutations that reduce NAb binding - probable mechanism of neutralization by $2 \mathrm{G} 12$ is unknown, but dependent on proper glycosylation and $2 \mathrm{G} 12$ is predicted to be oriented toward the target cell when bound, so neutralization may be due to steric hindrance - mutations in positions N 295, T 297, S 334, N 386, N 392 and N 397 HXBc2 (IIIB) decrease $2 \mathrm{G} 12$ binding, and the binding region is 25 angstroms from the CD4 binding site - probably the Ab binds in part to carbohydrates, which may account for both its broad reactivity and the scarcity of Abs in the same competition group [Wyatt1998a]

- 2G12: Enhances Hx10 binding to CD4 positive or negative HeLa cells, but inhibited binding to CD4+ T-cell line A3.01 - neutralizes Hx10 infection of the HeLa cells [Mondor1998]

- 2G12: Ab from gp120 vaccinated individuals prior to infection, who subsequently became HIV infected, could not achieve $90 \%$ neutralization of the primary virus by which the individuals were ultimately infected - these viruses were not particularly refractive to neutralization, as determined by their susceptibility to neutralization by MAbs 2G12, IgG1b12, 2F5 and 447-52D [Connor1998]

- 2G12: Does not compete with binding of MAb generated in response to gp120-CD4 complex, CG10 [Sullivan1998b]

- 2G12: A panel of MAbs were shown to bind with similar or greater affinity and similar competition profiles to a deglycosylated or variable loop deleted core gp120 protein ( Delta V1, V2, and V3), thus such a core protein produces a structure closely approximating full length folded monomer - MAb 2G12 was the only exception to this, showing reduced binding efficiency [Binley1998]

- 2G12: A wide range of neutralizing titers was observed that was independent of co-receptor usage [Trkola1998]

- 2G12: Notes that 2G12 and 2F5, potent neutralizing antibodies, were identified by screening for cell surface (oligomeric Envelope) reactivity [Fouts1998

- 2G12: Induces Complement-mediated lysis in MN but not primary isolates - primary isolates are refractive to CML [Takefman1998]

- 2G12: MAbs 2G12, 2F5 and b12 are broadly neutralizing, as are some human polyconal sera, but this paper describes a set of primary isolates that are resistant to all three MAbs and 2 broadly neutralizing sera - results indicate that resistance levels of pediatric isolates might be higher than adult isolates resistance in general did not seem to be conferred by a loss of binding affinity for gp120 or gp41, rather by a more global perturbation of oligomeric Envelope [Parren1998b]

- 2G12: Neutralization synergy was observed when the MAbs 694/98-D (V3), 2F5 (gp41), and 2G12 (gp120 discontinuous) were used in combination, and even greater neutralizing potential was seen with the addition of a fourth MAb, F105 (CD4 BS) [Li1998]

- 2G12: Discussed in a review of the antigenic and receptor binding-domains of gp120 in relation to the structure of the molecule - antibodies are discussed by category (anti-V2, anti-V3, CD4i, CD4BS...), however as $2 \mathrm{G} 12$ binds to a rarely immunogenic region, and it is dependent on glycosylation, it was discussed individually [Wyatt1998b

- 2G12: The complete V, J and D(H) domain was sequenced - unlike non-neutralizing anti-gp41 MAb 3D6, five neutralizing MAbs (2F5, 2G12, 1B1, 1F7, and 3D5) showed extensive somatic mutations giving evidence of persistent antigenic pressure over long periods $-2 \mathrm{G} 12 \mathrm{D}(\mathrm{H})$ has the best homology to a $\mathrm{D}(\mathrm{H})$ segment between D3-22 and D4-23, a region not usually considered for heavy-chain rearrangement because it lacks associated recombination signals in the flanking regions, Kunert et al. suggest this may be why Abs that compete with 2G12 are rare [Kunert1998]

- 2G12: In a study of the influence of the glycan at position 306 of the V3 loop on MAb recognition, 2G12 was found to neutralize an HIV-BRU mutant virus that lacks the V3 loop glycan and has a mutation at the tip of the loop more efficiently than it neutralizes HIV-BRU [Schonning1998] 


No. MAb ID HXB2 Location Author's Location $\quad$ Sequence $\quad$ Neutralizing Immunogen

- 2G12: Infection of dendritic cells cultured from CD14+ blood cells or from cadaveric human skin was blocked by neutralizing MAbs IgG1b12, or 2F5 and 2G12 delivered together, but not by control non-neutralizing anti-gp120 MAb 4.8D, indicating that NAbs could interrupt early mucosal transmission events [Frankel1998]

- 2G12: A meeting summary presented results regarding neutralization-MAbs 2G12 and 2F5 tested for their ability to neutralize primary isolate infection of genetically engineered cell lines (cMAGI and others, presented by T. Matthews, A. Trkola, J. Bradac) - an advantage of such cells lines over PBMCs is that markers (X-Gal) can be added for staining to simplify the assay - the consensus of the meeting was that these engineered cell lines did not improve the sensitivity of detection of primary isolate neutralization - D. Burton and J. Mascola presented results concerning passive immunization and protection of hu-PBL-SCID mice and macaques, respectively, and both found combinations of MAbs that were able to achieve $99 \%$ neutralization in vitro corresponded to efficacy in vivo Montefiori1999

- 2G12: rgp120 derived from a R5X4 subtype B virus was used to vaccinate healthy volunteers and the resulting sera were compared with sera from HIV-1 positive subjects and neutralizing MAbs - 2G12 was able to bind with low affinity to the rgp120 monomer HIV-1 W61D [Beddows1999]

- 2G12: A Semliki Forest virus (SFV) expression system carrying BX08 Env was used to study the conformation of gp120 Env - intracytoplasmic gp120 was recognized by the anti-V3 MAbs K24 and F5.5, while gp120 at the plasma membrane was detected only by conformation dependent MAbs 2G12, 670-D and 694/98D and not V3 MAbs - expression in rat brain also showed that surface expressed Env was recognized only by the conformation-dependent antibodies and not by anti-V3 antibodies [Altmeyer1999]

- 2G12: Hu-PBL-SCID mice were infected with HIV-1s JRCSF and SF162 to study the effect of NAbs on an established infection - no significant differences in the initial rate of decrease in viral load or the plateau levels of viral RNA between the b12 treated and control mice were seen - in most of the Ab treated mice b12 escape mutants were observed with varying patterns of mutations - a combination of b12, $2 \mathrm{G} 12$ and $2 \mathrm{~F} 5$ protected $1 / 3$ mice, and an isolate from one of the other two was resistant to neutralization by all three MAbs [Poignard1999]

- 2G12: Review of the neutralizing Ab response to HIV-1 [Parren1999]

- 2G12: Combinations of HIVIG, 2F5, 2G12 were administered in passive-transfer experiments 24 hours prior to challenge with pathogenic SHIV 89.6PD $3 / 6$ animals given HIVIG/2F5/2G12 were completely protected, the others had reduced viremia and normal CD4 counts - 1/3 monkeys given 2F5/2G12 showed transient infection, the other two had reduced viral load - all monkeys that received HIVIG, 2F5, or 2G12 alone became infected and developed high-level plasma viremia, although animals that got HIVIG or 2G12 had a less profound CD4 T cell decline [Mascola1999]

- 2G12: Because HIV-1 is most often transmitted across mucosal surfaces, the ability of passive transfer of infused HIVIG/2F5/2G12 to protect against mucosal exposure of macaques to pathogenic SHIV 89.6PD was studied - HIVIG/2F5/2G12 protected 4/5 animals against vaginal challenge, 2F5/2G12 combined protected 2/5 animals, and 2G12 alone protected 2/4 animals - in contrast, Mascola and co-workers had previously shown single MAbs could not protect against intervenous challenge - Ab treated animals that got infected through vaginal innoculation had low viral loads and only modest declines in CD4 counts - the infused Abs were detected in the nasal, vaginal, and oral mucosa [Mascola2000]

- 2G12: Combinations of HIVIG, 2F5, 2G12 were administered in passive-transfer experiments 24 hours prior to challenge with pathogenic SHIV 89.6PD $3 / 6$ animals given HIVIG/2F5/2G12 were completely protected, the others had reduced viremia and normal CD4 counts - 1/3 monkeys given 2 F5/2G12 showed transient infection, the other two had reduced viral load - all monkeys that received HIVIG, 2F5, or 2G12 alone became infected and developed high-level plasma viremia, although animals that got HIVIG or 2G12 had a less profound CD4 T cell decline [Mascola1999]

- 2G12: The MAbs with the broadest neutralizing activity, IgG1b12, 2G12 and 2F5, all have high affinity for the native trimer, indicating that they were raised in an immune response to the oligomer on the virion surface rather than dissociated subunits - a disulfide linked gp120-gp41 (SOS gp140) was created to mimic the native conformation of Env and explore its potential as an immunogen - SOS gp140 is recognized by NAbs IgG1b12, 2G12, and CD4-IgG2, and also by anti-V3 MAbs 19b and 83.1 - SOSgp140 is not recognized by C4 region MAbs that neutralize only TCLA strains, G3-42 and G3-519 - nor did it bind $\mathrm{C} 11,23 \mathrm{~A}$, and M90, MAbs that bind to gp120 C1 and C5, where it interacts with gp41 - MAbs that bind CD4 inducible epitopes, $17 \mathrm{~b}$ and A32 were very strongly induced by CD4 in SOS gp140 - anti-gp41 MAbs that bind in the region that interacts with gp120, 7B2, 2.2B, T4, T15G1 and 4D4, did not bind to SOSgp140, in contrast to 2F5, which binds to the only gp41 epitope that is well exposed in native gp120-gp41 complexes [Binley1999]

- 2G12: A mini-review of observations of passive administration of IgG NAbs conferring protection against intervenous or vaginal SHIV challenge, that considers why IgG MAbs might protect against mucosal challenge [Robert-Guroff2000] 


No. MAb ID HXB2 Location Author's Location Sequence $\quad$ Neutralizing Immunogen

- 2G12: A triple combination of 2F5, F105 and 2G12 effectively neutralized perinatal infection of macaque infants when challenged with SHIV-vpu+ - the mean plasma half-life was $14.0+/-7.9$ days, the longest of the three Abs [Baba2000]

- 2G12: To determine the antigenicity of virus killed by thermal and chemical inactivation, retention of conformation-dependent neutralization epitopes was examined, and exposure of CD4BS epitopes was found to be enhanced (MAbs IgG1b12, 205-46-9, and 205-43-1) - binding to 2G12 and 447-52D epitopes was essentially unaltered - the 17b CD4i epitope was also exposed [Grovit-Ferbas2000]

- 2G12: Six mutations in MN change the virus from a high-infectivity neutralization resistant phenotype to low-infectivity neutralization sensitive - V3, CD4BS, and CD4i MAbs are 20-100 fold more efficient at neutralizing the sensitive form - 2G12 was an exception and could not neutralize MN in either form [Park2000]

- 2G12: SHIV-HXBc2 is a neutralization sensitive non-pathogenic virus, and several in vivo passages through monkey's yielded highly pathogenic SHIV KU-1 - HXBc2 and the KU-1 clone HXBc2P3.2 differ in 12 amino acids in gp160 - substitutions in both gp120 and gp41 reduced the ability of sCD4, IgG1b12, F105 and AG1121 to Env achieve saturation and full occupancy, and neutralize KU-1 - 17b and 2F5 also bound less efficiently to HXBc2P3.2, although 2G12 was able to bind both comparably [Si2001]

- 2G12: Neutralization synergy between anti-HIV NAbs b12, 2G12, 2F5, and 4E10 was studied - a classic fixed-ratio method was used, as well as a method where one $\mathrm{Ab}$ was fixed at a low neutralization titer and the other was varied - using primary isolates, a two-four fold enhancement of neutralization was observed with MAb pairs, and a ten-fold enhancement with a quadruple Ab combination - no synergy was observed with any MAb pair in the neutralization of TCLA strain HXB2 - there was no evidence for cooperativity of binding between b12 and 2G12 to envelope spikes expressed on the cell surface of TCLA or primary isolates [Zwick2001c]

- 2G12: Review of studies in macaques that have shown immune control of pathogenic SHIV viremia, improved clinical outcome, and protection, and the implications of the observations for HIV vaccines [Mascola2001]

- 2G12: SF162DeltaV2 is a virus that has a 30 amino acids deletion in the V2 loop that does not abrogate its infectivity but renders it highly susceptible to neutralization - when incorporated into a codon-optimized DNA vaccine with a CMV promoter and delivered by gene gun, SF162DeltaV2 gave higher neutralizing Ab titers against SF162 than did SF162 itself, and Abs that cross-neutralized non-homologous primary isolates were obtained only when SF162DeltaV2, but not intact SF162, was used as the immunogen - Control MAbs 2F5 and 2G12 could neutralize all of the following primary isolates: 91US056(R5), 92US714(R5), 92US660(R5), 92HT593(R5X4), and BZ167(R5X4), while after the first protein boost, the sera from two SF162DeltaV2 immunized macaques could neutralize 91US056(R5), 92US714(R5), 92US660(R5) and ADA(R5), but not 92HT593(R5X4) or 92US657(R5) - the pattern of cross-recognition shifted after the second boost [Barnett2001]

- 2G12: Moore and colleagues review structural aspects of gp120 and how they relate to antigenic domains, and review the data concerning the lack of a clear relationship between genetic subtype and serotype - an exception exists for human MAb 2G12, which does not recognize CRF01 envelopes because of an unusual additional disulfide bond in the V4 loop region that appears to be unique to the subtype E, CRF01 gp120 protein [Moore2001]

- 2G12: Structural aspects of the interaction of neutralizing Abs with HIV-1 Env are reviewed - Env essentially has three faces, one is largely inaccessible on the native trimer, and two that exposed but have low immunogenicity on primary viruses - neutralization is suggested to occur by inhibition of the interaction between gp120 and the target cell membrane receptors as a result of steric hindrance and it is noted that the attachment of approximately 70 IgG molecules per virion is required for neutralization, which is equivalent to about one IgG molecule per spike - the 2G12, 17b and b12 epitopes are discussed in detail - although it is potently neutralizing, 2G12 does not interfere with CD4 and coreceptor binding, and this Ab specificity is uncommon in sera from HIV-1-infected individuals [Poignard2001]

- 2G12: Neutralizing synergy between MAbs 1b12, 2G12 and 2F5 was studied using surface plasmon resonance to determine the binding kinetics for these three MAbs with respect to monomeric and oligomeric Env protein gp160 IIIB - the 2G12 epitope is highly accessible on both monomeric and oligomeric Envs, $1 \mathrm{~b} 12$ is highly accessible on monomers but not oligomers, and 2F5 on neither form - binding of $2 \mathrm{G} 12$ exposes the $2 \mathrm{~F} 5$ epitope on gp160 oligomers 2G12-gp160 oligomer interactions were best fitted to a two state model, with the first complex having a high association constant and fast dissociation, that is stabilized by conformational changes induced by the binding of a second MAb [Zeder-Lutz2001]

- 2G12: A luciferase-reporter gene-expressing T-cell line was developed to facilitate neutralization and drug-sensitivity assays - luciferase and p24 antigen neutralization titer end points were found comparable using NAb from sera from HIV+ donors, and MAbs 2F5, 2 G12 and IgG1b12 [Spenlehauer2001] 


No. MAb ID HXB2 Location Author's Location $\quad$ Sequence $\quad$ Neutralizing Immunogen

- 2G12: A panel of 12 MAbs was used to identify those that could neutralize the dual-tropic primary isolate HIV-1 89.6 - six gave significant neutralization at 2 to $10 \mathrm{ug} / \mathrm{ml}$ : 2F5, 50-69, IgG1b12, 447-52D, 2G12, and 670-D six did not have neutralizing activity: 654-D, 4.8D, 450-D, 246-D, 98-6, and 1281 - no synergy, only additive effects were seen for pairwise combinations of MAbs, and antagonism was noted between gp41 MAbs 50-69 and 98-6, as well as 98-6 and 2F5 [Verrier2001]

- 2G12: A combination of MAbs IgG1b12, 2F5, and 2G12 was given postnatally to four neonates macaques that were then challenged with highly pathogenic SHIV89.6P - one of the four infants remained uninfected after oral challenge, two infants had no or a delayed CD4(+) T-cell decline [Hofmann-Lehmann2001]

- 2G12: Twenty HIV clade C isolates from five different countries were susceptible to neutralization by anti-clade B MAbs in a synergistic quadruple combination of mAbs IgG1b12, 2G12, 2F5, and 4E10 [Xu2001]

- 2G12: Chloroquine reduces the HIV-1-infectivity of H9 IIIB cells, apparently through altering the conformation of envelope - there is a reduction of reactivity of 2G12 to its epitope in chloroquine treated cultures [Savarino2001]

- 2G12: A phase I trial in seven HIV+ individuals was conducted with MAbs 2F5 and 2G12-no clinical or laboratory abnormalities were observed throughout the study—eight infusions were administered over a 4-week period (total dose $14 \mathrm{~g})$ —-the elimination half-life $\left(t_{-} 1 / 2\right)$ was calculated to be 7.94 (range, 3.46-8.31) days for 2F5 and 16.48 (range, 12.84-24.85) days for 2G12 [Armbruster2002].

- 2G12: The fusion process was slowed by using a suboptimal temperature (31.5 C) to re-evaluate the potential of Abs targeting fusion intermediates to block HIV entry - preincubation of E/T cells at $31.5 \mathrm{C}$ enabled polyclonal anti-N-HR Ab and anti-six-helix bundle Abs to inhibit fusion, indicating six-helix bundles form prior to fusion - the preincubation $31.5 \mathrm{C}$ step did not alter the inhibitory activity of neutralizing Abs anti-gp41 2F5, or anti-gp120 2G12, IG1b12, 48d, and 17b |Golding2002b]

- 2G12: The 2G12 epitope is composed of carbohydrates involving high-mannose and hybrid glycans of residues 295,332 , and 392, with peripheral glycans from 386 and 448 contributing on either flank, and with little direct gp120 protein surface involvement - these mannose residues are proximal to each other near the chemokine receptor binding surface [Sanders2002]

- 2G12: Alanine scanning mutagenesis used in conjunction with competition and replacement studies of $\mathrm{N}$-linked carbohydrates and sugars suggest that the 2G12 epitope is formed from mannose residues contributed by the glycans attached to N295 and N332, with the other N-linked carbohydrates in positions N339, N386, and N392 playing a role in maintaining conformation relevant to 2G12 binding - N295A and N332A mutants showed essentially unchanged anti-CD4BS NAb b12 binding affinities, while N339A, N386A and N392A mutants displayed significantly lowered b12 affinity, presumably due to conformational changes [Scanlan2002]

- 2G12: Ab binding characteristics of SOS gp140 were tested using SPR and RIPA - SOS gp140 is gp120-gp41 bound by a disulfide bond - NAbs 2G12, 2F5, IgG1b12, CD4 inducible 17b, and 19b bound to SOS gp140 better than uncleaved gp140 (gp140unc) and gp120 - non-neutralizing MAbs 2.2B (binds to gp41 in gp140unc) and 23A (binds gp120) did not bind SOS gp140-2G12 complexes with SOS gp140 or with gp120 had a very unusual linear structure [Schulke2002]

- 2G12: Uncleaved soluble gp140 (YU2 strain, R5 primary isolate) can be stabilized in an oligomer by fusion with a C-term trimeric GCN4 motif or using a T4 trimeric motif derived from T4 bacteriophage fibritin - stabilized oligomer gp140 delta683(-FT) showed strong preferential recognition by NAbs IgG1b12 and 2G12 relative to the gp120 monomer, in contrast to poorly neutralizing MAbs F105, F91, 17b, 48d, and 39F which showed reduced levels of binding, and MAbs C11, A32, and 30D which did not bind the stabilized oligomer [Yang2002]

- 2G12: Passive immunization of neonate macaques with a combination of F105+2G12+2F5 conferred complete protection against oral challenge with SHIV-vpu+ or - the combination b12+2G12+2F5 conferred partial protection against SHIV89.6 - such combinations may be useful for prophylaxis at birth and against milk born transmission - the synergistic combination of IgG1b12, 2G12, 2F5, and 4E10 neutralized a collection of HIV clade C primary isolates [Xu2002]

- 2G12: A modified gp140 (gp140deltaCFI), with C-term mutations intended to mimic a fusion intermediate and stabilize trimer formation, retained antigenic conformational determinants as defined by binding to CD4 and to MAbs 2F5, 2G12, F105, and b12, and enhanced humoral immunity without diminishing the CTL response in mice injected with a DNA vaccine [Chakrabarti2002 


No. MAb ID HXB2 Location $\quad$ Author's Location $\quad$ Sequence $\quad$ Neutralizing Immunogen

- 2G12: A combination of MAbs 2F5 and 2G12 given in multiple infusions was found to be safe and well tolerated even in high doses in a phase I study of seven HIV-1 infected healthy volunteers - the median elimination half-life was 7.94 days for 2F5, and 16.48 for $2 \mathrm{G} 12$ - no anti-2F5 or anti-2G12 IgM or IgG responses were detected - although there was some transient increases, overall plasma viral RNA levels decreased in 6/7 volunteers, by a median of 0.62 log_10 [Armbruster2002

- 2G12: Truncation of the gp41 cytoplasmic domain of X4, R5, and X4R5 viruses forces a conformation that more closely resembles the CD4 bound state of the external Envelope, enhancing binding of CD4i MAbs 17b and 48d and of CD4BS MAbs F105, b12, and in most cases of glycosylation site dependent MAb 2G12 and the anti-gp41 MAb 246D - in contrast, binding of the anti-V2 MAb 697D and the anti-V3 MAb 694/98D were not affected - viruses bearing the truncation were more sensitive to neutralization by MAbs 48d, b12, and 2G12 - the anti-C5 MAb 1331A was used to track levels of cell surface expression of the mutated proteins [Edwards2002]

- 2G12: HIV-1 gp160deltaCT (cytoplasmic tail-deleted) proteoliposomes (PLs) containing native, trimeric envelope glycoproteins from R5 strains YU2 and JRFL, and X4 strain HXBc2, were made in a physiologic membrane setting as candidate immunogens for HIV vaccines - 2F5 bound to gp160deltaCT with a reconstituted membrane ten-fold better than the same protein on beads, while such an affinity difference was not seen with F105 and 2G12-anti-CD4BS MAbs IgG1b12 and F105, A32 (C1-C4), C11 (C1-C5), and 39F (V3) MAbs bound gp160deltaCT PLs indistinguishably from gp160deltaCT expressed on the cell surface [Grundner2002]

- 2G12: Rhesus macaques were better protected from vaginal challenge with SHIV89.6D (MAb 2G12, 2/4; MAbs 2F5/2G12, 2/5; and HIVIG/2F5/2G12, 4/5 infected) than from intravenous challenge (MAb 2G12, 0/3; MAbs 2F5/2G12, 1/3; and HIVIG/2F5/2G12, 3/6 infected)- the animals that were infected by vaginal challenge after Ab infusion had low or undetectable viral RNA levels and modest CD4 T-cell decline [Mascola2002]

- 2G12: A rare mutation in the neutralization sensitive R2-strain in the proximal limb of the V3 region caused Env to become sensitive to neutralization by MAbs directed against the CD4 binding site (CD4BS), CD4-induced (CD4i) epitopes, soluble CD4 (sCD4), and HNS2, a broadly neutralizing sera - 2/12 anti-V3 MAbs tested (19b and 694/98-D) neutralized R2, as did 2/3 anti-CD4BS MAbs (15e and IgG1b12), 2/2 CD4i MAbs (17b and 4.8D), and 2G12 and 2F5 - thus multiple epitopes on R2 are functional targets for neutralization and the neutralization sensitivity profile of R2 is intermediate between the highly sensitive MN-TCLA strain and the typically resistant MN-primary strain [Zhang2002]

- 2G12: Review of NAbs that notes 2G12 alone or in combination with other MAbs can protect some macaques against SHIV infection, that it has strong ADCC activity, and that it is safe and well tolerated in humans [Ferrantelli2002]

- 2G12: Review of NAbs that discusses mechanisms of neutralization, passive transfer of NAbs and protection in animal studies, and vaccine strategies [Liu2002]

- 2G12: Alanine scanning mutagenesis was used to compare substitutions that affected anti-CD4BS NAb b12 - rec gp120s were engineered to contain combinations of Alanine substitutions that enhanced b12 binding, and while binding of b12 to these gp120 monomers was generally maintained or increased, binding by five non-neutralizing anti-CD4bs MAbs (b3, b6, F105, 15e, and F91) was reduced or completely abolished - 2G12 binding was largely unperturbed, indicating these proteins were not grossly misfolded [Pantophlet2003]

- 2G12: CD4BS MAbs b12 (neutralizing) and 205-42-15, 204-43-1, 205-46-9 (nonneutralizing) all cross-competed for binding to monomeric gp120, indicating the topological proximity of their epitopes, however, the nonneutralizing CD4BS MAbs did not interfere with the neutralization activity of MAb b12 - 2G12 was used to normalize and as a control in these experiments Herrera2003]

- 2G12: UK Medical Research council AIDS reagent: ARP3030

- 2G12: NIH AIDS Research and Reference Reagent Program: 1476 Env gp41 Ab type cluster I Donor Susan Zolla-Pazner (Zollas01@ @ mcrcr6.med.nyu) (NYU Med. Center)

References Nyambi1998, Gorny2000b Gorny2000a, Nyambi2000

- 1367: Using a whole virion-ELISA method, 18 human MAbs were tested for their ability to bind to a panel of 9 viruses from clades A, B, D, F, G, and H anti-gp41 Abs 98-6, 1367 and 1342 were not able to bind detectably with any of the viruses from any clade [Nyambi1998] 


\begin{tabular}{|c|c|c|c|}
\hline No. & MAb ID & Author's Location & Neutralizing Immunogen \\
\hline & & $\begin{array}{l}\text { - 1367: A cluster I epitope that binds to rgp41 } \\
\text { fusogenic form of gp41 - this MAb doesn't } \\
\text { [Gorny2000b] } \\
\text { 1367: Binding of panel of } 21 \text { MAbs to solub } \\
\text { MAb 50-69 bound with a } 5 \text { fold preference } \\
\text { - } 1 \text { Gorny2000a] } \\
\text { reactivity - } 1367 \text { weakly bound to the major } \\
\text { these isolates [Nyambi2000] }\end{array}$ & $\begin{array}{l}\text { 647, recognizes a peptide N51-C43 complex trimer of heterodimers that approximates the core of the } \\
\text { with either of the peptides N51 or C43 individually - MAbs 50-69 and } 1367 \text { had similar properties } \\
\text { gomeric gp140 versus gp41 or gp120 monomers was compared - no MAb was oligomer specific, but gp } 41 \\
\text { e oligomer, while other gp41 MAbs }(1367,98-6,167-\mathrm{D}, 1281,1342 \text {, and 1379) did not show a preference } \\
\text { were tested for binding to } 47 \text { MAbs, including } 5 \text { cluster I anti-gp41 MAbs which showed good cross clade } \\
\text { isolates - no neutralizing activity was observed when tested with } 5 \text { isolates, but } 1367 \text { did not bind well to }\end{array}$ \\
\hline 1047 & $\begin{array}{l}126-6 \\
(S Z-126.6)\end{array}$ & $\begin{array}{l}\text { Env gp41 (HXB2) } \\
\text { Ab type cluster II Donor Susan Zolla-Paz } \\
\text { References Robinson1990b Robinson1991 } \\
\text { - 126-6: No enhancing activity for HIV-1 IIIB } \\
\text { - 126-6: No enhancing or neutralizing activity } \\
\text { - 126-6: Specific for a conformational epitope } \\
\text { - 126-6: Called SZ-126.6 [Eddleston1993] } \\
\text { - 126-6: One of several anti-gp41 MAbs that } \\
\text { that the construct has retained aspects of nol } \\
\text { - 126-6: Discontinuous epitope recognizing re } \\
\text { binding [Binley1996] } \\
\text { - 126-6: This cluster II MAb binds to a confo } \\
\text { reacted similarly) it binds to a peptide N51- } \\
\text { nor to N51 alone - MAb 126-6 was biotinyl } \\
\text { liquid phase [Gorny2000b] } \\
\text { - 126-6: } 26 \text { HIV-1 group M isolates (clades A } \\
\text { and 1281 bound across clades, but usually } \\
\text { MAbs [Nyambi2000] } \\
\text { - 126-6: NIH AIDS Research and Reference }\end{array}$ & 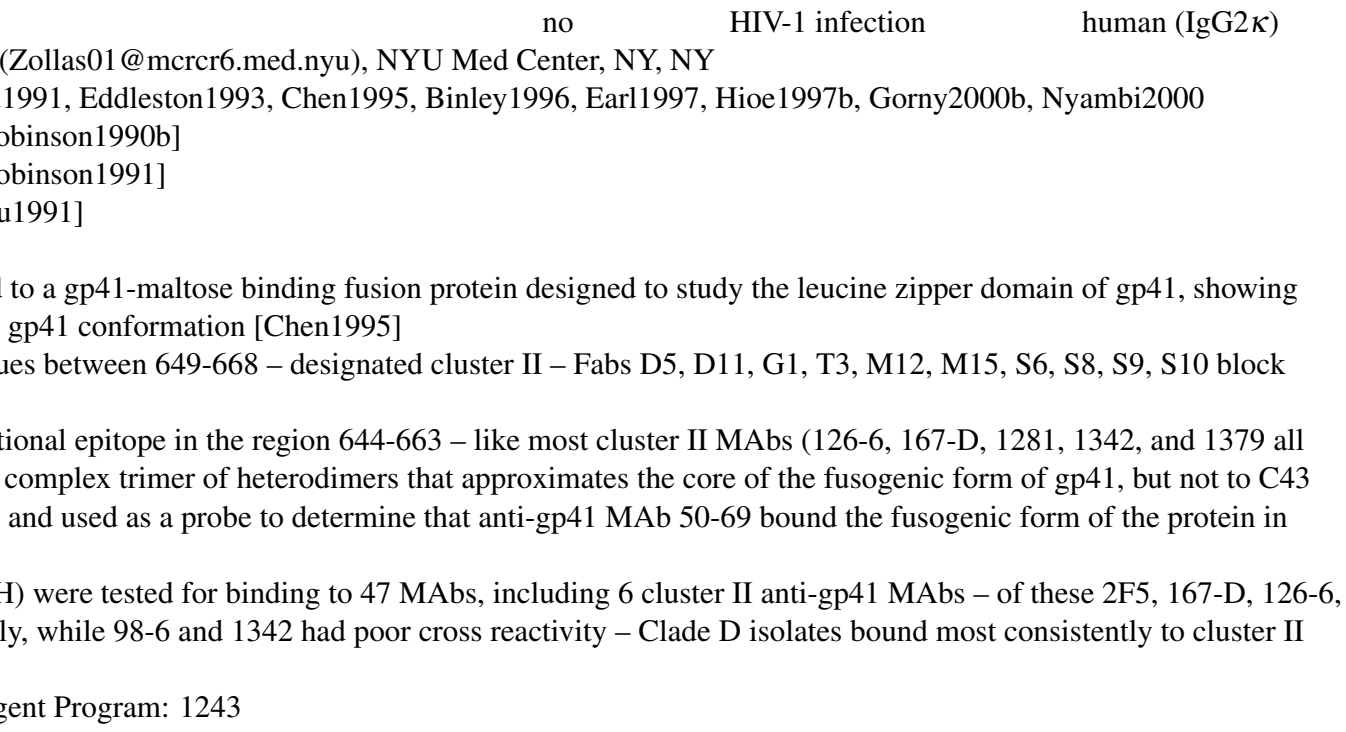 \\
\hline 1048 & 2 & $\begin{array}{l}\text { Env } \\
\text { Ab type cluster II Donor Susan Zolla-P } \\
\text { References Nyambi1998, Gorny2000b G } \\
\text { - 1342: Using a whole virion-ELISA metho } \\
\text { anti-gp41 Abs 98-6, } 1367 \text { and 1342 were } \mathrm{r} \\
\text { - 1342: This cluster II MAb is a conformati } \\
\text { reacted similarly) it binds to a peptide N51 } \\
\text { nor to N51 alone [Gorny2000b] } \\
\text { - 1342: Binds within the region gp41 647-6 } \\
\text { no MAb was oligomer specific, but gp41 } \\
\text { 1342, and 1379) did not show a preference }\end{array}$ & $\begin{array}{l}\text { no } \\
\text { ollas01@ mcrcr6.med.nyu) (NYU Med. Center) } \\
\text { 0a Nyambi2000 } \\
\text { man MAbs were tested for their ability to bind to a panel of 9 viruses from clades A, B, D, F, G, and H - } \\
\text { to bind detectably with any of the viruses from any clade [Nyambi1998] } \\
\text { ope that binds in the region 644-663 - like most cluster II MAbs (126-6, 167-D, 1281, 1342, and } 1379 \text { all } \\
\text { mplex trimer of heterodimers that approximates the core of the fusogenic form of gp41, but not to C43 } \\
\text { ding of panel of } 21 \text { MAbs to soluble oligomeric gp140 versus gp41 or gp120 monomers was compared - } \\
69 \text { bound with a } 5 \text { fold preference for the oligomer, while other gp41 MAbs (1367, 98-6, 167-D, 1281, } \\
2000 \text { a }\end{array}$ \\
\hline
\end{tabular}




\begin{tabular}{|c|c|c|c|c|c|}
\hline No. & MAb ID & Author's Location & Sequence & Neutralizing Immunogen & Species(Isotype) \\
\hline & & \multicolumn{4}{|c|}{$\begin{array}{l}\text { - 1342: } 26 \text { HIV-1 group M isolates (clades A to H) were tested for binding to } 47 \text { MAbs, including } 6 \text { cluster II anti-gp41 MAbs - of these } 2 \text { F5, } 167-\mathrm{D} \text {, } 126-6 \text {, } \\
\text { and } 1281 \text { bound across clades, but usually weakly, while } 98-6 \text { and } 1342 \text { had poor cross reactivity - Clade D isolates bound most consistently to cluster II } \\
\text { MAbs - no neutralizing activity was observed when tested with } 5 \text { isolates, but } 1342 \text { did not bind to these isolates [Nyambi2000] }\end{array}$} \\
\hline 1049 & 1379 & \multicolumn{4}{|c|}{$\begin{array}{l}\text { Ab type cluster II Donor Susan Zolla-Pazner (Zollas01@ @mcrcr6.med.nyu) (NYU Med. Center) } \\
\text { References Gorny2000b Gorny2000a } \\
\text { 1379: This cluster II MAb binds to a conformational epitope in the region 644-663 - like most cluster II MAbs (126-6, 167-D, 1281, 1342, and 1379 all } \\
\text { reacted similarly) it binds to a peptide N51-C43 complex trimer of heterodimers that approximates the core of the fusogenic form of gp41, but not to C43 } \\
\text { nor to N51 alone [Gorny2000b] } \\
\text { 1379: Binds within the region gp41 647-682 - binding of panel of } 21 \text { MAbs to soluble oligomeric gp140 versus gp41 or gp120 monomers was compared - } \\
\text { no MAb was oligomer specific, but gp41 MAb 50-69 bound with a } 5 \text { fold preference for the oligomer, while other gp41 MAbs (1367, 98-6, 167-D, 1281, } \\
\text { 1342, and 1379) did not show a preference [Gorny2000a] }\end{array}$} \\
\hline 1050 & Fab D11 & $\begin{array}{l}\text { Env } \\
\text { Ab type cluster II } \\
\text { References Binley1996 } \\
\text { Fab D11: Binds to cluster II region - comp }\end{array}$ & vith MAbs 1 & no HIV-1 infection & human $(\operatorname{IgG} 1 \kappa)$ \\
\hline 1051 & Fab D5 & $\begin{array}{l}\text { Env gp41 (LAI) } \\
\text { Ab type cluster II } \\
\text { References Binley1996 } \\
\text { Fab D5: Binds to cluster II region - compe }\end{array}$ & h MAbs 12 & $\begin{array}{l}\text { no HIV-1 infection } \\
\text { nation sensitive - variable regions }\end{array}$ & human $(\operatorname{IgG1} \kappa)$ \\
\hline 1052 & Fab G1 & $\begin{array}{l}\text { Env gp41 (LAI) } \\
\text { Ab type cluster II } \\
\text { References Binley1996 } \\
\text { Fab G1: Binds to cluster II region - compe }\end{array}$ & MAbs 12 & $\begin{array}{l}\text { no HIV-1 infection } \\
\text { nation sensitive - variable regions }\end{array}$ & human $(\operatorname{IgG} 1 \kappa)$ \\
\hline 1053 & Fab M10 & $\begin{array}{l}\text { Env gp41 (LAI) } \\
\text { Ab type cluster II } \\
\text { References Binley1996, Parren1997c } \\
\text { Fab M10: Binds to cluster II region - comp } \\
\text { Fab M10: Does not bind to MN native olige }\end{array}$ & $\begin{array}{l}\text { ith MAbs } 1 \\
\text { ut does bin }\end{array}$ & $\begin{array}{l}\text { no HIV-1 infection } \\
\text { rmation sensitive - variable regio } \\
0 \text { and rgp140 [Parren1997c] }\end{array}$ & d [Binley1996] \\
\hline 1054 & Fab M12 & $\begin{array}{l}\text { Env gp41 (LAI) } \\
\text { Ab type cluster II } \\
\text { References Binley1996 } \\
\text { Fab M12: Binds to cluster II region - comp }\end{array}$ & MAbs 1 & $\begin{array}{l}\text { no HIV-1 infection } \\
\text { mation sensitive - variable regio }\end{array}$ & human (IgG1 $\kappa)$ \\
\hline 1055 & Fab M15 & $\begin{array}{l}\text { Env gp41 (LAI) } \\
\text { Ab type cluster II } \\
\text { References Binley1996 } \\
\text { Fab M15: Binds to cluster II region - comp }\end{array}$ & vith MAbs 1 & $\begin{array}{l}\text { no HIV- } \\
\text { rmation sensitive - va }\end{array}$ & human $(\operatorname{IgG} 1 \kappa)$ \\
\hline
\end{tabular}




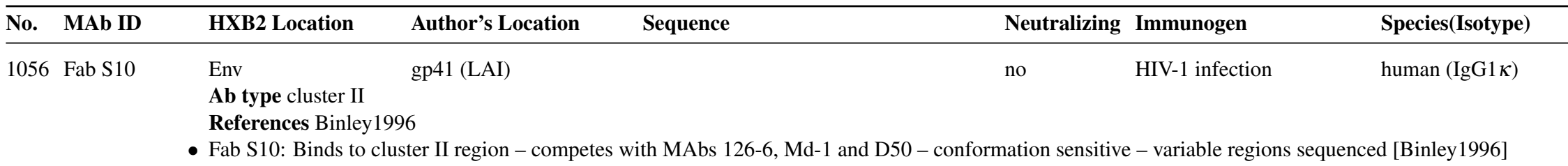

\begin{tabular}{llll}
\hline 1057 Fab S6 & Env gp41 (LAI) & no hIV-1 infection \\
& Ab type cluster II \\
& References Binley1996 \\
& - Fab S6: Binds to cluster II region - competes with MAbs 126-6, Md-1 and D50 - conformation sensitive - variable regions sequenced [Binley1996]
\end{tabular}

\begin{tabular}{lll}
\hline 1058 Fab S8 & Env gp41 (LAI) \\
& Ab type cluster II \\
& References Binley1996 \\
& - Fab S8: Binds to cluster II region - competes with MAbs 126-6, Md-1 and D50 - conformation sensitive - variable regions sequenced [Binley1996] HIV-1 infection \\
\hline
\end{tabular}

\begin{tabular}{llll}
\hline 1059 Fab S9 & Env gp41 (LAI) & no \\
& Ab type cluster II & References Binley1996 \\
& & - Fab S9: Binds to cluster II region - competes with MAbs 126-6, Md-1 and D50 - conformation sensitive - variable regions sequenced [Binley1996]
\end{tabular}

1060 Fab T3 Env gp41 (LAI) homan (IgG1 $\kappa$ )

Ab type cluster II
References Binley 1996

- Fab T3: Binds to cluster II region - competes with MAbs 126-6, Md-1 and D50 - conformation sensitive - variable regions sequenced [Binley1996] gp41

Ab type cluster II Donor R. A. Myers State of Maryland Dept. of Health

References Myers1993, Chen1995, Binley1996

- Md-1: Called MD-1 - discontinuous epitope that binds in the N-terminal region - reacts exclusively with oligomer [Myers1993]

- Md-1: Called MD-1 - one of several anti-gp41 MAbs that bind to a gp41-maltose binding fusion protein designed to study the leucine zipper domain of gp41, showing that the construct has retained aspects of normal gp41 conformation [Chen1995]

- Md-1: Discontinuous epitope recognizing residues between 563-672, does not recognize cluster I disulfide bridge region - reacts almost exclusively with trimers and tetramers on WB - designated cluster II - Fabs D5, D11, G1, T3, M12, M15, S6, S8, S9, S10 block binding [Binley1996]

- Md-1: NIH AIDS Research and Reference Reagent Program: 1223

$10621281(1281-D)$ Env gp41 Human (IgG1 $\lambda$ )

Ab type cluster II, six-helix bundle Donor Susan Zolla-Pazner (Zollas01@ mcrcr6.med.nyu) (NYU Med. Center)

References Hioe1997b, Gorny2000b, Gorny2000a, Verrier2001, Golding2002b

- 1281: Called 1281-D: Four primary isolates showed distinct patterns of sensitivity to neutralization by polyclonal sera or plasma and MAbs - BZ167 was the only isolate inhibited by all polyclonal sera and plasma tested, and was also neutralized by 8/17 MAbs, in particular anti-V3 loop (419-D, 447-52D, 782-D, and 838-D), anti-CD4bd (559/64-D, 654-D and 830-D and a cluster II of gp41 directed MAb (98-6) - isolates 92 HT593 and 91US056 were neutralized by V3 loop (419-D, and 447-52D)and cluster II gp41 (98-6) MAbs at higher concentrations - US4 was neutralized by some of the polyclonal sera/plasma tested and not at all by MAbs individually or by a cocktail of ten MAbs consisting of 419-D, 447-52D, 782-D, 838-D, 559/64-D, 654-D, 450-D, 670-D, 1281-D and 98-6 [Hioe1997b 


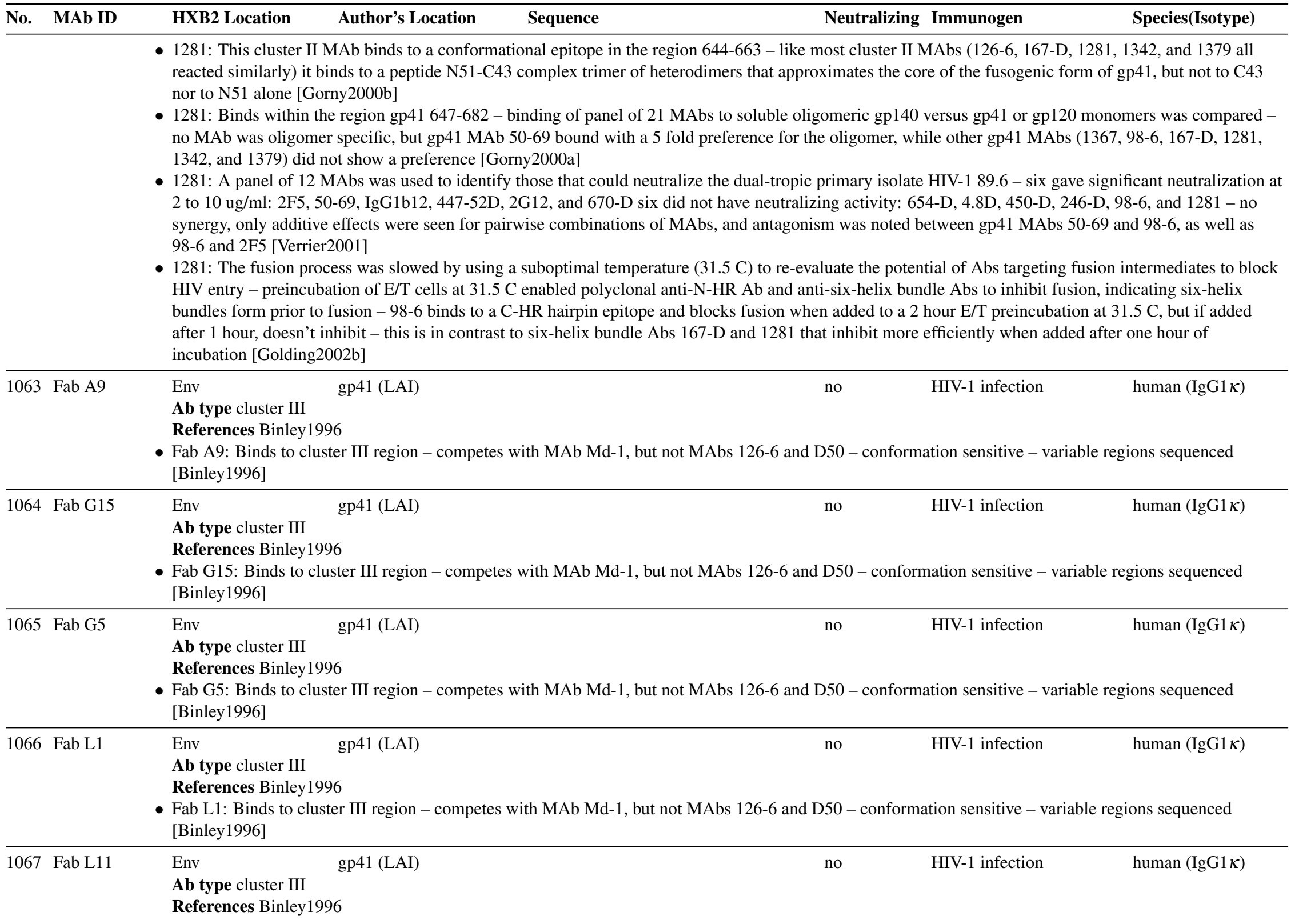




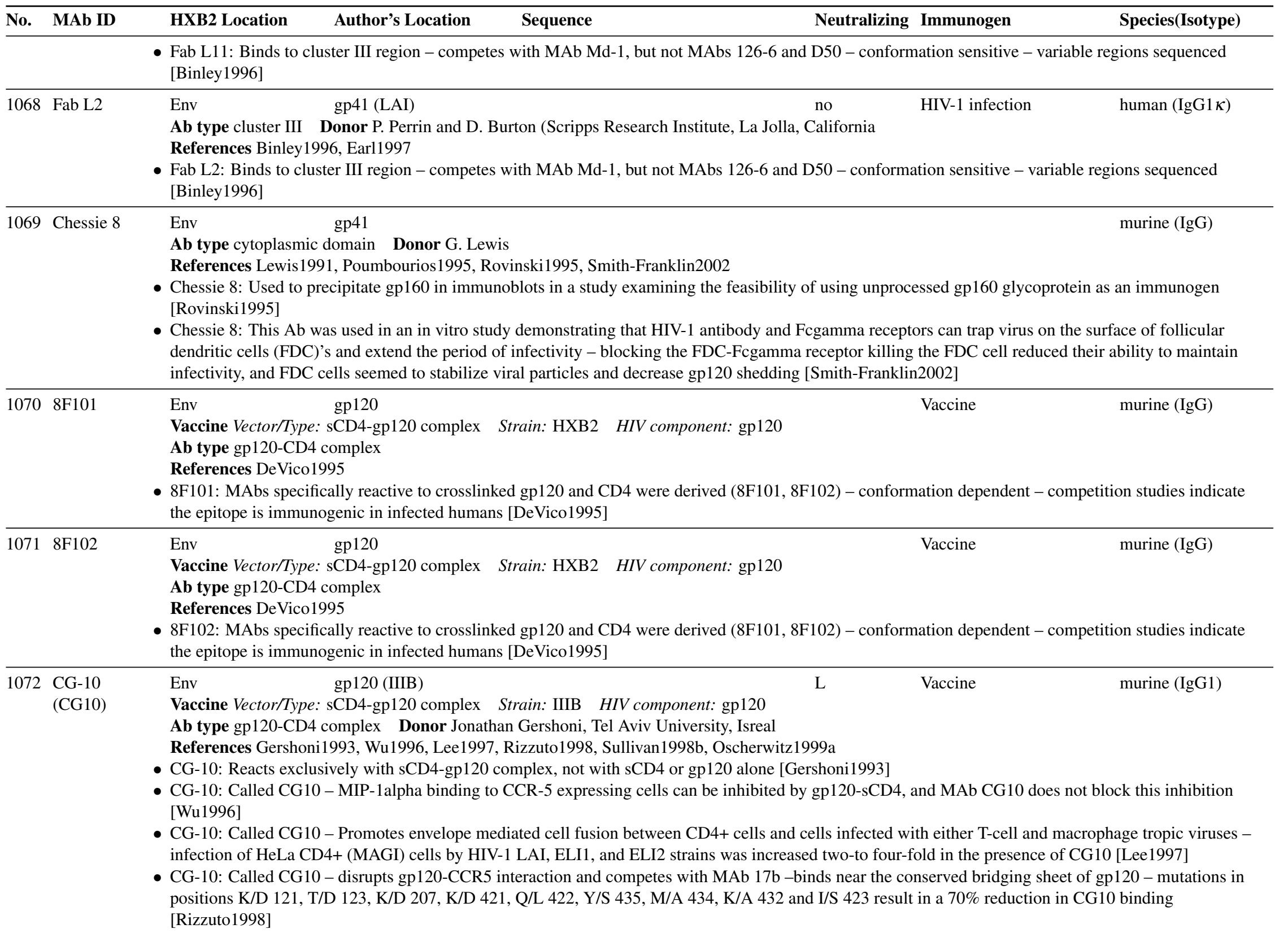




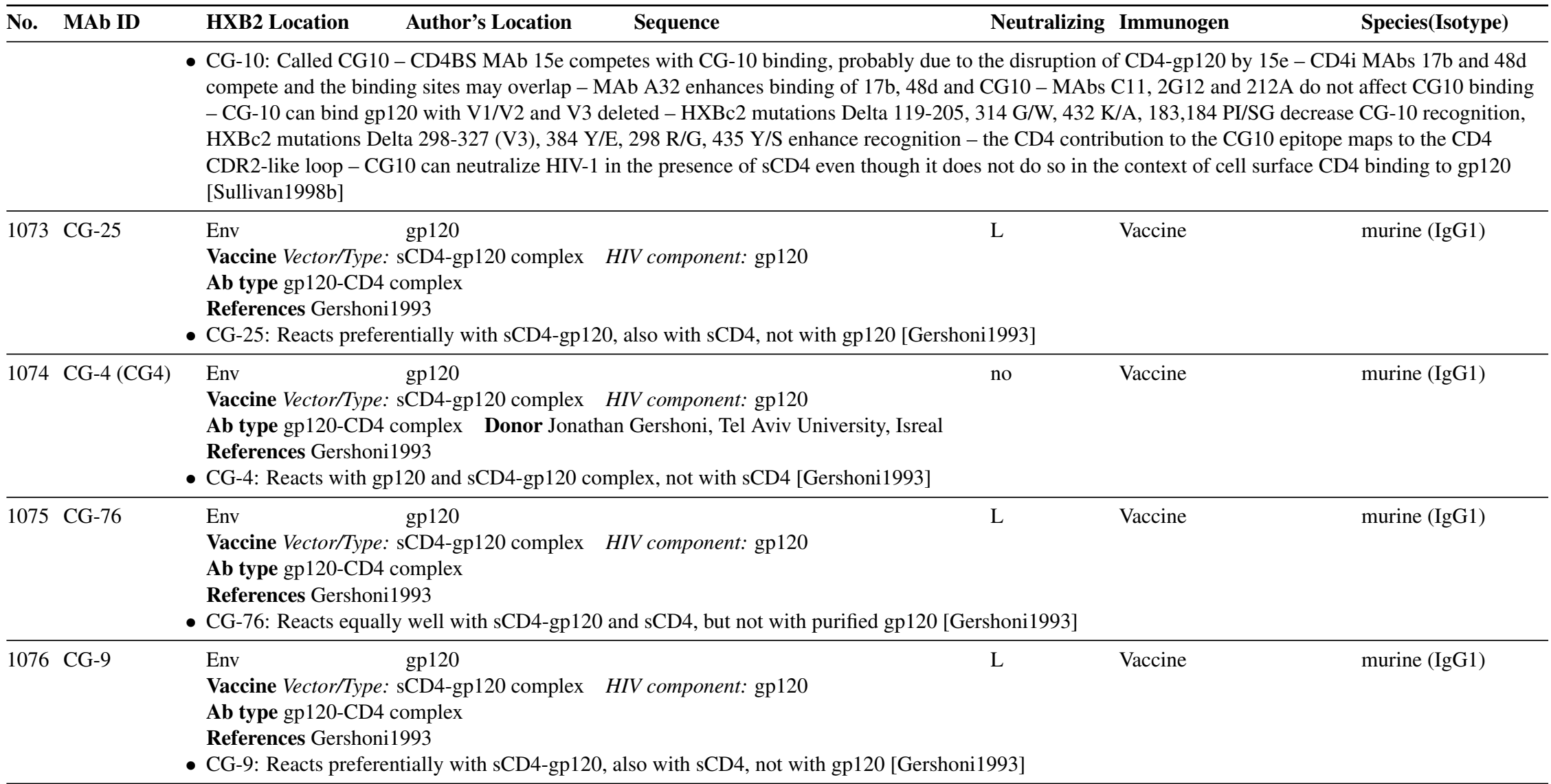

\begin{tabular}{|c|c|c|c|c|}
\hline \multirow{2}{*}{\multicolumn{2}{|c|}{$1077 \quad 105-518$}} & $\begin{array}{ll}\text { Env } & \text { gp41 (608-637 } \\
& \text { HAM112, O group) }\end{array}$ & Vaccine & murine $(\operatorname{IgG} 1 \kappa)$ \\
\hline & & $\begin{array}{l}\text { Vaccine Vector/Type: recombinant protein Strain: HAM112 (group O) } \\
\text { Ab type immunodominant region } \\
\text { References Scheffel1999 } \\
\text { 101-518: Overlapping peptides based on group O HAM112 Env were test }\end{array}$ & $\begin{array}{l}\text { HIV component: gp160 } \\
\text { d for MAb reactivity [Scheffel1999] }\end{array}$ & \\
\hline 1078 & 31A1 & $\begin{array}{l}\text { Env gp41 } \\
\text { Ab type p24+gp41 } \\
\text { References Pollock1989 } \\
\text { 31A1: Denatured virus was used for in vitro stimulation to generate Abs - }\end{array}$ & $\begin{array}{c}\text { no } \\
\text { in vitro stimulation } \\
\text { eacts with both p24 and gp41 [Pollock1989] }\end{array}$ & human $(\operatorname{IgM} \kappa / \lambda)$ \\
\hline
\end{tabular}




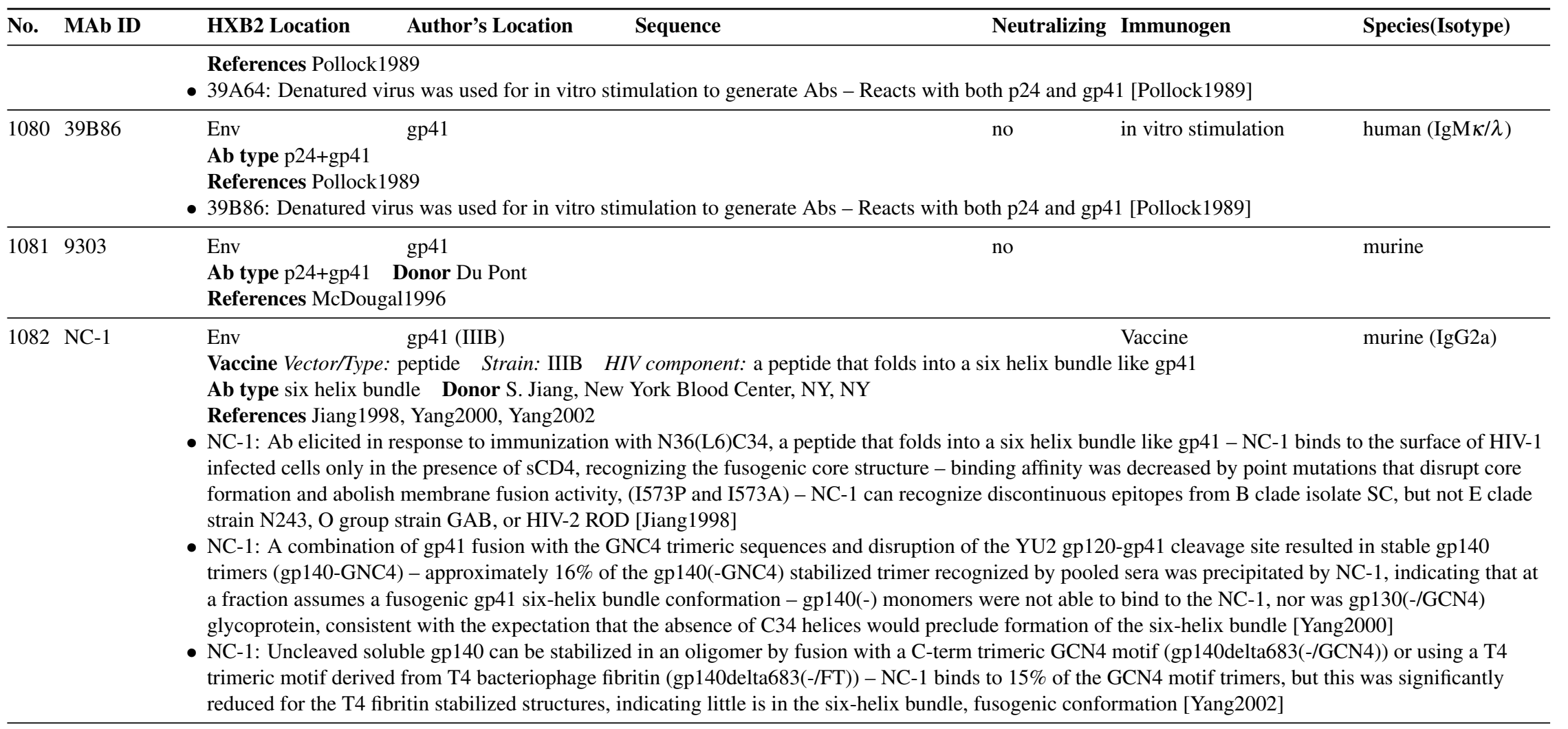




\section{IV-C-15 Nef Antibodies}

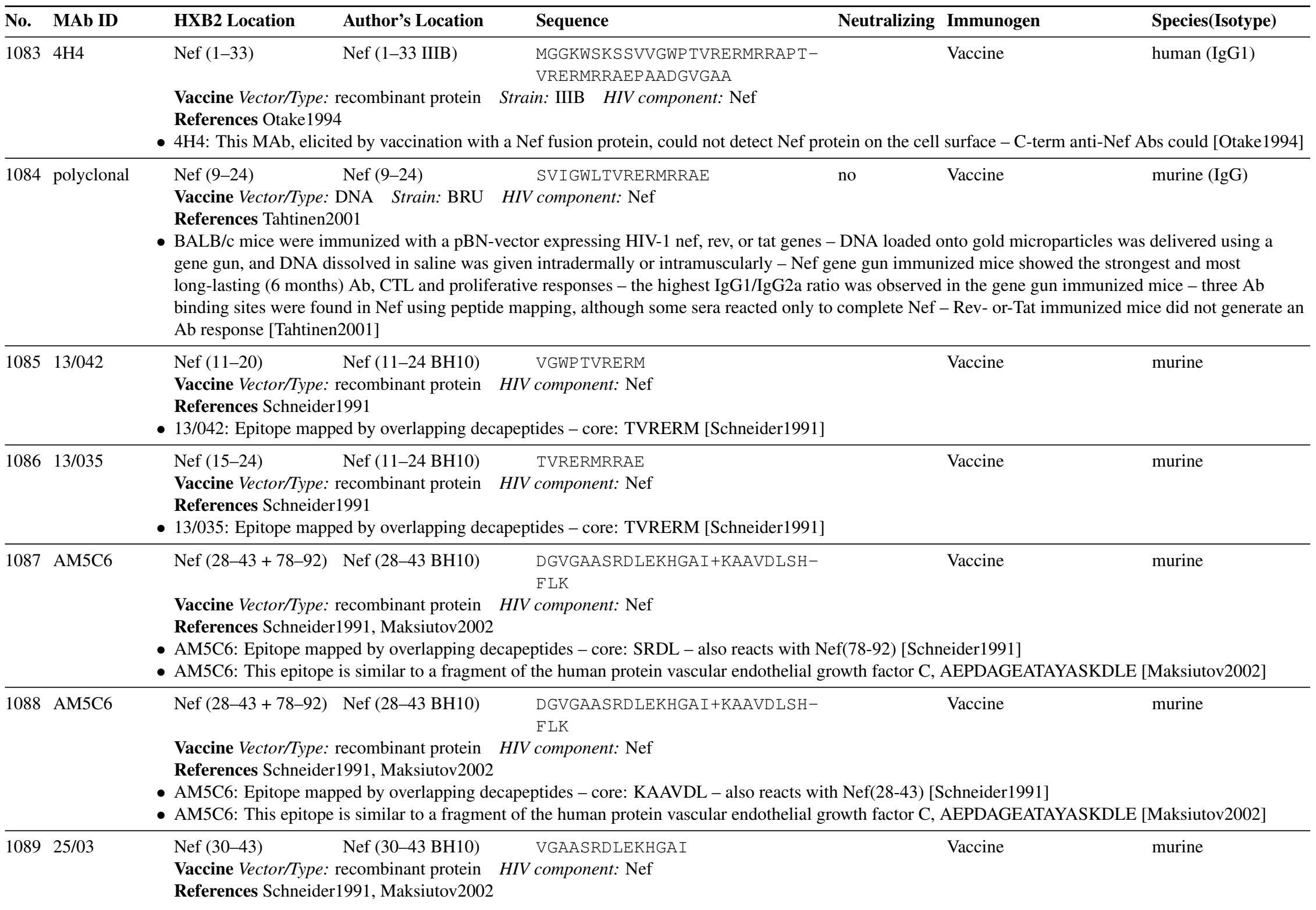




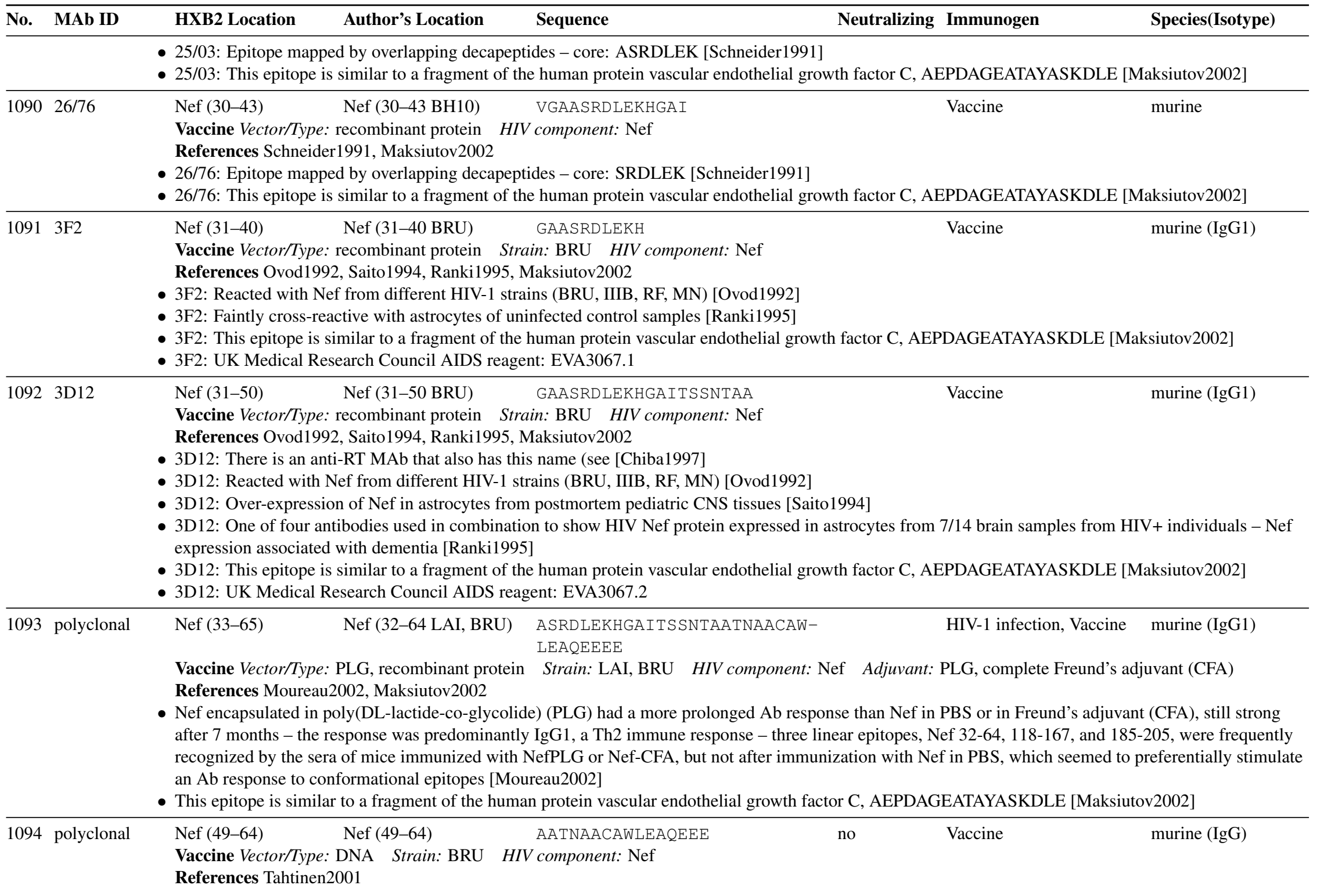




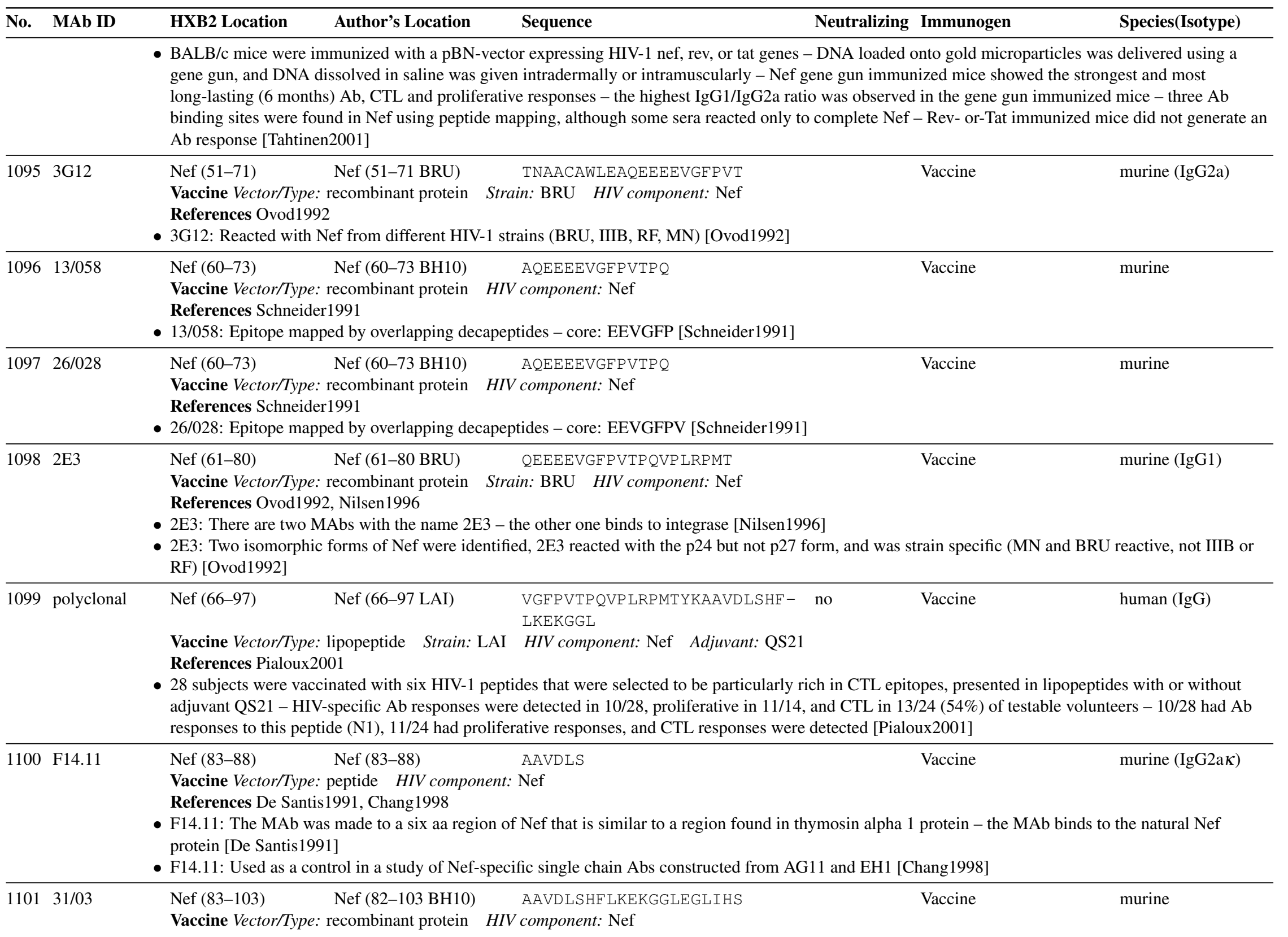




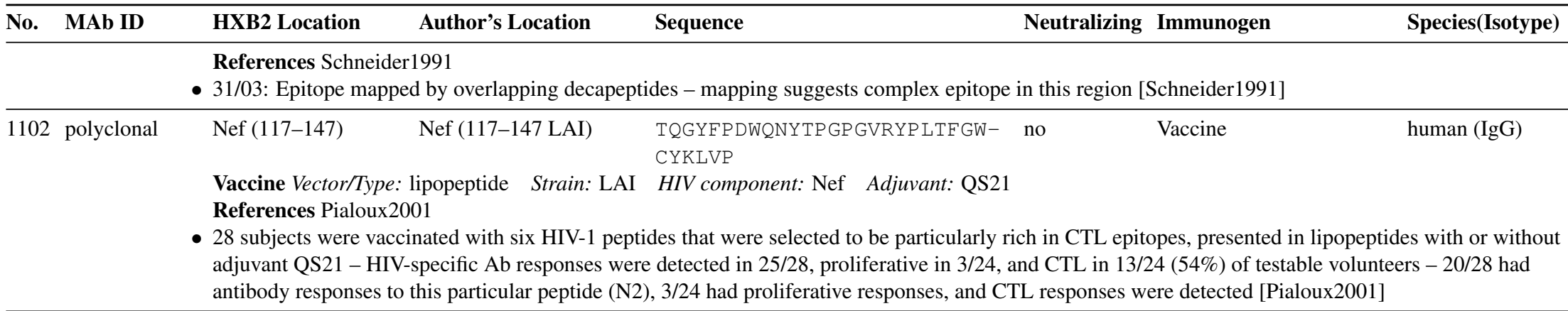

1103 polyclonal Nef(118-133) Nef (118-133) QGYFPDWQNYTPGPGV $\quad$ no

Vaccine Vector/Type: DNA Strain: BRU HIV component: Nef

References Tahtinen2001

- BALB/c mice were immunized with a pBN-vector expressing HIV-1 nef, rev, or tat genes—DNA loaded onto gold microparticles was delivered using a gene gun, and DNA dissolved in saline was given intradermally or intramuscularly-Nef gene gun immunized mice showed the strongest and most long-lasting (6 months) Ab, CTL and proliferative responses-the highest IgG1/IgG2a ratio was observed in the gene gun immunized mice- three Ab binding sites were found in Nef using peptide mapping, although some sera reacted only to complete Nef-Rev- or Tat-immunized mice did not generate an Ab response [Tahtinen2001].

Nef $(119-168)$

Nef (118-167 LAI

GYFPDWQNYTPGPGVRYPLTFGWCY-

KLVPVEPDKVEEANKGENTSLLHPV

HIV-1 infection, Vaccine murine $(\operatorname{IgG} 1)$

Vaccine Vector/Type: PLG, recombinant protein Strain: LAI, BRU HIV component: Nef Adjuvant: PLG, complete Freund's adjuvant (CFA) References Moureau2002, Maksiutov2002

- Nef encapsulated in poly(DL-lactide-co-glycolide) (PLG) had a more prolonged Ab response than Nef in PBS or in Freund's adjuvant (CFA), still strong after 7 months - the response was predominantly IgG1, a Th2 immune response - three linear epitopes, Nef 32-64, 118-167, and 185-205, were frequently recognized by the sera of mice immunized with NefPLG or Nef-CFA, but not after immunization with Nef in PBS, which seemed to preferentially stimulate an $\mathrm{Ab}$ response to conformational epitopes [Moureau2002]

- This epitope is similar to a fragment of the human protein Bone-derived growth factor, PLEPAKLEE, and to Hematopoietic progenitor cell antigen CD34, TSLHPVSQHG [Maksiutov2002]

murine $(\operatorname{IgM})$

References Fujii1993, Otake1994 Fujii1996c. Fujii1996b

- F1: The C-term end of Nef is accessible to Abs at the cell surface - stained IIIB/M10, but not MN/M10, cells [Otake1994] Fujii1993]

- F1: Insect cells expressing myristylated Nef proteins on their cell surface can induce cytolysis of unstimulated CD4+ cells - this response is not due to MHC restricted CTL activity - the cell surface of Nef expressing insect cells carry Nef that can be recognized by MAbs E7 and E9 but not F1 [Fujii1996c]

- F1: A carboxy-terminal domain of Nef on the cell surface induces cytolysis of CD4+ T cells [Fujii1996b]

Nef (151-170) Nef (151-170 BRU) DKVEEANKGENTSLLHPVSL

Vaccine Vector/Type: recombinant protein HIV component: Nef

References Ovod1992, Saito1994, Ranki1995, Maksiutov2002,

- 2F2: Strain specific (MN and BRU reactive, not IIIB or RF) [Ovod1992]

- 2F2: Over-expression of Nef in astrocytes from postmortem pediatric CNS tissue [Saito1994] 


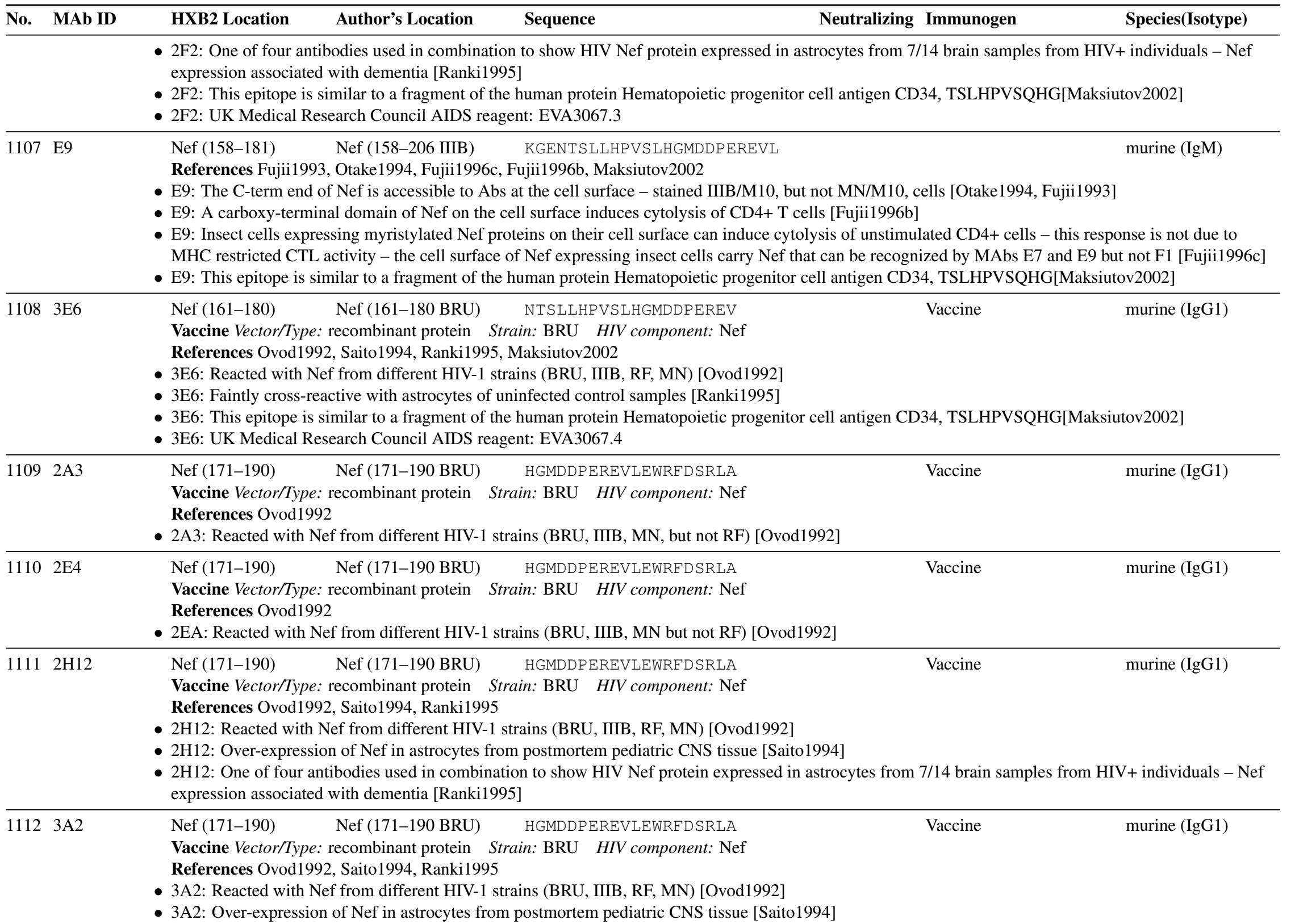




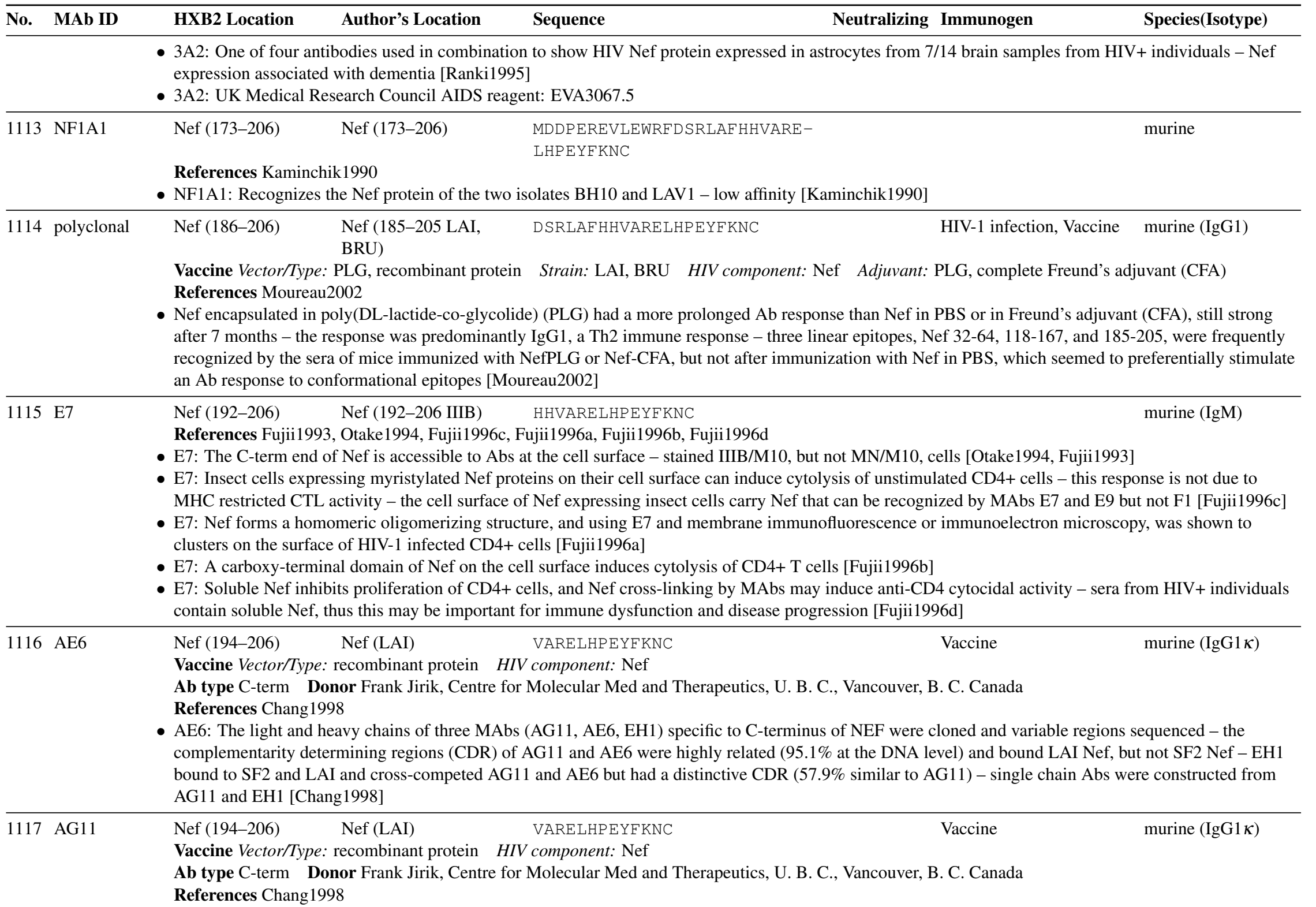




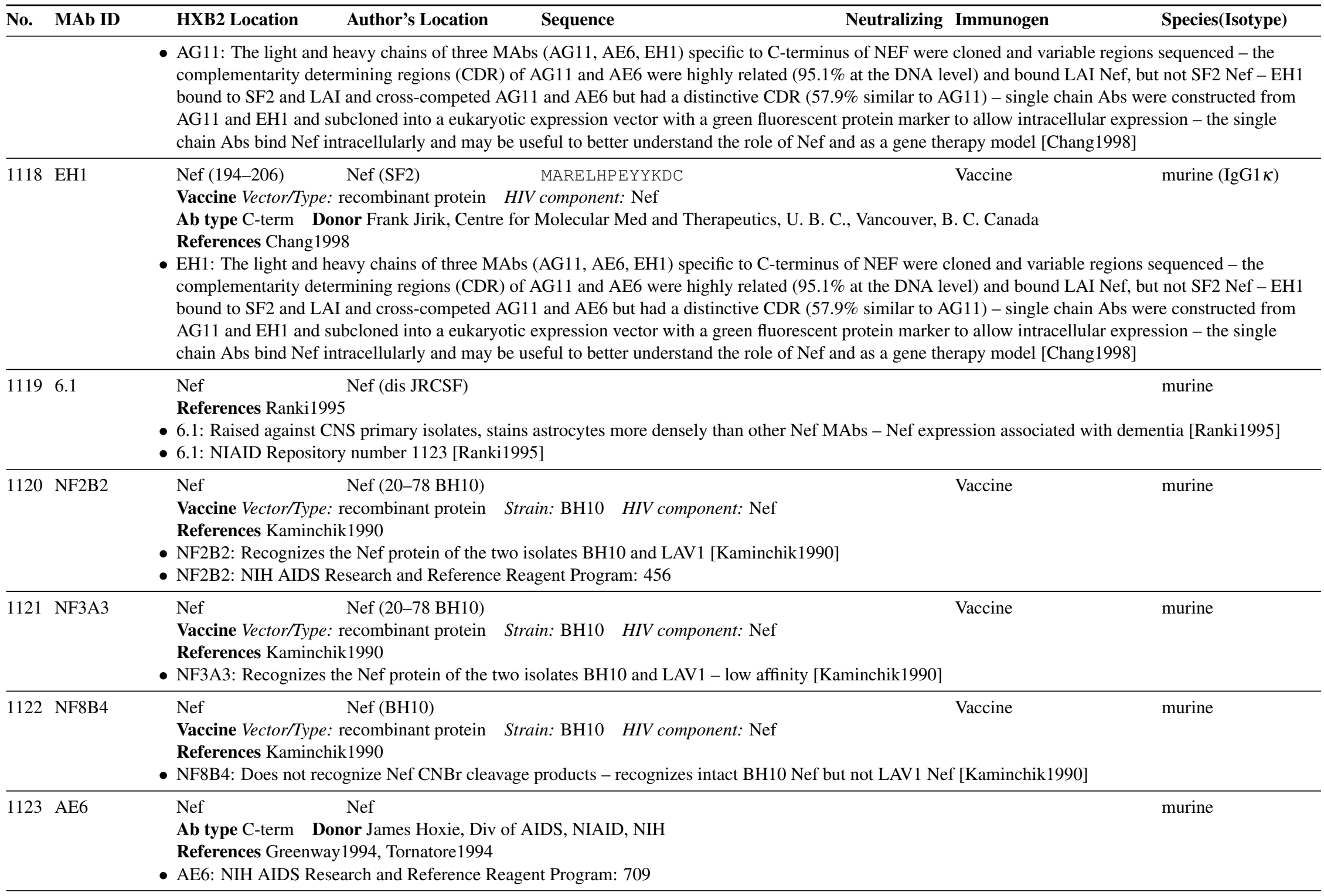




\section{IV-C-16 HIV-1 Antibodies}

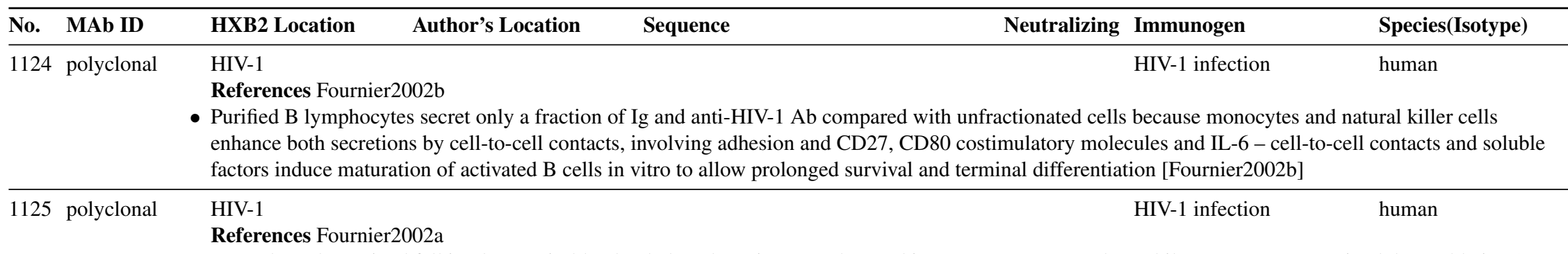

- An early and sustained fall in plasma viral load to below detection was observed in 17 HAART responders while HIV-1 RNA remained detectable in 13 incomplete responders - HIV-1 specific Ab secretion decreased in parallel with plasma viral load - HIV-1 specific Abs became negative in only six responders, and was correlated with greater increases of CD4 T-cell counts and higher levels of HIV-specific IgA secretion at baseline - persistent immune activation may be due to residual HIV antigen [Fournier2002a

\begin{tabular}{lll}
\hline 126 polyclonal & HIV-1 Human \\
& References Subbramanian2002
\end{tabular}

- Sera from 39 patients were used to study the relative prevalence of neutralizing Abs (NAbs), ADCC-Abs and enhancing Abs - 69\% of the sera were positive for NAbs but only $39 \%$ could neutralize in the presence of complement $-60 \%$ had ADCC Abs $-72 \%$ mediated the enhancement of infection in the presence of complement [Subbramanian2002]

1127 polyclonal $\begin{array}{ll}\text { HIV-1 Human (IgA, IgG1) } \\ \text { References Battle-Miller2002 }\end{array}$

- In a study of HIV-1 infected women, ADCC Abs were detected in 16\% (12/51) of cervicovaginal fluids, and 56\% (25/45) of serum samples - 3 women had ADCC in cervical lavage fluids, but not sera, suggesting local production [Battle-Miller2002]

References Wu2002

- IgA1 accounted for the majority of anti-HIV-1 IgA in the saliva in HIV-1 infected individuals - there was no anti-gp41 IgA in saliva, in contrast to plasma lower levels of IgA and IgM were found in saliva than in plasma [Wu2002]

\begin{tabular}{lll}
\hline 1129 polyclonal & HIV-1 \\
& References Hioe1997a \\
& Four primary isolates showed distinct patterns of sensitivity to neutralization by polyclonal sera or plasma and MAbs - BZ167 was the only isolate inhibited \\
& by all polyclonal sera and plasma tested, and was also neutralized by 8/17 MAbs, in particular anti-V3 loop (419-D, 447-52D, 782-D, and 838-D), \\
& anti-CD4bd (559/64-D, 654-D and 830-D and a cluster II of gp41 directed MAb (98-6) - isolates 92HT593 and 91US056 were neutralized by V3 loop \\
& (419-D, and 447-52D)and cluster II gp41 (98-6) MAbs at higher concentrations - US4 was neutralized by some of the polyclonal sera/plasma tested and not \\
& at all by MAbs individually or by a cocktail of ten MAbs consisting of 419-D, 447-52D, 782-D, 838-D, 559/64-D, 654-D, 450-D, 670-D, 1281-D and 98-6 \\
& [Hioe1997a] & HIV-1 infection \\
\hline 1130 polyclonal & HIV-1 HIV-1 infection & References Oelemann2002
\end{tabular}




No. MAb ID HXB2 Location $\quad$ Author's Location $\quad$ Sequence $\quad$ Neutralizing Immunogen

- A urine based commercial EIA kit from Calypte Biomedical Corporation, Berkeley, CA was found to work well as a primary screening for HIV in Brazilian samples - 76 HIV+ samples were correctly identified (100\% sensitivity), and 278/284 negative samples 97.9\% specificity Oelemann2002]

1131 polyclonal HIV-1

HIV-1 infection

human $(\operatorname{IgE})$

References Secord1996, Pellegrino2002

- HIV-specific IgE found in clinically healthy HIV-1 infected children [Secord1996

- Pediatric long term survivors (LTS) have been found to carry HIV-1 specific IgE - serum from these children inhibit HIV-1 production in culture, but this

inhibition did not seem to be due to neutralization, rather due to a cytoxic event - serum lost the HIV-1 inhibitory effect when depleted of IgE

|Pellegrino2002 



\section{IV-D Maps of MAb Locations Plotted by Protein}

Linear epitopes less than twenty-two amino acids long are shown with their IV-D-1 p17 Ab Epitope Map antibody ID and the experimental species.

\begin{tabular}{cl}
\hline Key & Species \\
\hline $\mathrm{h}$ & human \\
$\mathrm{p}$ & non-human primate \\
$\mathrm{m}$ & murine \\
$\mathrm{o}$ & other \\
\hline
\end{tabular}

Table IV-D.1: The species for which the epitopes react
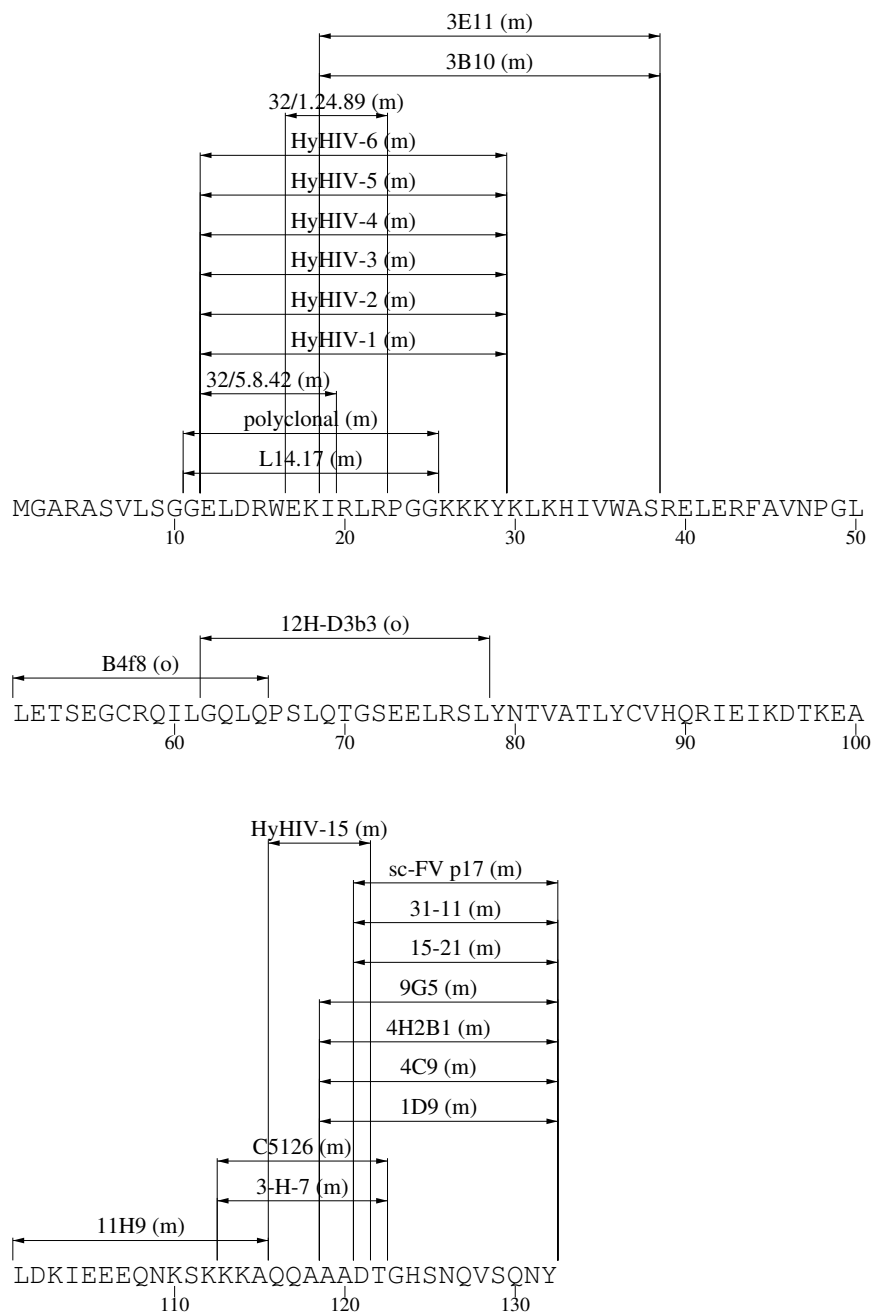


\section{IV-D-2 p24 Ab Epitope Map}

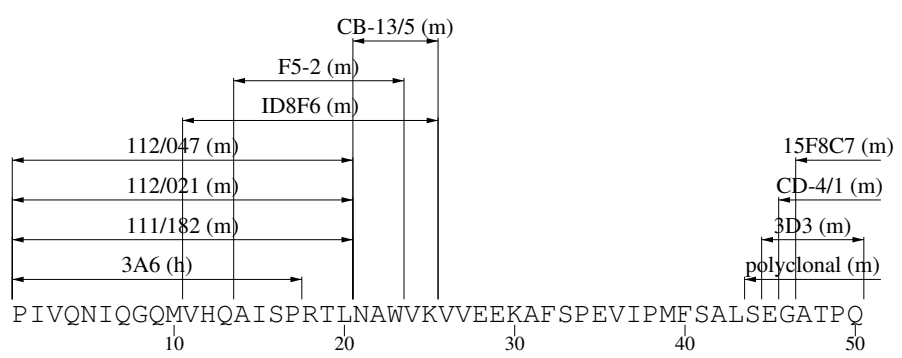

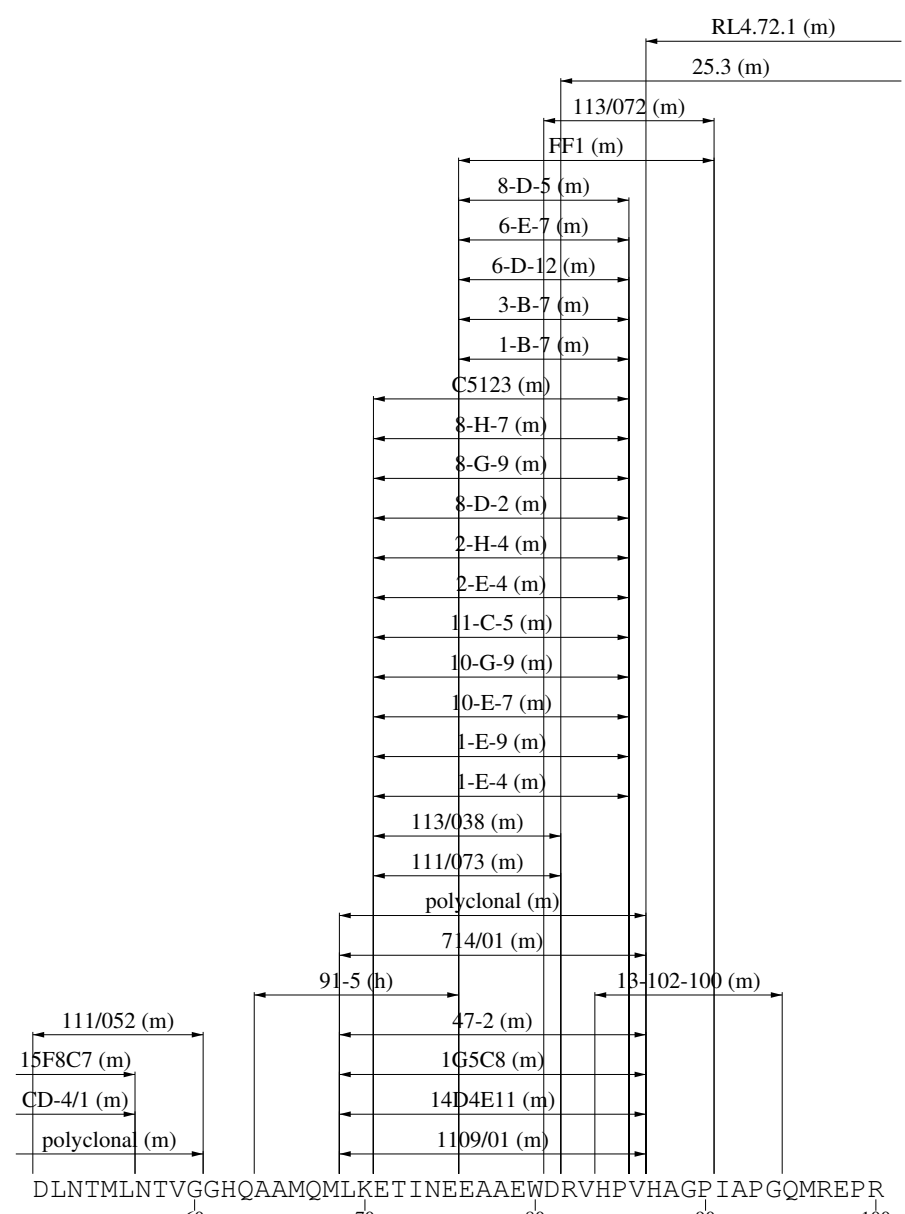

90

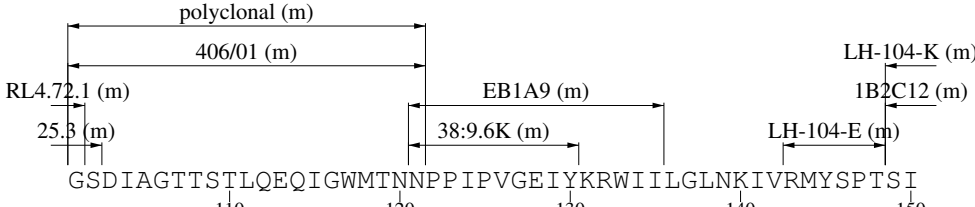

110 


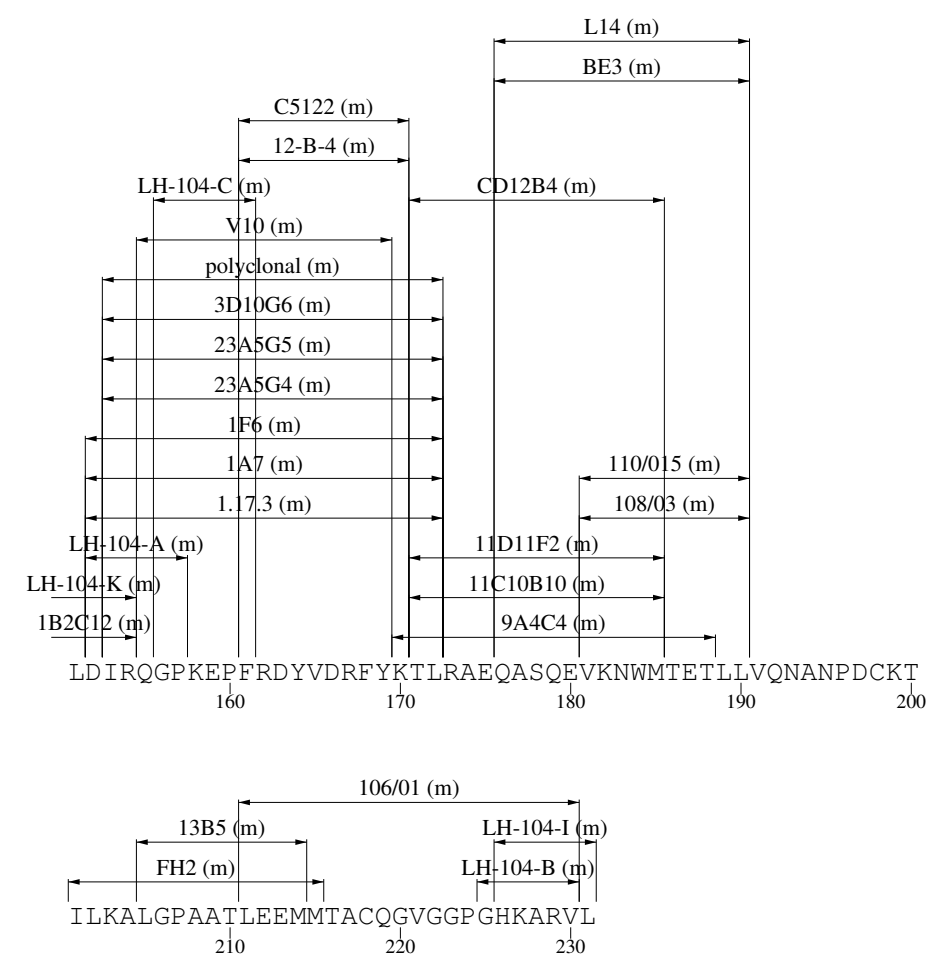

IV-D-3 p2p7p1p6 Ab Epitope Map

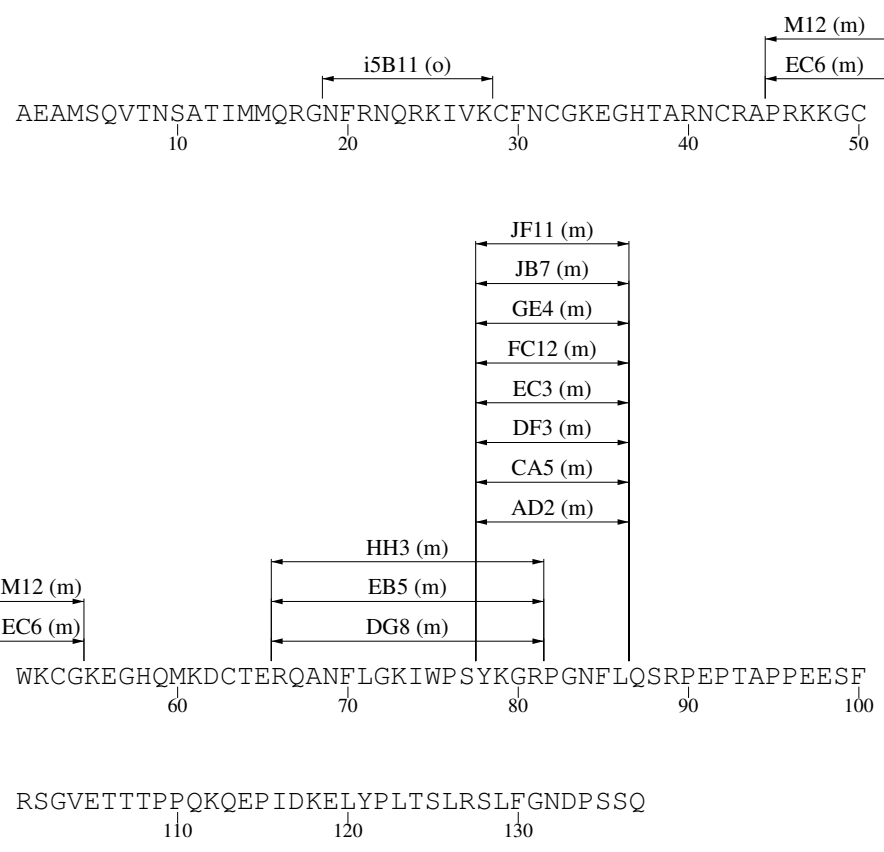




\section{IV-D-4 Protease Ab Epitope Map}

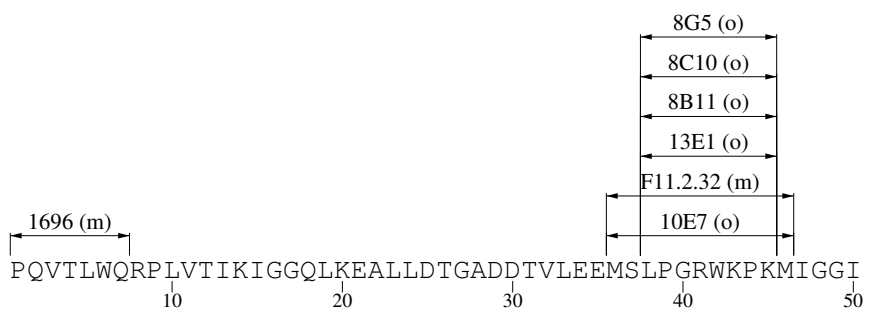

GGF IKVRQYDQI LIEICGHKAIGTVLVGP TPVNI I GRNLLTQIGCTLNF

$$
\begin{array}{llll}
60 & 70 & 80 & 90
\end{array}
$$

\section{IV-D-5 RT Ab Epitope Map}

P ISP IETVPVKLKP GMDGP KVKQWP LTEEKIKALVEICTEMEKEGKISKI

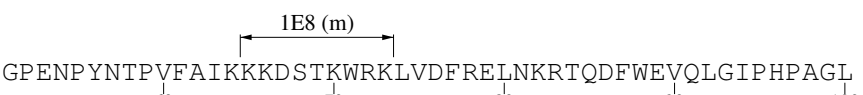
80 100

$\begin{array}{cccc}\text { KKKKSVTVLDVGDAYF SVPLDEDFRKYTAF T IPS INNETPGIRYQYNVLP } \\ 110 & 120 & 130 & 140\end{array}$

QGWKGSPAIFQSSMTKILEPFRKQNPDIVIYQYMDDLYVGSDLEIGQHRT $160-1700180$

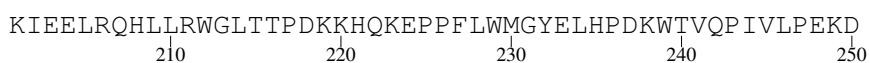

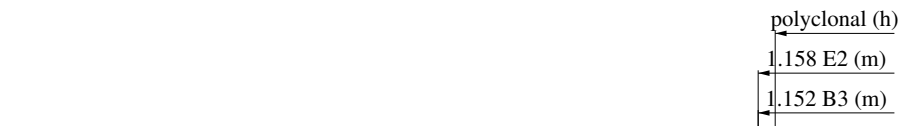

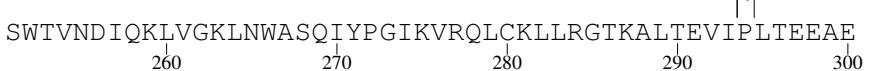

polyclonal (h)

$1.158 \mathrm{E} 2(\mathrm{~m})$

$1.152 \mathrm{~B} 3(\mathrm{~m})$

LELAENRE I LKEPVHGVYYDP SKDLIAEIQKQGQGQWTYQIYQEPFKNLK $\begin{array}{lllll}310 & 320 & 330 & 340 & 350\end{array}$

WWTEYWQATWIPEWEFVNTPPLVKLWYQLEKEPIVGAETFYVDGAANRET
410


KLGKAGYVTNRGRQKVVTLTDTTNQKTELQAIYLALQD SGLEVNIVTD SQ
460
470

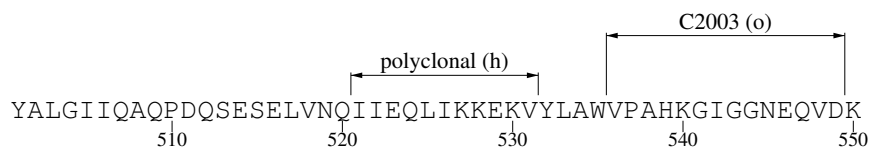

LVSAGIRKVL

\section{IV-D-6 Integrase Ab Epitope Map}

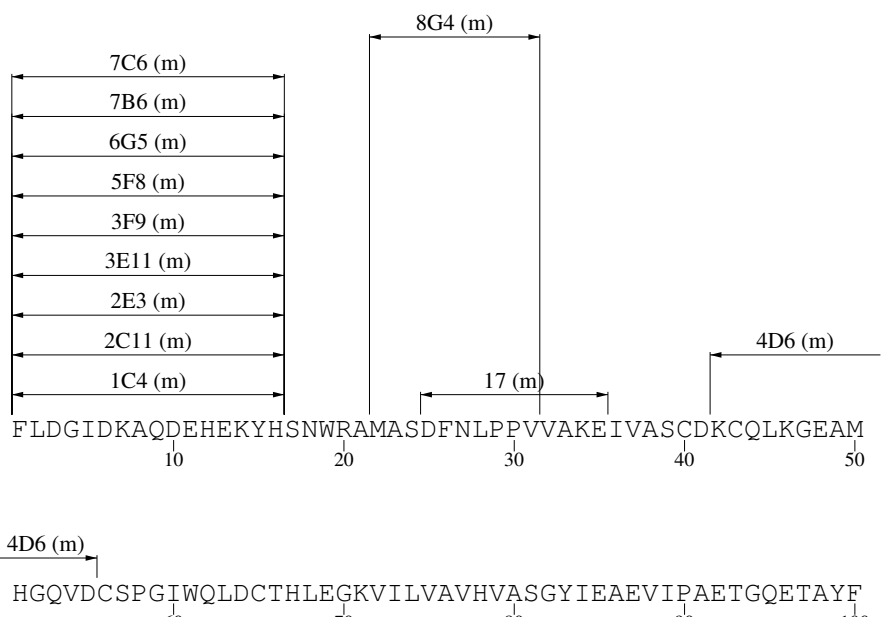

$$
\text { 60 }
$$
$\begin{array}{ccccc}\text { LLKLAGRWPVKT I HTDNGSNF TGATVRAACWWAGIKQEFGIPYNPQSQGV } \\ 110 & 120 & 130 & 140 & 150\end{array}$

VESMNKELKKIIG

QAEHLKTAVQMAVF I HNFKRKGGIGGYSAGERI

$$
160
$$

170

180

190
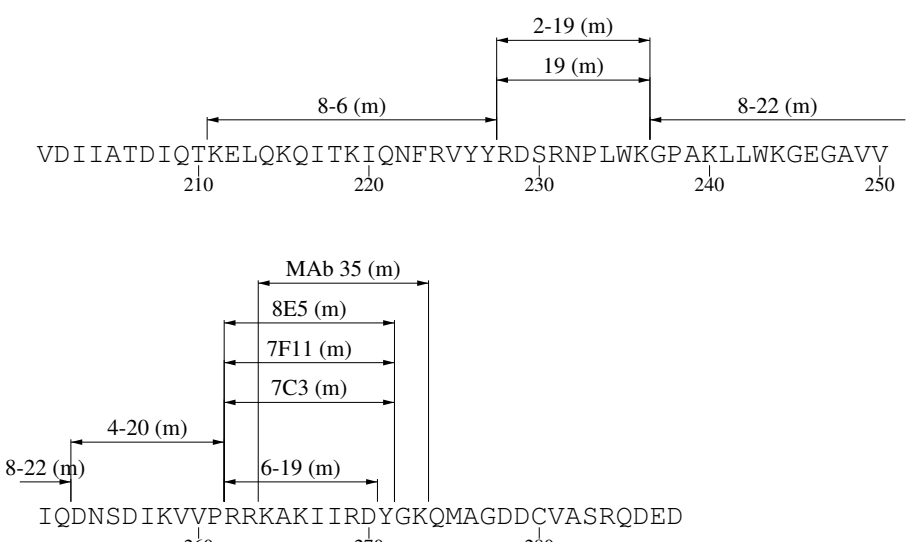


\section{IV-D-7 Rev Ab Epitope Map}
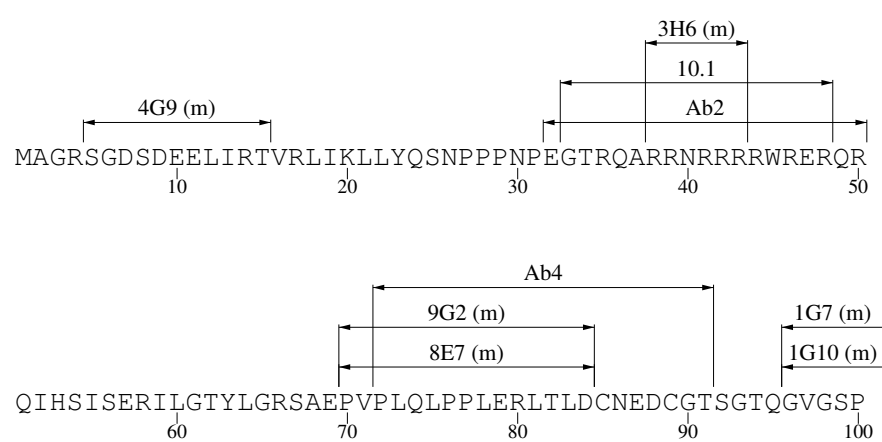

\section{IV-D-8 Tat Ab Epitope Map}

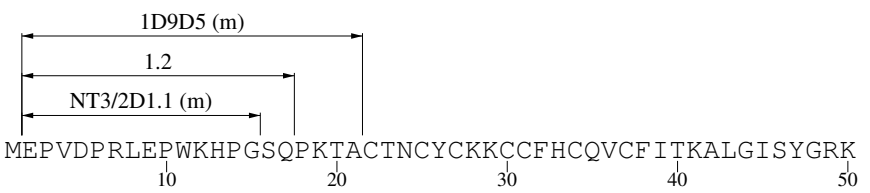

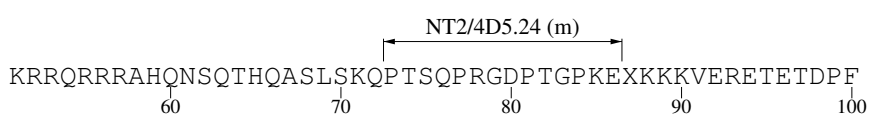

$\underset{101}{D}$

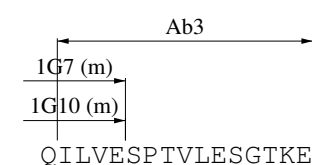

110 


\section{IV-D-9 Vif Ab Epitope Map}

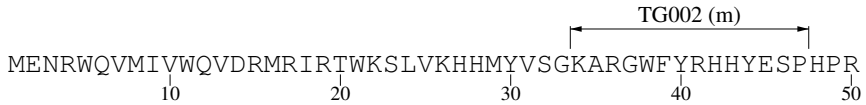

$$
\begin{aligned}
& \text { ISSEVHIP LGDARLVITTYWGLHT GERDWHLGQGVS IEWRKKRYSTQVDP } \\
& \begin{array}{llll}
1 & 1 & 1 & 1
\end{array} \\
& \begin{array}{cccc}
\text { ELADQLIHLYYFDCF SD SAIRKALLGH IVSPRCEYQA GHNKVGSLQYLAL } \\
110 & 120 & 130 & 140
\end{array} \\
& \underset{\substack{160 \\
\text { AALITPKKIKPPLPVTKLTEDRWNKP QKTKGHRGSHTMNGH }}}{170} \underset{180}{\mathrm{TG001}(\mathrm{m})}
\end{aligned}
$$

\section{IV-D-10 Vpr Ab Epitope Map}

MEQAPEDQGP QREP HNEWT LELLEELKNEAVRHFPRIWLHGLGQH I YETY

GD TWAGVEAI IRI LQQLLF I HFRIGCRHSR I GVTRQRRARNGASRS

$$
\begin{array}{cccc}
60 & 70 & 10 & 90
\end{array}
$$




\section{IV-D-11 Vpu Ab Epitope Map}

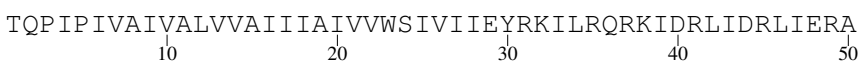

EDSGNESEGE I SALVEMGVEMGHHAPWDVDDI

\section{IV-D-12 gp160 Ab Epitope Map}
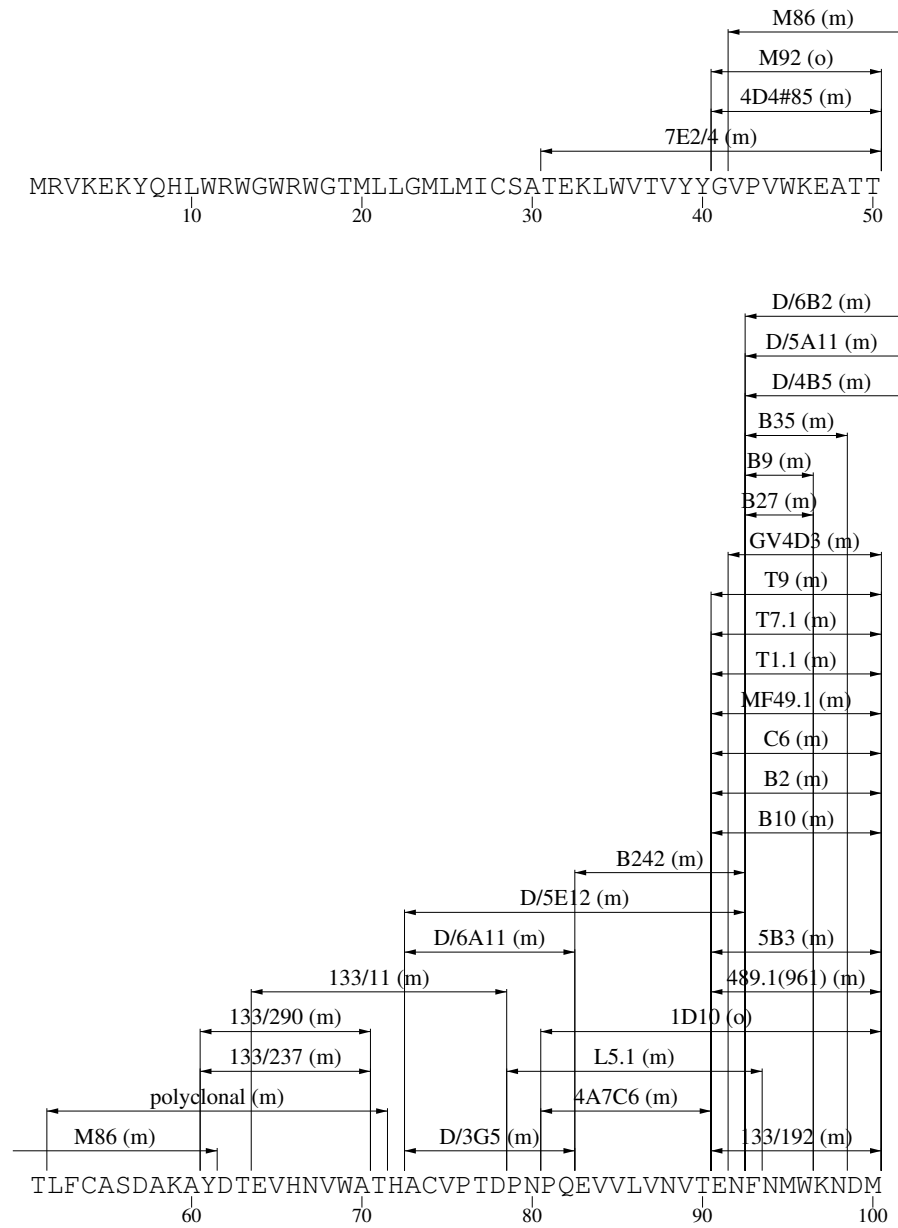

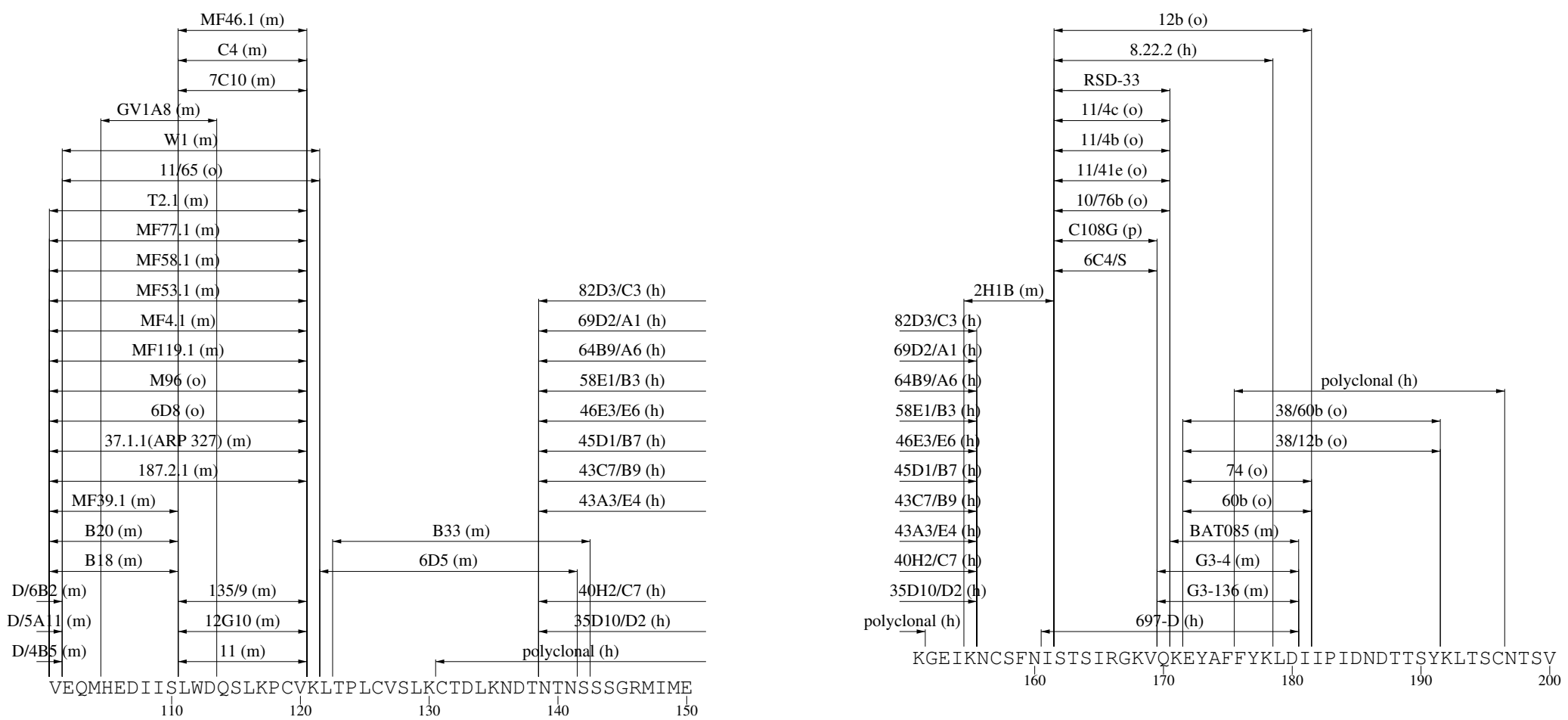


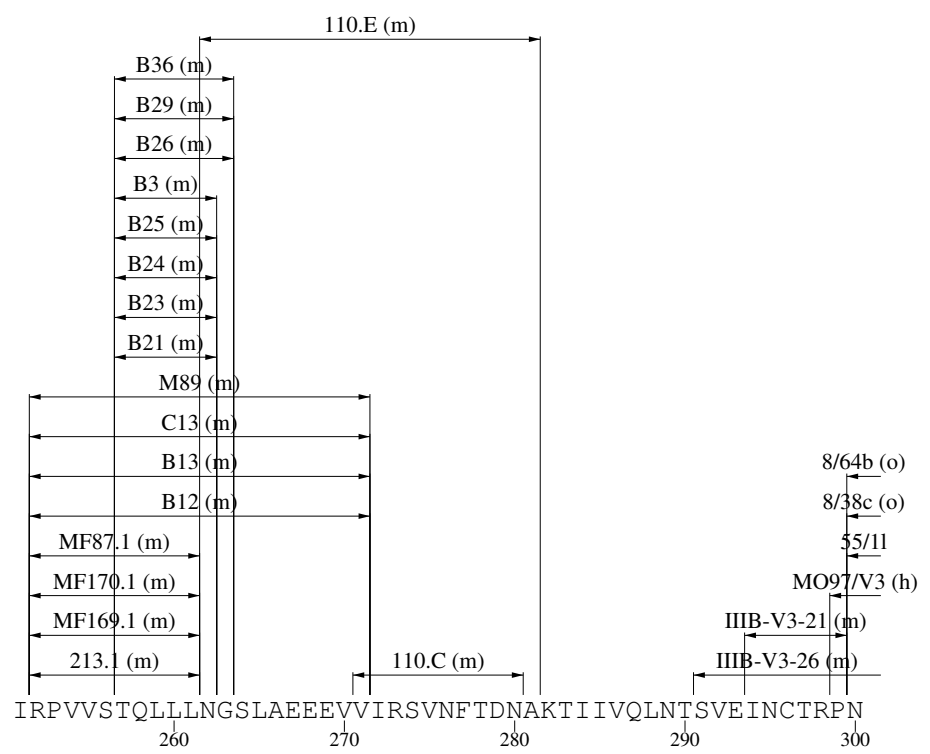

IIIB-V3-21 (m)

VEINCTRPN 


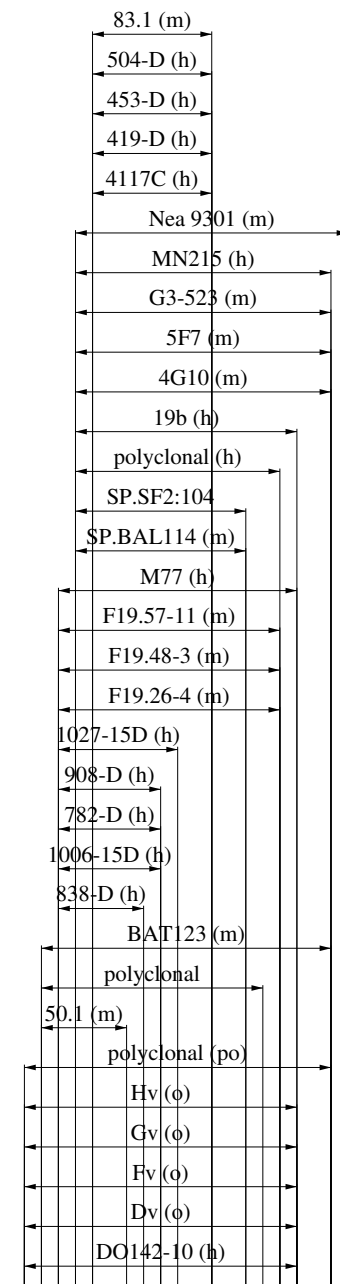

NNTRKRIRIQRGP GRAFVTIGKI GNMRQAHCN I SRAKWNNTLKQIASKLR

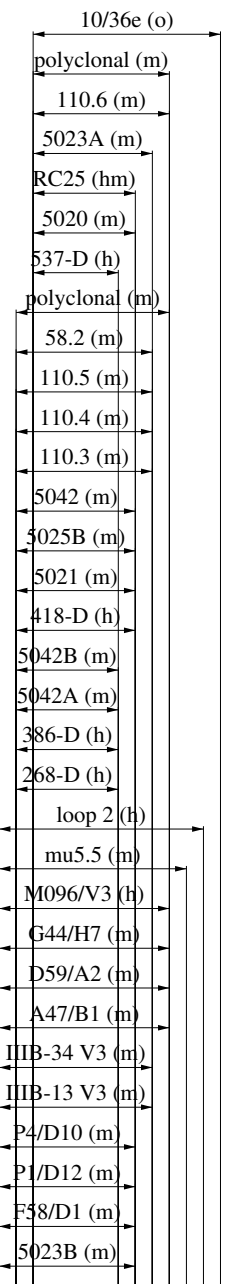

NNTRKRIRIQRGP GRAFVT I GKI GNMRQAHCNISRAKWNNTLKQIASKLR 
$9205(\mathrm{~m})$

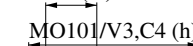

$694 / 98-\mathrm{D}-(\mathrm{h})$

No-1.9b (h)

$\overrightarrow{N 11} 1-20$ (m)

polyclonal (mo)

$10 \mathrm{E} 3(\mathrm{~m})$.

polyclonal ( (

$59.1(\mathrm{~m})$

$-1034(\mathrm{~m})$

$1026(\mathrm{~m})$

$\mathrm{NM}-01$ (m)

Cbetal, $0.5 b e t a(\mathrm{hm})$

o.

polyclonal (o)

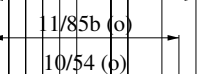

NNTRKRIRIORGP GRAFVTI IGKI GNMRQAHCNISRAKWNNTLKQIASKLR$$
320
$$

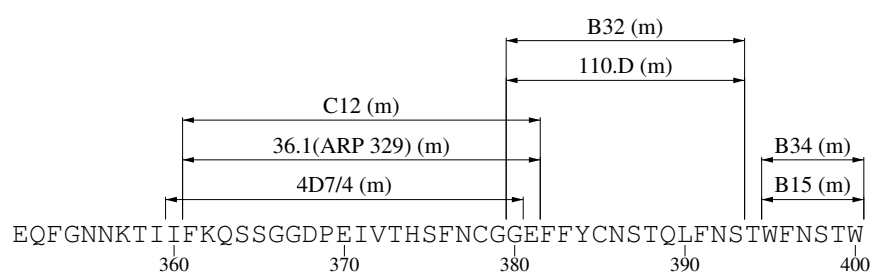

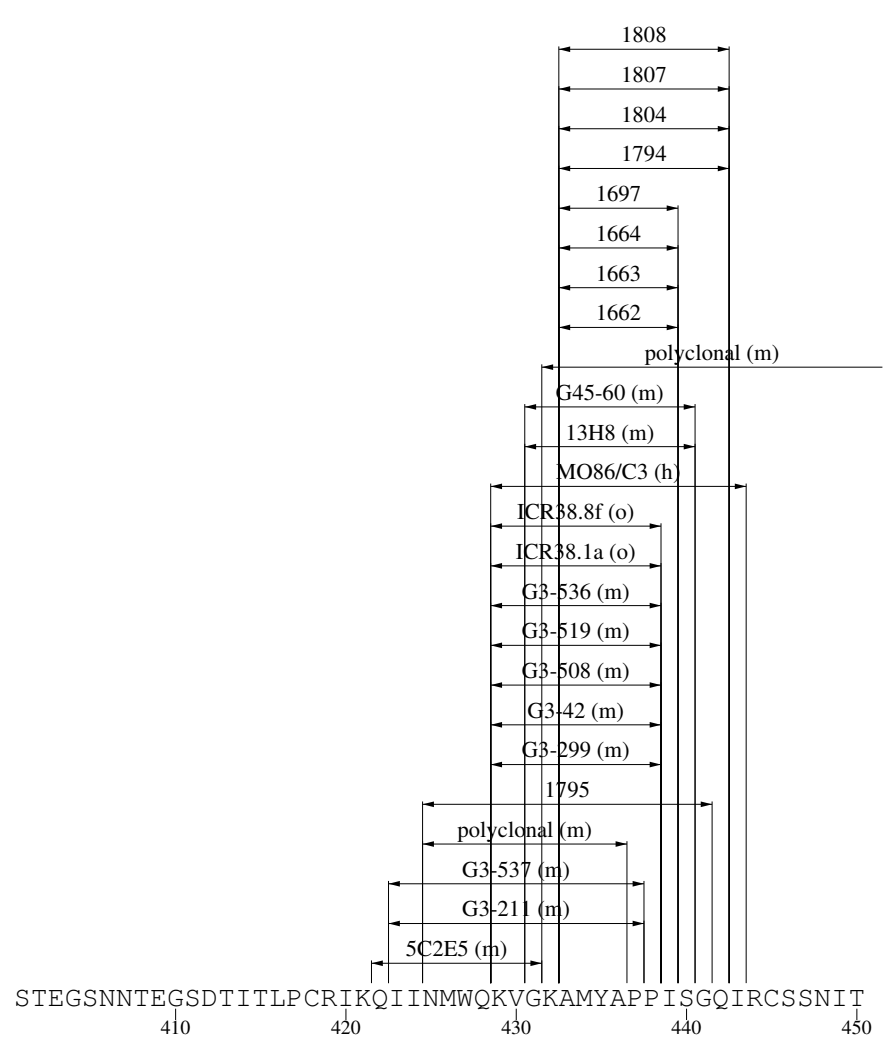

420
430 

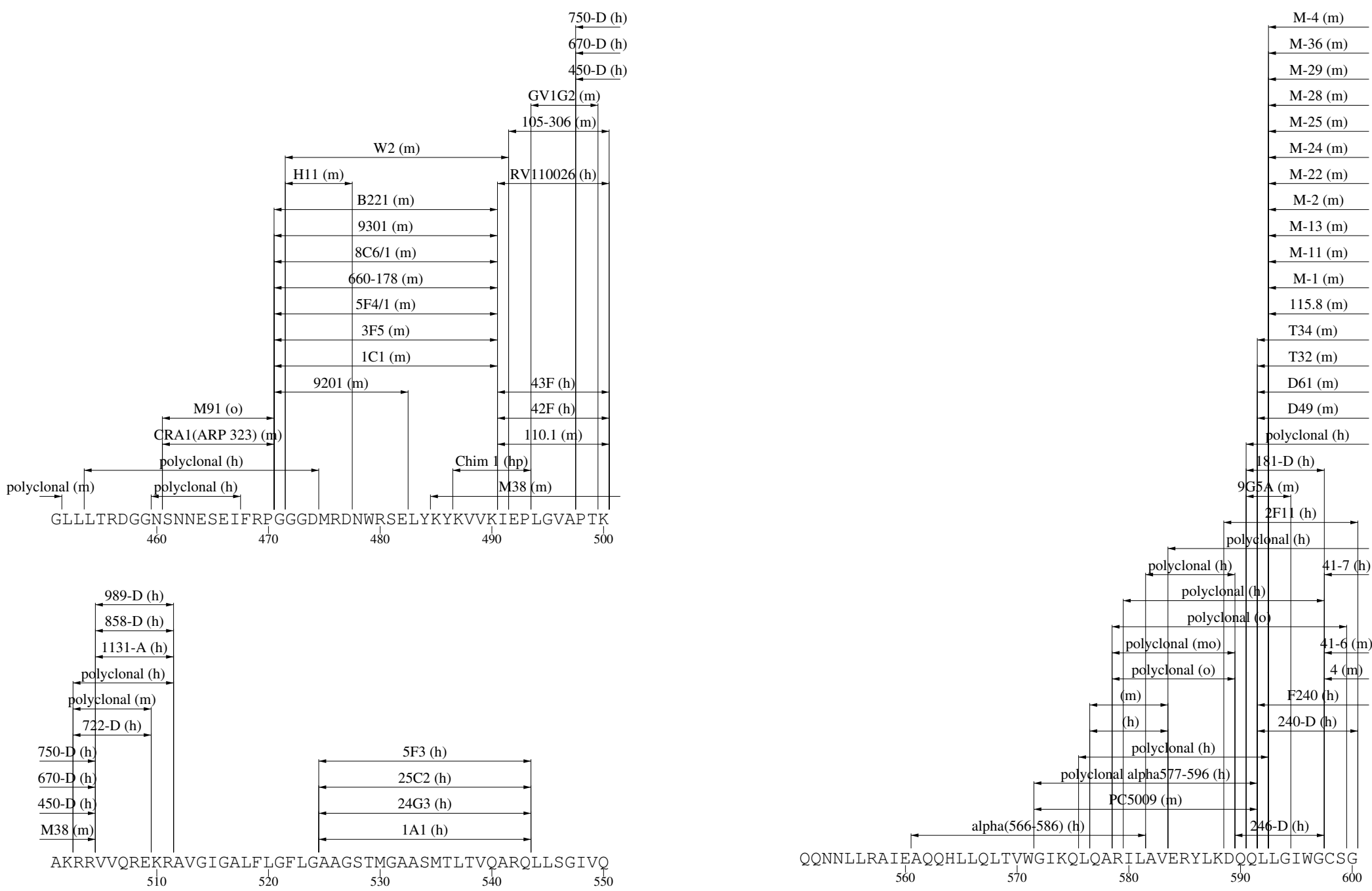


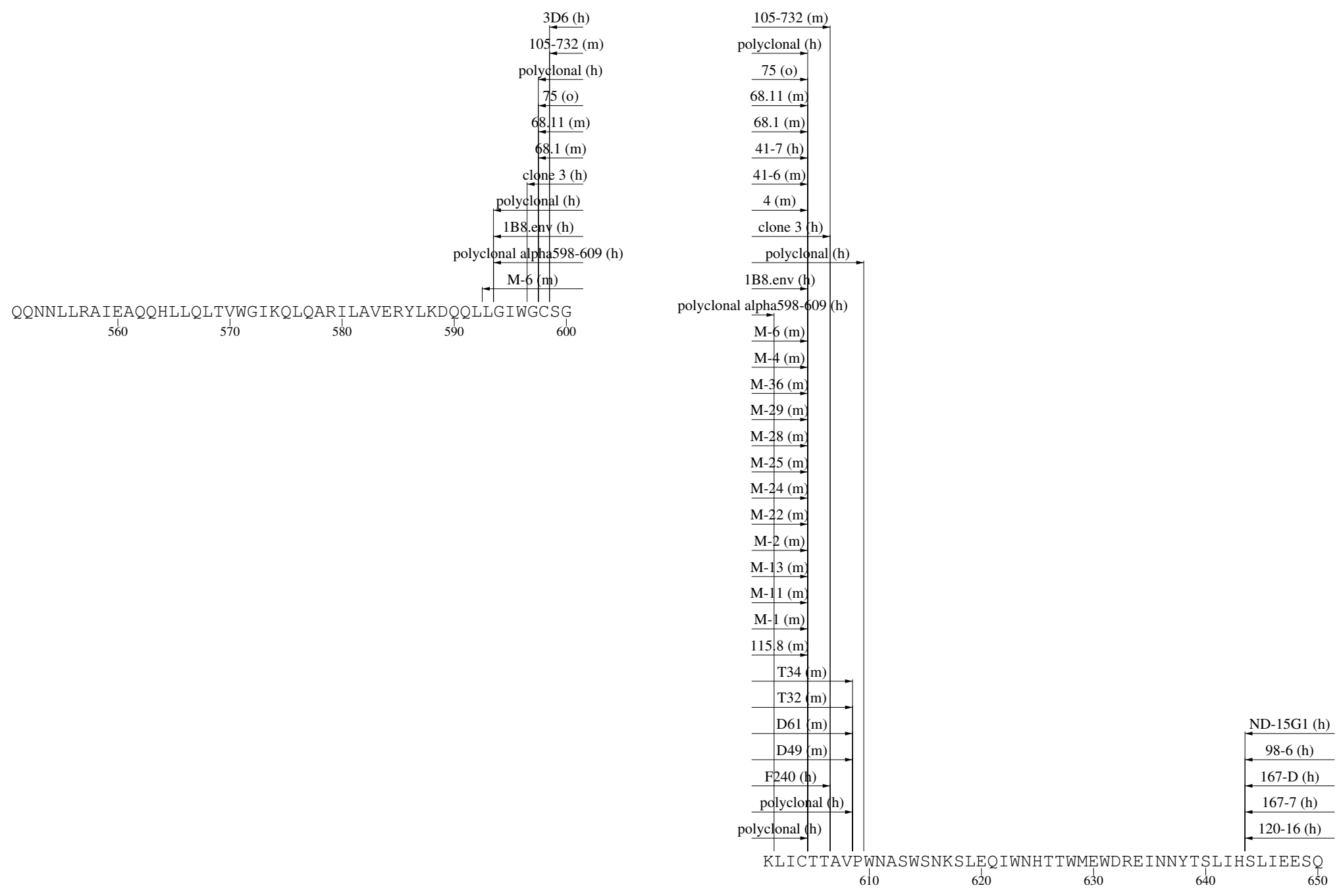



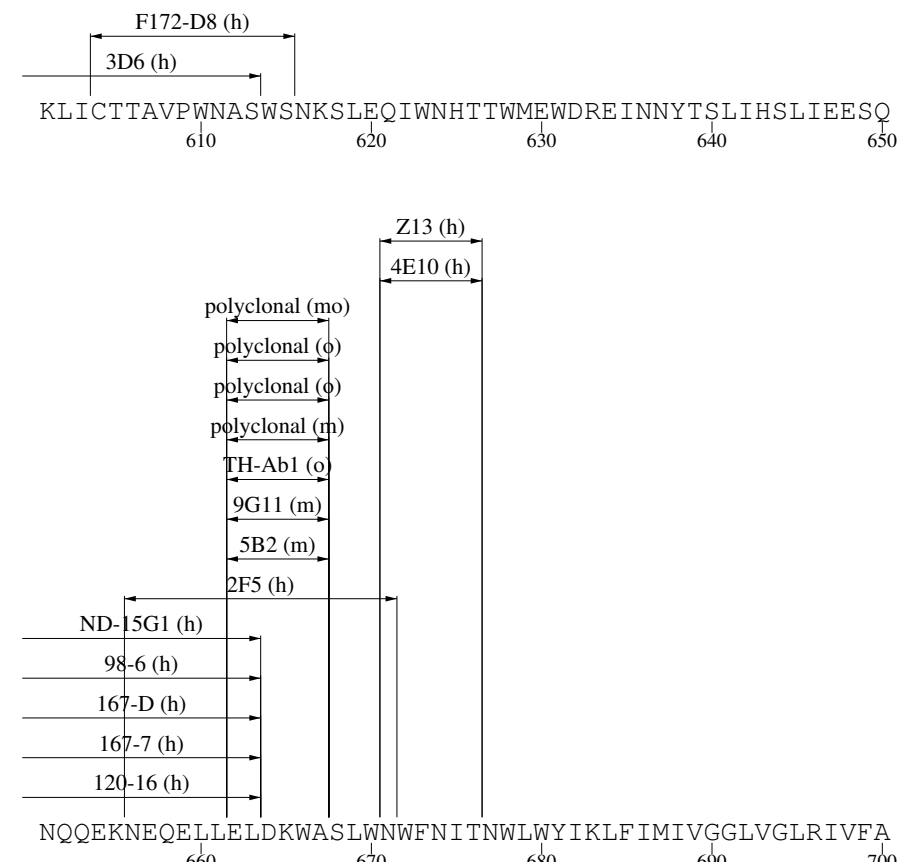

680

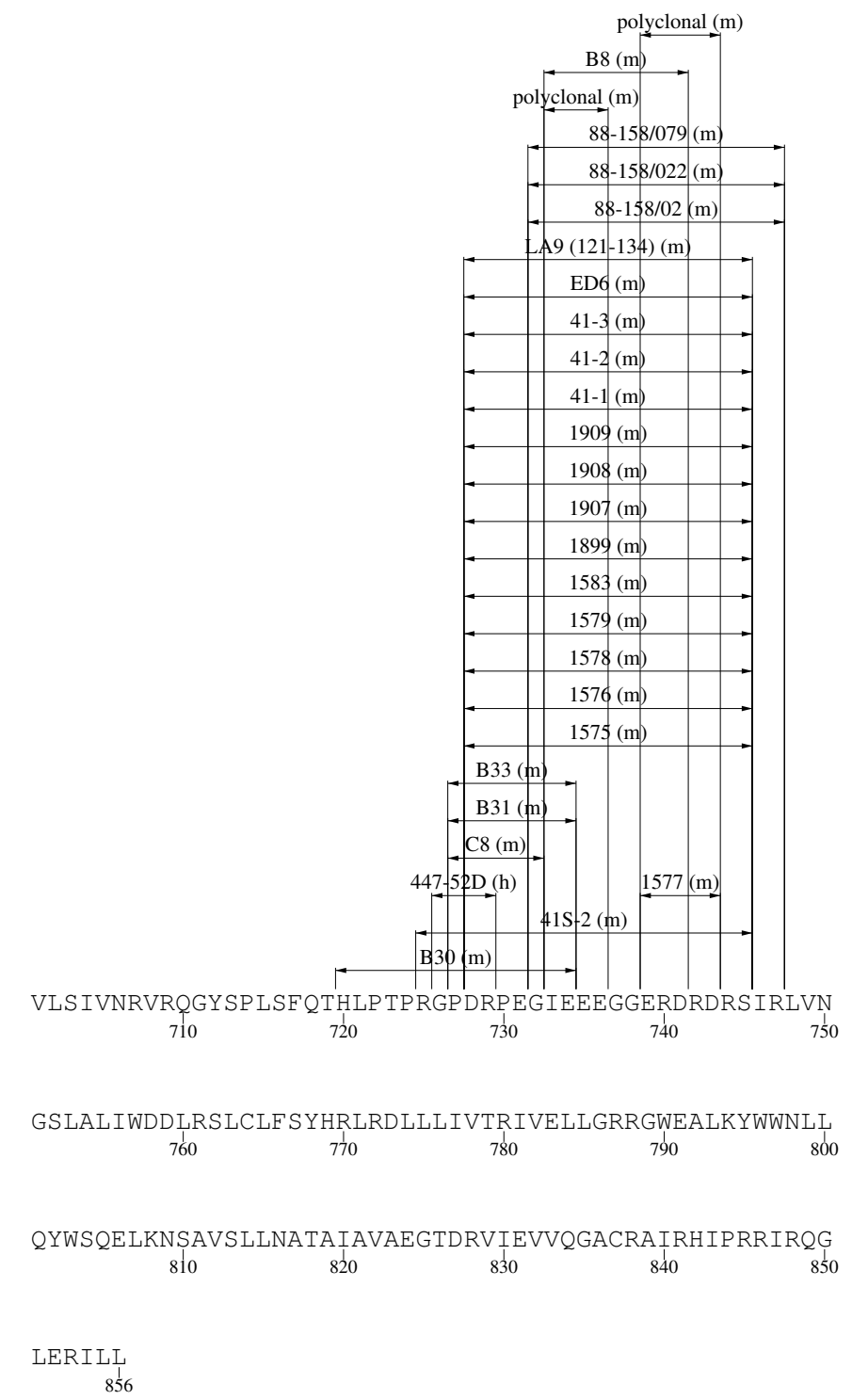

856 
IV-D-13 Nef Ab Epitope Map
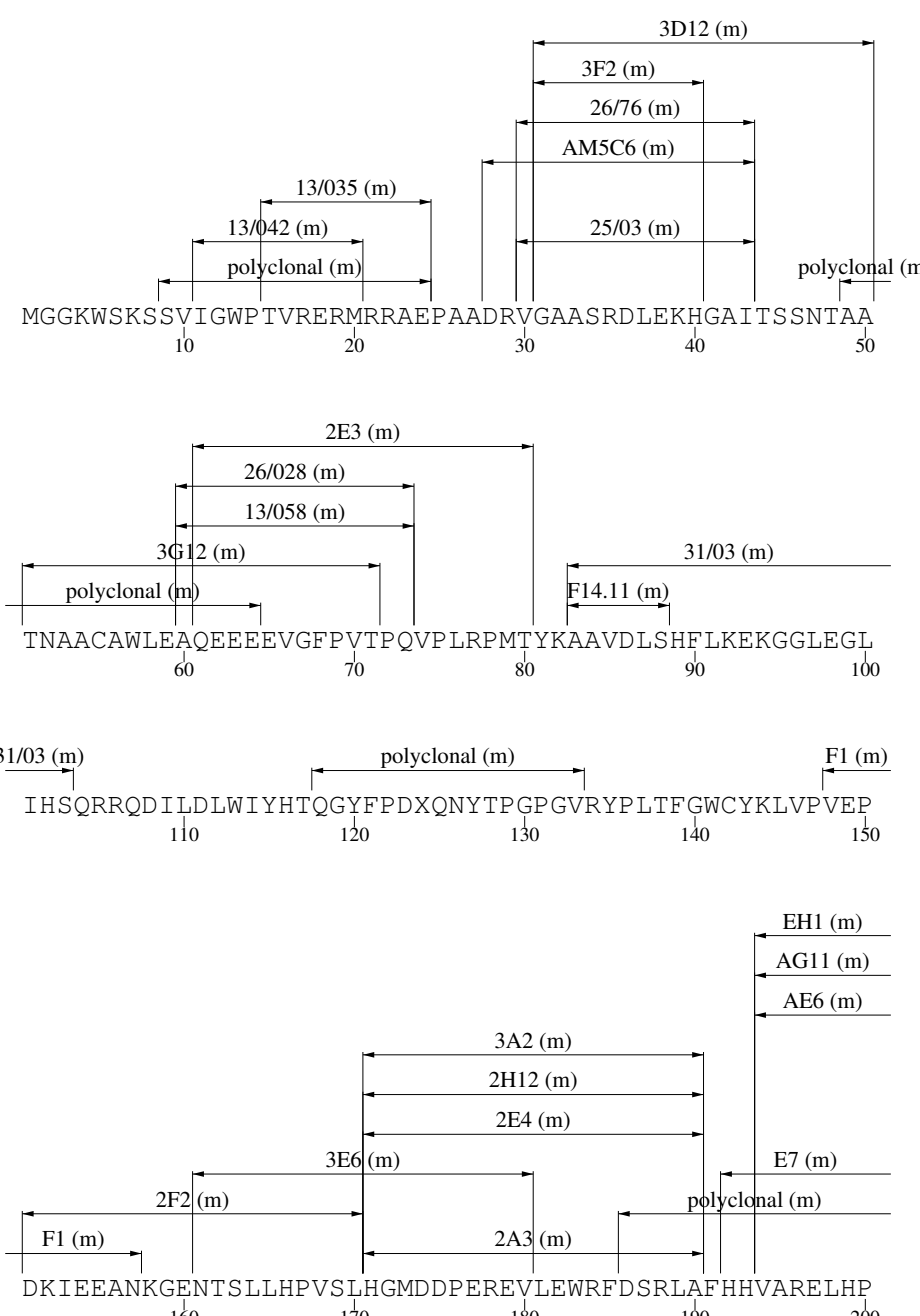


\section{Part V \\ HIV Immunology References}



[Abacioglu1994] Y. H. Abacioglu, T. R. Fouts, J. D. Laman, E. Claassen, S. H. Pincus, J. P. Moore, C. A. Roby, R. Kamin-Lewis, \& G. K. Lewis. Epitope Mapping and Topology of BaculovirusExpressed HIV-1 gp160 Determined with a Panel of Murine Monoclonal Antibodies. AIDS Res Hum Retroviruses 10:371-381, 1994. Thirty MAbs were obtained from BALB/c mice immunized with rgp160 LAI expressed in baculovirus. These antibodies map to 4 domains: gp120 C1, C2, $\mathrm{C} 3 / \mathrm{V} 4$, and the cytoplasmic tail of gp41. All epitopes were exposed on rgp160 without denaturing the protein, but $6 / 8$ epitopes mapped in gp120 are not exposed unless the protein is denatured

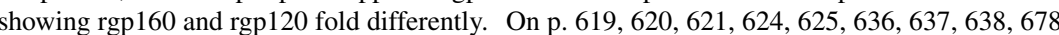
$679 \sqrt{719} 723,724,726$

[Acel1998] A. Acel, B. E. Udashkin, M. A. Wainberg, \& E. A. Faust. Efficient gap repair catalyzed in vitro by an intrinsic DNA polymerase activity of human immunodeficiency virus type 1 Integrase. J Virol 72:2062-71, 1998. Intrinsic polymerase activity that can catalyze gap repair was described for HIV Integrase. This activity was inhibited by the MAb 35, that binds to KAKIIRDYGK at the C-term end of Integrase. On p.604

[Achour1990] A. Achour, O. Picard, D. Zagury, P. Sarin, R. Gallo, P. Naylor, \& A. Goldstein. HGP-30, a synthetic analogue of human immunodeficiency virus p17, is a target for cytotoxic lymphocytes in HIV-infected individuals. Proc Natl Acad Sci 87:7045-7049, 1990. This epitope serves as a B cell epitope as well as an HLA-A2 T-cell epitope. The peptide is also called HGP-30. On p. 92

[Achour1993] A. Achour, O. Picard, J. P. M'Bika, A. Willer, R. Snart, B. Bizini, C. Carell, A. Burny, $\&$ D. Zagury. Envelope protein and P18 IIIB peptide recognized by cytotoxic T lymphocytes from humans immunized with AIDS virus envelope. Vaccine 11:699-701, 1993. Both HLA A2 and A3 class I molecule types can present this V3 antigen: RIQRGPGRAFVTIGK. On p. 288

[Achour1994] A. Achour, S. Lemhammedi, O. Picard, J. P. M'Bika, J. F. Zagury, Z. Moukrim, A. Willer, F. Beix, A. Burny, \& D. Zagury. Cytotoxic T lymphocytes specific for HIV-1 gp160 antigen and synthetic P18IIIB peptide in an HLA-A11-immunized individual. AIDS Res Hum Retroviruses 10:19-25, 1994. CTL restricted by several different class I molecule HLA types can present this V3 antigen: RIQRGPGRAFVTIGK, HLA A11. On p.287

[Achour1996] A. Achour, F. Bex, P. Hermans, A. Burny, \& D. Zagury. Induction of anti-gp160 cytotoxic T cells cross-reacting with various V3 loop P18 peptides in human immunodeficiency virus type 1 envelope-immunized individuals. $J$ Virol 70:6741-6750, 1996. On p.294

[Adams1997] S. L. Adams, R. A. Biti, \& G. J. Stewart. T-cell response to HIV in natural infection: optimized culture conditions for detecting responses to Gag peptides. J AIDS Hum Retrovirol 15:257-263, 1997. On p. 432 433 437 438 491

[Addo2001] M. M. Addo, M. Altfeld, E. S. Rosenberg, \& et al. The HIV-1 regulatory protein Tat and Rev are freqently targeted by cytotoxic T lymphocytes (CTL) derived from HIV infected individuals. ProcNatlAcadSci 98(4):1781-1786, 2001. On p. 256 260 $261|263| 264$

[Addo2002a] M. M. Addo, M. Altfeld, A. Rathod, M. Yu, X. G. Yu, P. J. R. Goulder, E. S. Rosenberg, \& B. D. Walker. HIV-1 Vpu represents a minor target for cytotoxic T lymphocytes in HIV-1-infection. AIDS 16(7):1071-1073, 2002. On p. 266

[Addo2002b] M. M. Addo, X. G. Yu, E. S. Rosenberg, B. D. Walker, \& M. Altfeld. Cytotoxic T-lymphocyte (CTL) responses directed against regulatory and accessory proteins in HIV-1 in-

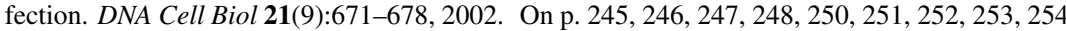
\begin{tabular}{|l|l|l|l|l|l|l|l|l|l|l|l|l|}
\hline 256 & 257 & 258 & 260 & 262 & 263 & 264 & 266 \\
\hline
\end{tabular}

[Ahlers1996] J. D. Ahlers, N. Dunlop, C. D. Pendleton, M. Neuman, P. L. Nara, \& J. A. Berzofsky. Candidate HIV type 1 multideterminant cluster peptide-P18MN vaccine constructs elicit type 1 Helper T cells, cytotoxic T cells and neutralizing antibody, all using the same adjuvan immunization. AIDS Res Hum Retroviruses 12:259-272, 1996. On p. 289

[Ahlers1997a] J. D. Ahlers, N. Dunlop, D. W. Alling, P. L. Nara, \& J. A. Berzofsky. Cytokine-inadjuvant steering of the immune response phenotype to HIV-1 vaccine constructs: granulocyte- macrophage colony-stimulating factor and TNF-alpha synergize with IL-12 to enhance induction of cytotoxic T lymphocytes. J Immunol 158:3947-58, 1997. On p. 289

Ahlers1997b] J. D. Ahlers, T. Takeshita, C. D. Pendleton, \& J. A. Berzofsky. Enhanced immunogenicity of HIV-1 vaccine construct by modification of the native peptide sequence. Proc Nat Acad Sci USA 94:10856-61, 1997. On p.288 509

Ahlers2001] J. D. Ahlers, I. M. Belyakov, E. K. Thomas, \& J. A. Berzofsky. High-affinity T helper epitope induces complementary helper and APC polarization, increased CTL, and protection against viral infection. J Clin Invest 108(11):1677-1685, 2001. On p. 286 508

[Ahluwalia1997] A. Ahluwalia, K. Gokulan, I. Nath, \& D. N. Rao. Modification of delivery system enhances MHC nonrestricted immunogenicity of V3 loop region of HIV-1 gp120. Microbiol Immunol 41:779-84, 1997. On p. 491644

Ahmad2001] R. Ahmad, S. T. Sindhu, E. Toma, R. Morisset, J. Vincelette, J. Menezes, \& A. Ahmad. Evidence for a correlation between antibody-dependent cellular cytotoxicity-mediating antiHIV-1 antibodies and prognostic predictors of HIV infection. J Clin Immunol 21(3):227-33, 2001. On p. 743

Akahata2000] W. Akahata, E. Ido, T. Shimada, K. Katsuyama, H. Yamamoto, H. Uesaka, M. Ui, T. Kuwata, H. Takahashi, \& M. Hayami. DNA vaccination of macaques by a full genome HIV-1 plasmid which produces noninfectious virus particles. Virology 275(1):116-24, 2000. On p. 164 335,526

Akerblom1990] L. Akerblom, J. Hinkula, P.-A. Broliden, B. Makitalo, T. Fridberger, J. Rosen, M. Villacres-Eriksson, B. Morein, \& B. Wahren. Neutralizing cross-reactive and non-neutralizing monoclonal antibodies to HIV-1 gp120. AIDS 4:953-960, 1990. On p.617 619 620 623 661 662663

[Akridge1999] R. Akridge, F. Hladik, J. Markee, C. Alef, H. Kelley, A. Collier, \& M. J. McElrath. Cellular immunity and target cell susceptibility in persons with repeated HIV-1 exposure. Immunol Lett 66:15-9, 1999. On p. 335

[Aladdin1999] H. Aladdin, H. Ullum, A. C. Lepri, H. Leffers, T. Katzenstein, J. Gerstoft, S. B Gjedde, A. N. Phillips, P. Skinhoj, \& B. K. Pedersen. Bulk culture levels of specific cytotoxic T cell activity against HIV-1 proteins are not associated with risk of death. Scand J Immunol 50:223-7, 1999. On p. 164 239335396

Aladdin2000] H. Aladdin, C. S. Larsen, B. K. Moller, H. Ullum, M. R. Buhl, J. Gerstoft, P. Skinhoj, \& B. K. Pedersen. Effects of subcutaneous interleukin-2 therapy on phenotype and function of peripheral blood mononuclear cells in human immunodeficiency virus infected patients. Scand $J$ Immunol 51:168-75, 2000. On p. 335

Alatrakchi2002] N. Alatrakchi, V. Di Martino, V. Thibault, B. Autran, \& the ALT and IMMUNEVIRC ANRS study groups. Strong CD4 Th1 responses to HIV and hepatitis C virus in HIVinfected long-term non-progressors co-infected with hepatitis C virus. AIDS 16(5):713-717, 2002. On p. 445

[Aldhous1994] M. C. Aldhous, K. C. Watret, J. Y. Mok, A. G. Bird, \& K. S. Froebel. Cytotoxic T lymphocyte activity and CD8 subpopulations in children at risk of HIV infection. Clin Exp Immunol 97(1):61-67, 1994. On p. 166242259337

Alexander-Miller1996] M. A. Alexander-Miller, K. C. Parker, T. Tsukui, C. D. Pendleton, J. E. Coligan, \& J. A. Berzofsky. Molecular analysis of presentation by HLA-A2.1 of a promiscuously binding V3 loop peptide from the HIV-1 Envelope protein to human cytotoxic T lymphocytes. In Immunol 8:641-649, 1996. On p.293

[Allaway 1993] G. P. Allaway, A. M. Ryder, G. A. Beaudry, \& P. J. Madden. Synergistic inhibition of HIV-1 envelope-mediated cell fusion by CD4-based molecules in combination with antibodies to gp120 or gp41. AIDS Res Hum Retroviruses 9:581-587, 1993. On p.641 677 710

[Alsmadi1997] O. Alsmadi, R. Herz, E. Murphy, A. Pinter, \& S. A. Tilley. A novel antibodydependent cellular cytotoxicity epitope in gp120 is identified by two monoclonal antibodies 
isolated from a long-term survivor of human immunodeficiency virus type 1 infection. $J$ Virol 71:925-33, 1997. On p. 690.

[Alsmadi1998] O. Alsmadi \& S. A. Tilley. Antibody-dependent cellular cytotoxicity directed against cells expressing human immunodeficiency virus type 1 Envelope of primary or laboratoryadapted strains by human and chimpanzee monoclonal antibodies of different epitope specifici\begin{tabular}{|l|l|l|l|l|l|l|l|l|l|l|l|l|l|}
\hline ties. J Virol 72:286-93, 1998. On p. 629 & 644 & 649 & 659 & 690 & 730 & 760 & 761 & 767 \\
\hline
\end{tabular}

[Altes2001] H. K. Altes, D. A. Price, \& V. A. A. Jansen. Effector cytotoxic T lymphocyte numbers induced by vaccination should exceed levels in chronic infection for protection from HIV. Vaccine 20(1-2):3-6, 2001. On p. 401

[Altes2002] H. K. Altes, D. Wodarz, \& V. A. A. Jansen. The dual role of CD4 Thelper cells in the infection dynamics of HIV and their importance for vaccination. J Theor Biol 214(4):633-646, 2002. On p. 400 536

[Altfeld2000a] M. Altfeld. Personal communication, 2000. On p.181, 208 $245[281$

[Altfeld2000b] M. A. Altfeld, A. Trocha, R. L. Eldridge, E. S. Rosenberg, M. N. Phillips, M. M. Addo, R. P. Sekaly, S. A. Kalams, S. A. Burchett, K. McIntosh, B. D. Walker, \& P. J. Goulder Identification of dominant optimal HLA-B60- and HLA-B61-restricted cytotoxic T-lymphocyte (CTL) epitopes: rapid characterization of CTL responses by enzyme-linked immunospot assay. \begin{tabular}{|l|l|l|l|l|l|l|l|l|l|l|l|l|l|l|}
\hline$J$ Virol 74:8541, 2000. On p. 87 & 91 & 93 & 108 & 109 & 110 & 147 & 203 & 204 & 312 & 322 & 323 & 330 & 354 \\
\hline
\end{tabular} 361371

[Altfeld2001a] M. Altfeld, M. M. Addo, R. L. Eldridge, X. G. Yu, S. Thomas, A. Khatri, D. Strick, M. N. Phillips, G. B. Cohen, S. A. Islam, S. A. Kalams, C. Brander, P. J. Goulder, E. S. Rosenberg, B. D. Walker, \& The HIV Study Collaboration. Vpr is preferentially targeted by CTL during HIV

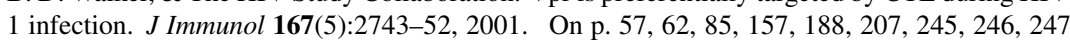

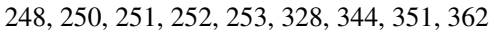

[Altfeld2001b] M. Altfeld, E. S. Rosenberg, R. Shankarappa, J. S. Mukherjee, F. M. Hecht, R. L. Eldridge, M. M. Addo, S. H. Poon, M. N. Phillips, G. K. Robbins, P. E. Sax, S. Boswell, J. O. Kahn, C. Brander, P. J. Goulder, J. A. Levy, J. I. Mullins, \& B. D. Walker. Cellular immune responses and viral diversity in individuals treated during acute and early HIV-1 infection. $J$ Exp \begin{tabular}{ll|l|l|l|l|l|l|l|l|l|l|l|l|l|l|l|l|l|l|l|} 
Med 193(2):169-80, 2001. On p. 59 & 63 & 67 & 69 & 70 & 73 & 86 & 91 & 92 & 95 & 98 & 99 & 106 & 119 & 123 \\
\hline
\end{tabular} \begin{tabular}{|l|l|l|l|l|l|l|l|l|l|l|l|l|l|l|l|l||l|l|}
\hline 127 & 129 & 136 & 140 & 146 & 152 & 156 & 158 & 178 & 182 & 185 & 189 & 192 & 198 & 204 & 219 & 224 & 225 & 226 \\
\hline
\end{tabular} \begin{tabular}{|l|l|l|l|l|l|l|l|l|l|l|l|l|l|l|l|l|l|l|l|l|l|}
\hline 254 & 263 & 267 & 274 & 281 & 284 & 301 & 312 & 324 & 326 & 330 & 343 & 347 & 348 & 354 & 359 & 361 & 364 & 368 \\
\hline
\end{tabular} \begin{tabular}{|l|l|l|l|l|l|l|l|l|l|}
\hline 370 & 372 & 375 & 378 & 381 & 383 & 389 & 394 & 444 \\
\hline
\end{tabular}

[Altfeld2001c] M. A. Altfeld, B. Livingston, N. Reshamwala, P. T. Nguyen, M. M. Addo, A. Shea M. Newman, J. Fikes, J. Sidney, P. Wentworth, R. Chesnut, R. L. Eldridge, E. S. Rosenberg, G. K. Robbins, C. Brander, P. E. Sax, S. Boswell, T. Flynn, S. Buchbinder, P. J. Goulder, B. D. Walker, A. Sette, \& S. A. Kalams. Identification of novel HLA-A2-restricted human immunodeficiency virus type 1-specific cytotoxic T-lymphocyte epitopes predicted by the HLA-A2 supertype peptide-binding motif. $J$ Virol 75(3):1301-11, 2001. On p. 78 [8| $159|175| 185|200| 218$

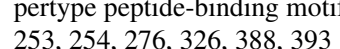

[Altfeld2002] M. Altfeld, J. van Lunzen, N. Frahm, X. G. Yu, C. Schneider, R. L. Eldridge, M. E Feeney, D. Meyer-Olson, H.-J. Stellbrink, \& B. D. Walker. Expansion of pre-existing, lymph node-localized CD8+ T cells during supervised treatment interruptions in chronic HIV-1 infec-

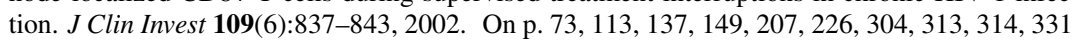
349382384

[Altman1996] J. D. Altman, P. A. H. Moss, P. J. R. Goulder, D. H. Barouch, M. G. McHeyzerWilliams, J. I. Bell, A. J. McMichael, \& M. M. Davis. Phenotypic analysis of antigen-specific T lymphocytes. Science 274:94-6, 1996. See comments in Science 1998 Jun 19;280(5371):1821. On p. 75,212

[Altmeyer1999] R. Altmeyer, E. Mordelet, M. Girard, \& C. Vidal. Expression and detection of macrophage tropic HIV-1 gp120 in the brain using conformation-dependent antibodies. Virology

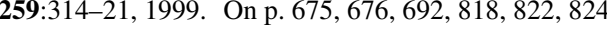

Anderson2001] D. E. Anderson, M. P. Carlos, L. Nguyen, \& J. V. Torres. Overcoming Original (Antigenic) Sin. Clin Immunol 101(2):152-157, 2001. On p.491

Andris1992] J. S. Andris, S. Johnson, S. Zolla-Pazner, \& J. D. Capra. Molecular characterization of five anti-human immunodeficiency virus type 1 antibody heavy chains reveals extensive somatic mutation typical of an antigen-driven immune response. Proc Natl Acad Sci USA 88:7783-7788, 1992. On p.707 708

Andrus1998] L. Andrus, A. M. Prince, I. Bernal, P. McCormack, D. H. Lee, M. K. Gorny, \& S. Zolla-Pazner. Passive immunization with a human immunodeficiency virus type 1- neutralizing monoclonal antibody in Hu-PBL-SCID mice: isolation of a neutralization escape variant. $J$ Infect Dis 177:889-97, 1998. On p. 653 675 710 712 822 823

[Angel2001] J. B. Angel, K. G. Parato, A. Kumar, S. Kravcik, A. D. Badley, C. Fex, D. Ashby, E. Sun, \& D. W. Cameron. Progressive human immunodeficiency virus-specific immune recovery with prolonged viral suppression. J Infect Dis 183(4):546-54, 2001. On p.443

[Appay2000] V. Appay, D. F. Nixon, S. M. Donahoe, G. M. Gillespie, T. Dong, A. King, G. S. Ogg, H. M. Spiegel, C. Conlon, C. A. Spina, D. V. Havlir, D. D. Richman, A. Waters, P. Easterbrook, A. J. McMichael, \& S. L. Rowland-Jones. HIV-specific CD8(+) T cells produce antiviral cytokines but are impaired in cytolytic function. $J$ Exp Med 192(1):63-75, 2000. On p. 69 $79 \mid 607$ \begin{tabular}{ll|l|l|l|l|l|l|l|l|l|l|l|l|}
\hline 131 & 133 & 205 & 213 & 234 & 352 & 368 & 379 \\
\hline
\end{tabular}

Appay2002] V. Appay, P. Hansasuta, J. Sutton, R. D. Schrier, J. K. Wong, M. Furtado, D. V. Havlir, S. M. Wolinsky, A. J. McMichael, D. D. Richman, S. L. Rowland-Jones, \& C. A. Spina. Persistent HIV-1-specific cellular responses despite prolonged therapeutic viral suppression. AIDS

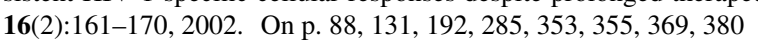

[Arai2000] H. Arai, K. Q. Xin, K. Hamajima, Y. Lu, S. Watabe, T. Takahashi, S. Toda, K. Okuda I. Kudoh, M. Suzuki, \& K. Okuda. 8 Br-cAMP enhances both humoral and cell-mediated immune responses induced by an HIV-1 DNA vaccine. Gene Ther 7(8):694-702, 2000. On p. 296529 [Arendrup1993] M. Arendrup, A. Sonnerborg, B. Svennerholm, L. Akerblom, C. Nielsen, H. Clausen, S. Olofsson, J. O. Nielsen, \& J. E. S. Hensen. Neutralizing antibody response during human immunodeficiency virus type 1 infection: type and group specificity and viral escape. $J$ Gen Virol 74:855-863, 1993. On p.657, 662 667

[Arendrup1995] M. Arendrup, L. Akerblom, P. M. Heegaard, J. O. Nielsen, \& J. E. Hansen. The HIV-1 V3 domain on field isolates: participation in generation of escape virus in vivo and accessibility to neutralizing antibodies. Arch Virol 140:655-670, 1995. The anti-V3 Ab titre in patient serum was generally low against autologous virus isolated later than the serum sample, in contras to a higher titre against peptides corresponding to virus isolated earlier than the serum sample. The authors conclude that the V3 domain is subject to immunoselection in vivo, and that V3 on early field virus is less accessible to NAbs than the V3 loop on laboratory strains. On p. 657

[Ariyoshi2002] K. Ariyoshi, N. Promadej, K. Ruxrungtham, \& R. Sutthent. Toward improved evaluation of cytotoxic T-lymphocyte (CTL)-inducing HIV vaccines in Thailand. AIDS Res Hum Retroviruses 18(10):737-739, 2002. On p.402

[Armbruster2002] C. Armbruster, G. M. Stiegler, B. A. Vcelar, W. Jager, N. L. Michael, N. Vetter, $\&$ H. W. D. Katinger. A phase I trial with two human monoclonal antibodies (hMAb 2F5, 2G12) against HIV-1. AIDS 16(2):227-233, 2002. On p.710 716822826827

[Armstrong1996a] S. J. Armstrong \& N. J. Dimmock. Varying temperature-dependence of postattachment neutralization of human immunodeficiency virus type 1 by monoclonal antibodies to gp120: identification of a very early fusion-independent event as a neutralization target. J Gen Virol 77:1397-1402, 1996. On p. 783 820

[Armstrong1996b] S. J. Armstrong, T. L. McInerney, L. McLain, B. Wahren, J. Hinkula, M. Levi, \& N. J. Dimmock. Two neutralization anti-V3 monoclonal antibodies act by affecting different functions of human immunodeficiency virus type 1. J Gen Virol 77:2931-2941, 1996. On p.820 
[Arora2001] A. Arora \& P. Seth. Immunization with HIV-1 subtype B gp160-DNA induces specific as well as cross reactive immune responses in mice. Indian J Med Res 114:1-9, 2001. On p.293

[Arp1999] J. Arp, B. Rovinski, S. Sambhara, J. Tartaglia, \& G. Dekaban. Human immunodeficiency virus type 1 envelope-specific cytotoxic T lymphocytes response dynamics after prime-boost vaccine regimens with human immunodeficiency virus type 1 canarypox and pseudovirions. Viral Immunol 12:281-96, 1999. On p. 292

[Arthos2002] J. Arthos, C. Cicala, T. D. Steenbeke, T.-W. Chun, C. Dela Cruz, D. B. Hanback, P. Khazanie, D. Nam, P. Schuck, S. M. Selig, D. Van Ryk, M. A. Chaikin, \& A. S. Fauci. Biochemical and biological characterization of a dodecameric CD4-Ig fusion protein: Implication for therapeutic and vaccine strategies. J Biol Chem 277(13):11456-11464, 2002. On p. 798 802 AVEG022PT2001] AVEG022PT. Cellular and humoral immune responses to a canarypox vaccine containing human immunodeficiency virus type $1 \mathrm{Env}, \mathrm{Gag}$, and Pro in combination with rgp 120 . J Infect Dis 183(4):563-70, 2001. AIDS Vaccine Evaluation Group 022 Protocol Team. On p. 336

[Ayash-Rashkovsky2002] M. Ayash-Rashkovsky, Z. Weisman, J. Diveley, R. B. Moss, Z. Bentwich, \& G. Borkow. Generation of Th1 immune responses to inactivated, gp120-depleted HIV-1 in mice with a dominant $\mathrm{Th} 2$ biased immune profile via imunostimulatory oligonucleotides-relevance to AIDS vaccines in developing countries. Vaccine 20(21-22):2684-2692, 2002. On p.536

[Ayyavoo2000] V. Ayyavoo, S. Kudchodkar, M. P. Ramanathan, P. Le, K. Muthumani, N. M. Megalai, T. Dentchev, L. Santiago-Barrios, C. Mrinalini, \& D. B. Weiner. Immunogenicity of a novel DNA vaccine cassette expressing multiple human immunodeficiency virus (HIV-1) accessory genes. AIDS 14:1-9, 2000. On p. 249 266 459 466 |534

[Baba2000] T. W. Baba, V. Liska, R. Hofmann-Lehmann, J. Vlasak, W. Xu, S. Ayehunie, L. A Cavacini, M. R. Posner, H. Katinger, G. Stiegler, B. J. Bernacky, T. A. Rizvi, R. Schmidt, L. R. Hill, M. E. Keeling, Y. Lu, J. E. Wright, T. C. Chou, \& R. M. Ruprecht. Human neutralizing monoclonal antibodies of the IgG1 subtype protect. Nat Med 6:200-6, 2000. On p. 710713 775777822825

[Back1993] N. K. T. Back, L. Smit, M. Schutten, P. L. Nara, M. Tersmette, \& J. Goudsmit. Mu tations in Human Immunodeficiency Virus Type 1 gp41 Affect Sensitivity to Neutralization by gp120 Antibodies. J Virol 67:6897-6902, 1993. Three closely related clones were derived from neutralization resistant IIIB isolate that had been passaged in a chimpanzee. gp41 mutations wer shown to profoundly alter the ability of V3 loop MAbs 5023 and 178.1 to neutralize. Critical substitutions in gp41 were 668 and 675, close to the immunogenic domain 662-668, or ELDKWAS. Less profound inhibition was observed for the anti-CD4 binding site MAb GP13. On p. 646 647669780

[Bagley1994] J. Bagley, P. J. Dillon, C. Rosen, J. Robinson, J. Sodroski, \& W. A. Marasco. Structural Characterization of Broadly Neutralizing Human Monoclonal Antibodies Against the CD4 Binding Site of HIV-1 gp120. Mol Immunol 31(15):1149-1160, 1994. This paper is a detailed study of the V-D-J heavy chain usage and V-J light chain usage for the three monoclonals that bind to the HIV-1 envelope CD4 binding site: F105, 15e and 21h. Different germline genes wer used, and there was evidence for antigen-drive clonal selection of somatic mutations. Eight positions in the heavy chain and two in the light chain complementarity determining positions were identical in the three Mabs. On p.762 764775776780781

[Bahraoui1990] E. Bahraoui, M. Yagello, J. N. Billaud, J. M. Sabatier, B. Guy, E. Muchmore, M. Girard, \& J. C. Gluckman. Immunogenicity of the human immunodeficiency virus (HIV) recombi nant nef gene product. mapping of T-cell and B-cell epitopes in immunized chimpanzees. AID Res Hum Retroviruses 6(9):1087-1098, 1990. On p.533

[Bai2000] Y. Bai, Y. Zhao, T. Yu, M. P. Dierich, \& Y. H. Chen. Antibodies to HIV-1 gp41 recognize synthetic peptides of human IFN-alpha and IFN-beta. Int Arch Allergy Immunol 121:170-2, 2000. On p.748 749
[Baier1995] G. Baier, G. Baier-Bitterlich, D. J. Looney, \& A. Altman. Immunogenic targeting of recombinant peptide vaccines to human antigen-presenting cells by chimeric anti-HLA-DR an anti-surface immunoglobulin D antibody Fab fragments in vitro. J Virol 69:2357-2365, 1995. On p. 494508

Bajaria2002] S. H. Bajaria, G. Webb, M. Cloyd, \& D. Kirschner. Dynamics of naive and memory CD4+ T lymphocytes in HIV-1 disease progression. J Acquir Immune Defic Syndr 30(1):41-58, 2002. On p. 536

Balla-Jhagihoorsingh1999a] S. Balla-Jhaghhoorsingh, P. Mooij, G. Koopman, T. Haaksma, V. Teeuwsen, J. Heeney, \& R. Bontrop. Differential cytotoxic T-lymphocyte (CTL) response in HIV-1 immunised sibling chimpanzees with shared MHC haplotypes. Immunol Lett $\mathbf{6 6}(1-$ 3):61-7, 1999. On p. 300

Balla-Jhaghhoorsingh1999b] S. S. Balla-Jhaghhoorsingh, G. Koopman, P. Mooij, T. G. Haaksma, V. J. Teeuwsen, R. E. Bontrop, \& J. L. Heeney. Conserved CTL epitopes shared between HIVinfected human long-term survivors and chimpanzees. J Immunol 162:2308-14, 1999. On p.97

Banapour1987] B. Banapour, K. Rosenthal, L. Rabin, V. Sharma, L. Young, J. Fernandez, E. Engleman, M. McGrath, G. Reyes, \& J. Lifson. Characterization and Epitope Mapping of a Human Monoclonal Antibody Reactive with the Envelope Glycoprotein of Human Immunodeficiency Virus. J Immunol 139:4027-4033, 1987. On p.705

Bandres1998] J. C. Bandres, Q. F. W. QF, J.O'Leary, F. Baleaux, A. Amara, J. A. Hoxie, \& S.-P. M. K. Gorny. Human immunodeficiency virus (HIV) envelope binds to CXCR4 independently of $\mathrm{CD} 4$, and binding can be enhanced by interaction with soluble CD4 or by HIV envelope deglycosylation. J Virol 72:2500-2504, 1998. On p.589 590693

Barbas III1992] C. F. Barbas III, E. Bjorling, F. Chiodi, N. Dunlop, D. Cababa, T. M. Jones, S. L. Zebedee, M. A. Persson, P. A. Nara, E. Norrby, et al. Recombinant human Fab fragments neutralize human type 1 immunodeficiency virus in vitro. Proc Natl Acad Sci USA 89:9339-9343, 1992. On p.784

Barbas III1993] C. F. Barbas III, T. A. Collet, P. Roben, J. Binley, W. Amberg, D. Hoekstra, D. Ca bana, T. M. Jones, R. A. Williamson, G. R. Pilkington, N. L. Haigwood, A. C. Satterthwait, I. Sanz, \& D. R. Burton. Molecular profile of an antibody response to HIV-1 as probed by combinatorial libraries. J Mol Biol 230:812-823, 1993. On p. 663664

[Barnett1997] S. W. Barnett, S. Rajasekar, H. Legg, B. Doe, D. H. Fuller, J. R. Haynes, C. M. Walker, \& K. S. Steimer. Vaccination with HIV-1 gp120 DNA induces immune responses that are boosted by a recombinant gp120 protein subunit. Vaccine 15:869-873, 1997. On p. 293

[Barnett2001] S. W. Barnett, S. Lu, I. Srivastava, S. Cherpelis, A. Gettie, J. Blanchard, S. Wang, I. Mboudjeka, L. Leung, Y. Lian, A. Fong, C. Buckner, A. Ly, S. Hilt, J. Ulmer, C. T. Wild, J. R. Mascola, \& L. Stamatatos. The ability of an oligomeric human immunodeficiency virus type 1 (HIV-1) envelope antigen to elicit neutralizing antibodies against primary HIV-1 isolates is improved following partial deletion of the second hypervariable region. $J$ Virol 75(12):5526-40,

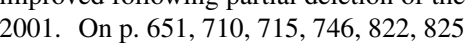

Barouch1998] D. H. Barouch, S. Santra, T. D. Steenbeke, X. X. Zheng, H. C. Perry, M. E. Davies, D. C. Freed, A. Craiu, T. B. Strom, J. W. Shiver, \& N. L. Letvin. Augmentation and suppression of immune responses to an HIV-1 DNA vaccine by plasmid cytokine/Ig administration. J Immunol 161:1875-82, 1998. On p. 289

[Barouch2000] D. H. Barouch, S. Santra, J. E. Schmitz, M. J. Kuroda, T. M. Fu, W. Wagner, M. Bilska, A. Craiu, X. X. Zheng, G. R. Krivulka, K. Beaudry, M. A. Lifton, C. E. Nickerson, W. L. Trigona, K. Punt, D. C. Freed, L. Guan, S. Dubey, D. Casimiro, A. Simon, M. E. Davies, M. Chastain, T. B. Strom, R. S. Gelman, D. C. Montefiori, M. G. Lewis, E. A. Emini, J. W. Shiver, \& N. L. Letvin. Control of viremia and prevention of clinical AIDS in rhesus monkeys by cytokineaugmented DNA vaccination. Science 290(5491):486-92, 2000. On p. 305 
[Barouch2001a] D. H. Barouch, A. Craiu, S. Santra, M. A. Egan, J. E. Schmitz, M. J. Kuroda, T. M. Fu, J. H. Nam, L. S. Wyatt, M. A. Lifton, G. R. Krivulka, C. E. Nickerson, C. I. Lord, B. Moss, M. G. Lewis, V. M. Hirsch, J. W. Shiver, \& N. L. Letvin. Elicitation of high-frequency cytotoxic T-lymphocyte responses against both dominant and subdominant simian-human immunodeficiency virus epitopes by DNA vaccination of rhesus monkeys. J Virol 75(5):2462-7, 2001. On p. 306

[Barouch2001b] D. H. Barouch, S. Santra, M. J. Kuroda, J. E. Schmitz, R. Plishka, A. BucklerWhite, A. E. Gaitan, R. Zin, J. H. Nam, L. S. Wyatt, M. A. Lifton, C. E. Nickerson, B. Moss, D. C. Montefiori, V. M. Hirsch, \& N. L. Letvin. Reduction of simian-human immunodeficiency virus $89.6 \mathrm{P}$ viremia in rhesus monkeys by recombinant modified vaccinia virus Ankara vaccination. $J$ Virol 75(11):5151-8, 2001. On p. 306 743

[Barouch2002] D. H. Barouch, S. Santra, K. Tenner-Racz, P. Racz, M. J. Kuroda, J. E. Schmitz, S. S. Jackson, M. A. Lifton, D. C. Freed, H. C. Perry, M.-E. Davies, J. W. Shiver, \& N. L. Letvin Potent CD4+ T cell responses elicited by a bicistronic HIV-1 DNA vaccine expressing gp120 and GM-CSF. J Immunol 168(2):562-568, 2002. On p. 293495

[Barsov1996] E. V. Barsov, W. E. Huber, J. Marcotrigiano, P. K. Clark, A. D. Clark, E. Arnold, \& S. H. Hughes. Inhibition of human immunodeficiency virus type 1 Integrase by the Fab fragmen of a specific monoclonal antibody suggests that different multimerization states are required fo different enzymatic functions. $J$ Virol 70:4484-4494, 1996. MAb 35 does not inhibit HIVIN, but Fab 35 inhibits 3'-end processing, strand transfer and disintegration. This appears to be through interfering with multimerization, and suggests that the C-terminal region is important for IN function. On p. 604606

[Bartlett1998] J. A. Bartlett, S. S. Wasserman, C. B. Hicks, R. T. Dodge, K. J. Weinhold, C. O. Tacket, N. Ketter, A. E. Wittek, T. J. Palker, \& B. F. Haynes. Safety and immunogenicity of a HLA-based HIV envelope polyvalent synthetic peptide immunogen. AIDS 12:1291-300, 1998 On p. 507 640 641

[Basmaciogullari2002] S. Basmaciogullari, G. J. Babcock, D. Van Ryk, W. Wojtowicz, \& J. Sodroski. Identification of conserved and variable structures in the human immunodeficiency virus gp120 glycoprotein of importance for CXCR4 binding. J Virol 76(21):10791-800, 2002. On \begin{tabular}{l|l|l|l|l|l|} 
p. 758 & 760 & 775 & 779 & 798 & 802 \\
\hline
\end{tabular}

[Battle-Miller2002] K. Battle-Miller, C. A. Eby, A. L. Landay, M. H. Cohen, B. E. Sha, \& L. L. Baum. Antibody-Dependent Cell-Mediated Cytotoxicity in Cervical Lavage Fluids of Human Immunodeficiency Virus Type 1-Infected Women. J Infect Dis 185(4):439-47, 2002. On p. 842 Immunodeficiency Virus Type 1-Infected Women. J Infect Dis 185(4):439-47, 2002. On p. 842
[Bauer1997] M. Bauer, M. Lucchiari-Hartz, R. Maier, G. Haas, B. Autran, K. Eichmann, R. Frank, Bauer1997] M. Bauer, M. Lucchiari-Hartz, R. Maier, G. Haas, B. Autran, K. Eichmann, R. Frank,
B. Maier, \& A. Meyerhans. Structural constraints of HIV-1 Nef may curtail escape from HLAB7-restricted CTL recognition. Immunol Lett 55:119-22, 1997. On p. 359

[Beddows1999] S. Beddows, S. Lister, R. Cheingsong, C. Bruck, \& J. Weber. Comparison of the antibody repertoire generated in healthy volunteers following immunization with a monomeric reantibody repertoire generated in healthy volunteers following immunization with a monomeric re-
combinant gp120 construct derived from a CCR5/CXCR4-using human immunodeficiency virus type 1 isolate with sera from naturally infected individuals. $J$ Virol 73:1740-5, 1999. On p. 647 \begin{tabular}{|l|l|l|l|l|l|l|l|l|l|l|l|}
\hline 648 & 664 & 665 & 710 & 713 & 720 & 722 & 747 & 784 & 787 & 822 & 824 \\
\hline
\end{tabular}

[Bedford1997] P. Bedford, L. B. Clarke, G. Z. Hastings, \& S. C. Knight. Primary Proliferative Responses to Peptides of HIV Gag p24. J Acquir Immune Defic Syndr Hum Retrovirol 14:301-

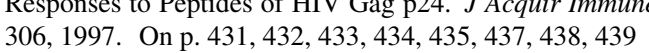

[Beirnaert2000] E. Beirnaert, P. Nyambi, B. Willems, L. Heyndrickx, R. Colebunders, W. Janssens, \& G. van der Groen. Identification and characterization of sera from HIV-infected individual with broad cross-neutralizing activity against group $\mathrm{M}$ (env clade A-H) and group O primary HIV-1 isolates. J Med Virol 62(1):14-24, 2000. On p.744

[Beirnaert2001] E. Beirnaert, S. De Zutter, W. Janssens, \& G. van der Groen. Potent broad crossneutralizing sera inhibit attachment of primary HIV-1 isolates (groups $\mathrm{M}$ and $\mathrm{O}$ ) to periphera blood mononuclear cells. Virology 281(2):305-14, 2001. On p.743,744

Bell1992] S. J. D. Bell, D. A. Cooper, B. E. Kemp, R. R. Doherty, \& R. Penny. Definition of an immunodominant T-cell epitope contained in the envelope gp 41 sequence of HIV-1. Clin Exp Immunol 87:37-45, 1992. Notes: This gp41 peptide consistently elicits both T-cell blastogenic and B-cell (antibody) responses in asymptomatic HIV-seropositive individuals but not in ARC and AIDS patients. gp41 epitope: LGIWGCSGKLIC. On p.516

Belshe1998] R. B. Belshe, G. J. Gorse, M. Mulligan, T. Evans, M. Keefer, J. Excler, A. Duliege, J. Tartaglia, W. Cox, J. McNamara, K. Hwang, A. Bradney, D. Montifiori, K. Weinhold, \& NIAID AIDS Vaccine Evaluation Group. Induction of immune responses to HIV-1 canarypox virus (ALVAC) HIV-1 and gp120 SF-2 recombinant vaccines in uninfected volunteers. AIDS 12:240715, 1998. On p. 163 332 747 748

Belshe2001] R. B. Belshe, C. Stevens, G. J. Gorse, S. Buchbinder, K. Weinhold, H. Sheppard, D. Stablein, S. Self, J. McNamara, S. Frey, J. Flores, J. L. Excler, M. Klein, R. E. Habib, A. M. Duliege, C. Harro, L. Corey, M. Keefer, M. Mulligan, P. Wright, C. Celum, F. Judson, K. Mayer D. McKirnan, M. Marmor, G. Woody, \& and. Safety and immunogenicity of a canarypoxvectored human immunodeficiency virus Type 1 vaccine with or without gp120: a phase 2 study in higher- and lower-risk volunteers. J Infect Dis 183(9):1343-52, 2001. On p.748

Belyakov1998a] I. M. Belyakov, J. D. Ahlers, B. Y. Brandwein, P. Earl, B. L. Kelsall, B. Moss, W. Strober, \& J. A. Berzofsky. The importance of local mucosal HIV-specific CD8(+) cytotoxic T lymphocytes for resistance to mucosal viral transmission in mice and enhancement of resistance by local administration of IL-12. J Clin Invest 102:2072-81, 1998. On p. 297

Belyakov1998b] I. M. Belyakov, L. S. Wyatt, J. D. Ahlers, P. Earl, C. D. Pendleton, B. L. Kelsall, W. Strober, B. Moss, \& J. A. Berzofsky. Induction of a mucosal cytotoxic T-lymphocyte response by intrarectal immunization with a replication-deficient recombinant vaccinia virus expressing human immunodeficiency virus 89.6 envelope protein. $J$ Virol 72:8264-72, 1998. On p. 296

[Benjouad1993] A. Benjouad, J.-C. Gluckman, L. Montagnier, \& E. Bahraoui. Specificity of antibodies produced against native or desialylated human immunodeficiency virus type 1 recombinan gp160. J Virol 67:1693-1697, 1993. MAbs raised against desialylated HIV-1 gp160 cross-react with HIV-2 gp140 due to the immunodominant epitope in gp41. On p.696

Beretta1987] A. Beretta, F. Grassi, M. Pelagi, A. Clivio, C. Parravicini, G. Giovinazzo, F. Andronico, L. Lopalco, P. Verani, S. Butto, F. Titti, G. B. Rossi, G. Viale, E. Ginelli, \& A. G. Siccardi. HIV env Glycoprotein Shares a Cross-Reacting Epitope with a Surface Protein Present on Activated Human Monocytes and Involved in Antigen Presentation. Eur J Immunol 17:1793-1798, 1987. The MAb M38 binds to gp120 and also to a human protein of $80 \mathrm{kd}$ that is expressed on a small fraction of mononuclear cells in the lymph nodes. M38 inhibits proliferation in autologous tetanus toxoid presentation, so is involved in antigen presentation. Suggested molecular mimicry. On p. 688

[Beretta1994] A. Beretta \& A. Dalgleish. B-cell epitopes. AIDS 8(suppl 1):S133-S145, 1994. On \begin{tabular}{ll|l|l|l|l|l|} 
p. 618 & 688 & 701 & 710 & 783 & 798 \\
\hline
\end{tabular}

Berman1991] P. W. Berman, K. Rosenthal, G. Nakamura, L. Riddle, J. P. Porter, D. Dowbenko, M. Hobbes, R. Byrn, J. Groopman, T. Gregory, \& B. Fendly. Monoclonal antibodies to gp160 of HIV-1 that neutralize HIV-1 infectivity, block the binding of gp120 to CD4, and react with diverse isolates. J AIDS 4:306, 1991. On p.618 734

[Berman1997] P. W. Berman, A. M. Gray, T. Wrin, J. C. Vennari, D. J. Eastman, G. R. Nakamura, D. P. Francis, G. Gorse, \& D. H. Schwartz. Genetic and immunologic characterization of viruses infecting MN-rgp120-vaccinated volunteers. J Infect Dis 176:384-397, 1997. On p. 652 673 \begin{tabular}{l|l|l|l|l|l|}
728 & 759 & 762 & 763 & 809 \\
\hline
\end{tabular}

Bernard1998] N. F. Bernard, K. Pederson, F. Chung, L. Ouellet, M. A. Wainberg, \& C. M. Tsoukas. HIV-specific cytotoxic T-lymphocyte activity in immunologically normal HIV-infected persons. AIDS 12:2125-39, 1998. On p.122 $136[154] 182[183$ 
[Bernaschi2002] M. Bernaschi \& F. Castiglione. Selection of escape mutants from immune recognition during HIV infection. Immunol Cell Biol 80(3):307-313, 2002. On p.536

[Berthet-Colominas 1999] C. Berthet-Colominas, S. Monaco, A. Novelli, G. Sibai, F. Mallet, \& S. Cusack. Head-to-tail dimers and interdomain flexibility revealed by the crystal structure of HIV-1 capsid protein p24 complexed with a monoclonal Fab. EMBO J 18:1124-36, 1999. On p. 585

[Bertoletti1998a] A. Bertoletti. Personal communication, 1998. On p. 110123

[Bertoletti1998b] A. Bertoletti, F. Cham, S. McAdam, T. Rostron, S. Rowland-Jones, S. Sabally, T. Corrah, K. Ariyoshi, \& H. Whittle. Cytotoxic T cells from human immunodeficiency viru type 2 -infected patients frequently cross-react with different human immunodeficiency virus type 1 clades. J Virol 72:2439-2448, 1998. On p. 123

[Berzofsky 1988] J. A. Berzofsky, A. Bensussan, K. B. Cease, J. F. Bourge, R. Cheynier, Z. Lurhama, J.-J. Salaun, R. C. Gallo, G. M. Shearer, \& D. Zagury. Antigenic peptides recognized by T lymphocytes from AIDS viral envelope-immune humans. Nature 334:706-708, 1988. Notes: Test of response to synthetic peptides of lymphocytes from 14 healthy human volunteers who had been immunized with a rec vaccinia virus containing HIV gp160, then boosted with a recombinant fragment containing the carboxyl-terminal 404/14 to T2. A reduced response to $\mathrm{T} 2$ in terms of both magnitude and frequency may have been because of the boost containing the region covering $\mathrm{T} 1$, but not $\mathrm{T} 2$, and because of the timing of sampling relative to immunization. Some HLA typing was done but no conclusive MHC restriction patterns were determined. Env epitopes: T1: KQIINMWQEVGLAMYA and T2: HEDIISLWDQSLK. On p. 471506

[Berzofsky1991a] J. A. Berzofsky, C. D. Pendleton, M. Clerici, J. Ahlers, D. R. Lucey, S. D. Putney, \& G. M. Shearer. Construction of peptides encompassing multideterminant clusters of human immunodeficiency virus envelope to induce in vitro $\mathrm{T}$ cell responses in mice and humans of multiple MHC types. J Clin Invest 88(3):876-84, 1991. On p.470 471 472 496 497|501 508

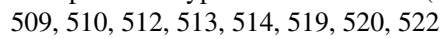

[Berzofsky1991b] J. A. Berzofsky, C. D. Pendleton, M. Clerici, J. Ahlers, D. R. Lucey, S. D. Putney, $\&$ G. M. Shearer. Peptides containing multideterminant clusters of human immunodeficiency virus envelope induce murine and human T-cell responses in diverse histocompatibility types.

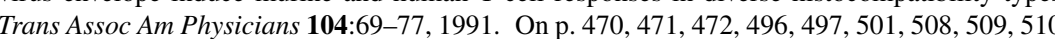
512513 514 519,520 522

[Betts 1997] M. R. Betts, J. Krowka, C. Santamaria, K. Balsamo, F. Gao, G. Mulundu, C. Luo, N. N'Gandu, H. Sheppard, B. H. Hahn, S. Allen, \& J. A. Frelinger. Cross-clade human immunodeficiency virus (HIV)-specific cytotoxic T- lymphocyte responses in HIV-infected Zambians. $J$ Virol 71:8908-11, 1997. On p. 163 240 334

[Betts1999] M. R. Betts, J. F. Krowka, T. B. Kepler, M. Davidian, C. Christopherson, S. Kwok, L. Louie, J. Eron, H. Sheppard, \& J. A. Frelinger. Human immunodeficiency virus type 1-specific cytotoxic T lymphocyte activity is inversely correlated with HIV type 1 viral load in HIV typ 1-infected long-term survivors. AIDS Res Hum Retroviruses 15:1219-28, 1999. On p.163 239 334

[Betts2000] M. R. Betts, J. P. Casazza, B. A. Patterson, S. Waldrop, W. Trigona, T.-M. Fu, F. Kern, L. J. Picker, \& R. A. Koup. Putative immunodominant human imunodeficiency virus-specific CD8+ T cell responses cannot be predicted by major histocompatibility complex class I haplotype. \begin{tabular}{|l|l|l|l|l|l|l|l|l|l|l|l|l|l|l|l|l|l|l|l|}
\hline$J$ Virol 74:9144-9151, 2000. On p. 61 & 64 & 75 & 97 & 98 & 99 & 106 & 108 & 128 & 132 & 153 & 187 & 193 \\
\hline
\end{tabular} \begin{tabular}{|l|l|l|l|l|l|l|l|l|l|l|l|}
211 & 304 & 307 & 320 & 324 & 343 & 355 & 363 & 366 & 374 & 375 \\
\hline
\end{tabular}

Beyrer1999] C. Beyrer, A. W. Artenstein, S. Rugpao, H. Stephens, T. C. VanCott, M. L. Robb, M. Rinkaew, D. L. Birx, C. Khamboonruang, P. A. Zimmerman, K. E. Nelson, \& C. Natpratan Epidemiologic and biologic characterization of a cohort of human immunodeficiency virus type 1 highly exposed, persistently seronegative female sex workers in northern Thailand. J Infect Dis 179(1):59-67, 1999. On p.751,752
[Billaut-Mulot2001] O. Billaut-Mulot, T. Idziorek, M. Loyens, A. Capron, \& G. M. Bahr. Modulation of cellular and humoral immune responses to a multiepitopic HIV-1 DNA vaccine by p. 141 258 381 447 463 (592 p. $141,258,381,447,463,592$

Binley1996] J. M. Binley, H. J. Ditzel, C. F. Barbas III, N. Sullivan, J. Sodroski, P. W. H. I. Parren, $\&$ D. R. Burton. Human antibody responses to HIV type 1 glycoprotein 41 cloned in phage display libraries suggest three major epitopes are recognized and give evidence for conserved antibody motifs in antigen binding. AIDS Res Hum Retroviruses 12:911-924, 1996. A panel of anti-gp41 human Fab fragments were generated by panning phage display antibody libraries prepared from HIV-1 positive donors with rgp41. Fabs tended to be directed against three epitopes, designated clusters I-III. None were neutralizing. A common CDR3 motif was found in several of the heavy

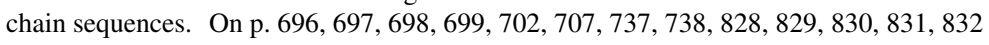

Binley1997a] J. M. Binley, H. Arshad, T. R. Fouts, \& J. P. Moore. An investigation of the high avidity antibody response to gp120 of human immunodeficiency virus type 1. AIDS Res and

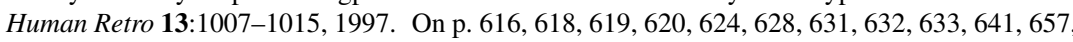
\begin{tabular}{|l|l|l|l|l|l|l|l|l|l|l|l|l|l|l|}
6688 & 681 & 682 & 689 & 720 & 753 & 756 & 758 & 762 & 764 & 798 & 803 & 822 \\
\hline
\end{tabular}

Binley1997b] J. M. Binley, P. J. Klasse, Y. Cao, I. Jones, M. Markowitz, D. D. Ho, \& J. P. Moore Differential regulation of the antibody responses to Gag and Env proteins of human immunodeficiency virus type 1. J Virol 71:2799-809, 1997. Retention of anti-Env antibodies and loss of anti-Gag antibodies during progression was studied, and suggested to be the result of the loss of T-cell help and the ability of Env to stimulate B cells even with declining CD4 cells, because of Env's unique ability to bind to the CD4 molecule. On p. 592 746 747

Binley1998] J. M. Binley, R. Wyatt, E. Desjardins, P. D. Kwong, W. Hendrickson, J. P. Moore, \& J. Sodroski. Analysis of the interaction of antibodies with a conserved enzymatically deglycosylated core of the HIV type 1 Envelope glycoprotein 120. AIDS Res Hum Retroviruses 14:191-8, 1998. This paper helped showed the biological relevance of a deglycosylated variable loop deleted

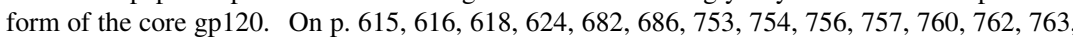
\begin{tabular}{ll|l|l|l|l|l|l|l|l|l|l|}
779 & 780 & 784 & 786 & 798 & 799 & 803 & 804 & 822 & 823 \\
\hline
\end{tabular}

[Binley 1999] J. Binley, R. Sanders, B. Clas, N. Schuelke, A. Master, Y. Guo, F. Kajumo, D. Anselma, P. Maddon, W. Olson, \& J. Moore. A Recombinant Human Immunodeficiency virus type 1 envelope glycoprotein complex stabilized by an intramolecular disulfide bond between the gp120 and gp41 subunits is an antigenic mimic of the trimeric virion associated structure. $J$ Virol \begin{tabular}{ll|l|l|l|l|l|l|l|l|l|l|l|l|l|} 
74:627-43, 1999. On p. 657 & 658 & 661 & 681 & 682 & 683 & 695 & 730 & 734 & 740 & 741 & 742 & 754 & 756 \\
\hline
\end{tabular} \begin{tabular}{ll|l|l|l|l|l|l|l|l|l|l|l|l|l|l|}
757 & 758 & 759 & 784 & 787 & 798 & 800 & 822 & 824 \\
\hline
\end{tabular}

Binley2000] J. M. Binley, A. Trkola, T. Ketas, D. Schiller, B. Clas, S. Little, D. Richman, A. Hurley, M. Markowitz, \& J. P. Moore. The effect of highly active antiretroviral therapy on binding and neutralizing antibody responses to human immunodeficiency virus type 1 infection. $J$ Infect Dis 182(3):945-9, 2000. On p.745

[Bird2002] T. G. Bird, R. Kaul, T. Rostron, J. Kimani, J. Embree, P. P. Dunn, J. J. Bwayo, F. A Plummer, S. L. Rowland-Jones, T. Dong, \& The Oxford-Nairobi HLA Collaborative Group. HLA typing in a Kenyan cohort identifies novel class I alleles that restrict cytotoxic T-cell responses to local HIV-1 clades. AIDS 16(14):1899-1904, 2002. On p.103 301

Birk1998a] M. Birk, J. I. Flock, A. Sonnerborg, \& M. Sallberg Coexisting members of HIV-1 p17 gene quasispecies represent proteins with distinct antigenicity and immunogenicity. AIDS 12:1973-81, 1998. On p. 442

[Birk1998b] M. Birk, A. Vahlne, A. Sonnerborg, \& M. Sallberg. Nonsynonymous mutations within the human immunodeficiency virus type $1 \mathrm{p} 17$ gene are clustered to sequences binding to the hos human leukocyte antigen class I molecules. AIDS Res Hum Retroviruses 14:241-8, 1998. On p. 60 \begin{tabular}{l|l|l|l|l|l|l|l|l|l|}
68 & 70 & 71 & 72 & 82 & 91 & 93 & 94 \\
\hline
\end{tabular}

[Bizub-Bender1994] D. Bizub-Bender, J. Kulkosky, \& A. M. Skalka. Monoclonal antibodies against 
HIV type 1 Integrase: clues to molecular structure. AIDS Res Hum Retroviruses 10:1105-1115, \begin{tabular}{l|l|l|l|l|l|l|} 
1994. On p. 602 & 603 & 604 & 605 & 606 & 608 & 705 \\
\hline
\end{tabular}

[Bjorling1992] E. Bjorling, L. Goobar-Larson, G. Utter, E. Norby, \& F. Chiodi. Four distinct antigenic regions are present in the primary structure of HIV-1 and HIV-2 proteinases. AIDS 6:157163, 1992. On p. 595

[Blankson2001a] J. N. Blankson, J. E. Gallant, \& R. F. Siliciano. Proliferative responses to human immunodeficiency virus type 1 (HIV-1) antigens in HIV-1-infected patients with immune reconstitution. J Infect Dis 183(4):657-61, 2001. On p. 443457

[Blankson2001b] J. N. Blankson \& R. F. Siliciano. MHC class II genotype and the control of viremia in HIV-1-infected individuals on highly active antiretroviral therapy. J Clin Invest 107(5):549-51, 2001. On p.435 436439

[Blazevic1993] V. Blazevic, A. Ranki, S. Mattinen, S. L. Valle, S. Koskimies, G. Jung, \& K. J. Krohn. Helper T-cell recognition of HIV-1 Tat synthetic peptides. J Acquir Immune Defic Syndr 6(8):881-890, 1993. On p. 461, 462

[Blazevic1995] V. Blazevic, A. Ranki, \& K. J. E. Krohn. Helper and cytotoxic T cell responses of HIV type 1-infected individuals to synthetic peptides of HIV type 1 Rev. AIDS Res Hum Retroviruses 11:1335-1342, 1995. The same set of four peptides from the Rev protein could stimulat proliferation of CD4+ cells and trigger CTL killing of autologous target cells transformed with EBV. On p.260 261464

[Blazevic2000] V. Blazevic, N. Sahgal, H. A. Kessler, A. L. Landay, \& G. M. Shearer. T cell responses to recall antigens, alloantigen, and mitogen of HIV-infected patients receiving longterm combined antiretroviral therapy. AIDS Res Hum Retroviruses 16(17):1887-93, 2000. On p. 444527

[Boe1998] S. O. Boe, B. Bjorndal, B. Rosok, A. M. Szilvay, \& K. H. Kalland. Subcellular localization of human immunodeficiency virus type 1 RNAs, Rev, and the splicing factor SC-35. Virology 244:473-82, 1998. On p.612

[Boehncke1993] W. H. Boehncke, T. Takeshita, C. D. Pendleton, R. A. Houghten, S. SadeghNasseri, L. Racioppi, J. A. Berzofsky, \& R. N. Germain. The importance of dominant negative effects of amino acid side chain substitution in peptide-MHC molecule interactions and $\mathrm{T}$ cell recognition. J Immunol 150:331-41, 1993. On p.508

[Boissonnas2002] A. Boissonnas, O. Bonduelle, A. Antzack, Y.-C. Lone, C. Gache, P. Debre, B. Autran, \& B. Combadiere. In vivo priming of HIV-specific CTLs determines selective cross-reactive immune responses against poorly immunogenic HIV-natural variants. J Immunol 169(7):3694-3699, 2002. On p.214 390

[Bojak2002] A. Bojak, D. Hammer, H. Wolf, \& R. Wagner. Muscle specific versus ubiquitous expression of Gag based HIV-1 DNA vaccines: A comparative analysis. Vaccine 20(15): 1975 1979, 2002. On p.589 593594

[Bolmstedt1990] A. Bolmstedt, S. Olofsson, E. Sjogren-Jansson, I. Sjoblom, L. Akerblom, J.-E. S Hansen, \& S.-L. Hu. Carbohydrate determinant NeuAc-Gal $\beta(1-4)$ of N-linked glycans modulate the antigenic activity of human immunodeficiency virus type 1 glycoprotein gp120. J Gen Virol 73:3009-3105, 1990. On p. 619 620 623 634

[Bolmstedt1996] A. Bolmstedt, S. Sjolander, J. E. Hansen, L. Akerblom, A. Hemming, S. L. Hu, B. Morein, \& S. Olofsson. Influence of N-linked glycans in V4-V5 region of human immunodeficiency virus type 1 glycoprotein gp 160 on induction of a virus-neutralizing humoral response. J AIDS Hum Retrovirol 12:213-220, 1996. Because N-linked glycans on viral glycoproteins can protect otherwise accessible neutralization epitopes of the viral envelope from neutralizing antibodies, the aim of this study was to explore the possibility of achieving a more broadly neutralizing immune response with a gp160 depleted of three N-linked glycans in the CD4-binding domain. Mutant and wild type gp160 were formulated into immunostimulating complexes (iscoms), and guinea pigs were vaccinated. Both preparations induced high serum antibody response to native gp120 and V3 peptides. The sera from animals immunized with the mutated glycoprotein lacking CD4 glycosylation sites did not neutralize nonrelated HIV strains better than did sera from animals immunized with wild type glycoprotein, but animals immunized with mutant gp160 neutralized mutant virus better than wild type virus, and vice versa. On p. 780

Bond2001] K. B. Bond, B. Sriwanthana, T. W. Hodge, A. S. De Groot, T. D. Mastro, N. L. Young N. Promadej, J. D. Altman, K. Limpakarnjanarat, \& J. M. McNicholl. An HLA-directed molecular and bioinformatics approach identifies new HLA-A11 HIV-1 subtype E cytotoxic T lymphocyte epitopes in HIV-1-infected Thais. AIDS Res Hum Retroviruses 17(8):703-17, 2001.

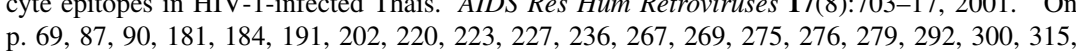
\begin{tabular}{|l|l|l|l|l|l|l|l|} 
p. 69 & 87 & 90 & 181 & 184 & 191 & 202 \\
\hline 320 & 3224 & 327 & 353 & 362 & 387 & 391 \\
\hline
\end{tabular}

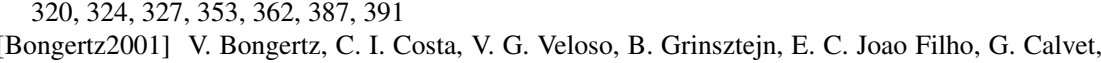
J. H. Pilotto, M. L. Guimaraes, \& M. G. Morgado. Vertical HIV-1 transmission: importance of neutralizing antibody titer and specificity. Scand J Immunol 53(3):302-9, 2001. On p. 642643 [Boots1997] L. J. Boots, P. M. McKenna, B. A. Arnold, P. M. Keller, M. K. Gorny, S. Zolla-Pazner, J. E. Robinson, \& A. J. Conley. Anti-human immunodeficiency virus type 1 human monoclonal antibodies that bind discontinuous epitopes in the viral glycoproteins can identify mimotopes from recombinant phage peptide display libraries. AIDS Res Hum Retroviruses 13:1549-59, \begin{tabular}{ll|l|l|l|l|l|l|l|l|} 
1997. On p. 657 & 658 & 696 & 697 & 720 & 721 & 756 & 757 & 784 & 785 \\
\hline
\end{tabular}

Borrow 1994] P. Borrow, H. Lewicki, B. H. Hahn, G. M. Shaw, \& M. B. Oldstone. Virus-specific CD8+ cytotoxic T-lymphocyte activity associated with control of viremia in primary human immunodeficiency virus type 1 infection. J Virol 68:6103-6110, 1994. On p.309

Borrow 1997] P. Borrow, H. Lewicki, X. Wei, M. S. Horwitz, N. Peffer, H. Meyers, J. A. Nelson, J. E. Gairin, B. H. Hahn, M. B. Oldstone, \& G. M. Shaw. Anti-viral pressure exerted by HIV-1-specific cytotoxic T lymphocytes (CTLs) during primary infection demonstrated by rapid selection of CTL escape virus. Nat Med 3:205-11, 1997. Genetic pathways of virus escape from CTL pressure resembled virus escape from antiretroviral therapy. On p. 268

[Borrow1998] P. Borrow \& G. M. Shaw. Cytotoxic T-lymphocyte escape viral variants: how important are they in viral evasion of immune clearance in vivo? Immunol Rev 164:37-51, 1998. On p. 268

[Botarelli1991] P. Botarelli, B. A. Houlden, N. L. Haigwood, C. Servis, D. Montagna, \& S. Abrignani. $\mathrm{N}$-glycosylation of HIV-gp120 may constrain recognition by T lymphocytes. J Immunol 147:3128-3132,1991. Notes: 20recombinant nonglycosylated form of gp120 failed to respond to glycosylated protein. The epitope for one such clone was mapped and contained two glycosylated asparagines. Thus $\mathrm{N}$-linked carbohydrates can abrogate antigen recognition by $\mathrm{T}$ lymphocytes. On p. 489

[Bou-Habib1994] D. C. Bou-Habib, G. Roderiquez, T. Oravecz, P. W. Berman, P. Lusso, \& M. A. Norcross. Cryptic nature of envelope V3 region epitopes protects primary monocytotropic human immunodeficiency virus type 1 from antibody neutralization. J Virol 68:6006-6013, 1994. This paper shows that antibodies to the tip of the V3 loop fail to neutralize primary isolate JR-CSF, and that the V3 loop is far more accessible on the JR-CSF derived T-cell tropic strain T-CSF. AntiV3 antibodies successfully neutralize T-CSF. Weak binding of anti-V3 antibodies to the primary isolate JR-CSF suggests the V3 loop is accessible only in a minor fraction of proteins. On p. 652 673

Boudet1991] F. Boudet, J. Theze, \& M. Zouali. UV-treated polystyrene microtitre plates for use in an ELISA to measure antibodies against synthetic peptides. J Immunol Methods 142:73-82, 1991. On p. 656

[Boudet1994] F. Boudet, J. Theze, \& M. Zouali. Anti-Idiotypic Antibodies to the Third Variable Domain of gp120 Induce an Anti-HIV-1 Antibody Response in Mice. Virology 200:176-188, 1994. On p. 656667671

[Boudet1995] F. Boudet, H. Keller, M. P. Kieny, \& J. Theze. Single peptide and anti-idiotype 
based immunizations can broaden the antibody response against the variable V3 domain of HIV1 in mice. Mol Immunol 32:449-457, 1995. Given the high degree of sequence variability of the V3 loop, the humoral response to this region tends to be type specific. An anti-idiotypic antibody could broaden the anti-V3 antibody polyclonal response in BALB/c mice, relative to the original $\mathrm{Ab}$ used to generate the anti-idiotype response. A synthetic peptide derived from the $\mathrm{V} 3$ determinant of HIV-1 MN induced an antibody response to multiple HIV-1 strains, but the exten of this cross-reactivity was inversely correlated with the binding affinity to V3 MN peptide. On p. 656

[Bouhdoud2000] L. Bouhdoud, P. Villain, A. Merzouki, M. Arella, \& C. Couture. T-cell receptormediated anergy of a human immunodeficiency virus (HIV) gp120-specific CD4(+) cytotoxic T-cell clone, induced by a natural HIV type 1 variant peptide. J Virol 74(5):2121-30, 2000. On p. 303

[Bouillot1989] M. Bouillot, J. Choppin, F. Cornille, F. Martinon, T. Papo, E. Gomard, M. C. Fournie-Zaluski, \& J.-P. Levy. Physical association between MHC class I molecules and immunogenic peptides. Nature 339:473-475, 1989. Describes an assay for binding radiolabelled MHC class I molecules to specific peptides that are bound to a solid phase support. Both presenting and non-presenting MHC molecules could bind, which suggested that quantitative difference in binding constants may ultimately determine in vivo MHC restriction. On $\mathrm{p} .138$

[Boyer1991] V. Boyer, H. Broly, S. Souche, P. Madaule, J. Rossier, D. Zagury, \& C. Desgranges. Characterization and large production of human monoclonal antibodies against the HIV-1 envelope. Clin Exp Immunol 83:452-459, 1991. On p.727.736

[Boyer1999] J. D. Boyer, M. A. Chattergoon, K. E. Ugen, A. Shah, M. Bennett, A. Cohen, S. Nyl, K. E. Lacy, M. L. Bagarazzi, T. J. Higgins, Y. Baine, R. B. Ciccarelli, R. S. Ginsberg, R. R MacGregor, \& D. B. Weiner. Enhancement of cellular immune response in HIV-1 seropositive individuals: A DNA-based trial. Clin Immunol 90:100-7, 1999. On p.525

[Bradney1999] A. P. Bradney, S. Scheer, J. M. Crawford, S. P. Buchbinder, \& D. C. Montefiori. Neutralization escape in human immunodeficiency virus type 1-infected long-term nonprogressors. J Infect Dis 179(5):1264-7, 1999. On p.747

[Bradney2002] C. P. Bradney, G. D. Sempowski, H.-X. Liao, B. F. Haynes, \& H. F. Staats Cytokines as adjuvants for the induction of anti-human immunodeficiency virus peptide immunoglobulin $\mathrm{G}$ (igg) and $\mathrm{IgA}$ antibodies in serum and mucosal secretions after nasal immunization. J Virol 76(2):517-524, 2002. On p. 820

[Brand1998] D. Brand, F. Lemiale, I. Turbica, L. Buzelay, S. Brunet, \& F. Barin. Comparative analysis of humoral immune responses to HIV type 1 envelope glycoproteins in mice immunized with a DNA vaccine, recombinant Semliki Forest virus RNA, or recombinant Semliki Forest virus particles. AIDS Res Hum Retroviruses 14:1369-77, 1998. On p. 775 777 784786

[Brander1995a] C. Brander, W. J. Pichler, \& G. Corradin. Identification of HIV-protein derived CTL epitopes for their potential use as synthetic vaccine. Clin Exp Immunol 101:107-113, 1995. On p.230 237, 279

[Brander1995b] C. Brander \& B. Walker. The HLA-class I restricted CTL Response in HIVInfection: Identification of optimal epitopes. HIV Molecular Immunology Database pp. IV-1 to IV-9, 1995. On p. 64 $91,188,193,231,271,328$

[Brander1996a] C. Brander, G. Corradin, T. Hasler, \& W. Pichler. Peptide immunization in humans: a combined $\mathrm{CD} 8+/ \mathrm{CD} 4+\mathrm{T}$ cell-targeted vaccine restimulates the memory CD4 $\mathrm{T}$ cell response but fails to induce cytotoxic T lymphocytes (CTL). Clin Exp Immunol 105:18-25, 1996. On p. 230279

[Brander1996b] C. Brander \& B. Walker. The HLA-class I restricted CTL response in HIV-1 Infection: Systematic identification of optimal epitopes. HIV Molecular Immunology Database pp.

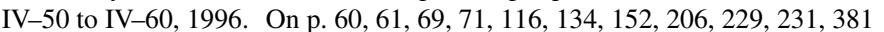

[Brander1997] C. Brander \& B. Walker. Systematic identification of optimal HIV-1 CTL epitopes.
HIV Molecular Immunology Database pp. IV-1 to IV-11, 1997. On p. 93 [101][149]189

Brander1998a] C. Brander, K. E. Hartman, A. K. Trocha, N. G. Jones, R. P. Johnson, B. Korber P. Wentworth, S. P. Buchbinder, S. Wolinsky, B. D. Walker, \& S. A. Kalams. Lack of strong immune selection pressure by the immunodominant, HLA-A*0201-restricted cytotoxic T lymphocyte response in chronic human immunodeficiency virus-1 infection. J Clin Invest 101(11):255966, 1998. On p.77 200 213393

[Brander1998b] C. Brander, B. D. Walker, \& B. Korber. Questionable HLA-A2 restriction of two HIV-1 Nef-derived CTL epitopes listed in the HIV Molecular Immunology Database. AIDS Res Hum Retroviruses 14(11):923-4, 1998. On p. 393

[Brander1999] C. Brander, O. O. Yang, N. G. Jones, Y. Lee, P. Goulder, R. P. Johnson, A. Trocha, D. Colbert, C. Hay, S. Buchbinder, C. C. Bergmann, H. J. Zweerink, S. Wolinsky, W. A. Blattner, S. A. Kalams, \& B. D. Walker. Efficient processing of the immunodominant, HLA-A*0201 restricted human immunodeficiency virus type 1 cytotoxic T-lymphocyte epitope despite multiple variations in the epitope flanking sequences. $J$ Virol 73:10191-8, 1999. On p. 74 140

Brander2001] C. Brander \& P. Goulder. The evolving field of HIV CTL epitope mapping: New approaches to the identification of novel epitopes. HIV Molecular Immunology Database pp. IV 1, 2001. This review article in the annual HIV Molecular Immunology Compendium presents the table of Optimal CTL Epitopes that has been curated by Brander and others for several years. On \begin{tabular}{l|l|l|l|l|l|l|l|l|l|l|l|l|l|l|l|l|l|l|l|l|l|} 
p. 57 & 61 & 64 & 67 & 68 & 70 & 72 & 78 & 81 & 91 & 92 & 93 & 98 & 99 & 101 & 104 & 105 & 108 & 110 & 111 & 113 & 115 \\
\hline
\end{tabular} \begin{tabular}{|l|l|l|l|l|l|l|l|l|l|l|l|l|l|l|l|l|l|l|}
\hline 116 & 119 & 122 & 124 & 125 & 126 & 130 & 132 & 133 & 139 & 140 & 142 & 143 & 145 & 149 & 150 & 151 & 152 & 154 \\
\hline
\end{tabular} \begin{tabular}{|l|l|l|l|l|l|l|l|l|l|l|l|l|l|l|l|l|l|l|l|l|l|l|l|l|l|l|l|}
\hline 155 & 157 & 161 & 172 & 173 & 177 & 179 & 180 & 181 & 182 & 184 & 186 & 190 & 195 & 199 & 204 & 205 & 206 & 208 \\
\hline
\end{tabular}

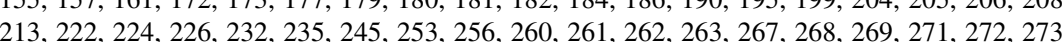
\begin{tabular}{|l|l|l|l|l|l|l|l|l|l|l|l|l|l|l|l|l|l|l|l|l|}
213 & 222 & 224 & 226 & 232 & 235 & 245 & 253 & 256 & 260 & 261 & 262 & 263 & 267 & 268 & 269 & 271 & 272 & 273 \\
\hline 281 & 284 & 294 & 301 & 303 & 304 & 307 & 310 & 314 & 316 & 317 & 319 & 321 & 322 & 323 & 328 & 343 & 346 & 348 \\
\hline
\end{tabular} \begin{tabular}{|l|l|l|l|l|l|l|l|l|l|l|l|l|l|l|l|l|}
281 & 284 & 294 & 301 & 303 & 304 & 307 & 310 & 314 & 316 & 317 & 319 & 321 & 322 & 323 & 328 & 343 \\
\hline 351 & 352 & 356 & 359 & 361 & 363 & 366 & 370 & 372 & 373 & 374 & 375 & 377 & 381 & 383 & 386 & 389 \\
\hline
\end{tabular}

Bristow 1994] R. G. W. Bristow, A. R. Douglas, J. J. Skehel, \& R. S. Daniels. Analysis of murin antibody responses to baculovirus-expressed human immunodeficiency virus type 1 envelope glycoproteins. J Gen Virol 75:2089-2095, 1994. BALB/c mice were immunized with baculovirus coproteins. J Gen Virol 75:2089-2095, 1994. BALB/c mice were immunized with baculovirus
expressed gp160 or gp120, and 15 MAbs were generated. No MAbs generated in this study neutralized reference strains, using a tetrazolium-based cytotoxicity assay to test for neutralization Ten of the Mabs were mapped by peptide ELISA, and seven reacted with the $\mathrm{C} 1$ region, one with \begin{tabular}{ll|l|l|l|l|l|l|l|l|}
$\mathrm{V} 2$, one with V4, and one with the C-terminal end. On p.617 & 618 & 620 & 621 & 625 & 678 & 688 & 724 \\
\hline
\end{tabular} Broder 1994] C. Broder P. Earl, D. Long, S. Abedon, B. Moss, \& R. Doms. Antigenic implication of human immunodeficiency virus type 1 envelope quaternary structure: Oligomer-specific and -sensitive monoclonal antibodies. Proc Natl Acad Sci USA 91:11699-11703, 1994. 35 anti-gp41 and 27 anti-gp120 murine MAbs generated by immunization with oligomeric HIV-1 IIIB envelope were studied. These MAbs tended to react with conformational epitopes. 21 of the anti-gp4 MAbs reacted preferentially with oligomeric env, while only 1 of the anti-gp120 MAbs reacted more strongly with the oligomer, and 14 of the anti-gp120 preferentially recognized monomeric env. On p.671 675 736 7417755 772

Brodie1999] S. J. Brodie, D. A. Lewinsohn, B. K. Patterson, D. Jiyamapa, J. Krieger, L. Corey, P. D. Greenberg, \& S. R. Riddell. In vivo migration and function of transferred HIV-1-specific cytotoxic T cells [see comments]. Nat Med 5:34-41, 1999. On p. 57] 103 149

[Brodie2000] S. J. Brodie, B. K. Patterson, D. A. Lewinsohn, K. Diem, D. Spach, P. D. Greenberg, S. R. Riddell, \& L. Corey. HIV-specific cytotoxic T lymphocytes traffic to lymph nodes and localize at sites of HIV replication and cell death. J Clin Invest 105:1407-17, 2000. On p. 58 $650101,103[149$

[Broliden1989] P. A. Broliden, V. Moschese, K. Ljunggren, J. Rosen, C. Fundaro, A. Plebani, M. Jondal, \& P. Rossi. Diagnostic implication of specific immunoglobulin G patterns of children born to HIV-1 infected mothers. AIDS 3:577, 1989. On p.705

[Broliden1990] P. A. Broliden, K. Ljunggren, J. Hinkula, E. Norrby, L. Akerblom, \& B. Wahren. A 
monoclonal antibody to human immunodeficiency virus type 1 which mediates cellular cytotoxicity and neutralization. $J$ Virol 64:936-940, 1990. On p. 619 662

[Broliden1991] P. A. Broliden, B. Makitalo, L. Akerblom, J. Rosen, K. Broliden, G. Utter, M. Jondal, E. Norrby, \& B. Wahren. Identification of amino acids in the V3 region of gp120 critical for virus neutralization by human HIV-1 specific antibodies. Immunology 73:371-376, 1991. On p. 661,662

[Broliden2001] K. Broliden, J. Hinkula, C. Devito, P. Kiama, J. Kimani, D. Trabbatoni, J. J. Bwayo, M. Clerici, F. Plummer, \& R. Kaul. Functional HIV-1 specific IgA antibodies in HIV-1 exposed, persistently IgG seronegative female sex workers. Immunol Lett 79(1-2):29-36, 2001. On p.751

[Brown1995] L. E. Brown, D. O. White, C. Agius, B. E. Kemp, N. Yatzakis, P. Poumbourios, D. A McPhee, \& D. C. Jackson. Synthetic peptides representing sequences within gp41 of HIV as immunogens for murine T- and B-cell responses. Arch Virol 140:635-54, 1995. On p. 515 516

[Bruce1999] C. B. Bruce, A. Akrigg, S. A. Sharpe, T. Hanke, G. W. Wilkinson, \& M. P. Cranage. Replication-deficient recombinant adenoviruses expressing the human immunodeficiency virus Env antigen can induce both humoral and CTL immune responses in mice. J Gen Virol 80( $\mathrm{Pt}$ 10):2621-8, 1999. On p. 297

[Buchacher1992] A. Buchacher, R. Predl, C. Tauer, M. Purtscher, G. Gruber, R. Heider, F. Steindl, A. Trkola, A. Jungbauer, \& H. Katinger. Human monoclonal antibodies against gp41 and gp120 as potential agents for passive immunization. Vaccines 92:191-195, 1992. On p. 575 694695 696710719

[Buchacher1994] A. Buchacher, R. Predl, K. Strutzenberger, W. Steinfellner, A. Trkola, M. Purtscher, G. Gruber, C. Tauer, F. Steindl, A. Jungbauer, \& H. Katinger. Generation of human monoclonal antibodies against HIV-1 proteins; electrofusion and Epstein-Barr virus transformation for peripheral blood lymphocyte immortalization. AIDS Res Hum Retroviruses 10:359-369, 1994. A panel of 33 human monoclonal antibodies were produced. Linear epitopes for some of this set of MAbs were mapped using peptide ELISA. Linear epitopes were mapped in gp41, and a single epitope was mapped in p24. While multiple gp120 specific MAbs were generated, all seemed to be conformational or carbohydrate dependent, or both. On p.575 693694695696 710719729731822

[Buchbinder1992] A. Buchbinder, S. Karwowska, M. K. Gorny, S. T. Burda, \& S. Zolla-Pazner. Synergy Between Human Monoclonal Antibodies to HIV Extends Their Effective Biologic Activity Against Homologous and Divergent Strains. AIDS Res Hum Retroviruses 8:425-427, 1992

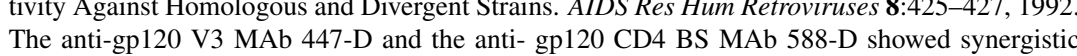
neutralization. On p.720 769

[Bugge1990] T. H. Bugge, B. O. Lindhardt, L. L. Hansen, P. Kusk, E. Hulgaard, K. Holmback, P. J. Klasse, J. Zeuthen, \& K. Ulrich. Analysis of a Highly Immunodominant Epitope in the Human Immunodeficiency Virus Type 1 Transmembrane Glycoprotein, gp41, Defined by a Human Monoclonal Antibody. J Virol 64:4123-4129, 1990. On p. 705

[Bukawa1995] H. Bukawa, K.-I. Sekigawa, K. Hamajima, J. Fukushima, Y. Yamada, H. Kiyono, \& K. Okuda. Neutralization of HIV-1 by secretory IgA induced by oral immunization with a new macromolecular multicomponent peptide vaccine candidate. Nature Med 1:681-685, 1995. Thi paper studies the anti-HIV-1 antibodies raised in response to a multicomponent peptide vaccine, given orally. It consisted of: V3 loop peptides based on sequences from cyclized B consensus sequence; a PND common in Japan; IIIB PND; Thai B strains PND; a CD4 binding site peptide and a Gag peptide, HPG30. BALB/c mice were immunized. Serum $\operatorname{IgA}$ and $\operatorname{IgG}$ and fecal $\operatorname{IgA}$ were detected. IgA from fecal samples was capable of neutralizing lab strains. On p. 572 642 680

[Buonaguro2001] L. Buonaguro, F. M. Buonaguro, M. L. Tornesello, D. Mantas, E. Beth-Giraldo, R. Wagner, S. Michelson, M. C. Prevost, H. Wolf, \& G. Giraldo. High efficient production of Pr55(gag) virus-like particles expressing multiple HIV-1 epitopes, including a gp120 protein derived from an Ugandan HIV-1 isolate of subtype A. Antiviral Res 49(1):35-47, 2001. On p. $591592,818,819$

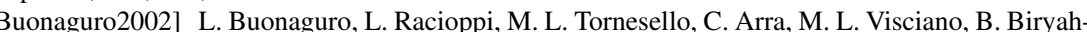
waho, S. D. K. Sempala, G. Giraldo, \& F. M. Buonaguro. Induction of neutralizing antibodies and cytotoxic T lymphocytes in Balb/c mice immunized with virus-like particles presenting a gp120 molecule from a HIV-1 isolate of clade A. Antiviral Res 54(3):189-201, 2002. On p. 402447 529728

[Buratti1997] E. Buratti, S. G. Tisminetzky, P. D’Agaro, \& F. E. Baralle. A neutralizing monoclonal antibody previously mapped exclusively on human immunodeficiency virus type $1 \mathrm{gp} 41$ recognizes an epitope in p17 sharing the core sequence. $J$ Virol 71:2457-62, 1997. On p.725 Burnett2000] M. S. Burnett, N. Wang, M. Hofmann, \& G. Barrie Kitto. Potential live vaccines for HIV. Vaccine 19(7-8):735-42, 2000. On p. 180,458607

Burton1991] D. R. Burton, C. F. Barbas III, M. A. Persson, S. Koenig, R. M. Chanock, \& R. A. Lerner. A large array of human monoclonal antibodies to type 1 human immunodeficiency virus from combinatorial libraries of asymptomatic seropositive individuals. Proc Natl Acad Sci USA 88:10134-10137, 1991. A panel of human monoclonal antibody Fab fragments was generated against the surface of the gp120 glycoprotein of HIV-1 by antigen selection from a random combinatorial library prepared from $5 \mathrm{ml}$ of bone marrow from an asymptomatic individual who had been HIV-positive for 6 years. These Fab variable regions were sequenced and were found to be diverse. Binding constants were measured and the Fabs generally bound gp 120 with high affinity. The methods used to obtain this panel could be used to obtain antibodies to test passive immunization as a therapy for AIDS. On p. 742 784

Burton1994] D. R. Burton, J. Pyati, R. Koduri, S. J. Sharp, G. B. Thornton, P. W. Parren, L. S Sawyer, R. M. Hendry, N. Dunlop, \& P. L. Nara. Efficient neutralization of primary isolates of HIV-1 by a recombinant human monoclonal antibody. Science 266:1024-1027, 1994. The MAb IgG1b12 showed very potent neutralization of a range of primary B subtype isolates. Binding with a variety of international isolates was tested; bound to most B isolates, 20with E clade. On p. 784

[Burton1997] D. R. Burton \& D. C. Montefiori. The antibody response in HIV-1 infection. AIDS 11 Suppl A:S87-S98, 1997. An excellent review of Ab epitopes and the implications for Envelope structure, neutralization of HIV, the distinction between primary and TCLA strains, ADCC and its role in clearance, and the $\mathrm{Ab}$ response during the course of infection. On p. 710,712756 757,784, 785, 822,823

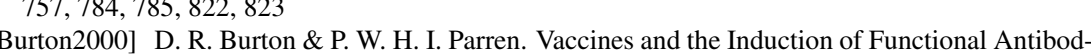
ies: Time to Look Beyond the Molecules of Natural Infection? Nat Med 6:123-125, 2000. On p. 728

Buseyne1993a] F. Buseyne, S. Blanche, D. Schmitt, C. Griscelli, \& Y. Riviere. Detection of HIVspecific cell-mediated cytotoxicity in the peripheral blood from infected children. J Immunol

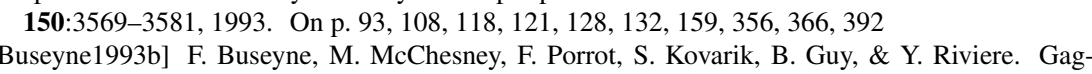
specific cytotoxic $\mathrm{T}$ lymphocytes from human immunodeficiency virus type 1-infected individuals: Gag epitopes are clustered in three regions of the p24 gag protein. $J$ Virol 67:694-702, 1993 . Using autologous Epstein-Barr virus-transformed cells that were infected with vaccinia constructs carrying p18, p24 and p55 proteins of LAI, or truncations of p24, it was shown that epitopes within p24 were most commonly recognized in a set of cell lines derived from 29 infected subjects. The autologous transformed cells coated with synthetic peptides were used to identify several regions of p24 where CTL epitopes tended to cluster. HLA restriction was determined CTL-responsive to four of the peptides. Among the four epitopes that had determined HLA specificities were the two peptides in the study that proved to stimulate CTL from the highest fraction of the cell lines: peptide p24(263-272) HLA-B27 and peptide p24(256-270) HLA-A33; these peptides were each 


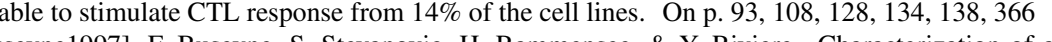

[Buseyne 1997] F. Buseyne, S. Stevanovic, H. Rammensee, \& Y. Riviere, Characterization of an HIV-1 p24 gag epitope recognized by a CD8+ cytotoxic T cell clone. Immunol Lett 55(3):145 149, 1997. On p. 151

[Buseyne1998a] F. Buseyne, M. Burgard, J. P. Teglas, E. Bui, C. Rouzioux, M. J. Mayaux S. Blanche, \& Y. Riviere. Early HIV-specific cytotoxic T lymphocytes and disease progression in children born to HIV-infected mothers. AIDS Res Hum Retroviruses 14:1435-44, 1998. On p. 163,239334395

[Buseyne1998b] F. Buseyne, M. L. Chaix, B. Fleury, O. Manigard, M. Burgard, S. Blanche, C. Rouzioux, \& Y. Riviere. Cross-clade-specific cytotoxic T lymphocytes in HIV-1-infected children. Virology 250:316-24, 1998. On p. 163239333.396

[Buseyne1999] F. Buseyne. Personal communication, 1999. On p. 151] 152 352

[Buseyne2001] F. Buseyne, S. Le Gall, C. Boccaccio, J. P. Abastado, J. D. Lifson, L. O. Arthur,

Y. Riviere, J. M. Heard, \& O. Schwartz. MHC-I-restricted presentation of HIV-1 virion antigen without viral replication. Nat Med 7(3):344-9, 2001. On p. 85. 151

[Cafaro2001] A. Cafaro, F. Titti, C. Fracasso, M. T. Maggiorella, S. Baroncelli, A. Caputo, D. Goletti, A. Borsetti, M. Pace, E. Fanales-Belasio, B. Ridolfi, D. R. Negri, L. Sernicola, R. Belli, F. Corrias, I. Macchia, P. Leone, Z. Michelini, P. ten Haaft, S. Butto, P. Verani, \& B. Ensoli. Vaccination with DNA containing tat coding sequences and unmethylated $\mathrm{CpG}$ motifs protects cynomolgus monkeys upon infection with simian/human immunodeficiency virus (SHIV89.6P) Vaccine 19(20-22):2862-77, 2001. On p. 259

[Calarota1996] S. Calarota, M. Jansson, M. Levi, K. Broliden, O. Libonatti, H. Wigzell, \& B. Wahren. Immunodominant glycoprotein 41 epitope identified by seroreactivity in HIV type 1-infected individuals. AIDS Res Hum Retroviruses 12:705-713, 1996. On p.710711

[Calarota1999] S. A. Calarota, A. C. Leandersson, G. Bratt, J. Hinkula, D. M. Klinman, K. J. Weinhold, E. Sandstrom, \& B. Wahren. Immune responses in asymptomatic HIV-1-infected patients after HIV-DNA immunization followed by highly active antiretroviral treatment. J Immunol 163:2330-8, 1999. On p. 258 264 395 462 465 533

[Calarota2001] S. A. Calarota \& B. Wahren. Cellular HIV-1 immune responses in natural infectio and after genetic immunization. Scand J Infect Dis 33(2):83-96, 2001. On p. 259 265 397| 462 465533

[Callahan1990] K. M. Callahan, M. M. Fort, E. A. Obah, E. L. Reinherz, \& R. F. Siliciano. Genetic variability in HIV-1 gp120 affects interactions with HLA molecules and T-cell receptor. Immunol 144:3341-3346, 1990. Notes: Synthetic peptides representing a defined CD4+ human T-cell epitope in gp120 were used to survey gp120 molecules from various HIV-1 strains for the capacity to be recognized in the context of a single human MHC molecule, DR4. gp120 epitope: GSDTITLPCRIKQFINMWQE. On p.505

[Callan1998] M. F. C. Callan, L. Tan, N. Annels, G. S. Ogg, J. D. K. Wilson, C. A. O'Callghan, N. Steven, A. J. McMichael, \& A. B. Rickinson. Direct visualization of antigen-specific CD8+ T Cells during the primary immune response to Epstein-Barr virus in vivo. J Exp Med 187:13951402, 1998. On p. 82

Cao1997a] H. Cao, P. Kanki, J. L. Sankale, A. Dieng-Sarr, G. P. Mazzara, S. Kalams, B. Korber, S. M'Boup, \& B. D. Walker. CTL cross-reactivity among different HIV-1 clades: Implications for vaccine development. $J$ Virol 71:8615-8623, 1997. On p. 62 85 145 192 216 272 310 321329

Cao1997b] J. Cao, N. Sullivan, E. Desjardin, C. Parolin, J. Robinson, R. Wyatt, \& J. Sodroski. Replication and neutralization of human immunodeficiency virus type 1 lacking the V1 and V2 variable loops of the gp120 Envelope glycoprotein. J Virol pp. 9808-12, 1997. An HIV-1 mutan lacking the V1-V2 loops can replicate in Jurkat cells and revertants that replicate with wild-type efficiency rapidly evolve in culture. These viruses exhibited increased neutralization susceptibility to V3 loop or CD4i MAbs, but not to sCD4 or anti-CD4BS MAbs. Thus the gp120 V1 and V2 loops protect HIV-1 from some subsets of neutralizing antibodies. On p.641 642 667. 775 . 777 798799817

[Cao2000] H. Cao, I. Mani, R. Vincent, R. Mugerwa, P. Mugyenyi, P. Kanki, J. Ellner, \& B. D. Walker. Cellular immunity to human immunodeficiency virus type 1 (HIV-1) clades: relevance to HIV-1 vaccine trials in uganda. J Infect Dis 182(5): 1350-6, 2000. On p.109 165 241281 329336397

Cao2002] H. Cao, D. Agrawal, N. Kushner, N. Touzjian, M. Essex, \& Y. Lu. Delivery of exogenous protein antigens to major histocompatibility complex class I pathway in cytosol. J Infect Dis 185(2):244-251, 2002. On p. 147 [268 281 280

Capon1989] D. J. Capon, S. M. Chamow, J. Mordenti, S. A. Marsters, T. Gregory, H. Mitsuya, R. A. Byrn, C. Lucas, F. M. Wurm, J. E. Groopman, \& et al. Designing CD4 immunoadhesin for AIDS therapy. Nature 337:525-31, 1989. On p.791.

Carcelain2001] G. Carcelain, R. Tubiana, A. Samri, V. Calvez, C. Delaugerre, H. Agut, C. Katlama, \& B. Autran. Transient mobilization of human immunodeficiency virus (HIV)-specific cd4 Thelper cells fails to control virus rebounds during intermittent antiretroviral therapy in chronic HIV type 1 infection. J Virol 75(1):234-41, 2001. On p.443

[Carlos1999] M. P. Carlos, Y. Yamamura, F. Diaz-Mitoma, \& J. V. Torres. Antibodies from HIVpositive and AIDS patients bind to an HIV envelope multivalent vaccine. J Acquir Immune Defic Syndr 22:317-24, 1999. On p. 625 634641679685

[Carreno1992] B. M. Carreno, S. Koenig, J. E. Coligan, \& W. E. Biddison. The peptide binding specificity of HLA class I molecules is largely allele-specific and non-overlapping. Molecular Immunol 29:1131-1140, 1992. Peptide competition experiments for presentation of viral peptide restricted by HLA-A 3 and HLA-B27 were performed to study the specificity of peptide binding to class I molecules. HIV-1 Nef (74-82) presentation by HLA-A3 was among the epitopes studied. On p. 134356

[Carruth 1999] L. M. Carruth, T. F. Greten, C. E. Murray, M. G. Castro, S. N. Crone, W. Pavlat, J. P. Schneck, \& R. F. Siliciano. An algorithm for evaluating human cytotoxic T lymphocyte responses to candidate AIDS vaccines. AIDS Res Hum Retroviruses 15:1021-34, 1999. On p. 82.270

Caruso1997] A. Caruso, S. Licenziati, A. D. Canaris, M. Corulli, M. A. D. Francesco, A. Cantalamessa, F. Fallacara, S. Fiorentini, A. Balsari, \& A. Turano. T cells from individuals in advanced stages of HIV-1 infection do not proliferate but express activation antigens in response to HIV-1 specific antigens. J Acquir Immune Defic Syndr Hum Retrovirol 15:61-69, 1997. On p. 473494 509520

[Casement1995] K. S. Casement, P. N. Nehete, R. B. Arlinghaus, \& K. J. Sastry. Cross-reactive cytotoxic T lymphocytes induced by V3 loop synthetic peptides from different strains of human immunodeficiency virus type 1. Virology 211:261-267, 1995. Seven diverse V3 peptides were found to induce CTL in immunized mice. All contained the $\mathrm{H}-2 \mathrm{D}^{d}$ binding motif $\mathrm{G}, \mathrm{P}$ and $\mathrm{R}$ a positions 2, 3 and 5. Only a CTL (no antibody response) was detected in immunized mice. On p. 290

Cavacini1993a] L. A. Cavacini, C. L. Emes, J. Power, A. Buchbinder, S. Zolla-Pazner, \& M. R. Posner. Human monoclonal antibodies to the V3 loop of HIV-1 gp120 mediate variable an distinct effects on binding and viral neutralization by a human monoclonal antibody to the CD4 binding site. J AIDS 6:353-358, 1993. On p.647 675 720775

[Cavacini1993b] L. A. Cavacini, C. L. Emes, J. Power, J. Underdalh, R. Goldstein, K. Mayer, \& M. R. Posner. Loss of serum antibodies to a conformational epitope of HIV-1/gp120 identified by a human monoclonal antibody is associated with disease progression. J AIDS 6:1093-1102, 1993. Serum from 100binding, while serum samples from 27binding. On p.775, 776

Cavacini1994a] L. A. Cavacini, C. L. Emes, J. Power, M. Duval, \& M. R. Posner. Effect of antibody valency on interaction with cell-surface expressed HIV-1 and viral neutralization. J Immunol 
152:2538-2545, 1994. On p.775 776

[Cavacini1994b] L. A. Cavacini, J. Power, C. L. Emes, K. Mace, G. Treacy, \& M. R. Posner. Plasm pharmacokinetics and biological activity of a human immunodeficiency virus type 1 neutralizing human monoclonal antibody, F105, in cynomolgus monkeys. Tumor Immunol 15:251-256, 1994. MAb F105 was administered intravenously to four cynomolgus monkeys. At 15 days post-dose total serum F105 was $230+/-79 \mu \mathrm{g} / \mathrm{ml}$ and F105 was immunoreactive with cells infected with the MN and IIIB strains of HIV-1 as determined by flow cytometry. On p.775 776

[Cavacini1995] L. A. Cavacini, C. L. Emes, J. Power, F. D. Desharnais, M. Duval, D. Montefiori, \& M. R. Posner. Influence of heavy chain constant regions on antigen binding and HIV-1 neutralization by a human monoclonal antibody. J Immunol 155:3638-3644, 1995. By changing the $\mathrm{IgG} 1$ constant region of MAb F105 from $\mathrm{IgG}_{1} \kappa$ to $\mathrm{IgG}_{3} \kappa$, dramatic strain specific increases in neutralization efficiency were obtained. On p. 7757776

[Cavacini1998a] L. A. Cavacini, C. L. Emes, A. V. Wisnewski, J. Power, G. Lewis, D. Montefiori, $\&$ M. R. Posner. Functional and molecular characterization of human monoclonal antibody. AIDS Res Hum Retroviruses 14:1271-80, 1998. On p.702 706 707|775|777

[Cavacini1998b] L. A. Cavacini, M. H. Samore, J. Gambertoglio, B. Jackson, M. Duval, A. Wisnewski, S. Hammer, C. Koziel, C. Trapnell, \& M. R. Posner. Phase I study of a human monoclonal antibody directed against the CD4-. AIDS Res Hum Retroviruses 14:545-50, 1998. In an immunotherapeutic study, administration of a single dose of F105 was non-toxic and the Ab persisted, yet no benefit was observed in 4 individuals. The authors suggest it may be more helpful in other settings, for example, patients with no pre-existing anti-CD4 BS Abs, or in combination with other MAbs. On p.706 775 .777

[Cavacini1999] L. A. Cavacini, A. Wisnewski, J. E. Peterson, D. Montefiori, C. Emes, M. Duval, G. Kingsbury, A. Wang, D. Scadden, \& M. R. Posner. A human anti-HIV autoantibody enhances EBV transformation and HIV. Clin Immunol 93:263-73, 1999. On p.706 707 $737 \mid 775$

[Cease1987] K. B. Cease, H. Margalit, J. L. Cornette, S. D. Putney, W. G. Robey, C. Ouyang, H. Z. Streicher, P. J. Fischinger, R. C. Gallo, C. DeLisi, et al. Helper T-cell antigenic site identification in the acquired immunodeficiency syndrome virus gp120 envelope protein and induction of immunity in mice to the native protein using a 16-residue synthetic peptide. Proc Natl Acad Sci U S A 84(12):4249-53, 1987. An algorithm based on a model of immunodominant helper T-cell sites forming amphipathic helices was used to identify for the first time two T-cell sites, env T1 and env T2. These two peptides were shown to stimulate proliferation of T-cells in mice immunized with a fragment of the env protein. Also, mice immunized with T1 were able to induce immunity to env gp120. Multiple haplotypes were responsive. Env epitopes: T2: HEDIISLWDQSLK and T1: KQIINMWQEVGKAMYA. On p. 305. 472508

[Chakrabarti2002] B. K. Chakrabarti, W.-p. Kong, B.-y. Wu, Z.-Y. Yang, J. Friborg, X. Ling, S. R King, D. C. Montefiori, \& G. J. Nabel. Modifications of the Human Immunodeficiency Virus Envelope Glycoprotein Enhance Immunogenicity for Genetic Immunization. J Virol 76(11):5357\begin{tabular}{l} 
5368, 2002. On p. $401 \mid$ \\
\hline 662
\end{tabular}

\begin{tabular}{rl|l|l|l|l|l|l|l|l|} 
5368, 2002. On p. 401,662 & 710 & 716 & 752 & 775 & 778 & 784 & 790 & 822 & 826 \\
[Chan1998] S. Y. Chan, M. C. Louie, J. R. Piccotti, G. Iyer, X. Ling, Z. Y. Yang, G. J. Nabel, \&
\end{tabular} D. K. Bishop. Genetic vaccination-induced immune responses to the human immunodeficiency virus protein Rev: emergence of the interleukin 2-producing helper T lymphocyte. Hum Gene Ther 9:2187-96, 1998. On p.464

[Chang1998] A. H. Chang, J. A. Hoxie, S. Cassol, M. O’Shaughnessy, \& F. Jirik. Construction of single-chain antibodies that bind an overlapping epitope of HIV-1 Nef. FEBS Lett 441:307-12, 1998. On p. 837. 840 841

[Chang1999] J. S. Chang, M. J. Choi, T. Y. Kim, S. Y. Cho, \& H. S. Cheong. Immunogenicity of synthetic HIV-1 V3 loop peptides by MPL adjuvanted pH- sensitive liposomes. Vaccine 17:1540 8, 1999. On p. 283

[Chanh1986] T. C. Chanh, R. C. Kennedy, B. E. Alderete, P. Kanda, J. W. Eichberg, \& G. R.
Dreesman. Human immunodeficiency virus gp120 glycoprotein detected by a monoclonal antibody to a synthetic peptide. Eur J Immunol 16:1465-1468, 1986. On p.753

[Chanh1987] T. C. Chanh, G. R. Dreesman, \& R. C. Kennedy. Monoclonal anti-idiotypic antibody mimics the CD4 receptor and binds human immunodeficiency virus. Proc Natl Acad Sci USA 84:3891-3895, 1987. On p.781

Chassin1999] D. Chassin, M. Andrieu, W. Cohen, B. Culmann-Penciolelli, M. Ostankovitch, D. Hanau, \& J. G. Guillet. Dendritic cells transfected with the nef genes of HIV-1 primary isolates specifically activate cytotoxic $\mathrm{T}$ lymphocytes from seropositive subjects. Eur J Immunol 29:196-202, 1999. On p. 354

Chattergoon2002] M. A. Chattergoon, H. H. Maguire, Jr., T. M. Robinson, E. Serrano, J. D. Boyer $\&$ D. B. Weiner. Plasmid immunization primes unique DTH responses to HIV-1MN envelope epitopes as compared to recombinant protein vaccination. Hybrid Hybridomics 21(2):117-122, 2002. On p. $4744481 \quad 496 \quad 500$ 501 513

[Cheingsong-Popov1992] R. Cheingsong-Popov, D. Callow, S. Beddows, S. Shaunak, C. Wasi, P. Kaleebu, C. Gilks, I. V. Petrascu, M. M. Garaev, \& D. M. Watts. Geographic Diversity of Human Immunodeficiency Virus Type 1: Serologic Reactivity to Env Epitopes and Relationship to neutralization. J Infect Dis 165(2):256-261, 1992. On p. 646

Chen1994a] S. Y. Chen, Y. Khouri, J. Bagley, \& W. A. Marasco. Combined intra- and extracellular immunization against human immunodeficiency virus type 1 infection with a human anti-gp120 antibody. Proc Natl Acad Sci USA 91:5932-5936, 1994. On p.775 776

[Chen1994b] Y.-H. Chen, A. Susanna, G. Bock, F. Steindl, H. Katinger, \& M. P. Dierich. HIV1 gp41 shares a common immunologic determinant with human T, B and monocyte cell lines. Immunol Letters 39:219-222, 1994. The MAb 3D6 binds to HIV gp41, and to a $43 \mathrm{kd}$ protein found in human T, B and monocyte cell lines. The authors suggest the possibility of molecular mimicry. On p.695, 706710

[Chen1995] C. H. Chen, T. J. Matthews, C. B. McDanal, D. P. Bolognesi, \& M. L. Greenberg. A molecular clasp in the human immunodeficiency virus (HIV) type $1 \mathrm{TM}$ protein determines the anti-HIV activity of gp41 derivatives: implication for viral fusion. J Virol 69:3771-3777, 1995. On p. 696 697 $708,828,830$

[Chen1996] J. D. Chen, Q. Yang, W. A. Marasco, \& S. Y. Chen. Intra- and extra-cellular immunization against HIV-1 infection with lymphocytes transduced with an AAV vector expressing a human anti-gp120 antibody. Hum Gene Ther 7:1515-1525, 1996. On p.775 777

Cherpelis2001a] S. Cherpelis, X. Jin, A. Gettie, D. D. Ho, S. W. Barnett, I. Shrivastava, \& L. Stamatatos. DNA-immunization with a v2 deleted HIV-1 envelope elicits protective antibodies in macaques. Immunol Lett 79(1-2):47-55, 2001. On p.748

Cherpelis2001b] S. Cherpelis, I. Shrivastava, A. Gettie, X. Jin, D. D. Ho, S. W. Barnett, \& L. Stamatatos. DNA vaccination with the human immunodeficiency virus type 1 SF162DeltaV2 envelope elicits immune responses that offer partial protection from simian/human immunodeficiency virus infection to CD8(+) T-cell-depleted rhesus macaques. J Virol 75(3):1547-50, 2001. On p. 748

Chesebro1988] B. Chesebro \& K. Wehrly. Development of a Sensitive Quantitative Focal Assay for Human Immunodeficiency Virus Infectivity. $J$ Virol 62:3779-3788, 1988. On p. 644675

[Chesebro1992] B. Chesebro, K. Wehrly, J. Nishio, \& S. Perryman. Macrophage-tropic human immunodeficiency virus isolates from different patients exhibit unusual V3 envelope sequence homogeneity in comparison with T-cell-tropic isolates: definition of critical amino acids involved in cell tropism. J Virol 66:6547-54, 1992. On p.589

[Cheynier1992] R. Cheynier, P. Langlade-Demoyen, M. R. Marescot, S. B. S., G. Blondin, S. WainHobson, C. Griscelli, E. Vilmer, \& F. Plata. Cytotoxic T lymphocyte responses in the periphera blood of children born to human immunodeficiency virus-1-infected mothers. Eur J Immunol 22:2211-2217, 1992. CTL effectors that killed HLA-matched HIV-1-infected H9 target cells 
or doubly transfected P815-A2-env, gag or nef mouse tumor cells, which expressed the vira antigens in association with HLA-A1/A3 or HLA-A2, were isolated in children born to HIV-1infected mothers. HIV-1-specific CTL were detected less than 2 months after birth, and declined with disease progression. CTL were detected in the PBMC of three children who subsequently became seronegative. On $\mathrm{p}$. 391

[Chiba1996] J. Chiba, A. Yamaguchi, Y. Suzuki, M. Nakano, W. Zhu, H. Ohba, A. Saito, H. Shinagawa, Y. Yamakawa, T. Kobayashi, \& T. Kurata. A novel neutralization epitope on the 'thumb' subdomain of human immunodeficiency virus type 1 reverse transcriptase revealed by a monoclonal antibody. J Gen Virol 77(12):2921-9, 1996. On p. 598 599

[Chiba1997] J. Chiba, M. Nakano, Y. Suzuki, K. Aoyama, H. Ohba, T. Kobayashi, A. Yasuda, A. Kojima, \& T. Kurata. Generation of neutralizing antibody to the reverse transcriptase of human immunodeficiency virus type 1 by immunizing of mice with an infectious vaccinia virus recombinant. J Immunol Methods 207:53-60, 1997. On p. 598 5996066

[Chiba1999] M. Chiba, H. Takahashi, K. Kato, Y. Nakagawa, T. Fukushima, H. Iinuma, \& K. Nerome. Recombinant vaccinia viruses expressing an immunodominant epitope of HIV-1 en velope protein within an influenza hemagglutinin cassette predominantly prime epitope-specific CD8(+) CTL. Arch Virol 144:1469-85, 1999. On p.290

[Chin1995] L.-T. Chin, A.-C. Malmborg, K. Kristensson, J. Hinkula, B. Wahren, \& C. A. K. Borrebaeck. Mimicking the humoral immune response in vitro results in antigen-specific isotype switching supported by specific autologous T helper cells: generation of human HIV-1 neutralizing IgG monoclonal antibodies from naive donors. Eur J Immunol 25:657-663, 1995. On p. 645

[Choppin2001] J. Choppin, W. Cohen, A. Bianco, J.-P. Briand, F. Connan, M. Dalod, \& J.-G. Guillet. Characteristics of HIV-1 Nef regions containing multiple CD8+ T cell epitopes: Wealth of HLA-binding motifs and sensitivity to proteasome degradation. J Immunol 166(10):6164-6169,

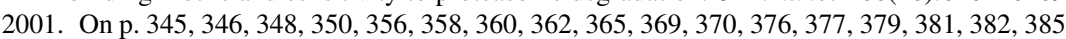
386387

[Chun2001] T. W. Chun, J. S. Justement, S. Moir, C. W. Hallahan, L. A. Ehler, S. Liu, M. McLaughlin, M. Dybul, J. M. Mican, \& A. S. Fauci. Suppression of HIV replication in the resting CD4 $\mathrm{T}$ cell reservoir by autologous CD8+ T cells: implications for the development of therapeutic strategies. Proc Natl Acad Sci U S A 98(1):253-8, 2001. On p.165

[Claverie1988] J.-M. Claverie, P. Kourilsky, P. Langlade-Demoyen, A. Chalufour-Prochnicka, G. Dadaglio, F. Tekaia, F. Plata, \& K. Bougueleret. T-immunogenic peptides are constitute of rare sequence patterns. Use in the identification of T epitopes in the human immunodeficiency virus gag protein. Eur J Immunol 18:1547-1553, 1988. Based on what was known about epitope structure and amino acid frequencies in 1988, the authors predicted epitopes in the gag protein Four peptides that were predicted to contain epitopes were found to specifically stimulate a HLA-A2 restricted polyclonal CTL cell line, when presented by mouse P815 target cells that had been transfected with HLA-A2. On p. 116,121$] 160$ [161

[Clerici1989] M. Clerici, N. I. Stocks, R. A. Zajac, R. N. Boswell, D. C. Bernstein, D. L. Mann G. M. Shearer, \& J. A. Berzofsky. Interleukin-2 production used to detect antigenic peptide recognition by T-helper lymphocytes from asymptomatic HIV-seropositive individuals. Nature 339:383-385, 1989. Notes: Investigation of the T-helper cell response of 42 asymptomatic HIVseropositive patients to four synthetic gp160 peptides and to influenza A virus. This paper suggests that a proliferative response is lost in HIV-1 infected individuals prior to the loss of IL-2

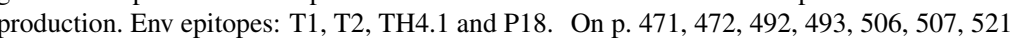

[Clerici1991a] M. Clerici, D. R. Lucey, R. A. Zajac, R. N. Boswell, H. M. Gebel, H. Takahashi, J. A. Berzofsky, \& G. M. Shearer. Detection of cytotoxic T lymphocytes specific for synthetic peptides of gp160 in HIV-seropositive individuals. J Immunol 146:2214-2219, 1991. Four peptides that could be used to stimulate helper T-cell function were also found to be reactive with MHC class
I-restricted CTL in infected individuals. 14 of 20 patients were responsive to at least one of the

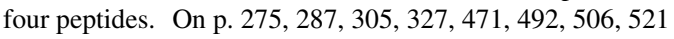

Clerici1991b] M. Clerici, C. O Tacket, C. S. Via, D. R. Lucey, S. C. Muluk, R. A. Zajac, R. N. Boswell, J. A. Berzofsky, \& G. M. Shearer. Immunization with subunit human immunodeficiency virus vaccine generates stronger $\mathrm{T}$ helper cell immunity than natural infection. Eur J Immuno 21:1345-1349, 1991. Notes: Immunization of uninfected individuals with an HIV subunit vaccine results in stronger Th cell immunity than does natural infection. Boosting enhances helper function. Env epitopes: T1, T2, TH4.1, P18. On p.471 492 506521

[Clerici1992] M. Clerici, J. V. Giorgi, C.-C. Chou, V. K. Gudeman, J. A. Zack, P. Gupta, H.-N. Ho, P. G. Nishanian, J. A. Berzofsky, \& G. M. Shearer. Cell-Mediated Immune Response to Human Immunodeficiency Virus Type 1 in Seronegative Homosexual Men with Recent Sexual Exposure to HIV-1. J Inf Dis 165:1012-9, 1992. Notes: Cell-mediated immune response to HIV-1 can be detected in the absence of a humoral immune response in individuals recently exposed to HIV-1. gp160 epitopes: T1, T2, TH4.1, P18-IIIb, P18-MN. On p.471 4924933506521

[Clerici1997] M. Clerici, S. Piconi, C. Balotta, D. Trabattoni, A. Capetti, M. L. Fusi, S. Ruzzante, R. Longhi, M. C. Colombo, M. Moroni, \& F. Milazzo. Pentoxifylline improves cell-mediated immunity and reduces human immunodeficiency virus (HIV) plasma viremia in asymptomatic HIV- seropositive persons. J Infect Dis 175:1210-5, 1997. On p. 471| 492 |506 521]

[Clerici2002a] M. Clerici, C. Barassi, C. Devito, C. Pastori, S. Piconi, D. Trabattoni, R. Longhi, J. Hinkula, K. Broliden, \& L. Lopalco. Serum IgA of HIV-exposed uninfected individuals inhibi HIV through recognition of a region within the alpha-helix of gp41. AIDS 16(13):1731-1741, 2002. On p. 694 695 710 716

Clerici2002b] M. Clerici, E. Seminari, F. Maggiolo, A. Pan, M. Migliorino, D. Trabattoni, F. Castelli, F. Suter, M. L. Fusi, L. Minoli, G. Carosi, R. Maserati, \& the Master Group. Early and late effects of highly active antiretroviral therapy: A 2 year follow-up of antiviral-treated and antiviral-naive chronically HIV-infected patients. AIDS 16(13):1767-1773, 2002. On p. 528

[Cleveland2000a] S. M. Cleveland, E. Buratti, T. D. Jones, P. North, F. Baralle, L. McLain, T. McInerney, Z. Durrani, \& N. J. Dimmock. Immunogenic and antigenic dominance of a nonneutralizing epitope over a highly conserved neutralizing epitope in the gp41 envelope glycoprotein of human immunodeficiency virus type 1: its deletion leads to a strong neutralizing response. Virology 266:66-78, 2000. On p. 725726727

[Cleveland2000b] S. M. Cleveland, T. D. Jones, \& N. J. Dimmock. Properties of a neutralizing antibody that recognizes a conformational form of epitope ERDRD in the gp41 C-terminal tail of human immunodeficiency virus type 1. J Gen Virol 81 Pt 5:1251-60, 2000. On p.726.727

[Coeffier2000] E. Coeffier, J. M. Clement, V. Cussac, N. Khodaei-Boorane, M. Jehanno, M. Rojas, A. Dridi, M. Latour, R. El Habib, F. Barre-Sinoussi, M. Hofnung, \& C. Leclerc. Antigenicity an immunogenicity of the HIV-1 gp41 epitope ELDKWA inserted into permissive sites of the male protein. Vaccine 19(7-8):684-93, 2000. On p.710

[Cohen2002] W. M. Cohen, A. Bianco, F. Connan, L. Camoin, M. Dalod, G. Lauvau, E. Ferriès, B. Culmann-Penciolelli, P. M. van Endert, J. P. Briand, J. Choppin, \& J. G. Guillet. Study of antigen-processing steps reveals preferences explaining differential biological outcomes of two HLA-A2-restricted immunodominant epitopes from human immunodeficiency virus type 1 . $J$ Virol 76(20):10219-10225, 2002. On p. 88 220

[Collado2000] M. Collado, D. Rodriguez, J. R. Rodriguez, I. Vazquez, R. M. Gonzalo, \& M. Esteban. Chimeras between the human immunodeficiency virus (HIV-1) Env and vaccinia viru immunogenic proteins p14 and p39 generate in mice broadly reactive antibodies and specific activation of CD8+ T cell responses to Env. Vaccine 18:3123-33, 2000. On p.616 684

[Collings 1999] A. Collings, J. Pitkanen, M. Strengell, M. Tahtinen, A. Lagerstedt, K. Hakkarainen, V. Ovod, G. Sutter, M. Ustav, E. Ustav, A. Mannik, A. Ranki, P. Peterson, \& K. Krohn. Humora and cellular immune responses to HIV-1 nef in mice DNA- immunised with non-replicating or 
self-replicating expression vectors. Vaccine 18:460-7, 1999. On p. 399

[Collins1998] K. L. Collins, B. K. Chen, S. A. Kalams, B. D. Walker, \& D. Baltimore. HIVNef protein protects infected primary cells against killing by cytotoxic T lymphocytes. Nature 391:397-401, 1998. On p. 83.216

[Conley1994a] A. J. Conley, M. K. Gorny, J. A. Kessler II, L. J. Boots, M. Ossorio-Castro, S. Koenig, D. W. Lineberger, E. A. Emini, C. Williams, \& S. Zolla-Pazner. Neutralization of primary human immunodeficiency virus type 1 isolates by the broadly reactive anti-V3 monoclonal antibody 447-52D. J Virol 68:6994-7000, 1994. On p.720

[Conley1994b] A. J. Conley, J. A. Kessler II, L. J. Boots, J.-S. Tung, B. A. Arnold, P. M. Keller A. R. Shaw, \& E. A. Emini. Neutralization of divergent human immunodeficiency virus type variants and primary isolates by IAM-41-2F5, and anti-gp41 human monoclonal antibody. Proc Natl Acad Sci USA 91:3348-3352, 1994. 2F5 is capable of neutralizing a broad range of primary isolates and lab strains. Susceptibility to neutralization was dependent on presence of a conserved antibody binding site. Kinetic studies were done, and $2 \mathrm{~F} 5$ has a very long $\mathrm{t}_{1} / 2$ of dissociation, 156 minutes for gp 41 . The authors point out that LDKW core is present in highly diverged international isolates. On p.710

[Conley1996] A. J. Conley, J. A. Kessler II, L. J. Boots, P. M. McKenna, W. A. Schleif, E. A. Emini, G. E. Mark III, H. Katinger, E. K. Cobb, S. M. Lunceford, S. R. Rouse, \& K. K. Murthy. The consequence of passive administration of an anti-human immunodeficiency virus type 1 neutralizing monoclonal antibody before challenge of chimpanzees with a primary virus isolate. Virol 70:6751-6758, 1996. The MAb 2F5 was infused into two chimpanzees which were then given an intravenous challenge with a primary HIV-1 isolate - both became infected, but with delayed detection and prolonged decrease in viral load relative to controls, indicating that preexisting, neutralizing antibodies (passively administered or actively elicited) affect the course of acute-phase virus replication and can be influential after the Ab can no longer be detected in the peripheral circulation. On p.710.711

[Connan1994] F. Connan, F. Hlavac, J. Hoebeke, J. G. Guillet, \& J. Choppin. A simple assay for detection of peptides promoting the assembly of HLA class I molecules. Eur J Immunol 24:777-780, 1994. Peptides from influenza and HIV-1 were tested for their ability to promote the assembly of HLA-A2 and HLA-B51 molecules in T2 cell lysates. HIV Pol 476-484 allowed sig nificant assembly of HLA-A2, and is a target for CTL. Nef peptide 186-194 produced significan assembly of HLA-B51. A hydrophobic anchor residue (V, L, I) at position 9 could occupy pocket $\mathrm{F}$, and a hydrophobic residue (V, L) at position 3 or 4 may anchor to hydrophobic pocket $\mathrm{D}$ of HLA-B51. Proline at position 2 increases HLA-B51 anchoring. On p. 217] 392,393

[Connelly1994] R. J. Connelly, M. Kahn, J. Blake, O. K. Haffar, \& E. K. Thomas. Dual specificity of a monoclonal anti-idiotypic antibody for HIV-1 neutralizing monoclonals 110.3 and 110.4 a well as the V3 loop of gp120. Virology 205:554-557, 1994. On p. 667

[Connor1998] R. I. Connor, B. T. Korber, B. S. Graham, B. H. Hahn, D. D. Ho, B. D. Walker, A. U. Neumann, S. H. Vermund, J. Mestecky, S. Jackson, E. Fenamore, Y. Cao, F. Gao, S. Kalams, K. J. Kunstman, D. McDonald, N. McWilliams, A. Trkola, J. P. Moore, \& S. M. Wolinsky. Immunological and virological analyses of persons infected by human immunodeficiency virus type 1 while participating in trials of recombinant gp120 subunit vaccines. J Virol 72:1552-76, 1998 No gp120-vaccine induced antibodies in a human trial of gp120 MN and SF2 could neutralize the primary viruses that infected the vaccinees. The primary isolates from the infected vaccinees were shown not to be particularly refractive to neutralization by their susceptibility to a panel of \begin{tabular}{ll|l|l|l|l|l|l|l|l|l|l|l|l|} 
neutralizing MAbs. On p. 636 & 710 & 712 & 720 & 721 & 784 & 786 & 822 & 823 \\
\hline
\end{tabular}

[Cook1994] D. G. Cook, J. Fantini, S. L. Spitalnik, \& F. Gonzalez-Scarano. Binding of human immunodeficiency virus type $1 \mathrm{HIV}-1$ gp120 to Galactosylceramide (GalCer): relationship to the V3 loop. Virol 201:206-214, 1994. Antibodies against GalCer can block infection of CD4 negative cells from the brain and colon that are susceptible to HIV infection. This paper explores the ability of a panel of MAbs to inhibit binding of gp120 to GalCer, and also of the binding of GalCer to inhibit MAb-gp120 interaction. MAbs to the V3 loop and GalCer showed mutual inhibition of binding to gp120, and anti-CD4 binding site MAbs showed reduced inhibition. Nand C-terminal MAbs didn't influence GalCer binding. On p.621 635 641 644 645 646 646 647 \begin{tabular}{ll|l|l|l|l|l|l|l|l|l|}
656 & 671 & 675 & 689 & 690 & 691 & 762 & 775 & 776 & 807 & 808 \\
\hline
\end{tabular}

Copeland2002] K. F. T. Copeland. The role of CD8+ T cell soluble factors in human immunodeficiency virus infection. Curr Med Chem 9(20):1781-1790, 2002. On p.401

[Cordell1991] J. Cordell, J. P. Moore, C. J. Dean, P. J. Klasse, R. A. Weiss, \& J. A. McKeating. Rat monoclonal antibodies to nonoverlapping epitopes of human immunodeficiency virus type I gp120 block CD4 binding in vitro. Virology 185:72-79, 1991. On p.668 679 680 683660 762783

Corey1998] L. Corey, M. J. McElrath, K. Weinhold, T. Matthews, D. Stablein, B. Graham, M. Keefer, D. Schwartz, G. Gorse, \& the AIDS Vaccine Evaluation Group. Cytotoxic T cell and neutralizing antibody responses to human immunodeficiency virus type 1 envelope with a combination vaccine regimen. J Infect Dis 177:301-9, 1998. On p. 334

[Corinti2002] S. Corinti, L. Chiarantini, S. Dominici, M. E. Laguardia, M. Magnani, \& G. Girolomoni. Erythrocytes deliver Tat to interferon-gamma-treated human dendritic cells for efficient initiation of specific type 1 immune responses in vitro. J Leukoc Biol 71(4):652-658, 2002. On p.462

Cotropia1992] J. Cotropia, K. E. Ugen, D. Lambert, K. Ljunggren-Broliden, S. Kliks, J. Hoxie, \& D. B. Weiner. Characterization of Human Monoclonal Antibodies to the HIV-1 Transmembrane gp41 Protein. Vaccines 92 pp. 157-163, 1992. Editors: F. Brown, H. S. Ginsberg and R. Lerner, Cold Spring Harbor Laboratory Press, Cold Spring Harbor, NY. On p. 705

Cotropia1996] J. Cotropia, K. E. Ugen, S. Kliks, K. Broliden, P.-A. Broliden, J. A. Hoxie, V. Srikantan, W. V. Williams, \& D. B. Weiner. A Human Monoclonal Antibody to HIV-1 gp41 with Neutralizing Activity Against Diverse Laboratory Isolates. J AIDS 12:221-232, 1996. On p. 705

Couillin1994] I. Couillin, B. Culmann-Penciolelli, E. Gomard, J. Choppin, J. P. Levy, J. G. Guillet, \& S. Sarasgosti. Impaired cytotoxic T lymphocyte recognition due to genetic variations in the main immunogenic region of the human immunodeficiency virus 1 NEF protein. $J$ Exp Med 180:1129-34, 1994. HIV-1 HLA-A11 and -B18 restricted epitopes were sequenced from donors who do and do not express the HLA-A11 and B18 molecule. Selective variations were only detected in virus isolated from individuals expressing the appropriate HLA type. Variant peptides with single substitutions within the minimal epitope did not always completely abrogate HLA binding, suggesting that multiple alterations within a particular epitope may need to accumulate during disease progression to allow viral escape. On p. $352,363,383$

[Couillin1995] I. Couillin, F. Connan, B. Culmann-Penciolelli, E. Gomard, J.-G. Guillet, \& J. Choppin. HLA-dependent variations in human immunodeficiency virus Nef protein alter peptide/HLA binding. Eur J Immunol 25:728-732, 1995. Viral sequences across this region were compared from 3 HLA-A11 positive and 10 negative donors. Substitutions that were found only in the 3 HLA-A11 donors did not promote HLA-A11 assembly. Substitutions that were found in both HLA-A11 positive and negative donors, however, did not markedly alter the reactivity of the peptides. This suggests that substitutions that result in loss of HLA-A11 occur mainly in HLA-A11 positive donors. On p. 352363

Cox1999] J. H. Cox, R. P. Garner, R. R. Redfield, N. E. Aronson, C. Davis, N. Ruiz, \& D. L. Birx. Antibody-dependent cellular cytotoxicity in HIV type 1-infected patients receiving VaxSyn, a recombinant gp160 envelope vaccine. AIDS Res Hum Retroviruses 15(9):847-54, 1999. On p. 743

[Croix 1993] D. A. Croix, H. Y. Yeh, J. Sedlacek, R. B. Luftig, \& P. D. Gottlieb. A dominant epitope of HIV-1 protease recognized by hamster monoclonal antibodies. J AIDS 6:558-566, 1993. On 
p. 595

[Cruikshank1997] W. W. Cruikshank, S. R. Doctrow, M. S. Falvo, K. Huffman, J. Maciaszek, G. Viglianti, J. Raina, H. Kornfeld, \& B. Malfroy. A lipidated anti-Tat antibody enters living cells and blocks HIV-1 viral replication. J Acquir Immune Defic Syndr Hum Retrovirol 14:193-203, 1997. A technique was developed to lipidate antibodies and allow intracellular delivery; lipidated anti-Tat inhibited viral replication of several HIV-1 isolates by approximately 85 of infected cells and decreased reverse transcriptase activity. On p. 611

[Culmann-Penciolelli1994] B. Culmann-Penciolelli, S. Lamhamedi-Cherradi, I. Couillin, N. Guegan, J. P. Levy, J. G. Guillet, \& E. Gomard. Identification of multirestricted immunodominan regions recognized by cytolytic T lymphocytes in the human immunodeficiency virus type $1 \mathrm{Ne}$ protein. J Virol 68:7336-43, 1994. See comments in J Virol 1995 Jan;69(1):618. On p. 363 383

[Culmann1989] B. Culmann, E. Gomard, M. P. Kieny, B. Guy, F. Dreyfus, \& A. G. Saimot. An antigenic peptide of the HIV-1 NEF protein recognized by cytotoxic T lymphocytes of seropositive individuals in association with different HLA-B molecules. Eur J Immunol 19:2383-2386, 1989. On p. 373

[Culmann1991] B. Culmann, E. Gomard, M.-P. Kieny, B. Guy, F. Dreyfus, A.-D. Saimot, D. Sereni, D. Sicard, \& J.-P. Levy. Six epitopes with human cytotoxic CD8+ cells in the central region of the HIV-1 Nef protein. J Immunol 146:1560-1565, 1991. Nef-specific CTL were generated from six seropositive donors. Six epitopes were defined, all localized to two regions in the central part of Nef. Some epitopes could be recognized in the contexts of several HLA class I molecules. Peptides were based on BRU epitopes: QVPLRPMTYK, HLA A3, A11, B35; AAVDLSHFLKEK, HLA A11; HTQGYFPQWQ, HLA B17;TQGYFPQWQNYT, HLA B17, B37, NYTPGPGVRYPLT, HLA B7; and GVRYPLTFGWCYKLVP, HLA B18). On p. 355 357 $363,374,375$ 381383

[Culmann1998] B. Culmann. Personal communication, 1998. On p. 224 356 375$] 376$ 382

[Currier2002a] J. R. Currier, M. deSouza, P. Chanbancherd, W. Bernstein, D. L. Birx, \& J. H. Cox. Comprehensive screening for human immunodeficiency virus type 1 subtype-specific CD8 cytotoxic T lymphocytes and definition of degenerate epitopes restricted by HLA-A0207 and -Cw0304 alleles. J Virol 76(10):4971-4986, 2002. On p.137] 144$] 308$

[Currier2002b] J. R. Currier, E. G. Kuta, E. Turk, L. B. Earhart, L. Loomis-Price, S. Janetzki, G. Ferrari, D. L. Birx, \& J. H. Cox A panel of MHC class I restricted viral peptides for use as a quality control for vaccine trial ELISPOT assays. J Immunol Methods 260(1-2):157-172, 2002. On p. 400

[Dadaglio1991] G. Dadaglio, A. Leroux, P. Langlade-Demoyen, E. M. Bahraoui, F. Traincard, R. Fisher, \& F. Plata. Epitope recognition of conserved HIV envelope sequences by human cytotoxic T lymphocytes. J Immunol 147:2302-2309, 1991. Using synthetic peptides, six conserved epitopes on gp120 Env were identified, recognized by polyclonal human CTL in association with HLA-A2 class I. Conserved epitopes: RIQRGPGRAFVTIGK, IIIB; LWVTVYYGVPVWKEATTTLFCA; TTSYTLTSCNTSVITQACPK; SVEINCTRPNNNTRKSI; PEIVTHS; KNCGGEFFYCNS; LPCRIKQFINMWQEVGKAMY VKIEPLGVAPTKAKRRVVQR. Control: gag, YKRWIILGLNKIVRMYSPT, HLA B27. On \begin{tabular}{l|l|l|l|l|l|l|l|l|l|l|l|l|} 
p. 132 & 269 & 279 & 283 & 287 & 301 & 303 & 304 & 307 \\
\hline
\end{tabular}

Dai1992] L. C. Dai, K. West, R. Littaua, K. Takahashi, \& F. A. Ennis. Mutation of human immunodeficiency virus type 1 at amino acid 585 on gp 41 results in loss of killing by CD8+ A24-restricted cytotoxic T lymphocytes. J Virol 66:3151-3154, 1992. An A24-restricted CD8+ CTL gp41 epitope was localized: YLKDQQLL, using a CTL clone from an HIV infected individual. Lys to (Arg or Gln) substitution in peptides used to pulse a target cell line eliminated killing. On p.315

[Dai2001] G. Dai, N. K. Steede, \& S. J. Landry. Allocation of helper T-cell epitope immunodominance according to three-dimensional structure in the human immunodeficiency virus type I envelope glycoprotein gp120. J Biol Chem 276(45):41913-20, 2001. On p. 467 468469473 \begin{tabular}{|l|l|l|l|l|l|l|l|l|l|l|l|l|l|l|}
476 & 478 & 486 & 488 & 490 & 494 & 497 & 501 & 502 & 504 & 513 & 515 \\
\hline
\end{tabular}

A. G. Dalgleish, T. C. Chanh, R. C. Kennedy, P. Kanda, P. R. Clapham, \& R. A. Weiss. Neutralization of Diverse HIV-1 Strains by Monoclonal Antibodies Raised against a gp 4 Synthetic Peptide. Virology 165:209-215, 1988. On p. 698 7257753

[daSilva1998] J. da Silva \& A. L. Hughes. Conservation of cytotoxic T lymphocyte (CTL) epitopes as a host strategy to constrain parasite adaptation: evidence from the nef gene of human immunodeficiency virus 1 (HIV-1). Mol Biol Evol 15(10):1259-68, 1998. On p. 396 533

[Day2001] C. L. Day, A. K. Shea, M. A. Altfeld, D. P. Olson, S. P. Buchbinder, F. M. Hecht, E. S Rosenberg, B. D. Walker, \& S. A. Kalams. Relative dominance of epitope-specific cytotoxic T-lymphocyte responses in human immunodeficiency virus type 1-infected persons with shared

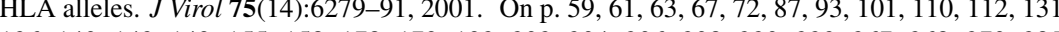

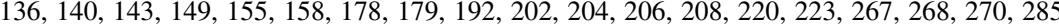

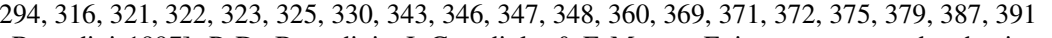

De Berardinis 1997] P. De Berardinis, J. Guardiola \& F Manca. Epitope context and reshaping of activated T helper cell repertoire. Hum Immunol 54:189-93, 1997. On p. 524

De Berardinis1999] P. De Berardinis, L. D’Apice, A. Prisco, M. N. Ombra, P. Barba, G. D. Pozzo, S. Petukhov, P. Malik, R. N. Perham, \& J. Guardiola. Recognition of HIV-derived B and T cell epitopes displayed on filamentous phages. Vaccine 17:1434-41, 1999. On p. 451

[De Berardinis2000] P. De Berardinis, R. Sartorius, C. Fanutti, R. N. Perham, G. Del Pozzo, \& J. Guardiola. Phage display of peptide epitopes from HIV-1 elicits strong cytolytic responses. Nat Biotechnol 18(8):873-6, 2000. On p. 222 451

De Groot1991] A. S. De Groot, M. Clerici, A. Hosmalin, S. H. Hughes, D. Barnd, C. W. Hen drix, R. Houghten, G. M. Shearer, \& J. A. Berzofsky. Human immunodeficiency virus reverse transcriptase T-helper epitopes identified in mice and humans: correlation with a cytotoxic $\mathrm{T}$ cell epitope. J Infect Dis 164:1058-1065, 1991. This peptide stimulates both murine helper and cytotoxic T-cells and was able to stimulate IL-2 producing T-cells from 9 out of 17 HIV seropositive humans. RT epitope: CTEMEKEGKISKIGP. On p. 180 449450

De Groot2001] A. S. De Groot, A. Bosma, N. Chinai, J. Frost, B. M. Jesdale, M. A. Gonzalez, W. Martin, \& C. Saint-Aubin. From genome to vaccine: in silico predictions, ex vivo verification

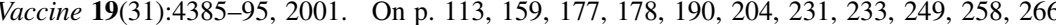
$282 \quad 283 \quad 306$

[De Lucca2002] F. L. De Lucca, V. S. F. Sales, L. R. Souza, \& M. A. E. Watanabe. Evidence for the involvement of the RNA-dependent protein kinase (PKR) in the induction of human cytotoxic $\mathrm{T}$ lymphocytes against a synthetic peptide of HIV-1 by regulatory RNA. Mol Cell Biochem 238(12):19-26, 2002. On p.221

[De Maria1994] A. De Maria, C. Cirillo, \& L. Moretta. Occurrence of human immunodeficiency virus type 1 (HIV-1)-specific cytolytic T cell activity in apparently uninfected children born to HIV-1-infected mothers. J Infect Dis 170(5):1296-1299, 1994. On p. 166 241 337, 397

[De Maria1997] A. De Maria, A. Ferraris, M. Guastella, S. Pilia, C. Cantoni, L. Polero, M. C. Mingari, D. Bassetti, A. S. Fauci, \& L. Moretta. Expression of HLA class I-specific inhibitory natural killer cell. Proc Natl Acad Sci USA 94:10285-8, 1997. On p.163 240 334395

[De Santis1991] R. De Santis, A. Anastasi, S. Marcolini, G. Valesini, M. Pezzella, N. Vonesch, E. Sturchio, \& A. Mele. Production of a Nef-specific monoclonal antibody by the use of a synthetic peptide. AIDS Res and Human Retroviruses 7(3):315-21, 1991. On p. 837

Dela Cruz2000] C. S. Dela Cruz, R. Tan, S. L. Rowland-Jones, \& B. H. Barber. Creating HIV-1 reverse transcriptase cytotoxic T lymphocyte target structures by HLA-A2 heavy chain modifications. Int Immunol 12(9):1293-302, 2000. On p.200 213

[deLorimier1994] R. de Lorimier, M. A. Moody, B. F. Haynes, \& L. D. Spicer. NMR-derived solution conformations of a hybrid synthetic peptide containing multiple epitopes of envelope protein gp120 from the RF strain of human immunodeficiency virus. Biochemistry 33(8):2055- 
2062, 1994. On p. 489507

[delReal1999] G. del Real, M. Llorente, P. Lucas, L. Kremer, J. L. Toran, \& M.-A. C. Antibody repertoire against HIV-1 gp120 triggered in nude and normal mice by GM-CSF/gp120 immunization. Mol Immunol 36:721-31, 1999. On p.735,737,738

[Deml1997] L. Deml, R. Schirmbeck, J. Reimann, H. Wolf, \& R. Wagner. Recombinant human immunodeficiency Pr55gag virus-like particles presenting chimeric envelope glycoproteins in duce cytotoxic T cells and neutralizing antibodies. Virology 235:26-39, 1997. On p. 288

[Deml1999] L. Deml, R. Schirmbeck, J. Reimann, H. Wolf, \& R. Wagner. Immunostimulatory CpG motifs trigger a T helper-1 immune response to human immunodeficiency virus type-1 (HIV-1) gp 160 envelope proteins. Clin Chem Lab Med 37:199-204, 1999. On p.286

[Deml2001] L. Deml, A. Bojak, S. Steck, M. Graf, J. Wild, R. Schirmbeck, H. Wolf, \& R. Wag ner. Multiple effects of codon usage optimization on expression and immunogenicity of DNA candidate vaccines encoding the human immunodeficiency virus type $1 \mathrm{Gag}$ protein. J Viro 75(22):10991-11001, 2001. On p.593

[Denisova1995] G. Denisova, J. Zwickel, \& J. M. Gershoni. Binding of HIV-1 gp120 to an anti-V3 loop antibody reveals novel antigen-induced epitopes. FASEB J 9:127-132, 1995. This pape describes the characterization of five antibodies that bind M77-epitopes that are only revealed upon M77-gp120 interaction. On p. 656

[Denisova1996] G. Denisova, B. Stern, D. Raviv, J. Zwickel, N. I. Smorodinsky, \& J. M. Gershoni. Humoral immune response to immunocomplexed HIV envelope glycoprotein 120. AIDS Res Hum Retroviruses 12:901-909, 1996. Mice were injected with the gp120 in different configurations: free, complexed with CD4, and as an immunocomplex bound to a V3 loop MAb (M77) of the protein. Polyclonal sera, as well as monoclonal antibodies produced in each case, were analyzed. The free gp120 and gp120-CD4 complex immunogens stimulated responses were directed mainly toward conformational epitopes, but gp120 immunocomplexed with MAb M77 also produced numerous and varied MAbs directed toward linear epitopes that were presumably inaccessible on the gp120, gp120-CD4 proteins. On p. 620 623 635 656 691 742 743

[Denisova2000] G. F. Denisova, M. Zerwanitzer, D. A. Denisov, E. Spectorman, I. Mondor, Q. Sattentau, \& J. M. Gershoni. Expansion of epitope cross-reactivity by anti-idiotype modulation of the primary humoral response. Mol Immunol 37:53-8, 2000. On p. 656 657

[deQuiros2000] J. C. de Quiros, W. L. Shupert, A. C. McNeil, J. C. Gea-Banacloche, M. Flanigan, A. Savage, L. Martino, E. E. Weiskopf, H. Imamichi, Y. M. Zhang, J. Adelsburger, R. Stevens, P. M. Murphy, P. A. Zimmerman, C. W. Hallahan, R. T. Davey, Jr., \& M. Connors. Resistance to replication of human immunodeficiency virus challenge in SCID-Hu mice engrafted with peripheral blood mononuclear cells of nonprogressors is mediated by CD8(+) T cells and associated with a proliferative response to p24 antigen. J Virol 74(4):2023-8, 2000. On p. 165

[deRosny2001] E. de Rosny, R. Vassell, P. T. Wingfield, C. T. Wild, \& C. D. Weiss. Peptides Corresponding to the Heptad Repeat Motifs in the Transmembrane Protein (gp41) of Human Immunodeficiency Virus Type 1 Elicit Antibodies to Receptor-Activated Conformations of the Envelope Glycoprotein. J Virol 75(18):8859-8863, 2001. On p.807

[DeSantis1994] C. DeSantis, L. Lopalco, P. Robbioni, R. Longhi, G. Rappocciolo, A. G. Siccardi, \& A. Beretta. Human Antibodies to Immunodominant C5 Region of HIV-1 gp120 Cross-React with HLA Class I on Activated Cells. AIDS Res Hum Retroviruses 10:157-162, 1994. On p.688

[DeVico1991] A. L. DeVico, T. D. Copeland, S. Oroszlan, R. C. Gallo, \& M. G. Sarngadharan Interaction of C-terminal sequences of human immunodeficiency virus reverse transcriptase with template primer. J Biol Chem 266:6774-6779, 1991. On p. 598

[DeVico1995] A. L. DeVico, R. Rahman, J. Welch, R. Crowley, P. Lusso, M. G. Sarngadharan, \& R. Pal. Monoclonal antibodies raised against covalently crosslinked complexes of huma immunodeficiency virus type $1 \mathrm{gp} 120$ and CD4 receptor identify a novel complex-dependent epitope on gp120. Virology 211:583-588, 1995. To explore the immunogenicity of regions of gp120 that are exposed due to conformational changes in gp120 upon CD4 binding, CD4 was covalently linked to gp120 and this complex was used as an immunogen for BALB/c mice. Two MAbs were produced, both of which bind preferentially to the gp120-CD4 complex, and are conformational. Competition assays indicate these MAbs bind to epitopes that are recognized by sera from HIV-1 infected humans. On p. 656754832

[Devito2000a] C. Devito, K. Broliden, R. Kaul, L. Svensson, K. Johansen, P. Kiama, J. Kimani, L. Lopalco, S. Piconi, J. J. Bwayo, F. Plummer, M. Clerici, \& J. Hinkula. Mucosal and plasma IgA from HIV-1-exposed uninfected individuals inhibit HIV-1 transcytosis across human epithelial cells. J Immunol 165(9):5170-5176, 2000. On p.751

[Devito2000b] C. Devito, J. Hinkula, R. Kaul, L. Lopalco, J. J. Bwayo, F. Plummer, M. Clerici, \& K. Broliden. Mucosal and plasma IgA from HIV-exposed seronegative individuals neutralize a primary HIV-1 isolate. AIDS 14(13):1917-1920, 2000. On p.751

Devito2000c] C. Devito, M. Levi, K. Broliden, \& J. Hinkula. Mapping of B-cell epitopes in rabbits immunised with various gag antigens for the production of HIV-1 gag capture ELISA reagents. $J$ Immunol Methods 238(1-2):69-80, 2000. On p.593

Devito2002] C. Devito, J. Hinkula, R. Kaul, J. Kimani, P. Kiama, L. Lopalco, C. Barass, S. Piconi, D. Trabattoni, J. J. Bwayo, F. Plummer, M. Clerici, \& K. Broliden. Cross-clade HIV-1-specific neutralizing IgA in mucosal and systemic compartments of HIV-1-exposed, persistently seronegative subjects. J Acquir Immune Defic Syndr 30(4):413-420, 2002. On p.751

[Dianzani2002] F. Dianzani, G. Antonelli, E. Riva, O. Turriziani, L. Antonelli, S. Tyring, D. A Carrasco, H. Lee, D. Nguyen, J. Pan, J. Poast, M. Cloyd, \& S. Baron. Is human immunodeficiency virus RNA load composed of neutralized immune complexes? J Infect Dis 185(8):1051-1054, 2002. On p. 750

DiBrino1994a] M. DiBrino, K. C. Parker, D. H. Margulies, J. Shiloach, R. V. Turner, M. Garfield, W. E. B. WE, \& J. E. Coligan. The HLA-B14 peptide binding site can accommodate peptides with different combinations of anchor residues. J Biol Chem 269, 1994. On p. 311

[DiBrino1994b] M. DiBrino, K. C. Parker, J. Shiloach, R. V. Turner, T. Tsuchida, M. Garfield, W. E. Biddison, \& J. E. Coligan. Endogenous peptides with distinct amino acid anchor residue motifs bind to HLA-A1 and HLA-B8. J Immunol 152:620-31, 1994. On p.93

[Dickey2000] C. Dickey, U. Ziegner, M. G. Agadjanyan, V. Srikantan, Y. Refaeli, A. Prabhu, A. Sato, W. V. Williams, D. B. Weiner, \& K. E. Ugen. Murine monoclonal antibodies biologically active against the amino region of HIV-1 gp120: isolation and characterization. DNA Cell Biol 19:243-52, 2000. On p. 807 808

diMarzo Veronese1985] F. di Marzo Veronese, M. G. Sarngadharan, R. Rahman, P. D. Markham, M. Popovic, A. J. Bodner, \& R. C. Gallo. Monoclonal antibodies specific for p24, the major core protein of human T-cell leukemia virus type III. Proc Natl Acad Sci USA 82:5199-5202, 1985. On p.739

[diMarzo Veronese1986] F. di Marzo Veronese, T. D Copeland, A. L. DeVico, R. Rahman, S. Oroszlan, R. C. Gallo, \& M. G. Sarngadharan. Characterization of highly immunogenic p66/p51 as the reverse transcriptase of HTLV-III/LAV. Science 231:1289-1291, 1986. This study identified the 66 and 51 kilodaltons bands in western blots as RT. Enzymatic activity was shown, and the protein was defined by Edmund N-terminal degradation and comparison to HIV-1 pol nucleotide translation. A mouse hybridoma was generated that inhibited enzyme activity. On p.607

[diMarzo Veronese1992] F. di Marzo Veronese, R. Rahman, R. Pal, C. Boyer, J. Romano, V. S. Kalyanaraman, B. C. Nair, R. C. Gallo, \& M. G. Sarngadharan. Delineation of immunoreactive, conserved regions in the external envelope glycoprotein of the human immunodeficiency virus type 1. AIDS Res Hum Retroviruses 8:1125-1132, 1992. On p. 615 616 622 637 656686 740.754

19. Marzo Veronese, M. S. Reitz, Jr, G. Gupta, M. Robert-Guroff, C. Boyer-Thompson, A. Louie, R. C. Gallo, \& P. Lusso. Loss of a neutralizing epitope by a 
spontaneous point mutation in the V3 loop of HIV-1 isolated from an infected laboratory worker. J Biol Chem 268:25894-25901, 1993. The MAb M77 cannot neutralize a virus isolated from a IIIB infected lab-worker that has a single point mutation in the defined linear epitope. M77 canno bind to the mutant native gp120, but can bind to a peptide that carries the substitution. On p. 656 659671

[diMarzo Veronese 1994] F. di Marzo Veronese, A. E. Willis, C. Boyer-Thompson, E. Appella, \& R. N. Perham. Structural mimicry and enhanced immunogenicity of peptide epitopes displayed on filamentous bacteriophage. J Mol Biol 243:167-172, 1994. On p. 506

[Dingwall1989] C. Dingwall, I. Ernberg, M. J. Gait, S. M. Green, S. Heaphy, J. Karn, A. D. Lowe, M. Singh, M. A. Skinner, \& R. Valerio. Human immunodeficiency virus 1 tat protein binds transactivation-responsive region (TAR) RNA in vitro. Proc Natl Acad Sci USA 86:6925-6929, 1989 On p. 610611

[Ditzel1995] H. J. Ditzel, J. M. Binley, J. P. Moore, J. Sodroski, N. Sullivan, L. S. W. Sawyer, R. M Hendry, W.-P. Yang, C. F. Barbas III, \& D. R. Burton. Neutralizing recombinant human antibodies to a conformational V2- and CD4-binding site-sensitive epitope of HIV-1 gp120 isolated by using an epitope-masking procedure. J Immunol 154:893-906, 1995. A panel of Fabs was obtained from a library prepared from the bone marrow of a long-term asymptomatic HIV-1 seropositive male donor. Four Fabs recognize the CD4BS. An additional four Fabs were retrieved after epitope masking gp120 with the CD4BS Fabs at the screening stage. 3/4 of these Fabs bind to a V2 dependent conformational epitope. On p.784 $792,811,812$ 813

[Ditzel1997] H. J. Ditzel, P. W. Parren, J. M. Binley, J. Sodroski, J. P. Moore, C. F. Barbas III, \& D. R. Burton. Mapping the protein surface of human immunodeficiency virus type 1 gp120 using human monoclonal antibodies from phage display libraries. J Mol Biol 267:684-95, 1997. (Genbank: U82767 U82768 U82769 U82770 U82771 U82772 U82942 U82943 U82944 U8294 U82946 U82947 U82948 U82949 U82950 U82951 U82952 U82961 U82962) Recombinant monoclonal antibodies from phage display libraries provide a method for Env surface epitope mapping. Diverse epitopes are accessed by presenting gp120 to the library in different forms, such as sequential masking of epitopes with existing MAbs or SCD4 prior to selection or by selection on peptides. Fabs identified by these methods have specificities associated with epitopes presented

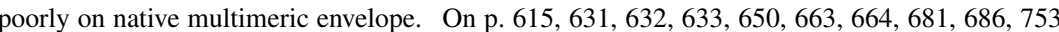
\begin{tabular}{|l|l|l|l|l|l|l|l|l|l|l|l|l|}
\hline 754 & 756 & 758 & 760 & 784 & 792 & 794 & 798 & 808 & 809 & 811 & 812 \\
\hline
\end{tabular}

[Doe1997] B. Doe \& C. M. Walker. HIV-1 p24 Gag-specific cytotoxic T-lymophocyte responses in mice. AIDS 10:793-794, 1997. On p.117

[Dong1998a] T. Dong. Personal Communication, 1998. On p. 96 [172] 225

[Dong1998b] T. Dong \& S. Rowland-Jones. Personal communication, 1998. On p.234

[Dong2001] X. N. Dong, Y. Xiao, \& Y. H. Chen. ELNKWA-epitope specific antibodies induced by epitope-vaccine recognize ELDKWA- and other two neutralizing-resistant mutated epitopes on HIV-1 gp41. Immunol Lett 75(2):149-52, 2001. On p.710 714] 718

[Donners2002] H. Donners, B. Willems, E. Beirnaert, R. Colebunders, D. Davis, \& G. van der Groen. Cross-neutralizing antibodies against primary isolates in African women infected with HIV-1. AIDS 16(3):501-503, 2002. On p.750

[Dorrel11999] L. Dorrell, T. Dong, G. S. Ogg, S. Lister, S. McAdam, T. Rostron, C. Conlon, A. J. McMichael, \& S. L. Rowland-Jones. Distinct recognition of non-clade B human immunodeficiency virus type 1 epitopes by cytotoxic T lymphocytes generated from donors infected in Africa $J$ Virol 73:1708-14, 1999. On p. 66
76

[Dorrel12001] L. Dorrell, B. E. Willcox, E. Y. Jones, G. Gillespie, H. Njai, S. Sabally, A. Jaye K. DeGleria, T. Rostron, E. Lepin, A. McMichael, H. Whittle, \& S. Rowland-Jones. Cytotoxic lymphocytes recognize structurally diverse, clade-specific and cross-reactive peptides in huma . p. $94\left[\begin{array}{l|l|l|l|l|l|}112 & 120 & 144 & 151 \\ \hline\end{array}\right.$
[Douek2002] D. C. Douek, M. R. Betts, J. M. Brenchley, B. J. Hill, D. R. Ambrozak, K.-L. Ngai, N. J.Karandikar, J.P. Casazza, \& R. A. Koup. A novel approach to the analysis of specifcity clonality, and frequency of HIV-specific T cell responses reveals a potential mechanism for control of viral escape. J Immunol 168(6):3099-3104, 2002. On p.73

Dowbenko1988] D. Dowbenko, G. Nakamura, C. Fennie, C. Shimasaki, L. Riddle, R. Harris, T. Gregory, \& L. Lasky. Epitope mapping of the immunodeficiency virus type 1 gp120 with monoclonal antibodies. J Virol 62:4703-4711, 1988. On p. 618 622

[Dowd2002] C. S. Dowd, S. Leavitt, G. Babcock, A. P. Godillot, D. Van Ryk, G. A. Canziani, J. Sodroski, E. Freire, \& I. M. Chaiken. Beta-turn Phe in HIV-1 Env binding site of CD4 and CD4 mimetic miniprotein enhances Env binding affinity but is not required for activation of coreceptor/17b site. Biochemistry 41(22):7038-7046, 2002. On p.798 801

[D’Souza1991] M. P. D'Souza, P. Durda, C. V. Hanson, G. Milman, \& C. Investigators. Evaluation of monoclonal antibodies to HIV-1 by neutralization and serological assays: an international collaboration. AIDS 5:1061-1070, 1991. On p.647 652 664 669 671673674626

[D’Souza1994] M. P. D'Souza, S. J. Geyer, C. V. Hanson, R. M. Hendry, G. Milman, \& C. Investigators. Evaluation of monoclonal antibodies to HIV-1 envelope by neutralization and binding assays: an international collaboration. AIDS 8:169-181, 1994. On p.633647 662 673682 710.719

[D'Souza1995] M. P. D'Souza, G. Milman, J. A. Bradac, D. McPhee, C. V. Hanson, \& R. M. Hendry. Neutralization of primary HIV-1 isolates by anti-envelope monoclonal antibodies. AIDS 9:867-874, 1995. Eleven labs tested the 6 human MAbs 1125H, TH9, 4.8D, 257-D-IV, TH1, 2F5, and also HIVIG for neutralization of MN, JRCSF, the two B clade primary isolates 301657 and THA/92/026, and the D clade isolate UG/92/21. 2F5 was the most broadly neutralizing, better than HIVIG. The other MAbs showed limited neutralization of only MN (anti-CD4BS MAb 1125H, TH9, and 4.8D), or MN and JRCSF (anti-V3 MAbs 257-D-IV and TH1). On p. 647

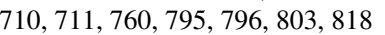

D'Souza1997] M. P. D'Souza, D. Livnat, J. A. Bradac, S. H. Bridges, the AIDS Clinical Trial Group Antibody Selection Working Group, \& C. Investigators. Evaluation of monoclonal antibodies to human immunodeficiency virus type 1 primary isolates by neutralization assays: performance criteria for selecting candidate antibodies for clinical trials. J Infect Dis 175:1056-1062, 1997. Five laboratories evaluated neutralization of nine primary B clade isolates by a coded panel of seven human MAbs to HIV-1 subtype B envelope. IgG1b12, 2G12, 2F5 showed potent an broadly cross-reactive neutralizing ability; F105, 447/52-D, 729-D, 19b did not neutralize the

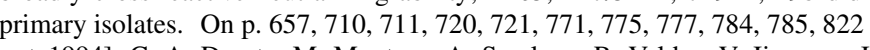

Duarte1994] C. A. Duarte, M. Montero, A. Seralena, R. Valdes, V. Jimenez, J. Benitez, E. Narciandi, J. Madrazo, G. Padron, G. Sanchez, G. Gilljam, K. Persson, S. Ojeda, A. Caballero, A. Miranda, M. C. Dominguez, B. Wahren, \& A. Menendez. Multiepitope polypeptide of the HIV-1 envelope induces neutralizing monoclonal antibodies against V3 loop. AIDS Res Hum Retroviruses 10:235-243, 1994. On p. 645

Duarte1996] E. A. Duarte, G. Eberl, \& G. Corradin. Specific tolerization of active cytotoxic lymphocyte responses in vivo with soluble peptides. Cell Immunol 169:16-23, 1996. Mice immunized with this peptide had an active CTL response that could be specifically tolerized with continued administration of soluble peptide; this was also observed with a malaria peptide. This suggests that soluble peptide may be useful for treatment of autoimmune disease. On p. 305

[Dupuis1995] M. Dupuis, S. K. Kundu, \& T. C. Merigan. Characterization of HLA-A*0201 restricted cytotoxic $\mathrm{T}$ cell epitopes in conserved regions of the HIV type 1 gp160 protein. Immunol 155:2232-2239, 1995. Five HLA-A2 HIV-1 seropositive HIV-1 MN rec gp160 vaccinees had their CTL activity assessed using peptides known to bind with high affinity to HLAA*0201. Four of the patients had specific CTL activity for a minimum of at least three epitopes, thus the response appears heterogeneous. One of the four peptides was confirmed to be HLA A2 


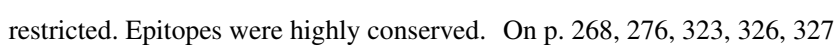

[Durali1998] D. Durali, J. Morvan, F. Letourneur, D. Schmitt, N. Guegan, M. Dalod, S. Saragosti, D. Sicard, J. P. Levy, \& E. Gomard. Cross-reactions between the cytotoxic T-lymphocyte responses of human immunodeficiency virus-infected African and European patients. J Virol 72:3547-53, 1998. On p. $83 \quad 135354386$

[Durda1988] P. J. Durda, B. Leece, A. M. Jenoski, H. Rabin, A. Fisher, R. Gallo, \& F. Wong-Staal. Characterization of murine monoclonal antibodies to HIV-1 induced by synthetic peptides. AIDS Res Hum Retroviruses 4:331-342, 1988. On p.666 667 691

[Durda1990] P. J. Durda, L. Bacheler, P. Clapham, A. M. Jenoski, B. Leece, T. J. Matthews, A. McKnight, R. Pomerantz, M. Rayner, \& K. J. Weinhold. HIV-1 neutralizing monoclonal antibodies induced by a synthetic peptide. AIDS Res Hum Retroviruses 6:1115, 1990. On p.666 667677

[Durrani1998] Z. Durrani, T. L. McInerney, L. McLain, T. Jones, T. Bellaby, F. R. Brennan, \& N. J. Dimmock. Intranasal immunization with a plant virus expressing a peptide from HIV-1 gp41 stimulates better mucosal and systemic HIV-1-specific $\operatorname{IgA}$ and $\operatorname{IgG}$ than oral immunization. $J$ Immunol Methods 220:93-103, 1998. On p.720

[Dyer1999] W. B. Dyer, G. S. Ogg, M. A. Demoitie, X. Jin, A. F. Geczy, S. L. Rowland-Jones, A. J. McMichael, D. F. Nixon, \& J. S. Sullivan. Strong human immunodeficiency virus (HIV)specific cytotoxic T- lymphocyte activity in Sydney Blood Bank Cohort patients infected with

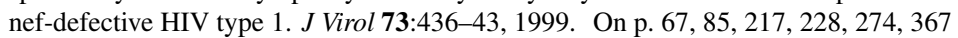

[Dzuris2000] J. L. Dzuris, J. Sidney, E. Appella, R. W. Chesnut, D. I. Watkins, \& A. Sette. Conserved MHC class I peptide binding motif between humans and rhesus macaques. J Immuno 164:283-91, 2000. On p. 342, 399

[Earl1994] P. L. Earl, C. C. Broder, D. Long, S. A. Lee, J. Peterson, S. Chakrabarti, R. W. Doms, \& B. Moss. Native oligomeric human immunodeficiency virus type 1 Envelope glycoprotein elicit diverse monoclonal antibody reactivities. $J$ Virol 68:3015-3026, 1994. In a study of the repertoire of response to oligomeric versus monomeric Env protein, 138 murine MAbs were generated in response to an immunogen that was a gp120/bp41 oligomeric molecule that was not cleaved due to a mutation in the cleavage site. The oligomeric molecule was found to elicit a respons that was very different than the monomer. Most MAbs were conformational, many were to gp4 or if in gp120, to the CD4 BS. Few MAbs to linear V3 epitopes were produced in response to oligomeric protein, though this was a common specificity in response to immunization with gp120

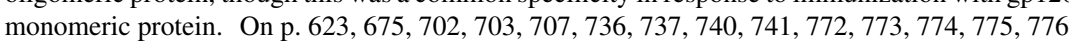
\begin{tabular}{ll|l|l|l|l|l|l|}
793 & 795 & 797,806 & 809 & 817 & 821 \\
\hline
\end{tabular}

[Earl1997] P. L. Earl, C. C. Broder, R. W. Doms, \& B. Moss. Epitope map of human immunodeficiency virus type $1 \mathrm{gp} 41$ derived from 47 monoclonal antibodies produced by immunization with

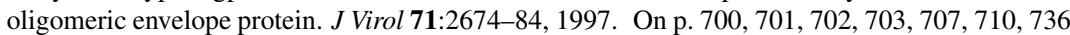
\begin{tabular}{|l|l|l|l|l|l|l|l|l|l|l|l|l|l|l|}
\hline 737 & 741 & 755 & 772 & 817 & 818 & 828 & 832 \\
\hline
\end{tabular}

[Earl2001] P. L. Earl, W. Sugiura, D. C. Montefiori, C. C. Broder, S. A. Lee, C. Wild, J. Lifson, \&

B. Moss. Immunogenicity and protective efficacy of oligomeric human immunodeficiency virus type 1 gp140. J Virol 75(2):645-53, 2001. On p.743

[Eaton1994] A. M. Eaton, K. E. Ugen, D. B. Weiner, T. Wildes, \& J. A. Levy. An Anti-gp41 Human Monoclonal Antibody That Enhances HIV-1 Infection in the Absence of Complement. AIDS Res Hum Retroviruses 10:13-18, 1994. On p.700

[Eddleston1993] M. Eddleston, J. C. de la Torre, J.-Y. Xu, N. Dorfman, A. Notkins, S. Zolla-Pazner \& M. B. A. Oldstone. Molecular Mimicry Accompanying HIV-1 Infection: Human Monoclona Antibodies That Bind to gp41 and to Astrocytes. AIDS Res Hum Retroviruses 10:939-944, 1993. In this paper, three anti-HIV-1 gp41 specific MAbs were found to react with astrocytes: 98-6, 167-7 and 15G1. Reactive astrocytes in the hippocampus were most prominently involved, and the antibodies stained no other cell type in the brain, kidney or liver. All three mapped to a conformationally dependent epitope between aa 644-663. On p. 696 697|700 $701|707| 708$ 709828

Edgeworth2002] R. L. Edgeworth, J. H. San, J. A. Rosenzweig, N. L. Nguyen, J. D. Boyer, \& K. E Ugen. Vaccine development against HIV-1: Current perspectives and future directions. Immunol Res 25(1):53-74, 2002. On p. 401

[Edwards2002] B. H. Edwards, A. Bansal, S. Sabbaj, J. Bakari, M. J. Mulligan, \& P. A. Goepfert Magnitude of functional CD8+ T-cell responses to the gag protein of human immunodeficiency virus type 1 correlates inversely with viral load in plasma. $J$ Virol 76(5):2298-2305, 2002 . On

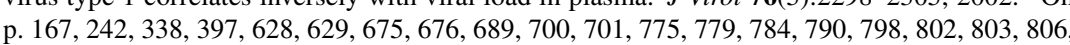
p. $167 \mid 242$

\section{7}

[Egan1999] M. A. Egan, M. J. Kuroda, G. Voss, J. E. Schmitz, W. A. Charini, C. I. Lord, M. A. Forman, \& N. L. Letvin. Use of major histocompatibility complex class I/peptide/beta2M tetramers to quantitate CD8(+) cytotoxic T lymphocytes specific for dominant and nondominant viral epitopes in simian-human immunodeficiency virus-infected rhesus monkeys. J Virol 73:5466-72, 1999. On p. 306

Ehrhard1996] B. Ehrhard, R. Misselwitz, K. Welfle, G. Hausdorf, R. W. Glaser, J. SchneiderMergener, \& H. Welfle. Chemical modification of recombinant HIV-1 capsid protein p24 leads to the release of a hidden epitope prior to changes of the overall folding of the protein. Biochemistry 35:9097-9105, 1996. On p.576

[Emini1992] E. A. Emini, W. A. Schleif, J. H. Nunberg, A. J. Conley, Y. Eda, S. Tokiyoshi, S. D. Putney, S. Matsushita, K. E. Cobb, C. M. Jett, J. W. Eichberg, \& K. K. Murthy. Prevention of HIV-1 infection in chimpanzees by gp120 V3 domain-specific monoclonal antibody. Nature 355:728-730, 1992. On p. 671 672

[Engelmayer2001] J. Engelmayer, M. Larsson, A. Lee, M. Lee, W. I. Cox, R. M. Steinman, \& N. Bhardwaj. Mature dendritic cells infected with canarypox virus elicit strong anti-human immunodeficiency virus CD8+ and CD4+ T-cell responses from chronically infected individuals. J Virol 75(5):2142-53, 2001. On p. 79213,446

Enshell-Seijffers2001] D. Enshell-Seijffers, L. Smelyanski, N. Vardinon, I. Yust, \& J. M. Gershoni Dissection of the humoral immune response toward an immunodominant epitope of HIV: A model for the analysis of antibody diversity in HIV+ individuals. FASEB J 15(12):2112-1220, 2001. On p.700 705706

[Ernst1998] W. Ernst, R. Grabherr, D. Wegner, N. Borth, A. Grassauer, \& H. Katinger. Baculovirus surface display: construction and screening of a eukaryotic epitope library. Nucleic Acids Res 26:1718-23, 1998. On p.710 712

Estaquier1992] J. Estaquier, C. Boutillon, J.-C. Ameisen, H. Gras-Masse, J.-P. Lecocq, B. Barbier, A. Dixson, A. Tartar, A. Capron, \& C. Auriault. T helper cell epitopes of the human immunodeficiency virus nef protein in rats and chimpanzees. Mol Immunol 29:489-499, 1992. Notes: Helper T-cell epitopes in nef were investigated using five synthetic peptides selected for thei amphipathic and alpha helix properties. One of the peptides, 45-59 was very immunogenic, and could induce functional T-cell help it in vivo. On p. 530 531

[Evans1989] D. J. Evans, J. McKeating, J. M. Meredith, K. L. Burke, K. Katrak, A. John, M. Ferguson, P. D. Minor, R. A. Weiss, \& J. W. Almond. An engineered poliovirus chimera elicits broadly reactive HIV-1 neutralizing antibodies. Nature 339:385-388, 1989. On p. 667 $724,725,726$

[Evans1999] T. Evans, M. Keefer, K. Weinhold, M. Wolff, D. Montefiori, G. Gorse, B. Graham, M. J. McElrath, M. Clements-Mann, M. Mulligan, P. Fast, M. Walker, J. Excler, A. Duliege, J. Taraglia, \& the NIAID AIDS Vaccine Evaluation Group. A canarypox vaccine expressing multiple human immunodeficiency vrus type 1 genes given alone or with Rgp120 elicits broad and durable CD8+ cytotoxic t lymphocyte responses in seronegative volunteers. J Inf Dis 180:290-8, 1999. On p. 164240334396

[Evans2001] T. G. Evans, M. J. McElrath, T. Matthews, D. Montefiori, K. Weinhold, M. Wolff, 
M. C. Keefer, E. G. Kallas, L. Corey, G. J. Gorse, R. Belshe, B. S. Graham, P. W. Spearman, D. Schwartz, M. J. Mulligan, P. Goepfert, P. Fast, P. Berman, M. Powell, D. Francis, \& \{NIAID AIDS Vaccine Evaluation Group.\}. QS-21 promotes an adjuvant effect allowing for reduce antigen dose during HIV-1 envelope subunit immunization in humans. Vaccine 19(15-16):208091, 2001. On p.745

[Faiman1997] G. A. Faiman \& A. Horovitz. Thermodynamic analysis of the interaction between the 0.5 beta Fv fragment and the RP135 peptide antigen derived from the V3 loop of HIV- 1 gp120. Biol Chem 272:31407-11, 1997. On p.671 672

[Fan1997] Z. Fan, X. L. Huang, L. Zheng, C. Wilson, L. Borowski, J. Liebmann, P. Gupta, J. Margolick, \& C. Rinaldo. Cultured blood dendritic cells retain HIV-1 antigen-presenting capacity for memory CTL during progressive HIV-1 infection. J Immunol 159:4973-82, 1997. On p.134 137,216

[Fanales-Belasio2002] E. Fanales-Belasio, S. Moretti, F. Nappi, G. Barillari, F. Micheletti, A. Cafaro, \& B. Ensoli. Native HIV-1 Tat protein targets monocyte-derived dendritic cells and enhance their maturation, function, and antigen-specific T cell responses. J Immunol 168(1):197-206, 2002. On p. 463

[Felgenhauer1990] M. Felgenhauer, J. Kohl, \& F. Ruker. Nucleotide sequence of the cDNA encoding the V-regions of the $\mathrm{H}$ - and L-chains of a human monoclonal antibody specific to HIV-1 gp41. Nucl Acids Res 18:4927, 1990. On p.706

[Fenoglio1999] D. Fenoglio, G. L. Pira, P. D. Berardinis, D. Saverino, M. P. Terranova, M. N. Ombra, L. Bracci, L. Lozzi, C. Viotti, J. Guardiola, \& F. Manca. Antagonistic activity of HIVT helper peptides flanked by an unrelated carrier protein [In Process Citation]. Eur J Immunol 29:1448-55, 1999. On p. 451, 485

[Fenoglio2000] D. Fenoglio, G. Li Pira, L. Lozzi, L. Bracci, D. Saverino, P. Terranova, L. Bottone, S. Lantero, A. Megiovanni, A. Merlo, \& F. Manca. Natural analogue peptides of an HIV-1 GP12 T-helper epitope antagonize response of GP120-specific human CD4 T cell clones. J Acquir Immune Defic Syndr 23:1-7, 2000. On p. 486

[Ferns1987] R. B. Ferns, R. S. Tedder, \& R. A. Weiss. Characterization of monoclonal antibodie against the human immunodeficiency virus gag products and their use in monitoring HIV isolate

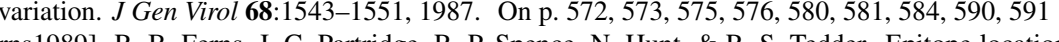

[Ferns1989] R. B. Ferns, J. C. Partridge, R. P. Spence, N. Hu of 13 anti-gag HIV-1 monoclonal antibodies using oligopeptides and their cross-reactivity with HIV-2. AIDS 3:829-834, 1989. On p. 572 573 575 $576|580| 584|590| 591$.

[Ferns 1991] R. B. Ferns, J. C. Partridge, M. Tisdale, N. Hunt, \& R. S. Tedder. Monoclonal antibodies define linear and conformational epitopes of HIV-1 pol gene products. AIDS Res Hu Retroviruses 7:307-313, 1991. 21 anti-RT MAbs were raised and characterized - three narrowly defined linear epitopes were mapped. The three linear and selected conformational MAbs are included in the database. On p. 598605607

[Ferrantelli2002] F. Ferrantelli \& R. M. Ruprecht. Neutralizing antibodies against HIV — back i the major leagues? Curr Opin Immunol 14(4):495-502, 2002. On p. 672 710 717 719 \begin{tabular}{ll|l|l|l|l|l|l}
723 & 775 & 779 & 784 & 791 & 822 & 827 \\
\hline
\end{tabular}

[Ferrari2000] G. Ferrari, D. D. Kostyu, J. Cox, D. V. Dawson, J. Flores, K. J. Weinhold, \& S. Osmanov. Identification of highly conserved and broadly cross-reactive HIV type 1 cytotoxic T lymphocyte epitopes as candidate immunogens for inclusion in Mycobacterium bovis BCG-vectored

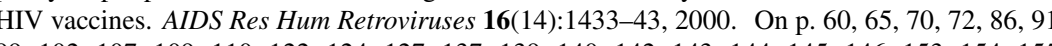
\begin{tabular}{|l|l|l|l|l|l|l|l|l|l|l|l|l|l|l|l|l|l|l|l|l|l|}
\hline 159 & 172 & 182 & 183 & 188 & 193 & 221 & 224 & 228 & 229 & 237 & 270 & 272 & 277 & 308 & 340 & 344 & 345 & 347 \\
\hline 3
\end{tabular}

[Ferrari2001] G. Ferrari, W. Neal, A Jones, N. Olender, J. Ottinger, R. Ha, M. J. McElrath, P. Goepfert, \& K. J. Weinhold. CD8 CTL responses in vaccines: Emerging patterns of HLA restriction and epitope recognition. Immunol Lett 79(1-2):37-45, 2001. On p. 80 168 340 [Ferris 1996] R. L. Ferris, C. Buck, S. A. Hammond, A. S. Woods, R. J. Cotter, M. Takiguchi, Y. Igarashi, Y. Ichikawa, \& R. F. Siliciano. Class I-restricted presentation of an HIV-1 gp41 epitope containing an N-linked glycosylation site. J Immunol 156:834-840, 1996. The class I processing pathway usually begins in the cytosol. Env proteins are co-translationally located in the endoplasmic reticulum, where they are glycosylated, and in general are not found in the cytosol. The N-linked glycosylation site was not occupied in the TAVPWNASW naturally processed peptide presented by $\mathrm{B} * 3501$, and the non-glycosylated form was the form recognized by an envspecific CTL clone. This suggests that there may be limited failure of translocation, resulting in synthesis and degradation in the cytosol, and entry in the normal class I processing pathway. On p. 317

Ferris1999] R. L. Ferris, C. Hall, N. V. Sipsas, J. T. Safrit, A. Trocha, R. A. Koup, R. P. Johnson, $\&$ R. F. Siliciano. Processing of HIV-1 envelope glycoprotein for class I-restricted recognition: dependence on TAP1/2 and mechanisms for cytosolic localization. J Immunol 162:1324-32,

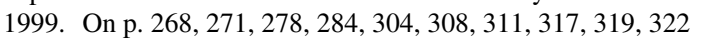

Fevrier1995] M. Fevrier, F. Boudet, A. Deslandres, \& J. Theze. Two new human monoclonal an tibodies against HIV type 1 glycoprotein 120: characterization and neutralizing activities agains HIV type 1 strains. AIDS Res Hum Retroviruses 11:491-500, 1995. On p.767

[Fidler2002] S. Fidler, A. Oxenius, M. Brady, J. Clarke, I. Cropley, A. Babiker, H.-T. Zhang, D. Price, R. Phillips, \& J. Weber. Virological and immunological effects of short-course antiretroviral therapy in primary HIV infection. AIDS 16(15):2049-2054, 2002. On p.445

FitzGerald1998] D. J. FitzGerald, C. M. Fryling, M. L. McKee, J. C. Vennari, T. Wrin, M. E. Cromwell, A. L. Daugherty, \& R. J. Mrsny. Characterization of V3 loop-Pseudomonas exotoxin chimeras. Candidate vaccines for human immunodeficiency virus-1. J Biol Chem 273:9951-8, 1998. On p. 639

Fomsgaard1998a] A. Fomsgaard, H. V. Nielsen, K. Bryder, C. Nielsen, R. Machuca, L. Bruun, J. Hansen, \& S. Buus. Improved humoral and cellular immune responses against the gp120 V3 loop of HIV-1 following genetic immunization with a chimeric DNA vaccine encoding the V3 inserted into the hepatitis B surface antigen. Scand J Immunol 47:289-95, 1998. On p.288

[Fomsgaard1998b] A. Fomsgaard, H. V. Nielsen, C. Nielsen, K. Johansson, R. Machuca, L. Bruun, J. Hansen, \& S. Buus. Comparisons of DNA-mediated immunization procedures directed agains surface glycoproteins of human immunodeficiency virus type-1 and hepatitis B virus. APMIS 106:636-46, 1998. On p. 289

Fontenot1995] J. D. Fontenot, T. C. VanCott, B. S. Parekh, C. P. Pau, J. R. George, D. L. Birx S. Zolla-Pazner, M. K. Gorny, \& J. M. Gatewood. Presentation of HIV V3 loop epitopes for enhanced antigenicity, immunogenicity and diagnostic potential. AIDS 9:1121-1129, 1995. On \begin{tabular}{ll|l|l|l|l|l|l|l|l|l|l|l|l|l|l|l|l|l|l|} 
p. 641 & 645 & 647 & 649 & 652 & 659 & 660 & 664 & 665 & 669 & 677 & 701 & 720 \\
\hline
\end{tabular}

[Forthal1995] D. N. Forthal, G. Landucci, M. K. Gorny, S. Zolla-Pazner, \& W. E. Robinson, Jr. Functional activities of 20 human immunodeficiency virus type 1 (HIV-1)-specific human monoclonal antibodies. AIDS Res Hum Retroviruses 11:1095-1099, 1995. A series of tests were performed on 20 human monoclonal antibodies to assess their potential therapeutic utility. An tibodies were tested for potentially harmful complement-mediated antibody enhancing activity (C-ADE), and for potentially beneficial neutralizing activity and antibody dependent cellular cy-

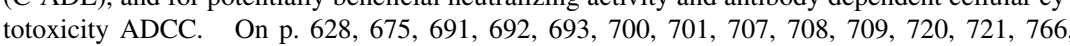
768

Fortin2000] J. F. Fortin, R. Cantin, M. G. Bergeron, \& M. J. Tremblay. Interaction between virionbound host intercellular adhesion molecule-1 and the high-affinity state of lymphocyte functionassociated antigen-1 on target cells renders R5 and X4 isolates of human immunodeficiency viru type 1 more refractory to neutralization. Virology 268:493-503, 2000. On p. 671 672 778803 804 
[Fournier2002a] A.-M. Fournier, V. Baillat, C. Alix-Panabieres, J.-M. Fondere, C. Merle, M. Segondy, M.-F. Huguet, J. Reynes, \& J.-P. Vendrell. Dynamics of spontaneous HIV-1 specific and non-specific B-cell responses in patients receiving antiretroviral therapy. AIDS 16(13): 17551760, 2002. On p. 842

[Fournier2002b] A. M. Fournier, J.-M. Fondere, C. Alix-Panabieres, C. Merle, V. Baillat, M.-F Huguet, J. Taïb, V. Ohayon, M. Zembala, J. Reynes, \& J. P. Vendrell. Spontaneous secretion of immunoglobulins and anti-HIV-1 antibodies by in vivo activated B lymphocytes from HIV-1 infected subjects: Monocyte and natural killer cell requirement for in vitro terminal differentiation into plasma cells. Clin Immunol 103(1):98-109, 2002. On p. 842

[Fouts1997] T. R. Fouts, J. M. Binley, A. Trkola, J. E. Robinson, \& J. P. Moore. Neutralization of the human immunodeficiency virus type 1 primary isolate JR-FL by human monoclonal antibodie correlates with antibody binding to the oligomeric form of the envelope glycoprotein complex. $J$ Virol 71:2779-2785, 1997. To test whether antibody neutralization of HIV-1 primary isolates is correlated with the affinities for the oligomeric envelope glycoproteins, JRFL was used as a model primary virus and a panel of 13 human MAbs were evaluated for: half-maximal binding to rec monomeric JRFL gp120; half-maximal binding to oligomeric - JRFL Env expressed on the surface of transfected 293 cells; and neutralization of JRFL in a PBMC-based neutralization assay. Antibody affinity for oligomeric JRFL Env but not monomeric JRFL gp120 correlated with

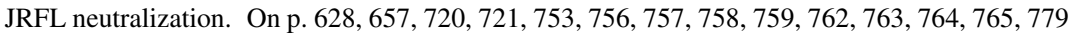
\begin{tabular}{ll|l|l|l|l|l|l|}
781 & 782 & 784 & 785 & 798 & 822 \\
\hline
\end{tabular}

[Fouts1998] T. R. Fouts, A. Trkola, M. S. Fung, \& J. P. Moore. Interactions of polyclonal and monoclonal anti-glycoprotein 120 antibodies with oligomeric glycoprotein 120-glycoprotein 4 complexes of a primary HIV type 1 isolate: relationship to neutralization. AIDS Res Hum Retroviruses 14:591-7, 1998. Ab reactivity to oligomeric forms of gp120 were compared to neutralization of the macrophage tropic primary virus JRFL, and did not always correlate. This builds upon studies which have shown that oligomer binding while required for neutralization, is not always sufficient. MAb 205-46-9 and 2G6 bind oligomer with high affinity, comparable to IgG1b12, but unlike IgG1b12, cannot neutralize JRFL. Furthermore, neutralizing and non-neutralizing sera from HIV-1 infected people are similar in their reactivities to oligomeric JRFL Envelope. On \begin{tabular}{l|l|l|l|l|l|l|l|l|l|l|l|l|l|l|l|} 
p. 710 & 712 & 762 & 763 & 764 & 765 & 766 & 779 & 780 & 781 & 782 & 784 & 786 & 822 & 823 \\
\hline
\end{tabular}

[Fouts2002] T. Fouts, K. Godfrey, K. Bobb, D. Montefiori, C. V. Hanson, V. S. Kalyanaraman, A. DeVico, \& R. Pal. Crosslinked HIV-1 envelope-CD4 receptor complexes elicit broadly crossreactive neutralizing antibodies in rhesus macaques. Proc Natl Acad Sci U S A 99(18):1184211847, 2002. On p. 752

[Franke1992] L. Franke, R. Grunow, R. Meissner, T. Portsman, \& R. von Baehr. Inhibition of HIVinfection in vitro by murine monoclonal anti-p24 antibodies. J Med Virol 37:137-142, 1992. On p. 575,576

[Frankel1998] S. S. Frankel, R. M. Steinman, N. L. Michael, S. R. Kim, N. Bhardwaj, M. Pope M. K. Louder, P. K. Ehrenberg, P. W. Parren, D. R. Burton, H. Katinger, T. C. VanCott, M. L. Robb, D. L. Birx, \& J. R. Mascola. Neutralizing monoclonal antibodies block human immunodeficiency virus type 1 infection of dendritic cells and transmission to T cells. $J$ Virol 72:9788-94, 1998. Investigation of three human MAbs to elicit a neutralizing effect and block HIV-1 infection in human dendritic cells. Preincubation with NAbs IgG1b12 or a combination of 2F5/2G12 prevented infection of purified DC and transmission in DC/T-cell cultures. On p.710 713784

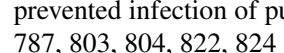

[Froebel1997] K. S. Froebel, J. Y. Mok, M. C. Aldhous, M. P. Armitage, M. Arnott, L. M. Reynolds, J. F. Peutherer, \& S. M. Burns. In vitro measurement of cytotoxic T cell activity does not predict clinical progression in pediatric HIV disease - two case studies. Clin Exp Immunol 110:15-21, 1997. On p.240.259

[Fujii1993] Y. Fujii, Y. Nishino, T. Nakaya, K. Tokunaga, \& K. Ikuta. Expression of human immunodeficiency virus type 1 Nef antigen on the surface of acutely and persistently infected human T-cells. Vaccine 11:1240, 1993. On p.838 839840

Fujii1996a] Y. Fujii, K. Otake, Y. Fujita, N. Yamaimoto, Y. Nagai, M. Tashiro, \& A. Adachi. Clustered localization of oligomeric Nef protein of human immunodeficiency virus type 1 on the cell surface. FEBS Letters 395:257-261, 1996. On p.840

[Fujii1996b] Y. Fujii, K. Otake, M. Tashiro, \& A. Adachi. Human immunodeficiency type $1 \mathrm{Nef}$ protein on the cell surface is cytocidal for human CD4+ T cells. FEBS Letters 393:105-108, 1996. On p. 838839840

[Fujii1996c] Y. Fujii, K. Otake, M. Tashiro, \& A. Adachi. In vitro cytocidal effects of human immunodeficiency virus type $1 \mathrm{Nef}$ on unprimed human CD4+ T cells without MHC restriction. J Gen Virol 77:2943-2951, 1996. On p. 838 839840

[Fujii1996d] Y. Fujii, K. Otake, M. Tashiro, \& A. Adachi. Soluble Nef antigen of HIV-1 is cytotoxic for human CD4+ T cells. FEBS Letters 393:93-96, 1996. On p. 840

[Fukada1999] K. Fukada, Y. Chujoh, H. Tomiyama, K. Miwa, Y. Kaneko, S. Oka, \& M. Takiguchi. HLA-A*1101-restricted cytotoxic T lymphocyte recognition of HIV-1 Pol protein [letter]. AIDS 13:1413-4, 1999. On p. 232 235

[Fukada2002] K. Fukada, H. Tomiyama, C. Wasi, T. Matsuda, S. Kusagawa, H. Sato, S. Oka, Y. Takebe, \& M. Takiguchi. Cytotoxic T-cell recognition of HIV-1 cross-clade and clade-specific epitopes in HIV-1-infected Thai and Japanese patients. AIDS 16(5):701-711, 2002. On p. 90 \begin{tabular}{l|l|l|l|l|l|l|}
\hline 155 & 190 & 207 & 232 & 236 & 280 & 363 \\
\hline
\end{tabular}

[Fukasawa1998] M. Fukasawa, Y. Shimizu, K. Shikata, M. Nakata, R. Sakakibara, N. Yamamoto, M. Hatanaka, \& T. Mizuochi. Liposome oligomannose-coated with neoglycolipid, a new candidate for a safe adjuvant for induction of CD8+ cytotoxic T lymphocytes. FEBS Lett 441:353-6, 1998. On p. 290

[Fung1987] M. S. C. Fung, C. R. Y. Sun, N.-C. Sun, N. T. Chang, \& T.-W. Chang. Monoclonal antibodies that neutralize HIV-1 virions and inhibit syncytium formation by infected cells. Biotech nology 5:940-947, 1987. On p. 633 653,735736

Fung1990] M. S. C. Fung, C. R. Y. Sun, R. S. Liou, W. Gordon, N. T. Chang, T.-W. Chang, \& N.C. Sun. Monoclonal anti-idiotypic antibody mimicking the principal neutralization site in HIV-1 gp120 induces HIV-1 neutralizing antibodies in rabbits. J Immunol 145:2199-2206, 1990. On p. 653

[Fung1992] M. S. C. Fung, C. R. Y. Sun, W. L. Gordon, R.-S. Liou, T. W. Chang, W. N. C. Sun, E. S. Daar, \& D. D. Ho. Identification and characterization of a neutralization site within the second variable region of human immunodeficiency virus type 1 gp120. J Virol 66:848-856, 1992. Two anti-envelope $\mathrm{V} 2$ antibodies were raised that neutralize virus in either a conformation dependent (G3-136) or conformation independent (BAT085) manner. G3-136 has diminished reactivity with deglycosylation or DTT reduced gp120, and sCD4 inhibits binding in a competition assay; BAT085 is not sensitive to these alterations in gp120. On p. 631 632633

[Furci1997] L. Furci, G. Scarlatti, S. Burastero, G. Tambussi, C. Colognesi, C. Quillent, R. Longhi, P. Loverro, B. Borgonovo, D. Gaffi, E. Carrow, M. Malnati, P. Lusso, A. G. Siccardi, A. Lazzarin, \& A. Beretta. Antigen-driven C-C chemokine-mediated HIV-1 suppression by CD4(+) T cells from exposed uninfected individuals expressing the wild-type CCR- 5 allele. J Exp Med 186:45560, 1997. On p.472 491505

[Gahery-Segard2000] H. Gahery-Segard, G. Pialoux, B. Charmeteau, S. Sermet, H. Poncelet M. Raux, A. Tartar, J. P. Levy, H. Gras-Masse, \& J. G. Guillet. Multiepitopic B- and T cell responses induced in humans by a human immunodeficiency virus type 1 lipopeptide vaccine. $J$ \begin{tabular}{ll|l|l|l|l|l|l|l|l|l|l|l|l|} 
Virol 74:1694-703, 2000. On p. 116 & 125 & 284 & 344 & 375 & 392 & 433 & 435 & 489 & 531 & 532 & 533 \\
\hline
\end{tabular}

[Gamberg1999] J. C. Gamberg, M. I. Bowmer, J. C. Trahey, C. M. Campbell, I. Pardoe, \& M. D. Grant. Functional and genetic integrity of the CD8 T cell repertoire in advanced HIV infection. AIDS 13:2043-53, 1999. On p. 171 332395 
[Gao2003] F. Gao, T. Bhattacharya, B. Gaschen, J. Taylor, J. P. Moore, V. Novitsky, K. Yusim, D. Lang, B. Foley, S. Beddows, M. Alam, B. Haynes, B. H. Hahn, \& B. Korber. Reply: Consensus and ancestral state HIV vaccines. Science 299(5612):1515-1518, 2003. On p. 400

[Garba2002] M. L. Garba, C. D. Pilcher, A. L. Bingham, J. Eron, \& J. A. Frelinger. HIV antigen can induce TGF-beta(1)-producing immunoregulatory CD8+ T cells. J Immunol 168(5):2247 2254, 2002. On p. 402

[Garboczi1992] D. N. Garboczi, D. T. Hung, \& D. C. Wiley. HLA-A2-peptide complexes: refolding and crystallization of molecules expressed in Escherichia coli and complexed with single antigenic peptides. Proc Natl Acad Sci USA 89:3429-3433, 1992. On p. 279

[Garcia1997] S. Garcia, M. Fevrier, G. Dadaglio, H. Lecoeur, Y. Riviere, \& M.-L. Gougeon. Potential deleterious effect of anti-viral cytotoxic lymphocyte through the CD95 (FAS/APO-1) mediated pathway during chronic HIV infection. Immunol Lett 57:53-58, 1997. On p. 351

[Gaschen2002] B. Gaschen, J. Taylor, K. Yusim, B. Foley, F. Gao, D. Lang, V. Novitsky, B. Haynes, B. H. Hahn, T. Bhattacharya, \& B. Korber. Diversity considerations in HIV-1 vaccine selection Science 296(5577):2354-2360, 2002. On p.400

[Gaudebout1997] P. Gaudebout, D. Zeliszewski, J. J. Golvano, C. Pignal, S. L. Gac, F. BorrasCuesta, \& G. Sterkers. Binding analysis of 95 HIV gp120 peptides to HLA-DR1101 and -DR040 evidenced many HLA-class II binding regions on gp120 and suggested several promiscuous regions. J Acquir Immune Defic Syndr Hum Retrovirol 14(2):91-101, $1997 . \quad$ On p. 473476 480

[Gauduin1995] M. C. Gauduin, J. T. Safrit, R. Weir, M. S. Fung, \& R. A. Koup. Pre- and postexposure protection against human immunodeficiency virus type 1 infection mediated by a monoclonal antibody. J Infect Dis 171:1203-1209, 1995. Passive protection against HIV-1 LAI wit MAb BAT123 was achieved in SCID mice reconstituted with human peripheral blood lymphocytes (hu-PBL-SCID) BAT123 is specific for the V3 loop gp120 of HIV-1 LAI. Animals wer protected against subsequent infection with LAI strain, but not other virus strains, when BAT123 was given 1 hour before virus inoculation, or up to 4 hours post-exposure. No therapeutic effect was observed when BAT123 was administered after infection had been established. On p. 653

[Gauduin1996] M.-C. Gauduin, G. P. Allaway, P. J. Maddon, C. F. Barbas III, D. R. Burton, \& R. A Koup. Effective Ex Vivo Neutralization of Human Immunodeficiency Virus Type 1 in Plasm by Recombinant Immunoglobulin Molecules. J Virol 70:2586-2592, 1996. Virus direct from plasma from six HIV-1 infected individuals was used for neutralization assay. MAb 19b could neutralize 2/6 plasma samples, while MAb IgG1b12 could neutralize 5/6 plasma samples. CD4based molecules were also tested: $\mathrm{CD} 4-\mathrm{IgG} 2$ was effective in the it ex vivo assay, but $\mathrm{SCD}$ was not. Thus, MAbs IgG1b12 and CD4-IgG2 have broad and potent it in vitro and it ex vivo neutralizing activities. On p. $657,784,785$

[Gauduin1998] M. C. Gauduin, R. Weir, M. S. Fung, \& R. A. Koup. Involvement of the complement system in antibody-mediated post-exposure protection against human immunodeficiency virus type 1. AIDS Res Hum Retroviruses 14:205-11, 1998. Post-exposure passive transfer of murine BAT123 can confer protection to hu-PBL-SCID mice challenged with HIV-1 LAI, and the mechanism is by complement-mediated cytolysis or virolysis. This protection was not conferred by CGP 47439 , a BAT123 chimera that has a human $\operatorname{IgG}_{1}$ Fc domain, substituted in place of a murine $\mathrm{IgG}_{1} \mathrm{Fc}$ domain, suggesting that the protection is mediated by complement. Furthe evidence was that the protective ability of BAT123 is lost when mice were treated with cobra venom factor, which inactivates serum complement. $\operatorname{IgG}_{1}$ does not fix complement efficiently, so an $\mathrm{IgG}_{2} \mathrm{MAb}$ might perform better. Therefore, in spite of the potential for enhancement in some circumstances, in this circumstance complement activation provided a protective advantage. On p. 646653

Gea-Banacloche2000] J. C. Gea-Banacloche, S. A. Migueles, L. Martino, W. L. Shupert, A. C. McNeil, M. S. Sabbaghian, L. Ehler, C. Prussin, R. Stevens, L. Lambert, J. Altman, C. W. Hallahan,
J. C. de Quiros, \& M. Connors. Maintenance of large numbers of virus-specific CD8+ T cells in HIV-infected progressors and long-term nonprogressors. J Immunol 165(2):1082-92, 2000. On p. 79213

Geffin1998] R. B. Geffin, G. B. Scott, M. Melenwick, C. Hutto, S. Lai, L. J. Boots, P. M. McKenna J. 2nd. Kessler, \& A. J. Conley. Association of antibody reactivity to ELDKWA, a glycoprotein 41 neutralization epitope, with disease progression in children perinatally infected with HIV type 1. AIDS Res Hum Retroviruses 14:579-90, 1998. On p.710 712

[Geretti1994] A. M. Geretti, C. A. Van Baalen, J. C. Borleffs, C. A. Van Els, \& A. D. Osterhaus. Kinetics and specificities of the T helper-cell response to gp120 in the asymptomatic stage of HIV-1 infection. Scand J Immunol 39(4):355-362, 1994. On p. 467 [468 469 470 473 474

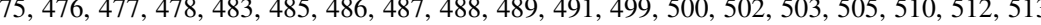

\section{3}

[Gershoni1993] J. M. Gershoni, G. Denisova, D. Raviv, N. I. Smorodinsky, \& D. Buyaner. HIV binding to its receptor creates specific epitopes for the CD4/gp120 complex. FASEB J 7:11851187, 1993. MAbs generated to a sCD4-gp120 complex, and the potential usefulness for vaccine design of epitopes specifically in the complex is discussed. On p. 832 833

Ghanekar2001] S. A. Ghanekar, S. A. Stranford, J. C. Ong, J. M. Walker, V. C. Maino, \& J. A. Levy. Decreased HIV-specific CD4 T cell proliferation in long-term HIV-infected individuals on antiretroviral therapy. AIDS 15(14):1885-1887, 2001. On p.536

[Gherardi2000] M. M. Gherardi, J. C. Ramirez, \& M. Esteban. Interleukin-12 (IL-12) enhancemen of the cellular immune response against human immunodeficiency virus type 1 env antigen in DNA prime/vaccinia virus boost vaccine regimen is time and dose dependent: suppressive effects of IL-12 boost are mediated by nitric oxide. J Virol 74:6278-86, 2000. On p. 295

Ghiara1993] J. B. Ghiara, E. A. Stura, R. L. Stanfield, A. T. Profy, \& I. A. Wilson. Crystal structure of the principal neutralization site of HIV-1. Science 264:82-85, 1993. Crysal structure of V3 loop peptides bound to Fabs 59.1 and 50.1 was determined. The GPGRAF motif forms a double turn. On p. 652673

Ghiara1997] J. B. Ghiara, D. C. Ferguson, A. C. Satterthwait, H. J. Dyson, \& I. A. Wilson. Structure-based design of a constrained peptide mimic of the HIV-1 V3 loop neutralization site J Mol Biol 266:31-9, 1997. On p.673

Gillespie2002] G. M. A. Gillespie, R. Kaul, T. Dong, H.-B. Yang, T. Rostron, J. J. Bwayo, P. Kiama, T. Peto, F. A. Plummer, A. J. McMichael, \& S. L. Rowland-Jones. Cross-reactive cytotoxic T lymphocytes against a HIV-1 p24 epitope in slow progressors with B*57. AIDS 16(7):961-972, 2002. On p.105

Gilljam1999] G. Gilljam, A. Svensson, A. Ekstrom, \& B. Wahren. Immunological Responses to Envelope Glycoprotein 120 from Subtypes of Human Immunodeficiency Virus Type 1. AIDS Re Hum Retroviruses 15(10):899-907, 1999. On p.813

[Giraud1999] A. Giraud, Y. Ataman-Onal, N. Battail, N. Piga, D. Brand, B. Mandrand, \& B. Verrier Generation of monoclonal antibodies to native human immunodeficiency virus type 1 envelope glycoprotein by immunization of mice with naked RNA [In Process Citation]. J Virol Methods 79:75-84, 1999. On p. 729 775784

[Glaser1996] R. W. Glaser \& G. Hausdorf. Binding kinetics of an antibody against HIV p24 core protein measured with real-time biomolecular interaction analysis suggest a slow conformationa change in antigen p24. J Immunological Methods 189:1-14, 1996. The MAb CD-4/1 and p24 have unusual biphasic kinetics of association. On p. 575 576

Goepfert2000] P. A. Goepfert, A. Bansal, B. H. Edwards, G. D. Ritter, Jr., I. Tellez, S. A. McPherson, S. Sabbaj, \& M. J. Mulligan. A significant number of human immunodeficiency viru epitope-specific cytotoxic T lymphocytes detected by tetramer binding do not produce gamm interferon. J Virol 74(21):10249-55, 2000. On p.78

[Goh1999] W. C. Goh, J. Markee, R. E. Akridge, M. Meldorf, L. Musey, T. Karchmer, M. Krone, 
A. Collier, L. Corey, M. Emerman, \& M. J. McElrath. Protection against human immunodeficiency virus type 1 infection in persons with repeated exposure: evidence for T cell immunity in the absence of inherited CCR5 coreceptor defects. J Infect Dis 179:548-57, 1999. On p. 163 240334

[Golding1995] B. Golding, J. Inman, P. Highet, R. Blackburn, J. Manischewitz, N. Blyveis, R. D. Angus, \& H. Golding. Brucella abortus conjugated with a gp120 or V3 loop peptide derive from human immunodeficiency virus type 1 induces neutralizing anti-HIV antibodies, and the V3-b. abortus conjugate is effective even after CD4+ T-cell depletion. J Virol 69:3299-3307, 1995. On p. 670

[Golding2002a] B. Golding, N. Eller, L. Levy, P. Beining, J. Inman, N. Matthews, D. E. Scott, \& H. Golding. Mucosal immunity in mice immunized with HIV-1 peptide conjugated to Brucella abortus. Vaccine 20(9-10):1445-1450, 2002. On p.292 669

[Golding2002b] H. Golding, M. Zaitseva, E. de Rosny, L. R. King, J. Manischewitz, I. Sidorov, M. K. Gorny, S. Zolla-Pazner, D. S. Dimitrov, \& C. D. Weiss. Dissection of Human Immunodeficiency Virus Type 1 Entry with Neutralizing Antibodies to gp41 Fusion Intermediates. $J$ Viro \begin{tabular}{ll|l|l|l|l|l||l|l|l|l|l||l|} 
76(13):6780-6790, 2002. On p.702 & 703 & 708 & 709 & 710 & 716 & 755 & 756 & 784 & 789 & 798 & 801 & 803 \\
\hline
\end{tabular} \begin{tabular}{ll|l|l|l|l|l|l|}
\hline 805 & 807 & 822 & 826 & 830 & 831 \\
\hline
\end{tabular}

[Goletz1997] T. J. Goletz, K. R. Klimpel, N. Arora, S. H. Leppla, J. M. Keith, \& J. A. Berzofsky. Targeting HIV proteins to the major histocompatibility complex class I processing pathway with a novel gp120-anthrax toxin fusion protein. Proc Natl Acad Sci USA 94:12059-12064, 1997. On p. 296

[Goncalves2002] J. Goncalves, F. Silva, A. Freitas-Vieira, M. Santa-Marta, R. Malhó, X. Yang, D. Gabuzda, \& C. I. Barbas. Functional neutralization of HIV-1 Vif protein by intracellula immunization inhibits reverse transcription and viral replication. J Biol Chem 277(35):32036-45, 2002. On p. 609

[Gonzalo1999] R. M. Gonzalo, D. Rodriguez, A. Garcia-Sastre, J. R. Rodriguez, P. Palese, \& M. Esteban. Enhanced CD8+ T cell response to HIV-1 env by combined immunization with influenz and vaccinia virus recombinants. Vaccine 17(7-8):887-92, 1999. On p.341.

[Goodman-Snitkoff1990] G. Goodman-Snitkoff, L. E. Eisele, E. P. Heimer, A. M. Felix, T. T. Andersen, T. R. Fuerst, \& R. J. Mannino. Defining minimal requirements for antibody productio to peptide antigens. Vaccine 8:257-262, 1990. Notes: In this study, mice were immunized with multivalent peptides anchored in a phospholipid complex; these peptides were able to stimulate potent antibody response. That a functional T-helper cell epitope is present within the peptide is inferred by the ability of B-cells to respond to these constructs. Using this system, adjuvant could be bypassed. On $\mathrm{p}$.491, 514,516

[Gordon2000] C. J. Gordon \& E. L. Delwart. Genetic diversity of primary HIV-1 isolates and their sensitivity to antibody-mediated neutralization. Virology 272:326-30, 2000. On p. 809

[Gorny1989] M. K. Gorny, V. Gianakakos, S. Sharpe, \& S. Zolla-Pazner. Generation of human monoclonal antibodies to human immunodeficiency virus. Proc Natl Acad Sci USA 86:1624 1628, 1989. This paper described immortalization of B-cells from HIV-1 positive individuals with Epstein-Barr virus, to produce seven stable antibody producing cell lines. On p. 576 590696698708

[Gorny1991] M. K. Gorny, J.-Y. Xu, V. Gianakakos, S. Karwowska, C. Williams, H. W. Sheppard, C. V. Hanson, \& S. Zolla-Pazner. Production of site-selected neutralizing human monoclona antibodies against the third variable domain of the human immunodeficiency virus type 1 envelope glycoprotein. Proc Natl Acad Sci USA 88:3238-3242, 1991. On p. 647 648 649660664666 675

[Gorny1992] M. K. Gorny, A. J. Conley, S. Karwowska, A. Buchbinder, J.-Y. Xu, E. A. Emini, S. Koenig, \& S. Zolla-Pazner. Neutralization of diverse human immunodeficiency virus type variants by an anti-V3 human monoclonal antibody. J Virol 66:7538-7542, 1992. On p. 669
[Gorny1993] M. K. Gorny, J.-Y. Xu, S. Karwowska, A. Buchbinder, \& S. Zolla-Pazner. Repertoire of neutralizing human monoclonal antibodies specific for the V3 domain of HIV-1 gp120. Immunol 150:635-643, 1993. Characterizaton of 12 human MAbs that bind and neutralize the MN isolate with 50neutralized IIIB: 447-52-D and 694/98-D; all others could not bind HXB2 peptides. All but two, 418-D and 412-D could bind to SF2 peptides. On p. 645 647 648 649 \begin{tabular}{l|l|l|l|l|l|l|l|l}
659 & 660 & 664 & 665 & 666 & 669 & 675 & 720
\end{tabular}

Gorny1994] M. K. Gorny, J. P. Moore, A. J. Conley, S. Karwowska, J. Sodroski, C. Williams, S. Burda, L. J. Boots, \& S. Zolla-Pazner. Human anti-V2 monoclonal antibody that neutralizes primary but not laboratory isolates of human immunodeficiency virus type 1. J Virol 68:83128320, 1994. Detailed characterization of the MAb 697-D. On p. 628 632 633675683684 \begin{tabular}{|l|l|l|l|l|}
\hline 691 & 720 & 769 & 811 & 812 \\
\hline
\end{tabular}

Gorny 1997] M. K. Gorny, T. C. VanCott, C. Hioe, Z. R. Israel, N. L. Michael, A. J. Conley, C. Williams, J. A. Kessler II, P. Chigurupati, S. Burda, \& S. Zolla-Pazner. Human monoclonal antibodies to the V3 loop of HIV-1 with intra- and interclade cross-reactivity. J Immunol 159:5114 5122, 1997. On p. 589 653 653 654 $655|692| 710|720| 721 \mid 769$

Gorny 1998] M. K. Gorny, J. R. Mascola, Z. R. Israel, T. C. VanCott, C. Williams, P. Balfe, C. Hioe, S. Brodine, S. Burda, \& S. Zolla-Pazner. A human monoclonal antibody specific for the V3 loop of HIV type 1 clade E cross-reacts with other HIV type 1 clades. AIDS Res Hum Retroviruses

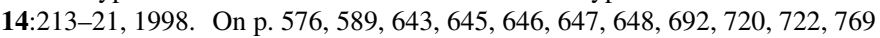

[Gorny2000a] M. K. Gorny, T. C. VanCott, C. Williams, K. Revesz, \& S. Zolla-Pazner. Effects of oligomerization on the epitopes of the human immunodeficiency virus type 1 envelope glycopro-

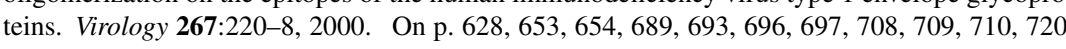
\begin{tabular}{|l|l|l|l|l|l|l|l|l|l|l|l|l|l|l|l|l|l|}
\hline 722 & 761 & 768 & 769 & 770 & 771 & 772 & 809 & 810 & 813 & 827 & 828 & 829 & 830 & 831 \\
\hline
\end{tabular}

Gorny $2000 \mathrm{~b}]$ M. K. Gorny \& S. Zolla-Pazner. Recognition by human monoclonal antibodies of free and complexed peptides representing the prefusogenic and fusogenic forms of human immunodeficiency virus type $1 \mathrm{gp} 41$ [In Process Citation]. J Virol 74:6186-92, 2000. On p.692 \begin{tabular}{|l|l|l|l|l|l|l|l|l|l|l|l|l|l|}
\hline 696 & 697 & 700 & 701 & 708 & 709 & 710 & 713 & 827 & 828 & 829 & 830 & 831 \\
\hline
\end{tabular}

Gorny2002] M. K. Gorny, C. Williams, B. Volsky, K. Revesz, S. Cohen, V. R. Polonis, W. J. Honnen, S. C. Kayman, C. Krachmarov, A. Pinter, \& S. Zolla-Pazner. Human monoclonal antibodie specific for conformation-sensitive epitopes of $\mathrm{V} 3$ neutralize human immunodeficiency virus type 1 primary isolates from various clades. J Virol 76(18):9035-9045, 2002. On p. 689|700|701 \begin{tabular}{l|l|l|l|l|l|l|}
720 & 723 & 769 & 771 & 814 & 815 \\
\hline
\end{tabular}

Gorse1999a] G. J. Gorse, L. Corey, G. B. Patel, M. Mandava, R. H. Hsieh, T. J. Matthews, M. C. Walker, M. J. McElrath, P. W. Berman, M. M. Eibl, \& R. B. Belshe. HIV-1MN recombinant glycoprotein 160 vaccine-induced cellular and humoral immunity boosted by HIV-1MN recombinan glycoprotein 120 vaccine. National Institute of Allergy and Infectious Diseases AIDS Vaccine Evaluation Group. AIDS Res Hum Retroviruses 15:115-32, 1999. On p.525.

[Gorse 1999b] G. J. Gorse, G. B. Patel, M. D. Mandava, \& R. B. Belshe. Vaccine-induced cytotoxic $\mathrm{T}$ lymphocytes against human immunodeficiency virus type 1 using two complementary in vitro stimulation strategies. Vaccine 18:835-49, 1999. On p. 239 332395

[Gosting1987] L. H. Gosting, J. McClure, E. S. Dickinson, S. M. Watanabe, K. Shriver, \& L. C. Goldstein. Monoclonal antibodies to gp110 and gp41 of human immunodeficiency virus. J Clin Microbiol 25:845-848, 1987. On p. 634 689698

[Gotch1990] F. Gotch, D. Nixon, N. Alp, A. McMichael, \& L. Borysiewicz. High frequency of memory and effector gag specific cytotoxic T lymphocytes in HIV seropositive individuals. In Immunol 2:707, 1990. On p. 124

[Gotch1993] F. Gotch, S. N. McAdam, \& C. E. A. et al. Cytotoxic T cells in HIV-2 seropositive Gambians. Identification of a virus-specific MHC-restricted peptide epitope. J Immuno 151:3361-3369, 1993. On p.112 
[Goulder1996a] P. Goulder, C. Conlon, K. McIntyre, \& A. McMichael. Identification of a novel human leukogen antigen A26-restricted epitope in a conserved region of Gag. AIDS 10(12):14411443,1996 . This paper is correspondence briefly describing the identification and characterization of an immuno-dominant A26-CTL epitope in an asymptomatic HIV+ individual. On p. 108

[Goulder1996b] P. J. R. Goulder, M. Bunce, P. Krausa, K. McIntyre, S. Crowley, B. Morgan, A. Edwards, P. Giangrande, R. E. Phillips, \& A. J. McMichael. Novel, cross-restricted, conserved and immunodominant cytotoxic T lymphocyte epitopes in slow HIV Type 1 infection. AIDS Res and Hum Retroviruses 12:1691-1698, 1996. HLA-B*57 is over-represented in slow progressors HLA $* 5801$ is a closely related molecule, and while the defined anchor residues of HLA*5801 can be used to predict epitopes in HIV-1 proteins, the CTL from HLA-B*57 positive individuals have limited cross-presentation capacity with HLA*5801 targets. In this paper five new HLA-B*57 epitopes were defined. On p. 100 [104 123 [124 150 [381

[Goulder1997a] P. Goulder, D. Price, M. Nowak, S. Rowland-Jones, R. Phillips, \& A. McMichae Co-evolution of human immunodeficiency virus and cytotoxic T-lymphocyte responses. Immuno

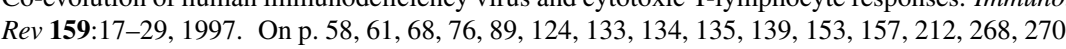
352 354 $363,367,371,383$

[Goulder1997b] P. J. Goulder, M. Bunce, G. Luzzi, R. E. Phillips, \& A. J. McMichael. Potential underestimation of HLA-C-restricted cytotoxic T-lymphocyte responses. AIDS 11(15):1884-1886, 1997. On p. 70108

[Goulder1997c] P. J. Goulder, A. Edwards, R. E. Phillips, \& A. J. McMichael. Identification of a novel HLA-B*2705-restricted cytotoxic T lymphocyte epitope within a conserved region of HIV-1 Nef. AIDS 11:536-538, 1997. On p. 133 372

[Goulder1997d] P. J. Goulder, A. Edwards, R. E. Phillips, \& A. J. McMichael. Identification of a novel HLA-B*3501-restricted cytotoxic T lymphocyte epitope using overlapping peptides. AID 11(7):930-932, 1997. On p.70

[Goulder1997e] P. J. Goulder, A. K. Sewell, D. G. Lalloo, D. A. Price, J. A. Whelan, J. Evans, G. P. Taylor, G. Luzzi, P. Giangrande, R. E. Phillips, \& A. J. McMichael. Patterns of immunodominance in HIV-1-specific cytotoxic T lymphocyte responses in two human histocompatibility leukocyte antigens (HLA)-identical siblings with HLA-A*0201 are influenced by epitope mutation. J Exp Med 8:1423-33, 1997. Primary human immunodeficiency virus (HIV) infection is controlled principally by HIV-specific cytotoxic T lymphocytes (CTL) to a steady- state level of virus load, which strongly influences the ultimate rate of progression to disease. Epitope selection by CTL may be an important determinant of the degree of immune control over the virus. This report describes the CTL responses of two HLA-identical hemophiliac brothers who were exposed to identical batches of Factor VIII and became seropositive within $10 \mathrm{wk}$ of one another. Both have HLA-A*0201. The CTL responses of the two siblings were very dissimilar, one donor making strong responses to two epitopes within p17 Gag (HLA-A*0201-restricted SLYNTVATL and HLA-A3-restricted RLRPGGKKK). The sibling responded to neither epitope, but made strong responses to two epitopes presented by HLA-B7. This was not the result of differences in presentation of the epitopes. However, mutations in both immunodominant epitopes of the p17 Gag responder were seen in proviral sequences of the nonresponder. We then documented the CTL responses to two HLA-A*0201-restricted epitopes, in Gag (SLYNTVATL) and Pol (ILKEPVHGV) in 22 other HIV-infected donors with HLA-A*0201. The majority (71responses to the Gag epitope. In the $29 \mathrm{Gag}$ epitope in standard assays, there was evidence of low frequency memory CTL responses using peptide stimulation of PBMC, and most of these donors also showed mutations

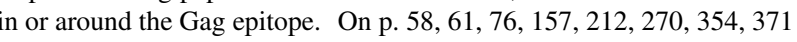

[Goulder1997f] P. J. R. Goulder, R. E. Phillips, R. A. Colbert, S. McAdam, G. Ogg, M. A. Nowak, P. Giangrande, G. Luzzi, B. Morgan, A. Edwards, A. McMichael, \& S. Rowland-Jones. Late escape from an immunodominant cytotoxic T-lymphocyte response associated with progression to AIDS. Nature Med 3:212-216, 1997. The CTL response was studied in six HIV+ individuals who make a strong immunodominat response to the same B27 epitope. In two donors an escape mutation arose after close to 10 years of epitope stability, around the time of progression to AIDS On p. 62135

[Goulder1997g] P. J. R. Goulder, S. W. Reid, D. A. Price, C. A. O'Callaghan, A. J. McMichael R. E. Phillips, \& E. Y. Jones. Combined structural and immunological refinement of HIV-1 HLA B8 restricted cytotoxic T lymphocyte epitopes. Eur J Immunol 27:1515-1521, 1997. On p.66

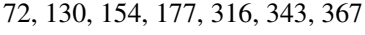

Goulder1999a] P. J. Goulder. personal communication. unpublished 1999. On p.157, 373

[Goulder1999b] P. J. Goulder \& B. D. Walker. The great escape - AIDS viruses and immune control [news]. Nat Med 5:1233-5, 1999. On p.69

Goulder2000a] P. J. Goulder, C. Brander, K. Annamalai, N. Mngqundaniso, U. Govender, Y. Tang, S. He, K. E. Hartman, C. A. O'Callaghan, G. S. Ogg, M. A. Altfeld, E. S. Rosenberg, H. Cao, S. A Kalams, M. Hammond, M. Bunce, S. I. Pelton, S. A. Burchett, K. McIntosh, H. M. Coovadia, \& B. D. Walker. Differential narrow focusing of immunodominant human immunodeficiency virus gag-specific cytotoxic T-lymphocyte responses in infected African and caucasoid adults

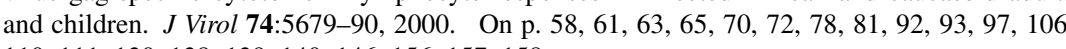

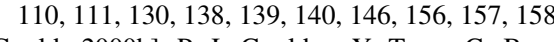

Goulder2000b] P. J. Goulder, Y. Tang, C. Brander, M. R. Betts, M. Altfeld, K. Annamalai, A. Trocha, S. He, E. S. Rosenberg, G. Ogg, C. A. O'Callaghan, S. A. Kalams, R. E. McKinney, Jr., K. Mayer, R. A. Koup, S. I. Pelton, S. K. Burchett, K. McIntosh, \& B. D. Walker. Functionally inert HIV-specific cytotoxic $\mathrm{T}$ lymphocytes do not play a major role in chronically infected adults and children. J Exp Med 192(12):1819-32, 2000. On p. 79] $111 \mid$ [131|369

[Goulder2000c] P. J. Goulder, Y. Tang, S. I. Pelton, \& B. D. Walker. HLA-B57-Restricted cytotoxic T-lymphocyte activity in a single infected subject toward two optimal epitopes, one of which is entirely contained within the other. J Virol 74:5291-9, 2000. On p. 62 64 $104 \quad 106$

[Goulder2001a] P. J. Goulder, M. M. Addo, M. A. Altfeld, E. S. Rosenberg, Y. Tang, U. Govender, N. Mngqundaniso, K. Annamalai, T. U. Vogel, M. Hammond, M. Bunce, H. M. Coovadia, \& B. D. Walker. Rapid definition of five novel HLA-A*3002-restricted human immunodeficiency virusspecific cytotoxic T-lymphocyte epitopes by elispot and intracellular cytokine staining assays.

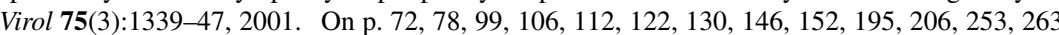
$311319322 \quad 324,367$

Goulder2001b] P. J. Goulder, C. Brander, Y. Tang, C. Tremblay, R. A. Colbert, M. M. Addo, E. S. Rosenberg, T. Nguyen, R. Allen, A. Trocha, M. Altfeld, S. He, M. Bunce, R. Funkhouser, S. I Pelton, S. K. Burchett, K. McIntosh, B. T. Korber, \& B. D. Walker. Evolution and transmission of stable CTL escape mutations in HIV infection. Nature 412(6844):334-8, 2001. On p. 61,63

Goulder2001c] P. J. Goulder, C. Pasquier, E. C. Holmes, B. Liang, Y. Tang, J. Izopet, K. Saune, E. S. Rosenberg, S. K. Burchett, K. McIntosh, M. Barnardo, M. Bunce, B. D. Walker, C. Brander, \& R. E. Phillips. Mother-to-child transmission of HIV infection and CTL escape through HLAA2-SLYNTVATL epitope sequence variation. Immunol Lett 79(1-2):109-16, 2001. On p. 87 123

Graham2002] B. S. Graham. Clinical trials of HIV vaccines. Аппи Rev Med 53:207-21, 2002. On p. 401

[Gram2002] G. J. Gram, A. Bolmstedt, K. Schonning, M. Biller, J.-E. S. Hansen, \& S. Olofsson. Detection of orientation-specific anti-gp120 antibodies by a new N-glycanase protection assay APMIS 110(2):123-131, 2002. On p. 677 760

[Grant2000] M. Grant, F. Smaill, S. Muller, H. Kohler, \& K. Rosenthal. The anti-idiotypic antibody 1F7 selectively inhibits cytotoxic T cells activated in HIV-1 infection. Immunol Cell Biol 78:20-7, 2000. On p. 729730

[Grassi1991] F. Grassi, R. Meneveri, M. Gullberg, L. Lopalco, G. B. Rossi, P. Lanza, C. DeSantis, 
G. Brattsand, S. Butto, E. Ginelli, A. Berretta, \& A. G. Siccardi. Human Immunodeficiency Virus Type 1 gp120 Mimics a Hidden Monomorphic Epitope Borne by Class I Major Histocompatibility Complex Heavy Chains. J Exp Med 174:53-62, 1991. On p.688

[Gray 1999] C. M. Gray, J. Lawrence, J. M. Schapiro, J. D. Altman, M. A. Winters, M. Crompton, M. Loi, S. K. Kundu, M. M. Davis, \& T. C. Merigan. Frequency of class I HLA-restricted antiHIV CD8+ T cells in. J Immunol 162:1780-8, 1999. On p.75 76 211 212

[Greenough1999] T. C. Greenough, D. B. Brettler, F. Kirchhoff, L. Alexander, R. C. Desrosiers, S. J. O'Brien, M. Somasundaran, K. Luzuriaga, \& J. L. Sullivan. Long-term nonprogressive infection with human immunodeficiency virus type 1 in a hemophilia cohort. J Infect Dis 180:1790-802, 1999. On $\mathrm{p} .163$

[Greenway1994] A. L. Greenway, D. A. McPhee, E. Grgacic, D. Hewish, A. Lucantoni I. Macreadie, \& A. Azad. Nef 27, but not the Nef 25 isoform of human immunodeficiency virustype 1 pNL4.3 down-regulates surface CD4 and IL-2R expression in peripheral blood mononuclear cells and transformed T cells. Virology 198:245-256, 1994. On p.841

[Griffiths1993] J. Griffiths, S. J. Harris, G. T. Layton, E. L. Berrie, T. J. French, N. R. Burns, S. E. Adams, \& A. J. Kingsman. Hybrid human immunodeficiency virus gag particles as an antigen carrier system: induction of cytotoxic $\mathrm{T}$ cell and humoral responses by a gag:V3 fusion. $J$ Viro 67:3191-3198, 1993. On p. 288

[Grimison1995] B. Grimison \& J. Laurence. Immunodominant epitope regions of HIV-1 reverse transcriptase: correlations with HIV-1+ serum IgG inhibitory to polymerase activity and with disease progression. J AIDS 9:58-68, 1995. On p. 598

[Grovit-Ferbas2000] K. Grovit-Ferbas, J. F. Hsu, J. Ferbas, V. Gudeman, \& I. S. Chen. Enhanced binding of antibodies to neutralization epitopes following thermal and chemical inactivation of human immunodeficiency virus type 1 . J Virol 74(13):5802-9, 2000. On p. $720|722| 764 \mid 784$ $787,798,800,822,825$

[Grundner2002] C. Grundner, T. Mirzabekov, J. Sodroski, \& R. Wyatt. Solid-Phase Proteoliposomes Containing Human Immunodeficiency Virus Envelope Glycoproteins. J Virol 76(7):3511-

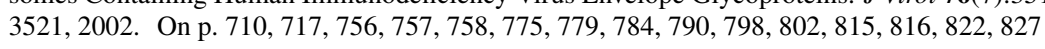

[Grunow1990] R. Grunow, R. Giess, T. Portsman, H. Dopel, K. Hansel, \& R. von Baehr. Development and biological testing of human and murine antibodies against HIV antigens. Z Klin Med 45:367-369, 1990. On p.575.576

[Gruters2002] R. A. Gruters, C. A. van Baalen, \& A. D. M. E. Osterhaus. The advantage of early recognition of HIV-infected cells by cytotoxic T-lymphocytes. Vaccine 20(15):2011-2015, 2002 On p.259 265

[Gu1996] Z. Gu, Z. Li, Y. Quan, M. A. Parniak, \& M. A. Wainberg. Studies of a Neutralizing Monoclonal Antibody to Human Immunodeficiency Virus Type 1 Reverse Transcriptase: Antagonistic and Synergistic Effects in Reactions Performed in the Presence of Nucleoside and Nonnucleoside Inhibitors, Respectively. J Virol 70:2620-2626, 1996. On p. 597 606 607

[Guardiola2001] J. Guardiola, P. De Berardinis, R. Sartorius, C. Fanutti, R. N. Perham, \& G. Del Pozzo. Phage display of epitopes from HIV-1 elicits strong cytolytic responses in vitro and in vivo. Adv Exp Med Biol 495:291-298, 2001. On p.214

[Guevara2002] H. Guevara, J. Casseb, L. S. Zijenah, M. Mbizvo, L. F. Oceguera III, C. V. Hanson, D. A. Katzenstein, \& R. M. Hendry. Maternal HIV-1 antibody and vertical transmission in subtype C virus infection. J Acquir Immune Defic Syndr 29(5):435-440, 2002. On p. 819

[Guillerm1998] C. Guillerm, V. Robert-Hebmann, U. Hibner, M. Hirn, \& C. Devaux. An antiCD4 (CDR3-loop) monoclonal antibody inhibits human immunodeficiency virus type 1 envelope glycoprotein-induced apoptosis. Virology 248:254-63, 1998. On p.667

[Guillon2002] C. Guillon, C. A. van Baalen, P. H. M. Boers, E. J. Verschuren, R. A. Gruters, \& A. D. M. E. Osterhaus. Construction and characterisation of infectious recombinant HIV-1 clones containing CTL epitopes from structural proteins in Nef. J Virol Methods 99(1-2):115-121, 2002

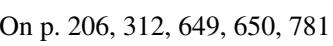

[Guimaráes2002] M. L. Guimaráes, A. S. Moreira, \& M. G. Morgado. Polymorphism of the human immunodeficiency virus type 1 in Brazil: Genetic characterization of the nef gene and implications for vaccine design. Mem Inst Oswaldo Cruz 97(4):523-326, 2002. On p. 348 366 373
[Gunthard1994] H. F. Gunthard, P. L. Gowland, J. Schupbach, M. S. C. Fung, J. Boni, R.-S. Liou, N. T. Chang, P. Grob, P. Graepel, D. G. Braun, \& R. Luthy. A phase I/IIA clinical study with a chimeric mouse-human monoclonal antibody to the V3 loop of human immunodeficiency virus type I gp120. J Inf Dis 170:1384-1393, 1994. On p.646

Gupta2001] S. Gupta, K. Arora, A. Sampath, S. S. Singh, A. Gupta, \& V. K. Chaudhary. Mapping of HIV-1 Gag epitopes recognized by polyclonal antibodies using gene-fragment phage display system. Prep Biochem Biotechnol 31(2):185-200, 2001. On p.592

Gupta2002] K. Gupta, M. Hudgens, L. Corey, M. J. McElrath, K. Weinhold, D. C. Montefiori, G. J. Gorse, S. E. Frey, M. C. Keefer, T. G. Evans, R. Dolin, D. H. Schwartz, C. Harro, B. Graham, P. W. Spearman, M. Mulligan, P. Goepfert, \& AIDS Vaccine Evaluation Group. Safety and immunogenicity of a high-titered canarypox vaccine in combination with rgp120 in a diverse population of HIV-1-uninfected adults: AIDS Vaccine Evaluation Group Protocol 022A. J Acquir Immune Defic Syndr 29(3):254-261, 2002. On p. 167 339 752

Guzman1998] C. A. Guzman, D. Saverino, E. Medina, D. Fenoglio, B. Gerstel, A. Merlo, G. L. Pira, F. Buffa, T. Chakraborty, \& F. Manca. Attenuated Listeria monocytogenes carrier strains can deliver an HIV-1 gp120 T helper epitope to MHC class II-restricted human CD4+ T cells. Eur J Immunol 28:1807-14, 1998. On p.485

Haaheim1991] L. R. Haaheim, J. P. Maskell, P. Mascagni, \& A. R. M. Coates. Fine molecular specificity of linear and assembled antibody binding sites in HIV-1 p24. Scand J Immunol 34:341 350, 1991. Seven murine MAbs to a 104-mer peptide spanning residues 270-373 of p24 gag were generated. On p. 5815582583.585586

Haas1991] G. Haas, R. David, R. Frank, H. Gausepohl, C. Devaux, J.-M. Claverie, \& M. Pierres. Identification of a major human immunodeficiency virus-1 reverse transcriptase epitope recognized by mouse CD4+ T lymphocytes. Eur J Immunol 21:1371-1377, 1991. Notes: RT peptides were recognized by several of the T-helper lines established from RT-primed mice. Further, Tcells from peptide-primed mice could be restimulated by native RT. RT epitope: KEKVYLAWVPAHKGIG. On p. $446,453,457,528,534$

Haas 1996] G. Haas, U. Plikat, P. Debre, M. Lucchiari, C. Katlama, Y. Dudoit, O. Bonduelle, M. Bauer, H. Ihlenfeldt, G. Jung, B. Maier, A. Meyerhans, \& B. Autran. Dynamics of viral variants in HIV-1 Nef and specific cytotoxic T lymphocytes in vivo. J Immunol 157:4212-4221, 1996. On p. 346378389

[Haas1997] G. Haas, A. Hosmalin, F. Hadida, J. Duntze, P. Debre, \& B. Autran. Dynamics of HIV variants and specific cytotoxic $\mathrm{T}$ cell recognition in nonprogressors and progressors. Immunol Lett 57:63-8, 1997. On p.203 $378 \mid 389$

[Haas1998] G. Haas, A. Samri, E. Gomard, A. Hosmalin, J. Duntze, J. M. Bouley, H. G. Ihlenfeldt, C. Katlama, \& B. Autran. Cytotoxic T cell responses to HIV-1 reverse transcriptase, integrase

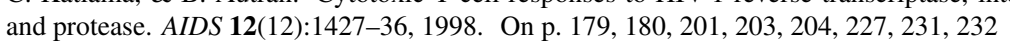

[Hadida1992] F. Hadida, A. Parrot, M. P. Kieny, B. Sadat-Sowti, C. Mayaud, \& P. Debre. Carboxylterminal and central regions of human immunodeficiency virus-1 NEF recognized by cytotoxic $\mathrm{T}$ lymphocytes from lymphoid organs. An in vitro limiting dilution analysis. J Clin Invest 89:53-60, 1992. HIV-1-specific CTL can be detected in lymph nodes and spleens. The carboxyl-terminal domain of Nef is recognized by CTL in association with HLA-A1 and B8, with clonal frequencies

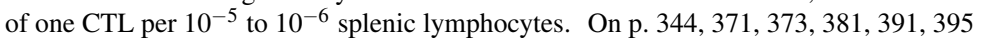

[Hadida1995] F. Hadida, G. Haas, G. Zimmermann, A. Hosmalin, R. Spohn, A. Samri, G. Jung, P. Debre, \& B. Autran. CTLs from lymphoid organs recognize an optimal HLA-A2 restricted and HLA-B52 restricted nonapeptide and several epitopes in the C-terminal region of HIV-1 Nef. $J$ 
Immunol 154:4174-4186, 1995. An in vitro limiting dilution analysis showed CTL recognition in the context of HLA B52 and A2.1, A2.2 and A2.4 in nanomolar concentrations. Molecula modeling suggests motifs important for peptide binding to the pocket of an HLA-A2.1 molecule On p. 391 392 $392393 \quad 394$

[Haglund2002a] K. Haglund, I. Leiner, K. Kerksiek, L. Buonocore, E. Pamer, \& J. K. Rose. Highlevel primary $\mathrm{CD} 8(+) \mathrm{T}$-cell response to human immunodeficiency virus type $1 \mathrm{Gag}$ and En generated by vaccination with recombinant vesicular stomatitis viruses. J Virol 76(6):2730-2738, 2002. On p. 118298

[Haglund2002b] K. Haglund, I. Leiner, K. Kerksiek, L. Buonocore, E. Pamer, \& J. K. Rose. Robust recall and long-term memory T-cell responses induced by prime-boost regimens with heterologous live viral vectors expressing human immunodeficiency virus type $1 \mathrm{Gag}$ and Env proteins. Virol 76(15):7506-7517, 2002. On p. 118.298

[Hale1989] P. M. Hale, K. B. Cease, R. A. Houghten, C. Ouyang, S. Putney, K. Javaherian, H. Margalit, J. L. Cornette, J. L. Spouge, C. DeLisi, et al. T cell multideterminant regions in the human immunodeficiency virus envelope: toward overcoming the problem of major histocompatibility complex restriction. Int Immunol 1(4):409-15, 1989. On p.470 472 4965501509510512 $5 1 4 \longdiv { 5 1 9 } [ 5 2 0 , 5 2 2$

[Halim2000] S. S. Halim, D. N. Collins, \& A. I. Ramsingh. A therapeutic HIV vaccine using coxsackie-HIV recombinants: a possible new strategy. AIDS Res Hum Retroviruses 16(15):15518, 2000. On p. 169447

[Hamajima1997] K. Hamajima, J. Fukushima, H. Bukawa, T. Kaneko, T. Tsuji, Y. Asakura S. Sasaki, K. Q. Xin, \& K. Okuda. Strong augment effect of IL-12 expression plasmid on the induction of HIV-specific cytotoxic T lymphocyte activity by a peptide vaccine candidate. Clin Immunol Immunopathol 83:179-84, 1997. We previously reported that repeated inoculation of VC1, a macromolecular multicomponent peptide vaccine emulsified with Freund's adjuvan (VC1-F), induced high cytotoxic T lymphocyte (CTL) levels and a substantial level of multivalent antibodies which neutralized various human immunodeficiency virus type 1 (HIV-1) isolates. In the present study, we report that inoculation of VC1-F plus interleukin (IL)-12 expression plasmid can induce a higher antigen-specific CTL response compared to that with $\mathrm{VC} 1-\mathrm{F}$ alone. VC1-F plus IL-12 expression plasmid or VC1-F alone were inoculated to BALB/c mice twice at interval of 2 weeks. Two weeks after the second inoculation, spleen effector cells from these mice were examined. Stronger CTL responses against target cells were observed from the inoculation of VC1-F plus IL-12 plasmid than from that with VC-1F alone, but there was no difference in antibody induction. The inoculation of VC1 plus IL-12 plasmid also produced higher CTL activ ity than the inoculation of $\mathrm{VC} 1$ alone. These augmented CTL activities were not observed using target cells pulsed with non-HIV-specific peptides and different class I haplotype cells. These data demonstrate that co-inoculation of cell-mediated immune potent antigen and IL-12 plasmids can enhance the antigen-specific CTL response. This may be a potential approach for the induction of cellular immunization against HIV-1 and other diseases. On p.92 296

[Hammond1991] S. A. Hammond, E. Obah, P. Stanhope, C. R. Monell, M. Str, F. M. Robbins, W. B. Bias, R. W. Karr, S. Koenig, \& R. F. Siliciano. Characterization of a conserved T cell epitope in HIV-1 gp41 recognized by vaccine-induced human cytolytic T cells. J Immunol 146:1470-1477, 1991. An HLA DPw4.2 human CTL epitope located in gp41 was described, recognized by CD4 CTL clones that were induced in seronegative humans by immunization with recombinant gp160 BRU. gp41 CTL epitope: GIKQLQARILAVERYLKDQ. On p. 309

[Hammond1995] S. A. Hammond, R. P. Johnson, S. A. Kalams, B. D. Walker, M. Takiguchi, J. T Safrit, R. A. Koup, \& R. F. Siliciano. An epitope-selective transporter associated with antigen presentation TAP-1/2-independent pathway and a more general TAP-1/2-dependent antigenprocessing pathway allow recognition of the HIV-1 envelope glycoprotein by CD8+ CTL. $J$ Immunol 154:6140-6156, 1995. Two peptide-processing pathways are utilized for MHC class I presentation of HIV-1 Env epitopes. The previously characterized TAP-1 and TAP-2 dependent pathway can generate all Env epitopes and uses Env protein mislocalized in the cytosol to produce peptides. The second, novel pathway uses a TAP-1/2 independent pathway, and allows a subset of MHC-restricted epitopes to be processed in the endoplasmic reticulum or a Golgi compartment.

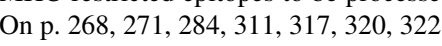

[Hanke1998a] T. Hanke, T. J. Blanchard, J. Schneider, G. S. Ogg, R. Tan, M. Becker, S. C. Gilbert, A. V. Hill, G. L. Smith, \& A. McMichael. Immunogenicities of intravenous and intramuscula administrations of modified vaccinia virus Ankara-based multi-CTL epitope vaccine for huma immunodeficiency virus type 1 in mice. J Gen Virol 79:83-90, 1998. On p. 199 211.295 296 367

[Hanke1998b] T. Hanke, J. Schneider, S. G. Gilbert, A. V. S. Hill, \& A. McMichael. DNA multiCTL epitope vaccines for HIV and Plasmodium falciparum: Immunogenicity in mice. Vaccine 16:426-435, 1998. On p. 1992211295367

Hanke1999a] T. Hanke \& A. McMichael. Pre-clinical development of a multi-CTL epitope-based DNA prime MVA boost vaccine for AIDS. Immunol Lett 66:177-81, 1999. On p. 297

Hanke1999b] T. Hanke, V. C. Neumann, T. J. Blanchard, P. Sweeney, A. V. Hill, G. L. Smith, \& A. McMichael. Effective induction of HIV-specific CTL by multi-epitope using gene gun in a combined vaccination regime. Vaccine 17:589-96, 1999. On p.297

[Hanke2000] T. Hanke \& A. J. McMichael. Design and construction of an experimental HIV-1 vaccine for a year-2000 clinical trial in Kenya. Nat Med 6(9):951-955, 2000. On p. 127128

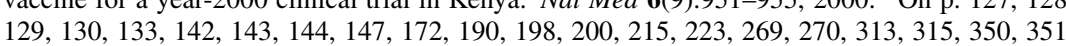
\begin{tabular}{l|l|l|l|l|l|l|l|l|l|l|l|l|l|}
\hline 129 & 130 & 133 & 142 & 143 & 144 & 147 & 12 \\
\hline 359 & 364 & 365 & 369 & 370 & 380 & 393 \\
\hline
\end{tabular}

Harcourt1998] G. C. Harcourt, S. Garrard, M. P. Davenport, A. Edwards, \& R. E. Phillips. HIV-1 variation diminishes CD4 T lymphocyte recognition. J Exp Med 188:1785-93, 1998. On p. 429 431

Harrer1993] T. Harrer, C. Jassoy, E. Harrer, R. P. Johnson, \& B. D. Walker. Induction of HIV-1 replication in a chronically infected T-cell line by cytotoxic T lymphocytes. J Acquir Immune Defic Syndr 6(8):865-71, 1993. On p. 146

Harrer1996a] E. Harrer, T. Harrer, P. Barbosa, M. Feinberg, R. P. Johnson, S. Buchbinder, \& B. D Walker. Recognition of the highly conserved YMDD region in the human immunodeficiency virus type 1 reverse transcriptase by HLA-A2-restricted cytotoxic T lymphocytes from an asymptomatic long-term nonprogresser. $J$ Inf Dis 173:476-479, 1996. The amino acid stretch YMDD is a critical functional domain of reverse transcriptase, and is highly conserved. This sequence is also part of an HLA-A2-restricted epitope. The substitution YMDD to YVDD confers drug resistance to FTC and dideoxyinosine, and also abolishes the CTL specific response. On p.148

[Harrer1996b] T. Harrer, E. Harrer, S. A. Kalams, P. Barbosa, A. Trocha, R. P. Johnson, T. Elbeik, M. B. Feinberg, S. P. Buchbinder, \& B. D. Walker. Cytotoxic T lymphocytes in asymptomatic long-term nonprogressing HIV-1 infection. Breadth and specificity of the response and relation o in vivo viral quasispecies in a person with prolonged infection and low viral load. J Immuno 156:2616-2623, 1996. On p. 145.226304

Harrer1998] T. Harrer, E. Harrer, P. Barbosa, F. Kaufmann, R. Wagner, S. Bruggemann, J. R. Kalden, M. Feinberg, R. P. Johnson, S. Buchbinder, \& B. D. Walker. Recognition of two overlapping CTL epitopes in HIV-1 p17 by CTL from a long-term nonprogressing HIV-1-infected individual. J Immunol 161:4875-81, 1998. On p. 85.90

[Haslett2000] P. A. Haslett, D. F. Nixon, Z. Shen, M. Larsson, W. I. Cox, R. Manandhar, S. M. Donahoe, \& G. Kaplan. Strong human immunodeficiency virus (HIV)-specific CD4+ T cell responses in a cohort of chronically infected patients are associated with interruptions in antiHIV chemotherapy. J Infect Dis 181:1264-72, 2000. On p.441

Haugan1995] I. R. Haugan, B. M. Nilsen, S. Worland, L. Olsen, \& D. E. Helland. Characterization 
of the DNA-binding activity of HIV-1 integrase using a filter binding assay. Biochem Biophys Res Commun 217:802-810, 1995. On p.600 601 602 602 603 604

[Hay 1999a] C. Hay. Personal communication, 1999. On p. 235

[Hay1999b] C. Hay, D. Ruhl, N. Basgoz, C. Wilson, J. Billingsley, M. DePasquale, R. D'Aquila, S. M. Wolinsky, J. M. Crawfod, D. Montefiori, \& B. D. Walker. Lack of viral escape and defective in vivo activation of human immunodeficiency virus type 1-specific cytotoxic T lymphocytes in rapidly progressive infection. $J$ Virol 73:5509-5519, 1999. On p.77] 188,329

[Hayball1997] J. D. Hayball, S. J. Fidler, D. Palliser, A. D. Rees, J. R. Lamb, \& R. A. Lake. Tandem peptide epitopes facilitate CD4-dependent activation of T cell clones. Immunol Cell Biol 75:148 153, 1997. On p. 494

[Haynes1993] B. F. Haynes, L. O. Arthur, P. Frost, T. J. Matthews, A. J. Langlois, T. J. Palker, M. K. Hart, R. M. Scearce, D. M. Jones, C. McDanal, J. Ottinger, D. P. Bolognesi, \& K. J. Weinhold Conversion of an Immunogenic Human Immunodeficiency Virus Envelope Synthetic Peptide to Tolerogen in Chimpanzees by the Fusogenic Domain of HIV gp41 Envelope Protein. $J$ Exp Med 177:717-727, 1993. Notes: In this study the immunogenicity of a T1-V3 loop hybrid peptide in chimpanzees was dramatically reduced by the addition of the gp41 fusogenic domain to the hybrid peptide. This was hypothesized to be the result of the HIV gp41 fusion domain having a immunoregulatory function it in vivo, that results in primate immune hyporesponsiveness to otherwise immunogenic peptides. On p. 506

[He1992] X. M. He, F. Ruker, E. Casale, \& D. C. Carter. Structure of a human monoclonal antibody Fab fragment against gp41 of human immunodeficiency virus type 1. Proc Natl Acad Sci USA 89:7154-7158, 1992. On p. 706

[He2002] Y. He, W. J. Honnen, C. P. Krachmarov, M. Burkhart, S. C. Kayman, J. Corvalan, \& A. Pinter. Efficient isolation of novel human monoclonal antibodies with neutralizing activity against HIV-1 from transgenic mice expressing human Ig loci. J Immunol 169(1):595-605, 2002

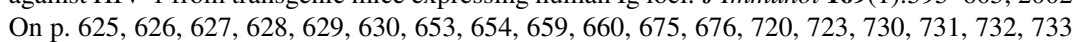

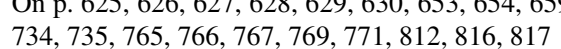

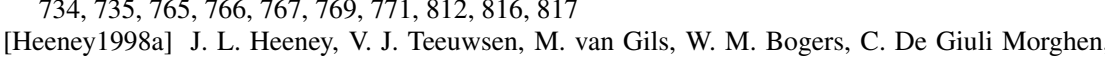
A. Radaelli, S. Barnett, B. Morein, L. Akerblom, Y. Wang, T. Lehner, \& D. Davis. Betachemokines and neutralizing antibody titers correlate with sterilizing immunity generated in HIV 1 vaccinated macaques. Proc Natl Acad Sci U S A 95(18):10803-8, 1998. On p.746

[Heeney1998b] J. L. Heeney, M. E. van Gils, P. van der Meide, C. de Giuli Morghen, C. Ghioni, M. Gimelli, A. Raddelli, D. Davis, L. Akerblom, \& B. Morein. The role of type-1 and type-2 T-helper immune responses in HIV-1 vaccine protection. J Med Primatol 27:50-8, 1998. On p. 525

[Heeney1999] J. Heeney, L. Akerblom, S. Barnett, W. Bogers, D. Davis, D. Fuller, G. Koopman, T. Lehner, P. Mooij, B. Morein, C. de Giuli Morghen, B. Rosenwirth, E. Verschoor, R. Wagner, \& H. Wolf. HIV-1 vaccine-induced immune responses which correlate with protection from SHIV infection: compiled preclinical efficacy data from trials with ten different HIV-1 vaccine candidates. Immunol Lett 66(1-3):189-95, 1999. On p. 443] 526

[Heeney2002] J. L. Heeney. The critical role of CD4+ T-cell help in immunity to HIV. Vaccine 20(15):1961-1963, 2002. On p. 536

[Henderson1997] B. R. Henderson \& P. Percipalle. Interactions between HIV Rev and nuclear import and export factors: the Rev nuclear localisation signal mediates specific binding to human importin-beta. J Mol Biol 274:693-707, 1997. On p.612 613

[Hernandez2000] M. Hernandez, L. Pozo, I. Gomez, \& A. Melchor. Chimeric synthetic peptide as antigen for immunodiagnosis of HIV-1 infection [In Process Citation]. Biochem Biophys Res Commun 272:259-62, 2000. On p. 689 700

[Herrera2003] C. Herrera, C. Spenlehauer, M. S. Fung, D. R. Burton, S. Beddows, \& J. P. Moore Nonneutralizing antibodies to the CD4-binding site on the gp120 subunit of human immunodefic- iency virus type 1 do not interfere with the activity of a neutralizing antibody against the same \begin{tabular}{ll|l|l|l|l|l|l|l|} 
site. J Virol 77(2):1084-1091, 2003. On p.760 & 781 & 782 & 784 & 791 & 822 & 827 \\
\hline
\end{tabular}

[Hewer2002] R. Hewer \& D. Meyer. Producing a highly immunogenic synthetic vaccine construct active against HIV-1 subtype C. Vaccine 20(21-22):2680-2683, 2002. On p.820

[Hickling1990] J. K. Hickling, C. M. Fenton, K. Howl, S. G. Marsh, \& J. B. Rothbard. Peptides recognized by class I restricted $\mathrm{T}$ cells also bind to MHC class II molecules. International Immunology 2:435-441, 1990. Peptides shown to be presented in the context of MHC class I protein by mouse or human CD8+ T lymphocytes could also bind to HLA-DR molecules on the surface of B lymphoblastoid cell lines (B-LCL). Four out of five class I-restricted T cell determinants bound, including the HIV-1 gp120 epitope. On p. 303

Hifumi2000] E. Hifumi, Y. Okamoto, \& T. Uda. How and why 41S-2 antibody subunits acquire the ability to catalyze decomposition of the conserved sequence of gp41 of HIV-1 [In Proces Citation]. Appl Biochem Biotechnol 83:209-19; discussion 219-20, 297-313, 2000. On p.720

Hill1997] C. M. Hill, H. Deng, D. Unutmaz, V. N. Kewalramani, L. Bastiani, M. K. Gorny, S. ZollaPazner, \& D. R. Littman. Envelope glycoproteins from human immunodeficiency virus types 1 and 2 and simian immunodeficiency virus can use human CCR5 as a coreceptor for viral entry and make direct CD4-dependent interactions with this chemokine receptor. J Virol 71:6296-6304, 1997. On p. 647 692 720 721

[Hinkula1990] J. Hinkula, J. Rosen, V.-A. Sundqvist, T. Stigbrand, \& B. Wahren. Epitope mapping of the HIV-1 gag region with monoclonal antibodies. Mol Immunol 27:395-403, 1990. Localiza-

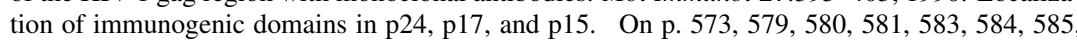
587

[Hinkula1994] J. Hinkula, G. Bratt, G. Gilljam, S. Nordlund, P. Broliden, V. Holmberg, E. Olausson-Hansson, J. Albert, E. Sandstrom, \& B. Wahren. Immunological and virological interaction in patients receiving passive immunotherapy with HIV-1 neutralizing monoclonal antibodies. J AIDS 7:940-951, 1994. Eleven HIV-1 infected patients in the late stages were treated with mouse MAbs that showed high HIV-1 neutralization. The amount of p24 in the sera of patients decreased in five patients, but remained the same or increased in six of them. The level of viral RNA in the plasma of patients decreased in four, showed no changes in another four and increased in the other three. By themselves, the MAbs did not appear to be efficient enough to decrease the virus burden in a permanent form in late-stage HIV patients. On p. 662

[Hinkula1997] J. Hinkula, C. Svanholm, S. Schwartz, P. Lundholm, M. Brytting, G. Engstrom, R. Benthin, H. Glaser, G. Sutter, B. Kohleisen, V. Erfle, K. Okuda, H. Wigzell, \& B. Wahren Recognition of prominent viral epitopes induced by immunization with human immunodeficiency virus type 1 regulatory genes. J Virol 71(7):5528-5539, 1997. On p.461 462 464530531 532533

Hioe1997a] C. Hioe, S. Burda, P. Chigurupati, S. Xu, \& S. Zolla-Pazner. Resting cell neutralization assay for HIV-1 primary isolates. Methods: A companion to Methods in Enzymology 12:300-5, 1997. A technique is described for detecting the activity of neutralizing polyclonal or MAbs against HIV-1 primary isolates, using unstimulated PBMC as the target cell. On p.720 721,768 842

Hioe1997b] C. E. Hioe, S. Xu, P. Chigurupati, S. Burda, C. Williams, M. K. Gorny, \& S. ZollaPazner. Neutralization of HIV-1 primary isolates by polyclonal and monoclonal human antibod\begin{tabular}{ll|l|l|l|l|l|l|l|l|l|l|l|l|} 
ies. Int Immunol 9(9):1281-1290, 1997. On p. 647 & 653 & 654 & 655 & 659 & 660 & 664 & 691 & 692 & 696 \\
\hline
\end{tabular} \begin{tabular}{l|l|l|l|l|l|l|l|l|l|l|l|l|l|l|l|l|l|l|l|}
\hline 700 & 708 & 720 & 721 & 768 & 769 & 770 & 771 & 828 & 830 \\
\hline
\end{tabular}

Hioe 1999] C. E. Hioe, J. E. Hildreth, \& S. Zolla-Pazner. Enhanced HIV type 1 neutralization by human anti-glycoprotein 120 monoclonal antibodies in the presence of monoclonal antibodies to lymphocyte function-associated molecule 1. AIDS Res Hum Retroviruses 15:523-31, 1999. On

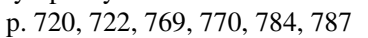

[Hioe2000] C. E. Hioe, G. J. Jones, A. D. Rees, S. Ratto-Kim, D. Birx, C. Munz, M. K. Gorny, 
M. Tuen, \& S. Zolla-Pazner. Anti-CD4-binding domain antibodies complexed with HIV type 1 glycoprotein 120 inhibit CD4+ T cell-proliferative responses to glycoprotein 120 [In Process Citation]. AIDS Res Hum Retroviruses 16:893-905, 2000. On p.628,635 664, 665, 691, 720 \begin{tabular}{l|l|l|l|l|l|l|l|}
7222 & 760 & 761 & 768 & 769 & 770 & 771 & 772 \\
\hline
\end{tabular}

Hioe2001] C. E. Hioe, M. Tuen, P. C. Chien, Jr., G. Jones, S. Ratto-Kim, P. J. Norris, W. J. Moretto, D. F. Nixon, M. K. Gorny, \& S. Zolla-Pazner. Inhibition of human immunodeficiency virus type 1 gp120 presentation to CD4 T cells by antibodies specific for the CD4 binding domain of gp120. J Virol 75(22):10950-7, 2001. On p. 691 768 769|770

[Hladik2001] F. Hladik, S. Bender, R. E. Akridge, Y. Hu, C. Galloway, D. Francis, \& M. J. McElrath. Recombinant HIV-1 glycoprotein 120 induces distinct types of delayed hypersensitivity in persons with or without pre-existing immunologic memory. J Immunol 166(5):3580-3588, 2001 On p. 527

[Ho1991a] D. D. Ho, M. S. C. Fung, Y. Cao, X. L. Li, C. Sun, T. W. Chang, \& N.-C. Sun. Anothe discontinuous epitope on glycoprotein gp120 that is important in human immunodeficiency virus type 1 neutralization is identified by a monoclonal antibody. Proc Natl Acad Sci USA 88:8949 8952 , 1991. A description of the neutralizing murine MAb G3-4. Evidence suggested that the G3-4 epitope was discontinuous, but later studies showed marginal peptide binding in the V2 region. On $\mathrm{p} .632$

[Ho1991b] D. D. Ho, J. A. McKeating, X. L. Li, T. Moudgil, E. S. Daar, N.-C. Sun, \& J. E. Robinson. Conformational epitope of gp120 important in CD4 binding and human immunodeficiency virus type 1 neutralization identified by a human monoclonal antibody. J Virol 65:489-493, 1991 A description of the neutralizing human MAb 15e. It binds to HIV-1 with a broad specificity, and blocks gp120 binding to CD4, and is a discontinuous epitope; DTT reduction of env abrogate binding. On p. 680683762764

Ho1992] D. D. Ho, M. S. C. Fung, H. Yoshiyama, Y. Cao, \& J. E. Robinson. Discontinuous epitopes on gp120 important in HIV-1 neutralization. AIDS Res Hum Retroviruses 8:1337-1339, 1992. Further description of the human MAb 15e and the murine MAb G3-4. gp120 mutants that affect 15 e epitope binding: $113,257,368,370,421,427,475$; four of these coincide with amino acids important for the CD4 binding domain. G3-4 is neutralizing and behaves like a discontinuous epitope, and partially blocks sCD4 binding. On p. 632 762 764

[Ho2002] J. Ho, K. S. MacDonald, \& B. H. Barber. Construction of recombinant targeting immunogens incorporating an HIV-1 neutralizing epitope into sites of differing conformational constraint Vaccine 20(7-8): 1169-1180, 2002. On p.710 716

[Hochleitner2000a] E. O. Hochleitner, C. Borchers, C. Parker, R. J. Bienstock, \& K. B. Tomer. Characterization of a discontinuous epitope of the human immunodeficiency virus (HIV) core protein p24 by epitope excision and differential chemical modification followed by mass spectrometric peptide mapping analysis. Protein Sci 9:487-96, 2000. On p.589

[Hochleitner2000b] E. O. Hochleitner, M. K. Gorny, S. Zolla-Pazner, \& K. B. Tomer. Mass spectrometric characterization of a discontinuous epitope of the HIV envelope protein HIV-gp120 recognized by the human monoclonal antibody 1331A. J Immunol 164:4156-61, 2000. On p. 689

Hoffman1999] T. L. Hoffman, C. C. LaBranche, W. Zhang, G. Canziani, J. Robinson, I. Chaiken, J. A. Hoxie, \& R. W. Doms. Stable exposure of the coreceptor-binding site in a CD4-independen HIV-1 envelope protein. Proc Natl Acad Sci U S A 96(11):6359-64, 1999. On p.652 798799 803804

[Hofmann-Lehmann2001] R. Hofmann-Lehmann, J. Vlasak, R. A. Rasmussen, B. A. Smith, T. W. Baba, V. Liska, F. Ferrantelli, D. C. Montefiori, H. M. McClure, D. C. Anderson, B. J. Bernacky, T. A. Rizvi, R. Schmidt, L. R. Hill, M. E. Keeling, H. Katinger, G. Stiegler, L. A. Cavacini, M. R. Posner, T. C. Chou, J. Andersen, \& R. M. Ruprecht. Postnatal passive immunization of neonata macaques with a triple combination of human monoclonal antibodies against oral simian-human
Hohne1993] W. E. Hohne, G. Kuttner, S. Kiessig, G. Housdorf, R. Grunow, K. Winkler, H. Wessner, E. Giessmann, R. Stieger, J. Schneider-Mergener, R. von Baehr, \& D. Schomburg. Structura base of the interaction of a monoclonal antibody against p24 of HIV-1 with its peptide epitope Mol Immunol 30:1213-1221, 1993. On p.576

[Horner2001] A. A. Horner, G. F. Widhopf, J. A. Burger, K. Takabayashi, N. Cinman, A. Ronaghy, H. L. Spiegelberg, \& E. Raz. Immunostimulatory DNA inhibits IL-4-dependent IgE synthesis by human B cells. J Allergy Clin Immunol 108(3):417-423, 2001. On p. 299

[Hosmalin1990] A. Hosmalin, M. Clerici, R. Houghten, C. D. Pendleton, C. Flexner, D. R. Lucey, B. Moss, R. N. Germain, G. M. Shearer, \& J. A. Berzofsky. An epitope in human immunodeficiency virus 1 reverse transcriptase recognized by both mouse and human cytotoxic $\mathrm{T}$ lymphocytes. Proc Natl Acad Sci USA 87:2344-2348, 1990. On p. 180

Hosmalin1991] A. Hosmalin, P. L. Nara, M. Zweig, M. W. Lerche, K. B. Cease, E. A. Gard, P. D. Markham, S. D. Putney, M. D. Daniel, R. C. Desrosiers, \& J. A. Berzofsky. Priming with T-helper cell epitope peptides enhances the antibody response to the envelope glycoprotein of HIV-1 in Primates. J Immunol 146:1667-1673, 1991. Notes: Induction of T-cell help in rhesu monkeys it Macaca mulatta by priming with peptides T2 or TH4.1 enhances antibody response to a subsequent suboptimal gp160 immunization. T1 alone failed to elicit a response in these experiments. Env epitopes: T1, T2, TH4.1. On p. 471 520

Hossain2001] M. S. Hossain, H. Tomiyama, T. Inagawa, B. Sriwanthana, S. Oka, \& M. Takiguchi. HLA-A*3303-restricted cytotoxic T lymphocyte recognition for novel epitopes derived from the highly variable region of the HIV-1 Env protein. AIDS 15(16):2199-2201, 2001. On p. 318327

[Huang 1997] X. Huang, J. J. Barchi, Jr., F. D. Lung, P. P. Roller, P. L. Nara, J. Muschik, \& R. R. Garrity. Glycosylation affects both the three-dimensional structure and antibody binding properties of the HIV-1IIIB GP120 peptide. Biochemistry 36:10846-56, 1997. Glycosylated analogues of the V3 loop of gp120 were studied using NMR and circular dichroism spectroscopies, and by $\mathrm{AB}$ binding properties to MAb $0.5 \beta$. A 24-residue peptide from the HIV-1 IIIB isolate (residue 308-331) designated RP135, was glycosylated with both N- and O- linked sugars. On p. 671 672

Huang2000] X. L. Huang, Z. Fan, C. Kalinyak, J. W. Mellors, \& C. R. Rinaldo. CD8(+) T cell gamma interferon production specific for human immunodeficiency virus type 1 (HIV-1) in HIV1-infected subjects. Clin Diagn Lab Immunol 7:279-87, 2000. On p. 74 $102|132| 210 \mid 279$

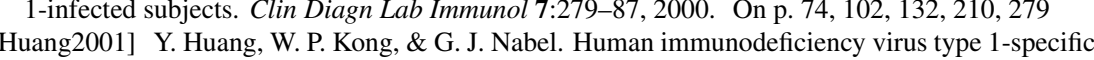
immunity after genetic immunization is enhanced by modification of Gag and Pol expression. $J$ Virol 75(10):4947-51, 2001. On p. 169244

Hunt1990] J. C. Hunt, S. M. Desai, J. M. Casey, T. J. Bolling, T. K. Leung, R. H. Decker, S. G. Devare, \& V. Sarin. Mouse monoclonal antibody 5-21-3 recognizes a contiguous, conformationdependent epitope and maps to a hydrophilic region in HIV-1 gp41. AIDS Res Hum Retroviruses 6:587-98, 1990. On p.707

Hunziker1998] I. P. Hunziker, A. Cerny, \& W. J. Pichler. Who is right? Or, how to judge the disagreement about HLA restriction of Nef peptides. AIDS Res Hum Retroviruses 14:921-4, 1998. On p. 352393

[Igarashi1999] T. Igarashi, C. Brown, A. Azadegan, N. Haigwood, D. Dimitrov, M. A. Martin, \& R. Shibata. Human Immunodeficiency Virus Type 1 Neutralizing Antibodies Accelerate Clearance of Cell-Free Virions from Blood Plasma. Nat Med 5(2):211-216, 1999. On p.752

[Ihata1999] A. Ihata, S. Watabe, S. Sasaki, A. Shirai, J. Fukushima, K. Hamajima, J. Inoue, \& K. Okuda. Immunomodulatory effect of a plasmid expressing CD40 ligand on DNA vaccination against human immunodeficiency virus type-1. Immunology 98:436-42, 1999. On p. 265495 [Ikeda-Moore1997] Y. Ikeda-Moore, H. Tomiyama, K. Miwa, S. Oka, A. Iwamoto, Y. Kaneko, \& 
M. Takiguchi. Identification and characterization of multiple HLA-A24-restricted HIV-1 CTL epitopes: strong epitopes are derived from V regions of HIV-1. J Immunology 159:6242-6252, \begin{tabular}{l|l|l|l|l|l|l|l|l|l|} 
1997. On p. 96 & 131 & 233 & 291 & 303 & 313 & 314 & 318 & 320 & 382 \\
\hline
\end{tabular}

[Ikeda-Moore1998] Y. Ikeda-Moore, H. Tomiyama, M. Ibe, S. Oka, K. Miwa, Y. Kaneko, \& M. Takiguchi. Identification of a novel HLA-A24-restricted cytotoxic T-lymphocyte epitope derived from HIV-1 Gag protein. AIDS 12:2073-4, 1998. On p.69

[Imami2002a] N. Imami, G. Hardy, A. Pires, C. Burton, J. Pido-Lopez, C. Mela, \& F. Gotch. Immune reconstitution in HIV-1-infected patients. Curr Opin Investig Drugs 3(8):1138-1145, 2002. On p.535

[Imami2002b] N. Imami, A. Pires, G. Hardy, J. Wilson, B. Gazzard, \& F. Gotch. A balanced type $1 /$ type 2 response is associated with long-term nonprogressive human immunodeficiency viru type 1 infection. $J$ Virol 76(18):9011-9023, 2002. On p. 81 446 528 534

[Inouye1998] P. Inouye, E. Cherry, M. Hsu, S. Zolla-Pazner, \& M. A. Wainberg. Neutralizing antibodies directed against the $\mathrm{V} 3$ loop select for different escape variants in a virus with mutated reverse transcriptase (M184V) than in wild-type human immunodeficiency virus type 1. AIDS Re Hum Retroviruses 14:735-40, 1998. The M184V substitution in RT yields high level resistance to 3TC and low level resistance to ddI and ddC, and alters the properties of RT. Virus containing the wt form of RT grown in the presence of the MAb 447-D develops 447-D resistance in 36 days, with the GPGR to GPGK substitutions (AGA(R) to AAA(K)). 447-D resistance took longer to acquire in virus with the M184V substituted RT, and had the form CTRPN to CTRPY (AAC(N) to $\mathrm{TAC}(\mathrm{Y})$ ) at position 5 of the V3 loop. On p. 720 721

[Iroegbu2000] J. Iroegbu, M. Birk, U. Lazdina, A. Sonnerborg, \& M. Sallberg. Variability and immunogenicity of human immunodeficiency virus type 1 p 24 gene quasispecies. Clin Diag Lab Immunol 7(3):377-83, 2000. On p. 169

[Ishii1997] N. Ishii, J. Fukushima, T. Kaneko, E. Okada, K. Tani, S. I. Tanaka, K. Hamajima, K. Q. Xin, S. Kawamoto, W. Koff, K. Nishioka, T. Yasuda, \& K. Okuda. Cationic liposomes are strong adjuvant for a DNA vaccine of human immunodeficiency virus type 1. AIDS Res Hum Retroviruses 13:1421-8, 1997. On p. 265 341

[Ishii2001] N. Ishii, Y. Sugita, L. J. Liu, S. Watabe, S. Toda, K. Q. Xin, \& K. Okuda. Immunologi characterization of HIV-specific DNA vaccine. J Investig Dermatol Symp Proc 6(1):76-80, 2001 On p. 338

[Ishikawa1999] T. Ishikawa, N. Okui, N. Kobayashi, R. Sakuma, T. Kitamura, \& Y. Kitamura Monoclonal antibodies against the minimal DNA-Binding domain in the carboxy-terminal region of human immunodeficiency virus type 1 Integrase. J Virol 73:4475-80, 1999. On p. 602 603 604

[Ishioka1999] G. Y. Ishioka, J. Fikes, G. Hermanson, B. Livingston, C. Crimi, M. Qin, M. F. del Guercio, C. Oseroff, C. Dahlberg, J. Alexander, R. W. Chesnut, \& A. Sette. Utilization of MHC class I transgenic mice for development of minigene. J Immunol 162:3915-25, 1999. On p. 222 270278

[Islam2001] S. A. Islam, C. M. Hay, K. E. Hartman, S. He, A. K. Shea, A. K. Trocha, M. J. Dynan, N. Reshamwala, S. P. Buchbinder, N. O. Basgoz, \& S. A. Kalams. Persistence of human immunodeficiency virus type 1-specific cytotoxic T-lymphocyte clones in a subject with rapid disease progression. J Virol 75(10):4907-11, 2001. On p. 86 [89 312 329

[Jackson1999] N. A. Jackson, M. Levi, B. Wahren, \& N. J. Dimmock. Properties and mechanism of action of a 17 amino acid, V3 loop-specific microantibody that binds to and neutralizes human immunodeficiency virus type 1 virions. J Gen Virol 80(Pt 1):225-36, 1999. On p. 661, 784, 787 [Jacobson1998] J. M. Jacobson. Passive immunization for the treatment of HIV infection. Mt Sinai J Med 65:22-6, 1998. Review. On p. 646662

[Jagodzinski1996] P. P. Jagodzinski, J. Wustner, D. Kmieciak, T. J. Wasik, A. Fertala, A. L. Sieron, M. Takahashi, T. Tsuji, T. Mimura, M. S. Fung, M. K. Gorny, M. Kloczewiak, Y. Kaneko, \&
D. Kozbor. Role of the V2, V3, and CD4-binding domains of GP120 in curdlan sulfate neutralization sensitivity of HIV-1 during infection of T lymphocytes. Virology 226:217-227, 1996. On \begin{tabular}{l|l|l|l|l|l|l|l|l|l|l|l|l|l|l|} 
p. 623 & 632 & 659 & 671 & 672 & 681 & 684 & 720 & 721 & 775 & 776 \\
\hline
\end{tabular}

Jagodzinski2000] P. P. Jagodzinski \& W. H. Trzeciak. Application of monoclonal antibodies to monitor the synthesis of a glycoprotein core of envelope glycoproteins of human immunodeficiency virus (HIV-1). Biomed Pharmacother 54:50-3, 2000. On p. 671 672 681682

[Janvier1990] B. Janvier, P. Archinard, B. Mandrand, A. Goudeau, \& F. Barin. Linear B-cell epitopes of the major core protein of human immunodeficiency virus types 1 and 2. J Virol 64:42584263, 1990. On p. 576 577 581 582584

Janvier1992] B. Janvier, P. Archinard, B. Mandrand, A. Goudeau, \& F. Barin. Linear B-cell epitopes of the major core protein of human immunodeficiency virus types 1 and 2 (Author's Correction). J Virol 66:613, 1992. On p. 576 577 581 582 584

[Janvier1996] B. Janvier, J. J. Lasarte, P. Sarobe, J. Hoebeke, A. B.-B. F. Borras-Cuesta, \& F. Barin. B-cell epitopes of HIV type 1 p24 capsid protein: a reassessment. AIDS Res Hum Retroviruses 12:519-525, 1996. The reactivity pattern of 45 overlapping synthetic pentadecapeptides, spanning amino acids 133 to 363 (p24) of HIV-1 p55 gag precursor, using sera from 20 HIV-1 positive and 8 HIV negative individuals was determined by ELISA. A peptide covering aa 178-192 was recognized by 40 covering $288-302$ of p 55 by 45 aa $272-322$ of p 55 was recognized by most human sera. A conformational epitope involving sequences from aa 183-186 and 289-292 was proposed, based by analogy to the structure of the Mengovirus VP2 protein. On p. 582

[Jardetzky1991] T. S. Jardetzky, W. S. Lane, R. A. Robinson, D. R. Madden, \& D. C. Wiley. Identification of self peptides bound to purified HLA-B27. Nature 353:326-9, 1991. A pool of endogenous peptides bound to the human class I MHC molecule, HLA-B27, has been isolated. Microsequence analysis of the pool and of 11 HPLC-purified peptides provides information on the binding specificity of the HLA-B27 molecule. The peptides all seem to be nonamers, seven of which match to protein sequences in a database search. These self peptides derive from abundant cytosolic or nuclear proteins, such as histone, ribosomal proteins, and members of the $90 \mathrm{~K}$ heat-shock protein family. On p. 137,300

[Jassoy 1992] C. Jassoy, R. P. Johnson, B. A. Navia, J. Worth, \& B. D. Walker. Detection of a vigorous HIV-1 specific cytotoxic T lymphocyte response in cerebrospinal fluid from infected person with AIDS dementia complex. J Immunol 149:3113-3119, 1992. CTL clones derived from CSF of individuals with AIDS dementia. HIV-1 specific CTL were detected in CSF from 5 out of 6 patients who were suffering from HIV-1 associated cognitive/motor complex disturbances. On \begin{tabular}{l|l|l|l} 
patients who were & \\
p. 60 & 309 & 376
\end{tabular}

[Jassoy1993] C. Jassoy, T. Harrer, T. Rosenthal, B. A. Navia, J. Worth, R. P. Johnson, \& B. D Walker. Human immunodeficiency virus type 1 -specific cytotoxic $\mathrm{T}$ lymphocytes release gamma interferon, tumor necrosis factor alpha (TNF-alpha), and TNF-beta when they encounter their target antigens. $J$ Virol 67:2844-2852, 1993. In this study the ability of HIV-1-specific CTL clones derived from seropositive persons to release gamma interferon (IFN- $\gamma$ ), tumor necrosis factor alpha (TNF- $\alpha$ ), and TNF- $\beta$ upon contact with target cells presenting viral antigen wa assessed. Epitopes: p17: KIRLRPGGKKKYKLKHIVWASRELE, A3; gp41: VERYLKDQQL, B14 and A28, ERYLKDQQL, B14; RT: AIFQSSMTKILEPFRKQNPDIVIYQ, A11; and Nef SQRRQDILDLWIYHTQGYFPDWQNY, B13. On p. 60 $194,309,311,372$

Jeffs 1996] S. A. Jeffs, J. McKeating, S. Lewis, H. Craft, D. Biram, P. E. Stephens, \& R. L. Brady. Antigenicity of truncated forms of the human immunodeficiency virus type 1 envelope glycopro\begin{tabular}{|l|l|l|l|l|l|l|l|l|l|l|l|} 
tein. J Gen Virol 77:1403-1410, 1996. On p. 640 & 668 & 671 & 672 & 680 & 684 & 760 & 766 & 768 & 769 \\
\hline
\end{tabular} 783.820

[Jeffs2001] S. A. Jeffs, M. K. Gorny, C. Williams, K. Revesz, B. Volsky, S. Burda, X. H. Wang J. Bandres, S. Zolla-Pazner, \& H. Holmes. Characterization of human monoclonal antibodies selected with a hypervariable loop-deleted recombinant HIV-1(IIIB) gp120. Immunol Lett 
79(3):209-13, 2001. On p. 761. 762

[Jelonek1999] M. Jelonek, J. Maskrey, K. Steimer, B. Potts, K. Higgins, \& M. Kellor Materna monoclonal antibody to the V3 loop alters specificity of the response to a human immunodeficiency virus vaccine. J Inf Dis 174:866-9, 1999. On p.661

[Jensen1997] T. H. Jensen, A. Jensen, A. M. Szilvay, \& J. Kjems. Probing the structure of HIV-1 Rev by protein footprinting of multiple monoclonal antibody-binding sites. FEBS Lett 414:50 4, 1997. Rev was mapped using MAb protein footprinting, which gave results that agreed well with peptide mapping, but was useful for identifying a discontinuous interaction between two regions. Footprints supported a previously proposed structure (Auer et al., Biochemistry, 33 (1994) 2988-2996) predicting that a helix-loop-helix motif in Rev brings the termini of the protein into proximity. On p. 610 612613

[Jeyarajah1998] S. Jeyarajah, C. E. Parker, M. T. Summer, \& K. B. Tomer. Matrix-assisted lase desorption ionization/mass spectrometry mapping of human immunodeficiency virus-gp120 epitopes recognized by a limited polyclonal antibody. J Am Soc Mass Spectrom 9:157-65, 1998. On p. 692

[Jiang1998] S. Jiang, K. Lin, \& M. Lu. A conformation-specific monoclonal antibody reacting with fusion-active gp41 from the human immunodeficiency virus type 1 envelope glycoprotein. $J$ Viro 72:10213-7, 1998. MAb NC-1 specifically recognizes the fusogenic core of gp41, which allows for analysis of CD4-induced conformational changes in gp120 and gp41 as well as identification of mediators for HIV-1 fusion. On p. 7107712834

[Jin1998a] X. Jin, C. G. Roberts, D. F. Nixon, Y. Cao, D. D. Ho, B. D. Walker, M. Muldoon, B. T. Korber, \& R. A. Koup. Longitudinal and cross-sectional analysis of cytotoxic T lymphocyt responses and their relationship to vertical human immunodeficiency virus transmission. ARIEL Project Investigators. J Infect Dis 178:1317-26, 1998. On p.241]335 396

[Jin1998b] X. Jin, M. Wills, J. G. Sissons, \& A. Carmichael. Progressive loss of IL-2-expandable HIV-1-specific cytotoxic T lymphocytes during asymptomatic HIV infection. Eur J Immunol 28:3564-76, 1998. On p.282 317,320

[Jin2000a] X. Jin, G. Ogg, S. Bonhoeffer, J. Safrit, M. Vesanen, D. Bauer, D. Chen, Y. Cao, M. A Demoitie, L. Zhang, M. Markowitz, D. Nixon, A. McMichael, \& D. D. Ho. An antigenic threshol for maintaining human immunodeficiency virus type 1-specific cytotoxic T lymphocytes. Mol Med 6(9):803-9, 2000. On p. 79 165 213 241 336

[Jin2000b] X. Jin, C. G. Roberts, D. F. Nixon, J. T. Safrit, L. Q. Zhang, Y. X. Huang, N. Bhardwaj, B. Jesdale, A. S. DeGroot, \& R. A. Koup. Identification of subdominant cytotoxic T lymphocyte epitopes encoded by autologous HIV type 1 sequences, using dendritic cell stimulation an computer-driven algorithm. AIDS Res Hum Retroviruses 16:67-76, 2000. On p.66 112 276389

[Jin2002] X. Jin, X. Gao, M. Ramanathan, Jr., G. R. Deschenes, G. W. Nelson, S. J. O’Brien, J. J. Goedert, D. D. Ho, T. R. O'Brien, \& M. Carrington. Human immunodeficiency virus type 1 (HIV-1)-specific CD8+-T-cell responses for groups of HIV-1-infected individuals with differen HLA-B*35 genotypes. J Virol 76(24):12603-12610, 2002. On p. 168 244 340,398

[Johnson1991] R. P. Johnson, A. Trocha, L. Yang, G. P. Mazzara, D. L. Panicali, T. M. Buchanan, \& B. D. Walker. HIV-1 gag-specific cytotoxic T lymphocytes recognize multiple highly conserved epitopes. Fine specificity of the gag-specific response defined by using unstimulated peripheral blood mononuclear cells and cloned effector cells. J Immunol 147:1512 1521, 1991. This study presented a detailed study of gag-specific CTL from HIV-1 seropositive individuals. Seven p24 and two p17 epitopes were described, that were recognized by class I-restricted CD3+CD8+ CTL. p17 epitopes: KIRLRPGGKKKYKLKHIVWASRELE and QTGSEELRSLYNTVATLYCVHQRIE; p24 epitopes: NPPIPVGEIYKRWIILGLNKIV, VHQAISPRTLNAWVKVVEEKAF, NAWVKVVEEKAFSPEVIPMFSA, SALSEGATPQDL NTMLNTVGGH, GHQAAMQMLKETINEEAAEWDR, and RAEQASQEVK. On p.61] 71
[Johnson 1992] R. P. Johnson, A. Trocha, T. M. Buchanan, \& B. D. Walker. Identification of overlapping HLA class I-restricted cytotoxic T cell epitopes in a conserved region of the human immunodeficiency virus type 1 envelope glycoprotein: definition of minimum epitopes and analysis of the effects of sequence variation. $J$ Exp Med 175:961-971, 1992. Fine mapping and mutational analysis of gp41 epitopes: ERYLKDQQL, HLA B14 and YLKDQQLL, HLA B8. On p. 115, 311 316

Johnson1993] R. P. Johnson, A. Trocha, T. M. Buchanan, \& B. D. Walker. Recognition of a highly conserved region of human immunodeficiency virus type $1 \mathrm{gp} 120$ by an HLA-Cw4-restricted cytotoxic T-lymphocyte clone. J Virol 67:438-445, 1993. The epitope sequence FNCGGEFF stimulates CTL response; the natural variants FNCRGEFF (SF2), TNCRGEFL (ROD) and LNCGGEFF (NDK) do not serve as epitopes. This was the first report of an HIV antigen specific target cells restricted by an HLA-C molecule, $\mathrm{Cw} 4$. On p. 301$] 302$

[Johnson1994a] R. P. Johnson, S. A. Hammond, A. Trocha, R. F. Siliciano, \& B. D. Walker. Epitope specificity of MHC restricted cytotoxic T lymphocytes induced by candidate HIV-1 vaccine. AIDS Research and Hum Retroviruses 10, Supp 2:S73-S75, 1994. Volunteers were immunized with recombinant vaccinia virus expressing HIV-1 gp160 (vac-env) and boosted with recombinan gp160 (rgp 160). CTL clones were analyzed for HLA restriction and specificity. An immunodominant HLA-A3.1 restricted epitope was observed that showed very little sequence variation amon B subtype sequences, (TVYYGVPVWK). Naturally occurring variants of this peptide were able to stimulate reactivity. Two additional CD8+ CTL epitopes from vaccinees were characterized, as well as two CD4+ CTL epitopes. On p. 268 271 280 2800 317

Johnson1994b] R. P. Johnson, S. A. Hammond, A. Trocha, R. F. Siliciano, \& B. D. Walker. Induction of a major histocompatibility complex class I-restricted cytotoxic T-lymphocyte response to a highly conserved region of human immunodeficiency virus type 1 (HIV-1) gp120 in seronegative humans immunized with a candidate HIV-1 vaccine. J Virol 68:3145-3153, 1994. In two volunteers, immunization with a single strain of HIV-1 induced CD4+ and CD8+ CTL that are specific for multiple conserved regions of HIV-1 and would be expected to recognize a broad range of viral isolates. The immunodominant gp120 epitope, gp120 TVYYGVPVWK, elicited CD8 HLA-A3.1 restricted CTL, and this epitope is highly conserved. CTL specific for this epitope could lyse target cells sensitized with all known natural sequence variants. Additionally, CD8+ HLA-B35 and CD8+ HLA-B18 restricted epitopes were defined as well as two CD4+ cytotoxic T-cell gp120 epitopes: ITQACPKVSFEPIPHYCAPAGFAI and NNTLKQIDSKLREQFG. On p. $269\left[\begin{array}{l|l|l|l|l|}280 & 300 & 317 \\ \hline\end{array}\right.$

[Johnson 1994c] R. P. Johnson \& B. D. Walker. CTL in HIV-1 infection: Responses to structura proteins. Curr Topics Microbiol Immunol 189:35-63, 1994. Review. On p.224

[Johnson2002] W. E. Johnson \& R. C. Desrosiers. Viral persistance: HIV's strategies of immune system evasion. Annu Rev Med 53:499-518, 2002. On p. 400

Johnston2001] M. I. Johnston \& J. Flores. Progress in HIV vaccine development. Curr Opin Pharmacol 1(5):504-510, 2001. On p. 401

[Jones1999] G. J. Jones, P. von Hoegen, J. Weber, \& A. D. Rees. Immunization with human immunodeficiency virus type 1 rgp120W61D in QS21/MPL adjuvant primes T cell proliferation and C-C chemokine production to multiple epitopes within variable and conserved domains of gp120W61D. J Infect Dis 179:558-66, 1999. On p.470 475 477 483 511

[Joyce2002] J. G. Joyce, W. M. Hurni, M. J. Bogusky, V. M. Garsky, X. Liang, M. P. Citron, R. C Danzeisen, M. D. Miller, J. W. Shiver, \& P. M. Keller. Enhancement of alpha -helicity in the HIV-1 inhibitory peptide DP178 leads to an increased affinity for human monoclonal antibody 2F5 but does not elicit neutralizing responses in vitro: Implications for vaccine design. J Biol Chem 277(48):45811-45820, 2002. On p.710 716 717

[Jubier-Maurin1999] V. Jubier-Maurin, S. Saragosti, J. L. Perret, E. Mpoudi, E. Esu-Williams, 
C. Mulanga, F. Liegeois, M. Ekwalanga, E. Delaporte, \& M. Peeters. Genetic characterization of the nef gene from human immunodeficiency virus type 1 group $\mathrm{M}$ strains representing genetic subtypes A, B, C, E, F, G, and H. AIDS Res Hum Retroviruses 15:23-32, 1999. On p. 348 366 374381

[Kahn2000] J. O. Kahn, D. W. Cherng, K. Mayer, H. Murray, \& S. Lagakos. Evaluation of HIV-1 immunogen, an immunologic modifier, administered to patients infected with HIV having 300 to 549 x 10e6/L CD4 cell counts: A randomized controlled trial. JAMA 284(17):2193-2202, 2000. On p. 535

[Kalams1994] S. Kalams, R. P. Johnson, A. K. Trocha, M. J. Dynan, H. S. Ngo, R. T. D’Aquila J. T. Kurnick, \& B. D. Walker. Longitudinal analysis of T cell receptor (TCR) gene usage by HIV-1 envelope-specific cytotoxic T-lymphocyte clones reveals a limited TCR repertoire. $J$ Exp Med 179:1261-1271, 1994. This paper presents an in-depth longitudinal study of T-cell recepto gene usage to a well defined HLA B14 restricted gp41 epitope. Ten CTL clones were derived from a single individual over 31 months. T-cell receptor V-D-J sequencing was performed on PCR amplification products. All ten clones utilized V $\alpha 14$ and V $\beta 4$ genes; observed limited T cell receptor diversity to an immunodominant epitope was suggested to facilitate immune escape gp41 epitope: ERYLKDQQL. An HLA B14 restricted RT epitope from this individual used V $\alpha 2$ and $\mathrm{V} \beta 14$, showing use of these genes was not a feature of all HLA B14 restricted clones from this individual. RT epitope: AIYLALQDSGLEVNIVTDSQYALGI. On p.230 311

[Kalams1996] S. A. Kalams, R. P. Johnson, M. J. Dynan, K. E. Hartman, T. Harrer, E. Harrer, A. K. Trocha, W. A. Blattner, S. P. Buchbinder, \& B. D. Walker. T cell receptor usage an fine specificity of human immunodeficiency virus type 1 specific cytotoxic $\mathrm{T}$ lymphocyte clones analysis of quasispecies recognition reveals a dominant response directed against a minor in vivo variant. J Exp Med 183:1699-1679, 1996. On p.311

[Kalams1998] S. A. Kalams \& B. D. Walker. The critical need for CD4 help in maintaining effective cytotoxic T lymphocyte responses. J Exp Med 188(12):2199-2204, 1998. On p.444

[Kalams1999a] S. A. Kalams, S. P. Buchbinder, E. S. Rosenberg, J. M. Billingsley, D. S. Colbert, N. G. Jones, A. K. Shea, A. K. Trocha, \& B. D. Walker. Association between virus-specific cytotoxic T-lymphocyte and helper responses in human immunodeficiency virus type 1 infection. J Virol 73:6715-20, 1999. On p. 162 444 527

[Kalams1999b] S. A. Kalams, P. J. Goulder, A. K. Shea, N. G. Jones, A. K. Trocha, G. S. Ogg, \& B. D. Walker. Levels of human immunodeficiency virus type 1-specific cytotoxic T- lymphocyte effector and memory responses decline after suppression of viremia with highly active antiretroviral therapy. J Virol 73:6721-8, 1999. On p. 77,310

[Kalland1994a] K. H. Kalland, A. M. Szilvay, K. A. Brokstad, W. Saetrevik, \& G. Haukenes. The human immunodeficiency virus type $1 \mathrm{Rev}$ protein shuttles between the cytoplasm and nuclea compartments. Mol Cell Biol 14:7436-7444, 1994. Ten anti-Nef MAbs were generated and mapped. Nef is expressed in two isomorphic forms, and was shown to be expressed mainly in the Golgi complex and at the nuclear membrane, but occasionally $\mathrm{x}$ in the nucleus, particularly in MT-4 cells. On $\mathrm{p}$ 612 613

[Kalland1994b] K. H. Kalland, A. M. Szilvay, E. Langhoff, \& G. Haukenes. Subcellular distribution of human immunodeficiency virus type 1 Rev and colocalization of Rev with RNA splicing factor in a speckled pattern in the nucleoplasm. $J$ Virol 68:1475-1485, 1994. On p.612

[Kaminchik1990] J. Kaminchik, N. Bashan, D. Pinchasi, B. Amit, N. Sarver, M. I. Johnston, M. Fischer, Z. Yavin, M. Gorecki, \& A. Panet. Expression and biochemical characterization of human immunodeficiency virus type 1 nef gene product. J Virol 64:3447-3454, 1990. On p. 840 841

[Kaneko2000] H. Kaneko, I. Bednarek, A. Wierzbicki, I. Kiszka, M. Dmochowski, T. J. Wasik,

Y. Kaneko, \& D. Kozbor. Oral DNA vaccination promotes mucosal and systemic immune responses to HIV envelope glycoprotein. Virology 267:8-16, 2000. On p.341

[Kang1994] C.-Y. Kang, K. Hariharan, P. L. Nara, J. Sodroski, \& J. P. Moore. Immunization with a soluble CD4-gp120 complex preferentially induces neutralizing anti-human immunodeficiency virus type 1 antibodies directed to conformation-dependent epitopes of gp120. $J$ Virol 68:5854 5862, 1994. Most of the MAbs generated in this study were conformational, but there were four that bound a V3 loop peptide. These four could neutralize lab strains with different efficiencies. These MAbs were very sensitive to substitutions in the V3 loop, but also to substitutions in the base of the V1/V2 loop structure (120/121 VK/LE), indicating an underlying conformational character. Additionally, many anti-CD4 binding site MAbs were described, that shared a sensitivity to substitutions at residues 368 and 370. Another class of MAbs was found that appeared to be conformationally sensitive, and shared a reduction in binding with the amino acid substitution $88 \mathrm{~N} / \mathrm{P}$ in the C1 domain. On p. 643739754755793794

Kang1999] C. Y. Kang, L. Luo, M. A. Wainberg, \& Y. Li. Development of HIV/AIDS vaccine using chimeric gag-env virus-like particles. Biol Chem 380:353-64, 1999. On p.286 [Karwowska1992a] S. Karwowska, M. K. Gorny, A. Buchbinder, V. Gianakakos, C. Williams, T. Fuerst, \& S. Zolla-Pazner. Production of human monoclonal antibodies specific for conformational and linear non-V3 epitopes of gp120. AIDS Res Hum Retroviruses 8:1099-1106, 1992 A single linear MAb was generated, to the immunodominant domain in the C-terminal portion of gp120. This antibody did not inhibit rCD4-rgp120 binding or neutralize IIIB or MN. Three conformational epitope binding MAbs were also described in this paper that could neutralize IIIB and MN. On p. 691 766, 768,769

[Karwowska1992b] S. Karwowska, M. K. Gorny, A. Buchbinder, \& S. Zolla-Pazner. Type-specific human monoclonal antibodies cross-react with the V3-loop of various HIV-1 isolates. Vaccines 92 pp. 171-174, 1992. Editors: F. Brown, H. S. Ginsberg and R. Lerner, Cold Spring Harbor \begin{tabular}{ll|l|l|l|l|l|l|l|} 
Laboratory Press, Cold Spring Harbor, NY. On p. 647 & 659 & 664 & 665 & 666 & 669 & 691 & 720 \\
\hline
\end{tabular}

[Karwowska1993] S. Karwowska, M. K. Gorny, S. Culpepper, S. Burda, S. Laal, K. Samanich, \& S. Zolla-Pazner. The similarities and diversity among human monoclonal antibodies to the CD4binding domain of HIV-1. Vaccines 93 pp. 229-232, 1993. Editors: F. Brown, H. Ginsberg and R. Lerner, Cold Spring Harbor Laboratory Press, Cold Spring Harbor, NY. 447-D was the most broadly cross-reactive anti-V3 human MAb tested. On p. 769

Kato2000] H. Kato, H. Bukawa, E. Hagiwara, K. Q. Xin, K. Hamajima, S. Kawamoto, M. Sugiyama, M. Sugiyama, E. Noda, M. Nishizaki, \& K. Okuda. Rectal and vaginal immunization with a macromolecular multicomponent peptide vaccine candidate for HIV-1 infection induces HIV-specific protective immune responses. Vaccine 18:1151-60, 2000. On p. 341

Kaul1999] R. Kaul, D. Trabattoni, J. J. Bwayo, D. Arienti, A. Zagliani, F. M. Mwangi, C. Kariuki, E. N. Ngugi, K. S. MacDonald, T. B. Ball, M. Clerici, \& F. A. Plummer. HIV-1-specific mucosal IgA in a cohort of HIV-1-resistant Kenyan sex workers. AIDS 13(1):23-9, 1999. On p. 472493 507, 521,641

Kaul2000] R. Kaul, F. A. Plummer, J. Kimani, T. Dong, P. Kiama, T. Rostron, E. Njagi, K. S MacDonald, J. J. Bwayo, A. J. McMichael, \& S. L. Rowland-Jones. HIV-1-specific mucosal CD8+ lymphocyte responses in the cervix of HIV-1- resistant prostitutes in Nairobi. J Immunol

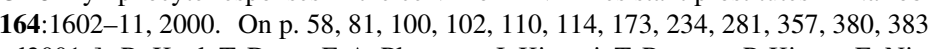

Kaul2001a] R. Kaul, T. Dong, F. A. Plummer, J. Kimani, T. Rostron, P. Kiama, E. Njagi, E. Irungu, B. Farah, J. Oyugi, R. Chakraborty, K. S. MacDonald, J. J. Bwayo, A. McMichael, \& S. L. Rowland-Jones. CD8(+) lymphocytes respond to different HIV epitopes in seronegative and

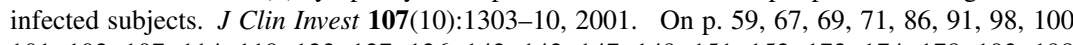
\begin{tabular}{|l|l|l|l|l|l|l|l|l|l|l|l|l|l|l|l|l|l|l|l|}
01 & 102 & 107 & 114 & 119 & 123 & 127 & 136 & 142 & 143 & 147 & 149 & 151 & 152 & 172 & 174 & 178 & 193 & 198 \\
\hline
\end{tabular}

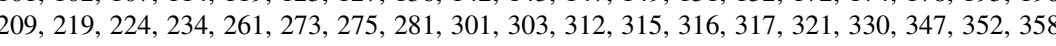

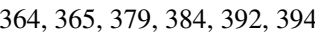

Kaul2001b] R. Kaul, F. Plummer, M. Clerici, M. Bomsel, L. Lopalco, \& K. Broliden. Mucosal IgA in exposed, uninfected subjects: evidence for a role in protection against HIV infection. AIDS 15(3):431-2, 2001. On p.745 
[Kaul2001c] R. Kaul, S. L. Rowland-Jones, J. Kimani, T. Dong, H. B. Yang, P. Kiama, T. Rostron, E. Njagi, J. J. Bwayo, K. S. MacDonald, A. J. McMichael, \& F. A. Plummer. Late seroconversion in HIV-resistant nairobi prostitutes despite pre-existing HIV-specific CD8+ responses. J Clin \begin{tabular}{ll|l|l|l|l|l|l|l|l|l|l|l|l|l|l|l|l|l|l|l|} 
Invest 107(3): 341-9, 2001. On p. 68 & 98 & 104 & 114 & 121 & 125 & 141 & 148 & 173 & 194 & 197 & 218 & 234 \\
\hline
\end{tabular} $315,346,377,385,392$

Kaul2002] R. Kaul, S. L. Rowland-Jones, G. Gillespie, J. Kimani, T. Dong, P. Kiama, J. N. Simonsen, J. J. Bwayo, A. J. McMichael, \& F. A. Plummer. Gonococcal cervicitis is associated with reduced systemic CD8+ T cell responses in human immunodeficiency virus type 1-infected an exposed, uninfected sex workers. J Infect Dis 185(10):1525-1529, 2002. On p. 88 $\mid 98$ |107||115

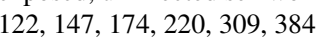

[Kawamura2002] M. Kawamura, T. Naito, M. Ueno, T. Akagi, K. Hiraishi, I. Takai, M. Makino, T. Serizawa, K. Sugimura, M. Akashi, \& M. Baba. Induction of mucosal IgA following intravaginal administration of inactivated HIV-1-capturing nanospheres in mice. J Med Virol 66(3):291298, 2002. On p. 819

[Kawana-Tachikawa2002] A. Kawana-Tachikawa, M. Tomizawa, J.-i. Nunoya, T. Shioda, A. Kato, E. E. Nakayama, T. Nakamura, Y. Nagai, \& A. Iwamoto. An efficient and versatile mammalian viral vector system for major histocompatibility complex class I/peptide complexes. J Viro 76(23):11982-11988, 2002. On p. 314 382

[Kawana1999] A. Kawana, H. Tomiyama, M. Takiguchi, T. Shioda, T. Nakamura, \& A. Iwamoto. Accumulation of specific amino acid substitutions in HLA-B35-restricted human immunodeficiency virus type 1 cytotoxic T lymphocyte epitopes. AIDS Res Hum Retroviruses 15:1099-107,

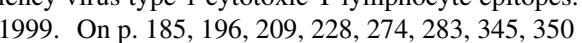

[Kelleher1998a] A. D. Kelleher, M. Roggensack, S. Emery, A. Carr, M. A. French, \& D. A. Cooper Effects of IL-2 therapy in asymptomatic HIV-infected individuals on proliferative responses to mitogens, recall antigens and HIV-related antigens. Clin Exp Immunol 113:85-91, 1998. On p. 442525

[Kelleher1998b] A. D. Kelleher, M. Roggensack, A. B. Jaramillo, D. E. Smith, A. Walker, I. Gow, M. McMurchie, J. Harris, G. Patou, \& D. A. Cooper. Safety and immunogenicity of a candidate therapeutic vaccine, $\mathrm{p} 24$ virus-like particle, combined with zidovudine, in asymptomatic subjects. Community HIV Research Network Investigators. AIDS 12:175-82, 1998. On p. 441 516

[Kelleher2001a] A. D. Kelleher, B. L. Booth, Jr., A. K. Sewell, A. Oxenius, V. Cerundolo, A. J. McMichael, R. E. Phillips, \& D. A. Price. Effects of retroviral protease inhibitors on proteasome function and processing of HIV-derived MHC class I-restricted cytotoxic T lymphocyte epitopes. AIDS Res Hum Retroviruses 17(11):1063-1066, 2001. On p. 87] 137, 202 220

[Kelleher2001b] A. D. Kelleher, C. Long, E. C. Holmes, R. L. Allen, J. Wilson, C. Conlon, C. Workman, S. Shaunak, K. Olson, P. Goulder, C. Brander, G. Ogg, J. S. Sullivan, W. Dyer, I. Jones, A. J. McMichael, S. Rowland-Jones, \& R. E. Phillips. Clustered mutations in HIV-1 gag are consistently required for escape from HLA-B27-restricted cytotoxic T lymphocyte responses. $J$ Exp Med 193(3):375-86, 2001. On p. 133

[Keller1993] P. M. Keller, B. A. Arnold, A. R. Shaw, R. L. Tolman, F. V. Middlesworth, S. Bondy, V. K. Rusiecki, S. Koenig, S. Zolla-Pazner, P. Conard, E. A. Emini, \& A. J. Conley. Identification of HIV vaccine candidate peptides by screening random phage epitope libraries. Virology 193:709-716, 1993. A library of 15 mers was screened for reactivity with 447-52D. 100s of 15 mers reacted, of which 70 were sequenced. All but one contained the motif GPXR. On p.720 721

[Keller1999] M. Keller \& Y. Arora. Inhibition of anti-V3 loop response to a recombinant gp120 SF2 vaccine be preexisting monoclonal Ab. AIDS Res and Human Retroviruses 15:855-60, 1999. On p. 661

Kent1997a] S. J. Kent, P. D. Greenberg, M. C. Hoffman, R. E. Akridge, \& M. J. McElrath. Antagonism of vaccine-induced HIV-1-specific CD4+ T cells by primary HIV-1 infection: potential mechanism of vaccine failure. J Immunol 158:807-15, 1997. A vaccinia-gp160 vaccinee made strong CD4+ T cell responses, including proliferative and cytolytic responses, but was infected anyway. The infecting virus had an escape mutant that could also serve as an antagonist. On p. 309

Kent1997b] S. J. Kent, A. Woodward, \& A. Zhao. Human immunodeficiency virus type 1 (HIV 1)-specific T cell responses correlate with control of acute HIV-1 infection in macaques. $J$ Infec Dis 176:1188-97, 1997. On p. 333524

Kent1998] S. J. Kent, A. Zhao, S. J. Best, J. D. Chandler, D. B. Boyle, \& I. A. Ramshaw. Enhanced $\mathrm{T}$ cell immunogenicity and protective efficacy of a human immunodeficiency virus type 1 vaccine regimen consisting of consecutive priming with DNA and boosting with recombinant fowlpox virus. J Virol 72:10180-8, 1998. On p. 164334442525

Kent2000] S. J. Kent, A. Zhao, C. J. Dale, S. Land, D. B. Boyle, \& I. A. Ramshaw. A recombinan avipoxvirus HIV-1 vaccine expressing interferon-gamma is safe and immunogenic in macaques. Vaccine 18:2250-6, 2000. On p. 171

Kessler II1997] J. A. Kessler II, P. M. McKenna, E. A. Emini, C. P. Chan, M. D. Patel, S. K. Gupta G. E. Mark III, C. F. Barbas III, D. R. Burton, \& A. J. Conley. Recombinant human monoclona antibody IgG1b12 neutralizes diverse human immunodeficiency virus type 1 primary isolates. AIDS Res Hum Retroviruses 13:575-82, 1997. Anti-CD4 binding domain antibodies generally do not neutralize primary HIV-1 isolates, with the exception of IgG1b12. Many primary isolates were shown to be neutralized by IgG1b12, including several non-B clade international isolates. Neutralization of a primary isolate with MAb IgG1b12 did not require continuous exposure to the antibody. A complete IgG1 molecule of a selected b12 FAb mutant with a $>400$-fold increase in affinity was assembled and evaluated in the infectivity reduction assay in comparative studies with the parent IgG1b12 antibody. The mutant did not retain the level of primary isolate neutralization potency of IgG1b12, despite the increase in affinity for gp120. On p. $710|711| 784 \mid 785$

[Kessler1995] J. A. Kessler, II, P. M. McKenna, E. A. Emini, \& A. J. Conley. In vitro assessmen of the therapeutic potential of anti-HIV-1 monoclonal neutralizing antibodies. Gen Meet Am Soc Microbiol 95:586, T-25, 1995. Aidsline: 96050622 Abstract. On p.710 $711|772| 784$

Khouri1995] Y. F. Khouri, K. McIntosh, L. Cavacini, M. Posner, M. Pagano, R. Tuomala, \& W. A Marasco. Vertical Transmission of HIV-1. Correlation with maternal viral load and plasma levels of CD4 binding site anti-gp120 antibodies. J Clin Invest 95:732-737, 1995. Differences in levels of Abs directed against the monomeric gp120 and against the V3 loop region of gp120 were not significantly different between transmitting and non-transmitting mothers. Differences were observed in the levels of CD4 binding site antibodies, as determined by the ability of diluted maternal plasma to inhibit binding of the CD4 binding site monoclonal antibody F105 (MAb F105) to monomeric gp120. On p.775 776

[Kim1997a] D. T. Kim, D. J. Mitchell, D. G. Brockstedt, L. Fong, G. P. Nolan, C. G. Fathman, E. G. Engleman, \& J. B. Rothbard. Introduction of soluble proteins into the MHC class I pathway by conjugation to an HIV tat peptide. J Immunol 159(4):1666-1668, 1997. A vaccine based on the conjugation of OVA to an HIV Tat peptide that enhances protein uptake by APC cells stimulated MHC Class I-restricted T cell response in vitro and CTL generation in vivo in a murine system. On p. 258

Kim1997b] J. J. Kim, V. Ayyavoo, M. L. Bagarazzi, M. Chattergoon, J. D Boyer, B. Wang, \& D. B. Weiner. Development of a multicomponent candidate vaccine for HIV-1. Vaccine 15:879 83, 1997. On p. 457, 607, 609 747

Kim1997c] J. J. Kim, V. Ayyavoo, M. L. Bagarazzi, M. A. Chattergoon, K. Dang, B. Wang, J. D Boyer, \& D. B. Weiner. In vivo engineering of a cellular immune response by coadministration of IL-12 expression vector with a DNA immunogen. J Immunol 158:816-26, 1997. On p.240 249333

[Kim1997d] J. J. Kim, M. L. Bagarazzi, N. Trivedi, Y. Hu, K. Kazahaya, D. M. Wilson, R. Cic- 
carelli, M. A. Chattergoon, K. Dang, S. Mahalingam, A. A. Chalian, M. G. Agadjanyan, J. D. Boyer, B. Wang, \& D. B. Weiner. Engineering of in vivo immune responses to DNA immunization via codelivery of costimulatory molecule genes. Nat Biotechnol 15:641-6, 1997. On p. 171 333457524

[Kim1998] J. J. Kim, L. K. Nottingham, D. M. Wilson, M. L. Bagarazzi, A. Tsai, L. D. Morrison, A. Javadian, A. A. Chalian, M. G. Agadjanyan, \& D. B. Weiner. Engineering DNA vaccines via co-delivery of co-stimulatory molecule genes. Vaccine 16:1828-35, 1998. On p. 164240335 443457526

[Kim2000] J. J. Kim, J. S. Yang, L. Montaner, D. J. Lee, A. A. Chalian, \& D. B. Weiner. Coimmunization with IFN-gamma or IL-2, but not IL-13 or IL-4 cDNA can enhance Th1-type DNA vaccine-induced immune responses in vivo. J Interferon Cytokine Res 20(3):311-9, 2000. O p. 458.529

[Kim2001] J. H. Kim, J. R. Mascola, S. Ratto-Kim, T. C. VanCott, L. Loomis-Price, J. H. Cox N. L. Michael, L. Jagodzinski, C. Hawkes, D. Mayers, B. L. Gilliam, D. C. Birx, \& M. L. Robb. Selective increases in HIV-specific neutralizing antibody and partial reconstitution of cellula immune responses during prolonged, successful drug therapy of HIV infection. AIDS Res Hum Retroviruses 17(11):1021-34, 2001. On p. 745

[Kimura2002] T. Kimura, K. Yoshimura, K. Nishihara, Y. Maeda, S. Matsumi, A. Koito, \& S. Matsushita. Reconstitution of Spontaneous Neutralizing Antibody Response against Autologous Human Immunodeficiency Virus during Highly Active Antiretroviral Therapy. J Infect Dis 185(1):53-60, 2002. On p. 669 672 751

[Kiszka2002] I. Kiszka, D. Kmieciak, J. Gzyl, T. Naito, E. Bolesta, A. Sieron, S. P. Singh, A. Srini vasan, G. Trinchieri, Y. Kaneko, \& D. Kozbor. Effect of the V3 loop deletion of envelope glycoprotein on cellular responses and protection against challenge with recombinant vaccinia virus expressing gp 160 of primary human immunodeficiency virus type 1 isolates. J Virol 76(9):42224232, 2002. On p. 277, 283 287 292,325

[Kitamura1999] Y. Kitamura, T. Ishikawa, N. Okui, N. Kobayashi, T. Kanda, T. Shimada, K. Miyake, \& K. Yoshiike. Inhibition of replication of HIV-1 at both early and late stages of the viral life cycle by single-chain antibody against viral integrase. J Acquir Immune Defic Syndr Hum Retrovirol 20:105-14, 1999. On p. 603

[Klasse1991] P. J. Klasse, R. Pipkorn, \& J. Blomberg. A cluster of continuous antigenic structure in the transmembrane protein of HIV-1: Individual patterns of reactivity in human sera. Mol Immunol 28:613-622, 1991. On p.700

[Klasse1993a] P. Klasse, J. A. McKeating, M. Schutten, M. S. Reitz, Jr., \& M. Robert-Guroff. An immune-selected point mutation in the transmembrane protein of human immunodeficiency viru type 1 (HXB2-Env:Ala 582( $\rightarrow$ Thr)) decreases viral neutralization by monoclonal antibodies to the CD4-binding site. Virology 196:332-337, 1993. On p.668, 671,710,775,776 781,783 820

[Klasse1993b] P. J. Klasse, R. Pipkorn, J. Blomberg, K.-Y. Han, B. Hilton, \& J. A. Ferretti. Threedimensional structure and antigenicity of transmembrane-protein peptides of the human immunodeficiency virus type 1. FEBS Letters 323:68-72, 1993. On p. 694

[Klasse1996] P. J. Klasse \& Q. J. Sattentau. Altered CD4 interactions of HIV type 1 LAI variants selected for the capacity to induce membrane fusion in the presence of a monoclonal antibody to domain 2 of CD4. AIDS Res Hum Retroviruses 12:1015-1021, 1996. On p. 696 697|783

[Klasse2002] P. J. Klasse \& Q. J. Sattentau. Occupancy and Mechanism in Antibody-Mediated Neutralization of Animal Viruses. J Gen Virol 83(9):2091-2108, 2002. On p.784]791

[Klein1997] M. R. Klein, J. Veenstra, A. M. Holwerda, M. T. Roos, I. Gow, G. Patou, R. A. Coutinho, F. De Wolf, \& F. Miedema. Gag-specific immune responses after immunization with p17/p24:Ty virus-like particles in HIV type 1-seropositive individuals. AIDS Res Hum Retroviruses 13(5):393-9, 1997. Gag-specific immune responses and changes in HIV-1 RNA levels were evaluated in eight HIV-1-infected persons, in order to assess the immunotherapeutic potential HIV-1 p17/p24: Ty virus-like particles (p24- VLP). All treated subjects showed transient and dose-dependent proliferative responses to the Ty-VLP carrier (stimulation index (SI), 2.0-119.5). Three of four individuals who received either 500 or 1,000 micrograms of p24-VLP also showed proliferative responses to $\mathrm{p} 17$ or $\mathrm{p} 24$ (SI, 2.0-15.7). In 2 subjects who were treated with either 500 or 1,000 micrograms of p24-VLP, enhanced Gag-specific CTL precursor (CTLp) frequencies were observed after immunization (10- to 14-fold). Both subjects had low baseline Gagspecific CTL activity $(<25 \mathrm{CTLp} / 10(6)$ PBMCs). In the other participants studied no significant boosting of preexisting Gag-specific CTL responses was observed. Short-term elevation of HIV-1 RNA levels at weeks 2 and 4 was observed in two subjects treated with the highest dose of p24VLP. However, HIV-1 RNA levels at week 24 did not significantly differ from those found in the placebo group. In conclusion, p24-VLP induced marginal Gag-specific immune responses in limited numbers of HIV-1-seropositive individuals, with some showing transient elevation of HIV-1 viral load. Further studies are needed to establish potential clinical effects of these observations.

$\begin{array}{rl}\text { On p. } 162 & 441 \\ \text { Klein } 1998] & 445 \\ \text { M. R. Klein, S. H. van der Burg, E. Hovenkamp, A. M. Holwerda, J. W. Drijfhout, C. J. }\end{array}$ Melief, \& F. Miedema. Characterization of HLA-B57-restricted human immunodeficiency virus type 1 Gag- and RT-specific cytotoxic T lymphocyte responses. J Gen Virol 79(Pt 9):2191-201, 1998. On p. 99 124 205225

Klenerman1994] P. Klenerman, S. Rowland-Jones, S. McAdam, J. Edwards, S. Daenke, D. Lalloo, B. Koppe, W. Rosenberg, D. Boyd, A. Edwards, P. Giangrande, R. E. Phillips, \& A. J. McMichael. Cytotoxic T cell activity antagonized by naturally occurring HIV-1 Gag variants. Nature 369:403-407, 1994. This paper documents that naturally occurring peptide variants can serve as antagonists, that is they can inhibit normal lysis of cells presenting the original epitope. The variants studied could serve as antagonists when they were processed from recombinant vaccinia, replicated HIV, or when they were synthetic peptides. Both agonist and antagonist sequences were found in the study subjects from whom the CTL clones were derived. On p.67 128134

Klenerman1995] P. Klenerman, U.-C. Meier, R. E. Phillips, \& A. J. McMichael. The effects of natural altered peptide ligands on the whole blood cytotoxic $\mathrm{T}$ lymphocyte response to human immunodeficiency virus. Eur J Immunol 25:1927-1931, 1995. This paper explores naturally occurring altered peptide ligands and their ability to sustain CTL, serve as antagonists to CTL specific for other variants, and to allow cell killing. The authors propose that a CTL response may be sustained in vivo that fails to recognize viral variants as they arise, proposing a mechanism for T-cell original antigenic sin. On p.67, 134,177

Klenerman1996] P. Klenerman, G. Luzzi, K. McIntyre, R. Phillips, \& A. McMichael. Identification of a novel HLA-A25 restricted epitope in a conserved region of p24 gag (positions 71-80). AIDS 10:348-350, 1996. On p. 119

Klenerman2002] P. Klenerman, Y. Wu, \& R. Phillips. HIV: Current opinion in escapology. Curr Opin Microbiol 5(4):408-413, 2002. On p.401

[Klinman1995] D. M. Klinman, B. F. Haynes, \& J. Conover. Activation of interleukin 4- and interleukin 6-secreting cells by HIV-specific synthetic peptides. AIDS Res Hum Retroviruses 11:97-105, 1995. Notes: Immunized mice activate IL-4 and IL-6 producing cells in a dose dependent manner. The $\mathrm{V} 3$ region epitope as well as the $\mathrm{T} 1$ epitope is able to activate cytokineproducing cells. The order of immunization of T1-SP10 peptides influences the magnitude and cross-reactivity of the response, where the SP10, V3 portion of the immunogen is varied. On p. 491508

[Kmieciak1998a] D. Kmieciak, I. Bednarek, M. Takiguchi, T. J. Wasik, J. Bratosiewicz, A. Wierzbicki, H. Teppler, J. Pientka, S. H. Hsu, Y. Kaneko, \& D. Kozbor. The effect of epitope variation on the profile of cytotoxic T lymphocyte responses to the HIV envelope glycoprotein. 
Int Immunol 10:1789-99, 1998. On p. 275 276$] 320 \mid 326$

[Kmieciak1998b] D. Kmieciak, T. J. Wasik, H. Teppler, J. Pientka, S. H. Hsu, H. Takahashi, K. Okumura, Y. Kaneko, \& D. Kozbor. The effect of deletion of the V3 loop of gp120 on cytotoxic T cell responses and HIV gp120-mediated pathogenesis. J Immunol 160:5676-83, 1998. On p.277 293326

[Kmieciak2001] D. Kmieciak, E. Bolesta, T. Naito, J. Gzyl, Y. Kaneko, \& D. Kozbor. Enhancemen of cellular and humoral immune responses to human immunodeficiency virus type $1 \mathrm{Gag}$ and $\mathrm{Po}$ by a G/P-92 fusion protein expressing highly immunogenic Gag p17/p24 and Pol p51 antigens. $J$ Hum Virol 4(6):306-316, 2001. On p. 88.221

[Koenig1990] S. Koenig, T. R. Fuerst, L. V. Wood, R. M. Woods, J. A. Suzich, G. M. Jones, V. F de la Cruz, R. T. Davey, Jr., S. Venkatesan, B. Moss, W. E. Biddison, \& A. S. Fauci. Mappin the fine specificity of a cytotoxic T cell response to HIV-1 Nef protein. J Immunol 145:127-135, 1990. A 10 residue peptide that triggers CTL in association with the HLA A3.1 molecule was studied. Human cell transfectants were used to map a critical residue in the HLA A3.1 molecule for recognition, amino acid 152, which is present on the alpha- 2 helix in HLA-A3.1 and is modified in the HLA A3.2 A3 allele. On p. 351.

[Koenig1995] S. Koenig, A. J. Conley, Y. A. Brewah, G. M. Jones, S. Leath, L. J. Boots, V. D. V, G. Pantaleo, J. F. Demarest, \& C. Carter. Transfer of HIV-1-specific cytotoxic T lymphocytes to an AIDS patient leads to selection for mutant HIV variants and subsequent disease progression. Nat Med 1(4):330-6, 1995. On p. 355

[Kolchinsky2001] P. Kolchinsky, E. Kiprilov, P. Bartley, R. Rubinstein, \& J. Sodroski. Loss of a single N-linked glycan allows CD4-independent human immunodeficiency virus type 1 infection by altering the position of the gp120 V1/V2 variable loops. J Virol 75(7):3435-43, 2001. On

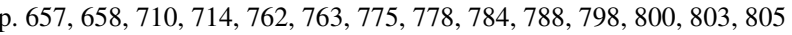

Kolowos 1999] W. Kolowos, M. Schmitt, M. Herrman, E. Harrer, P. Low, J. R. Kalden, \& T. Ha rer. Biased TCR repertoire in HIV-1-infected patients due to clonal expansion of HIV-1-revers transcriptase-specific CTL clones. J Immunol 162:7525-33, 1999. On p.216

[Konya1997] J. Konya, G. Stuber, A. Bjorndal, E. M. Fenyo, \& J. Dillner. Primary induction of human cytotoxic lymphocytes against a synthetic peptide of the human immunodeficiency virus type 1 protease. J Gen Virol 78:2217-2224, 1997. On p. 175 212

[Kostense2001] S. Kostense, G. S. Ogg, E. H. Manting, G. Gillespie, J. Joling, K. Vandenberghe, E. Z. Veenhof, D. van Baarle, S. Jurriaans, M. R. Klein, \& F. Miedema. High viral burden in the presence of major HIV-specific CD8(+) T cell expansions: evidence for impaired CTL effecto function. Eur J Immunol 31(3):677-86, 2001. On p. 85 $106,131,206$ 218 368

[Koup1991] R. A. Koup, J. E. Robinson, Q. V. Nguyen, C. A. Pikora, B. Blais, A. Roskey, D. Panicali, \& J. L. Sullivan. Antibody-dependent cell-mediated cytotoxicity directed by a human monoclonal antibody reactive with gp120 of HIV-1. AIDS 5:1309-1314, 1991. On p.762

[Kropelin1998] M. Kropelin, C. Susal, V. Daniel, \& G. Opelz. Inhibition of HIV-1 rgp120 binding to $\mathrm{CD} 4+\mathrm{T}$ cells by monoclonal antibodies directed against the gp $120 \mathrm{C} 1$ or $\mathrm{C} 4$ region. Immunol Lett 63:19-25, 1998. On p.624 680 681 775 777 784 787

[Krowka1990] J. Krowka, D. Stites, R. Debs, C. Larsen, J. Fedor, E. Brunette, \& N. Duzgunes. Lymphocyte proliferative responses to soluble and liposome-conjugated envelope peptides of HIVJ Immunol 144:2535-2540, 1990. Notes: Conjugation of HIV peptides or proteins to liposomes and stimulation with rIL-2 may enhance cell-mediated responses to peptides. gp120 epitopes: QIVKKLREQFGNNK, FRPGGGDMRDNWRSEL. On p.502 512

Kuhn2001] L. Kuhn, A. Coutsoudis, D. Moodley, D. Trabattoni, N. Mngqundaniso, G. M. Sheare M. Clerici, H. M. Coovadia, \& Z. Stein. T-helper cell responses to HIV envelope peptides in cor blood: protection against intrapartum and breast-feeding transmission. AIDS 15(1):1-9, 200 On p.472 493507521

[Kuhn2002] L. Kuhn, S. Meddows-Taylor, G. Gray, \& C. Tiemessen. Human immunodeficiency virus (HIV)-specific cellular immune responses in newborns exposed to HIV in utero. Clin Infect \begin{tabular}{|l|l|l|l|l|l|l|l|} 
Dis 34(2):267-276, 2002. On p. 166 & 241 & 242 & 259 & 337 & 397 & 401 & 535 \\
\hline
\end{tabular}

Kuiken1999] C. L. Kuiken, B. T. Foley, E. Guzman, \& B. T. M. Korber. Determinants of HIV-1 protein evolution. In K. A. Crandall, ed., The Evolution of HIV, chapter 13, pp. 432-68. The John Hopkins University Press, Baltimore, 1999. On p. 164396

[Kundu1998a] S. K. Kundu, M. Dupuis, A. Sette, E. Celis, F. Dorner, M. Eibl, \& T. C. Merigan Role of preimmunization virus sequences in cellular immunity in HIV- infected patients durin HIV type 1 MN recombinant gp160 immunization. AIDS Res Hum Retroviruses 14:1669-78,

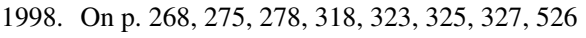

[Kundu1998b] S. K. Kundu, E. Engleman, C. Benike, M. H. Shapero, M. Dupuis, W. C. van Schooten, M. Eibl, \& T. C. Merigan. A pilot clinical trial of HIV antigen-pulsed allogeneic an autologous dendritic cell therapy in HIV-infected patients. AIDS Res Hum Retroviruses 14:55160, 1998. On p. 83 [183 216 237 277 324.

Kunert1998] R. Kunert, F Ruker \& H. Katinger. Molecular characterization of five neutralizing anti-HIV type 1 antibodies: identification of nonconventional D segments in the human monoclonal antibodies 2G12 and 2F5. AIDS Res Hum Retroviruses 14:1115-28, 1998. Study identifies five human MAbs which were able to neturalize primary isolates of different clades in vitro and reports the nucleotide and amino acid sequences of the heavy and light chain $\mathrm{V}$ segments of the

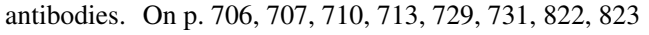

[Kunert2000] R. Kunert, W. Steinfellner, M. Purtscher, A. Assadian, \& H. Katinger. Stable recombinant expression of the anti HIV-1 monoclonal antibody $2 \mathrm{~F} 5$ after IgG3/IgG1 subclass switch in CHO cells. Biotechnol Bioeng 67:97-103, 2000. On p.710 714

Kunert2002] R. E. Kunert, R. Weik, B. Ferko, G. Stiegler, \& H. Katinger. Anti-idiotypic antibody $\mathrm{Ab} 2 / 3 \mathrm{H} 6$ mimics the epitope of the neutralizing anti-HIV-1 monoclonal antibody $2 \mathrm{~F} 5$. AIDS 16(4):667-668, 2002. On p.710 717

Kurane1998] I. Kurane \& K. West. Personal communication, 1998. On p. 98,116

[Kusakabe2000] K. Kusakabe, K. Xin, H. Katoh, K. Sumino, E. Hagiwara, S. Kawamoto, K. Okuda, K. Miyagi, I. Aoki, K. Nishioka, D. Klinman, \& K. Okuda. The timing of GM-CSF expression plasmid administration influences the Th1/Th2 response induced by an HIV-1-specific DNA vaccine. J Immunol 64(6):3102-11, 2000. On p. 495

[Kusk1988] P. Kusk, K. Ulrich, J. Zeuthen, \& G. Pallesen. Immunological characterization and detection of the major core protein $\mathrm{p} 24$ of the human immunodeficiency virus (HIV) using monoclonal antibodies. J AIDS 1:326-332, 1988. On p. 575, 583

Kusk1992] P. Kusk, T. H. Bugge, B. O. Lindhardt, E. F. Hulgaard, \& K. Holmback. Mapping of linear B-cell epitopes on the major core protein p24 of human immunodeficiency virus type 1. AIDS Res Hum Retroviruses 8:1789-1794, 1992. . The epitope for MAb F5-2 was found to be reactive with human sera from HIV-1 infected individuals, and reactivity to this epitope was associated with disease progression and low CD4 T-cell counts. On p.575 583

[Kuttner1992] G. Kuttner, E. Giessmann, B. Niemann, K. Winkler, R. Grunow, J. Hinkula, J. Rosen, B. Wahren, \& R. von Baehr. Immunoglobulin V regions and epitope mapping of a murine monoclonal antibody against p24 core protein of HIV-1. Mol Immunol 29:561-564, 1992. The nucleotide sequence of the $\mathrm{VDJ}_{H}$ and $\mathrm{VJ}_{L}$ regions of a murine MAb (CB-mab-p24/13-5) agains p24 was obtained. On p. 575

[Kwong 1998] P. D. Kwong R. Wyatt, J. Robinson, R. W. Sweet, J. Sodroski, \& W. A. Hendrickson. Structure of an HIV gp120 envelope glycoprotein in complex with the CD4 receptor and a neutralizing human antibody. Nature 393:648-659, 1998. Comment in: Nature 1998 Ju 18;393(6686):630-1. The X-ray crystal structure was solved at 2.5 A resolution of HIV-1 gp120 core complexed with human CD4 and the antigen-binding fragment of a neutralizing antibody that blocks chemokine-receptor binding. On p. 798779

[Laal1994] S. Laal, S. Burda, M. K. Gorny, S. Karwowska, A. Buchbinder, \& S. Zolla-Pazner. 
Synergistic neutralization of human immunodeficiency virus type 1 by combinations of human monoclonal antibodies. J Virol 68:4001-4008, 1994. Antibodies to the C-terminal part of gp120 and the V3 loop were shown to act synergistically with anti-CD4 binding site MAbs in terms of neutralization. C-terminal antibodies did not synergize V3 loop MAb neutralization. On p.675 \begin{tabular}{|l|l|l|l|l|l|l|l|l|l|l|l|}
6991 & 692 & 693 & 696 & 697 & 708 & 710 & 720 & 766 & 769 & 771 \\
\hline
\end{tabular}

[LaBranche1999] C. C. LaBranche, T. L. Hoffman, J. Romano, B. S. Haggarty, T. G. Edwards, T. J. Matthews, R. W. Doms, \& J. A. Hoxie. Determinants of CD4 independence for a human immunodeficiency virus type 1 variant map outside regions required for coreceptor specificity. Virol 73(12):10310-9, 1999. On p.736

[LaCasse1998] R. A. LaCasse, K. E. Follis, T. Moudgil, M. Trahey, J. M. Binley, V. Planelles, S. Zolla-Pazner, \& J. H. Nunberg. Coreceptor utilization by human immunodeficiency virus typ 1 is not a primary determinant of neutralization sensitivity. $J$ Virol 72:2491-5, 1998. A T-cell line-adapted (TCLA) derivative of SI primary isolate 168P acquired the ability to to be neutralize by anti-V3 MAbs 257-D, 268-D and 50.1. The primary isolate could use either CCR5 or CXCR4 and was not neutralized when infection was directed via either pathway, but the TCLA derivative uses CXCR4 only and is neutralized. Thus coreceptor usage is not the primary determinant of differential neutralization sensitivity in primary versus TCLA strains. On p.647 652 664

[LaCasse1999] R. A. LaCasse, K. E. Follis, M. Trahey, J. D. Scarborough, D. R. Littman, \& J. H. Nunberg. Fusion-competent vaccines: broad neutralization of primary isolates of HIV. Science 283(5400):357-62, 1999. On p.749 905

[Laisney1999] I. L. Laisney \& A. D. Strosberg. Dual specificity of a human neutralizing monoclonal antibody, specific for the V3 loop of GP120 (HIV-1). Immunol Lett 67:185-92, 1999. On p. 664 665

[Lake1989] D. Lake, T. Sugano, Y. Matsumoto, Y. Masuho, E. A. Petersen, P. Feorino, \& E. M Hersh. A Hybridoma Producing Human Monoclonal Antibody Specific for Glycoprotein 120kDa of Human Immunodeficiency Virus (HIV-1). Life Sciences 45:iii-x, 1989. On p. 729

[Lake1992] D. F. Lake, T. Kawamura, T. Tomiyama, W. E. Robinson, Jr., Y. Matsumoto, Y. Masuho, $\&$ E. M. Hersh. Generation and characterization of a human monoclonal antibody that neutralize diverse HIV-1 isolates in vitro. AIDS 6:17-24, 1992. On p.795

[Lalvani1997] A. Lalvani, T. Dong, G. Ogg, A. A. Patham, H. Newell, A. V. Hill, A. J. McMichael, \& S. Rowland-Jones. Optimization of a peptide-based protocol employing IL-7 for in vitro restimulation of human cytotoxic T lymphocyte precursors. J Immunol Methods 210:65-77, 1997. On p.76 $94[126[196,357$

[Laman1992] J. D. Laman, M. M. Schellekens, Y. H. Abacioglu, G. K. Lewis, M. Tersmette, R. A. M. Fouchier, J. P. M. Langeduk, E. Claassen, \& W. J. A. Boersma. Variant-specific monoclonal and group-specific polyclonal human immunodeficiency virus type 1 neutralizing antibodies raised with synthetic peptides from the gp120 third variable domain. J Virol 66:1823-1831, 1992. On p. 638639662663

[Laman1993] J. D. Laman, M. M. Schellekens, G. K. Lewis, J. P. Moore, T. J. Matthews, J. P. M. Langedijk, R. H. Meloen, W. J. A. Boersma, \& E. Claassen. A Hidden Region in the Thir Variable Domain of HIV-1 IIIB gp120 Identified by a Monoclonal Antibody. AIDS Res Hum Retroviruses 9:605-612, 1993. A peptide (FVTIGKIGNMRQAHC) induced MAb binds to the carboxy-terminal flank of the V3-loop, but the epitope is only exposed on gp120 when it is treated with SDS-DTT. On p. $6396062|663| 675 \mid 678$

[Lange2002] C. G. Lange, M. M. Lederman, J. S. Madero, K. Medvik, R. Asaad, C. Pacheko, C. Carranza, \& H. Valdez. Impact of suppression of viral replication by highly active antiretrovira therapy on immune function and phenotype in chronic HIV-1 infection. $J$ Acquir Immune Defic Syndr 30(1):33-40, 2002. On p. 445

[Langedijk1991] J. P. M. Langedijk, N. K. T. Back, P. J. Durda, J. Goudsmit, \& R. H. Meloen. Neutralizing activity of anti-peptide antibodies against the principal neutralization domain of human immunodeficiency virus type 1. J Gen Virol 72:2519-2526, 1991. MAbs were raised against RIQRGPGRAFVTIGK by immunizing mice. Fine structure of MAb binding sites was mapped using pepscan. Preservation of the $\beta$ turn at the tip of the loop was critical. On p.661 666667 669674

[Langedijk1992] J. P. M. Langedijk, N. K. T. Back, E. Kinney-Thomas, C. Bruck, M. Francotte, J. Goudsmit, \& R. H. Meloen. Comparison and fine mapping of both high and low neutralizing monoclonal antibodies against the principal neutralization domain of HIV-1. Arch Virol 126:129146, 1992. On p.667 668670

[Langedijk1995] J. P. M. Langedijk, G. Zwart, J. Goudsmit, \& R. H. Meloen. Fine Specificity of antibody recognition may predict amino acid substitution in the third variable region of gp120 during HIV Type 1 infection. AIDS Res Hum Retroviruses 11:1153-62, 1995. To investigate how HIV-1 escapes from recognition, a panel of V3 peptides based on sequences derived from 6 HIV-1 positive individuals was tested for reactivity with autologous sera sampled over time. The $\mathrm{V} 3$ region undergoes immune escape through mutation. On $\mathrm{p} .657$

[Lapham1996] C. Lapham, B. Golding, J. Inman, R. Blackburn, J. Manischewitz, P. Highet, \& H. Golding. Brucella abortus conjugated with a peptide derived from the V3 loop of human immunodeficiency virus (HIV) type 1 induces HIV-specific cytotoxic T cell responses in norma and in CD4+ cell-depleted BALB/c mice. J Virol 70:3084-3092, 1996. On p.297]

[Larsson1999] M. Larsson, X. Jin, B. Ramratnam, G. S. Ogg, J. Engelmayer, M. A. Demoitie, A. J. McMichael, W. I. Cox, R. M. Steinman, D. Nixon, \& N. Bhardwaj. A recombinant vaccinia viru based ELISPOT assay detects high frequencies of Pol-specific CD8 T cells in HIV-1-positive individuals. AIDS 13:767-77, 1999. On p. 77, 211

[Larsson2002a] M. Larsson, J.-F. Fonteneau, M. Lirvall, P. Haslett, J. D. Lifson, \& N. Bhardwaj Activation of HIV-1 specific CD4 and CD8 T cells by human dendritic cells: Roles for crosspresentation and non-infectious HIV-1 virus. AIDS 16(10):1319-1329, 2002. On p. 1682243

[Larsson2002b] M. Larsson, D. T. Wilkens, J.-F. Fonteneau, T. J. Beadle, M. J. Merritt, R. G. Kost, P. A. J. Haslett, S. Cu-Uvin, N. Bhardwaj, D. F. Nixon, \& B. L. Shacklett. Amplification of lowfrequency antiviral CD8 T cell responses using autologous dendritic cells. AIDS 16(2):171-180, 2002. On p. 167242338398

[Lasky 1987] L. A. Lasky, G. Nakamura, D. H. Smith, C. Fennie, C. Shimasaki, E. Patzer, P. Berman, T. Gregory, \& D. J. Capon. Delineation of a Region of the Human Immunodeficiency Virus Type 1 gp120 Glycoprotein Critical for Interaction with the CD4 Receptor. Cell 50:975-985, 1987. On p. 604679

$\begin{array}{rl}\text { On p. } 604 & 679 \\ \text { [Lauer2002] G. M. Lauer, T. N. Nguyen, C. L. Day, G. K. Robbins, T. Flynn, K. McGowan, E. S. }\end{array}$ Rosenberg, M. Lucas, P. Klenerman, R. T. Chung, \& B. D. Walker. Human immunodeficiency virus type 1-hepatitis $\mathrm{C}$ virus coinfection: Intraindividual comparison of cellular immune responses against two persistent viruses. J Virol 76(6):2817-2826, 2002. On p. 167 243$] 338$ 39

Lawson2002] V. A. Lawson, R. Oelrichs, C. Guillon, A. A. Imrie, D. A. Cooper, N. J. Deacon, \& D. A. McPhee. Adaptive Changes after Human Immunodeficiency Virus Type 1 Transmission. AIDS Res Hum Retroviruses 18(8):545-556, 2002. On p. 649650

[Layton1993] G. T. Layton, S. J. Harris, A. J. Gearing, M. Hill-Perkins, J. S. Cole, J. C. Griffiths, N. R. Burns, A. J. Kingsman, \& S. E. Adams. Induction of HIV-specific cytotoxic T lymphocytes in vivo with hybrid HIV-1 V3:Ty-virus-like particles. J Immunol 151:1097-1107, 1993. V3Ty-Virus-like particles can induce type specific CTL in mice in the absence of adjuvant. On p. 289

Le Borgne2000] S. Le Borgne, M. Fevrier, C. Callebaut, S. P. Lee, \& Y. Riviere. CD8(+)-cell antiviral factor activity is not restricted to human immunodeficiency virus (HIV)-specific T cells and can block HIV replication after initiation of reverse transcription. J Virol 74:4456-64, 2000 On p. 352 
[Leandersson2000] A. C. Leandersson, G. Gilljam, M. Fredriksson, J. Hinkula, A. Alaeus, K. Lidman, J. Albert, G. Bratt, E. Sandstrom, \& B. Wahren. Cross-reactive T-helper responses in patient infected with different subtypes of human immunodeficiency virus type 1. J Virol 74:4888-90, 2000. On p. 525

[Lebedev2000] L. R. Lebedev, L. I. Karpenko, V. A. Poryvaeva, M. S. Azaev, E. I. Riabchikova, I. P. Gileva, \& A. A. Il'ichev. [Design of virus-like particles, exposing HIV-1 epitopes]. Mol Biol (Mosk) 34(3):480-485, 2000. Article in Russian. On p. 593750

[Lee1995] C.-N. Lee, J. Robinson, G. Mazzara, Y.-L. Cheng, M. Essex, \& T.-H. Lee. Contributio of hypervariable domains to the conformation of a broadly neutralizing glycoprotein 120 epitope. AIDS Res Hum Retroviruses 11:777-781, 1995. Deletion of the V4 or V5 domains, in contrast to the V1, V2 and V3 domains of gp120, affect the broadly neutralizing epitope recognized by $1.5 \mathrm{e}$ by disturbing the overall conformation of the envelope protein. On p.762

[Lee1997] S. Lee, K. Peden, D. S. Dimitrov, C. C. Broder, J. Manischewitz, G. Denisova, J. M. Gershoni, \& H. Golding. Enhancement of human immunodeficiency virus type 1 envelope-mediated fusion by a CD4-gp120 complex-specific monoclonal antibody. J Virol 71:6037-43, 1997. On p. 803804832

Legastelois2000] I. Legastelois \& C. Desgranges. Design and intracellular activity of a huma single-chain antibody to human immunodeficiency virus type 1 conserved gp41 epitope. J Virol 74:5712-5, 2000. On p. 707

[Leggatt1997] G. R. Leggatt, M. A. Alexander-Miller, A. Kumar, S. L. Hoffman, \& J. A. Berzofsky. Cytotoxic T lymphocyte (CTL) adherence assay (CAA): a non-radioactive assay for murine CTL recognition of peptide-MHC class I complexes. J Immunol Methods 201:1-10, 1997. This pape describes a novel assay, the CTL adhesion assay (CAA), and uses an HIV epitope in a murine system as a model system. CAA is a rapid, simple screening method for identifying cytolytic epitopes for a given CTL line, and may also identify peptides that cause $\mathrm{T}$ cell activation and adherence but not cytolytic activity. Cytotoxic T lymphocytes (CTL) form an important immune surveillance system against intracellular pathogens. Here we describe a simple, visual assay for identifying peptides specifically recognized by CTL, based on the discovery that CTL develop increased adhesive properties upon TCR triggering. Several CTL lines were shown to pellet to the bottom of a round bottom 96-well plate in the absence of peptide. In contrast, these same CTL lines incubated with their cognate peptide, allowing them to present peptide to each other, adhered to the sides of the well and were readily distinguished by macroscopic visual examinatio of the plate after 4-5 h or overnight incubation. This CTL adherence assay (CAA) demonstrated peptide specificity and MHC restriction, and was titratable with peptide concentration. With thi technique, a minimal-sized, malaria CTL epitope was correctly identified from a panel of overlapping nonamers, although the adherence pattern of two mono-substituted, variant peptides was less. On $\mathrm{p} .180$

[Leggatt1998] G. R. Leggatt, A. Hosmalin, C. D. Pendleton, A. Kumar, S. Hoffman, \& J. A. Berzofsky. The importance of pairwise interactions between peptide residues in the delineation of TCR specificity. J Immunol 161:4728-35, 1998. On p. 180

[Legrand1997] E. Legrand, I. Pellegrin, D. Neau, J. L. Pellegrin, J. M. Ragnaud, M. Dupon, B. Guillemain, \& H. J. Fleury. Course of specific T lymphocyte cytotoxicity, plasma and cellular viral loads, and neutralizing antibody titers in 17 recently seroconverted HIV type 1-infected patients. AIDS Res Hum Retroviruses 13:1383-94, 1997. On p. 163333396

[Lekutis1997a] C. Lekutis \& N. L. Letvin. HIV-1 Envelope-specific CD4+ T helper cells from simian/human immunodeficiency virus-infected Rhesus monkeys recognize epitopes restricted by MHC class II DRB1*0406 and DRB*W201 molecules. J Immunol 159(4):2049-2057, 1997. On p. 487 502 514

Lekutis1997b] C Lekutis, J. W. Shiver, M. A. Liu, \& N. L. Letvin. HIV-1 Env DNA vaccine administered to rhesus monkeys elicits MHC class II-restricted CD4+ T helper cells that secrete
IFN-gamma and TNF-alpha. J Immunol 158:4471-7, 1997. The Th cell response elicited by an HIV-1 Env plasmid DNA vaccine was assessed in rhesus monkeys by isolation of gp120-specific, MHC class II- restricted CD4+ T cell lines from PBL of vaccinated animals. The Env- specific $\mathrm{CD} 4+\mathrm{T}$ cell lines recognized epitopes located in the second hypervariable region and in the carboxyl terminus of HIV-1 gp120. These cell lines proliferated in response to APC in the presence of recombinant gp120, as well as to APC expressing virally encoded Env. All of the CD4+ T cell lines responded to Env peptide by secreting IFN- gamma and TNF-alpha without appreciable IL-4 production. Recombinant gp120 stimulation of PBL from these vaccinated monkeys elicited the secretion of a similar profile of cytokines. Demonstration of a nucleotide vaccine eliciting Th1-like immune response is consistent with the well documented ability of naked DNA vaccines to induce durable CD8+ CTL responses. On p. 476 477,513

[Lekutis1998] C. Lekutis \& N. L. Letvin. Substitutions in a major histocompatibility complex clas II-restricted human immunodeficiency virus type 1 gp120 epitope can affect CD4+ T- helper-cell function. J Virol 72:5840-4, 1998. On p. 514

[Lescar1996] J. Lescar, R. Stouracova, M. M. Riottot, V. Chitarra, J. Brynda, M. Fabry, M. Horejsi, J. Sedlacek, \& G. A. Bentley. Preliminary crystallographic studies of an anti-HIV-1 protease antibody which inhibits enzyme activity. Protein Sci 5:966-968, 1996. On p.595

Lescar1997] J. Lescar, R. Stouracova, M. M. Riottot, V. Chitarra, J. Brynda, M. Fabry, M. Horejsi, J. Sedlacek, \& G. A. Bentley. Three-dimensional structure of an Fab-peptide complex: structura basis of HIV-1 protease inhibition by a monoclonal antibody. J Mol Biol 267:1207-22, 1997. (Genbank: U62632 U62633) F11.2.32 is a MAb raised against HIV-1 protease which inhibit proteolytic activity. The structure of the complex of the Fab fragment and the synthetic peptide that it binds to, residues 36 to 46 of protease, have been determined at $2.2 \mathrm{AA}$ resolution, and that of the Fab in the free state has been determined at 2.6 AA resolution. The conformation of the bound peptide shows no overall structural similarity to the corresponding segment in HIV-1 protease. On p. 595

[Lescar1999] J. Lescar, J. Brynda, P. Rezacova, R. Stouracova, M. M. Riottot, V. Chitarra, M. Fabry, M. Horejsi, J. Sedlacek, \& G. A. Bentley. Inhibition of the HIV-1 and HIV-2 proteases by monoclonal antibody. Protein Sci 8:2686-96, 1999. On p.595

Letvin1997] N. L. Letvin, D. C. Montefiori, Y. Yasutomi, H. C. Perry, M. E. Davies, C. Lekutis, M. Alroy, D. C. Freed, C. I. Lord, L. K. Handt, M. A. Liu, \& J. W. Shiver. Potent, protective antiHIV immune responses generated by bimodal hiv envelope dna plus protein vaccination. Proc Natl Acad Sci USA 94:9378-83, 1997. On p. 333 524

Levin1997] R. Levin, A. M. Mhashilkar, T. Dorfman, A. Bukovsky, C. Zani, J. Bagley, J. Hinkula M. Niedrig, J. Albert, B. Wahren, H. G. Gottlinger, \& W. A. Marasco. Inhibition of early and late events of the HIV-1 replication cycle by cytoplasmic Fab intrabodies against the matrix protein, p17. Mol Med 3:96-110, 1997. On p.573

Levine 1996] A. M. Levine, S. Groshen, J. Allen, K. M. Munson, D. J. Carlo, A. E. Daigle, F. Ferre, F. C. Jensen, S. P. Richieri, R. J. Trauger, J. W. Parker, P. L. Salk, \& J. Salk. Initial studies on active immunization of HIV-infected subjects using a gp120-depleted HIV-1 immunogen: Long-term follow-up. J Acquir Immune Defic Syndr Hum Retrovirol 11(4):351-364, 1996. On p. 535

[Levy-Mintz1996] P. Levy-Mintz, L. Duan, H. Zhang, B. Hu, G. Dornadula, M. Zhu, J. Kulkosky, D. Bizub-Bender, A. M. Skalka, \& R. J. Pomerantz. Intracellular expression of single-chain variable fragments to inhibit early stages of the viral life cycle by targeting human immunodeficiency virus type 1 Integrase. J Virol 70:8821-8832, 1996. See comments in J Virol 1998 Apr;72(4):3505-6. On p. 602 603 605 606 608

Levy1998] J. A. Levy, F. Hsueh, D. J. Blackbourn, D. Wara, \& P. S. Weintrub. CD8 cell noncyotoxic antiviral activity in human immunodeficiency virus-infected and -uninfected children. Infect Dis 177(2):470-472, 1998. On p.401

[Lewinsohn2002] D. A. Lewinsohn, R. Lines, D. M. Lewinsohn, S. R. Riddell, P. D. Greenberg, 
M. Emerman, \& S. R. Bartz. HIV-1 Vpr does not inhibit CTL-mediated apoptosis of HIV-1 infected cells. Virology 294(1):13-21, 2002. On p.64.

[Lewis1991] G. Lewis, Y. Abacioglu, T. Fouts, J. Samson, M. Mooreman, G. B. r, R. Tuskan, G. Cole, \& R. Kamin-Lewis. Epitope dominance in the antibody response to recombinant gp160 of HIV-IIIB. Vaccines 91 pp. 157-163, 1991. Editors: F. Brown, R. Chanock and H. S. Ginsberg and R. Lerner Cold Spring Harbor Laboratory Press, Cold Spring Harbor, NY. On p. 832

[Li Pira1998] G. Li Pira, L. Oppezzi, M. Seri, M. Westby, F. Caroli, D. Fenoglio, F. Lancia, A. Ferraris, L. Bottone, M. T. Valle, A. Kunkl, G. Romeo, A. G. Dalgleish, \& F. Manca. Repertoire breadth of human CD4+ T cells specific for HIV gp120 and p66 (primary antigens) or for PPD and tetanus toxoid (secondary antigens). Hum Immunol 59:137-48, 1998. On p.469]485

[Li1993] X. Li, E. Amandoron, M. A. Wainberg, \& M. A. Parniak. Generation and characterization of murine monoclonal antibodies reactive against $\mathrm{N}$-terminal and other regions of HIV-1 reverse transcriptase. J Med Virol 39:251-259, 1993. On p.606

[Li1997] A. Li, T. W. Baba, J. Sodroski, S. Zolla-Pazner, M. K. Gorny, J. Robinson, M. R. Posner, H. Katinger, C. F. Barbas III, D. R. Burton, T.-C. Chou, \& R. M. Ruprecht. Synergistic neutralization of a chimeric SIV/HIV type 1 virus with combinations of human anti-HIV type envelope monoclonal antibodies or hyperimmune globulins. AIDS Res and Human Retroviruse 13:647-656, 1997. Multiple combinations of MAbs were tested for their ability to synergize neutralization of a SHIV construct containing HIV IIIB env. All of the MAb combinations tried were synergistic, suggesting such combinations may be useful for passive immunotherapy or immunoprophylaxis. Because SHIV can replicate in rhesus macaques, such approaches can potentially be \begin{tabular}{ll|l|l|l|l|l|l|l|l|l|l|l|l|l|l|} 
studied in an it in vivo monkey model. On p. 675 & 691 & 710 & 711 & 756 & 762 & 763 & 764 & 765 & 766 \\
\hline
\end{tabular} \begin{tabular}{l|l|l|l|l|l|l|l|l|l|l|l|l|}
\hline 767 & 769 & 770 & 771 & 775 & 777 & 784 & 785 & 798 & 803 & 804 & 822 \\
\hline
\end{tabular}

[Li1998] A. Li, H. Katinger, M. R. Posner, L. Cavacini, S. Zolla-Pazner, M. K. Gorny, J. Sodrosk T. C. Chou, T. W. Baba, \& R. M. Ruprecht. Synergistic neutralization of simian-human immunodeficiency virus SHIV- vpu+ by triple and quadruple combinations of human monoclonal antibodies and high-titer anti-human immunodeficiency virus type 1 immunoglobulins. $J$ Virol 72:323540, 1998. On p. 675 710 712 775 777 7822 823

[Liang2002] X. Liang, T.-M. Fu, H. Xie, E. A. Emini, \& J. W. Shiver. Development of HIV-1 Nef vaccine components: Immunogenicity study of Nef mutants lacking myristoylation and dileucin motif in mice. Vaccine 20(27-28):3413-3421, 2002. On p. 344

[Liao2000] M. Liao, Y. Lu, Y. Xiao, M. P. Dierich, \& Y. Chen. Induction of high level of specific antibody response to the neutralizing epitope ELDKWA on HIV-1 gp41 by peptide-vaccine* [In Process Citation]. Peptides 21:463-8, 2000. On p.710,714,718

[Liao2002] H.-X. Liao, G. J. Cianciolo, H. F. Staats, R. M. Scearce, D. M. Lapple, S. H. Stauffer J. R. Thomasch, S. V. Pizzo, D. C. Montefiori, M. Hagen, J. Eldridge, \& B. F. Haynes. Increase immunogenicity of HIV envelope subunit complexed with alpha2-macroglobulin when combined with monophosphoryl lipid A and GM-CSF. Vaccine 20(17-18):2396-2403, 2002. On p. 752

[Lieberman1992] J. Lieberman, J. A. Fabry, M.-C. Kuo, P. Earl, B. Moss, \& P. R. Skolnik. Cytotoxic T lymphocytes from HIV-1 seropositive individuals recognize immunodominant epitope in gp160 and reverse transcriptase. J Immunol 148:2738-2747, 1992. This paper does not use T-cell clones to map epitopes, but rather T-cell lines from HIV infected donors. 20 amino acid peptides were used to map the region of the reactive epitopes. HLA restriction was not tested for

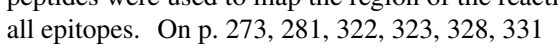

[Lieberman1995] J. Lieberman, J. A. Fabry, P. Shankar, L. Beckett, \& P. R. Skolnik. Ex vivo expansion of HIV type 1-specific cytolytic T cells from HIV type 1-seropositive subjects. AID Res Hum Retroviruses 11:257-271, 1995. Potent HIV-specific CTL lines were developed through culture of non-specific stimulation of $\mathrm{T}$ cell lines with autologous antigen presenting cells preincubated with HIV-1 peptides. On p. 107, $124 \mid$\begin{tabular}{|l|l|l|l|l|l|l|l|l|}
272 & 273 & 280 & 282 & 322 & 328 & 344 & 373 & 388 \\
\hline
\end{tabular}

[Lieberman1997a] J. Lieberman, J. A. Fabry, D. M. Fong, \& G. R. P. 3rd. Recognition of a small number of diverse epitopes dominates the cytotoxic T lymphocytes response to HIV type 1 in an infected individual. AIDS Res Hum Retroviruses 13:383-92, 1997 On p. 65 7102

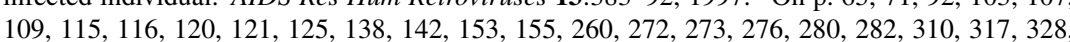
\begin{tabular}{l|l|l|l|l|l|l|l|l|}
\hline 344 & 351 & 362 & 371 & 373 & 376 & 389 & 391 \\
\hline
\end{tabular}

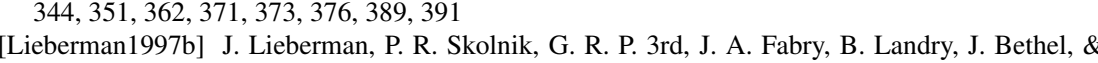
J. Kagan. Safety of autologous, ex vivo-expanded human immunodeficiency virus (HIV)-specific cytotoxic T-lymphocyte infusion in HIV-infected patients. Blood 90:2196-206, 1997. On p.91 \begin{tabular}{|l|l|l|l|l|l|l|l|l|l|l|l|l|}
108 & 109 & 125 & 143 & 153 & 272 & 280 & 282 & 344 & 351 & 373 & 389 \\
\hline
\end{tabular}

[Lieberman1998] J. Lieberman. Personal communication, 1998. On p. 322

[Lieberman2002] J. Lieberman. Defying death-HIV mutation to evade cytotoxic T lymphocytes. N Engl J Med 347(15):1203-1204, 2002. On p. 403

Lindenburg2002] C. E. A. Lindenburg, I. Stolte, M. W. Langendam, F. Miedema, I. G. Williams, R. Colebunders, J. N. Weber, M. Fisher, \& R. A. Coutinho. Long-term follow-up: No effect of therapeutic vaccination with HIV-1 p17/p24:Ty virus-like particles on HIV-1 disease progression. Vaccine 20(17-18):2343-2347, 2002. On p. 445

Linsley1988] P. S. Linsley, J. A. Ledbetter, E. Kinney-Thomas, \& S.-L. Hu. Effects of Anti-gp120 Monoclonal Antibodies on CD4 Receptor Binding by the env Protein of Human Immunodeficiency Virus Type 1. J Virol 62:3695-3702, 1988. On p. 689

[Liou1989] R. S. Liou, E. M. Rosen, M. S. C. Fung, W. N. C. Sun, C. Sun, W. Gordon, N. T. Chang, \& T. W. Chang. A chimeric mouse-human antibody that retains specificity for HIV-1 gp120 and mediates the lysis of the HIV-1-infected cells. J Immunol 143:3967-3975, 1989. On p. 646663 Littaua1991] R. A. Littaua, M. B. A. Oldstone, A. Takeda, C. Debouck, J. T. Wong, C. U. Tuazon, B. Moss, F. Kievits, \& F. A. Ennis. An HLA-C-restricted CD8+ cytotoxic T-lymphocyte clone recognizes a highly conserved epitope on human immunodeficiency virus type 1 gag. J Virol 65:4051-4056, 1991. Fine mapping of gag p24 epitope with HLA-C restriction: QAISPR, HLA, Cw3. On p. 97

[Litwin1996] V. Litwin, K. A. Nagashima, A. M. Ryder, C. H. Chang, J. M. Carver, W. C. Olson, M. Alizon, K. W. Hasel, P. J. Maddon, \& G. P. Allaway. Human immunodeficiency virus type 1 membrane fusion mediated by a laboratory-adapted strain and a primary isolate analyzed by resonance energy transfer. $J$ Virol 70:6437-6441, 1996. Fusion of primary (JRFL) and TCLA (LAI) strains of the virus were studied. The degree, kinetics, neutral $\mathrm{pH}$ and divalent cations requirements were similar for membrane fusion for both viruses. However, the inhibition of fusion by $\mathrm{SCD} 4$ and $\mathrm{CD} 4-\mathrm{IgG} 2$ occurred at virus neutralization concentrations for JRFL, but higher concentrations were required to inhibit LAI fusion than to neutralize LAI, suggesting that viral neutralization and fusion-inhibition are distinct. On p.775 777

[Liu1995] X. Liu, A. Ota, M. Watanabe, S. Ueda, A. Saitoh, H. Shinagawa, A. Nakata, T. Kurimura, $\mathrm{X}$. Wang, Y. Zhao, \& et al. Three antigenic regions in $\mathrm{p} 17$ of human immunodeficiency virus type 1 (HIV-1) revealed by mouse monoclonal antibodies and human antibodies in HIV-1 carrier sera Microbiol Immunol 39:775-85, 1995. On p. 569 570 571 574 591

Liu2002] X. S. Liu, W. J. Liu, K. N. Zhao, Y. H. Liu, G. Leggatt, \& I. H. Frazer. Route of administration of chimeric BPV1 VLP determines the character of the induced immune responses. \begin{tabular}{ll|l|l|l|l|l|l|l|l|}
\hline Immunol Cell Biol 80(1):21-9, 2002. On p.710 717 & 775 & 779 & 784 & 791 & 822 & 827 \\
\hline
\end{tabular}

[Livingston2002] B. Livingston, C. Crimi, M. Newman, Y. Higashimoto, E. Appella, J. Sidney, \& A. Sette. A rational strategy to design multiepitope immunogens based on multiple Th lymphocyte epitopes. J Immunol 168(11):5499-5506, 2002. On p.431 4494450453

Llorente1999] M. Llorente, S. Sanchez-Palomino, S. Manes, P. Lucas, L. Kremer, I. M. D. Alboran, J. L. Toran, J. Alcami, G. D. Real, \& M.-A. C. Natural human antibodies retrieved by phage display libraries from healthy donors: polyreactivity and recognition of human immunodeficiency virus type 1gp120 epitopes. Scand J Immunol 50:270-9, 1999. On p.749

[Locher1999] C. P. Locher, R. M. Grant, E. A. Collisson, G. Reyes-Teran, T. Elbeik, J. O. Kahn, \& 
J. A. Levy. Antibody and cellular immune responses in breakthrough infection subjects after HIV type 1 glycoprotein 120 vaccination. AIDS Res Hum Retroviruses 15:1685-9, 1999. On p.749 [Loemba2002] H. Loemba, B. Brenner, M. A. Parniak, S. Ma'ayan, B. Spira, D. Moisi, M. Oliveira M. Detorio, M. Essex, M. A. Wainberg, et al. Polymorphisms of cytotoxic T-lymphocyte (CTL) and T-helper epitopes within reverse transcriptase (RT) of HIV-1 subtype C from Ethiopia and Botswana following selection of antiretroviral drug resistance. Antiviral Res 56(2):129-142, 2002. On p. 242

[Loing2000] E Loing, M. Andrieu, K. Thiam, D. Schorner, K. H. Wiesmuller, A. Hosmalin, G. Jung, \& H. Gras-Masse. Extension of HLA-A*0201-restricted minimal epitope by N epsilonpalmitoyl-lysine increases the life span of functional presentation to cytotoxic T cells. J Immunol 164:900-7, 2000. On p.211

[Loomis-Price1997] L. D. Loomis-Price, M. Levi, P. R. Burnett, J. E. van Hamont, R. A. Shafer, B. Wahren, \& D. L. Birx. Linear epitope mapping of humoral responses induced by vaccination with recombinant HIV-1 Envelope protein gp160. J Ind Microbiol Biotechnol 19:58-65, 1997. On p. 686693

[Lopalco1993] L. Lopalco, R. Longhi, F. Ciccomascolo, A. D. Rossi, M. Pelagi, F. Andronico, J. P. Moore, B. T. Schulz, A. Beretta, \& A. G. Siccardi. Identification of human immunodeficiency virus type 1 glycoprotein gp120/gp41 interacting sites by the idiotypic mimicry of two monoclonal antibodies. AIDS Res Hum Retroviruses 9:33-39, 1993. The MAb M38 binds to the carboxy terminus of gp120, in a gp41 binding region. This MAb was used to create an anti-idiotype MAb, 9G5A, which can bind to gp41 at the base of the cysteine loop. The binding domains of these two monoclonals are consistent with the $\mathrm{C} 5$ domain of gp 120 being able to bind to the gp41 cysteine loop. The MAb M38 also binds to human HLA molecules, in antigenic homology or possibly molecular mimicry. On p. 688701

[Lopez2000] D. Lopez, B. C. Gil-Torregrosa, C. Bergmann, \& M. D. Val. Sequential cleavage by metallopeptidases and proteasomes is involved in processing HIV-1 ENV epitope for endogenous MHC class I antigen presentation. J Immunol 164:5070-7, 2000. On p.295

[Lori1999] F. Lori, H. Jessen, J. Lieberman, D. Finzi, E. Rosenberg, C. Tinelli, B. Walker, R. F. Siliciano, \& J. Lisziewicz. Treatment of human immunodeficiency virus infection with hydroxyurea, didanosine, and a protease inhibitor before seroconversion is associated with normalized immune parameters and limited viral reservoir. J Infect Dis 180:1827-32, 1999. On p.441

[Lotti2002] B. Lotti, T. Wendland, H. Furrer, N. Yawalkar, S. von Greyerz, K. Schnyder, M. Brandes, P. Vernazza, R. Wagner, T. Nguyen, E. Rosenberg, W. J. Pichler, \& C. Brander. Cytotoxic HIV-1 p55gag-specific CD4+ T cells produce HIV-inhibitory cytokines and chemokines. J Clin Immunol 22(5):253-262, 2002. On p. 429433439446

[Lu1999] Y. Lu, K. Q. Xin, K. Hamajima, T. Tsuji, I. Aoki, J. Yang, S. Sasaki, J. Fukushima, T. Yoshimura, S. Toda, E. Okada, \& K. Okuda. Macrophage inflammatory protein-1alpha (MIP1alpha) expression plasmid enhances DNA vaccine-induced immune response against HIV-1 Clin Exp Immunol 115(2):335-41, 1999. On p. 293.494

[Lu2000a] Y. Lu, R. Friedman, N. Kushner, A. Doling, L. Thomas, N. Touzjian, M. Starnbach, \& J. Lieberman. Genetically modified anthrax lethal toxin safely delivers whole HIV protein antigens into the cytosol to induce T cell immunity. Proc Natl Acad Sci U S A 97(14):8027-32, 2000. On p. 167,290397

[Lu2000b] Y. Lu, Y. Xiao, J. Ding, M. Dierich, \& Y. H. Chen. Immunogenicity of neutralizing epitopes on multiple-epitope vaccines against HIV-1. Int Arch Allergy Immunol 121:80-4, 2000. On p. 674696710714718

[Lu2000c] Y. Lu, Y. Xiao, J. Ding, M. P. Dierich, \& Y. H. Chen. Multiepitope vaccines intensively increased levels of antibodies recognizing three neutralizing epitopes on human immunodeficiency virus-1 envelope protein. Scand J Immunol 51:497-501, 2000. On p.674 696710 714 718
[Lubaki1997] N. M. Lubaki, S. C. Ray, B. Dhruva, T. C. Quinn, R. F. Siliciano, \& R. C. Bollinger Characterization of a polyclonal cytolytic T lymphocyte response to human immunodeficiency virus in persons without clinical progression. J Infect Dis 6:1360-7, 1997. Five individuals were studied who survived HIV infection in good health for over 5 years. A broad polyclonal response was found to multiple proteins. On p.60 113$] 139\left[\begin{array}{ll}149 & 354 \\ \hline\end{array}\right.$

[Lubaki1999] N. M. Lubaki, M. E. Shepherd, R. S. Brookmeyer, H. Hon, T. C. Quinn, M. Kashamuka, M. Johnson, R. Gottle, J. Devers, H. M. Lederman, \& R. C. Bollinger. HIV 1-specific cytolytic T-lymphocyte activity correlates with lower viral load, higher CD4 count, and CD8+CD38-DR- phenotype: comparison of statistical methods for measurement. $J$ Acquir Immune Defic Syndr 22:19-30, 1999. On p. 162 395

[Lubeck1997] M. D. Lubeck, R. Natuk, M. Myagkikh, N. Kalyan, K. Aldrich, F. Sinangil, S. Alipanah, S. C. S. Murthy, P. K. Chanda, S. M. Nigida, Jr., P. D. Markham, S. Zolla-Pazner, K. Steimer, M. Wade, M. S. Reitz, Jr., L. O. Arthur, S. Mizutani, A. Davis, P. P. Hung, R. C. Gallo, J. Eichberg, \& M. Robert-Guroff. Long-term protection of chimpanzees against high-dose HIV-1 challenge induced by immunization. Nature Med 3 No 6:651-658, 1997. On p. 275286

Lucchiari-Hartz2000] M. Lucchiari-Hartz, P. M. van Endert, G. Lauvau, R. Maier, A. Meyerhans, D. Mann, K. Eichmann, \& G. Niedermann. Cytotoxic T lymphocyte epitopes of HIV-1 Nef: Generation of multiple definitive major histocompatibility complex class I ligands by proteasomes. Exp Med 191(2):239-52, 2000. On p. 377, 383, 386,387

Lundin1996] K. Lundin, A. Samuelsson, M. Jansson, J. H. J, B. Wahren, H. Wigzell, \& M. A Persson. Peptides isolated from random peptide libraries on phage elicit a neutralizing anti-HIV1 response: analysis of immunological mimicry. Immunology 89:579-586, 1996. On p. 581

Luo1998] L. Luo, Y. Li, J.-S. Chang, S. Y. Cho, T. Y. Kim, M. J. Choi, H. S. Cheong, H. J. Kim, H. J. Ahn, M. K. Min, B. H. Chun, S. M. Jung, S. G. Woo, S. Y. Park, \& C. Y. Kang. Induction of V3-specific cytotoxic T lymphocyte responses by HIV gag particles carrying multiple immunodominant V3 epitopes of gp120. Virology 240:316-25, 1998. On p. 285

Luzuriaga1995] K. Luzuriaga, D. Holmes, A. Hereema, J. Wong, D. L. Panicali, \& J. L. Sullivan. HIV-1-specific cytotoxic T lymphocyte responses in the first year of life. J Immunol 154(1):433443, 1995. On p. 167339

[Luzuriaga2000] K. Luzuriaga, M. McManus, M. Catalina, S. Mayack, M. Sharkey, M. Stevenson, \& J. L. Sullivan. Early therapy of vertical human immunodeficiency virus type 1 (HIV-1) infection: Control of viral replication and absence of persistent HIV-1-specific immune responses. $J$ Virol 74(15):6984-6991, 2000. On p.74, 209

Ly2000] A. Ly \& L. Stamatatos. V2 loop glycosylation of the human immunodeficiency virus type 1 SF162 envelope facilitates interaction of this protein with CD4 and CCR5 receptors an protects the virus from neutralization by anti-V3 loop and anti-CD4 binding site antibodies [In

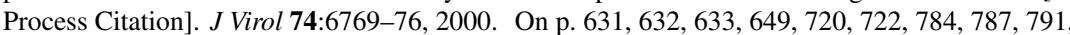

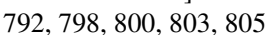

Macatonia1991] S. E. Macatonia, S. Patterson, \& S. C. Knight. Primary proliferative and cytotoxic $\mathrm{T}$ cell responses to HIV induced in vitro by human dendritic cells. Immunology 74(3):399-406, 1991. A primary CTL response in cells from uninfected donors was detected by using a system where peptide was presented by human dendritic cells. On p.275

MacGreoor 1998] R. R. MacGregor, J. D. Boyer, K. E. Ugen, K. E. Lacy, S. J. Gluckman, M. L. Bagarazzi, M. A. Chattergoon, Y. Baine, T. J. Higgins, R. B. Ciccarelli, L. R. Coney, R. S. Ginsberg, \& D. B. Weiner. First human trial of a DNA-based vaccine for treatment of human immuno deficiency virus type 1 infection: safety and host response. J Infect Dis 178:92-100, 1998. On p. 3335524

[MacGregor2002] R. R. MacGregor, R. Ginsberg, K. E. Ugen, Y. Baine, C. U. Kang, X. M. Tu, T. Higgins, D. B. Weiner, \& J. D. Boyer. T-cell responses induced in normal volunteers immunized 
with a DNA-based vaccine containing HIV-1 env and rev. AIDS 16(16):2137-2143, 2002. On p. 465528

[Maciejewski1995] J. P. Maciejewski, F. F. Weichold, N. S. Young, A. Cara, D. Zella, M. S. Reitz, Jr., \& R. C. Gallo. Intracellular expression of antibody fragments directed against HIV reverse transcriptase prevents HIV infection in vitro. Nat Med 1:667-673, 1995. Transduction of genes that encode and express anti-RT Fab inhibit the enzyme, making cells resistant to HIV infection by blocking an early stage of viral replication. The authors propose that if transduction of a vector into lymphohaematopoietic stem cells or mature lymphocytes becomes feasible, gene transfer therapy has promise for treating AIDS. On p.607

[Mackewicz2000] C. E. Mackewicz, S. Ridha, \& J. A. Levy. Fas and Fas ligand are not involved in the suppression of HIV replication by CD8 cells [letter]. AIDS 14:204-5, 2000. On p.400

[Maeda1992] Y. Maeda, S. Matsushita, T. Hattori, T. Murakami, \& K. Takatsuki. Changes in the Reactivity and Neutralizing Activity of a Type-Specific Neutralizing Monoclonal Antibody Induced by Interaction of Soluble CD4 with gp120. AIDS Res Hum Retroviruses 8:2049-2054, 1992. On $\mathrm{p}$.663 671

[Maino2000] V. C. Maino, M. A. Suni, S. B. Wormsley, D. J. Carlo, M. R. Wallace, \& R. B. Moss. Enhancement of HIV type 1 antigen-specific CD4+ T cell memory in subjects with chronic HIV type 1 infection receiving an HIV type 1 immunogen [In Process Citation]. AIDS Res Hum Retroviruses 16:539-47, 2000. On p.441

[Maksiutov2002] A. Z. Maksiutov, A. G. Bachinskii, \& S. I. Bazhan. [searching for local similarities between HIV-1 and human proteins. application to vaccines]. Mol Biol (Mosk) 36(3):447-459 \begin{tabular}{|l|l|l|l|l|l|l||l|l|l|l|l|l|} 
2002. Article in Russian. On p. 153 & 161 & 182 & 230 & 232 & 233 & 262 & 272 & 278 & 283 & 306 & 307 & 318 \\
\hline
\end{tabular}

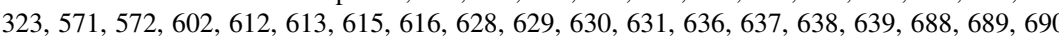
\begin{tabular}{l|l|l|l|l|l|l|l|l|l|l|l|}
\hline 693 & 694 & 835 & 836 & 838 & 839 \\
\hline
\end{tabular}

[Malhotra2001] U. Malhotra, S. Holte, S. Dutta, M. M. Berrey, E. Delpit, D. M. Koelle, A. Sette, L. Corey, \& M. J. McElrath. Role for HLA class II molecules in HIV-1 suppression and cellula immunity following antiretroviral treatment. J Clin Invest 107(4):505-17, 2001. On p.435, 436 439

[Manca1993] F. Manca, E. Seravalli, M. T. Valle, D. Fenoglio, A. Kunkl, G. L. Pira, S. Zolla-Pazner \& F. Celada. Non-covalent complexes of HIV gp120 with CD4 and/or mAbs enhance activation of gp120-specific T clones and provide intermolecular help for anti-CD4 antibody production Internatl Immunol 5:1109-1117, 1993. On p.484.

[Manca1995a] F. Manca, D. Fenoglio, M. T. Valle, G. L. Pira, A. Kunkl, R. S. Balderas, R. G. Baccala, D. H. Kono, A. Ferraris, D. Saverino, F. Lancia, L. Lozzi, \& A. N. Theofilopoulos. Human T helper cells specific for HIV reverse transcriptase: possible role in intrastructural help for HIV envelope-specific antibodies. Eur J Immunol 25:1217-1223, 1995. On p.449 451 691 \begin{tabular}{ll|l|l|l|l|l|}
\hline 696 & 697 & 700 & 708 & 709 & 766 & 767 \\
\hline
\end{tabular}

[Manca1995b] F. Manca, D. Fenoglio, M. T. Valle, G. L. Pira, A. Kunkl, A. Ferraris, D. Saverino, F. Lancia, L. Mortara, L. Lozzi, M. Pierres, A. G. Dalgleish, \& G. Lewis. Human CD4+ T cells can discriminate the molecular and structural context of T epitopes of HIV gp120 and HIV p66. AIDS 9:227-237, 1995. Notes: it in vitro priming with peptides often induced CD4+ T-cells that did not recognize whole protein. Priming with protein did not always induce T-cells that could be

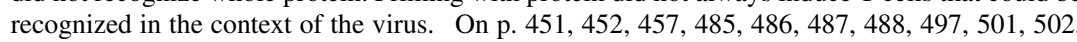
\begin{tabular}{lll|l|l|l|l|l|l|l|l|l|}
503 & 505 & 510 & 511 & 512 & 514 & 518 & 520 \\
\hline
\end{tabular}

[Manca1996] F. Manca, P. D. B. P., D. Fenoglio, M. N. Ombra, G. L. Pira, D. Saverino, M. Autiero, L. Lozzi, L. Bracci, \& J. Guardiola. Antigenicity of HIV-derived T helper determinants in the context of carrier recombinant proteins: effect on Thelper cell repertoire selection. Eur J Immuno 26:2461-9, 1996. Notes: A given Th epitope was recognized by a specific T cell clone only when it was inserted in a particular position of the carrier, and the permissive position was not the same for all epitopes. On $\mathrm{p}$ 451 485,486
[Mani1994] J.-C. Mani, V. Marchi, \& C. Cucurou. Effect of HIV-1 peptide presentation on the affinity constants of two monoclonal antibodies determined by BIAcore technology. Mol Immunol 31:439-444, 1994. Two MAbs are described; one 41-1 did not require the Cys-Cys disulfide bridge and loop formation, the other 9-11 depends on loop formation. On p. 698 725

[Marasco1992] W. A. Marasco, J. Bagley, C. Zani, M. Posner, L. Cavacini, W. A. Haseltine, \& J. Sodroski. Characterization of the cDNA of a Broadly Reactive Neutralizing Human anti-gp120 Monoclonal Antibody. J Clin Invest 90:1467-1478, 1992. On p.775

[Marasco1993] W. A. Marasco, W. A. Haseltine, \& S. Y. C. SY. Design, intracellular expression, and activity of a human anti-human immunodeficiency virus type 1 gp120 single-chain antibody. Proc Natl Acad Sci USA 90:7889-7893, 1993. Comment in: Proc Natl Acad Sci USA 1993 90:7427-8. On p.775 776

[Marks1992] J. D. Marks, B. Wahren, G. Gilljam, J. Hinkula, \& G. Winter. Cloning of an HIV1 neutralizing V3 specific monoclonal antibody and expression as a mouse-human chimaeric antigen binding fragment and antibody. J Acquir Immune Defic Syndr 1991:1162, 1992. Aidsline: 1116291Abstract: VIIth International Conference on AIDS, Florence, Italy, proceedings. On p. 662

Maroun1999] R. G. Maroun, D. Krebs, M. Roshani, H. Porumb, C. Auclair, F. Troalen, \& S. Fermandjian. Conformational aspects of HIV-1 integrase inhibition by a peptide derived from the enzyme central domain and by antibodies raised against this peptide. Eur J Biochem 260:145-55, 1999. On p. 602

[Marsac2002] D. Marsac, D. Loirat, C. Petit, O. Schwartz, \& M.-L. Michel. Enhanced presentation of major histocompatibility complex class I-restricted human immunodeficiency virus type 1 (HIV-1) Gag-specific epitopes after DNA immunization with vectors coding for vesicular stomatitis virus glycoprotein-pseudotyped HIV-1 Gag particles. J Virol 76(15):7544-53, 2002. On p. 117

[Mascola 1997] J. R. Mascola, M. K. Louder, T. C. VanCott, C. V. Sapan, J. S. Lambert, L. R. Muenz, B. Bunow, D. L. Birx, \& M. L. Robb. Potent and synergistic neutralization of human immunodeficiency virus (HIV) type 1 primary isolates by hyperimmune anti-HIV immunoglobulin combined with monoclonal antibodies 2F5 and 2G12. J Virol 71:7198-206, 1997. HIVIG derived from the plasma of HIV-1-infected donors, and MAbs 2F5 and 2G12 were tested against a panel of 15 clade B HIV-1 isolates, using a single concentration that is achievable in vivo (HIVIG, $2,500 \mathrm{microg} / \mathrm{ml}$; MAbs, $25 \mathrm{microg} / \mathrm{ml}$ ). While the three antibody reagents neutralized many of the viruses tested, potency varied. The virus neutralization achieved by double or triple combinations was generally equal to or greater than that predicted by the effect of individual antibodies, and the triple combination was shown to be synergistic and to have the greatest breadth and potency. Passive immunotherapy for treatment or prophylaxis of HIV-1 should consider mixtures of these potent neutralizing antibody reagents. On p.710 712822

[Mascola1999] J. R. Mascola, M. G. Lewis, G. Stiegler, D. Harris, T. C. VanCott, D. Hayes, M. K Louder, C. R. Brown, C. V. Sapan, S. S. Frankel, Y. Lu, M. L. Robb, H. Katinger, \& D. L. Birx Protection of Macaques against pathogenic simian/human immunodeficiency virus 89.6PD by passive transfer of neutralizing antibodies. J Virol 73(5):4009-18, 1999. On p. 7107713822 824

Mascola2000] J. R. Mascola, G. Stiegler, T. C. VanCott, H. Katinger, C. B. Carpenter, C. E. Hanson, H. Beary, D. Hayes, S. S. Frankel, D. L. Birx, \& M. G. Lewis. Protection of macaques against vaginal transmission of a pathogenic HIV-1/SIV chimeric virus by passive infusion of neutralizing antibodies. Nat Med 6(2):207-10, 2000. On p.710 713 822 824

Mascola2001] J. R. Mascola \& G. J. Nabel. Vaccines for the prevention of HIV-1 disease. Curr Opin Immunol 13(4):489-95, 2001. On p.710 715 822 825

[Mascola2002] J. R. Mascola. Passive transfer studies to elucidate the role of antibody-mediated protection against HIV-1. Vaccine 20(15): 1922-1925, 2002. On p.710 $717,822,827$ 
[Mata1998] M. Mata, P. J. Travers, Q. Liu, F. R. Frankel, \& Y. Paterson. The MHC class I-restricted immune response to HIV-gag in BALB/c mice selects a single epitope that does not have a predictable MHC-binding motif and binds to Kd through interactions between a glutamine at P3 an pocket D. J Immunol 161:2985-93, 1998. On p. 117 121

[Mata1999] M. Mata \& Y. Paterson. Th1 T cell responses to HIV-1 Gag protein delivered by a Listeria monocytogenes vaccine are similar to those induced by endogenous listerial antigens. $J$ Immunol 163(3): 1449-56, 1999. On p.432 435 438

[Mata2000] M. Mata \& Y. Paterson. Listeria monocytogenes as an alternative vaccine vector for HIV. Arch Immunol Ther Exp (Warsz) 48(3):151-62, 2000. On p. 170.447

[Mata2001] M. Mata, Z. J. Yao, A. Zubair, K. Syres, \& Y. Paterson. Evaluation of a recombinan Listeria monocytogenes expressing an HIV protein that protects mice against viral challenge. Vaccine 19(11-12): 1435-45, 2001. On p. 169447

[Matsuo1992] K. Matsuo, Y. Nishino, T. Kimura, R. Yamaguchi, A. Yamazaki, T. Mikami, \& K. Ikuta. Highly conserved epitope domain in major core protein p24 is structurally simila among human, simian and feline immunodeficiency viruses. J Gen Virol 73:2445-2450, 1992 Two MAbs are described that bind to a highly conserved region in $\mathrm{p} 24$, with antigenic conservation between FIV, SIV and HIV-1. The authors suggest this might be an immunodominant domain. On p. 583

[Matsushita1988] S. Matsushita, M. Rober-Guroff, J. Rusche, A. Koito, T. Hattori, H. Hoshino, K. Javaherian, K. Takatsuki, \& S. Putney. Characterization of a Human Immunodeficiency Virus neutralizing monoclonal antibody and mapping the neutralizing epitope. J Virol 62:2107-2114, 1988. On p. 659671

[Matsushita1992] S. Matsushita, H. Maeda, K. Kimachi, Y. Eda, Y. Maeda, T. Murakami, S. Tokiyoshi, \& K. Takatsuki. Characterization of a mouse/human chimeric monoclonal antibody (C $\beta 1$ ) to a principal neutralizing domain of the human immunodeficiency virus type 1 envelope protein. AIDS Res Hum Retroviruses 8:1107-1115, 1992. On p.671 672

[Matsushita1995] S. Matsushita, S. Matsumi, K. Yoshimura, T. Morikita, T. Murakami, \& K. Takatsuki. Neutralizing monoclonal antibodies against human immunodeficiency virus type 2 gp 120 J Virol 69:3333-3340, 1995. On p. 628,759

Mazzoli1997] S. Mazzoli, D. Trabattoni, S. L. Caputo, S. Piconi, C. Ble, F. Meacci, S. Ruzzante, A. Salvi, F. Semplici, R. Longhi, M. L. Fusi, N. Tofani, M. Biasin, M. L. Villa, F. Mazzotta, \& M. Clerici. HIV-specific mucosal and cellular immunity in HIV-seronegative partners of HIVseropositive individuals [see comments]. Nat Med 3:1250-7, 1997. On p.524

[Mazzoli1999] S. Mazzoli, L. Lopalco, A. Salvi, D. Trabattoni, S. Lo Caputo, F. Semplici, M. Biasin, C. Blé, A. Cosma, C. Pastori, F. Meacci, F. Mazzotta, M. L. Villa, A. G. Siccardi, \& M. Clerici. Human immunodeficiency virus (HIV)-specific IgA and HIV neutralizing activity in the serum of exposed seronegative partners of HIV-seropositive persons. J Infect Dis 180(3):871875, 1999. On p. 751

[McAdam1995] S. McAdam, P. Klenerman, L. Tussey, S. Rowland-Jones, D. Lalloo, R. Phillips, A. Edwards, P. Giangrande, A. L. Brown, \& F. Gotch. Immunogenic HIV variant peptides that bind to HLA-B8 can fail to stimulate cytotoxic T lymphocyte responses. J Immunol 155:2729-36, 1995. On p. 128.154

[McAdam1998] S. McAdam, P. Kaleebu, P. Krausa, P. Goulder, N. French, B. Collin, T. Blanchard, J. Whitworth, A. McMichael, \& F. Gotch. Cross-clade recognition of p55 by cytotoxic T lym-

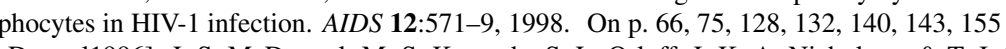

[McDougal1996] J. S. McDougal, M. S. Kennedy, S. L. Orloff, J. K. A. Nicholson, \& T. J. Spira Mechanisms of human immunodeficiency virus type 1 (HIV-1) neutralization: Irreversible inactivation of infectivity by anti-HIV-1 antibody. J Virol 70:5236-5245, 1996. Studies of polyclona sera autologous virus inactivation indicates that in individuals over time, viral populations emerge

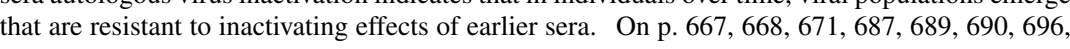

\section{762 763 $775,777,817,834$}

McElrath2000] M. J. McElrath, L. Corey, D. Montefiori, M. Wolff, D. Schwartz, M. Keefer R. Belshe, B. S. Graham, T. Matthews, P. Wright, G. Gorse, R. Dolin, P. Berman, D. Francis, A. M. Duliege, D. Bolognesi, D. Stablein, N. Ketter, \& P. Fast. A phase II study of two HIV type 1 envelope vaccines, comparing their immunogenicity in populations at risk for acquiring HIV type 1 infection. AIDS Vaccine Evaluation Group [In Process Citation]. AIDS Res Hum Retroviruses 16:907-19, 2000. On p.744

[McFarland1994] E. J. McFarland, P. A. Harding, D. Luckey, B. Conway, R. K. Young, \& D. R. Kuritzkes. High frequency of Gag- and envelope-specific cytotoxic $\mathrm{T}$ lymphocyte precursors in children with vertically acquired human immunodeficiency virus type 1 infection. J Infect Dis 170(4):766-774, 1994. On p. 166337

McGettigan2001] J. P. McGettigan, H. D. Foley, I. M. Belyakov, J. A. Berzofsky, R. J. Pomerantz \& M. J. Schnell. Rabies virus-based vectors expressing human immunodeficiency virus type (HIV-1) envelope protein induce a strong, cross-reactive cytotoxic T-lymphocyte response against envelope proteins from different HIV-1 isolates. J Virol 75(9):4430-4, 2001. On p. 341

[McInerney 1999] T. L. McInerney, F. R. Brennan, T. D. Jones, \& N. J. Dimmock. Analysis of the ability of five adjuvants to enhance immune responses to a chimeric plant virus displaying an HIV-1 peptide. Vaccine 17:1359-68, 1999. On p. 518

McKeating1992a] J. A. McKeating, J. Cordell, C. J. Dean, \& P. Balfe. Synergistic interaction between ligands binding to the CD4 binding site and V3 domain of human immunodeficiency \begin{tabular}{|l|l|l|l|l|l|l|l|} 
virus type I gp120. Virology 191:732-742, 1992. On p. 623 & 632 & 640 & 641 & 668 & 670 & 671 & 680 \\
\hline
\end{tabular} virus type

[McKeating1992b] J. A. McKeating, J. P. Moore, M. Ferguson, H. S. Marsden, S. Graham, J. W. Almond, D. J. Evans, \& R. A. Weiss. Monoclonal antibodies to the C4 region of human immunodeficiency virus type 1 gp120: use in topological analysis of a CD4 binding site. AIDS Res Hum Retroviruses 8:451-459, 1992. Antibodies were generated using an antigen poliovirus chimera, expressing aa430-446 of gp120. Results suggest that WQEVGKAMYA may be exposed on the surface of rec gp120. On p. 680683684685

McKeating 1992c] J. A. McKeating, M. Thali, C. Furman, S. Karwowska, M. K. Gorny, J. Cordell, S. Zolla-Pazner, J. Sodroski, \& R. A. Weiss. Amino acid residues of the human immunodeficiency virus type $1 \mathrm{gp} 120$ critical for the binding of rat and human neutralizing antibodies that block the gp120-sCD4 interaction. Virology 190:134-142, 1992. On p. 680|766 $768 \mid 783$

[McKeating1993a] J. A. McKeating, J. Bennett, S. Zolla-Pazner, M. Schutten, S. Ashelford, A. Leigh-Brown, \& P. Balfe. Resistance of a human serum-selected human immunodeficiency virus type 1 escape mutant to neutralization by CD4 binding site monoclonal antibodies is conferred by a single amino acid change in gp120. J Virol 67:5216-5225, 1993. On p. 629 670 680

[McKeating1993b] J. A. McKeating, C. Shotton, J. Cordell, S. Graham, P. Balfe, N. Sullivan, M. Charles, M. Page, A. Bolmstedt, S. Olofsson, S. C. Kayman, Z. Wu, A. Pinter, C. Dean, J. Sodroski, \& R. A. Weiss. Characterization of neutralizing monoclonal antibodies to linear an conformation-dependent epitopes within the first and second variable domains of human immunodeficiency virus type 1 gp120. J Virol 67:4932-4944, 1993. Substitutions in the V2 loop can result in complete dissociation of gp 120 and gp 41 , suggesting alterations in V2 can affect subunit assembly. Other substitutions allowed gp120-gp41 association and expression, but inhibite viral entry or syncytia. Binding of some neutralizing MAbs was altered by V2 substitutions. Fo MAb CRA-4, changes at residues 191/192/193 (YSL/GSS), and for 11/68b, changes at residues 183/184 (PI/SG), within V2, and for both MAbs a position $435(\mathrm{Y} / \mathrm{H})$ change in $\mathrm{C} 4$, abrogate binding. These MAbs can bind to V1 and V2 domains in the absence of $\mathrm{C} 4$ domain, so the $\mathrm{C} 4$

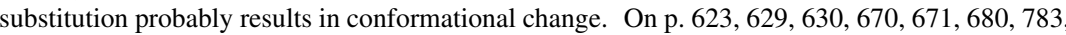
$808,811,820$ 
[McKeating1996a] J. A. McKeating. Biological consequences of human immunodeficiency virus type 1 envelope polymorphism: does variation matter? 1995 Fleming Lecture. J Gen Virol 77:2905-2919, 1996. On p. 710 784, 822

[McKeating1996b] J. A. McKeating, Y. J. Zhang, C. Arnold, R. Frederiksson, E. M. Fenyo, \& P. Balfe. Chimeric viruses expressing primary envelope glycoproteins of human immunodeficiency virus type I show increased sensitivity to neutralization by human sera. Virology 220:450 460, 1996. Chimeric viruses for HXB2 with primary isolate gp120 gave patterns of cell tropism and cytopathicity identical to the original primary viruses. Sera that were unable to neutralize the primary isolates were in some cases able to neutralize chimeric viruses, indicating that some of

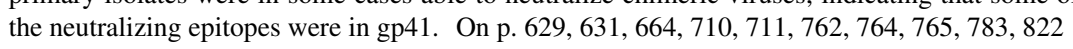

[McKinney1999] D. McKinney, D. Lewinson, S. Riddell, P. Greenberg, \& D. Mosier. The antivira activity of HIV-specific CD8+ CTL clones is limited by elimination due to encounter with HIV infected targets. J Immипо 163:861-7, 1999. On p.61

[McKnight1995] A. McKnight, R. A. Weiss, C. Shotton, Y. Takeuchi, H. Hoshino, \& P. R. Clapham. Change in tropism upon immune escape by human immunodeficiency virus. J Virol 69:31673170, 1995. On p. 650 651

[McLain1994] L. McLain \& N. J. Dimmock. Single- and multi-hit kinetics of immunoglobulin G neutralization of human immunodeficiency virus type 1 by monoclonal antibodies. J Gen Viro 75:1457-1460, 1994. On p. 783820

[McLain2001] L. McLain, J. L. Brown, L. Cheung, S. A. Reading, C. Parry, T. D. Jones, S. M. Cleveland, \& N. J. Dimmock. Different effects of a single amino acid substitution on three adjacent epitopes in the gp41 C-terminal tail of a neutralizing antibody escape mutant of human immunodeficiency virus type 1. Arch Virol 146(1):157-66, 2001. On p. 7237726727

[McMichael1994] A. J. McMichael \& B. D. Walker. Cytotoxic T lymphocytes epitopes: implications for HIV vaccine. AIDS 8S:S155-S173, 1994. Comprehensive review summarizing CTL epitopes that have known HLA type and are fine mapped to indicate epitope boundaries. Ancho residues are indicated when known for different HLA restricted epitopes. Includes a summary of the published literature, as well as much work that was in press or submitted for publication. On \begin{tabular}{l|l|l|l|l|l|l|l|l|l|l|l|l|l|l|l|} 
p. 65 & 84 & 94 & 115 & 126 & 134 & 139 & 191 & 196 & 223 & 322 & 357 & 359 & 363 & 365 & 375 \\
\hline
\end{tabular}

[McMichael2002] A. McMichael \& T. Hanke. The quest for an AIDS vaccine: Is the CD8+ T-cel approach feasible? Nat Rev Immunol 2(4):283-291, 2002. On p. 67, 402

[Meier1995] U.-C. Meier, P. Klenerman, P. Griffin, W. James, B. Koppe, B. Larder, R. E. Phillips, A. J. McMichael, \& R. E. Phillips. Cytotoxic T lymphocyte lysis inhibited by viable HIV mutants Science 270:1360-1362, 1995. HIV bearing mutations in epitope allowed transactive inhibition of specific CTL mediated lysis. Therefore, mutations in epitopes may not only allow escape from specific CTL, but enhance the ability of wildtype virus to persist. On p.177

[Meles2002] H. Meles, D. Wolday, A. Fontanet, A. Tsegaye, T. Tilahun, M. Aklilu, E. Sanders, \& T. F. Rinke De Wit. Indeterminate human immunodeficiency virus Western blot profiles in Ethiopians with discordant screening-assay results. Clin Diagn Lab Immunol 9(1):160-163, 2002. On p. 594

[Menendez-Arias1998] L. Menendez-Arias, A. Mas, \& E. Domingo. Cytotoxic T-lymphocyte responses to HIV-1 reverse transcriptase (review). Viral Immunol 11:167-81, 1998. On p. 177 \begin{tabular}{|l|l|l|l|l|l|l|l|l|l|l|l|l|l|l|l|l|l|l|}
\hline 180 & 182 & 184 & 187 & 188 & 189 & 191 & 196 & 197 & 199 & 200 & 203 & 204 & 205 & 206 & 208 & 209 & 211 & 212 \\
\hline
\end{tabular}

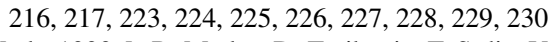

[Metlas1999a] R. Metlas, D. Trajkovic, T. Srdic, V. Veljkovic, \& A. Colombatti. Anti-V3 and anti$\mathrm{IgG}$ antibodies of healthy individuals share complementarity structures. J Acquir Immипe Defic Syndr 21:266-70, 1999. On p. 652

[Metlas1999b] R. Metlas, D. Trajkovic, T. Srdic, V. Veljkovic, \& A. Colombatti. Human immunodeficiency virus V3 peptide-reactive antibodies are present in normal HIV-negative sera. AIDS Res Hum Retroviruses 15:671-7, 1999. On p. 652 653
[Meyerhans1991] A. Meyerhans, G. Dadaglio, J. P. Vartanian, P. Langlade-Demoyen, R. Frank, B. Asjo, F. Plata, \& S. Wain-Hobson. In vivo persistence of an HIV-1-encoded HLA-B27restricted cytotoxic $\mathrm{T}$ lymphocyte epitope despite specific in vitro reactivity. Eur J Immunol 21:2637-2640, 1991. This study looked for the presence of CTL escape mutants in vivo in proviral DNA from an infected individual who had CTL activity; in 8 and 14 months escape mutants had not accumulated. On $\mathrm{p} .138$

Mhashilkar1995] A. M. Mhashilkar, J. Bagley, S. Y. Chen, A. M. Szilvay, D. G. Helland, \& W. A. Marasco. Inhibition of HIV-1 Tat-mediated LTR transactivation and HIV-1 infection by anti-Tat single chain intrabodies. EMBO J 14:1542-1551, 1995. Anti-Tat intrabodies specific for the N-terminal activation domain of Tat, block Tat-mediated transactivation of the HIV-1 LTR and intracellular trafficking of Tat in mammalian cells. Thus single chain intrabodies can effectively target molecules in the cytoplasm and nuclear compartments of eukaryotic cells and anti-Tat intrabodies may be useful for gene therapy of HIV-1 infection and AIDS. On p.610 611

[Michel1993] M. L. Michel, M. Mancini, K. Schlienger, \& P. Tiollais. Recombinant hepatitis B surface antigen as a carrier of human immunodeficiency virus epitopes. Res Virol 144(4):263-7, 1993. On p. 402728

Migueles2001] S. A. Migueles \& M. Connors. Frequency and function of HIV-specific CD8(+) T cells. Immunol Lett 79(1-2):141-150, 2001. On p. 80 |99|105| 121 |150|151

[Millar1998] A. L. Millar, N. A. Jackson, H. Dalton, K. R. Jennings, M. Levi, B. Wahren, \& N. J. Dimmock. Rapid analysis of epitope-paratope interactions between HIV-1 and a 17- aminoacid neutralizing microantibody by electrospray ionization mass spectrometry. Eur J Biochem 258:164-9, 1998. On p.661.

[Mills1990] K. H. G. Mills, A. L. Barnard, B. P. Mahon, P. A. Kitchin, S. E. Adams, S. M. Kingsman, \& A. J. Kingsman. Induction of HIV-specific immune responses in primates: fine specificity of antibody and helper T-cell recognition of the HIV p24 protein. Vaccines 90:213-218, 1990 Notes: Four cynomolgous macaques were immunized with 3 doses of p24 TY virus-like particles and their immune response was followed. Three 15 mer peptides stimulated CD4 T-cells proliferation and IL-2 production. Two of these responses were verified at the clonal level. B-cell responses were also studied in this paper. On $\mathrm{p} .433,434,436$

[Mitchell1998] W. M. Mitchell, L. Ding, \& J. Gabriel. Inactivation of a common epitope responsible for the induction of antibody-dependent enhancement of HIV. AIDS 12:147-56, 1998. On p.696 697699700702

[Mo1997] H. Mo, L. Stamatatos, J. E. Ip, C. F. Barbas, P. W. H. I. Parren, D. R. Burton, J. P. Moore, $\&$ D. D. Ho. Human immunodeficiency virus type 1 mutants that escape neutralization by human monoclonal antibody IgG1b12. J Virol 71:6869-6874, 1997. A JRCSF resistant variant was selected by culturing in the presence of IgG1b12. The resistant virus remained sensitive to $2 \mathrm{G} 12$ and $2 \mathrm{~F} 5$ and to $\mathrm{CD} 4-\mathrm{IgG}$, encouraging for the possibility of combination therapy. On p. 710 711784785822

Moja2000] P. Moja, C. Tranchat, I. Tchou, B. Pozzetto, F. Lucht, C. Desgranges, \& C. Genin Neutralization of human immunodeficiency virus type 1 (HIV-1) mediated by parotid IgA of HIV-1-infected patients [In Process Citation]. J Infect Dis 181:1607-13, 2000. On p.744]

[Mollet2000] L. Mollet, T. S. Li, A. Samri, C. Tournay, R. Tubiana, V. Calvez, P. Debre, C. Katlama, \& B. Autran. Dynamics of HIV-specific CD8+ T lymphocytes with changes in viral load.

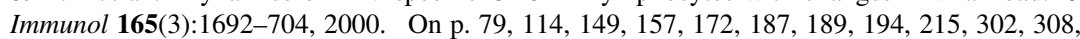
\begin{tabular}{ll|l|l|l|l|l|l|}
\hline 309 & 316 & 355 & 374 & 391 \\
\hline
\end{tabular}

Momany1996] C. Momany, L. C. Kovari, A. J. Prongay, W. Keller, R. K. Gitti, B. M. Lee, A. E. Gorbalenya, L. Tong, J. McClure, L. S. Ehrlich, M. F. Summers, C. Carter, \& M. G. Rossman. Crystal structure of dimeric HIV-1 capsid protein. Nature Struct Biol 3:763-770, 1996. On p. 580 [Mondor1998] I. Mondor, S. Ugolini, \& Q. J. Sattentau. Human immunodeficiency virus type 1 attachment to HeLa CD4 cells is CD4 independent and gp120 dependent and requires cell surface 


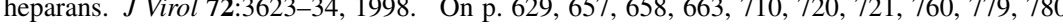
\begin{tabular}{ll|l|l|l|l|l|l|l|l|l|l|}
7884 & 786 & 803 & 804 & 822 & 823 \\
\hline
\end{tabular}

[Montefiori1991] D. C. Montefiori, I. Y. Zhou, B. Barnes, D. Lake, E. M. Hersh, Y. Masuho, \& L. B. Lefkowitz, Jr. Homotypic antibody responses to fresh clinical isolates of human immunodeficiency virus. Virology 182:635-643, 1991. On p.591

[Montefiori1993] D. C. Montefiori, B. S. Graham, J. Zhou, J. Zhou, R. A. Bucco, D. H. Schwartz L. A. Cavacini, M. R. Posner, \& the NIH-NIAID AIDS Vaccine Clinical Trials Network V3-Specific Neutralizing Antibodies in Sera From HIV-1 gp160-immunized Volunteers Block Virus Fusion and Act Synergistically with Human Monoclonal Antibody to the Conformationdependent CD4 Binding Site of gp120. J Clin Invest 92:840-847, 1993. On p.775776

[Montefiori1999] D. Montefiori \& T. Evans. Toward an HIV Type 1 vaccine that generates poten broadly cross-reactive neutralizing antibodies. AIDS Res and Human Retroviruses 15:689-98, 1999. On p.710 713 784 $787,822,824$

[Montefiori2001] D. C. Montefiori, T. S. Hill, H. T. T. Vo, B. D. Walker, \& E. S. Rosenberg. Neutralizing Antibodies Associated with Viremia Control in a Subset of Individuals after Treatment of Acute Human Immunodeficiency Virus Type 1 Infection. J Virol 75(21):10200-10207, 2001 On p. 593750

[Moog1997] C. Moog, H. J. A. Fleury, I. Pellegrin, A. Kirn, \& A. M. Aubertin. Autologous and Heterologous Neutralizing Antibody Responses Following Initial Seroconversion in Huma Immunodeficiency Virus Type 1-Infected Individuals. J Virol 71(5):3734-41, 1997. On p.749 750

[Moore1990a] J. P. Moore. Simple methods for monitoring HIV-1 and HIV-2 gp120 binding to soluble CD4 by enzyme-linked immunosorbent assay: HIV-2 has a 25 -fold lower affinity an HIV-1 for soluble CD4. AIDS NY 4:297, 1990. On p.760

[Moore1990b] J. P. Moore, J. A. McKeating, R. A. Weiss, \& Q. J. Sattentau. Dissociation of gp120 from HIV-1 virions induced by soluble CD4. Science 250:1139-1142, 1990. On p. 668

[Moore1993a] J. P. Moore \& D. D. Ho. Antibodies to discontinuous or conformationally sensitive epitopes on the gp120 glycoprotein of human immunodeficiency virus type 1 are highly prevalen in sera of infected humans. J Virol 67:863-875, 1993. CD4BS antibodies are prevalent in HIV1-positive sera, while neutralizing MAbs to C4, V2, and V3 and MAbs to linear epitopes are les common. Most linear epitope MAbs in human sera are directed against the V3 region, and crossreactive MAbs tend to be directed against discontinuous epitopes. On p. $617|621| 622|624| 631$

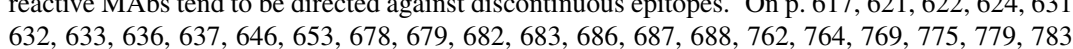
\begin{tabular}{r|r}
632 & 633 \\
\hline 803 & 811 \\
\hline
\end{tabular}

Moore1993b] J. P. Moore, Q. J. Sattentau, H. Yoshiyama, M. Thali, M. Charles, N. Sullivan, S.-W. Poon, M. S. Fung, F. Traincard, M. Pinkus, G. Robey, J. E. Robinson, D. D. Ho, \& J. Sodroski. Probing the structure of the V2 domain of human immunodeficiency virus type 1 surface glycoprotein gp120 with a panel of eight monoclonal antibodies: human immune response to the $\mathrm{V} 1$

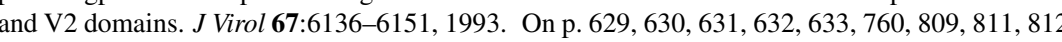

[Moore1993c] J. P. Moore, M. Thali, B. A. Jameson, F. Vignaux, G. K. Lewis, S.-W. Poon, M. S. Fung, P. J. Durda, L. Akerblom, B. Wahren, D. D. Ho, Q. J. Sattentau, \& J. Sodroski. Immunochemical analysis of the gp120 surface glycoprotein of human immunodeficiency virus type 1 : Probing the structure of the $\mathrm{C} 4$ and $\mathrm{V} 4$ domains and the interaction of the $\mathrm{C} 4$ domain with the V3 loop. J Virol 73:4785-4796, 1993. General observations: C4 and V3 MAbs are sensitive to the way the epitopes are presented, and this sensitivity cannot be correlated to peptide binding. Some V3-C4 domain interaction was indicated based on mutation and interference studies. On p.618 \begin{tabular}{|l|l|l|l|l|l|l|l|l|l|l|l|l|l|l|l|}
\hline 641 & 661 & 662 & 666 & 667 & 668 & 671 & 677 & 679 & 680 & 681 & 682 & 683 & 684 & 760 & 783 \\
\hline
\end{tabular}

Moore1993d] J. P. Moore, H. Yoshiyama, D. D. Ho, J. E. Robinson, \& J. Sodroski. Antigenic variation in gp120s from molecular clones of HIV-1 LAI. AIDS Res Hum Retroviruses 9:1185-1193, 1993. The binding of MAbs to four molecular clones of HIV-1 LAI: HxB2, HxB3, Hx10, and
NL4-3, was measured. Despite the close relationship between these clones, there is considerable variation in their antigenic structure, judged by MAb reactivities to the V2, V3, and $\mathrm{C} 4$ domain and to discontinuous epitopes. Small variations in sequence can profoundly affect recognition of gp120 by all five groups of defined anti-gp120 neutralizing antibodies. On p.798 803

Moore1994a] J. P. Moore, Y. Cao, D. D. Ho, \& R. A. Koup. Development of the anti-gp120 antibody response during seroconversion to human immunodeficiency virus type 1. J Virol 68:51425155,1994 . Three seroconverting individuals were studied. The earliest detectable anti-gp120 antibodies were both conformational and anti-V3 loop, and could be detected only after the peak viremia has passed. No uniform pattern of autologous neutralizing anti-CD4BS or anti-V3 MAbs was observed. On p. 657 720 762 764

Moore1994b] J. P. Moore, F. E. McCutchan, S.-W. Poon, J. Mascola, J. Liu, Y. Cao, \& D. D. Ho Exploration of antigenic variation in gp 120 from clades A through $\mathrm{F}$ of human immunodeficiency virus type 1 by using monoclonal antibodies. J Virol 68:8350-8364, 1994. Four of five anti-V3 MAbs were slightly cross-reactive within clade B, but not very reactive outside clade B. Two discontinuous CD4 binding site Mabs appear to be pan-reactive. Anti-V2 MAbs were only spo\begin{tabular}{lll|l|l|l|l|l|l|l|l|} 
radically reactive inside and outside of clade $\mathrm{B}$. On p. 632 & 652 & 657 & 663 & 664 & 668 & 756 & 762 \\
\hline
\end{tabular}

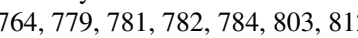

Moore1994c] J. P. Moore, Q. J. Sattentau, R. Wyatt, \& J. Sodroski. Probing the Structure of the Human Immunodeficiency Virus Surface Glycoprotein gp120 with a Panel of monoclona antibodies. J Virol 68:469-484, 1994. . This study compared a large number of MAbs that bind to linear epitopes of gp 120 , and compared binding affinities for: i) native and SDS-DDT denature gp120, (clone BH10 of the LAI isolate expressed in CHO cells); ii) recombinant gp120 lacking the V1, V2, V3 loops; iii) a panel of 20 mer peptides; iv) a panel of gp120 mutants; and v oligomeric versus monomeric gp120. The binding ratio of native versus denatured monomeric gp120 is included in the table in this database. These numbers should be considered with the following points in mind: a continuous epitope may be partially exposed on the surface; and a preparation of rgp120 is not homogeneous and contains fully folded, partly denatured, and some completely unfolded species, so the conformation of what is considered to be a native protein will not only reflect fully folded gp120. The authors suggest that a fivefold increase in the affinity for a MAb binding to denatured versus native gp120 indicates that the epitope is inaccessible in the native form. We also have included here information extracted from Moore et al's list of the gp120 mutations that reduced the binding of a particular MAb. In mapping of exposed regions of gp120, C2, C3, and C5 domain epitopes were found to bind preferentially to denatured gp120. $\mathrm{V} 1, \mathrm{~V} 2$ and $\mathrm{V} 3$, part of $\mathrm{C} 4$, and the extreme carboxy terminus of $\mathrm{C} 5$ were exposed on the native monomer. In the oligomeric form of the molecule, only V2, V3 and part of C4 are well exposed \begin{tabular}{ll|l|l|l|l|l|l|l|l|l|l|l|} 
as continuous epitopes. On $\mathrm{p} .615$ & 616 & 617 & 618 & 619 & 620 & 621 & 622 & 623 & 624 & 625 & 634 & 635 \\
\hline
\end{tabular}

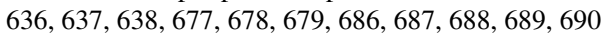

[Moore1994d] J. P. Moore, R. L. Willey, G. K. Lewis, J. Robinson, \& J. Sodroski. Immunological evidence for interactions between the first, second and fifth conserved domains of the gp120 surface glycoprotein of human immunodeficiency virus type 1. J Virol 68:6836-6847, 1994 Mutation 267N/Q in C2 region results in exposing the carboxy-terminal end gp120. On p. 615

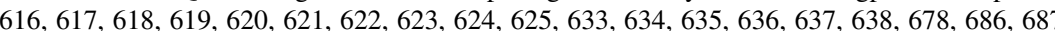
688690753758

Moore1995a] J. P. Moore, Y. Cao, L. Qing, Q. J. Sattentau, J. Pyati, R. Koduri, J. Robinson, C. F Barbas III, D. R. Burton, \& D. D. Ho. Primary isolates of human immunodeficiency virus type are relatively resistant to neutralization by monoclonal antibodies to gp120, and their neutralization is not predicted by studies with monomeric gp120. J Virol 69:101-109, 1995. A panel of anti-gp120 MAbs and sera from HIV-1 infected individuals was tested for its ability to neutralize primary isolates. Most MAbs bound with high affinity to gp120 monomers from the various isolates, but were not effective at neutralizing. The MAb IgG1b12, which binds to a discontinuous 
anti-CD4 binding site epitope, was able to neutralize most of the primary isolates. On p. 657 $720721,781,782,784$

[Moore1995b] J. P. Moore \& D. D. Ho. HIV-1 neutralization: the consequences of adaptation to growth on transformed T-cells. AIDS 9 suppl A:S117-S136, 1995. This review considers the relative importance of a neutralizing antibody response for the development of a vaccine, and for disease progression during the chronic phase of HIV-1 infection. It suggests that T-cell immunity may be more important. The distinction between MAbs that can neutralize primary isolates, and those that are effective at neutralizing only laboratory adapted strains is discussed in detail. Alternative conformations of envelope and non-contiguous interacting domains in gp120 are discussed. The suggestion that soluble monomeric gp120 may serve as a viral decoy that diverts the humoral immune response it in vivo is put forth. On p.628 657 $710,711,720,721$ $756757,784,822$

[Moore1995c] J. P. Moore, A. Trkola, B. Korber, L. J. Boots, J. A. Kessler II, F. E. McCutchan, J. Mascola, D. D. Ho, J. Robinson, \& A. J. Conley. A human monoclonal antibody to a complex epitope in the $\mathrm{V} 3$ region of gp 120 of human immunodeficiency virus type 1 has broad reactivity within and outside clade B. J Virol 69:122-130, 1995. The epitope was defined as including amino acids on both sides of the loop of the V3 loop: -I-G-FY-T, where the G is the second $G$ of the GPGR tip of the loop. This antibody bound well to gp120 molecules from clades A,B,C,E and $\mathrm{F}$, when the critical amino acids were present. Binding did not parallel neutralization however, $19 \mathrm{~b}$ could produce a 50 -fold reduction of infectivity in some primary B isolates, and in C clade isolates at low virus input concentrations, but not in isolates from all clades where binding could occur (A,E, and F). On p. 657

[Moore1996] J. P. Moore \& J. Sodroski. Antibody cross-competition analysis of the human immunodeficiency virus type 1 gp120 exterior envelope glycoprotein. J Virol 70:1863-1872, 1996. 46 anti-gp120 monomer MAbs were used to create a competition matrix, and MAb competition groups were defined. The data suggests that there are two faces of the gp120 glycoprotein: a face occupied by the CD4BS, which is presumably also exposed on the oligomeric envelope glycoprotein complex, and a second face which is presumably inaccessible on the oligomer an

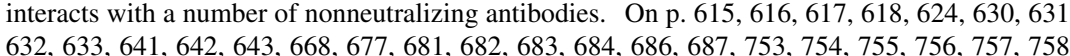
\begin{tabular}{|l|l|l|l|l|l|l|l|l|l|l|l|l|l|l|l|l|l|}
\hline 762 & 763 & 764 & 765 & 779 & 784 & 794 & 796 & 798 & 803 & 804 & 811 & 812 & 813 & 816 & 818 & 822 \\
\hline
\end{tabular}

[Moore1997] J. Moore \& A. Trkola. HIV type 1 coreceptors, neutralization serotypes and vaccine develop J. AIDS Res Hum Retriiuses 13:733-736, 1997. On p 710,712 784785] \begin{tabular}{ll|l|l|l|l|l|l|l|l|l|l|} 
development. AIDS Res Hum Retroviruses 13:733-736, 1997. On p.710 & 712 & 784 & 785 \\
\hline Moore1998] J. P. Moore \& J. Binley. HIV Envelope's letters boxed into shape. Nature
\end{tabular} 393:630-631, 1998. Comment on Nature 1998 Jun 18;393(6686):648-59 and Nature 1998 Ju 18;393(6686):705-11. On p.798.799

[Moore1999] J. P. Moore \& D. R. Burton. HIV-1 neutralizing antibodies: How full is the bottle? Nat Med 5(2):142-144, 1999. On p.744 752

[Moore2001] J. P. Moore, P. W. Parren, \& D. R. Burton. Genetic subtypes, humoral immunity, and human immunodeficiency virus type 1 vaccine development. J Virol 75(13):5721-9, 2001. On p. $71007150798 \quad 822825$

Moore2002a] A. C. Moore, W.-p. Kong, B. K. Chakrabarti, \& G. J. Nabel. Effects of antigen and genetic adjuvants on immune responses to human immunodeficiency virus DNA vaccines in mice. J Virol 76(1):243-250, 2002. On p. 287.

[Moore2002b] C. B. Moore, M. John, I. R. James, F. T. Christiansen, C. S. Witt, \& S. A. Mallal. Evidence of HIV-1 adaptation to HLA-restricted immune responses at a population level. Science 296(5572): 1439-1443, 2002. On p. 187] 190 [192] 195$] 244$

Moran1993] M. J. Moran, J. S. Andris, Y.-I. Matsumato, J. D. Capra, \& E. M. Hersh. Variable region genes of anti-HIV human monoclonal antibodies: Non-restricted use of the $\mathrm{V}$ gene repertoire and extensive somatic mutation. Mol Immunol 30:1543-1551, 1993. Sequenced variable regions from four human anti-HIV-1 MAbs: anti-gp120 13, S1-1 and HBW4; and anti-gp41 No.86. Extensive somatic mutation was observed and under-representation of $\mathrm{V}_{H}$ III usage. On p. 699 729 738 795

Morner1999] A. Morner, A. Achour, M. Norin, R. Thorstensson, \& E. Bjorling. Fine characterization of a V3-region neutralizing epitope in human immunodeficiency virus type 2 [In Proces Citation]. Virus Res 59:49-60, 1999. On p.677

[Morris2000] C. B. Morris, E. Cheng, A. Thanawastien, L. Cardenas-Freytag, \& J. D. Clements. Effectiveness of intranasal immunization with HIV-gp160 and an HIV-1 env CTL epitope peptide (E7) in combination with the mucosal adjuvant LT(R192G). Vaccine 18:1944-51, 2000. On p. 290 529

[Morris2001] M. K. Morris, D. A. Katzenstein, D. Israelski, A. Zolopa, R. M. Hendry, \& C. V. Hanson. Characterization of the HIV-1 specific humoral immune response during highly active antiretroviral therapy (HAART). J Acquir Immune Defic Syndr 28(5):405-415, 2001. On p.642 749

Moss1995] P. A. H. Moss, S. L. Rowland-Jones, P. M. Frodsham, S. McAdam, P. Giangrande, A. McMichael, \& J. I. Bell. Persistent high frequency of human immunodeficiency virus-specific cytotoxic T cells in peripheral blood of infected donors. Proc Nat Acad Sci USA 92:5773-5777, 1995. On p. 134217

Moss1997] R. B. Moss, R. J. Trauger, W. K. Giermakowska, J. L. Turner, M. R. Wallace, F. C Jensen, S. P. Richieri, F. Ferre, A. E. Daigle, C. Duffy, G. Theofan, \& D. J. Carlo. Effect of immunization with an inactivated gp120-depleted HIV-1 immunogen on beta-chemokine and cytokine production in subjects with HIV-1 infection. J Acquir Immune Defic Syndr Hum Retrovirol 14(4):343-350, 1997. On p. 535

[Moss1998] R. B. Moss, M. R. Wallace, P. Lanza, W. Giermakowska, F. C. Jensen, G. Theofan C. Chamberlin, S. P. Richieri, \& D. J. Carlo. In vitro p24 antigen-stimulated lymphocyte proliferation and beta- chemokine production in human immunodeficiency virus type 1 (HIV-1) seropositive subjects after immunization with an inactivated gp120- depleted HIV-1 immunogen (Remune). Clin Diagn Lab Immunol 5:308-12, 1998. On p.441,442

[Moss1999] R. B. Moss, M. R. Wallace, W. K. Giermakowska, E. Webb, J. Savary, C. Chamberlin Brandt, G. Theofan, R. Musil, S. P. Richieri, F. C. Jensen, \& D. J. Carlo. Phenotypic analysis of human immunodeficiency virus (HIV) type 1 cell-mediated immune responses after treatment with an HIV-1 immunogen. J Infect Dis 180(3):641-648, 1999. On p. 535

[Moss2000] R. B. Moss, J. Diveley, F. C. Jensen, E. Gouveia, J. Savary, \& D. J. Carlo. HIVspecific cd4(+) and cd8(+) immune responses are generated with a gp120-depleted, whole-killed HIV-1 immunogen with cpg immunostimulatory sequences of DNA. J Interferon Cytokine Res 20(12):1131-7, 2000. On p. 444592

[Moss2001] R. B. Moss, J. Diveley, F. C. Jensen, E. Gouveia, \& D. J. Carlo. Human immunodeficiency virus (HIV)-specific immune responses are generated with the simultaneous vaccination of a gp120-depleted, whole-killed HIV-1 immunogen with cytosine-phosphorothioate-guanine dinucleotide immunostimulatory sequences of DNA. J Hum Virol 4(1):39-43, 2001. On p. 444 [Moulard2002] M. Moulard, S. K. Phogat, Y. Shu, A. F. Labrijn, X. Xiao, J. M. Binley, M.-Y. Zhang, I. A. Sidorov, C. C. Broder, J. Robinson, P. W. H. I. Parren, D. R. Burton, \& D. S. Dimitrov. Broadly cross-reactive HIV-1-neutralizing human monoclonal Fab selected for binding to gp120CD4-CCR5 complexes. Proc Natl Acad Sci U S A 99(10):6913-6918, 2002. On p. 806

[Moureau2002] C. Moureau, P.-L. Vidal, Y. Bennasser, M. Moynier, Y. Nicaise, M. Aussillous, S. Barthelemy, L. Montagnier, \& E. Bahraoui. Characterization of humoral and cellular immune responses in mice induced by immunization with HIV-1 Nef regulatory protein encapsulated in poly(DL-lactide-co-glycolide) microparticles. Mol Immunol 38(8):607-618, 2002. On p.534 836838840

[Muhlbacher1999] M. Muhlbacher, M. Spruth, F. Siegel, R. Zangerle, \& M. P. Dierich. Longitudinal 
study of antibody reactivity against HIV-1 envelope and a peptide representing a conserved site on Gp41 in HIV-1-infected patients. Immunobiology 200:295-305, 1999. On p.710 713

[Muller1991] S. Muller, H.-T. Wang, S.-V. Kaveri, S. Chattopadhyay, \& H. Kohler Generation an Specificity of Monoclonal Anti-Idiotypic Antibodies Against Human HIV-Specific Antibodies. Immunol 147:933-941, 1991. On p.738

[Musey1997] L. Musey, Y. Hu, L. Eckert, M. Christensen, T. Karchmer, \& M. J. McElrath. HIV 1 induces cytotoxic T lymphocytes in the cervix of infected women. J Exp Med 185:293-303, 1997. Mononuclear cells in cytobrush specimens from the cervical samples were stimulated with antigen. Eight women with CD4 positive counts $>=500$ cells $/ \mu 1$ had HIV-1-specific CTL, but only $4 / 11$ with counts $<500$ cells/ $\mu$ l had HIV-1-specific CTL responses. On p. 97] 104] 141 217

[Muster1993] T. Muster, F. Steindl, M. Purtscher, A. Trkola, A. Klima, G. Himmler, F. Ruker, \& $\mathrm{H}$. Katinger. A conserved neutralizing epitope on gp41 of human immunodeficiency virus type 1. J Virol 67:6642-6647, 1993. Peptides containing the amino acid sequence LDKWAS or DKWASL showed reduced reactivity. The peptides LELDKW and KWASLW showed no significant reaction. These data suggest that the epitope of the MAb 2F5 comprises the amino acid sequence ELDKWA, with DKWA being the core sequence. On p.710

[Muster1994] T. Muster, R. Guinea, A. Trkola, M. Purtscher, A. Klima, F. Steindl, P. Palese, \& $\mathrm{H}$. Katinger. Cross-Neutralization activity against divergent human immunodeficiency virus type 1 isolates induced by the gp41 sequence ELDKWAS. $J$ Virol 68:4031-4034, 1994. On p. 710 718

[Muster1995] T. Muster, B. Ferko, A. Klima, M. Purtscher, A. Trkola, P. Schulz, A. Grassauer, O. G. Englehard, A. Garcia-Sastre, P. Palese, \& H. Katinger. Mucosal Model of Immunization against human immunodeficiency virus type 1 with a chimeric influenza virus. J Virol 69:6678-6686, 1995. On p.718

[Mutch1994] D. Mutch, J. Underwood, M. Geysen, \& S. Rodda. Comprehensive T-cell epitope mapping of HIV-1 env antigens reveals many areas recognized by HIV-1-seropositive and by lowrisk HIV-1-seronegative individuals. J Acquir Immune Defic Syndr 7(9):879-90, 1994. Notes: The proliferative T-cell response to pools of overlapping 17 mer peptides spanning Env were tested in both seronegative and low risk seropositive people. The pool that gave the greatest number of responders was pool 25, located in gp41. The 17 mer peptides used in this pool were tested individually for their ability to stimulate T-cell proliferation, and the most critical regions were found to be GIWGCSGKLIC and PWNASWSN. Mutch et al. suggest that the proliferative response in HIV-1 seronegative individuals is more likely due to cross-reactive, non-HIV induced memory cells than naive T-cells. On p.516 517

[Muthumani2002] K. Muthumani, D. S. Hwang, N. S. Dayes, J. J. Kim, \& D. B. Weiner. The HIVaccessory gene $v p r$ can inhibit antigen-specific immune function. DNA Cell Biol 21(9):689-695, 2002. On p. 171254398

[Myers1993] R. Myers, T. Meiller, W. Falkler, Jr., J. Patel, \& J. Joseph. A human monoclona antibody to a cryptic gp41 epitope on HIV-1 infected cells. Abstr Gen Meet Am Soc Microbio 93:444, 1993. Aidsline: 93291838 Abstract T70. On p. 830

[Nabel2002] G. J. Nabel. HIV vaccine strategies. Vaccine 20(15):1945-1947, 2002. On p. 166 337

[Nakagawa2000] Y. Nakagawa, T. Takeshita, J. A. Berzofsky, \& H. Takahashi. Analysis of the mechanism for extracellular processing in the presentation of human immunodeficiency virus1 envelope protein-derived peptide to epitope-specific cytotoxic T lymphocytes. Immunology 101(1):76-82, 2000. On p. 297

[Nakamura1992] G. R. Nakamura, R. Byrn, K. Rosenthal, J. P. Porter, M. R. Hobbs, L. Riddle, D. J. Eastman, D. Dowbenko, T. Gregory, B. M. Fendly, \& P. W. Berman. Monoclonal antibodies to the extracellular domain of HIV-1 IIIB gp160 that neutralize infectivity, block binding to CD4,
684 H. C. Wessling, M. A. Norcross, B. M. Fendly, \& P. W. Berman. Strain specificity and binding affinity requirements of neutralizing monoclonal antibodies to the $\mathrm{C} 4$ domain of gp120 from human immunodeficiency virus type 1. J Virol 67:6179-6191, 1993. Multiple CD4 binding domain antibodies are described; only one has a linear peptide reactivity (13H8). A V3 loop binding antibody is also described (1026). On p. 673684

Nakamura1997] Y. Nakamura, M. Kameoka, M. Tobiume, M. Kaya, K. Ohki, T. Yamada, \& $\mathrm{K}$. Ikuta. A chain section containing epitopes for cytotoxic T, B and helper T cells within a highly conserved region found in the human immunodeficiency virus type $1 \mathrm{Gag}$ protein. Vaccine 5:489-96, 1997. On p. 141] 438

[Nara1990] P. L. Nara, L. Smit, N. Dunlop, W. Hatch, M. Merges, D. Waters, J. Kelliher, R. C. Gallo, P. J. Fischinger, \& J. Goudsmit. Emergence of viruses resistant to neutralization by V3specific antibodies in experimental human immunodeficiency virus type 1 IIIB infection of chimpanzees. J Virol 64:3779-3791, 1990. On p.671.

[Nehete1993] P. N. Nehete, W. C. Satterfield, C. M. Matherne, R. B. Arlinghaus, \& K. J. Sastry. Induction of human immunodeficiency virus-specific $\mathrm{T}$ cell responses in rhesus monkeys by synthetic peptides from gp160. AIDS Res Hum Retroviruses 9:235-40, 1993. Notes: Three rhesus monkeys were immunized with eight synthetic peptides that induce $\mathrm{T}$ cell activity in mice. PBMCs from these monkeys were monitored every 2 weeks for 34 weeks for proliferative responses against individual peptides and gp160. On p.467, 473 479|486,491, 515 |516

[Nehete1995] P. N. Nehete, K. S. Casement, R. B. Arlinghus, \& K. J. Sastry. Studies on in vivo induction of HIV-1 Envelope-specific cytotoxic T lymphocytes by synthetic peptides from the V3 loop region of HIV-1 IIIB gp120. Cellular Immunology 160:217-223, 1995. On p.294

Nehete1998a] P. N. Nehete, D. E. Lewis, D. N. Tang, M. S. Pollack, \& K. J. Sastry. Presence of HLA-C-restricted cytotoxic T-lymphocyte responses in long-term nonprogressors infected with human immunodeficiency virus. Viral Immunol 11:119-29, 1998. On p. 271 $307 \mid 316$

[Nehete1998b] P. N. Nehete, S. J. Schapiro, P. C. Johnson, K. K. Murthy, W. C. Satterfield, \& K. J. Sastry. A synthetic peptide from the first conserved region in the envelope protein gp160 is strong T-cell epitope in HIV-infected chimpanzees and humans. Viral Immunol 11:147-58, 1998. On p.467 480 505. 515,516

Neidleman2000] J. A. Neidleman, M. Vajdy, M. Ugozzoli, G. Ott, \& D. O’Hagan. Genetically detoxified mutants of heat-labile enterotoxin from Escherichia coli are effective adjuvants for induction of cytotoxic T-cell responses against HIV-1 gag-p55. Immunology 101(1):154-60, 2000. On p. 116

[Neshat2000] M. N. Neshat, L. Goodglick, K. Lim, \& J. Braun. Mapping the B cell superantigen binding site for HIV-1 gp120 on a V(H)3 Ig. Int Immunol 12:305-12, 2000. On p.748

[Neurath1990] A. R. Neurath \& N. Strick. Confronting the hypervariability of an immunodominant epitope eliciting virus neutralizing antibodies from the envelope glycoprotein of the human immunodeficiency virus type 1. Mol Immunol 27:539-549, 1990. On p. 639

[Neurath1995] A. R. Neurath, N. Strick, K. Lin, \& S. Jiang. Multifaceted consequences of anti-gp41 monoclonal antibody 2F5 binding to HIV type 1 virions. AIDS Res Hum Retroviruses 11:687-96, 1995. On p.710 711

Newman1997] M. J. Newman, J.-Y. Wu, B. H. Gardner, C. A. Anderson, C. R. Kensil, J. Recchia, R. T. Coughlin, \& M. F. Powell. Induction of cross-reactive cytotoxic T-lymphocyte response specific for HIV-1 gp120 using saponin adjuvant (QS-21) supplemented subunit vaccine formulations. Vaccine 15:1001-1007, 1997. On p.290.

[Newman2002] M. J. Newman, B. Livingston, D. M. McKinney, R. W. Chesnut, \& A. Sette. Tlymphocyte epitope identification and their use in vaccine development for HIV-1. Front Biosci 
7:d1503-1515, 2002. On p. 400

[Nichols2002] C. N. Nichols, I. Bernal, A. M. Prince, \& L. Andrus. Comparison of two differen preparations of HIV immune globulin for efficiency of neutralization of HIV type 1 primary isolates. AIDS Res Hum Retroviruses 18(1):49-56, 2002. On p.594 738739

[Nickle2003] D. C. Nickle, M. A. Jensen, G. S. Gottlieb, D. Shriner, G. H. Learn, A. G. Rodrigo, \& J. I. Mullins. Consensus and ancestral state HIV vaccines. Science 299(5612):1515-1518, 2003 On p. 400

[Niedrig1988] M. Niedrig, J.-P. Rabanus, J. L. Stehr, H. R. Gelderblom, \& G. Pauli. Monoclona antibodies directed against human immunodeficiency virus gag proteins with specificity for conserved epitopes in HIV-1, HIV-2 and simian immunodeficiency virus. J Gen Virol 69:2109-2114, 1988. On p. 578 579583

[Niedrig 1989] M. Niedrig, J. Hinkula, W. Weigelt, J. L'Age-Stehr, G. Pauli, J. Rosen, \& B. Wahren Epitope mapping of monoclonal antibodies against human immunodeficiency virus type 1 structural proteins by using peptides. J Virol 63:3525-3528, 1989. Multiple linear MAb epitopes were described in p24 and p17. Several MAbs were able to react with HIV-2 ROD and SIV MAC in an immunoblot assay, as well as with HIV-1. On p. 573 578 57958

[Niedrig 1991] M. Niedrig, J. Hinkula, H.-P. Harthus, M. Broker, L. Hopp, G. Pauli, \& B. Wahren Characterization of murine monoclonal antibodies directed against the core proteins of huma immunodeficiency virus types 1 and 2 . J Virol 65:4529-4533, 1991. Multiple anti-HIV p24 MAbs were generated using HIV-1 IIIB p24 or HIV-2 ROD p26 as immunogens. The epitopes for these MAbs were mapped, and the cross-reactivity between HIV-1 IIIB, HIV-2 ROD and SIV MAC antigens were compared using multiple antibody binding assays. While some of the antibodies raised were cross-reactive by some or all of the assays, (ELISA, WB, immunofluorescence, immunoprecipitation and alkaline phosphatase anti-alkaline phosphatase assay), the different assays often gave different results. Only the antibodies raised to HIV-1 IIIB p24 are included in this database. On p. 575 [576 577 [58 580 584 585

[Niedrig1992a] M. Niedrig, M. Broker, G. Walter, W. Stuber, H.-P. Harthus, S. Mehdi, H. R. Gelderblom, \& G. Pauli. Murine monoclonal antibodies directed against the transmembrane protein gp41 of human immunodeficiency virus type 1 enhance its infectivity. J Gen Virol 73:951954, 1992. On p. 725726

[Niedrig1992b] M. Niedrig, H.-P. Harthus, M. Broker, H. Bickhard, G. Pauli, H. R. Gelderblom, \& B. Wahren. Inhibition of viral replication by monoclonal antibodies directed against human immunodeficiency virus gp120. J Gen Virol 73:2451-2455, 1992. On p.616 617 618 624

[Nietfeld1995] W. Nietfeld, M. Bauer, M. Fevrier, R. Maier, B. Holzwarth, R. Frank, B. Maier Y. Riviere, \& A. Meyerhans. Sequence constraints and recognition by CTL of an HLA-B27 restricted HIV-1 gag epitope. J Immunol 154:2188-2197, 1995. Single point mutations were introduced into this epitope in the viral strain LAI, and the ability of comparable peptides to sensitize target strains was tested. The change of anchor residue R 264 to (L or G), results in infectious virus, and corresponding peptide has reduced binding affinities for HLA-B27. The change of G 267 to K or E abrogated infectivity, and the peptide bound to HLA-B27, but did no serve as a target; thus nonrecognition of peptides derived from quasispecies analysis of a smal region might not really be associated with an escape mutant, but rather a non-viable mutant. On p. 135

[Nilsen1996] B. M. Nilsen, I. R. Haugan, K. Berg, L. Olsen, P. O. Brown, \& D. E. Helland. Monoclonal Antibodies against Human Immunodeficiency virus type 1 integrase: epitope mapping and differential effects of integrase activities in vitro. J Virol 70:1580-1587, 1996. In this study, 17 anti-integrase murine Mabs were generated and epitopes were mapped by deletion mutations and peptide scanning. The ability of MAb binding to inhibit (or stimulate) end-processing, DNA joining, reintegration, and disintegration enzyme functions it in vitro was determined. On p.571 \begin{tabular}{ll|l|l|l|l|l|l|l|l|l|}
6000 & 601 & 602 & 604 & 679 & 837 \\
\hline
\end{tabular}
[Nitayaphan2000] S. Nitayaphan, C. Khamboonruang, N. Sirisophana, P. Morgan, J. Chiu, A. M. Duliege, C. Chuenchitra, T. Supapongse, K. Rungruengthanakit, M. de Souza, J. R. Mascola, K. Boggio, S. Ratto-Kim, L. E. Markowitz, D. Birx, V. Suriyanon, J. G. McNeil, A. E. Brown, R. A. Michael, \& AFRIMS-RIHES Vaccine Evaluation Group. A phase I/II trial of HIV SF2 gp120/MF59 vaccine in seronegative Thais. Vaccine 18(15):1448-55, 2000. On p.746

[Nixon1988] D. Nixon, A. Townsend, J. Elvin, C. Rizza, J. Gallway, \& A. McMichael. HIV-1 gagspecific cytotoxic $\mathrm{T}$ lymphocytes defined with recombinant vaccinia virus and synthetic peptides. Nature 336:484-487, 1988. p24 KRWIILGLNKIVRMY. On p. [115][138

[Nixon1990] D. F. Nixon, S. Huet, J. Rothbard, M.-P. Kieny, M. Delchambre, C. Thiriart, C. R. Rizza, F. M. Gotch, \& A. J. McMichael. An HIV-1 and HIV-2 cross-reactive cytotoxic T cell epitope. AIDS 4:841-845, 1990. An HLA-B27 specific CTL clone from an HIV-1 infected individual that reacts with the Gag SF2 epitope KRWIILGLNKIVRMY also cross-reacts with the HIV-2 ROD analog RRWIQIGLQKSVRMY. The CTL also reacts with HIV-1 ELI KRWIIVGLNKIVRMY and SIVmm142 RRWIQLGLQKSVRMY, but only at very high concentration of peptide with SIVk6w78 RRWIQLRLQKSVRMY. The binding of the SIVk6w78 peptide to HLA-B27 does not seem to be reduced, so the authors suggest that the reduced ability to stimulate is in this case due to T-cell receptor interaction. On p. 138

[Nixon1991] D. F. Nixon \& A. J. McMichael. Cytotoxic T cell recognition of HIV proteins and peptides. AIDS 5:1049, 1991. p17: LRPGGKKKYKLKHIV, HLA B8 and p24: VQNANPDCKTILKAL, HLA B8. On p. 66153

[Nixon1999] D. F. Nixon, D. Douek, P. J. Kuebler, X. Jin, M. Vesanen, S. Bonhoeffer, Y. Cao, R. A. Koup, D. D. Ho, \& M. Markowitz. Molecular tracking of an Human Immunodeficiency Virus nef specific cytotoxic $\mathrm{T}$ cell clone shows persistence of clone-specific $\mathrm{T}$ cell receptor DNA but not mRNA following early combination antiretroviral therapy. Immunol Lett 66:219-28, 1999. On p. 361

Norris2001] P. J. Norris, M. Sumaroka, C. Brander, H. F. Moffett, S. L. Boswell, T. Nguyen, Y. Sykulev, B. D. Walker, \& E. S. Rosenberg. Multiple effector functions mediated by human immunodeficiency virus-specific CD4+ T-cell clones. J Virol 75(20):9771-9779, 2001. On p. 434445

[Notka1999] F. Notka, C. Stahl-Hennig, U. Dittmer, H. Wolf, \& R. Wagner. Construction and characterization of recombinant VLPs and Semliki-Forest virus live vectors for comparative evaluation in the SHIV monkey model. Biol Chem 380:341-52, 1999. On p. 335

[Novitsky2001] V. Novitsky, N. Rybak, M. F. McLane, P. Gilbert, P. Chigwedere, I. Klein S. Gaolekwe, S. Y. Chang, T. Peter, I. Thior, T. Ndung'u, F. Vannberg, B. T. Foley, R. Marlink, T. H. Lee, \& M. Essex. Identification of human immunodeficiency virus type 1 subtype C Gag-, Tat-, Rev-, and Nef-specific elispot-based cytotoxic T-lymphocyte responses for AIDS \begin{tabular}{|l|l|l|l|l|l|l|} 
vaccine design. $J$ Virol 75(19):9210-28, 2001. On p.72 $110 \mid 141$ & 144 & 160 & 257 & 265 & 396 \\
\hline
\end{tabular}

[Novitsky2002] V. Novitsky, H. Cao, N. Rybak, P. Gilbert, M. F. McLane, S. Gaolekwe, T. Peter, I. Thior, T. Ndung'u, R. Marlink, T. H. Lee, \& M. Essex. Magnitude and frequency of cytotoxic T-lymphocyte responses: Identification of immunodominant regions of human immunodeficiency \begin{tabular}{ll|l|l|l|l|l|} 
virus type 1 subtype C. J Virol 76(20):10155-10168, 2002. On p. 104 & 109 & 141 & 148 & 155 & 184
\end{tabular}

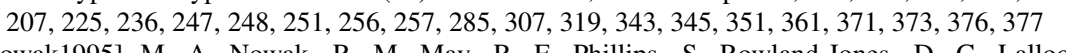

Nowak1995] M. A Nowak, R. M. May, R. E. Phillips, S. Rowland-Jones, D. G. Lalloo, S. McAdam, P. Klenerman, B. Koppe, K. Sigmund, C. R. M. Bangham, \& A. J. McMichael Antigenic oscillations and shifting immunodominance in HIV-1 infections. Nature 375:606-611, 1995. This paper presents longitudinal studies of epitope variation and corresponding CTL responses in two patients. A mathematical model was created to provide a framework to explain the observed shifts in epitope and CTLp frequencies. For discussion, see also: J. M. Coffin, Nature 375:534-535 (1995). On p. 67, 128 135] 154

[Nunberg2002] J. H. Nunberg. Retraction. Science 296(5570):1025, 2002. Retraction of 
LaCasse1999. On p.749

[Nyambi1998] P. N. Nyambi, M. K. Gorny, L. Bastiani, G. van der Groen, C. Williams, \& S. ZollaPazner. Mapping of epitopes exposed on intact human immunodeficiency virus type 1 (HIV-1) virions: a new strategy for studying the immunologic relatedness of HIV-1. J Virol 72:9384-91, 1998. 18 human MAbs binding to gp120 and gp41 were tested using a novel assay to test binding to intact HIV-1 virions. The new method involves using MAbs to the host proteins incorporated into virions to bind them to ELIZA plates. Antigenic conservation in epitopes of HIV-1 in clades A, B, D, F, G, and $\mathrm{H}$ was studied. MAbs were selected that were directed against V2, V3, CD4bd C5 or gp41 regions. Antibodies against V2, the CD4BS, and sp41 showed weak and sporadic reactivities, while binding strongly to gp 120 , suggesting these epitopes are hidden when gp 120 is \begin{tabular}{ll|l|l|l|l|l|l|l|l|l|l|l|l|l|} 
in its native, quaternary structure. On p. 628 & 645 & 646 & 653 & 659 & 660 & 675 & 689 & 692 & 708 & 720 \\
\hline
\end{tabular}

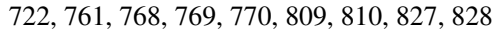

[Nyambi2000] P. N. Nyambi, H. A. Mbah, S. Burda, C. Williams, M. K. Gorny, A. Nadas, \& S. Zolla-Pazner. Conserved and exposed epitopes on intact, native, primary human immunodeficiency virus type 1 virions of group M. J Virol 74:7096-107, 2000. On p. 628 635643644 \begin{tabular}{|l|l|l|l|l|l|l|l|l|l|l|l|l|l|l|l|l|l|l||}
\hline 645 & 646 & 647 & 648 & 649 & 653 & 654 & 655 & 659 & 660 & 661 & 664 & 665 & 666 & $\frac{669}{675}$ & $\frac{676}{67}$ & 689 & 692 \\
\hline 693 & 696 & 697 & 700 & 701 & 702 & 708 & 709 & 710 & 714 & 720 & 722 & 761 & 766 & 767 & 768 & 769 & 770 & 784 \\
\hline 788
\end{tabular}

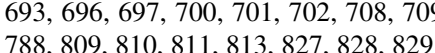

Oelemann2002] W. M. R. Oelemann, C. M. Lowndes, G. C. Veríssimo da Costa, M. G. Morgado, L. R. R. Castello-Branco, B. Grinsztejn, M. Alary, \& F. I. Bastos. Diagnostic Detection of Human Immunodeficiency Virus Type 1 Antibodies in Urine: A Brazilian Study. J Clin Microbio 40(3):881-885, 2002. On p. 842 [ 843

[Ogg1998a] G. S. Ogg, T. Dong, P. Hansasuta, L. Dorrell, J. Clarke, R. Coker, G. Luzzi, C. Conlon, A. P. McMichael, \& S. Rowland-Jones. Four novel cytotoxic T-lymphocyte epitopes in the highly conserved major homology region of HIV-1 Gag, restricted through B*4402, B*1801, A*2601, B*70. AIDS 12:1561-3, 1998. On p.141 144

[Ogg1998b] G. S. Ogg, X. Jin, S. Bonhoeffer, P. R. Dunbar, M. A. Nowak, S. Monard, J. P. Segal, Y. Cao, S. L. Rowland-Jones, V. Cerundolo, A. Hurley, M. Markowitz, D. D. Ho, D. F. Nixon, \& A. J. McMichael. Quantitation of HIV-1-specific cytotoxic T lymphocytes and plasma load o viral RNA. Science 279:2103-6, 1998. On p.76 211 273

[Ogg1999] G. S. Ogg, X. Jin, S. Bonhoeffer, P. Moss, M. A. Nowak, S. Monard, J. P. Segal, Y. Cao, S. L. Rowland-Jones, A. Hurley, M. Markowitz, D. D. Ho, A. J. McMichael, \& D. F. Nixon. Decay kinetics of human immunodeficiency virus-specific effector cytotoxic T lymphocytes after combination antiretroviral therapy. J Virol 73:797-800, 1999. On p.75 213 274

[Oggioni1999] M. R. Oggioni, D. Medaglini, L. Romano, F. Peruzzi, T. Maggi, L. Lozzi, L. Bracci,

M. Zazzi, F. Manca, P. E. Valensin, \& G. Pozzi. Antigenicity and immunogenicity of the V3 domain of HIV type 1 glycoprotein 120 expressed on the surface of Streptococcus gordonii. AIDS Res Hum Retroviruses 15:451-9, 1999. On p. 647 648 664665

[O'Hagan2000] D. T. O'Hagan, M. Ugozzoli, J. Barackman, M. Singh, J. Kazzaz, K. Higgins, T. C. Vancott, \& G. Ott. Microparticles in MF59, a potent adjuvant combination for a recombinan protein vaccine against HIV-1. Vaccine 18:1793-801, 2000. On p. 162 592 744

[O'Hagan2001] D. O'Hagan, M. Singh, M. Ugozzoli, C. Wild, S. Barnett, M. Chen, M. Schaefer B. Doe, G. R. Otten, \& J. B. Ulmer. Induction of potent immune responses by cationic microparticles with adsorbed human immunodeficiency virus DNA vaccines. J Virol 75(19):9037-43, 2001 On p. 592744

[O’Hagan2002] D. T. O'Hagan, M. Singh, J. Kazzaz, M. Ugozzoli, M. Briones, J. Donnelly, \& G. Ott. Synergistic adjuvant activity of immunostimulatory DNA and oil/water emulsions fo immunization with HIV p55 gag antigen. Vaccine 20(27-28):3389-3398, 2002. On p.117

[Ohba2001] H. Ohba, T. Soga, T. Tomozawa, Y Nishikawa, A. Yasuda, A. Kojima, T. Kurata, \& J. Chiba. An immunodominant neutralization epitope on the 'thumb' subdomain of human immunodeficiency virus type 1 reverse transcriptase revealed by phage display antibodies. J Gen Virol 82(Pt 4):813-20, 2001. On p. $598 \mid 599$

[Ohlin 1989] M. Ohlin, P.-A. Broliden, L. Danielsson, B. Wahren, J. Rosen, M. Jondal, \& C. A. K. Borrebaeck. Human monoclonal antibodies against a recombinant HIV envelope antigen produced by primary in vitro immunization. Characterization and epitope mapping. Immunology 68:325-331, 1989. On p.739

Ohlin1992] M. Ohlin, J. Hinkula, P.-A. Broliden, R. Grunow, C. A. K. Borrebaeck, \& B. Wahren Human MoAbs produced from normal, HIV-1-negative donors and specific for glycoprotein gp120 of the HIV-1 envelope. Clin Exp Immunol 89:290-295, 1992. On p. 639644663 676677684

[Ohno1991] T. Ohno, M. Terada, Y. Yoneda, K. W. Shea, R. F. Chambers, D. M. Stroka, M. Nakamura, \& D. W. Kufe. A broadly neutralizing monoclonal antibody that recognizes the V3 region of human immunodeficiency virus type 1 glycoprotein gp120. Proc Natl Acad Sci USA 88:1072610729, 1991. On p. 672

[Okada1994] T. Okada, B. K. Patterson, P. A. Otto, \& M. E. Gurney. HIV Type 1 Infection of CD4+ T-cells Depends Critically on Basic Amino Acid Residues in the V3 Domain of Envelope Glycoprotein 120. AIDS Res Hum Retroviruses 10:803-811, 1994. On p.641 671

[Okamoto1998] Y. Okamoto, Y. Eda, A. Ogura, S. Shibata, T. Amagai, Y. Katsura, T. Asano, K. Ki machi, K. Makizumi, \& M. Honda. In SCID-hu mice, passive transfer of a humanized antibody prevents infection and atrophic change of medulla in human thymic implant due to intravenous inoculation of primary HIV-1 isolate. J Immunol 160:69-76, 1998. On p.663

Okuda1997] K. Okuda, K. O. Xin, T. Tsuji, H. Bukawa, S. Tanaka, W. C. Koff, K. Tani, K. Okuda K. Honma, S. Kawamoto, K. Hamajima, \& J. Fukushima. DNA vaccination followed by macromolecular multicomponent peptide vaccination against HIV-1 induces strong antigen-specific immunity. Vaccine 15:1049-56, 1997. On p.293

[Oldstone1991] M. B. A. Oldstone, A. Tishon, H. Lewicki, H. J. Dyson, V. A. Feher, N. Assa-Munt, \& P. E. Wright. Mapping the anatomy of the immunodominant domain of the human immunodeficiency virus gp41 transmembrane protein: peptide conformation analysis using monoclonal antibodies and proton nuclear magnetic resonance spectroscopy. J Virol 65:1727-1734, 1991 Disulfide bond between cysteines and type I reverse turn about the residues SGKL are structural features of the immunodominant domain of gp41: CSGKLIC. On p. 606 703705706

[Orsini1995] M. J. Orsini, A. N. Thakur, W. W. Andrews, M.-L. Hammarskjold, \& D. Rekosh. Expression and Purification of the HIV Type 1 Rev Protein Produced in Escherichia coli and Its Use in the Generation of Monoclonal Antibodies. AIDS Res Hum Retroviruses 11:945-953, 1995. On p.612 613614,731

[Ortiz2001] G. M. Ortiz, M. Wellons, J. Brancato, H. T. T. Vo, R. L. Zinn, D. E. Clarkson, K. Van Loon, S. Bonhoeffer, G. D. Miralles, D. Montefiori, J. A. Bartlett, \& D. F. Nixon. Structured antiretroviral treatment interruptions in chronically HIV-1-infected subjects. Proc Natl Acad Sci U S A 98(23): 13288-13293, 2001. On p. 168 243 339 398

Ortiz2002] G. M. Ortiz, J. Hu, J. A. Goldwitz, R. Chandwani, M. Larsson, N. Bhardwaj, S. Bon hoeffer, B. Ramratnam, L. Zhang, M. M. Markowitz, \& D. F. Nixon. Residual viral replication during antiretroviral therapy boosts human immunodeficiency virus type 1-specific CD8+ T-cell responses in subjects treated early after infection. $J$ Virol 76(1):411-415, 2002. On p. 242

[Orvell1991] C. Orvell, T. Unge, R. Bhikhabhai, K. Backbro, U. Ruden, B. Strandberg, B. Wahren, \& E. M. Fenyo. Immunological characterization of the human immunodeficiency virus type 1 reverse transcriptase protein by the use of monoclonal antibodies. J Gen Virol 72:1913-1918, 1991. On p. 597598

[Oscherwitz1999a] J. Oscherwitz, F. M. Gotch, K. B. Cease, \& J. A. Berzofsky. New insights an approaches regarding B- and T-cell epitopes in HIV vaccine design [In Process Citation]. AIDS 13 Suppl A:S163-74, 1999. On p.775 $798 \quad 803832$ 
[Oscherwitz1999b] J. Oscherwitz, M. E. Zeigler, T. E. Gribbin, \& K. B. Cease. A V3 loop haptenic peptide sequence, when tandemly repeated, enhances immunogenicity by facilitating helper Tcell responses to a covalently linked carrier protein [In Process Citation]. Vaccine 17:2392-9, 1999. On p. 489

[Ostrowski2000] M. A. Ostrowski, S. J. Justement, L. Ehler, S. B. Mizell, S. Lui, J. Mican, B. D Walker, E. K. Thomas, R. Seder, \& A. S. Fauci. The role of CD4+ T cell help and CD40 ligan in the in vitro expansion of HIV-1-specific memory cytotoxic CD8+ T cell responses. J Immunol 165(11):6133-41, 2000. On p. 59 80 214 356366

[Ota1998a] A. Ota, X. Liu, H. Fujio, N. Sakato, \& S. Ueda. Random expression of human immunodeficiency virus-1 (HIV-1) p17 (epitopes) on the surface of the HIV-1-infected cell. Hybridoma 17:73-5, 1998. On p. 570 571 574591

[Ota1998b] A. Ota \& S. Ueda. Evaluation of the affinity measurement of anti-HIV-1 p17 monoclonal antibody by BIAcore. Hybridoma 17:471-7, 1998. On p.569 570

[Ota1999a] A. Ota, A. N. Bautista, M. L. Yadav, \& S. Ueda. Anti-P30-52 monoclonal antibody cross-reacted to Env V3 and inhibited the viral multiplication of HIV-1-infected MT-4 cells. Hybridoma 18:139-47, 1999. On p. 571]

[Ota1999b] A. Ota \& S. Ueda. Inhibitory mechanism of anti-P30-52 monoclonal antibody agains human immunodeficiency virus type 1 (HIV-1) multiplication in infected MT-4 cells. Hybridoma 18:235-41, 1999. On p. 571.

[Otake1994] K. Otake, Y Fujii, Y. Nishino, Q. Zhong, K. Fujinaga, M. Kameoka, K. Ohki, \& K. Ikuta. The carboxyl-terminal region of HIV-1 nef protein is a cell surface domain that can interact with CD4+ T cells. J Immunol 153:5826-5837, 1994. This study shows that the Cterminal end of Nef is accessible to Abs. This domain could bind in a soluble form to CD4+ uninfected cells, and this interaction is inhibited in the presence of the C-terminal specific antibodies. Syncytium formation was reduced by these Abs or peptides. Abs could stain IIIB/M10, but not MN/M10, infected cells, in a membrane immunofluorescence assay. On p. 587 835$] 838$ 839840

[Otteken1992] A. Otteken, S. Nick, W. Bergter, G. Voss, A. Faisst, C. Stahl-Hennig, \& G. Hunsmann. Identification of a gag protein epitope conserved among all four groups of primate immunodeficiency viruses by using monoclonal antibodies. J Gen Virol 73:2721-2724, 1992. Anti-SIVagmTYO-7 Mabs were obtained by intraperitoneal immunization of mice. Two reacted with $\mathrm{p} 17$ and three with $\mathrm{p} 24$. The anti-p24 MAbs recognized an epitope present in SIVagmTYO-7, SIVagmTYO-5, and HIV-2/SIVmac. The anti-p17 recognized an epitope present in SIVagmTYO7, SIVagmTYO-5, HIV-2/SIVmac, SIVagmTYO-1, HIV-1, and SIVmnd. This study shows that the matrix protein expresses at least one highly conserved epitope. On p. 571 582,600

[Otteken1996] A. Otteken, P. L. Earl, \& B. Moss. Folding, assembly, and intracellular trafficking of the human immunodeficiency virus type 1 Envelope glycoprotein analyzed with monoclonal antibodies recognizing maturational intermediates. J Virol 70:3407-15, 1996. On p. 736, 740

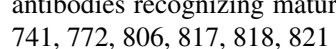

[Otten2000] G. R. Otten, B. Doe, M. Schaefer, M. Chen, M. J. Selby, C. Goldbeck, M. Hong, F. Xu, \& J. B. Ulmer. Relative potency of cellular and humoral immune responses induced by DNA vaccination. Intervirology 43(4-6):227-32, 2000. On $\mathrm{p} .170$

[Ovod1992] V. Ovod, A. Lagerstedt, A. Ranki, F. O. Gombert, R. Spohn, M. Tahtinen, G. Jung, \& K. J. Krohn. Immunological variation and immunohistochemical localization of HIV-1 Nef demonstrated with monoclonal antibodies. AIDS 6:25-34, 1992. Ten anti-Nef MAbs were generated and mapped. Nef is expressed in two isomorphic forms, and was shown to be expressed mainly in the Golgi complex and at the nuclear membrane, but occasionally $\mathrm{x}$ in the nucleus,

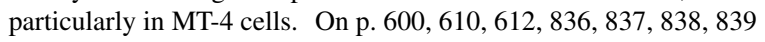

[Oxenius2000] A. Oxenius, D. A. Price, P. J. Easterbrook, C. A. O'Callaghan, A. D. Kelleher, J. A. Whelan, G. Sontag, A. K. Sewell, \& R. E. Phillips. Early highly active antiretroviral therapy for acute HIV-1 infection preserves immune function of CD8+ and CD4+ T lymphocytes. Proc Nat Acad Sci U S A 97(7):3382-7, 2000. On p. 68 71 99 100 106 107 122 [127 129 154 155 \begin{tabular}{|l|l|l|l|l|l|l|l|l|l|l|l|l|l|l|l|l|l|l|l|}
\hline 156 & 178 & 187 & 205 & 218 & 221 & 302 & 303 & 308 & 352 & 353 & 358 & 364 & 367 & 369 & 372 & 374 & 383 & 444 \\
\hline
\end{tabular} 457. 527 .534

Oxenius2001a] A. Oxenius, H. F. Gunthard, B. Hirschel, S. Fidler, J. N. Weber, P. J. Easterbrook, J. I. Bell, R. E. Phillips, \& D. A. Price. Direct ex vivo analysis reveals distinct phenotypic pattern of HIV-specific CD8(+) T lymphocyte activation in response to therapeutic manipulation of virus

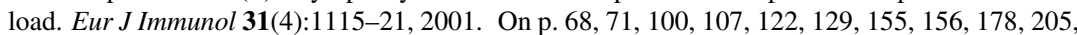
\begin{tabular}{ll|l|l|l|l|l}
221 & 303 & 353 & 364 & 367 & 369 \\
\hline
\end{tabular}

[Oxenius2001b] A. Oxenius, D. A. Price, S. J. Dawson, T. Tun, P. J. Easterbrook, R. E. Phillips, \& A. K. Sewell. Cross-staining of cytotoxic T lymphocyte populations with peptide-MHC class I multimers of natural HIV-1 variant antigens. AIDS 15(1):121-2, 2001. On p. 359

[Oxenius2002a] A. Oxenius, B. K. Jakobsen, P. J. Easterbrook, J. M. Boulter, T. Tun, A. Waters, J. Agudelo, M. Barnardo, R. E. Phillips, \& D. A. Price. Complete mapping of a novel HLA A*6801-restricted HIV-1 Tat epitope directly with a rapid modified enzyme-linked immunospot assay. AIDS 16(9):1285-1287, 2002. On p.257

[Oxenius2002b] A. Oxenius, A. R. McLean, M. Fischer, D. A. Price, S. J. Dawson, R. Hafner, C. Schneider, H. Joller, B. Hirschel, R. E. Phillips, R. Weber, H. F. Günthard, \& Swiss HIV Cohort Study Group. Human immunodeficiency virus-specific CD8(+) T-cell responses do not predict viral growth and clearance rates during structured intermittent antiretroviral therapy. $J$

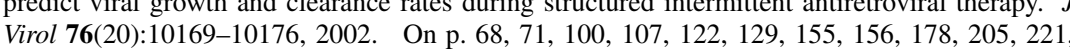
\begin{tabular}{|l|l|l|l|}
\hline 303 & 353 & 364 & 369 \\
\hline
\end{tabular}

Pai2002] E. F. Pai, M. H. Klein, P. Chong, \& A. Pedyczak. Fab'-epitope complex from the HIV-1 cross-neutralizing monoclonal antibody 2F5. U.S. Patent 6,482,928, WIPO Patent WO 00/61618, 2002. Filed USPTO Apr. 13, 1999. On p.710 714

[Pal1992] R. Pal, F. di Marzo Veronese, B. C. Nair, R. Rahman, G. Hoke, S. W. Mumbauer, \& M. G. Sarngadharan. Characterization of a neutralizing monoclonal antibody to the external glycoprotein of HIV-1. Intervirology 86:86-93, 1992. On p.656

[Paliard1998] X. Paliard, B. Doe, \& C. M. Walker. The T cell repertoire primed by antiviral vaccination is influenced by self-tolerance. Cell Immunol 188:73-9, 1998. On p.160

[Paliard2000] X. Paliard, Y. Liu, R. Wagner, H. Wolf, J. Baenziger, \& C. M. Walker. Priming of strong, broad, and long-lived HIV type 1 p55gag-specific CD8+ cytotoxic T cells after administration of a virus-like particle vaccine in rhesus macaques. AIDS Res Hum Retroviruses 16:273-82, 2000. On p. 162

[Palker1987] T. J. Palker, T. J. Matthews, M. E. Clark, G. J. Ciancolo, R. R. Randall, A. J. Langlois, G. C. White, B. Safei, R. Snyderman, D. P. Bolognesi, \& B. F. Haynes. A conserved epitope at the $\mathrm{COOH}$ terminus of human immunodeficiency virus gp120 envelope protein contains an immunodominant epitope. Proc Nat Acad Sci USA 84:2479-2483, 1987. On p. 693

[Palker1989] T. J. Palker, T. J. Matthews, A. Langlois, M. E. Tanner, M. E. Martin, R. M. Scearce, J. E. Kim, J. A. Berzofsky, D. P. Bolognesi, \& B. F. Haynes. Polyvalent human immunodeficiency virus synthetic immunogen comprised of envelope gp120 T helper cell sites and B-cell neutralization epitopes. J Immunol 142:3612-3619, 1989. Notes: Synthetic peptides containing type-specific neutralizing determinants of the V3 loop of gp120 were coupled to a 16 amino acid T-cell epitope (T1) of HIV-IIIB and used to immunize goats. The helper T-cell epitope T1 could induce both a proliferative response and a B-cell antibody response. Conversely, the B-cell epitope in the V3 region, SP10 was found to stimulate proliferative T-cell responses. On p. 489 506

[Palmer2002] B. E. Palmer, E. Boritz, N. Blyveis, \& C. C. Wilson. Discordance between frequency of human immunodeficiency virus type 1 (HIV-1)-specific gamma interferon-producing CD4+ T cells and HIV-1-specific lymphoproliferation in HIV-1-infected subjects with active viral repli- 
cation. J Virol 76(12):5925-5936, 2002. On p.446 457 528

Pancré2002] V. Pancré, B. Georges, G. Angyalosi, F. Castelli, A. Delanoye, M. Delacre, E. Hachulla, B. Maillere, A. Bouzidi, \& C. Auriault. Novel promiscuous HLA-DQ HIV Nef peptide that induces IFN-gamma-producing memory CD4+ T cells. Clin Exp Immunol 129(3):429437, 2002. On p. 530

[Pantaleo1997] G. Pantaleo, H. Soudeyns, J. F. Demarest, M. Vaccarezza, C. Graziosi, S. Paolucci, M. B. Daucher, O. J. Cohen, F. Denis, W. E. Biddison, R. P. Sekaly, \& A. S. Fauci. Accumulation of human immunodeficiency virus-specific cytotoxic T lymphocytes away from the predominan site of virus replication during primary infection. Eur J Immunol 27:3166-73, 1997. On p. 340

[Pantophlet2003] R. Pantophlet, E. O. Saphire, P. Poignard, P. W. H. I. Parren, I. A. Wilson, D. R. Burton. Fine mapping of the interaction of neutralizing and nonneutralizing monoclona antibodies with the CD4 binding site of human immunodeficiency virus type 1 gp120. J Viro \begin{tabular}{ll|l|l|l|l|l|l|l|l|l|l|l|l|l|} 
77(1):642-658, 2003. On p.762 & 764 & 775 & 779 & 780 & 784 & 791 & 796 & 797 & 822 & 827 \\
\hline
\end{tabular}

Papsidero1988] L. D. Papsidero, B. J. Poiesz, \& R. A Montagna Monoclonal Antibody Identifies a Highly Conserved and Immunodominant Epitope of the Human Immunodeficiency Virus Transmembrane Protein. Hybridoma 7:117-128, 1988. On p.729

[Papsidero1989] L. D. Papsidero, M. Sheu, \& F. W. Ruscetti. Human immunodeficiency virus type 1-neutralizing monoclonal antibodies which react with p17 core protein: characterization and epitope mapping. J Virol 63:267-272, 1989. Two Mabs with overlapping binding sites on p17 reduced the infectivity of free virus. A p24 monoclonal was not able to do this. On p. $569 \mid 570$

[Parekh2002] B. S. Parekh, M. S. Kennedy, T. Dobbs, C.-P. Pau, R. Byers, T. Green, D. J. Hu S. Vanichseni, N. L. Young, K. Choopanya, T. D. Mastro, \& J. S. McDougal. Quantitative detec tion of increasing HIV type 1 antibodies after seroconversion: A simple assay for detecting recen HIV infection and estimating incidence. AIDS Res Hum Retroviruses 18(4):295-307, 2002. On p. 701,702

[Park2000] E. J. Park, M. K. Gorny, S. Zolla-Pazner, \& G. V. Quinnan. A global neutralization resistance phenotype of human immunodeficiency virus type 1 is determined by distinct mechanisms mediating enhanced infectivity and conformational change of the envelope complex. \begin{tabular}{|l|l|l|l|l|l|l|l|l|l|l|l|l|l|} 
Virol 74:4183-91, 2000. On p. 647 & 648 & 649 & 652 & 657 & 658 & 664 & 665 & 675 & 676 & 710 & 714 & 720 \\
\hline
\end{tabular} \begin{tabular}{|l|l|l|l|l|l|l|l|l|l|l|l|l|l|}
722 & 762 & 763 & 775 & 778 & 784 & 788 & 798 & 800 & 803 & 805 & 822 & 825 \\
\hline
\end{tabular}

Parker1992] K. C. Parker, M. A. Bednarek, L. K. Hull, U. Utz, B. C. H. J. Zweerink, W. E. Biddison, \& J. E. Coligan. Sequence motifs important for peptide binding to the human MHC class molecule, HLA-A2. J Immunol 149, 1992. On p. 84 102 217 237, 319

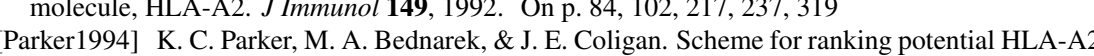
binding peptides based on independent binding of individual peptide side-chains. J Immunol 152 1994. The authors conclude that peptide amino acid side-chain binding to the HLA-A2 molecule is independent of the sequence of the peptide, and developed a table of coefficients that can be used to help predict peptide binding to HLA-A2. On p. 84 102,237

[Parker1996] C. E. Parker, D. I. Papac, S. K. Trojak, \& K. B. Tomer. Epitope mapping by mass spec trometry: determination of an epitope on HIV-1 IIIB p26 recognized by a monoclonal antibody. J Immunol 157:198-206, 1996. On p. 580

[Parker2001] C. E. Parker, L. J. Deterding, C. Hager-Braun, J. M. Binley, N. Schulke, H. Katinger, J. P. Moore, \& K. B. Tomer. Fine definition of the epitope on the gp41 glycoprotein of human immunodeficiency virus type 1 for the neutralizing monoclonal antibody 2F5. J Viro 75(22):10906-11, 2001. On p. 710.715

Parren1995] P. W. Parren, H. J. Ditzel, R. J. Gulizia, J. M. Binley, C. F. B. 3rd, D. R. Burton, \& D. E. Mosier. Protection against HIV-1 infection in hu-PBL-SCID mice by passive immunizatio with a neutralizing human monoclonal antibody against the gp120 CD4-binding site. AIDS 9:F1F6, 1995. The Fab b12, at $1.9 \mathrm{mg} / \mathrm{kg}$, was able to protect 25 mice from HIV-1 infection showing that complete protection against HIV-1 infection can be achieved in the hu-PBL-SCID model by passive immunization with physiologically relevant doses of antibody. On p.784 796

Parren1997a] P. W. Parren \& D. Burton. Antibodies Against HIV-1 from Phage Display Library: Mapping of an Immune Response and Progress toward Antiviral Immunotherapy. Chem Immunol 65:18-56, 1997. Editor, J. D. Capra. An excellent review of the potential for antiviral immune therapy using anti-HIV human monoclonal antibodies, emphasizing phage display library technology, and application to HIV. Fabs to gp120 and gp41 are summarized. The methodology of selection for enhanced affinity is discussed, and affinity shown to be related to neutralization. Fabs expressed in phage display libraries were generally converted to $\mathrm{IgG}$ molecules only if they show neutralization potential in vitro, and this conversion to an $\mathrm{IgG}$ enhances neutralizing potential for immunotherapeutics. The use of phage display libraries to assess vaccines is discussed. gp120, gp160 and gp140-oligomeric vaccines were compared as antigen for selection from phage display libraries. Despite the fact that CD4BS, V3 loop, and CD4BS-V2 loop directed Abs were obtained in vaccinees, none of these vaccines efficiently selected neutralizing Abs from long-term asymptomatic donors in phage display libraries. The protein with the best potential using this method was found to be native oligomeric HIV-1 Envelope expressed on infected cells. The possibility of using 2G12, IgG1 b12 and 2F5 in combination for immunotherapy is discussed. On p. 650663 664 756 784 [785 7896

[Parren 1997b] P. W. Parren, M. C. Gauduin, R. A. Koup, P. Poignard, P. Fisicaro, D. R. Burton, \& Q. J. Sattentau. Relevance of the antibody response against human immunodeficiency virus type 1 Envelope to vaccine design [corrected and republished in Immunol Lett 1997 Jul;58(2):125-32] Immunol Lett 57:105-12, 1997. On p.784 786

Parren1997c] P. W. Parren, M. C. Gauduin, R. A. Koup, P. Poignard, Q. J. Sattentau, P. Fisicaro, \& D. R. Burton. Erratum to Relevance of the antibody response against human immunodeficiency virus type 1 Envelope to vaccine design [corrected and republished article originally printed in Immunol Lett 1997 Jun 1;57(1-3):105-12]. Immunol Lett 58:125-32, 1997. On p. 628 650 657 \begin{tabular}{|l|l|l|l|l|l|l|l|l|l|l|l|l|l|l|l|l|l|l|}
\hline 658 & 663 & 664 & 720 & 721 & 753 & 756 & 757 & 758 & 759 & 762 & 763 & 764 & 765 & 771 & 775 & 777 & 784 & 786 \\
\hline
\end{tabular} \begin{tabular}{|l|l|l|l|l|l|l|l|l|l|l|l|l|l|l|l|l|l|l|l|l|l|}
\hline 796 & 797 & 798 & 799 & 803 & 804 & 808 & 809 & 812 & 822 & 823 & 829 \\
\hline
\end{tabular}

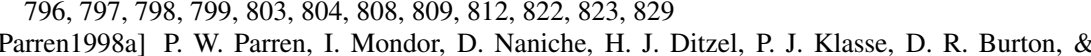

Q. J. Sattentau. Neutralization of human immunodeficiency virus type 1 by antibody to gp120 is determined primarily by occupancy of sites on the virion irrespective of epitope specificity. $J$ Virol 72:3512-9, 1998. The authors propose that the occupancy of binding sites on HIV-1 virions is the major factor in determining neutralization, irrespective of epitope specificity. Neutralization was assayed T-cell-line-adapted HIV-1 isolates. Binding of Fabs to monomeric rgp120 was not correlated with binding to functional oligomeric gp120 or neutralization, while binding to functional oligomeric gp120 was highly correlated with neutralization. The ratios of oligomer binding/neutralization were similar for antibodies to different neutralization epitopes, with a few

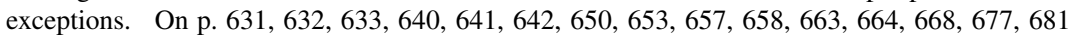

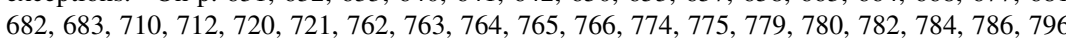
\begin{tabular}{|l|l|l|l|l|l|l|l|l|l|l|}
\hline 682 & 683 & 710 & 712 & 720 & 721 & 71 \\
\hline 797 & 803 & 804 & 812 & 822 & 823 \\
\hline
\end{tabular}

Parren1998b] P. W. Parren, M. Wang, A. Trkola, J. M. Binley, M. Purtscher, H. Katinger, J. P. Moore, \& D. R. Burton. Antibody neutralization-resistant primary isolates of human immunodeficiency virus type 1 . J Virol 72:10270-4, 1998. On p.710 712 784 $786 \quad 822823$

Parren1999] P. W. Parren, J. P. Moore, D. R. Burton, \& Q. J. Sattentau. The neutralizing antibody response to HIV-1: viral evasion and escape from humoral immunity [In Process Citation]. AIDS 13 Suppl A:S137-62, 1999. On p.710 $713|822| 824$

Parren2001] P. W. Parren, P. A. Marx, A. J. Hessell, A. Luckay, J. Harouse, C. Cheng-Mayer, J. P. Moore, \& D. R. Burton. Antibody protects macaques against vaginal challenge with a pathogenic R5 simian/human immunodeficiency virus at serum levels giving complete neutralization in vitro. J Virol 75(17):8340-7, 2001. On p.784 789

[Pastori2002] C. Pastori, C. Barassi, F. Lillo, R. Longhi, B. Capiluppi, S. Nozza, A. Galli, C. Uberti- 
Foppa, A. Lazzarin, G. Tambussi, \& L. Lopalco. The effect of HAART on humoral immune response in primary HIV-1 infected patients. J Biol Regul Homeost Agents 16(1):9-17, 2002. On p. 752

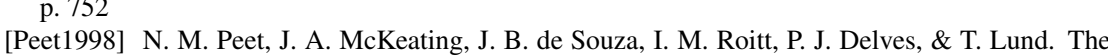
effect of low-profile serine substitutions in the V3 loop of HIV-1. Virology 251:59-70, 1998. On \begin{tabular}{l|l|l|l|l|l|l|l|l|l|l|l|l|l|} 
p. 623 & 630 & 640 & 670 & 680 & 760 & 783 & 808 & 816 & 820 & 821 \\
\hline
\end{tabular}

[Pellegrin1996] I. Pellegrin, E. Legrand, D. Neau, P. Bonot, B. Masquelier, J. L. Pellegrin, J. M. Ragnaud, N. Bernard, \& H. J. Fleury. Kinetics of appearance of neutralizing antibodies in 12 patients with primary or recent HIV-1 infection and relationship with plasma and cellular vira loads. J Acquir Immune Defic Syndr Hum Retrovirol 11(5):438-447, 1996. On p.728

[Pellegrino2002] M. G. Pellegrino, M. H. Bluth, T. Smith-Norowitz, S. Fikrig, D. J. Volsky, H. Moallem, D. L. Auci, M. Nowakowski, \& H. G. Durkin. HIV type 1-specific IgE in serum of long-term surviving children inhibits HIV type 1 production in vitro. AIDS Res Hum Retroviruses 18(5):363-372, 2002. On p.843

[Peng2001] B. Peng \& M. Robert-Guroff. Deletion of N-terminal myristoylation site of HIV Nef abrogates both MHC-1 and CD4 down-regulation. Immunol Lett 78(3):195-200, 2001. On p. 343.530

[Peter2001] K. Peter, Y. Men, G. Pantaleo, B. Gander, \& G. Corradin. Induction of a cytotoxic T-cell response to HIV-1 proteins with short synthetic peptides and human compatible adjuvants. \begin{tabular}{ll|l|l|l|l|l|l|l|} 
Vaccine 19(30):4121-4129, 2001. On p. 89 & 202 & 203 & 222 & 237 & 238 & 294 & 325 & 326 \\
\hline
\end{tabular}

[Peter2002] K. Peter, M. J. Brunda, \& G. Corradin. IL-12 administration leads to a transient depletion of T cells, B cells, and APCs and concomitant abrogation of the HLA-A2.1-restricted CTL response in transgenic mice. J Immunol 169(1):63-67, 2002. On p. 89 203 222 |238|326

[Petrov1990] R. V. Petrov, R. M. Khaitov, I. G. Sidorovich, S. P. Pavlikov, I. A. Nikolaeva, M. E. Ivachenko, S. M. Andreev, \& L. Y. U. Sklyarov. The use of synthetic peptides in the diagnosis of HIV infections. Biomed Sci 1:239-244, 1990. Peptides from 2 regions of Env were particularly immunoreactive in ELISA tests with human sera from HIV-1 positive individuals: 495-516 of gp120 (SP-III), peptide 584-602 of gp41 (LS-19), and peptide 601-616 of gp41 (SP-15). Uganda sera reacted with both SP-III from HTLV-III and SP-29 (gp41, 598-609) from the LAV-ELI isolate. HIV-1 peptides from gp32 were not cross-reactive. On p. 699705

[Phillips1991] R. E. Phillips, S. Rowland-Jones, D. F. Nixon, F. M. Gotch, J. P. Edwards, A. O Ogunlesi, J. G. Elvin, J. A. Rothbard, C. R. Bangham, C. R. Rizza, \& A. J. McMichael. Human immunodeficiency virus genetic variation that can escape cytotoxic $\mathrm{T}$ cell recognition. Nature 354:453-459, 1991. Fluctuations in the specificity of cytotoxic T-cells for HIV were correlated with variability in proviral gag (DNA) epitope sequences. On p. 68 6124$] 135][153$

[Pialoux 2001] G. Pialoux, H. Gahery-Segard, S. Sermet, H. Poncelet, S. Fournier, L. Gerard, A. Tartar, H. Gras-Masse, J. P. Levy, J. G. Guillet, \& \{ANRS VAC 04 Study Team.\}. Lipopeptides induce cell-mediated anti-HIV immune responses in seronegative volunteers. AIDS 15(10): 1239 49, 2001. On p. 576 581 639 637 638

[Pido-Lopez2002] J. Pido-Lopez, A. Pires, M. Nelson, E. O’Moore, M. Fisher, B. Gazzard, R. Aspinall, F. Gotch, \& N. Imami. Thymic activity in late-stage HIV-1 infected individuals receiving highly active antiretroviral therapy: Potential effect of steroid therapy. HIV Med 3(1):56-61, 2002. On p. 536

[Pilgrim1997] A. K. Pilgrim, G. Pantaleo, O. J. Cohen, L. M. Fink, J. Y. Zhou, J. T. Zhou, D. P. Bolognesi, A. S. Fauci, \& D. C. Montefiori. Neutralizing Antibody Responses to Human Immunodeficiency Virus Type 1 in Primary Infection and Long-Term-Nonprogressive Infection. J Infect Dis 176(4):924-932, 1997. On p.749

[Pincus1989] S. H. Pincus, K. Wehrly, \& B. Chesebro. Treatment of HIV tissue culture infection with monoclonal antibody-ricin A chain conjugates. J Immunol 142:3070-3075, 1989. On p.644 [Pincus1991] S. H. Pincus, R. L. Cole, E. M. Hersh, D. Lake, Y. Masuho, P. J. Durda, \& J. Mc-
Clure. In Vitro Efficacy of Anti-HIV Immunotoxins Targeted by Various Antibodies to the Envelope Protein. J Immunol 146:4315-4324, 1991. Six MAbs, (907, 924, 110.1, 41.1, 86 and P5-3) and polyclonal pooled serum antibodies purified on gp160 were coupled to RAC to create immunotoxins. Only 41.1-RAC, an anti-gp41 MAb-immunotoxin and the polyclonal immunotoxin showed direct activity against multiple strains, and activity of an immunotoxin was found not to \begin{tabular}{ll|l|l|l|l|l|l|l|} 
be directly correlated with cell surface binding. On p. 644 & 645 & 689 & 690 & 698 & 699 & 740 \\
\hline
\end{tabular}

[Pincus1993a] S. H. Pincus \& J. McClure. Soluble CD4 enhances the efficacy of immunotoxins directed against gp41 of the human immunodeficiency virus. Proc Natl Acad Sci USA 90:332-6, \begin{tabular}{ll|l|l|l|l|l|l|l|l|l|l|l|l|l|l|} 
1993. On p. 619 & 634 & 635 & 636 & 644 & 645 & 688 & 689 & 698 & 723 \\
\hline
\end{tabular}

[Pincus 1993b] S. H. Pincus, K. G. Messer, D. H. Schwartz, G. K. Lewis, B. S. Graham, W. A. Blattner, \& G. Fisher. Differences in the antibody response to human immunodeficiency virus1 Envelope glycoprotein (gp160) in infected laboratory workers and vaccinees. J Clin Invest 91:1987-96, 1993. On p.644 6450723 726 775 776

[Pincus 1996] S. H. Pincus, K. Wehrly, R. Cole, H. Fang, G. K. Lewis, J. McClure, A. J. Conley, B. Wahren, M. R. Posner, A. L. Notkins, S. A. Tilley, A. Pinter, L. Eiden, M. Teintze, D. Dorward, \& V. V. Tolstikov. In vitro effects of anti-HIV immunotoxins directed against multiple epitopes on HIV type 1 envelope glycoprotein 160. AIDS Res Hum Retroviruses 12:1041-1051, 1996. A pane of anti-gp160 MAbs to was used to construct anti-HIV immunotoxins by coupling antibodies to ricin A chain (RAC). The ability of the immunotoxins to kill HIV-1-infected cells was tested in tissue culture. Immunotoxins that bind epitopes on the cell surface killed infected cells, although killing was not directly proportional to binding. The activity of anti-gp41 immunotoxins was \begin{tabular}{ll|l|l|l|l|l|l|l|l|l|l|l|l|l|} 
markedly enhanced in the presence of sCD4. On p. 591 & 619 & 634 & 635 & 636 & 644 & 645 & 688 & 689 \\
\hline
\end{tabular} \begin{tabular}{l|l|l|l|l|l|l|l|}
698 & 710 & 711 & 760 & 761 & 767 & 775 & 777 \\
\hline
\end{tabular}

Pincus1998] S. H. Pincus, R. L. Cole, R. Watson-McKown, A. Pinter, W. Honnen, B. Cole, \& K. S Wise. Immunologic cross-reaction between HIV type $1 \mathrm{p} 17$ and Mycoplasma hyorhinis variable lipoprotein. AIDS Res Hum Retroviruses 14:419-25, 1998. On p.589 591644698

[Pinter1989] A. Pinter, W. J. Honnen, S. A. Tilley, C. Bona, H. Zaghouani, M. K. Gorny, \& S. ZollaPazner. Oligomeric Structure of gp41, the Transmembrane Protein of Human Immunodeficiency Virus Type 1. J Virol 63:2674-2679, 1989. On p.696 698 708

Pinter1993a] A. Pinter, W. J. Honnen, M. E. Racho, \& S. A. Tilley. A potent, neutralizing human monoclonal antibody against a unique epitope overlapping the CD4-binding site of HIV-1 gp120 that is broadly conserved across North American and African viral isolates. AIDS Res Hum Retroviruses 9:985-996, 1993. On p.659 767

Pinter1993b] A. Pinter, W. J. Honnen, \& S. A. Tilley. Conformational changes affecting the V3 and CD4-binding domains of human immunodeficiency virus type 1 gp120 associated with env processing and with binding of ligands to these sites. $J$ Virol 67:5692-5697, 1993. On p. 649 659 760

[Pinter1995] C. Pinter, A. G. Siccardi, \& A. Clivio. Production of human immunodeficiency virus by chronically infected cells grown in protein-free medium. Cell Biol Int 19:507-515, 1995. On p. 601 612 731

Pinto1995] L. A. Pinto, J. Sullivan, J. A. Berzofsky, M. Clerici, H. A. Kessler, A. L. Landay, \& G. M. Shearer. Env-specific cytotoxic T lymphocyte responses in HIV seronegative health care workers occupationally exposed to HIV-contaminated body fluids. J Clin Invest 96:867-876, 1995. Helper responses were detected in $75 \%$ of HIV sero

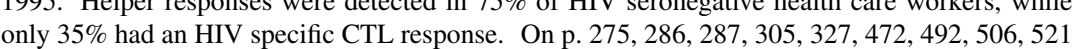
only 35\% had an HIV specific CTL response. On p. 275 286,287$] 305] 327] 472[492] 506] 521$
Pinto1999] L. A. Pinto, J. A. Berzofsky, K. R. Fowke, R. F. Little, F. Merced-Galindez, R. Humphrey, J. Ahlers, N. Dunlop, R. B. Cohen, S. M. Steinberg, P. Nara, G. M. Shearer \& R. Yarchoan. HIV-specific immunity following immunization with HIV synthetic. AIDS 13:2003-12, 1999. On p.286

[Pirofski1993] L.-A. Pirofski, E. K. Thomas, \& M. D. Scharff. Variable region gene utilization and 
mutation in a group of neutralizing murine anti-human immunodeficiency virus type 1 principa neutralizing determinant antibodies. AIDS Res Hum Retroviruses 9:41-49, 1993. Observed restricted subset of murine $\mathrm{V}$ heavy and light chain gene elements in a set of 5 antibodies that bin

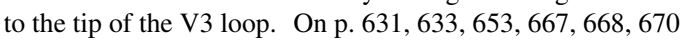

Pitcher1999] C. J. Pitcher, C. Quittner, D. M. Peterson, M. Connors, R. A. Koup, V. C. Maino, \& L. J. Picker. HIV-1-specific CD4+ T cells are detectable in most individuals with active HIVinfection, but decline with prolonged viral suppression [see comments]. Nat Med 5:518-25, 1999. On p. 442

[Plana1998] M. Plana, F. Garcia, T. Gallart, J. M. Miro, \& J. M. Gatell. Lack of T-cell proliferative response to HIV-1 antigens after 1 year of highly active antiretroviral treatment in early HIV 1 disease. Immunology Study Group of Spanish EARTH-1 Study [letter]. Lancet 352:1194-5, 1998. On p. 442524

[Pogue1995] R. R. Pogue, J. Eron, J. A. Frelinger, \& M. Matsui. Amino-terminal alteration of the HLA-A*0201-restricted human immunodeficiency virus pol peptide increases complex stability and in vitro immunogenicity. Proc Natl Acad Sci USA 92:8166-8170, 1995. In this mutationa study a substitution of $476 \mathrm{I}$ to $\mathrm{Y}$ did not increase affinity but did increase complex stability with HLA-A*0201. The altered peptide $(\mathrm{Y})$ provided a greater stimulation of wildtype pol-specific CTL response relative to the wildtype peptide (I), in three different seropositive individuals. On p. 212

[Poignard1996a] P. Poignard, T. Fouts, D. Naniche, J. P. Moore, \& Q J Sattentau. Neutralizing antibodies to human immunodeficiency virus type-1 gp120 induce envelope glycoprotein subunit dissociation. J Exp Med 183:473-484, 1996. Binding of Anti-V3 and the CD4I neutralizing MAbs induces shedding of gp120 on cells infected with the T-cell line-adapted HIV-1 molecula clone Hx10. This was shown by significant increases of gp120 in the supernatant, and exposure of a gp41 epitope that is masked in the oligomer. MAbs binding either to the V2 loop or to CD4BS discontinuous epitopes do not induce gp120 dissociation. This suggests HIV neutralization probably is caused by several mechanisms, and one of the mechanisms may involve gp120 dissociation. \begin{tabular}{l|l|l|l|l|l|l|l|l|l|l|l|l|l|l|l|l|l|l|l|l|} 
On p. & 631 & 632 & 633 & 641 & 642 & 653 & 668 & 677 & 681 & 682 & 683 & 696 & 697 & 762 & 763 & 764 & 765 & 784 \\
\hline
\end{tabular}

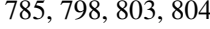

[Poignard1996b] P. Poignard, P. J. Klasse, \& Q. J. Sattentau. Antibody neutralization of HIV-1. Immunology Today 17:239-246, 1996. Comprehensive review of HIV envelope gp120 and gp41 antibody binding domains, and different cross-reactivity groups of MAbs ability to neutralize primary isolates. The distinction between neutralization of laboratory strains and primary isolates is discussed. The only three epitopes that have confirmed broad neutralization against a spectrum of isolates are gp120 epitopes for IgG1b12 and 2G12, and the gp41 epitope of 2F5. On p.710 711784785822

Poignard1999] P. Poignard, R. Sabbe, G. R. Picchio, M. Wang, R. J. Gulizia, H. Katinger, P. W. Parren, D. E. Mosier, \& D. R. Burton. Neutralizing antibodies have limited effects on the contro of established HIV-1 infection in vivo. Immunity 10:431-8, 1999. On p.710 713 784 787 822 824

[Poignard2001] P. Poignard, E. O. Saphire, P. W. Parren, \& D. R. Burton. gp120: Biologic aspects

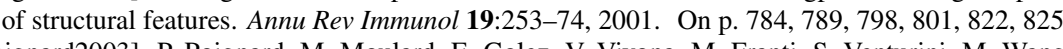

[Poignard2003] P. Poignard, M. Moulard, E. Golez, V. Vivona, M. Franti, S. Venturini, M. Wang, P. W. H. I. Parren, \& D. R. Burton. Heterogeneity of Envelope Molecules Expressed on Primary Human Immunodeficiency Virus Type 1 Particles as Probed by the Binding of Neutralizing an Nonneutralizing Antibodies. J Virol 77(1):353-365, 2003. On p. 657 658 720 723 760]779 784791797

[Pollock1989] B. J. Pollock, A. S. McKenzie, B. E. Kemp, D. A. McPhee, \& A. J. F. D’Apice. Human monoclonal antibodies to HIV-1: cross-reactions with gag and env proteins. Clin Exp Immunol 78:323-328, 1989. On p. 833834
[Poluektova2002] L. Y. Poluektova, D. H. Munn, Y. Persidsky, \& H. E. Gendelman. Generation of cytotoxic T cells against virus-infected human brain macrophages in a murine model of HIV-1 encephalitis. J Immunol 168(8):3941-3949, 2002. On p. 80.214

[Polydefkis1990] M. Polydefkis, S. Koenig, C. Flexner, E. Obah, K. Gebo, S. Chakrabarti, P. L. Earl, B. Moss, \& R. F. Siliciano. Anchor Sequence-dependent endogenous processing of human immunodeficiency virus 1 envelope glycoprotein gp160 for CD4+ T-cell recognition. J Exp Med 171:875-887, 1990. Notes: Human CD4+ T-cell clones and cell lines were shown to lyse recombinant vaccinia virus-infected cells that synthesize the HIV-1 envelope glycoprotein op 160 showing that endogenously processed antigen can be presented by class II MHC. gp160 epitope: GSDTITLPCRIKQFINMWQE. On p.505

Porgador 1997] A. Porgador, H. F. Staats, B. Faiola, E. Gilboa, \& T. J. Palker. Intranasal immunization with CTL epitope peptides from HIV-1 or ovalbumin and the mucosal adjuvant cholera toxin induces peptide-specific CTLs and protection against tumor development in vivo. J Immunol 158:834-41, 1997. To evaluate the ability of mucosal immunization protocols using peptide immunogens to induce CTL responses, BALB/c and C57BL/6 mice were immunized intranasally (i.n.) with peptides corresponding to a known CTL epitope in HIV-1 glycoprotein 120 or OVA, respectively, and the mucosal adjuvant cholera toxin (CT). Intranasal immunization of BALB/c mice with a 10- or 15-amino acid peptide corresponding to a CTL determinant in HIV-1 glycoprotein 120 and CT induced peptide-specific CTLs in spleen cells that persisted through 35 days after the last immunization. Intranasal immunization of C57BL/6 mice with the octameric OVA peptide and CT produced similar results with detectable peptide-specific CTL in both the cervica lymph node and spleen. To test whether CTL induced by i.n. immunization with OVA peptide and CT were functional in vivo, groups of C57BL/6 mice were injected with E.G7- OVA tumo cells that express the OVA protein and monitored for tumor growth. Animals immunized i.n. with OVA and CT were protected against tumor development as efficiently as animals immunized by the potent CTL induction protocol of i.v. injection with OVA-pulsed dendritic cells. Intranasal immunization with peptides corresponding to known CTL epitopes and CT provides a noninvasive route of immunization for the induction of CTL responses in vivo. On p. 290

[Posner1991] M. R. Posner, T. Hideshima, T. Cannon, M. Mukherjee, K. H. Mayer, \& R. A. Byrn. An IgG human monoclonal antibody that reacts with HIV-I/gp120, inhibits virus binding to cells, and neutralizes infection. J Immunol 146:4325-4332, 1991. Original paper describing the neutralizing MAb F105. On p. 775

Posner1992a] M. Posner, L. Cavacini, C. Emes, J. Power, M. Gorny, \& S. Zolla-Pazner. Human Monoclonal Antibodies to the V3 Loop of gp120 Mediate Variable and Distinct Effects on Binding and Viral Neutralization by a Human Monoclonal Antibody to the CD4 Binding Site. J Cell Biochem Suppl O(16 part E):69, 1992. On p. 775

[Posner1992b] M. R. Posner, H. S. Elboim, T. Cannon, L. Cavicini, \& T. Hideshima. Functiona Activity of an HIV-1 Neutralizing IgG Human Monoclonal Antibody: ADCC and ComplementMediated Lysis. AIDS Res Hum Retroviruses 8:553-558, 1992. On p.775

Posner1993] M. R. Posner, L. A. Cavacini, C. L. Emes, J. Power, \& R. Byrn. Neutralization of HIV-1 by F105, a Human Monoclonal Antibody to the CD4 Binding Site of gp120. J AIDS 6:7-14, 1993. On p.775

[Posner1995] M. R. Posner, L. A. Cavacini, J. Gambertoglio, C. Spino, E. Wolfe, C. Trapnell, N. Ketter, S. Hammer, \& M. Samore. An ACTG phase Ia safety and pharmacokinetic trial of immunotherapy with the anti-CD4 binding site human monoclonal antibody F105. Natl Conf Hum Retroviruses Relat Infect (2nd) 1995:150, 1995. Aidsline: 95920546 Abstract: Eight HIV-positive asymptomatic individuals were given F105 by intravenous infusion. There were no clinical side effects or changes in biochemical tests among the eight volunteers. The plasma half life of F105 had a range of 8.7-18.6 days. On p. 7755776

[Potts1993] B. J. Potts, K. G. Field, Y. Wu, M. Posner, L. Cavacini, \& M. White-Scharf. Synergistic 
Inhibition of HIV-1 by CD4 Binding Domain Reagents and V3-Directed Monoclonal Antibodies. Virology 197:415-419, 1993. Four anti-V3 loop MAbs, (59.1, 83.1, 50.1, and 58.2), were evaluated for their affinity, neutralization potencies, and their ability to synergize F105 or sCD4 neutralization. The most important parameter for synergy was the capacity to neutralize a given virus independently. On p. 652 6616686736756776

[Poumbourios1992] P. Poumbourios, D. A. McPhee, \& B. E. Kemp. Antibody epitopes sensitive to the state of human immunodeficiency virus type $1 \mathrm{gp} 41$ oligomerization map to a putative alpha-helical region. AIDS Res Hum Retroviruses 8:2055-2062, 1992. On p. 694 696|705

[Poumbourios1995] P. Poumbourios, W. E. Ahmar, D. A. McPhee, \& B. E. Kemp. Determinants of human immunodeficiency virus type 1 envelope glycoprotein oligomeric structure. J Virol 69:1209-1218, 1995. On p. 696 832

[Pozzi1994] G. Pozzi, M. R. Oggioni, R. Manganelli, D. Medaglini, V. A. Fischetti, D. Fenoglio, M. T. Valle, A. Kunkl, \& F. Manca. Human T-helper cell recognition of an immunodominant epitope of HIV-1 gp120 expressed on the surface of Streptococcus gordoni. Vaccine 12(12):10711077, 1994. On p. 485

[Price1995] P. Price, R. P. Johnson, D. T. Scadden, C. Jassoy, T. Rosenthal, S. Kalams, \& B. D. Walker. Cytotoxic CD8+ T lymphocytes reactive with human immunodeficiency virus-1 produce granulocyte/macrophage colony-stimulating factor and variable amounts of interleukins 2,3 , an 4 following stimulation with the cognate epitope. Clinical Immunology and Immunopathology 74:100-106, 1995. Cytokine release from stimulated CTL clones derived from either the peripheral blood or CSF of 3 patients was studied. HLA restriction was determined for two of seven clones. GM-CSF and TNF- $\alpha$ and IFN- $\gamma$ were produced by all clones; most clones produced low \begin{tabular}{ll|l|l|l|l|l|l|l|l|l|} 
amounts of IL-2, IL-3, and IL-4. On p. 148 & 194 & 223 & 230 & 309 & 327 \\
\hline
\end{tabular}

[Price1997] D. A. Price, P. J. Goulder, P. Klenerman, A. K. Sewell, P. J. Easterbrook, M. Troop, C. R. Bangham, \& R. E. Phillips. Positive selection of HIV-1 cytotoxic T lymphocyte escape variants during primary infection. Proc Natl Acad Sci USA 94:1890-5, 1997. Cytotoxic T lymphocytes (CTLs) are thought to play a crucial role in the termination of the acute primary HIV- 1 syndrome, but clear evidence for this presumption has been lacking. Here we demonstrate positive selection of HIV-1 proviral sequences encoding variants within a CTL epitope in Nef, a gen product critical for viral pathogenicity, during and after seroconversion. These positively selected HIV-1 variants carried epitope sequence changes that either diminished or escaped CTL recognition. Other proviruses had mutations that abolished the Nef epitope altogether. These results provide clear evidence that CTLs exert selection pressure on the viral population in acute HIV-1 infection. On p. 66.366

[Propato2001] A. Propato, E. Schiaffella, E. Vicenzi, V. Francavilla, L. Baloni, M. Paroli, L. Finoc chi, N. Tanigaki, S. Ghezzi, R. Ferrara, R. Chesnut, B. Livingston, A. Sette, R. Paganelli, F. Aiuti G. Poli, \& V. Barnaba. Spreading of HIV-specific CD8+ T-cell repertoire in long-term nonpro-

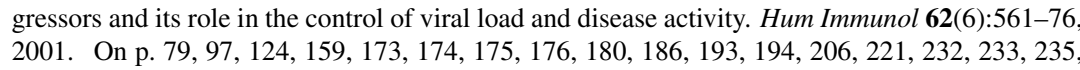

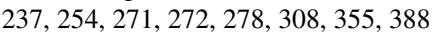

[Purtscher1994] M. Purtscher, A. Trkola, G. Gruber, A. Buchacher, R. Predl, F. Steindl, C. Tauer, R. Berger, N. Barrett, A. Jungbauer, \& H. Katinger. A broadly neutralizing human monoclon antibody against gp41 of human immunodeficiency virus type 1. AIDS Res Hum Retroviruses 10:1651-1658, 1994. On p.710 729,731.

[Purtscher1996] M. Purtscher, A. Trkola, A. Grassauer, P. M. Schulz, A. Klima, S. Dopper, G. Gruber, A. Buchacher, T. Muster, \& H. Katinger. Restricted antigenic variability of the epitop recognized by the neutralizing gp41 antibody 2 F5. AIDS 10:587-593, 1996. Binding and neutralization to gp41 ELDKWA variants by anti-gp41 MAb 2F5 were studied. LDKW is the core binding motif. On $\mathrm{p} .710711$

[Qian1998] X. H. Qian \& K. B. Tomer. Affinity capillary electrophoresis investigation of an epi- tope on human immunodeficiency virus recognized by a monoclonal antibody. Electrophoresis 19:415-9, 1998. On p.580

[Qiu1999] J. T. Qiu, R. Song, M. Dettenhofer, C. Tian, T. August, B. K. Felber, G. N. Pavlakis, $\&$ X. F. Yu. Evaluation of novel human immunodeficiency virus type $1 \mathrm{Gag}$ DNA vaccines for protein expression in mammalian cells and induction of immune responses. J Virol 73:9145-52, 1999. On $\mathrm{p} .116$

Qiu2000] J. T. Qiu, B. Liu, C. Tian, \& G. N. P. X. F. Yu. Enhancement of primary and secondary cellular immune responses against human immunodeficiency virus type 1 gag by using DNA expression vectors that target Gag antigen to the secretory pathway. J Virol 74:5977-6005, 2000 On p. 169446

Quayle1998] A. J. Quayle, W. M. Coston, A. K. Trocha, S. A. Kalams, K. H. Mayer, \& D. J Anderson. Detection of HIV-1-specific CTLs in the semen of HIV-infected individuals. J Immuno 161:4406-10, 1998. On p. 150

[Ranki1994] A. Ranki, A. Lagerstedt, V. Ovod, E. Aavik, \& K. Krohn. Expression kinetics and subcellular localization of HIV-1 regulatory proteins Nef and Tat in established lymphoid cell lines. Arch Virol 139:365-378, 1994. On p.612

[Ranki1995] A. Ranki, M. Nyberg, V. Ovod, M. Haltia, I. Elovaara, R. Raininko, H. Haapasalo, \& K. Krohn. Abundant expression of HIV Nef and Rev proteins in brain astrocytes in vivo is associated with dementia. AIDS 9:1001-1008, 1995. HIV Nef protein was found in the brain cells of infected individuals with clinical neurological disease. On p. $610,612,8 3 6 \longdiv { 8 3 8 } 8 3 9$ 840 841

Ranki1997] A. Ranki, J. Suni, V. Blazevic, P. Holmstrom, S. Mattinen, K. Krohn, \& S. L. Valle. T-cell recognition of HIV antigens in HIV-seroreverted persons. AIDS 11(1):132-133, 1997. On p. 4594661466533

[Ratto-Kim1999] S. Ratto-Kim, K. V. Sitz, R. P. Garner, J. H. Kim, C. Davis, N. Aronson, N. Ruiz, K. Tencer, R. R. Redfield, \& D. L. Birx. Repeated immunization with recombinant gp160 human immunodeficiency virus (HIV) envelope protein in early HIV-1 infection: evaluation of the T cell proliferative response. J Infect Dis 179:337-44, 1999. On p. 525

[Ray1998] S. C. Ray, N. Lubaki, B. R. Dhruva, R. F. Siliciano, \& R. C. Bollinger. Autologous strain-specific cytolytic $\mathrm{T}$ lymphocyte responses directed against human immunodeficiency virus type 1 Env. AIDS Res Hum Retroviruses 14:3-13, 1998. On p.304

[Rayevskaya2001] M. V. Rayevskaya \& F. R. Frankel. Systemic immunity and mucosal immunity are induced against human immunodeficiency virus Gag protein in mice by a new hyperattenuated strain of Listeria monocytogenes. J Virol 75(6):2786-91, 2001. On p. 117

[Reid1996] S. Reid, S. McAdam, K. Smith, P. Klenerman, C. O'Callaghan, K. Harlos, B. Jakobsen, A. McMichael, J. Bell, D. Stuart, \& E. Jones. Antagonist HIV-1 gag peptides induce structura changes in HLA B8. J Exp Med 184:2279-2286, 1996. On p.66 67

[Reitter1998] J. N. Reitter, R. E. Means, \& R. C. Desrosiers. A role for carbohydrates in immune evasion in AIDS. Nat Med 4(6):679-84, 1998. On p.745.

Reitz1988] M. S. Reitz, Jr., C. Wilson, C. Naugle, \& M. Robert-Guroff. Generation of a neutralization-resistant variant of HIV-1 is due to selection for a point mutation in the envelope gene. Cell 54:57-63, 1988. Growth of HXB2 in the constant presence of a neutralizing antiserum yielded a viral population resistant to the same serum. gp41 mutation 582 (Ala to Thr) conferred the resistant phenotype. On p. 668671820

Richardson1996] T. M. Richardson, Jr., B. L. Stryjewski, C. C. Broder, J. A. Hoxie, J. R. Mascola P. L. Earl, \& R. W. Doms. Humoral response to oligomeric human immunodeficiency virus type 1 envelope protein. $J$ Virol 70:753-62, 1996. An Env antigen capture enzyme-linked immunosorbent assay using a soluble, oligomeric form of HIV-1IIIB Env (gp140) that contains gp120 and the gp41 ectodomain was developed. The gp140, captured by various monoclonal antibodies (MAbs), retained its native oligomeric structure: it bound CD4 and was recognized by MAbs to confor- 
mational epitopes in gp120 and gp41, including oligomer-specific epitopes in gp41. On p.702

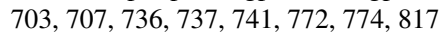

[Rinaldo2000] C. R. Rinaldo, Jr., X. L. Huang, Z. Fan, J. B. Margolick, L. Borowski, A. Hoji, C. Kalinyak, D. K. McMahon, S. A. Riddler, W. H. Hildebrand, R. B. Day, \& J. W. Mellors. Antihuman immunodeficiency virus type 1 (HIV-1) CD8(+) T-lymphocyte reactivity during combination antiretroviral therapy in HIV-1-infected patients with advanced immunodeficiency. $J$ Viro 74(9):4127-38, 2000. On p. 74 102 210. 279

[Rini1993] J. M. Rini, E. A. Stura, P. A. Salinas, A. T. Profy, \& I. A. Wilson. Crystal structure of a human immunodeficiency virus type 1 neutralizing antibody, 50.1, in complex with its V3 loop peptide antigen. Proc Natl Acad Sci USA 90:6325-6329, 1993. The V3 antigenic site is stretched out, not the $\beta$ turn seen as the primary determinant in other published anti-V3 peptide Fab structures. On p. 652

[Rizzuto1998] C. D. Rizzuto, R. Wyatt, N. Hernandez-Ramos, Y. Sun, P. D. Kwong, W. A. Hendrickson, \& J. Sodroski. A conserved HIV gp120 glycoprotein structure involved in chemokine receptor binding. Science 280:1949-53, 1998. This paper compares the epitope for CD4 inducible MAbs with the chemokine co-receptor binding site on the gp120 molecule. Site-directed mutagenesis of YU2 Env was guided by information obtained from the crystallized CD4-17bgp120 core structure, Kwong et al, 1998. YU2 is a primary macrophage tropic R5 isolate with high affinity for both CD4 and CCR5. A protein with the V1-V2 loops deleted, called wt $\Delta$ was the basis for the assay which detected binding of virus to cells expressing CCR5 in the presence of sCD4. Preincubaton with MAb 17b blocks binding, as did the natural ligand for CCR5, MIP-1 $\beta$ and anti-CCR5 MAb 2D7. Mutations 437 P/A and $442 \mathrm{Q} / \mathrm{L}$ increased CCR5 binding affinity. The region of gp 120 CCR5 binding is shown to be the highly conserved $\beta$-sheet bridging structure, located proximal to the V3 loop. On p. 7988799832

[Rizzuto2000] C. Rizzuto \& J. Sodroski. Fine definition of a conserved CCR5-binding region on the human immunodeficiency virus type 1 glycoprotein 120. AIDS Res Hum Retroviruses 16(8):741 749, 2000. On p. 798800

Roben1994] P. Roben, J. P. Moore, M. Thali, J. Sodroski, C. F. Barbas III, \& D. R. Burton. Recog nition Properties of a Panel of Human Recombinant Fab fragments to the CD4 binding site of gp120 that show differing abilities to neutralize human immunodeficiency virus type 1 . J Viro 68:4821-4828, 1994. On p. 784

[Robert-Guroff1994] M. Robert-Guroff, A. Louie, M. Myagkikh, F. Michaels, M. P. Kieny, M. E. White-Scharf, B. Potts, D. Grogg, \& M. S. Reitz, Jr. Alteration of V3 loop context within the envelope of human immunodeficiency virus type 1 enhances neutralization. $J$ Virol 68:34593466, 1994. MN-V3 loop inserted into a HBX2 background results in enhanced neutralization of anti-MN V3 MAb 50.1 and human HIV+ sera when the chimeric virus was compared to MN. Enhanced affinity, and greater proportions of labeled infected H9 cells by FACS analysis, were also observed using two anti-MN V3 MAbs, 50.1 and 83.1. On p. 652

[Robert-Guroff2000] M. Robert-Guroff. IgG surfaces as an important component in mucosal protection. Nat Med 6(2):129-130, 2000. On p.710,713 775 ,777, 822 824

[Robert-Hebmann1992a] V. Robert-Hebmann, S. Emiliani, F. Jean, M. Resnicoff, \& C. Devaux Clonal analysis of murine B-cell response to the human immunodeficiency virus type 1 (HIV-1) gag p17 and p25 antigens. Mol Immunol 29:729-738, 1992. On p.569 573 574 577578579 \begin{tabular}{|l|l|l|l|l|}
580 & 582 & 583 & 584 \\
\hline
\end{tabular}

[Robert-Hebmann1992b] V. Robert-Hebmann, S. Emiliani, M. Resnicoff, F. Jean, \& C. Devaux Subtyping of human immunodeficiency virus isolates with a panel of monoclonal antibodies: identification of conserved and divergent epitopes on p17 and p25 core proteins. Mol Immuno

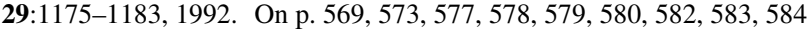

[Robertson1993] M. N. Robertson, F. Buseyne, O Schwartz, \& Y. Riviere. Efficient antigen presentation to cytotoxic $\mathrm{T}$ lymphocytes by cells transduced with a retroviral vector expressing the
HIV-1 Nef protein. AIDS Res and Hum Retroviruses 9:1217-1223, 1993. This paper presents a retroviral vector system for antigen presentation to CTLs. As part of the controls to test their system, they study the response to specific Nef peptides, which contain the dominant CTL epitopes in Nef in their study subject. On p. 352366

[Robinson1990a] J. E. Robinson, D. Holton, S. Pacheco-Morell, J. Liu, \& H. McMurdo. Identification of conserved and variable epitopes of human immunodeficiency virus type-1 (HIV-1) gp120 by human monoclonal antibodies produced by EBV transformed cell lines. AIDS Res Hum Retroviruses 6:567-579, 1990. On p.674,740 762

[Robinson1990b] W. E. Robinson, Jr., T. Kawamura, M. K. Gorny, D. Lake, J.-Y. Xu, Y. Matsumoto, T. Sugano, Y. Masuho, W. M. Mitchell, E. Hersh, \& S. Zolla-Pazner. Human monoclonal antibodies to the human immunodeficiency virus type 1 (HIV-1) transmembrane glycoprotein gp4 enhance HIV-1 infection in vitro. Proc Natl Acad Sci USA 87:3185-3189, 1990. Three gp41 MAbs out of 16 Env and Gag MAbs tested enhanced HIV-1 IIIB infection of MT-2 cells. The enhancing antibodies were competitive with the immunodominant epitopes of gp41 recognized \begin{tabular}{ll|l|l|l|l|l|l|l|l|l|} 
by sera from HIV-1 infected subjects. On p. 576 & 577 & 589 & 590 & 591 & 699 & 700 & 707 & 708 & 729
\end{tabular} 740.828

[Robinson1990c] W. E. Robinson, Jr., T. Kawamura, D. Lake, Y. Masuho, W. M. Mitchell, \& E. M. Hersh. Antibodies to the Primary Immunodominant Domain of Human Immunodeficiency Virus Type 1 (HIV-1) Glycoprotein gp41 Enhance HIV-1 Infection In Vitro. J Virol 64:5301-5305, 1990. On p. 699700

[Robinson1991] W. E. Robinson, M. K. Gorny, J.-Y. Xu, W. M. Mitchell, \& S. Zolla-Pazner. Two Immunodominant Domains of gp41 Bind Antibodies Which Enhance Human Immunodeficiency Virus Type 1 Infection In Vitro. J Virol 65:4169-4176, 1991. On p.589 590 696 700 701 $702707 \quad 708$ 729 828

[Robinson1992] J. Robinson, H. Yoshiyama, D. Holton, S. Elliot, \& D. D. Ho. Distinct antigenic sites on HIV gp120 identified by a panel of human monoclonal antibodies. J Cell Biochem Suppl 16E: 71,1992 . On p. 753758

Rodríguez1999] D. Rodríguez, J. R. Rodríguez, M. Llorente, I. Vázquez, P. Lucas, M. Esteban, C. Martínez-A., \& G. del Real. A human immunodeficiency virus type 1 Env-granulocytemacrophage colony-stimulating factor fusion protein enhances the cellular immune response to Env in a vaccinia virus-based vaccine. J Gen Virol 80(1):217-23, 1999. On p. 525 728 745

Root2001] M. J. Root, M. S. Kay, \& P. S. Kim. Protein design of an HIV-1 entry inhibitor Science 291(5505):884-8, 2001. On p. 710 714

Rosenberg1997] E. S. Rosenberg, J. M. Billingsley, A. M. Caliendo, S. L. Boswell, P. E. Sax, S. A Kalams, \& B. D. Walker. Vigorous HIV-1-specific CD4+ T cell responses associated with contro of viremia. Science 278:1447-50, 1997. Notes: Also see M. Balter, Science 278:1399-1400 fo comments. On p.431 $432434,435,437.524$

[Rosenberg1998] E. S. Rosenberg \& B. D. Walker. HIV type 1-specific helper T cells: a critical host defense. AIDS Res Hum Retroviruses 14 Suppl 2:S143-7, 1998. On p. 442

[Rosenberg1999] E. S. Rosenberg, L. LaRosa, T. Flynn, G. Robbins, \& B. D. Walker. Character ization of HIV-1-specific T-helper cells in acute and chronic infection. Immunol Lett 66:89-93, 1999. On p. 442

Ross2001] T. M. Ross, Y. Xu, T. D. Green, D. C. Montefiori, \& H. L. Robinson. Enhanced avidity maturation of antibody to human immunodeficiency virus envelope: DNA vaccination with gp120-C3d fusion proteins. AIDS Res Hum Retroviruses 17(9):829-35, 2001. On p.748

Rouaix 1994] F. Rouaix, H. Gras-Masse, C. Mazingue, P. R. Ridel, E. Diesis, M. Marguerite, J. Estaquier, A. Capron, A. Tartar, \& C. Auriault. Improvement of the T-cell response to a non mmunogenic peptide by its tandem association with a highly efficient T-helper peptide. Immunopharmacology 28(3):215-222, 1994. On p. 530

[Rovinski1995] B. Rovinski, L. Rodrigues, S. X. Cao, F. L. Yao, U. McGuinness, C. Sia, G. Cates, 
S. Zolla-Pazner, S. Karwowska, T. J. Matthews, C. B. McDanal, J. Mascola, \& M. H. Klein Induction of HIV type 1 neutralizing and env-CD4 blocking antibodies by immunization with genetically engineered HIV type 1-like particles containing unprocessed gp160 glycoproteins. AIDS Res Hum Retroviruses 11:1187-1195, 1995. On p. 669 $670 \mid 632$

[Rowland-Jones1993] S. L. Rowland-Jones, S. H. Powis, J. Sutton, I. Mockridge, F. M. Gotch, N. Murray, A. B. Hill, W. M. Rosenberg, J. Trowsdale, \& A. J. McMichael. An antigen processin polymorphism revealed by HLA-B8-restricted cytotoxic T lymphocytes which does not correlate with TAP gene polymorphism. Eur J Immunol 23:1999-2004, 1993. Individual fails to present HLA-B8-restricted influenza epitope, but can present an HLA-B8-restricted HIV-1 gag epitope. On p. 67

[Rowland-Jones1995a] S. Rowland-Jones. Personal communication, 1995. On p.194

[Rowland-Jones1995b] S. L. Rowland-Jones, J. Sutton, K. Ariyoshi, T. Dong, F. Gotch, S. McAdam, D. Whitby, S. Sabally, A. Gallimore, T. Corrah, M. Takiguchi, T. Schultz, A. McMichael, \& H. Whittle. HIV-specific cytotoxic T cells in HIV-exposed but uninfected Gambian women. Nature Medicine 1:59-64, 1995. Four HIV-1 and -2 cross-reactive epitopes that are presented to CTL from HIV-infected Gambians by HLA-35 were identified. These peptides could elicit HIV-specific CTLs from 3 of 6 repeatedly exposed but seronegative sex worker who carry the HLA-B35 allele. Most CTL derived from HIV-2 positive donors also recognized

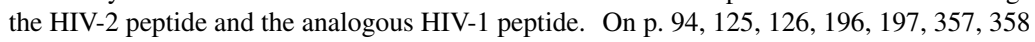

[Rowland-Jones1997] S. Rowland-Jones, R. Tan, \& A. McMichael. Role of cellular immunity in protection against HIV infection. Adv Immunol 65:277-346, 1997. An comprehensive, excellen review of CTL immunity. On p. 134

[Rowland-Jones1998a] S. Rowland-Jones, T. Dong, P. Krausa, J. Sutton, H. Newell, K. Ariyoshi, F. Gotch, S. Sabally, T. Corrah, J. Kimani, K. MacDonald, F. Plummer, J. Ndinya-Achola, H. Whittle, \& A. McMichael. The role of cytotoxic T cells in HIV infection. Dev Biol Stand 92:209-14, 1998. In this paper CTL response to previously defined conserved epitopes was found in exposed but uninfected prostitutes in Nairobi. Subtypes A and D are circulating in this regions, and the reactive epitopes tended to be conserved. Similarly previous studies in the Gambia showed that exposed but uninfected prostitutes tended to have B35 presented CTL epitope conserved between HIV-1 and HIV-2. It was suggested that what was special about B35 is simply

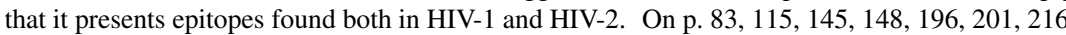
\begin{tabular}{|l|l|l|l|l|l|l|l|l|l|l|l|}
\hline 231 & 310 & 357 & 378 & 385 & 393 \\
\hline
\end{tabular}

[Rowland-Jones1998b] S. L. Rowland-Jones, T. Dong, K. R. Fowke, J. Kimani, P. Krausa, H. Newell, T. Blanchard, K. Ariyoshi, J. Oyugi, E. Ngugi, J. Bwayo, K. S. MacDonald, A. J McMichael, \& F. A. Plummer. Cytotoxic T cell responses to multiple conserved HIV epitope in HIV-resistant prostitutes in nairobi. J Clin Invest 102(9):1758-65, 1998. On p. 88 102 [103

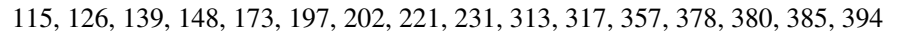

[Rowland-Jones1999] S. L. Rowland-Jones, T. Dong, L. Dorrell, G. Ogg, P. Hansasuta, P. Krausa

J. Kimani, S. Sabally, K. Ariyoshi, J. Oyugi, K. S. MacDonald, J. Bwayo, H. Whittle, F. A. Plummer, \& A. J. McMichael. Broadly cross-reactive HIV-specific cytotoxic T lymphocytes in highly- exposed persistently seronegative donors. Immunol Lett 66:9-14, 1999. On p. 67 94

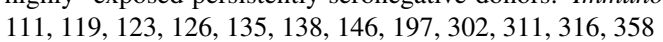

[Rowland-Jones2001] S. L. Rowland-Jones, S. Pinheiro, R. Kaul, P. Hansasuta, G. Gillespie, T. Dong, F. A. Plummer, J. B. Bwayo, S. Fidler, J. Weber, A. McMichael, \& V. Appay. How important is the 'quality' of the cytotoxic T lymphocyte (CTL) response in protection against HIV infection? Immunol Lett 79(1-2):15-20, 2001. On p. 165]241]336

[Ruiz2000] L. Ruiz, J. Martinez-Picado, J. Romeu, R. Paredes, M. K. Zayat, S. Marfil, E. Negredo,

G. Sirera, C. Tural, \& B. Clotet. Structured treatment interruption in chronically HIV-1 infected patients after long-term viral suppression [In Process Citation]. AIDS 14:397-403, 2000. O p. 441
[Saarloos1995] M. N. Saarloos, T. F. Lint, \& G. T. Spear. Efficacy of HIV-specific and 'antibodyindependent' mechanisms for complement activation by HIV-infected cells. Clin Exp Immuno 99:189-195, 1995. On p. 700 720

[Sabbaj2000] S. Sabbaj, M. J. Mulligan, R. H. Hsieh, R. B. Belshe, \& J. R. McGhee. Cytokine profiles in seronegative volunteers immunized with a recombinant canarypox and gp120 primeboost HIV-1 vaccine. NIAID aids vaccine evaluation group. AIDS 14(10):1365-74, 2000. On p. 527

Sabbaj2002a] S. Sabbaj, B. H. Edwards, M. K. Ghosh, K. Semrau, S. Cheelo, D. M. Thea, L. Kuhn, G. D. Ritter, M. J. Mulligan, P. A. Goepfert, \& G. M. Aldrovandi. Human immunodeficiency virus-specific CD8(+) T cells in human breast milk. J Virol 76(15):7365-7373, 2002. On p. 62 $64,183,188,198,385$

Sabbaj2002b] S. Sabbaj et al. Personal communication, manuscript submitted, 2002. On p. 57 \begin{tabular}{|l|l|l|l|l|l|l|l|l|l|l|l|l|l|l|l|l|l|l|}
62 & 70 & 73 & 82 & 95 & 100 & 101 & 104 & 105 & 107 & 111 & 118 & 119 & 120 & 124 & 127 & 130 & 136 & 150 \\
\hline
\end{tabular}

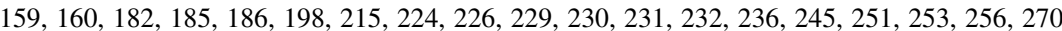
\begin{tabular}{|l||l|l|l|l|l|l|l|l|l|l|l|l|l|l|l|l|l|l|}
\hline & 274 & 292 & 331 & 352 & 358 & 366 & 370 & 372 & 373 & 374 & 376 & 377 & 383 & 386 & 392 \\
\hline
\end{tabular}

[Safrit1993] J. T. Safrit, M. S. C. Fung, C. A. Andrews, D. G. Braun, W. N. C. Sun, T. W. Chang, $\&$ R. A. Koup. hu-PBL-SCID mice can be protected from HIV-1 infection by passive transfer of monoclonal antibody to the principal neutralizing determinant of envelope gp120. AIDS 7:15-21, 1993. On p. 646653

[Safrit1994a] J. T. Safrit, C. A. Andrews, T. Zhu, D. D. Ho, \& R. A. Koup Characterization of human immunodeficiency virus type 1-specific cytotoxic T lymphocyte clones isolated during acute seroconversion: recognition of autologous virus sequences within a conserved immunodominan epitope. J Exp Med 179:463-472, 1994. HIV-1 specific CTL clones were isolated from two individuals at acute seroconversion. In one patient, two HLA A31-restricted clones recognized the same fragment of gp41, peptide RLRDLLLIVTR, but one was sensitive to a Thr to Val substitution, while the other was not. A CTL HLA A32-restricted clone from the other patient recognized the gp41 peptide VLSIVNRVRQGYSPLSFQTH. Autologous viral sequences from seroconversion were recognized by the CTL clones, but not the HIV-1 strain MN. On p. 319$] 32032$

[Safrit1994b] J. T. Safrit, A. Y. Lee, C. A. Andrews, \& R. A. Koup. A region of the third variable loop of HIV-1 gp120 is recognized by HLA-B7-restricted CTLs from two acute seroconversion patients. J Immunol 153:3822-3830, 1994. HIV-1 envelope-specific CTL clones were isolated from the peripheral blood of two patients within weeks of seroconversion. These clones were CD8+ and restricted by the HLA-B7 molecule. The minimum epitope was defined, RPNNNTRKSI, with anchor residues at the proline and isoleucine; the anchor residues are relatively well conserved. A Serine to Arginine change at position 9 of the epitope abrogated clone recognition in one of the patients. This amino acid change is one factor that has been associated with a change from a nonsyncytium-inducing to a syncytium-inducing phenotype of HIV-1. On p. $284[321]$

[Saito1994] Y. Saito, L. Sharer, L. Epstein, et al. Overexpression of Nef as a marker for restricted HIV-1 infection of astrocytes in postmortem pediatric central nervous tissues. Neurology 44:474481, 1994. On p. 836838839

[Sakaida1997] H. Sakaida, T. Murakami, S. Kawamata, T. Hattori, \& T. Uchiyama. V3 loop of human immunodeficiency virus type 1 suppresses interleukin 2 -induced T cell growth. AIDS Res Hum Retroviruses 13:151-9, 1997. See comments in AIDS Res Hum Retroviruses 13:633 (1997). On p. 675

[Salerno-Goncalves2000] R. Salerno-Goncalves, W. Lu, \& J. M. Andrieu. Quantitative analysis of the antiviral activity of $\mathrm{CD} 8(+) \mathrm{T}$ cells from human immunodeficiency virus-positive asymptomatic patients with different rates of CD4(+) T-cell decrease. J Virol 74(14):6648-51, 2000. On p. 165

[Salmon-Ceron1999] D. Salmon-Ceron, J. L. Excler, L. Finkielsztejn, B. Autran, J. C. Gluckman, 
D. Sicard, T. J. Matthews, B. Meignier, C. Valentin, R. El Habib, C. Blondeau, M. Raux, C. Moog, J. Tartaglia, P. Chong, M. Klein, B. Milcamps, F. Heshmati, S. Plotkin, The AGIS Group, \& L'Agence Nationale de Recherches sur Le Sida. Safety and immunogenicity of a live recombinan canarypox virus expressing HIV type 1 gp120 MN tm/gag/protease LAI (ALVAC-HIV, vCP205) followed by a p24E-V3 MN synthetic peptide (CLTB-36) administered in healthy volunteers low risk for HIV infection. AIDS Res Hum Retroviruses 15(7):633-45, 1999. On p. 162164 $239332,335,443,526$

[Salzwedel2000] K. Salzwedel, E. D. Smith, B. Dey, \& E. A. Berger. Sequential CD4-coreceptor interactions in human immunodeficiency virus type 1 Env function: soluble CD4 activates En for coreceptor-dependent fusion and reveals blocking activities of antibodies against cryptic conserved epitopes on gp120. J Virol 74:326-33, 2000. On p.798 $800|803| 805|817| 818$

[Samri2000] A. Samri, G. Haas, J. Duntze, J. M. Bouley, V. Calvez, C. Katlama, \& B. Autran. Immunogenicity of mutations induced by nucleoside reverse transcriptase inhibitors for human immunodeficiency virus type 1-specific cytotoxic T cells. J Virol 74(19):9306-12, 2000. On p. 179 \begin{tabular}{l|l|l|l|l|l|l|l|}
181 & 186 & 189 & 199 & 203 & 213 & 307 & 354 \\
\hline
\end{tabular}

Sandberg2000] J. K. Sandberg, A. C. Leandersson, C. Devito, B. Kohleisen, V. Erfle, A. Achou M. Levi, S. Schwartz, K. Karre, B. Wahren, \& J. Hinkula. Human immunodeficiency viru type 1 Nef epitopes recognized in HLA-A2 transgenic mice in response to DNA and peptide

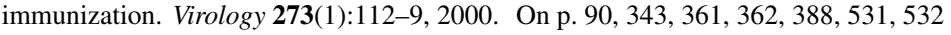

[Sanders2002] R. W. Sanders, M. Venturi, L. Schiffner, R. Kalyanaraman, H. Katinger, K. O. Lloyd, P. D. Kwong, \& J. P. Moore. The mannose-dependent epitope for neutralizing antibody $2 \mathrm{G} 12$ on human immunodeficiency virus type 1 glycoprotein gp120. J Virol 76(14):7293-7305, 2002. On \begin{tabular}{l|l|l|l|l|l|} 
p. 760 & 784 & 789 & 822 & 826 \\
\hline
\end{tabular}

[Sanhadji2000] K. Sanhadji, L. Grave, J. L. Touraine, P. Leissner, C. Rouzioux, R. Firouzi, L. Kehrli, J. C. Tardy, \& M. Mehtali. Gene transfer of anti-gp41 antibody and CD4 immunoadhesin strongly reduces the HIV-1 load in humanized severe combined immunodeficient mice. AIDS 14(18):2813-22, 2000. On p. 710714

[Santra1999] S. Santra, P. N. Fultz, \& N. L. Letvin. Virus-specific cytotoxic T lymphocytes in human immunodeficiency virus type 1-infected chimpanzees. J Virol 73:7065-9, 1999. On p. 100.114

[Saphire2001a] E. O. Saphire, P. W. Parren, C. F. Barbas III, D. R. Burton, \& I. A. Wilson. Crystallization and preliminary structure determination of an intact human immunoglobulin, b12: an antibody that broadly neutralizes primary isolates of HIV-1. Acta Crystallogr D Biol Crystallogr 57(Pt 1):168-71, 2001. On p. 784.788

[Saphire2001b] E. O. Saphire, P. W. Parren, R. Pantophlet, M. B. Zwick, G. M. Morris, P. M. Rudd, R. A. Dwek, R. L. Stanfield, D. R. Burton, \& I. A. Wilson. Crystal structure of a neutralizing human IGG against HIV-1: a template for vaccine design. Science 293(5532):1155-9, 2001. On p.784 788

[Saphire2002] E. O. Saphire, R. L. Stanfield, M. D. M. Crispin, P. W. H. I. Parren, P. M. Rudd, R. A Dwek, D. R. Burton, \& I. A. Wilson. Contrasting IgG structures reveal extreme asymmetry an flexibility. J Mol Biol 319(1):9-18, 2002. On p.784 790

[Sarmati2001] L. Sarmati, G. d'Ettorre, E. Nicastri, L. Ercoli, I. Uccella, P. Massetti, S. G. Parisi V. Vullo, \& M. Andreoni. Neutralizing antibodies against autologous human immunodeficiency virus type 1 isolates in patients with increasing CD4 cell counts despite incomplete virus suppression during antiretroviral treatment. Clin Diagn Lab Immunol 8(4):822-4, 2001. On p.748

[Sarobe1994] P. Sarobe, J.-J. Lasarte, I. Prieto, A. Gullon, M.-J. Soto, P. Labarga, J. Prieto, \& F. Borras-Cuesta. Induction of neutralizing antibodies against human immunodeficiency viru type 1 using synthetic peptide constructs containing an immunodominant T-helper cell determ nant from vpr. J AIDS 7:635-640, 1994. Notes: A vpr peptide was shown to stimulate a T-cell proliferative response in 37 epitopes, and immunized mice were capable of antibody production.
On p.460

Sasaki1998] S. Sasaki, K. Sumino, K. Hamajima, J. Fukushima, N. Ishii, S. Kawamoto, H. Mohri, C. R. Kensil, \& K. Okuda. Induction of systemic and mucosal immune responses to human immunodeficiency virus type 1 by a DNA vaccine formulated with QS-21 saponin adjuvant via intramuscular and intranasal routes. J Virol 72:4931-9, 1998. On p. 490642

[Sastry1991] K. J. Sastry \& R. B. Arlinghaus. Identification of T-cell epitopes without B-cell activity in the first and second conserved regions of the HIV Env protein. AIDS 5:699-707, 1991. Notes: Seven out of 19 peptides induced good T-cell proliferative response in mice representing four major histocompatibility complex haplotypes, without eliciting an $\mathrm{Ab}$ response. Eleven peptide were able to induce T-cells that could proliferate in response to recombinant gp160 (greater than or equal to 3 fold relative to unrelated peptides). Peptides were modified to generate polymers with disulfide bonds or micelles with palmitic acid residues attached to the amino-terminal lysine; in these configurations peptides were immunogenic without being coupled to a carrier molecule. $\mathrm{F}$ hybrid mice were used: ASW x BALBc F1 $\left(\mathrm{H}-2^{k} x b\right)$ and B6C3 F1 $\left(\mathrm{H}-2^{s} x d\right)$. On p. 467469 $470,480,482515$

Sastry1992] K. J. Sastry, P. N. Nehete, S. Venkatnarayanan, J. Morkowski, C. D. Platsoucas, \& R. B. Arlinghaus. Rapid in vivo induction of HIV-specific CD8+ cytotoxic T lymphocytes by a 15-amino acid unmodified free peptide from the immunodominant V3-loop of gp120. Virology 188:502-509, 1992. On p. 288

[Sattentau1991] Q. J. Sattentau \& J. P. Moore Conformational changes induced in the human immunodeficiency virus envelope glycoprotein by soluble CD4 binding. J Exp Med 174:407415,1991 . SCD4 binding to gp120 induces conformational changes within envelope oligomers. This was measured on HIV-1-infected cells by the increased binding of gp120/V3 loop specific MAbs, and on the surface of virions by increased cleavage of the V3 loop by an exogenou proteinase. On p. 641 668 696 697, 708 760

[Sattentau1993] Q. J. Sattentau, J. P. Moore, F. Vignaux, F. Traincard, \& P. Poignard. Conformational changes induced in the envelope glycoproteins of the human and simian immunodeficiency viruses by soluble receptor binding. J Virol 67:7383-7393, 1993. On p. 632 641

[Sattentau1995a] Q. J. Sattentau. Conservation of HIV-1 gp120 neutralizing epitopes after formalin inactivation. AIDS 9:1383-1385, 1995. On p. 657 720 784 803

[Sattentau1995b] Q. J. Sattentau \& J. P. Moore. Human immunodeficiency virus type 1 neutralization is determined by epitope exposure on the gp120 oligomer. J Exp Med 182:185-196, 1995. This study suggests that antibodies specific for one of five different binding regions on gp120 are associated with viral neutralization: V2, V3, C4, the CD4 binding site, and a complex discontinuous epitope that does not interfere with CD4 binding. Kinetic binding properties of a set of MAbs that bind to these regions were studied, analyzing binding to both functional oligomeric LAI gp120 and soluble monomeric LAI BH10 gp120; neutralization $\mathrm{ID}_{50}$ s were also evaluated. It was found that the neutralization $\mathrm{ID}_{50} \mathrm{~s}$ was related to the ability to bind oligomeric, not monomeric, gp120, and concluded that with the exception of the V3 loop, regions of gp120 that are immunogenic will be poorly presented on cell-line-adapted virions. Further, the association rate, estimated as the $t_{1 / 2}$ to reach equilibrium binding to multimeric, virion associated, gp120, appears to be a major factor relating to affinity and potency of the neutralization response to cell-line-adapted

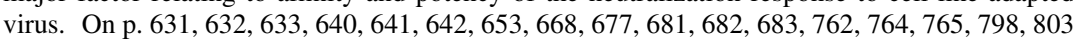

[Sattentau1995c] Q. J. Sattentau, S. Zolla-Pazner, \& P. Poignard. Epitope exposure on functional, oligomeric HIV-1 gp41 molecules. Virology 206:713-717, 1995. Most gp41 epitopes are masked when associated with gp120 on the cell surface. Weak binding of anti-gp41 MAbs can be en hanced by treatment with sCD4. MAb 2F5 binds to a membrane proximal epitope which binds in the presence of gp120 without sCD4. On p. 657 668 694 695 696 697 706 708 710 711 $720,724,725,784,803$

Sattentau 1996] Q. J. Sattentau. Neutralization of HIV-1 by antibody. Curr Opin in Immunol 8:540 
\begin{tabular}{ll|l|l|l|l|l|l|} 
545, 1996. Review. On p. 710 & 711 & 720 & 721 & 784 & 785 & 822 \\
\hline
\end{tabular}

[Savarino2001] A. Savarino, L. Gennero, H. C. Chen, D. Serrano, F. Malavasi, J. R. Boelaert, \& K. Sperber. Anti-HIV effects of chloroquine: mechanisms of inhibition and spectrum of activity. AIDS 15(17):2221-9, 2001. On p. 822 826

[Scala1999] G. Scala, X. Chen, W. Liu, J. N. Telles, O. J. Cohen, M. Vaccarezza, T. Igarashi, \& A. S Fauci. Selection of HIV-specific immunogenic epitopes by screening random peptide librarie with HIV-1-positive sera. J Immunol 162(10):6155-6161, 1999. On p.750

[Scanlan2002] C. N. Scanlan, R. Pantophlet, M. R. Wormald, E. Ollmann Saphire, R. Stanfield, I. A. Wilson, H. Katinger, R. A. Dwek, P. M. Rudd, \& D. R. Burton. The broadly neutralizing antihuman immunodeficiency virus type 1 antibody 2 G12 recognizes a cluster of alpha1 $\rightarrow 2$ mannose residues on the outer face of gp120. J Virol 76(14):7306-7321, 2002. On p.784 790 822 826

[Schafer1998] J. R. Schafer, B. M. Jesdale, J. A. George, N. M. Kouttab, \& A. S. D. Groot. Prediction of well-conserved HIV-1 ligands using a matrix-based algorithm, EpiMatrix. Vaccine 16:1880-4, 1998. On p.135.217

[Scharf2001] O. Scharf, H. Golding, L. R. King, N. Eller, D. Frazier, B. Golding, \& D. E. Scott Immunoglobulin G3 from polyclonal human immunodeficiency virus (HIV) immune globulin is more potent than other subclasses in neutralizing HIV type 1. J Virol 75(14):6558-65, 2001. On p.743

[Scheffel1999] J. W. Scheffel, R. Ziemann, D. Hawksworth, J. Tyner, R. K. Hickman, \& J. Hackett. Monoclonal antibodies to an HIV-1 group O envelope recombinant. J Acquir Immune Defic Synd

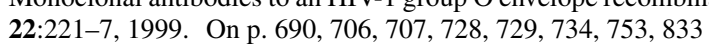

[Schiller2000] D. S. Schiller, J. M. Binley, K. H. Roux, C. S. Adamson, I. M. Jones, H. G. Krausslich, A. Hurley, M. Markowitz, \& J. P. Moore. Parameters influencing measurement of the Ga antigen-specific T- proliferative response to HIV type 1 infection. AIDS Res Hum Retroviruse 16:259-71, 2000. On p. 442

[Schito2001] A. M. Schito, E. Vittinghoff, F. M. Hecht, M. K. Elkins, J. O. Kahn, J. A. Levy, \& J. R. Oksenberg. Longitudinal analysis of T-cell receptor gene use by CD8(+) T cells in early human immunodeficiency virus infection in patients receiving highly active antiretroviral therapy. Blood 97(1):214-20, 2001. On p. 400

[Schmitt2000] M. Schmitt, E. Harrer, A. Goldwich, M. Bauerle, I. Graedner, J. R. Kalden, \& T. Harrer. Specific recognition of lamivudine-resistant HIV-1 by cytotoxic T lymphocytes. AIDS 14:653-8, 2000. On p. 201.

[Schneider1991] T. Schneider, H.-P. Harthus, P. Heldebrandt, M. Niedrig, M. Broker, W. Weigelt A. Beck, \& G. Pauli. Epitopes of the HIV-1-negative factor reactive with murine monoclona antibodies and human HIV-1-positive sera. AIDS Res Hum Retroviruses 7:37-43, 1991. Epitope for 9 murine MAbs were mapped, and found to be located in 4 immunogenic regions. 7/10 sera from HIV-1 positive individuals reacted to the four nef immunogenic regions. On p. 835836 837838

[Schönbach2002] C. Schönbach, Y. Kun, \& V. Brusic. Large-scale computational identification of HIV T-cell epitopes. Immunol Cell Biol 80(3):300-306, 2002. On p. 80 [168 $243 \mid 339$

[Schonning1998] K. Schonning, A. Bolmstedt, J. Novotny, O. S. Lund, S. Olofsson, \& J. E. Hansen. Induction of antibodies against epitopes inaccessible on the HIV type 1 envelope oligomer by immunization with recombinant monomeric glycoprotein 120. AIDS Res Hum Retroviruses 14:1451-6, 1998. On p.641 642 662 670|675|676|677|
169|

[Schonning 1999] K. Schonning, O. Lund, O. S. Lund, \& J. E. Hansen. Stoichiometry of monoclonal antibody neutralization of T-cell line-adapted human immunodeficiency virus type 1.J Viro 73:8364-70, 1999. On p. 590 662677

[Schrier1988] R. D. Schrier, J. W. Gnann, A. J. Langlois, K. Shriver, J. A. Nelson, \& M. B. A Oldstone. B- and T-lymphocyte Responses to an Immunodominant Epitope of Human Immunodeficiency Virus. J Virol 62:2531-2536, 1988. Notes: Characterization of murine T-lymphocyte dependent B-cell responses; also, T-cells from 7/29 HIV-1 positive people showed a proliferative response to this peptide. On p. 516

Schrier1989] R. D. Schrier, J. W. Gnann, R. Landes, C. Lockshin, D. Richman, A. McCutchan, C. Kennedy, M. B. A. Oldstone, \& J. A. Nelson. T-cell recognition of HIV synthetic peptides in a natural infection. J Immunol 142:1166-1176, 1989. Notes: The ability of 21 peptides to stimulate T-cell proliferation was tested in 30 HIV-infected donors in different clinical stages. T-cells from 27/30 donors were able to respond to at least one peptide. Two of the peptides were able to stimulate proliferation in 48it et al. did not write down the peptide sequences they used, but only provided the numbering of the boundaries on a reference sequence (LAI, Wain-Hobson it et al., Cell 40:9-17 (1985)). In our experience, such numbering is often imprecise, so the peptide assignments in this database may be off by several residues. Two epitopes that Schrier it et al. mistakenly labeled as p24 peptides are instead p15 peptides. On p. $429|434 \quad 438| 440|455| 456$

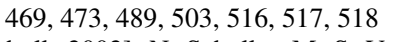

Schulke2002] N. Schulke, M. S. Vesanen, R. W. Sanders, P. Zhu, M. Lu, D. J. Anselma, A. R. Villa, P. W. H. I. Parren, J. M. Binley, K. H. Roux, P. J. Maddon, J. P. Moore, \& W. C. Olson Oligomeric and Conformational Properties of a Proteolytically Mature, Disulfide-Stabilized Human Immunodeficiency Virus Type 1 gp140 Envelope Glycoprotein. J Virol 76(15):7760-76, \begin{tabular}{l|l|l|l|l|l|l|l|l|l|l|l|} 
2002. On p. 657 & 658 & 710 & 716 & 730 & 759 & 784 & 789 & 798 & 801 & 822 & 826 \\
\hline
\end{tabular}

[Schutten1993] M. Schutten, A. McKnight, R. C. Huisman, M. Thali, J. A. McKeating, J. Sodroski, J. Goudsmit, \& A. D. Osterhaus. Further characterization of an antigenic site of HIV-1 gp120 recognized by virus neutralizing human monoclonal antibodies. AIDS 7:919-923, 1993. Three human anti-CD4 binding site MAbs were characterized. Amino acid substitutions that block $\mathrm{MAb}$ binding were similar but slightly different than those found in murine anti-CD4 binding site MAbs. On p. 780781

Schutten1995a] M. Schutten, A. C. Andeweg, M. L. Bosch, \& A. D. Osterhaus. Enhancement of Infectivity of a Non-Syncytium Inducing HIV-1 by sCD4 and by Human Antibodies that Neutralize Syncytium Inducing HIV-1. Scand J Immunol 41:18-22, 1995 . On p. 647, 739780 781

[Schutten1995b] M. Schutten, J. P. Langedijk, A. C. Andeweg, R. C. Huisman, R. H. Meloen, \& A. D. Osterhaus. Characterization of a V3 domain-specific neutralizing human monoclonal antibody that preferentially recognizes non-syncytium-inducing human immunodeficiency virus type 1 strains. J Gen Virol 76:1665-1673, 1995. Characterization of HuMAb MN215. On p. 647 659 739 780

[Schutten1996] M. Schutten, K. Tenner-Racz, P. Racz, D. W. van Bekkum, \& A. D. Osterhaus Human antibodies that neutralize primary human immunodeficiency virus type 1 in vitro do no provide protection in an in vivo model. J Gen Virol 77:1667-75, 1996. On p. 647, 739780

[Schutten1997] M. Schutten, A. C. Andeweg, G. F. Rimmelzwaan, \& A. D. Osterhaus. Modula tion of primary human immunodeficiency virus type 1 envelope glycoprotein-mediated entry by human antibodies. J Gen Virol 78:999-1006, 1997. A series of HIV-1 envelope glycoproteins from related primary virus isolates of different SI phenotypes, together with chimeras of these proteins, were tested in an envelope trans-complementation assay for their sensitivity to either antibody mediated inhibition or enhancement of HIV-1 entry. In contrast to the inhibition of HIV-1 entry, antibody mediated enhancement was not temperature dependent and could not be mediated by $\mathrm{F}(\mathrm{ab})$ fragments, implicating cross-linking as an important step. Enhancement or inhibition seemed to be determined by virus isolate rather than by the specificity of the antiserum used. $2 \mathrm{~F} 5$ \begin{tabular}{|l|l|l|l|l|l|l|l|} 
& was the only MAb that inhibited the entry of all viruses. On $\mathrm{p} .647$ & 710 & 711 & 739 & 780 & 781 & 784 \\
\hline
\end{tabular} 785

[Schutten2001] M. Schutten, C. A. van Baalen, C. Guillon, R. C. Huisman, P. H. Boers, K. Sintnicolaas, R. A. Gruters, \& A. D. Osterhaus. Macrophage tropism of human immunodeficiency virus type 1 facilitates in vivo escape from cytotoxic T-lymphocyte pressure. J Virol 75(6):2706-9, 
2001. On p. 263

[Scott-Algara2001] D. Scott-Algara, F. Buseyne, S. Blanche, C. Rouzioux, C. Jouanne, F. Romagne, \& Y. Riviere. Frequency and phenotyping of human immunodeficiency virus (HIV)-specific CD8+ T cells in HIV-infected children, using major histocompatibility complex class I peptide tetramers. J Infect Dis 183(11):1565-73, 2001. On p.74 210

[Scott1990] C. F. Scott, Jr., S. Silver, A. T. Profy, S. D. Putney, A. Langlois, K. Weinhold, \& J. E. Robinson. Human monoclonal antibody that recognizes the V3 region of human immunodeficiency virus gp120 and neutralizes the human T-lymphotropic virus type IIIMN strain. Proc Nat Acad Sci USA 87:8597-8601, 1990. On p. 657 674

[Scott2001] Z. A. Scott, E. G. Chadwick, L. L. Gibson, M. D. Catalina, M. M. McManus, R. Yogev, P. Palumbo, J. L. Sullivan, P. Britto, H. Gay, K. Luzuriaga, \& PACTG (Pediatric AIDS Clinical Trial Group) 345 Investigators. Infrequent detection of HIV-1-specific, but not cytomegalovirusspecific, CD8(+) T cell responses in young HIV-1-infected infants. J Immunol 167(12):7134 7140, 2001. On p.167, 243 339398

[Secord1996] E. A. Secord, G. I. Kleiner, D. L. Auci, T. Smith-Norowitz, S. Chice, A. Finkielstein, M. Nowakowski, S. Fikrig, \& H. G. Durkin. IgE against HIV proteins in clinically healthy children with HIV disease. J Allergy Clin Immunol 98(5 Pt 1):979-984, 1996. On p.843

[Selby1997] M. J. Selby, B. Doe, \& C. M. Walker. Virus-specific cytotoxic T-lymphocyte activity elicited by coimmunization with human immunodeficiency virus type 1 genes regulated by the bacteriophage T7 promoter and T7 RNA polymerase protein. J Virol 71:7827-7831, 1997. On p. 292

[Seligman1996] S. J. Seligman, J. M. Binley, M. K. Gorny, D. R. Burton, S. Zolla-Pazner, \& K. A Sokolowski. Characterization by serial deletion competition ELISAs of HIV-1 V3 loop epitope recognized by monoclonal antibodies. Mol Immunol 33:737-745, 1996. On p. 649650652 668673

[Seth2000] A. Seth, Y. Yasutomi, H. Jacoby, J. C. Callery, S. M. Kaminsky, W. C. Koff, D. F. Nixon, \& N. L. Letvin. Evaluation of a lipopeptide immunogen as a therapeutic in HIV type 1seropositive individuals. AIDS Res Hum Retroviruses 16:337-43, 2000. On p. 125

[Seth2001] A. Seth, J. Markee, A. Hoering, A. Sevin, D. E. Sabath, J. E. Schmitz, M. J. Kuroda M. A. Lifton, M. S. Hirsch, A. C. Collier, N. L. Letvin, \& M. J. McElrath. Alterations in T cell phenotype and human immunodeficiency virus type 1-specific cytotoxicity after potent antiretroviral therapy. J Infect Dis 183(5):722-9, 2001. On p. 58 68 66 , $95,178,218$

[Severino2000] M. E. Severino, N. V. Sipsas, P. T. Nguyen, S. A. Kalams, B. D. Walker, R. P. Johnson, \& O. O. Yang. Inhibition of human immunodeficiency virus type 1 replication in primary CD4(+) T lymphocytes, monocytes, and dendritic cells by cytotoxic T lymphocytes. J Viro 74(14):6695-9, 2000. On p. 59 312

[Sewell1997] A. K. Sewell, G. C. Harcourt, P. J. Goulder, D. A. Price, \& R. E. Phillips. Antagonism of cytotoxic T lymphocyte-mediated lysis by natural HIV-1 altered peptide ligands require simultaneous presentation of agonist and antagonist peptides. Eur J Immunol 27:2323-9, 1997. On p. 81,83

[Sewell1999] A. K. Sewell, D. A. Price, H. Teisserenc, B. L. Booth, U. Gileadi, F. M. Flavin, J. Trowsdale, R. E. Phillips, \& V. Cerundolo. IFN-gamma exposes a cryptic cytotoxic T lymphocyte epitope in HIV-1 reverse transcriptase. J Immunol 162:7075-9, 1999. On p. 199210

[Sewell2000] A. K. Sewell, D. A. Price, A. Oxenius, A. D. Kelleher, \& R. E. Phillips. Cytotoxic T lymphocyte responses to human immunodeficiency virus: control and escape. Stem Cells 18(4):230-44, 2000. On p.73

[Sewell2002] A. K. Sewell, B. L. Booth, Jr., V. Cerundolo, R. E. Phillips, \& D. A. Price. Differential processing of HLA A2-restricted HIV type 1 cytotoxic T lymphocyte epitopes. Viral Immuno 15(1):193-196, 2002. On p. 80.200214

[Shacklett2000] B. L. Shacklett, T. J. Beadle, P. A. Pacheco, J. H. Grendell, P. A. Haslett, A. S.
King, G. S. Ogg, P. M. Basuk, \& D. F. Nixon. Characterization of HIV-1-specific cytotoxic T lymphocytes expressing the mucosal lymphocyte integrin CD103 in rectal and duodenal lymphoid tissue of HIV-1-infected subjects. Virology 270:317-27, 2000. On p. 168 243 398

[Shafferman1989] A. Shafferman, J. Lennox, H. Grosfeld, J. Sadoff, R. R. Redfield, \& D. S. Burke Patterns of antibody recognition of selected conserved amino acid sequences from the HIV envelope in sera from different stages of HIV infection. AIDS Res Hum Retroviruses 5:33-39, 1989 On p. 700

Shang1991] F. Shang, H. Huang, K. Revesz, H.-C. Chen, R. Herz, \& A. Pinter. Characterization of monoclonal antibodies against the human immunodeficiency virus matrix protein, p17gag. identification of epitopes exposed at the surfaces of infected cells. J Virol 65:4798-4804, 1991 Six MAbs with linear epitopes were mapped. These Abs could only bind to HIV-infected cells that had been permeablized with acetone. Only G11g1 and G11h3, two antibodies that did not bind to peptides, but only to intact p17, could react with live HIV-1 infected cells. These antibodies were not neutralizing. On p. 571 572, 591

[Shankar1996] P. Shankar, J. A. Fabry, D. M. Fong, \& J. Lieberman. Three regions of HIV-1 gp160 contain clusters of immunodominant CTL epitopes. Immunol Lett 52:23-30, 1996. On p. 273 283314328331

[Shankar1998] P. Shankar, H. Sprang, \& J. Lieberman. Effective lysis of HIV-1-infected primary CD4+ T cells by a cytotoxic T-lymphocyte clone directed against a novel A2-restricted reversetranscriptase epitope. J Acquir Immune Defic Syndr Hum Retrovirol 19:111-20, 1998. On $\mathrm{p} .185$ [Sharon2002] M. Sharon, M. Görlach, R. Levy, Y. Hayek, \& J. Anglister. Expression, purification, and isotope labeling of a gp120 V3 peptide and production of a Fab from a HIV-1 neutralizing antibody for NMR studies. Protein Expr Purif 24(3):374-383, 2002. On p.720 723

[Shen2000] X. Shen \& R. F. Siliciano. Preventing AIDS but not HIV-1 infection with a DNA vaccine. Science 290(5491):463-5, 2000. On p. 305

[Shibata1999] R. Shibata, T. Igarashi, N. Haigwood, A. Buckler-White, R. Ogert, W. Ross, R. Willey, M. Cho, \& M. Martin. Neutralizing antibody directed against the HIV-1 envelope glycoprotein can completely block HIV-1/SIV chimeric infections of macaques monkeys. Nat Med 5:204-210, 1999. On p. 744

[Shiga1996] H. Shiga, T. Shioda, H. Tomiyama, Y. Takamiya, S. Oka, S. Kimura, Y. Yamaguchi, T. Gojoubori, H. G. Rammensee, K. Miwa, \& M. Takiguchi. Identification of multiple HIVcytotoxic T cell epitopes presented by human leukocyte antigen B35 molecule. AIDS 10:10751083, 1996. On p. $185\left[\begin{array}{l|l|l|l|l|l|l|l|l|}188 & 196 & 209 & 228 & 275 & 283 & 345 & 350 & 384 \\ \hline\end{array}\right.$

Shirai1992] M. Shirai, C. D. Pendleton, \& J. A. Berzofsky. Broad recognition of cytotoxic T cel epitopes from the HIV-1 envelope protein with multiple class I histocompatibiity molecules. $J$ Immunol 148:1657-1667, 1992. This paper explored the possibility that defined epitopes from HIV-1 Env might be presented by multiple class I genes to CTLs using a murine system, isolating CTL from mice immunized with gp160 expressing recombinant vaccinia virus. The CTL epitope at the tip of the V3 loop (P18) was found to be presented by class I MHC molecules from four of ten haplotypes tested. Peptides that had previously been defined as helper T cell determinants (T1 in gp120, and HP53 (also called TH4.3)) were also able to stimulate CTL from mice with multiple haplotypes. On p. $289,305,327$

[Shirai1993] M. Shirai, M. S. Vacchio, R. J. Hodes, \& J. A. Berzofsky. Preferential V $\beta$ usage by cytotoxic T cells cross-reactive between two epitopes of HIV-1 gp160 and degenerate in class MHC restriction. J Immunol 151:2283-2295, 1993. On p.289

[Shirai1996a] M. Shirai, M. Chen, T. Arichi, T. Masaki, M. Nishioka, M. Newman, T. Nakazawa, S. M. Feinstone, \& J. A. Berzofsky. Use of intrinsic and extrinsic helper epitopes for in vivo induction of anti-hepatitis C virus cytotoxic T lymphocytes (CTL) with CTL epitope peptide vaccines. J Inf Dis 173:24-31, 1996. On p.509

[Shirai1996b] M. Shirai, K. Kurokohchi, C. D. Pendleton, T. Arichi, L. F. Boyd, H. Takahashi, D. H. 
Margulies, \& J. A. Berzofsky. Reciprocal cytotoxic T lymphocytes cross-reactivity interactions between two major epitopes within HIV-1 gp160. J Immunol 157:4399-4411, 1996. On p.291 327

[Shirai1997] M. Shirai, S. Kozlowski, D. H. Margulies, \& J. A. Berzofsky. Degenerate MHC restriction reveals the contribution of class I MHC molecules in determining the fine specificity of CTL recognition of an immunodominant determinant of HIV-1 gp160 V3 loop. J Immunol 158:31818,1997 . The novel allogeneic presentation of an immunodominant determinant within the HIVgp160 V3 loop by three different class I MHC molecules to the same CD8+ CTL is used to study the influence of the MHC molecule on the fine specificity of CTL recognition. We previously the influence of the $\mathrm{MHC}$ molecule on the fine specificity of CTL recognition. We previously
reported that four distinct class I molecules of $\mathrm{H}-2 \mathrm{~d}, \mathrm{u}, \mathrm{p}, \mathrm{q}$ presented the V3 decapeptide P18-I10 (RGPGRAFVTI) to CTL. Surprisingly, we found that H- 2d,u,p cells mutually cross-present the P18-I10 peptide to allogeneic CTL clones of each of the other haplotypes, whereas none of these cross- presents to H-2q CTL, nor do H-2q targets present to CTL of the other haplotypes. Here, we explore the critical amino acid residues for the cross-presentation using 10 variant peptides with single amino acid substitutions. The fine specificity examined using these mutant peptides presented by the same MHC class I molecule showed striking similarity among the CTL of each haplotype, expressing either $\mathrm{V}$ beta 8.1 or $\mathrm{V}$ beta 14 . In contrast, the fine specificity is different between the distinct MHC class I molecules even for the lysis by the same CTL, as shown by reciprocal effects of the same substitutions. Thus, peptide fine specificity of a single TCR is influenced by changes in the class I MHC molecules presenting the Ag. On p.296

[Shirai2001] M. Shirai, R. Fujinaga, T. Masaki, \& J. A. Berzofsky. Impaired development of HIV-1 gp160-specific CD8(+) cytotoxic T cells by a delayed switch from Th1 to Th2 cytokine phenotype in mice with Helicobacter pylori infection. Eur J Immunol 31(2):516-26, 2001. On p. 296.529

[Shiver1997] J. W. Shiver, M. E. Davies, Y. Yasutomi, H. C. Perry, D. C. Freed, N. L. Letvin, \& M. A. Liu. Anti-HIV env immunities elicited by nucleic acid vaccines. Vaccine 15:884-7, 1997. On p. 333 524, 747

[Shotton1995] C. Shotton, C. Arnold, Q. Sattentau, J. Sodroski, \& J. A. McKeating. Identification and characterization of monoclonal antibodies specific for polymorphic antigenic determinants within the V2 region of the human immunodeficiency virus type 1 envelope glycoprotein. Virol 69:222-230, 1995. Anti-V2 linear and conformation dependent MAbs were studied. All V2 Abs studied could bind IIIB, but failed to neutralize non-clonal stocks. Epitope exposure different in rgp120 compared to native gp120. HXB2 V2-MAb neutralization escape mutant \begin{tabular}{ll|l|l|l|l|l|l|l|l|l|l|l|l|l|} 
were sequenced. On p. 629 & 630 & 631 & 633 & 634 & 808 & 810 & 811 \\
\hline
\end{tabular}

[Si2001] Z. Si, M. Cayabyab, \& J. Sodroski. Envelope glycoprotein determinants of neutralization resistance in a simian-human immunodeficiency virus (SHIV-HXBc2P 3.2) derived by passage

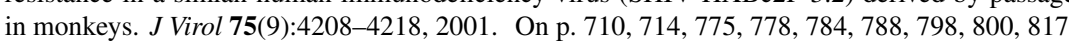
822825

[Sidorova1999] E. Sidorova. Human monoclonal antibodies to MN-24 peptide of gp 120 HIV-1. Hum Antibodies 9:107-10, 1999. On p.819

[Siliciano1988] R. Siliciano, T. Lawton, C. Knall, R. Karr, P. Berman, T. Gregory, \& E. Reinherz Analysis of host-virus interactions in AIDS with anti-gp120 T cell clones: effect of HIV sequence variation and a mechanism for CD4+ cell depletion. Cell 54:561-575, 1988. This article demonstrated that a class II HLA-DR4 restricted response can be stimulated by CD4 uptake of gp120, suggesting a mechanism for T-cell depletion in vivo. This peptide containing the epitope was also able to stimulate a class I restricted, CD8+ CTL response. On p. 304

[Singh2002] R. A. K. Singh, L. Wu, \& M. A. Barry. Generation of genome-wide CD8 T cell responses in HLA-A $* 0201$ transgenic mice by an HIV-1 ubiquitin expression library immunization vaccine. J Immunol 168(1):379-391, 2002. On p.81 214 287, 390

[Sipsas1997] N. V. Sipsas, S. A. Kalams, A. Trocha, S. He, W. A. Blattner, B. D. Walker, \& R. P. Johnson. Identification of type-specific cytotoxic T lymphocyte responses to homologous viral proteins in laboratory workers accidentally infected with HIV-1. J Clin Invest 99:752-62, 1997. To examine a situation where the autologous strain and the reference reagents would be the same, the CTL response of three lab workers accidentally infected with HIV IIIB was studied. Both group specific and type specific epitopes were targets for CTL clones. One subject had a broadening of CTL response over time, using a broad range of restricting HLA class I alleles. Characterization of the cytotoxic T lymphocyte (CTL) response against HIV-1 has been limited by the use of target cells expressing viral proteins from laboratory isolates of HIV-1. This approach has favored identification of group-specific CTL responses and precluded assessment of the extent of type-specific CTL responses directed against HIV-1. Using cells expressing viral proteins from the HIV-1 IIIB strain, we performed a detailed characterization of HIV-1-specific CTL response in three laboratory workers accidentally infected with HIV-1 IIIB. Eight of the epitopes identified were group specific, lying in relatively conserved regions of Gag, reverse transcriptase, and envelope. Three type-specific epitopes were identified, two of them in highly variable regions of envelope. In longitudinal studies in one subject, seven different epitopes and five different restricting HLA class I alleles were identified, with a progressive increase in the number of CTL epitopes recognized by this subject over time. Our data demonstrate that type-specific CTL responses make up a significant proportion of the host cellular immune response against HIV-1 an that a broadening of epitope specificity may occur. On p. $83 \mid$\begin{tabular}{l|l|l|l|}
820 & 156 & 185 & 187 \\
\hline
\end{tabular} $282,308,310$

[Sitz1999] K. V. Sitz, S. Ratto-Kim, A. S. Hodgkins, M. L. Robb, \& D. L. Birx . Proliferative responses to human immunodeficiency virus type 1 (HIV-1) gp120 peptides in HIV-1-infected individuals immunized with HIV-1 rgp120 or rgp160 compared with nonimmunized and uninfected controls. J Infect Dis 179:817-24, 1999. On p.473 475 478 488 495 501 511.

[Sjolander1996] S. Sjolander, A. Bolmstedt, L. Akerblom, P. Horal, S. Olofsson, B. Morein, \& A. Sjolander. N-linked glycans in the CD4-binding domain of human immunodeficiency virus type 1 envelope glycoprotein gp160 are essential for the in vivo priming of $\mathrm{T}$ cells recognizing an epitope located in their vicinity. Virology 215:124-33, 1996. Notes: An investigation of whether T cell responses to the HIV-1 gp160 were sensitive to deletion of three N-glycans of the protein. On p. 470478481510

[Skinner1988a] M. A. Skinner, A. J. Langlois, C. B. McDanal, J. S. McDougal, D. P. Bolognesi, \& T. J. Matthews. Neutralizing Antibodies to an Immunodominant Envelope Sequence Do Not Prevent gp120 Binding to CD4. J Virol 62:4195-4200, 1988. This report was an early suggestion that there are at least two classes of biologically active antibodies to HIV: one class is isolate restricted, primarily directed to a hypervariable loop structure of gp120 and not involved in CD4 binding; the second class is directed at more conserved structures that may directly block CD4 binding. On p. 641671

Skinner1988b] M. A. Skinner, R. Ting, A. J. Langlois, K. J. Weinhold, H. K. Lyerly, K. Javaherian, \& T. J. Matthews. Characteristics of a neutralizing monoclonal antibody to the HIV envelope glycoprotein. AIDS Res Hum Retroviruses 4:187-197, 1988. On p.641 671 675687

Skott1999] P. Skott, E. Lucht, I. Julander, J. Dillner, \& E. Björling. Salivary sIgA response in HIV-1 infection. J Acquir Immune Defic Syndr 21(2):73-80, 1999. On p. 821

[Smith-Franklin2002] B. A. Smith-Franklin, B. F. Keele, J. G. Tew, S. Gartner, A. K. Szakal, J. D. Estes, T. C. Thacker, \& G. F. Burton. Follicular dendritic cells and the persistence of HIV infectivity: The role of antibodies and Fc-gamma receptors. J Immunol 168(5):2408-2414, 2002. On p. 832

[Smith1996] K. J. Smith, S. W. Reid, D. I. Stuart, A. J. McMichael, E. Y. Jones, \& J. I. Bell. An altered position of the alpha 2 helix of MHC class I is revealed by the crystal structure of HLA B*3501. Immunity 4:203-213, 1996. The crystal structure of HLA-B*3501 complexed with Nef epitope VPLRPMTY was determined at 2 angstrom resolution, revealing details about binding such as the structural basis for the tyrosine specificity of the F pocket. On p. 356 
[Smith1998] A. D. Smith, S. C. Geisler, A. A. Chen, D. A. Resnick, B. M. Roy, P. J. Lewi, E. Arnold \& G. F. Arnold. Human rhinovirus type 14:human immunodeficiency virus type 1 (HIV-1) V3 loop chimeras from a combinatorial library induce potent neutralizing antibody responses against HIV-1. J Virol 72:651-9, 1998. The tip of the MN V3 loop, IGPGRAFYTTKN, was inserted into cold-causing human rhinovirus 14 (HRV14) and chimeras were immunoselected using MAb 447-52-D, 694/98-D, NM-01, and 59.1, for good presentation of the V3 antigenic region. The selected chimeric viruses were neutralized by anti-V3 loop MAbs. The chimeric viruses elicited \begin{tabular}{ll|l|l|l|l|l|} 
potent NAbs against ALA-1 and MN in guinea pigs. On p. 671 & 672 & 673 & 675 & 720 & 721 \\
\hline
\end{tabular}

[Sorensen1994] A. M. M. Sorensen, C. Nielsen, M. Arendrup, H. Clausen, J. O. Nielsen, E. Osinaga, A. Roseto, \& J.-E. S. Hansen. Neutralization epitopes on HIV pseudotyped with HTLV-I: Conservation of carbohydrate epitopes. J Acq Immune Def Synd 7:116-123, 1994. Pseudotype were formed with HIV and HTLV-I. MAb 9284, directed at the V3 loop of gp120, failed to inhibit the infection of CD-4 negative cells with pseudotypes, but anti-HTLV serum did inhibit infection. HIV and HTLV-I appear to induce common carbohydrate neutralizing epitopes. On p. 641642

[Soudeyns1997] H. Soudeyns \& G. Pantaleo. New mechanisms of viral persistence in primary human immunodeficiency virus (HIV) infection. J Biol Regul Homeost Agents 11:37-9, 1997. Reviews. On p. 340

[Soudeyns1999] H. Soudeyns, S. Paolucci, C. Chappey, M. B. Daucher, C. Graziosi, M. Vaccarezza, O. J. Cohen, A. S. Fauci, \& G. Pantaleo. Selective pressure exerted by immunodominant HIV1-specific cytotoxic $\mathrm{T}$ lymphocyte responses during primary infection drives genetic variation restricted to the cognate epitope. Eur J Immunol 29:3629-35, 1999. On p. 328

[Soudeyns2000] H. Soudeyns, G. Campi, G. P. Rizzardi, C. Lenge, J. F. Demarest, G. Tambussi, A. Lazzarin, D. Kaufmann, G. Casorati, L. Corey, \& G. Pantaleo. Initiation of antiretrovira therapy during primary HIV-1 infection induces rapid stabilization of the T cell receptor beta chain repertoire and reduces the level of T cell oligoclonality. Blood 95:1743-51, 2000. On p. 332

[Spear1993] G. T. Spear, D. M. Takefman, B. L. Sullivan, A. L. Landay, \& S. Zolla-Pazner. Complement activation by human monoclonal antibodies to human immunodeficiency virus. $J \mathrm{Vi}$ rol 67:53-59, 1993. This study looked at the ability of 16 human MAbs to activate complement. MAbs directed against the V3 region could induce $\mathrm{C} 3$ deposition on infected cells an virolysis of free virus, but antibodies to the CD4BS and C-terminal region and two regions in gp41 could induce no complement mediated effects. Pre-treatment with sCD4 could increase complement-mediated effects of anti-gp41 MAbs, but decreased the complement-mediated ef fects of V3 MAbs. Anti-gp41 MAbs were able to affect IIIB but not MN virolysis, suggesting spontaneous shedding of gp120 on IIIB virions exposes gp41 epitopes. IgG isotype did not appear to have an effect on virolysis or $\mathrm{C} 3$ deposition. On p. 589 645647648659664675 \begin{tabular}{|l|l|l|l|l|l|l|l|l|l|}
\hline 691 & 696 & 697 & 700 & 702 & 708 & 709 & 720 & 766 & 768 \\
\hline
\end{tabular}

[Spear1994] G. T. Spear, D. M. Takefman, S. Sharpe, M. Ghassemi, \& S. Zolla-Pazner. Antibodies to the HIV-1 V3 loop in serum from infected persons contribute a major proportion of immune effector functions including complement activation, antibody binding, and neutralization. Virolog 204:609-15, 1994. On p. 646

[Spenlehauer2001] C. Spenlehauer, C. A. Gordon, A. Trkola, \& J. P. Moore. A luciferase-reporter gene-expressing T-cell line facilitates neutralization and drug-sensitivity assays that use either R5 or X4 strains of human immunodeficiency virus type 1. Virology 280(2):292-300, 2001. On p.710 $715,784,789,822,825$

[Sperlagh1993] M. Sperlagh, K. Stefano, F. Gonzalez-Scarano, S. Liang, J. Hoxie, H. Maruyama, M. Prewett, S. Matsushito, \& D. Herlyn. Monoclonal anti-idiotype antibodies that mimic the epitope on gp120 defined by the anti-HIV-1 monoclonal antibody $0.5 \beta$. AIDS 7:1553-1559, 1993. On p. 671

[Spiegel1999] H. M. Spiegel, E. DeFalcon, G. S. Ogg, M. Larsson, T. J. Beadle, P. Tao, A. J.
McMichael, N. Bhardwaj, C. O'Callaghan, W. I. Cox, K. Krasinski, H. Pollack, W. Borkowsky, $\&$ D. F. Nixon. Changes in frequency of HIV-1-specific cytotoxic T cell precursors and circulating effectors after combination antiretroviral therapy in children. J Infect Dis 180:359-68, 1999. On p. 104 367

[Spiegel2000] H. M. Spiegel, G. S. Ogg, E. DeFalcon, M. E. Sheehy, S. Monard, P. A. Haslett, G. Gillespie, S. M. Donahoe, H. Pollack, W. Borkowsky, A. J. McMichael, \& D. F. Nixon. Human immunodeficiency virus type 1- and cytomegalovirus-specific cytotoxic T lymphocytes can persist at high frequency for prolonged periods in the absence of circulating peripheral CD4(+) T cells. J Virol 74:1018-22, 2000. On p.77, 210

Srivastava2002] I. K. Srivastava, L. Stamatatos, H. Legg, E. Kan, A. Fong, S. R. Coates, L. Leung, M. Wininger, J. J. Donnelly, J. B. Ulmer, \& S. W. Barnett. Purification and characterization of oligomeric envelope glycoprotein from a primary R5 subtype B human immunodeficiency virus

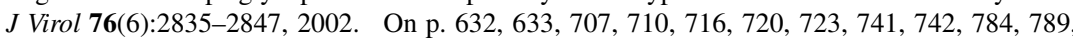
791792798,801

[Sriwanthana2001] B. Sriwanthana, T. Hodge, T. D. Mastro, C. S. Dezzutti, K. Bond, H. A. Stephens, L. G. Kostrikis, K. Limpakarnjanarat, N. L. Young, S. H. Qari, R. B. Lal, D. Chandanayingyong, \& J. M. McNicholl. HIV-specific cytotoxic T lymphocytes, HLA-A11, and chemokine-related factors may act synergistically to determine HIV resistance in CCR5 delta32negative female sex workers in Chiang Rai, northern Thailand. AIDS Res Hum Retroviruses

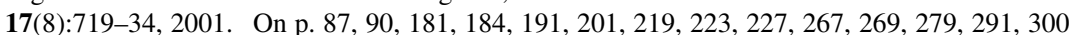
$319 \sqrt{324} 353362$

Staats2001] H. F. Staats, C. P. Bradney, W. M. Gwinn, S. S. Jackson, G. D. Sempowski, H.-X. Liao N. L. Letvin, \& B. F. Haynes. Cytokine requirements for induction of systemic and mucosal CTL after nasal immunization. J Immunol 167(9):5386-5394, 2001. On p.291]298

[Stamatatos1995] L. Stamatatos \& C. Cheng-Mayer. Structural modulations of the envelope gp120 glycoprotein of human immunodeficiency virus type 1 upon oligomerization and the differential V3 loop epitope exposure of isolates displaying distinct tropism upon viral-soluble receptor \begin{tabular}{l|l|l|l|l|l|l|l|} 
binding. J Virol 69:6191-6198, 1995. On p. 631 632 & 647 & 649 & 664 & 768 & 769 & 770 \\
\hline
\end{tabular}

[Stamatatos1997] L. Stamatatos, S. Zolla-Pazner, M. K. Gorny, \& C. Cheng-Mayer. Binding of antibodies to virion-associated gp120 molecules of primary-like human immunodeficiency virus type 1 (HIV-1) isolates: effect on HIV-1 infection of macrophages and peripheral blood mononuclear cells. Virology 229:360-9, 1997. On p. 631 632 647 649 664 696 697, 710 712 769 770 . 784

[Stamatatos1998] L. Stamatatos \& C. Cheng-Mayer. An envelope modification that renders a primary, neutralization- resistant clade B human immunodeficiency virus type 1 isolate highly susceptible to neutralization by sera from other clades. $J$ Virol 72:7840-5, 1998. On p. 628 631 \begin{tabular}{|l|l|l|l|l|l|l|l|l|l|l|l|l|l|l|l|l|l|l|l|l|l|l|l|}
632 & 647 & 648 & 649 & 769 & 770 & 784 & 787 & 791 & 798 & 799 & 803 & 804 \\
\hline
\end{tabular}

[Stamatatos2000] L. Stamatatos, M. Lim, \& C. Cheng-Mayer. Generation and structural analysis of soluble oligomeric gp140 envelope proteins derived from neutralization-resistant an neutralization-susceptible primary HIV type 1 isolates. AIDS Res Hum Retroviruses 16(10):98194, 2000. On p.741, 742798,800

[Stanfield1999] R. Stanfield, E. Cabezas, A. Satterthwait, E. Stura, A. Profy, \& I. Wilson. Dual conformations for the HIV-1 gp120 V3 loop in complexes with different neutralizing Fabs. Structure 7:131-42, 1999. On p.652 668673

[Stiegler2001] G. Stiegler, R. Kunert, M. Purtscher, S. Wolbank, R. Voglauer, F. Steindl, \& H. Katinger. A potent cross-clade neutralizing human monoclonal antibody against a novel epitope on gp41 of human immunodeficiency virus type 1. AIDS Res Hum Retroviruses 17(18): 1757 65, 2001. On p.710 715719822

Stigler1995] R. D. Stigler, F. Ruker, D. Katinger, G. Elliott, W. Hohne, P. Henklein, J. X. Ho, K. Keeling, D. C. Carter, E. Nugel, \& et al. Interaction between a Fab fragment against gp41 of 
human immunodeficiency virus 1 and its peptide epitope: characterization using a peptide epitope library and molecular modeling. Protein Eng 8:471-9, 1995. On p.706

[Stoiber1996] H. Stoiber, C. Pinter, A. G. Siccardi, A. Clivio, \& M. P. Dierich. Efficient destruction of human immunodeficiency virus in human serum by inhibiting the protective action of complement factor $\mathrm{H}$ and decay accelerating factor (DAF, CD55). J Exp Med 183:307-310, 1996 HIV and HIV-infected cells are not subject to efficient complement-mediated lysis, even in the presence of HIV-specific antibodies. HIV is intrinsically resistant to human complement. Decay accelerating factor (DAF) and human complement factor $\mathrm{H}(\mathrm{CFH})$, a humoral negative regulato of complement which binds to gp41 are critical for this resistance. MAb 2F5 can inhibit CHF binding and facilitate complement mediated lysis. On p.710,711

[Stuhler1997] G. Stuhler \& S. F. Schlossman. Antigen organization regulates cluster formation an induction of cytotoxic T lymphocytes by helper T cell subsets. Proc Natl Acad Sci USA 94:622627, 1997. Generation of cytolytic activity requires a three-cell type cluster consisting of APC's, Helper, and CTL's, and co-expression of helper and CTL epitopes on the same APC. On p. 84

[Subbramanian2002] R. A. Subbramanian, J. Xu, E. Toma, R. Morisset, E. A. Cohen, J. Menezes, \& A. Ahmad. Comparison of human immunodeficiency virus (HIV)-specific infection-enhancing and -inhibiting antibodies in AIDS patients. J Clin Microbiol 40(6):2141-2146, 2002. On p.842

[Sugano1988] T. Sugano, Y. Masuho, Y.-I. Matsumoto, D. Lake, C. Gschwind, E. A. Petersen, \& E. M. Hersh. Human monoclonal antibody against glycoproteins of human immunodeficiency virus. Biochem and Biophys Res Comm 155:1105-1112, 1988. On p.699

[Sugiura1999] W. Sugiura, C. C. Broder, B. Moss, \& P. L. Earl. Characterization of conformationdependent anti-gp120 murine monoclonal antibodies produced by immunization with monomeric and oligomeric human immunodeficiency virus type 1 envelope proteins. Virology 254:257-67,

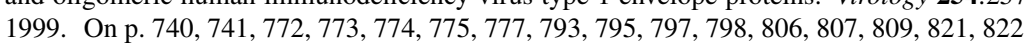

[Sullivan1993] N. Sullivan, M. Thali, C. Furman, D. Ho, \& J. Sodroski. Effect of amino acid changes in the V2 region of the human immunodeficiency virus type 1 gp120 glycoprotein on subunit association, syncytium formation, and recognition by a neutralizing antibody. J Virol 67:3674-3679, 1993. Recognition of neutralizing MAb G3-4 was altered by substitutions in 176
(irol to 184 in the V2 loop. Some changes in the V2 loop can affect subunit assembly; other change allow expression and CD4 binding but inhibit syncytium formation and viral entry, suggesting that V1/V2 may be involved in post receptor binding events. On $\mathrm{p} .632$

[Sullivan1995] N. Sullivan, Y. Sun, J. Li, W. Hofmann, \& J. Sodroski. Replicative function and neutralization sensitivity of envelope glycoproteins from primary and T-cell line-passaged human immunodeficiency virus type 1 isolates. J Virol 69:4413-4422, 1995. Three gp120 molecules derived from primary isolates were compared to T-cell adapted lines HXBc2 and MN. Complementation experiments showed viral entry into peripheral blood mononuclear cell targets was five-fold less efficient for primary isolates. Anti-CD4 binding site neutralizing MAbs were far less potent against primary isolates, and the single anti-V3 MAb tested was 3-fold less potent. The differences in neutralization efficiency could not be attributed to differences in affinity for monomeric gp120, but were related to binding to the oligomeric complex. Enhanced infectivity of primary isolates was observed using sCD4 and MAb F105, which can neutralize T-cell adapted strains. On p.775 776784817

[Sullivan1998a] N. Sullivan, Y. Sun, J. Binley, J. Lee, C. F. Barbas III, P. W. H. I. Parren, D. R. Burton, \& J. Sodroski. Determinants of human immunodeficiency virus type 1 envelope glycoprotein activation by soluble CD4 and monoclonal antibodies. $J$ Virol 72:6332-8, 1998. On p. 650 651 \begin{tabular}{|l|l|l|l|l|l|l|l|l|l|l|l|l|}
\hline 663 & 664 & 762 & 763 & 774 & 775 & 777 & 784 & 786 & 794 & 795 & 798 & 799 \\
\hline
\end{tabular}

[Sullivan1998b] N. Sullivan, Y. Sun, Q. Sattentau, M. Thali, D. Wu, G. Denisova, J. Gershoni, J. Robinson, J. Moore, \& J. Sodroski. CD4-Induced conformational changes in the hum immunodeficiency virus type 1 gp 120 glycoprotein: consequences for virus entry and neutralization. $J$ Virol 72:4694-703, 1998. A study of the sCD4 inducible MAb 17bi, and the MAb CG10 that recognizes a gp120-CD4 complex. These epitopes are minimally accessible upon attachment of gp120 to the cell. The CD4-binding induced changes in gp120 were studied, exploring the sequestering of chemokine receptor binding sites from the humoral response. On p.753 756757

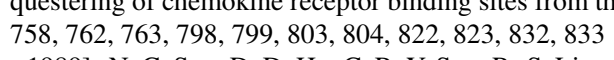

Sun1989] N. C. Sun, D. D. Ho, C. R. Y. Sun, R.-S. Liou, W. Gordon, M. S. C. Fung, X. L. Li, R. C Ting, T.-H. Lee, N. T. Chang, \& T. W. Chang. Generation and characterization of monoclonal antibodies to the putative CD4-binding domain of human immunodeficiency virus type $1 \mathrm{gp} 120$. J Virol 63:3579-3585, 1989. On p. 680 681 682 683684

[Surman2001] S. Surman, T. D. Lockey, K. S. Slobod, B. Jones, J. M. Riberdy, S. W. White, P. C. Doherty, \& J. L. Hurwitz. Localization of cd4+ T cell epitope hotspots to exposed strands of HIV envelope glycoprotein suggests structural influences on antigen processing. Proc Natl Acad Sci

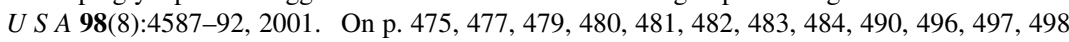
\begin{tabular}{l|l|l|l|l|l|}
499 & 500 & 504 & 511 & 517 \\
\hline
\end{tabular}

[Sutton1993] J. Sutton, S. Rowland-Jones, W. Rosenberg, D. Nixon, F. Gotch, X.-M. Gao, N. Murray, A. Spoonas, P. Driscoll, M. Smith, A. Willis, \& A. McMichael. A sequence pattern for peptides presented to cytotoxic T lymphocytes by HLA B8 revealed by analysis of epitopes and eluted peptides. Eur J Immunol 23:447-453, 1993. On p. 67 [128 $[154] 177] 316$

[Sykes1999] K. F. Sykes \& S. A. Johnston. Genetic live vaccines mimic the antigenicity but not pathogenicity of live viruses. DNA Cell Biol 18(7):521-531, 1999. On p. 81 214 287] 390

[Szilvay1992] A. M. Szilvay, S. Nornes, I. R. Haugan, L. Olsen, V. R. Prasad, C. Endresen, S. P. Goff, \& D. E. Helland. Epitope mapping of HIV-1 reverse transcriptase with monoclonal antibodies that inhibit polymerase and RNase H activities. J AIDS 5:647-657, 1992. 20 MAbs are described, only five are able to bind to short peptides. These five MAbs are insensitive to mutations through out the rest of RT. On p. 597,717

[Szilvay1995] A. M. Szilvay, K. A. Brokstad, R. Kopperud, G. Haukenes, \& K. H. Kalland. Nuclear export of the human immunodeficiency virus type 1 nucleocytoplasmic shuttle protein Rev is mediated by its activation domain and is blocked by transdominant negative mutants. J Virol 69:3315-3323, 1995. On p.612

Tahtinen2001] M. Tahtinen, M. Strengell, A. Collings, J. Pitkanen, A. Kjerrstrom, K. Hakkarainen, P. Peterson, B. Kohleisen, B. Wahren, A. Ranki, M. Ustav, \& K. Krohn. DNA vaccination in mice using HIV-1 nef, rev and tat genes in self-replicating pBN-vector. Vaccine 19(15-16):2039-47, 2001. On p. 835 836 837838

[Takahashi1988] H. Takahashi, J. Cohen, A. Hosmalin, K. B. Cease, R. Houghten, J. L. Cornette, C. DeLisi, B. Moss, R. N. Germain, \& J. A. Berzofsky. An immunodominant epitope of the human immunodeficiency virus envelope glycoprotein gp160 recognized by class I major histocompatibility complex molecule-restricted murine cytotoxic T lymphocytes. Proc Natl Acad Sci USA 85:3105-3109, 1988. Mice were infected with a recombinant vaccinia virus expressing the HIV gp160 envelope gene, and the primed lymphocytes were restimulated in vitro with a transfected histocompatible cell line expressing the same gene. $\mathrm{H}-2^{d}$ mice respond predominantly to a single immunodominant site represented by a 15 -residue synthetic peptide. On p.290

Takahashi1989a] H. Takahashi, R. Houghten, S. D. Putney, D. H. Margulies, B. Moss, R. N. Germain, \& J. A. Berzofsky. Structural requirements for class I MHC molecule-mediated antigen presentation and cytotoxic T cell recognition of an immunodominant determinant of the human envelope protein. J Exp Med 170:2

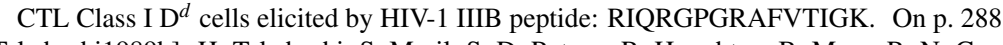

[Takahashi1989b] H. Takahashi, S. Meril, S. D. Putney, R. Houghten, B. Moss, R. N. Germain, \& J. A. Berzofsky. A single amino acid interchange yields reciprocal CTL specificities for HIV-1 gp160. Science 246:118-121, 1989. Murine BALBc CTL Class I D ${ }^{d}$ epitope elicited by HIV-1 IIIB and MN gp160 vaccinia construct, stimulated with peptides: RIQRGPGRAFVTIGK, IIIB and RIHIGPGRAFYTTKN, MN. These two peptides were non-cross reactive. Val/Tyr exchange 
was sufficient to interchange the specificities of the two peptides. On p. 288

[Takahashi1990] H. Takahashi, R. N. Germain, B. Moss, \& J. A. Berzofsky. An immunodominant class I-restricted cytotoxic T lymphocyte determinant of human immunodeficiency virus type induces CD4 class II-restricted help for itself. J Exp Med 171:571-576, 1990. Notes: This same epitope can be recognized in the context of a class I MHC $\mathrm{D}^{d}$, by CD4- CD8+ CTL, and in the context of a class II MHC A ${ }^{d}$ by CD4+ CD8- T-helper cells. On p. 494

[Takahashi1991] K. Takahashi, L.-C. Dai, T. R. Fuerst, W. E. Biddison, P. L. Earl, B. Moss, \& F. A. Ennis. Specific lysis of human immunodeficiency virus type 1-infected cells by a HLAA3.1-restricted CD8+ cytotoxic T-lymphocyte clone that recognizes a conserved peptide sequence within the gp41 subunit of the envelope protein. Proc Natl Acad Sci USA 88:10277-10281, 1991 Gp41 epitope: RLRDLLLIVTR, HLA A3.1 (NL43). Synthetic peptides of RF and CDC4 were recognized by CTL clone despite non-conservative Thr to (Val or Ala) change, but an MN peptide with four natural substitutions was not recognized. On p. 320

[Takahashi1992] H. Takahashi, Y. Nakagawa, C. D. Pendleton, R. Houghten, K. Yokomuro, R. N. Germain, \& J. A. Berzofsky. Induction of broadly cross-reactive cytotoxic T cells recognizin and HIV-1 envelope determinant. Science 255:333-336, 1992. Murine BALBc CTL Class epitope elicited by HIV-1 RF, IIIB and MN gp160 vaccinia construct, stimulated with peptides: SITKGPGRVIYATGQ, RF; RIQRGPGRAFVTIGK, IIIB; and RIHIGPGRAFYTTKN, MN. On p. 288

[Takahashi1993] H. Takahashi, Y. Nakagawa, K. Yokomuro, \& J. A. Berzofsky. Induction of CD8+ cytotoxic T lymphocytes by immunization with syngeneic irradiated HIV-1 envelope derived peptide-pulsed dendritic cells. Internatl Immunol 5:849-857, 1993. On p.294

[Takahashi1996] H. Takahashi, Y. Nakagawa, G. R. Leggatt, Y. Ishida, T. Saito, K. Yokomuro, \& J. A. Berzofsky. Inactivation of human immunodeficiency virus (HIV-1) envelope-specific CD8+ cytotoxic T lymphocytes by free antigenic peptide: a self-veto mechanism? J Exp Med 183:879889, 1996. On p.295

[Takahashi2001] M. Takahashi, Y. Nakagawa, J. A. Berzofsky, \& H. Takahashi. Counter-regulation of cytolytic activity and cytokine production in HIV-1-specific murine CD8+ cytotoxic T lymphocytes by free antigenic peptide. Int Immunol 13(1):43-51, 2001. On p.296

[Takahashi2002] M. Takahashi, E. Osono, Y. Nakagawa, J. Wang, J. A. Berzofsky, D. H. Margulies, \& H. Takahashi. Rapid induction of apoptosis in CD8+ HIV-1 envelope-specific murine CTLs by short exposure to antigenic peptide. J Immunol 169(11):6588-6593, 2002. On p. 299

[Takeda1992] A. Takeda, J. E. Robinson, D. D. Ho, C. Debouck, N. L. Haigwood, \& F. A. Ennis Distinction of human immunodeficiency virus type 1 neutralization and infection enhancemen by human monoclonal antibodies to glycoprotein 120. J Clin Inv 89:1952-1957, 1992. Complement receptors for IgG on monocytic cells can serve as a means for MAb mediated enhancement of HIV-1 infection. MAbs N70-1.5 and N70-2.3a bind distinct discontinuous epitopes in gp120. N70-1.5 is a potent neutralizing MAb with no enhancing activity, while N70-2.3a doesn't neutralize and mediates enhancement of HIV-1 infection. On p.740 762

[Takefman1998] D. M. Takefman, B. L. Sullivan, B. E. Sha, \& G. T. Spear. Mechanisms of resistance of HIV-1 primary isolates to complement- mediated lysis. Virology 246:370-8, 1998. On p.710 712 784 $786,822,823$

[Takeshita1995] T Takeshita, H. Takahashi, S. Kozlowski, J. D. Ahlers, C. D. Pendleton, R. L. Moore, Y. Nakagawa, K. Yokomuro, B. S. Fox, D. H. Margulies, \& J. A. Berzofsky. Molecular analysis of the same HIV peptide functionally binding to both a class I and a class II MHC molecule. J Immunol 154:1973-1986, 1995. Of RGPGRAFVTI, the upper case amino acids iGPgRaFvtI are critical for binding, consistent with $\mathrm{H}-2 \mathrm{D} d$ motif XGPX(RKH)XXX(X)(LIF) Stimulation of the HLA class II I-A ${ }^{d}$ required a longer peptide, IQRGPGRAFVTI or RIQRGPGRAFVTI, and riqrgPgRaFvti were essential for binding to the Class II molecule. On p.297 494
[Takiguchi2000] M. Takiguchi, T. Matsuda, H. Tomiyama, \& K. Miwa. Analysis of three HLAA*3303 binding peptide anchors using an HLA-A*3303 stabilization assay. Tissue Antigens 55(4):296-302, 2000. On p. 318 327

Tan1999] R. Tan, X. Xu, G. S. Ogg, P. Hansasuta, T. Dong, T. Rostron, G. Luzzi, C. P. Conlon, G. R. Screaton, A. J. McMichael, \& S. Rowland-Jones. Rapid death of adoptively transferred T cells in acquired immunodeficiency syndrome. Blood 93:1506-10, 1999. On p.75, 199

[Tanchou1994] V. Tanchou, T. Delaunay, H. de Rocquigny, M. Bodeus, J.-L. Darlix, B. Roques, \& R. Benarous. Monoclonal antibody-mediated inhibition of RNA binding and annealing activities of HIV type 1 nucleocapsid protein. AIDS Res Hum Retroviruses 10:983-993, 1994. On p.587 588

Tanchou1995] V. Tanchou, T. Delaunay, M. Bodeus, B. Roques, J. L. Darlix, \& R. Benarous. Conformational changes between human immunodeficiency virus type 1 nucleocapsid protein $\mathrm{NCp} 7$ and its precursor NCp15 as detected by anti-NCp7 monoclonal antibodies. J Gen Virol 76:2457-2466, 1995. On p.587 588 590 591

[Tani1994] Y. Tani, E. Donoghue, S. Sharpe, E. Boone, H. C. Lane, S. Zolla-Pazner, \& D. I. Cohen. Enhanced in vitro human immunodeficiency virus type 1 replication in B cells expressing surface antibody to the TM env protein. J Virol 68:1942-1950, 1994. The MAb 98-6 was expressed as a surface anti-gp41 monoclonal antibody receptor for gp41 (sIg/gp41) by transfection into a CD4-negative B-cell line. Transfected cells could bind HIV envelope, but could not be infected by HIV-1. When CD4 delivered by retroviral constructs was expressed on these cells, they acquired the ability to replicate HIV-1, and sIg/gp41 specifically enhanced viral replication. On p.708

[Taniguchi2000] Y. Taniguchi, S. Zolla-Pazner, Y. Xu, X. Zhang, S. Takeda, \& T. Hattori. Human monoclonal antibody 98-6 reacts with the fusogenic form of gp41. Virology 273(2):333-40, 2000 On p. 7087709

Tatsumi1990] M. Tatsumi, C. Devaux, F. Kourilsky, \& J. C. Chermann. Characterization of monoclonal antibodies directed against distinct conserved epitopes of human immunodeficiency virus type 1 core proteins. Mol Cell Biochem 96:127-136, 1990. On p. 569 580

Teeuwsen1990] V. J. Teeuwsen, K. H. Siebelink, S. Crush-Stanton, B. Swerdlow, J. J. Schalken, J. Goudsmit, R. van de Akker, M. J. Stukart, F. G. Uytdehaag, \& A. D. Osterhaus. Production and characterization of a human monoclonal antibody, reactive with a conserved epitope on gp41 of human immunodeficiency virus type I. AIDS Res Hum Retroviruses 6:381-392, 1990. On p.739

[Tewari1998] D. Tewari, S. L. Goldstein, A. L. Notkins, \& P. Zhou. cDNA encoding a single-chain antibody to HIV p17 with cytoplasmic or nuclear retention signals inhibits HIV-1 replication. $J$ Immunol 161:2642-7, 1998. On p.574

Thali1991] M. Thali, U. Olshevsky, C. Furman, D. Gabuzda, M. Posner, \& J. Sodroski. Characterzation of a discontinuous human immunodeficiency virus type 1 gp120 epitope recognized by a broadly reactive neutralizing human monoclonal antibody. J Virol 65(11):6188-6193, 1991. An early detailed characterization of the mutations that inhibit the neutralization capacity of the MAb F105, that binds to a discontinuous epitope and inhibits CD4 binding to gp120. On p. 762775

Thali1992a] M. Thali, C. Furman, D. D. Ho, J. Robinson, S. Tilley, A. Pinter, \& J. Sodrosk Discontinuous, conserved neutralization epitopes overlapping the CD4-binding region of human immunodeficiency virus type 1 gp120 envelope glycoprotein. J Virol 66:5635-5641, 1992. Maps the relationship between amino acid substitutions that reduce CD4-gp120 interaction, and amino acid substitutions that reduce the binding of discontinuous epitope MAbs that inhibit CD4 binding. On p. 7597600762764775

Thali1992b] M. Thali, C. Furman, B. Wahren, M. Posner, D. Ho, J. Robinson, \& J. Sodroski. Cooperativity of neutralizing antibodies directed against the V3 and CD4 binding regions of the HIV-1 gp120 envelope glycoprotein. J Acq Immune Def Synd 5:591-599, 1992. On p. 667

Thali1993] M. Thali, J. P. Moore, C. Furman, M. Charles, D. D. Ho, J. Robinson, \& J. Sodroski. Characterization of conserved human immunodeficiency virus type 1 gp120 neutralization epi- 
topes exposed upon gp120-CD4 binding. J Virol 67:3978-3988, 1993. Five regions are likely to contribute to the $48 \mathrm{~d}$ and $17 \mathrm{~b}$ discontinuous epitopes, either directly or through local conformational effects: the hydrophobic ring-like structure formed by the disulfide bond that links $\mathrm{C} 3$ and $\mathrm{C} 4$, the base of the stem-loop that contains V1 and V2, and the hydrophobic region in $\mathrm{C} 2$ from Arg 252 to Asp 262. Additionally changes in Glu 370, and Met 475 in C5, affected binding and neutralization. The hydrophobic character of these critical regions is consistent with the limited \begin{tabular}{ll|l|l|l|l|l|l|l|} 
exposure on gp120 prior to CD4 binding. On p. 616 & 617 & 619 & 631 & 632 & 633 & 641 & 653 & 667
\end{tabular} \begin{tabular}{|l|l|l|l|l|l|l|l|l|l|l|l|l|}
\hline 668 & 681 & 682 & 759 & 762 & 783 & 796 & 798 & 803 & 811 & 812 & 813 \\
\hline
\end{tabular}

[Thali1994] M. Thali, M. Charles, C. Furman, L. Cavacini, M. Posner, J. Robinson, \& J. Sodroski. Resistance to neutralization by broadly reactive antibodies to the human immunodeficiency virus type 1 gp120 glycoprotein conferred by a gp41 amino acid change. J Virol 68:674-680, 1994 A T- $>$ A amino acid substitution at position 582 of gp41 conferred resistance to neutralization to 30Virol 64:3240-48 (1990)). Monoclonal antibodies that bound to the CD4 binding site were unable to neutralize this virus, but the mutation did not reduce the neutralizing capacity of a V2 region MAb G3-4, V3 region MAbs, or gp41 neutralizing MAb 2F5. On p. 632 641 667 671 region MAb G3-4, V3 region MAbs, or
\begin{tabular}{|l|l|l|l|l|l|l|l|l|}
\hline 710 & 762 & 764 & 765 & 775 & 776 & 798 & 803 \\
\hline
\end{tabular}

[Thiriart1989] C. Thiriart, M. Francotte, J. Cohen, C. Collignon, A. Delers, S. Kummert, C. Molito D. Gilles, P. Roelants, F. V. Wijnendaele, M. D. Wilde, \& C. Bruck. Several antigenic determinants exposed on the gp120 moiety of HIV-1 gp160 are hidden on the mature gp120. J Immuno 143:1832-1836, 1989. On p.617 619621622623 624 625 636 646678

[Thomas1988] E. K. Thomas, J. N. Weber, J. McClure, P. R. Clapham, M. C. Singhal, M. K. Shriver, $\&$ R. A. Weiss. Neutralizing monoclonal antibodies to the AIDS virus. AIDS 2:25-29, 1988. On p. 667668670689

[Threlkeld1997] S. C. Threlkeld, P. A. Wentworth, S. A. Kalams, B. M. Wilkes, D. J. Ruhl, E. Kepgh, J. Sidney, S. Southwood, B. D. Walker, \& A. Sette. Degenerate and promiscuou recognition by CTL of peptides presented by the MHC class I A3-like superfamily. I Immunol 159(4):1648-1657, 1997. Similarities in peptide binding across A3-like superfamily results in 159(4):1648-1657, 1997. Similarities in peptide binding across A3-like superfamily
similar peptide-MHC complex structures engaged by T-cell receptors. On p. 191269

[Tian2001] H. Tian, Y. Xiao, M. Zhu, M. P. Dierich, \& Y. H. Chen. Induction of monoclona antibodies with predefined epitope-specificity by epitope-vaccines. Immunol Lett 75(2):161-2, 2001. On p. 597 674717718

[Tian2002] Y. Tian, C. V. Ramesh, X. Ma, S. Naqvi, T. Patel, T. Cenizal, M. Tiscione, K. Diaz, T. Crea, E. Arnold, G. F. Arnold, \& J. W. Taylor. Structure-affinity relationships in the gp41 ELDKWA epitope for the HIV-1 neutralizing monoclonal antibody 2F5: Effects of side-chain and backbone modifications and conformational constraints. J Pept Res 59(6):264-276, 2002. On p. 710716

[Till1989] M. A. Till, S. Zolla-Pazner, M. K. Gorny, J. W. Uhr, \& E. S. Vitetta. Human immunodeficiency virus-infected $\mathrm{T}$ cells and monocytes are killed by monoclonal human anti-gp41 antibodies coupled to ricin A chain. Proc Natl Acad Sci USA 86:1987-1991, 1989. On p. 696 708

[Tilley1991a] S. A. Tilley, W. J. Honnen, M. E. Racho, M. Hilgartner, \& A. Pinter. A human monoclonal antibody against the CD4-binding site of HIV-1 gp120 exhibits potent, broadly neutralizing activity. Res Virol 142:247-259, 1991. Characterization of human neutralizing MAb 1125H. On p. 760

[Tilley1991b] S. A. Tilley, W. J. Honnen, M. E. Racho, M. Hilgartner, \& A. Pinter. Human Monoclonal Antibodies Against the Putative CD4 Binding Site and the V3 Loop of HIV gp120 Act in Concert to Neutralize Virus. VII International Conference on AIDS 1991:p. 39, 1991. Aidsline: 1007091 Abstract 70. On p. 659760

[Tilley1992] S. A. Tilley, W. J. Honnen, M. E. Racho, T.-C. Chou, \& A. Pinter. Synergistic neutralization of HIV-1 by human monoclonal antibodies against the V3 loop and the CD4-binding site of gp120. AIDS Res Hum Retroviruses 8:461-467, 1992. On p. 659

[Tisdale1988] M. Tisdale, P. Ertl, B. A. Larder, D. J. M. Purifoy, G. Darby, \& K. L. Powell. Characterization of human immunodeficiency virus type 1 reverse transcriptase by using monoclona antibodies: role of the C terminus in antibody reactivity and enzyme function. $J$ Virol 62:36623667, 1988. On p. 598

[Tobery 1997] T. W. Tobery \& R. F. Siliciano. Targeting of HIV-1 antigens for rapid intracellular degradation enhances cytotoxic T lymphocyte (CTL) recognition and the induction of de novo CTL responses in vivo after immunization. J Exp Med 185:909-20, 1997. CD8+ cytotoxic $\mathrm{T}$ lymphocytes (CTLs) have the ability to recognize and eliminate virally infected cells before new virions are produced within that cell. Therefore, a rapid and vigorous CD8+ CTL response, induced by vaccination, can, in principle, prevent disseminated infection in vaccinated individuals who are exposed to the relevant virus. There has thus been interest in novel vaccine strategies that will enhance the induction of CD8+ CTLs. In this study, we have tested the hypothesis that targeting an antigen to undergo more efficient processing by the class I processing pathway will elicit a more vigorous CD8+ CTL response against that antigen. Targeting a type I transmembrane protein, the HIV-1 envelope (env) protein, for expression in the cytoplasm, rather than allowing its normal co-translational translocation into the endoplasmic reticulum, sensitized target cells expressing this mutant more rapidly for lysis by an env-specific CTL clone. Additionally, a greatly enhanced de novo env-specifc CTL response was induced in vivo after immunization of mice with recombinant vaccinia vectors expressing the cytoplasmic env mutant. Similarly, targeting a cytoplasmic protein, HIV-1 nef, to undergo rapid cytoplasmic degradation induced a greatly enhanced de novo nef-specific CD8+CTL response in vivo after immunization of mice with either recombinant vaccinia vectors or DNA expression plasmids expressing the degradation targeted nef mutant. The targeting of viral antigens for rapid cytoplasmic degradation represents a novel and highly effective vaccine strategy for the induction of enhanced de novo CTL responses in vivo. On p. 299

[Tomiyama1997] H. Tomiyama, K. Miwa, H. Shiga, Y. I. Moore, S. Oka, A. Iwamoto, Y. Kaneko, $\&$ M. Takiguchi. Evidence of presentation of multiple HIV-1 cytotoxic T lymphocyte epitopes by HLA-B*3501 molecules that are associated with the accelerated progression of AIDS. J Immunol

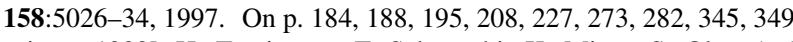

[Tomiyama1999] H. Tomiyama, T. Sakaguchi, K. Miwa, S. Oka, A. Iwamoto, Y. Kaneko, \& M. Takiguchi. Identification of multiple HIV-1 CTL epitopes presented by HLA-B*5101. Hum

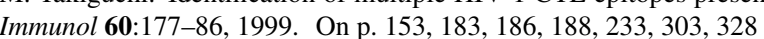

Tomiyama2000a] H. Tomiyama, S. Oka, G. S. Ogg, S. Ida, A. J. McMichael, \& M. Takiguchi Expansion of HIV-1-specific CD28- CD45RA- CD8+ T cells in chronically HIV-1-infected indi-

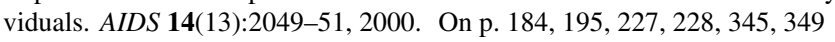

[Tomiyama2000b] H. Tomiyama, N. Yamada, H. Komatsu, K. Hirayama, \& M. Takiguchi. A single CTL clone can recognize a naturally processed HIV-1 epitope presented by two different HLA class I molecules. Eur J Immunol 30(9):2521-30, 2000. On p. 184 209

[Tomiyama2002] H. Tomiyama, H. Akari, A. Adachi, \& M. Takiguchi. Different effects of Nefmediated HLA class I down-regulation on human immunodeficiency virus type 1-specific CD8(+) T-cell cytolytic activity and cytokine production. $J$ Virol 76(15):7535-7543, 2002. On p. 339

[Toohey1995] K. Toohey, K. Wehrly, J. Nishio, S. Perryman, \& B. Chesebro. Human immunodeficiency virus envelope V1 and V2 regions influence replication efficiency in macrophages by affecting virus spread. Virology 213:70-9, 1995. On p.589

[Tornatore1994] C. Tornatore, K. Meyers, W. Atwood, K. Conant, \& E. Major. Temporal patterns of human immunodeficiency virus type 1 transcripts in human fetal astrocytes. J Virol 68:93-102, 1994. On p. 841

[Trabattoni2002] D. Trabattoni, S. Lo Caputo, M. Biasin, E. Seminari, M. Di Pietro, G. Ravasi, F. Mazzotta, R. Maserati, \& M. Clerici. Modulation of human immunodeficiency virus (HIV)- 
specific immune response by using efavirenz, nelfinavir, and stavudine in a rescue therapy regimen for HIV-infected, drug-experienced patients. Clin Diagn Lab Immunol 9(5):1114-8, 2002. On p. 338

[Trickett1998] A. E. Trickett, M. Kelly, B. A. Cameron, A. Lloyd, R. A. Ffrench, \& J. M. Dwyer. A preliminary study to determine the effect of an infusion of cryopreserved autologous lymphocyte on immunocompetence and viral load in HIV-infected patients. J Acquir Immune Defic Synd Hum Retrovirol 17:129-36, 1998. On p. 163240333

[Trickett2002] A. E. Trickett, Y. L. Kwan, B. Cameron, \& J. M. Dwyer. Ex vivo expansion of functional T lymphocytes from HIV-infected individuals. J Immunol Methods 262(1-2):71-83, 2002. On p. 167.243 338

[Trkola1995] A. Trkola, A. B. Pomales, H. Yuan, B. Korber, P. J. Maddon, G. P. Allaway, H. Katinger, C. F. Barbas III, D. R. Burton, D. D. Ho, \& J. P. Moore. Cross-clade neutralization of primary isolates of human immunodeficiency virus type 1 by human monoclonal antibodies and tetrameric CD4-IgG. J Virol 69:6609-6617, 1995. Three MAbs, IgG1b12, 2G12, an 2F5 tetrameric CD4-IgG2 were tested for their ability to neutralize primary isolates from clade A-F. 2F5 and CD4-IgG2 were able to neutralize within and outside clade B with a high potency IgG1b12 and 2G12 could potently neutralize isolates from within clade B, but showed a reduction in efficacy outside of clade B. 2F5 neutralization was dependent on the presence of the sequence: LDKW. On p.710, $7111784,785,822$

[Trkola1996a] A. Trkola, T. Dragic, J. Arthos, J. M. Binley, W. C. Olson, G. P. Allaway, C. Cheng Mayer, J. Robinson, P. J. Maddon, \& J. P. Moore. CD4-dependent, antibody-sensitive interaction between HIV-1 and its co-receptor CCR-5. Nature 384:184-187, 1996. CCR-5 is a co-factor for fusion of HIV-1 strains of the non-syncytium-inducing (NSI) phenotype with CD4+ T-cells. CD4 binding greatly increases the efficiency of gp120-CCR-5 interaction. Neutralizing MAbs against the V3 loop and CD4-induced epitopes on gp120 inhibited the interaction of gp120 with CCR-5,

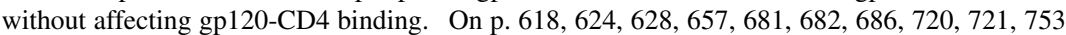

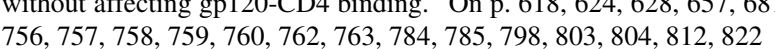

\begin{tabular}{l|l|l|l|l|l|l|l|l|l|l|l|l|l|l|l|l|l|l|l|l|l|}
756 & 757 & 758 & 759 & 760 & 762 & 763 & 784 & 785 & 798 & 803 & 804 & 812 & 822 \\
\hline
\end{tabular}

Trkola1996b] A. Trkola, M. Purtscher, T. Muster, C. Ballaun, A. Buchacher, N. Sullivan, K. Srin vasan, J. Sodroski, J. P. Moore, \& H. Katinger. Human monoclonal antibody 2G12 defines distinctive neutralization epitope on the gp120 glycoprotein of human immunodeficiency virus type 1. J Virol 70:1100-1108, 1996. On p.822

[Trkola1998] A. Trkola, T. Ketas, V. N. Kewalramani, F. Endorf, J. M. Binley, H. Katinger, J. Robinson, D. R. Littman, \& J. P. Moore. Neutralization sensitivity of human immunodeficiency viru type 1 primary isolates to antibodies and CD4-based reagents is independent of coreceptor usage.

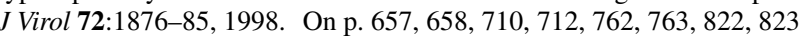

[Trujillo1993] J. R. Trujillo, M. F. McLane, T.-H. Lee, \& M. Essex. Molecular mimicry between the human immunodeficiency virus type $1 \mathrm{gp} 120 \mathrm{~V} 3$ loop and human brain proteins. J Viro 67:7711-7715, 1993. On p. 641 677

[Truong1996] C. Truong, D. Brand, F. Mallet, P. Roingeard, S. Brunet, \& F. Barin. Assembly an immunogenicity of chimeric Gag-Env proteins derived from the human immunodeficiency viru type 1. AIDS Res Hum Retroviruses 12(4):291-301, 1996. On p.593 797,819

[Truong1997] C. Truong, D. Brand, F. Mallet, P. Roingeard, \& F. Barin. Comparison of antibody responses to different forms of HIV-1 core antigens by epitope mapping. J Med Virol 51(3):145 51, 1997. On p. 569576576580582583

[Tsomides1991] T. J. Tsomides, B. D. Walker, \& H. N. Eisen. An optimal viral peptide recognized by $\mathrm{CD} 8+\mathrm{T}$ cells binds very tightly to the restricting class I major histocompatibility complex protein on intact cells but not to the purified class I protein. Proc Natl Acad Sci USA 88:1127611280, 1991. On p.217

[Tsomides 1994] T. J. Tsomides, A. Aldovini, R. P. Johnson, B. D. Walker, R. A. Young \& H. N. Eisen. Naturally processed viral peptides recognized by cytotoxic $\mathrm{T}$ lymphocytes on cells chron- ically infected by human immunodeficiency virus type 1. J Exp Med 180:1283-1293, 1994 Naturally processed peptides can be purified from trifluoroacetic acid lysates of HIV-1 infected cells. A gag and RT epitope were compared; both synthetic peptides are optimally active in CTL assays. The naturally processed gag peptide was more abundant than the RT peptide in HIV-1 infected HLA-A2 positive cells, and the gag specific CTL more effective, suggesting surface density of peptides may influence efficiency of CTL killing. On p. 84216

[Tugarinov1999] V. Tugarinov, A. Zvi, R. Levy, \& J. Anglister. A cis proline turn linking two betahairpin strands in the solution structure of an antibody-bound HIV-1 IIIB V3 peptide. Nat Struct Biol 6(4):331-335, 1999. On p. 671, 672

[Tugarinov2000] V. Tugarinov, A. Zvi, R. Levy, Y. Hayek, S. Matsushita, \& J. Anglister. NMR structure of an anti-gp120 antibody complex with a V3 peptide reveals a surface important for co-receptor binding [In Process Citation]. Structure Fold Des 8:385-95, 2000. On p. 671 672

[Tumanova2001] O. I. Tumanova, V. N. Kuvshinov, M. S. Azaev, A. E. Masharskii, N. A. Klimov, A. P. Kozlov, A. A. Il'ichev, \& L. S. Sandakhchiev. [Construction of peptide mimetics of an epitope of the human immunodeficiency virus (HIV-1) gp41 protein, recognized by virusneutralizing antibodies 2f5]. Mol Biol (Mosk) 35(1):146-51, 2001. Article in Russian. On p. 710 711

Turbica1995] I. Turbica, M. Posner, C. Bruck, \& F. Barin. Simple enzyme immunoassay for titration of antibodies to the CD4- binding site of human immunodeficiency virus type $1 \mathrm{gp} 120$. Clin Microbiol 33:3319-3323, 1995. On p.775, 776

[Turbica1997] I. Turbica, F. Simon, J. M. Besnier, B. LeJeune, P. Choutet, A. Goudeau, \& F. Barin. Temporal development and prognostic value of antibody response to the major neutralizing epitopes of gp120 during HIV-1 infection. J Med Virol 52:309-315, 1997. On p.710 712

Turner 1994] J. L. Turner, R. J. Trauger, A. E. Daigle, \& D. J. Carlo. HIV-1 immunogen induction of HIV-1-specific delayed-type hypersensitivity: Results of a double-blind, adjuvant-controlled, dose-ranging trial. AIDS 8(10):1429-1435, 1994. On p. 535

[Tyler1990] D. S. Tyler, S. D. Stanley, S. Zolla-Pazner, M. K. Gorny, P. P. Shadduck, A. J. Langlois, T. J. Matthews, D. P. Bolognesi, T. J. Palker, \& K. J. Weinhold. Identification of sites within gp41 that serve as targets for antibody-dependent cellular cytotoxicity by using human monoclonal

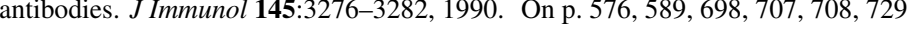

[Ueno2002] T. Ueno, H. Tomiyama, \& M. Takiguchi. Single T cell receptor-mediated recognition of an identical HIV-derived peptide presented by multiple HLA class I molecules. J Immunol 169(9):4961-4969, 2002. On p. 209

Ugen1993] K. E. Ugen, Y. Refaleli, U. Ziegner, M. Agadjanyan, M. A. R. Satre, V. Srikantan, B. Wang, A. Sato, W. V. Williams, \& D. B. Weiner. Generation of monoclonal antibodies agains the amino terminus of gp120 that elicit antibody-dependent cellular cytotoxicity. Vaccines $93 \mathrm{pp}$ 215-221, 1993. Editors: F. Brown, H. S. Ginsberg and R. Lerner, Cold Spring Harbor Laboratory Press, Cold Spring Harbor, NY. On p. 807

Ugolini1997] S. Ugolini, I. Mondor, P. W. H. I. Parren, D. R. Burton, S. A. Tilley, P. J. Klasse, \& Q. J. Sattentau. Inhibition of virus attachment to CD4+ target cells is a major mechanism of T cell line-adapted HIV-1 neutralization. J Exp Med 186:1287-1298, 1997. On p.629 657, 663 \begin{tabular}{|l|l|l|l|l|l|l|l|l|l|l|l|l|l|l|l|l|}
6664 & 668 & 710 & 712 & 721 & 760 & 764 & 765 & 784 & 785 & 803 & 804 & 820 & 822 & 823 \\
\hline
\end{tabular}

Uno-Furuta2001] S. Uno-Furuta, S. Tamaki, Y. Takebe, S. Takamura, A. Kamei, G. Kim, I. Kuromatsu, M. Kaito, Y. Adachi, \& Y. Yasutomi. Induction of virus-specific cytotoxic T lymphocytes by in vivo electric administration of peptides. Vaccine 19(15-16):2190-6, 2001. On p.289

Valenzuela1998] A. Valenzuela, J. Blanco, B. Krust, R. Franco, \& A. G. Hovanessian. Neutralizing Antibodies against the V3 loop of human immunodeficiency type 1 gp120 block the CD4 dependent and independent binding of the virus to cells. $J$ Virol 71:8289-8298, 1998. On p.634 \begin{tabular}{l|l|l|l|l|l|l|l|l}
638 & 639 & 667 & 674 & 678 & 689 & 690 & 784 & 786 \\
\hline
\end{tabular}

Valvatne1996] H. Valvatne, A. M. Szilvay, \& D. E. Helland. A monoclonal antibody defines a novel 
HIV type 1 Tat domain involved in trans-cellular trans-activation. AIDS Res Hum Retroviruses 12:611-619, 1996. CAT and beta-galactosidase assays, and immunofluorescence analysis were used to study the cellular uptake of the HIV-1 Tat protein. A MAb binding to the basic domain and the RGD sequence inhibits trans- activation by exogenous Tat. The inhibition of the cellular uptake of Tat by an anti-Tat monoclonal antibody and by the serum components implies specific binding of Tat to the cell membrane. On p.610 611

[vanBaalen1993] C. A. van Baalen, M. R. Klein, A. M. Geretti, R. I. P. M. Keet, F. Miedema, C. A C. M. van Els, \& A. D. M. E. Osterhaus. Selective in vitro expansion of HLA class I-restricted HIV-1 Gag-specific CD8+ T cells: cytotoxic T-lymphocyte epitopes and precursor frequencies. AIDS 7:781-786, 1993. Gag-specific epitopes and precursor frequencies were studied in seven individuals; for CTLs from one individual, fine mapping was done using peptides. PFA-fixed rVV-Gag-infected B-LCL cells were used as stimulator cells of bulk PBMC cultures to determine precursor frequencies and identify epitopes. On $\mathrm{p} .125] 138,155$

[vanBaalen1996] C. A. van Baalen, M. R. Klein, R. C. Huisman, M. E. Dings, S. R. K. Garde, A. M. Geretti, R. Gruters, C. A. van Els, F. Miedema, \& A. D. Osterhaus. Fine-specificity of cytotoxic T lymphocytes which recognize conserved epitopes of the Gag protein of human immunodeficiency virus type 1. J Gen Virol 77:1659-1665, 1996. On p.66.119

[vanBaalen1997] C. A. van Baalen, O. Pontesilli, R. C. Huisman, A. M. Geretti, M. R. Klein F. de Wolf, F. Miedema, R. A. Gruters, \& A. D. M. E. Osterhaus. Human immunodeficiency virus type $1 \mathrm{Rev}$ - and Tat-specific cytotoxic $\mathrm{T}$ lymphocyte frequencies inversely correlate with rapid progression to AIDS. J Gen Virol 78:1913-1918, 1997. CTLp frequencies to Rev an Tat were inversely correlated with rapid progression to AIDS, but not Gag, RT or Nef. 3/7 long term non-progressors and 0/5 progressors were positive for HLA-B57, so it was again found to be associated with long term survival. On p. 261

[vanBaalen1998] C. A. van Baalen, M. Schutten, R. C. Huisman, P. H. Boers, R. A. Gruters, \& A. D. Osterhaus. Kinetics of antiviral activity by human immunodeficiency virus type 1-specific cytotoxic T lymphocytes (CTL) and rapid selection of CTL escape virus in vitro. $J$ Virol 72:68517, 1998. On p. 263

[vanBaalen2000] C. van Baalen \& R. Gruters. Personal communication, 2000. On p. 262

[vanBaalen2002] C. A. van Baalen, C. Guillon, M. van Baalen, E. J. Verschuren, P. H. M. Boers,

A. D. M. E. Osterhaus, \& R. A. Gruters. Impact of antigen expression kinetics on the effectivenes of HIV-specific cytotoxic T lymphocytes. Eur J Immunol 32(9):2644-2652, 2002. On p. 206 263

[VanCott1994] T. C. VanCott, F. R. Bethke, V. R. Polonis, M. K. Gorny, S. Zolla-Pazner, R. R Redfield, \& D. L. Birx. Dissociation rate of antibody-gp120 binding interactions is predictive of V3-mediated neutralization of HIV-1. J Immunol 153:449-459, 1994. Using surface plasmon resonance it was found that the rate of the dissociation of the MAb-gp120 complex, but not the association rate, correlated with MAbs ability to neutralize homologous virus (measured by $50 \mathrm{in}-$ hibition of p24 production). Association constants were similar for all MAbs tested, varying less than 4-fold. Dissociation rate constants were quite variable, with 100-fold differences observed. \begin{tabular}{l|l|l|l|l|l|l|l|l|l|l|} 
On p. 641 & 645 & 647 & 652 & 660 & 664 & 665 & 669 & 675 & 677 & 720 \\
\hline
\end{tabular}

[VanCott1995] T. C. VanCott, F. R. Bethke, D. S. Burke, R. R. Redfield, \& D. L. Birx. Lack of induction of antibodies specific for conserved, discontinuous epitopes of HIV-1 envelope glycoprotein by candidate AIDS vaccines. J Immunol 155:4100-4110, 1995. The Ab response in both HIV-1 infected and uninfected volunteers immunized with HIV-1 rec envelope subunit vaccines (Genentech gp120IIIB, MicroGeneSys gp160IIIB, or ImmunoAG gp160IIIB) preferentially induced Abs reactive only to the denatured form of gp120. This may explain the inability of the vaccinee sera to neutralize primary HIV-1 isolates. On p. 641 642652675687670

[VanCott1999] T. C. VanCott, J. R. Mascola, L. D. Loomis-Price, F. Sinangil, N Zitomersky, J. McNeil, M. L. Robb, D. L. Birx, \& S. Barnett. Cross-subtype neutralizing antibodies induced in baboons by a subtype E gp120 immunogen based on an R5 primary human immunodeficiency virus type 1 envelope. $J$ Virol 73(6):4640-50, 1999. On p. 746

vanderBurg1995] S. H. van der Burg, M. R. Klein, C. J. V. de Velde, W. M. Kast, F. Miedema \& C. J. Melief. Induction of a primary human cytotoxic T lymphocyte response against a novel conserved epitope in a functional sequence of HIV-1 reverse transcriptase. AIDS 9:121-127, 1995. On p. 183212

[vanderBurg1996] S. H. van der Burg, M. J. W. Visseren, R. M. P. Brandt, W. M. Kast, \& C. J. M. Melief. Immunogenicity of peptides bound to MHC class I molecules depends on the MHCpeptide complex stability. J Immunol 156:3308-3314, 1996. Peptide-MHC dissociation rate is highly correlated with immunogenicity. In this study, HLA-A*0201 restricted epitopes from HPV, HBV and HIV were studied, some in the context of immunogenicity in peptide immunized HLA-A*0201/K $\mathrm{K}^{b}$ transgenic mice. On p. 76 183 212 237

[vanderBurg1997] S. H. van der Burg, M. R. Klein, O. Pontesilli, A. M. Holwerda, J. Drijfhout, W. M. Kast, F. Miedema, \& C. J. M. Melief. HIV-1 reverse transcriptase-specific CTL agains conserved epitopes do not protect against progression to AIDS. J Immunol 159:3648-3654, 1997.

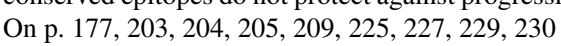

vanderBurg1999] S. H. van der Burg, K. M. Kwappenberg, A. Geluk, M. van der Kruk, O. Pontesilli, E. Hovenkamp, K. L. Franken, K. E. van Meijgaarden, J. W. Drijfhout, T. H. Ottenhoff, C. J. Melief, \& R. Offringa. Identification of a conserved universal Th epitope in HIV-1 reverse transcriptase that is processed and presented to HIV-specific CD4+ T. J Immunol 162:152-60, 1999. On p.450 451452

VanderRyst1998] E. Van der Ryst, T. Nakasone, A. Habel, A. Venet, E. Gomard, R. Altmeyer, M. Girard, \& A. M. Borman. Study of the immunogenicity of different recombinant Mengo viruses expressing HIV1 and SIV epitopes. Res Virol 149:5-20, 1998. Mengo virus did not prove to be a good background for eliciting a strong CTL response to HIV or SIV protein fragments, in Rhesus macaques or mice. On p. 391

[Vaslin1994] B. Vaslin, J.-M. Claverie, O. Benveniste, F. C. Barre-Sinoussi, \& D. Dormont. Nef and gag synthetic peptide priming of antibody responses to HIV type 1 antigens in mice and primates. AIDS Res Hum Retroviruses 10:1241-1250, 1994. Notes: Four Gag peptides, that when pooled are able to prime for subsequent antibody response to HIV in mice, were studied. These peptides were also able to prime it in vitro immunoproliferative responses. The two peptides of the four that were able to prime humoral responses to inactivated HIV-1 are included in the table (G2 and G4) - the other two are not included (G1 and G3). Three proposed nef helper T-cell epitopes are also not included in the table, but may be of interest. These nef peptides could prime the humoral response in mice, but not it it in vitro proliferation. Priming was also observed in baboons, using the pool of four Gag peptides. On p. 434440

[Vázquez Blomquist2002] D. Vázquez Blomquist, P. Green, S. M. Laidlaw, M. A. Skinner, P. Borrow, \& C. A. Duarte. Induction of a strong HIV-specific CD8+ T cell response in mice using a fowlpox virus vector expressing an HIV-1 multi-CTL-epitope polypeptide. Viral Immunol 15(2):337-356, 2002. On p. 300

[Vella1993] C. Vella, M. Ferguson, G. Dunn, R. Meloen, H. Langedijk, D. Evans, \& P. D. Minor Characterization and primary structure of a human immunodeficiency virus type 1 (HIV-1) neutralization domain as presented by a poliovirus type 1/HIV-1 chimera. J Gen Virol 7:15-21, 1993. This study elaborated on a set of antibodies first reported in Evans et al., 1989. Not all of the neutralization results are congruent between the studies. The antibodies in this study were raised to a region including the cytoplasmic domain of gp41 inserted into a poliovirus type 1/HIV-1 chimera On p.724 725 726, 727

Vella2002] C. Vella, N. N. Zheng, P. Easterbrook, \& R. S. Daniels. Herpesvirus saimiriimmortalized human lymphocytes: Novel hosts for analyzing HIV type 1 in vitro neutralization. AIDS Res Hum Retroviruses 18(13):933-946, 2002. On p. 648 664 665, 680 681 780 781 
783784790

[Venturini2002] S. Venturini, D. E. Mosier, D. R. Burton, \& P. Poignard. Characterization of human immunodeficiency virus type 1 (HIV-1) Gag-and Gag peptide-specific CD4+ T-cell clones from an HIV-1-seronegative donor following in vitro immunization. J Virol 76(14):6987-6999, 2002. On p. 433 434, 4364374,440

[Verrier2000] F. Verrier, S. Burda, R. Belshe, A. M. Duliege, J. L. Excler, M. Klein, \& S. ZollaPazner. A human immunodeficiency virus prime-boost immunization regimen in humans induces antibodies that show interclade cross-reactivity and neutralize several X4-, R5-, and dualtropic clade B and C primary isolates. J Virol 74(21):10025-33, 2000. On p. 819

[Verrier2001] F. Verrier, A. Nadas, M. K. Gorny, \& S. Zolla-Pazner. Additive effects characterize the interaction of antibodies involved in neutralization of the primary dualtropic human immunodeficiency virus type 1 isolate 89.6. J Virol 75(19):9177-86, 2001. On p. 691, 692 696697

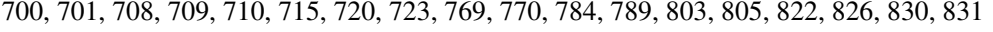

[Verschoor1999] E. J. Verschoor, P. Mooij, H. Oostermeijer, M. van der Kolk, P. ten Haaft, B. Verstrepen, Y. Sun, B. Morein, L. Akerblom, D. H. Fuller, S. W. Barnett, \& J. L. Heeney. Comparison of immunity generated by nucleic acid-, MF59-, and ISCOM- formulated human immunodeficiency virus type 1 vaccines in Rhesus macaques: evidence for viral clearance. J Virol 73:3292 300, 1999. On p. 526746

[Villacres1999] M. C. Villacres \& C. C. Bergmann. Enhanced cytotoxic T cell activity in IL-4 deficient mice. J Immunol 162:2663-70, 1999. On p.295

[Vinner1999] L. Vinner, H. V. Nielsen, K. Bryder, S. Corbet, C. Nielsen, \& A. Fomsgaard. Gene gun DNA vaccination with Rev-independent synthetic HIV-1 gp160 envelope gene using mammalian codons. Vaccine 17:2166-75, 1999. On p. 340

[vonBrunn1993] A. von Brunn, M. Brand, C. Reichhuber, C. Morys-Wortmann, F. Deinhardt, \& F. Schodel. Principal neutralizing domain of HIV-1 is highly immunogenic when expressed on the surface of hepatitis B core particles. Vaccine 11:817-24, 1993. On p. 658 659

[Wagner1996] R. Wagner, L. Deml, R. Schirmbeck, M. Niedrig, J. Reimann, \& H. Wolf. Construction, expression, and immunogenicity of chimeric HIV-1 virus-like particles. Virology 220:128 140, 1996. On p. 659687

[Wagner1998a] L. Wagner, O. O. Yang, E. A. Zepeda, Y. Ge, S. A. Kalams, B. D. Walker, M. S Pasternack, \& A. D. Luster. Beta-chemokines are released from HIV-1-specific cytolytic T cell granules complexed to proteoglycans. Nature 391:908-11, 1998. On p. 82 92 191 310

[Wagner1998b] R. Wagner, V. J. Teeuwsen, L. Deml, F. Notka, A. G. Haaksma, S. S. Jhagjhoorsingh, H. Niphuis, H. Wolf, \& J. L. Heeney. Cytotoxic T cells and neutralizing antibodies induce in rhesus monkeys by virus-like particle HIV vaccines in the absence of protection from SHIV infection. Virology 245:65-74, 1998. Self assembled non-infectious virus. On p. 103 305 607 747

[Wagner 1999] R. Wagner, Y. Shao, \& H. Wolf. Correlates of protection, antigen delivery and molecular epidemiology: basics for designing an HIV vaccine. Vaccine 17:1706-10, 1999. On p. 148 [Wahren1989a] B. Wahren, T. Mathiesen, J. Rosen, \& H. Wigzell. Common and unique T-ce epitopes of HIV-1. Vaccines 89:89-93, 1989. Notes: Using 15-amino-acid-long peptides that scanned all of gp41, the C-terminal half of gp120, and the gag proteins p17, p24, and p15, this study presents evidence that 18 envelope and 12 gag peptides could stimulate T-cell proliferative responses from multiple representatives among 99 HIV infected study subjects. Thirty-six

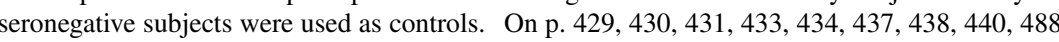

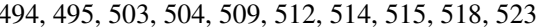

[Wahren1989b] B. Wahren, J. Rosen, E. Sandstrom, T. Mathiesen, S. Modrow, \& H. Wigzell. HIVPeptides Induce a Proliferative Response in Lymphocytes from Infected Persons. J AIDS 4:448 456, 1989. Notes: Using 15-amino-acid-long peptides that scanned all of gp41, the C-termina half of gp120, and the gag proteins $\mathrm{p} 17, \mathrm{p} 24$, and $\mathrm{p} 15$, this study presents evidence that 18 en- velope and 12 gag peptides could stimulate T-cell proliferative responses from multiple representatives among 99 HIV infected study subjects. Thirty-six seronegative subjects were used as \begin{tabular}{|l|l|l|l|l|l|l|l|l|l|l|l|l|l|} 
&
\end{tabular} $515,518,523$

Wainberg1995] M. A. Wainberg \& Z. Gu. Targeting HIV reverse transcriptase in novel ways. Na Med 1:628-629, 1995. Commentary on Maciejewski et al., 1995. On p.607

[Walker1989] B. D. Walker, C. Flexner, K. Birch-Limberger, L. Fisher, T. J. Paradis, A. Aldovini, R. Young, B. Moss, \& R. T. Schooley. Long-term culture and fine specificity of human cytotoxic T-lymphocyte clones reactive with human immunodeficiency virus type 1. Proc Natl Acad Sci USA 86:9514-9518, 1989. Seven HIV-1 reverse transcriptase-specific cytotoxic T-lymphocyte (CTL) clones from the peripheral blood of two seropositive subjects were generated. Five different HLA restricted CTL epitopes were identified by peptide mapping. On p. $177 \mid$\begin{tabular}{l|l|l|l|l|}
199 & 203 & 209 & 223
\end{tabular} [Walter1997] J. B. Walter, C. Brander, M. Mammen, D. N. Garboczi, S. A. Kalams, G. M. Whiteside, B. D. Walker, \& H. N. Eisen. Stimulation of human cytotoxic T cells with HIV-1-derived peptides presented by recombinant HLA-A2 peptide complexes. Int Immunol 9:451-9, 1997. HLA-A2 heavy chain and beta 2-microglobulin refolded in the presence of peptides became susceptible to lysis by HLA-A2-restricted cytotoxic T lymphocyte (CTL) clones specific for the peptides. Recombinant HLA-A2 peptide complexes covalently immobilized on microspheres stimulated the development of HLA-A2 peptide-specific CTL. On p.76 212

[Wang2002a] F.-X. Wang, T. Kimura, K. Nishihara, K. Yoshimura, A. Koito, \& S. Matsushita. Emergence of autologous neutralization-resistant variants from preexisting human immunodeficiency virus (HIV) quasi species during virus rebound in HIV type 1-infected patients undergoing highly active antiretroviral therapy. J Infect Dis 185(5):608-617, 2002. On p.759

Wang2002b] S. Wang, J. York, W. Shu, M. O. Stoller, J. H. Nunberg, \& M. Lu. Interhelical interactions in the gp41 core: Implications for activation of HIV-1 membrane fusion. Biochemistry 41(23): 7283-7292, 2002. On p. 636

Warren1992] A. P. Warren \& D. B. Thomas. Class II (II-A ${ }^{d}$ ) restricted T-cell recognition of the V3 loop region of HIV-1 gp120. AIDS Res Hum Retroviruses 8:559-564, 1992. Notes: The epitope defined here is the immunodominant epitope for a helper T-cell response to the gp120 vaccine in mice. On p. 500

[Warrier1994] S. V. Warrier, A. Pinter, W. J. Honnen, M. Girard, E. Muchmore, \& S. A. Tilley. A novel, glycan-dependent epitope in the V2 domain of human immunodeficiency virus type 1 gp120 is recognized by a highly potent, neutralizing chimpanzee monoclonal antibody. J Virol 68:4636-4642, 1994. On p. 629

Warrier1995] S. V. Warrier, E. Murphy, I. Yokoyama, \& S. A. Tilley. Characterization of the Variable Regions of a Chimpanzee Monoclonal Antibody with Potent Neutralizing Activity Agains HIV-1. Mol Immunol 32:1081-1092, 1995. On p.629

[Warrier1996] S. V. Warrier, A. Pinter, W. J. Honnen, \& S. A. Tilley. Synergistic Neutralization of Human Immunodeficiency Virus Type 1 by a Chimpanzee Monoclonal Antibody against the V2 Domain of gp120 in Combination with Monoclonal Antibodies against the V3 Loop and the \begin{tabular}{ll|l|l|l|l|l|l|l|} 
CD4-Binding Site. J Virol 70:4466-4473, 1996. On p. 629 & 644 & 671 & 672 & 760 & 767 \\
\hline
\end{tabular}

Wasik 1997] T. J. Wasik, P. P. Jagodzinski, E. M. Hyjek, J. Wustner, G. Trinchieri, H. W. Lischner, \& D. Kozbor. Diminished HIV-specific CTL activity is associated with lower type 1 and enhanced type 2 responses to HIV-specific peptides during perinatal HIV infection. J Immunol 158:602936, 1997. On p.492 505

Wasik1999] T. J. Wasik, J. Bratosiewicz, A. Wierzbicki, V. E. Whiteman, R. R. Rutstein, S. E. Starr, S. D. Douglas, D. Kaufman, A. V. Sison, M. Polansky, H. W. Lischner, \& D. Kozbor Protective role of beta-chemokines associated with HIV-specific Th responses against perinatal HIV transmission. J Immunol 162:4355-64, 1999. On p. 166 242 337493506

[Wasik2000] T. J. Wasik, A. Wierzbicki, V. E. Whiteman, G. Trinchieri, H. W. Lischner, \& D. Koz- 
bor. Association between HIV-specific T helper responses and CTL activities in pediatric AIDS. Eur J Immunol 30:117-27, 2000. On p. 162 239 332 395 392 402 505

[Watkins1993] B. A. Watkins, M. S. Reitz, Jr., C. A. Wilson, K. Aldrich, A. E. Davis, \& M. RobertGuroff. Immune escape by human immunodeficiency virus type 1 from neutralizing antibodies: evidence for multiple pathways. J Virol 67:7493-7500, 1993. A neutralization resistance poin mutation (HXB2 A281V) was studied using a variety of MAbs, and it was shown that this substitution affects a different epitope than a previously characterized neutralization escape mutant (A582T) (Reitz 1988, Wilson 1990). On p.656 662 671 $761|762| 775 \mid 776$

[Watkins1996] B. A. Watkins, A. E. Davis, S. Fiorentini, F. di Marzo Veronese, \& M. S. Reitz, Jr. Evidence for distinct contributions of heavy and light chains to restriction of antibody recognition of the HIV-1 principal neutralization determinant. J Immunol 156:1676-1683, 1996. On p.656 739

[Wee2002] E. G.-T. Wee, S. Patel, A. J. McMichael, \& T. Hanke. A DNA/MVA-based candidate human immunodeficiency virus vaccine for Kenya induces multi-specific T cell responses in rhesus macaques. J Gen Virol 83(Pt 1):75-80, 2002. On p. 127||128|[129|130|133|142 |143||144

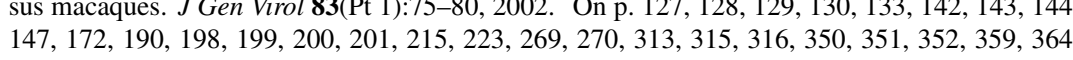
\begin{tabular}{l|l|l|l|l|l|l|l|l|l|l|}
\hline 365 & 369 & 370 & 380 & 393 \\
\hline
\end{tabular}

[Weekes1999a] M. P. Weekes, A. J. Carmichael, M. R. Wills, K. Mynard, \& J. G. Sissons. Human CD28-CD8+ T cells contain greatly expanded functional virus-specific memory CTL clones. Immunol 162:7569-77, 1999. On p.65 138 280 281

[Weekes1999b] M. P. Weekes, M. R. Wills, K. Mynard, R. Hicks, J. G. Sissons, \& A. J. Carmichael Large clonal expansions of human virus-specific memory cytotoxic T lymphocytes within the CD57+ CD28- CD8+ T cell population. Immunology 98:443-9, 1999. On p.65] 138 267]280 282

[Wehrly1997] K. Wehrly \& B. Chesebro. p24 antigen capture assay for quantification of human immunodeficiency virus using readily available inexpensive reagents. Methods: A companion to Methods in Enzymology 12:288-93, 1997. On p.589.

[Weinberg1997] J. Weinberg, H. X. Liao, J. V. Torres, T. J. Matthews, J. Robinson, \& B. F. Haynes Identification of a synthetic peptide that mimics an HIV glycoprotein 120 envelope conformational determinant exposed following ligation of glycoprotein 120 by CD4. AIDS Res Hum Retroviruses 13:657-64, 1997. On p.798 803 804

[Weissenhorn1996] W. Weissenhorn, S. A. Wharton, L. J. Calder, P. L. Earl, B. Moss, E. Aliprandis, J. J. Skehel, \& D. C. Wiley. The ectodomain of HIV-1 Env subunit gp41 forms a soluble, alphahelical, rod-like oligomer in the absence of gp120 and the N-terminal fusion peptide. EMBO J 15:1507-14, 1996. On p. 702 703 736 740.807

[White-Scharf1993] M. E. White-Scharf, B. J. Potts, L. M. Smith, K. A. Sokolowski, J. R. Rusche, \& S. Silver. Broadly neutralizing monoclonal antibodies to the V3 region of HIV-1 can be elicited by peptide immunization. Virology 192:197-206, 1993. Using a V3 loop peptide as immunogen, a panel of 50 anti-V3 neutralizing monoclonal antibodies were generated. Four of them were characterized in detail in this paper. On p. 652 661668673

[White2001] H. D. White, L. K. Musey, M. M. Andrews, G. R. Yeaman, L. R. DeMars, P. D. Man ganiello, A. L. Howell, C. R. Wira, W. R. Green, \& M. J. McElrath. Human immunodeficiency virus-specific and $\mathrm{CD} 3$-redirected cytotoxic $\mathrm{T}$ lymphocyte activity in the human female reproductive tract: lack of correlation between mucosa and peripheral blood. J Infect Dis 183(6):977-83, 2001. On p. 165. 241 336

[Wierzbicki2002] A. Wierzbicki, I. Kiszka, H. Kaneko, D. Kmieciak, T. J. Wasik, J. Gzyl, Y. Kaneko, \& D. Kozbor. Immunization strategies to augment oral vaccination with DNA an viral vectors expressing HIV envelope glycoprotein. Vaccine 20(9-10):1295-1307, 2002. O p. 298

[Wilkens1999] B. Wilkens \& D. Ruhl. Personal communication, 1999. On p.64
[Wilson1996] C. Wilson, B. Wilkes, D. Ruhl, \& B. Walker. Personal communication, 1996. Defined in the context of the Pediatric AIDS Foundation ARIEL Project, a mother-infant HIV transmission

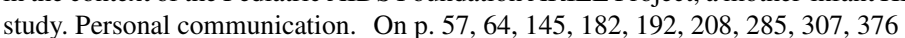

Wilson1997a] C. C. Wilson, S. A. Kalams, B. M. Wilkes, D. J. Ruhl, F. Gao, B. H. Hahn, I. C. Hanson, K. Luzuriaga, S. Wolinsky, R. Koup, S. P. Buchbinder, R. P. Johnson, \& B. D. Walker. Overlapping epitopes in human immunodeficiency virus type 1 gp120 presented by HLA A, B, and C molecules: effects of viral variation on cytotoxic T-lymphocyte recognition. $J$ Virol 71:1256-64, 1997. CTL clones were isolated from four individuals that interact with the same 25 amino-acid peptide using three different HLA presenting molecules. On p. 301302

Wilson1997b] S. E. Wilson, J. A. Habeshaw, M. A. Addawe, E. F. Hounsell, \& J. S. Oxford. HIV type 1 envelope glycoprotein 120 carboxy-terminal peptide-induced human $\mathrm{T}$ cell lines selectively suppress heterogeneous proliferative $\mathrm{T}$ cell responses to soluble antigens. AIDS Res Hum Retro 13(15):1313-1324, 1997. On p.515

[Wilson1998a] J. D. Wilson, G. S. Ogg, R. L. Allen, P. J. Goulder, A. Kelleher, A. K. Sewell, C. A. O'Callaghan, S. L. Rowland-Jones, M. F. Callan, \& A. J. McMichael. Oligoclonal expansions of CD8(+) T cells in chronic HIV infection are antigen specific. J Exp Med 188(4):785-90, 1998. On p.75] $134[211$

[Wilson1998b] S. E. Wilson, S. L. Pedersen, J. C. Kunich, V. L. Wilkins, D. L. Mann, G. P. Mazzara J. Tartaglia, C. L. Celum, \& H. W. Sheppard. Cross-clade envelope glycoprotein 160-specific CD8+ cytotoxic T lymphocyte responses in early HIV type 1 clade B infection. AIDS Res Hum Retroviruses 14:925-37, 1998. On p.284 329

Wilson1999a] C. C. Wilson, R. C. Brown, B. T. Korber, B. M. Wilkes, D. J. Ruhl, D. Sakamoto, K. Kunstman, K. Luzuriaga, I. C. Hanson, S. M. Widmayer, A. Wiznia, S. Clapp, A. J. Ammann, R. A. Koup, S. M. Wolinsky, \& B. D. Walker. Frequent detection of escape from cytotoxic Tlymphocyte recognition in perinatal human immunodeficiency virus (HIV) type 1 transmission the ariel project for the prevention of transmission of HIV from mother to infant. $J$ Virol 73:3975-

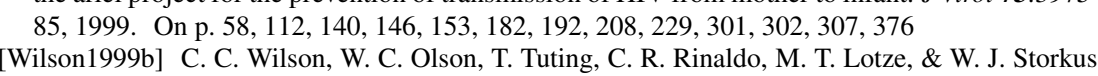
HIV-1-specific CTL responses primed in vitro by blood-derived dendritic cells and Th1-biasing cytokines. J Immunol 162:3070-8, 1999. On p.345 378 386 390393

[Wilson2000a] J. D. Wilson, G. S. Ogg, R. L. Allen, C. Davis, S. Shaunak, J. Downie, W. Dyer, C. Workman, S. Sullivan, A. J. McMichael, \& S. L. Rowland-Jones. Direct visualization of HIV1-specific cytotoxic T lymphocytes during primary infection. AIDS 14:225-33, 2000. On p.60 \begin{tabular}{l|l|l|l|l|l|l|l|l|l|l|l|l|l|}
\hline 62 & 89 & 94 & 126 & 132 & 194 & 197 & 210 & 228 & 271 & 274 & 356 & 357 & 378 \\
\hline
\end{tabular}

Wilson2000b] J. D. K. Wilson, N. Imami, A. Watkins, J. Gill, P. Hay, B. Gazzard, M. Westby, \& F. M. Gotch. Loss of CD4+ T cell proliferative ability but not loss of human immunodeficiency virus type 1 specificity equates with progression to disease. J Infect Dis 182(3):792-8, 2000. On p. 444 527. 534.

[Wilson2001] C. C. Wilson, B. Palmer, S. Southwood, J. Sidney, Y. Higashimoto, E. Appella, R. Chesnut, A. Sette, \& B. D. Livingston. Identification and antigenicity of broadly cross-reactive and conserved human immunodeficiency virus type 1-derived helper T-lymphocyte epitopes. $J$ Virol 75(9):4195-207, 2001. On p.431 436 449 450 452 453 454,456

[Wisnewski1995] A. Wisnewski, L. Cavacini, G. Kingsbury, D. Sadden, \& M. Posner. Anti-HIV human monoclonal antibody variable region gene usage. J Cell Biochem supple 21 B:229, 1995. On p.702 737738

Wisnewski1996] A. Wisnewski, L. Cavacini, \& M. Posner. Human antibody variable region gene usage in HIV-1 infection. J Acquir Immune Defic Syndr Hum Retrovirol 11:31-38, 1996. On \begin{tabular}{ll|l|l|l|l|l|l|l|l|l|l|l|l|l|l|l|l|l|} 
p. 647 & 664 & 699 & 702 & 706 & 707 & 708 & 729 & 737 & 738 & 762 & 763 & 764 & 765 & 775 & 777 & 780 & 781 & 795 \\
\hline
\end{tabular}

[Wodarz2002] D. Wodarz. The persistence of CTL memory. Neth J Med 60(7 Suppl):4-13; discussion 14-6, 2002. On p. 400535 
[Wolfe1996] E. J. Wolfe, L. A. Cavacini, M. H. Samore, M. R. Posner, C. Kozial, C. Spino, C. B. Trapnell, N. Ketter, S. Hammer, \& J. G. Gambertoglio. Pharmacokinetics of F105, a human monoclonal antibody, in persons infected with human immunodeficiency virus type 1. Clin Pharmacol Ther 59:662-667, 1996. On p. 775 776

Wolinsky1996] S. M. Wolinsky, B. T. M. Korber, A. U. Neumann, M. Daniels, K. J. Kuntsman, A. J. Whetsell, M. R. Furtado, Y. Chao, D. D. Ho, J. T. Safrit, \& R. A. Koup. Adaptive evolution of human immunodeficiency virus-type 1 during the natural course of infection. Science 272:537542,1996 . In a longitudinal study of six patients, epitope variation occurred only when there was a notable CTLp frequency and the individual had the appropriate HLA type. On p. 284 301

[Woodberry 1999] T. Woodberry, J. Gardner, L. Mateo, D. Eisen, J. Medveczky, I. A. Ramshaw, S. A. Thomson, R. A. Ffrench, S. L. Elliott, H. Firat, F. A. Lemonnier, \& A. Suhrbier. Immunogenicity of a human immunodeficiency virus (HIV) polytope vaccine. J Virol 73:5320-5, 1999.

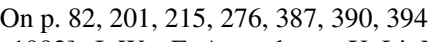

[Wu1993] J. Wu E. Amandoron, X. Li, M. A. Wainberg, \& M. A. Parniak. Monoclonal Antibodymediated Inhibition of HIV-1 Reverse Transcriptase Polymerase Activity. J Biol Chem 268:9980 9985, 1993. On p. 597

[Wu1995] Z. Wu, S. C. Kayman, W. Honnen, K. Revesz, H. Chen, S. V. Warrier, S. A. Tilley, J. McKeating, C. Shotton, \& A. Pinter. Characterization of neutralization epitopes in the V2 region of human immunodeficiency virus type $1 \mathrm{gp} 120$ : role of glycosylation in the correct folding of the V1/V2 domain. J Virol 69:2271-2278, 1995. Most epitopes based only on numbering. On \begin{tabular}{ll|l|l|l|} 
p. 629 & 630 & 632 & 633 & 634 \\
\hline
\end{tabular}

[Wu1996] L. Wu, N. P. Gerard, R. Wyatt, H. Choe, C. Parolin, N. Ruffing, A. Borsetti, A. A. Cardoso, E. Desjardin, W. Newman, C. Gerard, \& J. Sodroski. CD4-induced interaction of primary HIV-1 gp120 glycoproteins with the chemokine receptor CCR-5. Nature 384:179-183, 1996. Results suggest that HIV-1 attachment to CD4 creates a high-affinity binding site for CCR-5, leading to membrane fusion and virus entry. CD4-induced or V3 neutralizing MAbs block the interaction \begin{tabular}{ll|l|l|l|l|l|l|} 
of gp120-CD4 complexes with CCR-5. On p.657 663 & 664 & 756 & 757 & 758 & 759 & 798 & 832 \\
\hline
\end{tabular}

[Wu2002] X. Wu \& S. Jackson. Plasma and salivary IgA subclasses and IgM in HIV-1-infected individuals. J Clin Immunol 22(2):106-115, 2002. On p. 842

[Wyatt1992] R. Wyatt, M. Thali, S. Tilley, A. Pinter, M. Posner, D. Ho, J. Robinson, \& J. Sodroski. Relationship of the human immunodeficiency virus type $1 \mathrm{gp} 120$ third variable loop to element of the CD4 binding site. $J$ Virol 66:6997-7004, 1992. This paper examines mutations which alte $\mathrm{MAb}$ binding and neutralization. Anti-V3 MAb 9284 has enhanced binding due to a mutation in the $\mathrm{C} 4$ region that is also important for CD4 binding, and anti-CD4 binding MAbs F105, 1.5e and $1125 \mathrm{H}$ show increased precipitation of a gp120 from which the V3 loop was deleted, relative to wild type, in RIPA buffer containing non-ionic detergents. On p. 641 760 \begin{tabular}{l|l|l|}
762 & 775 \\
\hline
\end{tabular}

[Wyatt1993] R. Wyatt, N. Sullivan, M. Thali, H. Repke, D. Ho, J. Robinson, M. Posner, \& J. Sodroski. Functional and Immunologic Characterization of Human Immunodeficiency virus type envelope glycoproteins containing deletions of the major variable regions. J Virol 67:4557-4565, 1993. Affinity of neutralizing MAbs directed against the CD4 binding site was increased dramatically by deletion mutants across the V1/V2 and V3 structures, suggesting that these domain mask these conserved discontinuous epitopes. On p.762 764775776

[Wyatt1995] R. Wyatt, J. Moore, M. Accola, E. Desjardin, J. Robinson, \& J. Sodroski. Involvemen of the V1/V2 variable loop structure in the exposure of human immunodeficiency virus type 1 gp120 epitopes induced by receptor binding. J Virol 69:5723-5733, 1995. Deletions in the V1/V2 loops of gp120 resulted in the loss of the ability of sCD4 to induce binding of the MAbs $17 \mathrm{~b}, 48 \mathrm{~d}$, and A32. A32 can induce binding of $17 \mathrm{~b}$ and $48 \mathrm{~d}$; this induction does not appear to

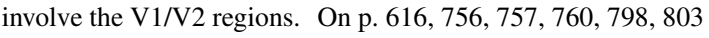

[Wyatt 1997] R. Wyatt, E. Desjardin, U. Olshevsky, C Nixon, J. Binley, V. Olshevsky, \& J. Sodroski. Analysis of the interaction of the human immunodeficiency virus type 1 gp120 Envelope glycoprotein with the gp41 transmembrane glycoprotein. J Virol 71:9722-31, 1997. This study characterized the binding of gp 120 and gp 41 by comparing Ab reactivity to soluble gp 120 and to soluble complex of gp 120 and gp 41 called sgp 140 . The occlusion of gp 120 epitopes in the sgp140 complex provides a guide to the gp120 domains that interact with gp41, localizing them in $\mathrm{C} 1$ and $\mathrm{C} 5$ of gp120. Mutations that disrupt the binding of the occluded antibodies do not influence $\mathrm{NAb}$ binding or CD4 binding, thus if the gp41 binding domain is deleted, the immunologically

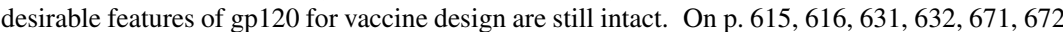

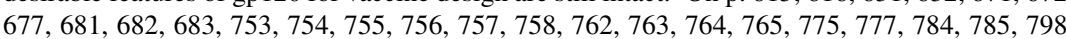
\begin{tabular}{llllll}
799 & 803 & 804 & 817 & 818 \\
\hline
\end{tabular}

Wyatt1998a] R. Wyatt, P. D. Kwong, E. Desjardins, R. W. Sweet, J. Robinson, W. A. Hendrickson, \& J. G. Sodroski. The antigenic structure of the HIV gp120 envelope glycoprotein. Nature 393:705-711, 1998. Comment in: Nature 1998 Jun 18;393(6686):630-1. The spatial organization of the neutralizing epitopes of gp120 is described, based on epitope maps interpreted in the context of the X-ray crystal structure of a ternary complex that includes a gp120 core, CD4 and \begin{tabular}{ll|l|l|l|l|l|l|l|l|l|l|l|l|l|l|l|} 
a neutralizing antibody. On $\mathrm{p} .760$ & 761 & 762 & 763 & 764 & 765 & 766 & 767 & 771 & 775 & 777 & 783 & 784 \\
\hline
\end{tabular} \begin{tabular}{ll|l|l|l|l|l|l|l|l|l|l|l|}
786 & 798 & 799 & 803 & 804 & 822 & 823 \\
\hline
\end{tabular}

Wyatt1998b] R. Wyatt \& J. Sodroski. The HIV-1 envelope glycoproteins: fusogens, antigens, an immunogens. Science 280:1884-1888, 1998. Review discussing of the mechanisms used by the virus to evade a neutralizing antibody response while maintaining vital Env functions of binding to target cells, and then entering through membrane fusion. On p. 822 823

[Xiang2002a] S.-H. Xiang, N. Doka, R. K. Choudhary, J. Sodroski, \& J. E. Robinson. Characterization of CD4-induced epitopes on the HIV type 1 gp120 envelope glycoprotein recognized by neutralizing human monoclonal antibodies. AIDS Res Hum Retroviruses 18(16):1207-1217, 2002. On p.756 760 798 802 803 805 806

Xiang2002b] S.-H. Xiang, P. D. Kwong, R. Gupta, C. D. Rizzuto, D. J. Casper, R. Wyatt, L. Wang, W. A. Hendrickson, M. L. Doyle, \& J. Sodroski. Mutagenic stabilization and/or disruption of a CD4-bound state reveals distinct conformations of the human immunodeficiency virus type 1 gp120 envelope glycoprotein. $J$ Virol 76(19):9888-9899, 2002. On p.710 717 762 763 764 \begin{tabular}{l|l|l|l|l|l|l|l|l|l|l|l|l|l|l|l|l|l|l|l|}
765 & 775 & 778 & 779 & 780 & 784 & 790 & 798 & 801 & 802 & 803 & 805 & 806 \\
\hline
\end{tabular}

Xiao2000a] Y. Xiao, X. N. Dong, \& Y. H. Chen. Induction of monoclonal antibody with predefined ELNKWA epitope specificity by epitope vaccine. Hybridoma 19(4):347-50, 2000. On p.718

[Xiao2000b] Y. Xiao, M. Liao, Y. Lu, M. P. Dierich, \& Y. H. Chen. Epitope-vaccines: a new strategy to induce high levels of neutralizing antibodies against HIV-1. Immunobiology 201:323-31, 2000 On p. 696718

Xiao2000c] Y. Xiao, Y. Zhao, Y. Lu, \& Y. H. Chen. Epitope-vaccine induces high levels of ELDKWA-epitope-specific neutralizing antibody. Immunol Invest 29:41-50, 2000. On p.710

Xin1998] K. Q. Xin, K. Hamajima, S. Sasaki, A. Honsho, T. Tsuji, N. Ishii, X. R. Cao, Y. Lu, J. Fukushima, P. Shapshak, S. Kawamoto, \& K. Okuda. Intranasal administration of human immunodeficiency virus type-1 (HIV- 1) DNA vaccine with interleukin-2 expression plasmid enhances cell- mediated immunity against HIV-1. Immunology 94:438-44, 1998. On p.495

[Xin1999] K. Q. Xin, K. Hamajima, S. Sasaki, T. Tsuji, S. Watabe, E. Okada, \& K. Okuda. IL15 expression plasmid enhances cell-mediated immunity induced by an HIV-1 DNA vaccine. Vaccine 17:858-66, 1999. On p. 295495

[Xin2001] K. Q. Xin, M. Urabe, J. Yang, K. Nomiyama, H. Mizukami, K. Hamajima, H. Nomiyama, T. Saito, M. Imai, J. Monahan, K. Okuda, K. Ozawa, \& K. Okuda. A novel recombinant adeno-associated virus vaccine induces a long-term humoral immune response to human immunodeficiency virus. Hum Gene Ther 12(9):1047-61, 2001. On p. 259]265] 341

[Xu1991] J.-Y. Xu, M. K. Gorny, T. Palker, S. Karwowska, \& S. Zolla-Pazner. Epitope mapping of two immunodominant domains of gp41, the transmembrane protein of human immunodeficiency virus type 1, using ten human monoclonal antibodies. J Virol 65:4832-4838, 1991. The 
immunodominance of linear epitope in the region 590-600 of gp41 (cluster I) was established, and a second conformational epitope was mapped that reacted with a region between amino acids 644 and 663 (cluster II). Titration experiments showed that there was 100-fold more antibody to \begin{tabular}{|l|l|l|l|l|l|l|l|l|l|l|} 
cluster I than cluster II in patient sera. On p. 696 & 698 & 700 & 701 & 702 & 707 & 708 & 709 & 729 & 828 \\
\hline
\end{tabular}

[Xu2001] W. Xu, B. A. Smith-Franklin, P. L. Li, C. Wood, J. He, Q. Du, G. J. Bhat, C. Kankasa, H. Katinger, L. A. Cavacini, M. R. Posner, D. R. Burton, T. C. Chou, \& R. M. Ruprecht. Potent neutralization of primary human immunodeficiency virus clade $C$ isolates with a synergistic combination of human monoclonal antibodies raised against clade B. J Hum Virol 4(2):55-61, 2001. \begin{tabular}{l|l|l|l||l|l|l|} 
On p. 710 & 716 & 719 & 784 & 789 & 822 & 826 \\
\hline
\end{tabular}

[Xu2002] W. Xu, R. Hofmann-Lehmann, H. M. McClure, \& R. M. Ruprecht. Passive immunization with human neutralizing monoclonal antibodies: Correlates of protective immunity against HIV. \begin{tabular}{ll|l|l|l|l|l|l|} 
Vaccine 20(15): 1956-1960, 2002. On p.710 716 & 719 & 775 & 778 & 784 & 790 & 822 & 826 \\
\hline
\end{tabular}

[Yamada1991] M. Yamada, A. Zurbriggen, M. B. A. Oldstone, \& R. S. Fujinami. Common immunologic determinant between human immunodeficiency virus type $1 \mathrm{gp} 41$ and astrocytes. Virol 65:1370-1376, 1991. On p.703 704 705

[Yang1996] O. O. Yang, S. A. Kalams, M. Rosenzweig, A. Trocha, N. Jones, M. Koziel, B. D. Walker, \& R. P. Johnson. Efficient lysis of human immunodeficiency virus type 1-infected cell by cytotoxic T lymphocytes. J Virol 70:5799-5806, 1996. On p. 84 [145|216 |310

[Yang1997a] O. O. Yang, S. A. Kalams, A. Trocha, H. Cao, A. Luster, R. P. Johnson, \& B. D.

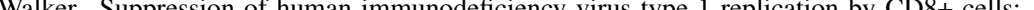
idence for HLA class I-restricted triggering of cytolytic and noncytolytic mechanisms. $J$ Viro 71:3120-8, 1997. Although CD8+ lymphocytes in human immunodeficiency virus type 1 (HIV 1)-infected individuals have been demonstrated to suppress viral replication, the mechanisms of inhibition have not been defined precisely. A large body of evidence indicates that these cells act via soluble inhibitory factors, but the potential role of HLA class I-restricted cytolysis has remained controversial. Here we demonstrate that HIV-1-specific cytotoxic T lymphocytes (CTL) mediate antiviral suppression by both cytolytic and noncytolytic mechanisms. The predominan mechanism requires direct contact of CTL with the infected cells, is HLA class I restricted, and can achieve complete elimination of detectable virus in infected cell cultures. Inhibition occur even at high multiplicities of infection or at ratios of CTL to CD4 cells as low as 1:1,000. The other mechanism is mediated by soluble inhibitory factors which are triggered in an antigenspecific and HLA-restricted fashion but then act without HLA restriction. On p. 84,145 310

[Yang1997b] O. O. Yang, A. C. Tran, S. A. Kalams, R. P. Johnson, M. R. Roberts, \& B. D. Walker Lysis of HIV-1-infected cells and inhibition of viral replication by universal receptor T cells. Proc Natl Acad Sci USA 94:11478-83, 1997. On p. 84]145

[Yang1997c] W.-P. Yang, K. Green, S. Pinz-Sweeney, A. T. Briones, D. R. Burton, \& C. F. Barba III. CDR walking mutagenesis for the affinity maturation of a potent human anti-HIV-1 antibody into the picomolar range. J Mol Biol 254:392-403, 1997. On p.784

[Yang1998] G. Yang, M. P. D’Souza, \& G. N. Vyas. Neutralizing antibodies against HIV determined by amplification of viral long terminal repeat sequences from cells infected in vitro by nonneutralized virions. J Acquir Immune Defic Syndr Hum Retrovirol 17:27-34, 1998. A neutralization assay was developed based on heminested PCR amplification of the LTR (HNPCR) LTR-HNPCR consistently revealed HIV DNA and was shown to be a rapid, specific and reliable neutralization assay based on tests with 6 MAbs and 5 HIV isolates. On p. 647 648 710712 \begin{tabular}{ll|l|l|l|l|l|l}
760 & 761 & 795 & 796 & 803 & 804 & 818 \\
\hline
\end{tabular}

[Yang2000] X. Yang, M. Farzan, R. Wyatt, \& J. Sodroski. Characterization of Stable, Soluble Trimers Containing Complete Ectodomains of Human Immunodeficiency Virus Type 1 Envelope

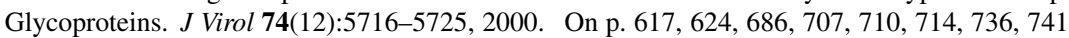

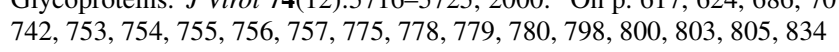

[Yang2001] X. Yang, R. Wyatt, \& J. Sodroski. Improved elicitation of neutralizing antibodies against primary human immunodeficiency viruses by soluble stabilized envelope glycoprotein trimers. J Virol 75(3):1165-71, 2001. On p.745 784 788

[Yang2002] X. Yang, J. Lee, E. M. Mahony, P. D. Kwong, R. Wyatt, \& J. Sodroski. Highly stable trimers formed by human immunodeficiency virus type 1 envelope glycoproteins fused with the trimeric motif of T4 bacteriophage fibritin. J Virol 76(9):4634-4642, 2002. On p. 88 |93 220

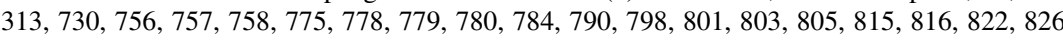

Yi2000a] J. Yi \& A. M. Skalka. Mapping epitopes of monoclonal antibodies against HIV-1 integrase with limited proteolysis and matrix-assisted laser desorption ionization time-of-flight mass spectrometry. Biopolymers 55(4):308-318, 2000. On p. 606 608

[Yi2000b] J. i. Yi, J. W. Arthur, R. L. Dunbrack, Jr., \& A. M. Skalka. An inhibitory monoclonal antibody binds at the turn of the helix-turn-helix motif in the N-terminal domain of HIV-1 integrase. J Biol Chem 275(49):38739-38748, 2000. On p.602

[Yi2002] J. Yi, H. Cheng, M. D. Andrake, R. L. Dunbrack, Jr., H. Roder, \& A. M. Skalka. Mapping the epitope of an inhibitory monoclonal antibody to the C-terminal DNA-binding domain of HIV1 integrase. J Biol Chem 277(14):12164-12174, 2002. On p.606 608

Yin2001] S. Yin, N. Okada, \& H. Okada. Elimination of latently HIV-1-infected cells by lymphoblasts armed with bifunctional antibody. Microbiol Immunol 45(1):101-8, 2001. On p.739

Yokosuka2002] T. Yokosuka, K. Takase, M. Suzuki, Y. Nakagawa, S. Taki, H. Takahashi, T. Fujisawa, H. Arase, \& T. Saito. Predominant role of T cell receptor (TCR)-alpha chain in forming preimmune TCR repertoire revealed by clonal TCR reconstitution system. J Exp Med 195(8):991-1001, 2002. On p.288 291

[York2001] J. York, K. E. Follis, M. Trahey, P. N. Nyambi, S. Zolla-Pazner, \& J. H. Nunberg. Antibody binding and neutralization of primary and T-cell line-adapted isolates of human immunodeficiency virus type 1. J Virol 75(6):2741-52, 2001. On p. 647 648 652664665668673 \begin{tabular}{|l|l|l|l|l|l|l|l|l|l|l|l|l|l|l|}
\hline 702 & 710 & 715 & 720 & 722 & 768 & 769 & 775 & 778 & 784 & 788 & 798 & 801 \\
\hline
\end{tabular}

Yoshida1997] K. Yoshida, M. Nakamura, \& T. Ohno. Mutations of the HIV type 1 V3 loop unde selection pressure with neutralizing monoclonal antibody NM-01. AIDS Res Hum Retroviruses 13:1283-1290, 1997. On p. 672

YYoshiyama1994] H. Yoshiyama, H.-M. Mo, J. P. Moore, \& D. D. Ho. Characterization of mutants of human immunodeficiency virus type 1 that have escaped neutralization by monoclonal antibody G3-4 to the gp120 V2 loop. J Virol 68:974-978, 1994. MAb G3-4 binds a conformationally sensitive epitope in the V2 loop of HIV-1 RF. RF was cultured in the presence of G3-4 to select for neutralization resistance. Three independent experiments yielded escape mutants, and sequencing revealed two V2 mutations to be responsible for the neutralization escape phenotype, $177 \mathrm{Y} / \mathrm{H}$ and $179 \mathrm{~L} / \mathrm{P}$. Experimental introduction of the $179 \mathrm{P}$ substitution resulted in non-viable virus, and $177 \mathrm{H}$ confirmed the resistance phenotype. On p. 631 632633612

[Young2001] J. M. Young, R. A. Ffrench, J. D. Clarkson, G. J. Stewart, T. Liang, R. L. Tideman, D. Packham, D. A. Fulcher, \& E. M. Benson. In vitro HIV-specific CTL activity from HIVseropositive individuals is augmented by interleukin-12 (IL-12). AIDS Res Hum Retroviruses 17(3): 233-42, 2001. On p. 165241336

Yu2000] T. Yu, Y. Bai, M. P. Dierich, \& Y. H. Chen. Induction of high levels of epitope-specific antibodies by epitope/peptide candidate vaccines against human immunodeficiency virus type-1 (HIV-1). Microbiol Immunol 44(2):105-10, 2000. On p.674

Yu2002a] X. G. Yu, M. M. Addo, E. S. Rosenberg, W. R. Rodriguez, P. K. Lee, C. A. Fitzpatrick, M. N. Johnston, D. Strick, P. J. R. Goulder, B. D. Walker, \& M. Altfeld. Consistent patterns in the development and immunodominance of human immunodeficiency virus type 1 (HIV-1)-specific CD8+ T-cell responses following acute HIV-1 infection, J Virol 76(17):8690-8701, 2002 . O \begin{tabular}{l|l|l|l|l|l|l|l|l|l|l|l|l|l|l|l|l|l|l|l|l|} 
p. 59 & 63 & 102 & 113 & 120 & 158 & 181 & 189 & 193 & 208 & 224 & 236 & 245 & 246 & 247 & 248 & 252 & 262 & 285 \\
\hline
\end{tabular} 
\begin{tabular}{|l|l|l|l|l|l|l|l|l|l|l|}
321 & 330 & 346 & 347 & 349 & 355 & 360 & 365 & 372 & 380 \\
\hline
\end{tabular}

Yu2002b] X. G. Yu, H. Shang, M. M. Addo, R. L. Eldridge, M. N. Phillips, M. E. Feeney, D. Strick C. Brander, P. J. R. Goulder, E. S. Rosenberg, B. D. Walker, M. Altfeld, \& HIV Study Collaboration. Important contribution of p15 Gag-specific responses to the total Gag-specific CTL responses. AIDS 16(3):321-328, 2002. On p. 160 161

[Yusim2002] K. Yusim, C. Kesmir, B. Gaschen, M. M. Addo, M. Altfeld, S. Brunak, A. Chigaev, V. Detours, \& B. T. Korber. Clustering patterns of cytotoxic T-lymphocyte epitopes in human immunodeficiency virus type 1 (HIV-1) proteins reveal imprints of immune evasion on HIV-1 global variation. $J$ Virol 76(17):8757-8768, 2002. On p. 166|242|245|246|247| 248 |250||251 \begin{tabular}{|l|l|l||l|l|l|l|l|l|l|l|}
252 & 256 & 258 & 260 & 261 & 262 & 264 & 338 & 397 \\
\hline
\end{tabular}

[Zafiropoulos1997] A. Zafiropoulos, E. Andersson, E. Krambovitis, \& C. A. Borrebaeck. Inductio of antigen-specific isotype switching by in vitro immunization of human naive B lymphocytes. I Immunol Methods 200(1-2):181-90, 1997. A two-step in vitro immunization protocol is described, which yields an isotype switch. On p.645

[Zarling1999] A. L. Zarling, J. G. Johnson, R. W. Hoffman, \& D. R. Lee. Induction of primary human CD8+ T lymphocyte responses In vitro using dendritic cells. J Immunol 162:5197-204, 1999. On p.57, $128[145] 177,217,277$

[Zavala2001] F. Zavala, M. Rodrigues, D. Rodriguez, J. R. Rodriguez, R. S. Nussenzweig, \& M. Esteban. A striking property of recombinant poxviruses: efficient inducers of in vivo expansion of primed CD8(+) T cells. Virology 280(2):155-9, 2001. On p. 335

[Zeder-Lutz2001] G. Zeder-Lutz, J. Hoebeke, \& M. H. Van Regenmortel. Differential recognition of epitopes present on monomeric and oligomeric forms of gp160 glycoprotein of human immunodeficiency virus type 1 by human monoclonal antibodies. Eur J Biochem 268(10):2856-66, 200 On p.710 $715 \mid$\begin{tabular}{l|l|l|l|l|l|}
784 & 789 & 822 & 825 \\
\hline
\end{tabular}

[Zerhouni1997] B. Zerhouni, K. Sanhadji, \& J. L. Touraine. Loss of T cell cytotoxic responses in the course of HIV-1 infection. Thymus 24:203-19, 1997. On p. 396

[Zhang1993] Q.-I. Zhang, R. Gavioli, G. Klein, \& M. G. Masucci. An HLA-All-specific motif in nonamer peptides derived from viral and cellular proteins. Proc Natl Acad Sci USA 90:2217 2221, 1993. On p.191, 359

[Zhang2001a] W. Zhang, A. P. Godillot, R. Wyatt, J. Sodroski, \& I. Chaiken. Antibody 17b binding at the coreceptor site weakens the kinetics of the interaction of envelope glycoprotein gp120 with CD4. Biochemistry 40(6):1662-70, 2001. On p. 798.801.

[Zhang2001b] Y. Zhang, M. Huber, B. Weissbrich, G. Voss, P. Langmann, H. Klinker, \& C. Jassoy Characterization of HIV-specific proliferative T cell responses in HIV-infected persons. AIDS Res Hum Retroviruses 17(7):623-9, 2001. On p. 443527

[Zhang2002] P. F. Zhang, P. Bouma, E. J. Park, J. B. Margolick, J. E. Robinson, S. Zolla-Pazner, M. N. Flora, \& G. V. Quinnan, Jr. A variable region 3 (V3) mutation determines a global neutralization phenotype and CD4-independent infectivity of a human immunodeficiency virus type envelope associated with a broadly cross-reactive, primary virus-neutralizing antibody response.

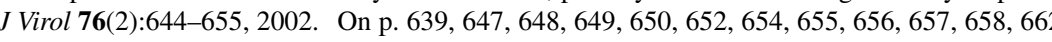

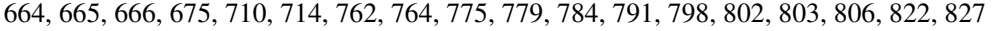

[Zheng 1999] L. Zheng, X. L. Huang, Z. Fan, L. Borowski, C. C. Wilson, \& C. R. Rinaldo. Deliver of liposome-encapsulated HIV type 1 proteins to human dendritic cells for stimulation of HIV type 1-specific memory cytotoxic T lymphocyte responses. AIDS Res Hum Retroviruses 15:1011 20, 1999. On p. 134 239 332

[Zinckgraf1999] J. W. Zinckgraf, J. M. Winchell, \& L. K. Silbart. Antibody responses to a mucosally delivered HIV-1 gp120-derived C4/V3 peptide. J Reprod Immunol 45:99-112, 1999. O p. 821

Zinkernagel2002] R. M. Zinkernagel. Immunity, immunopathology and vaccines against HIV? Vaccine 20(15):1913-1917, 2002. On p. 400
[Zolla-Pazner1995a] S. Zolla-Pazner, J. O’Leary, S. Burda, M. K. Gorny, M. Kim, J. Mascola, \& F. McCutchan. Serotyping of primary human immunodeficiency virus type 1 isolates from diverse geographic locations by flow cytometry. J Virol 69:3807-3815, 1995. A set of 13 human MAb to a variety of epitopes were tested against a panel of primary isolates of HIV-1, representing different genetic clades. The V3 loop tended to be B clade restricted, and a single gp120 Cterminus binding antibody was clade specific. Two other gp120 C-terminus binding antibodies were group specific. On p. $6476664675692693 \quad 720$

Zolla-Pazner1995b] S. Zolla-Pazner \& S. Sharpe. A resting cell assay for improved detection of antibody-mediated neutralization of HIV type 1 primary isolates. AIDS Res Hum Retroviruses 11:1449-1458, 1995. On p.720

Zolla-Pazner1997] S. Zolla-Pazner, C. Alving, R. Belshe, P. Berman, S. Burda, P. Chigurupati, M. L. C. ML, A. M. Duliege, J. L. Excler, C. Hioe, J. Kahn, M. J. McElrath, S. Sharpe, F. Sinangil, K. Steimer, M. C. Walker, N. Wassef, \& S. Xu. Neutralization of a clade B primary isolate by ser from human immunodeficiency virus-uninfected recipients of candidate AIDS vaccines. J Infect Dis 175:764-774, 1997. Comment in: J Infect Dis 1997 Nov;176(5):1410-2. Clade B primary isolate BZ167 was neutralized, using a new assay, by sera from HIV-uninfected volunteers in vaccine trials. On $\mathrm{p} .675$

Zolla-Pazner1999a] S. Zolla-Pazner, M. K. Gorny, \& P. N. Nyambi. The implications of antigenic diversity for vaccine development. Immunol Lett 66:159-64, 1999. On p. 643 644645646

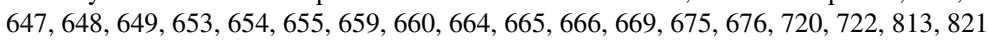

Zolla-Pazner1999b] S. Zolla-Pazner, M. K. Gorny, P. N. Nyambi, T. C. VanCott, \& A. Nadas Immunotyping of human immunodeficiency virus type 1 (HIV): an approach to immunologic classification of HIV. J Virol 73:4042-51, 1999. 21 human anti-V3 MAbs were studied with respect to cross-clade reactivity and immunological relationship to other human anti-V3 MAbs. Broad cross-reactivities were observed, and V3 peptides were grouped into immunotypes that \begin{tabular}{ll|l|l|l|l|l|l|l|l|l|} 
contained peptides from several clades. On p. 643 & 644 & 645 & 646 & 647 & 648 & 649 & 653 & 654 & 655 \\
\hline
\end{tabular} \begin{tabular}{|l|l|l|l|l|l|l|l|l|l|l|l|l|}
659 & 660 & 664 & 665 & 666 & 669 & 675 & 676 & 720 & 722 & 813 & 821 \\
\hline
\end{tabular}

zurMegede2000] J. zur Megede, M. C. Chen, B. Doe, M. Schaefer, C. E. Greer, M. Selby, G. R. Otten, \& S. W. Barnett. Increased expression and immunogenicity of sequence-modified human immunodeficiency virus type 1 gag gene. J Virol 74:2628-35, 2000. On p. 169

Zvi1995a] A. Zvi, I. Kustanovich, D. Feigelson, R. Levy, M. Eisenstein, S. Matsushita, P. RichaletSecordel, M. H. Regenmortel, \& J. Anglister. NMR mapping of the antigenic determinant recognized by an anti-gp120, human immunodeficiency virus neutralizing antibody. Eur J Biochem 229:178-187, 1995. On p.671

Zvi1995b] A. Zvi, I. Kustanovich, Y. Hayek, S. Matsushita, \& J. Anglister. The principal neutralizing determinant of HIV-1 located in V3 of gp120 forms a 12-residue loop by internal hydrophobic interactions. FEBS Lett 368:267-270, 1995. On p. 671

[Zvi1997] A. Zvi, D. J. Feigelson, Y. Hayek, \& J. Anglister. Conformation of the principal neutralizing determinant of human immunodeficiency virus type 1 in complex with an anti-gp120 virus neutralizing antibody studied by two-dimensional nuclear magnetic resonance difference spectroscopy. Biochemistry 36:8619-27, 1997. On p. 671 672

[Zvi2000] A. Zvi, V. Tugarinov, G. A. Faiman, A. Horovitz, \& J. Anglister. A model of a gp120 V3 peptide in complex with an HIV-neutralizing antibody based on NMR and mutant cycle-derived constraints. Eur J Biochem 267:767-79, 2000. On p. 671 672

[Zwick2001a] M. B. Zwick, L. L. Bonnycastle, A. Menendez, M. B. Irving, C. F. Barbas III, P. W Parren, D. R. Burton, \& J. K. Scott. Identification and characterization of a peptide that specifically binds the human, broadly neutralizing anti-human immunodeficiency virus type 1 antibody b12. J Virol 75(14):6692-9, 2001. On p.784.788

Zwick2001b] M. B. Zwick, A. F. Labrijn, M. Wang, C. Spenlehauer, E. O. Saphire, J. M. Binley, J. P. Moore, G. Stiegler, H. Katinger, D. R. Burton, \& P. W. Parren. Broadly neutralizing antibod- 


\section{HIV Immunology References}

ies targeted to the membrane-proximal external region of human immunodeficiency virus type 1 glycoprotein gp41. J Virol 75(22):10892-905, 2001. On p. 696 697 710 715 719 741 784 788

[Zwick2001c] M. B. Zwick, M. Wang, P. Poignard, G. Stiegler, H. Katinger, D. R. Burton, \& P. W. Parren. Neutralization synergy of human immunodeficiency virus type 1 primary isolates by cocktails of broadly neutralizing antibodies. J Virol 75(24):12198-208, 2001. On p. 7107715 $719784788,822,825$ 